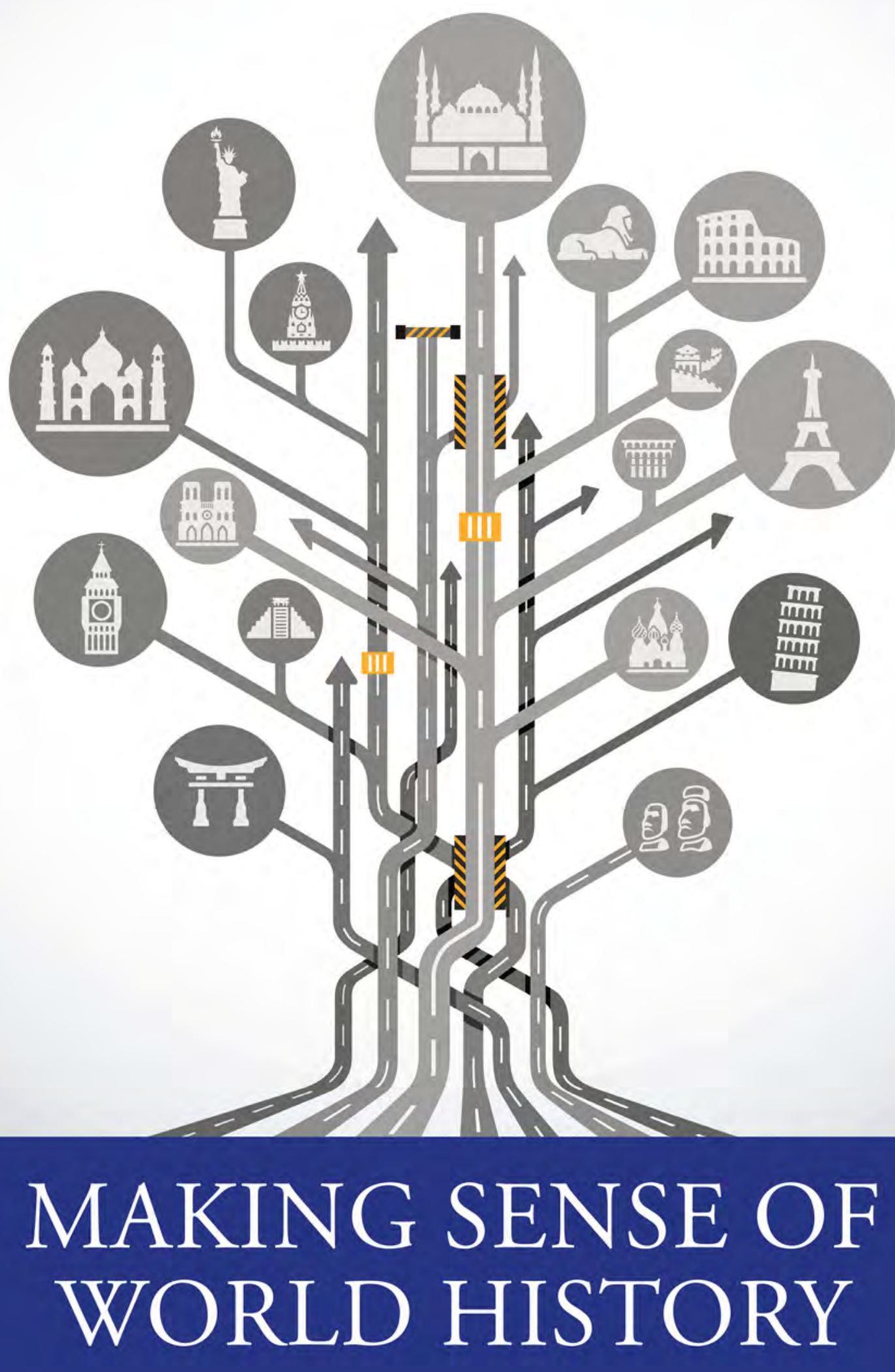

ใ

Rick Szostak

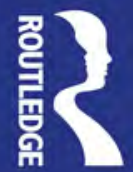




\section{MAKING SENSE OF WORLD HISTORY}

Making Sense of World History is a comprehensive and accessible textbook that helps students understand the key themes of world history within a chronological framework stretching from ancient times to the present day.

To lend coherence to its narrative, the book employs a set of organizing devices that connect times, places, and/or themes. This narrative is supported by:

- Flowcharts that show how phenomena within diverse broad themes interact in generating key processes and events in world history.

- A discussion of the common challenges faced by different types of agent, including rulers, merchants, farmers, and parents, and a comparison of how these challenges were addressed in different times and places.

- An exhaustive and balanced treatment of themes such as culture, politics, and economy, with an emphasis on interaction.

- Explicit attention to skill acquisition in organizing information, cultural sensitivity, comparison, visual literacy, integration, interrogating primary sources, and critical thinking.

- A focus on historical "episodes" that are carefully related to each other.

Through the use of such devices, the book shows the cumulative effect of thematic interactions through time, communicates the many ways in which societies have influenced each other through history, and allows us to compare and contrast how they have reacted to similar challenges. They also allow the reader to transcend historical controversies and can be used to stimulate class discussions and guide student assignments.

With a unified authorial voice and offering a narrative from the ancient to the present, this is the go-to textbook for World History courses and students.

Rick Szostak is a professor at the University of Alberta, Canada. He is the author of eighteen books and sixty journal articles spanning the fields of world history, economic history, history of technology, methodology, interdisciplinary studies, and knowledge organization. 


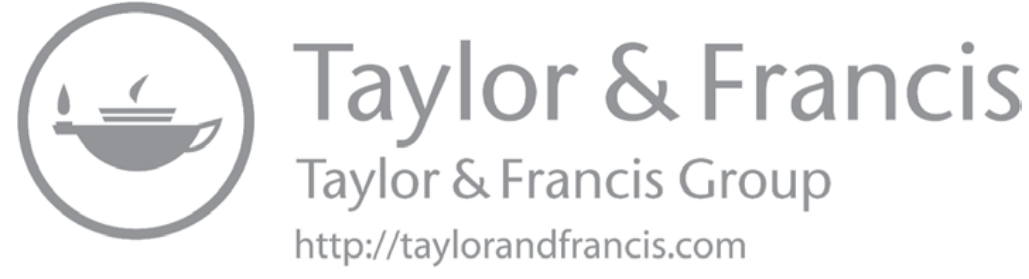




\section{MAKING}

SENSE OF

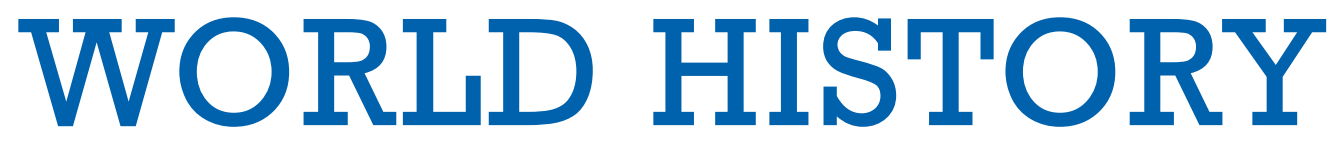

RICK SZOSTAK

(2) 
First published 2021

by Routledge

2 Park Square, Milton Park, Abingdon, Oxon OX14 4RN

and by Routledge

52 Vanderbilt Avenue, New York, NY 10017

Routledge is an imprint of the Taylor \& Francis Group, an informa business

(C) 2021 Rick Szostak

The right of Rick Szostak to be identified as author of this work has been asserted by them in accordance with sections 77 and 78 of the Copyright, Designs and Patents Act 1988.

The Open Access version of this book, available at www.taylorfrancis.com, has been made available under a Creative Commons Attribution-Non Commercial-No Derivatives 4.0 license.

Trademark notice: Product or corporate names may be trademarks or registered trademarks, and are used only for identification and explanation without intent to infringe.

British Library Cataloguing-in-Publication Data

A catalogue record for this book is available from the British Library

Library of Congress Cataloging-in-Publication Data

A catalog record has been requested for this book

ISBN: 978-0-367-82089-3 (hbk)

ISBN: 978-0-367-82088-6 (pbk)

ISBN: 978-1-003-01351-8 (ebk)

Typeset in Stone Serif, Avenir and Rockwell by Newgen Publishing UK 


\section{Contents}

List of figures $\quad \mathrm{xxV}$

List of tables $\quad$ xxvi

List of boxes xxvii

Challenges facing agents $\quad$ xxix

List of primary documents $\quad \mathrm{xxx}$

Preface xxxii

Acknowledgements xli

Note on the illustrations xlii

Glossary of regions and states xliii

PART I: Organizing world history 1

1 Making sense of world history 3

Guiding questions 3

Key organizing devices and principles 4

Placing this text within the evolution of world history 6

Implications for how the book is organized 9

Placing individuals in history 10

What is history about? The key themes of world history 10

Our themes and the world of today 13

The theme of culture 13

The theme of social structure 14

Understanding change in history through evolutionary analysis 18

Inter-societal transmission 19

Evolution in cultural values and practices 20

Evolution and progress compared 24

Philosophical orientation of this book 24

Interdisciplinarity and world history 25

Both/and thinking 26

Valuing multiple perspectives 27

Outline of this book 28

Questions 30

Readings 30 


\section{The Big History prelude: From the Big Bang}

\section{to hominids}

Guiding questions 37

The nature of Big History 38

The Big Bang 40

Stars and planets 42

The Earth 46

The theme of non-human environment 49

Life on Earth 51

Evolutionary theory revisited 54

Multicellular life 60

Mammals 63

Reprise 64

Questions 66

Readings 66

\section{Evolution of human nature itself in early}

\section{human history}

Guiding questions 69

The evolution of human nature 70

Environmental influences? 76

Culture and more 77

Language 78

Culture 81

Emotions 82

Altruism 83

The subconscious 84

Human activities 85

Tool-making 85

Fire 89

Economic activity 91

Incomes 92

Gender relations 93

Polity and social structure 94

Art 95

Migration 98

Population growth 104

Effects on the natural environment 105 
Connections across societies 106

Speculation on the nature of hominid life 106

The theme of common genetic inheritance 107

The theme of individual differences 108

Questions 109

Readings 109

\section{A critical transformation: The development} of agriculture, nomadism, and fishing

Guiding questions 111

The development of agriculture 113

Some preliminary considerations 113

Agriculture in the Middle East 118

A regional survey 120

Some effects 130

Agriculture means storage and control over land 130

Environmental impacts 131

Addressing the soil nutrient challenge facing farmers 132

Population and health 133

Human nature 135

Politics 139

Gender relations 140

Speculation on peasant behaviour 142

Nomadism 143

The nature and location of nomadism 143

The emergence of nomadism 145

Nomads and farmers 146

The domestication of the horse 148

Nomad life 148

Fishing 151

The theme of the economy 152

Questions 153

Readings 153

\section{Some early impacts of agriculture: Key technologies and trade practices}

Guiding questions 155

Agricultural technology 156

The diversity of agricultural practices 157

Irrigation 158 
Fermentation 158

Vegetable oils 160

Non-agricultural technologies 160

Pottery 161

Brick 164

Glass 164

Textiles 165

Metals and mining 167

Other technologies 172

The theme of technology and science 173

Trade 173

Money 175

Transport 177

Merchants 180

Artisans 186

Economic growth 187

Evolution of technology and science 192

Questions 197

Readings 197

\section{Grappling with "civilization": The} development of cities, states, and writing

Guiding questions 199

The earliest "civilizations" 201

Cities 202

Emergence of early cities 202

The nature of early cities 205

Effects of cities 208

Architecture 209

Organized states 214

Emergence and expansion of early states 214

Nature of early states 218

The rise of "kings" 219

Effects of states 223

Writing 223

Education 229

Laws 231

Gender relations 233

Science 235 
War 235

The birth of war 236

The common experience of war 237

Military leadership 243

Soldiers 243

War and social differentiation 245

The changing face of war: Technology, scale, and organization 245

The theme of politics 248

Questions 249

Readings 250

\section{Early civilizations around the world}

Guiding questions 251

City states and "empires" in Mesopotamia 252

Inscriptions 257

Anatolia 259

Egypt 260

Nubia 268

Africa beyond Nubia 268

The Levant 268

Greece 269

Indus Valley 272

The Ganges 275

China 277

Central Asia 282

Americas 283

Meanwhile elsewhere in the world 286

Trade expansion through time 286

Institutional evolution 289

Climate and civilization 293

Population 294

Migration 295

Travel 299

The theme of population and health 299

Families 300

Questions 301

Readings 301 


\section{Belief systems: The nature and development of early religions}

Guiding questions 303

Evolutionary processes 307

Historical analysis of religion 309

The earliest religions 310

Egypt 313

The Levant 316

Mesopotamia 316

Greece 317

Rome 318

Africa beyond Egypt 318

India 319

China 320

Mesoamerica 322

Nomadic religions 322

The nature of ethics 322

Reflections on religion and community 326

Religion and the family 327

Sacrifices and buildings 327

Art 329

The theme of art 334

Artistic evolution 336

Questions 338

Readings 339

PART III: Classical history

9 Political organization on an unprecedented scale: The classical empires

Guiding questions 343

From earlier large states to larger empires 345

The nature of empires 346

Imperial internal diversity and justification 348

Functions of empire 351

The expansion of empires 352

Administration of empires 353

The ends of empires 355

Particular empires 357

Persia 357 
Mauryan and Gupta Empires in India 363

The Kushan Empire 368

Axum 369

The Americas 370

Slavery 370

Trade 377

Currency 377

Finance 378

Sea trade 380

Trade networks 383

Silk Road 384

Cultural and health impacts 388

Ancient science 389

Libraries 392

Questions 394

Readings 395

\section{Similarities and differences: The Roman and Chinese Empires compared}

Guiding questions 397

Greece 398

The lasting impact of Greek civilization 404

Rome 409

The Republic 410

The Empire 412

Roman family life 417

China 421

Chinese philosophy 421

The Qin 424

The Han 427

Comparing China and Rome 431

Similarities and differences 431

Empire and trade 436

Imperial decline 438

The question of reconstitution 441

The legacy of empire 443

Political thought 443

China and bureaucratic theory 447

Democratic impulses 448

Jewish political thought 450 
Patriotism 451

Questions 451

Readings 451

\section{The birth of missionary religions: Why and} how did the world's major religions emerge?

Guiding questions 453

New religions from old 455

Religions and states 456

Why then? 457

Major religions 458

Hinduism 458

Jainism 459

Charvaka 460

Judaism 460

Zoroastrianism 463

Confucianism 465

Taoism 466

Buddhism 466

Christianity 472

Manichaeism 478

Islam 479

Shinto 485

Mayan religion 486

Some comparisons and reflections 487

Canon development 487

Religion and family values 487

Religion and trade 488

Faith 489

Religion and war 489

Religion and artistic representation 490

Relics 491

The geographical spread of religions 491

Conversion of rulers 492

Policies of rulers 493

Conquest 493

Political conflict 494

Missionaries and merchants 494

Migration 495

Financial resources 495 
Syncretism 495

Suitability 496

Other factors 496

Summing up 497

Evolutionary reflections 499

Questions 500

Readings 501

\section{A new force in world history: The Islamic} conquests

Guiding questions 503

Early days 504

Islamic political realities 511

Battles over succession 513

Umayyads 514

Abbasids 515

Abbasid cultural and scientific efflorescence 517

Islamic attitudes toward women 525

Trade and the movement of ideas 526

Islam in India 529

Mamluks 531

Islam in West Africa 531

Islam in Central Asia 533

Islamic art 533

Questions 536

Readings 536

\section{Eurasia in the centuries after the fall of the} classical empires

Guiding questions 537

Nomadic peoples of Central Asia 540

Nomads and sedentary societies 540

Nomadic military technology and organization 542

Particular nomadic groups 544

"Barbarian" incursions into Europe 548

The Germans 549

The Huns 551

The Slavs 551

Magyars 554

The Vikings 554 
Islam 557

The classic feudal system 558

The Byzantine Empire 565

Justinian and Theodora 566

Byzantine strategy 570

The nature of empire 571

The Huns 573

Islam 574

Slavs 575

China 575

The period of disunity 576

Sui 577

Tang 577

Korea 583

Japan 585

Southeast Asia 587

Tibet 589

India 590

Questions 594

Readings 594

14 Seeking global commonalities: Some key thematic trends 900-1500 and beyond

Guiding questions 597

Climate after 900598

Political consolidation after 900599

Courtly life 601

Cultural consolidation after 900603

Trade expansion 606

Trading cities 609

Travellers' tales 610

Porcelain 614

Agricultural expansion 617

Monuments 617

Family patterns 622

Population 624

Questions 625

Readings 625 
15 Regional developments: Eurasia after 900

Guiding questions 627

State formation in Europe 629

The Catholic Church 633

The Crusades 637

Trade and trading towns 642

Agriculture 646

Industry and guilds 650

The printing press 653

The Renaissance 654

China 658

Song politics 658

Economic dynamism under the Song 661

Landscape painting 665

The Yuan 665

Japan 667

India 670

Southeast Asian mainland 672

Southeast Asian islands 678

Questions 682

Readings 682

16 Regional developments: Polynesia, the Americas, and Africa

Guiding questions 685

Polynesian settlement 686

Easter Island 692

Australia 693

The Americas 694

Mesoamerica 695

Farther north 701

South America 703

Africa 709

West Africa 712

West African trading centres 716

East Africa 717

Kongo 717

Questions 719

Readings 719 


\section{The Mongols and the largest ever contiguous} empire

Guiding questions 721

Central Asia at the time of the Mongols 722

Chinggis Khan 722

Keys to Mongol success 726

Some speculations 730

The unfortunate logic of slaughter 730

The post-Chinggis Mongols 731

Mongol administration 734

The role of women 735

The Yuan Dynasty 736

Effects of the Mongol conquests 737

Trade and transport 737

Exchange of ideas 741

The Plague 743

Religion 746

After the fall 749

Muscovy 749

Timur 751

Questions 752

Readings 752

PART V: The Early Modern period

18 Thematic developments in the Early Modern period 1450-1800

Guiding questions 757

Climate 759

Political consolidation 759

Laws 760

Seventeenth-century disruptions 761

The Netherlands 764

Religious developments 764

Religious interactions 769

Witch hunts 770

Wars and gunpowder technology 772

Early development of gunpowder technology 772

The success of Europe 774

Trade 777 
Trading networks 777

Coffee, tea, and cocoa 779

Trans-Sahara trade 781

Collecting 784

The transmission of art 784

Capitalism 786

Population 791

Questions 791

Readings 792

\section{Exploration and trade: Linking the continents 795}

Guiding questions 795

The Spanish conquests in the western hemisphere 797

The Spanish discovery of America 797

Spanish conquests in the Americas 801

Silver and settlement 806

The Spanish and others across the Pacific 809

The Portuguese to the Indian Ocean 813

English and Dutch expansion 819

Russia into Siberia 822

Chinese expansion 825

The Columbian Exchange 827

The potato 830

An exchange of ideas 833

The slave trade 834

The Newfoundland fishery 839

The North American fur trade 842

Agricultural expansion 844

Questions 847

Readings 847

\section{Comparing new empires in Asia}

Guiding questions 849

Comparisons and contrasts 850

The Ottoman Empire 856

Ottoman administration 862

Reform initiatives 864

Ottoman decline 865

Ottoman culture 868

The Safavid Empire 869 
Safavid culture 872

The Mughals 873

Mughal culture 878

Caste 880

Mughals and British 881

The Ming in China 884

Ming society and economy 889

Ming naval expeditions 890

The Qing in China 893

Africa 898

Questions 900

Readings 901

\section{It seems so natural now: The emergence of} the modern nation state

Guiding questions 903

A historical survey 905

Europe in the fifteenth through eighteenth centuries 905

Europe in the nineteenth and twentieth centuries 911

Asia 916

Latin America 918

Nation states and empires 919

Settler societies 921

Africa 923

The Pacific 924

The nation state system 926

Ethnicity itself 928

Nationalism 931

Democracy 937

Questions 942

Readings 942

\section{The Great Divergence: The rise of the European economy and military}

Guiding questions 945

Roots of the Great Divergence 947

Comparing incomes 949

Natural resources and colonies 951

Trade and markets 954

Demography 955 
Political and economic institutions 956

Religion 961

Technology and technological innovation 961

Summing up 966

The scientific revolution 966

Changes in astronomical understanding 967

Changes in practice 971

The cultural environment of the scientific revolution 976

Non-European antecedents 977

Effects of the scientific revolution 977

Western Civilization revisited 978

Questions 979

Readings 980

\section{PART VI: The nineteenth and twentieth centuries}

\section{Key thematic transformations of the long nineteenth century}

Guiding questions 985

Colonization 988

South Africa and Algeria 1000

Japan and colonialism 1001

American imperialism? 1002

Mining 1003

Transport 1004

Changes in work 1009

Science 1015

Agriculture 1021

Questions 1023

Readings 1023

\section{Industrial revolutions: Innovation, factories,} and economic growth

Guiding questions 1025

The British Industrial Revolution 1026

Relationships among factories, technology, and economic growth 1031

Why? The European context 1032

Politics and property rights 1033 
The British Empire 1033

Transport 1035

Other factors 1038

The second generation of industrializers 1041

The Second Industrial Revolution 1045

The third generation of industrializers 1048

Demographic changes associated with industrialization 1050

Social and cultural changes associated with industrialization 1057

Questions 1058

Readings 1059

\section{Political revolutions around the world:}

\section{A diverse set of experiences with important commonalities}

Guiding questions 1061

The American Revolution 1063

To revolt or not 1063

The twenty colonies 1066

Forging a new state 1068

The French Revolution 1073

Haiti 1079

Latin American revolutions 1082

The Sokoto caliphate 1087

Other revolutions and revolts 1088

Questions 1092

Readings 1093

\section{A unique historical transformation: The abolition of slavery and serfdom}

Guiding questions 1095

The abolition of slavery 1097

Europe 1100

The Americas 1103

Elsewhere 1109

The aftermath of abolition 1112

Other types of coerced labour 1115

Racism 1117

The abolition of serfdom 1120

The abolition of seigneurial tenure in Quebec in 18541123 
Questions 1124

Readings 1125

\section{Key thematic transformations of the twentieth} century

Guiding questions 1127

Democratization 1131

Communism 1136

The growth of the state 1142

International organizations 1150

Rights 1154

Social change 1158

Religion 1163

Environment 1167

Questions 1171

Readings 1171

\section{Devastation and fear: War in the twentieth} century

Guiding questions 1173

Prelude to war 1174

World War I 1176

Linking the World Wars 1187

Fascism 1191

World War II 1194

Wartime atrocities 1202

Allied bombing 1203

The impacts of war 1205

The Cold War 1209

The Hot Cold War 1216

Korea 1216

Cuba 1217

Indonesia 1217

Afghanistan 1217

Terrorism 1218

New wars 1221

Trends in war-making 1222

Questions 1223

Readings 1223 
29 The worst of times and the best of times: The Great Depression and postwar recovery

Guiding questions 1225

The Great Depression 1226

Technological innovation and the Great Depression 1232

The postwar Golden Age 1238

Social policy revisited 1242

Convergence 1244

Consumer culture 1251

Globalization 1252

Questions 1262

Readings 1262

30 An unprecedented development: Postwar decolonization

Guiding questions 1265

A unique historical transformation 1266

Influences on the timing and nature of decolonization 1266

Challenges in transition 1271

The Middle East 1272

Asia 1274

Iran 1279

India 1280

China 1284

Vietnam 1287

Africa 1290

Latin America 1298

The Pacific 1300

Questions 1302

Readings 1303

\section{Population movements: Dramatic changes} in the numbers, location, and health of humans

Guiding questions 1305

Population growth and stabilization 1307

Life expectancy 1313

Migration 1315

Nineteenth-century migration 1316

Twentieth-century migration 1320 
Urbanization 1324

Tourism 1332

Epidemics and famines 1336

Obesity 1339

Questions 1341

Readings 1341

\section{Drawing lessons from history: Why, how,} and what

Why to draw lessons 1345

Some important general insights 1345

Challenges in drawing lessons 1347

How to draw lessons 1347

Institutions and values 1348

Human nature 1350

Do no harm 1352

Human challenges 1352

Cultural understanding 1354

Toleration 1354

Science 1356

Technology 1359

Energy, resources, environment 1362

Trade 1364

Politics 1366

War 1371

Changing paths 1373

Population 1374

History itself 1376

Thoughts on our organizing structure 1377

World history and the meaning of life: How this book might shape your view of life 1379

We can achieve meaning by striving to make the world a better place 1379

We can achieve meaning through celebrating group identity (within a common humanity) 1386

We can achieve meaning by celebrating personal identity 1387 
We can achieve meaning through balance 1388

We can achieve meaning through our own life story 1390

We can find meaning in religion or philosophy 1391 


\section{Figures}

1.1 Thematic interactions 12

2.1 Influences on and effects of star formation 46

2.2 Path dependence 56

3.1 Influences on and effects of language $\quad 80$

4.1 Malthusian population dynamics 138

4.2 Influences on and effects of agriculture 143

4.3 Influences on and effects of nomadism 150

5.1 Influences on and effects of metals technology 172

5.2 Influences on and effects of increased trade 185

5.3 The four stages of innovation 195

6.1 Influences on and effects of cities 209

6.2 Influences on and effects of states 235

6.3 Influences on and effects of war 248

8.1 Influences on and effects of the evolution of religion 337

12.1 Influences on and effects of Islamic conquests 509

12.2 Influences on and effects of Islamic science 522

13.1 Influences on and effects of feudalism 563

14.1 Influences on and effects of cultural consolidation 606

$\begin{array}{ll}14.2 \text { Influences on and effects of monuments } & 624\end{array}$

16.1 Influences on and effects of low population density in Africa 711

$\begin{array}{ll}17.1 \text { Influences on and effects of Mongol conquests } & 728\end{array}$

18.1 Influences on and effects of superior gunpowder technology 777

19.1 Influences on and effects of connecting the two hemispheres 832

21.1 Romanov Dynasty power profile 908

21.2 Influences on and effects of the nation state 927

22.1 Influences on and effects of the scientific revolution 978

24.1 Transport improvements and the Industrial Revolution 1039

24.2 Influences on and effects of the demographic transition 1055

26.1 Influences on and effects of the abolition of slavery 1119

27.1 Influences on and effects of expanded state size 1147

29.1 Influences on economic growth 1242

29.2 Influences on and effects of the Great Depression 1244

30.1 Influences on and effects of postwar decolonization 1272

31.1 Influences on and effects of population growth 1314 


\section{Tables}

1.1 Advantages of the five organizing devices

1.2 The phenomena

15

32.1 Human progress by theme 


\section{Boxes}

1.1 General education, world history, and this book 8

2.1 Path dependence $\quad 57$

3.1 Group selection $\quad 84$

3.2 Self-deception and historical research 86

3.3 Deception and hierarchy? 95

3.4 Sculpting the human body 99

3.5 Individual biographies 109

4.1 Malthusian population dynamics 136

4.2 Political leadership terminology 141

5.1 The nature of trade 175

5.2 The roles of money 176

5.3 Characteristics of transport 180

5.4 The reputation of merchants 183

5.5 Measuring economic growth 190

$\begin{array}{ll}5.6 \text { Increased complexity } & 197\end{array}$

6.1 Cities in history 206

6.2 Gender and stratification 234

6.3 The motives for war 238

6.4 The evolution of war 246

$\begin{array}{lll}7.1 & \text { Ecological collapse } & 276\end{array}$

7.2 Rules of succession $\quad 281$

7.3 The spread of languages 296

$\begin{array}{ll}7.4 \text { Migration decisions } & 298\end{array}$

8.1 The roles of religion 305

8.2 Magic and religion 323

8.3 The five types of ethical analysis 324

8.4 The purpose of artistic illustration 333

9.1 The economics of slavery 372

9.2 Shipwrecks 382

$\begin{array}{lll}10.1 \text { Sports and games } & 405\end{array}$

$\begin{array}{ll}10.2 \text { War elephants } & 411\end{array}$

$\begin{array}{lll}10.3 \text { Cuisine } & 420\end{array}$ 
10.4 Comparisons across societies 432

10.5 Expanding farm sizes 439

11.1 Religious choice $\quad 498$

$\begin{array}{ll}12.1 & \text { Decisive battles } \\ 12.2 & 511\end{array}$

12.2 Technology transfer 523

$\begin{array}{ll}12.3 \text { Travelling cuisine } & 528\end{array}$

$\begin{array}{ll}13.1 \text { Head-binding } & 545\end{array}$

$\begin{array}{lll}14.1 \text { Commodity history } & 616\end{array}$

$\begin{array}{lll}15.1 \text { Stocks and bonds and pensions } & 647\end{array}$

$\begin{array}{ll}15.2 \text { The history of timekeeping } & 666\end{array}$

$\begin{array}{ll}17.1 \text { Tales of leadership } & 725\end{array}$

$\begin{array}{lll}17.2 & \text { Epidemics } & 747\end{array}$

$\begin{array}{ll}18.1 \text { Artistic borrowing } & 788\end{array}$

$\begin{array}{llr}19.1 \text { Scurvy } & 810\end{array}$

$\begin{array}{ll}20.1 \text { Universal empire } & 857\end{array}$

$\begin{array}{ll}20.2 \text { Secluding rulers } & 867\end{array}$

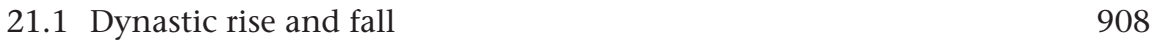

$\begin{array}{ll}21.2 \text { Cosmopolitan Vienna } & 921\end{array}$

$\begin{array}{ll}21.3 \text { State capacity } & 929\end{array}$

$\begin{array}{lr}23.1 \text { The history of rubber } & 991\end{array}$

23.2 The globalization of timekeeping 1009

24.1 International expositions 1043

24.2 History of childhood and ageing 1055

$\begin{array}{ll}25.1 \text { Revolutionary commonalities } & 1092\end{array}$

$\begin{array}{lr}27.1 \text { A third gender } & 1163\end{array}$

$\begin{array}{ll}28.1 \text { Ethical reflection } & 1220\end{array}$

$\begin{array}{lll}29.1 \text { Historiography } & 1238\end{array}$

$\begin{array}{ll}29.2 \text { Revisiting the ruler's challenge } & 1240\end{array}$

$\begin{array}{ll}29.3 \text { The causes of economic growth } & 1241\end{array}$

$\begin{array}{ll}29.4 \text { Air conditioning } & 1248\end{array}$

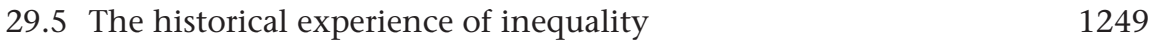

$\begin{array}{ll}29.6 \text { The historical novelty of globalization } & 1260\end{array}$

$\begin{array}{lll}30.1 \text { Poverty rates } & 1288\end{array}$

30.2 Resources and economic development 1292

31.1 The changing role of agriculture 1309

31.2 Population and economy in a post-Malthusian world 1313

$\begin{array}{ll}31.3 \text { The world's biggest cities } & 1325\end{array}$

31.4 Museums and historical sites and World History 1336 


\section{Challenges facing agents}

$\begin{array}{lr}\text { Hunter-gatherers } & 106\end{array}$

$\begin{array}{ll}\text { Farmers } & 131\end{array}$

$\begin{array}{ll}\text { Nomads } & 150\end{array}$

$\begin{array}{lr}\text { Fishers } & 152\end{array}$

$\begin{array}{ll}\text { Merchants } & 181\end{array}$

$\begin{array}{ll}\text { Artisans } & 187\end{array}$

$\begin{array}{ll}\text { Builders } & 214\end{array}$

$\begin{array}{ll}\text { Rulers } & 222\end{array}$

$\begin{array}{ll}\text { Soldiers } & 244\end{array}$

$\begin{array}{ll}\text { Priests } & 310\end{array}$

$\begin{array}{ll}\text { Slaves } & 375\end{array}$

Scientists $\quad 392$

Workers $\quad 421$

Women $\quad 526$

$\begin{array}{lr}\text { Explorers } & 690\end{array}$

$\begin{array}{ll}\text { Artists } & 785\end{array}$

$\begin{array}{ll}\text { Voters } & 942\end{array}$

Technological innovators $\quad 965$

$\begin{array}{lr}\text { Industrialists } & 1041\end{array}$

$\begin{array}{ll}\text { Modern parents } & 1053\end{array}$

$\begin{array}{ll}\text { Bureaucrats } & 1150\end{array}$

$\begin{array}{ll}\text { Migrants } & 1323\end{array}$ 


\section{Primary documents (by chapter)}

6. A Sumerian Schoolboy; Ibn Khaldun; The Art of War

7. Code of Hammurabi; Advice from an Egyptian scribal teacher

8. Creation stories (several from different societies); Hymn to the Nile

9. Cyrus Cylinder; Ashoka's Edicts; The Four Elements

10. Quotes from Cicero; Confucian quotations

11. Happiness of the Dhammapada; The Parable of the Pearl-Borer

12. The 1001 Nights; An anonymous account of Baghdad around $1000 \mathrm{CE}$

13. Ibn Khaldun's Theory of History; An Arab impression of the Slavic slave trade; Cast off the Yoke of Bondage

14. Excerpt from Tale of Genji; Marco Polo and Ibn Battuta

15. Anonymous in Wurzburg in 1147 disdains motives for Crusades; The Divine Comedy; Zhu Xi

16. How the Aztec raised sons as warriors; Inca social policy; Mansa Musa visits Cairo

17. Visiting the Mongols; Pegalotti's Advice to Merchants

18. Religious reform in many lands; Papal Bull of Innocent VIII on Witchcraft

19. The conquest of Tenochtitlan; Vasco da Gama on the natives of South Africa

20. Excerpt from Shaikh Hasan Al Kafrawi; Babur's Autobiography; An address to two hundred million fellow countrywomen

21. Excerpt from Muhammad Iqbal; Giuseppe Mazzini on nationalism

22. Adam Smith in the Wealth of Nations; Galileo's Recantation of Heresy

23. In Defence of Colonialism; Testimony of a Young Female Factory Worker in 1832

24. [Petition] To the Merchants, Clothiers...; Transport and industrialization

25. Declaration of the Rights of Man and of Citizens; Haitian Declaration of Independence; Excerpts from the Mexican Constitution

26. Decree of Simon Bolivar Emancipating Slaves in 1816; African slavery in America 
27. Declaration of Rights of Working and Exploited People; The Universal Declaration of Human Rights

28. Gas attack 1916; Treaty of Versailles

29. The Depression experience; Some quotes about globalization

30. A Plea for African Unity; The Peruvian Amazon Rubber Company

31. The dawn of restrictions on immigration; The slums of Manchester

32. Oswald Spengler on Human Progress 


\section{Preface}

This book had its genesis in a remark I came across some years ago: that students and instructors of World History courses found it hard to render the material coherent. They might enjoy tales of Babylonians and Aztecs and Polynesians but wondered why such different times and places were addressed in the same course. I set out to bring greater coherence to this subject matter through the use of a handful of organizing devices that are outlined in chapter 1. I stress here and there that these devices are innocuous in two important senses: They do not interrupt narrative flow, and they do not force the complexity of history into some simplifying perspective.

There are at least three key reasons why a coherent understanding of the history of the world is important:

- Humanity could benefit from a coherent sense of how we came to live in the sort of societies that we inhabit today. It might well prove that a better understanding of our past would aid humanity in charting its future. This is the hope of many world historians, and we will indeed suggest that this is so for the particular approach to world history advocated in this book and will devote a closing chapter to drawing such lessons.

- Humans wish to understand the meaning of life, and comprehension of our place in world history can potentially aid in our pursuit of metaphysical meaning. We will return to this question also in the closing chapter.

- We will suggest that world history encourages us to reflect on our common humanity. We might alleviate the strife that exists at present among different cultural groups if we appreciated that the world we live in was shaped by interactions across diverse societies over the last millennia, and if we likewise reminded ourselves of our common heritage and common responses to similar challenges through human history.

This book is written with a passion for the practice of world history. World history is, after all, an invitation to think big.

\section{An exhaustive and interactive thematic approach}

As we shall see in chapter 1 , world history texts often take a "thematic" approach, following themes such as "culture" or "environment" across time 
and place. This book takes a thematic approach also, but with two important differences:

- I will argue that we need to follow ten distinct themes through history. We gain a seriously biased and misleading view of history if we ignore "technology" or "health" or "gender relations" simply in order to make our story manageable in scope. We will, in particular, miss many important cases of cross-societal interaction if we arbitrarily limit the scope of our coverage. Happily, our organizing devices will allow us to treat all ten themes coherently.

- It is the interactions among themes that are central to world history. It is thus problematic to structure books or chapters around a single theme. We will highlight thematic interactions throughout this book. We will find that multiple themes play important roles in all major historical events and processes.

\section{Implications of these organizing devices}

The five organizing devices combine to achieve coherence in world history in four broad ways:

- To communicate the cumulative effect of thematic interactions through time (while stressing that cumulative change was never inevitable).

- To communicate the many ways in which societies have influenced each other through history.

- To communicate how all human societies have faced similar challenges, and allow us to compare and contrast how they have reacted to these.

- Indirectly these in turn support an appreciation that world history is a shared history that can only be understood by appreciating how themes, regions, and people interact.

\section{Timeliness of this book}

The timeliness of the book should be stressed. It comes at a point in time when:

- World history is growing in popularity.

- There is no consensus on how best to structure world history courses or texts. There is a great deal of experimentation.

- There is recognition that the material needs to be organized in some fashion to facilitate both teaching and learning. 
- World historians often claim that there are lessons in world history. But most texts make little effort to draw such lessons.

- Lessons regarding connections across societies over time and similarities in development across societies are potentially useful in a world that talks much about a "clash of civilizations."

\section{Achieving conceptual clarity}

We will in chapter 1 outline the themes and subsidiary phenomena that will guide us in this book. This exercise, importantly, allows us to achieve a considerable degree of conceptual clarity. Some of these phenomena should be easily understood: Who can doubt what "population" or "economic output" (measuring this is a different matter) mean in general? We will define other terms, such as "science" and "technology" carefully as the book proceeds. Of course, philosophers have long established that absolute precision in definition is beyond human capability. But we have achieved a level of precision that should allow different readers to have broadly similar understandings of what we are talking about. This is sadly often not the case in works of history or social science.

Table 1.2 provides further definitional clarity. We know best what a thing is if we know what sorts of things it subsumes and what sorts of things it does not. This is especially important with respect to culture. Table 1.2 provides an extensive list of the set of beliefs and practices that we embrace by the definition of culture employed in this book. Importantly, it also describes the set of things that are not culture: Notably, we treat art separately (for reasons we shall discuss in chapter 8 , but primarily because of the apparent existence of a shared aesthetic taste). To achieve further clarity we will strive in what follows to speak about particular attitudes or behaviours whenever possible rather than culture in general. Philosophers, it might be noted, have stressed that this "extensional" approach to definition in which one provides a list of "examples" of a thing is a useful complement to, if not substitute for, the "intensional" type of definition that tries to capture the essence of a term in a sentence or two. By providing an (fairly) exhaustive list of "types of culture" we achieve as much precision as may be possible.

Having achieved clarity in our definition of themes, we will not casually throw this clarity away by using other sorts of vague terminology in what follows. Our approach in this book is to break complex concepts - such as "globalization" or "modernization" - into their constituent parts. "Globalization" means for some the impact of expanding trade flows on employment, for others the increasing effect of American movies on French culture, and for still others the spread of democracy (a term we will use but carefully define in later chapters) globally. These are each important interactions among phenomena in their own right. And it is much easier to achieve a shared understanding of what we mean by "trade flows" or "American movies" than "globalization" itself. Yet 
the existence of a discourse around "globalization" suggests a belief that these economic, political, and cultural processes influence each other, and thus we should be sure to explore these thematic interactions as well. Likewise, the political, economic, cultural, and social aspects of "modernization" are best understood in isolation, though again these may interact. We should not assume at the outset that they interact in a particular way. Modernization theory also has had a tendency to imagine that all societies were tending toward Western outcomes, an assumption that can both bias historical understanding and offend non-Western sensibilities. "Civilization," "capitalism," "patriarchy," and "imperialism" are yet other ambiguous terms that we will carefully break into components that likely interact in what follows.

Note that there is a danger in eschewing such terminology. "Globalization" signals not just an interacting set of economic, political, and cultural processes, but also such messages as "This is something new," and "This is a problem." "Capitalism" and "imperialism" have similar connotations. "Civilization" often signals a sense that some societies are better than others. We thus lose some of the rhetorical, ideological, and theoretical meanings of such words when we simplify their meaning into sets of thematic interactions. But arguably for the purpose of this book that is no bad thing. We want not to assume the results of any historical process but to evaluate these. Terminology that clearly signals thematic interactions better serves our purposes. We will not be shy of clearly stating both negative and positive effects on other themes of these processes.

\section{Treating a multi-dimensional subject matter}

We can understand the challenge of teaching and learning world history as the challenge of multi-dimensional subject matter. The various themes or phenomena of interest to historians interact across both time and place. Tracing individual themes through time tends to push interactions into the background - and often makes it difficult to maintain temporal perspective. Focusing on particular regions diminishes one's ability to compare these and to appreciate spatial interactions. Endowing historical periods with unique characters associated with one or two themes abstracts away from both thematic interactions and historical continuity. The trick is to capture time, space, and themes simultaneously. This can only be done episodically, taking a change (or indeed stability) in one phenomenon - say, the development of agriculture, or the emergence of empire - as a temporary focus, examining its extent across time and space, and investigating how other phenomena influenced the change and were in turn affected by it. These episodes naturally feed into one another, as changes observed at one point in time and place set the stage for changes later in time and (often) in different places. The effects discussed in one episode can become influences or even the focus of analysis in another (though we must be careful not to imply that one historical episode inevitably leads to 
another). World history because of its multi-dimensionality places intellectual demands on both teachers and students. We can and should alleviate these challenges by providing linkages across historical episodes.

\section{Skill acquisition}

Universities are commonly urged these days to stress not just the information they impart to students but the skills that students master. World history courses, which often seek to prepare students to comprehend the world around them, are perhaps more open to explicitly fostering skill acquisition than other history courses. Some world history texts do speak to the skills that world history can generate, but these often parrot the sort of knowledge that these courses hopefully impart: Students will learn how to make comparisons across societies. Moreover, world history, like any decent university course, should have some beneficial impact on students' reading, writing, and reasoning skills. The approach to world history advocated in this book has some additional potential benefits in the area of skill acquisition:

- $\quad$ Readers in a world awash with information need to learn how to organize vast amounts of information in coherent (but flexible and as unbiased as possible) ways. This book provides organizing structures for understanding both the broad sweep of history and individual historical events and processes.

- The important skills around cultural sensitivity will be enhanced by regularly asking readers to place themselves in the positions of others.

- $\quad$ Students are often observed to perform comparisons poorly by comparing quite different elements of different societies. We need both to explicitly model appropriate comparisons and provide students with a clear set of phenomena and interactions to compare. We thus downplay the practice of many world history texts of organizing chapters around regions: a chapter on China followed by the Roman Empire, followed by India. We favour instead a more explicitly comparative treatment of themes and interactions across societies and over time (we will nevertheless still sketch an overview of major civilizations that emphasizes their unique characteristics).

- Our emphasis on flowchart diagrams will guide students to enhance their skills in appreciating and evaluating information that is communicated visually. This skill is often styled "visual literacy."

- World history has naturally focused on providing a broad overview, and is only slowly addressing a set of historical controversies. The emphasis of this book on thematic interactions inevitably exposes us to scholarly disagreement. We will seek in such cases not just to understand the sources of differences in opinion but to transcend these. We will do so not by imposing the author's opinion but by applying strategies of interdisciplinary integration (see chapter 1 ). Students can become understandably 
frustrated if regularly exposed to conflicts without any sense that a superior understanding can result from synthesizing opposing arguments. Readers should thus develop their critical thinking skills (and we can require students to hone these in written assignments). Note that we will not just expose students and readers to examples of critical analysis and synthesis but explain in detail how we go about this.

- More generally, students will gain important critical thinking skills. Our organizing devices provide us with a set of questions to ask throughout the book. Students will thus learn how to approach complex historical processes analytically. The questions asked at the end of chapters are designed not just to reinforce textual information but to encourage critical thinking.

\section{Use of primary sources}

As in most world history texts, this text will provide excerpts from primary documents. There are multiple textual excerpts in most chapters after the invention of writing occurs (see the Table of Contents for a list). For each excerpt, students are asked questions regarding how the excerpt should be interpreted and/or the challenges in doing so. Students should gain an appreciation both of how people of the time observed the world around them and how historians must strive to identify possible biases and errors in such accounts. Students are guided as to how to interpret such documents when the first documents are presented.

We also include many pictures in the text, of buildings, artefacts, and works of art. These also provide insights into particular times and places. Again, though, care must be taken in interpreting these. Box 8.4 discusses the purposes of the pictures in the text and how students should approach these.

\section{Some remarks regarding the table of contents}

- Many chapters are able to focus on (the influences on, characteristics of, and effects of) one historical transformation, notably 3, 4, 8, 11, 12, 17, 19, $21,24,26,29$, and 30. Several other chapters group together a handful of such episodes: 5, 6, 13, 15, 18, 23, 25, and 27. Yet the book still addresses each theme for each time period.

- There is nevertheless a clear chronological progression through the text. Since we discuss in all chapters interactions among themes we are thus able to pursue a thematic focus while addressing events in chronological order. While our main treatment of World War II comes in the chapter before our main treatment of the Depression, this does not at all prevent us from discussing how the Depression may have encouraged the path to World War II. 
- An explicitly comparative approach is taken throughout. Discussions of particular states or regions are always preceded and/or followed by comparative analyses. See for example chapters 6-7 and 9-10. A broad geographical coverage is pursued throughout. For example, chapter 4 surveys developments in all populated continents.

- There is thus a very balanced coverage of both regions and themes throughout the book. Economy, art, and climate are among the phenomena that receive more attention in this text than most others.

- The introductory and concluding chapters will add valuable material for many instructors and students but can be omitted by instructors wishing to focus exclusively on the historical narrative. It should be stressed that the concluding chapter is humble in tone: The reader is guided to ask their own questions and draw their own lessons.

- I address many topics that are not discussed in other world history texts. I devote chapter 2 to a discussion of the formation of the universe, stars, planets, life, and mammals. In chapter 3 I address the development of human psychology as well as physical capabilities. In chapter 4 I discuss the fishery along with agriculture and nomadism. The Big Bang, evolution of human emotions, the Newfoundland fishery, Costa Rican democracy, and obesity are among many topics that are addressed in this book but rarely treated elsewhere.

\section{Authorial voice}

I have striven to write this book in an even-handed fashion, trying to respect all sides in scholarly discourse (my philosophical approach is described in more detail in chapter 1). It may nevertheless be useful to reflect briefly on how this book reflects my previous research and teaching experience:

- I have co-authored with Gary Cross three editions of Technology and American Society: A History (the third edition was published by Routledge in 2018). I learned a lot about writing a history text in the process. And I draw on understandings of technology throughout the present book.

- I have written two books and several articles in economic history over the years. These address several different countries and time periods. I have found evolutionary analysis very useful in my research and teaching. Another of the organizing devices for this book - the descriptions of the challenges facing different historical actors - has its roots in my examination of the challenges facing entrepreneurs and innovators during the British Industrial Revolution, and Avner Grief's examination of the challenges facing merchants in the Middle Ages. More generally, I am able to treat economic issues authoritatively throughout the present text. 
- I have written several pieces of a methodological nature over the years. These variously urged attention to interdisciplinary linkages, theoretical and methodological flexibility, emphasis on careful comparisons across time and place, flowcharts, appreciation of the complexity of historical processes, and an emphasis on explanation rather than theory testing. These methodological considerations have also guided World History.

- I performed archival research in two languages in four countries. This gives me some appreciation for the challenges of the primary research on which our understanding of world history is largely based.

- I have often written about the implications for contemporary policy of historical understanding. I have thus stressed in this book the lessons we might draw from history.

- I have for decades been a scholar of the theory and practice of interdisciplinarity. I talk about the synergies between interdisciplinarity and world history in the last part of Chapter 1. In particular, I will employ interdisciplinary strategies for addressing conflicts in historical understanding. Two of this book's organizing devices - the flowcharts and in-text boxes reflect my research and textbook-writing in interdisciplinary studies (though I had also used flowcharts in my economic history work).

- I helped design a website on "Interdisciplinary General Education" and have had the fact that world history is often taught as a GenEd requirement much in mind as I drafted this book.

- My interest in interdisciplinarity guided me also to become a scholar of knowledge organization. This experience in turn encouraged me to insist on an exhaustive thematic treatment in this book, and to stress the interactions among themes. In my interdisciplinary texts we emphasize the importance of carefully defining terminology; in my knowledge organization research I have stressed how the act of classification clarifies meaning.

- The late Carolyn Neel invited me to make several contributions to the ABC Clio Encyclopedia of World History a few years ago. That experience also inspired me toward a broad thematic coverage in this book.

- My students have often praised my enthusiasm. I think that historical understanding is important. I hope that some of my passion shines through in these pages.

\section{More than a textbook}

We will in chapter 1 discuss which of the phenomena studied in the academy will receive most attention in this book, and why. Note that the phenomena that will receive less attention are not thus expunged from the history of the world. Any historian (or student) can continue to research these topics. If they can show important connections to the phenomena emphasized in this book, these topics will likely be more fully integrated into world history understanding in future. We will explore in chapter 32 how the organizing structure employed 
in this book can serve, among other things, to ensure that explorations on any topic or relationship can be found by subsequent researchers or students, and thus built into their understanding of world history. This book, then, provides a template for coherently organizing all world historical understanding, not just the understandings that can be squeezed into this book. Indeed it provides a template for organizing all scholarly understanding.

\section{A novel approach}

It is useful to summarize the key elements of novelty in this book:

- five innocuous organizing devices that lend greater coherence to world history - and potentially much more than world history

- $\quad$ an exhaustive and balanced treatment of themes (to avoid a biased history) and an emphasis on interactions among themes

- greater clarity in terminology

- a focus on historical "episodes" that are carefully related to each other

- use of interdisciplinary techniques for transcending historical controversies

- explicit (but subtle) attention to skill acquisition in organizing information, cultural sensitivity, comparison, visual literacy, integration, interrogating primary sources, and critical thinking more generally

- $\quad$ coverage of many important topics that are neglected in other texts.

- a sustained attempt to draw lessons from world history

- a unified authorial voice (most world history texts have multiple authors). 


\section{Acknowledgements}

I have presented material related to this book project at World History Association (WHA) conferences in Ghent, Honolulu, Boston, and Milwaukee. I received a great deal of advice and encouragement on each of these occasions. I would especially like to thank Marc Gilbert, who invited me to edit a Forum on "Organizing World History" in the online journal World History Connected (published in February 2018). I was joined in that Forum by Jonathan Reynolds, Stephen Morillo, and Mark Ciatola; they later also joined me in a joint session at the WHA conference in Milwaukee. I am grateful for the opportunity to interact with each of them. There are many others in the World History community that I would like to thank: I will mention here Jennifer McNabb, Paul Richgruber, Amy Manlapas, Sanford Holst, Maryann Rhett, Monika Johnston, Grace Chee, Bartholomew Zielinski, and the late John Langdon, but there are many others who I have interacted with profitably over the years. I thank Gary Cross for mentoring me over three decades in the task of writing a history text. I thank Miriam Davis for very helpful editorial advice. I thank the late Carolyn Neel for inviting me to write several entries in the ABC Clio Encyclopedia of World History. I thank the dozens of scholars who wrote referee reports about this project. I thank Pauline Gagnon, Camille Jasper-Fabiyi, Blake Lemay, Robert Smith, Valentina Galvani, David Sulz, Scott Auriat, and Suzanne Levesque for advice and encouragement along the way. I am very grateful to the many producers of open access illustrations employed in the text. I thank Peter Britton for permission to use several maps from TimeMaps.com. I would like to thank my children, Mireille, Julien, and Theo, who have brightened my life immeasurably while I have been writing this book. Julien read several chapters and gave very useful comments. Eve Setch at Routledge has been absolutely wonderful and imaginative in her approach. I am thrilled that we are able to provide this book in both traditional and open access formats. Sue Browning did a great job of copy-editing. Gail Welsh and her colleagues at Newgen did a fantastic job of creating the finished text. I thank Dean of Arts Lesley Cormack at the University of Alberta for financial support. 


\section{Note on the illustrations}

All images unless otherwise stated are in the public domain, either because they have been released into the public domain by their authors, their copyright has expired, or they are a work of government not subject to copyright. Other images are used and can be reused under their own creative commons licence, which is noted with each image. 


\section{Glossary of regions and states}

It is useful at the outset to clarify the regional terminology that will be used throughout this book. We have tried to limit the reader's need to master a lot of geographic terminology. Readers should have a basic familiarity with a modern map of the world.

\section{Continents}

We will follow common boundaries here. The Panama Canal separates North and South America. The Suez Canal and Red Sea separate Africa from Asia. The boundary between Europe and Asia follows the Bosporus, Black Sea, Caucasus Mountains, Caspian Sea, and Ural Mountains.

At times it is useful to speak of combinations of continents: The Americas (North and South America), Eurasia (Europe and Asia), or the eastern hemisphere (Europe and Asia and Africa). We will not, though, use the phrases "New World," and "Old World," for these privilege the history of the eastern hemisphere over that of the Americas.

\section{Regions}

The United States government during the Cold War fostered "Area Studies" programmes at American universities. It identified eight key regions: East Asia (originally called Far East), Southeast Asia, Africa, Middle East (originally Near East), Latin America, South Asia, Russia, and Europe. Russia would be expanded to include the Soviet Union and Eastern Europe. These regions are now fairly widely accepted within World History and beyond, though world historians note that the borders are often fuzzy and change through time. But of course when we refer to any region in the text the reader should generally appreciate that whatever point we are making is unlikely to apply everywhere within that region and nowhere outside of it.

East Asia generally comprises China, Korea, and Japan. Note that the borders of China have changed a lot during recorded history.

Southeast Asia comprises the nations of the Association of Southeast Asian Nations (ASEAN), stretching from Burma through Vietnam and including the island nations of Indonesia and the Philippines. 
The Middle East has vaguer boundaries. We will generally mean this to include only the present-day states of Lebanon, Israel, Syria, and Iraq, but at times the region has been thought to include parts of present-day Turkey, Iran, and Egypt. We will often refer to Anatolia, a region that largely comprises the Asian territories of modern Turkey. We will occasionally refer to the Levant, the eastern coastal area of the Mediterranean. We will also refer to Mesopotamia, the region comprising the lower reaches of the Tigris and Euphrates Rivers in Iraq.

South Asia refers to the area comprising the modern nations of India, Pakistan, and Bangladesh.

Latin America generally comprises all countries from Mexico to Argentina and Chile in the Americas - almost all of which have Spanish or Portuguese as national languages. The Caribbean islands are usually not included, though political references to Latin America may well apply equally well especially to the former Spanish colonies in the Caribbean. Central America refers to the region between Mexico and Colombia. Mesoamerica refers to Mexico and the northern parts of Central America; this term is applied earlier in history to refer to a region of cities and states such as those of the Maya and Aztecs.

We will not often refer to Eastern Europe, but when we do we generally mean the region of mostly Slavic-speaking nations (today) stretching from Poland and the Baltic states south to Montenegro, Serbia, and Macedonia, and eastward to Russia and Belorussia.

We will often refer to North Africa and sub-Saharan Africa, with the (shifting) area of the Sahara Desert serving as the boundary between them. We will occasionally refer to the Sahel, the region comprising the southern fringes of that desert.

One region that is missing from the government list but will figure often in these pages is Central Asia. This certainly comprises the area of the present-day "Stans": Kazakhstan, Kirgizstan, Turkmenistan, Uzbekistan, Tadzhikistan, and Afghanistan. But in earlier times nomadic peoples ranged over large areas of what are now Russia, the Ukraine, and China, and thus early references to Central Asia as an area of nomadic activity include large areas of these three modern states. Mongolia should also be considered part of Central Asia. Since this earlier sense of Central Asia includes the region north of the Black Sea now thought to lie within Europe, some scholars have lately begun to refer to "Central Eurasia." We will, though, maintain the more common terminology.

We will also often refer to the Pacific Islands. The Philippines are only included in this region if explicitly mentioned. All other islands east of New Guinea excepting those just off the coast of the Americas are included.

We will also refer often to the world's oceans and some of the larger seas and rivers and lakes and mountain ranges. Students should familiarize themselves with these features. 


\section{States}

For convenience, we will often identify a place of historical significance in terms of in which modern state that place now lies. The reader should of course remain cognisant that political boundaries have shifted often over time.

\section{Distance measures}

We will use kilometres rather than miles in this book to reflect its global reach. A kilometre is roughly six tenths of a mile. A mile is 1.6 kilometres. 


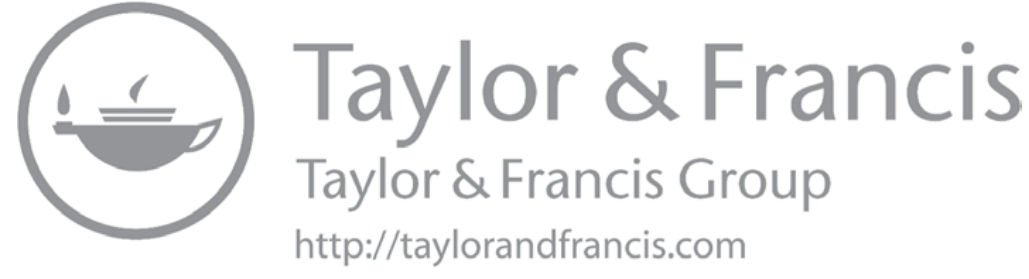




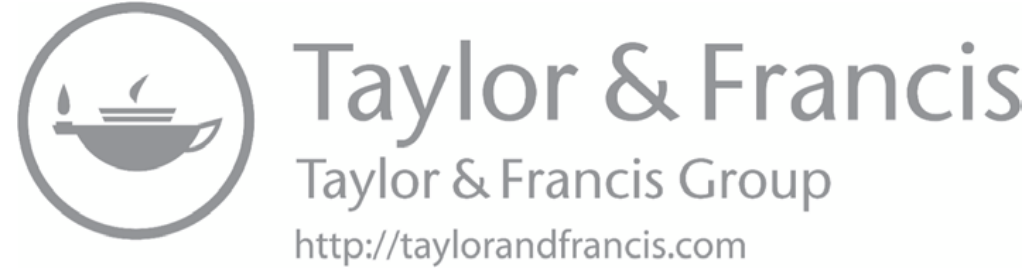




\section{Making sense of world history}

\section{Guiding questions}

Why study world history? What is the best approach to the study of world history?

How can we achieve a balanced coverage of the different themes and regions of the world?

How is historical analysis usually conducted? Are there better ways of performing historical analysis?

Relationship to other chapters: This chapter makes some important points regarding how we will approach the study of world history, and why we will take the particular approach that we do. Each chapter of the book will be grounded in the arguments we make in this chapter while expanding on and fleshing out those arguments. Since students are better able to appreciate discussions of how to study history while they are actually studying history, the key points made in this chapter will be reinforced when addressing particular historical events or processes in later chapters.

The study of world history reflects a keen desire among historians to bring some comprehensive understanding to the entire sweep of human history. There is a good reason for this. We could all benefit from a coherent sense of how we came to live in the sorts of societies that we do. Moreover, a better understanding of our past might help us chart our future. This is the hope of many world historians, and we will suggest that our particular approach to world history will help us do so. We will devote a closing chapter to drawing such lessons.

The danger, of course, is that in our desire to bring order to world history we become superficial. We seize on some organizing principle and ignore 
everything that does not fit. But we need to avoid a simplistic - and misleading "meta-narrative." The question is one of balance: Can we simplify world history enough to make it understandable while not ignoring crucial historical detail?

\section{Key organizing devices and principles}

Our focus will be on how ten key themes - political organization, social structure, economy, culture, art, the non-human environment, human genetic inheritance, individual differences, population and health, and technology and science - interact across time and place. We will not treat these themes in isolation but will focus on how they interact: How politics influenced the economy, which influenced culture (and vice versa). Most world history texts give a biased view of history by treating only a subset of these key themes, and often treat one theme at a time rather than stressing interactions. We will also carefully define each theme below and in later chapters.

We will also use five key organizing devices and principles to provide a broad and intelligible overview of the entire sweep of human history but also provide a place for the details generated by individual historical enquiries:

GUIDING QUESTIONS: We will start each chapter with a set of guiding questions and a brief discussion of how the chapter connects to other chapters in the book. So, we will begin with the Who?, What?, When?, Where?, Why?, and How? questions that motivate each chapter. A compelling historical account embraces all six questions, but we will place particular stress on Why? and How?

FLOWCHARTS: We will use diagrams to provide a visual summary of the thematic influences on and effects of particular events or processes. That is, we will show for many key "episodes" in history how elements of our key themes were interacting both within a particular society and across societies. Also, at the start of each chapter - or the beginning of each section in chapters that cover a range of topics - we will provide a visual indicator of the chapter's or section's key themes, with those that receive the most attention shaded in blue.

BOXES: We will also use "boxes" addressing material that transcends the time period of any one chapter. We usually introduce such boxes when a particular issue first becomes of significant importance. These boxes are of two broad types. One discusses processes that repeatedly occur in history, such as the epidemiological spread of disease. The second type briefly recounts a particular historical development, such as the history of rubber or of timekeeping. Such boxes serve as a microcosm of world history as a whole: We see how various types of human actor, various regions of the world, and various themes all interacted over a period of centuries to generate particular historical outcomes. Especially in the second half of the book, we will also employ smaller "postscripts" that briefly address how specific historical processes have played out into the present. 
EVOLUTIONARY ANALYSIS: Because we want to understand changes within our themes over time, we will apply evolutionary analysis to several key themes: culture, economic and political institutions, technology, science, and art. We will examine mutations (that is, new ideas), selection environments (how specific ideas are chosen), and transmission mechanisms (how ideas are communicated across generations and among societies). Evolutionary analysis helps us in organizing our understanding of history in two ways. Most obviously, it shows how one historical development builds upon what had happened before. Less obviously, it will guide us to often ask questions about mutations, selection environment, and transmission mechanism.

\section{CHALLENGES FACING HISTORI-}

CAL ACTORS: We will introduce the key challenges that faced different types of historical figures: farmers, rulers, merchants, soldiers, parents, voters, and many more. We will compare and contrast how these actors addressed these difficulties in different times and places. Farmers, for example, need to protect their harvest from theft. This simple challenge played an essential role in state formation, gender stratification, and the frequency of war in history. As we shall see, much of world history involves human actors facing a set of enduring challenges. Actors in different societies often, but not always, address similar difficulties in similar ways. The simple device of identifying key challenges thus allows us to understand better both similarities and differences across time and space.

You probably appreciate that historical enquiry involves answering a set of intertwined Who, What, Where, When, Why, and How questions. History courses in high school often focus on the first four. University courses - and this book

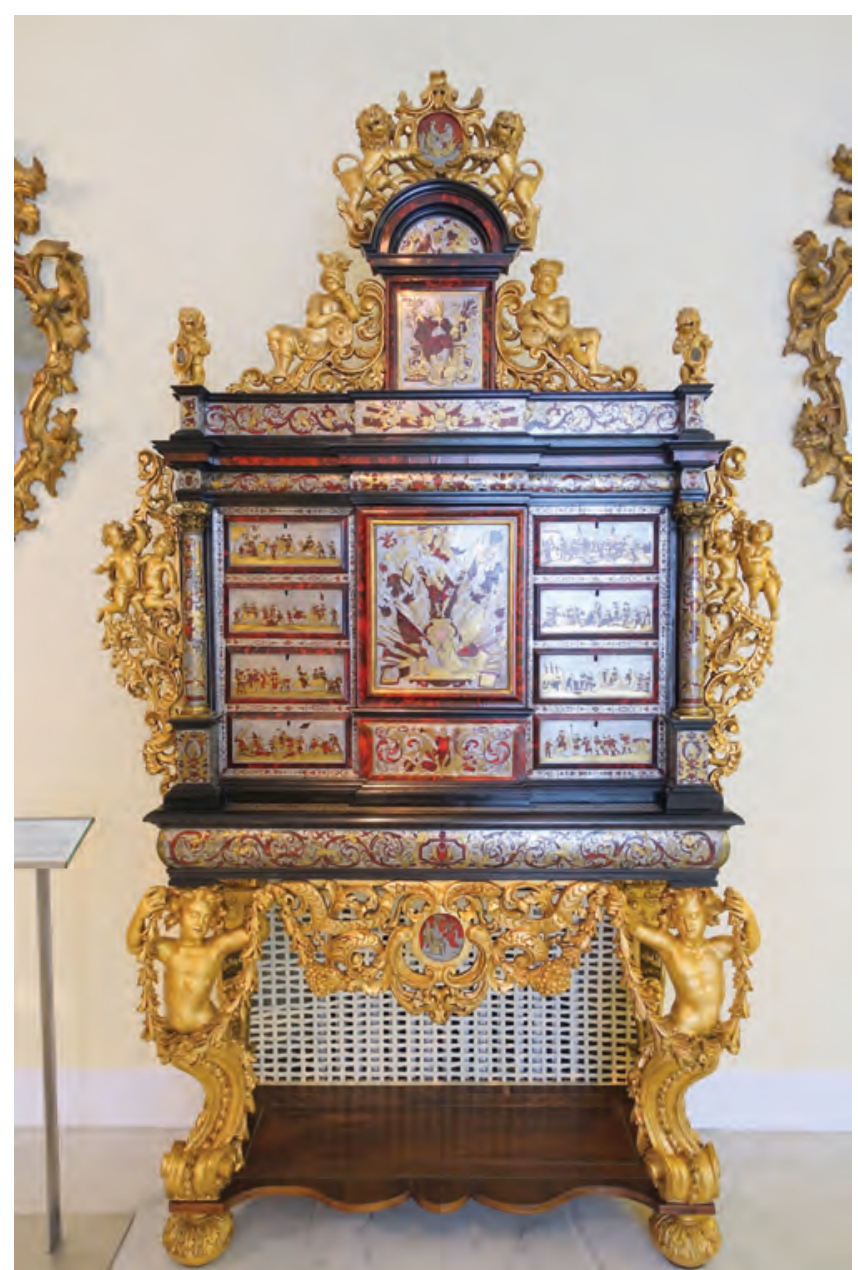

Illustration 1.1

Filing cabinet for King Philip V of Spain, 1713, Hendrick van Soest, Antwerp, Museo Nacional de Artes Decorativas, Madrid. Organizing information has long been a critical human activity

Source: Photograph by Daderot, CC0 1.0 
especially - tend to stress Why? and How? For each process or causal interaction that we address, we will organize our narrative around several fundamental questions: What is the nature of the process or interaction being discussed? How does it unfold through time? Who (or which) is the causal agent(s) or motors of change? (This may be a person, a group, or impersonal phenomena such as technological innovations or environmental changes.) Why does it occur? And When and Where does it happen? (questions for which there will often be multiple answers). We will also usually be interested in the impact of a given change and so will ask still other questions: What effects did the change have? How longlasting was the change?

Our five different organizing devices - (1) introductory guiding questions and discussion of how chapters are related; (2) flowcharts that illustrate crossthematic interactions; (3) boxes addressing issues that are not confined to the time period covered by any one chapter; (4) evolutionary analysis; and (5) discussion of the challenges facing different types of human actors - will help provide coherence to world history. Although students may be intrigued by tales of Persians and Polynesians, they can struggle to draw connections among such diverse societies. It is not always clear why we try to cover all of this material in one course or one book. Our organizing devices are intended to help you appreciate world history as a process with several essential elements:

- Influences of one society upon another

- Evolutionary developments within important themes such as political organization or the economy

- A cumulative process of thematic interaction in which changes in one theme trigger changes in another

- An ongoing process in which humans try to devise better solutions to an enduring set of challenges

Table 1.1 summarizes the key advantages associated with each of them.

\section{Placing this text within the evolution of world history}

The field of world history emerged in the postwar United States as a result of increased recognition of the importance of international connections and the increasingly multicultural American student body and professoriate. The earliest world history courses and texts had a strong "Western civilization" bias and mainly added discussions of other regions and how these had interacted with the West. Over time, such texts increased the amount of attention devoted to non-Western societies. In shifting their focus away from "Western civilization," such courses and texts became less "teleological" - i.e., they moved away from the implicit assumption that the politics, economy, and culture of 


\section{Table 1.1 Advantages of the five organizing devices}

\begin{tabular}{|c|c|}
\hline Organizing device & Advantages \\
\hline $\begin{array}{l}\text { Guiding questions } \\
\text { Discussing relations } \\
\text { between chapters }\end{array}$ & $\begin{array}{l}\text { Allows the student to appreciate why each chapter contains } \\
\text { the material it does, and also to see how chapters build on } \\
\text { what went before while setting the stage for what comes } \\
\text { after. }\end{array}$ \\
\hline Flowcharts & $\begin{array}{l}\text { See how themes interact in historical episodes; see how the } \\
\text { results of one episode become influences in later episodes. }\end{array}$ \\
\hline Text boxes & $\begin{array}{l}\text { Allow us to treat historical processes that operate over a } \\
\text { more extended time period than any one chapter embraces. }\end{array}$ \\
\hline Evolutionary analysis & $\begin{array}{l}\text { Guides us to ask about mutation, selection, and transmission; } \\
\text { guides us to appreciate how what happens in one time } \\
\text { period builds on what happened before. }\end{array}$ \\
\hline $\begin{array}{l}\text { Challenges facing types } \\
\text { of historical actor }\end{array}$ & $\begin{array}{l}\text { Allow us to compare reactions to common challenges } \\
\text { across time and place; allow us to treat (types of) individuals } \\
\text { prominently. }\end{array}$ \\
\hline
\end{tabular}

modern Western societies had been the goal toward which historical processes had always been tending. A world history focus instead seeks to understand how different outcomes have emerged in different societies and to identify global trends, useful comparisons, and important interactions across regions.

Also, over time, emphasis on connections among societies has been supplemented by a focus on common themes through world history. These themes are the primary phenomena that social scientists study: political structures, economic systems, culture (especially religion), social structure (ethnicity, class, gender, family organization and composition), technology, and the natural environment. We can compare and contrast themes (and especially changes within them) across societies: How did the Roman Empire differ from empires in China or India with respect to political organization or population growth or the effect of one on the other?

But at present, there is no consensus on what world history should stress or how to teach it. Some books emphasize the connections among societies. Some organize their chapters around different regions of the world; others strive for global coverage in most chapters. Some texts stress thematic comparisons. Yet they tend to focus on one theme at a time rather than highlighting crossthematic interactions. Many books - including this one - try to capture both themes and connections.

The organizing principles and devices of this book are designed to provide the desired coherence for world history and to illustrate how themes interact through time without interfering with the historical narrative, and, in fact, to link narratives. These organizing devices also allow us to enrich our narrative by guiding us to ask questions about the themes and how they interact, the 


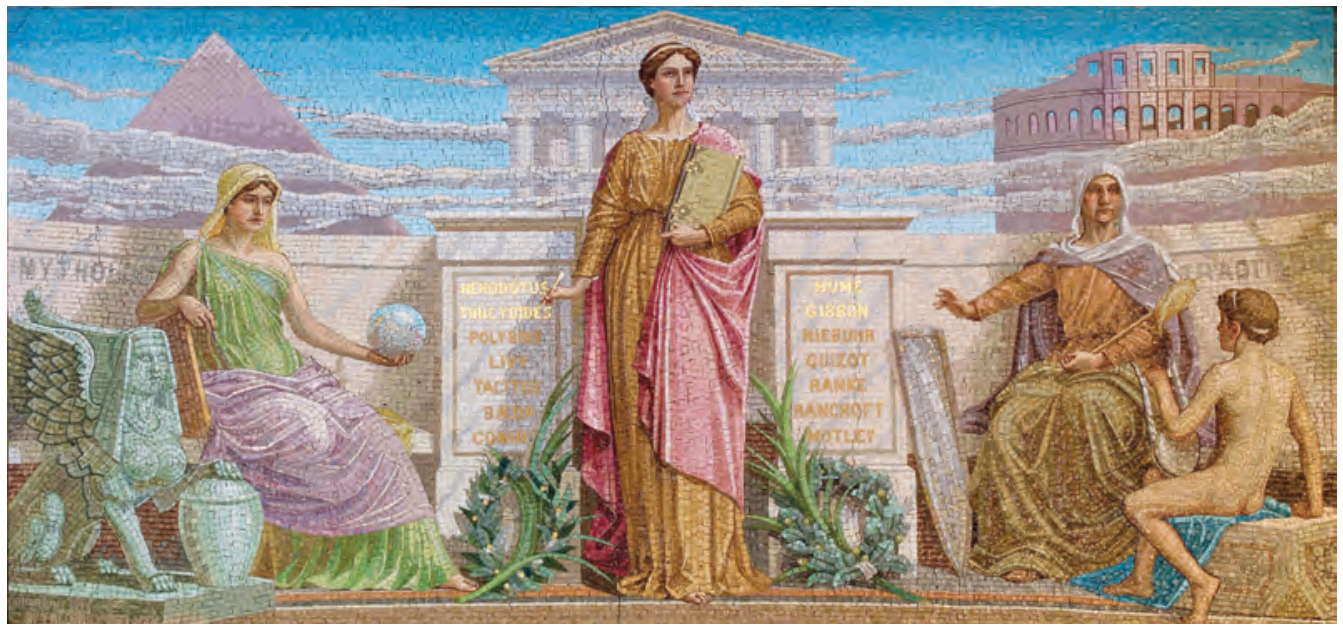

\section{Illustration 1.2}

History, a mosaic by Frederick Dielman. House Members Room, Library of Congress, Thomas Jefferson Building, Washington, DC. The figure of History is in the centre, flanked by figures representing mythology and tradition. The tablets in the walls list ancient and modern historians. The laurel wreath symbolizes peace and the oak wreath symbolizes war. The mosaic includes an Egyptian pyramid, a Greek temple, and a Roman amphitheatre, but no mention of Asia or sub-Saharan Africa. The mosaic was made in Venice

\section{BOX 1.1}

\section{GENERAL EDUCATION, WORLD HISTORY, AND THIS BOOK}

The boxes in this book generally capture material that transcends the time period or geographical scope of any one chapter.

University or college students are often required to take World History courses as part of General Education or core requirements. Since we will pursue a broad set of themes that capture what social scientists - and many natural scientists - study, this book ties World History to one of the standard goals of General Education: to give students some sense of how the academy as a whole is organized.We can briefly note here a variety of other critical General Education goals associated with skill acquisition. The organizing devices developed for this text hopefully enhance the student's general ability to organize the vast array of information they encounter in their studies and in life. Since each of our organizing devices encourages a set of questions, they should develop critical thinking skills. We stress two elements of critical thinking: By making explicit comparisons across regions, you will develop skills at comparing and contrasting complex cases; and by confronting historical controversies, you will develop integrative abilities (see below). We thus hope to facilitate lifelong learning - which requires both organization and critical thinking - regarding history in particular but also more generally. In this book, we will also address many other goals of General Education, including cultural sensitivity and ethical evaluation. 


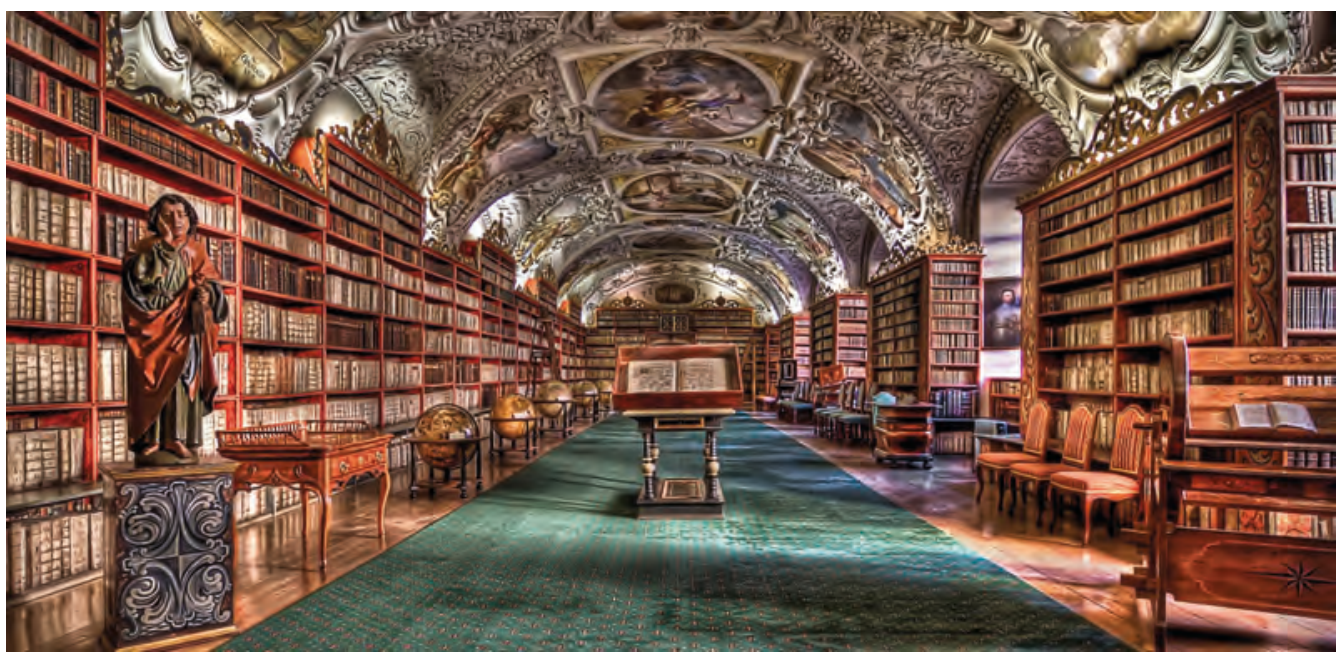

Illustration 1.3

Library, Prague Monastery

nature of selection environments, and how various individuals strove to engage age-old challenges.

It will be up to teachers and students of world history to decide if these ideas are useful. Congratulations! You are part of the selection environment. If any of these organizing devices are selected, then future texts can transmit these across generations.

\section{Implications for how the book is organized}

The characteristics of this work have some significant impact on its organization:

- There are implications for our approach to periodization. We will use some standard descriptors of periods - classical, early modern, and so on - for convenience. But do not make the mistake of thinking that any period has a particular ethos that distinguishes it from other periods. This is a particularly important attitude for world history, for periodization that makes sense for one region may make far less sense for another. We will be flexible in periodization, allowing for overlapping discussions that cross period boundaries. We can then appreciate that some historical processes unfold much more slowly than others. While we will recognize that there are "turning points" - or at least "inflection points" - where rates of change alter dramatically for some aspects of a society, we will also note that these may also be times of considerable continuity for other elements of the same society. As we have already indicated, we will use boxes (and postscripts) to address topics that span multiple time periods. 
- We will be sure to address all themes in all time periods, and we will stress the interactions among themes. While we cannot discuss all the significant interactions within and across every society, we will try to capture the major influences at work in any historical episode.

- This emphasis on interactions will affect the way we perform comparisons across societies, for we will explicitly look to these interactions across themes to explain both similarities and differences in experience among societies.

\section{Placing individuals in history}

In attempting to cover the entire history of the world in a few hundred pages, it is all too easy to push individuals into the background. This is especially the case for a text such as this that seeks a balanced coverage across ten themes and all regions of the world.

It is important to stress that two of the ten themes we embrace in this book operate at the level of individuals: "human genetic inheritance" and "individual differences." By showing how these two themes interact with themes that operate at the level of society, such as culture or the economy, we trace through history how individuals both shape and are shaped by their societies.

We will provide occasional biographical material on certain historical personalities. In order to appreciate more fully the role of individuals in human history, we will also:

- discuss how some aspects of human personality evolved during millennia as hunter-gatherers

- identify the challenges faced by various historical actors: farmers, artisans, parents. We can then compare how such actors attempted to meet these challenges in different times and places. History did not just happen to people but was shaped by individual or group attempts to improve their situation

- challenge readers to place themselves in specific historical situations, and ask how they would think and act.

\section{What is history about? The key themes of world history}

There are several good reasons to discuss in advance some of the themes - and subsidiary phenomena within those themes - that we will address in later chapters:

- Why did we choose particular themes? Our ten key themes capture elements of every category of phenomena studied within the social sciences and humanities (and address critical aspects of natural science as well). 
- There is considerable scope for vagueness in terminology unless we carefully define our themes. Culture, for example, looms large in thematic treatments of world history, but there are thousands of competing definitions of "culture" in the scholarly literature. Culture, as we shall see, involves a diverse set of beliefs and behaviours. Unless we identify these at the outset, the reader will not have a precise idea of what the "culture" of a particular group refers to.

- Clarity is particularly important given our emphasis on interactions. We will use the word "institutions" to refer to the formal rules of a society. We can note here that some define this word so broadly that it subsumes much of what we will call "culture." Unless we distinguish institutions from culture, we would be unnecessarily vague and confusing in our discussion of critical elements of politics and the economy, for institutions loom large in both spheres. And how could we outline the influences of culture on institutions if our definitions conflate the two?

Is it feasible to identify the full set of phenomena investigated by social scientists and humanists? It is actually straightforward to do so. Indeed, in Table 1.2 we provide a list of the central themes and subsidiary phenomena addressed across the social sciences and humanities. This table is organized around ten main themes.

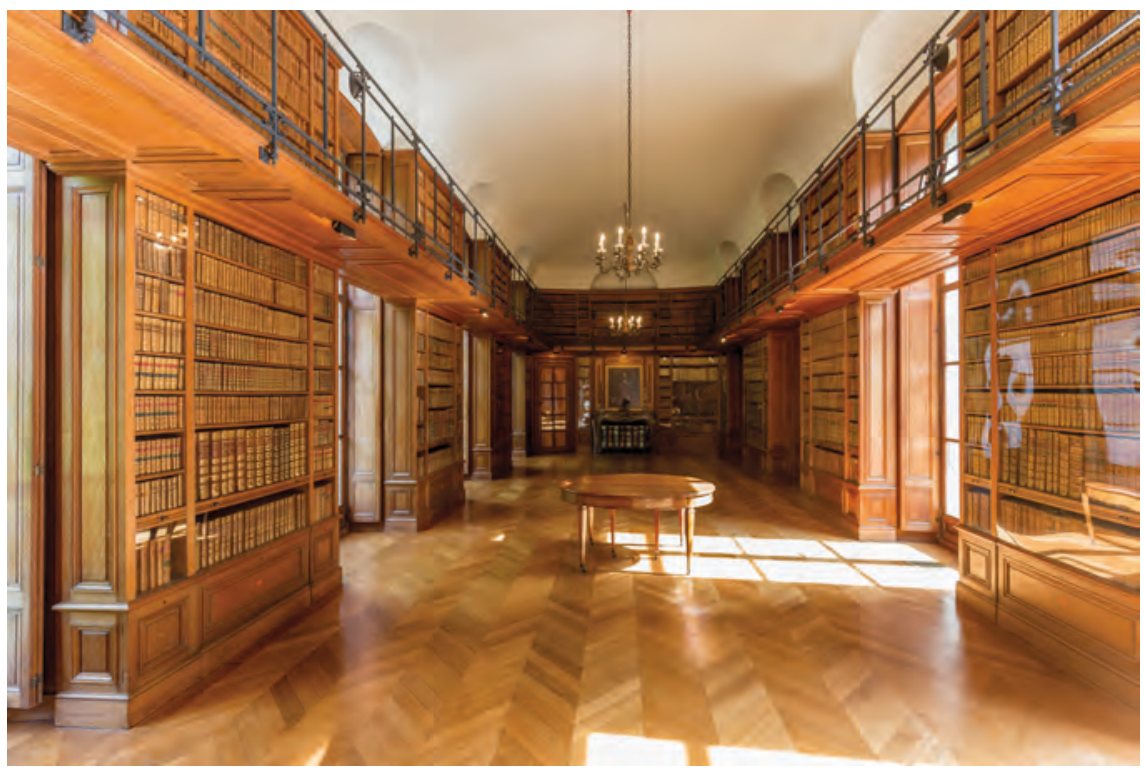

Illustration 1.4

Library, Castle of Beloeil, Belgium

Source: Photograph by Davidh820, CC BY-SA 4.0 
We have already noted that two themes capture individual-level phenomena: our shared genetic predisposition and individual differences in abilities and personality. There are also seven themes that operate at the societal level: economy, polity, culture, social divisions (by gender, occupation, ethnicity, and so on), technology and science, population and health, and art. To these, we can add a theme of the non-human environment that encompasses the physical world with which humans interact.

We will look at each of the main phenomena (that is, the second-level phenomena in Table 1.2) within each of the ten themes (these phenomena are each bolded at their first mention below) and ask of each phenomenon two questions: Is it important historically? Is it possible to trace that phenomenon historically? From this exercise, we will identify the phenomena that will be the focus of the book.

We discuss the cultural and social themes in detail below. We will discuss other themes in the next chapters: the non-human environment in chapter 2 , genetic predisposition and individual differences in chapter 3 , the economy in chapter 4 , technology and science in chapter 5 , politics in chapter 6 , population and health in chapter 7 , and art in chapter 8 .

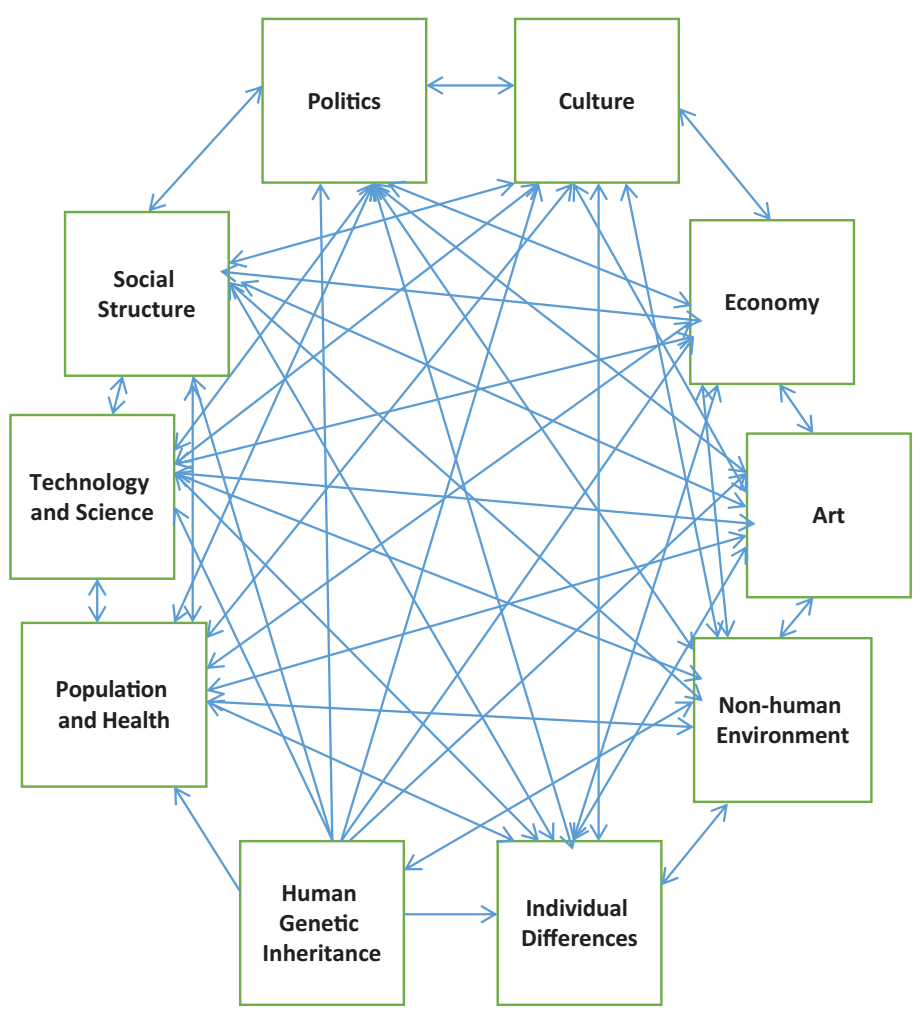

Figure 1.1

Thematic interactions

Note: There are potentially two-way arrows between any pair of themes, except for genetic inheritance. 
Does life really fit neatly into ten categories? Our sense of the world is often that life instead is a jumble of contradictory and inter-connected influences. But that is precisely the point: We can only hope to understand our lives - or the lives lived by others in history - by identifying distinct themes and how these interact.

\section{Our themes and the world of today}

We should be careful of approaching history only through a narrow lens that reflects modern circumstances and concerns; we should not ignore events and processes that were hugely important to the people in the past just because they fail to resonate in the present. Nevertheless, it is worth noting that the themes we will follow will help us to understand key features of the modern world: How did the global distribution of religions emerge? How did the global distribution of political institutions arise? How did the global distribution of incomes and wealth develop? How have genders and ethnicities, and classes/occupations interacted through history? What role has war played in human history, and what trends can we observe in its incidence and severity? Has humanity progressed in important ways? We will address these questions throughout the book, and then revisit them in the final chapter. A fundamental argument of this chapter - and of the book as a whole - is that there are many phenomena that we would wish to follow through history.

\section{THE THEME OF CULTURE}

All human societies - even that of the early hominids - are characterized by a set of "cultural" beliefs and practices. As we have already noted, the scholarly literature defines culture in literally thousands of ways. For our purposes, we provide a very precise definition: Culture comprises language, religion, stories, values, and expressions of culture such as rituals, dance, cuisine, etc. The most obvious element of culture is a set of values that includes attitudes toward wealth, power, love and friendship (enumerated in detail in Table 1.2.). Cultural values serve a purpose: They provide members of a society with a blueprint of how they, and others, are expected to behave. Since different values encourage different behaviours, values have a significant influence on all other social phenomena. Cultural values, in turn, will be influenced by these other phenomena, for societies will benefit if their values are well suited to their economy, political organization, and natural environment.

Cultural values are often transmitted between generations in stories. These stories often provide us with the best evidence of a society's values. While the stories themselves do not necessarily have a significant influence on a society, the values they communicate do.

Values are often associated with religion. We can define religion as a set of shared beliefs regarding mortality, nature, the supernatural, and community. Religion is ubiquitous in human society and has inevitably been a critical focus of world history. We will trace developments in religion in general and also particular religions. We will seek to understand how these both reflected and affected other phenomena. 
Values are often also associated with music, dance, clothing, and other expressive elements of culture. These expressions of culture serve the vital purpose of consolidating group identity. The way they reflect values is less evident than the way that, for example, stories do. It is not clear that differences in such expressions - though of great interest to the tourist necessarily reflect or influence other phenomena. While it is important that a society dances, perhaps that it dances in a particular way is not that important. So, we will not discuss different cultural expressions unless there are significant connections with other phenomena. One exception here is cuisine; from time to time, we will consider the inter-societal transmission of cuisine.

Language is usually treated as a component of culture. However, it is quite different from other aspects of culture. Language is necessary for the communication of values and religions and stories, so it is easier for a society with a shared language to have shared values. From time to time we will discuss how languages have spread.

\section{THE THEME OF SOCIAL STRUCTURE}

Human societies are never homogeneous. In addition to individual-level differences, there are also always distinct social groups. Two types of groups are ubiquitous: gender groupings and family. After the development of agriculture, social divisions by occupation are also common. So, too, are ethnic divisions. Other social divisions are also possible. We call the interactions among different social groups "social structure."

Recent trends in historical scholarship have widened the scope of enquiry beyond the study of politics and a narrow elite. In addition to integrating women into our understanding of history, we will try to capture also the experience of people in different classes or occupations, or of different ethnicities or religion within a society. What were they allowed to do and what did they do? In particular, we will discuss the challenges facing key occupational groups such as farmers, merchants, and artisans - and how these were addressed. We will note that one of the critical challenges confronting (most) rulers is managing ethnic diversity. We are unfortunately often limited in the documentary evidence regarding the lives of the relatively powerless.

When we can, we will record patterns of family life - nuclear, extended, and so on - and discuss their effects on other phenomena.We define "family" as "as a small group that cohabits for a while and is connected by descent, marriage, or adoption." Recent scholarship tries to understand the experience of children or the elderly in different historical societies, but we are still unfortunately very limited in our ability to discuss experiences by age group. We will also only rarely catch glimpses of the treatment of groups with a different sexual orientation.

One important consideration is whether people in inferior positions in society accepted their fate. Did they chafe under perceived injustice but feel powerless to object? Or did they accept the inevitability of their inferior status? Some institutions - police, armies, prisons, 
and so on - can force people to tolerate inequality of various types. But there are also subtler means to achieve social stability. Some of these are cultural; religious beliefs, for example, may encourage acceptance of misfortune, or even bestow godly approval on inequality. Ideologies are belief systems focused on justifying a particular allocation of social, political, or economic power and influence. Belief systems are often critical to the survival of particular societies, but also to their eventual collapse. Where possible, we will discuss the ideologies that supported, or challenged, social stability.

\section{Table 1.2 The phenomena}

\begin{tabular}{|c|c|c|}
\hline Themes & Second-level phenomena & Third-level phenomena \\
\hline \multirow[t]{3}{*}{$\begin{array}{l}\text { Genetic } \\
\text { predisposition }\end{array}$} & Abilities & $\begin{array}{l}\text { Consciousness, subconsciousness, } \\
\text { vocalization, perception (five senses), decision- } \\
\text { making, tool-making, learning, other physical } \\
\text { attributes (locomotion, eating, etc.) }\end{array}$ \\
\hline & Motivations & $\begin{array}{l}\text { Food, clothing, shelter, safety, sex, betterment, } \\
\text { aggression, altruism, fairness, identification with } \\
\text { group }\end{array}$ \\
\hline & Emotions & $\begin{array}{l}\text { Love, anger, fear, jealousy, guilt empathy, anxiety, } \\
\text { fatigue, humour, joy, grief, disgust, aesthetic sense, } \\
\text { emotional display }\end{array}$ \\
\hline \multirow{11}{*}{$\begin{array}{l}\text { Individual } \\
\text { differences }\end{array}$} & Abilities: & \\
\hline & Physical abilities & Speed, strength, endurance \\
\hline & Physical appearance & Height, weight, symmetry \\
\hline & Energy level & Physical, mental \\
\hline & Intelligences & $\begin{array}{l}\text { Musical, spatial, mathematical, verbal, kinaesthetic, } \\
\text { interpersonal }\end{array}$ \\
\hline & Personality: & \\
\hline & $\begin{array}{l}\text { Sociability } \\
\text { (Extro/introversion) }\end{array}$ & $\begin{array}{l}\text { Talkative, assertive, adventurous } \\
\text { enthusiastic vs. reserved, withdrawn }\end{array}$ \\
\hline & $\begin{array}{l}\text { Emotionality } \\
\text { (Stable/moody) }\end{array}$ & $\begin{array}{l}\text { Contentment, composure vs. } \\
\text { anxiety, self-pity }\end{array}$ \\
\hline & Conscientiousness & $\begin{array}{l}\text { Thoroughness, precision, foresight, organization, } \\
\text { perseverance vs. carelessness, disorderly, frivolous }\end{array}$ \\
\hline & $\begin{array}{l}\text { Affection } \\
\text { (Selfish/agreeable) }\end{array}$ & $\begin{array}{l}\text { Sympathetic, appreciative, kind, } \\
\text { generous, vs. cruel, quarrelsome, fault-finding }\end{array}$ \\
\hline & $\begin{array}{l}\text { Intellectual orientation } \\
\text { (Holistic/analytical) }\end{array}$ & $\begin{array}{l}\text { Openness, imagination, curiosity, } \\
\text { sensitivity vs. closemindedness }\end{array}$ \\
\hline
\end{tabular}




\begin{tabular}{|c|c|c|}
\hline Themes & Second-level phenomena & Third-level phenomena \\
\hline & Other dimensions? & $\begin{array}{l}\text { Dominant/submissive, in/dependent, strong/ } \\
\text { weak, future/present-oriented, humour, aggression, } \\
\text { happiness }\end{array}$ \\
\hline & Disorders? & Schizophrenia, psychoticism, ...? \\
\hline & Sexual orientation & \\
\hline & Schemas & View of self, others, causal relationships \\
\hline & Interpersonal relationships & $\begin{array}{l}\text { Parent/child, sibling, employee/r, romance, } \\
\text { friendship, casual }\end{array}$ \\
\hline \multirow[t]{4}{*}{ Economy } & Total output & $\begin{array}{l}\text { Price level, unemployment, trade, individual goods } \\
\text { and services }\end{array}$ \\
\hline & Income distribution & \\
\hline & Economic ideology & \\
\hline & Economic institutions & $\begin{array}{l}\text { Ownership (inheritance), production, exchange, } \\
\text { trade, finance, labour relations, organizations }\end{array}$ \\
\hline \multirow[t]{2}{*}{ Art } & Non-reproducible & Painting, sculpture, architecture, prose, poetry \\
\hline & Reproducible & Theatre, film, photography, music, dance \\
\hline \multirow[t]{5}{*}{ Social structure } & Gender & \\
\hline & Family types, kinship & Nuclear, extended, single parent \\
\hline & Classes (various typologies) & Occupations (various) \\
\hline & Ethnic/racial divisions & \\
\hline & Social ideology & \\
\hline \multirow[t]{5}{*}{ Politics } & Political institutions & $\begin{array}{l}\text { Decision-making systems, rules (including laws), } \\
\text { organizations }\end{array}$ \\
\hline & Political ideology & \\
\hline & Nationalism & \\
\hline & Public opinion & Issues (various) \\
\hline & Crime & Versus persons/property \\
\hline \multirow{6}{*}{$\begin{array}{l}\text { Technology and } \\
\text { science }\end{array}$} & Fields (various) & Innovations (various) \\
\hline & Recognizing the problem & \\
\hline & Setting the stage & \\
\hline & Act of insight & \\
\hline & Critical revision & \\
\hline & Diffusion/transmission & Communication, adoption \\
\hline \multirow[t]{2}{*}{ Health } & Nutrition & Diverse nutritional needs \\
\hline & Disease & Viral, bacterial, environmental \\
\hline
\end{tabular}




\begin{tabular}{|c|c|c|}
\hline Themes & Second-level phenomena & Third-level phenomena \\
\hline \multirow[t]{4}{*}{ Population } & Fertility & Fecundity, deviation from maximum \\
\hline & Mortality & Causes of death (various) \\
\hline & Migration & Distance, international?, temporary? \\
\hline & Age distribution & \\
\hline \multirow[t]{9}{*}{ Culture } & Languages & By descent? \\
\hline & Religions & Providence, revelation, salvation, miracles, doctrine \\
\hline & Stories & $\begin{array}{l}\text { Myths, fairy tales, legends, family sagas, fables, } \\
\text { jokes }\end{array}$ \\
\hline & Expressions of culture & $\begin{array}{l}\text { Rituals, dance, song, cuisine, attire, ornamentation } \\
\text { of buildings, games }\end{array}$ \\
\hline & Values & \\
\hline & (Goals:) & $\begin{array}{l}\text { Ambition, optimism, attitudes toward wealth, } \\
\text { power, prestige, honour, recognition, love, } \\
\text { friendship, sex, incest, marriage, time preference, } \\
\text { physical and psychological well-being }\end{array}$ \\
\hline & (Means:) & $\begin{array}{l}\text { Honesty, ethics, righteousness, fate, work valued } \\
\text { intrinsically, violence, vengeance, curiosity, } \\
\text { innovation, nature, healing }\end{array}$ \\
\hline & (Community:) & $\begin{array}{l}\text { Identity, family versus community, openness to } \\
\text { outsiders, egalitarianism, attitude to young and } \\
\text { old, responsibility, authoritarianism, respect for } \\
\text { individuals }\end{array}$ \\
\hline & (Everyday norms:) & $\begin{array}{l}\text { Courtesy, manners, tidiness, proxemics, cleanliness, } \\
\text { punctuality, conversational rules, locomotion rules, } \\
\text { tipping }\end{array}$ \\
\hline \multirow{12}{*}{$\begin{array}{l}\text { Non-human } \\
\text { environment }\end{array}$} & Soil & Soil types (various) \\
\hline & Topography & Land forms (various) \\
\hline & Climate & Climate patterns (various) \\
\hline & Flora & Species (various) \\
\hline & Fauna & Species (various) \\
\hline & Resource availability & Various resources \\
\hline & Water & Availability \\
\hline & Natural disasters & Flood, tornado, hurricane, earthquake, volcano \\
\hline & Day and night & \\
\hline & Transport infrastructure & Mode (various) \\
\hline & Built environments & Offices, houses, fences, etc. \\
\hline & Population density & \\
\hline
\end{tabular}

Source: Szostak $(2003,2004)$ 


\section{Understanding change in history through evolutionary analysis}

Evolutionary analysis plays a vital role in the story we will tell. We should stress that our focus on evolutionary processes provides an important complement to our focus on interactions among themes. Evolution, though conditioned by other phenomena, focuses on how individual phenomena change through time. It is the cumulative combination of changes within themes and interactions across themes that generates the majesty of history.

Evolutionary analysis involves three key components: mutations, a selection environment, and a transmission mechanism.

A mutation is an alteration of some aspect of a phenomenon. In biological evolution, mutations are random changes in genes. For the sorts of mutation addressed in this book, some kind of human initiative must be involved: some individual must write down a new rule, develop a new technology, or create an original painting. Such acts are almost certainly less random than genetic mutations, but, as we shall see, there is still a considerable degree of randomness.

The key to evolutionary analysis lies in a hypothesis that only a small subset of mutations survives. This hypothesis guides us to focus attention on selection mechanisms. Why were some mutations chosen and not others? As long as humans are experimenting with different technologies, values, rules, and types of painting, and only some of these experiments have lasting effects, we can reasonably apply evolutionary analysis to social processes.

The unit of social mutation is the "idea." We can identify one or more novel ideas at the heart of any of the types of evolution addressed in this chapter. In the case of culture, and especially art, it may not always be easy to describe the nature of the novel idea in words. But we can try to do so.

In biological evolution, the selection environment is the natural environment. A mutation that enhances an organism's ability to survive and have success in mating within its environment will increase the chances that the gene is transmitted to future generations. So, a mutation that enhances the organism's "genetic fitness" - a combination of survival and reproduction - will spread through a population.

The selection environment for human biological evolution, and for the various types of societal evolution addressed below, is the sum of the state of all other phenomena outlined in Table 1.2. Whether a particular technological innovation is selected will depend on whether it makes economic sense, is acceptable politically and culturally, fits with accepted social roles, fits with aesthetic appreciation, and so on. In studying the path of innovation, then, it makes sense to wonder why conditions were conducive to a particular innovation.

Students of biological evolution note that evolution may occur more quickly after an environmental change because of an increased likelihood that mutations will be beneficial for the organism. Likewise, we can appreciate that 
changes in the selection environment for social mutations will have an impact on the direction and speed of social evolution. In social evolution, changes in selection environment will also likely increase the rate of mutation, as humans struggle to cope with a new reality. We will thus devote particular attention in what follows to how selection environments themselves change through time.

The third essential element of evolutionary analysis is a transmission mechanism. How does a successful mutation get passed from generation to generation? In the case of biology, the answer is straightforward: genetically, from parent to child.

The transmission mechanisms for societal evolution are more complicated. Ideas can be transmitted in a variety of ways. This can have important effects on the rate of evolution: If young people absorb cultural ideas from each other rather than just their parents, culture can change very rapidly. In literate societies, ideas can be written down and transmitted across generations. Literacy, then, can increase the staying power of mutations. An idea that is written down has a better chance of being transmitted unchanged than an idea passed by word of mouth from generation to generation.

As we survey the various phenomena that are subject to evolutionary analysis, we have a set of questions to ask: How random are these mutations? Which other phenomena are most important in the selection environment? To what extent are ideas likely to be transmitted unchanged across generations?

\section{Inter-societal transmission}

Our discussion of transmission leads us to suspect that ideas - cultural, political, technological, and more - often change as they move from one society to another. First, communication itself is imperfect. This is especially likely to be the case in cross-societal transmission in which differences in language or culture or scientific understanding will encourage misunderstanding. There is then the challenge of adoption: For an idea to survive in its new home it must fit into the local selection environment, and will likely have to be adapted in some way. As they move around the globe, even religions and technologies change in meaningful ways to fit in with local cultures or environmental conditions or polities. Thus, transmission itself generally involves mutation.

So, evolutionary theory helps us to understand why different regions continue to have different cultures, institutions, and technologies despite millennia of contact. To be sure, communication encourages some degree of convergence as regions borrow from each other. But different societies follow different evolutionary paths (see chapter 2), and cannot easily switch from one to another.

We can apply evolutionary analysis to several social phenomena:

- cultural values and practices

- institutions (the formal rules we live by), both political and economic

- scientific understanding of both natural and human science 
- technology

- $\quad$ artistic styles and themes, including literature.

We will discuss cultural evolution here, institutional evolution in chapter 6, technological and scientific evolution in chapter 5, and artistic evolution in chapter 8 . We should also note that biological evolution itself is an integral part of our story. We will discuss human evolution in chapter 3, and analyse the selection environment in which this occurred. We will discuss other types of biological evolution in chapter 2 .

\section{Evolution in cultural values and practices}

It is easy to imagine that cultural mutations are usually fairly random. An individual alters their beliefs or behaviours in some trivial way, and others copy them. The person may not have thought very hard about the initial change. They may have made the change by chance and could as easily have done something else. Or, they may have sensed some advantage in the change, divining some aspect of the selection environment that would make the change beneficial to them.

Some cultural mutations are not at all random. In the modern era, advertisers devote vast sums deliberately trying to alter attitudes and behaviours. Throughout history, governments and religious leaders have attempted to encourage attitudes and behaviours conducive to their authority. But such efforts are not always successful. People do not always do as they are instructed. In other words, authorities sometimes either fail to appreciate the selection environment or fail to dominate transmission mechanisms. Even with purposeful mutation, then, it is still to the selection environment that we appeal for explanation and understanding.

What comprises the "selection environment" for cultural mutations? A key element is culture itself. Cultural attitudes are broadly complementary - that is, some cultural attitudes tend to go together. For example, you cannot value personal ambition without also valuing competition. An attempt to change one value may well fail if it does not align with other values. An attempt to change several values at once may fail because it is too confusing. The purpose of culture, after all, is to allow members of a society to know how to interact with each other. Cross-cultural contact is both frustrating and exciting precisely because we no longer know what to expect from others. At times of rapid cultural change, individuals often suffer severe emotional stress because they no longer know how others will behave and thus how they should behave. So, small changes are much more likely to succeed, but only if they fit with existing attitudes reasonably well. We might imagine an evolutionary process in which a change in one attitude increases the likelihood of changes in others. Increased respect for ambition, for instance, may lead over time to increased appreciation of competition. In this way, a complementary change in a set of attitudes might be achieved without the jarring effects of rapid cultural change. 
This evolutionary view of culture depends on several assumptions that are not universally accepted. The first is that culture is not monolithic - in other words, each cultural attitude is not precisely determined by the set of all other attitudes. Monolithic cultures would have difficulty changing over time, for any one change in attitudes would struggle to trigger a sudden change in all others. So, we assume there is some flexibility in cultures so that one attitude can change a little without requiring the sudden transformation of all other attitudes.

Indeed, it may be best to think of cultures as always possessing internal contradictions. Modern Western societies value both equality and freedom: Much social and political discourse involves decisions about which of these values to prioritize when they inevitably conflict. Given that any culture involves thousands of attitudes and behaviours, conflicts among these will occur regularly and provide an internal dynamic encouraging cultural evolution.

Such a view may suggest that gradual cultural change rather than stasis is the norm in human societies. "Traditional" societies are often assumed to be unchanging, but this may merely reflect the fact that they seem so different from us, and that contact with other societies encourages rapid change. We then imagine that only such contact causes change. The historical record, as we shall see, suggests that cultural change is far from uncommon.

A second - and complementary - assumption is that there is scope for individual diversity within cultures. Only then can one individual initiate a cultural mutation by changing an attitude or behaviour. Rather than assuming that everyone in a society has identical attitudes, we should think of cultural attitudes as tendencies from which individuals deviate to various degrees. Some cultures may be more tolerant of deviations than others. But no culture permits no degree of individual diversity. Individual differences in personality and ability probably play some role in cultural change. People who occupy positions of influence may find it is easier to initiate cultural change.

Cultures do not operate in isolation but interact with economic, political, social, natural, and artistic phenomena. We might anticipate that groups will choose (albeit subconsciously) cultural attitudes that reflect and support their economic environment. A group that must cooperate to chase buffalo over cliffs will likely have cultural values that favour cooperation and sharing; a group that relies on individual trap-lines may value individual initiative. Any group in which individuals are at the mercy of random events that threaten starvation will have attitudes that favour charity toward the deserving poor. But we should not embrace economic - or any other type of - determinism: Groups can and do choose to eschew eating pork or beef even though pigs and cows are well suited to their natural environment.

Among a society's elite, there will be some selection for attitudes that justify the unequal distribution of power, status, or wealth. But societal stability has its value even to those at the bottom, and those in subservient roles may (again, often subconsciously) opt for attitudes that support the status quo over social 
or political instability. At times of revolution, we need to understand how and why people change their attitudes in order to risk dramatic change.

Societies compete. So, we need to consider not just selection within groups but selection among groups. A society with attitudes that lead to economic growth may come to dominate societies with attitudes that discourage economic growth. A society with attitudes that encourage military cooperation may come to dominate pacifist societies. If defeated and destroyed, a society's cultural values may disappear. If its members are absorbed into the political or economic orbit of other societies, their values may become more like those of the dominant society. Nevertheless, the cultures of more dominant groups often absorb cultural attributes of the defeated or relatively powerless.

Cultural attitudes can be transmitted in any number of ways. Those who command respect tend to be more influential, so that transmission from old to young and from dominant to powerless may be particularly prevalent. But transmission in the opposite direction is possible. If young people influence each other, culture will change faster than if they merely follow their elders: Cultural attitudes toward the elderly may thus affect the rate of cultural change (as will other factors such as the age distribution of the population).

Writing is particularly important in cultural transmission and preservation. Preserving cultural attitudes in writing may serve to slow cultural change. This will be especially the case if a society values cultural integrity. If specific texts are endowed with religious significance, it becomes even harder to change the attitudes or behaviours advocated in those texts. As we have already pointed out, a society's stories may be a particularly powerful mechanism for the

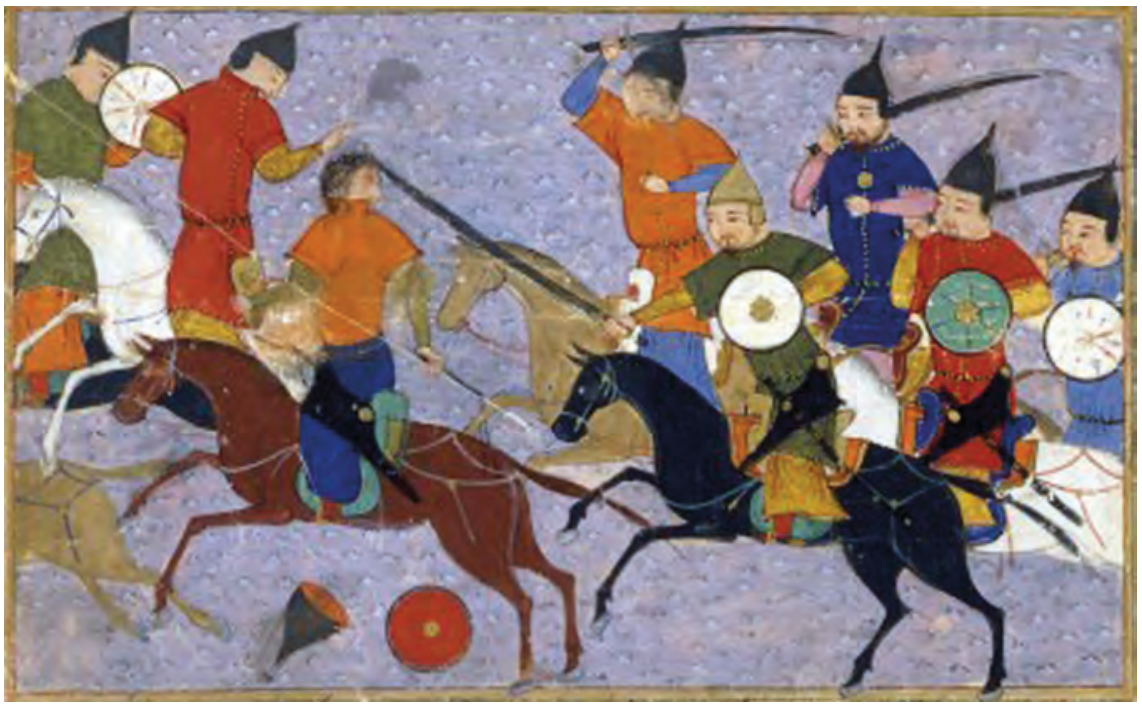

Illustration 1.5

Battle between Mongols and Chinese, 121 1, from Rashad al-Din's Jami' al-tawarikh 


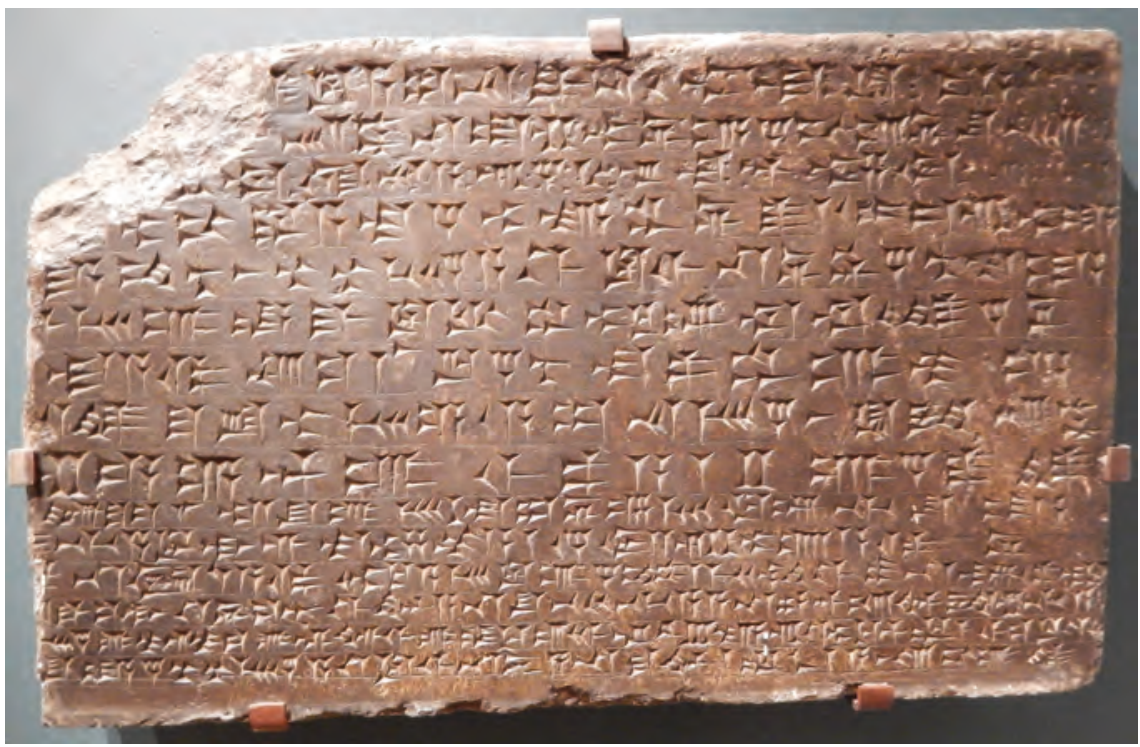

Illustration 1.6

Fragment of a stone relief in the Assyrian Northwest Palace, describing

Ashpurnasirpal's conquest of lands from Uratu to the mountains of Lebanon

Source: Photograph by BabelStone, CC BY-SA 3.0

intergenerational transmission of its values; if these stories are only preserved by repeated retelling, they may change as values change. A story that has been written down, however, is less likely to change.

Why do the world's cultures differ as much as they do - and did so even thousands of years ago? An evolutionary approach suggests four answers to this question. First, a cultural mutation may occur in one society but not others. Second, since cultures evolve slowly, building on what has gone before, any small initial difference may be magnified over time. That is, any cultural difference changes the selection environment for further mutations. For example, if a society develops a strong sense of identity, it may differentiate itself from others in a variety of ways. Third, the degree of cultural distinctiveness will depend on the degree of isolation. We have witnessed some convergence of culture in recent decades because of the ease with which ideas are can be transmitted around the world. Fourth, the selection environment for culture includes political, economic, environmental, and social phenomena as well as the preexisting culture on which mutations build. Any differences in these other phenomena will likely influence culture. Note that similarities in culture across societies can be attributed to similarities in selection environment or to transmission among societies of cultural ideas. Though most societies have some desire for differentiation, it is also common in world history to observe deliberate attempts to copy other cultures: Chinese practices were valued in Central Asia, Southeast Asia, Korea, and Japan; the Romans deliberately adopted Greek gods, philosophy, and art. 


\section{Evolution and progress compared}

Evolution does not have to be, but can be, "progressive" - that is, producing improvement over time. If a selection environment is stable for an extended period, we can expect selection for mutations that are an increasingly good fit for that environment. But in practice, selection environments change often, and mutations and selection move in a new direction. If the environment changes dramatically, culture or institutions or technology may seem for some time to be less well suited to the new environment than they were to the old.

Whether evolution results in outcomes that make most people better off depends on the selection environment. The most obvious consideration here is whether people in power dominate selection or whether the broader population does. Selection will often serve the interests of one group of people at the expense of others. Moreover, fear may drive selection: We may all prefer a world without atomic bombs, but fear even more a world where only our enemies have them.

World history must attempt to comprehend both stability and change: Empires, for example, will often survive for centuries and then collapse. Evolutionary analysis can be helpful here. The idea that evolution is a gradual process helps us comprehend lengthy periods in which phenomena appear to change little. But at the same time, the recognition that mutations are frequent, that changes in any one phenomenon alter the selection environment for others, and that rapid change is often observed when selection environments change, allows us to understand periods in which change is seen on multiple fronts - cultural, political, artistic, economic, etc.

\section{Philosophical orientation of this book}

You should be aware that in recent decades the discipline of history has been the scene of intense debate about the best way to study the past. These debates to some extent reflect the fact that history is part of both the social sciences and humanities, and has therefore absorbed influences from each.

One side in this debate - sometimes termed "postmodern" - has emphasized the importance of perspective: People from different countries, ethnicities, genders, time periods, or theoretical orientations are likely to see the past differently. Postmodernists are often sceptical of the notion that there is a unique historical "reality" we can discover. They instead emphasize the meanings that people of a time and place attached to the events they witnessed. Postmodernists also argue that each historical event is unique and comparison across time and place impossible. They have deep scepticism regarding over-arching "metanarratives" that attempt to simplify the course of history. Some have raised stylistic concerns. They argue that historians like to tell a "story" and so tend to adapt the facts to fit story-telling. 
The other side - the "modernists" - argues that historical research can lead to more accurate understandings of the past. If we can talk about accuracy, we must believe that there really is a historical reality, a set of events and processes that actually happened and for reasons that we can divine. This allows comparison of past events and processes to become an essential element of historical understanding. We can better understand what happened and why in one case by comparing it to similar situations.

This book seeks a middle ground. It assumes there is a reality out there, as modernists generally do, but also recognizes we are constrained in our ability to comprehend it by several concerns raised by postmodernists: not just our biases but also the limits to human understanding and communication. Historical scholarship advances by adding evidence and argument in order to generate increasingly compelling conclusions. Therefore, we will draw upon a wide array of perspectives. We will seek to evaluate the insights produced by different authors and explore whether we can integrate these into a more comprehensive understanding. We will also compare across a wide range of cases. We will address the meaning that individuals attach to events not as an alternative to explanation but as a component of explanation by seeking to integrate the understanding that comes from different perspectives. You should be comforted by the fact that there is a fair bit of consensus regarding what happened in history and (to a lesser extent) why it happened. We shall see if we can expand areas of agreement.

\section{Interdisciplinarity and world history}

This synthesis of modernist and postmodernist perspectives reflects thinking among scholars of interdisciplinarity - that is, the practice of combining several academic disciplines in the study of a subject in the hope that integrating insights from different disciplines will yield a better understanding. Scholars of world history often stress the advantages of an interdisciplinary approach. The idea is that a more accurate - more nuanced, more comprehensive - understanding of history can be gained by bringing different disciplinary perspectives and expertise to bear on historical issues.

The growing emphasis on interdisciplinarity also reflects the thematic focus that has come to characterize world history. If cultural, economic, political, social, and other forces are intertwined in history, it makes sense to draw on multiple disciplines to understand these interactions. Since this book stresses thematic interactions, it is particularly important that we adopt an interdisciplinary approach that will allow us to integrate the arguments of different scholars.

Interdisciplinary studies often rely on a two-way relationship between scholars who do specialized research and scholars who integrate such research into a broader analysis. Interdisciplinary scholars necessarily draw upon insights generated within disciplines. But in doing so, they suggest to disciplines new research questions, new theories, and new methods. Disciplinary scholars can also correct any misunderstandings that might have crept into an integrative analysis. 


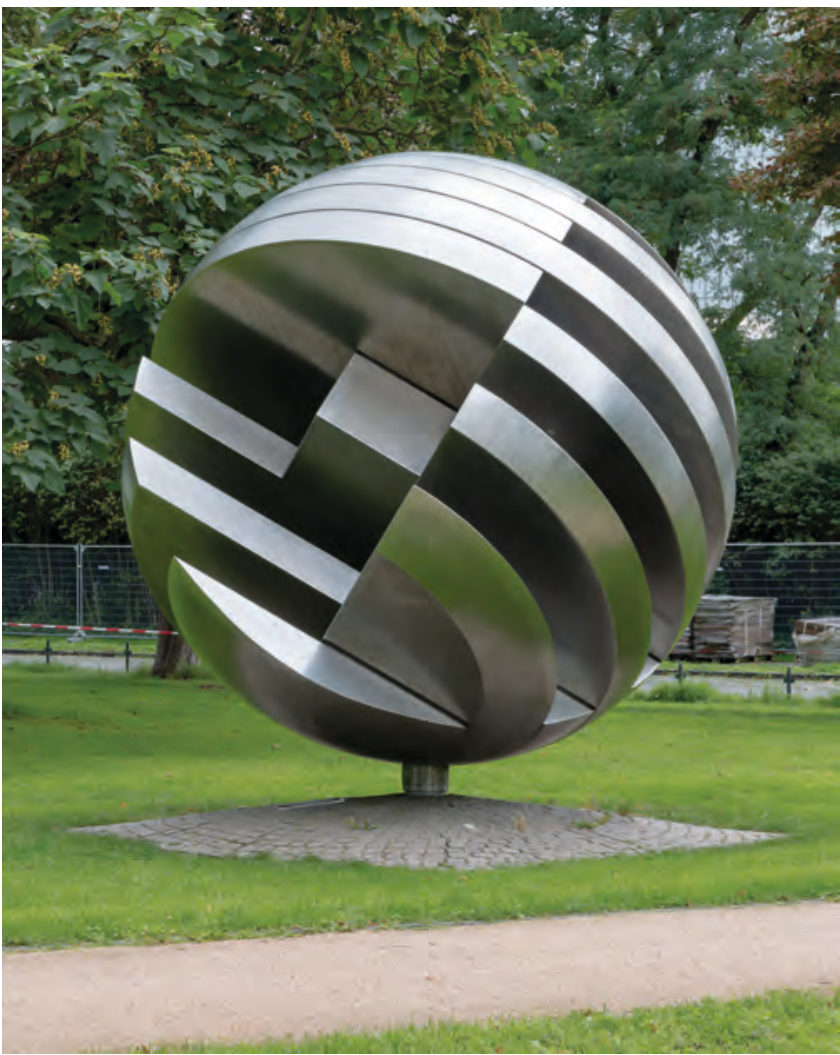

Illustration 1.7

Integration. Sculpture by Hans Dieter Bohnet, 1976, Bonn, Germany

Source: Photograph by Deitmar Rabich, CC BY-SA 4.0
The same sort of symbiotic relationship would be invaluable in history. World historians necessarily build on the work of other historians (and other disciplines). Other historians can learn much from world historians about how "their" time and place was affected by or can be compared to other times and places. They can also learn much about whether a particular thematic interaction they are examining-say the influence of authoritarian government on art has parallels elsewhere. A common language will facilitate this twoway conversation. Yet scholars of different lands and scholars of different fields each have their own terminology. The sort of approach advocated in this book, with its emphasis on interactions among carefully defined themes, can alert historians of particular times and places to when they are investigating a similar thematic interaction.

Happily for our project, the literature on interdisciplinarity has

come of age. There is now an extensive literature on how best to perform interdisciplinary analysis, and a fair bit of consensus on strategies that work well. We have in fact already employed some interdisciplinary strategies in this chapter. Our stress on clarity in terminology reflects a recognition that ambiguous terminology is one of the major barriers to cross-disciplinary communication and understanding. Our flowcharts reflect an interdisciplinary strategy of diagramming the critical relationships among phenomena involved in a particular issue or problem. In doing so, we can show that the phenomena emphasized by different scholars interact. Then, we do not have to choose between their explanations of events but can combine them. The next few sections expand on how we will apply interdisciplinary strategies in this book.

\section{Both/and thinking}

Interdisciplinary scholarship guides us to look past false dichotomies. Faced with an intellectual controversy, we prefer not to take sides but to look for the 
good in competing arguments. Even when we must reject certain conclusions (such as that the sun revolves around the earth), we can still respect the analysis on which the conclusion rested. We will not shy away from controversy in this book, but we will seek to transcend it through careful evaluation and synthesis.

We should realize that the alternative either/or approach - that is, choosing between different answers - is common both in academia and the outside world. Individuals are often applauded for taking a firm stand and derided for "sitting on the fence." Though a both/and perspective will guide us, this should not trouble the either/or thinker. We can combine ideas only after we have first evaluated them. The reader who wants a more straightforward historical narrative than we provide will have many opportunities to pick sides. Our approach is to invite discussion because by discussing possible alternative understandings the best understanding of the past often emerges.

The interdisciplinary strategy of "transformation" embodies a particular type of both/and thinking. We will employ this strategy when scholars make opposing arguments. For example, an economic historian studying migration may argue that migrants perform a "rational" calculation of costs and benefits. A cultural historian may stress "non-rational" elements of the decision such as feelings toward their society of origin and pressure from peers who may also be considering migrating. Rather than deciding that one is right and the other is wrong, we can appreciate that migrants are guided by a mix of rational and non-rational processes. We can indeed think of a continuum between perfect rationality and complete non-rationality and evaluate where certain migrants may have operated along such a continuum. We thus transform a seeming dichotomy into a balanced understanding.

\section{Valuing multiple perspectives}

We have stressed the value of integrating different perspectives, whether these perspectives are grounded in different disciplines or in social divisions such as gender or occupation or ethnicity. One implication of this approach is that we should strive to have a place for all types of people in our history. It should not just be a history of mostly male leaders of the most powerful societies. We should try to understand what life was like for a variety of people and how they exercised human agency. Unfortunately, we know far less about people without power and societies without power, for they leave fewer records. But we will try.

We will also include a wide variety of societies in our study. Historians of Oceania, Africa, and the pre-Columbian Americas complain that their histories receive little attention in many world history texts. Leaving these societies out not only leaves people from such lands feeling excluded from world history but makes world history seem more straightforward and more unidirectional than it was. It is useful to continually ask ourselves why people in different parts of the world were living such different lives at different points in history. Otherwise, Eurasian developments can seem inevitable rather than highly contingent. Polynesian sailors, Aztec warriors, and Bantu kings were as much 


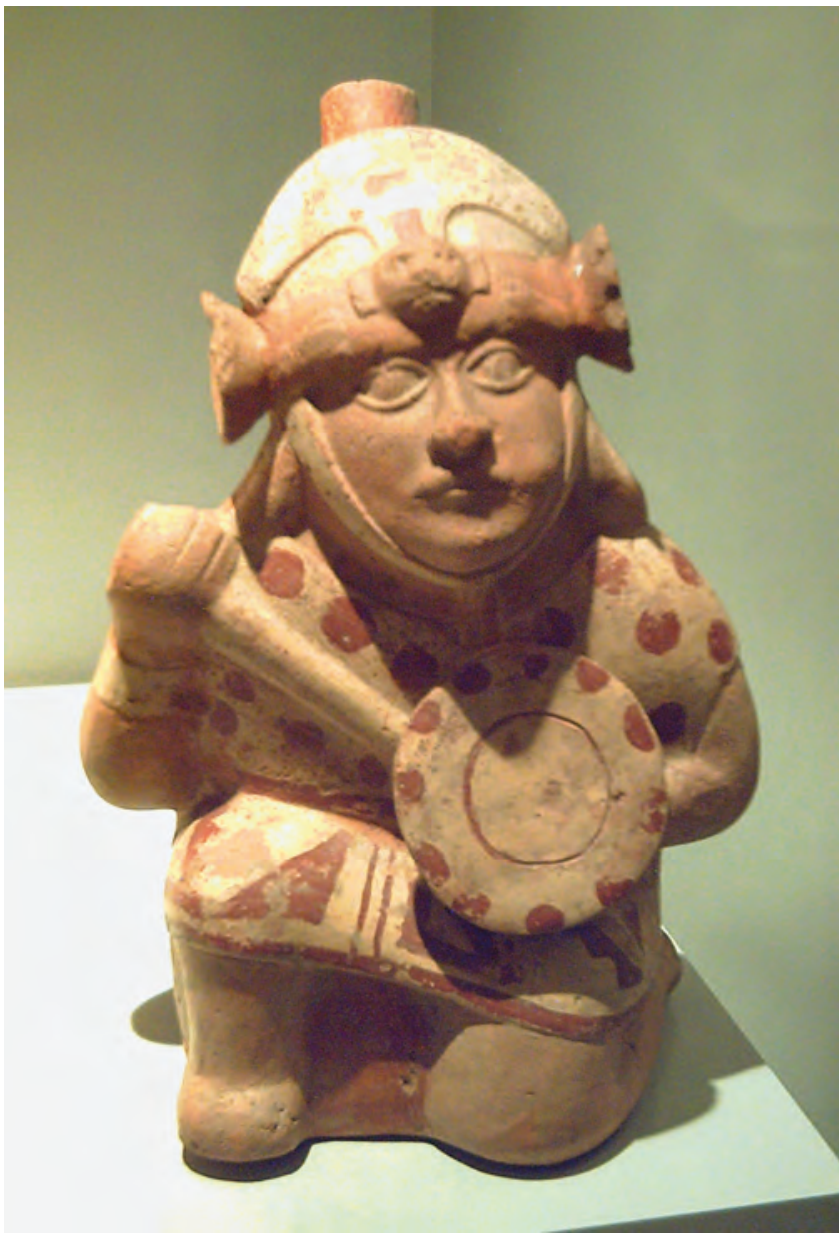

Illustration 1.8

Ceramic vessel representing a warrior, Moche Culture, South America, 100-700 CE, Museu America, Madrid

Source: Photograph by Luis García, CC BY-SA 3.0 a part of our collective history as Phoenician sailors or Chinese emperors. And, as we have already pointed out, less powerful societies can exert important influences on more powerful ones. Even our understanding of Eurasia will be limited if we do not address what was happening elsewhere.

So, the keys to our search for perspective are the inclusion of many different societies and social groupings; the search for connections in both directions between societies and between different social groups within a society; and an attempt to evaluate differing historical accounts in order to integrate the best elements of each.

\section{Outline of this book}

The second through sixth parts of the book provide our narrative of world history, following the rough chronological periods of most world history texts. But because we recognize that different historical processes operate at different rates, our time periods will not necessarily be precise and will

sometimes overlap. Within these periods, we will not follow a single dominant theme but will examine interaction among all the themes we have chosen. And we will recognize that different regions of the world may have quite different experiences.

Chapter 2 will address the "Big History prelude." Big History is a recent field of enquiry and is even more ambitious in scope than World History: It extends back to the Big Bang - the cosmic expansion that marked the beginning of our universe. So, it covers history both before and after humans came into existence. One popular way to make sense of Big History is to address threshold effects: When a certain threshold is reached, dramatic change ensues. The first such threshold occurred when the universe cooled enough that protons and electrons could bond to form chemical elements (see chapter 2). History then 
becomes a series of revolutions. In this chapter, we review the broad outlines of pre-human history with an eye to showing that it is also useful to look beyond the revolutions.

The next chapters address different historical episodes. Some focus on a particular historical transformation. Chapter 8, for example, examines the development of early religions. Other chapters deal with a handful of such transformations. Chapter 14, for example, addresses political consolidation, cultural consolidation, trade expansion, and monument building in the period after $1000 \mathrm{CE}$. Students should recognize that world history comprises a set of historical transformations, of which we generally address the most critical. The purpose of each chapter is to identify the most important processes at work as well as the most important thematic interactions and cross-societal connections. Key themes, interactions, connections, and evolutionary processes will change from one era to another although there may be elements of continuity. When scholars disagree about the relative importance of different processes and interactions and phenomena, we will show how these different insights might be integrated, and suggest avenues for further research. We will thus attempt to harness to the inherent excitement of history, the thrill of scholarly debate. Lack of scholarly consensus should not frustrate but stimulate.

Two of our organizing devices will be obvious when we employ them: the discussions of human challenges and the boxes. Our flowcharts will highlight the importance of thematic interactions, although we will not be able to diagram every thematic interaction or societal connection discussed in the text. As for evolutionary analysis, we will often be explicit and mention evolution or mutations or selection or transmission. At other times, however, we will not interrupt the narrative flow with explicit use of evolutionary terminology. So, we will open each chapter with a brief paragraph that will alert students to the themes, thematic interactions, connections across societies, and evolutionary processes that will be most important in that chapter. These themes will be highlighted visually: We will place small graphics at the start of chapters that indicate up to six themes that will receive the most attention. The themes that receive the most attention will be shaded blue. In chapters that address a number of historical episodes, this graphic appears at the start of each main section of the chapter.

Each chapter starts with our fifth organizing device: a set of guiding questions and discussion of how that chapter builds on preceding chapters and sets the stage for those to follow. This material allows students to appreciate better why we address specific topics in certain chapters.

We occasionally ask readers to place themselves in history because it is vital for them to see even distant history as their history. This is not always easy. Readers should remember that people were different from us even just centuries ago. They were, for the most part, closer to nature. They were also generally less well-nourished and more exposed to debilitating disease, and so weaker and smaller and perhaps less intelligent on average. It is a useful exercise for 
readers to imagine how they might have behaved in similar circumstances, and to reflect on why historical actors may have acted differently.

The seventh part addresses possible applications of the understanding of world history we have gained in previous chapters. Chapter 32 will examine whether we can draw lessons for the future from our knowledge of the past; we will argue that we would be foolish not to try but, given the ubiquity of "surprises" in history, we should be humble and cautious in doing so. This chapter discusses whether and how historical understanding can inform both public policy and individual behaviour. We will also briefly evaluate the usefulness of our organizing structure and suggest that it can be used in more than just historical study, including in the ways we organize our libraries and other information resources. Chapter 32 then discusses whether it makes sense to think of "progress" in history. We close with a discussion of how world history can inform the search for meaning.

\section{Questions}

1. Are you excited? Why?

2. Are there particular elements of the book's approach that you find particularly attractive (or troubling)? Why?

3. Why is it important to "organize" world history?

4. Are there particular lessons that you hope to learn from world history? It would be useful to reflect at the start of the book on what you wish to learn and return to those thoughts at the end.

5. How do processes of social evolution differ from biological evolution? Why is evolutionary analysis useful for the study of world history?

6. Do you prefer the modernist, postmodernist, or intermediate position? Why?

7. Why do we study world history?

\section{Readings}

The World History Association provides a very useful bibliography at: www.thewha. org/resource-links/bibliographies-recommended-books/

The resources below are a small sample of what is available.

Archer, Margaret, Roy Bhaskar, Andrew Collier, Tony Lawson, and Alan Norrie, eds. 1998. Critical Realism: Essential Readings. New York: Routledge.

Baker, David. 2016. "Collective Learning: A Potential Unifying Theme of Human History." Journal of World History 26:2, 77-104. DOI: 10.1353/jwh.2016.0006.

Harari, Yuval Noah. 2016. Sapiens: A Brief History of Humankind. New York: Random House.

Mitchell, Laura J., Ross E. Dunn, and Kerry Ward. 2016. The New World History. University of California Press. [Chapters 3 and 4 address the fuzzy nature of and 
changes in regional boundaries. The big lesson is that the historical question asked should determine the spatial area engaged. The first chapter recognizes the dangers of studying individual themes in isolation.]

Reilly, Benjamin. 2013. "Mapping Student Learning: Course Assessment Using Concept Maps in a World History Survey Course." World History Connected 10:2. [Reilly advocates concept maps. He gives students an assignment at the start and end of term where they are supposed to map relations among themes that comprise world history; he is pleased that they add more detail and increase stress on the environment.]

Sachsenmaier, Dominic. 2011. Global Perspectives on Global History: Theories and Approaches in a Connected World. New York: Cambridge University Press. [This book provides an international comparison of theories of and approaches to world history.]

Shreiner, Tamara L. 2020. “Turning on the Historian's Macroscope: A Call to Foreground the Teaching and Learning of Data Visualizations in World History Education." World History Connected 17:1.

Szostak, Rick. 2003. A Schema for Unifying Human Science: Interdisciplinary Perspectives on Culture. Selinsgrove, PA: Susquehanna University Press [This book shows how Table 1.2 can be derived, and how a broad range of works in the social sciences and humanities can be understood as drawing connections among phenomena in that table. It surveys a vast array of causal connections among the various phenomena in Table 1.2.]

Szostak, Rick. 2004. Classifying Science: Phenomena, Data, Theory, Method, Practice, Dordrecht: Springer. [This book reprises Table 1.2, and discusses how it is related to classifications of the theories and methods employed by scholars.]

Weller, R. Charles. 2017. 21st-Century Narratives of World History: Global and Multidisciplinary Perspectives. Cham, Switzerland: Palgrave Macmillan.

Xincheng, Liu. 2012. "The Global View of History in China." Journal of World History 23:3, 491-511. [Suggests that Chinese scholars value World History for decreasing - but not eliminating - Eurocentrism in the field of history.]

\section{Thematic histories with a global focus}

Goucher, Candice and Linda Walton, 2nd ed. 2012. World History: Journeys From Past to Present. New York: Routledge. [Chapters follow a different theme, such as population growth, through human history.]

McNeill, William. 1977. Plagues and Peoples. Garden City, NY: Anchor.

Ponting, Clive. 2007. A New Green History of the World: The Environment and the Collapse of Great Civilizations. New York: Penguin.

Smith, Bonnie, ed. 2005. Women's History in Global Perspective. 2nd ed. University of Illinois Press.

Spodek, Howard. 2011. The World's History. 4th ed. Upper Saddle River, NJ: PrenticeHall. [Addresses different themes at different points in time.]

Vanhaute, Eric. 2013. World History: An Introduction. London: Routledge. Translated by Linda Weix. [Chapters follow a different theme, such as population growth, through human history.] 


\section{With a focus on connections across societies}

Bentley, Jerry H., Herbert F. Zeigler, and Heather E. Streets-Salter. 2010. Traditions and Encounters: A Brief Global History. 5th ed. New York: McGraw-Hill. [The "traditions" of the title include economic, political, and social as well as cultural.]

Christian, David. 2004. Maps of Time: An Introduction to Big History. University of California Press.

Diamond, Jared. 1997. Guns Germs and Steel: The Fates of Human Societies. New York: Norton.

McNeill, John, and William McNeill. 2003. The Human Web. New York: Norton.

Stearns, Peter N. 2011. World History: The Basics. New York: Routledge.

\section{Civilizations}

Duiker, William J., and Jackson L. Spielvogel. 2008. The Essential World History. Belmont, CA: Thomson Wadsworth. [The majority of chapters focus on a particular region or civilization.]

Landes, David. 1999. The Wealth and Poverty of Nations: Why Some Are So Rich and Some So Poor. New York: Norton.

Roberts, J.M., and Odd Arne Westad. 2013. The History of the World. 6th ed. Oxford University Press. [A classic, and very detailed, but has been criticized as Eurocentric.]

\section{World systems or with a non-Western focus}

Abu-Lughod, Janet L. 1989. Before European Hegemony: The World System A.D. 12501350. New York: Oxford University Press.

Blaut, James. 1993. The Colonizer's Model of the World. New York: Guilford. [I reviewed this book rather harshly when it came out.]

Said, Edward W. 1978. Orientalism. New York: Pantheon.

\section{Modernism and postmodernism; interdisciplinarity}

Bergmann, Matthias, Thomas Jahn, Tobias Knobloch, Wolfgang Krohn, Christian Pohl, and Engelbert Schramm. 2012. Methods for Transdisciplinary Research: A Primer for Practice. Berlin: Campus.

Burke, Peter. 2005. History and Social Theory. 2nd ed. Ithaca, NY: Cornell University Press.

Evans, Richard J. 1997. In Defence of History. London: Granta.

Jenkins, Keith. 1991. Rethinking History. London: Routledge.

Lemon, M.C. 2003. Philosophy of History: A Guide for Students. London: Routledge.

Lloyd, Christopher. 1993. The Structures of History, Cambridge, UK: Blackwell. [Provides the list of dichotomies addressed above.]

Munslow, Alun. 2003. The New History. Harlow, Essex, UK: Pearson.

Repko, Allen, and Rick Szostak. 2020. Interdisciplinary Research: Process and Theory. 4th. ed. Thousand Oaks, CA: Sage.

Szostak, Rick. 2007. "Modernism, Postmodernism, and Interdisciplinarity." Issues in Integrative Studies 26, 32-83. 
Szostak, Rick. 2012. "Integrating Interdisciplinary Studies across the Humanities and Social Sciences," in Raphael Foshay, ed., Valences of Interdisciplinarity. Edmonton, Canada: Athabasca University Press, 165-88.

There was a special issue of the Journal of Global History in November 2019 on interdisciplinarity. The introductory essay notes that there is a synergy between a global approach to history and interdisciplinarity, since disciplinary approaches have often been national in focus, especially in history, art history, literary studies, and anthropology. It also discusses the problems with unclear terminology that afflict this new enterprise. A later paper argues that world history has borrowed ideas of culture from anthropology and techniques of cross-societal comparison from sociology, and a general appreciation of area studies. 


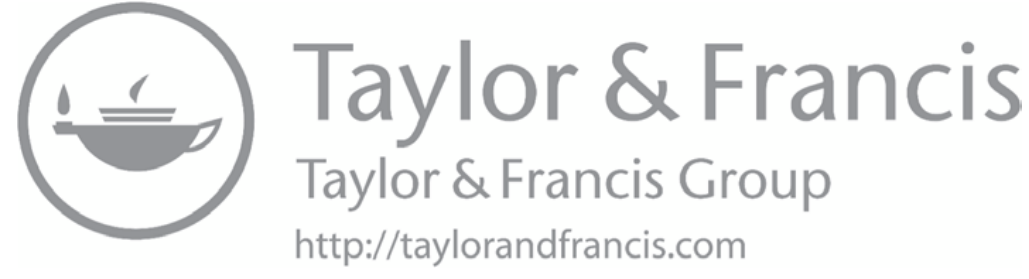


II Prehistory and ancient history 


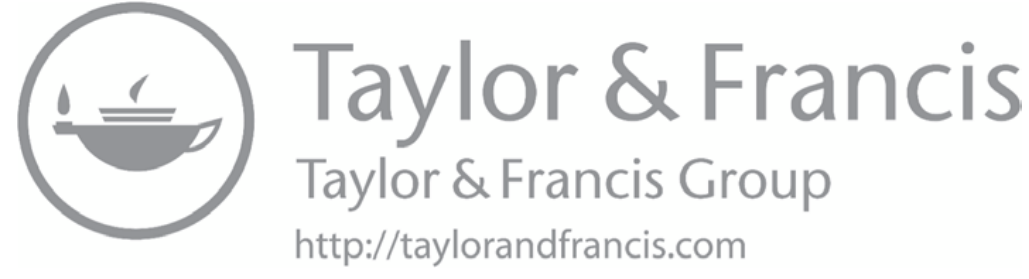




\section{The Big History prelude}

\section{From the Big Bang to hominids}

\section{Guiding questions}

How and why and when (and sometimes where) did the universe, stars, planets, life on earth, multicellular life, and mammals emerge? How did each of these developments set the stage for the next?

Why do stars, planets, and life-forms take the form that they do? What is the ideal place for these questions in a course in world history?

Relationship to other chapters: Chapter 2 sketches the emergence of the natural world with which humans will engage throughout the rest of this book. The story of biological evolution continues in the next chapter with the appearance of humans. This chapter also serves to clarify the evolutionary approach that we will take to several social phenomena in later chapters.

This chapter is unique in this book in focusing entirely on only one of our themes. Since this chapter addresses the time period before there were humans and human societies, we can discuss just the non-human environment. However, we will discuss many distinct phenomena within the non-human environment, including many non-human life-forms. Unusually also, we will employ theories of biological rather than social evolution. 


\section{The nature of Big History}

Most World History courses start with early humans: either the first hominids 5 million years ago or the first homo sapiens 200,000 years ago. We will pick up the story of the early hominids in the next chapter. There is an alternative approach to history: "Big History" begins with the "Big Bang" that (scientists think) initiated the universe some 13.7 billion years ago. Texts in Big History tend to devote equal space to history before humans and after the emergence of the earliest hominids. We pursue a middle ground here, providing only a brief survey of history before humans. We concur with those who teach "Big History" that it is useful for students of history to have some knowledge of this backstory to human history, the set of natural events and processes that unfolded before humans could emerge. As Big Historians have noted, most human societies have had a creation story (myth) that combines both the creation of the natural world and the development of human society. Such a combined narrative serves a human desire to understand how we came to be. Since one of the purposes of this book is to describe how contemporary human societies came to be, it makes sense for us to study both natural and social processes. Besides, an understanding of our shared natural history may encourage a sense of the shared place that humanity as a whole occupies in the universe. Nevertheless, we hold with world historians the belief that human history itself deserves centre stage in a work of world history.

In the first chapter, we developed a set of organizing devices for World History. We saw that world historians have struggled with how to organize their material. This issue of organization has been even more central in the field of Big History. From the outset, scholars of Big History have had to face the questions of both why we should and how we could treat natural and human processes together in the same book or course. The dominant approach in the field has been to focus on revolutionary events: points in both natural and human history when something demonstrably new emerges. The combined natural and human history can then be seen as a process of emergence of increasingly complex natural and then human systems of interaction.

There is, of course, a danger that the very pursuit of an organizing scheme that can encompass both natural and human processes will bias our treatment of human history. Why indeed should we expect that there must be any similarity between the two processes? To be sure, they are both historical in the sense of happening over time, but even there the timescales involved in Big History dwarf those in World History. We noted in chapter 1 the dangers of seeking analogies between natural and human processes (even if, as is the case, different Big Historians pursue different analogies). In particular, entropy - the tendency for energy levels to decrease through time - characterizes the natural world, but it may not make sense to apply entropy to human societies. (If we did so, it would be tempting to imagine that human efforts to utilize ever more energy in our lives must eventually lead to societal collapse, whereas we 
may achieve a less dramatic sustainable level of energy use.) We also noted in chapter 1 that we will cover periods of both change and (relative) stability in this book. We fight against the temptation to stress the former. This temptation exists even in World History but arguably even more so in Big History.

Of course, Big Historians appreciate that revolutions do not just happen. They inevitably discuss how the conditions necessary (and perhaps sufficient) for any revolution came together over time. As a result, they necessarily engage the history between revolutions: but only those elements of history that contributed to the next revolution. In a human view of natural processes spanning billions of years, this might be a practical focus. However, when we reach human history, do we want to ignore or downplay events and processes that were important at the time but do not seem to lead to the next revolution in human affairs? I think not. Doing so would risk leaving the mistaken impression that all of human history was devoted to the achievement of modern highly complex societies. This is simply untrue.

As we will see, natural processes are often characterized by substantial periods of (at least apparent) stability punctuated by dramatic changes. Stars, for example, burn in a broadly similar manner for billions of years before flaring out. It is only natural to focus then on the conditions driving revolutionary change. In contrast, human societies are always changing. Anthropologists increasingly appreciate that even "traditional societies" changed through time, though not so dramatically as after they encountered more technologically advanced societies. To focus only on dramatic changes would short-change the more gradual changes that might cumulatively be much more important historically. Historians of technology, for example, appreciate that millions of incremental innovations had a far greater historical impact than the dozens of major innovations that have received the bulk of (at least popular) historical attention. More generally, the history of smaller interactions among themes sets the stage for occasional "revolutionary" transformations in one or more themes. It therefore makes far more sense to describe stability in natural systems than in human societies. Human history guides us to an evolutionary understanding, albeit one with a place for revolutions.

We can often understand natural processes by looking at a minimal set of phenomena: A particular chemical reaction requires just a couple of chemicals and appropriate conditions of temperature and pressure. Human processes are often more complicated: Several conditions (perhaps each itself shaped by interactions among several phenomena) may be necessary for a political revolution (chapter 25). We can understand natural processes in terms of "regimes of order," relatively stable systems of interaction that nevertheless over long periods of time create the conditions for a new "regime of order." Human history may be much less orderly, with some processes or interactions pushing in opposite directions from others.

One purpose of this chapter will be to show we can apply to this pre-human history the same organizing strategy that we will apply to World History in later chapters. We should stress that our primary interest is World History: We begin 
with what we hope is an innocuous approach to organizing human history, and then see if we can extend this to earlier natural processes. We are not driven by a desire to link the two types of history. We will strive to organize our Big History around the interactions among (in this case natural) phenomena and the processes of change (sometimes evolutionary, sometimes not) associated with these phenomena.

We shall see that the historical paths followed in these natural processes could easily have been slightly different, and then the next revolution would have unfolded quite differently. Increased natural complexity may then not have occurred or may have taken an entirely different form. There is a great deal of contingency in this natural history: A planet such as Earth with conditions necessary to support life in general and human life in particular was in a sense highly unlikely (though there may well be billions of similar life-forms in the universe - or not). Moreover, the emergence to dominance of mammals was perhaps even more contingent on unpredictable events. It is likely that human history is similarly contingent: Small changes in historical processes might have yielded dramatically different outcomes. However, we should be careful of inferring that the contingency we observe in Big History must necessarily also apply to World History.

It is worth stressing that the scientific understandings we will review below are recent. Indeed, it is likely that these will be revised in important respects in future. Nevertheless, scientists now have plausible explanations for the course of Big History. Through most of human history, it would have been difficult for any human to imagine that the beautiful world around them had emerged through a set of natural processes. Some greater godlike being must have been at work. Modern scientific understandings do not force humans away from religion but do make it possible to imagine a world not guided by gods. We will encounter a variety of religious beliefs in the chapters that follow, and should never lose sight of the fact that at the times they were developed they were at least as plausible as any alternative explanation of the universe.

We should also remark on the timescales involved. Scientists estimate that the universe is 13.7 billion years old, the Earth itself 4.5 billion years old, and life on Earth has existed for 4 billion years. If we were to compare the history of the universe to a single earthly year, the human beings that will be the focus of the rest of this book emerge very late on the last day of the year. All of human history, that is, comprises a minute fraction of the history of the universe. The human mind, we should recognize, has trouble even conceiving of such lengthy timespans.

\section{The Big Bang}

Scientists think that the universe began about 13.7 billion years ago with what is commonly called the Big Bang. An immediate question arises: How 
can something arise from nothing? If there was previously no universe then how could a universe be created? There is, we should note, no very satisfying answer to such a question. Some cultures have viewed time as circular rather than linear, and the physicist Stephen Hawking has posited a circular approach to time. But to those of us who think that time must move in only one direction, an appeal to circularity is baffling rather than satisfying. (Yes, it is okay not to grasp what circular time might look like!) Others have appealed to the idea of infinity: If time is infinite, there is no necessary starting point. This idea also boggles the mind. Moreover, we must then face up to the problem of entropy: If the total energy within the universe must decline (even very slowly) through time, then an infinitely aged universe would be quiescent by now. Scientific understandings of the Big Bang steer us away from imagining infinite time toward trying to get our mind around a fixed starting point.

The Big Bang, as we shall see, generates mass and energy. Perhaps time and space were also produced at the same moment. But how and from what? What, if anything, existed before the Big Bang? Scientists have no idea. We can speculate if we wish, but there are no traces left of any pre-Big Bang reality. We could appeal to godly creation, but this raises the question of where god comes from. We will throughout this book try to explain events and processes in terms of previous events and processes; we simply cannot use this strategy when addressing the first creation. (We should note that concepts such as "mass" and "energy" prove difficult to define in practice - perhaps precisely because they are such fundamental concepts that it is hard to define them in terms of other concepts. We eschew an exercise in definition in the hopes that common-sense understandings will suffice for our purposes.)

We should admit, then, that the Big Bang is qualitatively different from every other transformation that we will address in this book. We can attempt to trace how the forces unleashed by the Big Bang set the stage for every subsequent development. Yet the Big Bang is a starting point for history beyond which we cannot see.

Scientists do provide a detailed description of the first fractions of a second of the history of the universe. Quarks, neutrons, and protons suddenly emerged and began to expand outward. Electrons were impossible at first but develop seconds later. Most of the particles generated were matched to anti-matter and became pure energy (which produces the cosmic background radiation that emanates throughout the universe and that radio astronomers first discovered by accident decades ago). However, about one billionth of the total did not combine - likely due to a very slight excess of matter over anti-matter - and was then available to form the matter that we observe.

About 300,000 years later the universe cooled such that protons and electrons could bond. The electromagnetic force would cause positively charged protons to attract negatively charged electrons. We can see this as the first post-Big Bang threshold: The conditions were then in place for the emergence of chemical elements, and thus matter as we know it. At first, only the simplest two elements, hydrogen (with one proton and one electron) and helium (with 


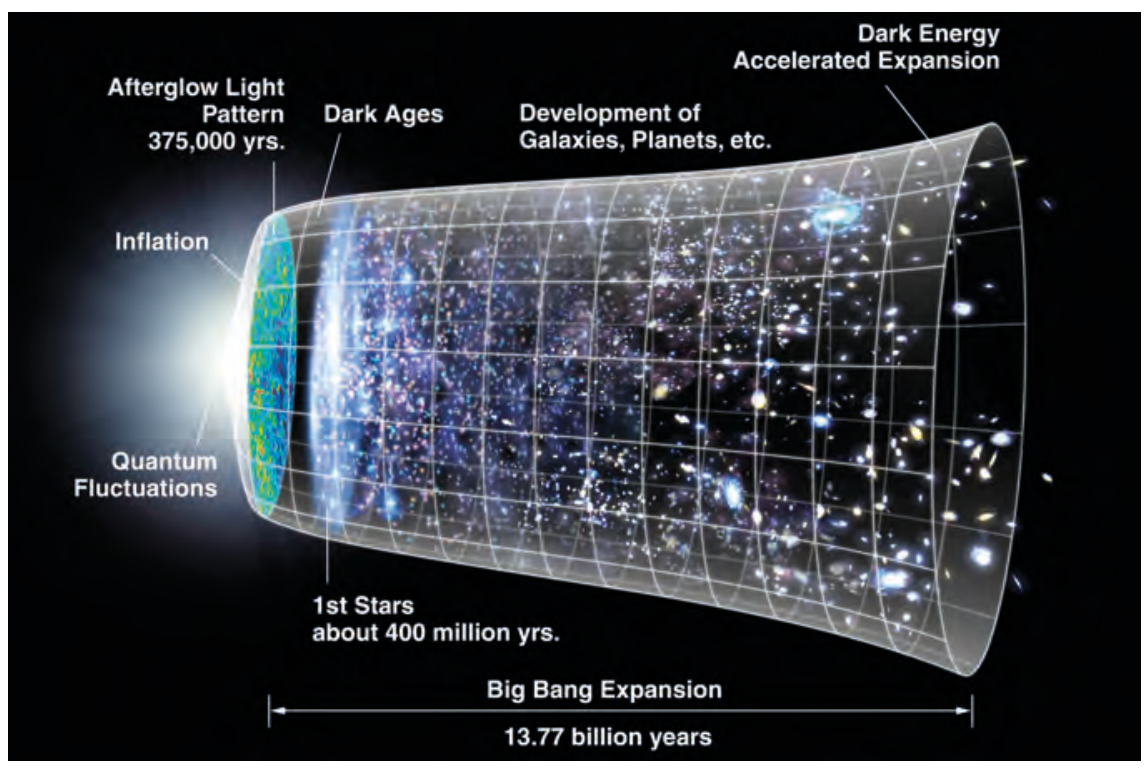

Illustration 2.1

The timeline of the universe

Source: NASA. Modified by Ryan Kaldari

two of each), could form. Only once stars began to form would they generate heavier elements (see below). Note that if the universe had expanded more slowly, heavier elements (iron being the most stable) might have predominated throughout the universe, and this would likely have rendered life impossible.

It seems bizarre that the universe has been continually expanding since the Big Bang. Just as the Big Bang raises uncomfortable questions about the nature of time, the idea of an expanding universe challenges our conceptions of space: What, after all, does the universe expand into? Nevertheless, astronomers observe (by examining the light spectrum emitted) that galaxies move away from each other and the farthest away move the fastest. It is indeed from reviewing the speed with which the universe seems to be expanding that scientists have estimated the age of the universe. The theory of relativity also suggests that the universe must either expand or contract - though Einstein when he first developed that theory was uncomfortable with the idea of an expanding universe and introduced an ad hoc constant in order to allow a stable universe. Astronomical observations later convinced him to accept the theoretical implication of expansion.

\section{Stars and planets}

Gravity plays a vital role in this next phase of our story. But for gravity to act, there must have been some unevenness in the distribution of particles in this 
expanding universe. That is, if the Big Bang had been perfectly symmetrical in all dimensions, then the gravitational pull of any particle would evenly match the gravitational pull of others, and they would all remain separate. If there was the slightest unevenness, however, then gravity will act over time to pull particles together. As more particles are drawn together, the gravitational force increases, allowing yet more particles to be drawn in. Yet there are limits, for the force of gravity, though positively related to mass, is inversely related to the distance between bodies. Rather than getting one massive body in the universe, then, we see over time the formation of billions of stars of different sizes. Indeed the visible universe - itself a fraction of the total universe - has 150 billion galaxies, each with a billion stars. Though these stars absorb the vast bulk of the matter in the universe - our sun, for example, has well over 99 per cent of the matter in our solar system - some particles remain free to form planets and comets and other non-stellar bodies. (There is also dark matter (or maybe dark energy) throughout the universe that scientists do not fully understand but likely represents $90-99$ per cent of the total mass of the universe. Dark matter may explain movements of stars and galaxies that scientists cannot comprehend in terms of their own gravitational forces. Dark energy may likewise explain peculiarities in the light emitted by some supernovas.)

The first stars formed some 700 million years after the Big Bang. We do not fully understand how gravity could have pulled so much matter together (dark matter is again invoked by some, while others revisit our understanding of gravity). However, scientists appreciate that as gravity pulled matter together, galaxies would form. They think that all galaxies emerged between 700 million and 2 billion years ago. Galaxies have black holes at their centre - bodies so dense that even light cannot escape their gravitational force. Just as the orbit of the earth around our sun prevents us from falling into the sun, the rotation of galaxies prevents stars from falling into these black holes. Note that stars and black holes co-evolve: They form over the same time period and interact.

Gravity strengthens as mass increases. Stars should then tend to collapse in size, for large stars pull mass powerfully toward their centres. But as the temperature and pressure at the heart of a star rise, fusion reactions occur. These have two important effects. Most obviously, perhaps, they counteract the force of gravity itself and allow stars to maintain a rough equilibrium in size and energy generation for billions of years. Gravity is at first stronger than fusion, and the star compresses, increasing the number of fusion reactions until a balance is achieved. This is a remarkable result: Only because gravity and fusion can balance each other do stars last so long. Eventually, stars will exhaust their nuclear energy and gravity will dominate in a final collapse of the star, generating a final intense flash of energy called a supernova (which we can often observe in the sky). For humans, the critical point is that this example of entropy - the final collapse reflects the gradual decrease in energy within the star over time - is delayed for billions of years. Our own sun is expected to last for another 5 billion years. 


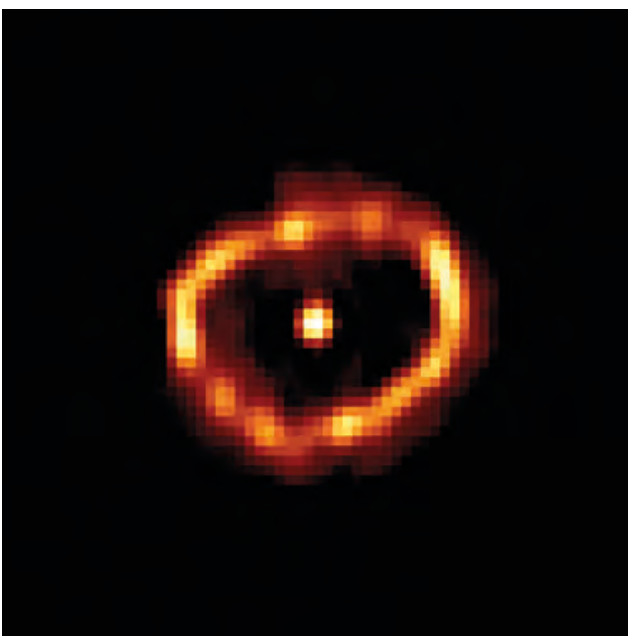

Illustration 2.2

Cygni Nova, 1992

Source: NASA, from Hubble telescope
The second effect of a fusion reaction is that it generates heavier elements. As noted above, the universe long contained only hydrogen and helium (and subatomic particles). Astronomers estimate that these still comprise some 97 per cent of the luminous matter in the universe. In the centre of stars, many other elements were created. Stars at first transformed hydrogen into helium. As energy declines, fusion generates heavier elements such as oxygen and carbon and iron. As stars die, they can eventually produce heavier elements than iron (up to uranium), but only for a relatively brief period: These heavier elements are therefore rare in the universe. When the star explodes as a supernova, these elements disperse.

The precise balance among different elements in the universe depended on the balance between physical forces. We might pay particular attention to carbon, an element that is essential in many ways to life as we know it on Earth. If gravitational forces had been weaker, then carbon would be much rarer in the universe. On the other hand, stronger gravity might have transformed carbon atoms into heavier elements.

We observe different types of star depending on how much matter gravity drew together. If there was very little matter, we get what is termed a brown dwarf star: These never really develop the characteristics of a star. Stars that are at least a large fraction of the size of our sun, or no greater than several times the size of our sun, will exhibit sun-like behaviour. They can last for hundreds of billions of years. Stars that are much bigger than the sun burn much faster: Some may last only hundreds of thousands of years. Such stars were much more common in the earliest period of star formation; billions of such stars had disappeared before our sun existed.

From the perspective of humanity, we have already observed what we might judge to be fortuitous circumstances. Stars exist only because of a rough balance between fusion and gravity. (Many of) The heavy elements on which life depends - we are carbon-based creatures that breathe oxygen and use iron were possible only because some stars burned fast and died, dispersing these elements into solar systems like ours that possess longer-lived stars.

Planets are also a common feature of the universe. High-powered telescopes have established in recent decades that most of the stars we can observe have planets orbiting them. The process of star formation everywhere seems to fail to absorb all matter in the vicinity, leaving some small amounts to form planets and other bodies. There are likely billions of billions of planets in the universe.

Humans have been shocked to learn over the last centuries that neither our planet nor even our sun lies at the centre of the universe. Indeed our sun is in an 
arm of our Milky Way galaxy that is itself on the fringes of a cluster of galaxies that is on the fringes of a super-cluster of galaxies. These various formations are all reflective of the workings of gravity over billions of years (against the pull of the expanding universe). Gravity pulls most galaxies into rotating discs with spiral arms surrounding a black hole. Earth was/is fortunately far enough from the centre of our galaxy to avoid being pulled in. The sun and earth are a fortuitous distance from the galactic centre: There was likely too much supernova activity near the centre of our (or any) galaxy for life to emerge, since supernovas send waves of radiation into the surrounding space - but we were not so far out as to fail to receive enough heavy elements from these supernovas.

Our sun is about 4.6 billion years old; it has another 5 billion or so years before it too will collapse. Our sun is relatively large. Perhaps 95 per cent of stars are smaller. It was created at a time when some heavier elements had already been generated elsewhere. As noted above, the sun comprises 99.9 per cent of the matter in the solar system. Among the matter not absorbed by the sun, lighter elements were more likely to remain farther out, and thus we see massive gassy planets like Jupiter and Saturn. Heavier elements were drawn closer to the sun, creating rockier planets such as Earth and Mars. It took millions of years for gravity (and electrostatic attraction) to pull planets together. There were likely once many more planets that combined over time into the eight present-day planets (and a host of asteroids and comets that never merged into a planet). As we all know, conditions conducive to life do not exist on all planets; we are still seeking to establish just how unlikely it was that a planet would form of the right size, composition, and distance from the

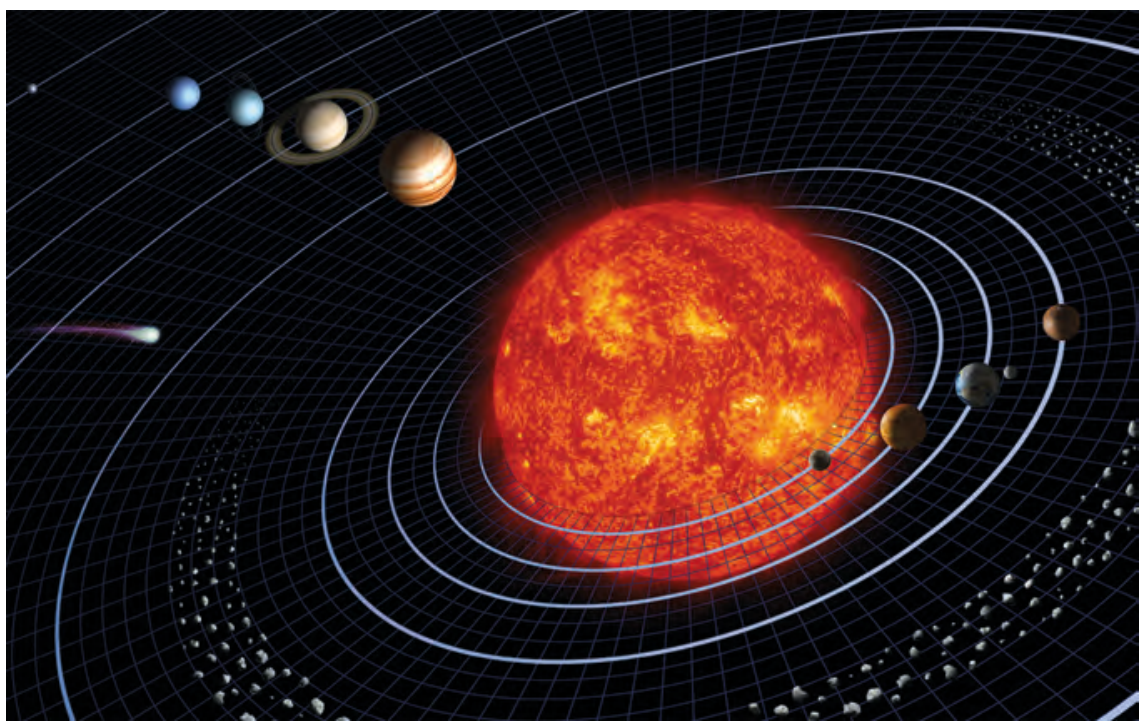

Illustration 2.3

Our solar system

Source: Harman Smith and Laura Generosa for NASA 


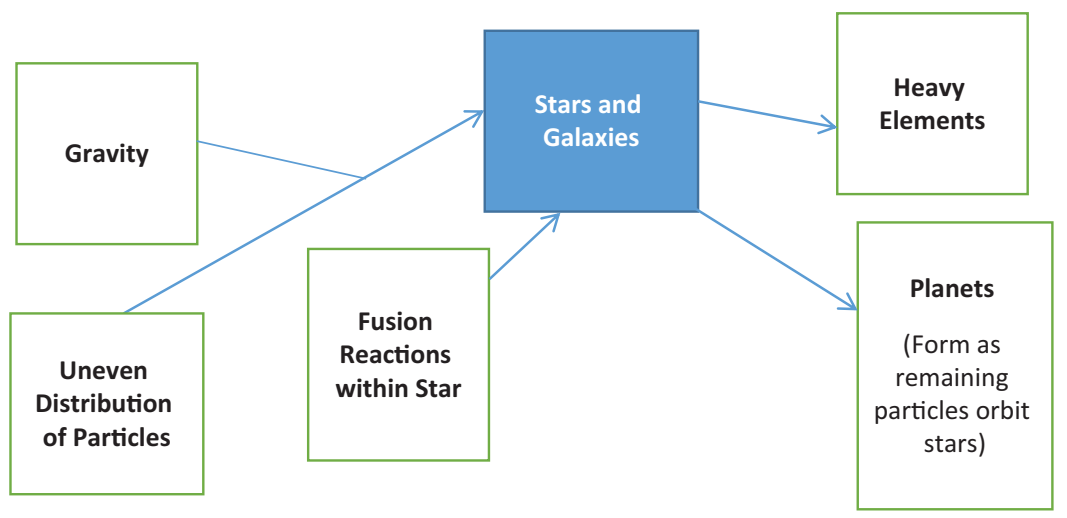

Figure 2.1

Influences on and effects of star formation

sun to support life. We can then better speculate on whether life does/did exist elsewhere in the solar system, and on the likelihood that it exists elsewhere in the universe. (We will be able to refine these probabilities as we develop our understanding of how life emerged on Earth below.)

\section{The Earth (4.5 billion years ago)}

As with stars, the force of gravity causes the cores of planets to heat - but not so much as to trigger fusion reactions. There is a tendency for gravity to pull heavier elements toward the molten centre. Luckily, this tendency is both limited and is counteracted by volcanic activity. As a rocky crust formed over the liquid core (equivalent in scale to an eggshell covering an egg), this crust contained a wide range of chemical elements. The most precious mineral resources on Earth are often in places of past or present volcanic activity. Lighter elements - usually emitted from volcanoes or absorbed from passing comets - formed an atmosphere above this crust. Oceans formed 3.5 billion years ago at least. It may be rare for planets elsewhere in the universe to have both water and an atmosphere. In our solar system, Venus is too hot for water to remain in liquid form, and Mars may have once had an atmosphere that it has lost over time.

The earth has undergone a variety of changes over these billions of years. There have been periods of massive volcanic activity. There have been dramatic changes in the nature of the earth's atmosphere: As we shall see in the next section, different compositions may have been necessary for different stages in the development of life. There have been several major asteroid impacts, each of which had dramatic effects on the earth and any life-forms inhabiting it. The earth today is much warmer than it was billions of years ago. Why did the climate warm? The sun has been getting more powerful - it may be 40 per cent more powerful than it was billions of years ago - but this is not likely the main cause. Changes in the earth's tilt relative to its orbit, and regular oscillations in 


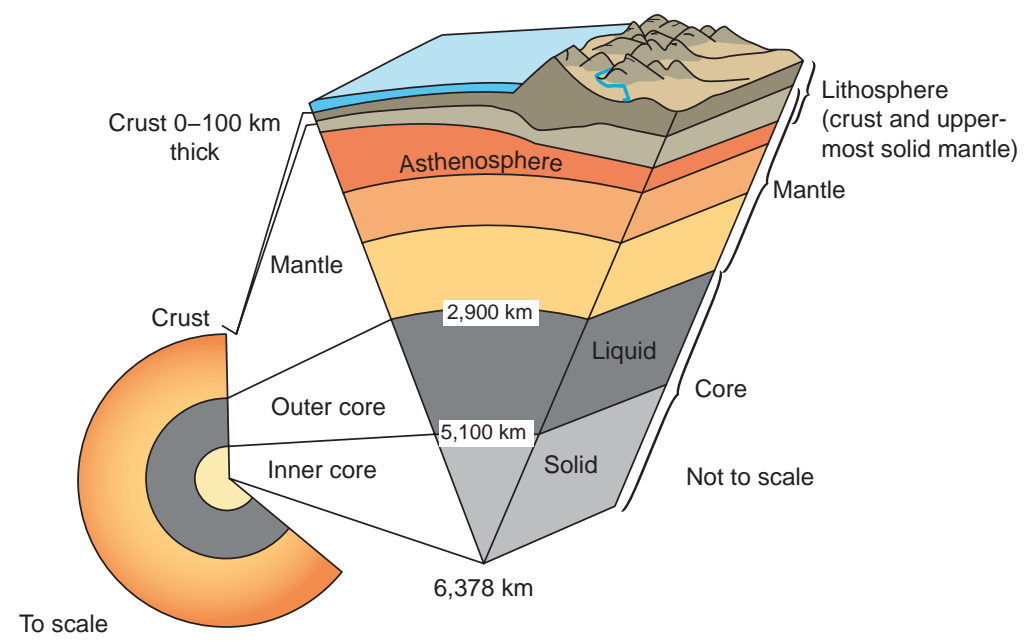

Illustration 2.4

Schematic diagram of the Earth's structure

how close its orbit is to the sun, are also likely not the main cause. It is more likely that volcanoes and natural processes created what we now call greenhouse gases over time, and as a result the earth's atmosphere retained more heat. As the earth warmed, ice would melt: The warming then became selfperpetuating since ice reflects heat. Yet in the millions of years of hominid history there has been no obvious trend (until recently) in average temperatures but rather fluctuations that can be attributed to various natural events. We will discuss one important phase of cooling below, and address changing climates again in the next chapter and some later chapters.

The earth's moon likely was created when some large body collided with the planet, throwing earthly material into space. Moon rocks have a similar chemical composition to the earth's surface. The moon serves to regularize the earth's orbit; the tilt of the planet as it orbits the sun might otherwise have frequently changed, causing massive climatic changes on the earth's surface.

When first suggested over a century ago, the idea that the earth's surface was composed of several plates that move slowly through time was generally dismissed. It is now generally accepted. The idea emerged from the casual observation that the east coast of the Americas matches the west coast of Europe and especially Africa. Empirically, scientists now appreciate that these are an even better fit if one looks at the respective continental shelves rather than present coastlines. Moreover, one finds similar rock formations where the continents would once have been joined. And we have identified a rift of volcanic activity in the mid-Atlantic that appears to be pushing the continents apart by a couple of centimetres a year. At this rate, it will only have taken 150 million years for the Americas and Europe/Africa to have separated this far. This movement in turn causes the "Ring of fire" of volcanic and earthquake activity around the Pacific Ocean as plates collide. Theoretically, scientists now appreciate that the 


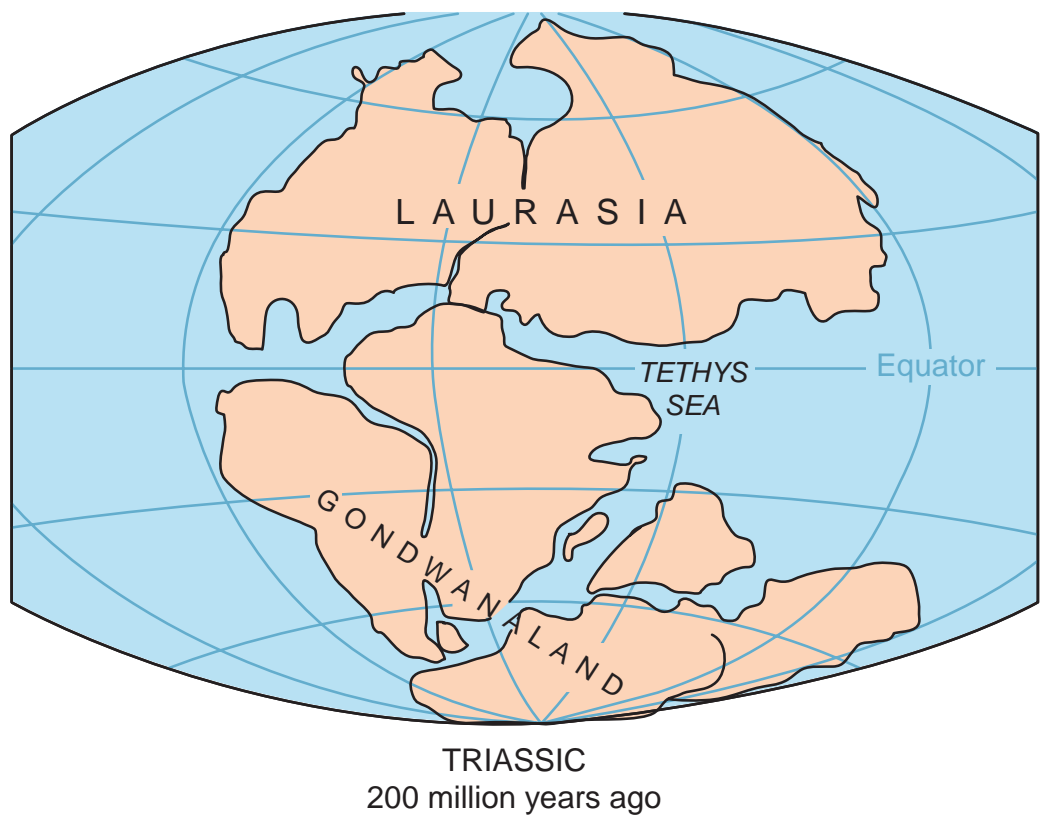

Map 2.1

The Earth's continents were once joined in a continent scientists call Gondwana

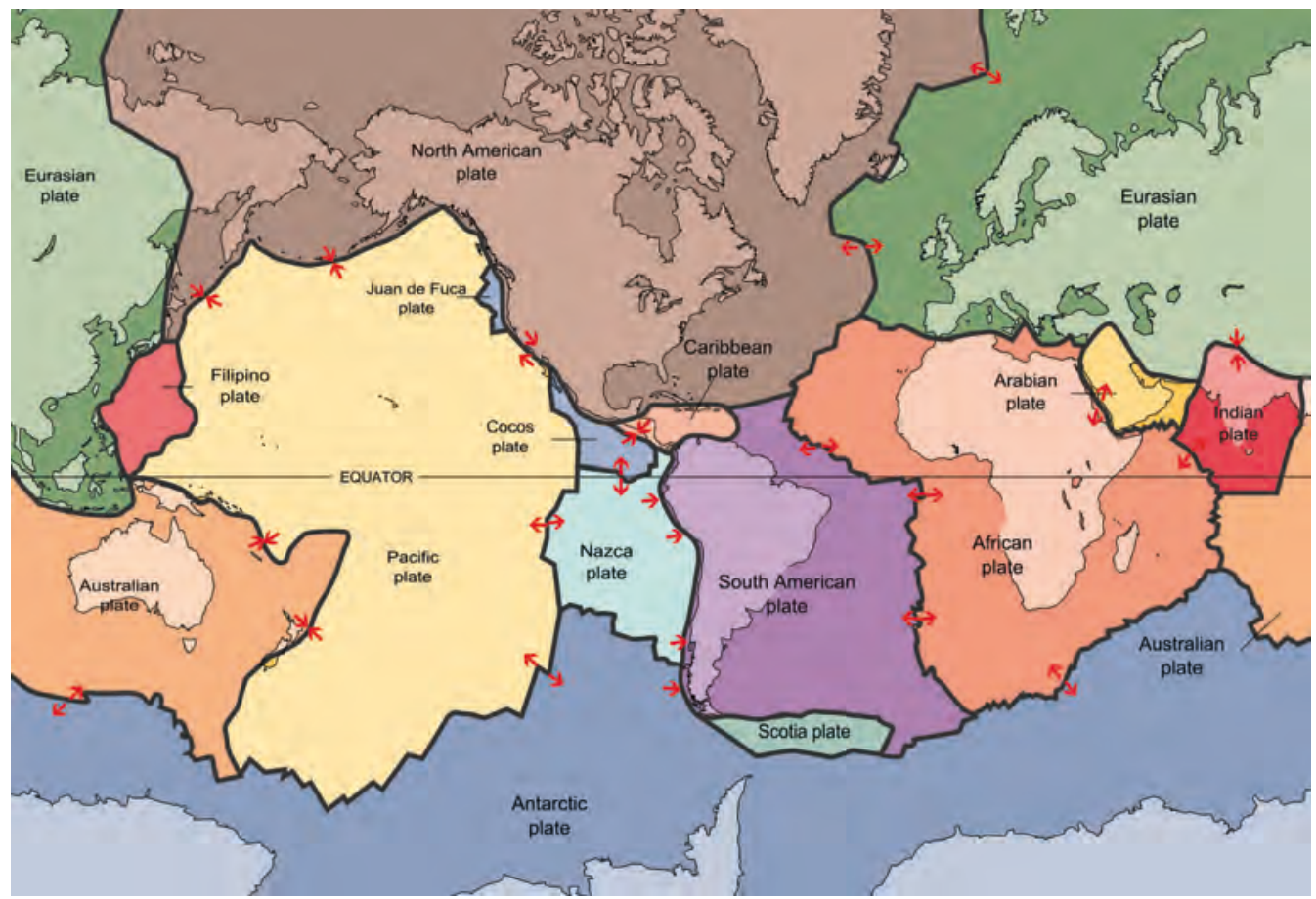

Map 2.2

The tectonic plates in the 21 st century 
earth's crust does indeed rest on a molten interior (see above). The molten core provides the energy that moves the plates.

These movements have naturally affected the earth's climate. If oceans can mix tropical and arctic waters, the overall result will be a warmer planet. The present configuration of continents - with Antarctica covering the South Pole, and North America and Eurasia limiting the connections between the Arctic and other oceans - has had an important cooling effect on the planet (which, as we will see in the next chapter, may have facilitated the emergence of humanity). One particularly dramatic event was the formation of the Isthmus of Panama. For millions of years, the Americas

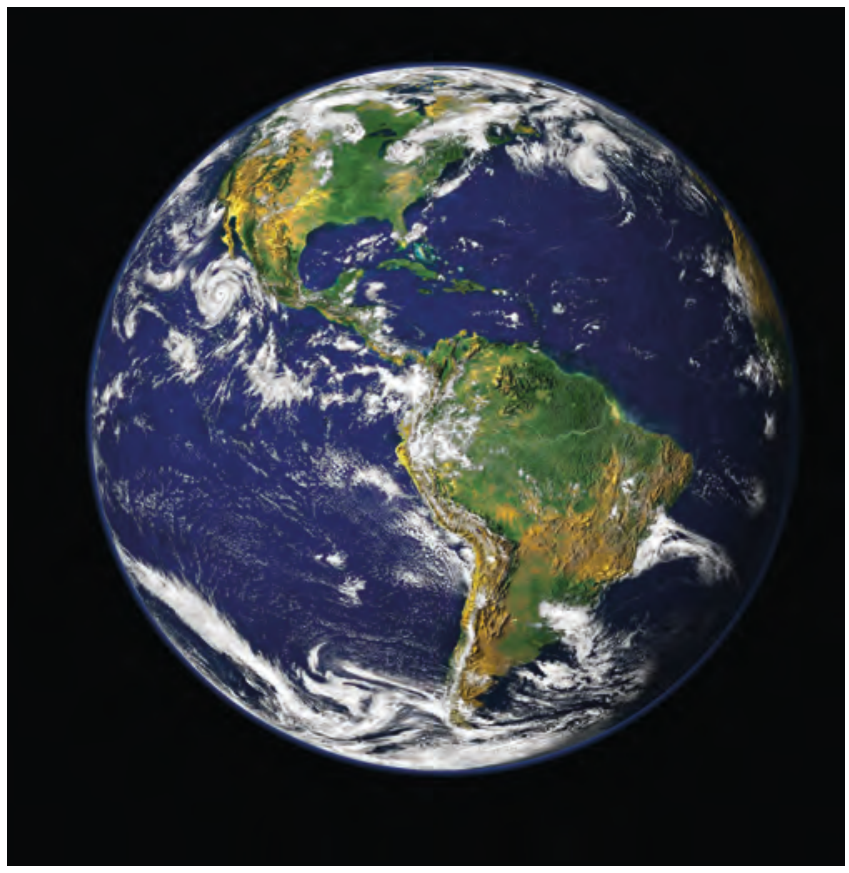

Illustration 2.5

Earth from space were separate, and the waters of the Pacific and Atlantic Oceans mingled. But once the two continents were connected, ocean currents operated in an entirely different fashion. This conceivably encouraged the retreat of forests in Africa at the time that humans began to wander the savanna.

The landscape on which human history takes place has been shaped over millions of years by a variety of forces. Mountains have been created by volcanoes - or by the collision of tectonic plates. The movements of ice sheets driven in turn by the warming and cooling of the planet through time - have created vast plains in many parts of the world. Water and ice have combined to crush rocks into the soils that humans use to grow various crops. Rivers have often gathered soils into particularly fertile floodplains. Winds and rain and tides have also sculpted particular landforms.

\section{THE THEME OF NON-HUMAN ENVIRONMENT}

We will not insist on a comprehensive coverage of all elements of the natural environment in each time period. However, we should note that soil, topography, climate, availability of fresh water, various natural resources, and incidence of various sorts of natural disasters differ greatly across the earth, necessitating quite different economic and other behaviours. 
We will make note of these various elements of the natural environment from time to time. Climates have also changed through time, causing changes in human behaviours and mass migrations. Historians have increasingly explored the effects of climatic change on human activity in recent decades, and so we will be able to reflect on a possible role for climate in a variety of human transitions. We would not call natural disasters "disasters" if their incidence and effects through time were regular and predictable:We will only rarely suggest that particular disasters exerted a major impact on world history.

Humans have altered the distribution of flora and fauna in the world, extinguishing many while encouraging others. Though the author agrees with those who value biodiversity, he confesses that this book will follow a human-centred approach, generally referring to these actions only as they affect humans themselves. The same is true of our efforts to change watercourses and flatten landscapes. Human effects on climate are long-standing but have increased in importance dramatically in recent decades and will only receive a lot of attention late in our story. Nevertheless, we will discuss earlier effects on both climate and more local types of pollution.

Transport infrastructure merits particular attention. Goods and ideas move physically through the world (ideas can now move electronically, but this is a historically recent change that we will also address). Roads and ports and canals or river improvements (and later railways and airports) are generally among the most expensive enterprises of any time period. Moreover, there are considerable challenges in obtaining and protecting the land necessary for such infrastructure. We will pay close attention to how particular societies were able to build transport infrastructure.

Early humans relied almost exclusively on the immediate products of solar energy: the plants that use photosynthesis to grow (see below) and the animals that eat these. Over time, humans came to utilize "stored" energy: They built fires that used energy stored in trees for decades or longer, and later came to utilize coal and oil, millennia-old sources of energy. It will be worthwhile, especially for later periods, to keep track of human energy usage. Although there has been a general tendency for energy use per person to expand over the course of human history, there are exceptions: Indeed, if small early human populations used fire to clear land they may have had a far higher energy consumption per person than contemporary humans. Energy use is just one way in which humans have over time come to extract a host of natural resources from their environment. We have discussed above why those materials were there to be extracted and will relate in later chapters how humans came to use these.

The natural sciences loom in the background of our discussion of the natural environment, and also of genetic predisposition and health. Just as it would be tedious to recount regularly how manual dexterity or cognitive capability was essential to each successive human accomplishment, it would bore the reader to talk at length about how each agricultural or industrial practice has to obey the laws of physics. We have reviewed some natural scientific material in this chapter. Otherwise, we will address natural scientific material only when this is necessary to comprehend particular social processes: certain sorts of technological innovation, maintenance of soil fertility, the spread of certain diseases, or human-caused climate change. 


\section{Life on Earth (4 billion years ago)}

It is worth first remarking again on timescales. It is thought that life emerged on earth about 3.6-3.8 billion years ago (depending on one's view of the fossil record - there are bacterial fossils from 3.5 billion years and perhaps chemical traces earlier - and very recent discoveries that point to a date more than 4 billion years ago). This is only a few hundred million years after the earth itself was formed - and long before its surface resembled that which we take for granted today. Therefore, if we place each of the transformations in this chapter on a timeline, the gap between "earth emerges" and "life on earth emerges" is short by comparison with all other transformations. It is even far shorter than the next gap: to the emergence of multi-celled organisms. We must be careful of using time as a proxy for the difficulty of transformation, but still the appearance of life after planetary formation was - within the timescales that we have had to wrap our mind around in this chapter - quick.

The emergence of life is still somewhat mysterious, but far less so than the Big Bang. Biochemists are steadily adding to our understanding of the chemical interactions associated with life. We can be optimistic that biologists will someday explain the emergence of life as a chemical process, and even create simple life from chemicals in the laboratory. Scientists have long been able to generate amino acids, one of the essential compounds in living organisms, by passing an electric current through a mixture of simpler chemicals thought to be common at the time life emerged (notably ammonia and methane, the former a compound of nitrogen and hydrogen, the latter a compound of carbon and hydrogen). The big question for us is how and why the requisite chemicals would have combined in just the right way without someone in a lab coat purposely guiding them.

We do not of course fully understand the nature of life. Life-forms are considered an "emergent property" of a particular set of chemical interactions: We cannot (yet at least) explain life in terms of the behaviours of specific chemicals. Yet the emergent nature of life need not mean that we cannot create it by combining chemicals in a certain way.

One remarkable characteristic of contemporary scientific speculation on the question of the origins of life is a tendency to suspect that the first life emerged at underwater sea vents. Mere decades ago, scientists thought that all life on earth received its energy from the sun: directly through photosynthesis for plants, and indirectly through eating plants for animals. The discovery of organisms thriving deep underwater where the sun's rays do not reach, fed by the abundant minerals spewed out from the earth's core through underwater vents, was itself a considerable surprise. Now it seems that these undersea vents may have played a crucial role in our story. This is, we might note, an intriguing episode in the history of science. If not for the discovery of these organisms, speculation on the origins of life would naturally have focused on the sunfuelled organisms with which humans have long been familiar. However, 
scientists now doubt that there was enough energy on the surface to guide the reactions necessary to the origin of life on earth. They suspect, though, that these underwater vents were far more active than today at the time that life emerged.

Some scientists have invoked an idea of chemical evolution. Chemical compounds that are robust in an environment will survive while others decompose. They may even encourage reactions that reproduce themselves: Chemists use the term "autocatalysis" to refer to the ability of some compounds to induce reactions that reproduce the compound. If the complex chemicals that constitute life were each likely to survive and reproduce in some early earth environment, we should not be surprised that they were available to participate in the reactions that initiated life.

Modern cells combine long strands of DNA that code genetic information, shorter strands of RNA that carry that information, and proteins developed from that information that guide chemical reactions. It is highly unlikely that these three emerged together. RNA can store information and induce reactions, so it seems plausible that it appeared first (note that RNA rather than DNA guides some contemporary viruses). Notably, modern cells contain ribozymes made from RNA that can perform some of the tasks of proteins; these may be an evolutionary remnant from the earliest cells. Perhaps, then, an early strand of RNA that reproduced itself was the starting point of life. One problem here is that RNA on its own is very error-prone in reproduction. While this enhances the possibilities for evolutionary experimentation, it also means that successful species might have failed to reproduce because of genetic errors.

Though scientists are naturally attracted to the idea that the simpler RNA emerged before the far-more-complicated DNA, there is debate as to whether RNA predates, antedates, or appeared simultaneously with the first cells. Cells without RNA might have difficulty reproducing accurately. RNA without cells would be particularly prone to errors in transmission. It would be quite a coincidence if the two emerged simultaneously. It is perhaps better to imagine them each struggling independently for a while - maybe millions of years - before combining. There is speculation that two symbiotic life-forms - one with cells, one with RNA - may have over time combined.

Cells themselves present a puzzle. As any high school biology student can attest, cells are complex structures, where several different parts interact. In addition to proteins and the nucleic acids of DNA and RNA, cells - at least today - contain two other key chemicals: carbohydrates (for storing energy among other things) and lipids (which form cell membranes). How did such a complex structure come to be - even allowing for the possibility that the earliest cells might have been far less complex? Sea vents again point to a possible answer, for the honeycombed mineral structures observed there might have mimicked a cell wall. More precisely, the first cells may somehow have developed around such mineral structures.

Some types of bacteria - archaebacteria are emphasized by some scientists (and often treated as a distinct species), eubacteria by others - display a very 


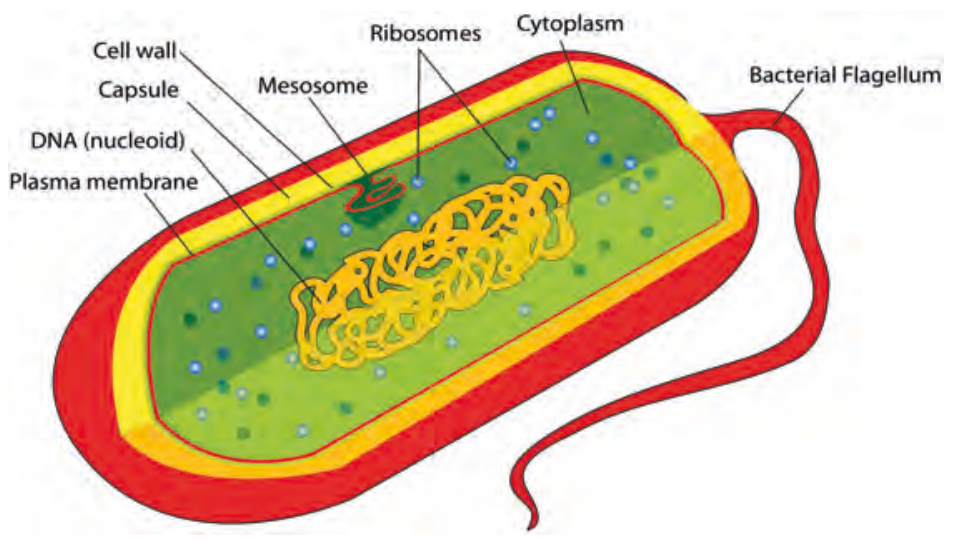

Illustration 2.6

Prokaryote cell of bacteria

strong resistance to heat, as well as ultraviolet light, and may thus have been the first ever life-forms. They could have thrived near sea vents and then prospered in the thin and toxic (by contemporary standards) atmosphere of early earth. Notably, the simplest bacteria can "borrow" genetic material from each other; this helps explain how a diversity of types of bacteria was able to emerge despite asexual reproduction (sexual reproduction generates genetic diversity because children inherit half of their genes from each parent).

Even if life first developed near sea vents, it must have at some point become adapted to surface living, or evolutionary processes would have been severely limited in extent. Here cyanobacteria may be of critical importance. These have the capacity for photosynthesis: using the energy of sunlight to stimulate life-sustaining chemical reactions. Not only are such bacteria perhaps the early ancestors of plants, but by performing chemical reactions on water molecules they emitted oxygen into the atmosphere, slowly (along with later organisms) over millions of years producing the oxygen-rich atmosphere we take for granted today. Oxygen comprises about 21 per cent of the contemporary atmosphere but comprised perhaps only 3 per cent a couple of billion years ago (scientists still debate the oxygen content of early atmospheres). Oxygen would have been toxic to many early life-forms but is essential to later life-forms, including ourselves. Note the contingency here: Early life developed - perhaps only could develop - in situations that humans cannot survive. Oxygen damages many single-celled organisms but creates the possibility of much higher energy absorption for multi-celled organisms. Moreover, the existence of an oxygen-rich atmosphere facilitates the formation of water droplets (which combine hydrogen and oxygen), contributing to the maintenance of a water supply on the earth's surface - itself essential to life (most of the water that Mars once possessed instead evaporated over time). (The Gaia hypothesis suggests that life-forms have somehow generated the conditions for 
their own existence over billions of years, but it is not clear how such a process could occur except by chance.)

The first life-forms were bacteria (or archaea if we treat this as a separate species). About 1.7 to 2 billion years ago, a different type of life-form, the eukaryotes, emerged. This second emergence has received far less scientific attention, though it will play a critical role in the next stage of our story. There is speculation that eukaryotes developed from combinations of bacteria. Eukaryote cells are larger and much more complicated, with nuclei and specialized compartments. These cells, with their specialized components, are likely a necessary precursor to multi-celled organisms in which different cells perform different functions. There is very recent speculation - and much consensus - that an archaeon may have absorbed (swallowed) a bacterium, and these then combined to form a more complex cell. This would most likely have occurred near an underwater vent. The bacterium - likely of the family Alphaproteobacteria - was the ancestor of mitochondria, a cell component (organelle) responsible for energy production. Scientists have recently discovered an archaeon that is closely related genetically to eukaryotic cells and interestingly different from most archaeons in containing actin, a protein important for cell walls.

Eukaryotes also, importantly, rely on oxygen as an energy source. They were only possible as the oxygen content of the sea and atmosphere expanded. Finally, yet importantly, eukaryotes reproduce in a manner that foreshadows sexual reproduction: Genetic material from two cells combines in generating a new cell.

One of the wonders of life is that individual organisms are short-lived but species, and especially types of species, can last for millions of years. Stars, as we have seen, are simple in form and can last for billions of years, but do not reproduce. The greater complexity associated with life seems to come at the price of a much shorter lifespan, but with a compensating capacity for reproduction.

\section{Evolutionary theory revisited}

We introduced evolutionary theory in chapter 1 and applied it to cultural phenomena. Yet of course, most people associate evolutionary theory today with its application to biology. In all life-forms, genes are transmitted between generations with occasional errors in transmission. These are the mutations at the heart of evolutionary biology. If a particular mutation aids the survival and reproduction of the organism, organisms possessing the mutated gene will become a more substantial proportion of the population over time. The natural environment in which the organism lives will determine which genes are selected.

For organisms that reproduce sexually, a species is defined as the set of organisms that can breed with each other (though this rule is often 
broken: Dogs, coyotes, and wolves are treated as separate species but can interbreed and have arguably generated an entirely new composite "species" in recent decades). Evolution may occur within a species. Over time, it may also produce new species. If the same species inhabits separate environments, it may evolve in entirely different ways in the two situations, perhaps becoming distinct species.

A species that has evolved so that it is well suited to a particular environment - it can gain nourishment, avoid predators, and survive climatic conditions - will prosper unless there is some change in environment (which might include the introduction of a new predator). We can imagine that a species' population will fluctuate around the maximum that their particular ecological niche can support. That is, there will be increased mortality if there are more organisms than the environment can support. (See our discussion of Malthusian population dynamics in chapter 4.)

Though mutations are random, the environment provides some "direction" to biological evolution, for the environment will reward certain types of capability. For example, we observe a similar diversity among marsupials in Australia as among mammals elsewhere: They each evolved in similar ways to fit similar but geographically distinct environments. Likewise, mammals and dinosaurs diversified in similar ways but in different time periods (see below). (Note that some very recent research in biology suggests that genes may be altered in some ways during an organism's life, and these genetic changes are passed along to children; if so this would alter our view of the randomness of genetic change.)

In the case of bacteria, their ability to borrow genetic material from each other created abundant opportunities for the emergence of new bacteria that could fit different ecological niches. These new species then reproduced rapidly until they reached some ecological limit. Note that life thus has an "evolutionary" advantage over non-life, for life can expand in extent quickly over time. Moreover, one of the defining characteristics of life is that it actively seeks to absorb energy from other sources for its survival. Once the first life-form emerged, it is not that surprising that living organisms expanded in both variety and total mass over time.

Sexual reproduction is standard among the multi-celled organisms that next arise (below). This allows a species to have individual diversity and lessens the risk of genetic disease (that is, harmful mutations). Mutations still occur frequently enough that species can both emerge and change over time. Scientists estimate that there is one error in transmission for every billion bits of genetic information transmitted. For bacteria with very short lifespans, humans can observe evolutionary processes, as when bacteria develop resistance to antibiotics because an antibiotic-rich environment selects for organisms with resistance. The longer a generation lasts for a species, the slower evolution will occur through time. Scientists therefore suspect that humans have not evolved (much) during historical time. However, we shall see in later chapters that 
humans have through selective breeding accelerated evolutionary processes among several plant and animal species.

The fossil record does not provide many examples of intermediate stages in the development of new species. This leads some to be sceptical of evolutionary biology. Biologists have two answers. One is just that fossilization occurs only under exceptional circumstances, and thus the historical record is inevitably limited. This problem of a limited historical record will often vex us in later chapters: We often lack documents of how certain polities, religions, or technologies emerged. The second answer provided by biologists is to suggest that evolution occurs unevenly through time. This answer may seem odd at first. Yet we need not posit that the rate of mutation changes through time (though this might happen). Instead, we can imagine that a species well adapted to a particular environment will exhibit little change: The vast majority of mutations do more harm than good. However, if that environment changes, or the species migrates to a new environment, then the probability that mutations aid the organism increases, for the simple reason that the organism is no longer as well suited to the environment it inhabits. We might then reasonably expect that there will be "punctuated equilibria": long periods of time in which little evolution occurs punctuated by periods of environmental change in which relatively rapid evolutionary change occurs. It is not that surprising, then, that the fossil record fails to capture many intermediate phases in evolution. While biologists debate the idea of punctuated equilibria, it is something we should keep in mind in addressing processes of social evolution: Changes in the environment will then not just encourage a greater tendency to select mutations but also to produce these.

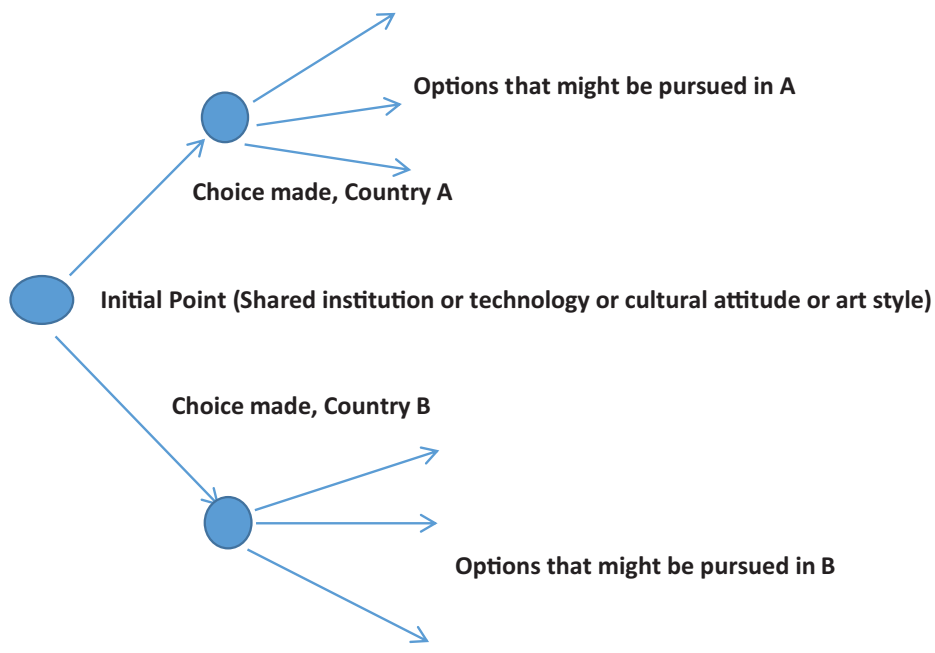

Figure 2.2

Path dependence

Note: The more decisions made since paths diverge, the harder it will likely be to switch paths later. 


\section{BOX 2.1}

\section{PATH DEPENDENCE}

One robust hypothesis for at least genetic evolution is that selection is much more likely for small mutations than big mutations. A genetic mutation that dramatically changes an organism will usually be destructive: Organisms are elaborate sets of interactions, and big changes are likely to destroy the delicate balance among different cells. Such mutations are a significant source of infant mortality in humans and other species. A small mutation has a much higher chance of aiding some of the organism's functions without doing severe harm to others. Most small mutations will still do more harm than good, but a minority will do more good than harm.

This celebration of small mutations causes the most significant controversies among students of biological evolution. How did complex structures such as eyes and hands emerge a little bit at a time? The general answer is that even a little bit of sight or manual dexterity might be useful, and so these complex structures could well have emerged gradually. One mutation in favour of sight might then encourage selection in support of other mutations that further enhance sight. Though biological evolution is a series of small mutations, a set of these may then combine over some set of generations to produce a significant change in the structure or functioning of an organism.

Is selection more likely for small than large mutations in social evolution as well? Human societies are, as we shall see, also elaborate sets of interactions: Cultural attitudes support political structures, which in turn reflect and structure economic interactions, and so on. Political revolutions often struggle because the economic, cultural, and social realities do not immediately react to work in concert with dramatically different political institutions. Yet some revolutions succeed (though these may on close examination be less revolutionary than they seem), and we might then hypothesize that large mutations have a somewhat higher chance of success in social evolution than in biological evolution. If so, we might attribute this difference, at least in part, to the fact that there is often some element of conscious thought involved in social mutations. Political revolutionaries might consciously strive to change the economy, culture, and social relations to align with the new political institutions. This is no easy task, to be sure, but genes have no ability whatsoever to fashion their selection environment purposely.

Nevertheless, the general case is that social evolution, like genetic evolution, involves an accretion of small mutations. As was suggested above with respect to sight, a mutation in a particular direction may encourage the selection of further mutations in that same direction. In the case of social evolution, we may also encourage mutations in that direction. At a temporal distance, we may think that there was a revolution - that the eye emerged in the blink of an eye - when there was, in fact, a series of small changes in a particular direction. We might think, for example, that agriculture emerged one day in Mesopotamia, when in fact agricultural practices slowly evolved over a period of centuries (chapter 4).

We can then expect evolutionary processes to exhibit "path dependence." Once a particular mutation has occurred, it changes the selection environment for future mutations (and perhaps the likelihood of certain types of mutation). In the year 1900, companies were 
producing petrol-powered, electric, and steam-powered automobiles. In the next years, petrol-powered cars gained a slight edge. For the next century, automobile manufacturers developed petrol-powered automobiles, and these both encouraged and were supported by advances in petroleum refining and the development of an infrastructure of service stations and repair facilities. Electric cars might prove superior in many ways. However, developers of electric cars struggle to compete: It is hard to match a century of technological development plus a vast infrastructure of service stations. It may yet prove possible to switch paths toward an electric car future - because the selection environment is turning against the environmental impact of petrol engines - but it will not be easy.

The implications of path dependence deserve emphasis. Foremost, path dependence means that random mutations can nevertheless generate a direction to history: Changes build upon each other along a particular trajectory. It is possible to change trajectories, but not easy. Indeed change is not simple even if clever humans can consciously recognize a better path. The computer keyboard that I am using as I type this has the standard English layout with the letters QWERTY across the top. It has often been noted that this layout places some of the most common letters in English - the 'e' and ' $a$ ' and ' $s$ ' - off to the side. We might well type faster if these letters were in the middle of the keyboard where stronger fingers could reach them readily. There is some empirical evidence that this is the case.The QWERTY keyboard was developed in the nineteenth century for manual typewriters: Typing speeds were limited by the technology of the day. There is even some speculation that the QWERTY keyboard was designed purposely to slow typing speeds in order to reduce the frequency with which keys jammed if struck too quickly. The keyboard solidified as typing schools and typing textbooks trained people how to type on the QWERTY keyboard. It would now be difficult to change precisely because of the body of people trained on the existing keyboard. This would be the case even if we were to agree that we could achieve a significant increase in typing speeds. (It is possible to download software that changes your keyboard layout, and even buy decals with which to change the lettering, but few people do this, most judging the likely gains not to be worth the hassle. It could be that they underestimate the value over an entire lifetime of a small increase in typing speed.)

The very idea of path dependence offends some scholars. They interpret this to mean that societies fail to achieve optimal outcomes even when human actors are aware of these. They doubt that there can be an important difference in typing speeds across computer layouts, for then society - in particular, entrepreneurs and consumers acting sensibly within markets - would have succeeded in popularizing the optimal keyboard. However, potential typing speeds are not the only determinant of what keyboard layout one chooses. I may know that I could type faster if I switched, but I have learned on a particular keyboard and will face costs in changing. I may wish my child learned on a better keyboard, but know that if I have my child trained in a new way they will often in life have to type on a QWERTY keyboard and be at a disadvantage. I may know that a different keyboard is locally optimal - it maximizes typing speeds, given appropriate training - but it may not be globally optimal within the complex web of interactions in which typing occurs in contemporary societies. 
Revolutionaries of various types may also dislike the idea of path dependence, and the more basic idea that small mutations are more likely to be beneficial, for it implies that we should focus on gradual change and be wary of switching paths. Yet while humans must take care in switching paths, it is useful to consult the historical record on whether path switching has sometimes proven both advantageous and important. This will be one of our tasks in later chapters.

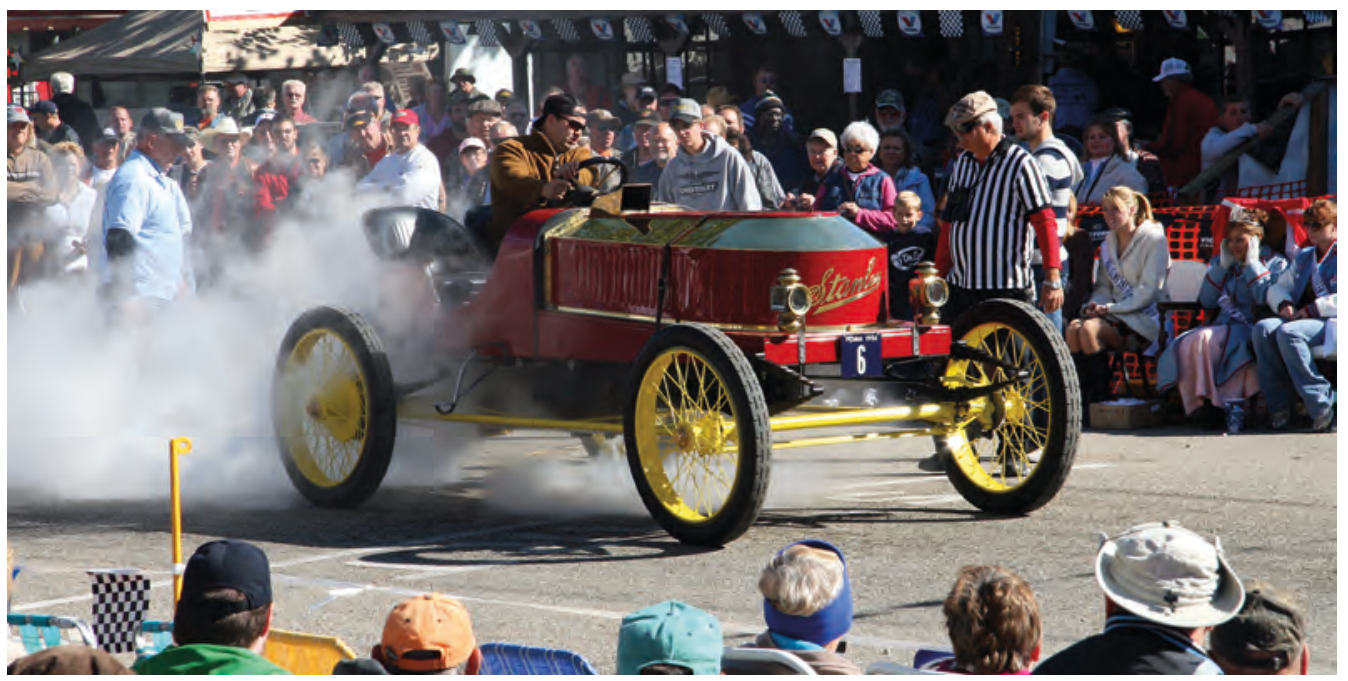

Illustration 2.7

Stanley Steamer at 2009 Newport Hill Climb

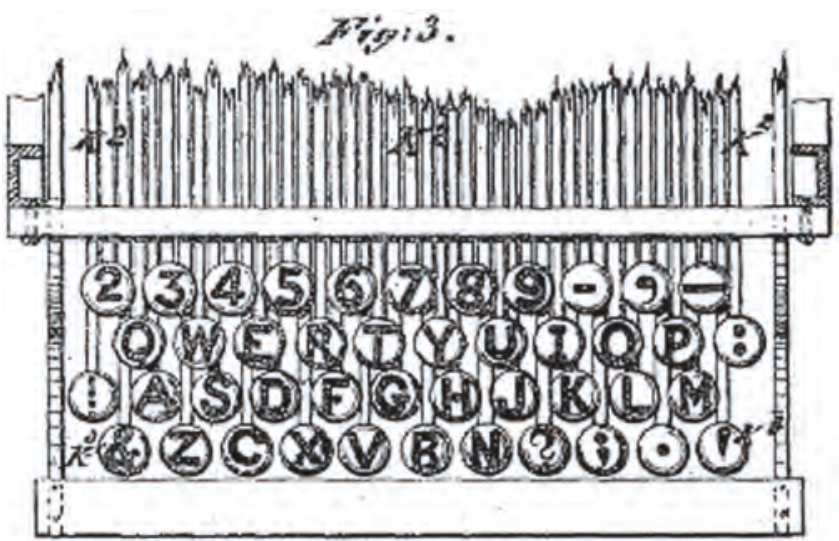

Illustration 2.8

QWERTY layout in Scholes' 1878 typewriter patent application 


\section{Multicellular life (600 million years ago)}

Single-celled organisms such as bacteria are still by far the most common lifeforms on earth: Their microscopic scale hides their presence from us. We should thus be careful of celebrating evolutionary processes as inherently producing greater complexity: The simplest life-forms have survived and prospered long after the development of multi-celled organisms. Yet humans are naturally intrigued by the development of more complex organisms such as ourselves.

After some 3 billion years in which life on earth comprised only singlecelled organisms, multi-celled organisms emerged some 600-700 million years ago. Climatic warming (for which scientists still debate the reasons) may have influenced the timing. Ice apparently covered much of the earth - both land and ocean - until just before this time. Once multi-celled life emerges, a variety of multi-celled creatures appears in the seas (but not yet on land) within mere tens of millions of years. These organisms came to possess a variety of organs: different cellular structures devoted to various tasks. Not all such organs were on the organism's surface and thus capable of absorbing energy directly; internal transmission of energy in the form primarily of oxygen was then critically important. Once multi-celled life was possible, selection pressures worked quickly - again within the sort of timescale we must embrace in this chapter - to generate life-forms that fit a variety of ecological niches. The fossil record shows at first various worm or jellyfish-like creatures followed (but not replaced) within millions of years by a variety of creatures with shells. These shells presumably represent an evolutionary selection in response to the fact that some multi-celled organisms ate other organisms. Some have speculated that both of these developments may have been aided by a climatic change that increased either the oxygen supply or the supply of calcium for shell-formation.

Different families of organisms emerged in these first millions of years. Plants are self-supporting in the sense that almost all of them extract all of their needs either from solar energy (producing the carbohydrate glucose through photosynthesis) or from inorganic substances in soil or water. All other organisms obtain some or all of their needs from other organisms. Animals can survive only by eating other organisms, mainly plants or other animals. But fungi (and a variety of microorganisms) also developed which live off animal and plant remains. This development was crucial because otherwise plant and animal wastes might eventually have destroyed the ecological niches in which plants and animals lived.

Palaeontologists speak of a "Cambrian explosion" from about 540 million years ago during which the ancestors of most if not all modern families of animal appeared. Though the explosion is associated with increased oxygen levels, it may have been triggered by periods of low oxygen that wiped out many preceding species, creating ecological room for new species to emerge (just as the disappearance of dinosaurs created room for mammals below). 
As noted above, the predatory nature of some organisms created evolutionary pressure for defensive capabilities in others. Shells were followed in time by camouflage, poisons, thorns, and a variety of other defensive mechanisms. Once predatory organisms existed, it may have become impossible for radically different types of life to emerge, for these would have been eaten before they could evolve defences. We should thus be careful of assuming that multicellular life evolved in some best (perhaps maximizing energy efficiency) fashion; there was path dependence such that once life evolved along a particular path, it prevented the pursuit of other paths.

Multi-celled organisms are less complicated than we might imagine at first. Thousands of genes guide single-celled bacteria. About 30,000 genes guide humans. The simplest multi-celled organisms have just a few cells and little more genetic material than single-celled organisms. We can then imagine an evolutionary process whereby genetic mutation and selection added new cells over time. Cells in multicellular organisms work together because they contain shared genetic material. As the organism develops, this common genetic material is triggered in different ways to form different types of cell.

About 400 million years ago multicellular life began to move from the sea onto land. This move might not have been possible if not for the development of the ozone layer - composed of an isotope of oxygen, and thus also generated by the previous increase in oxygen in the atmosphere - which protects the earth from ultraviolet radiation. To move from the sea to land, plants nevertheless had to develop exteriors that prevented them from drying out. Animals would need hardier skeletons to support their weight without the buoyancy of water. Four limbs and five digits are universal, suggesting a common ancestor. It was particularly challenging to manage reproduction in a non-liquid environment, and so animals developed first eggs and then, much later, embryos to provide a sheltered liquid environment for organism formation. While the land presented challenges, it also offered opportunities: abundant solar energy and - for a while - less competition from other organisms.

One legacy of these early land-based life-forms is the coal and petroleum resources of the earth. These were created hundreds of millions of years ago from the remains of carbon-based life-forms. (We do not fully understand why this process of transformation ended a hundred million or so years ago.)

Some 99 per cent of the species that have ever lived are extinct. Extinction is sometimes the result of competition for resources as a new species comes to dominate a particular ecological niche. More often, perhaps, extinction results from changes in the environment resulting from a variety of factors: climate change, volcanic activity, or (most dramatically) asteroid impact. The history of life on earth is a history of extinctions. Sometimes many species disappear at once. At other times, only one or a few species disappear. These extinctions remind us that evolutionary processes are unpredictable and largely directionless.

The most famous extinction is that of the dinosaurs. This is commonly attributed to an asteroid impact. Indeed the "fact" that an asteroid extinguished 
dinosaurs is one of the most widely accepted pieces of scientific "knowledge" among the public. Yet the historical record is much less clear than this common knowledge admits. The fossil record indicates that numerous dinosaur species had become extinct tens of millions of years before the asteroid, and some may have survived for some time afterward. It is apparently a mistake to imagine that one asteroid eradicated an otherwise healthy population. It seems instead that something else - perhaps the extensive volcanic activity associated with the time, or climatic cooling that also appears to have occurred - had already weakened dinosaur populations. The asteroid may only have accelerated a process that was already underway. Or perhaps the asteroid only had such a devastating effect because other factors had already diminished dinosaur populations.

There are many reasons that a more nuanced story of dinosaur extinction is not more widely appreciated. One is undoubtedly just the drama of the asteroid story: It is a simple explanation we can readily understand. And it ties into popular concerns that another asteroid could someday inflict similarly extensive damage on earthly creatures, including humans. We know from the craters they have left that large asteroids do periodically crash into the earth's surface, and there is clear evidence of an asteroid impact at around the time of (most) dinosaur extinctions that did indeed disturb the atmosphere for many years. Yet there is also at work a process of disciplinary miscommunication and disciplinary arrogance. Geologists, who pointed proudly to global evidence of

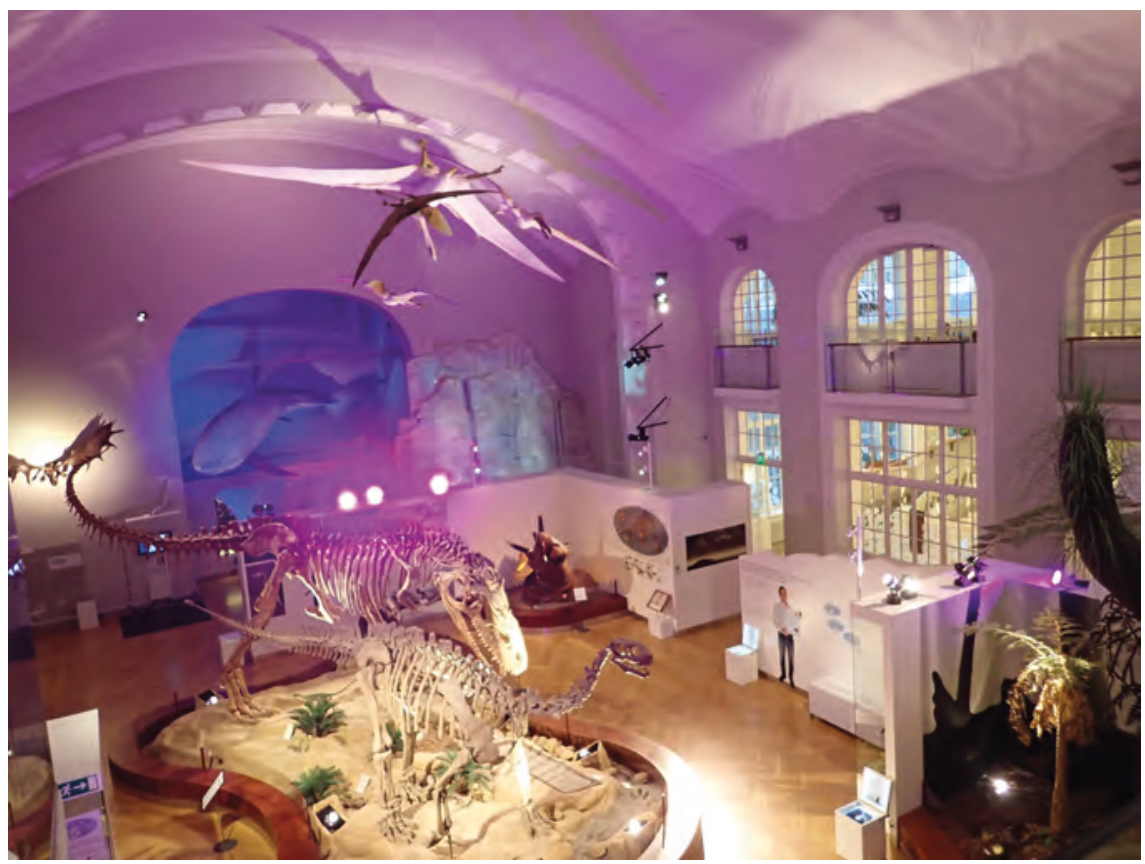

Illustration 2.9

Dinosaur skeletons, Natural History Museum of Helsinki

Source: Photograph by Sinikka Halme, CC BY-SA 4.0 


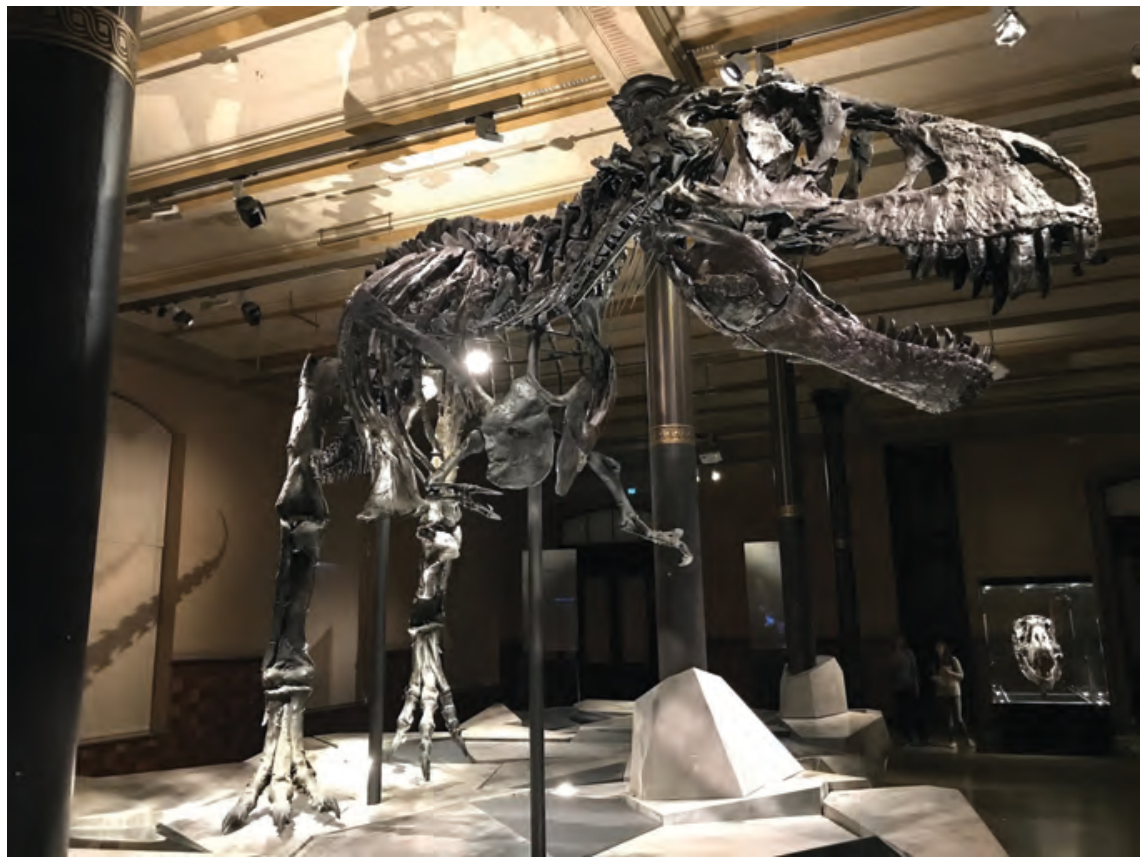

Illustration 2.10

Tyrannosaurus skeleton

an asteroid impact, put the asteroid hypothesis forward. Palaeontologists have slowly assembled the contrary evidence by painstakingly reconstructing the histories of individual dinosaur species. Advocates of the asteroid explanation have not wanted to admit that other factors may have been at work. One analogy used is that a fire in a seniors' residence can still be deadly; it does not matter that the residents may already have been near death. Yet, of course, it does matter: If younger and quicker, they might have escaped. Likewise, we should not leap to a conclusion that the asteroid impact would have killed all dinosaurs even if other circumstances were not already threatening them. After all, many species did survive the asteroid. We need to explore more carefully why certain species died - and precisely when - and others lived. We need an interdisciplinary approach that appreciates that the asteroid interacted with earth-based processes.

\section{Mammals (70 million years ago)}

Mammals are warm-blooded and furry and carry their young internally. Mammals were around during the time of the dinosaurs but occupied a tiny set of ecological niches while dinosaurs dominated the earth. Mammals were primarily burrow-dwelling animals at the time. This may have protected them both from dinosaurs and from the atmospheric catastrophes that extinguished 
dinosaurs. Being warm-blooded also may have proved advantageous in the difficult time in which the dinosaurs were extinguished (though there is speculation that some dinosaur species may have been warm-blooded). In any case, once dinosaurs disappeared, mammals expanded into these now-vacant ecological niches: Mammals roamed the earth, climbed trees, and came to eat a wide range of plants and animals. The evolution of these animals and eventually humans seems to have been entirely contingent on dinosaurs disappearing; previously there just was no room in the ecosystem for a variety of mammal species. We should thus be (yet again) careful of presuming that humans were a natural or inevitable result of evolutionary processes. It is entirely possible that warm-bloodedness is conducive to a more complex brain, for complex nervous systems react poorly to the changes in temperature that cold-blooded creatures experience. A planet dominated by cold-blooded dinosaurs may never have seen highly intelligent beings.

We have, then, an unpredictable and complex set of interactions without which humans would likely never have appeared. An asteroid impact combined with volcanism and perhaps other factors led to the extinction of dinosaurs. Mammals and a variety of plants managed to survive. Mammals then evolved to fit various ecological niches. One of these involved living in trees. Primates emerged soon after the dinosaurs disappeared, and needed both manual dexterity and good eyesight to navigate successfully from tree to tree. These qualities would later be important for humans. Humans evolved from these tree-dwelling primates (chapter 3). The evolution of humans, therefore, reflects both the contingent nature of evolutionary processes and a particular set of interactions among phenomena.

\section{Reprise}

It is perhaps too easy to tell the story of this chapter in terms of interactions among phenomena and evolutionary (and other) processes of change. Interactions were vital throughout, between matter and anti-matter, protons and electrons, mass and energy; the gravitational (and other) forces that formed stars and planets; RNA and cells combining to generate life; and archaeons and bacteria combining to set the stage for multicellular life.

Particular processes of ongoing change deserve mention also: the continued expansion of the universe, the slow work of gravity in drawing stars, planets, and galaxies together; the slow movement of plates across the earth's crust. Fusion reactions deserve special mention, for they remind us that this process of change - whereby light elements are transformed into heavier elements - is characterized by an amazing stability in that the same reactive process proceeds for billions of years within a star (changing very slowly with respect to which elements are transformed).

Evolutionary processes loom large in the latter part of our story, once life emerges. Yet we have seen that some have applied evolutionary theory to 
Genealogical Tree of Humanity.

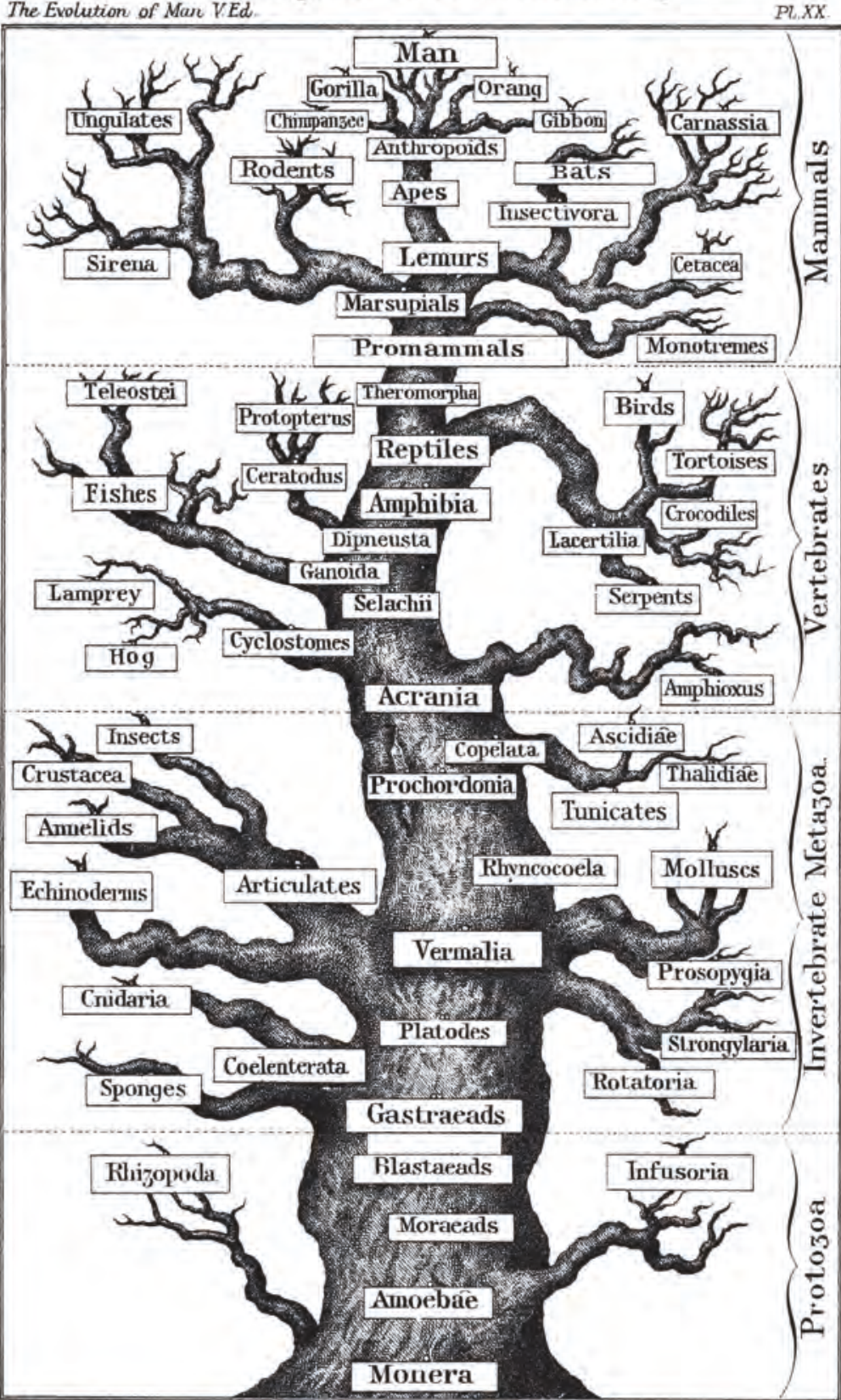

E. Haeckel del.

Illustration 2.11

Ernst Haeckel, Family Tree of the Human Race, c. 1877, an early attempt to comprehend human evolution 
chemicals and stars. Scientists have also applied evolutionary theory to the early formation of the universe in which the conditions "selected" lighter elements rather than heavier elements. We can note here that there is even some evolutionary speculation regarding universes themselves. We have eschewed above discussion of the possibility that there are multiple, perhaps infinite, universes, but can recognize here that many physicists (and science fiction writers) have speculated that this is so. If so, it is possible that not all universes achieve the conditions necessary for expansion or star formation. There may then be selection only for universes such as our own: universes that can create black holes and stars because of a particular balance among forces. If so, it becomes less surprising that all of the above interactions and processes unfolded in such a way as to allow humanity's story to unfold.

We should nevertheless close by remarking on the contingent nature of historical processes. A different distribution of mass in the early universe might have resulted in only massive fast-burning stars. Slight differences in chemical composition might have prevented life from emerging on earth. Survival of just some dinosaurs might have impeded the expansion of mammals. It will be interesting to explore whether we can identify similar contingencies in human history.

\section{Questions}

1. Should a world history text include a chapter (or more) that addresses the development of the universe before humans? Why or why not?

2. Is it important that our understanding of human history have analogies with our understanding of natural history?

3. Why would an interdisciplinary approach to dinosaur extinction be useful?

4. Define "path dependence." Why is it important for historical understanding?

5. Discuss how easily natural processes might have followed a different path such that humanity would not exist.

\section{Readings}

Brooke, John L. 2014. Climate Change and the Course of Global History: A Rough Journey. Cambridge University Press.

Christian, David. 2011. Maps of Time: An Introduction to Big History. 2nd ed. University of California Press. [Christian defends against the charge of meta-narrative by urging multiple meta-narratives that more focused histories can be related to.]

Christian, David, Cynthia Stokes Brown, and Craig Benjamin. 2014. Big History: Between Nothing and Everything. New York: McGraw-Hill Education.

Crofton, Ian, and Jeremy Black. 2016. The Little Book of Big History: The Story of Life, the Universe, and Everything. London: Michael O'Mara Books. 
de Queiroz, Alan. 2014. The Monkey's Voyage: How Improbable Journeys Shaped the History of Life. New York: Basic Books. [Discusses how species have spread into different habitats over time.]

Lane, Nick. 2015. The Vital Question: Why Is Life the Way It Is? London: Profile Books. [An accessible treatment of the emergence of life.]

Spier, Fred. 2010. Big History and the Future of Humanity. Malden, MA: Wiley-Blackwell.

Scientific thinking on many of the subjects addressed in this chapter is itself evolving rapidly. Students should here more than in any other chapter consult online sources and journal databases to establish the latest thinking on these issues. 


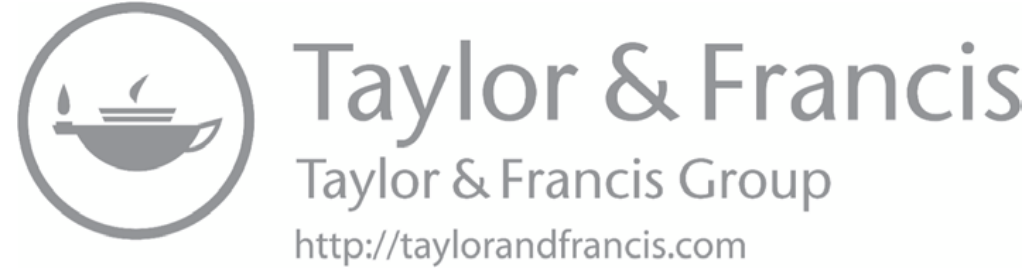




\section{Evolution of human nature itself in early human history}

\section{Guiding questions}

How did humans evolve as a species? How and why did critical physical and psychological characteristics emerge? How in particular did the natural environment and culture shape human evolution?

What sort of activities did early humans pursue and how and why did they develop the capabilities to perform these activities? What do we know or suspect about the beliefs and attitudes of early humans? In other words, who were they?

Relationship to other chapters: Chapter 3 resumes the story of biological evolution begun in Chapter 2, against the background of the physical environment examined in Chapter 2. It outlines the evolution of a set of physical and human capabilities (and limitations) that will apply throughout the rest of this book. It also discusses how humans spread across most of the globe, setting the stage for discussions of humans in different regions throughout the rest of the book. We will discuss further human migration in several later chapters, most notably Polynesian migration into the Pacific in chapters 7 and 16.

We focus in this chapter on the evolution of our basic human genetic composition. This theme will influence all other themes in the remaining chapters. In this chapter, it is primarily the non-human environment that shapes it. Yet human nature was also shaped in important ways by culture, and indirectly 
through culture by all other themes. We do address how all our themes operated during the millennia in which all humans hunted and gathered, but pay particular attention to culture (especially language), technology, economy, and art. Social structure and population are also addressed at some length. We apply evolutionary analysis to both human biology and culture, and indeed to how they evolved together.

Genetic

Predisposition

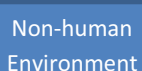

Environment

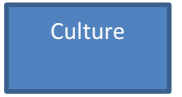

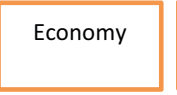

Art

\section{The evolution of human nature}

The big story in this chapter is the evolution of human beings. We provide an overview of millions of years of evolution in this section. Subsections discuss how both the natural environment and human culture may have shaped genetic evolution. In later sections, we discuss the evolution of particular human capabilities or traits. The latter part of the chapter then looks at a variety of human activities during the long period of human evolution.

Some four to eight million years ago, primitive hominids that walked upright first emerged. Over a period of millions of years, these early hominids evolved into modern human beings. There appear to have been at least several different species of hominid of which only one has survived. Anthropologists think that modern humans have existed in roughly their present genetic form - as homo sapiens sapiens - for about two hundred thousand years. The evolution of modern humans mainly took place in Africa, though remains of early hominids have also been found in Europe and Asia. Though anthropologists still debate the precise point in time when modern humans first appeared, and the degree to which homo sapiens bred with other hominids after leaving Africa, the broad outlines of when and where are widely accepted.

We can sketch in this chapter some of the critical stages in evolutionary development. We should stress at the outset that this history is based on mere dozens of archaeological sites; debate rages among anthropologists regarding the number of different species of hominid that existed at different points in time, which species evolved into more human-like form, how much interbreeding among species there may have been, why some species came to dominate over others, and what sort of capabilities different species possessed. (A new site recently discovered in South Africa, for example, suggests the discovery of a new species that does not comfortably fit into standard understandings of human evolution; readers can reasonably expect that our perceptions of early hominids will change with further discoveries of early hominid remains.) We often have very little evidence for particular species. The first hominids ape-like creatures that walked on two legs - likely emerged four to five million years ago, though this transformation may have occurred millions of years earlier than that. The earliest hominids are designated "Australopithecus" by 
anthropologists. Fossilized footprints from 3.6 million years ago in Tanzania suggest that Australopithecus Afarensis walked much as we do (though they were shorter and therefore took smaller steps) - though some anthropologists suspect that their gait was still similar in some ways to that of chimpanzees. To walk like humans would have required an interrelated set of evolutionary changes to the feet, legs, hips, pelvis, and spine (at least); this evolutionary process likely unfolded over a very long time period (by human standards, not Big History standards). Though Australopithecus may have walked like humans, it was unlike us in many meaningful ways: smaller stature, protruding jaw, and much smaller brain. The earliest hominid males were less than five foot tall, with females perhaps only three foot tall. This sexual dimorphism is common in ape species that operate in groups with one dominant male and where females join other groups at puberty. The stomachs of Australopithecus suggest that they primarily ate plant matter. From examining skulls, it seems that these early hominids had not developed the parts of the brain today associated with reasoning or language, and perhaps the asymmetry between hemispheres related to tool manufacture. We have no evidence of tool manufacture in the first 3.5 million years of hominid existence; this likely reflects limited cognitive capability, and perhaps limited manual dexterity.

The next evolutionary stage involves the genus "homo," of which several distinct species have been identified before the emergence of "homo sapiens." The several species dubbed "homo" have a larger cranial capacity, smaller teeth, and more human proportions compared to Australopithecus, as well as opposable thumbs. They resemble modern humans in significant ways. Homo habilis (some anthropologists think this was several species) and later homo erectus (sometimes distinguished from homo ergaster; sometimes the latter name is preferred) are each associated with increased brain size and smaller teeth. It is likely that homo habilis evolved from some branch of Australopithecus, and in turn evolved into homo erectus, but there may have been a more complicated evolutionary path. The more substantial brain size of these hominids allowed both a more extensive range of social interaction and the manufacture of simple tools. It is presumed

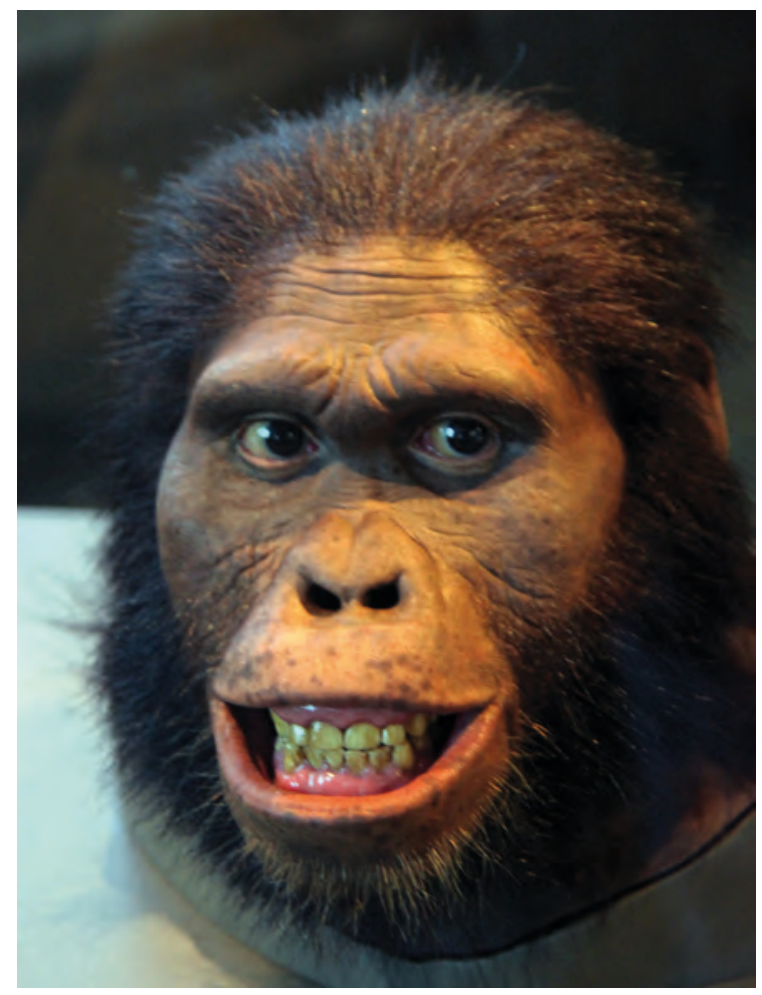

Illustration 3.1

Australopithecus female

Source: Smithsonian Museum of Natural History; reconstruction by John Gurche; photographed by Tim Evanson, CC BY-SA 2.0 
that Australopithecus lived in small groups (with a dominance hierarchy based on physical strength) and foraged individually, much like chimpanzees (the groups may have been larger than chimpanzee groups, which may have called for larger cranial capacity already; we discuss the connection between group size and brain size below); the homo species lived in larger groups and likely cooperated in many activities. Nevertheless, even homo erectus could probably make only limited sounds: It was capable of some forms of simple communication but not of the sort of language associated with homo sapiens. It may have been the first hominid species to control fire and was the first hominid species to spread out of Africa. It may have had some limited hunting capability associated with its tools. But in this as in language, it was still far short of modern human capabilities. Homo sapiens most likely evolved from homo heidelbergensis, which probably evolved in turn from homo erectus or ergaster, but again there may have been a different evolutionary path.

Hominids very similar to homo sapiens are found about 200,000 years ago (perhaps longer: a recent find in Morocco suggests 300,000 years, but this is controversial). Homo sapiens as a species recognizably similar to contemporary humanity can be dated to at least 125,000 years ago (There was at least one branch of homo sapiens that appears to have died out). DNA analysis of modern humans points to a common ancestral male and female between 200,000

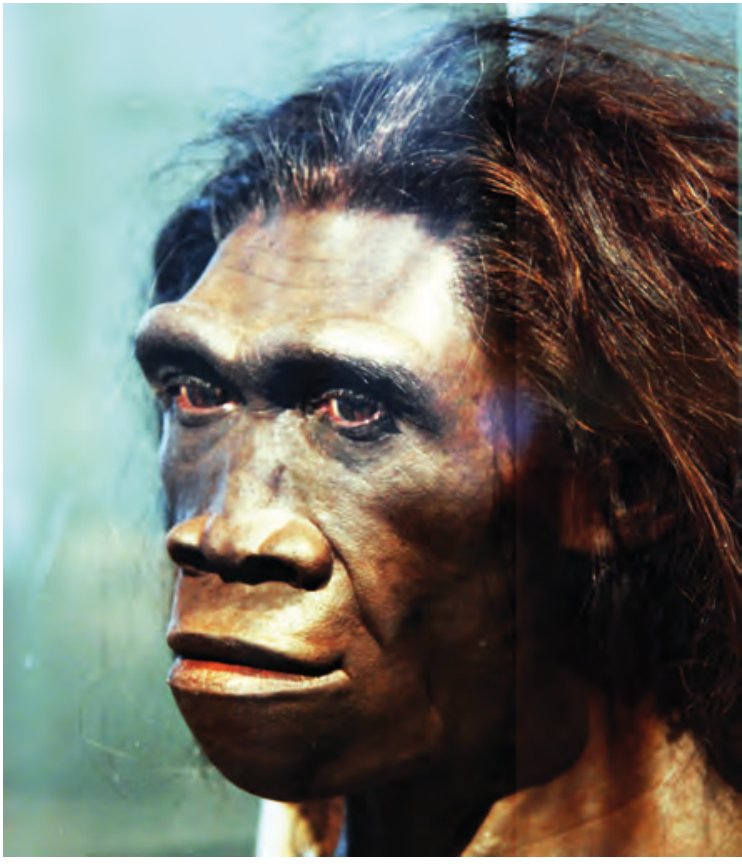

Illustration 3.2

Homo erectus female

Source: Smithsonian Museum of Natural History; reconstruction by John Gurche; photographed by Tim Evanson, CC BY-SA 2.0 and 60,000 years ago (when, as we shall see, homo sapiens began to migrate out of Africa). It is worth remarking on timeframes here: It took billions of years for the natural processes outlined in chapter 2 to unfold, and millions of years for homo sapiens to evolve from apes, but the single species of homo sapiens has accomplished all that we will recount in the remainder of this book in a mere 125,000 years. Put another way, there have only been some hundreds of generations of homo sapiens. Homo sapiens had yet larger brains (1,350 cc.) than previous species, and the ability to make the range of sounds associated with language. Homo sapiens had the abilities to affect their environment, plan, and express memories in words, pictures, and music.

It is generally thought that homo sapiens emerged first in Africa and spread to other continents, displacing other hominids. There is some speculation that homo sapiens evolved in multiple locales. There is genetic evidence that it interbred 


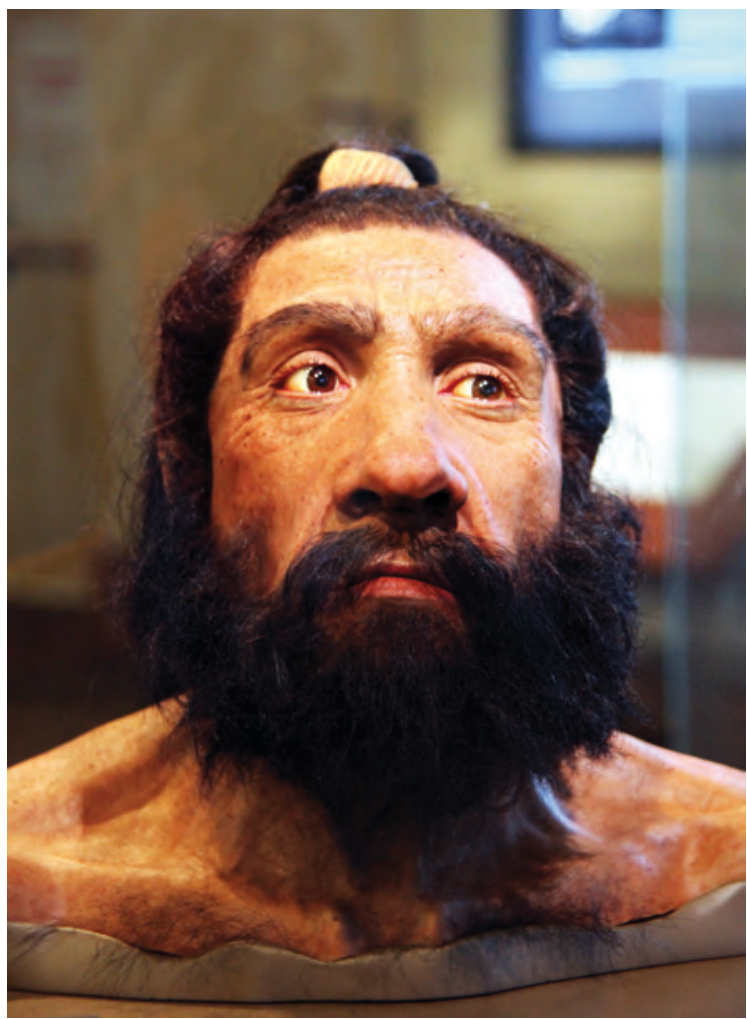

Illustration 3.3

Neanderthal male

Source: Smithsonian Museum of Natural History; reconstruction by John Gurche; photographed by Tim Evanson, CC BY-SA 2.0

with other hominid species (including the European neanderthal) as it spread across other continents. There is also evidence that the last survivors of another hominid species disappeared only 45,000 years ago on the Indonesian island of Flores (and recent discoveries on the island of Luzon in the Philippines suggest an entirely different species was alive at least 75,000 years ago). Recent analysis connects these to homo habilis but not homo erectus and raises the possibility of an even earlier first migration of hominids out of Africa - but no other fossil remains connect homo florensiensis to homo habilis across either half the world or millions of years.

Several elements of human evolution deserve emphasis:

- $\quad$ Bipedalism.

- Social cooperation in groups larger than are common in other animals, and of a complex nature.

- Increased cognitive capabilities in general.

- Linguistic capability in particular: For many, it is the ability to speak, and indeed to think in words, that most distinguishes humans from other animals. Note that hominids cooperated in groups long before they talked. 
- Increased manual dexterity.

- Dexterity and cognitive capability encourage and perhaps reflect toolmaking and fire-making (see below).

- Culture: Though often less stressed than the earlier bullet points, it is widely appreciated that human cultures emerged and evolved early in the course of human history (see below).

In addition to characteristics such as the above, the field of evolutionary psychology suggests selection for a host of psychological traits during early human history. It deserves to be stressed that humans operated as hunters and gatherers throughout this long evolutionary process. We can expect, then, that humans were selected to be successful hunters and gatherers. Evolutionary psychologists theorize that various characteristics of human psychology would have aided in hunting or gathering food or social interaction or (perhaps especially) success in mating. One notable aspect of this literature is a suggestion that we may have been selected for characteristics that are less well suited to modern society: It might be, for example, that heightened levels of aggression serve the needs of hunters more than office workers. Some of these conjectures are controversial; detractors suggest that plausible evolutionary stories can be told regarding any human characteristic. We lack the detailed understanding of early human history with which we might test competing hypotheses. The best we can do is use our judgement. We cannot in one chapter review the multiple hypotheses put forward in the evolutionary psychology literature, but we can get a flavour of the literature by examining a few key issues below.

As for "why" humans evolved as they did, much can be explained by appeal to nothing more than evolutionary processes. As noted in chapters 1 and 2, biological evolution is the most widely understood evolutionary process. Random mutations in genes are selected for if they aid the organism's survival and reproduction within some environmental niche. Genes that support the organism's genetic fitness (that is, survival and reproduction) are more likely to be passed on to later generations and thus over time become increasingly common. Random mutations might have enhanced brain size or manual dexterity or language capability, and these were selected for because they aided humans in one or more of hunting, gathering, impressing mates, and caring for children. As humans came to fashion tools and cooperate with others, the rewards of mutations that enhanced cognitive capabilities or manual capabilities were enhanced. There was thus some sort of path dependence in which enhanced mental capacity and manual dexterity increased the selection for further advances in cognitive capability and manual dexterity.

Note that increased brain size is not costless, for brains absorb a great deal of energy: If it were costless, we might have expected many animals to have evolved large brains. The modern human brain is about 2 per cent of body weight but absorbs 20 per cent of human energy (and half the oxygen we consume). There must then be something special about humans that made increased cognitive capability especially valuable. One of the great debates in 
anthropology is whether selection for increased brain size was driven more by the tool-making of humans or by the need/desire for human cooperation. Note that the number of relationships that a hominid strives to comprehend expands exponentially as group size increases - for we want to understand how our neighbours interact with each other. Of particular importance here is language, which is of obvious significance to complex social cooperation, but also contributes to shared knowledge of tool-making. And language requires substantial cognitive capability. Though the question of why increased brain size was selected for is usually approached at the level of individuals, it can also be argued that human groups with larger brains would outcompete other groups, and so there may have been group-level selection pressures as well. At the group level too, there are benefits of both greater cooperation and better tools. It may be that both tool-making and social collaboration contributed to selection pressure for larger brains; it is notable that these functions are located primarily in different parts of the brain and thus there may have been different reasons for selection of different cognitive capabilities.

We will discuss tool-making in more detail below. One interesting point to make here is that both cutting tools and the development of fire (also discussed below) effectively allowed humans to perform some of the work of digestion outside of the body. Cooking softens food and cutting of course further limits the need for chewing; humans then did not need to spend five hours a day chewing as chimpanzees do. By reducing the physical energy required to eat through cutting and cooking, the human body could devote greater energy to other tasks, including mental tasks. Cooking has further advantages in transforming both starches and proteins so that these are easier to digest, allowing us to eat harder plants, and enhancing nutritional value. Note that an animal's digestive system also utilizes a lot of energy: It is no easy task to transform eaten food into the nutrients the body requires. Once human brain capacity had expanded to allow cooking and cutting food, then, there could be further selection for even bigger brains that could be sustained by energy previously devoted to digestion. (We shall see, though, that there is debate as to whether fire-making occurred early enough in human history to have encouraged selection for brain size.)

Recent research suggests a third important consideration in selection for increased cognitive capability. As human brain - and head - volume grew, it became more difficult for women to give birth. Despite selection over time for wider hips among women, much of this brain/head development had to occur after birth. Taking care of helpless children is itself a challenging task both practically and in terms of parent-child communication. The need for extended periods of childcare itself encouraged enhanced cognitive capability. That is, selection for some degree of increased brain size necessitated an increased period of childhood dependency which in turn enhanced selection pressure for further increases in brain size.

Humans evolved physically as well as mentally. Walking upright has many advantages: The walker has a better view of both opportunities and dangers; 
there is more efficient energy use when crossing land (and shallow water, which some stress); and arms are free for carrying tools or babies and (later) for throwing weapons. On the other hand, the ability to walk upright came at the expense of ape-like abilities for climbing trees. Hominids were best suited to an environment of open savanna - treeless plains - rather than forest. It is not entirely clear how this transformation in capabilities occurred: There may have been an intermediate evolutionary step which mimicked the knucklecrawling gait of chimpanzees. In any case, it depended on the existence of large treeless areas. Note that many animals developed stiffer legs better suited to open savanna than forest, but only humans walk on two legs rather than four.

Shorter fingers and opposable thumbs also evolved as hominids became ground dwellers rather than tree dwellers. Again, the precise evolutionary path is unclear. There is a question as to the value of opposable thumbs before toolmaking. Yet hominids could hardly have been developing tools before having opposable thumbs. We might posit some co-evolutionary process in which once humans experimented with simple tools there was selection pressure for greater dexterity.

Humans also developed five reasonably good senses. It is worth noting here that some other animals exceed us in each of these senses. This should remind us that abilities come at a cost (the brain has to be fed, and so on) and that we have not been selected to be excellent in all respects. The simple fact is that we are capable of many things but incapable of many others.

Biological evolution occurs very slowly among species that live long lives. We should stress that there has been little change in historical time. The most important change here is selection for lactose tolerance in adults which could only occur after human farmers or nomads began keeping dairy animals (chapter 4) - though there is also some evidence of selection for height and other physical characteristics over historical time. For the most part, then, human nature was determined during humanity's millennia as hunters and gatherers. The attributes selected then may not be ideal for the very different circumstances in which most humans came to live after the development of agriculture and nomadism.

\section{Environmental influences?}

The natural environment in which selection occurs will, of course, shape biological evolution, including that of humans. If the environment remained the same throughout human history, we might attribute to it a passive albeit critical role in the course of human evolution. There is, though, good reason to believe that human evolution did not occur against a backdrop of an unchanging selection environment. It may well be, then, that changes in the natural environment were crucial influences on human evolution - just as changes in environment figured prominently in the evolution of species in chapter 2 .

The earth's climate has changed quite a bit over the millions of years of human evolution. As noted above, the first significant evolutionary change 
as humans evolved from apes was bipedalism, and this was advantageous only if there was non-forested land to walk about on, and plants growing on that ground to be consumed. It appears that there was climatic change that expanded savanna in Africa at the time that the first hominids appeared. Over time, these plains became home to large animals that could best be hunted cooperatively, perhaps creating selection pressures for increased human cognitive capability. The short and stocky stature of both homo erectus and homo neanderthalis may reflect the relatively cold temperatures of the time: They likely lacked the dexterity to make tight clothing and would have been selected for heat-preserving physical characteristics. Alternating periods of cooling and warming has characterized the era of homo sapiens. Their migration out of Africa appears associated with a warming period that may have facilitated movement northward. It may also have increased aridity in Africa, encouraging out-migration.

If the hypothesis of supportive environmental change is correct, then we are led to wonder why the environment changed so much. The earth's temperature does cool and warm over very long cycles. Changes in the earth's tilt and alterations in its orbit (it moves closer and farther away from the sun) were likely not the leading causes of climatic change over the last few million years. Volcanic activity could create clouds that trapped heat in the atmosphere (as carbon emissions do today). Warming trends could perpetuate as glaciers melted, for glaciers reflect heat while bare ground absorbs it. Changes in water currents can have substantial climatic effects. It is posited, in particular, that melting ice might have caused cold arctic water to spread south, attenuating or even reversing warming trends. We noted in the previous chapter that the formation of the Isthmus of Panama might have led to expanding savanna in Africa.

\section{Culture and more}

Though the non-human environment guided human evolution, it was almost certainly not alone in doing so. It was recognized above that "culture" likely emerged early in human history. Culture, as we noted in chapter 1, also evolves. There is an intriguing possibility that genes and cultures co-evolved in early human history. Indeed, we shall see below that this sort of co-evolution provides an answer to at least one key puzzle in the course of human genetic evolution.

We will discuss below why culture emerged early in human evolution. We can appreciate here that culture interacts with each of the other themes introduced in chapter 1: Humans needed culture because they interacted economically, politically, socially, and technologically. Culture would in turn shape these other activities - and genetic evolution itself. Humans cooperated in large part for economic reasons: They hunted and gathered and cooked and sheltered together. In doing so, they differentiated tasks by gender and perhaps in other ways, and had to develop mechanisms for decision-making and 
therefore some sort of politics. Hunter-gather groups likely tried to achieve consensus most of the time (groups might then have split if they disagreed over major decisions). As populations grew, they increasingly had to interact with other groups of humans. They migrated first within and then beyond Africa, forcing themselves to adapt to new environments. Necessarily they mated and cared for their children - which are unusually helpless for an extensive period by the general standards of animals. Culture is and likely always was important for mating and childcare. We can thus identify some scope for influence on evolutionary selection for culture of each of the main categories of human social phenomena defined in chapter 1 . These in turn shaped the selection environment for genes themselves.

We should make particular note here of "individual differences." Since early humans cooperated and specialized, there was likely some advantage to selection for differences in human abilities and personalities. Though science fiction occasionally celebrates the scope for cooperation among species of identical organisms, species characterized by internal diversity can accomplish things that draw on diverse capabilities and interests distributed across the population. Modern human societies benefit from having some people who are good at music, others who are good at maths, and still others with great social skills. We can expect, then, that evolutionary processes selected not only our average genetic propensities but the range of personality traits that were advantageous. Yet we, of course, know very little about the range of personalities that might have existed in our distant history as hunters and gatherers. Though personality diversity is observed in other animals, it would have complicated the cooperation within large groups that characterized early humans: Humans in large groups would then have to figure out how a large number of diverse personalities would interact. Personality diversity would have encouraged selection both for larger brains and for cultural values that urged different individuals toward common goals.

\section{Language}

Perhaps 150,000 to 200,000 years ago, early humans in Africa began to develop language. This required a physical mutation: the lowering of the larynx in the throat so that a variety of sounds could be produced. The parts of the brain associated with both making and hearing sounds likely developed as well, as also the parts related to reasoning. Language facilitated social cooperation of various types: hunting large animals, organizing a campsite, gathering various foods, mating, and childrearing. These effects would expand in scope long after the period in which language was selected for in order to facilitate trade, extensive division of labour (that is, different occupations), complex political systems, and a host of other human practices that require detailed communication. Importantly, language allowed the communication of ideas: If one human discovered any useful piece of information (this plant reduces the experience 
of pain), they could communicate this to others. Information could be passed along from one generation to another.

Other hominids such as the neanderthal exhibit some of the physiological changes associated with language capability. Nevertheless, scholars doubt that they had much if any language capability. They would perhaps have had a small set of noises such as our contemporary "hi" and "shhh." It is only homo sapiens that had full language capability. It seems likely that the capacity for language emerged slowly: As hominid communication aided group activities there would be selection for mutations that enhanced this. Linguists speculate that the evolutionary process may have been somewhat similar to the process by which young children acquire language. As the range of possible sounds expanded, a next step might have involved attaching words to objects or motions; this step could have occurred when humans could make far fewer distinct sounds than today. These words would later be combined in phrases; this would require not only linguistic ability but the cognitive capacity to understand causal relationships. Over time, the various types of words that we struggle to learn about in school but use without much reflection in daily life - nouns, verbs, adverbs, adjectives, conjunctions, prepositions - came into being among humans. All human languages use all of these and have broadly similar sentence structures (though there are differences regarding, say, whether nouns or verbs come first).

Why did language capability emerge (only) in homo sapiens? As with increased brain size in general, language capability is costly for it requires considerable mental energy. Nor is language capability just a side effect of greater cranial capacity, for it depended on the selection of a set of complementary cognitive and physical attributes. It is worth noting that changes in the human throat that facilitated speech also increased the likelihood of choking; the evolutionary advantage of the former must have exceeded the disadvantage of the latter. Among the many effects that language capability had on humans, scholars usually advocate two as critical sources of selection for language capability: increased social capability and increased tool-making. Both arguments were raised with respect to brain size in general above and are again plausible concerning language capability in particular. As human groups expanded in size, the grooming acts performed by other primates to maintain social cohesion became infeasible, and some other method of coordination was necessary. Since homo sapiens operated in larger groups than previous hominids, we can then understand why language emerged with that species. Large groups of humans require a set of cultural understandings and these need to be communicated with language. Yet humans were also developing tools that were more sophisticated at the time, and this suggests a communicative enterprise in which techniques were improved upon and passed along from generation to generation. So as tools became more important for human existence, there would have been selection for enhanced language capability. Simple tools might be explained with grunts and physical demonstrations, but tools that were more complex would be hard to explain without words. Again, the timing fits and the argument is plausible. We could add still other 
explanations: Enhanced language capability may have facilitated the hunting of bigger game. In addition, complex interactions within large groups require "politics": Decisions have to be made somehow about which group members perform which tasks. It could then be that language served more to establish a status hierarchy than to maintain social bonds. Notably, though each of these arguments differs in the relative importance ascribed to different phenomena, they are not really in conflict: It could be that there were a variety of selection pressures toward increased language capability. That is, we could pursue an interdisciplinary approach, as advocated in chapter 1, and recognize that (at least with our existing knowledge base) it is entirely possible that there were complementary selection pressures for language capability.

Scholars of language identify about a dozen major language groups in the world. These mostly emerged between 10,000 and 20,000 years ago. A couple, the Eurasiatic (extending from Europe to north India) and the Amerindian (extending through most of the Americas) were particularly extensive. But it should be stressed that all languages exhibit some similarities in grammatical structure. There is debate among linguists as to the degree to which these reflect our common genes rather than a common ancestral language.

The effects of language are immense in human history. Complex culture is impossible without language. Religion, in particular, would be impossible

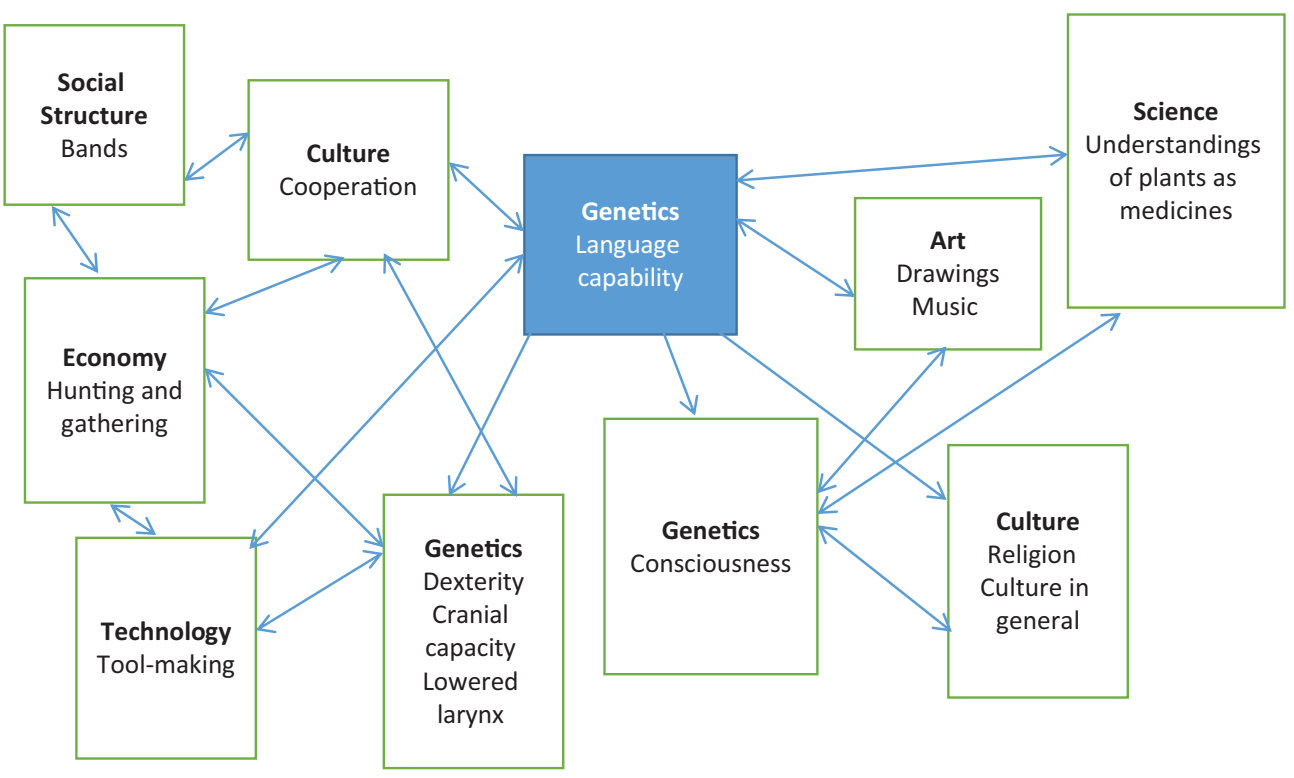

Figure 3.1

Influences on and effects of language

Note: The many two-way arrows reflect a co-evolutionary process. We have detailed here only the more immediate effects of language: Most of what happens in this book would have been impossible without language. It could be argued that tool-making and cultural cooperation (and other phenomena) only affect language capability indirectly through cranial capacity. 
without language (see below). Communication of technological innovations is impossible. Formal institutions are impossible. Trade would be immensely difficult. At the individual level, language allows us to reflect on who we are, what we want to be, and how to live our lives. It also allows us to theorize about the nature of our world, and evaluate our theories. Indeed human consciousness itself is impossible without language. This is indeed how we distinguish conscious from subconscious (below). Finally yet importantly, without language we could not study world history.

\section{Culture}

We argued above that culture emerged early enough to shape human evolution. What was that culture like, and why did it emerge? Chimpanzees lack both the communication skills and memory for culture. They manage interpersonal relationships primarily through mutual grooming. But this is timeconsuming and becomes infeasible as group size increases. At some point, then, hominids could only achieve larger group size through the development of culture (including language; see above). Cultural values allow group members to form reasonably stable expectations regarding how other group members will behave in a variety of situations. This, in turn, requires increased cognitive capability. There is speculation that culture emerged at about the same time as tools and thus with homo habilis. Hominid capacity for culture then expanded as species appeared with expanded cognitive ability.

We can only guess indirectly at the character of early human cultures from our understanding of other phenomena. We do not know their values but know that they cooperated and shared technologies both within and across groups. We do not know their languages because they did not write them down, but we know that they had these - though as noted above, hominid capability to make different noises evolved through time.

With respect to cultural expressions, we gain more insights once humans start living in structures, more carefully carving tools, and using these to fashion jewellery, ornamental clothing, and musical instruments. We will see that art emerged as a human activity at least 70,000 years ago.

Archaeologists have found in Iraq the buried remains of an older adult partblind, one-armed, and with impaired mobility, from at least 40,000 years ago. This is far from the only prehistoric burial of someone who must have relied on others for years before death. This indicates that at least some groups or families were able and willing to care for elderly that could not care for themselves.

Religion is a crucial aspect of culture that will receive much attention in later chapters. It requires a capacity for abstract thought: We are today alone among species in our capacity for both abstract thought and religion. Examination of possible brain configurations in skulls suggests that this capacity may have emerged with homo erectus. The emergence of works of art that seem to have no practical purpose, especially from about 70,000 years ago, is often ascribed 
a religious motive, but this may reflect a limited understanding of the role that art plays in human lives. Cave drawings of animals are assumed to reflect animist beliefs that plants and animals are imbued with spirits (a common belief system among hunters and gatherers to this day), and female figurines some sort of fertility cult (but some scholars suspect that these were more like dolls than religious figures). Cave drawings also seem to show people - perhaps shamans - in trance-like states. Evidence of human burial - which occurred among neanderthals from about 100,000 years ago - is also commonly taken as evidence of religious belief, but other explanations are possible: It may just have been the easiest way of removing decaying bodies from the community. Yet both neanderthals and homo sapiens often painted red ochre on dead bodies; there is no apparent non-religious justification of this practice.

What types of religious beliefs were pursued? Of course, we cannot know the exact thought processes of early humans. It is likely, though, that they grappled not only with their mortality, but with a sense of right and wrong and their ability to influence the world around them. Being dependent for sustenance on rain and the well-being of wild plants and animals, they must naturally have wondered about the forces at work behind such natural phenomena and whether they might influence these. It would be in the inherent nature of conscious human minds to question such things. How well could they have done these things at different stages of linguistic development? The reader can reflect on how they would likely think if they did not know modern science and had limited linguistic capabilities. The hunter-gatherer tribes that survived into recent times - albeit with sophisticated language capabilities tended to view the universe in terms of an extension of the tight kinship bonds that characterized their daily lives. We can speculate that early humans also drew analogies between their daily lives and the mysteries of the universe. Their ability to do so depended on the sophistication of their languages, and in particular their ability to speak symbolically about objects and actions that they could not directly observe. Cave drawings from 60,000 years ago suggest to some an animistic approach to understanding the universe: the sun may have been, for example, viewed as an animal spirit, reflecting the way that hunters knew how to interact with the world. (We might also wonder what influence gathering had on religion.) But the evidence is limited, and we should be careful of assuming that we understand these distant peoples.

\section{Emotions}

Culture exists because humans cannot adapt quickly enough genetically to changing circumstances; otherwise, cultural beliefs might be hardwired into our brains (chapter 1). Culture will only work if individual humans will be guided to follow cultural norms. Genes could then evolve in ways that encouraged individual adherence to cultural values. At some point, humans developed an expanded set of emotions: Social emotions such as shame 
and guilt joined individual emotions such as pain and fear and happiness. Moreover, humans came to display these emotions, primarily through facial expression. (See our discussion of the subconscious below.) Humans today exhibit considerable cross-cultural understanding of the meaning of a variety of facial expressions: These displays and perceptions are part of our shared genetic inheritance, and were presumably selected to facilitate human understanding of others and our capacity for cooperation. Emotions such as shame and guilt guide us to follow cultural norms; facial expression of such feelings exposes us to others when we fail to do so. In the latter case, anger can serve to discipline norm violators.

\section{Altruism}

Humans today almost all exhibit some degree of altruism: a willingness to help others even at personal cost. Indeed, we view an individual who displays no concern for the well-being of others with suspicion. We cannot know when altruism emerged as a human characteristic, but its universality across humanity suggests emergence at some point in our shared past as hunter-gatherers. We can, then, through all later chapters expect that humans will experience some (often limited to be sure) altruistic impulses. The process by which altruism emerged is a puzzle: Genetic selection operates at the level of individuals, and we might imagine that the first individual with altruistic impulses might suffer at the hands of selfish individuals. We can readily appreciate that a group characterized by altruism would be better able to cooperate - they would not have to evaluate suggestions for cooperation selfishly - and would then outcompete other groups both economically and militarily. However, such "group selection" arguments cannot explain the mystery of how a genetic disposition toward altruism might first become established within any group. Some have suggested that it may have emerged gradually via some sort of kin-based altruism: A tendency to help close relatives could be selected for if the benefit we gain from our cousins exceeds the costs we suffer in aiding them. But the first family to wish to help non-family members would still suffer.

Recognition that genes and culture co-evolved provides perhaps the most powerful possible explanation of altruism. Groups of selfish humans would develop cultural rules that encouraged and rewarded cooperation. Even selfish individuals would then find it useful to cooperate in many ways. But they would also have an incentive to cheat whenever they could get away with it. Issues of trust might then commonly arise: Will the person I help today help me tomorrow when I need it? (Only) In a cultural environment in which cooperation was valued, a person with a genetic mutation favouring altruistic impulses might be appreciated and thus prosper. Others would come to trust that individual and then be more willing to cooperate. The widespread existence of altruism thus provides a powerful reason to believe that human culture emerged early and shaped genetic evolution. 


\section{BOX 3.1}

\section{GROUP SELECTION}

Does selection occur at individual or group levels? Theories of biological evolution have tended to focus on individual-level selection. Human traits will spread through human populations if they enhance the fitness of individuals: These will then have more descendants than others, and therefore their genes will become increasingly common. But humans have long cooperated in groups, and these groups interacted with others. This raises the possibility that selection may occur at times at the group level: A trait that allowed a group to outcompete others (economically or politically) would allow that group to grow (and perhaps subdivide into many groups, each with a genetic advantage). But theories of group selection face a challenge: How does a trait become established within a group if it does not first bestow individual-level advantages?

Kin selection provides one possible answer. Say there was a mutation that encouraged us to help our cousins in some way. This mutation might be selected for in the next generations if the benefits our grandchildren received from their cousins outweighed the costs they incurred in helping them. However, kin selection cannot explain how genetic cooperation among non-relatives that does not enhance individual-level fitness might emerge.

Group selection may be of greater importance in cases of social evolution. A group may randomly adopt a cultural mutation, but this gives them an economic or political advantage over other groups. Their group then thrives, and the cultural attitudes of that group become more common than those of less successful groups. We can make similar arguments about art, institutions, or technology.

\section{The subconscious}

We have seen above that consciousness is associated with language. We can only reflect on the meaning of life - and a variety of other things, such as establishing our hopes and dreams - in words. Having evolved the capacity to think in words, we might have expected that all human mental activity would involve language. However, humans also have subconscious thought processes that operate sub-lingually, and that affect our behaviour as much or more than our conscious thoughts. While it makes sense in later chapters to study the conscious reasons that humans may have had for an action, it will also be useful to wonder about the selfish motives, fears, and other emotions that may have influenced them subconsciously.

Humans have an incredible capacity for self-deception. Our subconscious thoughts can guide us to act in ways that are cowardly, malicious, or jealous even if we would consciously disdain cowardice, malice, and jealousy. Why did a mental capacity for self-deception evolve in humans? One theory is that self-deception aids us in other-deception. Humans have been selected for 
cooperation, and therefore selected to give and observe cues regarding dishonesty. As discussed above, we will feel guilty for lying (that is, violating a cultural value favouring honesty), and display this guilt physically. Human cooperation would be difficult if we lacked any ability to discern when others were lying. While cooperation depends on some degree of confidence in the honesty of others, individual success can nevertheless rely on some ability to cheat. In the complex evolution of human beings, then, we can expect selection pressures for both (detection of) honesty and dishonesty. Difficulty in consciously lying would encourage the cooperation on which human societies depend. Ability to lie subconsciously would be individually advantageous, and the limits it imposes on human cooperation might not prevent collaboration in hunting or gathering or agreeing on group decisions. (A more controversial theory is that self-deception evolved so humans could cope with knowledge of their own mortality.)

We might then lie to ourselves that our selfish acts are actually in the interests of others. We will then be much better able to convince others of our good intentions, for we will not doubt them ourselves. We will not then give visual hints of deception. We will have much cause to speculate on the role of both self-deception and other-deception in the chapters that follow.

Though self-deception will guide many individual acts in history, it may have a limited impact on the broad course of human history. We should not lose sight of the role of evolutionary selection in human history. The institutions, art forms, and cultural values that are encouraged out of self-deception will often be selected against in the course of history. They will not prove as useful as their creators had deceived themselves into believing. Even if subconscious motives far outweigh conscious motives at the level of the individual (and even if conscious acts themselves are often misguided), selection may act such that the minority of sensible conscious actions have the most lasting effect.

This outcome depends, though, on the selection environment. If humans came to calm their subconscious fears by believing that they could influence rainfall or other natural processes, and this belief had no or little negative impact on their survival and reproduction, they would maintain such beliefs (see chapter 8).

\section{Human activities}

\section{Tool-making}

Though a few other animals occasionally engage in primitive tool-making activities, the variety of tools that we have come to fashion is one of the primary distinguishing characteristics of (some) hominids. Anthropologists have dated the first hominid-fashioned tools to about 2.6 million years ago. It is not entirely clear which species created these. There is more evidence for a set of simple but useful flaked rock chopping and cutting tools from about 1.8 to 


\section{BOX 3.2}

\section{SELF-DECEPTION AND HISTORICAL RESEARCH}

The human capacity for self-deception is particularly visible when humans describe events in which they were involved. We tend naturally to give ourselves credit for good outcomes but blame others or circumstances for adverse outcomes. We tend to forget unfortunate details. We forget details that conflict with our life story, whether harmful acts by ourselves or helpful acts by those we have come to dislike. We justify our own actions but may disdain the actions of others. We may be prone to seeing ourselves as victims.

Whether performing interviews or reading documents, the historian must be alert to these types of self-deception. The individual need not be consciously striving to mislead in order to provide a biased account. The student should keep self-deception in mind as they encounter primary documents in later chapters.

2.2 million years ago and associated with homo habilis (though australopithecus garhi may have first fashioned them). These are usually styled "Olduwan tools" (sometimes "mode 1") after the site in East Africa where they were first discovered. They were created by slashing rocks against a hard surface. We should recognize that even such tools required a hundred or more precisely aimed blows to make - and that most of us left to our own devices in the wild might struggle to do better. We should also appreciate that there may have been many more - and perhaps earlier - tools made out of wood and other perishable materials that have simply left no trace in the archaeological record. Tool-making thus predates homo sapiens by a couple of million years. Multiple hominid species are associated with tool-making. Note again that these must have for some reason developed hands capable of fashioning tools first, though there may then have been co-evolution once humans began creating tools.

The hominids at the time appear to have lived in even more extensive groups - of seventy to eighty - than the earliest hominids. They also had larger brains: $550 \mathrm{cc}$ rather than $450 \mathrm{cc}$. This increase in brain size would have allowed humans to manage more social interactions and to fashion tools, but we cannot be sure which of these was dominant in selection.

A more sophisticated set of tools - the "Acheulian" ("mode 2") - followed between 1.5 and 1.8 million years ago, and is more closely associated with homo erectus. This set of tools includes hand axes, cleavers, and knives. These tools are notable for having been worked on both sides rather than just one, and are as a result much sharper. One peculiarity of the historical record is that while Acheulian tools were carried out of Africa by homo erectus, they have not been found in East Asia. There is, then, relatively little innovation in tools for about a million years. About 600,000 years ago, tools are observed to become much more symmetrical and thinner, and thus sharper still (which some anthropologists speculate reflects the emergence of homo heidelbergensis). 


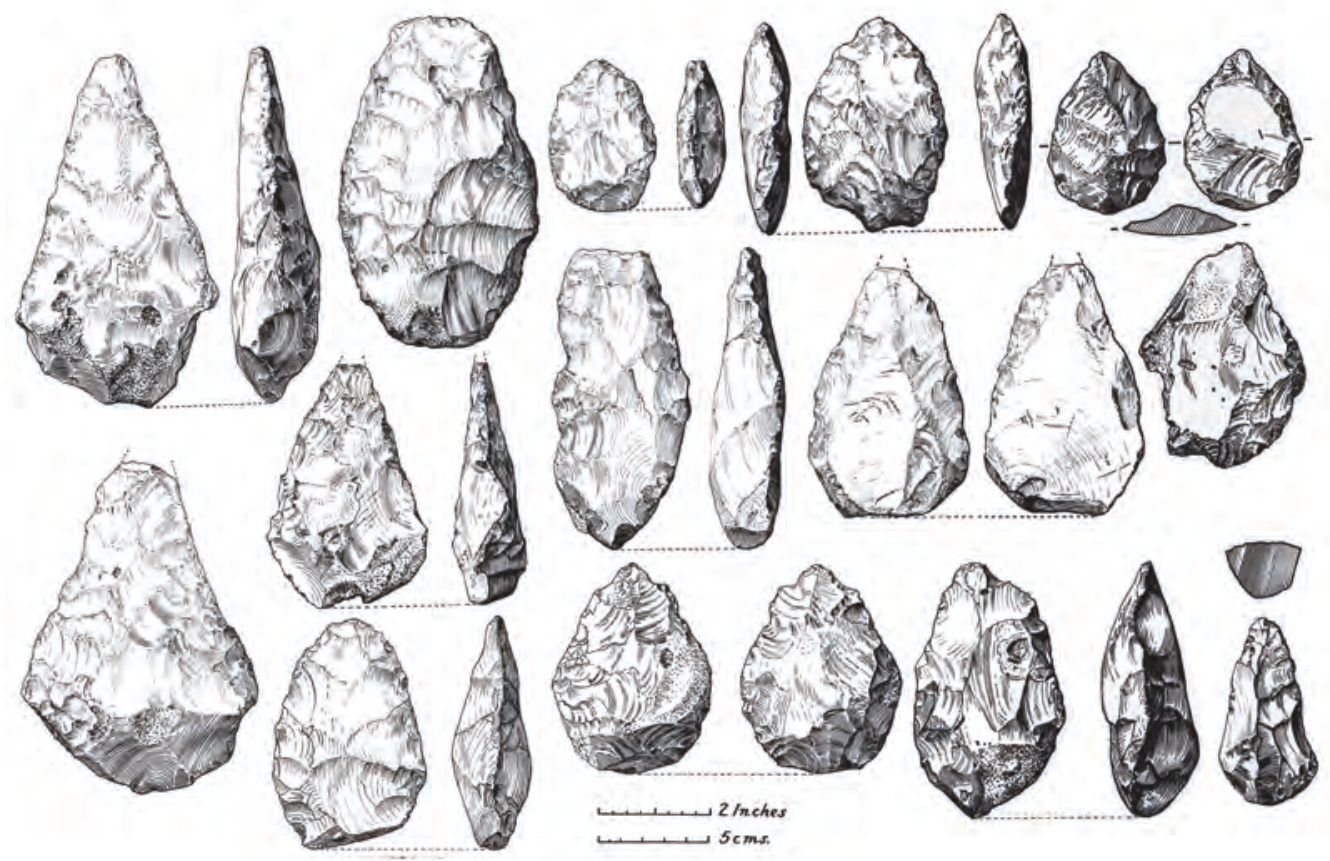

Illustration 3.4

Acheulian tools

Source: Wellcome Library, London, CC BY 4.0

With homo erectus, sexual dimorphism decreased to about the level of today. Increased female size may have been necessary for the birth of larger infants, for cranial capacity now doubled to over $1000 \mathrm{cc}$. These brains appear physically to have expanded in ways we today associate with reasoning, communication, and tool-making. It may then be that cranial capacity was selected at least in part for tool-making; it could indeed be the case that the larger brain was necessary to fashion these more complex tools. It is possible, as noted above, that developments in different parts of the brain were selected for different functions. Though hominids at this time likely still lacked language, they probably had the capacity to both communicate through gestures and mimic others. They would have been able to demonstrate simple tool-making skills to others. This would be essential to a tool-making society, for tool-making will advance as one person builds on the efforts of another. Without communication, any isolated development of a tool might be quickly forgotten.

The early part of our story above was a story of evolutionary genetic adaptation to the (changing) environment. With tool-making (and communication more generally), hominids developed a faster way of adapting both to environmental changes and to new environments they might visit. This allowed humans to be far more successful in a variety of settings than our basic physiology would facilitate. Hominid migration into different climatic zones might, 
in particular, have been impossible without tool-making capability: They needed to be able to fashion shelters and clothing suited to different climates. More generally, it is tool-making that has allowed humans over time to shape multiple environments to suit them.

The Acheulian set of tools is associated with hunting. Though hominids had been physiologically capable of consuming meat for millennia, and had indeed been meat eaters, it is likely that they had been only scavengers until the advent of Acheulian tools. We should not disdain scavenging - in general, hominids would still have had to beat other animals to dead carcasses and fend these off while eating. However, even the earliest of tools allowed hominids to get to nutritious bone marrow that was beyond the capability of other animals. Nevertheless, hunting allowed a much more regular and perhaps varied diet of meat. Moreover, the tools that facilitated hunting could also protect against other large animals. In biological terms, then, these tools moved hominids up the food chain from hunted to hunter. With the advent of hunting, hominids would have carried on an economic existence broadly similar to hunter-gatherer groups that survived into modern times.

Around 300,000 years ago species such as the neanderthal developed the first composite tools, with cutting devices of stone attached to wooden shafts or spears. This substantially increased the utility of tools. Neanderthal brain sizes were some $1400 \mathrm{cc}$. Yet again, increased brain size is associated with not only better tools but also increased group sizes. We also see semi-permanent dwellings at this time.

So far in our story, the pace of technological advance has been very slow: New tools emerge after hundreds of thousands of years and are associated with new species with bigger brains. The rate of technological progress would accelerate, and cease to depend on increases in brain size, some 100,000 years ago. Presumably, homo sapiens by that time had developed the cognitive capacity for a wide range of innovations and has continued to exercise this capability for ever-greater complexity to this day. One mystery is why this technological blossoming comes some 50,000-100,000 years after the emergence of homo sapiens, for there is no increase in brain size in the intervening years. There may have been subtle but significant changes in how different parts of the brain were connected.

From 50,000-100,000 years ago humans started to use a broader range of materials - antlers, bones - to fashion a much more comprehensive range of tools: needles (and thus ropes and new types of clothing), and spear-throwers among them. At least fifty-five distinct tools from this period have been identified. People even mined for chalk, flint, and obsidian to employ in tool construction. The bow and arrow emerged sometime between 15,000 and 30,000 years ago somewhere in Africa or Asia, and spread widely but not to Australia or the Americas; this greatly expanded human hunting possibilities. About 25,000 years ago the atlatl, a device for throwing spears or darts with much greater force, is first observed in northwestern Africa. About 20,000 years ago, at least in the Middle East, we also see devices for grinding hard grains such 
as barley; this may have been a critical advance in the slow movement toward settled agriculture (see chapter 4 ).

Humans also from at least 20,000 years ago fashioned from these materials what archaeologists refer to as "batons": "tools" not designed for work but to mark, say, the changes of seasons. Such batons represent the early human effort to understand and perhaps control the environment on which they depended. Such batons may have been associated with early religious belief; it may even be that individuals that possessed these exercised some religious authority. They may also have facilitated counting.

We have stressed through much of our story the connection between toolmaking and brain size. Tool-making is associated with other physiological changes. Most notably, human teeth and jaws contracted in size over the millennia. This change reflected perhaps many changes in selection environment, including changes in hominid diet. But it is widely speculated that our ability to cut our food reduced our need for strong molars and jaws. The control of fire (see below) may also have been important here, though it may have occurred too late in this evolutionary process. Another possible example of co-evolution is the increased hominid capability for throwing, which may have advanced as weapons for throwing were developed.

When did humans start building structures for habitation? Even hunters and gatherers might have needed periodic shelters, especially as they ranged from tropical into temperate climates. But since construction likely involved perishable materials such as wood, we may have little evidence of the first efforts. There has been speculation involving several early archaeological sites in which stones appeared to have been placed in a manner that might have provided a building foundation. But the earliest clear examples of human structures are no more than 130,000 years old, and these become common only around 50,000 years ago. They are primarily associated with homo sapiens. Portable tents may have been used beforehand, but these are difficult to document.

\section{Fire}

Some half a million years ago - we cannot be more precise - hominids in Africa learned how to make fire (though some suggest the earliest unequivocal evidence comes from Israel 780,000 years ago, and others point to Zhoukoutien in China as much as 670,000 years ago). It may be that fire was controlled earlier: There is evidence of occasional fires around human remains as much as 1.5 million

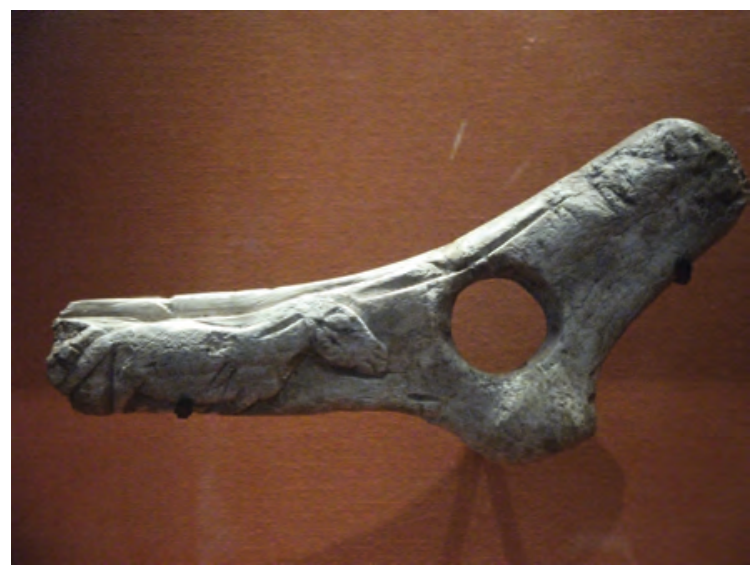

Illustration 3.5

Perforated baton with low-relief horse, about 12,500 years old, from the rockshelter of La Madeleine, Dordogne, France

Source: Photograph by Johnbod, CC BY-SA 3.0 
years ago, but it is possible that these fires were natural. There is widespread evidence of controlled fires from at least 130,000 years ago. Hominids may well have learned how to maintain naturally occurring fires before they learned how to make fire. If fires were difficult to make, then hominid communities would have struggled to maintain the fires they had. This would have required both communication and collaboration and would have made moving from place to place more challenging. Human communities would then have come to depend on personal behaviours, cultural attitudes, and social organization (someone had to watch the fire) that maintained fire.

Controlling fire is one of the great turning points in human history. It allowed food to be cooked, and so significantly expanded the human diet. It would later enable humans to burn vegetation in order to encourage the growth of edible plants and create pasture for large animals. Fires would also expose the burrows of small animals. Fires would also be used to drive animals into swamps or over cliffs. Polar ice strata provide evidence of human-based pollution from burning from at least 100,000 years ago. And fire offered protection against these same animals. It may indeed mark the historical turning point at which humans first gained superiority over large predatory animals. It may have given homo erectus an advantage over other early hominids. It also provided heat, allowing humans to expand geographically into colder climates. We can even speculate that fire served a social purpose as group members gathered around fires in the evening - and also gathered to eat cooked meals together (an important characteristic of human lives to this day). Moreover,

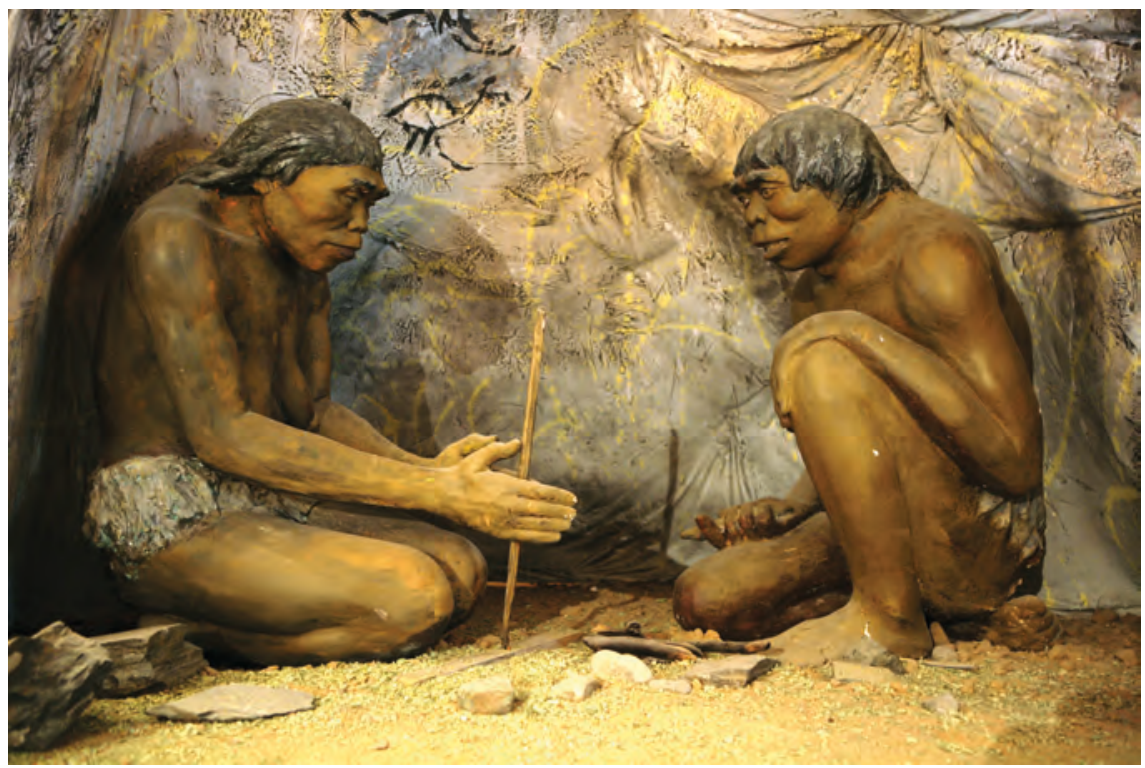

Illustration 3.6

Diorama of prehistoric fire-making, National Museum of Mongolian History 
harnessing fire can be seen as the first small step away from relying entirely on solar energy in satisfying human wants and needs.

We can further speculate that this ability to control fire reflected advances in both cognition - to understand the value of doing so - and manual dexterity. Though it is remotely possible that the first human smart enough to master fire did so, immediately ensuring selection for the necessary cognitive capability, it is far more likely that this cognitive capability was selected to serve other functions. If so, fire-making was a side effect of human evolution. It was of course conditioned by the natural environment, which made fire-making both feasible and useful. As noted above, the fact that fires occur naturally may have been critical in the development of this human capability.

\section{Economic activity}

As we have seen, the earliest hominids were foragers and (later) scavengers. Their diet consisted at first of plant materials growing wild on the savanna. Meat proteins gained from scavenging then supplemented this diet. With Acheulian tools, hominids moved toward a hunting and gathering lifestyle.

Hunters and gatherers engaged in two main economic activities. They hunted animals, and they gathered wild plants for both food and medicine. A minority engaged in fishing as well. The precise forms of organization depended on available animals and plants, and technology. Individuals or families might trap small animals. Large animals usually required cooperation by the entire band. Band members would share meat from large animals. This is the practice among modern hunter-gatherers, and the archaeological record suggests communal animal butchery among our ancestors. Plants are usually consumed within the family, though these may also be shared in times of hardship.

There is some evidence that small animals became increasingly important in human diets 50,000-70,000 years ago. This change may have preceded the development of snares with which to catch them. This diversification of the human diet may have reflected population pressure on resources. Or it may merely have been a practice that increased the stability of aggregate food intake. It may have both reflected and encouraged greater specialization within human groups (perhaps in turn reflecting and encouraging increased group size), as different skills are employed in trapping small animals versus hunting big animals.

One surprising characteristic of hunting and gathering is that hunters and gatherers (in recent history, at least) seem to have averaged about a five-hour workday. This may have increased when human populations expanded within a particular area, making it harder to achieve necessary food intake. Huntergatherers are thus affluent in terms of leisure time, especially compared to most farmers in world history and even to contemporary industrial or service workers. In terms of material goods, they were limited by a typically mobile lifestyle - though some later groups had semi-permanent dwellings. 


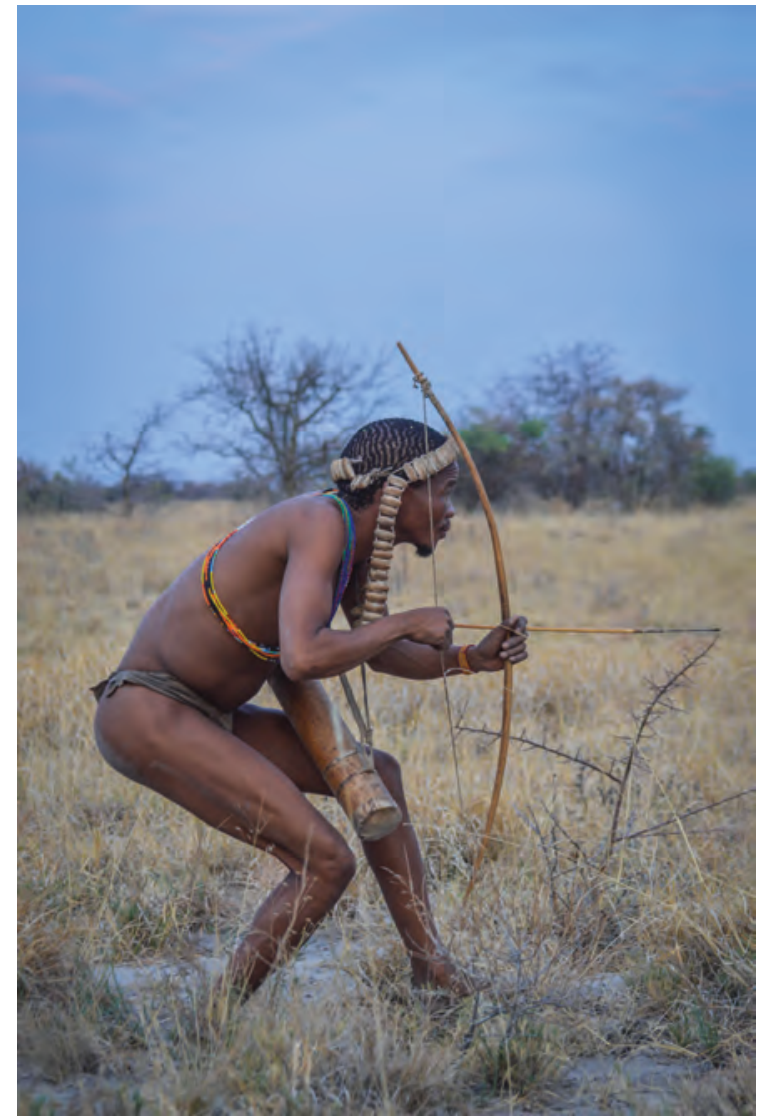

Illustration 3.7

Contemporary hunter, South African San

Source: Photograph by Andy Maano, CC BY-SA 4.0
We might think that hunter-gatherer groups were inherently self-sufficient. They consumed local food and had few possessions. However, there is evidence of short-distance trade as much as 120,000 years ago for items such as the stones used for stone tools (though it is possible that most/all bands obtained their own stone while hunting). There may have been long-distance trade for goods such as obsidian or even tin. At the close of our period, as humans moved toward the development of settled agriculture, we know that obsidian was traded from Anatolia into the Middle East; this trade may have begun much earlier. There also appears to have been a trade in human figurines - and perhaps even fragments of these - which seem to have had some religious significance. Even short-distance trade would have required communication across human groups. We do not know, of course, how they negotiated or achieved this trade.

\section{Incomes}

Hunter-gatherers are usually observed to have a better and more varied diet than pre-industrial farmers. They tend to be less subject to famine, because of the diversity of foods they consume and their mobility (unless surrounded by hostile neighbours). Their mobility tends, though, to mean that they have few other possessions and limited dwellings. Mobility may also have meant that they had to banish those too weak to survive on their own (but recall the one example above of a severely injured elderly person looked after for some time).

Hunter-gatherer groups are observed to have lower levels of income inequality than agricultural societies: They cannot carry many possessions as they travel and often (but not always) collaborate in hunting and gathering. Though there is evidence of inequality in some ancient burial sites, this is not the case most of the time. Notably, men and women appear to have been equally well fed. If ancient hunter-gatherers were in general egalitarian, this would distinguish them from most ape species in which "alpha males" are observed to have privileged access to both food and fertile females. The human capacity for cooperation (and the reduction in the difference in size between 
males and females) may have been critical here: Humans could appreciate the economic advantages of cooperating and sharing in economic activities and could collaborate against any one individual who might use physical strength to assert political power.

\section{Gender relations}

In most hunter-gatherer societies, there is a tendency for some gender specialization: Men do most of the hunting and women most of the gathering. Neanderthals appear to have been one exception to this general rule; women participated in hunting also in some historical hunter-gatherer groups. Evolutionary psychologists suspect that gender specialization may have led to differential selection pressures by gender: Men might have been selected for aggression, women for memory. Despite this economic specialization, there is considerable gender equality within hunter-gatherer groups. This may be because hunting and gathering were both essential to survival, though gathering likely contributed more to the group diet. It may also reflect low fertility: Mobile women who breastfeed for long periods tend to have few children. Arguably, women are more powerless when pregnant. There may still have been some gender inequality: Historical hunter-gatherer groups often have temporary male leaders, especially in times of conflict with other groups.

In very early hominid history, gender relations were likely far less equal. Indeed, there was a considerable disparity in the physical size of males and females among early hominids, which could well have contributed to male dominance as is observed in other ape species.

Since we lack direct information on gender relations or family structure during the period covered in this chapter, scholars have speculated based on either primate behaviour or the behaviour of contemporary hunters and gatherers. Primate groups exhibit male dominance. Primate species differ as to whether one male has many mates or there is monogamy. When monogamy is pursued, as with gibbons, it is serial in nature. Male primates rarely exhibit much interest in childrearing. Beyond the female-child bond, there is little sense of family. The earliest hominids were likely similar to other primates in this respect (some Australopithecines had one male mating with several females while others had a balance between males and females). Early homo sapiens instead likely had not just nuclear families but recognition of grandparents, aunts, uncles, and cousins, as is common in hunter-gatherer groups. Young children probably gathered plants alongside mothers, but at some point boys would have been trained to hunt. Adult initiation ceremonies are common in hunter-gatherer groups, especially for boys.

As hominid brain size has increased, the length of time it takes for humans to mature has also expanded: Most of the growth in human brains occurs after birth. This extended period of immaturity likely created selection pressure toward active paternal caring for infants. We cannot know if this selection pressure had much effect before the arrival of homo sapiens. 
One interesting question is whether our human ancestors practised outbreeding. We have no direct evidence of this. However, if small groups breed internally over several generations, genetic defects become much more likely for any child has a greater likelihood of receiving the same recessive gene from both parents. Contemporary hunter-gatherer groups do tend to practise some degree of out-breeding.

\section{Polity and social structure}

For early hominids, our best guess is that they operated like other apes. This likely would have meant dominance hierarchies based largely on physical strength, and in particular male dominance of women.

The typical homo sapiens group was a band of some dozens of people. There was no social stratification or rigid political hierarchy. Decisions were made communally (though of course some individuals would be more persuasive than others). There might be temporary leaders: say, to make decisions regarding a particular hunt or a particular move. Anthropologists use the term "band" to refer to a group with no leadership structure. However, some modern hunter-gatherer groups exhibit hereditary chiefs, and it is possible that this also occurred in the distant past. Archaeological investigations provide no clear evidence of social or economic inequalities before humans became sedentary though there is some speculation that the earliest works of art were not shared equally.

Tool-making may have been a specialized activity. If so - and this is much debated - then tool-making may mark the first real example of occupational specialization (beyond the broad and gendered division between hunting and gathering) in human history. Or at least semi-specialization: Tool-makers likely also continued to operate as hunters and gatherers. Skill at tool-making, just as talent at hunting or gathering, may well have earned some respect within the band, but again there is no evidence of a social hierarchy.

What about war and crime? There is evidence of physical trauma in a small minority of prehistoric remains that is hard to attribute to hunting, such as skulls that seem to have been broken by some weapon or bodies pierced by arrows. (But there is far more evidence of exchange of trade goods.) Still, we cannot rule out the possibility of accident or religious ritual as causes. One recent archaeological find in Sudan of many murdered victims in one place does suggest a small war 20,000 years ago. Chimpanzees will fight over territory, but other primates do not. About two-thirds of modern hunter-gatherer groups are observed to fight with other groups, but this may reflect increased competition over hunting grounds as agricultural societies have pushed huntergatherers off many lands. Wars are much more common among groups with leadership structures: tribes, chieftaincies, and states. It could be that war is unlikely when groups have consensual decision-making. If so, the hypotheses of some anthropologists that our ancestors may have warred frequently, often derived from the experience of contemporary tribes or chieftaincies, is likely 


\section{BOX 3.3 DECEPTION AND HIERARCHY?}

Intermittent exercises in leadership for purposes such as hunting may have had some limited effect on genetic selection: There is evidence that social success encourages enhanced levels of serotonin in humans, which in turn increases confidence. However, if hunter-gatherer societies were egalitarian, then humans will not have been selected for capacity to address hierarchy. The human capacity for self-deception (see above) may then encourage those who are powerless into self-deception about their situation. They may think that it is somehow justified or that it is due to some disliked external force (preferably one that it is not dangerous to oppose) or perhaps that it is only a passing inconvenience that prepares them for a better future. Any such self-deception will limit efforts at both individual betterment and societal reform. Among the powerful, it is often suggested that power corrupts. The evidence from experimental psychology suggests that powerful people do act less ethically in a range of situations (though some attribute this to ideological bias in the field), though survey data suggests otherwise (which is widely attributed to the powerful lying about their behaviour). We can imagine that it is much easier to convince oneself that one's acts are just if those around one are fawning and afraid to offend. One of the best antidotes to self-deception, after all, is to have others point out one's deceptions. Self-deceivers who lack political power may then develop mechanisms such as displays of anger that deter others from criticism. However, the powerful need make no special effort to discourage others from shattering illusions. They may indeed have to make special efforts to encourage others around them to speak their mind. Alternatively, institutions may be necessary that allow multiple points of view to be heard.

misguided. The archaeological evidence points toward infrequent wars that may only have emerged some 20,000 years ago. Wars might have been more frequent in some parts of the world than others. There may also have been violence between human groups and other early hominids (see below). One possibility is that humans migrated rather than fought until all habitable lands had been populated. Indeed one hypothesis for human migration, especially into less hospitable lands, is that some groups were forced to move.

Property crime is rare among modern hunter-gatherers, but murder and assault over sexual jealousy are common. This may have been the case with our distant ancestors as well.

\section{Art}

The earliest known cave drawings are only about 30,000-40,000 years old. They are usually attributed to homo sapiens, though it is possible that neanderthals produced cave drawings, and perhaps even the earliest ones. Cave art requires 


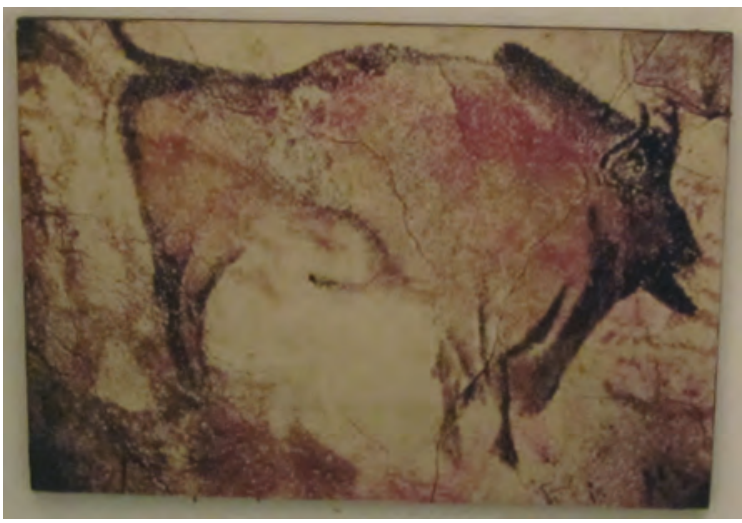

Illustration 3.8

Cave painting of bison, World Museum, Liverpool planning and the ability to recognize that certain plant substances can serve as paint. Most importantly, it requires an ability to think abstractly, to appreciate that a few spots of colour can represent some vital aspect of human life. While earlier hominids had the physical ability to paint, they likely lacked the necessary capacity for abstract thought. (The lack of burial sites for early hominids may also reflect a lack of abstract thinking.)

Particular attention might be paid to what are called "mirror neurons." Modern brain studies suggest that such neurons allow humans to replicate behaviours observed in others. They are critical to human learning processes. There is flexibility such that we can alter rather than blindly reproduce what we observe. Mirror neurons are likely essential for at least representative art. The capacity for art may thus in part reflect enhanced learning capability in general. Mirror neurons would allow representative art to serve an educational function for we could imitate the behaviours described in art. Neural networks strengthen in any human with repeated focus on particular objects: Artistic practice is therefore self-reinforcing at the individual level. It is not surprising, then, that hunter-gatherer art tended to focus on animals - though we might wonder why gathering received far less attention.

The Chauvet cave drawings in France from some 34,000 years ago - some of which are six feet or two metres long - are amazingly realistic. One gets a good sense not only of the way that animals looked but the way they moved. We can marvel at the artistic talent displayed, and reflect on how carefully artist/ hunters observed their subjects. (Arrows wound many of the animals depicted.) Later cave drawings are often more abstract. It has been hypothesized that this later art may be less realistic because artists were inspired by other art rather than directly by animals. Such a hypothesis presumes that there was a much greater body of cave art than has been discovered. (Note that it had long been hypothesized that cave art instead became more realistic through time - a hypothesis that fits well within a long tradition of European art history that stressed increased realism - but the discovery and dating of Chauvet art, and increased attention to abstract drawings that often accompany animal drawings, has dispelled that hypothesis.)

Cave drawings may have had religious significance (and their placing deep within caves reflects perhaps hidden places for worship). They may also have served an educational function, perhaps in communicating hunting strategies. But it seems clear that these drawings were not entirely practical. Early humans felt an impulse toward art. We can imagine that these people also told stories; these seem to be a universal means of transmitting culture, at least among 


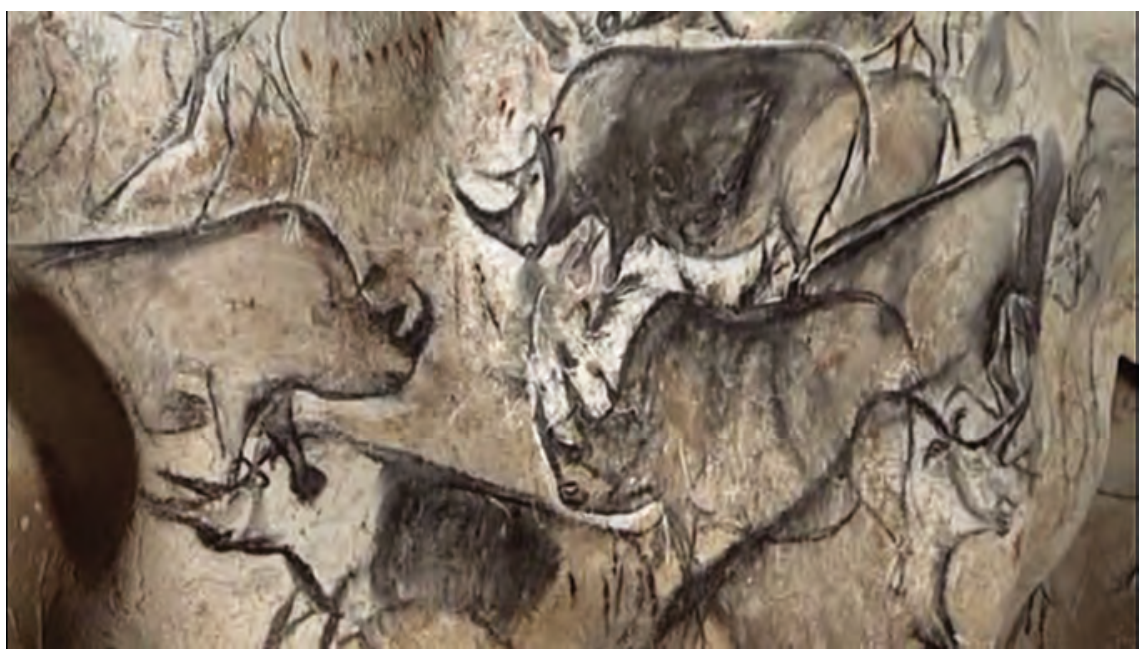

Illustration 3.9

Rhinoceros, Chauvet cave

modern humans. Contemporary hunter-gathering groups all have myths that justify their place in the universe and instil ethics; it is likely that early humans had myths also. The drawings may have reflected these myths. (Geometric shapes or other markings that presumably served some unknown symbolic purpose usually accompany cave paintings.)

After tool-making, art provides the densest and temporally most extensive record of human activity. Scholars of art hypothesize a variety of roles for art in human life: educational (perhaps of even greater importance before the development of writing), cathartic (that is, art allows emotional release), emotive, encouraging a sense of belonging (within and across generations by depicting ancestors, traditions, myths, rituals, or shared religious beliefs), and more. Art affects how we view the world, how we behave, and how we feel. Art is prevalent across historical human societies, and children everywhere appear to enjoy singing and drawing. It could then be that, as the human desire/need for art evolved, this also served to enhance the selection for cognitive and manual capabilities. One might imagine that the artist would have advantages in mating. Though we do not know when singing became common, its prevalence in modern societies suggests that it may well have emerged very early, and may even have predated language (like birdsong). Singing skills are observed to be useful in mating in modern societies. If artistic prowess enhanced mating in early human societies, then it would have been selected for genetically.

Cave drawings are the most famous but not perhaps the earliest examples of human art. Mobile human populations created small sculptures and ornaments that could be carried with them. This is thought to have occurred 50,000100,000 years ago, though some anthropologists have attributed aesthetic impulses to rare homo erectus artefacts from hundreds of thousands of years earlier. The symmetry of tools from 500,000 years ago or more may also reflect 
an aesthetic impulse; most human artefacts to this day blend economic and aesthetic functions. There have been multiple finds of shells drilled to be worn as beads from some 75,000 years ago. Art, then, was not just an activity performed in some special place but appreciated as having a place in daily life (tattoos, body piercings, and even skull deformation, also were common). Pipes for making music date from around 40,000 years ago (but musical notation is only about 2,000 years old, and thus we have a limited idea of what music sounded like through most of human history). Many figurines of animals and women (and some more substantial cave sculptures) dating from over 30,000 years ago have been found in Germany.

The fact that works of art are found in much larger numbers from about 50,000-70,000 years ago means that art blossomed at about the same time or shortly after humans were acquiring complex language capabilities (see above). This may be a coincidence, may reflect the fact that both language and art reflect abstract thinking, or may point to linguistic ability as facilitating artistic imagination.

\section{Migration}

It is thought that there were two main instances of migration of hominids out of Africa. The first homo erectus (or ergaster) left Africa sometime around 1.7 million years ago (perhaps much earlier): There is archaeological evidence from (European) Georgia about this time. Hominid remains from eastern Asia once known as "Peking man" and "Java man" - are now generally considered to be examples of homo erectus, though this is debated (there are morphological differences between finds in different areas, which may represent different species or different regional inheritances within one species). Much later, homo sapiens left Africa around 70,000 years ago. Homo sapiens would gradually displace other hominids in Asia and Europe. Homo sapiens would also spread to Australia, Oceania, and the New World, which other hominids had never reached. It is possible that in between these migrations, there was a migration of homo neanderthalis from Africa to Europe, but we do not know where neanderthals first evolved and from what other species.

How did these migrations occur? With homo erectus, it is assumed that it mostly/all happened overland, though they may have been able to craft primitive rafts as necessary to cross narrow waterways. With the much smarter homo sapiens it is far more likely that some of the migration occurred by water along the edges of the Indian Ocean and later perhaps the coast of the north Pacific. Certainly, the movement (very late) into Australia and Oceania required the use of boats.

Why did these migrations occur? In both cases, we can imagine that environmental conditions in especially the Middle East must have been propitious. This is especially important for homo erectus, which would not have been able to fashion tight clothing to protect themselves from extreme cold. There are some estimates - based on the genetic diversity of lice - that hominids may have first 


\section{BOX 3.4 SCULPTING THE HUMAN BODY}

One type of sculpture widespread both during this and later times is that of the naked human body. There is a crude female figurine found in Syria that has been dated to 300,000 years ago. Both male and female genitalia were often taken as symbols of fertility or of life more generally. Both were often portrayed out of proportion to the rest of the body. The prevalence of such sculptures throughout human history is notable (their absence in some societies can be attributed either to modesty or a distaste for capturing the human form). Female figurines are often particularly common, especially after about 25,000 years ago, perhaps indicating a tendency to associate females with fertility. Of course, particular cultures may have attached other meanings to the naked human form: sex itself, fear, temptation, or even the temporary nature of fertility and even death.
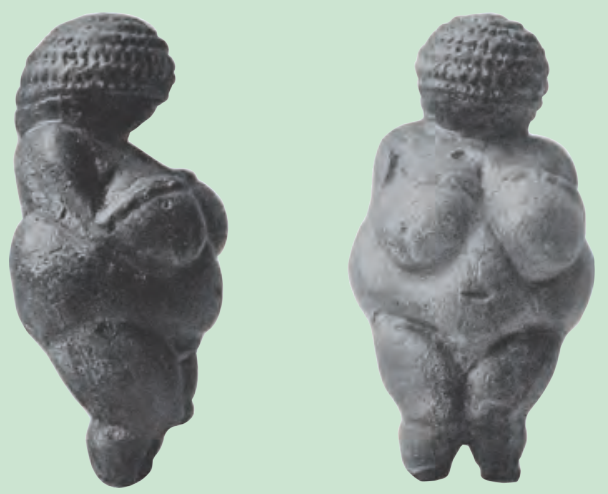

\section{Illustration 3.10}

Venus of Willendorf, 11.1-centimetre-high (4.4 in) statuette of a female figure estimated to have been made between about 28,000 and 25,000 BCE, Naturhistorisches Museum in Vienna, Austria Source: Wellcome Library, London, CC BY 4.0

developed loose clothing 1.1 million years ago; if so, this would have allowed expansion into mildly temperate zones. Homo sapiens could - and would eventually - prosper in much more severe climates than could homo erectus. It is possible in both cases that increased aridity in Africa encouraged the exploration of other lands: That is, climatic circumstances may have pushed hominids from Africa as well as pulled them into Asia.

Population pressure may have been a factor in both migrations. Hunters and gatherers need about two square kilometres per person to feed themselves, and so population growth - which itself might reflect nothing else but the human ability to breed far more than a replacement population (see chapter 4 ) - would require migration into new areas. If contiguous lands for settlement were 
available, the incentive to control population growth (which all human societies do to some extent) might have been very limited. Of course, humans may at times have migrated not because of population pressure but because of a calculation that life would be more comfortable in a new land - or because disagreements within a band induced some members to leave.

As noted above, increased brain sizes are biologically expensive. Homo erectus may have been forced to range widely in pursuit of food and especially meat. There is evidence that the proportion of meat in human diets tended to expand with brain size, though the precise causal connection (if any) between these two trends is debatable. Hominid lifespan also seems to have expanded: Grandparents could then care for the young while parents searched for food. Some have conjectured that these characteristics of homo erectus both encouraged and allowed the exploration of new lands for food. Note that migration would likely have occurred very slowly, with a particular band perhaps moving only a few kilometres during any lifetime. If there were a conflict between bands, then some may have been forced to flee longer distances to survive.

It is telling that the first migration seems to have occurred at the time of the development of Acheulian tools: This signals that homo erectus had the cognitive capacity to exercise some control of its environment at the time of migration. Nevertheless, the ability to both hunt and communicate was still limited. Some anthropologists, therefore, attribute the (likely very slow) migration of homo erectus more to conducive environmental conditions than the cognitive capabilities of homo erectus. Their ability to walk long distances efficiently may nevertheless have been of critical importance - note that apes that lived in trees did not migrate so far, and earlier hominids may have walked much more slowly.

Though one might think, looking at a map, that migration from Africa to Asia would have followed the Nile and Mediterranean coastline into what is now Israel, there is a consensus that 70,000 years ago homo sapiens instead boated across the southern Red Sea. Hominids could then migrate eastward along the coastline of Asia, occasionally using boats, and remain within a tropical climatic zone that did not require significant adaptations in clothing and dwellings. Spreading into temperate climates was more challenging due to both the climate and the different plants and animals to be gathered and hunted, and so was likely a much slower process. The fact that homo erectus never migrated very far into the temperate zone probably indicates that it lacked the mental capacity to develop either the clothing or the complex strategies required to eke a living from more demanding environments.

With respect to homo sapiens, one big question is why there seems to have been a gap of over 50,000 years between the emergence of the new species and its migration out of Africa. Though there is some evidence that some homo sapiens may have reached the eastern Mediterranean about 100,000 years ago (likely along the Nile route mentioned above), it is usually accepted that the primary out-migration occurred only 60,000-70,000 years ago. (Note: An article 
in Nature in 2015 reported a finding of homo sapiens teeth in Hunan province in China that were tentatively dated to 80,000 years ago. If this finding is verified, we would have to readjust our understanding of the timing of migration into Asia. The teeth themselves could not be dated, and thus the timing was attributed from nearby minerals and animal fossils.) Perhaps it just took time for the local population to grow and spread across Africa. Or perhaps there were climatic changes that facilitated movement into the Middle East or encouraged movement out of Africa. Maybe also the new hunting tools developed by the new species facilitated migration into new lands. Along with the development of new tools, homo sapiens are observed to develop covered camping sites (which would enhance the ability to migrate into colder regions) and personal ornamentation. It is possible that there was some critical genetic change during the period. Or it may just be that the same species developed advanced cultural practices and technologies over time. The more complex cultural practices may, in turn, reflect advances in human language. All of these advances may have facilitated the movement of human groups into new areas.

There does indeed appear to have been climatic warming about the time of the main out-migration of homo sapiens. However, the Middle East climate was still cold enough to have required tailored fur clothing, and heating of temporary homes with fire. The first example of tight clothing suitable to cold climates appears about 70,000 years ago. The technological capabilities of homo sapiens therefore appear to have facilitated migration.

Southeast Asia likely had a much more extensive coastline at the point in time when humans reached Australia about 50,000 years ago. Though Australia was at times linked by land to New Guinea, these were never connected by land to the Malaysian peninsula. Boats had to be used to reach Australia. It seems that the first humans reached Australia about 50,000 years ago, though some argue for an earlier date. The tools needed to build a dugout canoe were not available until tens of thousands of years later, and so these voyages likely took place on rafts. Sails were unknown until 5000 все, so the rafts were probably rowed. The trip to New Guinea would never have required being out of sight of land - though this would often be necessary during the much later settlement of Oceania. Manus, in the Admiralty Islands north of New Guinea, was reached 13,000 years ago and required sailing 50 kilometres out of sight of land. (We return to the saga of Oceanic exploration in chapters 7 and 16.)

The migration of homo sapiens into the Americas has received considerable attention. Recall that no earlier hominid had managed to do so. Homo sapiens had reached the eastern parts of what is now Russia some 30,000-35,000 years ago. Scholars had long presumed that they then migrated slowly overland into the Americas. Sea levels were lower at the time because there were still massive glaciers, making it possible to cross by foot what is now the Bering Strait. Indeed, there was likely a quite large land link containing edible vegetation between what are now two separate continents. However, the glaciers would then present a problem. There should have been a gap between two major ice sheets allowing movement into the centre of the continent about 


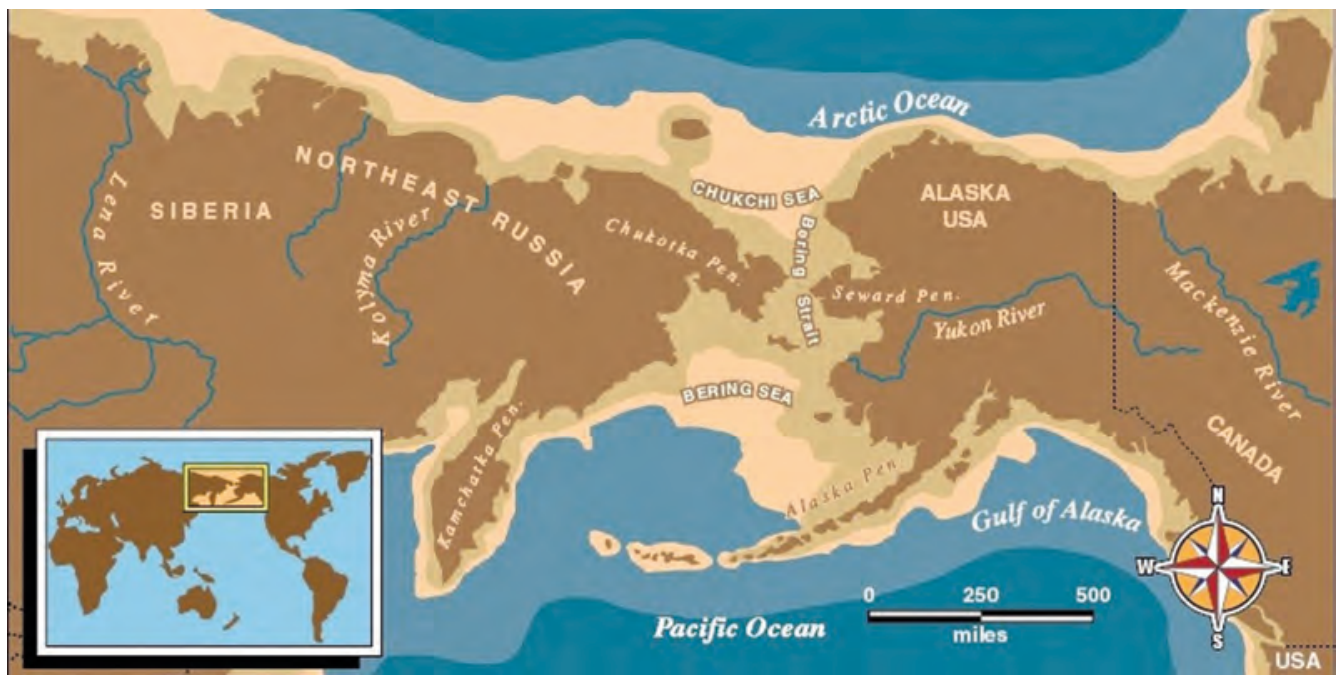

Map 3.1

Bering land bridge

12,000-14,000 years ago. Some archaeological finds along the west coast of North America suggest that humans instead (or also) migrated down the coast at least a thousand - perhaps several thousand - years earlier. While these early humans may (or not) have lacked the ability to boat across today's Bering Strait (a cold and often stormy body of water), they may well have had the ability to boat along the coastline. Even more confusingly, some recent finds in South America suggest humans may have arrived as much as 35,000 years ago, but it is unclear how. In any case, there is substantial evidence of humans throughout the Americas by about 11,000 years ago. Genetic analysis points to a common ancestor some 20,000 years ago (which may have been in Russia), but linguistic analysis suggest three waves of migration, one 35,000 years ago. There is some speculation that settlers may have arrived at some time from Europe perhaps along a northern ice sheet, or by boat from Southeast Asia.

As with the earlier migrations from Africa to Asia, or Asia to Australia, we can debate the relative importance of climatic change and human adaptability. The big question is whether humans fashioned boats and sailed past lengthy stretches of icebound coast. It is even possible that they sailed across open ocean from eastern Russia to western North America. They were certainly capable of building boats. Indeed the species had managed to reach Australia by boat across open water tens of thousands of years earlier. But sailing long distances along an inhospitable coastline would require confidence in one's ability to return if necessary.

By about 10,000 years ago, humans had inhabited virtually the entire planet though the settlement of Polynesia came only a thousand to three thousand years ago. This expansion was possible at least in part because humans had developed the ability to build tools, clothing, and shelters that allowed them 


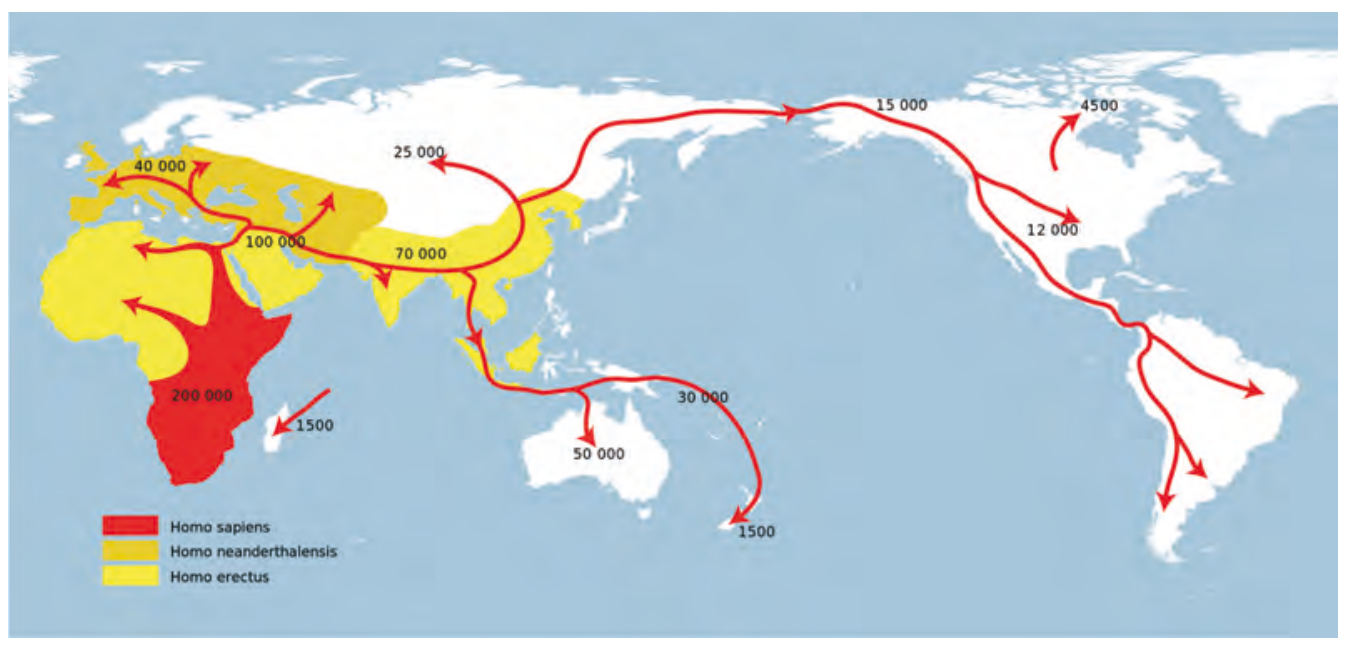

Map 3.2

Map of early human migration

to adapt to diverse physical environments. It was also possible because - given human adaptability - humans could eke out a living from local flora and fauna in much of the world. The much lower sea levels associated with the last Ice Age aided intercontinental expansion immeasurably; early humans might not have reached the Americas or Oceania under present climatic conditions.

We should not neglect the role of culture in human migration. As we have noted above, humans have culture because it allows human groups to adapt quickly to environmental changes. Hominids accustomed to life in one environment would have great difficulty moving to a different one, for this would likely require big changes in how group members functioned together. The evolved human capacity for culture facilitated migration into entirely different environments. Earlier hominids (with limited culture) had spread exceedingly slowly into adjacent areas of Asia and Europe, but homo sapiens would spread across the globe in a few tens of thousands of years.

One remaining question involves how homo sapiens came to replace homo erectus in Asia and homo neanderthalis in Europe. There appears to have been a lengthy period of cohabitation - some 10,000 years - at least in Europe. There is debate as to whether homo erectus was already extinct in Asia before the arrival of homo sapiens, though some period of cohabitation is widely assumed. Genetic analysis has established that modern humans - excepting those native to sub-Saharan Africa - have about 2 per cent of their DNA from neanderthals. This mating appears to have occurred about 45,000 years ago. This, of course, indicates that there was interaction between the two. It also shows that these two species were not so distant as to have prevented interbreeding (indeed some think that they were indeed the same species). Yet the 2 per cent figure indicates that interbreeding was uncommon and that homo sapiens were dominant. Was there military conquest (which tends, we might note, to be 
associated with involuntary sexual interaction throughout much of human history; chapter 6)? Alternatively, did homo sapiens for the most part merely outcompete these other species for resources? Both theories are plausible: Homo sapiens had better weapons that would have benefited them in both war and hunting. It is noteworthy, though, that neanderthals appear to have adopted some practices from homo sapiens, such as body ornamentation. Did they also adopt their tools? If so, they may still have been unable to throw weapons as well as homo sapiens. Homo sapiens likely also had a physical advantage in running, which would also have benefited them both in hunting and in war.

We have described migration above as if it were an impersonal experience. However, this global process was composed of thousands and thousands of group decisions. Many/most of these likely involved a conscious decision to move a few kilometres in search of more or better food. Much of the time, this would have required a conscious decision to split an existing band into two, one of which would move farther afield. Accidents may have played a role: A particular hunt took members of a band into new territory that seemed promising. Maybe hunter-gatherers had a natural curiosity about what lay beyond the next hill. Many times, there may have been less choice in the matter: Bands could be forced to move by other bands or by changes in the environment (natural disasters or more gradual changes in climate). Migrations by boat when these occurred likely required a degree of courage (or desperation). The first voyagers to a new land could not know what to expect. When one sets out across open water, one cannot be sure that winds and currents will allow a return trip. There may have been a special role in at least some episodes in migration history for individuals who were particularly curious or adventurous, or who perhaps were misfits within the society they left behind.

\section{Population growth}

Population density is severely limited in hunter-gatherer societies. Improved hunting tools and the harnessing of fire facilitated some increase in density as humans could obtain more food from a given resource base. More importantly, as the human population expanded geographically, it grew in size. Our best conjectures suggest that the homo erectus population peaked at between 500,000 and 800,000 about a million years ago. There may have only been 10,000 homo sapiens 100,000 years ago. This number is suggested by the observed genetic diversity in later populations; the population may have been as much as ten times higher, an amount consistent with archaeological understanding, if the bulk of the population left no surviving heirs. There were perhaps a few hundred thousand 30,000 years ago (a number which surely then declined with colder temperatures around 20,000 years ago), and maybe 6-10 million on the verge of the agricultural revolution (chapter 4). Human population, then, was only about a tenth of a per cent of its contemporary size before the development of agriculture. We should nevertheless appreciate the considerable population growth that accompanied the gradual spread of humans throughout the world. 
Population growth likely occurred slowly through this period. Women are thought to have breastfed for a long time after birth, in part as this would be the easiest way to feed infants in a geographically mobile population. Lengthy breastfeeding may also have reflected the lesser availability of foodstuffs appropriate for weaning small children off breastmilk. Women thus likely had only a handful of children during their lives. Many of these would have died before adulthood. It is difficult to carry small children while a band is on the move, and so there was likely cultural pressure to limit fertility. Among modern hunter-gatherers, the productivity of female gatherers is observed to fall when young children accompany them. Fertility was likely then only slightly above mortality (there is, as noted above, evidence of substantial death through violence, though we know not why).

Nevertheless, it would be a mistake to imagine that population always and everywhere slowly expanded upward. There would undoubtedly have been periods of population decline. Though human groups do indeed regulate fertility, they do so imperfectly. We have seen above that groups may have allowed population to expand more rapidly if aware of empty land. Nevertheless, migration might often have been an undesired response to population pressure on resources. Human groups may often reproduce beyond the carrying capacity (given the technology of the time) of the local environment (see Box 4.1). If migration is not an option, increased mortality will be necessary to bring the population back in line with resource availability. Moreover, there were significant climatic changes over the last hundred thousand years that would have at times decreased the resources available in environments inhabited by humans, and so increased mortality.

\section{Effects on the natural environment}

Though we have focused on human evolution in this chapter, we should confess that this emphasis reflects "history told by the victors." Even in prehistory, humans altered their environment. They used fire, as we have seen, to change landscapes. Human fires were extensive enough to show up in the atmospheric record as recorded in polar ice caps. At some point in human evolution, humans became omnivorous. The very hunting and gathering lifestyle that we have referred to above only made sense because humans could digest both plant and animal material. We are unusual among animals in being able to digest such a wide range of foods. Our omnivorous nature meant that we affected both the flora and fauna in our environment, shaping each to our purposes.

There is debate as to whether the extinction of mammoths and other large megafauna was due to hunting. Humans ate them and had developed pointed spears at the time, but it is not clear that they hunted them. Even if they did, humans hunted other animals that survived. The other likely cause is climate change. We saw in chapter 2 that climate-induced extinctions long predate humans, but also that many species can survive dramatic changes in climate. 


\section{Connections across societies}

In later chapters, we will devote much attention to connections across societies. How much did different hunter-gatherer bands interact? We have seen above that they may have traded and may have fought. We have evidence that neanderthals borrowed the practice of ornamentation from homo sapiens, and also that there was some limited interbreeding. If this occurred across distinct (sub-)species (as most think they were), then we might imagine that there was some mating and some transmission of cultural values across bands. We observe similar tools across vast distances; this may mostly reflect migration, but perhaps also sharing of information across bands. We noted above some evidence of trade in figurines with spiritual significance late in our period; this would make sense only if there were some sharing of religious beliefs.

\section{Challenges facing agents: Hunter-gatherers}

In the next chapter, we will identify key challenges faced by farmers, nomads, and fishers. These variously involve avoiding theft, control of particular land areas, and avoidance of agricultural degradation. Hunter-gatherers had little to steal. They may have battled over land, but their low population densities may have militated against such conflict most of the time. They did have an environmental impact in killing large animals, and in burning brush. All of these challenges were all likely slight compared to the challenges that faced later farmers. The challenge of hunting and gathering was then precisely hunting and gathering enough to feed one's family.

\section{Speculation on the nature of hominid life}

Imagine that neither you nor anyone you know has developed the capacity for language. Like some other animals, you may have a set of grunts that communicate certain basic ideas: run, danger, happy. You may even have a few gestures or hand signals. Think about how hard it would be to organize a hunt for a large animal. You may want one tribe-mate to chase the animal in a particular direction, and another to cut off its escape. This is easy with language but incredibly difficult without language. You may find it useful to draw a picture of the imagined hunt, but you may well lack the capacity for pictorial representation.

Imagine further how you mate. Humans, like many other animals with helpless offspring, tend to form monogamous bonds. How do you identify your life mate without language? On what basis do you decide that you are interested in another? How do you make your interest known? (Homosexuality is often observed among primates and may have been common among early hominids. This adds a further challenge.) 


\section{THE THEME OF COMMON GENETIC INHERITANCE}

The first category of individual-level phenomena involves the shared genetic inheritance of humanity (the second, treated below, addresses differences across individuals). Humans as a species have specific abilities, motivations, and emotions. History would have taken an entirely different course if we were all deaf or were incapable of fear or not terribly interested in sex. If we discover intelligent life on other planets that differs from us in important respects, such as, say, not having water-based biologies, we will likely find that their history followed a quite different course as a result. We have discussed in this chapter how humanity evolved, paying attention to psychological as well as physical characteristics. But it would be exceedingly tedious in later chapters to recount every time that, say, manual dexterity was essential to a particular historical process or event. So we will invoke our basic genetic composition only when its importance is not blindingly obvious. For example, we have discussed above how and why humans developed a capacity for self-deception and will suggest in later chapters when this capability appears to have been particularly important.

The ability to self-deceive is a characteristic of the human subconscious. We have seen above how both conscious and subconscious evolved. Among the world's species, humans (seemingly) have an exceptionally strong sense of self. We can reasonably examine our goals and our options and make choices that seem likely to achieve our goals. It is thus useful throughout history to analyse why humans made the decisions they did by exploring both their goals and their options. But we must also remember that our cognitive capabilities are both limited and slow; so humans often take cognitive shortcuts, such as following rules that have worked for them in the past (never trust strangers, say). When faced with unfortunate decisions by historical actors, we can then wonder what sort of guideline they might have been following. Then there is the subconscious: We can ask whether human actors deceived themselves into following basic emotions or motivations. Rulers may pursue war out of anger, but convince themselves that the war was justified.

Just as we need not repeatedly mention manual dexterity, we need not always remind the reader that humans have particular basic motivations: We seek food to eat, air to breathe, and shelter from wind, rain, extreme temperatures, and dangerous animals. We are (most of us at least) motivated to have sex and to care for children. However, humans also have less obvious motivations that deserve to be addressed as appropriate later. We seem to have a desire to be special, to succeed, and to have an impact on others. In situations where we have limited power or influence, we repress this motivation subconsciously, but in other situations we seek to make a difference. Since it is often easier to harm others than to help them, such a motive can lead us to have a bad influence if not mediated carefully by the conscious mind. This is especially so given a common predisposition (especially among men) toward aggression.

We also have motivations toward fairness and altruism and identification with social groups. Many important human actions in history are hard to comprehend in terms of naked self-interest or even love of family. Arguably, a critical task of world history is to give us 
a sense of how important altruism (or fairness) has been in human history. Have we often stepped back from selfish pursuits, and did we manage at such times to achieve useful ends for society? We will take special care in what follows to address this question. As for the human tendency to identify with social groups, it is useful to examine how often this led to improvements in the human condition and how often it supported cross-group strife.

\section{THE THEME OF INDIVIDUAL DIFFERENCES}

The second individual-level category captures individual differences. One set of phenomena here captures the schemas that we all develop to make sense of the world: We necessarily construct a view of our self, a view of others we deal with, and a sense of the causal relations (that is, thematic interactions) that operate in the world. We may not be entirely conscious of these schemas. But they will guide our actions in a host of situations: Do we feel confident? Do we trust the person we are dealing with? Do we think that being honest will be rewarded?

The second set of phenomena captures personality dimensions. There is now a fair bit of consensus among psychologists about at least the main dimensions such as (degrees of) emotionality and sociability and extroversion. Some psychological disorders may represent extreme positions along one or more personality dimensions; others may represent a genetic disorder. Individuals also differ in various mental and physical abilities, and in sexual orientation. They also differ in the relationships they have with friends and family.

We will often discuss particular historical actors in what follows. We can potentially apply our understanding of individual differences to individual historical actors. We will be limited here by the historical literature. Although psychological analysis of kings and generals is common, this is rarely performed with explicit reference to the personality dimensions identified by psychologists. It is as a result much harder than it need be to compare why different historical actors behaved as they did. We may nevertheless find it useful to speculate on why certain individuals acted in certain ways. Since individual differences reflect a combination of genes and upbringing, we can also sometimes speculate on why people raised in a certain way behaved as they did.

We will follow human beings through our collective history in what follows. We will see them act sagely and stupidly, kindly and meanly, selflessly and selfishly. We will see them celebrate good times and cope - or not - with war and disease and famine and natural disasters. We will see them try to understand - and laugh at, and find purpose in - the worlds in which they lived. We will see them often struggle to rise above the mundane facts of their daily existence in acts of self-expression, which we can often still admire and even emulate to this day. 


\section{BOX 3.5}

\section{INDIVIDUAL BIOGRAPHIES}

We will often refer to individuals in the chapters that follow. Care must be taken here, for every individual is more than just a creature of their time and place. Each may deviate in important ways from the cultural attitudes or social roles of their society. That is, individuals in the past exercised agency just as we (hopefully) do.

Each chapter in this book is followed by a set of questions that readers can pursue to expand their historical understanding. We can suggest here another assignment that can be applied to almost any time and place. Identify an individual that lived then and there. Strive to identify how the individual reflected or confronted the historical trends of their time and place. And also describe how they dealt with the challenges that type of human agent faced (e.g., if they were a farmer, how did they address the challenges faced by farmers? see chapter 4). Strive to describe their personality in terms of the personality dimensions identified by psychologists. If possible, provide some sense of their daily life. Discuss whether their lives seem to have elements that resonate with you.

This will prove a challenging assignment for these sorts of questions are only rarely asked.

\section{Questions}

1. Replicate Figure 3.1 for Emotions or Culture or the Subconscious. Explain each box and arrow.

2. Why do you think art is universal in human societies?

3. What do you think the religious beliefs of early hominids might have been?

4. Enlightenment philosophers (chapter 22) such as Locke and Rousseau found it useful to hypothesize that early humans were independent, but forged groups in order to achieve cooperative outcomes. Is this hypothesis reasonable?

\section{Readings}

Boone, Rebecca. 2017. Real Lives in the Sixteenth Century: A Global Perspective. London: Routledge. [This book compares biographies of pairs of people from quite different societies: Henry IV of France and Hideyoshi of Japan (both warlords that unify a state), Elizabeth I of England and Alfonso I of Kongo (who both grapple with the impact of increased global interaction), Hürrem Sultan and Lady Zheng (both powerful consorts of emperors who get blamed for court intrigue and imperial decline in the Ottoman and Ming Empires respectively), and Pope Clement VII and Moctezuma I (both of whom are blamed for losing power militarily due to weakness).] 
Dexter, Miriam Robbins, and Victor H. Mair. 2010. Sacred Display: Divine and Magical Female Figures of Eurasia. Amherst, NY: Cambria Press. [Discusses their prevalence at least in Afro-Eurasia.]

Shubin, Neil. 2008. Your Inner Fish: A Journey into the 3.5 Billion Year History of the Human Body. New York: Vintage.

Shryock, Andrew, and Daniel Lord Smail. 2011. Deep History: The Architecture of Past and Present. University of California Press.

Tavris, Carol, and Elliot Aronson. 2007. "Memory, the Self-Justifying Historian," ch. 4 in Mistakes Were Made (But Not By Me): Why We Justify Foolish Beliefs, Bad Decisions, and Hurtful Acts. New York: Houghton Mifflin Harcourt, 68-96. [This is the source for Box 3.2.]

Van der Lecq, Ria. 2012. "Why We Talk: An Interdisciplinary Approach to the Evolutionary Origin of Language," in Allen Repko, William H. Newell, and Rick Szostak, eds., Case Studies in Interdisciplinary Research. Thousand Oaks, CA: Sage, 191-224. [This chapter explicitly employs interdisciplinary techniques, and especially redefinition.]

Varki, Ajit, and Danny Brower. 2013. Denial: Self-Deception, False Beliefs, and the Origins of the Human Mind. New York: Twelve. [Posits that self-deception evolved so humans could cope with their mortality.] 


\section{A critical}

\section{transformation}

\section{The development of agriculture, nomadism, and fishing}

\section{Guiding questions}

How, when, why, and where did humans learn to farm, herd, and fish? How was each of these activities shaped by the local natural environment? In the case of farming, how did human activity affect that environment?

In what fundamental ways, and why, did early farming, nomadism, and fishing shape both individual behaviour and human society? How might attitudes and beliefs have differed from those of hunter-gatherers? (These questions inform our understanding of "Who were farmers, nomads, and fishers?")

How and why did early farming, nomadic, or fishing societies interact with each other and with hunter-gatherers?

Relationship to other chapters: These three practices farming, nomadism, and fishing - reflect interactions between humans in different regions and their local environments; we thus build on the story of human migration (Chapter 3 ) and the emergence of the natural environment (Chapter 2).We discuss why hunter-gatherers, as described in Chapter 3, came to adopt these new practices. These practices, and especially farming, 
allow and encourage more complex politics, social structures, economies, and cultures. We discuss these connections in this chapter, and will consider how polities, economies, social structures, and cultural practices (often but not in every time and place) become even more complicated in much of the rest of the book. The experience of highland New Guinea in this chapter reminds us that dramatically increased complexity was not an inevitable outcome of agriculture. Developments within nomadism - especially the domestication of the horse - also set the stage for many developments in later chapters. Nomads and farmers traded regularly and also fought against each other often over the millennia:We will frequently revisit this relationship in later chapters.

For over 100,000 years humans (homo sapiens) operated as hunters and gatherers. Only a dozen thousand years ago, humans took the first steps toward settled agriculture. Historians commonly view this as a major transformation in human history, setting the stage for urbanization, state formation, population growth, economic growth, and a host of other developments. We will explore in this chapter the causes, time path, and earliest effects of this transformation.

At a roughly similar time, other humans took steps toward a nomadic lifestyle of herding tamed animals. Since these societies remained mobile, this development has not seemed as dramatic to historians. Yet nomads were also responsible for significant developments in human history. Moreover, nomads and settled farmers would trade, fight, and learn from each other for thousands of years. Therefore, we will also treat nomads here, discussing how and why nomadism emerged, and the early effects it had. We will close with a brief discussion of a third form of food production: the fishery. Fishing also appeared thousands of years ago and remains an important form of food production to this day.

While we will treat these various forms of production separately, we should appreciate that during this lengthy period of transformation, human groups often moved between modes of production: They might farm part of the year but hunt and gather at other times. They might fish, herd, and farm at different times. They might become herders or hunters and gatherers if their crops failed.

As we shall see, these three activities - agriculture, nomadism, fishing - are both economic and technological in nature: Humans had to learn how to feed themselves in these ways. The natural environment profoundly conditions each of these activities; agriculture at least also has a significant impact on that environment. We also address effects of agriculture on politics, social structure, and population. We employ evolutionary analysis occasionally in our discussion of the human move toward agriculture. We discuss how agricultural 
practices were transmitted across societies, and how farmers, nomads, fishers, and hunter-gatherers interacted.

\begin{tabular}{|c|c|c|c|c|c|}
\hline Economy & Technology & $\begin{array}{l}\text { Non-human } \\
\text { Environment }\end{array}$ & Politics & $\begin{array}{c}\text { Social } \\
\text { Structure }\end{array}$ & Population \\
\hline
\end{tabular}

\section{The development of agriculture}

\section{Some preliminary considerations}

Agriculture involves an intense human interaction with the natural environment. We must first appreciate some key elements of this interaction before we can hope to appreciate the human adoption of agriculture. We first explore the nature and location of domesticable plants and animals, and then address the earliest human efforts to move toward farming.

Agriculture depends critically on the availability of crops that humans can take from the wild for planting. These crops must provide a yield that rewards the time and effort devoted to purposeful planting and tending of crops. It is notable that humans grew different crops in different places: wheat, barley, peas, and oats in the Middle East (olives and grapes were added about 6,000 years ago), rice in Southeast Asia, and corn, beans, and tomatoes in Central America. It has been vigorously argued that barley, wheat, and rice were simultaneously easier to plant and yielded more nutrients than corn, and much more than the plants available in sub-Saharan Africa (millet was grown in Ethiopia from early times, sorghum in the Sahel) and elsewhere in the world (taro was farmed in New Guinea but yields few nutrients). The Eurasian landmass thus provided a more auspicious natural habitat for the emergence of agricultural societies. Eurasia benefited further from a set of easily domesticable animals - cows, pigs, chickens, and more - that were absent on other continents. Of the fourteen most commonly domesticated animals in the world today, twelve originated in Asia (including sheep, goats, pigs, cattle, horses, and camels), with the only exceptions being the llama and alpaca of the Americas. The large domesticated animals are all herd animals in the wild, sociable and obedient in nature, with flexible non-carnivorous diets. The great animals of Africa have not proven easy to domesticate to this day. Not only did Eurasia benefit from a superior natural supply of both crops and animals but the geographically extensive temperate climate zone in Eurasia facilitated the transfer of agricultural advances from one region to another.

Plants seem everywhere to have been domesticated centuries or even millennia before animals. With the exceptions of Mesoamerica and subSaharan Africa, all areas that had domesticable plants also had domesticable animals. These could serve as a source of power - pulling ploughs to prepare the land - and fertilizer; in turn, they could be fed plant matter, and often plant matter such as oats that animals were more willing to eat than humans. There 


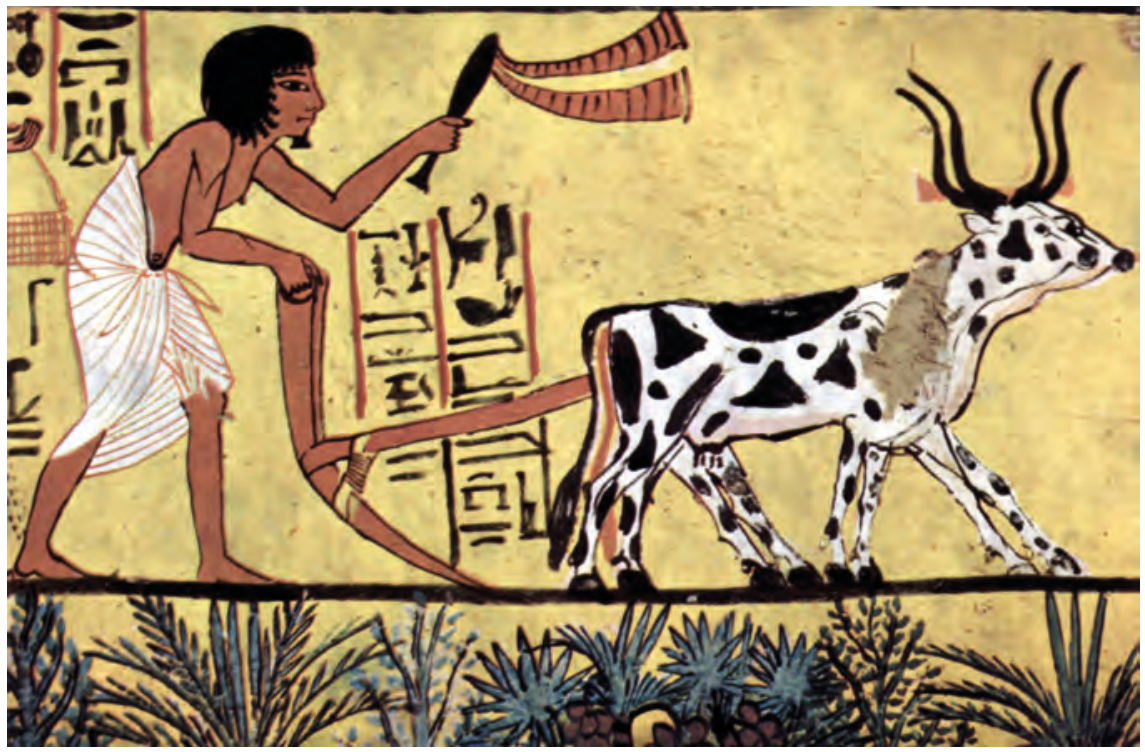

Illustration 4.1

Ploughing with horned cattle in Ancient Egypt;' painting from the burial chamber of Sennedjem, c. 1200 BCE

was, therefore, a potentially symbiotic relationship between growing plants and raising animals, and most agricultural societies would come to do both. Farmers in sub-Saharan Africa would over time import animals from Asia; in Mesoamerica, this process had to wait until the arrival of Europeans thousands of years later.

It is notable that the crops and animals we domesticate today are for the most part the ones domesticated thousands of years ago. Rare exceptions include rabbits and sugar beets, domesticated in the last centuries; fish farming is also for the most part novel. Wheat, corn, and rice comprise 80 per cent of human calories today. Add barley, millet, sorghum, oats, and rye, and that total reaches 92 per cent (some of these calories we obtain indirectly through meat). Of course, we have developed new strains of these crops. Also, we have extended their range geographically: We now grow wheat, corn, rice, and other crops worldwide. Nevertheless, humans have not developed entirely new crops for thousands of years. The most likely explanation is that these are simply the most suitable crops for human cultivation. If so, our ancestors likely experimented with many others. It is remarkable that early agriculturalists quickly - over a couple of thousand years at most - came to appreciate all the wild crops that they could usefully domesticate.

Humans must first have used wild seeds. It seems likely that they experimented with wild seeds for thousands of years before developing domesticable crops. Domestication required selective breeding - the purposeful selection of some seeds rather than others for planting - and resulted in plants (and later animals) that depend on humans for reproduction. Nature had selected 
wild cereals over time for brittle seedpods that naturally break and disperse seeds. However, these are harder to harvest. Humans over time selected seeds that were easier to harvest - but such seeds were also less suited to natural reproduction. Humans also understandably preferred larger seeds, as seeds were also food. The inevitable result was selection through time for crops that would not easily grow wild but with human intervention produced abundant food that was easier to harvest. There was a critical tradeoff involved: Greater yields depended on greater human effort.

There is a mistaken tendency to assume that the shift to agriculture must have occurred suddenly. You might think that people are either huntergatherers or farmers. Though it is convenient for us to classify humans into such groups, it is entirely possible to be a bit of both. A group that regularly perambulates through a particular area might decide to plant some seeds in a specific location, benefiting months later from a greater harvest. The group might then do some occasional weeding or divert a stream for primitive irrigation. A group might even spend a season tending crops and the rest of the year hunting and gathering (such a practice was often observed among the native peoples of North America when Europeans first arrived). The first "villages" might then have been seasonal affairs. In particularly favourable environmental and climatic conditions, hunters and gatherers might establish permanent settlements: Most gathering would occur locally, though hunters might range farther afield. Wetlands that provided a range of plants and also hosted bird and animal migrations might have been particularly conducive to permanent settlement. In such circumstances, gatherers might be especially likely to plant and weed crops near the settlement. Indeed some scholars argue that agriculture only emerged in places where permanent settlements had existed for millennia (but not all places of permanent settlement became agricultural - this was especially the case with the settlements of fishers we discuss later in the chapter). We could thus imagine two "revolutions" separated by perhaps thousands of years: toward settlements and later toward agriculture.

Note that with permanent settlements we could see some of the social characteristics of later agricultural communities: permanent structures, food storage, and even political and social stratification as some group members were able to appropriate buildings and food. We could also see developments of heavy tools for digging and grinding that would not be well suited to a mobile lifestyle. The archaeological record indicates a gradual increase of tools for grinding grains in particular locales, rather than a sudden shift. Grinding, we might note, is a crucial but time-consuming task: Women in remote Mexican villages still spend five hours a day grinding corn for their families. While we naturally associate agriculture with the key task of planting, sedentary populations would have developed skills and tools for other key agricultural tasks such as burning, weeding, pruning, watering, fertilizing, and harvesting before they started deliberately planting crops. Archaeologists have even discovered a granary in Jordan from 9300 все that might predate settled agriculture. Note that farming requires food storage, for humans do not eat the 


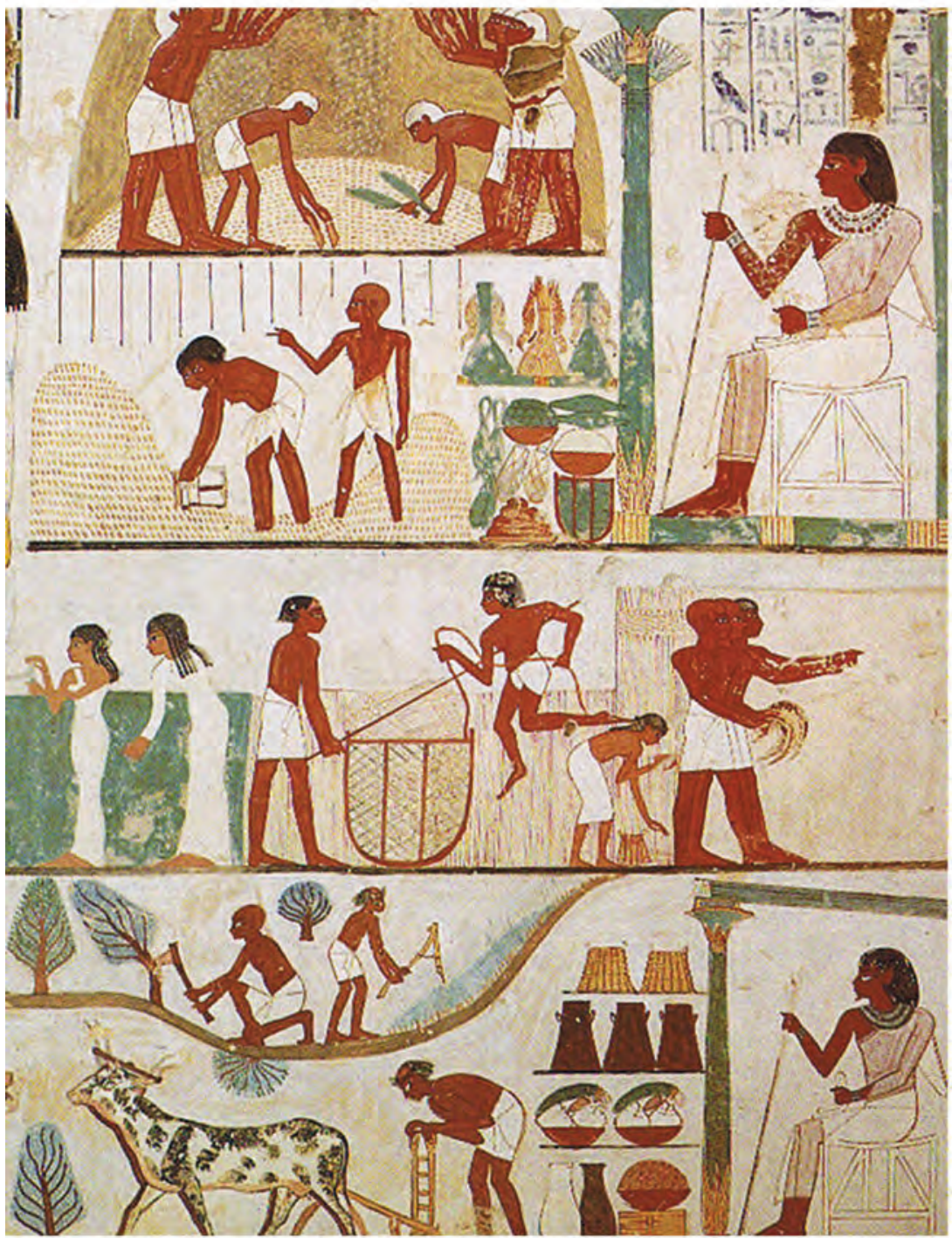

Illustration 4.2

Some of the many agricultural activities: threshing, storing, harvesting with sickles, digging, tree-cutting, and ploughing from ancient Egypt; Tomb of Nakht, fifteenth century BCE. Practices had already evolved from the early days of agriculture

entire harvest at once, but humans at first might have only stored food seasonally rather than from year to year.

In sum, humans did not just switch one day from a mobile hunter-gatherer lifestyle to a settled farmer lifestyle. It might take generations before fully settled agriculture came to dominate - and farmers might then continue to hunt for generations more. It was, then, an evolutionary process with revolutionary implications. We can tell from the bone stresses on skeletal remains that hunting continued for some time in some agricultural settlements but not 
in others. We can also say that there was intermarriage between agricultural and hunter-gatherer groups.

Particular note might be made of the general need to cook grains for human consumption. There are a dozen processes involved in cooking grains, from threshing and grinding through soaking to boiling or baking or fermenting: Humans developed these processes thousands of years before the move to settled agriculture. It would have made no sense to farm if we could not eat the grains we grew. Note that in consuming grains that were only palatable once cooked, humans gained an advantage of not having to compete with other animals for this food source - but only at the cost of cooking. As humans developed agriculture, they continued to refine cooking technology.

The gradual transition to settled agriculture depended on a range of technological innovations: in planting, tending, harvesting, storing, and cooking. These innovations in turn only made sense because of previous moves away from a mobile lifestyle. One critical reason why the transition to agriculture was so slow was precisely that humans had to develop a host of technologies slowly as they gradually became more sedentary and more dependent on farming.

The domestication of animals must also have occurred gradually. Animals with certain desired characteristics were kept, and over time these characteristics would become increasingly common. There need not have been a lot of intentionality. If humans only kept dogs that did not bite them, they would act without much thinking to select for docility. Animals could be selected in this way for fertility and for fearlessness toward humans. The dog may indeed have been the first animal to be domesticated, as long as 11,000 years ago (some think far before that, and by hunter-gatherers). Humans likely domesticated horses and camels 5,000-8,000 years ago. Donkeys were domesticated from 4000 вСе near Cairo. Domestication of animals may have been encouraged by population pressure that decreased access to wild animals. Yet domestication of animals would lead to improvements in agricultural productivity: The manure from animals could increase soil fertility, and farmers could apply animal labour to farming (at least once metal ploughs were developed; chapter 5).

It seems reasonable to speculate that women may have played a vital role in the development of agriculture. As we saw in the last chapter, women predominated in the gathering of wild plant material while men predominated in hunting. It seems likely that those who had come to recognize particularly advantageous plants and understand how these reproduced would have first engaged in the purposeful planting of seeds.

We might note before moving on that humans are arguably not the only species that farms. Some ants arguably practise agriculture: They construct nests or tunnels in which they care for fungi (mushrooms) - planting, feeding, and weeding - that they later eat. Other ants appear to have domesticated aphids. Of course, these "agricultural" practices are products of co-evolution of these species rather than learned behaviours, but it is nevertheless worth noting that humans are not unique in changing the nature of other species for their own purposes. Notably, humans were selected genetically for success as hunters and 


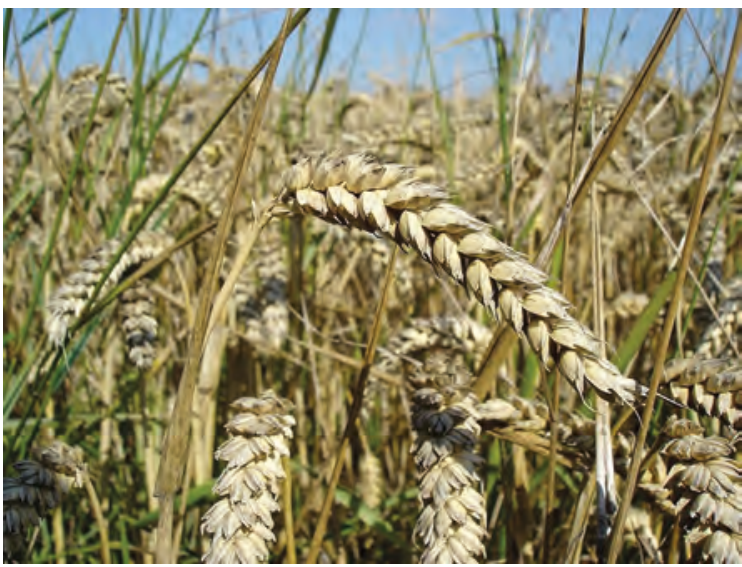

Illustration 4.3

Emmer wheat

Note:We picture modern plants in this chapter, though they will have evolved over the millennia.

Source: Bluemoose, CC BY-SA 3.0

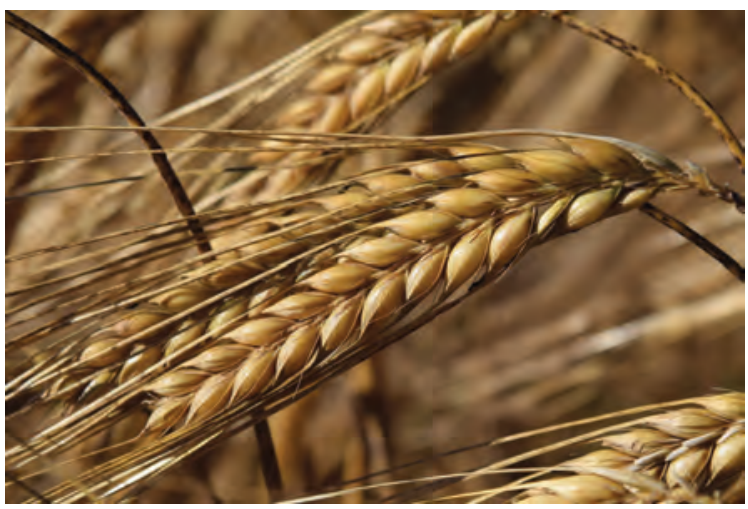

Illustration 4.4

Barley gatherers (chapter 3); our ability to farm was a side effect of the increased brain size associated with natural selection for hunting and gathering.

\section{Agriculture in the Middle East}

The first settled agricultural activity that we know of emerged perhaps 11,000 years ago - recall that we are speaking of a gradual process of change, and so precise dating is impossible - in the foothills of the Zagros and Taurus mountains in the Middle East near the Black Sea. Early crops included wheat, barley, oats, and rye; also lentils, chickpeas, broad beans, and (later) olives and figs. Sheep, goats, cattle, and pigs were domesticated between 7000 and 5000 BCE (perhaps much earlier). Agricultural practices then spread south into Mesopotamia (modernday Iraq). Barley was long preferred in Mesopotamia because it was resistant to salt. Unlike in Egypt (below), where regular Nile flooding washed salt into the sea, the slow flow of the Euphrates led to a salt buildup in the soil. The irrigation techniques practised in the area likely further encouraged salination.

Agriculture allows a much greater output from a given land area, but generally requires - at least at first - a greater expenditure of human effort. This work effort seems also more tedious than

hunting or gathering. Why then would humans ever develop agriculture? It is widely speculated that agriculture was a response to increased population density. It may also have been encouraged by extinction of large animals (due to either human hunting and/or climate change). Humans would not have taken on increased workloads unless they were facing difficulty feeding themselves from hunting and gathering. There was also it seems a period of climate cooling about 11,000 years ago which may have encouraged efforts to combat falling food intake as areas of wild grasses decreased. Humans may then have concentrated in smaller areas where wild grasses continued to grow (including the rich soils exposed as lakes decreased in size), and turned their attention to increasing output of these. We might wonder, though, how gradual climatic change would induce 
a particular group at a particular point in time to pursue agriculture, for the effect on any group of a gradual change would be slight - and humans might abandon experiments with agriculture if climate temporarily improved. It may be that the earliest agriculture was performed on floodplains in which relatively little labour was required, and the idea then spread over time to more challenging locales.

For several thousand years after about 9700 вCE, the climate was warmer both than it had been in the previous 100,000 years and than it would be until the modern era. The Sahara would be mainly grassland during this period, and there appears to have been herding of cattle there (later in this warm period). Wheat, barley, rice, and potatoes - but not corn - are plants that would have prospered from rising carbon dioxide levels associated with warming after 9700. This warming may have turned steppe into forest, reducing the animals that humans could hunt while increasing the possibilities for growing certain crops. This warmer climate allowed agriculture to spread to areas that would not have been hospitable to farming during the cooler period before 9700 BCE. (Some scholars have indeed speculated that it was warm climate rather than cold cli-

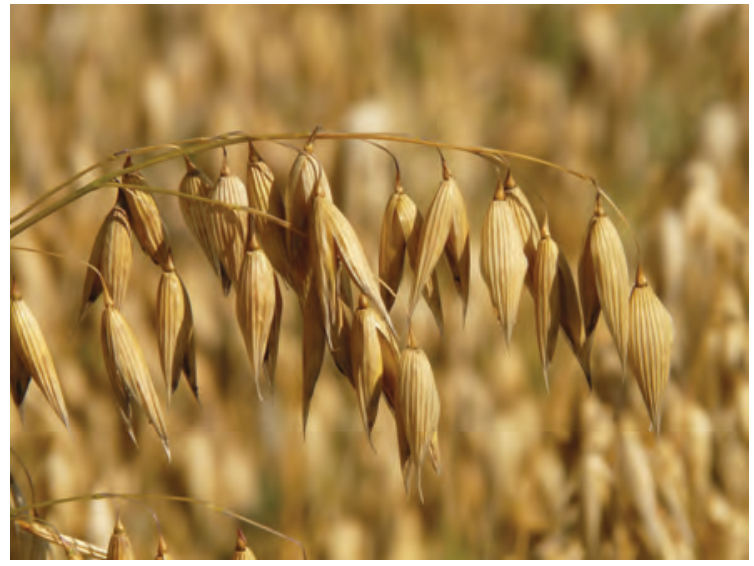

\section{Illustration 4.5}

Oats

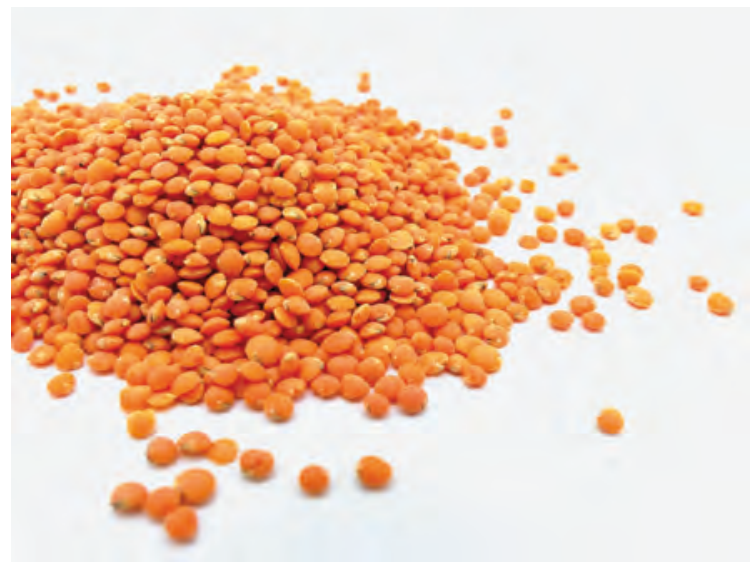

Illustration 4.6

Lentils mate that encouraged early agriculture though it seems that the earliest agriculture in the Middle East was associated with colder climate. The earliest agriculture in at least some of the other locales of independent agricultural development surveyed below may also have been associated with periods of somewhat cooler weather.)

Whatever the impact of climate, we might think of several stages in the development of agriculture in the Middle East: sedentary from the twelfth century вСЕ as groups came to live much of the year where wild grasses were still abundant; intentional planting of wild seeds from about the tenth century все; domesticated plants from about 8500; and domesticated animals from perhaps about 7500 .

We observe the earliest monuments - public structures that seem to serve a ritual purpose and were decorated with animal/human hybrids and at least 
one fertile woman - in various sites in Turkey from about the tenth century BCE. Before their discovery, it had seemed that the earliest monuments dated from only about the fifth century вСЕ and were associated with agriculture. It appears instead that these earliest signs of "civilization" (a word we will investigate further in chapter 6) predated a wholly agricultural society. Most early villages had just a couple hundred inhabitants. However, we know of at least three centres with thousands of inhabitants before 7000 вСE: Jericho and Ain Ghazal in Jordan and Catalhoyuk in Turkey. These must have served as regional centres for trade and perhaps social and political interaction. There is evidence of religious shrines in each centre. (At least one author has speculated that the earliest agriculture was designed to meet the needs of these early towns for a nearby source of food.) There then appears to be a period in which these larger centres contract. There seems to have been a roughly egalitarian society in Catalhoyuk, with homes and burial sites (inside dwellings) of similar size. There were no streets, but ladders up to walkways across roofs. Sheep, goats, grains, and legumes were grown nearby.

\section{A regional survey}

In how many different places did agriculture develop independently? We may never know the precise answer. We should be aware that those historians who like detailing the importance of cross-cultural transmission of ideas have tended to stress a small number of different sites of innovation: Most human groups then learned from others how to farm. On the other hand, those historians who like to describe human history in terms of the common progression of societies through distinct historical stages have been more open to the idea that agriculture developed independently in many different places. As noted in chapter 1, this book is open to exploring both information transmission across societies and similarities across societies. We can thus be appropriately dispassionate in assessing this question. Moreover, we can appreciate that even borrowing agricultural technology is not easy, as techniques tend to have to be adapted to local conditions. In any case, the answer to our question is undoubtedly at least two, for societies in the Americas developed agriculture without any knowledge of such developments in the eastern hemisphere. There are, as we shall see, debates as to whether India, China, Southeast Asia, and Africa developed agriculture independently from the Middle East. Agriculture may have evolved independently in as many as ten different regions of the world.

We should confess at the start of this regional survey that it is not always easy to discern what humans grew and when. Most food items leave limited archaeological remains. Archaeologists often draw inferences from remains of buildings and pots. New techniques, such as examining plant debris in bogs, examining human skeletons, and examining traces on old pots, promise to allow us better to establish what humans grew and ate where and when. Genetic analysis of modern plants can also provide information as to when these were likely first domesticated. 
There is little doubt that the idea of agriculture spread westward into Europe from the Middle East. It took four or five thousand years for this process to unfold, but by about 4000 вСE humans pursued agriculture in the western reaches of the European continent. One question that arises here and elsewhere is whether agriculture spread peacefully: Did neighbouring groups learn about farming and adapt it, or did agriculturalists conquer smaller hunter-gatherer (or later nomadic) groups and expand into their lands? We will see below that agriculture fostered dramatic population growth, and so we might well imagine the geographical expansion of groups that had mastered agriculture. Genetic analysis of European populations suggests that ethnic diversity was maintained during the transition to farming and thus points toward a mostly peaceful expansion of agriculture. Moreover, the archaeological record indicates that the spread of farming was not associated with substantial income disparities as elsewhere in the world. On the other hand, the spread of agriculture both westward and eastward was related to the spread of "Indo-European" languages: This suggests that people carried agricultural technology and language with them.

If there was a movement of agricultural populations away from the Middle East, this might have been encouraged by a more arid climate. There is also a hypothesis (supported by an analysis of sediments in the sea) that the Black Sea was flooded from the Mediterranean sometime around 5600 вCE. This flood event could have been the precursor of biblical and other mythical flood stories. However, the most recent scholarship suggests that any such flooding would have occurred very slowly, and so could not have suddenly displaced large numbers of farmers.

The gradual spread of agriculture across Europe is typical. Farming everywhere spread slowly from its areas of emergence. There are several reasons for this. Agriculture would only be adopted in areas facing population (or climatic) pressure. The idea of farming, and the technology of planting and harvesting, spread by word of mouth slowly, impeded wherever neighbouring groups were hostile. As noted above, we do not know if agriculture primarily spread between groups or by agricultural groups slowly encroaching on more and more land. Also, agricultural techniques need to be adapted for new regions with different soils and climates. But over a period of some thousands of years agriculture spread to virtually all suitable landscapes (covering about a third of the Earth's land surface), with groups of nomads and hunter-gatherers either becoming farmers or being squeezed out of existence or pushed onto lands ill-suited to agriculture.

Wheat and barley were grown in Egypt from at least 5000 BCE and perhaps much earlier. Cattle pulled ploughs there. It is not clear if Egypt borrowed the idea of agriculture from the Middle East or developed it independently: It may be that the crops grown in Egypt were not native and had to be imported from Asia. Though the use of the same crops might seem to suggest borrowing, we should recall that a limited number of crops were ever domesticated, and so separate discoveries regarding crops that grew wild throughout the region would not be surprising. It is also possible that some domesticated crops and animals 
in Egypt were borrowed from Nubia and beyond in Africa. Though Egypt is famous for its monumental remains, these were built on the banks of the Nile and out of stone; it is much harder to know what happened on the floodplain of the Nile where thousands of years of silting have destroyed or buried signs of agricultural production.

The Nile floods at the right time, just before the planting of winter crops. There is then little need for irrigation or weeding as weeds cannot survive the summer heat. The Nile brought nitrogen, potassium, and phosphorus to the Nile floodplain, and drained salt away. Note that agricultural labour had little to do during the dry season or the flood that followed: It could potentially be devoted to monument building.

We do not know if agriculture developed separately in sub-Saharan Africa. Sorghum and pearl millet were likely grown in several places in the vast strip of savanna between the Sahara and the equatorial rainforest in Africa by at least 5000 вСE and perhaps as early as 7500 вCE. Rice - an African strain different from that domesticated in Asia - was grown farther west, probably from about 3000 вCE. There may thus have been two separate independent developments of agriculture in sub-Saharan Africa. There is evidence of a different type of cattle from that common in the Middle East being domesticated by 6000 BCE - though for the most part Africans would later import cattle from Asia.

Postscript: Millet is used primarily as birdseed in North America and Europe in the early twenty-first century, but is still widely eaten by humans in parts of Africa and Asia. It is drought resistant and gluten free and may thus become more common globally in future.

The speakers of proto-Bantu on the borders of Nigeria and Cameroon were growing yams, groundnuts, beans peas, guinea fowl, and goats by 5000 вСE (and yams may have been domesticated there much earlier); the spread of Bantu languages may well have reflected the higher population densities that agriculture allowed. As in Europe, we do not know to what extent the spread of farming - and language - was the result of conquest or communication. Bantu agriculturalists traded pottery and stone axes with hunter-gatherers for meat and forest products. It seems that hunter-gatherer groups may have been absorbed into Bantu-speaking bands, which themselves subdivided as Bantu populations expanded. This might explain the diversity observed within the Bantu language families (there is a similar diversity in Indo-European languages). As agriculture spread into the tropics, it may have combined with a preceding hunter-gatherer economy to produce a mixed "horticultural" society of some cultivation and some gathering. Slash-and-burn agriculture, in which farmers burn wild foliage and move their farms every few years, was widely practised in Africa. After about 1000 BCE, Bantu-speakers became adept at iron production: This would have enhanced both agricultural productivity (iron ploughs and axes) and military 


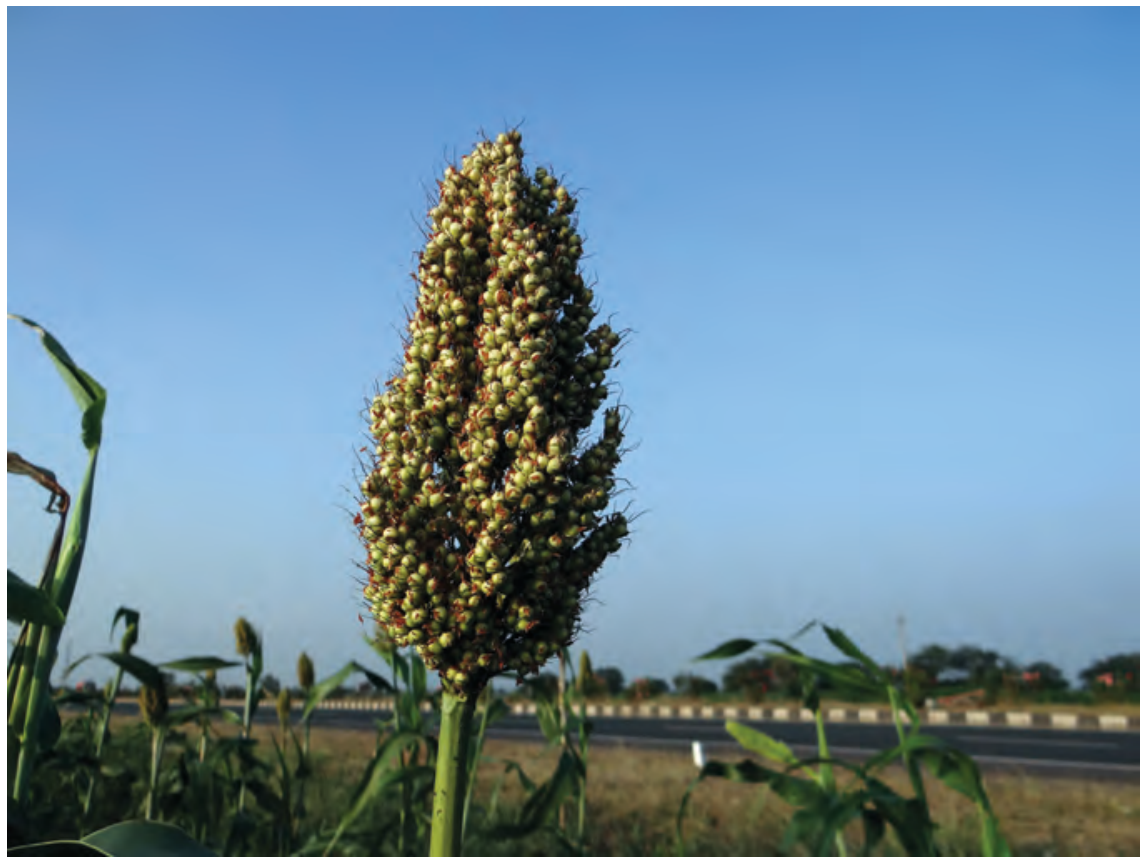

Illustration 4.7

Sorghum in Karnataka, India

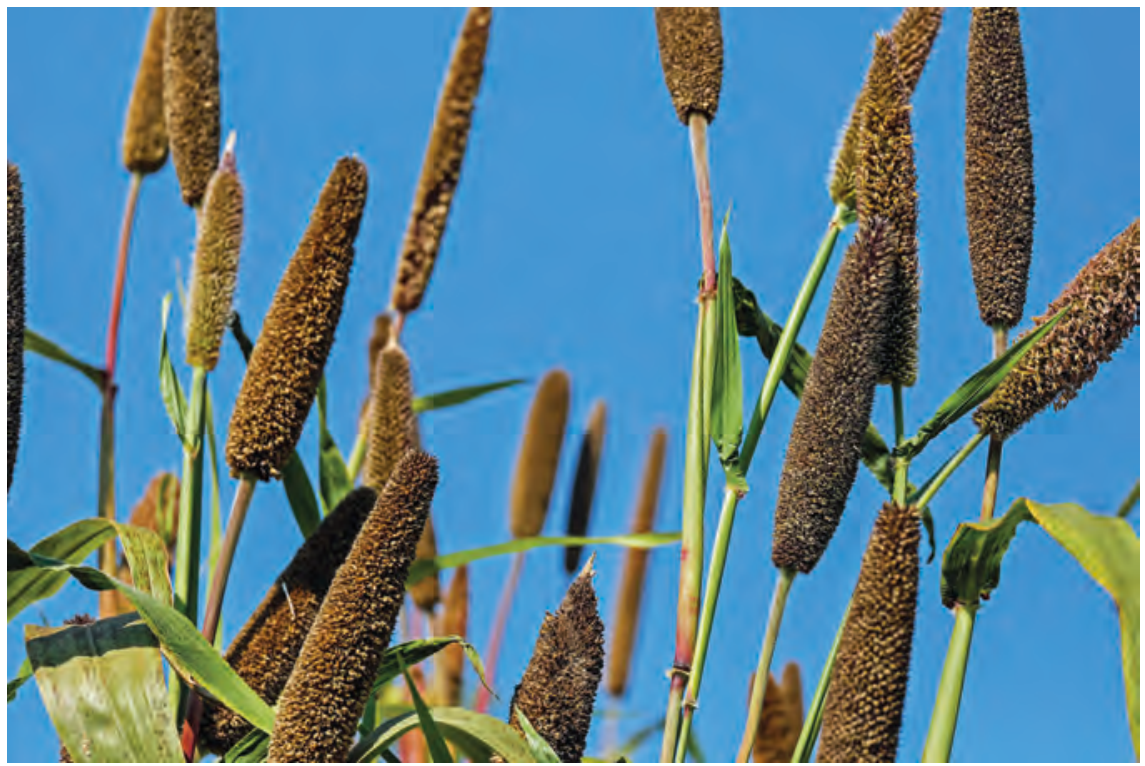

Illustration 4.8

Pearl millet 
prowess (iron weapons and shields); the speed of Bantu migration increases after that. The spread of agriculture (and language) in Africa would persist into modern times as Bantu agriculturalists expanded into South Africa.

Farther west in areas of present-day Ivory Coast and Ghana there is archaeological evidence of a sedentary society (the Kintampo Complex) from about 2250 to 750 всE; We know not where these people came from - though they were presumably hunter-gatherers - or what caused the society to decline. Sheep and goats were raised, and perhaps yams. Trade occurred over distances of hundreds of kilometres.

We do not know if agriculture emerged independently in the Indian subcontinent or was imported from the Middle East. Mehrgargh in Pakistan is the first known South Asian site with sedentary agriculture dating from at least 6500 вСE. This discovery suggests that agriculture developed independently there. Yet Mehrgargh grows the same plants and animals, and has the same female figurines, as in the Middle East.

This development long predates the Indus Valley civilization (see chapter 7). The Indus civilization grew wheat, barley, and pulses: These grew over the winter as in Mesopotamia; Rice, millet, and some vegetables that grew wild over the summer were later cultivated - by 2200 все at the latest. This involved more work for farmers but two harvests per year also meant a much more regular food supply. Pottery was produced from 5500 BCE. Cattle and chickens were domesticated: It seems that chickens were domesticated separately here and in China. By the last phases of the Indus civilization, it had imported rice and millet from the east, and sorghum and pearl millet from Africa.

Rice had been cultivated along the Ganges earlier (along with wheat and millet). This appears to have been a separate strain of rice from that grown in China; it seems that Indian and Chinese rice agriculture developed separately. However, knowledge of wheat and millet spread eastward from the Indus as rice cultivation spread westward.

There has been far less archaeological exploration and discovery in China than in the Middle East. We are as a result less sure about when agriculture began there. It is even unclear which came first: the cultivation of rice in the south or millet in the north. The idea of farming may have spread from one region to the other and then been adapted to entirely different crops. There are sites in the middle where both rice and millet were grown. Linguistic analysis suggests that languages of southern China may have spread outwards (as the Bantu language spread with African agriculture and Indo-European languages did in Europe). There is evidence for the gathering of wild rice and millet from 12,000 вС. Millet may have been domesticated by 7000 BCE; it was extensively farmed by 5000 BCE. Archaeologists have found stone spades, mortars for grinding grain, and sickles made from shell or stone from this era. Rice was domesticated in south China by 5000-6000 BCE at the latest (but maybe as early as 8000 вСЕ). Chickens were raised from 5400 вСе, but the primary farm animal was the pig (cows were likely imported later from steppe nomads). There is evidence of villages from 6500 все. These were at first egalitarian, but by 5000 вСе 


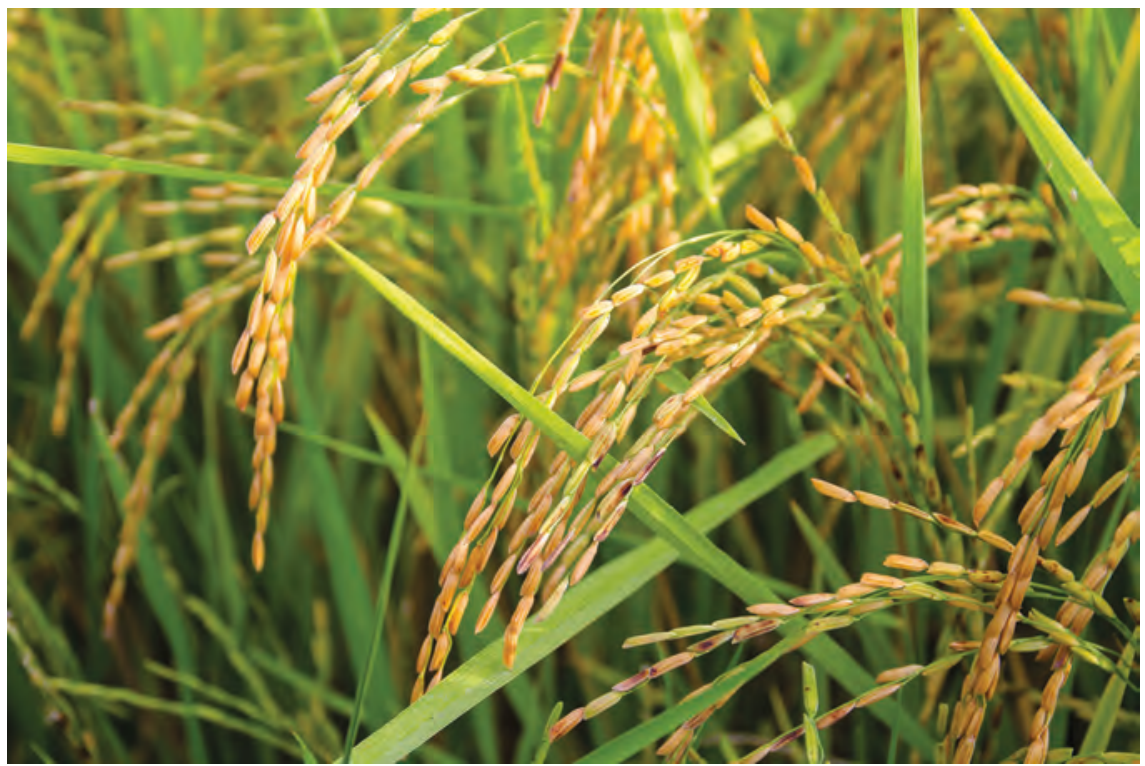

Illustration 4.9

Rice

clear evidence of social stratification (and increasing male power - male burials are more elaborate) emerges. As in Egypt, people often built homes on stilts to avoid flooding. As farther west, there is evidence of a semi-sedentary period in which groups planted rice but still hunted; it is not clear whether some people maybe children and the elderly and perhaps at least some women - stayed in villages year-round, or whether the entire group moved after the harvest. Rice was grown in the north alongside millet by 2500-3000 все at least. Ploughs were used, but there is no clear evidence of water buffalo pulling these until after 2000 все. Some scholars now think that the northern Chinese may have learned of millet-based agriculture from the Middle East through Central Asia but only some strains of Chinese millet seem to have a western origin.

Historians were long confident that rice agriculture emerged separately in Southeast Asia, but many now doubt this. The first evidence of farming in the region dates from about 2300 (maybe 3500) вCE, lending support to the idea that rice agriculture was borrowed from China. Rice agriculture was common by 1500 вСе. Surprisingly, the first agriculture in Japan from about 3500 вСЕ focused on yams and taro; it appears that rice was imported later from China.

There is evidence of cultivation of sugarcane, bananas, taro, and yams in the highlands of New Guinea and perhaps parts of Southeast Asia from about 8,000-10,000 years ago. Certainly, by 3000 BCE, agriculture, including the domestication of chickens and pigs, was widespread on the island (whereas neighbouring Australia was a land of hunting and gathering; it seems to have lacked domesticable crops). This agriculture may have been part-time as in tropical Africa. It likely involved slash-and-burn practices as in other tropical 


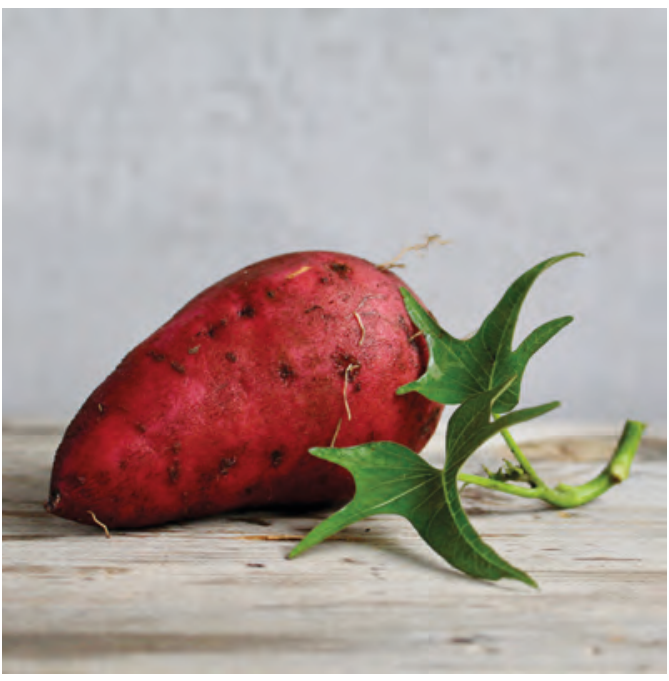

Illustration 4.10

Yam

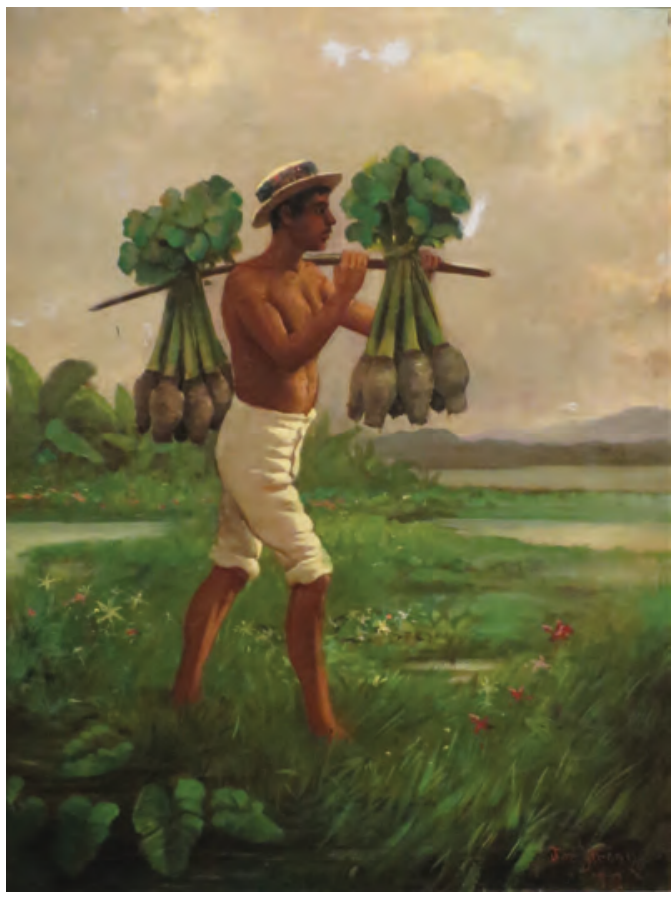

Illustration 4.11

Man with a Yoke Carrying Taro by Joseph Strong, oil on canvas board, 1880, Honolulu Museum of Art lands. These crops may (or not) have been brought to New Guinea by the Southeast Asian peoples who would later populate Polynesia (chapter 7). We might note that the highlands of New Guinea share some of the sub-tropical climatic conditions of other areas of early agriculture, but lacked access to the crops utilized in the Middle East or China. Note that we can identify the hard shells of domesticated grains, but that domesticated tubers such as yams and potatoes leave no remains that archaeologists can easily distinguish from wild cousins, so it is harder to date the first domestication of these.

It deserves emphasis that the highlands of New Guinea were likely one of the first areas to develop agriculture independently. However, as we shall see in later chapters, highland New Guinea remained isolated from the rest of the world into modern times (due to its mountains and dense forest) and did not advance beyond stone technology until interacting with outsiders in modern times. Nor do we observe large cities or states in highland New Guinea until modern times. The path from settled agriculture to modern industrial society is in no way guaranteed. Settled agriculture, in other words, may be necessary for the development of cities and states and advanced technologies, but it is hardly sufficient. Most of the regions surveyed in this section as early developers of agriculture are in the twenty-first century sites of developed economies and complex political institutions - but New Guinea remains poor and with limited state capabilities. The limited nutritional value of the plants of New Guinea, the harsh (but beautiful) topography which limited contact between groups and with the rest of the world, and limited mineral resources may all have played a role in limiting the process of innovation in New Guinea. We will see in this book many examples of regions that seemed to have more complex polities or economies than others did at one point in time, only to be surpassed by other regions in later centuries. None can match the record of 


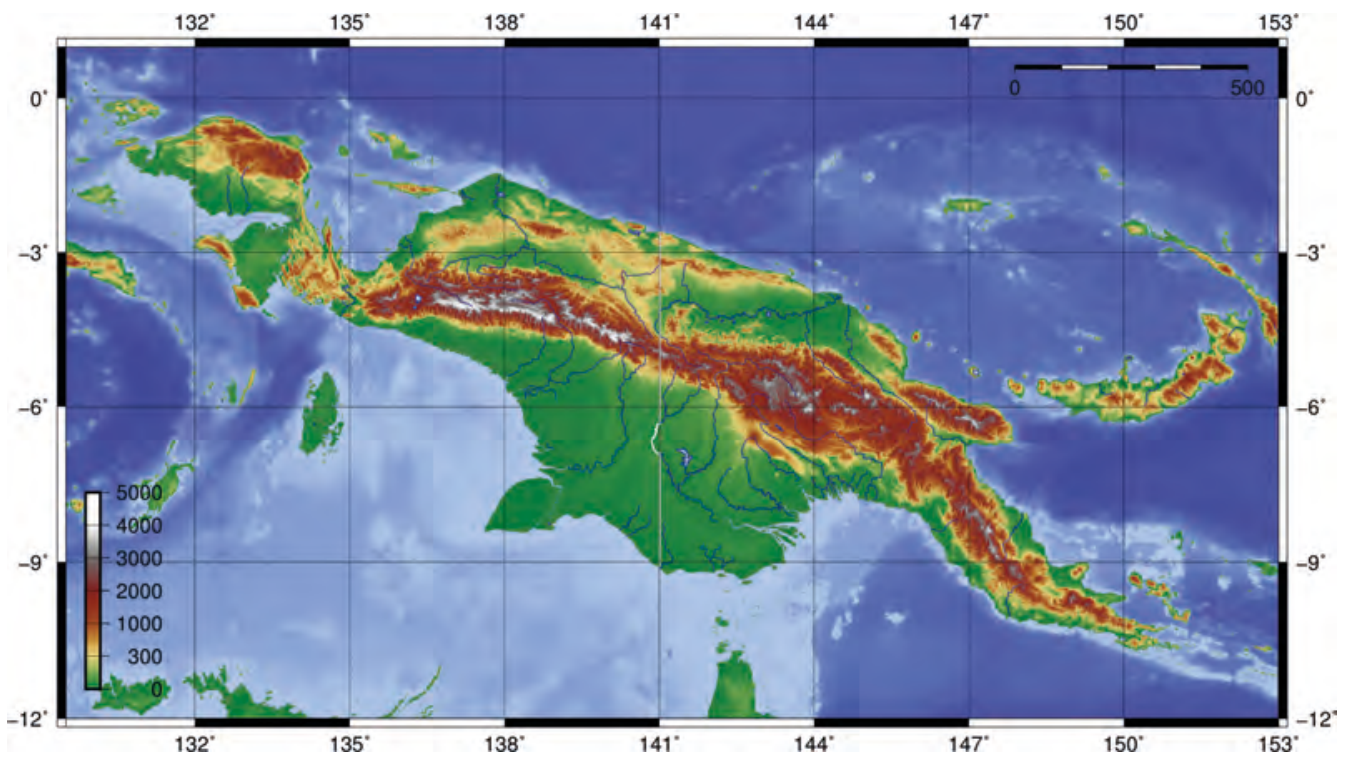

Map 4.1

New Guinea topography

Source: Map by Zamonin, CC BY-SA 4.0

highland New Guinea: one of the first human societies to develop settled agriculture, but one of the last to move past stone tools.

Corn was domesticated by about 7000 BCE in Guerrero in Mesoamerica (the region stretching from central Mexico to El Salvador). Since domesticated corn is so different from wild plants, it is not clear how it was first domesticated. Moreover, the domestication of corn may have been an unusually slow process because it has a high tendency to out-breed with wild varieties. Chilli peppers, beans, avocados, and squashes may have been cultivated even earlier. Chilli peppers do not have obvious nutritional value - we now know that they are rich in vitamins, but this would likely not have been obvious at the time - and so were cultivated primarily for their taste: It would be a mistake to think that early agriculturalists focused entirely on nutrition. Tomatoes would be domesticated later - the original tomatoes were the size of cherries but would be selected for size over time. Settled agriculture certainly emerged before 3000 BCE, and spread north into what is now the United States over the next centuries (reaching southern Canada by 500 BCE), and south into Central America.

It is remarkable that agriculture first developed in the Americas not long after it emerged in Eurasia. If population pressure was indeed a critical encouragement to the development of agriculture, one might have anticipated a much longer time lag before the newly settled Americas would face the same pressure as the Eurasian continent that had been peopled tens of thousands of years earlier. The timing may then indicate that perhaps climatic causes were important. As in the Middle East, there is evidence of millennia of unusually dry conditions on the eve of the development of agriculture in Mesoamerica. 


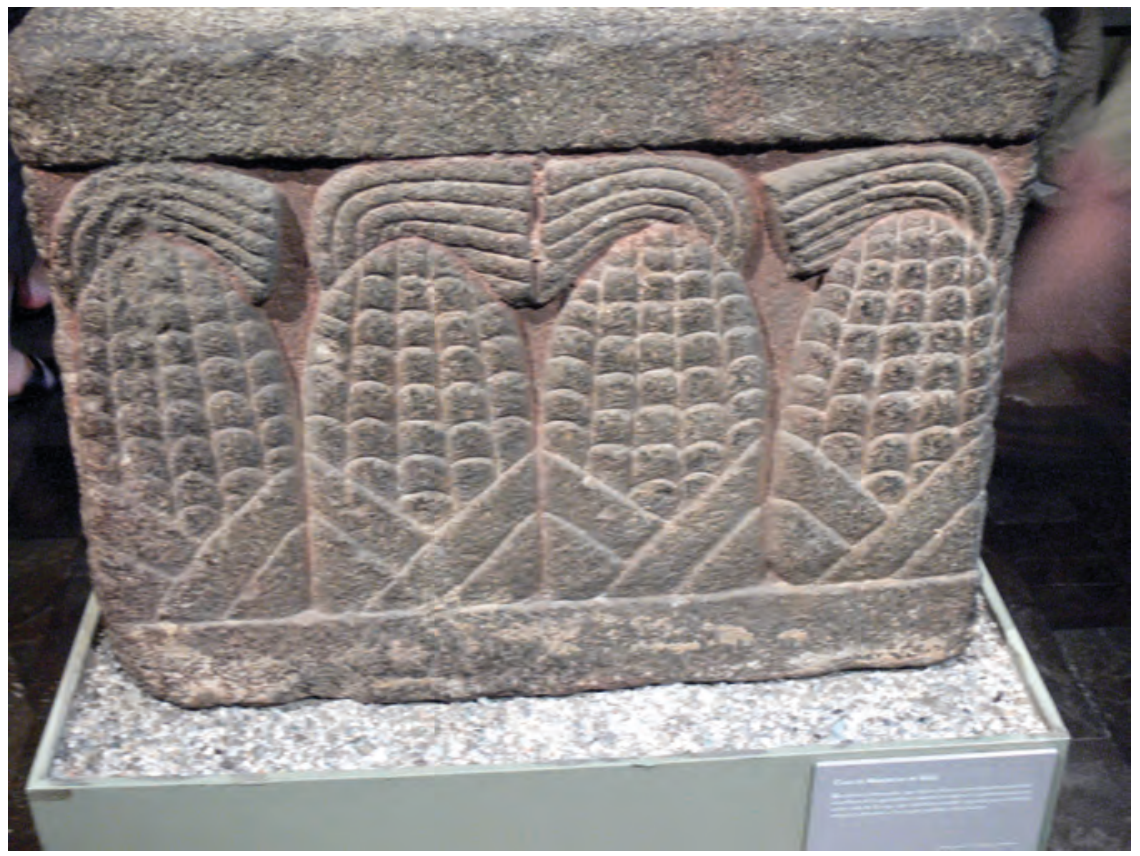

\section{Illustration 4.12}

Aztec stone for agricultural offerings decorated with corn cobs, National Museum of Anthropology, Mexico City, likely fifteenth century CE

Source: Photograph by Wolfgang Sauber, CC BY-SA 3.0

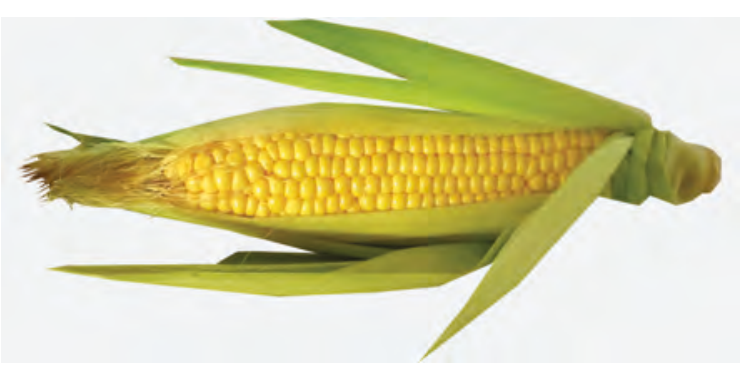

Illustration 4.13

Corn
One distinctive feature of Mesoamerica is the lack of domesticable animals: As a result, Mesoamericans performed more farm labour, and ate a less varied diet, than people in the eastern hemisphere.

Farther north, agriculture based on squash and sunflower and some other crops developed in the American Midwest from about 2000 BCE. It appears that growing such crops was always a secondary activity for the hunting and fishing populations of the area. Corn and other plants developed in Mexico would later replace this type of agriculture though a kind of corn better suited to northern climates seems to have been developed only around $800 \mathrm{CE}$.

Agriculture involving potatoes and chilli peppers developed by about 4000 вСE in the Andes of South America. It seems that this was an independent development from Mesoamerica. Corn somehow reached South America, perhaps by 3500 все. Squash may have been domesticated independently in the two regions. The rocky soils of the Andes were ill-suited to agriculture until the introduction of irrigation from about 4000 вСЕ. 


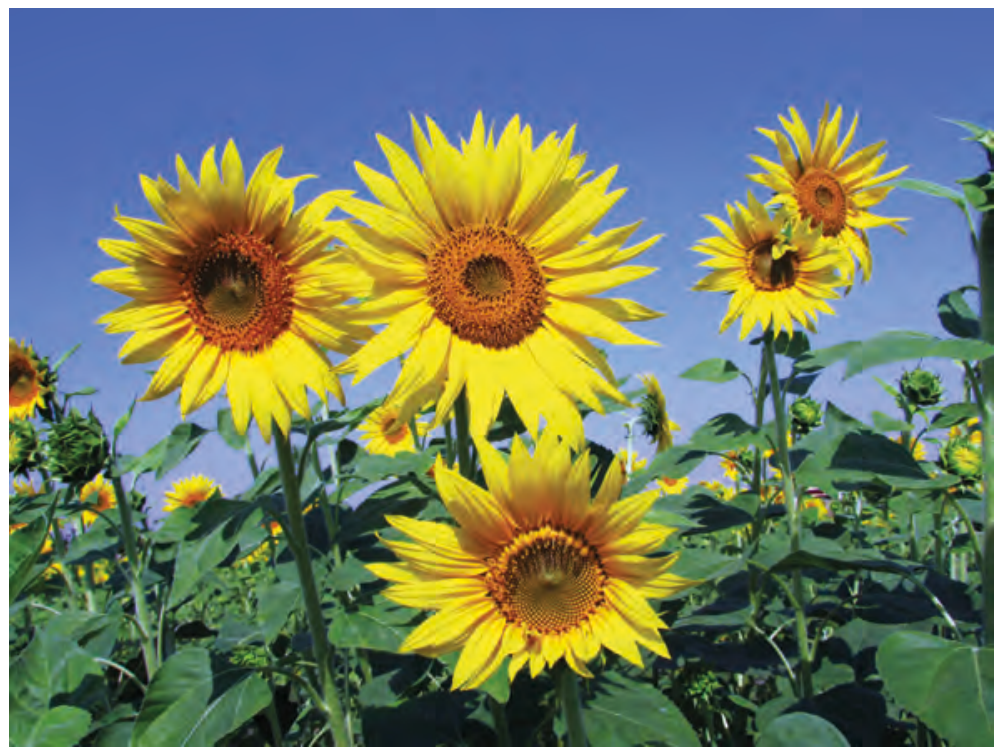

Illustration 4.14

Sunflowers

It had long seemed that there was no contact between ancient Mesoamerica and the Andes. However, there is evidence that there was contact by sea at least in the first millennium BCE and perhaps earlier, on boats developed by Peruvian sea peoples (see below). The northward voyage would have taken about fifty days and the southward journey almost twice as long, but it would have been possible to hug the coast of Central America most of the way (the southward trip would have required some open-water sailing).

Farther east, we tend to think of the Amazon as a region of primitive huntergatherers. Yet the earliest European explorers reported large societies with big boats, armies, and agriculture. Disease and European military efforts destroyed these societies. Though we know little about the timing (beyond finds of pottery from 6,000 years ago), we might speculate on agricultural practices similar to those in tropical Africa, but with the advantage of ready communication by river. There is evidence of very early slash-and-burn agriculture in the Amazon basin focused on the tropical plants manioc (also called cassava) and yams. This may also have been an independent development.

If there were independent agricultural development in the Amazon, this would be a unique case of the independent emergence of agriculture in a tropical climate. In Africa, agriculture developed on the savanna to the south of the Sahara and spread into the tropics. In Southeast Asia, scholars now think that agriculture was imported from temperate China. In New Guinea, agriculture developed in the temperate climate of high altitudes but not in lower tropical lands until imported from elsewhere. The rarity of independent tropical agriculture may in part be due to the challenges associated with domesticating tropical crops: These generally deliver a far lesser reward to farmer effort. (This 


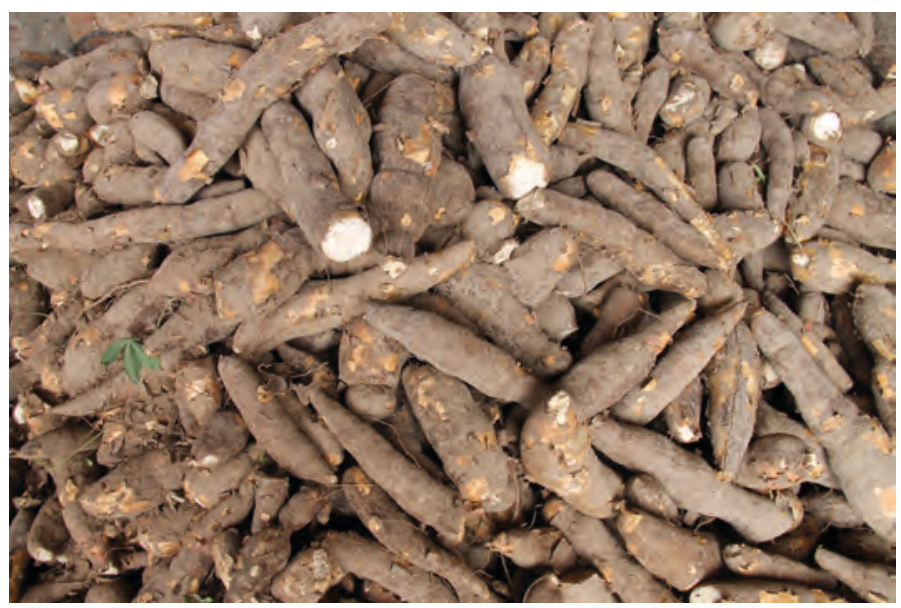

Illustration 4.15

Manioc (cassava)

difference has been exacerbated in the modern era as agricultural research has focused until recently on temperate crops.) It may be even more important that tropical areas provided abundant food to hunters and gatherers: There was less incentive to develop agriculture. The tropics were also (we think) far less subject to the sort of climatic shocks that may have played a critical role in the development of agriculture elsewhere.

There is archaeological evidence of settlement on Cuba and Hispaniola from the fourth millennium BCE. Scholars suspect that these islands were settled from South America (there are dugout canoes from perhaps 3000 вCE in Florida but for use on lakes rather than open water). The decimation of Caribbean populations by Europeans and the lack of any written script leaves us with little knowledge of how or why these migrations occurred. It is nevertheless worth noting that people had settled and farmed all of the habitable Caribbean islands long before the arrival of Europeans: the Windward Islands likely in the first millennium все.

\section{Some effects}

\section{Agriculture means storage and control over land}

Farming requires careful attention to particular plots of land. It inevitably involves concerns with who makes decisions regarding individual plots and who receives the output. These concerns need not (but often do) generate modern concepts of land ownership. Some mechanism has to be put in place that establishes plot-specific decision-making and sharing of resources. This, in turn, invites some political order that can make these decisions and adjudicate any disputes that may arise. 
The investment of considerable time and effort in a particular plot of land that will yield occasional harvests also dramatically increases the problem of theft: One can obtain the benefits of another's work by stealing their produce. The benefits of robbery in a hunter-gatherer world where little food was carried from day to day were much smaller. However, farmers necessarily stored food. Indeed, one of the main reasons that grains dominated in most human societies is that they were dry and could be stored for months or years. The problem of theft also invited a political order that could provide protection. Of course, those with power in such a political order would extract a surplus that might be more regular but otherwise similar in effect to theft.

\section{Environmental impacts}

Agriculture benefits humans in two ways. Most obviously, it increases the amount of food produced on a particular plot of land. Less obviously, humans can ensure that people receive the vast bulk of the food produced, whereas hunters and gatherers necessarily share the resources of their lands with other species. Humans shaped the landscape, preferred some plants and animals over others, and came over time to breed new plants and animals. Agriculture squeezes other species of both plants and animals into narrower ecological niches. Farming everywhere has a negative impact on biodiversity. Humans carried - either on purpose or by accident - crops long distances both within and across regions: Wheat, for example, was carried (we imagine by humans, likely gradually) from the Middle East to China some 1,500 years ago. We thus have fewer crops distributed far more widely.

Locally, by changing landscapes, and reducing forests, agriculture often causes soil erosion. This often changes watercourses, causes silting, and expands estuaries. Globally, agriculture may even have had a gradual but significant effect on climate as humans chopped down forests, releasing carbon dioxide into the atmosphere. Wet rice farming itself may have released methane into the atmosphere and caused minuscule amounts of global warming from 8,000 years ago.

\section{Challenges facing agents: Farmers}

The primary challenge facing farmers is to produce enough food to feed their families well enough that these can perform the necessary agricultural labour. This requires, among other things, that they have some control over a piece of land on which they farm.

It is in the nature of agriculture that food must be stored between harvests - and also between years as harvests are variable. Much of human history reflects the need to protect stored food: Walls, states, and armies are each at least in part a solution to this simple challenge. However, once there are states, farmers have to worry about these taking their food too.

Farmers may be less aware of another challenge: They must ensure sustainability by not robbing the soil of essential nutrients - nitrogen is of particular importance here. Only societies that addressed this challenge would prosper through time. 


\section{Addressing the soil nutrient challenge facing farmers}

There is a general challenge to agriculture in that cultivation of one crop will withdraw from the soil the particular nutrients that the crop requires, resulting in decreased soil fertility through time. The most critical nutrient in this respect is nitrogen, which all grain crops withdraw from the soil, though to varying degrees: Wheat has a 1.5 per cent nitrogen content, millet, corn, and sorghum significantly less. Settled farmers have recourse to various strategies:

- $\quad$ Flooding the fields with nutrient-rich water.

- Spreading manure on fields from farm animals who graze elsewhere (or, later in history, using natural nitrogen-rich fertilizers such as lime or bird droppings; or much much later using chemical fertilizers). The Chinese faced particular nitrogen-depletion problems in rain-fed northern agriculture, and thus spread human manure on their fields. Millet may have predominated there because it takes less nitrogen than wheat.

- Practising crop rotations that replenish the soil. The most important of these involves certain legumes, such as the beans and squash of Mesoamerica, which add nitrogen to soils that grain crops then absorb. Soybeans would be added to Chinese agriculture by 1000 BCE and were apparently appreciated for their ability to replenish the soil - the character for soy in the Chinese alphabet includes its nitrogen-fixing nodules.

- Growing symbiotic crops together. Tree crops can draw nutrients from deep in the soil, distributing these on the surface for surface crops.

- Practising "slash-and-burn" agriculture (as gatherers had), whereby crops are grown on one field for years, and then the farmer moves to areas of natural vegetation, cuts and burns this to release nutrients into the soil, and farms there for some years. This form of agriculture was practised throughout much of the world; it is limited today to the tropics where it still supports hundreds of millions of farmers. The Bantu agriculturalists of Africa often employed this method - likely because it is better suited to tropical conditions.

Note that many of these methods require regular access one way or another to nutrients from beyond the cultivated land. Humans would over time experiment with these various strategies, tailoring them to local resources. It should be stressed that farmers cannot just grow, say, wheat, but instead must engage in some set of practices beyond the wheat field that allow continued wheat production. We should appreciate farming as a complicated environmental process. Debate rages as to whether some human societies would fail to replenish their soils, resulting in the eventual collapse of their agricultural systems and societies (chapter 7). 


\section{Population and health}

Agriculture allowed much higher population densities: of about 100 times that of hunter-gatherers for early farming and 1,000 times today. The increase in nutrients per acre was even higher than the above ratios, for farmers needed more food since they worked harder than hunter-gatherers (though we might note that modern medicine still does not understand the precise link between exercise and calories burned). This increased food output, in turn, made possible villages, then towns, then cities, all fed by farms within range of the transport of the time. Historians estimate that the total human population was around 6 million when all humans relied on hunting and gathering; it had risen to 70 million six thousand years ago (before the rise of

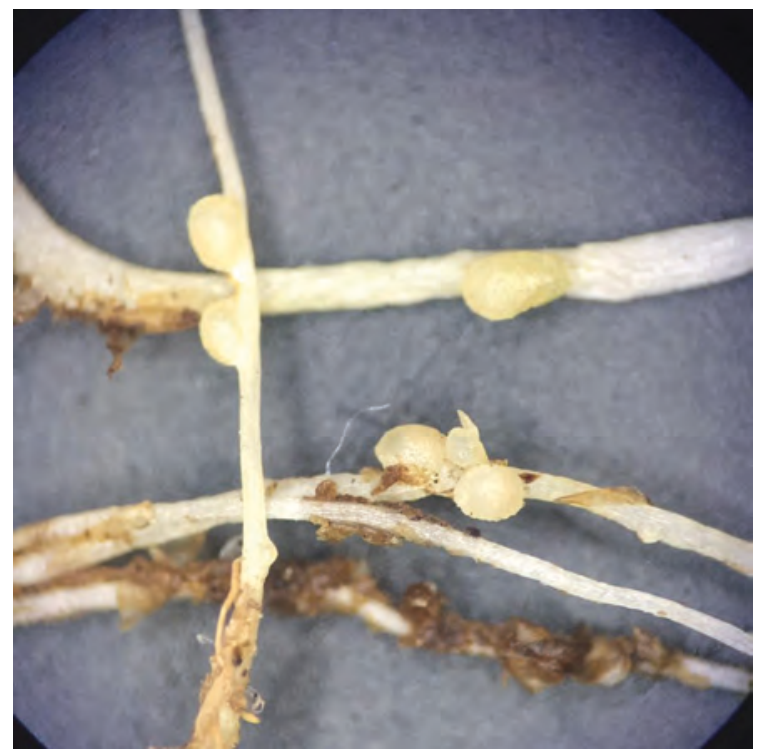

Illustration 4.16

Nitrogen-fixing nodules on the roots of clover Source: Photograph by Sebas l l tian, CC BY-SA 4.0 what has often been called "civilization"; see chapter 6), about 120 million three thousand years ago, and 250 million two thousand years ago. (Population collapsed to 200 million with the fall of empires (chapters 9 and 10), but rebounded.)

Over the next several thousand years most people - generally 80 per cent or more - in agricultural societies farmed. Yet if farmers could produce more than they ate, they could support a set of specialized occupations through trade or taxation: merchants, bureaucrats, priests, artisans, carriers, and others. Agriculture facilitated economic specialization and social differentiation. Politically, large centralized states were only possible with agriculture. Moreover, we have seen that farmers needed protection from theft and some control over land: The state was one possible solution to these challenges, as we shall see. Still, limited agricultural productivity nevertheless dictated that farming only freed a narrow elite from agricultural labour.

Though agriculture increased total food output dramatically, it was also problematic in several ways. Early farming is associated with both decreased diversity and increased variability in human food consumption. Examination of human remains establishes that agriculture had negative consequences for human health. We know that early farmers were significantly shorter than hunter-gatherers. This almost certainly means that they were less well fed. The less varied diet likely induced severe vitamin deficiencies. Grains variously are low in vitamin A (crucial for sight), B12 (associated with neurological 
problems), and C (scurvy) as well as calcium (rickets, a distortion of the bones) and sodium and iron (anaemia). (Jericho, Venice, and Salzburg were among historical centres that likely began as salt producers from water or mines to address sodium deficiencies.) Decreased diversity in food sources may also have reduced the enjoyment we associate with a varied diet (which itself may reflect the fact that the human body needs and thus appreciates various nutrients).

Though advanced agricultural societies can store food in order to survive bad seasons, the earliest farmers may have suffered severely if their crops failed. Mobile hunters and gatherers could more readily move or expand their food choices when faced with environmental shocks. Hunters and gatherers ate a few dozen different plants and animals distributed over a wide area. Farmers were more subject to famine or undernourishment because they depended on one or two crops from a confined area. Yet we should be careful here: Farmers could potentially avoid famine by storage of food or through trade. Examination of famines in the modern world suggests that these are generally not the result of an absolute shortage of food but of an inability of some of the population to access this. Famines, then, are more a failure of economic and political systems than a natural characteristic of agricultural communities. However, we must always be careful of projecting modern experience back into distant history: Early agriculturalists may have found it far more difficult to either store or trade their way through a bad harvest, and certainly a series of bad harvests. They likely produced only a small surplus even in good years, and transport was expensive. The earliest agriculturalists may have reverted to gathering wild insects and plants in times of trouble, but knowledge of what was edible in the wild would decline over time as agricultural communities established themselves and expanded in extent.

As humans came to live in proximity to livestock, several diseases - among them measles and tuberculosis and smallpox - evolved from animal diseases to become human diseases. Such diseases would weaken human populations in general, and will occasionally be the source of epidemics with dramatic effects on human history. However, livestock did allow some improvement in diet, especially as some human groups evolved to become lactose tolerant.

The decreased health associated with agriculture likely led to higher rates of (especially child) mortality. Life expectancy at birth was generally about 35, but if one reached 20 one could expect to reach 55 on average. The population increase mentioned above required, then, that fertility rise even faster than mortality. Agriculture likely both lowers the cost and raises the benefits of having children. These are likely easier to look after in one place than on the move. Also, there are many agricultural tasks that can be performed usefully by children. (Thousands of years later the rise of industrial and service employment would act in the other direction, increasing the costs and decreasing the benefits of children. See chapter 24.) The increased populations that resulted from this expanded fertility could be fed because of the increased food output per acre that agriculture allowed. Moreover, agricultural societies 
seem everywhere to be associated with a power imbalance between men and women (see below): Since women bear the costs and risks of pregnancy, their decreased power may have encouraged increased fertility. Agricultural societies usually produced both animal milk (once animals were domesticated) and easily digested cereals (once cooked); this would have allowed mothers to stop breastfeeding earlier, which in turn would increase the number of children they could have, since breastfeeding reduces fertility. Some scholars even hypothesize that the use of mush - a soft pulpy mass of grains and perhaps legumes - in weaning may have led to population growth during the transition to agriculture - and thus created further pressure for the adoption of agriculture.

High child mortality likely had cultural effects. It is hard to imagine what parenting was like when parents could anticipate that half of their children would die. This undoubtedly did not stop parents from being emotionally attached to children, but may well have had an impact on how much time and effort they invested in the very young. Readers can usefully speculate on how family life would have been different at a time of many children but very high levels of child mortality.

Large families, and the importance of child labour, generally led to an emphasis on strict child discipline in agricultural societies - at least this is observed across a wide range of historical agricultural societies and thus likely characterized early agricultural societies as well. The high rates of child mortality may have encouraged strict discipline by limiting the emotional bond between parent and child. Discipline may also have been encouraged by fear of both natural and human dangers - although the main threat faced by children was disease that they could neither see nor avoid. Children were often urged to behave like adults - contemporary appreciations of childhood and adolescence as important and (ideally) joyful periods of life were far less evident in agricultural societies.

Given the range of negative effects of agriculture on humans and the environment, some have termed agriculture a "big mistake." Yet without agriculture, humans would not have developed cities, states, or most of the technology that we now possess. To wish away agriculture is to wish away most of human history. To be sure, that history will prove to be both good and bad. Yet our ability to write about it, and to reflect on whether humanity should simply have accepted a hunter-gatherer lifestyle forever, is only possible because of agriculture.

\section{Human nature}

We discussed in chapter 3 how humanity evolved in prehistory. Scientists generally think that there has been limited genetic evolution during recorded human history: Genetic evolution is a glacial process. The major exception here is lactose tolerance: Farmers and nomads developed the ability to digest milk products as adults. (There may also have been some selection for height 


\section{BOX 4.1}

\section{MALTHUSIAN POPULATION DYNAMICS}

In the absence of migration, human fertility places an upper limit on how fast human populations can grow. Women are only fertile for a couple of decades, generally deliver only one child at a time, and usually require some recovery time after birth before they can again become pregnant (this becomes longer if the woman breastfeeds). Still, individual women can have as many as twenty children. It is thus possible for human populations to grow very fast. Yet we never have in human history observed population growth rates anywhere near the biological maximum - though when small numbers of people migrate to areas that have few or no people very high rates may be observed for a while. Since people are the basic building blocks of world history, we will strive to understand in what follows how and why people have acted to achieve population growth rates far below the maximum.

Population growth reflects not just fertility but mortality and migration. Here there are no theoretical limits: Entire populations can and have been wiped out or moved. Yet most of the time, rates of mortality and migration are less extreme. We will also keep a close eye in what follows on determinants of mortality and migration.

We should pay particular attention to a rough balance often achieved between mortality and fertility, such that population growth occurs slowly if at all. The concept of "demographic transition" refers to instances when a change in one follows a change in the other. Most famously, a drop in mortality rates in Western societies in the eighteenth and nineteenth centuries was followed decades later by a decline in fertility rates: The result was a period of rapid population growth that eventually transitioned to lower or no population growth (chapter 24).

In the late eighteenth century, Thomas Robert Malthus hypothesized that human populations would always expand until resource constraints were reached. Humans could and would breed much faster than they died. The only factor that prevented human populations from rising continually through human history was the limited ability to feed (and clothe and house) them. Malthus predicted inevitable poverty for the bulk of humanity, for we would always expand our population until we were just able to feed ourselves. If there were some sudden increase in food output - due perhaps to technological or organizational innovation, or climate change - poverty would be alleviated only temporarily until population expanded to absorb the increased food output. It is an irony of the history of ideas that Malthus developed this "Malthusian" theory on the eve of modern economic growth, a period in history in which economic output (including food output) grew far faster than population could (see later chapters). Still, Malthusian theory arguably is a good fit for both the hunter-gatherer and agricultural eras of human history. In both periods, human populations expanded until these 
could just feed themselves by existing modes of food production. The adoption of agriculture might have been a response to population pressure (see above), but in turn it set in motion continued population expansion. Improvements in technology or organization allowed first hunter-gatherers and then farmers to expand output. Over time, these advances in production simply allowed more mouths to be fed.

If human populations grew beyond the capacity of their environment to feed them, increased mortality would inevitably result. Malthus urged fertility restraint but was sceptical that this was feasible (he did not predict modern methods of birth control). Famine was one possibility, epidemic another (as weakened populations were more susceptible to disease). War was yet another, as an overpopulated society might be tempted to take resources from another: The mortality associated with war could lessen population pressure on resources. Malthusian theory thus provides a potential explanation for at least some of the major calamities in human history, though we must recognize other possible causes of particular famines, epidemics, and wars.

In its purest form, Malthusian theory would allow no increase in average incomes over time: These would all be eaten away (pun intended) by increased population. In reality, as we shall see, there likely was some slow if irregular increase in incomes. Humans became accustomed to better clothes, tools, or shelters that resulted from temporary increases in income. Population pressure did not cause these to disappear. There is thus some role for culture in the Malthusian picture. As Malthus himself recognized, human populations do limit fertility with rules - both institutions and cultural practices - governing sex and marriage and childrearing. And culture would affect what families thought was a minimal standard of life.

One important mechanism not stressed by Malthus would be family formation itself. As the most recent generation in human history often finds, the ability to leave one's parents' home and set up one's own household depends on one's income-earning ability. During periods of economic growth, it will be easier to do so, in part because of new job opportunities and in part as one's parents will have greater resources with which to subsidize such a move. The earlier that young people move out and get married, the more children they are likely to have. Note that this mechanism can still operate, albeit less powerfully, in societies with extended families, as long as parents' decisions regarding child marriage hinge on their sense of affordability of grandchildren. Such a mechanism can reduce the drama in the Malthusian picture: Marriage ages rise when population places pressure on resources, reducing the need for famine, epidemic, or war.

This Malthusian picture is not, of course, a description of population growth in isolation. Instead, Malthus described how population might interact with technology, economy, culture, and environment (among other things). As we have stressed before, developments within any one phenomenon or theme are conditioned by other phenomena. 


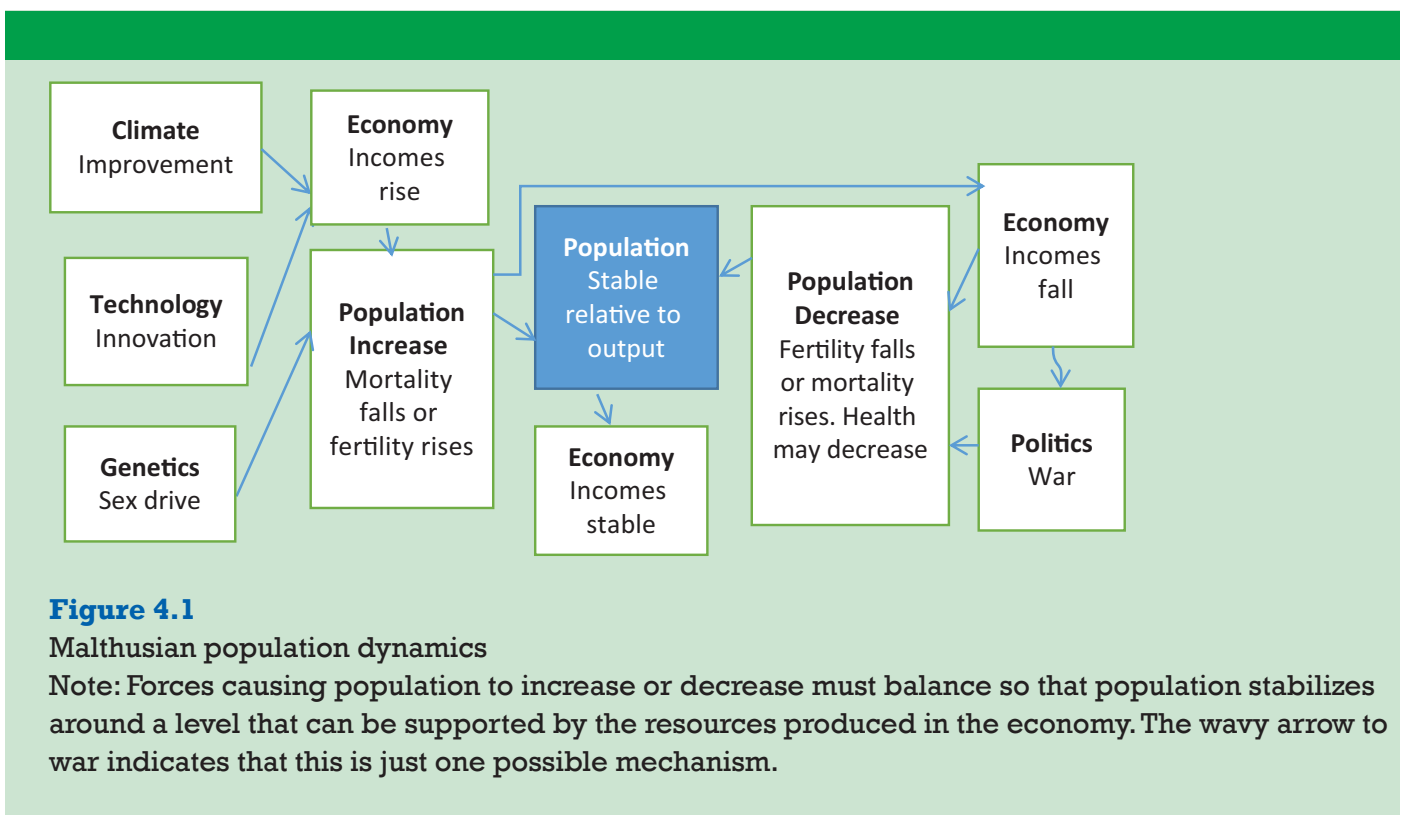

and perhaps markers of physical beauty in historical time; more controversially, there may have been selection for increased intelligence.) This does not mean that human nature has remained unchanged.

The nutritional intake of humans has changed immensely through history. So also has human work effort and the incidence of disease. Human bodies take in food energy and devote this energy to maintaining bodily systems, developing these while young, fighting diseases, and expending effort. Malnourished children do not grow as much as well-fed children. It appears that human growth processes protect the brain, but it is possible that brain development suffers in cases of severe malnourishment. There is some evidence from the modern era that brain development suffers if young people have to devote considerable energy to fighting infectious disease: This may indeed be the main reason for observed differences in intelligence across countries. Moreover, there is growing evidence that the brain, like other body organs, needs exercise to grow to its potential. We also know that hungry children are less attentive: We can then hypothesize that undernourished children will see less brain development (though here the effect is indirect through decreased levels of mental activity).

We also now know that brain development in a fetus suffers from drug use during pregnancy. We shall see in the next chapter that regular if smallish consumption of alcohol was common among all members of agricultural societies, including pregnant women and children. This may or may not have been at levels that would have interfered with brain development.

Though one purpose of this book is to comprehend our common humanity, it is nevertheless important to recognize that human actors were not always 
the same. We have already seen that humans in agricultural societies were on average shorter than they are today, or had been as hunter-gatherers. They may have been less intelligent. They had much lower life expectancies. They were more often afflicted with a debilitating disease. We should keep these facts in mind when we reflect on human behaviour during the thousands of years that agriculture would be the principal human occupation. The decisions we will analyse in the next chapters were made by people who were weaker and younger and sicker and perhaps not as bright as the average people of our day.

\section{Politics}

Agriculture requires coordination of activities over a fixed seasonal schedule. If one farmer does not weed, neighbours will suffer. Domesticated animals must be prevented from grazing on others' crops. Stored food must be protected from theft. (At times food was stored by families, at other times communally, and of course sometimes both.) This coordination could be achieved cooperatively, and this may have been the case in the earliest agricultural villages. However, some sort of leadership soon came to handle coordination in most places, and farming societies became stratified. Farmers would after that generally be a subordinate class, producing food for an elite group that exercised power.

Why did political stratification replace shared governance? As the population grew in agricultural areas, there was likely competition for the best land. This created an opportunity for the exercise of political power: Those who controlled good land could extract an income from the farmers that worked the land. If these farmers have nowhere else to go - if no free land is not subject to the depredations of bandits or local rulers - they will accept the necessity of supporting local rulers. (We return to this question in chapter 6.)

Whether governance is communal or authoritarian, agriculture requires clear rules regarding who farms which plots of land. This was not an institutional challenge that mobile hunter-gatherers faced: They collaborated as a group in both hunting and gathering. Farming requires instead that particular care is devoted to specific plots of land. The institutional solution that is generally though not always - observed in agricultural societies is that individual farm families gain control over particular parcels of land. This institutional innovation would not have been easy: How does a community that has tended to share its tasks and its food move toward the recognition that different families have different lands and food? How in practice is land divided in a way that is accepted? Though it will be less evident in the archaeological record than the gradual evolution of agricultural technologies, we might imagine a similar process of the gradual development of rules governing individual plots of land. These, in turn, would likely be encouraged by and depend upon changes in cultural attitudes toward land in general.

Most human societies would come to allow control over particular plots of land to pass from parents to children. This institutional innovation would encourage farmers to care for their land, but it also facilitated the development 
of sizeable differences in wealth and income over time as some families increased their land holdings. Luck, hard work, and different numbers of surviving children could all result in very different sizes of land holdings. Inequalities could grow over the generations if wealthy farmers acquired land from poor farmers.

The experience of early villages seems to reflect a hypothesis from the last chapter: Leaderless societies were less likely to go to war. The earliest villages in most parts of the world seem egalitarian. Moreover, they were not fortified. Human remains during this period show less signs of violent death than among either previous hunter-gatherers or later sedentary populations. Of course, the novelty of the first villages may have helped: They occupied limited space, and so could cohabit peacefully with hunters and gatherers. They may have had only small surpluses at first that did not tempt others. As populations increased within villages, and the number of villages in an area increased, competition over resources would increase. Anthropologists are divided among those who suspect that wars occur when a group is suffering from scarce resources (and so attack out of desperation) or when a group has abundant resources (and so can more readily afford a military adventure). The same human group might find itself in both situations as harvests varied through time. Yet if our opening hypothesis is correct - which we should hardly assume - then the main explanation of increased war over time between villages is that these came to have political elites who could benefit from conquest. (We address war in more detail in chapter 6.) Most anthropologists believe that leadership emerged to deal with the challenges of an increasingly complex society rather than initially to manage conflict. We see in many places - Jericho, Turkey, Peru, elsewhere - evidence of social stratification long before there is evidence of war. In any case, within a matter of centuries we see fortifications, evidence of burned villages, and new weapons such as slings and maces. War was an unintended but perhaps inevitable side effect of political stratification.

\section{Gender relations}

Agricultural societies decreased the relative power of women, though this may only have occurred after some millennia of agriculture. There are many possible explanations for this change. The gender division of labour within agriculture may have somehow changed the power balance between men and women. If so, gender inequality likely increased with the development of metal ploughs (chapter 5): Males tended to operate these, whereas previous implements had required less physical strength and were employed by women.

Agriculture, as we have seen, led to increased fertility. This - especially given high rates of infant and child mortality - required that women spend far more of their time in pregnancy or childcare (breastfeeding being one form of childcare that only women could perform). In both agriculture and hunting/ gathering, it could be that it was easier to combine childcare in general with the more locationally constrained productive activities of women than those of men. Increased frequency of children would then limit the activities of women 


\section{BOX 4.2}

\section{POLITICAL LEADERSHIP TERMINOLOGY}

Anthropologists have achieved some consensus on the terminology associated with political leadership. We spoke in the last chapter of hunter-gatherer "bands": Bands have no permanent leadership, though temporary leaders may guide a particular hunt or other activity. In "tribes," lineages (lines of family inheritance) dominate. The headmen of lineages have some real authority but can be disobeyed; they also necessarily compete with other headmen. Though tribes are small and largely self-sufficient, they can sometimes combine with other tribes (especially if these share a more distant ancestor) in times of war (see especially chapter 17). Tribes usually allocate farmland communally and often re-allocate when a tribe member dies. Tribes have no formal laws but may employ assemblies to mediate disputes rather than rely on duels or personal revenge. In "chiefdoms," one lineage becomes dominant, but competition may continue among lineages, making chiefdoms unstable. Finally, in "states," there tend to be rigid classes, codified laws, and recognized rulers. States exercise a monopoly over violence through armies and police forces. States are defined territorially rather than in terms of people. This typology is grounded in the study of contemporary societies or societies active in recent history. It seems likely that early agricultural societies may have moved from a "band" structure through "tribes" and "chiefdoms" to the states we will discuss in the next chapters. Political power, then, was first centred in families, but over time some families achieved a dominant position. We should note that even today there are societies that poorly fit this typology and so we should be careful of assuming that it also characterized early human history. Note also that many parts of the world, including many Pacific islands, much of Africa and the Americas, and parts of South and Southeast Asia, did not develop state structures until very late in history. We should be very careful of exaggerating the role of states in human history. We should also appreciate that each form of political organization has its benefits and costs, and is best suited to different circumstances.

We will occasionally use the term "polity" to refer to any of the above types of political organization. This practice will be especially convenient when there may be multiple types of political organization operating in a particular time and place.

more than men. There may thus have been a reciprocal relationship between increased fertility and the decreased power of women: Pregnant women were less powerful, and powerless women were more likely to be pregnant (arguably the power of women increased as birth rates fell later in history; chapter 24).

We have seen above that farmers had to worry about protecting stored food, and discussed how political stratification and armed conflict emerged in early agricultural societies. We shall explain in later chapters how war became a regular feature of agricultural societies. The military role of men should not be forgotten and perhaps deserves emphasis in understanding changes in gender relations. Agricultural societies (eventually) competed militarily with each other and with nomads. The physical dominance of male warriors could be 
reflected in gender relations. Though there is evidence of women serving as fighters in many nomadic societies, women only rarely serve in the armies of agricultural societies. This reflects perhaps both differences in physical strength and the high incidence of pregnancy. Internally as well, there was probably more opportunity for the exercise of physical force when farmers were tied to pieces of land than when hunters and gatherers roamed in collaborative groups. The selection that seems to have occurred for male aggression during hunter-gatherer times could have been crucial here in encouraging the exercise of force and perhaps even in seeking an outlet for aggression to replace hunting. Agricultural societies may have been inherently a bit less collaborative than hunter-gatherer societies, which would have increased opportunities for the exercise of power.

The gender imbalance had generational effects. In agricultural societies, rights over land had to be transmitted across generations. (The family arguably became a more important social unit as tribal loyalties were absorbed into the state, and families became associated with plots of land.) This was done primarily or exclusively through sons. Sons almost everywhere were as a result more esteemed than daughters. Inheritance systems were thus a transmission mechanism that reinforced male power. As inheritance became important, there were increased concerns about ensuring the paternity of children: This may also have served to encourage the control and seclusion of women. Inheritance would also be an important consideration among hereditary rulers: Such rulers would often limit the freedom of their wives to ensure paternity.

Though women were subordinated in all agricultural societies that we know of, there would be important differences in degree across societies. In some societies, women would be secluded and veiled. In others, they would participate actively in public life, and might even occasionally rule. In some societies, women could initiate divorce and inherit property, in others not. It is not always easy to judge the gender relations of past societies: Egypt (later) enshrined gender equality in law, but in practice women were prevented from political and economic power. Such differences seem to be rooted in culture or historical contingency rather than any stark differences in economy or polity.

\section{Speculation on peasant behaviour}

Did the typical peasant family work hard in order to produce a surplus with which to buy pretty pottery? Or did they rather decide to enjoy life, working just hard enough to provide a bare subsistence for themselves (and often not even that)? We can imagine that there was substantial personality diversity here. We should not forget that agricultural work was hard: We have noted before that farmers likely worked much longer days than hunter-gatherers. We can understand if the desire to work harder than necessary was not always evident. Through their taxation policies, states might serve to increase total economic output by forcing peasants to work harder than they wished (but states might then risk revolt). Cultural attitudes could loom large here: Villages 


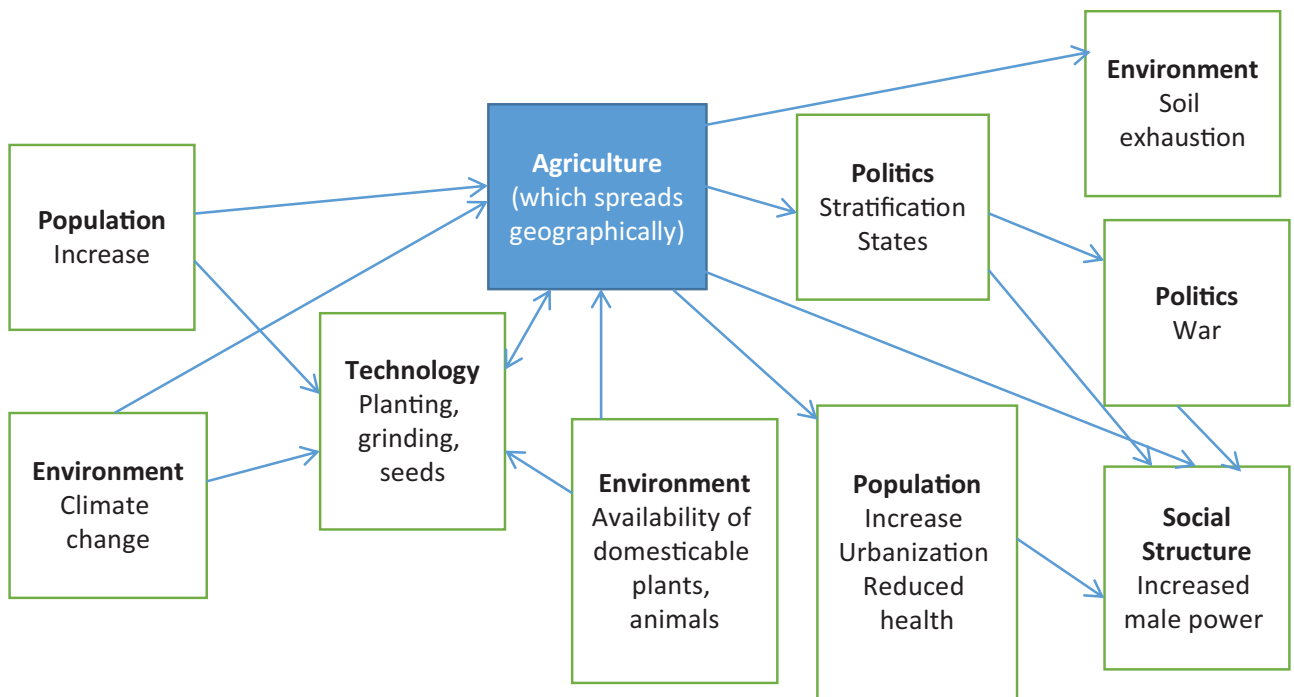

Figure 4.2

Influences on and effects of agriculture

Note: The arrows on the left side capture how population pressure and climate change encouraged a gradual process of developing the technology for agriculture (which we will develop further in the next chapter). This process depended on the availability of appropriate plants and animals (and rainfall etc.). The arrows on the right capture how agriculture then encouraged political consolidation and stratification, war, further population increases (but reduced health), and together these generated a change in gender relations. Soil exhaustion is possible. We will capture other effects in later chapters.

might come to celebrate an egalitarian ethos and disapprove of neighbours who displayed ambition for more. Since agricultural incomes are highly variable, small communities might develop expectations of sharing so that others would aid families suffering a bad harvest. This laudable sort of communitylevel insurance might nevertheless act to discourage anyone from working too hard if they might reasonably expect to have to share with others - unless of course the social prestige or sense of personal fulfilment that might be associated with giving served as a sufficient reward. Cultural attitudes might well evolve over time: It might come to be expected that every household would have a set of nice pots, and then every farmer would strive to buy these. As artisans developed better goods, the aspirations of farmers might rise in response.

\section{Nomadism}

\section{The nature and location of nomadism}

Historians heap deserved attention on agriculture, for agricultural societies are at the centre of much that happens in human history. However, hunting and 
gathering continued in some areas of the world until the present day (though hunting and gathering may disappear in the next century or so, except as a tourist attraction). Moreover, there were alternatives to both hunting/gathering and agriculture. One was fishing. In some parts of the world, a fishery could feed settled populations with little or no attention to growing crops (see below).

Another alternative was nomadism: Groups could travel with domesticated animals across broad areas as the animals grazed and humans continued to gather wild foods. Nomadism should be appreciated as an alternative set of practices for survival that developed over a roughly similar period as agriculture itself. It is similar to farming in that it involves the domestication of animals. Nomadism was most common in relatively arid regions that were not well suited to agriculture (at least given the technology of the time) but could sustain horses or camels or cattle or goats or sheep. Nomadism was prevalent on the margins of the deserts that spread from Africa through Arabia to Central Asia. Some nomads travelled long distances with their herds, while others followed a more constrained practice of moving between altitudes as the seasons changed. Group size ranged from ten to a hundred, generally with a common ancestor.

Nomadism inevitably was associated with far lower population densities than agriculture and little in the way of permanent settlements. This has meant that there are fewer archaeological remains to study and that we tend to know far less about nomads than about sedentary populations of the time. Despite the low population densities, nomadic groups would prove to have military advantages over farmers. Moreover, nomads traded with farmers and controlled critical trade routes between agricultural societies. For these reasons (and for a few others that we will treat from time to time) their interactions with agrarian societies would often prove to be of great historical importance.

Though nomads existed in many parts of the world (including the reindeer herders of Lapland, and nomads in Arabia and the Sahel), they were most common through history in Central Asia. The short growing seasons associated with the high altitudes of the Eurasian steppe, and the low precipitation associated with long distances from large bodies of water, combined to make agriculture challenging through most of the region. (The exceptions were the lands along the various rivers that crossed the steppe; agricultural oases would often emerge along these.) These extensive grasslands were well suited to grazing. Nomads in this area often controlled the overland trade route between China and the Middle East and Europe. Political consolidation within the nomadic population would occasionally facilitate this long-distance trade (see chapters 9, 17).

The Eurasian steppe - an area of natural grassland - stretches from the Hungarian plain in the west 9,000 kilometres to Manchuria in the east. Mountain chains occasionally interrupt the steppe, but mountain passes along which animals could graze punctuate each of these. We will find nomadic groups migrating long distances within this large steppe zone at many points in history, and interacting in various ways with neighbouring farmers: in Europe, the Middle East, Persia, India, and China. The most common direction of migration historically was from east to west, perhaps in part because the Mongolian 


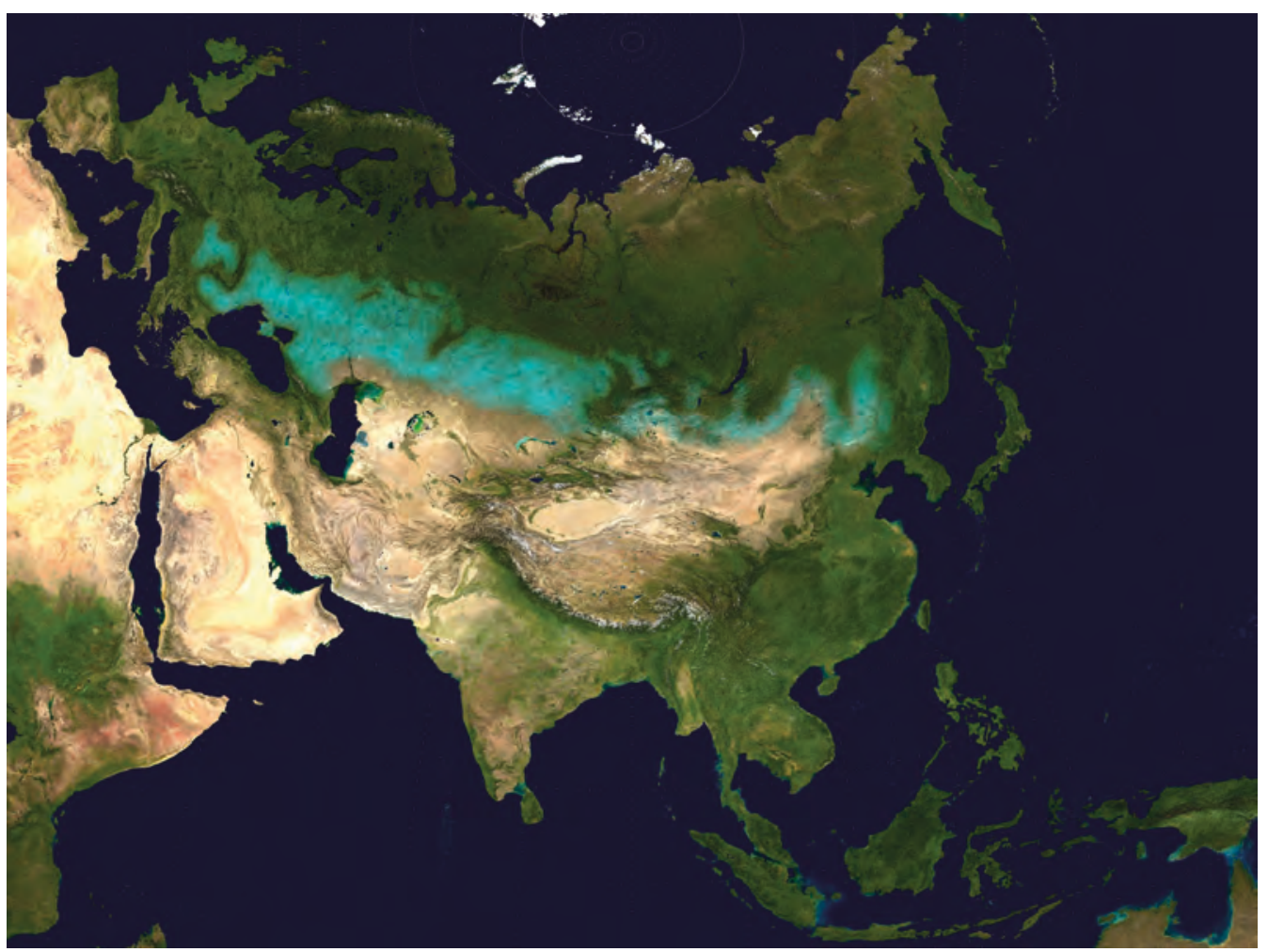

Map 4.2

The Eurasian steppe (in turquoise)

and Manchurian steppe lands are higher and colder than those to the west. Climate change may often have triggered these migrations, though losses in war might also trigger mass movements. In the earliest days, though, it appears that nomadic practices emerged in the western steppe and spread eastward.

Postscript: In 2008-9, a dry summer and cold winter caused some 8 million animals to die in Mongolia. Many animal herders moved into cities as a result. In an earlier era, nomads might have been tempted instead to migrate westward in search of pasture for their animals. There have been many bad summers since for nomads, though not quite as bad as 2008-9.

\section{The emergence of nomadism}

The two critical requirements for nomadism are wild grasses and domesticated animals. The natural environment in several parts of the world provided the first, but not the second. It is thus reasonable to ask whether farmers or nomads first domesticated animals. In the case of the horse, it seems that nomads first achieved domestication in Russia or Ukraine, and by 5000 BCE at the latest. 
Nomads farther south - in Arabia or Somalia - may have domesticated the dromedary camel by 3000 все; the Bactrian camel was domesticated hundreds of years later east of Mesopotamia. The general assumption is that farmers first bred most other animals. Farmers most likely first domesticated the goats grazed by nomads in Arabia, for example. If this was indeed the general case, and we accept that farmers domesticated animals long after they domesticated plants, then nomadism must have appeared a thousand years or more after agriculture. There is, though, some evidence of the herding of goats and sheep in the Middle East even before the advent of settled agriculture.

We should mention a third requirement of nomadism: lactose tolerance. Nomads tend to live off the milk and blood of their herds, butchering animals for meat only rarely. Adult humans did not initially have the physiological capability to gain nutrients from milk. Even more than sedentary farmers, nomads needed to develop lactose tolerance. This seems to have been a frequent genetic mutation, but still it must have taken many generations for communities to develop the physiological capability to live from milk.

We do not know if population pressure or climatic change propelled hunter-gatherers toward nomadism, as is conjectured for farmers. If agriculture predated nomadism, then at least some of the earliest nomads may have been former farmers. Population pressure or climatic change might have forced some farmers and their flocks out of regions that could support agriculture. The boundary between farming and nomadism shifted over time in both directions. About 5000 все farmers in western Ukraine started to spread into pastoralist lands farther east. Their villages soon were fortified. We also see migrations of nomads into Bulgaria and elsewhere over time.

By 1000 вСE at least, nomadism was practised not only across the Eurasian steppe but also in the semi-deserts of the Middle East and Africa and in parts of South America. As with agriculture, we thus know that nomadism developed separately at least twice and perhaps several times. It could not emerge in North America because of the absence of domesticable animals. In Eurasia, nomads herded cattle, sheep, and goats, often together. In the Middle East, sheep and goats were most common; there were also some water buffalo. In Africa, cattle dominated; it is not clear when and how these were first introduced from Eurasia. In Tibet, nomads would herd yaks (much later). In South America, the only available animals were the llama and alpaca (both of the camel family; the guinea pig was also later domesticated but had limited use).

\section{Nomads and farmers}

Nomads likely interacted with farmers as soon as there were both farmers and nomads. Nomads everywhere tended to trade with nearby settled societies; they also often facilitated long-distance trade across their territories. Egyptian kingdoms faced nomads in Sinai, Nubia (Sudan), and perhaps in western oases from the beginning of written Egyptian records. The Egyptians traded with them (agricultural goods for hides and milk at first; textiles and manufactured 
products for horses and perhaps metals and other materials later) but disparaged them as both uncivilized and dangerous. By the late Old Kingdom (third millennium BCE; chapter 7), some Medjay pastoralists from Nubia were employed in the Egyptian military as scouts and archers. We should be careful of assuming that just because texts from settled societies were often disparaging relations between nomads and farmers were always negative. Students should also appreciate that the historical record here is biased, as settled farmer societies left far more in the way of written records than nomads, who adopted writing millennia later (chapters 6, 17).

Nomads will often appear in history when they are at war with agricultural societies. We should stress, then, that there is far more trade than conflict between the two in history. Nomads had an interest in many goods that agricultural societies came to produce (chapter 5); they could trade the hides and milk from their animals, precious stones or metals from the lands they grazed, or goods from other agricultural societies. Nomads also engaged in manufacturing of products from leather, wood, and increasingly metals (see chapter 5). They fashioned tools - including the fearsome bows and arrows that they would come to use in hunting and war - from animal bones and wood, and fashioned clothes and tents from animal hides. By about 1000 все, Central Asian nomads were producing remarkable metal ornaments portraying animals. Nomads often traded these various manufactured goods as well. Nomads also continued to hunt wild animals and often fished. These activities provided further opportunities for trade. Hunting activities also proved useful training for war. Note that these trade opportunities reflected the fact that agricultural societies generally produced a fairly narrow range of goods and thus looked to nomads for many things.

With respect to conflict between farmers and nomads, it should be stressed that if nomads repeatedly attacked the same state they could soon destroy that state. Some nomad confederacies may indeed have fallen apart precisely because they destroyed their main source of income. Most, though, would reach some agreement with states such that the state made regular payments to avoid attack. Such payments could be used by nomad leaders to reward a large number of followers. Only if a confederacy could maintain control over large numbers of nomads would states continue to pay it large sums not to attack.

Just as hunter-gatherers had experimented with agriculture in many parts of the world, nomads often engaged in some agricultural activity. As noted above, some small oases with agricultural potential existed within nomadic territories, usually alongside rivers or lakes. Nomads might thus have semi-permanent settlements from which they left to herd. Nevertheless, nomads often disdained the agricultural life, and therefore these oases were generally farmed either by nomads who had lost their herds or by ethnically distinct (and often politically independent) groups. Note that nomads often indicated a disdain for farmers that matched the arrogance that farmers often voiced toward nomads. Yet oasis people naturally traded intensively with surrounding nomads. (Indeed some Central Asian oases would become major trading centres later in history, and 
would be noted for lavish houses and monuments; some were associated with mining, others celebrated for the melons they grew.)

We mentioned the spread of Indo-European languages with agriculture above. Indo-European languages mostly have similar terms for herding, indicating nomadic origins for the language group. That is, the original Indo-Europeans were likely nomads that adopted agriculture. They probably originated just north of the Black Sea.

\section{The domestication of the horse}

One crucial "technological" innovation associated with Central Asian nomads was the domestication of the horse some 5,000-8,000 years ago. This development is among the major transitions in human history. It is hard to imagine what world history would have looked like without the horse: It will often play a central role in both war and trade in subsequent millennia. It also enhanced agricultural productivity, since the horse is twice as strong (and fast) as an ox (a cow used as a draft animal, usually a castrated male). As we shall see, the domesticated horse provided nomads with at least a temporary military advantage, though agricultural civilizations soon copied the idea. For thousands of years, nomads would also export horses to neighbouring sedentary societies in exchange for agricultural and manufactured goods. The horse also later allowed nomads to pull tent hides and other possessions with them in wagons (chapter 5): Our romantic image of nomads as travelling with only the clothes on their backs is quite misleading.

Though we cannot know precisely how horses were domesticated, we can hypothesize a lengthy two-stage process. Hunter-gatherers had likely hunted horses for food for thousands of years. Nomads would then have appreciated the value of taming wild horses for use as a food source. Horses had an advantage over sheep and cows on the steppe for they could push away snow with their noses to graze on the grass underneath. At some point, perhaps centuries after they had begun to herd horses for food, nomads experimented with riding them. This might only have been possible after generations of selection for obedience. After nomads started riding horses, further selection for docility would occur.

In addition to their skills with horses, nomads had a further advantage in military conflict: Their very mobility allowed them to attack and flee, whereas settled agriculture societies must always provide a fixed target for attacks. (This advantage would diminish as towns fortified.) However, this advantage came with a matching disadvantage: The nomads' herds were much easier to steal once a nomad was defeated than were the grains stored by a farmer.

\section{Nomad life}

As noted above, we know little about the day-to-day lives of early nomads. We can speculate that the earliest nomadic societies would have exhibited similar 


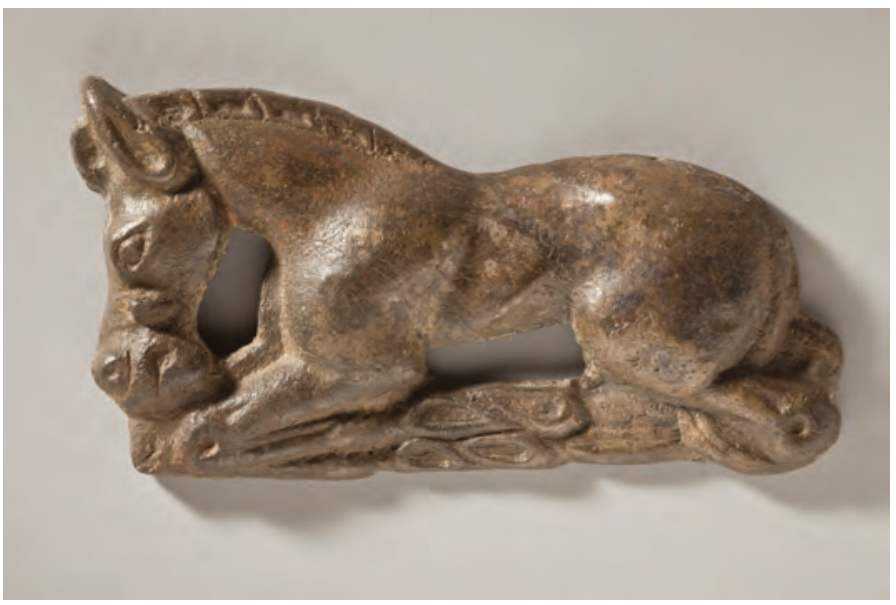

Illustration 4.17

Bronze belt buckle of horse, southern Siberia or western Inner Mongolia, fourth century BCE, LACMA

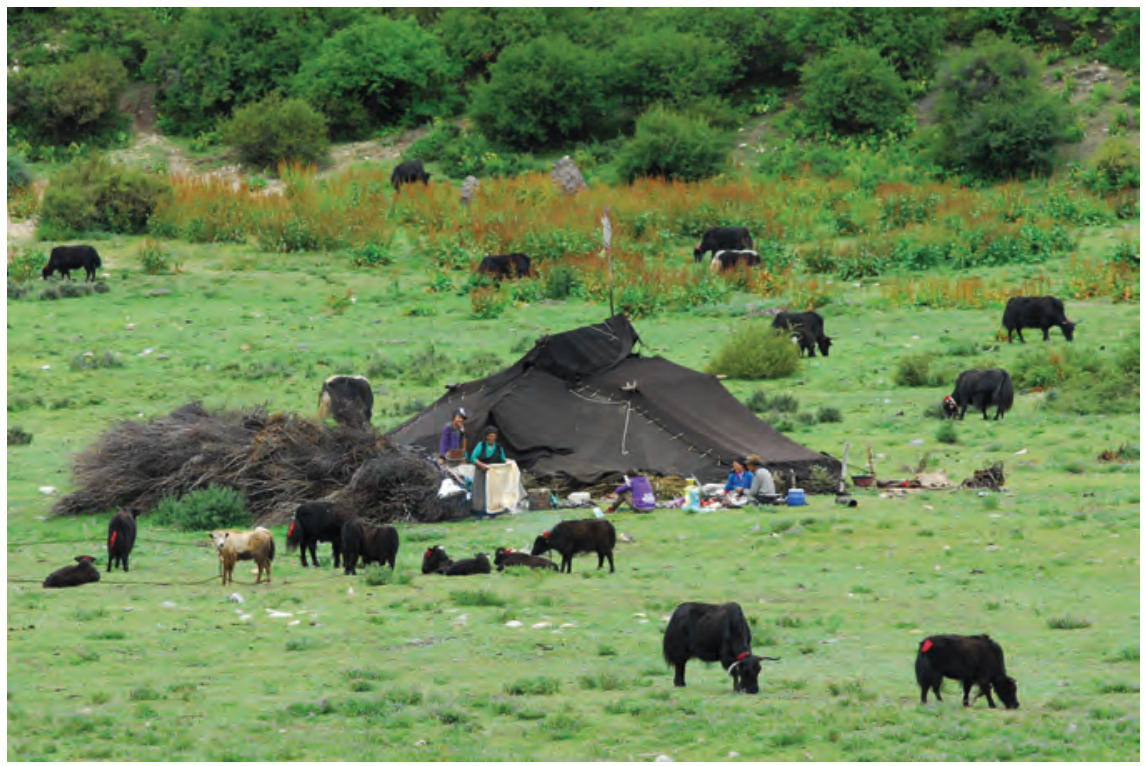

Illustration 4.18

Nomads in contemporary Tibet

characteristics to contemporary nomadic societies. Nomads today organize along patrilineal lines, with polygamy common. Men herd, while women milk and make clothing. Yet women are often more powerful than in agricultural societies. There is usually substantial inequality across families associated with different herd size. Note that herds - like land in agricultural societies - comprise a valuable resource that parents could easily pass to children, allowing 
inequality to increase over generations. It seems that early nomads were organized into clans, and these clans into tribes - but tribal boundaries were flexible such that charismatic leaders would often prove able to gather large military forces together when attacking agricultural populations.

Nomadic populations were likely healthier than agricultural societies. Their animals grazed on a variety of grasses and other plants, and the milk, blood, and meat they then obtained from these were probably richer in the range of nutrients that humans need than was the early farmer's typical diet. Since there were far fewer nomads than farmers, trade between the two did more to diversify the diet of the former than the latter.

\section{Challenges facing agents: Nomads}

The nomad does not have to worry (at least anywhere near as much) about environmental stability as the farmer does: Nomad herds graze lightly and move on, dropping manure as they go. Nor does the nomad have to worry about control over a particular piece of land in quite the same way, though they need some land on which to graze their animals. However, the nomad needs to worry about theft just like the farmer. Indeed the danger may be greater, for the nomad's stuff is mobile: Herds can merely be led away (and other goods were loaded on horses or, later, wagons). The nomad's paramount concern is maintaining and increasing the herd in the face of potential theft as well as environmental challenges.

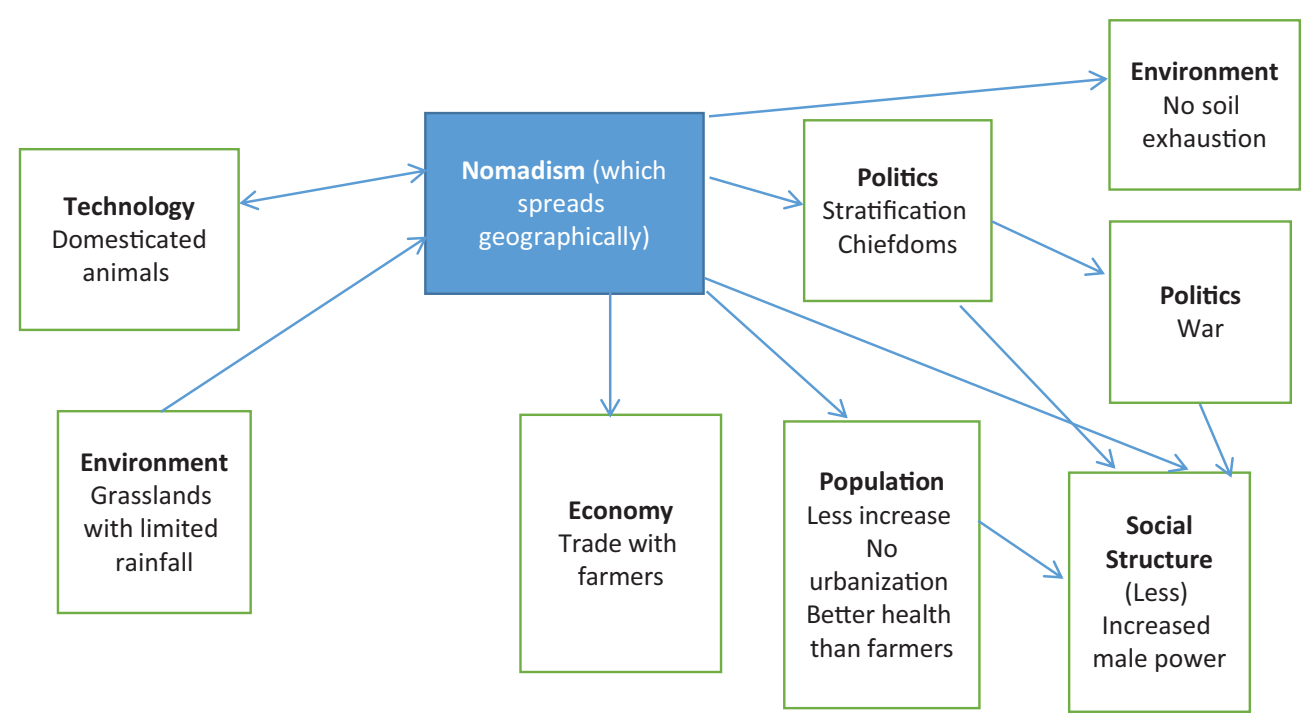

Figure 4.3

Influences on and effects of nomadism

Note: The diagram is designed purposely for the student to compare with that for agriculture. 
We suggested above that the responses to the farmer's challenges were of crucial importance in history. We will indicate in chapter 13 that the nomad's response to its challenges - a particular type of tribal organization - also had dramatic implications for the course of human history.

\section{Fishing}

Though we discuss fishing last in this chapter, humans undoubtedly engaged in the act of fishing long before they planted crops or herded tame animals. Fishing was an activity engaged in by hunter-gatherers when these had access to fishing stocks. Yet until humans developed good boats and fishing equipment, fishing likely provided a small part of human nutritional intake.

It is not clear when the first societies that relied heavily on fishing emerged. Some historians speculate that this could have been about 40,000 years ago (perhaps in Indonesia), others only 8,000 years ago. The first hard evidence we have of lines or nets for fishing comes from about 7,000 years ago in the Indian Ocean (and perhaps the Americas). Shellfish were likely harvested somewhat earlier and in more places. There is evidence that some 20,000 years ago, humans in the Cape region of South Africa spent part of the year fishing and part of the year inland hunting and gathering.

In the Pacific Northwest of North America, fishers used stands of giant cedar for dugout canoes large enough for ocean-going fishing and trading. Most fishing people in the world had to lash smaller boards together. In both the Pacific Northwest and along the coast of what is now Peru, fish were abundant, and fishing-based societies developed permanent settlements and much greater political, social, and economic inequality than surrounding groups of hunter-gatherers. In both the Pacific Northwest and Peru, fishers traded fish with inland peoples thousands of years ago (in the Pacific Northwest fish oil was traded for furs and amber). These Peruvian fishing settlements - which were building pyramids with bases the size of four football fields in the third millennium $\mathrm{BCE}$ - may have been the forerunners of later Andean civilizations (which themselves tended to a lucrative trade with coastal peoples of cotton used for fishnets in return for seafood).

Fishing - combined with local gathering - could in favoured locales such as these provide an abundant food source accessible from one locality, and so produce a surplus that supported an elite of non-fishers. It is possible that small fishing-based communities may have emerged while all other humans were still hunters and gatherers - that is, before agriculture or nomadism had emerged anywhere - and that these would have had more complex social, economic, and political structures (and thus more inequality) than other human groups of the time. Such communities would have been spatially limited. States covering large land areas were only feasible with the development of agriculture. Early fishing-based societies seem never to have developed agriculture autonomously, though many would later learn of farming from others. Given 
their abundant fish supplies, it is not surprising that they did not launch the laborious process of developing agriculture.

Special mention should be made of what is termed the Jomon culture in Japan from perhaps 11,000 to 400 BCE. This hunter-gatherer society relied heavily on fishing. Since fish are abundant in the rivers and coastline of Japan, these hunter-gatherers were able to form permanent settlements long before the development of agriculture in Japan. As noted in the next chapter, they created the earliest pottery that we know about. They used pots among other things to separate salt from seawater for food preservation. They also built permanent homes, burial grounds, and garbage pits. Trade for obsidian, jade, and artisanal goods over distances of hundreds of kilometres is evident. Economic specialization and political stratification are likely. The Jomon culture disappeared when agriculture was introduced to Japan from Korea: There is debate as to whether this reflected military conquest and ethnic dispersal, or whether the Jomon are in fact the ancestors (at least in large part) of the modern Japanese.

\section{Challenges facing agents: Fishers}

Fishers store food to a much lesser extent than farmers do, as they can usually catch fish regularly. They may store fish and other food items due to seasonal variability. They certainly have more to steal than hunter-gatherers that are more mobile. They also face less intense environmental challenges - at least until much later in history when new fishing technologies would make overharvesting a possibility (chapter 27). The primary challenge facing fishers, then, is catching enough fish, a challenging task with early technology.

\section{THE THEME OF THE ECONOMY}

We necessarily interact with each other and the non-human environment to create (and distribute) food, shelter, and other items of practical utility. We call this set of interactions "the economy." Among economic phenomena, world histories have tended to emphasize trade, for trade connects different societies and encourages the exchange of ideas as well as goods. We too shall speak much of trade but should appreciate here that trade often merits attention not for its direct economic importance - intercontinental trade, in particular, is usually very small relative to total economic activity until modern times - but as a conduit for information that influences other phenomena.

We will try to track changes in income and income distribution - in particular, how much of total income is absorbed by a narrow elite - through history. Though we of course lack official statistics on the gross domestic product or personal incomes for early human societies, there are various strategies for estimating what these might have been. For example, we can deduce food intake from skeletal remains and estimate consumption of pottery from 
archaeological digs. One danger is that "hourly wages," when these are known, may give a misleading impression of household income, which also depends on unemployment (and underemployment) rates, productive opportunities for all family members, and non-market income. As for unemployment or inflation rates, we are much more limited in our ability to discuss these before modern times, though some episodes of earlier unemployment or inflation are well documented.

We cannot of course track in detail the record of consumption of each good or service across time and space. We will trace the history of a few products of particular interest. We will from time to time refer to specific consumption items as evidence of either changing incomes or trade patterns. We will also occasionally discuss what humans ate and relate this to likely consequences for health. Luxury goods are often of disproportionate importance in long-distance trade (for only they can bear high costs of transport) and serve as evidence of income disparities.

As we shall see, a host of phenomena can influence economic growth. One of these is economic institutions: the rules that govern ownership, exchange, finance, production, labour relations, and any other economic activity. All agricultural and industrial societies necessarily have rules to guide economic activity. And some institutions encourage economic activity far more than others. It is highly useful in what follows to make note of key institutions and their likely effects. We can then discuss why those in power enacted these institutions.

\section{Questions}

1. Was agriculture a "big mistake" for humanity?

2. Some historians have speculated that human concern with heredity and lineages dates from the development of agriculture (and concern over control of particular plots of land). Does this seem reasonable to you?

3. How would you describe likely interactions between farmers and hunter-gatherers?

4. How do you respond to drought if you are transitioning from hunting/ gathering to farming? What if the only life you know is farming?

5. Would you prefer to have been a nomad, farmer, or fisher in early human history? Why?

6. In how many places do you think agriculture developed independently? Why?

\section{Readings}

Beckwith, Christopher I. 2009. Empires of the Silk Road: A History of Central Eurasia from the Bronze Age to the Present. Princeton University Press.

Cunliffe, Barry. 2015. By Steppe, Desert, and Ocean: The Birth of Eurasia. New York: Oxford University Press. [Stresses the interactions between nomads and sedentary.] 
Diamond, Jared. 1997. Guns, Germs, and Steel: The Fates of Human Societies. New York: Norton. [Discusses the advantages associated with the crops and animals that different human populations could domesticate.]

Golden, Peter B. 2011. Central Asia in World History. Oxford University Press.

Laudan, Rachel. 2013. Cuisine and Empire: Cooking in World History. University of California Press.

Mazoyer, Marcel, and Laurence Roudart. 2006. A History of World Agricultures from the Neolithic Age to the Current Crisis. New York: Monthly Review Press. [Identifies a set of agrarian systems and traces their development through history.]

Scott, James C. 2017. Against the Grain: A Deep History of the Earliest States. New Haven, CT: Yale University Press.

Tauger, Mark B. 2011. Agriculture in World History. New York: Routledge.

Some students may be interested in the efforts of Gregory K. Dow and Clyde Reed to formally model such developments as the human movement toward a sedentary lifestyle, the emergence of agriculture, and the emergence of warfare. They have published articles on these topics in several journals and hope to write a book. Even students who do not wish to wade through the formal models can appreciate the well-written introductions and conclusions. Though grounded in economic theory, the papers engage climate, population, and social stratification. 


\section{Some early impacts of agriculture \\ Key technologies and trade practices}

\section{Guiding questions}

How and why and where and when and by whom was a set of critical human technologies developed during the early millennia of agriculture? How did these technologies then spread across human groups?

How, what, why, and where did farmers, nomads, and fishers trade? How, where, and why did trade expand over time? Who was involved in trade?

How and why did average incomes and income distribution change through time?

Relationship to other chapters: This chapter continues the task begun in chapter 4 of investigating critical impacts of agriculture in particular but also nomadism and fishing. The technologies discussed in this chapter remain of vital importance to humans to this day. In many later chapters, we will discuss further developments in these technologies. With respect to trade, we will consider a set of institutions that remain important to this day, though these institutions will be refined in later chapters. We also discuss a set of transport technologies that humans will improve upon in later chapters. We close the chapter with a general discussion of how technology (and science) evolves through time; we discuss technological evolution in many later chapters. 
Average incomes grow - but exceptionally slowly - through much of human history. The discussion in this chapter shows how various developments surveyed in the last few chapters (agriculture, technology, trade, and more) allowed sluggish increases in incomes. We will discuss in many later chapters why incomes did or did not increase in various times and places. In chapter 24 (and briefly in chapter 15), we will discuss how and why and when and where much more rapid rates of economic growth became possible. We also survey trends in income inequality in this chapter, noting the common experience of increased inequality. We will discuss inequality briefly in many chapters but especially in Box 29.5, where we will consider how occasional crises have served to reduce disparities.

The chapter subtitle signals that both technology and economy will be key themes in this chapter. As in the preceding chapter, the non-human environment will often shape these (we devote particular attention to transport infrastructure below). We will discuss how specific technologies evolved. At several places, cultural attitudes will interact with these other themes. Many of the new non-agricultural technologies serve to create new occupations, and thus affect social structure. The expansion of trade facilitates many types of societal interaction, though we have limited knowledge of these effects for this time period.

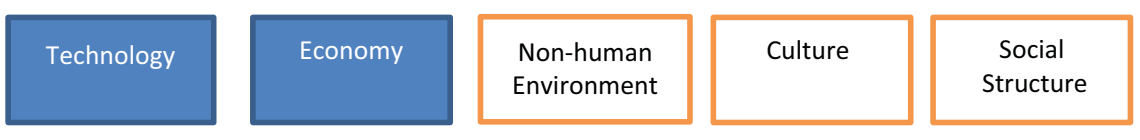

\section{Agricultural technology}

Agriculture was itself a technological innovation. More precisely, it was a set of technological innovations as different crops, soils, and climates dictate different techniques of cultivation. Agriculturalists built upon techniques used by hunters and gatherers to plant crops (digging sticks, soon supplemented by hoes), harvest grains (sickles), process these (fire, mortars), and likely store these. We have seen that this set of innovations was encouraged by population growth and perhaps animal extinction and climate change. In such a circumstance, many people may have experimented with purposeful efforts to plant wild crops. If successful, they then experimented with weeding, watering, and selecting particular seeds - and developing tools for each task. They could consciously examine which techniques worked best. This process of experimentation likely 
accelerated after the movement to settled agriculture. In Sumeria, we find sickles first made of clay, then flint, then copper. Evolutionary selection would also occur between groups: Techniques that allowed groups to feed themselves better would aid the group in competition with others.

We might devote special attention to ploughs. Turning the soil in preparation for planting was one of the most challenging agricultural tasks. Yet as humans domesticated plants it became increasingly important to plant seeds at appropriate depths. Metal ploughs could be hugely advantageous here, but only once metals were themselves produced (see below). Humans could and did plough

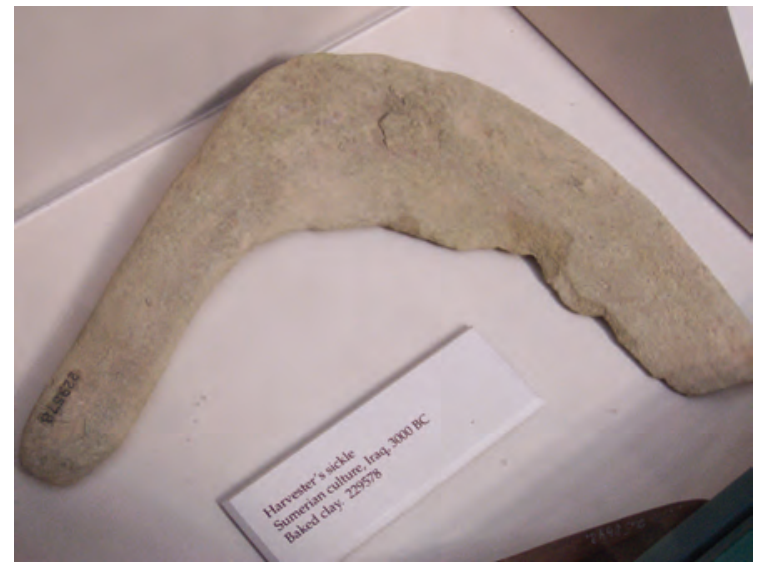

Illustration 5.1

Sumerian sickle made from clay, Field Museum, Chicago

Source: Photograph by Maksim, CC BY 2.0 themselves, but ploughing could be much more successful once horses, cows, or water buffalo were domesticated. The first ploughs merely scratched the surface but were superseded over centuries by mouldboard ploughs that turned the soil. Archaeologists have found ploughs pulled by cattle from at least 5000 вСE in the Middle East. A series of small improvements to ploughs occurred through time. Better harnesses for draft animals were also developed, which were more efficient in translating the energy of the animal into work. Though animals pulled ploughs in most parts of the world, considerable strength was required also in the human behind the plough who ensured that it dug into the earth: Men generally performed this work and this may well have contributed to the increased gender disparities noted in the previous chapter.

One indication of the importance of ploughs is that anthropologists often use the separate term "horticulture" to distinguish farming without ploughs from agriculture proper. We have not stressed this distinction in this book but can appreciate that the plough not only increases productivity but also encourages the use of animal power.

\section{The diversity of agricultural practices}

It will not be possible in this book to detail all of the different growing conditions under which humans have pursued agriculture in world history: Regions vary in rainfall, soil, topography, length of growing season, possibilities for irrigation, access to natural fertilizers, and in a variety of other ways. The reader well knows that each crop has its growing region: Wheat grows farther north than corn, and both farther north than bananas. We have noted in the preceding chapter which plants humans first domesticated where. We will detail in later chapters when and how humans moved some of the main plant and 
animal varieties across regions. We will also describe some climatic changes that affected what and how much humans could grow in particular times and places. We will discuss notable local practices from time to time, such as terracing in hilly regions. Moreover, we will consider how various technologies expanded the range in which certain crops could be grown. However, we do not have the space to detail the precise growing conditions required for each plant or animal, nor the particular practices of each locality. Even more so, we cannot specify the biological processes involved - though we can from time to time express the limitations that these impose on human actors. As was noted in the last chapter concerning nitrogen, though, the reader should be aware that agriculture everywhere requires an integrated set of practices tailored to particular plants and places: It is much more than just planting some seeds.

\section{Irrigation}

Agriculturalists first relied on rainfall, though very early they may have diverted small streams to water their fields. Diverting large rivers onto fields was a much more challenging task. It required not just a lot of labour but careful planning to achieve the desired water flow. The challenges increased the more extensive the area irrigated. However, irrigation potentially reduced harvest fluctuations quite dramatically and allowed agriculture to spread to areas that did not receive enough rainfall.

Scholars long thought that the extensive irrigation schemes of Mesopotamia, China, Egypt, and India required large states. It seems, though, that the earliest irrigation efforts - and even some extensive irrigation schemes - predated organized states. Nevertheless, the more substantial systems of irrigation did likely require a state with a bureaucracy to supervise the construction and maintenance of such schemes. We will say much more about irrigation in later chapters.

There was considerable regional diversity in irrigation schemes. The Sumerians had to hold water in canals for months, and only then flood fields after the summer heat had passed. It was hard to avoid retaining salt in such a situation. Northern China wrestled with both irrigation and flood control: Even during the later Han dynasty (chapter 10), the Yellow River flooded every six years on average.

\section{Fermentation}

One challenge faced by humans - and especially urban humans - throughout most of human history was obtaining clean drinking water. Human wastes polluted water sources near urban centres. There was then an important health advantage to treating beverages in some way. Early humans did not know the germ theory of disease but could have come to appreciate that they were healthier when they drank fermented beverages. They indeed celebrated the beneficial health effects of beer and wine across early human societies. Alcohol 
was also widely used as a painkiller. Alcoholic drinks also stored well. Of course, people also valued alcoholic beverages for other effects.

Alcohol appears to have been associated with agriculture from very early days. Archaeologists have discovered a pot with traces of wine from about 7000 BCE in the Zagros Mountains of southwestern Iran. Early farmers likely discovered fermentation by accident as they soaked grains for eating; ambient yeast would simply have dropped in. Humans then selected yeasts over time for both alcohol and bread making. Fermentation technology was likely first applied to make beers from grains, and later to grapes to produce wine. In a co-evolutionary process, humans must have developed an ability to metabolize alcohol over time - another rare example of human genetic evolution in historical time.

It is entirely possible that hunter-gatherers had fermented drinks, though we have no direct evidence. They could have discovered fermentation as they experimented with grains. This might even have encouraged them to grow grains. Certainly, nomads would develop intoxicating beverages by fermenting milk.

Barley is especially useful for making beer (and wheat for making bread). Early agriculturalists would have experimented and discovered which local crops were best suited to different purposes. It seems that barley first became popular because of its beer-making potential (though it was also resistant to salt and thus well suited to Mesopotamian soils). Later, Sumerians consumed 40 per cent of their barley crop as beer. The beer produced in Sumeria, and likely in most ancient societies, was lumpy, and they therefore drank it through straws. It would also have tasted quite different than it usually does today: Important ingredients in most modern beers such as hops were unknown at the time. The Chinese fermented millet.

Ancient texts in India (the Vedas) and Egypt have mixed opinions of wine, praising it but warning of drunkenness. Grapes were domesticated in many places by 3000 вCE for winemaking - even in places like Egypt where there were no wild vines.

The whole population, including children, drank beer and wine. The alcohol content was probably low in general. Pregnant women likely did not drink enough (usually) to trigger fetal alcohol spectrum disorder, though some adverse effect on fetal development is entirely possible.

Though we have stressed alcoholic fermentation here, we should appreciate that both bread making and cheese

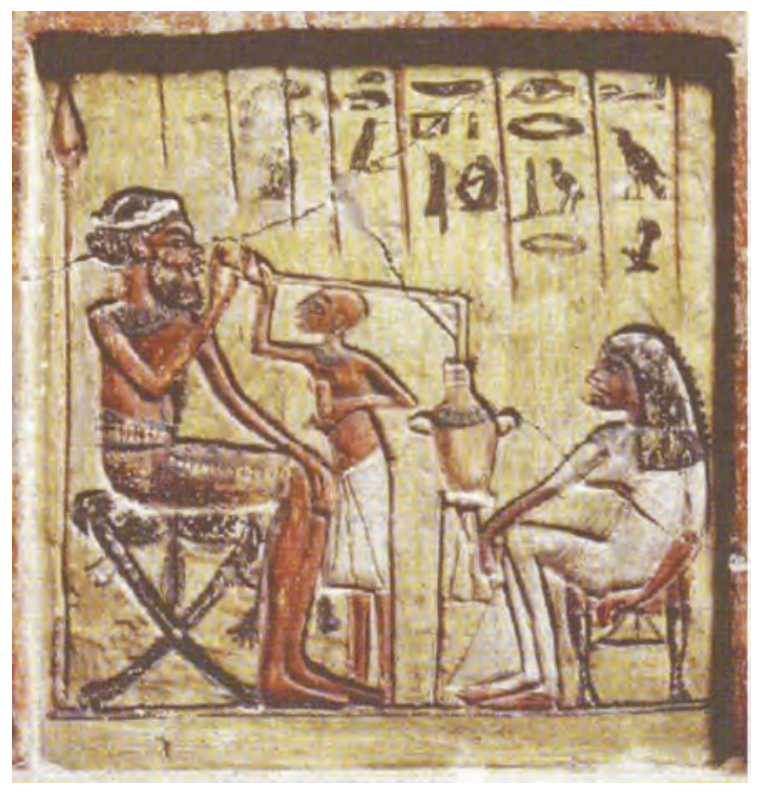

Illustration $\mathbf{5 . 2}$

Drinking beer through a straw, Ancient Egypt 


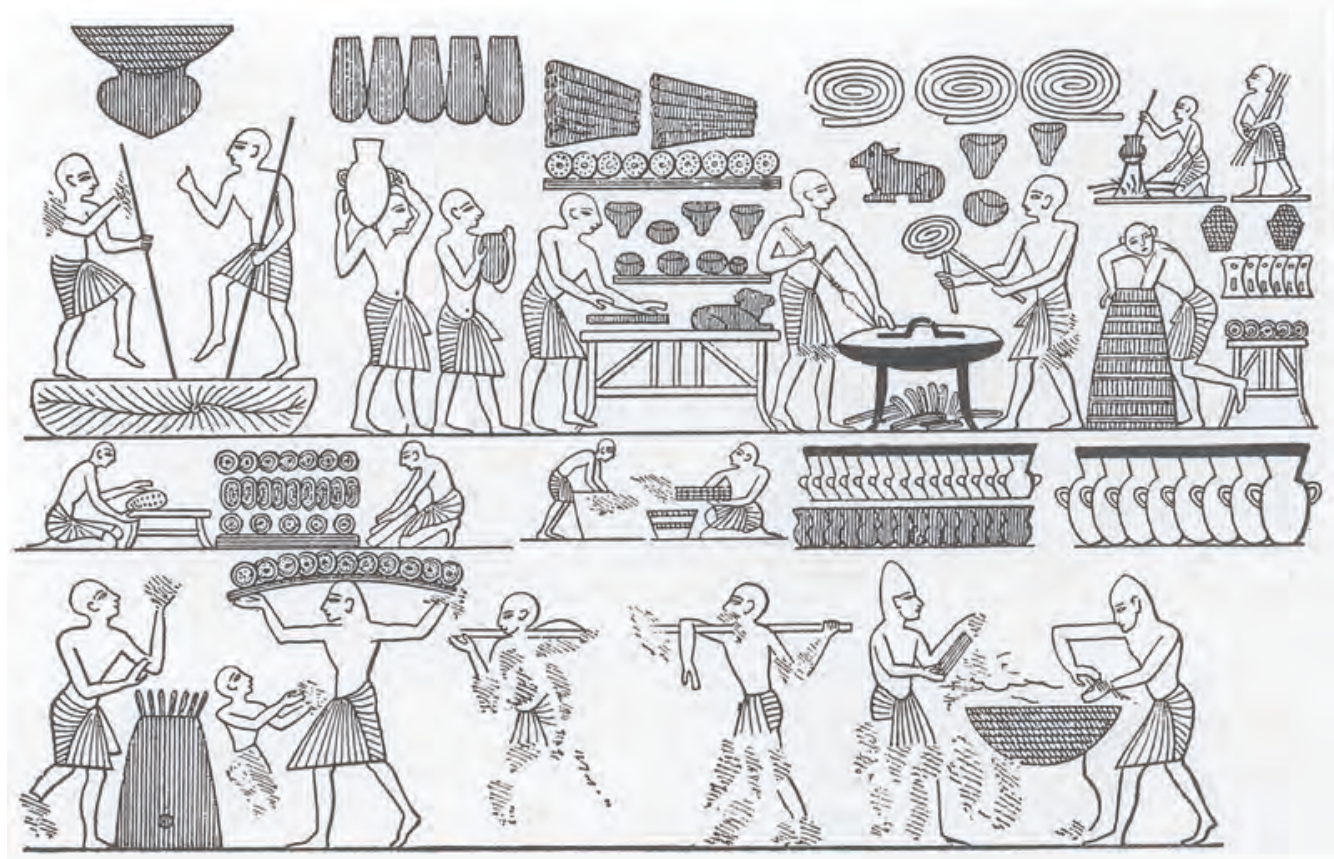

Illustration $\mathbf{5 . 3}$

Court bakery, from the tomb of Ramesses III in the Valley of the Kings, twentieth dynasty of Egypt Note that some loaves are shaped like animals

making are types of fermentation. The former was a way of making grains, and especially wheat, edible. The latter allowed storage of milk products for much more extended periods.

\section{Vegetable oils}

We noted in chapter 4 that most of the crops grown by humans were domesticated thousands of years ago. One of the later adoptions was the olive tree. These were domesticated in Jordan about 4000 всE. (Dates were domesticated at about the same time.) Olives grew wild in very few places. The oil of olives - and also oil from wild palm trees and sesame plants - was much valued as a fuel (likely its first use) and came over time to be used as a preservative, condiment, cooking oil, lubricant, perfume, and soap ingredient. As with textiles (below), the development of olive agriculture reflects the ability of agricultural societies to move beyond a focus on their most basic nutritional needs.

\section{Non-agricultural technologies}

Humans also developed an important set of technologies only indirectly related to agriculture during the first millennia of settled agriculture: pottery, bricks, 


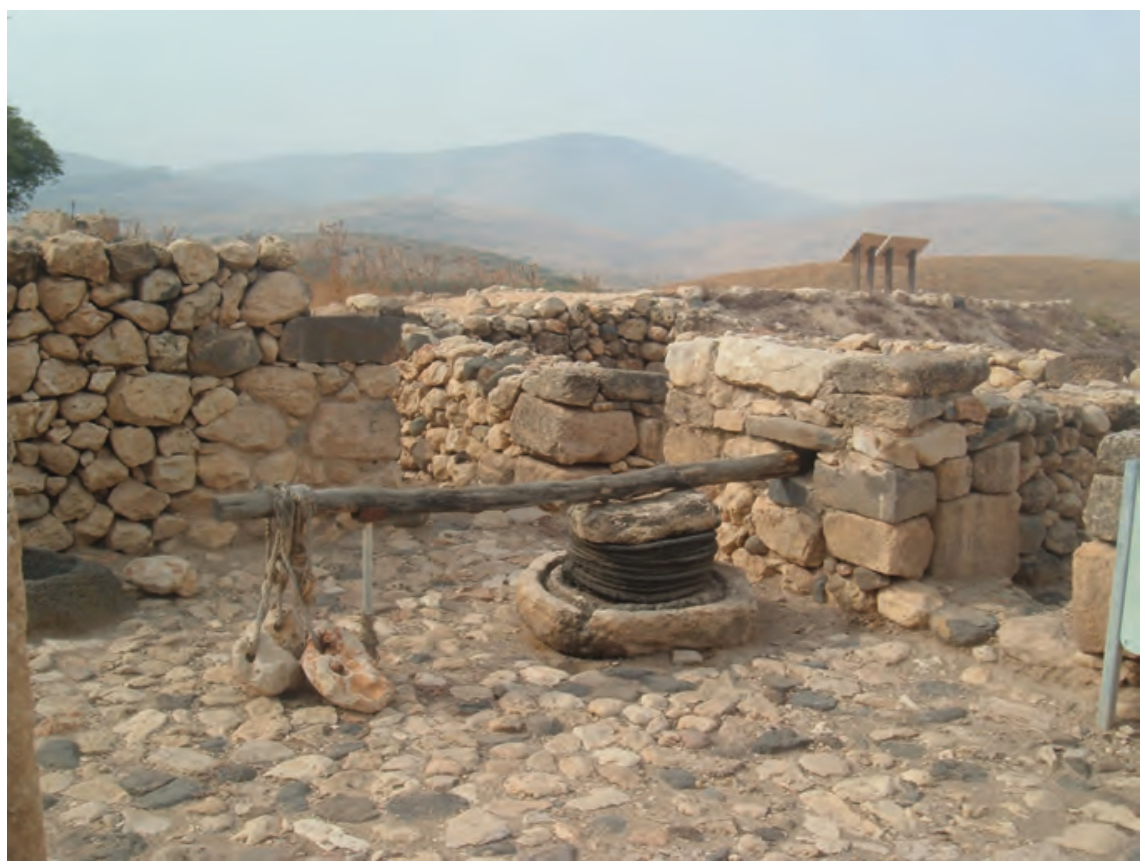

Illustration 5.4

Ancient oil press in Tel-Hatzor, Israel, an important trading centre in the first and second millennium BCE, mentioned in the Bible

glass, textiles, and metals. These technologies all reflect in part the existence of an agricultural surplus such that some human effort could be devoted to nonfood production. They also reflect in part a sedentary lifestyle: People could make use of a wider range of products than when they were mobile. All of the new technologies were employed in producing goods for personal consumption. They also had positive effects on agricultural production or the storage of agricultural goods. Though we know little about the details of the development of these technologies, we can be sure that these were gradual evolutionary processes in which the sharing and transmission of ideas between individuals was essential. A sedentary lifestyle facilitated the personal interactions necessary for technological evolution. Finally we can note the importance of trade (which we discuss below), for these technologies often depended on the movement both of raw materials and of finished products. Technological innovations likely flowed between societies with merchants and goods.

\section{Pottery}

Archaeologists study pottery in much detail for a variety of reasons. It is an important consumer item in early human societies. It was used to store a variety of foods, and for serving these. It lent itself to artistry: Indeed humans used fired clay for figurines long before they fired bowls. Decorated pottery 
combined use and aesthetics. Like any artwork, pottery also reflected culture: Archaeologists often trace cultural influences by identifying similarities in the designs on pottery. While it was once common to assume that differences in pottery styles reflected differences in culture more generally, it is now appreciated that groups that were quite different in other ways might borrow pottery styles or trade pottery. Archaeologists also trace the transmission of pottery-making technology, which provides evidence of the technical capabilities of different human groups, and the degree of their interaction. That leads us to perhaps the greatest single reason that archaeologists devote so much effort to pottery: Pottery survives very well, and often provides the greatest physical evidence of early human societies. Indeed, archaeologists only know certain societies by their pottery.

We might have expected that pottery would emerge soon after humans adopted a sedentary lifestyle. Mobile human populations would not wish to carry heavy pots, and in any case had little to store in these. On the other hand, sedentary populations, and especially those that harvested crops, had both the ability and the need to store foodstuffs both across seasons (the harvest must feed them for months) and years (for some harvests would always be better than others). Yet the historical record surprises us. In both East Asia and the Middle East, there is evidence of pottery long before sedentary agricultural lifestyles emerge. Indeed the first known ornamental pottery - baked clay figurines dates from 26,000 years ago in the Czech Republic, and the first utilitarian pottery vessels date from 10,000 BCE in Japan (maybe much earlier), and 8500 BCE in the Middle East. Nomads in what is now Russia seem to have had pottery from 7000 вСе, as early as farmers farther south. The earliest pottery in Thailand dates from before 3500 вСЕ, which may predate settled agriculture by a millennium. In the Americas, the earliest pottery is found in the Amazon (6000-5500 $\mathrm{BCE}$ ) and what is now South Carolina (2500 BCE) rather than in Mesoamerica (1000 BCE), where sedentary agriculture developed first. Even mobile humans often stayed in one place for a while, and thus had some time and incentive for pottery if suitable clays were found. The Jomon culture of Japan (see chapter 4), for example, involved hunter-gatherers who could stay in one place for long periods because of rich fishing grounds.

On the other hand, in both the Middle East and South Asia the archaeological record indicates that a thousand years or more might pass in particular localities between agricultural settlement and the use of pottery. Humans could and did store food in other receptacles. Natural gourds and coconuts or leather pouches could store liquids, and baskets could be used to store and transport grains. Nevertheless, pottery is particularly effective at protecting food from both pests and the weather. Pottery was also notable for getting stronger in fire, whereas fire destroys other materials: Storage in pots then safeguarded foods against fire. It is thus remarkable that agricultural societies did not adopt pottery quickly, especially given its existence for millennia in other places. It must then seem that the development of pottery making is no simple response 
to the need for storage: Human groups must find suitable clays and develop appropriate technologies for firing these.

To make pottery, one must first recognize that moulded wet clay will maintain its shape if heated (so that the water evaporated), and then one must develop techniques for achieving temperatures of at least 200 degrees Celsius but ideally 1200 degrees Celsius. The composition of the clay, the precise firing technique, and the addition of other materials will each have significant effects on the appearance of the final product. Many materials, including sand and flint, if added in small amounts, facilitate more even drying. Pottery makers experimented with different "recipes," and discovered a variety of new combinations over time. Ovens - first underground, then above ground - were developed from about 7500 все in the Middle East to achieve much higher temperatures than an open fire. Note that while pottery had existed before agriculture, significant advances in pottery making were associated with agricultural societies.

Pottery makers almost immediately decorated their wares. Though some undecorated pots were utilized for transport, most pottery destined for household

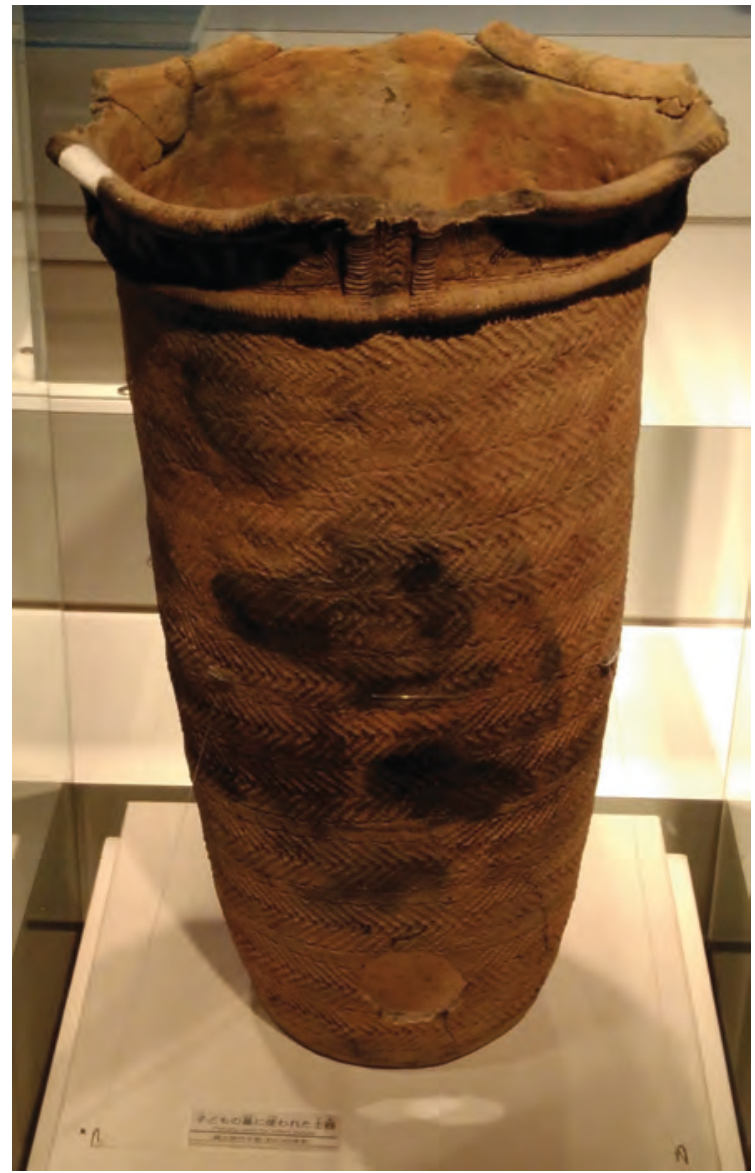

\section{Illustration $\mathbf{5 . 5}$}

Jomon pottery used for infant burial, Jomon Museum at Sannai Maruyama

Source: Photograph by G4lrn8, CC BY-SA 4.0 use received at least a little decorative effort. Some pottery bore elaborately coloured designs or pictures. Production of beautiful pottery in early societies is indicative of income inequality, for only a narrow elite could have afforded the best pottery. Decorated pottery of any sort required another set of technological developments: recognizing which substances would produce which colours under firing, developing glazes, increasing firing temperatures, and so on. The pottery wheel - on which wet clay rotated while being shaped - dates from the fifth millennium BCE in Mesopotamia and facilitated the production of round pots. Pottery wheels appear in China from at least the late fourth millennium вСE.

We should note that pottery was used for cooking as well as storing: Its resistance to heat made this feasible. Cooking with pots allowed humans to mix ingredients in a way that was not feasible if roasting meat on a spit over the fire. 


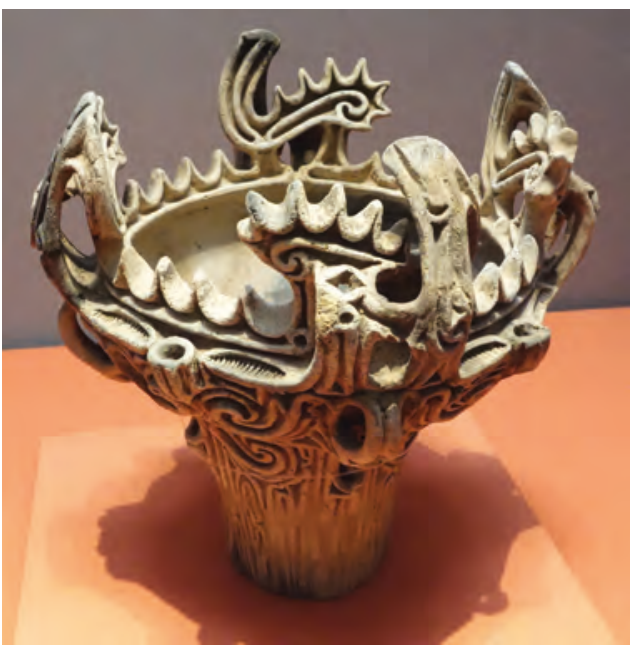

Illustration $\mathbf{5 . 6}$

Jomon pottery vessel with flame-like ornamentation, 3000-2000 BCE, Tokyo National Museum

Source: Photograph by Daderot, CC0

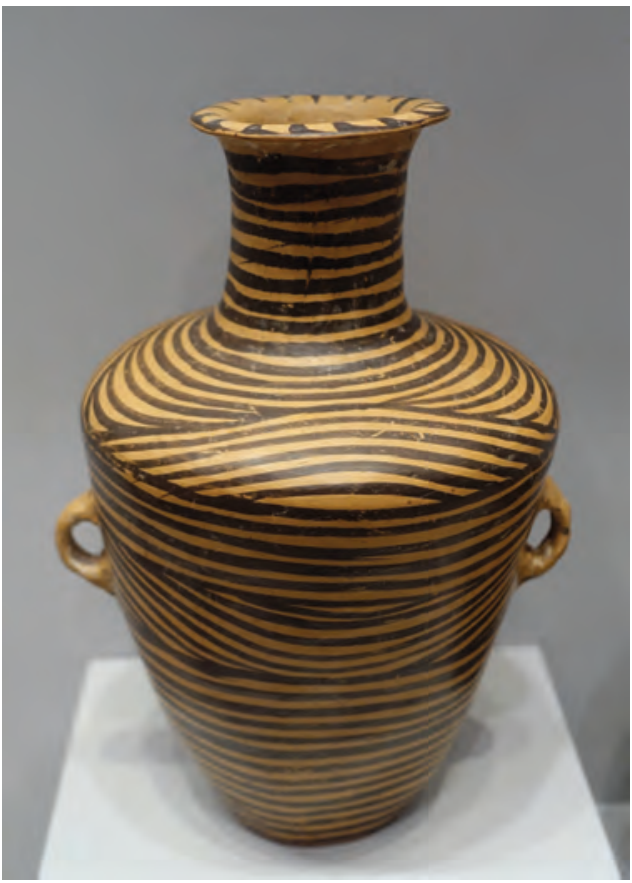

Illustration 5.7

Long-necked jar, Gansu, Qinghai, or Ningxia province, China, 3300-2900 BCE, Arthur M. Sackler Museum, Harvard University Source: Photograph by Daderot, CC0 1.0
Even early human societies had a few different foods they could mix. The development of pottery allowed humans to develop sophisticated recipes that could appeal to humans' natural appreciation of food mixtures (which may reflect humanity's diverse nutritional needs). Cuisine of any sort largely depended on cooking vessels of first pottery and later metal. The Jomon were not only the first potters, but analysis of ancient pots establishes that they used these pots to cook soups and stews (combining seafood, meat, vegetables, and nuts) long before any other human group could do so.

\section{Brick}

Mesopotamians along the Tigris River had built with sun-dried bricks from at least 7500 всE. They used straw or some other substance to bind the clay together. However, a much stronger brick was possible with clay that was fired as in making pottery. The first known use of fired bricks occurs in the Indus Valley around 3000 BCE, but we find fired bricks in the second millennium BCE in both Mesopotamia and China. Brick has remained a common building material to this day (with developments in the chemical composition to enhance strength and durability), especially in parts of the world with limited wood resources. Even where wood is abundant, brick has the critical advantage of being resistant to fire.

\section{Glass}

Glass is a solid that retains some of the qualities of a liquid when cooled. It is generally made from a mixture of sand, soda, and lime. It was discovered first in Egypt (perhaps Mesopotamia) before 3000 BCE, perhaps accidentally while making pottery. Glass was used first as a glaze. However, within centuries glass vessels were produced throughout the Middle East. Small windows were also then possible. Nevertheless, glass remained expensive and relatively rare until the first millennium вСE. The technique of glassblowing is observed 
from the first century $\mathrm{BCE}$ in Greece. Techniques for casting glassware also improve through time. Glass technology spread to China by 500 вСе at the latest.

\section{Textiles}

When humans first turned to agriculture, they naturally focused on plants - and then animals - that they could utilize for food. Over time, though, they recognized that they could use some plants and animals for the production of textiles. By spinning and weaving the fibres from particular plants or animals, humans could achieve clothing that both fit better and could breathe better than the animal hides they had previously worn. They could enhance both human comfort and human productivity by wearing such textiles. In addition, most of these fibres could be dyed: As with pottery, humans soon decorated their textiles.

Textile production requires an interrelated set of technologies: to first grow the plant or animal fibres, to then clean and prepare these, to spin, to weave, and perhaps to dye, and then produce items of clothing or furnishing.

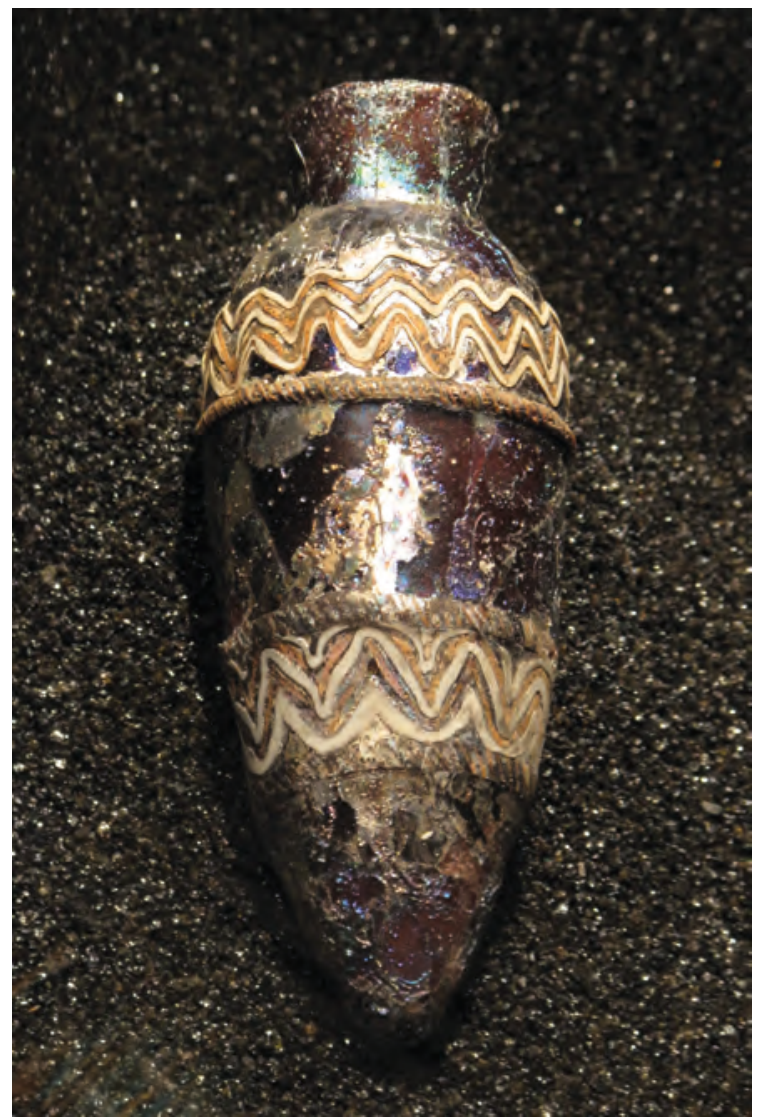

Illustration 5.8

Bottle, probably northwestern Iran, 6th century $\mathrm{BCE}$, the Landesmuseum, Württemberg

Source: Photograph by Rama, CC BY-SA $2.0 \mathrm{fr}$ How could this interrelated complex of technologies develop? Why develop any one technique if the others did not yet exist? The key may well have been spinning, and thus the appreciation that small natural fibres of wool or cotton or silk could be twisted together to form long but strong fibres. In this, early agriculturalists could build upon a rope-making practice using much larger plant fibres that dates to 30,000 years ago. It may then have been a small step to appreciate that such threads could be woven into cloth (basket weaving using reeds or rushes could provide an example here and had a long prehistory). Once humans appreciated the possibilities of spinning and weaving, then they would make small advances in each of the various stages in textile production.

The first woven textile was linen from flax; this was first produced in Israel or Turkey from about 7200-7000 BCE. Flax needs to be soaked and beaten to separate the fibres. It is a bit surprising that humans used flax before other fibres that require less initial processing. These beaten fibres then needed to be spun and woven. Linen was the source of most clothing in ancient Egypt, 
and Egyptian linens were prized elsewhere. Linen does not hold dye as easily as other natural fibres, and as a result, the classic Egyptian linen cloth was white. As humans domesticated flax to produce linen cloth, there would have been selection over time for plants that produced stronger and longer fibres.

Cotton grows wild in the Americas, India, and Africa. Its fibres can be spun after being cleaned. The South American variety of cotton now dominates in world markets, but it was the Indian variety that was first spun in Pakistan in the sixth millennium всE. Many different grades of cotton cloth were produced in the Indus Valley, differing in terms of the thickness of thread, tightness of weave, and quality of dyeing. Cotton cloth was likely exported to Mesopotamia; Mesopotamia would in turn export cotton cloth to Anatolia from at least the second millennium вCE. A new loom for weaving from about 2000 вСE in Anatolia allowed both much faster weaving and the creation of designs that were more complex. Knowledge of cotton spinning and weaving had reached China by the time of the Eastern Zhou dynasty (770-221 BCE; chapter 7), and would later reach Southeast Asia. Textiles do not preserve as well as pottery, and so we have far fewer specimens of early textiles. One exception here is cotton from the Andes, which was woven from at least the fourth millennium BCE, and where aridity has preserved considerable quantities of early textiles.

We might think that wool should have been the first textile: Surely, one merely had to shear the sheep in order to develop woollen textiles? Indeed, there are some suggestions that the first woollen cloths might have predated linen or cotton. Humans had domesticated sheep by 9000-7000 BCE. However, the first domesticated sheep had hair more like modern deer than like modern sheep. Humans had domesticated sheep for their meat and milk. Nevertheless, they used their hides in clothing. Over time, there was selection by farmers for softer, thicker hides and thus fibres. (Nomads may have played an essential role in this selection process, for they herded sheep in colder climates and also used hides for clothing.) It may have been only about 5000 вСе when wool fibres came to be spun and woven into cloth.

The Chinese may have spun hemp (from the cannabis plant) from 6500 вСе. They then wove these fibres into a cloth that somewhat resembled linen, and this would be the most common textile in China for millennia (hemp seeds were eaten as cereal and used as cooking oil). However, China would become famous for another fibre: silk. Silk cloth was produced from at least 3000 BCE in China. Silkworms grow wild throughout Eurasia, but silk fibres in the wild are short and hard. There was selective breeding of moths to produce higher-quality silk. Eggs were kept at an ideal temperature until they hatched; then worms were fed mulberry leaves to achieve a perfect weight before they began spinning a cocoon made of silk fibres. This complicated process could only have developed slowly over time, and silk always remained a more expensive product than other textiles. Silk fibres produced in this way were much softer than other fibres; Chinese silk would become famous throughout Eurasia for its quality.

Clothing styles differed across ancient societies. Within societies, the quality of their clothing distinguished elites from the masses: whiter linens in Egypt, 
fancier dyes in Mesopotamia, silk in China. Elite women everywhere came to wear more elaborate clothing than men; this was in many places accompanied by equally elaborate hairstyles. (Men shaved in Egypt, wore beards elsewhere, and wore long hair in some places.)

\section{Metals and mining}

Most metals are harder and sharper than almost any stone, and thus potentially far superior for a variety of tasks, such as chopping trees, ploughing soils, and killing other humans in war. Humans would also employ metals artistically, especially at first in human decoration. The discovery and application of metals is perhaps the most crucial set of technological developments in early human history.

The first uses by humans of metals occurred during the first thousands of years of settled agriculture. The first metals humans used (non-decoratively) were copper, tin, and especially bronze - an alloy of copper and tin that is much harder than either. We can date metal use for tools or weapons from about 6000 BCE (maybe as early as 7000 BCE), though humans had used copper for decorative purposes thousands of years earlier. Copper was utilized first in the Middle East; copper smelting spreads to Europe, Egypt, and other parts of Asia over the next millennia.

There are small surface deposits of copper in many parts of the world. Nevertheless, trade over hundreds of kilometres or more was often necessary. The agricultural heartland of the Middle East, in particular, lacked extensive mineral resources. Mesopotamia imported copper from both Persia and Anatolia. Archaeologists have investigated shallow mines dating from 3500 вСЕ in present-day Poland and Hungary; Eastern Europe seems to have soon become the primary source of metal for the Middle East. Oman and Cyprus would also become important sources of copper.

We do not know exactly how the metal deposits located among nonagricultural populations were first paired with their uses among agricultural communities. Members of sedentary societies may have sought these out. Alternatively, nomads may have first noticed brightly coloured rocks as they travelled, and discovered that they could pound these into shapes for ornamentation and useful items such as fishhooks. Beaten copper goods may have been traded for some time - much like precious stones such as lapis lazuli from Afghanistan that was highly valued in Mesopotamia - before humans discovered smelting: heating copper ores enough to separate metal from rock (below). Members of non-agricultural societies would then often take the lead in mining and even smelting. Note that smelting near the source of the metal would reduce the bulk that needed to be transported.

In some cases, agricultural societies may have established mining (and farming if possible) colonies near mineral resources (this may have been the case in the Rio Tinto region of southern Spain from 1700 BCE), but it seems that minerals were more often obtained by trade. Trade in metals encouraged extensive contact between agricultural and non-agricultural societies, and thus 


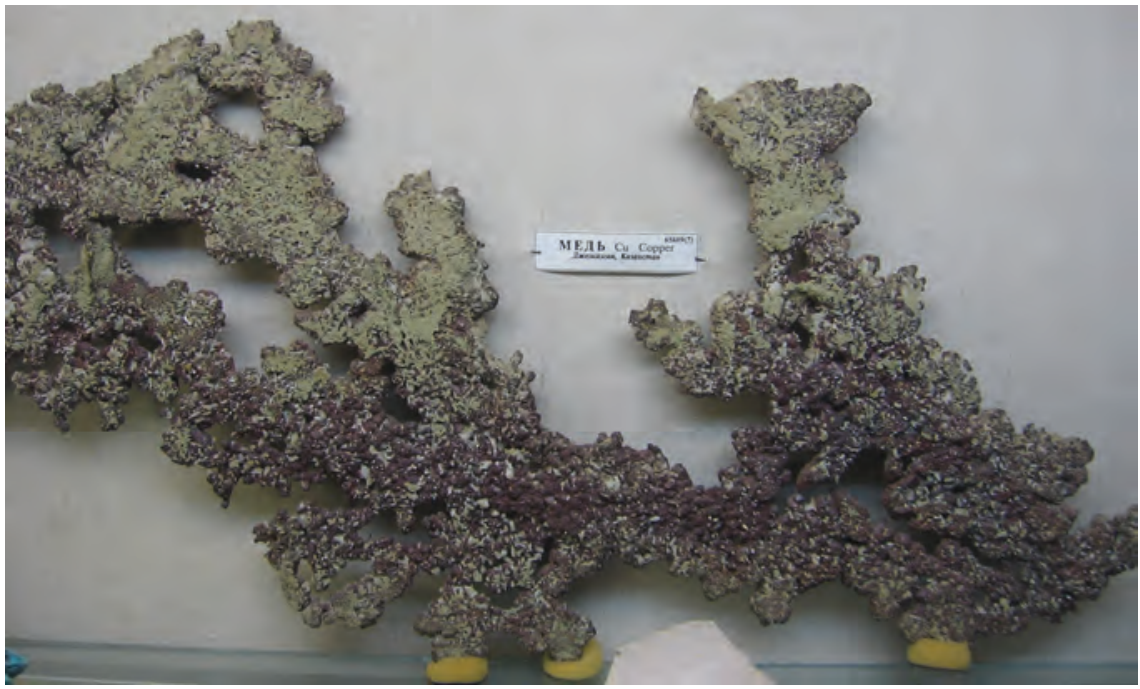

Illustration 5.9

Copper mineral

the sharing of both cultural and technological information: This exchange may well have facilitated the spread of agriculture itself. This last point deserves emphasis: Whereas natural resource exports in the late twentieth century $\mathrm{CE}$ have not always encouraged economic development (chapter 30), natural resource exports in the ancient world appear indeed to have helped resourceexporting societies to develop in the direction of resource-importing societies. Persia, Greece, and the Ganges region each enter world history exporting minerals to urban centres elsewhere. These resource-exporting societies were apparently able to retain much of the profit from this trade and devote some of this wealth to investment in industry or agriculture.

Although trade among societies predated extensive metal use, trade links would expand significantly as metal technology was developed. Merchant networks emerged to connect mines and markets (see below), and government bureaucracies to tax (and occasionally organize) this trade, negotiate with other polities involved, and ensure that the state had access to metals for military use. The movement of heavy metals over long distances likely inspired the development of both sturdier boats and wheeled vehicles (see below).

There is evidence of copper being heated from before 5000 BCE. From only after 4000 вСЕ is there evidence of mining for copper (rather than just using surface deposits) or of smelting. Copper needs to be heated to well above 1000 degrees Celsius before it becomes molten. Smelting could produce much higher-quality copper than merely beating unsmelted copper ores. Copper casting - pouring molten copper into moulds rather than hammering it into shape - also first occurs after 4000 вСE. Metallurgists may have used the oven technology developed for pottery to smelt copper and to heat it to liquid form for pouring into a mould. 
Though we cannot know the precise process by which humans first came to use metal, we can reasonably hypothesize a set of evolutionary steps. First, humans had to appreciate surface outcrops of copper as a material that was much harder and potentially sharper - or at least prettier - than stone, wood, or even obsidian. Then they had to recognize that by heating this substance it became much easier to form it into desired shapes. Increasing the heat - which required ovens rather than open fires - enhanced the quality of the metal, though these early metallurgists had no idea of the precise chemical reactions they induced. Early metallurgy was a process of experimentation. As higher temperatures were obtained, casting became possible.

By the early third millennium BCE, bronze was used in China, the Indus civilization, and the Middle East. Egypt used bronze by 2000 вСе, but its use was only widespread in Egypt after the invading Hyksos from the Middle East demonstrated the advantages of bronze weapons from 1700 вСЕ (see chapter 7). Bronze also was used in Southeast Asia from about 2000 BCE, though widespread use may have come only about 500 BCE, at which point finely decorated bronze drums could be found throughout much of the region. It is not clear if the people of Southeast Asia borrowed bronze technology from China or developed it independently.

One challenge in manufacturing bronze is that tin is much rarer than copper on the earth's surface. By 1500 вСЕ tin was imported to the Middle East from both Cornwall in England and Afghanistan in Central Asia. Humans must have discovered the value of tin before they would transport it hundreds of kilometres. It seems likely that small tin deposits nearer to copper deposits were exploited first for bronze manufacture. We cannot know why some early metallurgists experimented with heating copper and tin together; it may have been an accidental discovery. It may be that bronze was discovered independently in two or three places. Nevertheless, it may well have reflected the experimental orientation of metallurgists who were accustomed to trying different "recipes" and evaluating the results. Once they created bronze, metallurgists would have readily appreciated the fact that it

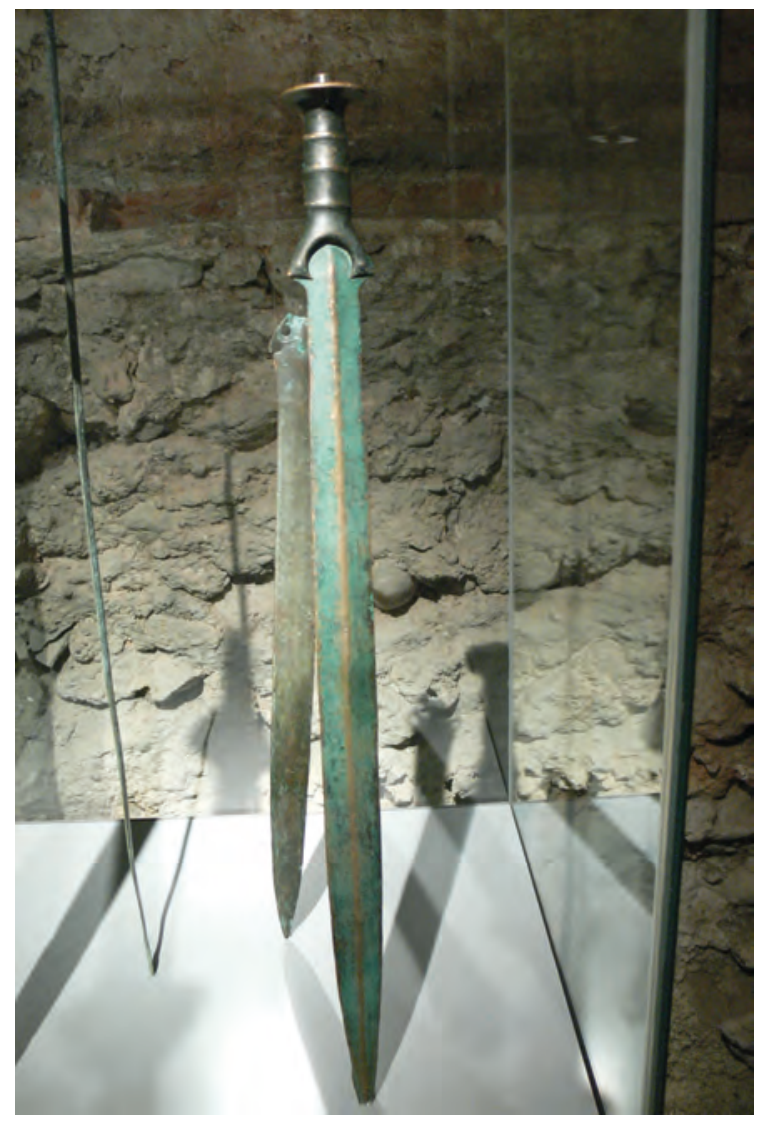

Illustration 5.10

Ancient bronze sword, City Museum, Wels, Austria Source: Photograph by Wolfgang Sauber, CC BY-SA 3.0 
was much harder than either copper or tin. This would have spawned further experiments to identify the best ratio of copper and tin.

The advances in agricultural and industrial practices - and military weaponry - associated with bronze tools are so significant that historians often celebrate a "bronze age." We avoid phrases such as "bronze age" and "iron age" in this book as they privilege one set of technological advances over all other historical processes. We shall see that societies in the Americas accomplished much with no or limited use of either bronze or iron. Nevertheless, we can appreciate that first bronze, and later iron, had a considerable impact both economically and militarily.

Across Eurasia, bronze came to be used for both spears and armour. Inevitably, some polities acquired bronze before others: The formation of the Akkadian Empire in Mesopotamia (chapter 7) may mostly reflect its early adoption of bronze weaponry. Bronze was also used for decorative purposes, such as bronze figurines or plaques. Since bronze was expensive, it was useful as a display of wealth. Nevertheless, artisans came over time to use bronze in artisanal tools, and farmers employed bronze as the cutting tool on ploughs. There is some evidence that human stature increased with the "Bronze Age," though perhaps especially among the elite. This could be indicative of the increased agricultural productivity associated with bronze tools. Population growth in this period (and later with iron) is further evidence of enhanced productivity.

In the Americas, indigenous groups near the Great Lakes first employed (but did not smelt) copper, perhaps as early as 5000 BCE. Knowledge spread south from there. Copper was known in Mexico, but there were limited copper deposits in Mesoamerica, and so it was used sparingly (and smelting dates from only about $800 \mathrm{CE}$ ). There were even more limited tin supplies - though the Spanish would use local tin to smelt bronze cannons in the early sixteenth century CE. It does not seem that Mesoamericans knew of bronze technology before the arrival of Europeans (chapter 19); if it was known, its use was infrequent and recent. Instead, Mesoamericans mined jade and obsidian and traded these long distances for use as tools, weapons, and decorations. The Mexican "failure" to develop bronze technology may reflect the abundance of obsidian: This can be sharper than steel, would be adopted by the Spanish for tools and weapons, and is even used in the twenty-first century for eye surgery. In South America, copper was employed from about 1400 BCE and smelted from 200 BCE, but generally used for small ornaments rather than tools and weapons. Again, there may have been rare use of bronze.

Note that with metalworking we change not just the shape but also the chemical composition of materials. Smelted metals are entirely different in form and function from the ores from which they are produced. Bronze is even more remarkable, being much harder than either copper or tin. Humans must have been quite amazed when they first discovered their ability to produce useful substances that did not naturally exist. Other people likely viewed early metallurgists with esteem and even attributed magical power to them. Though 
metallurgists did not understand the chemical processes they were inducing, they could through careful record-keeping establish precise relationships between raw materials, production techniques, and output. They often guarded this information closely within families or guilds (see our discussion of artisans below).

Humans smelted iron from meteorites from 2500 все, and from local ores from 1600 вСе. Iron is harder, stronger, and maintains a sharp edge better than bronze (bronze tools and weapons had to be regularly sharpened). However, these advantages were only apparent with the development of techniques for heating iron to high temperatures. Iron melts only at 1540 degrees Celsius, though this number falls to about 1200 degrees if the metal is heated in combination with carbon. Iron on its own becomes somewhat sponge-like at 1200 degrees and then could be hammered into various shapes - but the result was usually brittle. The Hittites in Anatolia from about 1300 все were able to smelt an iron that was not brittle; this technology would spread over time through Eurasia. There is debate as to whether West African metallurgists independently smelted iron from sometime after 2000 все. The Bantu may have produced iron as early as anyone - though the quality of their ores and scale of deposits may have limited the quality of their iron products. Iron was especially important in Africa because there seems not to have been a previous use of bronze. Chinese metallurgists smelted iron from at least 600 вСе, though iron supplants bronze only slowly over the next centuries. However, the Chinese may have more frequently achieved the high temperatures required for casting iron goods. Iron was widely used for weapons and tools in the first centuries BCE across Eurasia and parts of Africa. In addition to its other advantages, iron was far less expensive than bronze. Iron ore is also far more widespread on the earth's surface than copper and especially tin.

There were some experiments with steel from the tenth century всE. We now know that steel is iron with a 2 per cent carbon content, but metallurgists could only achieve this precise combination at the time by carefully mixing inputs in precise proportions determined by careful experimentation. Steel was prized for its particular sharpness, especially in weapons.

Harder, sharper, and cheaper than bronze, iron would have dramatic effects in both civilian and military uses. Indeed, historians often speak of an "Iron Age" from about 1000 вСЕ. We will discuss the military impact in later chapters but can note here that iron armour may have been an important factor in the development of classical empires (chapter 9). The Jewish conquests of Canaan celebrated in the biblical Old Testament (chapter 11) may also have owed much to superior iron weaponry. As for agriculture, iron ploughs were far better and far more widely employed than bronze ploughs, with even more dramatic effects on agricultural productivity. We will encounter iron (but also bronze) in myriad industrial uses in later chapters. We can note here that iron was combined with the idea of the wheel (see below) in industrial applications and in pumps for irrigation. 


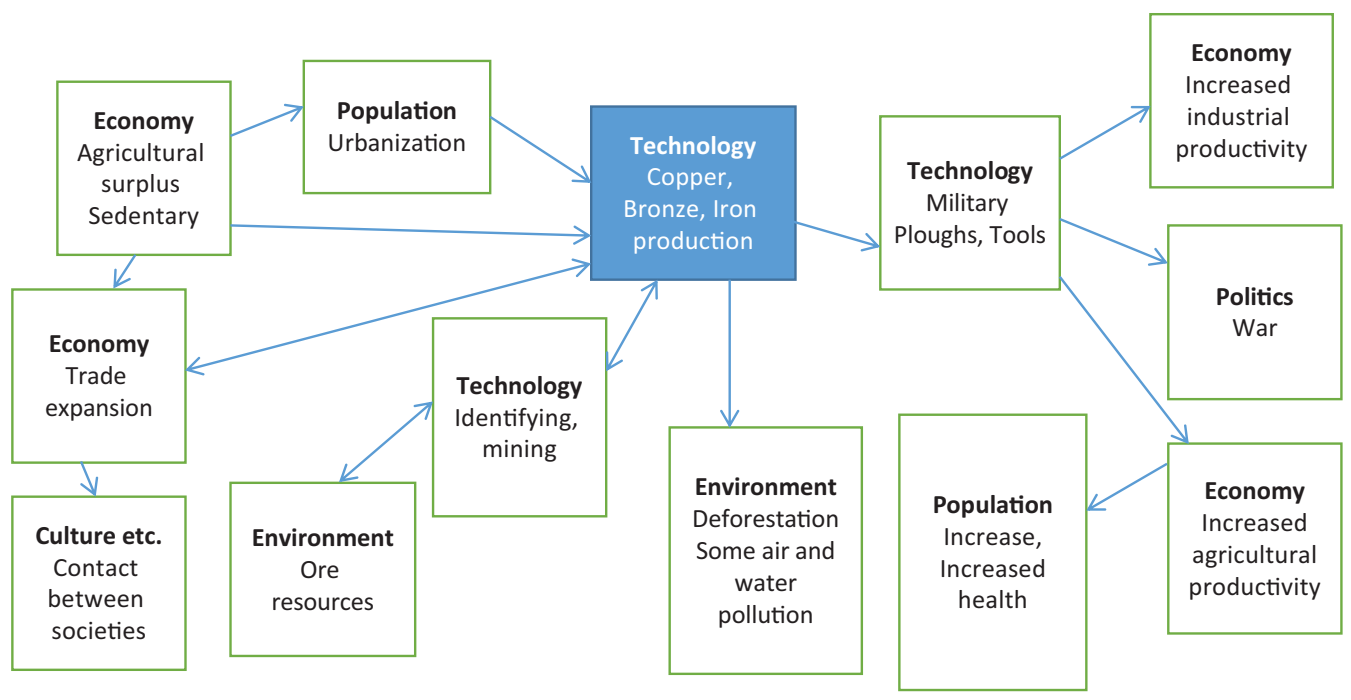

\section{Figure 5.1}

Influences on and effects of metals technology

Note: There are feedback effects on the lower left-hand side, especially between metals and trade. The arrows on the upper left capture relationships discussed at the opening of this section on nonagricultural technology. We could add a negative impact on health from local pollution.

Copper, tin, and iron were not the only minerals used but were by far the most important for agriculture and industry and the military. Gold and silver were of little use economically at first but were prized for their aesthetic value. They would become important for trade, though, because they came to be used as currency - precisely because they were expensive (see below).

Metal production, even in early days, had environmental impacts. As metal use expanded, there was an increased demand for wood to fuel the fires needed for smelting. In sites of heavy mineral production, this could cause deforestation. There was also local air and water pollution. For example, scientists have identified severe water pollution in a small stream in southern Jordan dating from some 7,000 years ago. Metal producers often located outside of towns to avoid polluting local air and water.

\section{Other technologies}

There were other technologies associated with settled agriculture. Most obviously, as humans settled in one place, they developed technological understandings of how to build permanent structures using mud brick, wood, and stone. We will talk a bit about this when we address cities in chapter 8 . There were also technologies associated with transport that we will discuss below. 


\section{THE THEME OF TECHNOLOGY AND SCIENCE}

Humans develop knowledge of how the world works and how we can best manipulate the non-human environment to suit various ends. General understandings of how the world works are "science" and understandings of how to achieve particular goals are "technology." Technology is of crucial importance throughout world history. We will also follow science through history, though its impact on other phenomena is moderate before the modern era. We should stress that we will deal with social science and philosophy as well as natural science.

Technology is often misunderstood as "applied science." If so, then we would have to understand the course of scientific innovation - changes in our understanding of how the world works - in order to understand the course of technological innovation - changes in our understandings of how to do practical things in the world. In practice, the relationship between technology and science is more complicated: Technological innovation often owes little to science; scientists may then struggle to understand new technology (as when metallurgists struggled to comprehend the nature of steel in the nineteenth century).

We will discuss a variety of innovations in a variety of fields, as well as how these diffused within societies and were transmitted across societies. We will consider the four stages of innovation referenced in Table 1.2 when we address evolution in technology and science below, but will not review each stage for every innovation we encounter in this book.

\section{Trade}

We see some evidence of short-distance trade for stones to make tools even 120,000 years ago (chapter 3). From 10,000 years ago, we see trade in obsidian from Anatolia to the Middle East. We saw above that copper and tin were transported hundreds of kilometres from perhaps 7000 BCE (and over $1000 \mathrm{~km}$ by the fifth millennium). Lapis lazuli and turquoise, both gemstones, were traded over long distances from at least 3500 все. Вy 4000 вСе, the Indus Valley trades regularly with the Middle East. Trade was likely particularly crucial for the Indus, which lacked metals but had some access to gold. The similarity in developments in pottery production between Egypt and the Indus Valley even before 4000 все indicates intense interaction. Highly decorative pottery was a luxury good that could be transported long distances profitably. Egyptians from before the days of the Old Kingdom were trading pottery and textiles (and other products) with Nubia to the south for ebony and gold and various skins and feathers from farther afield in Africa. In the "Bronze Age" (3000-1200 BCE), we see trade in a broader range of materials: indigo and other dyes, textiles from wool, flax, and cotton, salt, dried meats, copper, tin, gold, silver, implements and tools. There are even isolated cases of long-distance trade in grains by water: The Greeks imported grains from present-day Ukraine via the Black Sea by about 1000 BCE. Wood was another bulky good that was transported long 
distances: Egypt imported cedar from Lebanon and ebony from Nubia for construction, there being little native wood in the Nile Valley. A 150-room palace might require 2,000 trees. There is an Egyptian record of a forty-ship expedition to Lebanon. Mesopotamia also lacked wood; trees were floated downriver from Anatolia. There was trade between the Andes and the Peruvian coast from as early as 2500 все: Cotton to be used for fishnets was traded for seafood.

Trade expands with agriculture because farmers have occasional surpluses to trade. Also, settled communities can come to value a range of goods that it would be too difficult to carry with them over long distances. Moreover, they needed building materials for their houses. The technologies discussed above generated new possibilities for trade: Both the raw materials and finished products for pottery, textiles, and metals were carried long distances. Besides, agriculture allows urbanization, which in turn encourages trade, for towns and cities provide a place where merchants can meet and conduct business. Finally, the political consolidation and social stratification associated with agriculture promote trade because states and elites have the resources to buy both useful and ornamental items. Trade could occur with other agricultural communities or with neighbouring nomads: The latter had animal products and metals to trade for grains, textiles, pottery, and metals. In either case, traders had to overcome the natural human tendency to be suspicious of other groups.

We should not exaggerate the involvement of the typical farmer in trade. Students of history should never forget either that the vast bulk - 80 or even 90 per cent - of the population in these agricultural societies were farmers and that these farmers were mostly self-sufficient. They not only grew their food but also built their own houses (likely with help from neighbours) and made their own clothes. They came to pay taxes over time, and to buy pots and metal tools, but otherwise interacted little with the outside world. Most people at this time likely never left their village (except perhaps to marry). Still, just a bit of market interaction - or tax payment - among the agricultural population could create an economic opportunity for merchants and artisans. The agricultural surplus was not large enough to support a sizeable non-agricultural population. Nevertheless, this 10 per cent or so of the population was of particular importance in history, for it administered states, built public monuments, traded, and produced industrial goods. Many innovations would occur in all of these endeavours as some individuals focused their energies on them. These ideas then spread along trade routes.

The impacts of trade expansion depend quite a bit on what sort of trade expansion we see at a particular point in history. A small trade in luxury goods may be of limited economic impact, but may still allow transmission of cultural, political, and technological ideas. We shall see, for example (especially in chapter 11), that religions often spread along trade routes. An extensive trade in raw materials, finished goods, or services may have significant economic impacts, and further indirect effects on economic specialization and social stratification. Such trade may also intensify the sorts of cultural and political exchanges that luxury trade allows. 


\section{BOX 5.1}

\section{THE NATURE OF TRADE}

As noted in chapter 1, one of the sources of interest in world history is "globalization," the expansion in global trade and investment in the modern world, and its relationship to political and cultural changes. It is only natural that we will devote much space to trade connections in what follows. We should stress that trade can expand in three distinct ways:

- geographical extent, as new trade routes open

- intensity, as lower costs of trade allow a more extensive selection of goods (and eventually services) to be traded

- $\quad$ speed, as improvements in both trade institutions and transport infrastructure enable products and services to move more quickly.

It is often easiest to assess expansions in the geographical extent of trade: We usually know when one society begins trading with another. Nevertheless, we should always ask how intensive trade is, for the range of likely impacts is then much greater.

For this early period in history, it is hard to establish the intensity of trade flows. The growth of pottery, textile, and especially metals production tells us that there were substantial flows of bulky raw materials. Transport over some hundreds of kilometres was not limited to luxury goods, though trade over longer distances mostly was.

\section{Money}

One other development that encouraged trade was the development of "money." As noted above, traders employed gold and silver as money. The essential characteristic of money is that it be something whose value both a buyer and seller of goods or services can readily appreciate. The fact that people valued gold and silver across societies meant that it could serve this role. Money must usually also be easy to move. This means in turn that it should have a high value-to-bulk ratio. The fact that gold and silver were both rare but valued for their perceived beauty meant that smallish amounts of gold or silver could be used in trade. Gold was often too valuable to be useful in most exchanges, and so traders tended to use silver most often as money in this period.

Silver and gold were hardly the first monies, but they became common in Eurasia. Previously, other rare substances such as obsidian and amber had served as money in different parts of the world. In Mesoamerica, cacao beans operated as money right up until the time of the Spanish conquest (and would be employed as money in some parts of Mexico even in the early twentieth century). One advantage of cacao beans is that they come in a standard size; merchants had to carefully weigh gold and silver and evaluate these for mineral 


\section{BOX 5.2}

\section{THE ROLES OF MONEY}

We stress here the role of money in simplifying exchange. In the absence of money, people must barter. This has two disadvantages. First, it means that a pair of traders must each want what the other has. Second, they must agree on what each good is worth (at least relatively). Once money exists, a pair of traders needs only agree on the price of one commodity for an exchange to occur.

Once money exists, though, it serves many other purposes. It is a store of value: People can save money and spend this much later. Money also makes it easier to provide loans over long time periods - though loans predate the existence of money. We will encounter such uses often in later chapters.

content. This time-consuming process - which depended in part on trust among the merchants involved - would only be replaced when coins were introduced from the seventh century всE (chapter 9).

Cowrie shells from near the Maldives Islands in the Indian Ocean were used as currency throughout the eastern hemisphere as late as the second millennium BCE. Cowrie shells were scarce, durable, of standard size, and difficult to counterfeit. In India, cowries were then used for small purchases while silver was used for large purchases until perhaps the early centuries $\mathrm{CE}$, after which copper coins mostly (but not entirely) replaced cowrie shells. Indian states accepted cowries as tax payments. Thousands of cowries have been found in China from the third millennium BCE, and they are widespread from the second millennium BCE; they were used both as money and as decoration, with the cowrie shape often imitated in jade, bronze, and other materials. The use of bronze cowries as money from the first millennium BCE indicates that cowries themselves had long served as money; the substitution of bronze may reflect a limited supply of cowries in China. Some have thought that Chinese cowries came from the South China Sea, but the types of cowries used as money do not live there now and probably never did. Moreover, it seems that the use of cowries spread eastward through time from Central Asia (though Yunnan province gained cowries directly, and would later get its cowries from Southeast Asia). Cowries were used in Southeast Asia and much of Africa well into the early centuries CE. Indeed cowries were still being used in West Africa when Europeans arrived in the sixteenth century (chapter 19); the Europeans took advantage by importing 10 billion cowries from the Indian Ocean, destroying the cowrie as a local currency in Africa. Europeans also employed cowries in both Virginia and New Guinea. Cowries were also still in use in India at that time; the Europeans sought to dominate the cowrie trade there as well. Despite this ignoble end, we should appreciate that cowries were used across three continents for thousands of years; they are in some ways the most successful money in world history. 


\section{Transport}

Trade depends on the ability to move goods from one place to another. If the costs of transportation are high, then very few products - those that have much different prices in one place from another, and are reasonably easy to transport - are traded. Any innovation that decreases the cost of transport will tend to encourage trade. This simple relationship is perhaps too obvious, and can be easily under-stressed in historical accounts. However, some critical advances in transport occurred in the early millennia of agricultural production.

Boats would be the cheapest way of transporting goods until the development of railways in the nineteenth century. As a rough rule of thumb, transport by river was half the cost of transportation by wagon, and travel by sea half as expensive as that by river; there were, of course, important variations across time and place. (A decree of the Roman Emperor Diocletian in $300 \mathrm{CE}$ would suggest that the price of grain doubled even then if carried a mere 80 kilometres by wagon.) There is clear evidence of boats made of reeds and (later) wood thousands of years BCE. The oldest drawing of a boat dates from 4200 BCE in Norway, where it was used in a reindeer hunt, but boats undoubtedly existed long before that. The abundant fish in the Persian Gulf may have encouraged the development of the first boats; these would then have been adapted for longer trips to pursue trade. Over time, boats made of planks with mortise and tenon joints (where one piece of wood has a rectangular rod that fits into a hole cut in another piece of wood) replaced rafts; there were also various improvements in sails. Early sailors (as much later in Polynesia; chapter 7) used birds, clouds, and stars to navigate, but preferred to stay in sight of the coastline. There were already seafaring boats in the Mediterranean and Indian Ocean by 4000 все. The number of vessels expanded significantly with Egyptian and Mesopotamian "civilization" (see next chapters); river and canal traffic was substantial in both places. Egyptian boats could ride the current downstream on the Nile and use sails to ride the prevailing winds back upstream. Egyptians from about 3000 вСE mastered the challenge of sailing on the Mediterranean, and then the Red and Arabian Seas. The Great Pyramid (chapter 7) contains a boat 45 metres long, perhaps intended to carry the pharaoh to the afterlife. The Chinese also boated along the Yellow and other rivers.

Bronze tools facilitated the construction of better ships. Bigger and faster vessels facilitated trade throughout the Mediterranean and Indian Oceans. Bahrain, on the Persian Gulf, with harbours, fresh water, and dates, was a trading centre between Mesopotamia and the Indus Valley from the third millennium BCE. It is clear that boats and traders from the Indus went to Mesopotamia.

Though water transport was far cheaper than travel by land, the latter was often faster and more reliable: Boats were often dependent on winds and currents, and shipwrecks were common. Moreover, boats were only feasible where there was navigable water. There was a road connecting Egypt and the Levant (modern Israel) from 4300 BCE, though it is hard to know what kind of road this was. Land transport at first must have involved humans carrying goods 


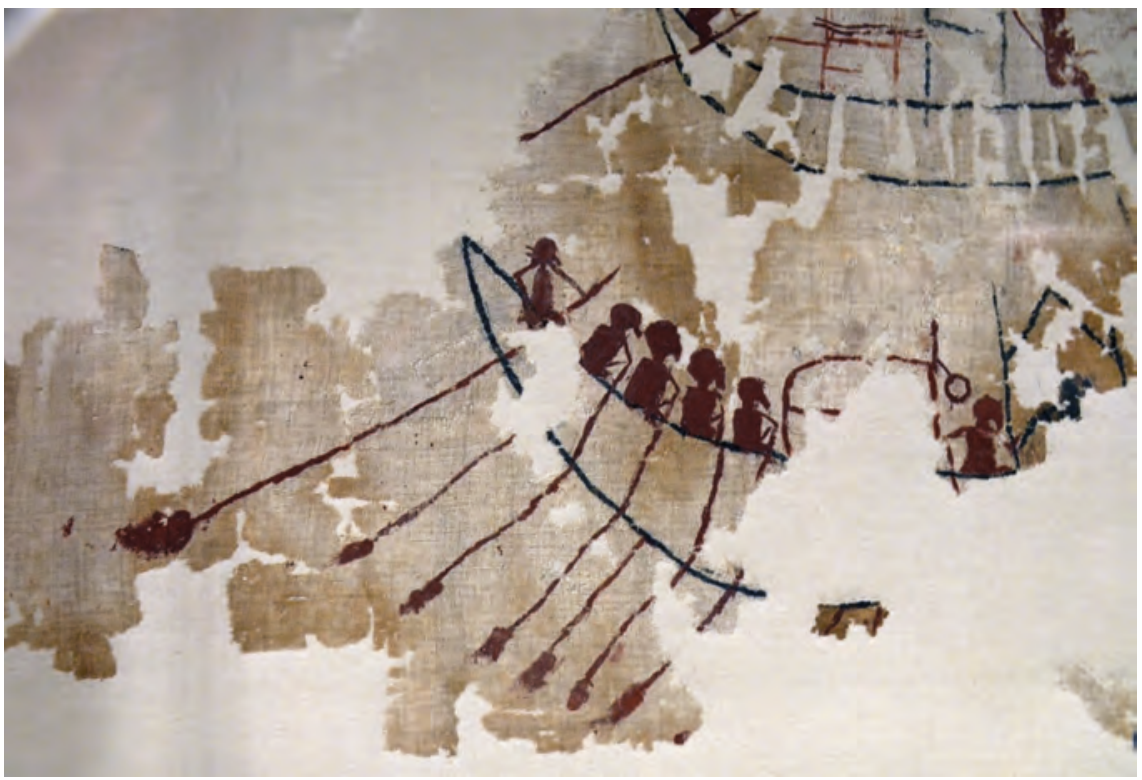

Illustration 5.11

Fragment of Egyptian linen picturing boats with rowers from about 3600 BCE, from a grave in Gebelein, about $40 \mathrm{~km}$ south of Thebes along the Nile, Torino Museo Egizio Source: Photograph by Vassil, CC0 1.0

on their backs. Animals such as donkeys, camels, and horses (used much later than donkeys for transportation; only from about 1600 BCE) would prove capable of carrying much more substantial burdens once these were domesticated. The donkey was domesticated around 4000 BCE, likely in a couple of places, Iraq certainly being one, perhaps Egypt also. Donkeys do not need much water and can carry large loads. Their domestication led to a significant expansion of trade from Egypt to Mesopotamia and beyond to Anatolia. Trading towns were established a day's donkey ride apart.

Animals (or indeed humans) can pull in a wheeled vehicle several times the weight that they can carry on their backs. However, wheels only make sense if there is a flat surface to travel along (and bridges across most if not all waterways along a route). Wheels, and wheeled vehicles, first seem to appear between 3500 and 3000 все. The first known picture of a wheeled vehicle on a piece of pottery dates to 3500-3350 вСE in southern Poland. They appeared at about the same time in Mesopotamia, the northern Caucasus, and central Europe, as well as in the nomadic steppe. The wheel's emergence in Mesopotamia may reflect expansion away from the rivers onto northern plains. It is possible yet again that there were separate discoveries. It may be that nomads were first to use wheels: They could then carry more household items across the flat dry grassland. The wheel may have been instrumental in allowing nomadic groups to travel long distances. Wheels are known in China (via Central Asia) by at least the fourteenth century вСЕ. 
Though attention naturally focuses on roads, wheels, and boats, decreases in transport costs occurred in humbler ways. Superior harnesses for draft animals increased the amount that these could pull. During the Roman Empire, the thickness of the pots or amphorae used to ship olive oil steadily decreased, lowering the weight and size of these pots and therefore the cost of moving the oils and wines that they contained.

Transport was essential to trade. It was also crucial for large polities. These struggled (see next chapter) to govern over long distances, and needed to be able to move information, goods, and people quickly. Assyria established regular resting posts along its highways in the third millennium BCE. Other states made provision for messengers to travel without local interference; bordering states often agreed to protect messengers carrying diplomatic communications. (We will discuss the political element of long-distance transport in detail in later chapters; we can note here that travellers needed political protection along with good infrastructure.)

By facilitating trade, transport promoted occupational specialization. Workers can only specialize in copper, pottery, or textile production if they can move their goods to market. Some specialization could occur within a single city, but much more was possible if cities traded (and individual cities themselves could only exist in the first place if food

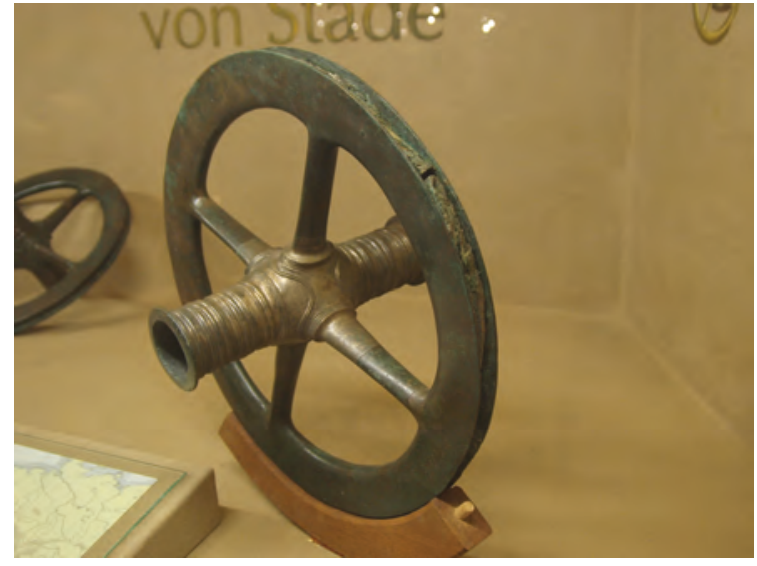

\section{Illustration 5.12}

Bronze wheel, c.700 BCE, Stade Museum, Stade, Germany

Source: Photograph by Bullenwächter, CC BY-SA 3.0

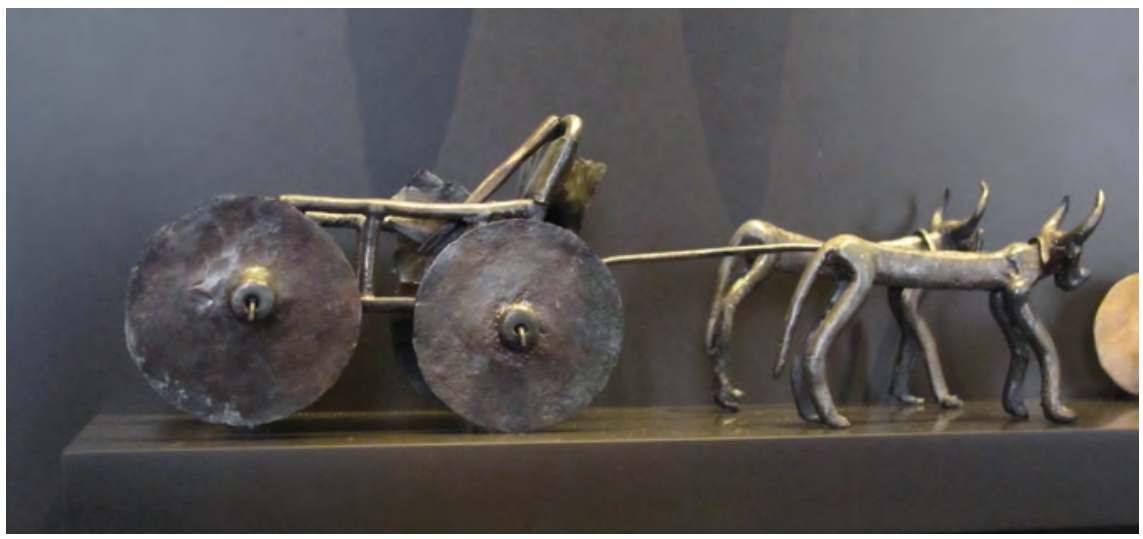

Illustration 5.13

Wagon model, central Anatolia, 4500-2200 вCE, Neues Museum, Berlin

Source: Photograph by Ismoon, CC BY-SA 4.0 


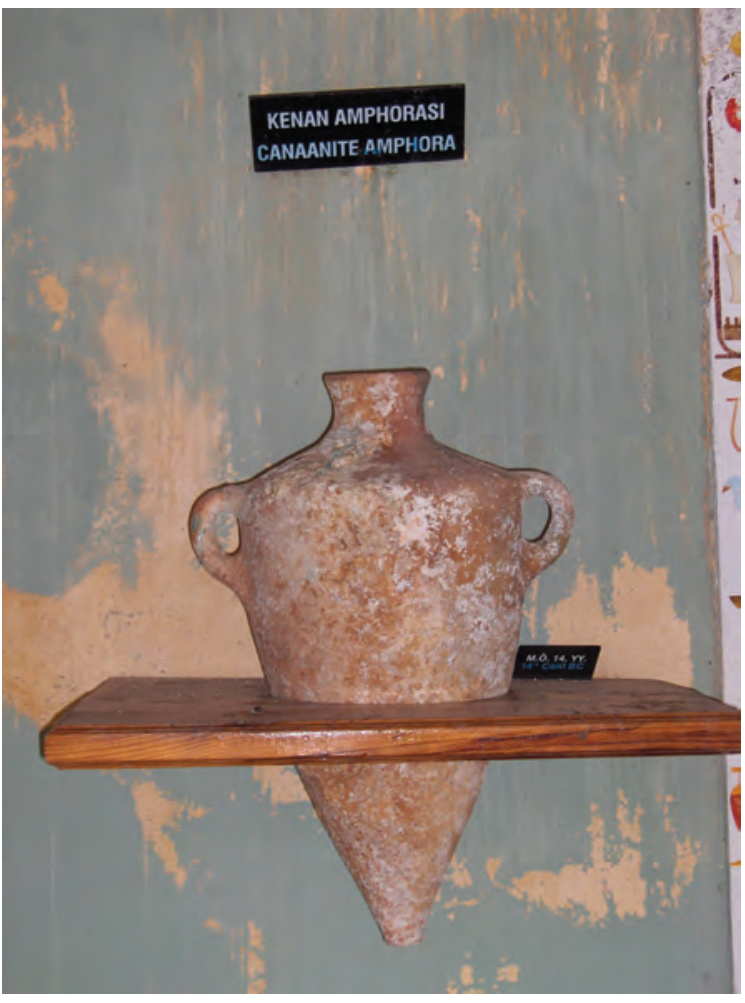

Illustration 5.14

Amphora, 14th century BCE, Canaan, Bodrum Museum of Underwater Archaeology

Source: Photograph by Georges Jansoone, CC BY-SA 3.0 and building materials were transported there). Recall that raw materials themselves often had to be moved long distances. Few artisans were fortunate enough to have both their materials and their markets nearby.

Finally, yet importantly, transport was essential to the movement of information. All of the contacts among societies that we will detail in this book were possible only because people (and eventually documents) could move between these.

\section{Merchants}

States can at times manage trade through their taxes and expenditures. However, we will find in later chapters that states often find it easier to contract with merchants to move goods around and even sometimes collect taxes. It is hard to ascertain precisely how trade was managed before there were written records, but it seems likely that merchants rather than states handled most of this. The earliest states had limited bureaucratic potential. Trade often, in any case, crossed the political

\section{BOX 5.3}

\section{CHARACTERISTICS OF TRANSPORT}

Since transport plays a critical role in world history, it is worth emphasizing some key points made above that will prove vital in later chapters:

- Before the railway, water transport was much less expensive than land transport.

- Land transport, though, was usually speedier and more reliable. Messages and highvalue goods were usually sent by land.

- Land transport was more extensive than water transport (though of course islands and the western hemisphere could only be reached by water).

- On land, wheeled transport is a fraction of the cost of carriage on the backs of animals, but this requires flat surfaces and bridges. 
boundaries of smallish states. We can suspect that there were merchants long before there were large states. Yet we mostly have to rely on later records to imagine how these operated.

\section{Challenges facing agents: Merchants}

Merchants need to sell goods for more than they pay for them, and also need to cover costs of transport and storage and any other expenses. One challenge for merchants is informational: They must be aware of opportunities to buy low and sell high. Their ability to profit will depend in large part on them being aware of opportunities that are not widely recognized.

We noted in the last chapter how farmers, nomads, and fishers had to worry about theft. Merchants, who are moving valuable goods between places, face even more significant concerns in this regard. They have to worry about bandits. They also have to worry about having their goods expropriated by states - primarily but not exclusively when crossing borders between states. States, as we shall see, find trade easy to tax (since valuable goods are concentrated in markets), and are often tempted to take merchants' goods. This is especially the case during times of war. Farmers also have to worry about states but are usually a much less tempting target.

Merchants make their living trading. As a result, they face a further challenge of not being taken advantage of by those they trade with. They must carefully evaluate the quality and quantity of goods transacted. It will often be useful to negotiate promises for delivery on some future date. Then issues of trust and dispute settlement become paramount: Will another merchant do as they promised, and what recourse does one merchant have if another does not do as promised? Accidents happen, and merchants die or go out of business; merchants need to negotiate how to deal with such surprises.

Finally, yet importantly, merchants need to finance their operations. There will necessarily be a time lag between purchase and sale. And there will always be some uncertainty regarding how quickly goods will be sold, at least at a price the merchant finds acceptable.

The merchant, then, faces a particularly complex set of challenges. The amount of trade we observe in different times and places in history will depend in large part on how well merchants can address these challenges.

There is, we should note, a historical debate between those who think that market exchange is typical in human history, and those who believe that other types of exchange such as ceremonial gift-giving or government redistribution might have mattered more in early history. In the latter case, political and cultural connections would have determined trade flows more than economic calculations. We can sketch a middle ground: Economists increasingly appreciate that market exchange, and especially long-distance trade, does indeed depend on institutions and culture. Merchants can operate more confidently if there are both formal rules and cultural attitudes that limit theft or deceit. The earliest trade likely then reflected a mix of the profit motive, cultural understandings, and political regulation. We can then speculate that both cultural norms and institutions co-evolved as trade grew. If rulers valued trade, they would adopt institutions that encouraged this. 
The very earliest "trades" may indeed have involved exchanges of gifts. Gift exchange was undoubtedly common among early rulers, though it is hard to judge the relative importance of such gifts. Nevertheless, we shall see that merchants - engaged in trade with the intent of making a profit - not only exist in the earliest written records but also played a crucial role in generating those earliest written records (chapter 6). It is reasonable to speculate that profitoriented merchants had become important long before we have records of these.

We suggested in the preceding chapter that early states formed in large part to protect farmers. They might also then protect merchants in return for taxes. States would then find that it was much easier to tax merchants who necessarily gathered in marketplaces than a dispersed mass of (generally much poorer) farmers. However, whereas it

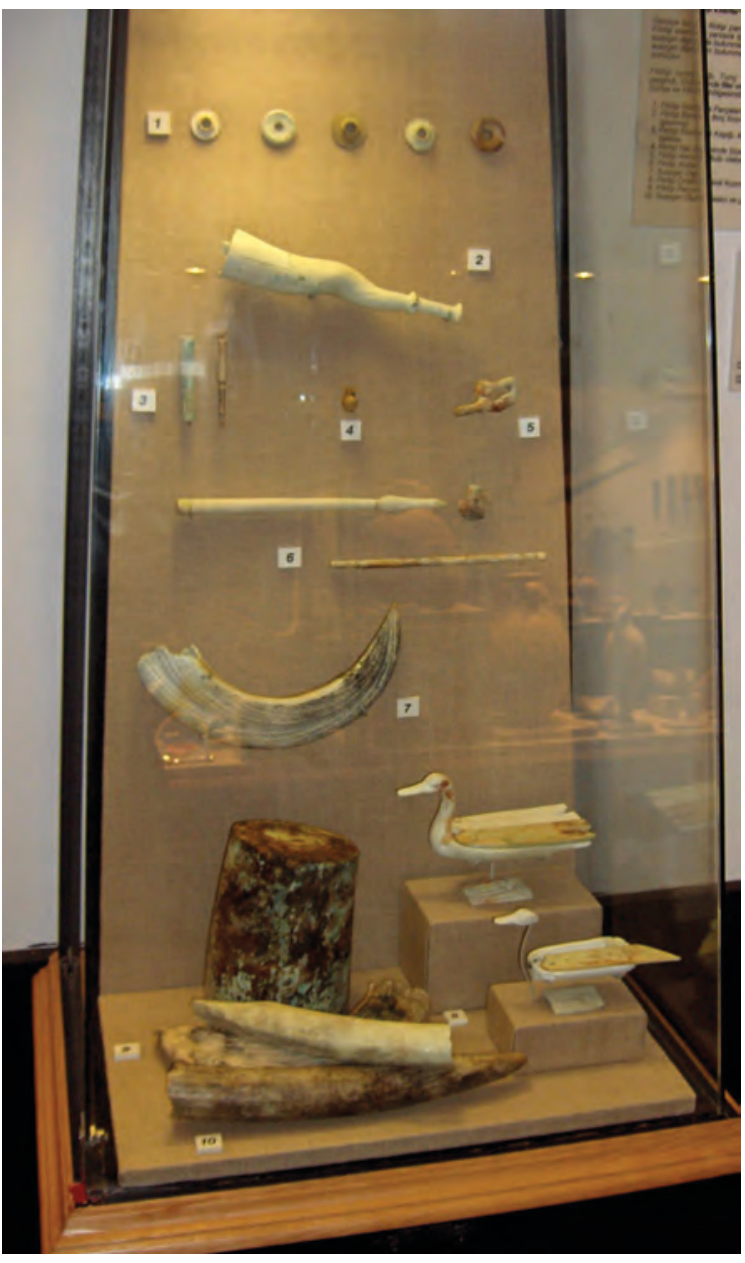

Illustration 5.15

Ivory, from shipwreck off Turkey, 14th century BCE, Bodrum Museum of Underwater Archaeology Source: Photograph by Georges Jansoone, CC BY-SA 3.0 made obvious sense to rulers not to tax farmers into starvation, it would be less obvious that taking a merchant's stuff was a worse policy than taxing them slowly over time. This would especially be the case for a merchant from another land. Merchants needed to negotiate carefully with states. In addition, we can suspect that they did not rely entirely on states for protection from bandits: Merchants would likely have travelled in armed groups whenever possible.

How did the earliest merchants address the merchant's challenges? There were Mesopotamian trading networks into Iran at least from the fourth millennium вСE. Networks are a useful strategy for merchants. They increase trust between merchants: You can be more confident in trading with someone you and your colleagues often trade with, for they will then care about their reputation for fair dealing. Networks can also increase the bargaining power of merchants with states: An isolated merchant is a tempting target, but a state might not wish to offend an entire network of merchants that it taxes regularly. We can imagine a slow process by which merchants formed networks, developed expectations of trust within these, and negotiated arrangements with states. As networks solidified, merchants could 
act as agents for each other, look after another's business when they were on the road (though wives or other relatives often performed this function), collaborate on risky but expensive trading ventures, and - importantly - aid in winding up a merchant's affairs upon death. Merchants always had to worry about what would happen if a merchant they were dealing with died before fulfilling commitments: Networks could alleviate this challenge.

Networks might also be sources of financing and information. With regard to the latter, we can imagine an evolutionary process of identifying new trade opportunities. Sometimes merchants would guess wrong about the likely market in one place for a good available in another. This was one of the costs of doing business. Nevertheless, over time, merchants would identify profitable opportunities. Of course, changes in politics or climate (including the alternation of good and bad harvests) or technology, or the discovery by other merchants of alternative sources of products, could render once-profitable ventures unattractive. The selection environment for profitable trade routes was always itself changing.

\section{BOX 5.4}

\section{THE REPUTATION OF MERCHANTS}

We will often find in history that states and farmers both viewed merchants with suspicion, even though the societies in which merchants operated might rely heavily on both the goods they traded and the taxes they paid. Even in the contemporary world, the business class is often viewed with suspicion. It is worthwhile to reflect a bit on what seems an enduring and common cultural attitude.

One crucial element here is undoubtedly just that it is less obvious exactly what the merchant does. The work of the farmer in the blazing sun is easy to observe and appreciate. The skilled work of the artisan in their workshop is less visible, and less valued by agrarian empires, but still easy to comprehend. The ruler issues orders, and the soldier engages in battles and staffs fortifications. The manual labour of the carter or sailor in moving goods from place to place is also visible. What exactly does the merchant add to all of this? The person buying a pot may remark on the skills of the potter and perhaps the care taken in transporting the pot without injury, but will be less likely to reflect on the fact that somebody paid the potter and carter in order to facilitate the sale. The merchant makes their money by financing the movement of goods from producer to consumer. In doing so, they bear the risks of theft along the way or that they have misjudged what consumers want (or how badly they want it). If not for the merchant, the artisan might not have made the pot, nor might the carter have moved it. However, the merchant's contribution is far less evident than that of the potter or carter.

States can and have substituted at times for merchants. Ancient states often taxed goods in kind and distributed these to bureaucrats and soldiers, or even the general populace. Yet 
even when the Roman state provided bread to the Roman masses, it usually found it easier to rely on merchants to provide this bread. Experiments in the modern world with state management of economic activity have likewise proven problematic, as we shall see in later chapters. The role of merchants may be invisible but it has proven hard to avoid.

People view merchants with suspicion not just for what they do but how they do it. The skills of the merchant are less valued than those of the farmer or artisan. The successful merchant has skills at haggling. They can both buy low and sell high. Merchants will often need to negotiate with producers who know more about the costs of production than the merchant and with consumers who know more about how much they are willing to pay. The merchant's skill in detecting how little the producer will take and how much the consumer will pay will determine the merchant's profit. A successful merchant may develop trading opportunities where a less perceptive merchant would lose money. Yet the artisan will still wish that they had exacted a higher price, and the consumer will wish that they had achieved a lower one. The merchant's income will seem to have come at the expense of others, even if producer and consumer could not have found each other without the merchant.

Other merchant skills - knowing who you can trust, identifying market opportunities, having a keen eye for the quality of particular goods - are easier to applaud, but less visible than their role in setting prices. Then there is the necessity of ensuring that the state does not steal all of the merchant's stuff, which requires that the merchant befriend or bribe the ruler and the bureaucracy, or form an alliance of merchants that can negotiate from some position of power with the state. Merchants will often benefit from unfair state preferences, and will be suspected of doing so even when they do not.

Merchants, we must admit, can make money by lying, cheating, or bribing. Yet they can also make money by facilitating transactions that benefit both producer and consumer. The fact that merchants can profit in both good ways and bad makes them similar to rulers - who can prosper through both encouraging prosperity in their realms or through more zealously taxing their citizens. However, it makes merchants dissimilar from farmers or artisans, who for the most part are rewarded for producing goods that others want (though they each have some scope for lying about the quality of their output).

None of this might matter if all merchants were poor. Many merchants are: Self-employment today as in the past can be a useful response to unemployment. However, some merchants make vast sums. Scorn is heaped on the merchant that does so by out-haggling poorer people, or through currying favour with government officials. However, the merchant who spots a previously unappreciated opportunity to link producers with consumers may also be scorned. So too with the merchant who decides that certain risks - most obviously the challenges of moving goods long distances - are worth bearing. Merchant wealth might have seemed especially suspicious in ancient human societies in which there was a tiny "middle class" between the mass of peasants and a small elite.

Farmers can only become wealthy by becoming large landowners; otherwise, the similarities in agricultural produce across farms limit the profitability of any one small farm. Artisans have a somewhat greater ability to distinguish their wares from others, but again artisans rarely grow rich (though later employers of large numbers of industrial workers could). 
Merchants, though, by monopolizing information, could become very wealthy. If only one merchant knows to buy a particular good cheaply in one place and sell it for a much higher price elsewhere, they can make a considerable profit. The same is true if they alone know how to transport the good cheaply and safely between the two places. Knowledge of what prices to negotiate, what government officials to persuade or bribe, or what traders to trust, can also generate enormous profits. And we have seen above that merchants managed issues of trust in part by forming networks: A network that uniquely connected any two points could potentially reap huge profits on the trade between these. Also, once merchants had amassed some funds, some of them combined these funds with their informational advantages to engage in borrowing and lending: This risky enterprise could also be immensely profitable, especially in times and places where few people were both willing and able to make loans.

The historian can benefit from a nuanced appreciation of merchants. The cultural suspicion of merchants, if embraced without question, would guide the historian away from appreciating the possible contributions of merchants. These, most obviously, identify trade possibilities, connecting producers and consumers in different places. Trade is of critical importance in all world histories, and would, in general, be impossible without merchants. Yet merchants facilitate the flow not just of goods but also of ideas: scientific, technological, and religious. The role of merchants in carrying religious beliefs in particular (chapter 11) would be incomprehensible if these were entirely the selfish and corrupt individuals imagined all too often in popular culture. The historian should also be guided away from lionizing merchants and believing that they always had kinder motives at heart. As with all historical players on our stage, merchants often had mixed motives and exerted unforeseen effects on the course of human history.

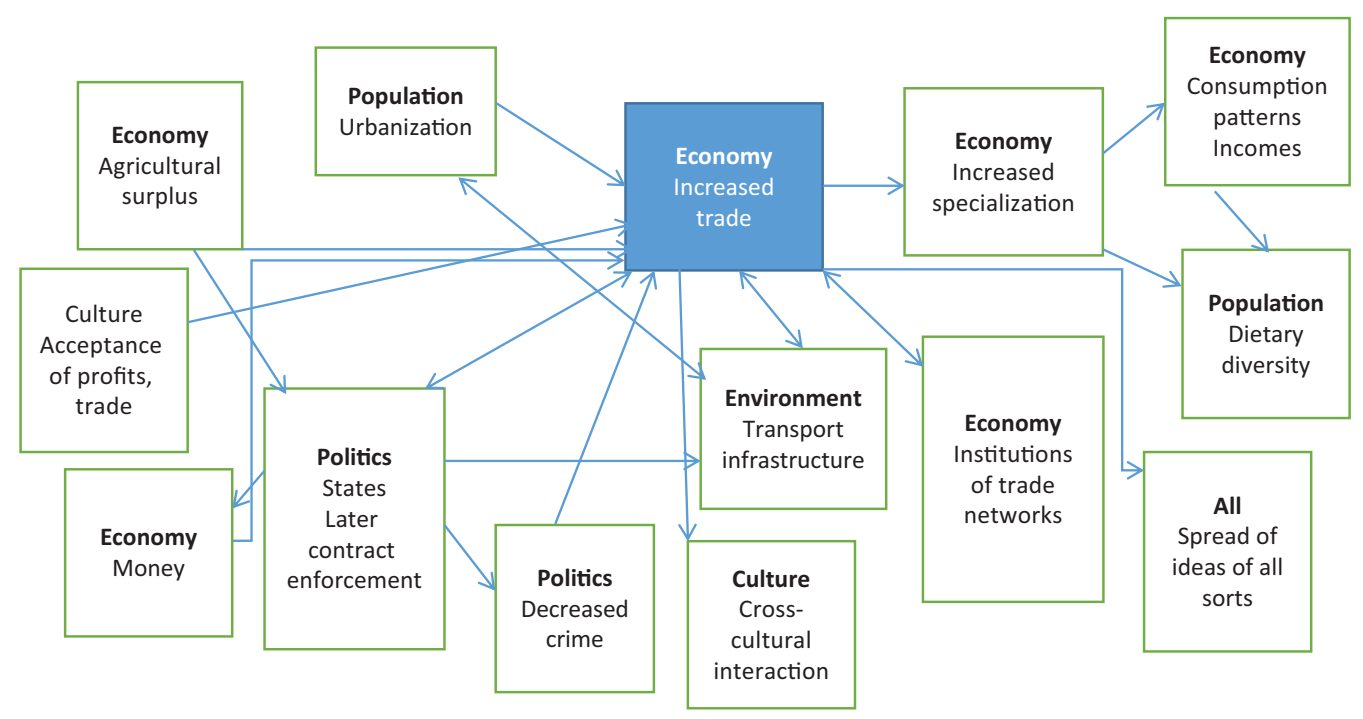

Figure 5.2

Influences on and effects of increased trade 


\section{Artisans}

We have very little direct knowledge of early industrial production. We have found a great deal of pottery. Some of this is utilitarian, but much of it is highly decorated and required great skill in production. We can also marvel at the capabilities of early architects and stonecutters, and also jewellers and metalworkers. In Megrargh in present-day Pakistan, we find evidence of a variety of crafts as early as the sixth millennium BCE: baskets; mats; copper, shell, and precious stone ornaments (beads were particularly popular); pottery and clay figurines; flint and bone and copper tools for scraping, cutting, harvesting, and other tasks; and various wood and leather goods. However, while we know that some degree of specialized industrial production emerges soon after humans become sedentary, archaeologists have identified very few early sites of industrial production. These tend to be small workshops with room for at most a handful of people. We are unaware of any schools for industrial production in early states. We presume then that there was some form of apprenticeship system where young people would learn a trade while assisting a master artisan. Styles of pottery and metalwork are often similar across vast distances, suggesting that there was a considerable exchange of

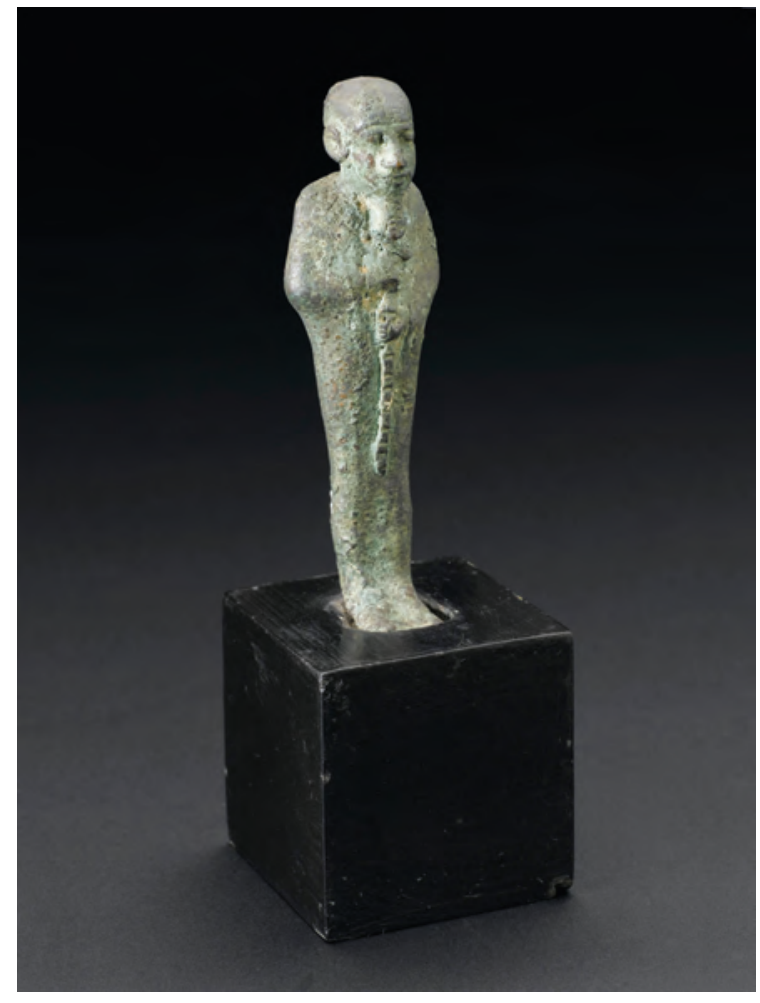

Illustration 5.16

Ptah was the Egyptian god of creation and artisans 800-100 вCE, Science Museum, London

Source: Wellcome Library, CC BY 4.0 production techniques across workshops. In (at least) Egypt and China, state sponsorship of certain crafts encouraged this coordination. Artisans in early China may have been in a servile position to the state; some may have worked in large workshops. In Mycenae in Greece, there is evidence of textile production in statemanaged workshops.

We know that there must have been pottery kilns and brickmaking and metalworking sites. These were probably most often established outside of towns. They required lots of fuel and released pollution into the air and water. Since archaeologists have focused on urban excavations, very few of these sites have been uncovered.

As with merchants above, we can only guess for the most part at how the earliest artisans addressed their various challenges. We have seen that states may have encouraged the sharing of information. We can expect that the standard workshop likely had a master and students (pursuing an apprenticeship), 


\section{Challenges facing agents: Artisans}

Artisans need to produce artefacts at a combination of price and quality that others will buy. If selling at a distance, they face the same challenges as a merchant. They must also negotiate the purchase of raw materials. They share with merchants and peasants the challenges of theft and confiscation. Reputation is of particular importance to the artisan, both for the quality of their goods and the honesty of their dealings. Artisans need to balance their own tastes with evaluation of what people want.

Artisans depend on specialized knowledge. They may wish to be secretive. However, they may need assistants and have some interest in passing their knowledge along to their children or others. They also want to remain competitive with other artisans. They then have some interest in sharing knowledge. It seems harder for artisans to obtain great wealth through specialized knowledge or skill than for merchants to do so.

Artisans depend for food on others (unless they farm part-time), and must always worry about forces beyond their control that affect the price of food. Most people take this dependence on others for granted today, but it would have been a much scarier proposition when food was a much more significant proportion of consumption expenditures, and harvest failures could have dramatic effects on prices. Artisans share this dependence with merchants and bureaucrats, but usually have a more tenuous economic position than those others have.

as would be common in later artisanal production. We can be impressed that ancient cities seem to have had hundreds (or more) artisans. There is evidence that these often did keep gardens and animals, and so were not utterly reliant on an external food supply. We have a surviving text from Middle Kingdom Egypt in which a father advises his son of the dangers of most crafts, and urges him to become a scribe instead (see chapter 7).

\section{Economic growth}

What happened to average incomes during the early millennia of agriculture and nomadism? Our discussion of Malthusian mechanisms in chapter 4 leads us to suspect that not much will have changed over much of human history. Developments in bronze and then iron implements in agriculture - and increased information flows between societies - may have boosted incomes temporarily. But these changes likely had their most significant long-run impacts on population growth - and thus the potential for the growth in the size of states - rather than average incomes. The population would expand until the typical farmer was again near subsistence with regard to food intake. Expanded trade may nevertheless have encouraged increased diversity in diet, though probably for the elite much more than the peasantry. This might have improved both the taste of food and the ability of the average person to satisfy the body's myriad nutritional requirements. Note that even today many people in developing countries suffer from deficiencies in iron or iodine or various 
vitamins: We can suspect that such deficiencies were far from unknown in ancient times. We will often have cause in this book to note how plants grown initially in one region were transplanted to another; this process could also enhance dietary diversity. If diets did improve because of these types of societal interactions, humans would have been healthier and lived longer. These would count as important improvements in the quality of life.

The above analysis may need adjustment in response to modern medical understandings. As we shall see in chapter 31, the fact that the human body adjusts how fast it burns calories in response to fluctuations in food intake or work effort complicates the modern fight against obesity. Would a society favoured by decades of prosperity experience physiological changes that would lead it to settle upon a new Malthusian equilibrium in which people ate more but burned calories faster? If so, Malthusian mechanisms might allow some increase even in average food intake through time.

Though food comprised the bulk of human consumption until the nineteenth century $\mathrm{CE}$, humans also built homes and produced or bought clothing, pottery, and a variety of other items. Developments in textile and pottery manufacture, in concert with developments in trade, likely meant that the average person gained nicer clothes and household goods over time. The development of cities, states, and writing, as we have seen, did support both trade and artisanal production. Advances in technology, trade, and institutions could increase the average individual's access to these goods. While much of this production served the elite, even the typical farmer likely benefited. Urbanization (next chapter) also generated a range of popular entertainments that were not feasible in isolated villages. Though occasional declines are observed with respect to non-food items - peasants in western Europe had fewer and inferior pots and roof tiles after the fall of the Roman Empire, for example (chapter 13) in general, non-food items tended to both increase in number and improve in quality through time. Despite Malthusian pressures, then, the average incomes of the bulk of humanity could and did creep upward over periods of centuries.

Looking ahead, we will find that average incomes probably were significantly higher during the classical empires than before: The trade and peace that characterized these empires (aided by good climatic conditions) did benefit the average person. These had better homes and pottery and likely diets than those before and after. Later, we will find evidence of economic growth in various parts of the world in the Middle Ages and after. There were also periods of economic decline - which may or not reflect Malthusian pressures - but there was in most of the world some degree of advance in average incomes over the thousands of years to come. (Economic growth will then accelerate dramatically from the late eighteenth century; chapters 22 and 24.) We will strive to identify and understand particular periods of economic advance and decline in what follows.

We do not have statistics on incomes for most of human history - though economic historians have attempted to estimate these. Note that we can gain some idea of nutritional intake by examining skeletal remains: In particular, 
changes in the average heights of a population (which armies or other organizations often record) can signal changes in nutritional status (but might also reflect changes in disease or work effort). Besides, archaeologists can tell us much about people's houses and pottery, though only rarely the clothes they wore (which biodegrade).

Though these various sources of evidence point to economic growth in many times and places, this was almost always so slow as to be imperceptible before the Industrial Revolution of the eighteenth century CE (chapter 24; Song China may be an exception; chapter 15). People died in a world that looked - at least economically - much like the world in which they were born. Most parents likely anticipated that their children - those that lived - would have a life much like their own. There were of course wars and harvest failures and epidemics: Life was far from boring, and incomes fluctuated from year to year. Nevertheless, there was little reason to discern any trend in economic (or indeed political) conditions. People tended toward a linear (the world is unchanging) or cyclical (the world experiences ups and downs) view of history (or in some cases a belief that humanity had fallen from a previous "Golden Age"). Few had reason to imagine that life was getting better in any important respect. However, even sluggish growth can yield over centuries a doubling or tripling of average incomes. An Egyptian peasant from the seventeenth century BCE might have been able to understand the life of an Egyptian peasant of the seventeenth century CE reasonably well, but would have marvelled at their tools and plates and homes and dietary diversity - and even more so the size of their cities.

What about income distribution? The earliest states were characterized by a small elite with incomes well above the average. There was what we might term a "middle class" of merchants, artisans, and bureaucrats whose incomes on average may have been a bit higher than farmers (and in the case of some merchants much higher). This middle class would remain very small until very late in history (chapter 27). Elite incomes would grow with increased state size (as one ruler could tax a larger population and achieve greater plunder in war) and expansions in trade (which produced both merchant profits and increased tax revenues). Kings and senior bureaucrats enriched themselves from both processes, and some merchants also profited immensely from expanded trade (such merchants would have had to maintain good relations with the political elite). There was also a tendency in most societies for land ownership to become concentrated through time (Box 10.5); landowners relied on strong states to enforce their control over land. Since we shall see that state size and the scope of trade expanded through most of the ancient period (and beyond), we can suspect that income inequality increased through time. A small elite saw their incomes grow more rapidly than the incomes of the mass of peasants. We see evidence of this process in elite burials and in elite households, both of which become more elaborate over time. The Akkadian elite, for example, was far more prosperous than previous Sumerian elites. The size of the wealth holdings of the wealthiest 1 per cent of the population in the Roman Empire may have 
expanded by seventy times between the second century BCE and the fifth century CE as empire and trade both expanded and land ownership became more concentrated. The top 1 (or 2) per cent may have earned a third of the total income in the empire.

Note that increased inequality provides yet another way in which average incomes might rise in a society despite Malthusian pressures: Elite incomes could grow even while the food intake of the bulk of the population was pushed toward subsistence levels. Economic growth in turn - whether from trade expansion or technological innovation or some other source - facilitated increased inequality because elites could only enjoy considerable incomes to the extent that the masses produced more than they needed for subsistence. Members of the elite were on average inches taller than the masses, reflecting the fact that the poor were often undernourished. While the general trend in income inequality was upward, there were occasional shocks that caused sudden reductions in inequality: The fall of the Roman and Han Empires (chapter 13) would cause elite incomes to collapse for centuries as state size and trade contracted dramatically. And occasional epidemics hurt elite incomes for a while by increasing the bargaining power of a depleted peasantry. In Eurasia, then, income inequality increased until late in the period of the Roman and Han Empires, then fell dramatically, and then rose again until the Great Plague of the fourteenth century (chapter 17), when levels of inequality again contracted, before rising again into the Early Modern period.

We have now outlined several ways in which average incomes might have risen over a period of centuries or more despite Malthusian pressures that should keep the bulk of the population near subsistence in the long run. Subsistence itself might become associated with a higher food intake; there might be increased dietary diversity; there might be improvements in both the quantity and quality of pottery, clothing, and a range of other non-food consumption items; and changes in income distribution might raise a minority of the population far above the level of subsistence.

\section{BOX 5.5}

\section{MEASURING ECONOMIC GROWTH}

Humans started out focusing like all other animals on food. They devoted some attention to shelters and clothing and primitive tools, but devoted the vast bulk of their work effort and consumption - to food throughout the millennia that humans operated as hunters and gatherers. We could speak of economic growth if they increased food intake or obtained a more diverse diet or increased their consumption of non-food items. We might also talk of economic growth if humans came to provide more services to each other.

As human societies have changed, human consumption "bundles" have changed dramatically. People in rich countries in the twenty-first century devote just a few per cent of their 
incomes on average to food. This alone distinguishes them qualitatively from the vast bulk of humanity before the nineteenth century, who still devoted most of their income to food. Moreover, humans today consume a wide variety of goods and services that were unknown to previous generations. It would be easier to talk about and measure economic growth if humans had consumed through time only more of the same goods and services that our distant ancestors had consumed. We could then just calculate how much more they consumed. We would still have to somehow weight increases in different goods and services: food, clothing, shelter, and so on. However, in the twenty-first century, we spend much of our income on products and services that even our great-grandparents could not have imagined; this creates challenges in trying to assess quantitatively differences in income across time for we are infinitely better off in our access to a wide range of goods and services.

We commonly measure economic output in a society with gross domestic product (GDP): the total value of all goods and services produced in a society in a year. GDP is often divided by the society's population to provide a measure of per capita GDP. It could be, but rarely is, divided by the average number of hours worked to provide a measure of GDP per hour worked. GDP calculations solve the challenge of weighing different goods and services (and in particular dealing with new goods and services) by valuing each at market prices. There is a value judgement involved - is one SUV equal in value to thousands of school lunches for hungry children? - but the justification is that prices realized in market exchange reflect the importance that buyers and sellers attach to a good or service. Economists know, though, that prices do not reflect value in any philosophical sense. Water is essential to human life while diamonds are for most a luxury, yet diamonds cost far more per ton than water. The reason is that water is abundant in most places (though that may change) while diamonds are rare. It would be folly to assume that a society with lots of diamonds but no water was better off - unless it could trade the diamonds for water. (Note that GDP is particularly difficult to estimate for countries that do not use money for then it is not apparent how to weigh the output of particular goods.)

Economists appreciate a host of problems with GDP. It does not account for environmental impacts: Polluting our air or water or utilizing non-renewable resources or deforesting our land has no negative effect on GDP (unless these cause reductions in goods or services production). Essential human activities such as childrearing are largely excluded, though these are paramount contributors to human well-being. There are many anomalies in GDP accounting: If I look after my children or clean my house myself, these activities are usually ignored in GDP, but if I pay someone to do this they are typically counted.We then exaggerate GDP differences between rich and developing countries because people in rich countries buy many goods and services that people in developing countries produce for themselves. Government expenditures receive special treatment: Since there is no market price for these, they are generally assumed to be worth precisely what governments spend on them. Political debate would be much less heated if this were obviously always the case. GDP treats many bad outcomes positively: Urban congestion is a bad thing but the fuel burned in traffic jams is included in GDP.

Over the broad sweep of history, GDP becomes even more problematic than it in the twenty-first century. Does it make sense to calculate that GDP per capita is 100 times greater 
today than it was during hunter-gatherer times? Are we really one hundred times better off? The lifestyles are so qualitatively different that it is perhaps silly to attempt to put a number on it. It may be just as well that hunter-gatherers - and indeed all societies until just a century ago - did not record GDP statistics. We are thus forced to make a variety of judgements about past societies: How well did people eat? What kind of clothes and pottery and other goods and services did they have (that is, what was their consumption bundle)? How hard did they work? We will refer at many places in our history to laudable efforts to estimate GDP for past societies, for these do provide a handy way of capturing how economic growth proceeded through human history, but we will also strive to look beyond these numbers to describe qualitative differences in work effort and consumption possibilities. What kind of pots, textiles, houses, and diets did people have access to?

In recent years, many scholars and international bodies have reacted to the limitations in GDP by suggesting other measures of economic welfare. The most well-known of these is the Human Development Index utilized by the United Nations. This provides some weight to GDP but also to measures of life expectancy and literacy. While we all value life expectancy and literacy, and can appreciate that these may be captured poorly by GDP, there is no obvious basis for attaching particular weights to these or any other societal outcome that we might wish to emphasize. Our strategy again will be to try to discuss advances in life expectancy, schooling, and other phenomena of interest as these occur, rather than only refer to some aggregate measure of diverse phenomena.

We will also strive to address income distribution, but this is not always possible. Average GDP tells us little about how the average person lived unless we know the income distribution in societies. Income distributions differ markedly in the modern world, with most European countries far more egalitarian than the United States. We may imagine that most of history is characterized by societies in which a small elite governed a mass of people in poverty, but there was almost undoubtedly considerable variety across societies.

\section{Evolution of technology and science}

It is widely appreciated that scientific insights and technological advances necessarily build on what people had understood before. Humans did not leap suddenly from stone tools to electric power generation but slowly accumulated an understanding of both how the world works (science) and how to produce goods and services (technology). In fact, technological and scientific innovations are usually a novel combination of pre-existing ideas. The airplane combined internal combustion engines and understandings of aerodynamics.

Even the big advances, the ones that inevitably receive the most attention, especially in popular histories - the steam engine, the theory of relativity, the printing press - appear on closer examination to build upon a set of small previous advances in understanding. Though it is common to portray these great discoveries as isolated acts of genius, they result from a lengthy process, usually 
involving multiple people, of gathering a set of ideas and carefully working out or experimenting on the implications.

We must nevertheless appreciate that both scientific and technological evolution are uneven processes temporally. Some innovations are quite simply much more important than others. The steam engine allowed coal (later oil) to power pumps in mines, then industrial establishments, and later agricultural implements and transport by rail and sea. Importantly, the first practical steam engine encouraged decades of efforts to improve its efficiency. The history of both science and technology are punctuated by significant discoveries that have lasting societal impacts and spawn a series of improvements. It is nevertheless likely that the combined effect of the set of minor little-heralded innovations is greater than the effect of the few that get the most attention. Both the big and little innovations are observed to cluster in particular times and places.

Technology must serve a purpose for it to be employed. It never makes sense, then, to say that technology was "wrong," though we may all wish that certain technologies had never been developed. When one technology is superseded by another, the selection environment must have found the latter to be superior. In the case of science, though, it is quite possible for an idea to be judged wrong when it is superseded by a quite different scientific theory. Famously, the idea that the sun revolves around the earth was rejected (though we can still admire the astronomical observations on which it was based). Much later, the theory of relativity showed that Newtonian physics, while a decent approximation in everyday life, was a woefully misguided approach to understanding subatomic particles. While the history of technology can be seen as a series of improvements to our collective ability to do things in the world (with occasional steps backward when certain technologies are forgotten), the history of science is more uneven, with occasional revolutions in which scientists reject some set of previous understandings. Yet this is still an evolutionary process, for the revolutions themselves reflect a growing dissatisfaction with previous ideas in the light of new information. Careful astronomical observations did not fit with an Earth-centred view of the universe (chapter 22). Revolutions in science as in institutions do not reject all that went before: Newtonian physics maintains its explanatory power in many realms.

As with institutions, both scientific and technological innovations require the conscious efforts of humans. Again, though, the history of both science and technology is littered with ideas that were not selected. For every advance, there were multiple failures. There is indeed cause for speculation that those who devoted time and energy to either type of innovation likely exaggerated their chances of success. We may owe our present state of understanding to a human tendency toward excessive optimism. In any case, while it makes sense to study why successful innovators did what they did (and also to examine why those who failed did what they did), it also makes sense to review the selection environment to determine why some innovations succeeded. Again, there is feedback, for the selection environment may encourage certain types 
of investigation, and so certain types of mutation in technology or science. If a large number of people are induced to explore a particular area of science or technology and put forward a diverse set of ideas, and only one or a few of these is selected and built upon by others, then evolutionary analysis will be essential to our understanding of the process of historical change.

It is best to think of two separate evolutionary processes. Technology evolves with selection for what seems most practical. Science develops with selection for what seems a better understanding of reality. We discuss the two evolutionary processes together here both because they are similar in many ways and because they interact. In the contemporary era, especially, technology builds on science: Biotechnology would be impossible without advances in biological understanding (indeed the boundary between theoretical understanding and practical application is often fuzzy in the field). In the nineteenth century, investigations into steam engine capabilities or chemical combinations were informed by the scientific understandings of the day (sometimes advances occurred despite building upon ideas we now consider mistaken). Before the nineteenth century, direct influences of science on technology are much rarer. We should also appreciate that science builds upon technology. Even today, and even more so in the past, scientists are often guided to ask why a particular technology works. Modern metallurgy grew to explain why iron- and steelmaking worked better in some places than others. Importantly, technology also creates better instruments with which scientists can study the world.

A scientific mutation is a new idea (generally a new combination of preexisting ideas) put forward by a scientist. A technological innovation is also a new idea (typically also a new combination of pre-existing ideas). Technological ideas are often encapsulated in a tool or machine or perhaps a recipe such as a chemical formula. Although accidental discoveries occur, both sorts of mutation generally result from a four-step process: The innovator recognizes a problem or question; sets the stage for innovation by gathering relevant information and exploring possible interrelations; develops a new idea; and then critically revises this idea. The first, second, and fourth stages rely heavily on conscious thought - though serendipity can play a role, especially in the second. The third step usually relies on the subconscious: As we all occasionally experience in life, the solution to a problem we have been wrestling with emerges suddenly in our conscious mind from our subconscious, often when we had temporarily set the issue aside. Popular histories often celebrate this "act of insight," encouraging the mistaken impression that innovations happen without much effort. However, these acts of insight would not have occurred if the problem had not been recognized and the necessary information gathered, and the inspiration almost always has to be further clarified before being useful (in technology) or persuasive (in science). As noted above, we can appreciate the conscious intent in innovation while still applying evolutionary analysis.

What then is the selection environment? For technology, usefulness for most innovations is determined economically. Can humans make money (or, before money, barter successfully) using the technology to produce goods or 


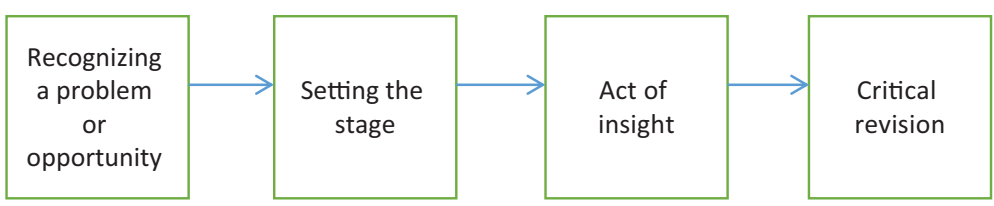

Figure 5.3

The four stages of innovation

services? Many a technique that proves physically feasible - you can indeed produce good X using technique $\mathrm{Y}$ - fails the further test of whether you can make money doing so. However, other phenomena influence economic decisions. The good or service has to be desired culturally. It has to fit within the social roles that people play. Technologies that challenge cultural values or social roles may sometimes survive and spread slowly as they encourage changes in these values or functions. Politics is also important: Governments have outlawed new technologies, often because they wish to ensure social stability. The most common case is one in which the new technology threatens some people's employment, and the government fears their unemployment.

The selection environment for science is entirely different. In the contemporary world, selection is mainly internal to science itself. The scientific community is large and in most countries can operate with considerable independence from political or economic elites (though there is understandable concern that these often influence the direction of research through funding decisions). In such a world, scientists themselves decide which of their ideas are best. Do scientific practices encourage the selection of ideas that capture reality instead of the selection of ideas that are congenial to the interests of scientists? Scientists aspire (consciously, at least most of the time) to select ideas by empirical exploration of reality. However, philosophers appreciate that there are always multiple possible ways of interpreting any piece of information, and so scientists must exercise judgement in deciding which ideas are best. It is theoretically possible that science merely evolves in ways that amuse scientists. Technology is important here in providing evidence that this is not the case: Successes in biotechnology suggest that biological theory is not entirely misguided, and the fact that airplanes do not regularly fall from the sky provides considerable support for theories of aerodynamics. However, in other realms of science and perhaps particularly the social sciences and humanities that are of central importance to this book - the direct influence of reality on scientific thinking is arguably far less.

Scientific selection of scientific ideas is relatively new in human history. In previous centuries, scientific ideas were often subjects of public debate. Without a large professional class of "scientists," political and especially religious authorities would often proclaim how the world worked. The Catholic Church's long defence of the Earth-centred view of the universe is but one example of this (and even today, of course, certain scientific ideas, such as certain applications 
of evolutionary theory, are subject to public scrutiny, though most people accept the judgement of scientific communities). Culture then could wield a tremendous influence on science. Even today, culture might influence how some scientists view some questions. Biologists may change their theories of sexual selection as cultural values come to support gender equality.

Finally, what are the transmission mechanisms? It is possible now to write down both scientific and technological ideas. Yet the history of technology tells us that technology only rarely is transmitted through written texts alone. It instead is transmitted by personal interaction and by the movement of the tools and machines that embody the technology. This was even more essential before the development of writing. The importance of human interaction means that it is quite easy for a society to forget how to do something if it ceases to do it for a generation or more. Tasmanian aborigines lost understanding of many of the tools used by their Australian forbears; thousands of years later, Europeans lost understanding of the architectural techniques of the Roman Empire. Written texts may help later generations recreate an idea but not without considerable difficulty. Transmission to other societies usually involves migration of people, at least temporarily. Scientific ideas travel better in written form, but here too personal contact is important: The public is often surprised at how much time scientists spend at conferences precisely because non-scientists think that written transmission works better than it does. Nevertheless, we can pay particular attention to how written texts have been produced, conserved, organized, and shared at different times and places in history: We will find that

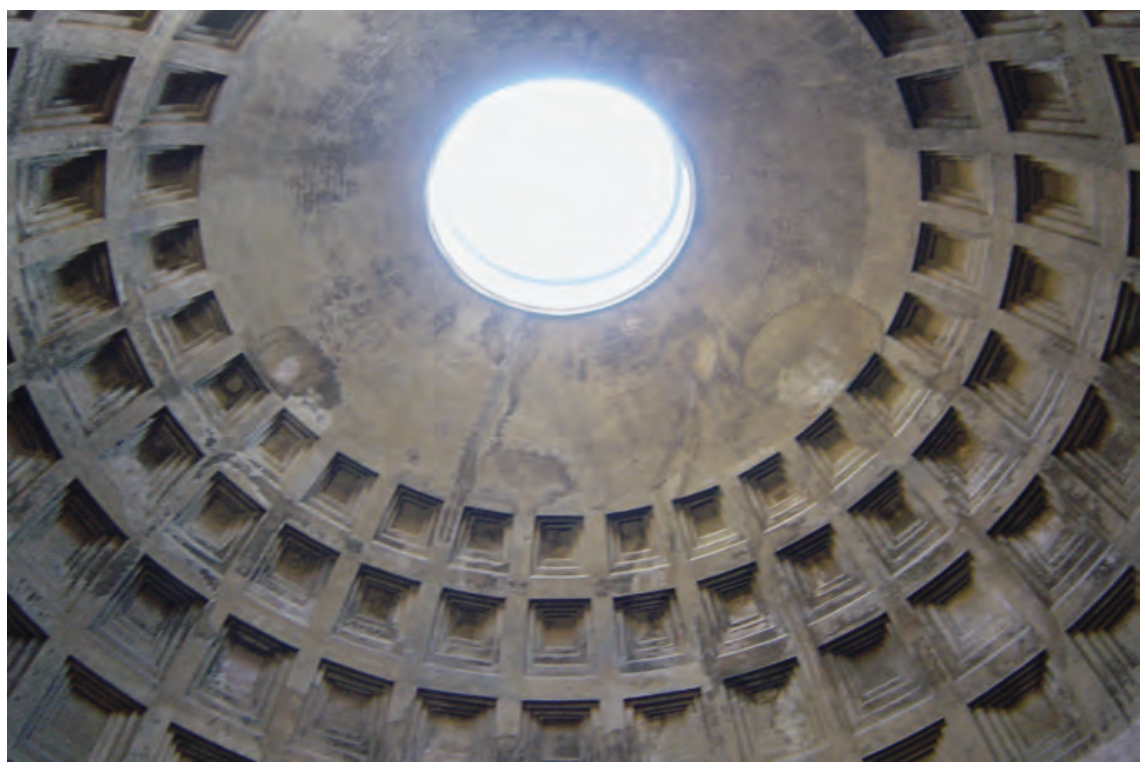

Illustration 5.17

Pantheon Dome built during the Roman Empire. Later Europeans marveled at its construction 


\section{BOX 5.6 INCREASED COMPLEXITY}

We should stress that evolutionary processes are unpredictable: One just cannot know what mutations may appear in future. However, if the selection environment favours certain sorts of mutations, we can have some confidence that - over an appropriate timeframe - some such mutations will appear in the population and be favoured by the environment. For biological evolution, the timescale is many generations - unless humans come to use genetic engineering - and so we can imagine a reasonably stable human nature going forward. The other types of evolution that we address in this book occur much more quickly.

It is often observed that evolutionary processes result in increased complexity. Life on Earth began with single-cell organisms (which are still the most common), and evolution generated much more complicated organisms (chapter 2). Contemporary economies are more complex than those of previous centuries, with a far more extensive range of goods and services, trade links (and supply chains) throughout the globe, a wide range of financial products, and so on. Nevertheless, the idea of increased complexity should not seduce us. It is too easy, for example, to tell a story of political evolution from simple tribal bands through to contemporary bureaucratic states. Yet often in history, simple political structures compete for influence: Think of the impact that non-governmental organizations exercise in contemporary societies or that city states exercised in an age of territorial states. We will in what follows often have cause to emphasize increased complexity, but also to recognize that very simple structures are often selected for - just as simple one-cell biological organisms have prospered to this day.

an array of technologies and institutions have been employed for this purpose with varying degrees of success.

\section{Questions}

1. How important do you think alcohol was in the history of agriculture?

2. Reproduce Figure 5.1 for alcohol, irrigation, or textiles. Explain each box and arrow.

3. Do you think merchants have been treated unfairly in history? Why?

4. We will often talk about roads and bridges in later chapters. Why?

5. We will also get excited about coinage. Why?

\section{Readings}

Barbier, Edward B. 2011. Scarcity and Frontiers: How Economies Have Developed Through Natural Resource Exploitation. New York: Cambridge University Press. 
Bostwick, William. 2015. The Brewer's Tale: A History of the World According to Beer. New York: Norton.

Goody, Jack. 2012. Metals, Culture and Capitalism. New York: Cambridge University Press.

Gordon, Stuart. 2018. There and Back: Twelve Great Routes of Human History. New Delhi: Oxford University Press.

Masschaele, James. 1993. "Transport Costs in Medieval England," Economic History Review 46: 266-79.

Yang, Bin. 2019. Cowrie Shells and Cowrie Money: A Global History. New York: Routledge. 


\section{Grappling with \\ "civilization"}

\section{The development of cities, states, and writing}

\section{Guiding questions}

What is "civilization"? Is this a useful concept?

How, why, when, and where did large cities and states emerge? What were the key characteristics of these early cities and states? Why, how, and where did kings assume power in these states? Who were these kings? How and why were particular institutions, including laws and schools, developed?

In particular, how, where, when, why, and by whom was writing developed?

What role did war play in human societies? Why was war so frequent? How did the technology and institutions of war change through time? How did war reflect and alter relations between societies? Who were the key actors in war-making and how did they behave and why?

Relationship to other chapters: In many parts of the world, the cities and states first mentioned in chapter 4 grew to an unprecedented size. These were all dependent on local agriculture (chapter 4) and trade (chapter 5). Cities and especially states will be of great importance throughout human history in most parts of the world; we discuss their early characteristics in this chapter, and how these change (or not) in later chapters. Writing is of central importance both to history itself and to the 
writing of history. Written documents are of crucial importance in most times and places addressed in this book; historical understanding generally reflects our interpretation of written records. We have mentioned war in several preceding chapters, especially 4; here we flesh out the causes and effects of war. Wars will prove to be a central element of many succeeding chapters; we can build on our understandings in this chapter to trace how the practice of war - and its effects - change across time and place.

"Civilization" is a vague and troublesome word. Given that "civilized" commonly signifies that a society (or person) is advanced and in some ways superior, it is potentially offensive to argue that only some historical societies represent "civilization." It is best to avoid a term that has such unfortunate connotations. For our purposes, there is a more central problem in that "civilization" conflates multiple themes. Our efforts to track the interactions among themes in this book will be defeated if we jumble these together. We should then identify the key themes generally captured within discussions of "civilization" and discuss why these appear to have emerged at about the same time. This will allow us to employ much clearer terminology and analysis than by addressing "civilization" itself.

There are three key phenomena generally implicated in historical discussions of "civilization." The first is the emergence of cities. The second is the emergence of states. The third is the development of writing and written records. Other criteria are sometimes mentioned, such as shared religion, social differentiation, monumental architecture, and occupational specialization; you will find that these are connected to the three transformations listed above. It is not hard at first glance to see a common cause of these three transformations: Only with a substantial agricultural surplus were cities, government bureaucracies and armies, and scribes possible. It is also straightforward to identify interactions among them: Governments needed to be based somewhere and needed to keep records. These developments in turn had consequences for other phenomena: They encouraged occupational specialization certainly, increased income inequality most likely, and increased trade necessarily (for cities and bureaucrats need to be fed, clothed, and sheltered). Traders, we shall see, often also kept records. We will flesh out these myriad thematic interactions (and more) in what follows. We will in particular discuss the frequency of war among states.

For some authors, "civilization" also implies cultural coherence. They then emphasize the differences between Egyptian civilization and Mesopotamian civilization. A world history text should always be alive to both interactions across and similarities between societies. We will occasionally use the word "civilization" in later chapters to refer to a particular society, but without making 
any assumptions about how similar or different it is to any other. That is, we will treat "civilization" as a synonym for "society."

Cities and states are each political in nature, but both are also situated physically and necessarily shape and are shaped by the natural environment. We will discuss several distinct political institutions in this chapter, often addressing how these evolve. We will explore how political institutions spread across societies. Writing is a technology. Other themes that receive significant attention in this chapter include economy, social structure, and science. Cultural attitudes are very important in justifying states. We employ evolutionary analysis in our discussion of the rise of kings.

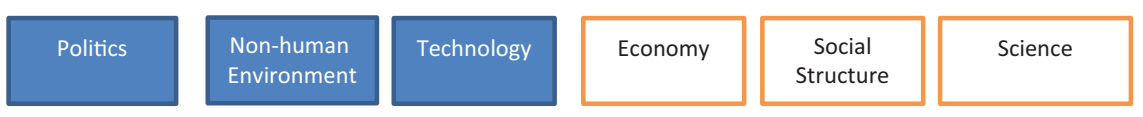

\section{The earliest "civilizations"}

The earliest society known to have had cities, states, and writing developed in Mesopotamia (present-day Iraq) about 5,500 years ago. Soon after that, we observe these three attributes in Egypt and in the Indus Valley region of presentday Pakistan and India. It could well be that the ideas associated with "civilization" had spread from Mesopotamia to these nearby locales. Later still, the three attributes are observed in northern China: This development was likely independent. Thousands of years later, the three attributes again emerge independently in Mesoamerica. As with the development of agriculture (chapter 4), then, there were at least two and likely several independent developments of "civilization."

The eastern hemisphere "civilizations" were all based along rivers and employed irrigation. This was not the case in Mesoamerica. Irrigation had the potential to increase agricultural productivity significantly, and thus support cities and states. But large-scale irrigation required cooperation that was hard to achieve in the absence of a government that could plan and force or encourage the construction of such systems (chapter 5). Planning and supervising construction required further occupational specialization. There is an obvious synergy between irrigation and the key themes associated with civilization. Yet we should appreciate that state systems of irrigation appear everywhere to have built upon early efforts at small-scale irrigation that had been organized cooperatively.

Why Mesopotamia? It had not been densely populated at the time that settled agriculture was being developed in the mountains to the north (see chapter 4), presumably because the land between the Euphrates and Tigris rivers was swampy and received limited rainfall. Nevertheless, as agricultural populations spread south (due likely to population pressure), the area proved advantageous in several respects. With irrigation, the land became very productive by the standards of the time. It also had fish to eat and mud 
well suited to brickmaking for buildings. The region was also fortunate externally, being close and connected by water transport to quite diverse ecological regions: There was sea-based trade in the Indian Ocean more than 6,000 years ago. Mesopotamia, with its rivers linking different ecosystems, was a natural trade centre. Geographers debate the relative importance of Mesopotamia's internal and external advantages, but agree that it is reasonable to stress both types of natural advantages.

It is important to note that the vast bulk of people at this time, even within civilizations, did not live a "civilized" life. Only perhaps 5 or 10 per cent of the population lived in the cities. Even fewer were likely literate. Most peasants may have had little contact with the state beyond paying taxes. Though there had to be steady migration into cities - since mortality rates were higher than fertility rates in cities (see below) - most people stayed on the land and probably never travelled more than a few miles in their lives. They devoted almost all of their income to the basics of life: food, clothing, and shelter - and the taxes required by the state. They nevertheless played an essential role in history, for it was only by producing a surplus that the specialized activities we focus on in this chapter were possible.

It was common just a century ago to tell the story of "civilization" as an exercise of human cleverness: Some societies had been smart enough to engineer the structures of modern states and others had not. Such a story is either ethnocentric - it praises certain cultures over others - or racist - it suggests that some groups had greater intellectual capability than others. The separate emergence of states in several quite different parts of the globe suggests otherwise (as does understanding of the common genetic inheritance of humanity). We need not prohibit cultural explanations - states everywhere were justified to some extent by cultural attitudes - but should recognize that cultures themselves evolve in response to changes in circumstance. We are guided then to seek an explanation in natural environment and economy, and to conclude that states emerged where agriculture could and did advance farthest. Since states require a steady stream of taxes, they were most likely in agricultural societies with regular harvests that were easy to tax. We should also appreciate that while states provided some public services, many peasants sought to escape states by fleeing to "barbarian" lands that did not collect taxes: States were not universally applauded as an advance in the human condition by those within them.

\section{Cities}

\section{Emergence of early cities}

Villages are a common characteristic of agricultural society. They allow farmers not only to socialize but to cooperate in such matters as digging wells for water. Villages must remain small in order for farmers to be able to walk to their fields. 
Only once farmers generate a surplus that can support a large non-agricultural population can larger towns or cities be created.

Catalhoyuk (just southeast of the modern city of Konya in southern Turkey) is considered by some to be the first city: It may have had 5,000 inhabitants 7,500-9,000 years ago. Artisans over time produced tools, textiles, pottery, and metal goods in this city. However, it was likely the practice of crafting products from local obsidian that first supported urbanization there. Jericho in the Middle East (north of the Dead Sea in the Palestinian Territory today) was another early small city. Interestingly, neither centre was surrounded by a rich agricultural area (as most later cities would be): They served as centres of trade and industrial production. Still, they must have had food sources nearby. There is evidence of hunting - but we have seen that this alone cannot support large population densities. There is no evidence of social stratification in either city, but there were religious murals in both.

The first cities in Mesopotamia emerged at least 5,500 years ago. Mesopotamian cities had 5,000-25,000 people by 3000 все. A thousand years earlier the largest urban centres in the region possessed no more than a few hundred.

Cities likely emerged separately in at least seven different places: Mesopotamia/Anatolia, Egypt, the Indus Valley, northern China, the Niger River in Africa, Mesoamerica, and the Andes (there were cities in the Andes and nearby coast from about $2500 \mathrm{BC}$ ). They absorbed 5-10 per cent of the population in each place; there simply was not a large enough agricultural surplus

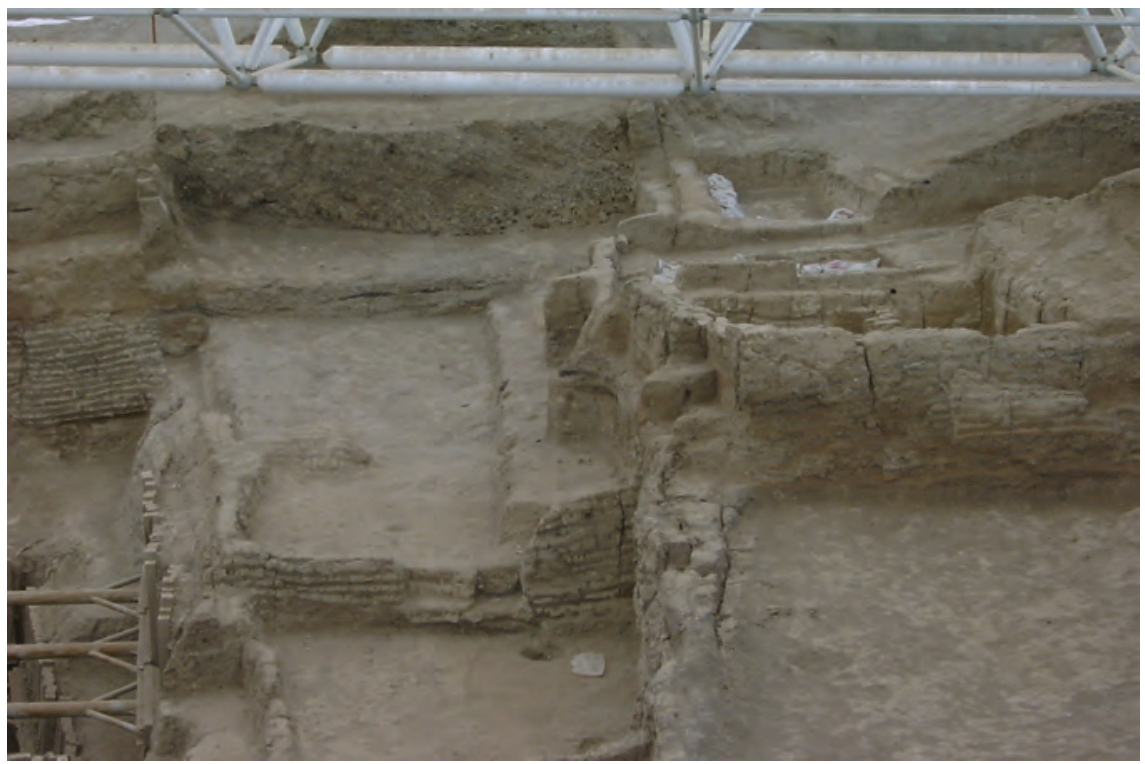

Illustration 6.1

Excavation at Catalhoyuk

Source: Photograph by Stipich Béla, CC BY 2.5 


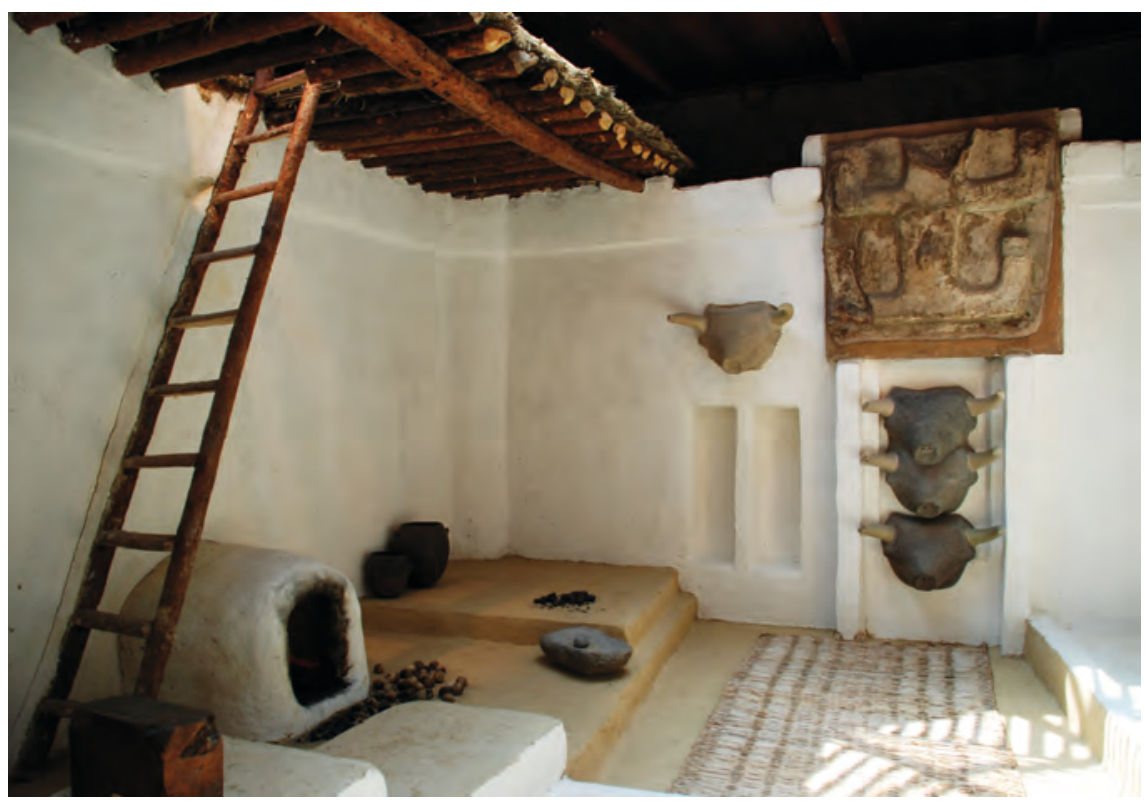

Illustration 6.2

Room reconstruction at Catalhoyuk

Source: Photograph by Elelicht, CC BY-SA 3.0

to support more. Note that cities predated the other key elements of "civilization." Some moderate degree of urbanization was possible without an elaborate state or written records.

Cities grew in size over time. The emergence of large cities in the eastern hemisphere may be associated with the use of ploughs pulled by animals, which increased agricultural productivity (recall that there were no draft animals in the Americas). The earliest cities seem to predate the use of bronze, but the development of bronze and its effect on agricultural productivity would encourage the expansion of cities. So also would the expansion of trade, for merchants tend to concentrate in cities. The rise of Mesopotamia and other civilizations around 3200 BCE may also have reflected a cooler dry climate, which encouraged human populations to concentrate geographically.

Only grains could support large cities (or armies) before the advent of railways in the nineteenth century CE. It would have been prohibitively expensive to transport the far larger mass of root crops required to feed people. Therefore, societies in the tropics that developed root-based agriculture around yams or taro did not develop large cities. We see here one of the reasons that highland New Guinea did not follow the historical path that other early centres of agriculture did (chapter 4). As for Africa, its agriculture mixed grains and tubers. The one glaring exception to the "only grains" rule is the potato, which would prove to be one of the most nutritious foods in the world and did provide the major food crop to many South American cities (though these generally also relied to a significant extent on corn). 
Cities also need a reliable source of water. Rivers and lakes could provide water, and also facilitate the transport of food and building materials to the city. Cities also drilled wells into underground aquifers. There are several natural springs near Jericho. Water sources often limited city size. As cities grew, they often polluted their waterways, forcing the city to (decline or) pipe in water from farther away.

The idea of cities spread, perhaps in large part as merchants from early cities sought to trade with nearby regions. There is at least one city of 170 acres in Central Asia along the Amu Darya (Oxus) River from 2200-1700 BCE. Irrigation was pursued there as well.

There were eight cities in the world with more than 30,000 people by 2250 BCE, but sixteen by 1500 вСE. These cities are small by our standards, but we should still marvel at the ability to provision that many people given the agricultural and transport technologies of the time. City size depended on the amount of territory from which a (small) agricultural surplus could be obtained. The first city of over 100,000 was Thebes in Egypt, Babylon in Mesopotamia reached 200,000, and Rome only surpassed 500,000 in the last centuries BCE. Each of these cities had access to extensive water transport. They also, as we shall see, exercised political control over the lands that fed them.

\section{The nature of early cities}

Cities from the beginning were sites of occupational specialization. Cities were the usual site of the pottery and metalworking that soon followed the development of agriculture (see previous chapter) - or nearby, if too much heat and pollution were involved. Excavations of Anyang, the last of the Shang dynasty (see chapter 7) capitals in China, have exposed two bronze workshops in the city and many artisanal workshops producing a range of goods. Artisans needed to sell their products, and therefore merchants also inhabited cities. Though merchants and artisans often farmed on the side, urban markets developed to feed those who did not feed themselves. Such markets usually operated daily since many foods did not preserve well. Urban riots were not uncommon if food prices rose.

Indus Valley cities flourished from 2300 BсE. There is evidence that these relied on animal herding as well as flood-based agriculture. The Indus cities were then abandoned about 1500 вСЕ. Floods that changed the river course may have ended the civilization. However, we do not know: The script employed in the Indus Valley has never been deciphered. Though we know that there was trade with Mesopotamia, Mesopotamian records tell us little about the Indus (it may be that Mesopotamian merchants did not travel there but traded through intermediaries in Bahrain and elsewhere). We must speculate on the basis only of archaeological remains. One heated debate involves what type of government there was, and what the degree of income inequality was. Some have claimed that there is no evidence of large state or religious buildings, though others have demurred. There do seem at least to have been large baths, 


\section{BOX 6.1}

\section{CITIES IN HISTORY}

You might marvel at the size of cities, for the simple reason that these require both a significant agricultural surplus and the ability to transport this (and building materials and industrial raw materials) to the city. The size of a society's cities gives a rough indication of the state of its agriculture, transport infrastructure, and trade networks or bureaucratic organization (for someone has to organize the movement of all that food). City size is thus a rough indicator of economic capability through much of human history (the most recent century being an exception in which many of the world's very big cities are in relatively poor societies; chapter 31). At different points the text will indicate where the largest cities in the world were (In $500 \mathrm{CE}$ some of the largest cities were in Italy, Mexico, and China; in 1000 in Central Asia, Iraq, and Peru; in 1500 in China, India, and Turkey).

It is important to remember, though, that cities serve a variety of roles. They are centres of trade, industry, religious observance, and government. Through most of world history, the largest cities in the world will be capital cities. This should serve as a warning that city size might reflect simply the way a state organizes its bureaucracy. Moreover, one city may be huge because it combines government, trade, and industry, while another society pursues these activities in separate cities. In sum, the distribution of the world's largest cities is a very rough guide to relative economic development across societies.

Trading cities tend to be organized in hierarchies. The largest city in a region is a centre of inter-regional trade. Trade goods then move outward to smaller cities and on to local towns. Government bureaucracies concentrate in capitals, but usually smaller numbers of bureaucrats work in smaller centres. The existence of one large city in a region commonly implies a hierarchy of smaller centres in that region. Zipf's Law suggests a tendency for the largest city to be twice the size of the second largest, three times the size of the third largest, and so on. But the relative size of the largest city can vary considerably across societies: Buenos Aires dominates the urban landscape in modern Argentina because it is both the capital and by far the major port.

which may have served a religious purpose. The Great Bath at Mohenjo-Doro measured 12 by 7 metres, but some think that this may have just been a reservoir. Tellingly, there is no evidence of elaborate burials.

With the possible exception of the Indus Valley, cities over time did become associated with substantial income inequality. Large temple and palace complexes characterized most cities. These were often built of stone. An elite of rulers and priests could live in relative luxury. In Anyang, even the palace complex was made of wood and mud with beaten-earth floors - but poorer people had below-ground pit dwellings. Burial sites displayed considerable differences in wealth. There were in Anyang burial chambers with jade, bronze, chariots, and evidence of human sacrifice: One tomb had three hundred skeletons of sacrificial victims. Human sacrifice accompanying death is indeed perhaps the 
most significant indicator of social inequality during this period (see our discussion of religion in chapter 8), and is found in Egypt, Mesopotamia, and China (and later in Mesoamerica). Human sacrifice in history is generally but not quite always inflicted on the poor and weak by the rich and powerful - though defeated warriors were also sacrificed.

In Shang and Zhou China, another marker of social inequality involved the use of bronze. The average person could not afford bronze, except perhaps for the cutting edge on a plough. However, bronze plates and figurines filled the homes and tombs of the rich. These works of art not only employed an expensive material but were intricately crafted by skilled artisans. One tomb of a provincial governor contained eleven tons of bronze materials.

As in most human societies, differences in wealth and power supported differences in social status. These were often recognized legally: The (legal) Code of Hammurabi from 1750 вCE establishes different penalties for crimes by social class. The societies of this time may have been the most unequal the world has ever seen. They were undoubtedly dramatically different in this respect from egalitarian hunter-gatherer and early agricultural societies.

We see records of slave trading as soon as there are written records, and so can presume that slavery was an early characteristic of cities. Slaves either were captured in wars, convicted criminals, or impoverished people who sold themselves into slavery. The first slaves seem to have been primarily women, and these often worked as prostitutes. Though slaves could buy their freedom, slavery was usually hereditary. The price of a slave was about double the annual unskilled wage in Mesopotamia: It was thus possible but far from easy to buy one's freedom. High rates of interest (of one-fifth or even one-third annually) meant that bad harvests or other economic misfortunes could quickly force the poor into slavery to feed their families. In Mesopotamia, the male head of household could sell his wife and children into slavery. Mesopotamian rulers would occasionally free debt slaves. It seems that the number of slaves was small, and that norms encouraged good treatment of slaves. Slavery was also practised in China, where it seems that most slaves were war captives. We will discuss slavery in more detail in chapter 9 when it becomes an even more pervasive feature of human societies.

In many ways, even the earliest cities would have had characteristics familiar to modern city-dwellers: economic, political, and social stratification; market transactions; production of goods and services; occasional festivals; religious establishments; probably prostitutes and taverns (alcohol, as we have seen, is observed in all early societies), and thieves. Since cities struggled to provide clean water, fermented beverages such as beer or wine were often particularly prevalent in cities. Cities allowed for (and indeed existed because of) considerable occupational specialization: traders, artisans, administrators, priests, and unskilled workers; with the dawn of writing (see below) scribes and schoolteachers joined the mix. At the bottom were those who had somehow to remove human and industrial wastes from the city: Such people were necessary but disdained in most societies. 


\section{Effects of cities}

Cities had many other effects beyond facilitating occupational specialization and income inequality. As centres of trade (and sometimes diplomacy), cities were places where people from different cultures would interact. We start to see a clear idea of "foreigner" during this period to refer to people with different language or dress or customs, from a different place, and often but not always from a different state. In Egypt there are many official documents espousing the view that foreigners are inferior, yet many other documents refer to marrying foreigners, freeing foreign slaves, and other friendly interactions. Likewise, the Rig-Vedas of India often disparage other groups but at times ally with them. Public disparagement of foreigners would allow states to justify both wars and slavery. Trading relations between cities would nevertheless require some accommodation with "foreigners."

Cities were very unhealthy until the development of modern water and sanitation systems just 150 years ago - though the Indus Valley is justly celebrated for early efforts at piping water into cities and sewage out. Cities thus had a direct negative effect on population growth. This was especially the case in early cities where humans lived near to animals (until the age of the automobile many horses or camels were required in cities for transport purposes). Removing human and animal wastes was always an urban challenge. If animals were slaughtered in or near a city, blood and carcasses had to be removed. Tanneries used urine and chemicals to treat hides. Metalwork polluted the air, as did fires for heating and cooking. Filth and pollution were a characteristic of urban lives from which even the rich could only partially escape. The excess of deaths over births in cities meant that cities survived only through continual migration from country to city. While all citizens suffered from the polluted city environment, artisans in particular occupations such as metalwork likely suffered more than average.

Once we have cities of thousands of people and trade over long distances, we also enter a new disease environment: Cities might develop immunities such that some diseases became childhood diseases (chapter 17), but trade would ensure occasional epidemics as new diseases entered. Diseases could spread quickly in a dense urban environment. War (see below) was another mechanism for transmission of disease for it brought populations together under unhealthy circumstances.]

In the extreme, cities could disappear due to war (with victors often enslaving those who survived an attack), epidemic, climate change, or perhaps environmental decline. Dozens if not hundreds of prehistoric cities disappeared in these ways. Some of these cities provide great resources to modern archaeologists. Many others are undoubtedly completely unknown. The rural populations surrounding these cities might (or not) survive even as the city fell.

Cities had many further effects. As nodes in trade networks, they were critical to the transmission of ideas about technology, culture, economy, politics, and art. They encouraged artistic production, in large part so that state and 


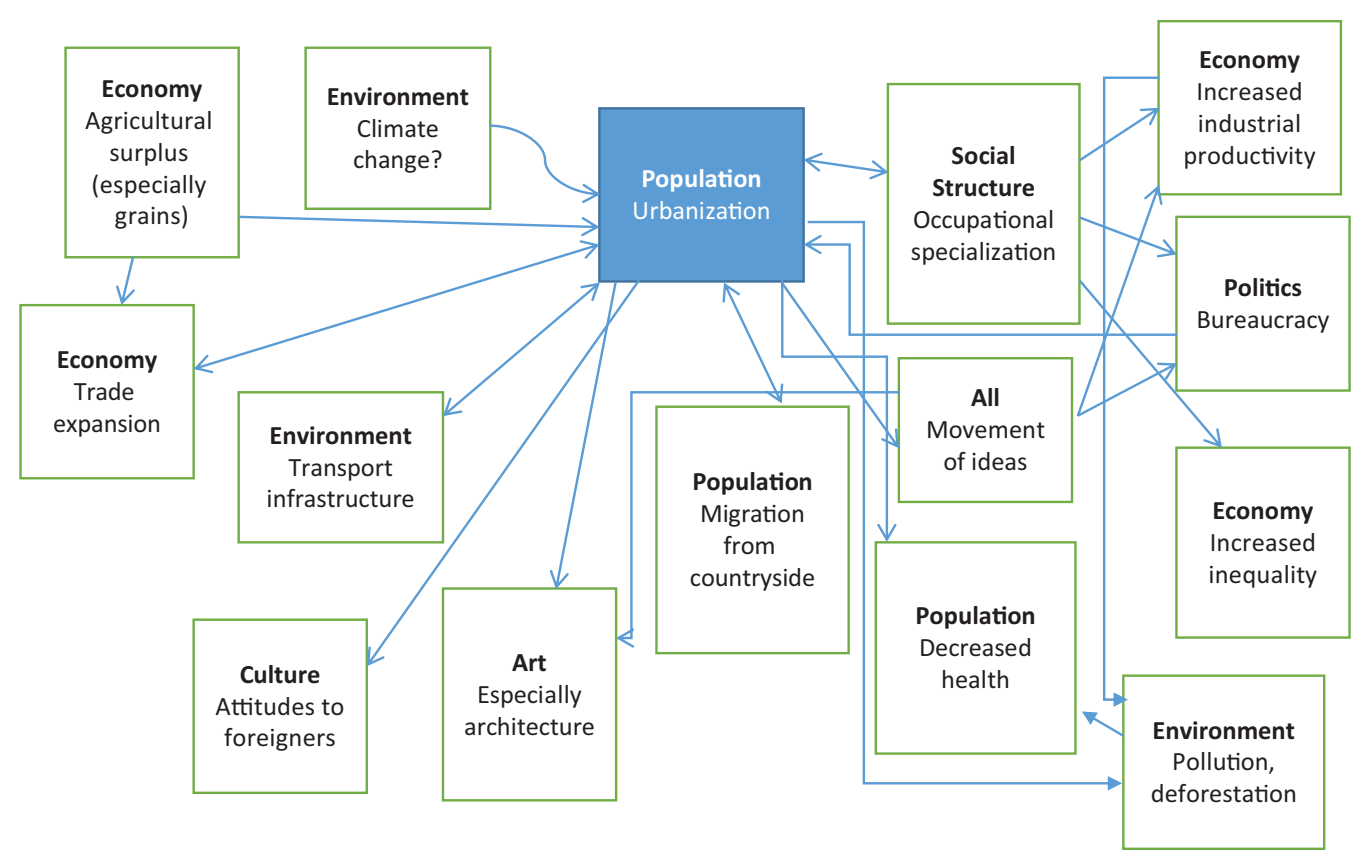

Figure 6.1

Influences on and effects of cities

Note: The wavy line again indicates a possible relationship. Slavery has not been included as it may not be an effect of cities themselves but rather of trade and war.

aristocracy could display their social superiority. Particular mention might be made of city design itself: Walls, roads, theatres, and aqueducts would be duplicated through the ages, with capital cities most likely to be copied. Ancient Anyang is notable here for the practice of geomancy: explicit urban planning to reflect divination from topographical features. Less favourably, cities were often associated with deforestation, as much wood was needed for both construction and fuel: As nearby wood resources were harvested, cities often had to seek wood from farther away at considerable expense.

\section{Architecture}

Monumental architecture, celebrating state or religion, was typical in the earliest cities as it is today. The state needed buildings to house rulers and bureaucrats. The religious authority needed buildings to accommodate priests and ceremonies. Both states and religious authorities almost everywhere built to a size and style that would awe and inspire. We cannot know to what extent this was conscious or subconscious: Self-aggrandizement may have guided authorities as much as a desire for social control. In any case, millennia later we can still be awed by these structures. As with other works of art, monumental architecture appeals to basic aesthetic tastes. We are also impressed technologically, for 
large-roofed buildings demand considerable engineering expertise. In addition, we are impressed economically that early societies, with limited agricultural surplus, could build such things. It is hard to measure the human hardship endured because of these building projects or to compare this to the pleasure that people through the ages have enjoyed as a result. It is also hard to estimate the role that these buildings played in encouraging loyalty to state and religion.

There are some examples of monumental architecture even in Catalhoyuk. These structures were likely temples: There is some speculation that these earliest cities were in large part built around religious shrines. These buildings would have required some expert builders. Yet the very earliest cities provide no evidence of significant income inequality: Both houses and burial sites seem broadly similar in size. These earliest monuments - unlike later monuments for which we have written records - may reflect collaboration rather than the command of a centralized state or religious authority.

Egyptian pyramids date from the third dynasty (twenty-seventh century BCE; see chapter 7) and almost a hundred were built. Most collapsed over time due to the placement of stone on top of mud brick in construction, and stones later being taken off by thieves. However, pyramid builders learned from their mistakes and built better pyramids with time. Pyramids were over ten times taller than any previous structure; the world would not witness another such leap in height until the skyscraper of modern times. Pyramids housed chambers in which treasures were deposited in honour of the dead. Theft was a constant problem, even at the time, and rulers developed many strategies for preventing this - which generally failed. Pyramids at first had a religious significance associated with sun worship, but became over time more a symbol of the ruler's wealth. The biggest pyramid is from the fourth dynasty, about $2500 \mathrm{BCE}$, for the pharaoh commonly known today as Cheops. It is estimated that 84,000 workers working eighty days a year would have taken twenty years to construct this pyramid of 2.3 million limestone blocks. Cheops and his son and grandson built most of the giant temples that still attract touristic attention. (The three largest pyramids from this time contain more stones than all other pyramids ever built by Egyptians.) Over time, pharaohs devoted more effort to temples and palaces than to pyramids; these have generally not survived but were likely very elaborate. Early Egyptian towns were also fortified, but these fortifications also have not survived.

Pyramids, we should note, were developed later but independently in both Mesoamerica and South America. This may simply be because it is easier to build tall structures in a (solid) pyramidal shape than in any other. Pyramids may also be aesthetically pleasing because they mimic mountains. The pyramids in Mesoamerica were richly decorated, perhaps because they were regularly employed in religious ceremonies.

Ziggurats - very tall brick structures with several levels and a flat top that likely housed temples (Herodotus, the ancient Greek historian, said so but there is no archaeological evidence of such temples) - became common in Mesopotamia from at least the third millennium всE. The highest was the Tower of Babel 


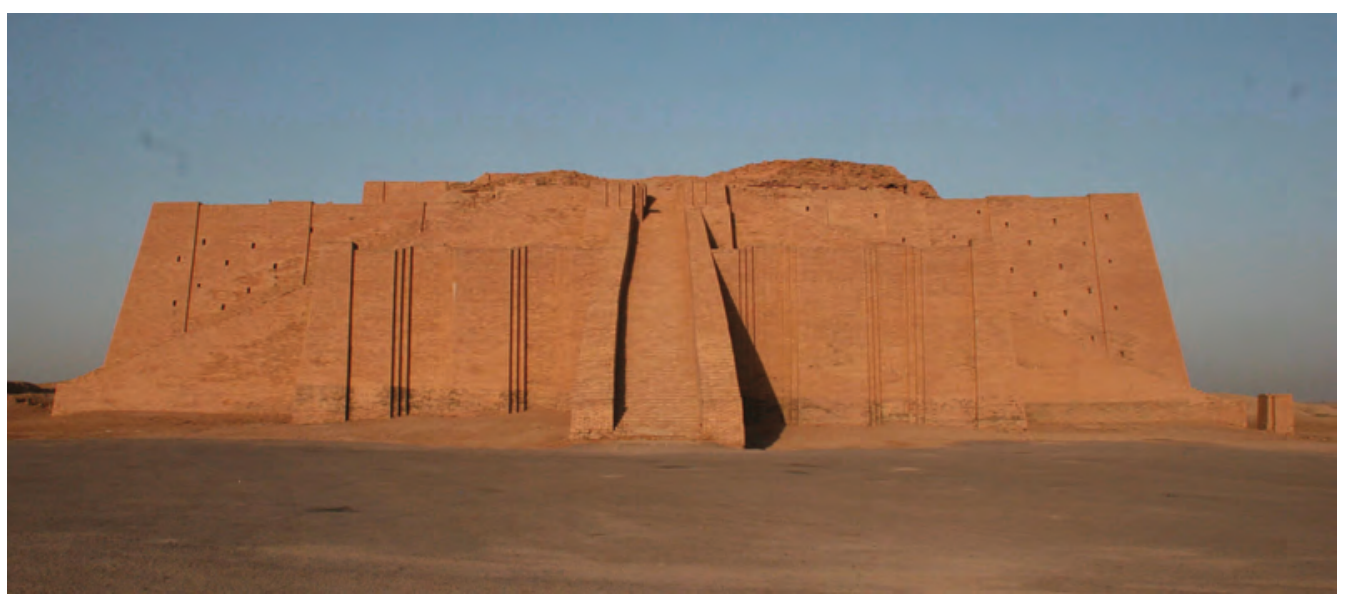

Illustration 6.3

Great Ziggurat at Ur in Mesopotamia

in Babylon (mentioned in the Christian Bible) from the first millennium всE. Mesopotamian authorities also valued urban gardens where fruits, vegetables, and herbs were grown under shade trees, usually date palms; these gardens were also a place for socializing. The "Hanging Garden" of Babylon - a garden built on raised terraces - was celebrated as one of the wonders of the ancient world.

The palace at Mari on the Euphrates River on the trade route from Mesopotamia to the Levant was famed at the time (2100-1750 BCE); its main floor had 250 rooms and courtyards. The king of Ugarit in the Levant asked the Mesopotamian king Hammurabi to take him there. Notably, a similar style in temples and palaces is observed throughout the Levant despite political disunity; authorities borrowed elements from both Egyptian and Mesopotamian architecture.

There were also massive palaces on the island of Crete (in present-day Greece). The palace at Knossos covered 3 acres, had 1,000 rooms, was made of stones without mortar, and had water and drainage systems and even some flush toilets. It thus exhibits excellent engineering skills. It is not entirely clear what its purpose was - maybe royal and religious functions along with state storage facilities. There were also later palaces in Mycenae on the Greek mainland.

There was less emphasis on monument building in Shang or Zhou China. Still, there was a large building in the centre of Anyang surrounded by columns that would likely have supported a thatched roof. It was presumably a palace or temple. However, like many other ancient edifices made of wood, only the foundations survive.

There was monumental architecture at this time in other parts of the world also. These indicate that cities were not necessary for monumental architecture. Stonehenge in England - one of hundreds of stone henges built at the time in northwestern Europe - is the most notable of these. Tourists can still visit the 


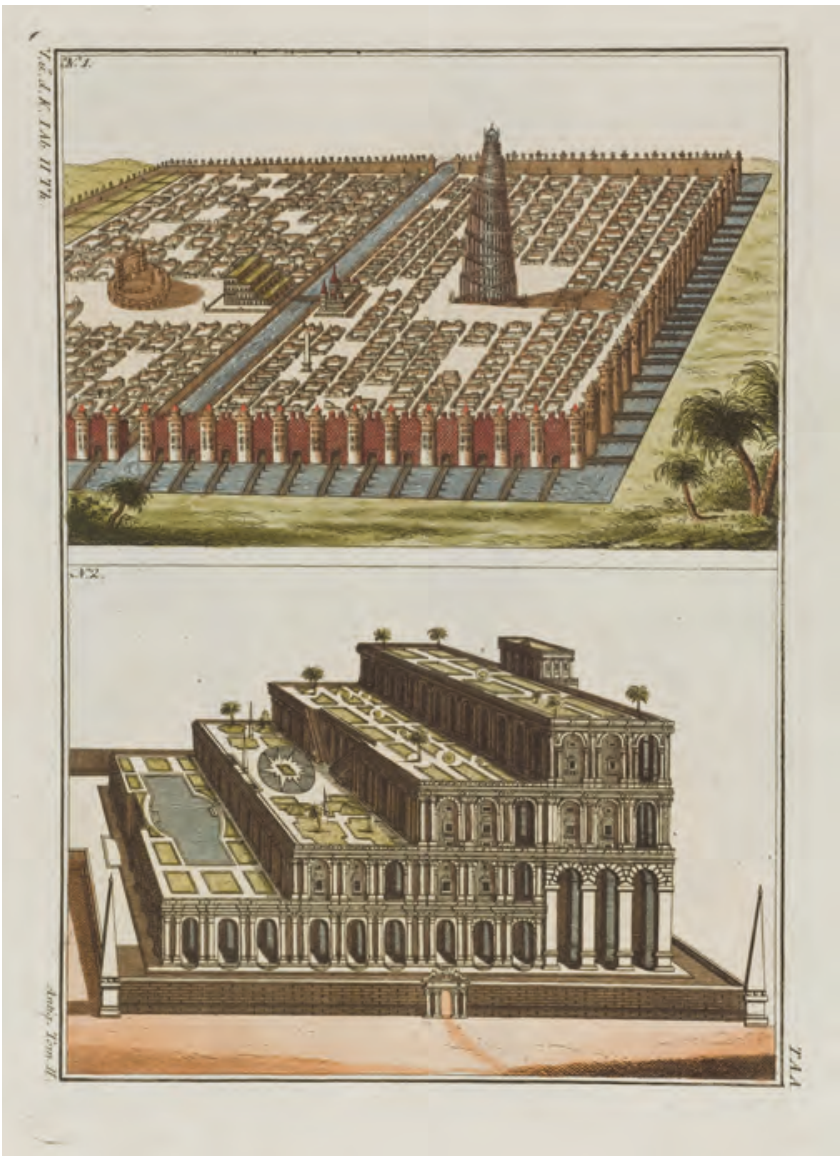

Illustration 6.4

Representation of Babylon and the Hanging Gardens

Source: Wellcome Trust, CC by-SA 4.0 circular rings of huge stones today. Stonehenge was likely constructed between 3100 and 1500 вСЕ; agriculture may have just reached the area as work began. We know nothing about the people who built Stonehenge: There was likely some political authority to organize the movement of massive stones (the biggest ranging from 30 to 50 tons) hundreds of kilometres and then into position. It is estimated that some 10,000 person-years in total would have been required but we do not know exactly how such huge rocks were manoeuvred into place (some lying on top of other upright stones). Stonehenge also speaks to very careful tracking of the movements of heavenly bodies, for the stones are aligned to capture the movements of sun and moon, and especially the winter and summer solstices (it being a matter of critical importance to early farmers in temperate climates that the days start getting longer again).

The builders of most ancient monuments are anonymous. These must have learned "on the job," experimenting with different designs and materials, trying to make structures just a bit taller or wider. Note that the pyramids and henges admired today were not the first to be built but benefited from decades or centuries of experiment. There must, then, have been some mechanism for transmission of building techniques across generations, either within families or via apprentices. There were no schools of engineering in ancient societies. Political authorities would turn to builders who had previously had success building or who at least had held senior positions on successful building sites. If war, epidemic, or just changing tastes should interrupt a building programme for a generation, the knowledge of how to build certain structures could easily be lost. There would be many centuries, for example, in which Europeans would marvel at the buildings of the Roman Empire but have no idea how to reproduce these. The greatest monuments tend to emerge, then, in times of political stability. 


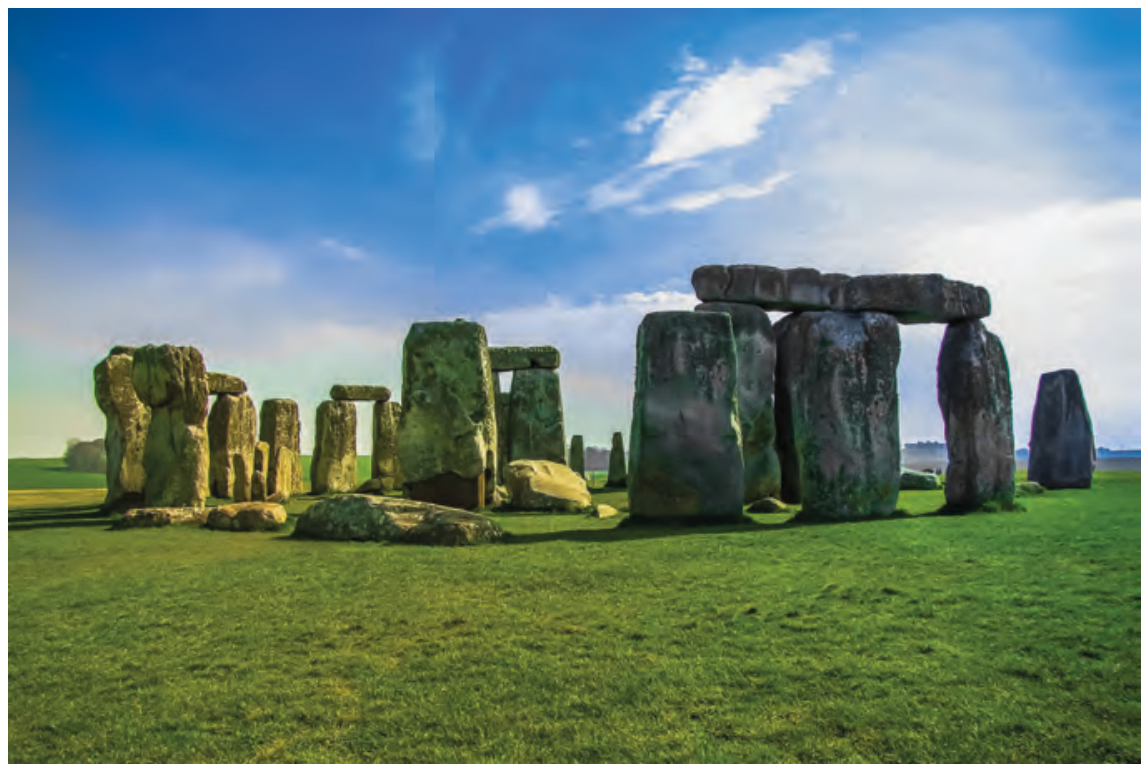

Illustration 6.5

Stonehenge

As builders experimented, there would be both technical and aesthetic selection. The technical selection is obvious: Buildings that collapsed needed to be redesigned. Yet aesthetic selection was equally important: These buildings were intended to awe and impress and therefore had to look good. There was likely also some degree of cultural selection and political selection for buildings that particularly appealed to local tastes (and religious beliefs), and political ideology.

The earliest roofed buildings employed the "post and lintel" technique in which horizontal beams were laid across vertical columns. Different styles of columns would be developed in different times and places, and often spread elsewhere (the Han Chinese - chapter 10 - appear to have borrowed fluted columns from the west). Though such buildings could be magnificent, there was a limit to the size of a room that could be roofed by beams without recourse to internal columns. Though there may have been experiments with vaulted ceilings in the third millennium BCE, builders in Mycenae around 1300 вСЕ appear to have established the possibility of domed architecture. Over the next centuries, builders would develop techniques for ever more substantial structures. The Roman development of concrete was an important advance. So also was the invention of the crane, without which some of the earliest wonders of the world such as the Colossus of Rhodes (a giant statue guarding the harbour) and the Lighthouse at Alexandria would have been impossible to build. 


\section{Challenges facing agents: Builders}

The builder wishes to gain a reputation for buildings that will both remain standing and look good. As political and religious authorities often want to outshine their predecessors, builders will experiment with different techniques and designs. Specialized knowledge regarding construction techniques is of paramount importance. Builders need somehow to transmit this specialized knowledge across generations. This is most likely to occur if there are a series of building projects pursued over a period of many decades or centuries.

Through much of history, states or religious authorities finance the largest monuments. The builder must maintain good relationships with these authorities.

We know more about palaces and temples - which occasionally survived than about the homes in which the bulk of the human population lived. Egyptian cities tended to move when these were rebuilt, but Mesopotamian cities instead just built on top of older structures (there was more room along the banks of the Nile for new cities). In both cases, little survives of the earliest structures.

Houses in the Levant at the time had earth floors. These were covered with stones or rugs. There were small windows in living areas. Homes had a small number of rooms, including storage space; they could have one large room with pillars to support short roof beams made of wood. Homes often had a central courtyard used for cooking and relaxing. Courtyards were even more common in Mesopotamia. We also see mud-brick houses with central courtyards in the Indus Valley. In northern China, semi-subterranean houses gave way to aboveground construction using wood: This must have been much drier. Houses in the south of China were often built on stilts as protection from flooding.

\section{Organized states}

\section{Emergence and expansion of early states}

Our best guesses at how organized states emerged come from our observation of contemporary societies (recall Box 4.2 on political leadership terminology). When the inland tribes of New Guinea - which engaged in a mix of agriculture and hunting/gathering, and were separated from each other by steep mountains - were first encountered by outsiders in the mid twentieth century, they were often observed to be characterized by "big men": One man would exercise some degree of political power in a small tribe of a couple of hundred people, influencing decisions such as whether/when to go to war. These tribes would inhabit either one village or a set of nearby villages. That man, and perhaps some advisers, would have access to greater economic resources than others. This meant greater political and economic inequality than within huntergatherer groups. However, the power of big men was transitory: Groups might 
rebel if they disliked the big man's decisions, and the position was not hereditary. Family lineages were often important: This could lead to conflict among lineages, but it also might support a more collaborative - some have even used the word "democratic" - type of leadership in which lineages collaborated in decision-making. In other areas of new contact, such as Oceania, hereditary chiefs ruled. Chiefdoms may reflect the difficulty of deciding on who should be "big man" as tribe sizes increased. Chiefdoms comprised one to a few thousand people. Such chiefs generally, but not always, exercised far more power than big men had. It was often the case that they, rather than the tribe, held property rights over the land. In many chiefdoms, chiefs could support a number of aides from their own large landholdings and contributions (taxes, that is) from others. Since chiefdoms were hereditary, some degree of social stratification necessarily emerged. Chiefdoms were characterized by specialized farmers, soldiers, administrators, priests, and artisans. In both cases - big men and chieftaincies - political dominance was usually decidedly male.

Organized bureaucracies and perhaps more explicit claims to sovereignty characterized the "states" that emerged 5,500 years ago. States generally presumed to have the sole right to exercise violence within their borders whereas chiefs might allow subjects to fight amongst themselves. We can see how these states might have emerged gradually from a large chiefdom where the chief had several aides. Organized states carry social and political stratification to new levels. This is possible because they embrace much larger populations: The kingdoms of the Nile would have 3 million citizens.

City states, with one city and surrounding territory, were likely the first states (City states have remained a factor through much of human history, though generally on the borders of larger territorial states.) These were then absorbed over time into kingdoms comprising multiple urban centres and much later - empires encompassing many (see chapter 9). City states apparently arose independently in many parts of the world. Indeed, city states appear to have emerged everywhere that there were cities and agriculture. Farmers producing regular harvests could be easily taxed by agents representing the city: Grain farmers were particularly easy to tax as harvests were predictable and short, and grain was storable. States for the most part could only directly control flat farmland with the military technology of the day; they tried to reach some accommodation with inhabitants of neighbouring mountains or swamps. These states were at first small in scale, with perhaps 5,000-20,000 people. City states necessarily controlled a hinterland that they relied upon for food and other resources. City states also tended to rely heavily on proceeds from taxation of the trade that flowed through the city.

Since states grew out of chieftaincies and gradually extended their territories, they were inherently ethnically diverse. They may seem otherwise to us: We may think of Egyptians as one ethnic group and imagine that this was the case thousands of years ago. Yet states often work to generate a sense of common identity. Individual Nile Valley villages previously ruled separately would not immediately feel a common identity when absorbed into a larger state. 
Why did states become bigger through time? One necessary condition for a large state or empire was a sufficiently large contiguous land mass that could support agriculture. The second condition was that agricultural techniques be employed that could produce a surplus in this land area. Yet these factors alone cannot explain why states grew in size. There are undoubtedly some "economies of scale" in providing military protection: For example, you can double a city's population without having to double the length of a defensive wall. Even without economies of scale, larger states could form larger armies than smaller states. In a battle, larger armies would usually be able to defeat smaller ones. If one state could gain more from a conquered territory in taxes than it would cost to administer it, then conquest would make sense, and the state could then afford a larger army. Yet conquest may often have been motivated more by war booty: the immediate windfall in terms of goods and people taken from the defeated (see below); some states may have grown in size just because armies pursued booty and found themselves able to fund even bigger armies from taxing lands they conquered. Nor should we neglect simple glory as a motive for conquest. Rulers dotted the ancient landscape with numerous monuments to successful wars: These were perhaps intended to awe the population into submission, but served also to symbolize the pride that rulers took in their military prowess. (We discuss the motives for war below in detail, but can note here that the history of states - and civilization - is inextricably tied to the history of war.) Still, rulers could only maintain their conquests if their bureaucrats could raise enough tax revenue from conquered lands to pay both soldiers and bureaucrats.

It might seem that a potent offence was the best defence against being invaded (and robbed) by others. If you are conquering others, they cannot be conquering you. Yet unless one state could exercise dominance over an entire region, its conquests were likely to stir antagonism and jealousy among other states, and thus invite attack.

Rulers may also benefit from conquest if trade is encouraged within the new larger state and the ruler can tax this trade. This benefit may be less immediate than others, for merchants must recognize new opportunities (or government officials: the state managed some types of trade). Yet it might become substantial over time. And it is one of the most obvious ways - coordination of irrigation works and transport infrastructure and systems of weights and measures being others - in which the ruler's mostly selfish quest for conquest might yield economic advantages for the society as a whole.

Urban agrarian societies at times expanded into territories still occupied by hunters and gatherers. Though the latter were stronger and healthier (see chapter 4), the former had larger numbers, specialized weapon-makers and soldiers, and often immunity to diseases that they would spread among hunters and gatherers (we discuss epidemiology in chapter 17).

We have analysed conquest above from the perspective mainly of rulers. We might also study conquest from the perspective of the residents of the conquering states themselves. We must be careful here of analysing early states 
through the lens of more recent entities such as the British Empire: Early rulers may have had a much different relationship with their citizens. Yet it is worth appreciating that (as we shall see much later) there is debate as to whether the average British citizen benefited from the British Empire. An elite profited, and those who served in the colonial civil service or army may have earned higher incomes than were possible at home. However, the average person who stayed in Britain may have gained little and even lost out due to higher taxes to support that army (and navy). The British Empire was not, in other words, an instrument of the British people but of a narrow elite that used British nationalism to achieve public support for self-serving foreign ventures. A similar argument might apply to these early states. Kings often assigned administrators and garrisons from their home city/region to rule conquered territories. But the taxes gained flowed to the ruler, not his city. Rulers, of course, wished to maintain loyalty both in their capital and in their conquered lands. They might (or not) privilege local merchants or artisans at the expense of those in areas they captured. We should be careful of assuming, then, that the mass of people in a conquering state gained much from conquest, but those who participated in the army or administration might stand to gain a great deal.

Though early states grew in size, they remained small by the standards of later empires. (Chapter 9; The Western Zhou in China from perhaps 1100 вСЕ is arguably the first of the large territorial empires.) This is likely because there are also challenges as state size increases. In particular, it was hard to communicate over long distances at the time. The ruler would have less control over distant bureaucrats, and thus be less likely to receive enough tax revenue to fund their efforts. Ethnic diversity would also increase as states expanded. We might hypothesize an optimal state size for any time and place that would depend on agricultural productivity, military technology, transport and communication technology and infrastructure, and ethnic and cultural diversity. Particularly talented rulers (or generals or bureaucrats) might temporarily extend borders, and poor rulers might lose territories. But there would be limits to what even the most talented ruler could achieve.

The fact that currency had not yet been invented also hampered the earliest states. Farmers paid taxes "in kind", in terms of grains and animals. The state (or the religious authorities associated with the state) then had to transfer these physical goods to those in the employ of the state. One of the reasons that palace and temple complexes were so big is that products had to be stored before being spent (and of course agricultural goods would be saved from harvest through the year, and from year to year). As states increased in size, the challenges of supporting the ruler and his advisers in the capital from taxes raised in distant lands would increase.

It was common at the time for weaker states to pay tribute to stronger states. They could then maintain some independence while gaining some protection - at least from the state to which they paid tribute. For the stronger state, tribute payments could be a significant source of income, and saved them from the costs of both conquest and administering tax collection. Egypt at 
times received wood from Lebanon and Syria, gold from Nubia, and grain from Palestine in tribute.

Once some states existed, neighbouring areas often copied the state form. Military incursions from the original state often provided an incentive for neighbouring groups to organize better for military defence. For example, the Nubian chieftaincies and the kingdom of Kush (from at least 1600 всE) in present-day Sudan may have formed to counteract Egyptian incursions. Nubia does not have the extensive floodplains unseparated by cataracts that Egypt possesses, and therefore political unity was likely harder to achieve. Archaeological explorations before the Merowe Dam flooded the area in 2007 found evidence of a vibrant Kush kingdom dating perhaps from 2000 вCE that had a centralized state and profited from mining gold. States in present-day Iran and Turkey may likewise have formed in response to Mesopotamian expansionism. Indeed, the early history of what is now modern-day (western) Iran witnesses periods of Mesopotamian conquest (in which local leaders may have maintained some local control) and of local independence and even military invasions of Mesopotamia.

\section{Nature of early states}

It is always tempting to imagine that hereditary rulers were all-powerful. But difficulties in communication required the decentralization of political control. That is, rulers had to cede administrative power to local authorities. The organizational challenge of large states was to encourage local administrators to serve the interests of the state. Different states tried different strategies, but all struggled to prevent their administrators from becoming wealthy at the ruler's expense. One critical decision was whether to send administrators from the capital or rely on locals. The former might be more loyal, but the latter had local knowledge. Locals could sometimes act to reduce local hostility to the state, but they would also often lead rebellions. Some states mixed these strategies, hoping that different officials would monitor each other's behaviour. It was of course particularly important to maintain the loyalty of the army that was necessary to ensure both domestic stability and foreign peace. But local armies might come to bestow loyalty on the local administrator that provisioned them rather than the distant ruler.

States provided some important services. The armies of the state could protect both farmers and merchants from theft - though these then had to pay taxes for the privilege, and had to fear theft by the state itself. Some states supported irrigation infrastructure or built bridges. Some would use state granaries to feed their populations in times of bad harvest. Less tangibly, states tried to foster some sense of community and purpose among those they ruled. At least some of the population likely valued the ceremonies, palaces, temples, and public works of art financed by states - though few may have judged them worth the cost. 
Though some states appeared very strong at the peak of their power, all states would eventually fall. In the Middle East and early China, different cities or regions might rise to power as others declined. In Egypt, instead, the most common outcome was for one dynasty to replace another in controlling the entire Nile Valley - though there were some periods of internal dispute. (Later Chinese history would also be characterized by one dynasty replacing another in controlling most/all of China.) The decline of states or dynasties was often attributed at the time to bad rulers, and it is certainly possible that a very bad ruler could do much damage. But we can also imagine that over time a state's bureaucrats might become more adept at siphoning off some of the agricultural surplus. Climatic shocks might also disrupt a state: A bad harvest would decrease tax revenues while perhaps increasing the costs of maintaining social peace. A series of bad harvests could spell disaster. In some cases these might reflect environmental degradation: Soil erosion from deforestation could clog waterways and irrigation might raise salt levels in the soil (see Box 7.1). Another common occurrence was kings dying without an (obvious) heir; a battle over the right to inherit the kingdom could destabilize the entire state. Last but not least, we should appreciate that epidemics might destroy the population and economy on which a state depended: Epidemics of diseases such as measles, smallpox, influenza, and cholera may have been particularly common in the early days of cities and states as diseases moved from animals to an unprotected undernourished human population living close to livestock and often with polluted water supplies (there may also have been diseases among the plants or animals on which humans had come to depend).

We have noted above that peasants often tried to escape state control. Though states did provide some useful services, we should be careful not to assume that the decline of states was always viewed in a negative light by residents of the state. If farmers could somehow collectively protect their stuff, they might be far better off without the exactions of the state.

\section{The rise of "kings"}

States throughout history have usually had one ruler. We apply words such as "king" to this person but should be aware that the degree of power exercised by a single ruler varied considerably across both time and space. The pervasiveness of kings (or "big men" or chiefs) implies that there is some critical advantage to having one explicit decision-making authority. As polities grew in size, it became ever more difficult to make decisions communally - though we will see notable examples of shared decision-making throughout history. Though individuals might make bad decisions, they at least could make these quickly. Moreover, advisers could surround rulers: The ruler might get good advice from these but could ignore them if he wished. If a ruler heeded good advice from advisers, one might have the advantages of both decisive rule and consultation 
in decision-making. But these advisers were generally drawn from an elite and likely did not always pursue the best interests of society as a whole. They were nevertheless guided to worry about social stability, and thus not to govern too selfishly.

There is an alternative explanation for the existence of kings. Kingship may merely reflect the exercise of power rather than a calculation of how to make the best decisions. Kings, that is, were kings because they could force others into obedience. Yet if kings on average made worse decisions than communal decision-making, we might have expected to observe far more communal decision-making in history.

In later chapters, we will discuss the conditions that would (much later) support democratic decision-making. We can note for now that this would have been particularly challenging in an age of poor communication, limited or no literacy, limited education, and likely far less leisure time for the bulk of the population to devote to political conversation.

We saw in chapter 4 that the earliest villages may (or not) have been organized communally. We saw above that even Indus cities might have been egalitarian. The essence of organized states is power imbalance: A few can make decisions regarding the many. Before organized states, power imbalances were much less severe. Small communities may have had leaders (big men or chiefs), but these had limited powers. Among hunter-gatherers, decisions tended to be communal, and group members had similar access to resources. We do not know precisely how and when early kingdoms emerged. An optimistic story could be told in which people came to appreciate the value of increased political coordination, and voluntarily agreed to cede power to some political authority in return for better economic outcomes (say, through better irrigation systems or more trade, or just protection from theft). There is some evidence of Mesopotamian city councils inviting leaders to guide them in war. On the other hand, one could tell a coercive story whereby some exercised force over others to compel obedience and could afford this exercise of power because of increased agricultural surplus. An evolutionary approach to political institutions can absorb elements of each story: Groups experiment with different political forms over time, and those that support economic and perhaps especially military success survive. Leaders in turn experiment with varying strategies of governance. Expanded bureaucracies (which must be paid for somehow) are selected for if the state can afford them and if they increase the state's power either through enhancing state power (by more than they anger the populace) or provide benefits to the population.

It might be thought that the coercive and voluntarist explanations of kingship outlined in the previous paragraph are so in conflict that they cannot be handled by the both/and approach recommended in chapter 1. It is, in fact, straightforward to do so. Even the most selfish of rulers have an interest in preventing social unrest, and should then be willing to enhance economic outcomes. They also should recognize that an increase in output could lead to a rise in tax revenues. On the other hand, a ruler put in place by the will of the 
community might become corrupted over time - or his children and grandchildren might - and operate more selfishly than the community might have imagined. Governments in our day pursue a mix of self-serving and publicserving goals, and so we should have little trouble believing (but be careful in assuming) that this was the case millennia ago. Evolutionary analysis reminds us that selection will be most directly determined by the elite in power, but over time, these can only maintain control if the populace does not successfully revolt.

Note that we have in the last couple of paragraphs reprised in an evolutionary context the arguments in the first paragraphs in this section. We have found that we need not choose between "kings rule because they are decisive," and "kings rule because they exert physical force." These two aspects of kingship reinforce each other.

An evolutionary approach can then see the details of state formation in different times and places as mutations, and examine why these might have been selected. Regarding the earliest states, one interesting hypothesis is that strong states were most likely if there was no easy escape for those who might lose in battle. Desert surrounds the Nile Valley. A ruler that lost to another had nowhere to run but would become subject to the power of the winner. In Mesopotamia, borders were porous, and so it was much easier for the defeated to escape and fight another day. As a result, we see one unified state through most of ancient Egyptian history but competing states in Mesopotamia most of the time.

Kings might exercise power through brute force, but could nevertheless appreciate that it was far less costly to rule a population that accepted kingly authority. Achieving economic prosperity could be important here. But all kings also sought to justify their rule culturally. They might invoke godly intervention: They ruled because the gods had selected them. They might celebrate their military successes and claim that they deserved to rule. Or, they might emphasize inheritance from respected ancestors. They might combine these strategies: Military victory might reflect both godly favour and the inheritance of martial skills.

In these early states, it was generally thought that all the land belonged to the king. It was up to the king to spend his "rent" as he saw fit. However, it was also widely assumed that the gods would withdraw their favour from "wicked" rulers, and so rulers were expected and encouraged to devote some attention to the well-being of their subjects. One notable characteristic of all early states was an official state religion that naturally served among other things to justify kingly rule (see chapter 8). In places such as Egypt and China where rulers were thought to be divinely chosen, new ruling families quickly asserted their divine legitimacy.

We should say a bit more about human psychology here. Humans have limited foresight. It is thus entirely feasible that one generation could set in motion a process of political stratification that later generations would regret. It is often said that power corrupts. An individual who either takes or inherits 
power will likely come to assume that their opinions are better than others' opinions, that their interests are more important than others' interests, and that they have some right to higher economic, social, and political status. It would be hopelessly naïve to imagine that rulers will not behave selfishly. However, humans are also selected for altruism. And rulers themselves will not be immune to the cultural values that will justify their rule in terms of some shared purpose that binds them to those they rule. Moreover, as noted above, their self-interest guides them not to annoy (most of) those they govern. Therefore, it would be equally naïve to imagine that rulers do not sometimes attempt to achieve good outcomes for society. Since the military prowess of a state will depend in part on its economic well-being and the loyalty of its subjects, there may be some selection pressure for institutions that do in fact have societal benefits. (We will find below that loyalty of soldiers is particularly important.) Citizens have a psychological incentive to internalize the legitimations that rulers provide for their rule: Citizens can see themselves as dutiful rather than cowardly when they obey the ruler and be proud rather than frustrated of their subservient social position. Both the powerful and powerless activate the human capacity for self-deception in situations of power imbalance.

It is notable that the Epic of Gilgamesh, the oldest human story that survives, from at least 4,000 years ago in Mesopotamia, speaks both of how the ruler Gilgamesh is admired by the people for his triumphs in war and his general character, but also about how he comes to oppress the people. The authors and audience appreciated, that is, that the same ruler might rule well at times and be corrupted by power at other times. It is noteworthy that political elites did not suppress a story that cast a ruler in an ambiguous light. The Epic of Gilgamesh would indeed serve as a base for discussion of ethical responsibilities of rulers but also of all people - for many centuries throughout Mesopotamia and beyond.

\section{Challenges facing agents: Rulers}

The ruler's primary challenge is to obtain more in tax revenue than it costs to administer the state. This requires sound administration. Rulers need to encourage bureaucrats to act in the ruler's interest; this challenge will recur throughout the history of states. Of particular importance are provincial governors: The ruler needs local officials, but these may use their local knowledge against the state. Rulers must also negotiate their relationship with religious or economic elites that might contest for power.

The ruler also needs to manage cultural diversity. The state's survival depends in large part on its people not being moved to rebellion. Rulers will wish to provide some justification for their rule.

The state also needs to manage its relations with other states. These will trade and war with each other.

Finally, yet importantly, the ruler (or at least the state) needs to devote some consideration to the question of succession: How will the next ruler be chosen?

These challenges may seem straightforward, but they will motivate much of world history. 


\section{Effects of states}

The emergence of states is one of the major turning points in history. By pooling the efforts of many people, states could achieve much that is important in history: irrigation systems, transport infrastructure, and public buildings and monuments (which contribute to the history of art and architecture). We will discuss below other contributions such as systems of writing and laws. Yet such states could also launch wars, treat lower classes despicably, and limit human freedoms and innovation. Where it was possible, peasants often escaped imperial control to live as nomads or form new settlements beyond the borders of the state. States typically employed physical force to prevent such escapes.

Cities and states both encouraged and reflected an expansion of trade both within and beyond the state's borders. The political elite needed merchants to supply them with both food and luxuries. States encouraged trade not just by building roads but also by punishing thieves and providing courts that might give merchants some recourse in trade disputes (recall the merchant's challenges from chapter 5). And trade (along with taxation and public expenditures) in turn encouraged occupational specialization. The cities, as we have seen, came to contain specialized builders, artisans, artists, and soldiers. Even the countryside might see dedicated fishers or brewers.

States reinforced some of the effects of cities by attracting people to their capitals. They then unintentionally encouraged the spread of ideas, caused reflection on the nature of other cities and states, and encouraged the spread of epidemic disease. However, states could exercise a positive effect on health by holding grain supplies between harvests. This was a common strategy of early states and likely reduced the incidence of famine. (The poor inevitably suffered more than the rich from food shortages.) States could also transfer food from a region of good harvest to an area of weak harvest - as long as the transport infrastructure was in place to allow this. In the face of severe food shortages, the state could become an actor in the Malthusian mechanism (Box 4.1) by trying to gain through war the food of others.

\section{Writing}

We noted above that the key challenge for states was to raise more in taxes from their territory than it cost to administer this. And early states needed to do so without recourse to currency, if not money itself. They thus faced an immense challenge in record-keeping: to ensure that everyone paid their taxes in kind, to ensure that local administrators did not confiscate an excessive proportion of tax revenues, and to ensure that appropriate quantities of grains and animals and other goods were transferred on time to the bureaucracy and army. Such an administrative process required some written record: No human brain(s) could keep track of thousands upon thousands of transactions. It is hard to imagine complex states without some form of record-keeping (though the Inca of South America, we shall find in chapter 16, managed without writing but 
with a system of knotted strings called quipu). But once states (and associated religions) had come to employ writing for record-keeping, they also began to use it to promote laws and religious beliefs.

States (and associated religious organizations) were not the only historical actors in need of record-keeping. Early merchants also had to keep track of their purchases and sales. Since trade was slow, months often elapsed between the purchase of goods and their disposal. Merchants often both made and took out loans. They entered into joint ventures with other merchants. They also - from at least the second millennium все in Mesopotamia - entered into what we now call "futures contracts" whereby one merchant agreed to buy or sell a fixed quantity of some good at a particular price at some point in the future. They needed some reliable way of keeping track of these diverse activities.

Sumerian merchants in Mesopotamia employed clay tokens from 10,000 years ago to keep track of their shipments. Over the next 5,000 years, these tokens became tablets on which elaborate sets of markings were made. There is a gradual transition from simple markings to something that more closely resembled written language. We can imagine that it was often worthwhile to record something more complicated than "received three goats from Igizi" such as "accepted two goats from Igizi because he has been sick; he promises two more next spring." Merchants wished to contract for a variety

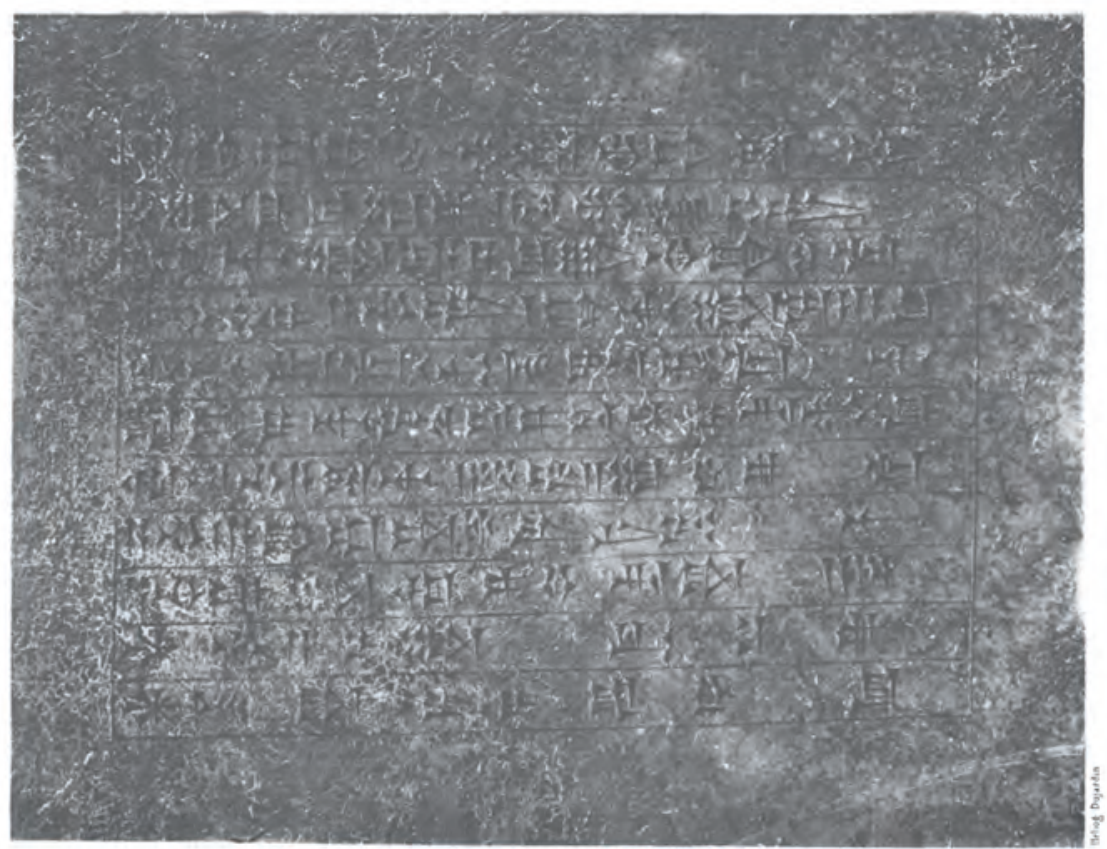

\section{Illustration $\mathbf{6 . 6}$}

The Tablet of Akaptaha, a Mesopotamian inscription recording a gift of land by Babylonian king, Kaštiliašu IV (c.1232-1225 BCE) 
of unforeseen circumstances that might affect one merchant's ability to meet commitments to another - illness, unexpected weather, crop failures, and so on - and would struggle to do so without a means of recording a variety of words as well as numbers. Moreover, merchants made loans and needed to calculate and indicate the interest due upon particular dates in future. Simple record-keeping might require only numbers and symbols for each person dealt with; more complex contracting leads us toward written language.

We should stress that the exercise of human deceit greatly enhances the record-keeping challenges of both states and merchants. States need to make sure that bureaucrats do not rob them and that taxpayers pay. Merchants need to make sure that both suppliers and customers do

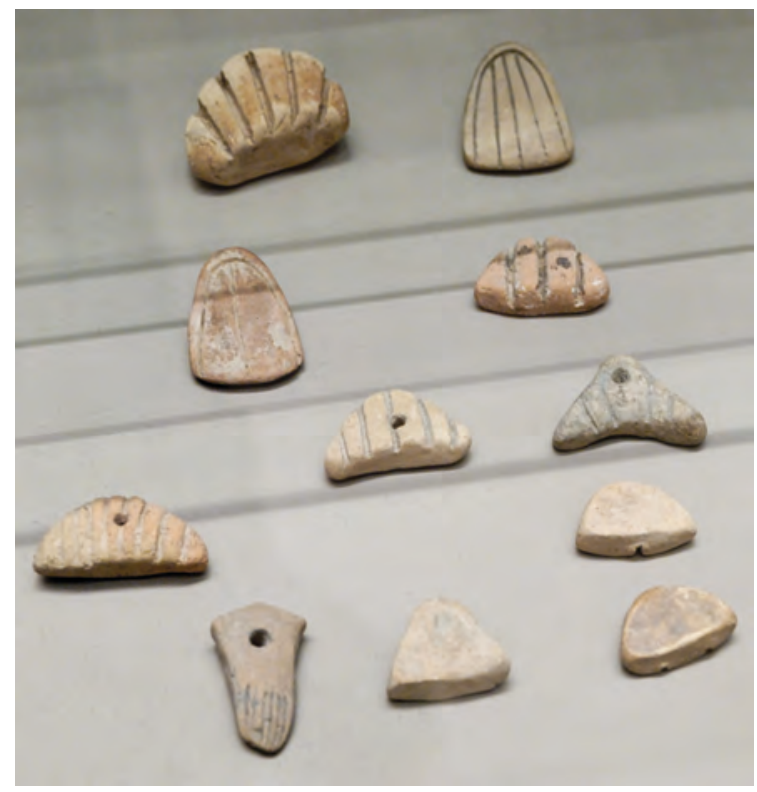

Illustration 6.7

Clay accounting tokens from Susa in Mesopotamia, Uruk Period, Louvre what they say they will. Both states and merchants have to attempt to keep track not just of what happened but to calculate what should have happened and compare the two. Then they need to keep track of whether transgressions were rectified. They will be robbed with regularity if their system of record-keeping does not expose theft. They might have moved beyond simple tally devices in any case, but the complications associated with human deceit would provide a powerful encouragement to the more complicated record-keeping that written language would allow. (We will find that the Incan quipu were likely up to this task as well.) Note in this regard that urbanization forces humans to interact regularly with strangers and thus itself increases the need for ways of reducing theft (in small villages one learns who can be trusted, and all villagers may wish that others regard them as trustworthy).

There is still debate about whether writing first developed in Egypt, Mesopotamia, or China; the answer depends on both archaeological finds and one's definition of writing. Egyptian hieroglyphs were used from about 35002900 вСE into the early years CE. (They were also used in Nubia for much of that period.) Only probably 2 per cent of the population could understand these. Scholars long thought that these were all "ideograms": stylized pictorial representations of particular words. But we now know that they were a mixture of ideograms and phonograms: symbols for sounds that could be combined to represent any spoken word. (There were also some symbols that clarified the nature of other symbols, given the complexity of hieroglyphs.) Hieroglyphs did have religious significance - aided by the fact that only a small minority could 


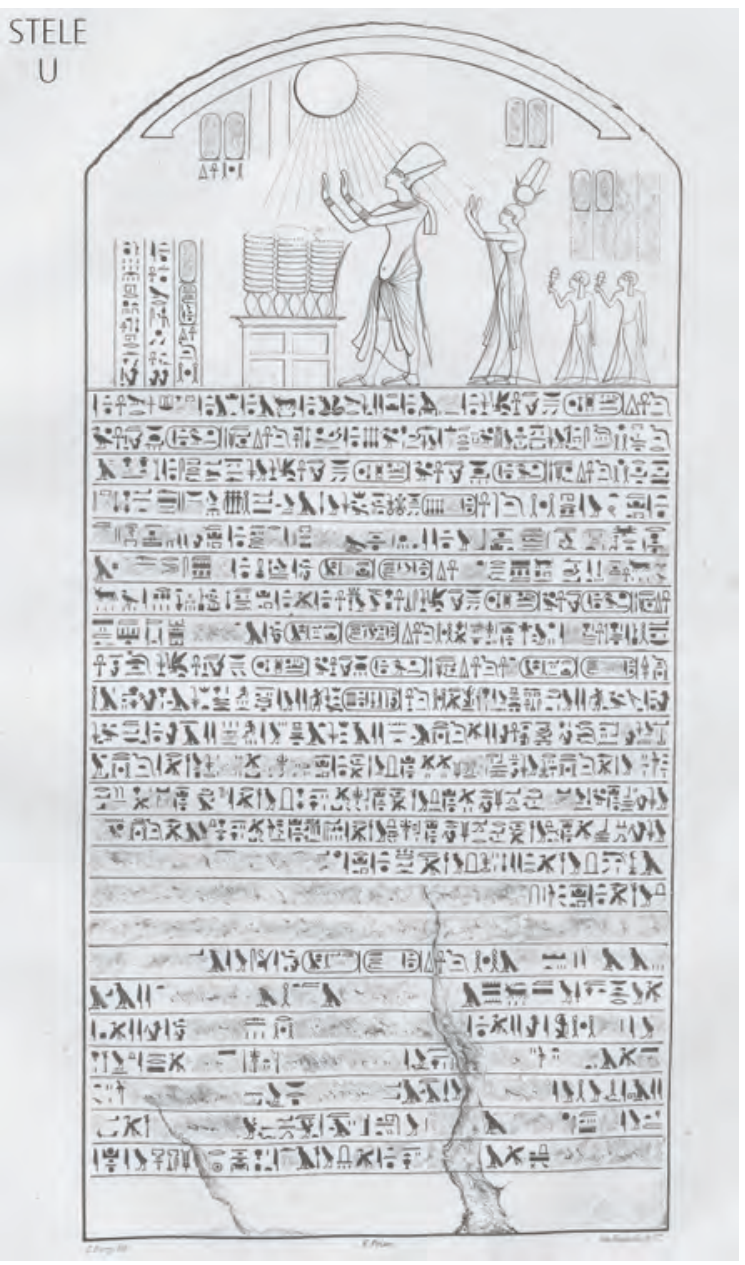

\section{Illustration 6.8}

Egyptian Amarna boundary stele with hieroglyphs, containing a lengthy proclamation of Akhenaten (husband of Nefertiti) declaring the enclosed area to be for religious purposes, including royal burials read them. Though most people today are familiar with hieroglyphs because of their use on temple walls, there was a simpler script used on papyrus (a paper-like substance made from plants).

Cuneiform writing is known from at least 3200 вСE in Uruk in Mesopotamia. Cuneiform involved pressing straight lines onto clay with a stylus. Several hundred symbols could be formed from combinations of straight lines, representing words or syllables, but over time seventy to eighty symbols came to dominate. This simpler version was widely employed by merchants, suggesting that literacy expanded significantly with the more straightforward script. Cuneiform soon spread through Mesopotamia, and on to the Levant, being applied to many languages. It was used in royal inscriptions celebrating wars or the construction of temples. Between 2900 and 2370 вСе - so only centuries after writing is first used for record-keeping - we see the first literature (from which we learn, among many other things, that there is not one standard creation myth in Mesopotamia but many). Also sometime after 2000 вСЕ, we see a numbering system based on divisions of 60 , from which the modern division of hours and minutes descends. We also see texts on mathematics and medicine. Though cuneiform survives for centuries, the alphabet gradually replaced it (see below; the two are used together in Ashurbanipal's famous library at Nineveh around $1180 \mathrm{BCE}$ ).

As we have noted before, we know far less about early China than about many other parts of the world. One peculiarity of Chinese history is that the earliest writing that we know of was employed for purposes of divination as much as or more than for record-keeping. Priests would write questions on bones or tortoise shells: Will there be a good harvest? Will the ruler have a son? These were then placed in a fire. The pattern of cracks that resulted from burning would provide the answer to the question. These divination shells have survived in much larger numbers than state records, but we know that records were kept on perishable pieces of bamboo or silk from at least the second 
millennium BCE. We see ideograms from at least 1600 все for both divination and record-keeping. These ideograms would develop over time into the written script employed today in China; later empires would standardize that script. Though modern ideograms are more stylized than those of the Shang period (much as western alphabets are more abstract than earlier ideograms), they clearly descend from early Shang ideograms. Chinese ideograms would influence writing practice throughout eastern Asia.

Though developed for official purposes, writing was employed for stories, songs, and manuals of divination, manners, and various other matters during the Zhou. Unfortunately, the first Qin emperor ordered all books without immediate utility to be destroyed (see chapter 10), and as a result, only a small fraction of this early literature survives. This includes, though, the writings associated with the name Confucius on a range of philosophical issues.

The Indus Valley civilization employed a recognizable script from at least 2500 все. Though it has never been deciphered, it seems to combine ideograms and other symbols that may or not have represented sounds. There are far too many symbols for it to have been an alphabet.

About 2000 BCE the Phoenicians - who had a small state along the Mediterranean coast in the Levant where present-day Lebanon is - created the first phonetic alphabet; their alphabet had twenty-two letters representing consonants. Such an alphabet was much easier to master than the hundreds of symbols of cuneiform. The Phoenicians were very active as traders around the Mediterranean. Their simplified alphabet may have reflected the need of merchants for a more straightforward script. The first alphabetic

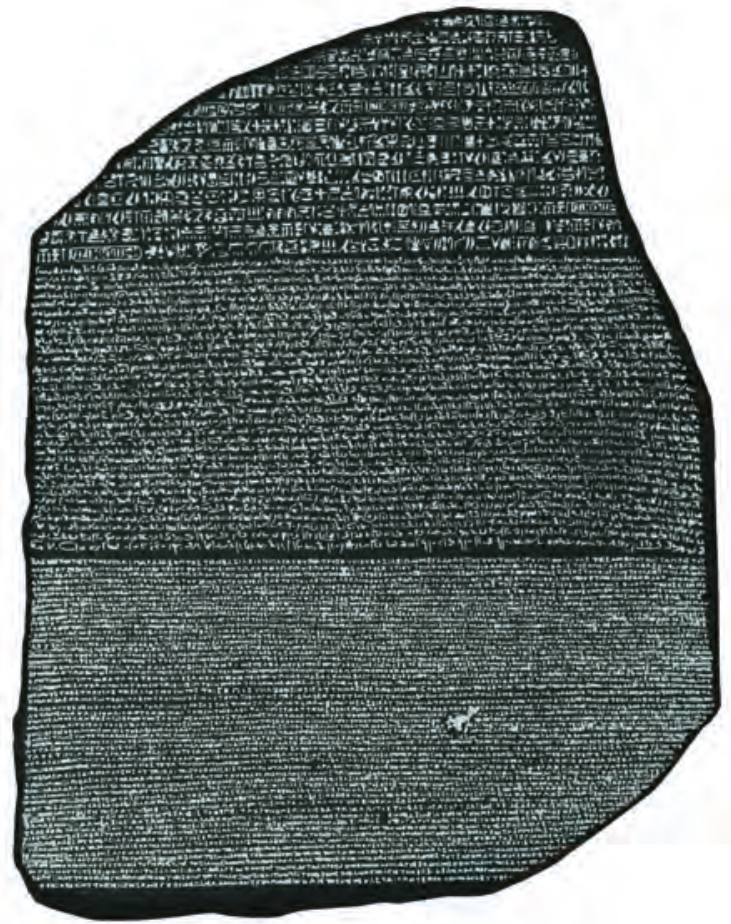

\section{Illustration 6.9}

The Rosetta Stone allowed Egyptian hieroglyphs to be translated. Top script: Egyptian hieroglyphs, middle: Demotic Egyptian; lower: Greek version of the same text, British Museum

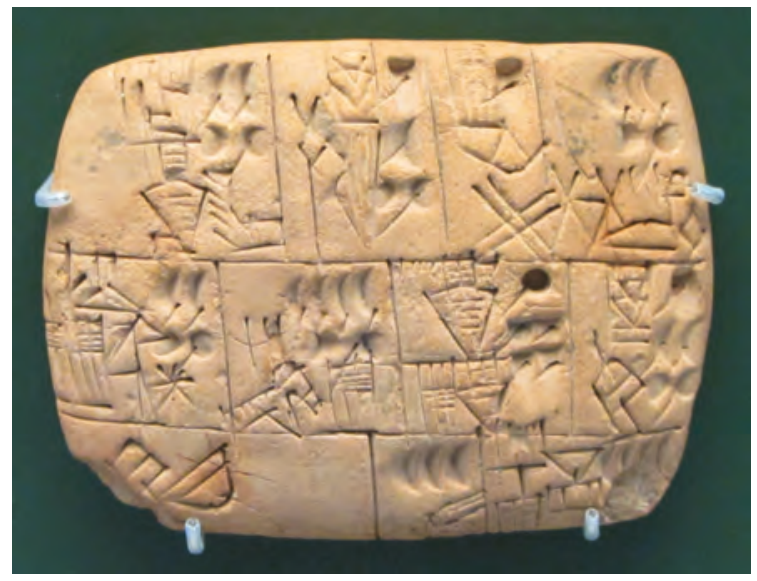

Illustration 6.10

Mesopotamian writing tablet recording the allocation of beer, 3000 BCE

Source: Photograph by BabelStone, CC BY-SA 3.0 


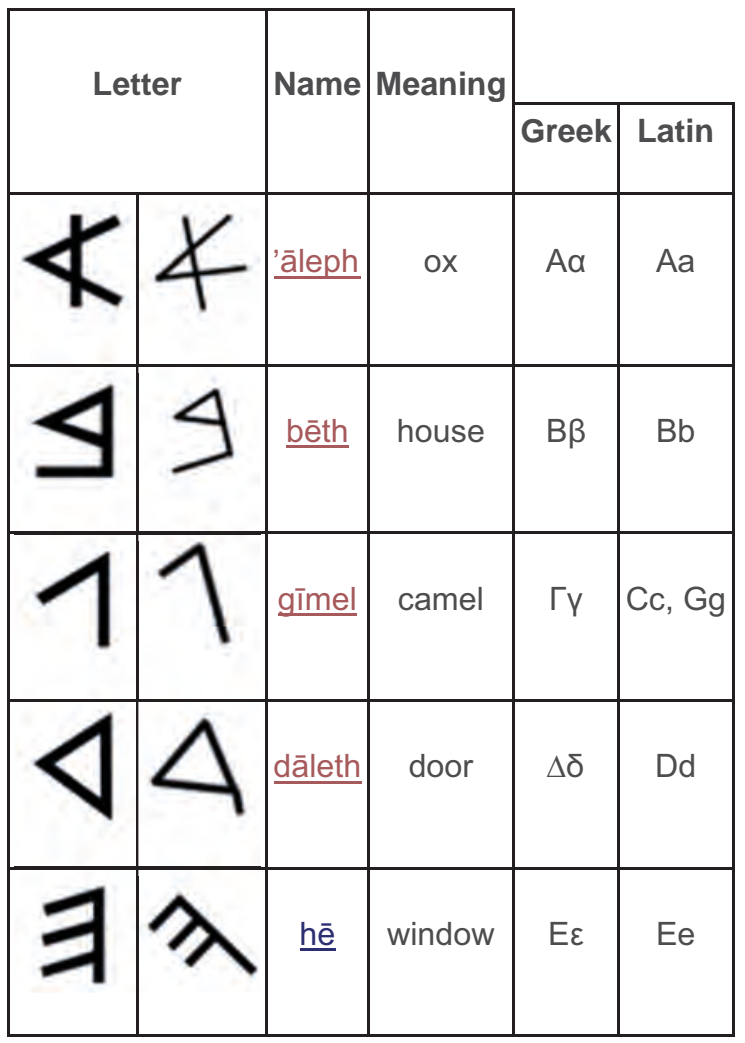

Illustration 6.11

The Phoenician alphabet and its relationship to other alphabets symbols were adapted ideograms: For example, a picture of a house represented the sound ' $b$ ' as the word for house started with that sound. These ideograms were simplified over time. This process was likely influenced by cuneiform, as complex symbols became simpler sets of lines. This idea of an alphabet would spread throughout the western world. The Greeks would add symbols for vowels. There was a similar process in Egypt whereby particular ideograms became associated with syllables. Indeed some think the earliest alphabet was Egyptian. Others trace the alphabet to the Sinai Peninsula. Sanskrit in India would also employ an alphabet during the second millennium BCE.

The Maya in the western hemisphere would later also develop a system that mixed alphabetic and ideographic elements. The ideograms likely had religious significance. The fact that phonetic elements did not entirely supplant these may have both reflected and reinforced the power of a priestly class.

Before the invention of writing, societies had transmitted stories and proverbs orally, often employing pictures and various mnemonic (that is, patterns that aid memory) devices to aid human memory. Writing freed humanity's collective memory from the limits of the human brain. It facilitated the ability of one person to build upon the ideas of another, even if the other was distant in time. Though literacy was limited, the literate proportion of the population came to both tell elaborate stories and collectively reason about all aspects of the human experience. Yet the literate minority - and those they served could also wield power through their control of the written memory of their group. (Communications theorists such as Marshall McLuhan hypothesize that writing changes the way that humans think. If so, only a small minority was so affected for thousands of years.)

Merchants and bureaucrats then as now often needed copies of records. And as manuals and stories were produced, these had to be copied many times. There are records from later Rome of manuscripts being read out while a dozen scribes copied them. As manuscripts were copied, libraries collected the most important of these. As libraries gathered hundreds or thousands of these, they needed librarians and systems of organization. The library of Ashurbanipal mentioned above had a system of subject headings and subheadings, including 
"sheep with arthritic hips." Still, writing was limited in its capacity for transmitting complex stories widely until literacy expanded.

\section{Education}

Education was mostly informal at home at this time, but we know of scribal schools in both Egypt and Mesopotamia. These seem to have been funded by pupils through tuition. The schools then tried to obtain jobs for their graduates in order to attract new students. Stories of Sumerian schooldays have survived which speak of harsh teachers and parents interceding on behalf of their children - who did not always follow their teacher's guidance. While these schools stressed reading and writing, they could also teach simple mathematics (such as computing compound interest) and came at least in Mesopotamia to also teach literature.

\section{PRIMARY DOCUMENTS: A SUMERIAN SCHOOLBOY}

A student in ancient Sumeria recorded his daily life. Much of the commentary involves the strict discipline at school:

My headmaster read my tablet and said: "There is something missing"; he caned me.

And later:

The person in charge of silence said "Why do you talk without permission." Caned me.

Teachers also caned the student for being late, being untidy, not standing at attention during assembly, leaving school without permission, and not speaking Sumerian.

He complains to his father that his teacher is not paying him enough attention. The father invites the teacher to the home and both flatters and bribes him:

You made wisdom enter there [the son]; you showed him all the fine points of the scribal art; you made him see the solutions of mathematical and arithmetical problems; ... give him [the teacher] some extra salary, put a ring on his hand.

The teacher responds that the father is too generous but promises to do all that he can for the boy.

Can you appreciate the schoolboy's narrative? Do you think he is exaggerating? In what ways is education similar to today and in what ways is it different?

We will include excerpts from primary documents in each of the remaining chapters. These documents shed light on the authors who wrote them and the times in which they were written. They also allow readers to hone the critical historical skill of placing documents in context. In this regard it is worth reprising the fourteenth-century Islamic historian Ibn Khaldun's thoughts on how and why such documents may be misinterpreted: 
All records, by their very nature, are liable to error... 1....Partisanship towards a creed or opinion... 2. ... Over-confidence in one's sources... 3....The failure to understand what is intended... 4....A mistaken belief in the truth... 5....The inability to place an event in its real context 6 ....The common desire to gain favour of those of high ranks, by praising them, by spreading their fame... 7....The most important is the ignorance of the laws governing the transformation of human society.

We might stress the last point:You should ask yourself how your understanding of history helps you to place documents in context. You cannot, for example, blame writers in the distant past from lacking modern scientific understandings. But you should also be wary of the danger of interpreting each document in a biased manner.

Note that Ibn Khaldun in his way guides us to ask: Who wrote a document (and what biases they may have had)? Why did they write it (and thus whether there were incentives to deceive)? Where and when was it written (so that we can place it in context)? Who was the intended audience (and would the author have tried to mislead them)? Only then are we in a place to focus on What does the document say? You should keep these questions in mind as you encounter primary documents through the rest of this book.

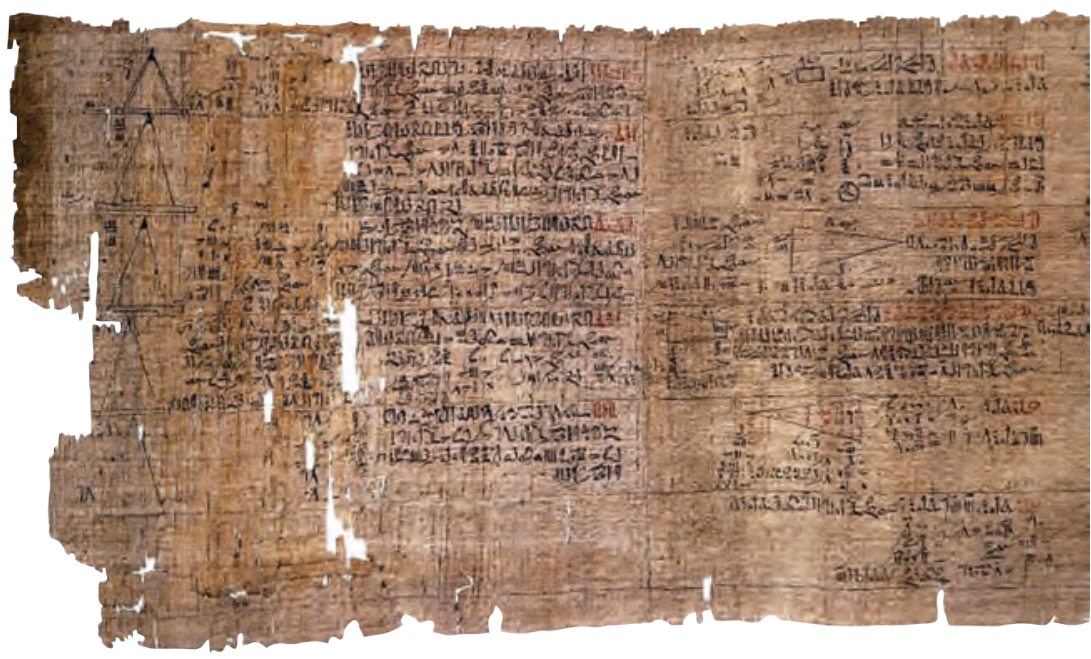

Illustration 6.12

Rhind Mathematical Papyrus, Thebes, end of the Second Intermediate Period (c. 1550 BCE). The Rhind Papyrus has eighty-four problems that a bureaucrat might have to solve, such as the slope of pyramid or the food required to feed birds (which Egyptians often kept). It is written in a script that is easier than hieroglyphs. It is the largest surviving ancient mathematical text. Similar problems would be taught in high school today. Egyptians were lauded by Greeks for their mathematical skills: There were likely more advanced texts that have been lost, British Museum 


\section{Laws}

We observe formal laws in both Egypt and especially Mesopotamia from the early third millennium BCE. There is some mentions of laws also in both the Indian Vedas and in the early Greek writer Homer. The widespread emergence of formal laws reflects multiple causes: the development of writing (laws could be formalized orally, but it is far easier to write them down); urbanization (strangers living together and interacting required formal rules, whereas small tribes could adjudicate disputes on the basis of cultural norms); and a state with the power to enforce laws. The state had multiple incentives to promote and enforce laws. It wished to maintain social peace and thus had an incentive to solve disputes among its citizens and punish thieves and murderers. Recall that states generally tried to monopolize the exercise of violence. It wanted to encourage trade and therefore had a particular interest in remediating disputes among merchants. Finally, it wished to provide public services that would encourage public support of the ruler. Recall that sedentary agricultural societies need somehow to allocate land (chapter 4): Systems of law played a critical role in most early states in adjudicating disputes over land, especially concerning inheritance.

There was a debate in early states regarding the tradeoff between codification of laws as opposed to relying on the wisdom of the ruler and the judges he appointed. Mesopotamian rulers decided that judges would make mistakes and be biased, and so developed the most complex of early law codes. The Chinese, who developed extensive bureaucracies before other states, tended instead to rely on the judgement of rulers and (generally educated) bureaucrats. This simple difference may have had ramifications over the millennia for whether the state and ruler would be viewed as the source of law (as in China) or would come to be viewed as to some extent governed by laws that could not be changed at the whim of the ruler. In principle, private property will be safer in a state in which the ruler cannot easily change or subvert laws. In practice, though, even rulers that felt themselves above the law might be motivated to respect laws of property in order to encourage economic activity and discourage public resentment. Such a ruler might, though, change their mind if facing financial difficulty.

The Code of Hammurabi of about 1754 вCE is far from the first law code in the world, but it was inscribed in stone and has thus survived to this day. It prescribed the death penalty for a variety of crimes. Penalties were smaller if the upper classes mistreated lower classes. Victims had a role in setting penalties: For murder, the victim's family could choose between execution or compensation. Oath-taking by witnesses was important (note that religion was invoked in the pursuit of justice): There being no police detectives to collect evidence, judges had to weigh the testimony of disputants and witnesses. The Code specified the roles of judge, jury, witnesses, plaintiffs, and defendants. (We reprise some clauses from the Code of Hammurabi in chapter 7.)

The Code addressed far more than criminal law. Notably, a third of the Code of Hammurabi addressed issues of the family, sex, inheritance, and dowries. 


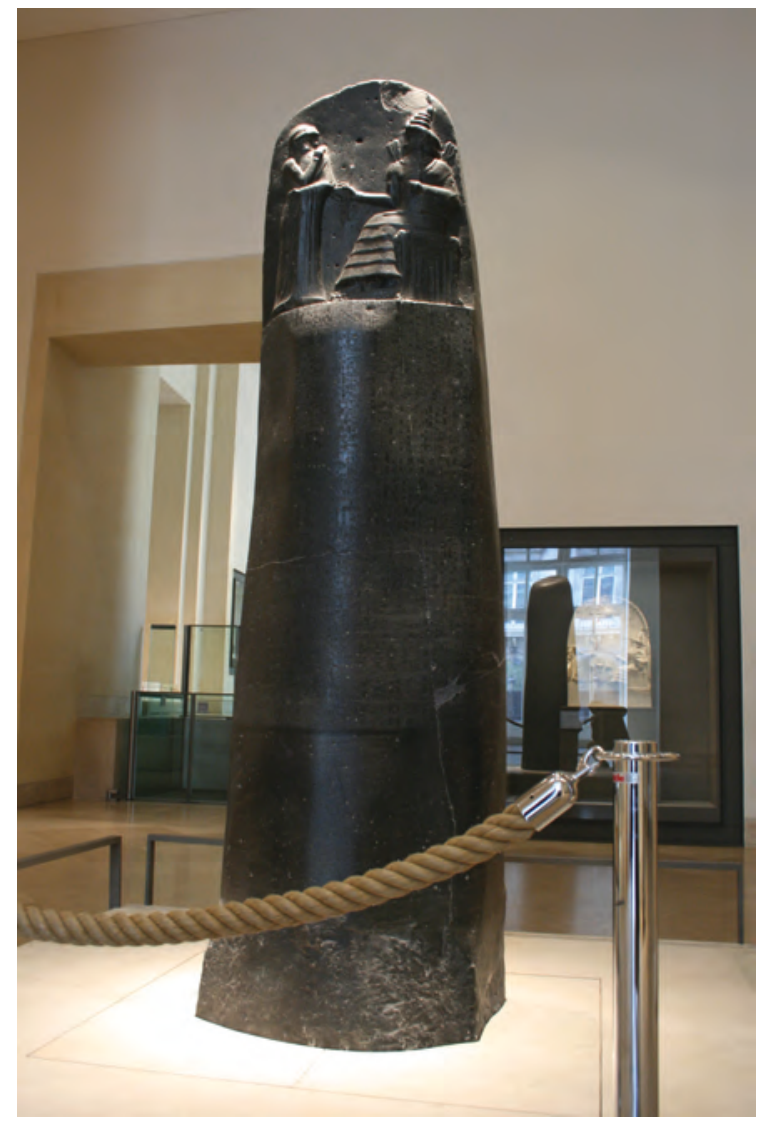

Illustration 6.13

Code of Hammurabi
It also discussed commercial law at some length. It recognized and elaborated rights of property ownership, including rights to lease and rights of eminent domain (when the state can force landowners to sell land needed for public works). It specified the role of written contracts, the importance of receipts, and how to proceed if receipts did not exist. It outlined the obligations of merchants, brokers, and agents and the limits to the liabilities of each in cases of attack or theft. It placed a three-year limit on debt servitude. In sum, it outlined how to deal with each of the major concerns in commercial law.

We should appreciate that there is a big difference between this law code and what actually happened in courts. It is clear that judges did not always follow the Code of Hammurabi - and other early law codes - in practice. Indeed, it would have been difficult to do so. These provided examples of appropriate penalties rather than abstract principles that judges could apply generally. That is, they followed a "case law" approach that provided examples of appropriate decisions. This is not surprising from an evolutionary perspective: States experimented with legal rules and decisions, and celebrated those that seemed to keep the peace. Judges then drew upon what appeared to be relevant examples in rendering judgements. Wise judgements were then incorporated in later codes.

We have noted above that rulers relied on local administrators to collect taxes. This was true also in administering the law. The ruler might only play a role in the most important cases, such as murder (though even here he would likely ask lesser officials to rule). Codes of law might be enacted to encourage local officials to judge as the king wished, but local courts would draw upon local customs as well as official laws in reaching judgements. It could be that laws were most often proclaimed soon after conquest in order to ensure some consistency - there was nevertheless respect for local customs. More cynically, the law codes might be viewed as propaganda to justify the king's rule.

The laws of the Bible would draw heavily on Mesopotamian precedent (though the Ten Commandments are arguably statements of principle rather than examples). However, there would be an essential difference. Mesopotamian rulers often claimed godly justification for their rule: The Code of Hammurabi 
proclaims that the gods wanted him to provide justice and it was inscribed on a column on which the king is shown receiving his powers from a god. But it was nevertheless the ruler who proclaimed the laws. Biblical laws instead were ascribed to God. The Bible was also more willing to recognize the exercise of personal vengeance, whereas the Mesopotamian state reserved punishment for itself. This latter difference may reflect the tribal society in which the early Bible emerged; there was a less developed state that could not exercise exclusive legal authority. One might speculate that the appeal to godly authority also reflected in part the limited power of the Jewish state.

In Egypt, there was a less codified system of law, but there were various royal decrees. There was a system of lower courts seemingly run by regular (that is, non-bureaucratic) people, and higher courts run by senior officials that dealt with crimes against the state. There was an intense cultural/religious worship of "ma'at," which can be variously defined as order, truth, and justice. Most surviving court records dealt with contracts and inheritance. There is some evidence of appeals to divine judgement through oracles.

The Hittite law tradition (1650-1180 BCE in Anatolia) relied on case precedents, and these could differ locally. There were, though, some general principles, including the idea of double repayment for both theft and manslaughter.

\section{Gender relations}

Male dominance is reflected in all early states. We have even used "he" once or twice above in describing rulers, for some 99 per cent of the known rulers in early states were men. We should note, though, that there were a couple of female pharaohs over the thousands of years of the Egyptian state.

Though men usually ruled, there were notable differences across states in the treatment of women. In Egypt, women had legal rights and occasionally served not just as pharaoh but more often (albeit still rarely) in other offices of state. Priestesses governed some temples. There were certain occupations associated with women, such as textile production and grinding grain. However, Herodotus of Greece would complain in the fifth century все that the Egyptians reversed standard practices by having women engage in trade while the men stayed home to weave. Women were blamed for adultery in many Egyptian texts. Nevertheless, women could move in public on their own and perform financial transactions.

In Mesopotamia, men had the power to beat and even disfigure their wives. Women could be killed for adultery (as could their partner), but men were free to consort with unmarried women. It seems that the power of women declined over time: They were restricted from interacting with men, and veils became common. But women still had legal status, and are often observed in written records running estates and business affairs.

It is not clear what gender relations were like in the Indus Valley. Some historians think that the earliest Vedas reflect life in the Indus, even though they were written down later. In these Vedas, women could pursue education 
and had to consent to marriage and indeed could actively choose suitors who they could meet socially (there were maybe even orgies), but once married they were subservient.

Texts criticize homosexuality and especially the passive partner in both Egypt and Mesopotamia. But some historians detect a degree of acceptance in these texts: The practices may not have been celebrated, but they need not be punished. Transgendered activities (males dressing and behaving as women) were important in some temples.

We noted in chapter 4 that the social position of women deteriorated with agriculture and especially early polities. States could codify gender imbalance in law, and perpetuate it in their choices of leaders and bureaucrats. There was also, it might be noted, a connection between gender stratification and the more general political and economic inequalities widely associated with cities and states (see Box 6.2).

\section{BOX 6.2 \\ GENDER AND STRATIFICATION}

There is always a gender aspect to social stratification. Men of upper social strata often exploit women of lower social strata sexually. Masters could sexually assault female slaves in most societies. This was not always legal but rarely punished. Similar practices occurred in non-slave societies. In European serfdom, the lord of the manor often had a right to sleep with serf women on the night of their marriage. Nor was this the only night on which assaults occurred. Men from higher castes often to this day prey upon women of lower castes in India. Female house servants have often had little recourse if assaulted by the men of the family they serve. Beyond cases of explicit assault, there is a long continuum of instances in which men use their power and wealth to persuade poor and powerless women to do their bidding.

While powerless women are the primary victims in these various scenarios, the social costs extend much farther. Men of low status had limited ability to protect their wives or daughters. Women of high social status were limited in their social interactions, even with their husbands. You can imagine yourself in any one of these social situations and reflect on how you would have responded psychologically.

Note that gender stratification also served to perpetuate other types of stratification. If elite men have greater access to women, then they can have more children. Modern Western societies are historically quite unusual in that rich and powerful men do not have more children than poor men on average. Most historical societies allowed wealthy men to have multiple wives or winked at children born to concubines. Though wealth can be dissipated if divided among many children, there are also potential advantages of large families if elite men can find places for sons in armies or bureaucracies, or forge alliances with other families by marrying off their daughters. 


\section{Science}

All of the early states displayed an interest in and public support for astronomy though this often reflected a common interest in astrology. Biology was limited to listing different plants and animals, with no attempt to categorize these. States also often supported medicine and mathematics. Though medical knowledge was limited, the Egyptians did know how to set broken bones, and knew that honey slowed the spread of gangrene. The Greeks would applaud Egyptian dentistry, but the skeletal remains examined to date suggest there was little good dental work performed (Egyptian teeth were likely ground down by bits of stone from the mortars used to grind grain). The Egyptian cure for snakebites involved drinking water poured over a carved stone thought to have magical powers.

\section{War}

We can usefully define war as a sustained and coordinated act of violence instigated by one political entity against another. These entities could be communal groups such as hunter-gatherer bands but are usually groups with some sort of authority structure. Some writers would include in the definition that war must serve a purpose, but the approach of this book is always to seek to

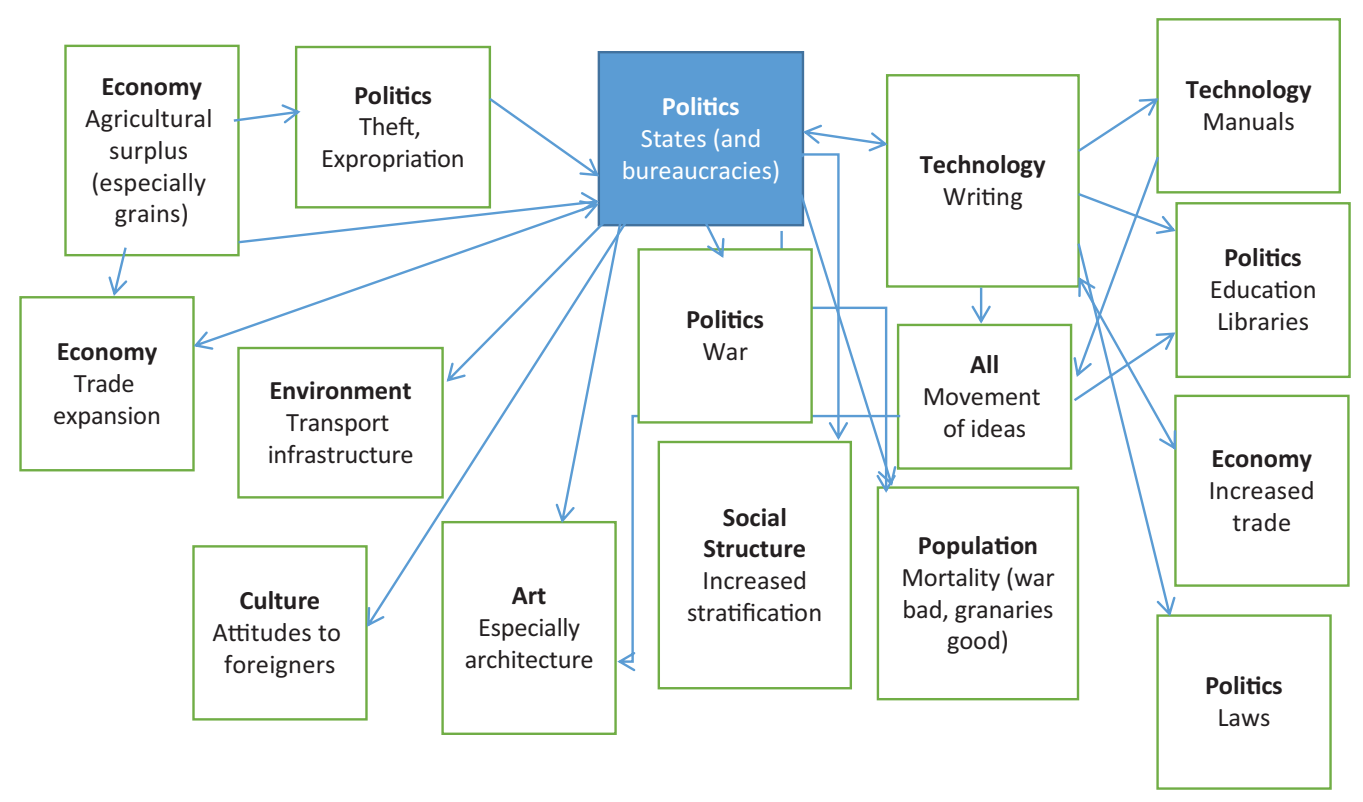

Figure 6.2

Influences on and effects of states

Note: Some of the links are the same as for cities. States and cities worked together.

We will add links from states to culture, and especially religion, in later chapters. 
distinguish what from why: It is best to identify what war is and only then seek explanations of why it is pursued.

\section{The birth of war}

As we saw in chapter 3, there is room for debate as to whether hunter-gatherer groups engaged in anything that would deserve to be called "war." They may have occasionally battled over resources, using the tools and aggressive instincts associated with hunting. However, they lacked specialized armies or weapons. Such battles, if they occurred, would have become more common as population pressure on resources increased. Wars might then have been a response to negative climate shocks or natural disasters. Wars might have been especially likely if one group suffered a natural calamity but nearby groups did not; the former group would then be tempted to attack others. But since hunter-gatherer groups were only a few dozen in size, the numbers killed might have been too small to provide clear archaeological evidence of war. We can also draw on the experience of modern hunter-gatherers. Some modern huntergatherer groups are warlike: One key factor that makes some warlike but others not seems to be a strong sense of belonging to a group much larger than one's family. Yet modern hunter-gatherers may be more disposed to war than our distant ancestors precisely because they have been squeezed onto small amounts of less desirable land.

From about 10,000 years ago there is clear evidence of war: mass burials of people with warlike injuries, fortifications around towns, burned towns, and occasional paintings or drawings of soldiers. There are rock paintings in Australia from 8000 вСе of military clashes in small groups. But the best evidence of warfare comes with city fortifications at Catalhoyuk, Jericho, and elsewhere in the Middle East from 7500-6500 BCE (and Yangshao in China from 5000 вCE, and Salinas de Santa in the Andes from 3500 BCE, and Oaxaca and elsewhere in Mesoamerica from $1800 \mathrm{BCE}$ ). It is quite possible that the very earliest centuries or even millennia of sedentary societies were fairly peaceful. Wars were rare at first - perhaps triggered by climatic shocks that impoverished some communities - but became more common over time.

The connection between urbanization - and thus places with stuff worth stealing - and war should nevertheless be appreciated. As noted in chapters 4 and 5, farmers and merchants need to worry about protecting goods from others. And settled populations with homes and goods to protect cannot quickly flee in the face of danger as hunter-gatherers could. Yet the incidence of war seems to have increased dramatically with the appearance of states. The first documented international conflict is a border skirmish between Lagash and Umma in Mesopotamia about 2500 все. When kings start to chronicle their exploits with the development of writing, wars loom large in their narratives. "War," then is as common a characteristic of "civilization" as cities, states, and writing.

While we do not know whether hunter-gatherers warred, we do know that they hunted. We saw in chapter 3 that humans developed spears, atlatls, and 


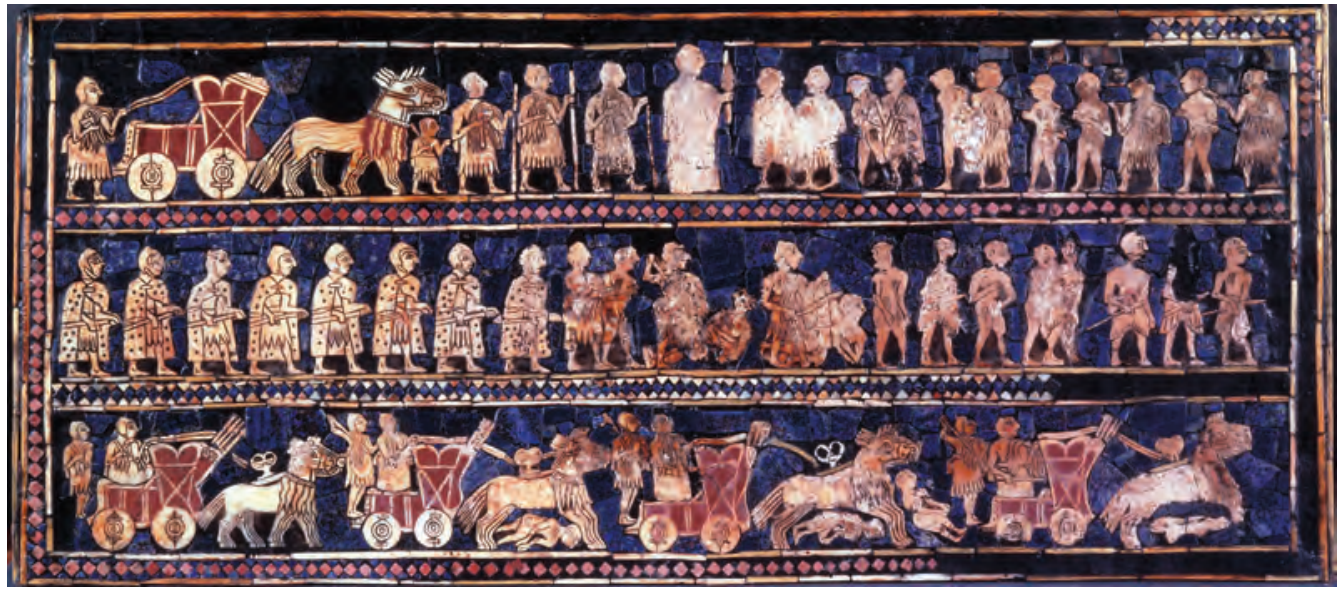

Illustration 6.14

Standard of Ur, 26th century BCE, "War” panel. The other side shows tax collection. Mosaic, British Museum

later bows and arrows for that purpose. So humans had the tools (and tactics) for war before they became sedentary. Early agricultural societies, we have seen, continued to hunt for millennia. Therefore, there was a long period during which humans combined knowledge of hunting with the incentives for war associated with villages. And of course, hunters and gatherers surrounded the earliest villages. There is some evidence that humans developed new weapons such as daggers during this period of transition to agriculture. In such an environment, the earliest farmers must have struggled to defend their harvests against neighbouring groups. Military defence capabilities might then be considered a necessary component of the human move to settled agriculture. We should be careful here, though, for there is certainly much evidence of early and unfortified villages. But farmers eventually found it necessary to defend their stored harvests. Once individuals were trained for defence, they might readily be employed in offensive capacities - and they might well be tempted to attack others at least in part to prevent these others from becoming powerful.

We lack reliable numbers on the death tolls associated with early wars. Given the size of armies, and the weaponry at their disposal, casualties in a typical battle may have numbered in the dozens or hundreds. The rewards for taking prisoners as slaves (see chapter 9) may have encouraged non-lethal injuries when possible.

\section{The common experience of war}

Why will war prove to be such a common feature of human history? It was suspended only in rare circumstances where one state came to so dominate a region that peace prevailed (as was the case in ancient Egypt for long periods, but not in Mesopotamia), and even rarer circumstances in which a balance 


\section{BOX 6.3}

\section{THE MOTIVES FOR WAR}

We can identify three key motives for war. The most obvious is the pursuit of wealth. Victors in war could steal goods from the losers - including, importantly, their animals. It would be common for victors also to enslave some or all of the losers, and it is plausible that this practice was common in the early history of warfare (as long as the victors had use for slaves). We must be careful to distinguish the short-run benefits of war - the immediate theft of goods and people - from the longer-run benefits of governing and taxing a subject group (or perhaps farming or pasturing on their land). The growth in state size that we observe indicates that the long-run benefits were seen by kings to be worth pursuing, at least until the state reached a size that strained the capacity for governance. And even if outright conquest would strain the ruler's resources, they could demand ongoing payments of "tribute" lest they were tempted to attack again: Payments of tribute were typical in the ancient world. Yet the shorterterm benefits of theft may often have loomed large in decisions to go to war. This may have been especially the case for individual soldiers, who were usually entitled to some share of the booty of war. As armies became institutionalized, though, success in war became one of the primary avenues for upward social mobility in many societies. Note that in a Malthusian world of slow economic growth, war is the easiest way for a state to gain wealth (though trade expansion may provide another avenue). Only when modern economic growth begins in the nineteenth century CE do states have an alternative strategy of fostering growth and thus their domestic tax base.

A second motive was security. As we know all too well in the modern age, even a polity with little interest in war itself may feel the necessity of a large army to defend itself against others. A threshold in which all polities in an area were militarized may have been reached in Anatolia before 5000 BCE, but perhaps not in Mesopotamia until about 3500 BCE. States in such an environment may at times decide that attacking others is a better strategy than waiting to be attacked. Some people worry today that those who profit from war benefit from emphasizing external dangers; in the ancient world, soldiers seeking booty may have exaggerated the danger of attack in order to urge kings to pursue an offensive strategy. But the simple fact is that once some political entities had decided to develop armies, other political entities would be forced to respond. The prevalence of urban fortifications almost everywhere in the world over a period of millennia is the most obvious symbol of this simple fact - only when a large state could achieve internal peace for a period of generations did towns cease to fortify themselves.

The third set of motives reflects some of humanity's less wonderful motivations and emotions. Rulers might seek power and glory for its own sake. The monuments which populated the ancient world on which rulers bragged of their military exploits may have served a practical purpose in encouraging the submission of conquered peoples, but seem 
to express rather blatantly the pride that rulers took in military conquest. Rulers often justified wars in terms of some sense of superiority or some imagined slight by the other group (maybe revenge for a previous attack, but perhaps just a failure to respond appropriately to demands for tribute). And basic human anger could then be lauded: The enemy deserved to be killed and enslaved. Individual soldiers might have been attracted to the possibility of exercising arbitrary power over those they conquered. (Rulers, it might be noted, face a hard choice here: If they try to limit their soldiers' behaviour in order to win favour among the newly conquered citizens, they risk reducing their soldiers' interest in war.)

Soldiers often sexually assaulted women among defeated groups or forced them into concubinage. Indeed this practice is so prevalent in the historical experience of warfare that we may wonder just how large it loomed in the overall motivation for war. Again it may have loomed larger for individual soldiers than for the state - but we will find some rulers who displayed great interest in gathering concubines (see for example Chinggis Khan in chapter 17). The frequency of sexual assault would have in turn increased the defensive motive to protect one's self and family from conquest.

We should also speak to the basic human capacity to identify with larger groups. Most humans to this day define themselves as members of some ethnic, religious, or political group. This capacity for group identification seems to have evolved in our days in small hunter-gatherer bands (chapter 3), but we can nevertheless identify with millions of other humans today. This capacity for group identification does much good in the world, for it encourages individuals to cooperate with others in a variety of projects. But it can easily be harnessed to the purpose of attacking other groups. We cannot know to what extent the earliest soldiers were motivated by the pursuit of booty versus a sense that their cause was just. But we do know that later rulers when writing of their conquests would appeal to a sense of group identity and the rightness of their cause. And the sad history of the implosion of the Yugoslav state in the twentieth century can remind us that in an environment of armed-group conflict individuals are encouraged to identify strongly with some group for mutual support. We can reasonably hypothesize that a sense of group loyalty motivated the earliest soldiers.

Soldiers motivated solely by booty will, it should be stressed, often prove of limited value. They will engage in battles only when confident of victory and will be tempted to retreat at the first sign of trouble. Soldiers motivated by a broader loyalty will stay and fight. They may fight if they think the security of their families depends upon it. But they may also fight if they feel an emotional attachment to their state or ruler or fellow soldiers. Victorious armies in history may often have been characterized more by a greater sense of loyalty than by superior tactics. A smaller army highly motivated could win over a much more substantial but unmotivated body. The victories of small nomad tribes over larger sedentary populations (chapter 12,17) may in part reflect differences in motivation. 


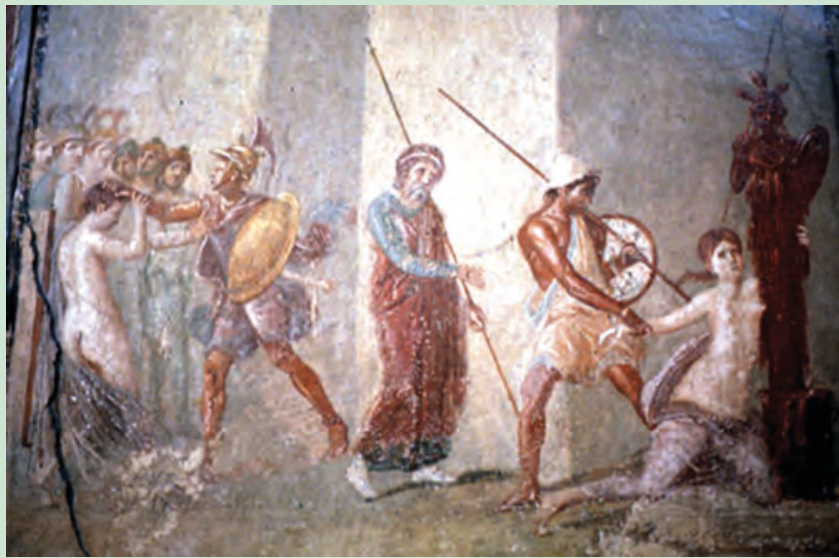

Illustration 6.15

Mural, from Pompeii, House of Menander, showing Ajax dragging Cassandra from Palladium before the eyes of her father. In tales of the Trojan War, he later assaults her

of power in a region dissuaded any polity from attacking another. Wars were often immensely profitable to both the conqueror and their soldiers. But many wars would prove ill-judged: The attacker would end up losing the war, and perhaps also their crown, their freedom, or their life. Such outcomes might be attributed to bad luck (for wars are unpredictable), bad judgement (the attacker misjudged their superiority), bad management (the attacker proved to be a weak general), or just bad circumstances (the attacker may have correctly judged that they were better off attacking than waiting to be attacked). Especially in cases where attackers lose, we can wonder to what extent anger and lust for power and glory got in the way of a reasoned evaluation of the likelihood of success.

With the advantage of hindsight, the thousands upon thousands of wars in human history must seem incredibly wasteful. Wars killed millions upon millions of soldiers - most often young men in the prime of their life - or gave them life-changing injuries. Millions upon millions of civilians have also been killed, injured, or raped. The energies put into building walls and weapons and armour could instead have been devoted to farming, trade, or industrial production, or just to having a good time. Wars often destroyed useful structures: bridges, homes, and grain storage facilities. Moreover, people living in fear of war would be much less likely to create structures or goods that might be destroyed or stolen. Humanity, it must seem, would have been much better off if it could have avoided war.

Yet humanity would only rarely find peace. To be sure, peace was often lauded. There is a Sumerian mosaic in which one panel celebrates victory in 
war while another celebrates the joys of peace. The Epic of Gilgamesh, while filled with tales of war, urges peace (the biblical Old Testament instead seems to take frequent wars for granted, though there are hints of a future age of peace). Rulers often proclaimed that their victories would usher in an era of peace - albeit on their terms. We can appreciate that such claims were self-serving and likely involved self-deception: Victories were more likely to encourage retaliation than reconciliation. Still, we can understand that the population, though often stirred to support war, valued these promises of eventual peace. Egypt - and to a lesser extent China - may have been exceptions here in that one polity did gain dominant status and so did achieve lengthy periods of peace (The Chinese Mandate of Heaven, with its support of one unified empire, can be seen as cultural support for a hegemonic peace.) There were peace treaties in Mesopotamia (and practices of royal intermarriage, gift exchange, and feasting to support these), though rulers broke these with some regularity. Fortifications there and elsewhere may have encouraged peace by raising the costs of conquest. The Indus civilization seems to lack extensive fortifications; again, we do not know how the Indus operated politically.

We should stress the complexity of human history. A balance of power might be achievable if all polities in a region could form alliances such that any attacker could be confident of defeat. However, if some groups had faster population growth than others, or some groups suffered harvest failures, or some groups developed better weapons, or idiots or sadists succeeded some rulers, the balance would be shaken. The frequency of change in human history - and indeed the complex set of interactions among themes around which this book is structured - guaranteed that peace would be fleeting, for the relative military strength of different political entities (and the personal relationships among rulers) would be far from stable.

Recall that war is among the mechanisms posited by Malthus to keep the population in line with food supply (chapter 4). A state that was facing difficulty in feeding its people - whether because of population growth or climatic shock or perhaps bureaucratic incompetence - would be tempted to go to war. This would not only divert the public's attention but would reduce the number of mouths to feed while hopefully allowing the theft of some other group's food.

One very pragmatic approach to appreciating the costs of war was to seek to avoid battle. Sun Tzu's The Art of War from the fifth century BCE in China a book still consulted by both military and business strategists - argued that the best outcome in international affairs was for an opposing side to concede without a battle. War was avoided, then, not by the direct pursuit of peace, but by a combination of overwhelming force, diplomacy, and espionage. Though The Art of War outlines this case most clearly, other societies, including the ancient Greeks, also appreciated the advantages of reducing the incidence of war by achieving hegemony. 


\section{PRIMARY DOCUMENTS: THE ART OFWAR}

These are some of the more famous quotes from Sun Tzu's The Art of War. Note that he stresses what happens off the battlefield, but still appreciates the importance of battlefield tactics:

The supreme art of war is to subdue the enemy without fighting.

Hence to fight and conquer in all your battles is not supreme excellence; supreme excellence consists in breaking the enemy's resistance without fighting.

For to win one hundred victories in one hundred battles is not the acme of skill. To subdue the enemy without fighting is the acme of skill.

All warfare is based on deception. There is no place where espionage is not used. Offer the enemy bait to lure him.

Pretend inferiority and encourage his arrogance.

Victorious warriors win first and then go to war, while defeated warriors go to war first and then seek to win.

The general who wins the battle makes many calculations in his temple before the battle is fought. The general who loses makes but few calculations beforehand.

Opportunities multiply as they are seized.

Strategy without tactics is the slowest route to victory. Tactics without strategy is the noise before defeat.

Do you appreciate Sun Tzu's wisdom? Can you understand why generals and managers still consult this work? Does Sun Tzu seem to speak from experience?

We have already seen one way in which war is reinforcing: The decision by one state to form an army forces others to respond. But there are others. Since success in war depends on being able to organize men and resources and lead them into battle, warring states concentrated power internally. Yet this concentration of power itself encouraged war, for powerful rulers gained much of the benefit of fighting for themselves (see below). Victory in a war was also self-reinforcing, for this would attract more soldiers to one's cause: Victory increased the soldier's booty (and exercise of arbitrary power) while decreasing their chances of injury or death.

We might also note that the outcome of a war is particularly challenging to predict. One cannot be sure of how particular technologies, organizational developments, battlefield strategies, or speeches of encouragement will work until these are applied. Small events on a battlefield may spell the difference between attack and retreat. Weather and disease may affect outcomes. If the results of war were more predictable, we might have witnessed far less of it. But the unpredictability of war - coupled with the immense rewards for victory and severe penalties for defeat - ensured that both sides in a conflict would usually decide that they should fight. 


\section{Military leadership}

Military or political leaders might seek glory, wealth, or power for themselves and then try to convince others that their motives were other-oriented. But they might well believe that their conquests were good for their city or ethnic group or god. These beliefs might be mostly subconscious and self-serving. Nevertheless, they might have some merit: Conquest might well allow them to reward many of their fellow citizens. And offence against others might - at least for a while - protect their hometown from attack by others.

There can be little doubt that leaders differed in abilities. Once large armies came to battle in fixed formations, strategy and tactics became of crucial importance: Choice of the battleground, placement of troops, and particular troop movements could all spell the difference between victory and defeat. There was also the critical matter of motivation: As noted above, a soldier that bolted at the first sign of trouble was far less valuable than one determined to battle even to their death. Leaders who could instil trust and confidence and awe in their soldiers could win even if the numbers were against them. Still, we can well imagine that many of the proclamations issued by victorious military leaders exaggerated their role in the outcome. Some of the time, though Sargon, who founded Assyria, or Thutmose (1479-1425 всE) who led seventeen Egyptian campaigns in the Levant, say - there is likely considerable merit in the self-congratulation. Still, while individuals may have won or lost specific wars, they may have had little influence on the more general history of war itself.

\section{Soldiers}

There was a historiography, now largely disdained, that celebrated a "Western" civic devotion that encouraged soldiers to engage in face-to-face combat. It

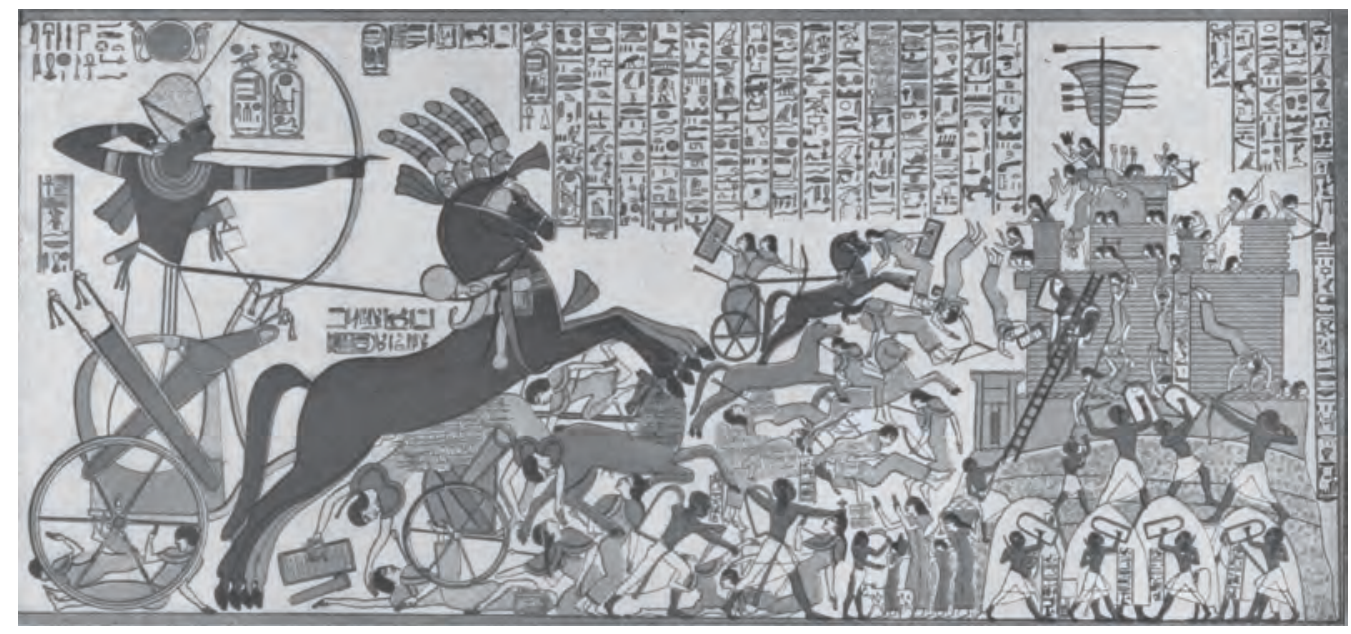

Illustration 6.16

Ramses II's victory over the Cheta people. From a mural in Ramses II's temple in Thebes 
is now appreciated that there are multiple exceptions on both sides of this argument. It is nevertheless still useful to examine soldier motives whenever possible.

We know relatively little about the lives of soldiers in early states. We can, though, gain some insight by looking ahead in our story to the experience of Roman soldiers. These soldiers received the same wage as labourers, but every day rather than intermittently. There were also bonuses from time to time (for example on the emperor's death) and at retirement - in land at first but after complaints about this, in money. Promotions led to even better incomes. There were frequent complaints that soldiers requisitioned from civilians without authorization, and extorted. The Roman army forced soldiers to save part of their income and maintained these savings for them (that is, they had a primitive form of a pension). There were also legal advantages: Soldiers gained control over their money against their father's wishes; charges against them were heard in military court; they could not be sued if away on duty; and they were excluded from forced labour requirements. Army camps had better sanitation and less crime than towns, though troops engaged in war generally experienced unhealthy living conditions. Soldiers ate well by the standards of the time. They, unusually for the time, had access to doctors. Soldiers could learn useful skills when not fighting or drilling. They were not - after the time of Augustus legally allowed to marry, but often did, or at least maintained concubines and children near the army camp. Retired soldiers were often recognized as leading citizens in their towns. In return for risking their lives and submitting to sometimes harsh army discipline, soldiers gained a significantly above-average income and experienced an unusual degree of social mobility.

The Roman army was, of course, a wholly different enterprise from earlier armies of a few hundreds or thousands. Personal ties between commanders and soldiers likely gave way to more bureaucratic structures as armies grew in size and became permanent. Regular salaries slowly replaced reliance on war booty to reward soldiers. Professionals replaced volunteers or conscripts. But the fact that soldiering offered an avenue to a better life was probably a common occurrence. Indeed soldiering was often almost the only available avenue to a better life.

\section{Challenges facing agents: Soldiers}

The soldier wants to gain an income while not suffering death or injury. Since war is risky, it is best to think in probabilistic terms: They want to increase their chances of gaining resources while reducing their chances of injury or death.

Students should note that (at least pre-modern) soldiers more often died of disease than in battle. Maintaining good health in unsanitary conditions was a critical, though likely less appreciated, part of the soldier's challenge.

Soldiers will also often be motivated by a desire to serve or protect. They will still be interested in achieving victory and avoiding defeat. 


\section{War and social differentiation}

We should also stress social differentiation. War generally made more sense for the ruler than for the ruled: The ruler took much of the booty and even more of any long-term benefit in tax and tribute, while the governed suffered most of the costs of war. Scholars often argue that wars are only common when there is an authority that can declare war. This may be why wars were rare in the early centuries of agriculture but became more common as political stratification emerged. And the ruler often only became the ruler because they had won some war. Rulers might justify their rule in various ways, but all depended in large part on their control of an army to maintain their position of power. Rulers would often force their subjects to fight, though these often proved all too willing to oblige.

As we know from the present day, wars can also prove convenient in diverting attention from domestic concerns. Ancient rulers, like modern counterparts, might not want their citizens focused too much on problems with taxation, corruption, or infrastructure. They would be tempted to blame other polities for any misfortune, and appeal to the potential gains from conquering others. Others - soldiers, weapons makers, bureaucrats seeking more power - who also saw personal benefits in going to war could join rulers in these efforts. Then as now, the interplay of groups within the state interacted with inter-state conflicts.

War likely made more sense for men than for women. To be sure, men were more likely to die in war, but they were also the ones that gained booty. Women in general gained from war only indirectly through their husbands or fathers. Armies commonly ill treated women. Across human history war was seen as an expression of masculinity: Men would be praised for displaying courage and athletic capability on the battlefield. The vast majority of soldiers were men though there are scattered examples of women soldiers across time and place. In ancient times these were known in Central Asia, South Asia, South America, and West Africa. Since men dominated armies, it is hardly surprising that societies that stressed war tended to be societies in which men were dominant.

\section{The changing face of war: Technology, scale, and organization}

We noted above that the incidence of war likely increased with the move to settled agriculture. The scale of war then advanced with the size of states and their ability to raise taxes. War both facilitates and is reinforced by increasing state size. As states grew, they could afford - and had the bureaucratic capability to organize - more massive armies, and armies comprised of military professionals. They needed larger armies to maintain control of conquered areas (in conjunction with a bureaucracy that could both provision the soldiers and identify trouble spots to which these might be moved). Whereas small groups of amateurs may have preferred to shoot arrows from a distance, 


\section{BOX 6.4}

\section{THE EVOLUTION OF WAR}

War will change through time as different weapons, strategies, and military organizations are developed. War will also change in nature due to a variety of other historical changes, such as state formation, increased taxation, and economic development.

War, we might stress, provides a very intense selection environment. The side with better military technology or organization will usually kill and conquer the side with less wonderful technology or organization. The intensity of the selection environment feeds back into motives for mutation: States have a more powerful and immediate incentive to identify (or copy) better technologies or strategies for war than they do for more humdrum activities like feeding their populations. The exceptions are states that achieve some degree of dominance in their part of the world: These may relax their search for military advances until new enemies appear (at which point they often find that they have fallen behind, and suffer the consequences). But where war is endemic, we can expect intense pressures for both mutation and selection of military technology and organization. There will then also be strong incentives for transmission: States will try to copy ideas from others, and they will devote resources both to spying and to training their armies and engineers.

There are occasionally useful spillovers into civilian life. Technologies for killing humans can be applied to killing animals (and vice versa). Much later, techniques for boring cannon will be applied to cylinders for steam engines (chapter 24). There can also be administrative spillovers as techniques for organizing armies are used in bureaucracies - these may occur most often where generals are also influential political figures.

large professional armies were drilled in formations and military strategies. Fortifications could slow such armies, but only an army of similar size and training could defeat them.

There were also critical technological shocks. The development of first bronze and then iron technology decreased the cost of both weapons and armour and thus encouraged much bigger armies. The first army of 5,000 is recorded in 2300 всE in Mesopotamia. The frequency of wars also seems to have increased at least in Mesopotamia, with its competing city states - with the introduction of bronze weapons from about 3000 все. Solid-wheeled battle carts were used as soon as wheels were known in Mesopotamia; these were replaced after 2000 вCE by war chariots with spoked wheels developed by nomads on the eastern steppe and pulled by horses. By lowering the cost of large armies, iron technology in particular may have encouraged an increase in the size of states - we will return to this issue in chapter 9. Iron armour was about 50 per cent stronger than bronze, and far cheaper to produce.

We can trace how armies grew as states grew and iron replaced bronze: Sumer was at most 400 kilometres across and had an army of slightly over 5,000. 


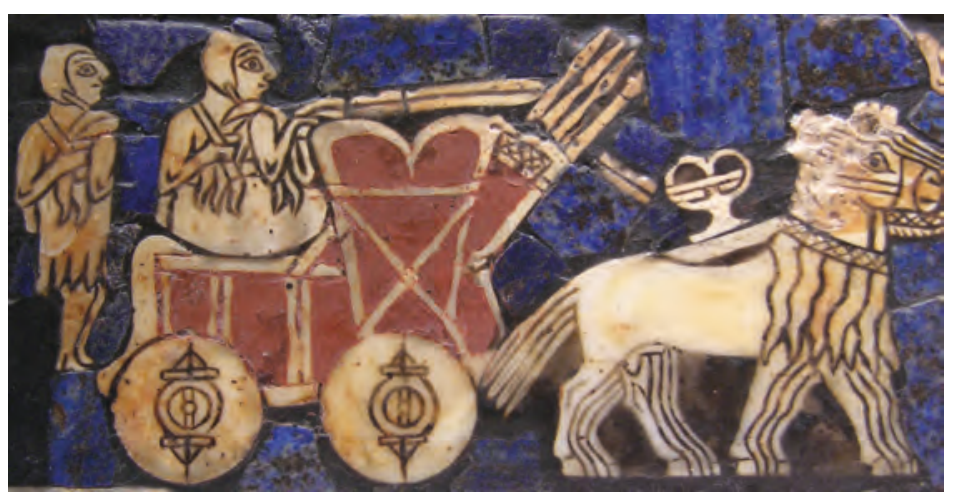

Illustration 6.17

Part of the Standard of Ur, 26th century BCE, depicting an early war chariot

As Egypt expanded from 1,000 to 2,000 kilometres in length, its army grew from 60,000 to 100,000. In the "Iron Age," Assyria, which was a bit bigger than Egypt, had an army of perhaps 200,000. Persia, about four times the size, had maybe as many as 400,000. Rome at its height would have 350,000. We can perhaps identify an early uptick in war associated with early states and bronze, and a later one related to larger states and iron.

Egypt when united was able to project force outward. Only when there was internal dissent could others attack it. This may have slowed the rate of advance in military technology relative to Mesopotamia. Egypt adopted bronze later than in Mesopotamia. The Hyksos during the second intermediate period (see chapter 7) used chariots and other weapons that were new to Egypt to invade the Nile delta. The Egyptians would later adopt these weapons and extend into Palestine and Nubia.

Chinese armies used bronze weapons after 2000 все; they may have been imported at first from Central Asia. Chariots were introduced through Central Asia and played a crucial role in the Shang's efforts to control areas hundreds of kilometres from the centre. (The Shang controlled the regions in which bronze was produced; this may have been instrumental in their rise to power.) War chariots would then become a common element of elite burials. Arrows were fired from chariots, which were supported by armoured infantry. The later Zhou dynasty came to stress infantry and iron weapons: They favoured swords, but crossbows became common also. Armies were at first about 10,000 but grew to 30,000 after about 500 BCE. After centuries of warfare during the Warring States period, centralization occurred with armies of hundreds of thousands of infantry and cavalry too. A bureaucratized state was required to support such armies.

There is less military development in Mesoamerica and South America because there was no metal weapons, wheels, or horses. But we nevertheless see changes in swords and armour. Armies still grew as states grew in size. 


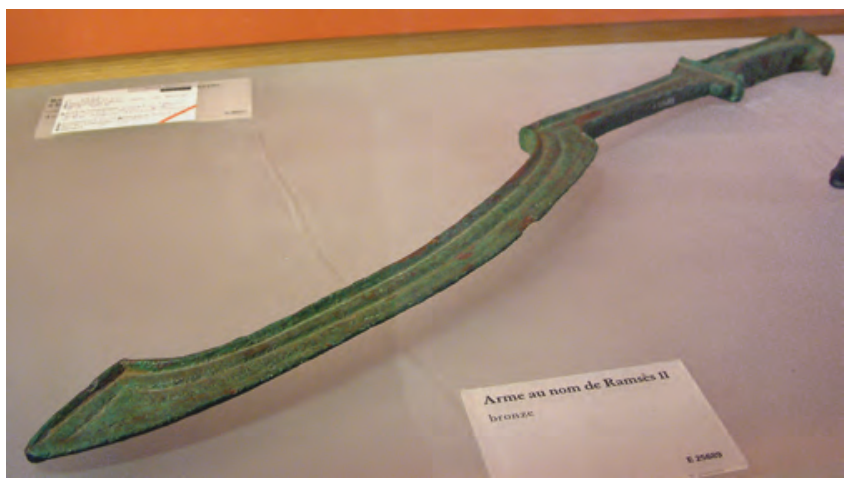

Illustration 6.18

Ancient Egyptian bronze sword, Louvre

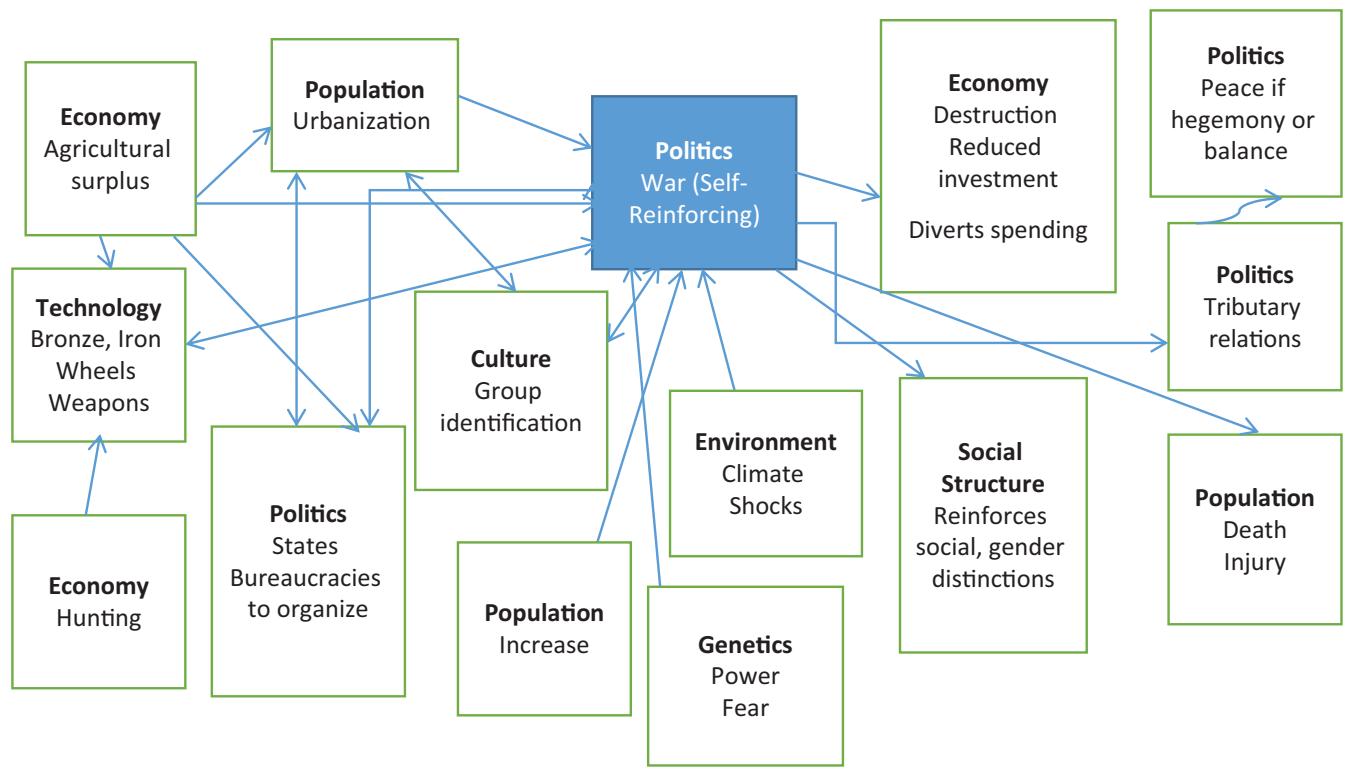

Figure 6.3

Influences on and effects of war

\section{THE THEME OF POLITICS}

Decisions must be made within any society.We must thus understand how power is distributed and exercised: This aspect of societies is termed "politics." The central phenomenon here is political institutions: the official rules that are in place within society. For institutions to be effective there must be penalties for breaking the rules and these rules must be enforced effectively. In literate societies, institutions are written down.We can still speak of institutions in 
preliterate societies if rules and penalties were clearly understood and regularly proclaimed. The most prominent institutions are the laws and regulations issued by political authorities. But any organization - a religious organization, a firm, a school - that can enforce penalties of some type can generate institutions. We will also include the organizations - including the state itself - that proclaim institutions within our definition of institutions. In understanding the exercise of power within a society, it is essential that we know its institutions, how well these are maintained or enforced, and why. We will pay particular attention in this book to how and why new institutions emerged, and how feasible it was to enforce these.

We can examine the psychologies of rulers and their advisers. But kings do not operate in a vacuum. They must worry about how their subjects will view their decisions. We should then strive whenever possible to assess public opinion and how this influenced (or reacted to) key decisions. Of course, we are quite limited in our ability to analyse public opinion in early human societies.

One all-too-frequent decision is to engage in war. (This is not treated directly in Table 1.2 but is a type of interaction between polities.) Wars always have certain effects: People die or are otherwise abused, the economic activities and often infrastructure of the war zone are destroyed, and peacetime political realities are shattered. Frequent wars have further effects on diverse phenomena, including investment, cultural values, and even fertility. Some wars serve to extend the political power of one society over others, and this process may also spread economic and cultural ideas. We will discuss of course those wars that altered the political landscape. It is also valuable to mention frequent wars that may not have moved borders much but certainly affected those caught up in them in important ways.

Wars often rely upon some sense of what in modern times is called nationalism. We have seen how the human tendency to identify with groups evolved during hunter-gatherer times. We will often see how group identity is employed to encourage hostility toward others. We will discuss when possible how states or societies fostered a sense of identity and the effects of this.

Finally, yet importantly, we will address crime. The incidence of crime has a significant impact on people's well-being. It affects and reflects social stability and the power of the state. As in the modern day, statistics on reported crime (when available) may understate the actual incidence of crime: We are thus not always able to speak knowledgeably about the level of crime in different societies. Yet one of the primary purposes of the state in human history is to protect individuals from crime.

\section{Questions}

1. If you were transported back to the third millennium BCE, would you prefer to live in a city or in the countryside? In a state or outside of state boundaries? Why?

2. Reproduce Figure 6.2 for writing.

3. What are the challenges facing scribes? Bureaucrats more generally? 
4. Do you lean toward a voluntarist or coercive view of kings? Why?

5. We have and will from time to time in the text invite the reader to place themselves within historical scenarios, as we did in Box 6.2. Reflect on the degree to which it is possible to do so.

6. What are the general's challenges?

\section{Readings}

Bauer, Susan Wise. 2007. History of the Ancient World: From the Earliest Accounts To The Fall Of Rome. New York: Norton.

Ferguson, R. Brian. 2002. "The History of War: Fact Versus Fiction," in William. L. Ury, Must We Fight? San Francisco, CA: Jossey-Bass. [A useful source on the very first wars.]

Fukuyama, Francis. 2011. The Origins of Political Order. New York: Farrar, Straus and Giroux.

Goetzmann, William N. 2016. Money Changes Everything: How Finance Made Civilization Possible. Princeton University Press.

Lee, Wayne E. 2016. Waging War: Conflict, Culture, and Innovation in World History. Oxford University Press.

Levy, Jack S., and William R. Thompson. 2011. The Arc of War: Origins, Escalation, and Transformation. University of Chicago Press.

Mazlich, Bruce. 2004. Civilization and Its Contents. Palo Alto, CA: Stanford University Press. [Mazlich is one who argues that we should cease to use the word "civilization."]

Scott, James C. 2017. Against the Grain: A Deep History of the Earliest States. New Haven, CT: Yale University Press. 


\section{Early civilizations around the world}

\section{Guiding questions}

How, why, and when did the general trends toward cities and states unfold in different parts of the world? How and why were institutions, cultures, architecture, and other characteristics of cities or states similar or different? What were the connections across societies, and (how) did ideas spread between them? (Since we survey different regions there is an important "where?" element to all of these questions.)

How, why, and when did climate change in early history, and what effect (and where) did this have on humans?

How, when, and where did trade expand during the period of early civilizations?

Why and how did population grow during this period?

Relationship to other chapters: This chapter is a companion to chapter 6. It examines how the development of cities and states occurred in different places, adding important detail to our understanding of these important developments. In surveying regions, we generally build on the brief histories of particular regions in chapter 4 . The various regions of the world that we survey in chapter 7 will reappear in later chapters. We will note both important similarities and differences across time in the politics, culture, economy, and so on of each of these regions, as well as how they influence each other across time. The disappearance of the Indus civilization reminds us that continuity is not inevitable in human history. We close the chapter with some more general discussion: of trade expansion, of how institutions evolve through time; of climate change; of population growth and migration; and 
of families. We discuss trade and institutional evolution in many later chapters. The discussion of climate links to a preceding discussion of climate in chapter 4 and to discussions of climate in many later chapters. Note that the discussion of the source and effects of climatic change remains important to this day.

Having outlined general trends in the preceding chapter, we can describe in this chapter how these processes unfolded in different ancient states. Our focus here will be on political organization, but we will discuss architecture (built environment), culture, and trade from time to time. Our discussions of buildings will inevitably touch upon both technology and art. We will often describe influences of one society on another.

We are guilty here of focusing on the most successful states, and thus those that are best known to historians. Readers should remember that there were myriad other states that lasted only a generation or two; many of these have left no historical record at all.

We close the chapter with brief coverage of some general topics: trade expansion, economic growth, trends in climate and population, and a discussion of ancient family life.

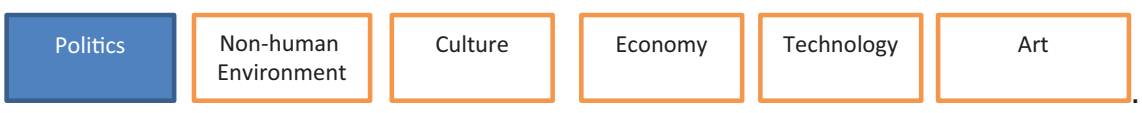

\section{City states and "empires" in Mesopotamia}

Mesopotamia, like Egypt and the Indus below, was not well suited to huntergatherers because of limited rainfall, but it had fairly stable water flow in its rivers during the ancient period (though there were of course periods of flood and drought over the millennia). The Tigris was generally too fast but the Euphrates was slow, meandering, and therefore well suited to gravity-flow irrigation (that is, irrigation that does not require pumps). Flood control was also necessary as crops were harvested during the spring run-off before the hot summer. Agriculture had emerged in the hills and mountains to the north where rainfall was greater (chapter 4); it could only spread southward with irrigation. Mesopotamia would prove to be very fertile once irrigated.

The Euphrates did provide some challenges. Since the winds generally blew in the same direction as the current, it was hard to navigate upstream. As we shall see, the Nile was much more suited to navigation, and so shipbuilding developed further and faster in Egypt. The Euphrates also shifted course several times during the ancient period. These changes resulted in migration of farmers and changes in which towns were dominant.

We know far more about Mesopotamia than any other early "civilization" due to an abundance of written records on both clay tablets and stone 
monuments in a script that has been deciphered. Yet there is still much that we do not know. Some sort of council may have ruled the earliest city states in at least southern Mesopotamia, though we do not know who chose these councils or how. Kingship may have emerged in early Mesopotamia in response to incessant military conflict. City councils may have selected kings to wage war. Kings were at first temporary, but later permanent. Religious authorities played an important role in recognizing the king's approval by the local god(s); later kings gained the power to appoint family members to the priesthood. As kings gained power relative to religious authorities, they came to play an important religious role themselves. Councils may have chosen the king's successor from among his sons (and perhaps brothers). These councils may have represented some sort of democratic control, at least by elites. All towns possessed councils, as did the wards of larger cities. Associations of artisans or traders also had councils. Records indicate that all males - and sometimes females - could participate in at least some councils.

Though there was no unified Mesopotamian state most of the time, there were shared gods and a shared sense of the duties of kings (though this evolved). This unity in religious and cultural practices implies that rulers did not dictate religious practice but rather strived to shape religious beliefs in their favour. We shall see in chapter 8 that Mesopotamian religion came to justify occasional shifts in political dominance among Mesopotamian cities by suggesting that a council of the gods occasionally decided that a different city god should lead. There was considerable in-migration of Semitic peoples at times, perhaps in response to the growing prosperity of Mesopotamia (or perhaps due to climatic challenges elsewhere); these seem to have integrated into the local population and adopted local customs and beliefs.

We should distinguish southern Mesopotamia - called Sumer and then Babylonia - from the north, which usually is called Assyria (there were also states beyond in Turkey about which we know far less). There were about thirty city states in Sumer between 2900 and 2700 все. The most prominent early city in the south was Uruk, on the banks of the Euphrates. Uruk was pre-eminent in the period 4000-3200 BCE. The city was almost a square mile in size, and probably had 20,000-40,000 people (whereas previous cities had only been about 25 acres, a fraction of this size). Archaeologists have found a list of 100 distinct offices or professions for Uruk at this time, indicating significant occupational specialization. A priest-king ruled Uruk. Uruk would be followed by several other dominant city states of a similar size. Legendary epics regarding this early period, notably the Epic of Gilgamesh, were recorded centuries later. Both the state and private individuals owned land.

Temples were the largest structures in these cities; Uruk had two main temples to different gods. These temples received vast quantities of grain. In Mesopotamia (and perhaps elsewhere) donations to temples were effectively taxes. Scholars had long thought that temples controlled most of the land because of the elaborate economic records found there, but it is now appreciated that both temples and states kept detailed records of tax contributions from 


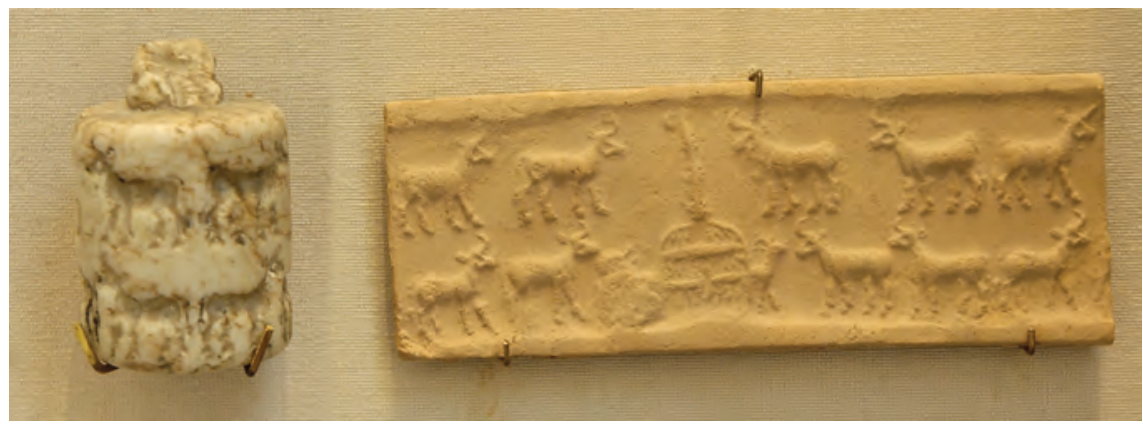

Illustration 7.1

Cylinder seal and impression, cattle and cowshed, Mesopotamia 4100-3000 вСE, Louvre. Such seals signified official approval of a document

other lands. States at times (for example under the king Shulgi of Ur, a city to the south of Uruk) owned most of the land, but taxing private owners became more common through time. Both practices required an extensive bureaucracy. In conquered territories, states granted military governors land on which they were to pay rent to the state. The ability of these early states to record taxes or revenues from each of thousands of little farm plots is truly remarkable - much larger states thousands of years later with access to much more sophisticated technology would not be able to tax incomes or land in the way that ancient societies in many parts of the world managed to do.

Mesopotamian states also taxed trade. There were Mesopotamian trading colonies in Syria, Iran, and elsewhere. Pottery wheels were developed, and southern Mesopotamia produced a great deal of undecorated pottery that was used throughout the Middle East. Mesopotamia obtained the stone for building monuments from Iran; we know little about who quarried or transported it or how.

In some ways the Akkadian Empire of Sargon from 2334 вСE, which captured much of southern Mesopotamia from the north, is the world's first multi-ethnic empire. It is intermediate in size and diversity between preceding large states (such as Egypt with 3 million) and the much larger later empires such as Rome with 60 million at its peak (chapters 9 and 10). In any case, later empires in the area would mimic Akkad. Sargon won (at least) thirty-four battles in creating his empire, according to Akkadian records. Sargon started as an administrator in the state of Kish, rebelled against Kish, and then attacked one city after another, destroying their walls. He seems to have been a gifted warrior. As noted in chapter 5, though, he may have had superior bronze weaponry. Sargon taxed trade routes, especially between Mesopotamia and Anatolia and Iran, making Akkad very rich. The Akkadian Empire employed regional governors that the state either sent or recruited among loyal locals. Military garrisons supported these governors. The governors and king visited each other regularly. Governors were given lands in other regions to avoid them developing a local power base. 
The Akkadian Empire expanded for a while after Sargon. One of his successors sent a flotilla to attack a state near the Straits of Hormuz: This is one of the earliest naval engagements in recorded history. The attack on Hormuz also suggests that trade by sea through the Persian Gulf was important at the time. However, the Akkadian Empire ended suddenly around 2200 BCE; there is (controversial) speculation that it collapsed because of climate change toward aridity in the area, perhaps triggered by a volcano. It could be, though, that Sargon and his successors relied too much on the plunder associated with conquest and failed to establish tax revenues that could support a non-expanding state.

A series of empires based in Ur followed Sargon's empire. The later empire based in Ur, called Ur III, employed a system within its Mesopotamian domains whereby local rulers governed in concert with a representative of the central state. As Ur III conquered beyond Mesopotamia, it instead installed military governors. Ur III is famous for its extensive bureaucracy and detailed recordkeeping (including the size of herds and agricultural yields on all state lands). Though successful militarily, Ur III seems to have relied on taxation far more than plunder. The second Ur III king Shulgi declared himself divine: This was a rare practice in Mesopotamia, though kings regularly claimed divine favour. He promulgated one of the first law codes, which provided some ethical justification for its rules; later kings would follow this example. The kingdom built roads and irrigation canals, standardized weights and measures, and regularized both language and calendar. The Ur kings claimed descent from Gilgamesh and popularized his stories. They taxed different goods in kind from different provinces and dispersed these.

One statistic may give a sense of the time: Around 2000 BCE, the markets of Ur processed 70,000 sheep annually. This indicates that there was a substantial textile industry in the area and also that the urban population, at least on average, had some meat in their diet. We know this number from the detailed records kept in the Ur markets. These records also tell us that there were contracts between merchants and shepherds (some of whom may have been nomads) that divided the risks of raising sheep for market. This single set of transactions gives us a glimpse of the complexities of provisioning a city with tens of thousands of inhabitants.

After the fall of Ur III, there was a lengthy period of conflict among states. Hammurabi, who we mentioned in chapter 6, ruled in Babylon from 1794 to 1750 вСе. During this period, there were also military incursions from areas outside Mesopotamia, though these peoples tended to adopt local ways once they settled in Mesopotamia. This period sees a decline in religious authority and a rise in private enterprise. No later kingdom was quite as powerful as Ur III, but there would often be dominant kings. There was a brief Babylonian Empire associated with the king Nebuchadnezzar (ruled 1125-1104 BCE). It is most famous for the Hanging Gardens of Babylon, terraced gardens with plants from the mountain homeland of one of Nebuchadnezzar's wives, which exemplified the vast wealth of Babylon. Babylon installed its god as the head of the Mesopotamian pantheon. Babylon was said to have massive walls (one could 


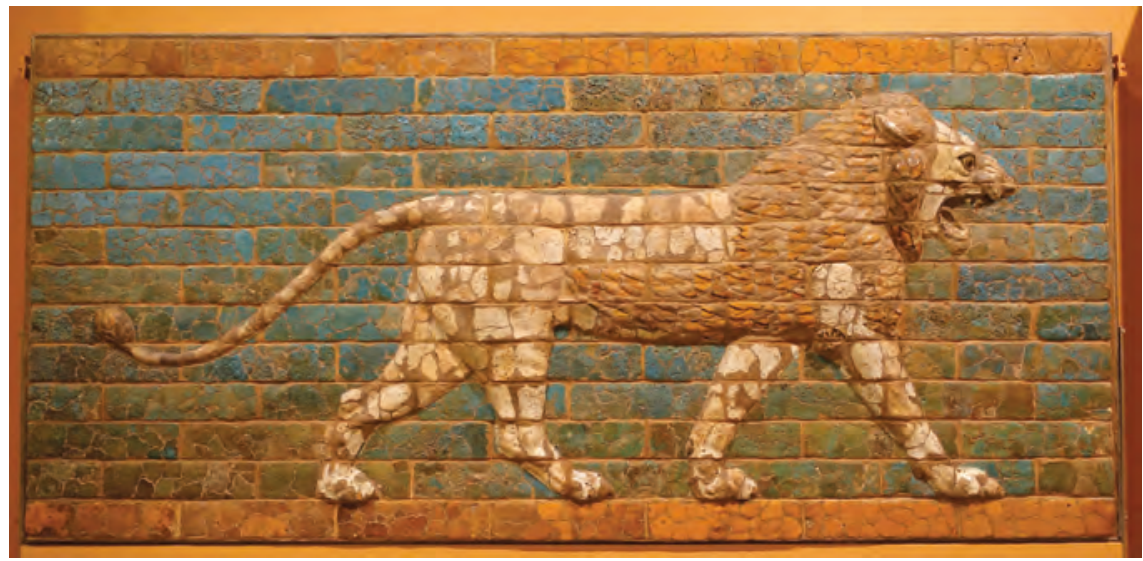

Illustration 7.2

Striding Lion, Processional Way, Babylon, Oriental Museum, University of Chicago

Source: Photograph by Daderot, CCO 1.0

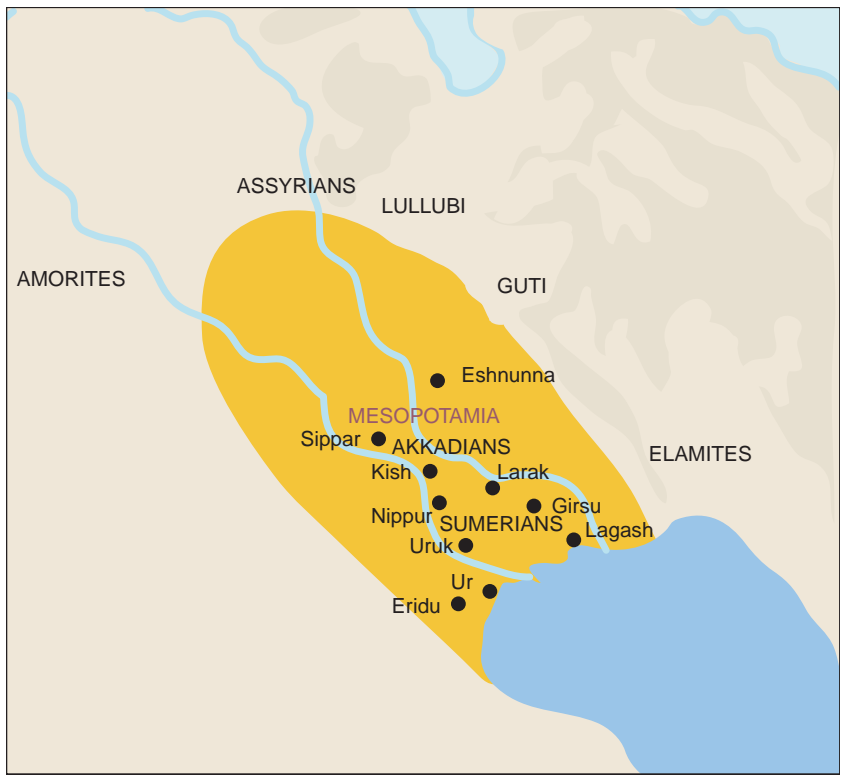

Map 7.1

Some major Mesopotamian cities in 3500 BCE. Note that the Persian Gulf extended much farther inland at the time

Source: TimeMaps.com supposedly turn a four-horse chariot on top) and many palaces and over a thousand temples.

Assyrians from around Nineveh (near the modern city of Mosul on the Tigris in northern Mesopotamia) built a state in the north from the nineteenth century BCE with a professional meritocratic army of mixed infantry and cavalry. They borrowed the idea of cavalry from the Hittites (see below) - a previous empire based in Anatolia that had briefly conquered Mesopotamia. Assyrians would conquer southern Mesopotamia from the thirteenth century (but suffer setbacks soon after) and rule until 612 все; by the eighth and seventh centuries, they controlled lands in Egypt and Anatolia. Much of the early Assyrian strength may have come as with the previous Akkadians -

from controlling trade routes between Mesopotamia and Anatolia. The Assyrians maintained Babylonian laws and preserved literature in huge libraries. Councils of leading merchants and landowners advised the ruler at the start, but these disappeared over time (there are three centuries after 1750 BCE where no records 


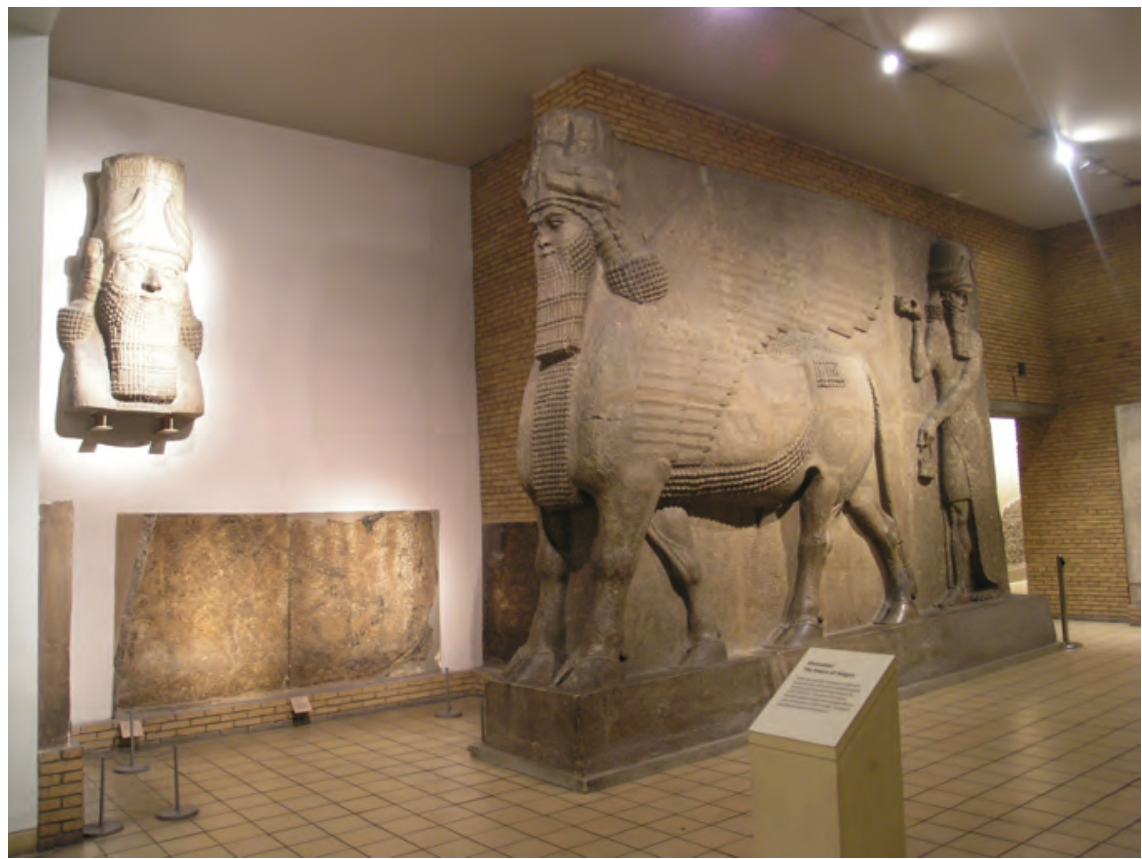

Illustration 7.3

Khorsabad Palace reliefs from the palace of Sargon II of Assyria (not to be confused with the much earlier Sargon of Akkad), British Museum

have survived, and in which long-distance trade declined). Nevertheless, aristocrats remained influential. From the early ninth century, a professionalized army led yearly campaigns. The ten lost tribes of Israel in the Bible were lost because they refused to pay tribute to the Assyrians, and were defeated and dispersed. Forced migration was a common Assyrian practice. Such dispersed peoples naturally had no loyalty to Assyrian state or culture. The Assyrian Empire would suffer a fate similar to the later Roman Empire: It was attacked from outside, and never rebuilt. In the case of Assyria, its language and religion would disappear. The harsh rule of the Assyrians, and their attempts to force their own religion on subject peoples, apparently inspired little affection among those they ruled.

\section{Inscriptions}

Mesopotamian inscriptions developed into lengthy narratives over time: Rulers provided justifications for wars and for usurping the throne; celebrated victories; celebrated construction of temples, and also canals and other public works; celebrated prosperity and trade, and trading expeditions; and sometimes celebrated their good works among their peoples. Over time, these narratives came to be written on clay tablets because they were too long to be inscribed on stone. Hundreds of thousands of clay tablets survive. We must, of course, 


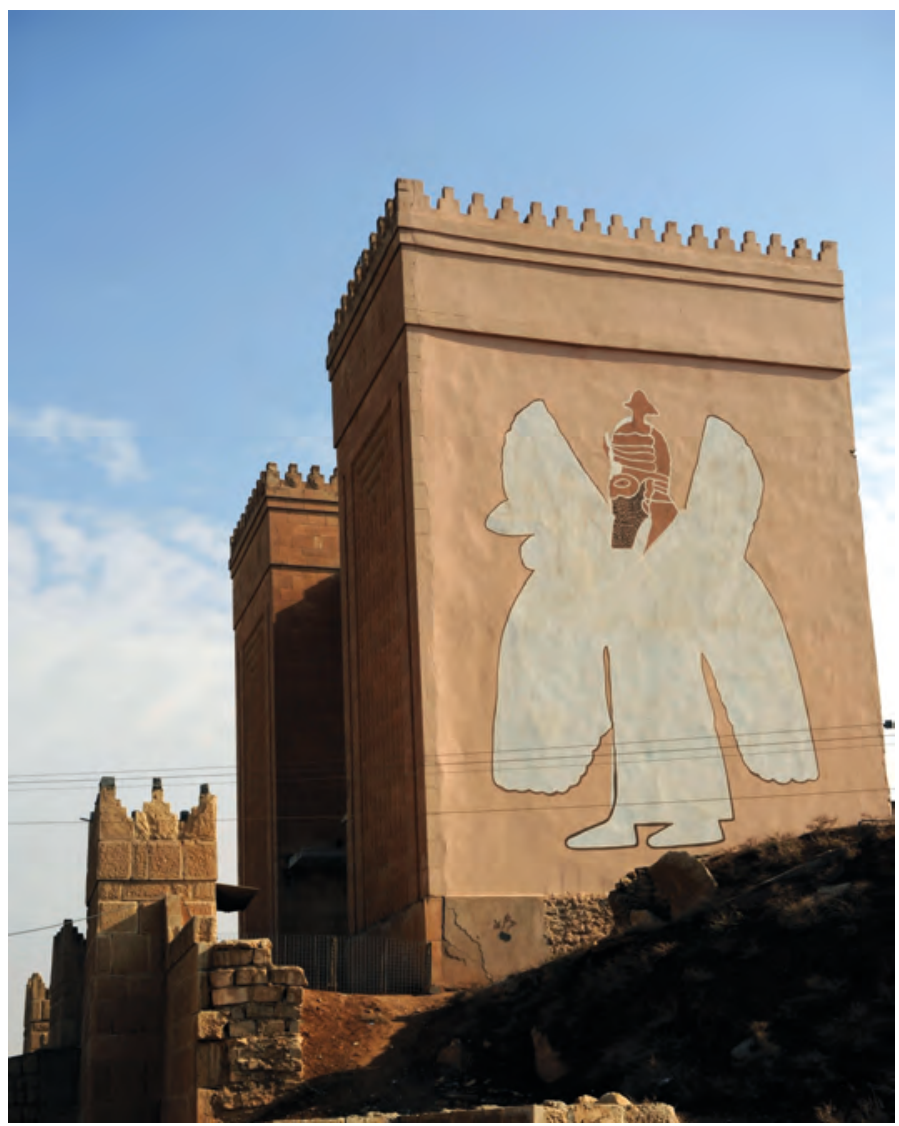

Illustration 7.4

Remains of the (reconstructed) Nergal gate in Nineveh, Iraq (Nov. 22, 2008). Nineveh, built between 704 and 681 BCE, was a capital of the Assyrian Empire and was surrounded by a 12-kilometre mud brick wall

view propaganda sceptically. Nevertheless, it is notable that rulers went to great lengths to advertise the accomplishments (allegedly) associated with their rule. They apparently thought it both valuable and possible to gain widespread support in this way. While these inscriptions cited military campaigns most frequently, kings saw value in advertising public works as well. These inscriptions are useful historically, for they tell us of ethnic and linguistic diversity and of constant tensions both internal and external to states.

Stelae - freestanding pillars with inscriptions - were built from the third millennium вCE. Such stelae were common also in Egypt, Europe, and Tibet, among other places. Inscriptions were not, then, just decorations for buildings, but merited construction in their own right. The Code of Hammurabi was inscribed on stelae, with a sculpture of Hammurabi gaining godly approval for his rule on top. 


\section{PRIMARY DOCUMENTS: EXCERPTS FROM CRIMINAL LAW IN THE CODE OF HAMMURABI}

1. If a man bring an accusation against a man, and charge him with a (capital) crime, but cannot prove it, he, the accuser, shall be put to death.

2. If a man charge a man with sorcery, and cannot prove it, he who is charged with sorcery shall go to the river, into the river he shall throw himself and if the river overcome him, his accuser shall take to himself his house (estate). If the river show that man to be innocent and he come forth unharmed, he who charged him with sorcery shall be put to death. He who threw himself into the river shall take to himself the house of his accuser.

9. If a man, who has lost anything, find that which was lost in the possession of (another) man; and the man in whose possession the lost property is found say: "It was sold to me, I purchased it in the presence of witnesses;" and the owner of the lost property say: "I will bring witnesses to identify my lost property": if the purchaser produce the seller who has sold it to him and the witnesses in whose presence he purchased it, and the owner of the lost property produce witnesses to identify his lost property, the judges shall consider their evidence. The witnesses in whose presence the purchase was made and the witnesses to identify the lost property shall give their testimony in the presence of god. The seller shall be put to death as a thief; the owner of the lost property shall recover his loss; the purchaser shall recover from the estate of the seller the money which he paid out.

14. If a man steal a man's son, who is a minor, he shall be put to death.

15. If a man aid a male or female slave of the palace, or a male or female slave of a freeman to escape from the city gate, he shall be put to death.

26. If either an officer or a constable, who is ordered to go on an errand of the king, do not go but hire a substitute and dispatch him in his stead, that officer or constable shall be put to death; his hired substitute shall take to himself his (the officer's) house.

What strike you as crucial differences between this code and contemporary codes? Why was this code different in these ways? Do you think these severe penalties were always applied?

\section{Anatolia}

Anatolia played an important role in ancient history. There had been trade between Anatolia and Mesopotamia from the earliest days of Mesopotamian civilization. During the early second millennium вCE, Assyrians traded Babylonian textiles and Iranian tin for Anatolian copper. There were Assyrian trading colonies in Anatolia until about 1830 вС. It seems that bronze metallurgy spread to Mesopotamia from Anatolia. 


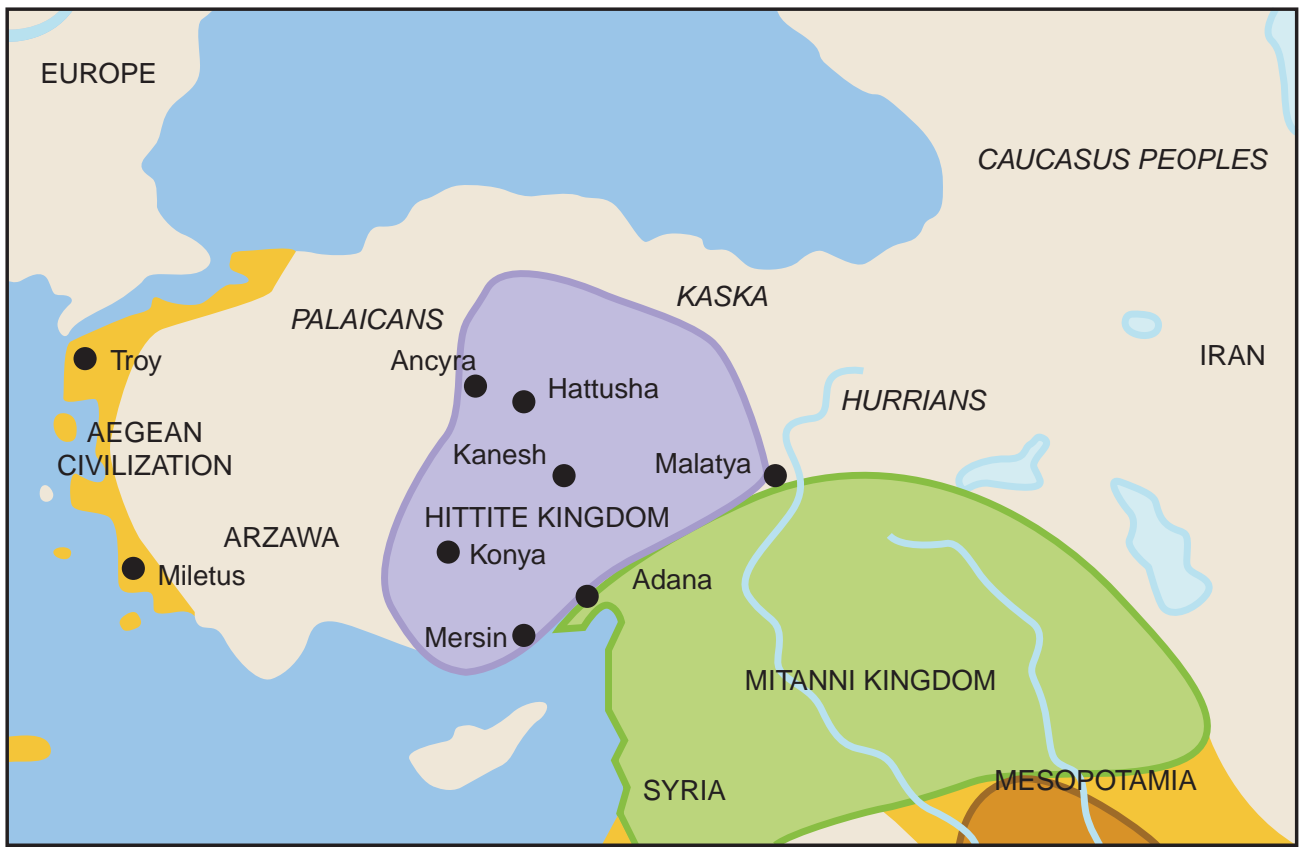

Map 7.2

Hittite kingdom in $1500 \mathrm{BCE}$

Source: TimeMaps.com

A bewildering array of peoples, with different languages, moved into and around Anatolia over the millennia, though it was mostly agricultural rather than pastoral (though much of the change in names of groups may involve a change in ruling elites far more than mass migration). One group of particular importance was the Hittites: These people spoke the first known Indo-European language. The Hittites produced bronze from 2000 все. We know little of any Hittite polity before $1650 \mathrm{BCE}$, but a Hittite kingdom would then last until about 1200 вСе, though there was a period in the middle of relative anarchy. Later kings would clarify the rules of succession to prevent earlier experiences of usurpation of power. The Hittites would win wars early against Babylon and much later against Egypt in Syria (actually a draw in the battle that forced the Egyptians to withdraw and sign a treaty). The Hittites would fight a variety of other states in all directions about which we know very little.

\section{Egypt}

The Sahara was wetter than today from 11,000 until 3500 BCE, and there is evidence of pastoralism there around 5000 все. By the time of the great Egyptian kingdoms, though, there was limited scope for raising or hunting food outside of the Nile floodplain. Egyptian society was dependent on the Nile to water its 
crops. The Nile has created over the millennia an extensive floodplain bounded by higher plateaus on both sides. Egyptians used irrigation, including a simple lever device that raised water from small pools to spread over crops. They built towns - and monuments - on the plateaus above the river or on small hills (reinforced to prevent flooding) within the floodplain.

Egypt was first united between 3200 and 3000 BCE, though regional differences between Upper (southern, since the Nile flows north) and Lower Egypt remained. From cultural evidence, it would seem that the south conquered the north: Southern art forms are found thereafter to the north. Iconography indicates a process of military conquest. Egypt was unified far earlier than other regions of the world. We can attribute this early experience of unification, at least in part, to two unique characteristics of the Nile Valley. First, it was unusually productive: The annual Nile flood deposited silt rich in nutrients. A larger agricultural surplus may then have facilitated the formation of more massive armies, which in turn could support larger states. Second, desert surrounds the Nile Valley: Defeated armies could not easily retreat and regroup, but would have to surrender (see our discussion of war in chapter 6). We should also note the ease of transport along the Nile which facilitated the administration of a large state. As elsewhere, the move to centralized states was associated with social stratification. There is clear evidence from cemeteries of a move from an egalitarian to a highly stratified society.

There was contact with Mesopotamia from at least 4000 BCE. These societies shared art and technology over the millennia and perhaps ideas of city and state (and there would later be intermarriage among Mesopotamian and Egyptian elites). Nevertheless, it seems likely that states emerged independently in the two regions. Certainly, military interactions between the two come long after states appeared in both.

Scholars divide Egyptian history into three "kingdoms," separated by "intermediate periods" of one to four centuries in which there were competing political entities within the Nile Valley. Each kingdom is divided further into dynasties: There are thirty-two numbered dynasties in Egyptian history. Although this periodization is useful for Egyptologists, it necessarily simplifies: There was political conflict even during the three kingdoms, the boundaries between dynasties are sometimes arbitrary, and it is unlikely that every dynasty represents hereditary rule within a single family. Nevertheless, the fact that a broadly similar form of government characterized the region for about three thousand years is remarkable.

Though popular imagination may conceive of an all-powerful Egyptian state, Egyptian rulers faced the same challenges of administration as any early state. They established a bureaucracy, which at first comprised members of the royal family. Note that since writing predates Egyptian unification, the earliest bureaucrats of a centralized state kept written records. By the fifth dynasty, it seems that those who were not royal family members could gain jobs in the bureaucracy through merit. Through much of Egyptian history, there appears to have been much greater opportunities for outsiders to rise through 


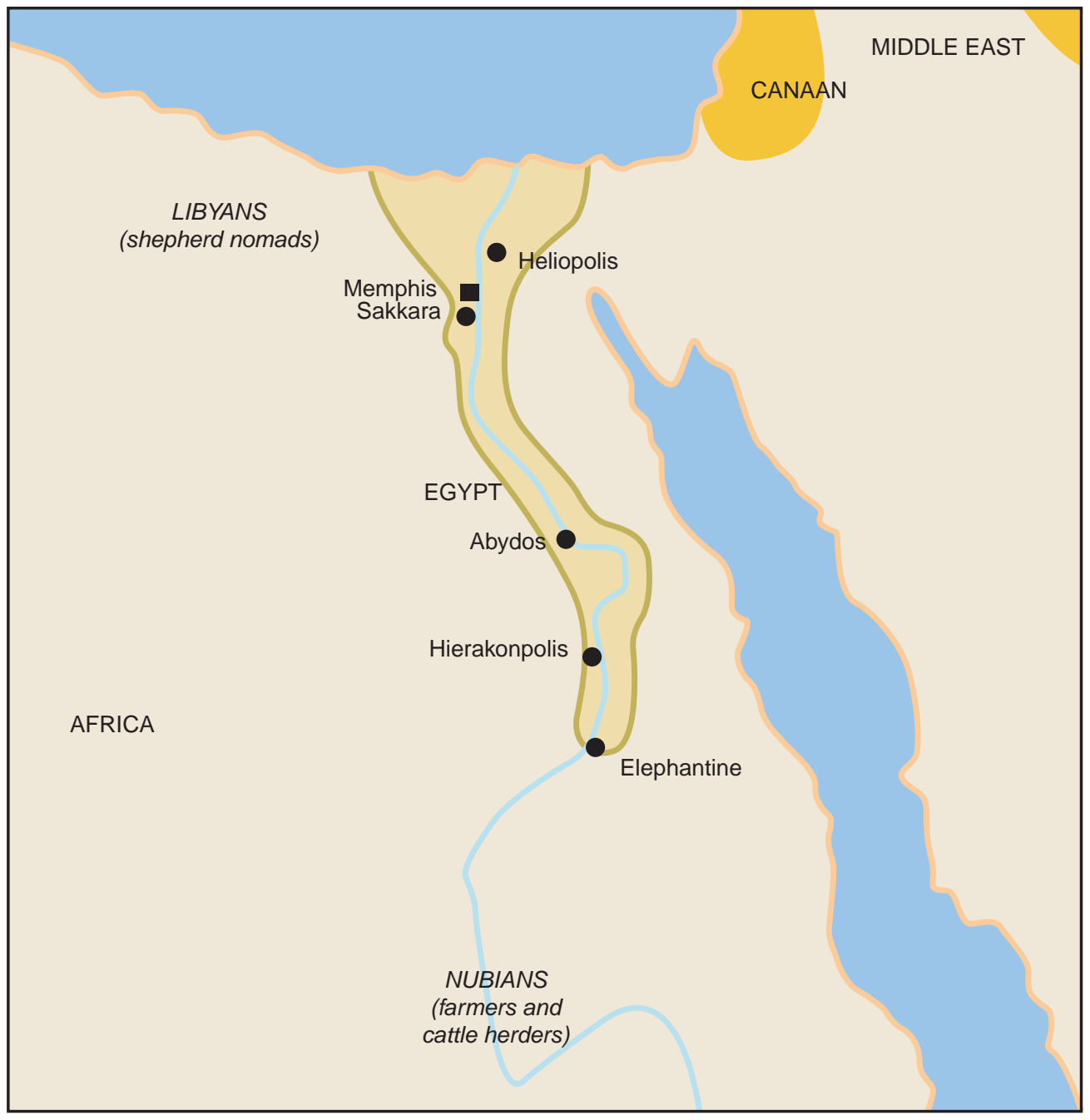

Map 7.3

Ancient Egypt, 2500 BCE

Source: TimeMaps.com

the bureaucracy than in Mesopotamia; this may reflect the larger size of the Egyptian state and bureaucracy and the Egyptian kings' desire to limit the power of local aristocrats. By the sixth dynasty, the office of provincial governor was established. These came to exercise hereditary power, and these hereditary governors overshadowed any titular kings that may have existed during the first intermediate period. Foreign incursions, excessive Nile flooding, and a lack of kingly heirs may have contributed to the dissolution of central power at this time. Behind these triggers, we can see the typical challenge of large states: that local authorities over time gained control at the expense of the centre. 
Central power was restored within about a century. The ideal of a centralized state was not forgotten during the time of conflict. Scholars often consider the middle kingdom the height of Egyptian civilization. There is evidence of a sizeable (by ancient standards) middle class of bureaucrats, merchants, and artisans. There was also military expansion into the Levant, Nubia to the south, and to the deserts to the west. Eventually, political turmoil resumed. There were sixty kings in 150 years in the thirteenth dynasty as different families fought for power. During the second intermediate period, the Hyksos ("foreign rulers"), a Semitic tribe from the Levant, dominated the Nile delta. The Hyksos used chariots and bronze weapons in their conquest, though there is speculation that the conquest may have been relatively peaceful in the face of a weak Egyptian state. The Hyksos exacted tribute from Egypt.

With the eighteenth dynasty and the new kingdom, we see Egyptian military expansion into Syria and to the Euphrates, and over the state of Kush to the south and desert oases to the west. It was during the new kingdom that pharaohs built the tombs in the Valley of the Kings.

Paintings associated with the very first Egyptian dynasty have elements that would remain common throughout ancient Egyptian history: They celebrate the ruler for uniting Lower and Upper Egypt, and both as a warrior and as nurturing the Egyptian soil; the ruler is associated with bulls and fake beards and falcons. Egyptian myths long spoke of a battle between chaos and order; the role of kings was to create order. Within centuries, kings claimed to be semi-divine, though later dynasties would not always stress this characteristic. Scholars now think that kings were believed to be divine only after death; gods only ordained the office of king. Indeed the social gap between ruler and at least some of the ruled closed through Egyptian history: The burial practices of rulers and aristocracy came to resemble each other. Priests came over time to exercise considerable political authority. These changes likely reflect the growing importance and size of the bureaucracy necessary to rule such an extensive state.

Currency did not exist in ancient Egypt - though the state and merchants used weights of gold and silver in exchanges of high value - and so taxes were paid in kind. The government would measure the flood, calculate how good the harvest should be in various areas, and assess taxes accordingly. An extensive bureaucracy was required to collect the taxes and move the goods to where they were needed. Forced labour was an important form of taxation. Egyptians could be required to work on pyramids or temples or roads - though most workers on these sites likely received wages. Forced labour has advantages: It allows individuals to pay some of their taxes without having to sacrifice real goods, and it enables the state to pursue public works without having to expend goods. However, forced labour must be supervised carefully and is found in many historical circumstances to be unproductive.

The dry conditions on the plateau above the river have meant that many Egyptian monuments have survived for thousands of years. The earlier pyramids and later elaborate tombs have both excited popular attention. Pyramids were pursued in different styles in the third through fifth dynasties of the first 
kingdom. Pyramid building would continue through the middle kingdom, but increasingly be replaced by the construction of temples (many of which were built on the floodplain and have not survived). Imhotep, the architect of the first pyramids, was originally from Sumeria, and Mesopotamian ziggurats may have inspired him. Egyptians quarried stone in the desert for their monuments and built paved roads through the desert to transport this (but used the river as much as possible). With primitive tools, it would have required a great deal of manual labour to cut and move large stones, much less build the actual monuments. Though the pyramids contained elaborate sarcophagi (coffins), archaeologists have not found mummified bodies in any of them: Tomb-raiders may have robbed richly decorated mummies, despite the precautions taken by builders in constructing hidden passages, or perhaps the pyramids never held actual bodies.

Many writers have suggested that slaves built the Egyptian pyramids. Egyptian slaves were mostly war captives, though, and the pyramids predate by centuries major Egyptian victories in Nubia or the Levant. (The Bible suggests that there were Jewish slaves at the time, but even so they would have been a small part of the workforce of 100,000.) The workforce was mostly Egyptian peasants, either pursuing their forced labour obligations or being paid by the state from tax revenues. Of course, one can still criticize even "voluntary" labour, for this was only feasible because the state had taxed so much away from the peasantry in the first place. It is hard to know the peasant mindset at the time: They may have seen working on the pyramids as a religious task (which would encourage the gods to control the Nile flood appropriately) rather than

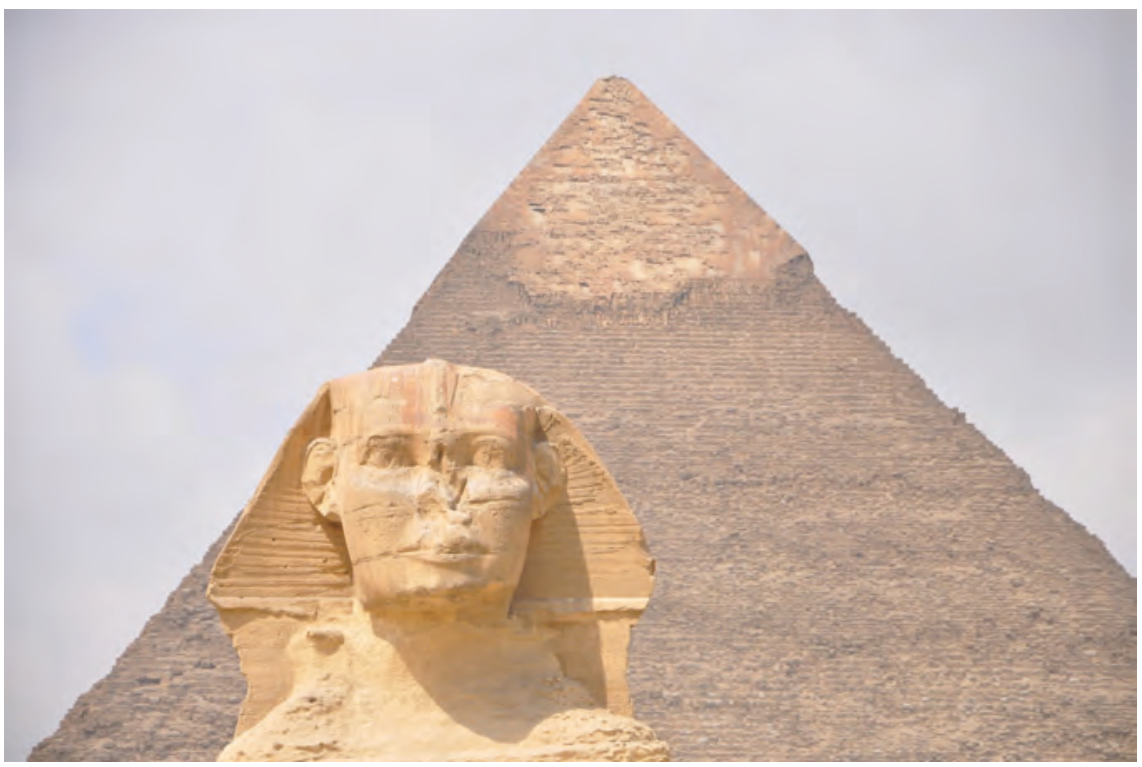

Illustration 7.5

Sphinx and Great Pyramid at Giza 


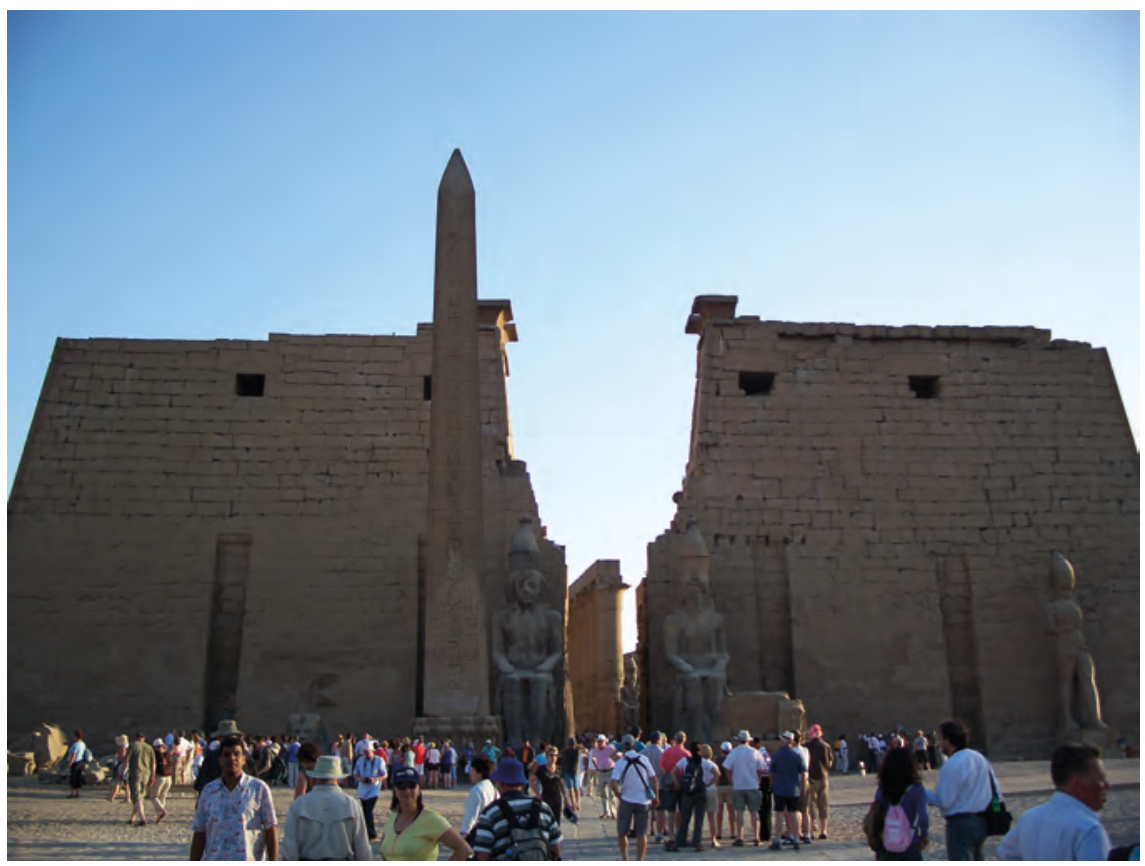

Illustration 7.6

Entrance to Luxor Temple, 1400 BCE

an obligation. As noted in chapter 4, there was little for Egyptian farmers to do in the fields during the hot summer or later flood; the state applied this labour to monuments on the plateau above.

There has been a historical controversy regarding the degree to which ancient Egypt borrowed important ideas from the black African populations to the south, or indeed was constituted in part by those peoples (and a related controversy about just how much Greece and then Europe borrowed from ancient Egypt). There is a natural attraction to the argument that Western civilization had its roots in sub-Saharan Africa. Moreover, some links undoubtedly exist: It could well be, for example, that African polities originated the idea of the divinity of the pharaoh. Yet the language of the Egyptians is related to neighbouring areas of Asia rather than Africa. In addition, critical Egyptian developments, including pyramids and hieroglyphs, have no known precursors anywhere. The point of view of this book (and the field of world history in general) - that all regions of the world have interacted in creating the world of today - should encourage a less emotional debate in which we carefully evaluate influences in all directions. That is, we should not look toward any one human society as the dominant source of human development. It is then noteworthy that there were indeed highly developed states to the south that interacted with Egypt through much of its history (see below). As for ethnicity, it is clear that merchants from Nubia lived in Egypt during times of peace, and that large numbers of Nubian slaves were captured during times of war. We know that some intermarriage 


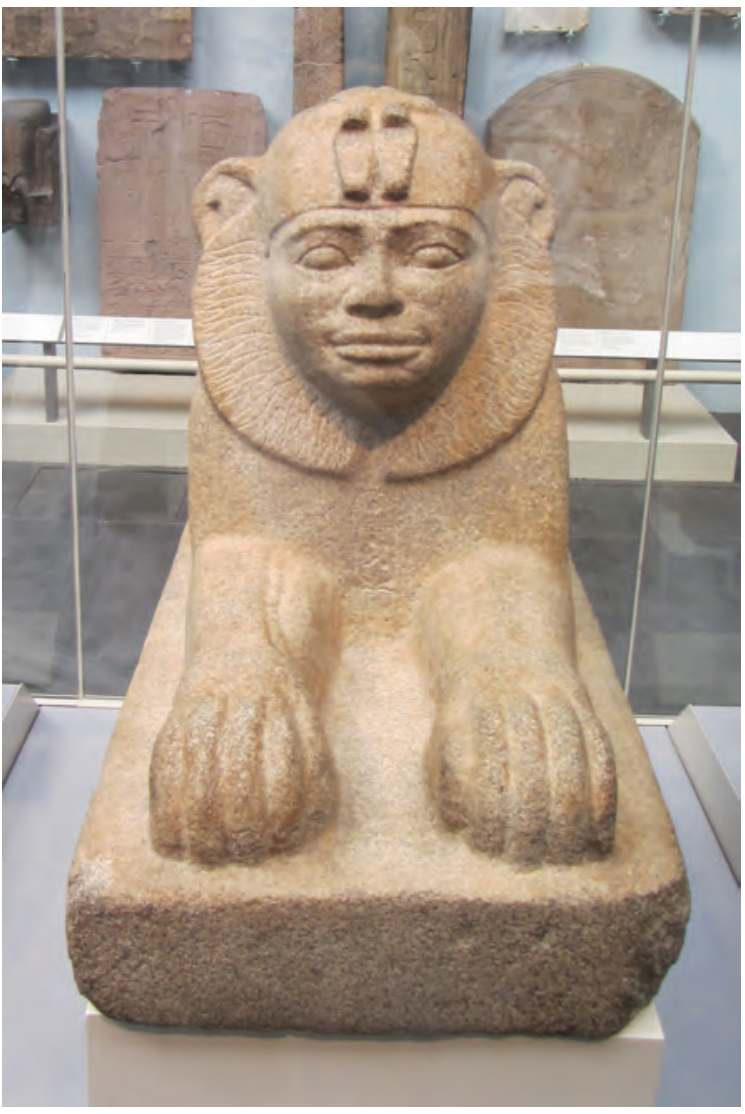

Illustration 7.7

Granite sphinx of Taharqo, twenty-fifth dynasty, from a temple at Kawa. His African heritage is clear, British Museum

Source: Photograph by BabelStone, CC BY-SA 3.0 occurred. As we shall see below, Africans from Nubia to the south governed Egypt during the twenty-fifth dynasty.

The role of water transport in Egypt deserves emphasis. The Nile flows northward into the Mediterranean for about 1,000 kilometres without any rapids. Floating downstream is easy. The prevailing winds for much of the year are from north to south. We find the first pictures of sails anywhere in the world in Egypt at about the time of the first Egyptian kingdom. Ships equipped with sails could easily move southward along the Nile. This was important administratively, for almost the entire Egyptian population lived within a few kilometres of the river. Travel by river was much faster and cheaper than travel by land. (Egypt was unusual in this respect; in most of the world, land transport is at least potentially faster.) It was thus easy to transport agricultural produce taxed from the population to the capital. It was along the river that the massive stones for the pyramids usually moved. Trade goods - including various minerals from the mountains to the east - also moved along the river. The river facilitated external trade, too. Nubia, to the south of the first cataract on the Nile, was a source for a variety of exotic African products. Having mastered sailing along the Nile (we presume), Egyptians soon ventured on to the Mediterranean, where they obtained cedar from Lebanon (of great importance since Egypt lacked wood for construction - the Nile floodplain may have been forested at one time but was cleared over the centuries of early Egyptian agriculture) and other goods. The Red Sea allowed trade with the exotic land of Punt and beyond - but the timber for ships had to be carried overland to the treeless shore of the Red Sea. Egyptian records report several expeditions to Punt, a land with a less developed polity but from which Egyptians obtained exotic goods such as ivory, spices, and myrrh (a perfume and medicine); Punt was perhaps along the Red Sea itself or in present-day Yemen or Somalia. Egyptians developed a wide range of boats for different purposes (the longest being metres longer than the ships used by Columbus). These developments would influence boat-building practices throughout the eastern Mediterranean and perhaps to the east as well. 


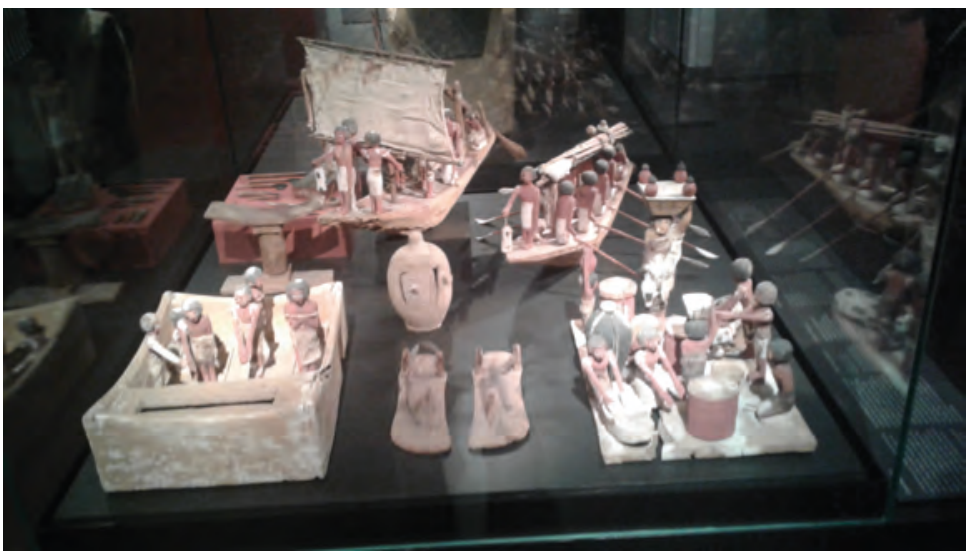

Illustration 7.8

Models from the tomb of Wadjet-hotep, c.2000 BCE, meant to ensure his well-being in the afterlife. One depicts a granary and another a bakery. There are two boats, one with sails up to move south against the flow of the river, Glyptoteket Museum, Copenhagen

Source: Author

Egyptian art is still widely admired. In part, this may be because Egyptian art seems to reflect a greater optimism than the art of Mesopotamia. This, in turn, may reflect the more benign nature of Nile floods or the lesser degree of warfare associated with a unified state. Egyptian religion (chapter 8) was perhaps also more optimistic in outlook.

\section{PRIMARY DOCUMENTS: ADVICE FROM AN EGYPTIAN SCRIBAL TEACHER}

The Egyptian scribe Nebmare-nakht advises his student Wenemdiamun (to encourage more study). He compares being a scribe with other occupations:

The maker of pots is smeared with soil, like one whose relations have died. His hands, his feet, are full of clay; he is like one who lives in the bog.

The cobbler mingles with vats. His odour is penetrating. His hands are red with madder, like one is smeared with blood. He looks behind him for the kite, like one whose flesh is exposed.

After criticizing the lives of washer, watchman, carpenter, sailor, merchant, farmer, soldier, and unskilled worker, he lauds the life of a scribe:

The scribe, he alone, records the output of all of them. Take note of it. and later

Be a scribe and be spared from soldiering ... You are safe from torments.

Can you appreciate why Wenemdiamun would be encouraged to apply himself by this advice? 


\section{Nubia}

We have mentioned Nubia a few times above. Nubia was the location of a series of black African kingdoms to the south of Egypt along the Nile from about 4000 BCE. There are more frequent cataracts along the Nile in Nubia than in Egypt, and so it was harder to fashion a sizeable contiguous state. Nubia had gold and copper, and obtained goods such as ebony and ivory from elsewhere in Africa. Egyptians esteemed Nubian skills in pottery and metalwork. Egypt traded with Nubia through most of its history. The Nubians used Egyptian hieroglyphs and blended Egyptian gods into their own pantheon. Egypt controlled northern Nubia through much of the old and middle kingdoms, but the kingdom of Kush survived in southern Nubia until conquered in the fifteenth century BCE. Kush would regain its independence in the eleventh century and in turn conquer Egypt in the eighth century BCE. Assyrians attacked the Kushites in Egypt and Egyptians then pushed the Kushites back in the seventh century. There was nevertheless almost 150 years (the twenty-fifth dynasty) in which the Egyptian pharaoh was black - though these pharaohs seem mostly to have adopted Egyptian ways. The Nubian kingdom after that moved farther south along the Nile and replaced hieroglyphs with a novel writing system. The new kingdom of Meroe continued to link Egypt and Africa. One unusual characteristic of Kush and Meroe is that women often ruled each of them.

\section{Africa beyond Nubia}

There is evidence of city states from about 2000 вСЕ in what is now Mauritania in West Africa. Such states would likely have had no connection with the states

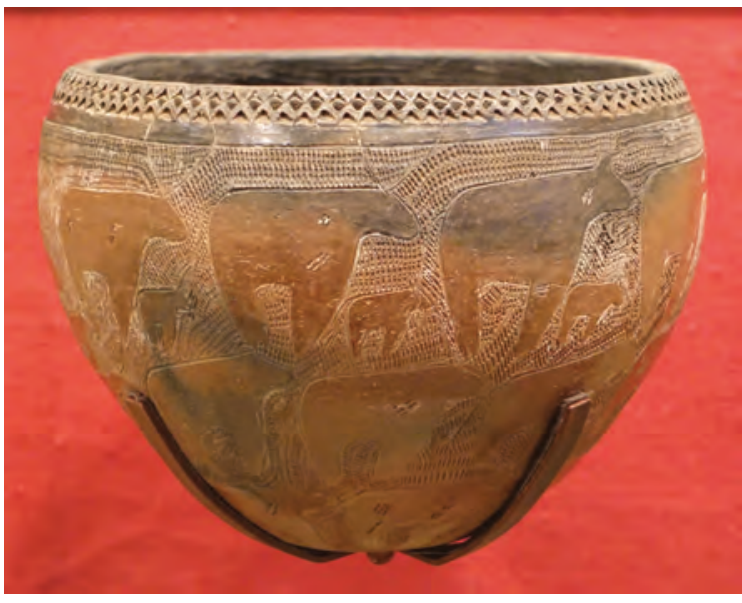

Illustration $\mathbf{7 . 9}$

Nubian pottery, with cattle, 1900-1750 BCE

Source: Photograph by Daderot, CC0 1.0 of Eurasia. If slash-and-burn practices (chapter 4) characterized agriculture in the area, then population densities would have been low, and it would have been particularly challenging to form large states.

\section{The Levant}

The Levant (present-day Israel, Lebanon, Jordan, and Syria; the eastern end of the Mediterranean Sea) will prove important in chapter 11 as the home of two of the world's religions: Judaism and Christianity (with Islam, in turn, drawing heavily on these). During the ancient period, small city states characterized the Levant: These 
states were rarely united and were often fought over by Egyptian, Mesopotamian, and Anatolian polities. The Levant likely received more rainfall during much of this period than it does today. Its cities could draw on supportive agriculture and benefit from trade between Mesopotamia and Egypt. Though not as magnificent as Mesopotamian or Egyptian capitals, the cities of the Levant nevertheless possessed public monuments and excellent works of art. Not surprisingly, Egypt and Mesopotamia both influenced architecture and art in the region.

In the Levant, we see the rise and collapse of cities between 3300 and 2300 BCE, a decline that may be due to climate change or war. There is a decline in population and the development of fortifications before a reversion to

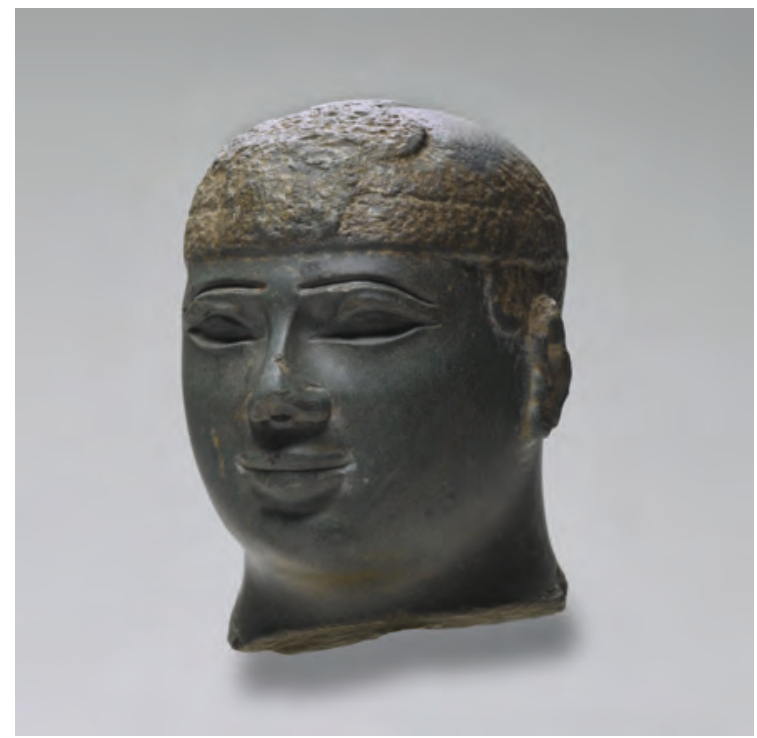

\section{Illustration 7.10}

Head of a Kushite ruler, late 8th century BCE, Brooklyn Museum nomadism. Cities may also have suffered as trade with Egypt declined. After an interlude, there is substantial urbanization and development of city states in the second millennium всE. There is much monumental architecture during this period. Egypt conquered the Levant in the mid fifteenth century все, despite an alliance among Levantine states. It seems that city states continued under Egyptian suzerainty.

In addition to religion, the Levant also played a critical role in the development of the modern alphabet. The Phoenicians, a small state in present-day Lebanon that traded throughout the Mediterranean from about 1500 вCE, first developed a system of phonetic letters - though their alphabet contained only consonants (chapter 6). We can speculate that Phoenician merchants needed an easily mastered script. The Phoenician state is notable for its reliance on taxation of trade - the other states addressed in this chapter relied primarily on revenues from agriculture. Phoenicians not only used galleys (boats powered by oars) to trade gold, tin, silver, glass, and many other goods but also established colonies throughout the Mediterranean. Phoenicians likely developed the bireme, or two-level galley, which was faster and more substantial than previous galleys. Also, the Phoenicians developed a purple dye from shellfish that because it was rare and expensive - became a marker of royalty in many lands.

\section{Greece}

There is debate over how much contact there was between the ancient societies surveyed above and ancient Greece. This debate in part reflects the simple 
fact that different scholars study these various regions. It also reflects a desire by some European historians to downplay "Asian" or "African" influences on European history - a peculiar project given that Greece, Mesopotamia, and Egypt certainly interacted with each other more than any did with distant parts of their respective continents. Unfortunately, we know far less about Greece before about 1000 все than after. The epic histories compiled by Homer in the late eighth or early seventh centuries BCE provide some guidance, but these characteristically mix history and mythology and so are not the most reliable of historical documents. Indeed, "Homer" may be not one person but a name affixed to a set of stories that evolved over many decades. Homerian epics suggest that kings were hereditary but subject to public approval, and also that there was some cooperation across city states.

Archaeological evidence suggests that agriculture had spread to the Danube region of Europe by 7500 BCE and that states with a writing system existed there during the ancient period. These peoples may then have been pushed south by Indo-European-speaking nomads. They may have then settled in Greece and played an important role in developing the later Greek civilizations. Traces of non-Indo-European language have survived in modern Greek.

The island of Crete was home to prosperous city states. This "Minoan" civilization seems to have started out egalitarian, but we see large palaces after about 2000 все (see chapter 6). There was apparent artisanal specialization in pottery and metals, and some trade with Egypt and Anatolia. Debate rages as to whether Minoan cities were ruled by king or queen or some sort of council. Our

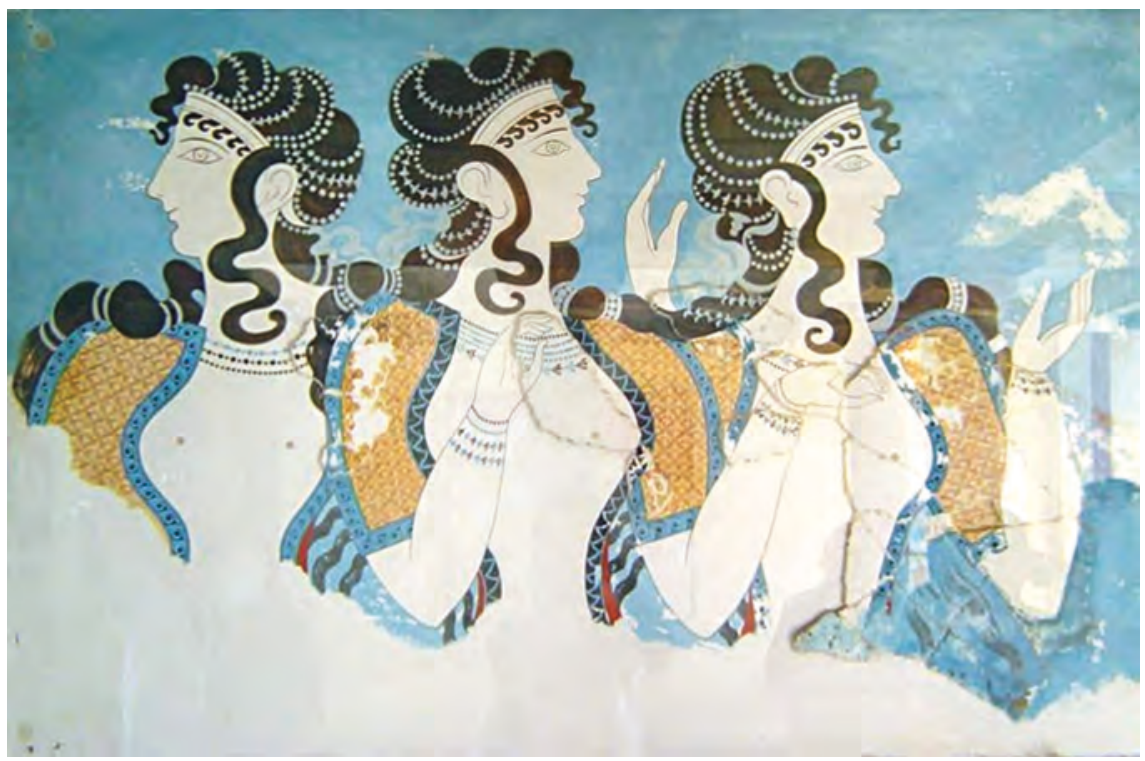

Illustration 7.11

Fresco of women, Knossos Palace, Crete

Source: Photograph by cavorite, CC BY-SA 2.0 


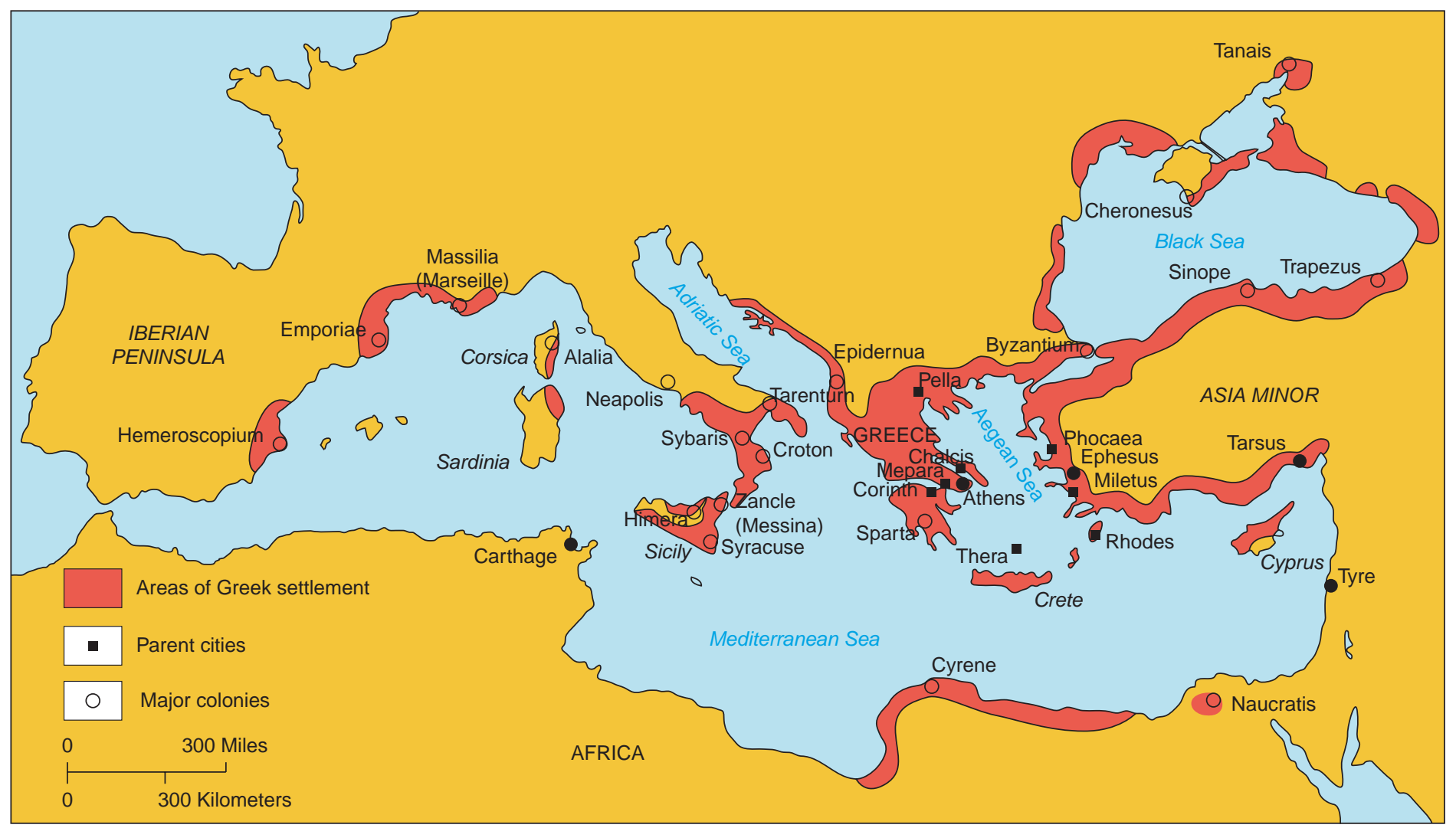

Map 7.4

Greek territories and colonies (750-550 BCE) 
primary evidence comes from palace frescoes, though these can and have been misinterpreted. There is pictorial evidence on the walls of queens or priestesses, so it seems that women held some positions of authority. The Minoan civilization ended suddenly. Perhaps its decline was a result of a nearby volcano on the island of Santorini (Thera) around 1600 вCE, but the timing seems inappropriate; Mycenaeans may have invaded Crete.

The Mycenaeans also seem to have operated in city states on the Greek mainland. They famously attacked Troy on the Black Sea coast of Turkey in a Homerian epic (supposedly hiding in a wooden horse to gain entry to the fortified city). Their civilization also in turn collapsed, perhaps due to nomadic incursions from the north. It is thought that some Mycenaeans then fled to the Levant, becoming the Philistines of biblical fame (they may have attacked the Hittites along the way).

\section{Indus Valley}

We know much more about Egypt and Mesopotamia than about the Indus Valley civilization. Historians have deciphered Egyptian and Mesopotamian scripts, and many of the texts of those civilizations survive on papyrus or clay. Egyptian stone - and inscriptions on stone - survived better than Mesopotamian and Indus mud brick. The few fragments of the unique Indus script may never be deciphered.

The Indus was a well-organized society. Its cities were planned with broad streets laid out in square grids - perhaps the first cities in the world organized in this way - and had extensive water distribution and large granaries. We have to guess at the form of government. There is debate as to whether there were administrative buildings, though there appears to have been monumental architecture. Scholars speculate that Indus cities were cooperative city states with some degree of shared governance. The existence of similarly sized cities some distance apart from each other suggests that the Indus was not unified politically. The walls around Indus cities seem to many scholars to be too small for defensive purposes; they may have facilitated tax collection at city gates. The existence of similar weights and measures across cities implies some degree of coordination. The similarity in town planning may suggest some degree of political unity (though we know that Mesopotamian city states borrowed townplanning ideas from each other). Some frescoes indicate processions like those associated with Mesopotamian rulers, but this is weak evidence of kingship. The right-angled streets and standardized bricks may reflect a common cultural preference for order.

The Indus civilization covered a far more extensive area than either Mesopotamia or Egypt, including most of modern Pakistan and much of northern India (with Pakistani and Indian scholars of today sometimes debating which of these cities were most important). Much of it was located along the Indus and its tributaries, which - like the Nile - have an annual flood that 
leaves rich soil well suited to agriculture. Nevertheless, Indus farmers may have depended on the monsoon rains. Though the region often receives only a few inches of rain annually today, it was likely wetter at the time. The Indus traded with the Hindu Kush, the Ganges, and Persia early by land, and later with Mesopotamia by sea. Even at this early time, we see the borrowing of cultural motifs from other lands, perhaps as artisans strove to serve foreign markets.

The most extensive city, Mohenjo Daro, had 35,000-40,000 people and covered 200 acres at its peak in 2500-2000 всE. There were definite social distinctions: Poor houses had one room, while the rich had three-storey houses with a dozen rooms and interior courtyards. These homes had private bathrooms with toilets and showers draining to sewers. Despite these differences in housing, Indus burials appear to have been surprisingly egalitarian: There is no evidence of the rich treasures associated with elite burials in other ancient societies. It thus seems that the Indus was more egalitarian than other "civilizations."

The Indus civilization was founded between 3200 and 2500 все. It declined between 1750 and 1000 все. We do not know why. It is not clear if there was an invasion (perhaps by the Indo-European language speakers we will encounter

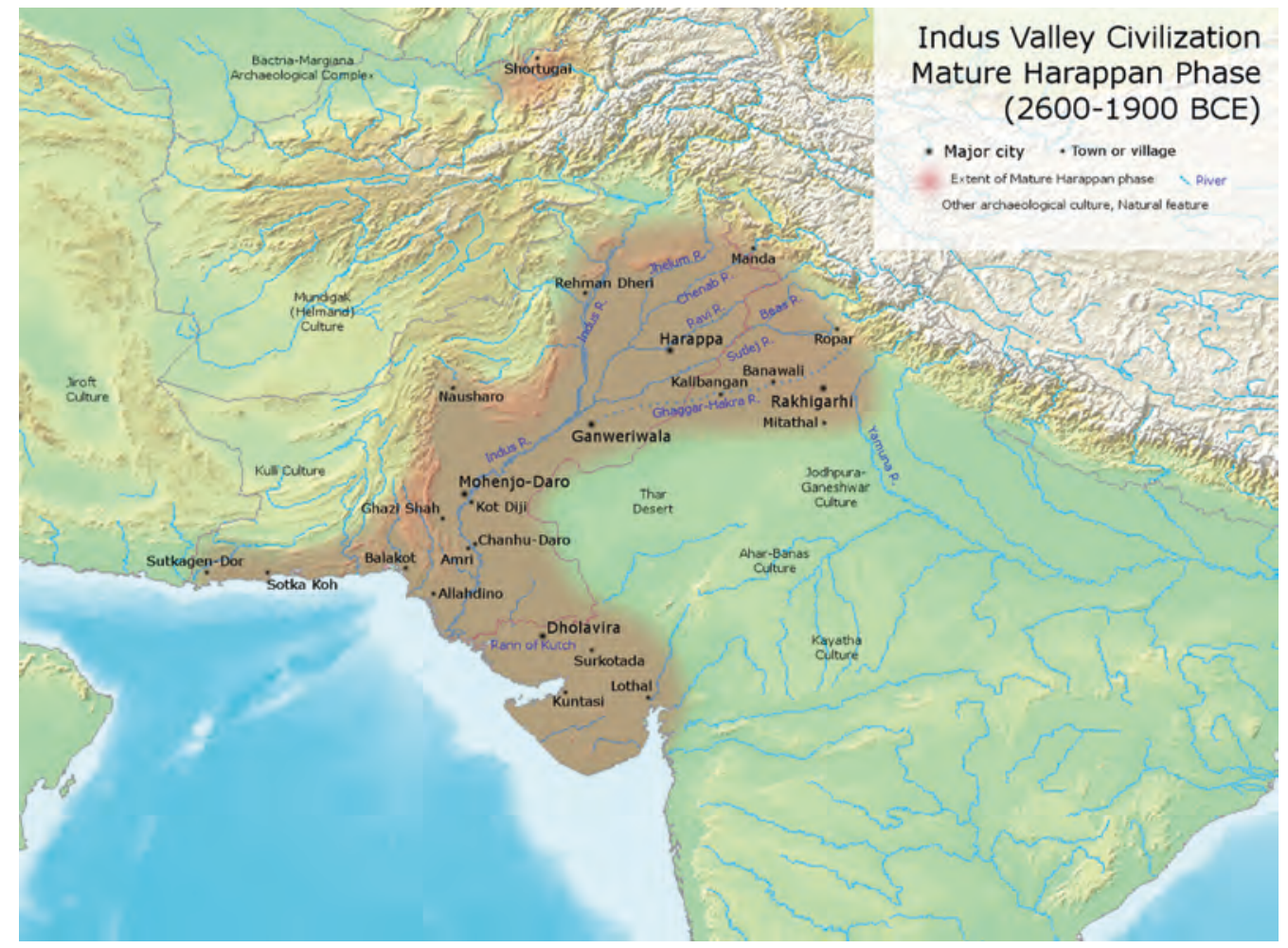

Map 7.5

The Indus Valley

Source: Map by Avantiputra7, CC BY-SA 3.0 


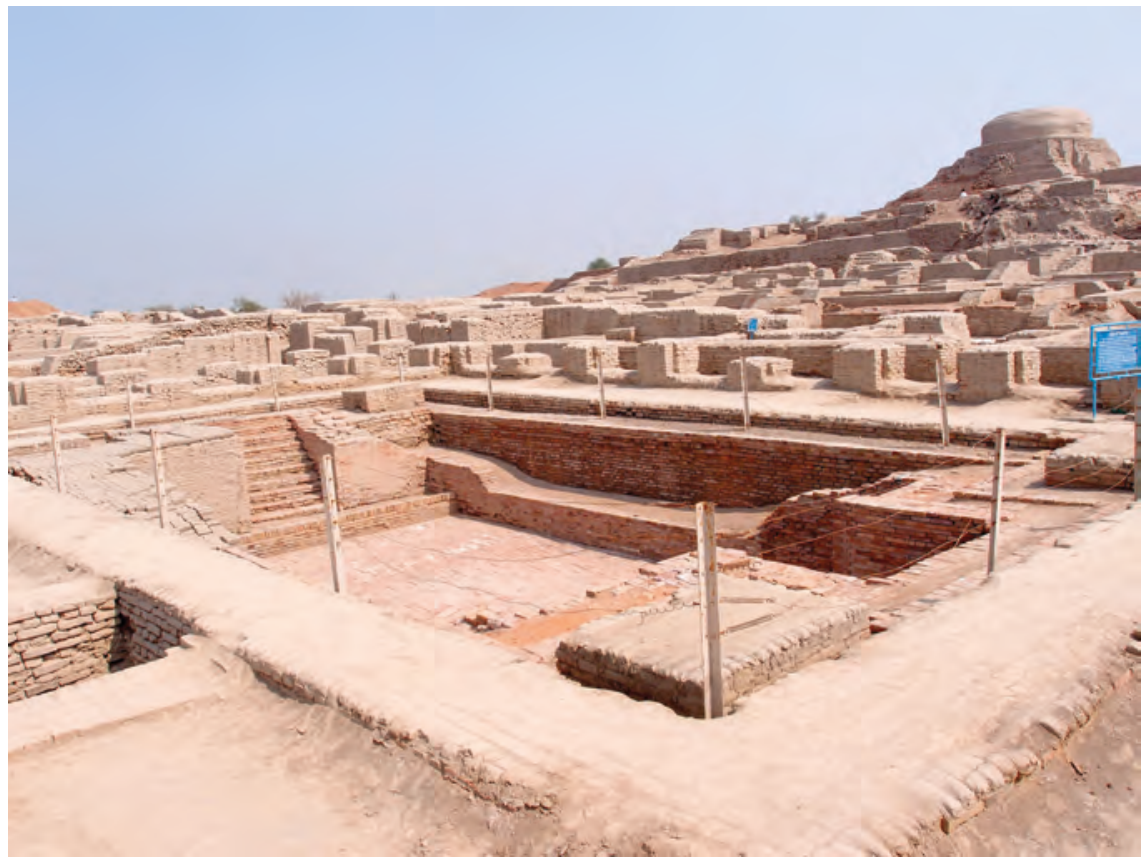

Illustration 7.12

Great Bath, Mohenjo Daro (much later Buddhist stupa in background). Some scholars think it may have been just a reservoir

Source: Photograph by Saqib Qayyum, CC BY-SA 3.0

in the Ganges below). One of the tributaries of the Indus, along which most cities lay, may have changed course due to earthquakes and landslides in the Himalayas. There may have been changes in rainfall, perhaps associated with changes in the monsoon weather pattern. Archaeological evidence suggests the movement of people from the Indus to the Ganges. The Vedas (Hindu religious texts; see chapter 8) came later, likely in the Ganges, but at times seem to refer to experiences in the Indus Valley.

There were trading colonies associated with the Indus civilization hundreds of kilometres both west and east along the coast. It is clear that there was an extensive seaborne trade not only with Mesopotamia and other lands to the west but with areas of the Indian subcontinent far to the east. The abundant fishing available in coastal waters may have encouraged the development of boats that were later applied to trade; vessels would then be built taller and stronger for longer voyages. For some reason we find Indus trade goods in Mesopotamia but not the reverse. As noted in chapter 5, the Indus played an essential role in the development of cotton textiles; such textiles were expertly woven and dyed and were much valued in Mesopotamia. Artisans in the Indus also were noted for jewellery crafted from both local and Afghan gemstones. We know that the Indus also supplied copper from Central Asia to Mesopotamia. The last mention of "Dilmun copper" in Mesopotamia around 1760 всE coincided with 
the first mention of Cyprus copper - the decline of the Indus civilization may have caused Mesopotamia to look west.

\section{The Ganges}

The Aryans were nomads who migrated into northern India from about 1500 BCE. They herded cattle with horses and had war chariots. Horses do not breed well in India, so they needed regularly to replenish their horse supply from Central Asia. It is not clear how much military conflict there was during this migration, but there was undoubtedly some. As they moved toward the Ganges, the Aryans became more agricultural. They also moved from a tribal to a state form of political organization, which would dominate Indian history after that. For a while trade with Central Asia declined, but after 1300 вСE the horse and camel were widely adopted, and trade expanded.

As noted above, there was also migration eastward from the Indus Valley. This seems to have involved the development of agriculture based on rice, millet, and sorghum, better suited to the monsoons of the Ganges than the wheat and barley of the Indus.

Castes likely first had an ethnic basis associated with skin colour (the term "Varna" now signifies caste but originally meant colour), but this did not prevent enough interbreeding to erase ethnic distinctions over time. There were originally four large castes: priests (Brahmins); warriors and aristocrats; peasants, artisans, and merchants; and landless peasants; later a fifth group of "untouchables," who performed undesirable duties such as handling human waste, was added. Thousands of occupational sub-castes emerged as society became more complex. Some mobility was possible for individuals (though this might require

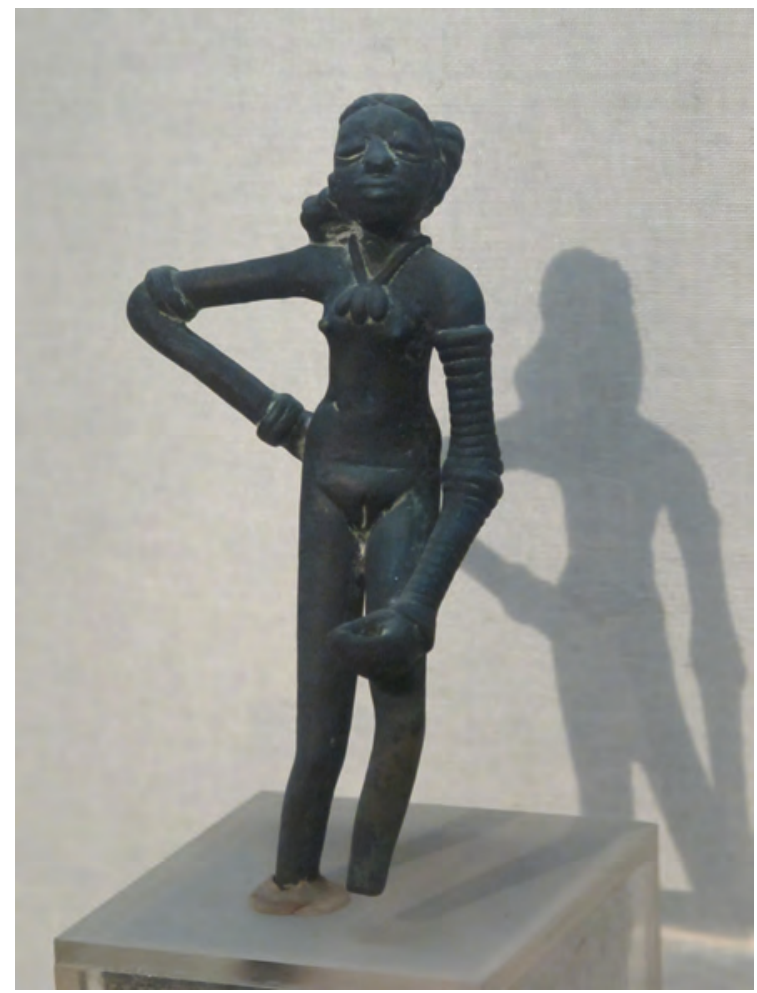

Illustration 7.13

Dancing girl, Mohenjo Daro

Source: Ismoon, CC0 1.0

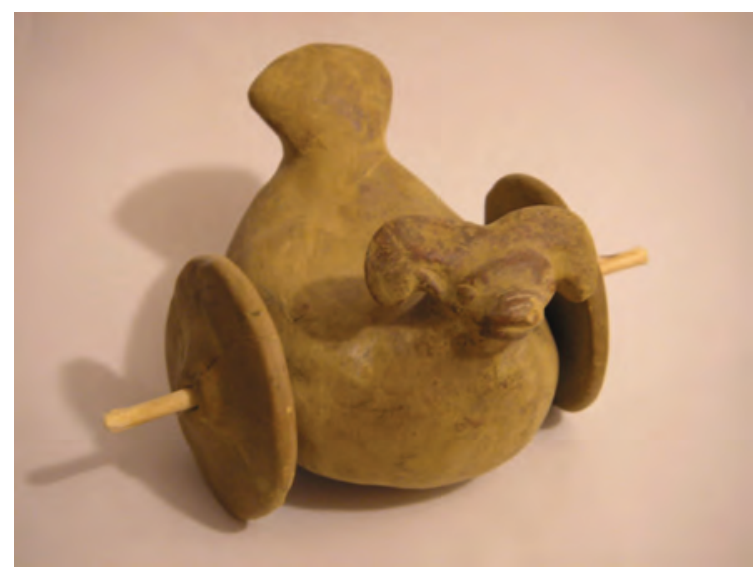

Illustration 7.14

Children's toy, Mohenjo Daro, Delhi Museum 


\section{BOX 7.1}

\section{ECOLOGICAL COLLAPSE}

Some historians have suspected that humans over time in a variety of early agricultural societies caused environmental damage so severe that it eventually led to a societal collapse. That is, they failed to employ one of the strategies for nitrogen fixation identified in chapter 4. Other types of environmental degradation are also possible. One oft-suggested possibility is that societies might fail to maintain irrigation systems, causing salt buildup in their soils. Deforestation might cause erosion that limits water retention. These explanations are plausible: Humans are short-sighted and quite capable of pursuing strategies with short-term benefits but long-term costs. Environmental degradation is such a slow process that humans might be unaware until it was too late. However, this very slowness might make us wonder if it is likely to trigger an ecological disaster over an extended area at a particular point in time.

Ecological collapse may have occurred in some of the areas where agriculture was first developed: It may explain the disappearance of the mysterious Indus Valley civilization, decline in Mesopotamia at about the same time (salt buildup being the main culprit), and the collapse of Mayan society in Central America (deforestation being the main problem). Scholars have also suggested ecological collapse for a variety of other societies, perhaps most famously Easter Island in the Pacific (chapter 16). However, plausibility does not mean that these tales of ecological collapse are necessarily true. Recent scholarship notes that Mesopotamian civilization survived in important ways until much later conquests by Greeks and Persians (see later chapters). And while the abandonment of Mayan cities in presentday Guatemala remains unexplained, Mayan cities farther north in Mexico not only survived but even prospered (perhaps receiving migrants from southern cities) and were absorbed into polities centred in central Mexico (see chapter 9). We know very little about the Indus civilization. It could be that there was deforestation or silting of irrigation works. Yet it is not obvious that such processes on their own would cause complete abandonment of such a widespread civilization. Even if problems with salt buildup occurred, this may have reflected climatic shocks: If rainfall levels decreased, salt levels would increase. A previously sustainable agriculture might then become unsustainable. The history of Mesopotamia and the Maya, and perhaps the Indus, is best seen as a case of changing relative fortunes regionally rather than of dramatic collapse.

We might also wonder why only some societies suffered environmental degradation. In Egypt and China, it could be that regular flooding served to wash excess salt to the sea. Perhaps irrigation systems were less intrusive and so did not increase soil salinity. However, it is not apparent that societies differed enough in environmental strategies to explain markedly different outcomes.

We should be careful here not to impose today's concerns on our understanding of the past. It is entirely possible to worry about the effects that humanity is having on the environment in the twenty-first century while remaining agnostic about environmental collapse in the distant past. Simply put, humanity exerts a much more considerable ecological impact 
today - because of industrialization and especially the burning of fossil fuels - than it ever has before. To be sure, farming changed landscapes in diverse ways, some deleterious. Nevertheless, human societies are resilient.

More centrally, we should be careful of conflating political and economic history. We will discuss in later chapters why many cities and states might fall; we will see that these usually are replaced in time by other cities and states, perhaps in slightly different locales. Their passing will have economic effects to be sure: In particular, trade may contract. However, the everyday lives of peasants may continue much as before. Some new polity may even maintain irrigation systems. Levels of population and degrees of social complexity may change little. The daily cultural practices of people may also change little, though major state-sponsored celebrations may suffer. The structure of this book guides us not to assume that "collapse" with respect to one theme necessarily means "collapse" for others.

Even when we do observe a clear case of agricultural decline, this need not reflect human activity. Agrarian populations would forever be subject to the whims of climate change and epidemic disease. We are in the early days of charting climate through human history, though this enterprise is progressing. We know for sure that climate has changed considerably over the millennia. Any time we see state decline or social unrest we should wonder if some change in climate or epidemic disease might be at work. As few as three harvest failures in a row could plunge a society into armed rebellion as desperate people fought over dwindling food supplies - and could reasonably presume that the gods no longer favoured the ruler and elite.

moving) or occupational groups as the income and prestige associated with different occupations changed. Foreign groups migrating into India could form new castes. India was also a very patriarchal society (recall that social stratification of other types is usually associated with gender stratification).

As noted above, the Vedas indicate some considerable degree of cultural continuity between the Indus civilization and the Ganges. Hinduism, the religion of the Vedas, would develop over time in the Ganges region and spread to other parts of India and Southeast Asia.

\section{China}

The Yellow River floods more than the Yangtze. The result is very fertile lands along the Yellow but a need for irrigation and flood control. Rainfall increases as one moves southeast in China. In ancient times, the land in the southeast was marshy; for the most part, these areas would be developed much later in Chinese history.

Chinese tradition had long spoken of a series of demi-gods followed by sage kings founding three dynasties - Xia, Shang, and Zhou - from about 2100 всE. 
Historians had (understandably) long thought that these early dynasties were perhaps mythical. Archaeology now supports the existence of states at that time but suspects that the earliest of the legendary dynasties, the Xia, controlled a small territory and was in competition with other states. The very early history of China, then, resembles that of Mesopotamia, and very early Egypt, with many small states jostling for power with each other.

Recent excavations have uncovered an elaborate palace dating from about 2000 BCE, generally attributed to the Xia. The Shang is the first dynasty for which we have written records. These are fragmentary, however, consisting mostly of oracle bones used for divination and some bronze engravings. The high incidence of inscribed oracle bones suggests that Shang rulers regularly consulted the gods before making decisions. Both dynasties were centred in northern China. The Shang may have governed from about 1500 to 1100-1050 BCE. Burial sites suggest a patrilineal society from very early. The royal family was heavily involved in state administration (though it is possible that local governors were

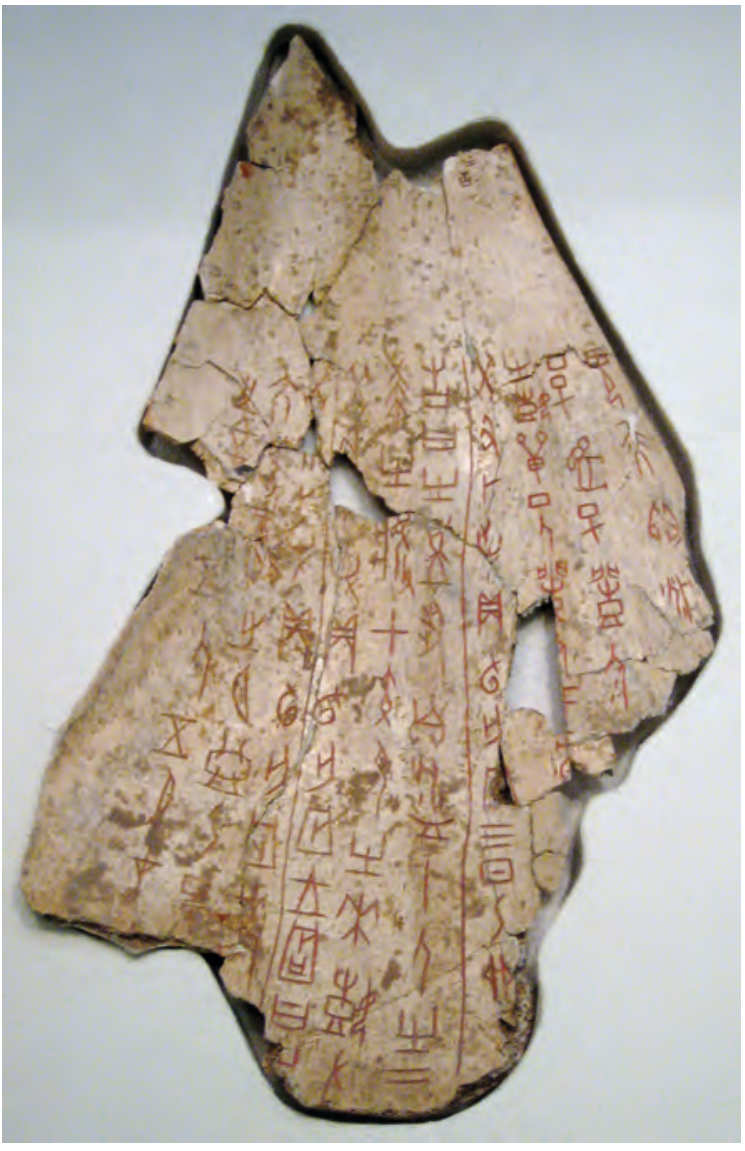

Illustration 7.15

Inscribed scapula for divination, Shang dynasty

Source: Photograph by BabelStone, CC BY-SA 3.0 simply addressed in filial terms). It is not clear how exactly it was decided which son would rule, but there is evidence of chaotic successions. Queens were highly respected but mostly for bearing children. In 1200 BCE the Shang capital of Anyang, with a population of 100,000 , was the biggest city in the world. Yet the Shang state was far from having complete control over Chinese territory.

The Western Zhou - apparently the rulers of a small state or perhaps province on the fringes of Shang rule - then conquered the Shang. They instituted a system of provincial governance with some hundred administrative districts. Members of the royal family governed these units in some cases, but in other instances, locals administered: The royal family then adopted these locals as kin. The Zhou may also have formed a standing army for the first time in China. The Zhou bureaucracy absorbed Shang officials, and the Zhou tried to encourage promotion by merit. The Zhou sent emissaries from the capital to observe local governors and these governors could be replaced, at least in principle. There are some examples of the Zhou exercising force against local authorities. 
The Zhou established control of a vast area from Inner Mongolia in the north to the Yangtze River in the south. This was likely by far the largest state by geographical extent of its time - and so a precursor of the empires we will engage with in chapter 9 . The Zhou tended to reward officials with land and people (these were more like serfs it seems, tied to the land, than like slaves who could be sold) rather than salaries; inevitably, the Zhou faced trouble in later centuries as it competed for power with substantial landowners.

Thousands of bronze ritual bowls survive from the Zhou period. These bear inscriptions as gifts or celebrations of important events. There is a marked similarity in style of these bronze vessels across Zhou domains, whereas pottery styles differed at the same time. This suggests that these bronze bowls were important signifiers of political status across a unified polity. There is far more in the way of bronze remains from Zhou China than any other society

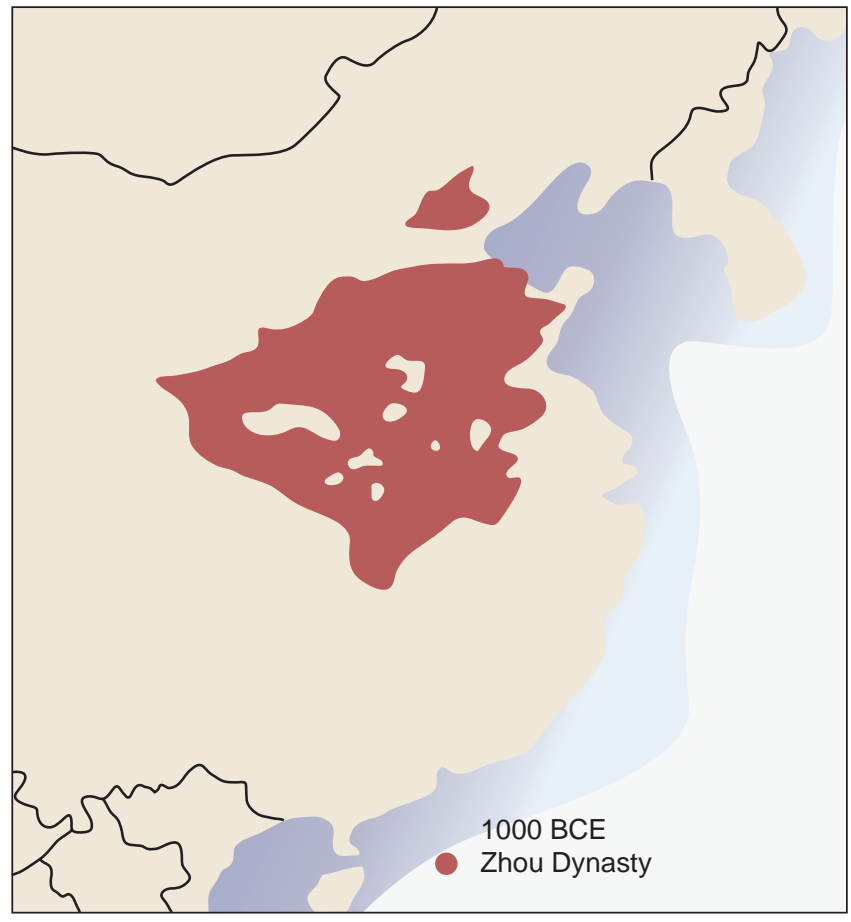

Map 7.6

Zhou state around 1000 вСЕ

Source: Map by Ian Kiu, CC BY-SA 3.0

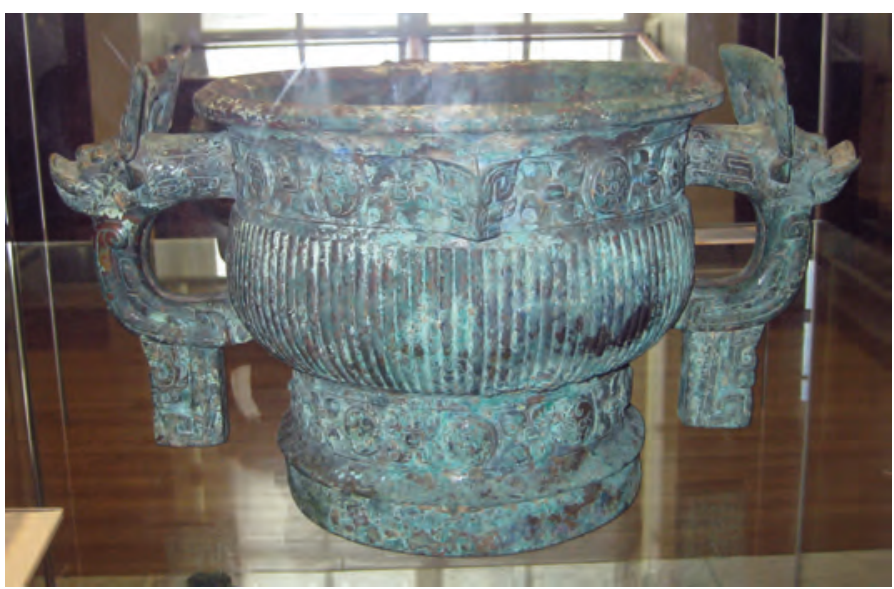

Illustration 7.16

Western Zhou bronze ritual vessel, known as the "Kang hou gui," British Museum Source: Photograph by BabelStone, CCO 1.0 


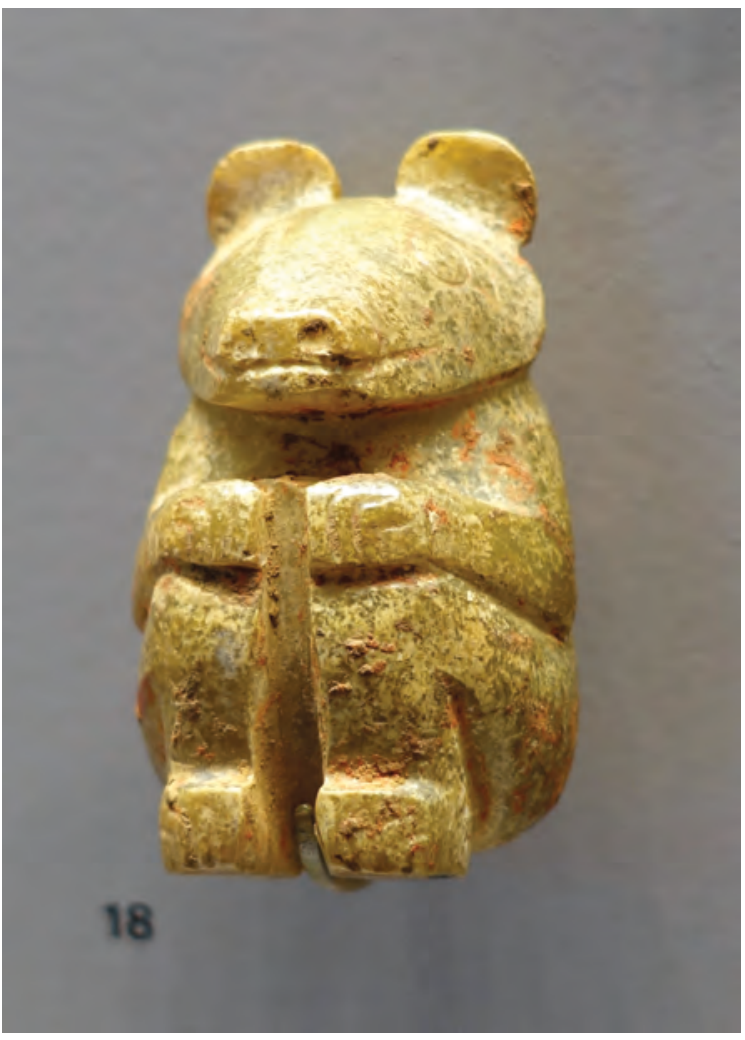

Illustration 7.17

Jade, seated bear, Zhou period c. 1050-771 BCE, Arthur M. Sackler Museum, Harvard University

Source: Photograph by Daderot, CC0 1.0 of that time. Nevertheless, it seems that Zhou agriculture rarely employed bronze.

The Shang and especially the Zhou were able to organize irrigation works. Although these irrigation systems were small in scale, they would lead to a considerable expansion of rice agriculture along the Yangtze. The Zhou controlled only the northern part of the Yangtze, but the Chu state to the south borrowed many customs and practices from the Zhou, paving the way for an even larger Chinese polity later.

The Shang had justified their dynasty by referring to corrupt practices of the last Xia ruler. The Zhou did the same, formalizing the idea of the "Mandate of Heaven." Rulers ruled with divine approval. If a ruler lost divine support through misbehaviour, the dynasty would be replaced by another that would govern with divine approval unless/ until its rulers also lost divine support. This belief system was at first associated with a god who may have been a revered Zhou ancestor but over time became depersonalized. This idea of a heavenly mandate would remain important in China into the twentieth century. Note that it not only provides religious support for dynastic change but for the idea that China should have one ruler: Another ruler that exercised power over the entire region should replace a displaced ruler. This result has indeed been prevalent throughout most but not all of recorded Chinese history. Nevertheless, the last two centuries of Zhou rule are called the Warring States period: The Zhou competed with many regional authorities for power during this period. The idea of a mandate of heaven also implies that the ruler is not supposed to rule selfishly but rather to act in the interests of the broader society. This idea is common in early states but is arguably stated most clearly in China. It is unclear, of course, to what extent rulers took this admonition - either consciously or subconsciously - to heart.

Forced labour was very common in ancient China. These workers built irrigation infrastructure, roads, and palaces and tombs for their rulers. Satellite polities also paid tribute in the form of labour for building, for the army, and perhaps for human sacrifice. 


\section{BOX 7.2}

\section{RULES OF SUCCESSION}

In both the Shang and Zhou, family members competed over succession; these contests often turned violent and verged on civil war. In later history, many kingdoms in the world, especially in Europe - would operate by primogeniture such that the eldest son always inherited the throne. But far from all: In Mughal India - and Muslim polities generally - sons fought over the succession, not always respecting the choice their father had made. The Ottomans even came to advocate fratricide so that the victorious son would kill his competitors (chapter 20). The Roman Empire would survive for centuries with an even less precise rule of succession where men from different families sought to be emperor. There is a tradeoff here: A chaotic battle over succession might be worth risking if a younger son might often govern better than the oldest. The simple fact of personality diversity - parents often remark at how different their children are - coupled with the power that the chosen king might exercise, encouraged some states to risk the chaos. Such a choice may make more sense in a polygamous society where the king might have many sons from which to choose. Notably it also makes sense in a society with a strong bureaucracy and identity; otherwise, each battle for succession risks fragmenting the state into smaller entities.

Most large states in human history follow rules of patrilineal descent in which sons (sometimes brothers) inherit rule from fathers. In smaller states in many parts of the world, descent is instead matrilineal through the ruler's sisters' children. Such systems were often also subject to intense succession battles.

Most systems of succession - even matrilineal - privileged men. Nevertheless, in times of succession crisis, women often asserted claims to rule. We will observe occasional female rulers - sometimes even series of female rulers - across almost all regions of the world. Since female rulers were rare and were most likely to emerge during times of crisis, it was easy for male successors to judge them harshly.

Walled cities appear along the Yangtze from around 5500 все. There seem to have been states in south China that we know little of at the time of the Shang in the north. The Chu in the south first developed the idea of rotating bureaucrats as local administrators. This was one possible approach to limiting the power of local administrators, for bureaucrats would not be in place (hopefully) long enough to establish a local power base. The disadvantage was that they would also not have detailed local knowledge of the region they administered.

Chinese polities interacted with nomads on their borders, generally peacefully. The Chinese adopted nomadic horse-riding trousers from the fourth century все (long before Europe did). They may have adopted the chariot and even the wheel in the fourteenth century BCE through Central Asia. Copper metallurgy was imported from the west in about 2000 вСЕ.

The early Zhou may have had a system somewhat like European feudalism (chapter 13). Confucius describes a "well-field system" where farmers 
farmed outer fields for themselves and an inner field for a landlord. The first documented land tax came in a successor state in 594 BCE, indicating that peasants had gained the ability to pay their own taxes. The development of oxpulled iron ploughs allowed new lands to be tilled, which may have increased peasant independence (and productivity), but such ploughs only became common from the third century BCE.

As the Zhou state declined in authority, independent city states emerged in many places. These often allowed much greater freedom to merchants and artisans. As states battled for power, armies grew in size and importance. Defensive walls became common across north China. The introduction of iron weapons (chapter 5) encouraged the use of peasant infantries rather than elite charioteers. Bureaucracies grew in size in order to tax and manage these armies. The greater agricultural productivity that iron implements allowed facilitated these bureaucracies in turn (though iron implements may not have been common until the fifth century вСЕ). States moved away from reliance on elites toward taxing small farmers and mobilizing these for war. This set the stage for the reunification of China under regimes with far greater administrative and military capabilities than the Zhou could have imagined (chapter 10).

The state of Wei from the fifth century всE introduced a policy of buying grain when prices were low and storing it until there were food shortages. This policy became a significant source of revenue, for the state could sell the grain when prices were high. It also served to support the population: Farmers received higher prices than they would have otherwise when they sold grain and faced lower prices when they had to buy due to harvest failure. It thus encouraged political stability, for hungry peasants might riot. Many later Chinese polities would follow this policy. Chinese states would often manage to administer such a policy better than anywhere else. It required a sophisticated bureaucracy and one not overwhelmed by corruption.

\section{Central Asia}

Central Asia was mostly nomadic during the period 4000-1000 BCE, but there was some agriculture. The area already served as a link between China and the West, though only little in the way of either goods or information passed through. (We will see later that even the Roman and Han Chinese Empires were only vaguely aware of the other's existence; chapter 10.)

It may be that speakers of an Indo-European language in the area north of the Black Sea first domesticated the horse. These same people then likely borrowed bronze technology to build chariots and wagons. The military superiority that resulted may explain the spread of Indo-European languages across Europe, Persia, and northern India. The Indo-European Hittites (see above) used war chariots in their conquest of Anatolia and wars in Mesopotamia from 1900 вСе (the Mesopotamians may have had war chariots before this, but the Hittites developed the technology). The Hittites then also developed superior 
iron metallurgy from 1300 BCE. It is unclear how much military conquest was involved in the spread of Indo-European languages into Europe, Persia, and India.

\section{Americas}

There is a debate about when the first Mesoamerican civilizations emerged. The Olmecs operated from 1800-150 BCE, with a capital near San Lorenzo in southeastern Mexico from 1200 to about 900 все. Southeast Mexico is an area of abundant rainfall in which river flooding deposits rich silt. The Olmecs left large pyramids as the most significant evidence of their cities. We know little of Olmec society but suspect it was authoritarian. There seems to have been human sacrifice. Their artwork celebrates war. Jade and obsidian traded for hundreds of kilometres. The Olmecs also produced sculptures of heads three metres high, made of giant stones ( 25 tons) that had to be moved eighty kilometres, mostly by water but the rest on land without the use of wheels (we think). Obsidian, which they obtained probably from Guatemala, was used to carve these stones, as well as jade jewellery. The Olmecs developed the Long Count calendar that adjusted accurately for the length of the earth year and dated everything precisely from a starting point of August 13, 3114 все. The Olmecs also developed the first known writing system in the Americas, which the Maya later extended. The Olmecs seem to have destroyed their own cities around 100 BCE. The Zapotec society of southwestern Mexico may have operated over a similar time period as the Olmecs; it built large ceremonial mounds rather than pyramids but also celebrated jaguars and practised some degree of human sacrifice.

The Mayan civilization - which operated in the southeastern part of contemporary Mexico and the northern part of Central America - also has the three characteristics of civilization: cities, states, and writing. There is debate regarding when the earliest Mayan civilization emerged; there were certainly cities by $750 \mathrm{BCE}$, but there is evidence of political organization from about 2000 BCE. Mayan society was organized in city states that battled with each other. There were as many as eighty separate city states, each with temples and palaces. One of these, Tikal, likely had 50,000 people. There were frequent wars, with captives

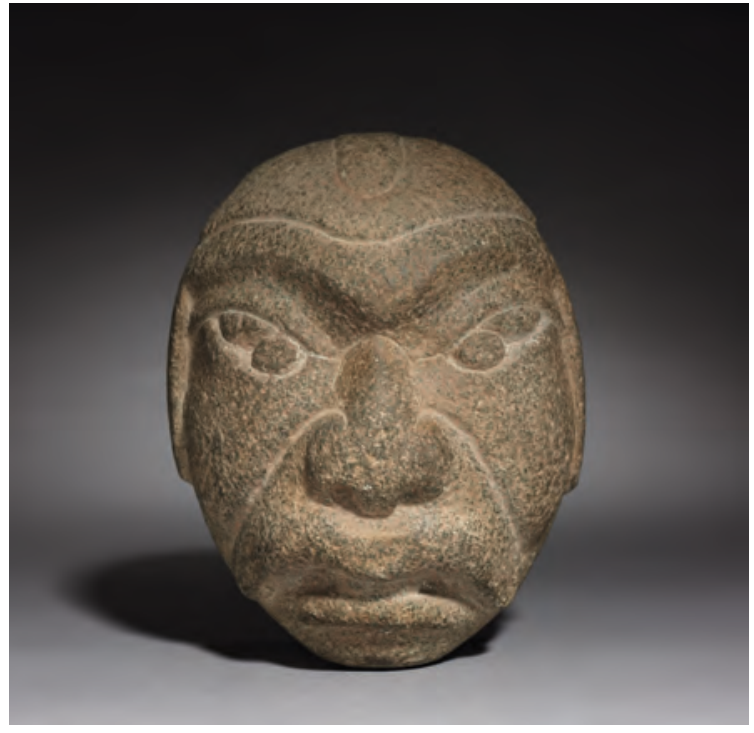

Illustration 7.18

Olmec Head, 1200-300 BCE

Source: John L. Severance Fund, Cleveland Museum of Art. CC0 1.0 
being enslaved or sacrificed. Slavery was hereditary, but slaves could buy their freedom. The Maya were like Mesopotamia (and perhaps the Indus) in having a common culture and religion across city states. Kings were seen as sacred. Female rulers, though a minority, seem to have been far more common than in most other societies of that time. Mayan society was highly stratified: Only the elite could consume the cacao bean (though this was also used as a currency). Merchants and builders may also have been esteemed, but were separate from the ruling class. Mayan monumental architecture took the form of pyramids on which religious rituals were performed. Its agriculture focused on corn and often involved creating raised fields within swamps to trap silt. The Maya also built agricultural terraces on hillsides. They grew squash, beans, chilli peppers (which are rich in vitamins), fruits, and cacao beans. The Maya had no farm animals but hunted deer and birds for meat; they used sea salt to preserve the meat. As in Egypt, there was a dry season with little labour requirement (outside of hunting); as in Egypt this labour was devoted to building pyramids and other monuments. The Maya mixed corn with lime while soaking it for eating: this may have unwittingly limited pellagra.

Historians have often exaggerated the demise of Mayan civilization. To be sure, the period of pyramid construction ended - but so did pyramid

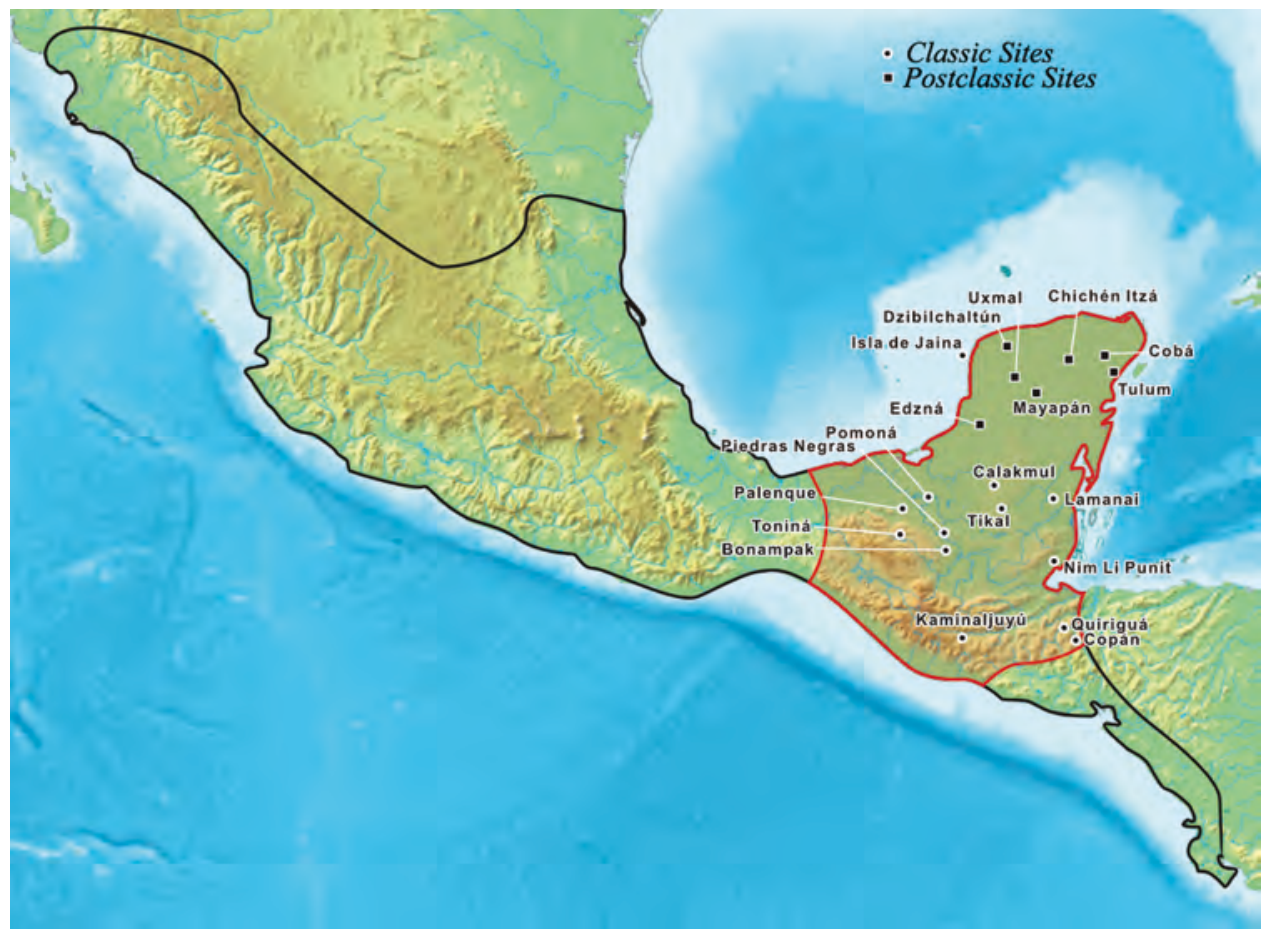

Map 7.7

Ancient Mayan cities

Source: Map by Kmusser, CC BY-SA 3.0 
construction in Egypt millennia before the Greeks conquered the Egyptians. There may just have been a decline in investment in monumental architecture as population centres changed. While cities in present-day Guatemala do disappear, Mayan cities in the Yucatan survive to interact with later empires in central Mexico (chapter 16). Historians have often attributed the decline of Guatemalan cities to ecological disaster resulting either from climate change or unsustainable farming practices. Disease is another possibility. However, the move north might reflect an increased elite interest in trade, which was easier along the northern coastline. This may reflect a change in political structures more than in per capita incomes.

The Maya adapted an accurate calendar from the Olmecs - it calculated the length of the year to within seconds. The Maya also developed the concept of zero (which Romans lacked). The Mayan script had both ideograms and symbols for syllables. The Maya had scribal schools as in other early states; their students likely came from the elite. Unfortunately, the Spanish (chapter 19) destroyed all but four Mayan books, so historians mostly have to rely on temple inscriptions.

The Maya played a ball game with a hard rubber ball in which players were supposed to kick the ball through a ring high on a wall without using their hands. The Olmecs had first developed this game, and later Mesoamerican societies would continue to play it. Historians long thought that losing sides were put to death, but it is now believed that losers were only sometimes killed.

Mayan society was clearly advanced and sophisticated, with social stratification, extensive trade, and impressive achievements in architecture, mathematics, and astronomy. There was an extensive literature that has unfortunately mostly been lost. Yet the Maya suffered from two disadvantages relative to societies in the eastern hemisphere: They had no domesticable animals and neither bronze nor iron. These deficiencies must have limited agricultural productivity and perhaps nutrition. They would also affect military practices. Given the critical roles that iron weapons and horses (and sometimes elephants) were playing in warfare elsewhere in the world, we might speculate that it was harder to support mass armies and as a result vast empires in Mesoamerica than in the eastern hemisphere (though the Aztecs and Incas would do so later).

We know less about South American societies of the time as these have not left written records. It is clear that there were cities and states linking the Pacific coast and the highlands of the Andes from 2500 все. The Caral state/society from 2500 to about 1600 BCE was followed by the Chavin state/society (1000200 ВСE), Moche (200 вСE-700 СЕ), and Tiwanaku (100 CE-1100 CE). The Chavin society is noted for finely crafted pottery with depictions of jaguars and birds of prey. There was also much textile production. The Moche built adobe pyramids. The Nazca (500 вС- -500 СЕ) in southern Peru also constructed pyramids but are better known for the Nazca lines: drawings - some of them over 350 metres in length - of geometric shapes and animals made on the arid Nazca plain by exposing light rocks under reddish pebbles. Though best observed from the sky, the drawings are (still today) visible to some degree from surrounding hills; 
they may nevertheless have been intended for the enjoyment of the gods or ancestors (or, some have speculated, visitors from outer space).

\section{Meanwhile elsewhere in the world}

Significant developments occurred in these millennia even in parts of the world not characterized by states. There are human remains from 9000 вCE at HeadSmashed-In Buffalo Jump in Alberta, Canada, where humans for millennia chased buffalo over a cliff. People also followed and hunted caribou from about 6000 вCE. Plains Indians may have employed a combination of chiefs and communal governance in which bands cooperated in certain activities like the buffalo hunt but smaller groups (or elders) made other decisions.

Marine mammals were hunted in the Arctic (following the extinction of earlier megafauna) from 6000 BCE. Political organization was minimal in the Arctic, with the family being critical (as in the vast desert basin of the American southwest). There was impressive seagoing technology in the form of different types of kayak built from animal skins over driftwood frames. Some of these could carry tons of cargo. Hunting expeditions involved many kayaks. Harpoons were developed from atlatls; they were attached to leather bladders that floated, tiring the sea animal struck by them. Stone lamps fuelled by walrus or seal oil were used from at least 500 BCE.

Fishing, hunting seals, and collecting shellfish allowed large villages in the Pacific Northwest of North America. Some fishing villages were independent, while others were chieftaincies (we think). We cannot be sure what the political organization was in this period, but stratified fishing societies are likely.

There were some towns along the Ohio and Mississippi with clear evidence of stratification from 1000 все, associated with the use of agriculture. Some of these towns had over a thousand people. Large mounds were built, presumably for ceremonial purposes. These would have required a lot of coordinated labour, which suggests, but does not entirely require, some hierarchical political organization and social stratification.

It is not clear exactly when the birch-bark canoe was developed, but Europeans would later marvel at its speed, capacity, and lightness. Note that canoes and kayaks are both copied throughout the world today.

\section{Trade expansion through time}

We outlined in chapter 5 the challenges faced by merchants. States could and did alleviate each of these challenges. "Alleviate" is the key word: States and merchants could not solve these challenges overnight, and a process of institutional change through history will slowly provide merchants with better protections. 
Courts that are trusted to render fair judgements and have the authority to enforce these can provide some protection from theft by other merchants. States can then encourage trade by establishing courts. All early states did create systems of courts and most promulgated laws that were to guide these. Some of these laws, we should note, explicitly addressed economic transactions. The Code of Hammurabi discusses in some detail how property owner and tenant should share the benefits of a long-term investment in an orchard. However, judges often did not behave as these law codes prescribed, and the earliest laws were in any case open to broad interpretation. Merchants certainly had recourse to such courts, but they could have only limited confidence in them. This would be especially the case if a merchant traded across political jurisdictions a common problem, especially in Mesopotamia.

Merchants continued to rely on networks. As noted before, merchants can best achieve trust through networks in which all will shun a merchant who steals from others in the network. Such networks tend throughout history to have a common ethnic or religious basis, presumably because networks can address accusations and penalties more easily if they share common cultural understandings. The Assyrian trading networks observed as early as 4000 BCE were among the first of what would become a common practice throughout human history.

We have documentary evidence - over 20,000 cuneiform tablets, mostly from a trading colony in Kanesh in Anatolia - of an Assyrian trade network based in Assur in northern Mesopotamia that traded tin from Afghanistan, textiles from southern Mesopotamia, and minerals from Anatolia, among other things, in the centuries after 2000 вС. It used large donkey caravans to travel over 1000 kilometres. Donkeys could carry up to $90 \mathrm{~kg}$; merchants likely often sold donkeys in Anatolia, as the returning silver weighed far less than the goods carried to Anatolia. Merchants clearly operated in a network; they used letters of debt - that is, promises to pay a particular debt at a specific time and place to transfer funds (one merchant could pay another by transferring debt from a third), and kept accounts in different places. They had mechanisms for sharing risk among themselves by cooperating in trade ventures. Merchants bought shares in trading ventures, even for a period of ten years or so. They used financial incentives to penalize thieves and reward honesty. Merchants could thus consign goods on the caravan without accompanying their products. Prices were stated in weights of silver, though caravans paid road taxes in copper and tin along the way. The merchants controlled the city assembly in Assur, which in turn negotiated treaties with Anatolian cities governing trade and taxation and regulation of trade. The city then taxed departing expeditions 1/120 of the value of their goods. The merchant colony in Kanesh in Anatolia charged another levy and then the king of Kanesh received a fifth of textiles but only 2/ 65 of incoming tin. Prices were so different between places (tin being twice as expensive relative to silver in Anatolia) that merchants could still make large profits. 


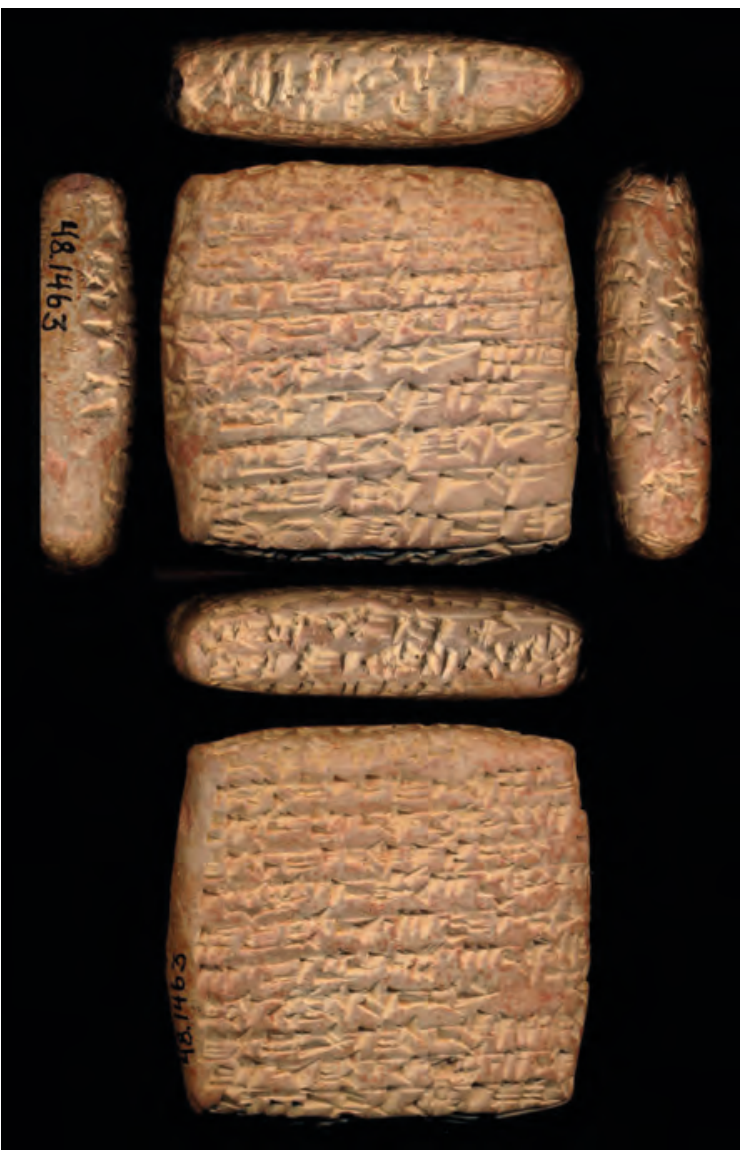

Illustration 7.19

Clay tablets from Kanesh

Source: Walters Art Museum, CC BY-SA 3.0
Beyond the network and its institutions, there are a few notable characteristics of this trade. Despite the distance, it was not just a luxury trade but involved industrial raw materials and finished goods - though seemingly a small range of each. The trade depended on political accommodation with a variety of states along the way. The merchants and states - by working together were able to negotiate a profitable arrangement for all. Trade would decline when political consolidation in Anatolia ended these arrangements (which is a bit surprising since political consolidation more often facilitates trade; see below).

The student should appreciate that this network not only addressed the problem of theft from other merchants but the issue of theft from states. The network had negotiated agreements with rulers regarding tax payments and non-confiscation. This was an extensive trade, and each state profited enough from taxing it that they had a clear incentive not to confiscate. When political circumstances changed, this confidence evaporated, and the trade declined.

Note that states will wish to tax merchants passing through their lands, even if they are not trading there. States that will see no other benefit from trade than the taxes they can raise will be tempted to charge very high taxes; they are limited only by the ability of merchants to pursue alternative trade routes. We can expect, then, that political consolidation will encourage trade, for one large state and one ruler will tax less than several states would have. However, note that the one ruler now has no competition: The merchant has no alternative route over which to transmit their goods. The benefits of political consolidation depend on the ruler perceiving that they can earn more by setting taxes at a level that encourages large trade volumes. A new ruler or state could destroy trade routes that prospered under a previous ruler. This appears to have happened in Anatolia. Trade may also have declined during the process of political consolidation. Political change is itself a threat to merchants, especially if it involves military conflict: Rampaging armies will view the goods of "enemy" merchants as booty, and desperate states may even view the goods of "friendly" merchants with avarice. 
We should stress that the trade was mutually advantageous to all. Since Mesopotamian states were more powerful than Anatolian states at this time, some scholars have speculated that the trade was to Mesopotamia's exclusive advantage. There is no evidence that Anatolian states were or had to be coerced to participate. It could be, of course, that Mesopotamian merchants were better at bargaining than Anatolian rulers.

Note also that the goods moved in large caravans. Such a practice not only raised organizational complications but also meant that the products of many merchants arrived simultaneously in markets (which would tend to depress prices). The use of large caravans was likely a protection against theft. Even though the merchants had arrangements with all states along the way, they could not be confident that bandits would not attempt to steal their goods.

By 1500 Bсе if not much earlier, there was regular trade between Greece, Anatolia, the Levant, Egypt (and likely beyond), and Mesopotamia. Greek and Cypriot pottery was much prized throughout this entire trade area. It was also common at this time for rulers in the region to exchange gifts. Over the next centuries, longer-distance trade emerged. Excavations of what may be thirdcentury все burial sites at Pazyryk in the Altai Mountains of Central Asia (near the modern border between Kazakhstan and Mongolia) find goods from Greece, India, and China.

Trade is important not just for its economic impact but because ideas spread along with goods. There is indeed much evidence of early interactions along trade routes. Wheat spreads from the Middle East to Europe and Africa. Writing spreads. Languages spread. Uruk-style city planning and architecture are found in Syria, Anatolia, and Iran, hundreds of kilometres from where they were first developed.

\section{Institutional evolution}

We can define institutions as the formal rules that govern us. In literate societies, we invariably write these rules down. In non-literate societies, one might still speak of institutions if people understand specific rules - perhaps the inheritance rules governing kings - widely and precisely. For rules to have meaning, there must be penalties for breaking them. Laws and constitutions are the most prominent institutions. Regulations are also institutions. But so too are the rules in a university calendar, for a recognized authority that can punish those who break the rules generates these. In complex modern societies, then, there are multiple institutions put in place by a variety of organizations, in addition to the institutions set in place by the government itself. The rules that determine who governs the state and various organizations, and those organizations themselves, are also institutions, for they decide who may put other institutions in place.

We should stress the condition that rule-breakers must be penalized. It is easier to put a rule in place than to enforce it. In societies both present and past, 
it is all too common to have rules that people just ignore. It is also common for a rule to cease to be enforced and yet survive on the books. In many modern societies, laws on drugs and on littering are commonly ignored. We should not judge unenforced laws to be institutions, though they may still affect human behaviour.

We care about institutions because they have a considerable impact on human behaviour. They encourage us to do some things and discourage us from doing others. They reflect and reinforce power relations in a society, for only those with authority to punish the disobedient can put institutions in place. One fundamental question for us in what follows is to ask whether a society's institutions also serve the interests of the society as a whole. It may be in the interest of both ruler and ruled to have laws against murder. Likewise, ruler and ruled may benefit from institutions that support commerce or protect the society from invaders or achieve political stability. Rulers will often claim that their rules help all. Yet we can suspect that many institutions serve the needs and desires of those that proclaim and enforce them more than the broader citizenry.

Does it make sense to apply evolutionary analysis to institutions? Given that these are formal and usually written down, it is clear that some human had to create each institution consciously. Indeed, we might attribute any institution to some committee or series of committees. It is an essential historical task to trace the influences on the development of any particular institution. The vast literature that discusses in intricate detail the framing of the United States constitution leaps to mind here. Yet even that document, venerated though it is, has been amended well over a dozen times and reinterpreted by the courts on numerous occasions. It has, in other words, been subject to an evolutionary process itself whereby it has changed over the years.

Institutions are amended through time. They are often repealed as well. Alternatively, the authorities that put them in place lose their power. We can spy an evolutionary process whereby institutions that "work" are maintained, while institutions that do not "work" either are rejected by those in power or weaken those who proclaimed them such that these lose power. Institutions mutate over time. This may occur officially by editing the form of the institution, or less officially by bureaucrats deciding to enforce them in a certain way. All of this suggests a process of "trial and error": People who lack perfect foresight design and amend institutions; the selection environment chooses some of these and rejects others. This selection may involve "learning": Those in power may come to appreciate that an institution is not achieving its goals. Alternatively, it may occur less consciously as those supporting an institution lose the strength or desire to enforce it.

As in biological evolution, we can expect that there will sometimes be "big" changes in institutions, though these will often turn out to be the result of a series of small mutations. The most obvious time for big changes is when there is some novel challenge. Constitutions are drafted to cope with the introduction of democratic decision-making. Regulations are written to deal with 
exchanges of shares in corporations. Earlier in time, the very first states or trading companies had to develop institutions. A point to stress here is that humans experiment with institutions for two reasons: to handle challenges better and to cope with new problems. We can expect lots of experimentation when a new challenge emerges.

What determines whether an institution "works"? The immediate selection environment is whether an institution serves the narrow interests of those with the power to enforce it. Does it help to maintain or enhance their power, wealth, and social status? Those in power will try to amend or even repeal institutions that do not seem to be serving their interests. They are likely to have an imperfect idea of how the broader society views an institution. As noted above, they will try to convince the less powerful that their institutions are in their interests as well. The historian can judge the verity of such claims. Those with power will also appeal if possible to ideas of fairness or justice or godly approval. These are all, we should note, elements of culture. Institutions that do not accord with cultural values - again think of laws against drugs or littering in contemporary societies - will prove difficult to enforce. The broader selection environment for an institution, then, is the interests and cultural attitudes of the broader population. These will seek to undermine institutions that they dislike.

There is a yet further source of selection. States compete with other states, at least through most of human history. Indeed, the fact that the Chinese Empire at times exerted undisputed control of Chinese territory, and so had no real competitors to fear, is such an unusual historical circumstance that it may have had a unique influence on the course of institutional change in China. Within states, different organizations may also compete. Institutions disappear when the states or organizations that proclaim them lose their ability to enforce them. Another critical element of the selection environment for institutions, then, is whether they strengthen the authorities that proclaimed them in competition with other states or organizations.

One crucial characteristic of institutional change is imperfect human foresight. Those in power can never know in advance the full impact of the institutions that they put in place (because of the complex set of causal relations in which human behaviour is nested). As we shall see, institutions often encourage behaviours that undermine the very authority of those that proclaimed them. If humans were prescient, then history would be both easier to explain and less exciting to study. There would be less need for evolutionary analysis, for we could just describe a set of rational responses to changing circumstances. The very fact that humans regularly misjudge situations makes evolutionary analysis valuable: It shifts some of our attention away from how institutions were created to wondering why some institutions survive through time. The institutions that exist in our contemporary world are the survivors in a millennia-long process of evolutionary selection. Humans have over the millennia attempted innumerable institutional innovations, some incredibly misguided, some unusually prescient, the vast bulk useful but imperfect in 
important ways. These have been subject to a complex and changing selection environment.

How are institutions transmitted? As noted, the vast bulk of institutions are written down. Unless repealed, they stay on the books as long as the state or organization that proclaimed them survives. They are, though, subject to continued modification as those in power recognize some of their imperfections and attempt to ameliorate these. They are also subject to less conscious forms of change as those that enforce them find it easier to patrol some aspects of the institution than others.

Institutions need not die with the authorities that proclaim them. Though the American Revolution changed the form of government in the United States, the vast bulk of English common law remained in force after the Revolution. New states do not tend to create an entirely new institutional structure but retain elements of the institutions that seemed to work for predecessor states. This is not just easier for them. As with culture, institutions serve to tell people both how they should behave and how they can expect others to act. A new ruler can then assure some degree of social stability by maintaining institutions with which the population is comfortable.

States and organizations may also borrow institutions that seem to work for other states or organizations. Newer democracies often copy clauses from the constitutions of the first successful democracies. Though copying is a conscious decision, we can see it as a strengthening of selection for institutions that prove useful. Yet care must be taken in copying institutions. It is not enough to borrow the rule; one must also be able to duplicate enforcement mechanisms. An institution that accords well with the culture (or economy or other key phenomena) of one society may prove a poor fit in another. A good example here is the sudden adoption of market institutions by former communist countries in Eastern Europe in the 1990s. Without bureaucracies to enforce contracts or complex stock market regulations, and with an inherited culture that expected bribery, widespread corruption was a perhaps inevitable consequence. Even when conscious borrowing occurs, there will still be selection for how well the institution fits the new environment. An institution that survives across a variety of societies will likely have some very positive attributes.

The fact that institutions are borrowed is particularly important for the project of world history. When acquired institutions work well, we can look for important similarities in selection environment. Note that there is then an evolutionary advantage to being in contact (and perhaps even in conflict) with other societies that are similar in at least some ways: Any of these may develop institutional "mutations" that prove useful to others. (In other words, there is a penalty for geographic isolation.) We are guided then to see institutional change in a multi-state and even multi-region context. It would make no sense, for example, to tell an exclusively European narrative of institutional change when we know that European polities were in regular contact with others; we should be surprised if there were no borrowing in all directions - unless the selection environment was so different that borrowing was not feasible. If 
selection environments did differ, we should expect to find examples of failed borrowing.

\section{Climate and civilization}

We mentioned in chapter 4 the possibility that climate change may have played a role in the human transition to agriculture. We noted above that the Sahara just before the advent of Egyptian civilization was far wetter than today. We should stress that the earth's climate has changed a great deal during human history. We now have a far better sense of global average temperatures through the examination of polar ice cores. Unfortunately, it is far harder to say how climate changed regionally. Still, it is useful to outline some broad trends.

Most broadly of all, though the climate has warmed and cooled noticeably over the course of human history, it has remained conducive in most parts of the world to the continuation of agriculture or nomadism or fishery. The fluctuations in climate have only rarely forced human populations to abandon particular localities entirely. It could be that humans themselves are partly responsible for this fortuitous outcome. The gradual spread of agriculture through much of the world (that we surveyed in chapter 4) meant that many forests became cropland. Trees absorb carbon much more than arable land, and so there may have been a significant "greenhouse effect" as humans inadvertently released carbon into the atmosphere (especially as humans often burned trees to clear land). The spread of nomadism would have enhanced this effect, for ruminant animals release methane into the atmosphere (as do microorganisms within rice paddies). In the absence of human activity, the world might have become much cooler - and more hostile to agriculture, nomadism, or fishing in most regions - than has been observed historically.

The thousand years or so after 3200 всE were very good climatically. Relatively warm and wet weather may have encouraged the emergence of states in at least Egypt and Mesopotamia. After 2200, a drier and colder period of a century or two followed. This is associated with the first intermediate period in Egypt, contraction of states in the Levant and northern Mesopotamia, and the invasion of the Akkadian Empire by waves of peoples from the north. Drought in the Middle East was apparently associated with flooding in north China reminding us that regional fortunes can be quite different - perhaps encouraging the celebration of early Chinese kings for flood control (chapter 9). The Middle Eastern drought may well have extended to the Indus and played a role in the dispersal of Indus populations to the east. Climate would then rebound and be good for many centuries in most of the world - but apparently not in the Indus, where drought conditions likely continued.

There seems to have been another dry period after about 1200 вСE. This can be connected to a period of decline in Mesopotamia and Egypt (but not the second intermediate period, which had occurred centuries earlier), which is in turn associated with mass migrations of peoples from farther north into 
their territories. Movements of peoples into the Ganges region (which had been mainly jungle before agricultural people settled it) at this time may have a climatic cause. Bantu migrations in Africa may reflect the expansion of the Sahara southward. The Greek historian Herodotus says the Etruscans migrated from Turkey to Italy at the time, and there is genetic evidence to support this. More generally, we see some evidence of change in house styles toward indoor hearths and less ventilation at the time; this could reflect colder weather.

The last centuries BCE, in which the classical empires emerged in Persia, Rome, China, and India (chapters 9 and 10) were good climatically. Notably, there was also state formation, albeit on a smaller scale, in the Peruvian Andes around 200 все. Teotihuacan, a huge city state in central Mexico, was established by 300 все. The global climate cooled significantly in the last centuries of the Roman and Han Chinese Empires.

Later, the climate warmed from after 500 to a peak in the eleventh to thirteenth centuries, after which global climate was cooler for a few centuries, and then warmed in more recent centuries - dramatically so, even against the backdrop of these historical fluctuations, in the last century. There were fluctuations within these broad trends. Chinese dynastic changes, in particular, may often have been associated with drier weather that would lower state revenues, threaten famine, and encourage nomadic incursions. We will often find states expanding or contracting simultaneously in different parts of the world.

We should avoid a crude climatic determinism. Our experience in this book suggests that major events or processes usually have multiple causes. Similar occurrences in different parts of the world may at times be pure coincidence especially as different regions may experience entirely different changes in climate. Nevertheless, agriculture and nomadism were the primary pursuits of the vast bulk of the human population through most of human history, and most states depended primarily on taxes on agriculture. Changes in average temperatures and especially precipitation patterns could, therefore, have dramatic economic and political repercussions.

\section{Population}

Population growth may have been slow in the early days of agriculture, but picked up later due to advances in agricultural productivity. The incidence of disease likely increased with early farming: Increased population density and living in close contact with animals would have combined to generate diseases. Human populations may have had to develop immunity to a set of animal diseases before population could grow. Population growth then accelerated with organized states. Populations doubled with the widespread use of bronze and doubled again with the widespread use of iron (these are, it should be clear, rough guesses). There was likely a setback in population growth trends associated with climatic shocks of 2200 and 1200 все. The "Iron Age" growth rate, if sustained, would have resulted in much larger human populations 
much earlier - a billion people in the Middle Ages. However, epidemics at the end of the period of classical empires (chapter 13) would result in substantial decreases in population across much of Eurasia. The world population likely first exceeded 100 million at some point in the last millennium BCE. It would rise to somewhere between 200 and 300 million toward the end of the period of classical empires in the fourth century CE. The population would decline somewhat in many post-imperial regions, but then slowly increase after that to some 300 million in the year 1000 (we return to population estimates after $1000 \mathrm{CE}$ in chapter 14).

\section{Migration}

One characteristic of the ancient period was mass migration. Tribes migrated into the Middle East from the Caucasus in the fourth millennium, and from Syria into Mesopotamia in the third. Nomads would much later attack the Akkadian Empire. The Kassites, probably from the Zagros Mountains, invaded Babylonia in the sixteenth century BCE. Anatolia, in particular, witnessed the arrival of several different groups from the north and east over the millennia. Records in Egypt and elsewhere speak of "sea peoples" who seem to have migrated from Greece and Libya to Anatolia and the Levant around 1200 все, then attacked Egypt and elsewhere. Five large states - Egypt, Hittites, Babylonia, Assyria (temporarily), and Elam in present-day Iran - fell in the thirteenth or twelfth centuries BCE as a result of these migrations. (Over the next centuries, Greeks, Hebrews, Persians, Phoenicians, and Nubians would form states in the power vacuum created by the fall of these earlier states.) These "sea peoples" may have been former Mycenaeans in part but also peoples pushing south into Mycenae. Scholars now suspect that a cooler climate impoverished farmers and set nomads on the move. Farther east, both central Asians carrying IndoEuropean languages and people from the Indus Valley moved into the Ganges plain over a period of centuries or more. The Indo-European language group seems to have emerged north of the Black Sea thousands of years BCE; the primary (but not only) wave of migration centred on the seventeenth century BCE and was associated with the use of war chariots. It is likely that these peoples moved as invited mercenaries rather than conquerors into many lands. (The Hittites in Anatolia were invited, and then later conquered the state that invited them, an occurrence often repeated in history.) The Zhou who attacked the Shang in 1045 BCE may have been a migrant group. There were likely many more migrations lost to the historical record: There have, for example, been recent excavations of people with Caucasian features in Xinjiang province in China dating from 2000 вСе; we do not know who they were or why they were so far east at the time. They may well have been part of the broader IndoEuropean migration, or peoples pushed by the movements of others.

One migration deserves particular attention. Humans began to spread into the Pacific Islands from about 1200 BCE. It is still unclear where Polynesians originally came from, though it is probably Southeast Asia or Taiwan. There 


\section{BOX 7.3}

\section{THE SPREAD OF LANGUAGES}

There are today five dominant language families in the world: the Indo-European of Europe and West and South Asia; Sino-Tibetan of East Asia; Niger-Congo of sub-Saharan Africa; AfroAsiatic of North Africa and the Middle East; and Austronesian of Madagascar and Southeast Asia. At roughly the same time that Indo-European languages spread from north of the Black Sea (we think) through the Middle East and across Europe and northern India, Niger-Congo (Bantu) languages spread from Nigeria through central and southern Africa, and Sino-Tibetan languages expanded geographically in East Asia.

Other important families include Dravidian of South Asia; Altaic in northern Asia (some doubt that Turkic and Japonic have a common Altaic ancestor); Austro-Asiatic and Tai-Kadai in Southeast Asia; Uralic in northern Eurasia; Eskimo-Aleut in the Arctic; several aboriginal language families in the Americas, and Nilo-Saharan and Khoisan in Africa. There are also thousands of smaller language families in places like New Guinea.

The spread of languages during the period from about 5,000 years ago tells us much about migration patterns, for groups do not tend to change languages unless overwhelmed politically, economically, or demographically by another group. Since language transmits cultural values and behaviours, these may be transmitted along with a particular language.

One challenge in reconstructing the history of languages is that languages borrow from multiple sources. It is common to think of a family tree whereby early languages are subdivided over time into many descendant languages. However, languages borrow words from outside their family with some regularity: English imported "algebra" from Arabic centuries ago, and "yin and yang" from Chinese more recently. If such borrowing were common, then it would be better to think of a web of language than a tree. Note that one's choice of analogy - tree or web - has a significant impact on how one approaches the history of language.

seems to be a physical resemblance, but genetic analysis is so far inconclusive. Distinctive "Lapita" pottery seems to have emerged in the Bismarck Archipelago northeast of New Guinea around 1200 вCE. The first human sites, and this pottery, are found in New Caledonia by 1000 все (2,200 km away) and Samoa and Tonga by 800 все (4,000 km away). The settlers had obsidian tools and farmed with pigs and chickens. We will pick up the story of the colonization of the Pacific Islands in chapter 16 and discuss how Polynesians accomplished this remarkable feat of discovery and settlement.

The migrations that we know the most about are migrations of groups of people. This is a particularly important practice among nomadic or seminomadic people. In such cases, some sort of group decision-making - perhaps communal, perhaps authoritarian - must be at work. We do not know if the 
entire group moved or if some stayed behind (the latter was the case in the Pacific). In the latter case, individual or family decisions may be important in determining who stays.

The mobility of groups during this extended period is astonishing - though keep in mind that we are talking about a period of thousands of years. It may reflect population pressures or climatic shocks or merely the attraction of the agricultural surpluses generated in the areas of agriculture. Note that while the "sea peoples" were blamed at the time and since for political and economic difficulties in Egypt and elsewhere, they may instead have been a symptom of climatic pressures affecting everyone. In any case, the migration of new peoples into areas with agricultural surpluses was often a source of increased conflict.

We have already encountered migration in this book in chapter 3, where human migrants over a period of tens of thousands of years populated most of the globe. People and peoples would continue to migrate throughout human history. This simple fact deserves emphasis: There has been a regrettable tendency in Western historiography to see the European migrations to the Americas after 1492 as the "migration" in

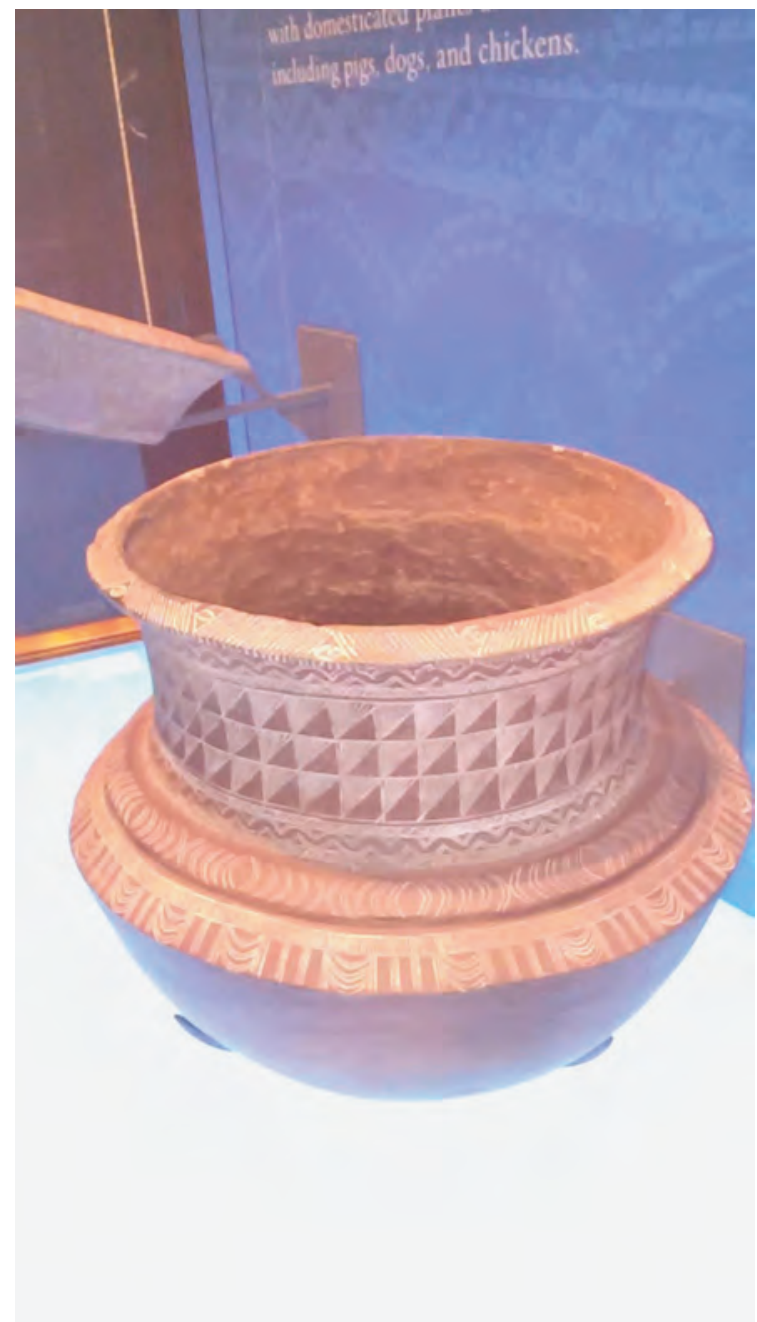

\section{Illustration 7.20}

Lapita Pottery, Bishop Museum, Honolulu human history (and non-historians often imagine that migrations that are more recent are a unique occurrence attributable to industrialization or "globalization"). It deserves instead to be seen as one - particularly important - instance of a recurring process in human history: large-scale movements of people from one place to another.

In addition to the dramatic movement of groups, there must have been a less-noted but important movement of individuals or families. Urbanization, as we have seen, requires continued migration from the countryside (because mortality exceeds fertility in urban centres). Trade also encourages migration as merchants form trading networks: Merchants from one town must operate in other towns, often permanently. 


\section{BOX 7.4}

\section{MIGRATION DECISIONS}

Why do migrants move? We can first distinguish forced from voluntary movements. Defeated peoples were often in history expelled or sold into slavery. Voluntary migrants judged that they could have a better life in another place. They might have been seeking higher incomes or a more benign political reality, or desired to live with others with similar cultural attitudes. In all cases, we can reasonably ask why one location seemed more promising than another. We can wonder how migrants gained information about their destination, and whether this proved accurate. We can also wonder how well they functioned in a new society where they did not know the rules or customs. For both forced and voluntary migration, we can wonder how the movement itself was financed and achieved. We can also recognize - importantly a middle ground between forced and voluntary migration in which a minority group is mistreated but not forced to leave, or there is such poverty that individuals feel compelled to try their luck elsewhere.

One huge advantage of a world history approach to migration is that we can consider the three combined acts of leaving, moving, and arriving together, whereas national or local histories tend to focus on only one.

Migration affects not only the migrant. Those they leave behind may view their departure with regret or relief (or neither). Those whose lands they pass through may see them with avarice, compassion, or suspicion. Those who live where they settle may be welcoming or hostile. In all three cases, a mixture of economic, political, social, and cultural forces will be at work: What happens to average wages and employment? Does migration aid or disrupt political stability, and are there legal barriers to migration, and are these enforced (these are rare before the nineteenth century $\mathrm{CE}$ )? Where do the migrants fit within social hierarchies? How do their beliefs compare to those of other groups?

In both group and family decision-making, we can recognize that the opportunities and costs associated with migration may differ by gender. The literature has often overemphasized the role of men in migration, but women's incomes were often significant too.

Population growth can encourage out-migration if it depresses incomes, especially for young adults (usually the most likely to move because they can hopefully recoup the cost of moving over a more prolonged timespan). Rigid social hierarchies may encourage outmigration of disadvantaged groups. Once some people leave a particular society they may encourage further migration if they remain in contact.

The preceding discussion leaves the impression that migrants always have a fixed destination. This is often not the case: Migrants may try man y locations before settling down, or may wander for their entire lives. Often, migrants view migration as temporary, with a plan to return to where one began after gaining wealth or perceiving welcome political or other changes back home. However, temporary migration often becomes permanent, and those who plan never to return sometimes do. Some early states, including Greeks and Incas, deliberately planted colonies of their citizens to establish influence in distant lands. 
It used to be asked whether migrants assimilated culturally. However, this question presumes both a clear dominant culture and that cultural transmission occurs in only one direction. A more likely outcome is some adaptation on both sides. Much will depend on how much interaction there is, and on the relative size of different groups.

\section{Travel}

We find the very earliest genre of travel writing in this early period. In $2300 \mathrm{BCE}$, the Egyptian traveller Harkhuf made four trips to Nubia, returning once with 300 donkeys laden with exotic goods: incense, ebony, panther skins, and elephant tusks. He did not, we should stress, travel alone. While he had a trading motive, this alone would not have driven him to recount his experiences in detail. Other societies amazed Egyptians, though they generally thought Egypt the best. The Greek historian Homer would celebrate those who travelled out of curiosity and maintained that such travellers were often treated as celebrities both when they travelled and when they returned.

\section{THE THEME OF POPULATION AND HEALTH}

We must perpetuate ourselves as a species (or this book would be of little use), and so "population" must be considered. Our ability to reproduce depends in turn on our ability to survive. We must also consider the related matter of "health"; this deserves more attention than it receives from either historians or social scientists. The simple fact is that humans were far less healthy through most of human history than they are today - due both to limited nutrition and endemic disease - and this had significant effects on their behaviour. We will often discuss epidemic disease, and will try to trace changes in nutrition. Note that the latter involves both how much and what kinds of food people eat.

The human population has expanded to several billion over the millennia. This is undoubtedly one of the critical elements of the human story. For each time period, we will discuss how much population growth occurred and why - appreciating that we often can only provide rough estimates of population. This will generally involve discussions of the determinants of fertility and mortality. It will occasionally also include discussions of the age distribution of the population. We will address the effects of population growth and increased population density (including urbanization) as well.

Human migration is of great importance for some periods, but not others. When people move in large numbers from one area to another, this migration has important effects on both places. 


\section{Families}

Families are universal in human societies. They have their most obvious role in the raising of children, but families also cooperate through most of human history in economic production. The nature of the family also has an important impact on cultural transmission, trade networks, and migration, among other phenomena. Given the importance of families, it is not surprising that early states sought to decree how these should operate.

Extended families - in which more than two generations cohabit - were most common in this period, judging from the size of households in the archaeological record (the cost of housing may be a significant cause of crossgenerational cohabitation). The oldest male usually had authority within the family. This was very explicit in China. Analogies were often drawn between male dominance in the family and the dominance of the male ruler in the state.

The Code of Hammurabi gives fathers the power to marry off their children and expects them to conserve their daughters' chastity. Daughters had some control over the dowry given in marriage, but the bulk of inheritance went to sons. The state thus reinforced male authority in families. Nevertheless, divorce seems to have been easy. Mesopotamian family law would influence other societies, including Greece and Rome. The experience of Penelope in the Odyssey (a Greek epic poem) indicates that widows could control estates, and even kingdoms, but only until they remarried (hence Penelope puts off diverse suitors in the hope that her husband will return).

There are many documents from the later Egyptian period that suggest that marriage was viewed as a contract whose primary purpose was children (it was also important to maintain parents in this life and the afterlife). Families do not seem to have had religious significance. The law did not limit whom one married. Nuclear families were more common than elsewhere. Divorce was again easy.

Polygamy was legal but rare beyond royalty in all ancient societies. Most men could likely not afford multiple wives, though they might take a second wife if the first wife did not bear children. Polygamy could become more common in societies at war, for there might then be an imbalance between the numbers of men and women of marriageable age. Widespread polygamy in the absence of male deaths in war would create a class of unhappy single men that would threaten social stability.

We know little about the lives of children. Many texts urge these to obedience - but perhaps these texts are not capturing reality but hoping to change child behaviour. There is some evidence of both toys and pets. A minority went to school to learn how to read or write or do simple arithmetic; a more substantial number were educated at home (but this required either a tutor or a literate parent with some free time). The main thing we know about childhood in this period is that levels of infant and child mortality were very high: Perhaps only half of those born survived to adulthood. 
It was typical for royal families in Mesopotamia, Mayan city states, and elsewhere, to marry royals from other cities to cement alliances. This will prove to be a common practice in history. It is not clear how common marriage outside a city was for lower classes. For smaller cities, there would be a genetic danger of inbreeding (where recessive genes with adverse effects are most likely to be inherited in pairs) if this was not done. DNA analysis of human remains shows that girls married out in ancient Yangshao in northern China.

\section{Questions}

1. Do human groups destroy their environments?

2. Did you detect important similarities or differences across the societies surveyed in this chapter? What were they?

3. Which of these societies would you most like to visit? Why?

4. Imagine you were trying to entice merchants or artisans to a particular city of your choice. What would you say?

\section{Readings}

Burstein, Stanley M. 2017. The World from 1000 bce to 300 ce. Oxford University Press. Diamond, Jared. 2005. Collapse: How Societies Choose to Fail or Succeed. New York: Viking.

Duindam, Jeroen. 2015. Dynasties: A Global History of Power, 1300-1800. Cambridge University Press. [Addresses the question of succession across many times and places.]

Harzig, Christiane, and Dirk Hoerder, with Donna Gabaccia. 2009. What Is Migration History? Malden, MA: Polity Press.

Livia-Bacci, Massimo. 2012. A Short History of Migration. Cambridge: Polity Press.

McAnany, Patricia A., and Norman Yoffee, eds. 2009. Questioning Collapse: Human Resilience, Ecological Vulnerability, and the Aftermath of Empire. New York: Cambridge University Press.

Manning, J.G. 2018. The Open Sea: The Economic Life of the Ancient Mediterranean World from the Iron Age to the Ascent of Rome. Princeton University Press. [Manning argues that there were many periods of growth in average incomes, often triggered by new technology or institutions, but never sustained for long, ended often by war or environmental shock. He tries to look beyond the moststudied civilizations in the area.]

Terpstra, Taco. 2019. Trade in the Ancient Mediterranean: Private Order and Public Institutions. Princeton University Press.

Thompson, William R., ed. 2001. Evolutionary Interpretations of World Politics. New York: Routledge. [Thompson says on page 2: "The point is that the evolutionary approach does not privilege a type of actor or a type of problem as the core foci. The other International Relations paradigms do." We can mix elements of various paradigms in pursuing evolutionary analysis.] 
Over a dozen volumes of the Cambridge Ancient History were published between 1970 and 2005, with detailed treatments of different ancient societies. The reader can also find many treatments of individual societies, such as John Romer's $2013 \mathrm{~A}$ History of Ancient Egypt (St. Martin's Press).

Readers may find much useful information on many of the societies surveyed above on the Ancient History Sourcebook at: http://sourcebooks.fordham.edu/Halsall/ ancient/asbook.asp. 


\section{Belief systems}

\section{The nature and development of early religions}

\section{Guiding questions}

Why and how did ancient religions develop? What roles did they serve in society? Who guided these religions, and how did individuals participate? What were the similarities and differences in religion across societies?

Why and how did artistic practices change in ancient times? What roles might art have played in human societies? Who were artists? What were the similarities and differences in artistic practice across societies?

Relationship to other chapters: We shall see that religions changed to reflect the development of agriculture and nomadism (chapter 4), and cities and states (chapter 6). We mentioned religions briefly in those chapters (and particular religions in chapter 7); this chapter clarifies the relationship between religion and cities and states and agriculture and nomadism. These religions would contribute during the classical period to a new generation of (generally universalist and monotheist) religions (chapter 11). Our discussion of ancient religions, and of religion in general, in this chapter sets the stage for our study of later religions in chapter 11 and again later in the book. This chapter also informs later discussions of families and architecture.

The chapter closes with a discussion of art. Art and religion are treated in the same chapter for convenience: Art and religion interact but no more than they do with other themes. Our 
treatment of art builds upon our analysis of the purposes of art in chapter 3 and discusses how societal changes detailed in the last few chapters influenced artistic practices. The discussion here informs discussions of art in later chapters. Students also receive advice on how they should approach the many artistic images throughout this book. We close with a general discussion of how art evolves through time; we discuss artistic evolution in many later chapters.

We begin this chapter with discussions of the roles that religion plays in human lives, evolutionary processes in the development of religions, and the historical approach to the study of religion. We then survey the earliest human religions, followed by some comparative analysis of these. This sets the stage for a survey of "modern" or "missionary" religions in chapter 11, most of which survive to this day. Though our primary focus is cultural, we relate religion to basic human psychology (genetic predisposition). Links are made between religion and politics, social structure, and philosophy (a kind of science). We also discuss religious buildings (built environment). We then discuss how religious ideas and practices moved across societies, but also differences across societies in religious practices. The chapter closes with a general discussion of art (which in turn interacts with technology and culture).
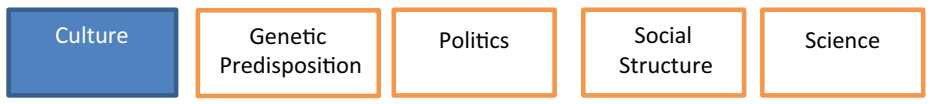

Non-human Environment

It seems that the very earliest religions focused primarily on the second of the three purposes noted in Box 8.1. Hunter-gatherers and the earliest farmers were intensely interested in understanding rainfall and river flow and other aspects of the natural world on which their lives depended. Though their religions also generally addressed questions of mortality and group cohesion, they focused on explaining and aspiring to control natural processes.

In addressing such immediate concerns, religion could build on a human tendency to see agency everywhere. A hunter-gatherer hearing rustling in the bushes benefits from imagining that a dangerous animal lurks within. It may be more accurate most of the time to assume that it is just the wind, but it is prudent to prepare for the worst. It may then be a small step to infuse nature with agency. Later, as religions became more concerned with how humans should behave in their daily lives, they could build on another basic human predisposition: our shared sense of justice (chapter 3 ). 


\section{BOX 8.1 \\ THE ROLES OF RELIGION}

Religions play three principal roles in life. We can define religions, then, as shared systems of belief that serve at least one of these roles. Most obviously, perhaps, religions provide a means of coping with the grief and fear associated with being self-conscious about our mortality. In so doing, religions also inevitably offer some answers to questions about the meaning of life.

Second, religions can provide explanations of natural phenomena that are important to human life, and perhaps offer the possibility of control: Why does it rain? Why do the rivers flood? Why do diseases strike? More generally, religion provides an understanding of the world and our place in it - and of our group as well. It is this long-standing role for religion that is most threatened by modern science, for science provides plausible explanations of the emergence of stars and planets and complex life-forms, as well as rain and disease (see chapter 2).

Third, religions everywhere provide a sense of community because people come to share answers to important questions. A set of cultural values reinforces this shared identity. Members of a group can then expect other group members to behave in certain ways - and they can impose penalties if they act in a manner considered unethical. This aspect of religion will prove of particular importance in chapter 11 . Rituals - patterned activities invoking religious symbols - are also universal features of religion: They are the means by which religious beliefs become active in the world. They are important in generating social cohesion.

Other effects are possible. Religions often seem to justify the existing social order. At other times, religions have been a motivation for social change. Moreover, religions seem to become more complex as human societies become more complicated. In part, this reflects merely specialization: One cannot have a priestly class without some degree of occupational specialization that itself depends on the production by others of a food surplus. However, it may also reflect the fact that religion serves additional purposes as societies become more complicated.

\section{PRIMARY DOCUMENTS: CREATION STORIES}

Ancient religions each grappled in their own way with the idea that the cosmos had emerged from nothingness. In Egypt there was first a universal god:

Thus said the universal lord after he came into being: I am the one who came into being ... When I came into being, being came into being. All beings came into being after I came into being. Manifold were the beings from that which came forth from my mouth.

He then creates other gods and then humanity out of his body. 
The Hebrew creation story in the Biblical book of Genesis starts with god:

In the beginning god created the heaven and the earth.... And god said "Let there be light" and there was light.

It describes how god created the world in six days and then rested on the seventh.

The Chinese P'an Ku creation myth also relies on one god, but notably this god dies in the process:

Earth with its mountains, rivers, and seas, Sky with its sun, moon, and stars: In the beginning all these were one, and the one was Chaos. Nothing had taken shape, all was a dark swirling confusion, over and under, round and round. For countless ages this was the way of the universe, unformed and unillumined, until from the midst of Chaos came P'an Ku. Slowly, slowly, he grew into being, feeding on the elements, eyes closed, sleeping a sleep of eighteen thousand years. At last, the moment came when he woke from his sleeping. He opened his eyes: nothing could he see, nothing but darkness, nothing but confusion. In his anger, he raised his great arm and struck out blindly in the face of the murk, and with one great crashing blow he scattered the elements of Chaos.

The swirling ceased, and in its place came a new kind of movement. No longer confined, all those things that were light in weight and pure in nature rose upwards: all those things that were heavy and gross sank down. With his one mighty blow, P'an Ku had freed sky from earth. Now P'an Ku stood with his feet on earth, and the sky rested on his head. So long as he stood between the two they could not come together again. And as he stood, the rising and sinking went on. With each day that passed earth grew thicker by ten feet and the sky rose higher by ten feet, thrust ever farther from the earth by P'an Ku's body which daily grew in height by ten feet also. For eighteen thousand years more, P'an Ku continued to grow until his own body was gigantic, and until the earth was formed of massive thickness and the sky had risen far above. Thousands of miles tall he stood, until the time when he could be sure that earth and sky were fixed and firm in their places.

When this time came P'an Ku, his task achieved, lay down on earth to rest, and resting died. Now he, who in his life had brought shape to the universe, by his death gave his body to make it rich and beautiful. He gave the breath from his body to form the winds and clouds, his voice to the rolling thunder, his two eyes to be the sun and moon, the hairs of his head and beard to be the stars, the sweat of his brow to be the rain and dew. To the earth, he gave his body for the mountains and his hands and feet for the two poles and the extremes of east and west. His blood flowed as the rivers of earth and his veins ran as the roads that cover the land. His flesh became the soil of the fields and the hairs of his body grew on as the flowers and trees. As for his bones and teeth, these sank deep below the surface of earth to enrich it as precious metals.

The Hindu creation myth begins with one god also:

At first there was only darkness wrapped in darkness. All this was only unilluminated water. That One which came to be, enclosed in nothing, arose at last, born of the power of heat. 
In the beginning desire descended on it - that was the primal seed born of the mind.

The myth then speaks of Purusa:

thousand-headed was the Purusa, thousand-eyed, thousand-footed.

All the animals and humans and the verses of the Vedas were made of Purusa. Purusa was sacrificed by the gods to create all that we know.

What is the significance of the fact that creator gods die in Chinese and Hindu creation stories?

\section{Evolutionary processes}

As a component of culture, religions are (potentially) subject to evolutionary processes. Before delving into the history of particular religions, it makes sense to address evolutionary processes in general. We should first appreciate that the religions that have survived historically are but a minute fraction of the religions that humans have created. Most religions fail within decades of their creation. However, if they can gain a large body of believers, then they can grow both through the example that these believers set and by providing a community that others can join. Since most religions disappear, it makes good sense to look not just at how particular religions originated but why they were selected (or not).

One fundamental way in which religions evolved was to take on new roles. Farmers had different interests from hunter-gatherers, and urban populations and especially merchants and artisans likewise had different needs and desires. A religion that had focused on affecting natural processes could add ethical precepts to guide human interaction. As societies grew larger, they needed to encourage members to act honestly and fairly toward each other. In small communities, we can develop reputations and learn whom to trust; in larger societies, we necessarily interact with many people that we do not know well. Both polity and economy would function better if people could trust each other. What sort of characteristics of religion might be selected for to encourage ethical behaviour? Religions that proposed watchful gods (or ancestors) that rewarded ethical behaviour would have a selection advantage: Societies with such religions would prosper. Religions that punished the unethical might be selected even more strongly, for punishment is often a more powerful motivator than reward. Note that religions need to encourage people to behave ethically even when nobody could see (or at least prove) unethical behaviour. Belief in vengeful gods can be very useful here. However, people who doubt the existence of such gods might pretend to be religious to deceive fellow community members into trusting them. There might then be selection for religions that required costly public rituals or acts of charity: These would make it difficult 
to pretend to religious belief. Since non-believers pose a threat to communal trust, there may also be selection for religions that treat non-belief, and especially false belief, harshly. Such an attitude would also be conducive to the prosecution of war against other societies (though the ethical precepts of religion might at times urge compassion toward others).

Such evolutionary changes might at times be so dramatic as to merit the recognition of a new religion. Yet we should appreciate that beliefs might change a great deal under the umbrella of a particular religion: Christianity and Islam and Buddhism have each changed a great deal over the centuries (chapter 11).

Transmission processes changed dramatically with the invention of writing (chapter 6). The earliest religions had to be transmitted orally between generations. It was then easy to amend received religion as circumstances changed or new religious roles emerged - though of course, we have little evidence of such a process precisely because beliefs were not written down (we can only strive to infer changing beliefs from religious practices for which there is physical evidence). Once religious beliefs or practices were written down which would in all religions lead to the veneration of certain texts - it became harder to change beliefs or practices. Explicit arguments needed to be made as to why these texts should be interpreted in new ways. Some textual elements proved particularly challenging to reinterpret.

We also need to recognize that ideas change as they move geographically. There is often a mistaken tendency to see world religions as the same everywhere. Even text-based religions change as they spread. Buddhism embraced violence from political necessity. Catholicism adapted in the Americas by incorporating local gods as saints within the Catholic tradition.

Though the written word clarifies religious doctrine, the inherent ambiguity of human language leaves considerable scope for interpretation of these texts. Doctrinal discussions and even disputes have been common in all world religions. Indeed, all of the world's major religions have fractured into different denominations that disagree about certain core beliefs or practices. These doctrinal disputes may at times emerge among the clergy at first, but clergy may often be reacting to changes in interpretation among believers. Though hard to trace through history, followers of a particular religion (often just in one specific region) might change their belief systems in subtle ways, generally in response to changes in their circumstances. Their clergy might complain at first but might seek over time to justify their flock's behaviour. We can think here of the fact that the majority of North American Catholics have come to ignore official church doctrine on birth control, and how some theologians seek to justify this decision.

Doctrinal disputes are no surprise within an evolutionary approach to understanding religion. Individuals with different interests and needs will posit subtle (or sometimes not-so-subtle) changes to a received faith. These will be selected for through the interactions of religious leaders, political leaders, private donors, and the community of faith itself. Believers will disagree regarding 
both what beliefs best serve the community and what changes in belief can be justified with respect to holy texts. Political and religious leaders may seek consensus but may not always be successful.

There can be evolution through time not just in beliefs but also in how and how fervently the faithful believe. It is tough to identify the intensity of faith historically. There are various degrees of religious adherence possible, and people might profess to a greater degree of belief than they feel. This would especially be the case when there was intense social or political pressure to conform. We should appreciate that actual religious practices might deviate quite a bit from official religious doctrines.

We have so far focused on religions as systems of belief. However, successful religions must be institutionalized: There must be priests and temples and rituals and - crucially - penalties for not following religious guidelines. These institutions also evolve. Importantly, religions, like any social practice, consume resources. The earliest religions might not have had dedicated priests or temples, but time and effort were still devoted to rituals, carvings, and cave paintings that are presumed to have had religious significance. Later religions would have both clergy and buildings dedicated to their cause. Such religions were only feasible once agricultural productivity had risen to a level that could afford to support religious activity on such a scale. The fortunes of individual religions would then depend to a considerable extent on the financial resources - usually some mix of private and public - at their disposal. To be sure, religions could sometimes survive on limited resources for extended periods, but religious expansion was usually financed in some manner. The evolutionary fitness of religions, in the end, depended critically on their access to resources.

\section{Historical analysis of religion}

World historians might be tempted to tiptoe around the question of religion lest they offend someone's beliefs. However, it is not the place of the historian to judge whether any beliefs are true. The historian can discuss how particular religions emerged - and if necessary record dispassionately the miraculous events that are said to have occurred. The historian can report the effects that religions have had on human societies. More controversially, the historian can investigate why particular religions emerged and spread where and when they did. This may offend the sensibilities of those who regard one religion as the true faith and attribute its emergence and spread to divine will. Yet the historical fact is that each religion has emerged in particular circumstances, and god(s) have propelled none to universal recognition. Indeed all religions have suffered "setbacks" in the sense of having lost ground in parts of the world in which they were once common. The global mosaic of religious beliefs in the contemporary world deserves a historical explanation. The historian seeks to 
understand why particular religions prospered in some places but not others. It is not the historian's role to speculate on whether there was divine intervention. Nor need the historian dismiss that possibility.

A century ago, the history of religion in the West pursued a teleological approach in which Christianity - and particularly Anglicanism - was effectively the point toward which religion had been tending all along. Historians have in response tended to shun historical generalizations of any sort regarding the evolution of religion, preferring to stress the differences between religions. Yet a world historian should not shy away from identifying similarities as well, both in beliefs and practices and in evolutionary trajectories.

\section{Challenges facing agents: Priests}

We have investigated in other chapters the challenges faced by rulers, merchants, farmers, and others. The priest faces two practical challenges: encouraging others to accept the priest's role as intermediary between human beings and the gods, and ensuring financial resources for the priestly class and religious buildings and rituals. The first task will be much easier if the priest is a believer in their religion in general and clerical ceremonies in particular. As we discussed in chapter 3, humans have been selected to detect deceit. It is tempting - especially when looking at the earliest religions with their close ties to states - to imagine that these were deliberate attempts to coopt the masses: to encourage docility while taking much of the agricultural surplus. We cannot know what was in the hearts and minds of the earliest priests, but can observe that deliberate manipulation on such a scale would be difficult. Priests can also ensure that they fulfil the roles outlined above in Box 11.1. Moreover, they can urge public disdain or worse for those who do not show appropriate devotion during public rituals.

Priests will always be tempted to compel financial support. Religions are often but not always allied with states in human history; they then gain the support that states can provide. States may fund religions directly or require adherents to do so. The priest can also urge followers to donate, telling them that gods look more kindly on those who make such a sacrifice.

\section{The earliest religions}

We speculated in chapter 3 that the religious views of hunter-gatherers were usually animist. Such beliefs would reflect their regular interaction with the universe as hunters or gatherers. The earliest works of art are often drawings or sculptures of animals, which may have had religious significance.

With the move to settled agriculture, religious priorities changed. Sedentary populations were much more susceptible to changes in the weather, and especially rainfall (and experience of frost in some regions). We see rain dances in many sedentary societies. Rituals commonly accompanied planting and harvesting. More generally, a series of annual ceremonies to mark such things as changing seasons becomes common. In areas where agriculture depended 


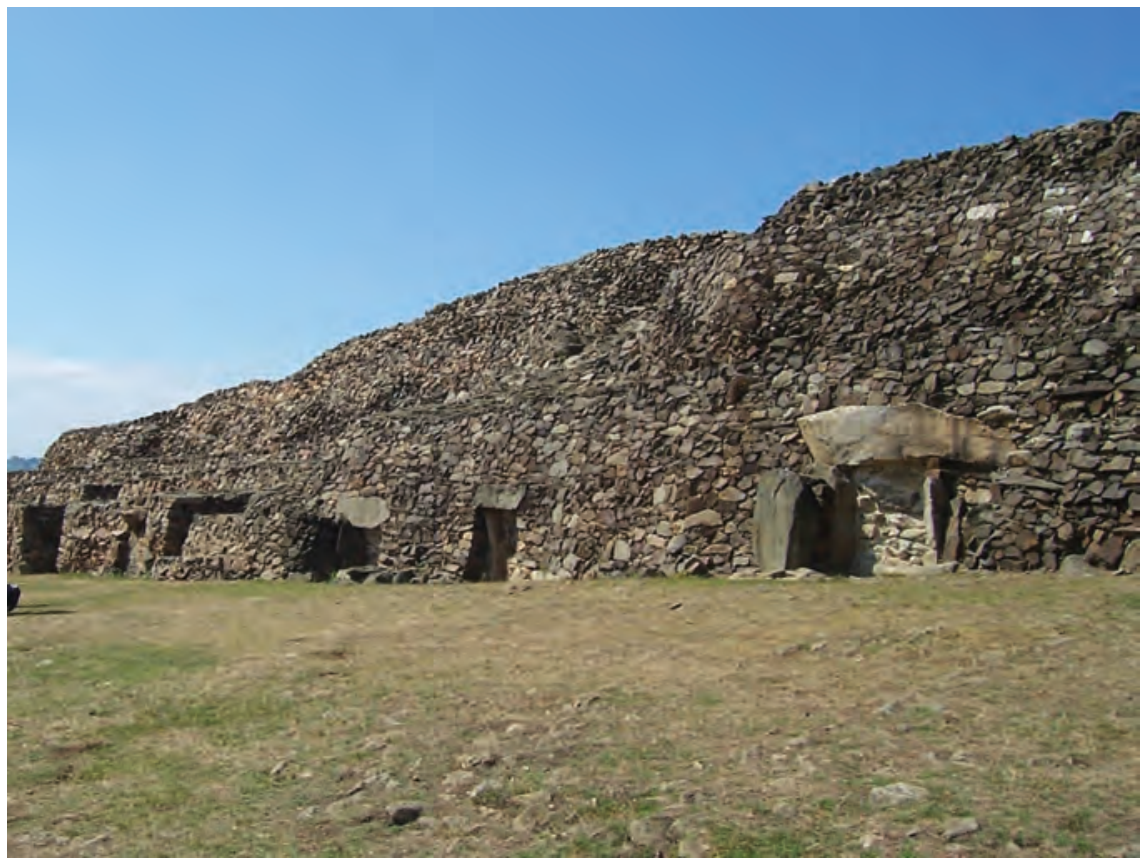

Illustration 8.1

Barnenez Cairn Tombs near the coast in Brittany, France. One of the oldest surviving structures in the world, it was built between 4800 and $4000 \mathrm{BCE}$, but reused in the third millennium BCE. It contains eleven separate burial chambers, reached from a common passageway. Tombs provide the most visible remains of the earliest religions

Source: Photograph by NewPapillon, CC BY-SA 3.0

on periodic flooding, such as the Nile and northern China, people came to expect that the rulers of the state would predict and control these floods. The Shang rulers in China claimed descent from ancestors who could intercede with $\operatorname{god}(\mathrm{s})$.

The sedentary life, and the fact that women were likely still most responsible for plant material in the early stages of the transition to agriculture, may have contributed to an increased focus on female fertility. Worship of female goddesses appears to be as old as agriculture and to have been common throughout the Middle East from at least 7000 все. Moreover, such goddesses remained important well into historical times: the Greek goddesses such as Aphrodite and Athena, the Anatolian Cybele, and the Phoenician Ashtart among many others (though these later religions are each identified primarily with male gods).

The religions of hunter-gatherers were often group-specific: The group had a special relationship with the universe. For example, myths may recount how the group came to be. The religions that emerged with large states from at least 3,000 years ago instead tended to be universalistic. Gods, to be sure, were often associated with particular cities or states, but these gods had interests beyond 


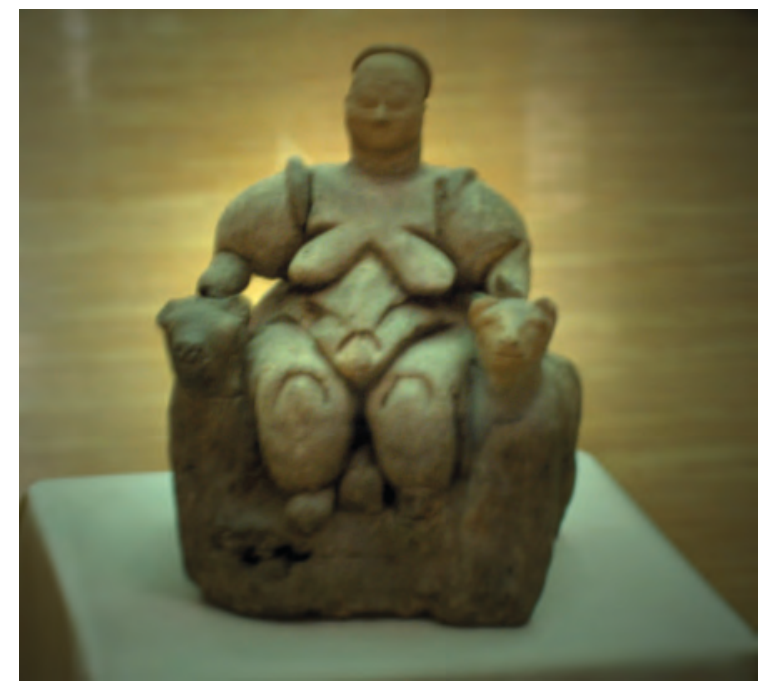

Illustration 8.2

Mother Goddess from Catalhoyuk the group. The Mesopotamian pantheon, in which different cities had gods but these interacted, is an example.

The development of centralized states, and the creation of a small elite that was not directly involved in food production, may have again changed attitudes toward the universe. This elite had the leisure time to reflect at length on such matters (recall that early farmers worked much harder than had hunters and gatherers). One notable characteristic of the religions associated with the earliest "civilizations" (chapter 6) is the powerful ties that usually existed between the state and religion. Only in India and Israel was early religion not closely associated with kings. Almost all states had an associated religion. Political authorities usually came to appoint priests. Temples were often really an arm of the state bureaucracy. Rulers played a crucial role in religious rituals, which served to justify the ruler's authority. Religions often viewed rulers as divine or semi-divine or at least as having an exceptional ability to intercede with the gods on behalf of the population.

It is tempting to imagine that states created these religions. However, since people must actually believe religions for these to serve to strengthen the state, it is perhaps likeliest that rulers sought to shape beliefs that already had some social acceptance. There is indeed much evidence that deities predated the states that would celebrate them. We can well imagine an evolutionary process in which ruling elites shaped existing religions to suit their needs. Looking ahead to our later discussion of "modern" or "missionary" religions (chapter 11), we will find that these all developed independently from organized states but came over time to associate themselves with states. A similar process may well have played out much earlier in human history.

Religions duplicated the political and social stratification associated with early states. Whereas hunter-gatherers may have shared religious duties (or recognized shamans who were also expected to hunt or gather), urban religions had hierarchies of priests supported by the same agricultural surplus that supported rulers. Indeed the head priests and priestesses were often members of the royal family.

We saw in chapter 6 that geographic expansion was a common characteristic of states. What could a state do if it conquered a state with different gods? If religion were a vital bulwark of the state, then it would be dangerous not to claim some affiliation with the gods of the conquered. Indeed, we commonly observe states associating themselves with multiple deities. The Assyrians 
upon conquering Mesopotamia proclaimed that they had the support of the Babylonian god, revered throughout Mesopotamia. The Egyptians combined southern and northern sun gods as the two kingdoms merged. This practice would later be pursued by the Romans (chapter 10), who regularly added the gods of conquered peoples to the Roman pantheon. This practice proved remarkably straightforward in a world of polytheistic religions: Since each group already recognized multiple gods, they could be convinced to accept also the gods of other cities. The problem would arise with monotheism: The Romans objected to both Judaism and Christianity because their followers refused to recognize other gods.

Note that while states attempted a religious monopoly, they would prove unable to stop the development of a later generation of "missionary" religions (chapter 11). It must seem that state religions were not quite as dominant as rulers might have wished.

The earliest religions were almost always polytheistic. That is, people worshipped multiple gods. The key advantage of polytheism is that different gods have different personalities and roles and can thus be asked for different favours: One might make it rain, another might keep animals healthy, a third might guarantee children to a couple. Different gods might require different rituals or priesthoods or places of worship. As we have seen, there was a further political advantage of polytheism: As states expanded they could absorb the gods of conquered peoples, achieving synthesis of religious beliefs that reinforced the power of the state. Polytheistic faiths might imagine a unified force greater than these gods - this is clearest in African religion (below) - but it was the diverse gods that received most religious attention.

\section{Egypt}

The sun played a significant role in Egyptian religion. Two gods from different cities - Amon and Re - that were each associated with the sun came over time to be revered as one. From the fourth dynasty (2600 BCE), kings (pharaohs) claimed to be descended from the sun god. There was also a river god, and indeed gods associated with a variety of natural forces. The pharaoh claimed to be able to intercede with the gods on his people's behalf.

The Egyptians had a well-known concern with the afterlife. Whereas Gilgamesh in Mesopotamia assumed that life ended with death, Egyptians imagined that pharaohs would join the gods after death. We may exaggerate the degree of Egyptian concern with immortality simply because Egyptian tombs are by far the most visible physical remains of Egyptian civilization. Yet it is nevertheless clear that substantial societal resources were devoted to building first pyramids and later cave tombs, and filling these with gold, silver, and even large boats to carry rulers (and eventually other members of the elite) into the afterlife. The pyramids may have served as symbols of political power but were nevertheless designed as tombs. Pictures in these tombs describe visions of the Egyptian afterlife, which is notable for being a continuation of this one 


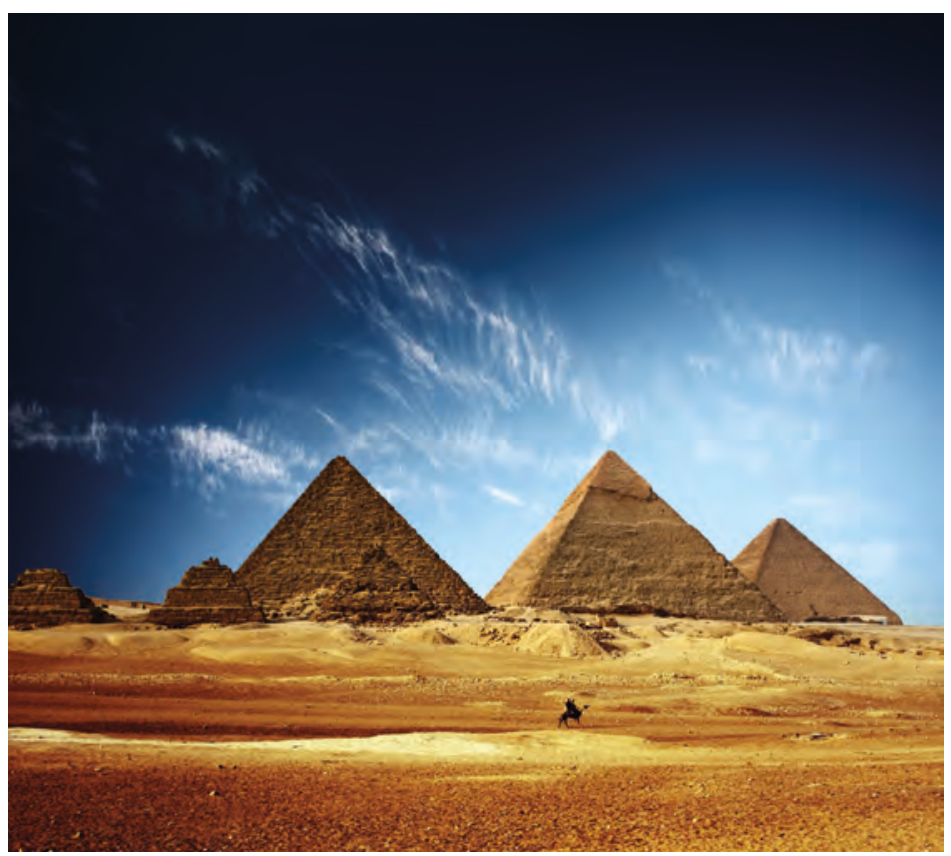

Illustration 8.3

Egyptian pyramids

but with no work. That is, the afterlife was the life of a wealthy landowner but without the vagaries of harvest. Mummification preserved the human body for such a life, and sacrificial goods would aid the deceased in the next life. It was thought that the dead intervened in this life; they then needed to be regularly honoured with offerings. There is debate as to whether there were human sacrifices, but it seems clear that some servants were killed alongside early royals to accompany them to the next life.

We observe two critical changes over time in tomb inscriptions: These come to speak of judgement in the afterlife for one's behaviour in this life. It also came to be accepted that non-royals also gained immortality. This second innovation would undoubtedly encourage greater devotion to the religion among the general population. The population might then be inspired toward ethical behaviour by the belief in godly judgement. The cult of Osiris in particular maintained that an afterlife was only possible for those who lived a moral life. Osiris would weigh the hearts of the dead against a feather; hearts heavy with guilt would fail the test. We see here more than a hint of the morality that would characterize later religions.

It was thought that mummification would ensure that the soul remained with the body in the afterlife. Mummification never became a general practice, perhaps because of the enormous expense involved: Priests extracted the brains of the corpse through a nostril, and internal organs through a small incision; they then filled the body with spices and soaked it in a salty substance for 


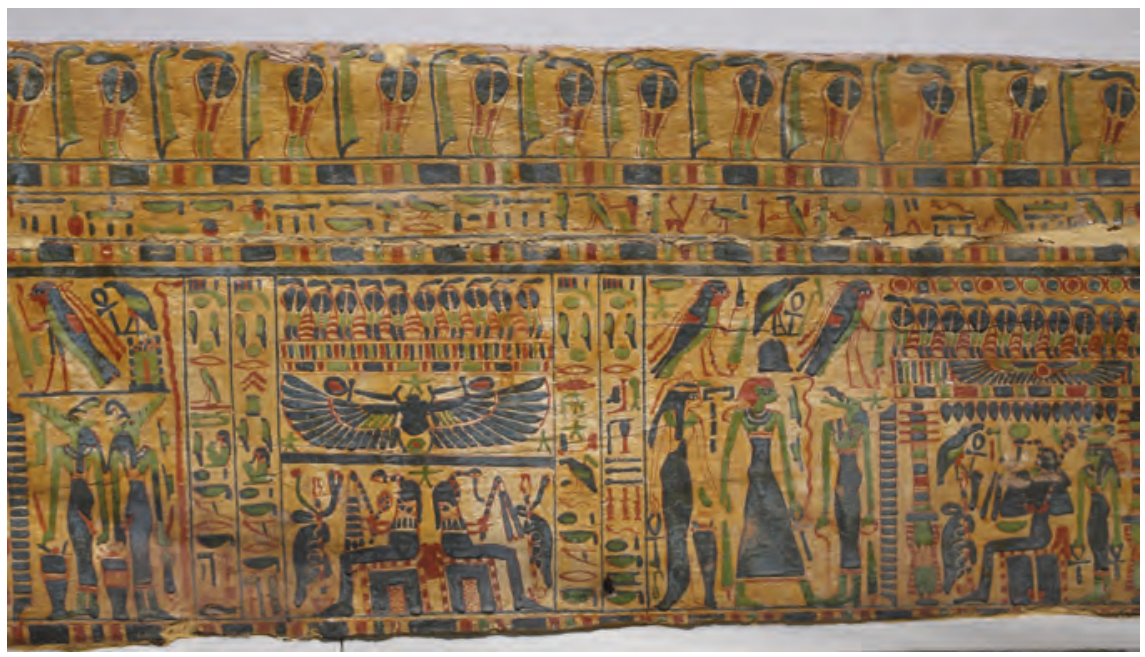

Illustration 8.4

Panel from human-shaped coffin, Thebes, twenty-first dynasty, 1070 to 945 BCE

Source: Walters Art Museum, CC BY-SA 3.0

months until completely dried out. Priests then wrapped it in fine linen soaked in natural resins and covered it with jewels. They then buried these mummies in elaborate tombs. Those who could afford it pursued mummification - and sometimes had pets mummified.

Egyptian religion did change over time. Amenhotep IV from 1353 всE urged the exclusive worship of Aten. This may be the earliest example of monotheistic religion. Upon his death, the priests of Egypt acted quickly to restore the pre-existing pantheon of gods. Amenhotep's son Tutankhamun would agree to the restoration of the pantheon of gods. This episode shows the power of a pharaoh to influence the society's religious beliefs but also the power over a more extended time period of a priestly bureaucracy to support the continued worship of traditional gods.

\section{PRIMARY DOCUMENTS: THE FIRST TWO VERSES OF HYMN TO THE NILE, 2100 BCE}

Hail to thee $O$ Nile! Thou showest thyself in this land, Coming in peace, giving life to Egypt: O Ammon, (thou) leadest night into day, A leading that rejoices the heart! Overflowing the gardens created by Ra. Giving life to all animals; Watering the land without ceasing:The way of heaven descending: Lover of food, bestower of corn, Giving light to every home, O Ptah! Lord of fishes, when the inundation returns No fowls fall on 
the cultures. Maker of spelt; creator of wheat:Who maintaineth the temples! Idle hands he loathes For myriads, for all the wretched. If the gods in heaven are grieved, Then sorrow cometh on men.

Is the Nile a god, or godlike, or a creation of gods? Can you see how early Egyptian religion cared about agricultural productivity?

\section{The Levant}

We know of the kingdom of Ugarit and its worship of Baal the storm (rain) god and other deities, both from critical mentions in the Bible and from a hoard of clay tablets discovered in the 1920s. The kingdom sat astride a major trade route between Egypt and Mesopotamia, and likely absorbed religious influences from both (especially as it tried to ally itself politically with its more powerful neighbours). We can expect that other societies that have disappeared without trace worshipped different though perhaps similar deities.

Were there human sacrifices? Scholars have sometimes interpreted elaborate child burials in the region as sacrifices, but these may not have been anything more than burials.

Judaism acts as a bridge between the early religions of the Levant - and by extension Egypt and Mesopotamia - and later missionary religions such as Christianity and Islam. Due to the close connection between Judaism, Christianity, and Islam, and the fact that Judaism itself is a prominent religion to this day, it will be easier to delay our discussion of Judaism until chapter 11.

\section{Mesopotamia}

Mesopotamian gods were associated with stars and planets but also with various purposes: the sun god with truth and justice (the sun god also allegedly patronized Gilgamesh and others), Venus with sex and war (usually separately), Mars with disease. These gods also had ties to political leaders. Ianna, the local goddess of Uruk in Sumeria, was said to have a relationship with the priest-king.

One remarkable feature of Mesopotamian religion is that it was both particularistic and universal. That is, each city state had its gods, but there was recognition of a broader pantheon. People revered the Babylonian god even in polities that were not subservient to Babylon. Kings called upon the local gods when Mesopotamian states battled, but then co-opted these if there was conquest. Religion could provide elements of a shared Mesopotamian identity despite political fragmentation. We should thus be careful of identifying state and religion, though appreciating that these were still tightly linked. It seems likely that the earliest Mesopotamian religion suggested harmony among the gods but over time came to stress godly conflict as Mesopotamian states fought each 


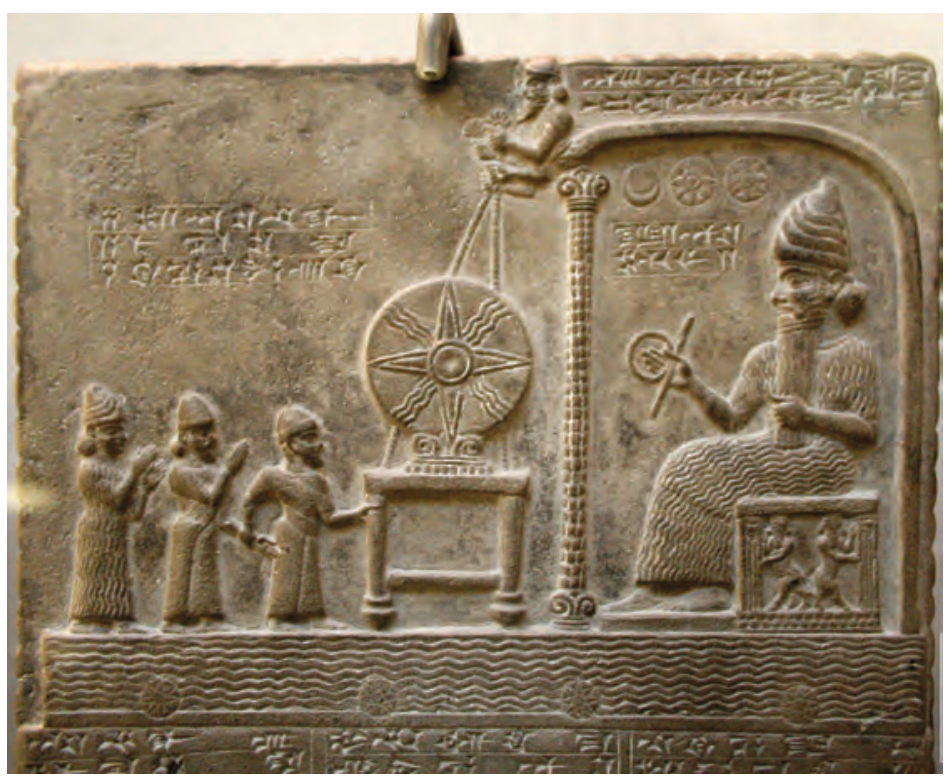

Illustration 8.5

Sun god Shamash on throne, 9th century BCE, Shippar, British Museum. The symbol in the middle is known to this day in the region as the star of Shippar Source: Photograph by Prioryman, CC BY-SA 4.0

other. It became accepted that Mesopotamian gods from time to time chose a different leader of the gods: A ruler who succeeded in war could then claim that the gods had selected "his" god to rule.

We have much less information regarding Mesopotamian conceptions of an afterlife than we do for Egypt. The belief in mortality espoused by Gilgamesh reflected the fact that Enki, the Sumerian god of wisdom, had decreed mortality as the human fate. Yet texts such as Gilgamesh also referred to a subterranean netherworld from which souls never returned. Though underground and dark, this netherworld was not a Hell that suffered in comparison with a more sublime Heaven (though it may have influenced later conceptions of Hell): All souls - or at least all those attended by appropriate rituals at and after death - went to the netherworld. The netherworld was initially a bleak place, but Mesopotamians came over time to celebrate the opportunity to reunite with ancestors. Notably, there was a social hierarchy in the netherworld, but this was determined not by one's ethical comportment in this world but by one's status in this life and the performance of rituals in one's honour. Still, such ideas may have influenced later religions' views of godly judgement.

\section{Greece}

There was considerable religious diversity in the Greco-Roman world, perhaps related to ethnic and class differences. The Greeks worshipped a polytheistic 


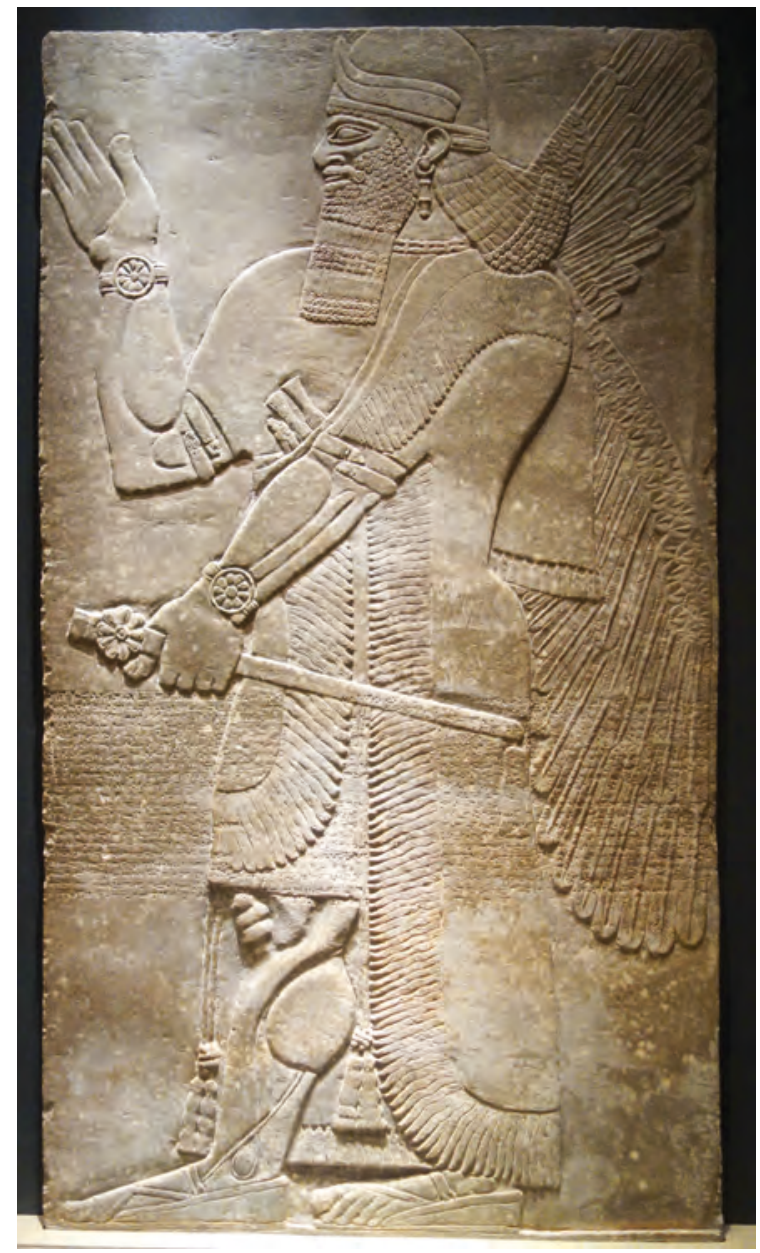

Illustration $\mathbf{8 . 6}$

Winged protective deity, Northwest Palace at Calakh, Northern Iraq, Assyrian, reign of Assurnasirpal II, 883859 вCE, alabaster, Museum of Fine Arts, Boston

Source: Photograph by Daderot, CC0 1.0 pantheon of gods, including Aphrodite, goddess of beauty and desire, Ares, god of war, Athena, goddess of wisdom, Dionysius (Bacchus), god of wine and theatre, Poseidon, god of the sea, and Zeus, king of the gods. This pantheon exhibited family relationships: Zeus had overthrown his father, and Athena was born from the forehead of Zeus. Many Greek gods can be traced to ancient Mycenae. These gods interacted with humans in a variety of myths. Greeks also worshipped Egyptian gods, often in a different form. The goddess Cybele was popular. Mithras, an Indo-Iranian sun god (which Zoroastrianism would develop; see chapter 11) was also worshipped.

There was considerable religious ferment during the period after about 500 все. This may reflect political fragmentation: No state could enforce any particular religion. As a trading region, Greece encountered a variety of religious ideas. The cult of Osiris from Egypt became popular with its notion of salvation through good behaviour. Monotheistic beliefs, and the idea of a saviour who was resurrected, were pursued by multiple cults.

\section{Rome}

Rome had many gods that were thought to intervene in human affairs. Mars affected war, Ceres grain. The Romans, like the Greeks, borrowed gods from others, including Minerva, goddess of wisdom, from the Etruscans. The cult of Isis, a benevolent goddess (and wife of Osiris) from Egypt, was perhaps the most popular. The Roman masses found comfort in new religions of salvation. Mithraism, imported from Anatolia but originally Persian, promised ecstatic union with its warrior god; it was popular with soldiers (and banned women from its services).

\section{Africa beyond Egypt}

African religions often receive less attention than they deserve in surveys of early religions. Though the earliest beliefs in Africa were likely polytheistic 
and animistic, as elsewhere, peoples of the region to the south of the Sahara recognized one divine spirit responsible for good and evil from about 5000 вCE. In the eyes of some scholars, these beliefs qualify as the first examples of monotheism in the world. To be sure, Africans still prayed to various spirits, but it was nevertheless felt that the one divine spirit made all major decisions. Farther south, in the Niger and Congo regions, it was felt that one god (Nyamba) had created the universe but then mostly left it to unfold as it would. It was still possible to pray to the spirits of one's ancestors. These religions, then, blend the idea of one dominant god with the idea of multiple spirits that might intercede on one's behalf. These spirits play a role somewhat similar to the saints that would characterize later monotheistic religions (see chapter 11). Priests attempted to intervene with these spirits on behalf of human groups, and tried to explain why good or ill fortune had befallen groups

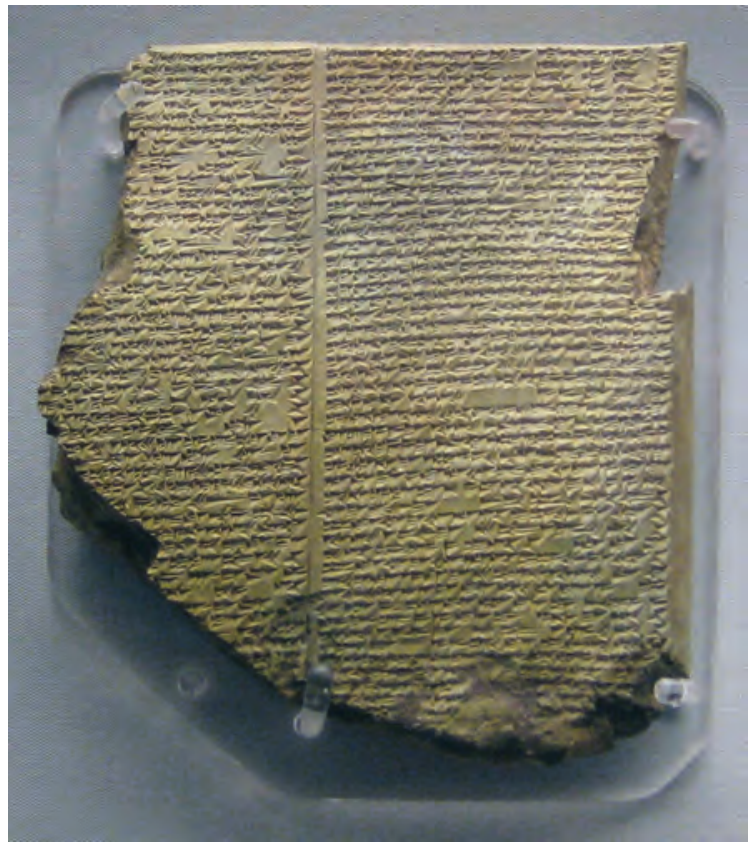

Illustration 8.7

Neo-Assyrian clay tablet. Epic of Gilgamesh, Tablet 11: Story of the Flood. Known as the "Flood Tablet," 700 BCE. It is thought to have influenced later flood epics in both Homer and the Bible, British Museum Source: Photograph by Babelstone, CCO 1.0 or individuals. After about 1000 BCE, Bantu speakers came to believe that the one divine spirit was inherently good. Though religious activity often focused on natural events, over time a set of ethical beliefs emerged which were thought to accord with the wishes of deities or spirits.

Though we know little about the nature of the earliest African states, we do know that later African kings would claim godly descent. It is entirely possible that earlier kings also did so.

\section{India}

The Indus civilization seems to have had multiple gods, one a fertility god, some associated with flora or fauna. It is widely thought that these were absorbed into the Hindu pantheon, as there are definite similarities. The Indus religion appears to have involved ritual bathing, animism, and a fertility cult.

The earliest Hindu texts, the Vedas, likely date from at least 1500 все, but were written down only about 600 все. There is evidence from geographical references that the first and longest of these (over 1,200 hymns), the Rig-Veda, was drafted along the Indus. However, the earliest surviving texts are in Indo-European languages that were unknown in the Indus civilization; Indo-European peoples 


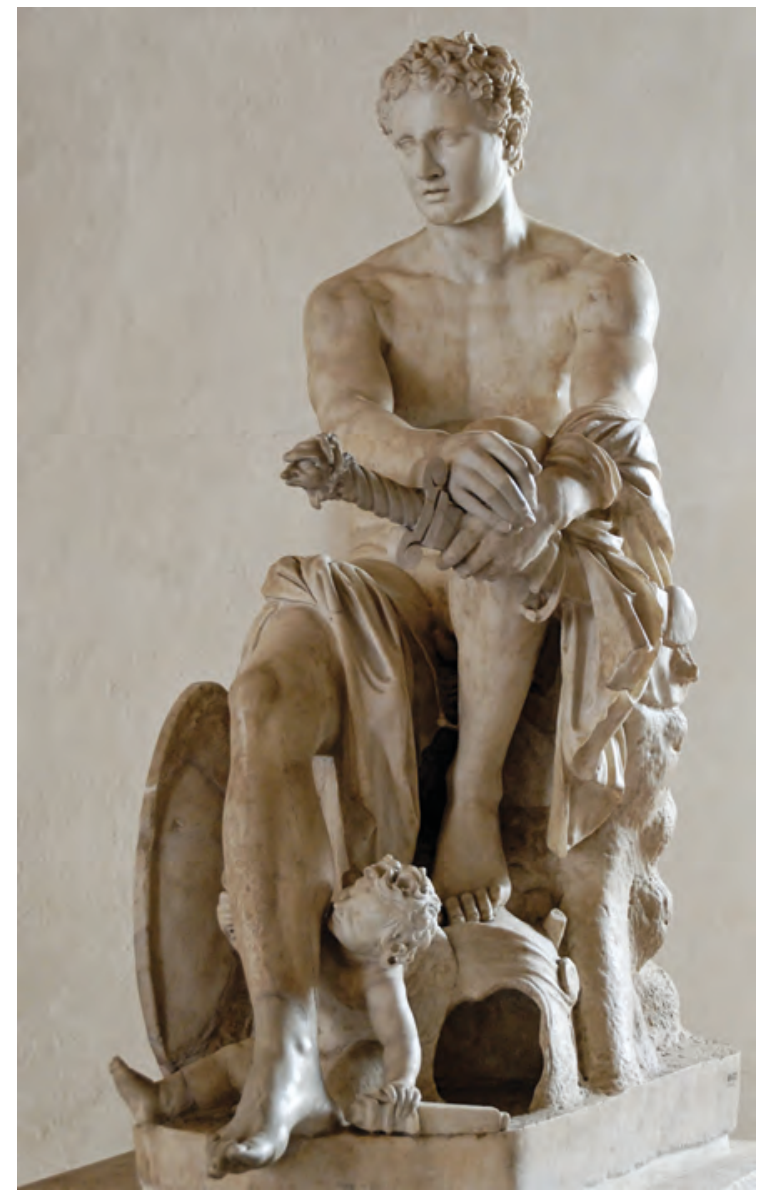

\section{Illustration 8.8}

The so-called "Ludovisi Ares." Pentelic marble, Roman copy after a Greek original from c.320 BCE. Some restorations in Cararra marble by Gianlorenzo Bernini, 1622, National Museum of Rome. Ares was the Greek god of war appear to have arrived after the Indus civilization declined. Later Vedas reference lands along the Ganges. The Vedas celebrate multiple gods. Indra, the war god, was particularly notable, with references to many battles among Indo-European tribes and with local Dravidians. Religion involved regular sacrifices to ensure success in herding or war: Priests could make five sacrifices per day on behalf of families.

Over time, there was a deep reflection on the nature of religion, especially among hermit sages who retreated to the forests from farming areas. Around 400 всE the Upanishads were written, generally in the form of dialogues involving questions to sages. These reflected on the Vedas but added new elements borrowed from Dravidian religious traditions. The Dravidians had long speculated on the possibility of reincarnation. The Upanishads developed the idea that individual souls separated from a collective soul to repeatedly inhabit earthly forms. One's behaviour in this life would affect the nature of one's next incarnation: This is the idea of karma. Individuals were therefore encouraged to behave ethically. They should also strive to free themselves from the pain and suffering associated with life by eventually severing themselves from earthly desires. Though the sages of the Upanishads were trying to understand the meaning of the universe, their ideas served to justify a castebased society.

Hinduism remains prominent today. The Upanishads are similar in significant ways to the holy texts of the missionary religions that will be the subject of chapter 11. We will revisit Hinduism in that chapter.

\section{China}

Early Chinese religious practices are different from other early religions in important respects. Although there were Chinese creation myths, these were 


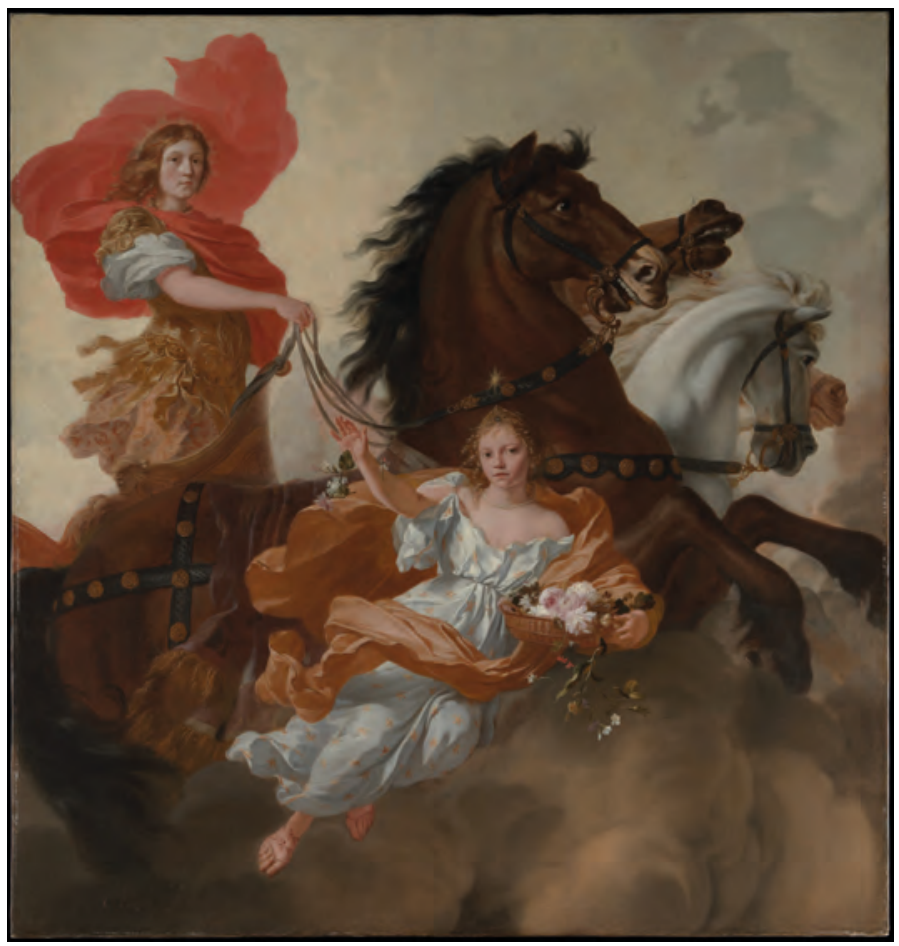

Illustration 8.9

Apollo and Aurora, Gerard de Lairesse, 1671. These were the Greek gods of the sun and dawn, Metropolitan Museum of Art

not organized into a coherent religion as elsewhere. There was some idea of a guiding deity, and that ancestors could intercede in human affairs. It is not clear when ancestor worship first emerges in China, but it seems always to have focused on male heads of families. Rulers would increasingly claim a divine "Mandate of Heaven" but without talking of a personal relationship with a particular god. There was not an extensive priestly class, though divination was commonly practised (chapter 6).

Chinese mythology celebrates three early rulers (and others, including model emperors), heroes with godly powers that variously invented hunting, agriculture, irrigation, fishing, and so on. We can note in particular the importance assigned to efforts to control floods. We might see these Chinese myths as recognition that humans had a role - but a limited one - in controlling natural forces. Perhaps these myths signalled the hope that human efforts to guide nature would receive support from ancestors or deities.

Early Chinese texts speak of a heaven and a god. The god, though anthropomorphic as in other religions, does not talk directly to humans but instead punishes these (and especially rulers) for bad behaviour with natural disasters or epidemics. Such a distant god likely set the stage for the impersonal god of Taoism (or indeed Buddhism when it arrives)(see chapter 11). 
Despite these many differences - lack of a coherent doctrine, limited priestly class, emphasis on ancestors, few myths of direct contact with gods - Chinese religion still provided some hope of affecting natural forces, justification of imperial rule, a sense of shared identity, and some sense of divine punishment for inappropriate behaviour. Different societies could apparently achieve these goals of early religions in entirely different ways.

\section{Mesoamerica}

Early Mesoamerican religions were also polytheistic, and involved a very close connection between religion and the state. Human sacrifice as a religious ritual was common in Mesoamerica from very early, maybe 30004000 все. Sacrifice was associated with pleas to the gods for the fertility of crops. We should stress that many societies practised human sacrifice at the time. In Ur, for example, it is clear that many were sacrificed to accompany royalty to the afterlife. More generally, elaborate elite burials signify religious approval of social inequality.

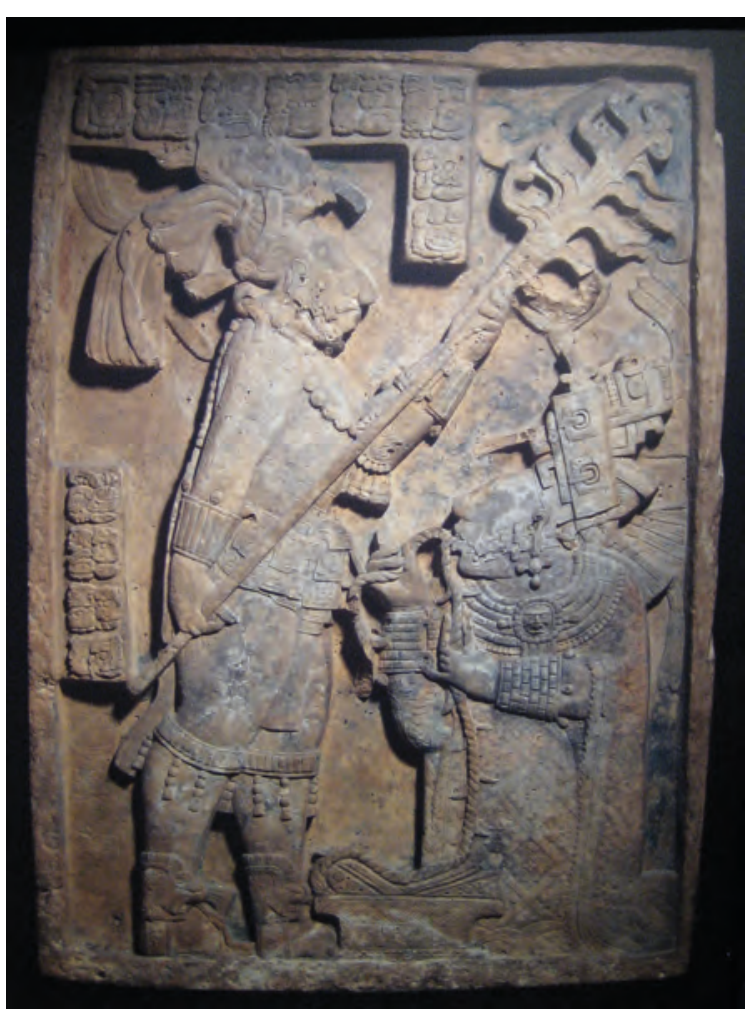

Illustration 8.10

Maya relief of royal bloodletting (Yaxchilan lintel 24), Mexico, about 600-900 CE. The Queen cuts her tongue Source: Photograph by BabelStone, CCO 1.0
The Maya also practised bloodletting, in which (sacred) kings and priests would cut themselves as a sacrifice to the gods. The Maya apparently believed that most people went to an underworld after death.

\section{Nomadic religions}

We know less about the religions of nomadic peoples. Such groups, given their small size and mobility, did not have elaborate priesthoods or religious edifices (though they built some shrines). It was likely common for such groups to have "shamans," individuals thought to have some ability to intercede on behalf of the group with gods or animistic spirits. These shamans might lead the group in rituals or engage in acts of divination. They would often enter a trance-like state to communicate with gods or spirits.

\section{The nature of ethics}

The earliest religions emphasized natural processes but came over time to justify 


\section{BOX 8.2 MAGIC AND RELIGION}

We can usefully distinguish shamanism from magic. Shamans appeal to the gods for their aid. While the group might criticize shamans if the gods do not provide, shamans are not expected to cause specific outcomes directly. Magic, on the other hand, does promise to achieve particular results. For example, magicians may promise that through certain spells they can move spirits to act in specific ways. (Such magic is of course entirely distinct from magic that explicitly employs sleight-of-hand techniques to achieve surprising outcomes; this other type of magic occasionally pretends to invoke spirits.) Magic of this type is widely observed in human societies, though less so in the modern era. For the most part, world religions have promised interaction with gods or the cosmos rather than direct control of these. Formal religions are usually hostile to magical practices such as casting the "evil eye" on others or employing voodoo techniques against others. It is shamans rather than magicians who are the precursors of priests, for both claim to intercede with god(s) or divine beings.

political and social stratification. All religions came to grapple with questions of mortality; tombs of one sort or another are often among the more visible physical remains of these religions. We also begin to see a religious concern with ethical behaviour during the ancient period. The Egyptian cult of Osiris captures the essential elements: the idea that gods will judge us on our behaviour in this life, and the suggestion that there are certain ethical precepts according to which they will judge us. Hinduism, especially with the Upanishads, also develops the idea of a reward for certain ethical behaviours.

We can reasonably hypothesize that as urbanization proceeds, people need increasingly to grapple with ethical considerations. Small hunter-gatherer or agricultural communities can self-regulate behaviour: The community can punish in a variety of ways individuals who gain a reputation for deceit or selfishness. However, in urban societies, one necessarily interacts with large numbers of people but only intermittently. It is much harder to know the character of each person one meets. It becomes increasingly valuable for societies to introduce both laws and cultural norms that encourage particular behaviours and discourage others. Religions can be particularly important here for they can provide an incentive to behave ethically even when no other human can witness or at least expose the behaviour.

The second generation of religions to which our attention turns in chapter 11 was even more concerned with ethics than the first generation of religions surveyed above. We will find in chapter 10 that ethical considerations also loomed large in philosophical conversations operating outside of organized religions (though influenced by these). We shall see that over the next thousands of years theologians and philosophers would interact in identifying commonalities and interactions among different conceptions of ethical 
behaviour. It is therefore useful at this point in our book to identify how ethical practices are both conceived and advocated.

We saw in chapter 3 that humans evolved to have some altruistic impulses. However, we are also both selfish and capable of self-deception. The challenge of ethics, then, is to encourage humans both to emphasize their altruistic impulses and not to deceive themselves into thinking that selfish acts are socially beneficial. We should stress the last point: Most of the evil in the world is perpetrated not by self-consciously evil people but by people who rationalize unethical behaviour.

Religions would draw on each of the five types of ethical analysis in Box 8.3, though different religions would have different emphases. Organized religions were each in effect trying to instantiate a new tradition among their followers. The fact that particular religions dominated particular localities, and were associated with group identity, meant that any ethical guidelines promulgated by the religion could set a powerful example for members of the group.

\section{BOX 8.3}

\section{THE FIVE TYPES OF ETHICAL ANALYSIS}

If people are to be encouraged to act ethically, they must know how to distinguish ethical from unethical acts. The better they can do so, the harder it will be to rationalize unethical actions. How in practice can people determine whether an act is ethical? It turns out that there are five broad ways in which humans can make such decisions, and that these five ways are all used - but with different emphases - across human societies. It is useful to review these briefly.

- Intuition. Our altruistic impulses guide us to feel good when we help others and bad when we hurt others. We can circumvent our natural inclinations through self-deception, but cannot erase them. Psychologists refer to people who cannot feel guilty as sociopathic; they are (fortunately) a small minority of human populations.

- Tradition. We often take our cues from the society in which we operate and judge acts to be unethical if our society disapproves of them. Much of this will occur subconsciously: We will absorb certain attitudes and apply them ourselves. The philosopher Socrates, as we shall see in chapter 10, would get in trouble for encouraging his students to interrogate received traditions. Recall from chapter 1 that cultural attitudes are selected in part - but only in part - to enhance the well-being of the society: We can then imagine that some but not all of these traditional attitudes will have good effects.

- Consequences. If we consciously ask ourselves if an act is ethical, we will often contemplate whether it has good effects. Stealing from another will almost certainly make them worse off, and so we will have to be particularly good at rationalization to convince ourselves that theft is justified. However, it is not always easy to calculate the likely effects of our actions, and we may not always have the time to do so. 
- Rules. It may, therefore, be advantageous to have some rules that guide ethical behaviour. The Golden Rule - treat others as you would like to be treated - is found in many times and places in human history. We will discuss appeals to various rights in later chapters: These also provide rules for ethical behaviour: Theft violates the right to property; murder violates the right to life. A variety of other rules is possible. Note that if we have formal rules to follow, it will be much harder to rationalize acts that clearly break these rules.

- Virtues. Rather than rules, one could stress values or virtues such as courage or honesty or responsibility. One could then decide that honest acts are ethical. As with rules, it may be tough to rationalize actions that clearly do not accord with the virtues we wish to pursue:You cannot self-identify as honest and then casually lie. Yet as we shall see in chapter 10, Aristotle would advocate a "Golden Mean" concerning virtues: One could be too courageous and thus foolhardy. This opens up considerable scope for us to rationalize unethical acts.

Philosophers tend to focus on the last three of these types of ethical analysis, for these have been the subject of formal philosophical theorizing across human societies over the last millennia. Individual philosophers tend to stress the advantages of one of these. Nevertheless, there is a fair degree of consensus that each of these five approaches has some merit. Moreover, they are interrelated: For example, we may follow specific rules precisely because we lack time to do detailed consequential calculations. Importantly, it is our intuition that can harness our selfish instincts to the pursuit of ethics for we all wish to avoid feeling guilty.

These traditions involved sets of rules or virtues that the religion sanctioned. Although religions would differ in whether they stressed rules or values, there is a considerable similarity in the rules or values recommended across religions. While we may associate early religions mostly with their advice to obey the ruler and priesthood, there was also an insistence on responsibility and honesty (both useful for societal cohesion in general but also likely to make the task of tax collection easier). This set of ethical precepts would expand in later religions.

Religions also could and did appeal to intuition. They wanted the believer to be infused with faith, and so guided intuitively toward good acts. If only guided consciously, the individual might misbehave when nobody was looking. Religions thus sought to blend subconscious and conscious: The person would want to be good and would know how to identify the good. In appealing to intuition, religions could appeal to our innate altruism. Some religions arguably appealed also to our capacity for self-deception, to believe things that are convenient for us.

Religions were less likely to exhort believers to perform consequential analysis. However, religious stories would often make implicit consequential arguments: These people acted ethically and achieved excellent results; these 
other people acted unethically and produced terrible results. One critical way in which religion could differ here from secular philosophy is that god(s) could play a key role in imposing unfortunate consequences on those who behaved unethically. Religions that embraced an afterlife could then hold out hope for godly justice - god would reward those who acted ethically - even if this lifetime seemed plagued by injustice. Religions could then bind selfish and altruistic motives: That which was altruistically beneficial in the short run would turn out to be selfishly helpful in the longer term.

Why be ethical? Secular philosophers have trouble with that one. They can in the end appeal to our intuition: We will feel bad if we misbehave. Religions could make the same point. However, they could also stress the advantages of pleasing an all-seeing god.

\section{Reflections on religion and community}

Rulers used armies to maintain their power. Yet it can be prohibitively costly to control a population through force alone (even in the twenty-first century). Rulers wished to encourage some sense of loyalty: to both the ruler as a person and the state as an entity. Individuals also had an incentive toward group identity: They needed protection from both theft and life's misfortunes, and so could benefit from a group that provided mutual support, especially if group members were urged to behave well toward each other.

Religions could play a crucial role in both enforcing group solidarity and justifying kingly rule. The gods in some way could sanction the king: This occurred almost everywhere. The king could intercede with the gods on behalf of the people: This makes the king appear useful by performing simple rituals but risks suspicion of the king in the event of adverse harvests or other natural disasters. A special connection between a god and a group of people reinforces group identity. It also encourages group members to behave as the gods wish (and perhaps allows priests to lay some of the blame for natural disasters on the group rather than the ruler). As religions came to focus more on ethics, rulers were urged to behave justly and benevolently - but their special relationship with god meant that they could exercise such virtues while enjoying power and wealth far above the average level.

As noted in chapter 6, victory in war is more likely if soldiers are devoted to the cause. And war, we appreciated, is a particularly intense selection environment. States whose citizens were motivated by religious fervour would be victorious much of the time. There would then be selection for religions that reinforced group identity and justified kingly rule. Such religions will necessarily encourage some hostility toward other groups. Nevertheless, the very best religion would be one that allowed conquered lands to be absorbed religiously.

We know in the twenty-first century that religion is not essential to group cohesion or respect for political authorities. However, the institutions and cultural beliefs on which modern group solidarity rests may not have been 
immediately available to early societies. Religion could simultaneously address some fundamental human concerns while allowing groups of people to behave collaboratively. Religion was thus a robust support to the earliest states.

\section{Religion and the family}

We noted above that the earliest religions were likely animist. Early humans infused the animals and plants on which their lives depended with religious significance. Another universal element of human existence is the family. It should not be surprising, then, that gods often fulfilled the role of mother or father, and polytheistic religions often described in detail the family relations among gods: Egyptian, Greek, and Mayan gods among others had explicit familial relationships. Moreover, one's human ancestors were often attributed some capacity to look after their descendants from the next world as they once had in this one.

Though we do not know the details of the earliest human religions, it is entirely possible that goddesses loomed at least as large as gods. The fertility symbols that dot the early human landscape mostly celebrate the female body. We saw above that goddesses remained significant in the earliest days of settled agriculture. It seems entirely possible that gods became more important than goddesses as gender relations evolved with settled agriculture (chapter 4). By the time of monotheism (see chapter 11), then, the one god was usually characterized as male. This vision reflected power relations on earth, perhaps especially among those who created these religions. Yet of course, such religious attitudes could, in turn, reinforce male power.

Families are critical in all human societies because human children are helpless for so many years. By celebrating families, religions shaped and encouraged strong family bonds that would support careful childrearing. Religions encouraged faithfulness - especially among women: This would serve to increase fatherly confidence that they were raising their own children.

It is noteworthy and surprising, then, that religion played little role in marriage ceremonies in these early societies. Marriage was usually a civil ceremony. Religious marriage ceremonies would be a standard feature of the next generation of religions. The idea of infusing marriage with religious significance was apparently a mutation that was selected for as religions took on an increased role in encouraging ethical behaviour. Opportunities for infidelity likely increased with urbanization and trade, which may have prompted a greater societal emphasis on the responsibilities associated with childrearing.

\section{Sacrifices and buildings}

One common religious attitude is a belief that god or gods want sacrifices. Even today, peoples around the world light candles, leave flowers or food, or make 
personal sacrifices (such as fasting) in the hopes that they will please a god or gods by the sacrifice. In earlier religions, these sacrifices were often much more significant in scope. Archaeologists have uncovered vast treasure troves of gold, silver, and ornate goods of many types buried under the temples of many ancient faiths: in China, Mesoamerica, Mesopotamia, Nubia, Greece, and elsewhere. The burials of more impoverished people also often contained simple tools that they might use in the next life. Many faiths also ritually sacrificed animals and even humans. We can speculate that if humans had dispassionately compared the fate of humans or human groups that sacrificed against control groups that did not they might have found little difference in their experience of rainfall or natural disaster. Humans, of course, did no such thing. They credited abundant rain to previous sacrifices and interpreted drought as a sign that the gods wanted more. We must recall that these early societies were poor by our standards. These sacrifices were not at all trivial. Yet humans entirely dependent on natural forces that they could not comprehend or predict - found the sacrifice justified by the resultant feeling that they could, in fact, exercise some influence over their fate.

Along with sacrifices, everywhere that they settled, humans built structures in which or with which to celebrate their gods. These were often the most elegant structures of their times - matched perhaps in some cases by edifices that celebrated state power. While sacrifices that are burned or buried had

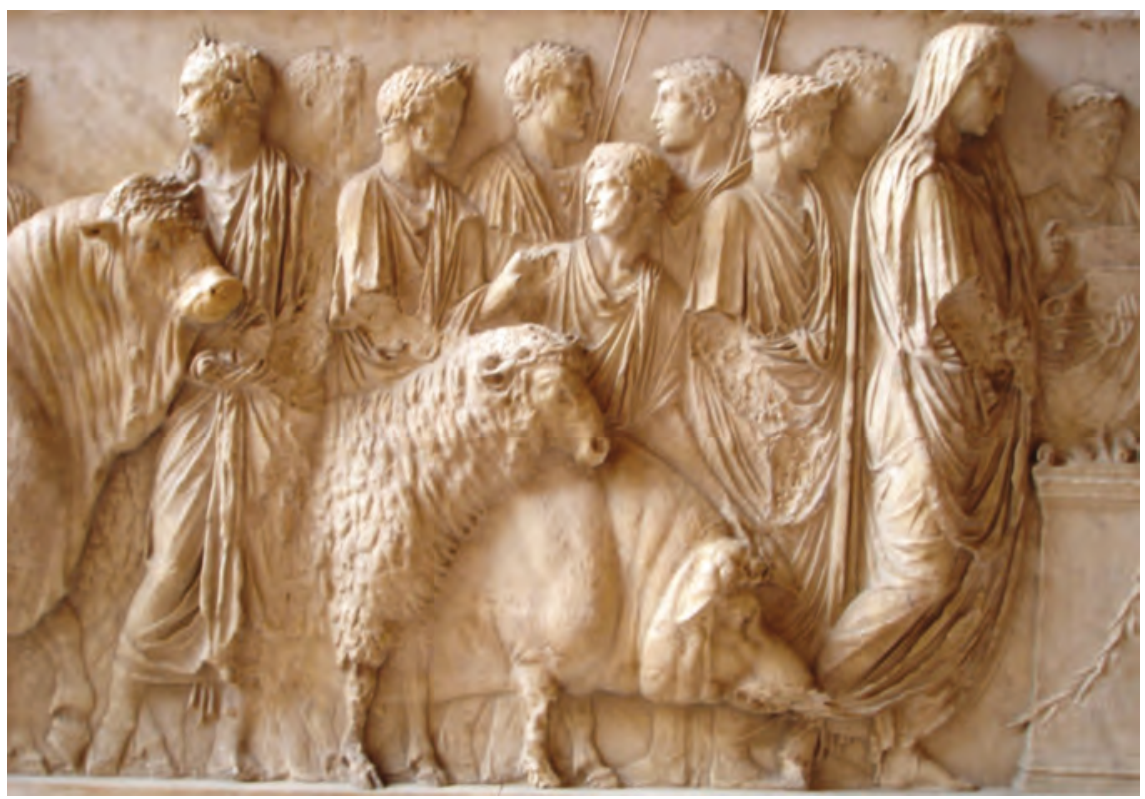

Illustration 8.11

The suovetaurilia was one of the most sacred rites of Roman religion, involving the sacrifice of a pig, sheep, and bull, Louvre. The name of the sacrifice embraces the names of the three animals 
but a transitory impact (at least until unearthed and placed in museums for us to enjoy), these structures often lasted for centuries or more. Indeed we still marvel at the Egyptian pyramids (which were both religious and state monuments) thousands of years later. We can well imagine that such structures signalled to people of the time the awesome power of their religion. Many of them - pyramids, ziggurats in Mesopotamia, the mounds of North America - were built high in order (we imagine) to signal an approach to the gods. It is less clear whether the average person thought them to be worth the trouble and expense of construction.

Religions also generally had shrines, places of particular religious significance, because it was felt that gods, spirits, or humans of particular religious importance had acted there and infused the place with special meaning. Such shrines might also be places of impressive architecture. Yet many shrines were more humble. Africa, for example, was dotted with small shrines to local spirits. Even natural sites could be revered: This practice was especially prevalent among the indigenous peoples of North America.

\section{Art}

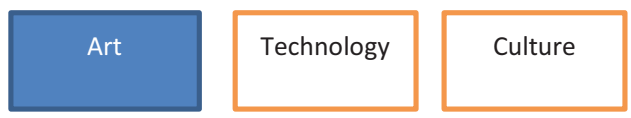

Works of art became highly developed in the period after about $4000 \mathrm{BCE}$, both technically and aesthetically. Many of the works of art produced in this time period are still highly valued today (though much Mesopotamian art appears

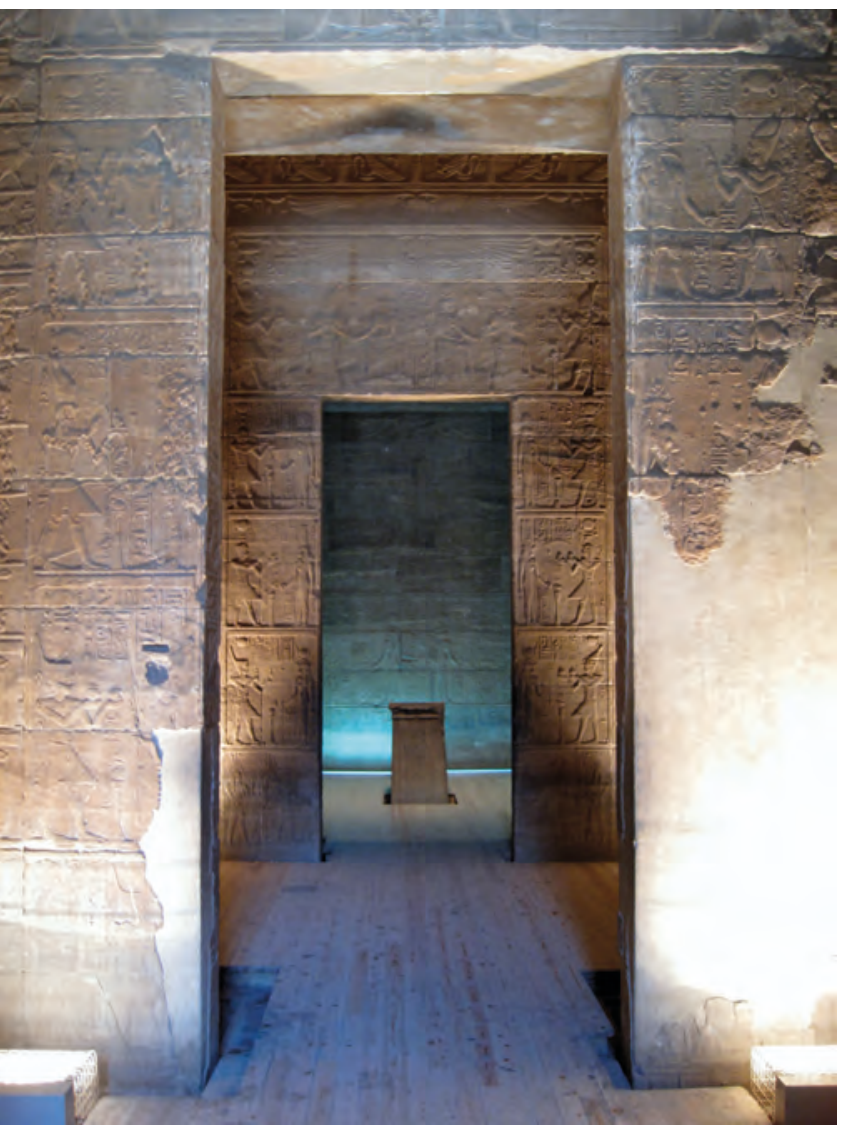

Illustration 8.12

Temple of Isis, Philae, The "Holy of Holies" with richly decorated walls leading to a square obelisk; 4th century BCE (but the entire temple was moved due to flooding after the Aswan Dam was built in the 20th century)

Source: Photograph by Olaf Tausch, CC BY 3.0 


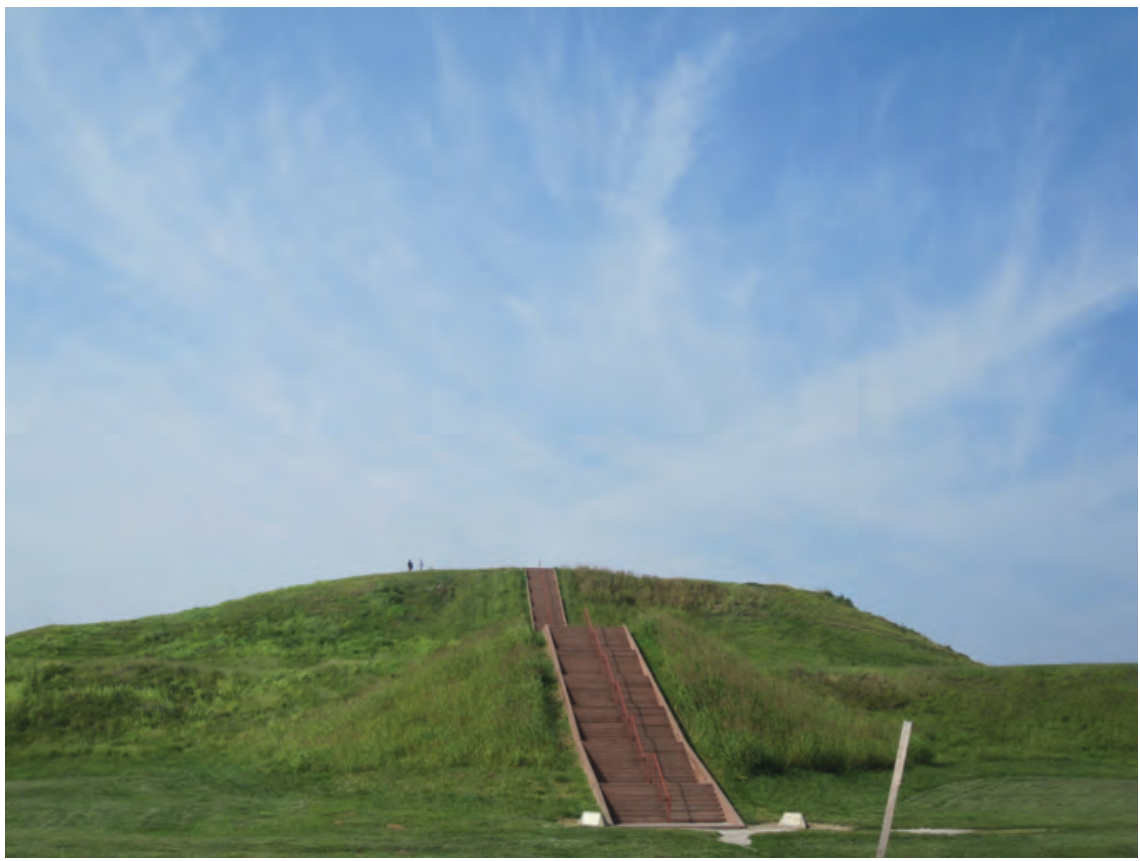

Illustration 8.13

Monk's Mound, Cahokia (Illinois). Cahokia was settled from about $600 \mathrm{CE}$

strange to modern eyes). This is testament both to the skill of the artists and to the general aesthetic appreciation that guides art and art appreciation. Yet art differed dramatically across human societies at the time, testifying to the importance of cultural influences on art, and to the cultural diversity of the time. There were also differences in materials: Though clay and then metals were widely employed, artists also used shells, coral, amber, jade, and other precious stones where available. Much of the art of the time blended utility and aesthetics: furniture or pottery for example. Nevertheless, there were also paintings and figurines that served only an aesthetic (and cultural) function.

We saw in chapter 6 that Catalhoyuk in Anatolia was among the earliest cities in the world. Archaeologists have uncovered large numbers of small female figurines there. There were also many large sculptures and paintings, though these tended, with some exceptions, to focus on men in activities such as hunting. Animals were still relevant in art, but would gradually be replaced by humans and symbols of agriculture in later cities. Catalhoyuk also produced impressive textiles and pottery.

We noted in chapter 3 that art could serve an educative purpose. As states grew in size, these everywhere sponsored art that celebrated the state and its rulers. The sheer height of Egyptian pyramids or Mesopotamian ziggurats awed the populace and served to symbolize the power of the state (and religion). Artists sculpted and painted rulers and state officials in ways that advertised 
their power and often their relationship with gods. In Egypt, there were sculptures of pharaohs, their wives, and senior bureaucrats.

Egyptian paintings are perhaps most familiar: These often take a "cartoon" format of panels in rows depicting important events or celebrations. This format may have served to symbolize political order. Artists pursued a recognizable Egyptian style throughout the millennia of ancient Egyptian history. Though Egyptian painters were capable of painting portraits from the front, the dominant fashion placed figures in profile - though the torso alone tended to be painted from the front. Artists integrated hieroglyphs into these paintings. Egyptian art often celebrated mastery of nature - reflective perhaps of cultural expectations that the pharaoh would control the all-important Nile flood. The importance of agriculture was signalled both by including many plants in the paintings and by the widespread use of rectangles that reflected the shape of fields. Egyptian art also often depicted an idealized afterlife.

Mesopotamian art was often religious in focus. Mesopotamian paintings, unlike those in Egypt, generally captured an important event in one frame; they may be the first paintings in the world to include background elements. Egypt and the Indus both influenced Mesopotamian art but this had little influence in return. Yet the ruler of the Hittites in Anatolia in about 1275 BCE wrote to the ruler of Babylon requesting that he lend him another sculptor. There has been less art found in the Levant than elsewhere; it reflected both Egyptian and Mesopotamian influences.

Archaeologists have discovered many small and beautiful figurines of animals and fertile women in the Indus - in copper, bronze, and especially terra cotta (hardened clay; see below) - but there is a notable absence of the massive public artworks of Mesopotamia and Egypt. The Indus civilization is also noteworthy for highly decorated seals. The Indus - and Mesopotamia - emphasized bulls in its artwork.

The advances in metalwork discussed in chapter 5 facilitated artistic expression (as well as the productive and military purposes emphasized in that chapter). Shang China would be particularly famed for works in bronze. Yet Chinese artisans also produced elaborate ceramics and jade carvings. Jade was thought to be a spiritual stone that communicated with the gods and was carved from the fourth millennium BCE.

Greek art celebrated the human form. Mycenae became famous for vases. These often pursued military themes, and are still noteworthy for their depiction of moving figures. Greeks borrowed the idea of large sculptures from the Egyptians after establishing a trading colony there in the seventh century всE. Greek statues of naked men (from the fifth century вСE) and women (only from the fourth century BCE) are celebrated to this day for their realism, beauty, and depiction of emotion. This humanistic approach to art would exert an influence over time across Eurasia. Greek sculptors developed an ideal human form with well-defined proportions among body parts; they often used more than one model for a particular sculpture. 


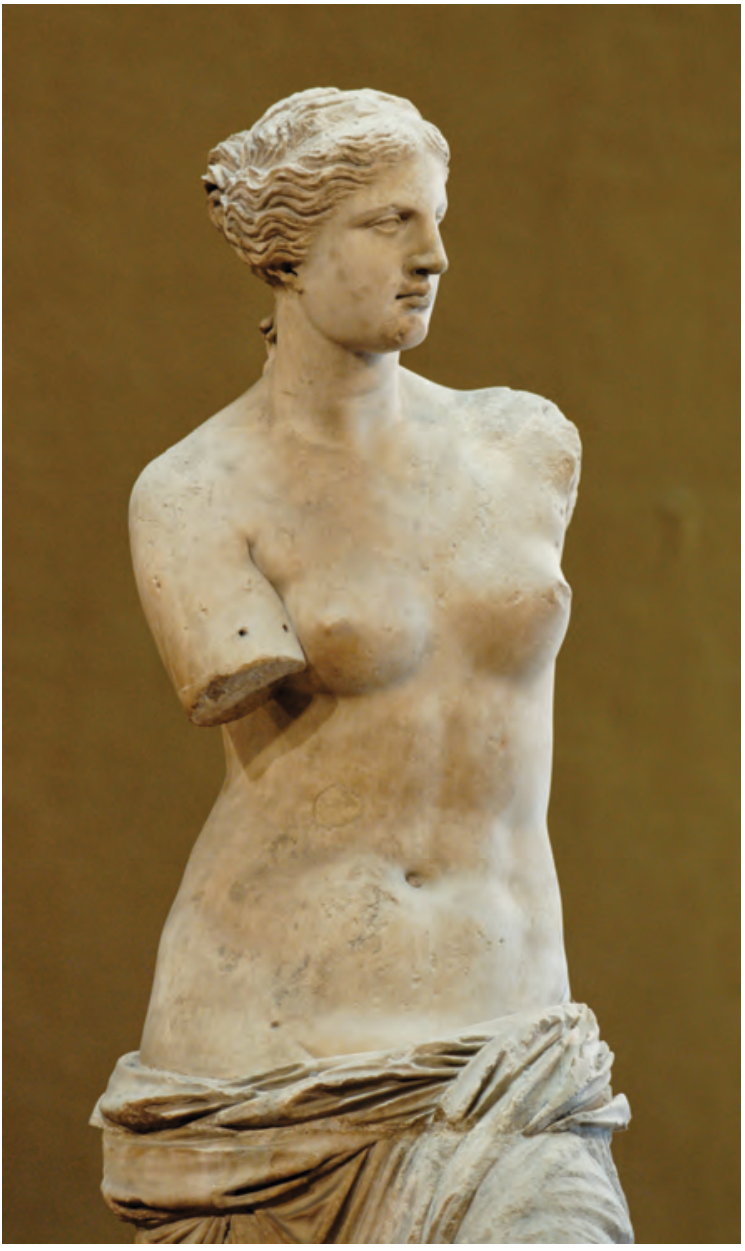

Illustration 8.14

Venus de Milo, Louvre
Elaborate pottery is also found in the Americas from at least $3000 \mathrm{BCE}$, as well as stone carvings and intricate textiles. The Olmecs produced jade chisels or axes often carved with jaguar figures. The Mayans depicted gods (which often combined animal and human elements), rulers, and sacrificial victims, as well as jaguars and birds. Notably, the Mayans, like the Egyptians, portrayed individual torsos frontally but legs and arms in profile. The sculptures of the Nok in Nigeria (800-200 BCE) already evince the oversized heads and geometrically shaped body parts that would characterize much later African sculpture. Terracotta sculptures were common in Africa. Elaborately carved bronze kettledrums were produced in what is now North Vietnam from at least the sixth century всE; the carvings provide evidence both of social stratification and of trade with New Guinea, for these carvings commonly feature bird-ofparadise feathers.

It seems, then, that all human societies produce sophisticated works of art once there is an agricultural surplus to support both a class of artists and an elite audience for their work. Nor is art limited only to agrarian societies: Recall from chapter 5 that decorated pottery was typical in many non-agricultural societies.

The nomadic Scythians (1000-200 вСе) produced and carried some remarkable animal figurines in both metal and stone.

We discussed in chapter 6 how the technology associated with architecture evolved through time. Several critical technological advances also facilitated the art of sculpture (there were also improvements in paints and painting surfaces). Each in its way allowed a sculptor to first work with a malleable substance such as clay but then replicate the sculpture in a much harder material. From the third millennium BCE in Mesopotamia, sculptors knew that one could coat a clay sculpture in wax, create a mould, and then drain the wax and replace it with molten bronze. Sculptors would employ this method for bronze figures throughout the eastern hemisphere. Alternatively, clay itself might be hardened to form what are termed terracotta sculptures: dried clay that is porous. The most famous terracotta sculptures are the terracotta warriors of the Qin dynasty 
(chapter 10), but terracotta sculpture is observed from the third millennium $\mathrm{BCE}$ in the Indus Valley and elsewhere. What, though, of large sculptures in stone? From the fifth century BCE in Greece, geometric methods were developed by which the outlines of a clay sculpture could be replicated on a block of stone so that workers would know how deep to drill or chisel in a particular location. The actual sculptor might then leave most of the work between fashioning the clay model and making the final adjustments in stone to less skilled workers.

It seems that Mayan rulers, and some

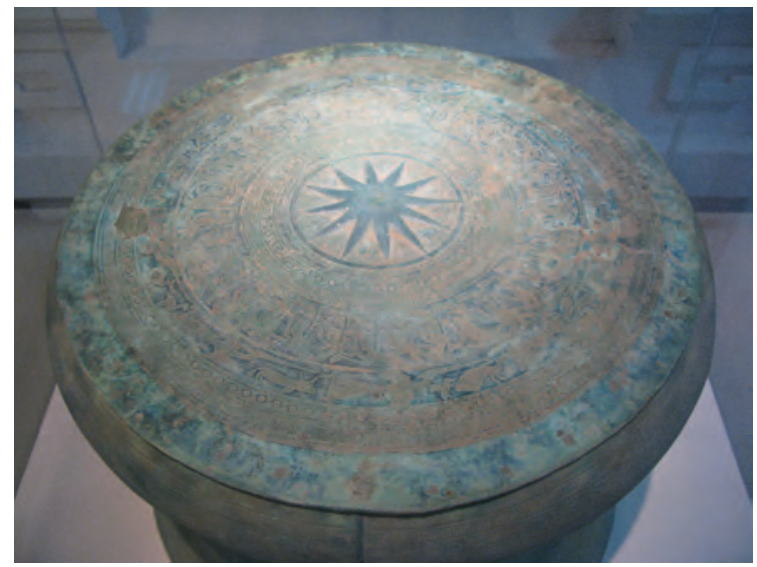

\section{Illustration 8.15}

Bronze drum, last centuries BCE, Vietnam other ancient rulers, were active producers of works of art. This should remind us not to assume that a specialized artistic community always produced art. People in positions of authority in many societies may have been expected to create art. Elsewhere, art may have been seen as a natural part of life in which all would participate.

\section{BOX 8.4}

\section{THE PURPOSE OF ARTISTIC ILUUSTRATION}

Many of the illustrations in this book are of works of art and architecture. Art thus comprises a far greater proportion of illustrations than of text in this book. This is a common characteristic of texts in world history. It reflects in part the simple fact that we can best appreciate art by looking at it rather than just describing it. Nevertheless, we can usefully appreciate several motives for including these many artistic illustrations.

First, they are - mostly, at least - aesthetically pleasing. They (hopefully) make the reading experience more satisfying while signifying in an important and moving fashion the common humanity of diverse human societies. We see in the totality of these illustrations both shared aesthetic tastes and universal human urges such as the desire of rulers to exalt their importance.

Nevertheless, works of art necessarily express the culture of the creator along with general aesthetic tastes. Art may also reflect local political imperatives, economic realities, and social structure, not to mention technological capability. We can hope to learn something of a society, then, by examining its art. Some of these linkages are obvious: The pyramids signal both the power and economic capability of the Egyptian state. Yet (as noted below) we are at an early stage of appreciating the role that art has played in human societies. Through looking at works of art, readers may well gain subconscious insights that no scholar has 
articulated consciously. Moreover, if readers can appreciate the art of diverse societies, they may indirectly achieve some respect for and even emotional attachment to those societies.

The economic and social implications of art deserve particular attention. Malthusian analysis (chapter 4) guides us to expect that most humans through most of history lived close to subsistence. Works of art, and especially works of architecture, remind us that there was nevertheless a substantial surplus generated in many times and places. This surplus was most likely to be applied to art if there were huge inequalities in income and power. While we may wince at the sacrifices made - how willingly we can usually only guess - by poor peasants to support massive palaces or temples, we can also see in these efforts some of the majesty of human history. Humans have always looked beyond food and shelter toward art, and in these works of art we can detect some element of the human spirit that resonates with us to this day.

We guide the reader, then, first to enjoy and marvel at the works of art in this book (and in the museums of the world). We ask the reader to remark on how humans on all continents have produced across thousands of years works that we admire today. Moreover, the reader is encouraged to appreciate both the similarities and differences across works of art.

We remind the reader that works of art before the modern era were intended for palaces and temples and public spaces rather than museums. Many art historians worry that works of art cannot fully be appreciated in museums: They were intended to be part of life, and were often designed with particular surroundings or activities in mind. Art historians also worry that the typical visitor to an art gallery spends less than a minute per work of art, and thus hardly has time to reflect on any work they view.

A final note: We will occasionally employ works of art produced in one period but that portray events of an earlier period. Such works of art may communicate something important about both time periods. They also tell us something important about historical sensibility: Humans have often looked to the past for inspiration.

\section{THE THEME OF ART}

We interact with each other and the non-human environment to create items desired primarily for their aesthetic appeal rather than their utility. We call this activity "art," and find artistic expression across all types of human society. Scholars often view art as a subset of "culture"; we treat it separately in this book because works of art, while they contain cultural elements, are defined in terms of an aesthetic effect that transcends cultural boundaries. How else could we today appreciate Ming vases, Incan jewellery, and ancient Greek sculpture? We cannot identify precisely what makes specific objects aesthetically appealing but can recognize works that were appreciated aesthetically at the time of their creation and also still today. Note that this definition of "art" embraces both the "fine art" that has traditionally been the focus of art historians and the "crafts" that have more often been studied by anthropologists. 
There is, to be sure, a scholarly debate regarding the degree of shared aesthetic taste. We can note, though, that the emerging field of "world art" or "world art history," which pursues a global approach to the study of art history, tends to presume or argue for a universal aesthetic taste.

We will often speak of works of architecture because many impressive architectural sites have survived into the modern day. Likewise, we can often treat paintings and sculptures. We should appreciate that many works of art in wood or textile format have not survived through the centuries. Many works of drama or literature have similarly disappeared, though we will often reference those that have survived. We very rarely know much about dance or even music in distant times.

We will often discuss the various influences on art through history. There has been much less analysis of the impacts of art on polity or other phenomena. States often try to encourage certain sorts of art, but there is scepticism that such efforts - for example in the Soviet Union - had the desired effect of encouraging support for particular political institutions. Nevertheless, the ubiquity of art in human societies from the cave paintings of early hominids suggests that art plays some vital role in our lives. Art likely serves an educational purpose - it exposes us to the lives of others and to nature. It may, as a result, encourage desirable behaviour. Art has an emotional appeal, surely, and perhaps then a cathartic effect in allowing us to transcend emotional tumult. Art may promote a sense of group belonging if art depicts important figures or events or if a group venerates particular art objects, or the group produces art collectively. Art can also accentuate status divisions within a group by celebrating these or just because only the rich or powerful will possess the most impressive art. Art - architecture most obviously, but even smaller works of art - can indicate the "specialness" of particular places or of particular rituals (much as "using the good china" can signal the importance of certain family celebrations). Note in this respect that the art we admire in museums was usually intended to play a role in the world, not just to be observed passively. Perhaps the most significant effect of art on individuals lies in exalting the best of the human spirit.

One influence deserves emphasis. We have separated economic activities from artistic activities in our categorization scheme. However, in practice, the boundary is quite fuzzy. Some works are intended entirely for artistic appreciation: Paintings and sculptures have little practical usage. However, pottery and textiles often serve both economic and aesthetic functions. Most items of utility - our clothes and buildings and furniture and plates and cutlery and bridges - are intended to be pleasing as well as useful. Very few things - usually those used out of sight - are designed entirely for usefulness. Humans could serve their economic needs much more easily if not for this common desire for aesthetic enjoyment. It still makes sense to distinguish items designed primarily for use from pieces designed mainly to be enjoyed aesthetically (while appreciating that pottery may serve both ends equally), but also to recognize that aesthetic motives influence economic activity and economic motives often affect artistic activity. In our evaluation of levels of income across societies, one useful question to ask is the degree to which individuals could satisfy their aesthetic taste in items of utility: People with lovely pottery are wealthier than people with plain pottery, even if they can eat equally well off each. 
We have noted that we will be limited in our ability to describe the effects of art. We will also be limited in our ability to draw comparisons across societies or sketch the influences of art in one society on art in another. The vast bulk of scholarship in art history has focused on tracing developments within one region of the world. In recent decades, art historians have increasingly appreciated the importance of comparison and cross-cultural influence, but the challenge of drawing comparisons and outlining channels of influence is in its early stages (notably in fields of "world art" and "world art history"). We cannot, then, integrate art into world history as we might like - but we will strive to identify certain vital developments and cross-cultural influences when this is possible.

Many scholars of "world art" stress how art appeals to our common humanity. They have found that it is hard to pursue comparison of artworks across time and place without being struck by similarities in how these appeal to human emotions. It is much easier when studying the works of only one small region of the world to focus on how art reflects culture. (And some art historians who just study one region have then been suspicious of the synthesis of art forms from different regions, whereas we in this book tend to look favourably on the interchange of artistic ideas across regions.) Yet there is scope for both approaches in art scholarship, for any work will reflect both our common humanity and the cultural (and economic and political and social) milieu in which the artist operates. We as students of world history can likewise value works of art for both their aesthetic and cultural elements. We may not be able to understand fully the lives of the artists that created them - we may often not even know who they were - but they can touch us both emotionally and intellectually by their attempts to grapple with their lives and find meaning in these. In doing so, we catch glimpses of our common humanity.

\section{Artistic evolution}

Each artist strives to produce unique works. They may do so due to a desire to express themselves or due to a strategic desire to differentiate themselves from other artists (or a mix of both). Nevertheless, the works they see around them influence artists. Moreover, the patrons for whom the artist works, or to whom the artist hopes to sell their art, will be influenced by these other works in choosing what they like. Art is a mostly subconscious process, both for the artist and audience. To be sure, the artist consciously thinks about what they are doing, and the audience consciously thinks about what they like, but the value of art seems to lie primarily in subconscious communication between artist and audience. Art then becomes perhaps the easiest social phenomenon to which we can apply evolutionary analysis: Individual artists will each try to innovate, often without really thinking about this, and audiences will choose works they like. As with biological evolution, small mutations are much likelier to succeed than large. The audience will be accustomed to a particular style and will usually find dramatic deviations from this jarring. 


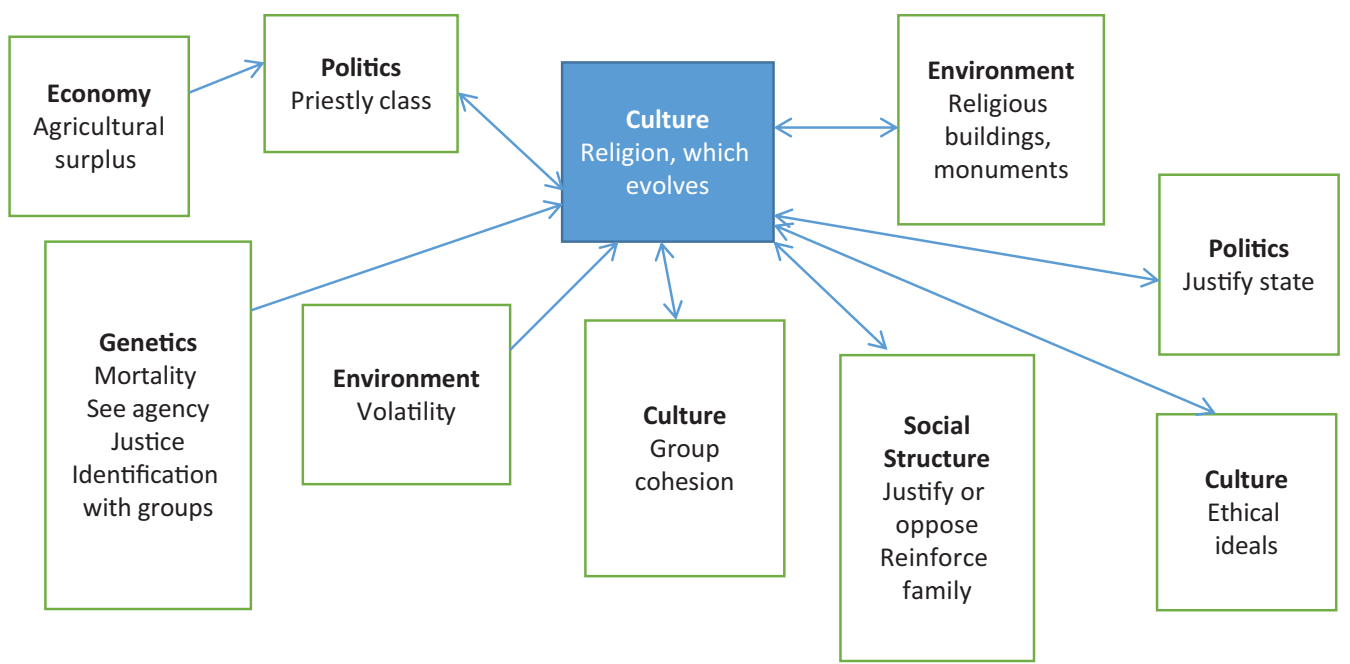

Figure 8.1

Influences on and effects of the evolution of religion

Note: Some effects, such as buildings and ethics, are not associated with the very earliest religions. We will explore further impacts of religion in later chapters.

The contemporary art world in rich developed countries is historically unusual. There is a variety of accepted styles for all types of art: painting, sculpture, music, dance, theatre, and so on. This reality likely reflects the size of the contemporary art market (which in turn reflects economic prosperity): It is so large that a variety of styles can each have an audience. Some of these audiences may value innovation and so be unusually open to dramatic change. Yet the general rule is still that small mutations are more likely to succeed than large.

In past societies, there was usually one dominant art style. This evolved through time as artists strove to distinguish themselves from others, and the audience appreciated some works more than others. Note that all societies distinguish good art from bad art (and often see the producers of good art as divinely inspired). Art historians have in the past devoted considerable effort to analysing individual artists and the innovations that these produced. Art history in more recent decades has dedicated more attention to what we would term the selection environment: How has art reflected the cultural or economic reality of its times? An evolutionary approach guides us to appreciate both types of analysis - but to understand that artists will have an imperfect understanding of the selection environment that determines their success. If the selection environment changes slowly, then artists operating within a particular style may gradually produce works that better satisfy their audiences, and perhaps better achieve aesthetic expression as well.

What is art? We have distinguished art from culture because of a belief accepted by most but not quite all who study art - that art appeals to basic aesthetic tastes in human beings. The evidence for this conjecture is that we 
can and do appreciate works of art from societies far different in both time and space. We may struggle to place ourselves in the lives of Roman peasants, medieval merchants, or fourteenth-century Japanese Buddhists but can marvel at the Venus de Milo or Mona Lisa or Golden Pavilion (Kinkaku-ji in Kyoto). It could be that the cultures of those times and places were less different from ours than we might imagine, but it seems more likely that art appeals somehow to something in human nature that transcends culture. The artworks of any time and place, then, may be selected in part just because they appeal to basic human nature.

While art appeals to human nature, it can also simultaneously reflect and support elements of the culture, polity, economy, and society of its time and place. Indeed, the very fact that art moves us creates the possibility that art may move us in particular directions. Given the subconscious nature of art, cultural influences on both artist and audience are likely. Both may celebrate cultural values and behaviours. Culture in turn, as we have seen, is influenced by economic, political, and social circumstances - and likely by art itself. A direct "economic" influence comes from the fact that those with atypically high incomes finance most art through most of history. It could be that art then reflects their values more than those of the ordinary people. Art might then act to justify their authority. An even more direct political influence is often observed: Rulers, recognizing (or at least assuming) the impact of art, encourage certain types of art and often prohibit other types. Such efforts are not always successful - for example, communist government efforts to influence styles of painting are widely disdained, at least by art critics.

Some works of art survive for thousands of years, many more for centuries. Transmission then is direct: The same work is viewed over an extended period and continues to influence artists and artistic appreciation. New works of art build on those that went before, generally in a prolonged evolutionary process. Transmission here occurs with previous works influencing both artists and audience in setting their tastes.

Transmission can occur across societies if artworks move across societies. Indeed, the cross-cultural merging of art styles is an important source of artistic mutation in history. In the contemporary world, where diverse styles exist within one society, these styles often borrow elements from each other. In all cases, such borrowings are only selected if they are not too shocking to the audience. If a society is impressed by the artworks of another, which will often depend on their attitudes toward the society in question, then efforts to merge elements of the domestic and foreign style are likely to be successful.

\section{Questions}

1. Did states "create" the earliest religions?

2. Why is sacrifice such an important part of early religions?

3. Do you favour a particular type of ethical analysis? Why? 
4. Do contemporary religious structures inspire awe as ancient structures did?

5. Why is it important to include art in world history? What challenges do we face?

\section{Readings}

Brancaccio, Pia, and Xinru Liu. 2009. "Dionysus and Drama in the Buddhist Art of Gandhara." Journal of Global History 4:2, 219-244. [Explores the Greek influence on Indian art.]

Carriere, David. 2008. A World Art History and Its Objects. Pennsylvania University Press. [Discusses why art history should take a world history perspective, and provides compelling examples of what this might look like.]

Johnson, Dominic. 2015. God Is Watching You: How the Fear of God Makes Us Human. Oxford University Press.

Kaufman, Thomas Dacosta. 2015. "Reflections on World Art History" in Thomas Kaufman, Catherine Dossin, and Beatrice Joyeux-Prunel, eds., Circulations in the Global History of Art. New York: Routledge. [The first half of the article defends the project of a world art history from various critiques that it is possible to escape one's own cultural or other perspective etc. He then suggests what some elements of such a world art history might be.]

McNeill, William H., et al. 2012. Art in World History. Great Barrington, MA: Berkshire. [The introduction provides a brief but good overview of the place of art in world history. The other chapters are area-specific encyclopaedia articles.]

MacGregor, Neil. 2010. A History of the World in 100 Objects. London: Penguin Books. [Discusses how objects from the British Museum illustrate and inform world history.]

Norenzayan, Ara. 2013. Big Gods: How Religion Transformed Cooperation and Conflict. Princeton University Press.

Slingerland, Edward. 2015. "Big Gods, Historical Explanation, and the Value of Integrating the History of Religion into the Broader Academy." Religion 45:4, 585-602. DOI: 10.1080/0048721X.2015.1073487 [Review of Ara Norenzayan's Big Gods.]

Super, John C., and Briane K. Turley. 2006. Religion in World History The Persistence of Imperial Communion. New York: Routledge.

Zijlmans, Kitty, and Wilfried Van Damme. 2012. "World Art Studies," in Matthew Rampley, Thierry Lenain, and Hubert Locher, eds., Art History and Visual Studies in Europe: Transnational Discourses and National Frameworks. Leiden: Brill, 21729. [Suggests three broad themes should motivate the field: when, how, and why art first emerged; cross-cultural comparisons; and causes and effects of cross-cultural influences.]

Volume 7, Issue 2 (2017) of World Art explores the relationship between aesthetics and ethics.

There was a Forum on "Art and World History" in World History Connected, October 2019. One article recommends asking students to analyse the purpose and meaning of particular works of art. 


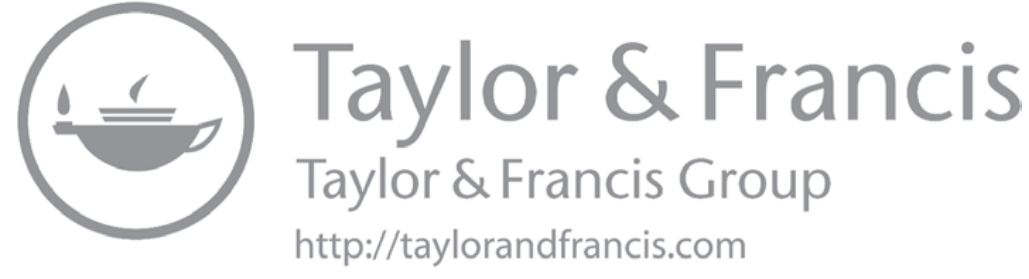


Classical history 


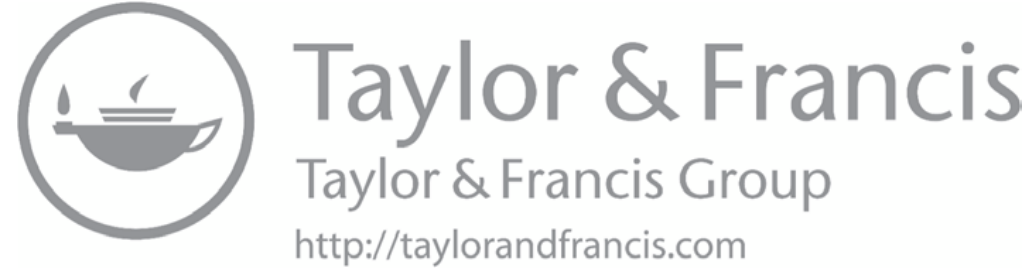




\section{Political organization on an unprecedented scale}

\section{The classical empires}

\section{Guiding questions}

What are empires? How, when, where, and why did empires first emerge in the world? What are the key similarities and differences across early empires? What were the main effects of empires?

How did empires change through time?

Who governed empires and how did emperors and bureaucrats and citizens interact?

Why was slavery so common? How did slaves and masters interact?

How, where, and why did trade expand during this period?

What were attitudes toward science and politics and how did these differ (and interact) across societies?

Relationship to other chapters: Empires represent a dramatic expansion in the size of states (and cities) discussed in chapter 6. They reflected - and reinforced - advances in trade and technology addressed in chapter 5 and military technology and organization in chapter 6 . They would encourage, interact with, and eventually adopt the new religions developed at about the same time (chapter 11). Empires would be the dominant form of political organization through most of human history (though other types of political organization always existed), and as a 
result, empires play a role in most later chapters. We will see that there are important commonalities in how empires operate. We must also appreciate the power relationship inherent in empire, which will prove to be a critical source of imperial tension and decline throughout history. Our discussions of particular empires in this chapter not only clarify our understanding of empire in general but also inform later discussions of regions such as Persia and Central Asia. Our extended analysis of slavery in this chapter will inform many later discussions of slavery.

Empires tended to encourage an expansion in trade:We shall see in many later chapters that information and goods both flowed more freely among societies during periods of imperial expansion. The considerable increase in long-distance trade during this "classical" period builds upon practices identified in chapter 5, but with advances in ships and currency and finance and the development of new routes; these set the stage for further reductions in the cost of trade in later chapters.

We have not often mentioned science previously, and most kinds of science will have limited impact for many centuries to come (though astronomy and mathematics were useful). Our discussion of science is nevertheless important both in better appreciating how people viewed the world at the time and in later appreciating critical changes in both scientific theory and practice (especially in chapter 22).

We saw in chapter 6 that the size of early states expanded through time. Yet the largest states were still small by modern standards. Egypt may have had 3 million people. Zhou China was an outlier with several million people. From about 600 BCE, much more extensive states would emerge in various parts of the world. These are usually termed empires. The first of these was in Persia, which we discuss below. The most dramatic cases were the Roman and Han Chinese Empires that at their peak may have had 60 million people each and jointly governed half of the world's population: We devote chapter 10 to a comparison of those two behemoths. In India and (to a lesser extent) the Americas and Africa, larger empires than ever seen before were also characteristic of at least some of this period. We will discuss these in turn below as well. In the cases of Persia and India, we can also examine how these empires interacted with other empires.

These several empires were, of course, different in many ways, but also had similarities. They each necessarily struggled with the challenges of administering 
a far-flung empire. They each nevertheless sought - for centuries - to expand that territory. They each had three primary sources of income: booty from conquest, taxes on agricultural output, and taxes on trade (and to a lesser extent industry). They each encouraged - and taxed - trade both within and beyond their borders. They each built roads and ports to facilitate both administration and trade. We therefore see an expansion in trade flows with empire. Each empire needed much larger bureaucracies than ever before, and each developed educational systems to train bureaucrats.

We discussed in Chapter 1 how we would not invest particular time periods with profound significance. We employ the term "classical" in this chapter and the next for the sake of convenience. It is a phrase commonly applied to this period: Indeed, many universities have Departments of Classics that study this particular time period (though often only with a focus on Europe). So for us, "classical" simply means "the period from about the fifth century BCE to the fifth century CE." We can note that this period is famous precisely because large empires encompassed most of the world's population for the first time. Nevertheless, the word "classical" need imply no judgement about the value or importance of these empires.

Thematically our focus in this chapter is politics, but we have already seen a vital role for economy and built environment. We will often discuss the relationships between polities and will compare different empires in both this chapter and the next. We shall see that all empires were ethnically diverse; we must thus also engage aspects of social structure and culture. All empires sought to justify themselves culturally. We close the chapter with a discussion of science.
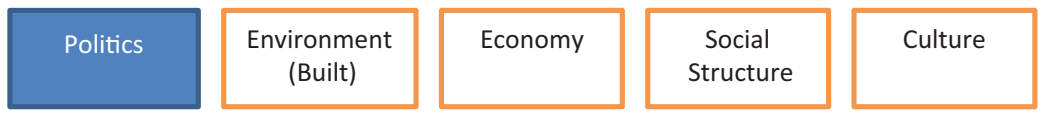

Science

\section{From earlier large states to larger empires}

If history were simple, we might have expected the large states discussed in chapters 6 and 7 to grow over time into the more extensive empires that we will consider here. We observe such a gradual and steady process of transition nowhere. Instead, the large states of chapters 6 and 7 either fractured internally or succumbed to foreign invasion. In China, the collapse of the Zhou state led to a centuries-long period of struggle between smaller states commonly referred to as the Warring States period. It is out of these smaller states that the Qin and Han empires would eventually emerge. The Greeks of Alexander and later the Persians would overrun Mesopotamia (see below); Mesopotamian states may (or not) have declined in wealth and power over the centuries preceding these conquests. The Greeks and then the Romans would conquer the great Egyptian kingdom. The Indus civilization disappears around 1000 BCE for unknown reasons (chapter 7); many inhabitants likely migrated to the Ganges 
River area where the Mauryan Empire would later emerge. We should stress that the trajectories observed between the earlier era of large states and the later era of empires are quite different across regions. It is thus remarkable that we will find so many similarities across these new empires.

It will prove common in history for empires to be created by societies that had been on the geographical fringes (or beyond) of previous large states or empires. Some have even suggested that there is a reason for this: Such societies are close enough to learn the arts of empire but distant enough to achieve military or administrative innovations that allow their success. In any case, each of the empires we discuss in this chapter or the next did rise outside the centres of earlier large states. Both the Qin and Han arise on the western fringes of Chinese "civilization." Rome is a small town far to the west of the centres of Greek society when it begins its rise to power. Mesopotamian states had dominated the Iranian plateau through most of its history, and the Persians were subjects of Mesopotamian rulers before rising to power. The relationship between the Indus Valley civilization and the people of the Ganges River is less well known, but all later Indian empires would concentrate on or near the Ganges rather than the Indus.

\section{The nature of empires}

We must first grapple here with the meaning of the word "empire." Scholars define this in multiple ways. However, these definitions usually include two elements: Empires are large, and the imperial state exercises power over diverse ethnic groups. Scholars tend to agree that the states discussed in previous chapters did not qualify: They were all too small, and many had a degree of ethnic unity - though we noted at the time that what appears to be ethnic homogeneity to our eyes might well have resulted from political consolidation. We will in this and later chapters confront states that are many times larger than the states dealt with previously - the Roman Empire at its height would control most of western Europe and North Africa. All told, there are about sixty empires in world history that comprised more than a million square kilometres (too many, indeed, for us to cover them all in this book).

Since empires are by definition ethnically diverse, they lend themselves to a third characteristic: Lacking borders defined by ethnicity, empires tend to expand in size over a period of decades or often centuries. Empires necessarily emerge and grow through the conquest of space and peoples. Most empires cease to grow before they fall, but celebrate the era of expansion and usually retain the idea that they might yet expand some more.

What do we mean by "exercises power"? We will limit our use of "empire" to direct political administration of conquered territories. We will therefore not speak later of an American Empire (beyond territories such as Puerto Rico and the Philippines, which were directly controlled), though we can and will examine other ways in which the United States exercised power (and thus 
look for ways in which it may have acted in a manner similar to empires). As outlined in chapter 1 , we seek precise and straightforward definitions of terms in this book. We can also note that empires can choose to accept tribute from another state rather than conquering and administrating it. We will record such tribute relationships when they occur, though they fall outside our narrow definition of empire. We stress yet again that empire is not the only way that a powerful state may exercise its power.

Empires exist through the exercise of force. Empires emerge through conquest - though many weaker states surrendered rather than face defeat in the face of a more substantial foe. Empires maintain large armies to both protect borders and repress rebellions. Empires also do useful things such as support trade and suppress crime, and they will devote considerable resources to providing justifications for their rule. In strictly economic terms, people were often better off inside empires than outside. Many citizens of empire may indeed have welcomed imperial government. Yet these positive characteristics of empire, vital though they were, should not blind us to the fact that empires always and everywhere relied on brute force, and usually proved ready to crush dissent violently.

Empires, we must stress, were not all-powerful, though their rulers liked to pretend so. The symbols of power developed over time by rulers can easily seduce students of history into attributing much greater authority to emperors than these ever exercised. The size of empires guaranteed that - even if the emperor's tax take per person was small - the empire could construct monuments to its glory both in the capital and throughout its territories. Empires could afford rich pageants, and emperors could dazzle visitors (intentionally) with the luxury of their palaces. Emperors could usually exercise absolute power over individual citizens. Nevertheless, these appearances of power and glory masked a reality of constant negotiation with local elites who also exerted considerable influence within all empires. We will devote much of our attention to the problems rulers faced in dealing with not just local elites but their own advisers and bureaucrats. These interactions would shape much of world history. Rulers could not treat too many of their citizens arbitrarily without risking revolt; they would instead regularly ally with some elites against others to ensure that no local elite became too powerful. In addition to the challenges of maintaining internal order, most empires had to cope with threats of external attack.

The ethnic diversity of empires encouraged political diversity. Empires governed different regions - and different ethnic groups - in different ways. These practices are so standard that some would include this diversity in political treatment as one of the defining characteristics of empire. Though empires treated different ethnic groups differently, they nevertheless sought to bind them together in a shared appreciation of empire: The resulting interactions among ethnic groups are one of the most important characteristics of empires.

Having defined empires and noted key similarities, we should also identify a host of ways in which empires differed: administrative practices, the involvement of members of diverse ethnic or social groups in positions of power, the 


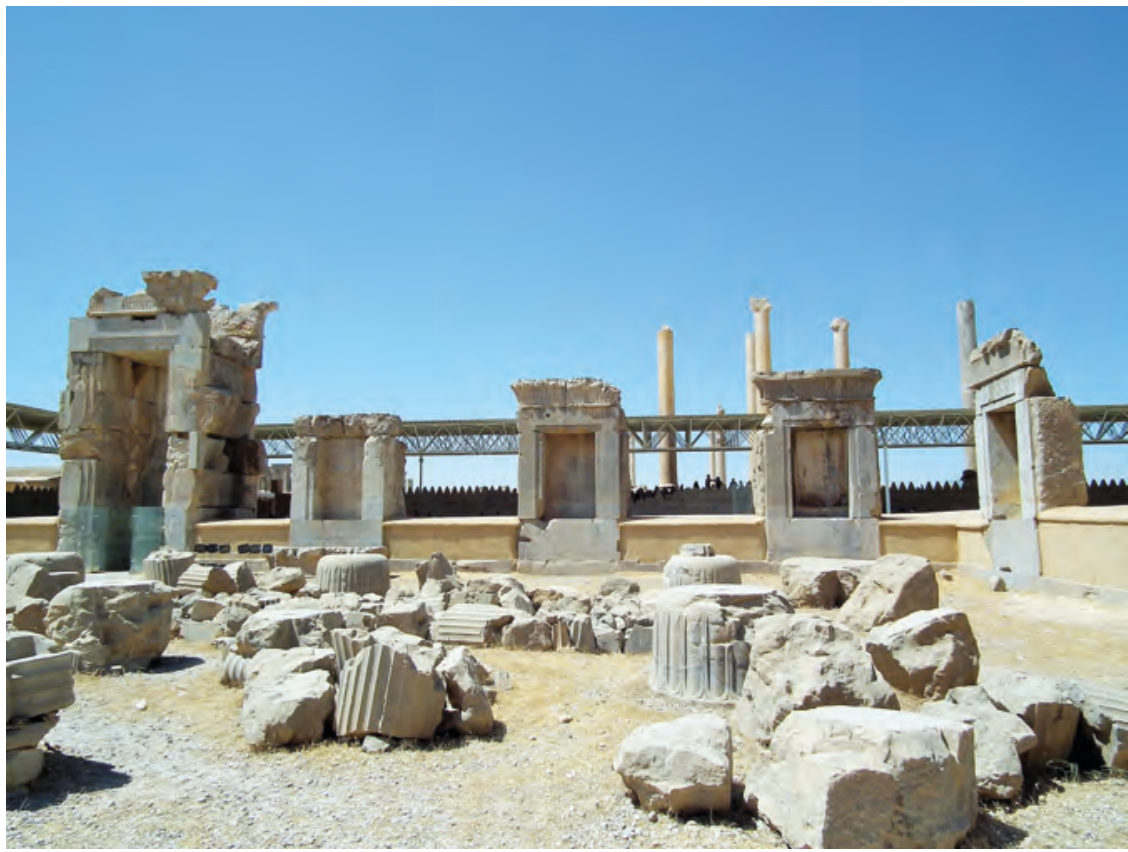

Illustration 9.1

Ruins of Persepolis, early capital of the Persian Empire

degree and type of ideological justification for empire, institutions governing trade, and so on. We should also stress the historical nature of empires. Empires were agents of history in the decisions they made to attack certain regions, raise certain taxes, build roads, proclaim laws, support or attack religions, and so on. Yet they also had to respond to historical circumstances beyond their control such as harvest failures, technological innovations, and attacks by others.

\section{Imperial internal diversity and justification}

Most empires sought to create a shared identity. Rome (eventually) granted conquered peoples the right of Roman citizenship, absorbed their gods into the Roman pantheon, and spread architectural and other ideas throughout the realm. Other empires were much more willing to embrace diversity, recognizing the different languages and religions of different provinces: As long as conquered peoples were loyal, the emperor was satisfied. Loyalty need not require a sense of shared cultural identity if the conquered people saw advantages in being members of the empire: protection from attack, competent administration, and opportunities to trade. Still, empires usually made some effort to draw at least local elites into some sense of imperial purpose. And conquered people often found it advantageous to adopt the language or religion or attire of the conquerors (the incentives to do so likely increased over 
the millennia as imperial bureaucracies and trade opportunities grew). Empires always inspired some degree of cultural consolidation, though local identities might remain very strong.

We must be careful of assuming that subject ethnicities were as horrified by empire as were most inhabitants of India or Africa during twentieth-century drives for decolonization (chapter 30). In the millennia before the idea of the nation state became commonplace (chapter 21), subject groups might find imperial rule unsurprising. That is, we can too easily take the contemporary nation state as "natural," and forget that most humans through most of history lived under empires in a world where rule over diverse subject groups was commonplace. We can also forget that modern conceptions of the nation state have often led to savage mistreatment of minorities. Though empires often also mistreated those they conquered, over the longer term they sought to reach an understanding with at least most subject groups. We shall also find in later chapters that ethnic identity tended to be more fluid in the past than it is today. Subject peoples might decide to identify with their conqueror. Nevertheless, when outsiders attacked empires, local peoples often aided the attackers - this indicates that it was force rather than love of empire that had kept them from rebelling earlier.

Even in recognizing diversity there could be differences: Some empires thought that subject peoples were inferior in some way (a standard view in the European empires of the nineteenth century) while others avoided such denigration. The Romans and Chinese tended to reserve pejorative terms such as "barbarian" for those beyond their borders. The Romans, indeed, went to some length to celebrate their "Asian" heritage and their extensive imperial holdings in Asia.

All empires sought to justify their rule. This in part may have reflected selfish psychological motives on the part of rulers: We all want to believe that we somehow deserve whatever power and wealth we receive. Yet these justifications also played a strategic role: The individuals within an empire were more likely to acquiesce in the exercise of imperial power if they believed that there was some useful purpose in the state. A large group can only share a sense of identity or loyalty if they share some common beliefs. One common though not universal form of justification was religious: Emperors could claim that they ruled in accordance with a preferred religion and perhaps even with godly approval. Such a strategy could strengthen the state - but it also raised the possibility that the religious might object to the behaviours of some empires or emperors. A claim that a ruler interceded with god or gods on behalf of the people was typical and a particularly potent form of justification, but carried the risk of revolt in the face of harvest failures or natural disaster. As we shall see in chapter 11, the decisions of particular empires to favour specific religions exerted a powerful influence upon the history of religion. We can note here that particular religions then influenced the behaviour and success of rulers.

Empires could also point to whatever services the state provided (see below). They could exalt the history of the state. They could work to ensure that the 
population had enough to eat. They could provide occasional celebrations that would impress and entertain the people. However, empires had to finance these services and ceremonies, and the state always faced a conflict between the desire/need to extract tax revenue from the population and the wish not to anger the people into rebellion.

We should stress that all historical empires devoted significant resources though usually a fraction of the sums spent on the military - to public rituals that celebrated the state and ruler. The Persians, for example, held a lavish annual or semi-annual celebration at the imperial palace which would be attended by thousands from throughout the empire and which involved banquets and music and a variety of entertainments: Both the palace and the pageantry awed visitors. Though we cannot entirely discount selfish psychological motives, the common experience of expensive ritual suggests that these did in fact "buy" the state some level of public support, especially from elites who might play a supporting role in or

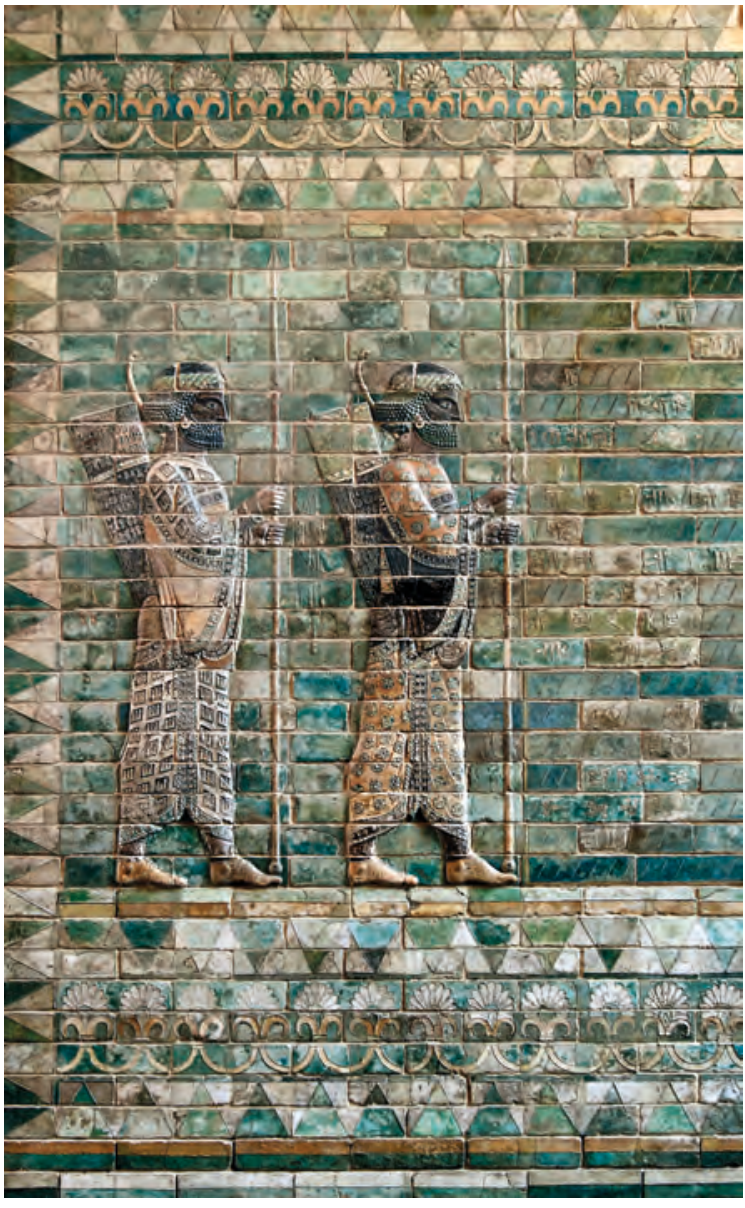

Illustration 9.2

Part of frieze showing archers, Persian emperor Darius's palace at Susa at least have privileged access to public ritual. This may have been particularly important for empires lacking the bureaucratic capacity to provide services of practical import (beyond an army) to their populations.

Emperors tended almost everywhere to claim universal authority. Their rulers were the "king of kings." The gods wished them to govern all of humanity in the interests of peace and prosperity. Even the Roman Empire - which as we shall see shunned the use of terms such as emperor or king, at least in Rome itself - still assumed a destiny of global domination. Such a claim justified - to themselves and others - continued conquest, though all long-lasting empires would cease to expand at some point. For the Chinese Empire, separated by desert and mountains from other large empires (until the rise of the Mongols in the thirteenth century; chapter 17), this idea of universal supremacy would remain influential well into the modern era. Empires in Africa and the Americas could also at times reasonably celebrate being the only empire around. From Europe through India, however, empires often bordered other empires. Hostility between Rome 
and Persia and India in this period (and much later the Ottomans, Safavids of Persia, and Mughals of India; chapter 20) was enhanced because each offended the other's claim to universal authority.

As just noted, empires differed in the competition they faced. The Chinese Empire through much of its history faced no other empire near its borders, though nomadic tribes were often a military threat. Rome also had periods where no large powers were threatening it. The type of military threat an empire faced - both when conquering and in defending its borders - conditioned both its military technology and organization and its approach to administering the realm. An empire facing few threats might become complacent and then be easier to attack should a new threat emerge.

\section{Functions of empire}

We discussed the nature of states in chapter 6. It is useful here to remind ourselves of what early states did. Most centrally, they provided protection. Once humans settled in one place and invested time and effort in producing crops that must be stored, they became targets for theft. The state provided protection both from small-scale robbery and from large-scale attacks. We can view this benignly as a necessary service for which otherwise defenceless peasants might willingly surrender their freedom. Less benignly, we must appreciate that the state is the foremost purveyor of force in the peasant's neighbourhood: The peasant often most needs protection from the actors in the state itself. We might then view the state as a protection racket.

Beyond providing the services of an army, the state might provide other services. It might build roads or ports or dredge rivers to improve navigability. We can indeed celebrate all empires of this period, albeit to varying degrees, for transport improvements, and for the increased trade that resulted from the combination of better infrastructure, unified political authority over trade routes, and reduction in banditry that empire afforded. Even the most impoverished peasant might enjoy a more varied diet or slightly better building materials because of the ease of trade. In many cases, the state built and maintained irrigation works. In such cases, the peasantry may have depended entirely on state success for their livelihood. Empires usually built public monuments, and these were often associated with public celebrations. The Roman population was hardly alone in swarming into places such as the Coliseum to watch gladiators battle animals and even to admire the re-enactment of sea battles (the arena of the Coliseum could be flooded). The primary purpose of other monuments may have been to inspire awe among the population. Royal palaces served a public function but also provided for personal enjoyment for the emperor's family and retainers.

Empires also proclaimed laws. Given the size and diversity of their populations, empires often codified these laws more carefully than had earlier states. Yet empires usually also respected diverse local legal traditions. 
Nevertheless, the existence of an empire-wide legal framework could facilitate trade and other interactions between regions of the empire. The biblical tale of Paul of Tarsus is instructive here: When he ran into difficulty with Jewish courts, he claimed his right as a Roman citizen to be tried by Roman courts. Though he lost there too, it is clear that he saw value in his ability to appeal to Roman law and judges.

\section{The expansion of empires}

How do empires expand? They expand by conquering neighbouring people. Though we can celebrate the advantages for trade of large states - merchants need not worry about crossing borders and can appeal to one legal authority in any dispute - we can be sceptical that neighbouring peoples often voluntarily submitted to a more extensive authority (except when surrendering to a dominant army). Empires gained in two ways from conquest. In the short term, there were the prizes of war: Winners took goods and even slaves from those they defeated. Over the longer term, there were tax revenues that the empire hoped would exceed the cost of administering the conquered territory. It may well be that the immediate gains from conquest often loomed larger than the long-term benefits in the calculations of potential conquerors. Empires might then conquer lands that they could not govern: Alexander the Great (chapter 10) and Chinggis Khan (chapter 17) are just two examples of rulers who conquered far more territory than their successors could manage to govern as one state.

The boundaries of some but not all contemporary nation states are broadly accepted: These states comprise either an ethnic group or a multi-ethnic community that recognizes a collective identity. Since empires comprised multiple ethnicities, the borders of empires were always subject to change. Any emperor might desire new conquests, or face rebellions. Most empires expanded through much of their history, but many declined in size for some time before collapsing. Empires differed in the degree to which they had "natural" boundaries of open water or mountains or deserts.

Once a territory was conquered, the state had conflicting motivations. On the one hand, it wished to extract resources from the conquered territory. On the other, it wanted to discourage rebellion. So one fundamental question was how much could the empire extract without inciting rebellion. Beyond this political calculation, there was a less obvious economic calculation. Empires can only raise taxes if there is economic activity to tax. However, citizens fearful that the state will take their stuff - in either taxes or outright confiscation may be wary of investing or trading in the first place. The wise state restrained its exploitative instincts enough to encourage economic activity. The state sacrificed what it could extract today to stimulate greater activity in future. However, states were always looking for funds (for wars are very expensive, as are transport and irrigation infrastructure, not to mention monuments), and so might sometimes be tempted to confiscate today. 
Empires must exercise control over their territories. This means being able to communicate with local government officials. Also, it means being able to deploy armies to quell any rebellions. In the days of early states, when communication was slow, and the state had a limited advantage in military technology over its peoples, states had difficulty governing over distances of more than a couple of hundred kilometres. By the nineteenth century, with steamships and telegraphs, and the advantages that gunpowder provided militarily, European empires could for decades control territories on the other side of the globe. During the period of classical empires, their realms could extend thousands of kilometres.

An empire might then prefer to exact tribute from a weaker neighbour on distant borders rather than go to the trouble of conquering and administering it. We must take care here, though, for sometimes what appear to be tributary relationships were something entirely different. The empire might also give gifts to a tributary state. If the empire feared the tributary state, these gifts might exceed the value of the tribute. The empire, rather than the tributary, was then trying to buy peace. We shall see that such a strategy often failed in the end.

This book provides maps of various empires. Students can thus gain a basic appreciation of where a particular empire operated. Nevertheless, students should appreciate that maps give a misleading impression of empires, for the empire's power varied from region to region. Bureaucrats may wield direct power in the capital but rely almost entirely on local elites in distant provinces. In borderlands, empires often relied on potential enemies for security. Empires might enforce their laws in the capital but default to local laws in the provinces.

\section{Administration of empires}

There was a further significant complication for the state: ensuring that government officials acted in the interests of the state. The bureaucracies of empires were necessarily much larger than those of earlier states, and so the ruler's challenge of encouraging bureaucrats to act in the ruler's interest (see chapter 6) was greatly exacerbated. This was a problem even in the capital, but the problem increased as the empire expanded. Corruption among public officials is not unknown in the modern world but was much harder to limit in the classical world. Bureaucrats tended then to have much greater flexibility and as a result more opportunity to act on their own behalf. Bureaucrats in the provinces might anger locals through excessive taxation; the ruler might be dimly aware at best of what was transpiring. We will see how particular empires struggled to put a variety of mechanisms in place to encourage bureaucrats to act in the interest of the empire rather than themselves. They could, for example, send inspectors or spies, or set up governance structures in which different officials kept an eye on each other. All empires punished officials that betrayed the state (if they could catch them) and rewarded others for, say, enhancing state 
revenues. Yet even rewards and penalties could only serve their purpose if the ruler first knew what their bureaucrats were doing.

Though empires depended on bureaucracies much larger than those of earlier states, these nevertheless pale in comparison to the size of modern bureaucracies. At the level of a local town, an empire might have one official or even none at all. Empires all relied heavily on local elites who collected taxes on behalf of the empire - and naturally kept a share of these revenues for themselves. Empires lacked the bureaucratic capability to do this for themselves and found it useful to co-opt local elites into mutually beneficial service to the empire. (Sometimes these elites were powerful before conquest; at other times empires would raise locals to positions of power.) Empires would differ considerably, though, in the balance struck between centrally employed bureaucrats and local elites. When there were local representatives of the imperial bureaucracy, these in some empires were also locals but in other empires would be individuals either sent from the capital or rotated from other provinces.

When speaking of the bureaucracies of this era, we should also be careful of assuming modern bureaucratic structures. Bureaucrats often obtained their posts through family connections, had vague duties, and were granted land or rights over some stream of taxes instead of a regular salary. Bureaucrats were expected to be loyal to the ruler and could be fired at will. We shall see below that there was likely a tendency through time for bureaucrats (and local elites) to hold on to an increasing share of the empire's tax revenues.

The arbitrary power of rulers over bureaucrats deserves emphasis. Rulers could, and often did, torture or kill bureaucrats who they suspected of wrongdoing. Emperors might even kill the bureaucrat's family or relieve them of their possessions. Kautilya, adviser to the Mauryan dynasty in India (below), indeed urged killing the families of disgraced bureaucrats. Though such penalties were intended to scare bureaucrats into subservience, they might instead encourage bureaucrats to amass independent power and to steal and hide resources that their family might use if the ruler turned against them. Bureaucrats might be tempted to protect themselves by spying on and making accusations against fellow bureaucrats. The very power of rulers, then, might encourage corruption and disobedience among fearful bureaucrats.

We should also speak briefly of the empire's armies. Empires needed vast armies but could also be threatened by these. Empires struggled to control their troops just as they struggled to control their bureaucrats. These might become more loyal to their generals than to the ruler. Throughout history, empires would often fall at the hands of their own generals.

Though empires faced more significant challenges in administration than earlier states, they would have one advantage. Lydia in Anatolia developed the first coin currency in the world in the seventh century все. Many of the classical empires would collect taxes in currency and pay soldiers and bureaucrats in currency. Payments in kind hardly disappeared overnight, but currency significantly reduced the challenges of managing the state bureaucracy. 


\section{The ends of empires}

Empires govern most of humanity for much of recorded human history. As a type of political organization, then, they are far more common and enduring historically than the contemporary nation state, which people often casually assume is the typical form of political organization. Yet while empire as a type has dominated human history for millennia, individual empires last usually just a few centuries: often less, very occasionally more. This mortality rate should signal to us that empires are not quite as all-powerful as they might seem at first glance.

When we study individual empires in isolation, we can attribute their demise to idiosyncratic causes: a particularly inept emperor, a series of harvest failures, a particularly adept usurper of the throne. All of these may well be important in various cases. However, the fact that most empires survive only a dozen generations or less suggests the existence of more general causes of imperial decline.

One of these may simply be the very logic of empire. Empires, as we have seen, wield power because of the breadth of their influence rather than its depth. They may rule through local elites in each province, but by extracting resources from many provinces, they can threaten any one provincial elite that tries to assert too much independence. If, though, many provincial elites simultaneously decide to exercise more authority, the empire may find itself unable to resist. Empires must always, then, pursue a "divide and control" policy of ensuring that provincial elites do not band together in opposition to the central ruler. This may be possible as long as local elites, in general, see advantages to maintaining their position within the empire. However, if the empire tries to increase its share of tax revenues, it risks annoying multiple elites simultaneously. Attacks by other empires, or succession crises at the centre, or many other circumstances may cause provincial elites to decide that they should seek to overthrow the existing dynasty. Once one rebellion gains traction, others will be tempted to rebel as well.

Nor is the interaction between ruler and provincial elite characterized by a simple dichotomy between loyalty and revolt. As we have seen, local elites (and state bureaucrats) are continually striving to achieve a higher share of local tax revenues. Rulers facing intermittent crises may find it necessary to grant new revenue streams in order to maintain loyalty. Later clawing these back risks offending multiple local elites. There may then be some natural tendency for the share of tax revenues captured by the central state to decline through time though our data on tax revenues for most empires is too limited to be sure. Particularly strong emperors might stem the tide, while weak rulers accelerated it. If there were such a tendency, then over time the emperor would become less and less able to oppose rebellious local elites. Once these elites sensed that the centre could no longer control them, they intensified their efforts to maintain resources locally. These efforts would further weaken the ruler's ability to support the necessary army and bureaucracy. 
Note also that economic growth was sluggish at best during the period of early empires. The formation of large empires usually encouraged trade and thus some increases in income, but over the course of centuries per capita incomes might rise only a few per cent in total. Alternatively, Malthusian forces might drive per capita incomes downward, reducing the ability of the empire to tax individuals. If rulers were losing resources to local elites, they could not replace these by taxing increased incomes. If Malthusian forces were strong, the empire might face a financial challenge even without rapacious local elites, for their tax take depended on farmers producing a surplus.

There is a further challenge for imperial finances. In the early days of conquest, empires gain booty from defeated foes: They steal the animals, land, and even people of those they conquer. This income from conquest may be more substantial at first than revenue from taxes. Although most emperors claim a universal authority, no human empire ever expands to cover the entire globe. All empires at some point cease to grow - presumably because the costs of conquest far from home start to exceed the potential benefits (but also perhaps because for the reasons above all empires become weaker over time) - and then the emperor is forced to rely entirely on taxes rather than booty. Though again we do not know as much as we would like about the relative importance of booty versus taxes for different empires at different times, we can imagine that at least some empires found it difficult to fund their operations from taxes alone, having become accustomed to the additional income stream from conquest. The transformation might prove manageable if the empire no longer needed a big army once it stopped expanding. However, empires must always be able to exercise force in the face of rebellion or social unrest. Moreover, a vast empire means a large border to defend - though empires differ in the degree to which some of their boundaries are oceans or scantily populated deserts or impenetrable mountains. The challenge for many empires, then, is how to support a large army that is primarily defensive and does not often yield booty.

This challenge helps to explain a recurring pattern in imperial behaviour. As we shall see, empires often come to hire the peoples it fights to patrol the empire's borders. The Romans employ Germanic tribes, the Han hire nomads, and so on. It is far cheaper in the short run to "buy off" those the empire might otherwise have to fight. The long-run danger - realized in empire after empire - is that these peoples, after learning the ways of empire, becoming familiar with the wealth associated with empire, and becoming ever more central to its defence, rebel against it. There is a parallel here to relying on local elites to collect taxes: This also makes sense in the short term but sets the stage for increasing provincial autonomy over time. The combination is particularly dangerous: When the frontier troops rebel, the centre no longer has the resources to fight them.

Having sketched some hypotheses regarding the ends of empires, we should remind the reader that history is complex. Changes in military technology or organization changed the balance of power between rulers and ruled. Improvements in imperial bureaucracy could counteract the rise of local elites. 
Conflicts between local elites might strengthen the power of the emperor. The benefits of trade within the empire might prove significant enough to deter local elites from rebellion. Cultural - and especially religious and linguistic diversity could prove a more significant barrier to elite cooperation than to imperial control. Nevertheless, all empires eventually collapsed.

\section{Particular empires}

\section{Persia}

The Persian plateau is not as well watered as Egypt, Mesopotamia, or China. Though agriculture developed there not long after it emerged in Anatolia, Persia was still primarily a territory inhabited by nomads long after Mesopotamia had become a land of agricultural societies. These nomads had likely arrived from Central Asia, there being a natural pass into northeastern Persia from that region (Persia is bounded otherwise by mountains or seas or desert). Over time, irrigation of streams flowing from the mountains permitted agriculture. Persian societies also developed mining and artisans.

As noted above, Persia was the site of the first world empire. During the period of the Roman and Han Chinese Empires, there would be four separate empires centred in Persia (though some of these placed their capitals in Mesopotamia): Achaemenids from 558-330 все, Seleucids from 323-383 все, Parthians from 247 вCE to $224 \mathrm{CE}$ (the temporal overlap will be explained below), and Sassanids from 224-651 CE. Each Persian empire would control territory from at least present-day Turkey to the Indus Valley. Though smaller than Rome or China, these Persian empires were nevertheless far more extensive than any previous states in southwestern Asia. They would have lasting effects on the region, though only rarely would Persia be the centre of later empires.

The Medes and Persians were Indo-European speakers from Central Asia who were mainly nomads but pursued some agriculture. They had migrated into Persia, and at first were subjects of Babylon and Assyria. Like most nomads, they were skilled with horses. They took advantage of conflicts within Mesopotamia to first gain their freedom and then attack Mesopotamia. In just twenty years, the Persian leader Cyrus, founder of the Achaemenid dynasty, conquered from India to the borders of Egypt and Europe; he was likely a military genius. His first success was in defeating his father-in-law, king of the Medes, who at that time dominated the Persians. Within decades, under Darius - a soldier who usurped the throne during a succession dispute - the empire was 3,000 kilometres wide from the Danube in Europe across the Indus, and 1,500 kilometres north to south from Armenia through to Egypt. With 35 million inhabitants at its peak, this empire was (for a century or two until surpassed by Rome and China) by far the biggest state the world had ever seen.

Herodotus, the ancient Greek historian, tells the story of Croesus, king of Lydia. Fearful of the steady conquests of Cyrus, he asks the Oracle of Delphi if 


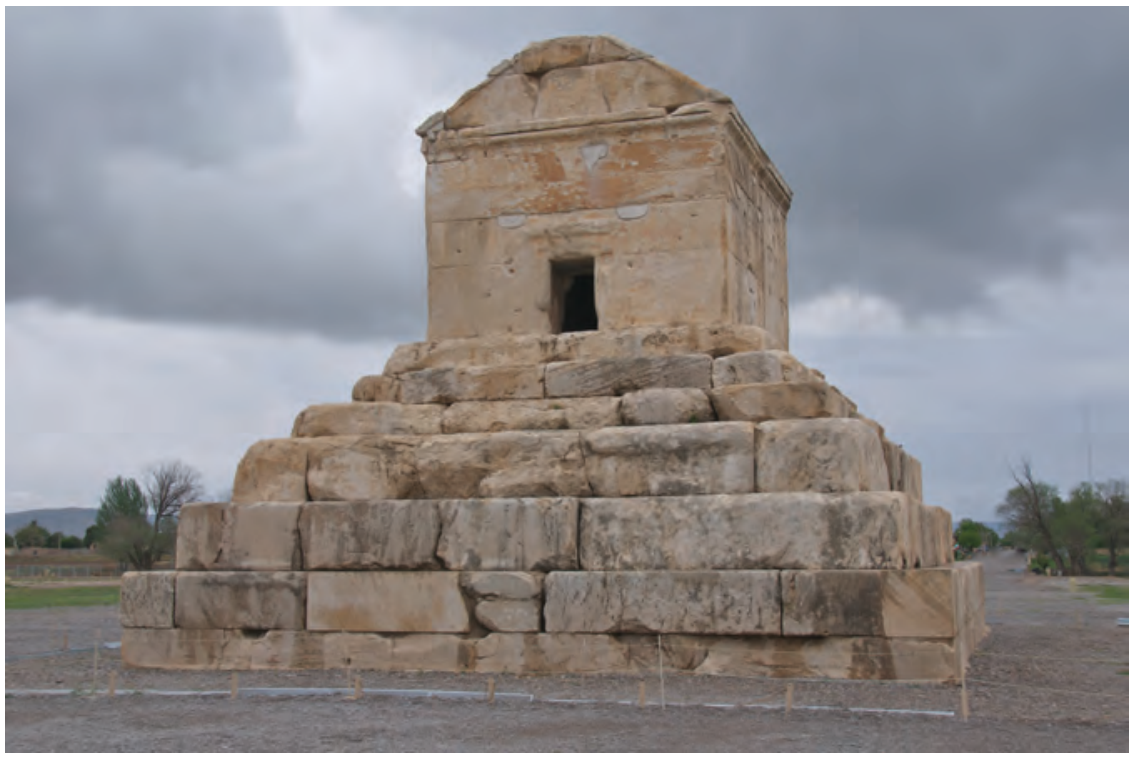

\section{Illustration 9.3}

Tomb of Cyrus, near Pasargades, Iran, thought to be the oldest base-isolated structure - that is, designed to resist earthquakes - in the world Photography by A. Davey, CC BY 2.0

he should attack Cyrus and Persia. He is told that the result will be the destruction of a great kingdom. Though the first battle was inconclusive, Cyrus, rather than retreating, attacked again and conquered Lydia - the camels of the Persians reportedly spooking the horses of the Lydians in battle. The result, then, was the destruction of Croesus' own kingdom: The Oracle, like many soothsayers, often gave ambiguous predictions. As we shall see in the next chapter, Persia's conquests over Greek states in what is now Turkey led to decades of war with Greek states in Greece.

Though successful in war, Darius is celebrated more for skill in administration. He built a grand new capital at Persepolis that was home to a large bureaucracy. He installed mostly Persian governors of twenty-three satraps or provinces, though locals performed most local administration. The Persians built upon existing Mesopotamian bureaucracies. Persian military garrisons and tax collectors kept a watchful eye on local administrators. In addition, the empire created a new group of what were effectively spies, who travelled the empire with guards gathering information and performing surprise audits. In place of irregular tributes from conquered lands, Darius established annual taxes and created coins for payment of these taxes.

Iron became common in agriculture (and in war). Increased productivity, as later in Rome and China, may have allowed an increased bureaucracy. Incomplete records show the imperial court receiving 800,000 litres of grain around 500 все, in addition to many other food items. The emperor Darius held banquets for as many as 10,000 guests. 


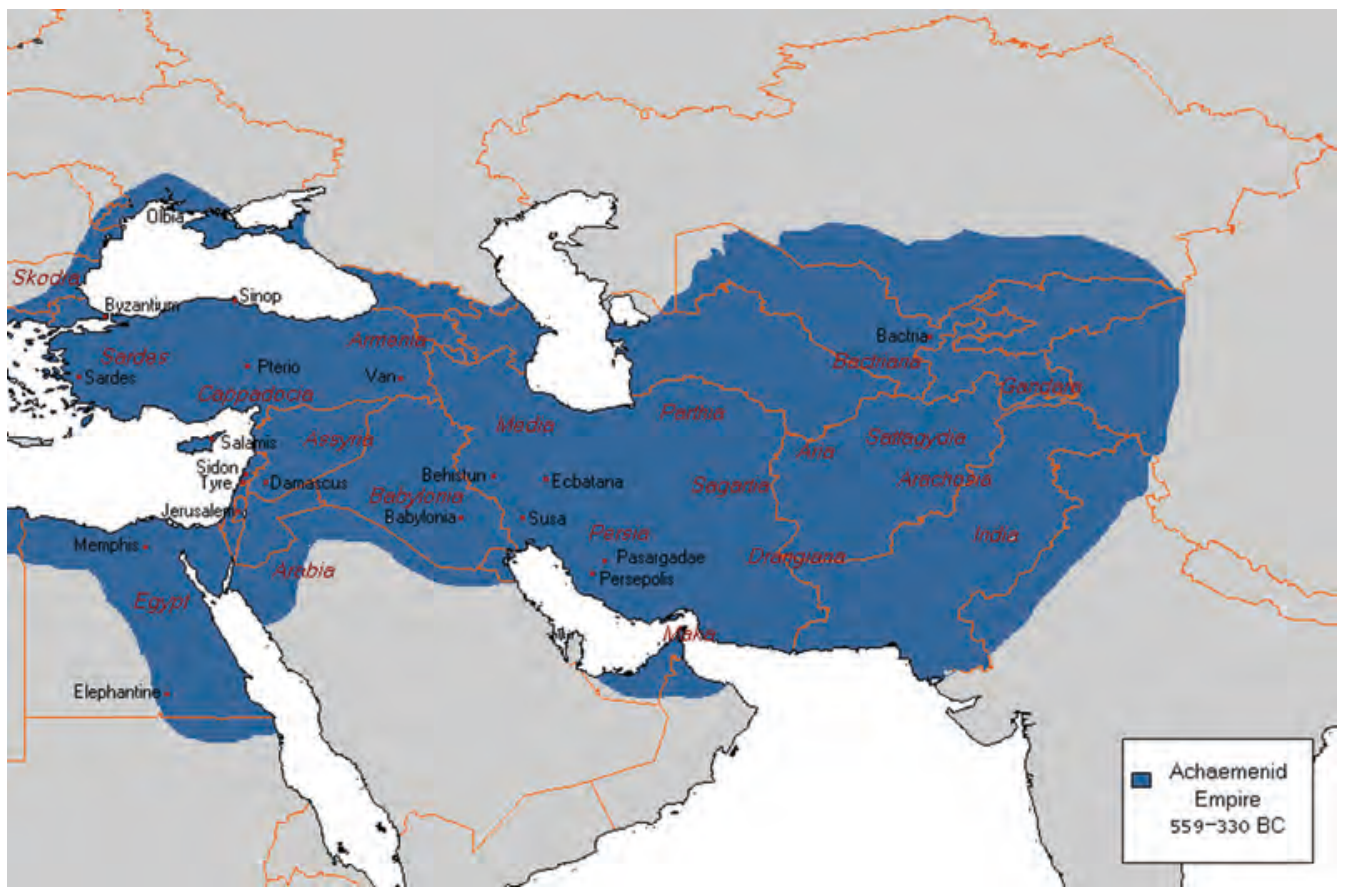

Map 9.1

The Achaemenid Empire at its greatest extent under Darius I

Governing such a large empire depended on an ability to communicate across its vast distances. The Persians not only built roads but also established a regular postal system - with postal stations every forty or fifty kilometres and a network of inns along these roads. The network of inns deserves emphasis: Travelling merchants must always have worried about the safety of goods and animals while they slept, and innkeepers carefully administered by the state could inspire confidence. The most famous road was the royal road from Ephesus on the western coast of Turkey through Anatolia and Mesopotamia to Persepolis. It would take a caravan ninety days to travel the 2,575 kilometres. There were also roads east to India and south into Egypt. All roads were well policed. As in Rome and China, these roads served administration but also significantly enhanced trade. The state maintained many stretches of road in a manner that allowed wheeled traffic. Trade also moved through waterways controlled by Persia, including the eastern Mediterranean (until later Roman conquests) and the Persian Gulf.

Darius and his successors essayed to standardize laws. They created an imperial judiciary. Darius issued a law code modelled on that of Hammurabi (Darius' code, unfortunately, does not survive, though it is mentioned in many documents). Cyrus and Darius practised toleration of different cultures and religions. Cyrus allowed the Jews to return to Jerusalem, from which the Babylonians had banished them. These rulers were also willing to leave local 


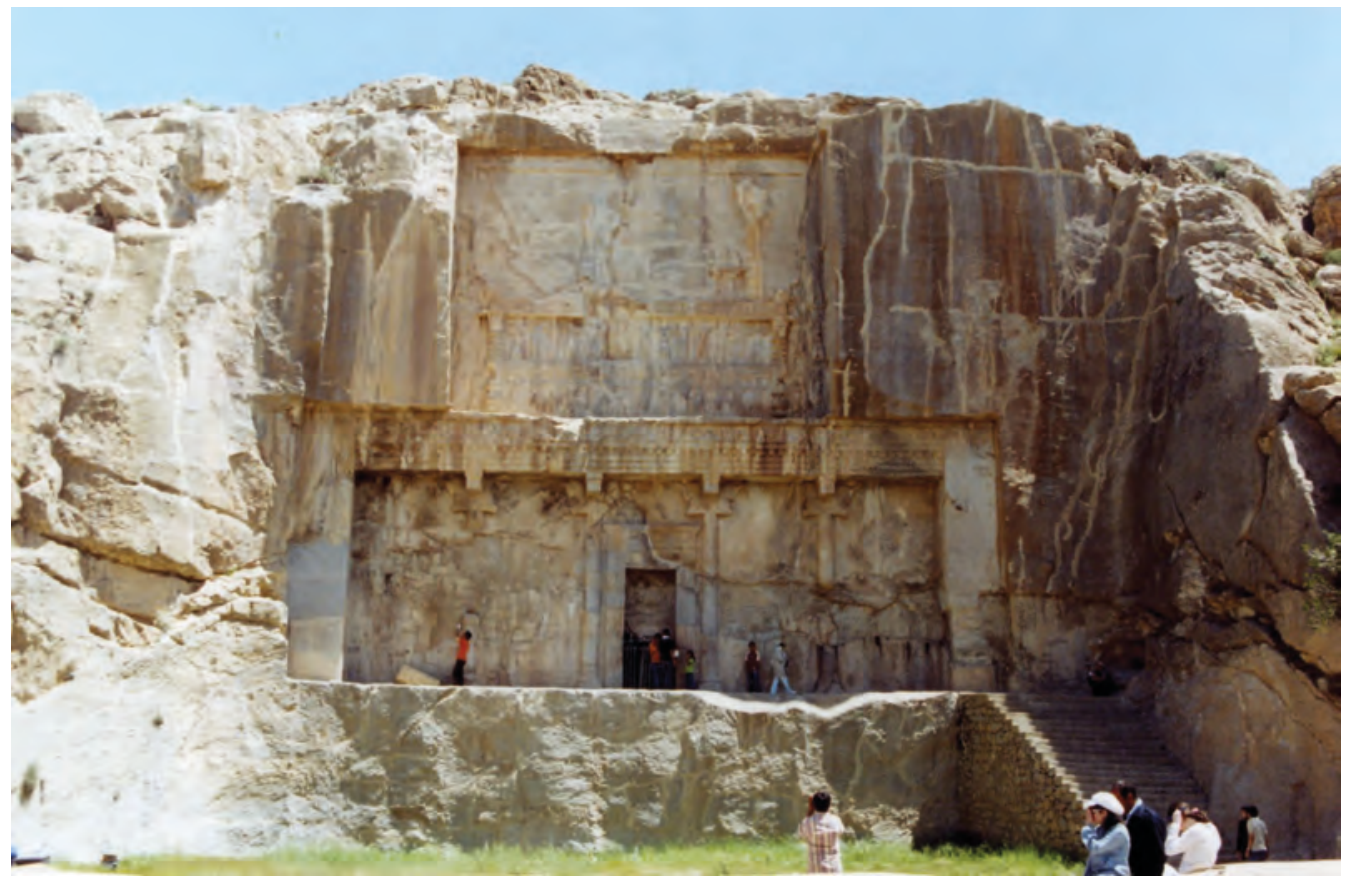

Illustration 9.4

Tomb of Ataxerxes II, Persepolis

elites in positions of influence as long as these cooperated with the Persian state. However, Darius' son Xerxes did not treat local elites or cultures with the same toleration. Local elites objected to Xerxes' efforts at centralization. They and the Persian satraps increasingly enriched themselves at the expense of the centre in the latter decades of the Achaemenid dynasty.

Persia was, like Rome and China, a slave-owning society - with slaves both taken in battle and sold into slavery. It was also a society dominated by men, and in which polygamy was legal (as long as the man could support multiple wives). The practice of secluding women in the home became common over time. Women, though, exercised control over businesses and estates while husbands were away. The semi-nomadic Persians soon developed tastes for architecture and exquisite works of gold and silver, which are admired to this day.

Alexander the Great of Macedon in Greece (see chapter 10) would conquer Persia. His army was much smaller (perhaps 40,000) but better trained and likely more loyal to its leader. Alexander would maintain the Persian administrative structure but burned the capital Persepolis to the ground. Upon Alexander's death, his general Seleucid gained most of Persia. The Seleucids had difficulties as foreign rulers. The semi-nomadic Parthians from northeastern Persia pushed them back (hence the overlapping dates for the two dynasties). The Parthians apparently fed alfalfa to their horses, such that these could then carry armoured 
knights. In any case, these knights proved to be a powerful military force. The Parthians governed in a more decentralized fashion and faced frequent local rebellions. The Sassanids conquered the weakened Parthians in 224 CE. The Sassanids came from Persia and claimed descent from the Achaemenids. They battled with Rome for centuries; Rome three times sacked their capital. Both the Parthians and Sassanids governed from Ctesiphon in Mesopotamia. The Sassanids, unlike their religiously tolerant predecessors, actively pursued Zoroastrianism (see chapter 11). The Sassanids also came into conflict with Hepthalite (White Hun) nomads from the north. They would in the fifth century build the Gorgan Wall, with a moat alongside, east from the Caspian Sea to the mountains of the Caucasus to defend against them.

There was considerable continuity in Persian art through the four dynasties. Religion and the state were the principal foci. Achaemenid art placed the king in place of local deities. The purpose was to show harmonious devotion to the king, so kings were often portrayed mastering animals. With the rise of Zoroastrianism, kings often appeared with Ahura Mazda, the Zoroastrian god. Sassanian coins often had the king on one side and Zoroastrian symbols like fire on the other. The Sassanians also produced large stone sculptures and statues. Sassanid Persia was widely esteemed for its textiles and metalwork. A recognizable Persian architectural style spanned the four different dynasties. It involved arches with fluted columns and reliefs of royal ceremonies. All Persian dynasties had capital cities graced with palaces and monuments. These were often massive brick structures.

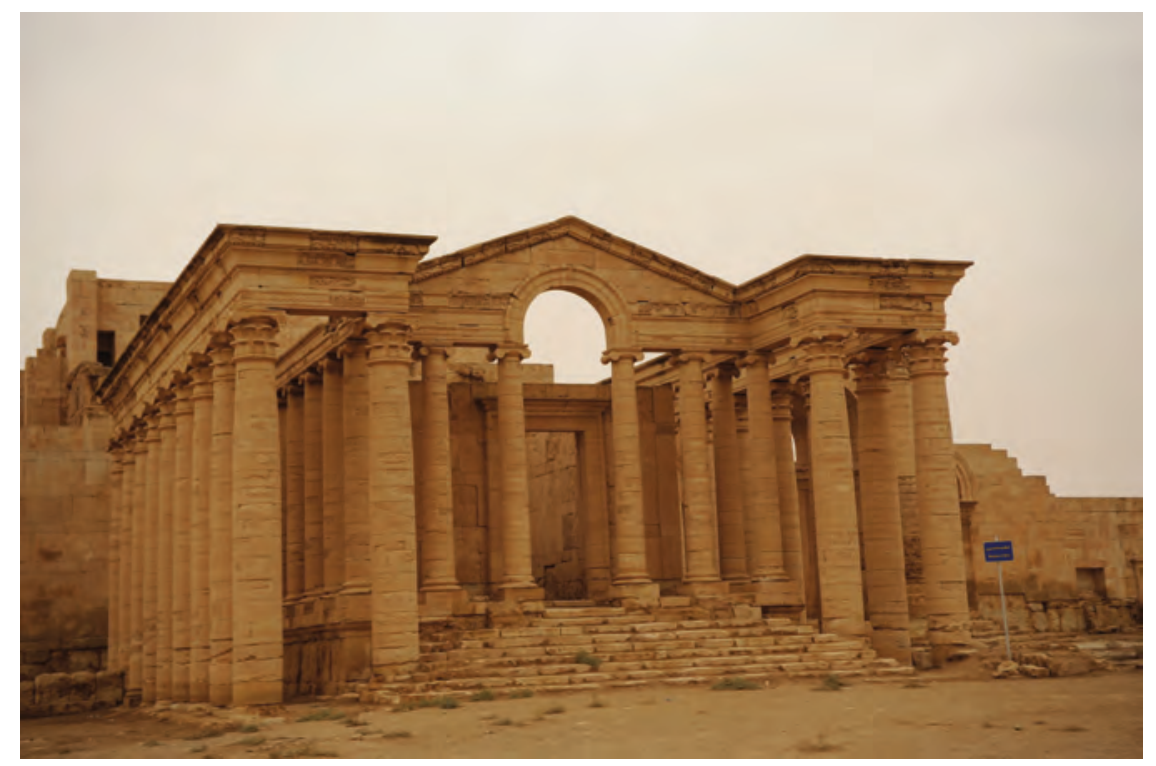

Illustration 9.5

Ruins of Hatra, Parthian trading city (attacked by ISIS in the 21 st century CE) 
The Persian Empire - through its four manifestations - was similar in many ways to Rome and Han China. It rose a little earlier and fell a bit later - and more dramatically to the new force of Islam (after being weakened by battles with the Roman Empire) that we will address in chapter 12. It also struggled with achieving a balance between centralized control and local control. The institution of an internal spy agency was an imaginative solution to the challenge of getting local administrators to act in the interests of the central state. The Persians built roads, established laws and courts, and encouraged trade both within and beyond the empire (Sassanid merchants may have introduced rice and cotton to the region from farther east). As in China, the Persians developed irrigation systems (building on a long Mesopotamian tradition of irrigation). Persians practised slavery, and this became more common over time, reflecting both conquests and poverty (as people sold themselves into slavery). Yet the bulk of the population was free. As in Rome and China, the bureaucracy over time became as or more important than the military in affairs of state.

\section{PRIMARY DOCUMENTS: EXCERPTS FROM THE CYRUS CYLINDER, DATED TO THE 530S BCE}

He [Cyrus] made the land of Guti and all the Median troops prostrate themselves at his feet, while he shepherded in justice and righteousness the black-headed people whom he had put under his care. Marduk, the great lord, who nurtures his people, saw with pleasure his fine deeds and true heart, and ordered that he should go to Babylon. He had him take the road to Tintir (Babylon), and, like a friend and companion, he walked at his side.

His vast troops whose number, like the water in a river, could not be counted, were marching fully-armed at his side. He had him enter without fighting or battle right into Shuanna; he saved his city Babylon from hardship. He handed over to him Nabonidus, the king who did not fear him. All the people of Tintir, of all Sumer and Akkad, nobles and governors, bowed down before him and kissed his feet, rejoicing over his kingship and their faces shone.

The lord through whose help all were rescued from death and who saved them all from distress and hardship, they blessed him sweetly and praised his name. I am Cyrus, king of the universe, the great king, the powerful king, king of Babylon, king of Sumer and Akkad, king of the four quarters of the world.

What strikes you most about this inscription? Did you notice that it celebrates war as a means to achieve peace? Does this seem like a reasonable argument? Why do you think this message was inscribed? Why did other lords surrender to Cyrus? 


\section{Mauryan and Gupta Empires in India}

The Mauryans were - like the Persians - originally nomads from Central Asia. Nomad conquerors had often replaced agriculture with nomadism in parts of Persia and Mesopotamia and the very northwest of India (often slaughtering rural populations in the process), but this did not happen as the nomads conquered the Ganges. The explanation may well be geographical: The Ganges was well suited to agriculture but not to nomadism whereas these other regions lay on the borders between agricultural and nomadic practices. Indeed nomadic conquerors in India would continually import horses from Central Asia as they found it difficult to raise these in India. As elsewhere, including most of Persia, conquering nomads soon adopted sedentary practices and supported monumental architecture and works of art.

We should stress that thick forest initially covered the Ganges region. Its development as a particularly fertile agricultural region owed much to the development of iron axes with which forest was cleared from at least 800 все. Yet iron was also used for weapons. As the nomadic Aryans became agriculturalists, they fought amongst themselves over land. The earliest Vedas celebrate Aryan victories over common foes, whereas later Vedas, such as the Mahabharata and Ramayana, grapple with questions of duty when brothers or cousins find themselves on opposite sides of a battlefield.

Pataliputra (near present-day Patna), capital of the Mauryan Empire, was perhaps the largest city in the world after the Mauryan Empire was founded in $322 \mathrm{BCE}$, and for two centuries after that. It was also a vital trading centre on the Ganges. It had massive wooden walls with 570 towers and 64 gates. It had a university and was a shipbuilding centre. The Greek ambassador Megasthenes described Pataliputra in glowing terms; he also celebrated reeds that produced honey without bees (sugarcane) and trees that provided wool (cotton) and spoke glowingly of the Mauryan roads. He maintained that soldiers did not ravage farmland during wartime in India; if true, this would be an unusual practice.

The Mauryan Empire was the first large empire in India and would be one of only a handful of large empires that ever existed there. Yet its founder, Chandragupta Maurya, had previously been an army officer for the state of Nanda, which was itself reputed to have an army of hundreds of thousands of soldiers. He employed Greek mercenaries in his rise to power, as had the Nanda before him; these presumably were inspired by booty. The Greeks with their military formations shattered the previous Indian practice of one-on-one combat. There is a tradition that, after his extensive conquests and administrative consolidation, Chandragupta then abdicated to become a Jain monk. His adviser Kautilya's administrative ideas survive in a book, Arthashastra. As in Persia, these ideas included spying on subordinates. Indeed, Kautilya urged spying on the royal family as well as bureaucrats, and envisioned a system of different spying operations, ignorant of each other, that would undertake different missions: stationary versus mobile; financial investigations versus 
personal investigations, and so on. Kautilya also urged rulers to kill sons that showed signs of disloyalty. Yet he also recommended the testing of bureaucrats for different skills and promotion based on merit. The Mauryan Empire modelled itself explicitly on the examples of Persia and Alexander the Great. Chandragupta's son and especially his grandson Ashoka expanded his empire. Ashoka estimated that 100,000 Kalingans died in his war against Kalinga (modern Orissa). However, Ashoka (like Darius above) is appreciated mostly as an administrator. He created an extensive bureaucracy that taxed agricultural production. Horrified by the slaughter of his Kalingan campaign, he became a devout Buddhist, urged Buddhism, and promised to be a just ruler. (Cynics note that he denounced violence only after defeating his rivals.) He discouraged (but far from eliminated) both meat eating and alcohol in his court. He built roads (one of 1,600 kilometres in length) and irrigation systems. Ashoka also installed polished stone pillars with Buddhist edicts throughout his realm - this practice echoed both Persian and Hindu precedents and employed Greek, Persian, and local styles of decoration. The stone for the pillars was quarried near the

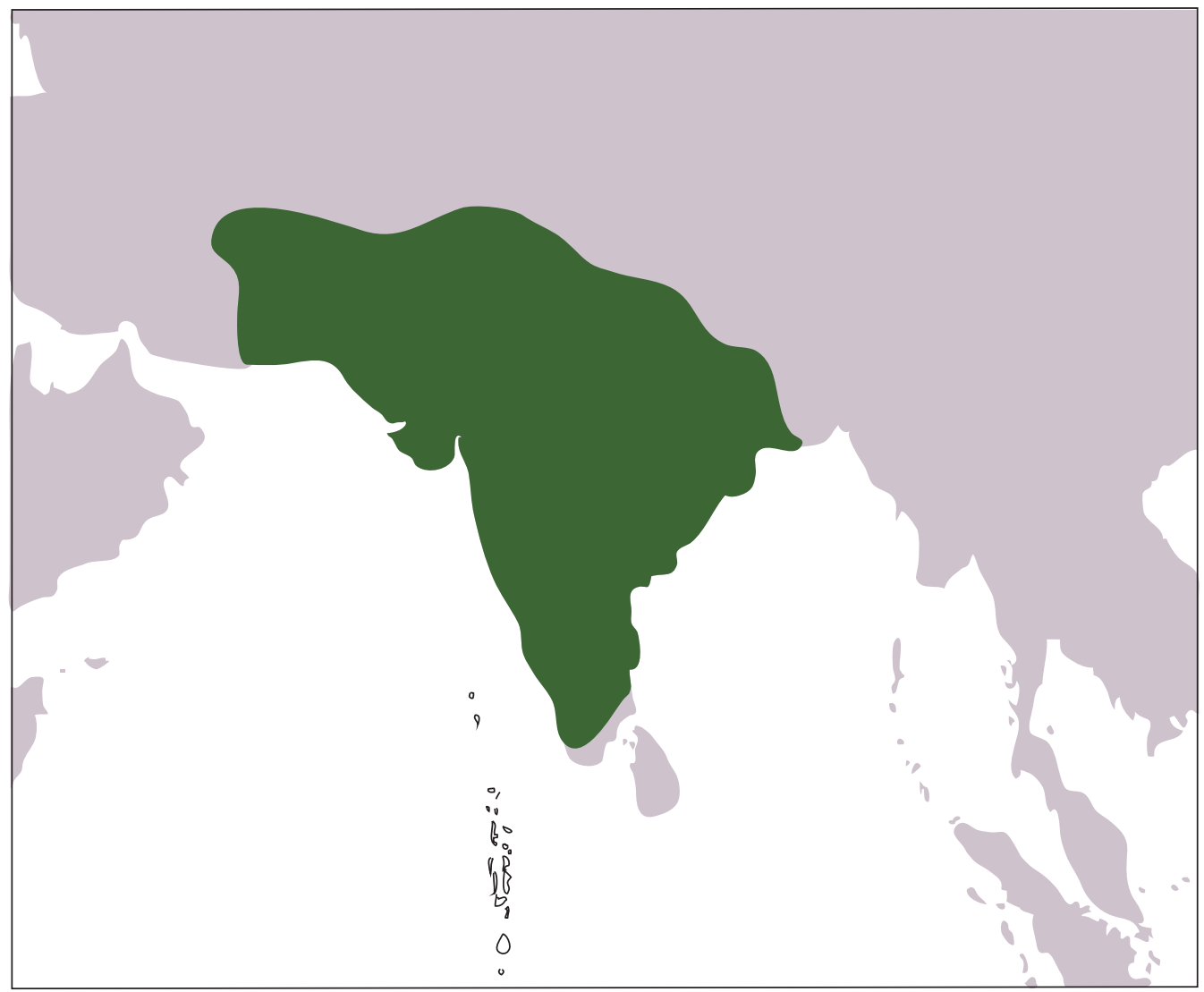

Map 9.2

The Mauryan Empire at its greatest extent; this map may exaggerate its southern extent 
ancient city of Varanasi (Benares). The pillars seem to have been intended more to encourage values such as honesty, compassion, and responsibility than to promote a particular religion.

\section{PRIMARY DOCUMENTS: ASHOKA'S EDICTS}

Ashoka had thirty-three edicts inscribed on pillars and rocks and caves, encouraging Buddhist principles but also religious toleration, promising fair punishment, celebrating pardons, and promoting animal welfare. In this edict, reproduced on many pillars, he regrets his conquest of Kalinga:

The Kalinga country was conquered by King [Ashoka], beloved of the gods, in the 8th year of his reign. One hundred and fifty thousand persons were carried away captive, one hundred thousand were slain, and many times that number died.

Immediately after the Kalingas had been conquered King [Ashoka] became intensely devoted to the study of dharma, and to the inculcation of dharma.

The beloved of the gods, conqueror of the Kalingas, is moved to remorse now.

Several more paragraphs elaborate on the suffering associated with military conquest and assert that moral conquest is the most important kind. Nevertheless, Ashoka asserts about forest peoples who resist his rule "that he exercises the power 'to punish, despite his repentance, in order to induce them to desist from their crimes and escape execution'" as he wants security in his realm.

He closes by explaining his edict:

This edict on Dharma has been inscribed so that my sons and great-grandsons who may come after me should not think new conquests worth achieving.

How do you think conquered peoples might have viewed this edict? What do you think of the stated motive?

Within fifty years of Ashoka's death in 232 вCE the Mauryan Empire was gone. It faced difficulty meeting its expenses and proved unable to prevent regional rebellions. Hindu leaders offended by Mauryan support of Buddhism often fostered rebellions. Given the relative brevity of the empire, we might speculate - but it is just speculation - that the empire had relied too much on the short-term gains associated with conquest and not established a stable administrative structure that could tax enough to support the bureaucracy and army on which the empire relied. As has been noted before, we tend to think of empires failing because of bad rulers or lost battles, rather than the boring failure to cover expenses. Given the fertility of the lands along the Ganges, the Mauryan Empire could potentially have exacted a large agricultural surplus.

The classical period in India is noted for its sculptures, often sensual with Hinduism, then spiritual with Buddhism. The Greeks and Persians had spread 


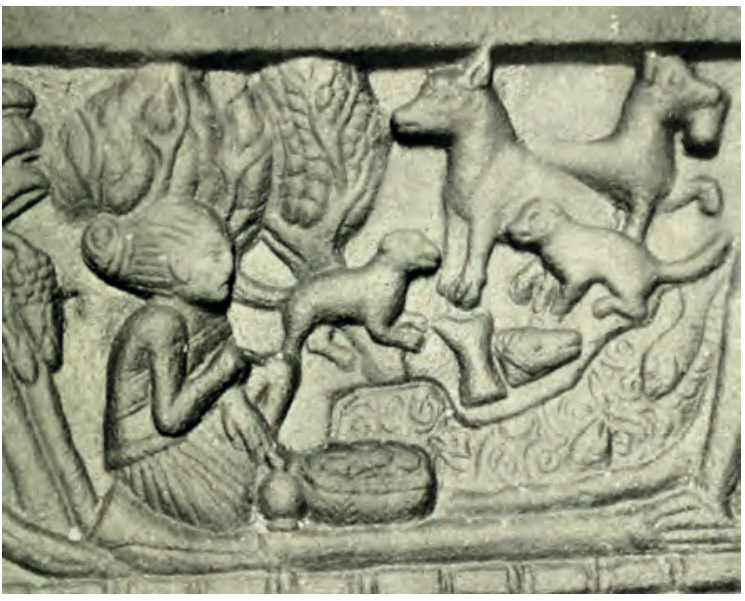

Illustration 9.6

Carving of the fable of the jackal and the otters, Bharhut, lst or 2nd century BCE. Ashoka may have built the Bharhut Stupa but much art was added later to India the idea and techniques of stone architecture. In particular, Ashoka famously sponsored the construction of many stupas: domed shrines for relics and religious ceremonies. These stupas would inspire centuries of development in Indian architecture. Stupas would be adorned with rich carvings over time.

After centuries of competition among regional kingdoms, from about $320 \mathrm{CE}$ the Guptas formed an empire also based in Pataliputra, but never quite as large as the Mauryan. The Guptas came from a region in northeast India with substantial iron deposits, which may have given them an advantage in both weapons and finance. The Gupta Empire was much more decentralized, with local elites very powerful. Though the Mauryan Empire had for a while favoured Buddhism, the Guptas would instead favour Hinduism. They usually practised tolerance, endowing Buddhist and Jain temples also but supported Hindu temples throughout their territory. Sizeable Hindu temple structures were something novel, and the Gupta period established an architectural template that would be embellished over the next centuries. Sculpture and painting both prospered. Hindu philosophy blossomed, and the school of yoga in particular developed. Hindus had borrowed from the Persians the practice of secluding wives, which spread during this period. It was also during this period that a system of numerals was developed that would later be borrowed by Arabs and then transmitted to Europe as "Arabic numerals." The first documented use of "zero" occurs in India in 499. Many Indian historians see the Gupta period as India's "Golden Age."

The Gupta Empire encouraged world trade through its ports on the Arabian Sea and Bay of Bengal and its access through the Punjab to Central Asia. It traded with Europe, China, and Southeast Asia, exporting pepper (much valued throughout the Mediterranean from the first century CE), cotton, wood (especially fine furniture), and ivory. The elite in the Roman Empire also valued exotic animals such as peacocks and tigers. The Guptas taxed both trade and agriculture (these were mostly taxes in kind). The Mauryans had obtained gold from Siberia, but disruptions in Central Asia caused later Indian merchants to work with Southeast Asian polities in Malaya and Indonesia to develop important gold deposits there.

We should pay particular attention here to textile production and exports: India gained fame for its cotton goods during the classical period and became a major exporter and perhaps the primary textile producer in the world. Indian cotton was much valued in Egypt by the first century $\mathrm{CE}$, reached East Africa 
and the Mediterranean soon after, was marketed widely in Southeast Asia by the fifth century, and entered China soon after. While Chinese silk receives far more historical attention, it was always a luxury good, whereas Indian cotton textiles served a broader market.

In about $500 \mathrm{cE}$, Gupta artisans developed granulated sugar from sugarcane syrup. This proved much easier to transport than the syrup itself. By 800, granulated sugar was known in both China and the Middle East. Europe would later learn of it during the Crusades (chapter 15); sugar plantations would then play a critical role in the discovery and settlement of the Americas (chapter 19).

The Gupta Empire came under increased pressure from Central Asian nomads in the late fifth century. The "White Huns" (Hephthalites) in particular broke through Gupta defences in the Khyber Pass. The Huns conquered the Punjab in 499, and by 550 the Guptas had fallen. The Huns received tribute from several small states that replaced the empire. There would not be another large empire in India for 1,000 years.

Although the Guptas came from a region with some iron, India as a whole had less iron ore than most other regions of the world. We shall see in the next chapter that iron weaponry was important in the expansion of Roman and Chinese armies and states. The fragmented political history of India may reflect in part its limited iron resources. Since iron ploughs also enhanced agricultural productivity elsewhere, India likely suffered economically as well.

Debate rages as to how robust the Indian caste system was during the classical period. It seems clear, though, that the priestly Brahmin class already was venerated. Though warriors were also highly valued, Brahmins were expected to play the leading role in establishing laws and adjudicating disputes. Warrior rulers (usually) appreciated that they were subject to laws proclaimed by others - whereas rulers elsewhere in the classical world could usually proclaim laws themselves. In practice, though, since there was no organized Hindu religious establishment, rulers could favour Brahmins whose legal interpretations they found congenial (though Brahmins, in turn, used their influence to support favoured rulers). Brahmins filled posts in imperial bureaucracies and were hired on personal ties rather than evaluations of merit. These bureaucracies may thus have been less effective than their Chinese counterparts (see next chapter). The influential Brahmins absorbed elements of Buddhist thinking (such as the emphasis on reincarnation) into Hinduism but otherwise urged rulers to foster Hinduism rather than Buddhism (we revisit Hindu borrowings from Buddhism in chapter 13).

Warfare tended to be the exclusive province of the warrior class. Peasants might serve in a supporting role, but Indian states after the Guptas tended to shun the mass peasant armies observed elsewhere. This situation may in part reflect the limited iron resources noted above. Indian armies also shunned cavalry, perhaps because of the difficulty of raising horses in India. Indian armies would often be defeated in history by invading nomads from Central Asia.

Though India most often comprised multiple states, the few empires that did exist played an important role in solidifying cultural traditions. The Gupta 
policy of supporting Hinduism was significant in that religion's ascendancy over Buddhism. Scholars once thought that the caste system also solidified during the Gupta dynasty, but recent scholarship questions this (see chapter 20). Gender stratification also increased; in particular, child marriage of girls was common by the Gupta period and expanded in popularity. The practice of sati - whereby widows killed themselves on their husband's funeral pyre - was known from the fourth century BCE but only became common among the warrior elite in the centuries after the fall of the Gupta Empire.

\section{The Kushan Empire}

As noted above, we can only survey some of the sixty historical empires in this book. We might have ignored the Kushan Empire, which occupied much of present-day Afghanistan, Pakistan, and northern India from the second century $\mathrm{BCE}$ through to the third century $\mathrm{CE}$ (and needs, of course, to be distinguished from Kush, the kingdom in Nubia discussed in chapter 7). Once it disappeared, it had no inheritors: No later empire referred back to the glory of the Kushan. Yet in its day it was, like other empires, noted for its arts and architecture, and for facilitating agriculture through irrigation. The reader should be aware that other empires that affected the lives of millions for centuries, and were famous in their day, receive little attention from historians because they had limited impact (apparently) on later history. In some cases, we may also have limited historical records. In the case of the Kushan, we have limited knowledge of the origins of the empire or its early days. We do know that the Kushan Empire had at least one significant impact on history.

The Kushan Empire was founded (we think) by nomads who had adopted sedentary ways. They may have been pushed southward as the Xiongnu were driven westward by the Chinese (chapter 10). They built upon an Indo-Greek kingdom called Bactria that in turn reflected the efforts of Alexander the Great to populate a region on the border between farmers and nomads. King Kanishka (dates uncertain; he ruled for decades from the first century $\mathrm{CE}$ ) expanded the state south into India and north into Central Asia. This expanded state then encouraged overland trade along the Silk Road to China. It encompassed Samarkand and Bukhara, famed trading centres along that route. Traders who would have had to negotiate with several nomadic or sedentary polities could now deal just with one. At one point, the empire reached the mouth of the Indus and could engage in sea trade with Roman outposts in Egypt, bypassing the Parthians in Persia. Archaeological excavations at the Kushan summer capital of Kapisa north of Kabul have found Roman bronzes, Indian ivory, Greek glass, and Chinese lacquerware. Kushan coins reflected this cultural interchange, sporting Greek, Roman, Buddhist, and Hindu imagery.

Kanishka actively supported Buddhism and sent Buddhist missionaries into Central Asia, from where the religion would spread eastward. Buddhism became firmly established throughout Central Asia at this time. Kanishka fostered Mahayana Buddhism, and so it would come to dominate in China, whereas the 
stricter Theravada Buddhism would eventually spread to Southeast Asia and Sri Lanka (see chapter 11). Buddhism would also reach China by sea via Southeast Asia but to a much lesser extent. The Kushan Empire was one of the last states in India to support Buddhism; the religion began a long slow decline in its homeland while expanding elsewhere. Though it is always dangerous to explore "what if?" questions in history, it could well be that Buddhism would never have become so popular in East Asia if not for the efforts of the Kushan Empire.

Buddhist art (and Indian narrative traditions) travelled with Buddhist religion. The Kushan Empire would excel in producing statues of the Buddha (chapter 11). These statues reflected traditions of Greek art but came to incorporate Indian sensuality: Some art historians speak of Greco-Buddhist art. The Kushan tradition would be carried east and would inspire centuries of Buddhist art in East Asia. The most notable early examples of this art were elaborate cave sculptures. The idea of large Buddhist cave sculptures had originated in India but was transmitted to China via Central Asia.

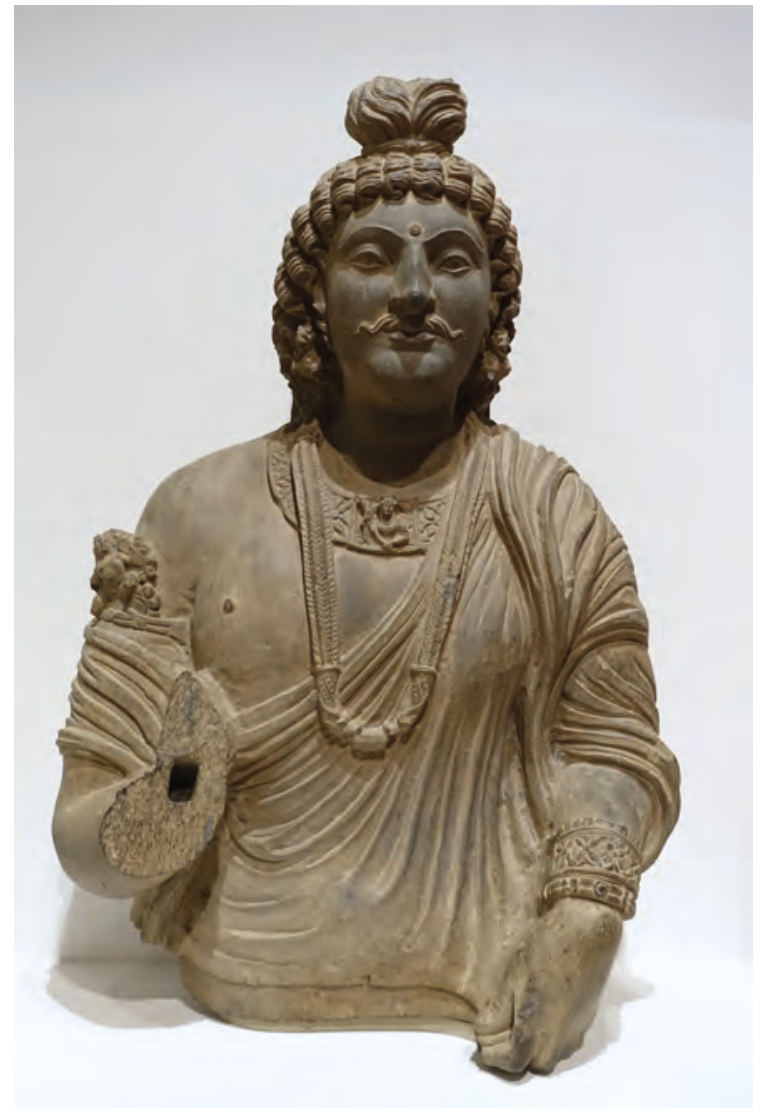

Illustration 9.7

Boddhisatva, Kushan Empire, 2nd century вCE, Dallas Museum of Art

Photograph by Daderot, CC0 1.0

The Longmen Cave near Luoyang alone

has over 100,000 statues and relief carvings produced over a period of centuries from about $500 \mathrm{cE}$. These were all elaborately carved and painted.

\section{Axum}

The Axum Empire was centred in modern Ethiopia but expanded into parts of modern-day Egypt, Sudan, Saudi Arabia, and Yemen. It was founded only in the first century $\mathrm{CE}$ but would last into the tenth century CE. It was the third or fourth largest empire geographically in the world through much of its history. Scholars once thought that Arabs from across the Red Sea founded the empire, but now appreciate that Axum was an indigenous African development. With its expansion along and across the Red Sea, the empire controlled one of the major trading routes between the Roman Empire and India. It also traded goods from elsewhere in Africa, notably ivory, into Eurasia. It appears to have gained much of its revenue through taxing trade (but we have limited records from 
Axum). It also produced its own coins; archaeologists have discovered some of these as far away as China.

Axum erected a granite stela 90 feet tall, the tallest such structure in the world. It would adopt Christianity in the fourth century CE, only a little later than Christianity was recognized in Rome (see chapter 10). It would also then play a role in the birth of Islam, providing refuge for some of the earliest adherents from persecution in Arabia around 615 CE. Later, though, it would suffer from attacks from the new Islamic Empire (chapter 12).

Postscript: Modern Ethiopia can claim to be the most long-standing state in sub-Saharan Africa, and the state with the longest history of Christianity and literacy. It also likely possesses the greatest heritage of art and architecture in sub-Saharan Africa. It also, likely coincidentally, has been most successful in exporting cuisine globally.

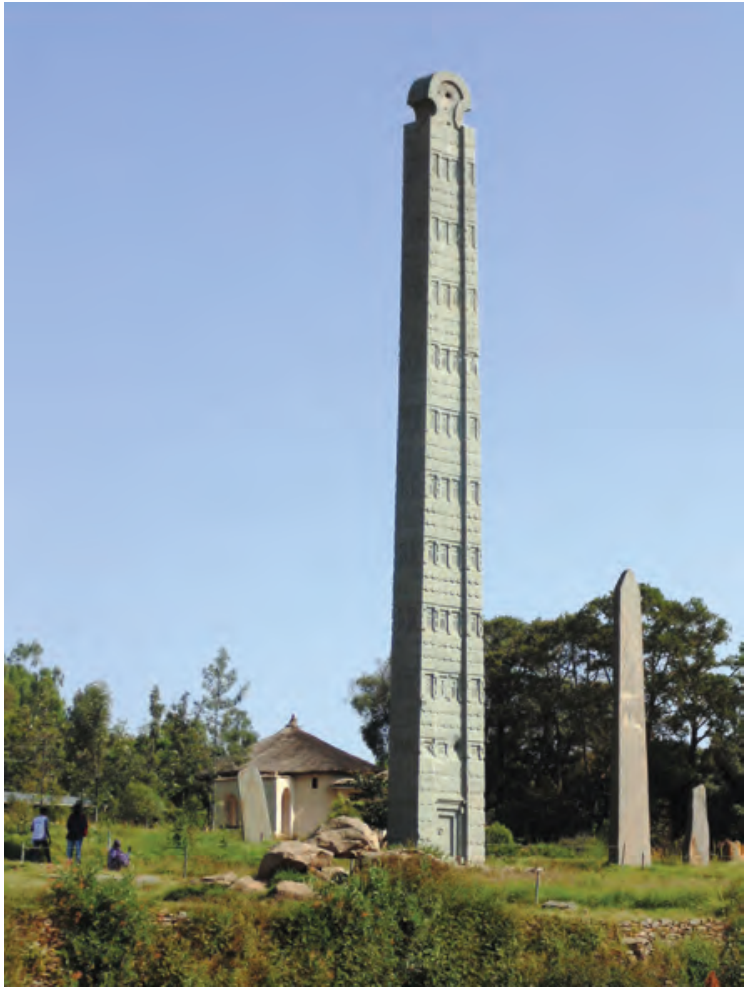

Illustration 9.8

Axum Stele, 4th century CE; it had broken three centuries ago. It was taken by Italians and rebuilt in Rome in the 1930s; in 2008, it was returned to Ethiopia and rebuilt

Photograph by O. Mustafin, CC0 1.0

\section{The Americas}

Teotihuacan near modern-day Mexico City had maybe 100,000 people around $100 \mathrm{BCE}$, and 200,000 by $400 \mathrm{CE}$. If so, it was one of the largest cities of its time. It was a centre of obsidian manufacture; its artisans also worked with onyx, jade, and shells. Archaeologists have discovered 400 workshops in the ruins, along with 600 temples. The city had no fortifications until very late in its history; this may indicate that it exercised political control over a large surrounding area. It clearly had an army, but we do not know its political structure. The city collapsed between 650 and $850 \mathrm{cE}$. Disease, volcano, earthquake, and attack have all been hypothesized as causes of societal collapse.

\section{Slavery}

Slaves are found across the ancient world from at least the first millennium BCE. Slaves came from three primary sources: captives in war, criminals, and people who sold themselves into slavery 


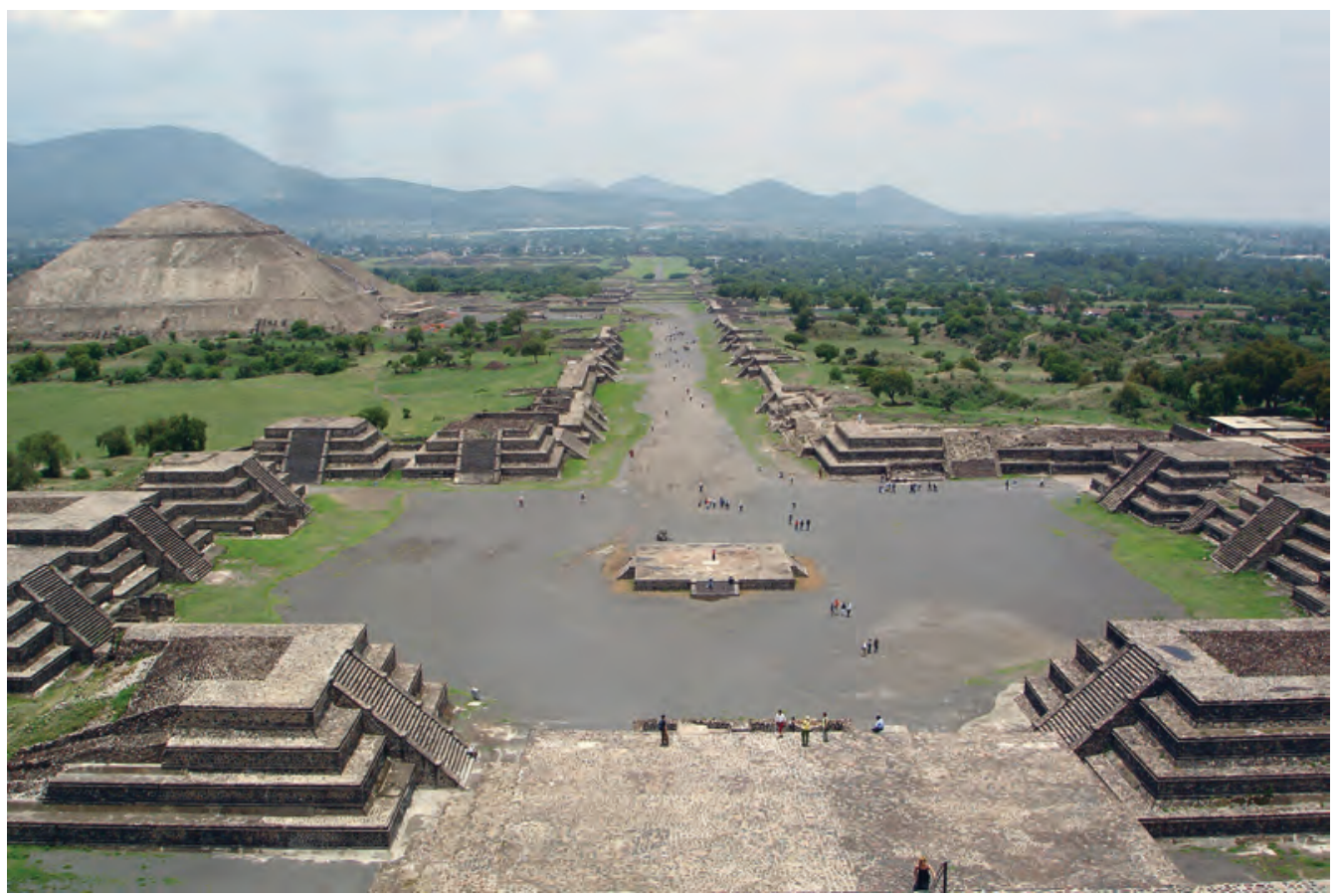

Illustration 9.9

The Avenue of the Dead and the Pyramid of the Sun, from the Pyramid of the Moon, Teotihuacan

because of indebtedness. Pirates or bandits also (illegally) captured free people and sold them into slavery. These could potentially free themselves if they could prove their free status, but this was no easy task with limited public records and judges who tended to favour slave owners over slaves. Abandoned free children were supposed to be raised free, but were often raised as slaves; they had virtually no chance of establishing free status even if aware of this. In Rome, at least, fathers could sell their children into slavery.

Slaves are most commonly associated in the modern mind with farm labour, and especially on large plantations. Here masters usually expected that slaves work from dawn until dusk with few breaks and only a few days off per year. Depending on the crop, there might be seasonal lulls in work effort. Slaves often worked in mines - a dirty and dangerous task where slaves might not see daylight for extended periods. Rowing galleys was common; this was hard work while the ship was at sea, but with less to do in the harbour. Slaves were also widely employed in cities as domestic servants of various types and in trade or industry. Such servants were often a sign of the wealth of their masters; these slaves usually had a much less severe work schedule than other slaves. Some of the poor may have sold themselves into urban slavery in the hopes of learning to be artisans or merchants: Such a practice would mimic an apprenticeship in which a student learns a skill while working for a master. Female slaves (sometimes male) were employed as prostitutes or concubines in many societies. 


\section{BOX 9.1}

\section{THE ECONOMICS OF SLAVERY}

The economics of slavery deserve close examination. It may seem obvious that there are benefits to masters of being able to force workers to work. Yet slaves still have to be fed and clothed. At a time when there were free workers earning wages that were not much more than was required for food and clothing, the benefits of slaves over free labour are less obvious. Masters could force slaves to work more extended hours, but the working week for free labour at the time was very long already. Masters could force slaves to do hard, dirty, and dangerous jobs that free labour might choose to avoid: mining or manning the oars in galleys for men, prostitution for women. Also, slaves could be sent to regions where there was a shortage of labour. If peasants were routinely fleeing a state to avoid taxes, slavery might appeal to both states and landowners. States might then be particularly interested in capturing slaves during wars. One distinct advantage of slaves is that they could not quit. Masters were then spared the costs of hiring and training new workers. They had, though, to bear the costs associated with preventing slaves from escaping. Moreover, they had to purchase slaves in the first place.

The existence of slavery across many societies and thousands of years of history suggests that it was indeed profitable. However, the estimates of economic historians suggest that it was not incredibly so: Incomes of masters were a few per cent higher with slaves than with free labour. The best evidence here comes from (the much studied) plantations in the southern United States after the Civil War (chapter 26): These mostly continued to be profitable because the former slaves continued to work for meagre wages. Yet we should not shrug at a difference in income of a few per cent; often in this book, we will celebrate technologies or institutions that boosted output by only a few per cent.

There were also cases where free labour was either unavailable or very expensive. The latter was especially likely on frontiers where land was abundant. If workers had the opportunity to move on to free land, then some coercive labour relationship such as slavery (or serfdom; chapter 13) might be the only way that landlords could gain labour at a nearsubsistence cost. Slavery may have been common in early Rome mainly because Rome was steadily expanding into new lands. Of course, the much later slavery in the Americas from the fifteenth century CE would also occur in an environment of an open frontier.

In addition to the income associated with slavery, there were non-financial benefits. Slavery was a way of punishing both captives in war and criminals. Slavery allowed one person to exercise raw power over others. One common occurrence within systems of slavery was male masters forcing themselves on female (and often male) slaves. If slavery had been entirely an economic proposition, then slaves might have maintained rights over their personal lives. However, a power relationship that served the economic motives of masters often was extended into all spheres of the slaves' lives.

In some cases, a strong bond of loyalty formed between master and slave. Indeed, we will find that several empires in world history purposely promoted slaves to positions of authority 
precisely because rulers hoped that these would be more loyal (in part as they had limited family ties).

Especially in classical times, slaves were often able to buy their freedom. Some former slaves rose to positions of wealth and influence. How could a slave amass the funds to buy their freedom? The answer lies in one of the challenges of slavery: It is hard to force a slave to work as hard as they might. It is therefore often useful to provide financial incentives for hard or skilful work. This is especially the case if the slave has skills that allow them to perform specialized tasks that are difficult to supervise. In such cases, the difference to the master's income between a slave and a free worker might be very slight.

Estimating the size of the slave population is hard. This undoubtedly fluctuated with the frequency of (victory in) war. Slaves may have comprised about 15 per cent of the population in the late Roman Empire. The number would have been higher in the years of expansion of the Roman Republic (we shall see in the next chapter that there might have been 60,000 slaves around 300 BCE when there were only 200,000 free males). But if slaves were a small part of the population, and if they were just fractionally more productive than free labour (see Box 9.1), then the common assumption that classical economies "depended" on slaves would seem to be erroneous. Slavery appears to have been an important convenience rather than an absolute necessity. The exception here would be in regions or activities for which free labour was unavailable.

If slavery was not essential, then it is remarkable that the institution of slavery was simply taken for granted at the time - and indeed for the next thousand years and more throughout the world. Notoriously, even Greek philosophers wondering about the nature of ethics accepted the existence of slavery without much question - though they did at

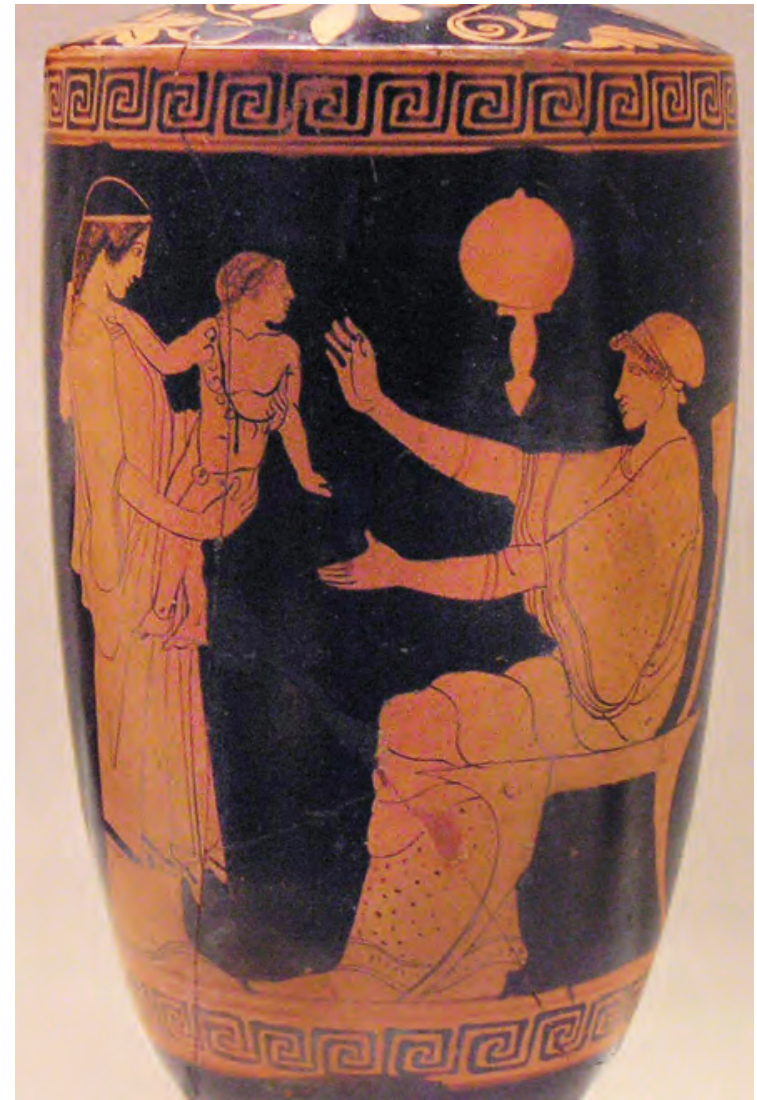

Illustration 9.10

Depiction on pottery of slave handing baby to its mother, 470-460 BCE, Eritrea, National Archaeological Museum, Athens

Photograph by Marsyas, CC BY-SA 2.5 
times urge masters not to abuse their power. Even slaves themselves seem to have accepted the existence of slavery, much as they may have regretted their own situation. Slave revolts occurred but were exceedingly rare - and states suppressed these harshly.

Some insight into why slavery was accepted may come from examining the sources of slaves. Many slaves came from military conquest. To oppose this kind of slavery would mean going against the justifications given for war: that the victims deserved their fate (Chapter 6). Slaves were an important component of war booty. Slaves need not all be captured by imperial armies: Neighbouring "barbarians" might desire Roman or Chinese goods and have little to trade for them, and so engage in wars with other "barbarians" to gain slaves to trade. Slaves were high-value "goods" that could transport themselves. We commonly observe political fragmentation in slave-exporting regions through history because of this dynamic: Frequent wars among small polities generated a regular stream of slave exports. These "barbarian" elites would value slavery as much as or more than imperial elites. A positive attitude was also possible toward enslaving criminals: At a time when legal penalties focused on death or mutilation rather than imprisonment, slavery might seem a means of punishment that was useful for society and fair to the victim. Also, some people became so indebted that they had to sell themselves or family members into slavery. If much of the population had recourse to loans from time to time to finance basic human needs, and their bodies were the main collateral they could provide, then the fact that many fell into slavery could appear as an unpleasant necessity among a population that was not rich and had limited access to charity. In 326 BCE, the Roman Republic had (as the masses gained political power) abolished formal "debt slavery" by which a free person pledged themselves to another as a slave in return for a loan. Nevertheless, it remained common for a person to become a slave or to sell their children into slavery if they defaulted on a loan. As for raising orphans as slaves, this might also seem better than the alternative at a time when there was limited charitable support for orphans. Of course, such conscious justifications of slavery might mask selfish subconscious desires: One might wish both to benefit from slavery and not to face the costs of alternative mechanisms of looking after the impoverished.

Slaves in this period were largely indistinguishable from the free population there was a mere smattering of black African slaves in Rome. This made it easier for slaves to escape, but also easier for free people to be sold falsely into slavery. Slaves were branded if caught after fleeing - and sometimes just if the master feared they might escape. Masters might also give slaves special haircuts to set them apart. It is not clear what the relationship was between slaves and the poor but free: Did the latter see the slaves as fellows or did they instead celebrate their superiority over mere slaves? Their own fear of becoming indebted and forced into slavery themselves might have encouraged a mixture of both disdain and fellow-feeling.

One exception to the observation that slaves generally resembled masters was the practice of transporting female slaves from the Mediterranean to 
India, where they served as prostitutes or concubines. These women were prized as exotic in Indian courts, and were seen as a sign of affluence. Many may have been kidnapped by Mediterranean pirates.

Masters were free to physically punish (assault) slaves as they saw fit. They were also, of course, free to inflict psychological abuse. Masters could sexually assault slaves. This was likely a typical experience for female slaves and reasonably common for male slaves. Female masters or the wives of masters might sexually assault male slaves, though there were laws in Rome against slave men having sex with free women. Some slave women became concubines; children of such unions, and their mothers, were often but not always freed. There were in Rome legal rules against killing and castration or recovering slaves that

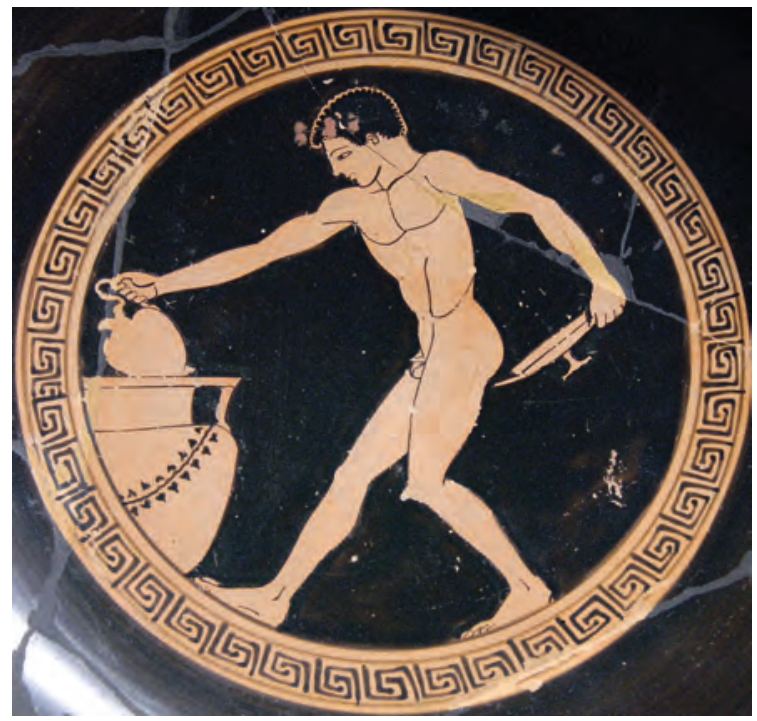

Illustration 9.11

Youth filling shallow wine cup from wine jug. His nudity indicates that he is a cup-bearer at a banquet, C. 490-480 BCE. Cup-bearers were often slaves, Louvre masters had abandoned to illness, but slaves would have faced difficulty in protesting even these offences. Slaves were often punished severely by courts even fed to animals - so would be wary of protesting even the most offensive behaviour by masters. Escape was a more likely option in the face of abuse.

\section{Challenges facing agents: Slaves}

The slave is under the control of the master, and so the slave's room for agency depends crucially on the slave-master relationship. A slave employed in a mine may have little scope to focus on anything beyond survival. In other pursuits, the slave had some scope for "negotiation" with the master. The slave's goal here would be to reduce punishment and enhance rewards: money, goods, or time off. The slave needed then to balance obedience - which would potentially limit punishment - with strategies such as shirking that might induce the master to provide incentives for harder work. For some slaves, freedom was an option that they might pursue. Again, though, it was entirely up to the master to grant freedom.

Female slaves in particular often had to negotiate the sexual advances of masters.

We might also mention a psychological challenge of endurance (or escape) in the face of injustice.

There is evidence of both camaraderie and competition among slaves. They shared common concerns, but each sought the master's favour. Slaves often supervised other slaves. These overseers usually had, and regularly exercised, the same rights as masters to abuse other slaves. 
Masters sought obedience and hard work. Manuals on slavery urged masters to be tough but fair: Slaves should be both punished and encouraged. Masters were encouraged to give slaves holidays (eight a year in Egypt) and even celebrate with them. We can hope that a wise master would appreciate that excessive punishment might achieve obedience on the surface but would encourage the slave toward acts of sloth or sabotage or theft or even violence as opportunities arose. (Violence against masters was not unknown; murder was rare and harshly punished.) For slaves, achieving the best accord with their masters must have dominated their thoughts. The literature on slavery of the time speaks of a difference between real obedience and pretend obedience. Since manuals warned slave masters of the possibility of pretence on the part of slaves, a slave might be better able to gain favour with the master if the slave accepted their position and developed some respect for the master. Even then, the slave would want to hold back some effort or initiative in the hopes that the master would reward them for trying harder.

Many urban slaves worked in trade or craft production. Some slaves took pride in having such an occupation. Here it often made sense for the master to share profits with the slave: Slaves then had an incentive to develop and employ skills and to report transactions honestly to the master. Such slaves were among the most likely to be freed. Masters might promise slaves freedom once they had earned a certain amount of money. Occasionally, though, they reneged on such promises. More often, former slaves continued to work with former masters: The master could finance them and could hope that gratitude for freedom would encourage the former slave to behave honestly toward them. Masters complained bitterly when freed slaves severed their business arrangement.

Most slaves, at least in Rome, had a "purse": an account with the master. Here the master might at least pay the slave bonuses on holidays or for special tasks. The slave could then buy little things in the market for themselves or others. Alternatively, if the sums were large and the master amenable, they might save toward buying their freedom or the freedom of others.

If freed, slaves usually gained that freedom late in life: Men were usually well into their 30s and women past their childbearing years. This in part reflected the time it would take to amass funds to purchase one's freedom. Yet it also meant that masters had received the best years of the slave's productive lives before freeing them. Some masters may have wished to free slaves before they became elderly. Freed slaves, at least in Rome, were barred from political office or serving in the main army, but otherwise had the rights of other free people.

Slaves generally could not marry, but did form unions and have children. The sexual interference of masters was one challenge to slave family formation. So also was the possibility of sale by the master. Masters rarely sold slaves in family units, and so there was always a risk that oneself, one's "spouse," or one's children would be sold to another master. Slaves would strive to negotiate with their master to prevent such sales. Masters had some incentives to encourage family formation: Slave families might be grateful and obedient, and their children would be the master's property. Slaves that gained their freedom would struggle to buy the freedom of family members. 


\section{Trade}

Trade expanded through much of the classical period. Vast empires significantly reduced the risks of trade within their borders. They also actively built roads and ports. They standardized weights and measures. Imperial cities could only survive if fed by the countryside; food was often moved long distances by water. Even the poor benefited from not only cheaper food but also access to pottery, textiles, and metal goods. Trade between empires was usually a luxury trade, but this facilitated the spread of information. Though the products might only serve the elite, technological and religious ideas could affect the entire population.

\section{Currency}

We discussed in chapter 5 the many roles that money plays in facilitating trade and economic activity in general. Before the classical period, cowrie shells and precious metals often served as money. Merchants had to carefully measure metals on scales and evaluate these for purity - a particular challenge since gold and silver usually occur together. The first known coins appear in Lydia in Anatolia in the seventh century BCE - thousands of years after humans had begun to experiment with types of "money." "Coins" in the shape of small knives or spades or cowries also appear independently in China at about the same time or a little after - with the different warring states sponsoring different currencies. (The Qin would later introduce round bronze coins with a hole in the middle, a form utilized for the next two millennia in China.) The emergence of bronze cowries may have reflected a shortage of natural cowries given growing volumes of trade. Coins also appear in India at about the same time. The different physical form of currency in different lands suggests that these were independent developments.

Such coins facilitated trade. They were particularly useful when strangers engaged in trade over small quantities, such as when a sailor wished to buy a meal or a drink in a distant port. It was not in such cases possible merely to keep a record of the transaction to be settled in future, and it was impractical to measure small quantities of precious metal. Coins had a recognized value, and so merchants need not measure them for their precious metal content. Yet this was only possible if states themselves had the technical capacity to produce coinage of uniform purity (we are not quite sure how they did this at first), and could be trusted to do so.

Coins were produced by states (at a small profit) and could be used to pay taxes to that state. Coins could then carry a value that exceeded their precious metal content. Coins traded far beyond the borders of the states that issued them. Other states soon recognized the advantages of issuing coinage. The Persian conquest of Lydia in the sixth century BCE served to spread the idea of coinage widely. In addition to the economic advantages of coins, there were also political benefits: By inscribing laudatory images of a present or past ruler 

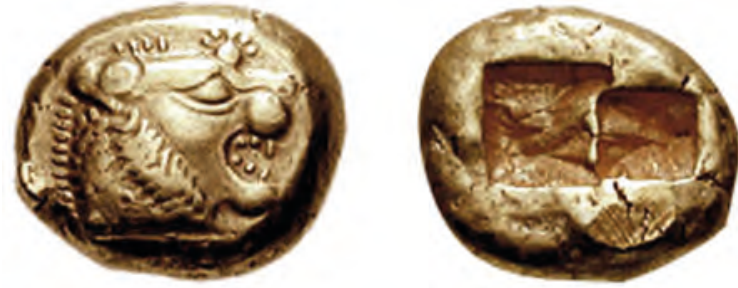

Illustration 9.12

Lydian coin, 6th century BCE, head of roaring lion with sun with multiple rays on forehead; punched on reverse

Classical Numismatic Group, Inc. www.cngcoins.com, CC BY-SA 3.0 on a coin, one advertised the power of the state. Coins were like little statues circulating within and beyond state borders. The financial and political advantages to states of minting coins gave them some incentive to protect the reputation of their currency - but there will be many examples in history of states trying to reduce the metallic content of coins.

\section{Finance}

We noted in chapter 6 that the recordkeeping needs of merchants played an important role in the development of writing. Most of the financial instruments with which we are familiar today - including loans, letters of credit (by which merchants could transfer loans to another, perhaps in a distant town), futures contracts, partnership agreements, deeds to land, and mortgages on property can be identified in Mesopotamia and elsewhere from the second millennium BCE. In ancient Ur, we even see contracts for a fixed annual payment for many years - perhaps a very early retirement pension. Ancient trade and ancient agriculture involved making commitments months or years in advance. States needed to coordinate tax payments and requisitions of supplies in advance also to ensure that they paid and provisioned armies on time.

In both Mesopotamia and ancient Greece, the word for "interest" derived from the word for "calf." If I borrow money to buy a cow, I reward the lender at some point in future with not just the price of the cow but also (some of) the value of the calf it produced as well. Notably, Christianity and Islam would later object to charging interest because money did not reproduce in the way that animals do. Still, the idea that borrowers paid interest to lenders was generally accepted in the ancient world. Yet while the principle of interest was accepted, states often worried about the level of debt incurred by citizens. (In Ur, interest rates were often in the range of 20 per cent per month, a level that would not be countenanced by today's legal authorities.) Mesopotamian kings periodically forgave all debts in their kingdoms - a policy which might relieve the poor temporarily but could be disastrous for merchants that had made many loans. There are two dozen documented cases of debt relief in the centuries after 2400 вСЕ in Mesopotamia. Merchants who feared that their ruler might forgive debts would charge higher interest rates to compensate for the increased risk; if rulers regularly forgave debts, then, they might serve to increase the suffering of borrowers. Ancient states also occasionally offered "bailouts" in which private debts were redeemed with state funds.

A succession crisis in Persia was resolved when the supporters of the man who would become Darius II mortgaged their properties in order to hire mercenaries to overthrow his relative Sogdianus. This single event signals both 
an important connection between kings and landowners and also the importance of financial institutions - and mercenary soldiers - in determining political outcomes.

The first "banks" appeared in ancient Greece. Though merchants (and temples and states) had been making loans for millennia, specialized institutions devoted to borrowing and lending first appeared in Athens. The word for "bank" may originate from a table at which bankers would sit, covered with a grid that facilitated arithmetic calculations: Bankers needed to be able to calculate interest payments on various sums for various time periods. Athens also developed a commercial court in which juries reached judgement within one day regarding contract disputes. Plaintiffs and defendants each were given a limited time period to make their case: They sometimes paid orators to draft their speeches to the court. Merchants from other locales often preferred the courts of Athens. (This legal system may also have facilitated trust and social peace and therefore Athenian democracy.) Athens occasionally prosecuted merchants in what we would now call "antitrust" proceedings: attempting to collude in determining prices. Rome would later allow lawyers to speak on behalf of clients. Rome would also allow an institution that looks like a modern limited-liability corporation: People could buy and sell shares in these and could not (it seems) be sued for more than the cost of the shares if the

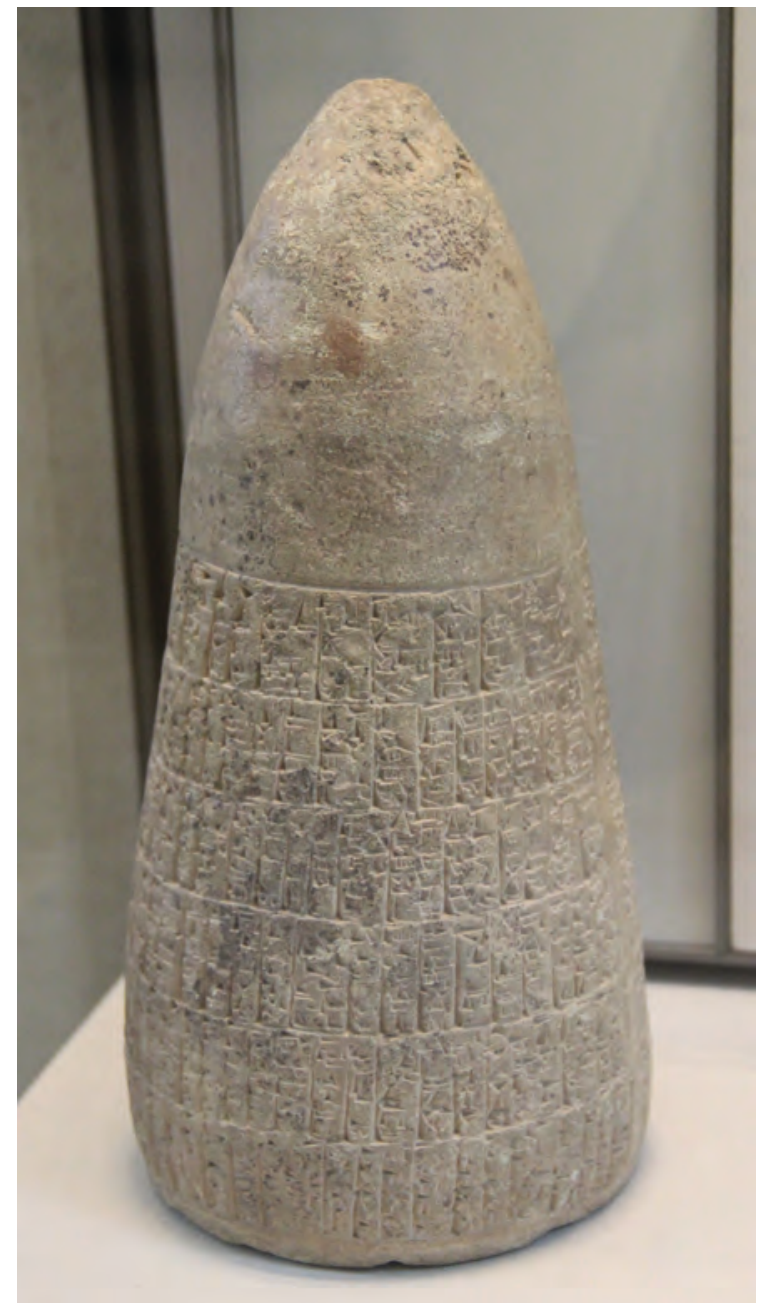

\section{Illustration 9.13}

Enmetena Cone; After a victory of Lagash over Umma, the cone awards reparations to Lagash - including calculations of compound interest - for lands taken by Umma two generations previously. The result was more than the entire barley harvest to date in Mesopotamia and thus justified conquest, Louvre Photograph by Gary Todd, CC0 1.0 corporation went bankrupt or committed fraud. Such corporations collected taxes or provisioned the army in certain regions, and may have pursued other tasks. In essence, they worked for the government.

Roman law limited the liability of a slave owner for the actions of a slave, as long as the slave was not acting on the direct instructions of the master. This allowed Romans an easy path to a limited-liability "company." They could finance a slave in business, and share in the profits (recall our discussion of 
slavery above). As long as the slave operated the business independently, the slave master could lose no more than the funds they had advanced to the slave. A wealthy Roman might have a diverse portfolio of slave-run businesses and need not worry about being sued because of the actions of any of these. Such an institutional setup might be particularly attractive to senators: These had to prove that they were wealthy to serve in the Senate but were not supposed to be directly involved in business (to limit conflicts of interest between their senatorial duties and private investments).

\section{Sea trade}

Though far less famous than the Silk Road (below), far more trade occurred, and for a much more extended period, by sea. The western half of the Indian Ocean was the primary hub of classical commerce. The Romans traded east to the Indian Ocean and even had trading colonies in India for pepper. Roman writers often complained of the unnecessary luxuries imported from the east and especially the precious metals sent east to pay for them. Indians traded pepper, cotton, and other manufactures to Southeast Asia for spices (essential for Buddhist healing practices, among other things), which were then often marketed further west. Persians, Arabs, Egyptians, and Romans all operated in the Indian Ocean, vying for dominance in its western half (but mostly leaving the eastern half to Indian merchants). The Byzantines (the Eastern Roman Empire; chapter 13) would attack Yemen in 525 CE in an attempt to pry trade away from the Persians. The Persian Gulf saw considerable traffic connecting Mesopotamia to India and the resources of Yemen.

There was less traffic on the Red Sea: It was a hostile environment with few trade goods of its own - but it served as a possible connection to the Mediterranean. The Red Sea is one of the hottest (and the saltiest) seas on the planet, and is mostly surrounded by deserts and often mountains also. The few ports that existed tended to be outposts of nearby societies in Egypt, Ethiopia, Arabia (which often imported food from Egypt across the sea), or Yemen. Coral reefs and shoals render navigation difficult. Winds from the north most of the year discouraged sailing, especially in the northern Red Sea, so merchants often transferred goods to the Nile or to Arabian caravans to be carried north. The Persian emperor Darius would build a canal to the Nile from the Red Sea, but this became impassable within a century. In $287 \mathrm{BCE}$ there is a recorded battle with pirates in the Red Sea, indicating that there was some traffic. This traffic seems to have expanded with Greek support after Alexander, and then again under the Romans. There is a report of 120 ships annually from the Red Sea to India after the Roman takeover of Egypt: This is a considerable but not huge number.

There was also trade southward along the coast of Africa; rhinoceros horn, tortoiseshell, ivory, and slaves were imported. Egypt itself exported metals, glass, tools, and textiles; it imported myrrh, frankincense, pepper, spices from Indonesia, precious stones, Chinese silk, and rhinoceros horn. Alexandrian 
merchants linked the Red and Mediterranean Seas. After the decline of Rome, merchants from Axum in modern Ethiopia became important in the western part of the Indian Ocean, from the port of Adulis on the Red Sea (Muslim Arabs would later eclipse them).

There were two significant advances in Mediterranean shipping technology (probably) in the late classical period. One was the lateen (triangular, with the broad part on top) sail, which allows sailing closer to the wind and requires fewer sailors. It was developed some time between the second and sixth centuries CE, and likely reflected preceding Arabic technology (which in turn probably borrowed from Southeast Asia; see below). The second is "framefirst construction" which involves building the ship frame and attaching the hull to this rather than starting with the shell. This approach rendered ships both cheaper and sturdier. It is hard to pin down the timing, but it was likely between the third and eleventh centuries CE.

We know little about the ships used in the Indian Ocean. None has been recovered, and textual references are vague. There appears to have been many types, some very large. They used both sails and oars. They seem to have relied on ropes to tie planks together rather than joints. Indian ship technology likely evolved independently from that in the Mediterranean. Southeast Asians also tied planks together, but in a different manner. The Chinese used joints.

Southeast Asians were also active in trade (and fishing). Somehow, sailors from Indonesia populated Madagascar, perhaps from the seventh century BCE. These likely also introduced the banana and taro to Africa, and the zither (a

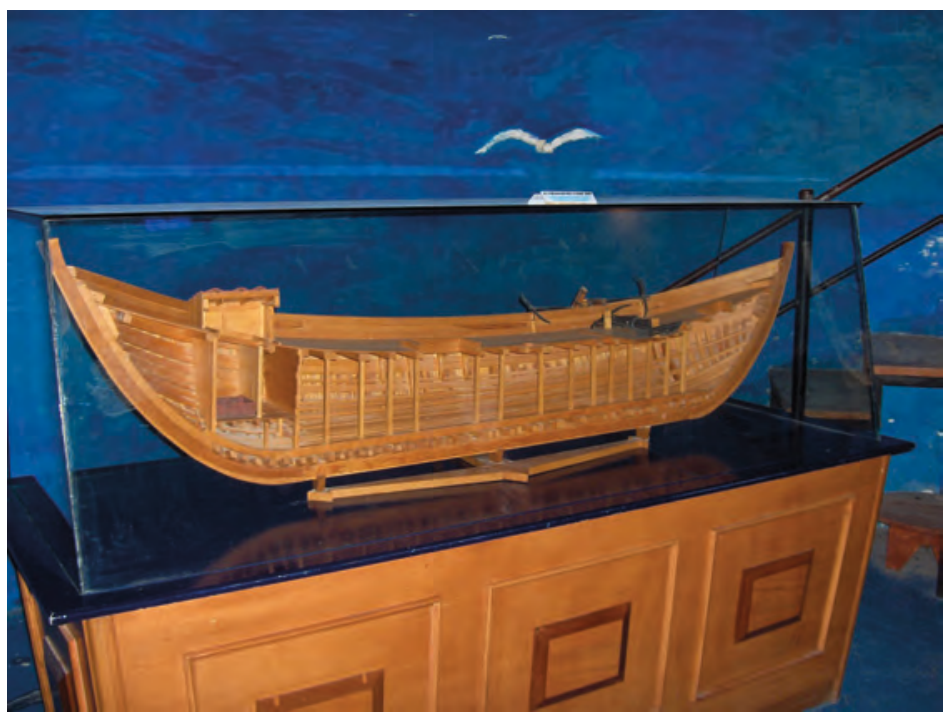

Illustration 9.14

Model of the Yassiada vessel (Byzantine shipwreck, 7th century CE), Bodrum (Turkey) Museum of Underwater Archaeology

Photograph by Georges Jansoone, CC BY-SA 3.0 


\section{BOX 9.2}

\section{SHIPWRECKS}

UNESCO estimates that some three million shipwrecks are lying at the bottom of the world's seas and oceans. Shipwrecks today are a rare tragedy, and are usually the subject of lengthy investigations. Shipwrecks were more common earlier in history. Ships were more fragile, and reliant on the wind, and sailors only slowly gained an appreciation of where and how to avoid rocks and shoals. Shipwrecks provide archaeologists with a good sense of the types of ships used in at least some times and places, and the goods that these carried.

musical instrument with many strings stretched across a flat surface). They likely did so in large ships with multiple sails; these ships with a cargo capacity of 500 tons may have inspired the later Chinese junks. Their sails may have been forerunners of both the Arabic lateen sail and (even more likely) the sails used by Polynesians in their Pacific explorations (chapter 16). They may have stopped in the Maldives and Seychelles on the way to Madagascar. They seem to have introduced cinnamon to Egypt, perhaps indirectly. They also traded north to China and Japan, and east to the Spice Islands (chapter 15) of eastern Indonesia.

In China, the first records of sea trade between south and north are from the sixth century вСе. In 219 вСе, while the Han were subduing the south, the Han cut a canal that linked the Yangtze and Pearl rivers, allowing inland travel for thousands of kilometres. That canal is still in use today. Trade by ocean through Guangzhou could then link China to other lands. There was undoubtedly regular communication by sea

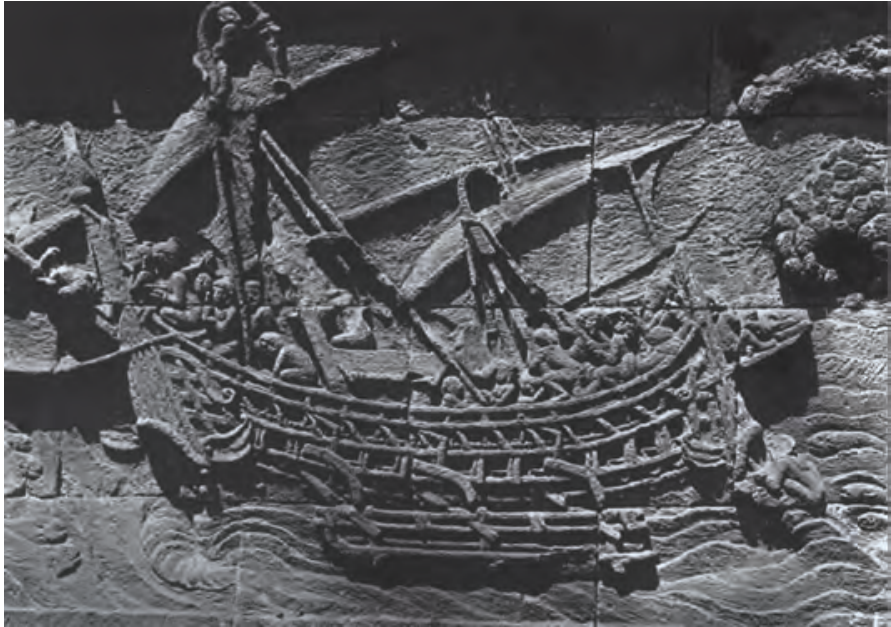

Illustration 9.15

Relief of a ship from Borodupur on Java (8th-9th century CE) Tropenmuseum, Amsterdam, CC BY-SA 3.0 with India by 87 BCE. Merchants (mostly Indian) at the time travelled by foot across the Malay peninsula, though from about the fifth century $\mathrm{CE}$ it became common to instead sail through the Straits of Melaka. Chinese merchants were known throughout the eastern Indian Ocean from at least the fourth century CE. Though longer than the overland Silk Road, the sea route may have been a safer means for Chinese trade with the west.

Spices from China (ginger, cinnamon), Southeast Asia (cinnamon, nutmeg, mace, 
cardamom, cloves), and India (pepper and sesame oil)) moved west, as did Chinese and Indian textiles. Glass, jewellery, pottery, and textiles moved east. Afghan gemstones were also traded. Note that spices were particularly important at the time both to flavour poorly preserved meats but also as aromas, drugs, reputed aphrodisiacs, and magic potions. (It may be that the supposedly magical properties associated with spices increased as contact with the lands they came from declined with the fall of classical empires: The lack of direct contact allowed scope for imagination.)

Monsoon winds governed shipping in both the Indian Ocean and the South China Sea. In the Arabian Sea, these blow steadily from the southwest from June to September/October, and from the northeast from November to April/ May, facilitating predictable, although not necessarily smooth or safe, passage between all ports of call in a year. The monsoon patterns meant that sailors often remained in ports for a month or two waiting for the winds to allow a return voyage. Goods usually moved in seasonal stages, with both southern India and Southeast Asia serving as trans-shipment points where products could be stored several months before the winds could carry them further. January was the best month for sailing around the southern tip of India. It appears that Greek sailors in Egypt discovered in the first century BCE how to sail straight across to India rather than hugging the coastline. Funan, a state in southeastern Vietnam and Cambodia, prospered in the second through sixth (at least) centuries $\mathrm{CE}$ as a transit point for sailors who might spend months there between monsoons, consuming the local rice surplus (generated by irrigation; see chapter 13). Archaeologists have found artefacts from as far as the Mediterranean there. Funan also controlled the portage route across the Malay peninsula.

\section{Trade networks}

The Periplous of the Erythraean Sea is a survey of trade and navigation in the Indian Ocean, written in Greek in the mid-first century CE by a merchant from Egypt, which was then a part of the Roman Empire. The book discusses how and when to sail to various ports and what to trade for there. It also addresses the political situation in different ports and suggests how merchants should conduct themselves. The book appears to be far more accurate in its discussions of ports nearer to Egypt, which is itself indicative of the fact that Indian Ocean trade proceeded in stages. The book describes networks of Greek merchants in some detail but also alludes to networks of Mesopotamian, north and south Indian, and Arabian merchants. It is hard to judge from this one source how strong the ethnic identity within these networks was; language may have been the most important bond, but religion may also have been critical. Archaeologists have found texts in twelve different languages in one Red Sea port of the period.

There are records of an extensive trade network based in Palmyra (in modern-day Syria) that spread from Rome to India. It certainly used a shared language - Aramaic, though this was not the most common language around 
Palmyra - and referred to a common pantheon of gods. This particular network appears to have stressed ties by lineage. The network fell apart when Rome attacked Palmyra, indicating yet again the importance of political circumstances for trade relationships.

\section{Silk Road}

The Han traded silk for horses with the nomadic Xiongnu Empire to the north and west - but also built the (first part of the) Great Wall to defend against them. Emperor Han Wudi in 139 BCE sent an envoy Zhang Qian west through Xiongnu lands to try to form alliances with other nomads. Though he was unsuccessful - and the Xiongnu captured him in both directions, holding him once for a decade - he reported that Chinese goods were reaching Central Asia via southwest China and India. This encouraged Han Wudi to increase efforts to find allies and push into Central Asia, which he did (after decades of battles) during 102-98 все. Once China established a state presence well into Central Asia, it became much easier for the Chinese to trade overland with Europe and the Middle East and northern India. Spices, animals, and a variety of other goods were transported along with silk.

Although there were multiple Silk Road routes, the most common focused on the Tarim Basin in present-day Xinqiang (the westernmost province of China). This is an area of sand dunes surrounded by mountains on three sides. Rivers flowing from the mountains provide oases that could support merchant caravans. The basin may have been the last part of Asia to be settled - and at

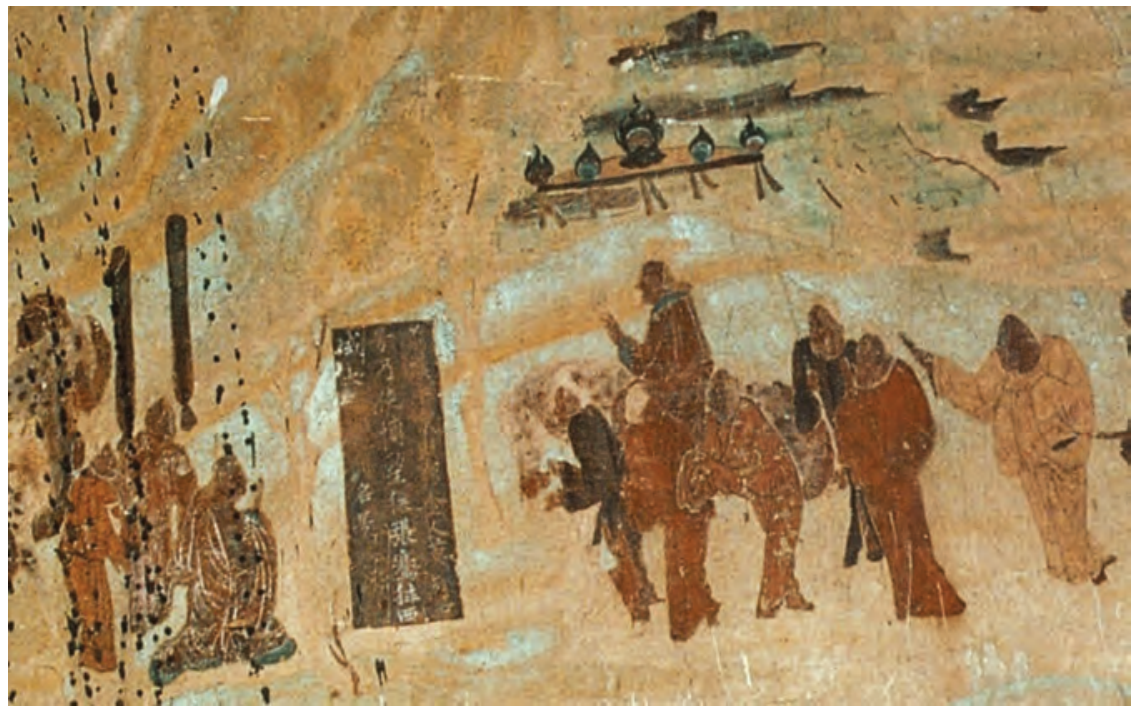

Illustration 9.16

Emperor Han Wudi and officials send Zhang Qian (kneeling at left) to the west. Mural in Mogao caves, 618-712 CE 
first by Caucasians from the west - some thousands of years BCE. The Xiongnu had taken control of the basin, but the Han replaced them. The Han also controlled the Gansu corridor, an 800-kilometre pathway to the Yellow River to the east, bordered to the north by the Gobi Desert and to the south by the Tibetan plateau. As in the Tarim, rivers flowing from Tibetan mountains provided occasional oases along the route. Though trade had passed through the Tarim and Gansu for millennia, and had been growing since the second half of the second millennium, Han control of these distant lands greatly facilitated this trade. To the west, the Kushan Empire expanded to, and later into, the Tarim Basin. Merchants trading between China and the west only had to negotiate passage with a couple of large empires rather than myriad small polities. After the fall of the Han, Hepthalite and Turkish nomadic polities would maintain trade routes through Central Asia in succeeding centuries; at this time a network of Sogdian merchants based in centres such as Samarkand dominated Silk Road trade and had representatives well into China (and sent trade missions to Persia and Constantinople). Indeed, the fifth through eighth centuries was a golden age for centres such as Samarkand and Bukhara, which were among the wealthiest cities on the entire continent. Still, merchants pursued trade through the Tarim only in large armoured caravans until the Sui dynasty (chapter 13) re-established Chinese control there centuries later.

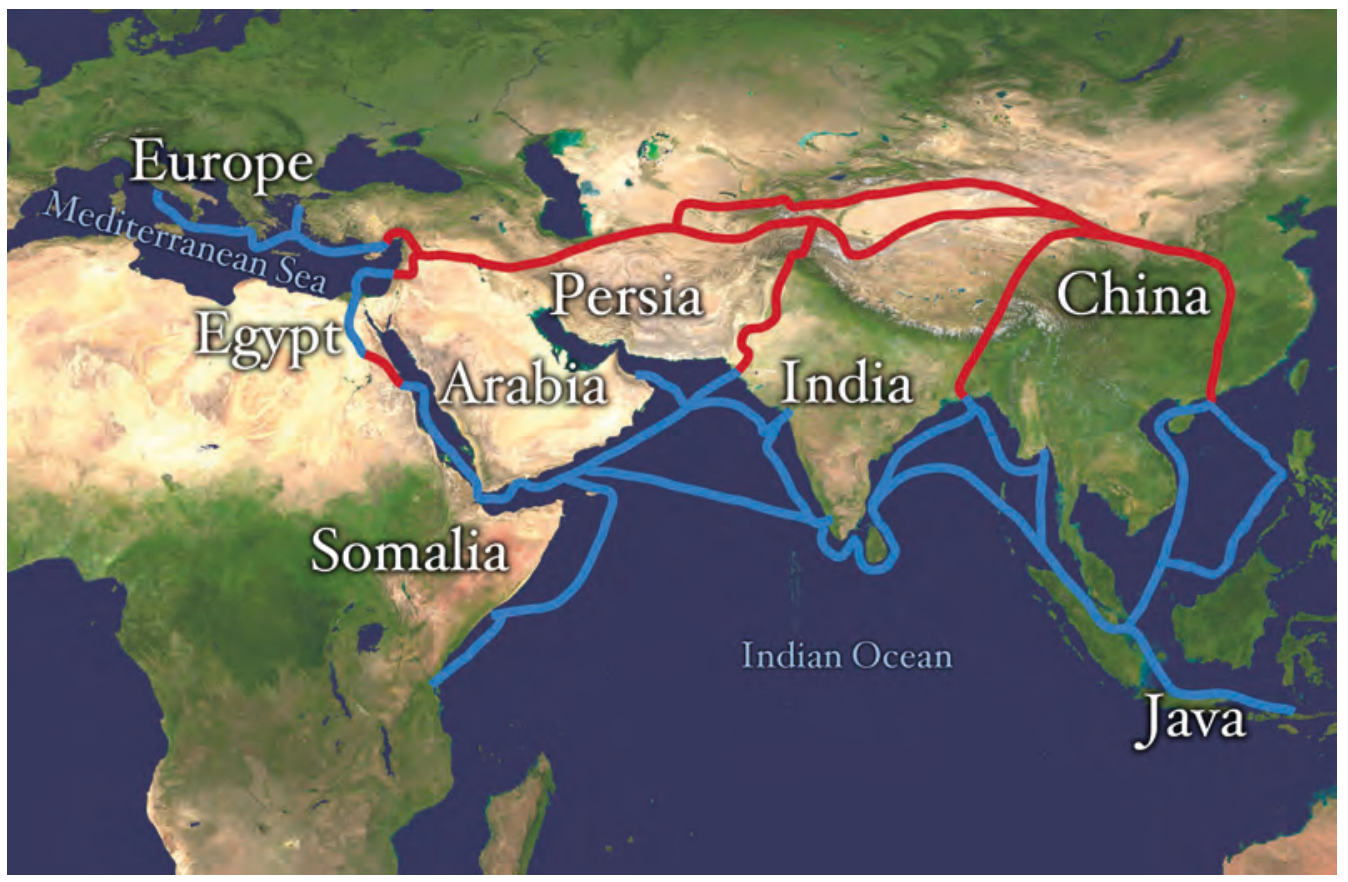

Map 9.3

The Silk Road and major water routes 
Silk Road trade usually occurred in stages. Trade goods passed from one ethnic network of merchants to other ethnic networks along the way. The nomadic and oasis peoples in between served as intermediaries but also added their own trade goods - animals and animal products, metals, furs, and melons, among others - to Silk Road trade. The Chinese, after conquering the Tarim, soon extended their influence into the Ferghana Valley to obtain the muchprized Ferghana horses. Information about more distant lands remained vague, though there was at least a dim awareness of these. The goods transported nevertheless had important cultural effects: Silk was a treasured luxury in the west, and Indian black pepper became a staple of Chinese cuisine.

Long-distance trade, we should stress, was not only international but internetwork. To facilitate this trade, merchants had to trade both within and beyond their networks. Merchants that could establish trust with a member of another network could become critical links in ongoing chains of commerce. Of course, merchants would only trade beyond their networks if they could earn a profit that rewarded them for the risk associated with doing so. The cost of trade would be higher the more networks that had to be involved.

Palmyra (in present-day Syria) marked a place where Roman merchants (often Jews or Armenians) traded with Persian merchants who in turn traded with Central Asian or Chinese merchants to the east. (We saw above that Palmyra was also the home of an Aramaic-speaking network.) The economic impact of

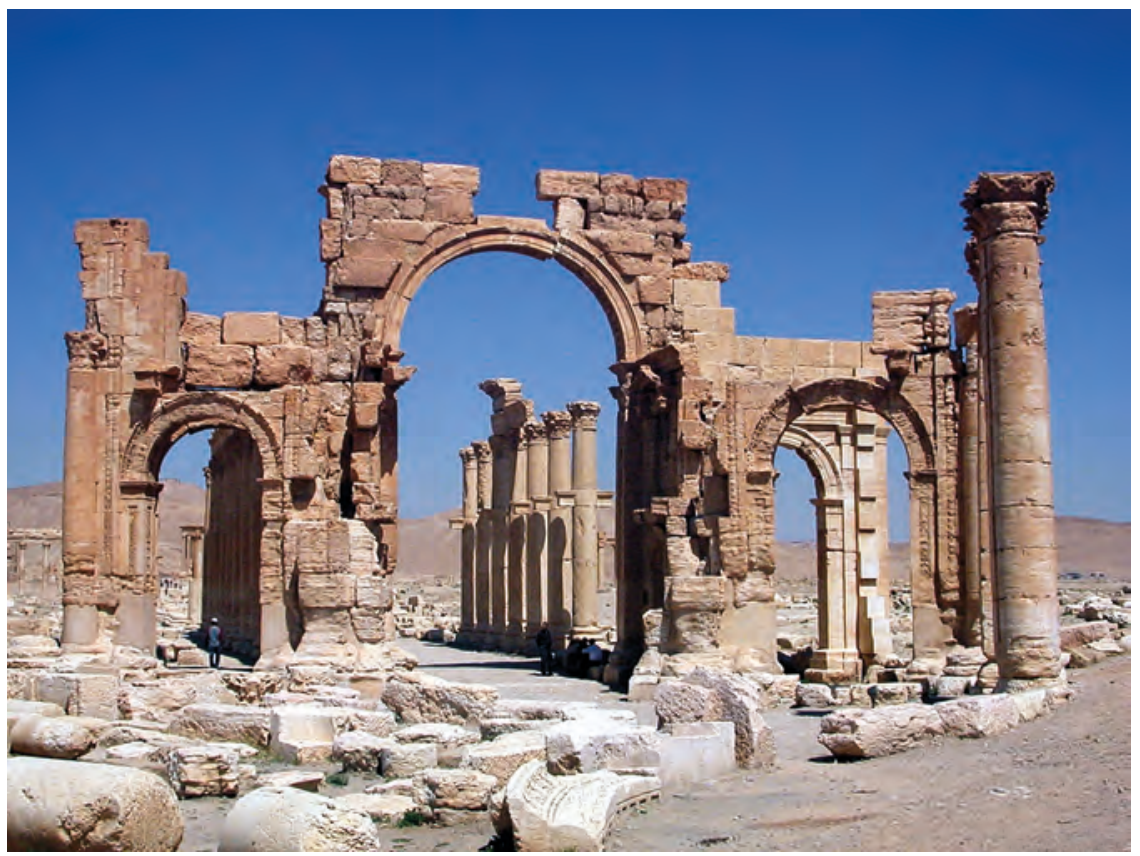

Illustration 9.17

Monumental arch, Palmyra (also called Hadrian's gate)

Photograph by O. Mustafin, CCO 1.0 


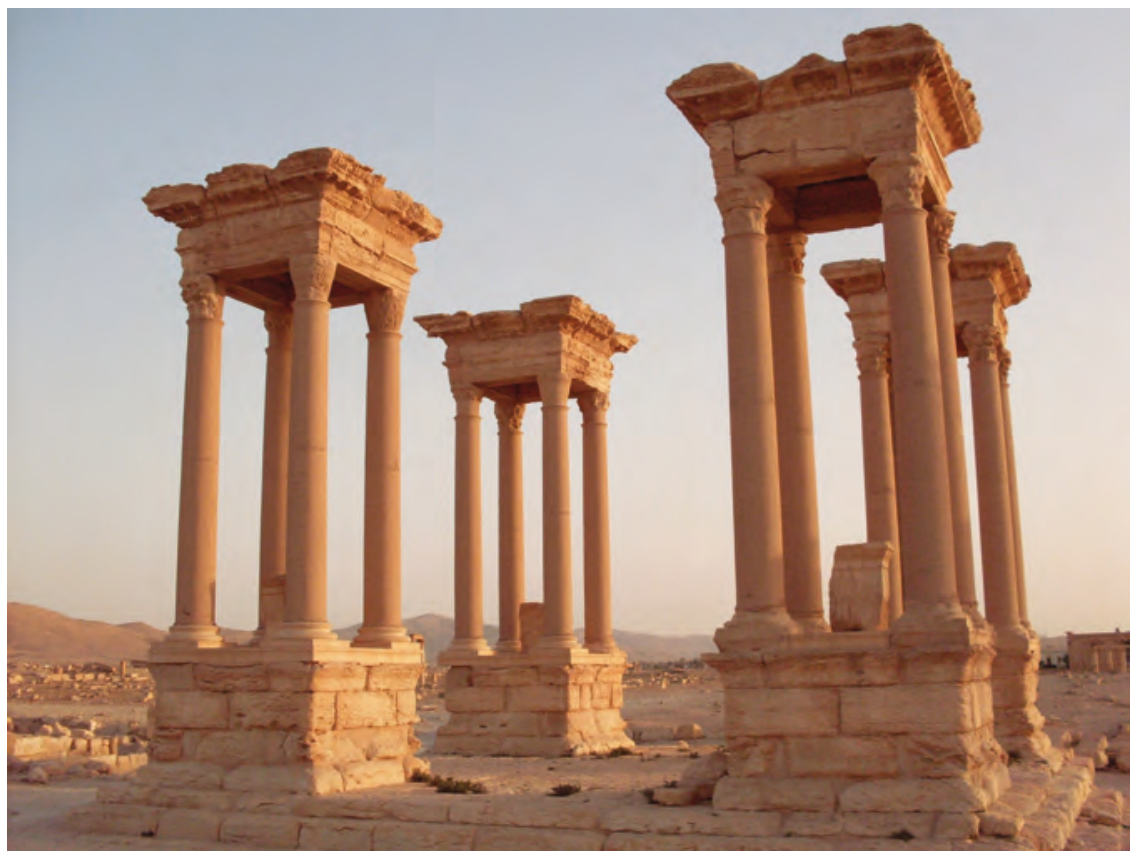

\section{Illustration 9.18}

Palmyra colonnades. These stood at an intersection; two have been destroyed in recent years

Silk Road trade on China or Persia or Rome as a whole was likely minimal, but its impact on trading centres such as Palmyra and Samarkand in Central Asia was dramatic. These (and many other centres along the way) became cities noted for public monuments and the wealth of their merchants. They were also cosmopolitan centres: They were, after all, places where different ethnic merchant networks intersected. Such centres were usually religiously tolerant. The Sogdian merchants of Samarkand and other trading centres in what is now Uzbekistan were known as far away as China and Europe for their sophistication in trade and finance, and played a significant role in the transmission not just of goods but of religions and technology. These trading centres were usually independent city states, though they often paid tribute to larger polities, whether nomadic or sedentary.

Trade expanded across the Sahara during the classical period also. Trade may have been common before 3200 BCE when the Sahara itself was wetter and therefore inhabited (chapter 7). Donkeys and even oxen likely traversed the desert, and coins from early Rome have been found south of the Sahara. Trade expanded with the introduction of camels into North Africa from Asia from at least the first century всЕ. Camels greatly facilitated travel across the desert. It was nevertheless a perilous journey with dust storms, days between oases, and thieves. Gold and salt moved north in return for the sorts of goods traded along the Silk Road. Trade networks and large armed caravans were of critical importance in this environment. 


\section{Cultural and health impacts}

The cultural - and especially religious - impact of inter-empire trade was likely far more significant than the economic impact. Buddhism was carried to Central Asia in large part by merchants and became the most common religion in the (cosmopolitan) oasis towns along the route in Central Asia from 200 BCE until the arrival of Islam. From there it reached China by about the first century $\mathrm{CE}$, but was mainly a merchant faith until about the fifth century $\mathrm{CE}$, after which it spread through East Asia. By at least the first century CE, both Buddhism and Hinduism had been spread by merchants to Southeast Asia where local rulers converted and built temples. Nestorian Christianity also spread along the Silk Road as far as China but in limited numbers (see chapter 11).

Epidemics of bubonic plague, smallpox, measles, and other diseases reduced the Roman population by about a quarter in the second, third, and fourth centuries. Similar epidemics occurred in China a little later in the third to sixth centuries. We can suspect that travellers along the Silk Road spread these epidemics. These epidemics in turn induced economic contraction in both of these empires and elsewhere. This economic contraction reduced imperial revenues, and

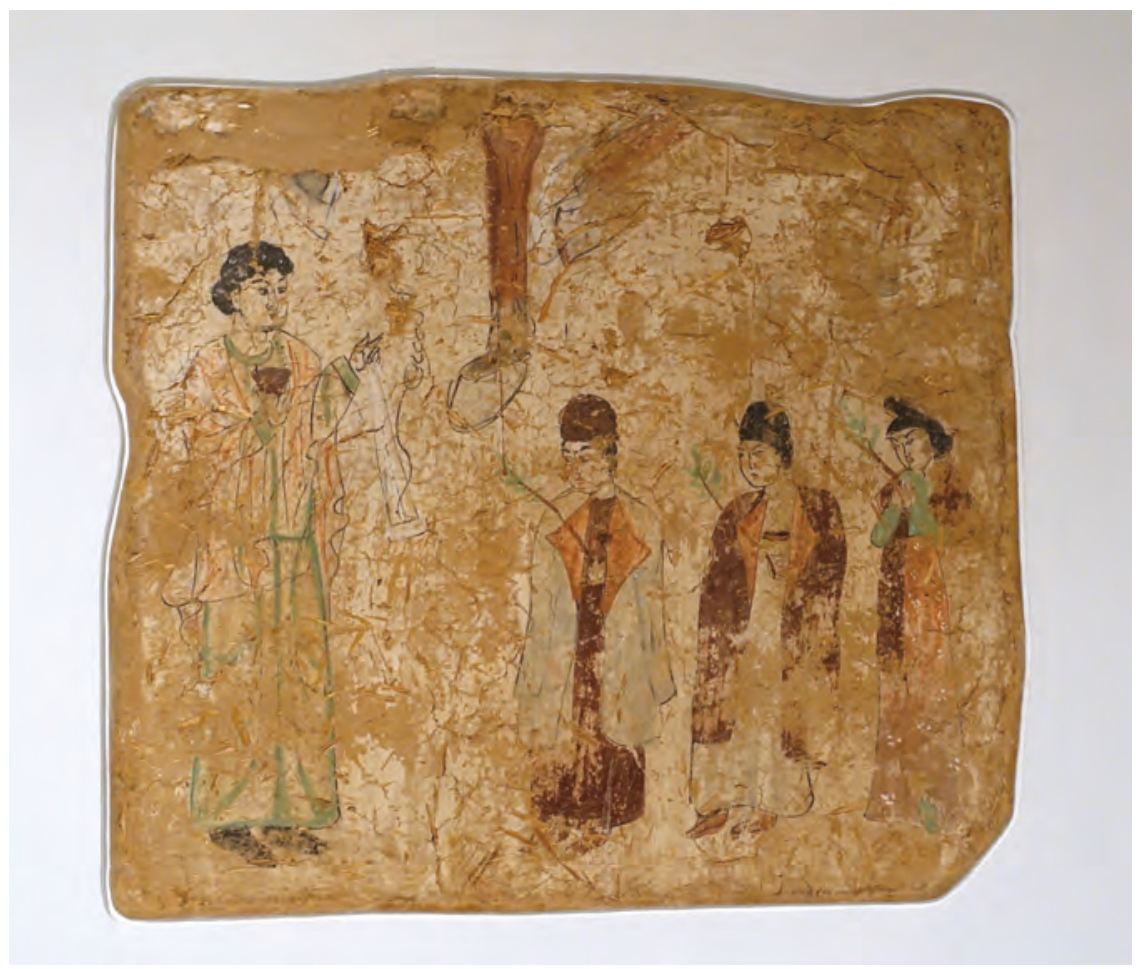

Illustration 9.19

Wall painting from a Nestorian Christian church, Gaochang, China, 683-770 CE, Ethnological Museum, Berlin

Photograph by Daderot, CCO 1.0 
might then explain (at least in part) the decline of classical empires. However, we know very little about the precise effects of these epidemics.

\section{Ancient science}

We should note that all early empires devoted state resources to the more practically oriented sciences: mathematics, astronomy, and medicine. Bureaucrats occasionally needed to perform complicated calculations, involving multiplication, exponents, and systems of equations. Astronomy itself demanded mathematical calculations. Astronomy was useful in determining the proper calendar and therefore when to plant and when to hold specific celebrations. Astronomy was often also used as astrology: to predict earthly events. Moreover, astronomy in at least some lands had some religious significance. Medicine, of course, might be useful to both the ruling elite and the general population - though the medicine of the time could do little more than suggest herbal remedies. We find astronomers and doctors on state payrolls in the Byzantine, Chinese, Sassanid, Gupta, and Abbasid empires, among many others. This was not, we should stress, a curiosity-driven science. States very much focused on practical aims. Of course, some government scientists may have pursued their curiosity, but such behaviour was not encouraged.

It was in India around 250 вСе that a system of numerical digits was first developed. These are known as Arabic numerals in the west because the idea was later transmitted westward by Arabs. The Chinese excelled at astronomical observations. For example, they had identified the regular appearance of Halley's Comet from 240 вCE. Europeans would celebrate the same feat two thousand years later and belatedly attach Halley's name to it.

The politically fragmented (before Alexander) Greeks produced the only curiosity-driven science in the ancient period. Notably, people (usually men) of independent means, or professionals pursued this science outside of state bureaucracies (of which Greece had little). Scientific practice was thus very similar to the Greek philosophy we will discuss in chapter 10, and indeed often was pursued by the same people - Aristotle was one who wrote about what we would now call science as well as philosophy. As with philosophy, we can identify a critical mass of scientists across Greece who communicated with each other. The earliest of these scientists were centred in Ionia - the western part of present-day Turkey - which would have facilitated communication. We know their names, whereas the scientists working for empires were typically anonymous. (We should note here that independent science and philosophy were pursued only because of basic human curiosity, the fact that at least some of us enjoy exploring the unknown.)

Empires saw little value in what we would now call "physics" and "chemistry." The Greek scientists grappled with questions of the nature of matter and earth. They famously came to argue that there were four essential substances: earth, air, water, and fire. This understanding may seem simplistic to modern students 
of atomic theory and the periodic table of elements; nevertheless, we might appreciate it as an advance over previous theories that had privileged one or the other of those four as the primordial substance. A minority school argued for something like the modern understanding of atoms. The Greeks, of course, lacked the scientific instruments with which to investigate atomic theory.

\section{PRIMARY DOCUMENTS: THE FOUR ELEMENTS}

Pliny the Elder, Roman aristocrat, in his The Nature Story, chapter 5, writes:

I do not find that anyone has doubted that there are four elements. The highest of these is supposed to be fire, and hence proceed the eyes of many glittering stars. The next is that spirit, which both the Greeks and ourselves call by the same name, air. It is by the force of this vital principle, pervading all things and mingling with all, that the earth, together with the fourth element, water, is balanced in the middle of space. These are mutually bound together, the lighter being restrained by the heavier, so that they cannot fly off; while, on the contrary, from the lighter tending upwards, the heavier are so suspended, that they cannot fall down. Thus, by an equal tendency in an opposite direction, each of them remains in its appropriate place, bound together by the neverceasing revolution of the world, which always turning on itself, the earth falls to the lowest part and is in the middle of the whole, while it remains suspended in the centre and, as it were, balancing this centre, in which it is suspended. So that it alone remains immoveable, while all things revolve round it, being connected with every other part, while they all rest upon it.

Between this body and the heavens there are suspended, in this aerial spirit, seven stars, separated by determinate spaces, which, on account of their motion, we call wandering, although, in reality, none are less so. The sun is carried along in the midst of these, a body of great size and power, the ruler, not only of the seasons and of the different climates, but also of the stars themselves and of the heavens. When we consider his operations, we must regard him as the life, or rather the mind of the universe, the chief regulator and the God of nature; he also lends his light to the other stars. He is most illustrious and excellent, beholding all things and hearing all things, which, I perceive, is ascribed to him exclusively by the prince of poets, Homer.

Later, he reasonably talks about how we see lightning before we hear thunder because of the relative speeds of sound and light - and notes that one is safe as long as the sound and light are quite distinct. Reflect on how his understanding of four elements made sense at a time when the instruments for studying atoms did not yet exist.

Greek scientists also grappled with medicine and astronomy. Hippocrates developed the oath "to first do no harm" still taken by doctors throughout the Western world. Yet classical medicine tended to be mostly theoretical: Its identification of a range of plausible causes of disease, including the psychological but 
also diet, exercise, and sexual activity, allowed learned men to give complicated advice to affluent patients - but it is far from clear that this advice had more than a placebo effect. There was no dissection of bodies, and little effort to track the effects of a range of natural remedies. As for astronomy, the Greeks posited circular orbits of the planets and sun around the Earth; astronomers in many lands would struggle to explain observations that deviated from this posited circularity for millennia (chapter 22).

Greek science would not disappear with political centralization after Alexander. Indeed, the state would come to sponsor the most famous scientific institution of the ancient world: the Museum and Library at Alexandria in Egypt. This was effectively a state-financed research centre with up to a hundred scholars at a time. It would survive for seven centuries. Rome, though less interested in science, would continue funding it after its conquest of Egypt. As with other state-sponsored science, the state hoped for practical results. Yet scholars exercised considerable independence (see below). Science in this part of the world would decline as Rome declined.

There is a potential conflict between science and religion: One of the purposes of religion, after all, is to provide an understanding of how the world works (chapter 8). Ancient science rarely confronted religious belief. Astronomers in the pay of the state were all too willing to apply their observations toward divination if the state asked. (As we shall see, this would be a characteristic of astronomical research for centuries: Kepler, who in the seventeenth century was the first to recognize that planets pursued elliptical orbits, financed much of his research by producing astrological charts; chapter 22.) Greek religion was polytheistic and flexible; this may be one reason why science flourished there. There is some speculation that the decline of science in the region is partly due to the rise of Christianity - Augustine, who did so much to connect Christian theology to Greek philosophy, disparaged science: All that we needed to know about the nature of the world was in the Bible.

Scientific ideas were transmitted across societies. Nestorian Christians fleeing eastward (chapter 11) carried Greek science to the Sassanid court. The Greeks had previously built upon Mesopotamian and Egyptian medicine. The Guptas in India would purposely borrow and develop ideas from Greece and Persia. Only Chinese science developed autonomously within Eurasia. Mayan science necessarily also developed in isolation. It too stressed mathematics and astronomy: The Mayans independently developed the important mathematical concepts of zero and place value. They may have produced the most complex calendars ever, with 260- and 365-day combinations. Mayan astronomy is acclaimed for detailed observations, which served both a religious and administrative role.

We should stress that science was limited in its impact during this period. Arithmetic, calendars, and some medical ideas were useful. However, theories of physics and chemistry were of little use to those building machines or smelting iron. The limited link from science to technology would remain in place until the eighteenth and especially nineteenth centuries CE. 


\section{Challenges facing agents: Scientists}

The scientist, like the priest, needs to convince others of the veracity of their ideas. They will be more convincing if they believe these ideas themselves. However, the scientist speaks primarily to other scientists - though in the early days of science a wider public might judge ideas. In talking to other scientists, the scientist needs to master the norms of the community: what questions they care about and what sorts of argument and evidence they find compelling. A considerable part of the story of science involves how these norms evolve toward recent insistence on evidence from some formal scientific method. It was still possible in the days of ancient Greece to engage in "armchair theorizing," though some observation of the world was typically expected. Even today, scientists may have to be as creative in their persuasive acts as in the development of new ideas.

Even "armchair theorizing" takes time. The scientist then always faces a challenge in financing their research. Here we should stress that science throughout most of history was linked weakly to technology: Science, the understanding of the world for its own sake, was therefore only rarely useful. This is why states sponsored only a minority of sciences. Scientists interested in pursuing their curiosity in other realms needed somehow to finance their activities. This simple but important constraint would limit the pursuit of science throughout pre-modern history.

We might also note the intellectual challenges. While some scientists may be most interested in fame, we can hope that scientists usually are motivated by a desire to understand. Even today, our understanding is limited. The scientist is always trying to understand something that humanity collectively has not yet understood. The scientist is always, then, confronting our collective ignorance. Yet the need to convince others encourages the projection of confidence (perhaps even arrogance) in scientific communication (though less so than in priestly communication) when humility might seem the more proper attitude.

Note that scientific innovation, like technological innovation, follows the four-step process of Figure 5.3. The scientist first needs to recognize a question that other scientists (and perhaps rulers and merchants) care about, and then gather relevant information; networking is typically vital in both steps. The act of inspiration can only follow much hard work, but itself requires that the scientist takes a break from work so their subconscious can thrive. The scientist must then put more effort into clarifying their idea, and then the critical task of persuasion begins.

\section{Libraries}

We can only speak of the sustained development of ideas - in science or politics or philosophy - if these ideas are transmitted across generations. Orally transmitted ideas tended to change with repetition. Writing allowed a particular author to clearly articulate ideas. Later writers could then build upon the previous body of writings, knowing what diverse predecessors had said. The more texts a given writer could read, the more connections that writer would be able to make. Though Socrates (chapter 10) preferred to communicate orally, Plato would show that Socratic dialogues were well suited to the written word. The Sophists he decried would develop the practice of carefully citing the works 
of others: This they could only do if there were authoritative written texts. Aristotle would read widely in producing his many works across the fields of philosophy, political thought, and science.

It is important, then, to look past the writers themselves to the critical but unassuming institution of the library. Libraries are expensive to maintain and challenging to organize. Yet libraries had existed for about as long as humans had written ideas down. There had been famous libraries in ancient Mesopotamia (chapter 7). Though the Qin notoriously burned books they deemed useless (and thus dangerous), the Han funded a central library and sought out copies of documents that had escaped the Qin. A classification system was developed that would also be employed by later dynasties. The Han also inscribed six Confucian classics (comprising some 200,000 characters) on a stone in Luoyang so that scholars from all over China could rub copies of these onto paper.

The most famous classical library, though, was that established by the Greek rulers of Egypt in Alexandria (mentioned briefly above). The Library at Alexandria hosted numerous famous scientists: Euclid (who worked on geometry); Archimedes (who investigated fluid mechanics among other things); Eratosthenes (who estimated the circumference of the earth reasonably accurately); and under the Romans, Galen (who wrote texts on medicine that would

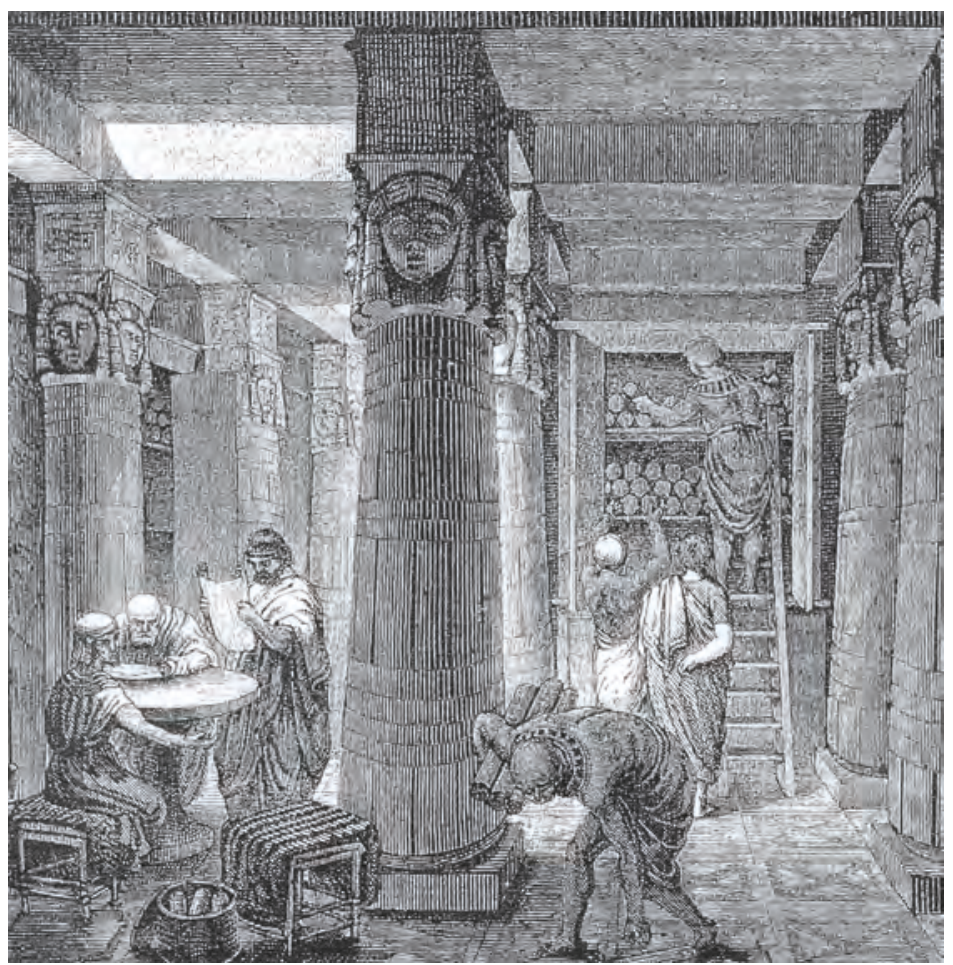

Illustration 9.20

Artistic rendering of the Library of Alexandria, based on some archaeological evidence, O. von Corven, 19th century CE 
be cited for centuries) and Ptolemy (who developed an explanation of an earthcentred solar system in which the orbits of the sun and other planets could be explained as circular orbits around circular orbits - these ideas would dominate astronomy in both Europe and the Islamic world for over a thousand years). Note that though they worked at a state-financed institution, we know the names of these researchers for they acted independently of state direction. The library may have had half a million scrolls, though one book might require two or three scrolls. The Greek rulers of Egypt had Persian, Egyptian, and Jewish texts translated into Greek - partly so that rulers could better understand subject peoples. (The Christian Bible would build upon Greek translations of Hebrew texts from Alexandria.)

A library with that many scrolls is overwhelming rather than useful unless readers can find what they seek. The library was at first organized alphabetically, but the librarian Callimachus developed extensive tables of what could be found where: references to particular rivers, metals, myths, customs, and so on. He tended to emphasize whatever was unusual in a work. Nevertheless, his classification of such a mass of literature encouraged many works of synthesis. Arguably, Euclid's Geometry gathered the ideas of others more than it generated new insights, but it would still be the core text in geometry for centuries.

Though scrolls remained the most common format of written work in the classical period, the book format emerged from at least the first century CE. As books were used - persecuted Christians valued them particularly it came to be appreciated that printing on both sides of a page and tying these tightly together allowed the same text to be communicated in a much smaller package. Readers soon recognized the advantages of quickly flipping between pages.

\section{Questions}

1. Did we somehow skip over several centuries of history in just a couple of paragraphs?

2. Are there new elements that would need to be added to Figure 6.2 on states to capture the case of empires?

3. What was the nature of group identity at the time that the earliest empires were forged?

4. What are the challenges and temptations faced by provincial governors?

5. What are the challenges faced by slave masters?

6. Why is the Silk Road more famous than the sea trade of the time?

7. Identify and map a trade route pursued by a particular merchant or merchant network. What goods did they buy and sell where, and what specific difficulties did they face? 


\section{Readings}

Bang, Peter Fibiger, and C.A. Bayly, eds. 2011. Tributary Empires in Global History. New York: Palgrave Macmillan.

Bang, Peter Fibiger, and Dariusz Kolodziejczyk. 2012. Universal Empire: A Comparative Approach to Imperial Culture and Representation in Eurasian History. Cambridge University Press.

Burbank, Jane, and Frederick Cooper. 2010. Empires in World History: Power and the Politics of Difference. Princeton University Press.

Burstein, Stanley M. 2017. The World from 1000 BCE to 300 CE. Oxford University Press.

Ede, Andrew, and Lesley B. Cormack. 2016. A History of Science in Society: From Philosophy to Utility. 3rd ed. University of Toronto Press.

Goetzmann, William N. 2016. Money Changes Everything: How Finance Made Civilization Possible. Princeton University Press.

Golden, Peter. 2011. Central Asia in World History. Oxford University Press.

Gordon, Stewart. 2015. A History of the World in Sixteen Shipwrecks. Lebanon, NH: University Press of New England.

Houston, Keith. 2016. The Book: A Cover-to-Cover Exploration of the Most Powerful Object of Our Time. New York: Norton.

McLellan James E., and Harold Dorn. 1999. Science and Technology in World History: An Introduction. 2nd ed. Baltimore, MD: Johns Hopkins University Press.

McNeely, William F., with Lisa Wolverton. 2008. Reinventing Knowledge: From Alexandria to the Internet. New York: Norton.

Miller, Joseph C. The Problem of Slavery as History: A Global Approach. New Haven, CT: Yale University Press.

Paine, Lincoln. 2013. The Sea and Civilization: A Maritime History of the World. New York: Alfred A. Knopf.

Parsons, Timothy H. 2010. The Rule of Empires: Those Who Built Them, Those Who Endured Them, and Why They Always Fall. Oxford University Press.

Rauh, Nicholas K., with Heidi E. Krauss. 2017. A Short History of the Ancient World. University of Toronto Press. [Explores the connections across developing urban societies in Europe, North Africa, and Asia.]

Seland, Eivind Heldaas. 2013. "Networks and Social Cohesion in Ancient Indian Ocean Trade: Geography, Ethnicity, Religion." Journal of Global History 8:3, 373-90.

There was a forum on the Silk Road in World History Connected in October 2016. The lead article by Timothy May includes a very useful bibliography. 


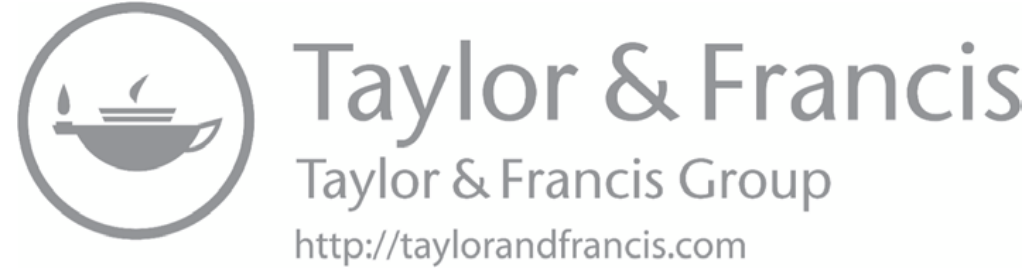




\section{Similarities and differences}

\section{The Roman and Chinese Empires compared}

\section{Guiding questions}

How, why, and when did the particular empires of Rome and Han China emerge and develop? What are the main similarities and differences between these two empires? Why, in particular, was the Chinese Empire later reconstituted but the Roman Empire never was?

What was life like during classical times? How did the average person think and behave?

How did people think about politics and about life at the time and why?

Relationship to other chapters: Chapter 10 is a companion to chapter 9. It not only reflects the general discussion of empire in chapter 9 but draws comparisons also with the empires surveyed there. The detailed comparison here informs our understanding of empire: Emperors who knew nothing of each other might act very similarly in some ways but quite differently in others. The memory of the Han Chinese Empire would reverberate through later Chinese history, and so we will often have cause to discuss how later empires built upon (or changed) Han practices. Though the Roman Empire was never reconstituted, we will nevertheless often have reason to refer to Roman precedents in later discussions of Europe and the Middle East. 
The attempt to understand what daily life was like should play an important if diffuse role in historical understanding. Students should appreciate that life was quite different in the past; this may help them to comprehend human actions that seem surprising to modern sensibilities. We can identify some of the ways in which life was different, but our information is limited regarding many important aspects of daily life.

We spoke of how states justified their power and conquests in chapters 6 and 9. The discussion of political thought in this chapter sheds further light on how people - some powerful, others not - thought about politics at the time; we will have much cause in later chapters to address both the theory and practice of autocracy and democracy.

In the millennium after about 500 BCE two empires of a size never before seen in the world rose - and fell - on opposite sides of the Eurasian landmass. Rome and Han China at their peaks each had populations of about 55-60 million. They may together have governed half of the world's population. Vast distances separated them and so they were never more than dimly aware of each other though luxury goods from each empire did reach the other, along with serious misinformation. The principal purpose of this chapter will be to understand comparatively - the rise and fall of these two empires. This will involve looking briefly at their precursors. We will also discuss the role that each would play in later history: We will devote particular attention to the question of why the Roman Empire was never recreated but the Chinese Empire would be many times.

We naturally address the same themes as in chapter 9 . We will also discuss at some length the development of philosophical thinking in both Greece/Rome and China. We can thus maintain science in the list of themes that received the most attention in the preceding chapter.

Politics
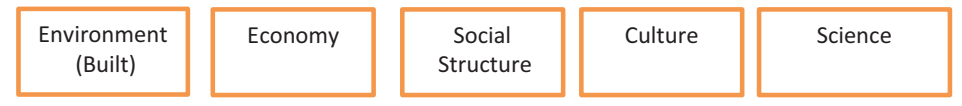

\section{Greece}

The story of the Roman Empire begins in important ways with Greek civilization. Competing city states characterized Greece throughout most of the centuries preceding the rise of Rome. These existed not only throughout much of present-day Greece but also the western part of modern-day Turkey. These city states were governed in a variety of ways. Athens, one of the most 
important - both at the time and historically - was for much of its time a democracy, though with limited voting rights. Its main competitor, Sparta, was instead an authoritarian state (though its leadership rotated).

The word "democracy" comes from the Greek words for "people" and "government." We should appreciate that Greek democracy was quite different from modern democracy. Most obviously, women (despite being citizens), slaves, and foreigners could not vote - and the status of foreigner was hereditary. The poor gradually gained the vote over time in response to civil strife. Athens was also a military state that received tribute from smaller city states that had no say in Athenian decisions. Nevertheless, free men did gather periodically both to elect officials and to debate legislation. All free men could speak and propose legislation. In practice, of course, a few loquacious speakers dominated proceedings. Moreover, one's work could easily limit one's ability to attend frequent meetings (there were forty Assembly elections per year in Athens): The rich could more easily participate than the poor. The Athenian political leader Pericles, who did much to consolidate democratic principles in Athens, celebrated the principles of freedom of expression and equality before the law that he felt made Athens stronger. He also introduced payments for attending meetings to encourage participation by the poor. Yet Pericles also proposed a law banning marriage to foreigners, to limit the size of the Athenian voting population. Pericles appreciated that Athenian democracy depended on a community that both shared values and knew each other; such a democracy could not serve a larger state. Pericles then fell in love with a woman from Miletus a year later; their son was (unusually) legitimized (gaining Athenian citizenship) on his deathbed. Athenian citizens at first volunteered for leadership positions but these became paid positions over time so that poorer Athenians could assume positions of responsibility. Pericles, like many other Greek thinkers, supported a meritocracy that selected men of superior talent and character to rule. In practice, an elite that could afford to educate their sons - in debating skills above all generally dominated Greek political life. Yet the Athenians also chose people for some important positions through a lottery where all free men had an equal chance of selection - an idea we might usefully consider in modern democracies (chapter 32).

Sparta forced neighbouring peoples to work the land on their behalf. These "helots" had a status between free and slave: They were required to give half of their output to Sparta. Sparta always feared rebellion and insisted on strong military training for its citizens. Interestingly, it sought to minimize social distinctions in order to encourage strong loyalty to the state, encouraging a "Spartan" lifestyle without conspicuous consumption. Athens might have evolved a similar political structure, but Solon's reforms in 594 BCE freed many of the poor from debt servitude and forbade enslavement of Athenians while employing slaves captured in wars on large estates. That is, Solon recognized the challenge to democracy of huge income disparities. Athens also encouraged trade and industry and therefore had a larger "middle class." 


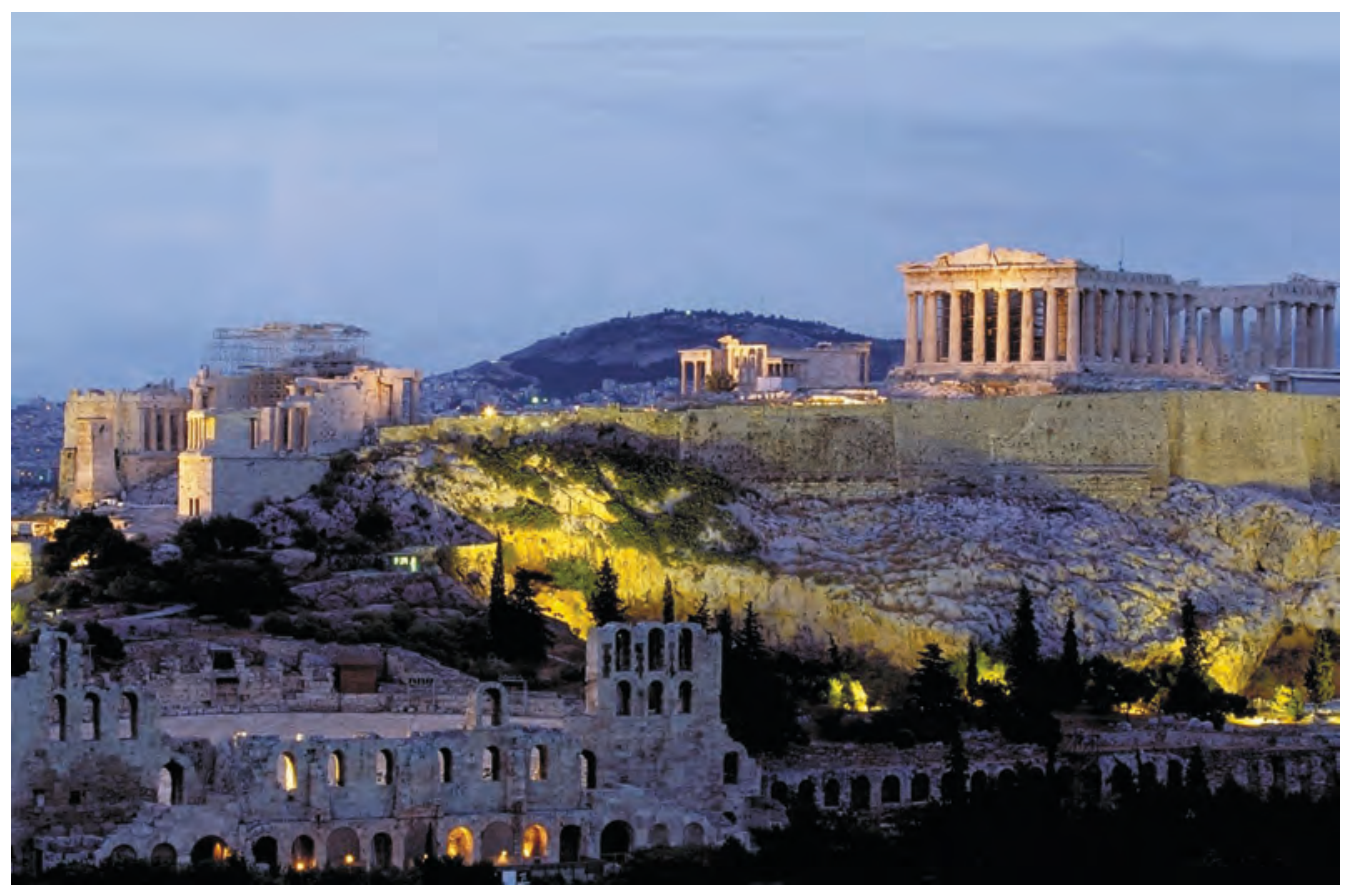

Illustration 10.1

The Parthenon above Athens

The Greeks, inhabiting a set of small agricultural enclaves divided by mountains, had become skilled at seafaring. The classic tales, the Odyssey and Iliad, indicate that Greeks had a well-developed knowledge of seafaring very early. They would establish trading colonies throughout the Mediterranean from the eighth century BCE and the Black Sea from the seventh century BCE. Though bounded on the east and southeast by mountains, the Black Sea was bordered by fertile lands to the north and west. In the Black Sea, the Greeks traded olive oil, wine, and cloth for grains, iron ore, timber, and fish (there were ready local supplies of salt to preserve fish). Note that this was far from just a luxury trade but involved movement of bulky goods such as grains and iron ore from the fringes of the Eurasian steppe. The Black Sea did provide some luxuries: Both the pheasant and the cherry take their name from regions along the Black Sea coast. Note also that the Black Sea was more challenging than the Mediterranean for sailors: It was stormier due to the mixing of temperate and sub-tropical climates, the water is usually murky enough to conceal the seabed, there were stronger currents, and the shoreline was rockier with few safe harbours. The Black Sea also brought the Greeks into contact with steppe nomads; since the latter lacked ships, they could only attack southward along the coast. Trade was a more common outcome of this interaction. The Greeks built outposts along the coast but did not venture too far into the steppe.

Greek colonies were politically independent. The Greeks had a trading colony in Egypt from at least the fifth century вCE through which they 
borrowed many architectural and other ideas from Egypt. The Roman Empire would later build upon this Greek trading network, and mimic the practice of importing grains from Egypt while exporting wine and olive oil. Some have accused the Greeks of exhausting their soils and needing to import grain, but it could just be that it was far more profitable for them to trade grapes and olives for grain. They had learned to process olives by at least 1000 всE. The Greeks also grew barley, lentils, apples, pears, and figs, and raised cattle, sheep, goats, and chickens (chickens had been carried from Asia by 1000 BCE). The Greeks developed port facilities. As Corinth rose to commercial prominence in the late eighth century BCE, the Corinthians built a track for hauling ships across the narrow Peloponnese isthmus, a track that was used until the ninth century $\mathrm{CE}$ (a canal would later be built). Despite relying on and developing infrastructure for trade, it seems that the people of at least some Greek cities viewed merchants with some suspicion.

Though the Greeks traded throughout the Mediterranean and Black Seas, they had limited knowledge of lands beyond these seas. The Greek writer Herodotus could not confirm the existence of the "tin islands" to the north from which tin was obtained. It is indeed remarkable that tin from Cornwall in Britain was reaching the Mediterranean at this time overland in a manner whereby those using the tin had only the dimmest idea of where it came from. Herodotus also repeats a likely apocryphal tale of a Carthaginian (Carthage was a Phoenician colony; see below) circumnavigation of Africa: Given the difficulties that the Portuguese would face in rounding Africa two millennia later (chapter 19), it is hard to imagine Carthaginian success so early. Yet Herodotus reports the Carthaginians' surprise that the sun should lie to their north as they rounded the southern cape.

The Greeks also developed what were perhaps the first specialized warships in the world. The trireme from the seventh century BCE - with three rows of rowers was much faster and stronger than earlier ships. It was also expensive to build and to train 170 rowers to work together and in concert with other boats. Still, naval historians credit it as one of the three main innovations in the history of maritime warfare. With the addition of the battering ram it would provide the basic form of naval conflict for over a thousand years (until flamethrowers were developed; see chapter 13). There were further improvements. A century or two later, either the Carthaginians or western Greek colonies developed polyremes with up to eight rowers per oar in one or two banks. These much larger ships had room for bigger weapons such as catapults.

As we have witnessed previously in Mesopotamia and China, there were often wars among city states in Greece. When the Persian Empire (see chapter 9) expanded into what is now Turkey, absorbing the Greek states there, the Greek cities joined them in fighting Persia. They struggled to a stalemate with Persia over a period of decades, with a crucial victory at Marathon on the Greek peninsula in 490 все. The Persians attacked again, but a Spartan force of just 360 delayed a Persian army of over 10,000 at the pass of Thermopylae long enough for Greek forces to gather which could defeat them. Persia would 
remain powerful in Turkey but cease to threaten Greece. We might attribute the Greek victories against a great empire to the fact that they were fighting on their own territory: They had more to lose, had much shorter supply lines, and were accustomed to fighting in mountainous terrain. When the Persian threat abated, Sparta and its allies fought Athens and its partners for decades, with Sparta eventually winning (with Persian support and with recognition of Persian superiority in the area). However, these wars did not result in political centralization. They did result in movements of an estimated 100,000 refugees.

Greek armies relied on plunder until about 500 BCE, when soldiers began to receive regular pay. The Greek phalanx formation - in which troops stood close together with interlocking shields and protruding spears - emerged over centuries of internal conflict. Sparta was unusual in Greece before about 500 BCE in providing extensive military training. After that time, states began to provide armour - likely in large part as costs fell with the introduction of iron. The philosopher Aristotle felt that the phalanx - with its need for many men, and its role in responding to an external threat - was instrumental in fostering democracy in Athens, but the phalanx had no such effect in Sparta nor most other Greek states. Military strategies changed when Macedonians - a northern Greek state - developed much larger armies. The Macedonians battled not only other Greeks but also nomads from the north; they likely learned from the Persians how to integrate cavalry and infantry. They also altered the Greek phalanx by employing long pikes rather than swords, which allowed them to attack the opposing line better.

Centralization would come at the hands of Macedon. Macedon under King Philip controlled all of Greece by 338 BCE. After his assassination in 336, his son, Alexander the Great (who had been, we might note, tutored by Aristotle in his youth), succeeded him. Alexander had taken all of the Persian Empire including Egypt - by 330. In one pivotal battle against a much more substantial Persian force, Alexander charged through the middle toward the Persian emperor; the emperor fled though he should have been able to regroup his forces. Alexander was almost certainly a talented general, but he was also able to finance a huge army, at first with the gold that his father had seized from Thrace (the region that borders modern Greece and Turkey), and then with an estimated 5 million kilograms of gold taken in the conquest of Persia. Alexander mostly left the Persian bureaucracy in place though he replaced the satraps (provincial governors) with his own men. Alexander proceeded to attack into India but soon died. Alexander - whose own son was a mere child when he died - ignored pleas to appoint a successor even on his deathbed. His generals then divided his empire in three: Greece and Macedon (which Rome would conquer in the second century вСE); the Ptolemaic Empire in Egypt (which the Romans took in 31 вCE) and Palestine, and the Seleucid Empire in Persia/ Anatolia (defeated by the Parthians in the second century всE). This Hellenistic period spread Greek (and other) ideas to west and east, and encouraged trade.

The Persians had previously pushed into Central Asia. Alexander would follow their example and then encouraged Greek settlers to move to the 


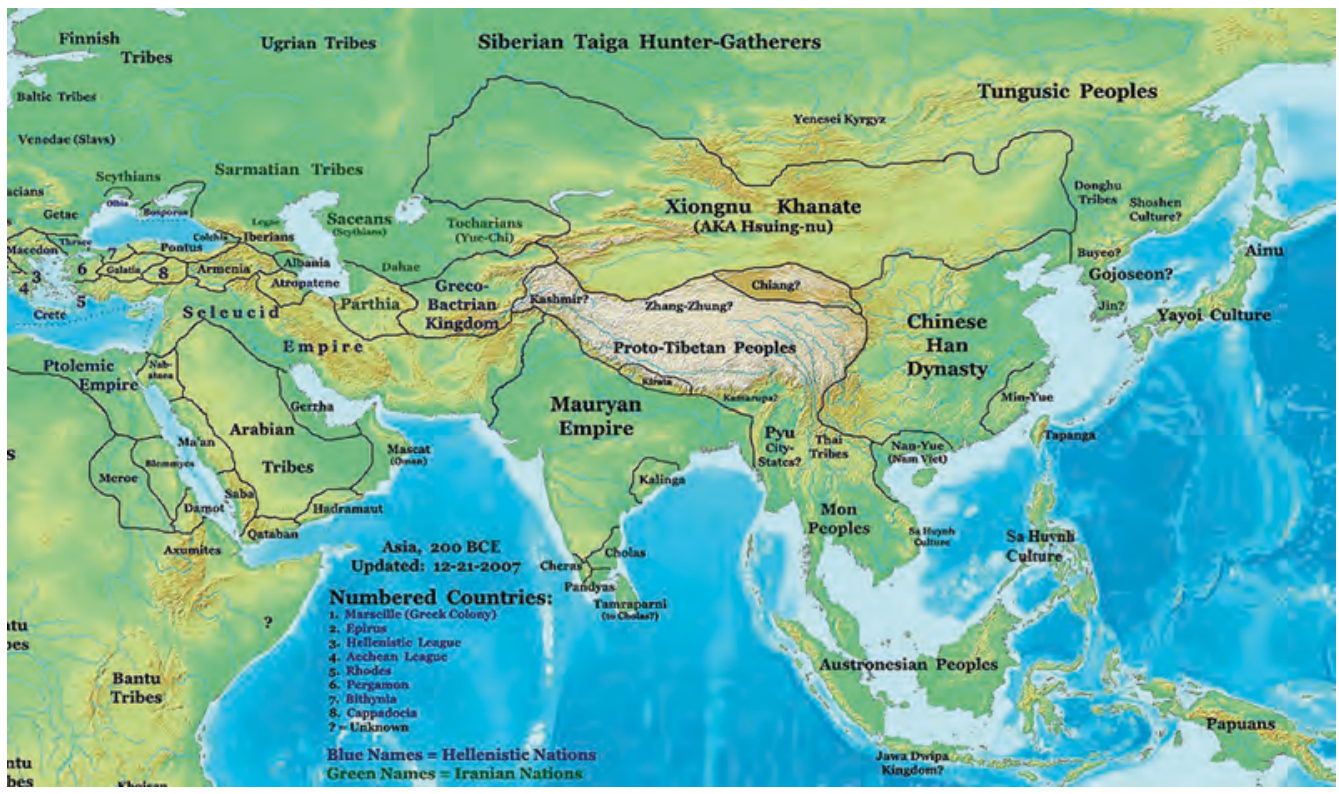

Map 10.1

Asia in 200 вСE, showing the Greco-Bactrian kingdom, and Han China before expansion into Central Asia

Map by Thomas A. Lessman, CC BY 3.0

frontier regions of Bactria and Sogdiana. The Greek cultural influence would last for centuries in the area. The Persians and Greeks both often fought against neighbouring nomads but also traded with them. Greek artisans crafted ornaments with nomadic themes that are often found in elite Scythian burials. ("Scythian" is a term applied by Greeks to nomadic groups for over 1,000 years; there was undoubtedly considerable change in group composition over that period.) Though Scythian consumption of both cannabis and fermented milk amazed the Greeks, artisans adopted Scythian animal motifs for Greek markets. The interactions between sedentary and nomadic populations would set the stage for the Kushan Empire (chapter 9).

There is a story that Alexander had the Persians bow to him in the traditional way that they had bowed before Persian kings. Alexander's Macedonian followers refused to bow in this way. If true, the story is indicative of how foreign conquerors would adopt local customs, especially if these could serve the conqueror's interests. It also symbolizes the difficulties of achieving common practices throughout a diverse empire, and the resulting incentives to respect local differences. An emperor's court could itself be a complex undertaking with different expectations regarding people from different parts of the empire.

The Ptolemaic Empire was perhaps the richest of the three successor kingdoms; it established a bureaucracy (primarily comprised of locals) and had state monopolies for beer, textiles, and salt. Alexandria, with its harbour for 
1,200 boats, became a cosmopolitan city, with a famed museum and library (chapter 9) and academics, the centre of the Late Greek world.

Like most ancient societies, Greece had a patriarchal structure. Women generally could not own land, though they sometimes ran shops. Sparta was a bit unusual in that it allowed women to travel unaccompanied, and to fight (and to wrestle naked like men and sometimes against men), and to own land - perhaps because the men were so often away training and fighting. The tale of Penelope in the Odyssey - who puts off suitors while her husband is presumed dead - does indicate that women could wield some considerable power in controlling their husband's affairs while these were away trading or fighting. Nevertheless, there are court cases in Egypt in the third century BCE in which women tried but failed to exercise rights they had held before the Greek conquest. Still, Greek inheritance practices allowed widows some control over estates, whereas in China and India these usually passed to sons who were expected to look after their mothers. Aristotle, among others, worried that women could not control their lust as well as men, perhaps because they were less adept at applying reason. One position of authority open to a woman was as priestess: They could then manage cults, perform ceremonies, assume prominent places at public ceremonies, and be exempt from tax.

\section{The lasting impact of Greek civilization}

Greek civilization is famous today for a few things. One is the Olympic Games, held every four years for over a thousand years. Men from all of the Greek states would travel to Olympia to compete in running, wrestling, and other sports; cities would celebrate their champions upon their return. The Olympics was one of several festivals of different types that brought a sense of community to the politically divided Greek states. The Olympics reflected respect for exercise and the training of the (male) human body: Training facilities for athletes were common in Greek cities.

Literature and the dramatic arts also blossomed in classic Greek civilization especially in the century after peace with Persia when resources once devoted to war were funnelled to arts and architecture. The dramatic forms of tragedy and comedy became popular in Greek cities. Such plays exposed the foibles and false strivings of everyday life. They served to both transmit an understanding of human lives and encourage the audience to reflect on the nature of their own lives. One common theme of Greek tragedy was cultural conflict: Playwrights appreciated that people with different values might struggle to comprehend the other, with potentially grave results. Ironically, the roots of Greek drama may lie in the choruses associated with celebrations of the god Dionysus by foreign residents of Athens: Athenian authorities at first viewed these celebrations negatively.

Ancient Greece is perhaps most famous for its philosophy. The three most famous names are Socrates, his student Plato, and his student Aristotle. Plato's works take the form of dialogues, usually featuring his master Socrates. These 


\section{BOX 10.1 \\ SPORTS AND GAMES}

Though the Olympic Games are notable, we should stress that sports were common across ancient societies. There are records in ancient Egypt of hunting, archery, wrestling, acrobatics, tag, and games with balls, among many other sports. Confucian scholars disdained ball games - which must thus have existed - but praised archery and other games with a military association. Mesoamerican societies had developed a ball game from about 1500 BCE in which a rubber ball - rubber was unknown in the eastern hemisphere - was handled with feet and elbows rather than hands. The idea of shooting the ball through a ring high on a wall may have come later. Cave paintings suggest that wrestling at least had been pursued thousands of years before there are written records.

Board games were popular in several societies from at least 3000 BCE. The rules of many of these have been lost, though some exhibit a resemblance to checkers or parchesi. The oldest game still in widespread use is the Chinese game of Go, which may date to 4000 BCE. (Chess would be developed only from about $500 \mathrm{CE}$ in India with pieces resembling war elephants instead of horses; it would later spread across the Islamic Middle East with abstract figures or viziers substituting for queens. Europeans would resuscitate queens but at first allow them to move only one space at a time.)

dialogues raised challenges to philosophical arguments and then addressed these. Socrates urged the examined life, and the active and careful questioning of received beliefs. Aristotle wrote treatises on an amazing array of scientific and philosophical subjects. Though his science had a limited empirical base, his philosophy has stood the test of time. He is perhaps most famous for advocating "Golden Means" in behaviour: for example, one should be courageous, but not timid nor foolhardy.

Although Socrates, Plato, and Aristotle are by far the best known of ancient Greek philosophers, a

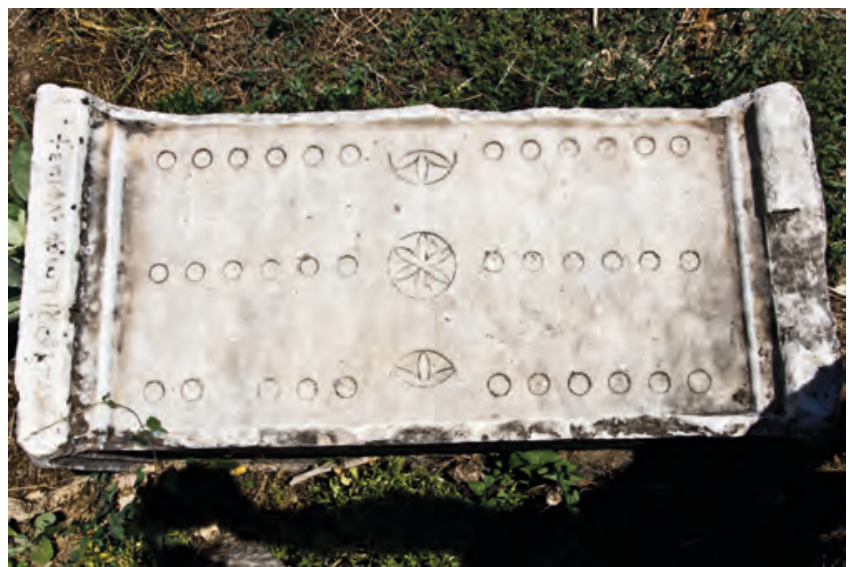

Illustration 10.2

Roman board game, from the market in Aphrodisias in the 2nd century CE; this game is thought to be a precursor to backgammon

Photograph by William Neuheisel, CC BY 2.0 variety of philosophical schools existed at the time. Special mention might be made of the Sceptics, who doubted the possibilities of both philosophical and scientific understanding. Philosophical schools of our day such as postmodernism echo many of 


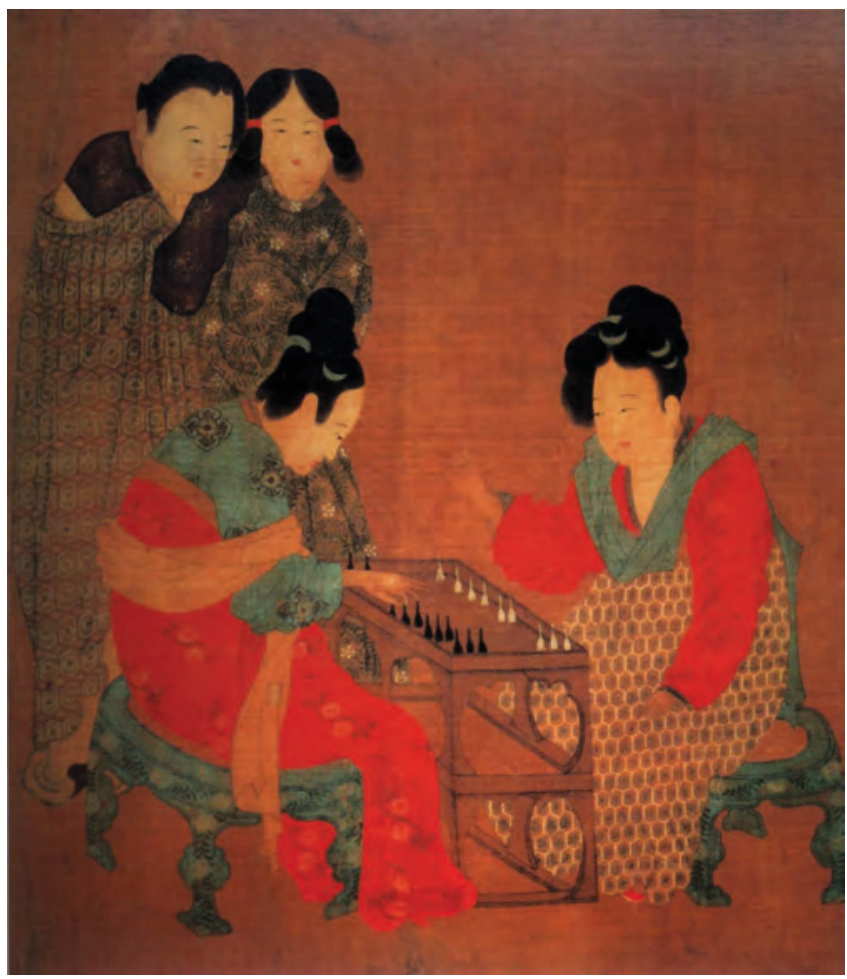

Illustration 10.3

Court Ladies Playing Double Sixes, Zhou Fang, 8th century painter. Ink and colour on silk, Freer Gallery of Art

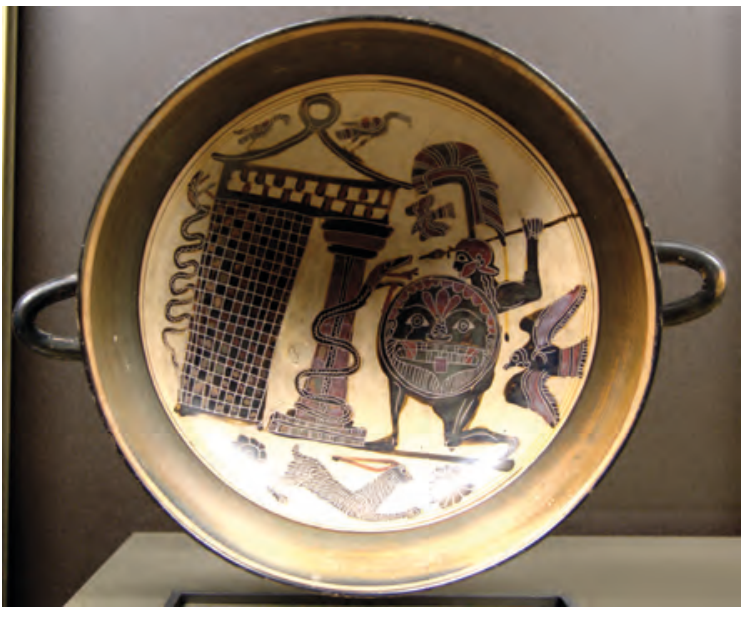

Illustration 10.4

Black-figure cup, c.550-540 BCE. Some say it is Achilles watching out for Troilus (this is the interpretation on the Louvre notice), but others think it is Cadmus fighting the Theban dragon, Louvre the arguments first made by Sceptics 2,500 years ago. Both may be reflective of a similarity in intellectual environment: When there are competing schools of thought and no obvious way to choose among them, it is tempting to throw up one's hands in despair and doubt that consensus can ever emerge. (We urged in chapter 1 some strategies for dealing more constructively with intellectual disagreements.)

Stoics were another important school of thought. They encouraged people to focus on how they lived their lives rather than on the results of their actions. Our success in life, they noted, was to a considerable extent beyond our control. We should be content if we lived honourably 
and tried to act ethically in the world, even if our efforts failed. The modern phrase "stoicism" - with its sense of remaining calm in the face of misfortune captures some of this ancient Stoic ideal. Stoicism would become the favoured philosophical school within the Roman Empire.

Greece built upon earlier advances in both philosophy and science. The Greeks learned of astronomy, mathematics, science, medicine, and magic from Mesopotamia, and geometry, medicine, and divination from Egypt. They adapted the Phoenician alphabet (chapter 6) and added vowels to this during the eighth and seventh centuries BCE. By the fifth and fourth centuries BCE, they began making a unique contribution to philosophy. We can even detect Indian influences: Both Socrates and Plato believed in reincarnation, and Plato's famous Allegory of the Cave closely resembles a tale in the Upanishads regarding illusion.

There is an understandable tendency to lionize Socrates, Plato, and Aristotle in the history of western philosophy. As any student who has taken some philosophy courses will know (and everyone should!), their thoughts still resonate in the contemporary academy. Yet every academic should recognize that scholarship is a conversation: We build upon the ideas of others and hone our thinking in reacting both to ideas that predate us and to critiques of our works. One of the mistakes that students commonly make (and our educational system often encourages this mistake) is to imagine that the books and articles they read are isolated acts of insight emanating solely from the mind of the author(s) of the work - though all of those pesky references should give a hint that this is not quite the case. And one of the mistakes that professors often make early in their careers - a career-ending error for many - is failing to place their ideas within the academic conversation, indicating how they respond to or add nuance to some idea(s) that are already out there. The Platonic dialogues themselves signal to us that Socrates did not just dream up all of these ideas on his own but responded to arguments made by others that forced him to clarify his thinking. The papers and books that scholars write today do not detail all of the mistakes and wrong turns we make during our research - but rather give the impression of a somewhat steady march from question through argument and evidence to conclusion. We can imagine that the Platonic dialogues reflect a distillation of many conversations among many people with Plato (or Socrates) choosing over time the most compelling lines of argument. In other words, these great philosophers could not have achieved all that they did without the community in which they operated and in which they could carefully hone their arguments - and perhaps even get credit for some ideas that had originated in the minds of others.

There may still be scope for analysing the psychology of figures such as Socrates. Yet as important as particular people undoubtedly were, we can focus our attention here on why a community of people - well, men, for the most part - was both able and willing to engage in philosophical discussion. We have already indicated that they could build upon pre-existing understandings. They could also build on a tradition of intellectual discourse. Mesopotamian and 
Egyptian advances in areas such as mathematics and astronomy were also not just the result of isolated brilliance but of interactions among people curious about such matters. These people needed to have not just curiosity but also the time to devote to thinking, conversing, and examining. Science and philosophy were only possible with an elite freed from the need to farm or perform bureaucratic tasks. In this respect, we should not neglect the fact that even Athenian democracy was a society of considerable income inequality. Slaves (who did not vote, it might be noted) performed much of the labour that allowed Socrates and others to spend much time in conversation.

Such an elite needed not just the time to contemplate science or philosophy but also the education and motivation to do so. Here Greek educational practices may have been critical. The Greek elite was not the first to seek formal education of their sons. Schools emerged for training the young wherever writing systems were developed (chapter 6). Yet Greek education extended far beyond writing skills. Philosophy, then as now, could be a career: In ancient Greece, students (or parents) could pay a famed thinker to teach. Much of the contest between Greek philosophical schools was indeed a contest for students. Some philosophers would decry the Sophists for their focus on debating skills rather than the ideas being debated (and in denouncing them attached a negative connotation to "sophistry" to this day), but some parents naturally thought that training in debating skills was a good idea, especially in an often raucous Athenian democracy.

States depended on organization, as we have seen, and so we can understand that Mesopotamian and Egyptian rulers had smiled on efforts to develop mathematical understanding or calculate the movements of the seasons. Philosophy was different: It was less obviously useful and might challenge beliefs that the state valued. An Athenian court indeed sentenced Socrates to death for corrupting the youth of Athens. (He famously argued at the time that death should not be feared (though not encouraged either): It would either mean a better afterlife, as promised by some religions, or it would mean nothingness, which he equated with an eternal good night's sleep, which all people treasure.) Socrates refused to escape his death sentence, noting that he had benefited from the laws and customs of Athens his entire life and could hardly shun them when they disserved him. Notably, Athenian authorities did not prevent Plato from writing down the ideas that Socrates had been condemned for; nor, critically were his works destroyed. Other philosophers also were free to argue in the marketplace, compete for students, and publish treatises and dialogues. Though Socrates' death must remind us that "freedom of speech" was not yet a robust defence against legal action, the Athenian democracy was willing to tolerate a lively discussion even if philosophers routinely questioned cultural beliefs and traditional practices. The furore over the death sentence on Socrates may have acted (ironically) to discourage attacks on other philosophers.

The Greek world, with its extensive trade among cities and states with quite different cultures and polities, was perhaps inevitably interested in questions 


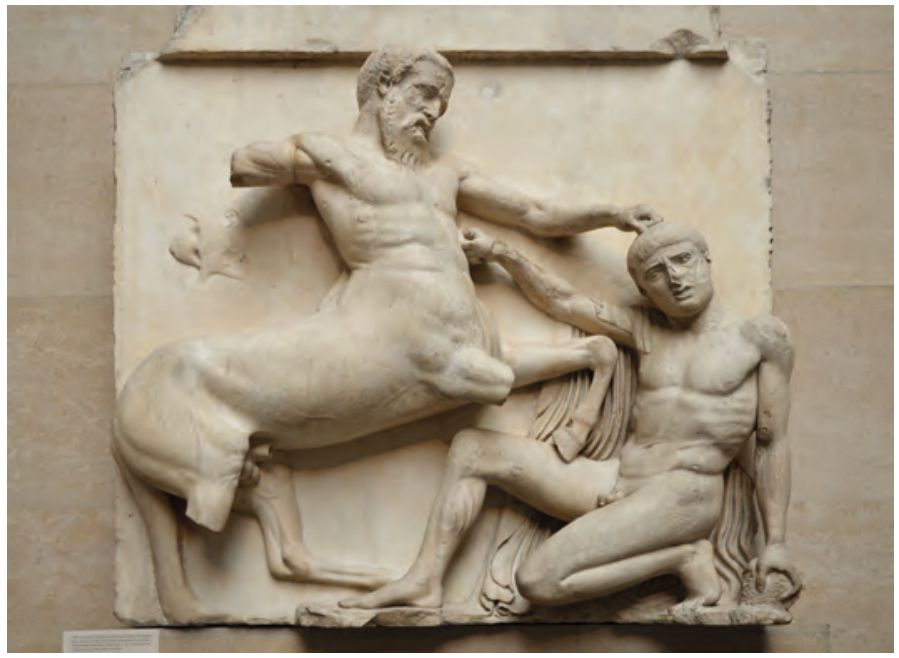

Illustration 10.5

This Parthenon sculpture of a centaur attacking a Greek is said to represent the challenge of protecting Greece from foreigners with different ways Photograph by Carole Raddato, CC BY-SA 2.0

of both how an individual should live and how to govern a community. Recall that Greek drama and literature engaged ethical issues also, though in an entirely different format. We have, then, all of the necessary ingredients for philosophy: a group of people with the means and freedom to engage in an examination of questions that were on the minds of many.

Later Christian and Islamic theologians struggled to connect their religious beliefs to the doctrines of writers such as Plato and Aristotle. That is, they sought to show that the ethical principles advocated in their holy scriptures could also be justified by the sort of logical argumentation at the heart of Greek philosophy. Although the task was not easy, it proved feasible. This may reflect the fact that both philosophers and prophets articulated these ethical principles to address the challenges of living in cities and organized states (recall our discussion of religion in chapter 8 ). There was much similarity across these ethical principles - as with those developed in religions and philosophies farther east.

\section{Rome}

Rome was founded in the eighth century всE as a city-state kingdom. It became a republic - albeit one with a restricted franchise - in 509 все, and remained a republic for five centuries. The city had an advantageous location, with ready access to the Mediterranean Sea via the Tiber River but being much harder to attack than if it had been located on the seacoast. Rome prospered even during times of political domination by the Etruscans in present-day Tuscany. 


\section{The Republic}

The Republic long sought to prevent political domination by any one family. It placed administrative power in the hands of two consuls so that these could monitor each other. The Senate could appoint dictators in a time of crisis, but only for a term of six months. As in Athens, aristocrats initially dominated the republic, but plebeian protests resulted in a widening of voting rights over time. Ordinary people gained the right first to elect tribunes (who could argue on behalf of individuals in legal difficulty and on behalf of ordinary people in general) and later to serve as one of two consuls. (They also gained the right to appeal death penalties to the people.) There was thus some popular voice and opportunities for social mobility.

The Republic first became dominant in Italy in the fourth century BCE. It then won wars with Carthage (264-146 BCE) and both the Seleucids of Persia and with Greece (215-148 BCE), coming to dominate the Mediterranean. It forced 50,000 Carthaginians into slavery. This rapid rise to power was in part due to military prowess, but also to the treatment of conquered territories: The Republic often allowed the vanquished to become Roman citizens or at least gain certain rights. Citizenship was first available only to Romans, then to all Latin peoples (Italian cities had rebelled 91-88 BCE to obtain citizenship), then to soldiers (if they served twenty-five years) and elites from conquered territories. (In $212 \mathrm{CE}$, during the Roman Empire, citizenship was extended to all male non-slaves.) Citizenship carried with it legal rights and privileges, and after the victory over Macedon in $167 \mathrm{BCE}$, the Senate abolished direct taxation of citizens. Local elites could continue to run local affairs in most conquered regions in return for taxes and military service. Rome might appoint governors, but these had limited powers (and were often locals themselves). This meant that the masses were less integrated into the empire than their leaders. Though local elites collected taxes for Rome, the Roman central bureaucracy then allocated these.

Rome did not depend entirely on the loyalty of local elites. It established Roman colonies in many conquered lands, granting land to citizens from Rome. These colonies effectively served as garrisons for Rome.

Rome had a powerful army. It initially was similar to Greek armies, being composed of farmer-citizens arranged in a phalanx formation. It developed over time a more complicated legion structure with three tiers of soldiers armed with different weapons, including javelins that soldiers hurled into the opposing army from a distance. The frequency of wars during the Roman conquests gave even its volunteer army a degree of professionalism. Over time, as the army grew in size and complexity, the ideal of the citizen-soldier was abandoned in favour of a permanent army (we described a typical Roman soldier of that era in chapter 6).

Rome was not above using terror as a tactic. It threatened to destroy local populations if they did not surrender. It carried through on this threat often enough to make the threat credible. We will discuss this strategy in more detail when we treat the Mongols in chapter 17, but should stress here that 


\section{BOX 10.2}

\section{WAR ELEPHANTS}

Alexander the Great was impressed by the use of war elephants by the Indian armies he faced. War elephants would later be used by the Seleucid and Ptolemy successors to his empire. The Ptolemy in Egypt would employ African elephants when they could not obtain Asian elephants, but these fared poorly in battle against their larger Asian cousins. Still later, the Romans and Carthaginians would use war elephants. The Carthaginian general Hannibal famously took war elephants through the western Alps in attacking Rome from Spain. Yet by the end of the first century BCE, war elephants had largely disappeared from Europe - even though the Persians would employ them in wars with Rome. Though valuable in battle, war elephants were very expensive to obtain, feed, and train. The political fragmentation that followed the decline of Rome likely rendered war elephants unaffordable by any state. War elephants remained in use in India into the nineteenth century. They were also employed for many centuries in Southeast Asia. In both places it was relatively easy to capture mature elephants in nearby forests. The experience of war elephants reminds us that successful technology transfer depends on many elements of the receiving society.

the Mongols seem to receive far more condemnation for a procedure that they were hardly the first to employ.

The Roman army is far more famous than its navy. Roman writers would often speak with disdain (or fear) of seafaring and seafarers. Nevertheless, a navy was essential to Roman success. They could hardly have attacked Carthage in Africa successfully without a navy, or indeed extended their power throughout the Mediterranean. Their ships not only moved troops and provisions more cheaply and quickly than could be accomplished by land but won many naval battles over the centuries. It was a naval battle - the Battle of Actium in 31 все, in which the forces of the future emperor Augustus defeated the navy of Roman general Marc Antony and the Egyptian queen Cleopatra - that inaugurated the Roman Empire. The Romans early emphasized the boarding of other ships and developed a hinged boarding ramp (with a spike at the end to hold it in place on the other boat) that allowed Roman troops to board enemy vessels quickly. However, these ramps likely made the ships susceptible to capsizing.

As the Romans overpowered the navies of other lands, they opened a space for piracy. Pirates even captured the Roman general Julius Caesar (see below) in his youth and held him for ransom for forty days. In 69 BCE, Pompey was given hundreds of ships and thousands of men over three years and successfully removed pirates from the Mediterranean. This success would be one of the great boons of Roman rule, for after that shipping within the Mediterranean could operate in much greater safety. Augustus, who defeated Egypt soon after Actium, inaugurating a period of centuries in which Rome controlled the entire Mediterranean, also created a standing Roman navy to maintain peace 
throughout that sea. One result was that huge ships carrying over 1,000 tons entered the grain trade from Egypt (Rome alone imported 150-300,000 tons of grain a year). Only 15-30 per cent of this trade involved the payment of taxes; merchants privately financed the rest. Rome was less powerful in the Black Sea: It never controlled more than half the shoreline, and relied heavily on local rulers (who were often insubordinate) even there. This may have encouraged Rome to rely more heavily on Egyptian grain as imports of grain from the Black Sea declined.

The Romans often left local laws and judges in place, though Roman citizens had recourse to Roman law. Rome only opposed local cultures or practices if these threatened the empire: In particular, it supported Jewish rituals in Jerusalem before the Jewish revolt in $70 \mathrm{cE}$.

The practice of respecting local laws created problems when a Roman and non-Roman had a contract dispute. Under pressure from merchants, the extent of Roman law expanded over time. Republican government encouraged respect for the rule of law, though corruption would always be problematic. A set of laws were debated and created in public forums and enforced by judges and lawyers with considerable independence. By the second century CE at least, there was training and a recognizable profession of jurists. Still, Roman law was only first codified in the sixth century CE in Constantinople (chapter 13).

\section{The Empire}

The Roman army would have 400,000 soldiers at its peak. Troops were paid by and therefore loyal to commanders rather than the state itself. Given the importance of conquest to the Republic, these commanders became politically influential. The Republic failed to establish institutions that prevented authoritarianism by making troops loyal to elected assemblies. As the Republic expanded, it faced increased challenges in administering a vast area. Julius Caesar, a military commander who had conquered much of Gaul (France) with tens of thousands in casualties - sought to centralize power in his hands. He thought that he could ease the suffering of the poor by doing so. The Roman elite killed him while he was consolidating his power. After a lengthy civil war, his nephew and chosen heir Octavian would take control of the state (after the Battle of Actium above). He claimed the titles Augustus and Princeps (first citizen), and commander (Imperator) of the army. He centralized power in his hands but maintained the appearance of republican institutions. The Empire retained Roman laws, and Romans continued to enjoy the freedom to debate the issues of the day and to pursue their own religions. In principle, the emperor was not above the law, though emperors in practice had few constraints on their power. The Roman Senate remained a place for debate even if emperors only consulted it on minor issues. It would agree over time to transfer many powers to the emperor, including the right to declare war. Augustus worked to absorb the elite rather than confront it, placing loyalists in key positions, and promoting people from lower classes into positions of authority. Augustus 
moved to establish both a more extensive bureaucracy and a more professional army that would be loyal to him; the republic had balked at both. The Romans decried what they saw as Persian-style despotism and avoided the term "king," but leaders of the empire were that in all but name. They were often called kings in the provinces but not in Rome.

The famous Roman orator and philosopher Cicero fought to maintain republican institutions. He thought that the solution to political problems lay in drawing merchants into power, and urging ethical behaviour on those with political power. He was less open to ideas of economic redistribution. Cicero valued liberty (at least for Romans; slavery was okay for those they conquered), but also stability. Agents of Marc Antony - who sought to be emperor - would kill Cicero in 43 BCE for his defence of the republic. Over time, most Roman citizens likely accepted the empire as the only way to have peace.

\section{PRIMARY DOCUMENTS: QUOTES FROM CICERO}

In a republic this rule ought to be observed: that the majority should not have the predominant power.

When you have no basis for an argument, abuse the plaintiff.

Nothing is so unbelievable that oratory cannot make it acceptable.

If you have a garden and a library you have everything you need.

Glory follows virtue as if it were its shadow.

Those wars are unjust that are taken without provocation. For only a war waged for revenge or defence can be just.

An unjust peace is better than a just war.

The only excuse for war is that we may live in peace unharmed.

The wise are instructed by reason, average minds by experience, the stupid by necessity, and the brute by instinct.

The higher we are placed the more humbly we should walk.

Ability without honour is useless.

To be ignorant of what occurred before you were born is to remain always a child.

Even if you have nothing to write, write and say so.

To what extent do Cicero's words still speak to us, and to what extent do they reflect his times? Is his oratorical skill reflected in his words? What is his attitude toward social structure?

Officially, the Senate was to appoint emperors. However, the military, and especially the elite Praetorian Guard stationed in Rome, often acted to select the next emperor. Assassinations were common, and there were frequent armed battles over the succession. There were twenty-six emperors between 235 and $285 \mathrm{CE}$, with only one dying in office of natural causes. 
Rome was able to survive struggles over succession because a bureaucracy remained in place and ready to serve whoever became emperor. There was perhaps also a widespread appreciation of the benefits of empire - such as the eradication of piracy and extension of Roman law. Other empires in history the Mongol Empire leaps to mind (chapter 17) - would rely to a much greater degree on personal ties between ruler and government officials; succession struggles in such an environment could easily fragment an empire. The Roman Empire would continue to expand for centuries, eventually stretching from England to Mesopotamia.

Trade expanded under both republic and empire. The transport infrastructure and the extensive network of merchants of the time would not be seen again for over 1,000 years in most of Europe. The Mediterranean Sea facilitated grain shipment to Rome, Constantinople, and to armies on some frontiers. Europe's rivers, notably the Rhine, promoted trade to the north. France exported wine, pottery, and glass. Britain sent tin, iron, grain, cattle, and slaves. Regions could increasingly specialize in wine or pottery and import grains by water from large estates geared to export. Merchants enjoyed trading across a vast territory governed by the same system of law and with a strong state that controlled piracy and banditry. Though the Roman state was notoriously corrupt, its reliance on trade and a tradition of judicial independence gave merchants some confidence that the state would not confiscate their goods - at least if they had the necessary bribe handy. The state often contracted road building and tax collection to merchants. The Romans financed a system of roads throughout the empire for administrative purposes; though these were often designed for messengers on foot or horseback rather than wagons, they nevertheless served to facilitate trade. Towns had considerable legal autonomy within the empire and usually acted to encourage and protect commerce. The corporate form was developed for towns and religious communities but was later applied to infrastructure projects: Private groups of builders and financiers could then manage public infrastructure projects. Extensive trade, coupled with institutions of urban governance, allowed Rome to achieve levels of urbanization not observed again in western Europe until the seventeenth century. Rome may also have reached a similar average standard of living to seventeenth-century Europe, though with a much less equal income distribution.

As is often the case, we know more about pottery than any other good. Roman pots of high quality were transported long distances and used by the poor. After the fall of the Roman Empire, pots would only be used locally and primarily by the rich. Though quality is hard to appreciate without seeing many pots, Roman pots were more attractive, lighter, easier to clean, and stackable because they were standardized. Since we find nice pottery in modest peasant houses, we can imagine that peasants also enjoyed good textiles, leather, and metal goods as well. Ceramic roof tiles were in widespread use during the Roman Empire but not after: These are durable, attractive, and prevent leaks much better than thatch. 


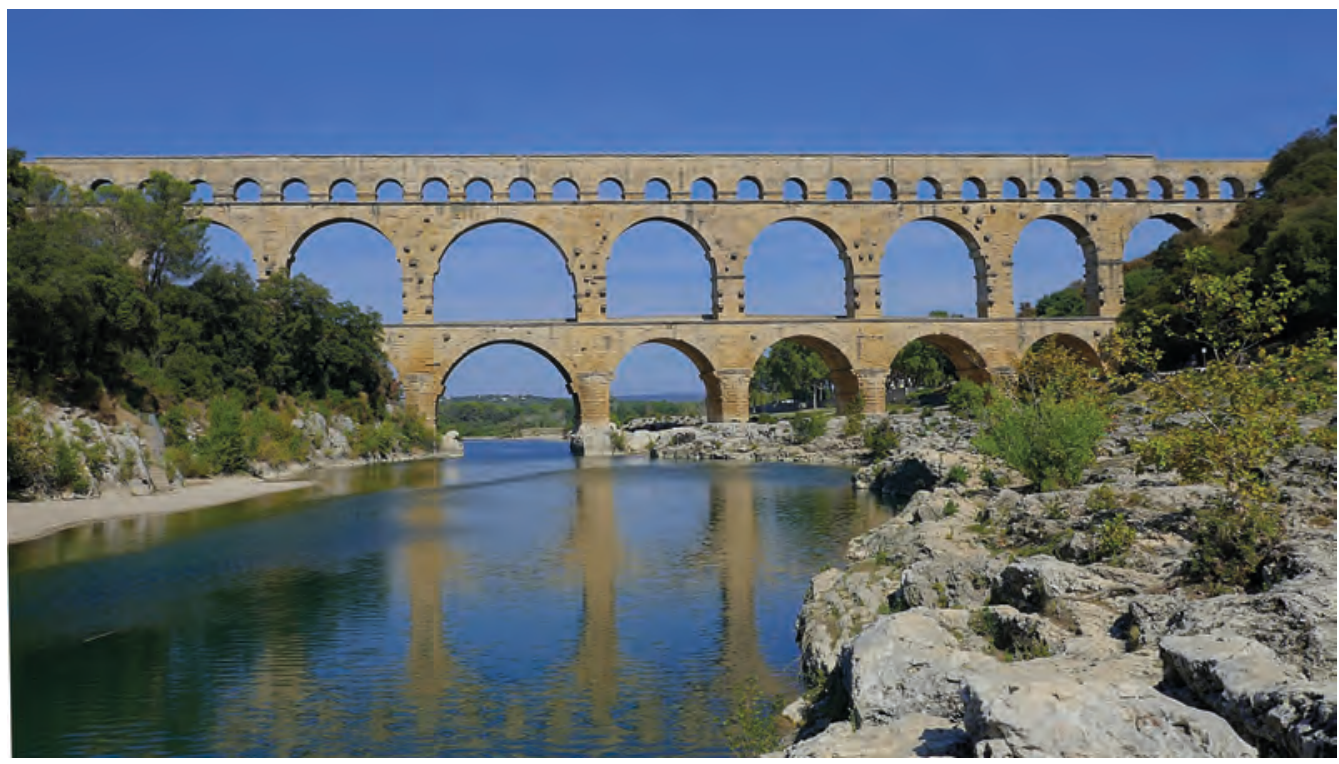

Illustration 10.6

Pont du Gard aqueduct, near Nimes, France, lst century CE

The Romans invented concrete. Though Roman concrete was far weaker than modern concrete, the use of volcanic ash as an ingredient appears to have protected concrete structures such as the Coliseum and Pantheon in Rome from the elements so that they could survive to the present. The Romans built aqueducts, triumphal arches, and domed buildings. There were 10,000 statues in Rome in the first century CE. There were water and sewage systems in all cities; public baths (five in Pompeii for a population of 20,000), stadiums and amphitheatres. Emperors and local elites built massive and highly decorated public buildings. Despite the savagery of the Coliseum - where condemned criminals battled lions - the Romans praised the ideals of education, familiarity with the classics, and living a good life. Though scholars long thought that the Romans cared little about agricultural technology, there were advances in ploughs, animal breeding, and irrigation. There were also improvements in industrial practices such as glass blowing. Because of these various advances in trade and technology, average incomes apparently rose during most of the Roman period - population also grew but not as fast as output.

From the third century $\mathrm{CE}$, the empire began to decline. It faced attacks by both "barbarians" and Persians, as well as difficulty in financing its army and administration. Part of the problem was a lack of booty as the empire stopped expanding. One key event here was the Battle of the Teutoburg Forest in $9 \mathrm{cE}$ : Germanic peoples destroyed three Roman legions (a Germanic officer working for the Romans apparently having lured them into a narrow forest path). After this defeat, the Roman Empire effectively accepted a boundary along the Rhine and Danube rivers. The decision to grant global citizenship, 


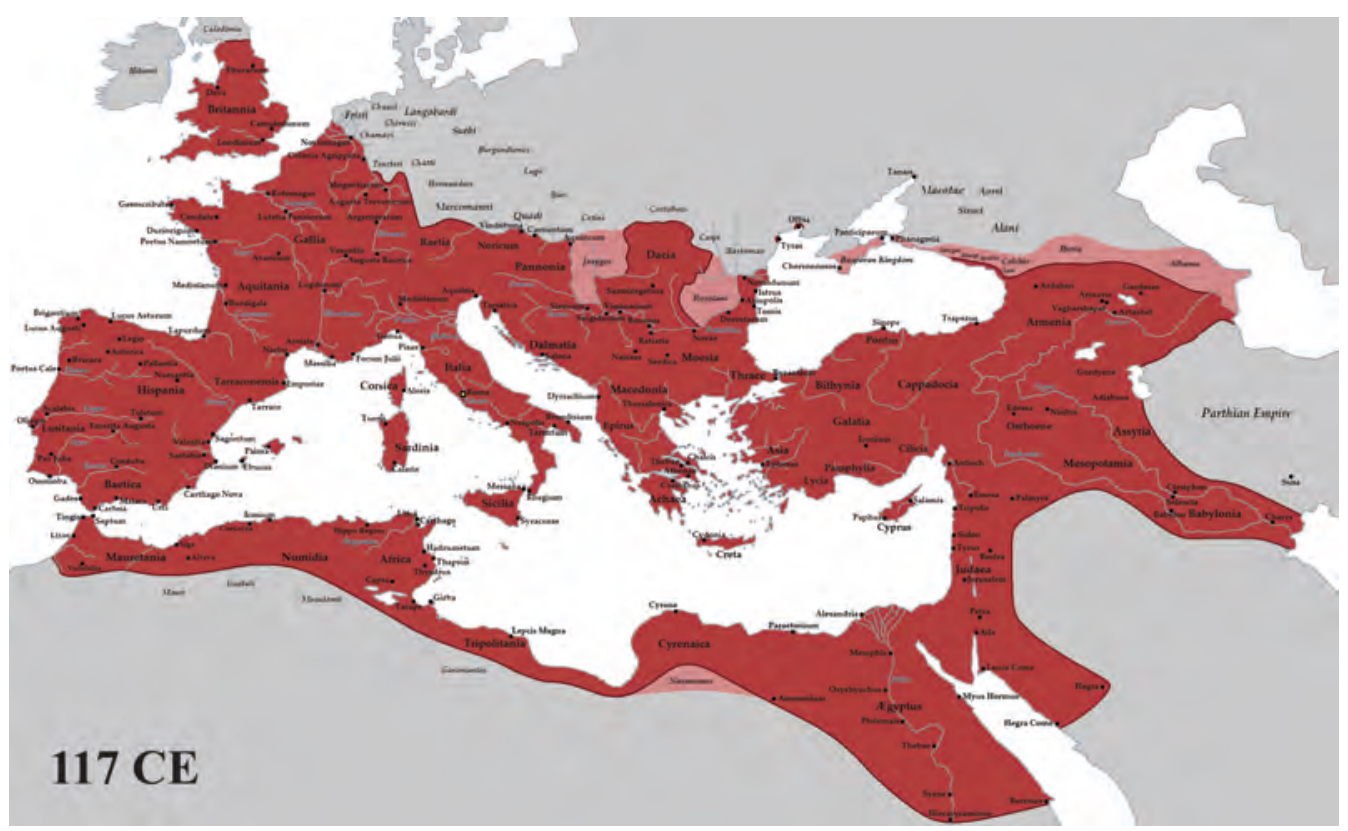

Map 10.2

The Roman Empire and tributary states in $117 \mathrm{CE}$

Map by Tataryn, CC BY-SA 3.0

and exempt citizens from taxation, limited the empire's revenues. Troops on distant borders proved hard to control. The Empire pursued a policy of coopting barbarian forces: This worked for a while, but these would eventually seek power. Emperor Constantine inaugurated a new capital in Constantinople (now Istanbul) in $324 \mathrm{CE}$, to strengthen the empire in the east and to distance himself from Roman elites. The empire would split in the fifth century, with capitals in both Rome and Constantinople. In the fifth century also, Rome itself would fall to Germanic groups it had long disdained as barbarians. The eastern part of the empire, though, would survive for centuries more. We will discuss both Germanic migrations and that Byzantine Empire in chapter 13.

We will see in chapter 11 that the Roman Empire came to adopt Christianity as its official religion. Christianity made it harder to absorb new peoples into Roman society: Their gods could no longer be incorporated into the Roman pantheon. Adoption of a monotheistic faith also exposed the empire to the dangers of schisms within the faith: These would prove particularly problematic for the Byzantine Empire (chapter 13).

Popular assemblies in Rome had long sought land reform, but the more aristocratic Senate opposed the idea. Large slave plantations had grown in importance during the Republic. When Rome had a population of 200,000 adult males just after 300 BCE, it had 60,000 slaves. Caesar gained much public acclaim for forcing the Senate to agree to land reform. However, Senators then killed him. Augustus gave lands to his army but did not redistribute beyond 
that. Large landholdings tended to specialize in wine, oil, grain, or livestock. Some plantations employed hundreds of free labourers and slaves. Slavery was particularly well suited to olive and grape production, but slavery declined in importance as conquests ceased. In the late Empire, free peasants were tied to the land by law, in a situation that looks much like serfdom (chapter 13).

\section{Roman family life}

This section deviates from the general narrative of this chapter and book to try to capture a sense of what daily life was like during this time period. We can take advantage here of some recent research that attempts to tease out what life was like for the typical Roman citizen. The reader will have to speculate as to what degree life would have been similar in Persia or India or China at the time.

The vast bulk of documents from classical times detail the thoughts and lives of the elite. Members of the elite often lacked a high opinion of the rest of society. The limited evidence we have from the rest (which has begun to receive much attention from historians) suggests that disdain was somewhat mutual. Though it was prudent to show respect for the elite in public, in private, people thought that the elite gained their wealth through a combination of corruption and abuse of power. The typical Roman would have doubted very much the possibility of achieving a victory in Roman law over a member of the elite who could hire expensive lawyers and bribe judges.

The records that do survive speak much about family life. Marriage and children were central concerns - as they are in most societies. Romantic love was often celebrated, but marriage was also seen in very practical terms as combining the productive capabilities of two people. Parents had a considerable say in the marriage of their children. Despite the legends of Roman orgies - much exaggerated by later Christians as evidence of previous debauchery - Romans were often quite prudish. Husband and wife were supposed to enjoy sex Roman physicians felt that there was a connection between female orgasm and conception - but not too much: Women especially might then become unfaithful. Men could exercise their more adventurous tastes with prostitutes or slaves rather than their wives. Homosexuality among both men and women was recognized and not usually disdained, though the heterosexual family was encouraged.

Fears regarding the health of children were paramount, as one might expect in a day when infant and child mortality claimed between a quarter and a half of all children. Parents also worried about having too few children to look after them in old age, or too many to feed. Roman physicians urged the rhythm method to avoid excess children - but they misunderstood the menstrual cycle and advocated intercourse on the most fertile days. Abortion was risky but practised; infanticide and child abandonment, especially of girls, was common. There were designated fields for abandoning unwanted babies; these were sometimes rescued. There were widespread concerns in the later Empire 
that children (and sometimes wives) were not as obedient as they had been in previous generations.

Petty crime was common in classical cities. There was little in the way of police (beyond the occasional night watchman), and these were not likely to concern themselves with small thefts. Vigilante justice was common.

Women concerned themselves primarily with the household and caring for children. Yet women went outside the house regularly to shop, perhaps sell bread or cloth, participate in religious ceremonies, and interact with other women. Women often travelled, and sometimes worked as nurses, midwives, priestesses, actors, or vendors. Women wore veils in some parts of the Roman Empire but not others - they were much more common in Persia.

Roman women had to have a male guardian until they had three (sometimes four) children. Roman law allowed for easy divorce (until Christianity became the state religion), and required that the bride's dowry be returned to the woman upon divorce. This must have given women some greater influence within marriage since they could threaten to not only leave but also take money with them. Women inherited half the estate if the husband died. In the countryside, women comprised one-third of landowners, accounting for 16-25 per cent of the land.

Roman cities had many entertainments that characterize modern cities, including taverns, theatres, and brothels. Religious celebrations were important

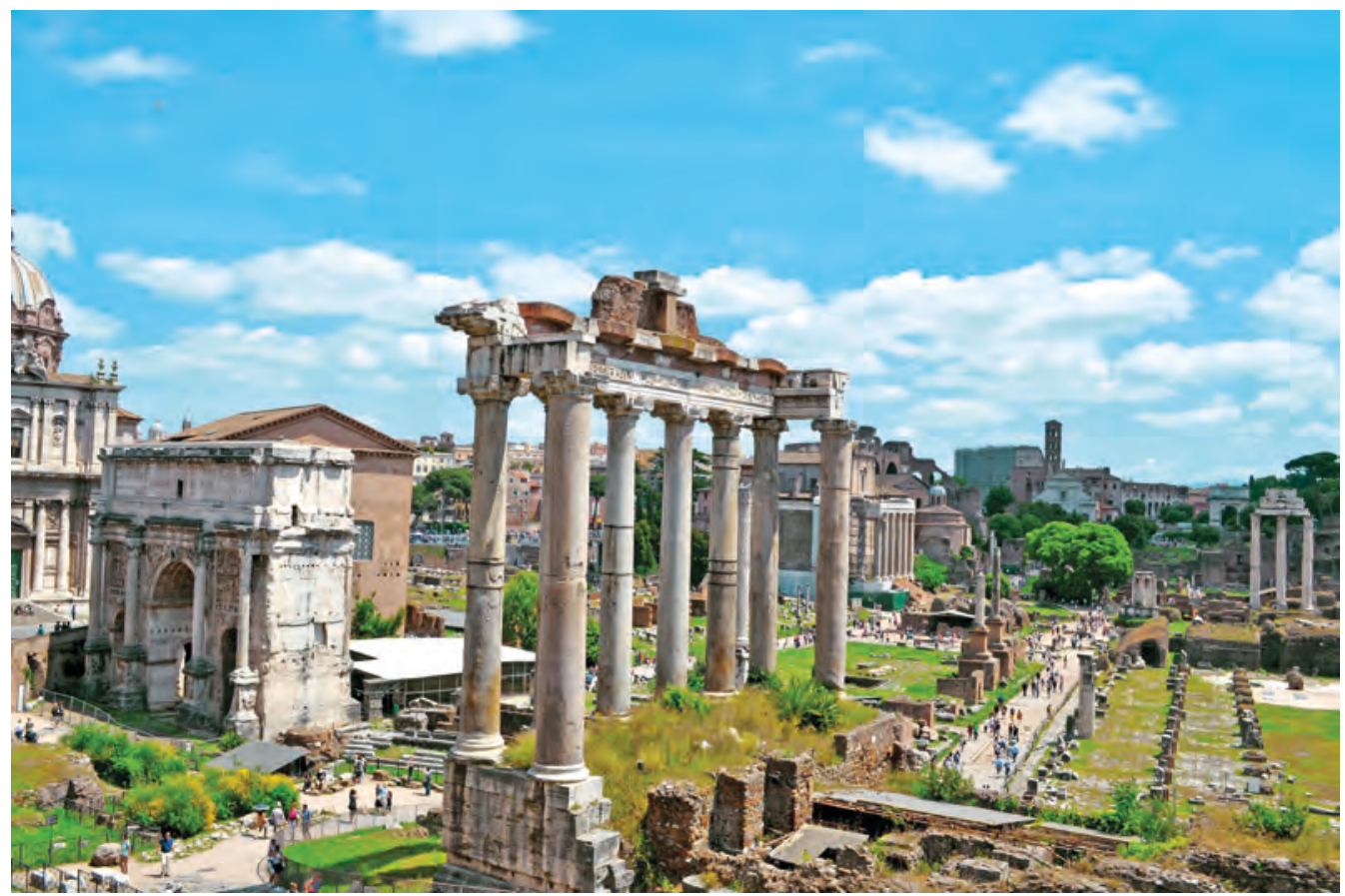

Illustration 10.7

The Roman forum 
social events. Public baths were important since people lacked facilities for cleanliness in their own homes. Yet Roman baths, even those of the elite, would undoubtedly fail modern standards of hygiene: Lacking knowledge of germs, they would not have treated or changed the water appropriately. Men and women usually, but not always, bathed separately. Roman cities also had brutal entertainments that offend modern sensibilities: gladiators fighting each other and wild animals to death, feeding condemned criminals to wild animals. These spectacles were not all about carnage: The Roman Coliseum could be flooded for the performance of mock sea battles.

The invention of concrete facilitated the construction of apartment blocks. More impoverished residents might have to carry water and food upstairs to a sixth or seventh floor. Though wealthy Romans could heat their floors through underground pipes, poorer Romans relied on open fires in their rooms. These were often a fire hazard.

Even poor families would have some plain ceramics with which to cook and eat and store foods. Plain glassware for storing liquids became increasingly common. Ceramic bowls with a rough interior were used for grinding foods. Metal cooking pots were expensive and thus were only used by richer households. Finer ceramics and glassware were also only found in richer households.

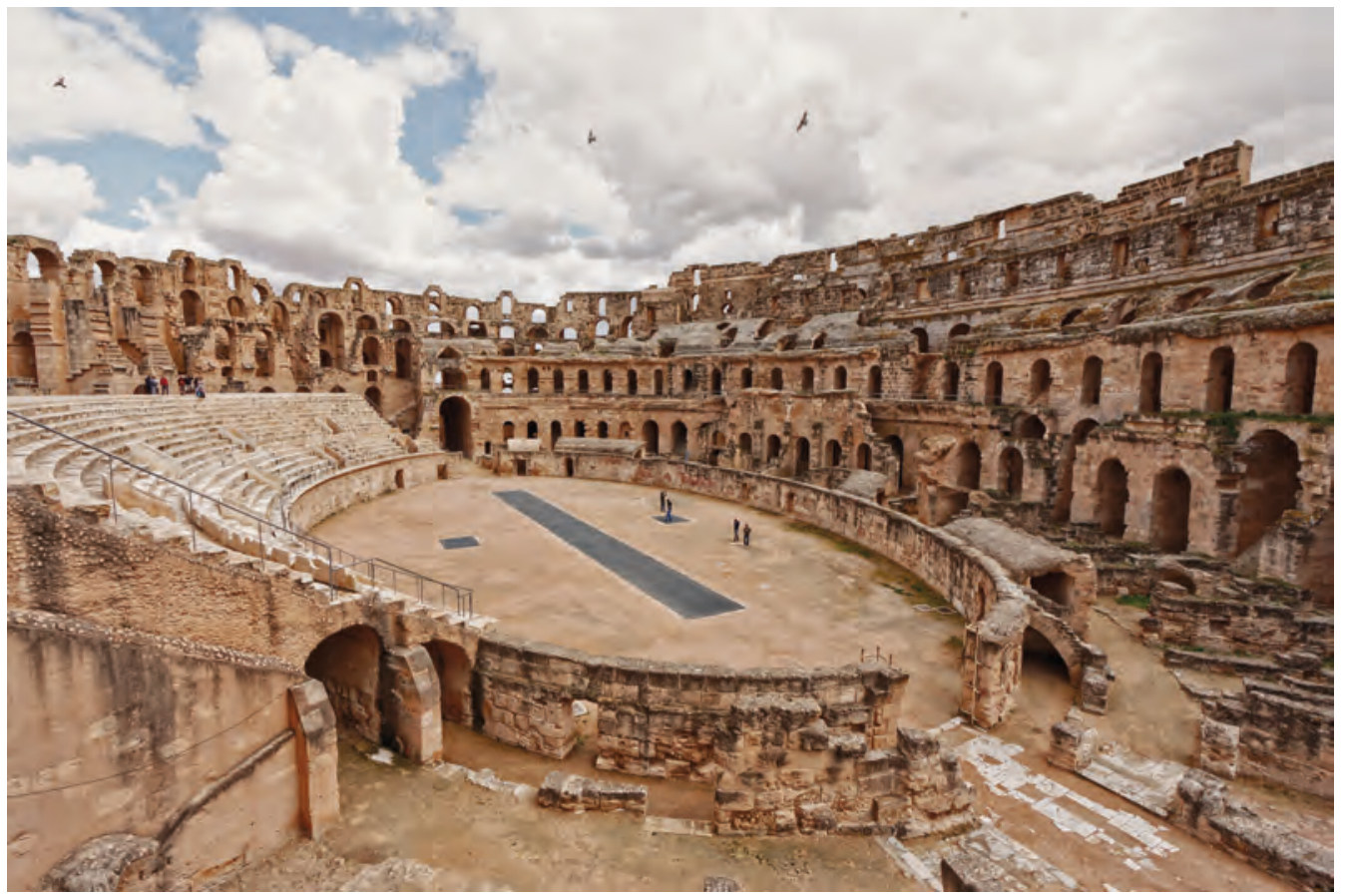

Illustration 10.8

The largest Roman coliseum in Africa in El Jem, Tunisia, dates from the 3rd century CE and could seat 35,000

Photograph by Agnieszka Wolska, CC BY-SA 3.0 


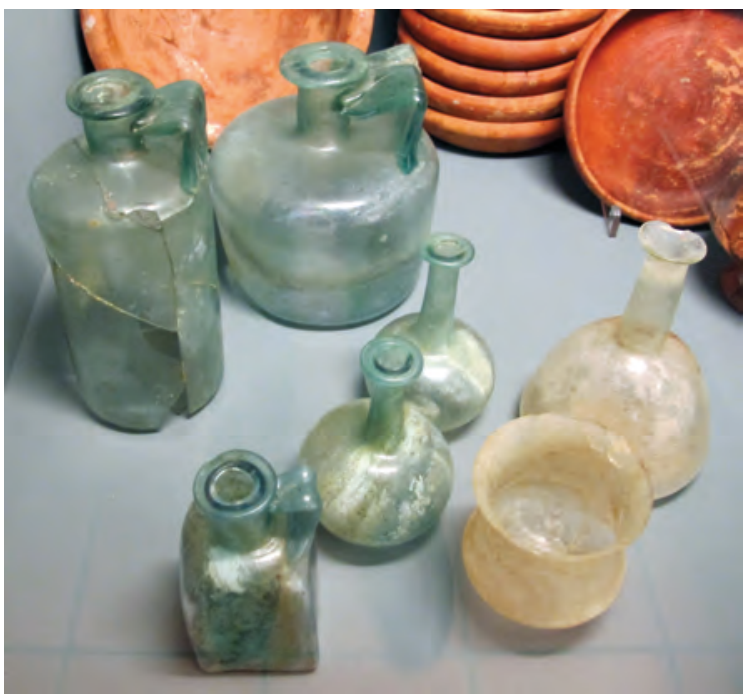

Illustration 10.9

Roman glassware, Historisches Museum, Bern Sailko, CC BY-SA 3.0
The majority of urban populations may have lived life on the edge economically. They lacked regular employment and struggled to feed and house their families. When they worked, they were often paid to perform a particular task rather than by the hour or day. These people were willing to work hard but had little hope of a better life - especially as they needed to protect from theft any wealth they did accumulate. To the extent that we can divine their attitudes, these seem to have been fatalistic. They did not question the existence of inequality but did complain about particular abuses that offended their sense of justice. Popular rebellions when they did occur focused on land redistribution or debt forgiveness, rather than extensive institutional changes. These rebellions did not seek to overturn the system but rather share the rewards of the system more equally.

There was also a mass of people with regular employment as merchants or artisans or bureaucrats. Different occupations had organizations that were active politically: In the ruins of Pompeii there is electoral graffiti in which fruitmongers pledged support for one candidate and millers for another (and worshippers of the goddess Isis for yet another).

\section{BOX 10.3} CUISINE

There was in many societies a quite different cuisine for rich and poor. Cuisine served as an important marker of status differentials. The cuisine of the rich usually varied far more across societies than the cuisine of the poor: French and German peasants ate very similar things just a few centuries ago. Law sometimes limited certain foods to the elite; at other times, they were just too expensive. There would also be a more complicated cooking process for the elite with multiple ingredients. The greater diversity in diet among the rich was usually healthier. Their food also tasted better. The rich in both Rome and Han China valued salt highly; this and various spices - was particularly important at a time when food storage techniques did not always prevent spoilage. The emphasis of missionary religions (chapter 11) on bread and wine or vegetarianism may have been in part a reaction to elite cuisine. 


\section{Challenges facing agents:Workers}

Given the diversity of non-agricultural and unskilled jobs (we have addressed farmers and artisans in earlier chapters), it is hard to summarize the challenges facing unskilled workers. We can appreciate that the uncertainty of employment was a significant challenge. Some unskilled workers might gain continued employment. Their challenge, then, was to continue to satisfy their employer. Through most of human history, employers could fire workers without any justification. Workers would also face the risk that employers would suffer a financial setback.

There was no social safety net in most times and places in human history (we will note some exceptions in later chapters). The Roman Empire was unusual in providing free bread to people in Rome in order to maintain social stability. Workers who only worked occasionally thus had to save when they had employment. This was no simple task when financial institutions served only the rich and petty theft was common. Workers who needed to borrow when unemployed faced very high interest rates. Repeated or long bouts of unemployment could easily force workers to sell themselves into slavery. Workers were also very sensitive to the prices of food. Some states notably the Chinese - would try to store grain to reduce the price volatility associated with good and bad harvests. Yet in many times and places workers would struggle to feed themselves in years of bad harvests.

Though we may think of them as "unskilled," most workers would strive to distinguish themselves from other workers: in strength, cleverness, trustworthiness, or perhaps brutality. Those who gained a reputation for performing some task well could hope for more steady employment and possibly higher wages.

\section{China}

\section{Chinese philosophy}

We saw above that Greek philosophy blossomed during a period of competing states. The Romans would build upon Greek ideas and apply these both to the workings of the state and to the lives of individuals. In China also, the Warring States period would produce a great deal of philosophical thinking that later empires would use to guide their polities and educate their young. In China, as in Greece, these philosophers could benefit from the previous development of a widely accepted script for writing.

Competition among smallish states may encourage philosophy in at least two ways. First, philosophers can move between states if their ideas cause offence. Second, the constant competition calls forth reflection on the nature of the state and of humanity. An itinerant class of bureaucrats who moved between states created much of Chinese philosophy.

We noted when discussing Greek philosophy the importance of interaction among philosophers. They could then hone their ideas in conversation. The same is true of Chinese philosophy: There was interaction among many over a period of centuries. This interaction was most formalized in an academy near 


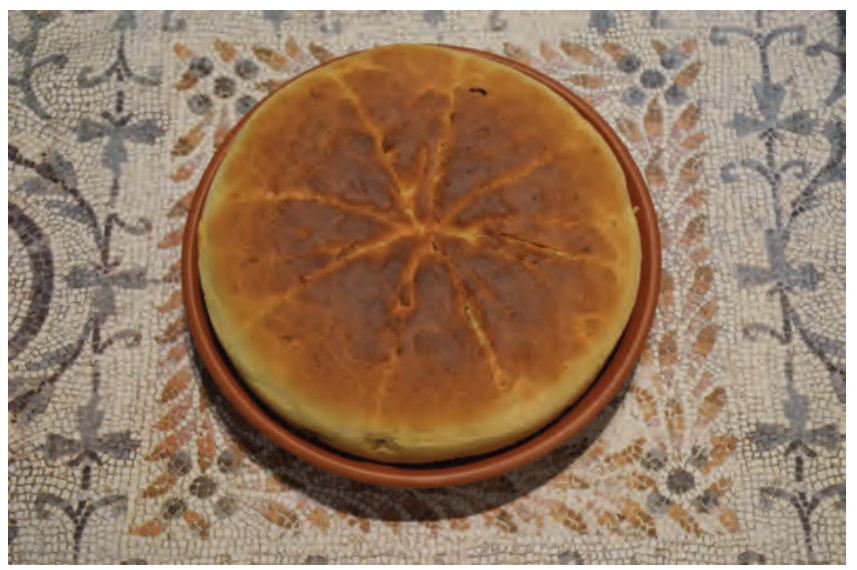

Illustration 10.10

Soft bread, from a Roman recipe

Photograph by Carole Raddato, CC BY-SA 2.0

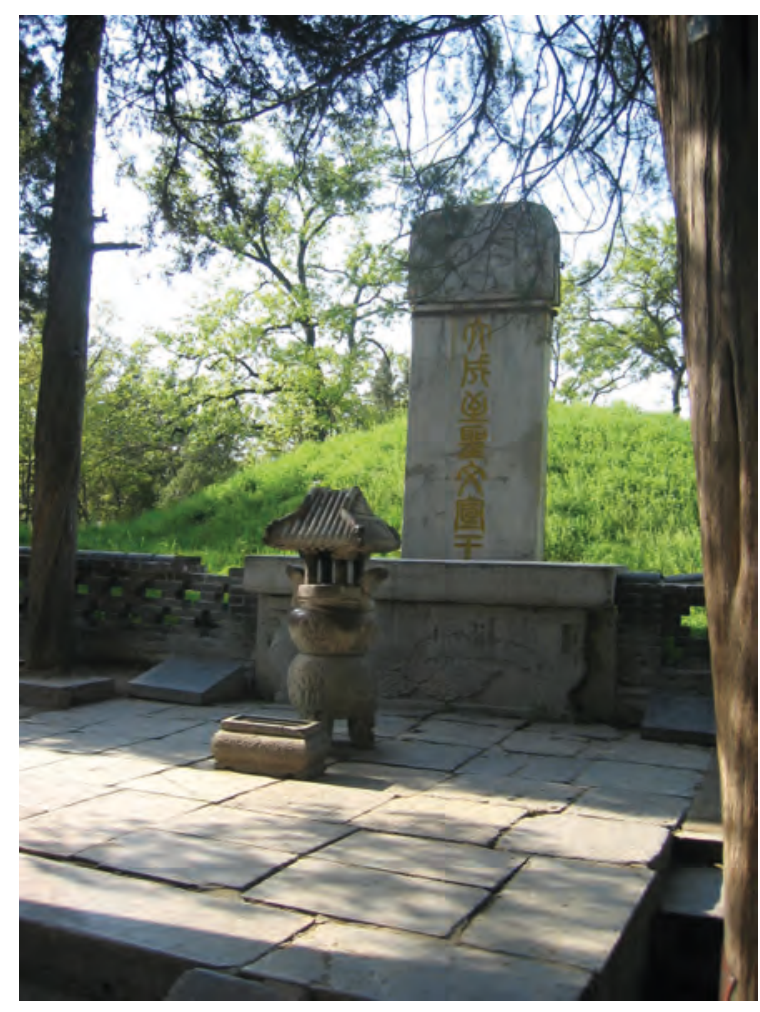

Illustration 10.11

Tomb of Confucius in Kong Lin. Many centuries of his descendants are buried nearby, likely the longest familial burial ground in the world. The cemetery received additional land grants from many emperors Photograph by Rolf Müller, CC BY-SA 3.0 the town of Jixia in the fourth and third centuries BCE. It is possible that thousands of scholars gathered there at a time.

The most famous Chinese philosopher is Confucius. Confucius sought but never received a post at any royal court because these thought him too hard to deal with. He nevertheless attracted many followers. He urged government by people of good education and character, and recommended the study of classical texts by this meritocratic elite. Bureaucrats should be diligent, courteous, loyal, and respectful. Later, the Confucian philosopher Mencius would argue that human nature was good, though he agreed that the proper education would encourage these good impulses. He urged low taxes and avoidance of war. Still later, Xunji was an administrator; He thought people were naturally selfish, and so recommended clear codes of bureaucratic behaviour (with penalties), but also felt that education could improve character. The Confucian tradition advocated a hierarchical society in which everyone knew their place: sons (and wives) obeyed 
their fathers, and bureaucrats their superiors, and all obeyed the emperor. We noted in chapter 4 that agricultural societies tended to encourage both gender inequality and strict child discipline: Confucian philosophy was typical in emphasizing strict hierarchy within the family, but formalized these familial relationships.

\section{PRIMARY DOCUMENTS: CONFUCIAN QUOTATIONS}

As with Sun Tzu in chapter 6, Confucian sayings lend themselves to brief quotation:

He who exercises government by means of his virtue may be compared to the north polar star which keeps its place and all the stars turn toward it.

By three methods we may learn wisdom: First, by reflection, which is noblest; Second, by imitation, which is easiest; and third by experience, which is the bitterest.

He who knows all the answers has not been asked all the questions.

The man who moves a mountain begins by carrying away small stones.

Study the past if you would define the future.

The man who asks a question is a fool for a minute, the man who does not ask is a fool for life.

Give a bowl of rice to a man and you will feed him for a day. Teach him how to grow his own rice and you will save his life.

Note that Confucius provided advice on statecraft but also ethics and the search for wisdom. We might make particular note of the importance he attaches to history. Do these sayings seem wise? Do they still resonate today? Did Confucius have personal motives for some of these sayings? Do Confucius and Cicero sometimes say similar things?

The Taoist philosophical tradition associated with Laozi instead urged harmony with the way (Tao) of the universe. It recommended a detachment from petty human concerns in order to achieve a transcendent sense of unity with the cosmos. The implication for the state was that we would get better government if we decreased human striving. Wars would cease, and bureaucrats could perform their duties without being tempted to steal.

The idea of "yin" and "yang" also emerged at this time. Yin represented such elements as darkness, coolness, and femininity, while yang represented light, warmth, and masculinity. Rather than being seen as in competition, each force contained important elements of the other. The idea of yin and yang, then, encouraged people to look past apparent dichotomies to see and seek cosmic harmony. The world needed male and female, sun and rain, night and day; it was when these worked together that the best results were achieved. (There is a similarity here to the "Both/And" approach urged in Chapter 1.) 
The most immediately effective school, though, were the Legalists, who promoted strict laws and punishments for even minor offences. As with Kautilya in India (chapter 9), they urged punishment of the entire family of a disloyal bureaucrat. They also stressed the value of the military and agriculture, downplaying the importance of merchants and artisans. The Qin Empire adopted Legalist practices. As we shall see, though, Qin practices promoted a backlash. The Han then officially adopted Confucianism as its favoured philosophy, though it continued to employ Legalist-inspired rules and punishments.

One shared aspiration of Chinese philosophy was an end to the endemic wars associated with political fragmentation. The Taoists sought an answer in withdrawal from earthly desires. Other schools supported the restoration of hegemonic empire. Confucians imagined that hierarchy and a sense of duty would contain violence within such a regime (Mencius was particularly hostile to war), while Legalists felt that only harsh punishments could maintain order. Even Sun Tzu's The Art of War, which is still millennia later consulted by military (and business) leaders around the globe, argued that the best victories were those won without violence, through negotiation and the mere threat of force (chapter 6). The ongoing efforts of the Han to purchase peace with nomads arguably reflected this shared philosophical outlook. However, the Romans likewise attempted to co-opt "barbarians" on their borders despite far less celebration of peace within the Greco-Roman tradition. (There was some: Plato decried the warmongering of tyrants, and Aristophanes wrote an entire play about peace.)

\section{The Qin}

We saw in chapter 7 how the Zhou dynasty had established the largest state of its time in northern China. As the Zhou state declined, north China experienced the "Warring States period" - so-called in large part because one vast empire characterized the bulk of later Chinese history. Historians estimate that there had been 256 wars during the Warring States period 656-221 BCE. When the Zhao state borrowed the idea of mounted and armoured archers from the nomads they also fought, other Chinese states added cavalry to their armies too. In 221 BCE one of the warring states, the Qin - from the western edge of the region - would unify northern China under its rule. Qin armies may have killed 400,000 Zhao soldiers in its conquest of that one state. A mere fifteen years later, the Han would replace the Qin, but the Han then unified the core territories of China for four centuries. The Qin and Han could build on the administrative practices of previous states. The idea that one state should rule this vast territory survives to this day.

The Qin state was very centralized. The Qin tried to replace local nobles with an extensive central bureaucracy. The Qin called local elites to the capital of Xianyang to live a genteel but powerless life. It decreed that their estates be divided among their children when they died in an effort to break up large landholdings. It appealed to peasants for loyalty by giving them land but then 
taxing it; it also sought to establish the rule of law. The Qin likely succeeded in taking a much larger share of economic output (perhaps 10 or 20 per cent) than the Romans ever did (maybe only about 1 per cent). A massive bureaucracy was necessary to raise taxes and to manage and provision a large army. The Qin also encouraged irrigation and the use of ploughs pulled by oxen. Moreover, the Qin sought to encourage investment by lending money to peasants at much lower interest rates than they could find elsewhere.

The Qin established seventeen ranks of society. Military success was the easiest way up the ranks: There were rewards for numbers killed. One could not gain high government office without military service and achievement. Constant warfare provided many opportunities for advancement. The Qin nevertheless claimed that the key to their success was agricultural productivity.

The Qin standardized weights and measures, the script used in writing, and even axle widths (to follow the ruts in the roads). There was a poll tax (tax per person) and a land tax; merchants paid a higher tax, and slave holders paid a tax per slave twice that of the poll tax. There were also labour requirements to work on the roads and public monuments (including the emperor's tomb). The Qin divided society into groups comprising five households: These had to report crimes, or the state would punish them jointly. The Qin also insisted on price tags in markets, eliminating the haggling that was common across many historical societies. Scholars often criticized Qin Shihuangdi during his rule; he responded by killing hundreds of them and burning any book that lacked an apparent practical value.

Qin strained its resources with its roads, walls, and canals. Historians estimate that between 500,000 and 700,000 worked on the tomb of the first emperor Qin Shihuangdi near Xian (about 220 BCE). This tomb was filled with jade and bronze goods and the bodies of hundreds of servants sacrificed to accompany the emperor into the next life - but is most famous for its massed army of thousands of terracotta soldiers. Some have speculated that the famous Terracotta Army reflected Hellenistic influences, as realistic sculptures were not part of previous Chinese tradition. There is no direct evidence of transmission of Greek ideas, though Greek influence on Buddhist art suggests a possible path of transmission. When the terracotta warriors were discovered in 1974, scholars were amazed by the lifelike features, expressiveness, and quality of the sculptures, as well as the sheer number. Though the soldiers were massproduced, each soldier has unique facial features. It is rare for such skill to be displayed in a work of art without a long preceding history of similar works.

Within a year of the first emperor's death, restive regions rebelled, and the Qin dynasty ended. Though short-lived itself, the Qin successfully established the ideal of a unified Chinese state. Its success in instituting a common written script is particularly noteworthy. No other empire of the time succeeded to the same extent in achieving a common written language. It is tempting to attribute this success to a greater cultural similarity within Chinese lands, but we must not assume that the sense of a shared cultural identity that exists today after over two millennia of (generally) political unity also characterized 


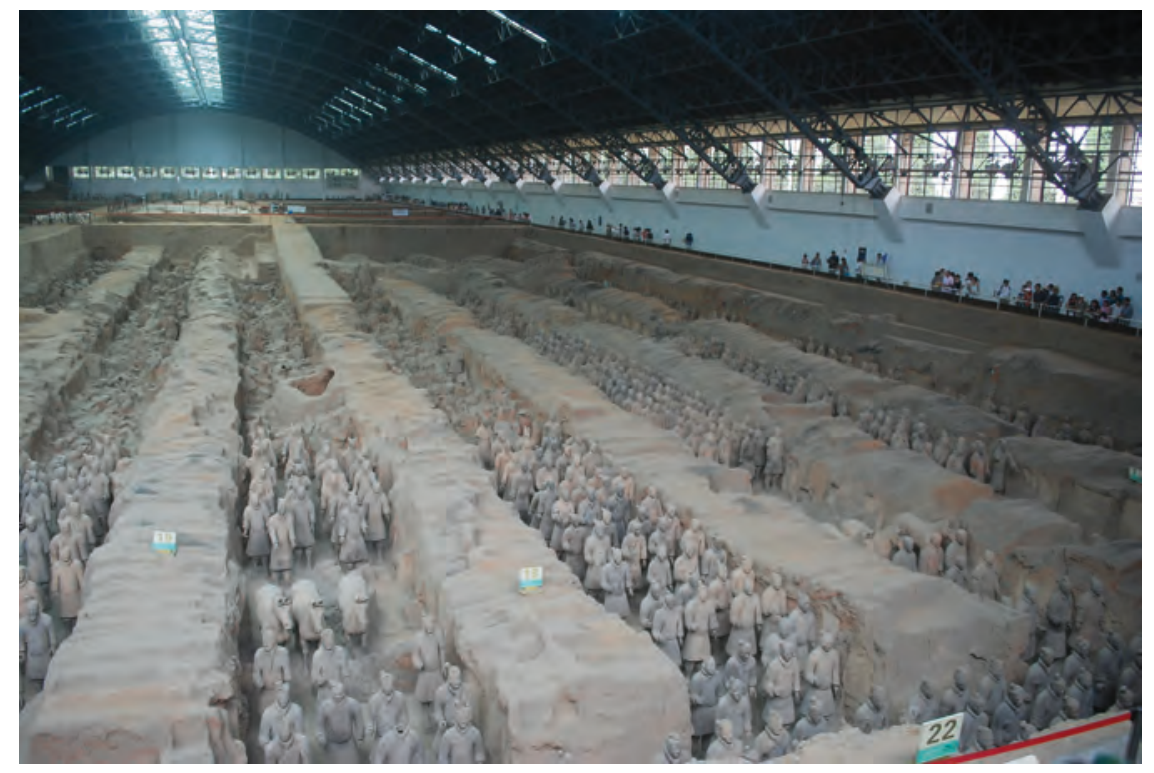

Illustration 10.12

Terracotta warriors from a distance

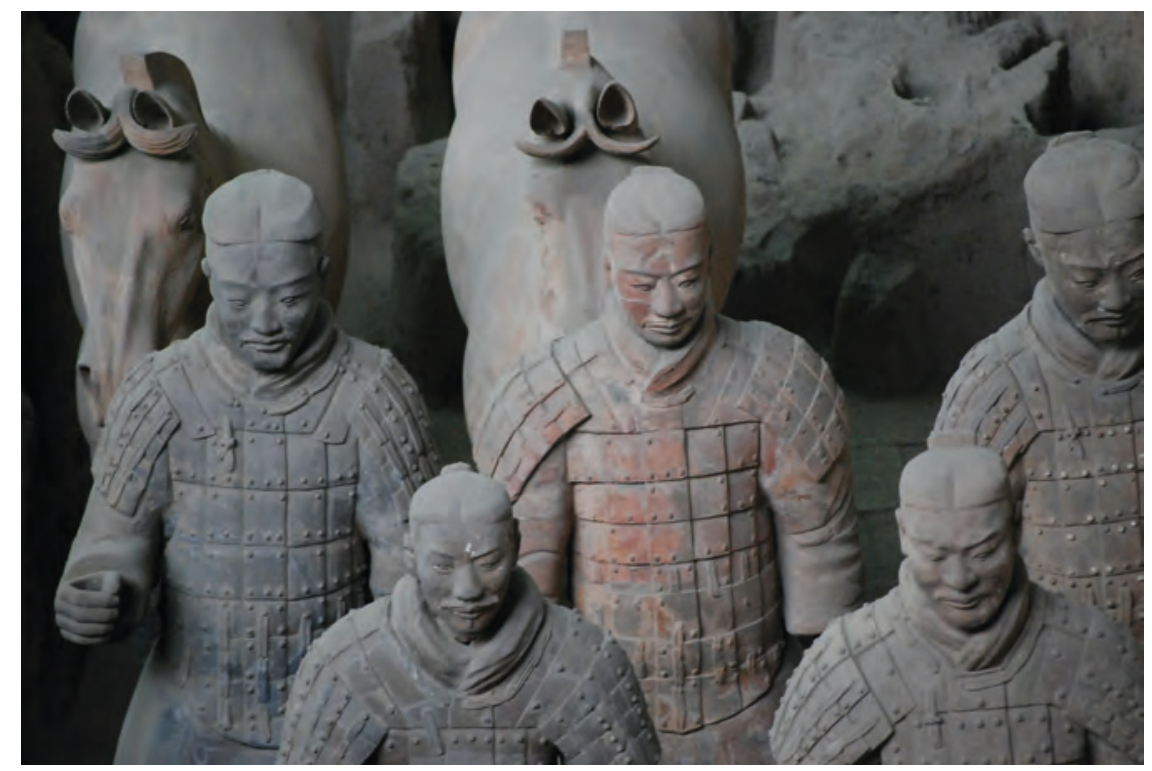

Illustration 10.13

Close-up of terracotta warriors

the distant past. The ideogram-based script employed in China may itself have been crucial here: Since most symbols represented words rather than sounds, entirely different dialects or languages could apply the same script. Educated 
Chinese from various provinces could read the same texts even if they had difficulty conversing.

\section{The Han}

The first Han emperor was a commoner; He would later claim that a god had fathered him. More importantly, he claimed the "Mandate of Heaven:" The Zhou had used this idea that "heaven" would bring down evil rulers and replace them with another dynasty, and new dynasties throughout Chinese history would invoke it. More pragmatically, the first Han emperor promised less severe punishments and lower taxes than had been levied by the Qin. The Han Empire at first tried to rule through local elites (on whose power it depended at first), who they expected to pass along tax revenues and provide military support. However, these elites provided limited military aid when Xiongnu nomads threatened the Empire. The Han responded with increased centralization, but never to the extent of the Qin. The capital city first moved to Luoyang in the south, then back to Chang'an near the Qin capital. It would later move back to Luoyang. The Han also in 198 BCE inaugurated a practice of seeking peace through marriage alliances when the emperor's daughter was married to the Xiongnu leader. Such marriage alliances would often be pursued in Chinese history, and the princesses then lobbied for peace and understanding in foreign courts.

One key emperor was Han Wudi, who ruled from 141 to 87 все. Han Wudi pursued land redistribution by arresting local leaders, confiscating property, and imposing high taxes on large landowners and merchants. Han Wudi, like the Qin, hoped for an egalitarian empire in which a poll tax (a uniform tax per person) could be the primary source of revenue. However, large landowners gained power again under later emperors. Also, many merchants found it prudent to buy land so that it was harder to tax their wealth.

Han Wudi extended the empire, conquering parts of Vietnam and Korea (and establishing Confucian principles there). Most critically, he attacked successfully into Central Asia against the Xiongnu nomads, reaching the Ferghana Valley in 101 все. The Xiongnu had won early battles against the Han and effectively received tribute from China for decades in return for a degree of peace. Both sides tried to encourage discontent among subjects of the other along the borderland. China would eventually win only by gaining nomadic allies hostile to the Xiongnu. Yet it would then - just as in Rome - incorporate nomads into the army in place of conscripted peasant infantry. This practice would weaken the state, as these nomads never developed a primary loyalty to the state. In the interim, China exercised control not just of China but also of much of the Central Asian steppe. This facilitated long-distance trade - albeit an expensive trade only suited to luxuries - between China and Rome, Persia, and India. There had been some movement of goods and ideas between China and these other regions even while the Xiongnu controlled the trade route - the Xiongnu were themselves willing to borrow useful ideas, after all - but this 


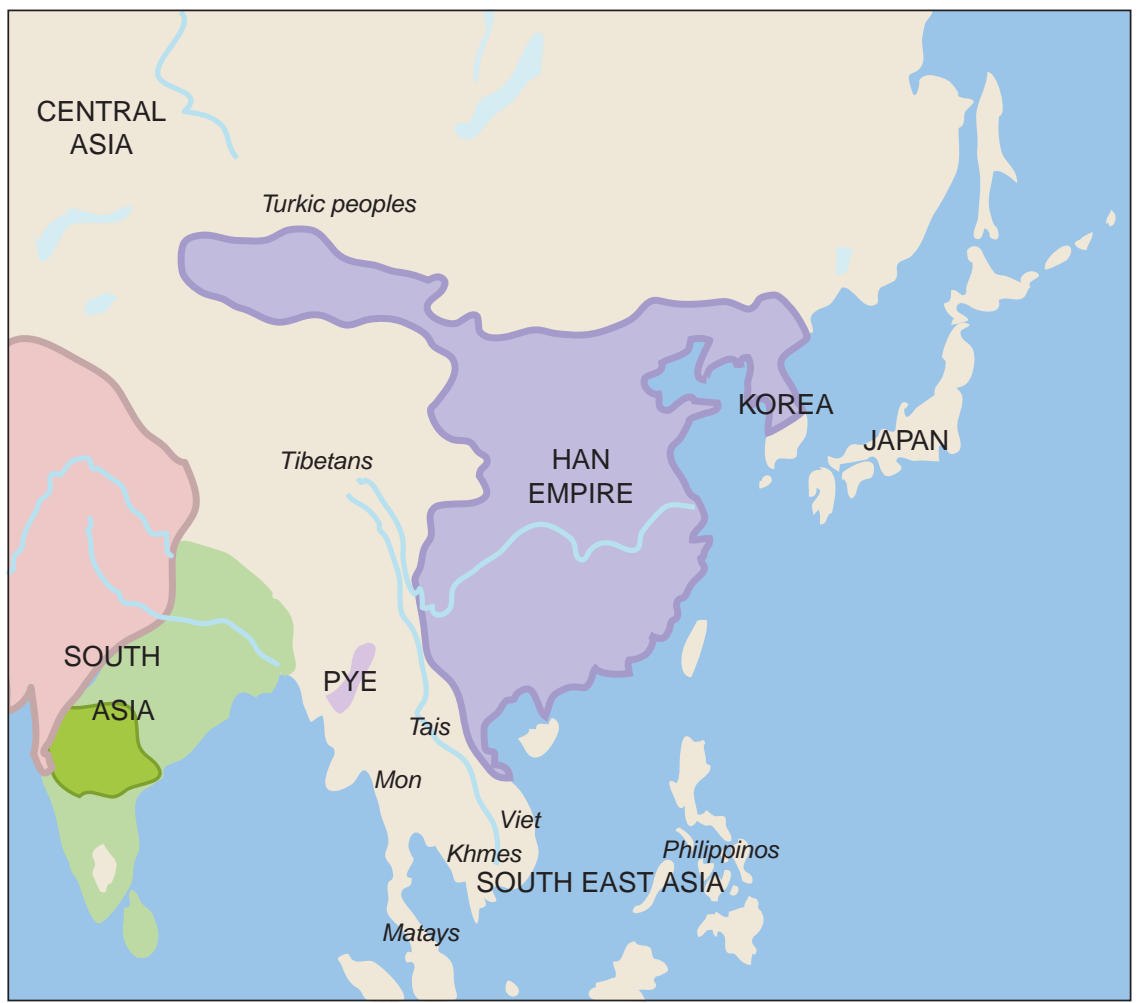

Map 10.3

The Han Empire, 30 BCE

TimeMaps.com

exchange of goods and ideas increased with direct interaction between large sedentary polities.

Employing thousands of bureaucrats, Han Wudi established an academy in 124 BCE that taught Confucian classics. The Confucian canon was far more extensive than the Legalist canon, though as noted above, the state continued many Legalist practices while officially embracing Confucianism. Confucian philosophy usefully encouraged integrity among elites.

Soon after the state formed the Confucian academy, it introduced a system of examinations for the selection of bureaucrats. This meritocratic examination system expanded during the Han and even more during later empires in Chinese history. There is no counterpart to this system anywhere else in the world until the modern era. The Han only selected a minority of bureaucrats in this way a hundred a year - though these occupied senior posts. The advantage of the system was that educated people rather than those with wealth or connections obtained positions of bureaucratic leadership. An oft-noted criticism was that Confucian classics were not obviously the best preparation for managing a complex state. This problem likely grew over time as bureaucracies became more complicated. Although the examination system could potentially foster 
social mobility, in practice, wealthy families devoted resources to educating their sons so that these had a much better chance of passing. Nevertheless, a broad section of the population could hope that their sons might advance in the bureaucracy. There were also - and this should be no surprise - frequent attempts at cheating on the exams.

Though the Han bureaucracy was less extensive than the Qin bureaucracy, it was far more extensive than that of Rome. There were some 130,000 bureaucrats in 5 BCE. Bureaucrats from one part of the empire were sent to work in quite distant districts. They thus had loyalty to the state rather than to local elites (though they needed to work with these). The efforts undertaken to train for and cheat on the examination signal the importance of the Chinese bureaucracy. This extensive bureaucracy was able to collect detailed information on individual farms, the skills available in each locality, the status of paupers, and availability of weapons. The state then employed auditors to identify bureaucrats who might be stealing from the state.

The idea of governance by an educated bureaucracy would be influential throughout Chinese history. It would replace the Qin practice of promotion based on military success. Chinese armies would, in general, remain subject to bureaucratic control. Only in times of dynastic collapse - usually triggered by peasant rebellions - would military prowess play a role in determining the next dynasty.

The Qin legal code had three punishments: death, mutilation, and forced labour. The Han softened the code, replacing most mutilations with corporal punishment. The guilty could often escape punishment by paying a fine.

We noted the Mandate of Heaven above. This vague divine mandate could be flexible and allowed the Chinese state to recognize many religions. Taoism was popular with some emperors. Buddhism arrived during Han times and was valued for its emphasis on ethical behaviour and acceptance of misfortune.

The population of the empire tripled from 20 million in 220 все to 60 million by 9 CE. Iron became common in weapons and agricultural implements. Iron technology had been transmitted from the west through Central Asia during the Warring States period. It had already then encouraged a dramatic expansion in the size of armies. Now its use expanded further. People who could not have afforded bronze pots and implements had iron. Iron ploughs and shovels boosted the productivity of agriculture and public works. As in Rome, though, income inequality grew through time, causing social unrest.

One key innovation of the Han period was paper, first developed around 100 все. This superior writing surface supported and may have encouraged the bureaucratization of the Chinese state.

In $6 \mathrm{CE}$, the regent Wang Mang took the throne, and attempted land redistribution. He was eventually toppled, and the Han restored. The later Han lasted centuries, maintaining control in Central Asia, but faced continuing problems of elite factions fighting for power. Local landowners and military commanders increased their power during the last centuries of Han rule. 
There were also many peasant rebellions as both the central state and local elites tried to increase revenues. The central state came to rely increasingly on taxes on trade - and especially monopolies on iron and salt - rather than taxes on agriculture: Confucian scholars usually objected to this sort of interference in the market. Though the Han were suspicious of the rising power of merchants, trade did expand and many merchants amassed considerable wealth. By the end, the Han emperor was merely a puppet of local elites. In 220 CE the Han dynasty fragmented into three kingdoms (See chapter 13). The power of local landowners would limit political centralization in succeeding centuries. The chaos that followed (which included nomadic incursions into the north) seems to have discredited Confucian teachings for a while: Taoism and especially Buddhism (popular already among nomads) prospered over the next centuries.

Classical China developed superior methods of bronze casting, including the lost wax method (chapter 8). It also produced enormous quantities of lacquerware (that is, wood decorated with lacquer), ceramics, and textiles. The motif of winged animals was borrowed from nomads. However, the central themes in Han art were paradise and immortality. The rich were buried with jade and silk items to employ in the afterlife.

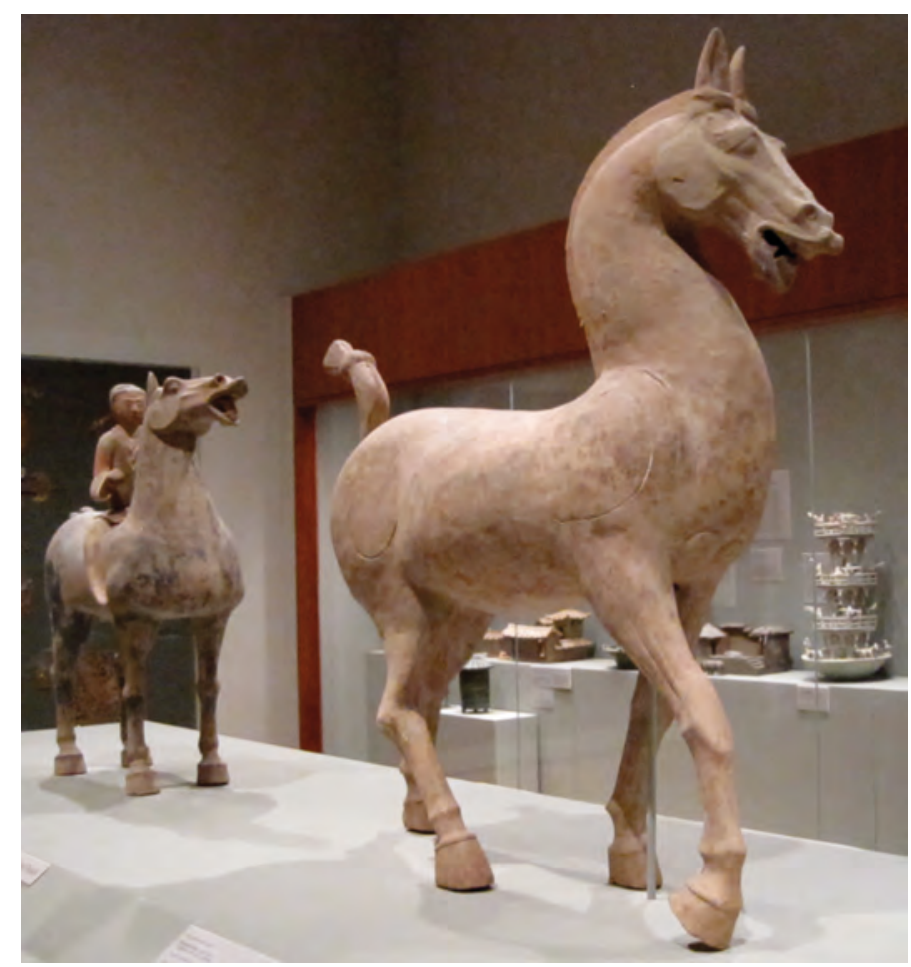

Illustration 10.14

Ceramic prancing horse, Late Han period, British Museum Photograph by Ellenm l, CC BY 2.0 


\section{Comparing China and Rome}

Han China and Rome traded indirectly with each other: Silk, jade, and iron products from China reached Rome, and linen, glass, and gypsum from Rome reached China. But the trade always was handled by intermediaries, and so the two empires had little knowledge of each other. (There may have been one group of intrepid travellers that went all the way from the Roman Empire to China, but they have not left detailed records as Marco Polo would a thousand years later.) Chinese writings in the first century CE referred to an empire in the far west with tall shaved people who had their own silk; whose ruler lived in a crystal palace; and which was hard to visit because of intervening lions and tigers. Romans, in turn, thought that people with blue eyes who did not talk to strangers and lived two hundred years ruled the Eastern Empire; they concurred (unknowingly) with Chinese writers that this other land was difficult to visit. Shielded from each other by mutual ignorance, both empires imagined that they ruled the world.

\section{Similarities and differences}

Given the minimal contact, the degree of similarity between these two empires is somewhat astonishing. If they had been in contact, we might well have speculated that they had learned from each other - a lesson we should keep in mind in later chapters when there is more contact between distant societies. Both empires moved from mass mobilization during their empire-building phase - with hundreds of thousands of soldiers at a time in both empires toward a later reliance on professional armies on their borders, often staffed by foreigners thought to be "barbarians." (One characteristic of professional soldiers was that they could prosecute war even during the harvest season in both empires.) Both came to celebrate "peace" as they established hegemony over vast areas - while continuing to fight on the borders and utilize violence to retain power internally. Both had bureaucracies that employed hundreds of thousands (though China's was much bigger). Both had currencies, state intervention in manufacturing and trade, censuses, capital cities that were the most extensive cities the world had ever seen (Rome being slightly larger), 1500-2000 administrative districts, similar populations of 55-60 million at the peak (both having experienced population growth), formal recognition of differences in social status, and a system of laws applied throughout the empire. Both experienced increased inequalities as land ownership consolidated and both pursued unsuccessful attempts at land reform. Both built massive monuments, developed an ideology to justify their rule, developed elite education around classic works of literature (Greek philosophy in Rome, Confucian works in China), and developed a court-centred historiography to celebrate their respective pasts. Both experienced in their later years a religious transformation that would generate an autonomous church (Christian or Buddhist) which would urge ethics and individual salvation. 


\section{BOX 10.4}

\section{COMPARISONS ACROSS SOCIETIES}

Comparisons across societies lie at the heart of world history. However, in the broader field of history, there is understandable concern about the very act of comparison. One source of concern is practical: Historians tend to specialize by time and place and thus will inevitably know more about one society under comparison than others. There is also a more philosophical concern that the act of comparison robs each case of its uniqueness. Historians naturally celebrate the uniqueness of each historical event or process.

We can only fully appreciated uniqueness, though, through comparison. Only once we have identified the common elements in a set of events or processes can we fully understand what was novel in each case. Once we appreciate the similarities in peasant behaviours in the French and Russian Revolutions, we can explore the differences. There is then no fundamental philosophical conflict between the pursuit of comparison and the pursuit of detailed understandings of particular events or processes: They should instead be seen as the opposite sides of the same coin. Comparisons inform our detailed understandings, and detailed understandings inform our comparisons.

The German philosopher Wilhelm Windelband distinguished a nomothetic search for theoretical generalizations and empirical regularities from an ideographic search for detailed understandings of particular objects or events or processes. The natural sciences are generally nomothetic, the humanities are generally ideographic, and the social sciences may be one or the other. History as a field straddles the social sciences and humanities. It is hardly surprising that it reflects both the nomothetic instinct to compare and generalize and the ideographic instinct to identify particularities. Historians can take heart in the fact that philosophers have long appreciated that there is a symbiotic relationship between the two approaches and that there is a continuum of research strategies between the two. As noted above, each informs the other.

We addressed in chapter 1 a particular concern of the ideographically inclined: That a broad comparative treatment may ignore individual human agency. We noted then that we would find a place for individuals in our story. We can see now that comparison can aid us in appreciating individual acts. By understanding the typical pressures of administrating a large state, we are better able to understand the extraordinary successes of some rulers and the failures of others. Moreover, we can only disentangle the personal sources of success or failure from the circumstantial sources if we know what the latter might be. A ruler might succeed through brilliance or because there were extraordinary harvests or a fortuitous technological innovation or some other force beyond their control. A comparison can alert us to these other forces at work.

We should also address the practicalities of comparison. Comparison requires an acceptance that there is scholarly value in integrating the insights of multiple historians. We should always celebrate the primary researches of individual historians in the archives or the archaeological dig, but can simultaneously recognize the value in tying their insights into a broader whole. Again, there is a symbiosis: The primary research of some historians 
provides the building blocks for the comparative efforts of others. These comparative efforts inform the further investigations of the first group, for they may look now for explicit similarities or differences with other cases. We can have more confidence in a historical account of a particular time and place if it is clear about the similarities with other cases as well as the differences.

The danger is that the historian pursuing synthesis may not fully understand the research on which they build. To some extent, this is unavoidable: The historian cannot record everything they find in the archives. The solution here is conversation: If a comparative work draws inappropriate inferences from the works of others, these can clarify what they had meant, or add details that had not appeared in their previous publications.

Conversation works best when people speak a common language. We noted in chapter 1 that our precise definition of themes would encourage clarity in expression. We can add in this chapter that an explicit focus on interactions between themes will further enhance clarity. If historians of different times and places are all clear about when they are discussing the influence of autocratic states on religious beliefs (and especially if they clarify which types of belief they address), it will be much easier for the comparative historian to identify similarities and differences across cases. Labour historians, economic historians, social historians, and cultural historians may all care about how workers viewed the earliest factories. African historians, British historians, and Indian historians may all care about the impact of the British Empire.Yet they will put different emphases on these questions, attend different conferences, and publish in different journals. A focus on interactions among themes makes it much clearer when different scholars are talking about the same thing - or not. Economic historians may view the factory favourably because they emphasize its future impact on average incomes, while cultural historians mourn the apparent loss of artisanal independence. They are talking about different links, and an appraisal of the role of the factory in human history should embrace but not confuse these. In this book, we will seek to enhance the historical conversation through the clarity of our organizing structure.

A final note: Comparison is facilitated if we are all clear about the time periods involved. A historian of one society may trace cultural changes over millennia while another looks at changes over decades. The comparativist can easily draw mistaken inferences if not aware that different timeframes are being pursued. Comparative research can usefully identify and seek to explain differences in the amount of time a particular process takes to unfold in different places, but only if these differences are clear.

Both Rome and Qin had risen on the western fringes of their respective regions, gaining regional power over the fifth and fourth centuries BCE before taking broader control of a massive empire in the third through first centuries BCE. In both, there was a significant political transition along the way: In Rome, the empire replaced the republic; in China, the more decentralized Han replaced the very bureaucratic Qin. Both thereafter had somewhat decentralized empire structures where local elites wielded some power (though China remained 
more bureaucratic and centralized than Rome). Geographic expansion slowed and ended over time in both empires. Local elites gained power. "Barbarians" attacked and destroyed the empires in the fourth century CE in China and the fifth in (western) Rome.

There were crucial differences too. The republican origins of Rome had no counterpart in China. It was at least in part because of its republican roots that Rome would remain suspicious of a too-powerful bureaucracy and preferred to rule through local elites much more than the Han did. The gradual ascension to power of Rome by conquering smaller states (the exceptions being Carthage and later Macedon) may have allowed it to succeed without relying on a similarly powerful bureaucracy. Yet the Roman bureaucracy was still large, hierarchical, professional, and powerful. And it grew in size over time, taking powers away from local councils. The Qin in China fought for centuries with states of similar size before coming to dominance: It could justify its centralizing tendencies with reference to these continued wars with threatening powers. The Roman Empire also allowed its various elected councils to develop laws, and trusted its judges to interpret these, whereas Chinese law was much more the exclusive province of the emperor and bureaucracy. The Legalist-Confucian tradition in Han China justified imperial centralization of both bureaucracy and law. The Roman census and even conscription were voluntary (though with penalties for evasion) whereas these were enforced bureaucratically by the Han. Slaves were more important in the Roman Empire, comprising a third of the population at times, when they were usually only 1 or 2 per cent of the Han population. Though Han emperors would claim the Mandate of Heaven, they did not claim semi-divine status as Roman emperors would.

Even the much more extensive Chinese bureaucracy depended on local elites; it just did not have enough workers to exercise local control, though it did have agents down to the county level. Over time, both empires suffered from granting tax exemptions to such elites and from locals creating locally supported government posts that contributed little to the empire.

The armies of both empires employed iron weapons. There is speculation that such weapons may have been critical in the development of vast armies and empires. Iron, as we have noted before (chapter 5), was both cheaper and stronger than bronze. It was much less expensive to arm a hundred thousand men in iron than it had been with bronze. Iron, of course, would aid both attackers and defenders. Nevertheless, if it assisted the formation of ever-bigger armies, these once formed could patrol a much more extensive empire than previously possible and scare off any outside threats. Agricultural implements also employed iron; the increased agricultural productivity that resulted may have been critical in financing armies and bureaucracies.

Though both empires relied on army and bureaucracy, both also sought to legitimize their rule. Emperors in both empires argued that they had a key religious role (though this would change dramatically with the rise of Christianity in Rome). Emperors in both presided over large and lavish public ceremonies which served to communicate their earthly and religious power. Both would 
draw upon ancient ideas in creating their own ceremonies. Yet of course the precise ceremonies employed differed greatly across empires. Emperor $\mathrm{Wu}$ in China performed the feng sacrifice, only allowed when the empire was united, a sage ruler was in charge, and the appropriate heavenly omens had been observed. Augustus in Rome presided over twelve days of games, sacrifices, feasts, and plays.

Both empires operated in the Eurasian temperate zone. Both were characterized by internal diversity in soil and rainfall and temperature: China had rice- and wheat/millet-growing regions and Rome developed wheat- and grape- or oliveproducing regions. Rome had one huge advantage in communication, for the Mediterranean Sea facilitated the transport of bulk products between many regions. China had navigable rivers to be sure, but contact between these rivers was far harder. China, in turn, had the advantage of being more self-contained by mountains and sea on three sides. This, plus its more compact - roughly hexagonal - shape meant that it had far less border to defend.

Both empires built extensive road networks. These served to facilitate administration and trade (which both empires taxed). The Chinese road network may have been twice as long as Rome's. Since these roads were designed primarily for messengers or troops moving by foot or on horse, they were not always well suited to the movement of wagons. Since animals can pull roughly eight times what they can carry on their backs, costs of transport did not fall as far as they might have. Both empires used forced labour in building projects, though China to a much greater extent than Rome (100,000 forced labourers may have died of exhaustion while building the Great Wall; Jewish captives after the rebellion of $70 \mathrm{CE}$ were put to work building the Roman Coliseum). Both also hired labourers to work on such projects.

Eunuchs (castrated men) played a role in both empires, though much more in China. They wielded influence because of close contact with ruler and wives, though officially they were excluded from power. They were usually loyal to the ruler because they had nowhere else to go, having no family and being viewed with suspicion in the broader society.

Both empires encouraged people to migrate from core areas to areas that they later conquered. The Greeks had long established trading colonies throughout the Mediterranean and Black Seas - but these operated independently of Greek city states. The Romans encouraged colonies. These would also be mostly self-governing but would serve as garrisons for the empire. The Chinese state encouraged movement from northern China, where Chinese empires had first emerged, into the newly conquered south.

Both empires would eventually fall, to be replaced by much smaller states. In China, though, the empire would be reconstituted centuries later, whereas in Rome this would never happen. We are curious below about the causes of imperial decline, and about why there would be an imperial rebirth in one rather than the other.

We should be careful in drawing comparisons. We could see many of the similarities described above also as differences in degree. Both empires had 
bureaucracies, but China's was much bigger. As we will see below, both this similarity and this difference may have been historically significant. It is still reasonable to assert that there was a strong family resemblance between these two empires. If it had been feasible for residents of one to visit the other, they would have been surprised by many details but would have found the overall pattern of life quite familiar.

\section{Empire and trade}

As noted above, both empires relied on and taxed internal trade. They supported this trade with laws and law enforcement, roads, and ports. Nevertheless, both empires were suspicious of merchants. Both empires glorified war and service to empire rather than commerce. Merchants were essential to the functioning of empire but operated outside of bureaucratic control and the ideals of empire. Who would devote their life to shipping wine and pottery when they could be killing barbarians? Nevertheless, elites in both empires would dabble in trade and welcome (perhaps grudgingly) wealthy merchants into their circle. Moreover, merchants arguably had a much more direct effect on everyday lives than bureaucrats did, providing even peasants with food and pottery and building materials and textiles.

To be sure, the state financed some internal trade. Famously, the Roman Empire provided grain to feed the masses of the city of Rome (lest these revolt). While some of this grain arrived as taxes or tribute, merchants seem to have imported most of it. Both empires also sought to monopolize specific economic activities. In practice, though, they usually contracted with merchants to operate these on their behalf. Rome at least also had tax farmers: private individuals paid to collect taxes in particular regions. Tax farmers limited both the need for and power of local bureaucrats.

Notably, neither empire seems to have seen its role as supporting a centralized industrial sector by securing raw material flows, as much later empires would. The vast bulk of their tax revenues came from taxing agriculture and trade. They needed industrial production just as they needed trade, but viewed it also with less respect than farming and war.

Agricultural productivity may have been significantly higher in China at the time. In the sixth century все, China was already growing crops in rows, paying attention to weeding, and frequently employing iron ploughs. Europe would not apply all of these innovations for centuries. The Chinese also by the third century всЕ used horse harnesses that placed pressure on the horse's shoulder rather than neck, avoiding the risk of strangulation of the horse and permitting them to pull ploughs and heavy equipment. The seed drill was in use by the second century вСе. In the first and second centuries вСЕ, types of mouldboard ploughs that became available in Europe only after about $800 \mathrm{CE}$ were already in use in China.

Both empires received taxes both in kind and in currency. China's tax system was more uniform, whereas Rome pursued different practices in different 
provinces. Rome had an advantage with respect to currency, having ready access to supplies of gold and silver for coinage. China's currency was bronze (though gold, silver, and even textiles were used in exchange). Both empires could debase the value of coins a little below the value of their metal content but ran into difficulty if they tried to reduce metallic content too much: Citizens would then balk at using the currency. The Roman economy was likely more monetized than China's. Yet currency was common in both, and so it is not entirely clear why some taxes were still collected in kind. Both empires sought at times to raise more taxes monetarily. There was clearly a market in which peasants could sell grain and other goods to pay taxes and those taxes were then used by the state to fulfil its various needs. It must have been easier in some cases to transfer agricultural produce directly from some farmers to some soldiers and bureaucrats. Nevertheless, the ability to collect some taxes in cash certainly facilitated the functioning of vast empires. Letters of credit were also common in Rome; we do not know about their use in Han China as a way of settling accounts.

One of the glories of empire and especially capital cities was that through tribute and trade, the products of diverse regional economies were interchanged. Writers in both Rome and Han China celebrated the diversity of products available in their capitals. Elites in both countries displayed exotic materials as a symbol of their affluence. Imports of foreign luxuries were particularly important in this regard. There was a luxury trade beyond the borders of each empire. Silk, incense, spices, and ivory reached Rome from Asia and Africa. (A pound of frankincense was worth a monthly labourer salary in Rome, yet this was the incense used in Roman temples. Myrrh was twice as expensive but the longest-lasting scent.) China imported from Southeast Asia and India and Europe (both Chinese and Southeast Asian ships moved between the two regions from the early Han). This trade was decried by writers in Rome especially for exporting silver (which was valuable as currency), but in China also. Elites in both empires developed a sense of taste: One should not be too vulgar in expressing one's wealth but nor should one be cheap. Newcomers to the elite needed to master this balance to avoid disdain.

In addition to buying luxuries, elites built large palaces and hired many servants. In the Roman Empire, it was common for the rich to build public monuments such as theatres in their name; in China, such monuments tended to be established by the state. Charity

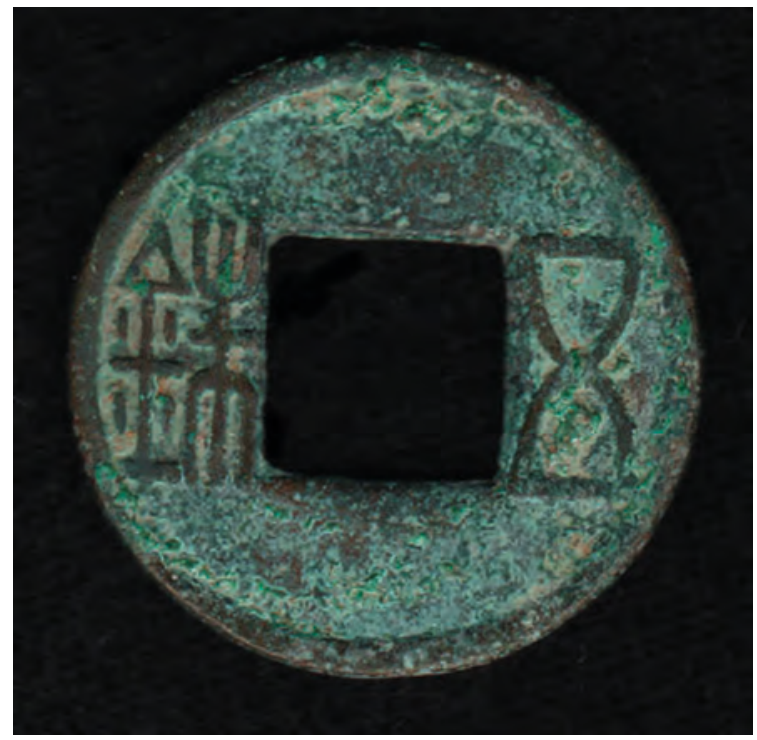

Illustration 10.15

Five Shu coin, lst century BCE, Han Dynasty 


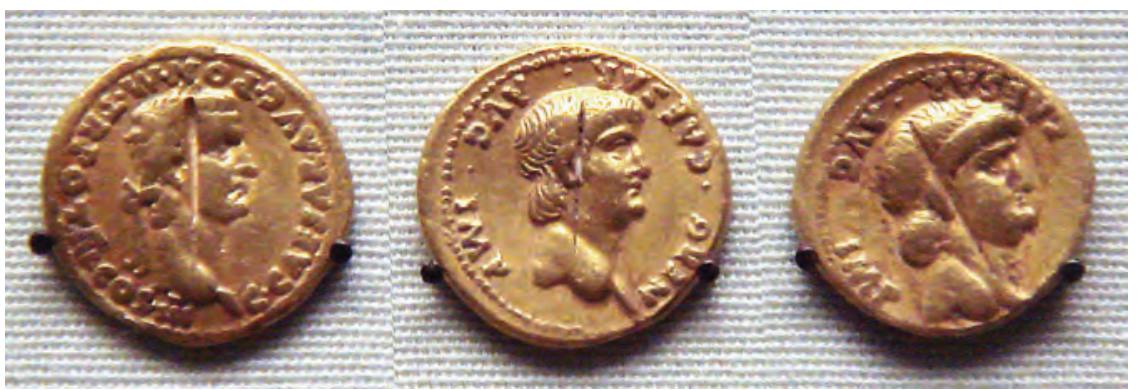

Illustration 10.16

Roman gold coins excavated in Pudukottai, India; first is the emperor Caligula; others are Nero, British Museum Photograph by Uploadalt, CC BY-SA 3.0

toward the poor was also encouraged, especially in the later period in both empires (when there was an increasing number of landless peasants who could no longer look to a large mass army for employment and advancement). Both Christian and Buddhist priests encouraged this.

Income inequality increased in both empires through time. This in part reflected the ability of local elites to extract incomes from the state. There was also a lengthy process of land consolidation - which both empires struggled unsuccessfully at times to arrest through land reforms (their lack of success here indicating the power of local elites to frustrate the wishes of the emperor). It seems that it was more profitable to operate large farms than small. This may have proved especially important as farms moved from self-sufficiency to involvement in market exchange. The institution of slavery may have encouraged consolidation: Large landowners with many slaves replaced family farms, especially in the Roman Empire. Large landowners also had an artificial advantage in that they often gained exemptions from taxation.

\section{Imperial decline}

There are again commonalities with respect to imperial decline. Both empires ceded control to local elites over time. We will observe this process many times in history, and it is perhaps hard to avoid. Empires have to rely on locals to some extent, and these will always scheme to achieve more power and higher incomes at the expense of the centre. Once a state cedes power or income to its regions, it becomes hard to take it back. However, as we have stressed above, the survival of the empire depends on it taking in more in taxes than it costs to administer. In both empires, the centre came to face financial difficulties over time.

Both empires, as noted above, developed professional armies (that is, paid mercenaries) to patrol their borders and attempted to co-opt nomadic or seminomadic peoples on their borders to serve in these armies. This policy made short-run sense: It was far cheaper to buy the support of these groups than to 


\section{BOX 10.5}

\section{EXPANDING FARM SIZES}

We have noted above that average farm size grew in both empires, despite the efforts of the empire to redistribute land. This process had an impact on income distribution - it was associated with a small but wealthy elite of large landowners and a large number of landless peasants and slaves. It also had political repercussions as this landed elite competed for power with the state. We will find the same process many times in history, and similar sustained but unsuccessful efforts by the state at land redistribution. It is, therefore, useful to remind ourselves of the forces that might have driven increased farm sizes:

- There may be "economies of scale" for some types of crop such that it is much cheaper per unit to produce these on large farms than small. If such economies are substantial, it will be difficult for the state to prevent consolidation. In the modern period, we see large farms in the production of grapes and olives, but family farms are typical for wheat and rice. Though the technology of wheat and rice farming was quite different two thousand years ago, we can wonder if there was a strong economic motive for very large farms. Indeed, in both Rome and China, large estates were often farmed in small plots rather than as one integrated entity.

- Slavery may tip the balance toward large farms. Though we should not exaggerate the cost advantage of slave over free labour (see Box 9.1), the ability to employ slaves certainly lowers the cost of production. There may be economies of scale in buying, keeping, and preventing slaves from escaping.

- Small farmers may often find themselves in debt because of harvest fluctuations. They may then sell their land (and sometimes themselves) to more substantial farmers. Slight differences in harvest fortunes might allow some farmers to expand at the expense of others. We may then see some consolidation even without any economies of scale. Note that land was the primary type of wealth holding at the time: A farmer that was gaining in wealth would naturally accumulate land.

- Those who agitated for land reform in Rome saw political corruption as the cause of land consolidation. Elites gained exemption from taxes. Since taxes on land were the primary source of imperial revenue, this gave elites a sizeable artificial advantage over small farmers. Peasants in both Rome and Han China often paid local landowning elites to intercede with tax collectors on their behalf. We might then again see consolidation that in no way reflected differences in cost of production.

- Expanding trade is important. Small farms may be mostly self-sufficient, but large farms are often oriented toward the market. Large landowners may be able to negotiate better prices for their output than small farmers.

Why were efforts at land redistribution usually unsuccessful? The obvious answer is that landowning elites were powerful enough to prevent redistribution. States might simply lack the physical power to remove many powerful property owners from their land (especially if 
these built fortifications). More subtly, states might lack bureaucratic capability. Registries of land ownership were often imprecise. Landowners could bribe bureaucrats to ignore the ruler's edicts or to lie about land titles. Exceptions might occur during times of political change: Each new dynasty in China tended to expropriate landholdings from existing elites. However, they then awarded these lands to those who supported the new dynasty, and the process of land consolidation would begin again.

fight them. However, neither empire instilled loyalty in these groups, and these groups would eventually turn against them (and use their knowledge of empire bureaucratic and administrative practices against them).

We should note that the story told in the last paragraph is far more dramatic than the story mentioned in the preceding one. Nomads brought into the bosom of the state by short-sighted leaders only to turn against them (or their descendants): It is a moving story and one that we can easily document. A slow process of financial strangulation because of a thousand efforts by local elites to wring power and money from the central state is both far less exciting and harder to document: We do not know the details of the budgets of either empire in a way that allows us to record decreased receipts reliably. Nevertheless, financial weakness may well have been the more central problem. Note that empires might have been more willing to conquer rather than co-opt their neighbours if they could easily finance wars.

If true, the financial decline of central authority occurred against a backdrop of economic prosperity. Though we should not discount the inequalities in power, status, and wealth associated with empire, we can also recognize that there were advantages. For centuries, these empires were mostly peaceful: There might be wars on the borders, but people far from the borders had little fear of invasion. Both empires enforced laws, and so people had some protection against crime. Both empires facilitated trade, both by building roads and ports and by establishing some set of legal prescriptions throughout their vast territories. Pottery manufacture prospered in both places, and even humble homes had decorated pots. Perhaps the best single indicator is tile roofs: These were common on homes throughout the Roman Empire, but thatched roofs would replace these in at least western Europe after the fall of the Empire. Tile roofs provide much better protection against both rain and fire. The diet of the average Roman or Han citizen may still have been poor by modern standards, but starvation was rare (both states stored grain to distribute when needed). As trade contracted after the fall of empire, diets became less diverse, and pottery became crude and local.

There are further shared elements. Both empires suffered from severe epidemics in their last centuries (see chapter 13). Such epidemics would reduce tax revenues while imposing costs on society, such as the disposal of bodies. 


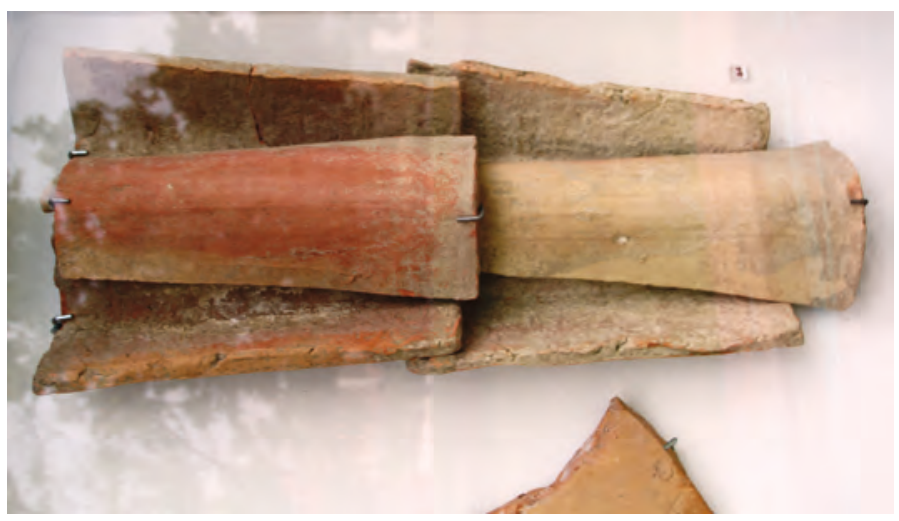

Illustration 10.17

Roof tiles from the Roman town of Aquincum (Hungary), lst or 2nd century CE Photograph by Bjoertvedt, CC BY-SA 4.0

These epidemics have received much greater historical attention in recent years. As well, the climate became somewhat drier in these centuries, which likely reduced agricultural output. The changing climate may also have encouraged nomadic incursions into both empires. These occurrences remind us that empires may decline due to a combination of internal forces and external shocks.

\section{The question of reconstitution}

Why would the Chinese Empire be often reconstituted (and even by foreigners) but Rome never? Though this question refers to later time periods, it makes sense to address it here if we think (many of) the roots of these different historical paths lie in the time of classical empires. As we have seen, these empires were similar in many important ways. Nevertheless, we can proffer a variety of explanations of differences in future paths.

One involves the external threat environment. China did not face Persians or Arabs and would deal better - for a while - with nomads from Central Asia than did Europeans. These differences in foreign pressure will indeed play an important role in later chapters. However, we have seen above that external pressure could justify centralization. Moreover, later Chinese success against nomads was arguably a result of centralization. Therefore, it is not entirely clear that this different threat environment necessarily prevented a resurgence of Rome.

China is likely a more manageable size and shape than the western section of Eurasia and North Africa dominated by Rome. It may then provide a more "natural" setting for one unified empire. The Roman lands would see many later empires with different centres and borders. The Byzantines would maintain an empire on the eastern territories of Rome for a thousand years, and Islamic empires would include Roman lands in North Africa and East Asia. It is in western Europe that the lack of a successor empire would be most remarkable. 
The Chinese tradition of Confucian thinking and educated scholars with a belief in one well-ordered state may have been critical in the periodic resurgence of a Chinese empire. We should be careful here that this tradition might have strengthened slowly through the last millennia precisely because empires were a recurring reality in China. In the west, in contrast, the Christian church may have worked against centralization, preferring to be the only central authority (chapter 15). Buddhism flourished in post-Han China just as Christianity flourished after the fall of Rome, but did not develop a hierarchical organization that could counter the power of states.

Our discussion of path dependence in chapter 2 may be useful here. A sizeable bureaucratic empire has a lot of staying power once in place but is hard to establish. China had a longer history of bureaucratic government before the Qin and Han than had (most parts of) the Roman Empire. The Qin could build on a much firmer basis of previous state bureaucracies than Rome. Its victory over other large states owed to its centralization of control within Qin lands. This allowed it to mobilize funds and labour for both army and public works. This centralization was easier to justify when competing with other states than after consolidation. Though the Han slowly decentralized, it retained more centralized bureaucratic control than did Rome. It selected senior administrators meritocratically and moved junior bureaucrats around the empire. These bureaucrats had loyalty to the centre rather than to local elites. When the Han fell, smaller states with bureaucracies replaced it and competed for centuries with the idea of regaining one empire.

Rome never achieved the same level of central control, perhaps because it had not faced the same threats as it developed. Moreover, republican forms of governance encouraged reliance on local councils to manage taxation; the Roman army was the only institution that required professionalization. When Rome fell, very localized and non-bureaucratic government replaced it. Though the memory of Rome never disappeared, within generations it would have been difficult to imagine its recreation. We will return to this theme in later chapters, discussing the operation of this highly localized system of governance (chapter 13) and how centuries later when centralization occurred, it did not proceed to the level of one large empire (at least within the region of the Roman Empire; chapter 21).

The greater importance of irrigation in China may play a role here. Irrigation improved agricultural productivity but required organization. There may, as a result, have been much more social support for bureaucracy in general.

We have stressed so far the fact that China had followed a path of central bureaucratic control. The competing states that replaced the Han would maintain centralized bureaucracies and the ideal of a uniform empire whereas the smaller polities that replaced western Rome would have small or no bureaucracies. The different paths of institutional inheritance intersected here with different behaviours of nomadic conquerors. The nomadic conquerors of the Han maintained bureaucracy but put nomads in senior posts. German tribes conversely took land as their prize. The new rulers in China thus had their 
own tax-funded armies, whereas German rulers relied on nobles for military support. The Chinese may have been encouraged to maintain bureaucracies by continued pressure from nomads. The critical point is that even during a period of political fragmentation, Chinese rulers continued with bureaucracies that could tax widely. We can reasonably ask to what extent the difference reflects the stronger bureaucratic traditions in China or the different behaviours of nomad conquerors.

There is also cultural path dependence. The Chinese people have come to see themselves as one people over time, whereas the people of the region of the old Roman Empire have diverse contemporary identities. We must be careful here that we do not backcast modern ideals of the nation state into the distant past: At a time when empires were the standard form of government, people would not necessarily imagine that every ethnic group should have its own state. Nevertheless, we can believe that it was much easier to pursue empire within a region if both rulers and ruled had some sense of commonality. It is harder to trace a cultural sense of belonging through history than to trace institutional forms. Yet it seems likely that the veneration of Confucian classics and the introduction of a common ideographic script were critical to the creation of a Chinese identity. Note that Chinese people to this day speak different languages but can read each other's messages because of a script that refers to things rather than sounds. The Roman tendency to rule almost entirely through local elites may have worked against the development of shared language or identity in the Roman Empire. It is likely that Chinese identity developed further during subsequent Chinese empires. Nevertheless, it seems likely that there was already a much stronger sense of cultural unity by the fall of the Han than characterized the Roman Empire.

\section{The legacy of empire}

We noted above that both Rome and Qin/Han built on the memory of precursors: notably Zhou in the case of China, and a variety of states but perhaps especially Macedon and Persia (see chapter 9) in the case of Rome. We have just seen that later Chinese empires would celebrate the Han idea of a unified empire. Though Rome was never recreated, its memory would also fire the imagination of empire builders for millennia. We shall see in later chapters that a variety of empires - the Byzantine most obviously but various Islamic and European polities - would seek to emulate Roman glory. In both cases, the Han and Roman examples provided not just an idea to imitate but a set of bureaucratic and ideological practices to either copy or transcend.

\section{Political thought}

In Greece and China, and to a lesser extent Rome and India, individuals with some independence from any state reflected on how to organize states. 
A hierarchical political organization was novel enough to merit justification, and hierarchical social organization meant that there were elites with the time to reflect on the nature of the state. In doing so, these thinkers developed some ideas that resonate in contemporary political philosophy and others that seem peculiar to modern sensibilities. Their works reflect the time period in which they were written but nevertheless provide some inkling of all later schools of political philosophy.

The most widespread idea is what can be termed "sacred monarchy," a sense that kings are selected by god(s) to rule. Greece and early Rome provided the only exceptions to this line of argument: The small size of most Greek states may have militated against seeing a king who was a familiar figure as having been ordained by the gods. The idea of a sacred monarchy would survive into modern times in some parts of the world.

There were differences in whether the king was seen primarily as a warrior or priest; in China, the king was a sage as well. There were also differences in how the relationship between king and god was imagined. Kings were most closely identified with gods in Egypt, where pharaohs were (often) considered divine or semi-divine. The most long-standing idea was the Mandate of Heaven in China: The Shang had been a sacred monarchy much like those farther west, but from the time of the Zhou, the notion that heaven would with some regularity replace misbehaving dynasties took hold.

The gods had given sacred monarchs unlimited power but also instructed these to use this power in the public interest. Kings were urged toward justice and benevolence, though these might be defined in different ways. It was common to urge kings to judge impartially, and especially to not favour the rich and mighty over the poor and weak. Laws were thought to have come from god in India and Israel, but from antiquity in Rome and China. Kings were also urged to care for the poor.

We can see some of these arguments as self-serving rationalizations of existing power relations. We might even see the emphasis on justice and caring for the poor - cynically perhaps - as arguments kings could use to limit the power of an aristocracy. Yet we have seen that state ideologies will be most successful if rulers themselves absorb these. Rulers might rationalize all sorts of selfish acts but might also strive some of the time actually to aid the poor or make just decisions. Moreover, the idea of sacred monarchy if widely shared could assist rulers in their challenge of getting bureaucrats to do what the ruler wanted.

We suggested in chapter 3 that humans were selected for a sense of justice. We saw in chapter 4 that agriculture raised concerns regarding who farms particular plots of land, and how to stop theft. We can see, then, that promising that a ruler would be just would appeal both to a critical shared element of human nature and to the primary concerns of an agricultural population.

Though religion infused ideas of sacred monarchy, the religious duties of rulers differed significantly. Kings in many places claimed divination powers or employed priests for this purpose: They then harnessed the state to the religious 
purpose of controlling nature. Divination was also a handy way of making and rationalizing decisions and sharing the blame when these went wrong. Priests in Egypt gradually wrestled religious power from the pharaohs, especially after Amenhotep's brief experiment with monotheism (chapter 8): Priests rather than rulers became the interpreters of the gods. Within Hinduism, warrior and priestly castes were separate, and the religious functions of the (warrior) ruler were limited.

One fundamental difference across societies was in their attitude toward social distinctions. India with its system of castes provided a religious justification for inherited social distinctions (it also provided for social stability in a region characterized by weak states), though the caste system was less rigid in classical times than it would become. Other states such as ancient Egypt were much more open to some degree of meritocracy: This was widely argued to support good governance. Chinese philosophers such as Confucius could, on the one hand, applaud merit, and on the other, encourage everyone to fulfil their role in a stratified society. His successor Mozi instead urged complete meritocracy (but still appreciated the need for a strong ruler surrounded by expert and influential advisers). Stability was widely recognized as an important feature of the state, one not to be casually set aside for egalitarian or meritocratic impulses. Indeed, in both the Jewish Old Testament and the Indian Vedas the people ask god for a king to ensure stability.

Whatever the precise attitudes toward social stratification, classical political philosophies urged charity on the rich and obedience and respect on the poor. They assumed that inequality was inevitable, but recognized it as worthy of some (appearance of) alleviation.

We should emphasize that the two regions, Greece and China, in which independent political philosophy blossomed - with competing schools fighting it out for generations - were characterized at the time by political fragmentation and war. In China, this philosophical reflection took the form of advocating a return to empire and reflecting on the form this should take. In Greece, with no ancient tradition of empire, and a variety of political systems across Greek city states, it took the form of reflection on the strengths and weaknesses of different kinds of states. The two regions (which had no intellectual contact with each other) nevertheless shared a concern with stability.

In China, philosophers used the idea of sacred kingship to justify a unified empire. In ancient Mesopotamia, through most of its pre-Persian history, writers instead celebrated the lived experience of changing power relations among city states. It was thought that a council of the gods - which may have mimicked to some extent advisory councils to Mesopotamian kings - decided from time to time to recognize a different god and thus king (since gods were associated with different cities) as the most powerful; this king might then justly settle disputes. It is worth note that city states shared this idea of a godly council. It may well have been hoped that it would reduce conflict since one king could be called upon to settle disputes among others. Yet, of course, it also encouraged kings to gain dominance and then claim that the gods chose them. 
One area of debate across all states, but especially in Greece and China, was how to balance ethics and expediency. It was fine to urge rulers to behave justly and benevolently, but these also had to rule. Philosophers wondered if rulers sometimes needed to act with harshness to ensure the stability from which all benefited. Kings, it was widely argued, were the alternative to chaos and anarchy. Egyptian writings in the Old Kingdom urged all people to behave ethically (they would be rewarded in this life and the next) - but the pharaoh was felt to be above the laws that might govern regular people. The Bhagavad Gita in India solved the dilemma for bureaucrats at least by prescribing a primary ethical obligation of duty itself: One would gain a better rebirth if one did what one was told without question. Kautilya, an Indian thinker who uniquely operated outside the Vedic tradition, is often called Machiavellian for his emphasis on practices such as spying, but he, in fact, urged a combination of pragmatism and ethics, and encouraged rulers to be righteous (Ashoka would proclaim himself to be righteous). Chinese philosophers also encouraged people to follow their duty, but the Confucian tradition - penned mainly by bureaucrats who moved between states - also argued that one should not work for a state that was not ethical. The Legalist tradition in China was instead in favour of harsh punishments for any disobedience. The Greek Stoics and the Roman Cicero argued that one's ethical duties always trumped expediency. There was thus a significant similarity between Stoic and Confucian thinking.

A related and more practical question involved war. It is noteworthy that peace was urged almost everywhere, and especially by those who operated independently of a particular state. It was widely appreciated that the costs of war often exceeded the benefits - for the people if not always for the ruler. Yet philosophers might approve war pragmatically if they thought that it could lead to eventual peace: The Legalists in China were particularly eager to see a renewed empire by conquest that would usher in a peaceful future (the Qin state followed their advice).

Sacred kings were often imbued with universal authority (Box 20.1). It could hardly then be surprising if these wished to conquer "their" world. At the same time, political philosophy was often ethnocentric, recognizing key differences between "us" and "them" (reflecting our selection for loyalty to groups; chapter 3 ). This could provide a different rationale for conquest: to have "them" work for "us." Mozi in China and the Stoics in Greece were unusual in the age in appealing to a common humanity. Mozi opposed war because it was an exercise in partiality: We should not view "them" as less deserving.

Darius of Persia was a typical sacred monarch. He claimed the support of the god Ahura Mazda due to his righteousness. He fought for light against darkness. He claimed to be destined to rule the world. He boasted of his good works, such as in developing infrastructure. Yet the Persians came over time to stress the consent of the conquered: They governed, that is, by popular recognition of their benevolent and stabilizing rule. This move away from sacred monarchy may have influenced later developments in Israel and Greece (see below). 


\section{China and bureaucratic theory}

The bureaucratic nature of Chinese states encouraged profound reflection on the role of the bureaucracy. Public service was widely considered the noblest path to self-realization. Confucians urged righteous government. This would promote righteousness in the broader society. Taoist philosophers were an exception: They reacted against the corruption and strife of the public sphere by urging withdrawal from human concerns (though some would urge rulers to govern in accord with the Tao). Confucians recommended, in addition to justice (which, as elsewhere, mostly meant impartiality) and benevolence, the pursuit of $l i$, a set of customs including ancestor worship that came over time to include how the ruler should interact with counsellors and how to manage the bureaucracy. We can see here recognition that cultural practices might encourage harmonious interactions. The Legalists responded that the masses were more likely to behave well if punished severely for even minor infractions. The Legalists also urged punishment of the powerful. (Sima Qian, historian of China who died in $86 \mathrm{BCE}$, suffered castration for publicly disagreeing with the emperor. Rather than kill himself, he wanted to finish his work, still the best primary source on early Chinese history.)

With their reliance on force, the Legalists saw little value in public discourse. The state should do as it saw fit. They felt that Confucian persuasion toward righteousness had failed. They worried that Confucian exhortations became less and less effective as population pressure on resources grew: The state must force hungry people to be good. They urged a system (adopted by the Qin) whereby groups of five families were jointly punished for not reporting any wrongdoing. The first Qin emperor would follow Legalist advice in destroying many classical texts that argued against authoritarianism. Note that the Legalists still wanted to pursue the public good - they just thought that the public would not understand that stability required obedience.

Postscript: The communist leader Mao would pursue the Confucian idea that righteous government would create righteous people thousands of years later. Though Mao disdained Confucianism, he argued that the people's behaviour would change if the correct institutions governed them. His experiments with collectivization would fail to eliminate selfish human impulses. The Communists celebrated the Legalist tradition, felt coercion was necessary, urged families to inform on themselves, destroyed texts, limited debate, and disdained intellectuals.

Legalist, Taoist, and Confucian principles would be combined to suggest an ideal sage ruler who was largely inactive, allowing his advisers to make the best decisions. This provided a solution to the "bad ruler" problem - but one that depended on even weak rulers having sound advisers. Han Feizi, who made 
such arguments, nevertheless argued that the ruler needed to be aware that his ministers had different interests; the ruler needed to check up on them. He recognized, that is, that his solution to the "bad ruler" problem needed to take into account that universal challenge of rulers to have bureaucrats do as they should.

\section{Democratic impulses}

Though the vast bulk of classical political thought stressed sacred monarchy, one can find signs of democratic thinking in many societies. Note that philosophers everywhere urged kings to govern in the public interest. They thus assumed the goal of democracy. There was also support for consultative decision-making. Though philosophers usually thought that strong leadership was necessary for stability and decision-making, they also appreciated that competent advisers should surround the king. As sacred as he might be, it was best to have some conversation before he made decisions. The king's advisers should then ideally reflect the public will. We might suspect that at least some philosophers were rationalizing the authoritarian rule under which they lived. Formal advisory councils existed in India and Mesopotamia. There is a debate about how influential Mesopotamian councils were and whether they became more or less powerful through time. It could be that they mainly addressed local concerns (there is a reference to a guild trying a murder case). Jewish tribal elders were expected to advise the king (or, earlier, rule collectively).

The Chinese idea of the Mandate of Heaven was in large part an expression of public will. Though heaven would "officially" determine when a dynasty needed replacement, in practice, peasant rebellions usually achieved this end. Such revolutions could be anticipated when rulers failed to be benevolent or just. Yet one should not prejudge heaven's wishes: The public should only exercise its power in dire circumstances. Of course, such rebellions would not usually change the institutional structure of the state, just the ruler: The idea was to place a just and benevolent ruler in charge and let him rule. The Mandate of Heaven was then entirely different from a modern sense of public sovereignty. Still, there was some recognition that in the end, a king's rule depended on the will of the people.

Though we can espy some democratic ideals in many places, it is only in Greece that these were practised. Interestingly, many Greek philosophers, including Plato and Aristotle, were not too impressed with the result. They worried that democracies were neither decisive nor stable, and wondered if a king or aristocracy that was well trained and virtuous might achieve better results. (Plato's Republic is, notably, the only work anywhere that gave women an important public role; for Plato, they could rule).

We should note that Greek republics were small, with only some thousands of people. Rugged mountain terrain and many islands militated against territorial consolidation (though Alexander the Great would achieve this). In such small states, citizens could potentially gather to debate the issues of the day. 
Proponents of democracy came to argue that the best decisions resulted from debate among the broadest range of views. This freedom - unusual in the classical world - would attract thinkers from other Greek lands to Athens (including Aristotle, originally from Macedon). We can note here that philosophers appreciated both disagreement and respect for those one disagreed with as essential characteristics of democratic governance.

Plays by Sophocles and Euripides addressed the challenges of choosing between competing arguments. Aeschylus' play the Eumenides celebrates and justifies the decision to let a representative body judge crimes. The play appeals to godly intervention in setting up this institution, which it is hoped will replace previous ideals of vengeance.

Education and literacy supported Athenian democracy. Nevertheless, there were intense debates regarding who, what, and how to teach. The Sophists offended philosophers such as Aristotle by emphasizing debating skills rather than the pursuit of truth. Protagoras - perhaps the first teacher to charge "tuition" - argued that democratic virtues and skills could and should be taught to everyone. Plato, in his Republic, claimed that they could be taught but only to a sage elite. He felt that the purpose of the state was to allow people with different skills and desires to specialize and interact (ideas that reflected an urban trading milieu); it then made sense to allow those with skills in governance to govern. A sage elite could better serve justice - which he, like others, appreciated was part of human nature - than treating everyone the same. Yet Plato in his later Laws would come to urge a broad ethical education as well but notably one that involved indoctrination rather than the sort of discourse that characterized most of Plato's philosophy. Such an education did not protect against the possibility that the sages might rule in their selfish interest.

Plato may have had more impact on Islamic thought than on later European political theory. Many Islamist thinkers and rulers liked the idea of indoctrination and seeing the state as prescribing moral laws (Chapter 12, 20). Islamists also liked the idea of a moral elite (think of modern-day Iran). The Catholic Church would occasionally also try to provide rulers with moral education.

Aristotle was an empiricist, collecting information on the varieties of forms of governance in the world. He liked the idea of democratic voting but worried like Plato that governing required particular skills. He argued that leaders often mastered such skills through practice: One huge advantage of monarchy was that the same person might rule for many years, becoming better and better at it. Aristotle recommended some combination of elite and democratic rule, and hoped that rulers could be urged to behave virtuously.

Most Greek states had limited bureaucracies. Modern democracies expect their very extensive bureaucracies to provide much of the skill that governance requires. Aristotle's concerns that the ruler must be highly skilled may be an expression (at least implicitly) of concerns that a bureaucracy could manipulate an unskilled ruler. These concerns are with us still today but were likely more significant at the time. (We will in chapter 27 discuss how modern technologies and institutions have transformed bureaucracy.) 
In any case, Alexander's conquests would bring to an end the golden age of Greek democracy and philosophy. These ideas would be resuscitated thousands of years later but would need to be revised extensively to fit much larger polities.

The Roman Republic had elements of democratic rule. The Roman Senate was an elite institution of former magistrates and their descendants; it generated most laws, but popularly elected assemblies ratified these and elected magistrates. Outsiders could be appointed as a magistrate and enter the Senate. Historians long thought that the Senate manipulated the popular assemblies, but now feel the latter exercised real power: The Roman Republic was then a mix of aristocracy and democracy. By appointing two consuls at a time and for only a year, the Republic could have the benefit of leadership while checking any individual's power. The Republic was an institutional expression of Greek concerns with achieving skilled rule balanced by the pursuit of the common good. Oddly, there was very little political philosophy during the Republic. What there was tended to stress the importance of ethics in government, and the role of religion in encouraging ethics. Cicero, the greatest of Roman political philosophers, would outline a voluntarist theory of the state: People were Romans because they chose to be out of respect for Roman institutions. Cicero would also describe the first theory of "just war" to justify Rome's conquests (but see his quotes above: He was wary of the idea of just war). The emperor Augustus would then claim that his wars were just. Later emperors would stress the meritocracy of their appointments and the fairness of their judgements, implying that they achieved better outcomes than the Republic. Stoic philosophers encouraged acceptance of the new reality while urging rulers to benevolence and others to duty. They thus reacted to authoritarian government in a manner broadly similar to Confucian thinking.

\section{Jewish political thought}

Judaism represents another deviation from the idea of sacred kings. Jewish political thought emphasized god, not king - no king could arguably represent such a god. It was the people, or at least tribal elders, then, that selected kings, and these could topple kings. Moreover, it was god, not kings, which proclaimed laws. Prophets with their special connection to god could and did criticize kings. Yet kings, in turn, used their power to encourage support from judges and prophets. Jewish defeat at the hands of the Babylonians forced another unique mutation: the idea of a nation (a people) without a state. Jewish leaders interpreted defeat as godly retribution for bad behaviour. Yet god had a mission for them, a purpose for them to help all people. The Arabs would later borrow this idea and associate it with Islam. The Jewish concept of a messiah that would liberate their people incorporated the "sacred king" expectations of justice and benevolence from a ruler; these ideas would, in turn, influence Christianity and Islam. 


\section{Patriotism}

Socrates' refusal to escape his death sentence is the first written reference to a "contract" between citizen and state. Socrates argued that he had benefited his entire life from Athenian citizenship and could hardly then ignore the state's wishes when he disagreed with them. It would make a mockery of his lifelong advocacy of ethics if he broke laws out of convenience.

Socrates is, among other things, advocating a kind of civic patriotism: One should be intensely loyal to a state whose institutions one admires. Cicero (above) later made a similar argument about Rome: One should choose to be a Roman and be proud of that choice. The Roman state would urge devotion to the state above all else (arguing that those who served would bring honour to their families).

It is noteworthy that these expressions of patriotism focused on loyalty to institutions rather than shared culture. The Greeks were often quite ethnocentric but emphasized identity with the state and its institutions rather than one ethnicity. As Rome conquered multiple ethnicities, it needed to encourage loyalty not associated with ethnicity. This was a common challenge of multiethnic empires.

\section{Questions}

1. What are the critical differences between Greek and Chinese philosophy, and why might these have emerged?

2. Do you think it would have been feasible to maintain the Republic as Rome expanded? How?

3. Compare Persia or Mauryan India to China or Rome.

4. What are the challenges faced by eunuchs?

5. Did political thought shape polities, or did polities shape political thought?

6. How does family life change when child mortality rates are high?

\section{Readings}

Beard, Mary. 2015. SPQR: A History of Ancient Rome. New York: Norton.

Black, Antony. 2009. A World History of Ancient Political Thought. Oxford University Press.

Burstein, Stanley M. 2020. "The War Elephants East and West." World History Connected 17:2.

Collins, Randall. 1998. The Sociology of Philosophies. Cambridge MA: Harvard University Press. [Collins makes two key points stressed above: that famous philosophers are often "emblems" of the ideas of their times, and that scepticism arises when there are many competing schools of thought.] 
Hansen, Valerie. 2011. The Silk Road: A New History. Oxford University Press.

Harper, Kyle. 2017. The Fate of Rome: Climate, Disease, and the End of an Empire. Princeton University Press.

King, Charles. 2005. The Black Sea: A History. Oxford University Press.

Knapp, Robert. 2011. Invisible Romans. Harvard University Press.

Lewis, Mark Edward. 2010. The Early Chinese Empires: Qin and Han. Harvard University Press.

Maynes, Mary Jo, and Ann Waltner. 2012. The Family: A World History. Oxford University Press.

Robinson, Rebecca. 2018. "Spectacular Power in the Early Han and Roman Empires." Journal of World History 29:3, 343-68.

Scheidel, Walter, ed. 2009. Rome and China: Comparative Perspectives on Ancient World Empires. New York: Oxford University Press. 


\section{The birth of missionary religions}

\section{Why and how did the world's major religions emerge?}

\section{Guiding questions}

How, why, when, and where did a new generation of (generally universal and monotheist or deist) religions emerge between about $600 \mathrm{BCE}$ and $650 \mathrm{CE}$ ? Why did most of the world's major religions to this day arise at this time? What were the fundamental similarities and differences across religions?

How did religions interact? How and why did some religions become popular in particular regions?

What were the main effects of these religions?

Relationship to other chapters: This chapter most obviously builds upon our discussion of ancient religions in chapter 8. We shall also see that there are significant synergies between the development of these new religions and the development of empires (chapters 9 and 10). The support of powerful empires encouraged each of the religions that have prospered the most in succeeding centuries. We will often have cause in later chapters to refer again to many topics addressed in this chapter, including the connection between state and religion, conflicts within and between religions, and changes within religions. We will also find in this chapter and later many examples of religious toleration and understanding. We can only appreciate 
these later experiences of change, conflict, and understanding if we first appreciate the main similarities and differences across religions, and early experiences of religious interaction. Our discussion of Manichaeism below reminds us that a religion can be popular at one point in time but eventually disappear. Moreover, all religions disappear from some regions in which they had once been familiar. Our understanding of why particular religions came to dominate particular regions will inform our discussion of those regions in later chapters.

Several new religions emerged - or at least codified their texts - between $600 \mathrm{BCE}$ and $650 \mathrm{CE}$, generally on the fringes of classical empires. These include Zoroastrianism, Manichaeism, Buddhism, Confucianism, Taoism, Judaism, Christianity, and Islam. To this list can be added Hinduism, which likely dates from about 1500 BCE (and so was discussed briefly in chapter 8) but was transformed so dramatically around 400 вСE that we might speak of a new religion. The vast majority of religious people in the world in the twenty-first century still adhere to one of these religions. The polytheistic religions that we discussed in chapter 8 have - except for the special case of Hinduism - largely been superseded by these new religions which are either monotheistic or deist (that is, with an impersonal guiding force rather than an anthropomorphic god). As we shall see, these new religions all tend to postulate one source of order in the universe, and promulgate a broadly similar set of ethical values. Repeatedly in history when a polytheistic religion encountered one or more of the new faiths, the new faiths would win out, though often absorbing elements of the preceding faith.

In this chapter, we will first discuss some commonalities in the development of these new religions, beginning with how they evolved from previous polytheistic faiths. We will then survey each religion in turn. We then provide some comparative analysis of these religions, and an analysis of their respective geographic spread.

The key theme of this chapter is culture. We will discuss how religion interacts with human nature (genetic predisposition), social structure, politics, economy, and art. There is also a brief discussion of migration. We discuss both how particular religions spread within and between societies and how religions borrowed ideas from other religions. We consider how individual religions evolved and close the chapter with a set of general evolutionary reflections.

\section{Culture}
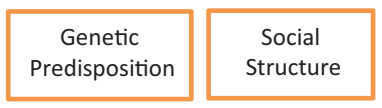

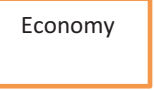

Art 


\section{New religions from old}

We should emphasize that the new religions did not start from scratch but built in important ways on the religions that they replaced. They could not so easily have replaced these earlier religions if they had not managed to serve the needs that these earlier religions had served. Yet their success was also predicated on responding to newer needs that previous faiths had not addressed very well. We can thus potentially understand the new religions as evolutionary developments, though each represented in its own way a considerable set of mutations from preceding faiths.

Indeed, one of the evolutionary successes of the new religions involved their openness to elements of polytheism. Saint-like figures could play at least some of the roles within a monotheistic or deistic faith that multiple gods had played within polytheism: People could pray in different ways to different beings for different outcomes. Missionaries would often find a place for polytheistic practices within the new faith, easing the conversion of peoples from one to another. Christianity, for example, co-opted pagan festivals, turned magic into miracles, and transformed gods into saints. Reverence for Jesus's mother Mary could at times substitute for goddess worship. Sufi Islam found synergies with the Hindu worship of personal gods. Importantly, the new monotheistic gods were anthropomorphic just as polytheistic pantheons had been. It was easier for those who had worshipped multiple human-like gods to conceive of one all-encompassing human-like god.

The gendered nature of monotheistic gods might indeed reflect evolution from a polytheistic past. The gendering of polytheistic gods makes some sense: The pantheons of polytheistic faiths usually include gods who were children of other gods (or sometimes of gods and humans). However, if there is only one god, there is by definition no other god to mate with. The founders of monotheistic religions did not contemplate an asexual god. This would perhaps have been an even more dramatic mutation than elevating one god above others, for where there had been multiple gods these had always been gendered (and generally of human-like form). Nobody questioned what use a monotheistic god would have for the physical paraphernalia associated with gender.

The gendering of gods reflects the broader personalization of monotheistic gods. Religions had long attributed the hidden workings of the universe to beings with characteristics much like humans. Even as many of these new religions imagined gods that were omniscient and omnipresent, they nevertheless told stories in which these gods were all too human: capricious, vain, or forgetful. Omniscience and omnipotence were crucial in encouraging ethical behaviour (for god knew everything and could punish or reward at will), but humans found it easier to relate to a god that had human failings. The god of the Jews would become less human and more omniscient as Jewish, Christian, and Islamic religions developed, but these would always strike a balance between human and omniscient characteristics. Note that the sacrifices that were so 


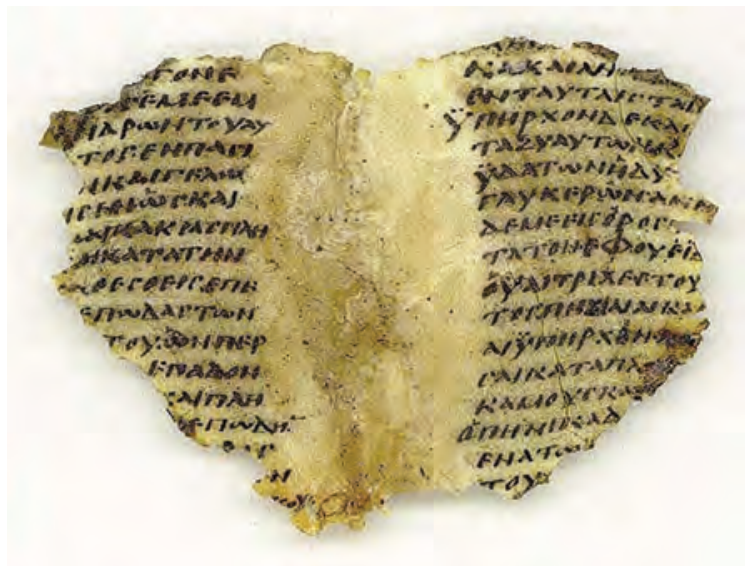

Illustration 11.1

Cologne Mani-Codex. Fragments of a Greek translation and commentary on early Manichaean texts that have otherwise been lost

Photograph by Qaramanoğlu, CC BY-SA 4.0

important within ancient religions make far more sense when seeking support from polytheistic gods that compete with each other and exhibit human frailties. As the idea of one omniscient god strengthened over time, the size and frequency of such sacrifices declined but did not disappear. The omniscient god was still human enough to wish some display of human affection and obedience.

While polytheistic gods were humanlike blends of good and evilinstincts, monotheistic gods were generally presumed to be good. Monotheistic religions often then developed or borrowed some idea of an evil "devil" to explain the existence of evil in the world - even if the existence of such a being contradicted the ideal of an all-powerful god. This evil force was strong but it was generally hoped that the good god could prove superior.

Deism is a more dramatic alteration to polytheistic beliefs than is monotheism. It is not surprising then that deism would take root most firmly in East Asia, where ancient religions had often referred to a vague heavenly force (recall the Mandate of Heaven from chapters 7 and 10) and had not developed the same elaborate pantheon as existed elsewhere in the world. In India, deism may have seemed at first to be compatible with polytheism in much the same way as African religions had long imagined one godlike force in the background of polytheistic gods (chapter 8).

Writing would play an important role in all of these religions, though only in the case of Manichaeism can we be sure that the religion's founder drafted the key texts. Writing gave these religions a complexity and detail that no oral tradition could match. Missionaries then had potential answers to a wide range of possible queries, while being able to suggest various limitations in previous belief systems.

\section{Religions and states}

States founded none of these new religions - though aristocrats established most of them. Nevertheless, all spread the fastest when associated with a state. Empires had good reasons to embrace religions that proclaimed one guiding force rather than a multiplicity of local deities. One all-powerful god was a useful ally for a ruler aspiring to be omnipotent. As empires expanded, they carried their religion to new lands: This process was especially important for Christianity and Islam. Yet these new religions often spread also beyond state 
boundaries, brought by merchants or missionaries to new lands. Still, their success in the end in these new lands was often dependent on the attitude of political authorities. Although religions fared best when supported by a state, they often also prospered during times of political conflict. Organized religions might then provide a source of stability in human lives.

\section{Why then?}

It seems unlikely to be coincidental that so many religions developed between 600 BCE and $650 \mathrm{CE}$. Scholars have proffered several possible explanations. We have hinted at the first of these in chapter 8: As urbanization proceeded in many human societies, it became increasingly important to encourage ethical behaviour, for individuals then faced an increasing need to interact with strangers (the somewhat lesser degree of urbanization in the Americas might help explain why similar new religions are less evident there). These new religions indeed spread faster in cities than in the countryside - though this might simply reflect the fact that knowledge of new religions spread along trade routes. Many scholars have noted a correlation between societal complexity and religions that threaten punishment for unethical behaviour. One apparent reason for such a connection is that complex societies will benefit from a religion that encourages people not to cheat strangers.

As empires grew in size, they also had to grapple with many impersonal relations. As we have seen, emperors needed to get bureaucrats and especially provincial governors to obey their commands. Emperors struggled to raise small amounts of tax from large numbers of people. Empires had long expanded by practising religious toleration and encouraging syncretic absorption of multiple deities into a common pantheon. Such empires would at first find monotheistic beliefs troubling, for monotheists refused to accept other gods. Over time, though, empires embraced religions that both aspired to universal truths and preached ethical values. Such a religion might encourage bureaucrats not to steal and peasants to pay their taxes. And such religions could potentially provide legitimation of kingly rule just as older religions had.

It is easier to imagine that there is a unique set of ethical precepts for people to follow if there is one god. Indeed Socrates inspired so much hostility in ancient Athens precisely because he argued that the Greek pantheon of gods acted in contradiction to the ethical guidelines that he thought should govern society: If gods could act in evil ways why shouldn't people? Diverse gods may disagree on ethical matters. One all-powerful god can prescribe ethical penalties and bestow rewards and punishments.

There may be a further connection between empire and the new religions. Empires were everywhere associated with emperors: one person, generally male, that officially ruled a vast territory. The existence of such individual power on earth may have encouraged a belief that one individual god ruled over the entire universe. Monotheistic gods - like emperors - were remote, powerful, 
and associated with symbolic tokens of their power and their right to rule. In turn, a monotheistic god might mandate earthly rule by an all-powerful male. Monotheism (or deism) could then be harnessed to imperial rule. That monotheist religions often incorporated polytheistic elements might then reflect in part the fact that emperors in practice depended on a bureaucracy to rule, and senior bureaucrats regularly interceded with the emperor to advocate for policies or people. We have seen that empires were associated with gender stratification; the new religions also reinforced gender roles.

Some scholars have stressed the individualism inherent in the new religions. Early religions had for the most part been rigidly hierarchical: A priestly class performed rituals on behalf of the population. The newer religions tended to stress a personal relationship with god. Individuals were expected to develop familiarity with religious doctrine and then a deep personal faith. In this way, these religions arguably reacted to the social and political stratification associated with empire. This might explain why these religions tended to develop on the fringes of empire and empires often viewed them with suspicion in their early days. Once empires recognized the advantages of these religions, they tried to decrease their individualist content by encouraging priestly authority and doctrinal purity. Some of the later new religions, including Christianity and Mahayana Buddhism, then perhaps attempted to reassert individualism. Indeed, the contest between an individualistic approach to religious faith and the assertion of priestly authority would play out in many religions over time.

\section{Major religions}

\section{Hinduism}

The oldest of today's major religions is Hinduism. As noted above, it differs from the others in being polytheistic in orientation. The Hindu pantheon likely drew in part on Middle Eastern and Indus Valley religions. The Vedas gave a priestly class a special role in dealing with this pantheon. Yet beyond the pantheon of gods, there was a sense of a cosmic reality termed "Brahman." Many Hindus see individual gods as the multiple faces of one divinity. Though Hinduism worshipped many gods, it nevertheless came to urge one ethical path to follow.

The Upanishads are often referred to as Vedanta, meaning the last or ultimate Vedas. There is intense scholarly debate regarding when these emerged. The earliest Upanishads can be dated to sometime between the ninth and third centuries BCE; most scholars are confident that they predated the rise of Buddhism, which then drew upon them. The later Upanishads may have been drafted as late as the early centuries $\mathrm{CE}$, in which case Buddhism may well have influenced them. The Upanishads are mainly a creation of the same time period that sees the rise of the new religions we address below and bear important similarities to these. The Upanishads differed from the Vedas in talking of how individuals could achieve release from the cycle of reincarnation by pursuing detachment 
from human desires (which would encourage ethical behaviour). They may have first appealed to lower castes. The Upanishads nevertheless reinforced caste distinctions: Those in lower castes were enjoined to appreciate that their low status must reflect misbehaviour in a previous life.

The Upanishads still suggested that release from the cycle of reincarnation was extremely difficult to achieve. From about 300 вСЕ, perhaps in response to Jainism and Buddhism, Hinduism evolved further. The Bhagavad Gita (also hard to date; it likely developed over the period from 300 BCE to $400 \mathrm{CE}$; it is considered part of the Vedanta by some) held out a new path to salvation that involved performing caste duties and pursuing absolute faith. Individuals were still supposed to seek detachment, but no longer needed to achieve a complete severance of human desires. Other texts of the time argued that release would come from a balanced pursuit of pleasure, economic well-being, and ethics. This new Hinduism encouraged people to enjoy themselves within their caste and was easier to follow even than Mahayana Buddhism (see below). Also, it allowed lower occupational castes to prosper economically.

By maintaining castes and social order, Hinduism may have appealed to the state, while at the same time giving the bulk of the population hope for a better future. In any case, the Gupta Empire would support Hinduism as the previous Mauryan Empire had supported Buddhism. Hinduism would gain in popularity relative to Buddhism in India after that (see chapter 13).

\section{Jainism}

Jainism was founded in the sixth century BCE, though some doctrines were likely developed earlier; it consciously built on the early Upanishads, and particularly the ideas of souls and reincarnation. It preaches an ascetic life, and non-violence. Jains even tried not to hurt insects as these also had souls. Mahariva, the effective founder of Jainism, was an aristocrat from the warrior class who abandoned his social position to pursue enlightenment. Though the ascetic life appealed to only a few, Jainism's disdain for castes was popular among lower castes. The doctrine of ahimsa or nonviolence that developed within Jainism would be influential within Buddhism

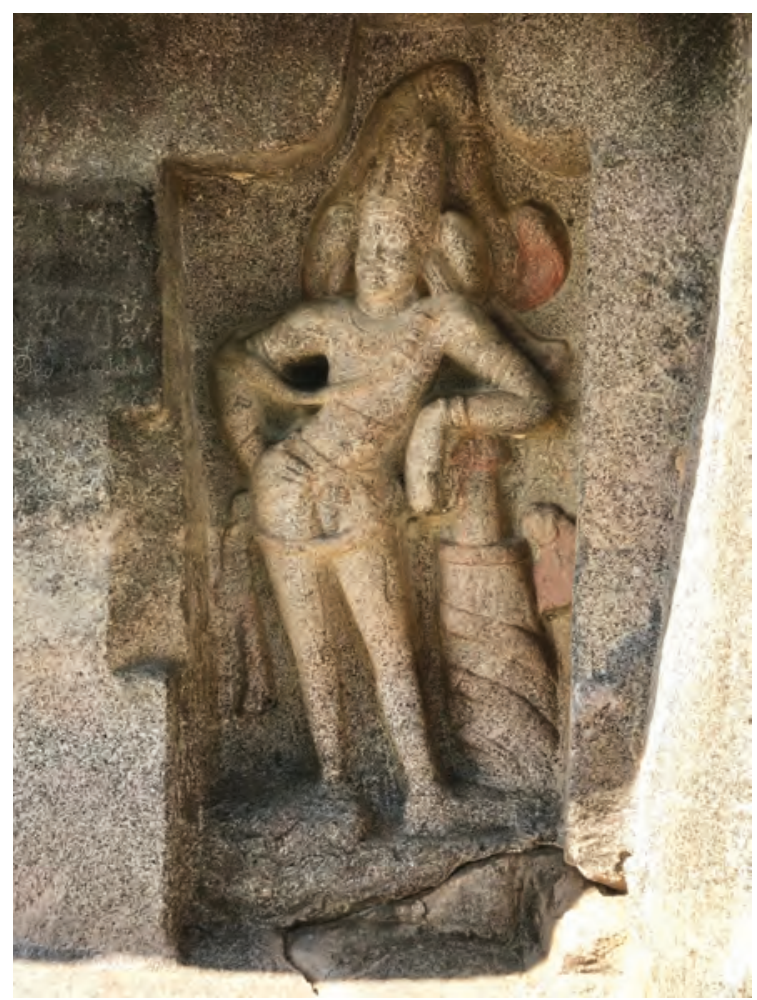

Illustration 11.2

Door guardian of 7th century Mandagapattu Trimurti Temple, rock-cut cave, Tamil Nadu, India Ms Sarah Welch, CC BY-SA 4.0 


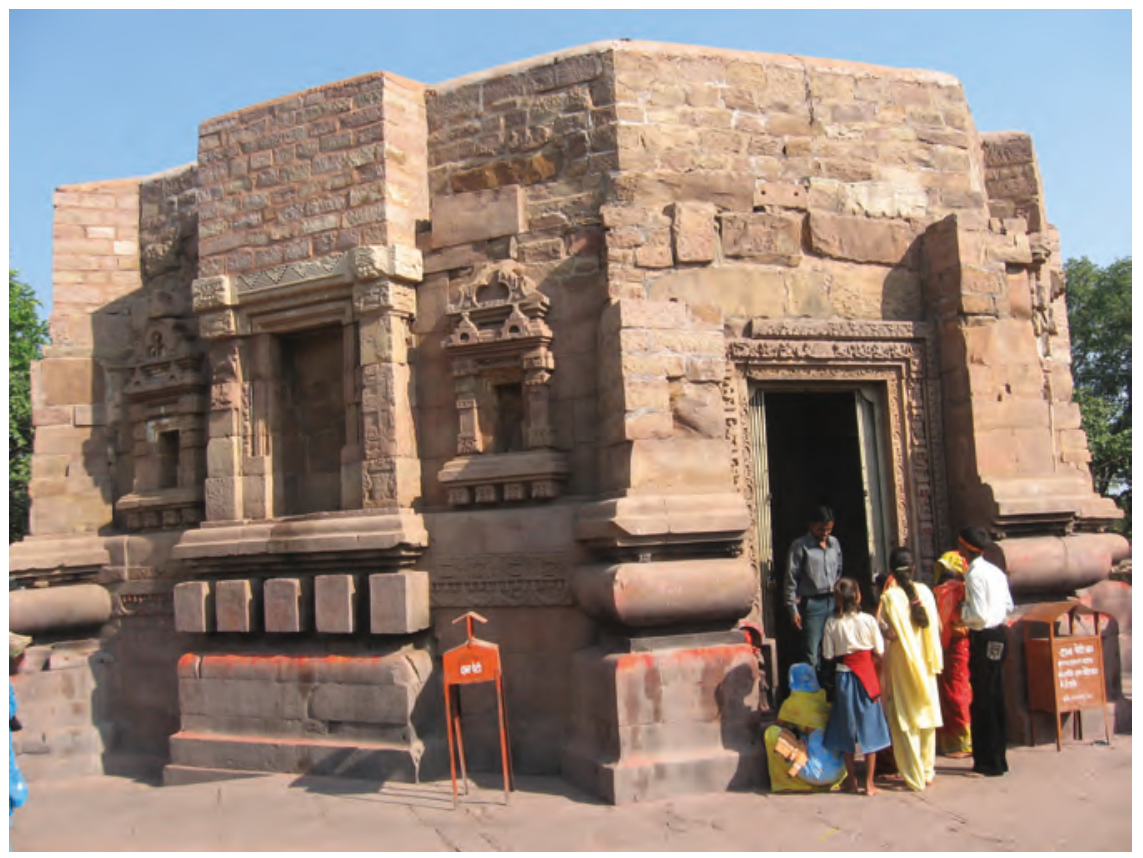

\section{Illustration 11.3}

Mundeshwari Temple, Bihar, India, dated to the 2nd through 4th centuries, considered by some to be the oldest surviving Hindu temple in the world Photograph by Lakshya2509, CC BY-SA 3.0

and Hinduism as well. Yet Jainism itself remained a minority religion, perhaps because it was too demanding. Jains often became merchants, in large part to avoid the violence associated with agriculture.

\section{Charvaka}

Charvaka developed from the sixth or fifth centuries BCE in India. Charvaka believed that gods were figments of the imagination and priests were charlatans. Other faiths naturally viewed Charvaka with hostility. Charvaka nevertheless provides evidence that agnostic beliefs also existed during this period of religious ferment.

\section{Judaism}

There is no independent evidence of the events described in the biblical book of Exodus (including the drowning of a Pharaonic army in the Red Sea), and it is doubtful that there were camels in Egypt at that time as Exodus describes. However, the Jews certainly existed as a distinct group from the twelfth century BCE. They were unusual in being literate but tribal. There is speculation 


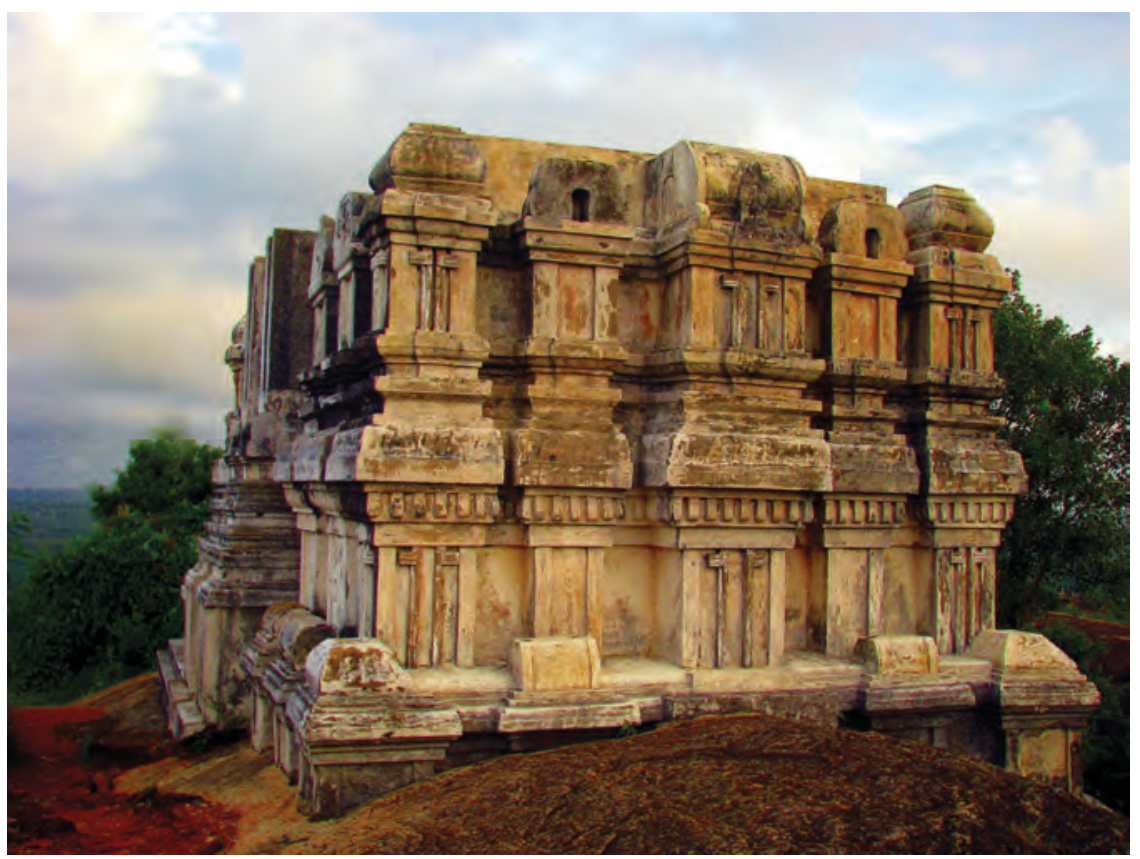

Illustration 11.4

Tower of Jain Temple, 9th century, Chitharal, Tamilnadu

Photograph by Santhoshj, CC BY-SA 3.0

that they were refugees from cities, perhaps due to the wars between Egypt and Assyria. The Jews were likely a semi-pastoral people of Iraq in the second millennium все. The patriarch Abraham might well have objected to idol worship in Ur, and moved to Palestine. Migration of groups from the Levant into Egypt and back was common at the time. Some of the stories in Genesis and Exodus Noah and the flood, Moses being set adrift in a basket as a baby - had precursors in the stories of other peoples of the area. The flood story in particular echoes one in the Epic of Gilgamesh. There are also clear links between Jewish law and the earlier Code of Hammurabi.

It is not clear exactly when the Jews became monotheistic. As we have seen in chapter 8, it was typical for groups in the Middle East to have a favoured god but still recognize multiple gods. It appears that the early Jews worshipped Mesopotamian gods. Many scholars argue that Jewish prophets expounded on how their god was the only god only after the Assyrians and then Babylonians destroyed Jerusalem. If so, then monotheism was a reaction to military defeat. (There is speculation that Amenhotep's earlier experiment with monotheism in Egypt (chapter 8) somehow influenced Judaism, but there is no evidence of such a link.) These prophets spoke of a covenant between the one god and the Jewish people in which god would care for them in return for belief and sacrifices. 
The Assyrian conquest of the northern Jewish kingdom led to the dispersal of the population (the biblical "lost tribes") and their disappearance as a distinct group from history. The Babylonian captivity of the remaining Jews from 598 всE - the conquering Babylonians moved the Jews to their capital; Cyrus of Persia allowed them to return to Jerusalem only in 538 BCE after he conquered Babylon - instead fostered rededication to their unique religion. Why did Judaism survive this second defeat but not the first? The codification of a detailed belief set in written texts must be part of the answer: These holy texts gave the Jews a unique identity and provided assurance that god favoured them even if he did not always reward them on earth.

Though some Jewish beliefs undoubtedly predate the Babylonian captivity, the codification of these beliefs occurs during the period of emergence of new religions. Judaism is unique in marrying the idea of a monotheistic god to the much earlier idea of an ethnically identified god. Nevertheless, Judaism would exert influence far beyond one ethnic group. Other groups - such as the Khazars of Central Asia in the eighth century CE (chapter17) and the rulers of Yemen in the sixth century CE (below) - would occasionally convert to Judaism. The two most successful monotheistic faiths, Christianity and Islam (and Manichaeism and perhaps Zoroastrianism), would build upon Jewish beliefs. Judaism combined the idea of a personal relationship with god with the idea that occasional prophets could serve as special interlocutors between the people and god; many later religions, including later versions of Buddhism, would adopt this dualism.

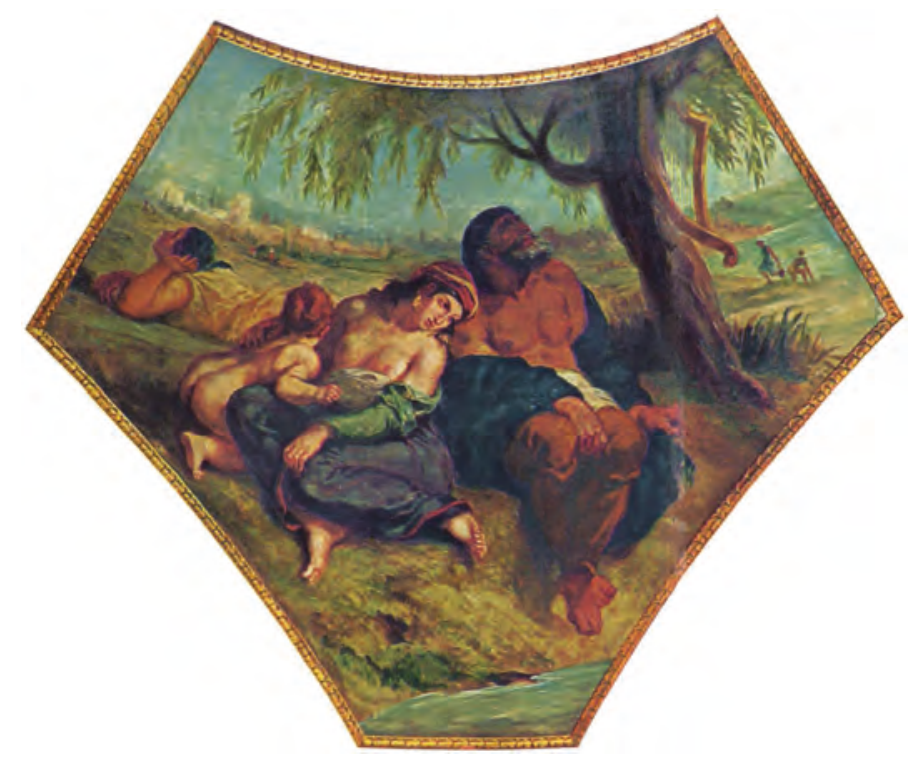

Illustration 11.5

The Babylonian Captivity, Eugene Delacroix (French Romantic School), mid-19th century 


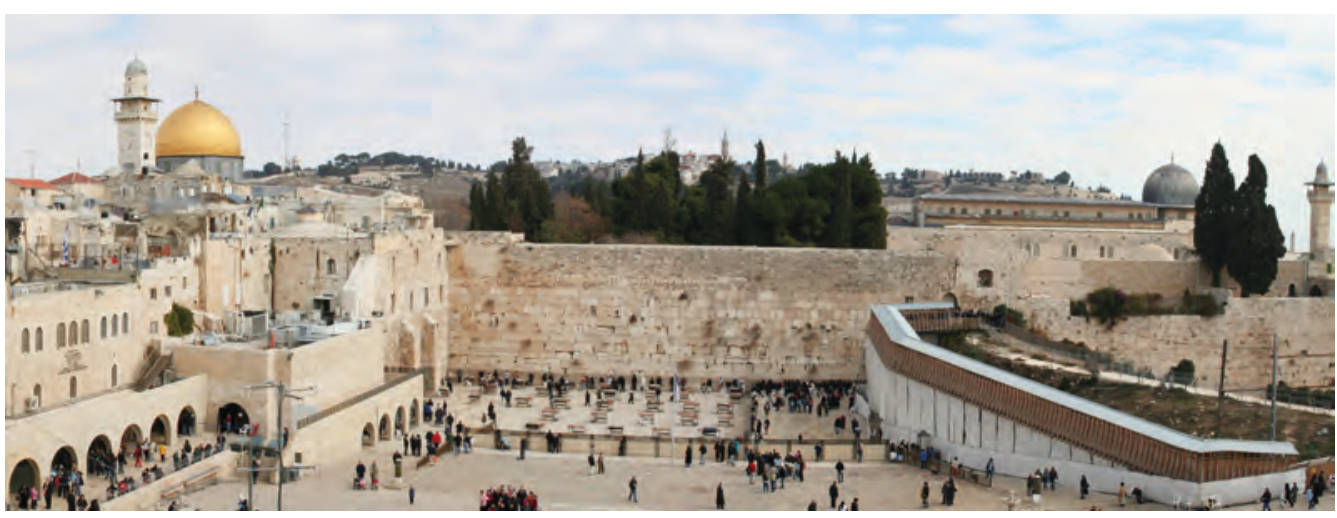

Illustration 11.6

Jerusalem:Western (Wailing) Wall, with Dome of the Rock in background left and Al-Aqsa mosque to the right. This area contains religious sites of great importance within Judaism, Christianity, and Islam

Photograph by Sheepdog85, CC BY-SA 3.0 de

\section{Zoroastrianism}

Persians in the seventh century всE mostly followed the Hindu pantheon (they were descended from similar Indo-European nomads). Zarathustra, the founder of Zoroastrianism, preached of conflict between a good god and an evil one. It is not clear exactly when Zarathustra lived; it was probably the late seventh century все but was perhaps centuries earlier. He was likely Persian, though perhaps from Central Asia. If the latter, Zarathustra may have built on the nomadic sense of good and evil. He may also have borrowed ideas from Judaism. He was an aristocrat and maybe a priest, who became disenchanted with existing beliefs, wandered for a decade, and had visions of a supreme god (but also lesser gods). His many hymns were transmitted orally for centuries before being written down. His followers wrote many poems, sayings, and moral treatises that clarified Zoroastrian beliefs. Zoroastrian texts were codified only during the Sassanid dynasty (226-641 CE). But much of this literature was lost with the arrival of Islam centuries later.

Zoroastrians believed that Ahura Mazda was the chief god, but recognized six other gods; they also believed that the chief god was good and fought with an evil spirit named Angra Mainyu. This battle would end after 12,000 years with the victory of good, at which point souls would be judged. Since Ahura Mazda was associated with light, Zoroastrianism adopted previous traditions of fire worship. Rather than urging asceticism, Zoroastrians were encouraged to enjoy earthly pleasures, albeit in moderation and with honesty. Indeed, the rich were urged to celebrate with the poor during several major annual celebrations. Zoroastrian priests were known as "magi," figures who would make an appearance in the Christian Bible to celebrate the birth of Jesus.

As would be the case with many other new religions, the Persian Empire viewed Zoroastrianism with suspicion at first, though it later became the 
official Persian religion. Darius adopted the religion and characterized himself as Ahura Mazda's agent on earth. Zoroastrianism then spread through the Persian Empire. Zoroastrianism encouraged people to be active in the world, behaving ethically and doing good works. The Empire urged its citizens and especially bureaucrats to live in accord with these principles, but did not force subject peoples to adopt Zoroastrianism. Zoroastrians were urged to be loyal to the emperor as long as he governed justly. As elsewhere, a religion that promised eternal bliss to the righteous, and that justified imperial rule, could lessen the chances of imperial revolt. Alexander the Great would disdain Zoroastrianism, but the Parthians and especially Sassanids supported it (see chapter 9). Though Islamic conquerors in the seventh century CE did not outlaw it, most Zoroastrians converted to Islam over the next centuries. Some fled to India, where they founded a community known as the Parsis (Parsi means Persian). Only a few thousand Zoroastrians remain in Iran, though they are guaranteed representation in the Iranian parliament.

Zoroastrianism was influential on later Judaism, Christianity, and Islam. One religious challenge is to understand why there is evil in the world; other faiths would replicate the Zoroastrian idea of a cosmic battle between good and evil forces. More generally, the concepts of an omnipotent creator deity, an evil

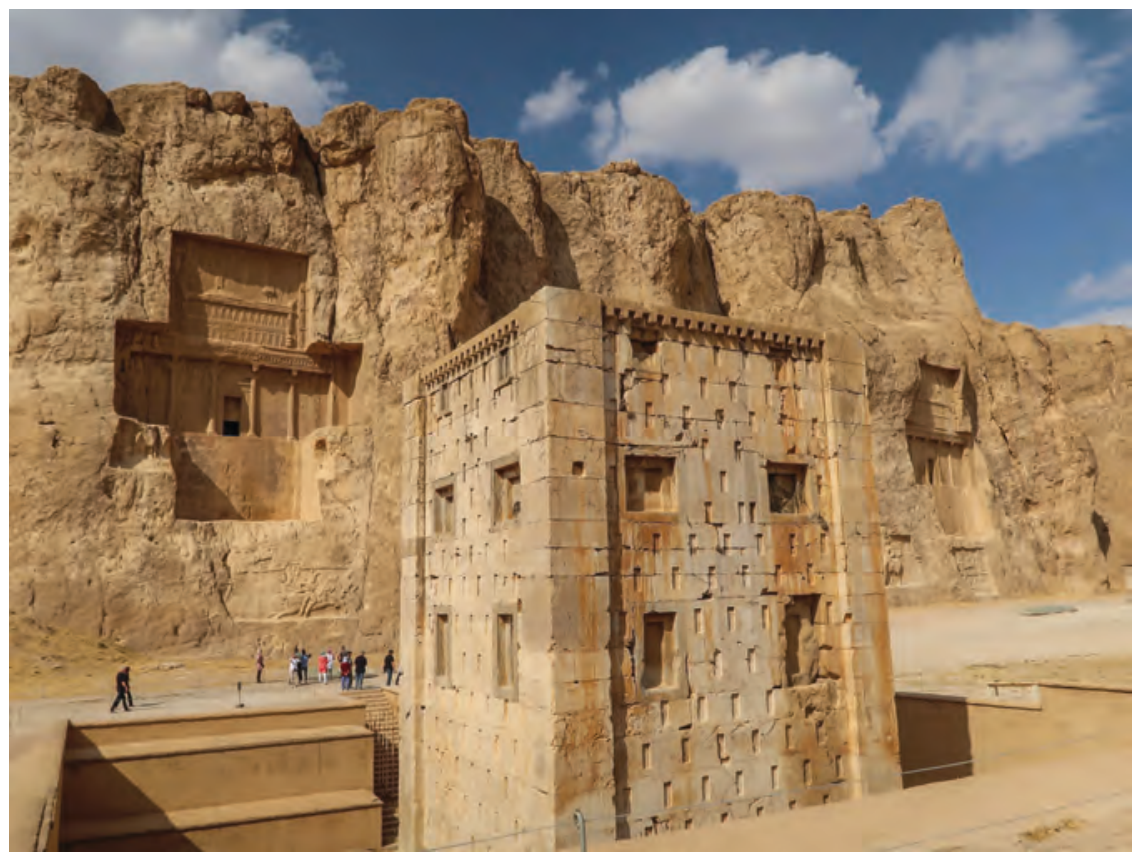

Illustration 11.7

Cube of Zoroaster (purpose unknown) in front of the tombs of Darius I and Darius II, Naqsh-e Rustam necropolis, Iran. Zoroastrian necropolises were generally located on heights

Maasaak, CC BY-SA 4.0 


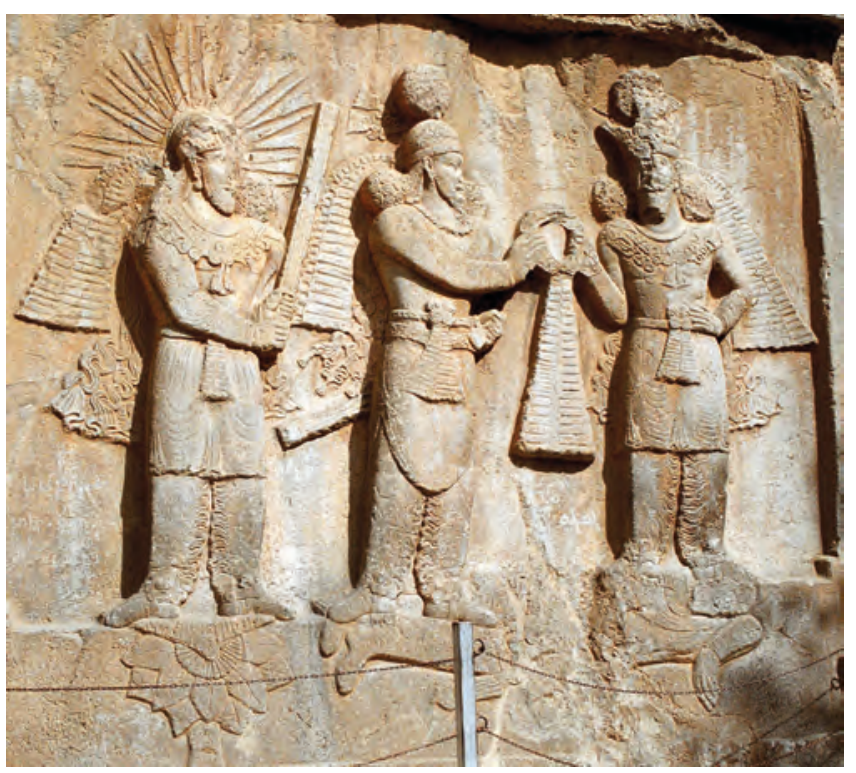

Illustration 11.8

Relief of Shapur II at Taq-e Bustan, Kermanshah Province, Iran. Ahura Mazda on right awards Shapur II, Sassanian ruler, the ribboned ring symbol of royal power while the god Mithra looks on; Shapur II stands atop the defeated Roman emperor

Photograph by Philippe Chavin, CC BY 2.5

adversary, that good will eventually triumph over evil, that humans should strive to be good, that humans had "free will" to choose, and that humans will undergo judgement, would all infuse later western religions.

\section{Confucianism}

Scholars debate whether Confucianism is a philosophy or a religion. We have already encountered it in our discussions of ethics and political philosophy in chapter 10. We have already seen, then, that it serves some of the purposes of religion by urging ethical behaviour and encouraging social stability. Though Confucianism often justifies these ethical and political principles without reference to heaven, there is nevertheless a core Confucian belief in a deistic force that governs the universe. The ethical and political precepts are consonant with this deistic force. Confucius himself was also a master of rituals designed to honour local spirits or ancestors; he encouraged others to pursue such ceremonies, though in part as a means to instantiate social harmony. Though its more practical focus can easily overshadow the strictly religious elements of Confucianism, it did encourage recognition of both a singular deity and a set of more approachable spirits and ancestors. There is, then, at least a family resemblance to both the monotheistic or deist gods and "polytheistic" saints of other religions. 
Confucianism would nevertheless react centuries later to the growing popularity of Taoism and Buddhism (both discussed below), each of which did make more explicit reference to humanity's relationship to this deist force. NeoConfucianism, popularized during the Song Dynasty (960-1279 CE), would explore the meaning of life: It argued that the purpose of life involved realizing one's true human nature, which was good, and using that nature in the service of humanity. It therefore urged action in the world whereas Taoism usually encouraged withdrawal from the world.

\section{Taoism}

Though Taoism builds upon much earlier thinking, it was formalized in the fourth century BCE in the Tao Te Ching, attributed to Laozi, who may or may not have been a historical figure. Its central tenet is a belief that one should attune oneself to the Tao or Way of the Universe. It is, like Confucianism, a deist faith: There is a god, but not one thought of in human terms or one likely to interfere in human lives. Still, humans should strive to understand and live in harmony with the universe. The Tao is usually conceived as being broadly cyclical (an idea that accords with the political view that dynasties rise and fall). Taoism (like Confucianism) also embraced the concepts of yin and yang: the recognition that seemingly contradictory forces such as light and dark are actually complementary, each containing an element of the other. Since human lives are an expression of the Tao, one can learn much by deep self-reflection. Taoism urged compassion, and frowned upon aggression and acquisitiveness. Taoism is often associated with limiting one's strivings and celebrating the glory of the universe. Some Taoists, though, could urge the application of compassion and non-aggression in the world.

Popular Taoist practices differ quite a bit from its theology. Just as Confucius could urge rituals that celebrated spirits and ancestors, Taoists came to venerate Laozi, mythical emperors, and others. Scholars often compare these practices to the Catholic veneration of saints. Conceptions of a deistic universe proved to be compatible with the worship of figures that could intercede on one's behalf, albeit in accord with the Tao. Though students should not underestimate the differences between deism and theism, it is notable that both types of religion found a place for the adoration of figures that bear some resemblance to polytheistic gods.

\section{Buddhism}

Buddhism was a reaction to Hinduism and was the first actively missionary world religion. There is no historical verification of the life of Siddhartha Gautama (the Buddha), the founder of Buddhism; there is even some doubt regarding the precise dates of his life. Like Mahavira, effectively the founder of Jainism (above), the Buddha was from an aristocratic/warrior caste but gave up his position to pursue enlightenment. There were undoubtedly advantages in 
coming from an aristocratic background: Aristocrats could more easily finance a life of wandering reflection, and had likely received an extensive education that gave them many ideas on which they could reflect. Modern scholars of creativity appreciate the importance to human creativity of this sort of combination of extensive learning and time to reflect.

The Buddha was born perhaps in 563 всE and was allegedly the son of the king of a small state in southern Nepal. His father kept him in the palace and gave him whatever he wanted, but he escaped and discovered human suffering. He then followed other "renouncers" of privilege but did not find the answer he sought. It is notable that there was a significant number of people seeking a new truth at the time; they may well have shared useful ideas with the Buddha. He then went on his own, achieving enlightenment in 528 вСE near Benares.

One element of his life would long be controversial. He was married and had a pregnant wife when he left the palace to seek the truth. This would strike many as an abrogation of one's duties as spouse and parent. Hinduism would argue, indeed, that a person should first perform familial responsibilities and only seek enlightenment after their children are grown. Buddhist texts would change over time to stress the Buddha's loving departure from his wife.

The Buddha taught that earthly suffering comes from human desires that one must seek to control. He rejected both luxury and its denial, and instead sought a life of balance. He urged the "eightfold path" of right belief, resolve, correct speech, right behaviour, right occupation, appropriate effort, contemplation, and meditation. The Buddhist faith focused on ethics rather than on ritual

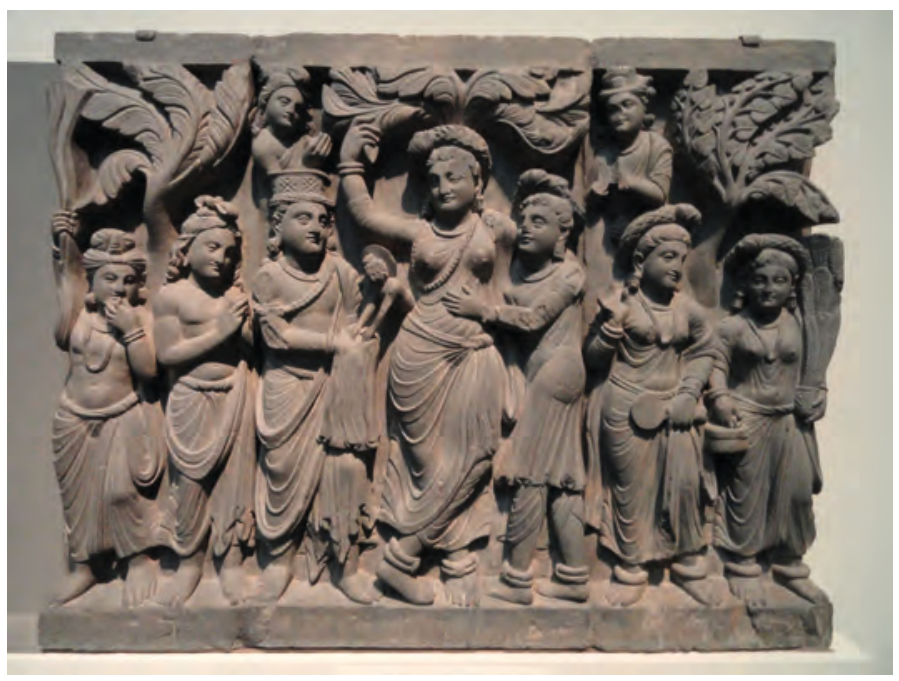

\section{Illustration 11.9}

Birth of the Buddha; Kushan dynasty, late 2nd to early 3rd century CE. One of four scenes from the life of the Buddha, Freer Gallery of Art, Washington, DC

Photograph by Daderot, CC0 1.0 
and ceremony. Like Jains, he sought relief from incarnations and suffering, but without denial of life's pleasures. He also disdained the caste system, arguing that all should pursue the eightfold path. Buddhism, as a result, became popular among lower castes. Buddhist texts were written in everyday languages rather than the scholarly Sanskrit employed in Hinduism. Devoted monks dedicated to preaching spread the faith. Yet Buddhism also often appealed to elites, perhaps as these had the time to study and meditate.

Though Buddhism tended to be critical of politics, it became the official Mauryan faith. Ashoka said he converted after witnessing the suffering of his Kalingan conquest (see chapter 9). He stopped hunting and banned most meat dishes from the palace. He probably also recognized that Buddhism was a religion that could unite his diverse realm. Yet it is not clear that the Empire actively encouraged the faith, though it did provide crucial financial support. During this time, Buddhism also spread to Sri Lanka and Southeast Asia. Ashoka supported missionaries in both places, and merchants also spread the faith. We saw in chapter 9 that the support of the Kushan Empire facilitated the spread of Buddhism into Central Asia and then China.

There were doctrinal disputes from the beginning, including about who or what is reincarnated. There were apparently four councils of Buddhist leaders held in early days, but these achieved no consensus. There were at one point sixteen recognized schools of Theravada Buddhism.

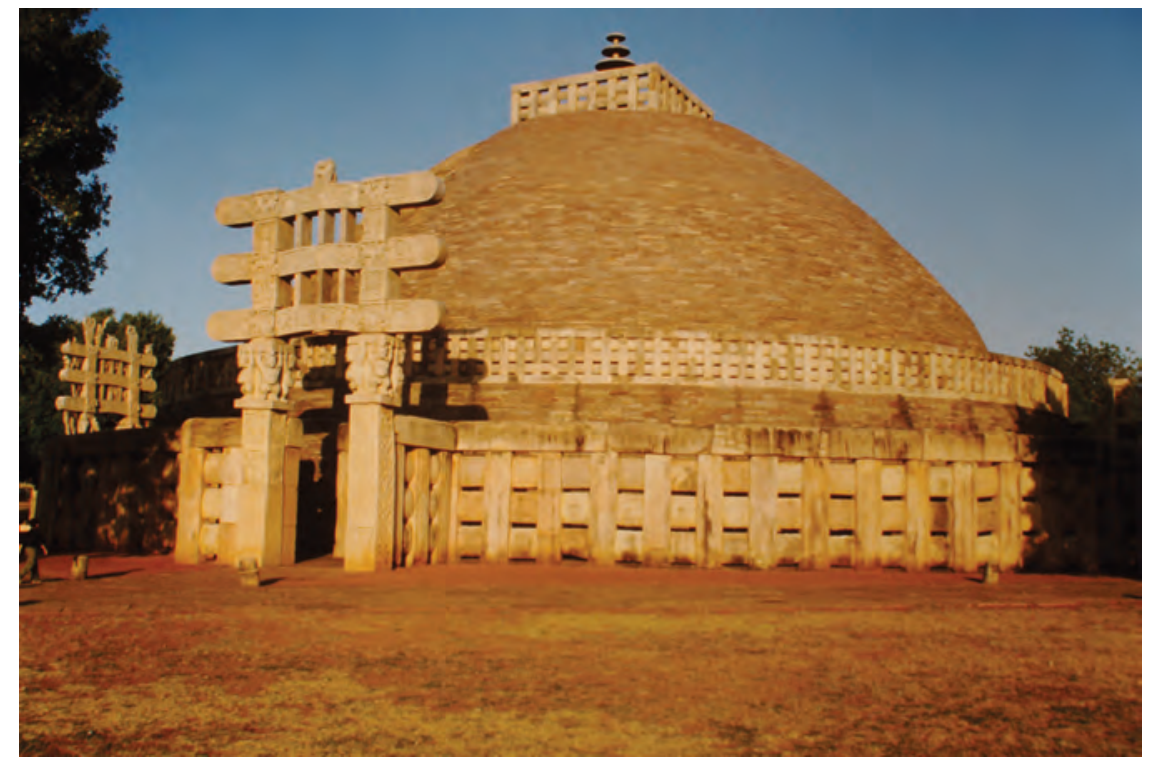

\section{Illustration 11.10}

The Great Stupa at Sanchi is the oldest stone structure in India, originally built by Emperor Ashoka in the 3rd century BCE. Ashoka's wife Devi was born nearby and they had been married in Sanchi. The four large ornamental gateways were added in the first century $\mathrm{BCE}$ 
Despite the Buddha's emphasis on balance, the eightfold path to enlightenment was still challenging to pursue. From the third century BCE (perhaps as early as the fifth) Mahayana Buddhism emerged; it treated the Buddha as divine, and spoke of bodhisattvas (saint-like figures), enlightened individuals who chose to stay on earth to help others; people could pray to these. Believers could also donate to monasteries to gain favour. Moreover, enlightenment could come through sudden inspiration rather than the lengthy pursuit of the eightfold path. All of these practices provided a more accessible path to follow than early Buddhism.

Buddhism arguably provided a way of coping with the urbanization, trade, and political centralization of the time in India. The fact that Buddhism encouraged believers to accept change may have been particularly useful. Confucians and Hindus were much less comfortable with social change, and each denigrated merchants. Mahayana Buddhism in particular may have been a response to increased social conflict within India.

Buddhism was especially popular with merchants. Recall that Buddhism urged a life of balance. The Buddha famously urged two merchants, Trapussa and Bhallika, who liked his teaching but did not want to become monks, to continue their trade honourably but make a donation. Such donations were critical to the growth of Buddhism: Early monks had relied on begging, but a larger religious establishment required a more substantial and steadier supply of funds. Notably, Buddhism did not see trade as a bad occupation. Networks of Buddhist merchants soon arose who could possess increased confidence in each other's honesty. Buddhism also had a practical implication in India in facilitating trade across caste boundaries (and would later be useful in China as a counterpoint to Confucian suspicion of merchants). Buddhism was often spread (as Islam would be; see below) by merchants along trade routes.

Donations financed temples and monasteries while providing some support to the poor. While Buddhism encouraged separation from life's strivings, it depended in practice, like all religions, on financial support from wealthy rulers, landowners, and merchants. In turn, Buddhist temples often served as banks where wealth could be stored and transactions facilitated.

In our discussion of merchants in chapter 5, we saw that there were both good and bad ways to make money; Buddhism may have encouraged the pursuit of ethical business practices. In any case, networks of Buddhist merchants would long be important throughout Asia. In particular, Buddhism would be the dominant religion of oases along the Silk Road for about 1,000 years from 200 все. These towns established Buddhist monasteries and schools. Since they were cosmopolitan centres, Buddhism could spread in many directions from these oases. By the fourth century $\mathrm{CE}$, there was a sizeable Buddhist community in Dunhuang in western China; they built hundreds of cave temples in the vicinity. They also produced Buddhist texts. Merchants also spread (Theravada) Buddhism and Hinduism to Southeast Asia during the classical period. Local rulers converted to one or the other. 


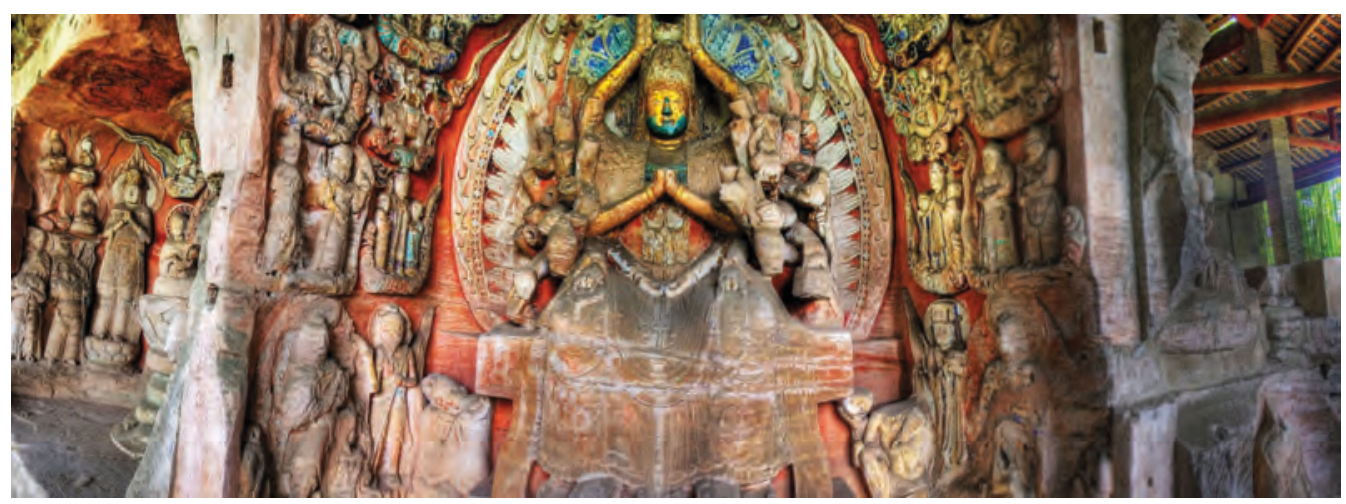

Illustration 11.11

Dazu Shike Rock Carvings (mostly Buddhist from the 7th century), Chongqing, People's Republic of China

Photograph by Davidmcbride, CC BY-SA 4.0

Buddhism reached central China by about 100 BCE, spread by Central Asian nomads. It remained small, though there were monasteries in major Chinese cities, until the fifth century $\mathrm{CE}$, after which it would become a dominant religion not just in China but also in Korea and Japan. It prospered in China in part as Confucianism suffered in prestige during the political disintegration following the Han, and especially in northern China that was at the time governed by nomadic peoples from Central Asia. (Taoism also prospered at the time.) The Sui and early Tang dynasties in China (chapter 13) would arguably adopt Buddhism precisely because both Chinese and non-Chinese could accept it.

Buddhism changed in important ways as it reached China. Buddhists in China borrowed Taoist terminology, including that of the Tao itself. They emphasized that while Buddhism applauded celibacy, it could be practised in concert with the family life venerated in China. They claimed that one Buddhist monk would benefit the entire extended family for generations. Chan (Zen) Buddhism blended Taoism and Buddhism; its emphasis on inspiration rather than the study of venerated texts reflects Taoist practices. Many Chinese saw little difference between Buddhist and Taoist practices or beliefs and indeed pursued both faiths. While Confucians typically abhorred Buddhism, its emphasis on charity and benevolence resonated with at least some Confucian precepts.

The later Tang persecuted all foreign religions, including Buddhism, but did not pursue these policies strenuously. The Song did not persecute Buddhism but favoured Confucianism. Confucianism itself, while hostile to Buddhism in principle, was inspired by Buddhist thought to develop a metaphysical underpinning for the practical Confucian tradition: This became known as neoConfucianism (see above).

In Central Asia itself, it seems that Buddhism had never put down deep roots beyond oases. Manichaeism had largely replaced it before the arrival of Islam. 
When the Uighur Empire adopted Manichaeism in $762 \mathrm{CE}$, many Buddhists migrated elsewhere, many perhaps to the Bay of Bengal. Buddhist migrations may also have resulted from changing trade routes as Central Asian nomads such as the White Huns attacked areas previously controlled by the Kushan Empire (chapter 9).

As with Confucianism and Taoism, we must be careful to distinguish philosophical Buddhism from religious practice. Though Buddhism is celebrated today in both west and east for being less "superstitious" and for referring to impersonal forces of the universe rather than human-like gods, in practice, there were a large number of anthropomorphic deities associated with the faith that could reward or punish, plus visions of heaven and hell. People often worshipped the Buddha himself as a god or godlike creature, even in nominally Theravada lands.

Buddhism also encouraged both vegetarianism and non-violence across much of Asia. Unfortunately, this did not prevent many Buddhist rulers from engaging in wars.

\section{PRIMARY DOCUMENTS: THE HAPPINESS OF THE DHAMMAPADA, ATTRIBUTED TO THE BUDDHA BUT WRITTEN DOWN CENTURIES LATER}

Let us live happily then, not hating those who hate us! among men who hate us let us dwell free from hatred!

Let us live happily then, free from ailments among the ailing! among men who are ailing let us dwell free from ailments!

Let us live happily then, free from greed among the greedy! among men who are greedy let us dwell free from greed!

Let us live happily then, though we call nothing our own! We shall be like the bright gods, feeding on happiness!

Victory breeds hatred, for the conquered is unhappy. He who has given up both victory and defeat, he, the contented, is happy.

There is no fire like passion; there is no losing throw like hatred; there is no pain like this body; there is no happiness higher than rest.

Hunger is the worst of diseases, the body the greatest of pains; if one knows this truly, that is Nirvana, the highest happiness.

Health is the greatest of gifts, contentedness the best riches; trust is the best of relationships, Nirvana the highest happiness.

He who has tasted the sweetness of solitude and tranquillity, is free from fear and free from sin, while he tastes the sweetness of drinking in the law.

The sight of the elect (Arya) is good, to live with them is always happiness; if a man does not see fools, he will be truly happy. 
He who walks in the company of fools suffers a long way; company with fools, as with an enemy, is always painful; company with the wise is pleasure, like meeting with kinsfolk.

Therefore, one ought to follow the wise, the intelligent, the learned, the much enduring, the dutiful, the elect; one ought to follow a good and wise man, as the moon follows the path of the stars.

Did you notice how this excerpt segues from preaching freedom from desire toward justifying a priestly class?

\section{Christianity}

As with the founders of most other religions, there is no independent evidence of the life of Jesus Christ. He was likely born in about 4 CE (medieval monks in establishing the western calendar probably miscalculated his date of birth) and died in his early 30s. He was born in Galilee, which, unlike Jerusalem, Rome ruled only indirectly at the time. The Christian Bible - likely set down about a century later - says much about his birth and his last years but little about his life in between. It claimed that the Holy Spirit impregnated his mother. Later in life, he would proclaim himself both the son of god and the Messiah promised in Jewish texts. He promised that all that would believe in him would achieve eternal life. Though faith was the key to the religion, believers were expected to follow a set of ethical behaviours.

Christianity, Manichaeism, and Islam all built on Judaism and Zoroastrianism. Christianity would proclaim a universal monotheist god, but also a malevolent devil that tempted people toward evil. There is also some speculation that Christian ideas of the separation of humans from nature echoed earlier concerns in both Egypt and Mesopotamia. Later Christian practices, such as fasting, monasteries, and saints, may each have borrowed from Buddhist examples.

One biblical version of Jesus's birth celebrates a visit by three "magi" from the east. "Magi" was the term used to designate Zoroastrian religious figures. These were noted for their astrological capabilities: It makes sense that they would have followed a star which others seem not to have noticed to the birthplace. Zoroastrianism had also prophesied a messiah and a virgin birth: The story of the visit by magi may have facilitated the conversion of Zoroastrians. In the early days of Christianity, Persia treated Christians better than Rome did; the magi reference (likely written down a century after the events described) may also have reflected a preference for rulers "from the east."

Christianity preached a common humanity (but at first also a unique destiny for Jews), and responsibility to aid the weak. It also respected women. It taught forgiveness (at a time when the pursuit of vengeance was common); this may have encouraged social peace, especially in societies characterized by ethnic diversity. It was nevertheless hostile to non-believers. Early communities were 
communistic, but Christians abandoned this practice over time. The emphasis on faith gave Christians a strong sense of community. Yet it also meant that once a church hierarchy existed, it would insist strictly on one set of beliefs: If there was a message that all must believe, there must be agreement on that message (see below).

At the time, Jews were sometimes fighting Roman rule and sometimes withdrawing from society. Jesus criticized Jewish priests and their reliance on ritual. Rome defeated a Jewish rebellion in 66-70 CE. This defeat may have encouraged the growth of Christianity and other sects that offered some relief from Roman rule.

The first followers of Christianity were Jews. From about 50 ce Paul of Tarsus preached to Greeks in Anatolia and Greece and then in Rome. Zealous missionaries would spread Christianity throughout the Roman Empire over the next centuries. Rome pursued a policy of religious toleration but was hostile to Christianity because Christians disdained other religions. Indeed, Christians occasionally engaged in violent conflict with these, and they refused to follow Roman religious practices or recognize the Roman pantheon. Christian theologians developed the intellectual underpinnings of the faith; it then spread from lower to upper classes. Augustine of Hippo (354-430 CE) was particularly important in connecting Christianity to Greek philosophy. Emperor

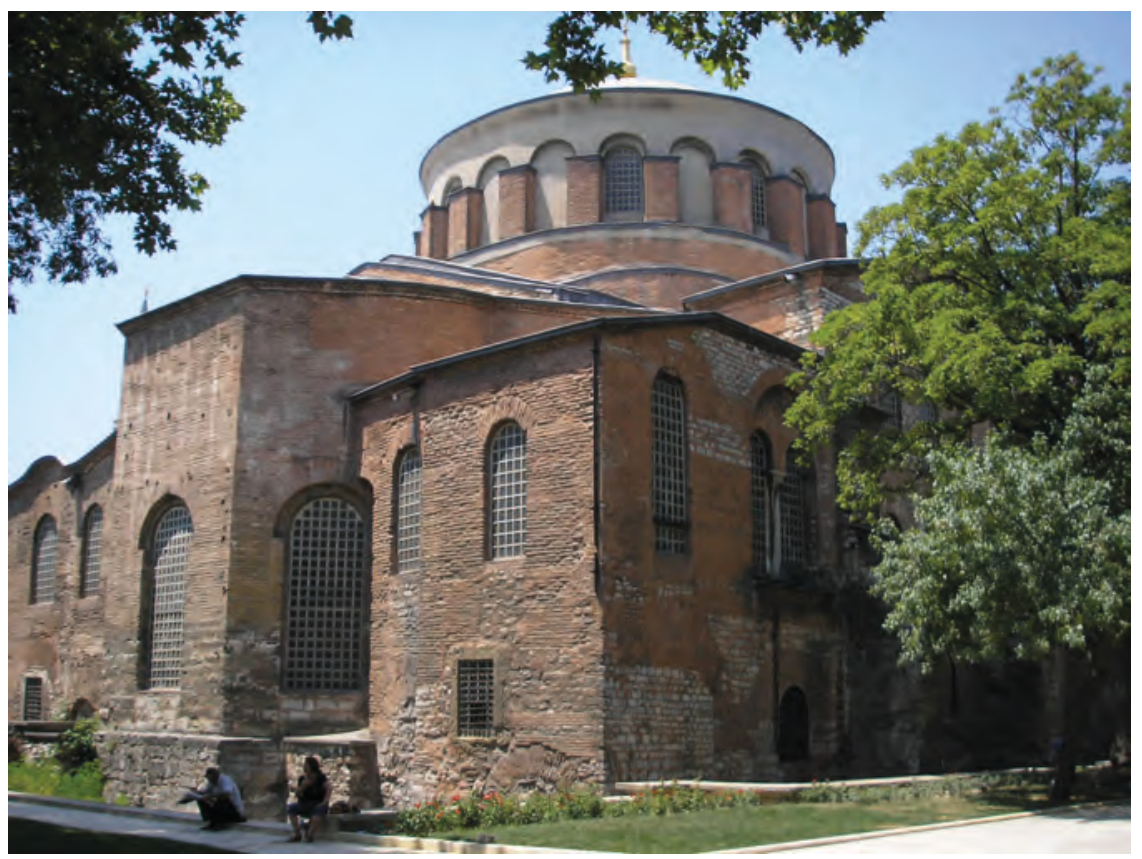

Illustration 11.12

Hagia Irene in Istanbul. Originally built by Emperor Constantine in the 4th century $\mathrm{CE}$, it was destroyed in riots under the Byzantine Emperor Justinian (chapter 17) and then restored. As part of the Topkapi Palace, it maintains much of its 4th- and 8thcentury heritage to this day 
Constantine legalized Christianity in 312, and Theodosius made it the state religion in 380. The empire then criticized other faiths as superstitions and declared them illegal. The empire banned the Olympic Games after a thousand years because these celebrated "pagan" gods. Rome came to see an advantage of collaboration with a religion with one god and a network of priests.

Christians also developed a formal church with recognized leaders in Rome, Antioch (in present-day Turkey), Constantinople, Alexandria, and Jerusalem. The bishop of Rome would claim precedence from the third century CE, but other bishops, especially in Constantinople, would contest Rome's primacy. The church in Constantinople would later ally closely with the Byzantine Empire - the part of the Roman Empire that survived after Rome itself fell (chapter 13). Both Rome and Constantinople would develop hierarchical church organizations that mimicked the structure of the Roman Empire. The bishoprics in Antioch, Jerusalem, and Alexandria would survive but decline in relative importance after Islamic conquests of those cities (chapter 12). Of the five original centres of Christianity only one - Rome - remains a predominantly Christian city today.

The Bible explicitly advocated separation of church and state - in sharp contrast to later Islam (below). Nevertheless, the eastern church would long live under the shadow of the Eastern Empire, with emperors often choosing the Patriarch of Constantinople (see below). The western church developed autonomously and came over time to have vast wealth from landholdings and tithes (taxes to the church). It perhaps inevitably suffered from severe corruption, with many popes and bishops pursuing personal gain rather than spirituality. That is, it experienced the challenges of any large bureaucracy, and reformers within the church struggled to reduce behaviours that threatened the integrity of the church (Pope John 12, pope from 955-64 was deposed for murder and adultery; the people chased Pope Benedict 9 from Rome in the mid eleventh century; Benedict returned and sold the office to another but claimed it back). In 1059, the church enacted the policy of having cardinals elect the pope and then having the church rather than secular rulers choose other officials. The practice of celibacy followed soon after. Though the celibacy rule discouraged the non-spiritual from entering the priesthood, it may have been even more critical in depriving priests of (legitimate) children to whom they might bequeath church properties. This newly independent church would launch the Crusades (chapter 15).

With the fall of the Western Roman Empire, the hierarchical Roman church remained and sought to provide spiritual unity where political unity had disappeared. In particular, the church tried to convert Germans and Celts, generally by first converting their leaders. Clovis, leader of the Franks, became Christian in about 496. The Irish and English were other early converts. In converting these peoples, the church proved open to syncretism: Their gods were associated with saints, and their religious customs - including holly and Christmas trees - with Christianity. The church also founded many monasteries devoted to maintaining and transmitting Christian teachings. 
The success of Christianity within the Roman Empire is well known. Christianity also spread east and south from its homeland in the Levant. Christianity would be widely adopted in Egypt and was common in Arabia. Missionaries also travelled east. By the second century $\mathrm{CE}$, there were many churches in Mesopotamia and Persia, and a couple as far as India. Christianity joined Judaism and Zoroastrianism as significant faiths throughout the Middle East. Early Christians in Mesopotamia and Persia popularized a hermit/monk life, an idea that then spread west into the Roman Empire. The eastward successes of Christian missionaries are little known today because Christianity (along with Manichaeism, Zoroastrianism, and Buddhism) would largely disappear from the region in the centuries following the Islamic conquest - though a few small Christian communities would survive into modern times in India. There were probably more Christians in Asia than in Europe until the ninth or tenth centuries.

There was also a critical doctrinal difference between eastern and western Christians. After the fifth century, eastern Christians tended to follow Nestorianism. Nestorian Christianity, though little known today, was common in Asia for centuries. The Roman Emperor Theodosius II had appointed Nestorius the bishop of Constantinople in 427 cE. The Empire, having only recently adopted Christianity, was trying to achieve doctrinal unity within the

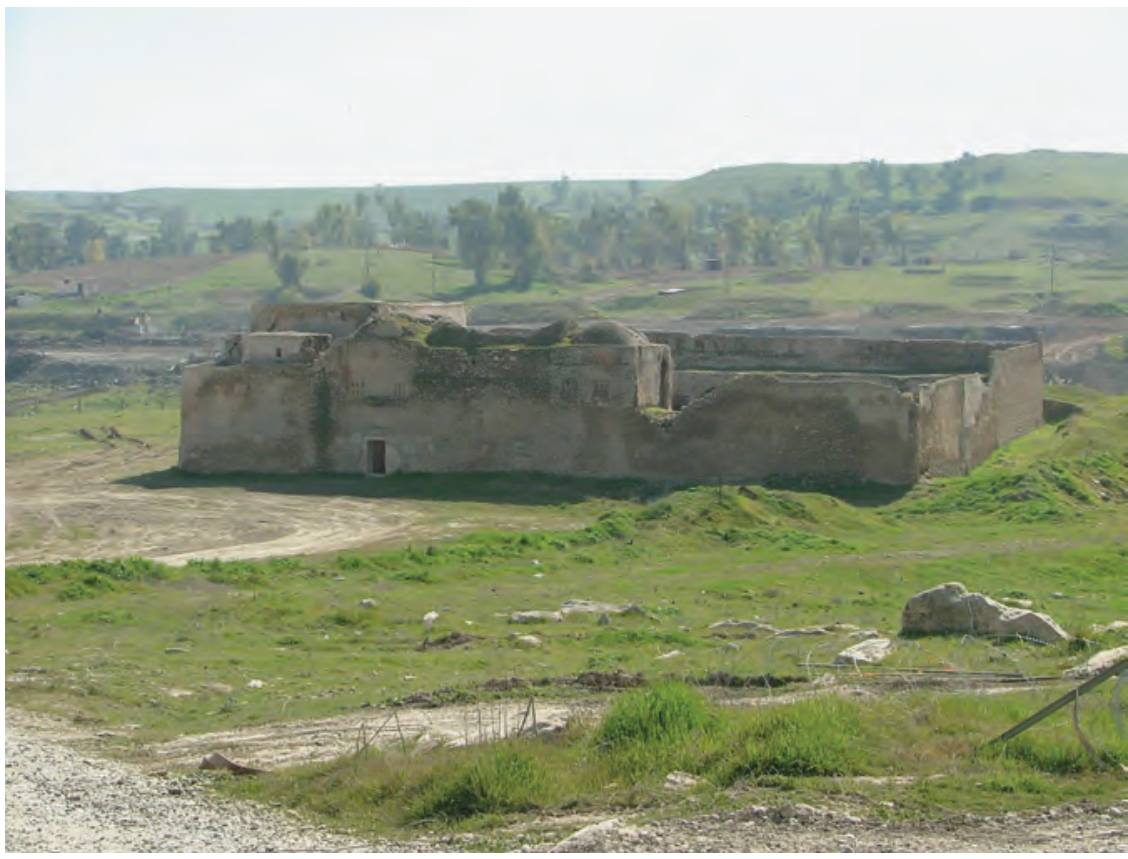

Illustration 11.13

Saint Elijah's Monastery near Mosul in Iraq, 6th century, one of the oldest surviving Christian monastery structures in the world until destroyed by Islamic State in 2014 Photograph by Doug, CC BY-SA 4.0 
church: A divided church could hardly provide religious solidarity and support for the empire. (Rome had earlier banished a popular "Arian" heresy that had questioned the nature of the holy trinity in the Council of Nicaea in $325 \mathrm{CE}$, convened by the emperor Constantine.) The New Testament describes Jesus as both human and godly; debate raged among church leaders as to how the human and godly combined. Some, drawing on Jesus's human activities in the Bible, stressed his humanity. Others instead stressed his divinity. Nestorius took the former view. Others, and in particular the bishop of Alexandria, accused Nestorius of denying Christ's divinity entirely. Nestorius was declared a heretic at the Council of Ephesus in 431, but primarily because he refused to appear and his allies from Antioch had not yet arrived. The Antiochenes would instead declare Cyril of Alexandria a heretic. The church would eventually achieve a consensus which some argue was far closer to the views of Nestorius than Cyril, but as part of the compromise Nestorius would remain a heretic but Cyril was restored. Historians debate to this day whether Nestorius was indeed a heretic. The point to stress here is that church councils at the time were still debating the nature of Jesus, and the point of view argued by Nestorius had substantial support. If he was a heretic, he was judged against standards that the church only instituted at that time. As the church persecuted followers of Nestorius, many fled to the east to avoid this persecution. The historical result was that after Islamic forces attacked Christian lands in the Middle East, it would be Nestorian Christianity that would survive in Asia for some centuries, acting in isolation from Christians in Europe.

Over time, Christians in Europe would also judge heretical the views of the Coptic Church in Egypt - which came to stress Jesus as a divine being, downplaying his humanity. The nature of Jesus could be understood in different ways, each of which attracted fervent devotion in different parts of the world.

Ethiopia adopted Christianity a little later than Rome. Axumite merchants in the Ethiopian highlands converted in the fourth century $\mathrm{CE}$, perhaps in part to strengthen trade with Rome; Local rulers were in turn converted, probably by visiting missionaries, only shortly after the conversion of Constantine. Monasteries were established, and the Bible translated into the local language. Christianity would retreat under pressure from Islam but would be encouraged as the national faith by later dynasties, especially in the twelfth through sixteenth centuries. Ethiopian Christianity evolved in isolation, with unusual practices including carving churches out of solid rock and borrowing a host of evil spirits from local religions.

We noted above that Christianity in Constantinople evolved in close contact with the imperial government (which claimed a semi-divine status for emperors). The Byzantine emperors would appoint the patriarch and guide the church to support imperial policy. There was inevitable conflict between church and state. When the emperor Leo III (717-41) attempted to have icons (religious images) destroyed in Byzantine churches, he was unsuccessful. Yet empire and church could agree that they need not heed commands from the Roman church. Over time, the Constantinople and Roman churches came to differ in 


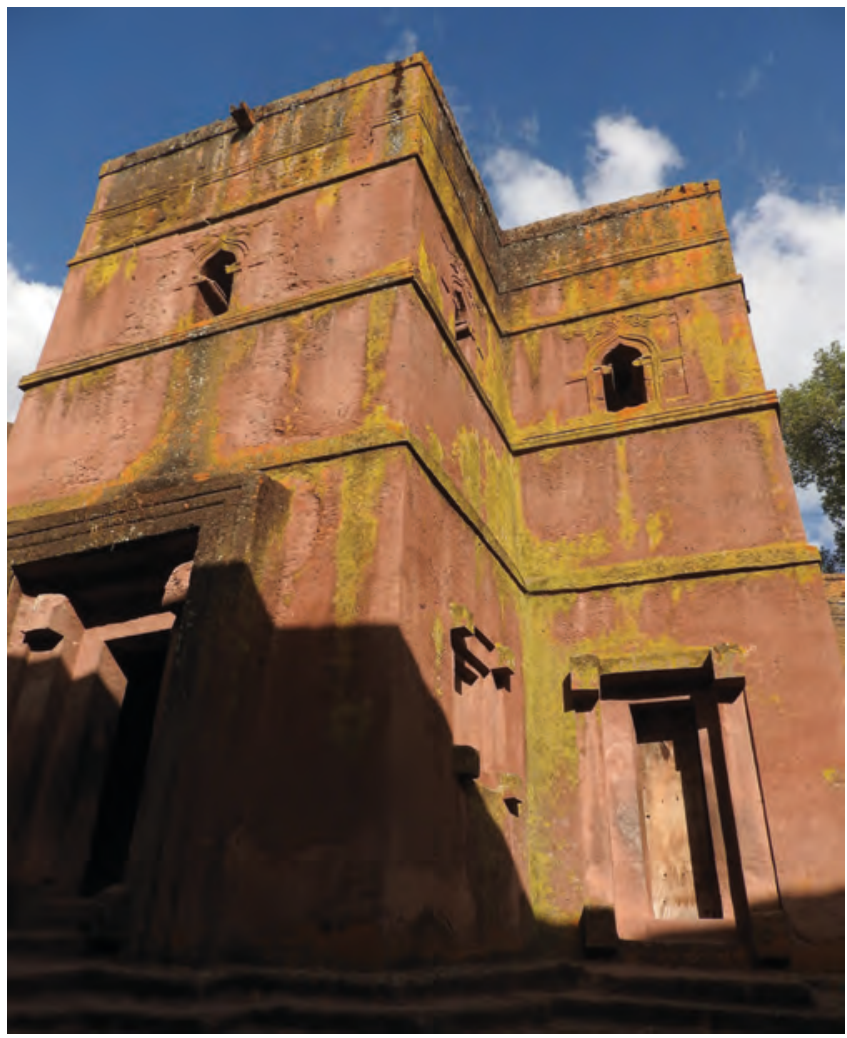

Illustration 11.14

Rock Church, Lalibela, Ethiopia. One of over a dozen churches hewn out of solid rock in this central Ethiopian town in the 13th century

both practices and doctrine. Constantinople was particularly concerned with drawing on Greek philosophy. There were also differences regarding the precise nature of Jesus. Constantinople used leavened bread in celebrating Mass whereas Rome used unleavened bread. The Constantinople Church allowed all who were baptized to take communion, whereas this involved a separate ceremony in Rome. Monasteries were common in the eastern Church. These provided social services, and were exemplars of religious devotion but did not play the educational role of western monasteries. Icons were felt to be infused with spirituality. Centuries of disagreement came to a head in 1054 when the two patriarchs excommunicated each other. After that, the two churches would be distinguished as Roman Catholic and Orthodox. (There are also "Eastern Rite" or "Uniate" churches that follow some Orthodox practices but recognize the authority of Rome.)

Orthodoxy would spread far beyond the borders of the empire - just as Roman Catholicism spread north into regions never conquered by Rome. The Byzantines had established ties with Slavs in Eastern Europe from the sixth century. Conversion of Slavic peoples to Orthodoxy by missionaries provided 
a robust cultural connection that supported political alliances. St. Cyril, one of these missionaries, developed the Cyrillic script, which would be replaced by the Roman alphabet in much of Eastern Europe but remains in use in much of the former Soviet Union. Russian merchants regularly visited Constantinople from the ninth century. Prince Vladimir of Kiev converted in 989. Russia would develop its own Orthodox Church and claim that this was the true Orthodox Church after Constantinople fell to the Islamic Ottomans in 1453 (chapter 20; note that Constantinople still has an Orthodox patriarch).

\section{Manichaeism}

Unlike the founders of other religions, Mani is undoubtedly a historical figure. He was initially from Babylon and lived in the third century Ce. Whereas the revered texts of other faiths have unclear authorship or timing, Mani clearly authored the critical texts of Manichaeism. Mani would indeed celebrate this fact, arguing that his religion was more authoritative than others. His followers need not worry that his disciples had misinterpreted his revelations. This was a claim that resonated at a time when priests of many religions (Christianity, Zoroastrianism, Buddhism, Jainism, and Confucianism) were worried about misinterpretation and were carefully writing down official texts. Though most of the new religions were founded centuries earlier, their official texts were written in the early centuries CE. Mani also travelled widely during his life and established a formal church with its own rituals.

Mani was born in Mesopotamia at the time of the Parthians (chapter 9); Zoroastrianism, Christianity, and Buddhism were all prevalent there at the time. He grew up among a group - the Elchasaites (a group now largely forgotten, but indicative of the religious ferment of the time and of the fact that most religions fail) - that mixed Judaism and Christianity and hoped to develop a new global faith. Mani drew on Zoroastrianism to suggest that humans were supposed to be with the light but had been drawn into darkness. He urged a Christian message of possible salvation. He then drew on Buddhism to suggest that humans could only obtain the light by shedding physical desires. He recognized Buddha, Zoroaster, and Jesus as prophets - but argued that their disciples had misinterpreted their teachings through time. Manichaeism was consciously a syncretic blend of Zoroastrian, Christian, and Buddhist elements.

Manichaeism had a priestly class, the Elect, which pledged poverty, celibacy, and vegetarianism. They could anticipate joining the light upon death. The followers, or Hearers, were urged to reject the pursuit of pleasure (including sex and procreation); but less zealous followers could live more normal lives but with a strict ethical code. They could hope to be reincarnated as Elect eventually. Manichaeism disdained science in favour of revealed truth.

Manichaeism was in a way the first global religion for it spread quickly to Spain and China. It was popular with merchants, who transmitted it along trade routes. Mani's texts were translated into many languages. The fact that Manichaeism had consciously drawn on multiple faiths aided its spread. 
Missionaries were often syncretic, associating local deities with the cosmic struggle at the heart of Manichaeism. However, Manichaeism does not seem to have gained as much financial support as other religions. Mani travelled to India and converted at least one Indian ruler. He received some support from the Sassanids in Persia, who may have seen his new religion as a means of uniting their diverse population, but in the end the Sassanids would favour Zoroastrianism. Mani himself died in a Sassanid prison, charged with apostasy. His followers fled to both Rome and Central Asia. However, Christian Rome persecuted Manicheans. Many Manicheans returned to Persia after the Islamic conquest, for the Umayyads were at first tolerant (chapter 12) but moved again as Islamic rule became less tolerant. The ruler of the large Central Asian state of the Uighurs adopted Manichaeism in 762, perhaps to strategically distinguish itself from other states. (Notably, some Manichaean monasteries within Uighur territories apparently acquired considerable wealth.) However, this state would soon dissolve, and no other state would ever pursue Manichaeism. Although Manicheans could interpret persecution as evidence of the cosmic battle between light and darkness, the religion gradually disappeared without state support (some small groups claim to follow the faith in the twenty-first century). It survived among Sogdian merchants in Central Asia for centuries, but these would later adopt Islam. It could be that Manichaean suspicion of procreation hastened its decline.

\section{Islam}

As with the Buddha and Jesus, there is no independent historical evidence of the life of Muhammad. He was likely born in Mecca in Arabia around 570 CE into a once-prominent merchant family. His parents both died while he was young, and relatives then raised and educated him. He followed desert trading caravans, married (first a widow he had once worked for), had children, and then sought meaning in life. About 610 he experienced visions of a monotheistic god, transmitted by Archangel Gabriel (also celebrated in Judaism and Christianity). He became unpopular in Mecca both for attacking polytheistic gods that many treasured and for criticizing the greed of fellow

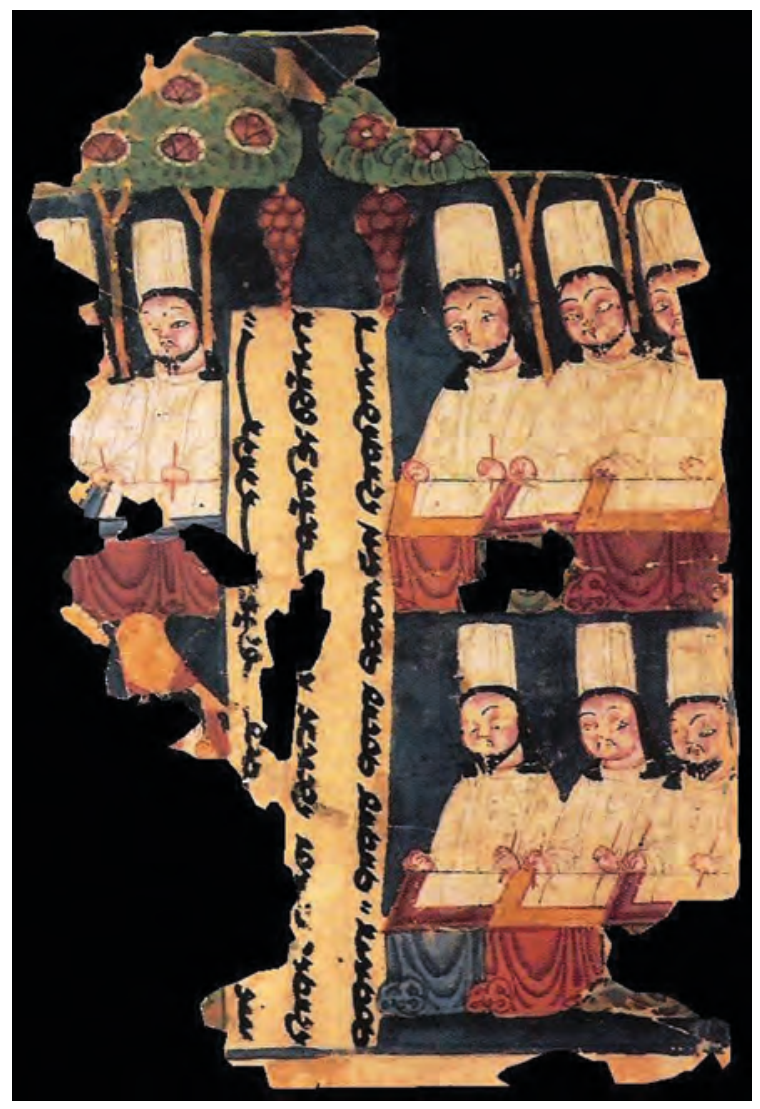

Illustration 11.15

Manichaean priests writing, from a Manichaean manuscript from Goachang, Ruin K. 8th/9th century CE Painting on paper 


\section{PRIMARY DOCUMENTS: THE PARABLE OF THE PEARL-BORER}

Despite Mani's efforts to create and maintain authoritative texts, most of his writings were destroyed, and only fragments of uncertain authorship remain. This parable was written first in Parthian and transcribed into Sogdian. Manichaean texts often appropriated myths from surrounding areas to their own purposes.

A merchant had many precious gems. To have them bored, he hired a man for hundred pieces of gold per day, and he went with him to his domicile. When he (the hired man) sat down, a lute happened to be there, and the worker looked at it.

When the merchant asked him if he knew how to play the lute, he answered, "Yes, very well." For he was skilled in this art. He (the merchant) said, "Then take it." He (the hired man) thus took it and correctly played beautiful melodies for the merchant the whole day long, leaving the box with gems open and beating the time with his hand and swaying his head to it, with great joy.

In the evening, the worker said to him (the merchant), "Have my wages given to me." When the other said, "Have you done anything to earn wages?," he answered, "You hired me, and I did what you told me to do." Thus, he urged him, until he received the hundred pieces of gold without any deduction, while the gems remained unbored.... There was a quarrel, it could not be settled.

So on the next day, they went before a judge for a trial. The owner of the pearls spoke thus, "My lord, when this gentleman saw me beside the bazaar, he asked me, 'Hey, what work can you do?' I replied, 'Sir, whatever work you may order me to do, I can do it all.' When he had taken me to his house, he ordered me to play the lute. Until nightfall, I played on the lute at the owner's bidding."

The judge pronounced this verdict, "You hired this man to do work for you, so why did you not order him to bore the pearls? Why did you bid him play on the lute instead? The man's wages will have to be paid in full. If again there should be any pearls to be bored, give him another hundred gold denares, and he shall then bore your pearls another day."

Thus under constraint, the owner of the pearls paid the hundred gold denares, his pearls remained unbored, left for another day, and he himself was filled with shame and regret.

The wise give this allegorical explanation: That man who understood all arts and crafts represents the body ... The pearl-borer is the body. The hundred denares represent a life of a hundred years. The owner of the pearls is the soul, and the boring of the pearls represents piety.

The parable proceeds to urge the Hearers to follow Mani's piety. They should feed their families and perform their duties to lords and the state but also pursue spiritual enlightenment. Can you appreciate why this parable was popular enough to be translated and copied many times over a period of centuries? 
merchants. There were polytheistic shrines in Mecca from which merchants profited. Townspeople forced Muhammad in 622 to move to a town he and his followers renamed Medina. There they established a community of the faithful with a strict legal code and emphasis on charity. He came to see himself as the final prophet with a more complete message than Judaism or Christianity but from the same god. In 630, he and his followers took Mecca, destroying all polytheistic shrines except the Kaaba, which they converted to their own use. He forced the local elite to convert. Islam had conquered most of Arabia by his death in 632. His followers recorded his sayings in the Quran from about 650.

Muhammad proclaimed five pillars of the Islamic faith: belief in one god; daily prayer facing Mecca; the hajj, or pilgrimage to Mecca at least once during one's lifetime; fasting during the day during the month of Ramadan, and providing alms to the poor. Some would add jihad to this list, a word that is interpreted variously to mean anything from holy war to efforts to spread the faith through conversation or simply to battling one's own temptations: The word translates best as "struggle." Sharia law developed over the first centuries of Islamic rule, and many have come to see it as central to the faith. Islam followed Judaism in prohibiting both pork and lending money for interest (usury).

Mecca at the time of Muhammad was a flourishing trade centre halfway up the Red Sea. Arabia sat astride major trade routes from the Mediterranean to the east via either the Red Sea or the Persian Gulf. Though he was successful as a merchant, Muhammad became critical of what he saw as immoral practices among the business elite. Much of the Quran is devoted to outlining appropriate

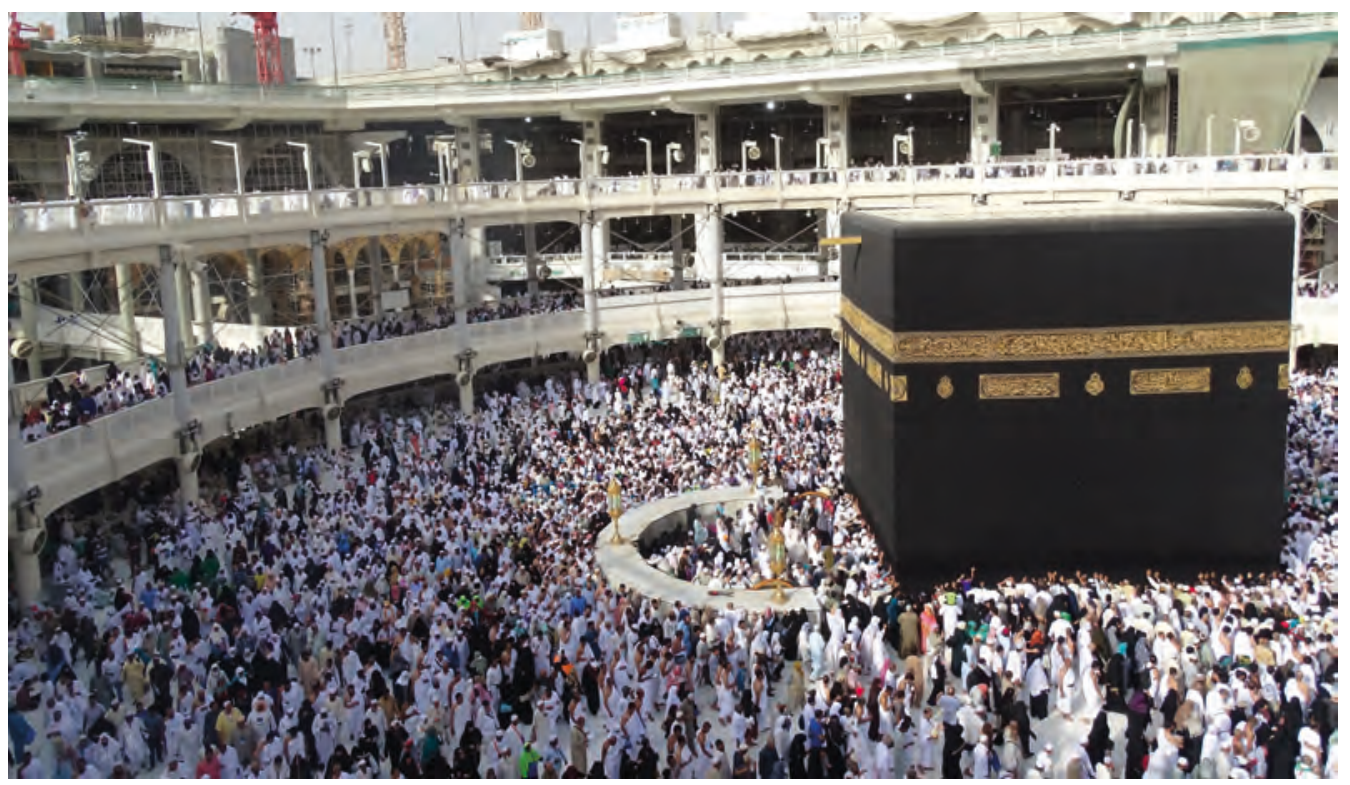

Illustration 11.16

The Kaaba in Mecca, the focal point of the hajj 
behaviours for merchants. This may have served to increase trust among Muslim merchants. More practically, the Quran and Islamic law provided very detailed guidance on how to address common issues in merchant contracts. Merchants were also encouraged to donate to Muslim institutions.

We noted in chapter 8 that religions serve to encourage ethical behaviour. However, religious people are usually not ethical every minute of every day. Particular religions may promote ethical behaviour in some situations more than others. Religions such as Buddhism or Islam, which spoke directly to the ethical challenges facing merchants, may then have been particularly appealing to merchants: Merchants could then be more confident that fellow believers would be ethical in trade.

Mecca was religiously diverse: Judaism and Christianity were both common, but so also were the more traditional beliefs of local nomadic populations. States in the well-watered southwest (present Yemen) - who had adopted Judaism in the fifth century - had long dominated the Arabian peninsula but their power had waned. At the time of Muhammad, Mecca was effectively selfgoverning, though Christian Byzantium, Christian Axum, and Zoroastrian Persia all coveted the peninsula. Islam would prove capable of uniting fractious Arab tribes against these various powers.

The nomadic nature of Arabia deserves mention. Islam would prosper throughout the Arab world and then later in Central Asia. It may be that Islam reflected nomadic beliefs and was in some ways especially attractive to nomadic peoples. Nomads may have appreciated its clear and sometimes harsh laws: Nomads were accustomed to settling disputes directly and physically. At a time when doctrinal disputes divided Christianity, Islam may have seemed a more straightforward faith with its five practices leading to paradise. As we shall see, though, Islam soon had its own doctrinal disputes. Islam would undoubtedly give nomads community and legitimacy and tools of statehood but other religions might have done that too. Islamic successes in nomadic regions might reflect historical contingencies rather than the nature of Islam. After all, Islam would also prosper in many sedentary societies. Indeed, Islam often expanded first along major trade routes into cities, and only slowly into the countryside.

Historical scholarship establishes that the Quran, like the Buddha's teachings and the Bible, built upon earlier texts and was compiled over a lengthy period. The Quran was not standardized for decades after Muhammad's death, and other Islamic texts were developed even later. Many practices today associated with Islam were only clarified in the ninth and tenth centuries. In particular, gender roles evolved. There are many egalitarian passages in the Quran. Muhammad valued his wives' input, but did have multiple wives and concubines, and did later in life practice some degree of seclusion of these. Arguably, Islam came to emphasize male power even more as it moved from its nomadic heartland into areas of more settled agriculture. Judaism, Christianity, and perhaps especially Zoroastrianism may have encouraged early Islamic scholars to interpret holy texts in a manner that exalted the role of men. It was common in the early days 
of Islam for widows to exercise power in the choice of a second husband, but within a century or two it was frowned on for men to marry non-virgins, and women played little role in choosing partners. The Quran gave women more rights than they may have had in Middle Eastern societies of the day, and was likely intended as liberating, but these rules hardened over time.

As with Buddhism, Islam accepted and addressed economic and political change, especially as conquest took the new faith to different economic and political realities. A message of fundamental equality, social justice, and ethics was useful at a time of social transformation and (probably) increasing inequality.

We will describe the dramatic military conquests of early Islamic polities in chapter 12. Within just a few decades, Islamic forces had conquered across North Africa and into Spain to the west, and across the Byzantine and Sassanid Empires to the Indus River to the east. These conquests meant that Islam, unlike most other missionary religions, did not have to develop over an extended period as a faith viewed with suspicion or even hostility by political authorities. After about a century Islamic polities came to focus on internal order rather than conquest. Central Asia was slowly absorbed into the Islamic orbit over the next centuries, India would be conquered centuries later, and merchants would spread the Islamic faith to Southeast Asia (as Buddhist and Hindu merchants had spread their beliefs before) and both West and East Africa, where local polities would eventually support it. As conquest slowed, it came to be accepted that Islamic states could sign treaties with non-Islamic states, and not pursue war against them. This was a significant change from the early days of the faith when global conquest was often urged (see chapter 12).

Many but not all modern Islamic polities pursue policies of religious orthodoxy: They see themselves as defenders of (a particular denomination of) the faith and tend to limit the activities of other religions. Yet the Islamic faith spread slowly in early Islamic polities and many of these practised religious toleration. In the very earliest days, Islam - like Judaism - was associated with a particular ethnic group, in this case the Arabs. Conquests beyond Arab lands were at first motivated by economic considerations more than religious ones. The small size of the conquering Arab population meant that religious toleration was necessary. However, Islam soon came to see itself as a universal faith.

Islam was probably not the majority religion even in Egypt until the tenth century; this was likely even more true on the Central Asian frontier. Muslim rulers encouraged conversion, supported the faith financially, and when feasible reserved government posts for believers. Nevertheless, the process of conversion took centuries.

In some places, authorities pursued toleration to such an extent that Islam never did become the majority faith. Spain in the west was one such place. Muslim rulers governed a state in which Muslims, Christians, and Jews cohabited for centuries. Though religious conflicts were frequent, so also were cross-religious friendships, business deals, and even marriages. Nevertheless, 
when, centuries later, Christian kings reconquered Spain they would expel both Muslims and Jews (chapter 15).

India in the east would be another case. Though Islam was the preferred faith of the later Mughal Empire, the Mughals would also sponsor Hindu temples though Buddhism was perhaps less well treated. The vast majority of the Indian population would practise Hinduism despite centuries of Mughal rule. Islam was itself changed in India and became most established in regions without a strong Hindu or Buddhist presence

When Muslim rulers expanded into Central Asia, they at first tolerated Buddhists and others, in large part because Buddhist merchants were central to the local economy. Over time, though, Muslim merchants replaced Buddhist merchants. Buddhism survived for centuries - indeed, it lasted centuries longer under Islamic rule than it did in neighbouring Hindu states. Yet Buddhism would eventually effectively disappear from Islamic states in Central Asia. Some Buddhists migrated to other lands. Others converted to join Muslim trade networks or to avoid special taxes imposed on non-Muslims.

Why did some Muslim states practise toleration? Part of the answer is that small Islamic elites would risk rebellion if they tried to force large subject populations to convert. Yet we have seen that in some conquered lands, subtle policies of religious preference could induce mass conversion over a period of centuries. In both Spain and India, Muslim rulers were in place for centuries. Perhaps in both India and Spain rulers saw advantages in pluralism: Empires had always governed diverse populations and had often pursued strategies of playing groups against each other. A ruler that arbitrated among religious groups might be stronger than one that ruled a more homogeneous state. Religious leaders indeed often harried Islamic rulers in Islamic-majority states to lead a life more congruent with holy texts.

The Pact of Umar - a supposed treaty between Islamic rulers and Christian subjects, attributed to various times and places in the ninth century or so - as well as later law codes, forbid refurbishment or construction of non-Islamic religious buildings in Islamic states. Many rulers ignored this. There were Buddhist monasteries well into the eleventh century in Islamic Central Asia, apparently with royal support.

Religious leaders criticized Islamic rulers from early days. Islam envisioned a state devoted to Islamic principles. Moreover, Islam encouraged egalitarianism and a strong sense of community. However, rapid conquest generated a political/military elite that could and did amass immense wealth. These rulers naturally emphasized those elements of holy texts that permitted individual success in life while downplaying emphases on sharing.

Muhammad had recognized the validity of Judaism and Christianity (the Quran briefly mentions Zoroastrianism under the title of "Magians"). The Quran urges respect for followers of these faiths. When Islamic conquerors encountered Manicheans, Buddhists, and Hindus, they had less guidance on how to treat them. In practice, some Islamic polities treated these religions better than other Islamic polities treated Judaism and Christianity. Christianity 
would become a small minority sect in the very lands in which it had first emerged.

Muhammad had made no provision for succession. When he died in 632, only years after he and his followers had taken Mecca, it was unclear who should lead. A dispute over the succession would eventually lead to the Sunni/Shia split that characterizes Islam to this day: Shias argued that only descendants of Muhammad's family should rule. Over time, a variety of differences in doctrine and ritual would further distinguish Sunni and Shia faiths (much as differences between Catholic and Orthodox Christianity accumulated over time). Sunnis would seek religious guidance from communities of scholars whereas Shias were more likely to see individual imams as divinely inspired. As within Christianity, these two denominations received support from different political entities. Since a denominational dispute within a faith is a direct challenge to belief systems, Islamic rulers would often discourage the alternative Islamic interpretation even more spiritedly than they discouraged non-Islamic faiths. The adoption of the Shia faith by later Persian states would solidify Shi'ism in that region, though Shia minorities would struggle to survive within many Sunni polities.

A third important denomination is Sufism. This also emerged early, and arguably in reaction to the wealth gained from conquests. Sufis lived an ascetic life. They also focused more on personal experiences of enlightenment than thorough mastery of holy texts. Sufis often earned the enmity of the leaders of other Islamic dominations whose authority came precisely from their familiarity with these texts. We can see parallels here with the treatment of mystics within Christianity, or the attitudes of Theravada Buddhists toward first Mahayana and especially Chan/Zen practices; in all these cases, those who revered and studied holy texts criticized those who downplayed these. Perhaps ironically, Sufis would prove to be very successful missionaries, especially in Central Asia: Their asceticism impressed others, and their emphasis on personal experience rather than textual orthodoxy allowed them to absorb local beliefs into Islam. There is speculation that Sufism borrowed from Buddhism since Sufism is mystical and speaks of the annihilation of the ego in order to know god, but there is no solid evidence of a connection. Sufis likely did benefit from seeming to behave like Buddhist bodhisattvas: people who had achieved enlightenment but remained on earth to aid others in their pursuit of enlightenment (and in India Sufis fit well with the tradition of Hindu ascetics who shunned wealth in their pursuit of religious revelation). Though Sufis converted millions, they did not develop religious institutions as Sunnis and Shias did, and so the regions they converted came over time to pursue orthodox Sunni or Shia beliefs.

\section{Shinto}

Shinto emerged during the same time period as the other faiths addressed in this chapter. Its texts were first written down in the fifth and sixth centuries CE (when Chinese writing techniques reached Japan), but it likely originated 
a millennium earlier. Shinto is polytheistic and animistic, but some gods are more important than others. The sun goddess is especially important, and Japanese emperors have claimed descent from the sun goddess. Shinto urges followers toward harmony with nature and other humans. It has thus been possible for adherents to accept other religious traditions, notably Buddhism. Shinto, like Judaism, is associated with one ethnic group, in this case the Japanese. It has often been employed in nationalist celebrations. Over a dozen separate denominations of Shinto religious practice emerged over the centuries. By some counts, Shinto is the fifth largest religion in the world with some 100 million adherents, but many of these are also practising Buddhists.

\section{Mayan religion}

The Spanish conquerors deliberately destroyed the religious texts of the Mayan people. However, through the efforts of a handful of Mayan and Catholic priests, Spanish translations of some documents exist. We cannot know how true to the original these are. The Mayan religion likely dates, like others discussed above, to the last centuries BCE. It embraces one central deity but also many demi-gods. In blending monotheistic and polytheistic elements, it was similar to many of the religions above - but with a more polytheistic balance. These gods demanded sacrifices from humans. The gods had purposely limited human understanding so that humanity would not gain power over gods. Yet individual humans could confront and trick particular gods, who were often capricious. Humans were thought to be immortal.

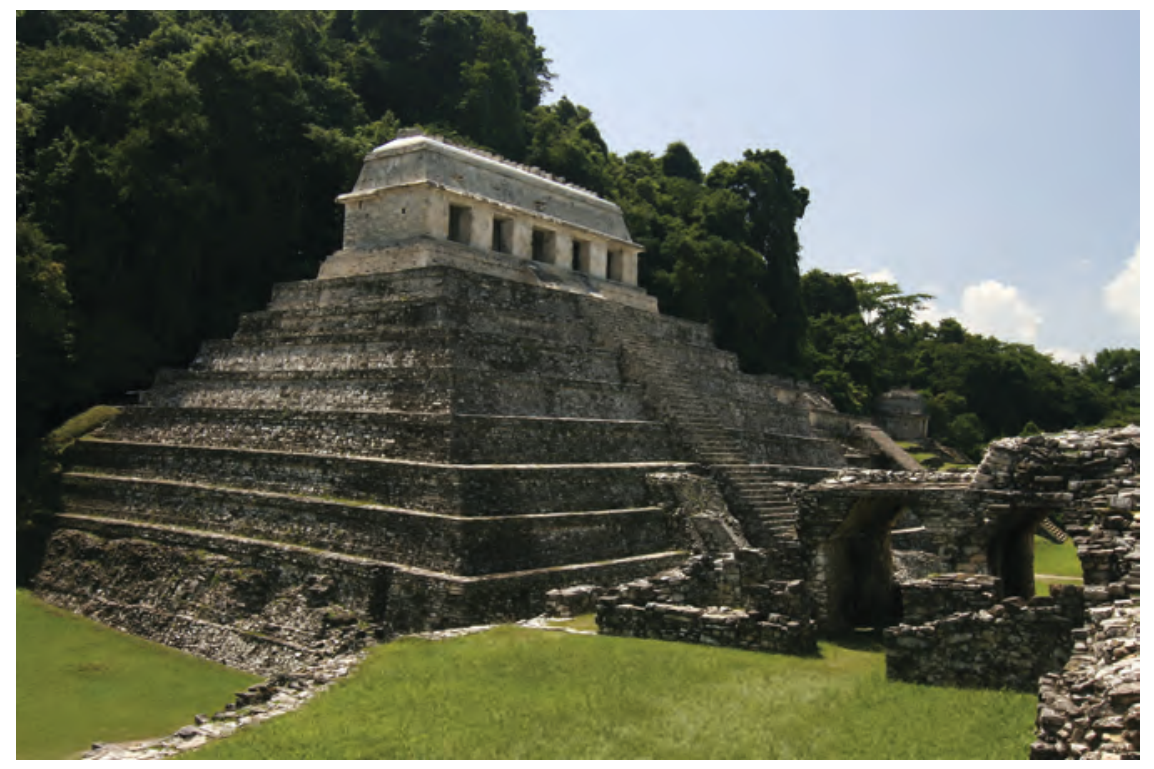

Illustration 11.17

Temple of the Inscriptions, Palenque, Yucatan, Mexico, 7th century CE 


\section{Some comparisons and reflections}

\section{Canon development}

We have seen that the holy texts of most new religions were developed over a period of centuries. Scholars have recognized several commonalities in this process. First, religious leaders usually disputed which versions deserved veneration. Second, authoritative bodies strove to establish an accepted canon, so that there could be one clear set of religious doctrines. Third, the recognition of a particular canon was often the result of a crisis within or external threat to the faith. Fourth, a group of textual scholars was required to establish and interpret these canons. Fifth, these canons then became a focal point of religious identity.

While all religions venerate a canon, there is diversity in how literally the texts in the canon are interpreted. Buddhism, on the one hand recognizes that texts require interpretation, but on the other hand advocates precise repetition of textual sayings. Christianity has been rocked in recent decades regarding how to interpret biblical passages regarding sex in general and homosexuality in particular. Islam professes that God gave the Quran to Mohammad, but Islamic scholars nevertheless debate the appropriate interpretation of specific passages.

\section{Religion and family values}

Religions that would advocate celibacy or monastic contemplation would need to negotiate their relationship with family values. Hinduism encouraged people to pursue a life of ascetic contemplation only after their children were grown. Many Buddhist texts address the fact that the Buddha had offended his father and wife and son with his pursuit of enlightenment; Buddhism would later urge that men needed the permission of their families to become monks. As Buddhism spread in China, where a celibate life seemed an insult to one's ancestors, it was argued that if one achieved enlightenment, one's entire family would benefit. Jesus recognized that his disciples must ignore familial duties to follow him; later Christian teachings would emphasize family but provide an opportunity for a minority of the population - both men and women - to pursue a life of sexual abstinence and therefore the absence of family responsibilities. This would be an unusual practice within world religions.

Religions had an ambiguous effect on the role of women. All of the new faiths appreciated that women also had souls. This made female lives equivalent to male lives in at least a spiritual sense. However, all of the new religions reserved the most important positions in religious hierarchies for men. There were almost no female sages in any of the new religions. Arguably, men created these religions with the interests of men in mind - though women over the centuries might exert some religious influence. Sometimes, as with Islamic inheritance laws, religions improved the legal status of women - but by inscribing 
such prescriptions in holy texts, religion could serve to limit improvements in female rights centuries later. Women were generally viewed as subservient to men across these religions.

\section{Religion and trade}

We noted in chapter 5 that merchants often operated in networks. We have since seen that such networks usually shared an ethnic or religious basis. In the era of early religions, ethnicity and religion often overlapped as each region worshipped its own gods. Networks associated with these early religions may then have been particularly tight.

The new religions severed the link between ethnicity and religion (with the exceptions of Judaism and Shinto and the very early days of Christianity and Islam). However, they also expanded the potential size of networks: Muslim merchants soon operated throughout the eastern hemisphere. Since these new religions preached a set of ethical practices, they might well have encouraged even greater trust within these networks. A Muslim merchant in China might feel a closer bond to visiting Muslims than to their neighbours. Islam in particular prescribed a set of acceptable business practices. While these might prove problematic in later centuries as trade and finance became more complex, they facilitated trade in earlier centuries: Merchants often called upon Muslim scholars to mediate trade disputes. Islam - and to a lesser extent other religions - then provided a supra-national set of legal principles that merchants engaged in international trade could employ.

Trade networks could mainly rely on self-interest: If all members of the network were profiting from trade within the network, they would be discouraged from mistreating other network members and risking ejection. However, times of trouble - a natural disaster or war or epidemic or just some severe decline in economic activity - might transform the costs and benefits associated with maintaining one's reputation. In such a case, religious belief might prove particularly important in binding merchants to their commitments.

We have noted above that merchants often spread religion. In part, this is merely an artefact of the fact that merchants travelled more than most people. Still, it is worth noting that merchants had an incentive: If religious belief supported the trust required in merchant networks, then merchants would benefit from converting those with which they wished to trade. Merchant networks could expand geographically through conversion as well as migration. If merchants could convince rulers of the places they traded in to convert, then they could not only be confident of fair treatment but could reasonably anticipate preferential treatment over merchants of other faiths. Nevertheless, we have noted often in this book that it is hard to convince others of beliefs that one does not actually hold; merchants would have been far less successful in converting others if they had not been believers themselves. 


\section{Faith}

It perhaps deserves emphasis that faith lies at the heart of all religions. In western religions, faith has tended to be a "test": only those with faith can achieve eternal salvation. In eastern religions, faith has tended to be rather the key to a "learning experience": only those who recognize the cycle of reincarnation and suffering can achieve release from this. Religions differ in who were/ are the prophets and how they came to their understandings. Religions vary also in the precise nature of the afterlife. Yet all religions concur that a deep and profound faith in one of these sets of beliefs is necessary for achieving the postulated afterlife.

\section{Religion and war}

All religions exhibited a mixed and changing attitude toward war and peace through time. Changing attitudes reflected conflicting impulses: Ethical prescriptions guided religions to shun violence toward others, while a desire to convert others might justify conquest (see below). Political realities encouraged change in emphasis: If associated with states that prosecuted wars, religions usually came to provide some justification for such wars. If instead associated with hegemonic states (those that dominated their region), or associated with peasants or merchants more than rulers, religions instead emphasized peace.

Hinduism in its earliest incarnation celebrated war. The warrior caste was at the top of the original caste hierarchy. Over time, Hinduism became less warlike, and the priestly caste gained precedence. Hinduism came to value non-violence toward both people and animals. Buddhism built upon Hindu ideas of non-violence. As we saw in chapter 9, Emperor Ashoka would forsake war after converting to Buddhism. Though his motives may have been complicated, his example would be celebrated by many who came after, including Mahatma Gandhi, the (Hindu but secular) Indian independence leader (chapter 30). Rulers may at times have rejected Buddhism because of its advocacy of peace. Nevertheless, over time many Buddhist rulers in China, Tibet, Japan, and Southeast Asia would claim that Buddhism did allow wars if protecting Buddhists or facing evil opponents. Buddhist monks would often serve as soldiers. They would often claim that their goal was to guarantee peace.

Judaism provided the most robust vision of godly-sanctioned war against heathens. Yet it envisioned a future peaceful golden age. Christianity, which favoured peace in its early days, would build upon the latter idea. As it gained state favour, ideas of just war emerged (much as in Buddhism); Augustine was one who urged just war after Christianity had become the Roman state religion. War against infidels was more easily justified. Later the church often tried to limit feudal conflict (chapter 13), even refusing to bury combatants early on, but these efforts mostly failed, and the church slowly came to accept war between believers. The Bible urged missionaries to be peaceful, but this 
ideal too was sacrificed over time in favour of forceful conquest of unbelievers. As for Islam, Muhammad described himself as a messenger of peace but was involved in military conflict from early on. Still, Islam argued that peace was something to work for, and placed limits on holy war (jihad): Christians and Jews and sometimes Zoroastrians were to be spared, and an imam had to make the declaration of holy war. As the Abbasids settled down and pursued peace with neighbours (chapter 12), Islamic thinkers such as Ibn Battuta and Averroes came to praise peace - though still allowing for just war. Muslims were therefore shocked when Christians launched the Crusades (though partly because of the brutality associated with this; chapter 15).

Postscript: In the period since the Second World War, there have been peace movements associated with all major world religions.

\section{Religion and artistic representation}

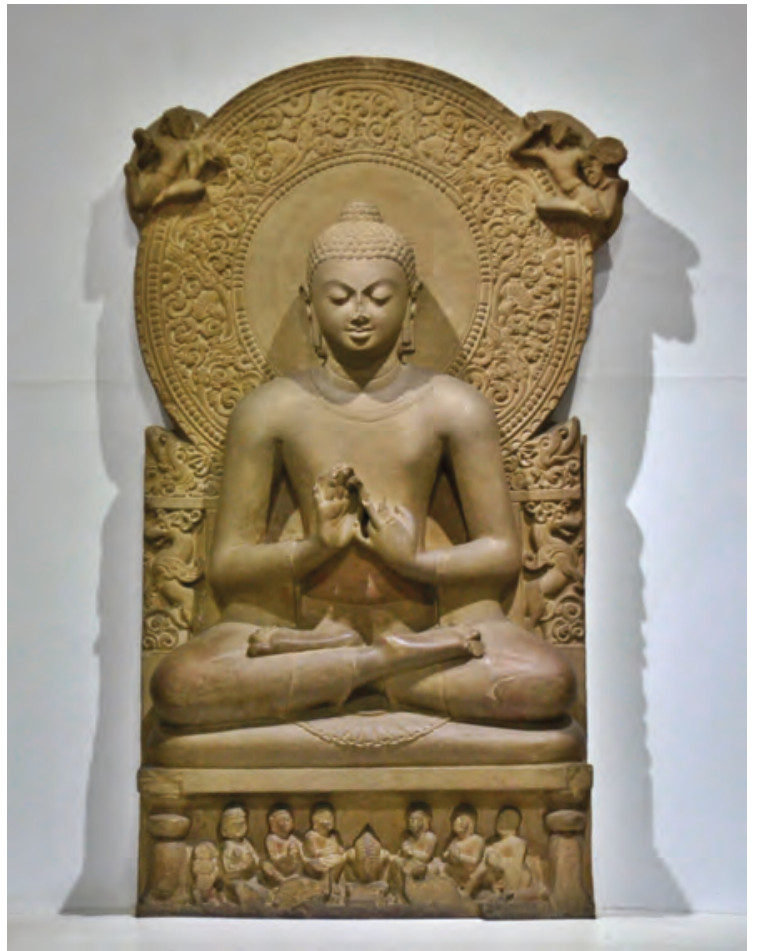

Illustration 11.18

Seated Buddha, Gupta period, Archaeological Museum (ASI), Sarnath, India

Photograph by Tevaprapas, CC BY-SA 3.0
Statues of the Buddha are now common. There are four common depictions: sitting, standing, lying, and walking. However, in the first few centuries of Buddhism, artists depicted the Buddha only symbolically. Then in the early centuries of the Common Era especially around $300 \mathrm{CE}$ - it became common to produce lifelike if idealized statues. This development seems to have occurred at roughly the same time in both the Kushan Empire of Central Asia and along the Ganges, though different styles were pursued (Kushan art reflecting a stronger Greek influence; chapter 9). Notably, at about the same time, each Hindu god came to be depicted in a particular fashion. During the Gupta era, there would be further standardization of the representation of specific gods. The early years of the Common Era were also, of course, the early years of Christianity which tended from its early days to produce lifelike depictions of religious figures (despite biblical injunctions against the worship of images). Greek 
statuary may have influenced each of these three religions. Paintings of Mary with baby Jesus may draw upon much earlier Egyptian paintings of the goddess Isis and her son Horus. Still, it is not entirely clear why three religions developed traditions of statues and paintings at about the same time. Islam would challenge this shared artistic desire to personalize the nature of religion. Islam would instead insist on only symbolic representation of Muhammad. However, there would be a brief period in Central Asia, when Muslims and Buddhists cohabited the area, when artists pursued pictorial representations of Muhammad.

Music of some sort is common in all religions. Islam has worried that music beyond the rhythmic chanting of holy texts might divert the believer's attention. Catholic popes have also occasionally worried that complex musical forms might be problematic. Yet in all religions, there is an appreciation that communal singing or chanting encourages both a sense of community and of joyful celebration.

\section{Relics}

Paintings or statues of religious figures were often thought to have captured some of the inner vitality of that figure. In addition, the bones, teeth, or hair of religious figures, or items they allegedly touched during their lives, were venerated in most religions. Believers sought to view or touch these "relics" in a somewhat similar way to how celebrity memorabilia is valued today. Such relics were often deposited in shrines that in turn became places of pilgrimage. Religious leaders often had a conflicted view of such relics, for their adoration suggested that physical objects possessed magical powers. Yet relics could serve useful purposes for organized religions: They caused believers to gather in one place and encouraged religious fervour. Religions often profited by charging for admission to shrines - or at least encouraging donations - or even selling relics.

\section{The geographical spread of religions}

The religions discussed above have each had successes and setbacks over the last millennia. Every religion has expanded at some times and places and contracted at others. There have been significant differences in the relative experience of expansion and contraction across religions. There are today about 2.2 billion Christians, 1.6 billion Muslims, 1.1 billion Hindus, 0.5 billion Buddhists, 12-173 million Taoists (depending on how you count), 100 million Shinto, 28 million Sikhs (see chapter 18), 14 million Jews, 4.2 million Jains, and 2.6 million Zoroastrians. Manichaeism has effectively disappeared from the world, and Confucianism is not usually considered a religion today.

The geographical distribution of religions in the world today reflects several historical processes. 


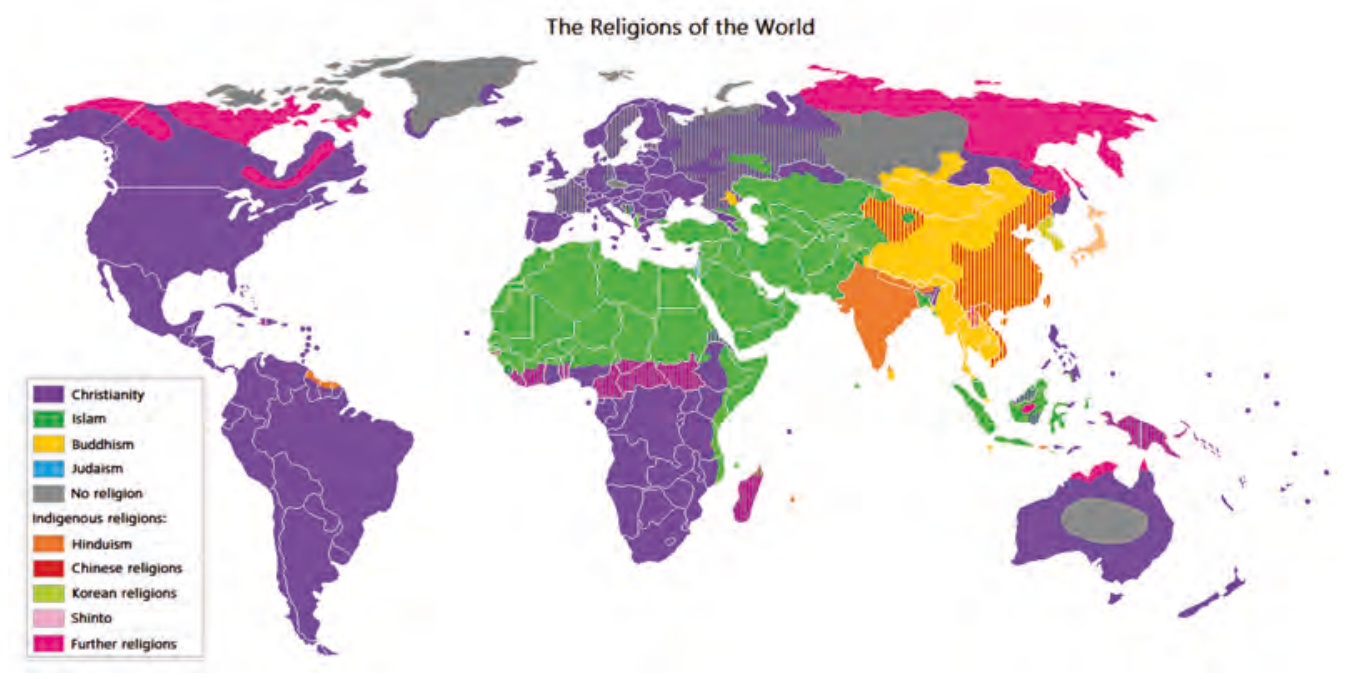

Map 11.1

Religions of the world in the 21 st century. The map shows the most common religion in each region

\section{Conversion of rulers}

Rulers can encourage or even force their subjects to accept a new religion. They can also provide financial and legal support to a particular religion. The conversion of Roman emperors to Christianity - and decisions to first stop persecuting it and later adopt it as the official Roman religion - undoubtedly greatly aided the spread of Christianity through Roman territories. The much later conversion of the kings of Poland, Denmark, and Norway likewise extended the reach of Christianity into regions never touched by Rome. The vote of the Icelandic Assembly in 1000 CE to adopt Christianity is an unusual example of a democratic conversion. In India, the support by the Mauryan dynasty of Buddhism encouraged that religion, whereas the later decision of the Guptas to favour Hinduism helped that religion to regain its dominance in India. The support of the Kushan king in northwest India of Buddhism, and especially his financing of missionaries, caused Buddhism to spread into Central Asia, from where it passed to China. Some, though not all, Chinese dynasties would embrace Buddhism, as would many Korean and Japanese rulers. Back in Central Asia, the conversion of Mongol khans to Islam would cement the role of that religion in the area (chapter 17). In Cambodia, the Khmer king converted to Buddhism in the twelfth century and built a large Buddhist temple complex next to the Hindu Angkor Wat. In India itself, most rulers would choose Hinduism over Buddhism, perhaps because the hierarchical outlook of Hinduism was more attractive to elites than Buddhist egalitarianism. 


\section{Policies of rulers}

Rulers often practised toleration. As we will see many times, rulers of large states face a dilemma: They can curry local favour by respecting local customs (and elites) but may also be tempted to create some collective cultural identity that can lend support to the state. Rulers desiring cultural unity were tempted to encourage one common religion. Yet in so doing they risked offending minorities. As a result, many rulers chose to be religiously tolerant. This decision may be primarily pragmatic, but rulers would often claim to be displaying respect for their people (the philosophical justification of religious toleration effectively comes millennia later; see chapter 27). It would become common among Buddhist rulers in especially Southeast Asia to argue that they ruled over people's bodies rather than their souls. One factor that may loom large in decisions regarding tolerance is how high a proportion of the subject population holds beliefs different from the rulers. The Ottomans were very tolerant when they ruled roughly equal numbers of Christians and Muslims, but as they conquered majority Muslim territories (and as some Christians rebelled), they came to stress more and more their Islamic character. Muslim rulers in both Spain and India also practised religious toleration. In both areas, there were many voluntary conversions to Islam over the years, encouraged no doubt by the access to positions in government bureaucracy associated with conversion. Yet the majority of the Spanish population remained Christian (though the size of the Muslim - and Jewish - community in Muslim-ruled Spain is obscured for us because Christian kings forcefully expelled these when they reconquered Spain in 1492). And the majority of the Indian population continued to embrace Hinduism.

The Ottomans and Mughals both managed to style themselves protectors of the Islamic faith while at the same time fostering other religions (chapter 20). They appointed the leaders of other religions and exempted them from taxes. Islamic religious authorities often urged them to be more forceful in their support of Islam. In this they were not unusual: Religious leaders in many lands urged tolerant rulers to be less tolerant.

\section{Conquest}

Islam spread rapidly from Arabia to Spain in the west and India in the east through military conquest. Most of this territory would become predominantly Muslim over the next centuries. When Charlemagne defeated the Saxons of northern Germany in 804, he demanded that they convert to Christianity. Centuries later, during the era of the Crusades (chapter 15), military religious orders would force conversions in northern Germany and the eastern Baltic region. Christianity would still later spread to the New World and to parts of Africa through conquest. As the first - and for a long time only - missionary religion to reach these places, it would soon become the dominant religion there. 


\section{Political conflict}

Though rulers play an important role in the history of religion, religions also often strengthen during times of political conflict. It may be that people cling to religious structures in the face of political trouble. Christianity and the Catholic Church became more powerful after the fall of Rome. Buddhism spread in China after the fall of the Han. Islam prospered in the Middle East as the Abbasids collapsed in civil war (chapter 12).

\section{Missionaries and merchants}

It would be a mistake to treat the history of religions as entirely reflecting the decisions of kings. Religion is, in the end, an intensely personal experience. While most people may follow the religion of their parents and community without much thought, they do not make decisions to change beliefs lightly. Christianity had while still outlawed spread through the Roman Empire through the activities of countless missionaries. The conversion of Arabian nomads preceded Islamic conquests; these nomads formed the first armies of conquest (chapter 12). Islam would then spread beyond the borders of Islamic polities into Central Asia and Southeast Asia and sub-Saharan Africa, and to merchant cities along the East African coast. Islamic merchants often played a crucial role in spreading the faith. Rulers often converted only after many/most of their people had. Still, the different choices of rulers between Buddhism and Islam exerted an important effect on the geographical distribution of those faiths in both Central Asia and Southeast Asia.

The Islamic conversion of the peoples of Central Asia occurred in the first instance through the actions of Sufi mystics. Islamic scholars debated for centuries whether and how Islam could embrace Sufism. Yet this more personalized vision of Islam proved very effective in drawing new people to Islam. It was easy for Sufis to respect local customs. And unlike orthodox Islam, which sought the creation of Islamic polities, the Sufis were quite content to encourage faith at an individual level while avoiding political activity (at first; Sufism itself would become more hierarchical and politically active for a time, especially in the Safavid Empire of chapter 20, though later Islamic states would prefer orthodox Islam to Sufism). The piety and abstemious behaviour of Sufi mystics proved attractive. Though Sufis may not have been experts on the details of Islamic teaching, they did profess the central tenets of the faith. Peoples that Sufi mystics converted were over time encouraged toward orthodox versions of Islam.

Missionaries sanctioned by Christian churches were also active in Central Asia but proved much less successful in their task than the Sufis. Christian missionaries had greater success in northern Europe at the same time, albeit without competition from other faiths. The Catholic Church also dispatched missionaries to China in the fourteenth century to little effect, perhaps because their numbers were small and other religions were already well established. 
Buddhism reached Sri Lanka in an embassy from Ashoka; Sri Lanka soon after that became a centre of Theravada Buddhism, even as Buddhism declined in northern India.

\section{Migration}

Religious minorities would often move from less tolerant states to states that were more tolerant. Zoroastrians, once the dominant faith in Persia, would face persecution in their homeland. Some would convert while others moved to India. In addition to these voluntary movements, states would often move peoples around for strategic reasons. For example, the Ottomans encouraged some Muslim populations to establish themselves on their European frontiers.

Religious minorities were often expelled. We have mentioned above that the Spanish deported Muslims and Jews from Spain in 1492. In the early twentieth century, millions of Christian Greeks were forced from Turkey and Muslims from Greece: there is now a sharp divide between Christian and Islamic territories where for the previous thousand years these two religions had cohabited the area.

Buddhists migrated from areas of Central Asia conquered by Islam into Tibet. As the fame of Tibet grew, Buddhists migrated there from India too, especially after Muslim conquests. The fusion of different Buddhist traditions would generate a unique Tibetan Buddhism that would survive for centuries (unlike Nestorian Christianity that would gradually fade from the scene in Central Asia). Tibetan Buddhism would cease to look to India for guidance but become a centre of world Buddhism.

\section{Financial resources}

Religions are both sets of beliefs and institutions. Religions rarely survive for long unless there is financial support for places of both worship and religious teaching, and for the production of religious texts and materials. States can be critical here in providing resources. Some states would support one religion while others would support several. States could also influence the amount of private giving: they might prohibit this (forcing religions underground), or they might encourage it by, for example, exempting all religious institutions from taxation.

While we understandably focus our attention on the beliefs associated with a religion, each religion (that survived for long) had to encourage at least some believers to donate. Religions such as Buddhism and Islam which were attractive to merchants may have raised funds more easily. Christianity, which at first appealed to the less prosperous members of society, instead pursued many small donations.

\section{Syncretism}

We should not leave the impression that world religions are or were pursued in a uniform fashion around the world. Missionaries might at times overlook 


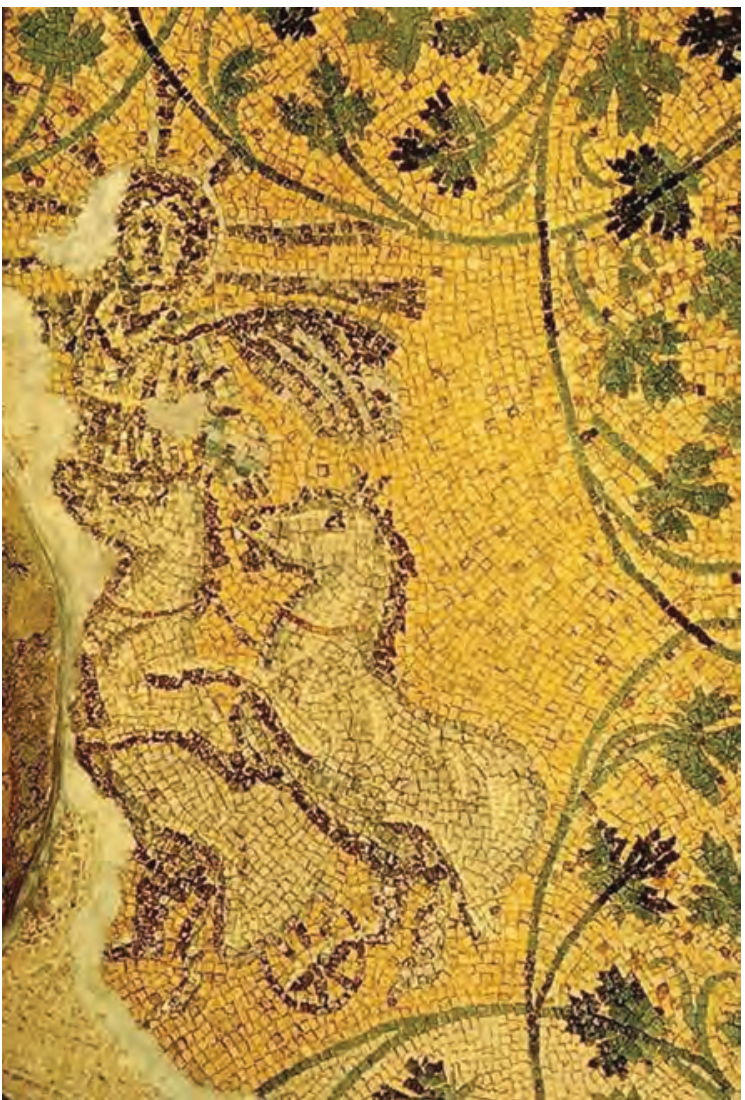

Illustration 11.19

Christ as a sun-god, a syncretic mosaic of the Vatican grottoes under St. Peter's Basilica, on the ceiling of the tomb of the Julii. Dated to the 3rd century CE practices - polygamy, nudity - that would be unacceptable back home. Missionaries often found it convenient to connect their religion to that of the locals they were trying to persuade. We have discussed this practice with respect to Sufis above. Catholic missionaries in Chiapas (in Mexico) in the sixteenth century CE related local gods to particular Catholic saints. Local people could then refashion many traditional celebrations of these gods to serve the new religion. Earlier, the conversion of the Aztecs was facilitated by the existence of a female Aztec god who was mother to all others; Mary was substituted. The resulting faith traditions are termed syncretic for they blend elements of two or more religions. Nor are missionaries necessary for syncretism: There is a minority tradition within Hinduism that claims the Christian Jesus as an avatar of the god Vishnu.

\section{Suitability}

The arguments above treat religious belief as a historical process. There is then a considerable degree of historical contingency involved. The actions of a particular ruler or missionary may cause one specific religion to prosper in a particular region of the world. This does not mean that there may not be reasons why specific religions were better suited to some places than others. Islam, a religion developed by merchants among nomads, may thus have been particularly attractive to merchants and nomads. Its spread along trade routes, and its success in Central Asia and parts of sub-Saharan Africa characterized by nomadism, may reflect in part its inherent nature (see above). Yet Islam also succeeded among some agricultural and urban populations, and so we should not push such an argument too far. Once a religion existed in a particular region for some time, it would naturally intertwine with the local culture and (often) political system. As it became solidified in this manner, it would become harder and harder for a new religion to gain hold.

\section{Other factors}

Religion was sometimes pursued as an act of defiance. Islam would gain strength in what is now Indonesia during centuries of Dutch rule. Judaism and 
Christianity may have been attractive to some precisely because the Roman Empire at first disdained these faiths.

Military defeat might be taken as a sign that the victor's gods were more powerful than one's own. Catholic missionaries would benefit from this attitude after Spanish conquests in the Americas (chapter 19). Some Persian armies adopted Islam in the face of the advance of Islamic troops in the seventh century.

The monuments built by all religions (chapter 8) served to awe believers and non-believers alike: Both their size and beauty could signal the power and glory of a particular faith. Miraculous stories (of healing or providing food or ending drought) played a critical role in the spread of many religions, including Christianity, Buddhism, and Islam.

\section{Summing up}

These few arguments allow us to understand much of the present world distribution of religions. Zoroastrianism and Manichaeism both had periods of success and occasions where they received state support, but both suffered after Islamic conquests in their areas of strength, and lacked state support after that. Zoroastrians would survive as a minority tradition in religiously tolerant India. Missionaries and merchants spread Buddhism to Central Asia, East Asia, Sri Lanka, and Southeast Asia; it would receive occasional support from states in all these places. Islamic conquest displaced it in parts of Central Asia. In India itself, it would slowly lose ground to Hinduism and Islam, in part as these other religions gained state support. Islam would first expand by conquest, but then merchants would carry the faith to Southeast Asia and subSaharan Africa, where local rulers later adopted it. Expansion into Central Asia combined missionary activity, ruler conversion, and conquest. The approval of the Roman Empire would boost Christianity, and both Roman Catholicism and Orthodoxy would later expand northward through missionary activity and ruler conversion. As noted above, the Christian identity of Spain reflects an unusual historical process of centuries of toleration followed by the expulsion of Muslims and Jews. The survival of Hinduism as the majority faith in much of India despite centuries of Islamic rule over most of the subcontinent is likewise remarkable. Christianity would thrive for a while in the Middle East and Central Asia but Islamic missionaries and conquerors would displace it. Conquest and missionary activity would then spread Christianity to the vast parts of the world that had not yet come in (extensive) contact with any of the new religions: the Americas, much of Africa, much of Oceania, and the northern Philippines. The survival of Judaism in many lands during thousands of years without a Jewish state, when so many other religions disappeared in such situations, is also remarkable. It would seem that ethnic identification with a monotheistic god and a set of holy texts (which provided some explanation of unfortunate political circumstances) allowed a minority religion to survive even in hostile conditions. In recent decades, religious toleration has allowed Christianity to spread in parts of East Asia at the same time that Muslims, 
Hindus, and Sikhs have migrated in large numbers into Christian lands. Muslim countries have been typically less welcoming of religious minorities except as temporary workers.

We should stress that all religions have suffered geographic setbacks. This is most obvious for Manichaeism and Zoroastrianism. Christianity and Buddhism would largely disappear from their lands of origin. Islam would be pushed out of Spain, Sicily, and parts of southeastern Europe. Buddhism or Islam would

\section{BOX 11.1}

\section{RELIGIOUS CHOICE}

When people grow up in an environment in which one faith tradition is dominant, they may adopt this faith without much reflection - or at least appear to the outside world to embrace it. To do otherwise is to risk social isolation or worse. If everyone that you know believes a certain thing, it will seem natural to believe it also.

In many times and places in history, people have grown up in religiously diverse communities. Many polities were too weak to enforce a single religion. Others made a strategic (or perhaps respectful) decision to pursue a policy of tolerance to encourage social stability. How, then, does a person decide which religion to choose? We can imagine both practical and spiritual influences on their decision. We should emphasize that the decision will be - for most people - mostly subconscious: They will decide at some point that they believe " $\mathrm{X}$ " and not be fully aware of their reasons for doing so. (Some manage to pursue two religions. This is especially common when a polytheistic faith first encounters a monotheistic religion. Rituals from the older religion may be continued alongside the doctrine of the new. Yet it is challenging to embrace two religions with conflicting doctrine - and such a practice may limit the practical benefits of belief.)

On the practical side, the beliefs of one's friends and families must loom large, for one can better maintain these important social ties if one pursues the same faith. Nevertheless, conversion has been common in human history, with individuals shedding the religion of their parents and peers. They often, but far from always, convert in the direction of a faith that is given preferential treatment by the state or provides entry to some profession or group of merchants. They may switch to a religion that has become common in their society; in such a case, conversion increases the possible social and economic ties they may pursue.

While some may be coldly calculating in their choice of religion, it is likely that most actually do believe what they profess to believe. Indeed, for most humans, it is difficult to pretend to believe something that one does not. While practical considerations may influence the subconscious, the attraction of religions extends far beyond these. A religion that holds out hope for a better life, and for some sort of cosmic justice (in place of the evident injustice of the world we inhabit), or provides merely some compelling explanation of life's vicissitudes, can prove very attractive. Still, when an individual faces a choice between two religions that each provide important spiritual benefits, it may be that practical considerations loom large in decision-making. 
supersede Hinduism in parts of Southeast Asia; Hinduism would become a minority faith in parts of the Indian subcontinent and largely disappear from Pakistan and Bangladesh after the Indian partition in 1945 (chapter 30).

\section{Evolutionary reflections}

As noted in chapter 8, it was common for European analysts a century ago to view modern Christianity as the endpoint toward which earthly religions had been tending all along. This practice has understandably generated some suspicion toward the evolutionary analysis of religion. However, we saw in chapter 1 that we need not and should not equate evolutionary analysis with any idea of progress. We should feel free to discuss how one mutation built on another, without assuming that the result was somehow better in some ethical or cosmic sense.

Faiths such as Judaism and Zoroastrianism set the stage for later universal monotheist faiths. Judaism pursued the idea of an ethnic god, and Zoroastrianism the concept of multiple gods serving under a dominant god. Both religions have survived to the present day. Both also grappled with the question of good and evil. Later religions would adapt Zoroastrianism's idea of an evil spirit battling the good god.

As noted above, one practical advantage of monotheism (or deism) was that it was then easier to justify a universal ethical code. One notable characteristic of the world's religions is a broad similarity in ethical systems: Every religion celebrates honesty, responsibility, humility, and kindness. This similarity may reflect in part the fact that these religions borrowed ideas from each other, but also the simple fact that these ethical values are ones that have the most obvious beneficial consequences within human societies. Faiths differed, though, in the relative importance accorded to duty. The Bhagavad Gita is unique in stressing this as a paramount virtue, but all religious and political leaders had an incentive to urge obedience.

While it is always dangerous to attribute any direction to evolutionary processes, we might suspect that there might be evolutionary selection through time for religious beliefs that are less demanding but yet manage to fulfil the primary roles of religion. The human and animal sacrifices associated with the earliest religions have largely disappeared over time (though we suggested above that these were better suited to polytheism). The new religions have tended to place greater emphasis on the profession of faith than the performance of elaborate rituals. We noted above that Mahayana Buddhism was easier to pursue than Theravada Buddhism, and that the Bhagavad Gita urged a more accessible path to release from suffering than earlier Hindu texts. Yet the new religions still urged regular prayers, charity, and ethical practices that are far from costless to individuals - though they may well provide societal benefits. There were apparently severe limits to selection for ease of religious observance. This may reflect the fact - observed in chapter 8 - that in the absence of some 
costs associated with religious practice, it will be too easy for non-believers to deceive believers by pretending to believe.

The vast majority of the world's population in 2015 adhered to religions first developed between 600 все and 650 сЕ. Though some new religions have emerged since then, they remain small relative to these previous faiths. Why? The selection environment in many parts of the world has favoured the maintenance of existing religions: Islamic, Christian, Buddhist, and Hindu rulers and religious authorities made it extremely difficult for other religions to gain a foothold in regions they controlled. However, there were also, we have seen, regions in which states practised religious toleration. There it must seem that existing religions served the roles of religion well enough that there was no felt need for an entirely new religion. Instead, there has been evolution of existing religions.

There have been doctrinal disputes within all religions. We have detailed the most important of these above: Catholic, Orthodox, and Nestorian Christianity (We will address Protestantism in chapter 18); Sunni, Shia, and Sufi Islam; Theravada and Mahayana Buddhism; preference for different gods within Hinduism. There are many more denominations within each of these religious traditions. Holy texts cannot settle all questions decisively, and so there is scope for disagreement within every religion. Once different denominations exist, they will evolve along different paths accumulating further sets of disagreement in belief and practice. The original dispute between Shia and Sunni focused on who should lead the community, but a host of differences in doctrine and ritual now distinguish the two. As with religions themselves, we can query whether denominational differences represent historical contingency different choices had broadly similar chances of selection - or whether certain denominations better served different communities. Note in the latter regard that some groups may have purposely favoured a particular denomination to distinguish themselves from co-religionists: Persians may have been attracted to Shi'ism in part because most Arabs were Sunni. The existence of doctrinal disputes challenges the belief that scriptures dictate a unique set of beliefs and practices: Intra-religious disputes are therefore often even more emotive than inter-religious disputes.

\section{Questions}

1. Readers can be asked to engage with a set of big questions regarding religion: Why do religions exist? Why do particular religions exist in particular places? What are the main similarities and differences across religions? Why have there been so few new religions in the last millennia? Why are there denominations within all religions? Why did all religions come to accept inequality?

2. Revisit Figure 8.1 and explore whether new elements are necessary to capture this later generation of religions. 
3. What are the challenges faced by missionaries?

4. Discuss how the global distribution of religions might easily have turned out quite differently.

5. Why are religions often but not always intolerant of other religions?

\section{Readings}

Elverskog, Johan. 2010. Buddhism and Islam on the Silk Road. Philadelphia, PA: University of Pennsylvania Press.

Hooper, Steven. 2014. "A Cross-Cultural Theory of Relics: On Understanding Religion, Bodies, Artefacts, Images and Art." World Art 4:2, 175-207.

Laine, James W. 2014. Meta-religion: Religion and Power in World History. University of California Press. [This book stresses how rulers have used religions to legitimate their rule. It also argues that religions are best seen as a source of group identity rather than individual faith.]

Super, John C., and Briane K. Turley. 2006. Religion in World History: The Persistence of Imperial Communion. New York: Routledge. 


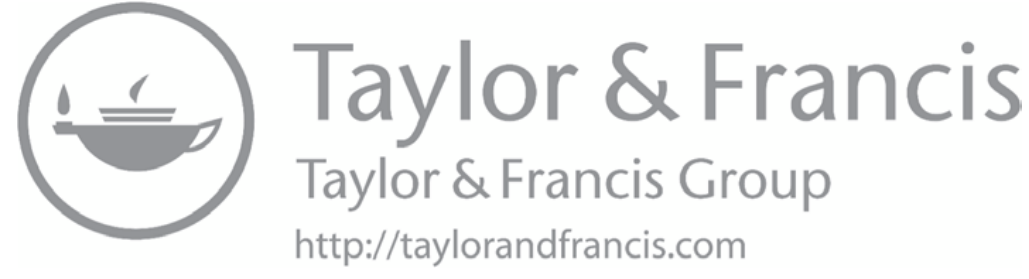




\section{A new force in world}

history

\section{The Islamic conquests}

\section{Guiding questions}

Why and how were Islamic armies able to conquer vast territories on three continents (where?) in less than a century? How were Islamic polities and armies organized and motivated? How did relationships between conqueror and conquered evolve?

What were the main effects of these conquests?

Why did these conquests come to an end?

Relationship to other chapters: This chapter draws heavily on our understandings of religion (chapter l1), empire (chapters 9 and 10), and war (chapter 6). It clarifies our understanding of one fundamental question from chapter 11: how religions become dominant in particular regions. It also provides a valuable case study of both religious toleration and religious conflict. Moreover, it powerfully illustrates how religion and political power can reinforce each other. We will have cause in many later chapters to discuss synergies between state, religion, and war. As in chapters 9 and 10, this episode of imperial expansion serves to encourage long-distance trade, and thus cross-societal contact. In addition to adding to our general understanding of critical historical processes, this chapter is crucial to later discussions of the various regions that we treat in this chapter. 
A certain unity was provided to the preceding "classical" period by the emergence and spread of empires in many parts of the world - and the increased trade links across societies that these encouraged. The next period in history experiences much greater political diversity around the world. This diversity is reflected, we might note, in the difficulty world historians face in attaching a catchy title to this period that does not seem to prioritize the experience of one region: They may have been "Dark Ages" in Europe, but this is hardly how Islamic historians see the period. The Islamic conquests that are the focus of this chapter instantiate new empires in large parts of Europe, North Africa, and Asia. The eastern Roman (Byzantine) Empire survives and occasionally prospers in parts of Europe and Asia and sometimes in Africa (chapter 13). However, in East Asia, Southeast Asia, India, and elsewhere political fragmentation characterizes these same centuries (chapter 13). Non-Byzantine Europe experiences an even greater degree of political fragmentation within the feudal system (chapter 13). There are of course some commonalities in climate and trade and technology during this period; we will address these as we discuss the histories of diverse regions in this and the next chapter.

The most important single development during the early post-classical period is the rise and spread of Islam. We have discussed Islam as a religion in chapter 11. Our concern here will be with the political manifestations of Islam, for rulers proclaiming the Islamic faith would come to power from Spain and Morocco in the west through to Indonesia in the east and from Central Asia in the north into sub-Saharan Africa in the south. This was the most geographically extensive religious expansion in history to that time (for Christianity and Manichaeism had not previously spread very far into Africa nor Buddhism into Europe or Africa) - though the much later spread of Christianity to the Americas and Africa and parts of Asia would surpass it.

Our thematic focus in this chapter is politics, though this inevitably interacts with culture. Particular attention is devoted to interactions with science, social structure, economy, and art. We are very interested throughout in how both political control and ideas spread geographically. There is less evolutionary analysis than in most chapters though we discuss how both political institutions and scientific ideas developed over time.
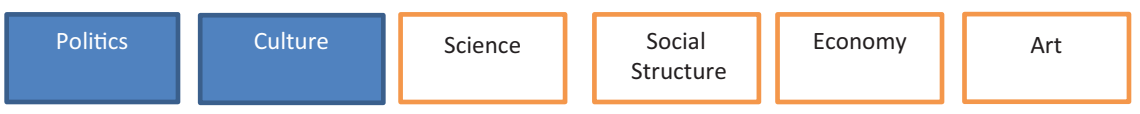

\section{Early days}

The Arabs of Arabia were nomads and merchants with a strong sense of clan loyalty that came from surviving in a harsh desert landscape. Arabia lay along important trade routes, with camel caravans carrying goods between the Mediterranean Sea and either the Red Sea or the Persian Gulf. The Red Sea, it 
might be noted, has few good ports, and so before the Suez Canal in the nineteenth century $\mathrm{CE}$, goods had to be carried a long distance by land to connect sea routes in Europe and Asia. This Arabian route increased in importance as the Silk Road across Central Asia became more difficult with the decline of the classical empires.

Many of the characteristics of a cosmopolitan trading region appalled Muhammad (likely 570-632). He thought that the merchants lived an immoral and luxurious life while others suffered in poverty. Moreover, he disliked the constant feuding between nomadic clans (kin-based societies). This feuding was encouraged by religious diversity: Judaism, Christianity, and polytheistic beliefs were all common in the peninsula. The Arabian peninsula had until recently been controlled by a Judaic kingdom based in what is now Yemen, and was being eyed by Christian Byzantium and Axum and Zoroastrian Persia; Ethiopian Christians had indeed toppled the Jewish ruler in what is now Yemen in the 520s in support of Christian residents of the area. Muhammad wanted to forge political and religious unity in Arabia. His emphasis on a shared Islamic community was perhaps reflective of the tribal disharmony he sought to overcome. After angry merchants forced his new congregation to leave Mecca, his followers took up arms to regain Mecca and then the entire peninsula (chapter 11).

The man who would succeed Muhammad, Muhammad's daughter, and two of his future wives fled to Axum during the early period of exile. Muhammad praised Axum for its righteousness. He later urged Muslims to leave Axum in peace, which they did for much of the next thousand years. Still the rise of Islam hurt Axum trade, and likely contributed - along with drought and other factors - to the decline of Axum over the next centuries.

Mecca was a place of pilgrimage already in Muhammad's time. Many of the merchants of Muhammad's tribe profited from the sale of religious icons to pilgrims. Muhammad's new faith was then a direct threat to an important line of business. Centuries earlier, angry merchants had likewise expelled the Christian missionary Paul from Ephesus on the coast of present-day Turkey in large part because his faith threatened the businesses associated with the local Greek deity.

We have and will have many occasions in this book to discuss nomadic conquests. Once-nomadic Germanic tribes had brought down the Roman Empire, and the nomadic Xiongnu had harassed Han China, while White Huns vanquished the Gupta Empire (Chapters 9 and 10). Many nomadic invasions were to come, including the dramatic conquests of the Mongols in the thirteenth century (chapter 17). The Islamic conquests were different in significant ways. Most obviously, the new and shared religion provided a uniting motive for conquest. Secondly, these nomads came from the Arabian deserts rather than the Eurasian steppes (and therefore rode camels rather than horses more than northern nomads). Thirdly, these nomads had begun to settle increasingly in oasis towns, though many still herded animals through the deserts, and the merchants in towns depended on trade caravans. 


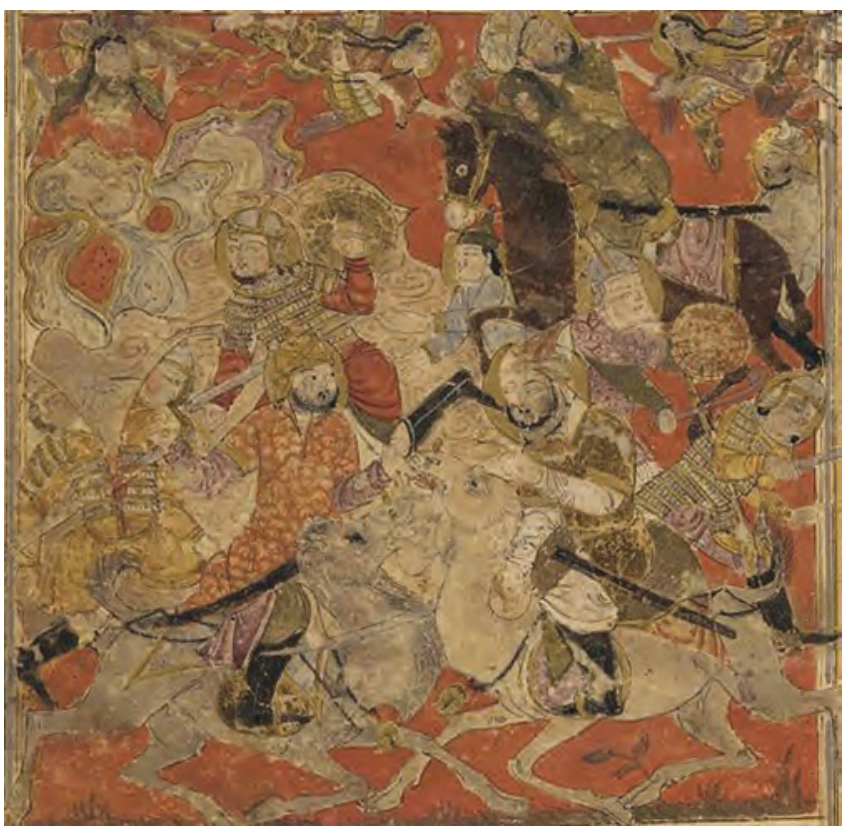

Illustration 12.1

Folio from a Tarikhnama (History) by Balima, early 14th century, depicting an angel sent to aid Muslim forces in an early battle (Battle of Badr)
Still, there are similarities with other nomadic conquests. Muhammad and his followers were able to weld together various clans that until recently had fought against each other. Clan loyalty was harnessed to the service of a much larger group guided by a charismatic leader. Nomadic men were accustomed to many days of travel by horse and camel, to combat, and to hunting. Moreover, these forces were able and willing to shrink back into the desert or steppe if they encountered an opposing force that they could not defeat.

In the next chapter, we will describe how a series of groups from the fringes of Europe or beyond would attack Europe through the first millennium CE. We can note a similarity here with the situation of Arabia. It operated on the fringes of three empires. It benefited enormously from their trade but could not in any way match the riches of Constantinople or Ctesiphon. There is always a temptation in such a situation for people on the edges of a wealthy civilization to coalesce into a military force that can procure some of those riches. The fact that the Byzantine Empire was resolutely Christian and the Persian Empire Zoroastrian may have encouraged Muhammad to urge a central political role for his new faith.

Islam is hardly unique among world religions in being associated with military conquest. Christianity would later spread militarily in eastern Europe, the Americas, and southern Africa. However, Islam is unique in having been associated with military conquest from its very earliest days. Christianity and Buddhism depended on missionary activity for centuries before gaining the support of polities that might fight for them. The difference here may have much to do with the simple fact that Islam's first converts were nomadic or semi-nomadic peoples. These were a natural military force, unlike the peasants and merchants first reached by Christianity and Buddhism. The fact that Islam was uniquely associated with conquest and governance from the outset meant that Islamic holy texts deal with such matters much more than the writings of other religions. As a result, there are many references in the Quran to "holy war" and "Islamic governance": Islamic scholars debate to this day the precise meaning of this terminology and whether these terms are of spiritual significance. 
Nomads often had their greatest successes when facing weakened empires. Though the Byzantine Empire would survive for several centuries and indeed prevent Islamic forces from entering southeastern Europe for centuries - it was far weaker than the Roman Empire of old. The Roman Empire at its prime had a vast and diverse territory which had potentially defensible borders (bordered by sea to the west and desert to the south, it had only to worry about the Persians to the east and defend the Rhine and Danube against Germanic tribes to the north). The Byzantines instead had a smaller territory with a much longer border, threatened on all sides. It would survive not by brute force but by playing one enemy against another (see chapter 13). It was ill prepared for a new and powerful enemy to emerge suddenly from a direction it had not previously much worried about defensively. The Persian Empire was also weaker at the time than it had been. Indeed, the Byzantines and Persians had just fought a war that had seen Persia take both Antioch and Egypt, but then the Byzantines had counter-attacked and invaded Mesopotamia in 622. Neither could know that they were weakening each other just as a new military force was emerging to the southwest. (Ironically, religious tension between Christians and Zoroastrians had fuelled this war; a Christian bishop had even destroyed a Zoroastrian temple in the Persian capital Ctesiphon.)

We will often find in this book that those who attack any empire find support from some of the empire's citizens who grate at imperial rule. The Islamic conquerors would gain much support within both the Byzantine and Persian empires. In particular, religious minorities objected to efforts to institutionalize one religion and proved open to Muslim promises of religious toleration. Byzantine efforts to impose Orthodox Christianity had offended both Jews and Coptic Christians, who then welcomed Arab invaders.

The result was a series of victories on the battlefield that saw Islamic forces within about a century reach Spain and Morocco in the west and the Indus River in the east. The Byzantines lost their holdings in Egypt and the Middle East but were able to defend Constantinople itself. The Persian Empire was utterly overwhelmed. Though weak, these empires could often bring larger and better-trained forces into battle but still lose. West of Egypt, conflict among the Berber tribes of North Africa aided Islamic forces. Feudal Spain (see chapter 15) provided limited opposition. This geographic expansion was much more rapid than that of the Roman Empire - though the Mongols would match it several centuries later.

The earliest battles against the Persians may have been of critical importance. If Islamic forces had lost then, they might not have been able to attract more recruits. The Persians seem to have been over-confident - as might be expected when battling a foe that had not previously provided a potent military threat. The Persians made tactical errors such as attacking across a river. The Arabs then proved to be a much larger and cohesive force than they had imagined. Faced with surprising defeats, the Persians themselves wondered if god was against them. 


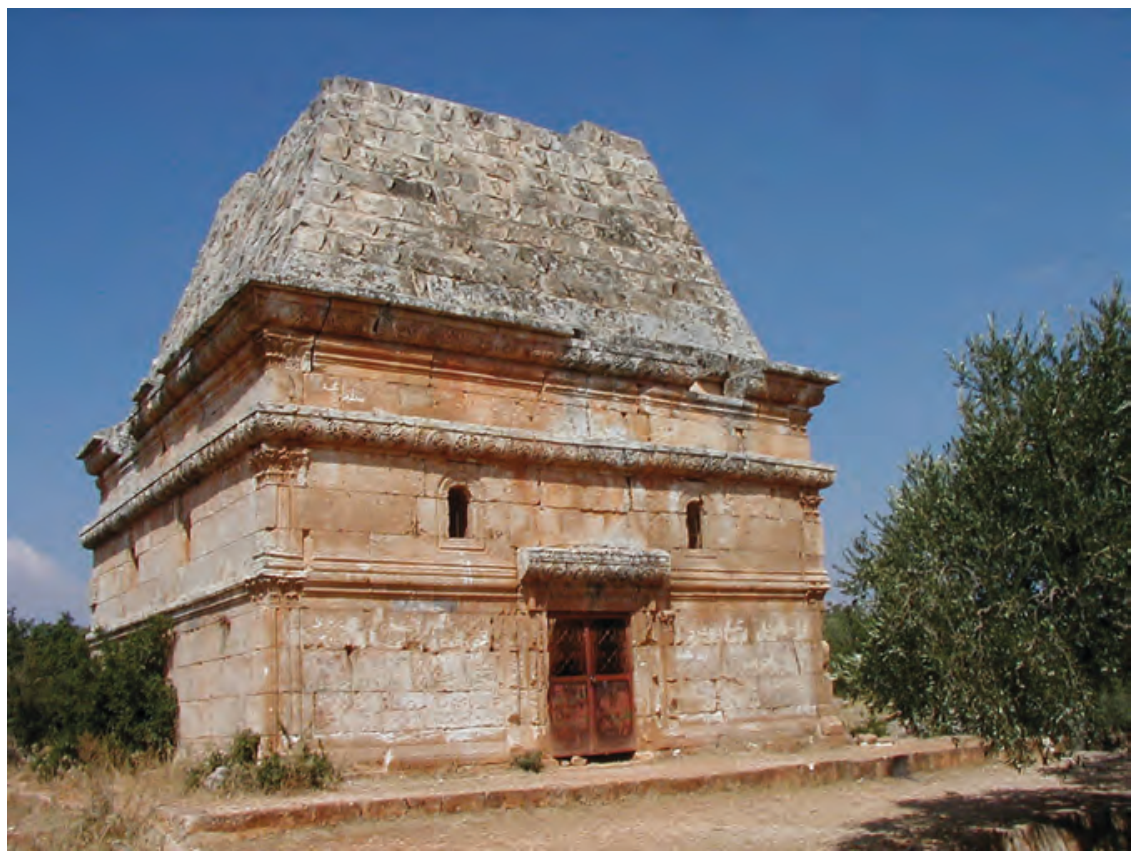

\section{Illustration 12.2}

Byzantine tomb, Al-Bara, Syria. Al-Bara survived for centuries as a Christian town after the Islamic conquest but would be used as a base during the Crusades and later subdued. Its final abandonment may have resulted from a 13th-century earthquake

Photograph by Syrian Inheritage, CC BY-SA 4.0

When something happens that is almost unique in history, it makes sense to look for multiple and perhaps unique causes. We have discussed a couple of these above: the nomadic nature of the conquerors and the weakness of their opponents. To this, we must add the religious element. Islamic soldiers believed that they were doing god's work and so god would reward them in the next life. This encouraged them to keep fighting when less devoted soldiers might have fled. Yet while motivated in part by rewards in the next life, soldiers also benefited in this life from the plunder associated with a string of victories. Victorious armies create their own momentum, as soldiers join a force that is likely to win. Rewards in both this and the next life were a profound combination. The leaders of Islamic armies successfully maintained soldier dedication for a century. Continued external conquest diverted the energies of warriors away from internal battles over succession.

Then it stopped. A century of conquest was followed by many centuries devoted to enforcing internal order. Islam would continue to spread over the next centuries - into Central Asia, India, Southeast Asia, and sub-Saharan Africa, and eventually even somewhat into southeastern Europe - but far more gradually and without the same exercise of massive military force. There were later military invasions of India and parts of Central Asia, but by individual Islamic 


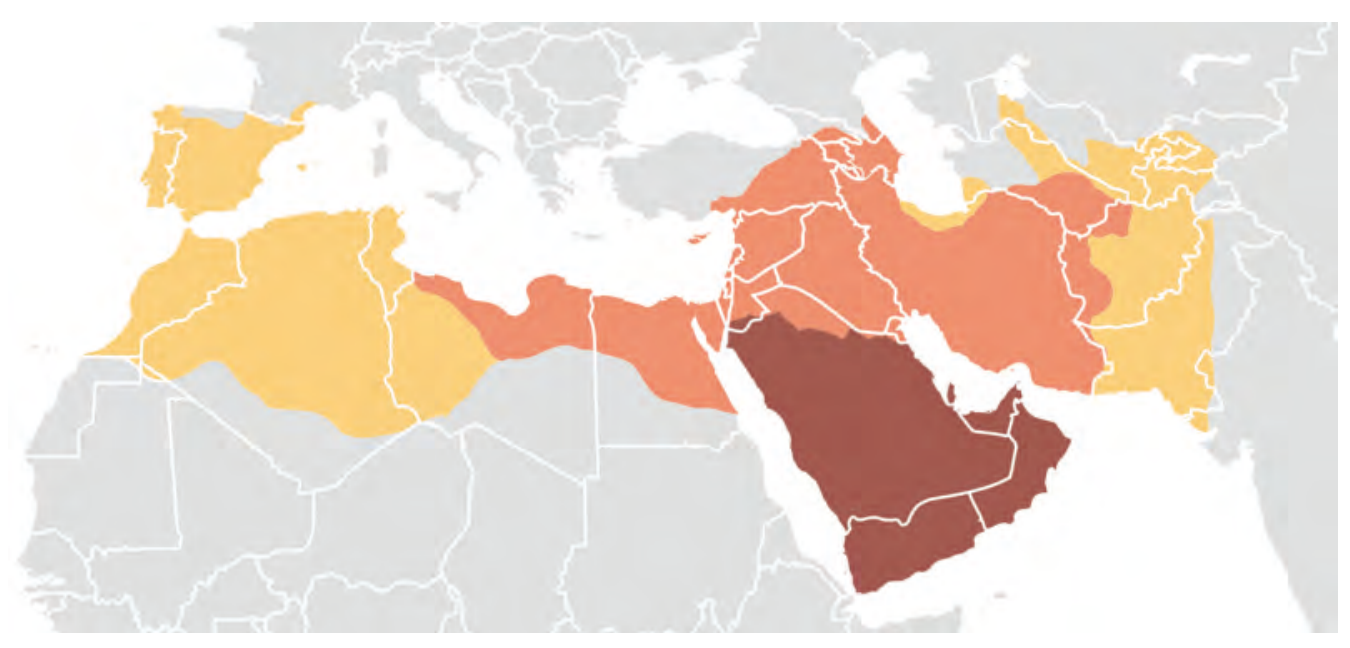

Expansion under M uhammad, 622-632

Expansion during the Patriarchal Caliphate, 632-661

Expansion during the U mayyad Caliphate, 661-750

\section{Map 12.1}

Early Islamic conquests

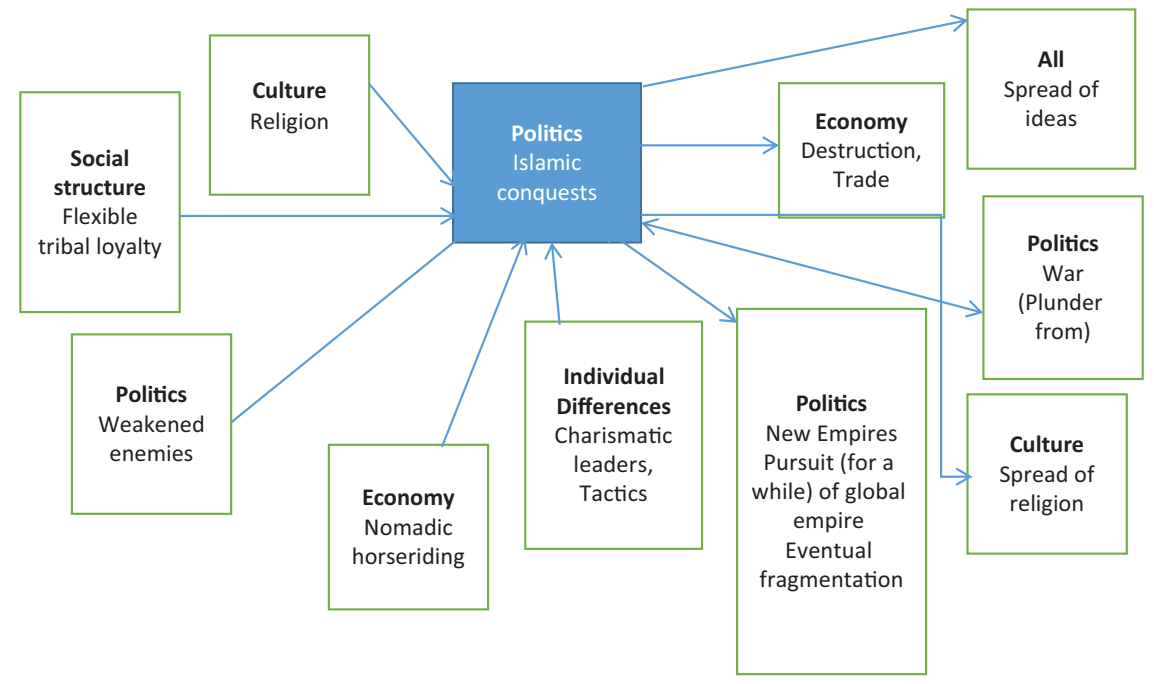

Figure 12.1

Influences on and effects of Islamic conquests

Note:We discuss the various results below.

leaders rather than by a united Islamic force. Islamic merchants and missionaries rather than armies would reach much of Central Asia, and all of subSaharan Africa and Southeast Asia, though local states would then encourage Islamic belief. 


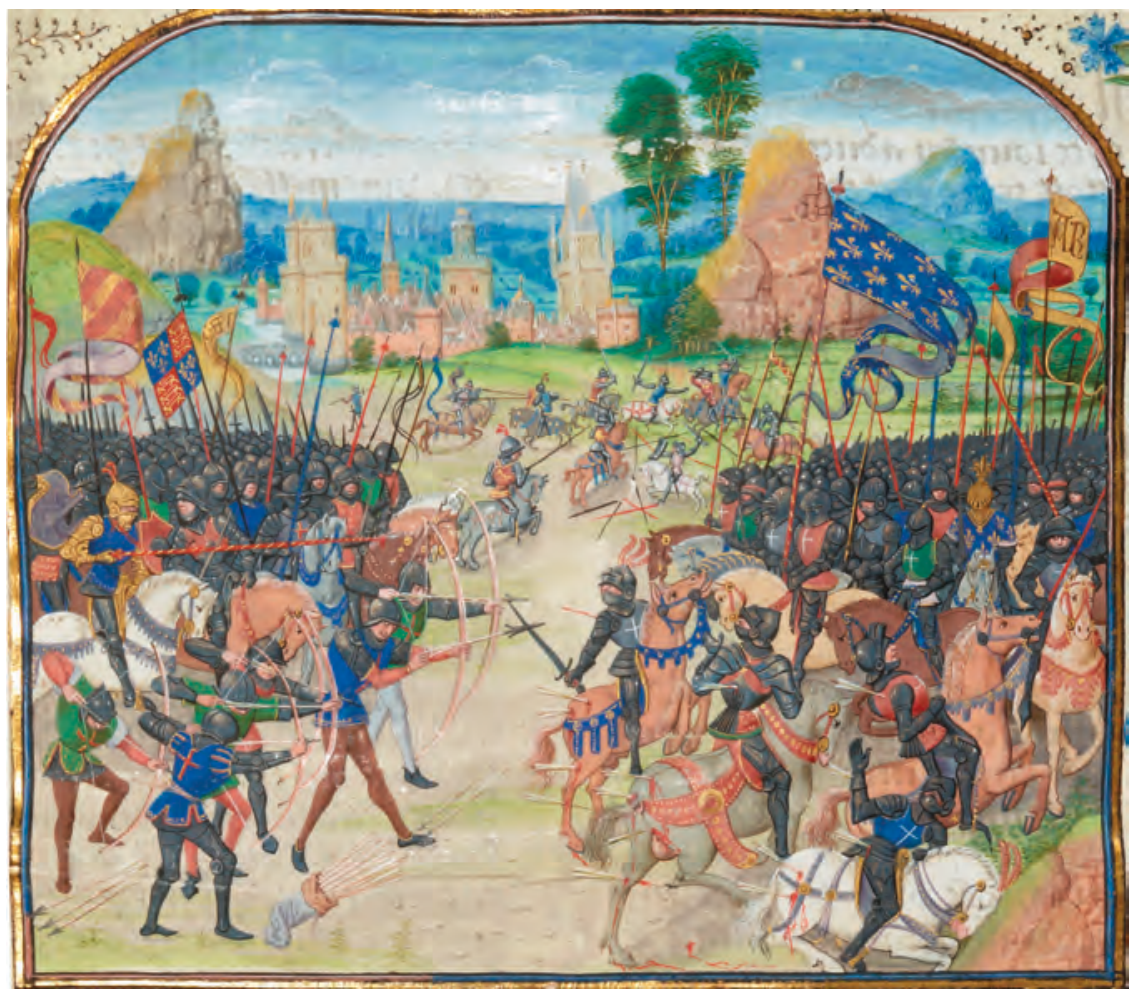

Illustration 12.3

Charles de Steuben (1788-1856), Bataille de Poitiers, en octobre 732. Over a thousand years later, the battle was still celebrated in Europe. The French king Charles Martel is in the centre

Scholars have often heralded the Battle of Poitiers in 732, in which French armies defeated Islamic forces from Spain, as a pivotal battle that prevented Islamic advance into the heart of Christian Europe. It could well be, though, that Islamic forces were over-extended and would have withdrawn even if victorious. Moreover, Poitiers comes toward the end of the century-long period of Islamic military expansion. It is quite likely that Islamic military expansion would have stopped in Europe just as it did in the east, even if Islamic forces had been victorious at Poitiers.

Why did such a successful series of conquests - motivated by promised rewards in this world and the next - not just continue until Islam had conquered the known world? It is certainly not the case that Islam faced tougher foes. While Constantinople was a challenge, there were weak states in Central Asia and India that Islamic armies could have invaded more easily than the Byzantine or Persian Empires. The answer, of course, is complicated, and involves a host of decisions made by individual Islamic leaders over a period of centuries (especially the Abbasid caliphs; see below). Yet part of it, at least, is just that empires always stop expanding at some point. Faith did not protect the Islamic Empire 
from the internal conflicts and bureaucratic challenges that afflict all empires. The caliphs, the early leaders to follow Muhammad, could not hold together such a vast territory for long and the Islamic Empire would splinter like other empires into smaller states that would often fight among themselves as much as they fought against non-Islamic states.

\section{Islamic political realities}

Muhammad's successors decided that the Arabs should stay in cities to serve the state in conquered lands; locals could keep their religions and land but then pay higher taxes. These higher taxes at first reflected the fact that only Muslims served in Islamic armies; later Islamic polities would sometimes waive the special

\section{BOX 12.1 DECISIVE BATTLES}

Not every battle is of major historical importance. If the result of a battle is that one ruler with a broadly similar world-view replaces another, then little may change. If one side in a war is much stronger than another, then victories in particular battles may have little impact on the outcome of the war. To change history, then, a battle needs to both have a decisive effect on who wins a broader war, and the victor needs to introduce changes in the conquered lands. Poitiers arguably fails on the first of these criteria even if Islamic rule would have differed in significant ways from Christian rule. Likewise, its enormous advantage in armaments may have guaranteed American victory in the Pacific in World War II; victories in particular battles may then have affected only (but importantly still) the time it took to achieve victory.

Some battles do meet both criteria. Here we might include some of the early Islamic victories against the Byzantines and Persians. These introduced an entirely different polity and religion to a large part of Asia. Moreover, defeats in these initial battles might have limited the ability of commanders to attract troops to their cause. The first victories of Alexander the Great in Persia (chapter 10) were surprising and allowed Greek cultural ideas to spread to India and Central Asia. The even earlier victory of a much smaller Greek force against the Persians at Salamis in $480 \mathrm{BCE}$ was also in some sense unlikely and served to ensure Greek independence for centuries. The victory of Germans over three Roman legions in 9 CE certainly surprised the Romans and discouraged Rome from expanding northeastward after that; the Germanic victory over Rome in 480 CE near Adrianople was very close but triggered the continued advance of Germanic tribes into the western Roman Empire. The much later victory of William the Conqueror over the English king Harold in 1066 was a close victory: The English had seemed to be winning early in the battle, but by the end most of the English ruling class was dead. This allowed William to institute a more centralized state in England and introduced continental culture to the island. These changes may have been critical to later developments of parliamentary governance and private property. 
tax and often employ non-Muslim troops. Conversion was discouraged at first, to maintain a strict distinction between conquerors and conquered. Over time, though, Arab soldiers received lands on which they employed captured peoples as slaves. The attackers soon became a landed elite. Muhammad had recognized Christians and Jews as followers of legitimate though superseded prophets; they had a higher status than polytheists. Nevertheless, many locals found it both useful and attractive to convert - especially as rulers began to replace local elites with Muslims in positions of power. In Egypt many Coptic Christians converted and then avoided the special tax.

Islam faced a unique challenge or opportunity in that Muhammad had urged the state to exercise religious authority. However, the skills and inclinations of the military general are not always the skills and inclinations of the priest. States that could inspire religious devotion - as states had tried to do as long as we have records - could inspire loyalty, but leaders that seemed to lack religious devotion could similarly attract hostility. The ulama, or leading religious figures, established themselves early as an independent source of theological interpretation, and thus of policy advice and criticism. Islamic states from the outset would seek legitimation from religious authorities but often receive severe rebukes for behaviours that religious authorities disdained. Yet the ulama had an interest in the political stability that supported their own positions, and this tempered their criticism.

Early Islamic states merged the bureaucratic traditions of the Byzantine and Persian Empires with their own ideas. They would over time often replace locals with slaves in their bureaucracy: Slaves were more often loyal than free people, but would occasionally rebel. Islamic states generally maintained strategies by which different arms of the bureaucracy spied on each other, and were not shy about torturing or killing bureaucrats thought to have been guilty of excessive bribery or treason.

Armies must be led, and there was almost immediately a conflict between the egalitarian ethos of Islam and the riches amassed by military leaders. This was not a challenge unique to Islam: All religions that preached equality before god had to reach some accommodation with earthly inequalities. Yet Islam stressed egalitarianism more than most, while a century of conquest inevitably enriched political leaders. Religious leaders might urge egalitarianism, but the elite that had fought in the name of that religion proved unwilling to sacrifice riches or power.

We have seen before that all empires expand to a certain point but no further. We have suggested that there are limits to the possibilities of imperial control set by the transport and military technology of the day. The goal of early Islamic conquest was to establish one global caliphate. However, it was not feasible at the time for one caliph to rule the world. When the Abbasids overthrew the Umayyad caliphate (see below), they abandoned the policy of continual conquest that had motivated the former. They focused instead on consolidating power within their domain. They acted as dozens of other empires had in history, recognizing that they had reached the geographic limits of imperial 
rule. They saw little advantage in further conquests if they could not rule a more extensive territory. They would engage in some battles, but for the most part would devote little effort to expanding their realm. They thus explicitly abandoned the goal of global Islam and appreciated that Islam would for some time cohabit with other religions in the world. This Abbasid decision would not mark the end of Islamic conquest, but the idea of a united Islamic community working together to spread the faith across the globe was abandoned. The further spread of Islam would be a far more decentralized effort.

\section{Battles over succession}

Muhammad had not designated who should succeed him as leader of the faith. He had no sons who might have been able to assert such a claim. There was therefore a battle over succession at Muhammad's death in 632 between his sons-in-law and other followers. A close friend was selected as the first caliph (the word means "successor"), a role that combined duties as general, judge, ruler, and priest. Muhammad's son-in-law Ali would serve briefly as the fourth caliph, but could not establish a dynasty. Disagreements over succession resulted in decades of conflict thereafter, with two caliphs assassinated along the way. Muawiyah would eventually win and establish the Umayyad caliphate from 661. Muawiyah convinced Muslim leaders that his descendants should rule, in place of the divisive tradition of electing caliphs among the group of leaders. This decision was widely accepted, but a group that would come to be known as the Shi'ites objected that only Muhammad's descendants should rule. This disagreement over succession would cause over time a significant division within Islam, as the Shias developed a religious hierarchy and distinct set of rituals (see chapter 11). It may then be the most important succession battle in world history. Both Ali and his son Husayn were killed while contesting the caliphate: Shias consider Husayn, in particular, to be a martyr and mark his death to this day with two weeks of ceremonies.

We have discussed succession crises at many points in this book. There appears to be no perfect mechanism for succession. Elite election as practised in early Islam and in Rome could be a recipe for political intrigue and armed conflict. Having the oldest son inherit limited scope for conflict but increased the chances of incompetent or malevolent rule. The general practice of Islamic empires would be for sons of the ruler to compete. This might ensure against incompetence (though not malevolence), but was also an invitation to continual conflict. And the switch from election to inheritance, which erased any pretence that the broader community should choose the ruler, may perhaps have encouraged centuries of military conflict over who should govern Islamic societies. If a vote among leaders could not settle disagreements over succession, political fragmentation would occur as different leaders established different regional power bases. However, all empires eventually fragment, and perhaps no institutional structure could have maintained a unified Islamic Empire for long. 


\section{Umayyads}

The Umayyads established their capital in Damascus, despite being from Mecca. They were a powerful family and solidified control of the growing Islamic Empire for a while. They reserved positions of power for Arabs, and distributed land to these, while imposing a special tax on non-Muslims. They then came to annoy fellow Arabs by living luxuriously. The caliphate would adopt Byzantine and Persian administrative practices in areas it conquered. It would come to employ non-Muslims in its service. It became hierarchical in structure, like all other states in human history, which further offended the egalitarian communitarian instincts that had first inspired Islam. Its practice of religious toleration - beyond the special tax imposed on non-Muslims - also offended some Muslims.

One fundamental question to ask of empires is whether the army critical to conquering remains powerful after conquest or whether a centralized bureaucracy gradually supplants it. For the Umayyads, the military stayed powerful. This was in part because the Umayyads were only in power for a bit over a century, and their empire was still expanding for most of this time. Yet the

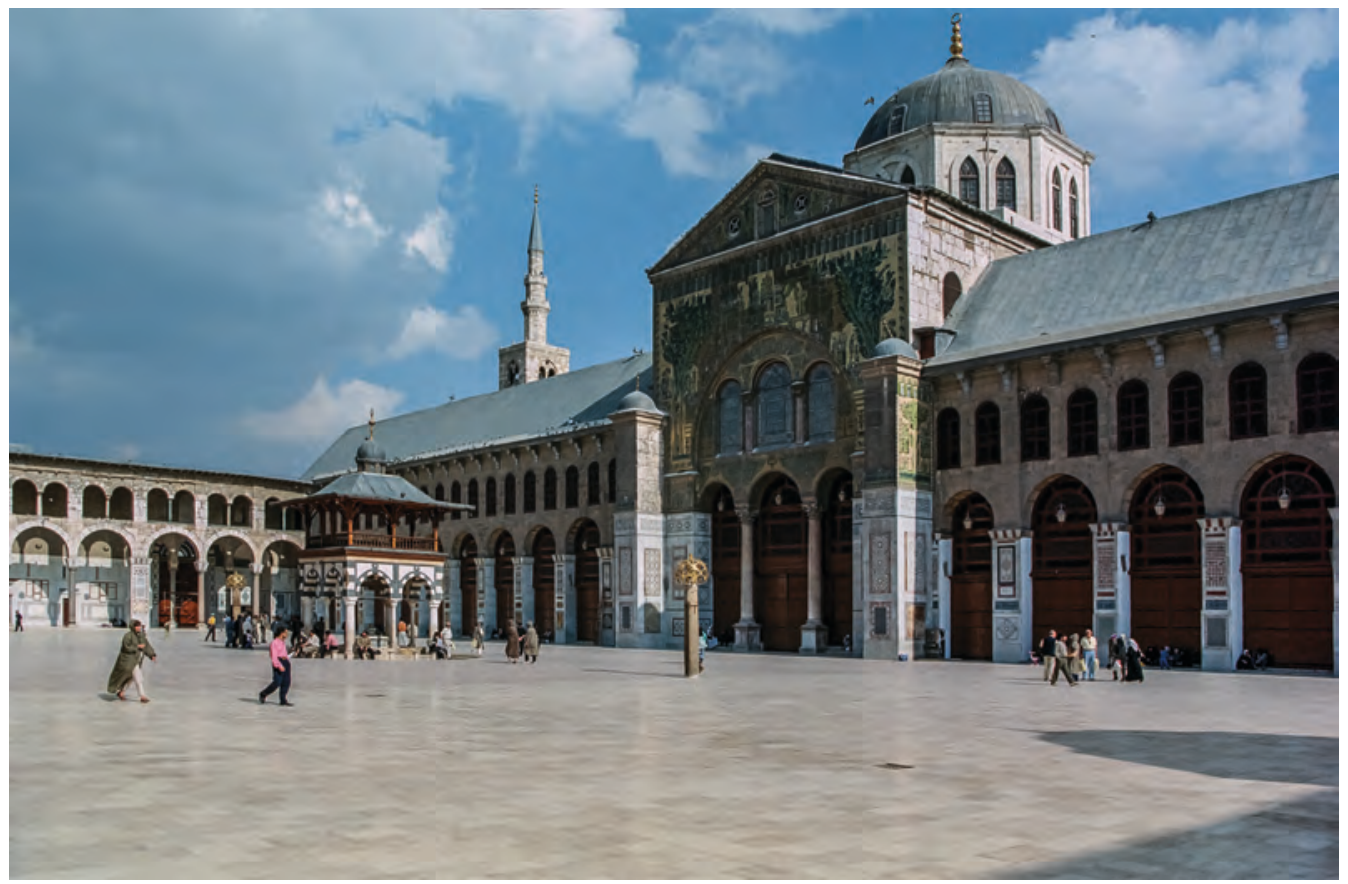

Illustration 12.4

Umayyad Mosque in Damascus, completed 715, considered by some the fourth holiest site in Islam. It had previously been the site of the Church of John the Baptist, and is said to contain his remains. It is one of the oldest surviving mosques in the world

Photograph by Jerzy Strzelecki, CC BY-SA 3.0 
strength of the army also reflected the fact that Christians and Zoroastrians dominated bureaucracy and even landowning. The Umayyads relied on local elites to raise the taxes used to support Arab armies. Local armies stationed in different regions absorbed most of these funds, with the centre often starved of funds. Some caliphs fought back by garrisoning Syrian armies in more distant regions and instructing them to forward funds to the capital. Nevertheless, the lack of a loyal bureaucracy that could raise taxes centrally was a weakness for the Umayyads, and local military commanders remained powerful.

The Umayyads would last longest in Spain, where a small conquering elite remained loyal, long after the Abbasids had replaced the Umayyads elsewhere (see below). The Spanish Umayyads respected the local Christian and Jewish religions, not attempting assimilation. They would rule for centuries. Later, Berber-based dynasties from North Africa would come to power in Spain: the Almoravids in 1086 and the Almohads in 1147. Nevertheless, Spain was ruled separately from the rest of Islam through most of its Islamic history. Though the Almohads preached holy war at times, religious toleration and cross-religious interaction characterized Spain throughout Islamic rule. Cities such as Cordoba became famed for libraries and scholarship, and Islamic, Jewish, and Christian thinkers interacted.

\section{Abbasids}

Abu Al-Abbas led a rebellion against the Umayyads in Persia, protesting that they were not governing as they should. Though a Sunni Arab, he allied with Shias and non-Arabs. Abu Al-Abbas could claim descent from Muhammad. His rise was in large part due to both the Sunni-Shia divide and ethnic conflict between Arabs and Persians that had emerged with the Islamic conquest of Persia. During the 740s, he and his allies seized Persia and Mesopotamia. Then under the pretext of a banquet invitation, he slaughtered the Umayyad ruling family. Unlike the Umayyads, the Abbasids did not pursue a policy of conquest, though they fought occasional battles (most importantly halting the Tang Chinese advance into Central Asia in 751 near Samarkand; chapter 13). They instead focused their energies on solidifying their power within territories conquered by the Umayyads, an effort that would prove fruitful for a couple of centuries.

The Abbasids developed a more centralized administration than the Umayyads had. They raised taxes and spent funds on public works such as roads. A hundred thousand workers built the new capital at Baghdad (not far from the old capital at Ctesiphon). The caliphs lowered trade barriers and encouraged trade across Asia and Africa. This policy not only raised tax revenue but also reflected an official recognition that Islamic states could and should interact with non-Islamic neighbours. The Abbasids employed members of various religions in their administration, even in senior posts (and in the army). It was thus a more multicultural administration than that of the Umayyads. 


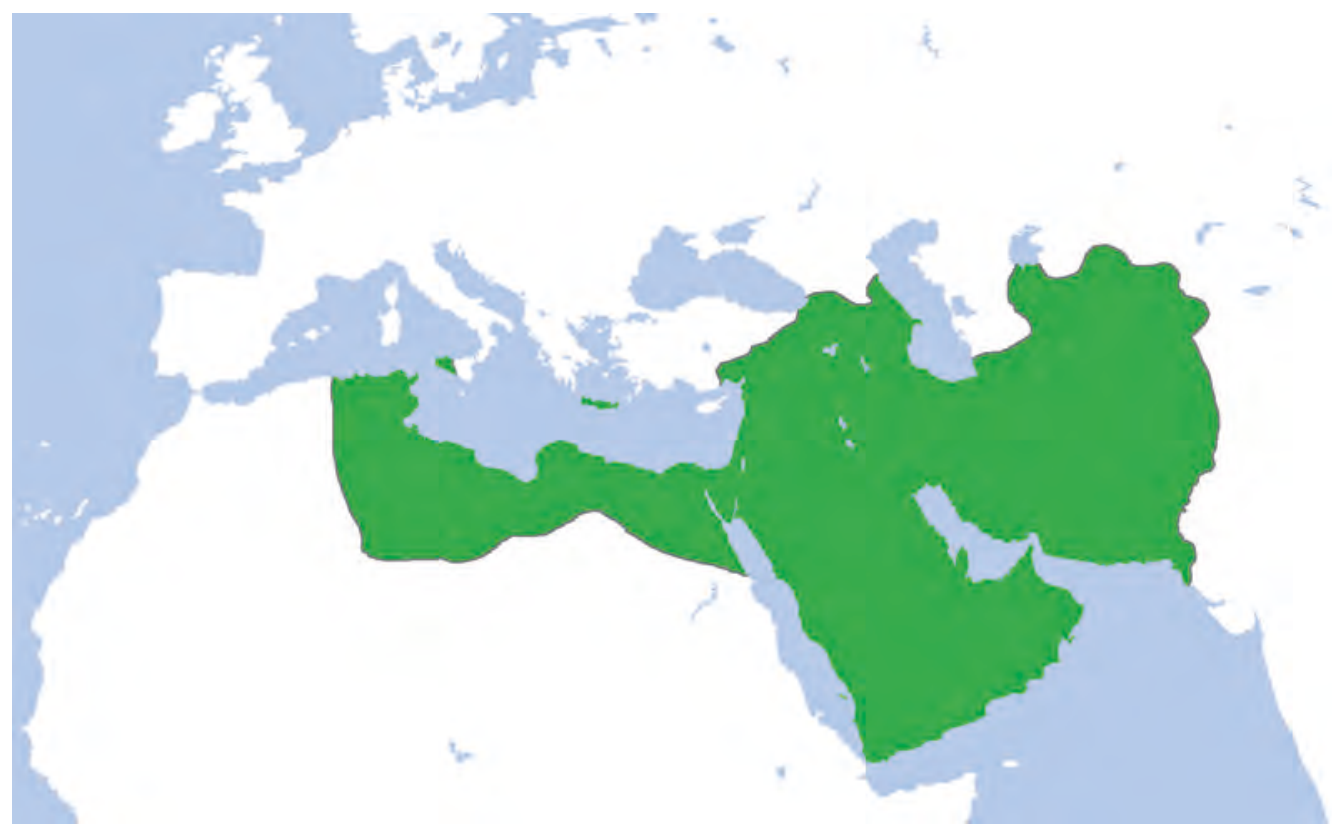

Map 12.2

The Abbasid Empire c.850

Map by Gabagool, CC BY 3.0

They employed local governors throughout their vast domain: Though they had lost Spain, they still ruled from Morocco to Persia.

Persian traditions of autocratic and centralized governance proved attractive to the Abbasids. Previous Persian rulers had exercised control over Zoroastrianism, appointing priests. The Abbasids exerted similar influence over Islam, rather than accepting the advice of religious leaders. Yet Islamic religious scholars still played an important role as judges and in formulating public policy.

The Abbasids often entrusted senior offices to slaves and eunuchs, whose loyalty was only to the caliph. These strategies they borrowed from Byzantines and Persians. The caliphate in this way mostly avoided the creation of a powerful aristocracy. It also developed a slave army that would be loyal to the caliph. Yet it was unable to prevent that other scourge of empires, as corruption became increasingly common in the Abbasid bureaucracy over time. This must have limited the financial capability of the caliphate. The Abbasids also suffered through a series of battles over succession. The Abbasids benefited from revenues from trade but then had to cope with the economic power of a trading elite that grew in wealth through time.

Like many empires before them, the Abbasids became reliant on neighbouring nomads - in this case Turkic tribes - as soldiers; these tribes then pushed their own choices for vizier (effectively the leading bureaucrat or prime minister) and killed a caliph in 869. In 1055 the Seljuk Turks took Baghdad and installed 
their ruler as sultan (the word means "chief"), leaving the caliph with only a religious role. The Abbasids had earlier lost power to the Fatimids in Egypt (who had Shia sympathies, and were named after Muhammad's daughter Fatima, who had married Ali) and others elsewhere who only nominally recognized Abbasid authority. These local powers had often gained authority at first by taking sides in succession battles.

\section{Abbasid cultural and scientific efflorescence}

Within fifty years of the founding of the Abbasid caliphate, Baghdad was maybe the largest city in the world outside China, with perhaps half a million people. It had numerous mosques, parks, baths, and markets. Baghdad was at the time a cosmopolitan city, its architecture reflecting a mixture of at least Arabic, Persian, and Roman elements. The caliphs were more interested in replicating Persian glory than Arab traditions, and invited entertainers from North Africa and India. Works of literature such as the 1001 Nights (often called the Arabian Nights) and the epic poems the Rubaiyat of Omar Khayyam were also penned at this time. They reflected an active culture that absorbed stories from throughout the known world. In particular, Arabic literature adapted Persian motifs and words.

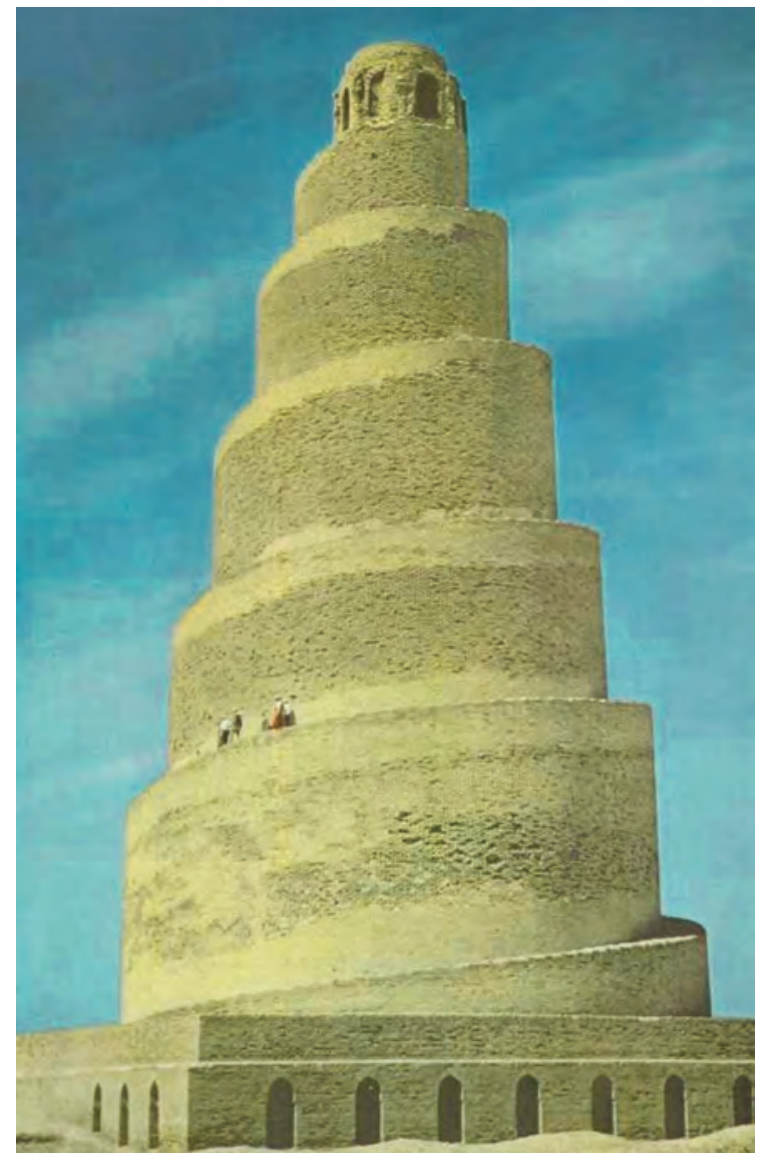

\section{Illustration 12.5}

Great Mosque of Samarra. Not all mosques followed a common design. The Great Mosque of Samarra is a 9th-century mosque located in Samarra, Iraq. The mosque was commissioned in 848 and completed in 851 by the Abbasid caliph Al-Mutawakkil, who reigned (in Samarra) from 847 until 861 Photograph by Izzedine

\section{PRIMARY DOCUMENTS: THE 1001 NIGHTS}

The pretext of The 1001 Nights is that a mighty king had learned of his wife's unfaithfulness from his brother, whose wife had also been unfaithful.

Mad out of anger, Shahryar at once rushed to his wife and chopped off her head. The angry Shahryar then took a wild oath, "Everyday I'll marry a maiden for a night. Next 
day, early in the morning, she will be killed. This way, I will never provide any opportunity to my wife to betray me." ...

Shahryar remained adamant on his insane oath. He called his Wazir and apprised him about his oath. Shahryar said, "You have to bring one young maiden every day and make her my bride for the evening. Make sure that she will be killed in the morning."

The king's cruel and crazy oath astonished everyone. People were frightened but what they could do? The cruel practice started. Each morning a young beautiful maiden was hacked to death. It went like this for years. Parents of the young girls started fleeing from the kingdom. Soon the kingdom was totally bereft of young girls.

One day, the Wazir returned home anxious. The Wazir had two beautiful daughters named Shahrazad and Dinarazad. Shahrazad, the elder daughter was exceptionally wise, humble and soft spoken. Besides this, she was a voracious reader and a learned person. When Shahrazad noticed her father's tensed and perturbed face, she asked, "Father, you are looking unusually disturbed. What's the reason for your worry?"

The Wazir said, "Dear daughter, the king has ordered me to bring a bride for tonight. However, not a single young girl is left there in our kingdom now. Shaken by the possible death, all the parents of the young girls had left the kingdom with their daughters. Now if I go to the king without any bride, he will chop off my head for not obeying his order."

Shahrazad said, "Father, please don't worry! Take me to the king as his bride."

Astonished to hear this, the Wazir exclaimed, "How can I do this? Don't you know what end do the king's brides meet?"

Shahrazad said calmly, "I'm fully aware of what fate the brides meet, my dear father! Even then, I emphasize you to produce me as the king's bride. I am confident that my wisdom will force the king to change his thought regarding women. In case I fail to do so, I'll have a satisfaction of dying for a good cause."

However, the argument laid by Shahrazad was not at all acceptable to the Wazir. He was not ready to send his daughter to meet such a cruel fate. He said angrily, "If you will not obey me, you will have to face the same misfortune, which was faced by the farm owner, the master of the bull and the donkey." Shahrazad asked, "O father, what's the tale of the farm owner?" The Wazir began the tale.

Shahrazad is able to keep the insane king so engaged in a series of stories that she tells that he can never have her killed. What insights might you (carefully) draw about the society from this story?

The Barmakid family were abbots of a Buddhist monastery in Balkh. One branch converted to Islam and became viziers to the Abbasid caliphate, even while others continued as Buddhist authorities. As viziers, they sponsored the translation of Sanskrit works on astronomy, medicine, and algebra (including the concept of zero, first identified in India). However, Islam would afterward turn back westwards and engage with Greek rather than Sanskrit philosophy. 


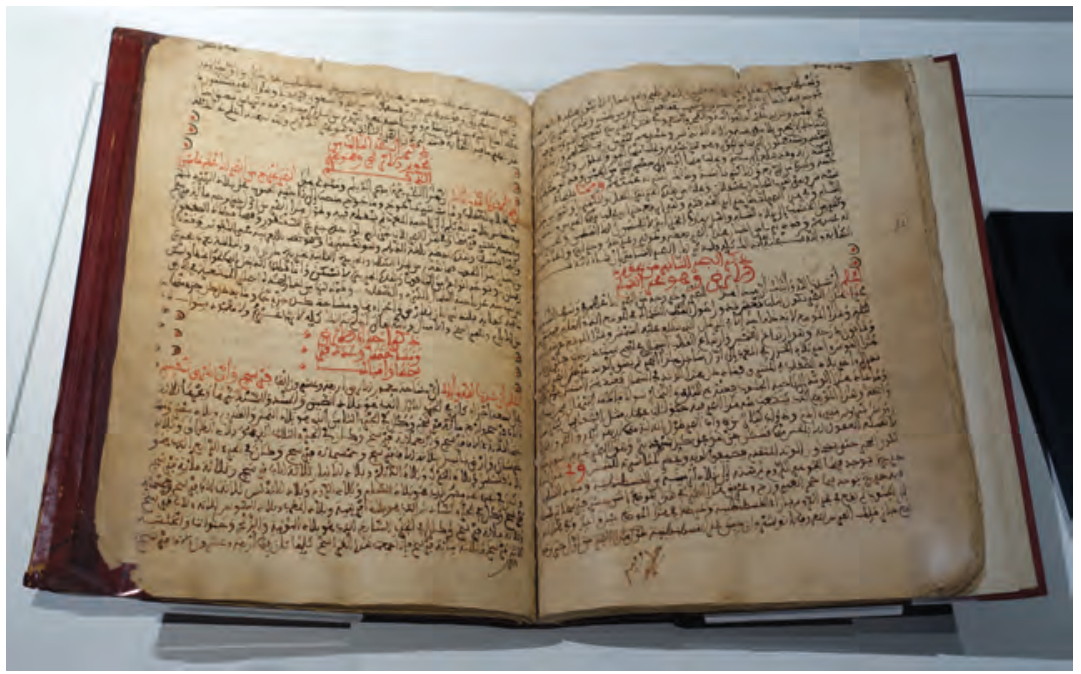

Illustration 12.6

Manuscript of the Arabian Nights copied by Abdullah al-Mawla an-Nujumi, Spain, $1235 \mathrm{CE}$. There are no illustrations as Islam had come to oppose pictorial representation by that time (see below), Aga Khan Museum, Toronto, Canada Photograph by Daderot, CC0 1.0

Yet the story of the Buddha's escape from imprisonment by his father (chapter 11) was translated into Arabic and would become a popular tale of the search for meaning. It would later spread to Christian Europe as the tale of Barlaam and Josephat but with the hero now discovering Christianity.

Baghdad would be a significant centre of philosophical and scientific investigation during the age of the Abbasid caliphs. The state or wealthy merchants endowed several schools, often attached to mosques. The Abbasids established an academy to study and translate the works of diverse civilizations. This encouraged the efforts of Islamic philosophers and scientists, who built among other things on the works of Aristotle. Averroes indeed attempted to align Greek philosophy with Islamic theology, an effort that would later inspire scholastic theologians in Europe, though such explorations fell out of favour in the Islamic world from the thirteenth century.

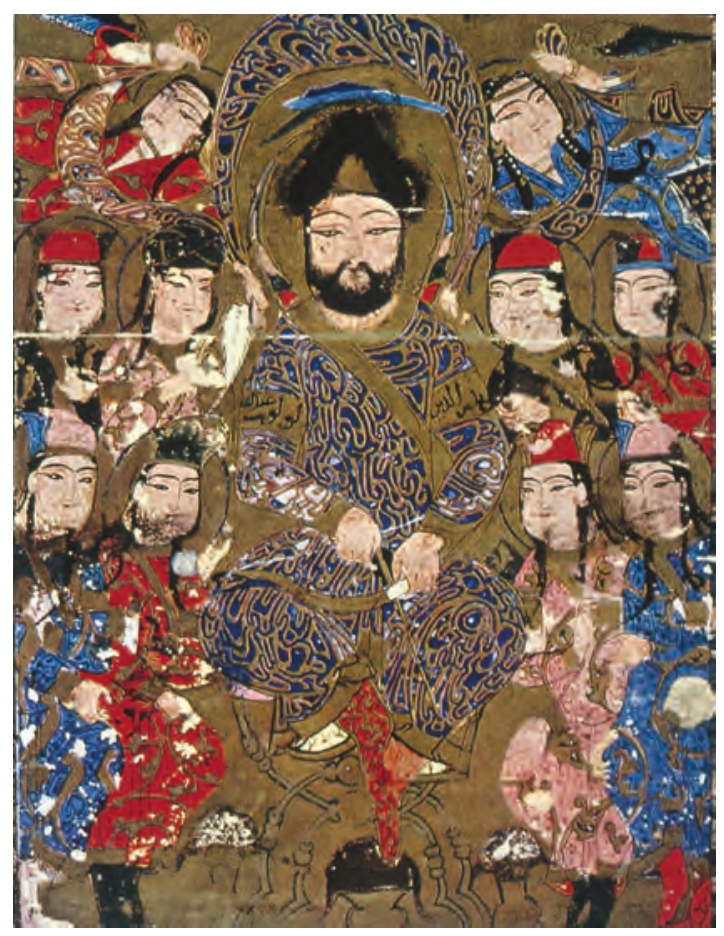

Illustration 12.7

Frontispiece of a manuscript of Kitāb al-Aghān̄̄ (Book of Songs) by Abu al-Faraj al-Isfahani, 1218-19 


\section{PRIMARY DOCUMENTS: AN ANONYMOUS ACCOUNT OF BAGHDAD AROUND $1000 \mathrm{cE}$}

The city of Baghdad formed two vast semi-circles on the right and left banks of the Tigris, twelve miles in diameter. The numerous suburbs, covered with parks, gardens, villas and beautiful promenades, and plentifully supplied with rich bazaars, and finely built mosques and baths, stretched for a considerable distance on both sides of the river. In the days of its prosperity the population of Baghdad and its suburbs amounted to over two millions! The palace of the Caliph stood in the midst of a vast park several hours in circumference which beside a menagerie and aviary comprised an enclosure for wild animals reserved for the chase. The palace grounds were laid out with gardens, and adorned with exquisite taste with plants, flowers, and trees, reservoirs and fountains, surrounded by sculptured figures. On this side of the river stood the palaces of the great nobles. Immense streets, none less than forty cubits wide, traversed the city from one end to the other, dividing it into blocks or quarters, each under the control of an overseer or supervisor, who looked after the cleanliness, sanitation and the comfort of the inhabitants.

The water exits both on the north and the south were like the city gates, guarded night and day by relays of soldiers stationed on the watch towers on both sides of the river. Every household was plentifully supplied with water at all seasons by the numerous aqueducts which intersected the town; and the streets, gardens and parks were regularly swept and watered, and no refuse was allowed to remain within the walls. An immense square in front of the imperial palace was used for reviews, military inspections, tournaments and races; at night the square and the streets were lighted by lamps.

There was also a vast open space where the troops whose barracks lay on the left bank of the river were paraded daily. The long wide plazas at the different gates of the city were used by the citizens for gossip and recreation or for watching the flow of travellers and country folk into the capital. The different nationalities in the capital had each a head officer to represent their interests with the government, and to whom the stranger could appeal for counsel or help.

Baghdad was a veritable City of Palaces, not made of stucco and mortar, but of marble. The buildings were usually of several stories. The palaces and mansions were lavishly gilded and decorated, and hung with beautiful tapestry and hangings of brocade or silk. The rooms were lightly and tastefully furnished with luxurious divans, costly tables, unique Chinese vases and gold and silver ornaments.

Both sides of the river were for miles fronted by the palaces, kiosks, gardens and parks of the grandees and nobles, marble steps led down to the water's edge, and the scene on the river was animated by thousands of gondolas, decked with little flags, dancing like sunbeams on the water, and carrying the pleasure-seeking Baghdad citizens from one part of the city to the other. Along the wide-stretching quays lay whole fleets 
at anchor, sea and river craft of all kinds, from the Chinese junk to the old Assyrian raft resting on inflated skins.

The mosques of the city were at once vast in size and remarkably beautiful. There were also in Baghdad numerous colleges of learning, hospitals, infirmaries for both sexes, and lunatic asylums.

Does this account make you want to visit Baghdad in 1000 ce? Why do you think such a description might have been written, and would it exaggerate the beauty of the city? What is missing from the report?

Islam would be the world leader in almost every area of science from 800 to 1300 (sadly, we have limited knowledge of this science since only a minority of Islamic scientific treatises have been published or translated). It first borrowed Indian science as it controlled parts of India; but actively sought out Greek science from the 830s, translating all Greek works of science. Indeed, Baghdad and other centres of learning had libraries with hundreds of thousands of volumes at a time when no library in (non-Islamic) western Europe would have had much more than a thousand. Yet, as with other empires, Islam emphasized practical sciences such as astronomy, medicine, mathematics, and logic.

Al-Khwarizmi developed algebra (integrating Greek and Indian ideas) and the (Indian inspired, and called by the Arabs "Indian numerals") Arabic numerals. Note that we know his name, and that of many other scientists of the time, unlike the case of classical science (chapter 9). Trigonometry was developed to aid astronomical calculations. The first formal astronomical observatory in the world opened in Baghdad around 838. (A larger and more famous observatory opened at Maraghah near the Caspian Sea from 1259, but only lasted sixty years.) The Abbasids sponsored treatises on agriculture, irrigation, and farm animals (which likely did serve to increase agricultural productivity). They developed optics for medical applications in treating disorders of the eye (which were common in desert areas).

Islam itself encouraged some types of scientific activity. Local mosques needed to have local experts to determine when to perform prayers, for the position of the sun and moon in the sky determined prayer times, and these positions varied with latitude and longitude. They also needed to know in which direction Mecca lay for this was the direction of prayer. Precise measurements of the angle of the sun were critical to both endeavours. The Islamic world would have the most detailed astronomical knowledge well into the sixteenth century. Beyond these religious tasks, though, religious fundamentalists saw little value in science. As with the Christian Augustine (chapter 11), they felt that scripture said everything one needed to know about the nature of the world. Even here, the value accorded to scripture encouraged an interest in literacy: The Islamic 


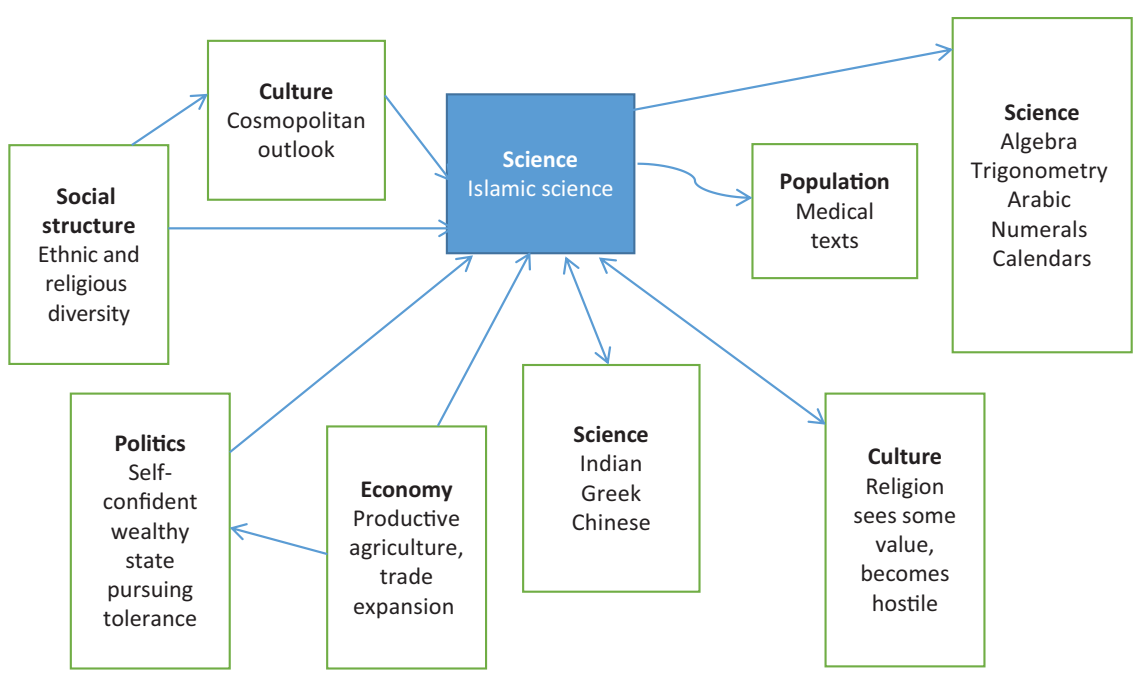

Figure 12.2

Influences on and effects of Islamic science

Note: There is little impact on technology at the time.

world likely had the highest literacy rates in the world in the early centuries of the second millennium. Still, Islamic science would decline in size and importance after 1300. Decreased government revenues from trade associated with the later arrival of the Portuguese in the Indian Ocean (chapter 19) may have further reduced the resources available for science.

There were also important technological advances. Windmills appeared in Greece from the first century BCE and China from the fourth century CE, and Greeks developed water mills from the third century BCE (there is some speculation that there were windmills in Mesopotamia in the time of Hammurabi, but little evidence), but both were really only developed in the Islamic world from the eighth century. Such mills could produce ten times the power of animal traction, without requiring that an animal be fed. Europe would borrow and further improve the technology from the twelfth century. Energy usage per person would rise significantly because of windmills and watermills.

Of particular note is paper: This had been invented by the Chinese in about the first century CE (chapter 10), and the Abbasids allegedly learned papermaking techniques from captured Tang soldiers. The Arabs improved the durability of paper (perhaps because they lacked the raw materials used by the Chinese) so that it was possible to write with pens rather than Chinese brushes. Paper factories were established in Samarkand along the Silk Road in 751, and in Baghdad in 793. The development of paper encouraged writing and the preservation of both scholarly thoughts and administrative edicts. In addition, books came to replace scrolls as paper replaced thicker parchments.

Though Baghdad was the centre of Abbasid culture and science, Cairo also grew as a cosmopolitan centre at this time, drawing on both the intellectual tradition of nearby Alexandria and its role as a significant trade centre linking 


\section{BOX 12.2}

\section{TECHNOLOGY TRANSFER}

Technology transfer requires two distinct processes. The most obvious is the physical movement of the technology. This generally involves the movement of one or more persons that understand the technology:Written instructions or blueprints do not usually convey how to do something well enough. The second process is adoption: The ideas transported only have a historical impact if the receiving society adopts them. This depends on local economic, political, and cultural circumstances. Mass-production technologies developed in the United States in the nineteenth century spread slowly in Europe where wages were lower and raw materials more expensive. Chinese emperors famously disdained European technologies when introduced to these in the sixteenth century. Adoption often means adaptation: The technology is changed and commonly used for different purposes in the receiving society. For example, Chinese paper was strengthened to meet the needs of Middle Eastern scribes.

east and west via the Red Sea. (Cairo would centuries later replace Baghdad as the Islamic centre of learning after the Mongols conquered Baghdad in 1258 and destroyed its massive library; Cairo would itself have half a million people in the fourteenth century.) Cordoba in Umayyad Spain was also a significant centre of philosophy and science at this time. Cordoba became a place where Jews, Christians, and Muslims could meet and discuss; and classics from many lands were translated into Latin, Arabic, and Hebrew. Perhaps the most famous Cordoban scholar is Maimonides (Moshe ben Maimon; 1135-1204), who was a scholar of Jewish law and ethics but also very influential in Islamic philosophy and science. Cordoba's most extensive library may have had 400,000 books. The library in Cordoba would indeed, after the conquest of Spain by Christian kings centuries later, become a source of manuscripts (including many from ancient Greece) otherwise unknown in western Europe. Smaller centres could also shine. Seraf in Persia, the port for the city of Shiraz, imported teak from Africa for opulent merchant homes.

Not all were happy with cosmopolitanism and displays of luxury. The wealth of Baghdad and other Islamic cities inspired both Sufi asceticism and Islamic fundamentalism. Though religious leaders were suspicious of Sufis, both would object in their own ways to the practices of their rulers. Baghdad at the time was likely characterized by a very unequal income distribution: Though we can celebrate the science and art financed by wealthy merchants, poorer members of society might easily take offence at displays of luxury.

This cosmopolitanism would not always characterize later Islamic polities. To some degree, it may merely reflect the personalities of the Abbasid rulers and the circumstances of their rise to power: They needed to respect the interests of the Shias and Persians that initially supported them but also the Sunnis and Arabs they came to rule. Cosmopolitanism may also reflect self-confidence: Though 


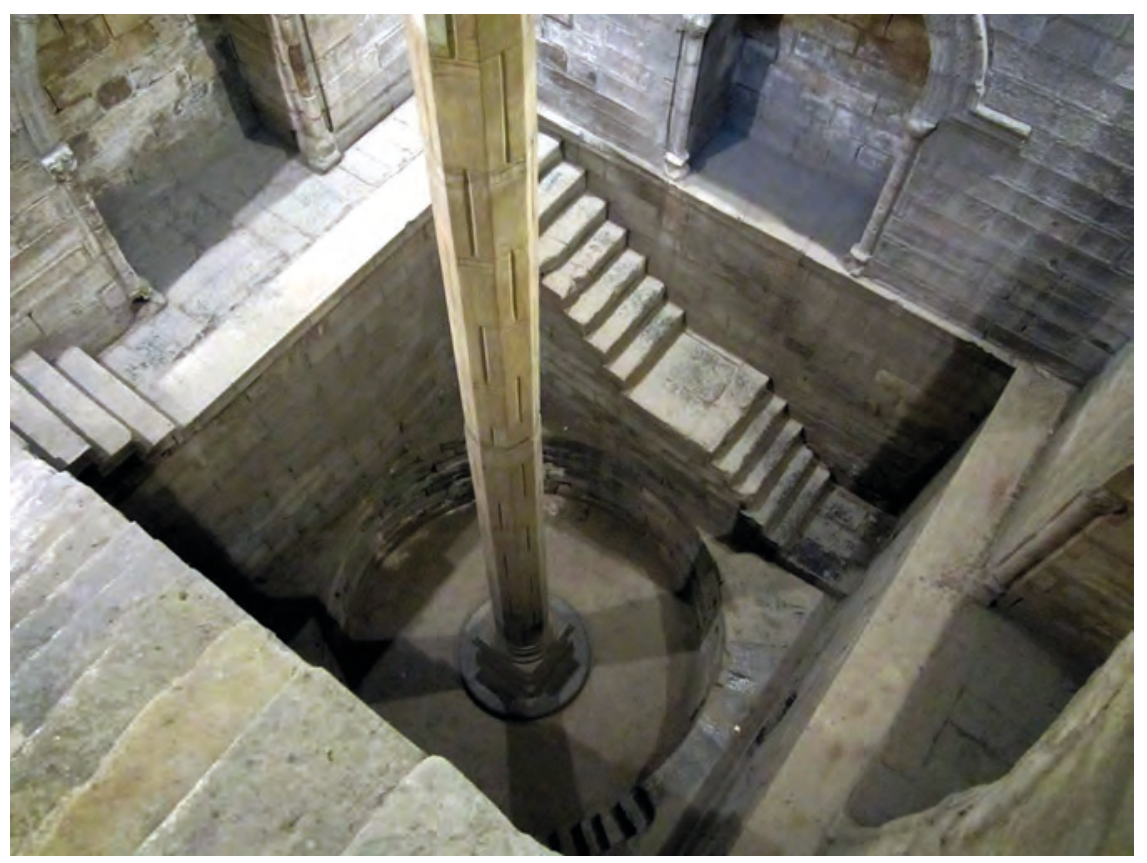

\section{Illustration 12.8}

Nilometer, Rhoda Island, Cairo, 861. The Egyptians had measured the depth of the Nile for millennia in order to predict the height of the annual flood. This is the oldest remaining nilometer

Photograph by David Stanley from Nanaimo, Canada, CC BY 2.0

the Abbasids had ended a policy of continued expansion, they stood unchallenged, much stronger than the Byzantines or the fragmented polities of Central Asia or India - and able to defeat distant China in battle. They could reasonably see themselves as the centre of the world, even if they had shelved the task of global military domination. From a position of strength, they could recognize the value of interacting - both economically and intellectually - with other societies. Cosmopolitanism might also reflect the power of the merchant elite: That elite made its money from trading across cultural boundaries and might then be predisposed to a cosmopolitan outlook. Finally, yet importantly, cosmopolitanism might merely reflect religious diversity at home: The process of conversion occurred slowly over the centuries, motivated in part by the special tax levied on non-Muslims. Turks and Persians gradually adopted Islam but still saw themselves as distinct from Arabs. Baghdad - and Cairo and especially Cordoba - were thus religiously diverse at the time. Whenever later Islamic states ruled substantial non-Islamic populations, they risked rebellion unless they practised some degree of toleration. Religious zeal sometimes guided them to intolerance, but often they embraced non-Muslim cultures. Abbasid policies may have served to limit ethnic and religious strife - though as noted above, they did stoke a religious reaction. 
We noted above that Islamic religious authorities often criticized rulers. These religious authorities operated largely independently from states in the early centuries of Islam, receiving much support from merchants. As a result, questions of theology and philosophy were debated openly. From about the tenth century, the Abbasids - perhaps guided mainly by the rising power of Shi'ism - moved to establish religious orthodoxy by absorbing a religious elite into the state bureaucracy, and transmitting this orthodoxy through statefinanced schools. Religious leaders then supported the state in return for exercising religious authority. Most Islamic polities would follow a similar strategy over the next thousand years, with religious orthodoxy serving to limit scientific, technological, and institutional innovation. Some historians argue that this institutional change is the primary reason why Islamic lands would gradually fall behind Europe both economically and politically over the next millennium. We can certainly note at this point that Islam operated differently in its first centuries from how it would function later. We should stress again that Islamic lands led the world in science, technology, and commerce for centuries, but that later Islamic polities would be less supportive of all three endeavours.

\section{Islamic attitudes toward women}

Attitudes toward women were ambiguous. The Quran provided that dowries go to the woman rather than the husband, and recognized women as equals before god: They had similar religious obligations and opportunities for an afterlife. Though daughters inherited only half as much as sons, and divorce was easier for men than women, these rules were more supportive of women than many classical law codes. The Quran likely gave women more rights than was typical in Arabia at the time. Yet attitudes toward women seem to have hardened in the early centuries of Islam. A concern with male inheritance encouraged severe limitations on female freedom (to ensure paternity). Expansion into new lands introduced new practices. The veil, though now commonly associated with Islam, had existed in Mesopotamia and Persia from perhaps the thirteenth century BCE (though most commonly worn by noble women) and had spread, though not to Arabia; this practice was adopted by Muslims as they expanded into the region.

Islamic inheritance laws may have had an unintended negative impact on women. If families were worried about maintaining their land holdings across generations, they would not wish to see daughters marry unrelated men. An institutional innovation intended to help women, introduced into a patriarchal society, may then have encouraged fathers to sequester their daughters and arrange marriages to cousins. The family land could then be kept in the family. In societies where only sons inherited, families had less financial reason to worry about daughters pursuing romantic attachments beyond the family. Rates of cousin-marriage remain much higher to this day in many Islamic 
countries (about half of all marriages in Pakistan and Afghanistan are to cousins) than in almost all other lands. If this conjecture is true, it highlights the challenges of trying to achieve social change - in this case improving the welfare and status of women - by introducing institutional changes that are in conflict with existing cultural expectations and social structure.

Polygamy was common in Arabia and Persia at the time. The Quran limited the number of wives to four and urged fair treatment of each. Muhammad himself did not marry again until his first wife had died.

There is an Islamic tradition of compiling biographical dictionaries of noted scholars. In the fourteenth and fifteenth centuries, women comprise about a tenth of the entries. In the nineteenth century, they are almost entirely absent.

\section{Challenges facing agents:Women}

The historical experience of women was almost as diverse as that of men. They gathered and occasionally hunted, and later worked as farmers, artisans, and occasionally artists, merchants, priests, scientists, and rulers. More often, they collaborated with or influenced their husbands and thus shared the challenges of farmer, artisan, merchant, and ruler, among others.

As we have seen, the legal position of women varied considerably across societies. In some, they had rights over marriage, divorce, inheritance, and property, but not in others. In many societies, they were entirely dependent on the decisions of husbands or fathers; these in some cases could even sell them into slavery. In cases where women were legally powerless, their challenges were broadly similar to those of slaves: They needed to encourage fathers or husbands to treat them kindly, and might withhold services to encourage better treatment. The many complaints through history about the malign influence of rulers' wives signals both that women were often successful in affecting their husband's behaviour but that there was cultural pressure to limit their influence.

Women generally had to worry about sexual assault, especially in times of war. Women in lower classes or castes were particularly subject to assault and harassment. Many women (and a much smaller number of men) would try to earn money from sexual services.

\section{Trade and the movement of ideas}

Trade expanded during the Abbasid period, though it later declined as the caliphate weakened. Cities grew as centres of administration, trade, and industry. The Abbasids traded with China, India, Russia, Europe, and Africa (both down the east coast and across the Sahara). There were institutional innovations to serve this trade. Banks with multiple branches facilitated the transfer of funds; these managed to cope with the Islamic prohibition of lending for interest. The idea that investors had to share risk, rather than just earn interest, inspired a form of contract that Venice would later copy as the "commenda," in which investors would join with merchants in financing trade ventures. (Of course, 
this was a formalization of practices common since at least the Assyrian trading missions to Anatolia in the fourth millennium вСE.)

In $900 \mathrm{cE}$, ibn Khordadbeh, a postmaster in Persia, described conditions as far as Korea in his Book of the Roads and Countries (8 vols.). This book reflects both the cosmopolitanism and importance of trade at the time: There was an audience for a book that described both transport infrastructure and customs of both near and distant lands. Ibn Khordadbeh could only compile such a book if information about distant lands was circulating. Muslim merchants were undoubtedly travelling to China in the tenth century.

One common item of trade was slaves. Islamic law forbade the enslavement of Muslims but permitted non-Muslim slavery. Such slaves had rights, could marry, and often held high office. Muslims were encouraged to free slaves, especially if these converted, and the children of a master and slave were to be freed, at least on the master's death. Still, the conditions in which slaves were transported were often miserable, and the terms of their ownership could be harsh. The Abbasids were not able to put down a slave revolt around Basra in Mesopotamia from 869 for fourteen years. The rule against Muslim slavery would often be broken in history, especially for black Muslims (though Islamic society was not particularly racist), but sources of non-Muslim slavery were generally preferred. East Africa and Europe were both important sources of slaves for the Abbasids. Two and a half million slaves were exported from East Africa between 850 and 1000, and another 10 million before 1900. The sale of Slavic peoples into slavery in the Middle East also occurred on a large scale (see chapter 13).

During the Abbasid period, a variety of crops from India, including sugarcane, rice, spinach, artichokes, oranges, and cotton were transplanted farther west. Many of these crops grew in the high heat of summer, and so allowed a second harvest in lands that could only produce grains in winter. Islamic agriculture, therefore, developed sophisticated rotations of new and old crops. The Abbasids also expanded irrigation systems. Islamic society could arguably never have developed sophisticated cities and polities if not for these simple developments

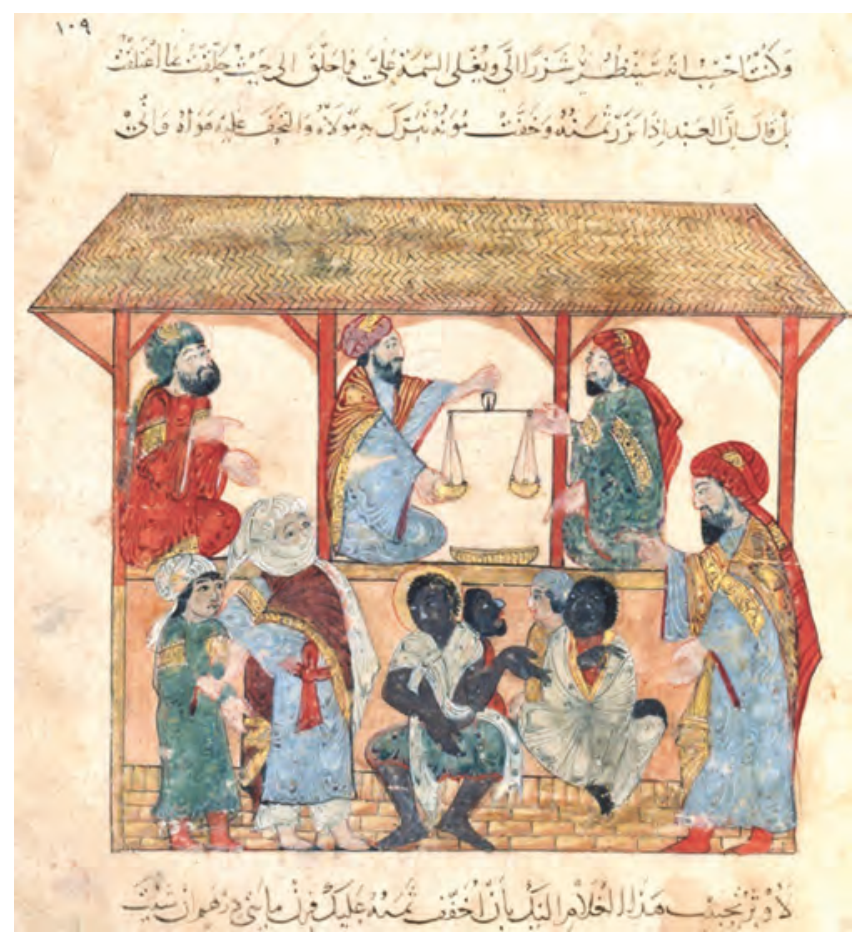

Illustration 12.9

Slave market, 13th century, Zadib, Yemen, Arabic manuscript, Bibliothèque nationale de France 
that significantly enhanced agricultural output in an arid part of the world. Islamic states also developed private property in land and water rights that should have encouraged farmers to put these to the most productive use. These new crops also expanded dietary diversity. Sugarcane would prove of great importance historically, for it would spread from North Africa to Europe and inspire Europeans - who had previously relied on honey as a sweetener - to develop slave-based sugar plantations first in Europe and later in the Americas (see chapter 19).

Merchants spread Islam itself into Central Asia, Southeast Asia, sub-Saharan Africa, and along the East African coast. Muhammad had been a merchant and, though the behaviour of some of his fellow merchants horrified him, the Quran respected merchants as long as these were faithful and charitable. Islam thus - as Buddhism had before - proved particularly popular with merchants. These merchants spread the word as they travelled. In Southeast Asia and subSaharan Africa (see below), some monarchs involved in trade would over time convert, in part to solidify trade relations with the growing body of Muslim merchants. This also occurred in small city states along the East African coast (from which flowed not just slaves but also gold, ivory, tortoiseshell, and iron), but Islam did not penetrate far inland. Islamic merchants, mostly from India, had arrived in Southeast Asia in the eighth century but only became prominent in the tenth century. When local rulers adopted the faith, they tended at first to allow multiple religions. This practice of toleration would change in later centuries. Melaka, originally a Hindu state, became a powerful Muslim state in the fifteenth century by controlling sea lanes. It sponsored Islamic missionaries throughout the region.

\section{BOX 12.3}

\section{TRAVELLING CUISINE}

Borrowing crops from another region will be a common practice in world history. Crops are adopted only when they are well suited to the local environment. We should appreciate, then, that natural evolutionary processes did not distribute wild plants into every ecological niche in which humans could domesticate them.

We should not ignore the cultural aspect of borrowing. Societies develop "cuisines," a set of practices regarding how to prepare local foods. Adopting a new crop then means adapting a region's cuisine. This will often happen in history - the tomato becomes a part of Italian cuisine only after Columbus (chapter 19). Yet we should nevertheless appreciate that cultural expectations regarding taste influence a decision to adopt a new crop. Tastes, in turn, connect with a group's sense of self, and may even be instantiated in religious beliefs regarding what one should eat. 


\section{Islam in India}

Islam's entry into India is a long and convoluted process compared to its rapid conquests up to the borders of India. Muslim merchants, who came to control trade westward from India from the seventh century until the arrival of the Portuguese in the fifteenth century, took the first steps. These merchants established coastal enclaves at various points along the Indian coast. When a pirate ship attacked an Arab merchant vessel in 711, the Umayyads responded by attacking and conquering Sind (Pakistan). However, Indian states combined to rebuff further advances until 998 (aided by the fact that the Abbasids were not expansionary). Between 998 and 1030, an Islamic state based in Ghazni in Afghanistan (one of three states that had arisen in the Abbasid heartland as that empire declined) repeatedly raided the Punjab. Temples were destroyed, riches were stolen, and women abducted. We should place these attacks in the context of the millennia of attacks suffered by India from nomadic or semi-nomadic peoples from Central Asia. In the end, the Ghaznavids conquered the Punjab and Indus Valley. As a slave elite, the Ghaznavids ruled harshly, treating non-Muslims with disdain and violent repression.

Another Turkic tribe, the Ghurids, succeeded the Ghaznavids. The Ghurids at first destroyed Hindu temples, but then chose a path of tolerance. A slave elite among the Ghurids established the Delhi sultanate from 1206, which would survive until 1526, albeit with many changes in borders, four dynastic changes, and multiple succession crises. While the sultans reserved the highest positions in the bureaucracy for Muslims, Hindus were able to serve as well. The sultans drew on both faiths to encourage loyalty to a ruler that promised stability to all. The sultanate was very decentralized, with local Hindu rulers maintaining considerable authority (chapter 15).

Southern India remained for the most part politically fragmented, though the Chola controlled large areas in the deep south from 850 to 1267 . The sultans of Delhi would dispatch an armed force under two brothers to the south, who would renounce Islam and form their own Hindu kingdom, Vijayanagar. Only many centuries later would the Islamic Mughal Empire conquer this.

The Ghurids and other Islamic rulers were often less charitable in their treatment of Buddhists. This may in part have been a matter of numbers: Hindus already far outnumbered Buddhists in India, and so it was far more critical for Islamic rulers to foster peace with Hindus. Yet rulers likely perceived Buddhism as more of a threat because it, like Islam, actively sought converts. The Buddhist advocacy of non-violence - not to be sure an attitude always pursued by Buddhist communities - may also have made it easier to attack Buddhists. In any case, Buddhism would continue to decline under Islamic rule in India.

The most famous story involves the Buddhist university of Nalanda in northeastern India, which attracted some 3,000 students at its peak, and was likely the 
greatest centre of learning (addressing far more than just theology) in Asia from the fifth century CE. It received endowments not only from Indian princes but from as far away as Sumatra and had special scholarships for Chinese students. It is said that Muslims brutally destroyed it at the start of the thirteenth century, with teachers and students killed on the spot, but in fact it survived into the fourteenth century.

Islamic conquerors tended not to place Indians in the most senior administrative posts even if they converted. This reduced the incentive to convert. Yet over time, a quarter of the population did convert, especially those from lower castes. It is unclear if they benefited economically from doing so. Sufis were, in India as elsewhere, important in encouraging conversion. They may have been most successful in lands that were marginal agriculturally at the time, for they carried with them not just religious ideas but hydraulic practices and plants well suited to drier climates (often termed by historians the "Islamic toolkit"). Local peoples that valued this new agricultural technology could also appreciate the religion with which it was associated.

The Bhakti movement in the twelfth century came to seek harmonization of Islamic and Hindu beliefs but flowered briefly. Harmony was likely limited by the sharp differences between the monotheism and egalitarianism of Islam and the polytheism and caste distinctions of Hinduism (but see our discussion of the later Sikhism in chapter 18).

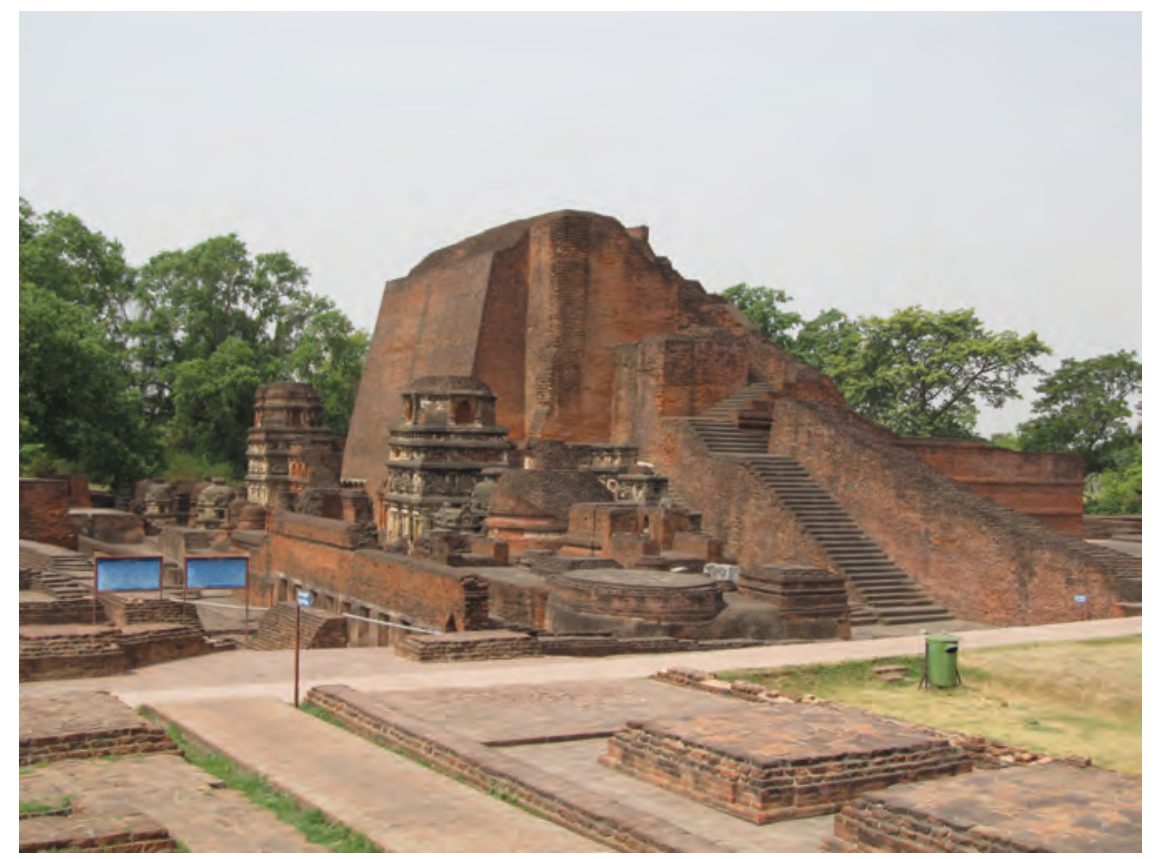

Illustration 12.10

Nalanda University ruins

Photograph by Mrityunjay.nalanda, CC BY-SA 3.0 


\section{Mamluks}

We mentioned briefly above that the Ghaznavids were a slave elite. It was common for Islamic rulers to employ slaves in senior posts in the bureaucracy and army: They hoped that these slaves, torn from their families, would be loyal to the ruler. However, the Ghaznavids were not the only slave elite eventually to seize power. The Abbasids themselves would have trouble with the slave elite they had developed from the ninth century. Slaves would overthrow the Fatimids in Egypt. These slaves were the Mamluks, military slaves of mostly Turkic origin, who rebelled and took power in Egypt and Arabia from 1250 as Egypt faced the Mongol threat (chapter 17). There had been a succession crisis among the Fatimids, and the Mamluks had supported Shajar al-Durr, the wife of a dead sultan. She eventually married a Mamluk, and the Mamluks killed the dead sultan's son by another wife (the tale of Shajar al-Durr is well known in Egypt; it sometimes celebrates and sometimes bemoans the political role that women can play). The Mamluks would win important battles against the Crusaders from Europe (chapter 15). In 1260, they stopped the Mongols who had taken Baghdad and were marching on Egypt (chapter 17) - though the Mongols would likely have withdrawn in any case due to a succession crisis of their own. The Mamluks would control Egypt (and sometimes beyond) until defeated by the Ottomans in 1517. They were thus the most successful slave elite in history. Yet of course, once they took power they ceased to act like slaves: They married, had families, and then fought amongst themselves over power and money. They would not be as loyal to each other as they had once been to sultans.

\section{Islam in West Africa}

West Africa had not been as involved in trade as had Europe and Asia. The Sahara was a massive barrier to overland trade. Some nomadic peoples had lived there since times when it was wetter, and these provided some limited indirect contact from ancient times between peoples to the south and north. Merchants occasionally organized travel across the desert in classical times (chapter 9). There were also geographical barriers to trade south of the Sahara. The African coastline has few natural harbours - and there would be limited trade along the coast before the arrival of Europeans in the fifteenth century. Most of Africa is elevated, and there are usually huge rapids or waterfalls on the rivers draining to the sea, severely limiting the possibilities of water transport inland. The Niger of West Africa is navigable in long stretches but often only seasonally.

The introduction of the camel from Arabia to North Africa from at least the first century BCE had dramatically increased the possibilities of trans-Saharan trade. Centuries later, a saddle was developed for use on camels. It took at least seventy days to cross the desert, and there were limited water sources along 
the way, but camels can go many days without drinking water. By the early centuries $\mathrm{CE}$, they were the primary draft animals for crossing the desert. From the eighth century, Muslim traders from North Africa intensified sub-Saharan trade as part of the global expansion in trade brought by Islam. West African gold was a particular attraction. Merchants could build upon existing trade centres such as Gao south of the desert, which connected desert routes with the Niger River. They encountered the state of Ghana in present-day Mali and Mauretania, which may have existed from the fourth century. Ghana traded for gold with peoples farther south and then sold this gold - along with ivory and slaves - northward in return for horses, cloth, manufactured goods, and salt. Ghana became rich and powerful as a result, with an army of perhaps 200,000 and a capital city of 15,000 people and many monuments built of stone.

By about the tenth century, the kings of Ghana had converted to Islam, though they also continued to practise local beliefs. They practised toleration, but locals, especially those involved in trade, converted over time. The state itself may have been encouraged to convert to achieve better relations with Muslim states and traders. Ghana would be overthrown by Mali in the thirteenth century (see chapter 16), which extended the boundaries of empire eastward and as a result controlled all of the growing trade northward across the Sahara. It also practised a tolerant form of Islamic rule. Trading centres such as Timbuktu grew large and were very wealthy. Mansa Musa who ruled from 1312 travelled to Mecca in 1324-5, with thousands of retainers and a hundred camels carrying gold. On his return, he built many mosques and established religious schools, importing teachers and sending students north.

Postscript: We can note here the heroic efforts in the early twenty-first century as Islamic insurgents stormed Timbuktu to rescue hundreds of thousands of manuscripts dating from the early second millennium. Hundreds of individuals smuggled documents southward to safety, evading jihadists and bandits and corrupt officials.

A syncretic Islam emerged at first in West Africa, combining Islam with local beliefs. In particular, it treated women quite differently, with women often socializing with men, and baring not just their faces but also their breasts. This behaviour scandalized the famous Muslim traveller Ibn Battuta (chapter 14) and many other visitors from the north. Over time, Africans were encouraged toward a more orthodox Islam. The Fulani, a nomadic group, would pursue a stricter form of Islam, and from 1680 to the nineteenth century fought to establish orthodox Islamic states throughout the region. 


\section{Islam in Central Asia}

The spread of Islam in Central Asia was an unusually long and drawn-out process we will indeed revisit it in chapter 17. At the time of the Islamic conquests, Central Asia was characterized for the most part by small states that tolerated a variety of religions: Buddhism was the most common, but Zoroastrianism, Manichaeism, Nestorian Christianity, and Judaism were also common. There is evidence of syncretism: Buddhists, for example, borrowed fire-oriented practices from Zoroastrians. Muslim merchants and Sufi mystics would each establish a presence in the region. Civic leaders often proved willing to change religions as political realities around them changed; Arab leaders played cities against each other as they moved into the region (and promised not to levy special taxes on nonMuslims). Though Islamic rulers would not rule the northern parts of Central Asia until after the Mongol conquests of the twelfth century (chapter 17), Islamic

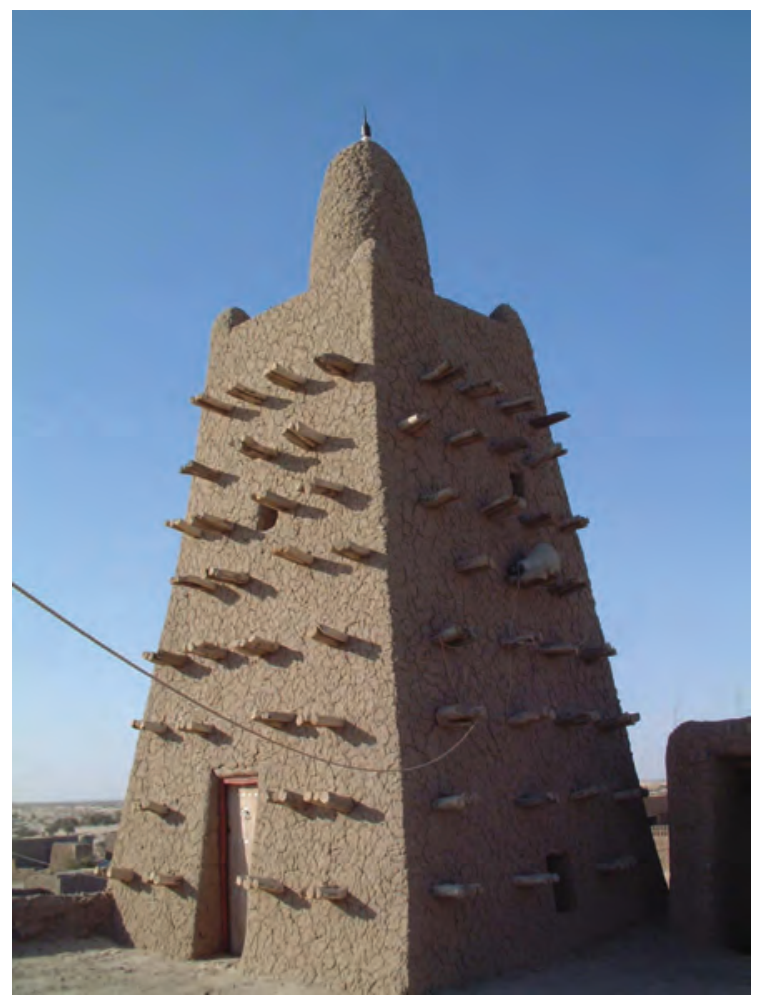

Illustration 12.11

The Djinguereber Mosque, Timbuktu, mud brick construction, 1327

Photograph by Taguelmoust, CC BY-SA 3.0 rulers conquered the southern parts of Central Asia much earlier (from whence the conquest of Punjab proceeded; see above). In these regions, conversion to Islam occurred over a period of centuries, with some choosing to migrate rather than convert. The vestiges of other religious communities remained for centuries: In particular, local wines continued to be produced, and local dancers remained famous as far as China. People around the world were horrified when the Afghan Taliban destroyed huge and ancient Buddhist statues at Bamiyan in Afghanistan in 2001. We should note, though, that these had survived 1,300 years under Muslim rule.

\section{Islamic art}

Due to military conquest, Islamic rulers governed vast lands filled with Christian and Zoroastrian art. The conquerors at first built upon these traditions: The Dome of the Rock in Jerusalem, one of the main Islamic sites in the world (from which Muhammad is thought to have ascended to heaven), clearly follows Byzantine architectural principles, albeit with the vital addition of Arabic 


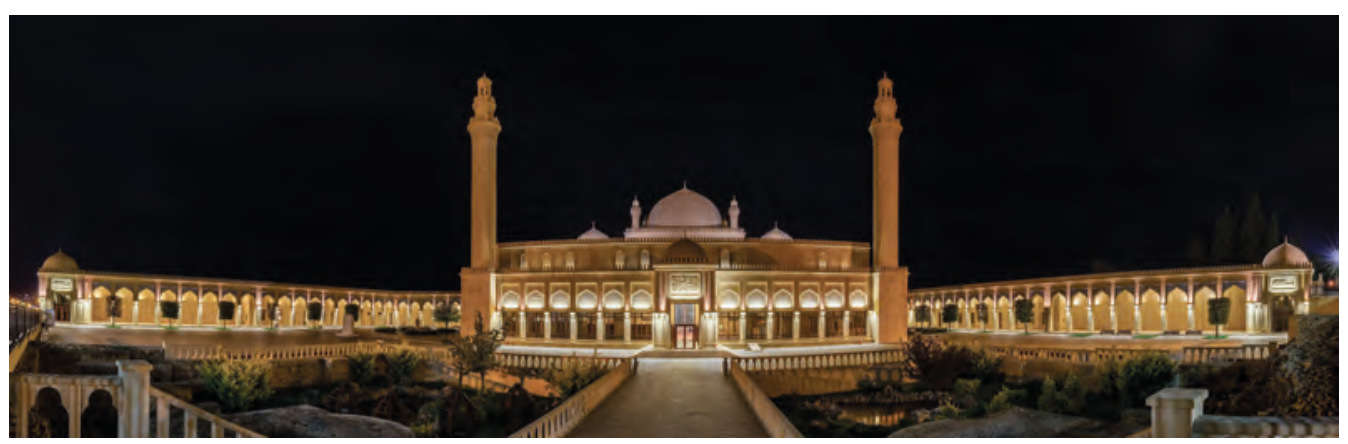

Illustration 12.12

Juma (Friday) Mosque, Shamakhi, Azerbaijan. This mosque, built in 743, is considered the first one in the Caucasus after the Friday Mosque of Derbent (constructed in 734). Reconstructed in 2009. (Note that Azerbaijan lies on what is generally considered the border between Europe and Asia) Photograph by Diego Delso, CC BY-SA 4.0

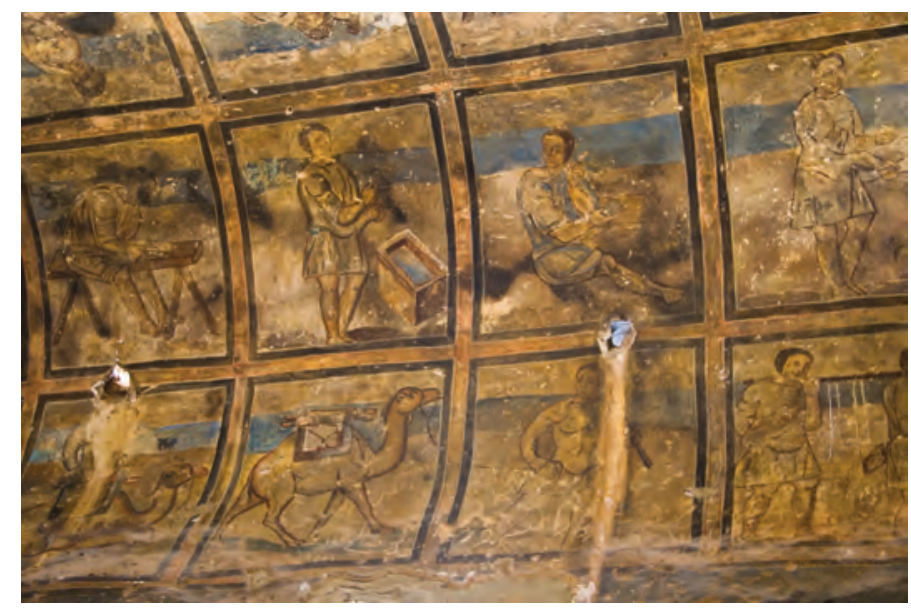

Illustration 12.13

Fresco, Audience Hall, Qasr Amra, Jordan palace bathhouse. The panels depict various artisans at work. Here we have camels transporting stone blocks and carpenters working above. The Islamic objection to figurative painting had not yet taken hold

Photograph by David Bjorgen, CC BY-SA 4.0

calligraphy. Over time, though, Islamic rulers sought to develop a distinct artistic tradition. We might then understand Islamic art as a reaction to these other artistic traditions. The Quran was critical of pictorial representations of religious figures, though this prohibition was ignored for a while in Central Asia (chapter 11). Moreover, pictorial representations of non-religious subjects, including naked women, were common in Umayyad and Abbasid palaces (the 


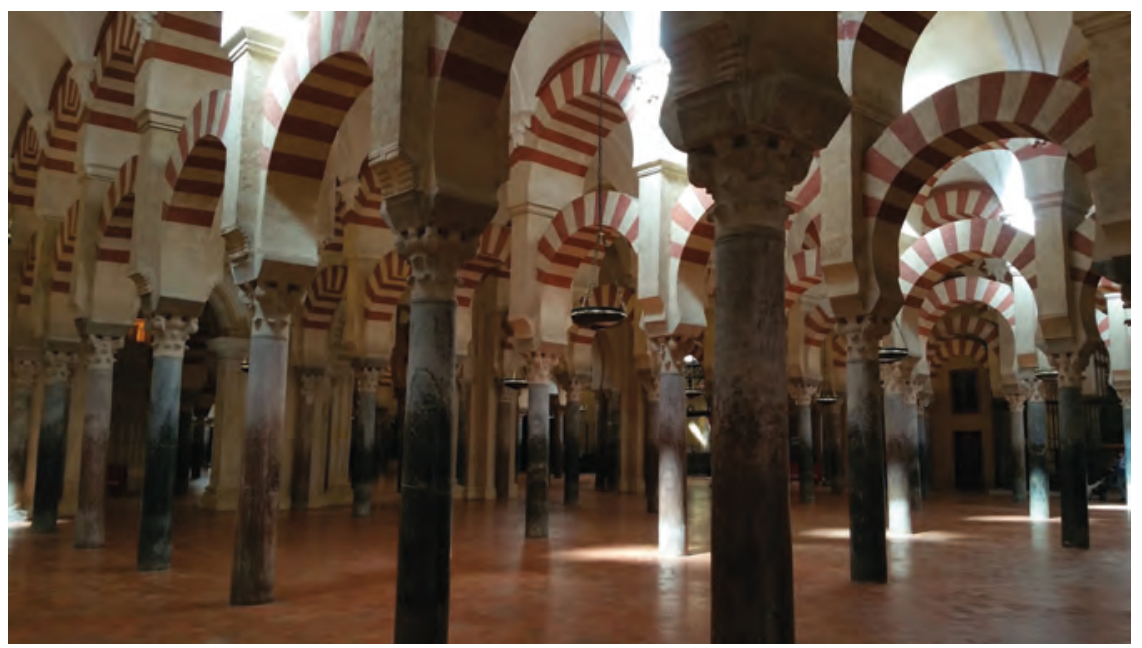

Illustration 12.14

Interior of the Great Mosque in Cordoba, built from the late 8th century and once the second largest mosque in the world. After Christian conquest in the 13th century it became a cathedral, and a large church has been built within the mosque

latter reflecting Persian influences). Over time, though, a preference for nonrepresentative art extended beyond the realm of the religious. Islamic art would focus on geometric designs and figurative depictions of plants. During Abbasid times, in which plaster-coated brick replaced Umayyad stone construction, a technique of shaping plaster into geometric designs was developed. Art scholars outside of Islamic lands often decried Islamic art as "merely decorative," and to this day it tends to figure less prominently in general histories of art. (There is debate as to how important Islamic art may have been in the emergence of abstract art in the West.)

Artistic borrowing nevertheless occurred. Islamic artists employed the mosaic techniques of others and applied Roman floral and geometric designs. In India, Islamic art incorporated elaborate local designs; Islam, in turn, introduced vaults and domes to India: The Taj Mahal (chapter 20) can be seen as the result of a centuries-long fusion of Indian and Islamic design. Middle Eastern carpets, which incorporated Islamic artistic designs, would be famous in Europe and elsewhere from the eleventh century, and would appear often in European works of art after that.

The earliest mosques duplicated church designs. However, the Great Mosque at Cordoba in Spain, dating from the eighth through tenth centuries, introduced horseshoe-shaped arches with alternating bands of light stone and dark brick. Such arches would become common in mosques worldwide. In the eleventh century, the four-vault (iwan) mosque - that is with large arched openings in four directions - was developed in Seljuk Iran; it would become the most common mosque design globally. 


\section{Questions}

1. How much do you think slaves benefited from Islamic rules regarding their treatment?

2. Refashion Figure 12.1 to capture the end of the period of Islamic conquest.

3. Discuss the similarities and differences between Islamic conquest and the expansion of earlier empires.

4. Why do we see religious diversity for so long in Central Asia, but the eventual dominance of Islam?

\section{Readings}

Bowersock, G.W. 2013. The Throne of Adulis: Red Sea War on the Eve of Islam. Oxford University Press.

Cowley, Robert, ed. 2001. What If?: Eminent Historians Imagine What Might Have Been. New York: Penguin, [Discusses how history might have unfolded quite differently if particular wars or battles had gone the other way, as they well might have.]

Elverskog, Johan. 2010. Buddhism and Islam on the Silk Road. Philadelphia, PA: University of Pennsylvania Press.

Kuru, Ahmet T. 2019. Islam, Authoritarianism, and Underdevelopment: A Global and Historical Comparison. Cambridge University Press. [Argues that the move toward state-supported religious orthodoxy from the tenth century hampered science, technology, and trade thereafter in Islamic lands. See also Jared Rubin, Rulers, Religion, and Riches, Cambridge University Press, 2017.]

Laudan, Rachel. 2013. Cuisine \& Empire: Cooking in World History. University of California Press.

Liu, Xinru. 2011. "A Silk Road Legacy: The Spread of Buddhism and Islam." Journal of World History 22:1, 55-81.

Marrozi, Justin. 2019. Islamic Empires: Fifteen Cities that Defined a Civilization. Penguin.

Safran, Janina M. 2013. Defining Boundaries in al-Andalus: Muslims, Christians, and Jews in Islamic Iberia. Ithaca, NY: Cornell University Press. [Explores the challenges of cross-religious interaction within a tolerant polity.]

Smil, Vaclav. 2017. Energy and Civilization: A History. Cambridge MA: MIT Press. [Celebrates the critical role of windmill and watermill technology.]

I am grateful to Duman Bahrami-Ram for sharing his research on the possible effects of Islamic inheritance laws on women. 


\section{Eurasia in the}

\section{centuries after the fall of the classical empires}

\section{Guiding questions}

What were the key political, economic, cultural, and other transformations in Eurasia after the fall of the classical empires? Why did these occur and what effects did they have?

What was the experience of nomadic societies of Central Asia in classical and post-classical times? How did nomadic groups form and migrate through time? How did they interact with neighbouring agriculture-based states? How did they prosecute both trade and war? How did nomadic military capability evolve?

In western Europe, history unfolded in an entirely different fashion. Why? Why and how did various groups of people migrate into western Europe at this time? What were the effects of these migrations? What is feudalism? Why did it emerge? How did it evolve over time? What effects did the feudal system have?

In the eastern part of the former Roman Empire, what is now commonly called the Byzantine Empire survived. In what ways did this differ from the Roman Empire? How did it survive for centuries? What influences did it exert on the course of history? 
What were the major changes in Asian societies in the centuries after the fall of the Han, Persian, and Gupta Empires? What sorts of states existed in different regions and what policies did they pursue? What were the key changes in religion, technology, social structure, and art in different regions? How did these societies interact?

Relationship to other chapters: The histories of different regions diverge more than converge in this period. The experience of the classical empires (chapters 9 and 10) provides an important point of comparison for these later historical developments. This chapter aids our general understanding of several issues, including imperial foreign policy, religion and state interactions and the spread of religion, and migration. Feudalism provides an important alternative to empire as a form of political organization. Our discussion of the causes and effects of feudalism thus indirectly informs our understanding of empire. Various issues in this chapter - the spread of Christianity northward, ethnic migrations, feudalism - would shape European history in succeeding centuries; the chapter will then prove particularly valuable in later discussions of Europe. Yet of course our discussions of Asia also inform later discussions of Asian regions.

One connection between Europe and Asia involves nomadic peoples: The actions of nomads in Central Asia pushed many groups to migrate into Europe. Our discussion of nomads in this chapter builds on our discussion in chapter 4; we shall find that interactions between nomads and agricultural societies - both trade and war - become increasingly important over time. This chapter informs many later discussions of such interactions but especially chapter 17 on the Mongol conquests.

The fall of the classical empires had quite different repercussions in different parts of the world. We saw in the preceding chapter how a new Islamic Empire displaced the old Persian Empire. In the eastern part of the former Roman Empire, the Byzantine Empire would survive for another thousand years though losing much of its territory to Islamic polities. In China, the reconstitution of a Chinese Empire would follow centuries of political fragmentation. In western Europe and India, though, political fragmentation would continue 
for several centuries. Small states competed in India. Europe would experience feudalism, a very decentralized form of political and economic organization.

We begin this chapter with a discussion of the nomads of Central Asia. Since we have not addressed nomads much since Chapter 4, we extend our analysis temporally to discuss both classical and post-classical developments. We shall find that nomads interact in important ways with most of the sedentary societies that we discuss later in the chapter. In particular, we turn next to a discussion of the influences on, nature of, and effects of nomadic migrations into Europe: These would change the political and cultural face of the continent over a period of many centuries.

We then pursue a discussion of the feudal system: This system is arguably unique in human history, reflected a peculiar set of historical circumstances, and would have a significant impact on European and world history. Since it lasts for over a thousand years, we carry the story of feudalism far beyond the temporal boundary of the rest of this chapter. Feudalism provides an alternative form of governance to centralized states; the differences become stark as we next turn our attention to the Byzantine Empire.

We noted in chapter 1 that we would be flexible in our treatment of time periods in this book. In the sections that follow on Asian societies, we pursue different time periods that make sense for particular regions. We trace Chinese history from the fall of the Han to the end of the Tang Dynasty in 907. We briefly outline Korean imperial history from the third century through to well after 1000. We also trace Japanese history from the third century but end at roughly 1000. Since we have not previously said much about Southeast Asia in this book, we provide a brief treatment of both pre-classical and classical developments, and then carry the story forward into the tenth century (and briefly beyond). For India, we begin with the fall of the Gupta Empire and carry our discussion of some Hindu kingdoms forward into the eleventh century so that we can then focus on Islamic incursions in chapter 15.

Politics is the central theme of this chapter. For feudalism especially, politics intertwines with the economy. Our lengthy discussions of migrations bring population and culture (in particular cultural identity) to the fore. Population is further implicated in a discussion of epidemics. In addition to its political and economic roles, the feudal system instantiates a set of social relationships. There are some brief but crucial mentions of technology and art. We employ evolutionary analysis most specifically in our discussion of Byzantine diplomatic strategy; this is also an important form of societal interaction. Though we address different societies in turn, we do discuss critical societal interactions: These are especially important in our treatments of Korea, Japan, and nomadic societies. Migration is one of the most potent forms of societal interaction.

Culture

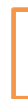

Social

Structure
Technology 


\section{Nomadic peoples of Central Asia}

We saw in chapter 4 that nomadism was practised as an alternative to agriculture from the early days of farming, and that agricultural and nomadic societies regularly interacted. We saw later that early and classical civilizations both traded and battled with nomads. Also, we noted in chapter 12 that the Islamic conquest of much of Eurasia benefited from the nomadic roots of the Arab people.

Nomadism is best suited to grasslands that do not receive quite enough rain to support agriculture but can provide a temporary food source for herds of animals. The Eurasian steppe is a massive grassland stretching from just north of the Black Sea in Europe through northern Iran to northwest of the Chinese heartland; after a break, it then resumes in Manchuria (see map in chapter 4). Though much of this land is farmland today, only small pockets oases - could be farmed with the technology of early times. These oases nevertheless generated some degree of urbanization within nomadic lands - though urbanites were often ethnically distinct. Beyond these oases, cattle, goats, yaks, and especially sheep (which provided wool as well as milk and meat) were herded by nomads riding either camels or - more commonly, and throughout the northern steppe - horses. These horses tended to be short and stocky, able to travel one hundred kilometres in a day, and able to dig for grass under snow (they were also a good source of milk).

\section{Nomads and sedentary societies}

This vast area of grassland gave access to most of the world's "civilizations": Nomads from the steppe could and did attack west into Europe, south into the Middle East and India, and east into China. The much larger agricultural populations

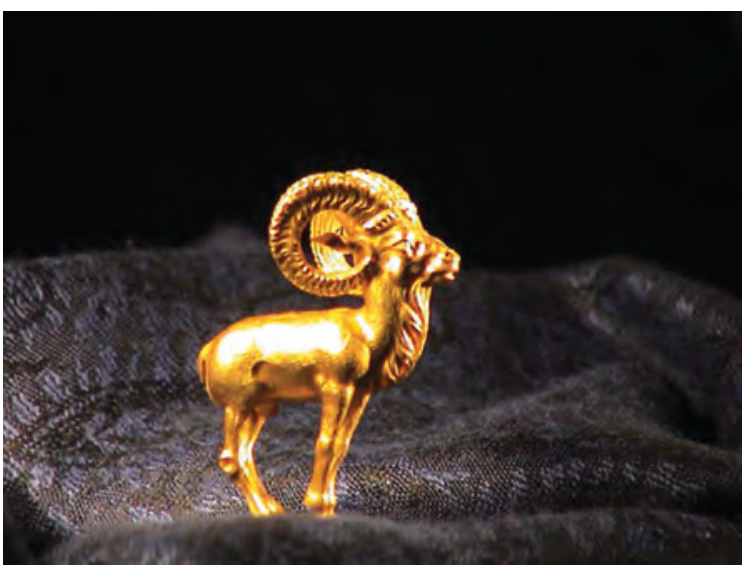

Illustration 13.1

Golden Ram, lst century BCE, Bactrian Afghan National Treasury that surrounded them attacked nomads in turn, especially if these sought to farm on some of the nomadic lands. The Chinese in the east, Arabs in the south, and Vikings and Slavs in the west were each expanding onto the steppe in the post-classical period. Nomads could be self-sufficient but traded for grains, some metals, and luxuries and ornaments like tea and silk. The nomads, in turn, exported animals and animal products, metals and metal goods (including swords), furs of various sorts (that they traded for with hunter-gatherers to the north), fish, and melons from Central Asian oases. Nomad peoples mastered metalwork, leatherwork, 


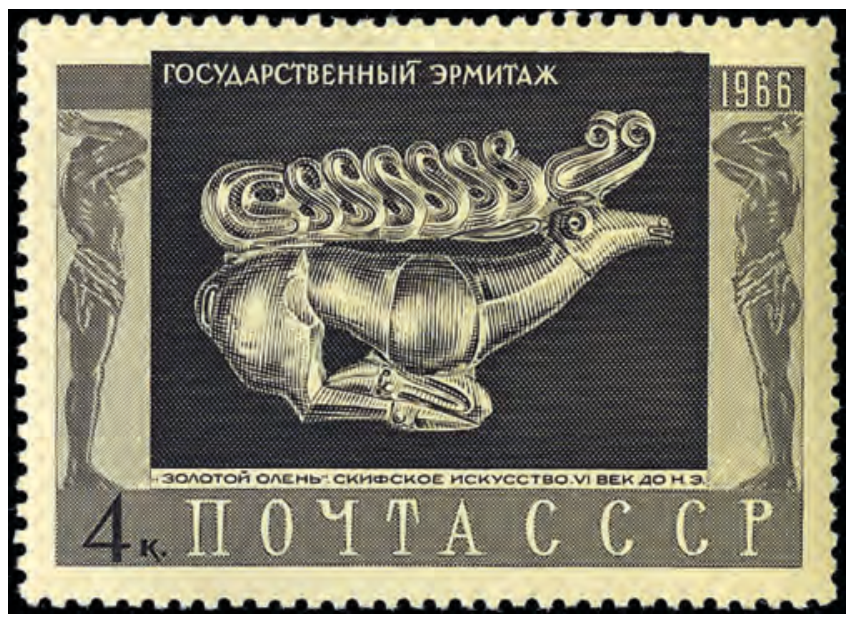

Illustration 13.2

Scythian (nomadic) art on Soviet stamp, 1966. The original gold ornament is in the National Museum in Prague and also the Hermitage

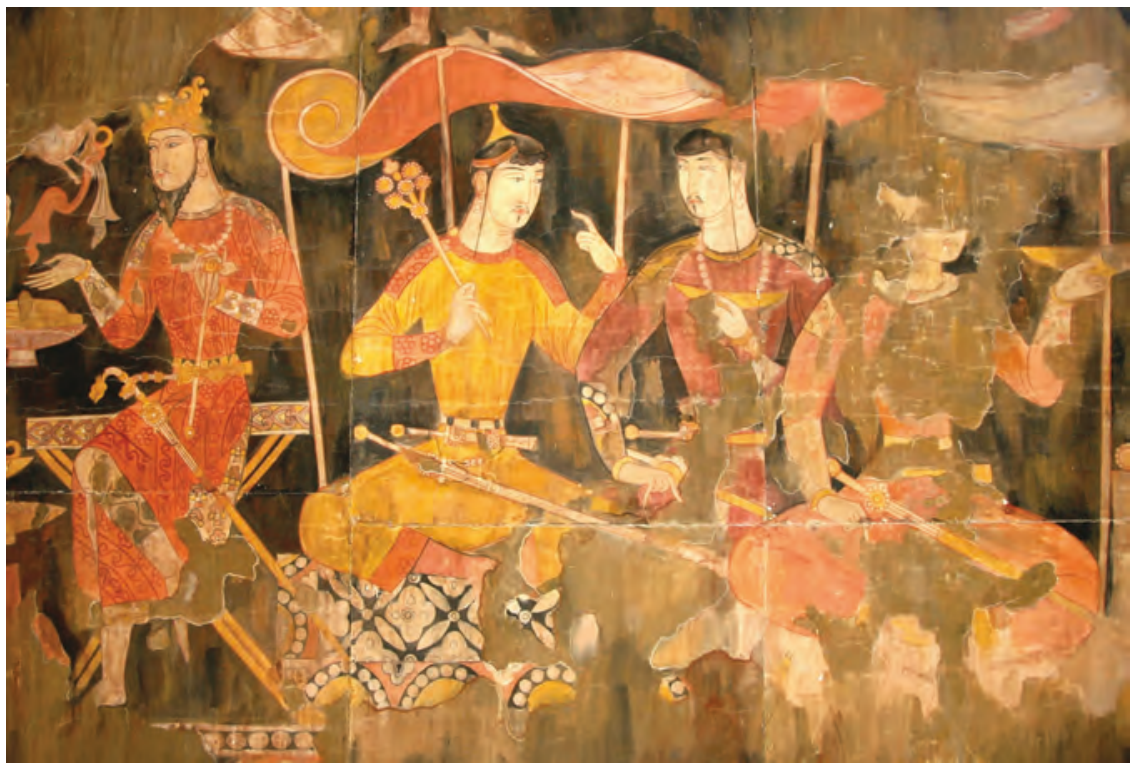

Illustration 13.3

Sogdian painting of Sogdian merchants, date unknown, Sogdia is the region surrounding the Central Asian trading centre Samarkand

and other artisan tasks, notably how to construct bows and arrows; they thus exported "industrial" goods as well as raw materials.

Special mention might be made of horses. Nomads traded tens of thousands of these with the Tang Chinese at a price each of twenty-five bolts of silk or more. India also regularly imported large numbers of horses from Central Asia 
for service in the military and transport. There was even trade in horses from Central Asia (and Arabia) to what is now Indonesia - a testimony to the strength of both the ships and trading networks of the time.

Nomads often raided agricultural societies for plunder. When they did conquer a sedentary society, they faced challenges in governance. Nevertheless, and despite the tendency of nomad societies toward fragmentation and succession disputes, nomad conquerors often formed stable polities in the lands they conquered. We have already described the vast polities established by Islam (chapter 12). Though the similarly vast Mongol Empire would be short-lived, the Mongols would found a dynasty in China that would survive for generations (and another nomadic people from Manchuria would later establish an even more long-lived Chinese dynasty; chapter 20). Descendants of Mongols and Turks would unify much of northern India (chapter 20). In India, at least, political fragmentation would likely have continued if not for nomadic conquest. Even in western Europe, the once-nomadic Germanic tribes established the national monarchies that replaced Roman rule.

Not surprisingly, writers in sedentary societies often sought to understand nomadic peoples. This they often did with a healthy dose of ethnocentrism. There was very little suggestion that nomads might somehow be educated toward more "civilized" behaviours. Instead, it was generally felt that nomads would always exist and always be different. Writers often then suggested some grudging toleration of cultural differences. This set of attitudes is evident in the ancient Greek Herodotus, Sima Qian in China (who knew nomads better than most; chapter 10), and the Islamic writer Ibn Khaldun.

\section{Nomadic military technology and organization}

The combination of skill with horses and hunting and especially the composite bow gave nomads a military advantage for centuries - until modern firearms would appear after 1600. Yet we should appreciate that the armies of agricultural societies often severely outnumbered nomadic forces. Nomads had attacked surrounding agrarian areas in the first century вСЕ, and significant incursions occurred in the fifth, tenth-thirteenth, and fifteenth-seventeenth centuries CE. There may have been a climatic impetus to at least some of these incursions: Nomadic life was tenuous, such that an unusually harsh winter or dry summer could impel a mobile population to look elsewhere for food. There were also technological roots of nomadic expansion: The first nomad outflow likely reflected the development of the war chariot around 2000 всE. Improvements in chariots and then the composite bow might have encouraged later nomadic incursions.

The composite bow dates from at least 1000 BCE and was likely developed first by agriculturalists. This bow would be a dominant weapon for thousands of years, and so merits some description. The key to the composite bow is a combination of horn, wood, and sinew, glued together. This combination provides many times the tension of wood alone. It could fire arrows with much 
greater force as a result. Composite bows also had some severe drawbacks. They were much harder to make. The glue the nomads used weakened if wet: Nomad archers were thus limited in wet terrain or rainy conditions. The composite bow was also hard to learn to use. It seems that nomads improved the composite bow considerably over time. It is hard, though, to trace its evolution, for bows rarely survive in the archaeological record, and textual descriptions are vague and often exaggerated.

Another key innovation was the stirrup: Riders are much more stable on horseback - and then far more able to fire an arrow or slash with a sabre - if they can solidly brace their feet in a stirrup. However, stirrups will put too much strain on a horse's back unless connected to a saddle that distributes the rider's weight. Though some sorts of loops for a rider's feet are found in India in the last centuries BCE, the stirrup seems to emerge in China in the fourth century CE and spreads to Central Asia by the sixth century.

Nomads by their nature are mobile. Whereas farmers organized geographically in villages, nomad society tended to be organized in clans based on descent from a common ancestor. However, nomads could be adopted by or pledge loyalty to a leader to whom they had no known relation. Clans could combine into tribes. These tribes might also reflect shared ancestry but might also emerge through shared attraction to a charismatic leader. Clan leaders chose tribal leaders from among the chiefly class, and usually because of success in battle. Even when tribal leadership was hereditary, sons contested it. There was always considerable scope for succession crises within nomadic societies - but also the potential for a charismatic leader with military skill to forge a sizeable tribal following quickly. Such tribes might take on some of the attributes of states: they might seek to control a large territory and might employ bureaucrats; courts and capitals were usually mobile.

We discussed in chapter 4 the challenges facing nomads. While nomads, who grazed over large areas, leaving manure as they moved, did not suffer the sustainability challenge that farmers faced, they most certainly had to protect their stuff. Indeed this concern was much more significant than for farmers, for nomadic herds were inherently mobile. An individual nomadic family could be easy prey to other nomads or hunter-gatherers or even groups of farmers. These might try to steal individual animals or the entire herd. Once bandits captured a herd, they could move it easily - much more easily than bushels of grain. The very mobility of nomads made it likely that they would cross paths with potentially hostile strangers from time to time. Nomads could not solve their protection problem in some of the ways that farmers could. Most obviously, a wall or a fort was of little use to those who wandered over large areas. Nor was a state defined territorially with an army on its borders a viable option most of the time for thin nomad populations. Flight was an option, but only if your attacker moved more slowly than the slowest member of your herd (and nomads after the invention of the wheel would also have wagons full of stuff). What then does a nomad family do? It binds itself to other families. Groups of families might find from time to time that they needed to combine into yet 
larger groupings to deal with potential threats. The nature of nomadic tribes as described above then provides a solution to the nomad's protection challenge. One may imagine a more egalitarian solution that does not insist on hereditary or charismatic leadership. The stratified solution not only reflects the inevitability that some nomads would end up with larger herds (through differences in skill, luck, and family size) but also provides a mechanism for organizing people for battle. In any case, the key point to stress here is that an idyllic landscape of independent nomadic families each mindful of their own herds was not an option. The basic nomadic challenge of protection necessitated some form of nomadic organization for defence. Of course, the defensive motive for tribal organization is hard to distinguish from the offensive motivation: Once a tribe formed, it could as easily attack as defend. Like the states of sedentary societies (chapter 6, 10) it might easily decide (convince itself) that another group was dangerous and stage a pre-emptive strike. And just as battles between sedentary states encouraged increasing army size and advances in military technology and organization, battles among nomads (or with sedentary neighbours) encouraged the formation of larger tribal confederacies and the development of nomads' own technology and strategy.

We noted above that there were social, economic, and political inequalities in nomadic society. It is hard to compare these to the inequalities associated with agricultural societies. It is also difficult to compare average incomes: Nomads and agriculturalists consumed different sets of goods, and we have little detailed description of nomad life. We can note that people often moved between the two worlds. Nomads would worry about adopting sedentary ways when they conquered sedentary societies. Poor peasants and slaves often ran from agricultural societies toward the seeming freedom of the steppe. The walls that the Chinese and others would build to keep nomads out often served also to prevent such escapes.

\section{Particular nomadic groups}

Since nomads had no written records until the ninth century CE (see below), and since nomadic tribes could readily coalesce around a new leader, historians are often unsure of the relationships among the nomadic tribes that appear from time to time in the histories of the surrounding societies, or of precisely where certain tribes came from. Was there a connection between the Xiongnu that harassed the Han dynasty and the Huns that a couple of centuries later attacked India and Europe? (chapter 10). This is indeed possible, for nomadic groups migrate over vast distances. However, beyond the similar sound of their name, and perhaps a few artefacts they shared such as cooking pots (but these were also shared with other nomadic groups), there is little evidence to support the conjecture. Given the tendency of nomadic groups to fragment and coalesce in a different form, it is unlikely that the Huns were in their entirety descendants of Xiongnu. The Huns were more likely another coalescence of clans around a charismatic leader. More generally, though linguistic and sometimes genetic or 
archaeological evidence can help us trace the origins and movements of particular groups, historians often find themselves in the same position as the agricultural societies of the day: New nomadic groups suddenly appear and trade and fight and then disappear.

We do know that the Xiongnu moved west after their defeat by the Han (and may have been expanding west even before). Whatever their relation to the Huns, the westward movement of one nomad group was likely to push others westward. These might then invade sedentary societies. Indeed, this westward nomadic movement may be associated with the founding of the Kushan Empire (chapter 9). It may be no coincidence that Lombards and Avars invaded Europe while the Tang Dynasty was strong in China; or that later the Slavs and Magyars invaded while the Song was strong, and still later the Turks moved into the Middle East while the Ming was strong (we describe some of these movements below).

Fights amongst nomadic peoples themselves could push some nomadic groups outward - as when the Huns pushed Germans into Europe (below), or Babur was forced from his Central Asian home toward India (chapter 20). Also, population growth or environmental misfortune within the steppe could push nomads outwards as well: This may explain why nomadic incursions were more severe in some centuries than others (see above). Drier weather may, in particular, have played a role in encouraging the Mongols (and their nomad collaborators) to move outward (chapter 17).

It is not clear where the Turks came from; they appear as a group from the sixth century $\mathrm{CE}$, though the Turkic language group that they share emerged earlier. They would be the first single nomadic group to link China and the west,

\section{BOX 13.1}

\section{HEAD-BINDING}

There are multiple reports that some early nomads such as the Huns (and some of the Germanic tribes that allied with these) bound the heads of the young to shape the skull as it developed. We must always be careful of descriptions of nomads that come only from the agricultural societies that often viewed them with a mix of suspicion, disdain, and fear (the nomadic Massagetae were alleged to practise ritual cannibalism of the elderly). Yet such a practice seems amazingly widespread in human history, and across human groups that had little or no contact with each other. Hippocrates described the practice among the "Longheads" in 400 BCE. In the Americas, the Olmec, Maya, Inca, and Choctaw, among others, practised head-binding. In the Pacific, it was observed among Australian aborigines and in Samoa, Tahiti, and Hawaii (and may still be practised in Vanuatu). These various societies differed in the desired skull shape: The Olmecs arguably sought to mimic ears of corn. None of these groups would have appreciated the importance, complexity, and fragility of the human brain when they pursued these practices. 
and facilitated trade flows between the two. Both the Byzantines and Chinese noted the wealth of their - mobile - court. Like many nomadic groups (and indeed sedentary states) both before and since, the Turks were fractious and often battled over succession. Tang expansion would topple Turkic authority in the eighth century.

The Turkic people would fragment into several distinct groups. Like the later Mongols, Turkish clans could be organized into large confederacies under a charismatic "khan." Although originally animist and shamanist in religion, they converted to Buddhism, Nestorian Christianity, or Manichaeism as they encountered these. One of these Turkic groups, the Bulgars, would conquer a Slavic people in southeastern Europe but then be converted to Orthodox Christianity and adopt a sedentary lifestyle. There would briefly be a large Bulgar empire in southeastern Europe in the ninth century; its capital was said to rival Constantinople in grandeur. Other Turkic groups would move into Anatolia (now part of "Turkey"), Turkmenistan, and many other areas, converting to Islam along the way and spreading that faith to new areas with their conquests (converting Christians in Anatolia and Hindus and Buddhists in India and parts of Central Asia); we will meet them in many other chapters. Despite adopting Islam, Turkic rulers often clashed with clerics.

The Khazars formed a large state in southern Central Asia (comprising present-day Afghanistan, the Caucasus, and some areas to the north) between the seventh and tenth centuries. Though the Khazars pursued many faiths, and the state practised toleration, Khazar rulers adopted Judaism from the eighth century. This decision served a strategic role: Khazari rulers avoided having to admit the suzerainty of either Muslim caliphs or Byzantine Christian authorities. Jewish scholars travelled from both Constantinople and Baghdad to instruct the ruling family in their new faith. It does not appear that large numbers of Khazars converted to Judaism, and scholars generally doubt that many if any modern Jews descend from Khazaris.

The Seljuk Turks, after conquests in nearby regions of Central Asia, would be invited to Baghdad by the Abbasid caliphs - as many empires before and after had done with nomads on their borders - with the unsurprising result that the Turks eventually came to control the caliphate. The caliphs became religious figureheads, and the Turks assumed the title of "sultan" ("chief"; chapter 12). Many Turkish groups would later align with the Mongols. The defeat in battle of the Byzantine Empire by the Seljuk-dominated Abbasid Empire in 1071 facilitated Turkish migration into Anatolia.

The Uighurs developed an empire in the eighth and ninth centuries in what are now Mongolia and Manchuria and parts of Siberia. They never controlled quite as much territory as the Turks, but unlike the Turks, they built a capital city: Karabalghasun, in present-day Mongolia a bit northeast of the later Mongol capital. They also adopted a script from the Middle East from the ninth century. Indeed Uighur society would produce an extensive literature; a third of the population may have been literate. The Uighur experience indicates that nomads could transition successfully to a more sedentary lifestyle. The 
Mongols would later employ Uighurs in their bureaucracy: They not only provided record-keeping skills but knowledge of both nomadic and sedentary ways (chapter 17). The period after the ninth century likely saw a more general appreciation of sedentary ways among nomadic peoples, as trade links between the two expanded.

Indeed, as noted above, there had long been oases within the steppe that practised agriculture. These often supported towns, especially the merchant towns along the Silk Road. These towns were often quite wealthy and celebrated for their architecture. We saw in chapter 11 that Buddhism had early spread along the Silk Road, nurtured by monasteries and schools. These same towns would later become Islamic centres. Indeed the famous Islamic scholar Ibn-Sina (Avicenna; 980-1037), who wrote treatises on philosophy and medicine (which were used as texts in medical schools in Europe into the mid-seventeenth century and also in China), long prospered in Bukhara - which had extensive libraries - before moving to Baghdad as a result of political uncertainty. Buddhism and later Islam would spread from these towns to the surrounding population.

The Qara Khitai (sometimes called the Western Liao) were a nomadic group that had ruled part of northern China but were pushed west by Manchurian nomads allied with the Chinese Song Dynasty (chapter 15). They would conquer Islamic polities in southern Central Asia, ruling there from 1124-1218. Unlike the Mongols, they would not convert to Islam as they had previously adopted Chinese culture, but they managed to maintain the loyalty of Muslim citizens who were tired of political conflict. The idea of just rule had emerged within the Islamic world, and a saying was attributed to Muhammad that a just infidel was better than a corrupt Muslim ruler. The resumption of larger trade flows with China also helped to encourage loyalty to the new rulers. The Qara Khitai generally practised religious toleration - though a later Buddhist ruler may have destroyed mosques and harassed Muslims. The idea that Muslims could accept the rule of non-Muslims would prove useful when the Mongols later moved into this region.

\section{PRIMARY DOCUMENTS: IBN KHALDUN'S THEORY OF HISTORY}

Though Ibn Khaldun (1332-1406) writes late in the time period addressed in this chapter, he attempted to understand the periodic conquests of nomadic peoples we have mentioned here. His resulting theory of the rise and fall of dynasties would become widely known, as would his emphasis on the group feeling which could cause people to set aside selfish quarrels for group purpose.

A dynasty can be founded and established only with the help of asabiya [group feeling] ... Royal authority and the foundation of dynasties are the goal of asabiya. A dynasty 
goes through different stages and encounters new conditions. The first stage is that of success, the overthrow of all opposition and the appropriation of royal authority from the preceding dynasty. The second stage is the one in which the ruler gains complete control over his people, claims royal authority all for himself ... and prevents them from trying to have a share in it. The third stage is one of leisure and tranquillity in which the fruits of royal authority are enjoyed ... the acquisition of property, creation of lasting monuments, and fame. The fourth stage is one of contentment and peacefulness. He adopts the tradition of his predecessors and follows closely in their footsteps. The fifth stage is one of waste and squandering ... the ruler wastes on pleasures and amusements ... acquires ... low class followers. In this stage, the dynasty is seized by senility and the chronic disease from which it can hardly ever rid itself, for which it can find no cure, and, eventually, it is destroyed. ... Provincial governors ... gain control over remote regions when (the dynasty) loses its influence there. Each one of them [attempts to found] a new dynasty ... to gain royal authority.

Ibn Khaldun, The Muqaddimah

How well did Ibn Khaldun understand historical processes? Would he have been surprised by the survival for six centuries of the originally nomadic Ottomans, who were rising to power as he wrote (chapter 20)? Does he exaggerate the role of royal complacency in imperial decline? Why might he have thought this way?

\section{"Barbarian" incursions into Europe}

The fall of the western Roman Empire was associated with migrations of several Germanic-speaking tribes into various parts of that Empire. These groups had practised shifting agriculture for centuries in the area to the east of the Rhine and north of the Danube. These movements were in turn encouraged by movements of the Huns into Europe. There would be two later nomadic incursions associated with the Avars and Magyars. There would also be the migration of another (shifting) agricultural people, the Slavs, who would come to dominate half the European landscape. In addition to these incursions from the east, there were also incursions southward out of Scandinavia by the Vikings. After about $1000 \mathrm{CE}$, though, these large-scale incursions largely cease, and the ethnic map of Europe changes relatively little after that (except for boundaries between Christianity and Islam).

Historians have sometimes thought that at least some of these migrations involved movements of millions of people. More recently, it has been common to doubt that large numbers moved at all. The truth likely lies in between: Most of these historical episodes involved tens of thousands of people moving at a time - with the Germanic and Slavic events involving several such migrations at different times by different groups. These movements were generally preceded by decades of raiding by smaller raiding parties. The larger group learned in this 
way of the attractions of the new land. Note that all of these migrations moved toward areas of the Roman Empire or lands that had been tributary to this: We discussed above critical "push" factors among nomadic groups on the steppe, there was always also the "pull" of relatively prosperous regions that were or had been within the Roman orbit.

Though "tens of thousands" is a large number it was in most cases small relative to the size of the populations into which these migrants moved. Only rarely were these new people invited, and the lands they moved into were hardly empty. The migrating groups, therefore, needed armies of thousands and came to dominate local peoples politically and economically, but over time intermarried until local and migrant were indistinguishable. This process would involve cultural synthesis: In some cases, the conqueror language became dominant (as when Slavs moved into Germanic areas) while in others the conquerors adopted local languages (as when the Germanic Franks adopted a Latin language).

Both successful raiding of wealthier foes and especially migrations of tens of thousands of people into generally hostile lands require leadership. Often smaller tribal groupings coalesced into larger entities around a war-leader: That is, a much larger kingship absorbed a set of minor tribal leaders or kings. The latter were enticed by the riches of the lands to be plundered or occupied. Ethnic identity was often forged during this process of coalescence, and it was far from unusual for nomadic and agricultural people to merge for the purposes of raiding and migrating.

\section{The Germans}

Germanic speakers had been interacting with the Roman Empire for centuries along its northern border. Germanic languages were at the time dominant in many areas - such as Poland to at least the Vistula and the Czech Republic - that now generally speak Slavic languages. The Romans traded and fought with the Germans. The trade - Roman legions had to be fed and otherwise provisioned, after all - undoubtedly meant that the Germanic tribes nearer the border had higher incomes than those farther away, but not so high as people within the Empire. Archaeological digs do indeed suggest that wealth declined as one moved north and east. There is also evidence that the Germans adopted Roman agricultural technology, including heavier ploughs and the use of animals and manure (which likely encouraged a more settled agriculture). Interactions with Romans helped produce the sort of surplus - in this case from both agriculture and trade - that finances a political elite. Moreover, the Romans often hired Germans as mercenaries or paid them not to attack. There is no sign of formal bureaucracies or taxation in Germanic lands: The primary sources of kingly income may well have been from plunder and Roman subsidies. These kings could compel forced labour, and often forced Germans to work for Romans. Germanic tribes too far from the border to benefit in other ways from Rome may have pursued a slave trade. 
Though German kingships were hereditary, kings were often overthrown, and there appears to have been a robust set of subsidiary kings and perhaps formal councils. Despite Roman efforts to discourage political consolidation the Romans would often hire one Germanic group to fight others - it appears that over the first through fourth centuries ever-larger political groupings emerged among the Germans. This increased their bargaining power with the Romans: A Roman legion could take care of smallish Germanic groups but would struggle if these could mount armies of several thousand.

The movement of the Huns into modern Hungary, and the raids these launched across Europe (see below) encouraged various Germanic groups to move. The relative importance of push and pull factors is unclear, but both were in play. It is quite likely that these groups achieved further consolidation as they moved: One does not move into richer farmland unless one has a formidable force. A group or leader that proved militarily successful would attract other followers.

We shall see below that the Byzantines encouraged the Huns to attack west rather than south. There is some suggestion that they did the same with the Germanic Goths, and may even have sought to weaken the western Empire in order to reassert eastern authority. Certainly, both Goths and Huns would decide to focus their energies on the western rather than eastern Empire. This may merely reflect the fact that the western Empire was weaker, or that it had a much longer border with Germanic peoples. The western Empire would face additional challenges in fending off Germans because it was devoting some troops to fighting with Constantinople at the same time. Whatever the reasons for the original Germanic choice to attack west, once one Germanic group achieved success in crossing the Roman border, others were encouraged to follow.

These groups often moved multiple times. Most famously the Vandals likely started in Slovakia (and their nomadic allies, the Alans, who spoke a language related to Persian, began thousands of kilometres farther east), moved into France and Spain, and then into North Africa, a migration of some 4,000 kilometres over a couple of generations. It is likely easier for a group that has moved once to move again (and the Germans had probably been practising shifting agriculture for centuries and so were somewhat mobile to begin with). The Goths moved into Italy and France, the Lombards into Italy, the Franks into France, and the Angles and Saxons into Britain ("France" and "Lombardy" and "Anglo-Saxon" being post-migration descriptors of these places). Such groups established themselves in positions of power over large regions but were ready to move if a more prosperous region presented itself or if Roman retaliation seemed imminent. It is not entirely clear how much the Vandals or any other Germanic group remained intact over decades: Some followers may have stayed behind along the way while new followers would be attracted to a successful group.

Notably, these Germanic groups did not set out to destroy Rome. It was far more profitable to plunder the Empire and negotiate subsidies for "loyalty" 
with the emperor (the modern connotations associated with "vandal" signals the importance of booty). Yet the fact that several Germanic groups moved into different parts of the western Empire dramatically reduced the Empire's income. Eventually, one group, the Goths, would take Rome itself, and attempt for a while to run the Empire themselves. By this point, decentralization of authority had likely proceeded too far. Different Germanic tribes would establish states in different parts of the old Empire. The eastern Empire would try to retake much of the former western Empire but would have only temporary success. The battles between Goths and Byzantines may have prevented either from being able to recreate the western Empire.

Though the western Empire fell apart, various Germanic groups maintained some authority in different regions. The most successful were the Franks, who not only consolidated power within the French parts of the old empire but also extended power eastward into lands that Rome had never conquered. These new Germanic polities were limited inheritors of Rome: They had limited bureaucracies with which to tax agriculture, and trade withered after the decline of Rome (see chapter 15). The rise of the Franks signals the increased importance of northern Europe relative to the Mediterranean region. Over time, the Frankish kingdom would suffer as local aristocrats gained lands previously held by kings and managed by counts (who shared the revenue with the king).

\section{The Huns}

Who were the Huns? As with other groups on the move, they were an agglomeration. It is clear that Goths and other Germans were part of the Hun forces, though not always voluntarily (indeed Germanic was the common internal language). The Huns thus combined nomadic and agricultural people. It appears that the Goths had earlier attacked the Huns only to be subdued by them. As to who the core Hun group was or where they came from we know little, for the Huns themselves left no written record and would soon disappear from history (itself evidence that the sense of shared identity among the Huns was limited). After decades of raids against both western and eastern Empires - and occasional employment as mercenaries - from a base around the Black Sea, the Huns would coalesce around Attila, move into Hungary, and attack for plunder deep into France. They may have been initially pushed westward by a drying climate on the steppe but would become attracted by the riches of the Mediterranean. Attila's rise to power may well have reflected his ability to exact resources from both Empires. As with the Germans, there was almost no Hunnic bureaucracy; the continuation of a Hun polity depended on the continued extraction of resources from other lands.

\section{The Slavs}

It is not clear where the Slavs came from, though likely somewhere to the east of the Carpathian Mountains. Historical records first mention them in the sixth 
century attacking outposts of the Byzantine Empire along the eastern Danube. Over the next centuries, they would first raid (and be employed as mercenaries by the Byzantines) and then migrate into the Balkans. We see in the Balkans a similar process to that observed among the earlier Germans by which Slavic groups coalesced into larger entities as they transitioned from raiding to conquering and migrating. Over the same period, but in a less documented process, Slavs would also spread northwest into modern Poland and the Czech Republic, absorbing Germanic speakers, and northeastward into Russia, incorporating speakers of Baltic languages. Slavic languages now dominate across half of the European landmass.

Just as Germanic groups had been pushed westward by the Huns, the Slavs were driven westward by the nomadic Avars, who in turn were pushed westward by the Turks (above). Just as Germanic groups were co-opted into the Hun forces, it could well be that Slavic groups occasionally were gathered into nomadic forces.

According to one Roman commentary, the Slavs were more willing than other groups to welcome those they defeated into Slavic society as equals. We cannot know if this was a general Slavic predilection. In any case, the Slavs, like the Germans before them, would become indistinguishable over time from those they conquered: the Latin speakers of the Balkans, Germanic speakers of what is now Poland, and Baltic speakers of parts of Russia. These subject peoples would come to speak Slavic languages over time, though they must have formed a majority of the population in many places. Expansion into Germanic areas was aided by the migration of Germanic speakers westward (above). The Balts may have suffered from a more egalitarian political structure that may have limited their ability to coordinate defence.

Just as a handful of Germanic states replaced the Roman Empire, a handful of states would emerge in these new Slavic territories: briefly Moravia, then Poland, Russia, and Bohemia. These states would slowly foster a shared sense of identity over time. It appears that the Slavs (and Balts) at first had similar gods to the Vikings (below), but they have left no written records of these beliefs. Their rulers would convert to Christianity, mostly in the tenth century. We will see below that the Orthodox Church would convert the Russians and some southern Slavs; Catholicism would reach the Poles and their neighbours first. While the process of conversion of rulers was underway, it was typical for Christian and non-Christian rulers and peoples to be found on both sides of battlefields. To be sure, some Christian rulers sought to spread the faith through conquest - and some non-Christian leaders converted to gain their support but most were willing to collaborate pragmatically with non-Christians. These early Slavic states, like their Germanic counterparts, had very limited bureaucracies, and kings could only rule through local elites; they travelled around to meet and exact support from locals. Yet these kings built impressive networks of castles (which dot the landscape to this day) and stationed permanent troops in these, and built roads and bridges. 
For the Russians and Bohemians, we know that the slave trade was an important source of kingly revenue; we suspect that the Polish state was also involved. (The Russians also profited from a fur trade to the north.) We further suspect that the primary sources of slaves were Slavic and other groups that lay between the Polish, Russian, and Bohemian states. Indeed some would characterize the frequent but inconclusive wars between Poland, Russia, and Bohemia in this era as concerned mainly with exacting slaves from the hinterlands between them while rarely threatening the core territories of the others. The size of this slave trade was such that the word for Slav would come to be associated with slavery itself. The primary market for slaves was the Islamic world, but Constantinople was also important, and some slaves were shipped west. This slave trade may have been far more lucrative than the earlier trade between Germans and Romans. Reports by visitors from both Islam and Constantinople establish that this northern slave trade was harsh, with rapes of women and castration of men frequent occurrences. The peasants in Slavic lands that were not sold into slavery were gradually enserfed (see below), while agriculture was slowly transformed by the increased use of heavier ploughs (perhaps only from the eleventh century).

\section{PRIMARY DOCUMENTS: AN ARAB IMPRESSION OF THE SLAVIC SLAVE TRADE}

The Arab diplomat Ibn Fadlan was sent as part of a mission to the Volga Bulgars in 921 ce. He may be describing here Viking slave traders:

They are the filthiest of all Allah's creatures: they do not clean themselves after excreting or urinating or wash themselves when in a state of ritual impurity (i.e., after sexual intercourse) and do not wash their hands after food. Indeed they are like asses that roam.

... They gather in the one house in their tens and twenties, sometimes more, sometimes less. Each of them has a couch on which he sits. They are accompanied by beautiful slave girls for trading. One man will have intercourse with his slave-girl while his companion looks on. Sometimes a group of them comes together to do this, each in front of the other. Sometimes indeed the merchant will come in to buy a slave-girl from one of them and he will chance upon him having intercourse with her, but will not leave her alone until he has satisfied his urge. They cannot, of course, avoid washing their faces and their heads each day, which they do with the filthiest and most polluted water imaginable. I shall explain. Every day the slave-girl arrives in the morning with a large basin containing water, which she hands to her owner. He washes his hands and his face and his hair in the water, then he dips his comb in the water and brushes his hair, blows his nose and spits in the basin. There is no filthy impurity which he will not do in this water. When he no longer requires it, the slave-girl takes the basin to the man beside him and he goes through the same routine as his friend. She continues to carry 
it from one man to the next until she has gone round everyone in the house, with each of them blowing his nose and spitting, washing his face and hair in the basin.

Historians debate whether Ibn Fadlan was engaged in purposeful exaggeration or even fabrication. Does his description seem plausible? How would the Arab world have received this description?

\section{Magyars}

From the late ninth century, this nomadic group, originally from the area of the Urals, invaded Europe. Like the Huns (above) and Vikings (below), they initially plundered, advancing far into central Europe. After the German king Otto (later crowned Holy Roman Emperor) defeated them, they settled in modern Hungary and converted to Christianity. While the Magyar incursion bears many similarities to earlier actions by Huns and Avars, it did not have the same effect: It did not set other groups on a rampage through other lands. Nor would the later incursions of the Mongols (chapter 17). It would seem that by the late ninth century, population densities and political development throughout Europe had reached a state where mass migrations were less feasible. The Magyars were quickly absorbed into the European state system without the massive disruption of earlier nomadic incursions.

\section{The Vikings}

The Vikings were peoples from Scandinavia who launched attacks against much of Europe over a period of centuries. They were also explorers and traders. Viking raids date from 793. Population growth at home, which encouraged a search for external resources, may have inspired these raids. The tribal structure of Scandinavian society allowed victorious warriors to gather men for foreign attacks and exploration. In the 840s, Viking raiding parties started staying for the winter outside Scandinavia, which allowed them to raid farther south. They could row their shallow-draft boats far upstream on many European rivers - but the boats had high sides also to withstand ocean waves. Though their boats were designed originally for rowing, they added sails as they covered longer distances. Vikings raided Seville in southern Spain in 844 - though the vast bulk of their attacks were in northern Europe. They were aided in these attacks by prevailing winds that blew toward the southwest in the spring and toward the north in autumn. They had a strategy at first of striking and fleeing quickly. They then slowly began to establish colonies in Ireland, northern Britain, Normandy (in France), and Russia by 900 . They also worked as mercenaries. Viking leaders then converted to Christianity as part of their negotiations with others.

The Vikings were also explorers. They landed in Iceland in 874; Irish monks had probably preceded them there (though the Vikings may not have known 
about this). Still, this is at least a six-day sail across open water in small ships. These Vikings may have been fleeing political centralization in Norway. They settled in Iceland, where the descendants of Vikings live to this day. Sailors overshooting Iceland then spotted Greenland. Eric the Red explored Greenland because both Norway and Iceland had banished him for murder. He convinced hundreds to join him in settling there. At the time, it seems that Greenland was unpopulated, though there is evidence of earlier indigenous settlement, and Inuit peoples would later settle Greenland. Vikings settled Greenland during a period of unusually warm temperatures; its eventual abandonment after five centuries in about 1410 might reflect the onset of the cooler temperatures of the Little Ice Age (see chapter 14). Attack by indigenous peoples, a return to vacant lands in Iceland or Scandinavia after the plague (chapter 17), and environmental decline are other possibilities. Another overshooting, this time of Greenland, encouraged some exploration of Newfoundland and brief settlement there. The role of overshooting in this tale of exploration may seem odd, but sailing across the North Atlantic would always have been tricky in the era before advanced navigational tools, as fog would have interfered with sailing by sun or stars, and frequent storms could have driven small Viking boats far off course. Newfoundland was valued and named Vinland for the wild grapes that grew there. As we shall see in chapter 19, Newfoundland has limited agricultural potential (due to an acidic and rocky soil); the Viking settlers likely relied on fishing as later colonists would. The settlement lasted only a few years - but probably produced the first European birth in the Americas - apparently never achieving a peaceful relationship with local indigenous peoples. Greenland's population itself never exceeded 2,500, so the Viking knowledge of North America remained limited. These Viking settlements were of necessity mostly self-sufficient, given the time it took to sail across the North Atlantic and the small size of Viking craft - but there was irregular contact between Greenland and Iceland throughout the period of Greenland settlement. It would not have been profitable to ship Newfoundland fish to Europe in small Viking vessels as ships from several European nations would do in the sixteenth century (chapter 19).

Postscript: The Danish/Norwegian state in the early eighteenth century was not sure what had happened on Greenland, and thought that there might be Vikings there still. An exploratory expedition discovered Inuit inhabitants instead, whom the Scandinavians began to convert. The Danes have continued to exercise sovereignty over the world's largest island to this day - though the Norwegians protested after they separated from Denmark that it should be theirs. The International Court in the 1930s recognized Denmark's claim over Norwegian protests. Greenland today mainly relies on the fishery - and Danish subsidies - though there is some herding of sheep and reindeer, and small gardens. 
Vikings from Sweden at the same time were moving down eastern rivers to control the eastern trade route with the Black Sea and Mediterranean (a fifth century statue of Buddha has been found in Sweden, suggesting long-standing trade links). Furs, honey, and slaves were traded for gold, silver, and silk, and sometimes spices. The Viking slave trade was as brutal as any, with many reports of family separation, rape of both sexes, and little regard for life. Rather than raiding, as in the west, the focus in the east was on establishing trading centres first on the Baltic, then at Novgorod and Kiev. But in 907 the Vikings attacked Constantinople, exacting a tribute of a million silver pieces. Vikings also attacked and destroyed in 965 the Khazars, a polity near the Caspian whose rulers had converted to Judaism (see above). In 988 Vladimir, the Viking ruler of Kiev, would convert to Orthodox Christianity on marrying the Byzantine emperor's sister. (See our discussion of Byzantine missionary activity among the Slavs below.) Over time, the Viking rulers in Kiev would adopt Slavic language and culture.

There were connections across these Viking enterprises: A tenth-century Viking buried treasure south of York in England contains coins from Samarkand in Central Asia (Vikings only started to mint their own coins in the twelfth century). The life story of Harald Sigurdsson (the ruthless) is notable in this regard. He had fled early in life from Norway to Kiev, and then was a soldier in the Byzantine army in both Europe and Asia, before returning to Norway to claim

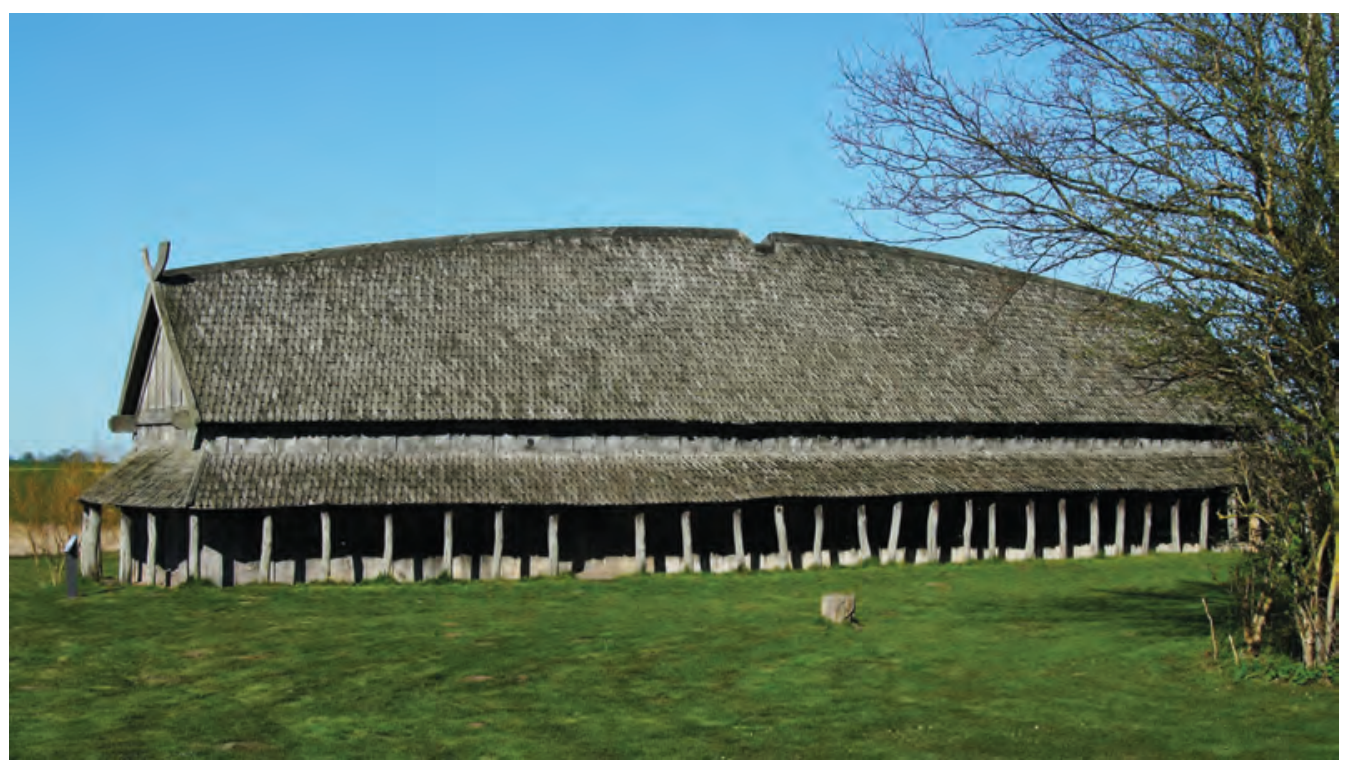

Illustration 13.4

Reconstructed Viking longhouse, Trelleborg, Denmark. In Iceland, these were built out of turf Photograph by Jens Cederskjold, CC BY 3.0 


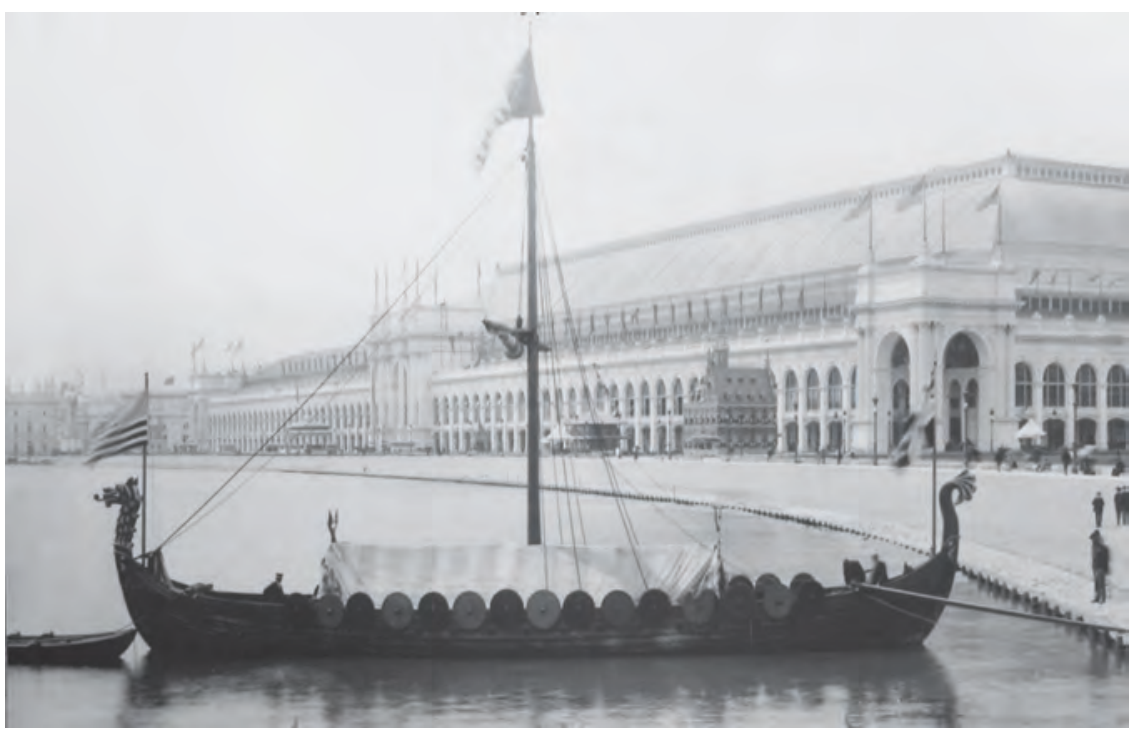

\section{Illustration 13.5}

Replica based on a 9th-century Viking ship found in a burial mound in Gokstad, Norway. It could be rowed or sailed, Chicago World Fair, 1893

the crown in 1047. His attack on England in 1066 failed but weakened the English king so that William of Normandy - a descendant of Vikings who had established a colony on the French coast - could successfully conquer England later that year. (Nor were they the first Vikings in England: King Knut or Canute (995-1035) was Danish).

\section{Islam}

We can briefly note here that the Viking raids from the north, and the various incursions from the east, were matched by Islamic attacks from the south. Muslims pillaged Rome in 846, for example. A fundamental difference here is that the raiders from north and east were generally associated with small polities whereas complex polities ruled Islamic North Africa. Islamic raiders could still be attracted to wealth that Europe was unable to protect.

A Byzantine governor invited Muslims into Sicily. Later Norman (Vikings who settled in northern France) rulers practised toleration at first and relied heavily on Muslim soldiers. Over time, they discriminated against Muslims. The Muslims rebelled in the 1220s. Frederick II, Holy Roman Emperor, attacked and then over the next two decades removed the entire Muslim population (killing many) to Lucera, a town 160 kilometres from Rome. For the next eighty years, they received protection and served as soldiers and weapons-makers. They were involved in most Italian wars of the century, sometimes in the employ of the Muslim-hating papacy. 


\section{The classic feudal system}

Feudalism was both an economic and a political system that operated for over a thousand years in Europe, though it changed in form and geographic coverage a great deal during that period. It developed as early as the seventh century $\mathrm{CE}$ in western Europe and would be extinguished in the late nineteenth century in eastern Europe. As we will see in chapter 15, something similar can be observed in Japan for a few centuries, but feudalism is arguably unobserved elsewhere in the world.

Politically, feudalism is a very decentralized form of governance. In principle, the king grants large plots of land to other individuals - given various grand titles such as duke or baron - who in return promise the king military service: They will provide (and generally lead) many armed knights should the king go to war. The dukes or barons, in turn, subdivide much of their lands among lesser nobility in return for similar though smaller promises of military service. The political structure is a hierarchical system of personal bonds of allegiance or loyalty; there were ceremonies to reinforce these bonds when a new lord inherited a title from his father: He would pledge obedience to his superior and receive pledges from his inferiors.

Our experience of emperors and bureaucrats, and the difficulties the former always faced in getting the latter to obey, will warn us that despite these solemn ceremonies, not every lord would abide by the call to arms from those above them. One could find an excuse if one deemed that a war was unwinnable and so it was perhaps best to switch one's allegiance or at least stay home. Also,

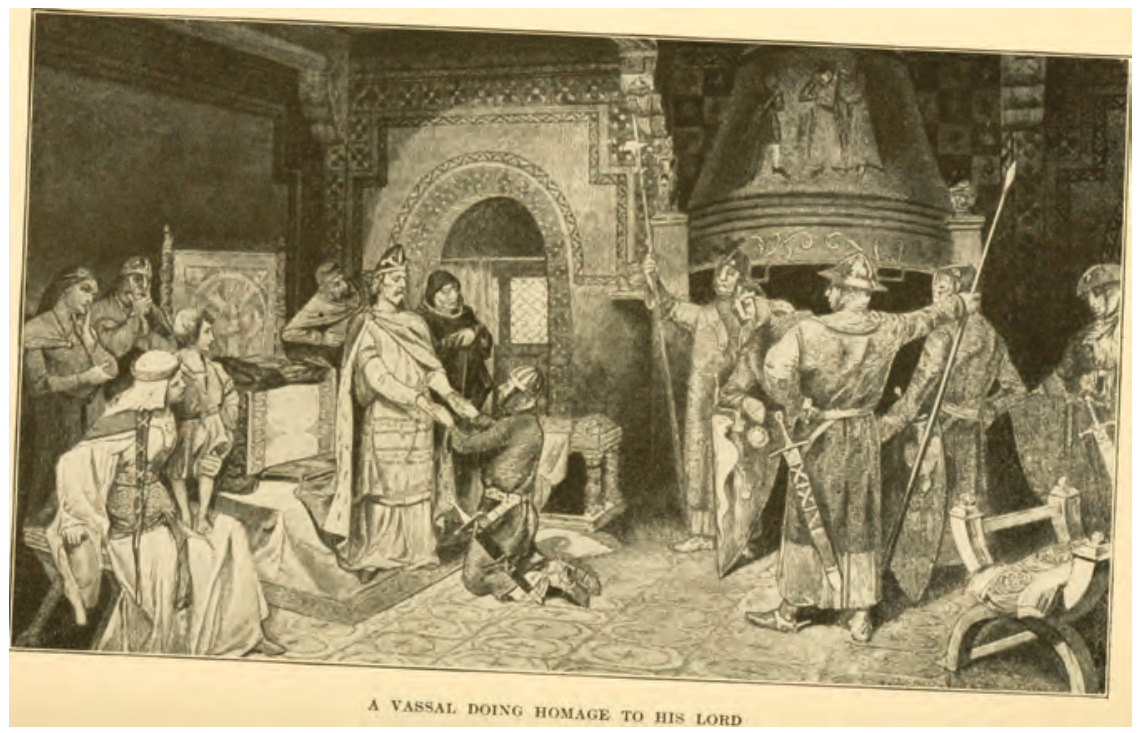

Illustration 13.6

A Vassal Doing Homage to His Lord, Louis-Paul Benezet, 1910 
there was always the temptation to move up the hierarchy through either strategic marriage or conquest. A king or duke judged to be incompetent or malicious could inspire disloyalty. Any number of imagined slights might justify ignoring one's vows of loyalty. We have seen that battles over succession often disrupted empires: In feudalism, there might be disputes regarding inheritance at any level of the feudal hierarchy which - given the practice of intermarriage among the nobility - could encourage battles among competing interests. Feudal kings, then, were much weaker than kings or emperors with their own bureaucracy and army; they could only win a war if their subordinates found it worthwhile to fight. Although the feudal system looks solid in a diagram of hierarchical relationships, in practice, it was riddled by conflict at each level. Incessant warfare characterized feudal Europe.

In classic feudalism, there was no massive bureaucracy. The king kept some lands for himself and lived off those. There might be some taxes on trade, but there were no taxes on land. The king, therefore, had limited revenues and limited expenses. The administration of justice was distributed hierarchically, with most matters dealt with by local lords. Even roads and bridges were mainly the responsibility of locals.

At the lowest level of the feudal hierarchy, the local lord would also divide the land on his manor, but this time in return for food rather than military services. Indeed, the lord would promise to protect the peasants and would provide both a small military force and a castle for that purpose. The peasants were mostly "serfs." Serfs were not slaves: The lord could not usually buy or sell serfs. Yet they were legally bound to the land, requiring the lord's permission to move. The serfs farmed small plots of land. These were usually long thin strips so that the serfs need not turn ploughs often. The serfs usually had multiple strips: These may have been accidents of inheritance, but likely also served to allow the serf to engage in different activities on different plots throughout the year (and may have served to reduce the risk of bad harvest on all of the serf's land). Serfs were usually required to share their output with the lord. In early feudalism, serfs were also required to work on "demesne" (domain) land that the lord kept for himself, often for three days a week. Later on, lords commonly divided demesne land among serfs in return for an increased share of their output. Later still, payments from serf to lord changed from in-kind to money payments. Most manors also had common land on which lords and peasants could graze animals.

Local lords faced challenges in getting serfs to work hard on demesne land. Lords generally employed overseers to keep an eye on them. However, the nature of agricultural work makes it hard to observe the work effort of a hundred peasants carefully. There was a logic to instead dividing the demesne land among serfs and taking a share of their output. Serfs were then working for both themselves and the lord, and could be expected to work hard for themselves. The lord then only had to measure the serf's output and take his share. Why did the practice of farming demesne land last so long? One argument is that it allowed lords to determine which crops serfs grew on the demesne, and 


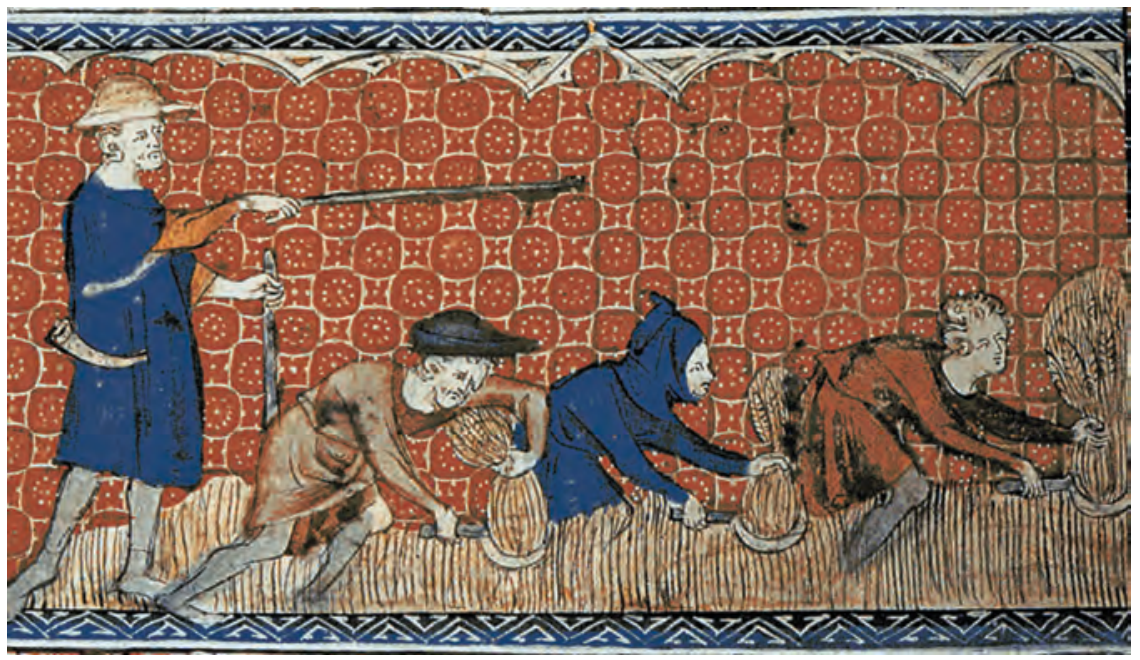

Illustration 13.7

Medieval illustration of men harvesting wheat with reaping-hooks, Queen Mary's Psalter, c.1310, British Library

that this became less important as trade expanded and they could buy food in markets. However, such an argument presumes that lords could not influence the crops that serfs grew or demand payment in certain crops.

We discussed in chapter 6 voluntarist and non-voluntarist explanations of the rise of kings. We can apply these two types of argument to the rise of serfdom also. Serfs might have willingly sacrificed their freedom in return for the protection a knight with castle could provide. Europe after the fall of Rome had no centralized state to punish thieves, and so peasants faced the age-old problem of protecting their harvest. This problem was exacerbated as Vikings, Muslims, and Magyars raided from north, south, and east (see above). Just as their distant ancestors may have seen value in early states that could protect them, serfs-tobe might have traded freedom for protection. However, there are, as with our earlier discussion of kings, some problems with a strictly voluntaristic explanation. The lords-to-be were likely among those whom the peasants needed protection from: Why would lords develop the military capability unless they were using it themselves? The lord-serf relationship was then to some degree like a modern protection racket where one pays the local bully to be left alone. Moreover, why did the serf have to surrender freedom? Why not just pay the lord for the right to hide in the local castle as necessary?

Although the serfs may have benefited from the availability of a castle to hide in and an armoured knight to attack thieves, there also seems an undeniable exercise of physical power at work. The local lord astride his horse was a fearsome force. Lords would combine to put down any serf revolt. One institution - not universally practised to be sure, but no figment of Hollywood imagination either - is the right of the lord to sleep with serf women on their 
wedding night. This is not an arrangement likely to have emerged through voluntary negotiation but rather through the exercise of raw physical power.

\section{PRIMARY DOCUMENTS: CAST OFF THE YOKE OF BONDAGE (1381), ATTRIBUTED TO JOHN BALI (1338-81), LEADER OF A SERF REBELLION}

When Adam delved and Eve span, Who was then the gentleman? From the beginning, all men by nature were created alike, and our bondage or servitude came in by the unjust oppression of naughty men. For if God would have had any bondsmen from the beginning, he would have appointed who would have had any bond and who free. And therefore I exhort you to consider that now the time is come, appointed to us by God, in which ye may, if ye will, cast off the yoke of bondage, and recover liberty. I counsel you therefore well to bethink yourselves, and to take good hearts unto you, that after the manner of a good husband that tilleth his ground, and riddeth out thereof such evil weeds as choke and destroy the good corn, you may destroy first the great lords of the realm, and after, the judges and lawyers, and questmongers, and all other who have undertaken to be against the commons. For so shall you procure peace and surety to yourselves in time to come; and by dispatching out of the way the great men, there shall be an equality in liberty, and no difference in degrees of nobility; but like dignity and equal authority in all things brought in among you.

How does the author explain feudal power relations? What is the author's motive? Does your attitude toward the author's motive affect your judgement of his words?

Of course, in practice, the feudal system did not always operate as described above. In particular, nobles often came through marriage and inheritance to hold different pieces of land at a considerable distance from each other. They might then owe loyalty to various lords. Moreover, they could not be on hand to supervise or protect the serfs on each plot of land.

Politically, the feudal system was well suited to a time when the dominant military power was a heavily armoured knight on a horse. Each local lord would provide himself and his sons with armour and horses, and follow calls to arms as he judged he should. Infantry was of little use against such knights until armour-piercing arrows (or much later, firearms) were developed. Lords at every level also built defensive structures such as castles behind which they could retreat if under attack. These were generally impregnable until the introduction of powerful cannons. As long as armoured knights and castles ruled militarily, and these were expensive but within the means of local lords, then a decentralized political structure followed where it was hard for any king to force his will on subordinates. 


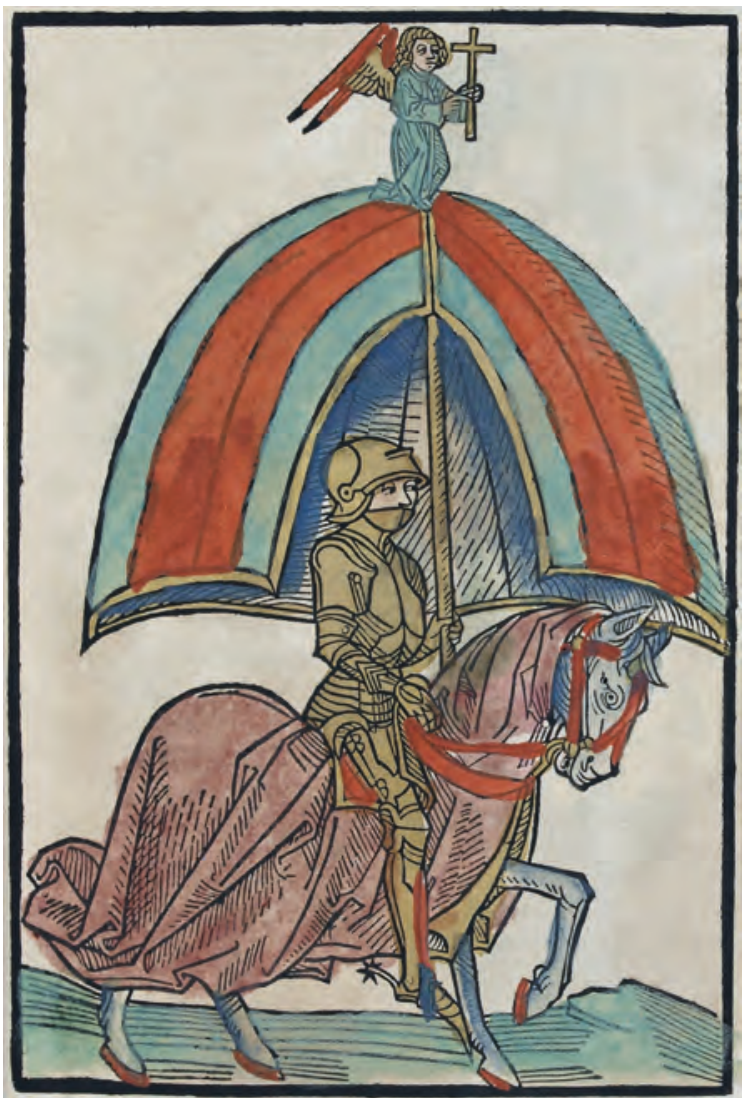

Illustration $\mathbf{1 3 . 8}$

Illustration of a knight in armour from a 15th-century woodcut, Concilium zu Constanz
Nevertheless, history mattered. The Byzantine Empire managed to maintain a centralized bureaucratic state for centuries in the east, despite the increased military importance of armour and cavalry. In the west, after the collapse of Rome, the conquering Germanic tribes lacked a tradition of strong centralized states. Early kings such as Charlemagne tried to establish such traditions (chapter 15), but once local power was entrenched in local lords with castles, it was hard for a centralized state to take control away from them. This would only happen centuries later with changes in both military technology and economic conditions.

Agriculture in western Europe was generally rain-fed. There were not extensive irrigation systems as there were in many other regions of the world. Though it is easy to exaggerate the connection between irrigation and political consolidation, it is nevertheless true that states elsewhere at least justified their rule with reference to the maintenance of irrigation systems. Lords and manors in western Europe need not negotiate with others over water supply.

Economically, the classic feudal system was well suited to a society that traded little. Local manors were mostly selfsufficient. The peasants ate food grown locally and made their own clothes, though they might barter for some consumer goods. The lord might have to buy armour, and maybe spices, but mainly ate the food of his manor(s). The primary "economic" task of empires - raising taxes to fund armies - was localized so that each manor directly supported local knights. There was no need to move tax revenues to a central place and disperse them. In such a world, there was little need for money. Note that in turn feudalism inhibited trade since merchants now had to engage with a host of local authorities rather than one large empire.

There was a dramatic decline in the use of coins in western Europe, and the roads and ports declined in quality. (Ice cores in Greenland indicate a sudden drop in lead pollution after the fall of Rome; lead was used in smelting silver for coins.) More seriously, the security that had allowed trade to prosper under Rome dissolved. Whereas the quality and quantity of pots would improve in the Byzantine Empire, these would fall dramatically in the west (see chapter 10). 
With limited bureaucracy and trade, literacy rates dropped from 10-30 per cent under Rome to almost zero. Only slowly over a period of centuries would processes of economic growth and political consolidation proceed in the west.

Over time, the idea of "chivalry" emerged in Europe, which urged certain ethical behaviours on knights. Chivalry is comparable to the cultural attitudes associated with empires that urged rulers and bureaucrats to act in the interests of the people. That is, we can see chivalry as a set of cultural attitudes that justified the exercise of power but also sought to channel that power in useful ways. Chivalry encouraged knights to value their honour, and especially those vows of fealty at the heart of the feudal system. If all knights honoured their pledges, stability might be achieved in which it made no sense for anyone to threaten the existing order - though such stability was never in fact achieved. If knights fought fairly, according to agreed rules, then there might be fewer imagined slights to justify knightly battle. An interesting characteristic of chivalry was its stress on loyalty to women, and on romantic love. While feudalism was a predominantly male institution, rooted as it was in promises of military service, women could sometimes inherit lands and even crowns (the latter was barred in France but allowed in most other lands), and were commonly expected to supervise estates while their husbands were at war. Chivalry celebrated the principle of defending a woman's honour. Knights could pledge loyalty to the woman they loved as well as to the man they served. Chivalry may thus have reduced the frequency of sexual assault after battle, at least of noble women.

As trade expanded (for reasons we discuss in chapter 15, but which inevitably involved institutional solutions to the feudal challenges to trade) and money spread through the European economy, it became easier to take cash rather than particular goods from the serfs. The serfs could then grow crops to sell in town. Once all of this happened, the economic logic of feudalism was

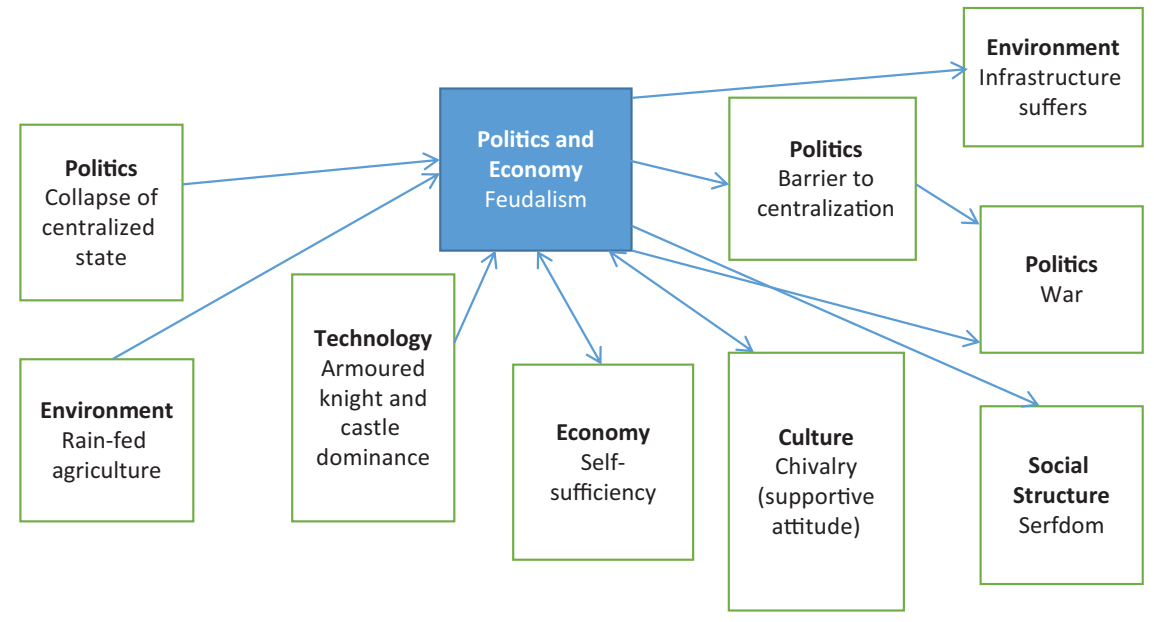

Figure 13.1

Influences on and effects of feudalism 
undermined, for market transactions were replacing feudal obligations. Serfs were effectively paying rents to a proprietor, and both serfs and lords looked to markets for an increased proportion of their consumption. Once serf payments became money payments, these were slowly inflated away as silver from the Americas reached Europe from the sixteenth century (though there is debate as to why lords were unable to raise these fees).

Note that lords themselves had agreed to these changes in feudal arrangements. We will see in chapter 15 that (some) lords also fostered trade by chartering trading towns and sponsoring fairs. In both ways, feudal lords themselves introduced institutional innovations (mutations) that made sense for them at the time but would in the end act to weaken feudalism itself. It is not unusual in history for the powerful to make decisions that serve to undermine their - or more often, their descendants' - power. We see it, for example, every time an empire hires neighbouring "barbarians" as soldiers. If not for the unexpected effects of institutional innovation, we might witness fewer transitions in societal power relations through history.

Trade (and lords) fostered urbanization. Urbanization gave serfs another option. It was easier to tie serfs to the land when there was nowhere for them to flee. As towns grew, serfs might be tempted to leave. This changed the relative power of lord and peasant: Lords might have the legal authority to prevent serfs from going, but this might not always be easy to exercise in practice.

Plagues, with their reduction in population, further increased the relative power of serfs, as lords struggled to keep serfs on their land. Indeed, we see widespread peasant revolts, and rising peasant incomes, after the great fourteenthcentury plague (chapter 17).

Though economic changes weakened the economic rationale for feudalism, feudalism as an exercise of power could only be erased through political means. This occurred differently in different lands. In England, feudalism ended over a period of centuries through a process known as enclosure. Lords recognized that they could make more money running the estate as a single farm (often raising sheep for wool) than from the payments they received from serfs. In the earliest cases, lords reached agreements with serfs where the latter gave up their rights in return for some payment. Later, Acts of Parliament would determine "appropriate" compensation for serfs on particular manors. In France, feudalism would disappear with the French Revolution (chapter 25), which gave the land to the serfs and no compensation to the lords. Small farms characterize France to this day as a result.

Though the path to the end of feudalism was different in England and France, in both countries it involved exercises of power by the state. This was the general rule: Feudalism was extinguished when states (kings and perhaps assemblies) had obtained the power to tell lords and peasants what to do (often allying with peasants against lords), and decided that feudalism was no longer in the interest of the state. We will turn to the question of state formation later (especially chapter 21), but can note here the importance of military 
technology: cannon that could destroy castle walls, and armaments that allowed infantry to kill knights from afar. The expanding trade also provided kings with new sources of revenue.

While feudalism was disappearing in western Europe, it strengthened in eastern Europe. This was a different kind of feudalism. Lords in places such as Poland and Russia were not just seeking self-sufficiency but instead exported 10 or 20 per cent of their grain to growing towns in the west. They found it advantageous - especially given the lower population densities in the east - to bind peasants to the land in order to increase their exports to the west. Kings in Russia and Austria who relied on noble support acted to entrench serfdom from the fifteenth century; they would centuries later come to see it as a barrier to economic development, but abolition would only come with massive payments from the state to buy out the rights of lords (chapter 26).

\section{The Byzantine Empire}

We should really refer to the Byzantine Empire as the Eastern Roman Empire. After all, the emperor Constantine had moved the Roman Empire's capital to Constantinople, though after that, Rome and Constantinople had often shared the duties of capital. When the Germanic tribes took Rome in the fifth century, then, they did not truly conquer the Roman Empire but only its homeland. Constantinople in 500 vied with Xian in China as the largest city in the world (Ctesiphon in Persia soon joined them). The Roman Empire would last another thousand years in the east. This institutional continuity in the east - reflected in a considerable degree of cultural and artistic continuity - stands in stark contrast to the dramatic change we have reviewed in western Europe. Historians of western Europe are so accustomed to distinguishing "classical," "feudal," and "early modern" periods that the Byzantine Empire - which spans parts of these three time periods - must seem a bit of an aberration. This may explain in part why historians based in western Europe have generally not employed the terminology of Eastern Roman Empire.

In any case, historians have called the Eastern Empire Byzantine for so long that this is now standard usage. The Byzantines certainly never called it that - Byzantium was the name of the smallish town that their grand capital of Constantinople had replaced. They were acutely aware that they had inherited Roman institutions, including ugly succession battles and a Senate filled with wealthy landowners. Most importantly, they had inherited a Roman bureaucracy. They were likely also aware of significant differences: The Byzantines soon came to operate primarily in Greek rather than Latin, and their capital was far more ethnically diverse than Rome had ever been. They used eunuchs as senior bureaucrats far more than the Romans had, an idea they had borrowed from Persia. Crucially, they had expanded the bureaucracy so that they were directly collecting taxes on land throughout their empire rather than relying on local elites to do so. 
There was also an important strategic difference. Rome was a vast empire that had borders that for centuries it could readily defend. It was bordered to the west and south by sea and desert. It faced the Persians to the east, to be sure. However, the Rhine and Danube together provided a defensive line in the north that held for centuries. Rome itself, and many other key cities of the empire, were a long way from the border. The Byzantines had a much smaller landmass that was bordered on all sides by threats - including from the peoples who now populated the former western empire. Though it is hard to measure these things, the Byzantines likely lived in one of the more hostile environments of any empire in history, especially after the rise of Islam (chapter 12). In its prime, Rome could boldly threaten the world. Necessity forced the Byzantines to a more strategic stance. They are often hailed as experts in diplomacy and spycraft, often managing to encourage their potential enemies to fight each other.

Constantinople was an odd choice as a capital. Byzantium had never previously been the capital of any large polity. Its location astride a waterway that connected the Mediterranean and Black Seas may seem advantageous today. However, neither the Romans nor Byzantines viewed Black Sea trade as valuable as the Greeks had considered it - though the Byzantines would come to rely on grain from what is now Romania and Bulgaria once they lost Egypt to Islam (below). Neither the Romans nor Byzantines ever controlled the entire Black Sea coastline. That sea would often prove an avenue for foreign attack, especially as a succession of nomadic groups arrived on its northern shores. The Byzantines would often buy the allegiance of polities along the Black Sea coast. The Kingdom of Georgia apparently prospered during Byzantine times (until defeated by the Mongols), controlling a trade route from Trebizond east, skirting to the south of the Caucasus Mountains (the other trade route east from the Black Sea was further north and followed a small gap carved through the mountains by the Rioni River).

The Byzantines would be a historical curiosity if only noteworthy for their diplomatic intrigues. Yet the Byzantine Empire had some vital roles to play in human history. We have already mentioned one in chapter 12: It would delay the Islamic advance into southeastern Europe for many centuries. And another was recognized in chapter 11: It would develop a quite distinct form of Christianity from that of Rome and then spread that faith to Slavic lands before either Catholic or Islamic missionaries much touched these. Like the Kushan Empire, which transmitted Buddhism to China (chapter 9), the Byzantine Empire would have a dramatic impact on the distribution of religions in the world (and like the Kushans, it would eventually be toppled and displaced by Islam).

\section{Justinian and Theodora}

The height of Byzantine power came in the sixth century, under emperor Justinian and his wife Theodora. They were able to retake Italy and North Africa, 
restoring much of the former Roman Empire. They also achieved a standoff and peace accord with Persia to the east. The empire (and its church) amassed great wealth during this period. The massive church of Hagia Sofia in Constantinople, the largest cathedral in the world for almost a thousand years (though turned into a mosque in 1453) until the cathedral of Seville was built in 1520, symbolized the wealth and power of the empire under Justinian and Theodora. The period is also famed for the elaborate mosaics in Ravenna, Italy: Mosaic was the dominant artistic format throughout the Byzantine period, and specialized glassworks produced the multi-coloured fragments essential to the art. (On the other hand, Justinian also closed the Academy in Athens, which had survived since the time of Plato.) However, these victories were short-lived. The fact that there was no significant threat from the north after the dissolution of Attila's Huns may have facilitated Justinian's success. In the decades after his death, Byzantium would face renewed conflict with Persia in the east, and Slavs and Germans to the north, followed by the eruption of Islam from the south.

Justinian was born poor but supported the rise to power of his uncle, who became Emperor Justin. Justinian married Theodora, an ex-actress, a decision that was only possible because Justin had loosened the laws governing who the ruling class might marry. Justinian's hold on power was initially weak. We noted in chapter 10 that sports were important across early societies. In Constantinople, there were colour-denoted teams - of which the blue and green teams were most prominent - who took part in sports and especially chariot races, but also played roles in politics and criminal activity. A riot and murder after one race in $532 \mathrm{cE}$ led to criminal charges against both blue and green

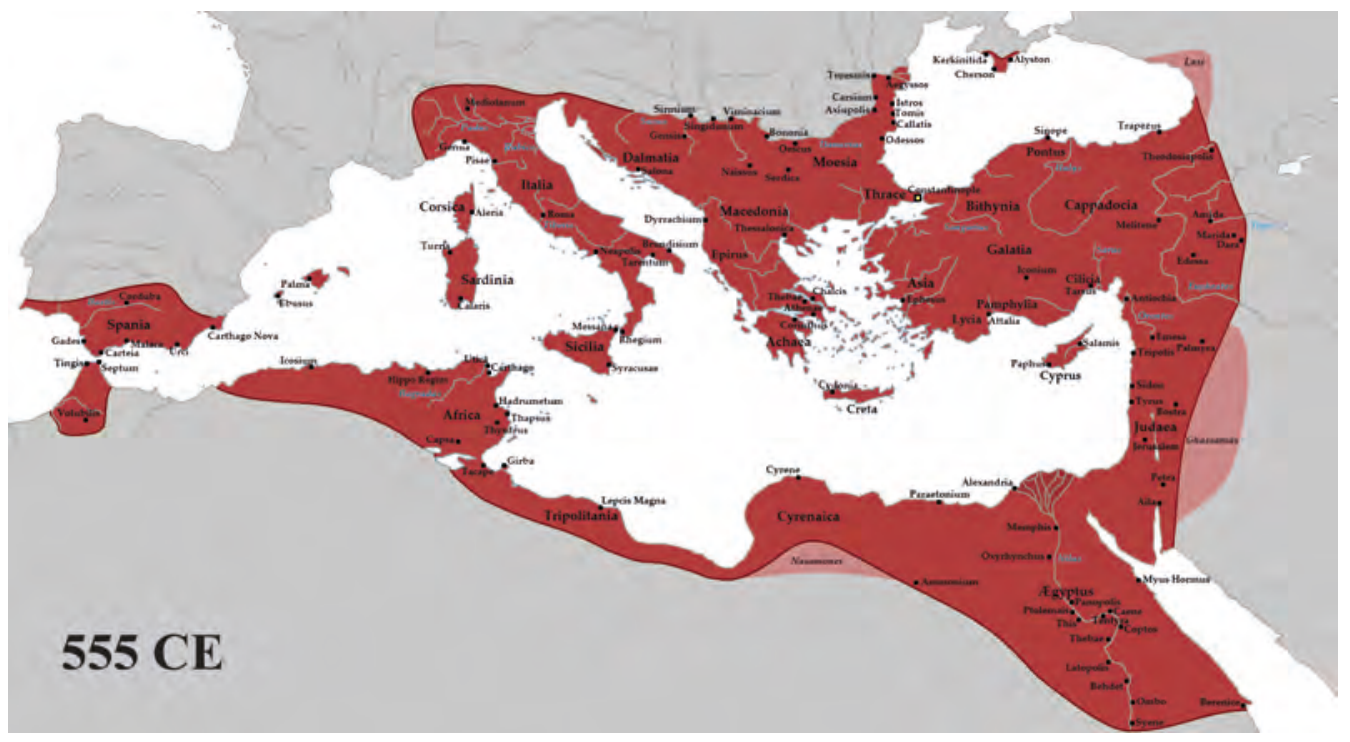

Map 13.1

Byzantine Empire under Justinian, $555 \mathrm{CE}$

Map by Tataryn, CC BY-SA 3.0 


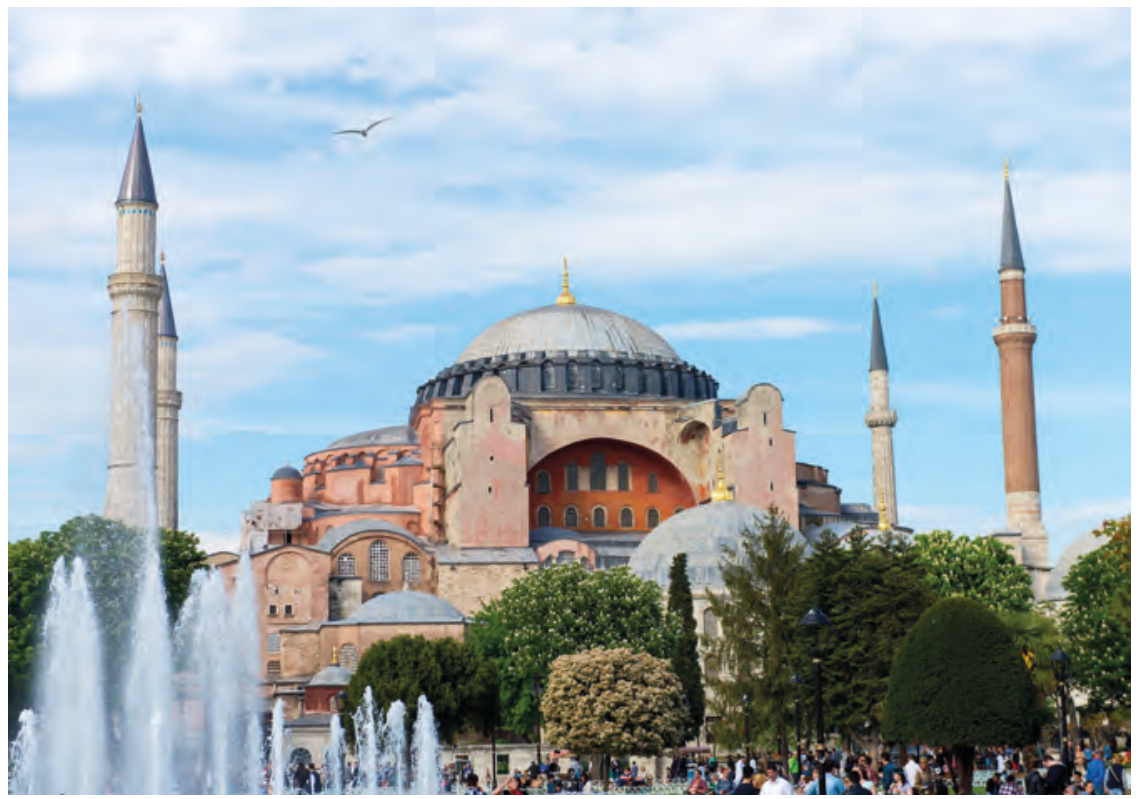

\section{Illustration 13.9}

Hagia Sofia, Istanbul; built 532-7 by Justinian; it became a mosque after Ottoman conquest, and later a museum. For centuries it was one of the biggest buildings in the world. In 2020, the Turkish government made it a mosque again

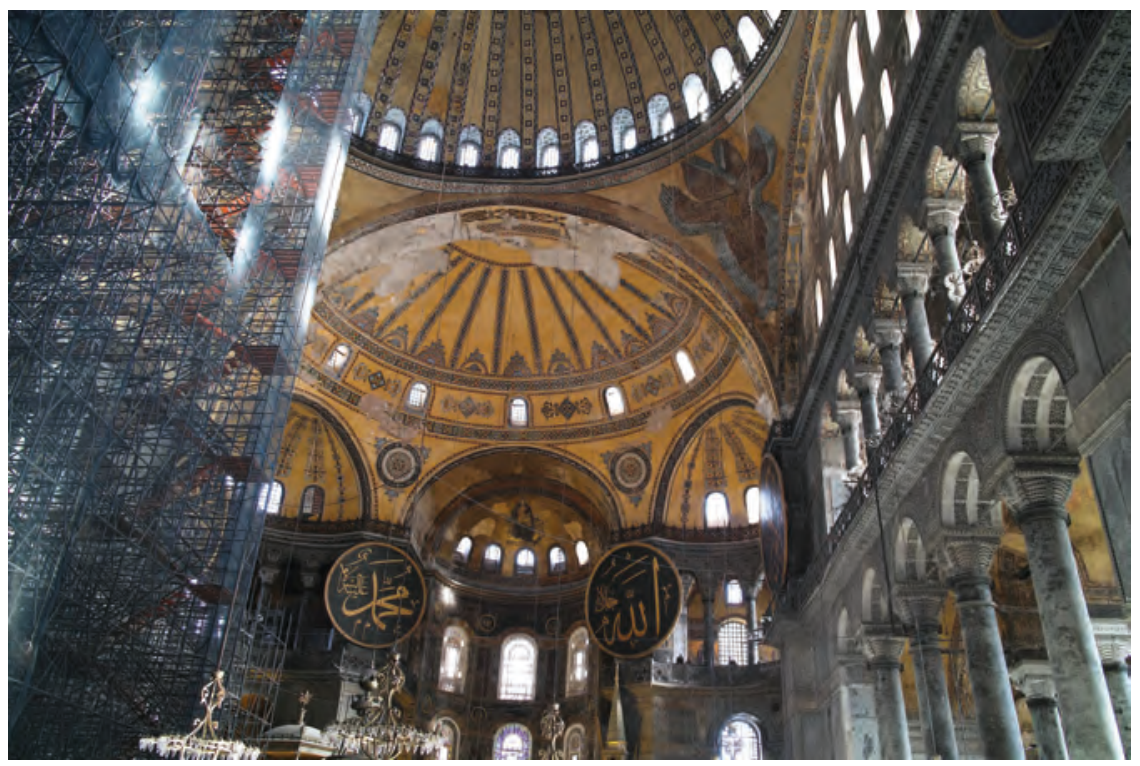

\section{Illustration 13.10}

Hagia Sophia interior 


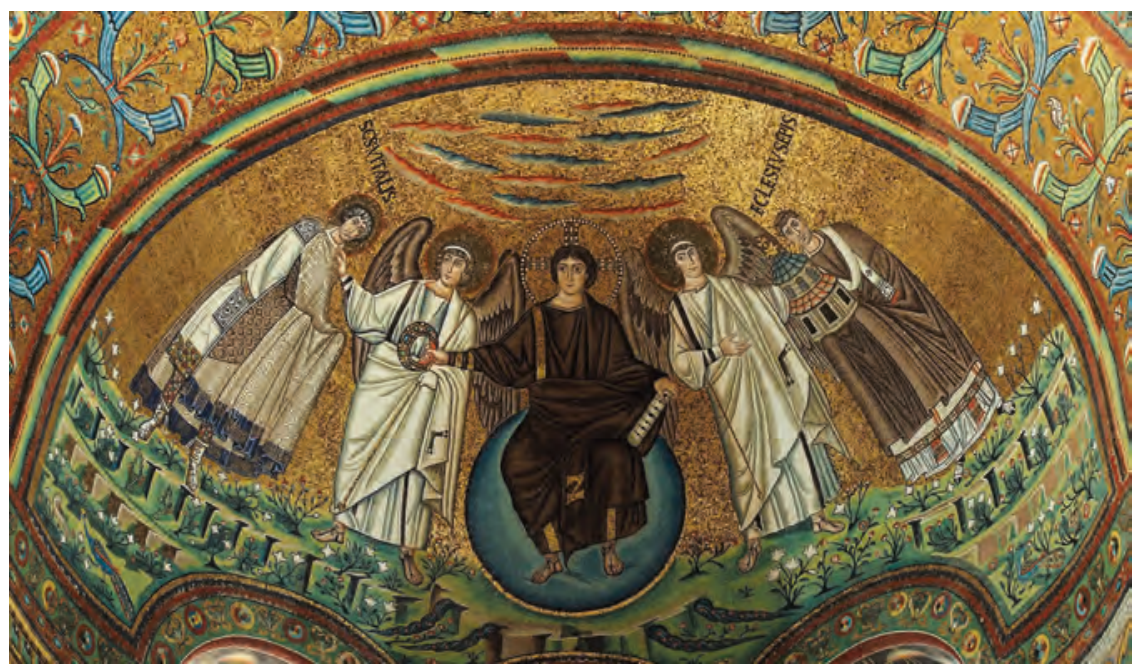

\section{Illustration 13.11}

Mosaic in the apse of the Basilica of San Vitale, Ravenna, Italy; built 547 CE; Christ with saint, bishop, archangels

Photograph by Petar Milošević, CC BY-SA 4.0

leaders, but these were later dropped. Blues and greens both agitated for full exoneration. This led to mass rioting - the Nika riots - in which many senators encouraged both blues and greens to attack the imperial palace. Records suggest that Justinian was tempted to flee but Theodora urged him to stand. It appears that Justinian, known to favour the blues, managed to encourage them to attack the greens instead of the palace. Some 30,000 may have died in the resulting conflict. Justinian then deported the senators who had supported the rioters. Justinian's power grew considerably as a result.

Historians have long assumed that Justinian over-extended the Byzantine Empire. It was unable to protect the extended territory he conquered against enemies on all sides. Of course, he could not foresee the range of enemies that would later harass his empire. Moreover, in recent years scholars have identified another explanation of Byzantine decline. One of history's great plagues descended on Eurasia in the mid sixth century. There is documentary evidence of widespread death. Indeed this may have been the third worst single epidemic in history in terms of global mortality rate after the Great Plague of the fourteenth century (chapter 17) and the Spanish influenza of the 1910s (chapter 28). Those documents also suggest that the epidemic arrived from the Red Sea: Scholars at first speculated that it originated in the Great Lakes region of Africa, where the plague is today endemic in certain animals, but recent analysis suggests that both Byzantium and Africa were infected at the time from India. In any case, polar ice cores indicate a massive reduction in atmospheric methane at the time, which signals a dramatic decrease in agricultural activity. Indeed, polar ice suggests that this was the most severe epidemic to that time. The plague would, of course, weaken both Byzantium and its 
foes, but it is likely that the heavily urbanized traders of the Byzantine Empire would suffer more than dispersed nomadic populations. Plagues are tremendously destabilizing forces that invite social instability: Small polities may be better able to survive than a vast empire dependent on a huge bureaucracy and army and especially the taxation of a diminished peasantry. Therefore, some now hypothesize that without the plague, Justinian's conquests might have indeed proved sustainable.

Justinian was more than a warrior and patron of the arts. He ordered the codification of Roman law. Roman law had long been one of the unifying features of the Empire, ensuring that Roman citizens were treated the same way throughout (though judicial corruption was a problem). However, nobody had ever gathered the various laws in one place. Justinian's Code, as it came to be known, did just that, and would be cited by legal authorities far beyond the borders of the Byzantine Empire for centuries.

As we saw in chapter 11, the Empire controlled the Orthodox Christian church. This control did not end doctrinal disputes. Justinian tried to get bishops to agree on doctrine. The church and state both proved unwilling to allow doctrinal conflicts to fester and often labelled the losers heretics. Although con-

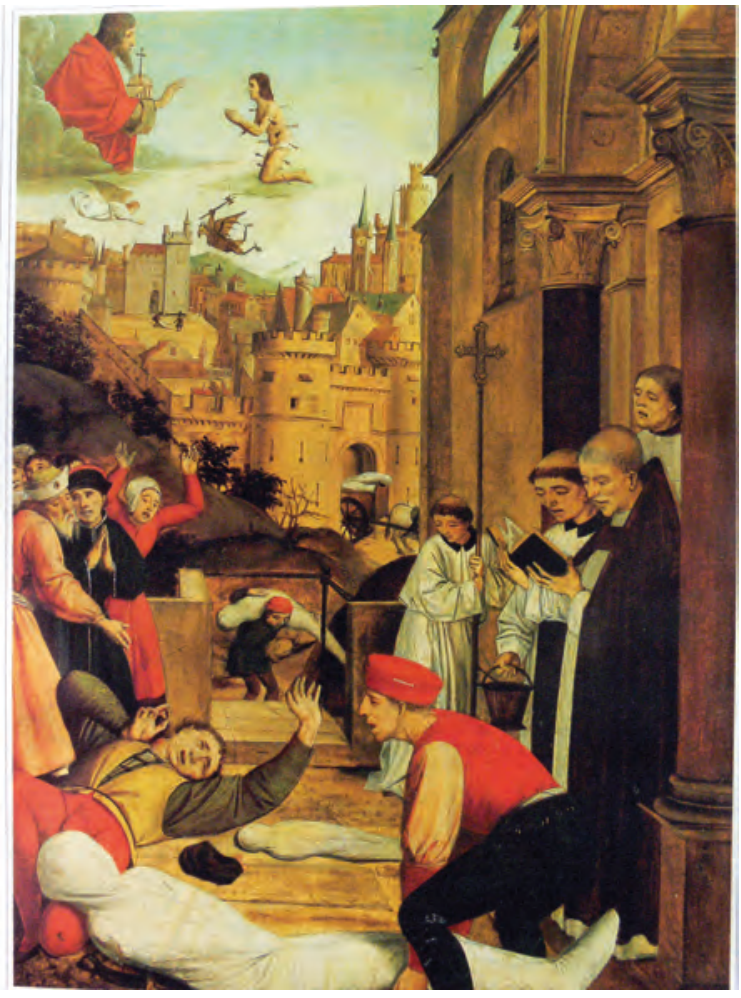

Illustration 13.12

St Sebastian pleading for the life of a gravedigger afflicted with plague during the Plague of Justinian, Josse Lieferinxe 1497-9, Walters Art Museum servative at home, the Orthodox Church proved willing to proselytize in Slavic languages, and thus Orthodoxy spread to the north far beyond the Empire's borders.

\section{Byzantine strategy}

Does it make sense to speak of a Byzantine strategy of diplomatic intrigue pursued purposely over a period of centuries? Many historians are sceptical of such a claim. They can indeed point to occasional emperors who sought - usually when Islam was weakened by internal strife - to recall the glory days of Rome (or Justinian) with campaigns of conquest that arguably strained the Empire's resources and opened it to attack. Nor is there some obvious Byzantine guidebook on diplomatic trickery that one can point to as evidence of a comprehensive strategy - though not surprisingly some writers sometimes urged particular diplomatic stratagems. An evolutionary approach can be helpful here. An oftenthreatened Byzantine state would have 
experimented with many policies over the years. Bureaucrats would remember those strategies that worked best - and advocates of such strategies would be rewarded within the bureaucracy. When the next crisis came - and these were frequent - bureaucrats would urge strategies that worked the last time. A set of simple policies could be pursued over time without ever being consciously recognized as a clear and interdependent strategy. These might include: Have an army large enough that others will think twice about attacking; be careful of weakening that army by fighting battles that can be avoided; try to encourage enmity among your enemies; forge strategic alliances; spy on others to find out their plans and design your own in response. However, transmission mechanisms are imperfect - in particular, emperors change and replace senior bureaucrats with people who say what they want to hear. As a result, these simple lessons may be occasionally forgotten and have to be remembered or even relearned.

Students can reflect on how the governments of our day struggle with challenges in foreign policy. Do they operate from a well-articulated playbook or do they often seem to patch together strategies that reflect what appears to have worked - often only in the limited sense of not having led to disaster - in the past? Though it may seem to us that the Byzantines faced a similar set of external threats over a period of centuries, to them it would have seemed that they faced an ever-changing set of new rulers and even new states whose motivations and capabilities needed always to be evaluated and then influenced. On the Byzantine side, each new emperor likely brought a slightly different perspective on the dangers and opportunities they faced - just as foreign policy changes in modern nations, at least a bit, with each new leader.

\section{The nature of empire}

The Byzantines could only successfully play their strategic games if they could maintain an army that others would not take lightly. Since they were not generally pursuing a policy of conquest that would bring them plunder, this meant that they needed a steady supply of tax revenues. Here the Byzantines - as talented as they may have proved in foreign policy - suffered over time in the same ways that other empires had suffered. The emperor favoured a broad distribution of land that he could tax, and that could provide the free peasants that were the backbone of the army. Soldiers were often allocated land in order to achieve these goals (and as a low-cost reward for services), but these former soldiers then became a local elite. There was consolidation of land into estates that gained exemption from tax from as early as the eighth century.

Decline, though, took place over centuries. The Islamic conquests of the early seventh century (chapter 12) had robbed the Byzantine Empire of perhaps two-thirds of its revenues. Its tax-raising strategies in the next decades were a profound reorientation of the Empire's finances. Though it would never again be quite as strong as it had been, it would manage to survive for centuries. 


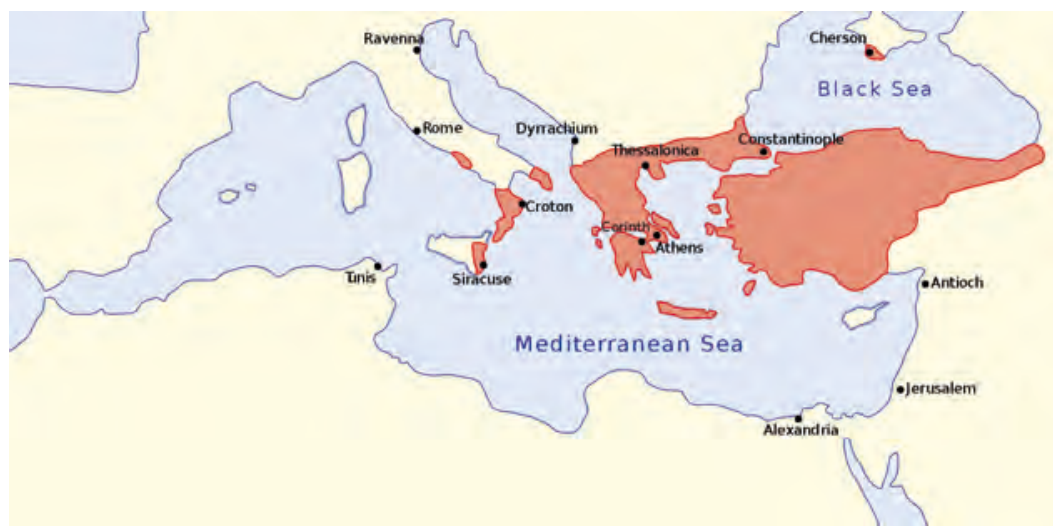

Map 13.2

Byzantine Empire in $867 \mathrm{CE}$

Map by Bigdaddy 1204, CC BY 3.0

The Byzantines, like so many empires before and after, came to employ peoples from outside the realm as soldiers. This may in part have reflected decreased internal supplies of free peasants. Yet it also reflected their policy of forging alliances with potential enemies. If so, this policy mutation would prove disastrous in the end for (yet again in history) these soldiers would have no great loyalty to the empire.

The Byzantine Empire also encouraged and then taxed both internal and external trade. The archaeological evidence is clear (and unsurprising) that there was far more trade in Byzantium than in western Europe and much bigger cities (and bigger houses). Egypt had been the granary of the empire; after the Islamic conquest, Anatolia and the lower Danube region of Europe played this role. Constantinople had hundreds of thousands of people (some think a million) from the fifth to the thirteenth centuries, and so a massive transfer of food was required to feed the city. (The Byzantines, like the Romans, subsidized the movement of grain to large cities to limit social discord.) In addition to being the capital, Constantinople was a major trading centre, situated as it was both on a major sea route to the east via the Black Sea and on the major land route from Europe to Asia. Its currency, the bezant, was the chief currency throughout the Mediterranean from the sixth through twelfth centuries. One of the primary sources of tension between Byzantines and Persians involved control of access to Central Asian trade routes.

Constantinople also became famed for artisans in textiles, glass, gold, and silver. It then became the western centre of silk production. The contemporary historian Procopius tells a story of Christian monks stealing silkworm eggs from China. This likely simplifies the story, for one needs more than just some eggs to copy a complex industry. Nevertheless, it is true that Byzantines mastered silk manufacture from the sixth century, displacing centuries of imports from China. We should note, though, that silk production also emerged in Iran 
and India at about this time. The Byzantine government strictly regulated every stage in silk production to prevent further dissemination of its secrets. Byzantine silks often incorporated Islamic designs.

Byzantine tax revenues were not just spent on the army, though this employed hundreds of thousands (down from perhaps 650,000 in the fourth century). The Byzantines were able for centuries to maintain the public works for which Rome is and was acclaimed, such as roads and aqueducts. They also funded extensive primary education, in part to provide the staff for their extensive bureaucracy with tens of thousands of bureaucrats (the finance ministry had eleven grades of bureaucrat) - though bureaucracy decentralized over time. There were elaborate ceremonies at the hiring of bureaucrats; the emperor officiated for the most senior posts. Advanced students studied the Greek classics (much as generations of Chinese students, angling for bureaucratic posts, studied Chinese classics), though basic literacy was all that was required for lower bureaucratic posts. It is again worth recalling that western Europe was largely illiterate at this time and that many classical texts would have been lost forever if not copied and protected in Byzantine and Islamic lands.

Constantinople was an impressive city. The palace had thousands of staff and a golden fountain shaped like a pineapple that sprayed wine. Emperors installed mechanical devices to awe visitors by having the throne raised or lion statues wag their tails. (The emperors also tended to dress in purple silk.) Aristocrats also had large palaces. Women were often secluded in these palaces. There were many theatres, baths, and taverns. The massive stadium, the Hippodrome, held a variety of shows. Emperors filled the city with monuments. Surrounded by water on three sides, a massive wall further protected the city on its land boundary. Moreover, the harbour was guarded by a long chain that when raised would not allow boats to enter. The site would prove impregnable for centuries.

\section{The Huns}

As noted above, the Byzantines faced many enemies. We can survey some of these here. The Romans first heard of a nomadic group called the Huns when, in the late fourth century, Germanic tribes - themselves fierce warriors - fleeing the Huns begged entry to the Empire. The Huns had composite bows, swords, and lariats, and were, as a result, the most potent fighting force around. The composite bow of the Huns could pierce armour from 60 metres. The Byzantines would over time train their soldiers in its use.

The Huns were skilled equestrians and so could travel very fast and attack without warning. They raided both Byzantine and Persian territories in the Middle East. The Byzantines tried to match Hun military tactics and engaged them in a few battles. Yet the strategy the Byzantines finally settled upon was to buy peace with the Huns. This encouraged the Huns to attack to the west. The Huns focused more on plundering than conquering. They employed fear as a weapon, threatening awful reprisals to those who did not submit to their demands. Note that in their use of the composite bow and their use of fear as 
a weapon, they presage the attacks of later nomads, including the Mongols who we shall study in chapter 17. The Byzantine strategy worked in this case because the Huns - though fearsome when guided by a charismatic leader such as Attila - fought amongst themselves after his death in 453 and ceased to be a significant military threat.

\section{Islam}

In the first eruption of a united Islam into Byzantine territories, war was the only recourse - though by putting up a strong defence, the Byzantines did encourage Islamic armies to focus their efforts elsewhere. Although the Byzantines lost much territory to Islam, they did usefully employ certain weapons against Islamic forces. One of these was "Greek fire," a mix of sulphur, lime, and petroleum that could even burn on water and was launched - through bronze tubes with the aid of an air pump - at enemies on both land and sea. The Byzantines first employed Greek fire in the sixth century, and they kept the recipe secret for centuries.

Once Islamic polities fragmented, the Byzantines could employ their diplomatic strategies. So too could Islamic states, including the one that would finally topple them, the Ottomans (chapter 20). It is too simplistic to see the Byzantine-Islamic contest in strictly religious terms, for Christians and Muslims fought on both sides of battlefields during centuries of conflict. Indeed, when Seljuk Turks had first arrived in Anatolia from the eleventh century (see above), they were often welcomed by the Christian citizenry tired of Byzantine oppression. Leaders on both sides pursued shifting alliances that often crossed religious boundaries. Then, when the Byzantine emperors fatefully appealed to the pope in Rome for help, and popes responded with the Crusades, the Fourth Crusade would attack Constantinople itself in 1204, ruling it for sixty years and weakening it severely. (See chapter 15 for the Crusades and chapter 20 for a discussion of the Ottoman conquest of Constantinople in 1453. We can note here the irony that, after centuries of assaults from Persians, Muslims, and nomads, western Europeans would fatally weaken the Byzantine Empire.)

With Islam fragmented, Emperor Basil II (990-1025) would see an opportunity to take some of the former Byzantine territories back. He made so much in plunder from conquests in Syria and Bulgaria that he cancelled taxes for two years. Yet he also used diplomacy to forge strategic alliances. Nevertheless, the Empire would not be able to hold Syria for long.

We saw in chapter 12 that many Persians felt that god had abandoned them when faced with the sudden eruption of Islamic forces. Faced with early defeats at the hands of Islamic armies, Byzantine citizens wondered if god preferred the abstract representations of god pursued by Muslims. For some 150 years, emperors suppressed the rich iconographic tradition of Orthodox Christianity. Yet this tradition would eventually be revived, though with an emphasis on very standard forms of iconic representation. When, many 
centuries later, the Byzantines faced the final onslaught of the Islamic Ottoman Empire, emperors invoked restored icons in efforts to mobilize the population.

\section{Slavs}

The Byzantines had established ties with Slavs from the sixth century as these migrated into Eastern Europe. The Byzantines sought to have these new peoples as allies rather than enemies. Church and state agreed on a policy of missionary outreach to these polytheistic peoples. Most Slavs converted to Orthodox Christianity (see chapter 11). Though religious agreement was no guarantor of political alliance (see above), it did provide a strong cultural connection which would often support political alliances.

\section{China}

Much of China's political history accords with the ideal of dynastic cyclicality. Dynasties would rise and fall and generally be replaced by another dynasty. Comparisons of political and climate data have established that revolts against dynasties were more likely during colder and drier climates. There would sometimes be periods of disunity between dynasties, but these would be of decreasing duration over time. In this, China would be like many other regions of the world, in which political consolidation would become increasingly common through time. While the trend was similar, the relative stability of the Chinese polity was unique. Moreover, the Chinese state was for many centuries by far the largest in the world in terms of both population and land area.

This stability should not mask important changes. Geographically, the Chinese state expanded over time, pushing farther and farther into Central Asia, Manchuria, and the southwest. This process was uneven, to be sure, and accelerated after 1650, but characterized a much more extended period. Even within China, there was an important migration southward over time so that the majority of the population came to live in southern rice-growing regions. This migration also accelerated in later periods. While expanding westward, northward, and southward, China did lose its power in northern Vietnam, especially after 939 . 
There was also a long and slow process of cultural homogenization. A billion people may self-identify as Chinese today; their ancestors thousands of years ago would not have shared the same sense of community. A shared government through most of history encouraged a sense of community (though we should not over-stress this; the Hindu religion would promote a sense of community in India despite political fragmentation). That government supported the use of a common script throughout the land - and to a lesser extent expanded the use of a common spoken dialect. This meant that Chinese people could easily share literature and philosophy. The fact that most dynasties - even those dominated by external conquerors - employed a formal examination system for jobs in its bureaucracy meant that aspiring bureaucrats throughout the land mastered the same set of classic Confucian works. The fact that bureaucrats were often sent to posts far from their homes also worked against strong regional identities. Many dynasties moreover moved populations around to reduce regional diversity. Also, the building of the Grand Canal, in combination with China's long rivers, ensured a national market that exposed Chinese from any region to the goods produced and consumed elsewhere.

\section{The period of disunity}

The period of disunity from 220 to 589 saw China divided for the most part into two or three competing kingdoms that battled with each other. None gained the upper hand despite many internal fissures. Nomadic groups dominated the northern kingdom. At first, they ruled as outsiders, but later they adopted Chinese bureaucratic practices. Indeed they would develop an even more centralized and bureaucratic state in the north than generally existed in the south. China would never again be so divided for more than a few decades after 589, so this period is quite unusual in Chinese history (being matched only by the lengthy period of turmoil before the classical Qin and Han dynasties). Nevertheless, the Han ideal of one unified state was maintained during this protracted period.

We saw in chapter 10 that Han China likely had higher output per person in agriculture than the Roman Empire. This gap probably widens after the fall of those empires. Much of Europe slipped toward local self-sufficiency after the fall of Rome (above). There would be important agricultural improvements in Europe, but they mostly came later (chapter 15). In China, practices pioneered during the Han became increasingly prevalent during the period of disunity. Farmers alternated grains with soybeans or turnips; both of these fixed nitrogen in the soil whereas grain crops took nitrogen from the ground. Such rotations addressed one of the farmer's critical challenges of not depleting soil fertility over time. Curved iron ploughs and better harnesses so that oxen could pull these became widely used. The Northern Wei dynasty worried indeed that farmers with oxen and ploughs had much higher incomes than farmers without; they encouraged more impoverished farmers to share these.

In southern China, an even more momentous change began toward the end of the period of disunity. Farmers had long grown rice in much the same way 
as wheat or millet and with a similar output per acre. Farmers of all of these crops can increase production by careful seeding and weeding. Alternatively, wet rice agriculture allows a much higher - likely at least 50 per cent more output per acre. However, it requires more labour effort. Farmers first plant rice in seedbeds and then transplant the small plants into fields that they then flood. The rice matures in a flooded field, but farmers must drain the field for harvesting. Wet rice agriculture had been known for thousands of years but had only been practised in small plots that were easily flooded and drained. Wet rice agriculture would become common in southern China and much of Southeast Asia over the next centuries, allowing the population to expand significantly. Its adoption may have in turn reflected population pressure, but also developments in irrigation.

\section{Sui}

China was unified again from the late sixth century. The Sui dynasty would last for thirty years (589-618), followed by the much longer Tang. There are interesting parallels between the experience of the Sui and the much earlier experience of the Qin (chapter 10). Most obviously, both dynasties were brief, but each ended centuries of internal conflict and passed power to dynasties that would last for centuries. Yang Jian, the first Sui emperor, echoed the first Qin ruler in establishing tight bureaucratic control in his original state before extending his authority to all of China. Yang Jian had begun as a governor under a Turkic ruler in the north but rebelled. He limited the power of local elites and rotated bureaucrats through different administrative regions. He, again like his distant predecessor, levied high taxes to support his army and build public works such as walls and roads. Yang Jian's most notable public work was the Grand Canal: He extended this north-south waterway to 2,000 kilometres by connecting many earlier canals. This integrated the economies of northern and southern China (each served by mighty rivers that flow from west to east) and is used to this day. It is by far the longest artificial waterway in the world, and one of the most impressive engineering projects of all time. Over a million workers, many forced, were involved. The Sui required some two million workers overall to perform forced labour. The Sui also extended the Great Wall, and built numerous roads and bridges. As with the Qin, the Sui's high taxes and extensive use of forced labour provoked tension within the realm. The Sui did pursue policies that should have earned them some public support, such as distributing land to up to 200,000 soldiers and restoring a national system of granaries to cope with famines. Nevertheless, one of his ministers assassinated the second Sui emperor.

\section{Tang}

The Tang Dynasty lasted from 618 to 907 . The second emperor, Tang Taizhong (627-49), murdered two brothers and pushed his father out. He built a 


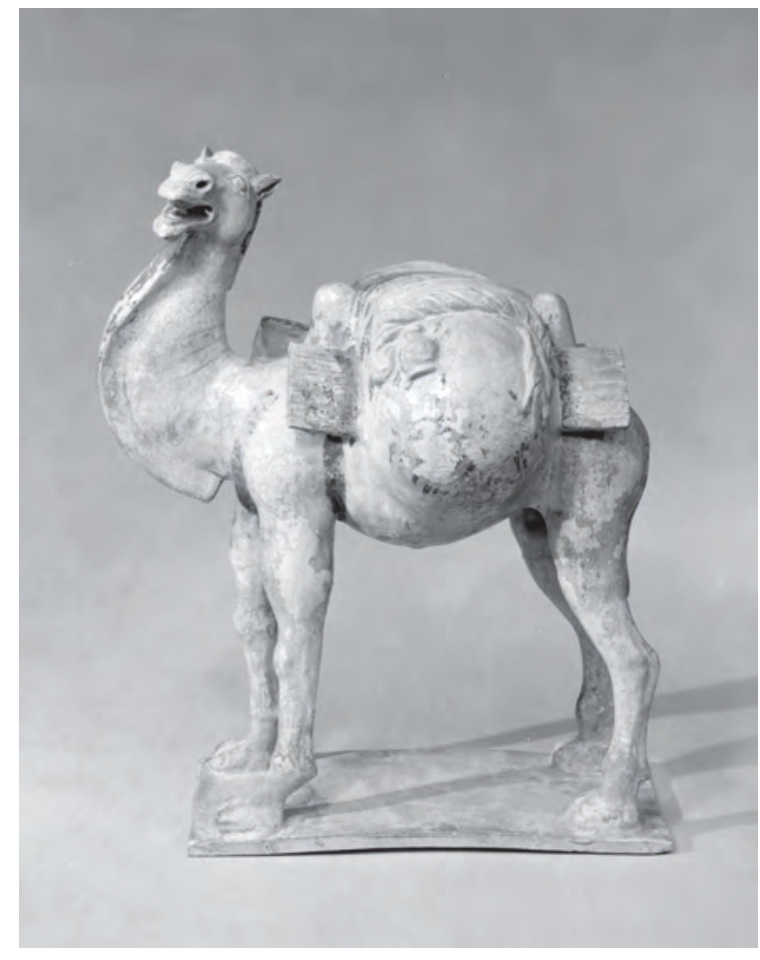

Illustration 13.14

Ceramic camel tomb pottery, Sui dynasty, Metropolitan Museum of Art magnificent new capital at Chang'an. Chang'an would grow to about 2 million people during the Tang Dynasty, by far the largest city in the world. Tang Taizhong deliberately followed Confucian precepts of government (after his exercise in fratricide and filial disloyalty, that is). $\mathrm{He}$ lowered levels of taxation from those collected by the Sui: He only collected 2.5 per cent by some reports. The Tang initially relied on poll taxes rather than income-based taxes, and so the burden could fall more heavily on the poor. Some of the tax had to be paid in woven cloth, stimulating each family to produce cloth (a task usually performed by women). While the Tang did not pursue grand public works on the scale of the Sui, Tang Taizhong developed a system of roads and posts such that it was possible to communicate anywhere in the empire in eight days. Such a system would be useful for trade as well as administration. His police allegedly eliminated banditry, further facilitating trade. Though not requiring forced labour for massive public works, labour was compelled to serve in military garrisons and the lowest ranks of the bureaucracy.

The Tang also expanded militarily into Manchuria and even Korea, and west to the Aral Sea and much of Tibet, and into northern Vietnam (which had been held by the Han long before). Plunder may have financed these military successes in large part, if indeed the reports of low levels of taxation at home are accurate. The Tang would, though, lose a major battle to the Abbasids in Central Asia in 751 (chapter 12). The Tang would also exact tribute from weak states on its borders. Yet the Tang gave these states gifts in return - it is not entirely clear who gained more from the interaction. Perhaps the Tang were buying peace. Japan paid tribute. Even Indian princes sent some fifty tribute missions to the Tang court over the centuries. Again, these received gifts in return.

The Tang, like the Sui, repeatedly tried to redistribute land. It for some time followed the "equal-field system" whereby most land was regularly redistributed to needy families - though it took care not to offend large landowners, especially in the south. While such a system has excellent egalitarian qualities, it also discouraged farmers (who might reasonably fear that the state would redistribute some of their land away from them) from investing in their property or worrying about the environmental sustainability of their agricultural practices. 


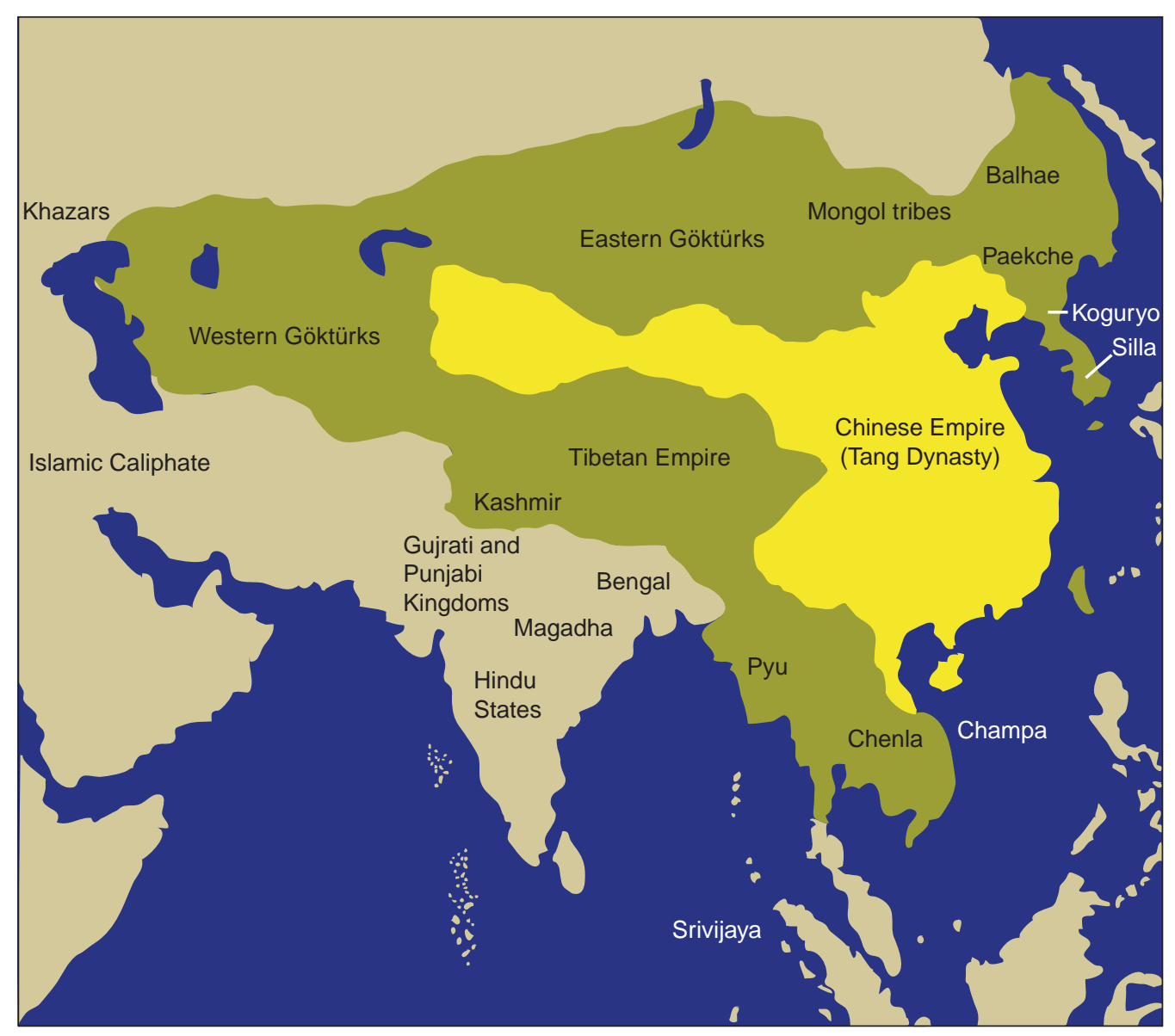

Map 13.3

The Tang Empire

Map by Peppe64, CC BY-SA 3.0

It is not that surprising that this system broke down over time. Large holdings increased, especially after the Tang stopped giving land to soldiers in 749 . The Tang then faced a problem common to empires of the rising power of large landowners.

The Sui had reintroduced the national examination system for the bureaucracy in 605. The Tang expanded this. However, neither dynasty relied exclusively on the examination system to fill bureaucratic posts.

Empress Wu Zitian ruled from 690-705. Though the mothers and wives of Chinese emperors often exerted a powerful influence in Chinese history, she was the only woman to rule in her own right. She had long exercised power as the favourite consort over Emperor Gaozong, who had had a debilitating stroke in 655. She may have poisoned her own son to maintain her influence. She then displaced another of Gaozong's sons after Gaozong's death. She suppressed a rebellion of Tang aristocrats. Later male rulers heavily criticized her rule. 
From 755, the Tang general An-Lushan rebelled, amassing some 100,000 troops. This rebellion is evidence of the power that Tang generals had come to wield. The rebels forced the Tang emperor to flee the capital. The Tang survived but mainly through the support of Uighurs from Central Asia. These exacted a hefty payment from the Tang for their services. The Tang lost control over much of Central Asia and Tibet at the time. Internally, the Tang could not reassert control over generals who remained powerful in different regions of the empire.

An-Lushan's father was Sogdian (that is, of Central Asian heritage). His rebellion split the Sogdian community in China. After his defeat, Sogdians in China tended to hide their identity. They mostly disappeared from Chinese history thereafter.

The Tang period saw a resurgence of Confucianism. The Tang had initially favoured Buddhism, yet they came to harass Buddhism in particular, but also Nestorians, Manicheans, Muslims, and Zoroastrians. They destroyed thousands of shrines. Buddhist monasteries had earlier gained an exemption from taxes, citing Indian policies in this regard. The Tang later confiscated the wealth of many monasteries.

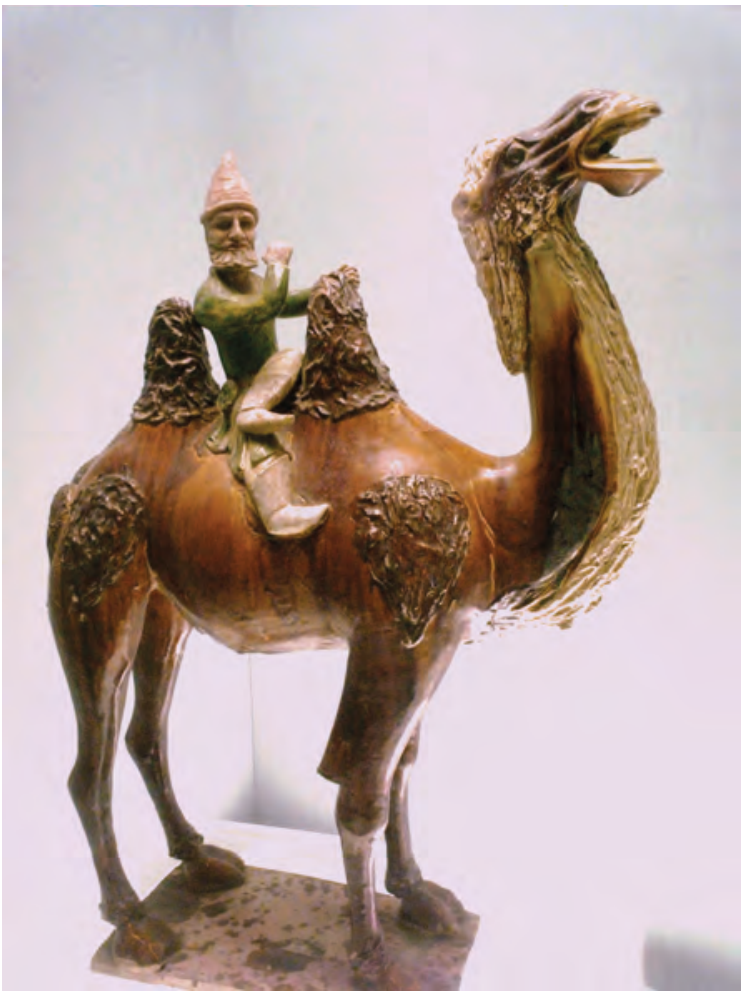

Illustration 13.15

Sogdian on a camel, Tang Dynasty, likely 7th century, Shanghai Museum
The Tang also introduced a ban on travel outside China. Xuanzang, a Chinese Buddhist monk, decided to ignore this ban to study in India. He almost died on the way, but a Buddhist ruler of the oasis town of Turpan gave him riches and letters of introduction to twenty-four rulers along the road to India. He studied at Nalanda - a major Buddhist-run university (see below and chapter 12) - and elsewhere in India for twelve years, returning to China with hundreds of books to a hero's welcome. Even the emperor welcomed him, ignoring the fact that he had broken the empire's travel ban and was encouraging Buddhism.

Despite the ban on travel, Chinese elites - including emperors - displayed an interest in exotic goods. Sandalwood carvings from India and Indonesia were particularly prized. Some Tang elite members even wore foreign clothing. International trade was not generally encouraged by the Tang, but toward the end they may have become more open, perhaps because they needed additional 
sources of revenue to support their ongoing fight with nomads. They also, though, occasionally discouraged trade by impounding merchant goods.

One important import during the Tang period was indigo. India had produced and traded this natural blue dye for a thousand years. Imports of both indigo and cotton expanded during the Tang period. Increasingly, they came to be produced locally. Chinese peasants would wear blue-green clothing for centuries thereafter; this was only possible because of these imported ideas.

Historians often portray the Tang Dynasty as a "Golden Age" in Chinese history. Despite its occasional travails, and its losses of territory, the Tang period is celebrated for its cultural achievements. As the largest city in the world, Chang'an attracted artists and poets from throughout the Empire. The poetry of the time is widely viewed as the best produced in any Chinese empire. Painting also flourished. The art market expanded as merchants and landowners sought to express their wealth with works of art. Many works of history, science, and technology were published. The early development of woodblock printing from the eighth century aided these latter endeavours - Buddhist monasteries were critical in this development. The Tang

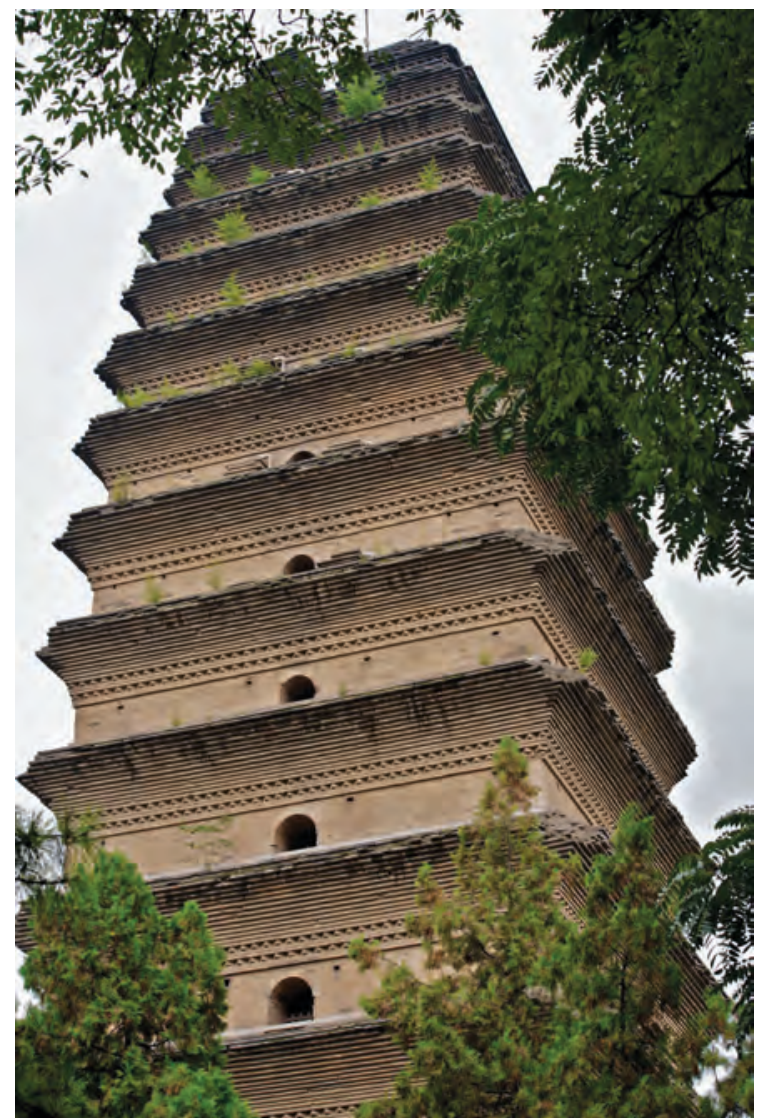

Illustration 13.16

The Small (Little) Wild Goose Pagoda was built between 707 and 709 under Emperor Zhongzong of

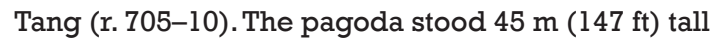
until the 1556 Shaanxi earthquake. It now stands $43 \mathrm{~m}$ (14l ft)

Photograph by kevinmcgill from Den Bosch, Netherlands, CC BY-SA 2.0 also pursued urban planning, urging an orderly grid-like development of its cities.

The Sui and Tang Empires impressed their neighbours. Korea, Japan, and Tibet duplicated the institutions of the Chinese Empire. They also absorbed Chinese literature and elements of the Chinese writing system. They admired and in some cases reproduced the quality of Chinese ceramics and textiles. Chinese motifs (and indirectly Indian and Central Asian elements) infused Korean and Japanese art.

Though the capital city celebrated and exported a unified Chinese culture, the process of cultural consolidation in China was far from complete. China was a land of ethnic and cultural diversity. Practices such as the examination 

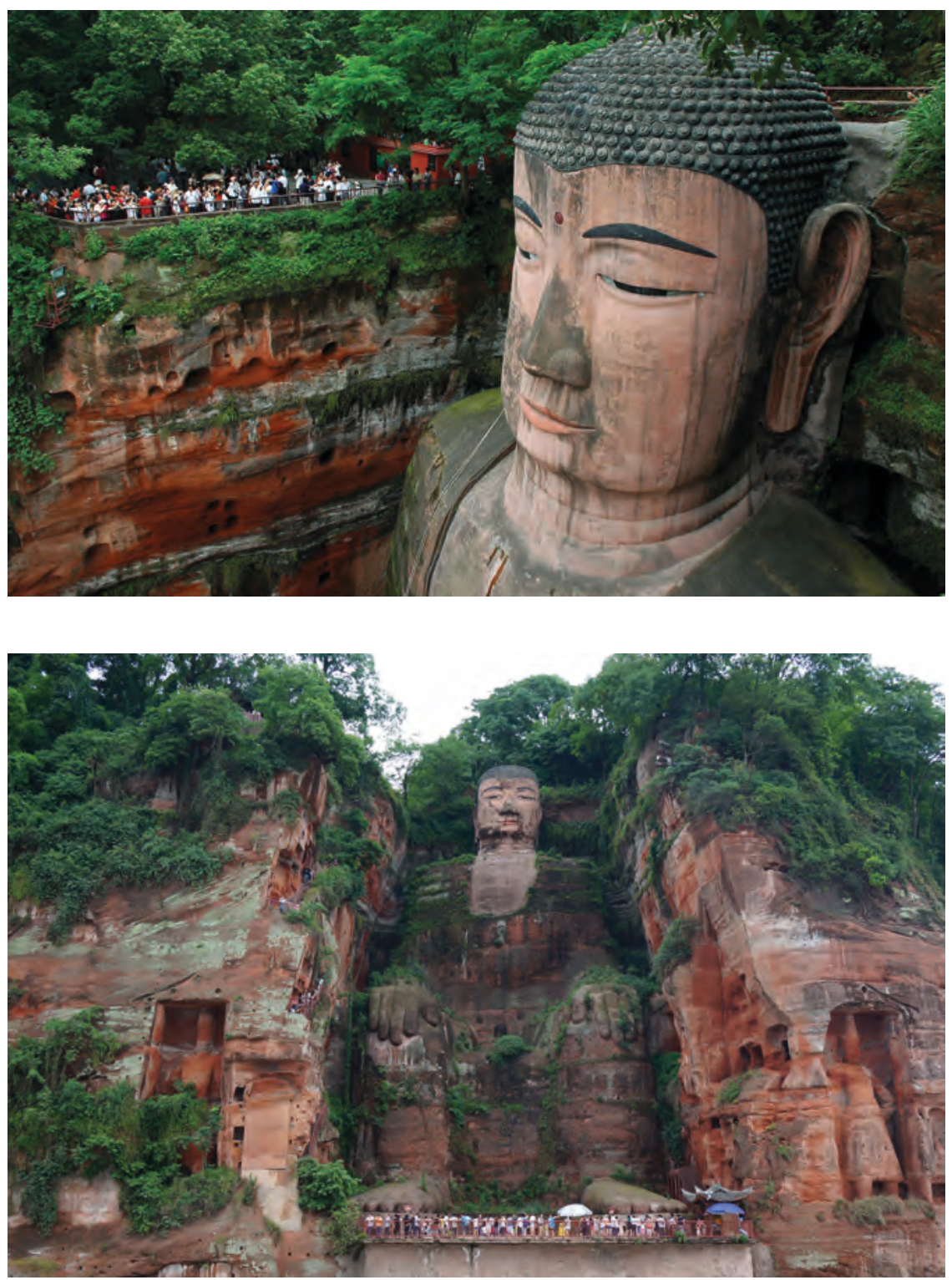

Illustration 13.17

Leshan Buddha. The Leshan Giant Buddha is a 71-metre (233 ft) tall stone statue near the city of Leshan in the southern part of Sichuan province in China. Built between 713 and 803 (during the Tang Dynasty), it is the largest stone Buddha in the world and by far the tallest pre-modern statue in the world

system and rotation of bureaucrats encouraged common cultural attitudes, but only slowly. It is hard for scholars to gauge the degree of cultural diversity because the vast bulk of surviving records come from the capital.

Tang provincial governments maintained offices in the capital through which they lobbied the central government (much as the modern taxpayer 
pays for university bureaucrats whose primary job is to squeeze money from state bureaucrats). This symbolizes the decentralized nature of governance. Such offices also came to serve an economic role, for merchants could transfer funds from the capital to the provinces through these offices without having to carry money.

The decline of the Tang is widely attributed to a series of weak emperors. The idea of the Mandate of Heaven would suggest this sort of explanation. Whether the emperors were weak or not, we see characteristics associated with the decline of many empires. We have mentioned the increased power of both local landowners and generals above. These often worked together to assert power against the central state. The Tang attempted to raise taxes, but these efforts encouraged rebellions. The final Tang emperor abdicated in 907.

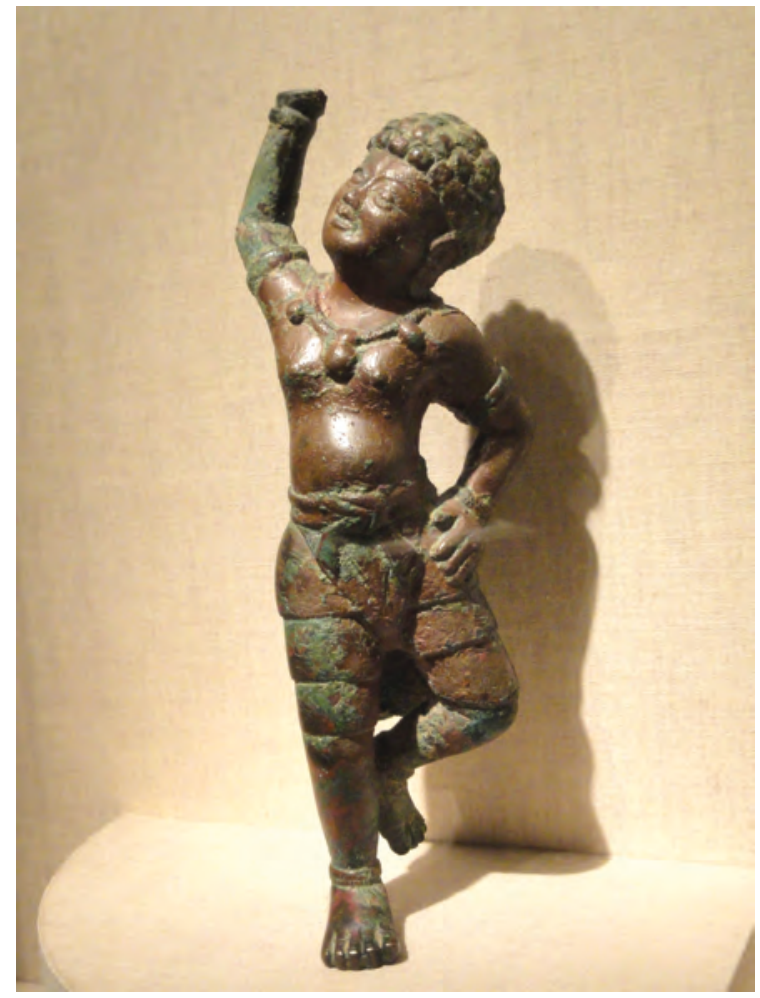

Illustration 13.18

Bronze of foreign dancer, Tang Dynasty, Sackler Gallery, Washington

Photograph by Daderot, CC0

Postscript: The examination system, and bureaucracy more generally, would encourage among other things the use of a common written language throughout China. Yet the ideographic nature of Chinese writing meant that a common written language could co-exist with multiple spoken dialects. The Chinese Republic from 1912, and Communist China from 1949, declared the dialect of Beijing the official language, but at least a half dozen quite different dialects are still widely spoken in China (Cantonese being the best known of these outside of China). The government insists that these be termed dialects rather than distinct languages.

\section{Korea}

The Chinese influence in Korea dates from the third century BCE when refugees from the warring states took agricultural techniques and writing there. The Han conquered Korea, imposing their administration. Korea was itself characterized 
by warring states after the fall of the Han. More Chinese refugees arrived during China's age of disunity despite Korea also being divided. The Sui and early Tang were unable to conquer Korea. Yet the Tang then mounted successful offensives against both Korea and Vietnam. The Tang conquered much of Korea but then reached an agreement with the local Silla Dynasty, which recognized the Chinese as overlords. The Silla rulers performed the kowtow before the Chinese emperor (touching their head to the floor), and paid tribute, but likely received even greater gifts in return. Much trade followed. The Silla controlled about two-thirds of the Korean peninsula.

Silla would be semi-autonomous and imitate the Chinese administration for centuries. The Korean dynasty modelled its new capital of Gyeongju (in southeastern Korea along the coast) and administration on the Chinese. Korean scholars travelled to China and returned with texts. Both Confucian and Buddhist ideas became common in Korea. However, Korea had a powerful aristocracy that prevented selection on merit for the bureaucracy. The Silla nevertheless introduced an examination system and the Chinese writing system. Korea also gained fame for its ceramics.

The Silla would decline at about the same time as the Tang. The Koryo Dynasty - from which the modern countries of North and South Korea derive their names - would replace it in 935; this dynasty would last until 1392. The Koryo continued the policy of borrowing Chinese ideas; it also used an exam

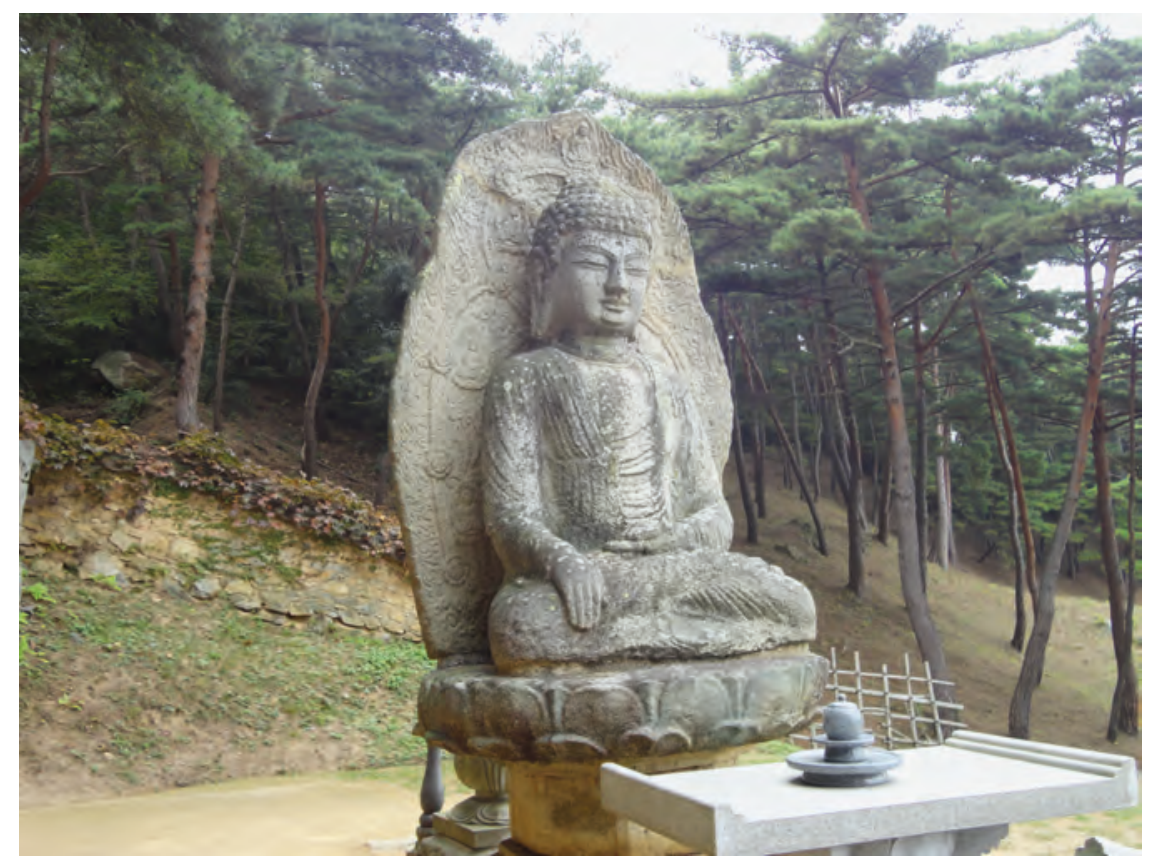

Illustration 13.19

Sitting Buddha in the Mireuk valley, Namsan (Gyeongju), South Korea; built during the Silla Dynasty

Photograph by Pldx 1, CC BY-SA 3.0 


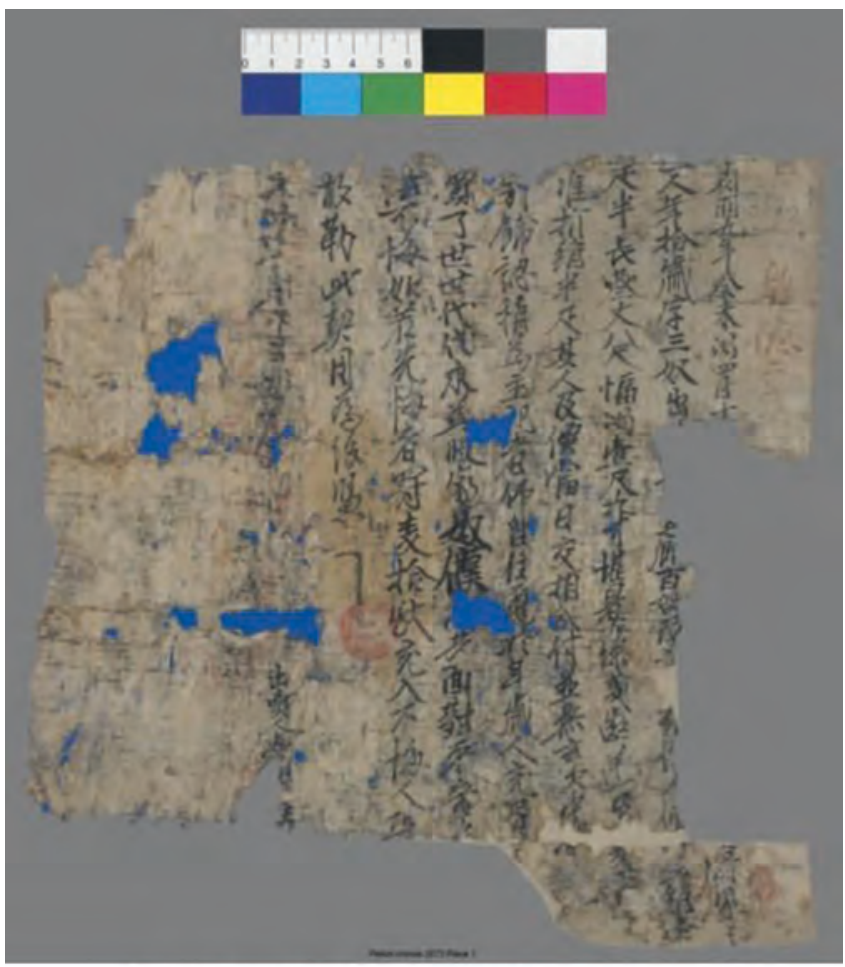

Source gallica.bnf.fr / Bibliothèque nationale de France

\section{Illustration 13.20}

Contract for the sale of a slave, 9th-century China, Bibliothèque nationale, Paris. The Tang allowed slavery of foreigners and criminals. Free people could sell themselves into slavery

system, though it still reserved the top bureaucratic posts for the aristocracy. The Koryo even built a wall to defend against nomads.

Jang Bogo (Gungbok; 787-846 probably) would become famed as a merchant and shipmaster during the later Silla period. There were at the time pirates in the Yellow Sea between China and the Korean peninsula. These often caught Koreans, many of whom had settled in neighbouring areas of China, and sold them as slaves. Jang Bogo encouraged the Silla to fight the pirates and gained a prestigious post as a Maritime Commissioner from the Silla to do so. He then almost married his daughter into the royal family. He traded actively with both China and Japan. He also endowed Buddhist monasteries.

\section{Japan}

We have not discussed Japan much in this book since our discussion of the Jomon culture in chapter 4 . Japan was late to develop agriculture, but by the third century $\mathrm{BCE}$, farmers grew rice and employed both bronze and iron. Koreans fleeing Chinese incursions may have carried these innovations to Japan; if so, 
then Japan might have moved quite suddenly from hunting and gathering to the use of iron. The Shinto religion developed over time with its worship of spirits and ancestors and gods and emphasis on fertility and purification rituals (chapter 11). The Yamato emperors from about the fifth century ce claimed descent from the sun goddess; their descendants still rule, the longest dynasty anywhere in the world - though their power would be nominal in many periods of Japanese history. It is possible that the Japanese royal family has Korean roots.

The Chinese were never able to invade Japan successfully. The Sea of Japan is difficult to cross, though less so at the straits that separate Japan from the Asian mainland. Yet Japan nevertheless absorbed many ideas from China due to long-standing links with Korea. Buddhism arrived in the sixth century CE. Some members of the aristocracy adopted it while others remained Shintoist in orientation. The conflict was resolved with the installation of Prince Shotoku as regent from 593 to 622; he sent missions to China, installed a bureaucracy based on merit, and actively promoted Buddhism. Japan would actively borrow - but adapt - Chinese culture and institutions for centuries. Nevertheless, Shinto remained popular. Many Japanese would pray at both Buddhist and Shinto temples, seeing these as complementary religions.

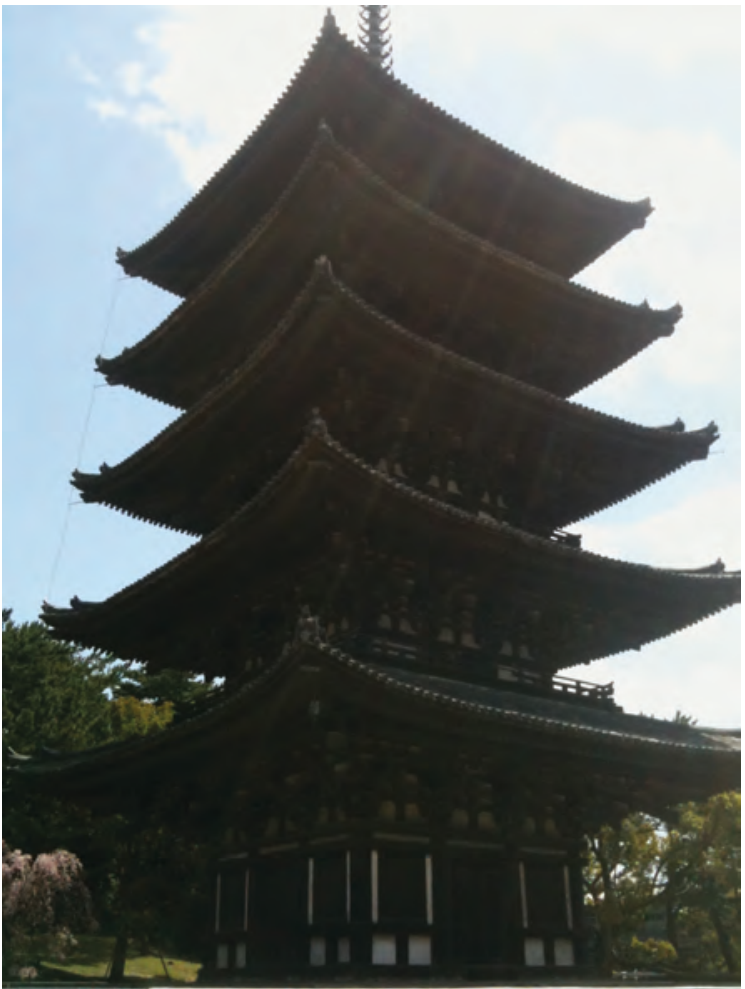

Illustration 13.21

Kofukuji five-storey pagoda, Nara, first built in 730 , and most recently rebuilt in 1426. It is the second tallest pagoda in Japan
The Sui/Tang example arguably inspired centralization of the state in Nara (710-94; Nara is south of Kyoto). The new Japanese state supported both Confucianism and Buddhism, and instituted a Chinese style of bureaucracy. Chinese classics were the key to elite education, and court records employed Chinese. Although the Japanese adopted many Chinese ideograms, they maintained a different writing script. As in China, the Japanese state pursued efforts to distribute land equally, but these failed through time.

After the capital moved to what is now Kyoto in 794, Japan began to develop more distinctly, with emperors encouraging a blend of Buddhism and Shinto. They developed a new writing system in the ninth century that simplified characters to produce a phonetic alphabet: Men still learned Chinese but women learned the new script. The role of women likely declined in Japan under the influence of Confucianism. Nevertheless, women were influential in literature. 
A woman used the simplified script to write the Tale of Genji of 1008, a story of court romance and intrigue that may be the world's oldest novel.

\section{Southeast Asia}

Mainland Southeast Asia (from Burma to Vietnam) is a rugged land. Much of it was too steep for the agriculture of pre-modern times (but would be settled in the nineteenth century; chapter 31). The main exceptions were the valleys of the Irrawaddy (in Burma), Chaopraya (in present-day Thailand), Mekong, and Red (in northern Vietnam) rivers, and along the coast, especially in Vietnam. As a result, population density was much lower than in India or China throughout most of history (in 1800 it was only about 10-20 per cent). The hills and jungles that separated these different river valleys impeded political centralization: There would always be at least three polities in the region and often many more.

Ancestors of the Austronesian speakers that later dominate the region had likely migrated from south China through Taiwan and the Philippines over tens of thousands of years, reaching Southeast Asia in the second millennium BCE. Scholars debate when agriculture first emerged in the region and whether it emerged there independently (chapter 4). There is evidence of trade with Southeast Asian islands and even to India from 600 все.

The first large state (that we know of) was Funan between the first and fifth centuries CE. Though centred in Cambodia and southern Vietnam, Funan grew wealthy by controlling the portage across the Malay peninsula, which was preferred at the time to sailing through the Straits of Melaka (likely because of piracy in the region, but also because ships hugged the coastline until bigger vessels were developed from the sixth century). The Funan state profited from the growing sea trade between China and India. Funan then invested in irrigation at home, increasing agricultural output and taxing this. Food surpluses were sold to visiting merchants and sailors, who often stayed for months waiting for monsoon winds to change. The Funanese rulers purposely modelled themselves on Indian royal houses, adopted Hinduism as justification for their rule, and employed Sanskrit for their records. Nevertheless, in 243 the local ruler sent gifts to the Chinese court. Archaeologists of Funanese sites have found Roman coins and glass, Chinese bronzes, many Indian religious amulets, some Chinese Buddhist figurines, and locally produced glass beads and tinwork. Funan declined as Chinese sailors pioneered the route through the Straits of Melaka, reaching agreements with local polities in southern Malaya and Sumatra.

The Chinese controlled northern Vietnam during most of the Han, Sui, and Tang dynasties. Vietnam absorbed Chinese institutions and culture, including Mahayana Buddhism, whereas the rest of the region absorbed Indian ideas and Theravada Buddhism. Some Vietnamese students took the Chinese examinations. A syncretic combination of Buddhism, Taoism, and Confucianism 


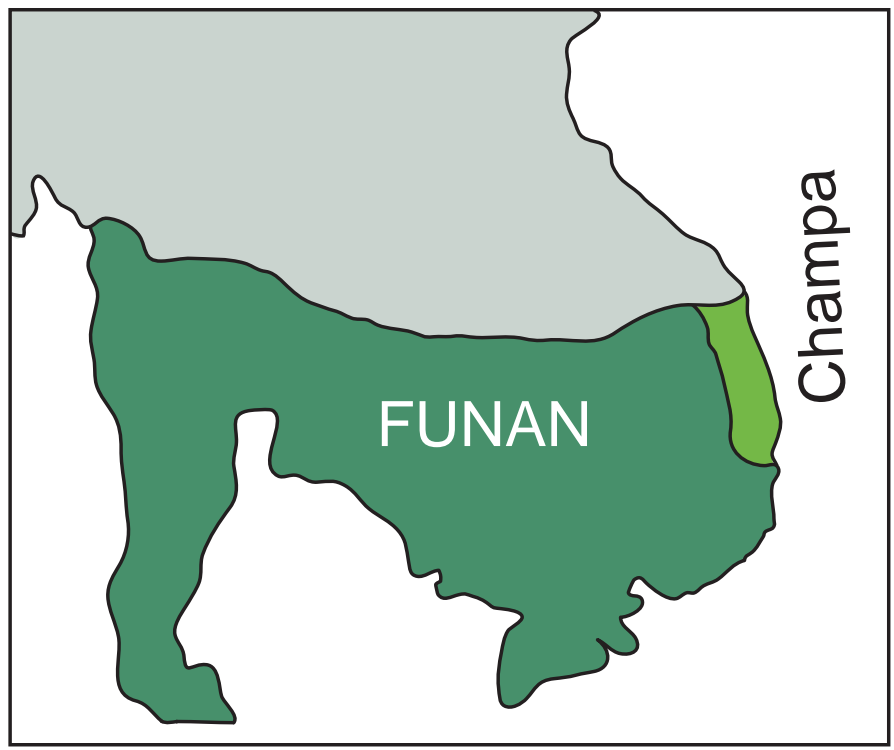

Map 13.4

Funan, 3rd century CE

became common in Vietnam. Women in Vietnam had a higher position than in China, playing a role in choosing spouses, inheriting, and pursuing divorce. These practices predated Chinese conquest and would survive centuries of Chinese influence. Vietnam gained independence in 939 as the Tang Dynasty declined. The Vietnamese would pay tribute to the Song (chapter 15), but the Song would mostly leave them alone. They would nevertheless continue to imitate the Chinese bureaucracy. As in China, the Vietnamese came to expect a process of dynastic cycles. Trade between Vietnam and China fostered the continued interchange of ideas: The Chinese would benefit from Vietnamese strains of rice during the Song Dynasty (chapter 15). Northern Vietnam would also gradually absorb the state of Champa to the south over the next centuries, which India had previously influenced. The people of Champa initially spoke a language related to Malayan and were predominantly Hindu (some small Hindu communities remain in the region).

Though Chinese influence came through both trade and conquest, the Indian influence in the region had come exclusively through trade. Political fragmentation characterized India itself through much of its history and Indian states would only once attack any part of Southeast Asia (below; chapter 15). Indian merchants had been more common than Chinese merchants in most of the region. They had carried both Hinduism and Buddhism with them. It should hardly be surprising that Funan and later states in the region outside of Vietnam borrowed the religious justifications that Indian states employed to justify their own rule. There were likely also advantages in dealing with 
Indian merchants of accepting their religions. As Muslim merchants came to supplant Hindu and Buddhist merchants in later centuries, the incentives changed for states along the main trade routes (chapter 12, 15).

\section{Tibet}

Like Southeast Asia, Tibet would interact with both China and India. Unlike Southeast Asia, though, Tibet would pursue military conquests in both China and India (and in Central Asia) during this period. Indeed, the Tibetan Empire from the seventh to ninth centuries extended far beyond the Tibetan plateau. The Tang would invade Tibet in the seventh century but withdraw. The Tibetans, in turn, would briefly attack the Tang capital Chang'an in 763 at the end of the An-Lushan rebellion (above). At its height in the ninth century, the Tibetan Empire controlled lands in Bengal in India and Yunnan in China. It also controlled areas south into Nepal, north into Mongolia, and west as far as Samarkand and perhaps

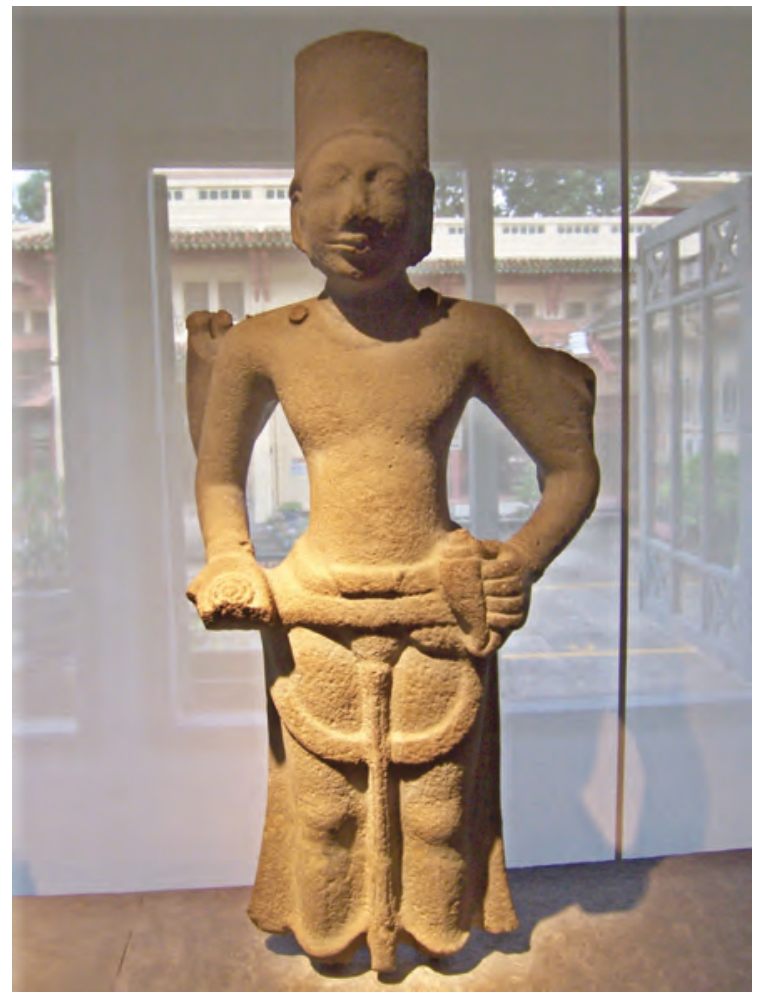

Illustration 13.22

Stone statue of the Hindu god Vishnu found at Óc Eo in An Giang Province, Vietnam. It is dated to the 6th or 7th century CE, Museum of Vietnamese History, Ho Chi Minh City Kabul. The Tibetan Empire is remarkable for incorporating people from Chinese, Indian, Middle Eastern, and Central Asian societies. The Tibetans fought with not just the Tang but also the Uighurs to the north and the Abbasids to the west, among many others.

Tibet had known of Buddhism for centuries but this was not the dominant faith before the rise of the Tibetan Empire. There had long been a significant Christian community in Tibet - both Nestorian and Orthodox - though the Islamic invasions would sever this from Christian Europe. The main religion was a local animistic faith called Bon that still survives as a minority religion in the region. Buddhism would gain royal favour after the Tibetan emperor married a Tang princess in the early seventh century. Later emperors would import Buddhist scholars from India (see below). Like most of Southeast Asia, it would pursue Theravada Buddhism rather than the Mahayana Buddhism prevalent in China (both the Mongols and Manchus that later conquered China - chapters 17 and 20 - would favour Tibetan Buddhism, but not change the general orientation of Chinese Buddhism). 
Postscript: A distinct Tibetan Buddhism was codified during the "Tibetan Renaissance" of 1300-1500, though critical texts were often published in Beijing. Though ideas of reincarnation had been associated with particular people beforehand, Tibetan Buddhism would from the fifteenth century become unique in proclaiming that its lamas (religious leaders) were reincarnations of previous lamas in an unbroken line.

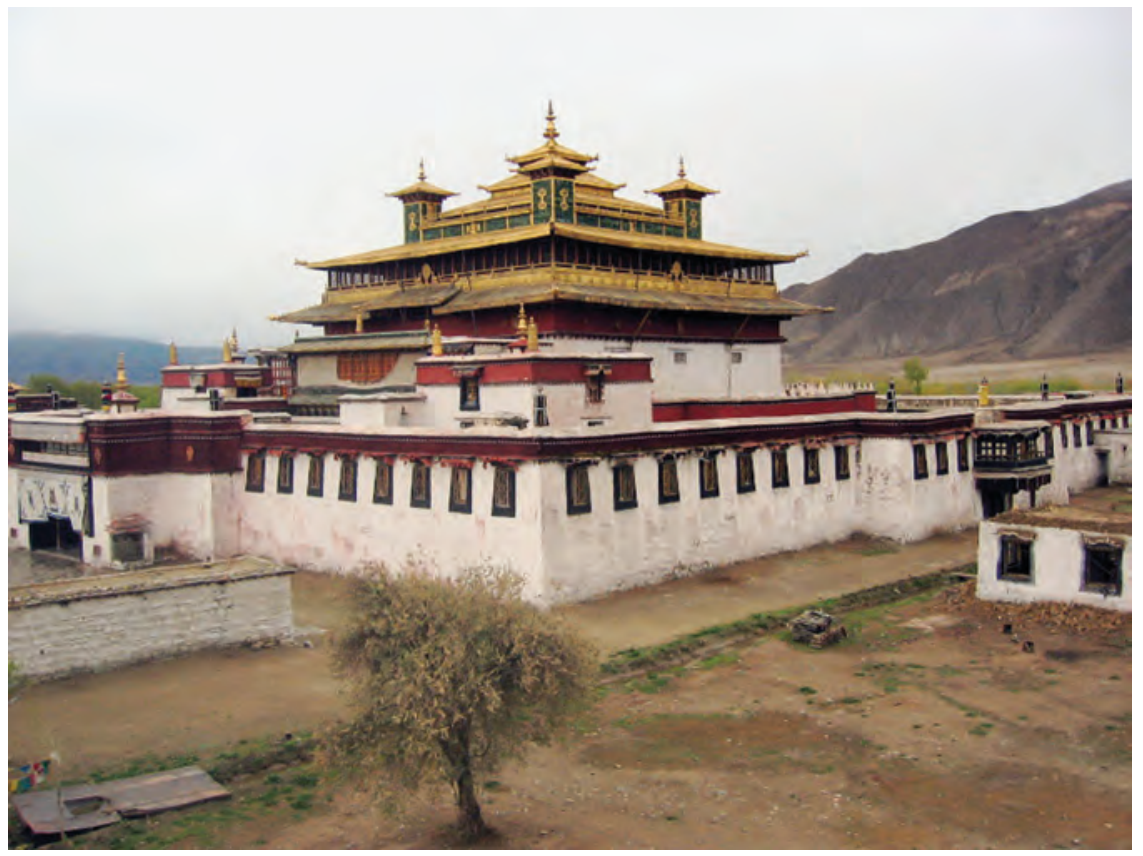

Illustration 13.23

Samye temple in Tibet has been rebuilt many times since being founded in the 8th century

Photograph by Tsui, CC BY-SA 2.0

\section{India}

Nomads from Central Asia assailed the Gupta Dynasty (chapter 9). For the next several centuries, political disunity characterized India. Some histories of India pay much less attention to this period of disunity than to earlier and later empires. Yet such a treatment exalts empire above all other considerations. There were notable developments in trade, religion, architecture, sculpture, painting, and science during this period, and also the only example of foreign conquest (that is, beyond the subcontinent itself) by an Indian state. Indeed, Arab writers 
would celebrate India during this period for its sculptures, paintings, poetry, medicine (especially for counteracting poisons), and astronomy.

The religious transformation is perhaps of greatest historical importance. After the decline of the Hindu Guptas, both Buddhism and Jainism gained influence in the courts of Indian states. The brief Hartha Empire of the seventh century (which was praised by the Chinese visitor Xuan Jang for being both cosmopolitan and just) would expand northward into the Himalayas; it was at this time that Buddhism spread most intensively to Tibet where it would grow and prosper over the next centuries (above). Buddhism, Jainism, and Hinduism would all witness the clarification of practices and beliefs in the sixth through ninth centuries. From the eighth century or so, however, Hinduism became by far the most common official religion in India. For example, the Shahi Dynasty, which ruled in parts of present-day Afghanistan and Pakistan, converted from Buddhism to Hinduism in 870. (It would later lose militarily to invading Muslim forces; see below.)

The caste system absorbed both migrants and new occupations by creating new sub-castes. The caste system spread to southern India during the postclassical period. Many scholars argue that it was far more flexible at this time than it would become in later centuries.

The rise of Hinduism may in part reflect the attractiveness of a hierarchical religion to authoritarian states. Hindu leaders also revitalized the faith in response to the influence of Buddhism and Jainism. Kumarilla Bhatta in the seventh century and then Adi Shankara in the eighth century each developed Hindu philosophical responses to Buddhism that proved very successful. Bhatta stressed the ritualism of the early Vedas while Shankara emphasized the spirituality of the Upanishads. Notably, Shankara drew on the Upanishads to speak of a process for achieving liberation/release from the cycle of reincarnation that resembles in essential respects the Nirvana of Buddhism. Shankara thus pulled together the critical elements of modern Hinduism. Buddhism lost ground steadily after that. It seems that the caste system likely became more important after Shankara, though historians debate this.

One exception to the general decline of Buddhism was the state of Pala

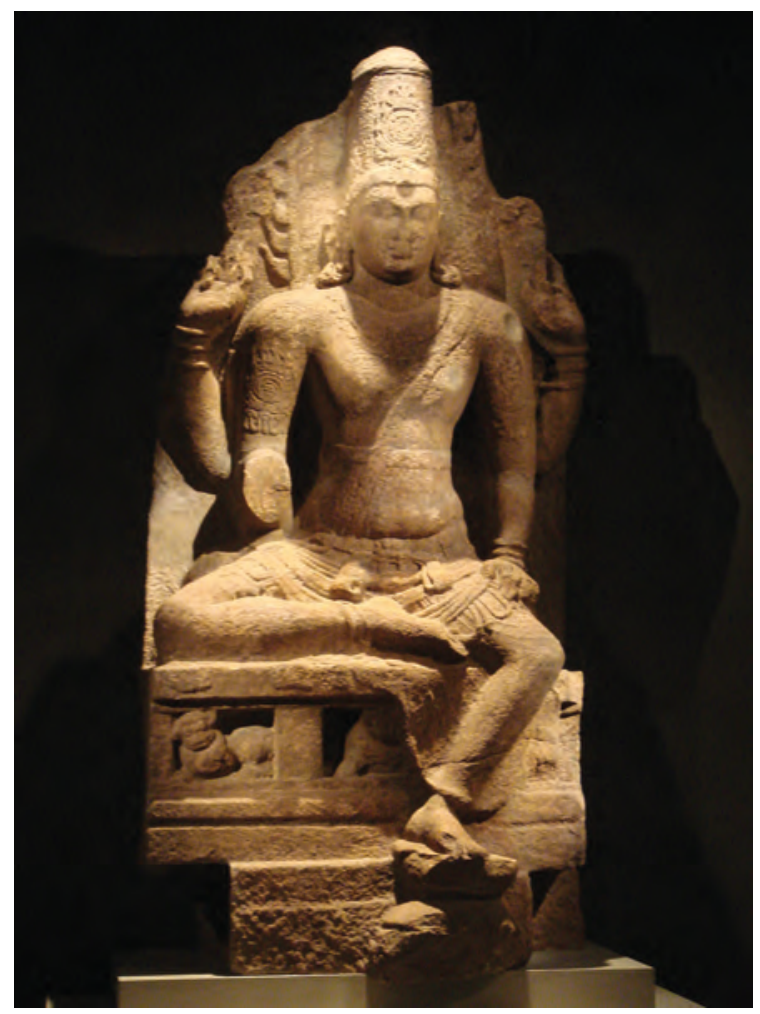

Illustration 13.24

Four-Armed Vishnu, Pandya Dynasty, 8th-9th century, Metropolitan Museum of Art

Photograph by PHGCOM, CC BY-SA 3.0 
(750-1154), which centred on Bengal in the northeast but sometimes extended far beyond this. Pala remained a Buddhist state throughout. It supported the famous university at Nalanda, one of the first universities in the world and a centre of Buddhist teaching (but it also addressed a range of secular subjects such as astronomy and medicine) that attracted students from both Southeast Asia and China. The Pala also built many Buddhist monasteries.

Postscript: The ruins of Nalanda were rescued from tropical overgrowth in the nineteenth century and are an important tourist attraction, especially among Buddhists. The government of India decided in 2010 to found a new Nalanda University in Rajgir near the site of the original Nalanda. Its founders hope that this university also will be international in both its funding and student body. They hope that it will develop a global academic reputation and encourage interaction among students from different lands. The new Nalanda welcomed its first students in 2014.

Buddhist decline occurred most rapidly in the parts of the northwest that were conquered by first the White Hun nomads from Central Asia and then Islamic forces in the eighth century. The polytheistic White Huns adopted Hinduism, and Islamic conquerors seem to have found Hinduism less offensive than Buddhism. There are records of hundreds of Buddhist temples transformed into mosques.

We cannot of course survey every Hindu kingdom that rose and fell over a period of centuries. We can note that all of these states tended to duplicate critical practices of the Guptas: drawing legitimacy from close association with Hinduism, believing that all land belonged to the ruler, and developing some bureaucratic capability. India was not, then, as politically fragmented as feudal Europe (above), but rather characterized by moderately sized states that competed militarily. These states often rewarded soldiers and bureaucrats with land rather than salaries and then had a continuing need to try to gain new lands.

The Rajputs, a set of clans within the Hindu warrior caste, emerged in the post-Gupta period. There would be numerous states ruled by Rajputs over the next centuries, and Rajputs were widely renowned for their military prowess. They would fight against invasions from Central Asia, often with great success. Some would convert to Islam and lead states within the areas of present-day Pakistan that Islamic forces conquered in the eighth century. They maintained their Rajput identity after converting.

The Rashtrakuta Empire in east India ruled from the Ganges south for two centuries from the eighth century. Rulers were originally Hindu but later converted to Jainism. Rulers of both faiths sponsored mathematics, architecture, and sculpture. 


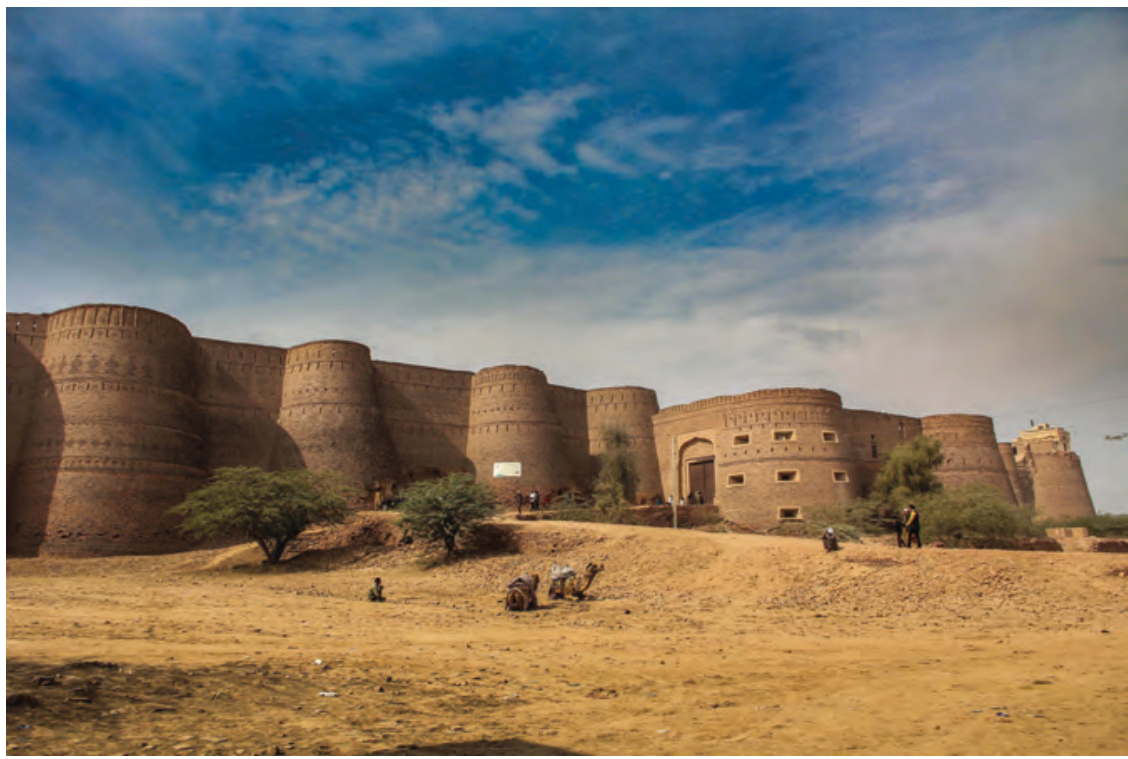

Illustration 13.25

Derawar Fort, Rajasthan; built by Rajput rulers in the 9th century. It has forty bastions, a 1500 metre perimeter, and is 30 metres high

Photograph by Fazal Khaliq, CC BY-SA 4.0

The most successful states during the five or six centuries after the fall of the Guptas were in the south of India, far from nomadic incursions. The Pallava Dynasty in southern India from the fourth to ninth centuries encouraged temples and poetry, and increased trade with Southeast Asia. The Chola built on the Pallava legacy. The Chola would come to control most of southern India and would dominate Sri Lanka for centuries. They would successfully invade parts of Southeast Asia in the eleventh century. For a time they controlled parts of Sumatra (chapter 15), Malaya, the Andaman Islands, and the coastline between Burma and Vietnam. The Chola traded with Arabia to the west and China to the east, and sent many embassies to the Chinese court.

In central India, there were three dynasties of the Chalukyas between the sixth and twelfth centuries. Political consolidation was associated with expanded trade and the development of a unique architecture.

Islam made smallish advances. As noted above, there were conquests in Sind (Pakistan) during the initial Islamic expansion that we traced in chapter 12. There were many battles over the next centuries between Islamic and Hindu rulers, but Hindu rulers often successfully repulsed Islamic invaders. There was also a growing Muslim merchant presence along the southern coast. The Islamic presence would expand dramatically with attacks from Central Asia from the eleventh century (chapter 15). 


\section{Questions}

1. Discuss the differences between serfs and slaves.

2. What are the serf's challenges?

3. Why do "barbarians" blend into local populations?

4. Evaluate the relative importance of "pull" and "push" factors for any one of the migrant populations.

5. Was the Byzantine Empire a success?

6. Why do you think histories of India often downplay centuries of political fragmentation?

7. Compare the experience of Buddhism, or of government bureaucracies, across Asian regions in this time period.

8. What do you think were the most important interactions among these regions in this time period?

9. Were you surprised that some peasants sought to join the nomads? Why?

\section{Readings}

Beckwith, Christopher. 2011. Empires of the Silk Road: A History of Central Eurasia from the Bronze Age to the Present. Princeton University Press.

Ferguson, Robert. 2009. The Vikings: A History. London: Penguin Books. [There are many books written about the Vikings. This one likely exaggerates the homogeneity of Viking society.]

Heather, Peter. 2010. Empires and Barbarians: The Fall of Rome and the Birth of Europe. Oxford University Press. [This book discusses the movements of ethnic groups in detail.]

Herrin, Judith. 2013. Margins and Metropolis: Authority across the Byzantine Empire. Princeton University Press.

King, Charles. 2005. The Black Sea: A History. Oxford University Press.

Lieberman, Victor. 2003 and 2009. Strange Parallels: Southeast Asia in Global Context, c. 800-1830. 2 vols. Cambridge University Press. [A very comparative work that surveys all regions of Eurasia.]

Ludden, David. 2013. India and South Asia: A Short History. London: Oneworld Publishers.

Luttwak, Edward N. 2009. The Grand Strategy of the Byzantine Empire. Cambridge, MA: Belknap Press of Harvard University Press.

Murphey, Rhoads, with Kristin Stapleton. 2016. A History of Asia. 7th ed. New York: Routledge.

Von Glahn, Richard. 2016. The Economic History of China: From Antiquity to the Nineteenth Century. Cambridge University Press.

There was a forum on the Vikings in the February 2020 issue of World History Connected. 
IV The Middle Ages 


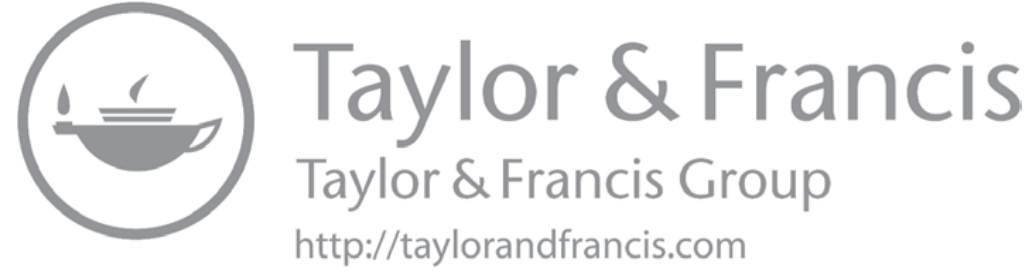




\section{Seeking global commonalities}

\section{Some key thematic trends 900-1500 and beyond}

\section{Guiding questions}

Can we observe common trends across multiple regions in the centuries after about $900 \mathrm{cE}$, and if so, why and what effects did they have?

Why, where, and in what ways do we see political consolidation? Why, where, and in what ways do we see cultural consolidation? Why, where, when, and how does trade expand in this time period?

Where, why, and how are new monuments built? How do family patterns differ across regions? Why, where, and when does population expand?

Relationship to other chapters: The chapter addresses a wide range of topics, in each case examining similarities and differences across regions. Later discussions of particular regions often reference these differences. The similarities often prove to be of critical importance to later developments: In particular, the political and cultural consolidation observed in this time period is crucial to the development of nation states in chapter 21. The discussion of political consolidation naturally builds on treatments of states and empires in earlier chapters. Our discussion of culture builds on previous discussions of religion but broadens to aspects of culture that 
we have not addressed at length before. We naturally compare the monuments of this period to the monuments of earlier chapters (especially chapter 7). We expand our discussion of how architectural understandings develop through time and spread across regions - but recognize that these can be lost if not applied for generations. Our review of trade introduces a couple of new topics: trading cities and travellers' tales; it therefore both broadens and updates our understanding of trade. The brief discussion of population picks up the story where chapter 7 left off and will segue into a further discussion of population trends in chapter 18. We know less about family patterns than about other phenomena addressed in this chapter; we try here to sketch some key similarities and differences across societies.

It is much easier to identify some common trends across regions of the world in the period after 900 than immediately before 900. After briefly reviewing climatic trends, we examine the general experience of both political and cultural consolidation. We note along the way similarities in courtly behaviour across societies. We then turn to trade, emphasizing that there was a general expansion of trade in at least the eastern hemisphere. The early centuries of the second millennium witness the building of monuments in many lands. We discuss the similarities in these building programmes. Of course, in all of these discussions, we are careful to note critical cross-regional differences as well. Differences across regions may outweigh similarities with respect to family patterns, though we shall see below that scholars have sometimes exaggerated these differences. We close this chapter with a brief discussion of population trends. We then explore in more detail in the next two chapters the particular paths taken in different regions of the world.

This chapter is unusually diverse and comparative. Different sections of the chapter are devoted primarily to the environment (both natural and built), politics, culture, economy, social structure, and population - though each section engages multiple themes. We often mention technology and art. Evolutionary analysis is most evident in our discussion of porcelain, one of the most important trade goods of the time.

\section{Climate after 900}

The period from 900 to 1300 was likely unusually warm and wet throughout most of Eurasia. These climatic conditions encouraged increased agricultural output and population throughout those two continents at least (on average; 
there were still yearly variations). These favourable climatic conditions may well have contributed to two of the trends we will discuss below: both political consolidation and expanded trade may have in part reflected the increased agricultural surplus that a warm and wet climate induced.

The fourteenth century was cooler and drier, however. This may have played some role in both inducing and slowing the Mongol advance and in spreading the plague (chapter 17).

\section{Political consolidation after 900}
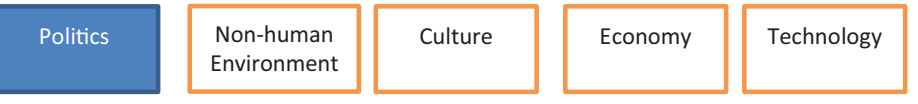

Population

Though there would be occasional periods of fragmentation, the world would comprise far fewer states in 1500 than in 900. Moreover, these states were generally more centralized. Though the trend was the same in all regions, regions differed quite a bit in their degree of centralization in 900 and thus how large and centralized their states were by the end of the period. Notably, there is some correlation across regions in the periods in which fragmentation interrupted the general trend of consolidation; this correlation may reflect climatic shocks (or perhaps epidemics) - but it may also be a coincidence.

There were some regions - including northwestern Europe, most of Southeast Asia, Japan, and Korea - that had for the most part been characterized previously by political fragmentation. Large states would emerge in each of these regions from about $900 \mathrm{CE}$. In each case, these new states would draw on the experience of preceding empires in neighbouring regions. These new states would each set important precedents: Later states in the same region would claim a connection to them to enhance their legitimacy (much as later Chinese empires would refer to the Han and even the Shang). Recall in this regard that Korea is named for the Koryo Dynasty that gained power in 935 (chapter 13). Yet these tenth-century states were both less centralized and more culturally diverse than later states. They were also smaller and less centralized than the states elsewhere that inspired them. The France of the Capetian kings was a shadow of the Roman Empire, but later French states would celebrate it for governing a similar area to modern France. Likewise, Japan and Korea at this time were far less centralized than China, but would also be invoked as precursors by later Japanese and Korean states. The Khmer state in Cambodia, Dai Viet in northern Vietnam (which gained independence from China in 939), and Pagan in Burma were each much more extensive than any previous state in the area and would set the stage for later and even more centralized states. In Russia also, a state loosely modelled on Byzantium began to emerge that later Russian rulers would herald. There was also political consolidation in Polynesia (as well as continued exploration) during this period, though 
geography limited the size of states (we return to these various developments in the next three chapters).

Consolidation also occurred in the regions of classical civilizations. In China, the centuries of political conflict after the fall of the Han had already given way to a series of dynasties that would govern China with only brief interludes of conflict into the twentieth century (chapter 13); there is a general trend of both geographic expansion and bureaucratic centralization across these dynasties. In northern India, centuries of political disunity gave way to the Delhi Sultanate and then the much more centralized and expansionist Mughal Empire. The Safavids in Persia would recall the ancient glory of the Achaemenids. The Ottomans would slowly build an empire across three continents, and when they took Constantinople in 1453 would proclaim themselves the inheritors of Rome. Though the Italian heartland of the Roman Empire remained fragmented (if somewhat less so over time) a unified Spanish state would emerge in 1492 after centuries of warfare on the peninsula. (We return to China and India in chapter 15, and the Safavids and Ottomans in chapter 20; we explore European developments in chapter 15.)

In West Africa, the Mali Empire from the thirteenth century was much more extensive than its Ghana precursor, controlling at its height the vast bulk of West Africa. Farther south the Kongo Empire would come to control much of central Africa in the fifteenth century. To the southeast, the mysterious state centred on Great Zimbabwe seems to have expanded from the eighth through the fifteenth century (chapter 16).

In Mesoamerica, the Toltecs from the eighth century ended a period of fragmentation following the decline of Teotihuacan. The Aztecs (Mexica) from the thirteenth century would found a much more extensive empire. Farther north, the record is mixed. There were centralized polities that rose and then declined both in the American southwest and along the Mississippi. In South America, the vast and centralized Inca Empire superseded from 1438 a series of smaller polities (chapter 16).

Why do we see political consolidation around the world during this period? As noted above, beneficial climate may have helped, though there were undoubtedly regional differences here. For example, the centuries after 1100 were likely unusually dry in West Africa (but this allowed Mali to employ horses far more than their predecessors, which may itself have encouraged military dominance and political centralization). In any case, there appears to have been increasing agricultural productivity and population density through much of the world. There were also undoubtedly advances in administrative techniques. These may have been shared among polities in western Eurasia, but China would only join such a conversation after the Mongol conquests (chapter 17) - and of course, polities in the Americas and much of Africa developed independently. A contemporaneous process of cultural consolidation (see below) aided (and was encouraged by) the process of political consolidation. Last but far from least, we should talk about military technology. Late in our period, the development of gunpowder weapons across Eurasia tipped the balance away from 
mobile cavalry toward large ranks of armed infantry, while also allowing rulers to destroy the castles of rebellious nobles. Many of the centralized states of this later period would be styled "gunpowder empires." (We pick up the story of gunpowder technology in chapter 18.)

\section{Courtly life}

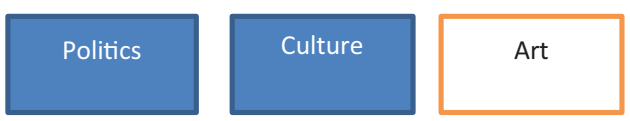

These states that grew in size and power during this period were all monarchies. These rulers, as had classical predecessors, wished to awe and impress both foreigners and their citizens. They had a particular interest in impressing local elites with their power. One way to awe and impress was to build monuments (see below). Yet another was to organize elaborate processions in which people could observe their ruler and the ruler's entourage in a manner that would signal both the wealth of the state and its military and administrative power. Such processions were common in all centralized states, and often occurred in provincial cities as well as the capital. Another strategy for awing and impressing especially local elites was to host a lavish courtly life. This had the added advantage of drawing nobles to the royal court, limiting their ability to plot against the ruler. Attendance at court generally required elegant clothing

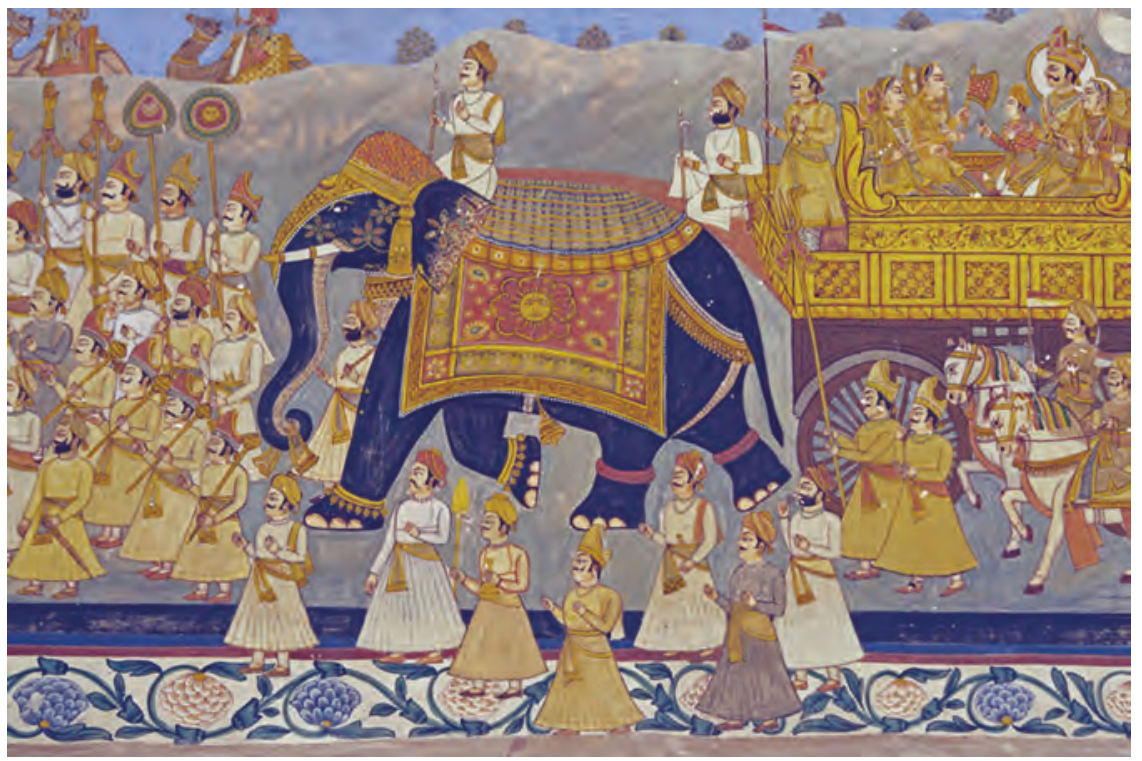

\section{Illustration $\mathbf{1 4 . 1}$}

Rajput royal procession, mural from Mehrangarh Fort in Jodhpur, Rajasthan. The fort, built in 1460, is one of the largest in India 
and sophisticated manners. The common people might over time try to copy both dress and manners: The English language thus speaks of behaviour that is ladylike, gentlemanly, or courteous.

Courts were not just centres of lavish dinners and entertainments. Rulers throughout the world patronized the arts - and often even practised these. This was yet another way that rulers might both advertise the value of a centralized state and signal their fitness to rule. Nobles attending at court were expected to display both an understanding of classical texts and an appreciation of art.

One less obvious aspect of courtly life was an emphasis on romantic love. It was perhaps inevitable that if numerous people with time on their hands gathered in one place, romantic intrigue would ensue. We have discussed the European celebration of chivalric love in chapter 13. Historians suspect that this European tradition borrowed from the Islamic Middle East: The 1001 (Arabian) Nights provide many tales in which romantic love was celebrated. The Tale of Genji of 1008, thought to be the first Japanese novel, and perhaps the first novel in the world, is a tale of courtly love and intrigue written (we believe) by a woman of the Japanese court. If the general population echoed upper-class manners and dress, it is likely that this increased emphasis on romantic love may also have spread through the community. Certainly, literate people read tales of chivalry or the 1001 Nights, and not just in the lands in which these were written.

\section{PRIMARY DOCUMENTS: EXCERPT FROM}

\section{TALE OF GENJI}

In the Twelfth Month, there was a royal outing to Oharano. All the ministers and councillors and the whole court had turned out for the occasion. The princes and high officials were beautifully fitted out. Their guards and grooms, very good-looking, had put on special dresses. The princes and high courtiers in charge of falcons were in fine hunting dress. The falconers from the royal guards were even more interesting, all in printed robes of most fanciful design. Even the skies seemed intent on favouring the occasion, for there were flurries of snow. The procession left the palace at six in the morning and proceeded south along Suzaku Avenue and west on Gojo. Carriages of viewers lined the streets all the way to the river Katsura. The princes of Rokujo and Tamakazura were among the spectators. Tamakazura paid special attention to the Emperor Reizei, her father, To-no-Chujo, Prince Hotaru and General Higeguro. The emperor in his red robe, who resembled Genji, attracted Tamakazura. It was her opinion that no one compared with him.

What is the primary purpose of this outing? Do you think the description is accurate? 


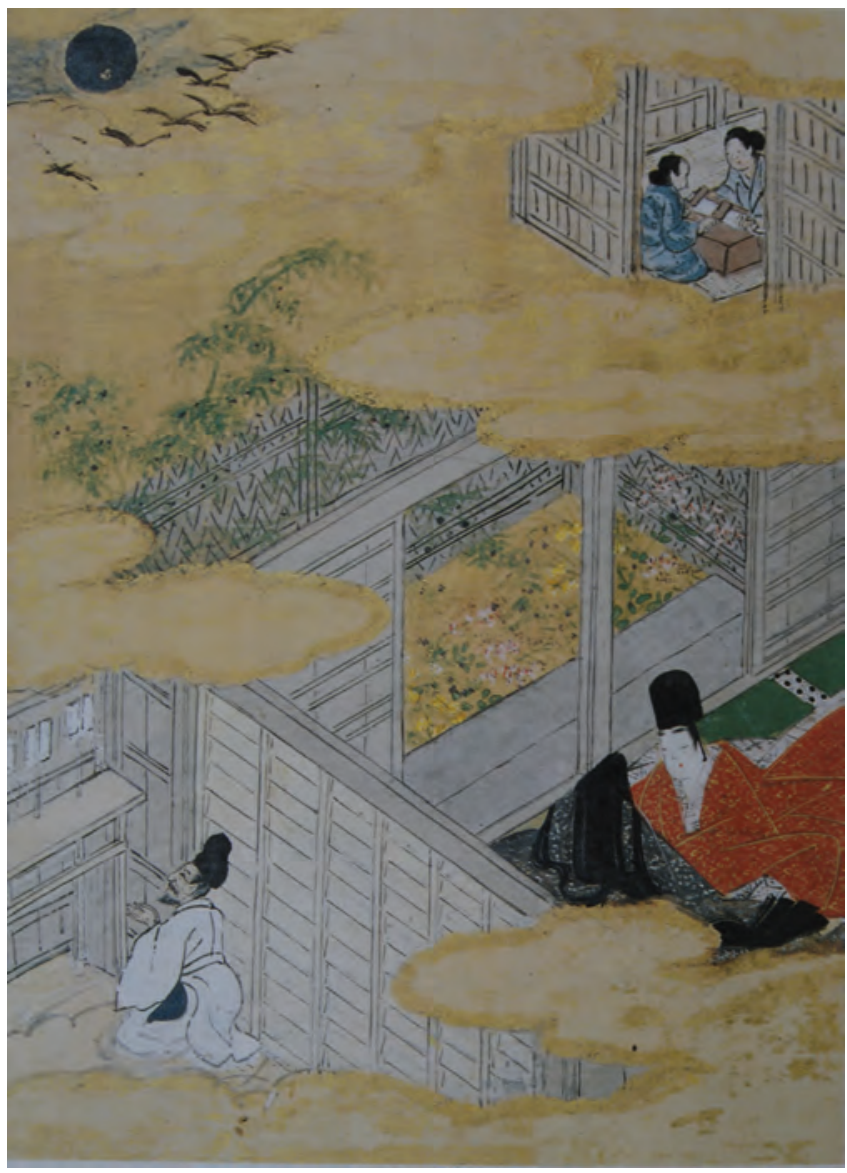

Illustration 14.2

Illustrated scroll of the Tale of Genji, 16th century

\section{Cultural consolidation after 900}
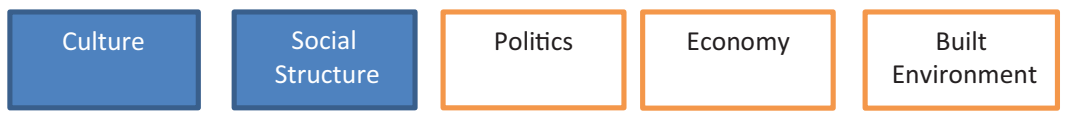

States during this period were still willing to embrace cultural diversity, especially in newly conquered territories. However, they could also see the value in appealing to a shared identity (which would, in turn, simplify social structure by lessening ethnic divisions). This was especially the case in the area of religion. With the rise of missionary religions (chapter 11), it was no longer possible to syncretize the diverse faiths of conquered peoples as the early Romans and Persians had done. States in this era often associated themselves with a particular religion - though many would practise toleration if they ruled a 


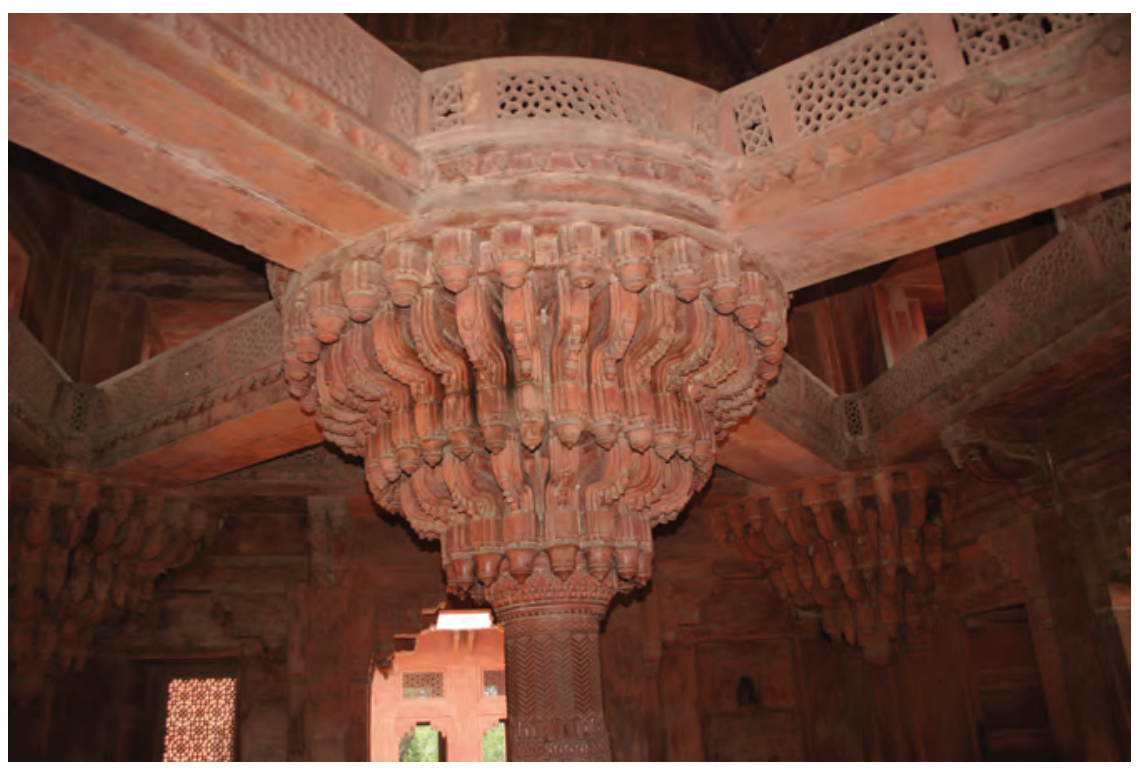

Illustration 14.3

The private audience hall in the Mughal Emperor Akbar's fort Fatehpur Sikri near Agra, India. Muslim, Hindu, Buddhist, and Christian iconographic elements are integrated together in the column

religiously diverse population. The Mughals in India would manage the difficult task (not always well) of proclaiming themselves protectors of the Islamic faith while also supporting other religions.

Political consolidation was far from the only source of cultural consolidation. Increased state power and increased trade (see below) combined to encourage improved transport infrastructure, which further intensified contact between people. Economic growth funded an enlarged religious apparatus, which supported the spread of particular religions within particular states. Religions were also throughout most of the world responsible for increases in literacy: Religious leaders then hoped that people would read religious texts. Literacy was advantageous also as both trade and government bureaucracies expanded. With expanded literacy came a blossoming of works of both fiction and non-fiction in local vernacular languages - rather than ancient languages like Latin and Sanskrit known only by a small elite. This literature, in turn, provided a further incentive toward linguistic standardization. Not only did people learn the language of the capital in order to read the vernacular literature but in doing so they experienced the lives lived by others in their region. We noted in chapters 1 and 6 that stories have always been an important means by which societies transmitted cultural values and practices; the spread of regional vernacular literatures could then serve as a critical source of shared cultural values and practices. (In particular, this literature could acquaint ordinary people with the courtly practices we discussed above.) 
Local peoples faced increased exposure to the language, religion, and cultural practices of the capital (or other nearby cities). If they wished to engage in trade or interact with the bureaucracy or religious authorities, they had an incentive to adopt the language and cultural practices of the capital. Slowly over time, then, we see the adoption of a broader cultural identity. This process would not be universal. Many groups would remain hostile to central authorities, celebrating cultural differences. Centralized states would, in turn, be hostile to minorities that they saw as a threat: Huguenots (Protestants) in France, Muslims in Europe, and Christians in Japan. Nevertheless, throughout much of the world, we see in this period the development of large groups with a shared ethnic identity, and shared language and religion and literature. Persecuted minorities might either leave or assimilate.

Though cultural consolidation proceeded most easily within states ruled by local elites, it also occurred in states ruled by foreign elites. Such states naturally had a sceptical attitude toward a strong sense of cultural identity among those they ruled, since the governed would then view rulers with enhanced suspicion. One strategy in such a situation was to go local, adopting local culture and even language. This would be the strategy of the Mongols and (especially) Manchus in China, and was (perhaps less consciously) the strategy of Franks in France and Angles and Saxons and then Normans in England. The second strategy - pursued by the Mughals in India and at least for a while by the Ottomans in the Middle East - was to celebrate ethnic and religious diversity. Such a policy might slow but would not prevent cultural consolidation.

Ethnic identification was fluid in this period. Millions of people around the world would change their ethnic identity in ways that reduced the ethnic diversity of the world. Such a process of ethnic consolidation would become harder in most places in later centuries as people became more self-conscious of their ethnic identity and less willing to adopt new languages or religions (with the notable exception of lands of new settlement such as the United States, where immigrants from many lands might forge a new identity). Much of the global map of ethnic identities and religious affiliation was therefore solidified during this period.

We saw in chapter 13 how a process of ethnic consolidation occurred as groups coalesced while attacking and migrating in Europe. We see here a similar process throughout the world in which successful ethnicities or cultures - those associated with cities and states and trade and literature - attracted individuals from other groups. Some groups would "win" over larger areas and populations than others, depending on differences in state size, religious organization, trade intensity, and linguistic diversity. Once a particular language and culture came to dominate a specific region, there would be powerful incentives for others to adopt it.

We should not exaggerate the degree of consolidation achieved during this period. Later nation states (chapter 21) would still have much work to do in forging national identities: As late as 1789, perhaps only a third of the French population spoke Parisian French. Still, the consolidation achieved before 1500 


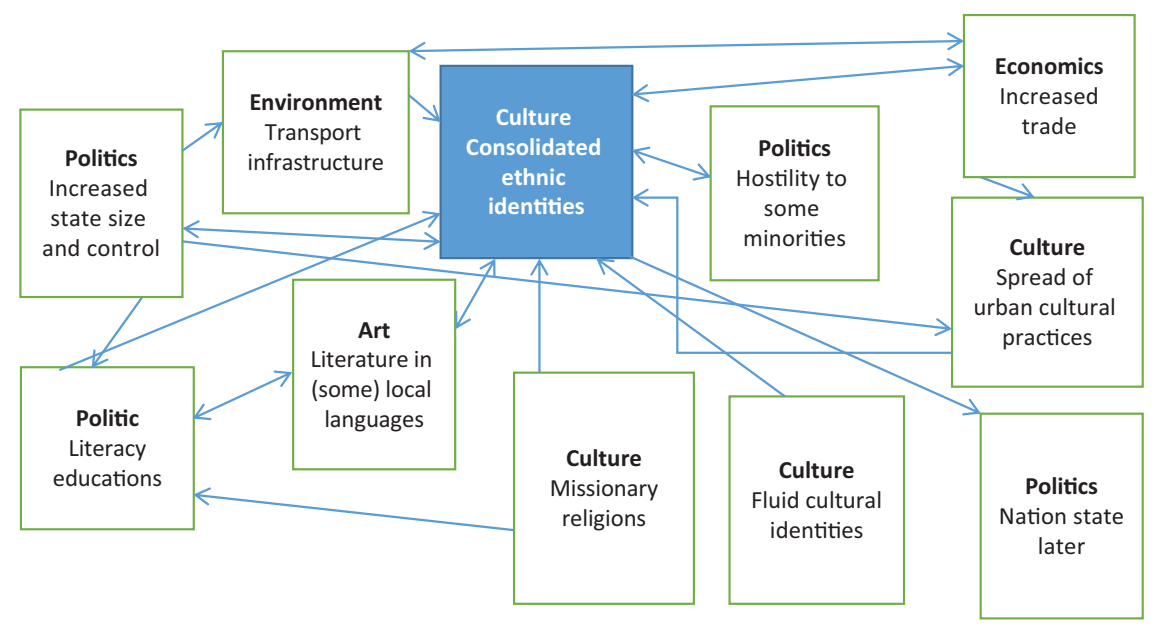

Figure 14.1

Influences on and effects of cultural consolidation

would set the stage for this later achievement of national identities in many parts of the world. Indeed, it is regions of the world that did not see extensive cultural consolidation during this earlier period - much of Africa, the hill country of Burma - that tend to have weak national identities to this day (see chapter 30).

\section{Trade expansion}
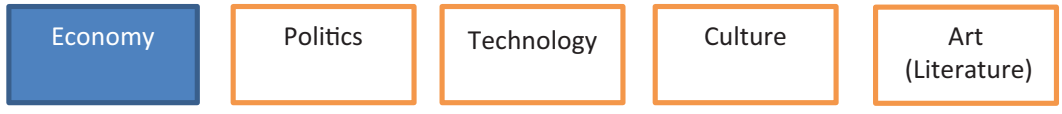

Trade expanded through most of this period - though there would be occasional setbacks associated with the intermittent periods of political fragmentation noted above, and with the fourteenth century plague (chapter 17). Indeed, it is common to speak of a "commercial revolution" across Eurasia from about 1100 - though significant trade expansion first occurred in the Arab world from the eighth century.

In the Islamic world and elsewhere, the political consolidation that we spoke of above tended to encourage trade by reducing the number of states that merchants had to engage. As states developed greater bureaucratic capabilities, they could provide superior adjudication of merchant disputes and invest in transport infrastructure. In particular, the development of large tradeoriented Islamic polities (chapter 12) and the steady expansion of the Chinese state encouraged trade both within and beyond those regions, both by land and by sea. The Silk Road trade revived, though seaborne trade between China and the west expanded even faster. 
Trade also intensified along the East African coast, across the Sahara, between northern and southern Europe, and between China and Japan. (We will discuss in chapter 15 a set of institutional innovations that facilitated trade in the relatively fragmented political environment of Europe.) Muslim traders appeared from Africa to China, with communities of thousands of such traders dotting the Indian coast. Swahili - a Bantu language with many Arabic words - emerged from the eleventh century as a trading language in Islamic trading centres that developed along the East African coast as far as present-day Mozambique. These centres would export gold, slaves, ivory, rare woods, and iron from Africa while importing Chinese porcelain, Arabic manufactures, and glassware. By the fourteenth century, West Africa was producing and exporting more gold than anywhere else in the world, providing metal for court luxuries and commercial uses in both Europe and the Islamic world (the gold was traded in part for cowrie shells that were used as a currency in West Africa).

There were extensive Jewish and Armenian trade networks throughout Eurasia. Records survive of a Jewish network based in Cairo from the eleventh and twelfth century that extended from Spain to western India (the records were saved on religious grounds because they mentioned god). These records indicate that the network would expel any member found to have traded unfairly within the network. They also indicate that the network negotiated as a group with rulers in the lands in which they traded. This network thus sought collectively to reduce two of the main challenges faced by merchants: theft and deceit by those they traded with and robbery by the states they traded in. They were not always successful: These documents criticize the ruler of Yemen for impounding their goods and charging exorbitant taxes. Gujaratis (both Hindu and Jain) and Fukienese Chinese formed smaller networks. Yet the biggest networks were Muslim, mostly Arabs and Persians. As these Muslim networks grew in importance, many Gujarati merchants converted, especially in the thirteenth century. So did merchants in Southeast Asia and China. The expansion of Muslim traders meant that Indian and Chinese merchants were less active in the Indian Ocean by the time Europeans arrived in the sixteenth century (chapter 19; we shall see in chapter 15 that the Ming dynasty discouraged Chinese from trading abroad). Still, while some networks were extensive geographically, most merchant networks focused on a particular region, such as the western Indian Ocean: Long-distance trade then still often had to pass through the hands of multiple networks.

Trade by sea benefited from better ships and better navigation tools. European, Arab, and Chinese shipbuilders all built larger ships with better sails. Trade between China and India primarily moved on Chinese boats, especially early in this period. The Chinese developed the first compass, which spread through the Indian Ocean in the eleventh century and into the Mediterranean in the twelfth century. The Chinese compass utilized a floating needle; Europeans would later develop a solid compass. Lighthouses were built on the Shatt-alArab and elsewhere so that boats could avoid numerous sandbars. (There were fewer improvements on the Red Sea; local captains were necessary to avoid 


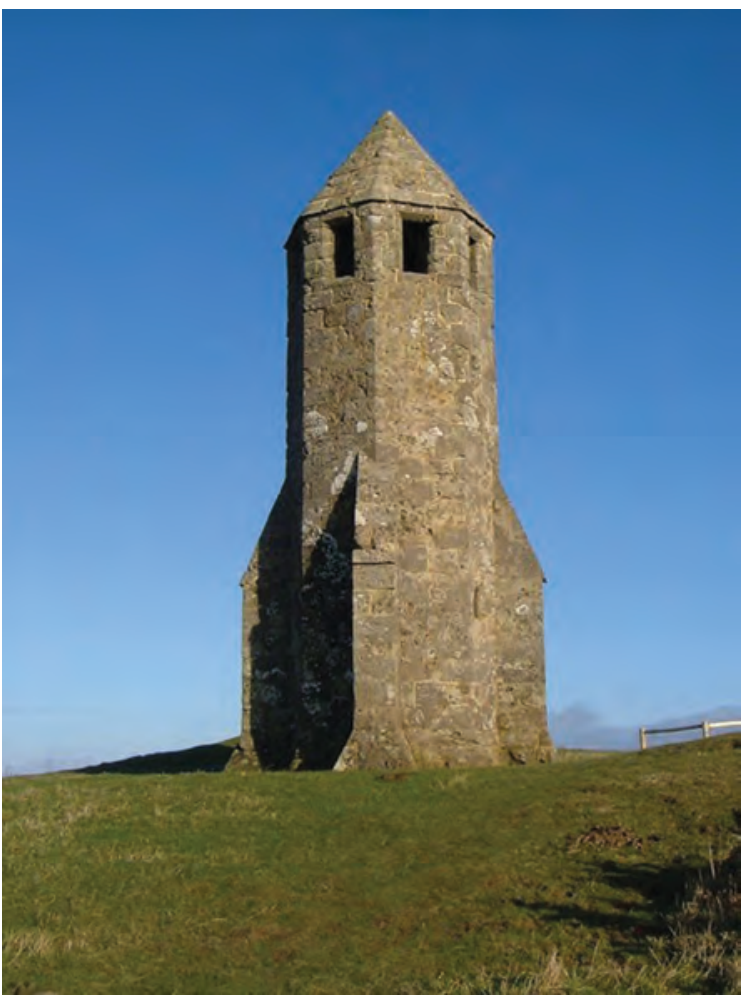

Illustration 14.4

Tower of St Catherine's Oratory, built as a lighthouse in 1328 after a nearby shipwreck in 1314. The eight windows at the top emitted light from a fire. A monk maintained the fire and prayed for lost seamen. This is a rare surviving medieval lighthouse Photograph by Derek Harper, CC BY-SA 2.0 rocks and manage fog and storms; nevertheless, trade increasingly went by water through Cairo rather than overland from Baghdad.) We see the first maps of hazards in many parts of the Indian Ocean from the eleventh century (perhaps earlier). Guides to navigation - detailing ports as well as dangers - were published across Eurasia at the time.

Expansion in trade encouraged and was in turn facilitated by the increased production of currency. In Europe, Florentine and Genoan currencies supplemented Byzantine and Egyptian gold coins. Chinese copper currency traded for more than its metallic value because of the solidity of the state: Merchants could be confident that they would receive face value when paying taxes with such coins. Paper currency also emerges in China as early as the ninth century but becomes much more important under the Song Dynasty (chapter 15). Merchants in all regions, but especially in Asia, also developed credit instruments through which funds could be transferred between distant lands without moving currency (that is, a loan made by one merchant could be transferred to another merchant in a distant locality).

Although intercontinental trade expanded markedly, it remained more important culturally than economically during this period. World trade was minuscule compared to what it would become after the fifteenth and especially nineteenth centuries - but it was also far more extensive than ever before. Costs of transport were high enough that merchants who were engaged in longdistance trade focused on profit-per-unit in the exchange of luxuries rather than bulk trade in necessities. The goods involved in long-distance trade were either rare raw materials such as spices or finished products such as porcelain or certain textiles with a high price to weight ratio. Religious and technological ideas continued to spread along trade routes. So did culture: The 1001 Nights became popular in Europe during this period.

We should not entirely discount the economic effects of intercontinental trade. Though this comprised a small part of global output, it loomed larger in the tax revenues of states. States, in turn, were encouraged to develop transport infrastructure and legal systems to further facilitate trade. One type of raw 
material traded between regions was agricultural: Transplanted food crops often enhanced agricultural productivity. Finally yet importantly, growing intercontinental trade spurred efforts to develop new trade goods and trade routes. Southeast Asian countries were by the fifteenth century planting peppers and other spices that they sold to both China and the Islamic world. In Europe, the Spanish and Portuguese began to wonder late in the fifteenth century if there were easier ways to trade with Asia than dealing with Islamic and Italian intermediaries (see chapter 19).

\section{Trading cities}

Cities along trade routes could grow rich through both the activities of their merchants and the taxes on trade imposed by city governments. Though world trade might have a limited effect on the global economy, it did exert a significant influence on such cities (on which world trade in turn depended). Changes in trade routes could have dramatic effects on cities: Cairo grew at the expense of Baghdad as trade followed the Red Sea rather than overland (though merchant guides in the Middle Ages spoke of no ports north of Jeddah on the Red Sea, due presumably to the dominant northerly winds farther north). Cities that could monopolize a particular trade route - such as Constantinople, Corinth, Melaka, and Samarkand at times - were able to charge higher taxes than those that competed with other cities. Cities could encourage trade by establishing markets, providing legal protection, and building ports or bridges or roads. Melaka quickly grew to 50,000 people as it came to control trade through the Straits of Melaka. Samarkand and Timbuktu became famous throughout the eastern hemisphere because of their riches and their situation far from other societies but along vital trade routes. Ibn Battuta (below) would judge Caffa in Crimea to be one of the world's great ports as Black Sea trade (including Silk Road trade, but also an extensive slave trade) rebounded after centuries of relative neglect. Cities such as Melaka or Cairo, which connected different regional trading networks (see above), were of particular importance. Such cities were inevitably cosmopolitan: Visitors often remarked at the dozens

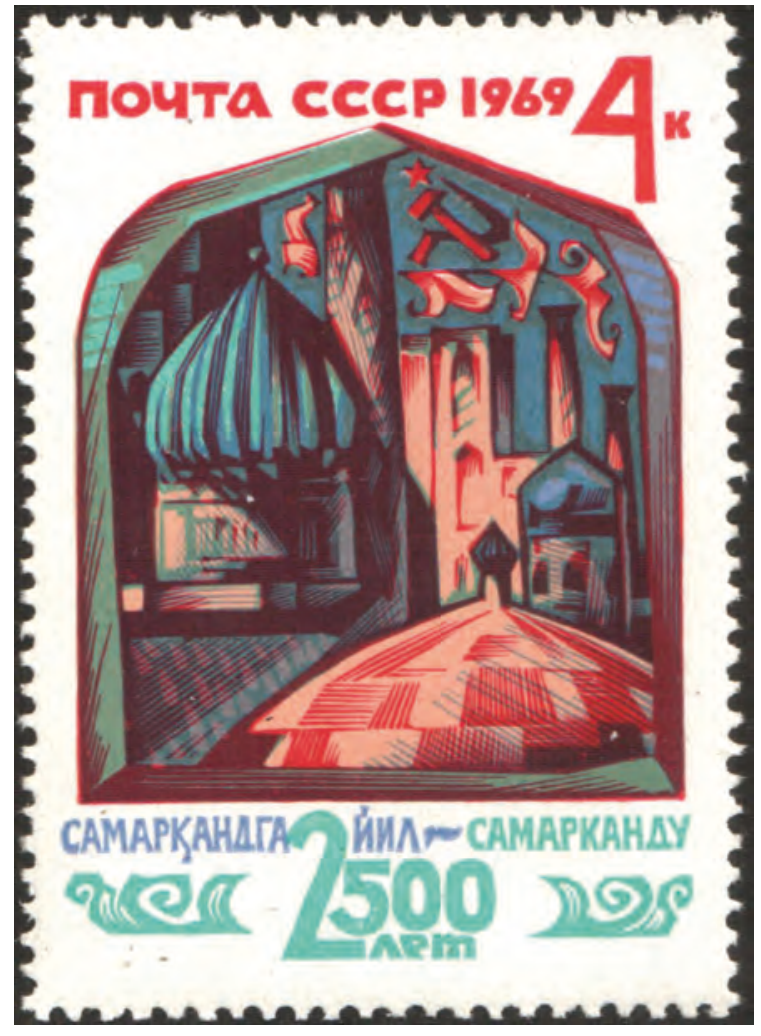

Illustration 14.5

Registan Square, Soviet Union stamp, 1969, celebrating the 2500th anniversary of Samarkand 
of languages heard in such places. Red Sea ports were noted for intermarriage between Arab and African residents.

\section{Travellers' tales}

Trade, as we have often noted, requires the movement of people as well as goods. Trade does not occur anonymously, but through merchants who strive to know those they deal with. Networks handle most trade; these networks require that many merchants live in foreign communities. As trade expanded, and as literacy and publishing expanded (see above), the genre of travel writing blossomed. In the Islamic world, there was a further impetus: Those with the means to do so were expected to perform the hajj - visit Mecca - at least once during their lives. Those planning such a trip were curious to read about what to expect, and those who could not afford it wished to have the experience vicariously. Travel writing was not a new genre: We saw examples of travel writing in ancient Egypt in chapter 6. Yet there were now many more works, and these could describe lands that were more distant.

The two most celebrated travel writers of this era were Marco Polo of Venice (1253-1324) and Ibn Battuta of Morocco in the early fourteenth century. Marco Polo's family was involved in trade with Asia. Polo himself would travel with his father and uncle to the Mongol capital in Central Asia (see chapter 17). The Mongols would then employ him for several years, during which he was able to visit many parts of Asia, including Tibet and many parts of China. There is, we should note, some scepticism regarding Marco Polo's story - written by a cellmate in an Italian jail late in Polo's life. Though Polo may well have embellished his role in the Mongol court, it is true that the Mongols liked to employ foreigners in their service (for these could be more loyal than those with local connections), and many of Marco Polo's observations ring true. Polo had travelled overland to China but returned by sea via Melaka and the Arabian and Black Seas. He returned by sea on ships escorting a Yuan (Mongol) royal bride to Persia. It is noteworthy that she went by sea rather than land: The Yuan apparently viewed the route by sea to be safer and more comfortable than travel overland. This voyage home comprised a quarter of Polo's tale, and western merchants would use it as a guide to Asian trade routes by sea for centuries. Greater scepticism was exhibited at the time for his descriptions of the glories of China. Marco Polo would say, for example, that Quanzhou had many times the traffic of Alexandria, the main port of the Mediterranean at the time. He described Quanzhou as the world's grandest city. As historical scholarship expands our understanding of the Chinese Empire through the ages, Polo's descriptions of its riches seem more believable than they appeared at the time.

Ibn Battuta was an Islamic legal scholar from Tangier (in present-day Morocco) born in 1304. He made the hajj to Mecca in 1325-6. He then travelled south to Kilwa on the East African coast and east to India (by way of Constantinople and the Black Sea and Central Asia; he was surprised to find Genoese and Venetian traders in Tabriz in present-day Iran). He then worked 


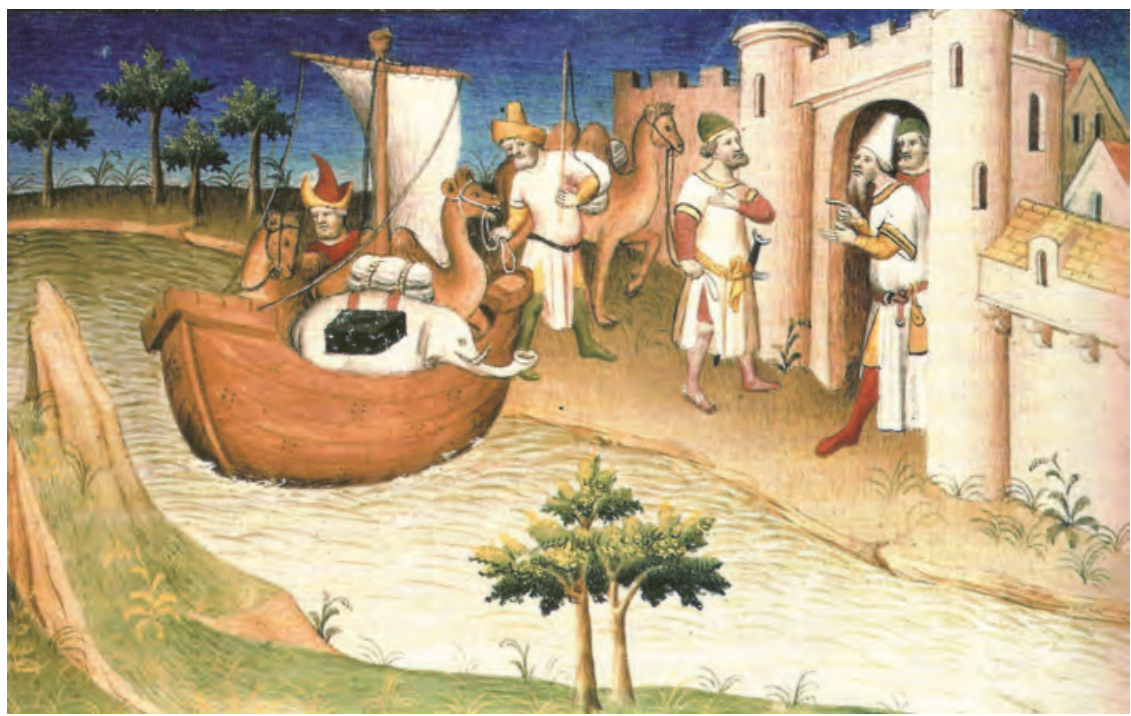

Illustration 14.6

Miniature from the book The Travels of Marco Polo ("Il milione"), originally published during Polo's lifetime (1254-1324)

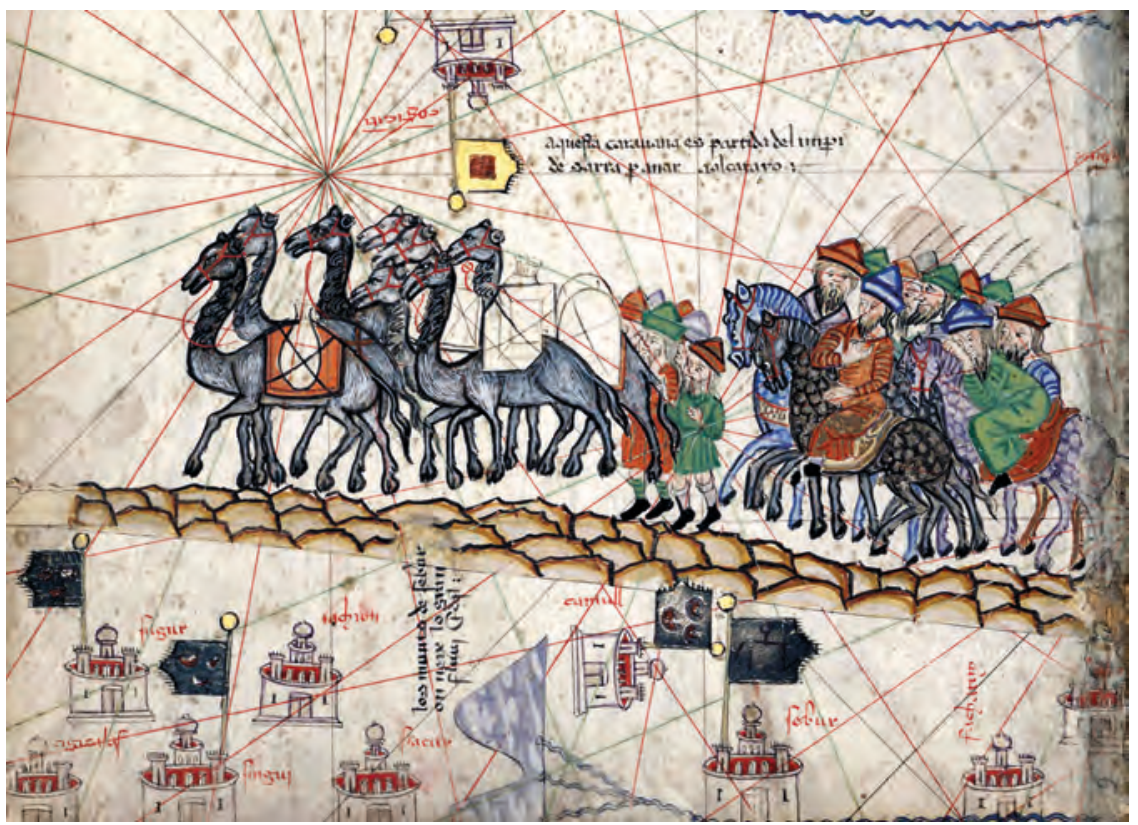

Illustration 14.7

Marco Polo's caravan, from Coureurs des mers, Abraham Cresques 1375 


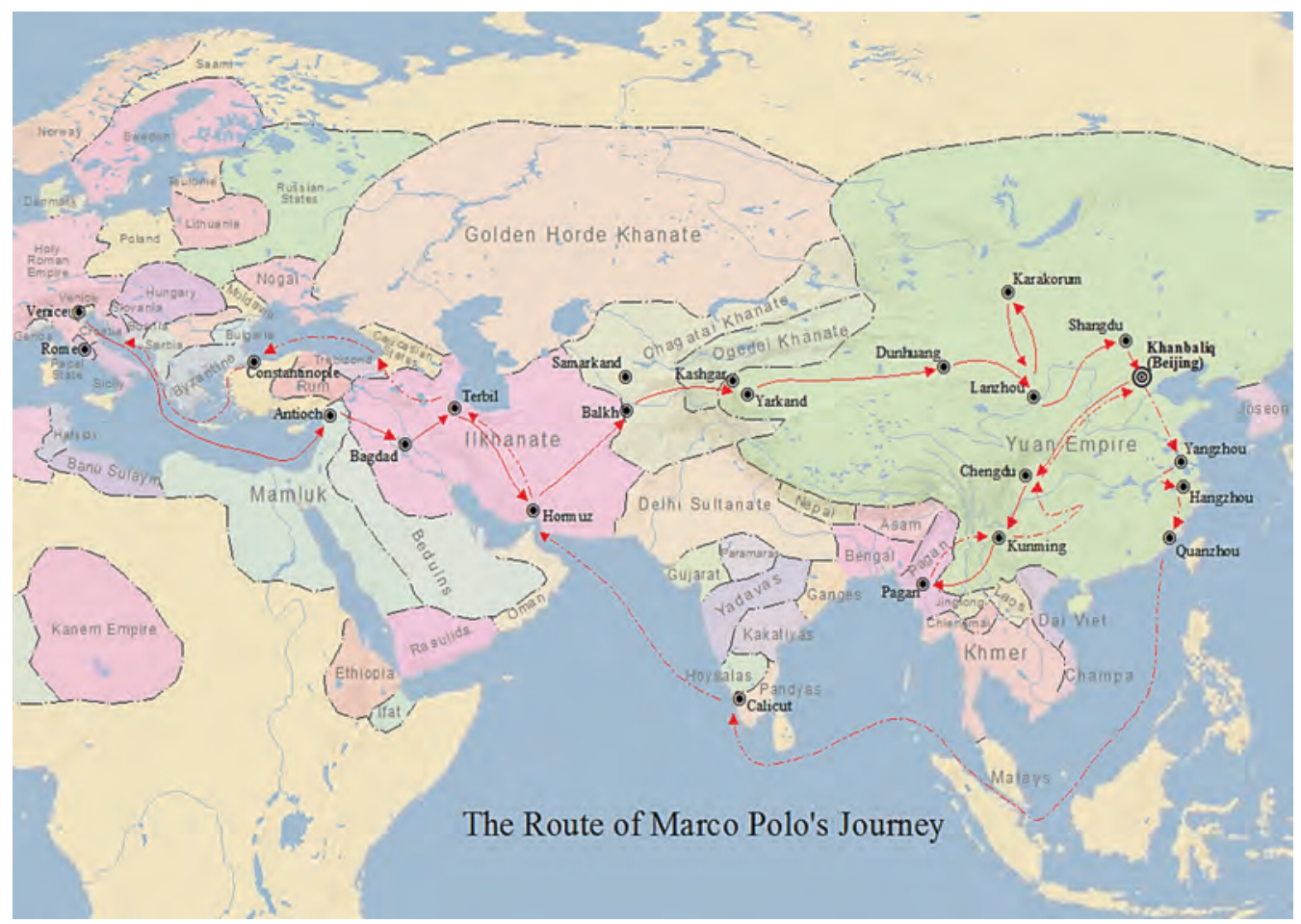

Map 14.1

Marco Polo's journey

Map by SY, CC BY-SA 4.0

as an Islamic judge in India for several years. His services as a legal scholar and judge had previously been valued in East Africa, and would later prove useful in West Africa. He was a very orthodox legal scholar and judge: He once sentenced a man to eighty lashes for having drunk alcohol eight years earlier. From India, he went by sea to southern China and back, and then returned to Morocco (this time through Syria). Later in life, he would travel to southern Spain and across the Sahara to the Mali kingdom. He would be scandalized in West Africa by the behaviour of women who not only socialized with men in public but also often bared their breasts - though most other elements of West African society impressed him. He recorded his observations along the way. He is perhaps the greatest pre-modern travel writer due to travelling well over 100,000 kilometres and keenly observing life around him. He was able to travel so extensively because Islamic legal scholarship was widely appreciated, especially in lands only recently converted; he was warmly greeted wherever he went. Even in China, communities of Muslim merchants welcomed him.

There were many other works written by travellers - or merely the wellinformed - from many lands. Zhao Rugua from his role at the port of Quanzhou described trade in forty-six goods with ports as far as the Mediterranean and East Africa. Modern historians much value these various travel descriptions for their 
insights into politics, culture, trade, and everyday life. They were important at the time in inspiring curiosity about distant lands, which in turn encouraged both trade and travel.

\section{PRIMARY DOCUMENTS: MARCO POLO AND IBN BATTUTA}

Marco Polo on Kublai Khan's palace:

Built for the present Great Khan, Kublai Khan, here there is a stone and marble palace with gilded rooms wonderfully decorated with magnificent and delicate paintings of birds, trees and flowers of every kind. Leading away from the palace is a wall which encloses sixteen miles of fertile ground with rivers and streams running through it. Here the Great Khan raises every sort of animal, including fallow deer and roebuck which are fed to the many gerfalcons and falcons, and at least once a week he goes to look at them. He often rides through these gardens with a leopard squatting on his horse's hindquarters...

Marco Polo on the use of coal and frequency of baths:

It is a fact that all over the country of Cathay there is a kind of black stone existing in beds in the mountains which they dig out and burn like firewood. If you supply the fire with them at night and see that they are well kindled you will find them still alight in the morning, and they make such fine fuel that no other is used throughout the country. It is true that they have plenty of wood also but they do not burn it because those stones burn better and cost less. [Moreover with the vast number of people and the number of baths they maintain - for every one has such a bath at least three times a week, and in winter if possible every day, while every nobleman and man of wealth has a private bath for his own use - the wood would not suffice for the purpose.]

Ibn Battuta meets the Byzantine Emperor:

After this I reached a great pavilion, where the Emperor was seated on his throne, with his wife, the mother of the khatun, before him. ... The Emperor signed to me, before I had saluted and reached him, to sit down for a moment, in order that my apprehension might be calmed. After doing so I approached him and saluted him, and he signed to me to sit down, but I did not do so. He questioned me about Jerusalem, the Sacred Rock, the Church of the Holy Sepulcher, the cradle of Jesus, and Bethlehem, and about the city of Abraham [Hebron], then about Damascus, Cairo, Iraq, and Anatolia, and I answered all his questions about these, the Jew interpreting between us. He was pleased with my replies and said to his sons 'Treat this man with honour and ensure his safety.' Then he bestowed upon me a robe of honour and assigned me a horse with saddle and bridle, and an umbrella of the kind which the king has carried above his head, that being a sign of protection. I requested him to designate someone to ride in the city with me every day, that I might see its marvellous and rare sights and tell of them in my own 
country, and he appointed a man as I had asked. They have a custom that anyone who wears the king's robe of honour and rides his horse is paraded round with trumpets, fifes and drums, so that the people may see him. They do this mostly with the Turks who come from the territories of Sultan Uzbeg, so that the people may not molest them, and I was paraded in this fashion through the bazaars.

Ibn Battuta on crossing the Sahara:

From this point the "takshif" is dispatched. The "takshif" is a name given to any man of the Massufa tribe who is hired by the persons in the caravan to go ahead to Iwalatan, carrying letters from them to their friends there, so that they may take lodgings for them. These persons then come out a distance of four nights' journey to meet the caravan, and bring water with them. Anyone who has no friend in Iwalatan writes to some merchant well known for his worthy character who then undertakes the same services for him.

It often happens that the "takshif" perishes in this desert, with the result that the people of Iwalatan know nothing about the caravan, and all or most of those who are with it perish. That desert is haunted by demons; if the "takshif" be alone, they make sport of him and disorder his mind, so that he loses his way and perishes. For there is no visible road or track in these parts, nothing but sand blown hither and thither by the wind.You see hills of sand in one place, and afterwards you will see them moved to quite another place.

Ibn Battuta in sub-Saharan Africa:

The local people possess some admirable qualities. They are seldom unjust, and have a greater abhorrence of injustice than any other people. Their sultan shows no mercy to anyone who is guilty of the least act of it. There is complete security in their country. Neither traveller nor inhabitant in it has anything to fear from robbers or men of violence....

Among their bad qualities are the following. The women servants, slave-girls, and young girls go about in front of everyone naked, without a stitch of clothing on them. Women go into the sultan's presence naked and without coverings, and his daughters also go about naked. Then there is their custom of putting dust and ashes on their heads, as a mark of respect, and the grotesque ceremonies we have described when the poets recite their verses. Another reprehensible practice among many of them is the eating of carrion, dogs, and asses.

Note how both travellers marvel at both emperors and daily life. Which elements of these excerpts would have most surprised their readers then and now?

\section{Porcelain}

We noted in chapter 5 that archaeologists study pottery in general because it is useful and common, and because it survives very well. The same is true of a particular ceramic: Chinese porcelain. As we noted in chapter 5, pottery makers 
throughout the world would experiment with local clays, additions of non-clay materials, and firing temperatures. Porcelain is generally defined as a ceramic that contains kaolin - a type of clay that is almost white and composed of very fine particles - and is fired to temperatures of 1200 to 1400 degrees Celsius. Manufacturers of porcelain generally fire kaolin in combination with petuntse, often called porcelain stone. For many centuries, China - and indeed the town of Jingdezhen near Canton in southern China - dominated porcelain manufacture. This town had ready supplies of both kaolin and petuntse, and developed over centuries precise techniques of porcelain manufacture. The combination of rare raw materials and very high kiln temperatures would prove extremely hard to duplicate. Jingdezhen was the most substantial industrial town in the world for centuries.

As such, it was the scene of extensive division of labour: Over a hundred separate hands might play a role between the digging of clay and the final firing of a particular piece: digging, cleaning, kneading, painting (with many specializations), calligraphy, polishing, glazing, and more. Over a thousand kilns specialized in different types of pottery (bowls, platters, and so on) and different designs. Porcelain would first find a market within China, where Chinese emperors prized it. Less expensive porcelain was also produced: Marco Polo marvelled that Chinese peasants ate from porcelain bowls. Chinese porcelain would during our period become appreciated first throughout the Middle East and then in both Europe and along the East African coast. In the seventeenth century, English speakers came to refer to porcelain tableware as "China." Many scholars herald porcelain as one of the first manufactured items of global consumption.

Potters in many parts of the world would strive for centuries to match the quality of Chinese porcelain. Europeans would achieve success only from the seventeenth century (due in part to good fortune in having similar clays, and in part to applications of the scientific method of carefully recorded experimentation, and perhaps to some explicit spying in Jingdezhen). This was mostly a process of technology transfer by conscious imitation rather than the actual movement of technology. The Chinese successfully kept the recipe for porcelain a secret for centuries: Jingdezhen thus likely achieved the most extended industrial monopoly in world history (Koreans and Japanese produced some porcelain under Chinese guidance). Nevertheless, other potteries could also find a place in world markets, albeit at lower prices. In East Africa, a great deal of less expensive pottery from Southeast Asia and Africa itself is found alongside Chinese porcelain (though some of the broken pottery found there may merely have been ballast on incoming ships).

Chinese porcelain with its higher price and quality was generally a sign of affluence, though cultural meanings naturally varied across time and place. Filipino tribes imbued "dragon jars" with magical powers while Shah Abbas of the Safavids (chapter 20) donated over a thousand pieces of Chinese porcelain to an Islamic shrine in a display of piety. Chinese porcelain also inspired an interest in Chinese motifs (and Chinese culture more generally) in 


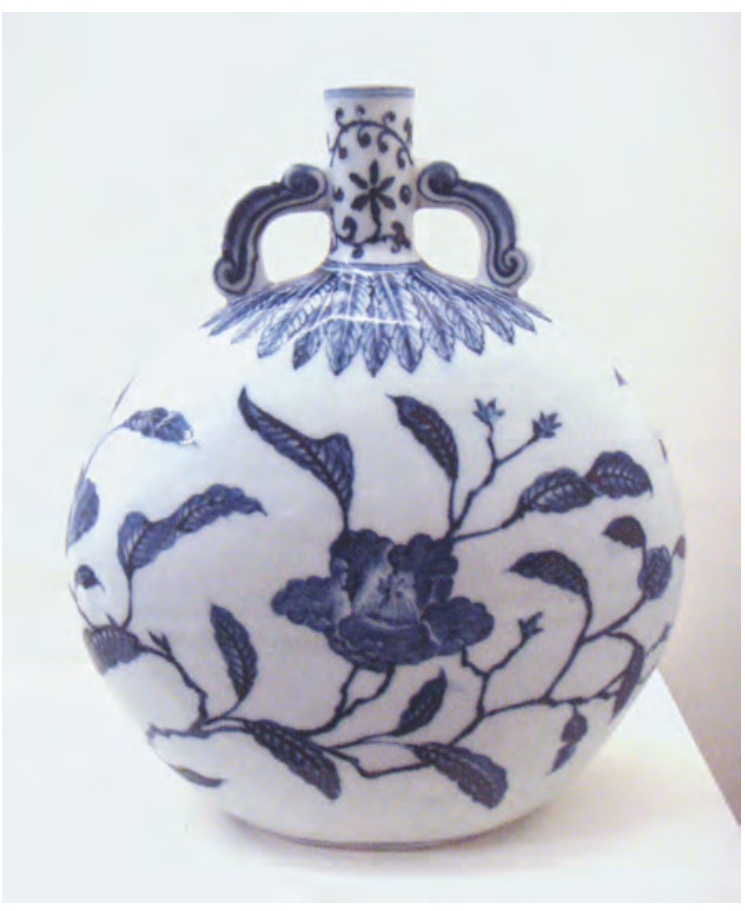

Illustration 14.8

Blue and white jar from Jingdezhen, Ming era, 1403, Musée Guimet

Photograph by World Imaging, CC BY-SA 3.0 other parts of the world - but over time Chinese manufacturers also tailored their output to the tastes of particular markets. Porcelain came to exhibit designs inspired by India, Central Asia, and the Middle East. By the sixteenth century, it was possible for European aristocrats and rulers to special-order porcelain with family crests and other favoured design elements (though Chinese artisans occasionally printed instructions such as "place red here" on the porcelain). European trading companies would establish offices in Jingdezhen in the seventeenth century to process such orders. Three hundred million pieces of porcelain were exported to Europe alone in the three centuries after the arrival of the Portuguese in China. Blue and white ware gained particular esteem, though scholars now appreciate that many other glazes were employed. As we shall see in chapter 17, the Chinese adoption of blue glaze owed much to the contact with the Middle East forged by the Mongol conquests. Blue and white ware would later become a staple of European porcelain production.

\section{BOX 14.1 COMMODITY HISTORY}

World historians have traced in recent decades the history of a variety of commodities. Some of these are natural or agricultural products: cod, corn, salt, spices. We will ourselves treat the potato in chapter 19 , rubber in chapter 23 , and coffee, tea, and cocoa in chapter 18. Some, like porcelain, are manufactured goods. Commodity history provides insights into international trade and cultural interaction; connections between workers, merchants, and rulers; the influences on and effects of technological and institutional innovation; and various other topics of interest to world historians. By tracing how the role of a particular commodity changes through history - who produces and uses it, how and where, and the meanings attached to it - we have a microcosm of world history itself. 


\section{Agricultural expansion}

Trade expansion was associated with agricultural expansion. As noted above (and elsewhere in this book), extensive trade is only possible if there is an agricultural surplus to support this. Yet trade itself could encourage agricultural changes, as when Southeast Asians grew spices for export (above). Trade allowed crops to be raised in new regions: These were often better suited to particular soil and climate niches than local crops. (We will see even more of this sort of beneficial transmission of plants when the eastern and western hemispheres come into contact after 1492; see chapter 19.) Partly because of the infusion of new crops, we see a gradual process throughout the world of extending agriculture both into new regions and into marshes and forests. Increased trade opportunities and increased population pressure were important motives. Improved techniques for draining soils and cutting trees supported agricultural expansion. Agricultural productivity was also enhanced by the development and extension of iron ploughs, replacing oxen with horses (which are faster and more efficient consumers of food energy), and new crop rotations (sometimes involving new crops). The spread of windmills and watermills allowed grains to be ground without the use of massive amounts of human or animal power.

\section{Monuments}

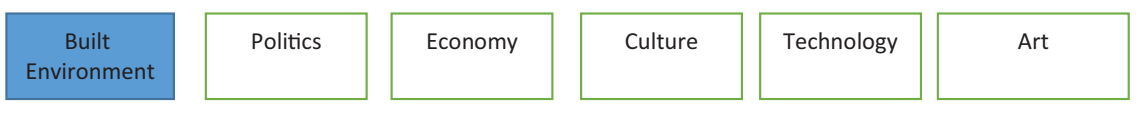

Several of the trends we have discussed above - especially political consolidation, increased state revenues from expanding trade and agriculture, and a desire among rulers and religious authorities to display publicly the glory of their rule - combined to generate a massive expansion in the building of public monuments during this period. Since cultural consolidation was often associated with missionary religions, these monuments usually but not always were religious in nature. As during the classical period, the building of many monuments over periods of centuries allowed builders to develop superior techniques and a superior appreciation of (artistic) architectural possibilities. These monuments thus expressed not only the wealth and vanity of states and religions but also the enhanced understandings of builders themselves. Most but not all of these monuments were built of sturdy materials such as stone or brick and survive to this day.

In Europe, the primary type of monument of this period was the Gothic church: These were built across several European countries from the twelfth through sixteenth centuries. Church construction had expanded from the eleventh century in Europe, and the Gothic form slowly emerged. Whereas previous churches had needed strong support walls for their roofs, the builders of Gothic churches realized that they could support roofs on columns instead of 
entire walls: This allowed them to place large windows between these columns, flooding their churches with light and creating an opportunity for artistic experimentation with stained glass. Internal columns allowed for vast interior spaces. As churches were built taller and taller - the tallest internal vault is 48 metres in Beauvais - the weight of the roof tended to push the outer columns outward (some early Gothic churches, therefore, require sophisticated engineering in the present to keep them standing). A significant innovation was the "flying buttress:" Pillars outside the church structure itself connected by slanted beams to the pillars in the church wall such that they would absorb the sideways pressure of the roof. Though cathedral builders had some rules of thumb to follow, they experimented regularly in order to build both taller and wider and to adapt to local conditions (different soils required different foundations and different building materials had distinct advantages and disadvantages). Over the decades of construction of a single cathedral, changes in design would be made, and different experts consulted.

In China during the Song Dynasty (960-1279), many pagodas and pagoda towers were built. As with Gothic churches, builders often achieved new records for the heights of pagoda towers. Although pagodas originally were constructed of wood, stone or brick construction became increasingly common: While most wooden pagodas would burn down at some point over the centuries, many of the stone pagodas survive to this day. The wooden pagoda of the Fogong

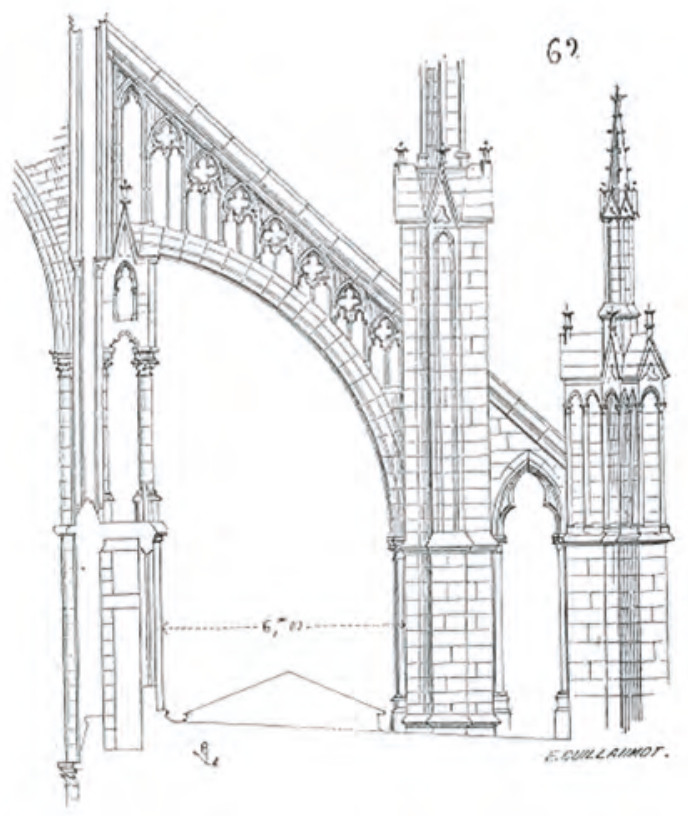

Illustration 14.9

Diagram of flying buttress, Cathedral of Amiens, from Dictionary of French Architecture from 11 th to 16 th Century (1856) by Eugène Viollet-le-Duc 


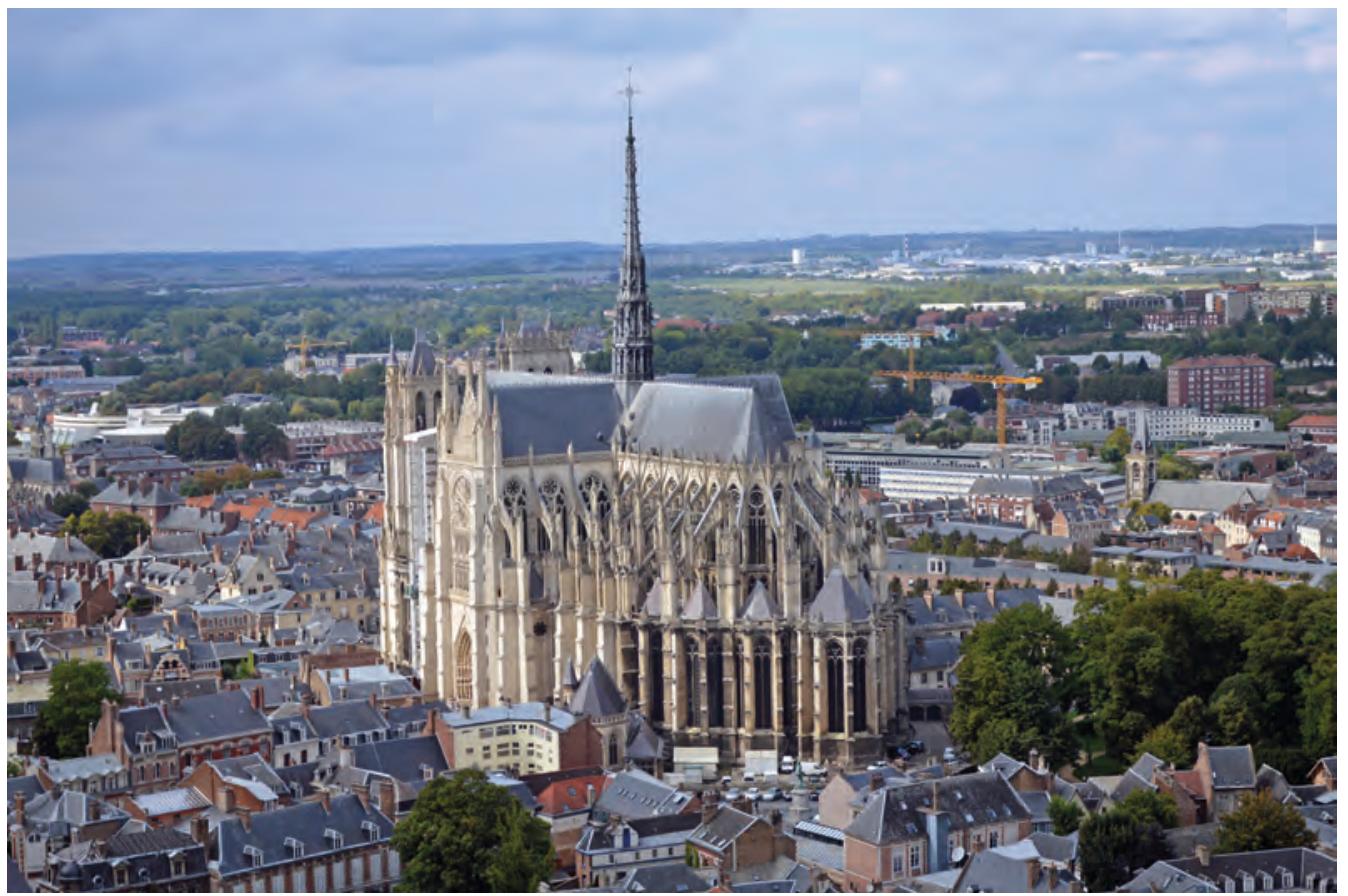

Illustration 14.10

Amiens Cathedral, Somme, Picardie, France: the chancel, 1220-70; the tallest cathedral in France

Photograph by Thierry80, CC BY-SA 4.0

temple in Shanxi rose 67 metres. The so-called "Iron Pagoda" of Kaifeng - in fact built with grey glazed bricks - is 57 metres tall, and the Liuhe (Six Harmonies) Pagoda in Hangzhou, also made in brick, stood 60 metres tall. The tallest still standing is the Liaodi Pagoda from 1055 at 84 metres. The broad eaves that add beauty to these structures also served to stabilize them against both wind and earthquake, while protecting the beams from the rain. Curved roofs also aided wind resistance. Heavy roof tiles added further stability.

In Cambodia from the eighth to the thirteenth centuries, the Khmer state built numerous temple complexes: first Hindu and later Buddhist. The Angkor Wat temple complex is the most extensive religious complex in the world. It and the other Khmer structures are notable both for their sheer size and for the intricate carvings that decorate almost every inch of these temples. Elaborate Hindu and Buddhist temples were also built on Java and other Indonesian islands at the time (chapter 16).

As we saw in chapter 13, the Byzantines had continued the Roman tradition of monument building, notably with the massive Hagia Sophia (Aya Sofya) church in Constantinople that would be the largest domed building in the world for centuries. In the Islamic world, a great many impressive architectural monuments date from the first half of the second millennium. These include several mosques and madrasas in Cairo, the Great Mosque in Bursa, Turkey, and 


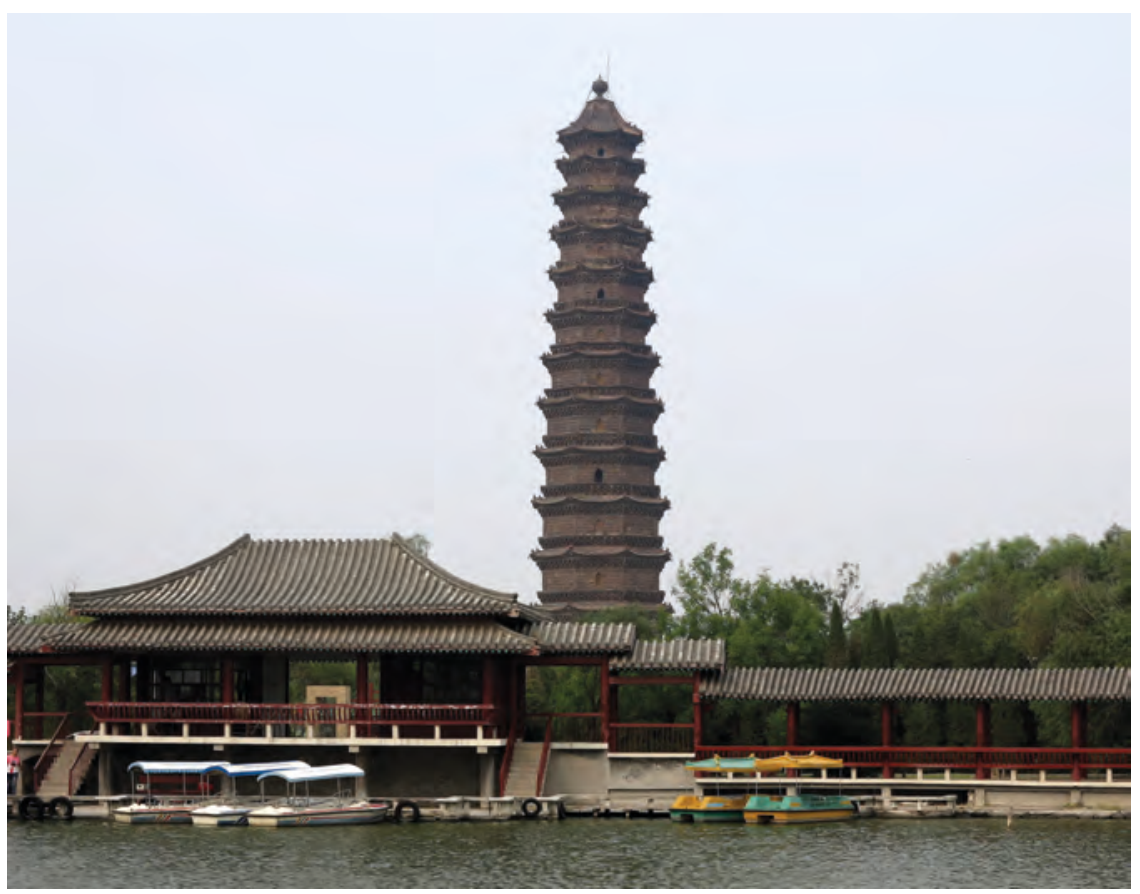

Illustration 14.11

The "Iron Pagoda" of Kaifeng, Henan, China, built out of glazed brick in $1049 \mathrm{CE}$ during the Chinese Song Dynasty (960-1279)

Photograph by Robinlun, CC BY-SA 4.0

the 72.5-metre-high Qutb minaret in Delhi, as well as non-religious buildings such as the Alhambra palace complex in Granada, Spain. Impressive structures were built throughout the Islamic world: Timbuktu on the fringes of the Sahara in present-day Mali would build three famous and remarkable mosques in the thirteenth through fifteenth centuries, while several centuries of monument building in Samarkand (in present-day Uzbekistan) were capped by Timur's construction of a massive mosque and mausoleum around 1400. These various structures are notable not just for size and height but for the exquisite nonrepresentational carvings that came to characterize Islamic architecture globally. Yet there was also diversity as Islamic architecture blended with local designs throughout the Islamic world. Though Islamic builders long marvelled at the Hagia Sophia, they did not build a larger dome until the Blue Mosque of the sixteenth century, built near the Hagia Sophia in the newly renamed Istanbul.

Important monuments were built elsewhere in the world as well. Great Zimbabwe (in present-day Zimbabwe), a walled town and palace, was constructed from the eleventh through fifteenth centuries. The Toltecs and then Aztecs built massive pyramidal temples in Mesoamerica. The Incas built the massive temple complex of Machu Picchu, with its polished stone walls, from about 1450 . 
Pagodas could take about ten years to build, churches and mosques multiple decades. Builders employed dozens or even hundreds of workers at a time on construction sites - a small number relative to the pyramids or the tomb of the Qin emperor, but still an impressive organizational achievement. Construction was not constant: There would be periods of months in which mortar hardened, and then cracks were repaired, before the next stage of construction began. There was considerable occupational specialization among the workforce. Some of these workers were hired locally: Carpenters were generally available in any large city. However, stonemasons were rarer and often moved from one monumental building site to another. In Europe, they often moved between countries in the pursuit of their occupation. On Islamic building sites, ethnic diversity was also typical. In China, the state regularly forced specialized builders to work on distant building sites. As noted above, movement of skilled builders from one location to another would allow understandings of building techniques to be shared and enhanced through time. If this had not occurred, we might instead

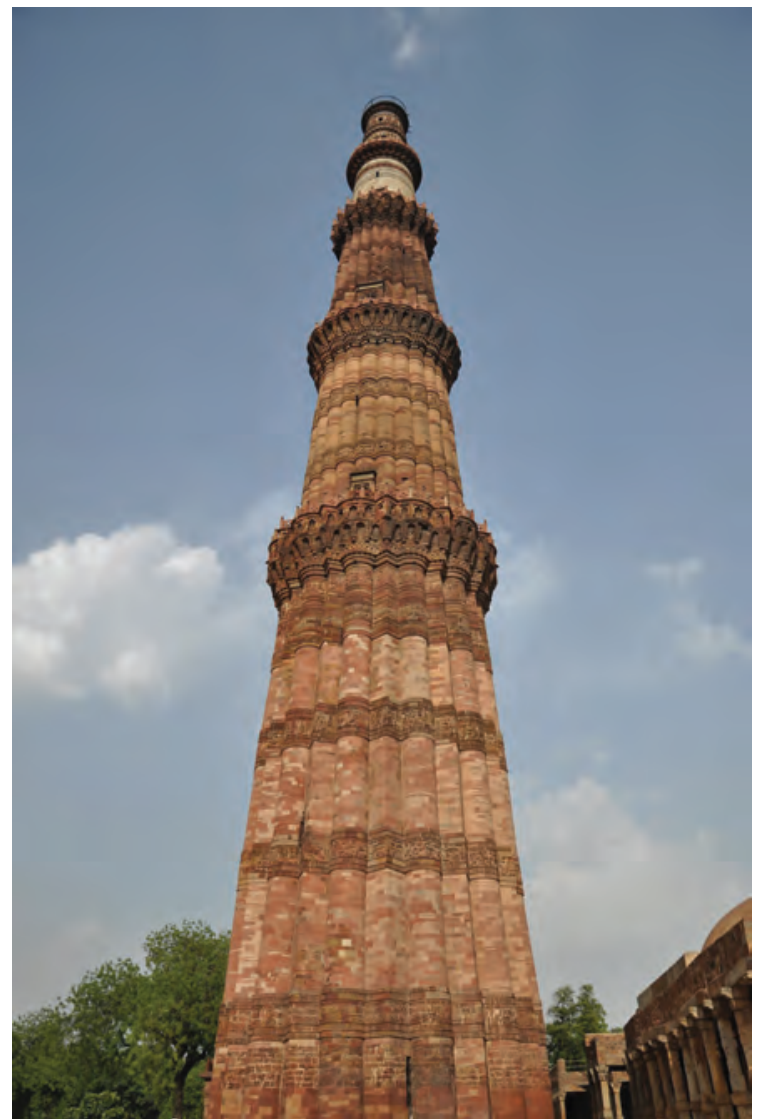

Illustration 14.12

Qutb minaret, Delhi, the tallest stone minaret in the world, established from 1192 using materials from Hindu and Jain temples (with their inscriptions intact); Persian design with Indian influences have seen innovations not duplicated due to a failure in technological transmission across space.

There were some sorts of apprenticeship among skilled builders, though the details are often obscure. Families were likely important for the intergenerational transmission of technical understandings. Yet it seems clear that outsiders often obtained apprenticeships - perhaps especially when the demand for builders outstripped the supply that families alone could provide.

There was apparently transmission of building knowledge and architectural and artistic tastes across long distances within societies. While there are obvious regional differences in the mosques of Timbuktu and Samarkand, they are still recognizably examples of Islamic architecture. English Gothic cathedrals might stress length and French cathedrals height, and Spanish cathedrals have more complex designs, and eastern European cathedrals stress towers at the entrance, but they are nevertheless clearly of the same family. There are also, at least in 


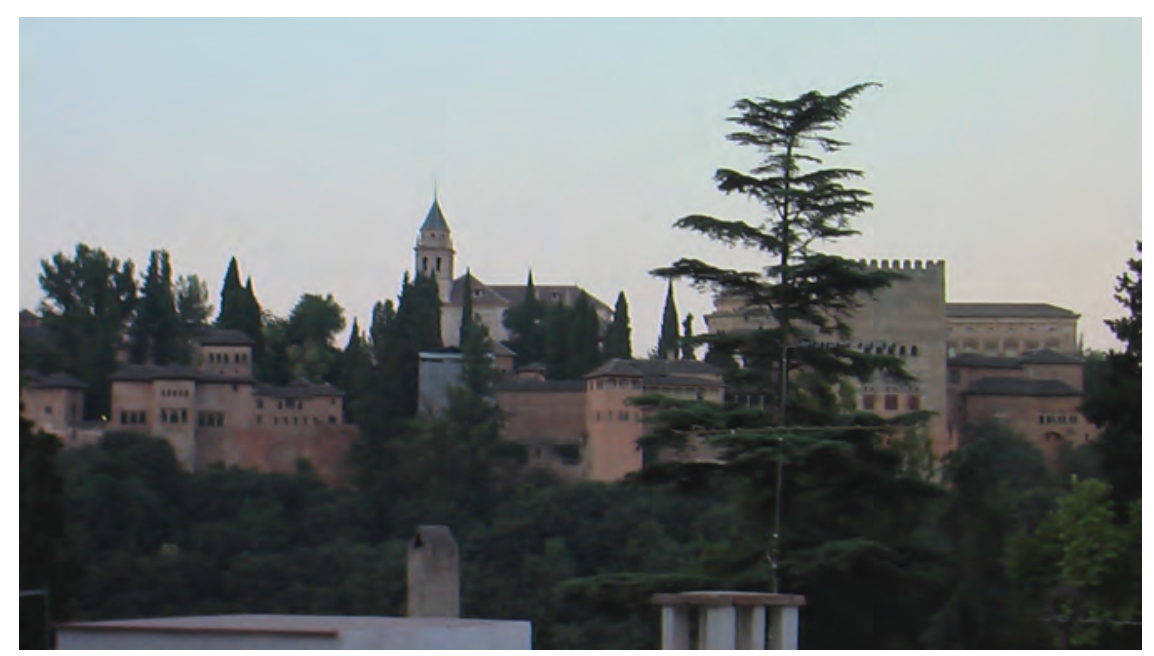

Illustration 14.13

The Alhambra in Granada

Author

the west, signs of the spread of ideas between different societies. The pointed arch that characterizes Gothic churches seems to draw on Islamic precursors. Islamic builders, in turn, borrowed both the style and construction techniques of the Byzantines.

One challenge in stone construction was obtaining the stone itself. Local sources were preferred if possible. Stone could be moved long distances by water if necessary, but overland travel was prohibitively expensive. Notably, stone tended to be cut and trimmed, and even cut to fit a particular architectural purpose, near the source of the stone, as it was cheaper to move finished stones. This meant that skilled masons were required both at the source of the stone and again at the building site to put the stones together.

There is no evidence from any of these sites of the sorts of detailed plans we would expect on a contemporary construction site. There were instead simple sketches of certain parts of the building and applications of simple geometry. There was a modular approach characterized by fixed proportions between different elements such as length and width of a particular room. Rules of thumb regarding, say, how thick walls should be, developed over time.

\section{Family patterns}

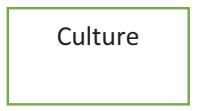

Politics

Though we can detect some commonality in political and economic trends in this period (above), there is considerable diversity in family patterns. These 
differences then generate differences in cultural attitudes. We record in this section a variety of observations of different aspects of family behaviour from different regions of the world. It is unfortunately difficult to draw detailed global comparisons of family patterns for this time period. The challenge here is that it is hard to establish what sorts of arrangements were typical given the lack of records about everyday family life.

In Europe, the church encouraged the practice of primogeniture in which the oldest son inherited most of the family's wealth. As a result, land was less fragmented over the generations than elsewhere. The church also encouraged celibacy among a substantial minority (about one-fifth on average in this period) of the population; most other societies viewed celibacy with suspicion - as Luther and other Protestant reformers later in Europe (chapter 18). The church also urged voluntary marriage: In 1556 in Bologna, a 13year-old girl protested in court against an arranged marriage. This shows both that consent was a legal right but that parents did not always respect this right.

It used to be common to stress the importance of nuclear families within Europe as opposed to the popularity of extended families - in which more than two generations inhabit one household in much of Asia. Scholars now appreciate

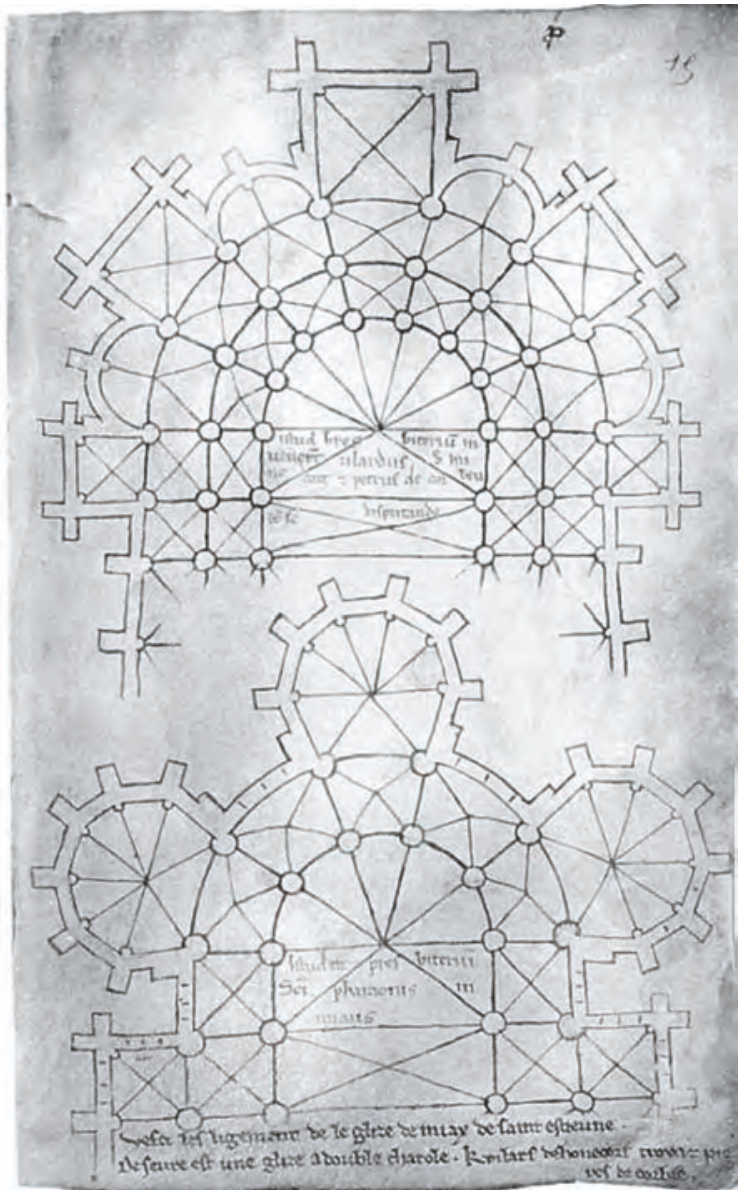

Illustration 14.14

Page from the Sketchbook of Villard de Honnecourt, 1230. Villard de Honnecourt may have been an itinerant master-builder in northern France. He left 33 sheets of parchment containing about 250 drawings for buildings or sculptures, Bibliothèque nationale, Paris that there was if anything a difference of degree: Nuclear families often appear in Asia, and extended families in Europe. Nor do differences in family structure or differences in the incidence of celibacy seem to have resulted in dramatic differences in levels of fertility (see chapter 22).

In Africa, polygamy would become increasingly common in societies in which many young men were sold into slavery. Polygamy was also legal in China, India, and Islamic lands, though generally only the elite could afford multiple wives. Christianity banned polygamy, though wealthy males often took concubines.

In both China and Africa, ancestor worship, and thus one's lineage, remained of great importance. In West Africa, though, one's maternal, rather 


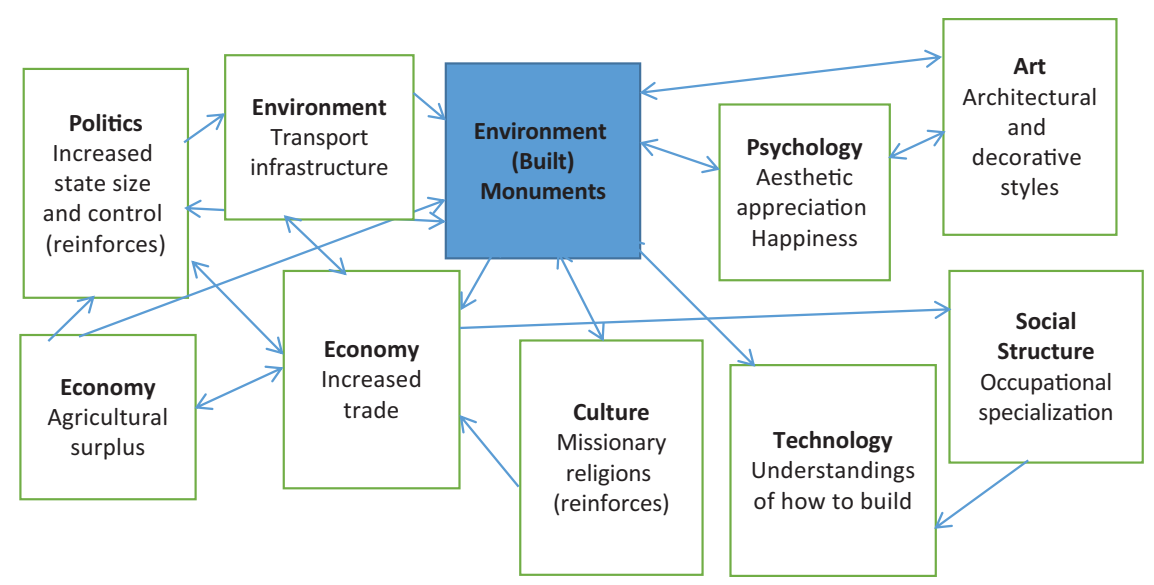

Figure 14.2

Influences on and effects of monuments

Note:We did not stress above that monument building reflects the basic human aesthetic sensibility.

than paternal, lineage was often stressed. (This practice shocked Ibn Battuta, though he had previously observed a similar practice among "heathens" in India.) Africans also imagined that ancestors might be reborn within their lineage.

The position of women varied a great deal across Islamic lands. Ibn Battuta was shocked by female behaviour in West Africa, and surprised by relative gender equality in Turkey. In Southeast Asia, wives and daughters retained considerable power as Islam spread. Chinese merchants in Southeast Asia married local women but maintained Confucian patriarchal ideals. Over time, Chinese women also moved to Southeast Asia, and Chinese men married them instead.

There were diverse family types in the pre-contact Americas. Pueblos in the southwestern United States were both matrilineal and matrilocal: Men married into and lived with the woman's family. The Aztecs and Incas instead emphasized male power and inheritance.

\section{Population}
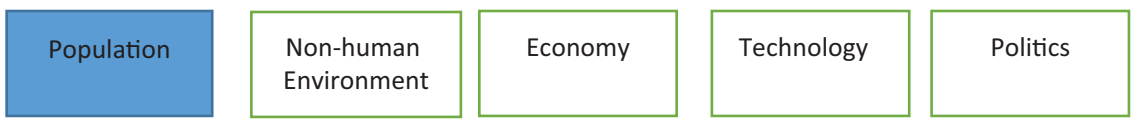

The population of the world was somewhere around 300 million in the year 1000: Estimates range from 250 to 350 million. The population would rise by about 100 million over the next three centuries or so, reflecting and encouraging the increased agricultural output noted above. There would then be a 
decline of some tens of millions associated with the great Eurasian plague of the mid fourteenth century (chapter 17). Population would recover after that, and may have reached 500 million by 1500 (almost certainly at least 450 million). This increase of some 50 per cent in the global population represents perhaps the most important trend of the entire period. It, in turn, reflected favourable climate, expansion of agriculture into new areas, increased productivity from new crops and rotations, and the continued expansion in use of (and improvements in) iron implements. The relative peace that political consolidation brought to at least some lands, and the increased dietary diversity associated with increased intra-regional trade, may also have supported population increase. In a Malthusian environment, though, increased food output was necessary to sustain increased population.

\section{Questions}

1. Compare the reasons given for state consolidation during this period with the reasons given for early states and early empires in previous chapters. Place the arguments made above on a diagram.

2. How can historians judge the reliability of travellers' tales? What motives did travellers have for misleading their audience?

3. How does the history of porcelain inform our understanding of world history more generally? (It may be helpful to think in terms of evolutionary processes, interactions among themes, connections across societies, and so on.)

4. What to you was the most surprising development in this chapter? Why?

5. Do the monuments of this time period impress you? Why?

\section{Readings}

Abu-Lughod, Janet. 1989. Before European Hegemony. Oxford University Press.

Campbell, Bruce. 2016. The Great Transition: Climate, Disease and Society in the LateMedieval World. Cambridge University Press. [Though he stresses Europe and especially England, he surveys the impacts of climate and epidemics across Eurasia over four centuries, drawing heavily on natural science insights into climate.]

Finlay, Robert. 2010. The Pilgrim Art: Cultures of Porcelain in World History. University of California Press. [See also From Object to Concept: Global Consumption and Transformation of Ming Porcelain, by Stacey Pierson, Hong Kong University Press, 2013, which discusses the meanings that were attached to porcelain. There was also a Forum in the Journal of World History in March 2012 about porcelain.]

Power, Timothy. 2012. The Red Sea from Byzantium to the Caliphate AD 500-1000. Cairo: American University in Cairo Press. 
Prak, Maarten. 2011. "Mega-structures of the Middle Ages: The Construction of Religious Buildings in Europe and Asia, c.1000-1500." Journal of Global History 6:3, 381-406.

Readers interested in commodity history have many books they might consult, including Sven Beckert's Empire of Cotton (Vintage, 2015), Betty Fussell's The Story of Corn (University of New Mexico Press, 2005 reprint of 1992 edition), Mark Kurlansky's Cod: A Biography of the Fish That Changed the World (Walker, 1997), Kurlansky's Salt: A World History (Walker, 2002), Andrew Dalby's Dangerous Tastes: The Story of Spices (University of California Press, 2000), Ken Albala's Beans: A History (Bloomsbury, 2017). Gregory T. Cushman's Guano and the Opening of the Pacific World: A Global Ecological History (Cambridge University Press, 2013), Paul Gootenberg's Andean Cocaine: The Making Of A Global Drug (University of North Carolina Press, 2008), and John Soluri's Banana Cultures: Agriculture, Consumption, and Environmental Change in Honduras and the United States (University of Texas Press, 2005). Erika Rappaport's A Thirst for Empire: How Tea Shaped the Modern World (Princeton University Press, 2017) details how companies and nations fought for market share, how tea was advertised, how tea became primarily associated with women, and how British firms urged empire loyalty, changing preferences from Chinese green tea to Indian black tea. For a more general treatment of commodity history, see The Matter of History: How Things Create the Past, by Timothy J. LeCain (Cambridge University Press, 2017).

There have been many translations of the travels of both Marco Polo and Ibn Battuta. 


\section{Regional}

\section{developments}

\section{Eurasia after 900}

\section{Guiding questions}

How and why did the processes of political consolidation and trade expansion unfold across Eurasia?

How did the unique experience of the Catholic Church shape European developments?

What were the key developments in European agriculture and industry and how and why did these occur?

How, why, and where was the printing press developed, and what effects did it have at the time?

What was the Renaissance? Why, when, and where did it occur? Why is it important?

How dynamic was the Song Chinese economy, and why?

What were the attitudes of Asian states toward international trade and information flows?

What were the origins of Chinese landscape painting, and of substantial temple complexes in Southeast Asia?

Relationship to other chapters: Chapter 15 is a companion chapter to chapter 14, fleshing out the details of political consolidation and trade expansion across Eurasia. It discusses the unique role played by the Catholic Church in Europe, and especially its interaction with states. Earlier understandings of relationships between religions and states (chapters 8,11 , and 12) inform this discussion, which will inform later discussions of European states and religion, especially in chapter 21. 
The analysis of the Crusades enhances our understanding of relationships between religions and between states; the Crusades turn out to have inspired cross-societal information flows of considerable importance - such as the spread of sugar to Europe, which would later encourage sugar colonies in the Caribbean (chapter 19). Developments in European agriculture and industry during this period arguably set the stage for the revolutionary economic transformations of chapter 24 . The printing press had been known in Asia for centuries, but its development in Europe would lead to a dramatic expansion in printing - eventually worldwide. We place the Renaissance in a global context, noting influences from other societies and global effects: The way (some) humans saw the world changed in important ways. We are also able in this chapter to expand and update our understanding of Chinese history, noting similarities and differences across Chinese dynasties. Yet we also devote detailed coverage to developments in Japan and Southeast Asia, regions that have only received brief treatments in earlier chapters. While we treat different regions in turn, the student can usefully compare across regions. We compare the economic dynamism of Song China to the British Industrial Revolution of chapter 24. The chapter, therefore, informs our general understanding of both economic development and exploration. (Note that we say little about the Islamic Middle East in this chapter since this received extended treatment in chapter 12.)

This chapter's treatment of Europe follows a significantly different format from the ensuing discussion of Asia. This deviation from this book's general practice of carefully comparing the same elements of different societies deserves justification. In both cases, we will devote considerable attention to political developments and especially the strengthening of state authority. We will trace economic developments as well. Yet the fact that Europe was more politically fragmented than Asia in general and China in particular means that political and economic development proceeds quite differently. The state wields a great deal of economic influence in China. In Europe, political fragmentation both encourages and allows merchants and artisans to develop a set of institutions that guide economic activity. After first addressing state formation in Europe, we devote much of our attention in this chapter to these institutions. The independence of the Catholic Church in Europe is also quite unusual, and we 
therefore devote some attention to the political role of the church. We also trace important developments in agriculture. We then discuss the development of the printing press: This had appeared first in China but would have a more dramatic impact in Europe. We close with a discussion of the Renaissance, a period of intellectual and artistic ferment in Europe.

We extend our discussion of Southeast Asia well past 1500. The processes of political and cultural consolidation in Southeast Asia before the arrival of Europeans deserves treatment in one place. On the other hand, our discussion of India in this chapter is somewhat truncated as we carried the story well beyond 1000 in chapter 13 and will begin again with the Mughals shortly after 1500 in chapter 20. Likewise, we treat mainly the Song Dynasty in China in this chapter, relegating detailed treatment of the Mongol Yuan Dynasty to chapter 17 and the Ming dynasty from 1344 to chapter 20. It makes little sense arbitrarily to divide our discussion of the Ming dynasty, which lasted until 1644.

Thematically, then, we have sections devoted primarily to politics, culture, economy, technology, science, and art. Inevitably, we also engage social structure. We occasionally discuss the built environment and climatic impacts. We engage in an evolutionary analysis of financial institutions and later of art in the Renaissance. We compare political developments across states. The Crusades are an important episode of cross-societal interaction. We also discuss Chinese military adventures and scientific and religious interactions. In other sections, we discuss how technologies, art, and certain institutions were transmitted across societies.

\section{State formation in Europe}

\section{Politics}
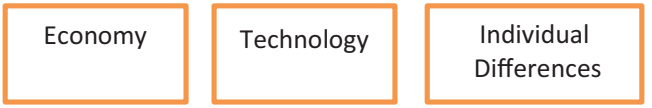

Culture

We described the decentralized nature of the European feudal system in chapter 13 . We saw in chapter 14 that there was a global tendency toward political centralization from about 900. European kings struggled to assert power over subsidiary lords throughout the feudal period. They had some success early, before local lords strengthened their power, and would much later finally succeed in establishing centralized states. The earliest successes occurred in France. Charles Martel defeated a Muslim army at Poitiers in 732; this battle was the only large-scale incursion of Islamic forces north from Spain. Some have seen this outcome as having been critical for the future history of Europe, while others doubt that overstretched Islamic forces would have pushed far into France even if they had won. Nevertheless, the victory certainly gained Charles Martel considerable acclaim. His son Pippin was declared king of the Franks and Pippin's son Charles - generally known as Charlemagne or Charles the Great - aspired to empire. He did assemble a vast territory that included most of modern France and much of modern Germany. Charlemagne established a 
capital at Aachen in Germany, but he spent most of his time travelling. This fact indicates that it was his personal presence rather than a centralized bureaucracy that held his empire together. He did establish "counts" and bailiffs to collect taxes for every county (usually a town and surrounding area) in his realm; these reported to his court twice a year, and Charlemagne dispatched auditors to review their accounts. Counts and bailiffs needed to be literate and numerate, and were thus generally drawn from the economic elite, though promising boys of humble birth might be taught to read by local priests. Charlemagne also sent slaves to the Muslim world. Harun-al-Rashid of Cordoba gave Charlemagne an elephant, which he used often. He responded with less notable gifts.

The pope proclaimed Charlemagne emperor in 800, an act that much annoyed the Byzantines, who aspired to be emperors of all of Christendom. However, Charlemagne's three sons would divide the empire, which was in any case internally weak and externally attacked by Vikings, Magyars, and Muslims. His descendant Otto would be proclaimed Holy Roman Emperor in 962. The empire at the time comprised much of modern Germany and some of northern Italy. One limitation to the emperor's power was the papacy itself (see below), which crowned emperors but guarded its own religious (and temporal) authority jealously. The Church would act with various Christian rulers to limit imperial power. Emperors were always weak relative to local lords. Yet the emperor was not entirely powerless and could work to limit - but not eliminate - internecine wars.

Postscript: The Holy Roman Empire at first comprised most of modernday France and Germany and later would encompass most of Germany and parts of northern Italy. The title of Holy Roman Emperor would survive for over a thousand years, but the region was politically fragmented after Charlemagne's death. A set of kings and bishops would come to elect emperors. It was famously said that the Holy Roman Empire was neither holy nor Roman nor an empire. Nevertheless, its loose confederal structure appeals to some modern historians as a way of achieving some shared goals while respecting local cultural preferences.

In France, the lords elected a minor noble, Hugh Capet, as king in 987. He controlled only a small area around Paris, but his descendants gradually extended their power. Though these early kings and emperors were not strong - they relied on a feudal hierarchy - the memory of these states would inspire later state builders. Although some leaders in Europe might from time to time dream of recreating Rome, those in France would generally concentrate on recreating the France of Pippin or Hugh Capet, though in a far more centralized form. 
One of the French state's vassals, Duke William of Normandy (later William the Conqueror), a descendant of Viking invaders, conquered England in 1066. (The English king Harold had been weakened by an earlier attack that year from Norway; chapter 13.) The conquest allowed considerable centralization as Anglo-Saxon lords lost their lands. Yet William would then reward his own followers with grants of land. The Norman conquerors compiled the Domesday Book, an extensive survey of the properties and peoples of the new kingdom. This book, reminiscent of the efforts of the Chinese and other Asian bureaucracies, indicates both some central bureaucratic capability and a desire to understand the tax-paying capacity of each part of the realm.

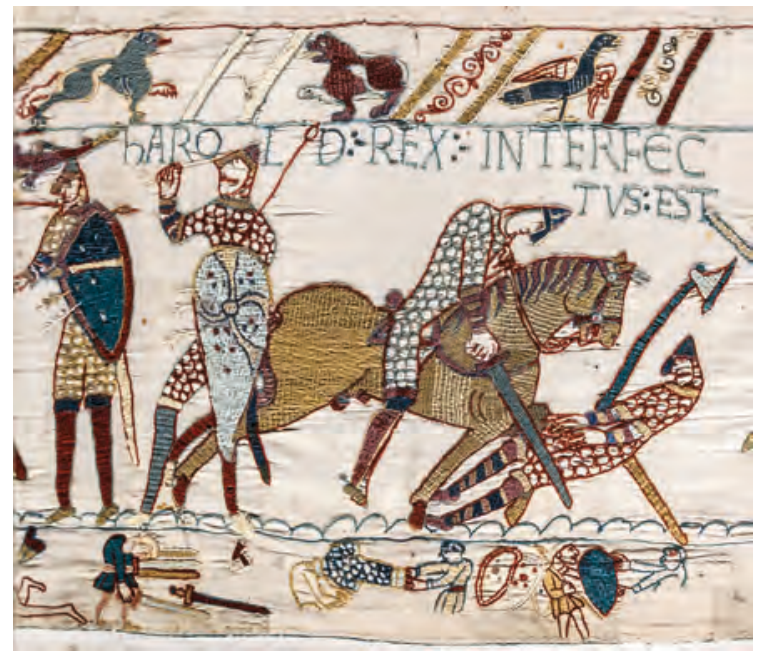

Illustration 15.1

The Bayeux Tapestry, Scene 57: The death of King Harold at the Battle of Hastings. The Tapestry is 68 metres in length, depicting scenes from the Norman Conquest of England

In the eleventh and twelfth centuries, English kings tried to extend tax-collecting and law-providing powers, but local lords were still powerful. In the Magna Carta of 1215, English lords forced the (unpopular) English king to recognize a set of lordly prerogatives. State formation would accelerate in later centuries. European city states (notably in Italy) grew wealthy on trade from the thirteenth century, but these states also expanded and introduced taxes on farmers. From the fourteenth and fifteenth centuries, the English and French kings levied new taxes to fund a royal army. The Spanish state also strengthened, though it was mostly dependent on a sales tax.

Two developments were critical here. One was expanding trade (which we discuss below). This allowed kings - often in association with towns and cities to gain new sources of revenue with which to fund their armies. The other was military technology and in particular the cannon. Early cannons were not much of an advance over the trebuchets (a kind of catapult) which for centuries had hurled rocks at castle walls. However, smoothly bored cannon that fired spherical iron balls were a much different weapon. These exerted much greater force and could destroy even a thick castle wall in a matter of days or weeks. Recalcitrant lords could previously hide from an angry king for months - as long as their food and water lasted. Now a king could issue royal decrees and send an army with cannon to ensure that these were enforced. The combination of revenue and enforcement tipped the power balance toward kings.

The speed with which kings asserted this potential for new power differed across Europe. The kings of France and England and Spain were already in place 


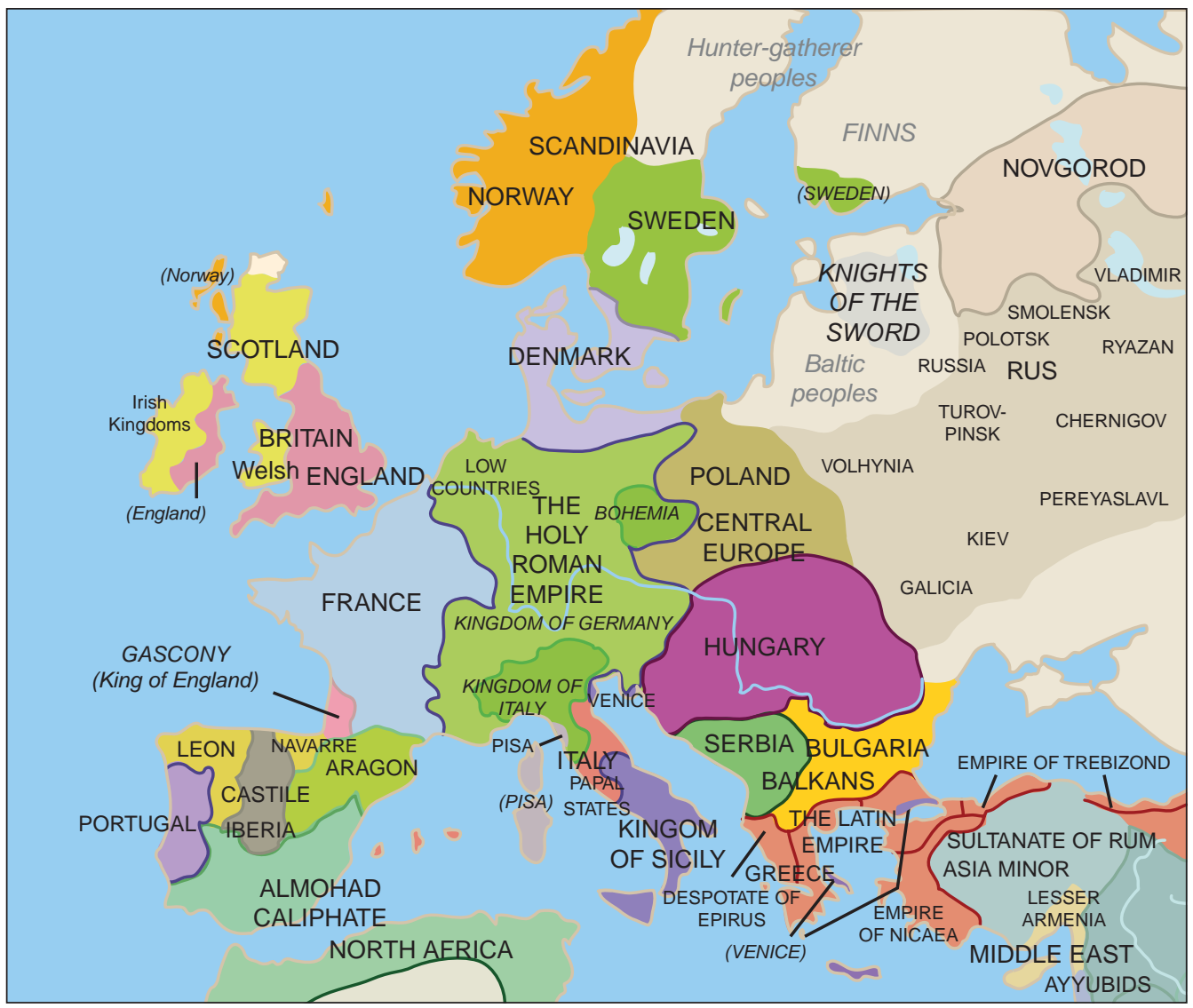

Map 15.1

Europe in 1215. The later nation states of England, France, and Spain exist but will expand in later centuries. Islamic rulers still controlled much of Spain at the time

when the iron cannonball was developed. They could expand their previously constrained authority. The Holy Roman Emperor lacked a similar authority on which to build; an array of states of varying size would characterize Germany for centuries until one of these managed to absorb the rest (chapter 21). In Italy, the cannon allowed northern city states to expand and solidify their rule, but there was no exemplar of Italian unity save the very early days of the Roman Empire on which to build. Moreover, these very rich trading city states could develop sophisticated fortresses with cannon emplacements that could prevent an attack. (Such defensive fortifications were far too expensive for feudal lords to build.)

From the fourteenth century, the European aristocracy came to emphasize ceremony, tournaments, and chivalry as their military role and political power declined. We will see later a similar process unfold for Japanese samurai as political consolidation proceeded from the sixteenth century. 


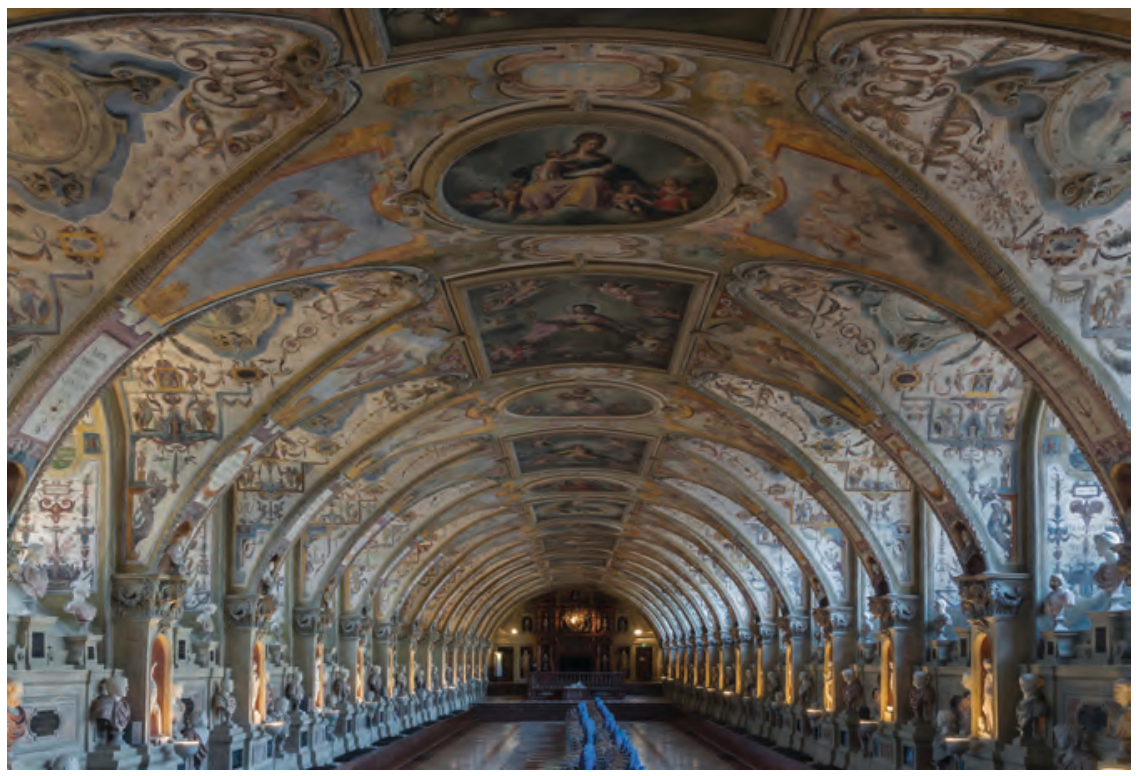

Illustration 15.2

The Antiquarium in the Residenz, the Palace of Bavarian kings in Munich, a Renaissance design to house the king's collection of antiquities. The Residenz was built from 1385

Photograph by Jebulon, CC0

\section{The Catholic Church}
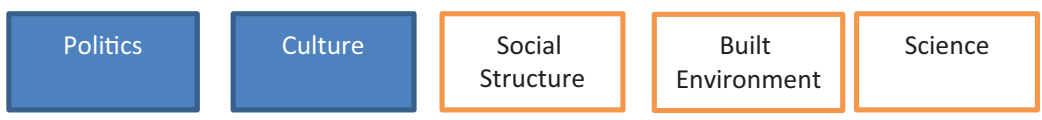

The fragmented political authority of feudal Europe allowed the Roman Catholic Church an unusual and perhaps unique degree of independence. Emperors would guide the Orthodox Church in Constantinople (chapter 11, 13). Political authorities also influenced the Catholic Church: Italian city states often succeeded in installing their choices as pope, and French kings would later exert much influence on the choice of popes. However, there was no one political authority to dominate the church (it certainly ignored the Byzantines), and the church was generally able to play on the enmity between European rulers to assert its independence. Pope Gregory I (590-604) was critical in asserting Rome's authority, emphasizing the importance of confession (which made priests powerful in local life by establishing the penance that churchgoers had to make for the sins that they had confessed), and dispatching missionaries to distant lands.

The authority of the Church waxed and waned with the degree of corruption within it. The Church was wealthy from both extensive landowning and the tithes (taxes) that people paid to the church. There was always a temptation 
for religious authorities to divert some of that wealth to personal use. The fourteenth-century church reformer John Wycliffe argued that no large bureaucracy could ever be free of corruption - a point that the history of empire provides much justification for - and so could never reflect the purity of the faith. Popes themselves were often corrupt, but even popes with the best intentions would face the ruler's challenge of getting thousands of priests to do as the church wished. The key innovation here was the idea of priestly celibacy. Though one might think today that the purpose of priestly celibacy is to encourage (and select for) unusual devotion to the faith, a more compelling motive at the time was inheritance: If priests could have no legitimate children, they would have far less incentive to steal lands or gold from the Church.

The Church also cared about inheritance patterns in the wider society. By prohibiting divorce and polygamy, insisting that widows inherit, and discouraging widows from remarrying, the Church had the practical impact of increasing the numbers of both men and women that died with an estate but no heir. Such people were encouraged to bequeath their estate to the church. As the church asserted its authority over marriage, it decreased the power of parents over their children and especially daughters. These Church policies had a particular effect on royalty: Royal families were smaller in Europe than in lands where polygamy was allowed (which may have reduced the financial pressure on royal treasuries), but this increased the possibility that a king would die without a child. While Asian succession crises might often be attributed to having too many sons, European succession crises often reflected having too few (or there being debates regarding legitimacy). Moreover, since royal families habitually intermarried, battles between competing cousins often had an international component. It is worth noting that those on the losing side in European succession battles were often killed - most famously the nephews of Richard III of England, who stood in the direct line of inheritance but were (apparently) killed by agents of either Richard or his successor Henry VII - just as losers were often killed in Asia.

Christianity attacked homosexuality, which Greece and Rome had accepted to some extent. There is a scholarly debate - rooted in limited documentary evidence - about whether this occurred from the early days of Christianity or only from the eleventh century. There is also debate as to why: Was it just a literal reading of specific scriptural texts, or was it connected to the Church's efforts to decry sexual activity beyond the purposes of procreation? Note that the Church might have benefited financially from accepting homosexuality if this would have increased the number of people who died without an heir.

We discussed monument building across Eurasia in chapter 14. The wealth of the Catholic Church supported the construction of massive Gothic cathedrals throughout Europe. Their height and huge windows filled with stained glass must have inspired awe among the population. Though architecturally novel, these cathedrals borrowed the design of arches from the Islamic world.

Monasteries were important for both copying rare manuscripts and spreading the faith. They naturally at first focused on copying religious texts 
but also copied some works of practical importance such as in medicine. Monks also sometimes played administrative roles for rulers who lacked a dedicated bureaucracy. The church developed a variety of monastic orders (some for men, some for women) over the centuries; these placed different emphases on contemplation versus working in the community. Many orders became large landowners over time - they often received donations and were generally exempt from taxation. They may have played an important role in developing agricultural technology for they had land with which to experiment, access to such manuscripts as existed on farming practices, and a cadre of monks with the capability to keep detailed records and the desire to increase the output of the monastery.

The Church would play an important educational role, as did Islam and Buddhism in their parts of the world. In the eleventh and twelfth centuries, cathedral schools expanded into early universities that provided training not just in theology but in law and medicine. By 1500, there would be over a hundred universities in Europe. These pursued a guild-like structure, and so had some independence from the Church. Indeed, groups of students or teachers rather than the Church developed these universities: "University" was a Latin term for a society of people with shared interests. Students in Bologna in 1088 organized to set standards for their teachers: Students could fine instructors for being late. In Paris, the instructors formed a guild around 1170, and then organized separate faculties such as theology and law. Though originally focused on professional training of priests, physicians, and lawyers, these universities soon appreciated the importance of education in philosophy and science. Europe was unique in having independent (and largely secular) universities such as these. Some of them were large: Bologna had 10,000 students in the thirteenth century and Paris 7,000. "Colleges" were endowed as places for students and instructors to live - in part to concentrate and possibly control the rowdiness of students. These universities would play an important role in translating ancient Greek and Arabic texts into Latin. Universities also encouraged analysis of and debate regarding these texts. The Church only occasionally interfered. In 1277, the Bishop of Paris, backed by the pope in Rome, would condemn 1,267 "errors" in Aristotle, but this sort of action was rare.

Scholars in the west learned of Greek philosophy through contacts with Byzantium and Muslim lands. (The scholar Petrarch and his associates also visited western monasteries in search of rare manuscripts to copy.) Europeans read Averroes' commentaries on Greek philosophy, Avicenna's treatises on medicine, and Al-Khwarzimi's works on mathematics. In the thirteenth century, the works of Aristotle, in particular, were translated into Latin and widely copied. This encouraged the development of scholastic philosophy that sought to connect Christian theology to Greek thought. We saw in chapter 12 that scholastic philosophy could build on the efforts of Averroes to link Greek philosophy to Islamic belief. Thomas Aquinas (1225-74) is the most noted of these scholastic philosophers and urged the linking of reason and faith. In 
this, he was similar to earlier Byzantines, neo-Confucians, and many Islamic philosophers of his time. The Church did not accept at first this deviation from relying exclusively on scripture: His works were banned for decades before becoming orthodoxy.

It would be a mistake, though, to equate the scholarly musings of learned men such as Aquinas with how religion was practised on the ground. Popular Christianity likely revolved much more around ritual than textual analysis, and reverence of particular saints that believers could pray to for specific purposes. Relics - bits of wood or bone or other materials allegedly associated with biblical figures or saints - and pilgrimages are examples of this popular faith. The Church itself would prosper by selling both relics and indulgences (promises of godly forgiveness). Reverence for Jesus's mother Mary became increasingly common from the twelfth century; this may have reflected and reinforced a slightly increased role for women in society, at least among the nobility (recall our discussion of chivalry in chapter 13).

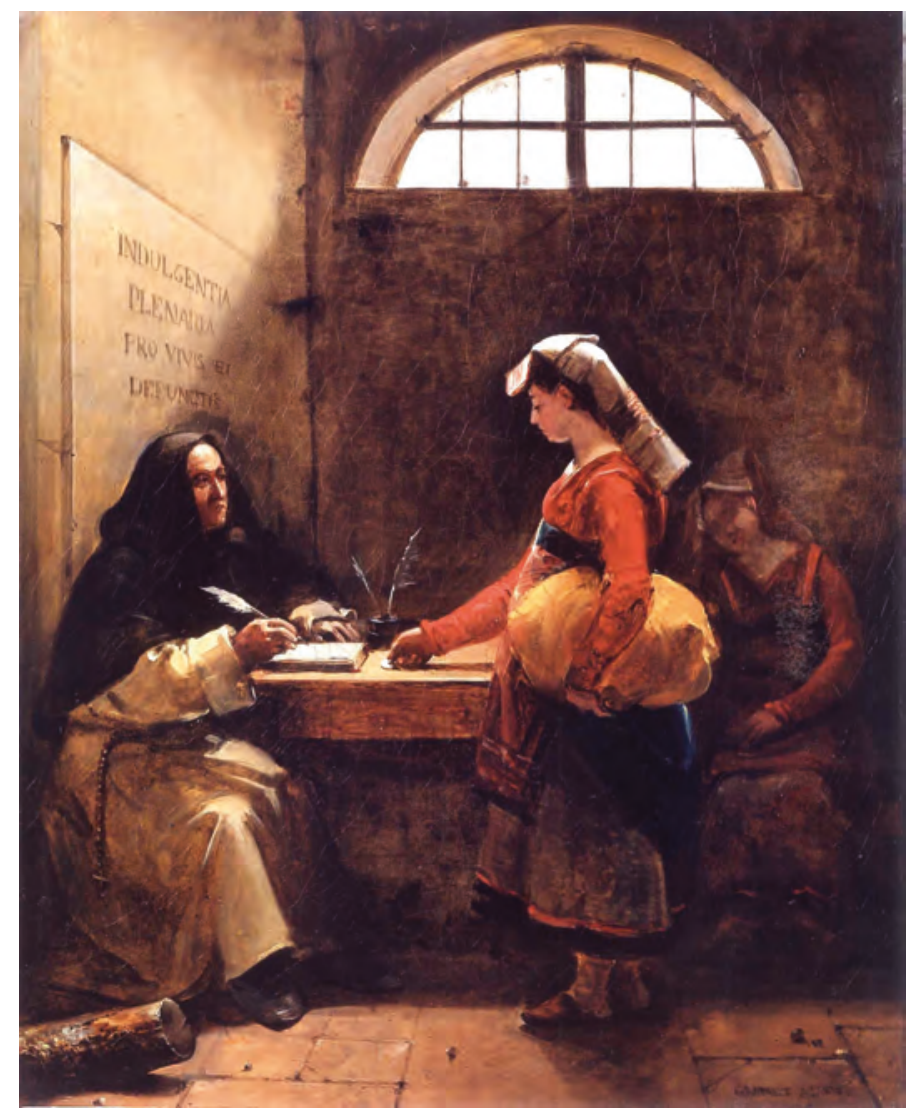

Illustration 15.3

A Peasant Girl Buying an Indulgence, painting by François-Marius Granet, 1825, but set in medieval times 


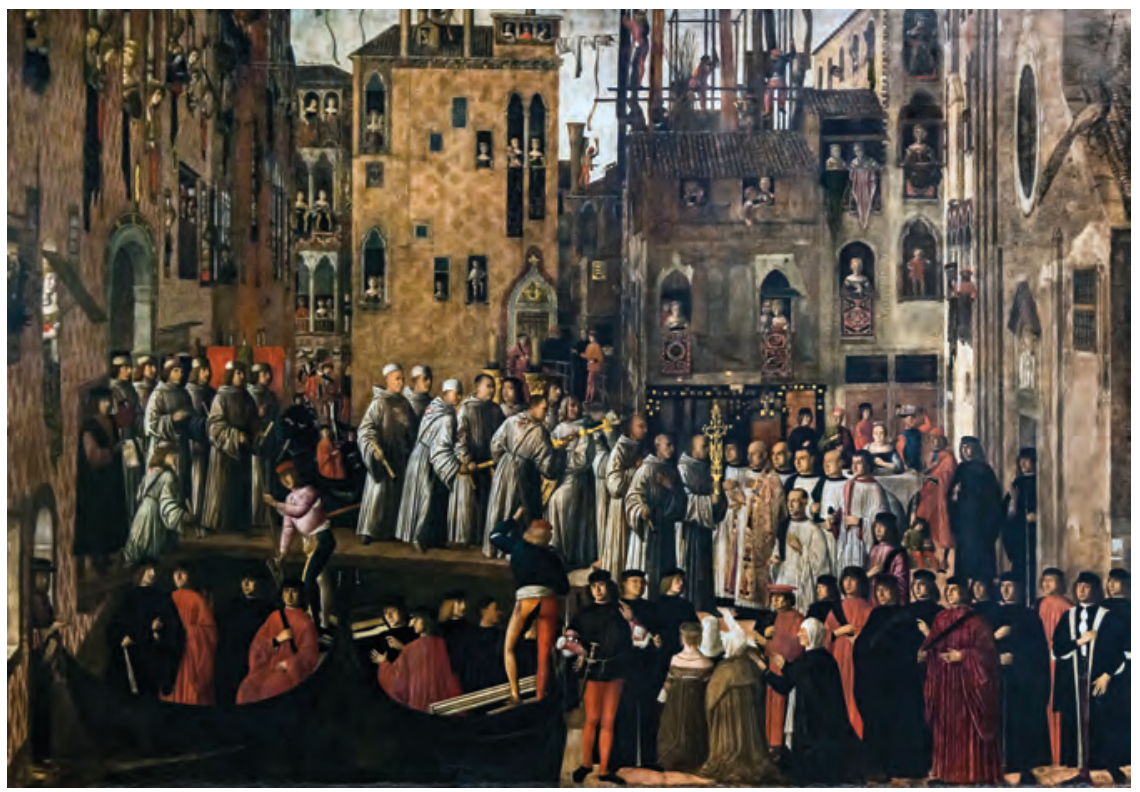

\section{Illustration 15.4}

Miracle of the Relic of the Holy Cross in Campo San Lio by Giovanni Mansueti, 1494. It is said that the reliquary containing the relic became too heavy to carry into the church for the funeral of a sceptic of its power in 1474, Gallerie dell'Accademia in Venice

Photograph by Didier Descouens, CC BY-SA 4.0

A dispute between Philip the Fair, King of France, and Pope Boniface regarding the taxing of clergy by the king (and their relative power) resulted in the French king kidnapping the pope in 1302, and later French kings encouraging popes to live in Avignon in southern France from 1309-77, where the French king could better influence them. When the papacy moved back to Rome, there was conflict between Italian and French cardinals that resulted in their being two popes (briefly three), one in Avignon and the other in Rome, until 1417. The authority of the Church naturally suffered at this time (and faith was in any case shaken by the Plague; chapter 17). This weakening of the Church relative to kings arguably set the stage for the Protestant Reformation (chapter 18).

\section{The Crusades}
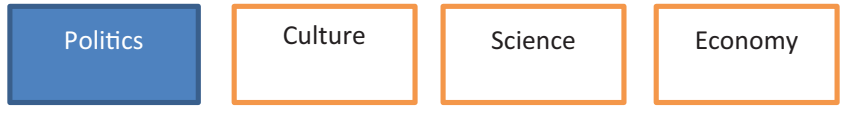

Between the late eleventh and early thirteenth centuries, the Catholic Church encouraged four "Crusades" to retake the Holy Land - modern-day Israel, 
Lebanon, Syria, and Jordan - from Muslim forces. Though the Church itself had limited military forces, it could make a compelling promise to both kings and peasants: that any martyrs in these Crusades would gain eternal salvation (a promise often made to suicide bombers in the twenty-first century, we might note). Pope Urban II launched the First Crusade of 1096-9 in response to a request from Constantinople for help against Turkish attacks. This was a rare attempt at collaboration between Orthodox and Catholic Christians, coming a half-century after the two churches had formally split in 1054 (chapter 11). It reflected the fact that the Byzantine Empire was steadily losing ground to the Turks (chapter 13). These advances had limited the ability of Catholic pilgrims to reach the Holy Land. Crusaders hoped to not only retake the Holy Land but also reunite Christianity. The Crusaders would take advantage of Muslim infighting at the time to establish a small kingdom that included Jerusalem.

The Second Crusade (1147-9) may have mostly reflected the desire of King Louis VII of France to make a pilgrimage to the Holy Land. The Crusaders retreated after a battle near Damascus. The Third Crusade (1189-92) responded to the loss of Jerusalem to Saladin (Salah-al-din) in 1187. King Richard of England led this Crusade after the Holy Roman Emperor drowned and the French king turned back. The Europeans won some battles but did not take Jerusalem. The Fourth Crusade (1202-4) was a debacle. The Crusaders were diverted to Constantinople - perhaps to collect monies promised by

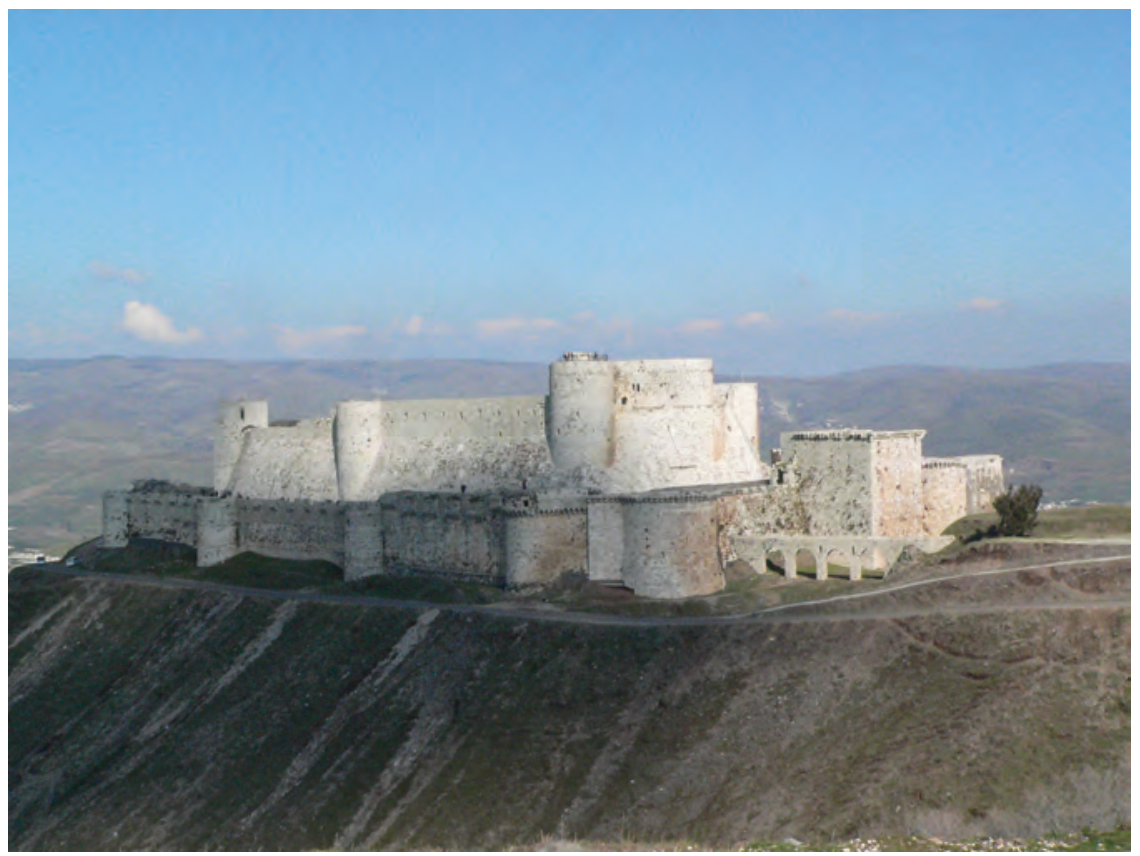

Illustration 15.5

Krak des Chevaliers Crusaders Fort, 12th-13th centuries; one of the best-preserved medieval forts, Syria 
Constantinople but not paid. The Crusaders rampaged through that city, killing many Christians who merely looked foreign. This was, of course, an ironic end to the Crusade saga initiated by Constantinople. This attack severely weakened Constantinople. It lost further ground after that to the Ottoman Turks - though Constantinople itself would not fall to them until 1453. For the Crusaders themselves, Orthodoxy could be as suspicious as Islam. (We saw in chapter 11 that denominational battles are often harsher that battles between religions.) The attack on Constantinople - though condemned by the pope increased the suspicion between Orthodox and Catholic Churches. (Crusaders often attacked Jews in Germany along the way, increasing hostility between Christians and Jews as well.)

The Crusades would score occasional victories and establish a Christian kingdom of Jerusalem for several decades. In time, though, Muslim armies crushed the Crusaders. They conquered the last Christian holdings along the coast of the Levant in 1291. The Catholic Church would have better luck elsewhere. It made similar promises of salvation at times to those who joined Christian kings in Spain against Muslim rulers in Spain: Indeed, Pope Alexander II (1061-74) established the precedent later used in the Crusades when he promised to absolve the sins of French and Norman soldiers fighting in Spain. Most of Spain (and Portugal) fell by the mid thirteenth century, and Christian kings would take all of Spain by 1492. Farther north, the Teutonic Knights - a Christian fighting order - moved from the Levant to northeastern Europe and forcefully converted multitudes of Slavs in the twelfth through fourteenth centuries, while absorbing some of the lands of these peoples (who were at the time still hunting and gathering or practising shifting agriculture). The church also dealt harshly with heretics within Europe at this time.

The Crusades were an expression of religious zeal. They allowed warriors to combine subservience to the church with secular ideals of chivalry that were often in conflict with Christian ideals. It might be ethically questionable to slaughter another Christian prince, but the church applauded attacks on "infidels" occupying the homeland of Christianity. As in any war, pecuniary motives were significant for most if not all soldiers. The Holy Land had riches to plunder. Moreover, it lay astride important trade routes to Asia. The European policy of primogeniture, by which the eldest sons of kings and lords inherited the bulk of the estate, meant that younger sons could be enticed to seek their fortune in foreign conquest. Plunder in this life and salvation in the next: It proved a powerful combination for potential Crusaders, as it had for the early Islamic armies that had first taken the Holy Land (chapter 12).

We should also recognize European ignorance of the wider world at the time. When the Crusades were launched, it was widely thought in Europe that Christians vastly outnumbered Muslims. Only after repeated defeats at the hands of Islamic armies, and then the later incursions of the Mongols (chapter 17) did Europeans come to appreciate that they were a minority on the Eurasian landmass. European Christians knew that there had been substantial Christian populations in the Holy Land and farther east before the 
Islamic invasion; there was a long-standing myth in Europe that there was a mighty Christian king on the other side of Islamic lands. Europeans dreamed of squeezing Islamic armies between Christian forces. This myth would only be thoroughly shattered when the Portuguese sailed around Africa to Asia in the sixteenth century (chapter 19) - though the tales of travellers such as Marco Polo (chapter 14) inevitably weakened it.

The Crusades, of course, looked quite different from the perspective of Muslims in the Middle East. To them, Europe was an economically and technologically backward region gripped by religious fanaticism. The Crusader belief that it was laudable to kill in the name of religion was hardly unknown among Muslims of the day, but they often were shocked by the barbarity that Crusader troops showed toward Muslim civilians. (Recall from chapter 12 that Islamic rulers long pursued a policy of religious tolerance in the region.) However, the fact that Seljuk Turks had taken Aleppo in 1064 meant that conflict between the Turks, Abbasids, and the (Shia) Fatimids of Egypt gripped the Levant at the time. The Abbasid caliph was reluctant to retake territories taken by Europeans from the Fatimids, and even at one point indicated that he would rather see Jerusalem in European hands than Fatimid ones. Over the next centuries, Muslim rulers would often collaborate with Crusader kingdoms against other Muslim rulers. Once Muslims in the area achieved political unity, especially under Saladin, they placed Crusaders on the defensive.

The Crusades did lead to some more benign forms of cross-cultural contact. We saw in chapter 12 that sugarcane had spread across Islamic lands after the conquest. Though Europeans had likely had some contact with sugar before, the Crusades acquainted many Europeans for the first time with this sweet substance. Shortly thereafter, Europeans developed sugar plantations on Mediterranean islands. Unfortunately, from the outset these plantations tended to rely on slave labour - usually Slavs in the beginning. More generally, the Crusades exposed Europeans to a variety of goods available in the east, including coffee and tapestries, encouraging trade and the rise of trading cities such as Venice and Genoa. Europeans came to export woollen goods, glass, and gems to the Middle East in return. The increased contact between Christian and Muslim worlds facilitated the transmission of Arabic and ancient Greek (and some Indian) philosophy and science to Europe (see above).

Though Crusaders would be no match for Islamic forces on land, the Crusaders were generally able to transport troops to the Levant without interference (though many travelled overland). Already by the time of the First Crusade, cities such as Venice, Genoa, and Pisa had ships that could rival those of Islamic powers. These cities profited both from transporting troops and from the trade that expanded during and after the Crusades. The Venetians are widely blamed for diverting the Fourth Crusade to Constantinople; Venice thereafter absorbed trade routes and colonies that Constantinople had previously dominated. 


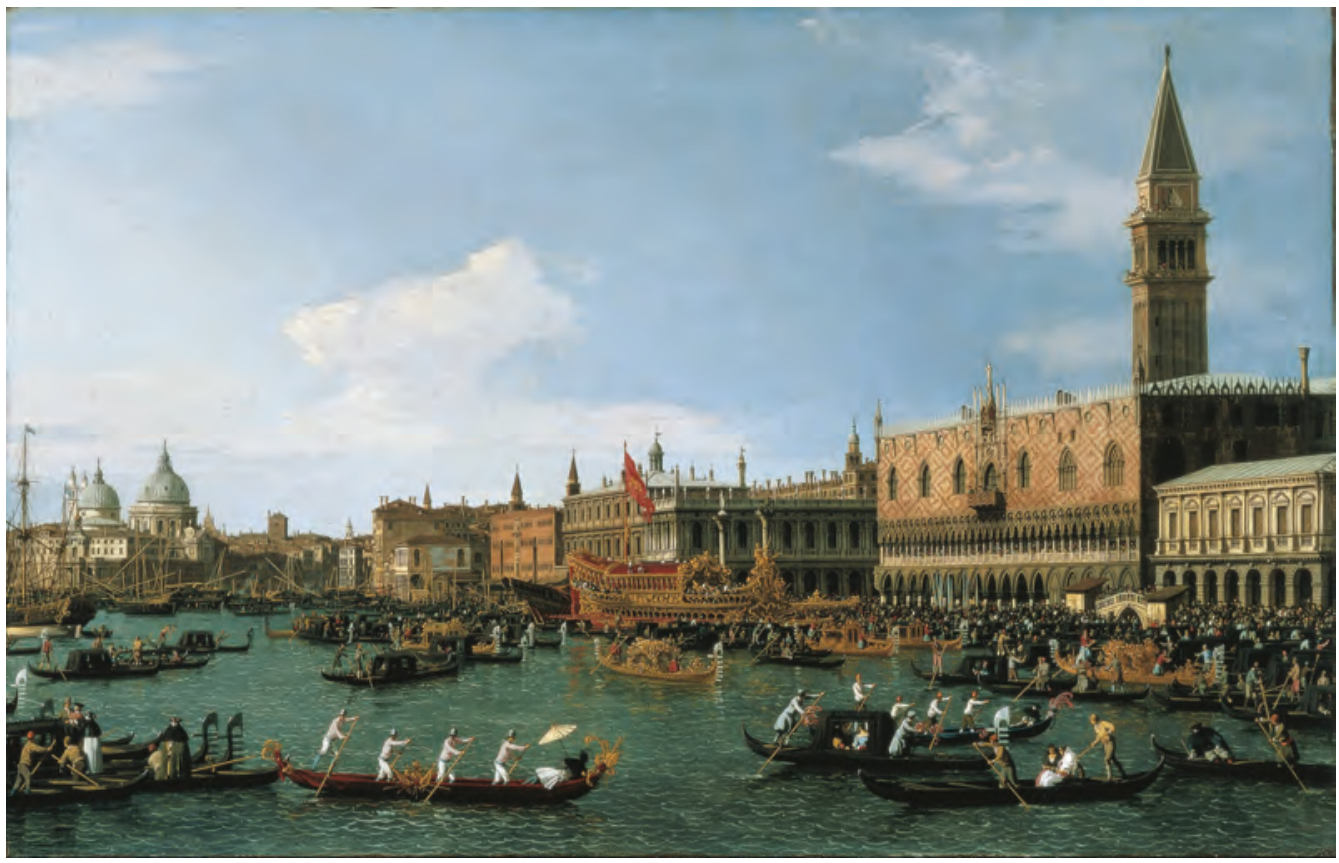

Illustration 15.6

Return of 'Il Bucintoro' on Ascension Day, Venice, 1745-50, Giovanni Antonio Canal, known as Canaletto

Website of the Museu Nacional d'Art de Catalunya of Barcelona at www. museunacional.cat/en/colleccio/return-il-bucintoro-ascension-day/giovanniantonio-canal-canaletto/212851-000

\section{PRIMARY DOCUMENTS: ANONYMOUS IN WURZBURG IN 1147 DISDAINS MOTIVES FOR CRUSADES}

God allowed the Western church, on account of its sins, to be cast down. There arose, indeed, certain pseudo-prophets, sons of Belial, and witnesses of anti-Christ, who seduced the Christians with empty words. They constrained all sorts of men, by vain preaching, to set out against the Saracens in order to liberate Jerusalem. The preaching of these men was so enormously influential that the inhabitants of nearly every region, by common vows, offered themselves freely for common destruction. Not only the ordinary people, but kings, dukes, marquises, and other powerful men of this world as well, believed that they thus showed their allegiance to God. The bishops, archbishops, abbots, and other ministers and prelates of the church joined in this error, throwing themselves headlong into it to the great peril of bodies and souls... The intentions of the various men were different. Some, indeed, lusted after novelties and went in order to learn about new lands. Others there were who were driven by poverty, who were in hard straits at home; these men went to fight, not only against the enemies of 
Christ's cross, but even against the friends of the Christian name, wherever opportunity appeared, in order to relieve their poverty. There were others who were oppressed by debts to other men or who sought to escape the service due to their lords, or who were even awaiting the punishment merited by their shameful deeds. Such men simulated a zeal for God and hastened chiefly in order to escape from such troubles and anxieties. A few could, with difficulty, be found who had not bowed their knees to Baal, who were directed by a holy and wholesome purpose, and who were kindled by love of the divine majesty to fight earnestly and even to shed their blood for the holy of holies.

Would the writer have been so hostile if the Crusade had been more successful?

\section{Trade and trading towns}
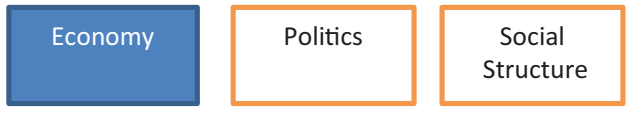

Built

Environment

Trade never wholly disappeared from western Europe after the fall of Rome. In particular, some Italian cities survived in a smaller form and rebounded in later centuries. Cities like Venice traded salt, olive oil, wine, wool textiles, leather, and glass for spices, silk, and other eastern luxuries. Merchants from Italy operated throughout the Mediterranean and Black Seas; some, like the Polos (chapter 14), ventured further.

We saw in chapter 14 that trade expanded throughout Eurasia from about the eleventh century. Though the luxury trade with Asia and across Europe is best known, there was also growing regional trade in grain and timber and wool, goods that could not bear the transport costs associated with long overland distances. We should also note an expansion in trade between the Mediterranean region and northern Europe. This trade generally proceeded overland through Alpine passes and then down the Rhine River. Cities arose in what are now Belgium and later the Netherlands to manage trade that connected the Rhine and Baltic. There were other routes: to the west along French rivers and in the east between rivers that flowed into the Baltic and rivers that flowed into the Black Sea. Increased trade between north and south Europe led to the first trip on the North Atlantic by a Genoese ship in 1277. The Genoese had been sailing south along the Moroccan coast for years (and would later perhaps fail in an attempt to circumnavigate Africa). The Atlantic Ocean had long worried sailors accustomed to the calmer waters of the Mediterranean Sea but proved not so challenging once a clear economic incentive existed. Practice in sailing along the Atlantic coast would prepare Europeans for longer ocean voyages in later centuries (chapter 19). Larger taller sailing ships were developed for the Atlantic trade. 
In Europe, political fragmentation complicated trade expansion. Despite the ongoing process of political consolidation (above), merchants - especially in the eleventh century itself - had to engage not only with a large number of kingdoms but also with powerful lords within these. The story of trade expansion in Europe, then, depends on a set of institutional innovations designed to address the merchant's challenges in an environment of political fragmentation.

We have often seen in this book that trade expanded when large empires ruled but contracted with political fragmentation. Empires could alleviate the three main challenges faced by merchants. Most obviously, they could police their territory and limit banditry. They could also protect merchants from each other by providing courts to settle trade disputes. Perhaps most importantly, merchants needed protection against the power of states themselves, who might always be tempted to plunder the merchant's goods. Empires could develop a reputation for protecting trade and would be wary of destroying that reputation for the sake of isolated plunder (unless they could concoct an explanation as to why they robbed some merchants while others should feel safe).

Trade in politically fragmented Europe would be a far greater challenge. Merchants would deal across many borders. There was no obvious venue in which to settle trade disputes. Petty nobles with small plots of land, and perhaps with debts from their latest exercise of war, might be all too tempted to shred their reputation in return for the instantaneous benefit of theft. They might promise to be honourable, but merchants could be reasonably sceptical.

Institutions, including many that seem peculiar to modern eyes, could alleviate these challenges. One of these was the merchant networks that we have often encountered in this book: If merchants traded primarily with members of a narrowly defined group, and it was profitable to remain a member of the group, then members would not cheat each other for fear of expulsion. We see many networks in Europe. Trading cities such as Venice had networks, and ethnic or religious groups such as Jews had networks. Shared religious or cultural norms could strengthen these networks, but their essence was the fear of expulsion for misbehaviour.

Such groups could also strengthen the bargaining power of merchants with rulers. If a small state benefited from trade with Venice, it would think twice about interfering with the activities of the Venetian merchant guild. Independent merchants would be tempting targets for arrest and abuse, but a local ruler might credibly promise not to molest members of important merchant guilds. Their promise could be trusted for they would not wish to lose the tax revenues they obtained from trade with a large group of merchants. Merchants, therefore, developed formal guild organizations that then signed treaties with political authorities.

One peculiar institution was the medieval "fair." Though we now associate the word "fair" with entertainment, the original purpose of fairs was trade. Fairs occurred at various levels; the dukes of Champagne in France held the largest in Europe and these attracted merchants from throughout Europe. One purpose of these fairs was informational: Merchants could get in one place a 
sense of the goods available throughout Europe. These fairs also had courts and could banish from the fair a merchant found guilty of dishonest trading. As long as it was important for merchants to attend these fairs, they would be wary of behaving dishonestly. The fair thus provided a solution to the fact that no European court had authority over any large territory. The courts of the fair could likewise only impose penalties on the fairground itself, but these penalties mattered to merchants from all over the continent.

The dukes of Champagne did not run the fair courts. Instead, these awarded charters to merchants to govern the fairs in return for tax revenues from the fair. Lesser fairs duplicated this mutually beneficial arrangement between lords (occasionally religious authorities) and merchants. There was a large and growing set of regional fairs. In England alone, the number of fairs may have almost tripled to around 150 in the twelfth century and would increase even more in the thirteenth century. These later additions were generally smaller, but they nevertheless provide evidence of a denser network of trade. The incomes earned by local lords or bishops in sponsoring these fairs often matched all other income from their estates.

A similar process allowed the emergence of independent towns throughout Europe. In some places such as Italy, towns had never been subject to feudal

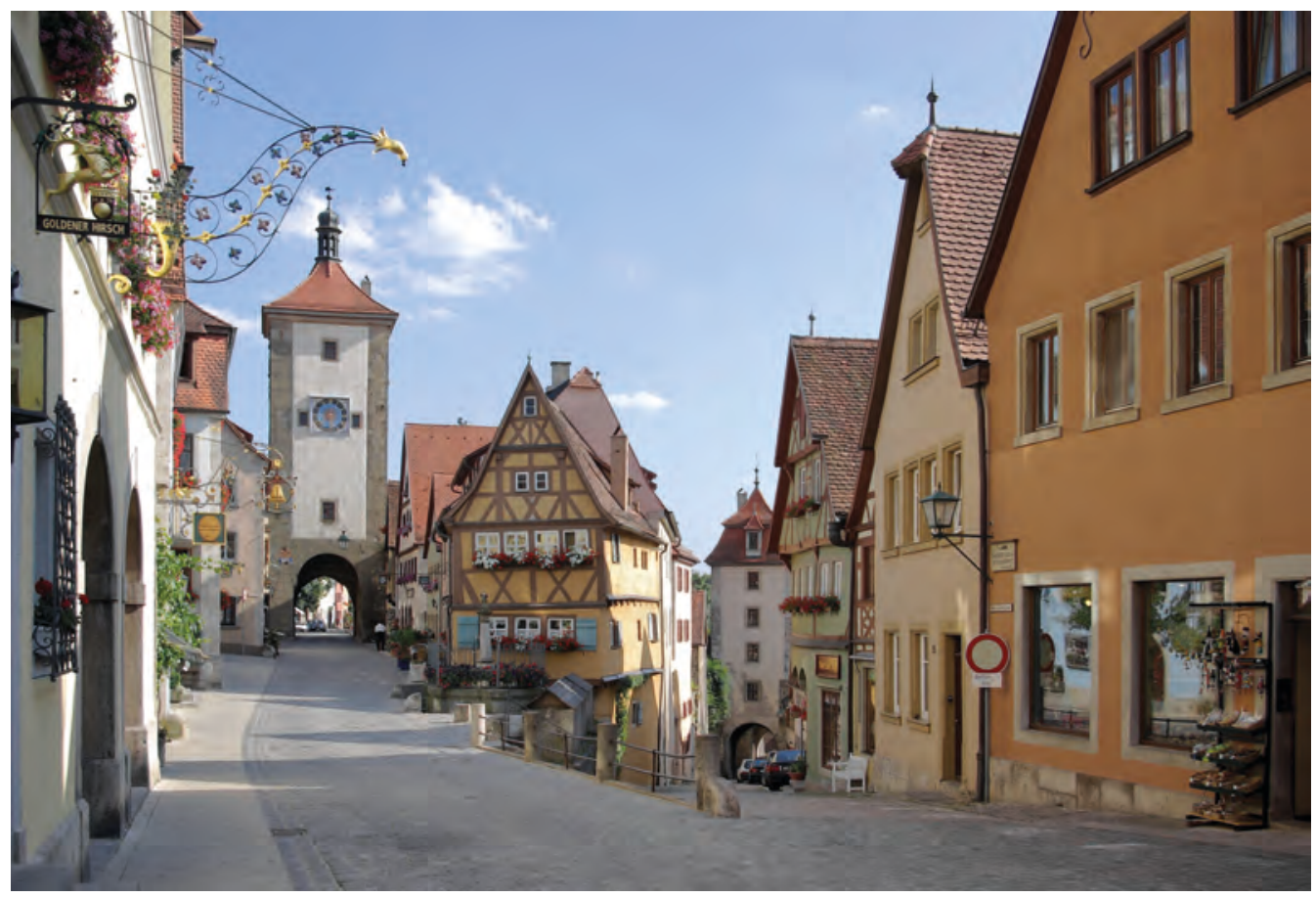

Illustration 15.7

Rothenburg in Bavaria; the walled town has many medieval buildings. Unusually, the wall surrounding the town has survived and can still be walked on around the town

Photograph by Berthold Werner, CC BY-SA 4.0 
authority but had acted instead as city states. In other parts of Europe, local lords or kings granted charters to merchant guilds to establish trading centres. These towns, of course, paid taxes. As long as the stream of taxes was significant, the lord could be trusted not to interfere with the town's activities. Merchants were free to run their own affairs.

Merchant guilds and networks, though critical to the expansion of trade, could also act in ways that limited trade. A prosperous trading town could exclude others from trading there merely to enhance the prosperity of the local merchants. A thriving merchant network could likewise limit membership in order to maximize individual profits. After all, merchants were trying to make money. Their institutions benefited others by reducing the costs of trade, but merchants tried to keep much of the benefit of lower costs for themselves.

One notable development was the Hanseatic League from 1241. This was a league of German-speaking trading cities in northern Europe and around the Baltic. The Hanseatic cities in the Baltic often replaced Slavic trading centres; some barred Slavs from guilds but others pursued collaboration. (Both Hanseatic merchants and the Teutonic Knights (above) sometimes encouraged German migration eastward, resulting in considerable ethnic diversity in

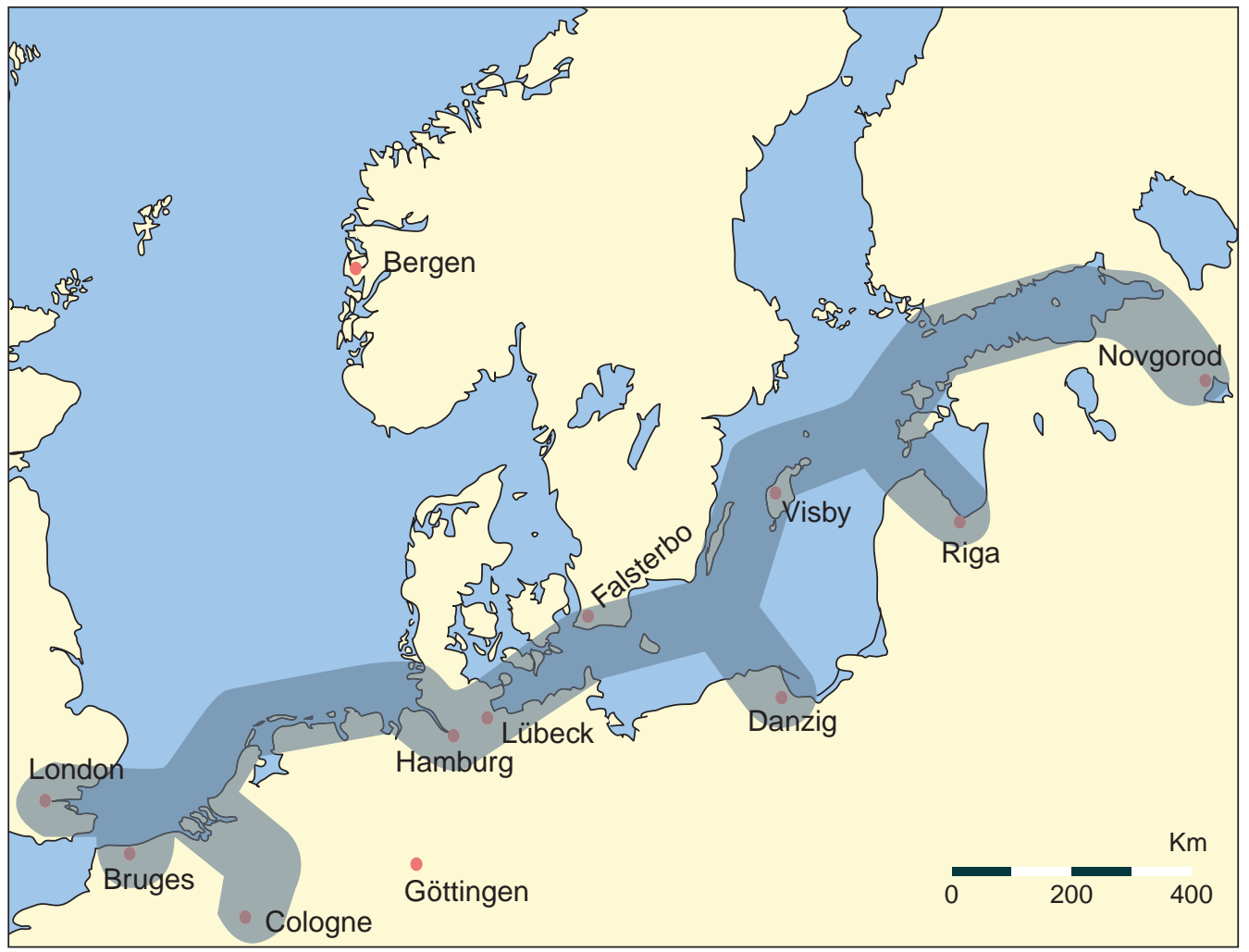

Map 15.2

The main trading routes of the Hanseatic League 


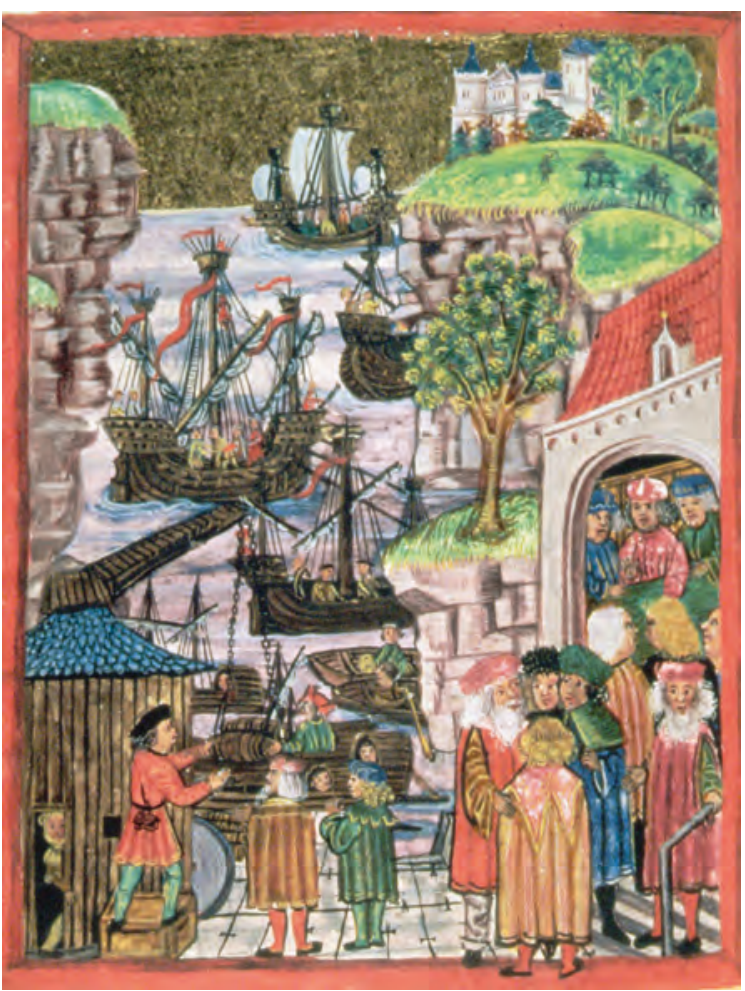

Illustration 15.8

The Hanseatic League Is Formed in Hamburg; unknown painter c. 1497 the Baltic region.) The League also had trading "colonies" farther west in centres such as London and Bruges in Belgium. The League had very strong bargaining power with the rulers with which it interacted, because the Baltic trade in grain and timber (and fur and honey) toward western Europe was lucrative and the League sought to control it. As with other merchant guilds, the League acted both to reduce trading costs and to monopolize trade. The Baltic cities also traded south to the Black Sea and beyond: This trade route shifted west through Krakow after Mongols destroyed Kiev in 1240. The Hanseatic League would have over a hundred member cities in the fourteenth century, but would decline in importance thereafter as political consolidation proceeded. Lubeck, the central city of the League, had 20,000 people by 1400 . Its skilled artisans gained fame, especially in the production of altarpieces. Red-brick architecture had been introduced from Italy in the eleventh century, and many Hanseatic cities built red-brick Gothic churches from the thirteenth century.

We might also mention double-entry bookkeeping. This was developed in the fourteenth century in Italy and systematized in the fifteenth century. It both reflected and reinforced the increased size and complexity of trade. Though elements of double-entry bookkeeping had been employed in Islamic lands and East Asia for centuries, its principles had never been so carefully articulated. There were also improvements in physical infrastructure. In particular, many bridges were built in the twelfth and thirteenth centuries that would not be bettered for centuries. Political authorities financed these bridges sometimes, but charity or the church also supported bridge construction.

\section{Agriculture}
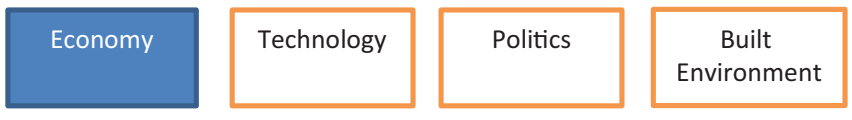

Expanded trade and urbanization both encouraged and reflected increased agricultural productivity. Scholars have studied this relationship in detail in 


\section{BOX 15.1}

\section{STOCKS AND BONDS AND PENSIONS}

Venice forced a loan on its citizens while fighting the Byzantines in 1172. Its army became ill on the way, and so Venice could not repay the loan with war booty. It transferred the debt into government bonds with an effective interest rate of 5 per cent annually. A market for these bonds soon emerged, and their value would fluctuate with estimates of the financial solvency of the Venetian state. The idea of government bonds would quickly spread in Europe (long before these emerged elsewhere): They were an effective means by which governments could borrow, and they provided citizens with an opportunity to purchase a fixed income stream (that is, $x$ units of a particular currency per year in perpetuity). Such an income stream might be particularly useful to retirees. Parents might assign a fixed income stream to children who might squander a lump-sum bequest. Though the original Venetian bonds never expired, northern European polities pioneered the idea of bonds that expired on the death of the bondholder. Amazingly, governments for centuries charged the young and old the same price for such securities, despite the fact that young adults could expect to have more years to live: Some of the financial difficulties of the eighteenth-century French kings (chapter 25) reflected the mispricing of such bonds. Eventually, though, states would estimate age-specific life expectancies and price these securities accordingly.

Tontines were developed in the seventeenth century. A group of people would collectively purchase a security. They would each receive an income stream. As members of the group died, surviving group members received their share of the income stream. Tontines served as a sort of insurance against old age: Those who lived longest received most of the proceeds. Tontines remained popular through the nineteenth century. They would become less popular as private and public pension plans emerged in the nineteenth and twentieth centuries. Such pension plans built on the previous experience of - and interest in - bonds and tontines. By the end of the twentieth century, virtually every country in the world had a public pension plan, though coverage in poorer countries tended to be limited to a small proportion of the population (and there are concerns that even rich countries will face difficulties in meeting their obligations as populations age). Bonds - now usually for a fixed period of years - remain an important source of both government and corporate financing worldwide, and remain useful for people who desire a (more) predictable return on their investment than buying stocks.

We mentioned in chapter 10 that Rome allowed some kinds of "corporations." Corporations were less common in later Roman history and disappeared from western Europe with the fall of Rome. It is not quite clear when they reappeared. There were corporations in activities such as milling and mining in the fourteenth century. The corporate form allows many investors to pool their funds in the pursuit of expensive projects - but each investor is not legally liable for debts incurred by the corporation beyond what they have invested. The earliest corporations could ask investors to provide more capital to the corporation, but investors could always 
decline or sell their shares to others. The corporate form would prove especially useful in financing intercontinental trade as new trade routes were developed to Africa, Asia, and the Americas from the late fifteenth century (chapter 19). Shares were initially sold in individual trading ventures (which could take years to complete) and then trading companies were developed to manage trade in particular regions in perpetuity. These corporations no longer requested additional funds from shareholders; they instead sold bonds or issued new shares when they needed additional funds. Stock markets emerged to facilitate trading in corporate shares. These markets soon began also to trade in "options": One could promise to buy or sell a particular stock at a specific price at some point in the future (a useful transaction for investors worried about future fluctuations in price). Stock markets allowed people of limited means to participate in merchant and industrial investment but exposed them also to fraudulent sales practices and periodic financial crises. Interest in corporations fell and legal restrictions enhanced - after the South Sea Bubble in Britain in the 1720s when predictions of massive profits from trade with South America proved exaggerated and share prices collapsed. One key innovation of the later twentieth century was the mutual fund, which allowed small investors to purchase a basket of shares in different corporations, reducing (but not eliminating) the risk associated with investing in stocks. Yet mutual funds were occasionally fraudulent and charged management fees that significantly reduced the return on investment. More recently, index-funds have been introduced that merely buy all of the stocks that are in a stock exchange index (such as the Dow Jones index) and charge minimal fees to do so.

The corporate form did not emerge in China until the late nineteenth century. However, at that time, corporations and stock markets on the western model were developed and quickly gained popularity. Chinese investors had gained experience investing in western corporations operating in China. The Qing government worried about the power that Chinese corporations might wield and often proved more willing to deal with foreign investors. Relying on the precedent of the imperial salt monopoly, the Qing first insisted on a corporate form in which government representatives sat on corporate boards; complaints of their interference and corruption led to a new corporate law in 1905 that no longer required government representation. Government bonds were also unknown in China until the modern period.

We saw in chapter 9 that even ancient economies needed sophisticated financial institutions. We see here that financial institutions evolved to aid both governments and citizens in the pursuit of a variety of goals. These solutions were always problematic, and institutions tended to become more complex over time to address the challenges of fraud and financial speculation. Yet financial institutions would come to shape people's lives. We shall see in chapter 24 that people confident of a pension could be tempted to have fewer children, for they would no longer be dependent on children in old age. The reader can reflect on how financial institutions shape their own lives, and marvel at how long most of these have been around. 
Europe, but it likely characterized trade expansion in Asia as well. Farmers with access to urban markets produced more than isolated rural farmers: They could sell food in towns and buy industrial goods. Increased output was possible through labour-intensive but straightforward practices such as careful seeding or weeding, or more expensive and long-term investments such as draining soils, fencing in animals, building barns, or spreading fertilizers such as lime on the ground. For reasons that are not clear, the cost of borrowing, at least in England, appears to have fallen from about 10 per cent per year in 1100 to under 5 per cent centuries later; this would have encouraged such investments. Recall from chapter 13 that the feudal system gradually decayed as trade and urbanization proceeded: Individual farmers gained increased ability over time to make planting and investment decisions. Such farmers produced not only more food but also industrial raw materials such as wool, flax, leather, and wood.

Farmers near Italian cities like Venice had long faced incentives to increase their output. As trade and urbanization expanded in northern Europe, agricultural productivity and population advanced fastest there. Slowly over the centuries, northern Europe became more economically vibrant than the Mediterranean region. The increased economic importance of the north was aided by the development of the mouldboard plough. - developed in the sixth century, perhaps by Slavs, it became common in the eleventh and twelfth centuries - and the importation of Asian horse collars from the ninth to tenth centuries. These were both well suited to the heavier soils of northern Europe. The mouldboard (curbed) plough could dig deep in heavy soils: This allowed deep planting of seeds while uprooting weeds. The horse collar allowed horses to pull hard without choking themselves (yes, the earliest collars actually went around the neck and choked the horse). Horseshoes also became common from about 1000: These allowed horses to work hard in wetter and colder climates than their Central Asian homeland without suffering splitting of the hooves. Also, new rotations were introduced in which nitrogen-fixing crops (that is, they put nitrogen in the soil) such as peas and beans alternated with grains.

Horses slowly replaced oxen as draft animals. Although horses were stronger and faster, this transition was challenging. While oxen had often grazed wild, horses ate grains. Oats became a common crop as a result.

Another significant innovation was milling. We saw back in chapter 4 that grinding grain was a critical step in the agricultural process. To this day, hand grinding of grains can take several hours a day in developing countries without mechanized grinding. Europeans had adopted both windmills and watermills from the Middle East. There were over 10,000 mills for grinding grain in England alone by 1300. The spread of mills is a case of technological transmission in which skilled millwrights moved between localities to build new mills. Yet the popularity of mills reflects the growth of markets: Farmers - and especially farmwomen, who tended to do most grinding - with access to markets could more productively spend their time than by hand grinding grain. They then encouraged local lords to build mills. Both lords and peasants could then 


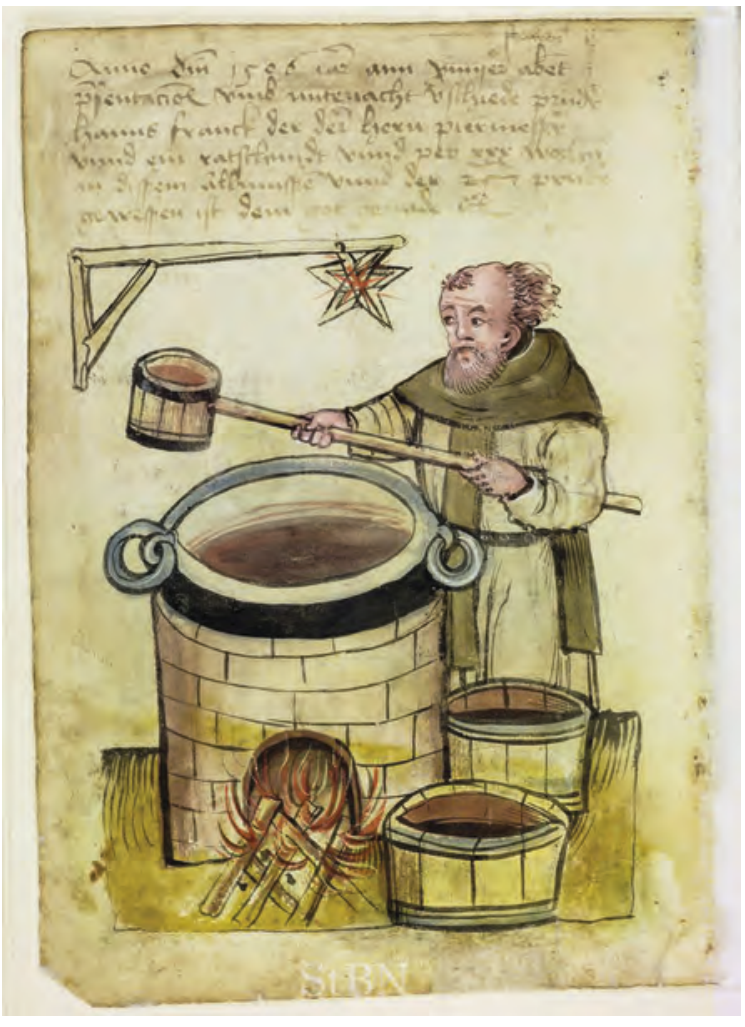

Illustration 15.9

Brewer, 1506, unknown artist. In Hausbuch der Mendelschen Zwölfbrüderstiftung. Nuremberg 14261549, Stadtbibliothek Nürnberg benefit from the use of mills. Farmers paid a fraction of the grain milled to the mill owner. This fraction was set by local custom and changed little over time, but both sides seem to have found it beneficial. Farmers often complained of thievery by millers; there may perhaps have been unofficial fluctuations in the real price of milling associated with the incidence of theft.

We should not leave the impression that hunger disappeared with these agricultural advances. It has been estimated that as late as 1700 , one-fifth of French peasants were not getting enough food to be able to work hard all day. Slowly over the centuries, the incidence of hunger and malnutrition fell among both rural and urban populations. This meant that both incomes and work effort increased.

Mills - and the bridges that we noted above - were significant capital projects for the time, surpassed only by churches and palaces. A surge in mill or bridge construction would reflect economic prosperity. It would, in turn, stimulate economic activity as workers were hired in construction. This "construction

boom" might last for decades in a region but would subside as all major rivers were bridged and most farmers had access to a mill.

We mentioned fermentation in chapter 5 and noted that ancient beers tasted quite different from most modern beers because they did not use hops. The hop plant is first documented in Europe in the eighth century. Scholars suspect that it was employed in beer making soon after. Hops reduce spoilage by attacking bacteria. They also have a marked effect on flavour. Though generally bitter, different varieties of hops impart different flavours to beer. The widespread use of hops replaced a variety of herbs. Nevertheless, beers without hops continued to be brewed; in Europe, these are known as ales.

\section{Industry and guilds}

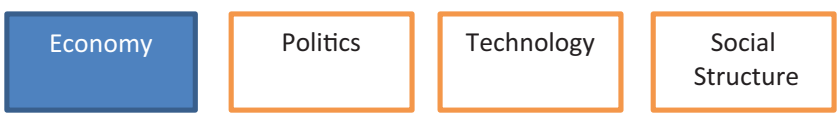


We have seen above that towns became centres of trade and encouraged agricultural developments in nearby agricultural areas. Towns also became centres of industry. Textiles were woven and metals shaped into tools and ornaments. Some towns served only local markets. However, textile manufacturers in Florence or Flanders (the Dutch-speaking part of modern Belgium) came to sell their goods across Europe and beyond. As trade expanded, artisans became increasingly specialized.

Guilds came to dominate this industry. Guilds had long existed in China and Byzantium. However, in western Europe, they were rare between the fall of Rome and the eleventh century. Yet guilds would come to be more formalized and powerful there than anywhere else in the world. Indeed artisan guilds would often work with merchant guilds in town governance. Town councils, in turn, respected the power of guilds to restrict entry to a particular occupation in a specific locality: The wool weaver's guild of Bruges in Flanders could determine who could weave wool there.

We saw above that merchant guilds sought both to lower trade costs and to monopolize trade flows. There has been an intense debate among historians as to whether artisan guilds served social or selfish motives. The answer is clearly "both," though there is room for debate as to which was more important. We have identified challenges facing artisans in chapter 6. Artisans needed to worry about their reputation for quality work. If they sold their goods locally, they would strive to produce quality goods to enhance their reputation. If they sold their goods at a distance through merchants, how could they achieve and maintain a reputation that would strengthen the value of their products? Artisans also had to worry about technology. How might they ensure that they had access to the best techniques, while perhaps limiting access by their competitors to such methods? Last but not least, what of training? How could artisans learn their crafts and train their assistants?

Artisan guilds provided solutions to each of these challenges. While an individual artisan might struggle to gain a reputation in distant lands, an artisan guild could nurture such a reputation over a period of decades. The Bruges artisan guild stamped their woollen products so that buyers throughout Europe would know that a particular piece came from Bruges. Such a stamp would be meaningless unless the guild ensured that its members produced to an acceptable level of quality. One bad artisan could ruin the reputation for all. Each guild then endeavoured to ensure that its members delivered quality goods. In practice, guilds tended not just to inspect the output of guild members but to insist on the use of shared techniques.

As historians, we must look past a simple observation that a lot of wool was woven in Bruges to reflect on the challenges of selling this cloth over great distances at a time when transport and communications were slow. Consumers did not just buy "cloth" but pieces of cloth of a certain style and quality - just as we today do not just buy "clothes" but particular pieces of clothing. We may come to trust certain manufacturers or retailers. Consumers in the twelfth century might also come to trust a specific artisan guild. Note that a guild's 
reputation served to lower the costs of trade: Merchants need not convince other merchants or customers of the quality of particular pieces. Artisan guilds, like merchant guilds, were therefore an institution that encouraged trade. They did so by providing a valuable and reliable source of information about the quality of goods from distant places.

Training occurred through the practice of apprenticeship: An apprentice agreed to work for an artisan for a fixed period - seven years was common in return for learning the artisan's craft. The artisan's guild then admitted successful apprentices. Apprenticeship solved the problem that most aspiring artisans lacked the financial resources to pay for years of training. The work the apprentice did for the artisan was effectively the apprentice's tuition. The artisan paid - or at least fed and housed - the apprentice while they gained knowledge. The apprentice became more useful as they learned. Yet the more they learned, the less they needed to learn. The artisan might then worry that an apprentice might leave his service once he had learned enough but before having repaid the artisan through work for his teaching. The guild prevented this, for only those who completed an apprenticeship could enter the guild.

Guilds had a mixed impact on technological innovation. On the one hand, the extensive training provided to apprentices ensured that best practices were transmitted without error from one generation to the next. Guilds had a powerful incentive to ensure that all guild members used the best techniques. However, the desire for shared standards in output could also militate against innovation: The guild could prevent an artisan from experimenting with a new method. Guilds might also be secretive, limiting the spread of ideas between towns. In practice, though, European apprentices were often encouraged to visit artisans in other towns. This practice naturally encouraged the transmission of best practices between towns.

The advantages of guilds with respect to reputation, training, and perhaps technology came with a social cost. Artisan guilds, just like merchant guilds, restricted entry. They only accepted so many apprentices. If a particular locale had advantageous access to raw materials or markets, its artisans might be able to profit handsomely by limiting their numbers. Customers would end up paying higher prices than if more artisans could enter the trade.

Apprenticeship could be abused. How hard was it to teach specific trades? How much of their time did apprentices spend learning as opposed to sweeping the shop floor and carrying materials? University students in the twenty-first century may pay tuition more for the financial value of the degree they receive than for the value of what they learn. If it were profitable to enter a particular guild, apprentices would likewise have been willing to "pay" through hard work for the privilege of guild entry even if they learned little during their apprenticeship.

In addition to their strictly economic role, guilds also played a social function. They held regular banquets at which guild members and their families interacted. Women entered many guilds and dominated some, though 
generally not the most prestigious or lucrative. Guilds often provided an insurance function also: The guild would care for the families of artisans that died young. Guilds also had a political role: Though merchant guilds tended to be the dominant political force, artisan guilds were often part of town governance. Artisans and merchants sometimes contested for power: There were artisan revolts in thirteenth-century Flanders demanding a greater say in town government.

Guilds would dominate European industrial production until the sixteenth century. At that point, some industry migrated into the countryside. There is debate again as to whether this occurred to escape guild controls or to take advantage of lower wages in the country (chapter 22). It is in the countryside that large industrial firms would emerge.

\section{The printing press}

Technology
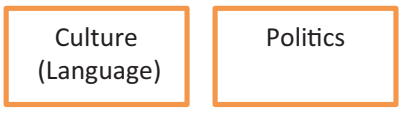

Art

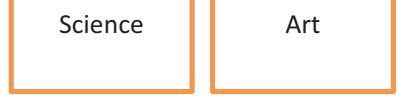

Humans had used block printing for centuries: Block printers carve an entire page into wood or metal. This expensive process can only be justified if very many copies of that page are wanted. Movable type involves the development of individual components - most commonly the letters of an alphabet - that are then combined to produce any desired page. Though simple in principle, the challenge is to achieve a great deal of standardization in the height and width of each component. Otherwise, the printed page will have uneven letter spacing or uneven letter strength and may then be illegible. Movable type required a set of interrelated inventions: a means to cast individual letters with precision; oil-based ink that would not blot or spread; paper on which to print; and the press itself that transferred the printed word onto paper.

Movable type was introduced in China in 1040, four centuries before it appeared in Europe, but was ill-suited to the ideographic written Chinese language. We shall see below that printing does nevertheless expand in scope and importance in Song China. It is likely, though far from certain, that knowledge of the Chinese press reached Europe during Mongol times (chapter 17). Though Europe was a relative latecomer to the printing press, printing would become far more common in Europe. This may in part reflect the freedom of expression that resulted from political fragmentation: Printers could relocate if they offended any ruler. Yet it is likely reflective for the most part of the simple fact that an alphabet with just twenty-six letters was easy to mechanize in this manner.

The Chinese had also invented paper, as early as the first century cE. Papermaking technology had spread to the Middle East by the ninth century (where the quality of the paper was improved; chapter 12) and reached 


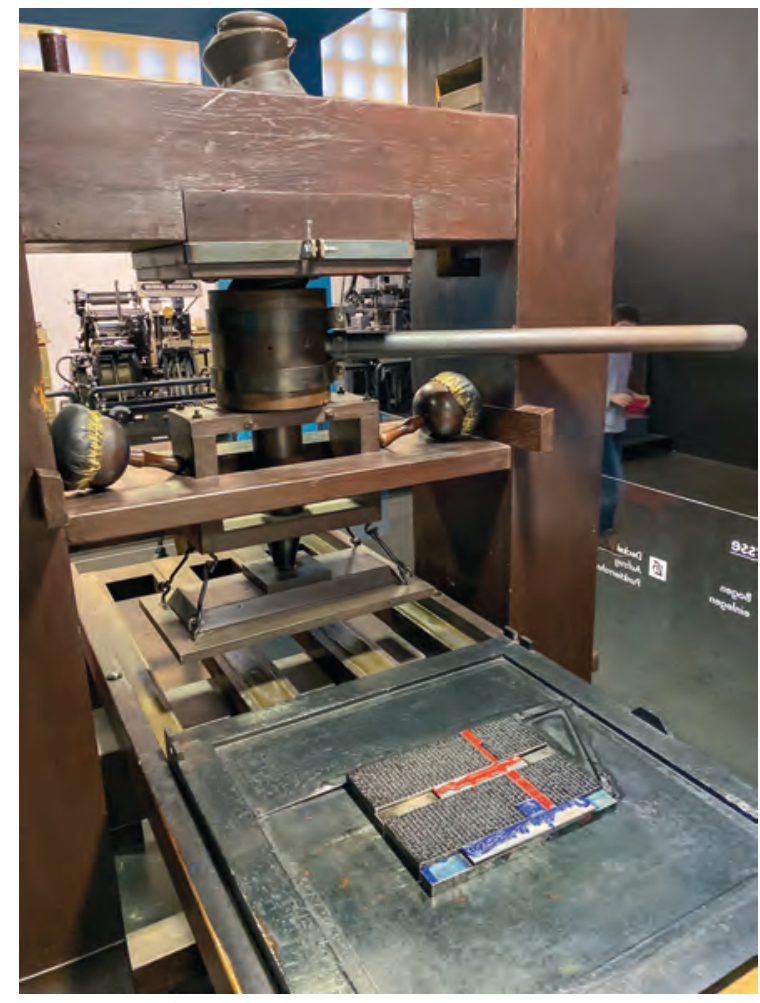

Illustration 15.10

Gutenberg's Press in the Gutenberg Museum in Mainz, Germany

Photograph by dronepicr, CC BY 2.0
Europe from there by at least the twelfth century. Paper after that replaced both papyrus and calfskin, both of which were expensive and ill-suited to use in a printing press.

Johannes Gutenberg around 1450 would become the first European to employ movable type successfully. It took him several years to develop his printing press. Notably, Gutenberg's financer sued him for not succeeding within the five years he had initially promised, and bankrupted Gutenberg. This is a useful cautionary tale about the risks associated with inventing - though Gutenberg did become one of the most famous names in the history of technology. The earliest printed works were religious texts in Latin, but the printing press soon produced a variety of works in diverse languages. It both encouraged and served a larger literate population. There were immediate fears that scribes would lose their jobs, and that lies, mistakes, and propaganda could spread too quickly. Nevertheless, the printing press would also facilitate the spread of science, technology, music, literature, and philosophy.

\section{The Renaissance}
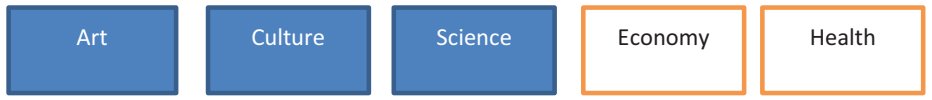

Social
Structure

The Renaissance was a period of artistic and intellectual creativity in Europe from the fourteenth to the sixteenth centuries. The name "Renaissance" or "rebirth" signals that this involved to a considerable extent the rediscovery of the works of classical Greece and Rome. Nevertheless, just as classical Greece and Rome had built on ideas from Egypt and Mesopotamia (chapter 10), the Renaissance drew inspiration from the intellectual and artistic ferment that had characterized Baghdad, Cairo, and Cordoba in preceding centuries. Indeed, as we have noted before, the rediscovery of Greek and Roman classics was only possible in large part because these had been copied, studied, and analysed 
in Islamic lands. The painter Bellini had spent a year in Istanbul painting the sultan.

The embrace of Greek philosophy meant that Christian teachings no longer dominated intellectual life. The efforts of Aquinas (1225-74) and other scholastic philosophers to link theology and Greek philosophy, although greeted with scepticism at first (above), eventually provided an opening for the pursuit of secular philosophy. Philosophers could apply reason to the analysis of human affairs without necessarily always connecting their musings to Christianity as the scholastic philosophers did. Philosophers built on classical philosophy to outline a conception of the good life that was still in line with Christian teaching but did not stress faith and asceticism. This approach is often termed "humanism" for it emphasized the responsibility of humanity to chart its own course. In this, some comparison might be drawn (and indeed has been) to the neo-Confucian revival in China in the eighth through twelfth centuries (chapter 8, and below) that sought to reinterpret classical Confucian thought to provide a compelling alternative guide to how people should live their lives.

Renaissance painting is famous for three-dimensional representation - which was both a technological breakthrough and a reflection of cultural comfort with idealizing the human form. The first notable painter in this style was Giotto in Florence 1267-1337. Dutch painters would later employ shading of colours to give a greater sense of depth to their paintings (one key development here was oil paints that took a while to dry). Art historians celebrate both painting and sculpture for the realistic portrayal of both the human form and human emotion. At the same time, architects relearned how to build massive domed buildings.

Renaissance art would first flower in Florence (Donatello and Botticelli being just two of the famous names), where it had a market among wealthy merchants: The subject matter moved away from religious imagery toward realistic portrayals of humans. Donatello's David in the 1440s was the first nude male statue produced in Italy since classical times. Popes in Rome then sought to use this new art to glorify the church, employing Michelangelo, Leonardo da

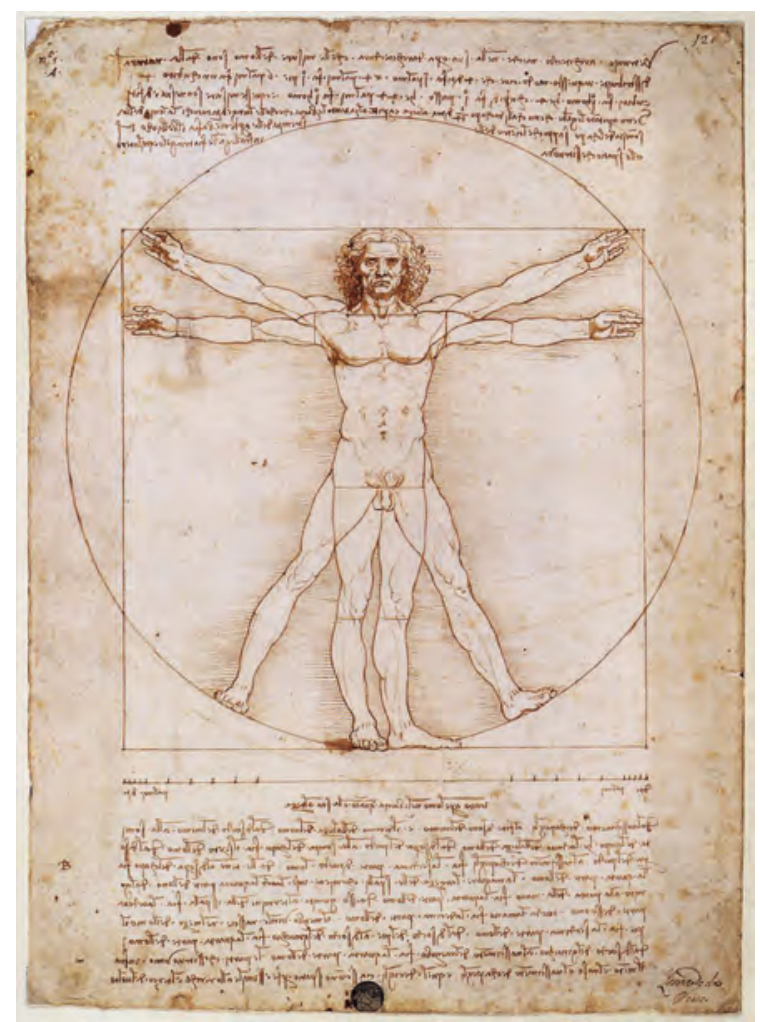

Illustration 15.11

Vitruvian Man, Leonardo DaVinci, 1492. Renaissance art drew on an enhanced understanding of the human body. DaVinci was both artist and scientist 
Vinci (who also worked in Florence, Milan, and France), and Raphael, among others. Other Italian cities, including Milan and Venice, also came to support leading artists. In the fifteenth century, Renaissance art spread to Flanders, where van Eyck (1395-1441) emulated the realism of Giotto.

There was a long tradition in European art history of describing a gradual process of achieving increased realism over a period of centuries. However, in the late nineteenth and twentieth centuries, artists in Europe would turn toward impressionism, cubism, and abstract art forms that moved away from realistic portrayals. We should apparently be careful of assuming that realism is necessarily "better" than alternatives. Artists in many times and places may have been much more interested in indicating the relative importance of the figures in their paintings than in placing these in three-dimensional perspective. It is nevertheless the case that this European artistic realism would have a global influence in later centuries.

We noted in chapter 3 that earlier cave art depicted animals very realistically because the artists observed animals very carefully - but that later cave art was more stylized because artists drew on the observation of cave art as well as animals. Art historians have made a similar argument about the Renaissance. Early Renaissance artists devoted considerable effort to studying the human form; Michelangelo and others even engaged in dissection. Later artists, though, examined other works of art. There may have then been subtle mutations away from realistic portrayal.

Dante's (1265-1321) Divine Comedy was written in Italian rather than Latin, and though religious in theme (it involves travels through hell, purgatory, and heaven), it satirized many church and political leaders. Later, Petrarch and Boccaccio also wrote in Italian as they ushered in the Renaissance: Boccaccio wrote a realistic novel, the Decameron, which dealt with everyday matters, including sex and love. The idea of writing in vernacular languages that people could read, rather than in Latin, proved popular. In England, Geoffrey Chaucer would write the Canterbury Tales in English, using a religious pilgrimage as a setting in which people from various occupations could tell their stories. Christine de Pasan is notable both for writing novels in French and for being a woman; women were not often celebrated in the Renaissance but did play a vital role in the intellectual life of the times. (Artist studios often employed women, though only the name of the male owner of the studio would appear on the final work; it is therefore hard to evaluate the female contribution to Renaissance art.) Other famous northern writers included Erasmus, who urged a simple piety, and Thomas More, whose Utopia satirized the inequalities of the world in which he lived.

Why did the Renaissance occur? The economic bases are easy to identify. It was through trade - and conquest - that western Europeans became acquainted with the classics. A very wealthy merchant class financed this art, first in Italian city states and later in the growing merchant cities of Flanders and elsewhere 
in northern Europe. The wealth of the Church also played a role. Wealth also financed the universities in which philosophers and other scholars debated the good life. Growing incomes encouraged growing literacy and these combined to generate a market for works of both fiction and philosophy. Yet while average incomes likely grew in this period, this was almost certainly a period in which income inequality widened. It was the elite rather than the average person that financed the Renaissance.

While wealth may be necessary for a flowering of art and philosophy, it is hardly sufficient. Wealth can instead endow monasteries or finance armies. The Renaissance, though hardly a direct attack on Christianity (indeed, much of Renaissance art celebrated Christian themes and was financed by the Church), did reflect a willingness to question the authority of both the church and state. The crises of the Church, noted above, likely contributed to this. The recurring experience of plague (chapter 17) likely also encouraged spiritual reflection the power of the Church did little to stop these random scourges. Yet as the plague receded in Europe, people may have turned from fear toward optimism. And the Renaissance occurred at a time of social change - expanding trade, growing inequalities, growing state powers - which may have both encouraged and allowed a culture of artistic and philosophical reflection.

\section{PRIMARY DOCUMENTS: THE DIVINE COMEDY}

Dante, in The Divine Comedy, describes the entrance to Hell:

Through me you pass into the city of woe:

Through me you pass into eternal pain:

Through me among the people lost for aye.

Justice the founder of my fabric moved:

To rear me was the task of power divine,

Supremest wisdom, and primeval love.

Before me things create were none, save things

Eternal, and eternal I shall endure.

All hope abandon, ye who enter here.

The last line above has often been quoted in the centuries since. So also has:

Midway upon the journey of our life, I found myself within a forest dark, for the straightforward pathway had been lost.

Why do you think - some of - Dante's words are still valued? 


\section{China}

Politics
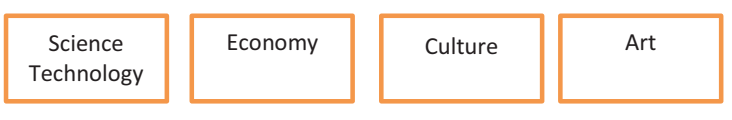

Social Structure

\section{Song politics}

The Song Dynasty from 960-1279 was never militarily as strong as the Tang in its prime, though it did have perhaps a million paid soldiers. The Song emperors distrusted the military and placed the army under tight civilian control. They were probably all too aware from the experience of the later Tang that troops could be disloyal. The Song Empire perhaps also concluded that foreign expansion was costly and focused its efforts internally. The Song suffered militarily, losing land to nomads in the west, and then northern China to the Jurchens, nomads from Manchuria, from 1127. The Jurchens were initially allies of the Song against other nomads to the north but then turned against them. The Tang had used gunpowder for fireworks; the Song developed grenades and crude cannons, though these had limited military impact at the time.

Internally the Song expanded both the examination system and the bureaucracy. There would be about 100,000 bureaucrats. These strove to enforce common laws and taxes throughout the empire. At the local level, two or three officials rotated from other regions every three or four years would supervise hundreds of local clerks about whom we know little. Local officials had to file regular reports with central authorities, and their performance was regularly evaluated. Books were published providing advice to local bureaucrats: These were urged in particular toward multiple assessments of taxes owed in order to reduce opportunities for fraud. Detailed records were kept of both people and lands. This more extensive bureaucracy centralized authority but generated financial challenges. Though the Song's loss of territory (above) was an exception to the general trend toward political consolidation in this time period (chapter 14), its bureaucratic developments were very much in accord with that trend. There were a series of local, regional, and national examinations. The Song reduced opportunities for cheating. Those who passed the local exams escaped requirements for forced labour. Scholars have estimated that it would take six full years of memorizing 200 Chinese characters a day to master the Chinese classics in preparation for these examinations. Students commonly took the exams several times before passing. This large professional and merit-based bureaucracy both reflected and reinforced the decline of the Tang-era aristocracy. Wang Anshi, chief minister from 1071, would create new bureaucratic divisions focused on particular tasks (similar to modern government departments); he would also add some social science elements to the examinations - later emperors would repudiate his policies.

It was during the Song period that Confucianism responded vigorously to the popularity of Buddhism. Building on the works of some Tang-era philosophers, Zhu Xi (1130-1200) added metaphysical elements to Confucianism. His 
metaphysics had a broad similarity to the Taoist emphasis on the Way of the Universe (chapter 11). He used this metaphysics to provide a reasoned justification for Confucian ethical precepts. The resulting synthesis, known as neoConfucianism, would become Confucian orthodoxy. Neo-Confucian texts joined classical texts in the examination system during the later Song period; this would remain the case through later dynasties until the examination system was abolished finally in 1905. Even those who failed the examinations tended to value the Confucianism they had learned in preparation. Confucianism, in turn, influenced Buddhism: Chinese Buddhism stressed the importance of hard work far more than was the case in Southeast Asia.

\section{PRIMARY DOCUMENTS: ZHU XI}

Zhu Xi devoted much thought to the nature of the universe:

Heaven-and-Earth has no other business but to have the mind to produce things. The qi [material force] of the origination [the supreme ultimate] revolves and circulates without a moment of rest, doing nothing except creating myriad things. Heaven-andEarth reaches all things with this mind. When human beings receive it, it becomes the human mind. When things receive it, it becomes the mind of grass, trees, birds, and animals. All of these are simply the one mind of Heaven-and-Earth.

He then reflected on how humans might best absorb the ideas immanent in Heaven-andEarth. He urged being active in the world over the meditation urged by others:

From the time one has life, one has some kind of knowledge. Affairs and things come into his life, and he responds to and is in contact with them without a moment's rest. ... Yet sages and superior men have spoken of ... the state of total stillness without movement. ... When I tried to think of it in this way, I only found moments without awareness, during which false and dark motions would clog up my mind, hardly the substance of pure consciousness responding to things.

He arrived at a view of the state similar to that of classical Confucianism:

Among the pressing matters faced by the state, there is none more important than comforting and providing for the people. The foundation of comforting and providing for the people lies in the emperor's upright heart and proper, skillful enactment of laws, regulations, and moral standards.... Moreover the successful implementation of such regulations cannot rely solely on the work of an emperor, but instead necessitates the participation of virtuous ministers.

Can you appreciate how he linked metaphysics and ethics?

Like other empires, the Song encouraged the practical sciences. These included astronomy, mathematics, some chemistry, the study of plants, and the development of the first forensic science for solving crimes in the world. 


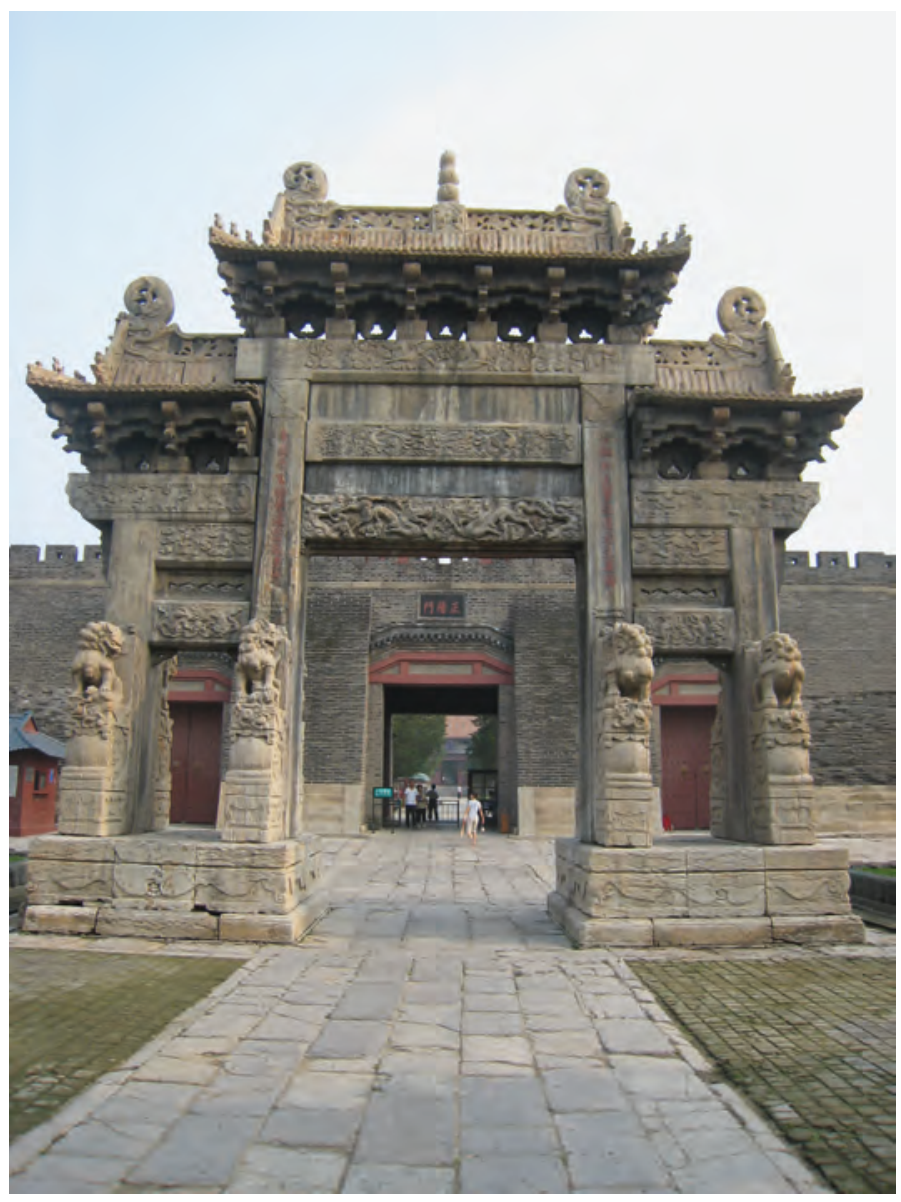

\section{Illustration 15.12}

Gateway to Dai (Taoist) Temple in Mount Tai, Tai'an, Shandong, China, 1489. The temple complex was founded during the Han dynasty but was expanded and rebuilt during later dynasties. It is one of the most renowned architectural sites in China. Its centrepiece is the Palace of Heavenly Blessings, built by the Song Emperor Huizong; this contains a 62-metre long mural

Science, though, was part of the bureaucracy; there were no independent universities or guilds as were beginning to emerge in Europe at this time. Chinese science was not completely isolated from that of the rest of the world. Indian science had reached China during the Tang as Buddhist monks travelled to India. The Chinese had developed decimals from the fourth century BCE; by the thirteenth century $\mathrm{CE}$, China possessed the best algebra in the world. This was a very practical algebra: Texts did not so much expound theory but provide answers to challenges that bureaucrats might face.

The Song slowly eliminated national requirements for forced labour, though local authorities continued to require forced labour on a temporary basis. The national government increasingly relied on a progressive income tax: They established nine income grades and levied taxes accordingly. The system worked 
well, but over time provincial authorities retained a larger and larger share of tax revenue. Another problem was that the income grades were determined based on cultivable land and so missed most types of urban income. The Song did introduce a set of sales taxes and monopolies on salt and tea, which did serve to capture some portion of urban incomes. These taxes came to comprise a third of Song revenues. Historians had long thought that the Song impoverished the peasantry with its taxes but now generally appreciate that the overall tax take was similar to that of the Tang. Indeed, the Song may have taxed merchants more severely than peasants. Moreover, we shall see below that incomes may have risen quite a lot during the Song.

The loss of northern China to the Jurchens decreased Song revenues while increasing their military needs. We can then attribute the eventual decline of the Song in part to the usual combination of foreign threats, grasping provinces, and financial challenges. However, the Song, in the end, would fall to the Mongols, one of the most successful military enterprises in world history (below; chapter 17).

\section{Economic dynamism under the Song}

Though the Song Dynasty experienced the loss of considerable territory in northern China to nomadic incursions, it would be a mistake to judge the Song harshly on that basis alone. Indeed, there is much evidence that there was significant economic growth during the Song period. Some have even spoken of a Song Industrial Revolution and wondered why the Chinese economy did not sustain economic growth as Britain would from the early nineteenth century (chapter 24). Although it is, of course, difficult to estimate economic growth rates for the Song period, there is evidence of many of the characteristics of the much-later British Industrial Revolution: growth in industrial output, technological innovation, large industrial enterprises, and institutional change. Song China, that is, experienced far more than the trade expansion observed across Eurasia in this time period (chapter 14). Song China may indeed have had the highest average incomes on the planet (though this is very hard to measure). It may even have had the highest average incomes ever experienced in imperial China: It could be that China did not reach the same income levels again until the twentieth century.

The Chinese population likely doubled to 115 million in 1200, twice that of Europe and about 40 per cent of the global population. Hangzhou, the capital of the Southern Song (that is, after they lost north China to the Jurchen), had one million people. Some say that 20 per cent of the Song population was urban, a level not seen in Europe until the nineteenth century (Europe was still under 10 per cent urban in 1500).

Some of this prosperity may reflect the transport developments of the Sui and Tang. The Song also invested in canal construction. The canal lock was invented during the Song period: A lock raises and lowers ships by raising and reducing the water level in an enclosed chamber that the boat enters and exits at different levels. Before the canal lock, canals were limited to flat terrain. The suspension bridge was also invented during the Song period. The abacus - a 


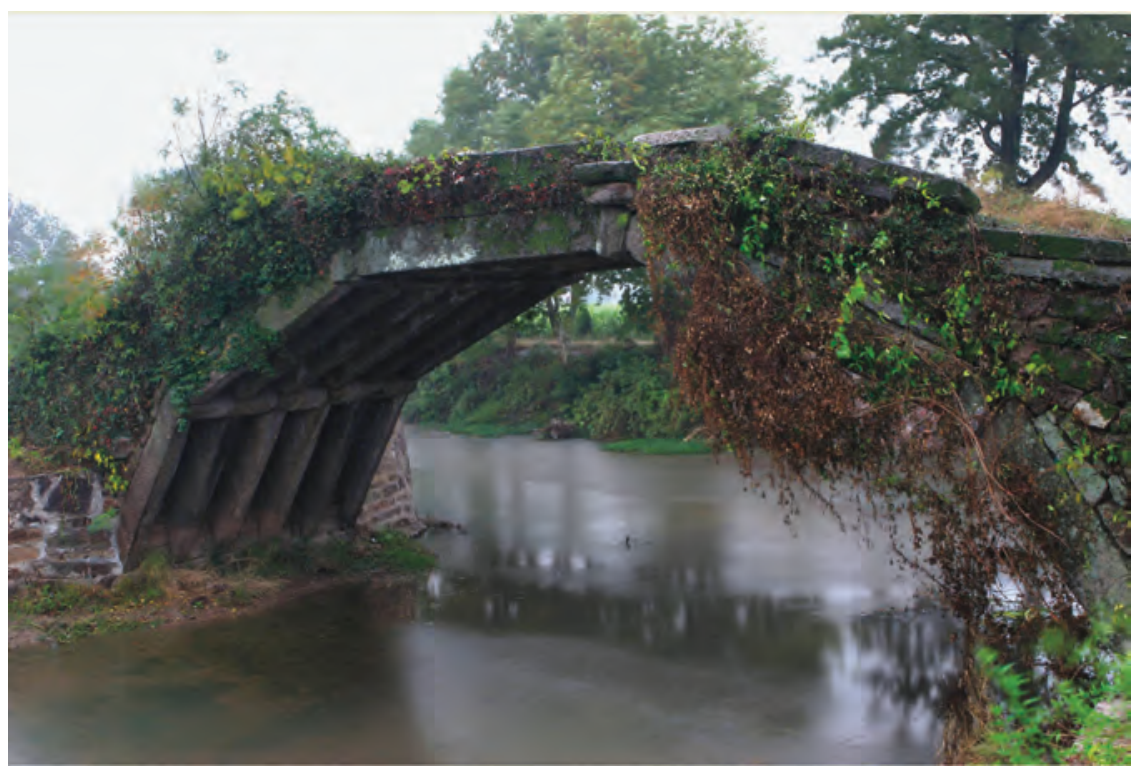

Illustration 15.13

13th-century Guyue Bridge. A stone arch bridge built during the Song Dynasty in 1213. Yiwu, Zhejiang province, China

mechanical device for the performance of simple algebraic calculations - was another Song-era innovation.

Internal trade was further encouraged by expansion in coinage and the development of paper money. Paper money would amaze Marco Polo. Private banknotes (that is, currency backed by reputable merchants) had emerged during the Tang. The first governmental paper currency in the world was introduced in Sichuan in 1024. Sichuan had a particular need for paper currency: The Song did not allow copper currency in the region for fear that it would leave the country to buy goods from nomads to the west (a bulky iron currency was employed instead). When the Sichuan provincial government failed to honour its paper currency (that is, trade precious metals for it at the face value of the currency), the central government began to print its own paper money. Sichuan may have had problems with counterfeiting; the central government used four-colour printing - the first time this was employed anywhere in the world - to produce its currency. Both governments had the benefit of paper from mulberry trees that was strong enough to be printed upon. The Song could ensure the viability of paper currency by promising to honour the currency as payment for taxes.

The Song loosened restrictions on foreign trade, and would eventually receive 20 per cent of government revenue from tariffs. Self-governing communities of Muslim merchants mainly handled external trade, though there were also many Indian merchants and a few merchants from as far afield as Europe. Muslim merchants had come to dominate trade during the Tang; though the 
Muslim merchants of Canton were killed in the late Tang, the Muslim merchant community rebounded during the Song. It was in Song China that the world's first compass was developed to aid sailors (though prototypes of the compass had been around for centuries). The Song also supported mapmaking. "Fuzhou" ships of hundreds of tons were built: They were not just big but fast, being able to reach Japan in a week. Chinese merchants occasionally sailed as far as Africa, but only rarely traded beyond Sumatra.

There were several key developments in agriculture. The most famous was the adoption of Champa rice from Vietnam. This grew faster than previous strains of rice - in 100 rather than 150 days - allowing multiple crops annually (or the alternation of summer rice and winter wheat). It was also drought resistant and so grew in areas that other rice strains could not. Extended irrigation systems employed primitive pumps. There was increased use of (improved) iron ploughs; increasingly, oxen pulled these in the north and water buffalo in the south. The Song encouraged terracing, which created fields that trapped fertile silts so that they were not washed away. Farmers spread manure more consistently as fertilizer. The Song government utilized Tang-era printing technology to produce manuals of agricultural techniques, though it is hard to judge how widely farmers consulted these. The government appointed "master farmers" from local communities, who were to disseminate new farming techniques and knowledge of new tools, fertilizers, and irrigation methods. As transport costs fell, there was regional specialization in Chinese agriculture, with each area producing what it was best suited to: Oranges and sugarcane traded for grains. Wang Anshi, chief minister from 1071, and a determined economic reformer (he urged the end of forced labour and the expansion of the currency), encouraged rural relief efforts and made loans to small farmers and merchants: Farmers borrowed money before planting and could repay in grain after the harvest. The 20 per cent interest charged by the government was far less than farmers could obtain elsewhere but allowed the state to profit from the transaction. The reform fell into disuse, perhaps as later governments focused more on revenue than aiding farmers. It seems that many Song farmers achieved levels of output not surpassed until the eighteenth or nineteenth centuries; it is harder to establish just how widespread these impressive yields were. The Song struggled to reduce the power of property owners over rural workers. With per capita food output growing, more and more land was devoted to tea production: This became an element of mass consumption in the eleventh century, with elaborate tea ceremonies spreading beyond the elite. Tea was sweetened with sugar imported from Southeast Asia.

Though the Song lost land militarily in the north, it steadily expanded into new areas in the south along the Yangtze River. These proved amenable to irrigation and new crops. Expansion into new lands not only raised incomes but also shifted the balance of the Chinese population southwards. Whereas twothirds of Chinese people lived in the northern wheat-growing region in 750, this ratio had reversed by 1100 . 
Industrial output expanded. Iron output reached 125,000 tons per year (compared to about 20,000 under the Tang), a figure not seen in Europe for centuries (England would smelt only 68,000 as late as 1788). Coal was used as a fuel for iron manufacture during the Song; the adoption of coal as a fuel would later be one of the signal innovations of the British Industrial Revolution. Nevertheless, China experienced severe deforestation from the use of wood as a fuel. Production of textiles and porcelain and printed books also expanded (there was some experimentation with movable type but Chinese characters rendered this problematic). It was during the Song that Chinese porcelain (chapter14) became a significant export, reaching Africa and the Middle East. The spinning wheel was developed from 1035; there was also an elaborate machine for unwinding silkworm cocoons. Spinning mills powered by water wheels were introduced; this also would be a key innovation of the muchlater Industrial Revolution (chapter 24). Significant technological advances also occurred in papermaking, printing, and food processing.

We can imagine that these various developments were interrelated. Advances in agricultural productivity freed labour for industrial work at the same time that it created markets for industrial products. The availability of such products likely in turn encouraged farmers to increase output in order to buy textiles or ceramics. Trade expanded with these increases in production and with increased regional specialization. (Recall that European farmers also expanded output to reach urban markets.) Merchants and artisans organized but mainly to collect taxes from their members on behalf of the state: Their guilds were far less powerful than in Europe.

Developments in agriculture and industry in turn led to increases in what we now call the service sector. Large firms emerged to produce and distribute industrial and agricultural goods. These needed to develop bureaucratic practices. Banks emerged to transfer funds (but did not make loans). A variety of occupations such as notary and real estate agent grew in importance.

Though porcelain and silk exports grew, rising incomes in China also stimulated imports. Indian cottons, in particular, became popular in China. Interestingly, the Song took steps to discourage the outflow of silver to buy foreign goods. This policy should be recalled when we later discuss the massive Chinese imports of silver from the Americas from the sixteenth century onward (chapter 19): We are guided to view with suspicion later arguments that China always had more to sell to the rest of the world than it wished to buy from elsewhere.

Why did this Song-era economic dynamism not continue? Perhaps it just ran out of steam. Maybe the institutional structure was not quite good enough to support continued growth. Perhaps it ended because the later Ming and Qing dynasties pursued less enlightened policies. (The Ming certainly restricted foreign trade; they may also have been unenthused about innovation and have viewed merchants with suspicion.) Even the Song stumbled in later years: Faced with rising military expenditures in the north, they over-printed paper currency, generating inflation of some 60-75 per cent. The Song had 
monopolized many industries, including silk and ceramics. While it seems to have encouraged innovation in these, we shall see in later chapters that states have often had difficulty in promoting innovation in state-owned enterprises. The intriguing counterfactual question is whether the Chinese economy might have entered a sustained trajectory of economic growth as Britain would in the nineteenth century. If so, then world history would have unfolded in a dramatically different fashion, and we might today be wondering if Europe would ever amount to much.

\section{Landscape painting}

It was from the tenth century that the Chinese tradition of landscape painting emerged (or at least became prominent). Art elsewhere in the world would continue to emphasize people, animals, or religious imagery for many centuries. The economic prosperity of the Song period provided a large private market for works of art. Whereas wealthy merchants and landowners in some other parts of the world favoured portraits, the influence of Taoism and Buddhism which both urged humans to live in harmony with nature - may have been critical in guiding Chinese tastes toward landscape art. Though humans sometimes appeared in Chinese landscapes, they were generally dwarfed by nature. Guo Xi, in his eleventh-century book Advice on Landscape Painting, argued that people are naturally attracted to nature but congregate in cities by economic reality; the landscape painter reconnected them to nature.

Chinese landscapes were generally painted on paper scrolls (which in turn reflected the early development of paper in China; chapter 10). Though scrolls were sometimes hung, the general expectation was that the scrolls would be slowly unrolled. This distinct medium may have encouraged a "textual" approach to painting, whereas European canvases encouraged a window-like realistic depiction of the world. As porcelain output expanded (chapter 14), landscapes were painted on porcelain as well. Sales of porcelain in other lands would in later centuries inspire an interest not just in Chinese landscape art but also in Chinese gardens (which were particularly popular in eighteenth-century England).

\section{The Yuan}

We will discuss the Mongols and the Yuan Dynasty they established in China in chapter 17. The southern Song had hired the Mongol leader Chinggis Khan as "bandit suppression commissioner." When his son Ogedei later attacked China, one weapon used by the Song in a land of rivers and canals was humanpowered paddlewheel boats with up to seven wheels, which fired explosives. The Mongols responded with their own paddleboats. After taking China, the Mongols took Korea in 1231-70. The Mongols allowed Korean students to take the Chinese examinations; this encouraged the further spread of Confucian ideas to Korea. The Mongols also attacked Japan, but unsuccessfully. The 


\section{BOX 15.2}

\section{THE HISTORY OF TIMEKEEPING}

The ancient Egyptians and Sumerians had divided the day into hours and minutes. However, it had long proven difficult to measure these accurately. Through most of human history, humans relied on observations of the sun in the sky to roughly establish what time of day it was. Sundials could formalize these observations by having the sun cast shadows on a clock face, and were used in Egypt from 3500 BCE. Sundials were useless at night or on cloudy days and had to be recalibrated as seasons changed. An alternative was to utilize some substance that flowed or changed at a constant rate. The hourglass, in which sand flowed through a small hole, was perhaps the most common such device. The Egyptians had water clocks, and knowledge of these spread to Zhou China. Ancient China and Mesopotamia also employed candle clocks.

The ancient Greeks experimented with mechanical clocks. The challenge here is the tendency of any mechanical device to slow through time. Song mechanics built a huge mechanical clock in the eleventh century that was marvelled at for its accuracy - and the sounds it made on the hour. The invading Jurchens dismantled it in 1127. It was never rebuilt, arguably because nobody knew how, despite the survival of a written treatise by the builder. Though the Chinese continued to rely mainly on sundials and water clocks, there were occasional mechanical clocks during the Ming and Qing periods, though apparently of less impressive design than that of the Song. Note that scholars long thought that the Chinese had no mechanical clocks at all when Europeans arrived in the sixteenth century.

Mechanical clocks would be developed in Europe only from the fourteenth century. Advances in escapement mechanisms - the mechanism that ensures stable movement - would allow accurate mechanical clocks to be produced much less expensively. Some historians suggest that the Chinese trajectory of a few very complicated clocks reflected imperial rule, while the European path of many smaller clocks reflected demand for clocks from a host of towns with some degree of political independence. Indeed, towns across Europe would erect clock towers in the decades after the technology was developed; such towers would become common in much of the rest of the world only in the nineteenth century. The development of the mainspring in the early fifteenth century - which stored energy in a small metal ribbon when wound - allowed small portable clocks, and from the seventeenth century pocket watches (and even later smaller wristwatches). Digital watches would supersede these in the late twentieth century. Ironically, the atomic clocks that provide the most accurate time in the twenty-first century have returned to the principle of constant flow, now of electronic waves.

Accurate timekeeping would become increasingly important over time. It would allow transport companies to schedule arrivals and departures precisely (and would enable precise measurements of longitude on ships; see chapter 19). Perhaps most importantly, it would allow and require workers to show up at factories or offices at fixed times to work a specified number of hours. It has been argued that the development of timekeeping in Europe was one key reason why it pulled ahead of Asia economically by at least the nineteenth century (chapter 22). 


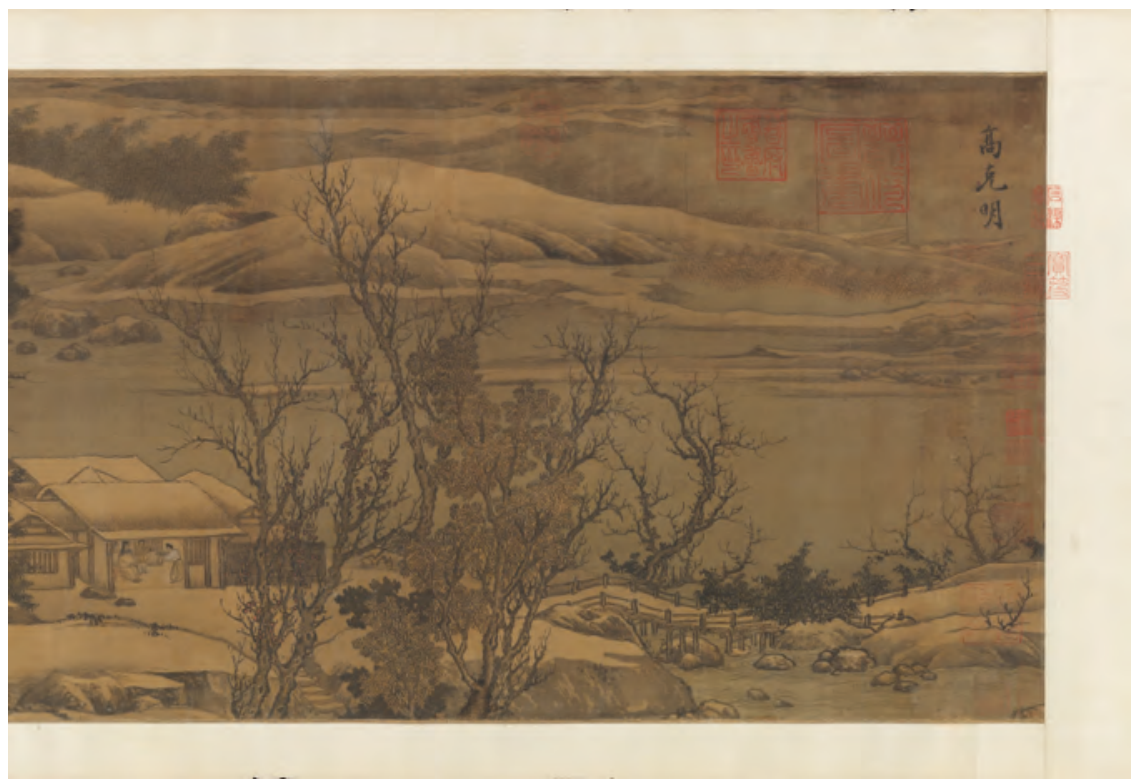

Illustration 15.14

Streams and Mountains under Fresh Snow, late 12th century, possibly Liu Songnian, Metropolitan Museum of Art

Mongols even struck distant Java because of fears that this was becoming too powerful (due to its control of the spice trade and influence over the Straits of Melaka). However, they were effectively tricked into aiding political consolidation of the new Majahapit kingdom (which lasted from the late thirteenth to late fifteenth centuries and may have collected tribute from Sumatra to New Guinea, but documentary evidence is limited).

\section{Japan}

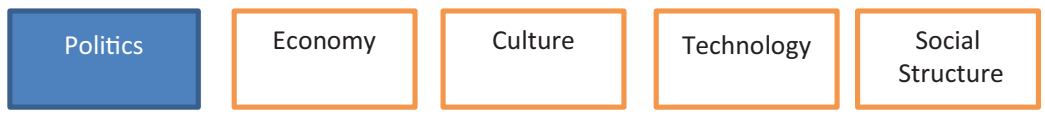

We described in chapter 13 how a centralized empire had developed in earlier centuries with capitals at Nara and then Kyoto. The central state then lost power from the ninth century. The powerful Fujiwara clan forced emperors to marry Fujiwara women and then retire so that Fujiwara could serve as regents. Power ebbed from the central state to local lords and their samurai warriors. These lords gave peasants small plots of land and required them to work for a lord: These may have comprised half of the population. The system has similarities with feudalism, and the samurai developed a code of honour (which included ritual suicide if dishonoured) which is comparable to chivalry in feudal Europe. Peasants were tied to the land like European serfs. Yet political 
leaders did not forget the ideal of a unified Japanese state. Yoritomo became the first shogun in 1185: Shoguns were the real central political authority but kept the emperor in place for symbolic purposes. For centuries, shoguns had limited power over local lords.

Despite political disunity, the Japanese were able to rebuff Mongol attacks from Korea and China in 1274 and 1281. There were thousands of ships on each side in these naval battles, though most of them were probably small boats with perhaps ten sailors. The Japanese boats reflected a thriving inland trade in Japan. Estimates of the Mongol attack force vary from a few thousand to tens of thousands of soldiers. The Mongols likely had much larger ships than the Japanese. The Mongols landed and won some land battles during the first invasion. In both attacks, the Mongols lost many boats and thousands of men at sea in storms. (The Japanese phrase "kamikaze," or divine wind, emerged at this time, and was later applied to suicide pilots in World War II.) Some scholars hypothesize that the Mongols, with limited understanding of the ocean, had employed riverboats ill-suited to ocean waves. Japan would not face foreign invasion again until Americans and Europeans arrived in force in the nineteenth century; its security as a set of islands likely served to make Japanese history somewhat more stable than the histories of other lands at the time. The challenges of launching a naval invasion also deterred the Japanese from foreign conquest: Except for one brief attack on Korea in the sixteenth century (below), Japan would not engage in foreign conquest until the late nineteenth century.

Japan unified in the sixteenth and seventeenth centuries after a lengthy period of conflict. There had been civil war through much of the sixteenth century. The Portuguese had introduced gunpowder weapons to Japan, and these increasingly replaced samurai swords in this civil strife. Toyotomi Hideyoshi (1537-98) would consolidate power in the sixteenth century, employing infantry units drilled in the use of gunpowder weapons. After his victory, he took swords away from the peasantry and turned the samurai class into paid retainers of local elites. The national army became the only effective fighting force. He is considered a "great unifier" in Japanese history; Japan has operated as a unified state ever since. However, a failed attack on Korea weakened him, and the Tokugawa displaced his son. The Tokugawa shoguns would rule until the late nineteenth century. (There are obvious parallels here to the brief rule of Qin before Han and Sui before Tang in Chinese history.) The rise to power of the Tokugawa depended primarily on careful alliances and military victories, but they did employ cannon against the castles of opponents - just as European kings used cannon against recalcitrant lords (above). Although the Japanese knew of cannon technology for centuries, cannon were only widely used after the arrival of the Portuguese in the mid sixteenth century.

The Tokugawa moved the capital to Edo (present-day Tokyo), which grew within a century to be one of the world's largest cities. (Indeed, in the late seventeenth century, five of the twenty largest cities in the world were in Japan, and Edo might have briefly been the largest.) The Tokugawa required daimyo, 


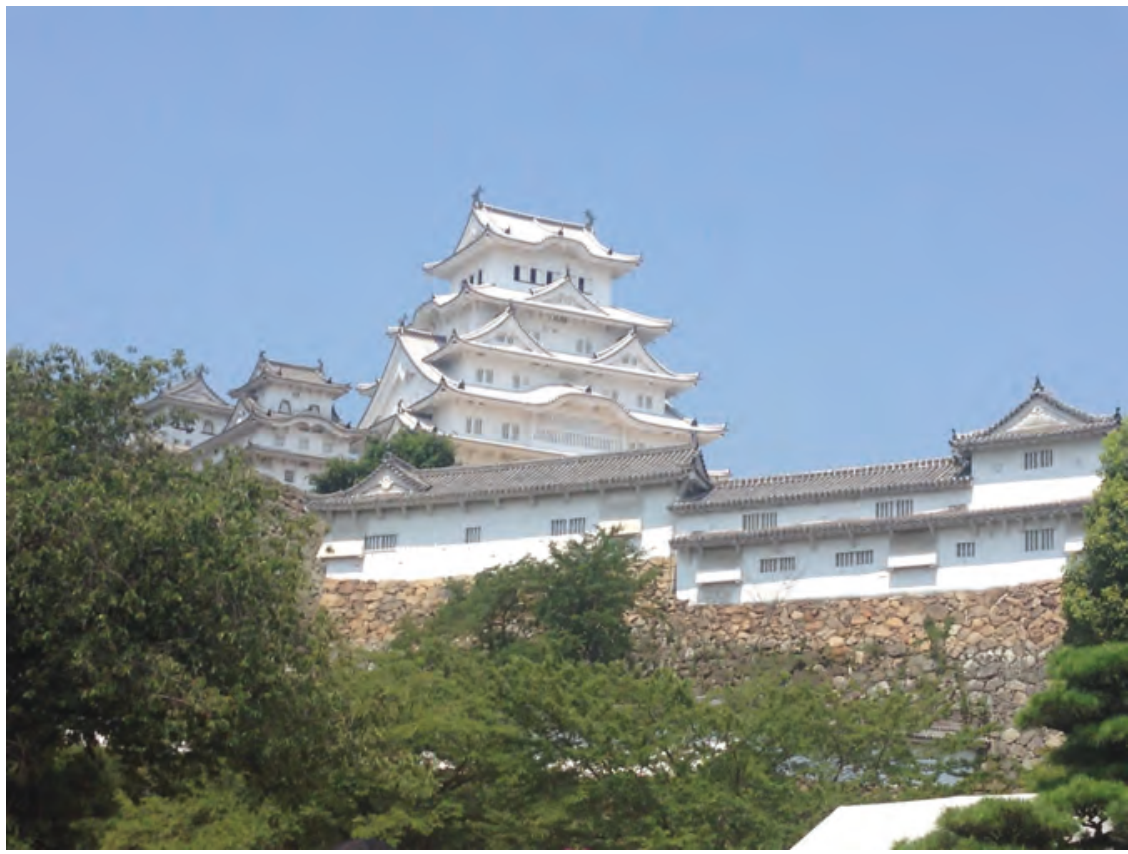

Illustration 15.15

Himeji Castle, the largest and most-visited castle in Japan. It dates from 1333.

Hideyoshi expanded the castle keep. The Tokugawas awarded it to a supporter, who made further additions

Mireille Szostak

powerful lords, to spend every second year at court in Edo (this policy is similar to that of French kings requiring nobles to live at Versailles; chapter 21). The Tokugawa instituted detailed taxation based on small private farms. Afraid that the daimyo might gain foreign weapons or alliances, they restricted international trade, with Europeans (the Dutch) only allowed to trade at Nagasaki.

We saw in chapter 13 that Japan had long borrowed cultural and political ideas from the Chinese. Trade with China expanded from the twelfth century. Neo-Confucianism gained official recognition, and Chinese was the language of scholarship. Yet increasingly, some scholars urged a glorification of Japanese history and identity. The fall of China to the Mongols (chapter 17) encouraged many Japanese to think that Japan was now the centre of world civilization. Popular novels were written in Japanese and kabuki and puppet theatre developed as uniquely Japanese entertainments. In 1720, the Tokugawa lifted a ban on foreign books, and European ideas became lively topics of conversation.

Catholic missionaries converted many daimyos, and by 1615 there were some 300,000 Christians in Japan. This prompted a backlash from both intellectuals and the government. Missionaries were expelled and locals forced to convert. Tens of thousands died in the process - with obvious parallels to the Spanish Inquisition of the fifteenth century that forced non-Christians to convert or 


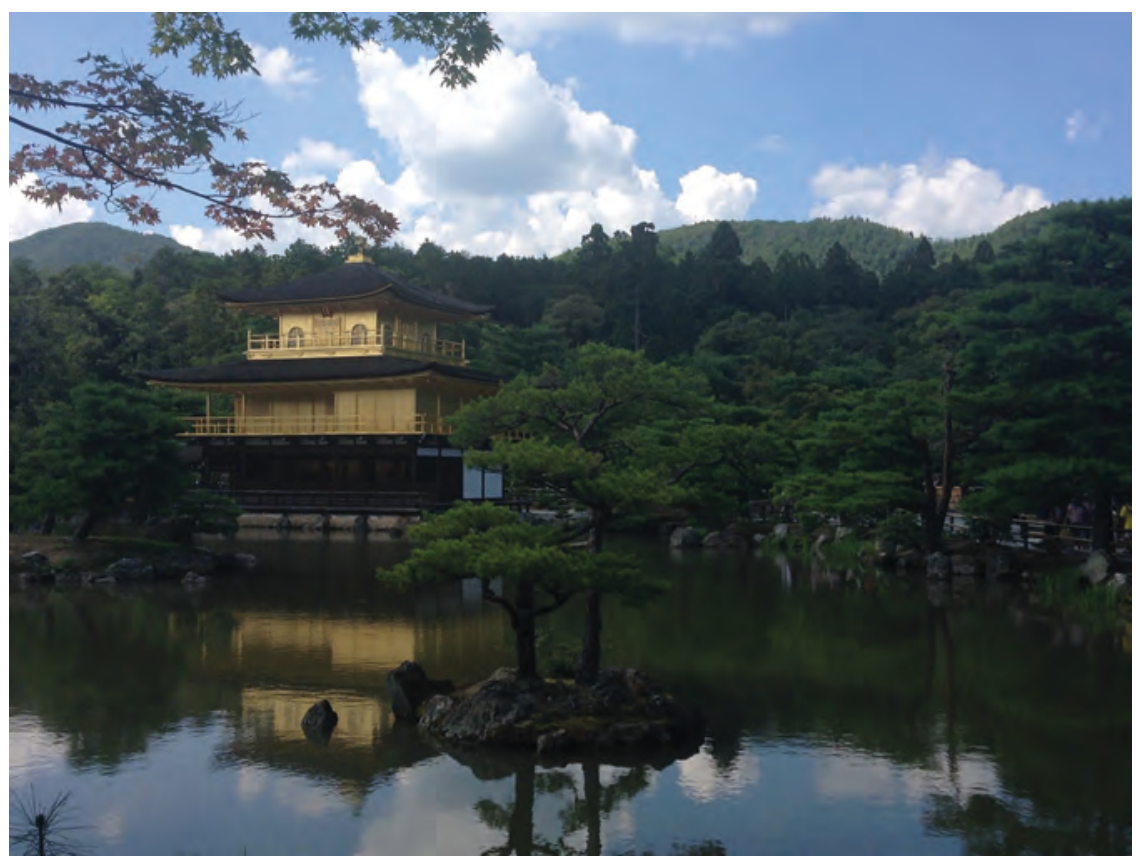

\section{Illustration 15.16}

Golden Pavilion (Kinkaku-ji), Buddhist temple in Kyoto; originally completed in 1397, it was rebuilt in 1955 after a deliberate fire Mireille Szostak

leave Spain. By the late seventeenth century, Christianity was a small secret religion in Japan.

The Tokugawa stressed stability, much like the Ming and Qing dynasties in China. There were nevertheless significant economic and social transformations. Increased agricultural production during the seventeenth century fostered population growth from 21 to 29 million. Internal trade also increased. Many merchants grew rich. At the same time, though, many daimyo became indebted due to changes in agricultural relations and the costs of maintaining appearances in Edo.

\section{India}

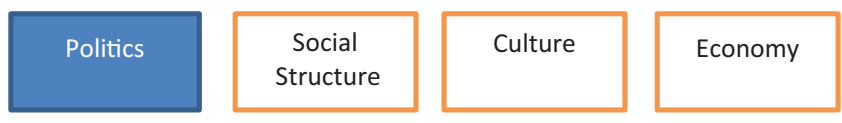

As we saw in chapter 12, Muslim Turks from Afghanistan had established the Sultanate of Delhi from the early eleventh century. This nominally held most of northern India but was generally a weak polity. Strong local Hindu rulers exerted considerable independence. The sultanate suffered from severe 
succession crises and often had to re-establish control after periods of civil war. The sultanate relied heavily on both slaves and foreigners - Arabs, Persians, and Central Asians - to fill its bureaucracy. Its slave holdings expanded from 50,000 in the early thirteenth century to 200,000 in the late fourteenth century.

The Delhi sultan Iletmish had - unusually - named his daughter Radiya as heir, but when he died in 1236, her brothers attacked. Hindu forces killed her as she fled Delhi in 1240. The sultanate survived, though weakened, but then fell apart under Timur's attacks in the fourteenth century (chapter 17). Northern India was then comprised of many competing states when Babur attacked and created the Mughal Empire in 1526 (chapter 20). Southern India mostly remained politically fragmented throughout this period, though the Chola controlled large areas in the deep south from 850 to 1267 (and even attacked Southeast Asia; see below, and chapter 13).

Agricultural productivity expanded in India as elsewhere in the post-classical period, allowing expanded trade and industry. There was substantial development of irrigation. The population was 53 million at the end of the Gupta dynasty, 79 million in 1000, and 105 million in 1500. Delhi had 400,000 people in the fourteenth century, making it the second largest "Muslim" city in the world. Delhi and other Indian cities were characterized by large urban parks surrounding temples or palaces. Temples employed hundreds, and they organized important irrigation works in the countryside.

Though politically fragmented, India benefited from the increased trade associated with political consolidation in Islam and China. Coastal India was a staging post in trade between east and west, as traders travelled by monsoon winds. India's cotton goods, in particular, were valued in diverse foreign markets.

Jainism and Buddhism declined while Hinduism and Islam expanded, especially after 1000 . As

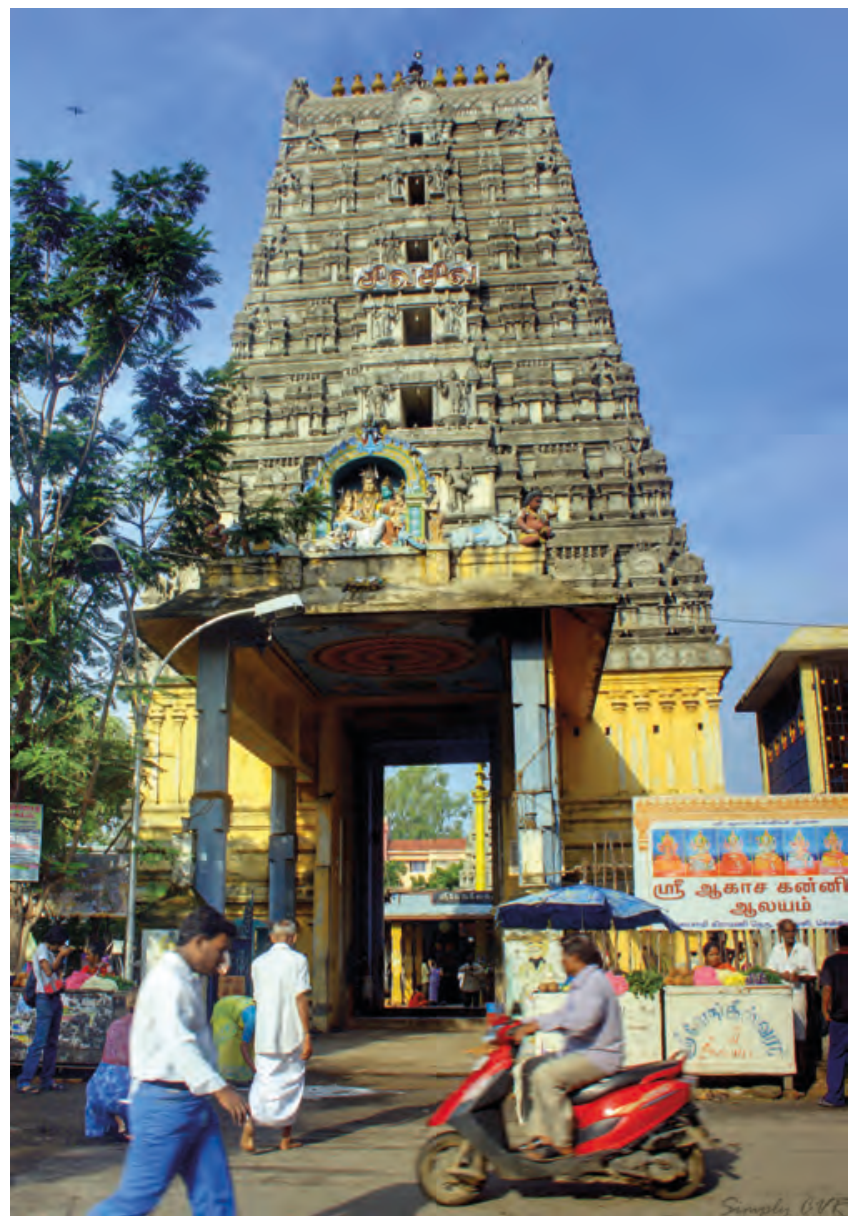

Illustration 15.17

The Vengeeswarar Temple is a Hindu temple located in Chennai, India; the temple structure is over 1,000 years old and one of the oldest Hindu temples in Chennai Photograph by Simply CVR, CC BY 2.0 
we saw in chapter 12, Islamic rulers may have been harsher in their treatment of Buddhism than Hinduism. Hinduism itself developed in directions that were similar to monotheistic religions. In particular, Hindu devotional cults, especially to Vishnu and Shiva, promised personal salvation. As elsewhere, there were impressive attempts to blend philosophy and theology - though in India these generally involved justifying philosophical arguments in terms of passages from the Vedas.

\section{Southeast Asian mainland}

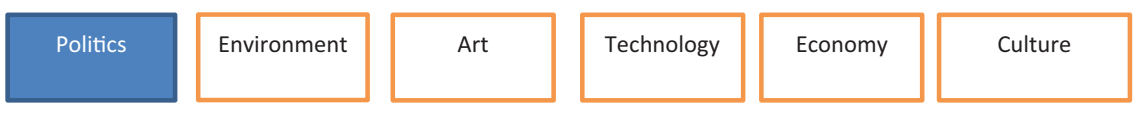

Between 900 and 1350, there were four large polities in mainland Southeast Asia: Angkor (along the mid and lower Mekong in present-day Cambodia, with some extension into the Chao Praya region of present-day Thailand), Pagan (in upper Burma), Dai Viet (along the Red River in northern Vietnam), and a smaller Champa state in southeast Vietnam. Dai Viet was a former Chinese province that had gained independence in 939. The other three states reflected Indian cultural influences. In all four states, political consolidation and increased rainfall led to increased output and population growth. All four built religious monuments as well as infrastructure for water control. We see some degree of geographic expansion in all four states, though each still controlled only areas close to rivers and coastline.

Angkor is the most famous of these states today because of the elaborate temple complexes built there at the time. The Khmer kingdom of Angkor (8021432) likely had about a million people at its peak. Unlike the earlier Funan, its rulers were not much involved in trade (which now largely bypassed its coastline) but instead gained their tax revenue from agriculture. There is nevertheless architectural continuity between Funan and the Khmer state. The Khmer also built canals and reservoirs to support this agriculture. The Khmer, like Funan, absorbed Indian religions and used these to unite their people. Khmer kings justified their rule in terms of their relationship with the gods and constructed a fantastic series of temples in celebration of that relationship.

Angkor Wat is the most extensive religious complex in the world. Construction began in the twelfth century as a Hindu temple in what was then the capital. As with many other religious monuments in the world, it was organized along astronomical lines, this time to emphasize the spring equinox (the winter solstice which was the focus farther north likely attracted less interest in a land so near the equator). The temple complex combines massive size with careful attention to detail, including thousands of carvings. We should stress, though, that impressive temples had been built in the region for centuries. Builders had honed their skills across these generations. Although influenced by Indian temples, there are unique Khmer elements, such as lotus-shaped towers and 


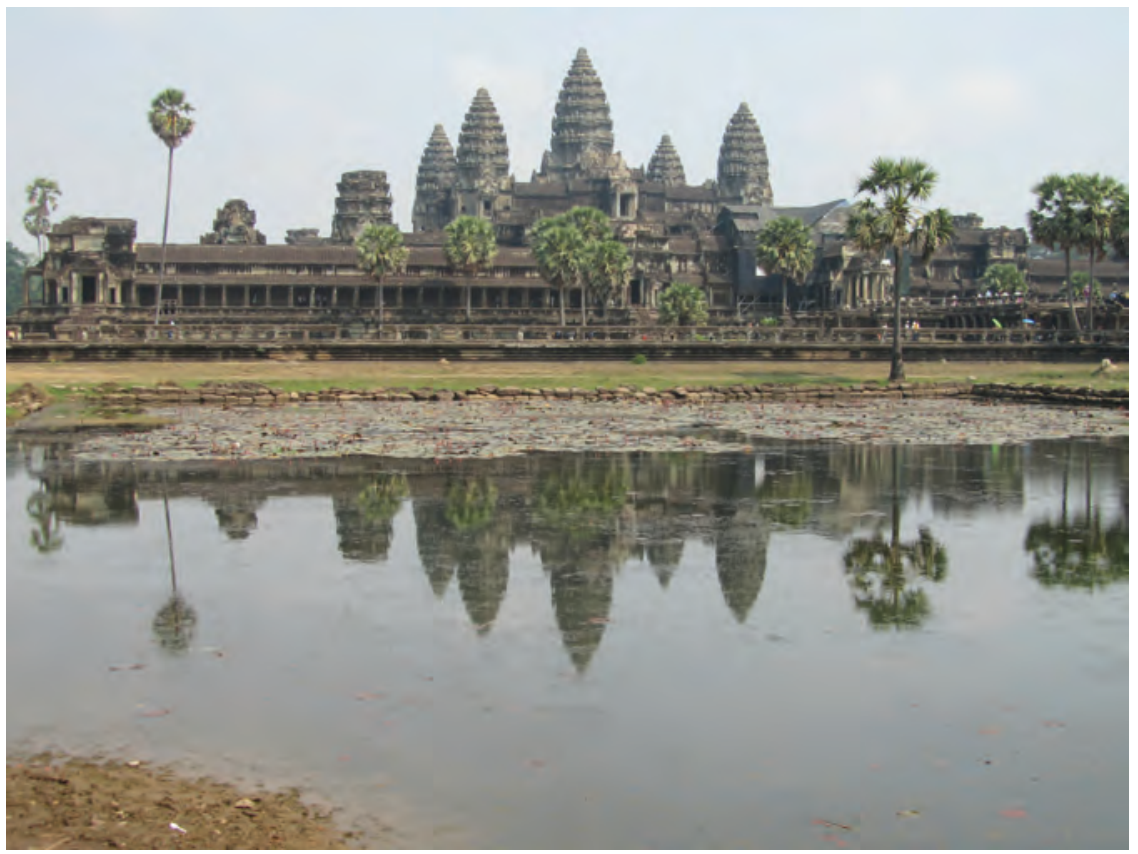

Illustration 15.18

Angkor Wat, Cambodia

figures with square chins, thick lips, and bow-shaped single eyebrows. Later rulers made Buddhism the state religion and built Buddhist temples on this site as well as a large Buddhist temple complex a few kilometres away. These temples fell into relative disuse as political power migrated to Thailand (see below), but have been restored in recent decades. The moat around Angkor Wat may have prevented the jungle from covering the complex as occurred with some other temples in the region.

These many colossal temple complexes indicate both that there was a considerable agricultural surplus in the Khmer kingdom and that the state was able to appropriate much of this. Indeed, the Khmer possessed a very advantageous geographical situation for irrigating rice cultivation. Not only did the Mekong River flood (somewhat like the Nile) but also the large lake Tonle Sap served as a natural reservoir along an important tributary of the Mekong. The Khmer built irrigation systems that controlled flooding and distributed lake water when needed. They also developed a highway system to integrate their kingdom. Angkor Wat was itself near Tonle Sap and linked to it by canals.

The Khmer at different times controlled parts of modern-day Vietnam, Thailand, and Malaya - though they tended to rule distant provinces through local elites. Later invasions by Thais and Chams destroyed the Khmer irrigation system - a strategy that weakened the Khmer in the short run but also limited tax revenues for the conqueror in the longer term. Unfortunately, the only written records beyond inscriptions on buildings that remain are from a few 


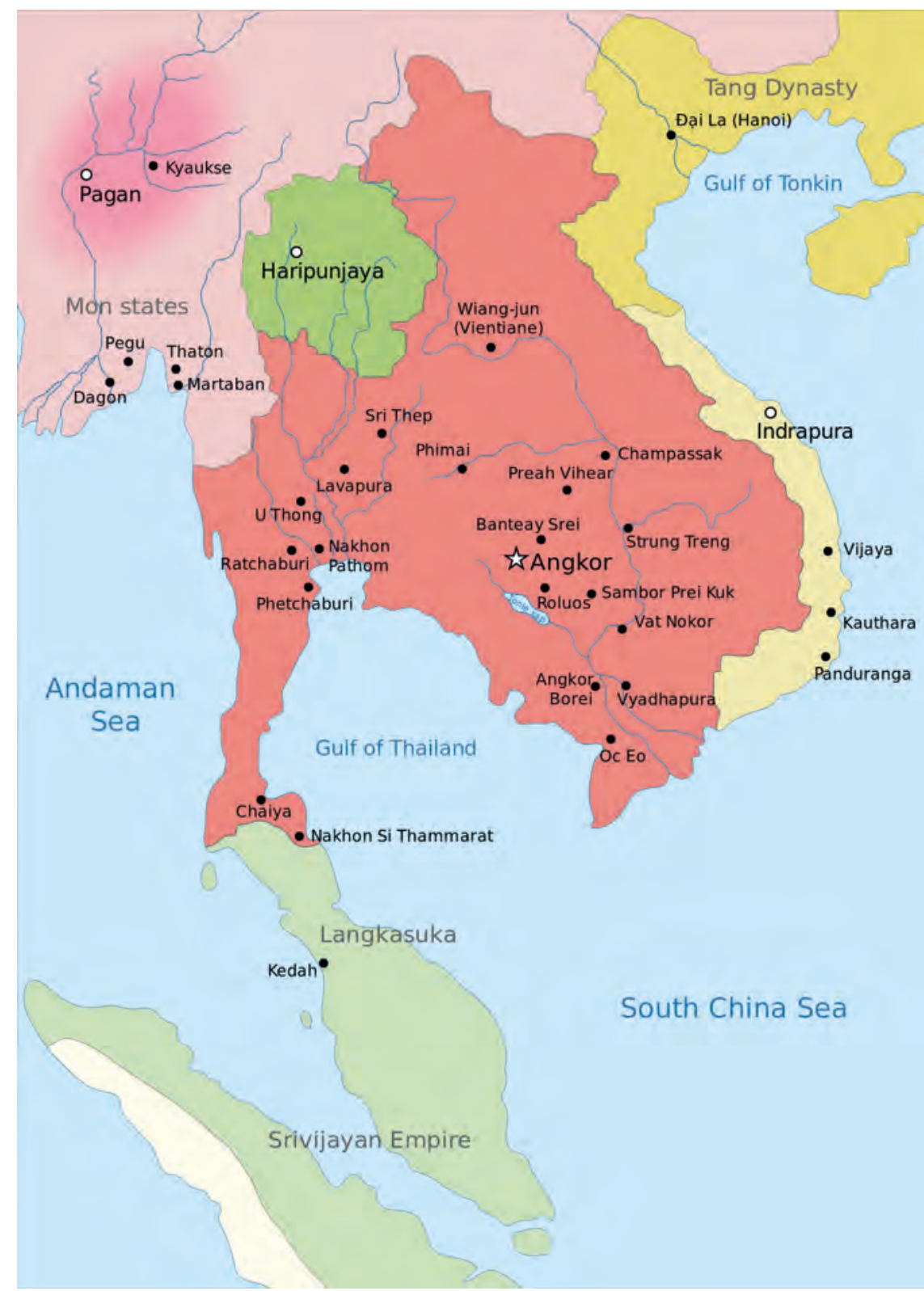

Map 15.3

The Khmer Empire, $900 \mathrm{CE}$

Jembezmamy, CC0 1.0

Chinese travellers, and so we lack detailed knowledge of how the Khmer state operated.

In the late thirteenth and early fourteenth centuries, the three larger states Angkor, Pagan, and Dai Viet - all collapsed. There may have been some common ecological problems. Strong provinces seceded from all three states. All three 
had inland capitals and suffered as maritime trade strengthened the relative position of coastal areas. Although Southeast Asia was spared the full brunt of Mongol expansionism (see chapter 17), there were Mongol incursions into the region and resulting Thai migrations into Burma and especially Angkor. By 1340 , there were twenty-three independent kingdoms in the region.

When political unification later occurred, we would eventually see three large states in Burma, Thailand (absorbing previously dominant Cambodia), and Vietnam. Each of these three states would fragment in the late sixteenth century, and even more briefly in the mid eighteenth, but in each case a strong central state would re-emerge. It is, of course, tempting to posit some common climatic cause of these similar processes of fragmentation and reconstitution. Relative to other regions of the world, periods of political fragmentation in Southeast Asia were brief.

Toungoo Burma had not only consolidated its Burmese territories by 1574 but also invaded deep into Siam; it seems to have over-extended itself and soon collapsed. As a result, the Burmese capital was moved northward by the next dynasty; Burmese cultural unity may not have been achieved if not for this move, for there had previously been tension between northern and southern Burmese. North and South Vietnam also united in this period. These regions had previously been governed separately but would thereafter generally be administered in concert. A process of Vietnamese cultural consolidation then unfolded (see below), though with significant regional differences.

Why did Thailand (Siam) rise relative to Cambodia? It may have had both better agricultural potential and better ports from which to pursue trade. Also, conflicts within the Khmer elite in Cambodia appear to have weakened the Khmer response. We have discussed many times how succession battles could undermine a state: Succession in Angkor was generally contested whereas it was usually peaceful elsewhere in Southeast Asia. The effect of Thai political dominance was to allow Thai cultural influence to spread inland, and the Vietnamese to exercise political and cultural authority in the Mekong Delta. The ethnic and political map of Southeast Asia might look quite different today if Thai states had not gained power at the expense of Khmer polities.

We can see a process of increased central control over this entire period. The earlier large states had tributary relations on their fringes, and ruled through either local elites or members of the royal family in provinces closer to the capital. The king directly ruled only a radius of 100 kilometres or so from the capital. There were also influential monasteries that performed some administrative tasks. Over time, but especially in the eighteenth century, states in Siam and Burma regularized taxes in the central zone and extended bureaucratic control outwards, diminishing the status of local elites. These bureaucracies were nevertheless quite limited in comparison to later colonial bureaucracies. Bureaucratization was less successful in Vietnam, where local loyalties remained strong. However, the Vietnamese state introduced civil service examinations on the Chinese model from the late fifteenth century. Though states gained power across the region, local elites maintained some role 
in government while prospering in trade: They thus tended to collaborate with rather than oppose the process of state building.

The early states were religiously diverse, with animism and different brands of Hinduism and Buddhism common - and Confucianism in Vietnam. There was also linguistic and ethnic diversity. With political consolidation came cultural consolidation. From the fifteenth century, and especially in the eighteenth century, there was considerable linguistic and religious consolidation (though some regional elites battled this). States encouraged a core language and religion (and were as elsewhere drawn into religious disputes), though they tolerated diversity, especially in newly conquered areas. Increased internal trade also served to spread cultural practices. Individuals adopted the culture of the capital because of snobbery or because it aided success in business or government. This process of consolidation should not be confused with nationalism (but rather with incentives to curry favour with the king) but paved the way for it.

Cultural consolidation was closely associated with the spread of literacy, which was in turn associated in most states with Theravada Buddhism but in (especially northern) Vietnam with Confucianism. The fact that it was Confucians rather than Buddhists that organized schools in Vietnam accentuated differences between Vietnam and the rest of Southeast Asia. While monks may have dominated teaching (except in Vietnam), literacy was also valued for trade and for dealing with the expanding government bureaucracy.

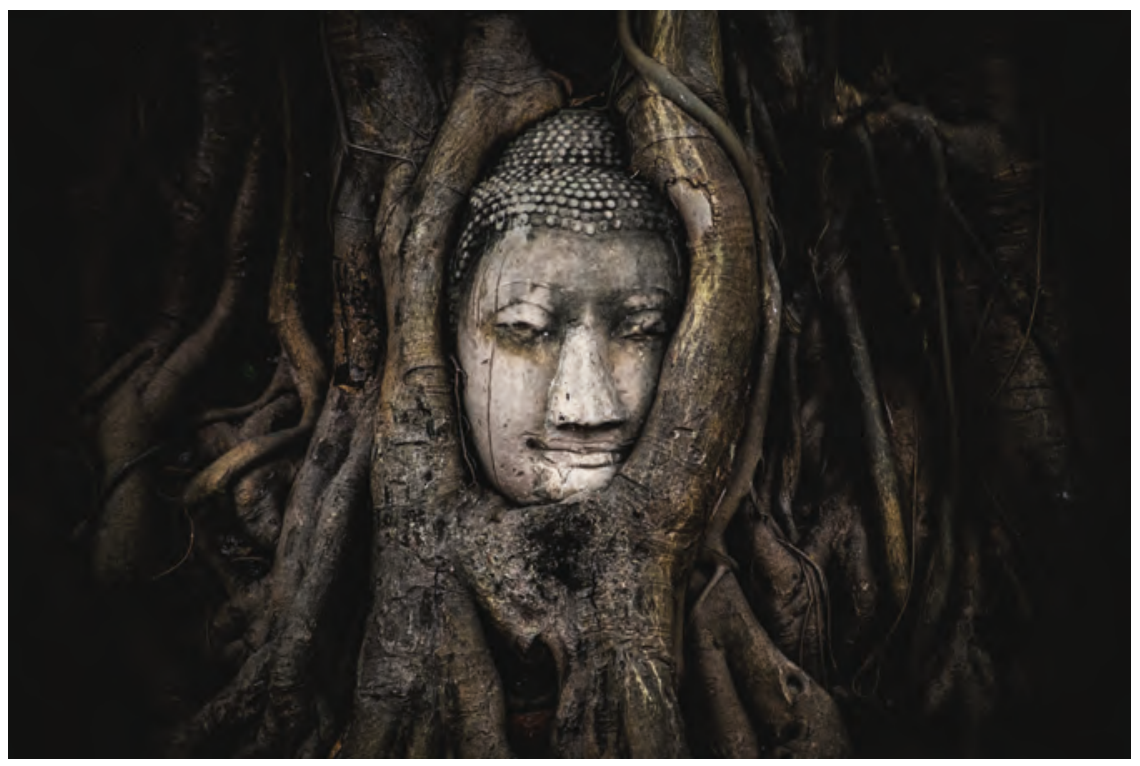

Illustration 15.19

The head of a sandstone Buddha statue nestled in the tree roots at Ayutthaya, capital of Siam from 1350 to 1767

Photograph by Siripatwongpin, CC BY-SA 4.0 
As literacy spread, a vernacular literature (that is, literature in the languages employed within each state) developed within each state, which reinforced the value of literacy and further spread cultural norms and especially a common language.

Religion became increasingly orthodox. Locals could read religious texts rather than follow local customs. They interacted with monks - and merchants who preached the faith as practised in the capital. There was increased income inequality, and many of the wealthy supported monasteries, which in turn spread the faith (and literacy). States exempted these monasteries from tax. Theravada Buddhism and neo-Confucianism gained state support and in turn justified the power of the state.

There were, though, also increased foreign influences associated with the increased trade. These included the arrival of Christian missionaries and contacts with Buddhists from Sri Lanka. Islamic merchants first arrived in the eighth century but only became prominent in the tenth century. Most were from India. They naturally concentrated where trade opportunities were greatest, and particularly on either side of the Strait of Melaka. Some local rulers adopted the faith, perhaps to facilitate trade. They tended, though, to allow multiple religions. Islam spread in the thirteenth century as locals embraced the religion of their rulers, and Muslims from other lands migrated to these states. Melaka became a powerful Muslim state in the fifteenth century by controlling the sea lanes through the Strait of Melaka, but gaining a reputation for dealing fairly with traders (the state had initially been Hindu-Buddhist and is discussed briefly below; the Malayan peninsula had before that generally been independent or subservient to more northern states). Melaka then sponsored Islamic missionaries throughout the region. Yet Islam would be adopted syncretically: Most obviously, locals (in Malaya and Indonesia) showed little interest in veiling women.

Trade expanded throughout the region from the fifteenth century (with a brief downturn in the late seventeenth century). This mainly but not entirely involved exporting raw materials (from mining, and increasingly rice and timber and raw cotton) and importing manufactured goods. Though this trade would later be associated with European colonization, it occurred for centuries during which Southeast Asian states were politically independent and saw advantages in this trade for themselves. Growing Chinese merchant communities managed much of the trade. As the Chinese settled Yunnan in southwest China (chapter 19), some trade moved overland, but most travelled by sea.

Rulers encouraged trade. They established currencies, and standardized weights and measures. Siam built some canals to connect the interior to trade opportunities. Some states reduced royal tolls. Peasants in the interior benefited from new markets for their goods. They may have responded by increasing output, especially as they came to desire foreign goods. Requirements that peasants pay taxes in money may have encouraged/forced peasants to produce for the market. Trade, in turn, monetized the economy, making it easier to raise taxes. 
Improved climate may have encouraged increases in both output and trade, but these seem to have expanded even as climatic conditions moderated. Political centralization may have reflected the increased agricultural surplus and perhaps especially the ability to tax increased trade flows. States based near the coast also benefited from new European firearms, which were much better than the older firearms (obtained from Muslim traders) available in the interior. There were advances in irrigation, which some historians have associated with migration of peoples from areas where irrigation was more common. There was also experimentation with different rice varieties for different regions. There was likely a tripling of the Vietnamese population between 1400 and 1800 and a doubling elsewhere in Southeast Asia.

Warfare was less important than elsewhere in the world due to the low population densities and the geographical barriers separating population centres. We have seen that Thais invaded Cambodia and the Burmese attacked Siam. Such incursions were rare - much rarer, for example, than the warfare that characterized Europe in this period. Rugged mountains separated the three primary centres of population: Burma, Thailand and Cambodia, and Vietnam. Once the Thais had come to dominate Cambodia, the region's natural barriers limited the opportunities for external warfare. Internally, though, the ability of rulers to mount large infantries and to destroy castles allowed and encouraged political centralization here as elsewhere.

There has been a tendency in historiography to over-stress borrowing from India and China. We should emphasize, then, that there was little overt political interference by outsiders from the time of Viet independence in 939 until later episodes of European colonization. For the most part, people native to the region pursued the political and economic developments traced above. Yet Vietnam certainly learned of civil service exams and other political practices from China, and Burma and Siam to some extent learned from Indian states, though there were few prosperous Buddhist states at the time to mimic. Chinese and Muslim traders played critical roles in linking the region to external markets.

\section{Southeast Asian islands}
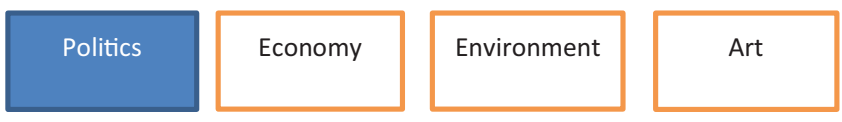

Culture

We have seen above that low population densities and geographical barriers limited the size of states on the mainland of Southeast Asia. This was even more the case on the islands that comprise the modern states of Indonesia and the Philippines. These generally had fragmented polities before the arrival of Europeans from the sixteenth century (chapter 19). Yet earlier states did grow in size as agricultural productivity expanded and as larger boats for travel between islands were developed. 
The most important state was the Srivijaya kingdom on Sumatra (670-1025, and then again into the thirteenth century). Srivijaya, as Melaka would later (see above), prospered by dominating the sea route through the Strait of Melaka. By bringing stability to this region, it may have encouraged traders to use the strait rather than the portage across the Malayan peninsula (chapter 9). Srivijaya, like Funan earlier, provisioned traders passing between China and India, and provided a safe place to await monsoon winds. Srivijaya occasionally expanded political control into both the Malay peninsula and the island of Java. Java, in particular, was able to produce a sizeable agricultural surplus, and thus states such as Srivijaya could tax agriculture as well as trade. Sumatra itself was the source of the spice camphor as well as minerals and wood products. There is very little surviving documentary evidence for Srivijaya, and so we know little about how it governed its provinces. The Chola kingdom from Tamil Nadu in southern India attacked Sumatra in the late eleventh century and disrupted trade for a while. Chola was the only Indian state that ever launched military

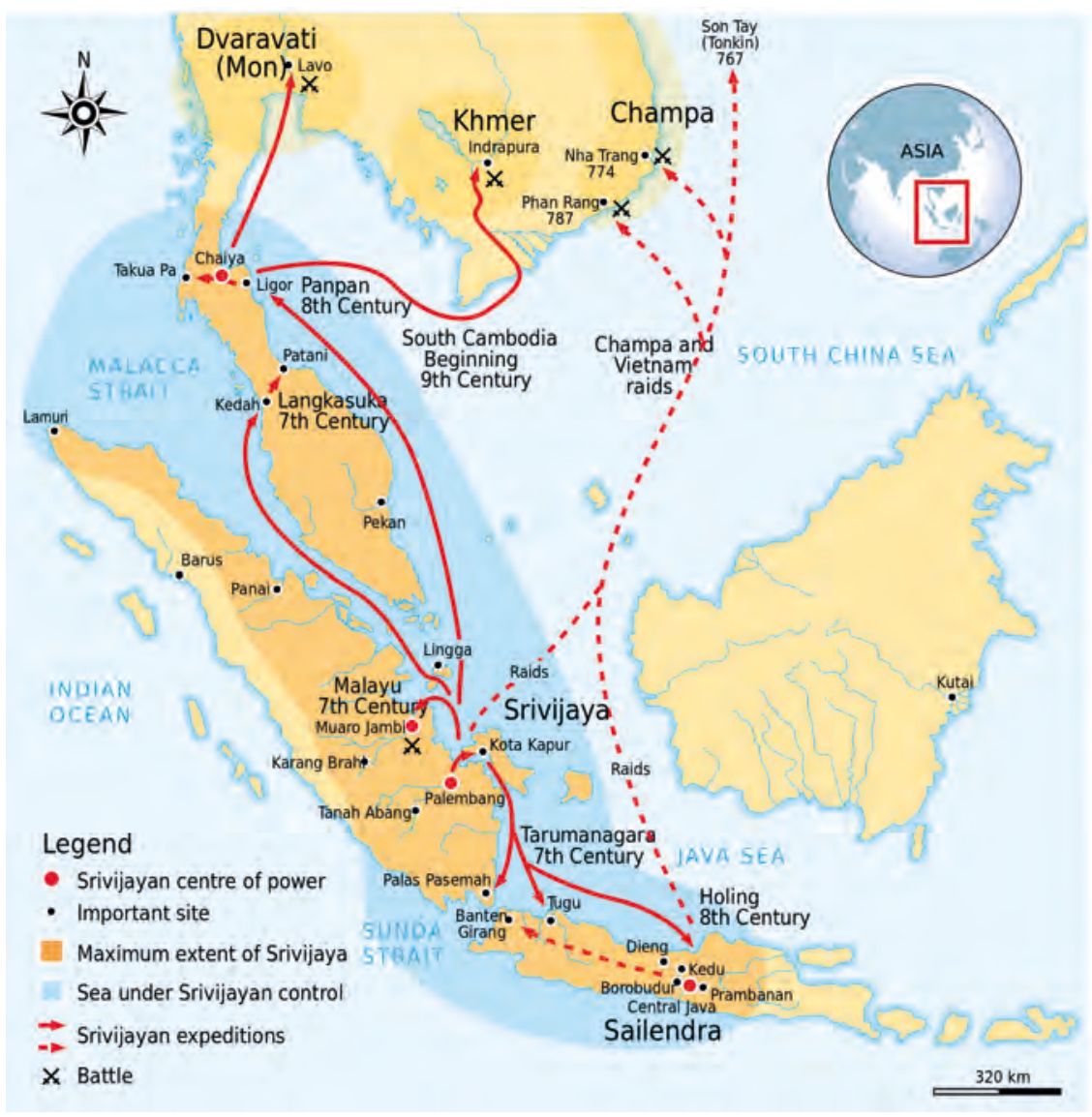

Map 15.4

Srivijaya, 8th century CE

Map by Gunawan Kartapranata, CC BY-SA 3.0 
expeditions into any part of Southeast Asia. Though the motives for this invasion are unclear, it is likely that the riches of Srivijaya and its attempts to control the Straits of Melaka both provided incentives. Srivijaya would recover from the Chola attacks and govern into the thirteenth century. The Srivijaya state was generally Buddhist and spread the Buddhist religion - though few traces of this remain in the lands that it ruled (Muslim states later conquered the area).

Srivijaya's primary capital, Palembang, is today a city of some 2 million surrounded by Sumatran rain forest (a later capital, Jambi, is a smallish city today of about 500,000). There is little sign that it once ruled a vast area. As the kingdom declined, one of its princes would establish a state across the strait in Melaka (and claim to be descended from Alexander the Great, as well as Sumatran, Javan, and Indian royalty). This place would prove even better suited to dominating the trade of the strait - though Melaka too after its heyday as capital of a Muslim state would decline over time to become a small provincial town (trade through the strait today concentrates in Singapore).

In addition to facilitating trade between China and the west, Sumatra - and Javanese polities to the east - benefited from trade for spices. Incredibly, at the time, three of the most desired spices in the world - cloves, nutmeg, and mace grew only on different tiny islands in eastern Indonesia. Cloves came from a handful of islands, including Ternate and Tidore (much smaller than New York's Manhattan), and nutmeg and mace from the same plant - mace comes from the outer shell of the nutmeg - on ten volcanic Banda Islands to the south of them. The fact that two spices with entirely different flavours came from one plant, and that this plant only grew on ten islands in the entire world - though later experience would show that it could grow in many places - is remarkable. For many centuries, these tiny islands supplied the entire Eurasian market for these three important spices. Though local political organization in these islands seems to have been simple, with councils of elders making decisions, these islands successfully prevented the transplantation of these plants until the arrival of Europeans from the sixteenth century. Yet the islanders had to share the profits from this monopoly with the various merchants who carried the spices to distant markets.

It is not clear when spice exports from these islands began. There is archaeological evidence of trade from the second century BCE, and documentary evidence of exports to China from the sixth century CE. Malay sailors (chapter 9) seem to have traded with the islands in the early centuries CE, and Arab traders certainly brought nutmeg to Constantinople in the sixth century. Yet there is a reference to the nutmeg tree by the Roman Pliny the Elder in the first century, some documentary references to cloves in China in the third century BCE, and a tantalizing archaeological discovery of traces of cloves in Syria in the seventeenth century BCE. The opening of traffic through the Strait of Melaka accelerated spice exports by bringing Chinese and Indian (and later Muslim) merchants in contact with Javan and Sumatran polities. Merchants from the Spice Islands brought the spices to Java at the time. In the Middle Ages, at 


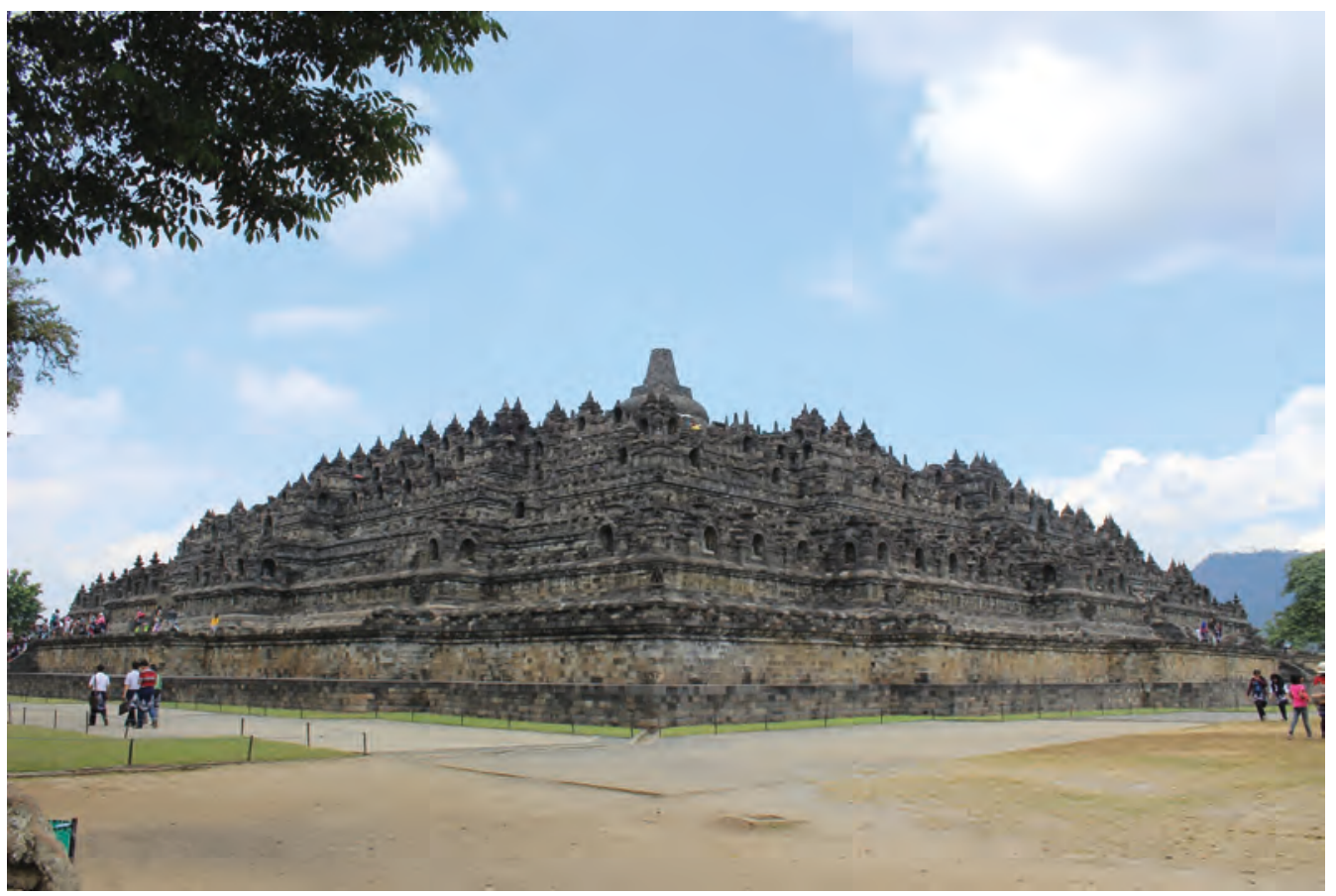

Illustration 15.20

Borobudur

least, merchants carried the spices to Java on the monsoon winds, and so foreign traders coming in the opposite direction mistakenly thought for centuries that the spices were local to Java. In the fourteenth century, one could trade a kilogram of nutmeg for two cows in Europe: This high price reflected both the cost of transporting the spice halfway around the world and the monopolized production of nutmeg.

We spoke at some length of Angkor Wat above. Hindu and Buddhist temples were built on Indonesian islands from the eighth century as well. The most notable of these, the Buddhist Borobudur in central Java, likely was constructed over a period of some seventy-five years beginning in the eighth century. The Mataram kingdom, a vassal state of Srivijaya, most likely financed it. The excellence of its construction is particularly notable given that it appears so early in the history of temple construction on Java. The carvings on the temple trace the several stages of enlightenment of the Buddha, but relate these to scenes of everyday life on Java (employing gentler poses and expressions than was common in India). The viewer progresses from the Buddha's early life at the lower entrance toward enlightenment at the top. Art historians often hail Borobudur as the most extensive unified work of art in the world. It is also the largest Buddhist temple in the world. It has over 2,500 relief panels and over 500 Buddha statues on a series of nine platforms topped by a dome. 
Postscript: Like Angkor Wat, Borobudur was abandoned. This appears to have happened centuries before Islam came to dominate Java. Jungle and volcanic ash would cover the temple. Yet locals would remember its existence. Europeans would uncover it from the eighteenth century. It is the most-visited tourist site in contemporary Indonesia.

\section{Questions}

1. Diagram the influences on and effects of either merchant or artisan guilds.

2. Why do we not see a powerful and independent religious authority like the Catholic Church elsewhere in the world?

3. How might you apply evolutionary analysis to the history of the printing press?

4. Why did the Crusades happen (only) when they did?

5. Is there one criterion by which we should evaluate the performance of empires?

6. Is it surprising that the most extensive Buddhist and Hindu temple complexes in the world are in Southeast Asia? If so, why?

7. What regional comparisons could you draw from the material in this chapter?

8. The Tokugawa displaced the Hideyoshi family rule, just as the Han had replaced the Qin and the Tang the Sui. Do you think it is just a coincidence that when a large state is unified after centuries of disunity, the unifiers rule for mere decades before power is passed to a dynasty that lasts much longer?

\section{Readings}

Bisson, Thomas N. 2008. The Crisis of the Twelfth Century: Power, Lordship, and the Origins of European Government. Princeton University Press. [Stresses how power was exercised first by local lords then by kings. Emphasizes the use of violence.]

Cobb, Paul M. 2014. The Race for Paradise: An Islamic History of the Crusades. New York: Oxford University Press.

Duindam, Jeroen. 2015. Dynasties: A Global History of Power 1300-1800. Cambridge University Press. [Examines the challenges of succession in global comparison.]

Ebrey, Patricia. "China as a Contrasting Case: Bureaucracy and Empire in Song China," in Peter Crooks and Timothy H. Parsons, eds., Empires and Bureaucracy in World History: From Late Antiquity to the Twentieth Century. Cambridge University Press, pp. 31-53.

Gilbert, Marc Jason. 2017. South Asia in World History. Oxford University Press. 
Goetzmann, William N. 2016. Money Changes Everything: How Finance Made Civilization Possible. Princeton University Press.

Goldthwaite, Richard A. 2009. The Economy of Renaissance Florence. Baltimore, MD: Johns Hopkins University Press. [Stresses the role of institutions and entrepreneurship. See also Samuel Y. Edgerton, The Mirror, the Window, and the Telescope: How Renaissance Linear Perspective Changed Our Vision of the Universe, Ithaca, NY: Cornell University Press, 2009.]

Haldén, Peter. 2011. Stability Without Statehood; Lessons from Europe's History Before the Sovereign State. New York: Palgrave.

Langdon, John, and James Masschaele. 2005. "Commercial Activity and Population Growth in Medieval England." Past and Present 190:1, 35-82. [Explores the development of milling, among other things.]

Lieberman, Victor. 2003 and 2009. Strange Parallels: Southeast Asia in Global Context, c. 800-1830. 2 vols. Cambridge University Press. [This work compares the experiences of other Eurasian regions with that of Southeast Asia.]

Ludden, David. 2013. India and South Asia: A Short History. London: Oneworld Publishers.

Murphey, Rhoads, with Kristin Stapleton. 2016. A History of Asia. 7th ed. New York: Routledge.

Von Glahn, Richard. 2016. The Economic History of China: From Antiquity to the Nineteenth Century. Cambridge University Press.

Wilson, Peter H. 2016. The Holy Roman Empire: A Thousand Years of Europe's History. London: Allen Lane. [Argues that consensual government within a confederation has advantages.]

Readers may find much useful information in the Medieval Sourcebook at: http:// sourcebooks.fordham.edu/Halsall/sbook.asp. 


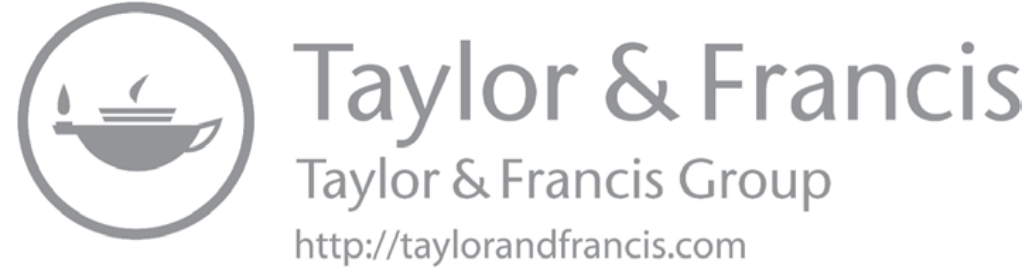




\section{Regional}

\section{developments}

\section{Polynesia, the Americas, and Africa}

\section{Guiding questions}

How did the various processes outlined in chapter 14 (but especially political consolidation) unfold across the Pacific, the Americas, and Africa?

As in chapter 15, there are a set of subsidiary questions of particular regional importance:

How, why, and when did Polynesians discover and settle a set of islands across the Pacific Ocean? What sort of societies did they establish, and how similar were these across islands?

How did the different natural environments of the Americas and Africa influence political, economic and other developments there?

More generally, what similarities or differences can we identify in societies that had little contact with other regions of the world?

Relationship to other chapters: Chapter 16 joins chapter 15 in fleshing out our understanding of the trends outlined in chapter 14.This chapter is most obviously important for treating regions that have received less treatment in preceding chapters than Eurasia. In the case of Polynesia, we address human expansion into a new area, picking up the story of the human settlement of the globe from chapters 3 and 7. In the case of Africa 
and the Americas, we have been limited by the lack of written records for earlier time periods. We can say much more about these continents in this chapter. The expanded geographical coverage that this chapter provides increases our scope for historical comparison. We wonder, for example, about the degree to which the relative lack of draft animals affected political and economic developments in the Americas. We also examine the influences on and effects of much lower population densities in Africa than in most of Eurasia. More generally, we can explore in this chapter how regions with limited or no contact with Eurasia developed in ways that were similar to or different from Eurasia. Note that connections across all regions of the world become much tighter after 1500, as we shall see in chapter 19, and so this is our last opportunity to compare large societies that are not in direct contact. This chapter will naturally inform later discussions of the Pacific, Americas, and Asia, but also through comparison strengthens our understanding of more general historical processes.

In both the Americas and Africa during this period, we trace political consolidation, increased agricultural output, population growth, and monument building. As we saw in chapter 14, these trends are visible in much of the world in this time period. We observe similar trends in Polynesia, but they are overshadowed by the dramatic geographical expansion of human populations into previously unpopulated islands across a vast ocean. It is during this period that almost every habitable point on the earth's surface is settled. Though the three regions addressed in this chapter had no connection with each other until the very end of this period (when crops were exchanged between Africa and the Americas), we will trace significant trade and cultural interactions within these regions. We will also explore the relationship between humans and the environment in our discussion of Easter Island.

\section{Polynesian settlement}

Population (Migration)
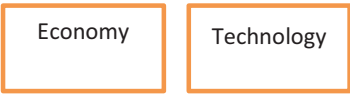

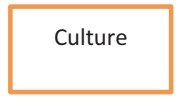

Social Structure

If one's focus is entirely on the development of modern industrialized states with vast bureaucracies, then Polynesia may seem unimportant. However, one of the unique characteristics of humanity is that it has settled almost the 
entire planet. The last part of the planet humans settled (excluding some scientific stations in the Antarctic) comprised the small islands sprinkled across the Pacific Ocean. The distances involved are immense: It is 10,000 kilometres from New Guinea to Easter Island, and 8,500 kilometres from Hawaii to southern New Zealand. The Pacific Ocean is larger than the Earth's entire landmass and contains the vast majority of its islands. The settlement of this area by Polynesian seafarers in double-hulled sailing canoes relying on stars and clouds and birds for navigation is an amazing feat of human ingenuity.

Europeans when they first arrived in the Pacific thought the natives were primitive: They had no metalwork, no writing system, and no compasses or charts to guide them across the oceans. Why would they? Small islands spread across vast distances did not lend themselves to the large states that were associated with the development of writing. Though there was extensive trade between some islands, this trade involved a small number of goods over a small number of routes; extensive record-keeping was not necessary. Much of this trade likely involved networks of gift exchange governed as much by cultural expectations as economic calculation; such networks in Melanesia spanned hundreds of kilometres. Societies, of course, need metal to engage in metalwork. Though generally unimpressed by Polynesian economy and politics, European explorers were very impressed with the islander's boats: These are often called canoes, but they could be large enough to carry a hundred people, and were built to withstand rough oceans. They employed a lateen sail likely derived from that of Malay sailors during the classical period (chapter 9).

People from somewhere in Southeast Asia - Taiwan seems likely - seem to have arrived by sailing canoe on the northern coast of New Guinea by 5000 все. Over the next millennia, they gradually inhabited nearby island chains such

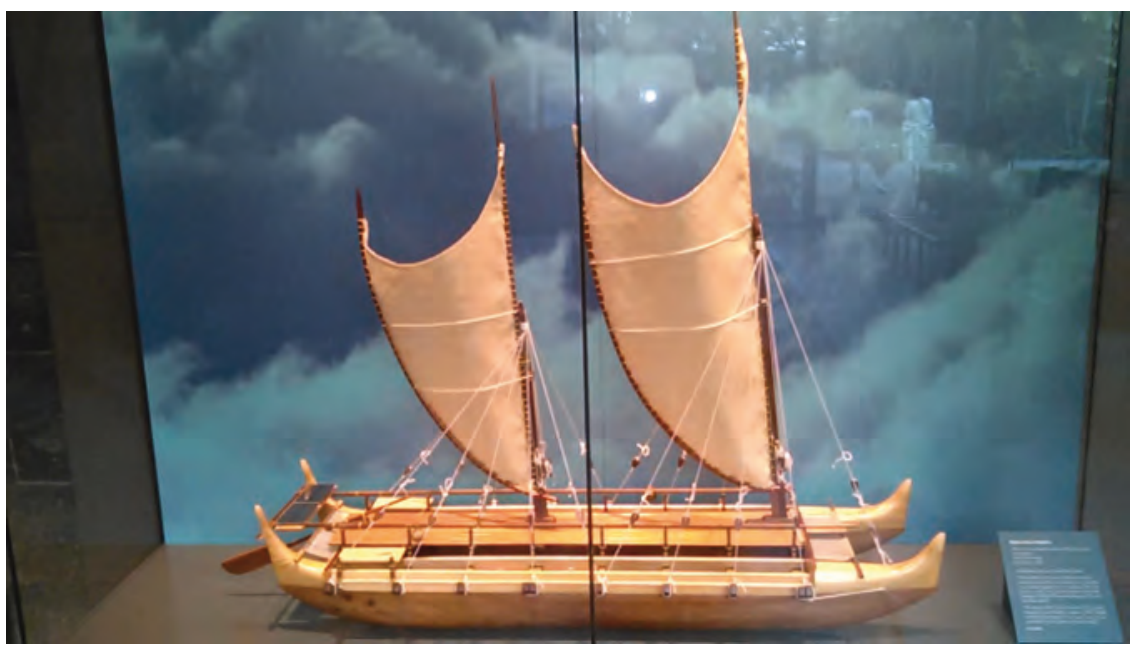

Illustration 16.1

Model of Polynesian canoe, Bishop Museum, Honolulu

Author 
as the Bismarcks and Solomons. These islands are close enough together that sailors could reach them without being out of sight of land for long. Sailors had reached Vanuatu by 1500 BCE, Fiji by 1300, Tonga by 1000, and Samoa about 800 BCE. (Micronesian islands to the north may have been settled instead from the Philippines.) Trade links occurred throughout this vast region: Settlers remained connected to the islands of their ancestors. (The westernmost islands traded with Asia too.) Obsidian and pottery and stone tools were carried long distances. Settlers carried pigs, chickens, and the seeds for the crops domesticated in New Guinea; they supplemented their diet by fishing. Settlers also took - and likely traded - a distinctive "Lapita" pottery.

Scholars once thought that Polynesians had pushed farther north and east soon after reaching Samoa by 800 вCE. However, more recent archaeological studies suggest that there may have been a gap in exploration between 800 вСЕ when Samoa was reached and $800 \mathrm{CE}$ when Polynesians pushed farther into the Pacific. It had been thought, for example, that Easter Island was settled as early as $400 \mathrm{CE}$, but it is now considered likely that this occurred after 1200 . If the latter timing is correct, then the Cooks, Society (Tahiti), Marquesas, and Hawaii were likely first reached between 800 and $1000 \mathrm{CE}$, and New Zealand around 1100. Whatever the dates, New Zealand was the last sizeable habitable area on the planet that humans settled.

Why would exploration have resumed after such a significant gap in time? One possibility is population pressure: As we have seen, the population was rising in many parts of the world at about this time. Yet travelling long distances in boats that could carry only a hundred people would be a challenging solution to population pressure. We might speculate that there was political con-

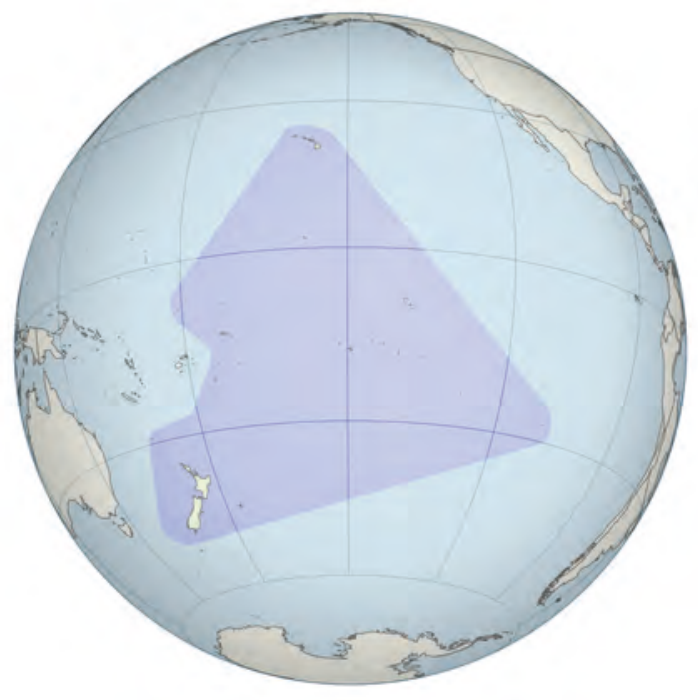

Illustration 16.2

The Polynesian triangle flict: Losing groups might choose or be forced to emigrate. We shall see below that island societies became highly stratified: It is possible that disadvantaged people decided to migrate, but these must then have had the ability to finance such a voyage. Another possibility is that there was a critical technological development. Polynesians may have developed the double-hulled canoe at this time. As noted above, this was an impressive craft capable of transporting many people and their supplies long distances in challenging conditions.

As settlement expanded beyond Samoa to eastern Polynesia, the distances became larger and navigation more difficult. Lacking compasses and astrolabes, the Polynesians could not establish latitude or longitude. However, they knew 


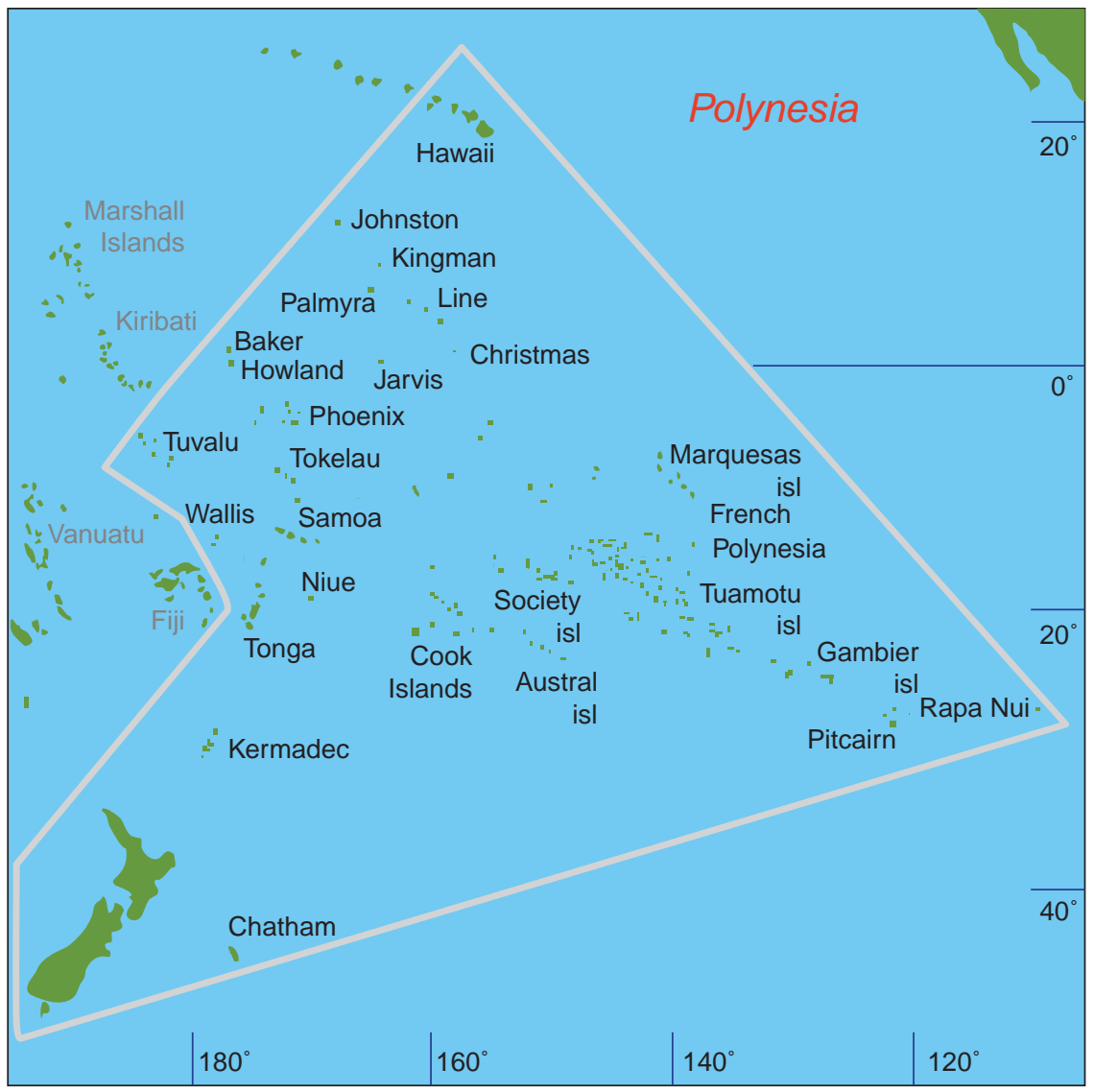

Map 16.1

Polynesia

the stars well and could use these both to determine their direction of travel and to achieve an estimate of latitude. Clouds gather over land and take on a different form. Birds nest on land, and fish prefer the shallows that often surround land. Even small landmasses perturb ocean waves. Careful observation of clouds and birds and even fish and waves could guide the sailor to land across distances of at least 50 kilometres. Ocean currents provided yet another marker for navigation. If the sailor was seeking an archipelago (that is, a group of many islands), they could miscalculate by hundreds of kilometres and still arrive safely.

The winds within some 20 degrees of latitude of the equator generally blow from east to west. Sailors heading eastward into the ocean could then be confident of being able to sail home again. However, Hawaii is too far north and New Zealand too far south: Sailors heading to these places had to master new winds. Sailors heading to Hawaii also had to learn the stars of the northern hemisphere. Easter Island, hundreds of kilometres from the nearest island, was a much harder target to hit than an archipelago. 
The alternative to deliberate navigation is accident: Storms might carry boats and their passengers to remote islands hitherto undiscovered. Boats do from time to time drift in this way, to be sure. Moreover, it is reasonable to wonder why Polynesians would ever have purposely looked for Hawaii or Tahiti or Easter Island, islands thousands of kilometres from anywhere else. Nevertheless, these drifting canoe discoveries would have had to be repeated on purpose, unless the drifting canoe itself had contained women as well as men and all of the seeds and animals that accompanied Polynesians to other islands. Even if accident played some role in some discoveries, exercises in deliberate sailing had to follow. We might draw a comparison here with the accidental Viking discoveries of Greenland and Newfoundland: These were followed by deliberate attempts to reach these islands (chapter 13).

Admittedly, some seeds might have blown with the wind. This may indeed be how some plants from South America such as sweet potatoes reached some Polynesian Islands - though recent genetic analysis suggests that eastern Polynesians interacted with people in South America. Chickens, though, had to be carried purposely between islands. It is clear that Tahiti and Hawaii did trade with other islands, at least for a while (see below).

\section{Challenges facing agents: Explorers}

The explorer needs somehow to finance their exploration. This usually at least means accumulating supplies of food and tools to take along. In the Pacific, there also had to be a boat that was seaworthy - but this then provided a means of carrying people, food, and supplies long distances. On land, while early human explorers walked, later explorers utilized draft animals.

Explorers will generally wish to provide for the possibility of return. Except if banished (and maybe even then) they will likely envision some trade with their homeland (explorations that fail to do so may be forgotten). On land, return generally requires just careful attention to the route followed. At sea, the explorer must worry about winds and currents.

Explorers necessarily face the unknown. There may be hostile humans or animals or diseases or not enough food in the new land. The explorers of the Pacific did not have to worry about the first three, and generally not the fourth, but they could not know that in advance.

The Pacific Islanders fished. Their art tended to focus on fish and birds, just as that of hunters tended to focus on animals (chapter 3). The Hawaiians even constructed fishponds into which small fish could swim, but larger fish could not swim out as they grew. The Pacific Islanders also practised agriculture, growing taro, yams, bananas, breadfruit, and coconuts (and sweet potatoes in the east). Europeans would later prize breadfruit as a productive tropical crop and transplant it to the Caribbean to feed slave populations. By combining fishing and agriculture, the islands could support large populations - not anywhere near as large as those in Eurasia or even Africa and the Americas, but 
large enough for chiefdoms, armies, public works, priests, social stratification, and artisans. Hawaii alone may have had half a million people by the time of European contact.

Chiefdoms were the dominant political form; chiefs tended to come to view themselves as semi-divine. Chiefs had advisers. On the largest islands at least, societies were highly stratified. Marriage was often forbidden between members of different classes: In Tahiti, children of mixed-class parentage were killed. Chiefly palaces were built of stone on even some smallish islands such as Pohnpei in the Caroline Islands. The chiefly elite wore special clothes and ate special foods, and contact with commoners was severely limited. Religion tended to be polytheistic and diverse though gods of agriculture and war were common; temples (sometimes open-air) were built for religious ceremonies. One common element of Polynesian religions was the idea that specific objects or behaviours were "taboo" - a word that would be absorbed into English and other languages.

As elsewhere in the world, social stratification in general was accompanied by gender stratification in particular. In Hawaii, men and women could not eat together, or prepare food for the other sex. Women could not eat many foods, including pork, bananas, coconuts, and certain types of fish. However, both unmarried women and men were generally allowed sexual freedom. Polygamy was common throughout Polynesia; unusually, polyandry - where women can take multiple husbands - was practised in the Marquesas.

When Europeans arrived in Polynesia, they found a common culture across distant islands but no evidence of recent trade between Hawaii or Tahiti and places such as Fiji. We do not know how or why trade between the more distant islands stopped. Perhaps after a while, few goods were desirable but not available locally such that they could bear the cost of transport over such long distance. (Note that even those engaged in ceremonial "gift exchange" will evaluate whether a trip is worthwhile.) There was little in the way of metals on the islands that might have encouraged trade, and most islands grew the same crops and caught similar fish. There certainly had at one time been trade between Tahiti and Hawaii: Oral tradition speaks of trade between the two, and both fishing technology and words seem to have moved between the two. Basalt from the Tuamotus is found in Hawaii, thousands of kilometres away. There was undoubtedly regular trade among closer islands in the western Pacific: Axes, pottery, shells, and foods moved between islands. There was even intermarriage between the peoples of Tonga, Samoa, and Fiji.

Polynesians achieved impressive societal outcomes within the ecological niche that they inhabited. We should not criticize them for lacking large landmasses or deposits of iron ore. It is useful for the reader to engage a different type of speculation here: If you had lived during this time period would you rather be a Polynesian or a citizen of some Eurasian empire or European feudal manor? There is, as with most speculations, no right answer, but the question can guide an appreciation that a society might be valued along many dimensions. 


\section{Easter Island}

Easter Island (Rapa Nui) has been the subject of intense historical speculation. When Europeans first arrived, they noticed two things: the many large monolithic sculptures of human heads (Moai) that had been there for centuries, and the clear evidence that the island had once had much more extensive forest cover. It was reasonable to hypothesize that the island had suffered a civilizational decline, and that some environmental crisis may have precipitated this. The Islanders had cut down their trees, in part to move their monolithic sculptures into place, suffered soil erosion as a result, and then experienced a Malthusian population decline and decreased social complexity.

If so, the Easter Islanders would not be the only humans to damage their environment through short-sighted practices. Nevertheless, destruction of a local ecosystem is rare (at least before modern times), and many have wondered if the Easter Islanders were quite so short-sighted. The rats that travelled with them may have attacked the forest: The brush that remains has seeds that these rats will not eat. The population may not have in fact declined much: There is a debate about whether it was ever much higher than three or four thousand, and the population may have still been about that level when Europeans arrived. We do not know for sure since European arrival was devastating: Many

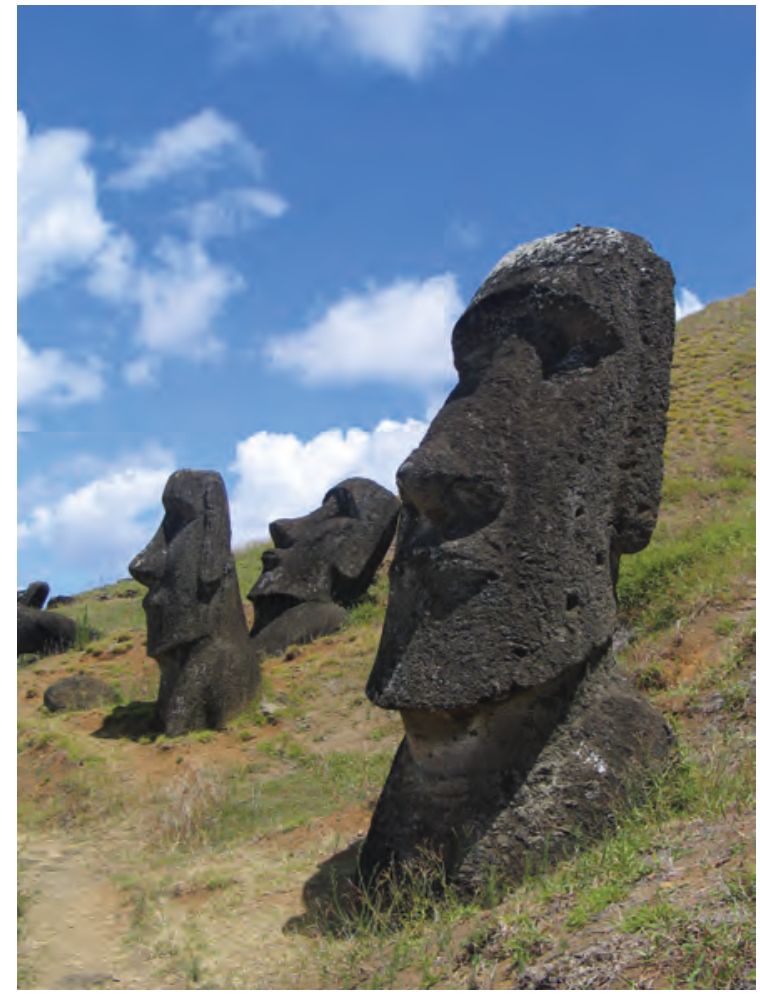

Illustration 16.3

Moai at Rano Raraku, Easter Island islanders died of disease, and others were captured for plantation labour elsewhere. The soil on the island had never been particularly good, rainfall is barely adequate, and Easter Island is too far south for some of Polynesia's staples such as breadfruit. The island may have had limited agricultural potential from the outset, though soil erosion surely made this worse. As to why the Islanders stopped building giant heads - and even destroyed some that they had created - societal collapse is but one possible explanation. History is full of peoples that build large structures or statues for centuries and then stop. The heads appear to be arranged to capture critical astronomical movements: It could be that some design plan had been fulfilled. The heads may have served as homes for ancestral spirits; a change in religious practices may have discouraged monolith construction. In any case, these monoliths were impressive both technically and aesthetically: Easter Islanders only had stone tools but carved broad 
but expressive features onto their monoliths - and then moved these huge sculptures long distances.

\section{Australia (No; it's not part of Polynesia, but it is in the same hemisphere)}

Though Pacific Islanders were agriculturalists, Australian peoples remained hunters and gatherers throughout tens of thousands of years of habitation. However, they did pursue "firestick agriculture," which involved burning forests to generate savanna suitable for animals such as kangaroo that they hunted. There were likely only a few hundred thousand people in Australia before the arrival of Europeans. Why was there no agriculture before European arrival? The aboriginal Australians must have known of agriculture. Their neighbours in New Guinea and the Torres Strait (to whom they are related genetically) had turned to farming several thousand years BCE. There was some limited trade with both Southeast Asia and New Guinea: Australian spears and shells traded north, while some iron axes traded south to a continent that otherwise had no metal tools. However, the tropical crops of New Guinea or Southeast Asia were not well suited to temperate Australia. Australia lacked indigenous crops suitable for cultivation: Europeans would later farm with seeds brought from the temperate zone of the northern hemisphere. Even with such seeds, much of Australia remains unsuitable for agriculture to this day. Moreover, Australia lacked the animals - horses, sheep, and cattle - employed today on the vast ranches that cover much of the continent.

It would be a mistake to imagine that aboriginal life was unchanging over this extended period. Like peoples elsewhere, they adjusted to dramatic changes

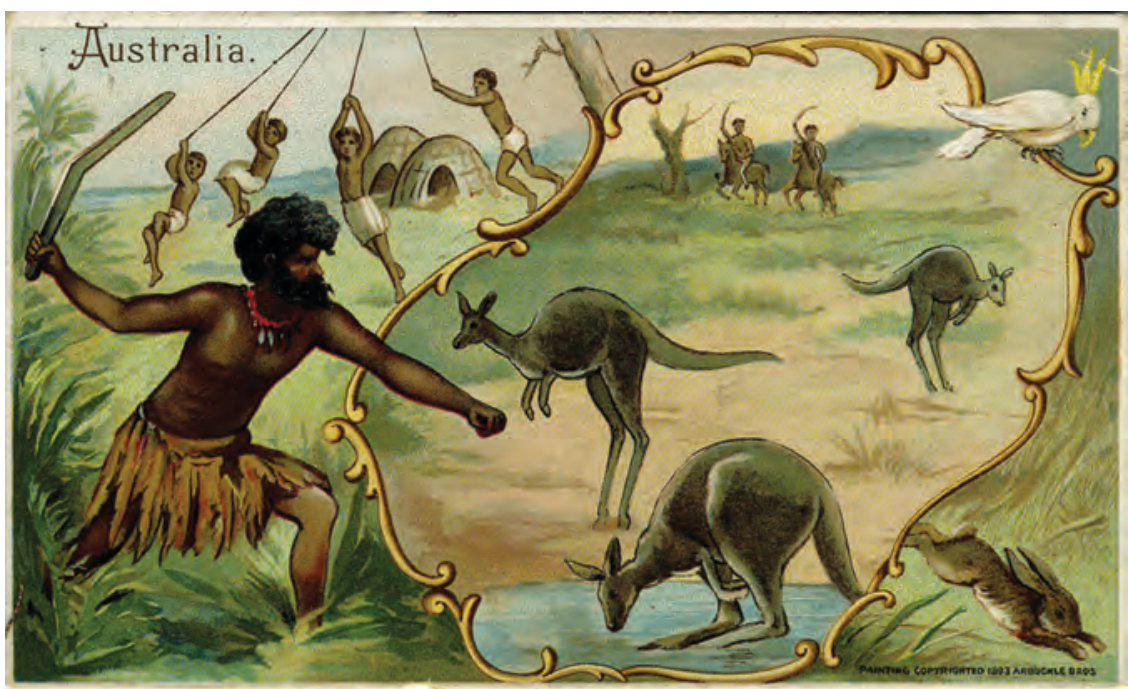

Illustration 16.4

19th-century trading card depicting aboriginal use of a boomerang 
in climate over time. They developed digging sticks and spears (and spearthrowers) for hunting. They may not have been the only people to invent the boomerang, but they made it a crucial part of their hunting arsenal: There is cave art that seems to show boomerangs some 30,000 years ago. They domesticated the dingo (a type of dog). They may have played a role in the extinction of megafauna. Two aboriginal groups built elaborate eel ponds in southeastern Australia. There was extensive trade within Australia: Oyster shells are found 1,600 kilometres from their source. Religious practices were diverse but mostly animistic. Analysis of rock art suggests important changes in cultural practices over time. There is also some evidence of increased social stratification.

\section{The Americas}

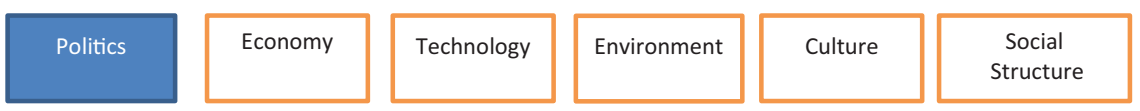

The rather rapid conquest of the Americas by Europeans from the fifteenth century has led many to assume that societies in the Americas were far less "advanced" than those of Eurasia. We shall see in chapter 19 that epidemic disease played a critical role in European conquest: It is hardly surprising that societies facing mortality rates of 50 to 90 per cent put up feeble resistance. Moreover, we shall see that Europeans often took advantage of regional conflicts within empires to topple these; they then built their new administrations on the base of previous imperial bureaucracies. European conquest of the Aztecs and Incas resembles in this way the overthrow of many Eurasian empires in history: All empires have internal tensions that a new force that grows or appears on its borders can utilize.

We should stress that the pre-contact Americas had productive agriculture, extensive trade links, large states, and large cities. The Aztecs of Mesoamerica were not alone in the Americas in having a writing system - though the Incas of South America ran by far the largest state in human history that did not employ a writing system. The Spanish were impressed by some characteristics of the societies they conquered, such as the cleanliness and order of the Aztec capital of Tenochtitlan.

There were, to be sure, some significant differences between the western and eastern hemispheres. The Americas lacked draft animals. The one exception was the camel: The llama and alpaca of South America are members of the camel family. Though camels served an important niche in desert areas of Eurasia, horses and other animals were much preferred elsewhere in Eurasia. Llamas were far slower than horses and notoriously stubborn. The rugged terrain of the Andes further limited the usefulness of camels in South America. Still, historians estimate that the Inca employed some 100,000 llamas as draft animals. Llamas unlike their Eurasian cousins - could only carry about 30 kilograms: They could not move adults but could transport various goods. Llamas were also used in 
war: Some 15,000 llamas were abandoned after one battle with the Spanish. In North America, there were no draft animals at all. As a result, wheels were rare throughout the Americas - some have even argued that they were unknown. Overland transport must then have been far more expensive than in most of Eurasia (though we have seen that camel caravans could engage in profitable trade across both the Sahara and Central Asia). Agricultural productivity was likely far lower than in societies where animals could pull ploughs and produce manure.

The Americas had also not developed iron technology, and there was little if any use of bronze. This would also have limited agricultural productivity we have seen that iron especially was associated with significant advances in Eurasian and African agriculture. Of course, the lack of both draft animals and metal would put American armies at a severe technological disadvantage militarily once Europeans arrived.

The crops of the Americas were entirely different from those of the eastern hemisphere. The eastern crops may have had a slight advantage on average in the food value provided for a particular work effort. Yet corn has spread worldwide - and today more corn is grown in the world than either wheat or rice (though some corn is used for ethanol production rather than food). The potato has been adopted extensively: It may indeed provide the easiest source of protein of any crop in the world. South American cotton has largely supplanted other strains worldwide. While imported crops would revolutionize agriculture in parts of the Americas, plants from the Americas would have a similar impact elsewhere (see chapter 19).

Though there was considerable trade in the Americas, there was not the extensive intercontinental trade that characterized the eastern hemisphere. There were a smaller number of civilizational centres. There was limited if any contact between South and North America - though corn somehow reached Peru by 1500 BCE. We have often seen in this book how Eurasian societies borrowed ideas from each other. This process occurred to a much lesser extent in the Americas.

Agriculture had emerged a little later in the Americas. Yet the Americas had been settled tens of thousands of years later than Eurasia. If farming was a response to population pressure, and requires a deep familiarity with local wild plants, then we might have expected agriculture to have emerged far later in the Americas. The broad similarity in timing may again suggest that there is some climatic process at work in encouraging people to adopt agriculture (chapter 4). Given the size of cities and states in the Americas, it is clear that American agriculture - despite its later start and different crops and lack of iron or draft animals - was able to produce a substantial surplus.

\section{Mesoamerica}

There is a long history of cities and states in Mesoamerica, the region comprising modern Mexico and the northern parts of Central America. We discussed 


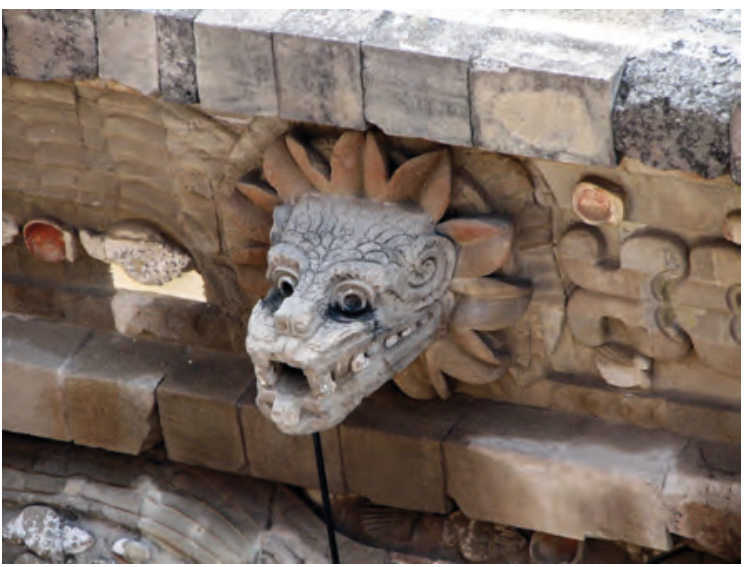

Illustration 16.5

Bust of Quetzalcoatl at Teotihuacan james, CC BY 2.5 ES the Maya in chapter 7, and Teotihuacan in chapter 9. Teotihuacan - located just northeast of modern-day Mexico City was probably the fifth largest city in the world in 500 CE. Teotihuacan was unusual (compared to the competing city states of the Maya at least) in its dominance of its surroundings. It relied on local irrigated agriculture and an extensive trade in obsidian. We do not know quite what sort of political system it possessed or the precise political relationship the city had with surrounding territories. Teotihuacan maintained large pyramidal temples dedicated to the sun and moon; these were decorated internally with storytelling paintings (somewhat akin to the art of Egyptian temples).

After Teotihuacan fell, there was a period of conflict in Central Mexico. The Toltecs brought some unity from the eighth century, though the Toltec state only consolidated from the late ninth century. Their capital of Tula, near Mexico City, had about 60,000 people surrounded by irrigated fields. They exacted tribute from neighbouring peoples. They traded with the Maya, and borrowed architectural ideas from them. The Toltecs built the biggest pyramid ever at Cholula - though this was made of stone piled on dirt rather than a complete stone construction. Toltec artisanship is widely admired. The Toltecs borrowed and expanded Mayan traditions of human sacrifice. Toltec society collapsed around 1200 for unknown reasons, long before the arrival of the Aztecs.

The Aztecs (Mexica) would arrive in the thirteenth century from the northwest, and would travel around Mesoamerica for decades stealing land and women. They eventually settled in Tenochtitlan (present-day Mexico City). There is an Aztec myth that the god of sun and war Huitzilopochtli had told them to seek an eagle sitting on a cactus and eating a snake, and they had found exactly that when they reached Tenochtitlan. This myth has often been depicted in later Mexican art, and an eagle with a snake appears in the Mexican coat of arms today.

In Tenochtitlan, the Aztecs developed an irrigated agriculture using soil dredged from the lake bottom. (That lake no longer exists, but has been replaced by one of the largest cities in the world.) This proved very productive: Some claim that seven crops per year could be harvested. Since the lake had no drainage, it was quite salty. The Aztecs had to use dams to separate fresh from salt water before they could use the water for agriculture.

The Aztecs built a mighty army and exacted tribute from neighbours. Tribute had to be paid in quantities of both food items and manufactured items such 


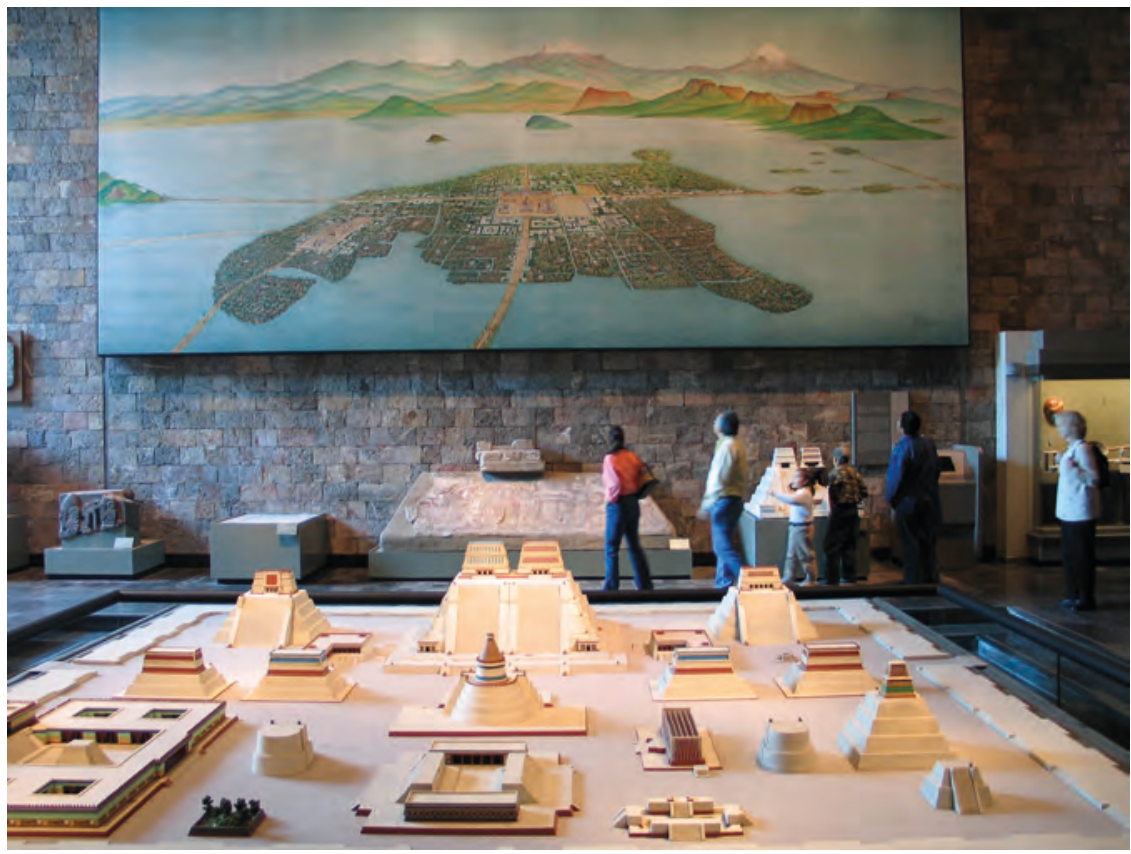

Illustration $\mathbf{1 6 . 6}$

Model of Tenochtitlan

Photograph by Luidger, CC BY-SA 3.0

as clothing. The Aztecs developed a writing system that was more pictographic than that of the Maya. The lake gave them a natural defence against attack, and by the middle of the fifteenth century, they had come to dominate almost all of Mesoamerica from the northern deserts to the southern rainforest. They left local governance in the hands of local tribes. They did not even maintain military garrisons but used the threat of severe reprisals if tribute was not paid. Subject peoples, therefore, received no government services in return for their tribute, though the Aztecs did achieve a kind of peace. Tenochtitlan prospered, growing to about 200,000, with another 300,000 nearby, one of the largest urban areas in the world. The Spanish celebrated Tenochtitlan as a wellplanned, clean and orderly city on islands with canals and causeways to the shoreline. The Spanish conqueror Cortes sent Aztec works of art back to the Spanish king, who displayed them throughout Europe.

When the Spanish arrived in Tenochtitlan, they observed markets that compared well with those in Europe. These markets possessed a wide variety of goods, including various foods from different regions, animals, textiles, a kind of paper, jewellery, tools, hides, and more. Cacao beans were used as a currency. Aztec society had a specialized class of traders, who travelled across Mesoamerican societies and knew many languages. They seem to have had their own hierarchies and religion. They also appear to have limited entry to this trading class. They were perhaps similar to merchant networks in Europe and 


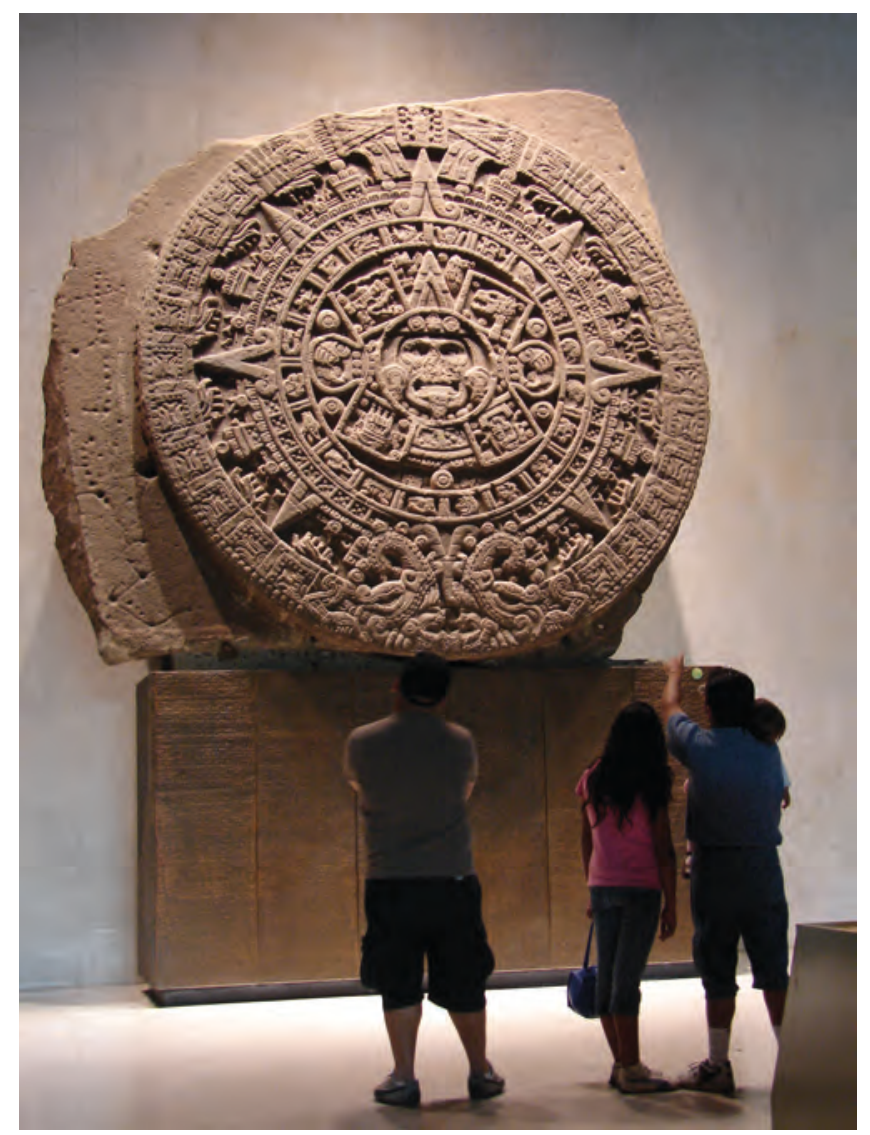

Illustration 16.7

The Stone of the Sun, also called the Aztec calendar stone, National Museum of Anthropology and History, Mexico City Antony Stanley, CC BY-SA 2.0

elsewhere: They could have established internal relationships of trust grounded in fear of rejection from the group for malfeasance. They seem, though, to have distinguished themselves culturally from the broader population to a greater degree than was the case in Eurasia. This may have been a reaction to the militarist ethos of Aztec society. As elsewhere, these merchants must have negotiated some guarantees of safety with Aztec rulers. These rulers appear to have recognized the importance of trade. Some historians even speculate that the state employed them. In any case, this merchant class seems quickly to have disappeared after the Spanish replaced Aztec governance.

Though the Spanish destroyed much, some Aztec books survive. Also, the Spanish would interview many people in the early decades of the conquest. We thus know that Aztec society was very hierarchical. Politically it was an autocracy without any ruling council. Warriors were especially prized, and poor people might gain social status as warriors, though the aristocracy received the 


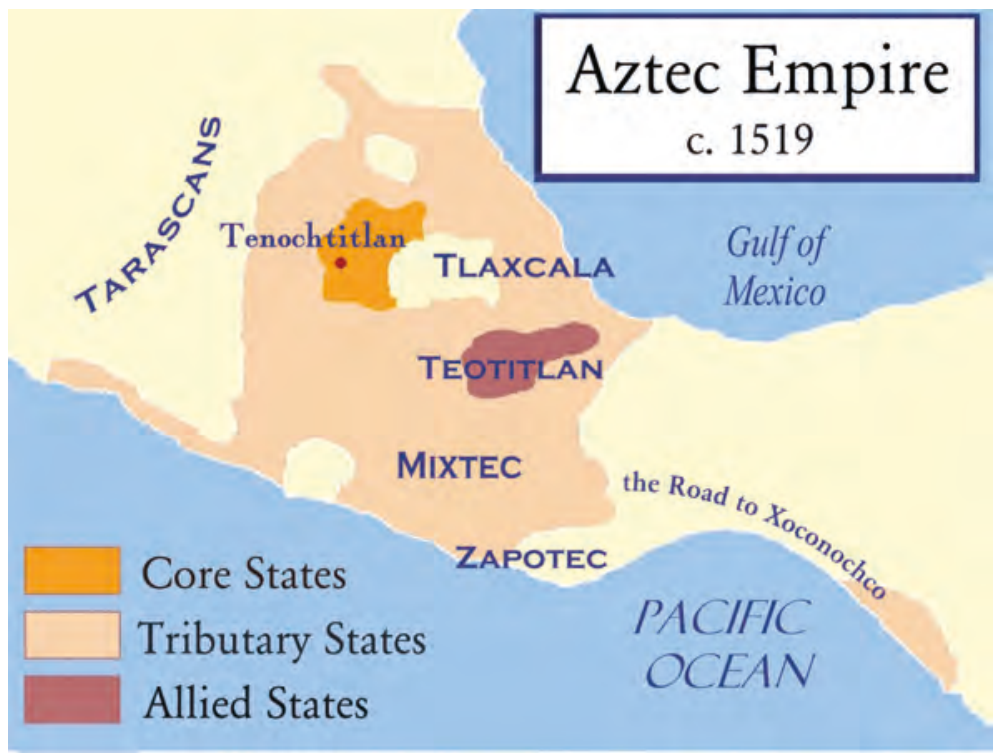

Map 16.2

The Aztec Empire in 1519

Map by Badseed based on work by historicair, which in turn was based on Madman2001, CC BY-SA 3.0

most military training. Aristocrats and especially warriors were allowed to wear better clothes (cotton rather than a kind of burlap, and brightly coloured). As we have observed in many other warrior societies, women played a limited public role and were valued mainly as mothers. Yet they did operate as traders and in textile manufacture. A priestly class performed rituals and advised rulers on what the gods wanted; some even became rulers, including Moctezuma, who the Spanish defeated. Skilled artisans were appreciated. Commoners paid taxes and provided forced labour for public works.

The Aztecs as they arrived and conquered had adopted local deities such as the rain god, known since Mayan times, and the religious significance attached to jaguars and eagles and snakes. Human sacrifice had been practised in Mesoamerica for centuries before the Aztecs. The Aztecs substantially increased the frequency of sacrifice - of criminals, captured warriors, and slaves given in tribute. Indeed the Aztecs may have taken human sacrifice to a scale never seen elsewhere in human history. The blood-encrusted temples they encountered, and the hundreds of skulls of sacrificial victims on display in these, understandably appalled the Spanish. Though the Spanish had an incentive to exaggerate to justify their own brutal conquest, archaeology supports the existence of large-scale sacrifice. We do not know much about what the Aztec people themselves thought of the practice - but one can imagine that in a society that sacrifices many people it would be incredibly courageous to speak out against the practice. 


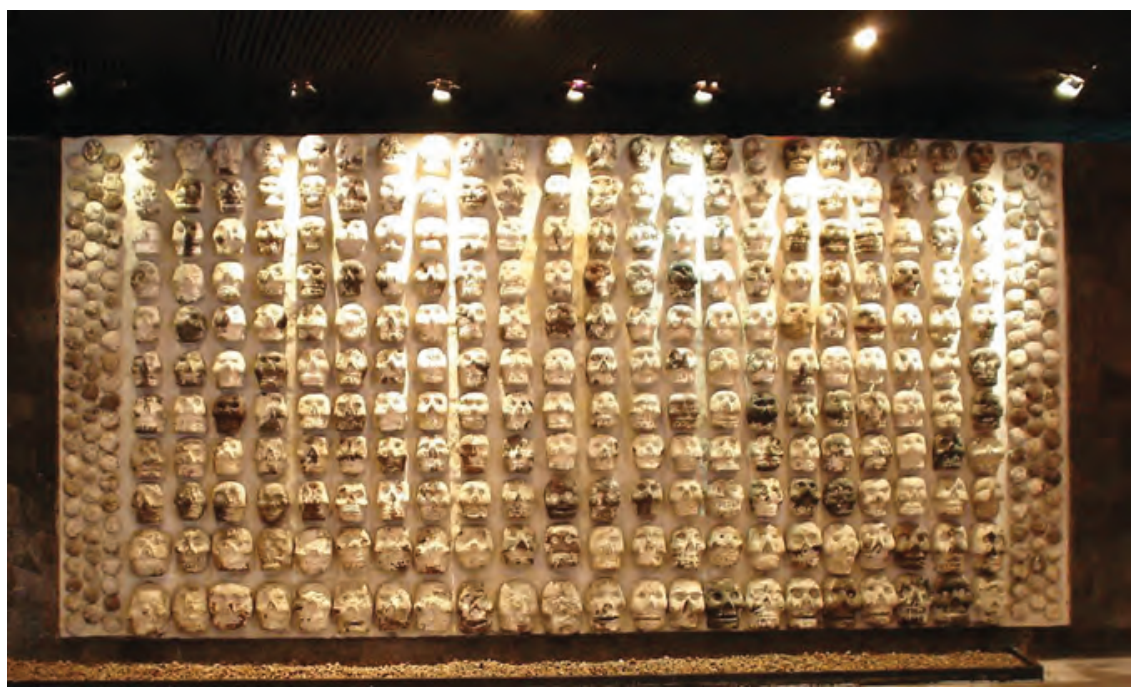

Illustration 16.8

Aztec Wall of Skulls, Templo Mayor, Mexico City

Photograph by Iris from Culemborg, The Netherlands, CC BY 2.0

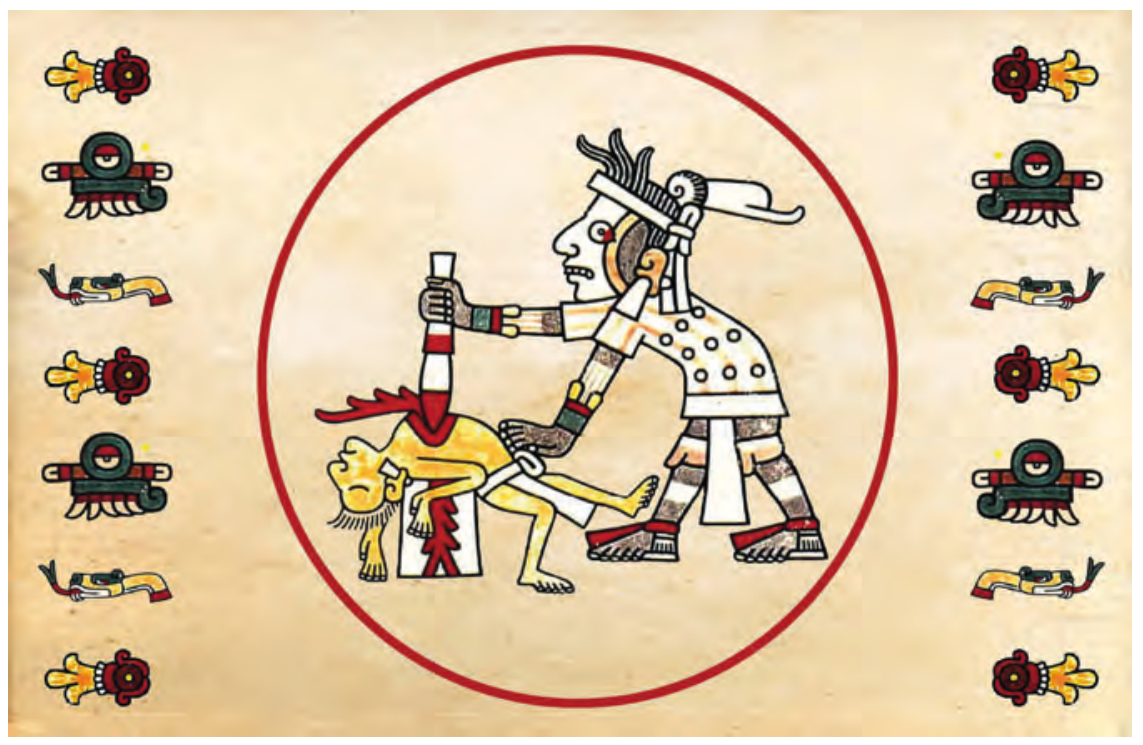

Illustration 16.9

Aztec human sacrifice (from 16th-century codex)

Though we do not know as much as we would like to about the Aztecs, the reader can still usefully imagine themselves in Aztec society. If you are a male commoner, the only way ahead is as a warrior. If you are female, your options are even more limited. What is your attitude toward human sacrifice? 


\section{PRIMARY DOCUMENTS: HOW THE AZTEC RAISED SONS AS WARRIORS}

The Franciscan friar Bernardino de Sahagún arrived in Mexico in 1529 and interviewed locals about pre-conquest life. The following is an excerpt from his Florentine Codex: General History of the Things of New Spain, Book 8 - Kings and Lords, Chapter 21 (translated by Charles E. Dribble and Arthur J.O. Anderson). Note that the Spanish government suppressed this work for centuries. (From Children and Youth in History, Item \#441, http://chnm.gmu. edu/cyh/items/show/441)

And behold how began the life of the young boy. At first, while still a small boy, his hair was shorn. And when he was already ten years old, they then let a tuft of hair grown on the back of his head. And when he was fifteen years old, then the tuft of hair became long. [This was] when he had nowhere taken captives.

And if he took a captive with the help of others, - perchance doing so with the aid of two, or of three, or four, or of five, or of six, at which point came to an end [the reckoning] that a captive was taken with others' help-then the lock of hair was removed. And this was the division of their captive: in six parts it came. The first, who was the real captor, took his body and one of his thighs-the one with the right foot. And the second who took part [in the capture] took the left thigh. And the third took the right upper arm. The fifth took the right forearm. And as for the sixth, he took the left forearm.

And when the tuft on the back of his head was removed, he was shorn so that he was left [another] lock: his hair dress kept, on the right side, the hair hanging low, reaching the bottom of his ear; to one side [only] was is lock of hair set. When this [was done], he assumed another face, he appeared otherwise, so that it might be seen that he had made a captive with the help of others [and that] the tuft of hair on the back of his head had been removed.

And then his grandfather, or his beloved uncle, addressed him. He said to him: "My beloved grandson, the sun the lord of the earth, hath washed thy face. You have taken another face; and you have gone to throw yourself against the foe. Let them take you if, without profit, once more you take a captive with the aid of others. What would you be? Would you have a young girl's lock of hair? Take care lest you again take a captive with others' help. Cast yourself against our foes..."

How reliable are Spanish reports of Aztec behaviour? If this report is accurate, what does it tell you about Aztec childhood?

\section{Farther north}

Scholars long assumed that the northern landmass occupied today by the United States and Canada had always been characterized by hunting and gathering groups, with perhaps some limited agricultural activity. However, there is clear archaeological evidence of substantial towns supported by 


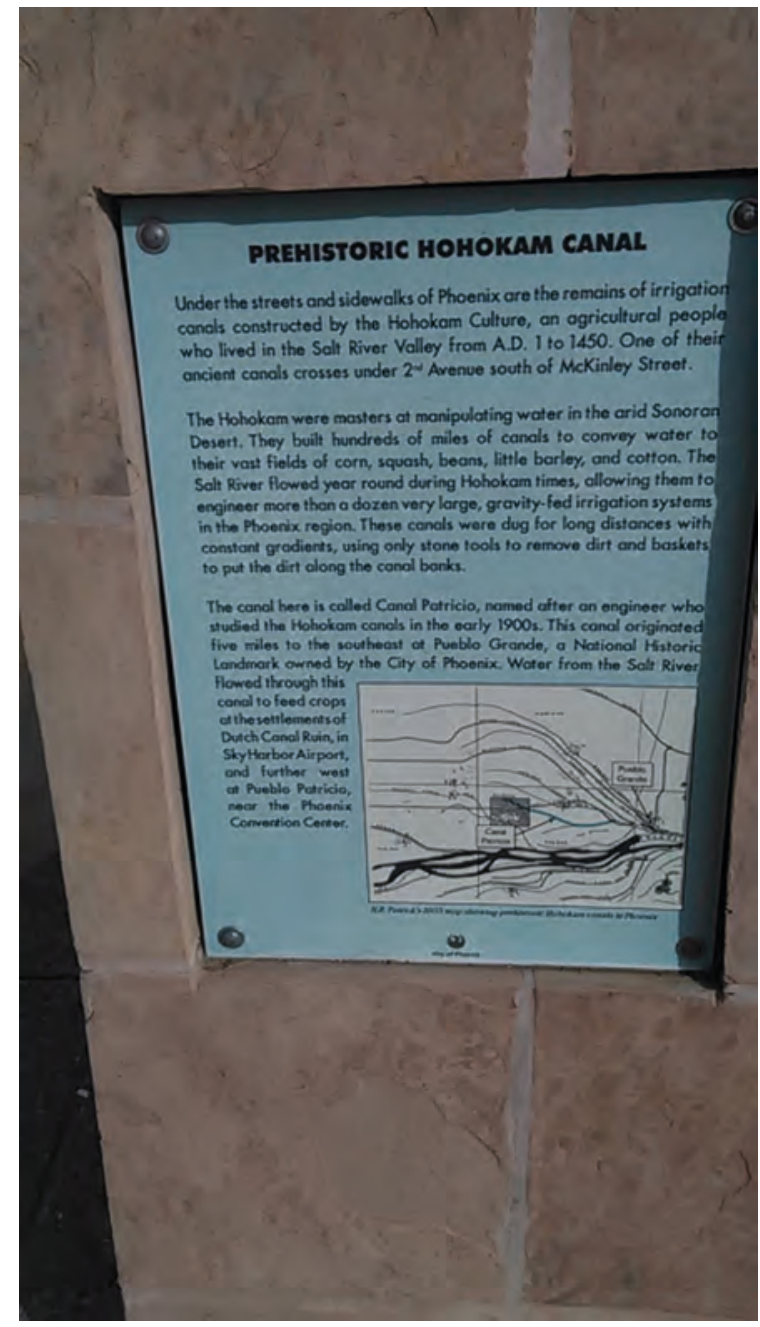

Illustration 16.10

Hohokam canal marker, downtown Phoenix. The Hohokam culture existed to the southwest of the Anasazi. History is all around us Author agriculture and trade in many places. Cultivation of corn, beans, and squash had spread north from Mexico into northern North America. The Anasazi employed irrigation in the United States southwest between the third and thirteenth centuries. The Pueblo (who were likely descendants of the Anasazi) lived in towns for several centuries (and apparently used smoke signals to communicate between towns). Turquoise from the area is found throughout Mesoamerica, indicating some sustained trade relationships. The Iroquois of the Great Lakes region from about 1400 built on a legacy of agriculture in the area that may have been a thousand years old; they had towns of at least hundreds of people.

Cahokia, near East St. Louis, likely had 15,000-35,000 people at its height between 900 and 1250. This was the largest city north of Mexico before the arrival of Europeans. It is famed for its large earthen mounds, which presumably served ritual purposes, but were perhaps also the site of the homes of the elite. Such mounds were common throughout eastern North America, indicating a cultural network likely knit together by trade. Archaeologists indeed have discovered minerals from the Rockies and upper Mississippi and seashells from Florida at many sites in the region. This "Mississippian" civilization may have employed canoes that were 50 feet long on major rivers. They appear to have traded to Hudson Bay in the north, the Atlantic in the east, and Oklahoma in the west, but there is no clear evidence of sustained contact with Mexico to the south. Cahokia, lying near the junctions of the Mississippi, Missouri, and Ohio Rivers, may have been a major trading centre. There is evidence of a complex society of priests and artisans and soldiers. Beautiful pottery and small stone carvings were produced. Since they had no written script, we have to rely on archaeology for evidence. Populations began to decline from 1200 or 1300 for reasons we do not fully understand (there was a severe earthquake around 1250, but this alone should not have 
destroyed such a civilization) and Cahokia and the cities it traded with were abandoned. The history of the United States might have unfolded quite differently if European settlers had confronted this civilization.

\section{South America}

There is a long history of states and cities in South America - but the rugged Andes tended to limit the size of these more than in Mesoamerica. Remarkably, South Americans never developed writing systems. This may reflect, at least in part, the smaller size of states and trading networks in the region. Yet we should not discount historical contingency: As we shall see, South Americans would develop an ingenious alternative method of record-keeping. The fact that there was little or no direct contact between South and North America - separated by thousands of kilometres of ocean or jungle - meant that Mesoamerican writing systems were not transferred south. The lack of written records means that we know less about South America than about most other parts of the world. There is, though, clear evidence of cities and states with social distinctions, textiles, and pottery from the last centuries все (chapter 7). The artistic achievements of many of these societies were remarkable.

Tiahuanaco near Lake Titicaca had a population of 30,000-40,000 from about 500 ce. The city had running water and a sewer system, and impressive public buildings. An elaborate drainage system turned surrounding swamps into farms. It was the largest city in South America at the time, though much smaller than Teotihuacan in Mesoamerica.

The Chimu state/society would emerge on the coast of Peru around 900, and would survive until conquered by the Incas in the fifteenth century. Its capital city (present-day Trujillo) likely had about 50,000 people at its peak. The Chimu employed irrigation to deploy the waters of Andean rivers across strips of the coastal desert plain. They produced elegant pottery and works of copper, bronze, gold, and silver. They had a religion that seems to have focused on the worship of the moon.

Though these earlier cities and states are notable, the extent of the Inca Empire dwarfed them all. Indeed, the Inca Empire was the largest empire in the world by land area in 1500 . The Inca in only a century conquered a vast territory extending 4,000 kilometres and including most of modern Peru, Ecuador and Bolivia, and parts of Chile and Argentina. Inca kings were expected to finance themselves (like medieval European kings; chapter 13), but did not inherit their father's land holdings and therefore had to conquer new lands to support their administration. There was a population of about 10 million at the empire's peak. The Incas held hostages of conquered groups to ensure loyalty. The Incas also moved peoples around their empire to discourage a sense of regional identity. Their army and bureaucracy were mostly composed of conquered peoples. Members of the royal family occupied the senior posts. The Inca employed spies to check up on local officials, who were often drawn from 


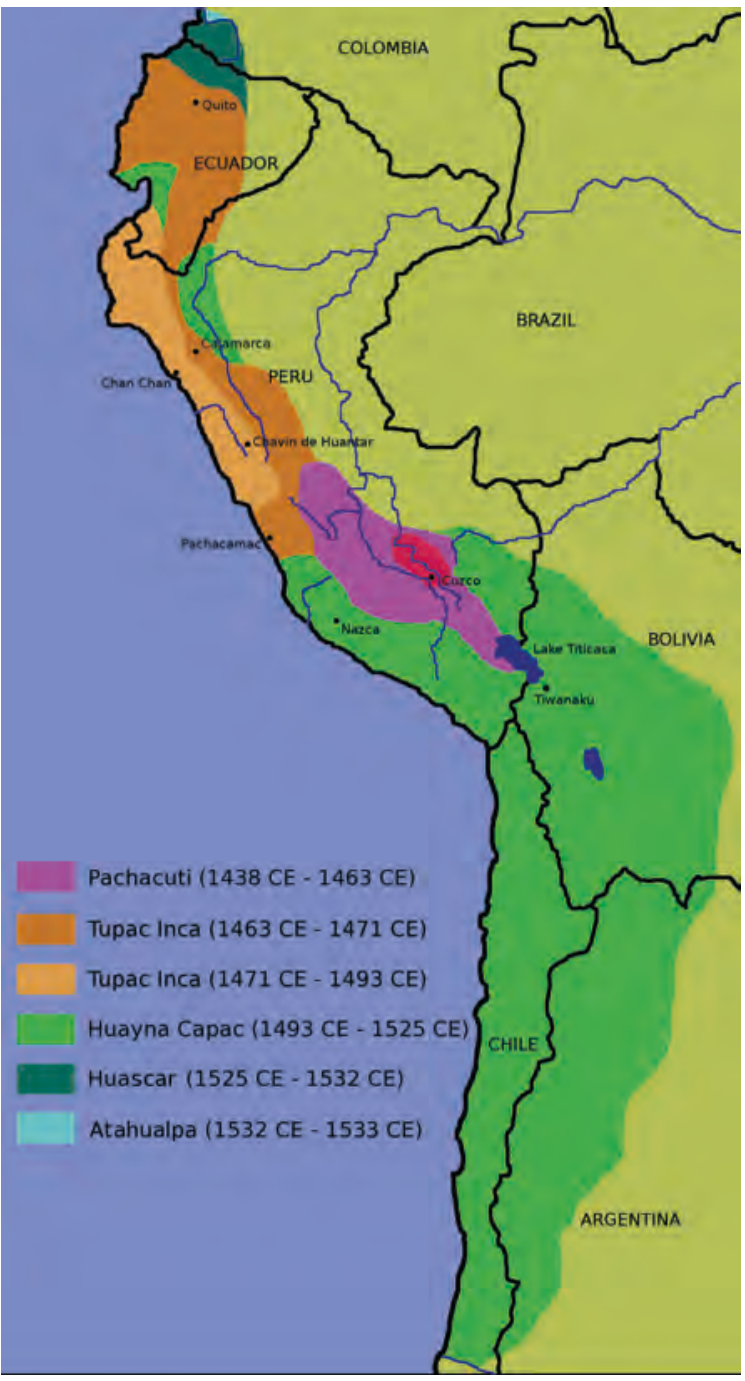

Map 16.3

Expansion of the Inca Empire under different emperors local elites. They may have practised a system of dual offices in which a bureaucrat from the centre would shadow a local official. Certainly inspectors from the centre were sent to audit local accounts.

The Incas were (almost certainly) the largest empire in world history to operate without a system of writing. How did they manage a vast bureaucracy and extensive inter-regional trade without writing? Recall that the earliest writing systems likely evolved from simple tally records that recorded amounts (chapter 6). The Inca employed a memory-aiding device called the quipu that consisted of up to a hundred or so coloured cords that could be knotted at various lengths. It is easy to see how such a device could work like an abacus to add even very large numbers. Yet quipu also stored other sorts of information, such as the history of the Inca though in this case quipu may have only served as mnemonic device to trigger the memory of a story-teller. We do not know precisely how quipus were utilized, but there have been archaeological finds of multiple quipus in state storehouses, suggesting that they were broadly used in the bureaucracy to keep track of goods and likely taxes, and thus also land and population. Different colours could signal different commodities or purposes. The patterns of knots in cords represent a type of "code": The messages a quipu might contain are unintelligible without knowing the code employed.

Though archaeologists have found a limited number of quipus, it is notable that these often occur in matched pairs. This might merely represent something akin to the copying of manuscripts in societies with written scripts. Yet it appears to represent how the Incas dealt with the possibility of bureaucratic deceit or incompetence. Different administrators regularly compared quipus to ensure, say, that tax receipts counted by one official matched transfers to the state counted by another. One bureaucrat might do inventory in a state storehouse, and then compare the result with the quipu of an administrator who had been subtracting outflows and adding inflows since the last inventory. 
As we noted in chapter 6, states needed some way of tracking whether what happened was the same as what was supposed to have occurred.

The Incas are by far the largest political entity to have relied on knotted cords for record-keeping, but this approach - and a somewhat similar approach involving notches on sticks - has been observed among non-literate societies in various parts of the world, including west and central Africa, various Pacific Islands, and North America. The Luba people of central Africa used the lukasa, a board

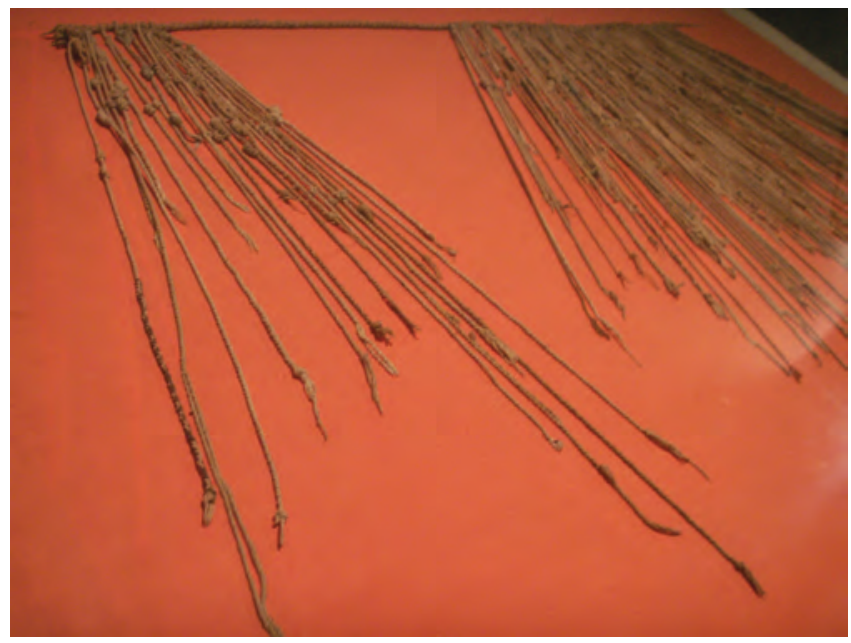

Illustration 16.11

Quipu, Museo De La Nacion, Lima, Peru with pins, strings, and beads, as a device to help oral historians remember the detailed history of their people. It seems human societies can record information in a limited number of ways. Writing is likely the most efficient but is not essential to detailed record-keeping or detailed histories.

Postscript : San Andrés de Tupicocha in Peru uses quipus in ceremonies that install new officials. As recently as the nineteenth century, quipus were used to record attendance at meetings. Harvard University has digitized over a thousand quipus and hundreds are on display in museums around the world.

The Inca capital at Cuzco may have reached 300,000 people by the late fifteenth century. Buildings were of red stone, many decorated in gold. The Spanish would later marvel at the rich silver and gold ornamentation of Inca buildings - but would melt it all down for bullion. The Spanish conqueror Pizarro's secretary said that Cuzco was so large and beautiful that it would be praised in Spain. Incan art often celebrated the state and military victories, but also agriculture and fertility.

To support their capital, the Inca developed an extensive road system, with a combined length of about 16,000 kilometres (some say twice as much). They employed runners to carry messages; these could reach any point in the empire in a matter of days. They moved four times as fast as Spanish on horses later would. The roads were paved with stone, and rope bridges crossed rivers. Llamas were used extensively as pack animals (see above), but wheels for wagons seem 


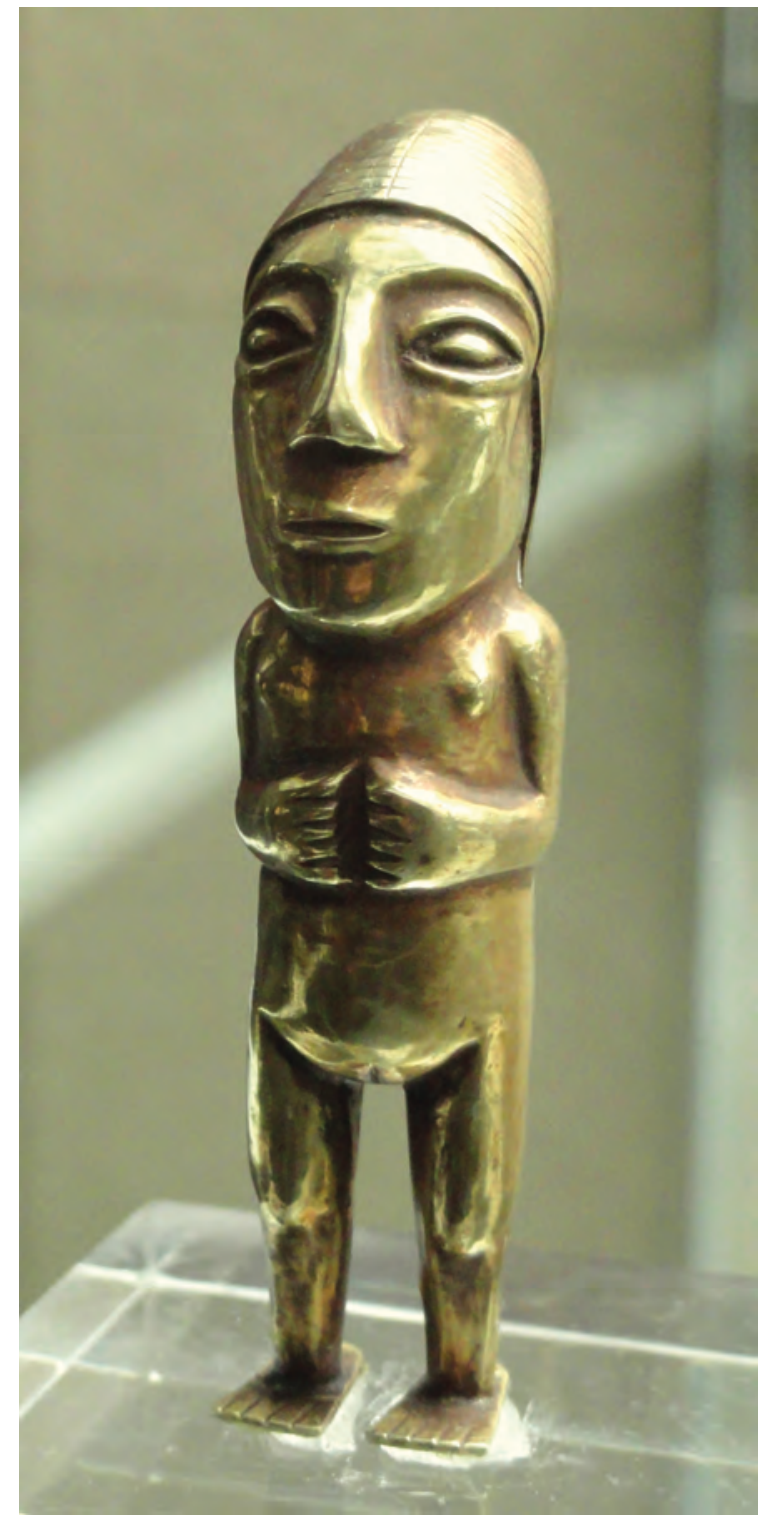

Illustration 16.12

Inca gold figure, 1450-1532, Staatliches Museum für Völkerkunde, Munich, Germany not to have been employed. These would likely have had trouble crossing rope bridges, or coping with steep inclines. Still, there was extensive trade: The Andes have several microclimates with different crops, and the mountain regions traded with coastal areas for seafood and shells and with tropical lowlands. It seems that this trade was mainly if not entirely managed by the state rather than independent merchants: If so, this sort of "command economy" is a remarkable achievement, especially for a state with no system of writing.

The Inca Empire had extensive irrigation works; it also had followed earlier societies in building agricultural terraces. The Incas had discovered the fertilizer capability of guano (manure from birds). People had enough food, in part because of state storehouses (the Incas succeeded here better than most empires, perhaps in part because potatoes are easy to grow and store), and adults all had a plot of land. Much land was controlled and distributed by kinship groups. The Inca often gave gifts to widows and orphans. The Inca state was tyrannical but arguably better ordered than the Spanish that would follow. The Incas forced labour among men on state lands and public works and in the military and postal system. Women generally paid tribute in goods instead, though some were likely compelled to serve as concubines or weavers. The Inca may have produced the most elaborately woven textiles in the world at the time. Slavery itself was unknown, perhaps because the lands of the aristocracy also employed forced labour. Though the Inca relied on violence as did all other empires, it arguably provided more in the way of public services than most other empires; many subject peoples voluntarily accepted Inca rule rather than fight them. There is some evidence, nevertheless, that this young empire was already plagued by rising local disenchantment (perhaps as the Inca attempted to increase extraction) and the rising power of local elites. Some 
subject peoples would welcome the Spanish, though others would remain loyal for decades, especially as they witnessed Spanish rule.

The Inca had an elaborate calendar of twelve months of thirty days. Quipu were used to track the movements of the sun and moon. The famous ruins at Machu Picchu are oriented toward the summer solstice - an orientation toward the sun's path that figures in many ancient monuments. Like Angkor Wat (which stressed the spring equinox; see chapter 15), Machu Picchu is not oriented toward the winter solstice as structures in northern temperate zones generally were: The change of seasons was still meaningful, but the "return" of the winter sun not as critical as in the temperate zone.

Rulers were mummified, with these mummies later used in rituals and asked for advice. Rulers claimed to be divine. They thus married their sisters (as Egyptian pharaohs often had) and chose the next ruler from among their children - though there were often succession battles among sons. There was also an aristocracy and a priesthood that was likely chosen from the aristocracy. There was no legislature or even formal advisory council to rulers. The sun and planets were worshipped, but the sun dominated; there was an even

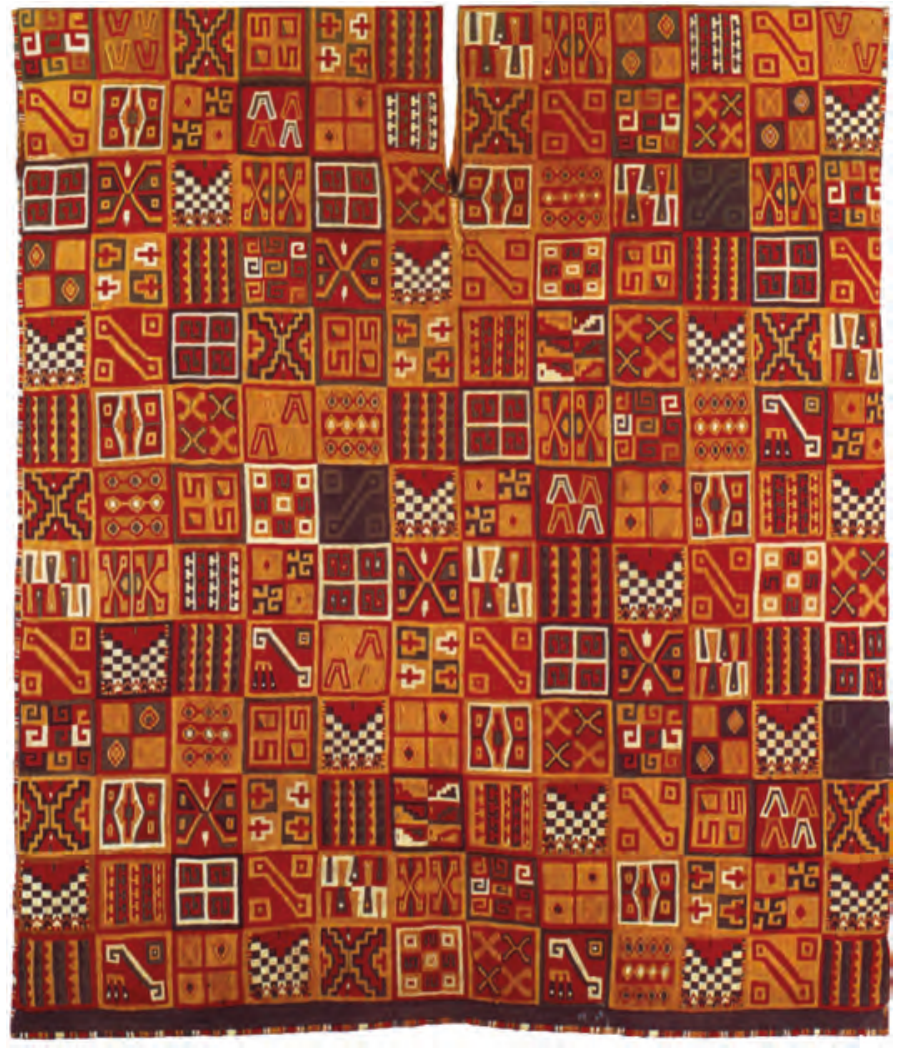

Illustration 16.13

Incan tunic, between 1450 and 1540, Dumbarton Oaks Library, Washington 


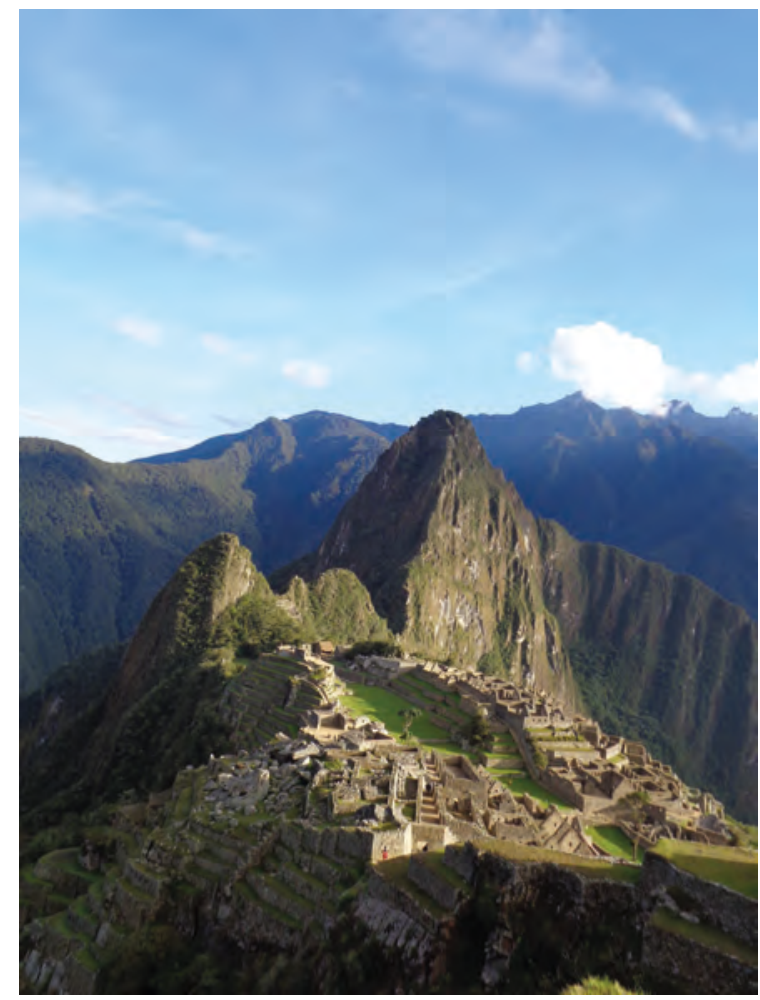

Illustration 16.14

Machu Picchu higher but invisible creator of everything. Like the Romans (chapter 8, 10) the Incas absorbed the gods of those they conquered into their pantheon. Yet all were expected to worship the sun. The religion had an ethical element whereby gods would punish individuals and communities in this life and the next for misbehaving. The definition of misbehaving included not respecting one's place in the social order. The Incas occasionally sacrificed animals (including llamas) or even children in a crisis, but far less than the Aztecs.

Pre-marital sex was apparently common. Some priestesses were nevertheless virgins. There would occasionally be mass marriages supervised by the state where bureaucrats would match unmarried men and women. Outside of these mass marriages, people could choose their mates. This may have been one of the few areas of personal choice for the people of the empire.

\section{PRIMARY DOCUMENTS: INCA SOCIAL POLICY}

Pedro Cieza de Leon, The Second Part of the Chronicle of Peru, translated by Clements $\mathrm{R}$ Markham, London: Hackluyt Society, 1883 (reproduced in Bonnie Smith, Modern Empires: $A$ Reader). After describing how government officials used quipu to keep track of taxes and expenditures, he talks about social policy:

At the beginning of the new year the rulers of each village came to Cuzco, bringing their quipus, which told how many births there had been during the year, and how many deaths. In this way the Inca and the governors knew which of the Indians were poor, the women who had been widowed, whether they were able to pay their taxes, and how many men they could count on in the event of war, and many other things they considered highly important....

As this kingdom was so vast, in each of the many provinces there were many storehouses filled with supplies and other needful things; thus in times of war wherever the armies went they drew upon the contents of these storehouses ... If there came a lean year, the storehouses were opened and the provinces were lent what they needed 
in the way of supplies; then in a year of abundance they paid back all they had received. No one who was lazy or tried to live by the work of others was tolerated; everyone had to work. Thus on certain days each lord went to his lands and took the plough in hand and cultivated the earth, and did other things. Even the Incas themselves did this to set an example. And under this system there was none such in all the kingdom, for, if he had his health, he worked and lacked for nothing; and if he was ill, he received what he needed from the storehouses.

This description was written less than a decade after conquest, when the Spanish still employed Incan institutions, and memories were vivid. Nevertheless, we can wonder if the description is accurate. How does this description compare with the situation in most other world empires?

\section{Africa}

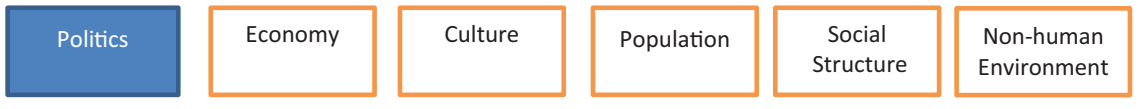

We have spent much time in this book discussing trade links and information flows across the Eurasian landmass. Relations with Africa were much less intense through much of history. The Sahara Desert was a severe barrier to interaction. To the east, we have seen that the Nile River afforded some opportunity for trade between Egypt and sub-Saharan Africa. Farther east still, there were trade links along the eastern coast of Africa. Traders from Asia or Europe had not ventured farther along that coast than Mozambique before the arrival of the Portuguese (chapter 19). Trade inland was handled mainly by Africans. Eurasian societies, as a result, knew very little about sub-Saharan African societies through most of human history.

Moreover, these societies lacked written records. It was then easy for historians from Eurasia to imagine even mere decades ago that there had been little "civilization" in sub-Saharan African history. It is now widely appreciated, though, that there were indeed empires, cities, and networks of internal trade. For some societies, we must rely on archaeological investigation. For others, we can combine this with oral history and with the reports of outsiders that did occasionally trade with them.

By the earliest centuries $\mathrm{CE}$, agriculture and nomadism had replaced hunting and gathering through most of sub-Saharan Africa (the exceptions occurring within the deepest rainforest and the Kalahari Desert). As noted in chapter 5, Africans were among the earliest to use iron, and so iron tools were utilized widely for clearing land and farming (ironworking was a prestigious skill passed from father to son). The banana, which had reached Africa from Southeast Asia likely via Madagascar by about $500 \mathrm{cE}$, allowed agriculture to spread into 
forest areas where millet and sorghum and yams did not prosper. As agricultural activity intensified and spread, the population grew from only 3.5 million in 400 CE to 17 million by 800 and 22 million by 1000 .

As population density increased, so too did political complexity. Clans grew into chiefdoms, some of which expanded to become kingdoms or even empires with dedicated bureaucracies. As elsewhere, kingdoms and empires exhibited social stratification and income inequality. One unusual feature of much African political and social organization was/is the importance of groupings of people of similar ages, generally accompanied by elaborate rites of passage. Different age groups performed different societal functions. Slavery was common, with slaves being primarily captives in war or people sold into slavery because of debt. From the eighth or ninth century, slaves were sold to Muslim traders along the east coast (as demand for slaves in the Middle East outstripped the ready supply of Europeans; see chapter 12); this encouraged slave raiding in the interior. Ten million slaves were exported via the east coast between 750 and 1500 .

Communal management of land seems to have been important throughout sub-Saharan Africa. Indeed, historians suggest that private land ownership was mostly unknown. Throughout history, the move from communal to private property has generally been associated with increased productivity: Peasants prove willing to work harder for themselves than for others, and it is easier to innovate. Why, then, did Africa retain communal ownership for centuries? This practice likely reflects the long history of slash-and-burn agriculture: a form of shifting agriculture in which brush is burned before new land is farmed. This clears the ground while leaving nutrients from the burned bush in the soil. Groups of farmers would move to new areas every few years. Even where settled agriculture prevailed, communal practices might continue for similar reasons as those observed in feudal Europe: Villagers needed to cooperate lest one's animals or weeds invade another's fields. Yet European peasants still had individual plots of land. It is not entirely clear how village leaders allocated land in African villages.

We should stress that population densities remained lower in Africa than in much of Europe and Asia (Southeast Asia may have had comparable population density to Africa much of the time). This suggests that agricultural productivity per acre was lower. This, in turn, may reflect several interrelated causes: soil conditions, the crops grown, the use of slash-and-burn agriculture (which is much more common in tropical and semi-tropical areas than in temperate climates), and the reliance on communal land ownership. Diseases ravaged draft animals in tropical regions, limiting the ability to apply these to agriculture as was done throughout Eurasia. Though much of sub-Saharan Africa is tropical rainforest, much instead receives too little rainfall to support annual agriculture. Lower levels of agricultural productivity would, in turn, limit the development of complex polities, trade, and industry. We do see the development of states in areas with favoured agricultural conditions, such as in the area 


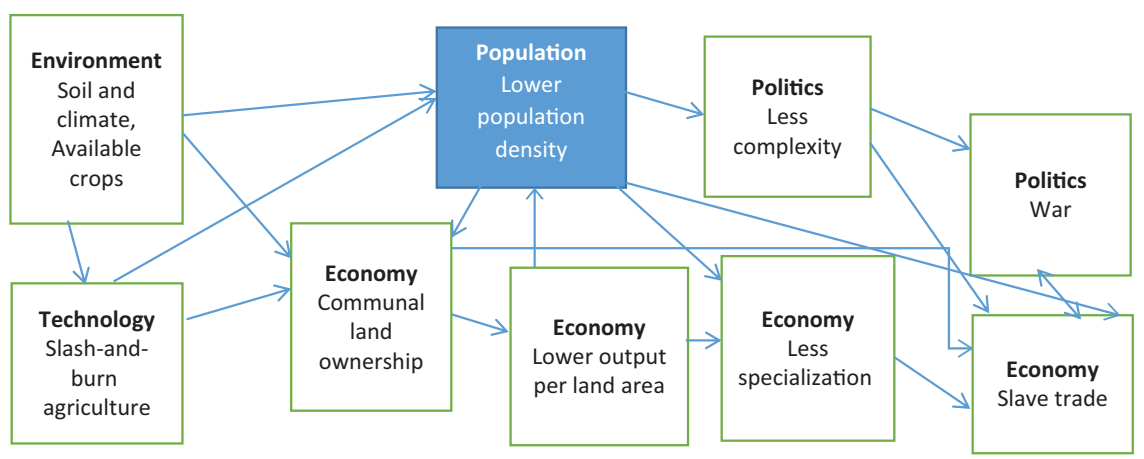

Figure 16.1

Influences on and effects of low population density in Africa

Note: The diagrams in this book capture important sets of influences. But there are always other processes at work in any time and place. The lower population density was an important factor in African development but did not act in isolation.

around the Great Lakes and in the Rift Valley. Yet the availability of open land nearby could limit the coercive capabilities of such states.

It is noteworthy that one could own slaves but not land. This arguably made slavery even more important within African societies. The rich could not use their wealth to buy property but could buy people. The allocation of land would then reflect the possession of slaves. Since slaves, as we have seen, are often war captives, the prevalence of slavery encouraged systems of small competing states rather than one dominant empire. The export of slaves from Africa then built upon the internal slave trade; it would, in turn, be encouraged by relatively weak African states. It would also reflect the lesser degree of economic specialization in Africa: Africa wished to import a range of industrial goods for which it had limited goods to export (beyond the exotic animal products and gold in some areas that had always been staples of African trade).

Difficulties in transport exacerbated the challenges to both trade and state formation associated with low agricultural productivity. The topography of subSaharan Africa meant that most rivers were navigable for only short distances, and rarely were navigable to the sea. Africans thus developed limited seagoing shipping: Throughout most of history, trade by water with other continents occurred in ships from other places. Mountains and rainforest further limited transport links. We can note here that slaves - who can be marched to markets if necessary - may be a particularly attractive "commodity" when it is hard to transport other goods.

Postscript: Though agricultural output has quadrupled since 1960 in Africa, output per farmer and per acre remain far below levels on other continents. Forests are still cut down to create new farmland in many 
African countries. There seems to be much scope for the increased use of fertilizer, but it is still a challenge to transport inputs and outputs to/ from many African farms. As noted in chapter 31, agricultural research in the twentieth century tended to focus on the crops and conditions of other continents, though this has begun to change.

Though rulers were usually male, women exerted significant influence both politically and as merchants in most African societies. Matrilineal and matrilocal practices were common in Africa (and parts of the Americas). Inheritance passed through a man's sister's children rather than his own. In addition, a man would often leave his village to join his wife's family. The arrival of Islam did not reduce, at least immediately, the ability of women to interact with men. As we saw in chapter 12, though, over time attitudes within Muslim polities hardened.

The Columbian exchange of crops between continents (chapter 19) significantly affected sub-Saharan Africa. The Portuguese brought important new crops from the Americas, including manioc and peanuts that thrived in tropical areas. Corn was also widely grown: Note that it grows well with bananas, a crop imported earlier from Asia. The productivity of manioc in particular, which could be made into bread, helped the sub-Saharan population to grow (despite the slave trade) from 34 million in 1500 to 44 million in 1600 and 60 million by 1800 .

\section{West Africa}

Trade across the Sahara was facilitated before 200 CE with the arrival of camels from Central Asia. Berbers then began to explore routes south across the desert, and trade had become regular by the time of the arrival of Islam. Muslim merchants would over time introduce Asian rice (there had previously been an African version grown in West Africa), citrus fruits, and cotton to West Africa. Rice and fruit improved diets. Cotton became the most common form of clothing by the fifteenth century. Merchants also introduced bricks, which replaced mud construction.

West Africa would witness three large empires: Ghana, Mali, and Songhay. All three empires had dynastic rule with succession struggles among royal family members. The modern nations of Ghana and Mali took the names of two of these at the time of independence; students should appreciate that these new countries have entirely different boundaries from their namesake empires. All three empires operated along the Niger River and to the south of the Sahara.

The Ghana Empire was likely founded in the second century $\mathrm{CE}$, predating extensive trade across the Sahara. Over the next centuries, it would come to profit as an intermediary as gold from farther south was traded for salt from 


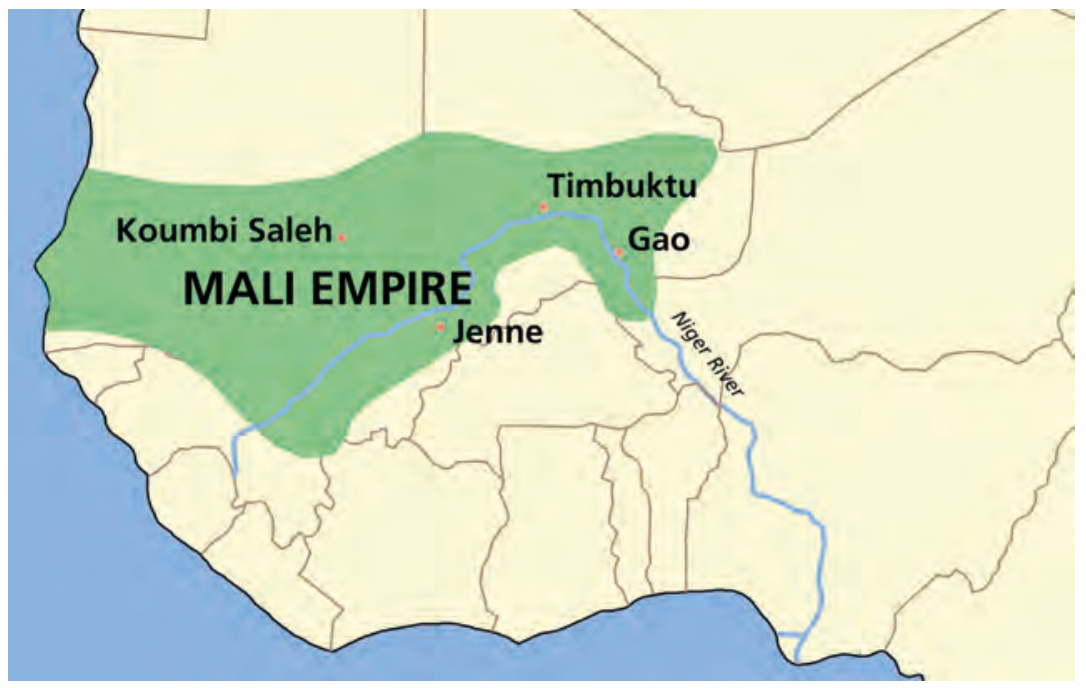

Map 16.4

Mali Empire, 15th century

Map by Astrokey44, CC BY-SA 3.0

north of the Sahara. Muslim traders gave the king bureaucratic advice. However, since the king was a religious leader, he was hesitant to convert. Conversion occurred when the Almoravids, the same militant Islamists who would conquer southern Spain (chapter 12), conquered Ghana in 1076. It is not clear how much force was involved in converting the population after that.

The Mali Empire from the thirteenth to sixteenth century came to cover much of West Africa, and especially the Niger River basin. It comprised both herders and agriculturalists, and many languages. According to oral tradition, the founder was Sundiata, who had been forced into exile when young but returned as a conqueror. He was Muslim, though he practised toleration. He invited Muslim merchants from the north. The empire managed the Sahara trade, but also internal trade between savanna and desert. It relied heavily on horses, which thrived in a dry period from 1100-1500 that limited the tsetse fly (the carrier of sleeping sickness, a danger to both humans and animals). Wetter

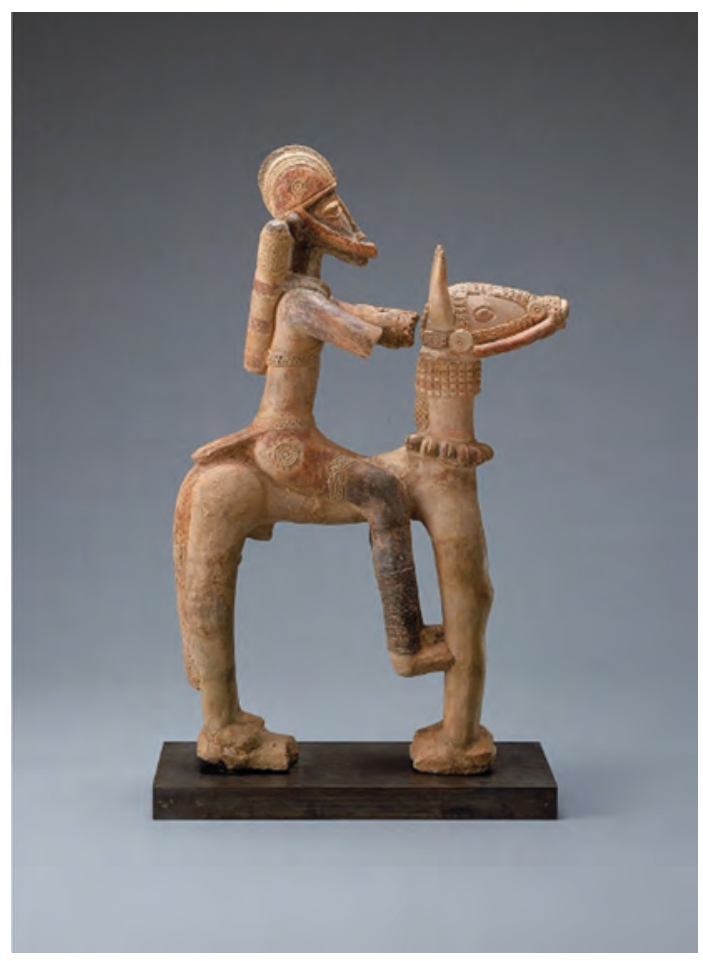

Illustration 16.15

Terracotta equestrian figure from Mali, 13th-15th century 


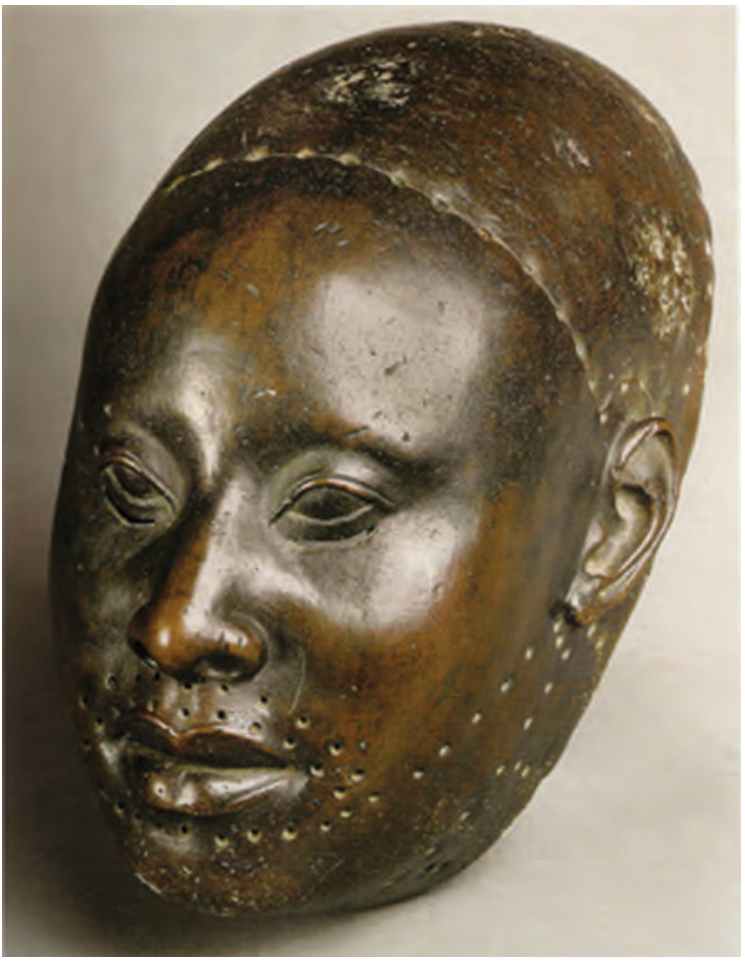

Illustration 16.16

Bronze head, 12th-15th century, Nigeria sailko, CC BY-SA 3.0 conditions later may have contributed to imperial decline. Horses had been in West Africa since the last centuries BCE but only became important militarily in the thirteenth century.

The Songhay Empire from the fifteenth century took over from Mali. It had previously built a flourishing city state around the trading centre of Gao from the eighth century. In 1464, this state conquered the surrounding area, and especially Timbuktu and Jenne (see below). The king, Sunni Ali, developed an extensive army, and a navy on the Niger. The empire had a sophisticated administration. It often employed slaves as administrators, distrusting others. Later kings extended the empire east to Lake Chad and west to the upper reaches of the Niger. The capital had 75,000 people. The rulers were Muslim and supported mosques and schools, and an Islamic university at Timbuktu that attracted students from throughout West Africa. The Songhay Empire would remain dominant through the sixteenth century,

but in 1591 an army from Morocco arrived with muskets. Local peoples then rebelled.

Though these three empires were likely the largest ever seen in sub-Saharan Africa, there were dozens of smaller kingdoms that operated in various parts of the region between the Sahara Desert and the equatorial rainforest. An elaborate ninth-century burial site in Nigeria with ivory, amulets, and 100,000 glass beads suggests the existence of a large state about which we know little. The region near Lake Chad was home to the Kanem and later Bornu Empires that together lasted for over a thousand years; Kanem became Islamic by the thirteenth century. These smaller kingdoms also generally profited from some role in trade across the Sahara.

Ocean trade replaced Sahara trade from the late fifteenth century (chapter 19) and extinguished this long era of empires and smaller kingdoms controlling Saharan trade routes. City states instead prospered over the next centuries (We discuss the later Sokolo caliphate in chapter 20). Political fragmentation was undoubtedly encouraged by the growth of the Atlantic slave trade, for slaves were captured in battles between competing polities. 


\section{PRIMARY DOCUMENTS: MANSA MUSA VISITS CAIRO}

The following description of the visit to Cairo in 1324 by the King of Mali, Mansa Musa, was written by Al-Umari, who visited Cairo several years after Mansa Musa's visit.

From the beginning of my coming to stay in Egypt I heard talk of the arrival of this sultan Musa on his Pilgrimage and found the Cairenes eager to recount what they had seen of the Africans' prodigal spending. I asked the emir Abu... and he told me of the opulence, manly virtues, and piety of his sultan. 'When I went out to meet him \{he said\} that is, on behalf of the mighty sultan al-Malik al-Nasir, he did me extreme honour and treated me with the greatest courtesy. He addressed me, however, only through an interpreter despite his perfect ability to speak in the Arabic tongue. Then he forwarded to the royal treasury many loads of unworked native gold and other valuables. I tried to persuade him to go up to the Citadel to meet the sultan, but he refused persistently saying: "I came for the Pilgrimage and nothing else. I do not wish to mix anything else with my Pilgrimage." He had begun to use this argument but I realized that the audience was repugnant to him because he would be obliged to kiss the ground and the sultan's hand. I continue to cajole him and he continued to make excuses but the sultan's protocol demanded that I should bring him into the royal presence, so I kept on at him till he agreed.'

When we came in the sultan's presence we said to him: 'Kiss the ground!' but he refused outright saying: 'How may this be?' Then an intelligent man who was with him whispered to him something we could not understand and he said: 'I make obeisance to God who created me!' then he prostrated himself and went forward to the sultan The sultan half rose to greet him and sat him by his side. They conversed together for a long time, then sultan Musa went out. The sultan sent to him several complete suits of honour for himself, his courtiers, and all those who had come with him, and saddled and bridled horses for himself and his chief courtiers....

This man [Mansa Musa] flooded Cairo with his benefactions. He left no court emir or holder of a royal office without the gift of a load of gold. The Cairenes made incalculable profits out of him and his suite in buying and selling and giving and taking. They exchanged gold until they depressed its value in Egypt and caused its price to fall....

Gold was at a high price in Egypt until they came in that year. The mithqal did not go below 25 dirhams and was generally above, but from that time its value fell and it cheapened in price and has remained cheap till now. The mithqal does not exceed 22 dirhams or less. This has been the state of affairs for about twelve years until this day by reason of the large amount of gold which they brought into Egypt and spent there."

Cited in Levitzion and Hopkins, Corpus of Early Arabic Sources for West African History (Cambridge University Press, 1981, 269-73.)

What do we learn about Mansa Musa, Cairo, and the challenges of cross-cultural contact? 


\section{West African trading centres}

Jenne-Jeno, in present-day Mali, played an important role in trade and agriculture from the late centuries BCE (though the city may have relied on hunting and fishing at first). It thrived from 500-900 CE with a population of 7,00010,000. Rice, sorghum, and millet were cultivated nearby. There was no monumental architecture (that we know of) before Islam - though there was a wall around the city. Jenne-Jeno seems to have been a set of clusters of occupationally specialized towns rather than one centralized city. It owed its importance as a trade centre to its position at the highest point of navigation on the Niger River.

Timbuktu emerged around 1100 as a trading centre. It soon also became famed for its schools and libraries, as well as its general wealth and monuments. Major trading families, which dominated city governance, endowed these schools, libraries, and monuments. These merchant families seem to have been ethnically diverse. Timbuktu often had considerable independence from West African empires. Its population was 30,000 people in 1300 and grew to some 50,000 people. Its governance was likely similar to that in the Hanseatic cities (chapter 15): Merchant families might rise and fall in commercial fortunes and thus political influence.

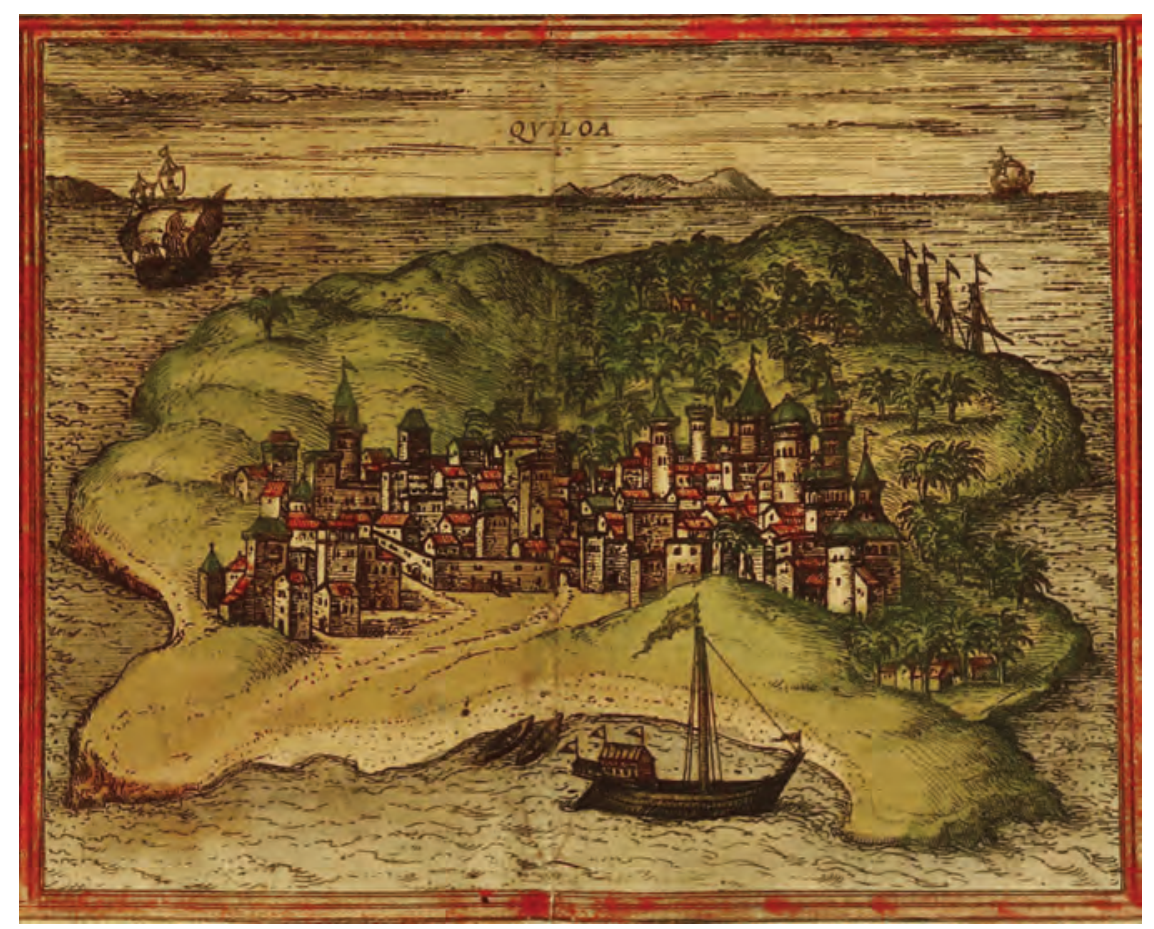

Illustration 16.17

View of the city of Kilwa from Georg Braun and Franz Hogenberg's atlas Civitates orbis terrarum, vol. I, 1572 (slightly later than our time period) 


\section{East Africa}

Swahili is a Bantu language with many Arabic words, used along the east coast of Africa. (It is often mistakenly assumed to be an Arabic language.) Swahili traders were drawn into Muslim networks early, trading gold, ivory, and slaves for pottery, glass, and textiles. Trading ports became wealthy city states, and buildings of stone and coral became common. Kilwa in modernday Mozambique had about 15,000 people, and archaeologists have unearthed staggering amounts of Chinese porcelain there. Swahili merchants and rulers often converted to Islam, but the religion spread slowly into the interior. The Portuguese would subdue these wealthy Swahili city states in the early sixteenth century and they would never fully recover.

Historians long presumed that there was less political development in the interior. However, the town of Great Zimbabwe, near Nyanda in modern Zimbabwe, likely had 18,000 people in the fifteenth century living in stone buildings behind stone walls 32 feet tall. The rulers of Great Zimbabwe controlled the trade (especially in gold) to the coast. It seems that Great Zimbabwe grew over a period of centuries, beginning in the eighth century. It may have controlled an empire as large as those in West Africa. There are no written records that can tell us - even by those who traded with it. Although early European archaeologists hypothesized that the buildings were modelled on those in the Middle East (the ethnocentrism of the time encouraged doubt that Africans could have built such a place on their own), it appears that they were a local architectural development. Great Zimbabwe declined well before European colonization: Scholars hypothesize that there was an environmental crisis as population grew, but perhaps there was simply a decline in gold output.

\section{Kongo}

The kingdom of Kongo came to control much of modern Congo and Angola from the fourteenth century until the seventeenth century, when the Portuguese disrupted it. Cowrie shells were used as currency, and there was substantial trade between coast and interior. The kingdom had six provincial governors who supervised local officials, though most local governance usually remained in the hands of local chiefs. The Portuguese had arrived in 1483, traded, and provided advisers to the king. The kings later converted to Christianity and invoked the divine right of kings. Christian saints were associated with local gods. Yet the Portuguese, while they traded for copper and ivory, were most interested in slaves, and dealt with local slave traders that worked outside state control. Portugal would later attack Kongo from its Angolan colony (see chapter 20). 


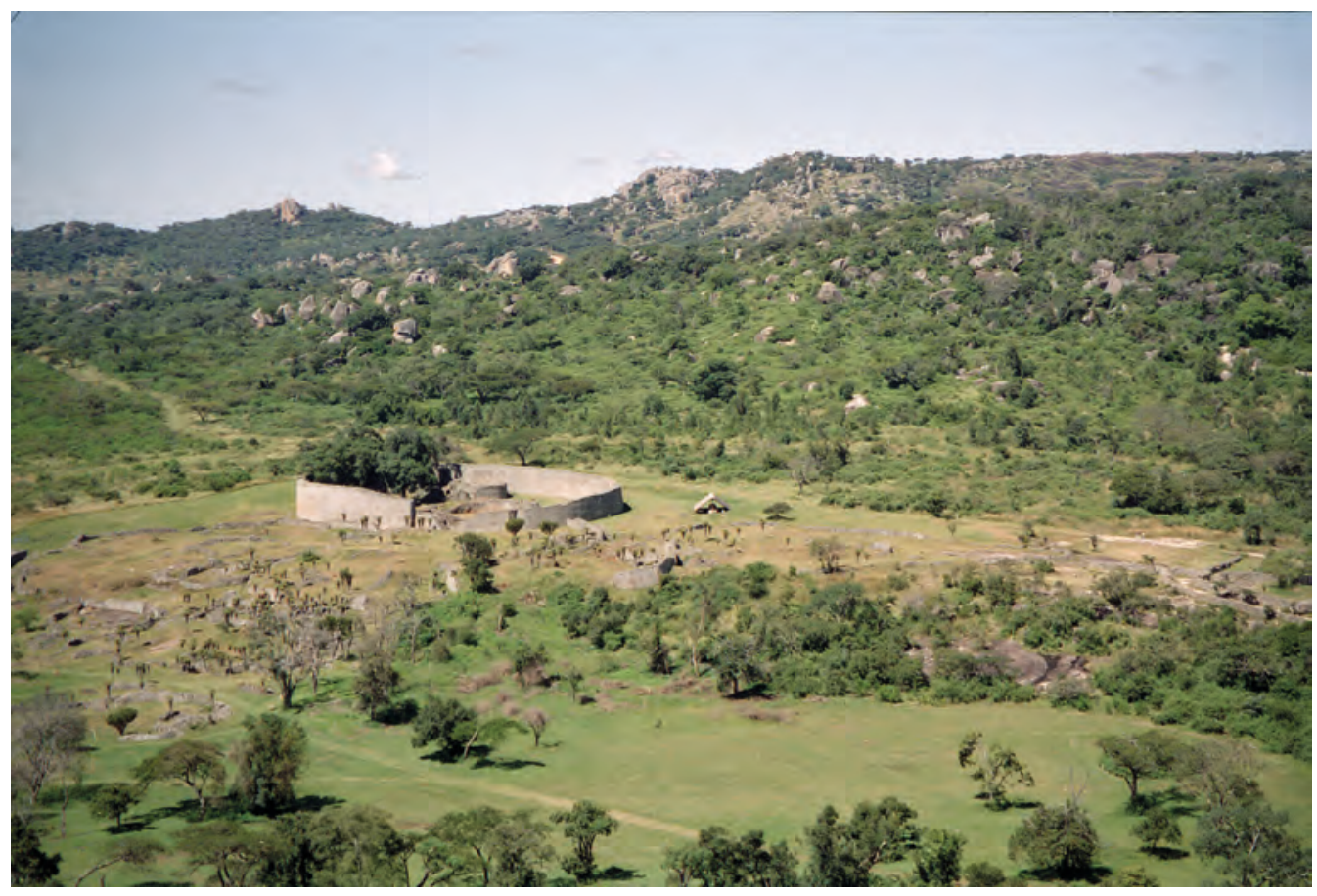

Illustration 16.18

Great Zimbabwe

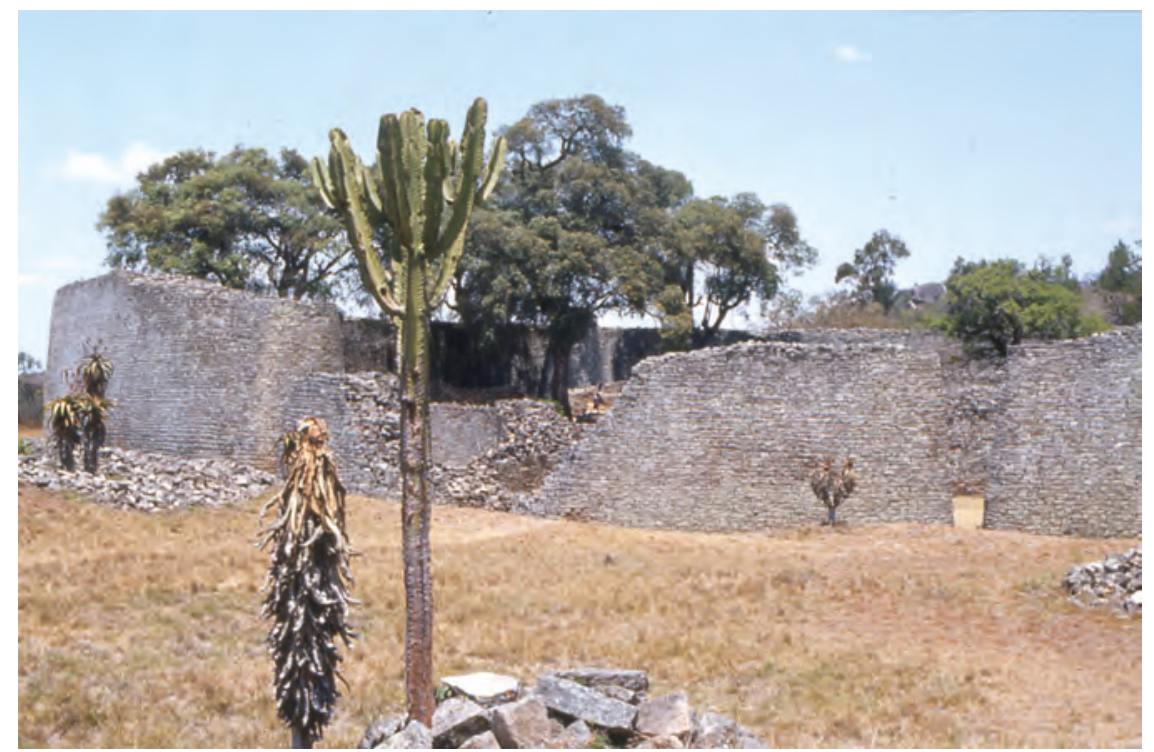

Illustration 16.19

Wall of Great Zimbabwe 


\section{Questions}

1. This chapter surveys quite different parts of the world. Compare the cultures or polities of the three regions studied. Recall that the key to good comparison is to be precise about what you are comparing: Do not compare religion in one society to politics in another.

2. In what ways does the Inca use of quipu inform our understanding of the emergence of writing elsewhere in the world?

3. Do you have enough information to make a decision regarding which region of the world you might have preferred to live in 1200 ?

4. Do Inca and Aztec religions seem to fulfil the roles of religion outlined in chapter 8?

5. One connection among the three regions surveyed in this chapter was that Europeans would disparage each in some way. Why?

\section{Readings}

Cochrane, Ethan E., and Terry L. Hunt, eds. 2018. The Oxford Handbook of Prehistoric Oceania. Oxford University Press.

D'Altroy, Terence N. 2014. The Incas. Hoboken, NJ: Wiley-Blackwell.

Fernández-Armesto, Felipe. 2006. Pathfinders: A Global History of Exploration. Oxford University Press.

Gilbert, Erik T., and Jonathan T. Reynolds. 2012. Africa in World History. 3rd ed. London: Pearson.

Given-Wilson, Chris. 2016. "Bureaucracy without Alphabetic Writing: Governing the Inca Empire, c.1438-1532," in Peter Crooks and Timothy H. Parsons, eds., Empires and Bureaucracy in World History, Cambridge University Press, 81-101.

Gomez, Michael A. 2018. African Dominion: A New History of Empire in Early and Medieval West Africa. Princeton University Press.

Smith, Michael. 2009. The Aztecs. 2nd ed. Malden, MA: Blackwell Publishing. 


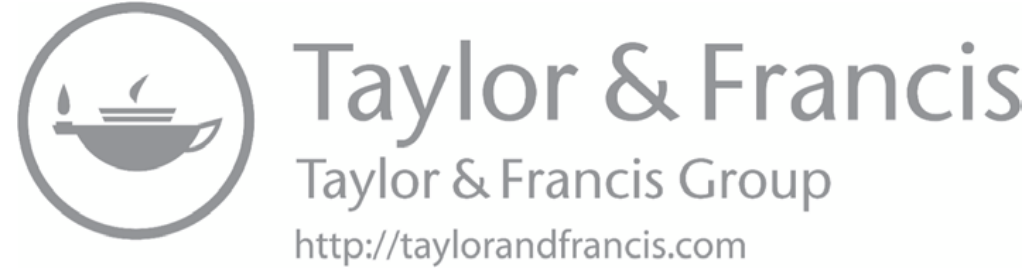




\section{Guiding questions}

How, why, and when did the Mongols create the world's largest ever contiguous empire? Why did they conquer some parts of Eurasia but not others?

What were the effects of the Mongol conquests? On trade? On information flows? On political developments in various regions? On religions? On disease transmission?

Relationship to other chapters: We devote this entire chapter to one historical transformation. In studying the influences upon Mongol conquests, we most obviously draw upon the discussion of nomadic political organization and technology in chapter 13, but also on the development of the states that the Mongols attacked in various other chapters. We draw explicit comparisons with the Islamic conquests of chapter 12. Many effects of the Mongol conquests will reverberate through remaining chapters (see the last set of guiding questions above). The Mongol conquests also place in greater context some of the events addressed in chapter 15: how Europe might have learned about printing and gunpowder; how Mongol conquest fit within the Chinese dynastic cycle; how Southeast Asia and most of Europe escaped direct Mongol conquest and may have benefited as a result. Beyond its treatment of the Mongols, this chapter enhances our general understanding of the challenges of administering a vast empire, the role of credible threats of 
slaughter in war, the benefits of expanded trade and information flows, and the sources and effects of epidemics. We will also reference these general lessons in later chapters.

The Mongol Empire would become the largest land-based empire in history. It emerged in a matter of decades and fell apart almost as fast. It linked most of Eurasia economically and politically, and would have lasting effects throughout Eurasia. We will devote this chapter to discussing its development, its nature, and its consequences.

The sheer vastness of the Mongol Empire has proven a challenge in reconstructing its history: There are useful sources in a variety of languages in archives strewn across Eurasia. It is increasingly possible, though, to integrate the insights of many scholars with different language skills and access to various documents.

We will have cause to engage every one of our key themes in this chapter (making it particularly challenging to list only six in the graphic below): The unparalleled success of the Mongol Empire reflected diverse influences and had various effects. We will trace how, by linking most of Eurasia politically, the Mongol Empire dramatically increased the flow of goods and ideas of all types across societies. We will employ evolutionary analysis most explicitly in our discussion of military technology.

\begin{tabular}{|c|c|c|c|c|c|}
\hline Politics & $\begin{array}{l}\text { Individual } \\
\text { Differences }\end{array}$ & $\begin{array}{c}\text { Social } \\
\text { Structure }\end{array}$ & $\begin{array}{l}\text { Non-human } \\
\text { Environment }\end{array}$ & Technology & Culture \\
\hline
\end{tabular}

\section{Central Asia at the time of the Mongols}

Central Asia was politically fragmented at the time of Chinggis (Genghis) Khan. Manchurian tribes - Khitans and later Jurchens - controlled much of north China. The Khitans controlled the Silk Road route to the west (hence, Europeans called China "Cathay" - from "Khitai" - for centuries). The Mongols had moved from Manchuria to what we now call Mongolia. There were only a few hundred thousand Mongols when Chinggis Khan became their leader. The Mongols were under pressure from the Manchurians on one side and the nomadic Tatars to the west. Yet they were skilled equestrians who loved to hunt and had a fearsome composite bow. Unlike infantry, they could fight far from any supply line for they could live off the land and drink the blood of their horses.

\section{Chinggis Khan}

Chinggis Khan is one of the more controversial figures in human history. The many peoples he conquered often reviled him. Nevertheless, Mongolia 
celebrates him to this day as the father of the Mongolian state (after decades in which the Soviet Union pushed Mongolia to treat him critically) - and Kazakhstan also claims a Chinggis heritage. His face is on the Mongolian currency, the main airport in Mongolia bears his name, and there is a 40-metre statue of him just outside the capital of Ulan Bator.

We know some critical details of Chinggis Khan's early life. He was born (and named Temujin) around 1160 into a respected family, and would likely have learned to ride a horse at the age of four. At the age of nine, he and his mother and siblings fled when Tatars killed his father. He murdered his elder half-brother as an adolescent; he may well have done this to establish a claim as head of the family. His early childhood experiences may have equipped him with a strong desire both for power and for the punishment of those who offended him. He was illiterate.

As a young adult, he pledged allegiance to others but often rebelled against his masters in his quest for personal advancement and power. He made alliances carefully and broke promises when necessary. He was employed by Chinese rulers for a while to suppress nomadic incursions. He experienced many military defeats as he gathered power, occasionally retreating to Manchuria or China, but managed to attract and keep many followers - in part because he seemed un-beholden to the traditional chiefly class. He was a person of great charisma and a skilled warrior. Some Mongol tribes elected him khan in 1190, but others then defeated him. He took the title of Chinggis ("fierce ruler," a title much like "Augustus") finally in 1206 when he was proclaimed great khan of the Mongols.

Chinggis Khan knew how to solidify victory. When he won against other Mongol tribes, he killed their leaders but absorbed their members into his tribe. He later did the same with Tatars and other nomads of the steppe, swelling the ranks of those who identified themselves as Mongols. He then mixed soldiers from different clans, replacing clan leaders with commanders loyal to himself. He trained his army to pursue enemies until they had destroyed their leadership, rather than pausing to collect booty. When Tatars or others refused to submit, he killed them. As more groups joined the Mongols - some by force, others voluntarily - he reinforced group identity by mingling the peoples who swore allegiance to him while ruthlessly attacking others. Perhaps all too aware that he had not always been devoted to the service of others to whom he had sworn allegiance, he insisted on complete loyalty and killed followers who betrayed him in any way.

Chinggis Khan was famously brutal - even allowing for tasteful exaggeration among those he conquered (who were, we should note, much more willing to ignore the brutality of "civilized" rulers). When attacking in the Middle East, he used prisoners both as human shields and to fill the moats. He regularly told towns under attack that he would kill them all if they did not surrender - and carried out this threat on multiple occasions. Late in his life, he apparently ordered the destruction of an entire people - the Tangut - who he thought had reneged on an agreement: Tens of thousands died, though 
others were absorbed into the Mongol people and army. Chinggis is reported to have been a serial rapist, taking (perhaps tens of) thousands of captured women and distributing tens of thousands more among his supporters (genetic analysis suggests that he may have millions of descendants across Eurasia).

Some have called him a psychopath. However, his brutality was not random but instead harnessed to the purpose of conquest. Though we know little of his thoughts, it is not hard to speculate that he felt justified in his conquests. He had grown up in a harsh environment in which battles for power were frequent, and he may have learned that the alternative to conquest was destruction. The armies he directed would kill millions of people.

It is not clear what his original goal was. Some have thought that he only sought to control the tribes of the steppe. As we have noticed previously, military success can have its own momentum. As the enlarged Mongol army began to plunder neighbouring societies, its success attracted even more fighters. Chinggis supposedly had a vision that it was his destiny to rule the entire world. If so, he would hardly be the first or last victorious warrior to imagine this. However, it may also be that it was only with his son Ogedei that the idea of conquest far beyond the Mongol homeland took shape.

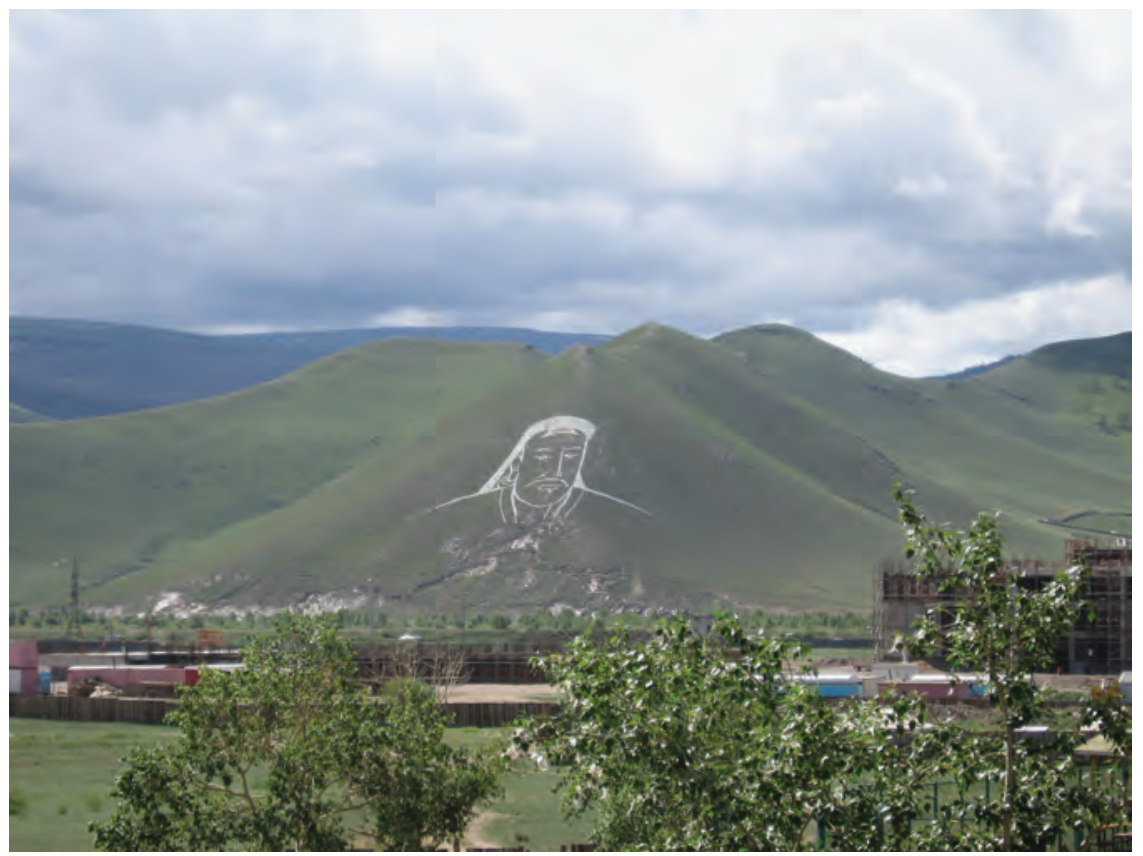

Illustration 17.1

Portrait of Chinggis Khan on Mongolian hillside in 2006 


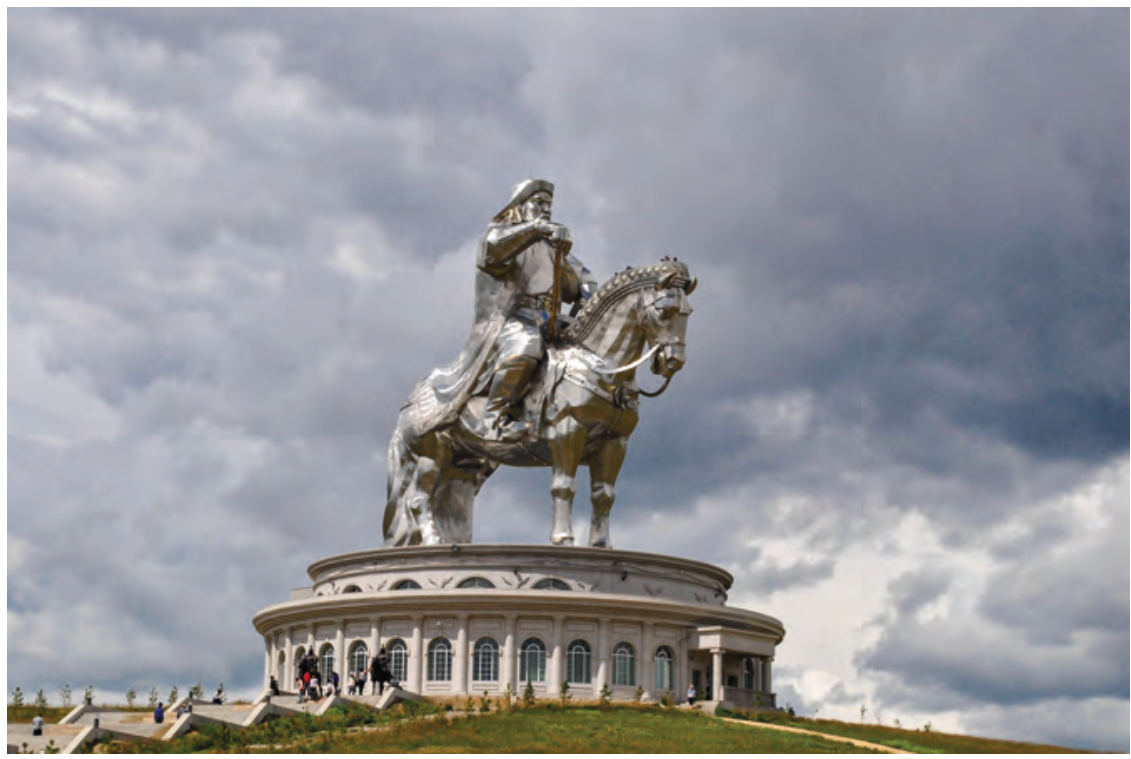

Illustration 17.2

Giant statue of Chinggis Khan near Ulan Bator

\section{BOX 17.1}

\section{TALES OF LEADERSHIP}

There is a mythical story common among both nomadic and sedentary peoples through much of history. A son is born from the coupling of god and a mortal woman. The son is feared and banished as an infant by an unjust ruler but is raised by animals. The unfair ruler attacks the son, but the son gathers righteous followers, wins great battles, and establishes a just rule. Nomadic peoples tell this story from at least classical times. Chinese celebrations of the first Zhou emperor (chapter 7), the Roman tale of Romulus and Remus (who were raised by animals and founded the city of Rome), the biblical Moses, and stories told by Sassanids, Turks, and others, all display elements of the same story. It is possible both that some of the biographies of Chinggis were infused with aspects of this myth and that Chinggis saw himself as replacing undeserving rulers.

The idea of gathering a cadre of devoted followers deserves emphasis. We have seen that rulers must strive to get their bureaucrats and governors to act in their name. A leader attempting to replace a ruler is in a particularly vulnerable position and needs to gather supporters who will be devoted to their leader. The leader then rewards these followers if victorious. Chinggis would gather first a handful and then larger numbers of devoted followers who he would indeed shower with riches. 


\section{Keys to Mongol success}

The Mongol conquests were rapid. Chinggis became great khan in 1206. By 1241, Mongol armies were approaching Vienna in the west. In 1258, they took Baghdad. Within decades, they had conquered China. It took only seven decades to form the largest land empire in human history (though admittedly the core territories of that empire had low population densities). Along the way, the Mongols destroyed some twenty sizeable states and numerous smaller polities. To what can we attribute this fantastic military success?

We have identified some reasons already above and in chapter 13. Nomads had a military advantage in their horsemanship and composite bow. The wood and antler construction of the composite bow provided both tension and compression; it was much more powerful than any other bow of the time, and yet Mongols could fire accurately from a galloping horse. The Mongol bow could pierce chain mail at 300 metres. The stirrup (chapter 13) and the composite bow are innovations that are easily comprehended (though the composite bow, in particular, was tricky to make), but would have profound effects. They are, that is, technological mutations with substantial unforeseen effects. The Chinese who first developed stirrups would hardly have imagined the impact these would have on their nomadic foes. Nomads themselves as they developed the composite bow would likely not have recognized that they were forging global domination.

The usual Mongol strategy was not to engage opposing troops frontally but instead to harass them from afar, charging toward them and then wheeling away while launching a barrage of arrows. Mongol riders could shoot while facing backward. They staged false retreats to entice enemy forces into ambushes. When they did engage in close combat, they employed a curved

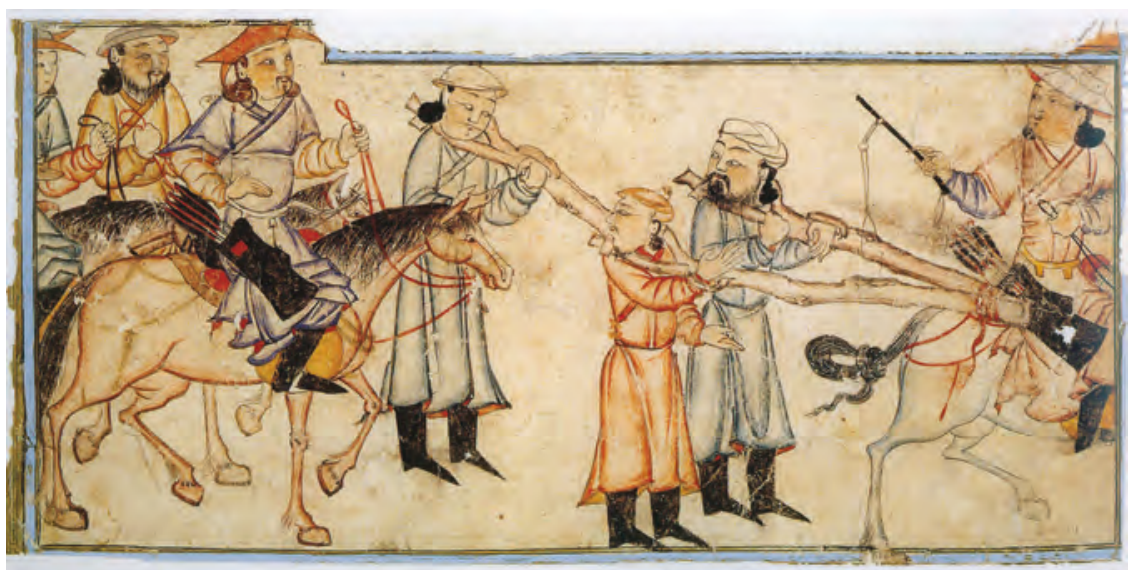

Illustration 17.3

Mongol riders with prisoners, early 14th century, from Rashid-ad-Din's Compendium of Chronicles, Berlin State Library 
sabre that was far better suited to slashing from a moving horse. They often defeated much larger forces. Yet the Mongol success also involved incorporating conquered peoples into their army and attracting volunteers through repeated success. At their height, the Mongols would have a million soldiers. They were a fearsome force: States and cities fell to them without a fight because resistance seemed futile and punishment for resistance severe. They were far from the first to employ fear as a weapon - the Romans had also destroyed entire cities on their way to empire - but arguably used it far more than any other conqueror in history. They deployed survivors of their attacks to sow fear as they advanced.

The Mongols also proved flexible. They adopted catapults and eventually

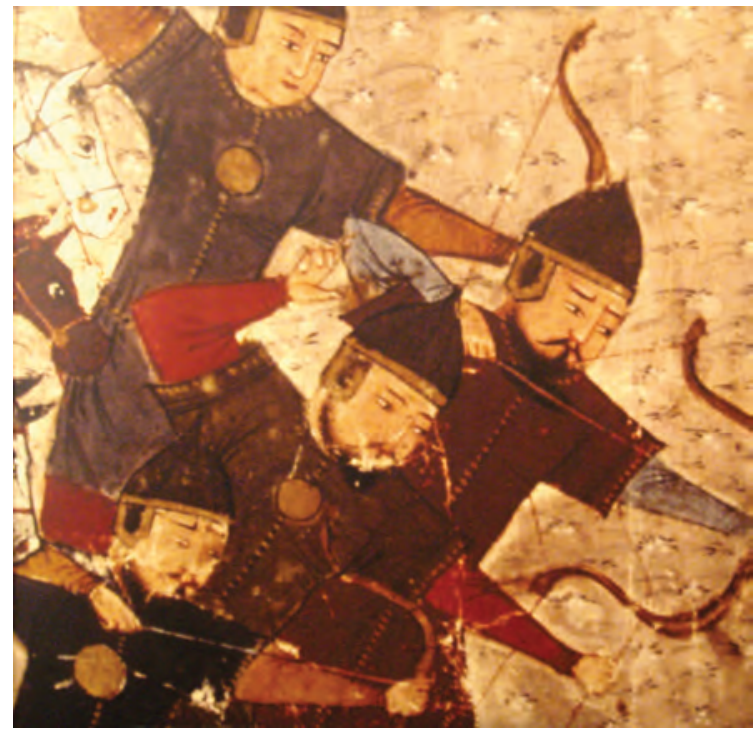

Illustration 17.4

Mongol archers featured in Compendium of Chronicles of Rashid-al-din, 1305 the European trebuchet - a massive sling for hurling rocks at walled cities - and employed experts in their use from among the peoples they had defeated. Later in China, they adopted the use of firebombs, again employing experts in their use. The Mongol army employed both Muslim and Chinese engineers. The Mongols were then able to attack walled cities when necessary, even though they had no experience of these in their homeland. The Mongols even attempted biological warfare, hurling diseased corpses over city walls on more than one occasion.

Nor did they rely on brute force. As in Chinggis's initial rise to power, the Mongols always sought to harass opposing leaders and then invite their disorganized troops to surrender. The Mongols took advantage of conflicts within the societies they attacked, portraying themselves as liberators of minority groups. They employed clever tactics on the battlefield: using dummies to give the appearance of greater numbers, or herding oxen and horses into enemy lines. They used merchants as spies on the lands they were attacking. Although their advance was rapid, they were careful not to over-extend themselves, but to always be able to supply their army (though nomad horsemen could survive for weeks on the blood of their horses) and govern the lands they conquered. They pulled back at the Indus in the 1220s after victories in Iran and Afghanistan, rather than press on into India.

The Mongols were a less complicated society than those they conquered in many ways: less social complexity, less occupational specialization, far simpler governance structures, much less industrial technology, and so on. They nevertheless did prove able to govern more complex societies - albeit usually only for decades. They tended, not surprisingly, to employ existing 


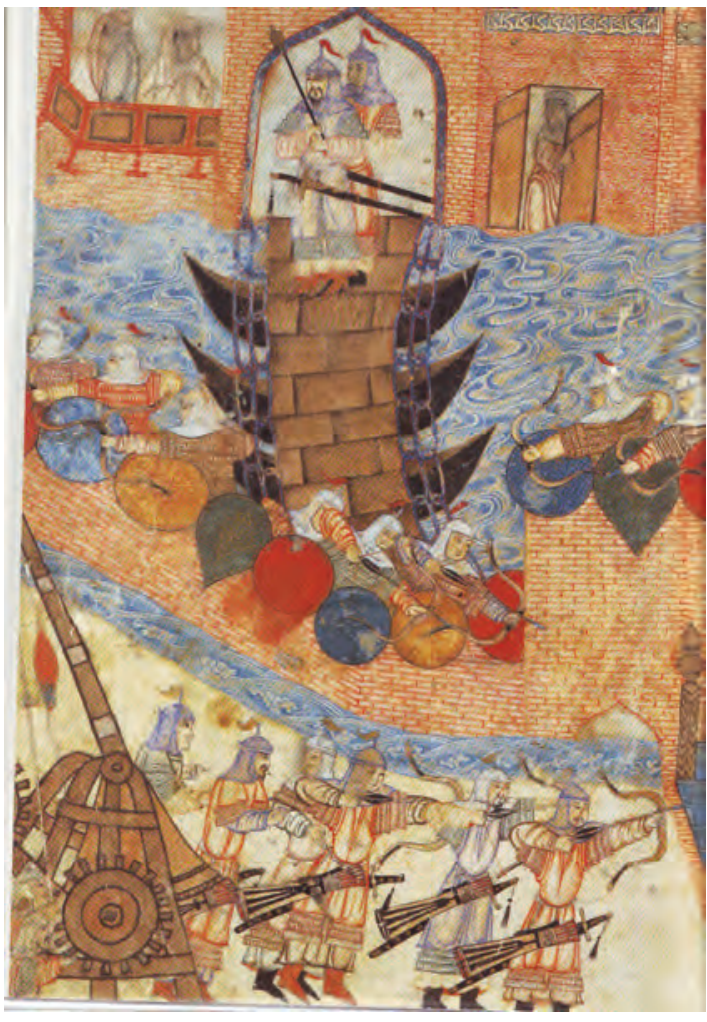

Illustration $\mathbf{1 7 . 5}$

Persian painting of Mongol army besieging a city. Note the use of a catapult bureaucracies wherever possible. Moreover, they were quite willing to accept tribute from areas that surrendered rather than go to the trouble (at least immediately) of governing these.

We noted in chapter 12 that the early success of the Arabs owed much to the weakened state of both Persia and Byzantium. The Mongols also benefited from facing opponents that were weaker than they once had been. This was especially the case in Central Asia: States there were more fragmented than during the golden age of the Silk Road. They may have suffered as intercontinental trade shifted toward the ocean over the centuries. The Abbasids had gradually lost power to Turks, Fatimids, and others. The Byzantine Empire was far smaller and weaker than when it had first faced the Arab invasions. Chinggis Khan in expanding authority over the nomads of the steppe had behaved much like many earlier nomadic leaders; if he had met tougher opposition from sedentary states in Central Asia, the Mongols

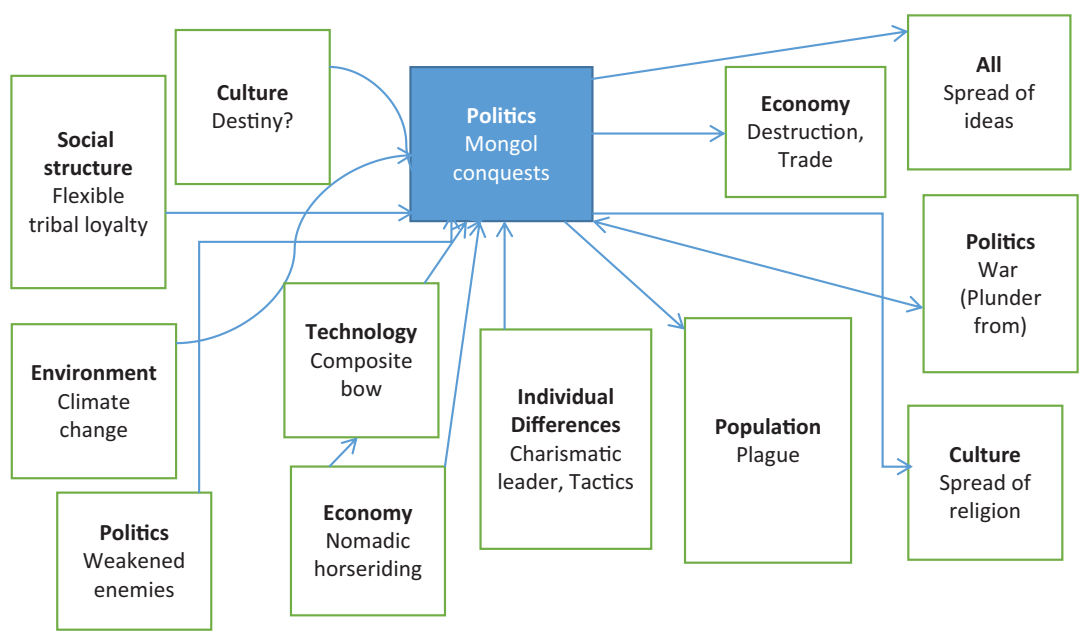

Figure 17.1

Influences on and effects of Mongol conquests

Note the many similarities between this diagram and Figure 12.1 regarding the Islamic conquests. We will discuss the various effects below 
might not have gained the momentum required to seize greater prizes in Persia and China.

\section{PRIMARY DOCUMENTS: VISITING THE MONGOLS}

John Pian del Carpini (about 1182 - 1252) was sent by Pope Innocent IV to visit the Mongols. This is part of his report:

The Tartars [Mongols] have a very different appearance from other peoples. There is more space between their eyes and between their cheekbones than is true of other men... They have flat noses and rather small eyes... Almost all of them are of medium height. Their men have only sparse growths of beard, some letting their wispy moustaches droop long... I will tell something of their good characteristics and then of their bad ones... They show greater respect to their superiors than any other people in the world... They deceive their own masters rarely or never with words and never at all with deeds... They do not fight among themselves: Internal warfare, brawls and assaults do not occur... They respect each other quite well enough and ... throw frequent banquets in spite of the scarcity of good things to eat among them. At the same time, they are so hardy that they can go a day or even two without eating and still sing and joke around as if they had had plenty to eat... Tartar women are chaste: There are not even rumours of immodest female behaviour among them, although the women do sometimes use filthy language... Even though the Tartars get quite drunk often, this does not lead to hostile words or actions among them. Having said this much about their good side, let me go on to their bad one. Their pride is terrible when they confront non-Tartars - nobles and commoners alike - whom they are apt to despise... They show their angry and totally condescending natures to foreigners, to whom they habitually lie. When Tartars speak to non-Tartars the truth is seldom in them. When they start off, their conversation is nice enough, but they sting like scorpions before they are through talking. They are cunning, crafty, and very elusive with their falsehoods. When they have hostile plans toward foreigners, they are experts in concealing them so that the foreigners will not know to be on guard... They are very greedy and shameless with their outrageous demands, while they hold fast to what is theirs and are unbelievably stingy givers. Killing off foreign peoples simply does not bother them.

[...]

Thus, if Christians wish to defend themselves, their countries, and Christianity, it will be necessary for kings, princes, barons, and other chiefs of the lands to cooperate as one and to send men under a consolidated command into battle against them before they have so drained the earth of men that there will be nowhere to draw aid from.

Does he provide an unbiased report? 


\section{Some speculations}

As noted in chapter 10, the Mongols were far from alone in employing fear as a weapon, but they arguably applied this more than most. Certainly, those that they attacked viewed them with horror. Even allowing for the likelihood that it was in the interest of both attacker and defender to exaggerate Mongol atrocities, news that the Mongols were approaching must have been terrifying. We can personalize the experience with a couple of examples: The Mongols allegedly demanded 3,000 virgins as part of their tribute when they took Antioch. Thousands of virgins reportedly leaped to their death in Zhongdu rather than be captured.

What do you do if you are the ruler of Antioch? What do you do if you are a virgin? (You can indeed speculate on the pros and cons of quickly losing your virginity if that is an option.) What if you are a parent? Can you imagine the absolute horror of those in a community that may face a choice between death or enslavement? There may well have been rulers that were better than most rulers and who had governed a reasonably happy polity by the standards of the time who were suddenly faced by the Mongol onslaught. What do they think of their attackers? And what about the commander of the Mongol forces and their soldiers: Are they guided mostly by greed and lust, or a belief that the gods have ordained military domination? Are they thinking of their own families? Do they feel empathy for those on the other side? Are they thinking at all of the potential benefits that may come from Mongol hegemony (see below)?

\section{The unfortunate logic of slaughter}

Chinggis Khan (and his successors) is (in)famous for on more than one occasion slaughtering the entire population of a defeated city or small region. He was hardly the first or last historical figure to act in ways that we would now call genocide or ethnic cleansing. (Women were sometimes killed along with men, at other times taken as wives or concubines; skilled artisans were generally spared.) Yet he may well have been responsible for more indiscriminate killing of this type than any previous conqueror. Allegedly, Mongols killed some 700,000 in Merv and perhaps a million in Nishapur. When they took Baghdad in 1258, the Mongols massacred the caliph and probably 200,000 citizens of Baghdad.

It is common to attribute this killing to the violent and vindictive nature of Chinggis and other such killers. It may well be correct that he and they took some pleasure in wholesale murder. Moreover, there was generally some pretext, some sense of ethnic or religious inferiority or some imagined slight to the conqueror's sense of how wars ought to be fought.

It is also common to appreciate the strategic value of occasional slaughter. Chinggis or his armies could arrive at the walls of a town or city and threaten 
the inhabitants with death should they not immediately surrender. The inhabitants would know that the threat was credible precisely because the armies of Chinggis had done this before. The rapid geographical spread of the Mongol Empire would not have been possible if many peoples had indeed not surrendered without a fight.

Slaughtering a population means that one cannot extract taxes from them in future. The tactic may appear to be costly - unless the conqueror has compatriots to put in their place. Conquest may often be encouraged by population pressure on resources at home, which may in turn reflect either population growth or environmental change. (We noted in chapter 13 that there might have been climatic pressure in Mongolia.) In such a circumstance, there is both an incentive to take lands from others and an abundant supply of young men seeking fortune through military conquest. If a conqueror wants to give lands to his followers, he will be tempted to kill the original inhabitants - perhaps keeping some as slaves and concubines.

When nomads attack agricultural settlements, there is an additional calculation. Can the nomads earn more from their conquest by taxing the locals or by pasturing the nomads' animals on their lands? If the conquered territory is well suited to agriculture, as in China and most of India, it will be most profitable to leave the farmers in place and tax the surplus that they produce. Some Mongol leaders would nevertheless suggest turning northern China into pasture (and the Chinese population may have fallen by as much as 10 million during the Mongol conquest). In marginal agricultural areas, including parts of Iran, Iraq, and northwest India, the nomads might reasonably conclude that they would be better off obliterating the farmers and turning the land to pasture. At least the tradeoff might seem close enough that they could pursue an exercise in bloodlust or in righting perceived wrongs at little cost. Some estimates suggest that the Persian (Iranian) population fell from 2.5 million to just a couple of hundred thousand during the Mongol conquest. It is clear that farmland was turned into pasture, though it is less clear if this was only for military horses.

\section{The post-Chinggis Mongols}

Chinggis divided his empire among his sons, choosing his third son Ogedei to be the primary leader. There would after that be loose cooperation among the different parts of the Mongol Empire. It could be that the empire was too vast and diverse to manage centrally even if not divided among sons. One interesting stratagem for ensuring cooperation is that Mongol leaders were often entitled to incomes from lands in different parts of the empire: This provided a strong incentive toward peace among the Mongols. The Mongols also came to respect a tradition that only descendants of Chinggis should rule (and only, it might be stressed, descendants through his recognized wives). This substantially reduced but hardly eliminated the possibilities for succession struggles. 
The Mongols seemed to be conquering deep into the heart of Europe when Ogedei died without naming a successor. The Mongols withdrew from Hungary as the succession struggle proceeded and their leaders headed back to the Mongolian heartland to stake their claim. The Mongols after that were never quite the expansive force that they had been before. Yet the Mongols did hold together and continue expansion under the regency of Ogedei's sixth wife, the brief and controversial rule of his son Goyuk, and then the regency of Goyuk's wife (during which she and her sons issued contradictory orders). Chinggis's grandson Batu would attack into Eastern Europe and Russia, forming the khanate of the Golden Horde. There was then a Mongol resurgence when Chinggis's grandson Mongke became great khan in 1251. He removed corrupt officials and purged those who opposed him. This was when the Mongol army had a million men: the famed cavalry, but also its siege engineers and infantry (mostly for garrisons). Mongke ordered attacks in the Middle East, defeating many states, including the shrunken Abbasid caliphate, and attacked the Song in China. Mongke's brother Khubilai led one of the armies in China. As noted above, the Mongols took Baghdad in 1258; formally ending the Abbasid caliphate, though the caliphs had been puppet leaders to the Turks for some time. The Mongols then moved across Mesopotamia toward Egypt but were stopped by the Mamluks (a slave elite that took power as the Mongols advanced toward them; see chapter 12).

After Mongke's death in 1259 - perhaps from an arrow or from dysentery while fighting the Chinese - central control of the Mongol Empire dissolved.

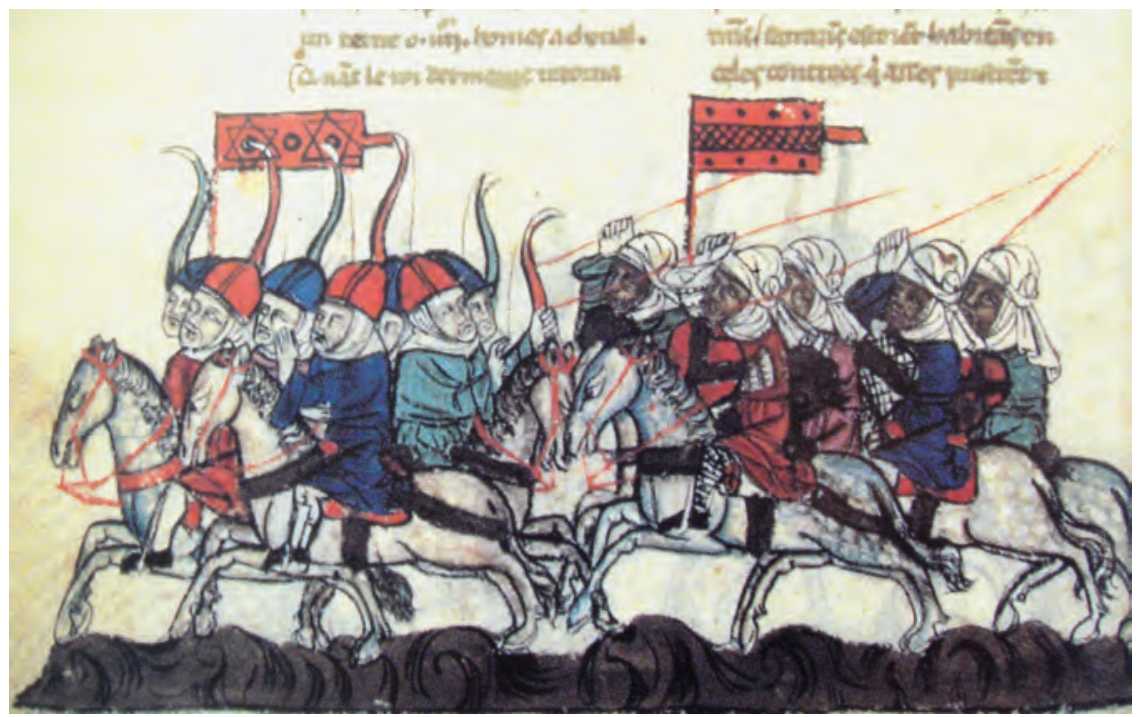

Illustration 17.6

Mongols defeated by Mamluks at Battle of Homs, 1281, from the Histoire des Tartares, Hayton of Coricos, 14th century, Bibliothèque nationale de France 
Khubilai won a battle with another brother for succession, but effectively only had power in Mongolia and China, where he founded the Yuan dynasty. Khubilai then moved his capital into northern China: Mongolia remained an essential source of cavalry but ceased to be the centre of the empire. The movement of the capital from Karakorum in Mongolia reflected in part the attraction of Chinese culture and economy, in part safety from other polities to the west, and in part the perpetual difficulty of provisioning Karakorum. Khubilai then extended the Grand Canal 200 kilometres to ease the provisioning of his new capital (he had two) at Daidu near modern-day Beijing.

Though western khans generally maintained the fiction that Khubilai was the great khan of all, they would abandon this pretence after his death. There were then competing khans in Persia, Central Asia, and on the borderlands between Europe and Asia. There were often wars both within and between these entities. The Mongol khanates in the west all experienced succession disputes. Since Mongol government relied on personal ties between a khan and his followers, succession struggles often led to political fragmentation. As the process of political fragmentation unfolded, Mongol expansion mostly ended in the west.

We noted above that Mongol leaders often had incomes from lands in distant parts of the original Mongol Empire. Khubilai and others would allow those they were fighting to continue to collect revenues. Yet over time, the holdings of individual Mongols became concentrated. Moreover, a further source of disunity emerged as the eastern Mongols adopted Buddhism while the western Mongols adopted Islam (see below).

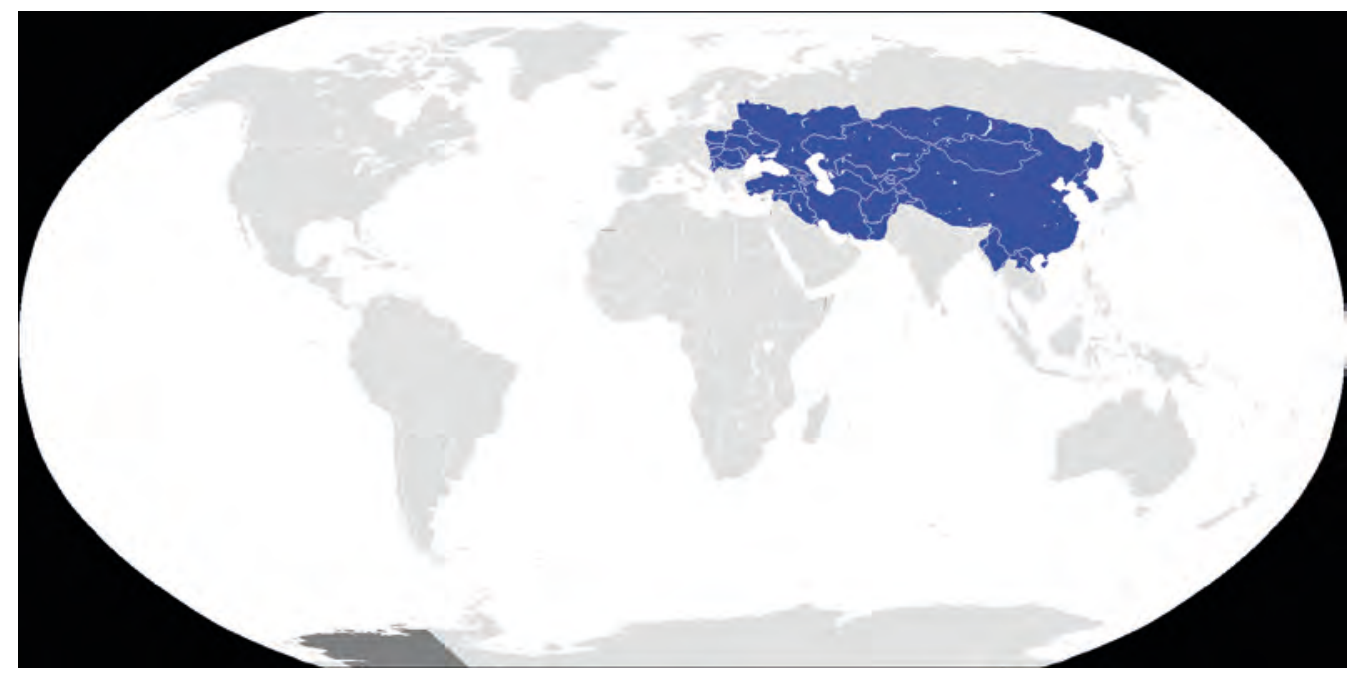

Map 17.1

The Mongol Empire at its greatest extent

Map by Onetwol, CC BY-SA 3.0 


\section{Mongol administration}

Even in the twenty-first century, we can appreciate that it can be much easier to conquer a territory than to administer it. This is especially the case if one destroys local institutions of governance. The Mongols tried not to do so. Though perhaps driven by booty at the outset, they nevertheless ensured that they could tax conquered lands over the longer term. By at least the time of Mongke, the taxation motive dominated the plunder motive. Mongke urged armies to cause little damage as he wanted a prosperous economy to tax. Even under Ogedei, the Mongols had begun to recognize the advantage of regular tax over irregular plunder. Territories that voluntarily submitted to Mongol rule could maintain local elites that raised supportive armies and taxes. In other regions, the military would govern at first but eventually be replaced by a civilian governor. Where possible, these worked through pre-existing administrations. Sometimes the Mongols employed tax farmers, locals who collected taxes in return for a share of these.

There were attempts to homogenize administrative structures across regions. However, Mongol administration was generally quite diverse. The Mongols charged different taxes in different regions: in Persia, a progressive poll tax (that is, a simple form of income tax) was instituted. Land taxes stayed in place where these had been common before conquest. In China, the Mongols pursued monopolies on salt, tea, and iron. Sales taxes of 5-10 per cent were common - but overall the cost of trading was far lower than the previous need to pay customs and tolls in each region (see below). The Ilkhanate (or sub-khanate) in Persia mostly maintained the existing Persian bureaucracy, though Mongols occupied the highest positions. The children of Mongols and Persians were educated together at court, providing some opportunity for mutual understanding. The Golden Horde in Eastern Europe mainly limited themselves to pastoral activities and exacting tribute from neighbouring societies such as the Russians.

The Mongols had adopted a Uighur writing system before the conquest. Though local administrations generally operated in local languages, the central bureaucracy functioned in Mongol and kept records in the Mongol language. The Mongols sponsored the publication of dictionaries that would translate across the diverse languages of the realm. In 1360 in Yemen a dictionary was published that engaged Arabic, Persian, Turkic, Mongol, Greek, and Armenian.

The Mongol capital at Karakorum (in the days before Khubilai moved the capital and Mongol cooperation ceased) was a cosmopolitan place, attracting people from not only throughout various Mongol territories but beyond. A Parisian goldsmith captured by Mongols in Hungary supervised a large staff of artisans there and gained fame for designing both a tree made of silver for the courtyard and a fountain in the tree that supplied alcoholic beverages. Though Marco Polo likely exaggerated his role in the Mongol court, there is little doubt that he was there and that he saw much of what he reported. Both the riches assembled there and the diversity of peoples that he encountered dazzled him. 


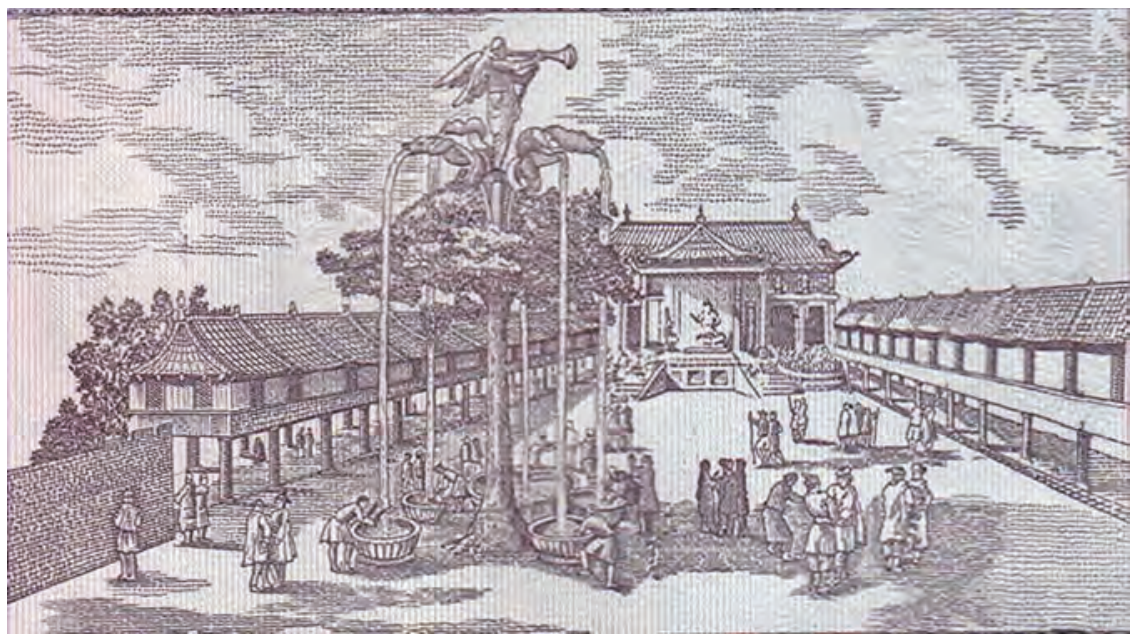

Illustration 17.7

18th-century engraving of the silver tree and drink fountain in the courtyard of the palace in Karakorum, later employed on Mongolian currency

The army remained firmly under Mongol control. Turks and other nomads were often trusted with leadership positions in the army. The army was stationed throughout Mongol lands, and local peoples could be confident of severe punishment for failure to obey authority or pay taxes.

Despite these administrative strategies, most Mongol states lasted only a few decades (though the last Mongol polity in the Crimea was defeated only in the eighteenth century). Their decline seems to have been primarily a result of succession battles. Nevertheless, it could be that Mongol administration did not manage the transition from plunder to taxation effectively enough to allow rulers to maintain the loyalty of their subordinates in the Mongol hierarchy. It is perhaps not a coincidence that the Golden Horde - which mostly pursued pastoralism while exacting tribute from neighbouring farming populations proved among the most durable of Mongol polities, defeated finally by the Russians only in the sixteenth century. The Mongols would also stay in power in the Mongolian heartland, from which they would harass China for centuries.

\section{The role of women}

Women occasionally ruled the Mongol Empire, and generally participated in councils and supervised their own officials. Upon Ogedei's death, his widow and his brother's widow contested for several years to determine whose son should rule: Mothers and sons had separate bureaucracies and gave conflicting orders. The role that women played in Mongol society often astonished travellers from Europe, China, and Islamic lands. Women's visible exercise of influence likely reflects a tendency for women to play a more significant 
role on average in nomadic than in sedentary societies. With men often away hunting or fighting, women had to be able to manage the family's herds. This legacy would survive in Mongol lands but weaken after the Mongol fall. One lasting incentive was for those who could not claim Chinggis as an ancestor to marry a female descendant of Chinggis to strengthen their claims to authority. The respect accorded Mongol women unfortunately contrasts sharply with the disdain often shown to conquered women.

\section{The Yuan Dynasty}

There would be conflict throughout the Yuan Dynasty between those Mongols who favoured the adoption of Chinese practices and those who wished to preserve nomad ways. The Mongols of necessity took over the administrative apparatus of the Song but viewed this with suspicion - and for a while they had a parallel bureaucracy in the original Mongol lands staffed by Mongols and Persians. In the earliest days, the conquerors disdained Chinese agriculturalists, forbade intermarriage, and even forbade the Chinese from learning the Mongol language (these practices would be followed centuries later when Manchurians established the Qing dynasty in China; chapter 20). Some of the conquerors even urged the slaughter of much of the population so that their lands could be grazed. The conquerors reserved the senior positions in the Chinese bureaucracy for themselves (and sometimes outsiders from other Mongol lands). Nevertheless, over time, the Yuan came to adopt Chinese ways and employ Chinese in senior posts. Khubilai himself would have Chinese sages proclaim that he had received the Mandate of Heaven. Khubilai's grandson would reintroduce the examination system in 1315. When the last Yuan emperor fled China after 1368, he was greeted with hostility and civil war in Mongolia, where he and his troops were viewed as not really Mongol any more.

Khubilai had moved his capital to China, eventually to near present-day Beijing. Marco Polo would glorify the Yuan summer palace at Shengdu, which centuries later inspired the mythical Xanadu of Samuel Taylor Coleridge,

The Mongol victory effectively extended the size of the Chinese state. This now included Tibet for the first time. The Yuan also exacted tribute from Korea and Vietnam, though they failed in military expeditions against both Japan and Java. (The Mongols had little previous need for a navy, but drew on Chinese knowledge to carry their troops to distant shores.) When the Yuan reinstated the exam system, they allowed Koreans to study and take exams. Many returned to Korea, where they appear to have changed Korean ideas. Although from the steppe, the Yuan encouraged an expansion of Chinese trade by sea.

The Yuan adopted and encouraged Buddhism, though they were generally tolerant of all religions. The monks he supported recognized Khubilai as a bodhisattva: an enlightened being who guides others to enlightenment. 
Though the Yuan was one of the more lasting Mongol conquests, it too suffered from succession battles. Two emperors would be assassinated, one by a half-brother. The Yuan also faced financial difficulties from very early on. Some of these difficulties reflected the challenges the Mongols faced in absorbing the Chinese institutional structure.

\section{Effects of the Mongol conquests}

The Mongol conquest was harsh. It is estimated that 40 million may have died over the decades of Mongol conquests, or roughly one tenth of the global population at the time. If so, this would be the deadliest conflict in world history in terms of the proportion of the population killed, and it would not be surpassed in terms of total deaths until the World Wars of the twentieth century. In addition to the millions of lives lost, there was considerable destruction of infrastructure: Cities and irrigation systems were often destroyed. The immediate effect of conquest was therefore quite negative. Nevertheless, the establishment of a vast empire had the usual impact of stimulating trade and the exchange of ideas, this time on a particularly grand scale.

\section{Trade and transport}

The Mongols appreciated not only the value of taxing local output and people but of facilitating and then taxing trade. The Silk Road was not merely a conduit by which Chinese goods traded for those of Europe, India, and the Middle East. The nomads in between were active participants who contributed their own trade goods to Silk Road trade (see chapter 13). Nomadic peoples had thus long valued the possibilities of trade. Yet the Silk Road trade had been declining from the ninth century as both the Tang and Abbasid Empires contracted. Trade appears to have expanded significantly with the Mongol conquest until about 1350 - though we should note that trade by sea was expanding at the same time as trade overland through Mongol territories. The Mongols encouraged greater exports of silk, porcelain, and other goods from China. Flemish and Islamic textiles moved east. Mongol conquests also expanded trade to the north, which exported furs and amber. Marco Polo was only one of many Europeans attracted to stories of abundant spices and other goods in China. Even when successor khanates fought each other, they upheld the principle of free-ish trade across borders. Merchants such as the Polos often, though, had to change route to avoid wars.

In 1234, Ogedei instituted a system of posts 40-50 kilometres apart where couriers could gain new horses. These couriers could potentially travel hundreds of kilometres in a day. Merchants could also use these posts. They at first carried a medallion (that they had to pay for) that indicated they had the right to 


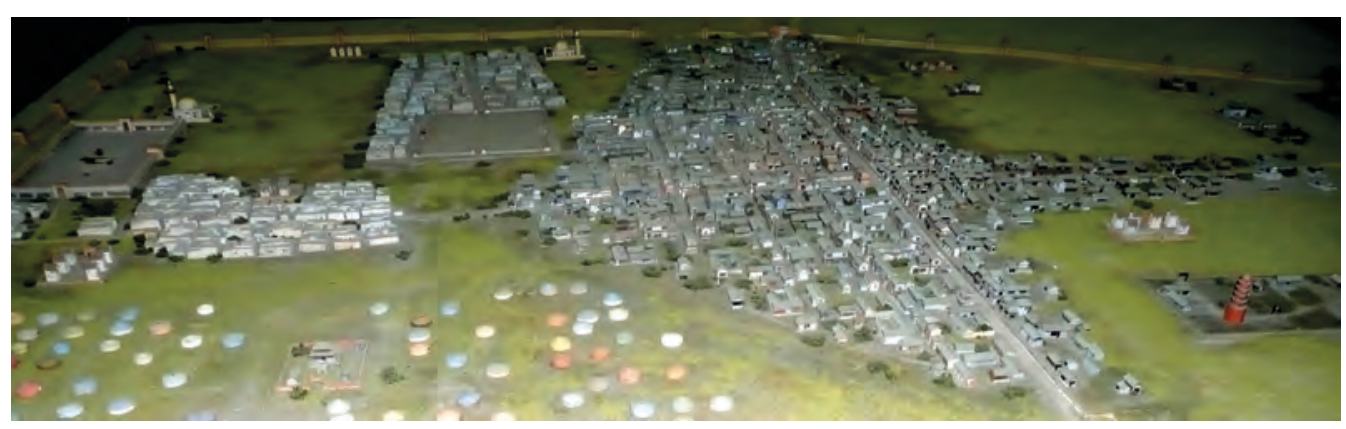

\section{Illustration 17.8}

Model of the city of Karakorum. Note the yurts on the outskirts - which can still be found in Ulan Bator as people migrate from the countryside, National Museum of Mongolian History, Ulan Bator

pass - this may be the origin of the modern term "passport." The duties of locals to support the posts became onerous under some khans as usage of the posts increased. Mongke cancelled the passports so that merchants had to pay for the services of each post. Yet merchants still benefited from access to these posts at a reasonable cost.

The Mongols also policed their empire. It was said at the time that a virgin carrying a gold urn filled with jewels could walk unmolested across the realm. Chinggis had posted guards at trading centres from 1206. The massacre of a trading caravan by the sizeable Khwarzmian empire (which operated from the Zagros mountains to the Syr Darya river) had instigated (or at least rationalized) the Mongol attack and destruction of that state. Yet decades later (after considerable fragmentation of the Mongol realm), Marco Polo would write that, though the Mongols did much to deter attack, travellers still needed to protect themselves from murderous brigands. The Mongols also introduced a paper currency, though this was not accepted everywhere.

As in other trading empires, the Mongol capital at Karakorum became a place where a vast array of goods was available. Karakorum may have only had about 10,000 people (in part because officials camped outside) but it attracted a cosmopolitan population of merchants and artisans. It had Buddhist temples, Muslim mosques, and one Nestorian Christian church. Ogedei built massive storage facilities there, which was a novelty for nomads. Rice wine from China, grape wine, and mead from Russia were all available at the Mongol court, as was the local airag made from fermented milk.

The Mongols not only reduced transport costs but addressed a couple of the challenges that had always worried merchants: depredations by bandits and by petty local rulers. The decentralized nature of Mongol administration, though, must have limited their ability to enforce contracts uniformly across the empire. 


\section{PRIMARY DOCUMENTS: PEGALOTTI'S ADVICE TO MERCHANTS}

Francesco Pegalotti, a fourteenth-century Florentine merchant based in Cyprus, gave advice to merchants heading toward China (called Cathay at the time) based, we think, on the reports of merchants who had been there. This is chapter 2 of his Pratica della Mercatur

Things needful for merchants who desire to make the journey to Cathay above described.

In the first place, you must let your beard grow long and not shave. And at Tana you should furnish yourself with a dragoman (translator/guide). And you must not try to save money in the matter of dragomen by taking a bad one instead of a good one. For the additional wages of the good one will not cost you so much as you will save by having him. And besides the dragoman It will be well to take at least two good men servants, who are acquainted with the Cumanian (Tatar) tongue. And if the merchant likes to take a woman with him from Tana, he can do so; if he does not like to take one there is no obligation, only if he does take one he will be kept much more comfortably than if he does not take one. Howbeit, if he do take one, it will be well that she be acquainted with the Cumanian tongue as well as the men.

And from Tana travelling to Gittarchan you should take with you twenty-five days' provisions, that is to say, flour and salt fish, for as to meat you will find enough of it at all the places along the road. And so also at all the chief stations noted in going from one country to another in the route, according to the number of days set down above, you should furnish yourself with flour and salt fish; other things you wilt find in sufficiency, and especially meat.

The road you travel from Tana to Cathay is perfectly safe, whether by day or by night, according to what the merchants say who have used it. Only if the merchant, in going or coming, should die upon the road, everything belonging to him will become the perquisite of the lord of the country in which he dies, and the officers of the lord will take possession of all. And in like manner if he die in Cathay. But if his brother be with him, or an intimate friend and comrade calling himself his brother, then to such an one they will surrender the property of the deceased, and so it will be rescued.

And there is another danger: this is when the lord of the country dies, and before the new lord who is to have the lordship is proclaimed; during such intervals there have sometimes been irregularities practised on the Franks, and other foreigners. (They call Franks all the Christians of these parts from Romania westward.) And neither will the roads be safe to travel until the other lord be proclaimed who is to reign in room of him who is deceased.

Cathay is a province which contained a multitude of cities and towns. Among others there is one in particular, that is to say the capital city, to which is great resort of merchants, and in which there is a vast amount of trade; and this city is called Cambalec. 
And the said city hath a circuit of one hundred miles, and is all full of people and houses and of dwellers in the said city.

You may calculate that a merchant with a dragoman, and with two men servants, and with goods to the value of twenty-five thousand golden florins, should spend on his way to Cathay from sixty to eighty sommi of silver, and not more if he manage well; and for all the road back again from Cathay to Tana, including the expenses of living and the pay of servants, and all other charges, the cost will be about five sommi per head of pack animals, or something less. And you may reckon the sommo to be worth five golden florins. You may reckon also that each ox-wagon will require one ox, and will carry ten cantars Genoese weight; and the camel-wagon will require three camels, and will carry thirty cantars Genoese weight; and the horse-wagon will require one horse, and will commonly carry six and half cantars of silk, at 250 Genoese pounds to the cantar (a Genoese pound was apparently about 12 ounces). And a bale of silk may be reckoned at between 110 and 115 Genoese pounds.

You may reckon also that from Tana to Sara the road is less safe than on any other part of the journey; and yet even when this part of the road is at its worst, if you are some sixty men in the company you will go as safely as if you were in your own house.

Anyone from Genoa or from Venice, wishing to go to the places above-named, and to make the journey to Cathay, should carry linens with him, and if he visit Organci he will dispose of these well. In Organci he should purchase sommi of silver, and with these he should proceed without making any further investment, unless it be some bales of the very finest stuffs which go in small bulk, and cost no more for carriage than coarser stuffs would do.

Merchants who travel this road can ride on horseback or on asses, or mounted in any way that they list to be mounted.

Whatever silver the merchants may carry with them as far as Cathay the lord of Cathay will take from them and put into his treasury. And to merchants who thus bring silver they give that paper money of theirs in exchange. This is of yellow paper, stamped with the seal of the lord aforesaid. And this money is called balishi; and with this money you can readily buy silk and all other merchandise that you have a desire to buy. And all the people of the country are bound to receive it. And yet you shall not pay a higher price for your goods because your money is of paper. And of the said paper money there are three kinds, one being worth more than another, according to the value which has been established for each by that lord.

And you may reckon that you can buy for one sommo of silver nineteen or twenty pounds of Cathay silk, when reduced to Genoese weight, and that the sommo should weigh eight and a half ounces of Genoa, and should be of the alloy of eleven ounces and seventeen deniers to the pound.

You may reckon also that in Cathay you should get three or three and a half pieces of damasked silk for a sommo; and from three and a half to five pieces of nacchetti of silk and gold, likewise for a sommo of silver.

What can we learn about the Mongol Empire from such a source? What can we learn about the life of a merchant? 


\section{Exchange of ideas}

The most obvious exchange was in goods. Chinese porcelain had been exported previously but became particularly prized in the Middle East and parts of Europe during this period. The Chinese learned from the Middle East of the blue glaze that came to characterize much of their porcelain: The cobalt used to produce blue would be known in China as "Muslim blue." Mongol bureaucrats encouraged the production of blue glaze for markets farther west. Western producers were inspired to try to emulate Chinese porcelain. Porcelain also gained its English name through the Mongol-era travels of Marco Polo: He compared its sheen to that of cowrie shells, known in Italian as porcelanna for "little piglets." The Mongols moved weavers from Central Asia to China to produce "cloth of gold" textiles.

Agricultural products and foodstuffs were exchanged. Chickpeas moved east from the Middle East to China. The Persian elite came to value rice. Noodles, long a staple of the Chinese diet (millet is particularly suitable for making noodles), moved west. Hard wheat moved eastward into China: This proved particularly useful in the making of dumplings.

The Mongols pursued a deliberate practice of identifying skilled people and moving these to other parts of the realm where their skills might be useful. Allegedly, 30,000 artisans were shipped eastward when Samarkand was taken, though this number seems very high. The Mongol capital, in particular, became a place where skilled artisans from across Eurasia gathered. Though these interactions are hard to trace, industrial techniques undoubtedly were shared and combined across societies.

There was also mingling of artistic traditions: We see styles and objects of art move between regions. Persian art and literature in particular spread throughout Mongol lands (and beyond). Chinese scenes and styles also became common in the western parts of the empire. Some art historians detect eastern influences on the later art of the European Renaissance (and that art was financed in part by the profits European merchants made trading to the east).

Islamic and Chinese astronomy interacted at the Mongol observatory at Marageh. (Some scholars claim that Tuli there established the sun-centred solar system centuries before Copernicus, though this is controversial.) Medical ideas (and many doctors) also moved between Islam and China. Avicenna's book on medicine from the early eleventh century was in the Chinese imperial library in 1273. The Mongols sponsored cartography as well as astronomy and developed much better maps of Eurasia.

There is no direct evidence of Gutenberg in Europe learning of eastern printing presses, but his design was very similar. People in the Middle East certainly knew about Chinese printing presses but did not adopt these, perhaps because the Arabic script was challenging. (The wheelbarrow is another Chinese invention that appears in Europe at this time.)

The case for the transfer of knowledge about gunpowder is somewhat clearer. The Chinese used primitive gunpowder weapons from the tenth century (it 


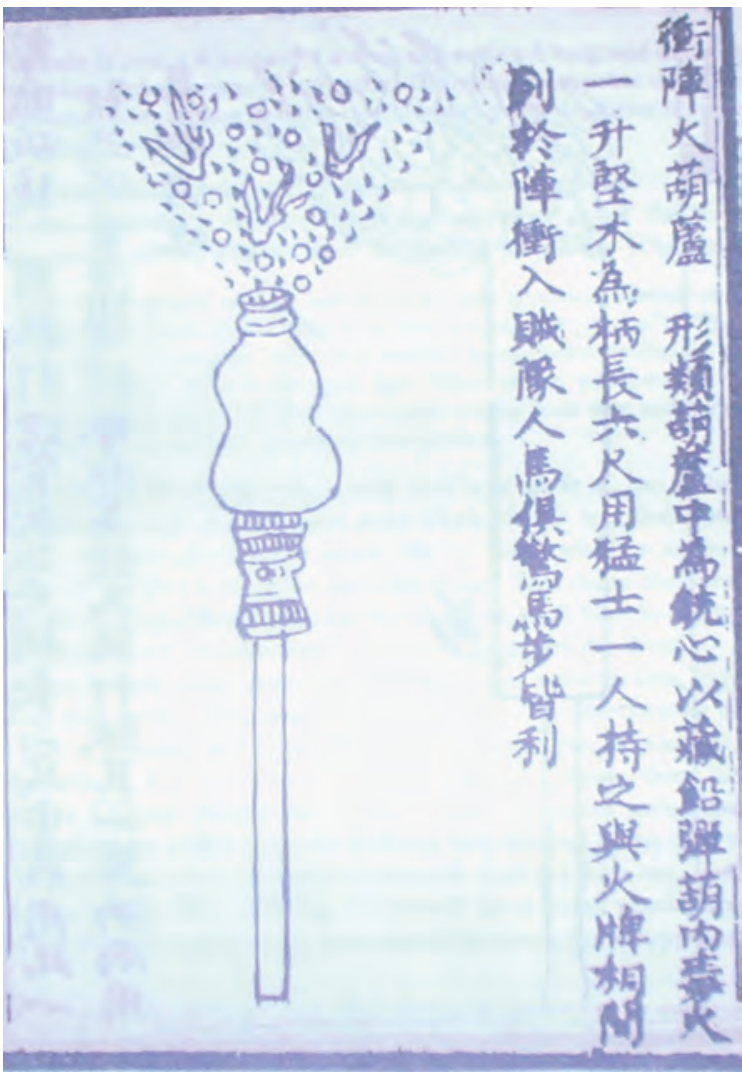

Illustration 17.9

An illustration of a flask-shaped Chinese gunpowder fire-lance shooting a blast of flame and lead pellets. The weapon was called the "phalanx-charging firegourd" (chong zhen huo hu-lu). This illustration is from the Huolongjing, a 14th-century military treatise compiled by Liu Ji and Jiao Yu is a mistake to think that the Chinese only used gunpowder for fireworks, as is often alleged). The Mongols certainly used gunpowder within China itself, and seem to have fired gunpowder "bombs" into besieged cities in the Middle East. We can be confident - though documentary evidence is lacking - that knowledge of the recipe for gunpowder passed through the Mongol Empire to Europe. This certainly fits with the timing of the first use of gunpowder in Europe. It is also entirely possible that the idea of the cannon, developed by the Mongols in China, also spread. The Mongols would not have worried too much about transferring gunpowder technology. Early firearms were too limited in range, accuracy, and speed to be much use against mounted archers. Firearms would, centuries later, prove of much greater use in the infantry wars of western Europe (chapter 18). Gunpowder technology then developed away from the steppe over the centuries to be quicker, mobile, more accurate, and more powerful; it would eventually erase the military advantage of horse archers.

Rashid-al-Din converted from Judaism to Islam as an adult and rose in the bureaucracy to become vizier. He was later executed, as was all but one vizier in the history of the Ilkhanate. He hoped in his Compendium of Chronicles (completed between 1307 and 1316) to capture the history and knowledge of all human groups, now that the Mongol peace encouraged interaction across these. His work reflects the cosmopolitan ethos of the time. The book was only possible because he could indeed gain information about virtually every group of people that then inhabited Eurasia. Although he could not know about the Americas, Oceania, or most of Africa, his work was the first world history with an extensive geographical coverage - though it tended to treat each group separately rather than to stress their interactions or similarities.

The existence of the Mongol Empire, its administrative diversity, and efforts to regularize administrative practices, must have encouraged the sharing of 


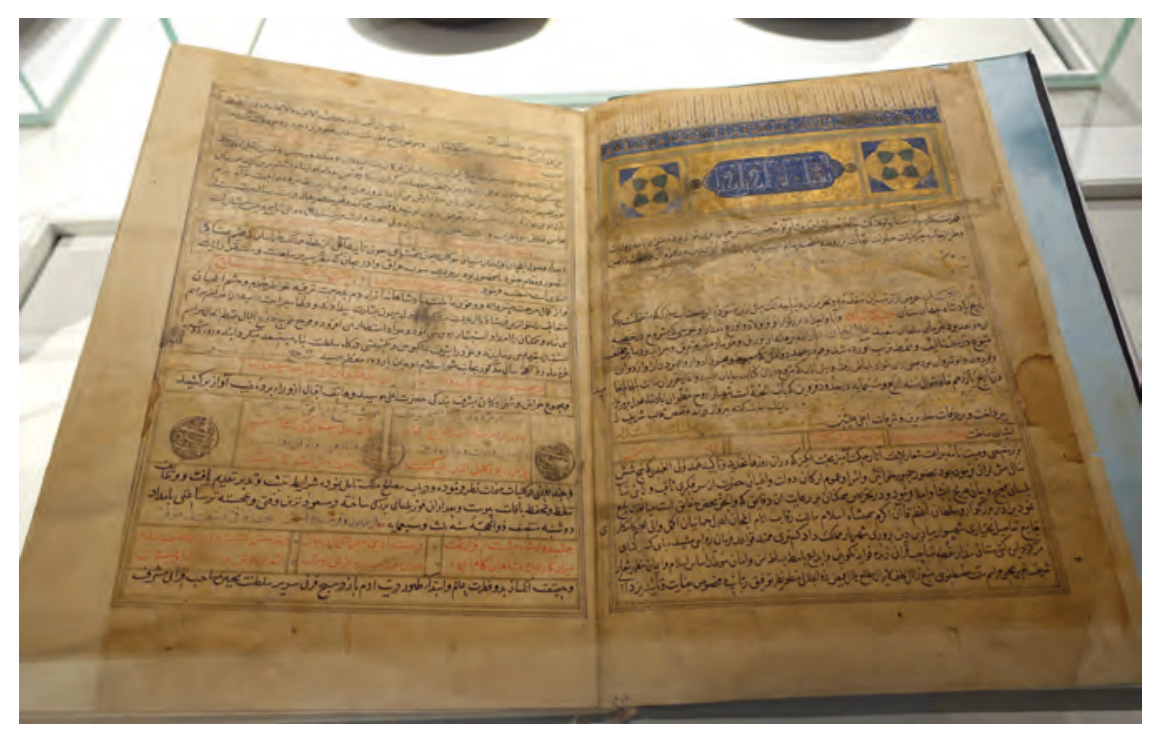

Illustration 17.10

Compendium of Chronicles, copy of manuscript by Rashid al-Din, Fazlullah, Iran, early 15th century CE, ink, watercolour, and gold on paper, Aga Khan Museum, Toronto, Canada

Photograph by Daderot, CC0 1.0

administrative practices across regions. There were also diplomatic contacts between the Mongols and other states in Europe, North Africa, Japan, and Southeast Asia. This sharing of administrative ideas may have been particularly important for China: The earliest Chinese empires had necessarily developed autonomously, but now there was direct contact between Chinese bureaucrats and bureaucrats from other lands.

\section{The Plague}

The Great Plague of the mid fourteenth century seems to have started on the steppe - though some speculate that it emerged farther south in Yunnan in China, where the disease may have been endemic. In either case, local peoples may have developed immunity to the disease. The plague spread across Eurasia along Mongol trade routes. Fleas from marmots or some other animal transferred the plague; humans in other regions had no resistance to the disease. There may also have been a climatic influence at work: A cold climate that hurt the rodent population might have encouraged fleas to seek human hosts. The plague was first recorded along the northern caravan route in present-day Kyrgyzstan in 1338. It reached Crimea in 1346, and spread from there into Europe. Mongols besieging the city of Caffa over a dispute 


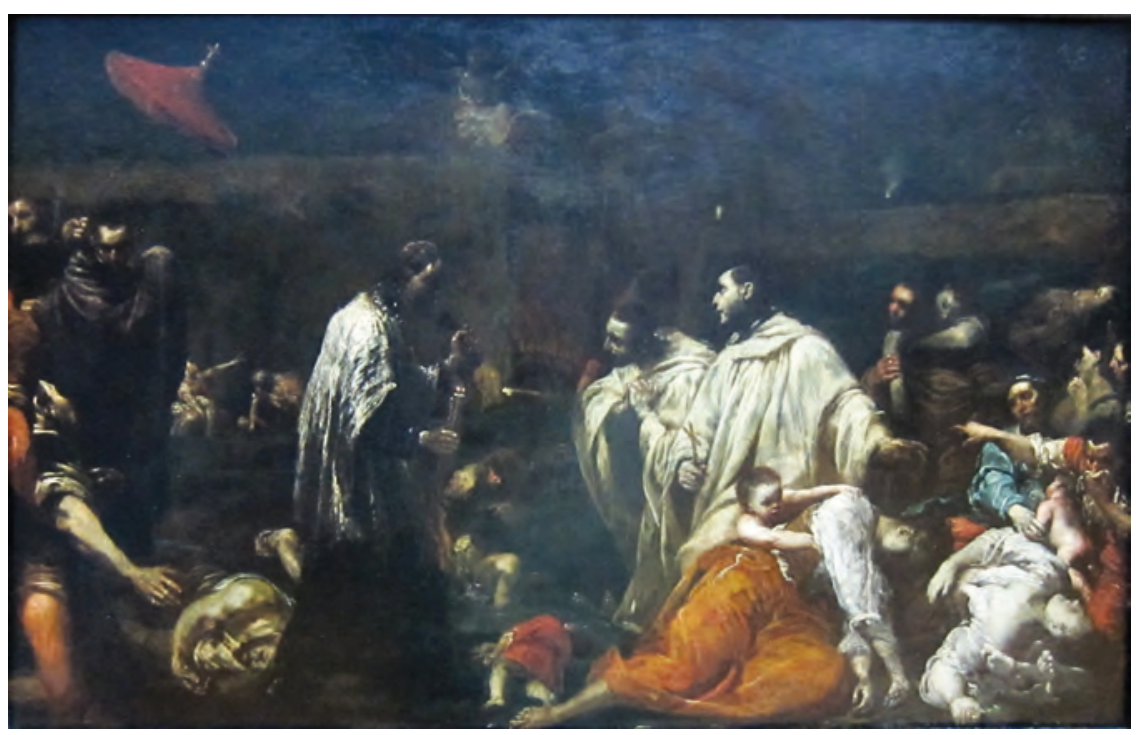

Illustration 17.11

Bernardo Tolomei among Victims of the Black Plague in Sienna, 1348, Giusseppe Crespi, 1735

Photograph by Wmpearl, CC0

with Venetians launched infected body parts into the town. (Since fleas soon abandon the dead, rats likely carried the infection into town - but some fleas may have travelled with body parts.) Escaping merchants carried the disease westward (Venice refused them entry, but they sailed on). As often in epidemics, then, both trade and war encouraged the spread of disease. The plague in Europe caused the questioning of religion, introduced women to crafts such as beer production as their husbands died, and may have encouraged labour-saving technology. A third of the population is thought to have died (some recent estimates, based on careful examination of parish or city records, say over half), though death rates were far higher in some localities. Scandinavia escaped, presumably because the disease-carrying fleas could not survive harsh winters. There were likely similar death tolls across Mongol lands; these may even have contributed to the breakup of Mongol states by introducing social disruption. Mortality in China was less severe, though about one-eighth of the population likely died. Some 50 million may have died globally, making this the second most deadly single epidemic in history after the Spanish influenza of the 1910s, and by far the worst in terms of global mortality rate. Trade across Eurasia contracted as economic activity declined and as cities limited the access of outsiders that might carry disease. It is unclear why India and sub-Saharan Africa were spared, though heat may be a factor. 
Postscript: Between 2010 and 2015, the World Health Organization (WHO) reported 3,248 cases of human plague worldwide: 96 per cent of these were in Africa - with Madagascar particularly hard hit - but there were also five deaths in the United States where the plague became endemic in rodents after transmission to San Francisco in 1900. Hundreds more died in Madagascar in 2017. With signs of antibiotic-resistant strains, plague can be considered a re-emerging infectious disease and global threat. More generally, historical understanding of the high mortality associated with the plague informs modern reactions to various epidemics: People often compare these to the plague and are aware that death tolls in the tens of millions or more are possible. Though modern quarantine methods have limited Ebola mortality to the thousands, it is noteworthy that Ebola, like the plague of the fourteenth century, was a surprise with no cure and an extended incubation period that facilitated transmission. The Covid-19 pandemic that began in 2019 provided the world with a further reminder that new diseases could kill many people across diverse lands; it also showed the economic and political disruption that such a pandemic could cause.

Fleas were likely the primary transmission mechanism, though human-tohuman transmission is possible (especially in colder and humid climates). The plague seems to have spread faster than either transmission mechanism can explain. Though Europe, in particular, was utterly unprepared for the plague, it would have success over the next decades and centuries with quarantine measures that tried both to limit travel from infected regions and to place infected people in isolation wards. Such actions would only have been effective to the degree that humans rather than fleas were the primary transmitters.

The plague at first killed indiscriminately: Young and old, and rich and poor, and men and women had very similar death rates. When the plague returned periodically, the young might suffer disproportionately merely because the old had achieved immunity during previous periods of plague. The rich over time became adept at limiting their contact with the sick. Women in some localities suffered disproportionately if they cared for or otherwise interacted with the sick.

The Plague tended to raise wages everywhere as lords and merchants competed over a diminished labour supply. Wages doubled in many European cities. Many states introduced laws enforcing "traditional" wage rates (a practice often observed in history after epidemics, including after the Justinian plague of the sixth century and by the Spanish in the Americas), but these were hard to enforce. Some states in eastern Europe, as well as Mamluk Egypt, used force to tie peasants to the land, but in western Europe, feudal relations weakened in the aftermath of the plague. The value of land fell if lords had to compete for 
labour. As noted in chapter 5, the general effect of the Plague was a dramatic reduction in income inequality across much of Eurasia.

There were interesting cultural responses to the Plague. Muslims were far more willing than Christians to understand the Plague as godly punishment for misbehaviour. This limited - but hardly eliminated - an appreciation of contagion. Religious authorities often viewed flight from the disease negatively: One should accept god's will. Yet over time, some Christian religious leaders came to argue that it was indeed acceptable and even laudable to try to save one's life by fleeing from the disease. What, though, to do when friends or family fell ill? Many people across Eurasia made the difficult decision to abandon sick family members to their fate rather than risk infection.

\section{Religion}

The Mongols were unlike most other empires of their time in not being guided by a missionary religion. The Mongols themselves were mostly animists, though some had converted to Buddhism or Nestorian Christianity (Ogedei's chief bureaucrat was a Nestorian). Animism may have encouraged them not to want to offend any spirits. Religious toleration was common on the steppe, and the Mongols practised toleration during their conquests. They recognized a strategic value in getting local religious authorities on side and spared these from taxes. Their toleration extended to diverse religions: Christians, Jews, Muslims, Zoroastrians, and Buddhists (and of many different sects within these faiths). Some Muslim clerics in conquered lands even praised the just rule of non-Muslim Mongols.

Especially as the Mongols advanced, they were the subject of missionary efforts. Popes and European kings hoped to convert the Mongols, especially as these came to threaten European lands, and felt that the presence of Nestorian Christians among the Mongol leadership was a good sign (even if Nestorianism was considered a heresy). They had trouble getting missionaries to visit the Mongols. The Polos carried a message from Khubilai asking for a hundred Christian missionaries, but Rome only sent two and these quickly returned. Ironically, the Mongols may have disdained Christianity in part because they thought it was opposed to alcohol and suspicious of trade and luxury - problems arising from contact mainly through a small number of atypical missionaries.

Buddhists also tried to convert the Mongols. Buddhism spread in parts of the Empire, like Persia, where it had previously disappeared (the Mongols at times moved groups around purposely, but Buddhism may have spread voluntarily in Persia). The Uighurs had converted to Buddhism before the rise of the Mongols and were influential in Mongol administration (recall that they had developed the written Mongol script). Khubilai in China would come to adopt and support Buddhism, though doctrinal disputes, especially between Tibetan and Chinese versions of Buddhism, would trouble him. The first rulers of the Persian Ilkhanate also pursued Buddhism. 


\section{BOX 17.2}

\section{EPIDEMICS}

The Great Plague is neither the first nor the last epidemic that we shall encounter. We have already seen that epidemics gripped Eurasia in both the third and sixth centuries (these may have combined plague, influenza, and measles). Epidemics (mainly of smallpox, measles, and influenza) will later decimate populations in the Americas and Oceania when these first encountered people from Eurasia (chapter 19). The connection between epidemic and cross-cultural contact is not accidental. Human populations in regular contact with a disease may develop immunity. Immunity may be hereditary, but for many diseases, it is (before the modern age of vaccinations) something that each generation must acquire. Children may die from the disease, but those who survive will have developed antibodies that protect them through life. High rates of infant and child mortality were a characteristic of most human societies in history, in part because of deaths through infectious disease. When a population with immunity to a disease encountered a community without immunity, high rates of adult mortality in the second population could result. The early Silk Roads, the Mongol caravan routes, and later European explorations would bring immune communities into contact with others lacking immunity. In the case of the Plague and some other epidemics (including Covid-19), there is also transmission from animal hosts with immunity to human hosts without immunity; this often requires some genetic transformation in the disease itself, as well as close contact between humans and animals.

Epidemics may be especially severe in weakened populations, but this depends on the nature of the disease: The Plague killed the well fed and the hungry alike. On the other hand, tuberculosis was far more dangerous in poor and overcrowded circumstances. Epidemics are thus only sometimes a truly "Malthusian" force reflecting population pressure on resources. Yet the widespread mortality they unleash can reduce population pressure on resources for decades.

The Plague was not the first epidemic to afflict multiple continents. However, with increased trade links this would become the norm. The further connections between continents that emerged in the late fifteenth century (chapter 19) would only increase the tendency for epidemics to be transmitted across continents.

We should close with a clarification of terminology. "Plague" is sometimes used in common parlance as a synonym for "epidemic." However, plague is a particular type of disease, caused by a specific pathogen, the bacterium Yersinia pestis, and with a unique set of symptoms.

There is a story that Khubilai was offended when Muslim guests refused his food; he then declared it a capital crime to perform halal (the Muslim ritual slaughter of animals). (Rashid al-Din blamed Christian advisers for this decision.) Many Muslim merchants then left China. The Chinese Empire eventually reversed this decree. We need to understand this story in the context of the prominence of Muslim merchants and tax collectors in Yuan China, used by the Yuan precisely because they lacked local ties. While non-Chinese Muslims 
would leave, an ethnic Chinese Muslim population remained in western China and was prominent in trade with Muslim nations.

Islam at first had little success in converting Mongols in Central Asia - indeed Mongol conquests caused a crisis of faith in Islam for that religion had always seemed to expand before. In the late thirteenth and early fourteenth century, though, the Mongol khans in the west converted to Islam. Notably, these conversions only occurred after the Mongol Empire fractured into pieces, perhaps because rulers then needed greater public support, especially in fighting against other Muslim powers such as the Mamluks in Egypt. Maybe also the previous confidence that "god" favoured the Mongols had dissipated. Islam was usually the majority religion in their realms by the time the rulers converted, having been spread beyond the borders of earlier Islamic states by merchants and missionaries. Mongol soldiers often converted before their leaders as they married local women. After conversion, Mongol rulers showed a religious preference. Buddhist temples often became mosques, though rulers still usually practised toleration, at least for a while. This Mongol conversion would solidify the position of Islam in Central Asia (see chapter 11).

Ghazan Khan of Persia would convert to Islam in 1295. Historians suggest that he did so to appease rebellious Muslim leaders. His attack on Buddhists after that may have been motivated in large part by booty. Buddhists were allowed to practise but in a weakened financial state. When Rashid al-Din (see above) was asked to write first a Mongol and then a world history, he was able to talk to many Buddhists and describe elements of many types of Buddhist practice. Moreover, he did so in a positive way that drew parallels with Islam (treating Buddha as a prophet, emphasizing judgement for sins). One unique characteristic of this period of Muslim-Buddhist cohabitation is that Muslims often portrayed Muhammad pictorially, a practice generally shunned within Islam: Pictorial representations of the Buddha likely influenced Muslims.

While most Mongol leaders converted to Islam after much of their population had, the rulers of Chaghatai to the north (stretching from Samarkand to China) converted while most of the population was Buddhist (albeit concentrated in the eastern part of the realm). They practised toleration and even supported the building of Buddhist monasteries. They may have converted to placate local Muslims and encourage trade to the west. Over time, they became less tolerant.

The Oirads, nomads who operated in Mongolia as the Yuan state was imploding, were Muslim when their main trade links were with Islamic states to the west but became Buddhist in the fifteenth century as they came to trade more with China. They are one of the very few groups historically to convert in that direction. They would come to identify their state with Buddhism to distinguish it from Muslim states to the west. Though the Oirads were soon defeated (for a while at least), later Mongol and Manchu rulers in the region would also identify with Buddhism. As a result, Mongolia would be a mostly Buddhist region thereafter. The later Mongol leader Altan Khan would meet with the third Dalai Lama, and Mongols would consequently emphasize Tibetan Buddhism. The Ming dynasty in China encouraged the spread of Buddhism 
among the Mongols, hoping that this would make them less warlike.

We described the emergence of Buddhism in Tibet in chapter 13. The high altitudes and harsh winters of Tibet appear to have protected it from successful attack (both humans and horses suffered from altitude sickness) by Islamic rulers. Sa'id Khan declared holy war against Tibet in 1532. This was one of the first times in centuries that jihad had been invoked against Buddhists. Tibet retained its independence.

By the sixteenth century, one can identify distinct Muslim and Buddhist polities in Central Asia, with clear borders. Over time, religious minorities would move or convert.

\section{After the fall}

The political fragmentation that

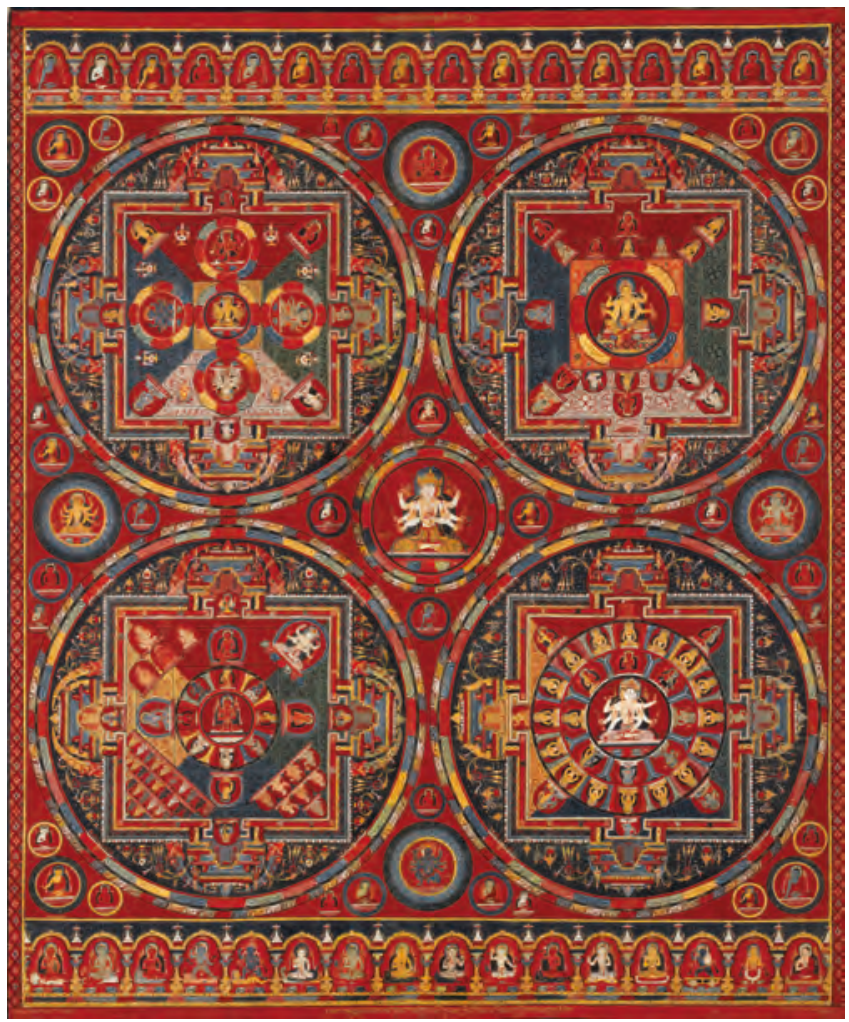

\section{Illustration 17.12}

15th-century Tibetan painting of mandalas, a Hindu-Buddhist religious symbol

resulted from the fall of the Mongol Empire would lead to a contraction in trade and the flow of ideas. The English playwright Shakespeare (active 1585-1613) would imagine that the khans still ruled China centuries after the Ming had chased the Yuan emperor back to Mongolia. Nevertheless, we shall see in later chapters that rulers across the former Mongol territories would draw on both Mongol practices and Mongol symbolism to fashion empires that, though far smaller than the Mongol Empire, would also last much longer.

\section{Muscovy}

As noted above, the Mongols ruled lightly in many lands. The Golden Horde, in particular, was often satisfied with payments of tribute. Arguably, then, Russia was not as affected by the nomads as were China, India, and the Middle East, though Russians were for centuries captured in raids and sold south into slavery. Russian princes could maintain traditional political institutions - though many came to adopt Mongol attire. Some have wondered if Russian traditions of autocracy also reflect, at least in part, the experience of Mongol rule. Russians were among those who learned horse archery to oppose the Mongols, in part 
while serving in Mongol armies. Although Mongol conquests at first disrupted Russian trading networks, Russians later participated in growing Mongol trade.

Muscovy gained power by collecting taxes/tribute for the Mongols; it grew in importance relative to more established entities like Novgorod. It would slowly

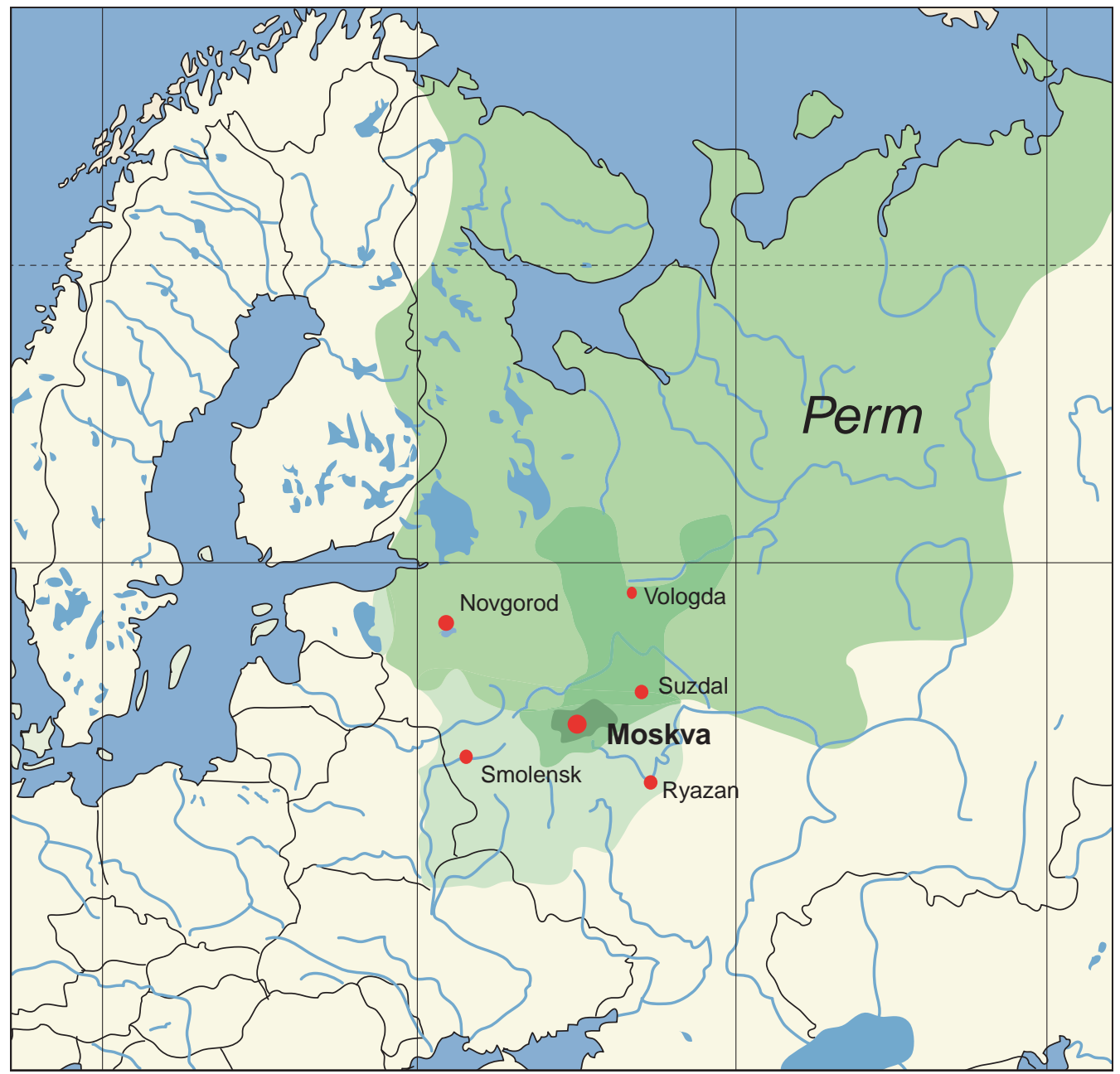

Grand Duchy of Moscow (Muscovy) between 1390 and 1547.

Core territory of Muscovy, AD 1300

Territory of Vladimir-Suzdal, acquired by Muscovy by 1390

Territory acquired by 1505 (Ivan III)

Territory acquired by Vasili III (r. 1505-1533)

Modern national borders are shown for orientation.

Map 17.2

The expansion of Muscovy 1390-1550

David Liuzzo 
conquer other Russian polities while also holding off expansionary Catholic polities to its west such as Lithuania and Poland. It would take Russian-speaking territories from Lithuania. Ivan III built a large army with which he could defeat the Mongols; he also married the niece of the last Byzantine emperor and proclaimed himself Tsar (Caesar) and the inheritor of the Roman/Byzantine tradition. Russia would soon begin to expand south and east into lands still governed by nomads. These lands had few people at the time but now could be transformed into farmland. These lands were often settled by religious or political dissidents, sometimes independently and sometimes with state encouragement. The Russian state was like the British in North America, who were happy to see religious minorities settle, but unlike the French, who feared disloyalty from such a policy. This tradition of using dissidents of various types as frontier settlers would continue well into the days of the Soviet Union.

\section{Timur}

In the late fourteenth century, Timur (often called Tamerlane in the west, or Timur-the-lame since he walked with a limp) would create an empire from Baghdad to Delhi and north to the Aral Sea. His is a story with many parallels to that of Chinggis, full of shifting and strategic alliances and considerable violence. Like Chinggis, he conquered in many directions. Several European

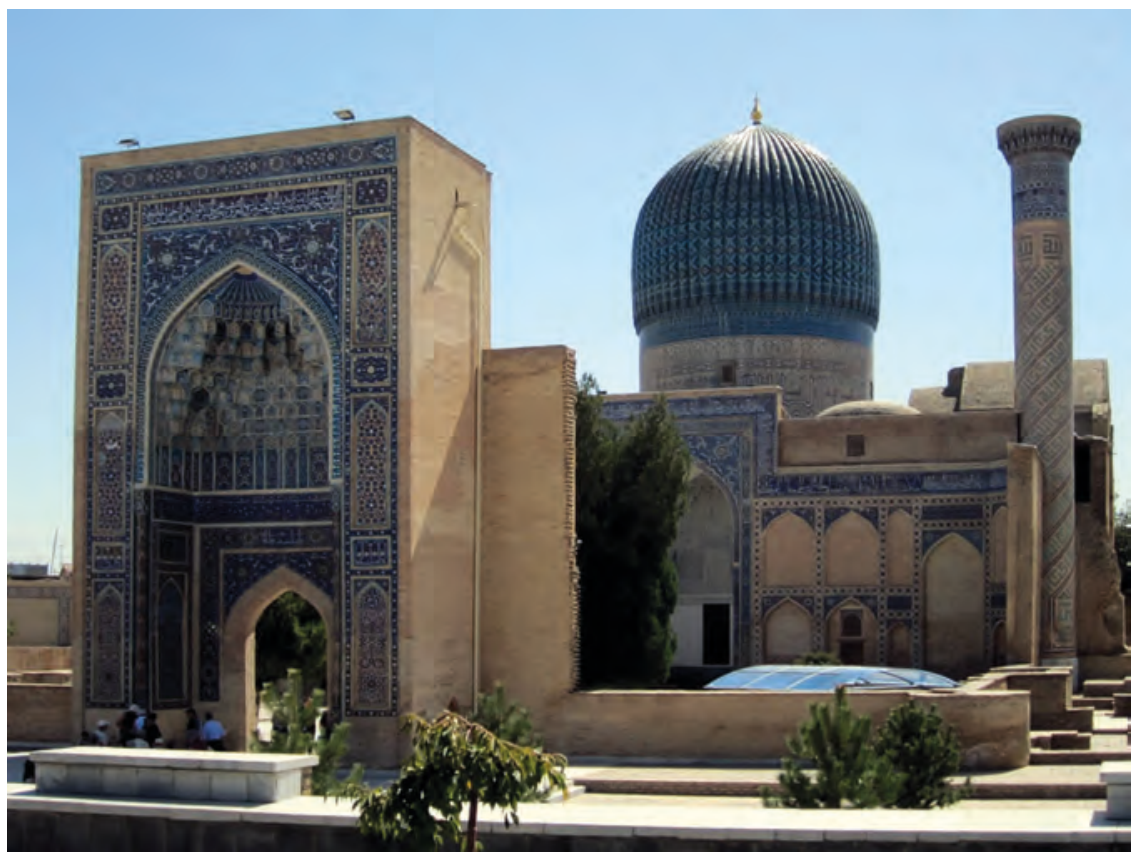

Illustration 17.13

Timur is buried in the Guri Amir Mausoleum (1404) in Samarkand, Uzbekistan Photograph by David Stanley from Nanaimo, Canada, CC BY 2.0 
kings congratulated him for winning battles against the Muslim Ottomans in Anatolia and the Golden Horde north of the Black Sea. He was heading to attack China when he died in 1405.

Timur, though Turkic rather than Mongol, compared himself explicitly to Chinggis: hard childhood, overcame many defeats, touched by the gods. He had married into the Chinggisid line to justify his rule (though his father had claimed descent from Chinggis), and one of their descendants, Babur, would later found the Mughal Empire in India (chapter 20). He also developed a reputation for brutality. He enslaved Indians to build a grand mosque in Samarkand and built piles of the skulls of less fortunate Indians, but he also slaughtered fellow Muslims. He allegedly used the captured Ottoman sultan as a footstool and imprisoned him in a cage too small to stand or lie down in, while forcing his favourite wife to serve meals naked. He might have continued into Europe after defeating the Ottomans, but he chose instead to attack China. His empire fragmented after his death. He had appointed relatives as governors of various parts of his realm, and they saw little reason to cooperate after the end of the constant stream of booty associated with conquest.

\section{Questions}

1. Compare Figures 12.1 and 17.1. What are the critical differences between the two conquests?

2. Evaluate Chinggis or Timur according to the personality dimensions identified in Table 1.2.

3. What are Rashid al-Din's challenges?

4. The Mongol conquests brought destruction but then increased trade flows within and beyond their borders. Is it likely that regions such as Europe or Southeast Asia that were mostly untouched by the Mongols would have developed economically or politically relative to the lands conquered by Mongols?

5. How does an understanding of historical pandemics affect our attitude toward possible future pandemics?

\section{Readings}

Aberth, John. 2011. Plagues in World History. Lanham, MD: Rowman \& Littlefield. [Discusses the plague as well as epidemics of cholera, smallpox, tuberculosis, influenza, and AIDS; in all cases discusses social processes that often involved blaming outsiders and suspicions of government efforts to control disease.]

Alfani, Guido, and Tommy E. Murphy. 2017. "Plague and Lethal Epidemics in the Pre-Industrial World." Journal of Economic History 77:1, 314-43. [Provides a great overview of the influences on and effects of epidemics in world history.] 
Beckwith, Christopher. 2011. Empires of the Silk Road: A History of Central Eurasia from the Bronze Age to the Present. Princeton University Press.

Campbell, Bruce M.S. 2016. The Great Transition: Climate, Disease and Society in the Late-Medieval World. Cambridge University Press. [Argues that the Plague set northwest Europe on course to the Great Divergence (chapter 22).]

Delgado, James P. 2008. Khubilai Khan's Lost Fleet: In Search of a Legendary Armada. University of California Press. [Puts failed attempt to conquer Japan in context.]

Elverskog, Johan. 2010. Buddhism and Islam on the Silk Road. Philadelphia, PA: University of Pennsylvania Press.

May, Timothy. 2012. The Mongol Conquests in World History. London: Reaktion.

Rossabi, Morris. 2011. The Mongols and Global History. New York: Norton.

White, M. 2011. Atrocities: The 100 Deadliest Episodes in Human History. New York: Norton. [He claims that the Mongol attacks are among the deadliest, and then attributes another 17 million deaths to Timur. The 100 deadliest events are listed at: https://en.wikipedia.org/wiki/The_Great_Big_Book_of_Horrible_ Things] 


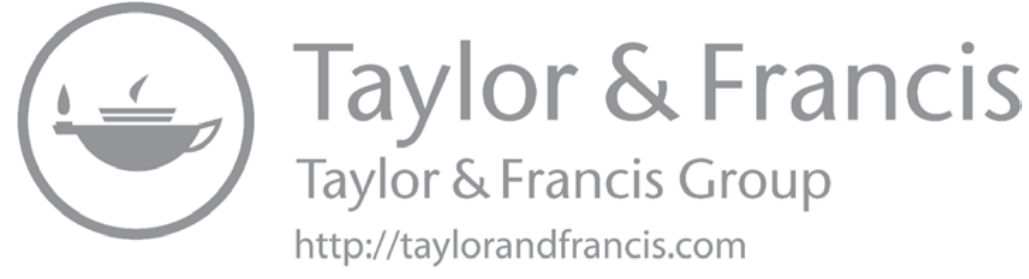


The Early Modern period 


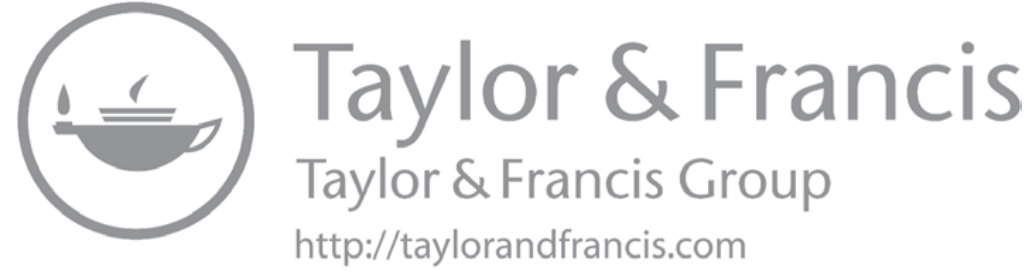




\section{Thematic}

\section{developments in the Early Modern period $1450-1800$}

\section{Guiding question}

Can we observe common trends across multiple regions in the centuries after about $1450 \mathrm{CE}$ ? If so, why and what effects did these trends have?

How did the climate change in this period and what effects did these changes have?

How, why, when, and where did political consolidation proceed in this time period?

Why do we see important changes in most of the world's religions during this period? What are the main similarities and differences in these changes? How do religions interact?

How and why is gunpowder technology developed in different parts of the world? Why in particular do some regions develop more powerful weapons than others? What are the effects of this divergence in gunpowder technology?

How, why, and where does trade expand in this period?

What is capitalism? When, where, how, and why does it develop?

What effects does it have?

Why, where, and when does population expand?

Relationship to other chapters: This chapter most obviously picks up where chapter 14 left off, in particular in tracing 
continued developments in political consolidation, trade, and population. We update our general understandings of these themes but also engage in more detailed discussions of laws, trading networks, and the transmission of artistic practices. Our discussions here naturally inform later chapters since political consolidation, trade expansion, and population growth continue and indeed accelerate in later time periods. Our examination of religion naturally builds on our preceding discussions, especially in chapter 11 . We also in this chapter introduce some important new topics. We have briefly mentioned gunpowder before but here for the first time discuss it in detail but drawing on previous discussions of military technology and war. We shall see that the divergence across regions in gunpowder technology has immense implications for political and military interactions between regions. We shall see that capitalism is a multifaceted term: Some characteristics of capitalism have emerged in previous time periods, but others become prominent only in this time period.

The period from about 1450 to 1800 is often termed "Early Modern," for it has struck many historians that the stage was set during this time for the dramatic changes that would take place in the nineteenth and twentieth centuries. We should, of course, strive to avoid a teleological approach to history that treats only those topics that directly inform our understanding of the present. We thus start this chapter with a brief review of the climatic situation in the Early Modern period.

Political consolidation is an important characteristic of both this and later periods in history (as it was of the last period; chapter 14). We describe some characteristics of consolidation in this period below. We then address the global emergence of the nation state in chapter 21. But empires remain important throughout this period. We discuss comparatively the main empires of Asia in chapter 20 .

The most dramatic story during the Early Modern period is the development of trade links among all continents (excepting Antarctica). We discuss some general trends in trade later in this chapter. In chapter 19, we describe the development of new trade routes and the effects of these. These new trade routes may (or not) have had an impact on one critical characteristic of the nineteenth century: the widening gap in per capita incomes between Europe and the rest of the world. We explore in chapter 22 the question of whether we can identify sources of this "Great Divergence" in the Early Modern period. 
Europe is also militarily dominant by the nineteenth century. We discuss in this chapter developments in gunpowder technology during the Early Modern period. Though gunpowder was invented and employed in Asia for centuries before it was used in Europe, European nations would develop more powerful gunpowder weapons. We explore the possibility that this was because of more extensive warfare in Europe. We also note the more general fact that gunpowder technology aided political consolidation.

We also discuss some critical religious developments below. And we address the origins of "capitalism." Since that word has multiple definitions, we consider several distinct characteristics of capitalism. We close with a brief review of trends in population.

This chapter will address diverse themes as it proceeds, and will often draw comparisons across societies. We will also discuss how different religions interacted in this period, and how gunpowder technology and artistic styles spread globally. We will employ evolutionary analysis most explicitly in our discussions of laws, gunpowder, and artistic styles.

\section{Climate}

Climatically, this was not a particularly favourable period. Colder than normal temperatures across the northern hemisphere (at least) from the fourteenth through nineteenth centuries, but especially the late seventeenth and early eighteenth centuries, have caused this period to be referred to as the Little Ice Age. There were more frequent crop failures and famines as a result. In 1709 ice on French rivers prevented famine relief. Yet despite this negative climatic influence, this was a period of political and economic transformation.

\section{Political consolidation}

\section{Politics}
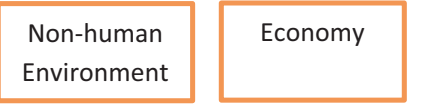

Culture

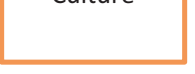

In all regions of the world, territorial consolidation - that is the expansion of states geographically - would accelerate from the fifteenth or sixteenth centuries, continuing into the nineteenth century. Likewise, there would be considerable internal political consolidation, as these states developed more extensive bureaucracies that were capable of exercising similar degrees of effectiveness throughout the state's territory (whereas preceding empires often governed through local elites in distant provinces). Censuses and detailed maps were among the characteristics of this period of bureaucratic development. These bureaucracies often pursued policies of cultural and especially linguistic 
consolidation (see chapter 21) - though this was still a period when empires sought geographic expansion with limited concern about ethnic boundaries.

\section{LaWS}

As bureaucracies expanded and sought uniformity in governance, they codified systems of law. As we have seen in preceding chapters, institutions in general and laws in particular evolve over time. Bureaucrats could not just create whatever laws they liked but instead built on existing precedent.

A fundamental difference would emerge across states concerning the settlement of contract disputes. In some lands, there was a tradition of mediation in which those involved in the dispute were expected to find their way to a mutually satisfactory solution. In other lands, it had become common instead to pursue adjudication in which a judge would make a decision that would generally satisfy one party in the dispute more than the other. Mediation was slow and uncertain though it probably on average generated results that were fairer than adjudication. Yet the speed and greater predictability of adjudication - since judges relied on precedent and law codes in rendering decisions - may have been more conducive to commerce. Europe and Japan leaned toward adjudication while China and the Islamic world relied heavily on mediation.

These different preferences were associated with a distinction between "private" and "public" law. In China and the Islamic world, laws were decreed by the state. In Europe and Japan, some laws were allowed instead to emerge organically through the accumulation of precedent. A professional class of lawyers and judges emerged that over time would develop principles to guide adjudication. This similarity in Japanese and European practice likely facilitated Japanese adoption of particular European laws in the nineteenth and twentieth centuries. More generally we might imagine that private law provides a superior selection environment in which laws might be selected for the general good (or at least the good of the social class from which most judges and lawyers came) rather than the good of the state.

Political fragmentation in Europe encouraged competition for laws that would promote innovation, investment, and commerce. Though laws evolved through precedent, they often were borrowed if thought to be superior. As it commercialized from the thirteenth century, the Netherlands adopted laws from the Flemish and English. Importantly, the long tradition of granting foreign merchants the right to adjudicate their own cases gradually disappeared in Europe: As legal systems converged, merchants no longer saw the need for this sort of protection.

There were other differences in legal traditions. Islamic courts preferred witnesses whereas European courts preferred written testimony. Confucian tradition limited an individual's ability to sell family property. As a result, it became commonly accepted - from the time of the Song (chapter 15), who wished to facilitate land sales - that if the price of land increased soon after a 
sale, the original seller received some of the benefits. Such an outcome might be considered fair and facilitate transactions (for the seller would not worry as much that they should wait for prices to rise before selling), but it might discourage investment by the new owner. It is doubtful, though, that this one institution had a huge impact on economic decisions in China.

In early Islamic states, religious scholars fiercely defended their right to set laws. The Ottoman Empire (among others) would assert the state's authority to establish laws and appoint judges - though in practice they generally negotiated with religious leaders. Though Islam itself is sometimes blamed for conservatism in commercial law - opposing the corporation and the charging of interest in particular - it appears that the Ottomans objected to the corporate form not because of religious scruples but because they appreciated that this might result in non-governmental concentrations of power. Recall from chapter 12 the hypothesis that the Abbasid decision to draw previously independent religious authorities into government would generate conservative economic policies; the Ottomans, like most later Islamic polities, followed this Abbasid precedent and limited legal debate in order to pursue their own interests - which they then used religion to justify.

We have seen in previous chapters (especially chapter 9) that the indebted sold themselves into slavery in many lands. The idea of personal bankruptcy by which an individual could declare an inability to pay their debts - was, therefore, a dramatic change in human relations. The primary purpose of bankruptcy laws was to encourage trade and investment by limiting the downside risk to those who borrowed funds. Bankruptcy laws had to be carefully administered: If people could purposely borrow and then declare bankruptcy, the price of loans would rise. Both Judaism and Islam had allowed some capacity for bankruptcy. But it was Early Modern Europe in which these laws first became formalized.

In sum, the Early Modern period is characterized globally by the codification of systems of law. There were significant differences in the nature of these laws, which in turn reflect path-dependent processes. These differences, in turn, had political implications for whether rulers could legislate as they wished and economic consequences for how and how well commercial disputes were settled and whether corporations, interest, and bankruptcy were allowed.

\section{Seventeenth-century disruptions}

Though the general trend was toward political consolidation, there were social/ political disruptions in many parts of the world in the seventeenth century. These have been variously attributed to colder climate, a decrease in trade associated with declining silver output in the Americas (chapter 19), or just the increasing power of merchants being asserted against rulers. Political upheavals occurred in the 1640s in the Spanish, Portuguese, and Ottoman Empires, and in England, Scotland, Ireland, France, and Naples: In all cases, there is mention of extremely low temperatures and resulting food shortages. Frozen 
bodies of water or unprecedented droughts often amazed commentators at the time. Drought may have been particularly extensive in Africa and may have decreased population levels even more than the slave trade had (though the import of crops from the Americas alleviated the damage; chapter 19). Though destabilizing, these disturbances may have accelerated the development of bureaucracy as states sought to both suppress rebellion and develop better means of coping with harvest shortfalls.

In China, the Ming Dynasty (chapter 20) had come to face financial difficulties. The scholar-bureaucrats in their service seemed unable to alleviate this financial challenge - perhaps in part because Confucian philosophy urged low taxes. The Ming increasingly turned to eunuchs for advice, even though the eunuchs were supposed to have influence only in the royal harem. Many bureaucrats resigned in protest. When the Ming closed rural postal stations as a cost-cutting measure in the 1620s, those who lost their jobs rebelled. At the same time, indebted peasants attacked lords. Rebels would take Beijing, inducing the emperor to kill himself in 1644. The Manchus - semi-nomadic peoples from what is now Manchuria - were invited in to fight the rebels, and chased them from Beijing within ten weeks. The Manchus declared a new Qing dynasty in 1650, though they had to fight Ming remnants and warlords for years - massacring tens of thousands in Yangzhou. In 1645, the Qing forced all Chinese men to adopt the Manchu hairstyle: shaved in front with a long braid in the back. The Qing Dynasty (chapter 20) would survive until overthrown by revolution in the twentieth century. Though it would prove weak in the face of European and Japanese encroachments in later centuries, it would expand the borders of the Chinese state deep into Central Asia (chapter 19).

In Continental Europe, the Thirty Years War raged from 1618 to 1648 . This is commonly portrayed as a religious war between Protestant and Catholic German rulers. However, Catholic France often gave support to Protestant rulers to thwart the territorial ambitions of Catholic Austria. The Habsburg family that ruled both Austria and Spain had been elected as Holy Roman Emperor continually since 1279 - a position that gained them additional wealth, power, and prestige. The seven authorities that chose the emperor were three Catholic bishops, three German princes, and the elected ruler of Bohemia (roughly the modern Czech Republic). When it seemed that Bohemia might elect a Protestant, the Austrians invaded. The war was thus really about how the religious divide in Europe (which we address below) affected power relations. The war was brutal: Poorly trained troops attacked peasants and towns if not paid on time. As much as a third of the German population may have died. Disabled veterans begged on street corners throughout German lands. By the end of the war, though, the idea of religious toleration was grudgingly accepted.

The English Civil War (1642-51) began as a battle between Parliament and the king. The king needed money and Parliament wanted recognition of its 


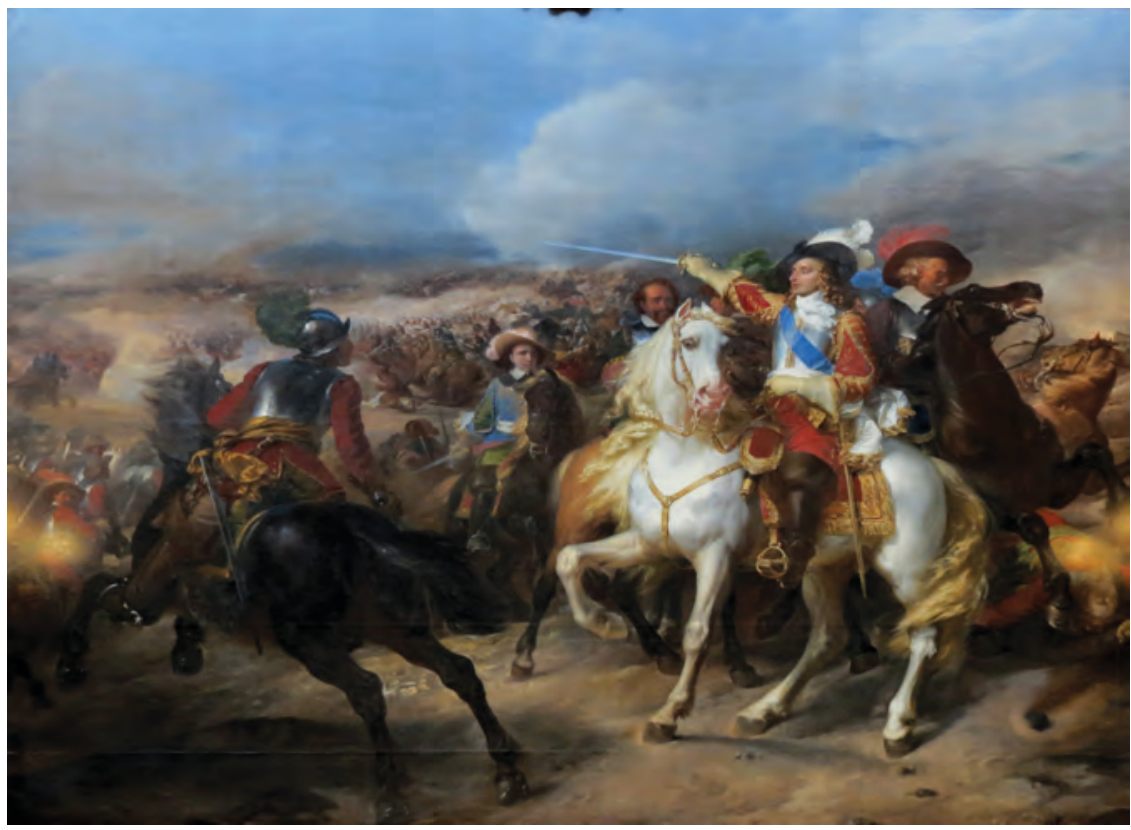

Illustration 18.1

Pierre Franque, Battle of Lens, 1648. This was the last major battle of the Thirty Years War

Photograph by Giogo, CC BY-SA 4.0

powers in return. As often in civil wars, though, the result was not what either side at first imagined. The Parliamentary army triumphed (and the king was beheaded) but its commander Oliver Cromwell then dismissed Parliament and ruled as a military dictatorship. Though a Puritan (a strict Protestant faith), Cromwell generally pursued religious toleration, though he did discourage social customs (such as dancing around a maypole) that had pre-Christian origins. Soon after Cromwell died, the kingdom was restored but with increased powers to Parliament. Decades later, Parliamentary concern regarding the succession led to an invitation to William of Orange from the Netherlands and his wife Mary (second in line to the British throne) to take the throne with an expectation that they would respect Parliamentary powers.

The Russian state had learned authoritarian ways from the Byzantine and Mongol Empires. When Constantinople fell, Russia saw itself as the protector of the true faith. It tended for centuries to avoid contact with Catholic Poland to the west. It instead expanded south and east, employing a system in which elites called "boyars" were granted land and serfs in return for military service. Serfdom was institutionalized in a royal decree of 1649. Many fled to the forests as Cossacks - militaristic and egalitarian communities. Boyars became more powerful than the tsar, but under foreign attack chose the Romanov dynasty in 1613. This dynasty would face peasant rebellions in 1648 and respond by strengthening both tsarist power and boyar power over serfs. 


\section{The Netherlands}

The Netherlands would successfully revolt against Spain in the seventeenth century, after decades of rebellion. There were a variety of motives: the idea of national sovereignty (chapter 21); religious differences as the Netherlands became increasingly Protestant; fears that the Spanish Inquisition (in which "heretics" against Catholicism were severely punished) might be applied in the Netherlands; and growing economic prosperity as the Netherlands became an important trading and fishing centre (a quarter of the population was in the herring fishery). Economic developments provided not just an incentive but the means to revolt. Dutch merchants and city governments felt that they could expand their prosperity if freed from Spanish taxes and regulation. Though the rebellion itself was an example of political fission, it transformed the Netherlands from a distant holding - through an accident of marriage and inheritance - of the Spanish crown to a strong state. National and local assemblies developed which exercised considerable power: They even displaced the stadtholder - a king-like figure - and governed on their own for decades. The trade-oriented Netherlands pursued religious toleration and attracted a large Jewish community. The Netherlands would become a dominant force in the trade to the Baltic, and then in international banking and finance. It would later become a significant colonial power. It would also develop the most productive agriculture in Europe, and drain low-lying areas to expand the area of farmland dramatically: This plus the import of food from the Baltic allowed the Netherlands to become heavily urbanized.

\section{Religious developments}

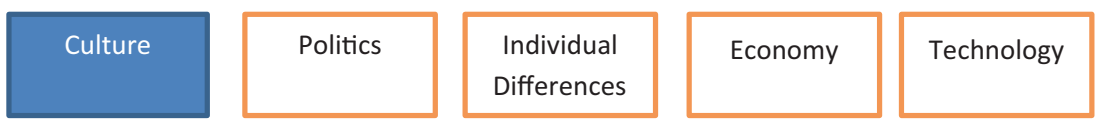

As noted in chapter 11, all successful religions make compromises over time as they interact with rulers. As a result, religions may experience periodic efforts to regain the supposed purity of earlier days. Reformers may attempt to reinterpret religious doctrine or transform the bureaucratic structures associated with the religion. An alternative is to emphasize the individual believer's religious experience, which decreases the importance of religious authorities. Reform movements emerged across the world's major religions during the Early Modern period.

We discussed in chapter 12 the vital role of Sufi mystics in the process of Islamic conversion in Central Asia and elsewhere. Religious authorities nevertheless viewed Sufism with suspicion precisely because it promised a personal religious experience without their intermediation. Sufism proved popular in succeeding centuries and began to attract large donations. The period from the 
thirteenth to sixteenth centuries is considered the Sufi Golden Age. Funds at first supported simple lodges in which Sufi mystics could gather. Over time, these grew into substantial structures with kitchens and libraries. These Sufi institutes became common throughout the Islamic world. Ironically, by requiring some organization, they may have had the unintended effect of reducing some of the attraction of Sufi simplicity.

Within Judaism, the Kabbalah is a set of mystical teachings that also encourage an individualistic pursuit of the spiritual. Although there are claims that the Kabbalah predate the period of missionary religions, the first documentary evidence of Kabbalah practices dates from the twelfth century in southern Europe. These practices would flower within the Ottoman Empire in the sixteenth century and would exert a significant influence both within and beyond Judaism in later centuries. In the 1660s, Sabbatai Sevi of Izmir (on the west coast of Anatolia) proclaimed that he was the Jewish messiah. Jewish religious authorities denounced him, arguing that his mystic visions were no substitute for religious education. His teachings were nevertheless popular throughout the Jewish diaspora. The Ottomans viewed him as a threat to social peace and arrested him. He then offended his followers by converting to Islam.

In the late fifteenth and early sixteenth century in Bengal, a religious teacher who is known as Sri Krishna Caitanya advocated a more individualistic form of Hindu worship. Any individual could celebrate the god Krishna (a manifestation of the god Vishnu) through dancing and chanting, regardless of the individual's caste or gender. Sri Krishna Caitanya built upon a tradition of individual devotion that can be traced to the Bhagavad Gita, but that had grown in importance in the fourteenth and fifteenth centuries. His followers would develop the movement known as Hare Krishna after its most common chant; this movement was unusual for Hinduism in actively seeking converts.

Sikhism is a much later creation than the religions surveyed in chapter 11 , being founded only in the fifteenth century CE. It is a monotheistic religion like Christianity or Islam but speaks of reincarnation and eventual release from suffering, as do Hinduism and Buddhism. Nanak, the founder of Sikhism, often described a "deist" deity (rather than the anthropomorphic deity of Christianity and Islam) that was infinite and formless. Religious scholars suspect that Sikhism represents a fusion of these different religious traditions. Sikhism developed in the Punjab in northern India at a time when Muslims ruled the mostly Hindu region. Followers of Sikhism are - like followers of most religions - sceptical that the faith represents anything other than divine revelation to its founding gurus. Yet we can note here that Sikhism seems a reaction against the power of both Islamic judges and Hindu priests. As with Sri Krishna Caitanya, Nanak broke with caste traditions. He would eat with people from any caste or religion. Communal eating remains an integral part of Sikh rituals. Although much smaller in size than Christianity, Islam, Buddhism, or Hinduism, which between them have over 4 billion adherents, Sikhism is one of the ten largest religions in the world with some 26 million followers. It is by far the largest religion founded after Islam. 
Postscript: A couple of additional religions emerged in the nineteenth century. Mormonism - which builds upon Christian teachings - has some 15 million adherents, and the Baha'i faith - which extends pacifist components of Islam, stresses equality of both genders and all ethnicities, and sees value in all religions - about 6 million. Members of other faiths have often persecuted these nineteenth-century faiths, with Mormons migrating westward for safety in the United States in the late nineteenth century and the Baha'i fleeing Iran for the Middle East, Europe, and the Americas.

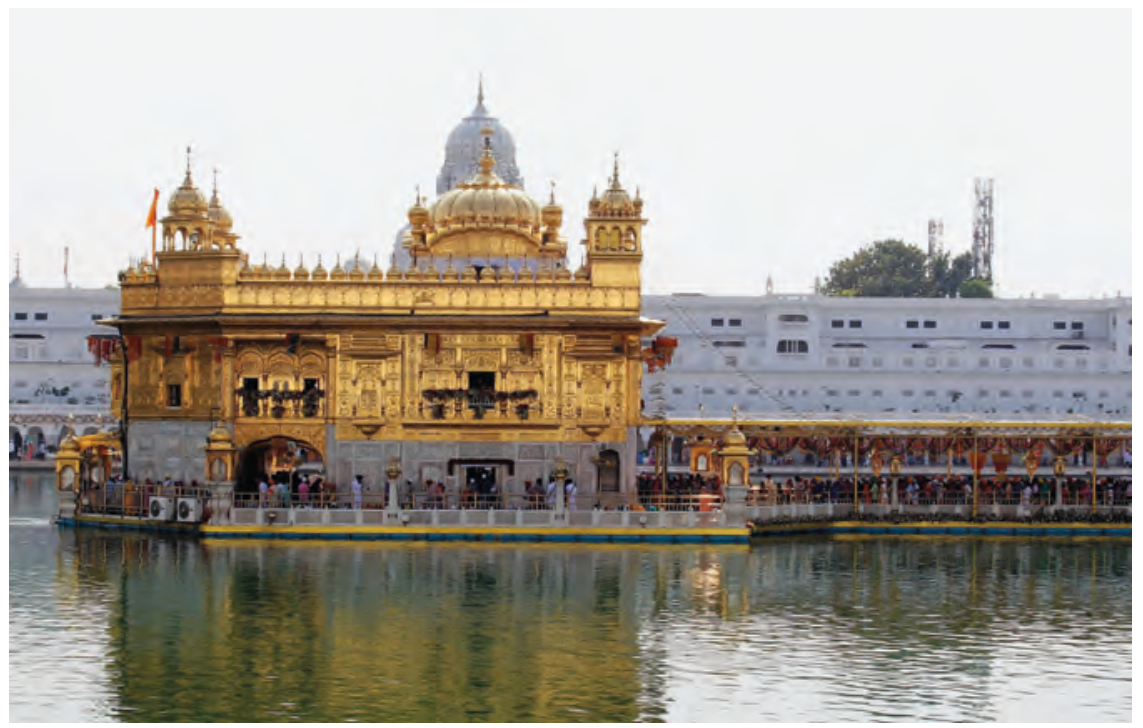

Illustration 18.2

Golden (Sikh) Temple (Harmandir Sahib), Amritsar, Punjab, India

Wang Yangming (1472-1529) was a Confucian scholar-bureaucrat in Ming China who had been exiled to the southwest because he spoke up about abuse of power by palace eunuchs. He naturally reflected on how to restore Confucian virtues. He came to emphasize the importance of self-knowledge: The truth was within us if we would confront our selfish desires. (We have spoken of the dangers of self-deception at many points in this book.) As with religious reformers elsewhere, then, he sought a spiritual awakening grounded in individual effort. He and his followers preached to the masses rather than just fellow bureaucrats. Importantly, he urged a life that combined reflection with action: One can only truly understood how to behave by being active in the world. 
Postscript: Chinese and Japanese reformers would draw upon Wang Yangming's emphasis on individualism in the late nineteenth and twentieth centuries as they sought to match Western powers while maintaining

a Confucian emphasis on civic duty. That is, they did not merely adopt Western individualism.

In Europe, a monk by the name of Martin Luther (1483-1546) complained about corruption in the Catholic Church. His main objection was to the widespread sale of indulgences, by which the Church promised godly forgiveness of sins in return for a monetary payment. Luther argued that there was no biblical justification for such a practice; the Church responded that it could establish such practices through its own authority and tradition. Luther was not the first to make such objections: Complaints in previous centuries by John Wyclif in England and John Hus in Bohemia had been repressed, and advocates of reform burned alive. Why then did Luther have far greater success? Luther's arguments were spread across Europe by the newly invented printing press (he probably did not nail them to a church door as has long been suggested) and reached a receptive audience, especially in lands distant from Rome. The great danger they faced from the Ottomans at the time likely muted the reaction of Catholic rulers to reformers (chapter 20). Nationalist sentiment was employed in opposition to Rome. When the Church excommunicated him, Luther translated the Bible into German; it would soon be translated into other European languages thereby encouraging the development of a range of written languages such as Swedish, Lithuanian, and Estonian. Luther argued that each Christian should read the Bible for themselves. This emphasis on individualism would invite fragmentation within Protestantism, with a variety of beliefs and national churches. Many Germanic princes found it financially useful to renounce Catholicism: They could then claim church lands and the flow of funds that went to Rome. Despite his praise of individual spirituality, Luther would side with such princes when they brutally crushed peasant revolts. The English king would break with Catholicism a century later in order to divorce his first wife, but would soon after that use accusations of corruption as a pretext for taking the lands of Catholic monasteries. The Catholic Church would respond to the rise of Protestantism with efforts to reform the Church. The Jesuit monastic order, in particular, would espouse pure devotion to the faith. As noted above, Europe would experience decades of war that was at least in part motivated by religion. Though religious toleration would slowly come to be valued in many parts of Europe, religious minorities were, in the Early Modern period, repressed and killed in many countries, including England (where Catholic royalty killed Protestants and vice versa), France (where thousands of Protestants gathered for a wedding were massacred in 1572), and Spain (where the Inquisition, a process of religious interrogation initially designed to root out Jews and Muslims was 
turned against Protestants). Nevertheless, the eventual acceptance of toleration in many lands as an alternative to religious strife would create an environment that allowed and even encouraged the rise of secularism and agnosticism. Though toleration was at first extended only to other Christian denominations, it would over time be extended to believers in other faiths and even agnostics.

Why might we see an individualistic type of religious reform across so many religions at a similar time? The printing press is implicated in some but not all of these movements. Rising literacy rates may have been significant more generally. These movements may all have been reactions to social change (and especially urbanization) and to the growing wealth and power of religious authorities. Though they may have been responses to similar forces, they nevertheless were shaped by the religions in which reformers operated. These reformers would likely not have had much interest in each other's ideas.

\section{PRIMARY DOCUMENTS: RELIGIOUS REFORM IN MANY LANDS}

Most of Luther's 95 Theses focus on the church practice of selling "indulgences" which allegedly served to forgive sins:

No 32. Those who believe that they can be certain of their salvation because they have indulgence letters will be eternally damned, together with their teachers.

No. 43 Christians are to be taught that he who gives to the poor or lends to the needy does a better deed than he who buys indulgences.

No. 54 Injury is done to the Word of God when, in the same sermon, an equal or larger amount of time is devoted to indulgences than to the Word

No. 75 To consider papal indulgences so great that they could absolve a man even if he had done the impossible and had violated the mother of God is madness.

Luther nevertheless seeks to make a broader point about personal salvation that does not rely on a corrupt bureaucracy.

Caitanya stresses the path to personal salvation of chanting:

In this Age of Kali, the holy name of the Lord, the Hare Krishna mantra, is the incarnation of Lord Krishna. Simply by chanting the holy name, one associates with the Lord directly. Anyone who does this is certainly delivered.

Regardless of time or place, one who chants the holy name, even while eating or sleeping, attains all perfection.

Wang Yangming stresses personal responsibility differently:

The sages do not consider that making no mistakes is a blessing. They believe, rather, that the great virtue of man lies in his ability to correct his mistakes and continually make a new man of himself. 
If you really want to find out something about immortality, you have to live in the mountain forests for 30 years. If you succeed in perfecting your eyes and ears there, if you harmonize the heart and the will so that your mind becomes clear and pure and free of all that is evil, you will be able to discuss the matter.

Nanak also values individual piety:

Even Kings and emperors with heaps of wealth and vast dominion cannot compare with an ant filled with the love of God.

But he still values religious authority:

Let no man in the world live in delusion. Without a Guru, none can cross over to the other shore.

What similarities and differences do you observe across these quotes?

\section{Religious interactions}

As noted above, hostility between religions might accompany reform within religions. Yet there were also many signs of cross-religious cooperation in this period. Two of the great Islamic dynasties of the period - the Ottomans in the Middle East and the Mughals in India - would generally pursue policies of religious toleration (chapter 20). Jews fleeing the Spanish Inquisition usually headed for the Ottoman Empire (though some went to the Netherlands; see above).

We noted above that it would be a mistake to see the Thirty Years War as entirely a religious conflict. Catholic rulers fought on both sides. Mercenary soldiers often switched sides. The same lesson applies to wars between Christians and Muslims. In both the centuries-long Christian re-conquest of Spain and the centuries of war involving the Ottomans in southeastern Europe, Christian and Muslim princes and soldiers could be found on both sides of a battlefield on literally hundreds of occasions. In both the Ottoman victory over the Byzantines in 1453 and the siege of Vienna in 1683, the Ottomans had almost as many Christians as Muslims in their attacking armies. Pragmatic considerations could easily trump religious loyalty. Nor was it all merely pragmatic: There were genuine friendships between both rulers and soldiers that crossed religious divides. In India too, the Mughals attracted Hindu soldiers to their service in battles against Hindu polities. Even in Central Asia, Buddhist and Islamic rulers and soldiers often appeared on the same side during centuries of conflict there (chapter 17).

Although the general attitudes of Christians and Muslims toward each other might best be described as a mixture of ignorance and cultural superiority, there was also a genuine curiosity about the cultural practices of the other. Trade regularly brought Christians and Muslims together, and socialization often followed. 


\section{Witch hunts}

In the sixteenth and seventeenth centuries in Europe (and European colonies such as Massachusetts), 100,000 alleged witches were tried and 60,000 were killed. Though Europeans had long feared witches, these became strongly associated with the devil in this time of religious conflict. A book published in 1486 by Dominican friars argued that witches were in league with the devil, with whom they had sex. Luther and the Catholic Church both believed in burning witches, and witches may have been associated with what each considered the

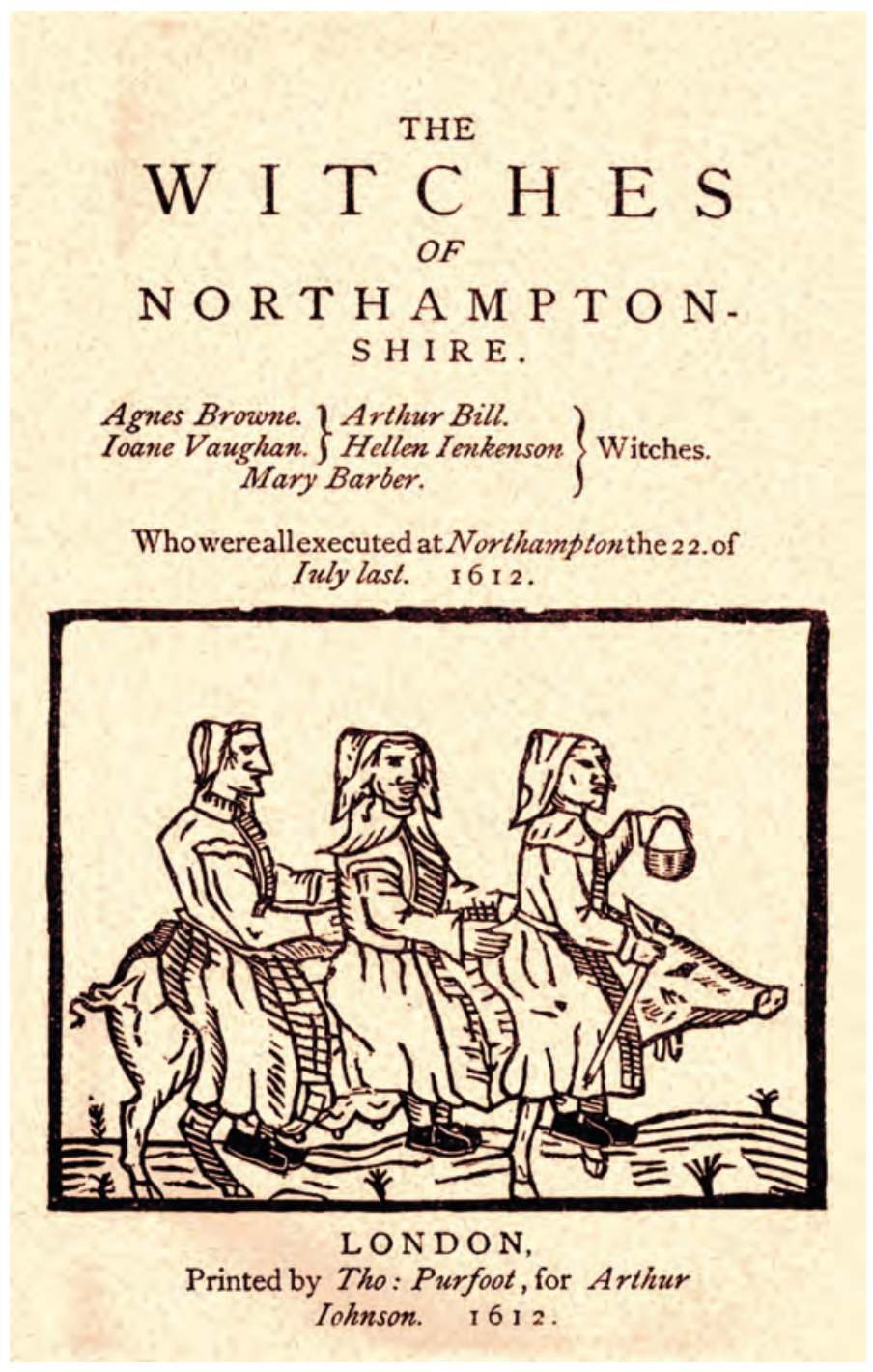

Illustration 18.3

Cover of the pamphlet "The Witches of Northamptonshire," 1612, showing three witches riding on a pig 
heresy of the other. Witch trials were more common in areas where Catholics and Protestants were competing for converts. Nevertheless, it would be a mistake to see witch trials as merely a side effect of religious conflict. There is an important gender aspect. Some wonder if witch hunts were not motivated to an even greater degree by new job opportunities that allowed women to gain occupational independence. The association of the word "spinster" with unmarried women indicates the connection between being able to make money spinning and choosing not to marry. Unattached women were often viewed with suspicion in lands where traditionally only nuns remained unmarried. It was then easy to blame unattached women for any evil that befell a community. After over a century of hunting witches, though, both Protestants and Catholics came to doubt that the devil would work through powerless women.

\section{PRIMARY DOCUMENTS: PAPAL BULL OF INNOCENT VIII ON WITCHCRAFT}

It has recently come to our ears, not without great pain to us, that in some parts of upper Germany ... many persons of both sexes, heedless of their own salvation and forsaking the Catholic faith, give themselves over to devils male and female, and by their incantations, charms, and conjurings, and by other abominable superstitions and sortileges, offences, crimes, and misdeeds, ruin and cause to perish the offspring of women, the foal of animals, the products of the earth, the grapes of vines, and the fruits of trees, as well as men and women, cattle and flocks and herds and animals of every kind, vineyards also and orchards, meadows, pastures, harvests, grains and other fruits of the earth; that they afflict and torture with dire pains and anguish, both internal and external, these men, women, cattle, flocks, herds, and animals, and hinder men from begetting and women from conceiving, and prevent all consummation of marriage; that, moreover, they deny with sacrilegious lips the faith they received in holy baptism; and that, at the instigation of the enemy of mankind, they do not fear to commit and perpetrate many other abominable offences and crimes, at the risk of their own souls, to the insult of the divine majesty and to the pernicious example and scandal of multitudes.... nevertheless certain of the clergy and of the laity of those parts, seeking to be wise above what is fitting ... do not blush obstinately to assert that these are not at all included in the said parts and that therefore it is illicit for the aforesaid inquisitors to exercise their office of inquisition in the provinces, cities, dioceses, territories, and other places aforesaid, and that they ought not to be permitted to proceed to the punishment, imprisonment, and correction of the aforesaid persons for the offences and crimes above named. Wherefore in the provinces, cities, dioceses territories, and places aforesaid such offences and crimes, not without evident damage to their souls and risk of eternal salvation, go unpunished.

We see here that Catholic clergy disagreed on the issue of witchcraft. Why was it important to the pope to overrule local clergy and send "inquisitors" to punish witches throughout Europe? 


\section{Wars and gunpowder technology}
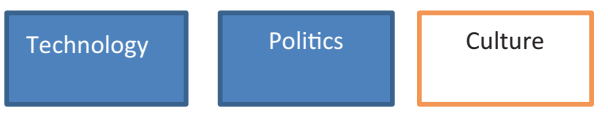

Economy

Wars were endemic through much of the world in the Early Modern period. The main exceptions occurred where large empires achieved peace for a while. Gunpowder technology changed the face of war in many ways. War became far more deadly. Firearms turned the military advantage toward well-drilled infantry and thus permanent professional armies, while cannon enhanced the ability of centralized states to discipline local elites in their castles. Though gunpowder encouraged political consolidation everywhere, there would be an uneven global development of gunpowder technology such that European states would gain a decided military advantage over polities in the rest of the world.

\section{Early development of gunpowder technology}

It is useful to distinguish two main types of gunpowder weapon. One is the cannon: large pieces of artillery that fire very large objects. They are used on land primarily to destroy fortifications, though they can also cause much damage if fired against an opposing army. They are also used at sea to destroy other ships. The second type of weapon is the firearm employed by individual soldiers. These go under a variety of names through time, with new names reflecting significant advances in the speed of use or range or accuracy.

It is well known that gunpowder was first discovered in China. Taoist alchemists apparently made the discovery. It is often mistakenly thought that gunpowder was only used for fireworks there, but it is now appreciated that the Chinese were also the first to employ gunpowder as a weapon. The Song developed grenades and primitive cannons. The Ming Dynasty founded in 1368 arguably was successful because of its use of firearms.

As we saw in chapter 17, gunpowder technology likely reached Europe during the time of the Mongol Empire. The Mongols had employed it in China, and then apparently applied gunpowder bombs launched by catapults in their attacks on Middle Eastern cities in the mid thirteenth century. Gunpowder was used against Crusaders by Islamic forces in 1249 .

Cannons were first employed in Europe from the 1310s. Cannon were especially useful in a decentralized world of local lords and castles and so were steadily improved over time in Europe. If local knights could defy the king by retreating to their castle, kings before gunpowder could only punish them by placing the castle under siege for many months. With well-designed cannon, kings could instead destroy castles in a matter of days. The earliest cannons were massive and therefore difficult to move and use. Iron cannons replaced bronze from 1541. A critical technological advance was the development of well-bored 
cannon and round cannonballs: These could be fired much more powerfully than rough or stone projectiles from a poorly bored cannon.

New fortifications were developed in response to cannons. Star-shaped plans, with earth packed around walls, allowed those within forts to fire their own cannon from multiple angles at anyone setting up cannons outside. However, such fortifications were hugely expensive. They could be employed by Italian city states but were generally beyond the means of feudal lords.

European cannon also proved dominant at sea. European navies defeated the Ottoman navy in the Battle of Lepanto in 1571. Though this battle may have had limited political consequences, it did establish that sailing ships with cannon could wreak havoc on the "traditional" Mediterranean navy of galleys.

Personal gunpowder weapons also changed the face of war. Even muskets an early firearm that took two minutes to load and was inaccurate - could potentially pierce metal armour. (So could the

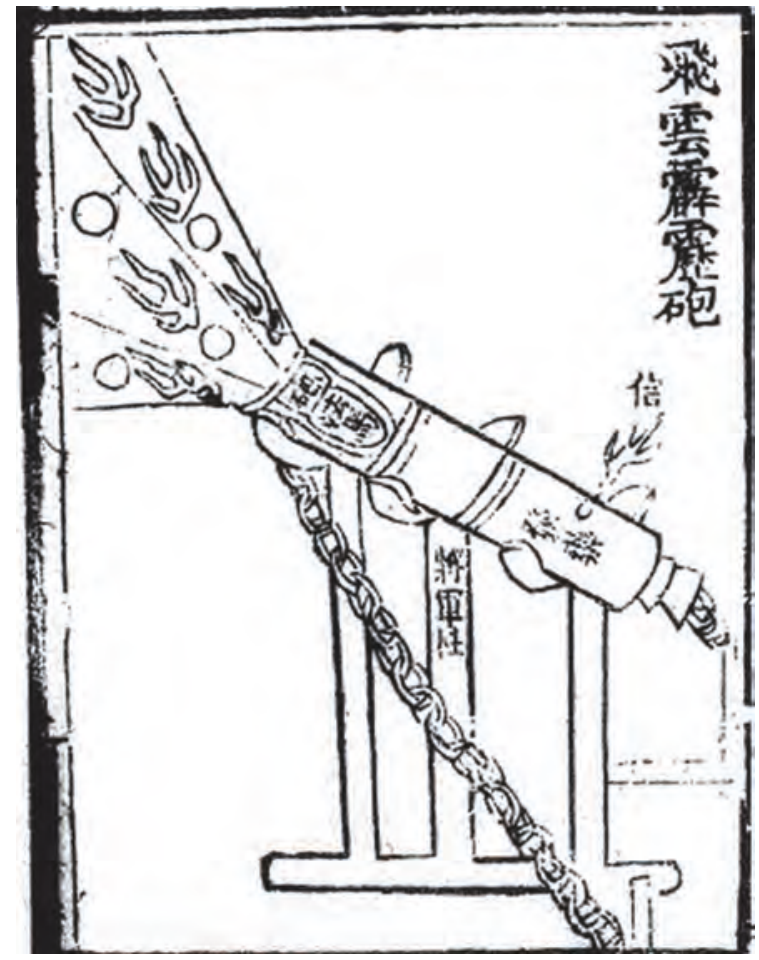

Illustration 18.4

An illustration of a cannon-like "eruptor," from the 14th-century Ming Dynasty book Huolongjing. The cannon was capable of firing cast-iron bombs filled with gunpowder longbow, developed in Europe from the fourteenth century; the decline of armoured knights thus predates gunpowder but would be accelerated by this.) This technology encouraged the employment of large infantry formations. These would form lines that were much longer and thinner than those of earlier infantries armed with swords and shields, who had had to engage faceto-face with their enemies. These new lines could fire in rotation, maintaining steady fire despite the time it took to reload an individual weapon. By firing many guns at once, the significance of the inaccuracy of individual weapons was reduced: Many bullets would inevitably strike the enemy. Such formations required a great deal of training. The musket, therefore, favoured large states that could amass and train large armies. This was not the first time in history that military technology favoured consolidation: Iron technology had done so thousands of years earlier, for example (chapter 5).

We should, as always, avoid simplistic historical analysis. Gunpowder encouraged but was not the only cause of large armies or large states. Much later, the introduction of conscription in Napoleonic France would cause army sizes to increase even more (chapter 25). 


\section{The success of Europe}

Gunpowder weapons were employed worldwide. The Ming and Qing would use them as they consolidated power in Central Asia. We saw in chapter 15 that gunpowder weapons were employed in the unification of Japan. The Ottomans, Safavids, and Mughals are often termed "gunpowder empires" precisely because they used muskets and cannons against their rivals (Chapter 20). When the British arrived in India, they admired Indian cannons made of brass and iron but these proved unable to prevent British conquest. African kingdoms traded slaves for guns that they then employed in slave raiding (chapter 19). Aboriginal peoples in the Americas also traded for guns, which were sometimes then used in battles with European settlers and their armies.

Though gunpowder was known across Eurasia by the early fourteenth century, differing political situations influenced how it was used. Europe was far more politically fragmented than other regions of Eurasia at the time. Its states had a far greater incentive to develop better weapons. We have seen above that these were employed internally against local elites. They would also be employed externally in battles between European states. The much later global military dominance of Europe - European countries would control an astounding 84 per cent of the surface of the globe at some point between the fifteenth and twentieth centuries, and exercise influence over the remainder (primarily China, parts of the Middle East, and Thailand) - would result from centuries of military conflict within Europe. That is, Europeans spent centuries developing better gunpowder weapons to (mostly) kill each other before they began to shoot non-Europeans with these on a large scale.

It would be a grave mistake, though, to imagine that gunpowder technology was being ignored elsewhere in the world at the time. There is clear evidence, for example, that cannons were being used in the Indian Deccan in the 1460s before there was much Portuguese presence in the area. These had likely been bought from Egypt. By the early sixteenth century, the Portuguese were impressed by the quality of local cannon production. Portuguese technology was then adopted. The Deccan, it might be noted, was also politically fragmented, whereas the Mughals dominated northern India. Cannon technology advanced most rapidly where there was frequent warfare. Many hills in southern India overlooked surrounding plains, and forts were built on these with large granaries and cannon emplacements. The Ming in China could likely also match European firepower into the sixteenth century, but after that had fewer towns to attack and so little cause to employ cannon. China won a war with the Dutch over the island of Taiwan in 1622: It appears that the Chinese guns were a match for the Dutch at the time, though the Chinese may already have been at a naval disadvantage. (This brief war may have been decided by Dutch strategic blunders rather than relative technological prowess.)

The Ming, Mughals, and Ottomans all sought and praised peace after their initial conquests. This may have been strategic: In so doing, they discouraged attacks on their empires. Monarchs in Europe were far less likely to praise peace. 
There were periods of peace in Europe, to be sure - though even these were characterized by state-sponsored piracy that encouraged the development of naval weaponry - but rulers could never be confident that these would last. Some intellectuals did urge peace and especially disdained religious justifications for war. Erasmus and Thomas More were among them. Radical Protestant sects like the Quakers also opposed war. The fact that peace was a radical idea in Europe but trumpeted by emperors in Asia is symbolic of the different war environments of the two continents.

We noted above that armies had to be trained carefully in how to employ firearms to best advantage. There is evidence that appropriate strategies may have been pioneered in China and were applied widely in East Asia in the sixteenth century. Their first documented use in Europe is in the Netherlands in the 1590s. Though relative peace during the Ming Dynasty slowed the further development of both weaponry and strategy there, Japan at this time was riven by conflict and employed muskets from the mid 1400s to the early 1600 s. Korean sources tell us that the Japanese army used musket drills also in the 1590s when they invaded Korea. The Japanese were repelled from Korea by Chinese Ming units using cannon, but their musketry was unchallenged. The Koreans then replaced their previous reliance on cavalry with infantry units drilled to use muskets. Over the next century, they developed drilling techniques that seem similar to those developed in Europe. The Koreans fared well in some smallish battles against Russian forces in the 1650s along the Amur River. (The Russian expansion across Siberia had itself depended greatly on musketry).

The Ottomans used musket volleys even before the 1590s. It is possible that they also had learned these from the Chinese. Separate development of military strategies appropriate to muskets is also possible. The slow loading of muskets demanded careful drilling of units, and there may have been a difference in strategy since Asians used more accurate but slower muskets and as a result emphasized quality over quantity of shots. The Ottomans may have matched European powers in the quality and quantity of their manufacture of both cannons and muskets well into the eighteenth century. After all, they were engaging with European powers with considerable success until that time - their European enemies may indeed have been motivated in part by Ottoman successes to develop better arms. But from the eighteenth century, it is clear that European weapons were superior. The Ottomans came to import both weapons and military experts from Europe - despite occasional objections by religious authorities - but were unable to maintain a competitive armaments industry of their own.

The Mughals and Safavids also employed gunpowder weapons. Indeed the strength of these empires owed much to their use of these weapons. Nevertheless, their political situation was entirely different from the Europeans. The Mughals had no competitors that could come close to matching the size of their armies. The Safavids in Persia had to worry about the Ottomans and Mughals and nomads from the north but did not face the array of competitors that European states did. 
Europe had been exporting weapons and expertise from the sixteenth century, and the size of these exports would grow through time. If a country that was importing weapons and military expertise wished to develop a competitive armaments industry, it needed to import both iron-making and weaponsmanufacturing technology. The Russian state focused on just such technology from the time of Peter the Great. As the financial capacity of the Russian state expanded, it was able to defeat the Ottomans in the nineteenth century. It was nevertheless at a severe armaments disadvantage against Germany in World War I (chapter 28). The Qing readily adopted Western weapons in the sixteenth and seventeenth centuries but then stopped trying to keep up, perhaps because they did not for a while face external enemies.

The nomads of Central Asia lacked the industrial base to compete in gunpowder technology - though they, like the Plains Indians of North America, would buy guns and learn to fire from horseback. As gunpowder weaponry was developed, nomads would slowly lose the military advantage they had held for centuries. The earliest slow-to-load muskets were of limited use against fastmoving nomads, but as guns became faster and more accurate, they became an effective weapon. Still, the fact that nomads themselves could not compete in weapons manufacture meant that fighting nomads provided less incentive for continual improvements in firearms than did fighting other sedentary states. States that spent much of their time fighting nomads - China, the Ottomans, the Safavids, and Russia - fell behind western Europe militarily even as they scored many victories over nomadic forces.

Of course, the development of superior gunpowder weapons depended not just on the number and type of enemies a state faced. The state also had to be able to finance large armies and arms production. Here one might have thought that Asian empires would have had an advantage: They had extensive bureaucracies that were able to tax agriculture. European states were much less capable of this but were able to tax trade very effectively. Asian empires followed a familiar historical pattern of facing financial challenges as the empire aged and ceased expansion. European states, in contrast, strengthened their financial capability as they consolidated power. There is an important feedback effect here: Gunpowder weapons supported centralization (because local elites could no longer hide in castles), and centralization supported the development of better weapons. The result was that European states became able to tax a much more significant share of economic output than Asian empires. The development of "nation states" - in which states became firmly linked to particular communities of identity (chapter 21) - further enhanced the financial capability of states. This greater financial capacity, coupled with incessant warfare, encouraged the more rapid development of gunpowder technology in Europe. When India was riven by inter-state conflict as the Mughals declined in the eighteenth century, Indian states had much less money to spend on weapons than did European states.

Though states elsewhere in the world could match European gunpowder technology into the sixteenth century - and in the case of the Ottomans 


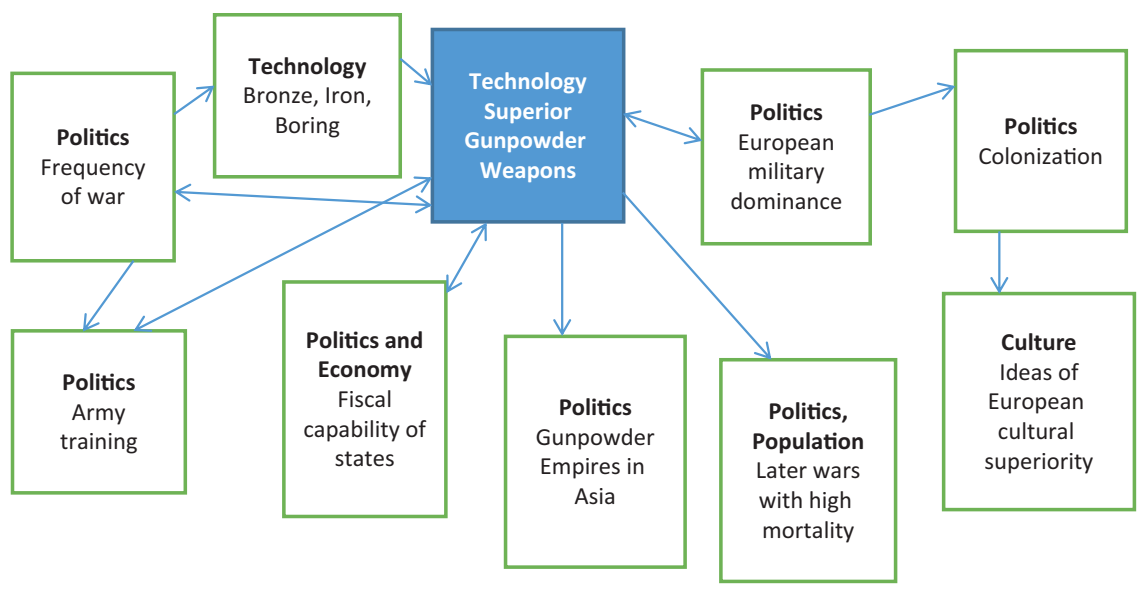

Figure 18.1

Influences on and effects of superior gunpowder technology Note:We discuss later wars in detail in chapter 28.

perhaps into the eighteenth century - by the nineteenth century, the European technological edge was huge. The gap would further widen as Europe developed huge iron and armaments industries in the nineteenth century. China had no effective response to European gunboats, and the Ottomans steadily lost ground to European powers. There would be important further technological advances in the nineteenth century: Rifling enhanced the accuracy of firearms, and the machine gun from 1862 dramatically increased the speed with which guns could be fired; steam power and iron hulls made navies both quicker and stronger. Each of these new technologies extended the European military advantage.

\section{Trade}

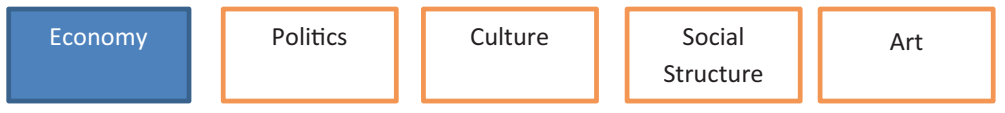

We will discuss in the next chapter both how the Americas became part of global trade networks, and how Europeans developed a new trade route to Asia by going around Africa. Yet trade intensified along overland trade routes within Eurasia as well.

\section{Trading networks}

Ethnic or religious trading networks remained important in this period. Chinese, Indian, Jewish, and various Muslim trading networks continued to operate throughout the eastern hemisphere. As noted in chapter 9, networks 
with an extensive geographical range could serve to reduce the costs of trade significantly. An Armenian trade network would expand internationally during this period but then largely dissipate. Armenians had first entered trade because of their location between the Caspian Sea to the east and the Black and Mediterranean Seas to the west. Armenians thus sat astride one of the leading trading routes between Asia and Europe. Over time, Armenian networks expanded far afield, as Armenian agents were dispatched to distant lands. In the fifteenth century, Armenian traders could be found in both Belgium and China. In 1506, the Safavids conquered Armenia and moved merchants to near the Safavid capital of Isfahan. After that, Armenian merchants worked closely with the Safavid rulers and came to dominate many trades. They were especially prominent in the silk trade - in large part because Armenian farmers who also had been moved by the Safavids produced much raw silk. The Armenian trade network expanded further - to London at least and perhaps West Africa. However, Armenian traders would inevitably suffer as the Safavid Empire declined (chapter 20).

There were numerous regional trading networks. For example, there was a Wajorese trade network based in south Sulawesi (in present-day Indonesia) which spread throughout island Southeast Asia and was famed for its trading skills by Malays and others. The Wajorese diaspora increased in size after the Dutch conquest of Sulawesi in the 1660s. The dispersed Wajorese maintained cultural practices and stayed in close contact with their homeland. Some even returned after 1730 to fight unsuccessfully for independence. Scholars are just beginning to analyse surviving documents in the Wajorese language.

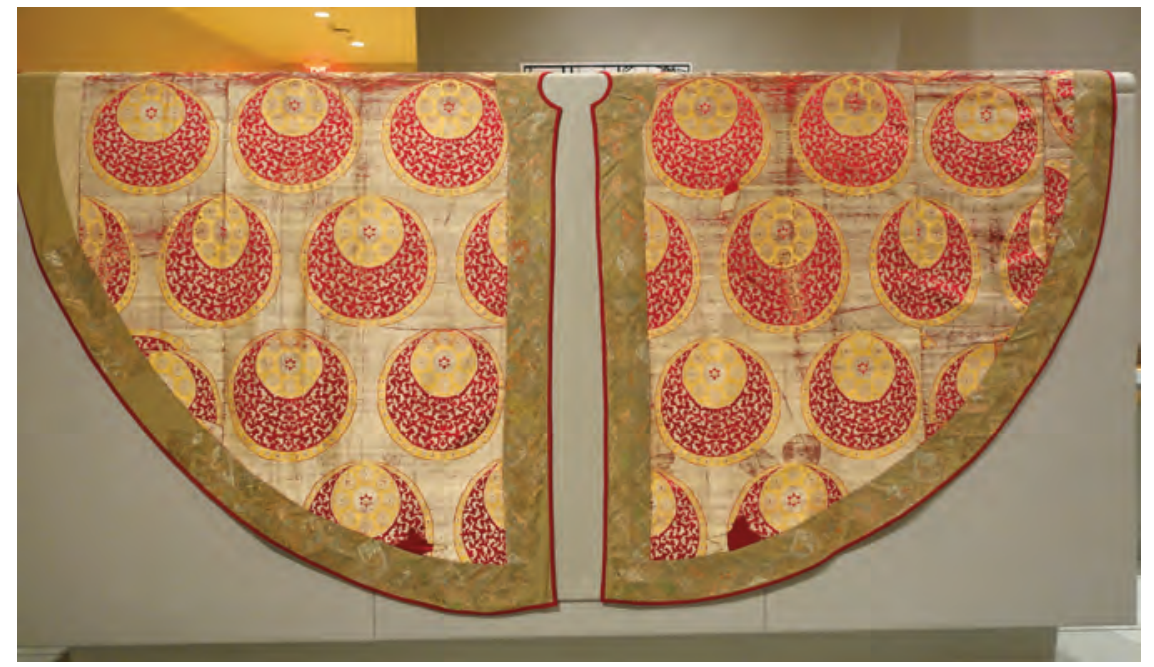

\section{Illustration 18.5}

Silk garment, 16th century. It is likely an Armenian ecclesiastical garment made near Isfahan, George Washington University Textile Museum

Photograph by Daderot, CC0 1.0 


\section{Coffee, tea, and cocoa}

We have seen in chapter 5 that beer and wine were safer to drink than untreated water: Fermentation killed many bacteria. Distilling - which further purifies alcoholic beverages while increasing alcohol content - was known from the twelfth century in both Italy and China and was practised worldwide by the sixteenth century. Since coffee, tea, and cacao were boiled, they also were healthy beverages, and could replace beer or wine as an everyday drink. This, in turn, increased global demand for sugar, for unsweetened coffee or tea or cacao had a bitter taste.

Coffee plants originated in Ethiopia but were first grown commercially in Yemen around 1400. Coffee drinking thereafter spread through the Muslim world (in part as pilgrims to Mecca learned of it), aided by Muslim prohibitions on consuming alcohol. Mocha, a port on the Yemen coast, monopolized the coffee trade for over a century (ironically, the name "Mocha" became associated with coffee mixed with chocolate). Coffee was known in Europe but was expensive until the Dutch started coffee plantations in Java in the early seventeenth century with seeds smuggled out of the Islamic world. Coffee production then spread to Central America, Brazil, and East Africa. Louis XIV introduced coffee to the French aristocracy in the early eighteenth century. However, the British provided the first European mass market, before tea overtook coffee as a national drink.

Tea had been known in China since the sixth century CE. It had soon spread to Korea and Japan and also became popular with the peoples of Central Asia. Tea use then spread from Central Asia to India, the Middle East, and Russia. The Chinese forbade the export of tea plants, making China the sole exporter of tea (Japan was self-sufficient). European imports blossomed in the eighteenth century, likely because of the availability of sugar from Caribbean

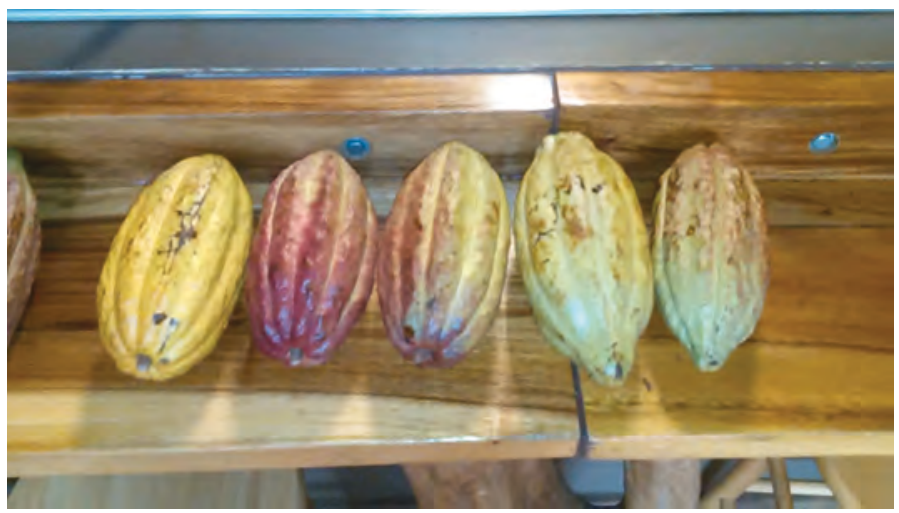

Illustration 18.6

Cacao pods; cacao beans are found inside Author 


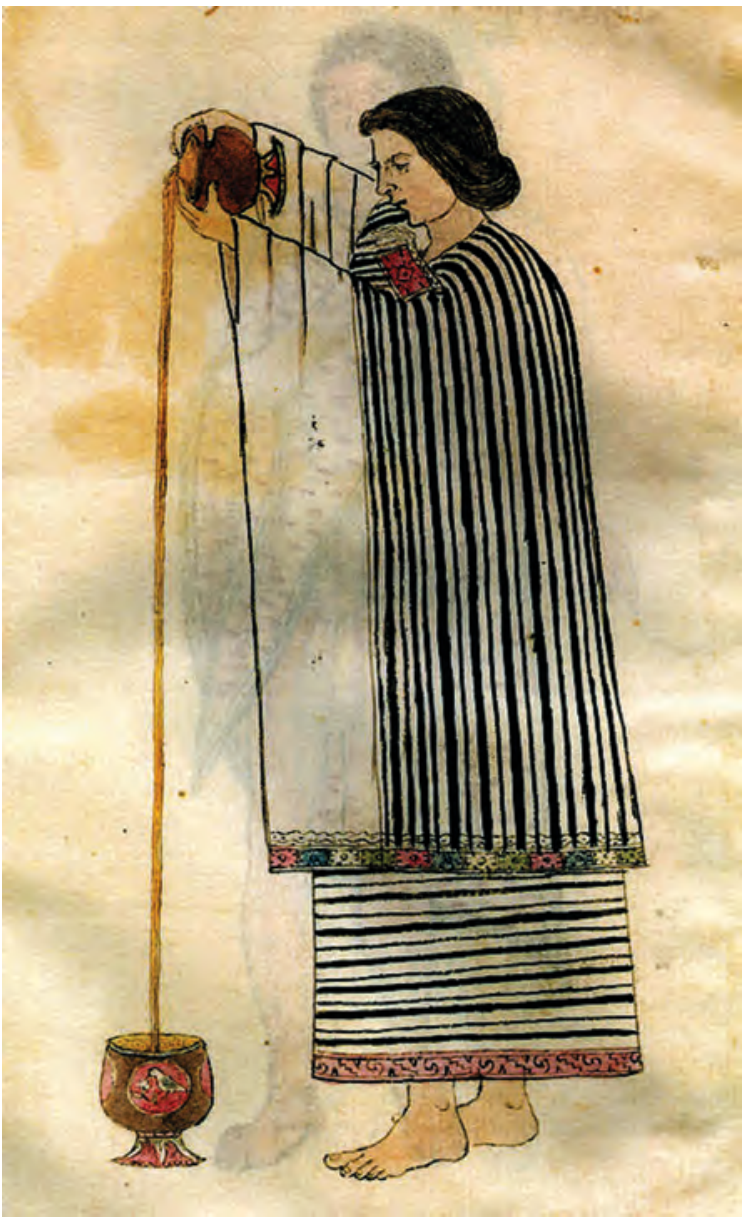

Illustration 18.7

Making chocolate, Codex Tudela, 1553, Museum of the Americas, Madrid plantations. Tea plants were likely too fragile for a long sailing-ship transport. Only in the early nineteenth century were tea plants moved to Java. Later in the nineteenth century the British developed extensive tea plantations in Sri Lanka and Assam in India: The latter was at the time lightly populated and forested, but indigenous people rebelled as their forests were cleared. British tea companies appealed to a sense of empire loyalty in encouraging British consumers to switch from Chinese green tea to Indian black tea.

Coffee and tea had both long been associated in Asia with religious ceremonies in Sufi Islam and Buddhism respectively. Cacao was an elite good among the Maya and Aztec, which had long been associated with warriors and religion. Aztec warriors consumed a sticky solidified form of chocolate before battle to gain energy. As with coffee and tea, Europeans would spread the cultivation of cacao beans globally, and make chocolate beverages an item of everyday consumption. Chocolate was consumed almost exclusively as a beverage before the invention of cocoa powder in the early nineteenth century in the Netherlands; after that, hard chocolate became increasingly common.

Europeans transformed the social meaning of coffee, tea, and chocolate, while eliminating profitable local monopolies in the Middle East, China, and Central America. Most of the profits from trade in these goods thereafter went to Europeans.

Coffee, tea, and cocoa were originally elite beverages in Europe but soon gained a mass market. This was only possible because most Europeans had incomes well above subsistence. Yet many scholars have suggested that coffee, tea, cocoa, and sugar instigated a "consumer revolution" in which Europeans came to identify themselves as consumers of non-subsistence goods. Europeans may have been inspired to work harder in order to afford these goods. 
Postscript: Consumption of coffee, tea, and chocolate is now global but uneven. Travellers often complain of the difficulty of finding "good" coffee or tea in various locales. Coffee is not widely consumed in some countries in which it is produced such as Uganda. Chocolate is popular in Japan and Taiwan, but per capita consumption in China and India is a fraction of the global average. Growing use in China and India may affect price and quality in world markets - but there are tropical lands that seem well suited to cacao cultivation that at present produce little chocolate.

\section{Trans-Sahara trade}

We have occasionally mentioned trans-Sahara trade in preceding chapters (especially chapter 9). The Sahara was a forbidding barrier to trade through most of history: Daytime temperatures can exceed 50 degrees Celsius (130 Fahrenheit) in places, but night-time temperatures can approach freezing. The Sahara has likely become hotter and drier during historical time: Buffaloes and tortoises are mentioned in the eighth century but are not seen today. The Sahara, with its sand and rocky outcrops punctuated by occasional oases, can be compared to an ocean dotted with islands: It was a barrier, but it also allowed trade to be pursued across its expanse by peoples on either side. It is clear that Jewish and Arab trade networks had prosecuted this trade for centuries before the arrival of Islam in the seventh century. Trade expanded as Islam entered North Africa from the seventh century and merchants transmitted the religion across the desert (chapter 12).

With the brief exception of a few decades of French colonial rule, transSaharan trade has always meant moving across political boundaries on either side of the desert. These polities would each levy tolls or taxes - and of course merchants needed to negotiate safe passage. The Sahara itself was often not subject to strong political authority of any sort, and so merchants generally had to protect themselves against bandits. In such an environment, both trade networks and sizeable armed trade caravans were of crucial importance. (Caravan sizes would decrease in the nineteenth century as firearms became common on caravans.) Traders would often hide gold on their bodies in fear of thieves.

The magnitude of trans-Saharan trade and the range of goods carried expanded in the Early Modern period. Dates from Saharan oases were valued on both sides of the desert. Copper and cowrie shells moved south from the eleventh century. The latter quickly became the leading currency south of the desert (gold being too valuable for small transactions). The long-standing northward movement of gold, ivory, and slaves was supplemented from the fifteenth century by trade in leather - valued by European bookbinders - and an extract from the testicles 
of civets that was much prized as a perfume. Acacia gum from Senegambia (the westernmost area of West Africa) came to be used in Europe for papermaking and textiles. West African textiles also came to be valued both within the desert and in North Africa: The famous "blue men" camel caravanners of the western Sahara were blue precisely because of the indigo dye in the African textiles they wore.

The Songhay king (chapter 16) forbade Jewish traders from the mid-fifteenth century. This decision likely reflected both religious zeal and lobbying by Muslim merchants. Jewish traders remained active in financing Sahara caravans after the expulsion, but Muslim traders managed those caravans. These Muslim merchants often turned to Muslim scholars (who were also often merchants) to settle trade disputes. These scholars, notably, often proved willing to rely on written contracts rather than oral testimony as Islamic law prescribed: It was impractical to demand oral testimony across the desert. Still, the Islamic preference for oral testimony may have hindered trade. Scholars also often disagreed: Islamic law did not erase all sources of possible dispute. Islamic law and scholars were particularly crucial in determining inheritance. There is also evidence of the transfer of debts across the desert through paper transactions as early as the tenth century. The Sahara trade was apparently as sophisticated as any of its time. [But paper tended to be expensive in the region. Arguably trade would expand again in the nineteenth century as the cost of paper fell.]

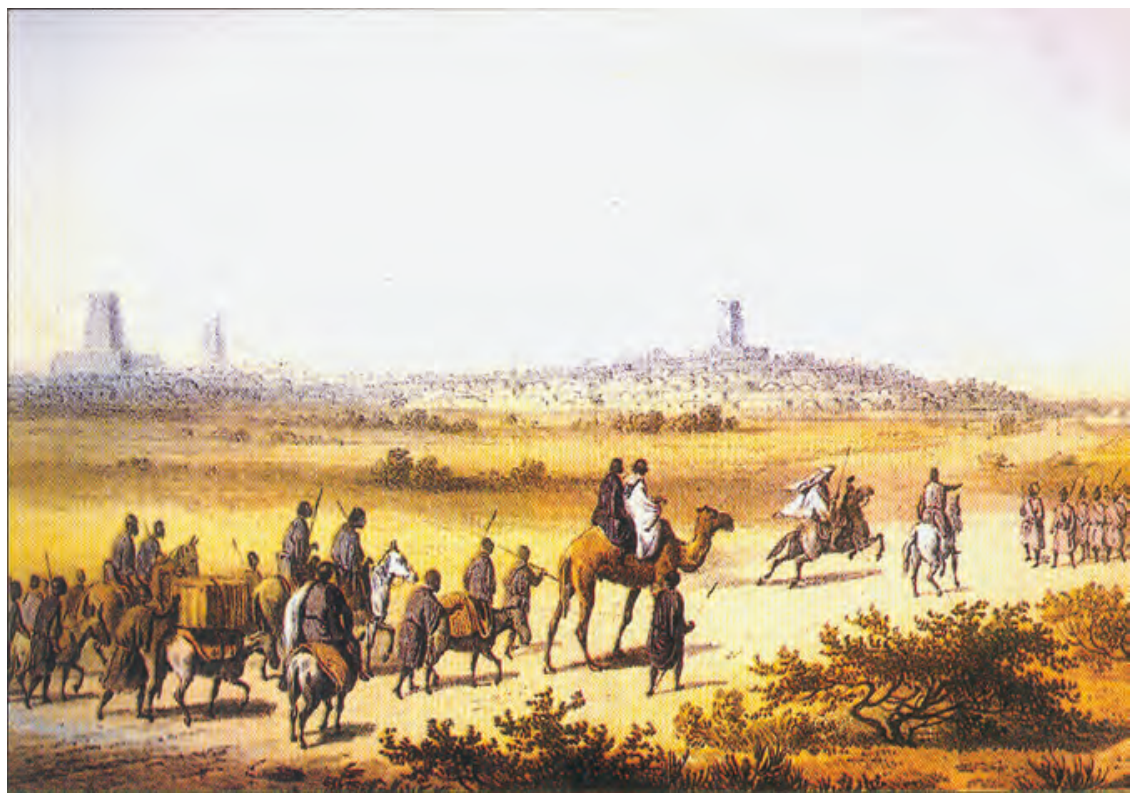

Illustration 18.8

Caravan approaching Timbuktu in 1853, from Travels and Discoveries in Northern and Central Africa by Prof Dr Heinrich Barth, vol. iv, London 1858. Caravans became smaller with the use of firearms in the 19th century 


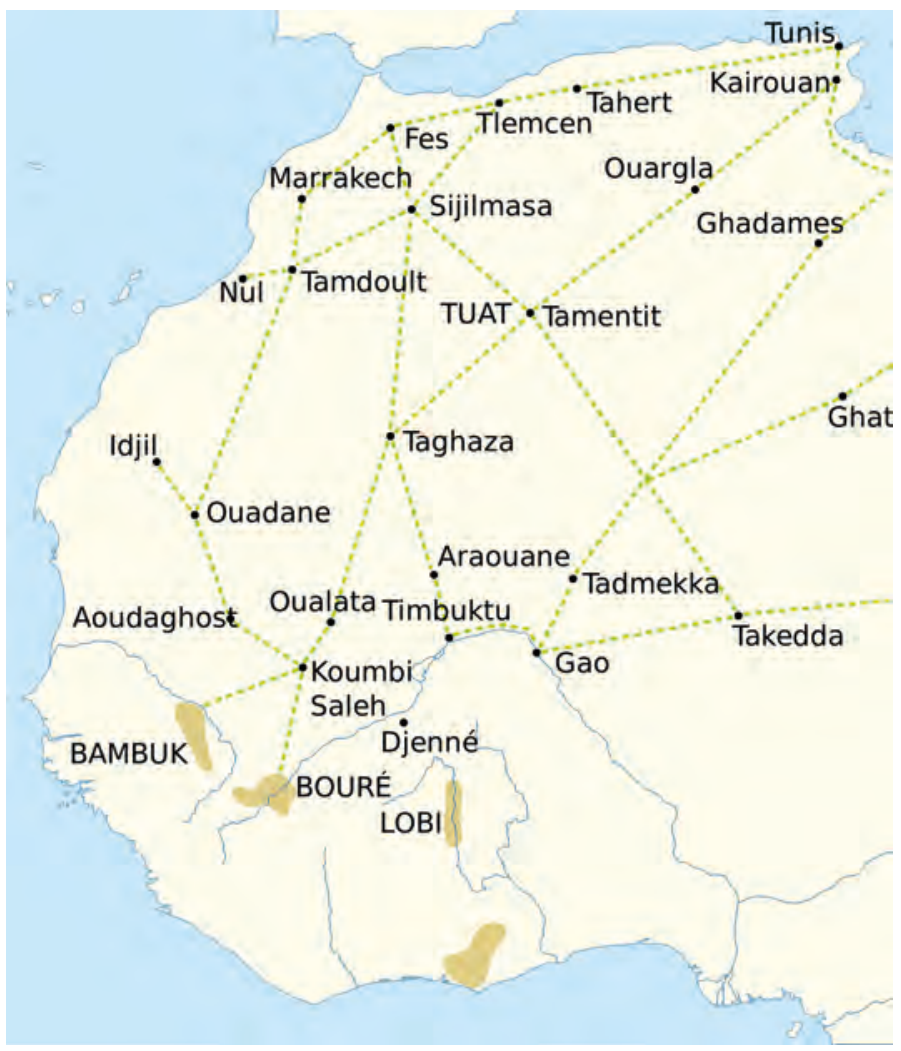

Map 18.1

Trans-Saharan trade routes before 1500. The shaded areas represent the major gold fields of the time

Map by Aa77zz, CC0

Women played a crucial role in some Muslim trade networks (looking after their husbands' affairs) but were secluded in others.

It would be a mistake to see Saharan trade as entirely a North African initiative. The trans-Saharan trade built upon a more local trade south of the desert that was generally larger in volume if not profits. In particular, peoples south of the Sahara had long valued salt that they could obtain within the desert. The desert also had metal deposits that could be traded for grains.

Sources speak of a Wangara trade network south of the desert for centuries. Little is known of this network except that it was of sub-Saharan origin. The Wangara operated within the southern reaches of the desert, gathered goods from south of the desert to trade with merchants from north of the desert, and occasionally crossed the desert to trade directly with North Africa.

Trade links that existed for centuries inevitably had cultural impacts. The most obvious of these is the spread of Islam southward. However, music and cuisine and clothing from south of the desert moved northward at the same time. 


\section{Collecting}

The wealthy had always liked to show off exotic goods. As costs of trade fell, and as Africa and the Americas were drawn increasingly into international trade, it became feasible for a much more substantial proportion of the population to collect and display items from distant lands. Ostentation was far from the only motive. Curiosity was important. For many, exotic goods had religious significance as examples of the wonders of creation. Exotic plants and feathers had an aesthetic appeal as well. Such items were often displayed in elaborate cabinets.

\section{The transmission of art}

We are in the early days of appreciating how the artistic traditions of different regions influenced each other over time. Recall from chapter 8 that artistic styles tend to evolve slowly, for the audience must become accustomed to a new style. This suggests that cross-cultural transmission of art will (usually) only happen when interaction is sustained: If only one work of art is transmitted it will likely shock rather than excite new audiences. There may, then, have been more limited cross-societal influences on art before the fifteenth century than there were influences on technology. Alexander's conquests exposed India to Greek art and inspired humanistic sculpture in India. Buddhism then carried a tradition of Buddha sculpture into China - though Chinese tastes rendered their Buddhas less sensual, more rigid, and more fully clothed. Later the global trade in Chinese porcelain (chapter 14) would provide a nexus not only for Chinese art styles - and Chinese motifs such as stylized dragons - to reach the wider world but also for astute porcelain manufacturers to learn Middle Eastern, Indian, and eventually European styles. It is less clear if a global taste for Middle Eastern carpets - which expressed the decorative designs characteristic of Islamic art - exerted much influence on artistic tastes in the many lands in which they were purchased. Such carpets appear in many European paintings of the Renaissance, but generally in the background: European artists remained focused on realistic portrayals of nature and people.

We will explore in the next chapter how intercontinental trade and cultural interaction was facilitated from the fifteenth century. As might have been expected, the initial reaction to the art of distant lands was often negative. Europeans thought that Indian art was pornographic (having conveniently forgotten the explicit nature of some ancient Greek art). The Chinese thought that Europeans were perhaps good with portraits but poor at capturing nature. Over time, though, artistic influences flowed in all directions. Artists came to appreciate that all artistic traditions - including those of traditional societies - might have useful insights that the artist could incorporate. Islamic artists began to adopt the representational art of Europeans after centuries of focusing on abstract designs (chapter 20). European artists would absorb Indian sensuality, Chinese nature painting, and the emotive art of many traditional societies. Japanese printmakers would adapt techniques for capturing 
three-dimensional perspectives pioneered in Europe. The style and subject matter (both of nature and of everyday life) of Japanese printmakers would later exert a powerful influence on European impressionist painters.

In the late sixteenth century, well over a dozen books were produced in Europe describing the clothing worn in various parts of the world. These books were part of a broader literature that described the arts and crafts of different peoples. This early interest in "world art" would fade over time with the rise of nationalism and a celebration of local traditions (chapter 21), but it did reflect a recognition that other regions of the world had something to contribute.

We spoke of collecting above. Gradually from the fifteenth century, a global market for art would emerge. In the sixteenth century, it was primarily wealthy Europeans who provided a market for art from all continents. Ivory carvers in Benin in West Africa changed both the shape and motifs of their carvings to serve European markets. Artisans in Ceylon (Sri Lanka) created works of ivory, metal, and gems in styles to please the Portuguese; Portuguese rulers often gave such items as diplomatic gifts in Europe. Notably, the Ceylonese often mixed Christian, Buddhist, and Hindu elements.

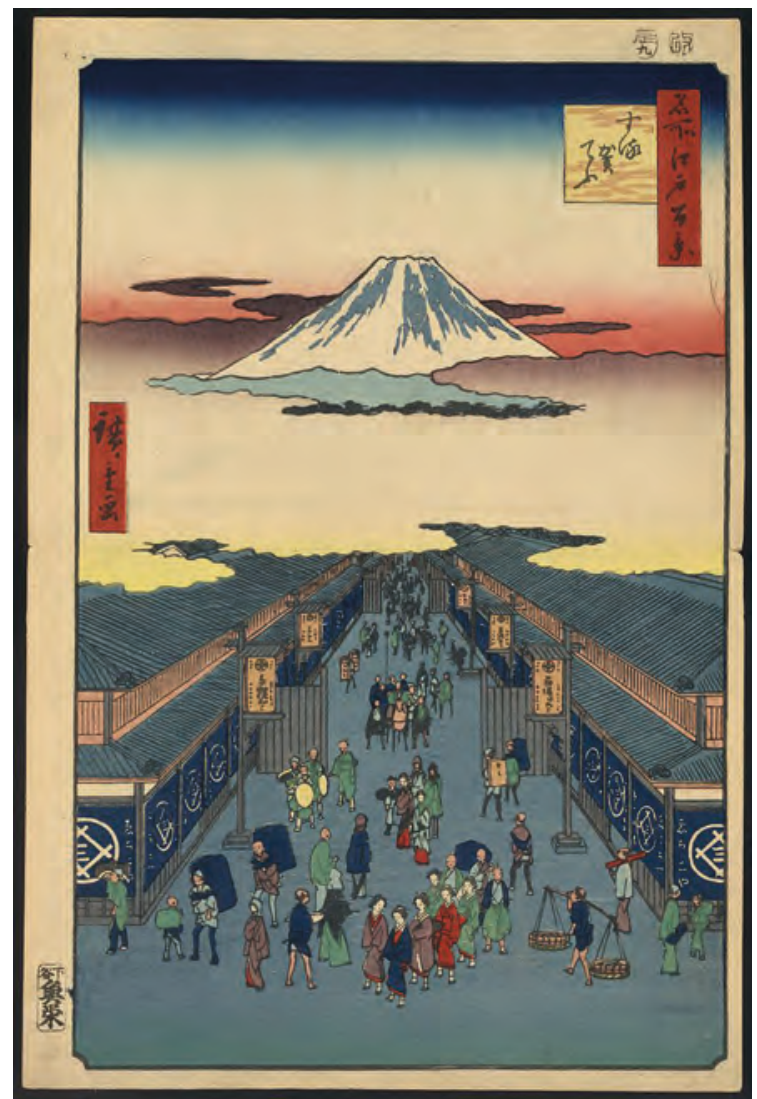

Illustration 18.9

Woodblock print from Hiroshige's Hundred Famous

Views of Edo (present-day Tokyo), 1856, a great example of employing perspective

\section{Challenges facing agents: Artists}

The artist is a kind of artisan and therefore faces the artisan's challenges (chapter 5): They must worry about quality, cost, reputation, and theft, and about feeding themselves. Indeed, in this book we have purposely blurred the distinction between artist and artisan for both of them appeal to our aesthetic tastes. Artists can be distinguished in part by generally producing works that are valued entirely for their aesthetic value. Perhaps a more important distinction is that artists are expected to create works that are somehow unique. Artists then must grapple with a critical challenge of balance: They want to introduce elements of novelty into their work but cannot deviate too much from the dominant style of their time and place for fear of offending their intended audience. 


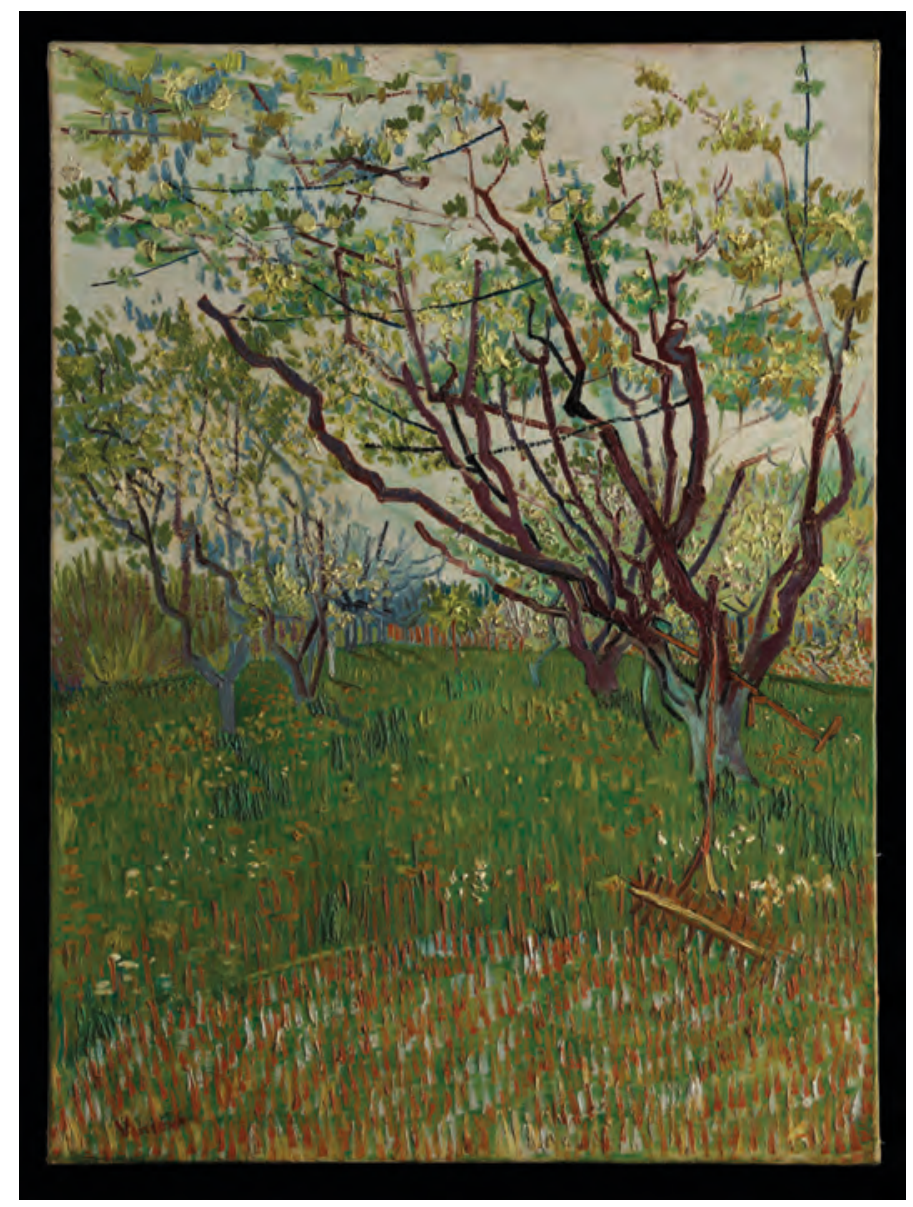

Illustration 18.10

Vincent Van Gogh, The Flowering Orchard, 1888. This composition, and especially the elongated branches, reflects Van Gogh's admiration of Japanese prints, Metropolitan Museum of Art

\section{Capitalism}

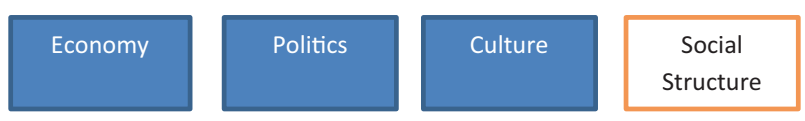

Capitalism is one of those vague terms that can confuse historical analysis if not handled carefully. Dictionary definitions of capitalism tend to stress interactions in the marketplace, private ownership of the means of production, and the pursuit of profits. Capitalism, then, is the antithesis of state control of economic activity. It can also be contrasted with the existence of mostly self-sufficient local communities. Thus defined, we can see that capitalism 


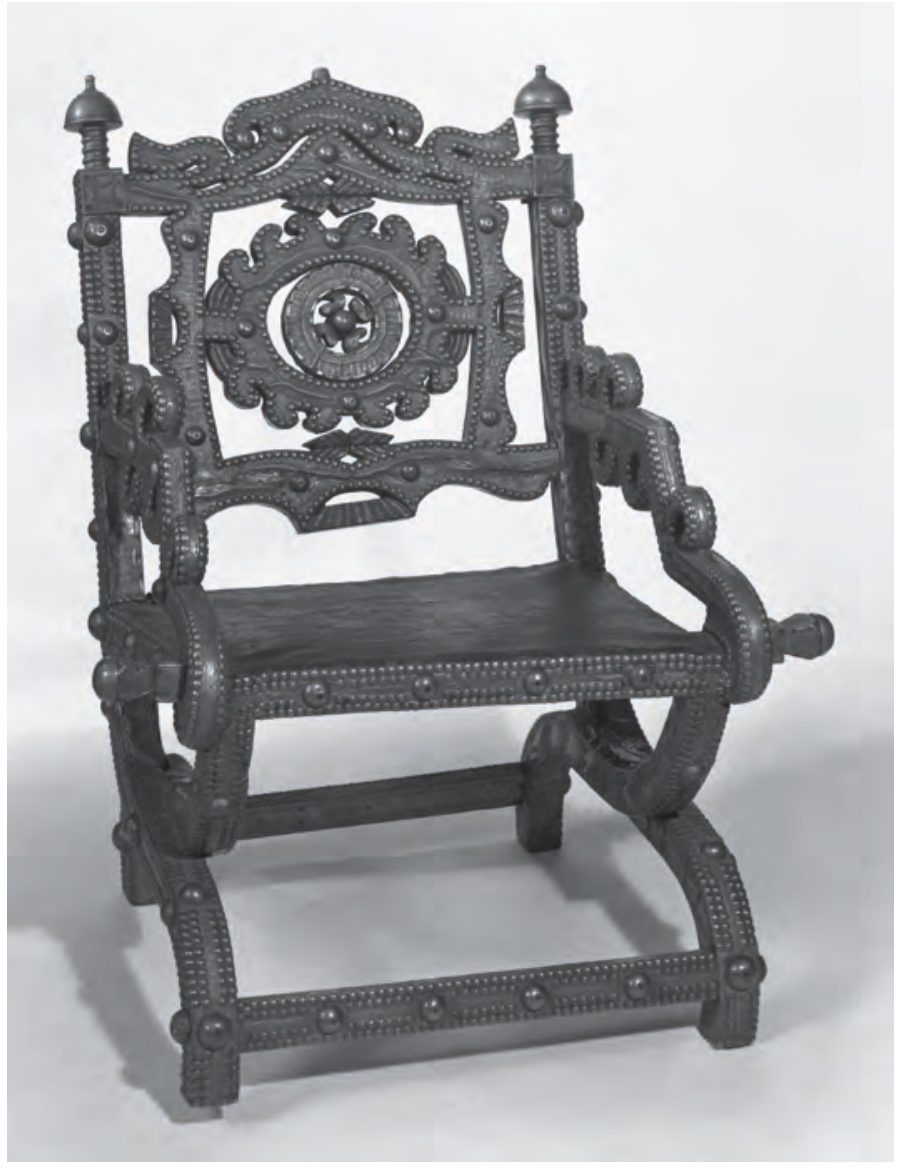

Illustration 18.11

Chairs with backs were unknown in West Africa before the arrival of Europeans, but soon Africans began carving chairs for European markets (and African kings came to demand them in place of traditional stools). Though this chair dates from the 19th century, it reflects 17th-century European styles

Brooklyn Museum, CC BY 3.0

is not something that emerged suddenly one day but rather a form of economic activity that waxed and waned through human history. There had been merchants and artisans operating independently of the state in most human societies. Their economic importance expanded as increases in agricultural productivity allowed for a more substantial non-agricultural population. Trade expanded with improvements in transport and with institutional developments (especially political consolidation) that lowered the costs of trade. Nevertheless, artisans and especially merchants were often viewed with suspicion both culturally and politically.

Capitalist activities gained economic and political importance in the Early Modern era in a variety of ways, none of them entirely new: 


\section{BOX 18.1}

\section{ARTISTIC BORROWING}

Increased artistic borrowing in all directions after 1500 reflected a variety of motives. Art styles were sometimes adopted along with religions. Art was sometimes adopted to please foreign conquerors or reach international markets. In some times and places certain foreign locales were viewed as exotic or admirable; copying artistic styles from such areas might then appeal to local audiences and bring international prestige to the artist.

The process of borrowing in most cases proceeded slowly. (One exception was when local art traditions were denigrated in favour of "modern" western art; this would occur later in Japan and elsewhere.) Artists first had to appreciate foreign styles. They then had to be careful of not transforming local styles beyond recognition. In borrowing, as in any artistic innovation, artists had to balance the potential rewards from novelty against the potential penalties for offending local tastes (recall our discussion of artistic evolution and transmission in chapter 8). Artists would struggle to predict which elements of foreign art styles could best be employed in achieving this balance.

- Farmers, especially those with access to expanding cities, were increasingly drawn into market exchange as opposed to mainly pursuing a self-sufficient lifestyle. But of course farmers had almost always produced more than they consumed, or the development of cities and states and trade would have been impossible (though their surplus was often extracted by taxes rather than via trade).

- Industry grew in scale so that private industrial producers could make large profits.

- Trade continued to expand, and so opportunities for merchants increased.

- In some states far earlier than others, merchants and industrialists gained political power and social acceptability. This had previously been rare outside of trade-oriented city states.

- Though some merchants had been wealthy in many previous human societies, wealth had most generally been associated with land ownership (or herd ownership among nomads) and the exercise of political authority. Merchants and industrialists would over a period of centuries first join landowners in the economic elite and eventually supplant them in many countries. Note that both commercial and industrial cities tended to be very unequal, with small elites having very high incomes and wealth holdings and often exercising much political power.

- Largely as a result of changes in elite composition, governments came to provide credible legal protections for market activity. This, of course, had been achieved to varying degrees by states throughout history.

- Markets for both land and labour were freed from a variety of restrictions, again earlier in some states than others. 
- It came to be accepted, though often grudgingly, that the fortunes of people depended on a plethora of market exchanges beyond their control. This had almost always been the case to some degree, but as people left selfsufficient agriculture behind, they increasingly depended on movements of prices in local economies, which came over time to be influenced more and more by movements in international trade and finance.

- As trade expanded, new business practices emerged. Of particular note was the corporation, but many advances in finance - and especially of longterm funding for industrial investments - should also be noted. Likely, the prevalence of corporations and financial institutions in modern societies most supports the idea of a new capitalist era.

- From the early nineteenth century, first in some countries, then others, economic growth began to occur at a rate that was noticeable in human lives. This changed people's expectations for their lives and those of their children, and transformed attitudes toward merchants and innovators.

While capitalism can be contrasted with state control of the economy, few have argued that capitalism has waned as governments in the twentieth century came to tax and spend 40-50 per cent of national income in most developed countries. It is appreciated, that is, that private market exchange is still the dominant form of economic activity and that such societies are still correctly termed capitalist (though some admittedly suggest that capitalism might function better if taxes were lower).

Particular note should be made of cultural attitudes. Capitalism may make the most sense as a historical concept if it is viewed as primarily cultural: the acceptance and even celebration of industrialists and merchants and the profit motive, and thus of resulting economic realities such as business cycles and income inequality. Those of us who live in capitalist societies may have to make a special effort to imagine a society in which the pursuit of profit for its own sake would have been widely questioned. However, in earlier days when the bulk of the population mostly pursued self-sufficiency, they could easily view profit-seeking with suspicion. If we define capitalism culturally, then it likely does make sense to suggest that capitalism emerged in the Early Modern period. There may have been city states pervaded by a capitalist ethos beforehand, but large capitalist societies arguably were something new.

Some of the bulleted processes above occurred slowly and globally, while others were more concentrated both temporally and geographically. We will see how corporations first became common in the pursuit of foreign trade in the next chapter, and how elected assemblies gained power in some countries in the succeeding chapter. Both transformations occurred first in Europe.

So also did the development of very large industrial firms - considered by many an essential element of capitalism (see chapter 22). To be sure, some industrial practices such as shipbuilding always had of necessity occurred on a large scale. What was new in this period was the organization of industrial activities such as spinning or weaving that had long been performed on a small 
scale (though yet again we should be aware of historical exceptions such as the thirteenth-century Belgian wool industry that was characterized by much strife between workers and merchants). Often called "cottage industry" or the "putting-out system," this involved merchants taking raw materials to rural workers and paying these for spinning or weaving or some other act (though in some places rural workers bought and sold on their own account). Some merchants came to employ hundreds or even thousands of workers in this manner. They might also supply their workers with machines as well as raw materials. Historians have often argued that merchants pursued rural industry to escape the guild controls of cities (chapter 15). However, rural workers were also less expensive: It was easier to move yarn to and from them than move food from the countryside to the city. Moreover, rural workers, at least at first, could work in agriculture during the busy harvest season and focus their industrial efforts during the agricultural off-season. This also reduced the amount that they needed to be paid for industrial work. Rural industry tended to concentrate in regions where land was of inferior quality. These large-scale industrial firms became common from the sixteenth century; factory organization would supersede them from the eighteenth (chapter 24) but especially during the nineteenth century. Large industrial firms were only feasible once merchants had access both to a substantial market and to financing to cover the weeks or months between the purchase of raw materials and the sale of finished goods. Beyond their economic impact, these firms had an important social impact: Young people married earlier in areas of rural industry because industrial jobs allowed them to establish an independent household without waiting to inherit farmland; fertility rates tended to rise as a result.

The rise of capitalism in this period, and the increased trade and incomes associated with this, were in turn connected to a transformation in consumption itself. Through much of human history most humans spent the vast bulk of their income on the basic necessities of life. To be sure they sought out nice-looking clothes and plates to eat off when possible. We have seen that average incomes often increased slowly in history, allowing people to buy better pottery and textiles and food. In the fifteenth through eighteenth centuries, though, the idea of consumption - of consuming a variety of goods and services beyond food, clothing, and shelter - gained hold in the broader population of many lands. This both reflected and encouraged increases in income and trade. And capitalists encouraged and then served the mass markets that emerged for various goods and services. This new consumption seems everywhere associated with displays of social status: Many goods and services were purchased in order to impress others. There is a question as to whether elite practices of public display of wealth were echoed by others, or whether emerging middle classes developed their own ideals of display. At times, elites disparaged consumerism in others, but over time most societies accepted an ideal that all should be consumers. Sumptuary laws in some lands - decreeing that only elites could wear certain clothes - were widely evaded and fell into disuse. 


\section{Population}

Population
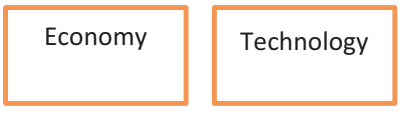

Built

Environment

The global population was around 450-500 million in 1500 (chapter 14). This was a bit higher than it had been before the Great Plague of the fourteenth century. Population growth would accelerate in the Early Modern period: Population was 550 million in 1600, 650 million in 1700, and 950 million by 1800. The global exchange of plants and animals facilitated much of this population growth. As we shall see in the next chapter, plants from the Americas prospered in the eastern hemisphere and vice versa. Often new plants grew where farmers had struggled to grow anything before. The dramatic increase in land under cultivation was aided by cutting down forests and draining swamps. There were also advances in output per acre.

Population growth patterns differed regionally. The most dramatic story unfolded in the Americas and Pacific, where diseases from Eurasia killed up to 90 per cent of local populations. High birth rates and mass migration - accompanied by the adoption of horses and other Eurasian techniques in agriculture in the Americas - soon caused populations in the Americas to rise far above previous levels. In Europe population expanded from 80 million in 1500 to 120 million in 1700 and 180 million by 1800 . Population growth was much lower in some parts of the world, including North Africa. Africa had likely had about 20 per cent of the world's population in 1500 but only 11 per cent in 1800 and 8 per cent in 1900. (It is projected to have 20 per cent again by 2050, with some of the highest population growth rates in the world.)

Population growth was accompanied in most of the world by urbanization. Both London and Paris had half a million people by the mid eighteenth century. Urbanization in this period would set the stage for more dramatic developments in the nineteenth and twentieth centuries (chapter 31).

\section{Questions}

1. Were there reform movements in all world religions in the Early Modern period?

2. Why do some scholars discuss capitalism without carefully defining the term?

3. Why must we be careful about giving a time period a title such as "Early Modern"?

4. Compare the trans-Saharan trade discussed in this chapter with the Silk Road trade addressed in chapter 9.

5. What are the most important differences between this time period and the preceding time period addressed in chapters 14-17? 


\section{Readings}

Almond, Ian. 2011. Two Faiths, One Banner: When Muslims Marched with Christians across Europe's Battlegrounds. Harvard University Press.

Andrade, Tonio. 2016. The Gunpowder Age: China, Military Innovation, and the Rise of the West in World History. Princeton University Press. [Provides some comparative data on the frequency of wars in China and Europe; argues that China emphasized rockets over cannon.]

Appleby, Joyce. 2010. The Relentless Revolution: A History of Capitalism. New York: Norton.

Burke, Edmund III. 2012. "Toward a Comparative History of the Modern Mediterranean, 1750-1919." Journal of World History 23:4, 907-39. [This article argues that there are many commonalities between Christian and Muslim societies around the Mediterranean in the Early Modern period: sluggish population growth compared to the rest of the world; silting of irrigation systems; depletion of forests; increased raising of sheep; economic decline due to shifting international trade routes (later to be reversed to some extent with the Suez Canal and railway projects); efforts to convert religious minorities; challenges of self-determination associated with the decline of the Ottoman Empire; and arguments over gender rights.]

Goodman, Ruth. 2017. How to Be a Tudor: A Dawn-to-Dusk Guide to Tudor Life. New York: Norton. [See also her How to Be a Victorian.]

Hoffman, Philip T. 2015. Why Did Europe Conquer the World? Princeton University Press.

Kagan, Richard L., and Philip D. Morgan, eds. 2009. Atlantic Diasporas: Jews, Conversos, and Crypto-Jews in the Age of Mercantilism, 1500-1800. Baltimore, MD: Johns Hopkins University Press.

Kaufmann, Thomas Dacosta, Catherine Dossin, and Béatrice Joyeux-Prunel, eds. 2016. Circulations in the Global History of Art. New York: Routledge.

Kocka, Jürgen. 2016. Capitalism: A Short History. Princeton University Press.

Lydon, Ghislaine. 2009. On Trans-Saharan Trails: Islamic Law, Trade Networks, and Cross-Cultural Exchange in Nineteenth-Century Western Africa. Cambridge University Press. [Though the focus is on the nineteenth century, the book surveys earlier developments and also addresses the relevant theories of networks and institutions.]

Ma, Debin, and Jan Luiten van Zanden, eds. 2011. Law and Long-Term Economic Change: A Eurasian Perspective. Stanford University Press.

McCabe Ina B. 2015. History of Global Consumption 1500-1800. London: Routledge.

Marks, Stephen G. 2016. The Information Nexus: Global Capitalism from the Renaissance to the Present. New York: Cambridge University Press. [Recognizing that none of the characteristics of capitalism were entirely new, he argues that the key to capitalism was the control of information, due to publishing, double-entry bookkeeping, and so on. But one might argue that the control of information has always been critical to merchant success.]

Neal, Larry, and Jeffrey G. Williamson, eds. 2014. The Cambridge History of Capitalism. 2 Vols. Cambridge University Press. 
Ogborn, Miles. 2008. Global Lives: Britain and the World, 1550-1800. New York: Cambridge University Press. [This book provides biographies of more than forty individuals around the world who negotiated a variety of contradictions during the 250 years after about 1600 . Students of biography could also consult Ouladah Equiano's autobiography about his life as a slave (though it is now thought that he may have made up the part about being born in Africa). Emma Rothschild traces eleven Scottish siblings who seek fortunes with some success throughout the Empire.]

Parker, Geoffrey. 2013. Global Crisis: War, Climate Change and Catastrophe in the Seventeenth Century. New Haven, CT: Yale University Press. [The book describes the nature and effects of the seventeenth-century political crises. There was a Symposium on the book in the Journal of World History, June 2015.]

Pomeranz, Kenneth, and Stephen Topik. 1999. The World that Trade Created: Society, Culture, and the World Economy 1400 - the Present. Armonk, NY: ME Sharpe. [There are multiple editions of this book, each containing many essays on trade-related topics.]

Rappaport, Erika 2017. A Thirst for Empire: How Tea Shaped the Modern World. Princeton University Press.

Tolan, John, Gilles Veinstein, and Henry Laurens. 2013. Europe and the Islamic World: A History. Translated by Jane Marie Todd. Foreword by John L. Esposito. Princeton University Press.

Wills John E. 2009. The World from 1450 to 1700. Oxford University Press. [See also his 1688: A Global History, New York: Norton, 2002.] 


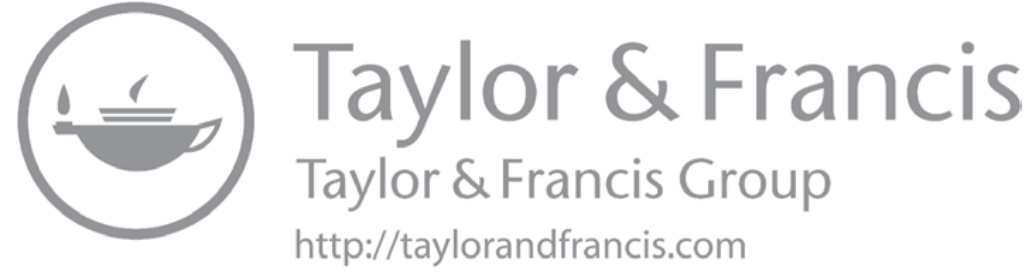




\section{Exploration and trade Linking the continents}

\section{Guiding questions}

How, why, when, and where did Europeans first explore and then colonize the Americas? How and why did Europeans expand across the continents, and Russians and Chinese into Central Asia and northeastern Asia? In particular, what role did fish, fur, and agriculture play in these expansions?

How and why did Europeans establish a new trade route to Asia around Africa?

What were the key effects of the increased connectedness of continents? On attitudes? On agricultural practices? On slavery? On trade itself?

Relationship to other chapters:We draw heavily on preceding discussions of trade and naval technology in understanding European exploration. In particular, we have often seen how merchants preferred engaging with as few states as possible. We then apply our understanding of various polities and of gunpowder technology to understanding European conquests. The exchange of ideas, goods, diseases, plants, and animals between the Americas and the eastern hemisphere represents a dramatic acceleration and reorientation of processes of interaction within the eastern hemisphere (and to a lesser extent within the Americas) that we have discussed in several preceding chapters. We naturally compare the Atlantic slave trade with earlier cases of slavery.

As noted in our introduction to chapter 16, the world changes after 1500. All large societies were connected thereafter-though 
small isolated communities in places like the Amazon and New Guinea remained unconnected with the broader world well into the twentieth century. This chapter sets the stage for many later discussions of how ideas, objects, and people move globally - and generally much faster than they had before.

The processes addressed in this chapter transform the history of most, maybe even all, regions of the world. Much of the Americas, but also large swathes of all other continents, was farmed for the first time as new crops were introduced. European colonization affected all aspects of life in its colonies, and the slave trade had dramatic effects on both African and American societies.

In the late fifteenth century, new sea routes connected the eastern and western hemispheres, and connected Europe to Asia around Africa. Over the next centuries, the Americas and Africa would increase their trade with Europe and Asia. One significant result of this trade was the spread of crops from one region of the world to others. European states would also establish colonies throughout much of the world. These political and economic connections on a global scale represent one of the most important transformations in world history.

We begin this chapter with Spanish exploration and conquest westward and then turn to the contemporaneous development by Portugal of a sea route to Asia around Africa. We then discuss how other European powers entered international naval commerce. While western Europeans were exploring the world, the Russians and Chinese were expanding into Central Asia, destroying the independence of the nomadic polities that had played such a critical role in the preceding history of Eurasia. We also discuss the new trade connections that emerged, the exchange of diseases and plants and ideas, and the development of the Atlantic slave trade. We close with a discussion of European settlement of the temperate parts of the Americas.

We will spend much of the chapter discussing interactions across societies, and most often of societies that had not been in contact previously. In addressing how these affected each other, we naturally compare how they differed before contact. Thematically, exploration is itself diverse: It is scientific in the sense of expanding human knowledge of the world, but also inherently political. Though financed for the most part by states, these voyages of discovery nevertheless had powerful economic motives. Particular individuals loom large in both exploration and colonization. Societal interaction transformed cultural beliefs and practices. (We discussed art and population in chapter 18.) The slave trade transformed aspects of social structure. There is less evolutionary analysis in this chapter than most, but some concerning 
the development of shipping technology and later the institution of trading companies.

\section{The Spanish conquests in the western hemisphere}

\section{The Spanish discovery of America}

We have traced in earlier chapters the rise and fall of trade between Europe, Asia, and parts of Africa. With the rise of Islam and fall of the Mongol Empire, the vast bulk of intercontinental trade passed through the Islamic Middle East. European traders naturally sought alternative routes that would decrease the bargaining power of Middle Eastern merchants and rulers. This desire encouraged exploration in two directions: southward around Africa, and westward across the Atlantic (and then the Pacific). However, the success of both of these avenues of exploration reflects historical contingency: The southward journey long focused on the African trade before the possibility of reaching Asia became clear, and a miscalculation of the circumference of the earth (see below) famously triggered the westward voyages. If the earliest adventures in both directions had not proven successful, the desire for a cheaper route to Asia might have remained unsatisfied.

Moreover, European mastery of the Atlantic reflected trade within Europe rather than any desire to reach Asia. Genoan galleys had begun to explore the north Atlantic from the late thirteenth century because of difficulties the Italians were facing in the overland route through France to northern Europe, and the decline of the Champagne fairs (chapter 15). Over the succeeding centuries, shipbuilders integrated Mediterranean shipping technologies especially the "caravel" employing lateen sails borrowed from the Arabs - and northern European shipping technologies to develop ships well suited to ocean travel. The resulting "carrack" used both square and lateen sails: The lateen were superior for sailing across the wind, while sailors could raise and lower square sails quickly, allowing fast sailing with the wind, and providing more control. The carrack used by Columbus (below) had five sails. Sternpost rudders had been added from the twelfth century, another innovation (like the compass) that had spread west from China. Such rudders made it far easier to steer a sailing ship.

The Canary Islands (named after dogs living there rather than birds) had been known since antiquity but were not often visited despite being only eighty kilometres off the coast of Africa. There was a small indigenous population. These islands were "rediscovered" by Genoese ships sailing for Portugal in the mid fifteenth century. A treaty of 1480 gave the Canaries to the Kingdom 


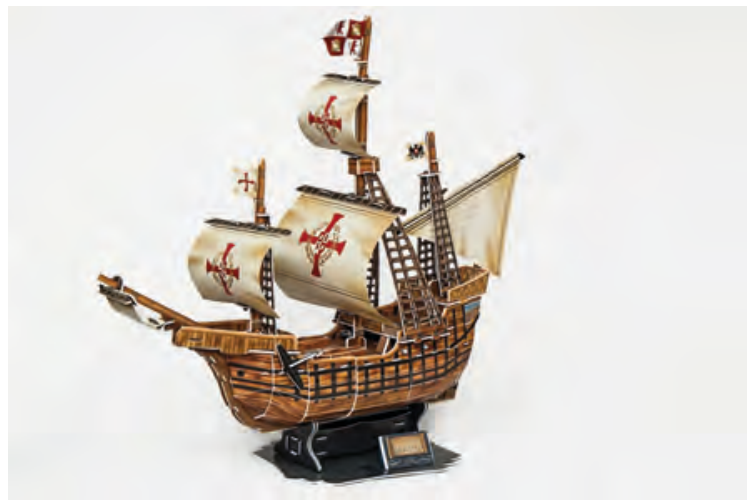

Illustration 19.1

Rigging for a carrack, combining square and lateen sails

of Castile in Spain, but Madeira, the Azores, and Cape Verde - other islands off the coast of Africa discovered as the Portuguese sailed south - to Portugal. This division encouraged Spain to look west and Portugal to look south. The Canaries were, it turned out, a great launching point from which to reach the Americas. In the meantime, Europeans used all of these islands for sugar plantations that employed African slaves - and obtained these slaves through trade with West African states. (Recall that Europe had learned of sugarcane from the Middle East, and had produced sugar in the Mediterranean using Slavs as slaves; chapter 15.)

Christopher Columbus had sailed multiple times to the Canaries, Madeira, the Azores, and West Africa, and north to Ireland and possibly Iceland. He could draw on centuries of European experience in sailing on the (eastern edges of the) Atlantic Ocean. Importantly, he knew how to use a compass (employed in Europe from at least 1180) to establish the direction of travel and to use an astrolabe (used by the Portuguese for about a decade) to ascertain latitude. He lived in Lisbon at a time when people were excited about discoveries in Africa and there was widespread speculation about the existence of unknown lands. There were tales of lands to the west, and of sailors seeing land birds heading west, and of corpses and plants and canoes of unknown origin washing up on the western shores of these Atlantic islands.

Postscript: Though Columbus had instruments that could establish direction and latitude, he had no means of determining longitude. Reckoning longitude would be increasingly important as ships traversed vast oceans. The solution would only come in the 1760s with the development of clocks that could maintain accurate time on a bouncy ship: Captains could then compare the clock time (of their home port) with the observed time (derived from the rise of the sun) to establish longitude. 
Of course, Columbus sought not the new lands that he discovered but a new route to Asia. There was a large and growing market for pepper, ginger, nutmeg, and cloves in Europe, as well as manufactured goods such as silk and porcelain, but prices had risen with the decline of the Mongol Empire. It was widely accepted by this time that the earth was round, and that it should then be possible to reach Asia sailing west. Columbus famously underestimated the circumference of the planet. He also thought that China was farther east than it is, and believed Marco Polo's estimate that Japan was 1,500 miles further east than China. He then underestimated the distance to Asia by a factor of four. Some have speculated that Columbus deliberately underestimated this distance to obtain funding. Certainly, his fame owes more to persistence in securing financing than in navigational skill. (The Chinese and Vikings and Polynesians deserve at least as much fame for their voyages, but these had much less historical impact.) The Portuguese lost interest in his plans after Diaz's success in rounding the southern cape of Africa (see below): They saw little value in a risky westward voyage to Asia when they were confident that they could sail east around Africa. The Spanish

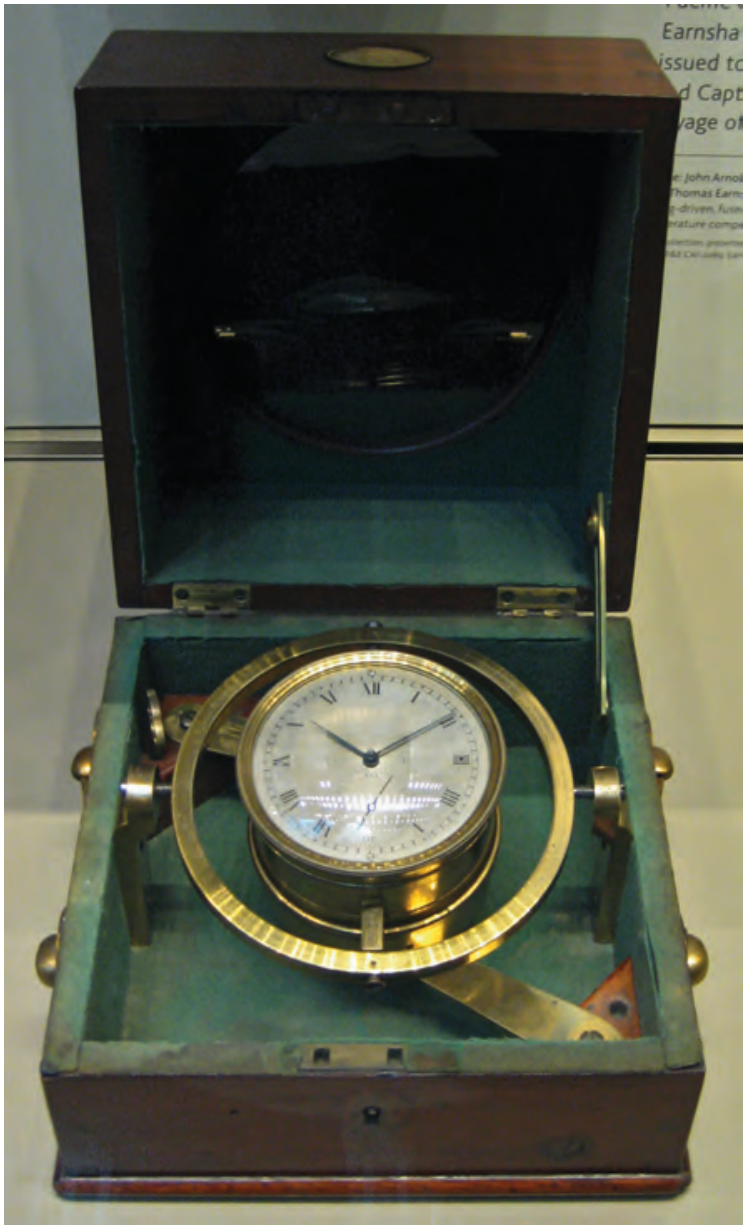

Illustration 19.2

Ship's clock from HMS Beagle, 19th century, British Museum

Photograph by BabelStone, CC0 1.0 were also sceptical that Columbus could reach Asia. Yet they worried that he might sail for France or even England, and so decided that a smallish outlay was worth the risk. If he died, they would lose little but if he succeeded - in either reaching Asia or making discoveries along the way - Spain might become rich. They were far less confident than Columbus, which may have encouraged them to meet his demands to be appointed admiral and governor-general, with the right to name governors, of any new lands he discovered.

Columbus somehow financed about a quarter of the cost of the voyage himself. His ships were not large, the largest being 27 metres long. This was perhaps one-quarter the size of the main ships on the Chinese voyages of discovery generations earlier (chapter 20). His "fleet" of three ships stopped in the Canaries for a few days. The fleet carried perhaps enough food for a year-long 
voyage. It then took thirty-three days more to cross to landfall in what was probably the Bahamas. His sailors were ready to mutiny despite sightings of land birds, and despite possessing more than enough food to return to Europe as long as they could find eastward winds. After sailing around the Caribbean searching for gold and spices and China, they headed back. The lead ship, the Santa Maria, had run aground and so thirty-nine volunteers stayed behind in a fort. The rest belatedly headed north to catch winds blowing east, almost died in storms, and ended up first in the Azores and then Lisbon. The Portuguese did not exactly welcome them, but they might have treated them worse. The trip lasted seven months in total.

Columbus would make three more trips. Each of these was more than a couple of years long and sought both to explore and to establish an administration. Parrots, feathers, tobacco, and rubber were among the exotic goods carried to Europe by Columbus. The second expedition had seventeen ships and discovered that indigenous peoples had killed the thirty-nine volunteers left behind. His Spanish associates disliked Columbus as a governor, and he was sent back in chains after the third trip. He managed a fourth voyage, but Spain thereafter turned to others for governors.

There were perhaps 4 million Tainos or Arawaks in the Caribbean when the Spanish arrived. They had little in the way of trade goods that interested the Spanish. The Spanish forced them to work in gold mines or on plantations. The Spanish treated them harshly, despite royal orders to convert them to Christianity. Then a smallpox epidemic after 1518 further reduced numbers. They

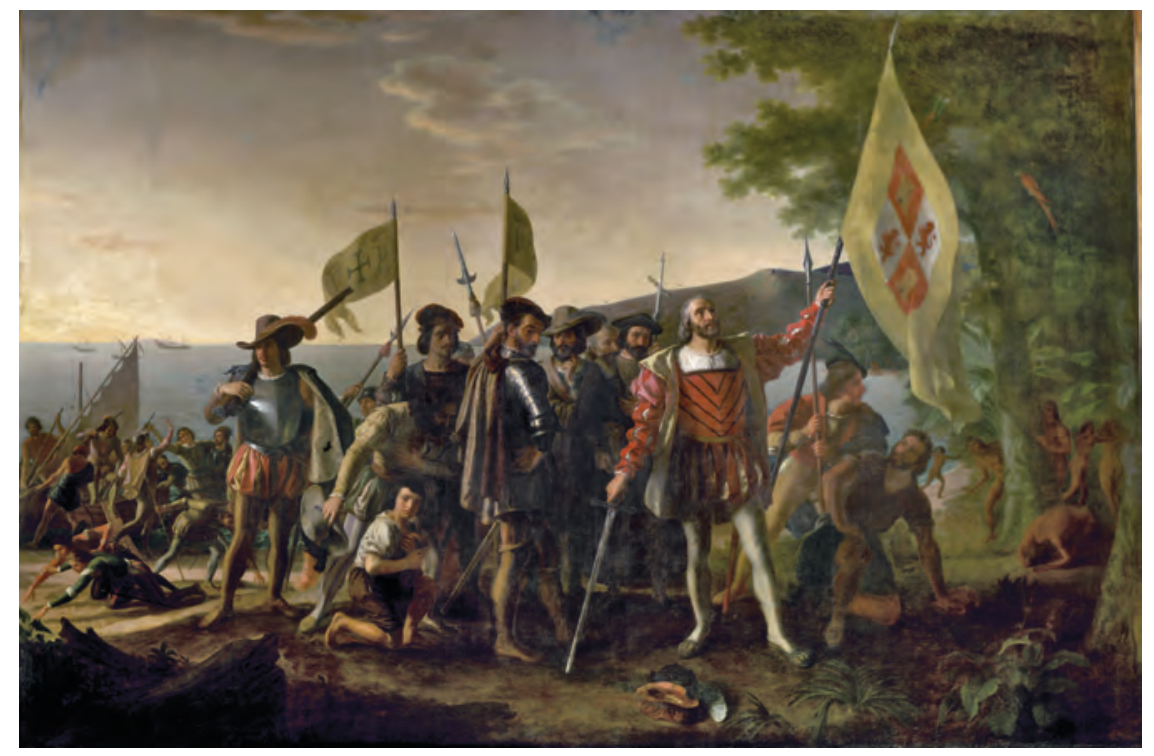

Illustration 19.3

John Vanderlyn, Landing of Columbus, 1847. Columbus drops his hat in a gesture of sacredness, while some of his men search for gold. Local people watch from behind trees 
had effectively disappeared by the late sixteenth century. Some of their words, such as "hurricane," survive. The Spanish came to focus their efforts elsewhere.

\section{Spanish conquests in the Americas}

As the Spanish explored the mainland of the Americas, they discovered not only the precious metals that they had long sought but also organized societies. These societies could both point them to sources of silver and provide a labour force for extracting this. Students should appreciate that the Americas would have been much less enticing if not for the existence of the Aztec and Inca Empires. These were well-developed states with organized markets and agriculture and systems of tribute (chapter 16). Once conquered, their institutions and infrastructure served Spanish needs. In the absence of such empires, the Spanish would have had to develop the necessary institutions and infrastructure for resource extraction from scratch.

The Spanish conquest of the Americas - the Inca and Aztec Empires, most of South America except Portuguese Brazil, and about a third of the modern United States - happened in mere decades. This is one of the most amazing expansions of empire in human history, comparable to the early Islamic conquests or the Mongol empire. The Spanish were even more of a surprise to those they conquered than these earlier conquerors had been.

Between 1519 and 1521, Hernán Cortés and a small group of perhaps 500 men destroyed the Aztec Empire, aided by local groups tired of Aztec rule. The Spanish had previously learned how to communicate with Maya speakers along the coast, but they then needed local translators to engage with the Aztecs and other peoples of central Mexico. As we have seen in chapter 16, Aztec rule was harsh, and so it is hardly surprising that many subject groups proved willing to join this small group of outsiders in attacking the Aztecs. In pursuing a "divide and conquer" strategy in Mexico, Cortés may have been inspired by similar tactics employed by Spanish Christian forces in their wars against Islamic rulers in Spain itself in preceding centuries (chapter 15).

The Spanish, of course, had a huge technological advantage: They had gunpowder and horses and iron armour. Even with these advantages, the sheer force of Aztec numbers might have defeated the small initial force of Cortés, especially if ambushed in some place where they would not have been able to shoot their attackers from a distance. However, the technological edge was enough to attract locals to their cause. Moreover, this new enemy understandably bewildered the Aztecs themselves.

The spread of disease aided the Spanish in this and other conquests. A smallpox epidemic raged through Aztec territories and especially the capital while these were under attack. Smallpox may have devastated a third of the population over the next year.

The size of Tenochtitlan (80,000) and the Aztec Empire (one million) surprised Cortés. He had amassed allies on the way to Tenochtitlan, but the Aztecs invited him into the city without them. Moctezuma, the Aztec ruler, might 
have attacked the Spaniards then but chose not to. Though we do not know Moctezuma's motives, it could be that he recognized superior force and wished to rule in concert with the Spaniards. The Spanish later alleged that the Aztecs thought that the Spanish were gods, but historians now suspect that the Aztecs were well aware that they were human. The Spanish imprisoned Moctezuma and other leading Aztecs in the palace. The Aztecs later revolted. It is not clear whether Aztecs or Spanish killed Moctezuma. In any case, the Spaniards had to fight their way from the city. Aztec weapons were no match for muskets and iron shields and swords, but the Aztec numbers were overwhelming. The Aztecs likely killed two-thirds of Cortés' men as they fled Tenochtitlan. Cortés received reinforcements from the Spanish garrison in Cuba, and won over many other locals to his battle against the Aztecs. The reinforcements may have been sent to replace Cortés, but he had achieved enough success that they opted to join him. Tenochtitlan fell to this force, but only after a four-month siege.

We should pause for a moment with Cortés and his men as they first enter Tenochtitlan. Their chief translator - an indigenous woman who the Spanish named Dona Marina and who would later have a child with Cortés and then marry one of his men - had likely given them some description. However, the

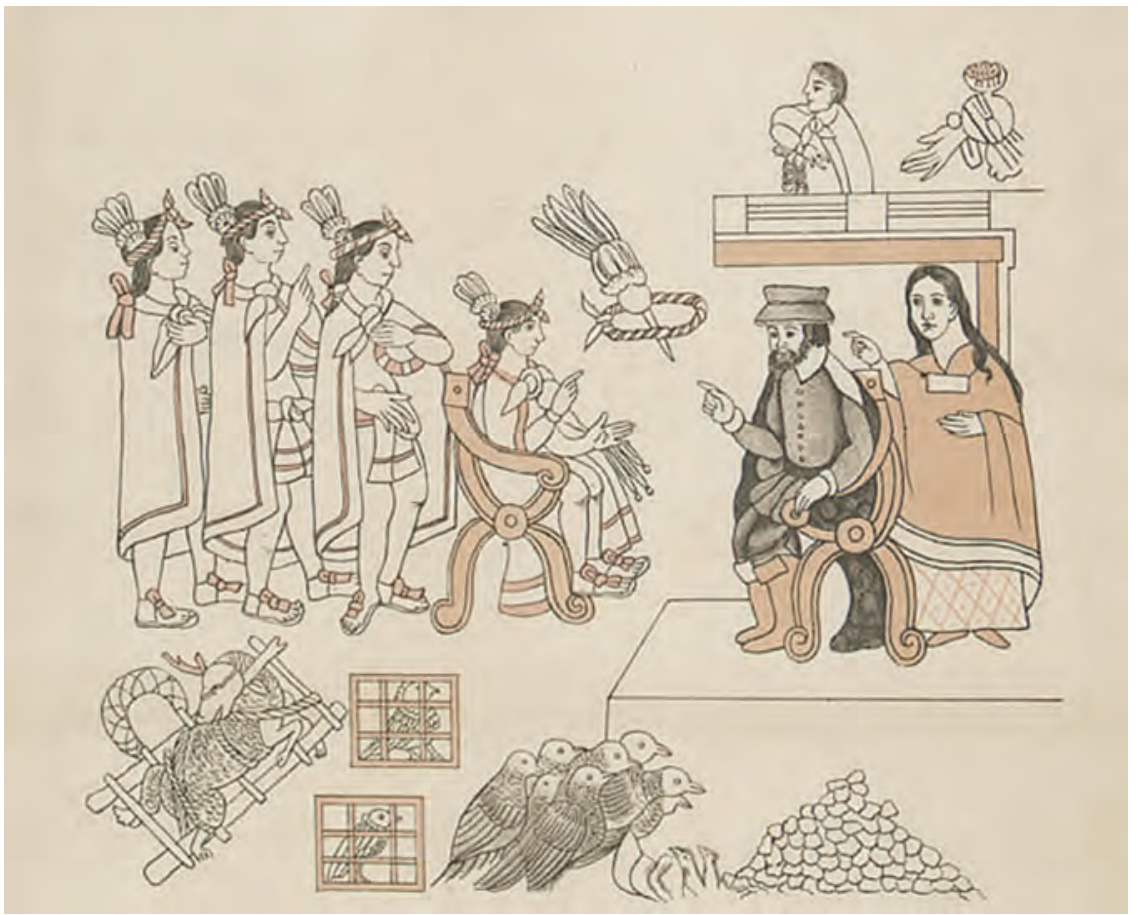

\section{Illustration 19.4}

“'Tenochtitlan, Entrance of Hernán Cortés," from the Lienzo de Tlaxcala, c. 1550, which was created by Tlaxcalans to remind the Spanish of their support of the Spanish conquest. The drawing contains historical inaccuracies such as the use of European chairs, Bancroft Library 
Spanish experience in the western hemisphere so far had been of small villages and polities. Suddenly they were presented with one of the biggest cities in the world, majestically situated on a set of islands linked to the shoreline by causeways, comprising palaces and temples that compared with any in Spain, watered by aqueducts, surrounded by verdant farms, and with streets and neighbourhoods that struck them as cleaner and more orderly than those back home. They had truly stumbled upon a "New World" that they had not at all expected. Their immediate reaction was awe. This awe blended with fear, and was soon transcended by thoughts of conquest. After all, they had travelled to the Americas in pursuit of fortune. Nevertheless, we should pause with them as they collectively think "Wow!" and before they move on to "We should kill some of these people and take over." We can also imagine a somewhat similar sense of awe among the indigenous peoples observing them, though fear likely loomed even larger in their immediate thought processes.

\section{PRIMARY DOCUMENTS: THE CONQUEST OF TENOCHTITLAN}

Cortés wrote to Emperor Charles V of his victory:

The following morning, they came out of the city to greet me with many trumpets and drums, including many persons whom they regard as priests in their temples, dressed in traditional vestments and singing after their fashion, as they do in the temples. With such ceremony, they led us into the city and gave us very good quarters, where all those in my company were most comfortable. There they brought us food, though not sufficient. On the road we had come across many of the signs which the natives of that province had warned us about, for we found the highroad closed and another made and some holes, though not many; and some of the streets of the city were barricaded, and there were piles of stones on all the roofs. All this made us more alert and more cautious.

There I found several of Moctezuma's messengers who came and spoke with those who were with me, but to me they said merely that they had come to discover from those others what they had agreed with me, so as to go and inform their master. So after they had spoken with them, they left; and with them went one of the most important of those who had been with me before. During the three days I remained in that city they fed us worse each day, and the lords and principal persons of the city came only rarely to see and speak with me. And being somewhat disturbed by this, my interpreter, who is an Indian woman from Putunchan, which is the great river of which I spoke to Your Majesty in the first letter, was told by another Indian woman and a native of this city that very close by many of Moctezuma's men were gathered, and that the people of the city had sent away their women and children and all their belongings, and were about to fall on us and kill us all; and that if she wished to escape she should go with 
her and she would shelter here. ... Because of this and because of the signs I had observed, I decided to forestall an attack, and I sent for some of the chiefs of the city, saying that I wished to speak with them. I put them in a room and meanwhile warned our men to be prepared, when a harquebus was fired, to fall on the many Indians who were outside our quarters and on those who were inside. And so it was done, that after I had put the chiefs in the room, I left them bound up and rode away and had the harquebus fired, and we fought so hard that in two hours more than three thousand men were killed. So that Your Majesty should realize how well prepared they were, even before I left my quarters they had occupied all the streets and had placed all their people at the ready, although, as we took them by surprise, they were easy to disperse, especially because I had imprisoned their leaders. I ordered some towers and fortified houses from which they were attacking us to be set on fire. And so I proceeded through the city fighting for five hours or more, leaving our quarters, which were in a strong position, secure. Finally all the people were driven out of the city in many directions, for some five thousand Indians from Tascalteca and another four hundred from Cempoal were assisting me.

In Hernán Cortés: Letters from Mexico. Translated and edited by Anthony Pagden, New Haven:Yale University Press, 1986, 12-4.

The Florentine Codex, compiled shortly after the Conquest, describes how locals perceived the Spanish entry into Moctezuma's palace (Book 12, chapter 18):

When the above had been done, [the Spaniards] attempted to find out about the special storehouse of Moctezuma, and he took them to his storehouse, named Totocalco, which means "bird house." The Spaniards went along very joyfully, thinking that they would find much gold there; on arrival they took everything out of Moctezuma's own storehouse, where there were many precious items of gold, silver, and precious stones, and they took it all. They removed all the gold and stones from the rich feather-pieces and put the feathers in the middle of the courtyard for their friends to take. Then Captain don Hernando Cortés gave orders through Marina, who was his interpreter-she was an Indian woman who knew the languages of Castile and Mexico; they took her in Yucatan. She began to call loudly to the Mexica tecutles [lords] and piles [nobles] to come to give the Spaniards the necessary food. But no one dared to come into their presence or approach them; they were all terrified and frightened. They sent them the necessary food, but those who carried it went trembling; when they put the food down, they tarried no longer, but immediately left, almost fleeing.

Bernardino de Sahagún. "Of How the Spaniards Entered Moteucçoma's Private Home, and What Happened There." Book 12, Chap. 18, p.125 in

Florentine Codex. c. 1570-85. In We People Here, translated and edited by James Lockhart. University of California Press.

Do victor and vanquished see similarly? 
Pizarro, the conqueror of the Inca Empire, may have learned from Cortés' example. If so, he knew what smallpox and internal divisions could do. Pizarro had explored along the Pacific coast, heard of a rich inland empire, and applied for royal permission to conquer this. Pizarro's force crossed the Isthmus of Panama and then rebuilt ships to sail south to Peru. He soon learned after arrival in Inca territory that a civil war over succession between two royal brothers had been raging for four years. He may have seen an opportunity to avoid the months of conflict that Cortés had faced. In 1532, Pizarro, with about 600 soldiers, met Atahualpa, the Inca ruler, at Cajamarca in Peru. It was not a first contact characterized by mutual respect or understanding. Atahualpa had come into town with an unarmed entourage. The Spanish gave him a bible to read; he had no idea what it was and threw it down. The Spanish then killed his entourage. His armed escort outside town fled. The Spanish held Atahualpa for a ransom in gold, and then killed him anyway after the Inca delivered the payment. This is at least the version of events recorded by the Spanish (who would also report later examples of Inca trickery).

Inca kings continued for forty years as either puppets or rebels. Some Incas retreated into the mountains and held out until 1572. Yet in general, the conquest of the Incas proceeded even faster than the conquest of the Aztecs. This might be because the Spanish had learned from the earlier experience. It may be because they successfully used deceit. Perhaps the lack of a system of writing (chapter 16) limited the Inca ability to communicate about how they should react to this novel threat. It may also be because smallpox had reached the Incas even before the Spanish. Maybe the Inca's subjects - forced to provide labour - were even more anxious to see them overthrown than the subjects of the Aztecs - who were only forced to pay tribute for the most part (but were occasionally used in human sacrifices).

The Spanish thereafter operated through a mostly indigenous bureaucracy, reorganized but built upon Inca precedents. The Spanish also continued the Inca practice of forced labour. Small numbers of Spanish troops supported local bureaucrats. Local elites, whether we choose to see them as collaborators or intermediaries, mostly continued as before to take their share of local resources. Their standing in the community became dependent on Spanish rule. They intermarried with the Spanish. If local chiefs - many only recently conquered by the Inca - hoped to gain autonomy by allying with the Spanish they miscalculated, but they were generally able to maintain considerable local influence.

Why were these attacks on both Aztecs and Incas so successful? The obvious answer is that the Spanish had a huge advantage in military technology: They had firearms, and their opponents did not even have iron weapons. The Incas had bronze weapons and cotton armour, while the Aztecs used obsidian for weapons. This advantage did undoubtedly allow small squads of Spanish troops to kill large numbers of locals on occasion. However, given that the original Spanish expeditions were small in number, it should still have been possible for them to be overwhelmed by sheer force of numbers. This would especially 
have been the case on occasions when the Spanish troops were isolated from each other, such as when Cortés and his men fled Tenochtitlan. It is not clear whether the Spanish would have responded with larger armies if the first expeditions had been rebuffed.

In addition to military technology, the Spanish had two other advantages. First was the spread of epidemic diseases. These wiped out perhaps two-thirds or more of the indigenous population within a few years (see below). This not only weakened the physical capability of these societies to resist but had a psychological effect: They thought that their gods had abandoned them. Europeans, in turn, were convinced that god was on their side. Second, the Spanish did not operate alone but found many allies among groups that had disliked rule by Aztec or Inca. Most battles during the conquest involved mainly indigenous peoples with their own weapons, though the presence of some Spanish soldiers was important.

The Spanish conquest of the Aztec and Inca Empires is therefore similar in an important respect to the defeat of many other historical empires. The Aztecs and Inca were widely disliked. A new force appearing on the borders could enlist a great deal of internal support for rebellion. These peoples were likely to see the new conquerors as liberators.

\section{Silver and settlement}

The Spanish first stole precious metals from locals, but then developed silver mines around Zacatecas in Mexico and Potosi in Peru. Potosi was developed from 1545; by 1600 , there were 150,000 people there and it was the largest city in the Americas; by 1800, it was a ghost town, as the silver had been mined out. Both mines relied mostly on paid labour, but forced labour was also common. Mortality rates especially for forced labour were high. The Spanish crown took one-fifth of all silver. Such mines produced 80 per cent of the world's silver between 1550 and 1800. There were also gold mines in the Americas that provided some 70 per cent of the world's gold. Large agricultural estates provided food for the miners. Workers on these estates were at first legally tied to the land in a system with some resemblance to European feudalism (chapter 13). Later, indebtedness to landowners often kept peasants on the land. Despite these agricultural enterprises, Potosi may have had the highest food prices in the world due to a problematic mountain location. Local peoples occasionally rebelled.

Arica (in northern Chile) was the port for Potosi. Spanish silver from Peru moved across the Isthmus of Panama to reach Europe. This route was much preferred to the dangerous voyage by sea around the southern tip of South America (though the choice was made politically and may not have reflected the actual relative costs of the two routes). However, the Panamanian route had its own challenges. Many died of disease crossing the isthmus or just in the ports on both sides of it. Nevertheless, Panama City on the western side would become one of the wealthiest towns in the Americas due to its role in organizing the 


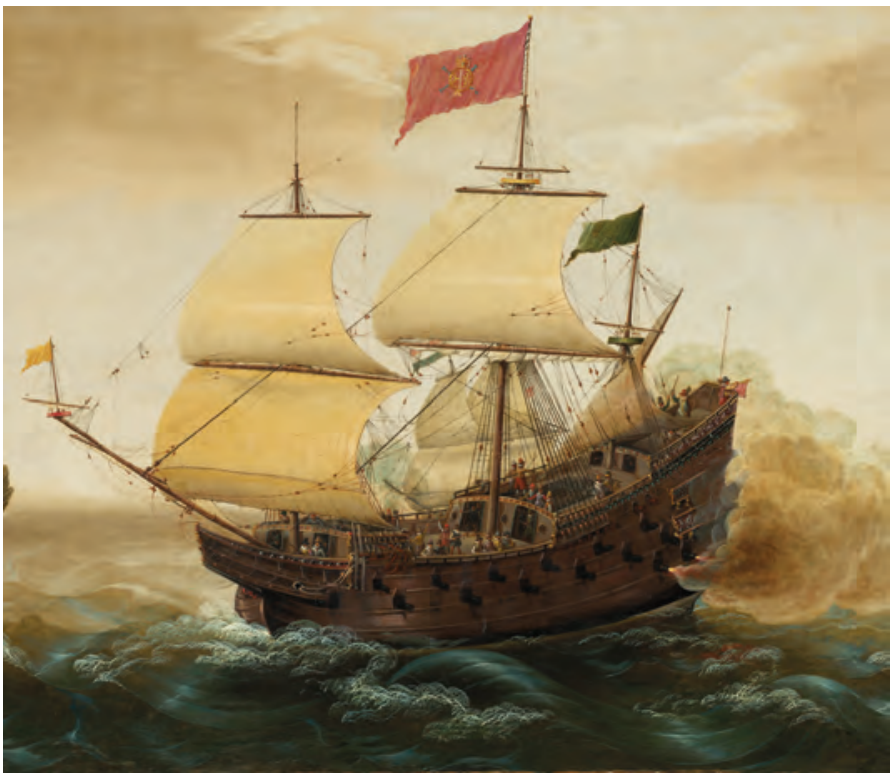

Illustration 19.5

Spanish Galleon Firing Cannon, Cornelis Verbeeck (c.1590, after 1637), National Gallery of Art

shipment of silver by land to the Caribbean. Cartagena just eastward of Panama in Colombia had a deep and well-protected harbour in which ships could gather to sail to Spain. Havana in Cuba was another place where ships often gathered for the trip to Spain. Veracruz was the main port for silver exports from Mexico to Europe (though much of this and soon a majority was instead shipped across the Pacific to Asia; see below). From 1564, Spanish shipping travelled in two convoys to avoid pirates from other European countries. These various ports were crude for some time, coming to life only briefly when ships were there. Guayaquil in Mexico was used for shipbuilding.

Though Peru and Mexico were each closely linked to Spain, they had limited contact with each other. Indeed, Seville merchants lobbied against direct trade between Peru and Mexico. They wished to encourage both colonies to trade instead with Spain.

While Spanish adventurers initiated the conquests of both Mexico and Peru, the Spanish crown welcomed these conquests as it became evident that there was vast wealth in both places. The Spanish within decades appointed viceroys in Mexico City (built on the ruins of Tenochtitlan, the Aztec capital), and Lima on the coast of Peru (this second viceroyalty would later be split in two). Cortés would become a prosperous landowner; Pizarro would be killed by the brother of a man who had been murdered by Pizarro for rebelling against him. The Spanish also appointed auditors to keep an eye on the viceroys and hear public complaints against them. A board back in Spain set official Spanish policy for the colonies. Nevertheless, viceroys operated with considerable independence 
because of the time it took for communications between Spain and its territories. Though Spain itself had an elected assembly with limited powers, it introduced no such body in its colonies; there were only town councils charged with local public infrastructure.

Between 1500 and 1800, half a million Spanish people moved to the Americas. The vast majority of these were men, and so a substantial mestizo population emerged as Spanish men married local women. Yet people from Spain long remained at the top of the social hierarchy. Mestizos were even barred from guilds in the 1540s and the priesthood from the 1550s.

The Spanish explored northward along both coasts, establishing trading posts as far north as Virginia in the east and Vancouver Island in the west. However, northern Europeans would later push them out of these distant territories (see below).

The Spanish justified their conquest from its earliest days with an appeal to the importance of converting local people to Catholicism. Missionaries focused at first on converting tribal leaders and local elites. They were aided by the widespread local belief that the Spanish gods must be superior since the locals had been devastated by war and disease. Missionaries also emphasized similarities with local beliefs: In particular, local gods were associated with Catholic saints. The popularity of Christianity expanded in Mexico after Mary (mother of Jesus) appeared in a vision to a local peasant in 1531; the shrine in Guadalupe near Mexico City became a major pilgrimage destination. When locals refused to convert, as when some Incas returned to their traditional faith, the state harassed them. The Jesuits soon became the dominant religious organization, establishing schools and two universities in 1551 (the Portuguese did not establish a university until the nineteenth century in their Brazilian colony), and amassing great wealth as landholders.

The Spanish rulers saw their conquests in the western hemisphere as a way to finance Spanish wars against both Islam and Protestants. Others (especially those Protestants, but also the French) soon criticized Spanish behaviour in the western hemisphere, noting that the lust for riches interfered with just treatment of natives. Some Catholic missionaries also complained of the mistreatment of local peoples. Other states then used these arguments to justify piracy: French ships soon attacked Spanish and Portuguese ships. Meanwhile, tales of western hemisphere riches inspired the French and English to take their own colonies in the Americas. Rivalry among European states likely increased the rate of colonization in the Americas.

The Portuguese had become acquainted with Brazil on their way to the Indian Ocean (see below). They discovered there brazilwood from which a red dye could be made. Only from the mid sixteenth century did they start establishing sugar plantations in the land they named after this wood. A hundred thousand Portuguese would eventually settle in Brazil. The vast majority were men, even more so than in Spanish territories, and so a substantial mestizo population emerged there too. Over time, Portuguese and mestizos also mated with slave populations, producing a very diverse population. Millions of 
slaves were imported. Slave mortality may have been 5 per cent or more annually, but nevertheless Africans became the dominant ethnic group.

What should Europeans call these new lands? Amerigo Vespucci, a Florentine sailor at the time in the employ of the Spanish, wrote a letter to the Medicis of Florence in 1503 about the new lands he was helping to map in what is now called South America. Unlike Columbus, who had died thinking that he had reached Asia, Vespucci appreciated that he was describing lands of continental extent that had previously been unimagined. The letter and a later one were reprinted many times in many languages: They provided some of the first details of these new lands to European audiences. The German mapmaker Martin Waldseemuller attached the name "America" to the new lands, thinking that Vespucci was the discoverer. The mistake was soon appreciated, but the name stuck.

\section{The Spanish and others across the Pacific}

The Spanish explorer Balboa had crossed Panama in 1513 and sighted the Pacific. As the Spanish became active in Peru, they became curious about whether it was possible to cross this vast ocean. Hundreds of sailors would die in a decades-long search for the winds that would allow ships to cross the Pacific. (Meanwhile, farther north, hundreds more died in the failed search by northern European states for northwest - around the northern edge of North America - and northeast - around the northern coast of Asia - passages, impossible with the ships and climate of the time.)

Ferdinand Magellan was Portuguese and had been involved in the taking of Melaka (see below). Like Columbus, he approached Spain when Portugal would not support his plans to find a western route across the Pacific. (The Portuguese likely refused because they already had developed a route to Asia.) He sailed in 1519 with five ships and 237 men provisioned for two years. His Spanish crew resented him, and he killed one ship captain and marooned another. It took them five weeks to get through the strait now known as the Strait of Magellan at the southern tip of South America. Then they spent fully fourteen weeks without sight of land while heading west. During this period, sailors died of scurvy and hunger. Rats and worms had eaten much of their food. Scurvy, resulting from a deficiency of vitamin $\mathrm{C}$, had not been a big problem for sailors until very long voyages of exploration occurred. It causes the gums to rot, teeth to fall out, haemorrhaging, and death. Twenty-nine of Magellan's crew died of scurvy. The rest finally reached Guam in the Marianas Islands. There they killed and burned houses in response to (alleged) theft. In the Philippines, it turned out that Magellan's Malay slave could understand the local language. They then reached the island of Cebu, where they converted the local ruler and subjects to Catholicism. Magellan was killed while fighting for the local ruler.

The two remaining ships carried on to the Spice Islands, where they traded cloth and hatchets for spices. The crew argued over what to do next: sail east 


\section{BOX 19.1}

\section{SCURVY}

Humans share with primates and a few other animals an inability to synthesize their own vitamin C. Human populations that do not regularly ingest vitamin C therefore suffer from scurvy, a deadly and debilitating disease. Scurvy has been known since classical times, and was often observed during the Crusades, but its most frequent incidence would occur on long sea voyages. Ships on such voyages naturally carried foods that would not spoil. Citrus fruits, a significant source of vitamin C, were not included. This was not a problem if ships regularly stopped at ports where sailors might have a more varied diet. However, in crossing oceans, sailors might go many weeks without vitamin C.

Magellan was far from the only explorer to be plagued by scurvy. Da Gama's sea voyage along the African coast in 1499 (below) lost 116 of a crew of 170 to scurvy. In all, some 2 million sailors may have died of scurvy between 1490 and 1800.Yet the cure was repeatedly discovered. The Portuguese planted vegetables and fruits on the island of St. Helena along their African sea route to care for sick sailors. Indigenous people near modern Montreal introduced Jacques Cartier to the use of boiled cedar needles in 1534, which saved many of his crew from death. Still, it was only at the very end of the eighteenth century that the medical community accepted the evidence that fresh vegetables and (especially) fruit could prevent scurvy. The simple nutritional "cure" for scurvy had simply not fit with accepted medical theories of the cause of disease.

or west. Forty-seven Europeans and thirteen Malays in one ship would voyage across the Indian Ocean and around Africa, reaching Cape Verde in twentyone weeks. Twenty-one crewmembers died along the way. The Portuguese then arrested thirteen. Eighteen Europeans and three Malays reached Spain, becoming the first humans ever to circumnavigate the globe (another seventeen eventually returned by other means). The entire trip took two years, eleven months and two weeks. The other ship tried to sail east across the Pacific but was blown back, and the Portuguese arrested its crew. The Spanish fleet sent to rescue them was also captured; a second turned back.

Though the trip proved that one could sail from Europe to Asia westward, it also established that it was easier to get there going east. The Portuguese had been correct in concluding that the route to Asia around Africa was superior to that imagined by Columbus - though of course they did not know at the time that the Americas stood in the way. Nevertheless, Magellan's voyage established the possibility of linking America to Asia directly (and thus indirectly Europe to Asia). It would turn out indeed that the trip west from Mexico to the Philippines was reasonably easy with prevailing winds. However, it would take forty years of searching before in 1565 the Spanish discovered that by sailing north they could find an easterly wind that allowed a speedy return to Mexico. The Spanish established a colony in the Philippines and interacted 
well with Chinese traders who were active there. The Spanish took the task of conversion very seriously in the Philippines, making it over time the only Christian-majority nation in Asia. They may have done so in part strategically to counter Chinese influence. In any case, missionaries again emphasized elements of Catholicism that resonated with local religious traditions

The Spanish traded silver from the Americas in the Philippines for silk, porcelain, and spices; these would be carried to Mexico, and some would be transported on to Europe. Only four ships would ever be attacked (by the English) on this lucrative route between Mexico and the Philippines. Note that more silver passed west from Potosi to China through Manila than east to Europe by the end of the sixteenth century. Tea became an important Chinese export in the seventeenth century. Overall, about half of New World silver found its way to China, either directly across the Pacific or indirectly via Europe.

The Spanish were satisfied with their route from Mexico to the Philippines. They occasionally encountered other Pacific islands but remained focused on trading silver for Asian goods. Their route passed far south of Hawaii (but north of most other Pacific islands) going west and far north of Hawaii going east. They did stop at Guam for provisions, generally trading peacefully with local people. Then in the 1670s the Spanish decided to conquer Guam, and were aided by a smallpox epidemic that reduced the local population from 50,000 to 5,000. The Spanish also colonized neighbouring islands in the Marianas. The Spanish in Peru also occasionally visited what is now French Polynesia.

Other European countries led centuries of effort to explore the Pacific. Drake circumnavigated the globe for the British in 1577-80, attacking Spanish ships off Cape Verde and again in Peru (taking 80 pounds of gold and 20 tons of silver) and sacking Spanish cities. He discovered that open sea lay south of South America. He stopped in the Spice Islands and then sailed non-stop from Java to Sierra Leone in West Africa. The English government's take from this journey of trade and looting was half their annual revenue. The Spanish claimed that the booty was at least twice what Drake reported. Drake would also chart the west coast of North America as far as Vancouver Island. James Cook for the British much later in 1728-99 charted lands from New Zealand and the east coast of Australia (declaring these lands suitable for settlement) north through New Caledonia, Vanuatu, and Hawaii to the Arctic. The Portuguese had reached the western and northern coasts of Australia perhaps as early as the 1520s. The Dutch visited in 1606 but concluded that it was a barren land inhabited by savages. (Abel Tasman twice circumnavigated Australia for the Dutch in the mid seventeenth century, but did not explore the continent. He thought aboriginal peoples were miserable whereas Cook pronounced them happy and noble.) The British established a penal colony in Sydney, Australia, from 1788 but the population grew slowly after that for decades.

The Russians hired the Dane Vitus Bering to explore the Pacific coast of their newly gained Siberian territories (see below). This would in time lead to expansion into Alaska. In two voyages in the 1720s and 1730s, Bering passed through 
the strait that now bears his name into the Arctic, establishing that Asia and North America were distinct continents.

It would be a grave mistake to view the Pacific as merely a transport route between Asia and the Americas. Though the Pacific has a very limited landmass, it nevertheless had products that locals could trade with the rest of the world. The ocean itself was important here. Whalers were enticed by the reports of Cook about the Pacific, and soon there were hundreds of whaling ships from many nations hunting whales in the Pacific - mostly for whale oil, a vital lubricant and illuminant at the time. Whalers would kill tens of thousands of whales in the next decades. Otters were found in abundance along the northwest coast of North America and the Aleutian Islands of Alaska. Indigenous people were encouraged to catch these (and other furs), and the furs were shipped via Hawaii to Asian markets. Shark fins, sea cucumbers (not usually eaten by locals), seaweed, precious shells, and abalone were other maritime products that were valued in China and shipped there from especially the westernmost Pacific islands.

The islands themselves had resources. Hawaiian kings organized large groups of workers to cut sandalwood for export. Fijian kings also deployed large workforces, which depleted Fijian sandalwood by around 1800; they thereafter focused on sea cucumbers. Australians traded sandalwood from Melanesian islands (previously not reached by the sandalwood trade) for Chinese tea in the 1840s and 1850s. Guano - an accumulation of bird droppings - was mined on several Pacific islands for use as fertilizer. The Philippines (and adjacent

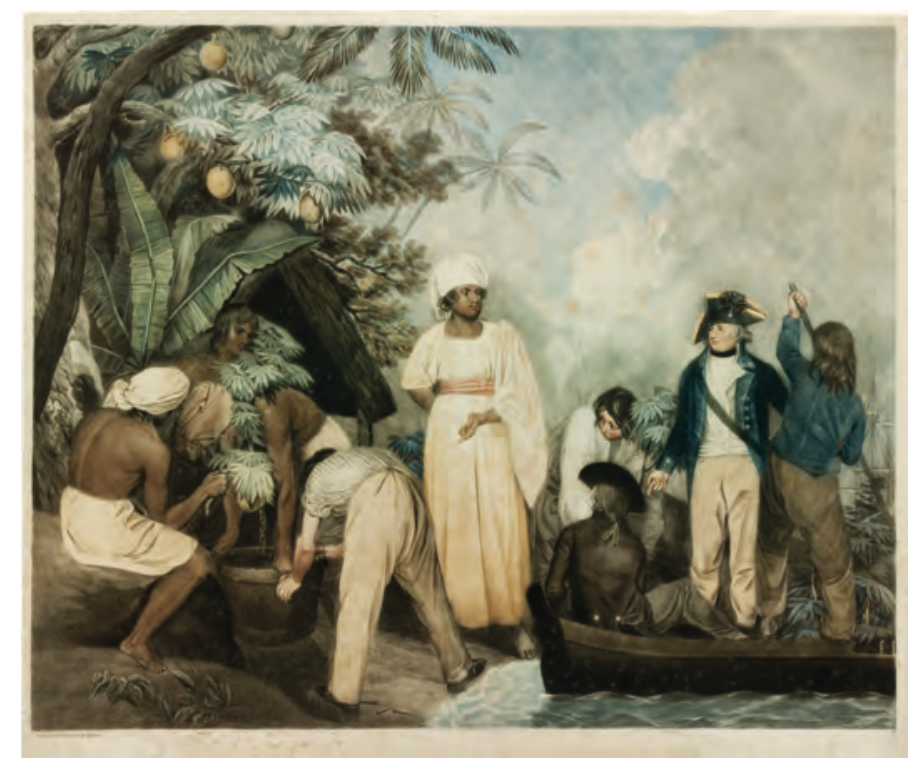

Illustration 19.6

Transplanting Breadfruit from Tahiti, Thomas Gosse, 1796, State Library of New South Wales 
parts of Indonesia) traded a variety of local items - among them mother-ofpearl, seaweed, precious shells, pepper, betel nut bird's nests, and beeswax - for Chinese porcelain, silk, iron, and rice.

The exploration of the Pacific was significant in many ways. In particular, these voyages meant (as other cases of cross-societal contact had) that crops from elsewhere were planted in the Pacific, and Pacific crops such as breadfruit were planted elsewhere. The voyages also introduced epidemic diseases to which islanders had no immunity. There was also cultural exchange: Christianity would spread across the Pacific, while idealized stories of sexual permissiveness and tropical paradise would excite western imaginations and inspire western authors and artists. We might, note, though, that Polynesian society was understood poorly: There was less sexual permissiveness than was generally thought by Europeans, and the sexual access European males gained to local females was often part of a deliberate local strategy of forming alliances with Europeans against other Polynesians. Polynesia nevertheless proved a convenient argument for many Europeans who were becoming hostile to the conservatism of European religious figures.

\section{The Portuguese to the Indian Ocean}

We have seen in earlier chapters (especially chapter 15) how sugarcane, originally from New Guinea, had been carried west by Muslim traders and had come to replace honey as a sweetener in Europe. As the Portuguese discovered uninhabited or barely inhabited islands off the west coast of Africa, they developed sugar plantations to serve the growing European market (the demand for sugar rose further as coffee and tea became common beverages in Europe; see chapter 18). Sugar production requires a great deal of labour in cutting the cane and carrying this, as well as burning fields; refining then requires expensive equipment as well as labour. Sugar, as a result, is generally produced in large plantations. The Portuguese worked these Atlantic island plantations with African slaves, setting the stage for the much later trans-Atlantic slave trade. Senegal was an important slave-trading locale: it was farther south than Arab traders operated along the coast, and local rulers raided inland with the help of Portuguese weapons. Madeira was the largest sugar exporter in the world by 1500. The Portuguese settled the Azores from 1439. Both had been unpopulated before the arrival of the Portuguese.

The Portuguese would round West Africa and reach the Gulf of Guinea by the 1470 s, but this was a slow process of slowly extending their reach farther south and trading for gold, ivory, and slaves - products long consumed in Europe via trans-Saharan trade. Being able to tap the Ghanaian goldfields - which had long supplied the bulk of Europe's gold via the Sahara (chapter 18) - from the south proved particularly profitable. Henry the Navigator, the Portuguese ruler, carefully organized these trips, insisting on both profits and careful mapping of the route. Once the Portuguese passed the limits of Arabic settlement, they 
faced challenges in communication and limited local knowledge of the seas. Though Henry is justifiably celebrated for sponsoring exploration, it is doubtful that he was thinking of reaching Asia: His focus was on Africa itself. He wanted to convert the locals while enslaving some of them.

One interesting question is why the Arabs of Morocco had not extended their presence south along the Atlantic. They certainly had access to sound ships and navigational devices. The answer may be that they felt less need, as they had caravan routes across the desert. Both Arabs and Portuguese had to appreciate that prevailing winds blew toward the south; they would face difficulty in returning, though lateen sails did allow sailing into the wind. The Portuguese would only slowly move down the coast, carefully gathering information. The Arabs may not have seen the advantage of engaging in such a process when they already had access to slaves, gold, and ivory. The Portuguese had the added incentive of wanting to cut the Arabs out as intermediaries in the African trade.

There is some evidence that Arab sailors - likely from the Indian Ocean - may have circumnavigated Africa centuries before the Portuguese. Arabs seemed to know about the Atlantic coast of Africa, but there are no records of particular voyages. It is possible that they found little use for such information: They could gain African trade goods from both North and East Africa

As the Portuguese moved east along the southern coast of West Africa, they expanded their exploratory goals. Henry's grandnephew Joao II recognized the possibility of reaching Asia. He would build a fort in Guinea and encourage exploration to the south. Joao also sent emissaries by land to East Africa to report on the trade possibilities. Along the way southward from Guinea, the Portuguese encountered the Kingdom of Kongo, from which millions of slaves would later be dispatched (see below). The Kongo king ruled through local elites, but rotated nobles from the centre through the kingdom's provinces as governors. The Portuguese converted the Kongolese king in 1506. The Portuguese then sent missionaries to cement their ties with the kingdom, but slave trading undermined the kingdom by the 1660s. As elsewhere, the profits of the slave trade trumped desires for peaceful commerce and saving souls.

Bartolomeu Diaz would round the Cape of Good Hope and reach the Indian Ocean in 1491. His sailors forced him to turn back. Vasco da Gama would be the first to reach the markets and trade routes of the Indian Ocean. His ships were provisioned for three years. He had a crew of 150 or so, including ten convicts to be left along the way to learn local languages; they would be freed if they survived. The Portuguese by this time knew much about the prevailing winds in the southern hemisphere. Da Gama sailed west from Cape Verde, and then southeast to South Africa (knowing from Diaz the latitude of the cape), spending ninety-five days out of sight of land (hence the high incidence of scurvy noted in Box 19.1). His ships then travelled for months along the East African coast interacting with Muslim traders, not always peacefully. 


\section{PRIMARY DOCUMENTS: VASCO DA GAMA ON THE NATIVES OF SOUTH AFRICA}

The inhabitants of this country are tawny-colored. Their food is confined to the flesh of seals, whales and gazelles, and the roots of herbs. They are dressed in skins, and wear sheaths over their virile members. They are armed with poles of olive wood to which a horn, browned in the fire, is attached. Their numerous dogs resemble those of Portugal, and bark like them. The birds of the country, likewise, are the same as in Portugal, and include cormorants, gulls, turtle doves, crested larks, and many others. The climate is healthy and temperate, and produces good herbage. On the day after we had cast anchor, that is to say on Thursday (November 9), we landed with the captain-major, and made captive one of the natives, who was small of stature like Sancho Mexia. This man had been gathering honey in the sandy waste, for in this country the bees deposit their honey at the foot of the mounds around the bushes. He was taken on board the captainmajor's ship, and being placed at table he ate of all we ate. On the following day the captain-major had him well dressed and sent ashore.

On the following day (November 10) fourteen or fifteen natives came to where our ship lay. The captain-major landed and showed them a variety of merchandise, with the view of finding out whether such things were to be found in their country. This merchandise included cinnamon, cloves, seed-pearls, gold, and many other things, but it was evident that they had no knowledge whatever of such articles, and they were consequently given round bells and tin rings. This happened on Friday, and the like took place on Saturday.

On Sunday (November 12) about forty or fifty natives made their appearance, and having dined, we landed, and in exchange for the [copper coins] with which we came provided, we obtained shells, which they wore as ornaments in their ears, and which looked as if they had been plated, and foxtails attached to a handle, with which they fanned their faces. The captain-major also acquired for one [copper coins] one of the sheaths which they wore over their members, and this seemed to show that they valued copper very highly; indeed, they wore small beads of that metal in their ears.

Do you think that this is an accurate portrayal? Why?

Da Gama reached India in 1498 - with the aid of a pilot he had picked up in Africa - just six years after Columbus reached America, and ten months after leaving Lisbon. He famously asked for Christians and spices - Christians in Europe had wondered for centuries what had happened to Christian communities in Asia after the Islamic conquests. It is notable that he earned a hefty profit on return to Europe with a cargo of spices (he had carried gold and wool with him to trade, though the wool attracted little interest in tropical India). Over half of his crew died during the voyage. It is also noteworthy that he used force in trading for those spices. A local Jewish merchant in India was able to serve as translator. 
The Portuguese would discover some Christians on their second voyage to India led by Cabral; these provided useful information. They also discovered Brazil along the way (though the Spanish had been there months earlier), but they did not realize that it was part of a continent. This voyage would involve violent exchanges in both Africa and India. Between 1510 and 1515, the Portuguese captured various strategic outposts: Goa in India (massacring 6,000 Muslims); Melaka (which controls the Strait of Melaka); Hormuz (the entry to the Persian Gulf); Colombo (on Sri Lanka, a key transit point between the western and eastern parts of the Indian Ocean); and several others. However, they failed in attempts to enter the Red Sea. They also gained trading posts by participating in local political conflicts: One local ruler would grant them land in return for support against others. They reached the Spice Islands in 1511 but did not conquer them at the time. They sailed to Macau in China in 1513. They smuggled in the area for a half-century (with perhaps unofficial Chinese government support) before China allowed an official trading colony. The Chinese rulers may have refused at first to deal with the Portuguese because of their reputation as smugglers. The Portuguese would transport Jesuit missionaries, who found it much easier to convert locals if these could retain Confucian ancestor worship practices. Authorities in Rome would later challenge this pragmatic decision. In the 1540s, the Portuguese established trade in Japan, where they mainly traded Chinese goods. (The Japanese government, pushed by Buddhists, disliked Christian missionaries and expelled the Portuguese in 1639.) They depended heavily on local knowledge in exploring and attacking throughout the Indian Ocean and beyond: Note how much faster they progressed there than they had moved along the west coast of Africa (there were also, of course, much greater economic rewards to be had in the Indian Ocean and the South China Sea).

The Portuguese did not at first seek large colonies in Asia but strategically located trading posts. By the 1570s, the Portuguese had forty trading posts from East Africa to Japan and China. These forts operated peacefully when possible, but were defended militarily when necessary. The Portuguese were the first to attach cannons to ships in the Indian Ocean, which gave them a substantial military advantage. These cannons and forts were employed in an effort to monopolize trade: Traders from other nations were encouraged to obtain licences from the Portuguese or risk capture. They charged other merchants a 5 per cent fee for using Portuguese ports, and tried to force them to use these ports. Both the Ottomans and Egypt fought the Portuguese for naval supremacy, but the Portuguese prevailed over a period of decades. Still, though the Portuguese would trade large volumes both within the Indian Ocean itself and back to Europe, they were never able to monopolize either trade; four times as much spice travelled to Europe via the Red Sea (recall that the Portuguese had not succeeded in entering the Red Sea). There just were not enough Portuguese to achieve a monopoly. Portugal had a population of only 1.4 million in 1525 . There may never have been more than 10,000 Portuguese in all of Asia (only a fraction of whom ever returned to Portugal due to death or poverty). 
Trade also continued on the old Silk Road routes for some time. Even with the arrival of the British and Dutch in the Indian Ocean from the seventeenth century (below), the overland trade continued. The Silk Road, though, did not leave behind the archives that East India Companies bequeathed to the historical profession, and so it has often been imagined that the new sea routes quickly eclipsed overland trade. Trade also continued across the Sahara, though much was diverted toward ports along the coast.

The new route around Africa did lead to an expansion in trade between Europe and Asia, and drew parts of Africa into that trade network. We noted in chapter 9 that the cost of doing trade increases with the number of networks engaged: By extending a European trade network throughout the eastern hemisphere, the Europeans could lower the cost of trade significantly. Yet this was still a luxury trade. Competition between trade routes may have reduced costs (the price of pepper in Lisbon fell to a fifth of what it had been before the

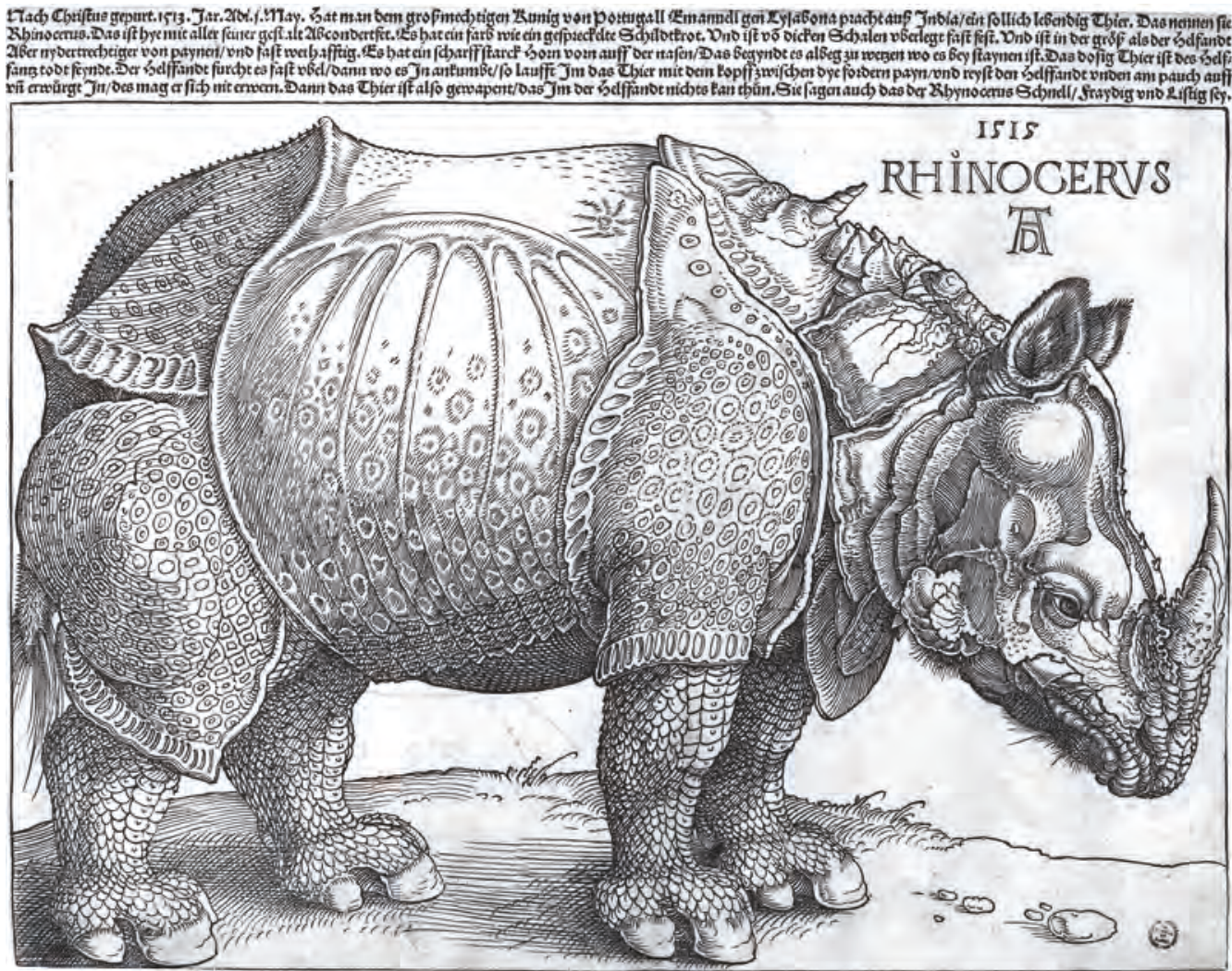

Illustration 19.7

Alfonso de Albuquerque, Portuguese naval commander, sent a rhinoceros to Portugal in 1515. The rhinoceros later drowned in a shipwreck on the way to Rome. Rhinoceros had been known in classical times but were unknown in Early Modern Europe. Albrecht Dürer produced this woodblock in 1515 on the basis of reports of the animal. This would be the dominant European image of rhinoceros for centuries 


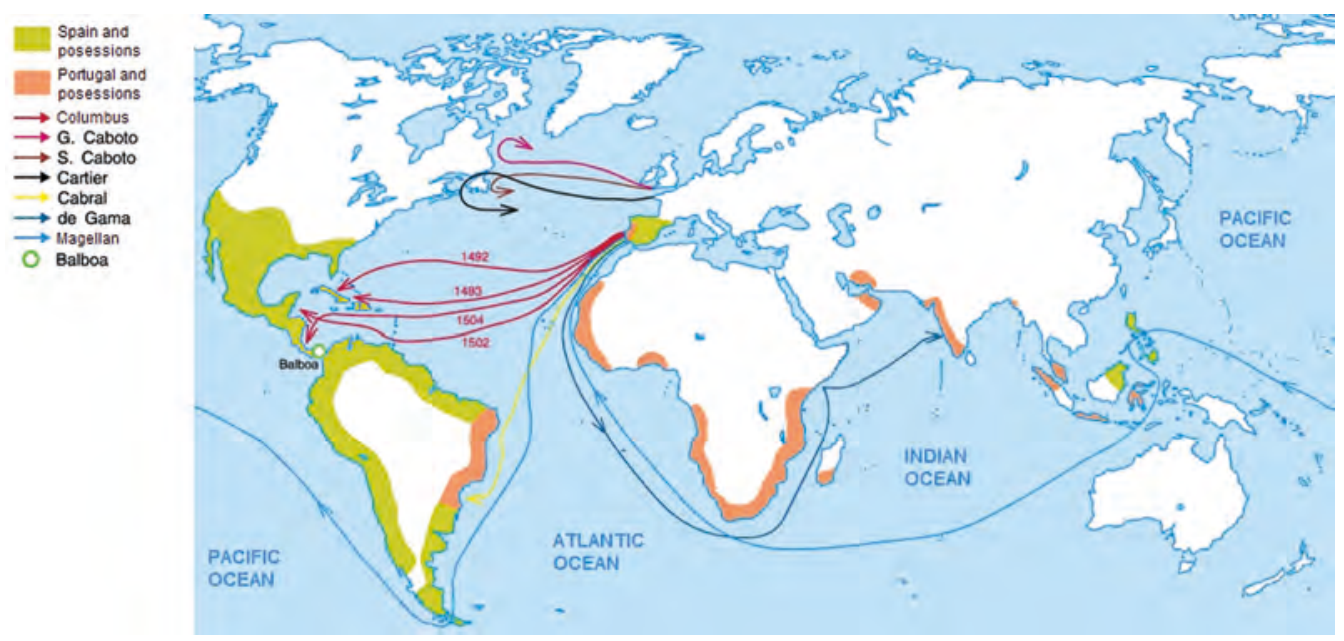

\section{Map 19.1}

Key European voyages of exploration (Giovanni Caboto is discussed below). Spanish and Portuguese colonies are shaded on the map

Map by Universalis, CC BY-SA 4.0

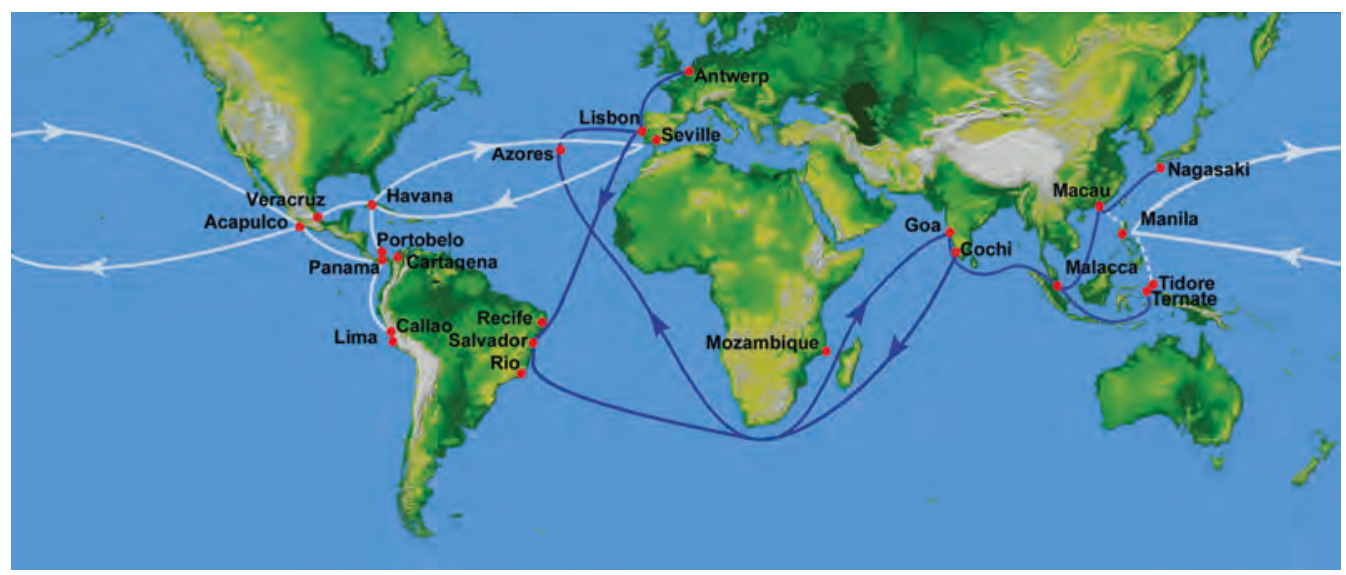

Map 19.2

Main Spanish and Portuguese trade routes in the 16th and early 17th centuries

circumnavigation of Africa), but it was still only high-value goods such as silk or silver or spices that moved between continents.

Expanded trade did inspire curiosity. Texts from both Europe and Asia were translated and read in distant lands. Christianity would grow in southern Africa as it had in the Americas - repeating the oft-observed success of text-based monotheistic religions when in contact with societies characterized by earlier religious traditions - but would spread much more slowly in parts of Asia that already had text-based religions and religious institutions. 
The Portuguese did have a lasting effect on Asian cuisine. Vindaloo, an Indian translation of "vinha d'alhos," was derived from Portuguese sailor provisions of meat marinated in wine and garlic; Indian cooks replaced the wine with vinegar and added a variety of spices. Tempura was an adaptation of a long Japanese tradition of deep-frying with a Portuguese batter made from flour and eggs.

\section{English and Dutch expansion}

The Portuguese had tried to limit knowledge of their trade route to the east. However, in 1595, Jan Linschoten published a guidebook based on his work for the Portuguese in Lisbon and Goa. This work would guide both Dutch and British entry into the Indian Ocean. The English and Dutch would follow the Portuguese in building trading posts but did not attempt to control local shipping. They had better ships than the Portuguese and also joint stock companies - merchants rather than bureaucrats then made trading decisions. They traded peacefully for the most part but armed themselves against the Portuguese in particular.

Joint stock companies (early corporations) deserve some discussion. Longdistance trading voyages were a risky proposition. As we have seen above, states could sometimes support these - though the Spanish crown only partly financed even the famous voyage of Columbus. Individual merchants could not bear the cost and risk associated with trips that took months or years and often ended in shipwreck or piracy. Merchants instead formed associations where they could pool expenses and risk. Joint stock companies provided an umbrella and set of legal provisions under which groups of merchants could finance trading ventures that often took years to complete.

Besides, there was the age-old problem of negotiating safe passage, for longdistance trade might be subject to the depredations of multiple polities. Travel by sea was somewhat easier than travel by land in this respect, but ships still had to land along the way to obtain supplies. Moreover, pirates or navies might seize ships in open waters. All too often, vessels were blown ashore in lands they had not intended to visit. As merchant networks had done for millennia, trading companies could negotiate safe passage with ports and navies, but only if the polities involved benefited enough from the overall trade with the company in question to reduce the temptation to confiscate. There was thus an advantage to forming large trading companies that handled all of English or Dutch - trade to the Indian Ocean. The differences between sea and land transport allowed trading companies to improve on the long-standing efforts of merchant networks in two important ways. They could build trading posts that provided safe places for their ships to gather provisions and trade. They could then threaten military repercussions for states that attacked their ships.

As noted above, these joint stock companies did employ military force. This force could oppose Portuguese efforts to monopolize trade, and strengthen 
bargaining positions with local rulers. Only a large organization that shared the profits of many merchants could bear these military expenses. Moreover, if the company was to set up a series of forts and negotiate with several states it needed to have permanency of its own: These companies were therefore designed to continue in perpetuity. While the military costs are the most obvious, the costs of diplomacy itself were not trivial. These joint stock companies would often rage against (and try to audit) the entertainment expenses that their local agents felt were necessary to impress local rulers.

The British initiated the modern joint stock company. The first joint stock company was the Muscovy Company in 1555, which sailed into the Arctic to trade for furs, and sought a northeastern passage to China. The Levant Company followed in 1581, financing trade to the eastern Mediterranean (still at the time the main trade route to the east). The British East India Company was founded in 1600. The Dutch East India Company was initiated in 1602 (amalgamating previous trading ventures), borrowing the institutional form pioneered by the British.

We can understand joint stock companies as an institutional mutation to preceding merchant networks. This mutation reflected the differences in trade by sea versus land. Though merchant networks had often negotiated independent trading colonies in foreign trading cities (where they could adjudicate their own disputes), they had not established forts along trade routes. They had necessarily negotiated safe passage when moving goods overland. However, trade by sea meant that there was much value in a combination of a few forts and the threat of naval violence. Europe's gunpowder advantage also encouraged European merchants to employ force rather than rely on negotiating safe passage.

The Dutch and then English would later employ this military advantage to form colonies. The Dutch would take advantage of political fragmentation in Indonesia to conquer parts of the archipelago. They expelled the Portuguese from the Spice Islands in 1605 and signed treaties with locals. They then decided, though, that they could not achieve their goal of monopolizing the spice trade through treaties with independent headmen. They invaded the Spice Islands in 1621, killing many of the locals. After that, European settlers with slaves from Asia moved in.

Postscript: As a result of migration and missionary work by both the Portuguese and Dutch, and the previous missionary work of Muslim merchants, the Spice Islands developed a mixed population of Christians and Muslims. These communities developed a communal relationship among villages known as "Pela Gandong" which mostly maintained peace for four centuries. However, communal violence killed thousands and displaced hundreds of thousands from 1999. 
In about 1620, the Dutch founded Batavia (now Jakarta) on Java, where a Dutch minority and a large group of Chinese traders prospered for centuries (though there would be huge problems with malaria). They established tributary relationships elsewhere in the archipelago (and eradicated spice plants from islands they did not control) - in the seventeenth and eighteenth centuries. They were thus able to monopolize the spice trade from the Spice Islands. Yet the cultural impact of the Dutch was limited: Indeed Islam may have strengthened in the region in reaction to the Dutch conquest. The Dutch were also the only Europeans allowed to trade with Japan after 1639, but they were barred from China. (The Dutch also briefly replaced the Portuguese

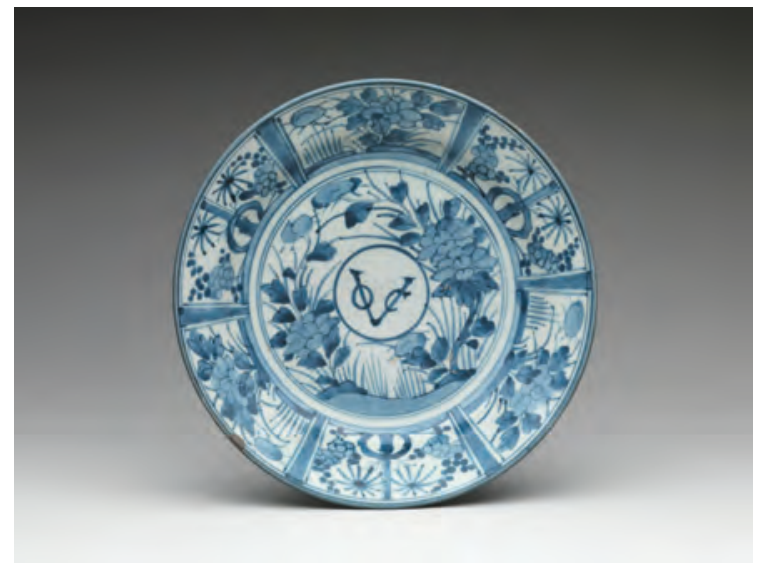

\section{Illustration 19.8}

A plate manufactured around 1660 in Japan for officers of the Dutch East India Company (VOC), with the company logo in the middle. The Japanese were asked to duplicate the Chinese style of the time, Metropolitan Museum of Art in both Angola and parts of Brazil in the early seventeenth century.) They and other north Europeans also took islands in the Caribbean and land in the Guianas on the coast of South America.

The Dutch followed one Portuguese practice: marrying local women with trading connections. This was most obviously a means of dealing with an under-supply of European women, but it had the further advantage of combining European political contacts with local knowledge of trading networks. Both husband and wife might bring capital into the marriage. Such marriages were far less common in other European empires: The apparent trading advantages of these trading alliances did not always lead to supportive cultural attitudes.

Both the Dutch and English came to focus on inter-Asian trade - including the trade in African and Indian slaves. Europeans would come to control almost half of the inter-Asian trade. Yet they employed their military advantage much more selectively than the Portuguese had. The prominence they gained in inter-Asian trade may reflect in large part the advantages of the joint stock company institutional form itself.

The entry of Europeans into the Indian and Pacific Oceans lowered the cost of trade between Europe and Asia, and often within Asia itself. Yet while European success is noteworthy, we should appreciate that most intra-Asian trade remained in the hands of Asian merchants and ships. Muslim traders in particular remained important throughout the region. Though they did not produce an institutional innovation as dramatic as the joint stock company, these also created sophisticated multi-generational contracts that facilitated long-distance trade. 


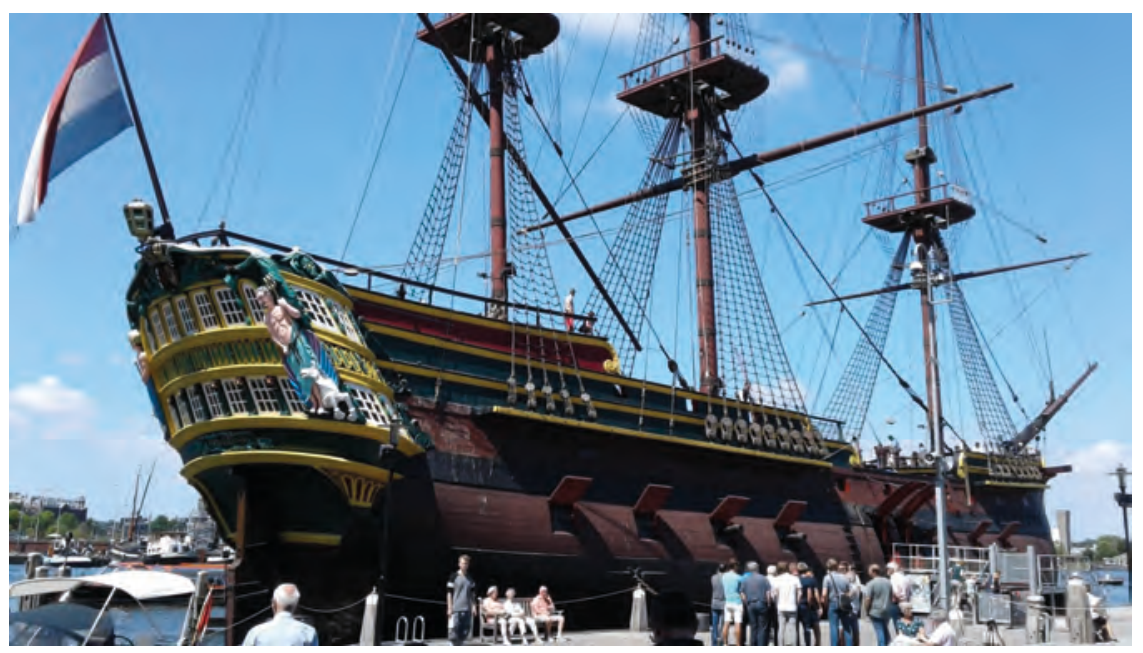

Illustration 19.9

An East India Company ship replica, Amsterdam Maritime Museum Author

\section{Russia into Siberia}

At the same time that western Europeans were colonizing the Americas and parts of Africa and Asia, the Russians were actively colonizing a vast territory in central and eastern Asia. This exercise in colonization is much less famous globally. It lacks the dramatic sea voyages we have recounted above - though, as we have seen, the Russians would sponsor some of these too, but only after they had expanded across the Asian landmass to the Pacific. These eastern territories remain a part of the Russian state to this day: It is by far the largest state in the world, sprawling across eleven time zones.

The Russian state had emerged as a tributary state to the Mongols, but as the Mongol Empire fragmented, the Russians were able to assert authority. Especially from the sixteenth century, the Russians went on the offensive against nomadic peoples to the south and east. One motive for this offensive was that these nomads still staged slave raids and sold Russians south into the Middle East. At the time, firearms were still at best an even match for the composite bow of the nomads. Over the next century or two, though, the military balance would shift decisively toward firearms, and centuries of nomadic military dominance in Central Asia would end. Russia and China (see below) would end up dividing Central Asia among themselves. When the Russians reached the Caspian Sea, they opened new trade routes to the Ottoman Empire, Iran, and India.

As the Russians took steppe lands from nomads, they were able to transform some of this into farmland. (Climatic conditions on the steppe may have been warm and dry at the time, unlike the Little Ice Age conditions in western Europe.) They did not do so by developing family farms as would later occur in 
North America. Instead, the institution of serfdom tied peasants to the lands of particular nobles. We saw in chapter 9 that some sort of coercive labour relationship may be highly advantageous to lords in a situation where free land is available.

The Russians had some success in converting people to Christianity in some of the northernmost areas they conquered. Russians also colonized these regions. The north shore of the Black Sea had been sparsely populated before Russian expansion, though there were Muslims in Crimea. The Russians would invite settlers from all over Europe and create towns such as Odessa with names that evoked Greek civilization. Islam remained dominant farther east and south. The Russians justified their conquests of quite different peoples in the Caucasus as bringing political stability to the region. By the end of the nineteenth century, Russia had 20 million Muslim subjects, more than the Ottomans (chapter 20). Only Afghanistan remained as an independent state governed by a Central Asian ruler (though influenced by the British from India). The Russians ruled these regions through local elites. They hoped Islam would wither, but it strengthened, perhaps as a form of identity among subject peoples. As educational systems developed in these regions, they communicated local language, religion, and history and thus strengthened (some would say created) local ethnic identities. The Russians (and later the Soviet Union) may at times have encouraged competing ethnic identities as part of a "divide and rule" strategy.

By the end of the sixteenth century, Russian-speaking people had also expanded eastward beyond the Ural mountains. This northern area of forests and tundra was lightly populated. Indigenous peoples there hunted, trapped, and herded reindeer. These people had long dealt with Mongol and Muslim trade networks (chapter 17) and exported fur and amber (and had imported Buddhism and Islam). Just as fur traders in North America pushed ever westward in search of furs (below), Russian fur traders pushed ever eastward across Siberia, reaching the Pacific by 1639. The Russian state did not at first authorize this movement. Rather, it was pursued by escaped serfs, Cossacks (an intriguing group with aggressive and democratic tendencies that often sought freedom from the tsar), and religious minorities. The vast region of Siberia takes its name from Sibir, a Mongol successor state east of the Urals that Cossacks defeated late in the sixteenth century. This geographical expansion of the fur trade began earlier than that in North America and moved faster. The Russians were aided by the flat terrain and the existence of several major rivers that flowed through this vast territory (as would be the case for the fur trade operating from Hudson's Bay). They were hampered by one of the harshest climates in the world - far harsher than the core areas of the North American fur trade but this climate had provided selection pressure for the thick furs of the mink, ermine, and other animals.

Over time, the Russian state became attracted to the potential profits from taxing the lucrative fur trade. Indeed, Russia did not just trade but exacted tribute in the form of furs from local peoples. (It also began a centuries-long 
process of banishing political and other prisoners to Siberia.) When the Yakuts of central Siberia rebelled in the 1640s, Russia responded by destroying their settlements over a period of decades; two-thirds of the Yakut population may have died. As in the Americas, the introduction of new diseases also reduced local populations - though the indigenous peoples of Siberia had not been entirely isolated from Eurasian diseases. Small agricultural settlements grew where possible around trading posts. By the late eighteenth century, there were half a million Russians in the area to the east of the Urals, whereas the indigenous population was perhaps half as large. Russia would continue expanding across the Bering Sea into Alaska. Russian fur traders in Alaska kidnapped women and children to force local men to catch otters for their fur.

In North America, one key impetus for the westward expansion of the fur trade was overharvesting: As beaver populations declined in one region, traders looked farther afield for more. Overharvesting is short-sighted for trading companies: They suffer higher transport costs as they are forced to travel farther. It is even more short-sighted for indigenous peoples who lose

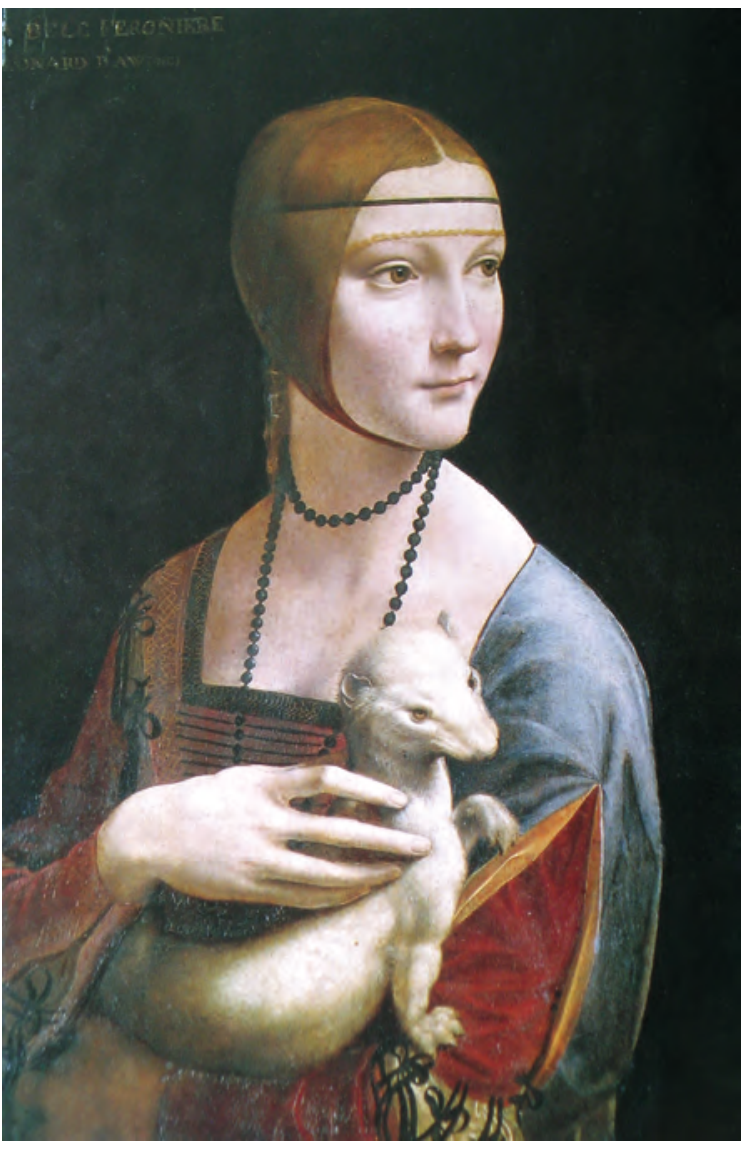

Illustration 19.10

Lady with an Ermine, Leonardo da Vinci, 15th century a source of income. Overharvesting will be more likely if those harvesting fur today will not benefit from the preservation of fur stocks for the future. If there is competition among both fur traders and indigenous peoples for the fur in a particular region, then nobody has an incentive to conserve. The independent nature of the earliest Russian fur traders likely encouraged overharvesting. The fact that Russians forced indigenous peoples to pay tribute in furs may have limited indigenous ability to manage local resources, further encouraging the rapid geographical expansion of the Russian fur trade.

Just as fur traders in North America traded with indigenous groups farther south for food - primarily pemmican, a dried meat from buffalo - and occasionally sought to establish agricultural production, Russian fur traders in the Far East tried to expand into the agricultural lands of the Amur River. However, this area was already part of Qing China. Though the Russians scored some early victories, the Qing repelled them: It was far easier for the Chinese to supply armies in the region than for the Russians to do so. 


\section{Chinese expansion}

We should be careful not to leave the impression that colonization was entirely a European exercise during this period. Just as Russia is the largest state in the world today because of its expansion across Siberia in the Early Modern period, China is the third largest state in the world today because of expansion into Central Asia at roughly the same time. (The second and fourth largest of today's states, Canada and the United States, were created through geographic expansion across North America a bit later.) The Chinese Qing Empire defeated the last of the great nomad empires in Central Asia, the Zunghar (1671 to 1760) in 1760 (massacring much of the population). It is noteworthy that the Zunghar also (like peoples of the Americas) suffered from a severe smallpox epidemic at the time that may have killed 40 per cent of the population. In general, Qing China discouraged migration into their westernmost provinces of Xinjiang and Tibet in the eighteenth and nineteenth centuries to avoid social conflict, though they sent some convicts to Xinjiang soon after the conquest.

Though the Chinese state would prove militarily weak relative to Europeans as these began to arrive off its eastern coast (chapter 20), it was able to assert military superiority over nomads on its western border. This should remind us that military might is relative: The Chinese state looked weak only because Europeans had developed even more deadly firearms over a period of centuries (chapter 18). If one of the soothsayers consulted by the Han had predicted the eventual subjugation of Central Asian nomads, Han rulers would likely have been much pleased and might have devoted little thought to the possibility of a greater threat from beyond the land of the nomads.

Postscript: With the collapse of the Soviet Union, there are now a handful of independent Central Asian states. These have generally been ruled in an autocratic fashion. This may reflect in part an autocratic past. It also reflects the difficulty of forging a nation state in lands with ethnic diversity. China has recently promised to invest or lend billions of dollars for building infrastructure for a controversial new "Silk Road" that would stimulate trade with and across Central Asia. The province of Xinjiang remains a part of China today, though scarred by conflict between the indigenous and mostly Islamic population (called Uighurs, though the connection with that ancient polity may be tenuous) and migrants from the rest of China. Migration into both Xinjiang and Tibet has been encouraged by the Communist government.

In treaties of 1689 and 1727, the Russians and Chinese divided northern Central Asia among themselves, limited trade in the region, and insisted that 
Russian and Chinese merchants perform this trade. The political eclipse of Central Asia - as noted above outside forces conquered all but Afghanistan - is intertwined with the declining importance of trade in the region. Gunpowder weaponry was important to be sure. Yet we have seen in chapters 13 and 17 how vital trade had always been to the peoples and rulers of Central Asia. The Russian/Chinese decision to limit trade reflected and reinforced the fact that intercontinental trade increasingly moved by sea. Central Asia's longstanding role in Eurasian history as both a region of commerce and of conquest disappeared, then, as military technology changed and trade routes shifted.

The Chinese also expanded toward their southwest. While the western hemisphere was slowly populated by Europeans in the sixteenth through eighteenth centuries (only between one and two million Europeans had migrated by 1800), some four million Chinese moved southwest between 1500 and 1800, pushing indigenous peoples from previously uncultivated lands. Millions also went to Manchuria and Taiwan. Some were soldiers given land on the frontier, and the state subsidized others, but in Manchuria and Taiwan the government discouraged migration in fear of local rebellion.

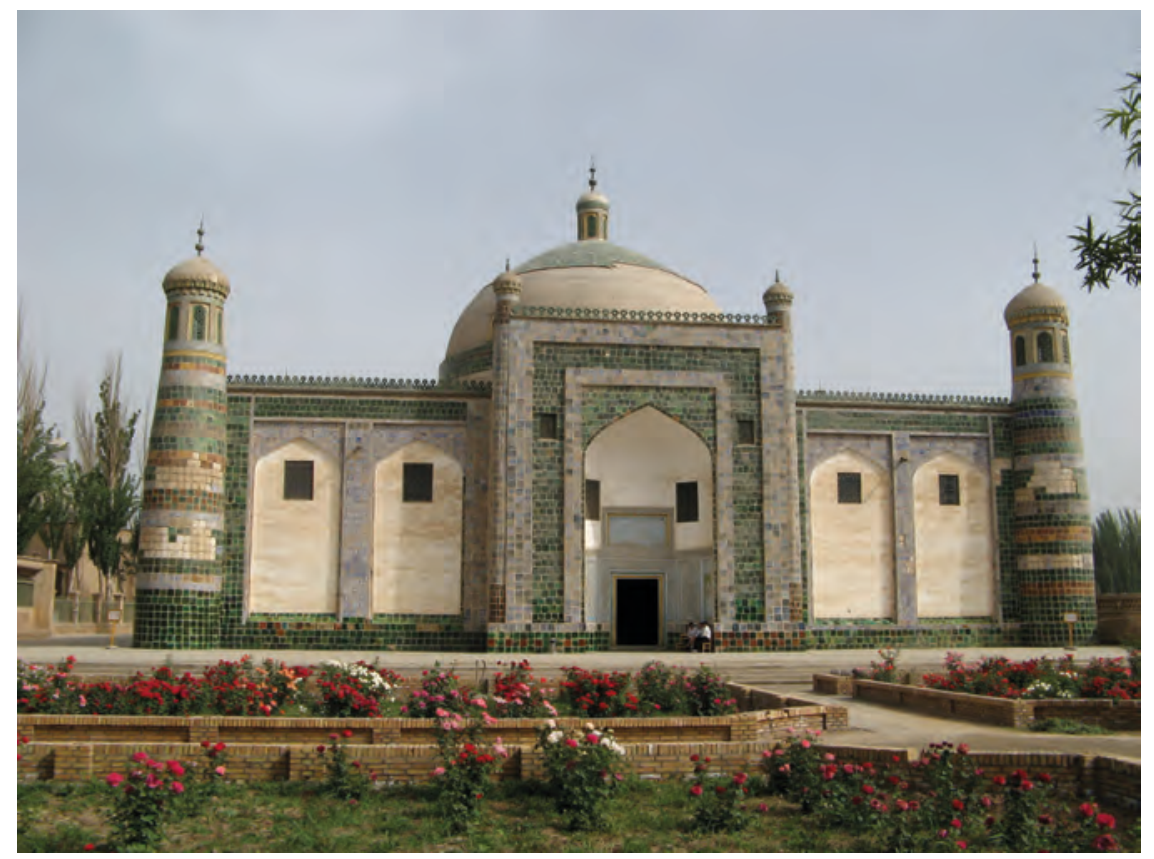

Illustration 19.11

Aba Khoja Mausoleum near Kashgar in Xinjiang, China; built from 1640 for generations of Sufi masters, it is widely thought to be the finest example of Islamic architecture in Xinjiang

Photograph by G4lrn8, CC BY-SA 4.0 


\section{The Columbian Exchange}

The "Columbian Exchange" is a phrase widely used to capture the range of diseases and foodstuffs that moved between hemispheres after the European arrival in the Americas. We have had many occasions to recognize the spread of disease to the Americas above. The diseases involved were mostly those for which people acquire immunity during their lifetime. These were childhood diseases in Europe because children either died or developed resistance. Smallpox likely killed 10-15 per cent of Europeans at the time, but as children; in the western hemisphere smallpox would devastate adult populations. Along with smallpox, significant killers in the western hemisphere included measles, diphtheria, whooping cough, and influenza. These diseases likely killed 50-90 per cent of the indigenous population; this is thus the worst medical disaster in history by mortality rate (edging out the Great Plague of the fifteenth century; chapter 17). All told, 100 million may have died in the Americas and Pacific from epidemic disease between 1500 and 1800. The indigenous population of the Aztec area may have fallen from 17 million to only 1.3 million. Though these diseases were most dangerous in densely populated areas, they nevertheless spread faster than Europeans across the Americas. Smallpox is contagious for a week before there are any symptoms and so it was entirely possible for merchants or others to carry the disease hundreds of kilometres unwittingly. Many parts of the Americas would as a result be underpopulated when Europeans first arrived. Densely populated areas would eventually develop immunity of necessity; sparsely populated areas would witness occasional epidemics for centuries. In all cases, poorly nourished people, or those under attack, were more likely to die.

Such a massive population decline undoubtedly had an environmental impact: Much farmland reverted to forest in both North America and the Amazon. There is controversial speculation that this transition may have occurred on a large enough scale to have had a global environmental impact. Since forests - and especially young trees - absorb carbon, we may have witnessed a "reverse greenhouse effect." This reforestation may have contributed to - in concert with volcanism and reduced sunspot activity - the Little Ice Age of the next centuries (see chapter 18). Ironically, the Little Ice Age may have then slowed the rate of European settlement of the Americas by making the new land less hospitable.

The Americas apparently harboured few diseases for which Eurasian populations lacked immunity. There was an epidemic of syphilis in Europe soon after Columbus, but this may have had domestic rather than western hemisphere origins. It is likely that at least one particularly virulent strain of syphilis came from the western hemisphere.

Why were there so many diseases from the eastern hemisphere that would devastate populations in the Americas but few or no diseases in the Americas that would devastate the eastern hemisphere? The two hemispheres had 
achieved similar levels of urbanization, and extensive trade characterized both. The most likely answer is the lack of domesticable animals in the Americas. Measles was initially a disease of chickens. The similarity between smallpox and cowpox suggests that smallpox was originally a disease of cows. Influenza likely came from pigs, and even malaria may have originally afflicted cattle. The fact that some humans in the eastern hemisphere had been living in close contact with pigs, chickens, and cows for millennia (chapter 4) resulted in the exposure of Eurasians to several potentially epidemic diseases. These diseases would have emerged in the eastern hemisphere after rising sea levels had separated the human populations of the two hemispheres. The limited contact between Vikings and indigenous peoples (chapter 13) had not transmitted these diseases to the Americas.

Postscript: Though these diseases generally spread faster than Europeans, isolated groups might not be touched for centuries. There were major epidemics in the eighteenth century among indigenous groups in the Dakotas and the interior of Oregon. In the Amazon, some groups suffered severe epidemics when first coming in contact with outsiders in the twentieth century.

Malaria had been known since classical times in Europe, but was less common in most parts of Europe than in sub-Saharan Africa. Malaria appears to have been unknown in the Americas until the arrival of Europeans and (perhaps especially) African slaves. As malaria became endemic in the warmer parts of the Americas, this created a further incentive to employ slaves, for these often had immunity. Some scholars have argued that the Spanish and Portuguese gained a significant advantage by being more willing to marry/mate with people of African descent who had resistance to malaria (and yellow fever) than British and Dutch colonists who were more hostile to such relationships.

The exchange of plants and animals is a happier story (for the most part that is; recall for example our discussion of slavery on sugar plantations above). Horses, wheat, cattle, pigs, goats, chickens, barley, oats, rice, coffee, vines, and many fruits and vegetables, were transplanted to the Americas (there were many other transplants about which we know far less: onions, peas, yams, okra, sorghum, and coconuts). Corn, potatoes, tomatoes, tobacco, turkeys, chilli peppers, cocoa, peanuts, avocados, papayas, guavas, pineapples, manioc, and a superior strain of cotton were transported to the eastern hemisphere. Cocoa and tobacco gained immediate popularity in Europe - though each became an item of enjoyment shorn of the role in religious rituals that they had played in the Americas. In both directions, new crops would prove better suited to particular ecological niches than local plants, and so agricultural output expanded. Farmers everywhere were able to diversify, and as a result, diets became more diverse in much of the world. The Columbian Exchange may be (one of) the 
major causes of world population growth from about 450 million in 1500 to 550 in 1600,650 by 1700, and 950 by 1800 . Taking China as an example, production of corn, potatoes, and peanuts on lands ill-suited to rice or wheat facilitated migration into areas at high altitudes and with sandy soils, and encouraged a doubling of the population between the Mongol conquest and 1600 .

It is hard today to imagine Italy without tomato sauce or Ireland without the potato or Colombia without coffee or Costa Rica without bananas or Szechuan cooking without chilli peppers. Likewise, the wheat, rice, oat, and barley fields that characterize much of North and South America today were only possible because of the Columbian Exchange. Tropical areas in the eastern hemisphere benefited from peanuts and manioc, while the potato would prove in time to be universally valuable, and corn would be grown in many lands.

We have described in earlier chapters how the crops domesticated in various parts of Eurasia had slowly spread throughout that continent and North Africa over the millennia - and into parts of subSaharan Africa. We have had less opportunity to describe the movement of crops within the Americas, but we have seen notable examples such as the adoption of corn in both northern North America and South America. The Columbian Exchange is far more rapid. Quite distinct ecologies were linked suddenly by a system of maritime transport that could easily transmit plants or animals around the world.

Particular note might be made of the horse. The absence of draft animals shaped the pre-contact history of the Americas (chapter 16). The horse would allow duplication of Eurasian agricultural practices in the Americas. The horse would also be central to land transport in the Americas for centuries as it had been for millennia in the eastern hemisphere. Horses reached the indigenous peoples of the North American plains before Europeans did. We have a limited understanding of what pre-horse societies were like in this region, but it is likely that horses encouraged at least some Plains Indians away from small agricultural plots toward hunting large animals.

The Columbian Exchange had effects beyond food production. Of special note here is the red dye (carmine) long

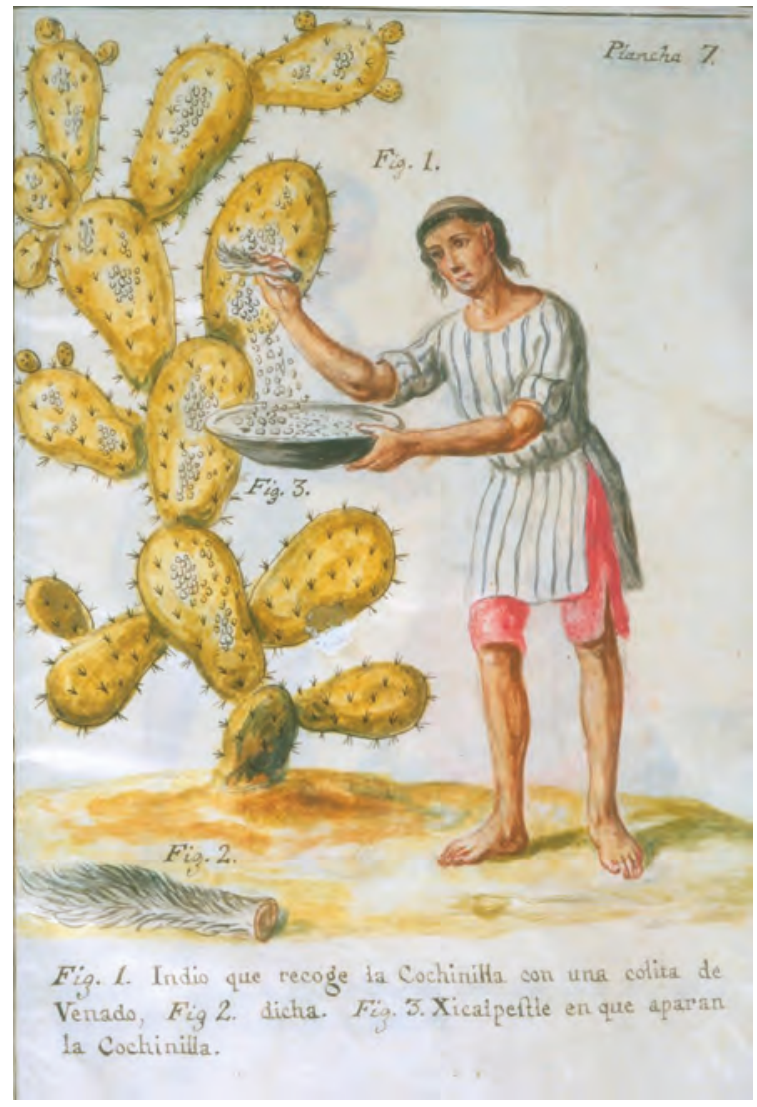

Illustration 19.12

"Indian Collecting Cochineal with a Deer Tail" from Memoria sobre la naturaleza, cultivo, y beneficio de la grana (...), José Antonio de Alzate y Ramírez (17371799), Newberry Library 
extracted in Mexico and Central America from the cochineal insect. This was found to be superior to red dyes employed in Europe, and would be a major export for centuries until synthetic dyes were developed in the mid nineteenth century (chapter 24). Since the insects were "farmed" on the nopal cacti, there were no advantages in large-scale production, and small indigenous farmers rather than large plantations dominated cochineal production.

\section{The potato}

It is too easy to forget that trade involves the movement of particular goods. Each of these goods has its own story. It is impossible in one book to report all of these. Nevertheless, it is useful to tell the story of particular products occasionally. We will spend a little time here on the potato, one of the key items in the Columbian Exchange. The potato is an excellent source of both calories (and starch releases energy gradually unlike sugar) and protein (it is lower than grains in total but particularly abundant in several amino acids that the body needs); it is also rich in vitamin C, most B vitamins, calcium, potassium, and phosphorus. Tests have shown that people can live for months on potatoes alone (with some margarine). Moreover, potatoes can be stored for months (like grains but unlike most tubers). Two or three times as many people can be sustained on a field planted with potatoes compared to grains - and it takes less effort to grow potatoes. In addition, potato crops on average are more resistant to climatic variations and disease than are grains. Potatoes are now grown almost everywhere that agriculture is pursued in the world; only three food crops - wheat, rice, and corn - are grown more (measured by total tonnage produced), but notably, potatoes are grown on less land area than are several other crops with a far lower output. NASA plans to grow potatoes on long manned missions into space.

Since wild potatoes are small, bitter and somewhat poisonous (presumably a mutation selected to ward off predators), it is not clear why they were ever domesticated. Nevertheless, this likely happened 6,000 years ago. Eating potatoes with clay limits the absorption of toxins; it is possible that humans first domesticated potatoes after discovering this fact. Freeze-drying potatoes by leaving them out in the cold works also to detoxify.

Potatoes would become over time a major component of South American diets, especially at higher altitudes. It was apparently the main crop supporting Tiwanaku, a city of 100,000 at its peak between 800-1200 CE on the south end of Lake Titicaca (an amazing population for such an altitude). The Incas would mostly grow corn below 2,500 metres but continued to rely on the potato above that level: They levied taxes in frozen potatoes there. The Spanish exploitation of Potosi (above) would have been far more difficult if the workforce could not eat potatoes grown nearby.

We do not know who first transported the potato to Europe or when this occurred. Ship captains may have fed potatoes to Spanish sailors on the trip 
home - despite the disdain that the Spanish at first displayed toward the potato. Potatoes are found in the Canary Islands from the 1560s and Spain from the 1570s. We do know that the potato met significant cultural resistance at first: It was odd looking, grew in the ground, and was associated with poor and backward peoples. In time, its many advantages would overcome these cultural misgivings. Basque fishers carried potatoes for food when they set out for the Newfoundland fishery (discussed below), and likely introduced these to Ireland on their way home. Irish peasants were attracted to the potato in the aftermath of devastating wars with the British in large part because it required little investment to grow or to store. Governments in eastern Europe encouraged potato cultivation as the cheapest way to maintain peasant populations. The Chinese found the potato particularly valuable as they advanced into the drier lands of Central Asia: Potatoes could be grown where nomads had once pastured. The potato also, of course, proved useful in highland areas of every continent. Populations expanded almost everywhere because of the simple potato.

Though grains have to be harvested at a particular time of year, potatoes can much more easily be left in the ground until needed. This reduced storage costs and also the risk of theft. Armies on the move would not harvest potatoes where they would steal grain. Peasants in central and eastern Europe may have better survived centuries of war because of their adoption of the potato.

It is possible, though, to have too much of a good thing. Historians estimate that the Irish in the mid nineteenth century were eating ten potatoes a day and gaining some 80 per cent of their caloric intake from this one crop. There had likely been a positive correlation between potato growing and population growth in previous centuries. The potato allowed people to survive on smaller farms, and so encouraged early marriage and population growth. Population growth, in turn, encouraged devotion to this one crop with its high caloric output per acre. When potato blight from South America struck Ireland in 1845 and 1846, a million died and another million migrated to other lands.

Postscript: It is useful to reflect on how much of the food we consume today is possible only because of the Columbian Exchange. Indeed a useful class project might involve having each student produce a recipe that involves ingredients that originated in different hemispheres. Provide some historical information on the recipe: where did it originate and how has it spread? Where did each ingredient originate? What does the recipe tell us about global cultural interaction? See Zara Anishanslin, "A Recipe for Teaching Atlantic World History: Food and the Columbian Exchange," The Recipes Project, 26 November 2015, https://recipes.hypotheses.org/7091. 


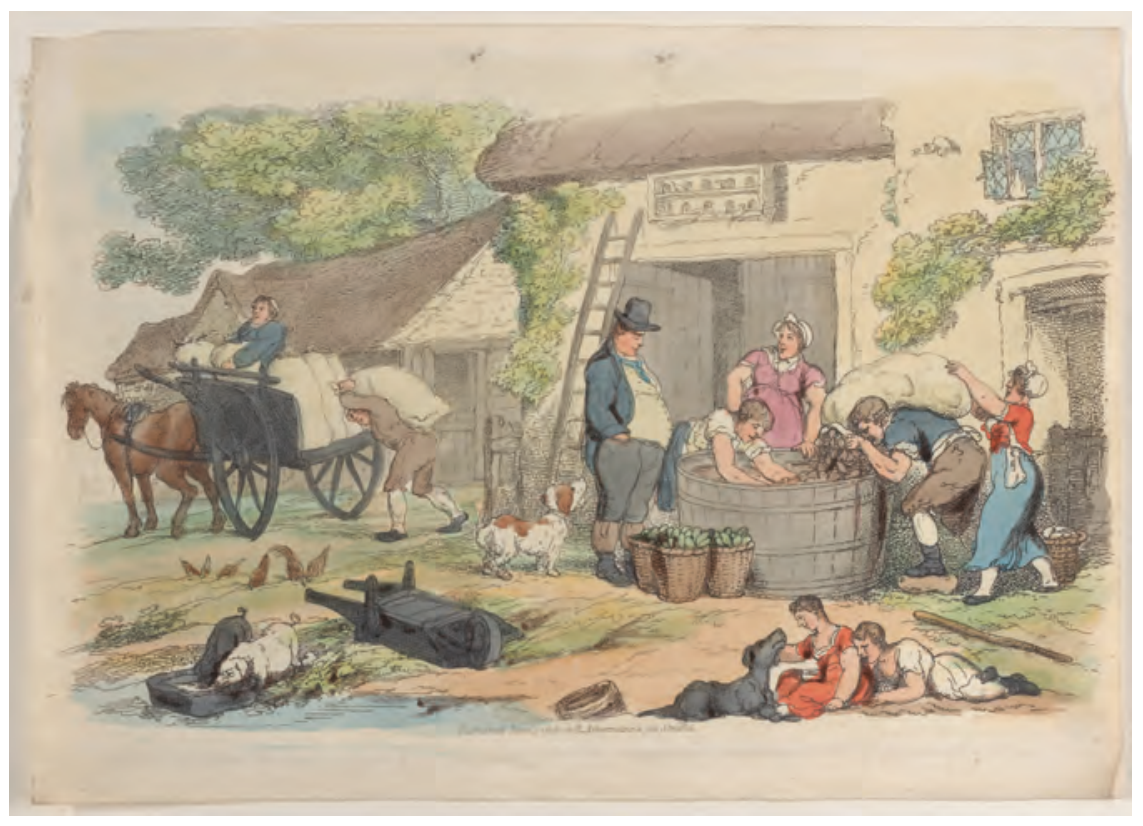

\section{Illustration 19.13}

Thomas Rowlandson, "World in Miniature," Plate 16, 1816, shows a potato harvest, with potatoes loaded on a wagon to the left and poured into a barrel on the right, Metropolitan Museum of Art

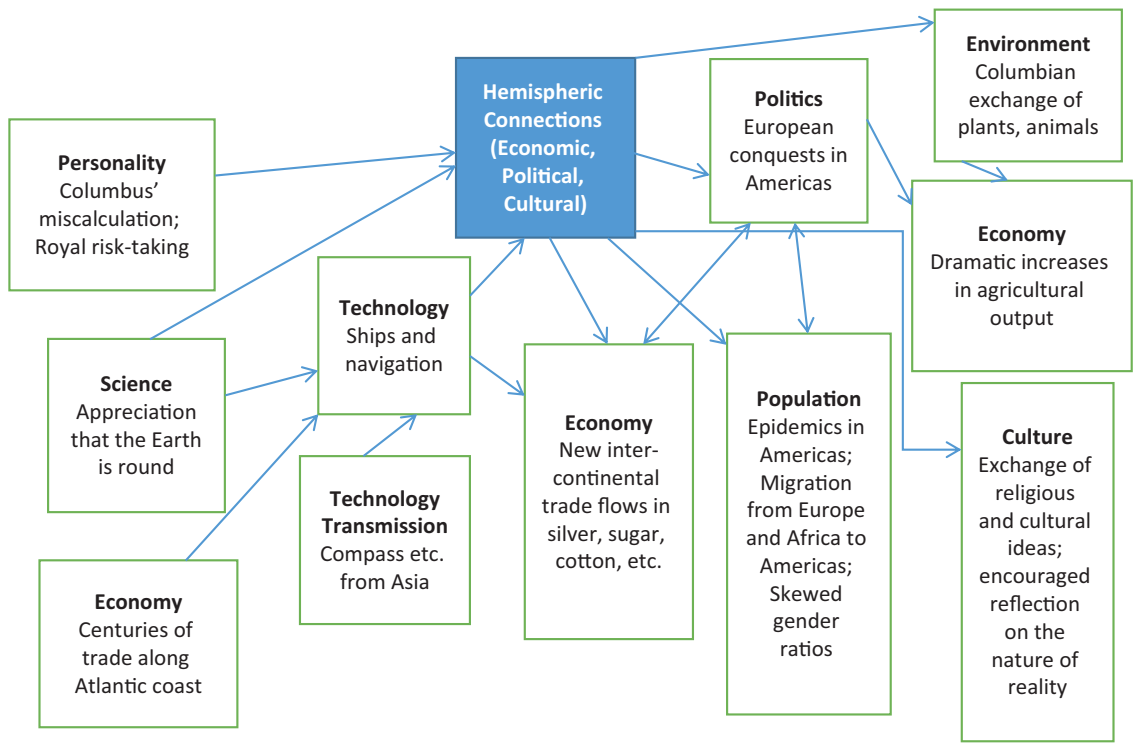

\section{Figure 19.1}

Influences on and effects of connecting the two hemispheres

Note:We discuss in this chapter many influences on the European conquests in the Americas. We will address in later chapters the effects these interactions may have had on economic and political developments in these continents and beyond. 


\section{An exchange of ideas}

The "discovery" of the Americas shocked Europeans (and others). Over the next decades, Europeans thrilled to tales of new peoples possessing a variety of unimagined customs, beliefs, and institutions. This shock might be comparable to what would happen if we discovered extraterrestrial life: life that looked like us (as it so often seems to in science fiction), and could come to visit, but seemed different from us in a variety of ways. However, in a way their shock was greater than ours would be. We have been warned for decades of the possibility of extraterrestrial life; they had no inkling that a pair of continents lay out there (well, very little indication: as noted above, those on the Atlantic littoral occasionally found unexplained objects in the sea). Moreover, they received information very slowly, and in a manner that made it hard to distinguish truth from fiction. The fact that explorers searched for a fabled El Dorado (a city of gold) or the Fountain of Youth symbolizes the wonder associated with those first decades of discovery. If cities, vast silver mines, and horrific human sacrifices could be found in these new lands, who knew what else might exist? Even hardened mariners managed to be shocked by casual nudity, occasional cannibalism, and ignorance of Jesus or Muhammad.

The discoveries forced reflection on god's plan - just as we might wonder what the discovery of extraterrestrial life with different sets of religious beliefs could mean. It made sense to wonder at first if these "new" peoples had souls of their own - and if so why god had not bothered to share his plan with them as he had in the eastern hemisphere. The religious beliefs encountered in the Americas were quite different from any that were known in the eastern hemisphere. Perhaps god had placed these people there for the convenience of Europeans? It would take a while for Europeans to construct self-serving answers to both halves of the question: These people indeed had souls to be saved but could also be used as Europeans saw fit.

Europeans drew selectively on classical and medieval ideas to define the new peoples as barbarians who needed European guidance on the ways of civilization and especially Christianity. They conveniently ignored writers such as Aristotle, who had equated civilization with cities: The Aztecs and Incas at least would then have deserved respect as civilizational equals. They likewise ignored the humanistic currents of the Renaissance (chapter 15) which exalted individual differences. This decision was far from unanimous: The missionary Las Casas, among others, complained bitterly of Spanish mistreatment of indigenous peoples. As so often in history, those with power convinced themselves that they were right to exercise this in ways that were beneficial to themselves.

We know far less what the indigenous peoples of the Americas thought about Europeans. We have some examples of surprise at European customs, such as the harshness with which Europeans disciplined their children. The indigenous Mikmaq of Nova Scotia would wonder why, if Europe was as wonderful as Europeans claimed, Europeans endured costly and risky voyages to the 
Americas. We have suggested above that the rapid conversion of indigenous peoples may reflect to some extent a belief that their gods had abandoned them. However, we have seen polytheistic peoples convert to monotheism after conquest or contact in many parts of the world. Still, we can well imagine that the indigenous peoples of the Americas had cause also to reflect on god(s)' plans.

We might close with one other observation. As the sixteenth century progressed, global histories were written in many lands. These reflect curiosity about the various regions of the world that were now drawn into much closer contact. Yet like the histories encouraged by the Mongols (chapter 17), these global histories tended to stress the differences among regions of the world rather than their similarities.

\section{The slave trade}

Slavery had a long history in Africa as elsewhere. Slaves sometimes achieved high positions; the Songhay, for example, employed them as administrators. Slaves had long been exported to the Muslim Middle East (and some as far as China), mostly along the east coast but also across the Sahara. Within Africa, slaves were an important sign of wealth since individual landowning was generally shunned. Those with many slaves could farm more land. It had become common to raid villages to obtain slaves.

European trade with Africa focused on goods such as gold and ivory in the first centuries after the Portuguese discovered a trade route south. Yet there was some interest in slaves from the start. The Portuguese very early on found it easier to trade for slaves than try to capture slaves themselves. A few slaves were taken to Portugal itself in the fifteenth century, but from the 1520 s slaves were carried to the Azores, Cape Verde, and other Atlantic islands (see above), and by the 1530 s to Brazil. Over 150,000 slaves were likely transported to Brazil in the sixteenth century alone, though perhaps only 15,000 survived there in 1600. Spain also sent African slaves to the Caribbean from 1518. Britain and France entered the slave trade decades later. From the 1640s, the French, British, and Dutch established colonies in the Caribbean to grow sugar or tobacco, and imported millions of African slaves to work on these plantations. It is estimated that some 12.5 million slaves left Africa over the centuries, with some 10.7 million arriving in the Americas. An infamous "triangular" trade developed: Firearms and other manufactured goods went to Africa (including a great deal of Indian cotton; see chapter 15) along with gold and silver, slaves were sent to the Americas, and sugar or tobacco were transported back to Europe. Rice and indigo were other main plantation crops, joined later by coffee and cotton; note that the goods produced on slave plantations were generally luxuries rather than necessities. This unfortunate trade is nevertheless remarkable for the volume of products and people that were moved among three different continents (four if we distinguish North and South America) over a period of well over three centuries. 


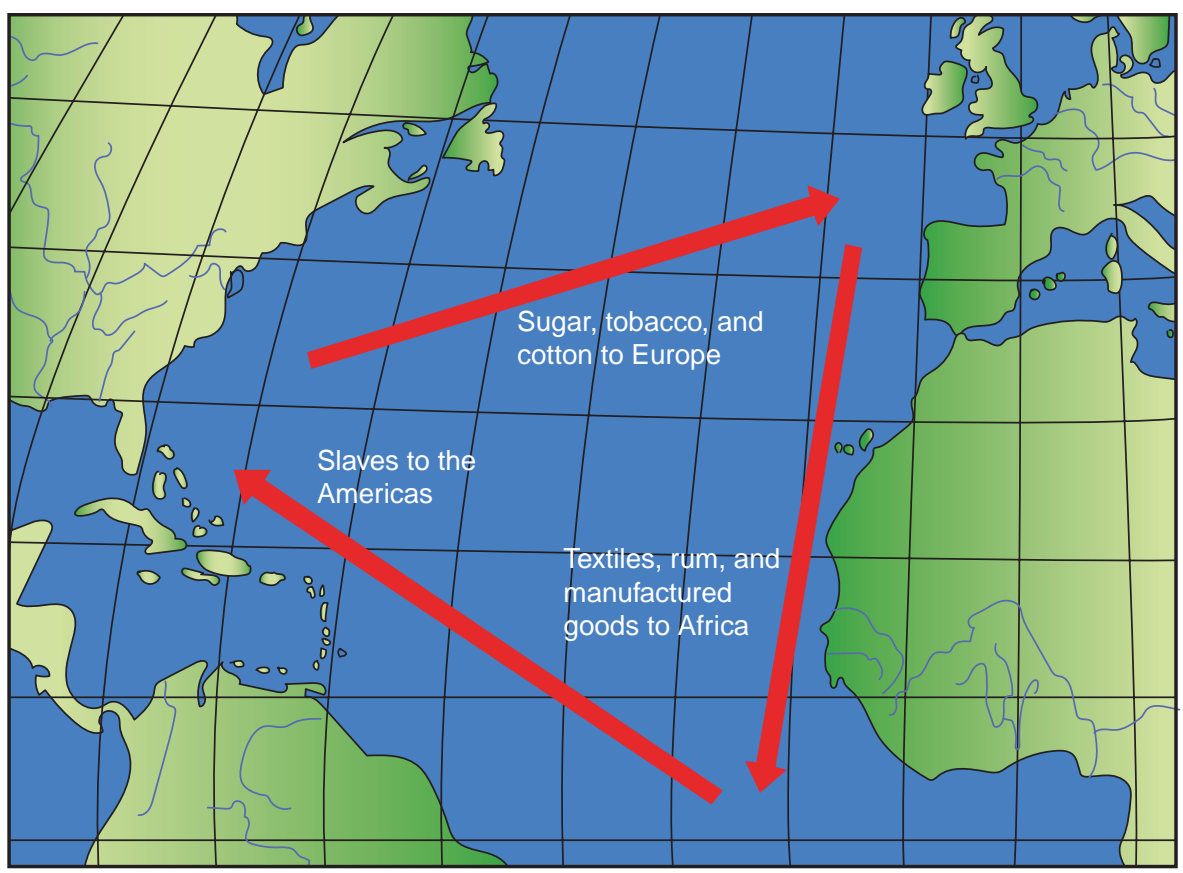

Map 19.3

The Triangular Trade

Adapted from map by SimonP, CC BY-SA 3.0

States within Africa that controlled the slave trade gained firearms and expanded: These included the Assante, Dahomey, and Oyo in West Africa, and the Kongo in central Africa (though, as we saw above, this state soon dissolved). The slave trade likely increased state conflict within Africa, as wars allowed states to gain captives that they could trade for European guns and goods. (We made a similar argument regarding competition among Slavic states in chapter 13.) This offensive motive is difficult to distinguish from a defensive purpose: Fear of being captured by slave traders provided a powerful incentive for people to form large polities. People might admire even a corrupt ruler if they felt protected. It is noteworthy in this respect that many of those captured were the children of wealthy families and even rulers (these were very occasionally redeemed and sent back to Africa): It was no simple matter to avoid being captured by slave traders. There was then a cycle of reinforcement: Success in war allowed states to trade slaves for guns, which increased their (offensive and defensive) military might. It is a mistake to see Africa as merely a victim of the Atlantic slave trade: Many African states were active participants in what was indeed a "trade" and these states profited handsomely from this. The Kongo would even participate in European rivalries, encouraging the Dutch to attack the Portuguese in Angola (who were bypassing the Kongo rulers in the slave trade) during the Thirty Years War in Europe (chapter 18). Of course, these polities would develop a strong sense of identity and use this to justify enslaving members of other groups. 
Postscript: Some scholars are sceptical that the slave trade encouraged conflict, noting that the frequency of wars did not rise when the price of slaves did (note that decreased wars might have pushed up the price of slaves). In any case, those regions that were heavily involved in the slave trade centuries ago are observed to have lower average incomes than other parts of Africa today. This correlation may reflect the fact that slavetrading polities did not introduce institutions that supported economic growth. There may also be a cultural link: Levels of trust are lower today in areas that were heavily involved in the slave trade.

Africans who found themselves on the outside of these powerful states suffered grievously. We should be careful not to equate the slavery long practised within Africa with that practised across the Atlantic. The rising European slave trade disrupted the traditions and institutions of African slavery. Slaves within Africa had usually had some rights and could often expect that they or at least their children would be freed. Those sold into the Atlantic slave trade had no such rights. Slaves themselves often reported good treatment by African captors before being traded to Europeans. There are multiple reports of Africans being sold into debt slavery in Africa, hopeful of eventually buying their freedom, but instead being sold to Europeans. Even those who avoided capture suffered: They retreated into deep jungle or desert to avoid capture.

Mortality on the four-six week passage from Africa to America may have been as high as 50 per cent at the start, but this fell to 5 per cent over time. Death, after all, was not in the ship captain's interest. Captains nevertheless kept slaves below decks and fed them poorly. There were 20,000 slaves per year on average crossing the Atlantic in the seventeenth century, and well over 50,000 per year in the eighteenth century (6-7 million in the century as a whole). It may surprise American students that only about 5 per cent of transported slaves went directly to continental North America. Transported slaves were mostly young adults. Two-thirds were male, distorting sex ratios in both Africa and the Americas. This encouraged polygamy in Africa, but perhaps also encouraged women to play roles otherwise reserved for men. The demographic effect of the slave trade on Africa was limited by the facts that mostly men were enslaved, the men left behind took multiple wives, and the Columbian Exchange (especially manioc) enhanced the productivity of many African farmers and thus the number of children they could support.

Such large numbers were required because of high mortality in tropical plantations due to disease and overwork (and low fertility since there were fewer women). (These harsh conditions also meant that those local indigenous people that had not been killed avoided plantation labour by retreating into the wilderness.) Farther north, mortality was lower and - perhaps as a result women were imported, and slave families encouraged. The lower mortality rate 
may have at first merely reflected a different disease environment. However, once slave owners appreciated that slave children reduced their need to purchase slaves, they would see greater value in maintaining the health of slave populations.

Despite high mortality, slaves came to form some 90 per cent of the population in Jamaica and a majority in some mainland colonies. Planters fearful of slave rebellions often encouraged immigration of Europeans to rebalance the racial ratio in the population. The Royal African Company in the seventeenth century advocated a ratio of less than 8 to 1 . Planters then employed racial discrimination in hiring to drive a wedge between poor whites and even free blacks. (The Spanish and Portuguese would allow slaves to buy their freedom in the eighteenth century; the British and French would introduce such a possibility only in the nineteenth century.)

Students should appreciate that transported slaves came from societies that practised agriculture, had artisans of various types, and engaged in activities such as mining and fishing. The transported slaves, therefore, had a set of useful skills. In particular, they had familiarity with farming under tropical or semitropical conditions. Many of the crops grown on slave plantations had long been farmed in Africa: cotton and indigo for centuries, and tobacco from the early fifteenth century. Rice was of particular importance. This had long been a key crop in parts of West Africa, and some scholars have argued that Africans were primarily responsible for rice cultivation in the Americas. It was African rice that was first planted in the Americas. We have noted in earlier chapters that there had been a long tradition of iron manufacture in Africa: African slaves with experience in iron-making were widely employed in iron manufacture in the Americas. Some ironworks employed hundreds of such slaves. These often considered themselves to be a slave elite. Slave traders were aware of and often advertised the skills of the slaves they transported.

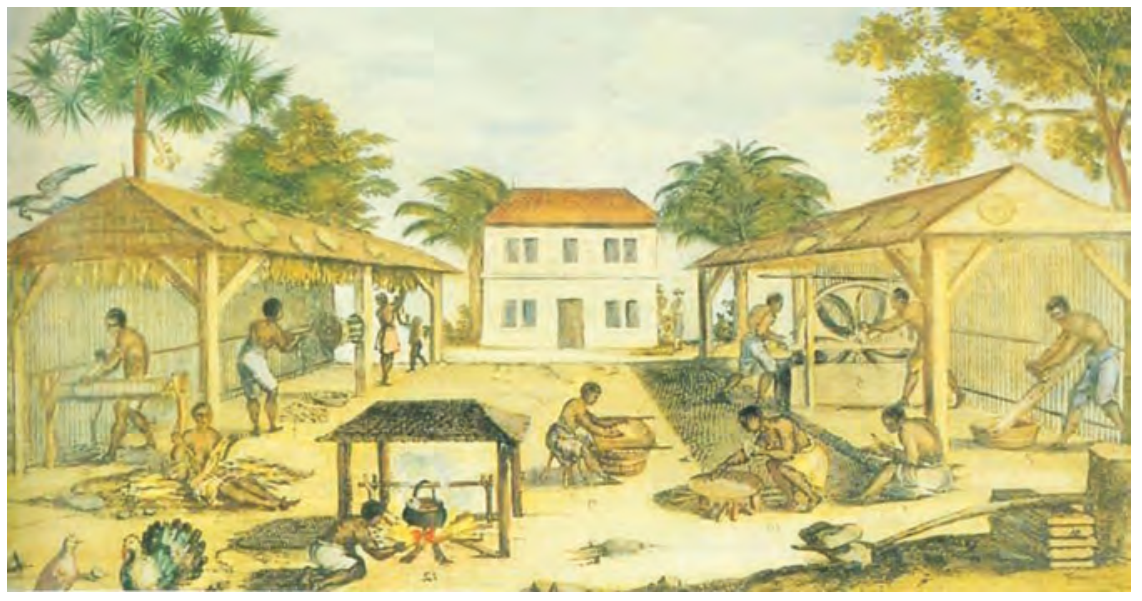

Illustration 19.14

Anonymous painting of slaves in tobacco sheds on Virginia plantation, 1670 
Slaves shirked when they could, and many escaped to form "maroon" societies in the forests or mountains that might border plantations. In Palmares in Brazil, escaped slaves built a community that survived for a century in the late sixteenth and early seventeenth centuries. The Maroons in Jamaica were among other long-lasting communities of escaped slaves. Slaves often revolted but only the revolt in Haiti between 1793 and 1804 (during the French Revolution, which temporarily decreased the French will to resist; see chapter 25) was successful.

Slaves sought to fashion lives as best they could. Slaves in the Americas developed syncretic religions that combined Christian and diverse African elements. One-fifth of slaves transported to the Americas may have been Muslim: This religion disappeared among the slave population but likely influenced the syncretic religions that developed. Europeans at first had often justified slavery in part by arguing that Africans were incapable of understanding Christianity, but over time Christian missionaries had reached out to slaves, and some slave owners encouraged or coerced conversion. Slaves also combined African musical traditions with European music. They used African okra to make gumbo.

Why did Europeans transport slaves across the Atlantic at considerable expense rather than employ them on plantations in Africa? As we shall see in chapter 23, in the nineteenth century Europeans would establish plantations in Africa itself. One part of the answer is just that there was lots of suitable land in the Americas, but epidemics had so devastated indigenous populations that there was little local labour. The potential return to forced labour in the Americas was therefore high. To have instead established colonies in Africa would have required (and would later require) armed conflict since the land in Africa was already being farmed. African polities had long employed iron weapons and soon became familiar with firearms. Europe would have developed a much more considerable military-technological advantage (chapter 18) by the time it colonized Africa in the nineteenth century. Moreover, the disease environment of much of Africa would limit European colonization for centuries until drugs were developed to address malaria in particular. Finally, the geography of Africa - with a high plateau separated from the coast by un-navigable rivers was less amenable than American geography to export-oriented agriculture before the development of the railway.

How profitable was the slave trade? Since it lasted for over four centuries, we can presume that some interests made money from it. Indeed, those in the trade must have profited. Plantation owners must have also benefited from the supply of slaves, or they would not have kept buying them. However, unless we imagine that these sectors were utterly uncompetitive (as some have), we might suspect that profits will not have been spectacularly high. In shipping especially the trade was open to ships from several countries. These had to compete in Africa when purchasing slaves and in the Americas when selling these. There have been estimates that have borne out the existence of good but not extraordinary profits in the slave trade, and moreover indicate that profits in the slave trade were highly variable. 
Governments bore some of the costs of the slave trade by protecting shipping and capturing escaped slaves. What was profitable for some may not have been advantageous for the society as a whole. The typical European citizen may (or not) have paid more in taxes to support the slave trade than they benefited from cheap sugar or cotton. (We can make a similar argument concerning empire itself; see chapters 23 and 30). Societies in the Americas also supported slavery financially by capturing and returning escaped slaves. Moreover, there are dynamic effects such that reliance on slaves, and thus a very unfree labour market, may have slowed urbanization and industrialization in the Americas. Nevertheless, cultural abhorrence rather than strict economic calculation would end the slave trade in the nineteenth century (chapter 26).

\section{The Newfoundland fishery}

While the Spanish investigated their new colonies in Central and South America, other European powers investigated the lands farther north. Though paling in comparison to Mexican and Peruvian silver mines, the first lucrative discovery to the north would be the abundant fishery of the Newfoundland Grand Banks - an extended piece of the continental shelf to the east of Newfoundland where the fish were so plentiful that early visitors described them as leaping into the ships of European fishers. John Cabot (Giovanni Caboto), sailing on behalf of the British (though Italian by birth), found the Grand Banks in 1508, only sixteen years after Columbus had first reached the Americas. He, like Columbus, imagined he was on the fringes of Asia, but appreciated that no Asians were fishing there. Basque fishers from northern Spain may have been active in the area over the previous century, but kept the fishery a secret.

There was a thriving trade in fish in Europe at the time. Catholic Europe had some hundred days a year when Catholics could not eat meat. Fish was, in any case, a valued source of protein as population growth threatened the average meat intake of Europeans. Fish caught in the North Sea were eaten throughout Europe. Aware of this ready market, ships from Britain, France, Spain (especially the Basque region), and Portugal converged on the Grand Banks.

Given the international conflict associated with the movement of Spanish silver, one remarkable characteristic of the Newfoundland fishery was that it would remain mostly peaceful for centuries. This is perhaps easiest to understand in the earliest days when the fishing was prosecuted offshore: Fish were caught and loaded straight onto ships and coated with salt for the return trip to Europe. Since there was enough fish for all comers, there was little motivation for conflict - though each nation harboured some hope of monopolizing the fish trade.

Fishers soon recognized, though, that they could obtain a much higher quality of fish if they landed on Newfoundland, lightly salted the fish, and dried these in the sun. Now there was a potential problem, for Newfoundland's 
shoreline provides only a few sheltered harbours with flat land suitable for drying fish. We might well have expected military conflict over this scarce shoreline. Instead, the international body of fishers agreed - informally to be sure - to what is commonly called the "Admiral System." The first ship into a harbour in a year would choose the best piece of shoreline. The captain also became the "Admiral," entrusted with settling any disputes among fishers in that harbour for that fishing season - aided by the captains of the second and third ships (who also received the second and third best pieces of shoreline). Sailors from different countries tended to prefer different harbours, but they did often move around, and so individual harbours often had an international character for a season. The decisions of admirals were nevertheless accepted. Though the European powers themselves were involved in many wars, the fishers in Newfoundland themselves rarely battled.

Britain would eventually gain control of much (eventually all) of the Newfoundland shoreline. Yet the Admiral System would remain in place. Indeed the merchants that sponsored the fishing fleet fought hard in Parliament to ensure that private property over shoreline was not instituted in Newfoundland. When Lord Baltimore won the right to establish a Catholic colony in a place called Ferryland in Newfoundland, the fishers ensured that the colonists were forbidden from engaging in the fishery. Since Newfoundland to this day has limited agricultural potential due to a rocky and acidic soil, and short growing season, the colony failed. Lord Baltimore was forced to look farther south to a place he called Maryland to plant his Catholic colony.

Why was the Admiral System maintained? It was a costly system. As the number of fishing ships increased, these began to race for shoreline, reaching Newfoundland months before the fish did. Since the crew had to be fed during this period - recall that there is little food on Newfoundland - this was an expensive proposition. Since the fishers all came from a small region in southwestern England, they should have been able to reach some agreement that shared out ownership of scarce shoreline. The answer may well lie in the very scarcity of good shoreline: In a world where ships often wrecked and ship captains might be lured away from the fishery by opportunities elsewhere in the world, the Admiral System had the unique advantage of ensuring that the best pieces of shoreline were used each year.

Then a second mystery arises: How was the Admiral System eventually replaced by private property, despite the fact that the politically powerful fishing interests remained implacably opposed to this? There is no record whatsoever of political or military conflict over this issue. For decades, the fishing interest succeeded in their goal of maintaining the Admiral System. Note that, as a result, the Newfoundland fishery was for long a European fishery: Fishers from Europe spent the fishing season in Newfoundland, but there was virtually no settlement on Newfoundland itself. Ironically, then, Newfoundland, the first part of northern North America to be encountered by Europeans, would be the last part of the eastern seaboard to be settled by Europeans. 
We saw in chapter 13 that feudal lords made decisions that set in train a historical process which replaced feudalism. A similar process occurred in Newfoundland. At some point - there are no records of any official agreement - it became accepted that a man wintering in Newfoundland could exercise the rights of a ship arriving in the harbour the next year. A captain confident of returning could leave a man behind and not have to race the next year. The advantage of doing so would increase as more ships entered the fishery. At the same time, Newfoundland experienced deforestation which some blame on the fishers and others on the local Beothuk people. The fishery needed wood for buildings and for the structures on which fish were dried and for the boats from which the actual fishing was done. These structures were rebuilt every year, in large part because the Beothuk would scavenge for nails after the Europeans left. A man left behind could both guard structures and boats and gather wood (which could be moved more easily on snow than rock).

Once it became accepted that a man left behind controlled shoreline the next year, the stage was set for de facto private property: A man who decided to live permanently in Newfoundland could exercise power over shoreline forever. Although fishing captains may have at first wished to leave employees behind, they soon discovered that this was a fraught practice: An employee out of sight for several months could easily claim that bad weather had prevented them from gathering wood or guarding boats and structures. It was much better to leave an independent agent behind who had their own need for boats and wood; their performance on their own behalf could then be compared to their performance on behalf of others. Over time, such agents gradually became landowners. The final nail in the coffin of the Admiral System came once Irish women started arriving on the island. The fishing ship captains had long known that their situation was secure as long as only men lived on Newfoundland. These Newfoundland women had very high birth rates, and a North American population came over a period of decades to dominate the Newfoundland fishery. Ironically, British merchants soon found that they could profit as much by provisioning these fishers as they once had by prosecuting the fishery themselves.

The experience of the Beothuk deserves attention here. Unlike aboriginal populations everywhere else in the western hemisphere, these avoided contact with Europeans. They could gain what they wanted by scavenging rather than trading. This practice continued after Europeans came to settle permanently on the island. As settlement spread along the coastline, the Beothuk were forced inland where food was scarce. When Europeans started pursuing the limited fur resources of Newfoundland, they trapped themselves whereas everywhere else on the continent aboriginal peoples did the trapping and traded with Europeans. The sad outcome for the Beothuk was that they disappeared as a people in the early nineteenth century. Though aboriginal groups elsewhere in the Americas suffered grievously through interactions with Europeans, the Beothuk suffered an even worse fate by not interacting. 
Fishing would prove important along the northern shoreline of North America. A large cod statue hangs prominently in the Massachusetts state house, for example, in recognition of the critical role of that fish in the development of that state. It replaces a cod statue that had hung in the colonial assembly until destroyed during the American Revolution (chapter 25).

\section{The North American fur trade}

As in Russia, the fur trade caused European exploration and eventual conquest of vast territories. In North America, the most important fur by far was the beaver pelt. Amazingly, the primary market for this for centuries was to make hats in Europe: That is, beaver fur was not used to keep people warm. Instead, the trade depended for centuries on a fashion for hats made of beaver pelt. Fur traders had at first travelled outward from North American centres such as Montreal and New York. As the fur trade moved farther west, trading companies were developed that could finance a series of trading forts. When the fur trade crossed the Rocky Mountains, it became easier to supply fur traders from the Pacific coast; Furs could then also be traded west to China (see above). Some of the earliest European settlements on the Pacific coast were fur

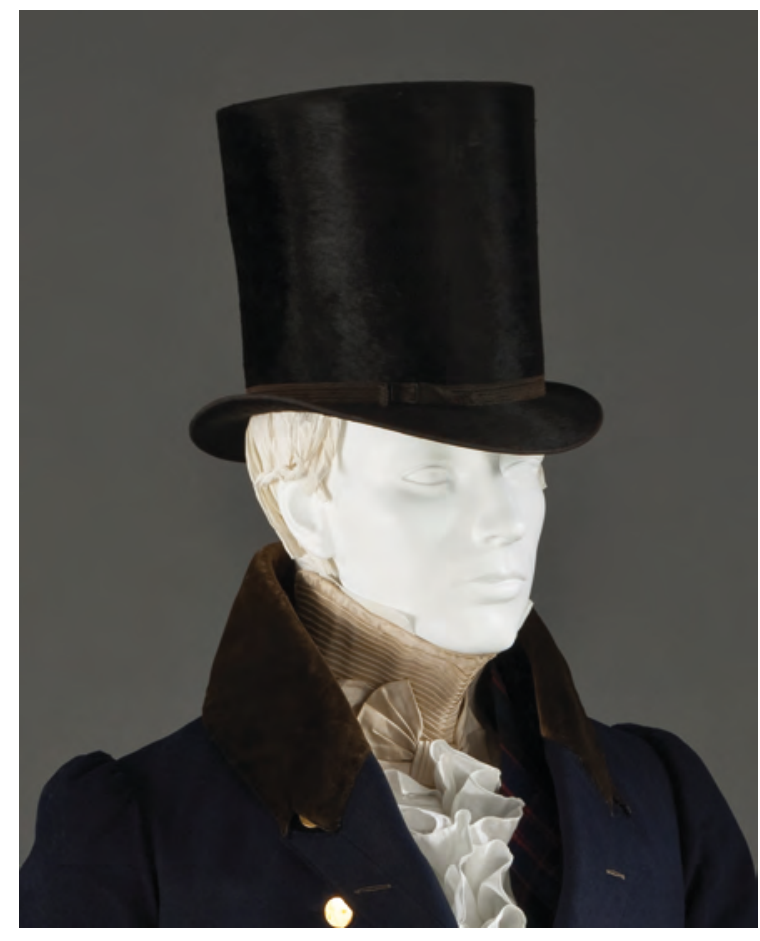

Illustration 19.15

Beaver felt top hat, Paris, c. 1815, LACMA trading posts.

One significant development occurred in 1670. Advised by two French fur traders, the British government created the Hudson's Bay Company (HBC). These fur traders had recognized that there was a potentially much quicker route between furs and European markets via Hudson's Bay than by canoeing through the Great Lakes to/from Montreal. Whereas other British-chartered trading companies had been empowered to establish forts - and the East India Company would later rule much of India (chapter 20) - the HBC was granted from the outset all territories that drain into Hudson's Bay. This vast land grant comprised the bulk of Western Canada east of the Rockies and a significant part of the United States (for the Red River flows north and drains into Hudson's Bay). The HBC would compete with Montreal-based fur traders for decades after the British conquest of 


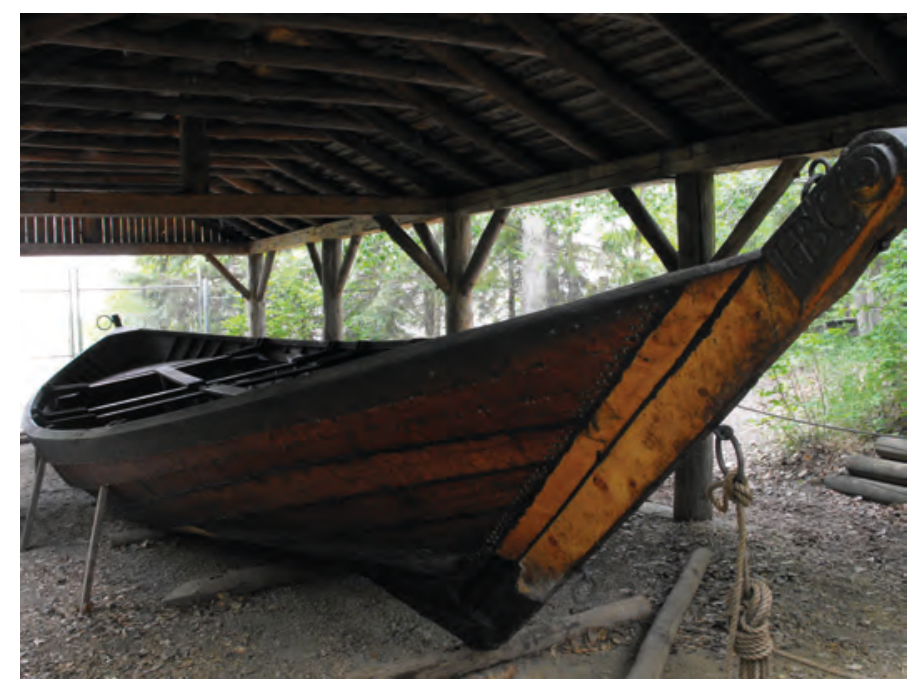

Illustration 19.16

York Boat replica, Fort Edmonton Park. The Hudson's Bay Company used York boats for centuries on Canadian rivers

Verne Equinox, CC BY 3.0

Montreal in 1760, but in 1821 the HBC would absorb these. As noted above, overharvesting and westward expansion were encouraged by this competition. Thereafter the fur trade would almost entirely flow through Hudson's Bay: Like the early Newfoundland fishery, it was tied more closely to the European economy than the broader North American economy. Though most fur traders were British, there were Norwegians, Germans, and even some African Americans involved in the trade. In 1871 the HBC would sell most of its land to the newly created country of Canada, allowing Canada to spread westward from its base in eastern Canada. The HBC would later transform itself into a retail chain that still exists.

The fur trade was indeed a trade. Europeans did not trap the animals except in Newfoundland where there was little contact with indigenous peoples. It was the indigenous peoples who traded furs (Plains Indians supplied fur traders with dried buffalo meat also) for a variety of goods. These trade goods - metal pots, axes, knives, guns, blankets, beads for decoration, and, most controversially, alcohol - came mostly from Europe (some had come from settler societies in North America when trade operated from Montreal or New York). Spices from Asia did reach distant trading posts in the interior of North America in the nineteenth century, and were employed by indigenous peoples. Though these trade goods were carried across oceans in large ships, they were then transported thousands of kilometres on small boats that could be carried around rapids. 


\section{Agricultural expansion}

The fur trade alone required a relatively small population. It would be agriculture (and the occasional mining frenzy) that would drive the settlement by Europeans of the Americas - and also Australia, New Zealand, and parts of Africa. We have space here only to record certain aspects of this centuries-long process:

- The British were unusual in allowing religious minorities to form colonies: Puritans in Massachusetts, Quakers in Pennsylvania, and Catholics in Maryland. This flexibility allowed the British to establish colonies that were barely viable economically. The French were not willing to let religious minorities migrate and ended up with a much smaller colonial population. As early settlers established agriculture, and the costs of intercontinental transport fell, the British colonies soon lost their religious character, coming in most cases to celebrate religious tolerance.

- The British, like the Russians, found distant lands a convenient place to dump prisoners. They did so in their American colonies, and turned to Australia after American independence. It is not clear that transporting prisoners all of the way to Australia made financial sense; the British may have been even more interested in pressing a claim to a potentially valuable land. Prisoners were (like slaves) useful in building forts and port facilities in an area with a limited labour force.

- There were very high mortality rates among early settlers in Virginia: Only 60 of the first 900 survived, and only 2,000 of the next 9,000. Likewise, half of the first 120 pilgrims in Massachusetts died in the first year. It took Europeans a few years to develop the ability to farm successfully in the Americas. In the meantime, they received much aid and advice from local indigenous peoples. The indigenous peoples also occasionally attacked, killing one-third of settlers in the Chesapeake in 1622.

- Though some religious minorities might wish to distance themselves from Europe, most immigrants hoped to trade. They wanted to be able to import European manufactures. They then needed something to export. For some, this could come from participation in fur or timber trades. Eventually wheat would become a significant export - encouraging expansion into the western plains. The first significant agricultural export was Virginia tobacco: Early settlers developed a hybrid tobacco that proved hugely profitable.

- Women were a much higher proportion of European migration to the United States and Canada than to the tropics. Intermarriage with indigenous people was relatively rare and frowned upon. Birth rates were high: The 1 million total immigrants before 1800 had produced a European population of 5 million. 
- Europeans could often not afford passage to the Americas. They often travelled as indentured servants: They promised to work for seven years for the person that financed their voyage, after which they were free. This practice fell away as transport costs dropped.

- Though immigrants came from many European lands - and at times from Asia - immigration rates were moderate enough that the colonial language remained dominant. Italians learned Spanish in Argentina and Germans learned English in the United States - even though there would in time be more migrants from Germany than Britain.

- Indigenous peoples were repeatedly removed by force from lands coveted by European settlers. In 1838-9, the United States government forced indigenous groups to migrate west of the Mississippi; many died on the way along what the Cherokee named the Trail of Tears. Canadian settlement in the west was less violent than American settlement but still resulted in indigenous peoples being forced onto reservations. In Chile and Argentina as well, firearms were used to push indigenous peoples off agricultural lands. These acts were justified in myriad ways. One peculiarity of this centurieslong practice is that many indigenous peoples had been (at least partly) agriculturalists, but efforts were rarely made to encourage these to engage in farming for export. Note that the indigenous population of what is now the United States had dropped precipitously from 5-10 million in the sixteenth century due to epidemic disease. It would fall further as European colonists took land: The indigenous population was perhaps 600,000 in 1800.

- Much of the territory of the modern United States was purchased or taken from other countries. The Mississippi Valley was purchased from Napoleon. Texas had separated from Mexico with American support before joining the United States. The ensuing Mexican-American War of 1845-8 would result in the United States purchasing California and other western states which at the time comprised half of Mexican territory but very little of its population. The United States failed, though, to take Canada in the war of 1812.

- $\quad$ River and canal transport, and later railways, facilitated westward settlement. Canals were built around rapids on the St. Lawrence River and around Niagara Falls in the 1820s. The Erie Canal of 1825 connecting Lake Erie to Albany meant that it was easier to ship through New York than Montreal. Many other eastern cities would fail in attempts to finance alternative canals through the Appalachians. The advent of railroad technology would allow several links across those mountains and later across the Canadian Shield and the Rockies. Roads were also important for transporting people but were generally too expensive for transporting grains before internal combustion.

- A combination of the relative quality of different lands and access to transport infrastructure largely determined when particular areas were 
settled. The rate of settlement was closely associated with rates of immigration.

- The experience of the Americas was reprised in Australia and New Zealand a little later. There were barely 1 million people in Australia as late as 1860, but the discovery of gold in the 1850s and 1890s and developments in shipping and especially refrigeration (chapter 23) encouraged immigration after that. Three and a three-quarter million Europeans moved to Australia in the nineteenth century, and 750,000 went to New Zealand. Sadly, the indigenous population in Australia fell from 650,000 in 1800 to 90,000 in 1900 and in New Zealand from 200,000 to 45,000. Though the Maori of New Zealand were promised equal rights in 1840, they were pushed off the best lands.

Postscript: Exploration has continued through later centuries. The interiors of North America, Africa, and Australia would be explored well into the nineteenth century - and some parts of the Amazon are still largely untouched. The Northeast Passage connecting Europe and Asia by Siberia was charted in 1903, and the Northwest Passage through Canadian islands in 1903-6. The melting of Arctic Ice has induced small numbers of cargo ships and cruise ships to pursue these routes in the twenty-first century. Antarctica was first sighted only in 1820, and was not landed upon until 1895. The South Pole was reached only in 1912.

The dramatic first contacts between Europeans and the peoples of the Americas would be replicated in the twentieth century in the Highlands of New Guinea. Australian explorers first reached the Highlands in the 1930s but new groups were still being contacted in the 1990s. The Australians were not surprised that there were people in the Highlands, though they had no foreknowledge of their ways. The peoples of New Guinea were as surprised by the arrival of outsiders as had been the Aztecs and Incas. However, the technological gap in the New Guinea contact was far greater: New Guineans had not developed the use of metals but were suddenly exposed to cars and planes. In the Americas too, indigenous peoples deep in the Amazon met outsiders for the first time in the twentieth century. It is possible that some groups remain to be contacted in both Amazonia and New Guinea.

Human exploration extended into space in the twentieth century. Yet most of the ocean floor remains to be mapped in the early twenty-first century. There are flora and fauna in the ocean depths still to be discovered. 


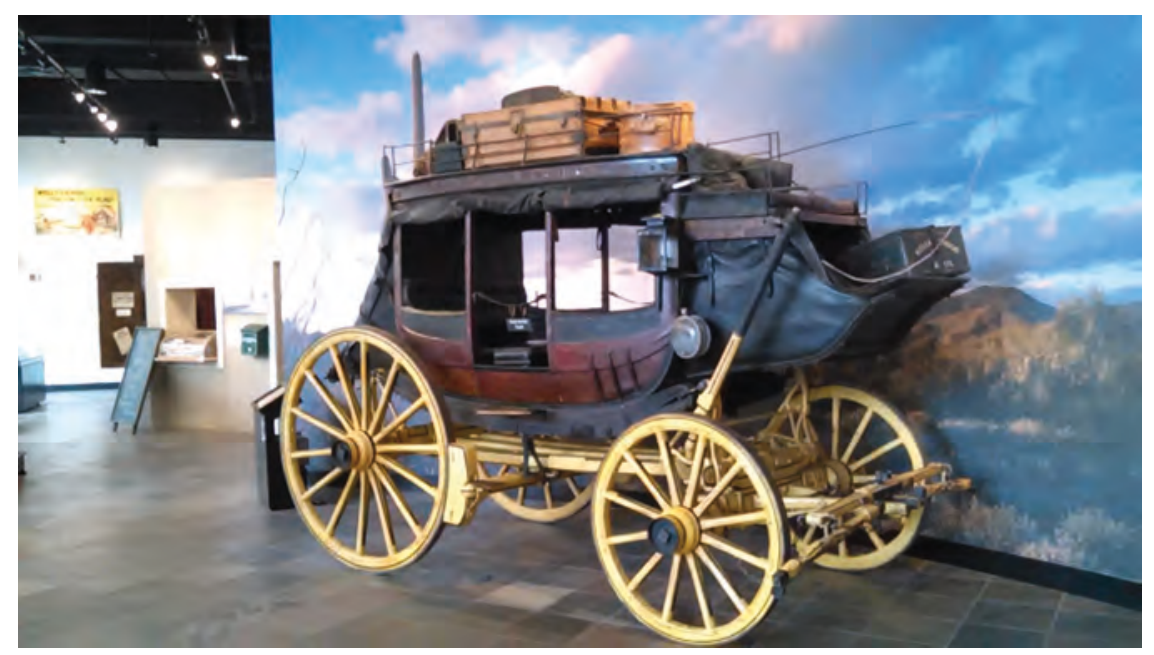

Illustration 19.17

Stagecoach, Wells Fargo Museum, Phoenix. Such stagecoaches were the main means of passenger transport for decades in the western United States and Canada Author

\section{Questions}

1. Why did Indian or Chinese merchants or states not seek to reach Europe by new routes?

2. Pick one of the many plants or animals transplanted during the Columbian Exchange, and examine its impact on the lands to which it was transferred.

3. Diagram the influences on and effects of the Columbian Exchange.

4. The Spanish conquest of the Americas lends itself to a host of counterfactual questions. If Cortés had been defeated at first, would the Aztecs have mounted a more effective defence in time? Would the rate at which epidemic disease spread have lessened? Would Spain have turned back to attacking Muslims in the Mediterranean if not for easy successes in the Americas?

5. Where did most African slaves shipped to the Americas come from? Where did they go in the Americas? On ships from what countries did they travel? [Students may usefully be asked to explore Voyages: The Trans-Atlantic Slave Trade Database, which provides records from almost 35,000 slave trading voyages.]

\section{Readings}

Abulafia, David. 2008. The Discovery of Mankind: Atlantic Encounters in the Age of Columbus. New Haven, CT: Yale University Press. [Explores the cultural results of European exploration of the Americas.] 
Appleby, Joyce. 2014. Shores of Knowledge: New World Discoveries and the Scientific Imagination. New York: Norton.

Armitage, David, Alison Bashford, and Sujit Sivasundaram, eds. 2017. Oceanic Histories. Cambridge University Press. [The book surveys the history of many seas and oceans across many time periods.]

Beckwith, Christopher. 2011. Empires of the Silk Road: A History of Central Eurasia from the Bronze Age to the Present. Princeton University Press.

Bishara, Fahad Ahmad. 2017. A Sea of Debt: Law and Economic Life in the Western Indian Ocean, 1780-1950. Cambridge University Press. [Explores the legal documents employed by Muslim traders over the centuries.]

Knight, Frederick C. 2012. Working the Diaspora: The Impact of African Labour on the Anglo-American World 1650-1850. New York University Press.

Konadu, Kwasi. 2015. Transatlantic Africa: 1440-1888. Oxford University Press. [This book reports the thinking of slaves about the Atlantic slave trade.]

Norrie, Kenneth, and Rick Szostak. 2005. "Allocating Property Rights over Shoreline: Institutional Change in the Newfoundland Inshore Fishery." Newfoundland Studies 20:2, 27-56.

Reader, John. 2009. Potato: A History of the Propitious Esculent. New Haven, CT: Yale University Press.

Salmond, Anne. 2009. Aphrodite's Island: The European Discovery of Tahiti. Berkeley: University of California Press.

Watts, Sheldon. 2003. Disease and Medicine in World History. New York: Routledge.

There was a Symposium in the Journal of World History in September 2011 on the cultural and religion interactions associated with European discoveries of traditional peoples. 


\section{Comparing new empires in Asia}

\section{Guiding questions}

Why, when, and how did new empires emerge in Asia during this period? What were the similarities and differences among these empires? What effects did they have on the areas they governed? In particular, how did they shape culture?

Why, when, and how did they decline?

Relationship to other chapters: The Mongol conquests discussed in chapter 17 influenced the early histories of each of the empires addressed in this chapter. We revisit and expand our discussion of religious toleration and conflict, especially from chapter 12. We also connect to the history of gunpowder in chapter 18. This chapter will naturally inform later discussions of the regions these empires governed. We also deepen our general understanding of the challenges of governing large and diverse populations. We see that new transport and military technology can ameliorate these challenges, while an increased sense of ethnic and religious identity complicated matters. These empires provide a useful contrast to the nation states that will be the subject of chapter 21. The Ming naval explorations discussed in this chapter can also usefully be contrasted with the European explorations addressed in chapter 19.

We discussed in chapter 19 the emergence of the new oceanic empires of the Spanish, Portuguese, British, French, and Dutch. We also recognized in that chapter that Russia and China had expanded overland at the same time. These empires were far from the only empires of the time. This chapter focuses 
attention on three great land-based empires in the Middle East, Persia, and India (we also discuss the Ming and Qing dynasties in China). The Ottoman Empire would at its height rule a vast swathe of southeastern Europe as well as most of the Middle East and Egypt. The Safavid Empire to the east would rule what is now Iran as well as much of Central Asia. The Mughal Empire would rule most of the Indian subcontinent. These three empires collectively ruled hundreds of millions of people for several centuries. In doing so, they shaped ethnic and religious boundaries that remain in place today. They also interacted in many ways with the empires discussed in the last chapter.

The Ottoman Empire would collapse in the early twentieth century to be largely divided up by European powers. The Mughal Empire would dissipate centuries earlier under pressure from the British in concert with local princes. It is tempting to imagine that these empires were always weak relative to European polities. This would be analogous to judging the Roman Empire by its fifth-century defeat at the hands of Germanic tribes (chapter 10). All historical empires have risen and fallen, and all naturally seem more impressive when judged at their height than at their point of dissolution. The Ottomans had in their earliest centuries conquered deep into Europe. They were both feared and admired in Europe at that time. The Mughals formed an empire with a far more substantial population than any European empire of their time. As with other historical empires, they exerted important effects on art, architecture, and literature.

We begin by discussing some of the fundamental similarities and differences in the development of these three empires, before proceeding to a more detailed history of each. Throughout we stress interactions among these empires and with the rest of the world. We close with briefer discussions of the Ming and Qing in China and the political situation in Africa. We devote special attention to Ming naval explorations in the Indian Ocean.

Thematically, the focus in the chapter is political. We also devote considerable attention to cultural - and sometimes artistic - developments. Economic developments interact with both politics and culture. We discuss social structure, and gender relations in particular. We occasionally address technology especially gunpowder technology. One of the author's favourite applications of evolutionary analysis occurs in our discussion of an unusual approach taken by the Ottomans (and Safavids) to limit succession crises.

Politics
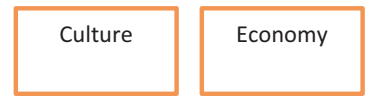

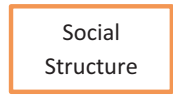

Technology
Art

\section{Comparisons and contrasts}

These three empires are often termed "gunpowder empires." They each utilized cannon to neutralize the castles of local elites. They were then able to concentrate power centrally more easily than had earlier empires. We should 
appreciate that only a well-organized state could employ expensive cannon against an array of local elites. We saw in chapter 18 that political consolidation characterized the Early Modern period globally. The organized use of gunpowder may well have contributed to - and required - large and enduring states on all continents. We suggested in chapter 18 that these three empires were likely too successful: Having eliminated local sources of power, they had far less need to continually develop gunpowder technology than did smaller European states - though the Ottomans and Safavids often battled each other. In later centuries, all three empires would import European guns and cannons. They may each have faced challenges from the eighteenth century in financing the purchase of armaments that had become much more expensive over time.

Nomadic or semi-nomadic peoples founded all three empires. In chapter 17, we noted the relatively brief rule of nomadic Seljuk Turks, Mongols, and Timur. The fourteenth-century Arab historian Ibn Khaldun had posited a theory in which conquering nomads lost their vigour over a couple of generations to quickly be conquered in turn (see the primary document in chapter 13). It is remarkable that these three later empires would each last for centuries. Though we will recount the early rise to power of each below, we should appreciate that their extraordinary accomplishment was to forge a lasting bureaucracy and military structure.

All three empires had Turkic origins. That is, all three ruling dynasties descended from nomadic peoples who had at some point moved south out of Central Asia and adopted Islam as a religion. All three would blend - as had Mongol conquerors before them (chapter 17) - elements of nomadic governance with local bureaucratic traditions. In all three cases, the rulers were considered to own all of the lands in the empire. All three empires experienced nasty succession crises as all sons (and sometimes other relatives) competed for the throne, not always waiting for the father to die. (Islam in general disdains primogeniture.) All three would experiment with problematic mechanisms for limiting succession battles. In all three cases, the mothers and wives of rulers exercised a significant influence on policy despite a general practice of female seclusion. The exemplar here is Nur Jahan, wife of the Mughal emperor Jahangir, who was likely the primary power in the Mughal Empire for many years.

All three empires sought to exercise control in part by employing outsiders in senior bureaucratic positions. The Mughals regularly imported Persians for this purpose. The Ottomans and Safavids often employed slaves in senior posts. Such people would not generally be able to develop an autonomous power base with which to threaten the ruler. Nevertheless, all three empires relied on local elites to administer some of their lands. They attempted to maintain personal relations with these by visiting them, inviting them to court, and sometimes intermarrying (even across religious lines). Especially among the Mughals, it was also common to spy on local elites and hold their sons hostage.

While all three were agrarian empires with bureaucracies for taxing farmers, all three also promoted and taxed trade, though perhaps not always as enthusiastically as they might have. Oddly, despite the fact that Islam had long been 
associated with commerce, all three empires tended to encourage non-Islamic traders: Jews and Christians (mainly Armenians and Greeks) were prominent in the Ottoman Empire, Armenians played a special role among the Safavids, and Hindu merchants dominated many trades in the Mughal Empire. Foreign merchants could govern their own affairs. One advantage of relying on "outsiders" as merchants was that these could not threaten imperial power in the same way that merchants who were members of the same ethnic and religious group as the emperor might.

This commercial policy reflected a more general practice of religious toleration. All three empires had substantial non-Islamic populations. The Ottomans made little effort to convert the Christian societies they conquered in Europe, though Sufi missionaries over time had some success (but a census of 1520 found that only one-fifth of the European population was Islamic). There were substantial Jewish and Zoroastrian minorities in the Safavid Empire, joined by Armenian Christians after the Safavid conquest of Armenia. The Mughal Empire was predominantly Hindu: Only about a quarter of the population converted to Islam. These non-Islamic populations could generally pursue their own religious practices and settle legal matters internally. The three empires allowed local religions as long as these paid taxes and did not rebel. They generally appointed the leaders of non-Islamic faiths and exempted these from taxes. Although all three states celebrated Islamic holidays, they also created secular celebrations of their empires that all could enjoy. The Ottomans would, for example, celebrate the circumcision of royal sons. As in earlier Islamic empires,

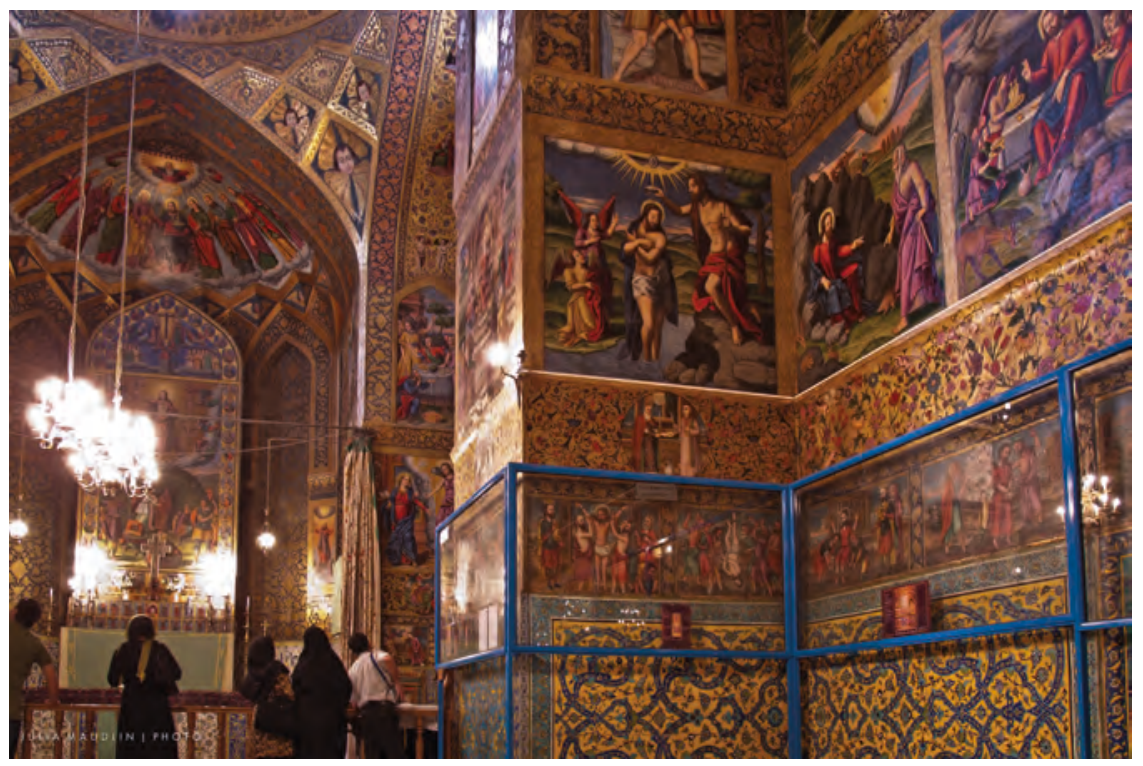

Illustration 20.1

Armenian All Savior's Cathedral, also known as Vank Cathedral, built in 1606, Isfahan, capital of the Safavid Empire

Photograph by Julia Maudlin, CC BY 2.0 
some religious authorities viewed toleration with suspicion. Some later rulers in all three realms would encourage Islam. Persecution of minorities proceeded farthest among the Safavids, with Zoroastrians fleeing to India as a result. Ottoman conquests of Arabian holy sites encouraged Ottoman leaders toward a more Islamic outlook. Aurangzeb, one of the last Mughal rulers, would reintroduce a tax on non-Muslims that had been waived for over a century (though applied by the Safavids next door), inspiring a series of local rebellions.

\section{PRIMARY DOCUMENTS: EXCERPT FROM SHAIKH HASAN AL KAFRAWI, THE SHAFIITE [PROFESSOR OF CANON LAW IN CAIRO], 1772 CE, ON THE TREATMENT OF JEWS AND CHRISTIANS}

They should not be permitted to employ mounts like the Muslims. They must use neither saddles, nor iron-stirrups, in order to be distinguished from the true believers. They must under no circumstance ride horses because of the noble character of this animal. The Most-High has said [Quran 8:62]: "And through powerful squadrons [of horses] through which you will strike terror into your own and God's enemies."

They should not be permitted to take Muslims into their service because God has glorified the people of Islam. He has given them His aid and has given them a guarantee by these words [Quran 3:140]: "Surely God will never give preeminence to unbelievers over the true believers." Now this is just what is happening today, for their servants are Muslims taken from among men of a mature age or from those who are still young. This is one of the greatest scandals to which the guardians of authority must put an end. It is wrong to greet them even with a simple "how-do-you-do"; to serve them, even for wages, at the baths or in what relates to their riding animals; and it is forbidden to accept anything from their hand, for that would be an act of debasement by the faithful. They are forbidden while going through the streets to ape the manners of the Muslims, and still less those of the cities of the religion. They shall only walk single-file, and in narrow lanes they must withdraw even more into the most cramped part of the road.

One may read that which follows in Bukhari and Muslim [religious authorities of the ninth century]: "Jews and Christians shall never begin a greeting; if you encounter one of them on the road, push him into the narrowest and tightest spot." The absence of every mark of consideration toward them is obligatory for us; we ought never to give them the place of honor in an assembly when a Muslim is present. This is in order to humble them and to honor the true believers. They should under no circumstances acquire Muslim slaves, white or black. Therefore they should get rid of the slaves which they now have for the), have no right to own them. If one of their slaves who was formerly an infidel, becomes a Muslim, he shall be removed from them, and his master, willingly or unwillingly, shall be compelled to sell him and to accept the price for him. 
It is no longer permitted them to put themselves, with respect to their houses, on an equal footing with the dwellings of their Muslim neighbors, and still less to build their buildings higher. If they are of the same height, or higher, it is incumbent upon us to pull them down to a size a little less than the houses of the true believers. This conforms to the word of the Prophet: "Islam rules, and nothing shall raise itself above it." This is also in order to hinder them from knowing where our weak spots are and in order to make a distinction between their dwellings and ours.

They are forbidden to build new churches, chapels, or monasteries in any Muslim land. We should destroy everything that is of new construction in every place, such as Cairo, for instance, founded under the Muslim religion, for it is said in a tradition of Umar: "No church shall be built in Islam." They shall no longer be permitted to repair the parts of these [post-Islamic] buildings which are in ruins. However, the old buildings [of pre-Islamic times] which are found in a land whose population had embraced Islam need not be destroyed. They shall not, however, be enlarged by means of repairs or otherwise. In case the tolerated peoples act contrary to these provisions we will be obliged to destroy everything that has been added to the original size of the building.

Some would say that he deliberately misinterprets the Quran. What do you think? How could a sultan inclined toward toleration address the advice provided here?

All three empires blended Islamic and local architectural traditions and built elaborate capital cities, palaces, and mosques. The Taj Mahal in India - a mausoleum for the ruler Shah Jahan's favourite wife - is perhaps the most famous building in the world today. The central square in Isfahan, the Safavid capital, was and is widely hailed as one of the most beautiful public spaces in the world. The seventeenth-century Blue (Sultan Ahmed) Mosque in Istanbul was one of the largest mosques in the world well into the twentieth century and is still celebrated for its internal and external beauty. As always, these structures were not isolated achievements but were instead part of a centuries-long process of architectural innovation.

Support for a variety of arts matched architectural efflorescence: Painting (especially miniatures to adorn texts), carpet making, and a variety of artisanal crafts were encouraged. However, none of these three empires duplicated the scientific and intellectual glory of Abbasid Baghdad. Despite their religious toleration, these empires did not wish to offend Islamic religious authorities suspicious of science. The Ottomans supported mathematics and astronomy in their early days but later declined to do so. Religious authorities pressured the Ottomans to destroy an observatory in 1580 and a printing press in 1742. (Jews within the Ottoman Empire had been printing in Hebrew for centuries, but it was only possible to publish in Turkic or Arabic from 1729; the ban on printing would only fully be lifted in 1784.) 


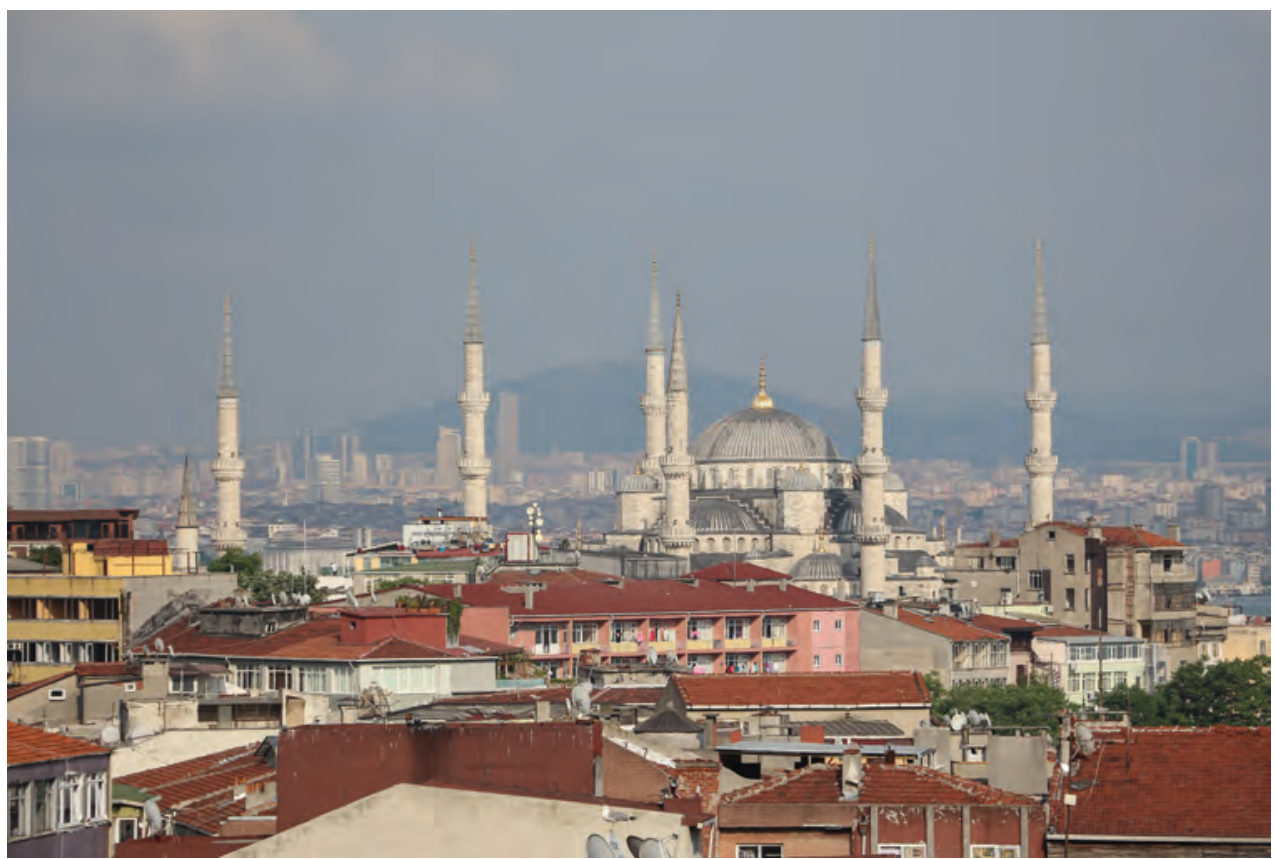

Illustration 20.2

The Blue Mosque

Photograph by Bernard Gagnon, CC BY-SA 3.0

We should note one critical difference among the three: The Mughal Empire was far larger, having 150 million people at its peak. The Ottoman Empire had 24 million in 1800, and the Safavids only 8 million at their greatest extent.

There were also quite different marriage practices among the rulers in the three empires. While the Ottomans were consolidating power through alliances, they had married their daughters to Christian princes. But from the fifteenth century, feeling that they were now masters of the world, Ottoman sultans stopped marrying but instead took slave concubines; their children were still considered legitimate. Since it was illegal to enslave Muslims, the concubines were usually Christian (and these exerted influence on their sons: Ottoman sultans proved less likely to invade Europe when their mothers

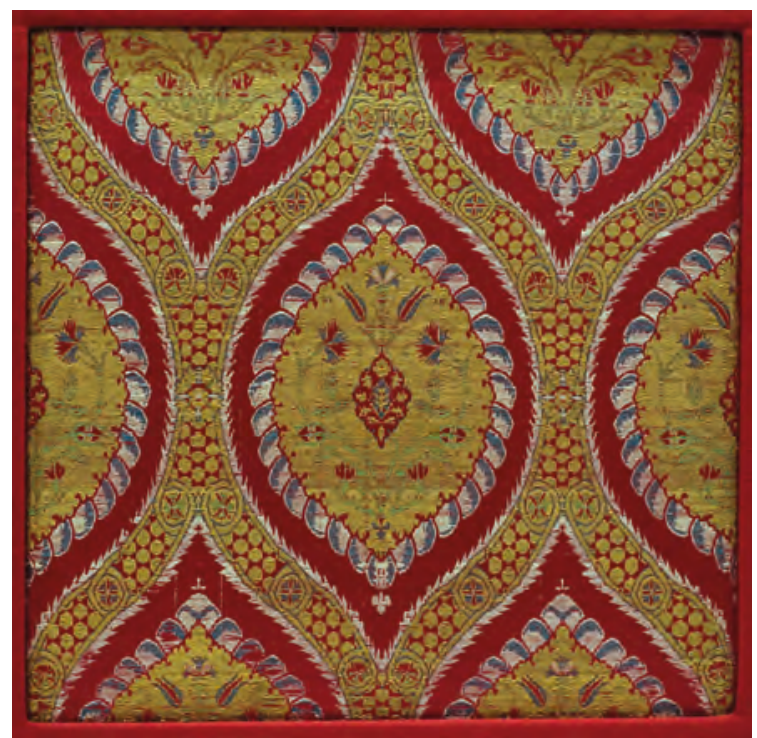

Illustration 20.3

Ottoman textile fragment with ogival pattern, probably Istanbul, 1570-80, silk, metal-wrapped thread, velour, Aga Khan Museum, Toronto, Canada Photograph by Daderot, CCO 1.0 


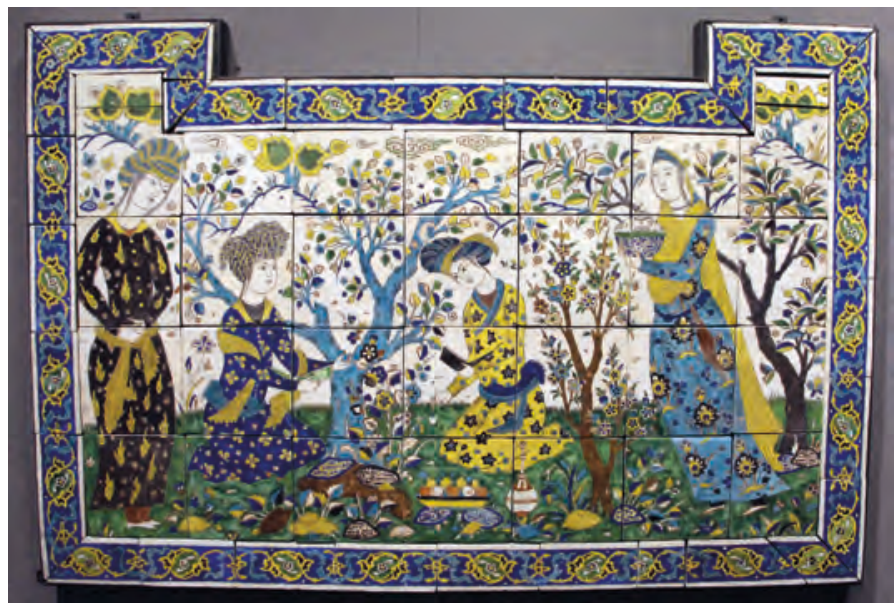

Illustration 20.4

Ceramic tiles, Isfahan, mid-17th century, in the Louvre

Photograph by Sailko, CC BY 3.0

were European - but European states used such practices to incite their citizens to support war against the Ottomans). Daughters were married to ambitious men within the royal household who often became grand vizier (chief minister). The Safavids had at first married daughters to local chieftains, but as these chiefs lost power they instead married daughters to local aristocrats. The Mughals, in contrast, followed an Indian belief that marrying daughters was a sign of inferiority and only rarely did so; these unmarried daughters could be quite influential at court, and many were active in public works or the arts. In all three empires, some women in the harem were accused of exercising malign influence over emperors; it is hard to know how to judge these complaints since the women in question often had little opportunity to defend themselves.

\section{The Ottoman Empire}

The Ottoman Empire lasted for over six centuries, making it one of the most enduring empires in world history. Indeed, it could be considered the most successful dynasty in history. It conquered lands on three different continents during this period, forging one of the more ethnically and religiously diverse empires ever. This would prove problematic as nationalist sentiments strengthened in the Early Modern period.

The Ottomans - named for the dynasty's founder Osman - had stepped into a political vacuum in Anatolia. The Byzantine Empire (chapter 13) was in decline and the Mongols had destroyed the power base of the Seljuk Turks. Historians suspect that Osman's people were refugees from Mongol rule who had arrived in the 1220s. Osman established a small state in northwestern Anatolia and in 1299 declared independence from the Seljuk Sultan. Osman and his heirs 


\section{BOX 20.1}

\section{UNIVERSAL EMPIRE}

Both the Ottomans and Mughals would at times claim to be the rulers of the entire world. The Safavids with their religious fervour could also dream of eventual universal dominion. In this belief, these empires were hardly unusual. The early Islamic caliphs had aspired to world domination. The Mongols at times felt that this was their destiny. Chinese empires could often reasonably claim to rule the "civilized" world; as far as they knew, there were only "barbarian" nomads - who they tried to draw into tributary relations - beyond their borders.

It is in the nature of empires to conquer diverse peoples. There is, therefore, no obvious limit to imperial rule. We know with hindsight that no one empire would ever achieve global domination, but the idea that they could only extend their power so far might be lost on emperors. Imperial vanity might then encourage the pursuit of tributary relations with groups that they could not directly control.

Emperors could generally develop some religious justification for aspiring to global domination: God or the heavens wanted universal rule. The idea of the Mandate of Heaven was regularly invoked in China in support of universal empire. This sort of justification was particularly easy for Islamic rulers as Islamic texts indeed urged one united Islamic community.

A claim to universalism could have adverse effects on international relations, as when both Rome and Persia thought that the world was theirs to rule. Chinese universalism would later hinder ties with other "civilized" states. The aspiration to universal rule also often encouraged emperors to conquer more than they could rule. The brief but extensive conquests of Alexander the Great are perhaps the most potent example here.

In practice, though, most empires after an initial period of conquest necessarily became satisfied with less-than-universal rule. They might then focus their visions of grandeur internally, erecting the most magnificent public monuments in the world. All three of our empires would do so. Indeed, since all three objected to the universal pretensions of the others, they consciously sought to outshine them architecturally.

formed alliances with various local princes as they rose to power. The Ottoman cavalry was often paid by gaining rights to collect taxes in conquered lands (a practice borrowed from the Byzantines); land grants to soldiers became common over time, though the land reverted to the sultan when the soldier died. As with other victorious conquerors, the Ottomans attracted new followers with each military success. Yet the Ottomans relied in early days on strategic marriages as much as conquest, even to Serbian Christian princesses.

The gradual victory of the Ottomans over the Byzantine Empire may seem a religious conflict. However, the Ottomans allied with many Christian leaders as they rose to power. As in all empires, not all subject peoples loved the Byzantines; Christian subjects often proved willing to ally with the Ottomans against them. The Ottomans cleverly took sides in Byzantine battles over succession. Even in the final assault on Constantinople in 1453, there were vast numbers of 
Christian soldiers - Greeks, Serbians, Hungarians - in the Ottoman armies, including European cannon and gunners. The conquest of Constantinople long the largest Christian city in the world - was a shock to both Catholic and Orthodox Christians but reflected the fact that these had not collaborated in protecting it.

The Ottomans laid siege to Constantinople for months, battering its massive walls with cannon and using carts to move Ottoman ships past the great chain that guarded the harbour. Upon taking the city, the Ottoman sultans proclaimed themselves the inheritors of Rome and added Caesar to their title. They could justify this claim not just through conquest but also with reference to the many Byzantine princesses married by previous sultans. The Ottomans would briefly attack Italy and threaten the pope in the next years. The appropriation of Roman heritage and title may have been particularly important in establishing some legitimacy in the eyes of non-Muslim subject peoples over the following centuries.

Though the victory in 1453 over the Byzantines in Constantinople - which they renamed Istanbul - represents an important transition between empires of quite different form, it was not the last victory over Byzantines. A powerful family from Constantinople, banished during the empire's continual power struggles, had established in the thirteenth century an independent state (with the aid of Georgians) in Trebizond, an important port in the southeastern Black Sea. The Ottomans would take Trebizond in 1461. The Ottomans would then force the Crimean khanate - a polity descended from the Mongol Golden Horde - to accept Ottoman suzerainty in 1478. With these victories, the Ottomans gained control of the entire Black Sea (albeit ruling through locals on the northern, eastern, and western shores), a feat never managed by Romans or Byzantines. They would erase piracy in that sea for the first time, and force all ships leaving that sea to pay taxes in Istanbul. This dominance would become a target for the growing power of the Russian state (chapter 19; Poland was also at times a threat). The Ottomans lost Crimea to the Russians in 1783.

The Ottomans had encircled Constantinople in previous decades by expanding from Anatolia into Europe. They had entered Europe in the 1340s encouraged by the Byzantines themselves, who were hoping to regain lands there - and won a significant victory over the Serbs in 1389 (a defeat still emphasized in Serbian history). From the 1370s, the Ottomans formed one of the most unusual and feared military forces in history: the Janissaries. The Ottomans had long followed Seljuk and Abbasid precedents in employing slave soldiers. These were usually Slavs from north of the Black Sea. As they consolidated power in Christian Europe, Christian families were required to surrender sons to the Ottomans: These were converted to Islam and trained as soldiers or bureaucrats. Separated from their families, the Janissaries had a fierce loyalty to the sultan - though they would over the centuries become powerful in their own right. The Janissaries would be the Empire's elite military force for centuries. This small standing army long predates the standing armies of European rivals. Nevertheless, conflict with the Austrian Empire would cause 
the size of the Janissary force to rise through time: There were only a couple of thousand by $1389,5,000$ by the mid fifteenth century, 10,000 or so through the sixteenth century, but 37,600 in 1609 and 54,000 in 1660 .

Raising the Janissary army was itself a bureaucratic challenge. Every four years in the sixteenth century, bureaucrats would look for teenage boys in the Balkans. Local priests were required to inform bureaucrats of all boys between 12 and 20. The bureaucrats would identify boys that seemed well suited to either the army or senior bureaucracy. The minority chosen for the bureaucracy would then receive years of training by palace eunuchs in calligraphy, languages, and basic mathematics. The others would receive extensive military training. The practice of recruiting Janissaries was, of course, a source of tension within the Christian populations of the Empire. Yet some more impoverished families may have appreciated that their sons would gain greater wealth and power as Janissaries than could their siblings left at home. The careful selection and training of bureaucrats did introduce an important element of meritocracy into the Ottoman bureaucracy - though selection occurred only among a segment of the overall population. (Yet whereas the Chinese examination system can be considered a forerunner of civil service examinations in many contemporary states, the practice of training a slave elite to govern would necessarily disappear with slavery itself.)

The Janissaries were at first forbidden to marry. As their numbers grew in the sixteenth century, they were allowed to have families, and it became common for their sons also to become Janissaries. This practice proved dangerous: The Janissaries might then choose familial loyalty over loyalty to the sultan. The Janissaries did come to participate in Ottoman succession struggles and even assassinated multiple sultans in the sixteenth century.

As noted above, the Ottomans were a gunpowder empire. Indeed, they had adopted both cannons and guns from the fourteenth century, when both were still mostly unknown in Europe. The Ottomans would be self-sufficient in gun and cannon manufacture well into the eighteenth century, after which point the European technological advantage widened. Musket-armed Janissaries and 150 cannons likely decided an Ottoman victory against the Safavids in 1514. Within three years, the Ottomans used the same weapons to take Egypt from the Mamluks.

Sultan Suleiman focused efforts in Europe, retaking Hungary and threatening Vienna in

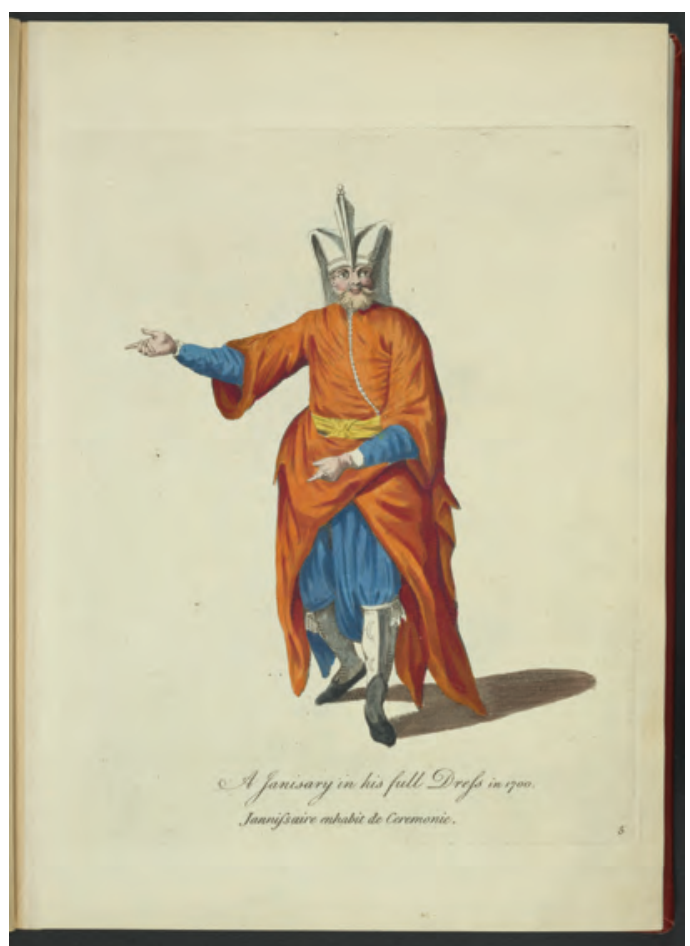

Illustration 20.5

Janissary in full dress, c. 1700 
1529. He allied with France and Protestant princes against Austria. He might have taken Vienna if heavy rains had not slowed his advance and forced him to leave heavy artillery behind. Although focused on Europe, he also won again against Safavid Persia, establishing a border that would largely last until World War I. Suleiman also codified secular laws and harmonized these with Islamic sharia law. Indeed he is often called Suleiman the lawgiver, and his reign from 1520-66 is often thought to have represented the golden age of Ottoman rule. The Ottomans had admired the Byzantine strategy of linking political and religious authority; in doing so, Suleiman clarified an earlier Ottoman argument that the sultan needs only consult religious scholars on matters clearly addressed in sharia law.

We saw in chapter 12 that the Abbasids had absorbed religious authorities into the state bureaucracy. Islam became a more conservative faith thereafter, with debates among religious leaders replaced by state authority pursuing orthodoxy. Many scholars feel that the Islamic world became less successful after that decision - militarily, economically, and scientifically. The Ottomans were not alone in following the Abbasid precedent in pursuing orthodoxy. The Safavids and Moghuls would each also encourage religious orthodoxy much of the time. The Ottomans thus limited scientific discourse and also the adoption of institutions that would allow Muslim merchants to prosper (see below). We should recall, though, that other empires such as the Byzantine also pursued religious orthodoxy.

The Ottomans would cease expansion in southeastern Europe after about 1606 (though they remained active in the Ukraine), with the singular exception of an attack in 1683 that laid siege to Vienna itself and was only repulsed by an alliance of Catholic rulers led by the

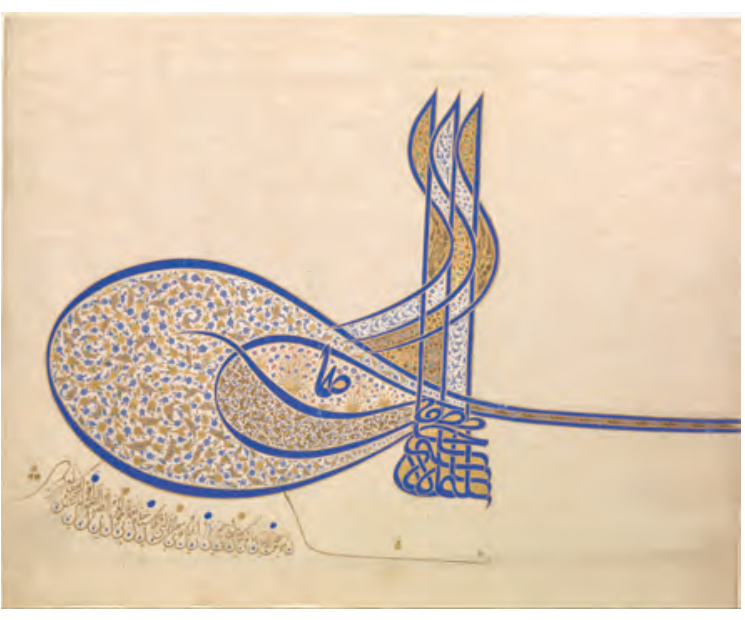

Illustration 20.6

Tughra of Suleiman the Magnificent's signature. This calligraphic monogram served like letterhead and signified the sultan's power, Metropolitan Museum of Art king of Poland. After that, the Ottomans lost more often than they won in Europe. Indeed, the Ottomans faced challenges in expanding in any direction by the late sixteenth century, facing the Austrian Empire to the northwest, the Russians to the north, and the Safavids to the east.

The Ottomans would slowly develop naval capability in the eastern Mediterranean and, to a lesser extent, the Indian Ocean. This naval force was important especially after the conquest of Egypt, for Istanbul (the former Constantinople) came to rely on grain imports from Egypt - as it had in the early days of the Byzantine Empire centuries before (chapter 13). The Ottomans would lose a massive naval battle at Lepanto in 1571 to an alliance of Christian forces. 


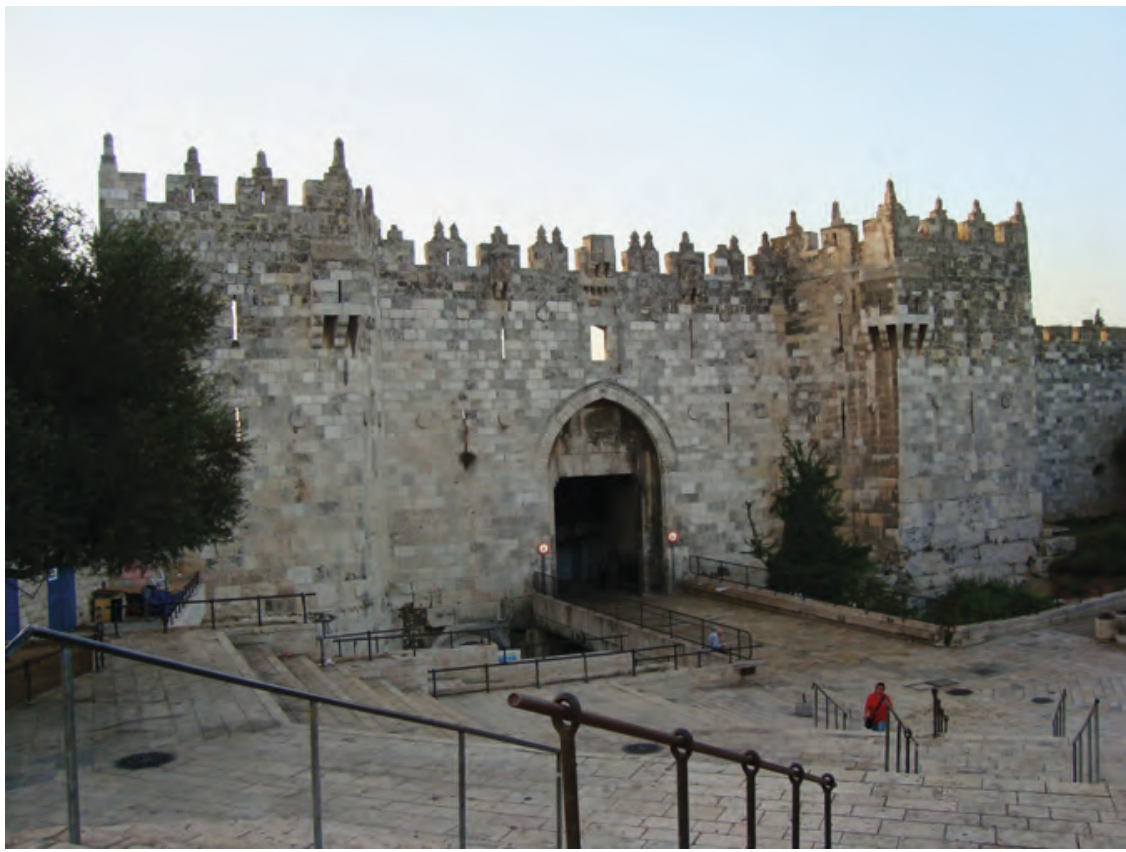

Illustration 20.7

Damascus Gate, Jerusalem, built by Suleiman the Magnificent in 1537 on top of an old Roman gate

They lost 200 ships and suffered 30,000 casualties. This battle is significant in military history for establishing that ships armed with cannons could destroy a navy of traditional Mediterranean galleys. It also signalled that Europeans, at least at sea, were surpassing the early Ottoman advantage in gunpowder technology. But the Ottomans rebuilt their navy - 150 ships within a few months and soon reasserted control in the eastern Mediterranean. The Ottomans continued to rely on galleys - perhaps in part because they had a steady supply of slaves, but also because they faced less naval warfare - while Europeans forged ahead with sailing ship technology.

The Ottoman conquest of Egypt in 1517 solidified their control of trade routes between Europe and Asia. As noted in chapter 19, the rising power of the Ottomans in the Middle East motivated in part the explorations of the Spanish and Portuguese. As the Portuguese and other Europeans began to arrive in the Indian Ocean, the Ottomans tried to match their naval capability. However, as at Lepanto, the Ottomans struggled to match European gunboats in the Indian Ocean.

The Ottoman sultans would proclaim themselves caliphs from about 1520 (until 1924). Recall from chapter 12 that the caliph presumes to be the legitimate ruler of Islam. Nobody had claimed the title of caliph since the fall of the Abbasids (chapter 12). This proclamation, plus their control of the holy cities of Mecca and Medina (also gained in 1517), did encourage greater efforts 


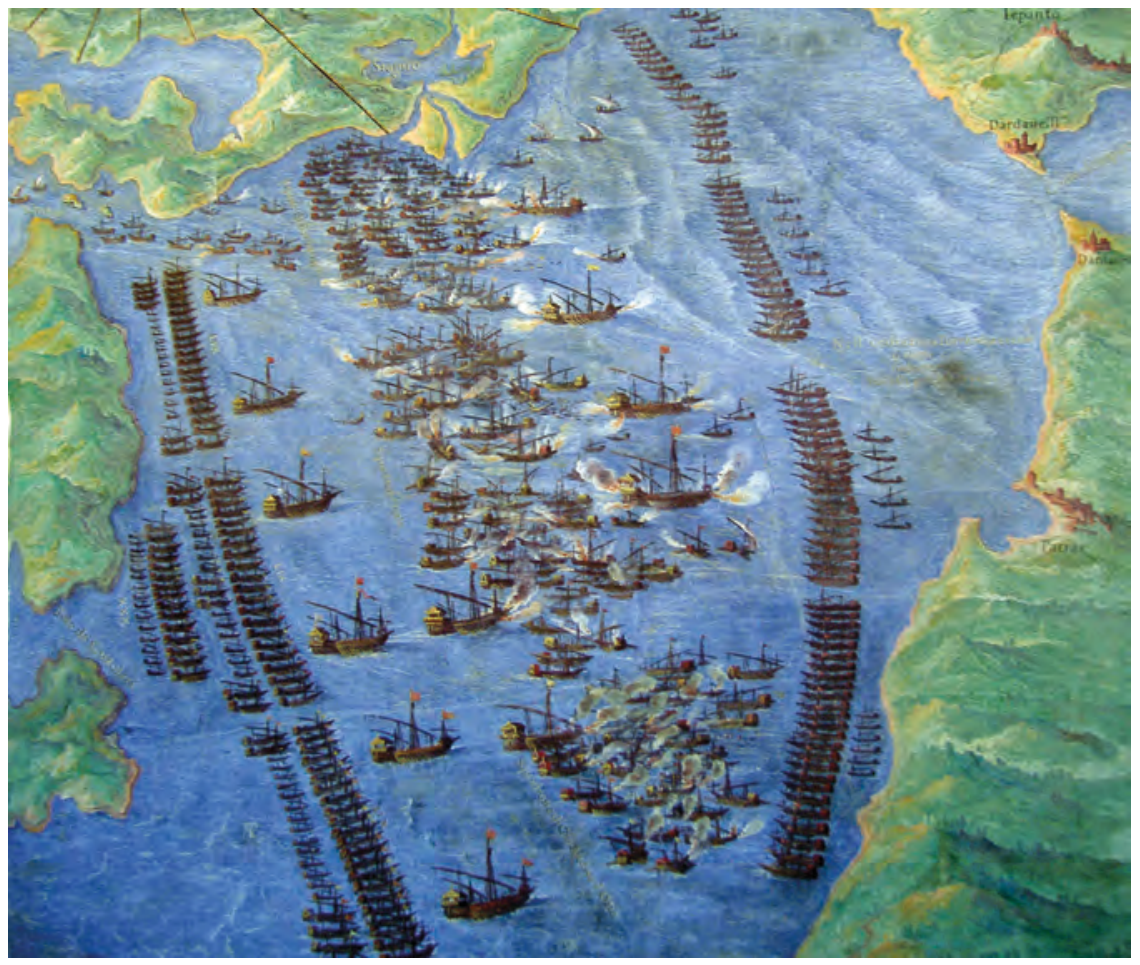

Illustration 20.8

Fresco of the Battle of Lepanto, Vatican Museum, Vatican City

at Islamization, and limits on Shia and Sufi practices, but a broad tolerance of Christians and Jews survived. Certainly, Ottoman practice compares well with Spanish treatment of Jews and Muslims after 1492.

\section{Ottoman administration}

In early centuries, the sultan convened a governing council comprised of elite, military, and religious leaders, plus key bureaucrats. Over time, the grand vizier (the head of the bureaucracy) became more important than other elites. Though merit played a role in selection for the bureaucracy, and especially promotion within the bureaucracy, high offices often went to those with connections. As the viziers squeezed out the power of other elites, they also decreased the influence of wives/concubines or mothers in the harem.

The Ottoman bureaucracy was minimal, comprising only about 1,500 people into the nineteenth century. They did organize land surveys every ten to thirty years to establish the tax that each landowner should pay but mostly left tax collection itself to local commanders and soldiers. Soldiers were often "paid" by being granted land to tax (a practice borrowed from the Byzantines). The Orthodox Church also raised taxes for the Empire. In Hungary and eastern Anatolia especially, the Ottomans relied on local elites to raise taxes. In distant 


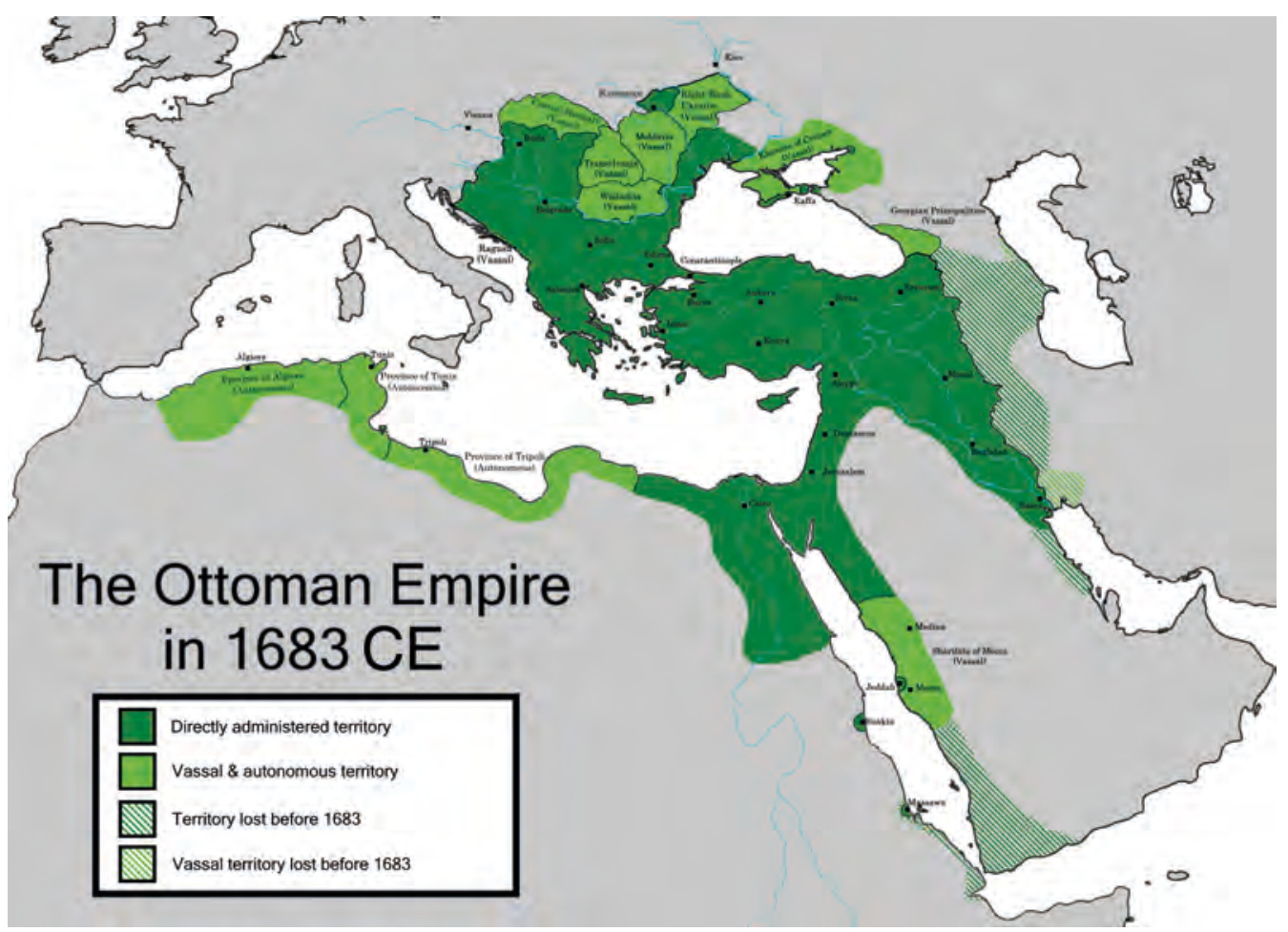

Map 20.1

The Ottoman Empire in 1683

Map by Chamboz, CC BY-SA 4.0

provinces, they also used tax farmers - individuals, often merchants, who paid a fixed sum for the right to collect and keep taxes in a particular region. They also had tributary states beyond their borders that paid to avoid Ottoman aggression. Nevertheless, Machiavelli - the Renaissance writer on politics and history - remarked that the Ottoman state was far more centralized than that of France: The Ottomans rotated provincial governors, for example, which the French could not do at the time. Machiavelli felt that the Ottoman state was richer and more powerful as a result of centralization. He and many other European thinkers urged European kings to copy the Ottoman example of centralized power (Such exhortations may have encouraged the centralization we will indeed observe in European states in chapter 21.) As occurred in myriad empires through history, though, local elites gained more power in the Ottoman provinces, especially in the seventeenth and eighteenth centuries. By the nineteenth century, local warlords had seized governmental control in many parts of the empire - but they did not rebel, in part to maintain common cause against Russia.

The Ottoman bureaucracy proved strong enough to survive the attacks of Timur (chapter 17). Timur had captured the sultan Bayezid. However, Timur had then turned his attention eastward to attack China. A decade-long succession 
struggle convulsed the Ottomans. After Timur's death, the Ottomans were able to reclaim the territory they had lost.

\section{Reform initiatives}

In the eighteenth century, the Ottomans lost ground to both Russia and Austria. By the 1820s, Serbia, Greece, and Egypt were effectively independent. The Ottomans imported Western military advisers to close the gap in military technology. Attempts by the sultan Selim to institute reforms in the early nineteenth century met resistance from Janissaries, religious leaders, and the masses. His efforts did at least open a debate about change. The Janissaries were abolished in 1826 after losing wars to both Russians and Greeks. The "Tanzimat" or reordering of 1839-76 sought to catch up to European polities both militarily and financially. New government departments were created in areas such as education and health that had previously been entrusted to religious authorities. Commercial law was revised along European lines - in part so that the Empire would no longer need to grant foreign merchants the right to settle their own disputes according to their own laws. Public education expanded. Religious freedom was proclaimed - though there were also efforts to suppress Christian minorities. A professional army backed by military and engineering schools was created. There were also some brief experiments with elected councils, but in general the reforms served to strengthen autocracy rather than borrow European ideas of democracy. The Ottomans had 35,000 bureaucrats by 1908, and tighter control of their territory due to railway and telegraph construction - though they still had difficulty controlling local elites, especially on their borders. Bureaucrats were well educated.

Napoleon (chapter 25) had attacked Egypt in 1798; this attack weakened Ottoman rule, allowing an Albanian general called in to fight the French, Mehmet Ali, to seize power. The Ottomans named him viceroy in 1807, but he was effectively independent. He increased cotton production with irrigation and then expanded textile manufacture, but under state control. He also established a printing press that translated European works. He introduced secular schools to train bureaucrats in Western ways. He modernized the army and captured Sudan and Mecca. He tried to reach an agreement with mild Islamists. He might have attacked Istanbul itself, but Britain and France discouraged this in fear that Russia and Austria would benefit from Ottoman collapse. His son engaged with the French to build the Suez Canal. Though Mehmet Ali showed that economic development and military prowess could be achieved in the region, debts would force his heirs (like the Ottomans; see below) to cede powers to Europeans; Britain would become the power behind the throne into the 1950s.

The "Young Turks" was the name given to an organization founded by exiles in Europe who were inspired by Enlightenment ideas to introduce public sovereignty and reduce the power of the sultan. They called for universal suffrage, secularism, women's rights, freedom of religion, and improved and expanded 
public education. An army coup in 1908 effectively reduced the sultans to puppet status and established constitutional government. These ideas would continue to inspire the new Turkish government that emerged after the Ottoman Empire collapsed in World War I (chapter 21).

\section{Ottoman decline}

We can attribute the Ottoman decline to a mix of typical and novel imperial challenges. As noted above, local elites gained power through time. Local revolts and mutinies then plagued the Empire. The Ottoman bureaucracy was not up to the task of providing famine relief (as many other empires could), and thus suffered political tensions during famines. The Empire also faced financial difficulties in financing its new army and bureaucracy. Financial challenges can, as with other empires, in part be attributed to both the end of conquest and the rising power of local elites. There were also decreased trade revenues due to European arrival in the Indian Ocean. Some of the government debt was incurred in financing railway construction in an attempt to modernize the economy and reinvigorate trade. One element of novelty was that the Ottomans borrowed heavily abroad since European countries were wealthier and had more developed capital markets. European creditors would gain influence on Ottoman decisions as a result, seeking to ensure debt repayment by controlling specific streams of government revenue such as tariffs. The other significant novelty was increased nationalist aspirations: Armenians, Kurds, Slavs, Greeks, Egyptians, and Arabs all began to clamour for independence. As we will see in chapter 21, the emerging nation states in Western Europe found it both congenial and convenient to encourage these various movements for national liberation.

We will discuss in chapter 22 the different institutional structures that governed commerce in different parts of the world. The Ottomans had long allowed merchants from different lands to operate according to their own laws. Since European laws supported corporations and lending for interest more than Islamic laws, Christian and Jewish merchants gained an institutional advantage within the Empire. Muslim merchants chafed at the growing wealth of Christian and Jewish communities. Even when the Ottomans belatedly reformed their economic institutions, the inherited wealth of Christian and Jewish communities gave them a continued advantage. These wealth disparities aggravated communal tensions.

As often in history, the weakened Ottomans fell to attack from outside. The growing European advantage in military technology then proved critical. Nevertheless, we must also appreciate the impact of the Great Divergence (chapter 22). Over the nineteenth century, economic growth proceeded at a much faster rate in Europe than in Asia. The result was that by the end of the century, per capita incomes were several times higher especially in Western European countries than they were in the Ottoman Empire. The Ottomans would have struggled to match the military expenditures of European countries 
even if not riven by internal conflicts. They were simply unable to buy the latest weapons in the same quantities as their foes.

Angered by interference by Britain and France, especially in supporting the independence of Slavs and Greeks in Europe, the Ottomans decided to ally with Germany and Austria against them during World War I. Though the Ottomans had long battled the Austrians in Europe, they were by the twentieth century facing even greater conflicts with Russia, losing land to Russia in both Central Asia and the Caucasus. They hoped that an alliance with Germany and Austria would allow them to push back Russian advances. In the end, though, they ended up on the losing side of World War I, and their empire - like that of the Austrians - was dismembered (chapter 28).

Historians often speak of a series of weak sultans (notably Selim the Sot 156674 and Ibrahim the Crazy 1640-8). We must be careful here: The Ottomans may well have declined even if cleverly ruled. A sultan may look bad just because he faces insurmountable difficulties. However, there is an institutional innovation that may serve to explain weak leadership among the Ottomans. As noted above, the Ottomans suffered from frequent battles over succession. In the early centuries of Ottoman rule, the sultan's sons often served as provincial governors. This gave them leadership experience but also allowed them to develop a local power base. Sons did not always wait for their father to die before attempting to seize control. The Ottomans, unlike the Mongols, urged the inheritance of the entire empire rather than its division: Sultans indeed urged fratricide among their sons to ensure one clear ruler. Mehmet III in 1595 killed nineteen brothers and several expectant mothers. He then decided to change Ottoman succession practices: Sons were kept in the harem and deliberately prevented from meeting those in positions of influence (except their father). When the sultan died, a chosen son would replace him and be transferred directly from the harem to the throne.

Students may be surprised by a practice that seems purposely designed to ensure ineffectual leadership of the empire. Yet this is an institutional mutation designed to address one of the ruler's challenges: to keep potential heirs to the throne from fomenting civil war, often before the existing sultan is dead. Why would this mutation be designed and selected? The sultan benefits from not having to worry about being attacked by his sons. And the sultan's advisers not only avoid the dangers inherent in civil war - where bureaucrats can lose their lives by choosing the wrong side - but are assured of a pliant ruler who will be dependent on advisers for knowledge of the outside world for at least the first years of their rule. The sultan and advisers are the selection environment for this mutation, at least in the short term. And they each transmit this practice to their descendants - along with stories of the internal conflicts over succession that had plagued the earlier empire. In the longer term, though, another sort of selection environment comes into play. The Ottoman Empire falls behind other empires both militarily and economically. Though there are many reasons for this, a series of incompetent Ottoman rulers likely contributed. In particular, this institutional mutation likely accelerated the dissipation of the power of 
the sultan, since sultans were at a severe informational disadvantage relative to all other elites. Sultans might have obtained book learning in the harem but would have limited experience of human duplicity. To be sure there was plenty of intrigue within the harem, but it was still likely not quite the preparation that being a provincial governor provided. Sultans would be poorly prepared for one of the ruler's other key challenges: getting bureaucrats to behave in the interests of the state. The institutional mutation whereby generations of sultans were thrown unprepared onto the throne may thus have placed the Empire at a competitive disadvantage with other empires, and led (at least in part) to competitive selection for the eventual eclipse of the Ottoman Empire itself. The losses to Russia and Austria (where succession occurred through primogeniture) may reflect in part poor leadership. Perhaps better leaders could have encouraged economic growth and technological advance. Certainly, the final fateful decision to join Germany and Austria in World War I appears with hindsight to have been ill-advised - but Kaiser Wilhelm of Germany thought that war was a good idea too, despite a great deal of worldly experience (chapter 28). If later sultans (or their advisers) became conscious of the challenges they faced by their secluded upbringing, they would hesitate to change the practice out of both fear for their own life and familiarity with the history of earlier succession battles. As a result, this institutional mutation could be transmitted through

\section{BOX 20.2}

\section{SECLUDING RULERS}

Though the Ottoman practice of raising sons in isolation is extreme, we should appreciate that ruling families often had limited contact with the populations they ruled. The exemplar here may be the Forbidden City in Beijing from 1420, a mass of buildings designed for the ruling family and their servants, and decidedly off-limits to the average citizen. The ruling family only rarely left this enclosure, and then generally to visit another palace. Such practices in part reflected concerns over security. A more important motive was to maintain public awe of rulers. It is much harder to think that one's ruler is special or perhaps even semi-divine if one regularly sees them in the market or possibly sneaking away from brothels. Indeed observers of Greek city states often commented that their rulers could not inspire awe because their daily habits were too well known.

Secluded rulers had to rely entirely on the knowledge of others. That is, they governed a state of which they had no personal experience. In the twenty-first century, leaders are often criticized if they seem to avoid contact with voters or journalists, yet even the most secretive of contemporary leaders likely has far more contact with the people they lead than did the leaders of many of the world's empires. The temptation of emperors' advisers to give misleading advice was often great. This may be one reason that empires decline over time: The founder of a dynasty necessarily has knowledge of the world, but each succeeding generation may have less direct understanding of the state and its people. 


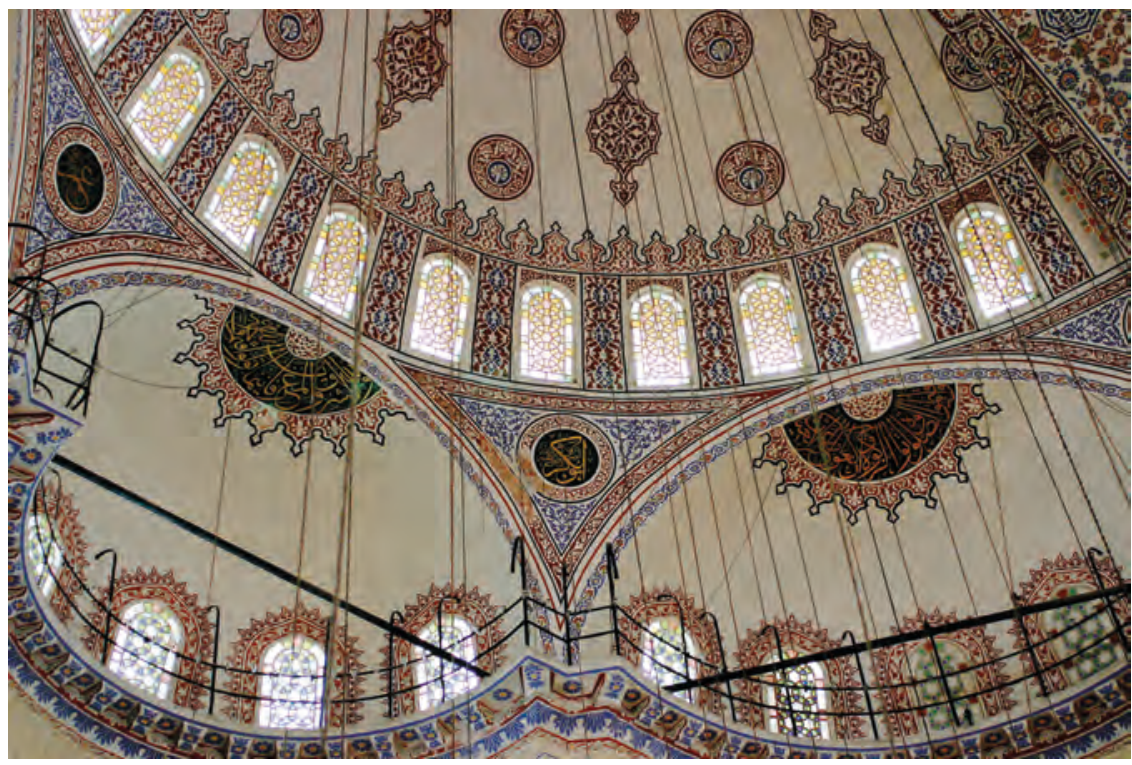

Illustration 20.9

The Blue Mosque interior

time even if its costs were appreciated. Evolutionary analysis allows us to place a seemingly bizarre practice in context, recognizing it as a problematic response to a problem (of succession) faced by all states. Note that evolutionary analysis does not demand or encourage a belief in either historical determinism or inevitable progress; it merely guides us to ask useful questions about historical transformations.

\section{Ottoman culture}

Europeans much admired both the Topkapi Palace in Istanbul and many mosques built by the Ottomans. These buildings indeed matched any in Europe both technically and aesthetically. It had taken Ottoman builders a while to figure out how to create a dome as large as the Byzantine Hagia Sofia (chapter 13), but the sixteenth-century Blue Mosque would surpass it in size. The Ottomans also fostered a famous ceramic tile industry that built on Chinese and Central Asian ideas; it was world famous by the sixteenth century. Textile production was also notable from the fifteenth through seventeenth centuries. Carpets were the most important output, but there was also silk and velvet production.

By 1600, Istanbul was the largest city in Europe, with 700,000 people. Its population had collapsed during the final decline of the Byzantine Empire and had only been 80,000 in 1478 . It served both as the administrative centre of the Empire and as a significant trading centre sitting astride the water link between the Mediterranean and Black Seas and the land link between Europe and Asia. 


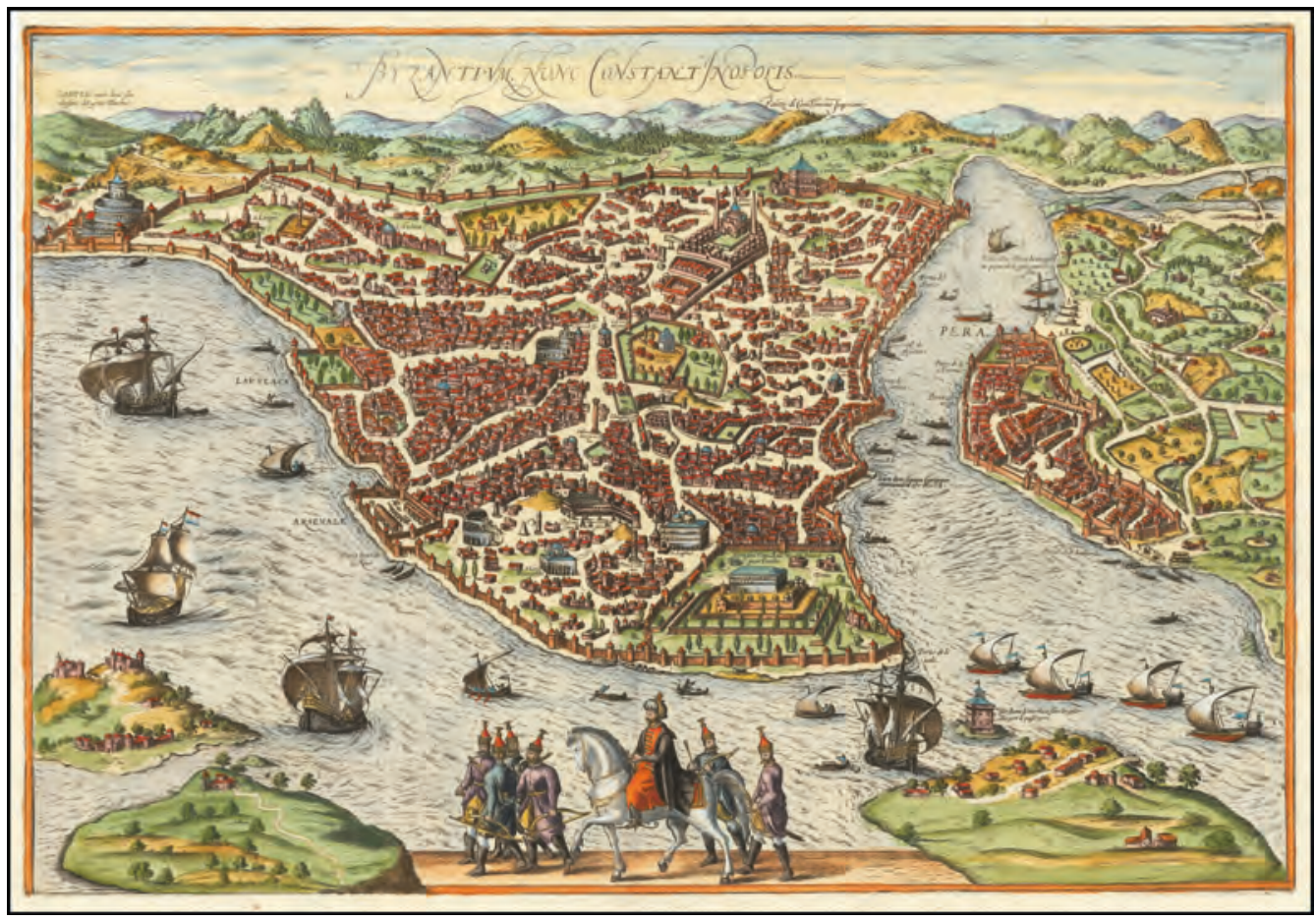

Illustration 20.10

Map of Istanbul, 1660

Istanbul was a very cosmopolitan city at the time with about 40 per cent of its population being Christian or Jewish in the late fifteenth and sixteenth centuries. Many Jews had moved to Istanbul when expelled from Spain in 1492. Istanbul was famed not just for its architecture but for its many gardens and markets (bazaars). It would lose some of the most lucrative trade to Europeans sailing around Africa but overall trading volumes still increased.

There seems to have been a great deal of cross-religious contact in Istanbul and other cosmopolitan cities such as Aleppo. There were cross-religious friendships, and people of one faith often gave gifts on the holy days of another. Groups borrowed the cuisines and music of others. Multilingualism was common. There were even shared celebrations of particular saints.

\section{The Safavid Empire}

Persia had been devastated by the Mongols and then again by Timur. It is possible that the majority of the population died or fled during these incursions. Much agricultural land became pasture under nomadic invaders (chapter 17). Turks came to comprise about a quarter of the population as they do today. Yet Turkic conquerors all tended to adopt Persian bureaucratic practices over time. 
Shah Ismail founded the Safavid Dynasty in 1501. He was the hereditary leader of a Sufi Shia religious order in what is now Azerbaijan. There is a debate about his ancestry but some consensus that this was mixed: Turkic, Kurdish, Greek, Azerbaijani, Georgian, and Persian are all possibilities. His ancestors had collaborated with the Mongols but later turned against them as the ancestors adopted Shi'ism. Shah Ismail claimed descent from Muhammad, in line with Shia belief that only descendants of Muhammad should rule. Most historians view this claim with scepticism, yet Shah Ismail inspired great devotion among his followers. Ismail allowed his nomadic Turkish followers to believe that he was the twelfth imam revered in Shi'ism, who had been forced into hiding in 874 but would one day return. He began by fighting against efforts to repress his religion. As with the earliest Islamic conquests, he could call upon religious devotion but also provide his followers with booty as conquest followed conquest. He could promise those that he conquered the peace associated with religious commitment in place of the political chaos that had characterized the region for some time. When his forces took Tabriz in 1501, he claimed a divine right to rule (but justly), and invoked the memory of ancient Persian rulers. He thus melded Persian traditions of all-powerful rulers to Shia visions of a just hereditary kingship. From his capital in Tabriz, he then advanced eastward, taking advantage of conflicts between Uzbeks and the descendants of Timur in the region. He proclaimed himself ruler of the world.

Ismail nevertheless developed a decentralized form of rule. Turkmens from his military coalition dominated governance and even raised Safavid princes. Ethnic Persians generally held only lower posts in Ismail's bureaucracy.

Nevertheless, the Safavids came to adopt the Persian language. The Shia faith would spread in part as a way in which Persians, dominated politically for centuries by Arabs and others, could distinguish themselves. Shi'ism had been common but not dominant in Persia before the Safavids. As noted above, its ideas of governance fit well with Persian traditions. The Safavids did occasionally force conversions, but the transformation of Persia into a predominantly Shia society occurred gradually over centuries as the Safavids supported Shi'ism financially and favoured the Shia in bureaucratic appointments.

The Shia identity of Persia would invite conflict with the Sunnis of the Ottoman Empire - though peace generally was maintained with the Sunni Mughals to the east. As noted above, all three empires generally pursued religious toleration. However, Shia and Sunni could often be more hostile to each other for they viewed the other as a direct insult to their faith - than to Christians and Jews. Hostility between Ottomans and Safavids encouraged internal religious hostility: Shias in the Ottoman Empire and Sunnis in the Safavid Empire were viewed with suspicion. Just as the Ottomans encouraged Sunni orthodoxy in their lands, the Safavids would encourage Shia orthodoxy in theirs. There were conversions and migrations over time as a result of these competing orthodoxies. The border that would be determined by wars between Ottoman and Safavid Empires separates Sunni and Shia populations to this day. Ethnic Persians and neighbouring Arab communities controlled by the Safavids are Shia. 


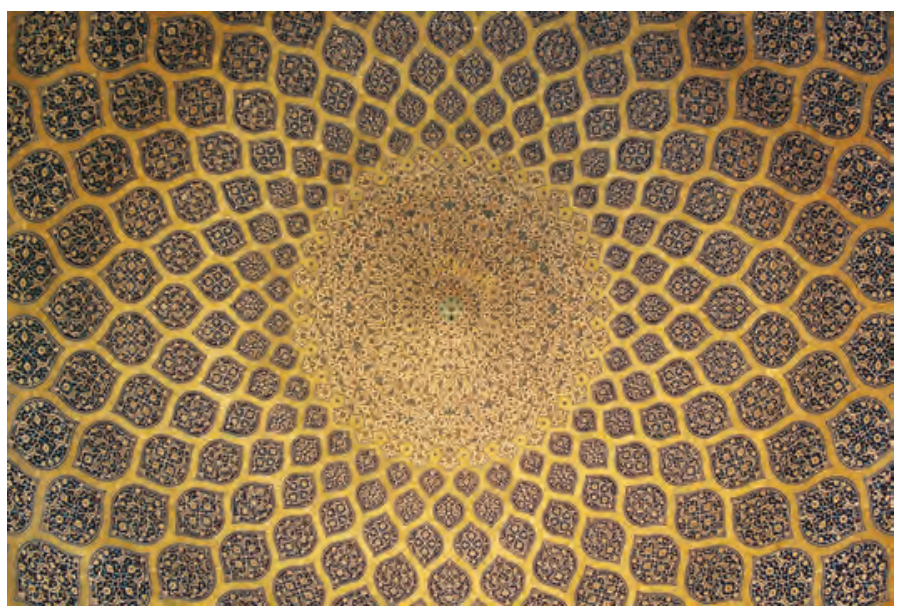

Illustration 20.11

Ceiling of the Safavid Sheik Lotf Allah Mosque, Isfahan

Photograph by Phillip Maiwald, CC BY-SA 3.0

Ismail himself was defeated by the Ottomans in 1514 (see above); they even occupied his capital of Tabriz, inspiring later Safavids to move the capital eastward. The Safavids thus lost eastern Anatolia forever. Upon Ismail's death in 1524 , there was a protracted civil war over the regency of his young sons that only ended in 1533. His son Tahmasp would transfer military and bureaucratic power to the Persian nobility. Tahmasp also inaugurated a slave army of captured and converted Georgians and Armenians and Circassians - an army that bears considerable similarity to the slave armies of the Ottomans. With the Ottomans still threatening Tabriz, he built a new capital.

Shah Abbas (1588-1629) reformed the Safavid army around musketeers with some English advice. His muskets and cannon could match the Ottomans. He expanded the Empire into both Central Asia and Mesopotamia (extending Shi'ism to Arab populations in what is now southern Iraq). He was also able (again with English support) to remove the Portuguese from forts in the Persian Gulf. He managed to take provincial control away from governors by utilizing superior gunpowder technology. He then entrusted more authority to a slave elite. He also employed Georgians, Circassians, and Armenians in key positions; these too would become powerful over time. To ensure his authority, he killed all of his male relatives and confined other family members to the palace. Like the Ottomans after Mehmet III (above), he raised his sons in the harem, where they were prevented from interacting with leaders of state (and, yes, later Safavid rulers are generally considered to have been ineffectual). Just as the Ottomans allied with some European states against others, the Safavids under Shah Abbas forged alliances with Europeans against the Ottomans.

After Shah Abbas, local elites and bureaucrats gained power. The Safavids found it challenging to fund the armies they needed for both internal and external purposes (including battles against an expanding Russia to the 


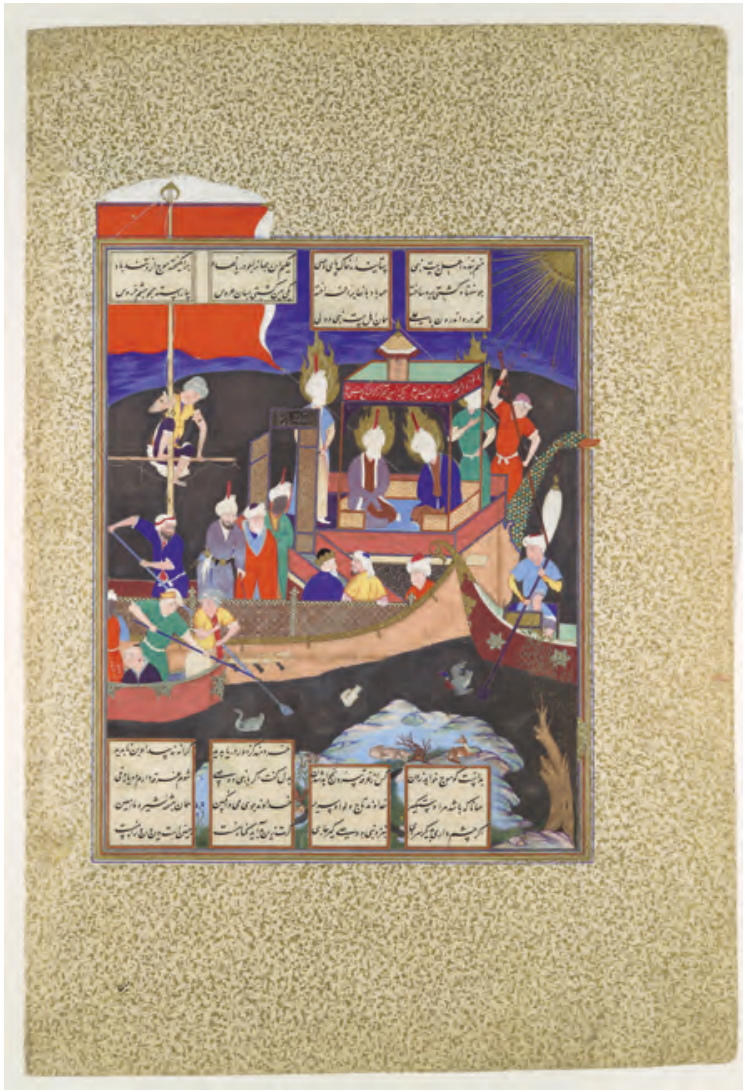

Illustration 20.12

Shahnama (The Book of Kings) of Shah Tahmasp, Abu'l Qasim Firdausi; Mirza Ali 1530s, Metropolitan Museum of Art north). Religious leaders came to assert more authority. Christians, Jews, and Zoroastrians were pressured to convert. Many chose instead to leave the country. This caused trade flows to decline, further weakening Safavid finances. In 1722, Afghan tribesmen conquered Isfahan and massacred the locals.

\section{Safavid culture}

Tabriz had long been a centre of painting and textiles. During Mongol times, artisans had borrowed styles and techniques from China. These crafts would be encouraged by the Safavids under Ismail. Ismail's son was less enthused about the arts, and many artists from Tabriz took Persian motifs to the Ottoman and Mughal courts, where they became influential. The early Safavids were too focused on war to build many monuments. It was only after Shah Abbas moved the capital to Isfahan that the Safavids gained fame for monumental architecture. The new city was carefully planned and would be famed for its many gardens. In the centre, Shah Abbas built massive monuments two mosques, a bazaar, and a palace around the biggest public square in the world, complete with extensive lawns and elaborate fountains. This square still exists, and only Tiananmen Square in Beijing is bigger today. The placement of the palace and the royal mosque within sight of the public reflected Shia expectations of a close public relation to their king. Shah Abbas often hosted multicultural feasts; it was rare among rulers of the day to eat with others. Regular markets and performances took place in the square.

Isfahan had maybe half a million people at its prime. Shah Abbas invited merchants and artisans from the whole world to visit. Chinese potters were especially prized. Isfahan gained fame for its textiles and especially carpets. Persian ceramics (especially tiles) and carpets would be sold in both Europe and Japan in the seventeenth century. Armenian, Persian, and Indian merchants interacted in the city's markets with a smattering of Europeans and East Asians. Trade with Mughal India was particularly prevalent.

One intriguing development involved the adoption of European representational art in the seventeenth century. Islamic art had tended through the 


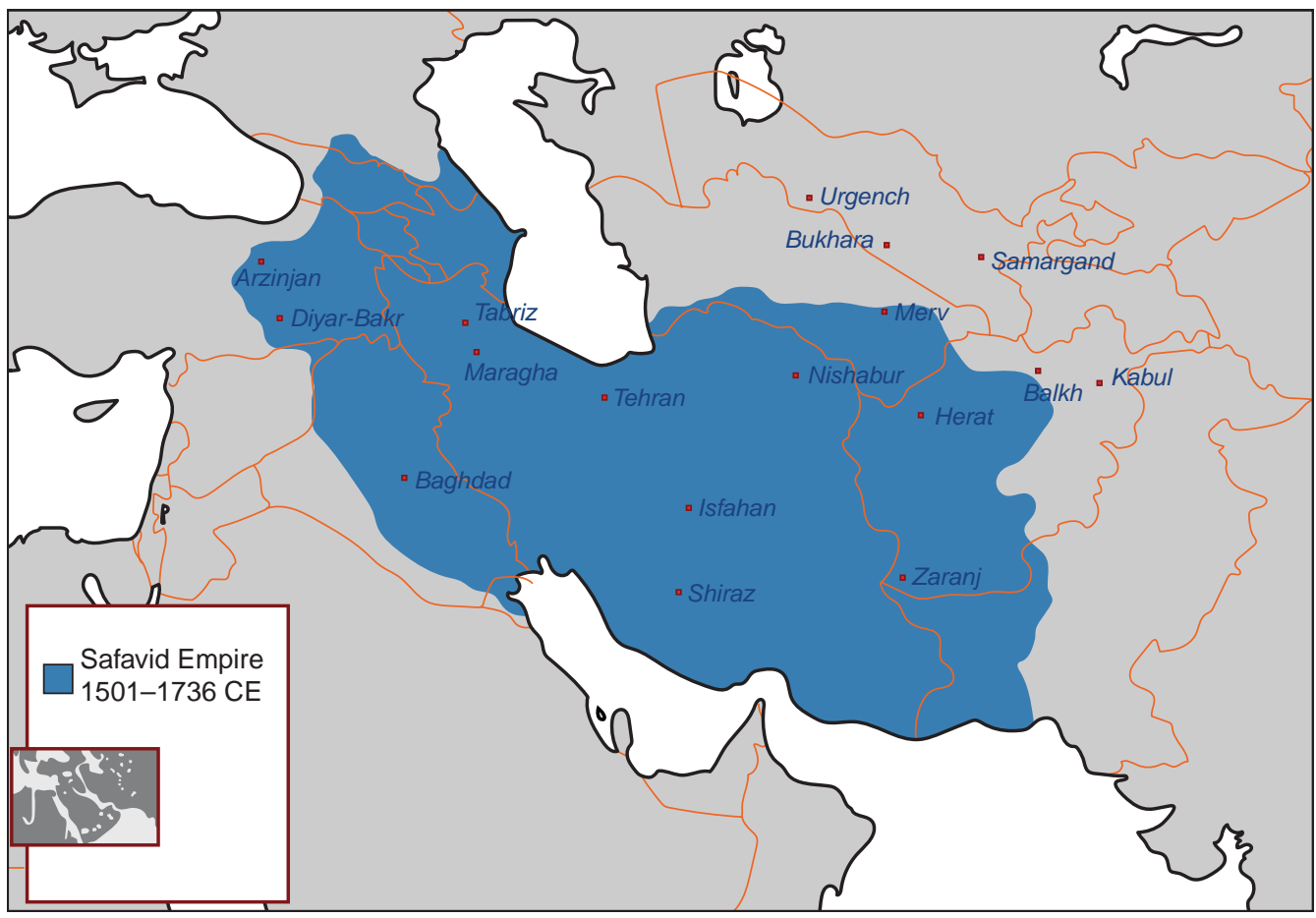

Map 20.2

The maximum extent of the Safavid Empire under Shah Abbas

centuries to be abstract - except for a brief period in Central Asia inspired by Buddhist influences (chapter 12). However, the Safavids liked the idea of portraits and encouraged this art form. Many European painters moved to Isfahan.

Ancient Persia had limited the role of women even more than Arabia, and Islamic attitudes toward women had hardened under the Abbasids (chapter 12). The Safavids also followed Persian traditions that limited the legal rights of women.

\section{The Mughals}

Babur, the founder of the Mughal Dynasty, was a descendant of both Chinggis Khan and Timur (and the Mughals would add to this ancestry by over time developing tales of a miraculously impregnated forbear). Indeed, "Mughal" is a Persian word for Mongol. Babur fought with limited success for decades in Central Asia - eventually losing the lands he had inherited from his father around Samarkand and briefly retaken. Babur then decided that India provided a more profitable target. A group trying to overthrow a local ruler invited him into India. He conquered for himself, defeating a much larger force of the Delhi 

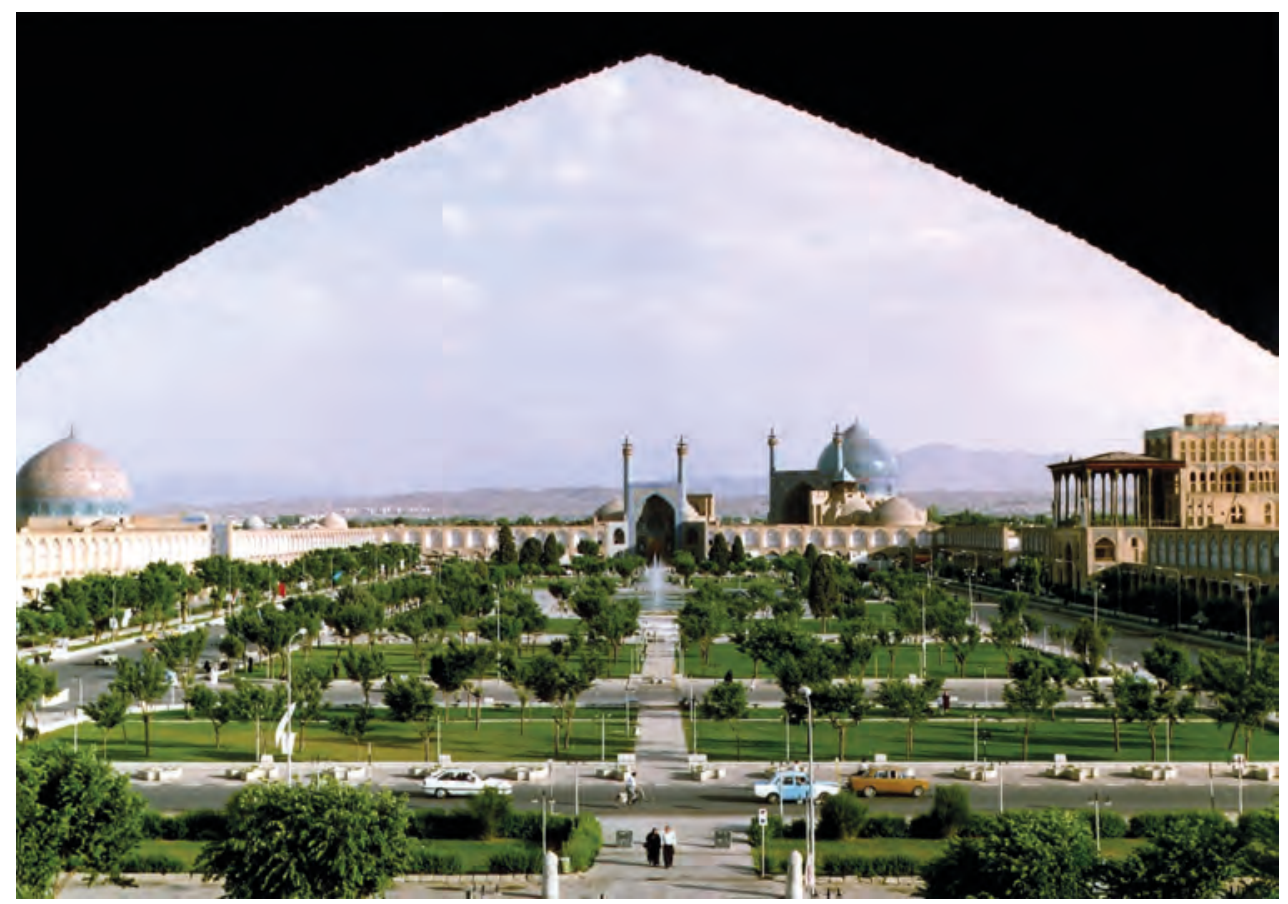

Illustration 20.13

Main Square, Isfahan

Photograph by Arad Mojtahedi, CC BY-SA 3.0

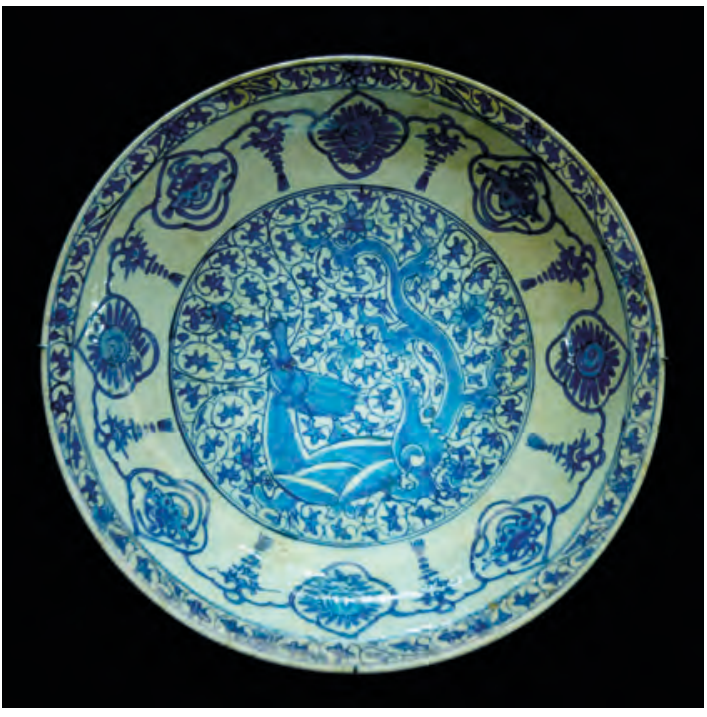

Illustration 20.14

Plate with dragon, 17th-century Safavid artwork inspired by 15th-century Chinese blue and white ceramic, Louvre
Sultanate near Delhi in 1556 with the use of cannon. He had obtained the cannon and training on how to use these - from the Ottomans in return for a promise not to attack them. He also allied himself with the Safavids, despite the hostility between Ottoman and Safavid. Many of his followers wanted to return to Central Asia with their booty, but Babur decided to stay. He was a prince without a land in Central Asia and welcomed the opportunity to rule.

One of his generals temporarily overthrew Babur's son Humayun, but he regained power after the Safavids sheltered him. Then an alliance of Hindus and Muslims unhappy with Mughal rule challenged his succession (nearly successfully): India might have received a Hindu ruler. However, Babur's grandson Akbar (1556-1605) inherited the crown at 12 and threw off an unpopular 
regent at 16; he then ruled for half a century. He would create a centralized bureaucracy and extend power to the seas east and west, north to Kabul, and south into the Deccan. Hindu princes that surrendered could administer territories on behalf of the Mughals. Those who did not surrender suffered: Akbar killed 30,000 when he took the fortress of Chitor in 1568. The population of his empire was probably about 150 million, similar to that of China at the time, and perhaps the largest empire in the world. Akbar and his chief administrator Todor Mal had the lands of the empire surveyed to establish the taxes due (in cash). These taxes were based on an estimate of the average productivity of land over a tenyear period: This is an amazing bureaucratic achievement for the time. Such a tax was easier to monitor and encouraged increased work effort more than a tax based on annual output. Akbar separated administrative from financial control in his bureaucracy to reduce opportunities for bureaucratic corruption; Western bureaucracies would not pursue this practice for centuries. Akbar may have had epilepsy, and was proud of his illiteracy - but enjoyed having books read to him (see

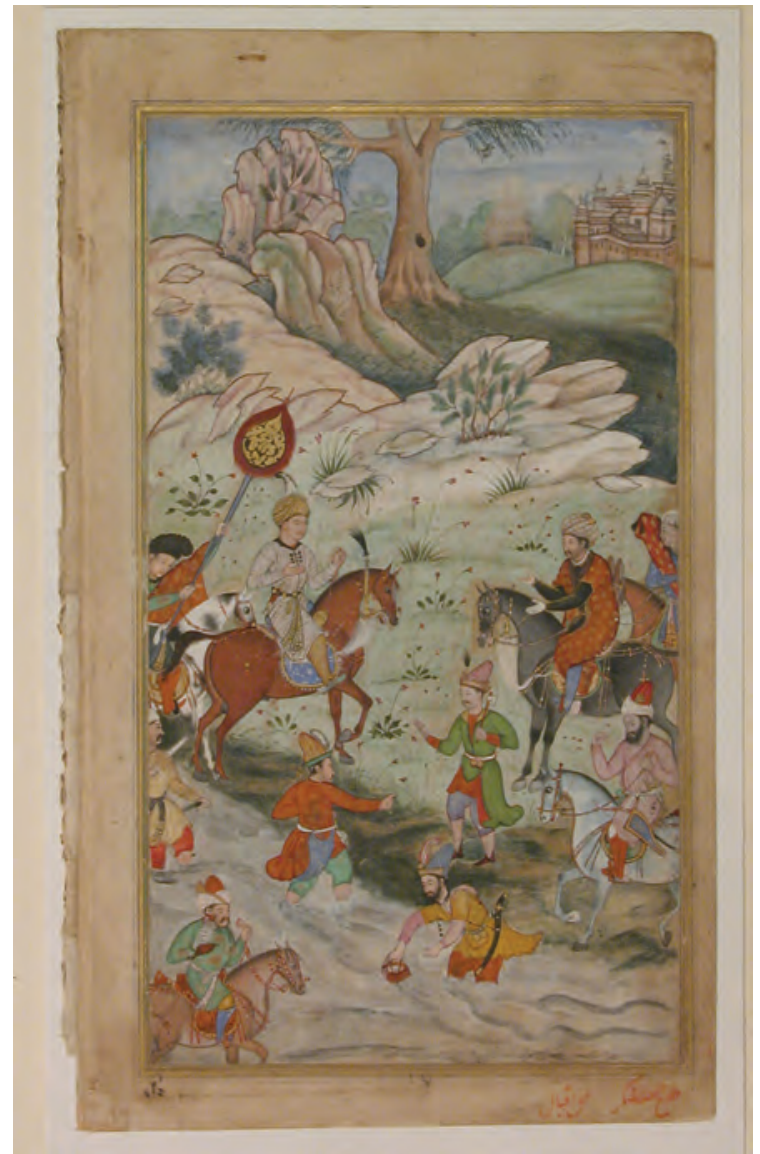

Illustration 20.15

Meeting between Babur and Sultan Ali Mirza near Samarkand; illustration for the Baburnama created for Babur's grandson Akbar below).

One-third of average grain output was taken in taxes; taxes were even higher on other crops (which included by this time chilli peppers and corn from the Americas). This heavy taxation might have been devoted to developing large grain reserves. Instead, it was spent on armies and public monuments (below). India suffered frequent famines during the period of Mughal rule. Collecting a tax based on average productivity during years of below-average output could exacerbate food shortages, though taxes were often reduced during severe droughts. The Mughals occasionally devoted public funds to famine relief.

Unlike the Ottomans and especially the Safavids, Babur downplayed religious motives for conquest. Akbar practised religious toleration. He officially banned forcible conversion as well as the enslavement of prisoners of war, and abolished the special tax on non-Muslims. Early Mughal rulers did see themselves as protectors of Islam but also supported Jainism and Hinduism. Hindus could administer their own courts. Attracted to Sufi mysticism, Akbar 


\section{PRIMARY DOCUMENTS: BABUR'S AUTOBIOGRAPHY}

\section{$B A B U R N A M A$}

Babur reports on his early difficulties:

During my stay in Tashkent, I endured much poverty and humiliation. I had no country or hope of one! Most of my retainers dispersed; those who remained were unable to move about with me because of their destitution. This uncertainty and want of house and home drove me at last to despair. I thought, "It would be better to go off by myself than live in such misery; better to go as far as my feet can carry me than for others to see me in such poverty and humiliation."

He describes victories against Afghans:

We had been told that when Afghans are powerless to resist, they go before their foe with grass between their teeth, this being as much as to say, "I am your cow." Here we saw this custom; Afghans unable to make resistance came before us with grass between their teeth. Those our men had brought in as prisoners were ordered to be beheaded and a pillar of their heads was set up in our camp.

I first heard the word sangur after coming to Kabul where people describe fortifying themselves on a hill as making a sangur. Our men went straight up, broke into it and cut off a hundred or two of insolent Afghan heads. There also a pillar of heads was set up.

After dismounting in Bannu, we heard that the tribesmen in the Plain (Dasht) were for resisting and were entrenching themselves on a hill to the north. A force headed by Jahangir Mirza, went against what seemed to be the Kiwi sangur, took it at once, made general slaughter, cut off and brought in many heads. Much white cloth fell into (their) hands. In Bannu also a pillar of heads was set up. After the sangur had been taken, the Kiwi head-man, Shadi Khan, came to my presence, with grass between his teeth, and did me obeisance. I pardoned all the prisoners.

Perhaps Babur's most famous statement - much quoted in India in the centuries since records his impressions of India itself:

Hindustan is a place of little charm... There are no good horses, meat, grapes, melons, or other fruit. There is no ice, cold water, good food or bread in the markets. There are no baths and no madrasas. There are no candles, torches, or candlesticks.

Why did Babur then stay in India? What is his general attitude toward those he fights?

regularly gathered Muslims, Hindus, Jains, Zoroastrians, Sikhs, and occasionally Catholics to discuss religion. He developed a ritual of light and fire for his court - Islamic clerics viewed this with suspicion. It is debatable as to whether he tried to found a new religion (that would be monotheistic like Islam but borrow Zoroastrian and Hindu elements) or whether he just sought recognition 
as a saint by all. Abu al-Fazl, a Sufi associated with the court, celebrated him as semi-divine - endowed with god's light. This view also drew on an Indian tradition of seeing the ruler as a father figure.

One-third of Akbar's bureaucracy was Hindu (though Hindus were threequarters of the population) but the rest were Muslim, mostly immigrants from Persia and Central Asia. Persians or Hindus tended to occupy the key posts; as "outsiders," these were less of a threat. Akbar deliberately tried to reduce the power of the Central Asian nobility that had arrived with his grandfather. He rotated these nobles through provincial governorships so that they could not establish regional power bases. The Hindu Rajputs (chapter 13) became a critical element of the Mughal military; they provided tribute from lands they administered and married their daughters into the royal family. They proclaimed Akbar to be an incarnation of the god Ram. Akbar more generally encouraged intermarriage among Hindu and Muslim elites.

Although primarily an agricultural empire, the Mughals did encourage and tax internal trade. Trade networks remained important, and specific castes were devoted to trading. There were also merchant (and artisan) guilds similar but less powerful than those found in Europe. Indians also developed bills of exchange that allowed merchants to make paper transfers of funds across time and place. Foreign commerce was more of a challenge. As we have seen, Indian merchants were familiar in Persia. Yet the Mughals had no navy and states that could protect their merchants tended to dominate commerce by sea (see our discussion of European trading companies in chapter 19). India exported spices, silk, and cotton textiles worldwide through foreign merchants.

The Mughals throughout allowed all male relatives of the emperor - brothers, sons, uncles - to compete for the throne. Sons occupied important provincial posts with which they could build a local support base. The result was a series of brutal succession crises. Still, the Mughals did not follow the Ottoman and Safavid precedent of raising sons in isolation in the harem.

Later rulers were less religiously tolerant. Though Akbar had viewed Sikhism positively (likely because it seemed to combine Islamic and Hindu elements), Jahangir (who rebelled against his father Akbar) killed the fifth Sikh guru because he thought he was becoming too powerful. The Mughals would after that face hostility from Sikhs. Jahangir also imprisoned Sheikh Ahmad Sirhindi, who wrote popular treatises that complained that the Mughals did not follow sharia law. The Mughals thereafter would pursue a supportive religious orthodoxy. Though Shah Jahan's son wrote with his father's approval that Hinduism and Islam had many similarities, Shah Jahan built many mosques and tore down some temples. He also favoured Persian over Hindu administrators.

Aurangzeb (1659-1707) was a more devout Muslim than his predecessors. He also built mosques and destroyed temples - but the latter perhaps only as revenge against disloyal nobles. More importantly, he sought to encourage Islam by reintroducing the special tax on non-Muslims familiar in many other Muslim polities. This incited numerous regional revolts. Aurangzeb may have killed as many as 2 million Hindus and Sikhs in quelling such uprisings. 


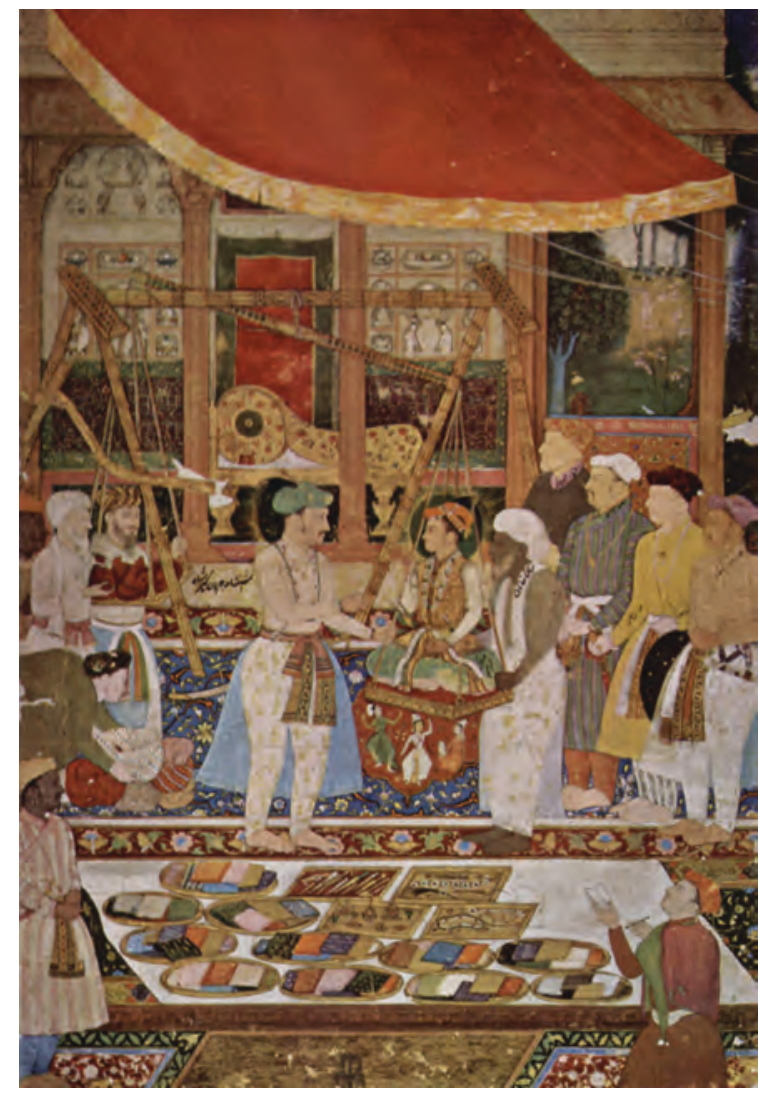

Illustration 20.16

Emperor Jahangir weighing his son Prince Khurram (later crowned Shah Jahan), in gold in 1607, from the Tuzk-e-Jahangiri (Jahangirnama) by Manohar, c. 1615
Assertions of independence by local princes characterized the last decades of Mughal rule. The British found it useful to ally with these local princes against the Mughals. The Mughals found it difficult to finance their bureaucracy and military despite the fact that trade and industry were growing. Since they were no longer expanding through conquest, they could no longer reward supporters with land.

\section{Mughal culture}

Though Central Asia had become politically fragmented after Timur, centres such as Samarkand and Herat were still heralded for their luxury and architecture: These were perhaps the most magnificent centres in the Muslim world at the time. The Mughals set out to copy and then outshine these Central Asian sites. They introduced to India Timurid walled gardens with running water and pools. Mughal architecture melded the styles of Central Asia with the red sandstone and marble trim long pursued in India. As the Ottomans and Safavids developed Istanbul and Isfahan, the Mughals strove to achieve even greater architectural glory. The wealth and ostentation of Mughal rulers would become famous: The English word "mogul" derives from "Mughal."

Humayun had escaped to Persia when temporarily evicted from his throne. When he returned to retake the crown, he brought back Persian painters. These supervised local painters, primarily in manuscript illustration, an art form popular in India since the eleventh century. Thereafter there would be a strong Persian influence on Mughal art. The Persian language was preferred at Akbar's court to both Arabic and Babur's Turkic. It was a well-developed language not closely associated with religion. Indeed the Mughals would increasingly see and advertise themselves as the inheritor of Persian cultural traditions and even the great Persian empires of the classical period. Many Hindus learned Persian to gain jobs in the lower bureaucracy.

Akbar fostered the arts, especially illustrated manuscripts and textiles, in government-sponsored workshops. These produced very expensive works but of impressive quality. Akbar had two Hindu classics, the Mahabharata (which 


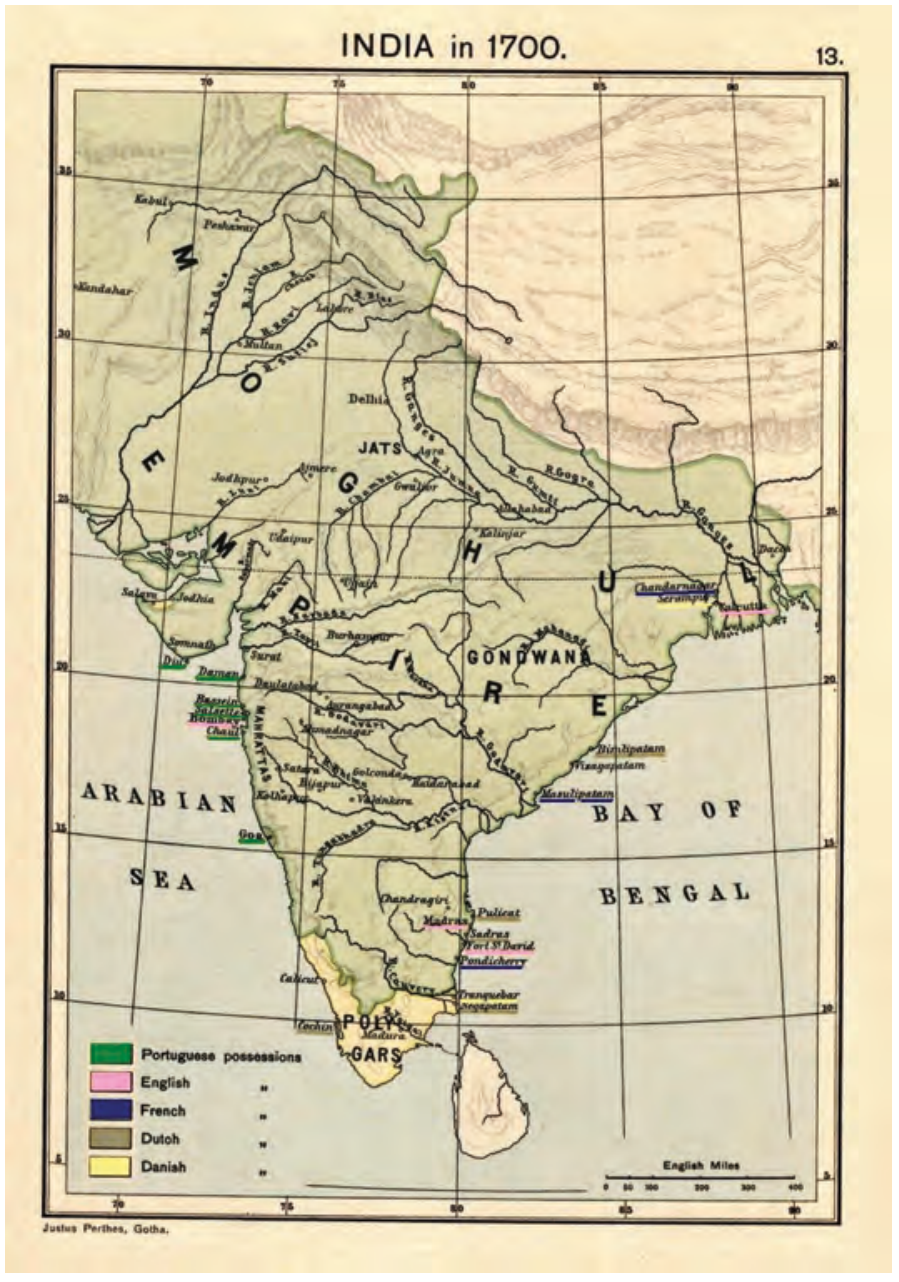

Map 20.3

The Mughal Empire c. 1700 CE

encompasses the Bhagavad Gita) and Ramayana, translated from Sanskrit into Persian and richly illustrated. He also commissioned many illustrated manuscripts of stories about and histories of the Mughals themselves. These illustrations blended Persian and Indian elements, and would later adopt the perspectivism of European art. The pictures were encouraged in part by the fact that Akbar admired these while being read to.

Akbar appeared every morning in a palace window. This practice followed the Islamic custom that the leader should be close to the people - a tradition pursued by the Safavids but less so the Ottomans. It also reflected an Indian tradition of viewing the ruler in an auspicious light. Aurangzeb would end the practice decades later, deciding that it did not accord with Islamic practice. The Mughals also held public audiences for at least members of the nobility through which the emperors might gain some appreciation of life beyond the palace. 


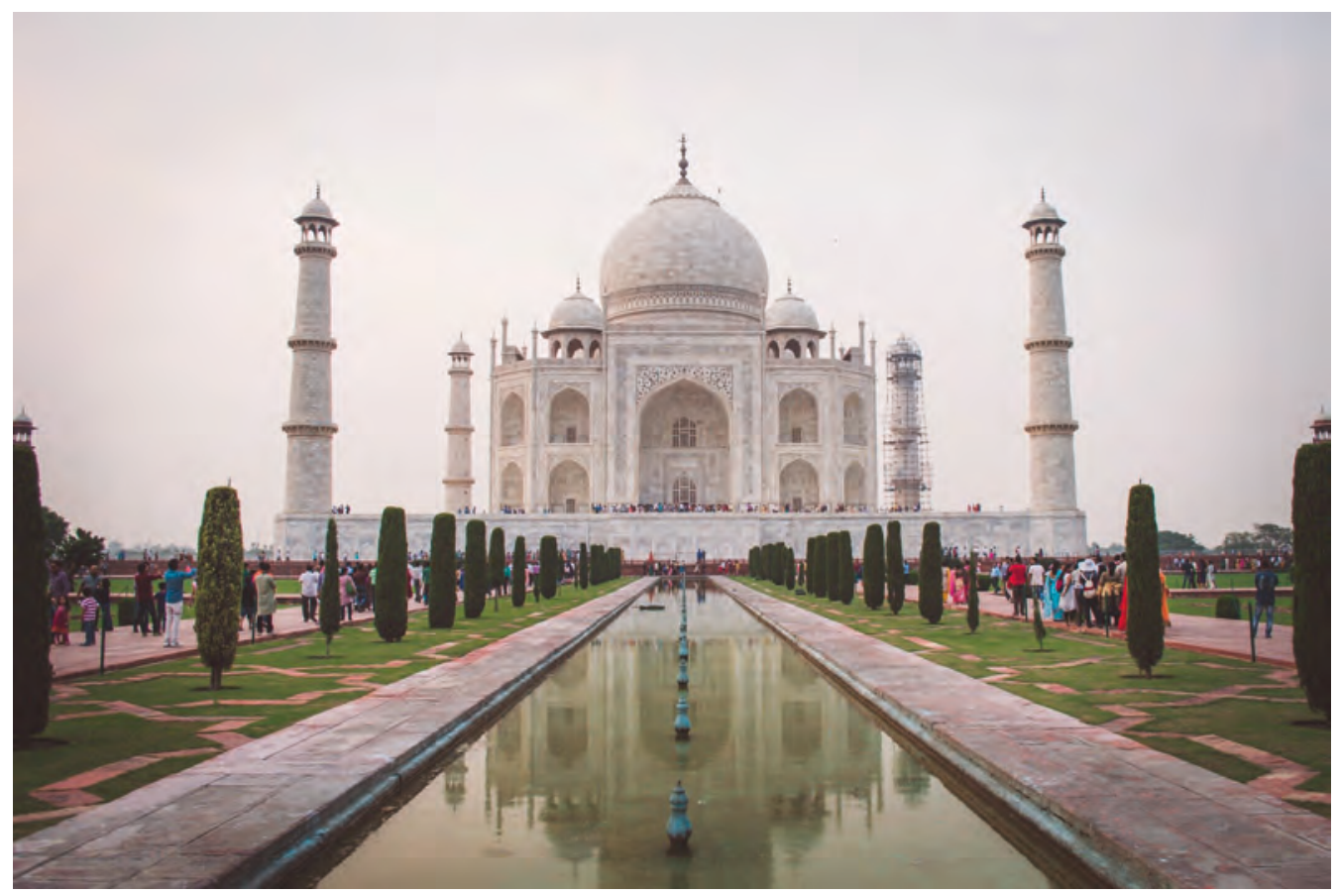

Illustration 20.17

Taj Mahal with reflection

All Mughal rulers supported the arts. Jahangir and Shah Jahan favoured painting. Shah Jahan famously supported architecture, but his architects could build on the achievements of generations of Mughal architecture. Though the Taj Mahal - a blend of Persian design with Indian elements and skills - that he constructed in honour of his favourite wife was initially a private monument, he also built magnificent mosques. He was inspired in part by competition with Safavids and Ottomans. Women, though guarded in the harem, were very influential in court. They also organized the construction of many mosques and gardens. Nur Jahan, Jahangir's wife, had a beautiful white marble tomb built for her parents.

Shah Jahan raised taxes by as much as 50 per cent to finance construction of a new capital at Delhi. Delhi had maybe 2 million people by the mid seventeenth century. The inner city had a six-kilometre stone wall that was eight metres high and three and a half metres thick. Shah Jahan also decreased expenditures on such things as famine relief to finance building projects. The Taj Mahal itself was built during a famine with funds that might have been devoted to famine relief.

\section{Caste}

We have seen that states are everywhere associated with social stratification. States must then decide how to address this stratification. Some rulers 
encourage some degree of social mobility, both to enhance the quality of bureaucracy and to limit the power of hereditary elites. In other states, hereditary stratification instead receives justification, usually religious in nature. In such instances, there will generally be expectations that people do not marry outside of their hereditary social status.

The most famous system of hereditary social stratification is the Indian caste system. There were initially four castes: warriors, priests, farmers, and merchants and artisans. A fifth caste was added of "untouchables," who performed undesirable tasks such as dealing with human wastes. The earliest castes may have reflected ethnic divisions between a conquering warrior elite and local populations. Over time, hundreds of new sub-castes emerged within these broad categories. These generally reflected new occupations, but could also serve to absorb new ethnic groups into society. The relative status of some castes might change as different occupations prospered or declined. Occupational castes could regulate the occupation, in the manner of artisanal guilds.

There is intense scholarly debate

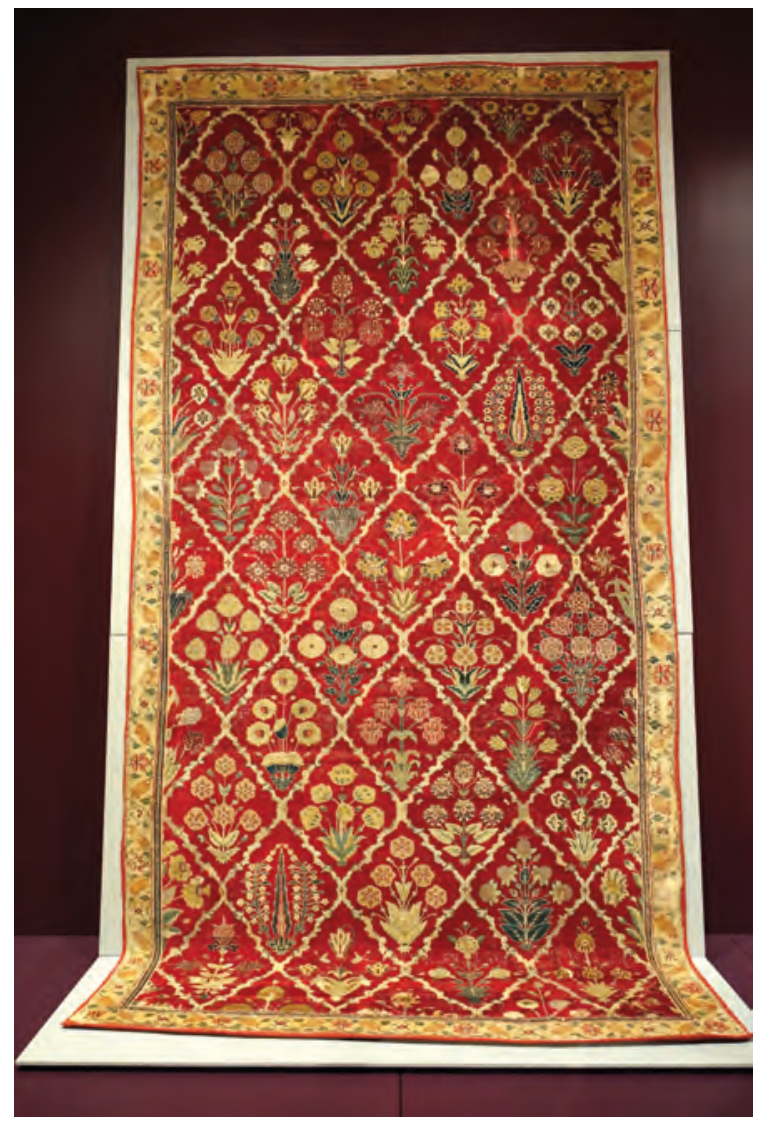

\section{Illustration 20.18}

Carpet, Lahore, Mughal Empire, 17th century, cotton, silk, and wool, Textile Museum, George Washington University

Photograph by Daderot, CC0 1.0 regarding when and why the caste system emerged. Though early Hindu texts speak of the four castes, it is not at all clear that society was organized around these until very late in history. It may have been during the Mughal Empire, or even the British Empire, that castes became both rigid and of central importance socially and economically. In either case, the caste system might have been convenient for rulers in limiting the ability of Hindus to combine in opposition to rule by non-Hindus.

\section{Mughals and British}

The Mughals had allowed the British East India Company to establish trading posts along the Indian coast. The British traded at first for pepper and Indian cotton textiles. During the eighteenth century, tea and coffee became the most prominent exports (chapter 18). Other European countries also operated posts along the Indian coast. With British victory over France in the Seven Years War 
of 1756-63, Britain became the dominant European power in the area. From the 1750s, the Company began to conquer inland, facilitated by the Mughal decline after Aurangzeb - and at times encouraged by attacks on their trading posts by the new polities rising in place of the Mughals. Through conquest, they could protect their sources of trade goods. They also - like all other empires sought to finance their administrative costs through taxation. About a century elapsed between the death of Aurangzeb and the consolidation of British rule over India. During this period, various Indian princes allied with the British in opposition to the Mughals. We have seen this sort of coalition between regional authorities and a new conqueror against an old empire many times in history, most recently in the Americas (chapter 19). The British promised Hindu princes a share of taxes, and Indian troops were trained in the use of British weapons. Hindu princes were mostly left alone in the less profitable regions of the subcontinent. Yet the British long retained the Mughal emperor as a puppet figure. The British claimed for decades to be merely collecting taxes on behalf of the emperor. This century of political conflict likely disrupted trade less than one might expect because networks of merchants that relied on trust more

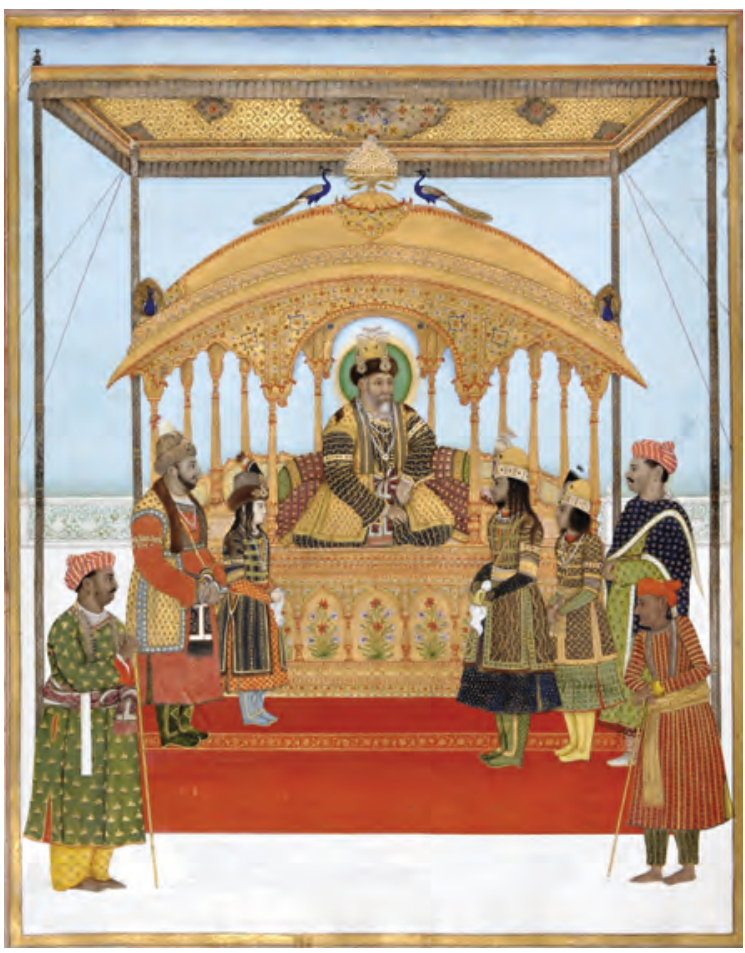

Illustration 20.19

Akbar II holds court on an elaborate throne, flanked by his sons. Painting likely by Ghulam Murtaza Khan, about 181 1. Although the British had conquered Delhi in 1803 , the emperor ruled symbolically into the 1840s. One of Akbar II's sons would be the last than institutional support handled trade within India.

It was the Company rather than the British government that at first ruled most of India, though the British parliament came over time to exercise influence over the choice of governor. A rebellion in 1857 (often termed just a mutiny; chapter 25) which was cruelly opposed - there were thousands of Indian deaths - induced the British government to take charge. The British ruled much of India directly but also maintained over 500 semi-autonomous princely states under British supervision. One of their earliest acts was to make English the official language. British citizens occupied the senior positions in bureaucracy and army, but locals filled the lower ranks. A local elite would be educated in English and come to ask how European ideals of popular sovereignty (chapter 21) could be reconciled with control over a land with thousands of years of history and accomplishment. The British showed little interest in spreading Christianity, and so practised religious tolerance, but did implement important changes in family 
law: Sati, a practice in which widows committed suicide after their husband's death, was outlawed and women gained a right to inherit and remarry (though these laws only slowly affected lower castes). The British also banned slavery (see chapter 26) and infanticide.

The East India Company had run an authoritarian government focused on profit. Yet it had appreciated the commercial advantage of mixing with locals and there was considerable social interaction between British and local elites. The British administration then debated the appropriate balance between authoritarianism and the rule of law. Governor Cornwallis (who had previously served in the Americas during the American Revolution) worried about abuse of power and introduced an extensive system of courts. These tended to follow British procedures but enforced Indian laws - which then required the employment of numerous Brahman experts in Hindu legal traditions. Over time, the British became frustrated that local elites were not encouraging economic growth or increased tax revenue; they then came to stress the rights of peasants over their land. Though the British likely exaggerated their effect on property rights, it did become easier to buy and sell land under British rule. The British would also introduce freedom of the press and - belatedly - legislative assemblies with some limited powers. The Indian army was trained not to usurp civilian authority. Though the British retained the most senior positions in government - and there was much less social contact between British and Indian elites during the later decades of British rule - India was better prepared for independence than most European colonies (chapter 30).

In the early days of British conquest in India, there was much curiosity about Indian culture and philosophy. The British appreciated that India had a much longer written history than Britain itself. They celebrated the Upanishads for their rationality and incipient monotheism. The Upanishads would influence European and American philosophers and writers such as Schopenhauer, Emerson, and Whitman. Many Indian words - including bungalow, khaki, and pyjama - were absorbed into the English language. In turn, Indian intellectuals sought to merge Indian and European thought: They detected in the Upanishads support for not just monotheism and rationality but monogamy, the end of caste distinctions, and the end of the practice of sati. Over time, though, British rulers came to stress cultural superiority and ignored or denigrated Indian history. Indian intellectuals came in turn to stress differences rather than similarities between Indian and European thought. They would absorb the emerging European ideal of the nation state (chapter 21) but wed this to a sense of a unique and shared Indian identity.

British rule had a mixed economic impact. The period of British rule was much more peaceful than Mughal rule, reflecting the much greater superiority of British arms. This peace did encourage the expansion of internal trade. However, British tariff policies encouraged industrial production in Britain rather than India. India came to provide raw cotton and tea in exchange for manufactured textiles and iron goods. Indian taxes paid for extensive railway construction - 56,000 kilometres by 1914 - but rails, railcars, and locomotives 
were built in Britain. The British supported roads, harbours, and irrigation systems. Over time, some industrial textile and steel production employing European technology did emerge in India.

\section{The Ming in China}

Hongwu, who had been a beggar early in life but had risen through the military ranks, founded the Ming Dynasty. The Ming Dynasty would last from 1368 to 1644 . Hongwu had joined a group of "Red Turban" Buddhists in rebellion against the Yuan (and specifically against efforts to conscript for dredging the Grand Canal), but severed his ties from them as his power grew. He proved to be an astute military commander, winning battles against both the Yuan and Red Turbans. After executing his chief minister for alleged treason, he abolished the position. Later emperors would interact with multiple bureaucrats rather than one key figure. Hongwu also executed elite households, but later Ming emperors would collaborate with local elites.

The Ming encouraged Confucian education and strengthened the examination system. The examinations became the only real path to high bureaucratic office. Gentry families hired tutors or sent their sons to elite schools from childhood to prepare for these exams. These students memorized Confucian works but also practised calligraphy and both poetry and prose. There were quotas for passing examinations at local, provincial, and national levels; students often took the exam many times before passing. Those who passed the local exam in their towns were then eligible for a prefectural exam held every two or three years; they also (as under the Song) gained an exemption from forced labour. Only one-half of one per cent who took the prefectural exams gained the "juren" status that entitled them to a post in the bureaucracy. A million degree holders competed for 20,000 positions. The highest exam was held every three years in Beijing; those who passed were "jinshi" and eligible for 2,000-4,000 senior posts. When no northerners achieved the highest grade in 1397, Emperor Hongwu established regional quotas. Once hired, promotion through bureaucratic ranks depended on formal evaluations by supervisors the very essence of a modern bureaucracy. The Ming also created a "censorate" or secret police that watched over other bureaucrats.

Hongwu had a detailed census taken of both people and landholdings. He created a system of joint responsibility among groups of 110 families for the payment of taxes. Nevertheless, as in other empires, the Ming from the early sixteenth century faced chronic financial difficulties. One reform (from 1531) was to translate tax payments in kind into payments in silver, which might have decreased administrative costs substantially, but this reform was introduced slowly and only in some regions.

We saw in chapter 15 that the Song had come to rely on sales taxes and tariffs for close to half of government revenues. The Ming returned to an emphasis 


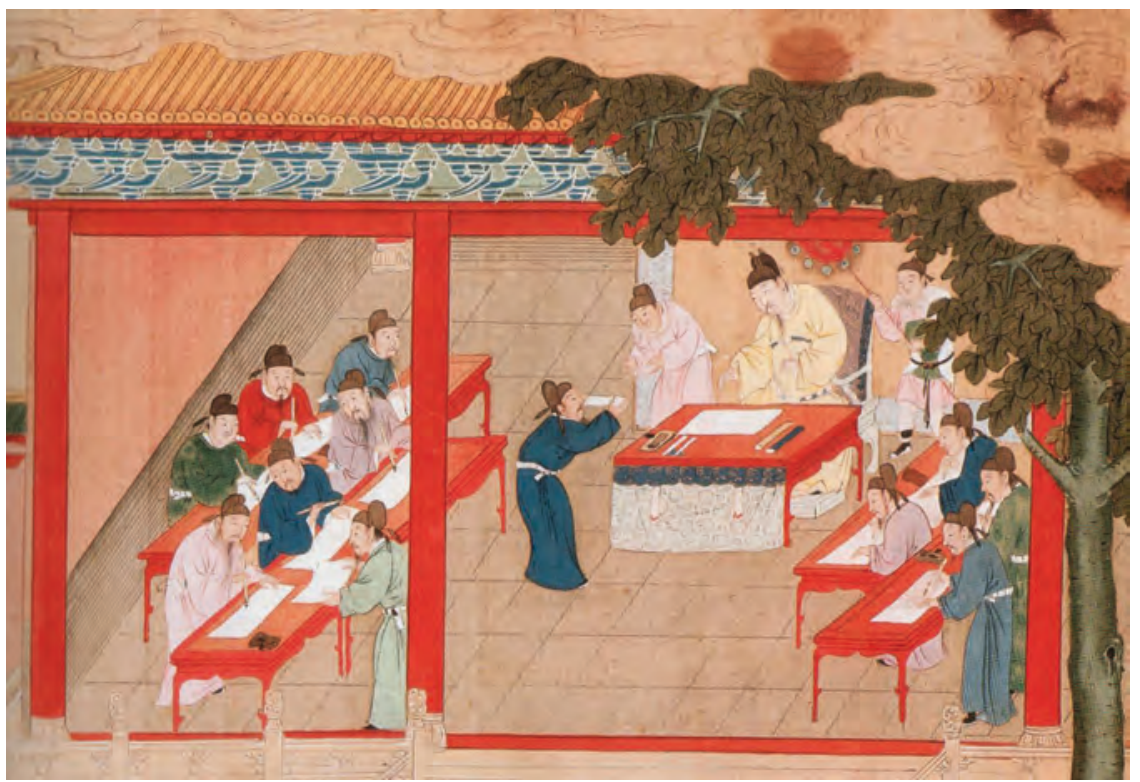

Illustration 20.20

Ming Dynasty painting of examination under the Song Dynasty

on taxing agriculture. This may have made them less interested in encouraging trade. Moreover, the Ming often pursued a policy of limiting disparities in wealth or income. They cooperated with local landowners of necessity but worried about the power that wealthy merchants might wield. It is generally accepted by economic historians that the Ming were much less supportive of merchants and innovators than the Song had been, and that Ming policies caused per capita incomes to fall below levels achieved during the Song. There is debate as to how severe the effect was (and how much economic decline should be attributed to the Mongols rather than the Ming). There is further debate as to whether Song levels of prosperity were regained by 1500 or even 1600. The Ming strengthened the system of government granaries, and generally succeeded in keeping grain prices from (more than) doubling during bad harvests; grain prices often quadrupled or more during bad harvests in France at the time. Nevertheless, frigid weather between 1626 and 1640 would lead to widespread famines and peasant rebellions that would topple the dynasty.

The Ming had difficulties with succession struggles. Hongwu's grandson succeeded him, but his uncle, Yongle, in turn deposed him. Yongle moved the capital to Beijing where his power base was. (The Ming was the first ethnic Chinese dynasty to control the Beijing area since the tenth century.) A million forced labourers and 100,000 artisans built the Forbidden City (with 9,000 rooms) over a period of fourteen years to 1420 , with materials from distant provinces, such as hardwood from Sichuan 1,500 kilometres away. The Ming dredged and extended the Grand Canal to feed this new capital. 


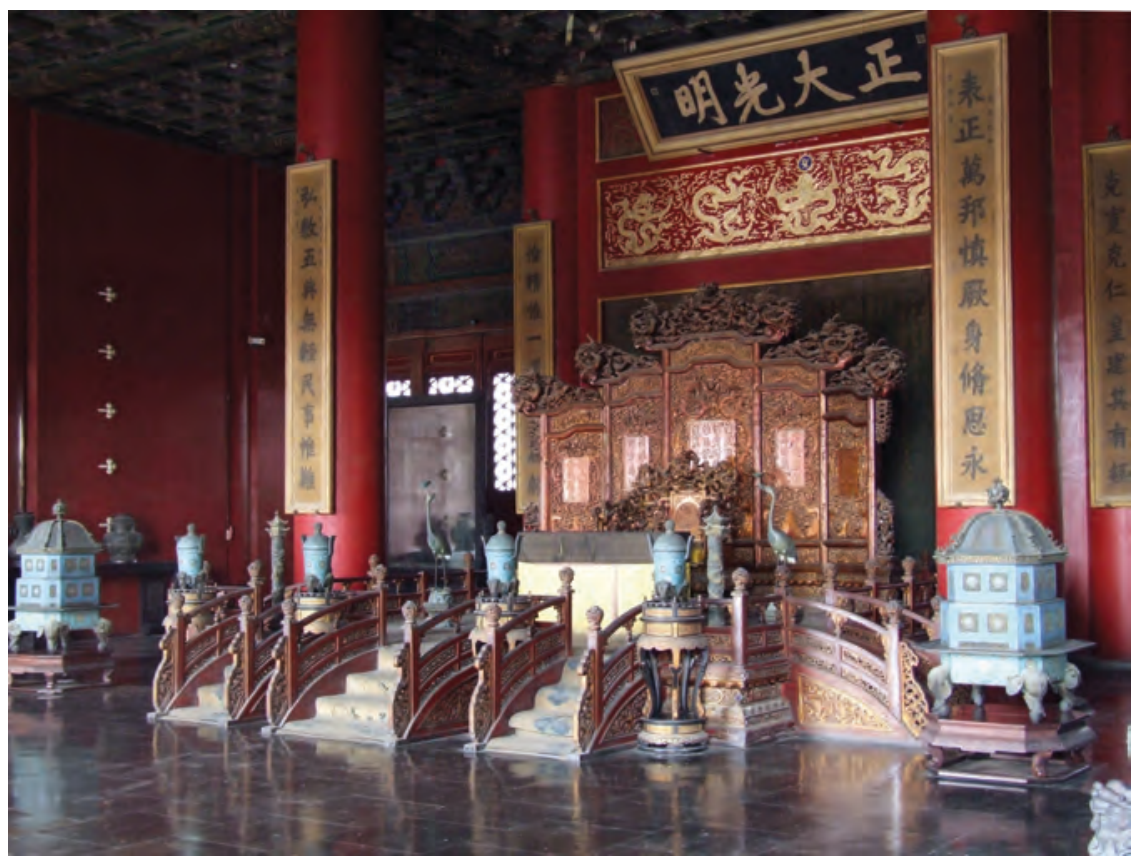

Illustration 20.21

Throne Room, Palace of Heavenly Purity, Forbidden City Photograph by Nicor, CC BY-SA 3.0

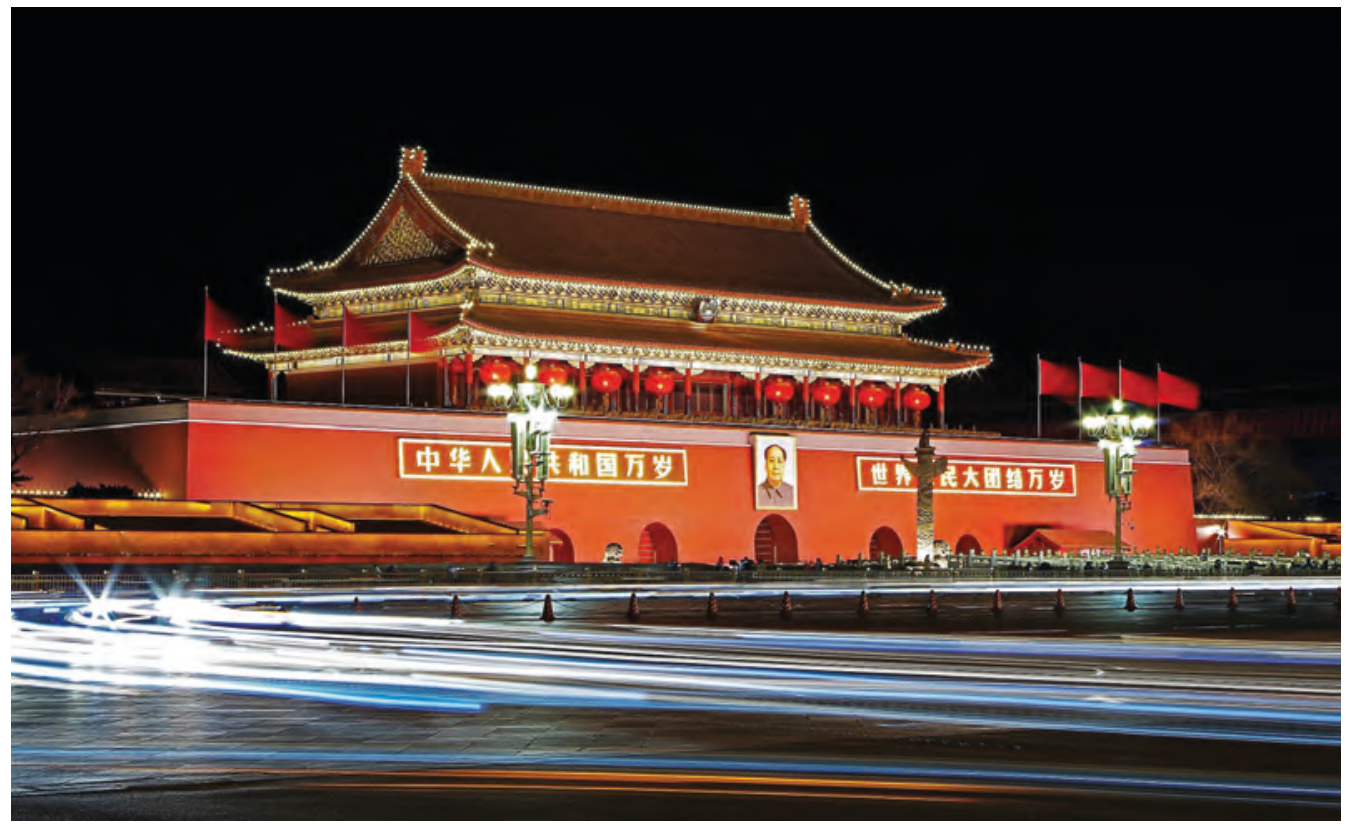

Illustration 20.22

Gate of Heavenly Peace, Forbidden City 
The ruling family thereafter rarely left the Forbidden City, leaving governance in the hands of eunuchs much of the time. Shenzong (the Wanli emperor) 1572-1620 simply refused to make decisions. The Ming relied more heavily on eunuchs than had previous dynasties. Though there were only a few eunuchs at the start of the Ming, there may have been 100,000 by the end. Eunuchs ran the secret police and served as the emperor's private secretary. A couple of these eunuchs were called "eunuch dictators" for the power they wielded. Though the Ming bureaucracy was professional, it reported to an emperor with autocratic powers and limited knowledge of the world beyond the Forbidden City; this created an opportunity for both incompetence and abuse of power. Emperors punished those found guilty of transgressions harshly: Hongwu had one official accused of bribery decapitated and his head publicly displayed. It was much more common to humiliate or ignore bureaucrats. Such actions, we might note, were common in Europe and elsewhere at the time, but also accorded well with the Legalist philosophy we discussed in chapter 10 .

Although the government spied on bureaucrats, the point of the spying was to decrease theft from the state rather than limit bureaucratic abuses against citizens. The average person had little recourse against local bureaucrats. There was no formal mechanism for complaints. Nor did China have much "civil society": Organizations of merchants or artisans were weak and (as

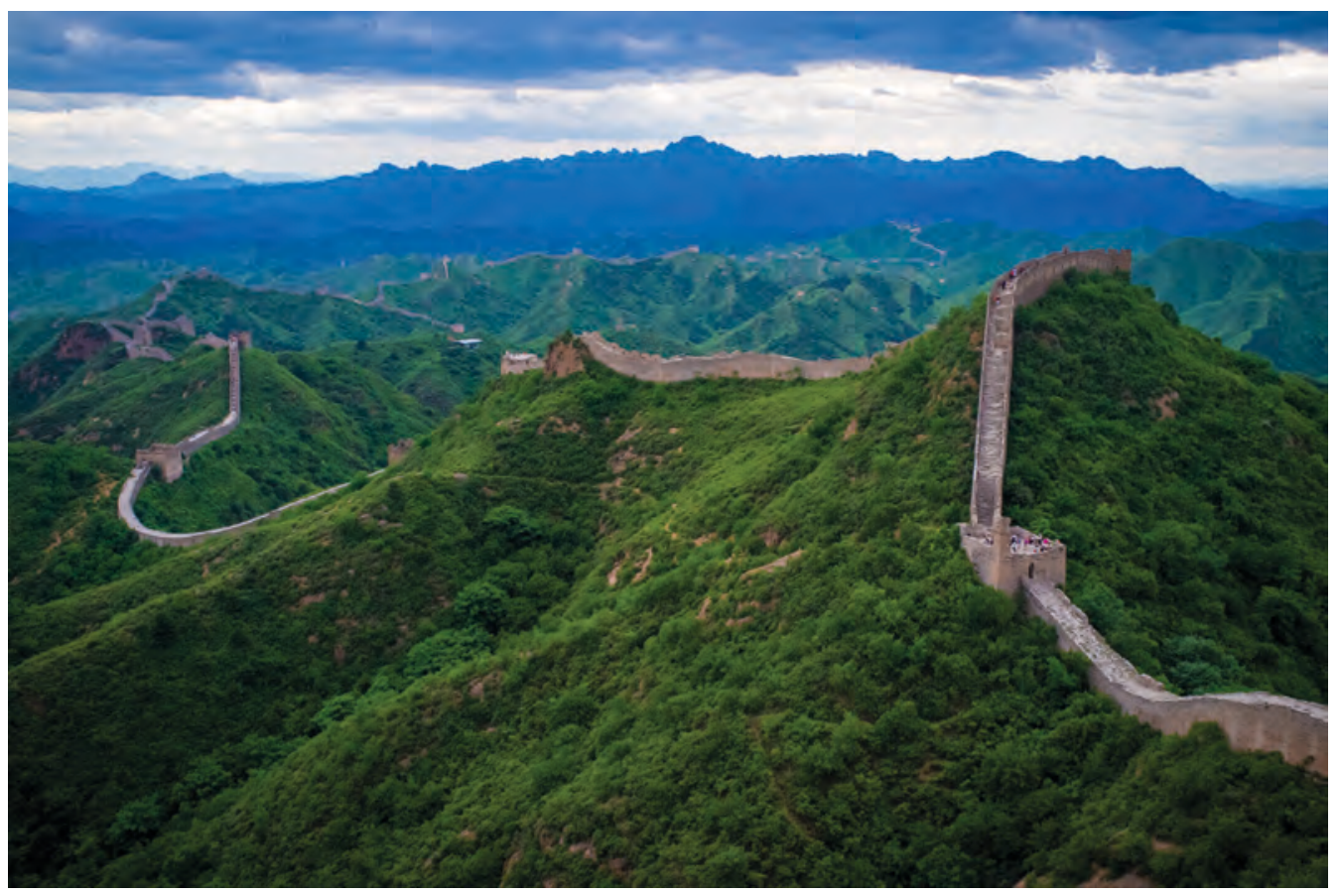

Illustration 20.23

The Great Wall of China at Jinshanling

Photograph by Severin.stalder, CC BY-SA 3.0 
noted above) local government was an arm of the central state. Arguably, the Ming achievement of an effective bureaucracy with limited transparency set a precedent for later authoritarian governments in China and elsewhere in East Asia.

Early Ming military victories gave way to defeat in 1449. Nomadic Mongolian troops from Oirat in the northwest surrounded an imperial army and took the emperor prisoner. Friction within the nomads themselves prevented a greater disaster. Though Chinese empires had been building walls against the nomads for centuries, it was the Ming who in the 1470s built almost 1,000 kilometres of the Great Wall. This was expanded in the 1550s to its present length, 2,500 kilometres, 10 to 15 metres high, with watchtowers. This required hundreds of thousands of workers. There is an irony here in that the military balance of power would turn against the nomads over the next century and independent nomadic states would disappear from the map after that (chapter 19, below).

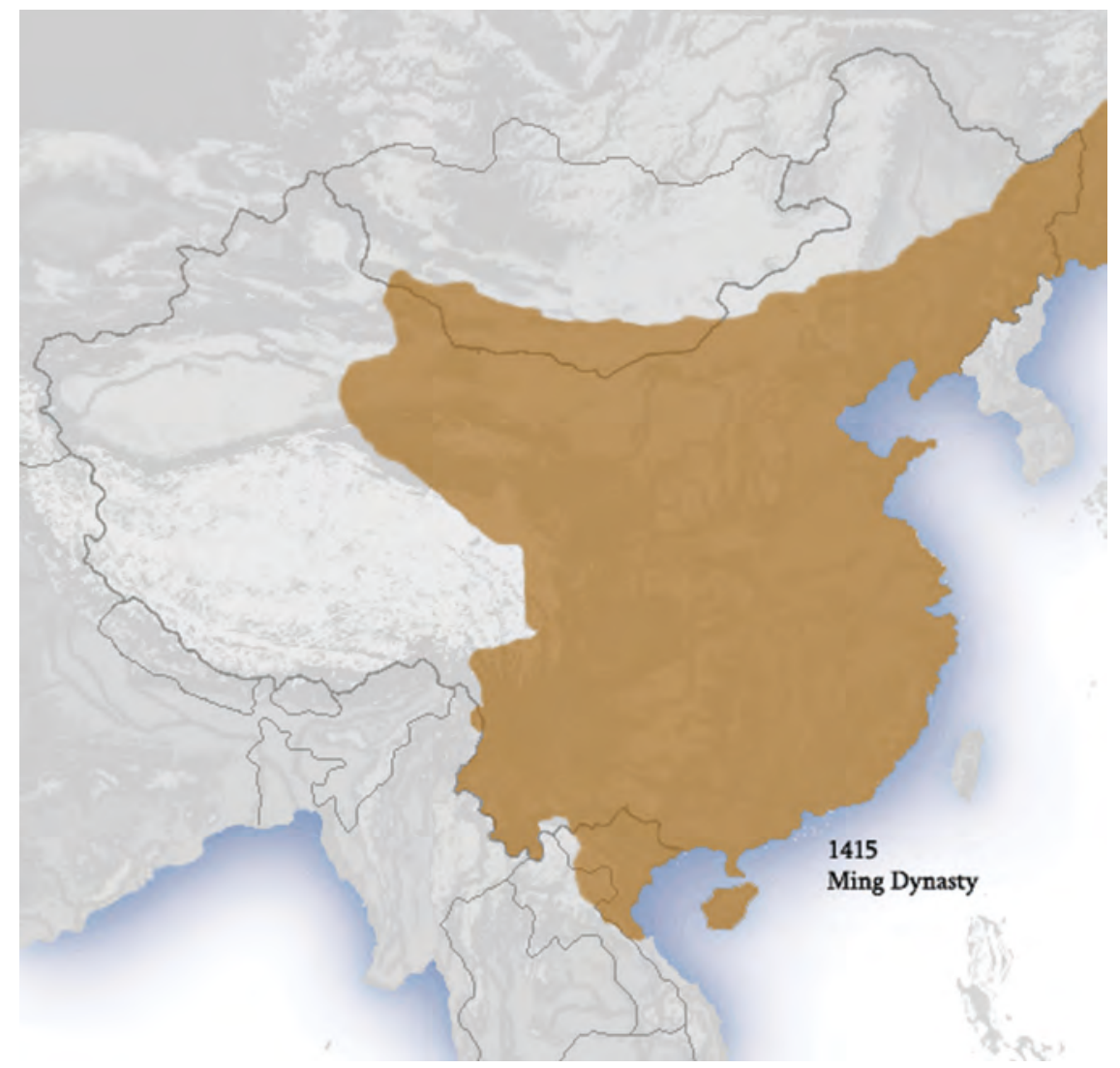

Map 20.4

Ming Empire C. 1415

Map by Michal Klajban, CC BY 3.0 


\section{Ming society and economy}

The Ming allowed Catholic missionaries. These had had some success during the Yuan but converted few during the Ming period. They did acquaint the Chinese with Western technology such as clocks (see Box 15.2), and European thinkers with Confucianism. Catholicism largely disappeared from China until Jesuit missionaries arrived in the sixteenth century.

The Ming (and later the Qing) reinforced patriarchy. This is exemplified by the practice of foot binding, where the feet of young girls were bound tightly so that they remained small. Some elite families had practised foot binding during the Song, but it became more popular during the Ming and Qing. The practice so deformed feet that adult women had trouble walking. The practice was limited mainly to the elite, for ordinary women had work to do. Some commoners nevertheless followed the practice in hopes of arranging upward marriages for pretty daughters. Some women may have come to accept the practice as a kind of virtuous self-sacrifice. More generally, women were expected to focus on home life, though there were occasional pleas that women should be educated.

The Ming was also a stratified society in which the local gentry wore special clothing (as the emperor did). They owned land but mostly made money by working for the government. Local bureaucrats worked with local landowners in providing local governance. Though the exam system allowed some upward mobility - and the Ming had instituted a policy of public elementary schooling for every boy, and secondary schools in every county - most bureaucrats themselves came from gentry families who could afford to tutor their sons in Confucian classics. Local gentry generally organized government schools.

The population had fallen dramatically during the Yuan conquest and later defeat. It increased from 65 million in 1368 to about 150 million in 1644 . This was partly the result of further agricultural expansion into new lands in the southwest. There were no dramatic changes in agricultural technology as during the Song but rather incremental advances on these. One significant change involved the introduction of New World plants such as corn, sweet potatoes, and peanuts that grew well on lands poorly suited to domestic crops. The Ming also used conscript labour to rebuild irrigation systems.

As under the Song (but less dramatically), expanded internal trade and urbanization encouraged many farmers to increase output. Silk, cotton, paper, and porcelain industries also expanded. The Ming is often blamed for standing in the way of technological innovation, but in fact the Ming interfered little in economic activity, relying primarily on land taxes and a salt monopoly for revenue. Ming experiments with paper currency were unsuccessful, and they became reliant on silver and copper coins.

Many citizens were literate but lacked Confucian education. Expansion in printing meant, among other things, that treatises on agricultural and industrial technology were distributed widely. There was also an encyclopedia of 12,000 volumes in 1407 embracing geography, history, and political institutions, and a compendium of medicines with 1,900 entries - including ephedrine for 
dealing with asthma - in the sixteenth century. As elsewhere, vernacular literature expanded with printing, with the first Chinese novels and short stories appearing. These, notably, often featured women in more active roles than were common in Chinese society.

External trade also expanded. Chinese silk and porcelain gained increased fame throughout the world. The Portuguese had begun purchasing porcelain in the late sixteenth century; when the Dutch captured two shiploads and sold the porcelain in Amsterdam in 1602 and 1604, Chinese porcelain immediately became sought after by the elite throughout Europe. The Ming discouraged Chinese from trading but allowed foreign merchants into the southern ports of Quanzhou and Guangzhou. China imported spices and hardwood from Southeast Asia. Rising wood prices in China meant that Chinese ships were often built in Southeast Asia.

\section{Ming naval expeditions}

With respect to foreign contact, the other big story (besides building the Great Wall) during the Ming Dynasty was the seven massive naval expeditions launched during the period 1405-33. Initiated by the emperor Yongle, these expeditions involved hundreds of ships, some thirty (maybe sixty) metres in length and big enough to carry 500 people. These were likely the largest ships of the day and perhaps among the largest wooden ships ever built. Zhen He, a eunuch from Yunnan, led the expeditions. There were 28,000 soldiers on the first expedition. These voyages were both military and commercial: Zhen He attacked pirates along the way, and then attacked states in Sri Lanka and Sumatra (the former attacked first; the latter harboured pirates). Zheng He gave gifts to local rulers and received tribute in return. Envoys from thirty states returned with him on the fourth voyage.

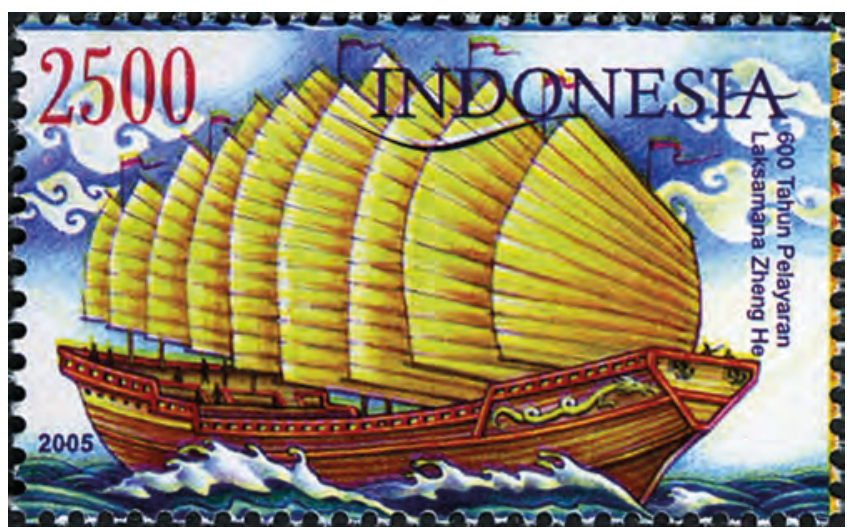

Illustration 20.24

Indonesian stamp from 2005 celebrating the 600th anniversary of Zheng He's first voyage 
Though Yongle's immediate successor sponsored the seventh expedition, that would be the last of these voyages. The cessation of these voyages is widely viewed as symbolic of an inward turn among the Ming. It is true that the Ming did become less interested in foreign trade. However, the fact that the expeditions ceased may have a far more prosaic explanation. The voyages must have been hugely expensive - and the Ming had nomadic incursions to deal with, and other demands on public finances. The returns from these trips may have been negligible. The tribute received from distant states was mostly symbolic, and these states received gifts that were likely of similar value. It is not entirely clear how much trade occurred along the way, but it does not seem that trade was the primary motive. Having hundreds of large ships arrive in port simultaneously would not be the ideal strategy for achieving the best prices for the goods one sought to sell or buy.

The expeditions provide evidence of the seafaring capabilities of the Chinese. They exemplify the power of the Chinese empire. They can be usefully compared with the voyages of discovery that were launched a couple of generations later by the Spanish and Portuguese (chapter 19). These latter voyages involved mere handfuls of much smaller ships. However, they had specific trading objectives. Both the Spanish and Portuguese rulers expected to earn a profit (the first expedition of Columbus being much more of a gamble, but one where the limited expense was set against the potential for massive profit). The Chinese fleet undoubtedly awed those it visited more than did the Spanish or Portuguese, but one can wonder whether awe is worth such an expense.

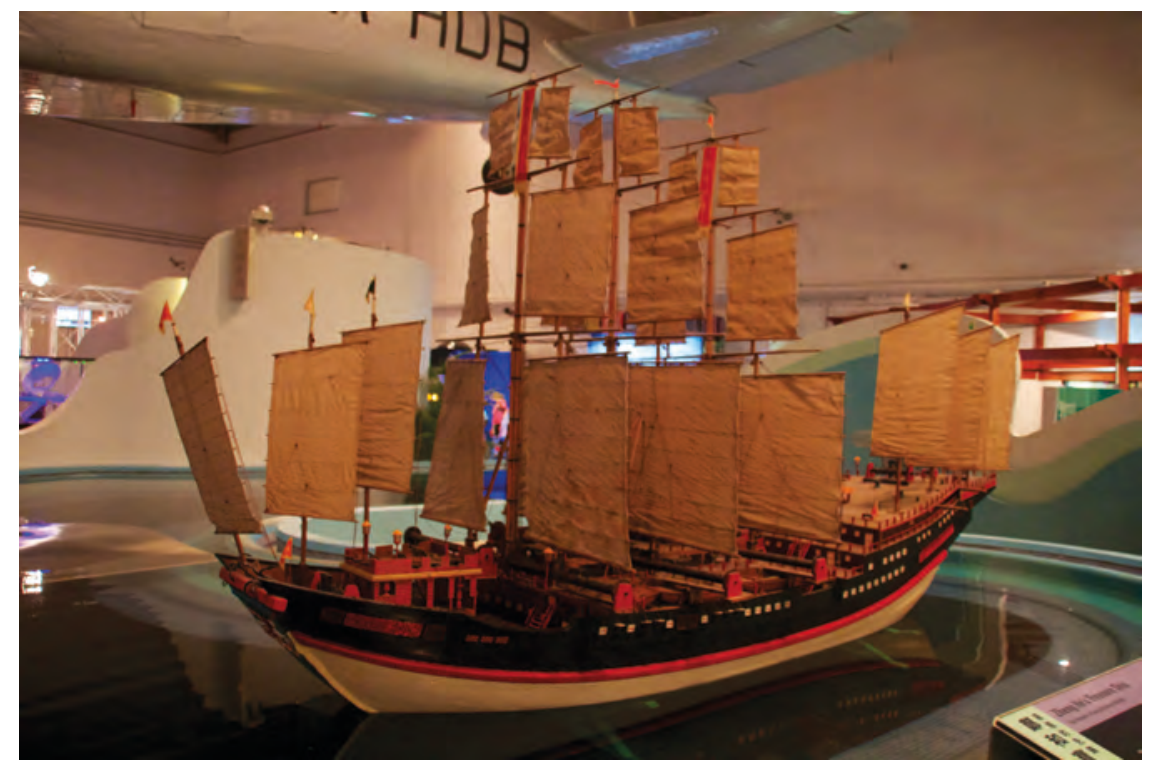

Illustration 20.25

Model of Zheng He ship, Hong Kong Science Museum

Photograph by Mike Peel (www.mikepeel.net), CC BY-SA 4.0 


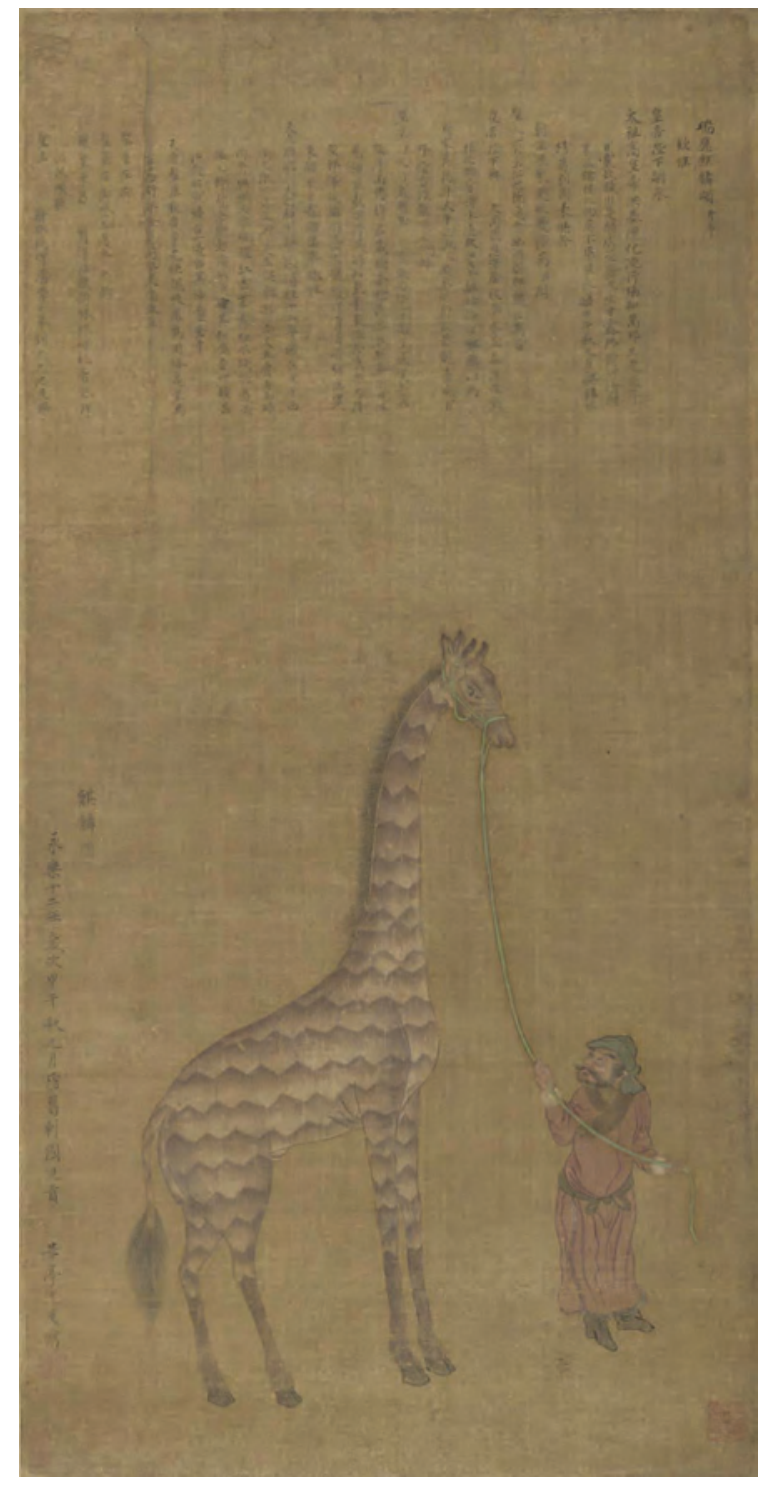

Illustration 20.26

Giraffe and attendant; perhaps the original by Shen Du, Ming calligrapher, 1414. The giraffe was a gift to the Ming court
The most significant difference between the Chinese and European voyages is in their aftermath. The Chinese must have gained valuable information about trading opportunities throughout the Indian Ocean. The Ming, having sated their curiosity, decided not to pursue these. (The movement of the Ming capital northward to Beijing may have further diverted Ming attention from trade from its southern ports.) Nor did they allow Chinese merchants to do so. Note that no one state in Europe could have enforced such a decision: Even if Spanish or Portuguese monarchs had tired of foreign voyages, others would have stepped in. The Ming even destroyed the charts of international waters developed during the expedition and allowed the ships to rot.

The Ming did allow foreign traders to operate in China, and Muslim merchants did continue to form a merchant community in southern China to take advantage of these opportunities. This indicates that these trade routes were indeed profitable. The prohibition on Chinese merchants trading abroad meant that the Indian Ocean lacked Chinese traders when the Portuguese arrived, except for the Chinese merchants who settled in the area to escape the Ming prohibition. Many of these were or became Muslim.

While the decision to end the voyages may merely reflect their huge cost and meagre return, these other decisions destroying charts and banning Chinese from foreign trade - do not have simple economic explanations. They may in part reflect the Confucian orientation of the Ming state: The Confucian tradition looked down upon merchants. Also, the Ming may have been more interested in stability than growth, and fearful that foreign contact would expose Chinese to dangerous foreign ideas. 


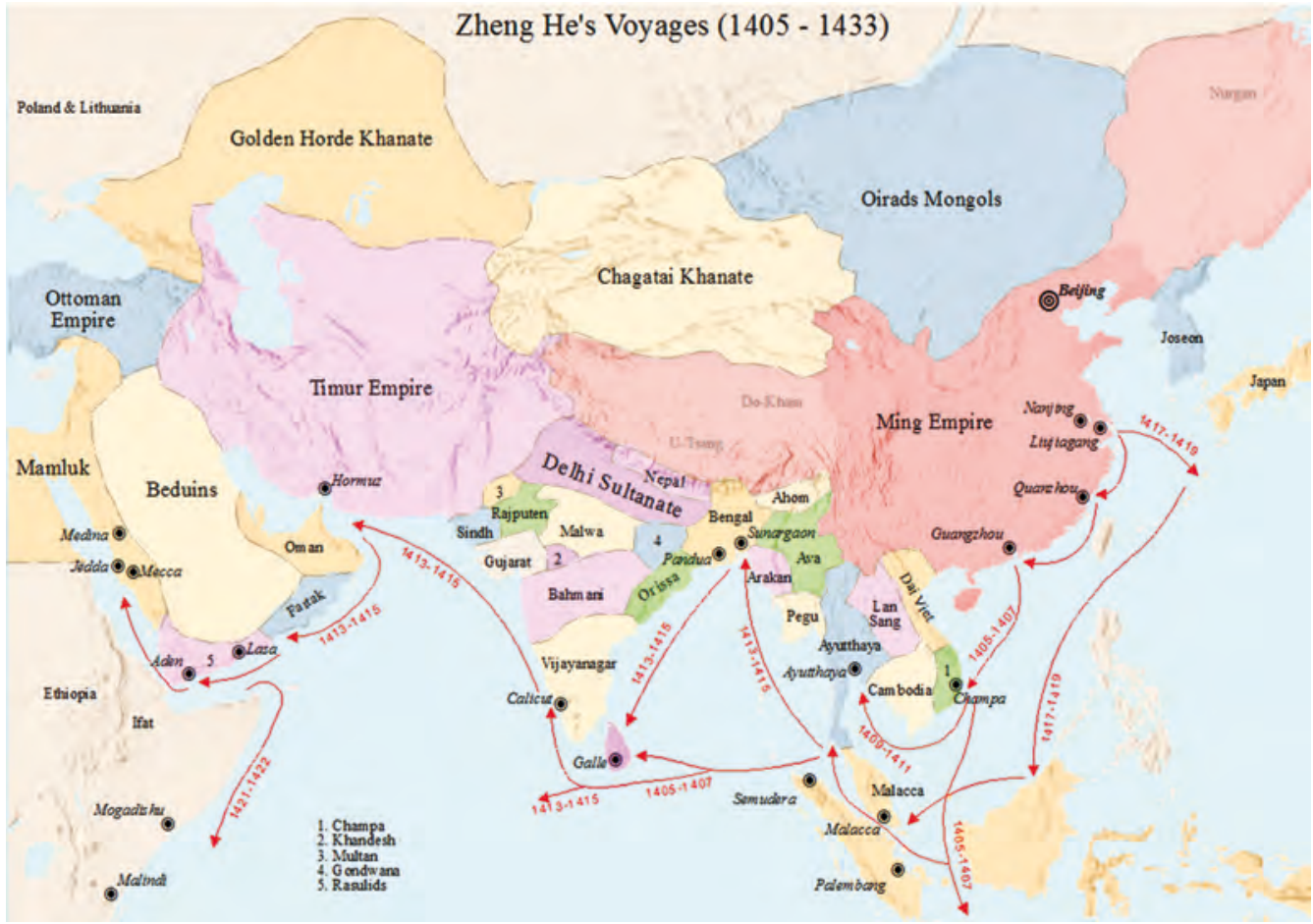

Map 20.5

Voyages of Zheng He 1405-33

Map by SY, CC BY-SA 4.0

\section{The Qing in China}

As noted above, the Ming failed to address famines in the early seventeenth century. The Ming army then struggled to put down local revolts. The Manchus, a nomadic people from Manchuria descended from the Jurchen tribes that had taken northern China from the Song centuries earlier, took advantage of these local revolts to invade and establish the Qing dynasty. The Qing dynasty, China's last, would rule from 1644 to 1911. As in other nomadic conquests, the Manchus had coalesced around a charismatic leader - Nurhaci - in the early seventeenth century. They had first attacked Mongolia and Korea, and then took Beijing in 1644; they would take decades more to conquer southern China. Famines and the Manchu conquest caused the Chinese population to fall yet again by tens of millions of people.

Although Chinese migrants would flood Manchuria in the late nineteenth century (chapter 31), it was in the seventeenth century ethnically distinct. Many Chinese bureaucrats who had come to disdain the Ming nevertheless 


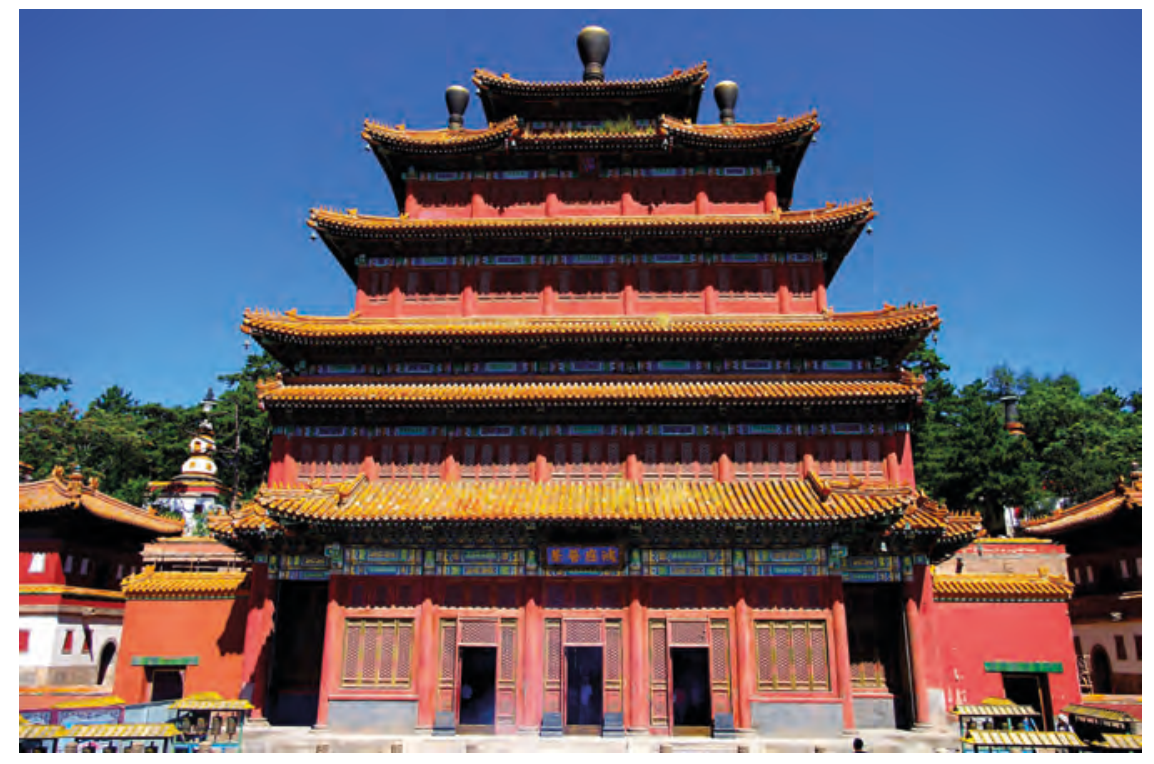

Illustration 20.27

Pu Ning temple 1755, Chengde Mountain Resort (summer palace), built by the Qing in the 18th century and one of the most famous tourist sites in China today

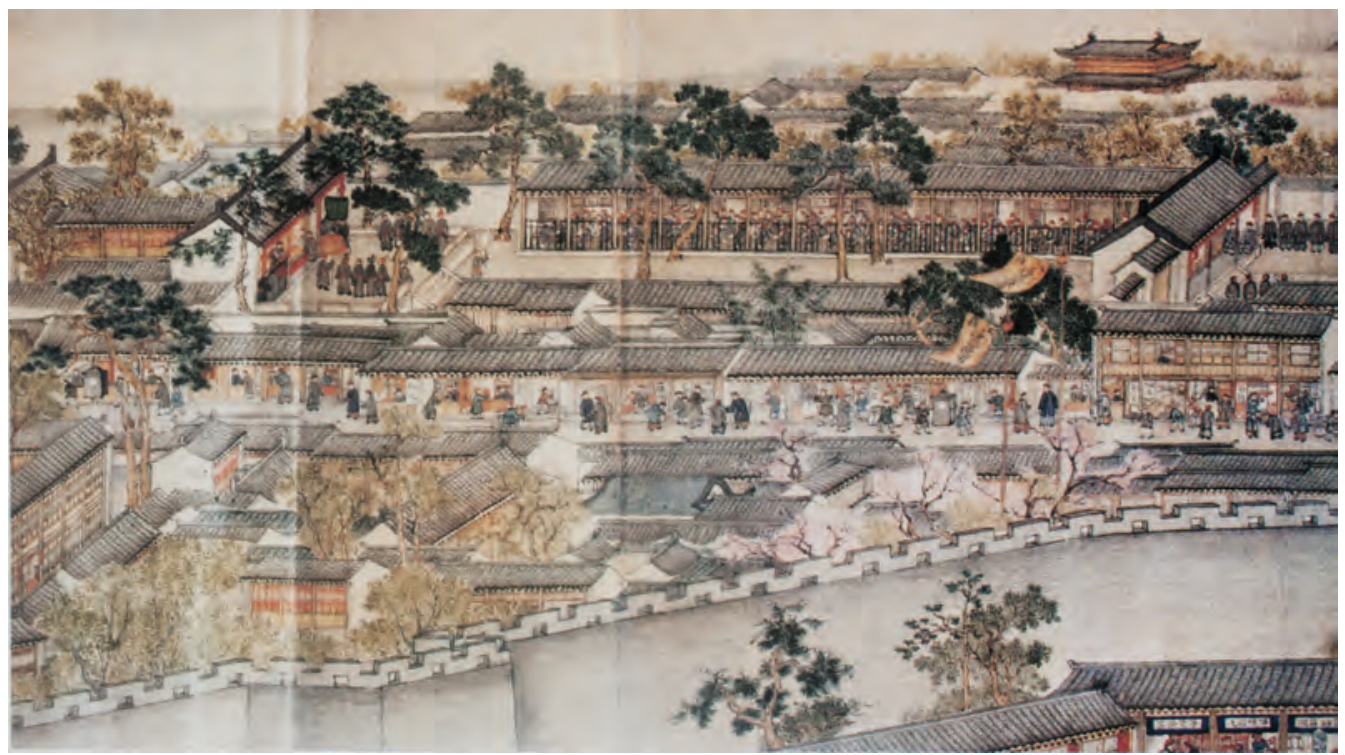

\section{Illustration 20.28}

Examination Hall, Suzhou, 18th century; Scroll by Xu Yang on order of Qianlong Emperor 
welcomed the Manchus. Indeed, the Manchus had been invited to quell peasant rebellions against the Ming. Like the Mongols before them (chapter 17), the Qing would develop a system of government that utilized the Chinese bureaucracy but sought to maintain control by a conquering ethnic elite. The Qing maintained the Ming administrative structure but placed Manchus in the most senior administrative positions. They maintained and later expanded the examination system.

The Qing would rule China for far longer than the Mongols had. Factional conflicts within the Qing court, often pitting Manchu against Chinese advisers, would weaken state authority. The Qing, though they claimed the Mandate of Heaven, did not have as much popular support as a local dynasty might have had.

The Qing at first wanted to prevent the conqueror elite from adopting Chinese cultural practices. They forbade intermarriage between Manchu and Chinese and made it illegal for Chinese to learn the Manchu language. Nevertheless, over time the elite did adopt Chinese ways. Kanxi, emperor from 1661 to 1722 , won favour by being a Confucian scholar, and by building irrigation and flood control systems. His grandson Qianlong was also a Confucian scholar. Though it is common in the west to think of China as monocultural, the Qing Empire comprised multiple languages - Manchu, Mandarin, Mongol, Korean, Arabic, Tibetan, Uighur, Miao, and Zhuang - and religions - Confucianism, Buddhism, Taoism, Tibetan Buddhism, Shamanism, Islam, and Christianity. The Manchus themselves practised Tibetan Buddhism, and encouraged this, but pursued a general policy of religious toleration.

The early Qing had followed the Ming policy of limiting trade. They soon changed strategy. Chinese merchants could trade abroad and became active throughout Southeast Asia. The Portuguese could trade at Macau and the English at Guangzhou. Porcelain, silk, tea, and lacquerware were exported; the main import from the west was silver.

Historians often see silver imports as a sign that China wanted little from the west. This argument is supported by the fact that emperors often disdained western manufactured goods. Yet there is a simpler explanation, masked by the fact that silver served as a currency. China had very little silver of its own. With discoveries in the Americas, Europeans had lots of silver. Therefore, the "price" of silver in terms of what could be bought with it was simply much higher in China than in the west. Silver would naturally be the good that was most profitable to trade in China - especially given that transport costs were low relative to the value of a ton of silver. The price differential would fall over time as silver was traded to China and as mines in the Americas were mined out. (Recall that the Song had centuries earlier worried about silver exports; chapter 15.)

China benefited from the Columbian Exchange. From the seventeenth century, farmers grew corn, peanuts, and sweet potatoes on lands poorly suited to rice or millet. Peanuts were well suited to sandy northern soils and encouraged migration into new areas. The Chinese population grew again, from 100 million in 1500 to 160 million in 1600 and 225 million in 1750. 
Scholars debate the economic record of the Qing. Recall that Song China had been the wealthiest and most dynamic economy in the world (chapter 15). By the late nineteenth century, the Chinese economy had fallen far behind Europe. We will discuss many possible reasons for this Great Divergence in chapter 22. Some but far from all of these reflect governmental decisions. It is common, nevertheless, to blame the Ming or Qing dynasties for having somehow arrested Chinese economic development. As in British India, the industrial sector contracted in Qing China as manufactured goods were imported from Europe. Yet the Qing government did build roads, establish schools, and protect property rights through a system of laws and courts. It continued a long Chinese tradition of suspicion of merchants but did introduce laws that governed markets and settled disputes. And it strengthened the system of national granaries to limit the effects of harvest failures. We can debate the effectiveness of its various actions, but it clearly tried in many ways to stimulate economic activity. It was likely less supportive of innovation than the Song had been, being primarily interested in social stability.

The Qing did try from 1860 to encourage scientific/technical education and the development of shipyards and armaments industries. However, these reforms were too late to stop European and Japanese encroachment. In 1898, Emperor Guangxu introduced a constitutional monarchy, guaranteed rights, and sought to transform education and the military, but pressure within the imperial household forced him to withdraw these reforms. The Boxer Rebellion

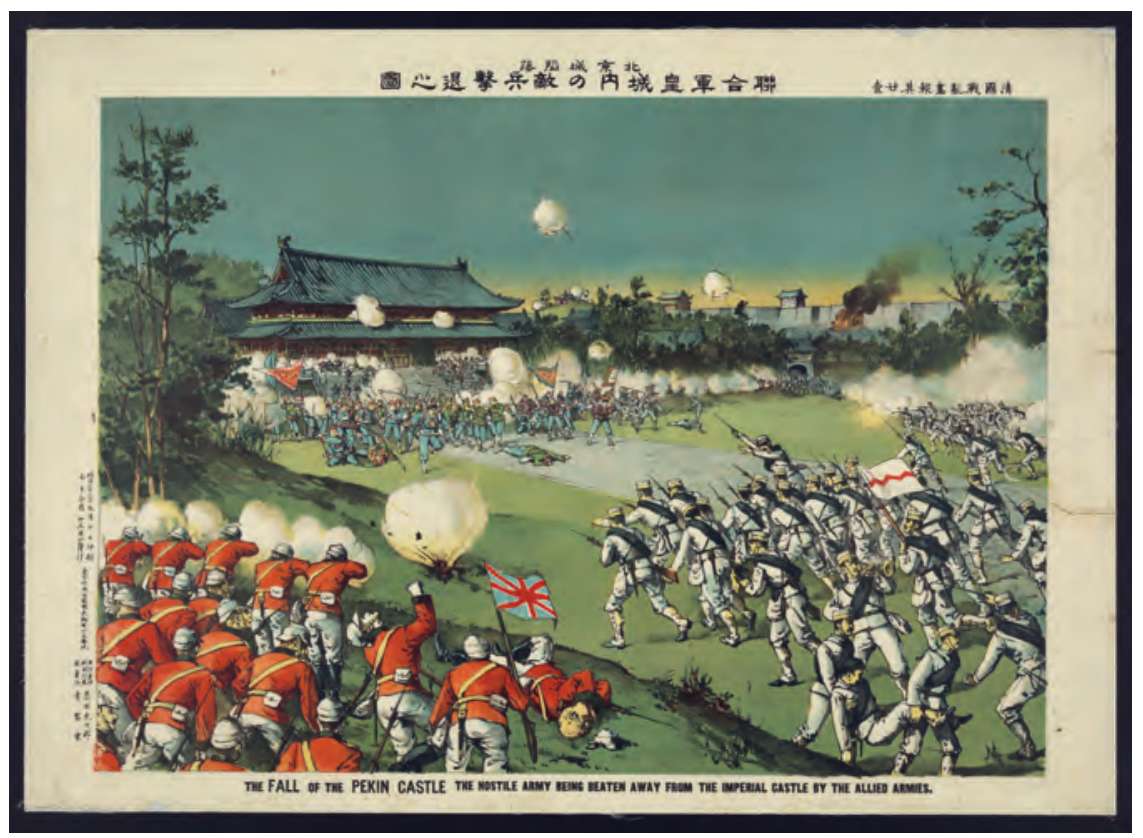

Illustration 20.29

Allied armies attack Beijing Castle during the Boxer Rebellion, Torajirō Kasai, 1900, Library of Congress 
of 1899-1900 sought to scare Europeans from China but instead encouraged a military reaction and the stationing of European troops at embassies. In 1912, the emperor abdicated in the face of a revolution.

Like the Ottomans and Mughals, the Qing dynasty would prove unable to compete with European powers militarily. It would lose multiple wars to Europeans and the Japanese over the course of the nineteenth century. Though not officially colonized by Europeans - excepting small enclaves such as Hong Kong and Macao - it would grant Europeans trading rights and spheres of influence on Chinese territory. The Opium Wars of 1839-42 and 1856-60 resulted in the British gaining the right to market Indian opium in China, an activity the Chinese government opposed due to concerns over the effects of drug addiction. Popular resentment at the relative weakness of the Chinese state resulted in a rebellion in 1911-12 that would end thousands of years of imperial rule in China. Still - as with the Ottomans and Mughals - we should

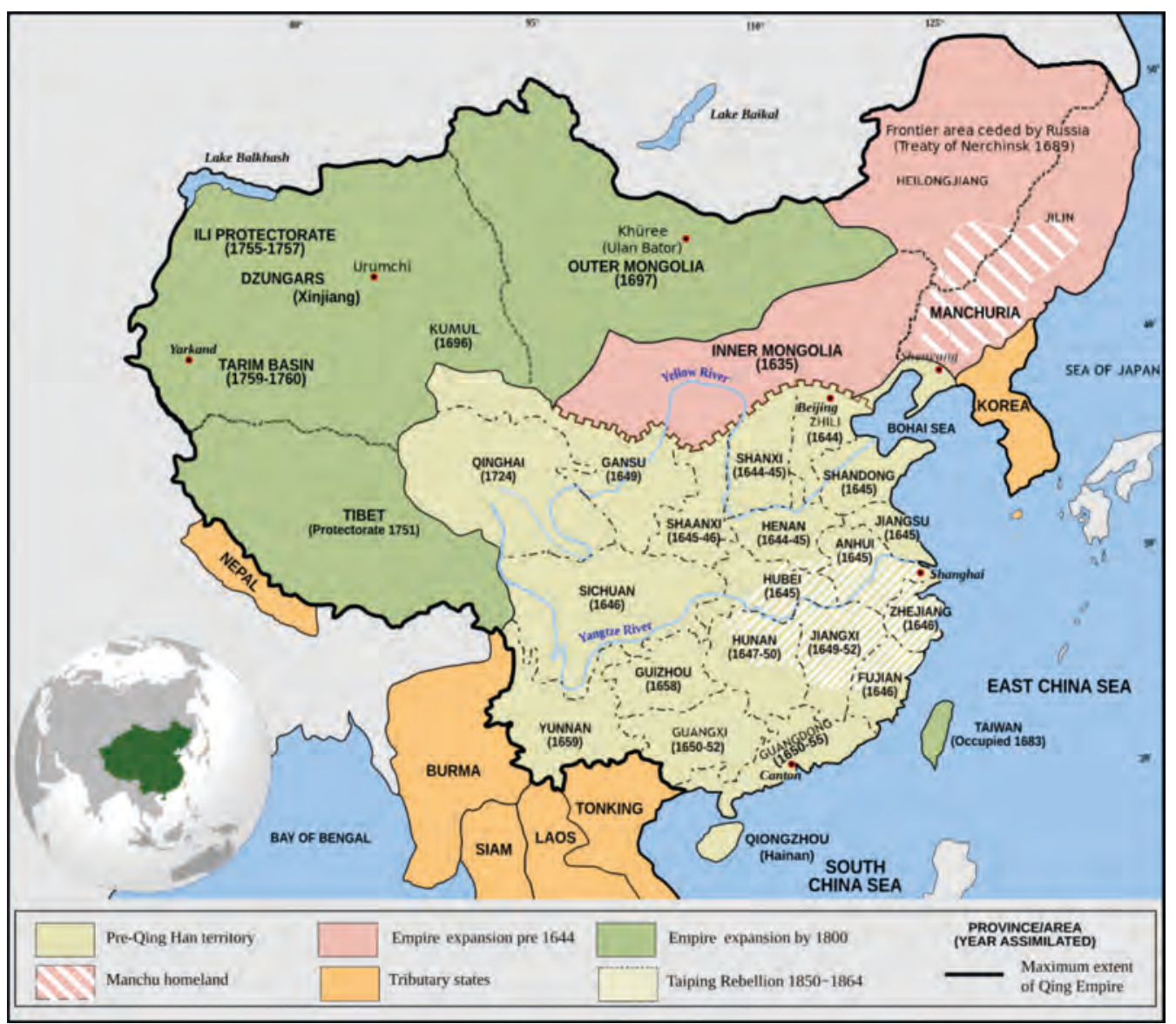

Map 20.6

The geographical expansion of the Qing Empire Map by Philg88, CC BY 4.0 
appreciate that the Qing ruled a vast territory for centuries before succumbing. Indeed, we saw in chapter 19 that the Qing expanded into Central Asia at the expense of nomads there, ending millennia of conflict between the Chinese state and Central Asian nomads. It was indeed during the time of the Qing that China doubled to its present size, adding much of Mongolia, Tibet, and Xinjiang. Qing China also established protectorates over Vietnam and Nepal.

\section{PRIMARY DOCUMENT: AN ADDRESS TO TWO HUNDRED MILLION FELLOW COUNTRYWOMEN}

Qiu Jin d. 1907, from Chinese Civilisation and Society: $A$ Sourcebook, Patricia B. Ebrey ed., New York; The Free Press 1981, translated by Nancy Gibbs (reproduced in Bonnie Smith, Modern Empires: A Reader).

Alas! The greatest injustice in the world must be the injustice suffered by our female population of two hundred million. If a girl is lucky enough to have a good father, then her childhood is at least tolerable. But if by chance her father is an ill-tempered and unreasonable man, he may curse her birth: "What rotten luck, another useless thing." Some men go as far as killing baby girls while most hold the opinion that "girls are eventually someone else's property." ... In a few years, without thinking about whether it is right or wrong, he forcibly binds his daughter's soft white feet with white cloth so that even in her sleep she cannot find comfort and relief until the flesh becomes rotten and the bones broken.

...[says that women should never have accepted footbinding, subservience]...

Let us all put aside our former selves and be resurrected as complete human beings.... After your sons are born send them to school. You must do the same for your daughters and, whatever you do, don't bind their feet. ... Those of you who are rich, persuade your husbands to open schools, build factories, and contribute to charitable organizations. Those of you who are poor work hard and help your husbands. ... You must know that when a country is near destruction, women cannot rely on men any more because they aren't even able to protect themselves.

How is her vision for women conditioned by the world in which she lives? Westerners at the time often disparaged the treatment of women in the east. Were they justified?

\section{Africa}

European colonizers of Africa (chapter 23) pretended that the societies encountered there were tribal, despotic, poor, and lacked cities. Europeans then proclaimed that it was necessary to bring civilization to Africa. While it is true that African polities were smaller and less complex than those in Asia, it is important to recognize that there had been considerable political 
and economic consolidation in many parts of Africa in the Early Modern period. The slave trade, while it ravaged certain populations, also often encouraged centralization: A strong central authority could attack others for slaves and use the income from selling these to support an army and bureaucracy (chapter 19). Although slaves were the primary African export, trade in a variety of other goods also expanded - though imported manufactures often replaced locally produced goods. Africa would contribute natural lubricants to the Industrial Revolutions (chapter 24), especially palm oil (over 20,000 tons annually by 1850) but also peanut oils (over 10,000 tons). Large Africanrun plantations as in the Sokoto Caliphate in West Africa often produced these new export crops.

The Sokoto caliphate was the most extensive state south of the Sahara before colonization. Some half of its population was slaves - though these were tied to plots of land and might better be thought of as serfs. The caliphate was actively involved in the export of agricultural products.

The Assante (of present-day Ghana) had long been organized in clans of matrilineal descent: These clans (as elsewhere in Africa) organized the periodic burning of forests for shifting agriculture and then assigned the cleared land to families. Profits from slaves and gold and kola nuts allowed powerful chiefs to emerge among the Assante. From about 1700, there was a centralized state and a creation myth involving a royal stool on which the king sat. Local chiefs remained important, but the power of the central state grew as trade expanded.

The Igbo of Nigeria lacked a sophisticated state but a shared religion guided collective efforts among lineages. There also seem to have been artisanal organizations somewhat similar to the craft guilds observed in Eurasia. Igbo nobles sponsored markets which held their own courts and revered a market god. There was a clan-like merchant network among the Igbo. The similarities to practices when Europe was politically fragmented (chapter 15) are notable.

Uganda was too far inland to be devastated by the slave trade. There also the Bugandan kings gained authority, though local clans retained more power than among the Assante. Bugandan kings profited from the increased productivity associated with new crops such as the banana. They encouraged intermarriage among clans to foster unity.

The Xhosa of southern Africa were an interesting mixture of Khoisan and Bantu peoples who focused on raising cattle. The Xhosan king was relatively powerless compared to local chiefs. The chief's sons did not inherit his lands: The sons were therefore encouraged to conquer other lands. We noted a similar practice among the Inca in chapter 16.

The coast of East Africa had for centuries been dotted with city states that traded with the Middle East. These had long relied on Africans from the interior to bring trade goods to the coast. In the nineteenth century, it became increasingly common for traders from the coast to establish inland networks.

In the seventeenth century, the Kongo kingdom tried diplomatically to use the Spanish and Dutch to maintain independence from an ambitious 
Portugal. This strategy prompted a Dutch invasion of the Portuguese colony in Angola. The Kongo strategy is not unusual: Many African polities, and many indigenous groups in North America also, sought to ally with some Europeans against others. They were not, that is, passive victims of European colonialism, but actively sought to utilize European rivalries to their own ends. This was always a risky strategy, for if the power balance among European powers shifted, a loss in local independence generally resulted.

In Angola itself, the Portuguese had first helped the kingdom of Ndongo gain independence from Kongo but eventually colonized the area. The Ndonga were led by a formidable queen named Nzinga from 1623-63, but she was unable to dislodge the Portuguese.

Islam south of the Sahara was in 1700 a religion mainly of traders, rulers, and nomadic pastoralists. Since African faiths generally also recognized a supreme deity, a syncretic Islam would spread in the next centuries among agricultural populations. Farther south Christian missionaries arrived, often intent on disrupting the slave trade. Their early converts would then spread the religion; syncretism was again common. Resettled slaves from the Americas were also important missionaries in West Africa. Missionaries often stressed the few biblical mentions of Egypt and Ethiopia.

Many African leaders had come to appreciate that European powers had superior military capabilities grounded in advanced industrial technologies. There were debates in many African polities about whether - and if so how to copy European ways. Only Ethiopia and Morocco (and for a while Egypt; see above) would be able to prevent European colonization in the nineteenth century. Ethiopia would defeat the Italians in 1896, using European weapons, but fall to them in the 1930s (chapter 28). The Moroccan sultan developed a tolerant state with a strong enough bureaucracy to stand up to Europeans. The French and Spanish would establish protectorates in Morocco in the twentieth century.

\section{Questions}

1. Discuss how the policies of the three Muslim empires shaped the geographic distribution of religions in the modern world.

2. Discuss the effects of gunpowder technology on the three empires.

3. Diagram the influences on and effects of any one of the empires discussed in this chapter.

4. Imagine you are a member of some society that is failing for some reason; do you lash out at those you have trusted with power?

5. The Ming Empire is generally far more well known in the West than the Song Empire (or indeed the Sultanate of Delhi). Why? 


\section{Readings}

Bang, Peter F. and Dariusz Kolodziejczyk, eds. 2012. Universal Empire: A Comparative Approach to Imperial Culture and Representation in Eurasian History. Cambridge University Press.

Dale, Stephen F. 2010. The Muslim Empires of the Ottomans, Safavids, and Mughals. New York: Cambridge University Press.

Desnoyers, Charles A. 2020. Patterns of East Asian History. Oxford University Press. Gilbert, Marc Jason. 2017. South Asia in World History. Oxford University Press.

Rogan, Eugene. 2015. The Fall of the Ottomans: The Great War in the Middle East. New York: Basic Books.

Roy, Tirthankar. 2013. An Economic History of Early Modern India. New York: Routledge. Rubin, Jared. 2017. Rulers, Religion, and Riches: Why the West Got Rich and the Middle East Did Not. New York: Cambridge University Press. [This book argues that Muslim rulers relied more heavily on religious authorities to legitimate their rule than did Christian rulers in Europe, and that conservative religious authorities impeded economic and technological development.] 


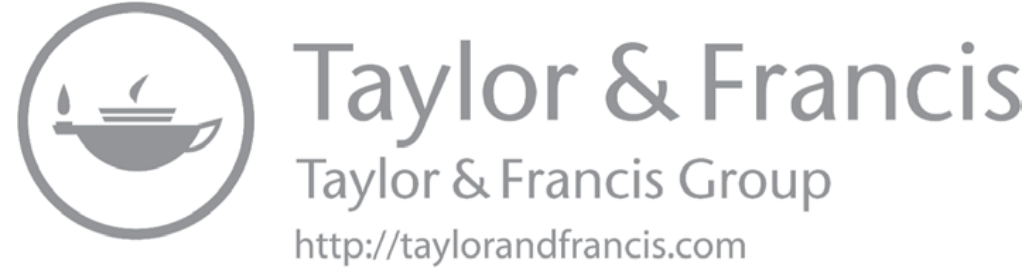




\section{It seems so natural now}

\section{The emergence of the modern nation state}

\section{Guiding questions}

What is a nation state? How, where, when, and why did the modern nation state emerge? How in particular was a sense of nation forged? (Who formed the nation and how?) What were the key characteristics and effects of nation states?

What is nationalism and when, where, why, and how did it develop during this period?

How and why did ethnic identities harden during this period?

What was the connection between ethnicity, nationhood, and nationalism?

What happened to multi-ethnic empires as ideas of ethnic identity and nationalism spread?

How and why did democracy emerge in some nation states but not others?

Relationship to other chapters: We can only appreciate the nation state by contrasting this with other political forms and especially the empire. Equally, we can only understand how particular nation states emerged because of our understanding of the previous political realities in different regions of the world. We shall see that the emergence of nation states reflected changes in trade and military technology that we have addressed in chapter 18 and elsewhere. We can only appreciate hardened ethnic identities in contrast to the more fluid identities we encountered in chapter 13 and elsewhere. The printing 
press of chapter 14 will prove important to the strengthening of ethnic and national identity. We shall see that nation states could potentially tax their population more than preceding empires usually could: This had significant implications for armies, transport infrastructure, education, and eventually a range of social services. We will often discuss in later chapters new governmental roles that became feasible because of nation states. We will also see how nationalism destroyed many multi-ethnic empires (especially in chapter 30) and encouraged horrific world wars (chapter 28). We will resume the story of democracy in chapter 27 , tracing how an institution that characterized only a few nation states in the early nineteenth century spread across much of the world in the later twentieth century. Our discussion in this chapter naturally informs our later analysis of why democracy has taken hold in some places but not others.

We must again warn the reader of the dangers inherent in viewing the past through the eyes of the present. "Nation states," independent polities with ideally at least - a shared sense of identity, govern much - but far from all - of the world of the twenty-first century. A shared ethnicity, language(s), religion, and/or history is often critical to national identity. It is all too easy for the modern person to assume that the nation state is the obvious form of human governance, and look to history for evidence of the inevitable spread of the nation state once it first emerges on the scene. They may indeed wonder why it took so long.

It is thus worth remembering at the outset that the nation state is problematic. Minority groups of many types may feel left out of the "nation." In many parts of the world, and especially much of sub-Saharan Africa, polities lack a shared sense of identity. Rather, different ethnic groups with a long history of mutual suspicion populate these states. Some scholars have speculated about the potential advantages in such places of a "return to empire," recalling the toleration of diversity (but perhaps downplaying the autocratic and self-interested exercise of power) that characterized the majority of empires in world history.

As we shall see, the nation state emerged within a particular set of historical circumstances. It is useful for us to explore dispassionately what these were. The path to the nation state was nowhere smooth nor seemingly inevitable: There were always political actors suggesting entirely different political outcomes. The modern nation state developed over a period of centuries. We therefore extend our treatment in this chapter into the nineteenth and occasionally twentieth centuries.

We should stress the composite nature of the term "nation state." "State" is a word we have often employed in this book to indicate a form of political 
organization involving a monopoly on violence, the control of territory, and a formal bureaucracy. "Nation" refers instead to the people that together comprise the state. A "nation state," then, is simultaneously an idea and a description of political reality: It makes sense only if people come to see that a particular nation should comprise a particular state. Not surprisingly, we shall find that the idea of the nation state and the political reality of nation states emerged together, with each encouraging the other. Once the idea was instantiated in reality in many states, it spread across the globe.

Much of our discussion in this chapter involves interactions among different sets of political phenomena. Though we shall stress that the idea of popular sovereignty does not necessitate a sense of cultural identity, we shall find that most nation states did indeed pursue an ideal of cultural identity. Culture then plays a vital role in this chapter, as does the ethnic dimension of social structure. Since philosophers as well as politicians espoused the ideal of popular sovereignty, we can consider it both a scientific and political idea. The development of strong centralized states reflected both economic and technological opportunities. Last but far from least, strong personalities generally forged nation states. We shall see that once some nation states existed, they encouraged nation-state formation elsewhere, in part by destabilizing multi-ethnic empires. We thus devote much attention to interactions among states. In discussing particular nation states, we will, of course, consider institutional evolution - but the most explicit applications of evolutionary analysis occur in later discussions of state capacity and democracy.

\begin{tabular}{|c|c|c|c|c|c|}
\hline Politics & Culture & $\begin{array}{c}\text { Social } \\
\text { Structure }\end{array}$ & $\begin{array}{c}\text { Science } \\
\text { Technology }\end{array}$ & Economy & $\begin{array}{l}\text { Individual } \\
\text { Differences }\end{array}$ \\
\hline
\end{tabular}

\section{A historical survey}

\section{Europe in the fifteenth through eighteenth centuries}

From the late fifteenth century, monarchs in England, France, and Spain centralized their states with new taxes and national armies: sales taxes in Spain; sales, household, and salt taxes in France; fees for services in England plus large revenues from dissolving the Catholic monasteries. We have discussed earlier (chapter 14) how military technology and expanding trade made this centralization of power possible.

It had seemed that an even greater degree of centralization of power might be possible. Charles V (1519-56) briefly looked capable of achieving empire, with the title of Holy Roman Emperor and vast lands in Spain (and Spanish colonies in the Americas), the Netherlands, and Austria, Bohemia, and Hungary. However, the French allied with both the Ottomans and the Lutheran princes of northern Germany against him, and his sons divided his lands upon his death. After that, a set of regional states of varying sizes jostled for power in Europe. 
The military technology that encouraged centralization discouraged the sort of loose assemblage of territories that strategic marriages had bequeathed to Charles V. Yet the forces of consolidation were not so great as to ensure that the largest European state would swallow the rest. The twin facts that the ideal of a pan-European empire had long been forgotten (chapter 10) and that regional states were putting together their own centralized bureaucracies may have militated against the formation of a continental state.

Regional states battling against each other sought to justify themselves. Religion was one source of identity: the Protestant rulers of England and the Netherlands against the Catholic rulers of France and Spain. The Thirty Years War - which primarily though not always pitted Catholic against Protestant states in Germany (chapter 18) - ended with the Treaty of Westphalia in 1648, which recognized the sovereignty of independent states, including in the religious realm.

Political institutions became another source of identity. In England and the Netherlands, kings shared power with elected assemblies. In both cases, there had been long battles over the relative powers of kings and assemblies. We saw in chapter 18 how civil war in England led to kingly recognition of the powers of Parliament in the seventeenth century. The Dutch Republic emerged

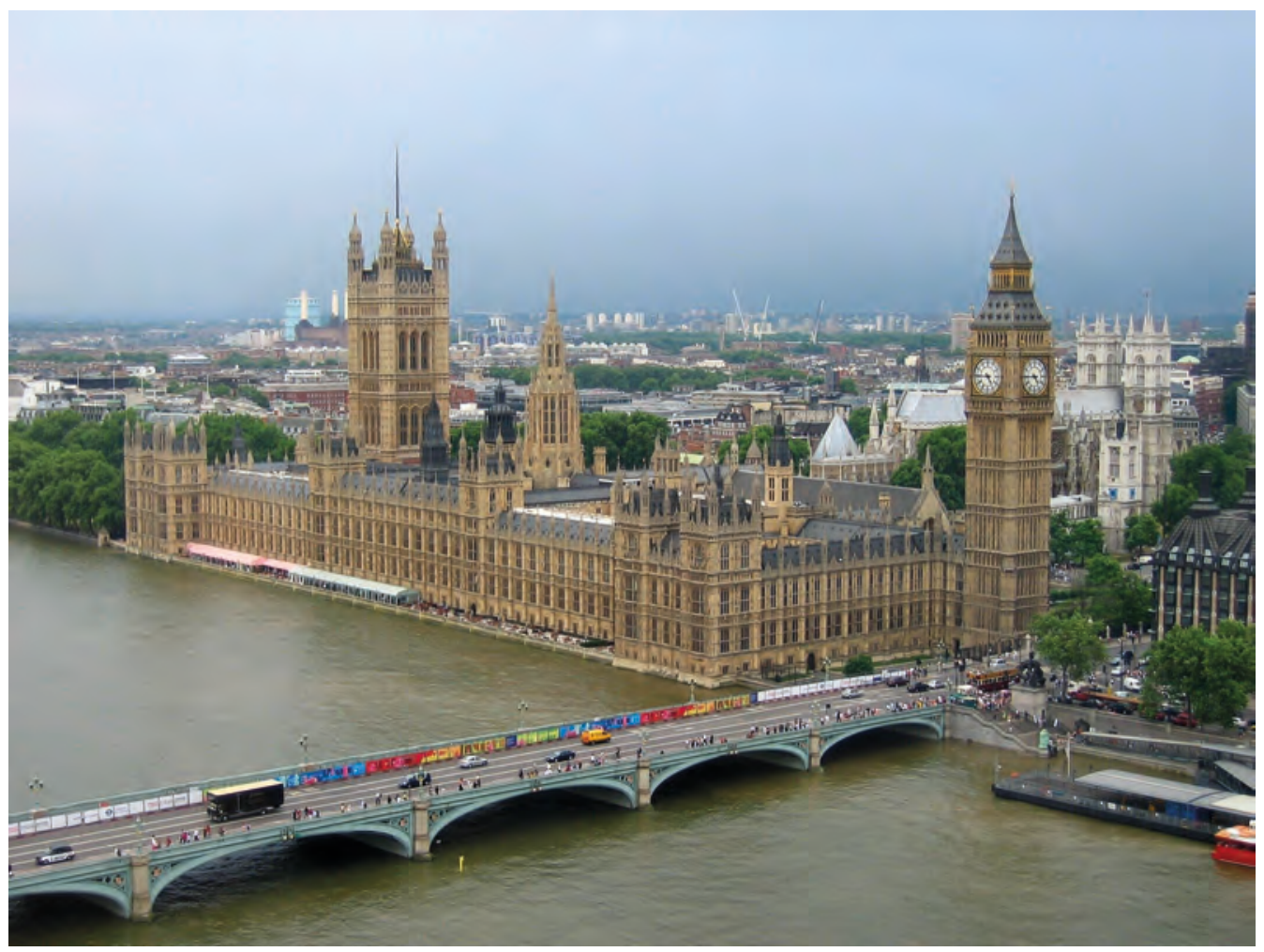

Illustration 21.1

Parliament buildings, London. These replace an earlier structure destroyed by fire in 1834 
as it achieved independence from Spain: Both mercantile and religious motives played a role in the Dutch rebellion and resulted in a state where elected assemblies were powerful (chapter 18). These assemblies, comprised of landowners and merchants, could reliably proclaim individual rights to property that in turn encouraged trade and investment.

Elsewhere, absolutist governments invoked the divine right of kings to justify their rule. This appeal to Christian doctrine resembled the Chinese Mandate of Heaven, but without the recognition of the inevitable overthrow of every dynasty. Louis XIII of France in the early seventeenth century reduced noble power, often by destroying castles, while developing a large bureaucracy. Louis XIV built the Palace of Versailles, the largest building in Europe, and invited nobility to live there where they could be watched (a practice, we have seen, pursued in many parts of the world; chapter 14).

Farther east, Peter the Great (1682-1725) imported Western administrative and military practices into Russia and created a centralized state. He established a new westward-facing capital in St. Petersburg on the Baltic after taking the region in a war with Sweden (the city grew to 220,000 by the end of the eighteenth century, by far the largest on the Baltic). The tsar and nobility had jostled for power in the centuries after the rise of Muscovy (chapter 17), but the need for a common defence of the vast steppe lands plus opportunities for foreign conquest (chapter 19) had allowed tsars to gain power over the nobility. The tsars had, in turn, supported the aristocracy in their enserfment of peasants.

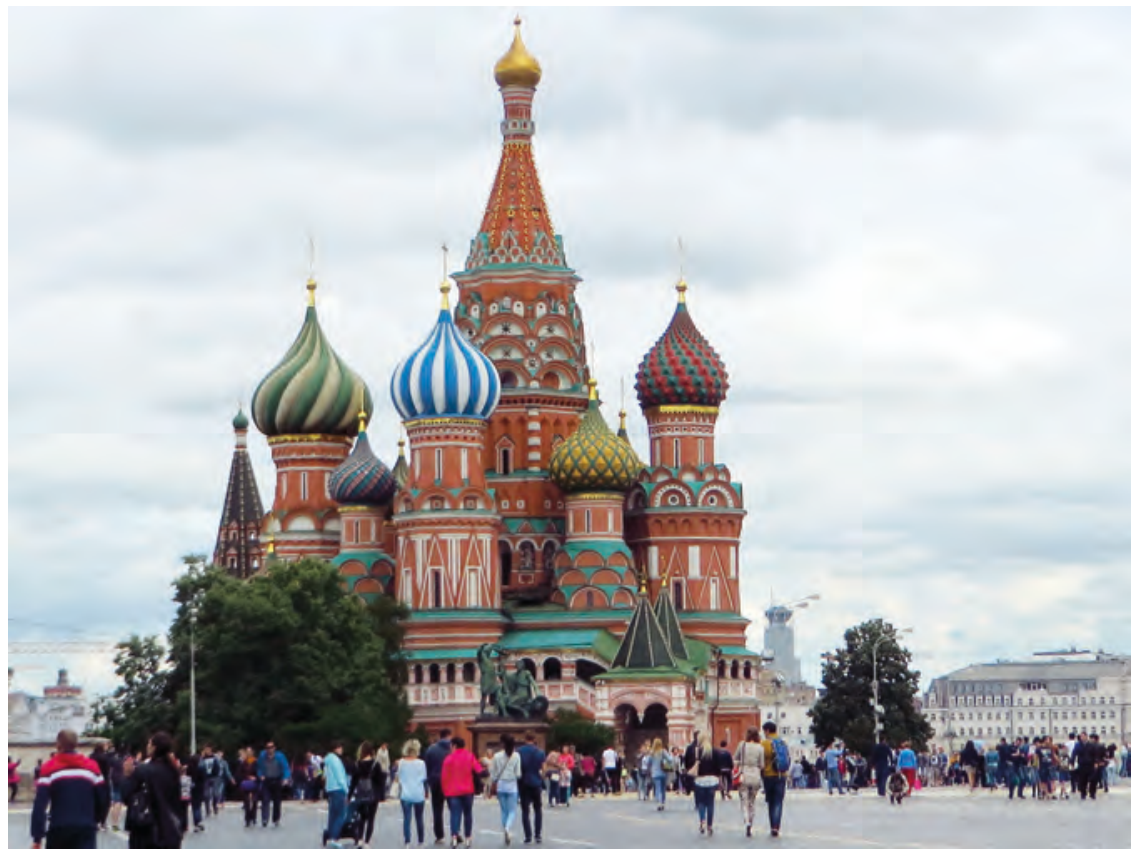

Illustration 21.2

Basil's Cathedral, late 16th century, Red Square, Moscow 
Peter would expand the Russian state into Ukraine and southeastern Europe. Though Russia had long seen itself as culturally distinct from the rest of Europe, Peter sought explicitly to copy the emerging ideal of a nation state. However, this would not prevent either him or his successors from conquering vast territories in Asia inhabited by peoples of entirely different languages and religions (just as England, France, and Spain would colonize much of the world). These conquests allowed the tsars to strengthen their authority: They appointed loyalists as nobles in the new lands and drew old noble families into military service. Peter also sought to borrow Western military and iron-making technologies in order to compete with other European polities militarily. Catherine the Great (1762-96) pursued similar policies. Catherine tried to limit noble power over serfs, but after the Pugachev serf rebellion of 1773-4, which killed thousands of nobles and bureaucrats, focused instead on strengthening absolutist power. In the artistic realm too, tsars sought to borrow from the West while at the same time enhancing a Russian sense of identity. The state

\section{BOX 21.1}

\section{DYNASTIC RISE AND FALU}

Mark Ciotola of San Francisco State University produced the following diagram (see references). It is a provisional attempt to graph dynastic "power." Power in this sense can capture both the social power that rulers exert over their subjects and the physical power employed within the state. Though it is difficult to estimate power precisely, the shape of the curve makes sense: The Romanovs, like many dynasties, accrued power over a period of decades or centuries, but lost this power much more quickly toward the end. Note that Romanov power peaked during or slightly after the reign of Catherine the Great.

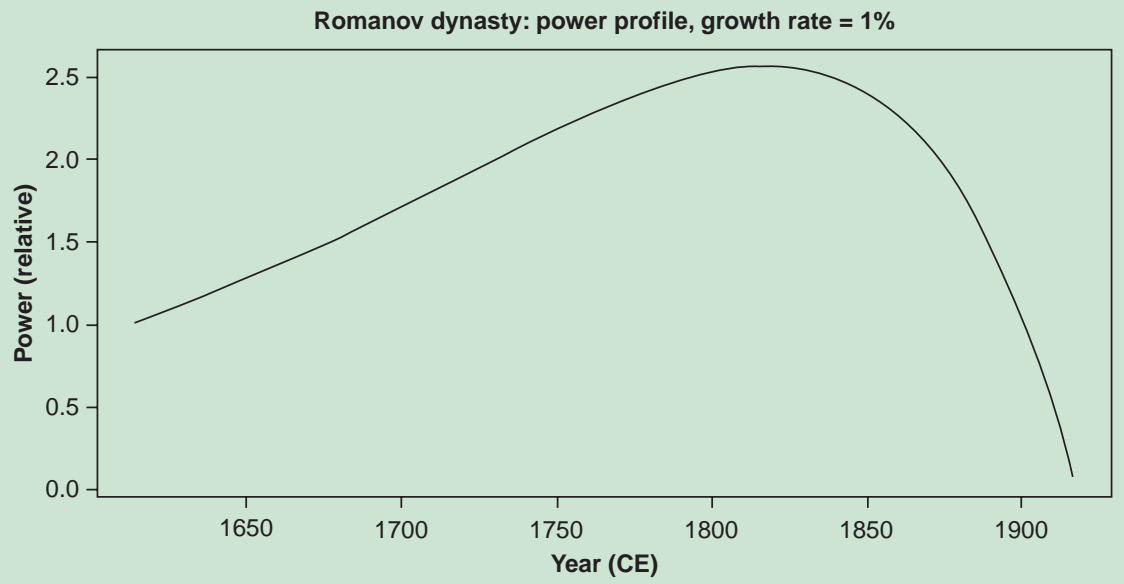

Figure 21.1

Romanov Dynasty power profile 
imported Italian architects to build national monuments: The result was a syncretic merging of local artistic sentiments (including the onion dome, which seems to have originated in Russia in the thirteenth century or earlier) with Western architecture. Russia would resume a tradition of iconic art but also borrow Western art forms such as ballet. A conservative peasantry was often hostile to imports of culture by the nobility.

In the various lands around the Baltic, ethnic intermingling and (in many cases) the lack of a strong sense of national identity hindered efforts to develop nation states. Rulers in both Norway and Sweden sought to codify languages that were distinct from Danish - though some intellectuals in all three lands urged a pan-Scandinavian identity. In Finland, nationalism was associated with an effort to replace the Swedish economic elite. In Latvia and Estonia likewise, nationalists faced a German economic elite: They would seek economic autonomy but decide over time to recognize minority rights to language and

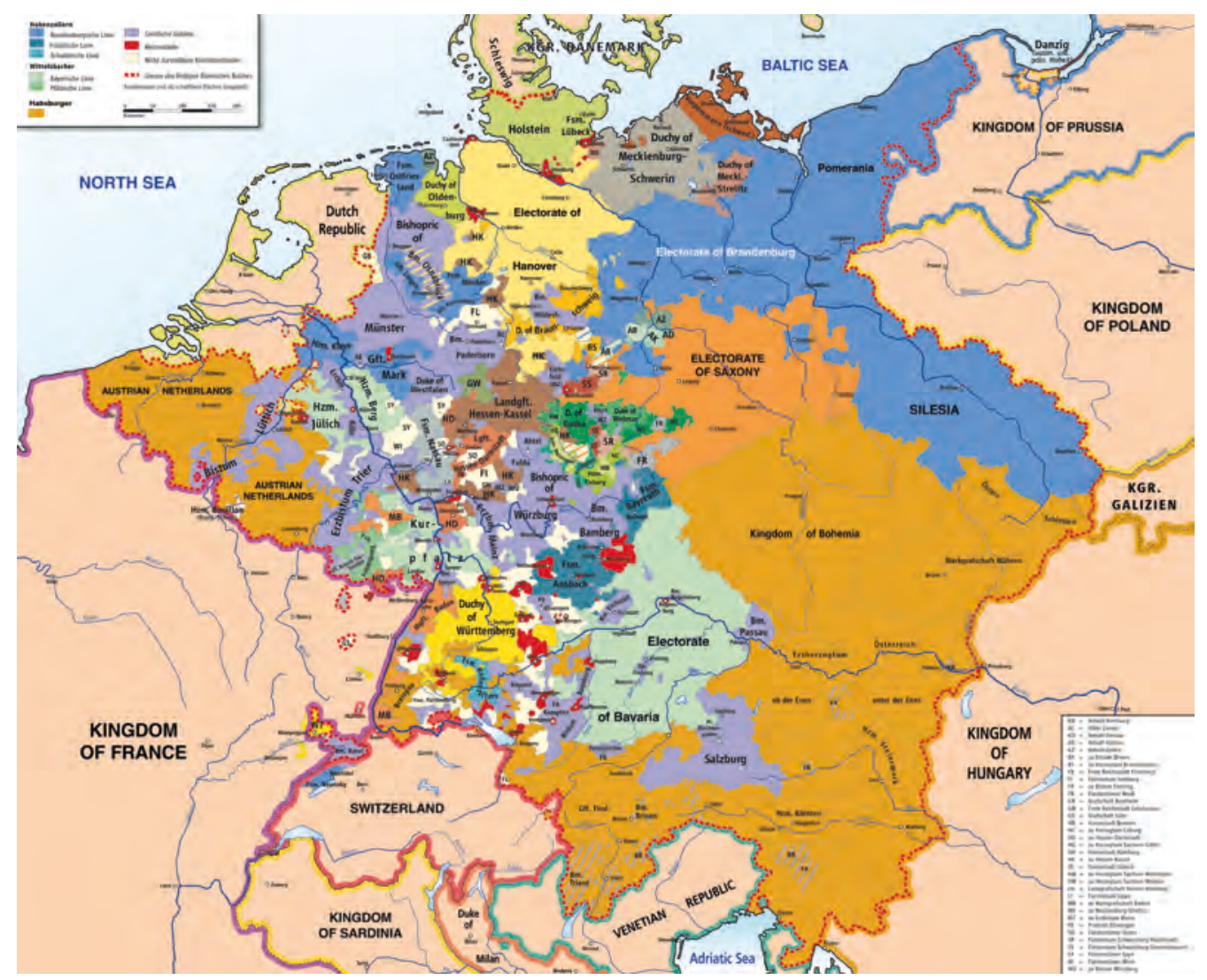

Map 21.1

Map of the Holy Roman Empire in 1789. Note the large Habsburg (Austrian) holdings (orange) and Prussia (blue) but also the many other small states that comprise Germany today. Many states were too small to show on the map Map by Robert Alfers, kgberger, CC BY-SA 3.0 
religion. (The Latvian state would later expropriate lands from minorities in the 1930s.) Though Scandinavian nationalists could revere a long history of Viking sagas, a distinct literature celebrating Latvian and Estonian nationality emerged only from the eighteenth century. As earlier in Scandinavia, governments would strive to codify the Latvian and Estonian languages.

As the idea of national sovereignty emerged in Europe, it reflected the reality on the ground of a set of regionally based states. In states with popular assemblies, intellectuals argued that peoples with a shared identity deserved to guide their collective destinies. There was, though, an intense debate as to whether the shared identity should primarily be ethnic or institutional: Should one be proudly English because of one's language or because one valued Parliament and the rights it guaranteed? (It was recognized that the latter attitude might make more sense if people generally chose which state they inhabited.) Leaders and intellectuals have invoked these two entirely different types of national identity ever since in states around the world. States with autocratic kings sought to argue that the king expressed the will of a collective people. The people were urged to value a collective identity and its expression in the institution of monarchy.

The intellectual currents of the seventeenth and eighteenth centuries in Europe are often termed the "Enlightenment": Thinkers at the time sought to provide a logical basis for societal institutions and behaviours. They began to expound the idea that sovereignty lay in the people rather than a king. This idea was not entirely novel: One can identify precursors such as Athens (chapter 10) and the Swiss cantons in Europe, and even the right of Chinese peasants to rebel against an emperor who had lost the Mandate of Heaven. The Enlightenment extended and clarified arguments for popular sovereignty. Enlightenment thinkers put forward the idea of a social contract between rulers and ruled: The ruled had certain personal rights, and the right to replace bad rulers. Freedom of speech and religion were advocated. Increasingly, Enlightenment figures argued for some sort of democratic decision-making though not usually for women, slaves, or sometimes even the poor. Nor did these thinkers generally grapple with the question of the geographic boundaries of the state, though their emphasis on freedom of religion guided them away from the narrower ethnic identities that would become associated with most nation states (see our discussion of nationalism below).

We stressed at the start of the chapter that the idea of the nation state emerged in concert with the political reality of nation states. These ideas of popular sovereignty both reflected the development of strong regional states and could be extremely useful to such states. These newly centralized states with their growing bureaucracies had both practical and ideological reasons for encouraging a common language and culture. It was much easier to administer a state with a shared language and customs. As educational systems developed, they tended to teach the language of the capital. They also tended to teach a shared national history. This shared history provided a compelling justification for the state. France, we might note, was once characterized by a set of regional 
dialects or languages that were almost mutually unintelligible. The region of "Languedoc" (the language of "Oc") in southern France is so called because the locals used to say "Oc" for yes rather than the "Oui" of Paris. However, over the centuries, Parisian French became dominant everywhere in France, and with it a stronger sense of French national identity. As late as 1789, only a third of the French may have spoken Parisian French. Yet Parisian culture and language had and would spread for centuries.

As nation states developed a strong sense of identity, they found it easier to raise taxes. The state now fought foreign wars on behalf of the nation rather than the ruler. National identity facilitated the practice of conscription and provided widespread support for war. Citizens with a shared identity also more readily supported the monuments, schools, and roads that nation states built.

At the same time that European states were forging common identities internally, they were conquering diverse territories abroad (in part because of their advantages in gunpowder technology and in part because of their ability to fund large armies). The emerging ideology of nation states did not dissuade states from conquering culturally distinct peoples. To be sure, they justified these conquests in terms of spreading "civilization" to the rest of the world. Yet they would each have far more success in entrenching their languages and (sometimes) religion abroad than their political institutions (chapter 23, 30). Many European intellectuals pointed to a sharp conflict between ideals of national sovereignty and the realities of imperial conquest. Many scholars today would argue that we cannot really speak of European nation states until they shed their colonies.

The French Revolution (chapter 25) proclaimed the ideal of national sovereignty. Revolutionaries generally assumed that there was a shared French history and vision on which to build a new republic. This idea of shared national destiny, especially in combination with external threats from other states, led to the practice of national conscription and the national projection of military force. France developed more substantial military forces than ever seen before in Europe, for unlike China, Europe had not practised mass conscription in preceding centuries. The French emperor Napoleon's conquests then spread the idea of national sovereignty further. When Napoleon was defeated, the victorious multi-ethnic empires to the east attempted to quell nationalist sentiments. Yet ideas of national sovereignty spread despite imperial opposition. Indeed the Prussian Empire would develop a vision of a united Germanic polity. Germany and elsewhere - including the Ottoman Empire and Japan - came also to practise conscription in the nineteenth century.

\section{Europe in the nineteenth and twentieth centuries}

We have seen so far how political and intellectual developments interacted in the formation of several early European nation states. Most of Europe's nation states would be created only in the late nineteenth or twentieth centuries. We 
discuss these below. In such cases, the idea of the nation state predated the political reality and provided a powerful incentive for the formation of these new nation states. Yet there too, political realities determined when and how nation states formed.

Various dialects of German were spoken in central Europe. Prussia used this common language as a pretext for conquests of most of these Germanic-speaking lands and the formation of the German state in 1871. It fought not only dozens of smaller German states but also the Austro-Hungarian Empire (which though headquartered in a German-speaking land, governed mostly Slavs and could therefore not make the same claim to celebrate German nationality), France, and Denmark. Along the way it absorbed areas of mixed ethnicity/language on its borders with both Denmark and France. Thereafter, this German state would encourage standardization of the German language and a celebration of the (supposedly) shared history of Germanic peoples. Notably, these appeals would prove stronger than the religious tension between Protestants and Catholics that had devastated the region just two centuries earlier.

To the south, many dialects of a common language likewise characterized the Italian peninsula. Political consolidation in Italy was a more convoluted process. King Victor Emmanuel of Piedmont and Sardinia, with the help of his Prime Minister Camillo di Cavour, allied with France: The French expelled the Austrian Empire from northeastern Italy in return for Victor Emmanuel renouncing his French territories. Assemblies in central Italy voted to join this new state. Giuseppe Garibaldi led 1,000 redshirted soldiers in a revolution

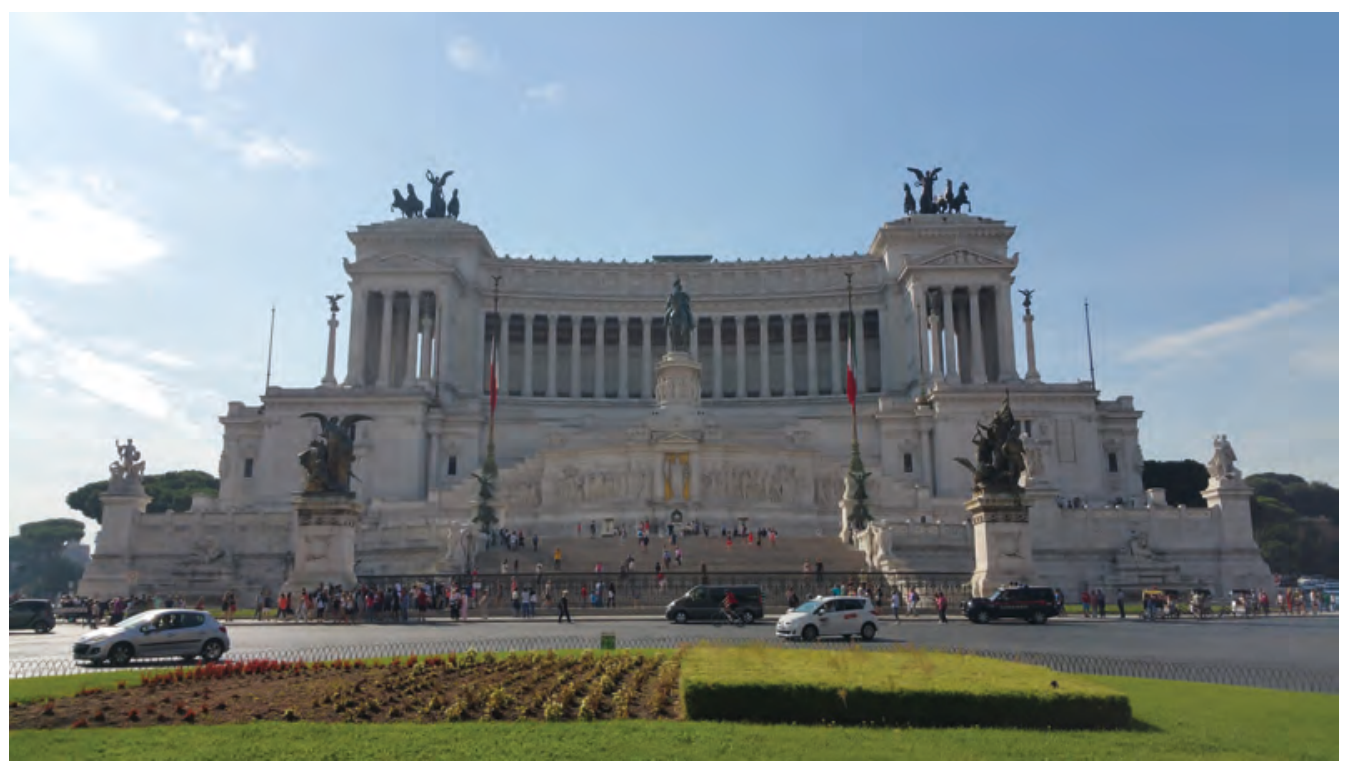

Illustration 21.3

Monument to Victor Emmanuel II, the first king of a unified Italy, in Rome, also called Altare della Patria (Altar of the Fatherland), built between 1895 and 1927 
against the Kingdom of Two Sicilies, which controlled much of southern Italy; he then turned this territory over to the new state as well. Though Garibaldi and others had sought to forge a democratic Italy, the result was instead a new Italian monarchy proclaimed in 1861 (though it would add Rome and Venice later). As in Germany, the new Italian state sought to reduce regional differences in dialect. It could recall the glories of both the Roman Empire and Italian Renaissance in celebrating the history of the Italian people. Yet it was famously (likely first by the Marquis d'Azeglio) and repeatedly said in 1861: "We have created Italy; now we must create Italians." Italian dialects were so distinct as to be almost mutually unintelligible. At the time, perhaps one-tenth of the population spoke the official Italian dialect of the new state.

Note that the creation of Italy and Germany did not occur in isolation. France was actively involved in the creation of Italy. Britain tacitly approved. Both recognized the principle of a sovereign Italian state. German unification involved military confrontation with other European states - but these were battles over particular territories rather than objections in principle to German unification (though Prussian military might was viewed with suspicion). These wars reflected the fact that ethnic groups were interspersed across the European landmass: The borders between nation states were far from obvious. Leaders did not so firmly hold to the ideal of popular sovereignty that local peoples could vote to determine boundaries. Military might instead drew borders. This would prove to be an enduring challenge within a system of nation states.

National competition was a characteristic of nation-state formation from the beginning. For example, German identity was contrasted with the identity of neighbouring groups. Yet there were also crucial examples of cross-national cooperation as when France supported the idea of Italian unification. It was possible as the nation state developed to both fear a future of conflict but hope for a future of cooperation. The simple fact that ethnicities were not always concentrated geographically enhanced the likelihood of conflict.

The Austro-Hungarian Empire (the inheritors of Charles V's holdings in Austria, Bohemia, and Hungary) would remain a multi-ethnic polity while the rest of western Europe was developing into nation states with a dominant cultural identity. Why? One can certainly argue that the population was more ethnically diverse. Moreover, there was not a long tradition of being in a shared polity as there was in England and France (see chapter 13). However, recall that the shared identities in France and elsewhere were to a significant extent created rather than inherited by those states. There may be an important role here for historical timing: The various ethnicities of the Austrian Empire had gained a strong sense of identity before the Empire could seek to instil a collective identity.

The Austrian rulers also suffered as existing nation states encouraged ethnic self-identification in the Austro-Hungarian Empire. It would be a mistake to forget the "state" part of nation state, and that states are about power. One way in which nation states exercised power was by encouraging ethnic selfdetermination among the peoples of multi-ethnic empires with which they 
competed. That is, once the original nation states in western Europe created a sense of national identity, they used this idea in their foreign policy. This behaviour could be simultaneously ideological and pragmatic. As we have seen with respect to the formation of Germany and Italy, existing nation states could recognize the value of self-determination. England and France also had strategic reasons for wishing to weaken the Austrian Empire or the Ottoman Empire or the Russian Empire. With respect to the Ottomans, there was, of course, a further ideological argument for freeing Christian populations from Islamic rule. England and France then pursued a strategy in eastern Europe of encouraging ethnic rebellions, but then ensuring that one of the other empires did not appropriate any rebellious territory. The British and French supported the sovereignty of Greeks and Slavs in the Balkans, and Armenians, Georgians and others in the Caucasus. Nor did France and England only engage in Europe: They supported Egyptian and Arabian independence from the Ottomans as well.

Greece would achieve independence in 1827 with the aid of Britain, France, and Russia - though the original Greek state was limited to the southern portion of modern-day Greece. Despite the glory of ancient Greece, it would take decades to forge a common Greek national identity. Western European powers also encouraged a significant degree of self-government within the Ottoman Empire for Serbia, Wallachia, and Moldavia (the latter two are parts of modern Romania). Ironically, these three areas would become fully independent after Austria invaded during the Crimean War (1853-6), but Western

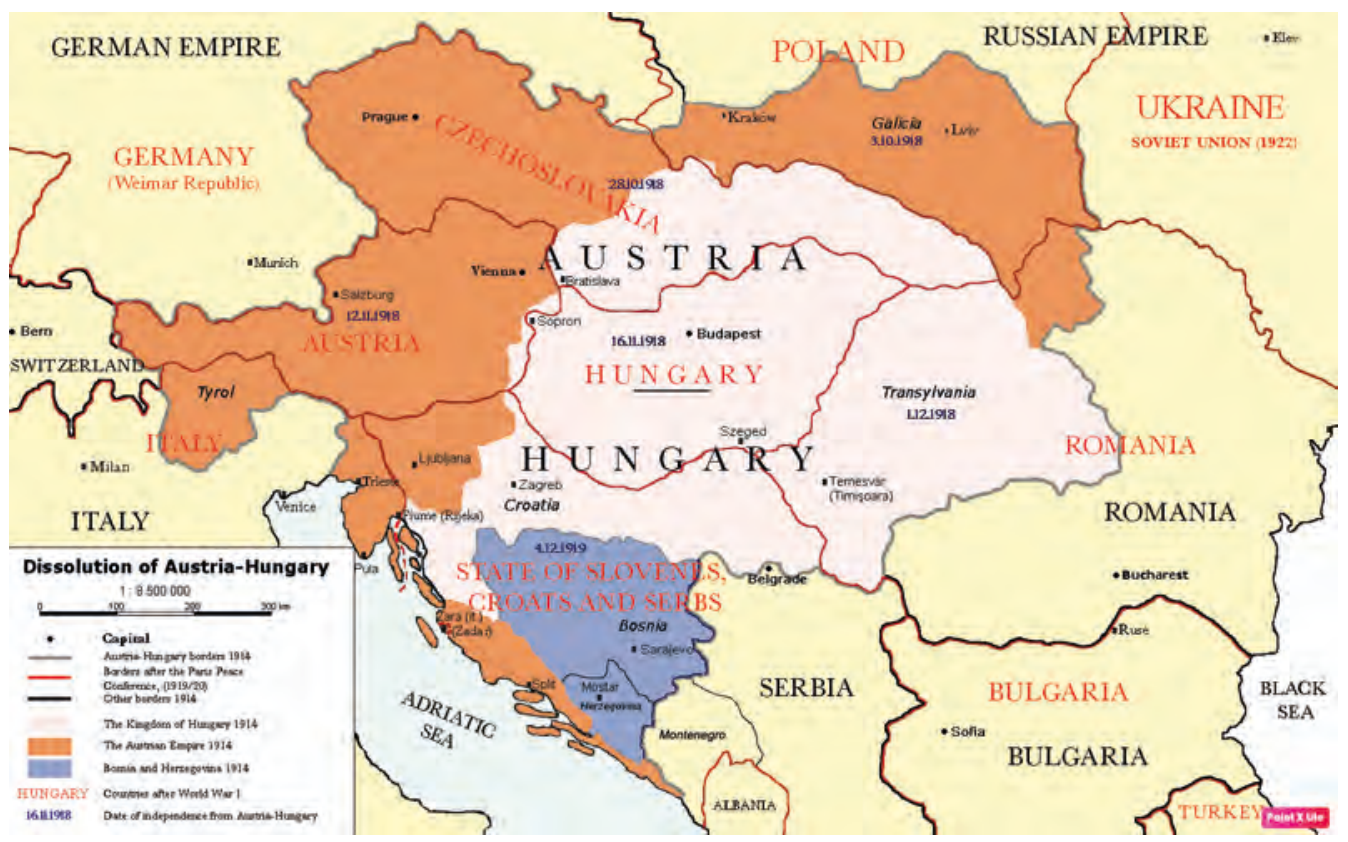

Map 21.2

The dissolution of the Austro-Hungarian Empire

Map by AlphaCentauri, derivative work by P. S. Burton, CC BY-SA 3.0 
powers supported the Ottomans in that war because they wished to limit Russian expansion.

Unfortunately for both the locals and Western powers, these areas in southeastern Europe were far from ethnically homogeneous. Fully one-quarter of the citizens of new states were minorities. Moreover, Western powers proved quite willing to see a new Armenian polity expand far beyond areas in which Armenians were a majority: Popular sovereignty could be set aside when it seemed useful.

The greatest surge in European nation creation occurred at the end of the World War I. Several new nation states emerged in Eastern Europe at that time. We should stress that this happened only because Austria and the Ottomans lost the war - which was nearly not the case (chapter 28). We should not presume that these multi-ethnic empires were doomed to disintegrate. If the Ottomans had stayed out of the war, and if the modernization programme of the Young Turks had been successful (chapter 20), the Ottoman Empire might have remained viable. Most Bulgars at the time could read only Turkish and often called themselves Greek for religious reasons. Bulgar national identity was primarily forged after the creation of Bulgaria. Even as the Ottoman Empire fell, many intellectuals called for at least pan-Islamic identity rather than the formation of Turkish and Arabic nation states.

\section{PRIMARY DOCUMENTS: MUHAMMAD IQBAL, ISLAM AND NATIONALISM, 1936}

I have used the world "millat" in the sense of "qaum" (nation). No doubt, the word millat has been used to mean law and religion in Arabic, and especially in the Holy Quran, but there exists in modern Arabic, Iranian and Turkish languages considerable evidence to show that the word millat is also used in the sense of nation. In my writings I have generally used the word in the latter sense. But in view of the fact that the meaning of the word millat does not, to any extent, affect the issues under consideration, I, leaving aside the controversy altogether, take it that Maulana Husain Ahmad said that "nations are formed by lands". As a matter of fact, I have nothing to say even against this statement of the Maulana. Objection must, however, be raised when it is contended that in modern times nations are formed by lands and the Indian Muslims are advised to accept this view. Such advice brings before our minds the Western modern conception of nationalism, to one aspect of which it is absolutely essential for a Muslim to take exception. It is a pity that my objection has led the Maulana to think that what I had in mind was to propagate the cause of some political party. Far from it, I have been repudiating the concept of nationalism since the time when it was not well known in India and the Muslim world. At the very start it had become clear to me from the writings of European authors that the imperialistic designs of Europe were in great need of 
this effective weapon-the propagation of the European conception of nationalism in Muslim countries-to shatter the religious unity of Islam to pieces. And the plan did succeed during the Great War. It has now reached its climax inasmuch as some of the religious leaders in India lend their support to this conception. Strange, indeed, are the vicissitudes of time. Formerly, the half-Westernized educated Muslims were under the spell of Europe: now the curse has descended upon religious leaders. Perhaps modern conceptions of Europe seem attractive to them but alas!

Can you appreciate how Iqbal would see the idea of the nation state as a tool of imperialism?

\section{Asia}

As the Ottoman Empire collapsed at the end of World War I, General Ataturk sought to forge a new state in the heartland of that empire. Ataturk arguably only became a nationalist because this allowed him to press territorial claims to Anatolia. European powers that had occupied the region at the end of the war did not put up a spirited opposition to the creation of the state of Turkey but did assume control of the Arabic parts of the Ottoman Empire. Ataturk relied heavily on Soviet cooperation against the British and French, and agreed that the Soviet Union could conquer the (Turkish) Azeris in return for that cooperation: He thus proved willing to sacrifice ethnic loyalty for pragmatic considerations of power. There was then a lengthy internal process of encouraging Turkish identity among Muslims in Anatolia, many with no Turkish heritage. The Kurds in southeastern Turkey were too concentrated for this policy to succeed. (The Great Powers had divided Kurdish lands among several states at the end of the war despite celebrating national sovereignty.) Elsewhere in Turkey, government action encouraged ethnic homogeneity. The Turks and Greeks would agree to a massive citizen swap, in which Turkey expelled 1.2 million Greeks and imported 400,000 Turks from Greece. Western European states had promised Armenians a homeland but did little to achieve this as the war ended. Armenians were at the start of the war widely dispersed across what is now Turkey. Historians estimate that a million Armenians died as the Ottoman Empire imploded. There was undoubtedly mass deportation and at least some purposeful killing by government troops though the Turkish state still denies this.

We discussed above how European states encouraged a common language and identity. We have seen in earlier chapters a similar process over a much more extended time period in China. Imperial bureaucracies encouraged both a common written script and a shared understanding of classical Chinese texts throughout their vast empire. The fact that one sizeable bureaucratic empire has ruled the Chinese landmass over most of the last two thousand years meant that a shared "language" and history could characterize a much more substantial population than was the case in Europe. Both the Mongol Yuan and 
Manchu Qing dynasties had mixed feelings about Chinese cultural identity, but over time even these foreign conquerors generally came to adopt Chinese ways and see advantages in a shared language in particular. Nevertheless, rising Chinese nationalist sentiment in the nineteenth century proved problematic for the Qing, which had always stressed its ethnic distinctiveness (even as Han Chinese migration into Manchuria in the late nineteenth century (chapter 31 ) swamped the Manchurian population). Nationalism would reinforce concerns about Qing corruption and incompetence in the face of European interventions, resulting in the overthrow of the Qing in 1911-12 (chapter 20).

In Southeast Asia, we also see cultural consolidation over the seventeenth through nineteenth centuries. As in Europe, though, states were smaller than in China, with mountains serving as barriers between river valleys. The Siamese state spread Thai language and culture, while the Vietnamese state encouraged Vietnamese language and culture. These states then came to see themselves as expressions of national identity. We saw above that war often strengthened national sentiments in Europe: Cultural consolidation may have been slowed in Southeast Asia because the mountain barriers between states limited inter-state conflict (chapter 16). Moreover, Southeast Asia did not face external conquest after the Chinese left Vietnam in the tenth century, and the Chola of India abandoned their conquests in the eleventh century, until the arrival of Europeans. This relative peacefulness, plus the rugged terrain itself, meant that many small minority ethnic groups survived into modern times on the fringes of Southeast Asian states.

Farther north, Korea and Japan both also achieved high degrees of political and cultural consolidation in these centuries. Japan - and to a lesser extent Thailand, which Europeans also never conquered would adopt the ideals and institutions of the nation state in a deliberate attempt

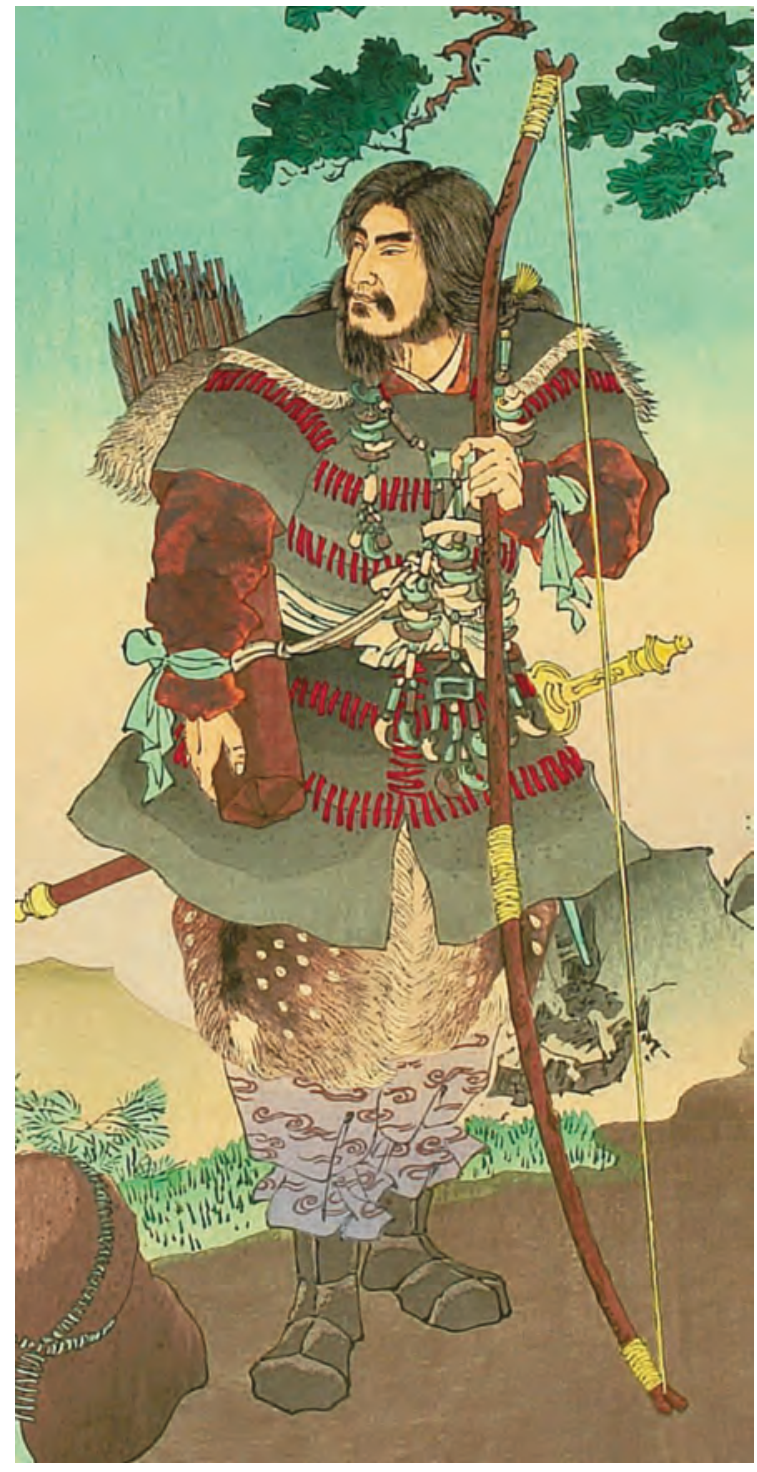

Illustration 21.4

Emperor Jimmu, legendary first emperor of Japan (supposedly from $660 \mathrm{BCE}$ ), 19th-century woodblock by Ginko Adachi. The Japanese imperial house has been an important symbol of the Japanese nation state 
to counter the imperial ambitions of European nation states. Japan had the benefit of shared language, religion, and history: With the critical exception of the indigenous Ainu, Japan was arguably one of the more "natural" nation states in the world. It self-identified as a nation state before Germany or Italy achieved that status. Japan then served as a model for nascent nation states elsewhere in Asia.

States were not the only source of shared identity. Hinduism could provide a shared identity to Indians living under different polities. As resistance to British rule grew in the late nineteenth century, various intellectuals celebrated a common Hindu heritage as a means of developing common cause in opposing British rule. They wedded the European ideal of the nation state to an appreciation of thousands of years of Indian "civilization." They often after 1870 cited Italy as an exemplar of a nation state forged from many distinct polities (and with a fair bit of linguistic diversity) based on an appeal to millennia-long history. In Asia, as in Europe, political leaders harnessed nationalism to the task of achieving political transformation.

\section{Latin America}

We should pay particular attention to Latin America. Almost all Latin American states successfully rebelled from Spain (or Portugal) between 1810 and 1825, aided by Napoleon's conquest of Iberia in 1807 (see chapter 25). Though revolutionary leaders such as Simon Bolivar hoped for one united state, the result of these revolutions was a set of nation states that closely followed the boundaries of Spanish administration. This outcome was far from obvious beforehand: Not only had revolutionaries such as Bolivar envisioned unity across former Spanish colonies but their shared language and religion seemed to support this vision. Local elites, though, found it advantageous to establish local autocratic governments rather than cede power to a more extensive state. We will find a similar experience a century and a half later in Africa: Elites in newly independent states would ignore calls for pan-African unity in order to forge states within the boundaries of European colonies (chapter 30).

Rulers of smaller states would encourage a strong sense of nationhood. Relative to later African states, Latin American states had an advantage of greater ethnic and religious homogeneity - though indigenous peoples were often excluded from nation-building projects. Yet this shared ethnicity and religion could have justified mergers among Latin American states. The Spanish had previously encouraged loyalty to the king and the king's appointed viceroy rather than to a particular colony. Independence leaders struggled to identify the common identity of the new states they forged. The ideal of the nation state was borrowed from Europe, but without the centuries-long process of forging common identities that had characterized the earliest European nation states. In 1830, there were more nation states in Latin America than in any other region of the world, including Europe. Latin American states would play an important role in urging an international system of nation states over the 


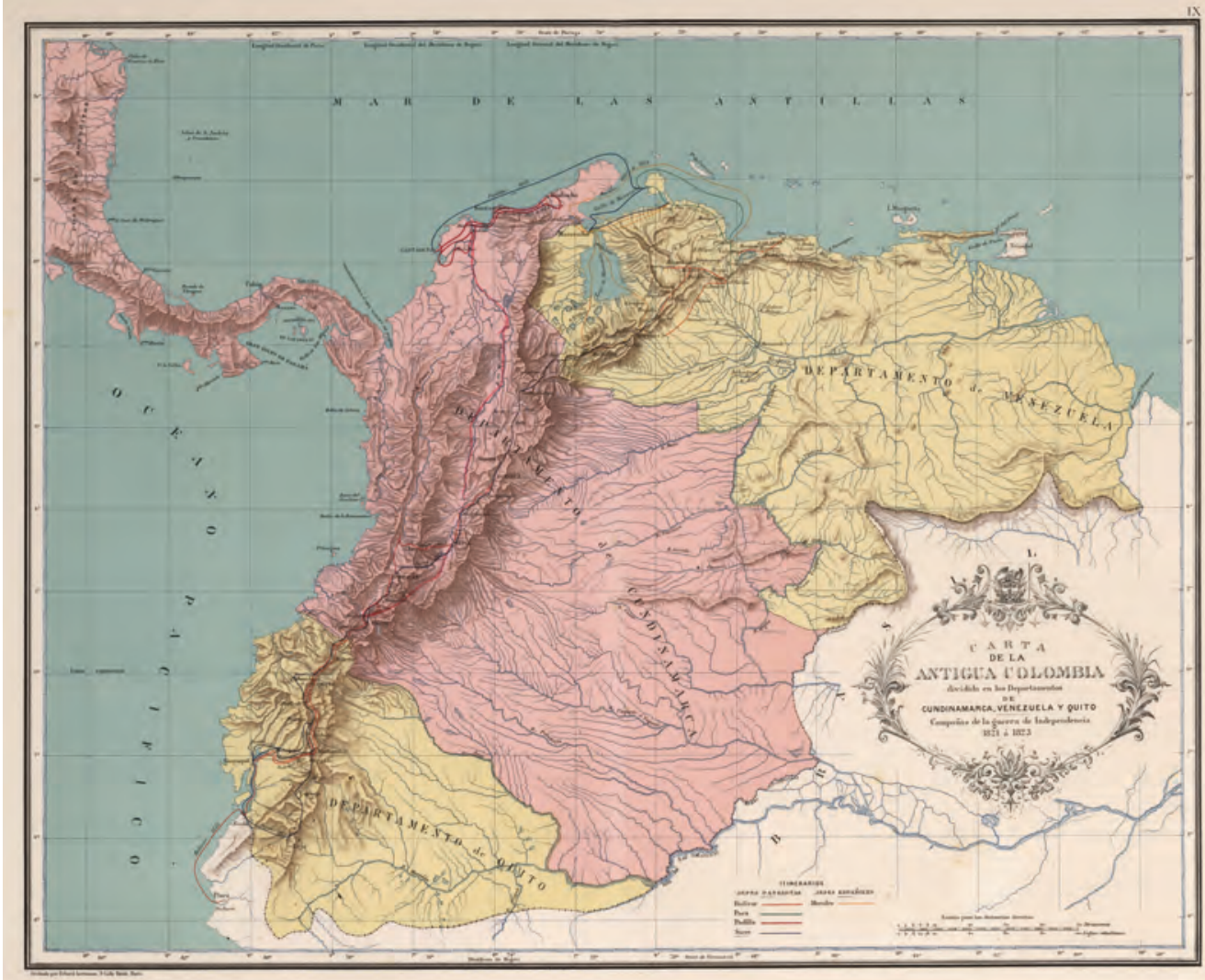

Map 21.3

The departments of Gran Colombia in 1820. This newly independent state would soon fracture into Colombia, Venezuela, and Ecuador; Panama would secede from Colombia with American aid in the twentieth century

next centuries. They would, with only a couple of exceptions, pursue peaceful coexistence within Latin America, and thus establish that a system of nation states could be stable. Yet they would struggle internally: Huge inequalities in power and wealth interfered with efforts to achieve democracy, and the region would largely be governed by military dictators for the next decades. These states would nevertheless develop over time a shared sense of national identity (despite waves of immigration from diverse lands). Lacking the shared histories that European states could emphasize, Latin American states (like the United States to the north) instead emphasized their novelty and potential shared future.

\section{Nation states and empires}

In many parts of the world, then, states became associated with one language, religion, history, or set of customs. Elsewhere, though, empires continued 
which either did not or could not instil a sense of shared identity. The Mughals in India valued diversity of necessity, for barely a quarter of the population of their empire had converted to Islam. An appeal to the shared history of the subcontinent would be a reminder that the Mughals were a conquering elite from Central Asia. The Ottomans likewise governed large numbers of both Christians and Muslims, and their empire comprised a dizzying array of languages and customs. They also would celebrate diversity as long as they ruled.

Emperors, as we have noted before, face a choice between cultural consolidation and celebration of diversity. The first option facilitates both administration and loyalty to the state but risks inflaming rebellion. An emperor facing only limited diversity, or fired by religious zeal, may pursue a policy of encouraging a dominant culture. Such policies may have a noticeable effect only over a period of generations. Emperors facing a great deal of diversity or with a paramount interest in political stability will instead embrace toleration. They would indeed then justify their rule to their subjects in terms of stability and toleration. Some intellectuals today also argue that a return to empire may offer some solution for multi-ethnic realities.

The idea of national sovereignty as it spread from states that had achieved a shared sense of identity challenged the remaining multi-ethnic empires. They were tested internally by ethnic rebellion, and they were challenged externally by the greater ability of nation states to mobilize resources for war (especially as internal divisions weakened empires). They often resorted in the short term to a divide-and-rule strategy of pitting minority ethnicities against each other, but such an approach tended to undermine the state over time. The transition from multi-ethnic empire to nation state was nowhere pretty. In Europe, ethnic objection to the Austro-Hungarian Empire triggered World War I (see chapter 28). The Ottoman Empire would crumble at about the same time, in part because its various ethnic groups rebelled. We can wonder if some less violent outcome might have been possible for these empires if not for that war. Russia would lose some of its ethnic minority populations in Eastern Europe after the war also, but the Soviet Union would hold on to multi-ethnic lands in Central Asia; these would gain independence with the dissolution of the Soviet Union in the 1990s but generally lacked the cultural homogeneity on which most of the world's nation states rely. The Mughals and later the British - had managed to suppress but not erase the mutual suspicion between Hindus and Muslims in India; tens of millions would die when the British decided in the mid twentieth century to divide the subcontinent into Muslim and Hindu polities despite the extensive intermingling of the two groups. The state of India remains divided over the precise question of whether to stress a civic multiculturalism or to emphasize the cultural heritage of the Hindu majority. A Hindu nationalist would kill the revered independence leader Mohandas Gandhi for espousing a more secular vision of the Indian state. 


\section{Settler societies}

As we shall see in chapter 31, settler societies such as the United States, Canada, Australia, and Argentina would absorb huge waves of immigration from diverse European countries over the nineteenth and twentieth centuries. They ended up with populations far more ethnically diverse than the Austro-Hungarian Empire. They would develop identities that in part celebrated a shared

\section{BOX 21.2 COSMOPOLITAN VIENNA}

We should not leave the mistaken impression that multi-ethnic empires exhibited a clear trajectory of political and cultural decay as nation states rose to prominence. Vienna, the capital of the Austro-Hungarian Empire, attracted people from throughout its realm and beyond in the decades just before and after 1900: Poles, Czechs, Slovaks, Ukrainians, Italians, Romanians, Germans, Jews, Slovenes, and many others. It was one of the most cosmopolitan cities in the world in the late nineteenth and early twentieth centuries. The emperor encouraged ethnic and religious tolerance. This cultural tolerance, in turn, encouraged intellectual innovation. This one city was the birthplace of the new Freudian psychology (which encouraged investigation of subconscious thoughts through the analysis of dreams, and stressed repressed sexual desires); the "Vienna Circle" in philosophy (which argued that science should advance through disproving testable hypotheses); the "Austrian school" in economics (which stressed the many advantages of market exchange and worried that economic planning must decrease both innovation and consumer choice); and many other innovations in not just the social sciences and humanities but also art and architecture. Though these various innovations have each been critiqued over the succeeding century, they are still appreciated as major intellectual developments. No other city can point to the same degree of intellectual ferment at the time. We discussed back in chapter 10 how periods of intellectual innovation reflect a set of favourable political, economic, and cultural circumstances: Despite the challenges facing the Empire, Vienna provided the appropriate environment. Note that ancient Greek and Chinese philosophy also prospered in a political environment characterized by freedom rather than imperial stability.

Most German-speaking citizens of Vienna were unenthusiastic about the increasingly cosmopolitan nature of their city. They were quite conscious of the foundation of a new German state to the north that celebrated the glories of German culture and history. They particularly disdained both Czech workers, who they accused of stealing their jobs, and Jewish merchants, who seemed far too wealthy. They elected an anti-Semitic city council and mayor - which the Emperor delayed recognizing for years in the interests of tolerance. In this environment, a young Hitler would begin to develop the fascist ideas that he would later employ in Germany and across much of Europe (chapter 28). When fascism gained strength in Austria itself in the 1930s, most Viennese intellectuals fled to Britain or the United States, where many of them continued to be intellectual leaders. 


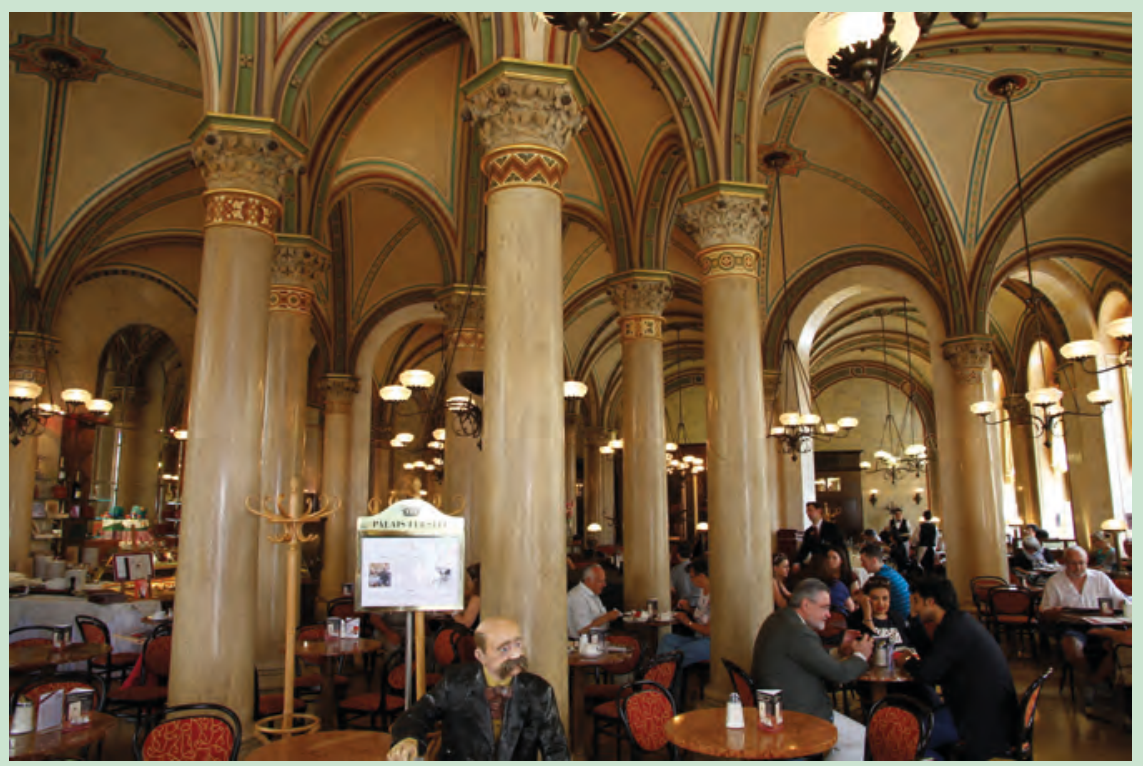

Illustration 21.5

Central Café, Vienna, opened 1876. The Vienna Circle often met there; Freud visited often

Clayton Yang, CC BY-SA 3.0

"European" heritage (a shared heritage, we might note, that did not excite the different ethnicities within the Austro-Hungarian Empire). They would also encourage celebration of shared ideals and a shared history in the new land. It is remarkable, for example, how the American Revolution anchors a shared sense of American identity even though most Americans have no ancestors that were in America at the time. Most of these countries achieved a considerable degree of linguistic unity: Italians learned Spanish in Argentina and Germans learned English in the United States. There are more people of German heritage than English heritage in the United States, but succeeding waves of German immigrants found it useful to learn English (and the two World Wars encouraged many of German heritage to adopt English language and customs).

The process of nation-building in these countries suggests that narrower ethnic identities can be surmounted. American identity indeed depended in part on a rejection of the "petty" ethnic loyalties of Europe. Yet there have been challenges. Indigenous groups in all of these countries have no obvious place in an identity that stresses European roots. The same is true of the descendants of African slaves. In Canada, the existence of two dominant languages has been a constant source of tension: The discourse of national sovereignty encourages many French Canadians to seek their own state to this day. The British government had indeed agonized about what to do with its French Catholic colonial 
subjects in Canada from the time of the conquest of Quebec in 1760: The population was too large and concentrated to be easily assimilated once many Englishspeaking settlers started to arrive. The British and later Canadian compromise generally involved a large degree of local self-government and efforts to forge a common attachment to Canada (which had one surprising early success when French Canadians showed no interest in joining the American Revolution; chapter 25). As Canada and other countries have increasingly received immigrants from Asia or Africa, the continued celebration of European heritage is problematic. As noted in chapter 1, this is, in fact, one of the key motives behind the development of "World History" courses. Nevertheless, the question remains of how well an appreciation of institutions and world history can substitute for a European identity.

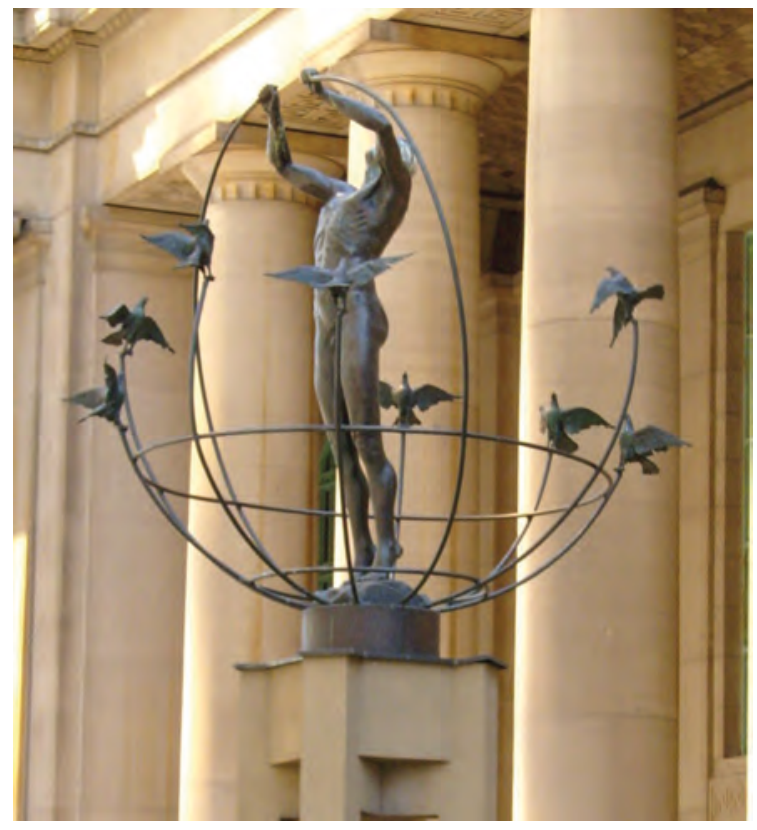

Illustration 21.6

Monument to Multiculturalism by Francesco Pirelli, Union Station, Toronto

Photograph by Robert Taylor from Stirling, Canada, CC BY 2.0

\section{Africa}

Sub-Saharan Africa was not well prepared for the age of nation states: It had not been characterized for the most part in recent centuries by large polities that could encourage common languages or histories over large areas. European colonial powers often drew borders that divided the ethnic groups that did exist, and they often found it useful to encourage hostility among subject groups. The Zulu conquests in southern Africa on the eve of colonization did forge a sense of identity among a large group, though this group would not gain its own state. Even when there was a shared language - as among the Yoruba and Igbo in West Africa - people were more aware of local dialects than a common language or identity: Efforts of missionaries to develop dictionaries in Yoruba and Ibo would help forge a common identity, and newspapers and schools also encouraged this (a common identity was also useful as a counterpoint to colonial authority). In the nineteenth century, pan-African sentiment was usually stronger than nationalist sentiment. Many argued that all subSaharan Africans shared a common history, including lengthy exposure to the slave trade and more recent colonialism. As in Latin America 150 years earlier, though, independence occurred within colonial administrative boundaries, and the resulting states would struggle to forge a national identity. Intellectuals, even as they disdained other elements of European thought, came to support the ideal of the nation state in the twentieth century; as in Europe, this support 


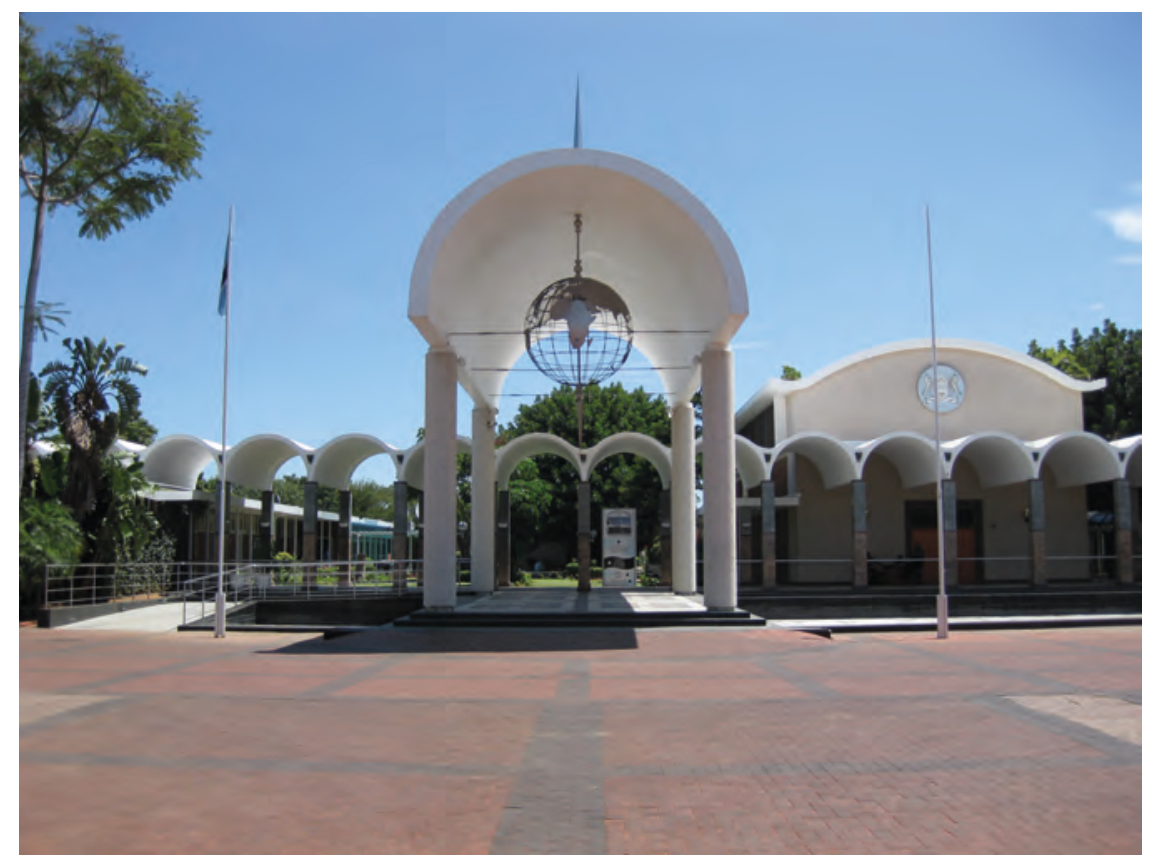

\section{Illustration 21.7}

Botswana Parliament, Gaborone

reflected the existence of first independence movements and then states that claimed national legitimacy. These states faced a challenge of ethnic diversity far greater than in most of Latin America or Europe. Botswana, one of the few African countries with one dominant ethnicity, has experienced both political peace and economic growth (vast diamond resources have undoubtedly helped, but resource revenues have encouraged massive corruption rather than good governance elsewhere). In other countries, military dictatorships were common since the army was often the only well-developed national institution. Democracy has spread in recent decades but has been hampered by ethnic rivalries (see chapter 27).

\section{The Pacific}

As the ideal of the nation state spread internationally, some smaller polities adopted the institutions of the nation state in part to forestall colonization by larger polities. This process is perhaps most visible in the Pacific. When Tāufa'āhau (King George Tupou I) unified Tonga after decades of civil war in 1845 , he established a constitutional monarchy with freedom of the press. He also abolished serfdom (or at least a system with some resemblance to serfdom) - in line with the actions of many states in the mid nineteenth century (chapter 26). He introduced a law code and a land tenure system grounded 
in private property. He and his successors then expressed disdain for European claims that a country such as Tonga could not effectively govern itself. Tonga would sign a treaty of friendship and protection with Britain in 1900 after disputes arose between local elites and British settlers. Nevertheless, Tonga would always maintain considerable political independence. In 1970, the British would agree to end the protection arrangement. Power has devolved over the years from the hereditary monarchy to the elected assembly.

Kamehameha I unified Hawaii in 1795 and his descendants would rule until deposed in the late nineteenth century. As in Tonga, the Hawaiian monarchy developed the institutions of a nation state, including a code of laws, elected assembly, and bureaucracy. Special mention might be made of the Iolani Palace in Honolulu, built in 1845. It was intended explicitly to compare with the residences of foreign rulers. Hawaiian rulers often used the palace to impress foreign dignitaries. Good food was ostentatiously served on fine imported dishes. A statue of King Kamehameha was explicitly modelled on a statue of Caesar to impress visitors with the authority of the Hawaiian king. Hawaiian rulers often also travelled. Indeed King Kalākaua in 1881 became the first ruler to travel around the entire world. He sought new sources of labour for Hawaiian plantations but also pursued treaties of friendship in Europe, Asia (he tried to marry his niece to a Japanese prince), and America. As with the palace, the travels were an attempt to advertise the power and solidity of the Hawaiian state to the world - though some observers sensed that the king was desperate for foreign support. At a time when European powers justified colonialism by

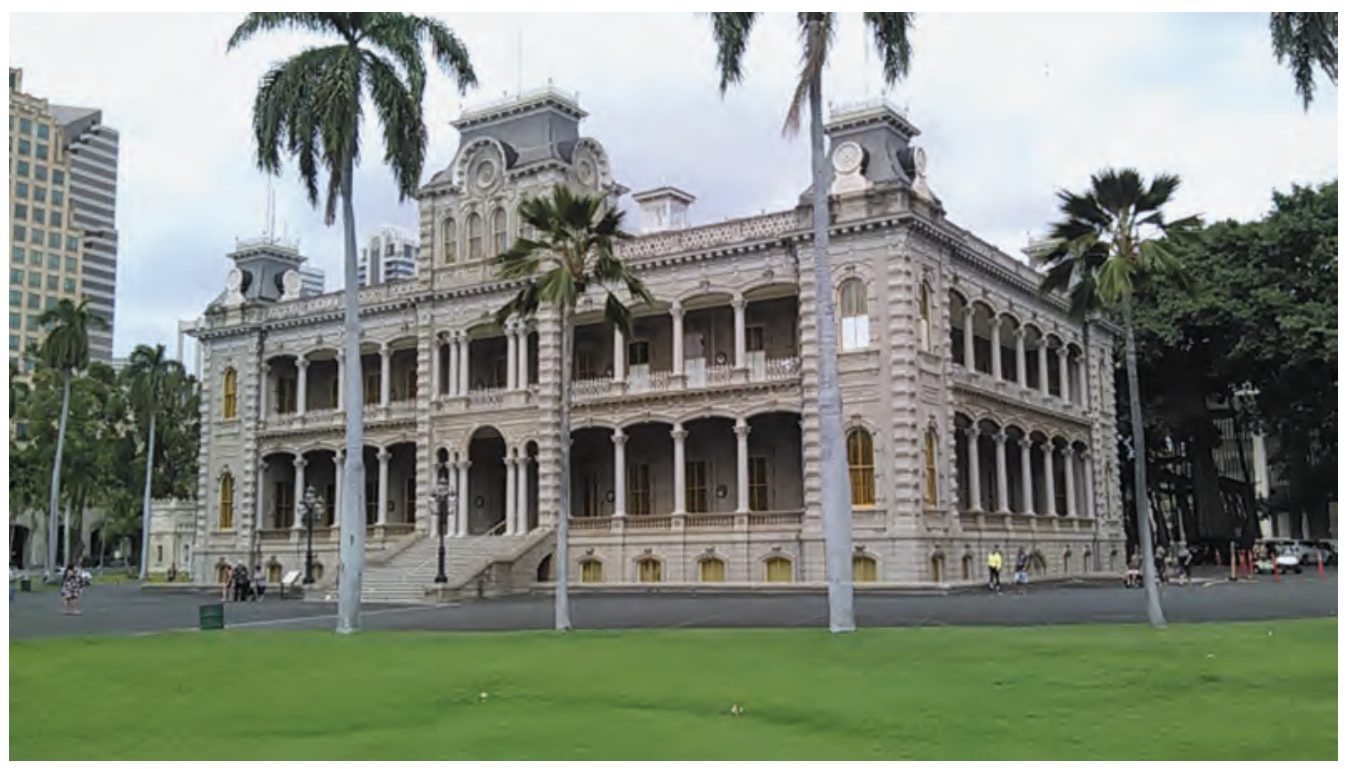

Illustration 21.8

Iolani Palace, Honolulu 
arguing that other nations were less "civilized," it seemed a reasonable strategy to the Hawaiian royalty to imitate the ways of European monarchs. As in Tonga, however, hostilities arose between locals and foreigners. American business interests, especially in the sugar trade, had become both economically and politically influential. The American navy had come to appreciate the potential of a naval base in Hawaii. Despite Hawaiian efforts to establish legitimacy on the world stage, American interests would overthrow the Hawaiian monarchy in 1893, with some assistance from the American navy. The United States would annex Hawaii five years later.

\section{The nation state system}

Though the nation state has proven problematic, the system of nation states is reinforced by an international legal and organizational framework. This system has some advantages: It urges respect for borders, which has deterred (some of) the sort of battles that characterized the period of nation state solidification in Europe. Yet the question remains of whether some looser political structure may be better suited to countries that lack a strong sense of shared identity.

One of the ironies of the system of nation states is that each proclaims its uniqueness in a very similar set of ways. Each celebrates shared languages, religions, cultural practices, and institutions. Schools teach histories that emphasize past glories. Each nation state develops museums, libraries, archives, government buildings, and monuments that celebrate the nation and its history. (Passports become common in the nineteenth century as nation states developed the bureaucratic ability to identify their citizens amidst fears of both mass migration and revolutionary agitators.) This similarity in the trappings of the nation state has hardly prevented hostility among nation states, for it has always proved tempting to celebrate one language or religion or set of institutions as better than another, or to emphasize real or imagined historical slights (recent or ancient) perpetrated by other nations.

One other commonality of the nation state was that each contained minority groups characterized by a different language or religion or ethnicity or some other distinguishing feature. Even nation states such as the United States or Canada that emphasized shared institutions rather than shared ethnicity would not readily embrace indigenous peoples or visible minorities within a shared sense of nationhood. The size of minority groups varied considerably across states. There were usually efforts at assimilation by the majority. These were sometimes successful if minority groups were small. More often, such efforts only encouraged a stronger sense of a distinctive identity among minorities themselves. Leaders of the majority and minority groups struggled to achieve accommodations, but the very idea of popular sovereignty made this problematic. Over time, though, many democratic nation states would develop constitutional protections of minority rights. 


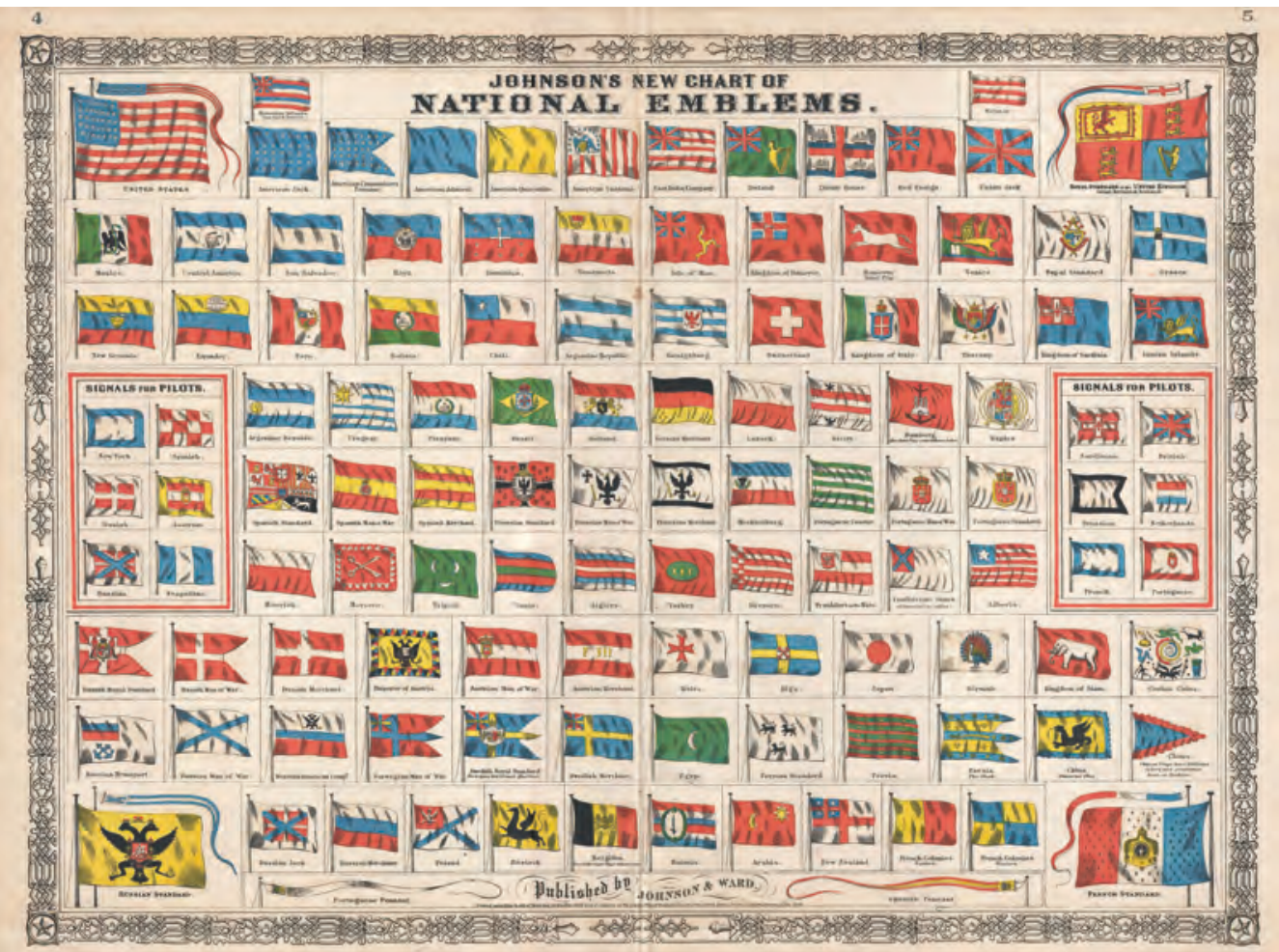

\section{Illustration 21.9}

Johnson's Chart of the Flags and National Emblems of the World, 1864, Johnson's New Illustrated (Steel Plate) Family Atlas of The World

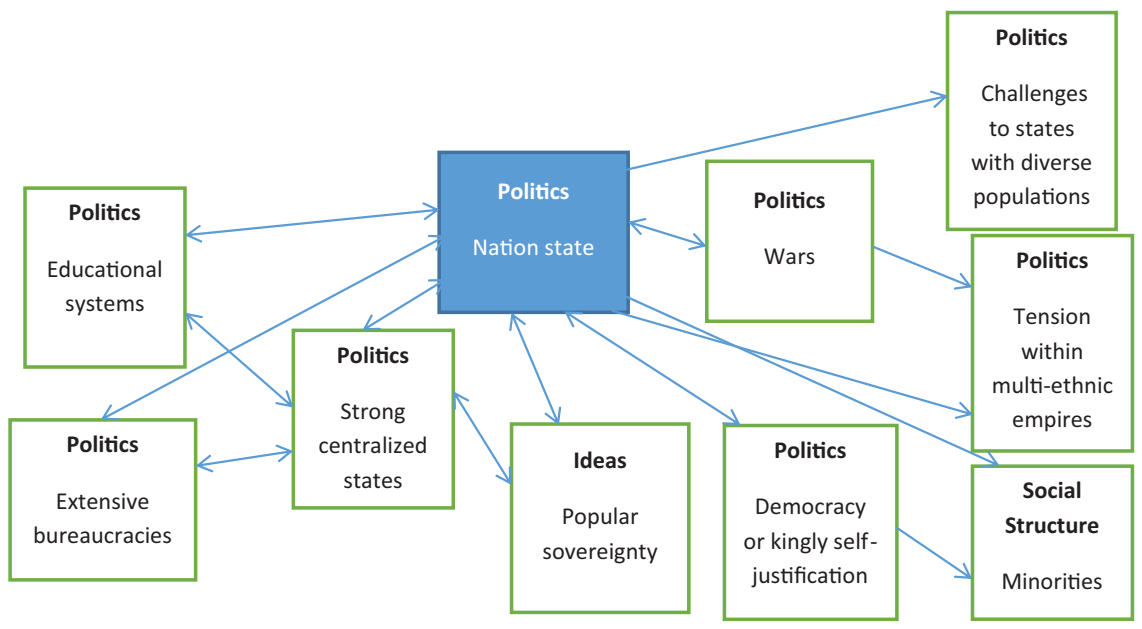

Figure 21.2

Influences on and effects of the nation state

Note the prevalence of two-way arrows, especially on the left side. We have discussed the causes of political centralization elsewhere. 


\section{Ethnicity itself}

We have seen above in various places that ethnic identity is fungible. The people of Languedoc are now (almost) just as French as Parisians. German Americans are largely indistinguishable from English Americans. Just as ethnic groups can grow by attracting members of other groups, they can splinter. Serbs and Croats speak much the same language but are divided by religion between Catholicism and Orthodoxy.

The experience of nomads is instructive here. We have seen (chapter 17) that new nomadic "peoples" often emerged as warriors were attracted to the service of a charismatic leader. The Huns emerge and disappear from history in this way. Chinggis Khan caused many to self-identify as Mongols for the first time. There is a broader lesson here: Ethnic groups that are successful or dominant attract "converts" who learn their language and adopt their customs. It is a severe over-simplification to assume that a shared genetic inheritance characterizes members of an ethnic group (such an assumption also neglects the frequency of both forced and voluntary migration in human history). It is better to understand ethnicity as a historical process whereby state formation, state policy, trade relationships, and various other factors determine the global distribution of ethnicities.

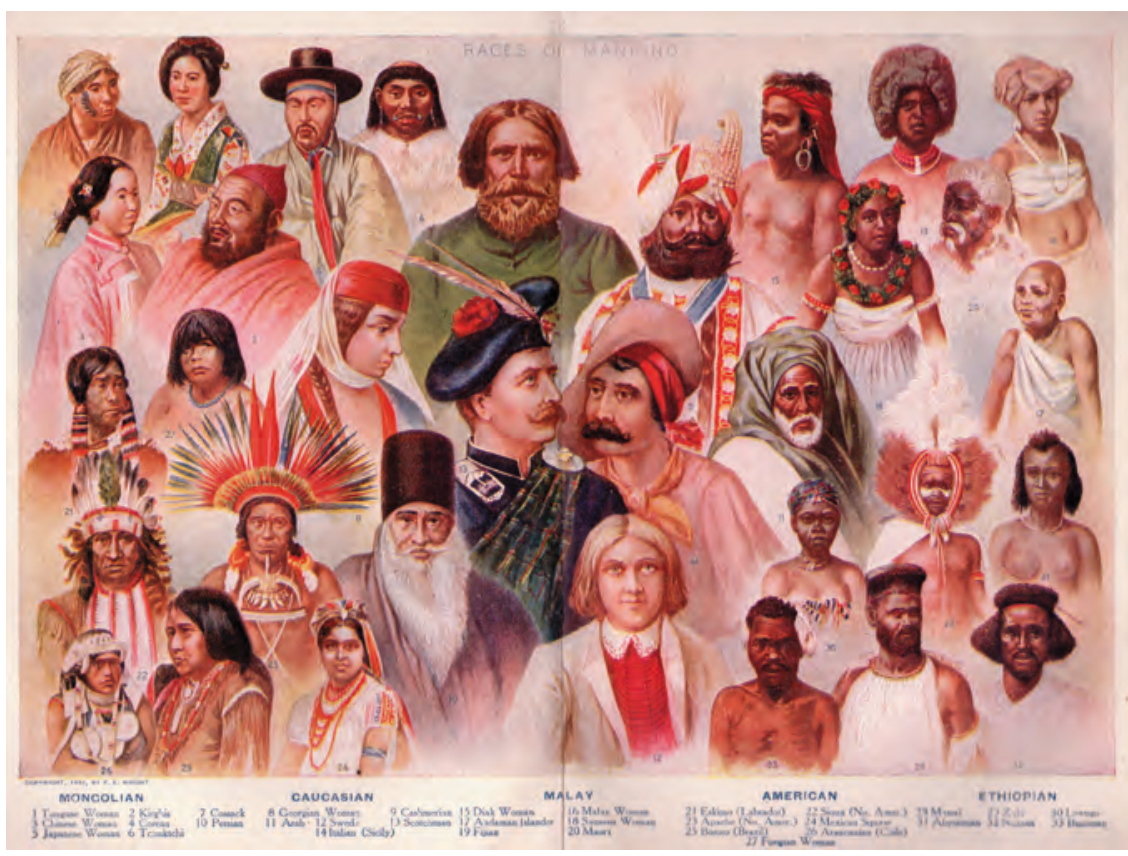

Illustration 21.10

"Races of Mankind,"'Webster's New Illustrated Dictionary, New York, 1911. Note the implicit assumption of natural "races"

Photograph by Sue Clark, CC BY 2.0 
In particular, in parts of the world rarely if ever drawn into large polities or extensive trade relationships, we see the survival of numerous ethnicities that are often small in numbers. The Burmese state encouraged cultural consolidation in its agricultural heartland but had little impact on tribal societies in the surrounding mountains, who maintain distinct identities to this day. Tribal ethnicities also characterize much of sub-Saharan Africa. The discourse of national sovereignty has ambiguous implications for ethnic groups that may be too small or too dispersed to form viable states.

The process of ethnic consolidation was transformed from the eighteenth and especially nineteenth centuries as people become more self-conscious about their ethnic identity. Ethnic identities had hardened as education and vernacular literatures had expanded (chapter 14). To be sure, immigrants to new lands often still embraced new identities. More generally, though, members of minority groups have become less likely to adopt the language, customs, and religion of a more powerful group.

\section{BOX 21.3}

\section{STATE CAPACITY}

The idea of "state capacity" has emerged in sociology and political science in recent decades, and has now become commonplace in the field of economic development and elsewhere in the social sciences. The basic argument is that modern economic growth is only possible within states that can enforce law and order - and ensure the provision of certain other services - throughout their domain; states, in turn, need to be able to tax a significant portion of national income to perform this role. The literature on state capacity often notes that the wealthiest countries in the world today - such as the United States, several countries in Western Europe, and Japan - tend to be those that formed strong nation states earlier than elsewhere in (or before) the eighteenth and nineteenth centuries. Some scholars extend the importance of history even further: State capacity today is strongest in regions of the world characterized by strong states in 1500. Many scholars worry that regions of the world that lack a history of strong states will struggle to develop state capacity.

We have often spoken in earlier chapters of the importance of institutions, and in particular those that protect property and enforce contracts. We have also seen that the ability of a state to maintain peace within its borders was critical for economic activity. We have often referenced the importance of transport infrastructure, and recognized the role that many states have played in providing this. From the nineteenth century, public education became of increasing importance as complex technologies and institutions necessitated a workforce with literacy and numeracy skills.

The costs of providing institutions and infrastructure have risen as trade has expanded and technology has become more complicated. Successful states in the twenty-first century generally tax between 20 and 50 per cent of national income. Most states before the eighteenth 
century rarely managed more than 5 or perhaps 10 per cent - though there were occasional exceptions such as Qin China (chapter 10). Though some states have relied heavily on coercion, state capacity today generally reflects some acceptance by the bulk of the population that a strong state is necessary. There may still be intense debates about the precise level of taxation or the benefits of certain types of government expenditure - but few would urge a return to pre-modern levels of taxation.

Some states have used their powers of taxation unwisely: both Russia and the Soviet Union were only intermittently successful in attempts to direct economic activity centrally. State capacity may then be essential for sustained economic growth, but state capacity alone does not guarantee economic success. States that are unconstrained in their exercise of power neither institutions nor practices of popular sovereignty such as elections prevent them from doing as they wish - may be more likely to apply state power in an unhelpful manner.

We have also had many occasions in this book to observe local elites capturing both tax revenues and governmental powers. States that can collect substantial revenues centrally can potentially employ these in ways that strengthen both economy and polity. They may, in particular, prevent local elites from attempting to obstruct developments in technology or institutions that threaten their position. One early action of centralized states such as France was to eliminate the tolls charged by local lords that discouraged internal trade. While such efforts to limit selfish exercises of power by local elites are notable, we have also seen that states themselves may be tempted to limit economic transformation in the interest of social stability.

The case of China is particularly interesting. As we have often seen in this book, China for millennia tended to have the most extensive bureaucracy in the world. In the eighteenth century, the Chinese state did effectively provide institutions that encouraged economic development. In the nineteenth century, though, as nation states elsewhere increased their tax take, China's did not rise much above 5 per cent. This may in part reflect Confucian arguments that a state should tax as little as possible. In any case, it meant that the Chinese state invested less in railways and schools than other lands. State capacity would be developed after the communist revolution of 1949. However, as in the Soviet Union, attempts to direct economic development centrally were not particularly successful. Since the 1980s, though, the Chinese state has developed institutions and infrastructure that have facilitated rapid economic growth. Many scholars would argue that the success of China in recent decades builds in important ways on the millennia-long tradition of effective bureaucracy.

It is widely appreciated in the state capacity literature that a strong sense of nationalism tends to characterize prosperous states, and that nationalism in turn tends to reflect a substantial degree of ethnic homogeneity (lands of new settlement such as the United States and Canada being exceptions that achieve a multicultural nationalism). Citizens are only willing to pay high taxes to a state with which they identify. The long history of ethnic identity formation may, therefore, exert a powerful influence on state capacity to this day (though social scientists appreciate that both the slave trade and colonial experience - and especially colonial borders that ignored ethnic divisions - exacerbate the challenges of building state capacity in sub-Saharan Africa). 
Though nationalism is important, states must also win popular acquiescence to taxation by providing valued services. There was an evolutionary and path-dependent process by which states that provided decent institutions, transport infrastructure, schools, and other services (including armies - nationalist fervour was often most supportive of these) gained public support that allowed them to expand taxation and service provision. For early nation states, growth in the level of taxation and services continued into the postwar decades but has stabilized more recently. In many other parts of the world, rates of taxation and the provision of institutions, infrastructure, schools, and other services remain at much lower levels. Such states often lack popular support.

One widespread problem is corruption. We have seen that corruption has been a problem throughout world history: Rulers always struggled to encourage bureaucrats to act in the interests of the state. As modern nation states expanded their bureaucracies in the nineteenth and twentieth centuries, they moved against corruption in many ways. Increased powers of taxation allowed them to pay bureaucrats better salaries: Bureaucrats then faced less incentive to steal and were more worried about losing their jobs. Competitive civil service examinations meant that employees were hired based on ability rather than political connections. The nature of these tests, and the expansion of public education, meant that bureaucratic jobs were open to the bulk of the population rather than just an educated elite as in pre-modern China. As governments came to pursue a range of valuable services, bureaucrats were encouraged to dedicate themselves to public service. We can again detect an evolutionary process by which corruption was slowly replaced (but not eradicated) by the development of a professionalized and reasonably well-paid bureaucracy infused with a sense of public service. This process unfolded over a period of centuries. Countries without a long history of professional bureaucracy may struggle to discourage corruption. (Note that both China and Turkey struggle with corruption today: State capacity may benefit from a history of pre-modern bureaucracy in the Chinese and Ottoman Empires, but the elimination of corruption may hinge instead on a history of modern bureaucracy.)

\section{Nationalism}

As noted above, Enlightenment thinkers recognized the value of popular sovereignty but were suspicious of the idea of identifying the state with a particular ethnicity. They were justifiably worried that this might jeopardize personal freedoms, especially the freedom of religion. Nationalism - the identification of the state with ethnicity - is neither a necessary nor an obvious outcome of the idea of popular sovereignty. However, supporters of the concept of popular sovereignty always had to grapple with the question of where to draw state boundaries. Unless one was to strive immediately for a global state or merely accept historical borders, there was a danger - often realized - that states would compete militarily over boundaries. There was then a practical advantage in urging the recognition of political communities with some shared sense of purpose. 


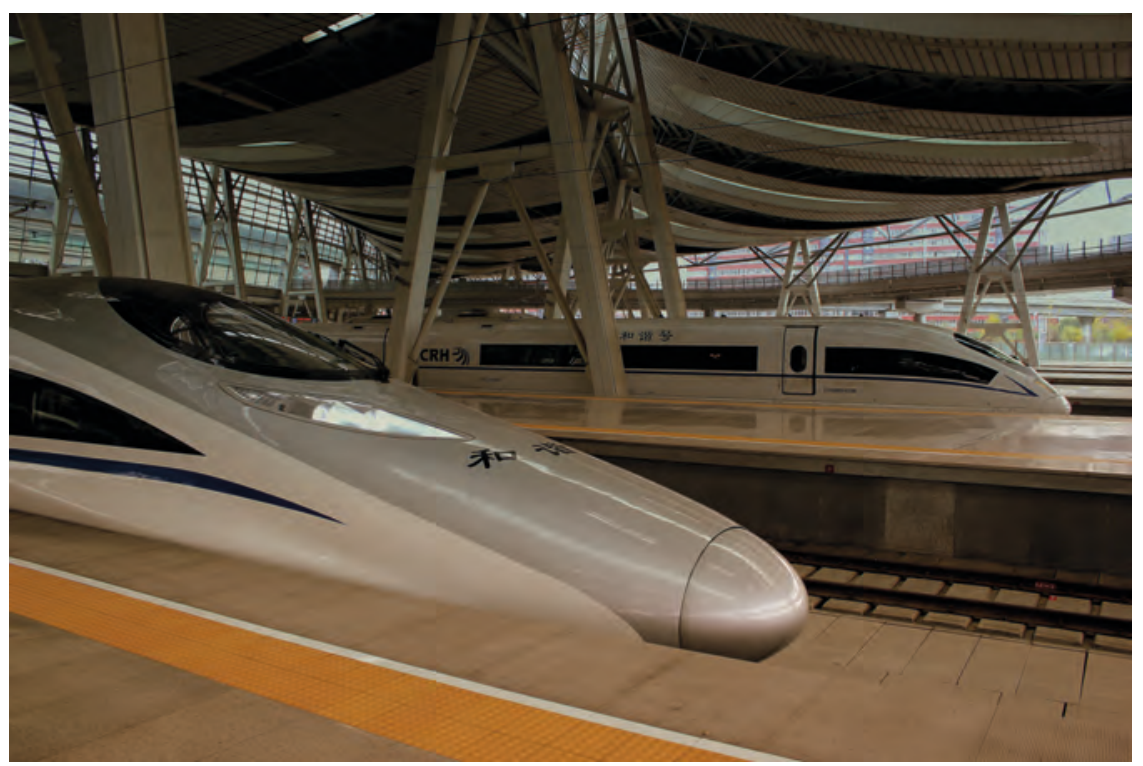

Illustration 21.11

High-speed (bullet) trains, Shanghai South railway station, 2012

Photograph by calflier001, CC BY-SA 2.0

This might suggest where to draw boundaries - though as we have seen, the reality of ethnic inter-mixing complicated the situation quite a bit. There was a further pragmatic advantage of a common language as state bureaucracies and educational systems expanded. As a result, the development of the nationalist idea that since some groups shared a cultural identity they should also share a political destiny was a mutation that had a strong chance of selection.

Important thinkers in each nation state came to express cultural nationalism. Johann Herder celebrated German history and language, but very similar ideas were expressed by Mazzini in Italy, Gregoire and Michelet in France, Mickiewicz in Poland, Scott in Britain, and Emerson and Cooper in the United States. Indeed one of the ironies of nationalist thinking is that very similar ideas about the uniqueness of the history and culture of each land emerged in different countries. One further commonality was that each "nation" developed a history that emphasized the glories in its past while excising negative or embarrassing aspects of that past: The nineteenth-century French writer Ernest Renan observed that "Forgetting, I would even say historical error, is an essential factor in the creation of a nation."

In addition to solving a conundrum in thinking about popular sovereignty, nationalism could also appeal to humans' natural instinct for group identity. We suggested back in chapter 3 that there was likely genetic selection for group loyalty. Hunter-gatherers needed to cooperate in many ventures, including hunting and fire-making, and perhaps in inter-group rivalry. It is remarkable that this genetic selection for identity with groups of a hundred or so extends 
so easily to much more substantial groups - a billion people may self-identify as Chinese. Our genes then place virtually no limit on the size of the groups with which we identify, but encourage each of us to identify with some group. The only limit to group size is that national identity is necessarily expressed in contrast to others: There must be other nations to which one can be compared. (A strong sense of global identity will then be more likely if there is extraterrestrial contact, and especially competition.)

From the late nineteenth century, rulers faced a further incentive to encourage nationalism: as a bulwark against socialism. Socialist thinkers, in turn, argued that workers would soon recognize that their shared identity as a global working class was far more important than imagined nationhood. In the wars of the twentieth century, though (chapter 28), most workers identified with their nation. Even states that proclaimed themselves socialist in the twentieth century (chapter 27) found it useful to justify their rule with appeals to nationalism.

Nationalism allied itself with religion wherever a nation had a dominant religion: This could then join language and history as markers of national identity. Yet nationalism itself had some striking

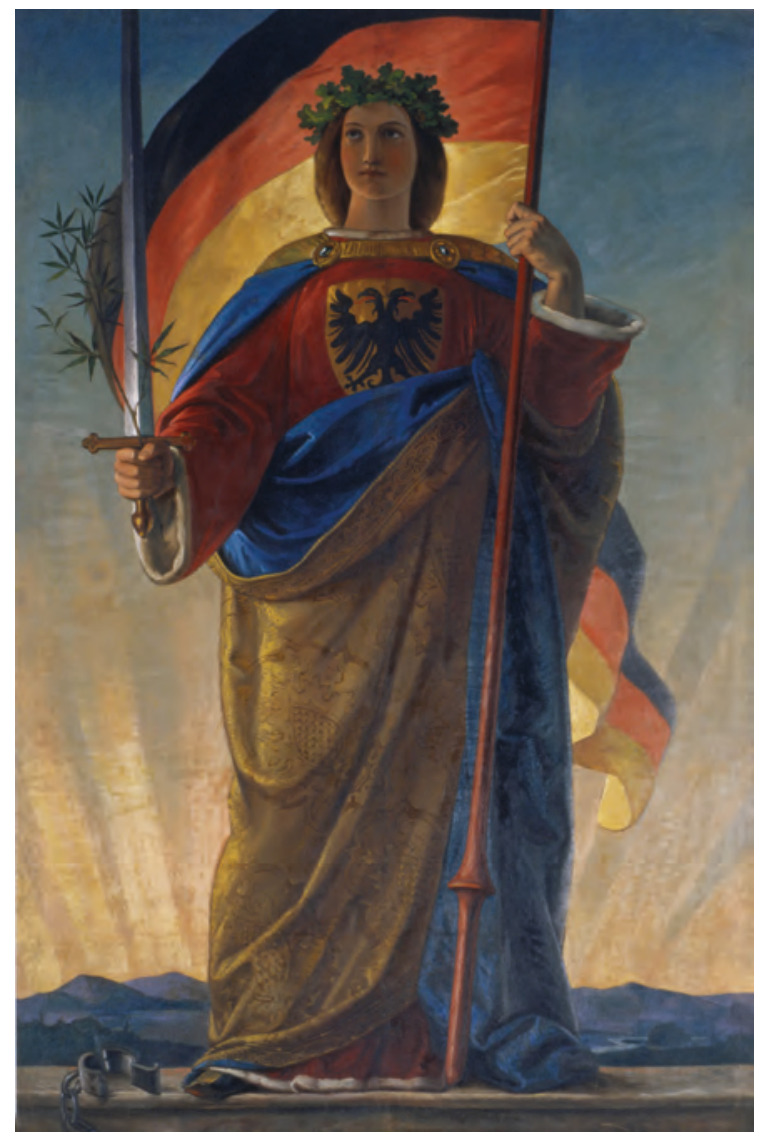

Illustration 21.12

Germania, wall fresco, St. Pauls Church, Frankfurt am Main, designed to cover the organ during the Frankfurt Assembly/Parliament, 1848-9, Germanisches Nationalmuseum, Nuremberg. A sense of national identity aided the formation of the German state similarities with religion. National constitutions became revered texts. Citizens were urged to sacrifice for a force greater than themselves, especially in times of war. Nations, like religions, had martyrs and shrines. Nationalism, like religion, would be instantiated in monumental architecture, songs, stories, works of art, and rituals. Perhaps most important of all, nationalism, like religion, provided an answer to the question of meaning in life: Each person inherited an identity from their ancestors and a sacred duty to celebrate and if necessary fight for that identity. (We return to questions of meaning in chapter 32.)

A common language could be particularly important in the nineteenth century as literacy and printing presses spread: A people who read the same books and newspapers could come to see the world similarly. There were 31,000 newspapers in the world by 1900, including almost 200 in Africa and over 600 


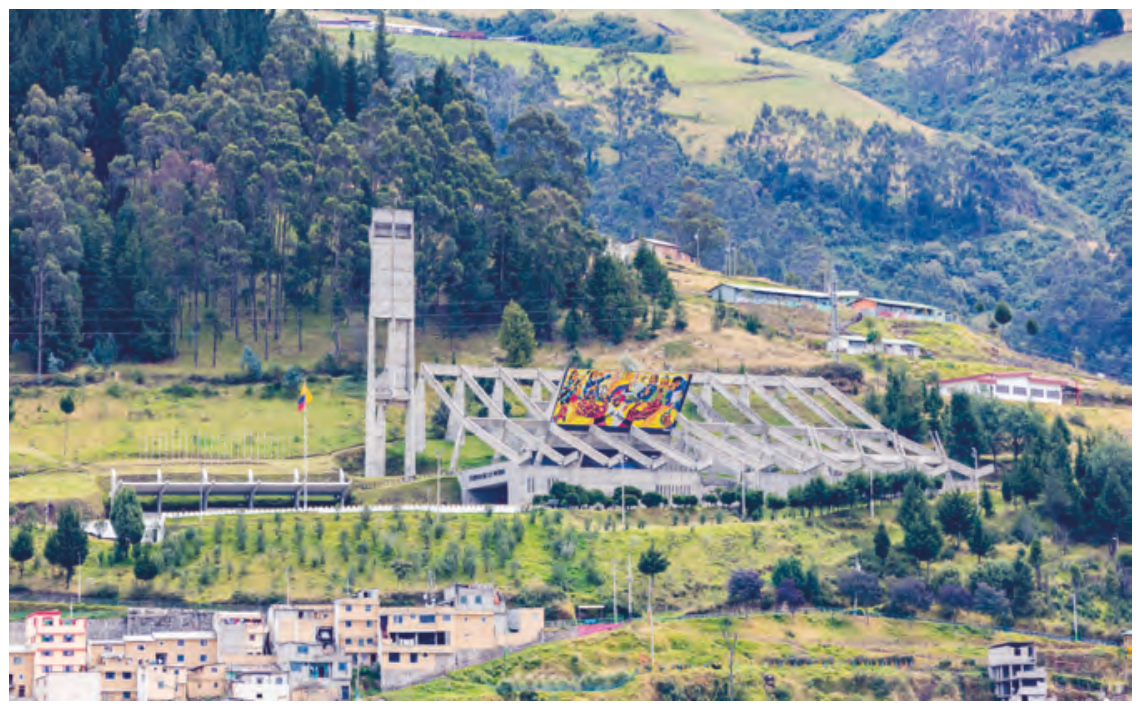

Illustration 21.13

Templo de la Patria (Temple of the Fatherland), Quito, Ecuador Photograph by Diego Delso, delso.photo, CC BY-SA 4.0

in India. These encouraged nationalism while at the same time informing people of global events and intellectual currents (especially as the telegraph and then international news services such as Reuters and Associated Press provided newspapers with quick and easy access to global news) - but first a common language was required. As states became increasingly complex, organizations such as political parties or professional/worker organizations naturally organized along national lines to interact with the state (albeit often with international connections), reinforcing a sense of national identity.

As we saw above and in earlier chapters, ethnic identity had been more fluid through most of human history than it has become in recent centuries. Nationalism further hardened ethnic identities: These now had a political as well as cultural dimension. Through most of history, multi-ethnic empires were the most familiar form of governance. One could change ethnic identity without changing or challenging one's political situation (though improving one's chances in the state bureaucracy might be a motive). With nationalism, this was no longer possible. This was especially the case when nationalism encouraged war: Ethnic identity then determined which side you were on. Whether fearful of defeat or avaricious toward victory, war inspired citizens to emphasize their differences from those they fought. As we saw when the Yugoslav state imploded in the 1990s, people who might otherwise have not felt a strong ethnic identification had to identify themselves strongly with a particular group for political and military protection.

Just as war could foster nationalism, nationalism could provide yet another justification for war. We have seen that warmongering has been a powerful force in human history. Nationalist warmongering could be particularly compelling, 
especially in circumstances where leaders might promise the group independence from others or make some claim to the resources of others. Given that human groups have migrated a great deal through human history, and that human history is full of conflict and real or imagined slights, it is generally entirely feasible to argue that one's nation has legitimate claims on the territory or treasure of another. Nationalist fervour was particularly important in justifying conscription, which in turn meant that wars could be particularly devastating.

The nationalist ideal of the nation state grounded in cultural unity has had some great successes - nations that forged a sense of unity and achieved economic and political success and cultural efflorescence - but has proven problematic from the outset. National feeling often supported foreign aggression or penalization of internal minorities: Jews, Roma, and many others. Small or intermingled ethnic groups characterized much of the world; autocracy is prevalent in such areas as democrats struggled to forge a sense of shared public sovereignty. The survival of democracy in India despite considerable ethnic diversity is remarkable if not always pretty.

The path forward is unclear. As separatist groups around the world have noted, international trade agreements increase the economic feasibility of very small states, for states are no longer as reliant for economic prosperity on a large internal market. Yet such states would then be very dependent on the continuation of such trade agreements. The sort of multicultural nationalism pioneered by countries such as the United States and Canada - which stress a shared history and institutions more than shared cultural practices - may provide an answer. However, the United States and Canada as lands of massive immigration could more easily achieve a multicultural sense of shared purpose than can countries characterized by groups that may have been in conflict for centuries.

Intermingled ethnicities cause some to celebrate the days of multicultural and tolerant empires. However, these were ruled autocratically for the primary benefit of the rulers themselves. The autocratic states that have so often emerged in multi-ethnic polities in recent centuries are laudable only if the alternative is political chaos. Some sort of federal structure can in some instances allow a degree of cultural autonomy for minorities - but this is most feasible if such groups are geographically distinct rather than intermingled (unless non-geographical forms of governance are possible). Some celebrate the messy confederal structure of the Holy Roman Empire as a template: It did, after all, survive for centuries. However, it did not have to contend with nationalist sentiments.

The horrors of the First and especially Second World Wars (chapter 28) did cause a widespread appreciation of the dangers of nationalism. This re-evaluation naturally proceeded farthest in Europe itself. The European Community and then European Union would emerge in large part as an effort to diminish nationalist sentiments. Germany, in particular, welcomed European integration as part of a strategy to step away from the excesses of nationalism in recent 
German history. On the global scale, the United Nations was also intended to at least diminish the effects of nationalist rivalry.

Some had urged a universalist appreciation of our common humanity even while nationalist sentiment was growing in the nineteenth century. This universalist voice has perhaps gained increased recognition since the Second World War. Nationalist tendencies may increase, though, as the memories of that war fade. World history may (or not) serve to establish that the individual histories celebrated by nationalists are intertwined.

\section{PRIMARY DOCUMENTS: GIUSEPPE MAZZINI ON NATIONALISM}

Mazzini was an eloquent advocate of nationalism:

Love your country. Your country is the land where your parents sleep, where is spoken that language in which the chosen of your heart, blushing, whispered the first word of love; it is the home that God has given you that by striving to perfect yourselves therein you may prepare to ascend to him.

Without country you have neither name, token, voice, nor rights, no admission as brothers into the fellowship of the Peoples. You are the bastards of Humanity. Soldiers without a banner, Israelites among the nations, you will find neither faith nor protection; none will be sureties for you. Do not beguile yourselves with the hope of emancipation from unjust social conditions if you do not first conquer a Country for yourselves.

The epoch of individuality is concluded, and it is the duty of reformers to initiate the epoch of association. Collective man is omnipotent upon the earth he treads.

Yet he appreciated some of the dangers inherent in nationalism, urging a forward-looking and respectful nationalism:

Slumber not in the tents of your fathers. The world is advancing.

A Country is not a mere territory; the particular territory is only its foundation. The Country is the idea which rises upon that foundation; it is the sentiment of love, the sense of fellowship which binds together all the sons of that territory.: God has given you your country as cradle, and humanity as mother; you cannot rightly love your brethren of the cradle if you love not the common mother.

The honour of a country depends much more on removing its faults than on boasting of its qualities.

Does Mazzini capture a healthy balance between nationalism and respect for a wider humanity? In what ways do his words reflect the times in which he wrote (as Italy was first unifying)? 


\section{Democracy}

Nation states need not be democratic. Through most of the last couple of centuries, most were not. However, the democracies that have emerged in the last centuries have been nation states. Democracy requires a community willing to collaborate; this collaboration has been easier to achieve within a "nation" with a strong sense of shared purpose. Democratic governance also requires that the will of the people be exercised: It therefore depends on the existence of a "state" with bureaucratic capabilities. The democratic challenge is, then, to assure that the power of the state serves the interests of its citizens. Sadly, democracies have often transitioned into autocracies - from the rise of Napoleon during the French Revolution to the recent transformations in Egypt. Democracies require state power, but also the means to limit arbitrary exercise of that power.

While the idea of popular sovereignty could support democracy, it could also justify autocratic rule if the autocrat promised to act - at least to some extent - in the interest of the people. In particular, given the importance of warfare in human history, the autocrat might proclaim a willingness to fight wars on behalf of the nation. As noted above, such an appeal might be especially attractive if a specific group of people felt a historical claim to lands or goods possessed by others.

Ideas alone, then, did not determine whether a nation state became democratic or autocratic. This outcome instead reflected the process of institutional evolution. Democracy depended most obviously on the creation of assemblies that exercised important legislative powers. Such assemblies themselves had to represent more than a narrow elite - though the percentage of the population represented in these assemblies could expand through time. Although the creation of powerful and representative assemblies is the obvious requirement for democratic governance, it was hardly sufficient. A set of institutions had to be put in place that prevented a ruler or army from merely dissolving the assembly, that prevented a narrow elite from dominating the assembly, and that ensured that bureaucracy and military mostly obeyed the will of the assembly. This set of institutions tended to emerge slowly over time, and tended to reflect a rough balance of power within a society. Countries that in recent times have tried to introduce democracy quickly have often found this to be difficult.

Since democracies everywhere evolved from systems of kingly (or other kinds of autocratic) rule, the most obvious challenge was for kings to come to accept the power of elected assemblies. This tended to occur when kings needed more money than they could raise on their own. They then traded power for finance. The financial stresses that we have recorded in polity after polity through human history - and that expanded in the Early Modern period as the costs of warfare increased, and as states expanded activities in areas such as transport infrastructure and education - were an important contributing factor in the evolution of democracy. 
Kings might always be tempted to reassert their power once a financial crisis had passed. Here a tradition of recognizing that even a ruler was subject to the laws of the state might be important. We have seen that many polities in history accepted in principle that rulers were not above the law: Islamic rulers appreciated to varying degrees the authority of Islamic law, Indian warrior elites recognized the legal jurisdiction of Brahmins, and European kings grudgingly accepted the authority of the church in at least some matters. The stronger institutional structure of Catholic (and much Protestant) Christianity may have served to strengthen this recognition in Western Europe, whereas the fact that Orthodox Christianity was governed mainly by states may have weakened it in Eastern Europe.

Russia had at times experimented with assemblies: An assembly of nobles had elected the Romanov dynasty of tsars. Tsars came over time to cooperate with the landed nobility in exercising power over peasants. Rather than trading power for money in assemblies, then, the tsar agreed to enforce serfdom, allowing the landed elite to profit from a peasantry that was bound to their land. The nobility, in turn, agreed to a strong centralized state that could both punish escaped serfs and organize the defence of the vast Russian steppe. The tsar and nobles placed the tax burden on peasants but denied them political representation. The tsar was not limited by the laws of any other body. (The Russian state controlled the local Orthodox Church; the latter might have maintained greater independence if not for the fall of Constantinople to the Ottomans in 1453.) A flat topography surrounded by enemies and a shared interest in enforcing serfdom meant a shared interest in a strong state.

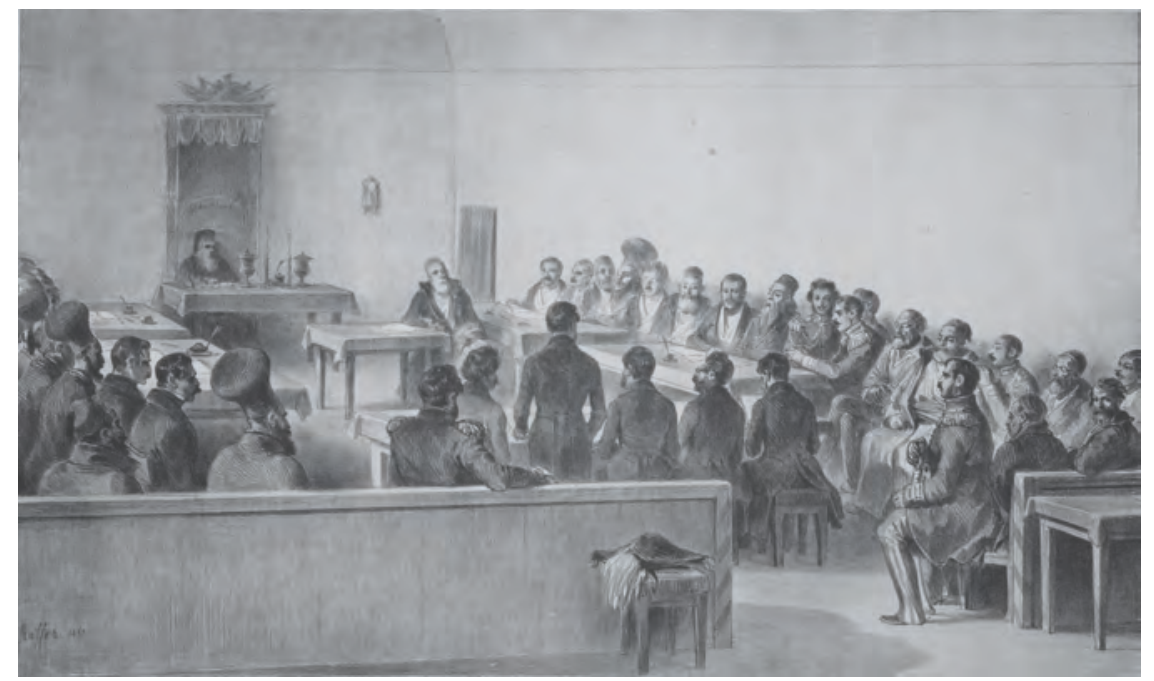

Illustration 21.14

Hungarian assembly, 1837, Budapest. The assembly was dominated by the nobility and senior clergy, though some wealthy merchants might participate. Painting by Auguste Raffet 
In Poland and Hungary also, rulers and nobility combined to enforce serfdom. There the aristocracy emerged with the most power, reserving the right to elect rulers that would respect elite privileges. Although these states were less centralized than Russia, there was still no interest in extending power beyond a small elite. The Hungarian assembly was a tool of oligarchic control rather than a stepping-stone to democratic governance.

France, as we have seen, developed a strongly centralized state. Though a national assembly - the Estates General - existed, the French king had been able to govern without convening this for a century and a half before the late eighteenth century. As we will see in chapter 25, that assembly then proceeded to demand dramatic changes in French governance. However, the assembly itself proved unable to prevent the emergence of new types of autocratic rule, since there were no institutions or cultural traditions in place to limit the authoritarian tendencies that emerged within the assembly or later the army.

In Britain, a quite different history unfolded. Parliament had gradually acquired increased powers over a period of centuries. Kings had had regular recourse to Parliament when they needed additional funds. The membership of Parliament had slowly expanded to include not just major landowners but smaller landholders and merchants from the cities. Perhaps 5 per cent of the population was represented by the seventeenth century (and maybe 20 per cent were involved in local governance). Kings had long ago granted powers to cities to gain an ally against nobles (chapter 15), but nobles too had come to appreciate the value of a merchant community with which they could interact. Such a parliament would not serve just a small oligarchy as in Hungary. Instead, it could focus on shared interests such as ensuring that the king respected private property and enforced contracts, and encouraging efficient and noncorrupt royal administration (since they all paid taxes). Here it could build on a long tradition of "common law." Over the centuries, the British crown had firmly established the precedence of royal courts over the feudal courts of local lords: In doing so, they sought to gain the support of the peasantry. A profession of educated judges and lawyers had emerged, and these argued cases based on precedent: A decision made by one judge anywhere in the kingdom would be cited in similar cases elsewhere and even centuries later. Such a system of law may be evolutionarily advantageous (though only if judges are not entirely corrupt or biased toward the interests of some minority): Decisions that seemed to have beneficial outcomes could be repeated while those that caused difficulties could be re-evaluated. In any case, centuries of common law served to establish that the king could not change the laws of the land unilaterally.

Even with these historical precedents, the path to democratic governance in Britain was far from smooth. The English Civil War (1642-51) pitted Parliament against the king (it also had religious overtones) but resulted in a decade of autocratic rule by Parliament's military commander Oliver Cromwell. Shortly after his death, Parliament agreed to restore the monarchy but with additional protections of Parliamentary power. Then, in the Glorious Revolution (1688), Parliament would disdain a king, James II, whose Catholic tendencies 
they feared, handing the crown to his sister Mary and her husband William of Orange (of the Netherlands), again with an insistence on the rights of Parliament. A slow process of institutional evolution had created the potential for a democratic outcome, but an autocratic result had still been possible.

One irony of the British case is that taxes rose as Parliamentary power increased: from an estimated 11 per cent of gross domestic product (GDP) in $1689-97$ to 17 per cent $1741-8$ and 24 per cent $1778-83$. This was a much larger tax take than autocratic rulers could generally achieve, and strengthened Britain's position in the world. There is a valuable lesson here about legitimacy: The willingness of people to pay taxes depends on whether they respect their government and its priorities. Parliament was far from perfect in this period: Only a minority could vote, and many constituencies had a small electorate that politicians bribed for votes. Nevertheless, it achieved far greater legitimacy than most autocrats.

In the Netherlands, merchants and landowners had combined in the lengthy effort to win independence from Spain in the sixteenth and seventeenth centuries. Rulers thereafter had frequent recourse to assemblies that jealously guarded their powers. As in England, then, assemblies that represented more than a narrow elite came to solidify their control over time. These assemblies, as in Britain, pursued goals such as property protection and contract enforcement that were in the general interest.

Denmark would pursue a different path: Strong rulers had created a nobility of service (that is, one could gain noble status through service to the king) in the eighteenth century that soon outnumbered the hereditary nobility. Freed of the need to compromise with their nobility, Denmark's rulers would create an assembly in which (free) peasants could participate. Widespread literacy facilitated this move; this, in turn, had been encouraged by the Protestant emphasis on personal revelation. This path to democracy would become more common as literacy rates rose elsewhere in the world.

One advantage of the slow evolution of democratic institutions was the recognition by those designing such institutions that democratic decision-making was often misguided or corrupt. Voters sometimes exercised good judgement but sometimes elected charlatans or thieves. Nationalist sentiment could support mistreatment of minorities. Politicians could work in favour of some segments of the population at the expense of others. It came to be appreciated, then, that democracies required "constitutions" that placed limits on democratic power and protected minorities and individuals. In Britain, these "constitutional" protections themselves evolved over the centuries in the common law and rules of Parliament. In later democracies, they would be enshrined in written constitutions that were difficult for later legislators to amend or ignore. (We discuss the American constitution, and some other constitutions, in chapter 25.) Like any institution, though, such constitutions were only valuable if the judiciary, bureaucracy, and army were dedicated to their enforcement.

China lacked both a tradition of elected assemblies and a tradition that the ruler was subject to laws generated elsewhere. The bureaucratic state had 
reduced the need for rulers to negotiate with other elites. Though we have seen that Chinese dynasties also struggled to limit the power of local elites, they often co-opted these into local bureaucratic governance. Also, the ruler and bureaucracy created laws as they saw fit. China inherited a powerful bureaucracy capable of pursuing the public will, but the very power of the Chinese bureaucracy worked against the creation of either assemblies or the rule of law.

In both India and the Islamic world, there was a limited appreciation that laws generated by religious authorities bound rulers. In practice, though, rulers often governed as they wished. They often were able to appoint religious authorities that respected state power. The theoretical recognition of limits to authoritarian rule did not much weaken authority in practice.

In Latin America, very unequal distributions of incomes, wealth, and power generally characterized newly independent states. The result was that small elites dominated assemblies and there was frequent recourse to military dictatorships aligned with these elites. (We discuss the main exception, Costa Rica, in chapter 25, noting that income distribution there was less unequal.)

Though the earliest democracies developed slowly, it has been possible for later democracies to copy the institutions established in these first democracies. Yet this path is difficult: One cannot just borrow the words on paper but needs to develop bureaucracies and armies that will enforce those institutions (for rules are meaningless without enforcement). Cultural attitudes that

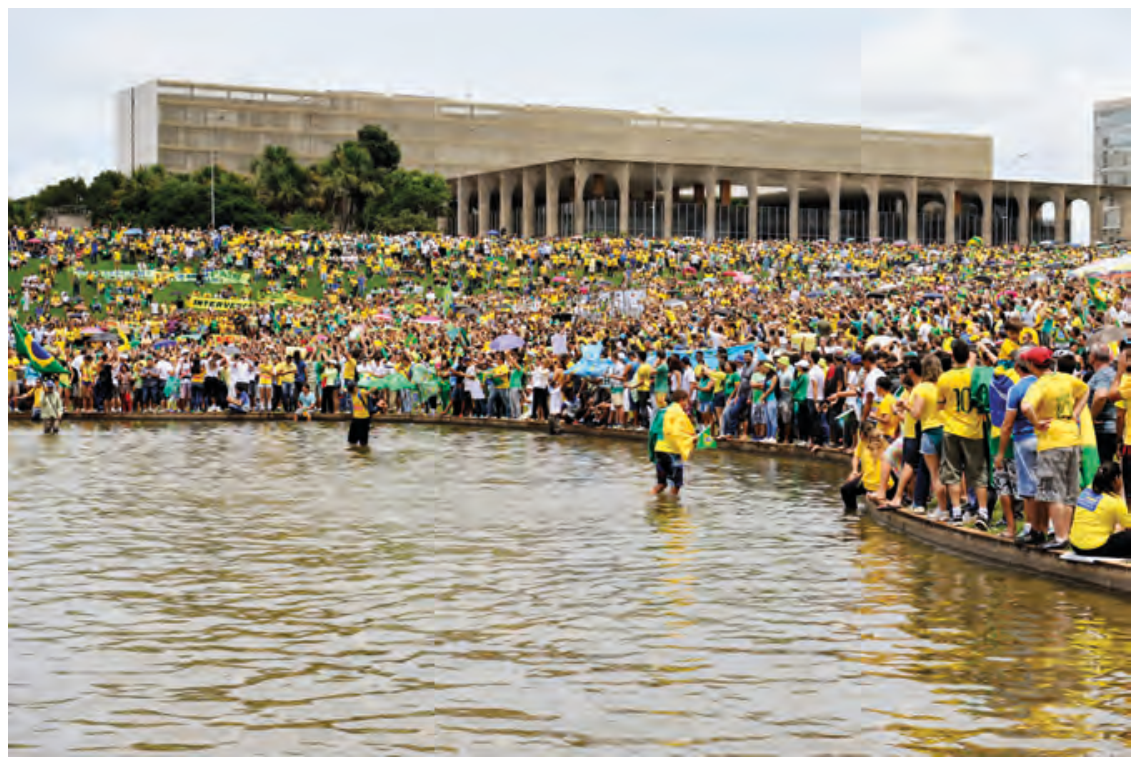

Illustration 21.15

Some 45,000-80,000 people protest against corruption in front of the National Congress in Brasilia in 2015

Senado Federal, CC BY 2.0 
support respectful discourse and disdain corruption are also critical and cannot be generated overnight. We will resume the story of democratization in chapter 27.

\section{Challenges facing agents: Voters}

The voter's fundamental challenge is to make a reasoned choice based on limited information. This challenge increases in scope as governments take on more responsibilities. The voter's incentive to gather relevant information decreases as the number of voters increases. The voter must judge both policies and personalities.

The voter must be aware that politicians will deceive them, whether deliberately or subconsciously. Politicians will at times suggest that there are simple solutions to complex challenges, but perhaps at other times ignore possible solutions.

Voters, like politicians, can easily self-deceive. They should be honest with themselves about whether they wish to vote based on self-interest or also for what is best for the broader community. They should be aware of the role that fear or jealousy or anger may be playing in their decisions.

The voter needs also to understand democracy's strengths and weaknesses. They cannot expect perfection but should appreciate their ability to encourage change in particular directions. They should strive to respect the views they disagree with - for democracy depends on a shared respect for democratic institutions and fellow citizens. They should recognize that democracy requires constitutional protections of both minorities and individuals, and be wary of politicians who might weaken these.

\section{Questions}

1. Would China have been better able to respond to European interventions in an age of nationalism if led by a domestic dynasty?

2. Is it advisable to urge the migration of ethnic groups as Turkey and Greece did in the 1920s?

3. Describe the temptations faced by nationalist leaders.

4. Diagram the influences on and effects of democratization.

5. Should nation states be encouraged everywhere?

6. Does the power profile in Box 21.1 fit our description of the rise and fall of other empires in this book?

\section{Readings}

Ciotola, Mark. 2018. "Navigating the Currents of World History." World History Connected February 2018.

Fukuyama, Francis. 2011. The Origins of Political Order: From Prehuman Times to the French Revolution. New York: Farrar, Strauss, and Giroux.

Johnson, Noel D., and Mark Koyama. 2017. "States and Economic Growth: Capacity and Constraints." Explorations in Economic History, April. [This article provides 
an excellent overview of the cross-disciplinary literature on state capacity, and of the critical importance of historical processes.]

Kramer, Lloyd. 2011. Nationalism in Europe and America: Politics, Cultures, and Identities since 1775. Chapel Hill, NC: University of North Carolina Press.

La Croix, Sumner. 2019. Hawai'i: Eight Hundred Years of Political and Economic Change. University of Chicago Press.

North, Michael. 2015. The Baltic. Cambridge, MA: Harvard University Press. [North notes that ethnic diversity delayed a sense of shared history in the Baltic region until relatively recently, despite the efforts of many rulers or merchant networks to dominate the region over time. North sees the region primarily as a venue for cultural and economic exchange.]

Reynolds, Michael. 2011. Shattering Empires: The Clash and Collapse of the Ottoman and Russian Empires, 1908-1918. Cambridge University Press.

Skwiot, Christine. 2018. "To 'Live Like King George' in Hawai'i and the United States: Entangled Exceptionalisms and Sovereignty Struggles in an Age of Revolutions, 1776-1819." World History Connected, October 2018.

Yun-Casalilla, Bartolomé, and Patrick K. O’Brien, with Francisco Comín Comín. 2012. The Rise of Fiscal States: A Global History 1500-1914. Cambridge University Press. [Stresses the development of abilities to tax the whole population; recognizes that China developed these capacities centuries before Europe.] 


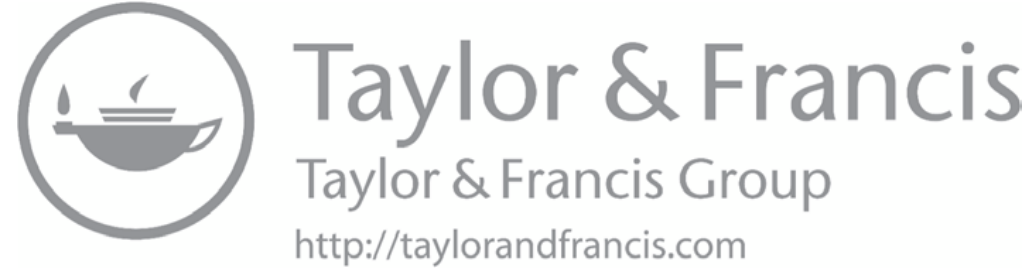




\section{The Great Divergence}

\section{The rise of the European economy and military}

\section{Guiding questions}

When, how, and why did Europe become both the wealthiest and most militarily powerful region in the world? What is the connection between economic growth and military conquest? How (if at all) and why did Europe in 1800 differ from other regions with respect to a wide range of phenomena that might have contributed to its later economic and military success?

What was the "scientific revolution"? When, where, and why did it occur? What were its effects? Did it, in particular, contribute in an important way to the divergence between Europe and other regions addressed above? Did science have much impact on technology at this time?

Does it still make sense to talk about "Western Civilization"?

Relationship to other chapters: We will compare the regional experience of a wide array of phenomena that might have affected economic and military success, a task that would be infeasible if we had not already familiarized ourselves with both the phenomena and the regions that we will be comparing. We must also revisit more than in most chapters some of our discussion in chapter 1 regarding historical methodology. More subtly, we can appreciate this particular historical episode better because we have observed countless cases in earlier chapters where one region outperformed another with respect to one or 
more of our key themes. It is important to remember that this is not the only occasion in world history where one region of the world seemed to pull ahead in important ways. European economic and military dominance will be a crucial feature of many developments in the nineteenth and twentieth centuries that we will address in later chapters, but most obviously colonization, war, and economic development. The discussion of the scientific revolution builds upon and contrasts with earlier discussions of science. It will naturally influence later scientific developments, which in turn influence technology. In addition to its historical contribution, this chapter represents the most rigorous exercise in historical comparison in this book. It is hoped that it thus contributes to the development of skills in drawing comparisons.

The "Great Divergence" is a term used to describe the widening gap in per capita incomes between Europe (and European settler societies such as the United States) and most of the rest of the world. It is not entirely clear when this divergence began - and therefore where best to place this chapter in this book. It is clear that the gap widened dramatically in the nineteenth and early twentieth centuries: Per capita incomes in European countries were several times greater than per capita incomes in Asian countries in 1900 or 1930. Debate rages as to how different per capita incomes were between Europe and Asia in 1800. Even if incomes were not much different in 1800, it could be that other factors technology or institutions being obvious possibilities - already differed by that time in a way that set the stage for differing economic performance after 1800. We will therefore attempt an intercontinental comparison for the late eighteenth century of most of the key causes of economic growth (see Figure 29.1) in what follows.

Since this chapter necessarily looks ahead - it seeks, that is, to explain changes that become obvious in the nineteenth century - it is perhaps useful to look briefly even farther forward. At least some of those Asian nations that had fallen behind Europe with respect to per capita incomes in the nineteenth century have managed a fair bit of "catch-up" in the late twentieth century: South Korea, Singapore, Hong Kong, and especially Japan from the early postwar period, and China, India, and much of Southeast Asia more recently. We should see the Great Divergence, then, as a particular historical episode that may well give way to the Great Convergence (see chapter 29).

The reader should also reflect upon one "big question" in advance. Is it more likely that the enormous differences that emerge in per capita incomes in the nineteenth century reflected either historical contingencies circa 1800 or more long-standing differences between Europe and Asia? The evidence should 
guide us in answering this question. Nevertheless, the reader can appreciate that scholars have debated the relative likelihood of these two possible broad explanations of the Great Divergence: a sudden shock of some kind propelling Europe forward or a more gradual process of transformation. Indeed the twin questions of when and why Europe pulled ahead of the rest of the world economically provide the greatest area of controversy in the entire field of world history.

Though our focus in this chapter is economic, we will also engage the question of why Europe simultaneously achieved military dominance. In exploring both economic and military divergence, we will look at many political and economic phenomena but also elements of the non-human environment, population, culture, and technology and science. Our purpose throughout the chapter is to compare different regions of the world. We will briefly apply evolutionary analysis to institutions, scientific standards of evidence, and the idea of Western Civilization.

\begin{tabular}{|c|c|c|c|c|c|}
\hline Economy & Politics & $\begin{array}{l}\text { Non-human } \\
\text { Environment }\end{array}$ & Population & Culture & $\begin{array}{c}\text { Technology } \\
\text { Science }\end{array}$ \\
\hline
\end{tabular}

\section{Roots of the Great Divergence}

It is understandable that this debate has excited the world history community. The fact of European economic - and military - success in the nineteenth century has had repercussions to our day. We will discuss in later chapters shifts in political power among regions of the world, "world wars" fomented by European powers, the spread of democracy and socialism from European roots, European colonialism and decolonization, and other such topics. The question of "Why Europe?" is then of substantial historical import. There are, as we shall see, many possible answers to this question. Answering this question requires the sort of detailed comparisons of Europe (or parts thereof) with other parts of the world that world historians generally advocate. We can celebrate the many efforts at comparison that scholars continue to make in an attempt to comprehend the Great Divergence.

We can also bemoan some unfortunate sources of disagreement that have rendered the debate less constructive than it might have been. During the heyday of European dominance in the late nineteenth century, before two world wars shattered the idea that European civilization was morally superior, it was common for Europeans to attribute their success to ethnic or cultural superiority. This idea is rarely (explicitly) voiced today. Yet historians understandably still wish to distance themselves from any sense that "European society - or people - was/is better." For some, this has encouraged a desire to show that Europe was not "special" in any way. We should as historians strive to engage in a detached and reasoned debate about causal forces in history without having strong preferences for what we might find. 
This book reflects the twin premises that there is a large (though manageable) set of phenomena or themes of historical importance, and that these interact in myriad ways. We can presume that there were several differences between Europe and any other region of the world, and that any of these differences may have exerted surprising (both to contemporaries and to historians) effects on economic growth or military prowess. In this sense, every region of the world is exceptional for each has unique combinations of institutions, attitudes, economic output, family relationships, and so on. The fact that Europe's unique combination resulted in dramatic economic growth and military conquest after 1800 need not imply that Europe was "superior" in the ethnocentric manner commonly expressed by European writers of the time. This is true whether we find that European success reflected developments in the fifteenth or even tenth centuries, or was instead contingent on more fleeting causal forces in the late eighteenth century.

Europeans, we might note, had predicted neither dramatic rates of economic growth nor military domination of the globe, and so we need not worry that they had set these processes in motion deliberately. Industrialization in particular only became a goal of public policy after several European countries had already industrialized; we should be careful of blaming those who fell behind for not having pursued a goal that was nowhere appreciated or consciously pursued.

Just as we should not worry that any claim of European exceptionalism that is, that Europe was different - implies an old ideal of European superiority, we should not pursue a desire to attribute European success to "luck." In a complex system in which many phenomena interact in diverse ways, outcomes are always unpredictable to a considerable extent. Nevertheless, we can seek after the fact to determine which causal interactions proved most important. Unfortunately, many scholars have thought that the only response to the claim that "Europe was superior" was to argue that "Europe was lucky" and thus sought to identify transitory and external causes of European success. The viewpoint of this book is that both propositions (superior or lucky) are nonsensical within an understanding that history always represents a complex interaction of causal forces. Some particular constellation of phenomena facilitated dramatic and unexpected economic growth in Europe. We can investigate this set of phenomena, just as we have been able to understand every transformation addressed in this entire book in terms of the interaction of multiple phenomena, without ever employing words like "superior" or "lucky."

We will proceed calmly to examine various possible differences that might have existed between Europe and other advanced regions of the world, and the effects that these might have had on economic growth or military success. Happily, this approach seems to reflect the most recent trends in historiography: Much of the heat has gone out of the debate, and attention has turned to detailed intercontinental comparisons and an appreciation that multiple forces were likely at work. The primary challenge now is empirical: How exactly did per capita incomes compare across regions at different times? How widely 
were particular technologies employed? How well were specific institutions enforced? Often in such cases, further research is called for to clarify empirical questions. We will in what follows reach conclusions when these seem warranted by the evidence but merely record arguments on both sides when the empirical debate seems unsettled. We will compare as widely as possible but can note here that most research to date has focused on examining Europe and China, though scholars are paying increased attention to India and the Middle East.

Note that we are often trying to identify degrees of difference. It matters a great deal whether we find that per capita incomes were roughly equivalent in 1800 or were instead even 10 per cent higher in Europe: The latter finding would force us to look well before 1800 for understanding of the Great Divergence. For technology, we need identify not just which techniques were known but how widely they were applied: Finding one windmill or steel mill in a region is entirely different from finding thousands, and we should be careful of assuming that a region with one is as advanced technologically as a region with thousands. Concerning institutions, we need to look beyond the rules that might exist on paper to ascertain how well they were enforced. The reader should appreciate in advance that such questions of degree are difficult to answer for a time period in which governments did not routinely collect data on incomes or technologies employed. Nevertheless, detailed research in the archives of many countries can steadily illuminate these comparisons (though limited documentary evidence may always leave room for doubt).

\section{Comparing incomes}

The most obvious way to compare incomes in 1800 - since governments were nowhere collecting these statistics for us is to look at wage rates. Then one has also to compare prices: The nominal wages of Indian workers were lower than European workers but so too was the price of food. Still, the most recent efforts to compare the purchasing power of wage income do suggest that real wages in Western Europe were higher than those in (the most economically dynamic regions of) India or China, and even farther above those in Africa, by 1800 . Wages in London may have been double those in Istanbul by

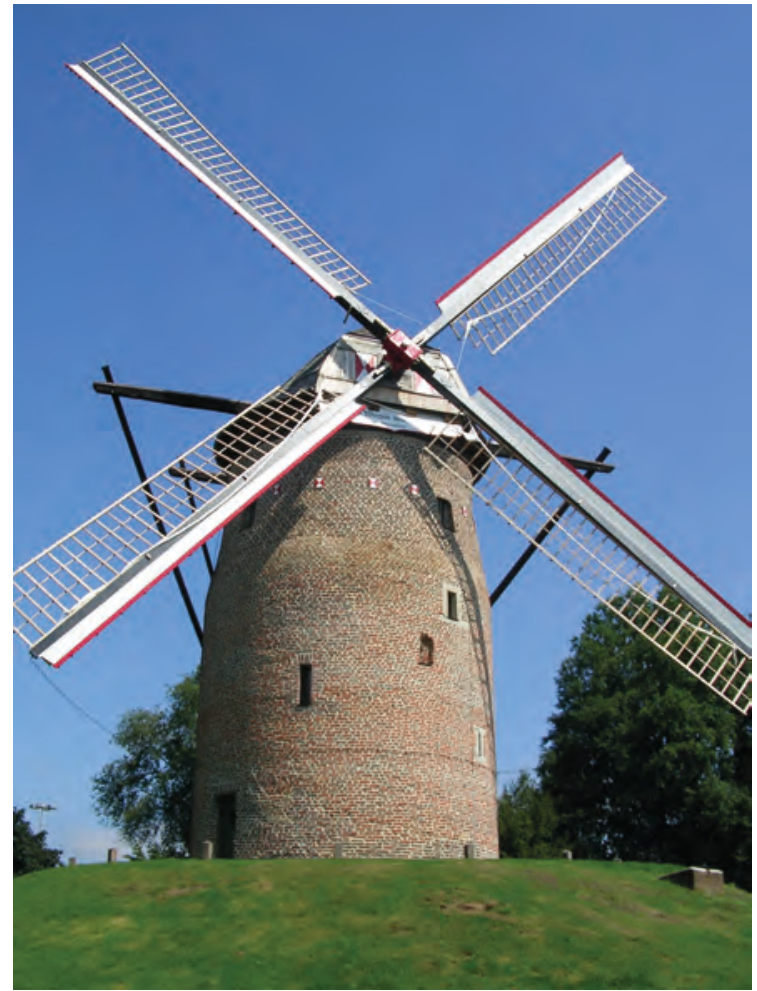

Illustration 22.1

Krefeld-Oppum windmill, Germany, first built in 1575 
1750. Yet many scholars question the validity of such estimates. Much of the world population comprised farmers or self-employed artisans or merchants that did not earn wages. Wage labour may have been far more important in Britain than elsewhere. Also, wage rates reflect in part the shape of institutions governing labour markets. Therefore, differences in wage rates may not reflect differences in average incomes.

An alternative approach is to look at food intake. Since food still comprised the majority of consumption expenditures for the majority of the population, a difference in food per person might well signal differences in income per person. Here again, there seems to have been an advantage in favour of Europe over China already by 1800 and likely by 1700 . Growing population had apparently counteracted the advances in agricultural productivity in China associated with the application of New World crops (chapter 19). It appears that Britain, at least, had a significantly more substantial proportion of its population outside agriculture in 1800 than even the most advanced regions of China: This is indicative of higher incomes and may have set the stage for later divergence. There is some evidence that Europeans had a higher caloric intake than (most) others; if so, this would have facilitated greater work effort and thus potentially supported higher incomes. Much less research has been performed on dietary diversity: Europe may not have been at an advantage here with implications regarding both the taste and enjoyment of food and European ability to achieve the various nutritional needs of the human body.

For non-food consumption, there is evidence that consumption was diversifying in the eighteenth century in both Europe and Asia, and even to a lesser degree in Africa. That is, peasants and workers were buying more and likely better textiles, pottery, and metal goods. They had become "consumers" in the modern sense that their consumption of non-food items had become a defining part of their lives. Even for food, an increasing proportion of the population in both Europe and China had come to appreciate the idea of cuisine, and so value complex food preparation. It is much harder, though, to quantify these trends in order either to estimate changes in income or to compare incomes across regions. It could be that "consumerism" had advanced farthest in Europe and that people there had decided to work longer hours in order to purchase consumer goods (many of them imported; see chapter 18); if so differences in income might reflect differences in work effort.

The differences identified in 1800 are small relative to the differences that existed by 1900 or 1950. Clearly, most of the Great Divergence occurred after 1800. Nevertheless, these small differences do lend support to the idea that at least some of the sources of the Great Divergence existed well before 1800 . Moreover, as noted above, even transformations in incomes after 1800 may reflect changes in other phenomena before 1800 .

Still, we should appreciate that much of the world experienced some economic growth both before and after 1800 . These rates of growth were impressive by historical standards: Growth was imperceptible or non-existent through much of world history. Tokugawa Japan may have experienced more rapid 


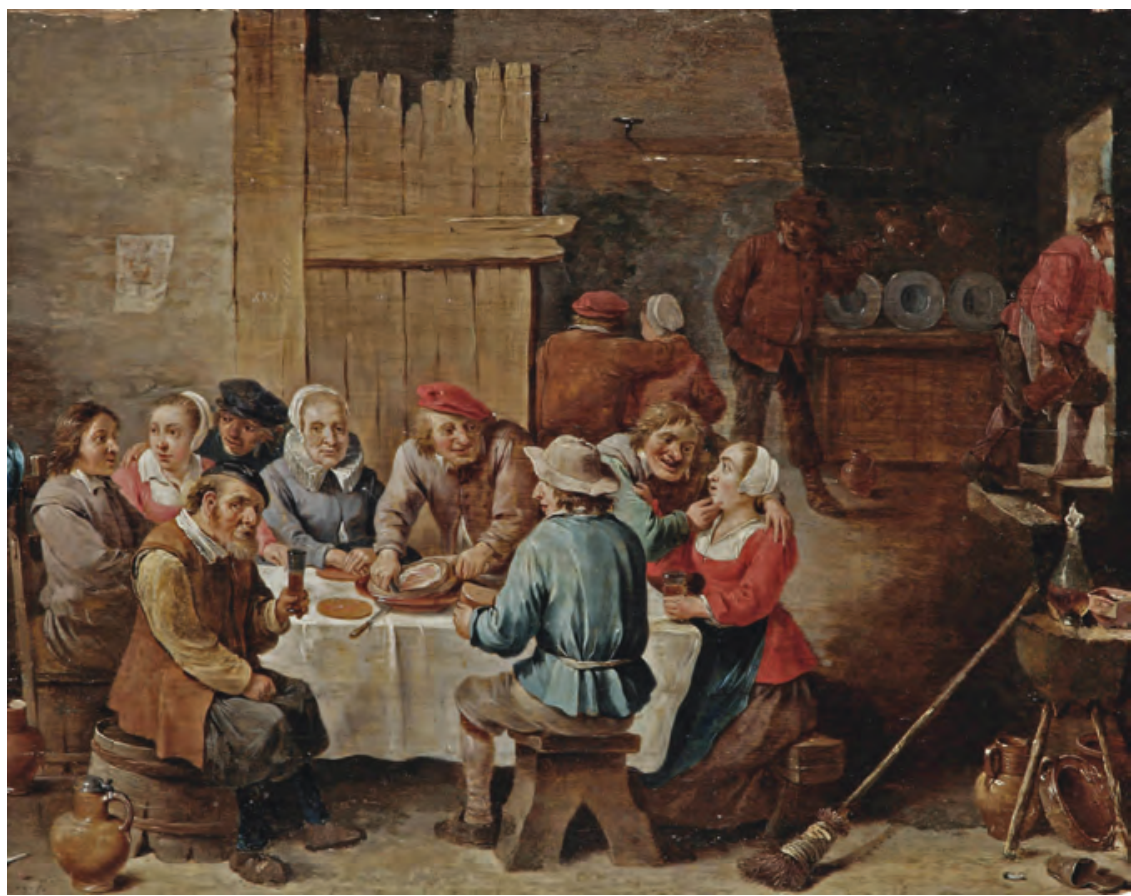

Illustration 22.2

Peasants Eating and Drinking in an Interior, Abraham Teniers, 1662. Clothes, dishes, and food would all improve over the next centuries

increases in per capita incomes than Europe in the eighteenth century (but started from a lower base). Per capita incomes in the Middle East likely grew by at least two-thirds in the century after 1820: This was faster than ever before but very slow relative to European incomes that may have increased eightfold in the same period.

\section{Natural resources and colonies}

As recognized above, the Great Divergence has both economic and political aspects. Europe conquers much of the world between the fifteenth and nineteenth centuries. It pulls ahead economically of the rest of the world sometime during the same period. It would seem a fantastic coincidence if there were no relationship between these two processes. How might they be related? There are three broad possibilities: Economic growth encouraged colonization; colonization promoted economic growth; or some earlier events or processes assisted both. The middle argument has generally received the most attention. It allows scholars to attribute Europe's economic success entirely to the suffering of those it colonized. However, all three lines of argument are plausible.

Could growth and conquest reflect a similar set of causes? Of course. One obvious candidate is technology. Europe's military conquests owed much to 


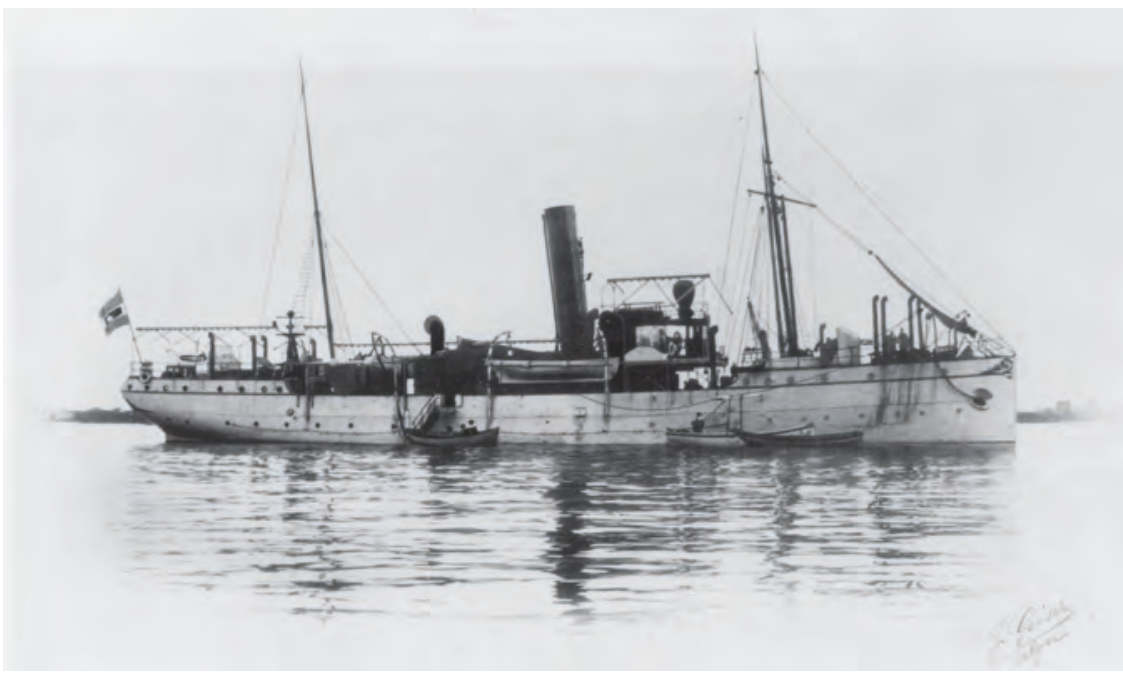

Illustration 22.3

Gunboat Siboga built in 1898 and used by the Dutch in what is now Indonesia

advances in gunpowder technology (Chapter 18). We will argue below (and have suggested in previous chapters) that technological innovation is a significant source of economic growth. If for some reason Europe had become more innovative in general over previous centuries then both growth and conquest would be likely outcomes. Institutions are another possibility: Strong states that respect individual freedoms and property might foster both growth and conquest. We have spent much of this book discussing the challenges of administering large empires, and so can appreciate that European states did not just stumble upon distant lands but proved able to administer these for centuries. Moreover, we have and will discuss at many points in this book the importance of economic institutions in fostering investment, trade, and growth. We might sketch various similar lines of argument. Note that such differences in technology or institutions or other phenomena - if they existed need have no strong connection to the cultural differences that nineteenthcentury European exceptionalists trumpeted and which generally offend contemporary sensibilities.

Could growth encourage colonization? At least in the nineteenth century, Europe's industrial might would allow it to produce massive amounts of firearms and steamships with which to subdue the world. Its need for raw materials to power its industry may also have encouraged colonization - though it received most of these most of the time from independent countries, and would grow even faster after shedding its colonies after the Second World War.

Could colonization facilitate growth? This is also entirely possible. Economic growth, especially in the nineteenth century, was associated with industrialization and this requires industrial raw materials and food for industrial workers. 
How much of each did Europe obtain from colonies? How much of this could it only acquire (at similar prices) through colonization? Note that even today developed countries import only a few per cent of their GDP from less developed countries. Europe's import of food was moderate until well into the nineteenth century but then became substantial. We might view these imports as results rather than causes of industrial expansion in Europe, but it is true that food imports into Europe facilitated the latter stages of the Great Divergence.

Even if colonization did not have a great impact on growth in Europe, it might have served to slow growth in colonies. Perhaps India was not far behind Europe economically in the eighteenth century but fell far behind in the nineteenth because British colonial authorities hampered economic development? It may be that differences in income between the most advanced parts of India and Europe were not huge in the eighteenth century, but there do seem to have been great differences in technology and institutions predating British colonization (discussed below). Debate rages about the effects of colonization on the colonies; the Indian economy grew sluggishly at best in the nineteenth century but it is hard to know to what degree this was due to colonization.

Another possible link is through profits. If European states or individuals made massive profits in their colonies, and invested these profits in industry or agriculture, then - since investment is one cause of growth - colonization might drive growth. Though some individuals did make huge profits in the colonies, detailed calculations suggest that average profits were not much higher than those obtained elsewhere. Moreover, those engaged in colonial investment were merchants rather than industrialists; studies of European industrialists find that they financed their enterprises locally. There may be indirect links, of course; colonial profits may have played an important role in funding of the transport developments discussed in chapter 24 that in turn facilitated industrialization. Nevertheless, at present, the case for the linkage between colonial profits and industrialization appears weak.

The Russian revolutionary Lenin argued that colonialism was essential to capitalism: Profits in capitalist countries depended on exploiting colonies. If so, the success of capitalist economies (so far) decades after decolonization (chapter 30) is puzzling.

As for domestic natural resources, coal has received much attention, since it was the primary industrial fuel of the nineteenth century. As we shall see in chapter 24, early industrializers in Europe tended to have access to coal, which was a source of both heat energy and mechanical power. Other regions such as India had far less coal. China also has abundant coal resources, though perhaps not as conveniently located. Some have argued, however, that the fundamental difference between China and Europe was not the location of Chinese coal but the fact that China had not developed access to the coal it possessed.

There may be some truth in all three possibilities. Perhaps Europe had some characteristics - technological, institutional, or other - that encouraged both 


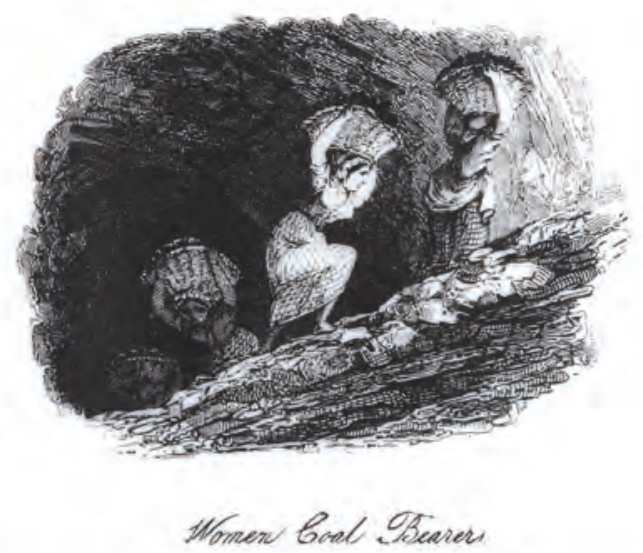

From: Taylor (I.), The Mine, 1829.

The usual method of carrying the coal from the place where it was dug to the entrance of the mine was by means of sledges drawn by horses up a gentle slope. Where the mine was not large enough for horses to work, men pushed the coal on a low carriage: where the ascent is too steep for this method, women bearers were employed carrying buckets on their backs.

\section{Illustration 22.4}

Women coal bearers, England, 1829. When passages were too narrow for horses, and too steep for men to push wagons, women carried coal up in baskets, Wellcome Library, CC BY 4.0

military success and economic growth. Thereafter, industrialization facilitated colonization and colonization made industrialization easier. It would appear to be a serious exaggeration, though, to attribute European economic success entirely to colonization.

\section{Trade and markets}

We traced in chapter 19 the entry into Asian markets of European ships after 1500. This has caused some to wonder if Europe did not come to appropriate most of the gains from trade over the next centuries. However, Asian trade networks remained vibrant through this period. Trade expanded worldwide in these centuries. Nor did Europe's strength in shipping (nor its efforts at colonization) lead to a concentration of industrial production in Europe until well into the nineteenth century. India exported cotton goods to Europe in large quantities, and these comprised over half of the textiles traded by Europeans in Africa for slaves, until late in the eighteenth century: Africans at the time preferred the lightness and colours of Indian cottons. China and India together likely accounted for half of global industrial production in the eighteenth century, though less than 5 per cent after 1900; this dramatic transformation partly reflected deindustrialization in Asia but mostly resulted from dramatic 
expansion in European production in the nineteenth century.

The main export from European countries to China before the nineteenth century was not manufactured goods but rather silver from the Americas. Many scholars have taken this fact as evidence of the superiority of Chinese manufactured goods. Europeans might value Chinese silk and porcelain, but the Chinese were unimpressed with European products. However, the flow of silver reflected also the low transport cost relative to value of moving silver, and the huge differences across continents in the value of silver that naturally emerged with the discovery of massive silver deposits in Peru and Mexico (chapter 19). China lacked large internal sources of silver but had long relied on silver for large coins (since copper currency was ill-suited to large transactions). Earlier Chinese empires had fretted that silver was exported to buy foreign goods. With the discovery of massive silver deposits in the Americas the "price" of silver suddenly became much lower there and in Europe than it was in China. It should be little surprise that so much of the silver found in the Americas found its way to large Asian economies.

One possible advantage that accrued to

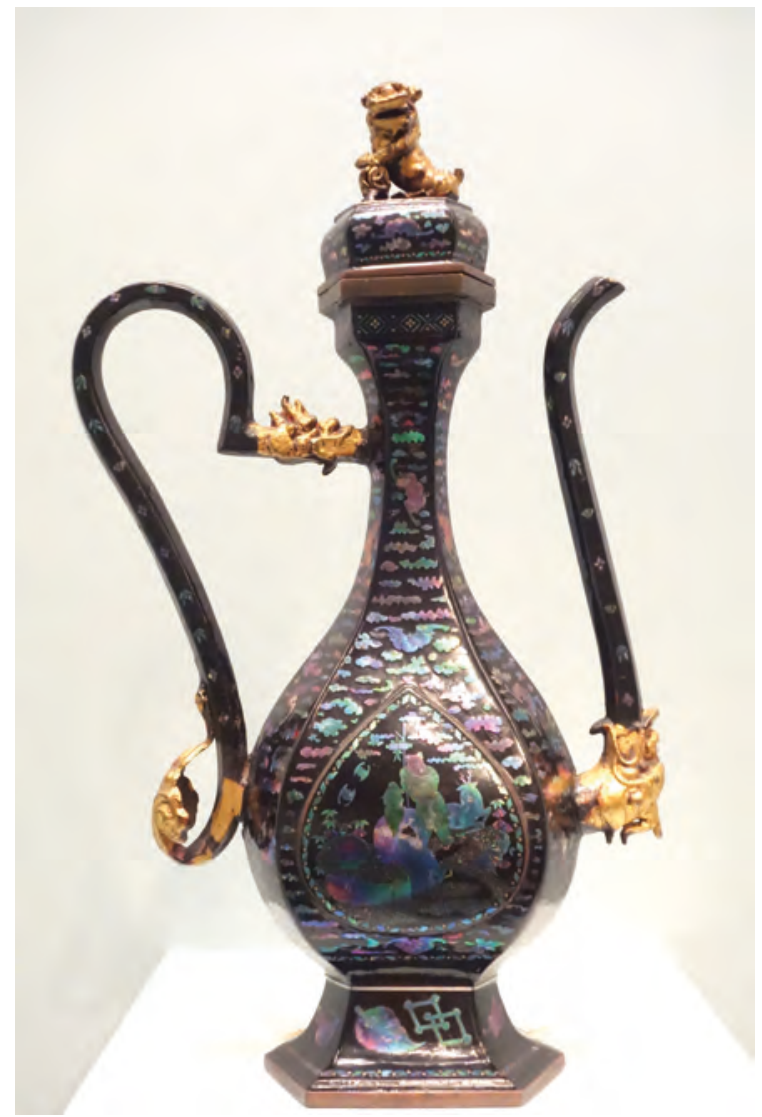

Illustration 22.5

Qing dynasty ewer with figures in a landscape, metal with black lacquer and mother-of-pearl inlays, Asian Art Museum of San Francisco Photograph by Daderot, CC0 Europe as its ships sailed throughout the world was better access to the world's technologies. Many European inventions of the Early Modern period built upon earlier Asian technologies. Europeans, in particular, sought to duplicate both Chinese porcelain and Indian cotton. The Dutch and British East India Companies made a point of transmitting scientific, medical, and technological understandings from their colonies: This provided good publicity for the colonial project.

In sum, while Europe undoubtedly benefited from its role in international trade, it is far from clear that this alone would have propelled its massive industrial expansion.

\section{Demography}

We noted above that increases in population might have swamped gains in Chinese agricultural productivity. There has been a long history of speculation 


\section{PRIMARY DOCUMENTS: ADAM SMITH IN THE WEALTH OF}

\section{NATIONS}

The great extent of the empire of China, the vast multitude of its inhabitants, the variety of climate, and consequently of productions in different provinces, and easy communication by means of water carriage between the greater part of them render the home market of that country of so great extent, as to be alone sufficient to support very great manufactures, and to admit of very considerable subdivisions of labour.

Smith had earlier criticized the lack of foreign trade in China but now explains it. How much would Smith have known of China when writing in 1776 ?

that Chinese population grew faster than European population, and that this difference reflected in turn differences in family structure. The latest research suggests, though, that population growth rates were broadly similar in the centuries before 1800, and that nuclear families were about equally common as well. (Within Europe, higher incidence of nuclear families is found both in some parts of the continent that would experience rapid growth early in the nineteenth century and in others that were much more sluggish economically.) Europe had more people that never married, but European celibacy balanced Chinese practices in which widows did not remarry. If so, then any advantages Europe had concerning growth in per capita incomes must reflect faster advance in aggregate economic output rather than slower increase in population.

One possible explanation of early European success deserves attention here. We noted in chapter 17 that the Plague had a much higher mortality rate in Europe than in China in the mid fourteenth century, and that India was mostly untouched. The Plague would return periodically for centuries (and then largely disappear for centuries for reasons we do not fully understand: changing trade routes, quarantine procedures, changes in the pathogen itself, and developing human immunity are all possible). As noted in chapter 17, though the Plague caused much disruption, it likely served to raise real wages in Europe. Arguably, the mortality rate was so high, and the recurrence of plague so frequent, that Malthusian mechanisms could not push incomes back near subsistence for centuries. If so, then the roots of the Great Divergence may lie (in part) in the far higher rates of epidemic mortality experienced in Europe. Europe may have had higher per capita incomes from the fourteenth century.

\section{Political and economic institutions}

European institutions differed from those elsewhere in many ways. We can debate both how great these differences were and whether they were important: 
Political fragmentation. Vast empires dominated Asia through most of the Early Modern period (chapter 20). The Spanish or Portuguese colonized the densely populated parts of the Americas (chapter 19). Political fragmentation instead characterized Europe and Africa. In Europe, emerging nation states and declining multi-ethnic states competed economically and militarily (chapter 21). Such competition discouraged states from opposing technological or institutional innovation. If they did so, the innovators often took their ideas to other countries. Jewish refugees from Spain in the early sixteenth century would make significant contributions to the merchant community of the Netherlands. Centuries later, Protestant refugees from France would infuse textile manufacture in England with new ideas. An empire that faced little foreign competition would instead be tempted to ban innovation to maintain social stability. The Ottomans would ban the printing press for decades, slowing the flow of all sorts of new ideas (chapter 20).

The argument that political fragmentation might have been beneficial is noteworthy in terms of the regrettable dialogue regarding Western exceptionalism discussed above. Europeans did not pursue political fragmentation in recognition that it had economic benefits. Indeed, political fragmentation was widely viewed with regret: Europe suffered much more from war in the Early Modern period than did Asian empires (this encouraged the development of gunpowder technology; chapter 18). Nor should we forget the economic disincentives associated with war: Merchants and farmers are much less likely to invest if they fear marauding armies. Moreover, political fragmentation was in no way a celebration of Europe's Roman heritage but rather its antithesis. If political fragmentation encouraged European technological and institutional innovation, this was an unexpected outcome of a generally undesirable political situation.

As we saw in chapter 21, though, some of these new nation states were able to develop centralized and effective - though still often quite corrupt bureaucracies, and to tax more than Asian empires. They could then better support legal systems and transport infrastructure on which merchants relied. As noted in Box 21.3, they may have developed the "state capacity" that was critical for modern economic growth.

Property rights. In some but far from all European states, we see during this time period the emergence of politically powerful elected assemblies, and the entrenchment of the idea that even kings are subject to the laws of the land. The exemplars are Britain and the Netherlands (chapter 21). In China, there were no assemblies, and the bureaucratic tradition was that the ruler was the source of all law. The Ottomans recognized the jurisdiction of religious authorities in some matters, but increasingly asserted the right of the state to legislate as it saw fit (chapter 20). We should be careful not to exaggerate this difference. One of the lessons of this book, after all, is that emperors were not as free to do as they pleased as we might 
have thought. They had to act through bureaucrats and governors with their own interests. There was, as noted above, extensive trade pursued by merchants in Asia as well as Europe. Merchants managed everywhere to cope with the property rights regime they faced. Yet on the margin, more secure property rights may have encouraged entrepreneurs in some parts of Europe toward risky investments in large factories and machines (see chapter 24; we will discuss there the links between property rights and transport infrastructure and economic growth).

Rural industry. We have discussed in earlier chapters the typical industrial structure through most of world history: small urban workshops, often associated with some sort of guild that limited entry and tried to protect the quality of output. In Europe from about the sixteenth century, much industry migrated to the countryside. Historians long thought that this migration occurred mainly to escape guild controls but now appreciate that lower wages in the countryside - because rural workers had access to cheap food and often still worked part-time in the agricultural sector were an even more significant incentive. Often rural workers performed tasks requiring less skill while urban guilds continued to perform work that was more skilled. Such an industrial migration depended, of course, on the ease of movement of materials between town and country. Some of these rural workers operated independently: Weavers in the wool trade around Leeds would bring their cloth to a weekly market to sell. However, many/most of these rural workers were employees: Entrepreneurs would bring them raw materials and pay them by the piece for finished goods. Entrepreneurs sometimes also financed whatever tools or machines the worker used. Some of these "putting-out" entrepreneurs came to employ hundreds or even thousands of such workers. There does not seem to have been much if any development of what are in fact large-scale industrial firms (albeit with a dispersed labour force) elsewhere in the world. When the factory emerged during the British Industrial Revolution (chapter 24), entrepreneurs did not need to invent the large industrial firm but only decide to gather workers in one place.

Financial institutions and laws. Did Europe have more sophisticated banks? Financial institutions poorly served the Islamic world until the nineteenth century. However, India had banks that could transfer funds between regions for a small fee. Europe transcended the Christian/Islamic distaste for charging interest earlier than the Islamic world, but there had always been low-cost methods of evading laws against charging interest (usury).

Bankruptcy laws developed in Italy in the thirteenth and fourteenth centuries and spread geographically across Europe while being further codified over the next centuries. Bankruptcy law would then spread to other continents. Bankruptcy laws are of crucial importance when multiple investors have competing interests (shares, bonds, or loans) in large 
industrial concerns. Historians tend to focus on the winners, but investors have to worry about what happens when a firm or person has trouble paying their bills. Bankruptcy law allows investors to know how the assets of a failed business will be divided.

Europe in the Early Modern period also benefited from clarifications of dispute settlement mechanisms and the development of regulations for stock markets. All of these evolved slowly over centuries. So too did the corporate form itself. The Ottomans in contrast effectively outlawed private corporations until the nineteenth century in favour of the waqf, a charitable endowment endorsed by Islamic law (one problem with the waqf was that administrators were required by law to follow to the letter the instructions associated with the original bequest; this reduced flexibility and prevented waqf mergers). China also did not allow corporations until very late in the nineteenth century (Box 15.1). Note that there is a symbiotic evolutionary process: If institutions such as corporations or stock markets facilitate impersonal economic interactions, those interactions will, in turn, create selection pressure for further institutional development. A small initial difference may yield a substantial institutional divergence over time.

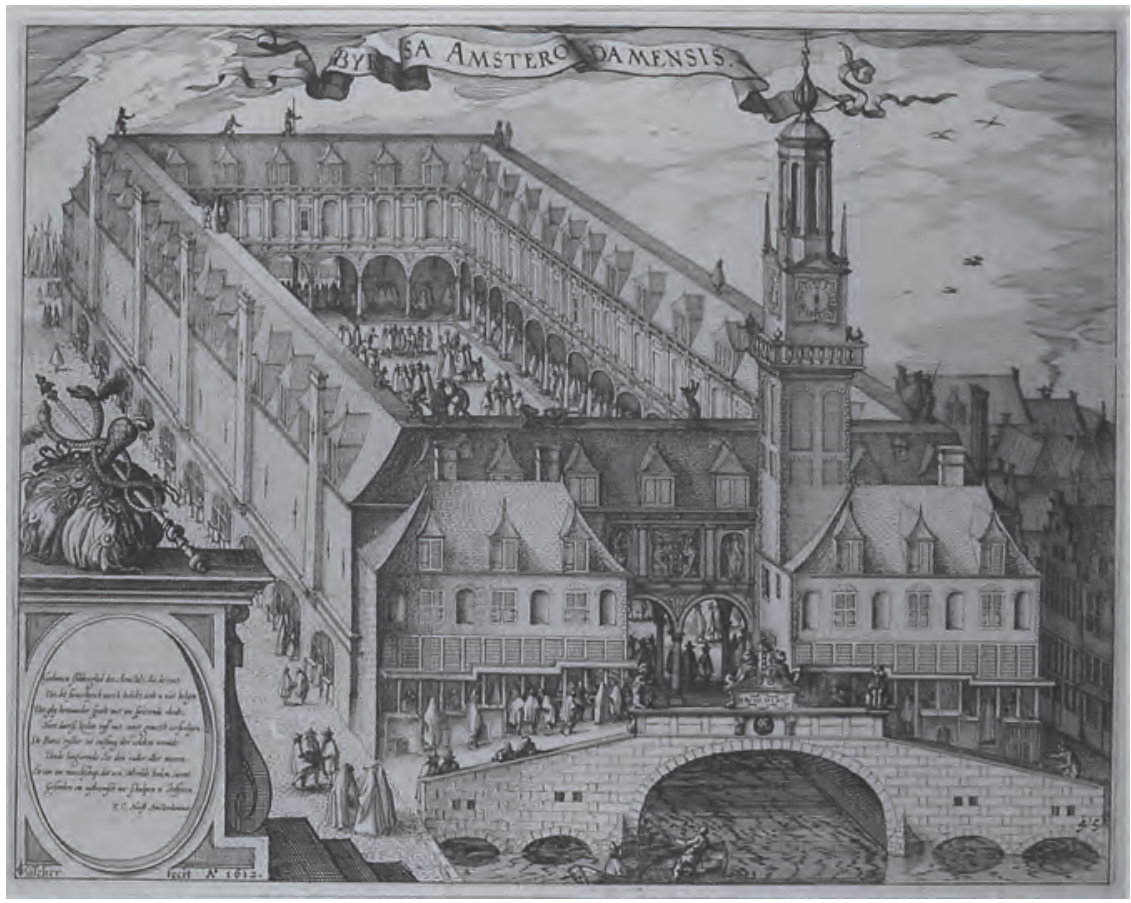

Illustration 22.6

Amsterdam Stock Exchange, 1612, engraving 
Postscript: Corporations were only legally required to publish audited accounts in the twentieth century in Britain and elsewhere. Firms often misled investors about their financial health until this crucial institutional mutation occurred. Countries - notably former communist countries that quickly adopted private enterprise in the 1990s - that have introduced corporations or stock markets without laws and bureaucracies to ensure that these operate honestly have tended to experience massive corruption.

One interesting distinction between European and (many) Asian systems of law is that the former came to emphasize arbitration and the evaluation of written testimony, while the latter stressed mediation and personal testimony (chapter 18). Both systems have advantages and disadvantages. Mediation is likely fairer: The two sides come to some compromise that both can abide. We have often noted that humans have difficulty being openly deceptive; face-to-face adjudication may then discourage dissembling. However, mediation typically takes longer than arbitration: The arbitrator can render a decision once they have assembled the arguments on both sides. It is likely easier to develop a sense of legal precedents over a body of formal adjudicated decisions. As trade through networks gradually gave way to impersonal trade, merchants especially valued a legal focus on the written contracts they had agreed to - especially if governed by clear precedents. European legal traditions may then have been conducive - in a manner unforeseen as the system evolved - to the expansion of commerce.

We should also mention inheritance laws. Islamic insistence - in the interest of fairness - that all children share in inheritance meant that commercial concerns were often wound up after one generation: Children could only rarely agree on how to share management and rewards. Partnerships had to close on the death of a partner, to protect heirs from abuse. The European practice of primogeniture may be much less fair - and as with kings often meant that incompetents succeeded to firm management - but did at least allow family firms to survive for generations, reinvesting profits in the firm. These differences in inheritance pattern became less important as the corporate form became common.

We should take care in assuming that any observed difference in institution implies superiority on one side. As we have repeatedly seen in this book, institutions are selected to fit within a particular environment. We often observe different institutional solutions to similar challenges. Still, there seems reason to believe that at least some European institutions were more supportive of economic activity well before 1800 . 
Postscript: Though the later Ottoman Empire and then Turkey would adopt European economic institutions, they have struggled to administer these and develop supportive cultural attitudes and educational systems. It could be that these are hard to generate quickly. One result has been corruption, which has been difficult to weed out. This decreases the level of trust in society.

\section{Religion}

There is a long tradition of celebrating the beneficial impact of Christianity on economic development. Such celebrations have not always been clear about the precise mechanisms by which religion influenced the economy. Note that it was common mere decades ago for authors to suggest that Confucianism was inimical to economic growth; after decades of rapid growth in China, however, one can now find many books that suggest that Confucianism encourages economic growth. We must be careful not to leap from coincidence to assumptions of causation. We must also appreciate that religions evolved through time (Chapters 8, 11). In the Middle Ages, Christianity may have seen nature as god's gift for humanity to do with as it saw fit, but early Christianity tended much more toward an attitude of reverence toward nature. Max Weber argued (controversially) in the nineteenth century that Protestantism encouraged work effort; if so then this is another mutation in Christian thought with economic implications. It may well be that such changes reflect rather than cause economic transformation: If humans were transforming nature or working harder, religions could evolve to reflect this reality. It could also be that religion does not have its most significant effect through beliefs but rather through practices: Those religions that taught adherents to read holy texts inadvertently encouraged both economic and political changes associated with literacy, and the very existence of monasteries may have encouraged agricultural innovation (because they joined educated people to agrarian pursuits). With respect to literacy rates, Europe may have developed a slight advantage as Protestantism spread - for Protestants urged all to read the Bible - but literacy rates may not have been crucially important economically until well into the nineteenth century (chapter 24).

\section{Technology and technological innovation}

We noted in Chapter 15 that China had been technologically innovative during the Song dynasty. We have indicated in various chapters that China had developed many vital technologies long before any other part of the world, including the spinning wheel, gunpowder, iron smelting, printing press, plough, and harness. India too had made impressive advances: Its cotton 


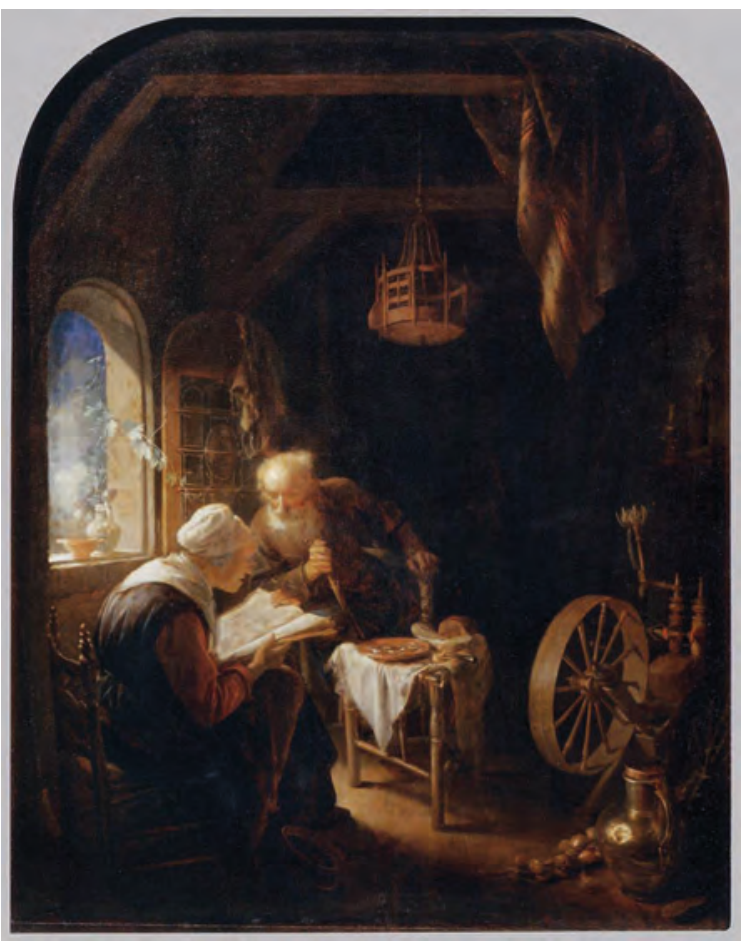

Illustration 22.7

Reading the Bible, Gerard Dou, c. 1645 technology was the envy of the world into the eighteenth century. We saw in chapter 12 that the Islamic Middle East had once been a world leader in both science and technology. The question here is whether Europe had come to surpass China and other parts of the world in the centuries before 1800. It is - widely but not unanimously - thought that the rate of innovation in China slowed under Mongol, Ming, and Qing. One oft-cited piece of evidence here is the way that Chinese emperors repeatedly showed disdain for Western technologies; this may be a sign both of a general indifference to innovation and perhaps of an empire more interested in social stability than innovation.

Europe absorbed many technologies from Asia over the centuries: In addition to the list just above we can mention windmills, the compass, lateen sails, and the stirrup. We noted above that European trading companies sought out technological and scientific understandings that they could transmit back to Europe. There were also more direct contacts: Dyers from the Ottoman Empire migrated to France in the seventeenth century, carrying an understanding of a dye that became popular as "Turkey Red," though its origins were in India. Europeans often then improved on imported technologies. We discussed the critical case of gunpowder in Chapter 18, suggesting that political fragmentation and resulting wars encouraged the development of superior weapons. Advances in printing technologies in part reflected the fact that European alphabets were better suited to movable type than Chinese ideograms, but also the freedoms of expression that Europeans were coming to enjoy (due in part to political fragmentation). Inexpensive printing was particularly important in facilitating the spread of ideas. The clock (see chapter 15) likely facilitated a more regimented approach to the workday. Europeans also developed ships better suited to the rough waters of the Atlantic (chapter 19). In addition, Europe also began to create unique technologies. Eyeglasses were a boon to those with vision problems. The earliest steam engines from the late seventeenth century set the stage for many later innovations that derived mechanical energy from fossil fuels.

What, though, of technology in areas such as iron production and textile manufacture? These would be critical sectors in the British Industrial Revolution and European industrialization in the nineteenth century (chapter 24). It 
has proven far harder to establish how extensively these sectors employed advanced techniques in different regions of the world in the eighteenth century, and whether there was any European advantage before the British Industrial Revolution of the late eighteenth century.

Why might Europe have become more technologically innovative than Asia? (We discuss science below.) The competitive pressures associated with political fragmentation may have encouraged innovation far beyond gunpowder; imperial governments in Asia could and did more readily ban innovations that might threaten social tranquillity. Imperial bureaucracies everywhere, and the Chinese examination system in particular, may have encouraged the "best and brightest" to pursue careers in bureaucracy rather than innovation; cultural attitudes that disdained commerce may also have been important in this respect. European cultural attitudes of the time may have been more open to foreign ideas than Asian especially Chinese - attitudes (though Europeans would become increasingly self-celebratory as they rose to military and economic dominance). Note that these are all arguments of degree: Europeans still valued landholding more than trade, and were developing their own state bureaucracies - political centralization occurs across Eurasia in this period and European states had conservative tendencies too.

Europe appears to have had much more in the way of "how to" manuals for industrial production. These attempts to capture "best-practice" techniques could

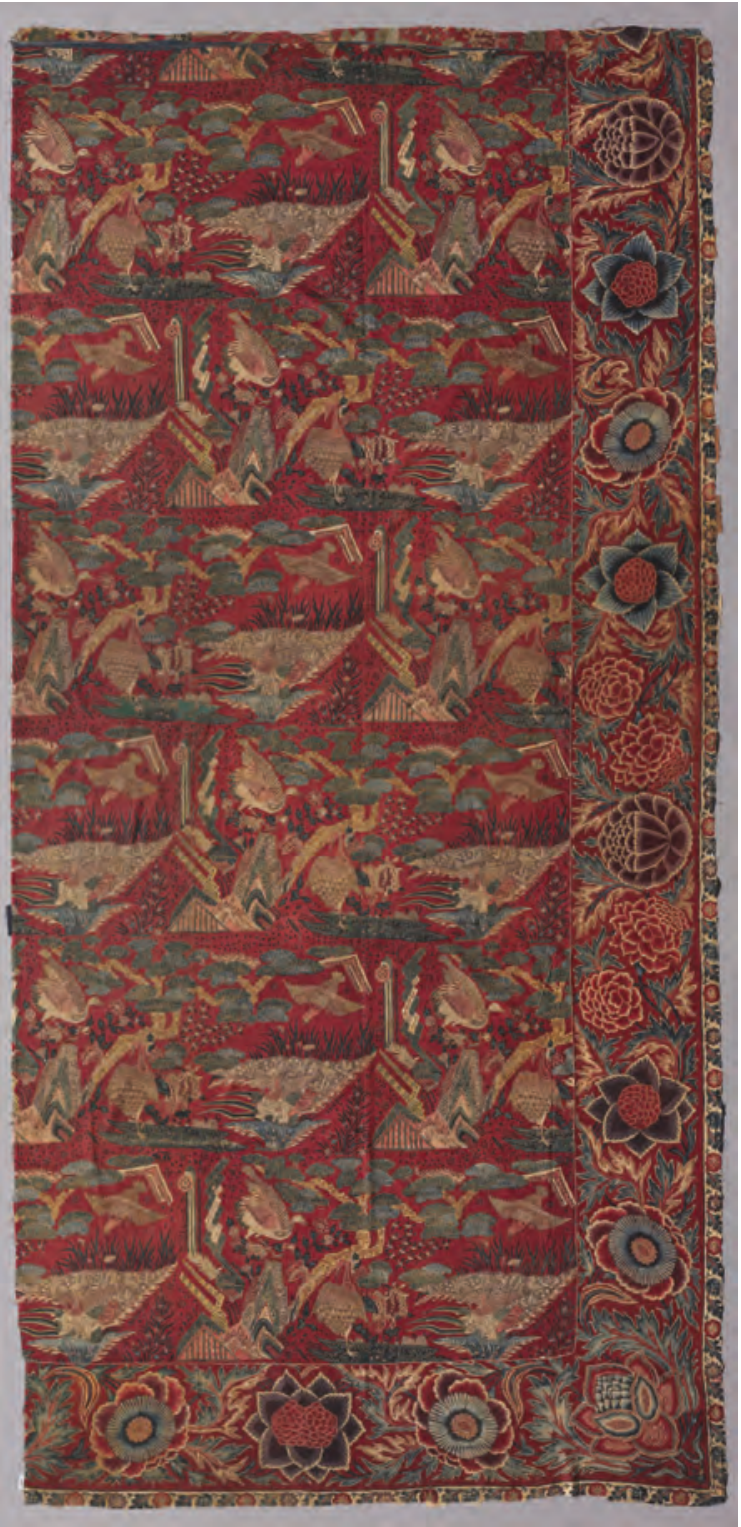

Illustration 22.8

Chintz bed cover or wall hanging with a Japaneseinspired pattern, right half, Coromandel coast, India, first half of the 18th century. Bed covers such as this were an important export item to Europe and to what is now Indonesia, Cleveland Museum of Art, CC0 make it easier for newcomers to learn a craft and for anyone to suggest improvements. An updated manual could then communicate these improvements widely. The prevalence of these manuals in Europe likely reflects both greater literacy and the far lower costs of printing that resulted from the printing press. It may 


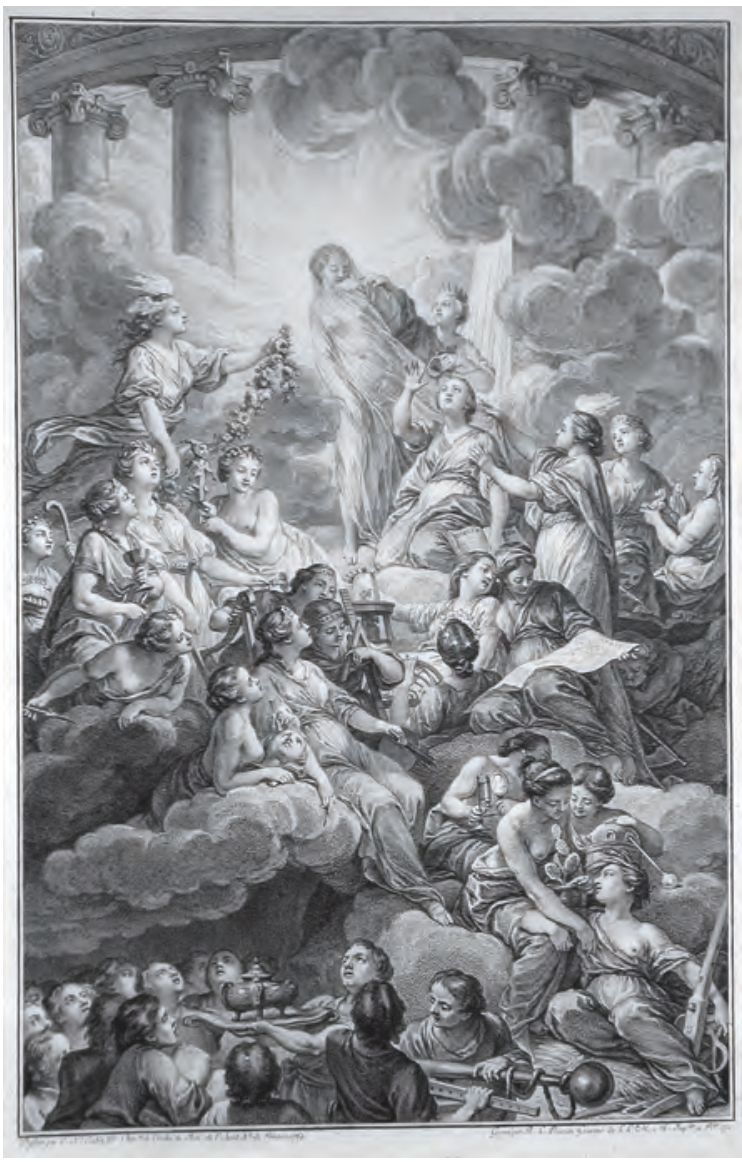

Illustration 22.9

Full frontispiece of the Encyclopédie, ou Dictionnaire raisonné des sciences, des arts et des métiers (1772). The Royal Society argued that technology would advance fastest if best practices were documented also reflect a scientific ethos of careful observation (see below). Indeed, the French Royal Academy of Sciences would sponsor a series of works on practical crafts, mostly in the eighteenth century, to spread best practices and stimulate innovation. We should be careful of exaggerating the importance of printed works, though: Apprentices generally learned their craft on the job and new techniques usually spread through faceto-face contact.

It could be that any advantage that Europe possessed was specific to particular technologies. That is, it may be that Europe was not generally (much) more innovative but happened to innovate in areas of particular - and perhaps surprising - importance. Shipbuilding is interesting in this respect: European "fluyts," Chinese "junks," and Indian "dhows" operated side-by-side in Asian waters for centuries, often constructed in the same shipyards. This suggests that there was no overwhelming technological advantage for any particular type of ship. The Chinese may have excelled in porcelain and Indians in cotton textiles, but the Europeans may have pulled ahead in machine-making (and printing). We discussed in chapter 18 how and why Europe pulled ahead with respect to gunpowder technology: It would turn out that the technology of boring smooth holes in cannon was very similar to the technology required for boring smooth cylinders for steam engines. One fired cannon balls with greater power; the other allowed pistons to operate with much greater efficiency due to a tighter fit. Mechanization more generally might have been encouraged by higher wage rates in Europe: It was more advantageous to replace expensive workers with machines.

Industrial technology and institutions intersect with respect to guilds. Some have argued that guild organizations in Europe were flexible in their recruitment strategies, and so encouraged inter-regional flows of knowledge. Though guilds could be conservative, they could also promote the flow of new ideas if they judged these to improve quality or lower costs without threatening employment. China and India also had guild-like institutions, but these were 
much weaker. Comparative studies suggest that these may also have been more rigid in recruitment: Workers were local and in India divided by caste. European guilds recruited to a much greater extent from outside guild families: European travellers remarked on the family orientation of especially Indian craft production. European guild members might have anticipated greater profits from innovation since they could restrict entry to their trade to a greater degree than Asian guilds could. In Europe - and Japan - teenage children of artisans were often urged to travel for a year or more to observe customs and practices of other regions: This intriguing cultural practice may have encouraged technology transmission. European guilds appear to have had practices that were more formal for training apprentices in the latest techniques. On the other hand, and as noted above, European guilds seem to have lost their ability to restrict innovation as rural industry increased in importance. Perhaps Europe benefited early from guilds that were stronger but more innovative, but later from guilds that were weaker.

\section{Challenges facing agents: Technological innovators}

The innovator's most obvious challenge is to be creative, and this means to produce an idea that is both novel and useful.

We diagrammed a four-stage process of innovation in Figure 3.1. The first stage involves recognizing a problem or opportunity. The successful innovator needs, then, to have enough understanding of some sector of the economy to know what kind of innovation might be useful. The second stage involves gathering relevant information and experimenting with possibilities. This generally requires networking, even in a literate age. More importantly, this is usually a time-consuming process that is also often financially costly. It is not, that is, an enterprise to be undertaken lightly, and will depend on attitudes toward work, risk, and the meaning of life.

The third stage is the act of insight, which generally emerges subconsciously when the mind is at rest. This act of insight often gets the bulk of attention in popular histories of innovation but is only possible after the collection of relevant information. Nevertheless, it is true that innovators can work too hard; the mind needs a break to be its most creative. Creative ideas are rarely fully formed: the fourth stage requires more effort and expense to produce a working machine or recipe.

The successful innovator then faces challenges in profiting from their innovation (A minority are happy to share their ideas with the world.). They need to protect their idea from being stolen through secrecy or patenting - theft once again becomes an integral part of the human story. Then they need to market it. They then face the entirely different challenges of the merchant (chapter 5) - and often find it advisable to team up with a merchant.

The innovator must also cope psychologically with the uncertainty associated with innovation. Among other things, they need to estimate their chances of success. The fact that history is littered with failed innovators - who contributed nevertheless to the successful innovations of others - suggests that humans may not be very good at this, and may be biased toward optimism. Humanity may have been far more innovative than would have been the case if humans were better at estimating their probability of success. 
For present purposes, the table on the challenges facing technological innovators suggests some additional questions. Were there important cultural differences across societies in attitudes toward work or risk or achievement? Was intellectual theft more problematic in some places than others? Was it easier to commercialize new ideas in some places than others? Was it easier to network in some places? Yet the central message of the table is that technological innovation does not just happen but is instead influenced by a diverse set of decisions: These might be far more likely in some societies than others.

\section{Summing up}

Though further research is much needed, it seems at this point that (parts of) Europe likely were a little richer than the rest of the world already by 1800, and also had advantages in terms of certain institutions and at least some technologies. There may or not have been advantages in terms of demography, religion, or resources. The economic benefits of colonies may have been small; colonies may have been a reflection of the institutional and technological advantages that also encouraged economic development.

\section{The scientific revolution}

We should warn the reader that we will question far below whether science mattered much for economic growth before the mid nineteenth century. Nevertheless, the story of science is of intrinsic importance. Moreover, the science of later centuries - which builds on the science of the Early Modern period - will have a substantial economic impact (chapter 24). The history of science may also tell us something useful about general intellectual currents that may have encouraged the Great Divergence.

Historians have long talked of a "Scientific Revolution" in Early Modern Europe. This revolution used to be primarily associated with developments in astronomical understanding, and especially the appreciation that the earth orbited the sun. This is certainly a critical development, for it changed the way humanity viewed its place in the cosmos. It also involved intense conflict between religion and science. The victory of science in this instance had effects on science more broadly: The Church would be careful of attacking scientific conclusions after that. Nevertheless, historians have over time come to stress something else: a "revolution" in scientific practice and standards of evidence. This is a quite different revolution, one with both different causes and a more dramatic effect on the course of science. Within an evolutionary perspective, we can see that the first approach focuses on a particular set of mutations in scientific thinking, while the latter approach suggests a change in the selection environment for scientific mutations. Both can be important, but a change in selection environment may have effects that are more lasting. In our discussion of evolutionary theory in chapter 1 , we recognized that evolutionary processes 
could sometimes yield dramatic change; we will essay to show below that both "revolutions" can indeed be appreciated as evolutionary processes.

\section{Changes in astronomical understanding}

Nicolai Copernicus of Krakow in Poland did not set out to transform human understanding. With his university education and affiliation, he was trying to solve a problem set by Plato and clarified by Ptolemy well over a millennium earlier. How could he square the received understanding of circular orbits with detailed astronomical observations that established without a doubt that the planets and sun did not orbit the earth in circles? Ptolemy had hypothesized circles on circles (chapter 9). As astronomers collected more data, it became clear that a quite messy system of circles on circles on circles was required to rescue the Platonic ideal. This problem had much challenged Islamic astronomers, and Copernicus began from an Islamic understanding of just how messy a system of such circles had to be. He cited several Islamic astronomers in his work despite a general tendency among European astronomers not to cite the Islamic astronomers that they drew upon - and followed them in rejecting a common European hypothesis that planets simply slowed and accelerated during their orbits. How could he save the idea of circular orbits without recourse to such a "monster" of complicated explanations? Careful scrutiny of the data over a period of decades led him to the conclusion that one gained a far better fit to the data if the earth and planets orbited the sun instead. This was an idea that astronomers had occasionally expressed in human history, but it had generally been dismissed based on simple logic: If the earth were flying through space why did we not fly off when we jumped in the air? The answer to that question - theories of gravity - would only come much later in our story. Copernicus held off publishing his results, perhaps fearful of embarrassment more than religious hostility. The data did not fit his new hypothesis perfectly, though they fit it much better than an earth-centred hypothesis. Also, he had no compelling answer to the question of why we did not fly off a moving earth. He recognized that convincing others would be no easy task. His hypothesis did

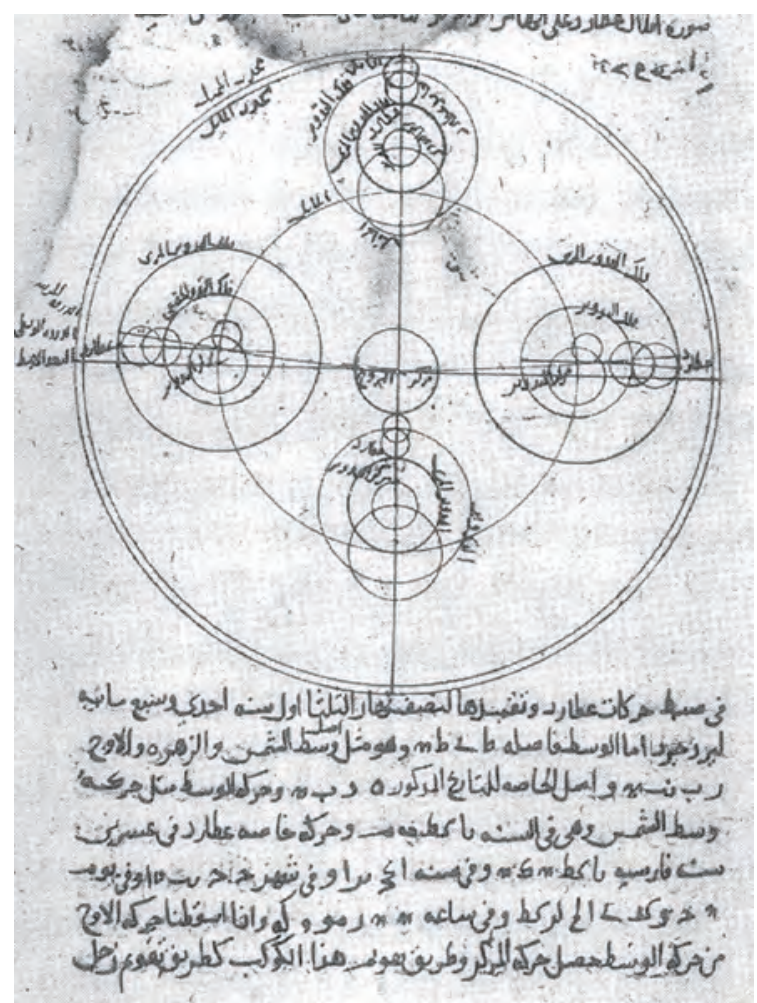

Illustration 22.10

Al-Shatir's model of the Ptolemaic epicycles of Mercury, 14th century CE 


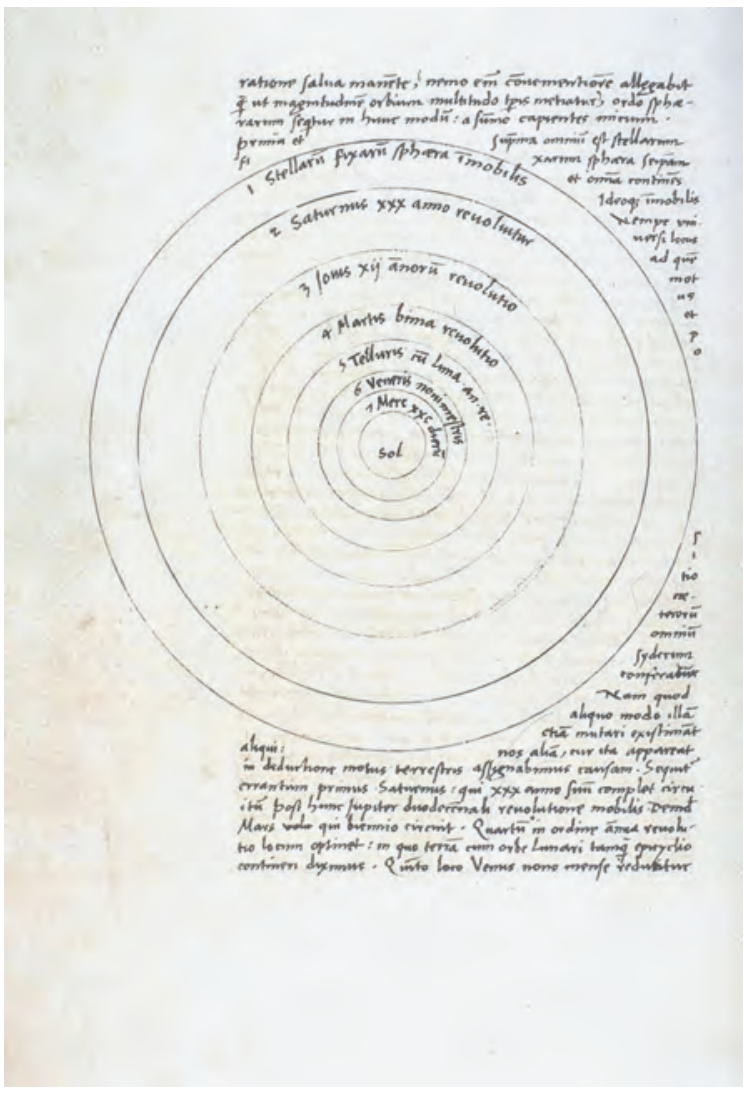

Illustration 22.11

Nicolaus Copernicus, solar system, from his “De revolutionibus," p. 9 verso explain why we always see Mercury and Venus close to the sun (because they are between the sun and Earth); indeed, his approach allowed the order and distance of the planets to be established. However, his approach raised another problem: The stars do not change their position in the sky much as the earth orbits the sun, and so would have to be much farther away than anyone had previously imagined was possible.

Galileo is the next key player in our story. He did not invent the telescope but improved it and was the first to use it to examine stars and planets. He had worked at a university in Venice when developing the telescope, but then parlayed his fame into a court appointment with the Medicis (a wealthy merchant family) in Florence - who may have had him do some practical work but mostly wanted to bask in reflected fame. He showed that there were moons around Jupiter, that the earth's moon had mountains and craters, and that Venus had phases, which indicated that it orbited the sun. His fight with the Church is complicated and took place over decades, but certainly involved the Church objecting to his arguing in favour of the Copernican view. Galileo argued that theology should adjust if observation differed clearly from scripture, but his own texts did not claim that he had "proof" of a sun-centred system.

Under pressure from the Church, Galileo would recant his Copernican beliefs. The Church's actions scared scientists into focusing on other subjects after that: Later developments in astronomy would occur farther north where the Catholic Church wielded less power. Galileo would later, while under house arrest, make his famous argument that falling bodies accelerate (and the argument that weight did not affect speed in a vacuum). Though hailed as an experimentalist, he used experiments to demonstrate rather than test his hypotheses, and did not report actual experimental findings (and there is a question as to whether the crude devices he employed could provide the accuracy he needed to measure acceleration). Though Galileo spoke of practical applications, such as calculating the path of projectiles, engineers and soldiers had already developed empirical rules of thumb that his theoretical analysis did little to aid. 


\section{PRIMARY DOCUMENTS: GALILEO'S RECANTATION OF HERESY}

This is the last part of Galileo's recantation of 1633 :

I, Galileo, son of the late Vincenzo Galilei, Florentine, aged seventy years, arraigned personally before this tribunal, and kneeling before you, Most Eminent and Reverend Lord Cardinals, Inquisitors-General against heretical depravity throughout the entire Christian commonwealth, having before my eyes and touching with my hands, the Holy Gospels, swear that I have always believed, do believe, and by God's help will in the future believe, all that is held, preached, and taught by the Holy Catholic and Apostolic Church. But whereas - after an injunction had been judicially intimated to me by this Holy Office, to the effect that I must altogether abandon the false opinion that the sun is the center of the world and immovable, and that the earth is not the center of the world, and moves, and that I must not hold, defend, or teach in any way whatsoever, verbally or in writing, the said false doctrine, and after it had been notified to me that the said doctrine was contrary to Holy Scripture - I wrote and printed a book in which I discuss this new doctrine already condemned, and adduce arguments of great cogency in its favor, without presenting any solution of these, and for this reason I have been pronounced by the Holy Office to be vehemently suspected of heresy, that is to say, of having held and believed that the Sun is the center of the world and immovable, and that the earth is not the center and moves.

Therefore, desiring to remove from the minds of your Eminences, and of all faithful Christians, this vehement suspicion, justly conceived against me, with sincere heart and unfeigned faith I abjure, curse, and detest the aforesaid errors and heresies, and generally every other error, heresy, and sect whatsoever contrary to the said Holy Church, and I swear that in the future I will never again say or assert, verbally or in writing, anything that might furnish occasion for a similar suspicion regarding me; but that should I know any heretic, or person suspected of heresy, I will denounce him to this Holy Office, or to the Inquisitor or Ordinary of the place where I may be. Further, I swear and promise to fulfill and observe in their integrity all penances that have been, or that shall be, imposed upon me by this Holy Office. And, in the event of my contravening, (which God forbid) any of these my promises and oaths, I submit myself to all the pains and penalties imposed and promulgated in the sacred canons and other constitutions, general and particular, against such delinquents. So help me God, and these His Holy Gospels, which I touch with my hands.

I, the said Galileo Galilei, have abjured, sworn, promised, and bound myself as above; and in witness of the truth thereof I have with my own hand subscribed the present document of my abjuration, and recited it word for word at Rome, in the Convent of Minerva, this twenty-second day of June, 1633.

I, Galileo Galilei, have abjured as above with my own hand.

Note the significance of "without presenting any solution of these": It is heresy because he cannot prove his conjectures. Note also the promise to turn in any other scientists who might argue for a sun-centred view. 
Tycho Brahe's astronomical instruments cost 1 per cent of the Danish state's revenues. However, they allowed him to produce much more precise observations of planetary orbits. He showed that comets passed through planetary orbits: This observation was a challenge to the idea that orbits were movements along some fixed crystalline sphere (an idea neither Copernicus nor Galileo rejected). He posited that the sun revolved around the earth, but the planets around the sun. Such a hypothesis still faced empirical problems but had the advantage of "bringing" the stars closer than in the Copernican system.

Johannes Kepler, in turn, strove to explain the precise data collected by Brahe. He worked for a while for Brahe but later found employment as a court astronomer. He also prepared astrological charts (as had Brahe). It took years of calculations before he induced the ellipse. Note the word "induce": he did not deduce the ellipse from logical principles - astronomers had merely assumed circular orbits for millennia - but instead induced it from a mass of data. With Kepler, we finally get a hypothesis regarding orbits - around the sun - that precisely fits the data. However, he could not explain why planets followed elliptical orbits. Kepler's discovery - in its way a more shocking revision of the Platonic system than that of Copernicus - was important evidence that very careful scientific practices (in this case observation) could lead to understandings that could never be arrived at deductively from classical texts.

The task of explaining elliptical orbits would fall to Isaac Newton (16421727). Newton was reclusive, superstitious, and an alchemist. He was also very religious (though he doubted the Christian trinity and refused an Anglican

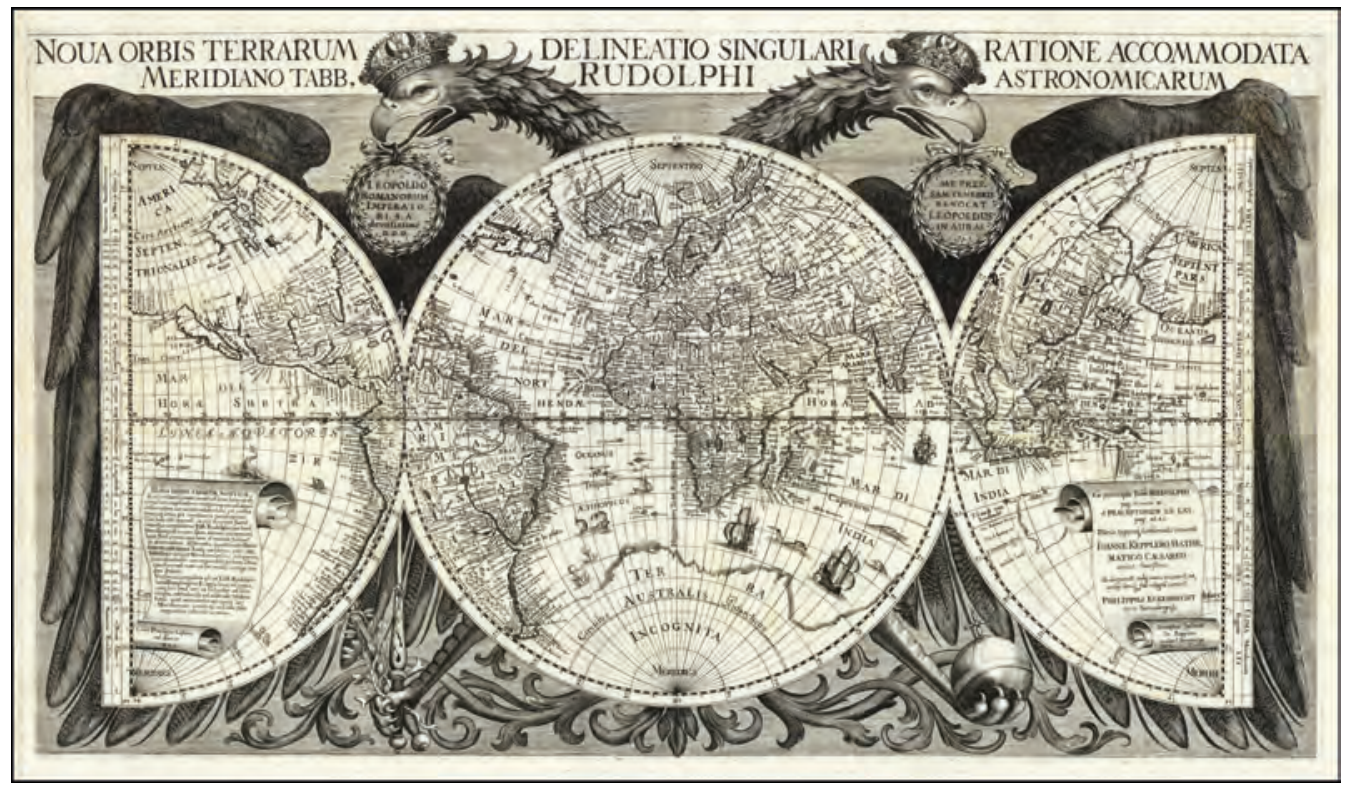

Illustration 22.12

Kepler, Map of the World, 1627 
burial), and thought he was exposing the works of god. Newton had faced vigorous critiques early in his career when he published a theory of optics. He would then outline a theory of gravity that explained behaviour both on earth and of planets: A force attracted objects to each other proportional to their combined mass but inversely proportional to the square of the distance between them. Planets could maintain stable orbits because the force of gravity weakened with distance enough to be balanced by the lateral motion of planets. The orbit that resulted was an ellipse. (Newton also co-discovered calculus - Leibniz developed this idea independently while Newton was hesitating to publish - and applied this new mathematical technique to calculate elliptical orbits.) Newton hesitated to publish his theory because he had disliked his earlier experience of scholarly dispute. However, Halley (the astronomer for whom Halley's Comet is named - though unbeknownst to him, the Chinese had identified it centuries before) was able to encourage him to publish.

We have recounted a story that involves several minds across several countries pursuing over a period of centuries a connected line of enquiry. We have told the story above as a history of ideas: We can see how each scientist built on preceding ideas. In its initial stages, this process combined insights from both Greek and Islamic astronomy. Yet we should also understand this story as a social process: These scientists were part of a community with shared interests. Scientists would hardly have pursued this line of research for centuries otherwise. While they faced some religious hostility at times, they also received support from states and aristocrats - and could earn additional funds through astrology. This social aspect will loom even larger when we turn below to the other element of the scientific revolution, the formalization of standards of evidence.

We should stress here that science is a very wasteful evolutionary process. We can see in the story above how certain ideas supplant earlier ideas. The handful of ideas we have recounted above were the successful ideas (at least for a while) within an intellectual conversation that involved many minds. We have, like most histories, focused on the scientists that made the big discoveries. These necessarily were part of a much larger community, most of whom are unknown even to historians of science. These other scientists either made less important discoveries or failed to convince others of the value of their insights. Science advances in communities that produce many mutations, most of which are not selected. The existence of a few winning ideas reflects an intellectual ferment in which many losing ideas (such as Brahe's own suggestion that the sun orbit the earth while everything else orbits the sun) were also put forward.

\section{Changes in practice}

As elsewhere in this chapter, the ghosts of historiography have impeded careful examination of the role of science in history. It was all too common in the past for European triumphalists to celebrate advances in Western science and credit these to a rationalism that they traced unerringly to the days of ancient 
Greece. Superstition might guide other societies, but Europe ruled the world through rational thought. The reaction has been all too predictable: Some have denigrated science itself, and others have denied any differences between Europe and Asia.

When we discussed Greek and Chinese philosophies in chapter 10, we noted that it is essential to recognize these as group processes rather than the coincidental insights of a few great minds. Greek philosophers learned from each other and honed their arguments in debate with each other. The same argument applies to periods of philosophical or scientific efflorescence in China, India, or the Islamic Middle East. These were social processes dependent on the interactions among a substantial body of individuals.

These social processes are arguably even more important for science than for philosophy. Philosophers in many societies were able to develop principles of rational argumentation: deductive arguments from logical premises, inductive arguments from examples, and so on. Scientific communities needed to establish standards of evidence. In early days, these standards were lax. The ancient Greeks and others investigated scientific questions in much the same way as they approached philosophical questions. The Greeks used casual empiricism to conclude that the cosmos was composed of four substances and the sun revolved around the earth. Scientific advance did occur in areas like (what we now call) the field of mechanics in physics, but it was slow. It may be that a much larger community is necessary for science than for philosophy, for scientific standards are more complicated (and scientific advance would soon become associated with specialization).

If we accept that science is a social practice, and that we need a community to both build on each other's ideas and establish guidelines for acceptable enquiry, then we might wonder why some times and places have more scientists than others. Note that we potentially have another critical difference of degree here: A society with even just a few more scientists may develop far better guidelines for judging scientific arguments. Individual scientists might gradually become increasingly specialized to distinguish their investigations from those of other scientists. Such a community might then defend the value of science in the broader society. The emergence of modern science - which can be distinguished from earlier efforts precisely because it developed standards of acceptable evidence and argument - need not then (but could - we should not rule that out a priori) depend on a dramatic difference in a region's culture.

Note that there are important network effects here: The value of being a scientist depends critically on how many others there are. Your science will be better if you can bounce ideas off many others. Your fame will be greater if there are others who care and will listen. A growing scientific community generates its own momentum, attracting ever more scientists. A small initial difference in the size of scientific communities might result in far greater differences over time. 
The turning point in Europe occurs between the time of Copernicus and Newton. The Church roundly criticized arguments of Copernicus that the earth orbited the sun. Newton's explanation of the earth's orbit in terms of his theory of gravitation was accepted. The bulk of the European population likely cared little about these arguments. It was therefore of critical importance that there be a sizeable and interconnected body of individuals with interest in performing science who could do battle with religious authorities wary of any endeavour that might diminish scriptural authority.

Historians of science speak of a "Republic of Letters" in Europe: Scholars from across Europe communicated with each other - in Latin - by letter. There were already some hundred scholars in this loosely defined network during the Renaissance, and its numbers would swell to well over a thousand later. Entry to the network was informal and occurred when existing members thought a newcomer had something to say. Members were expected to copy letters and pass these to others. This scholarly correspondence would often lead to the publication of books and pamphlets that would receive even wider circulation. These letters tended to move quickly past the niceties common in correspondence of the time to address matters of scholarly debate. This international network connected the activities of local clubs and societies, and linked scholars within and outside of universities. While scientists and philosophers in other parts of the world might also correspond, there does not seem to have been a network of correspondence of such a size anywhere else. Scholars in China did organize to push for changes in how examinations were graded, but do not seem to have developed nearly as intense a network of scholarly conversation as that observed in Europe. Governments in India had long hired Brahmins to provide advice on a range of subjects grounded in ancient texts, but these had tended to rely on received wisdom transmitted orally; only when the British employed them to interpret Hindu law did they begin to publish books that reflected received wisdom.

European scientists were far from the first individuals in history to employ careful observation of the world around them. Examples of such individuals can be found in many times and places. European scientists were the first to form a community large enough to overwhelm the forces of religious orthodoxy and gain a cultural acceptance of the value of science. In doing so, they formulated a robust set of internal values. Galileo could be casual about his experimental "evidence." Kepler provided precise observational support for his elliptical conjecture, as did Newton (who, for example, collected global data on tides to support his argument that the moon's gravity influenced these). We should not exaggerate the degree of change: Casual empiricism maintained a place in European science into the nineteenth century. However, the direction of change is evident.

Along with this "empiricist" emphasis on careful observation and experiment, the scientific community came to value mathematical expression. The laws of motion of planets, or Newton's theory of gravity, were expressed 
in precise formulae, and these then were precisely compared to empirical observations. (We can recall again that Europe had borrowed much mathematical understanding from India and the Middle East over the centuries.) Scientists of the day were careful, though, to explain their mathematical insights in words also.

Why did Europe develop a more substantial scientific community? One possible explanation is economic. Science takes time and energy and often research funding. It requires the production of an agricultural surplus that can support individuals dedicated to scientific enquiry. As noted earlier in this chapter, there is still debate as to when European incomes began to pull ahead of average incomes elsewhere. It is possible that Europe was better able than other regions to support a body of scientists well before 1800 .

Not everybody freed from backbreaking agricultural labour decides to pursue science. Some degree of economic prosperity may be necessary for science but is not sufficient. Culture is important here in affecting how people choose to spend their time. The Scientific Revolution might never have happened if science were universally disdained. Or if other pursuits were much more prestigious: As noted above, the Chinese examination system may have had the unintended consequence of diverting the cleverest into the bureaucracy.

We appreciated earlier in this chapter that European states were less likely to prevent technological innovation than Asian empires. We can make a similar argument concerning science. States might worry that scientific advances would yield technological advances to their competitors (though the link between science and technology was weak at the time). Scientists - or ideas that were unpopular in one state could move elsewhere. Investigation of suncentred systems proceeded in northern Europe, and with state support, despite Catholic objections in the south.

The European university may have been important in encouraging science. We noted above that Copernicus and Galileo had university connections; Newton was a Cambridge professor. European universities had first developed as centres of professional training of priests, doctors, and lawyers. They had provided a general education that included what the Greeks had called natural philosophy: speculation on the nature of the natural world. These universities gained considerable independence over time from both Church and state (chapter 15). They provided locales where educated people had both the means and freedom to pursue natural science. The state tended to control academies in China and elsewhere, and these then focused on teaching and researching subjects of direct use to bureaucrats. The focus of Chinese academies was on preparing students for government examinations.

The Renaissance era had many clubs devoted to science; Galileo was a member of one in Rome. Why this public interest? Scholars have posited various explanations: the shock to conventional views of discovering the Americas, the Renaissance itself with its dramatic changes in the arts, the Protestant Reformation and its re-evaluation of received wisdom, and the printing press 
that facilitated the transmission of scientific debates to a broader public. Membership in such clubs still represented a small minority of the population, but these were often people of means with social influence.

These clubs set the stage for royal societies in England from 1662 and France from 1666 and soon elsewhere. The first two started journals from 1666. States gave these societies independence but expected practical results. States also sponsored observatories and botanical gardens, and employed some scientists. Nevertheless, state expenditures on science were small - scientific societies received far less funding than artistic societies. The arts served a purpose in lending prestige to the state and its ruler(s). Science might also lend prestige, but had almost no impact on technology during this period (one exception being cartography, which benefited from developments in mathematics).

The European community of scientists, with significant public support and the development of standards of evidence, produced developments across many fields of science. Newton developed theories of mechanics. Others developed an understanding of atmospheric pressure. "Biologists" lacked a theory, but developed detailed classifications of (the characteristics of) plants and animals. There were also advances in chemistry and anatomy. Over time, the community would fissure into what we now recognize as "disciplines," each with their own evolving set of research questions and theories and methods. This would prove a useful way to organize science at the time but would limit human understanding of complex problems that require insights from more than one discipline: This challenge would not be widely appreciated until the late twentieth century.

Indian scientists would collaborate with European scientists in the classification of species. Yet the collaboration focused on the species of the Indian subcontinent: This may reflect a larger or better-funded community in Europe, or merely the fact that Europeans had come to know more about India than the reverse. While Indian scientists proved quite adept at observing species, there is little evidence of experimental science, though perhaps some signs of a desire for change in scientific practice in the eighteenth century.

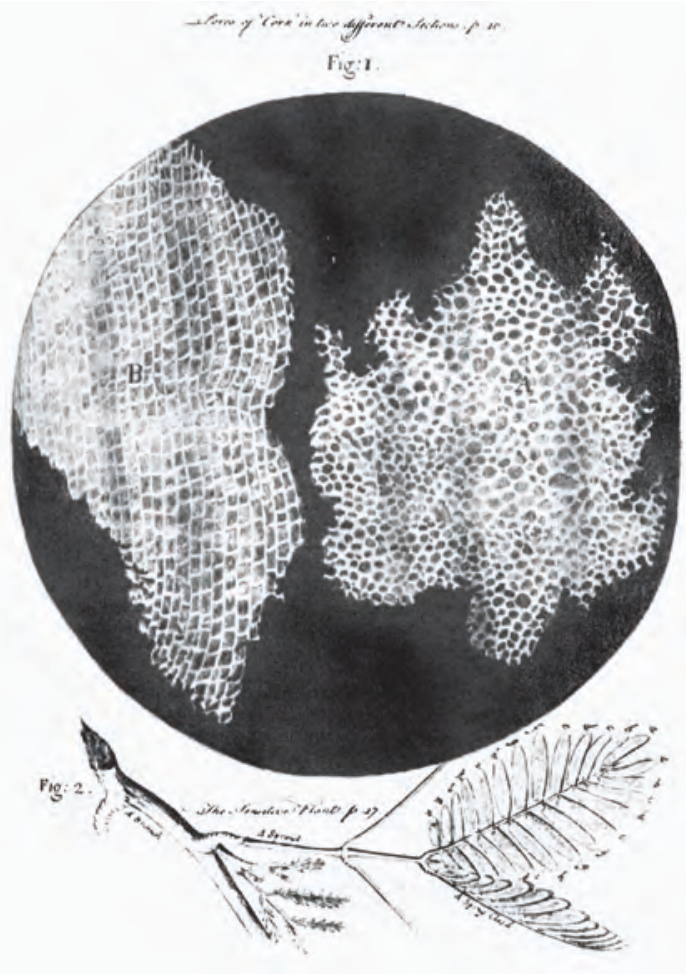

Illustration 22.13

Suber cells and mimosa leaves described in "Micrographia" by Robert Hooke, 1665 


\section{The cultural environment of the scientific revolution}

We should not assume away the possibility that European culture encouraged science just because we may find such an argument uncongenial. Many scientists during the Scientific Revolution - including Newton - declared that they saw themselves as uncovering god's plan. Such an attitude might be traced to Aquinas, who had sought to blend rational Greek philosophy with Christian theology (chapter 15). He believed that people could appreciate the works of an omniscient god not just through scriptural study but also through careful thought and examination of the world around them. Scholastic philosophers had argued that the Christian god had set in motion a set of laws of the universe (which he only occasionally disobeyed). Yet just as Islamic fundamentalists had railed against the science and philosophy of Baghdad and Cairo (chapter 12), many in the Catholic Church urged study of the Bible as the one true path to knowledge. The victory of rational enquiry was hardly inevitable, but in the end the value of philosophy and science was accepted.

Monotheism may be important here. It is reasonable to seek to understand one "god's plan." However, a cosmos governed by multiple gods or spirits might be chaotic in form. We should be careful of exaggerating this argument. Hunter-gatherers had discovered a great deal about the medicinal uses of various plants while practising animist religions. Greek philosophy advanced against a polytheistic backdrop. The deism prevalent in East Asian religions such as Buddhism and Taoism would also seem to lend itself to the idea of certain identifiable laws of the universe.

The Scientific Revolution unfolded in concert with - influencing and being influenced by - a more general Enlightenment, which elevated reason and observation above received texts as a source of authority. The Enlightenment was at least in part inspired by the discovery of the Americas and the expansion in intercontinental trade that served to challenge the authority of received wisdom. There was clearly a large set of societies that had existed for millennia but were not mentioned in the Bible. Enlightenment thinkers felt that through logic and careful analysis humans could fashion better societies (chapter 21). They often thought that they had much to learn from other societies - though this attitude ebbed as Europe developed economic and military superiority. Enlightenment thinkers often celebrated advances in natural science as an indicator of what reason and empiricism might achieve within the realm of social science. One key Enlightenment thinker was Voltaire (Francois-Marie Arouet, 1694-1778), who argued for individualism and against the powers of church and king (he was a deist). The Enlightenment sought to ground both ethics and politics in reason. René Descartes (1596-1650) had attempted to provide logical proofs of both human and godly existence, but succeeded only with the former. His "cogito, ergo sum" - "I think, therefore I am" - was a philosophical advance, showing that the very fact that we think is proof that we (at least one of us) exist.

Though this European celebration of reason would be used by many to justify colonization of "superstitious" societies (see above), several Enlightenment 
thinkers, including Diderot, Kant, and Adam Smith, made reasoned arguments against conquest. Decisions to colonize were likely determined by the incentives to war and empire that we have often discussed in this book, rather than by the reasoned arguments of philosophers. Yet, as we saw in chapter 21, the idea of popular sovereignty advocated by Enlightenment thinkers would play a role in the development of nation states. It could well be that this more general emphasis on careful and logical argument also encouraged the development of modern science.

\section{Non-European antecedents}

Even those who might emphasize the European cultural roots of modern science can appreciate that European science borrowed much from other lands. The Islamic world had more libraries and books in 1500 than anywhere else in the world. Copernicus drew on Islamic astronomy. Algebra was developed in the Middle East, and "Arabic" numerals came from India. Newton used data on tides from Asia collected by the East Asia Company. The printing press and paper were each important in allowing an international community of scientists to interact; both of these technologies had originated in China.

\section{Effects of the scientific revolution}

We have noted above, and in earlier chapters, that scientific ideas moved between continents. Nevertheless, there was scope for substantial regional differences as when European and Islamic astronomers pursued different hypotheses regarding planetary orbits. In the twenty-first century, though, there is one global scientific enterprise. Scientists disagree, but it is rare for scientists in one country to disagree with scientists in another (even when scientists in some countries have been required to salute Marx in their opening paragraphs). This global community of science reflects advances in communications technology in recent centuries. It also reflects the worldwide adoption of the standards of argument and evidence grounded in careful empiricism and experimentation developed during and since the Scientific Revolution.

What were the effects of the Scientific Revolution on technology? As we noted above once or twice, the direct links were quite limited at the time. Science would come to have a substantial direct impact on technology only in the late nineteenth century (chapter 24). Science may well have exerted a significant indirect effect before then, however. We recognized above the association between Scientific Revolution and Enlightenment. The emphasis on careful observation and recording of results associated with both Scientific Revolution and Enlightenment could be beneficial also in the realm of technological innovation. A community of technological innovators devoted to careful measurement and perhaps sharing a belief that reason and empiricism could improve the world might prove far more innovative. Several scholars have suggested that what might be termed a scientific ethos did come to infuse 


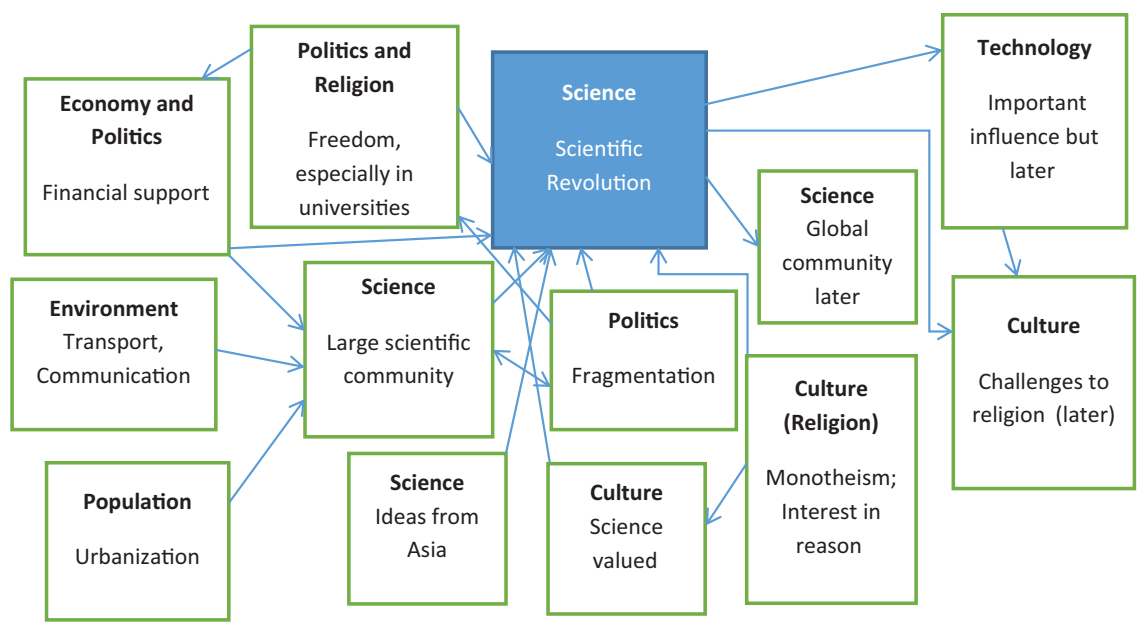

Figure 22.1

Influences on and effects of the scientific revolution Note:We will address challenges to religion in chapter 27.

the European community of technological innovators in the eighteenth century. They note in particular that some innovators were members of the scientific clubs mentioned above. They may not have found the science particularly useful, but they might have usefully absorbed the emerging scientific method of careful testing of hypotheses. There may then be an important link from Scientific Revolution to the Industrial Revolution of the late eighteenth century (chapter 24).

\section{Western Civilization revisited}

As noted in chapter 1, there has been a tension between world history - as taught in Europe and its former settler colonies - and a previous tradition of courses and books that addressed the evolution of "Western Civilization." World history indeed sprang from a desire to downplay the idea that Europe was somehow special. Sometimes "Western Civilization" books and courses start with early civilizations in Mesopotamia. We have seen that these early empires likely had as much influence on Asian society as on European. Sometimes such books and courses start with Greek and Roman states and empires. These, while primarily situated in Europe, both had Asian and African territories and traded and fought with Asian powers. Moreover, later European understandings of Greek and Roman societies depended on the survival of texts within the Islamic world that had been lost in Europe. Sometimes the essence of Western Civilization is sought in the Middle Ages, when Christianity - itself an import from the Middle East - spread throughout what is now Europe. Yet we have also seen that Europe interacts in powerful ways with the rest of the world in this 
period: trading, fighting, and borrowing technology. While there are significant developments internal to the West, we would lose much that is important if we failed to place these within the context of interactions with the rest of the world.

One of the challenges of speaking of Western Civilization is that the region "the West" is ever moving, albeit usually westward: from Mesopotamia to Greece to Rome to northwestern Europe to the Americas. The very idea of Western Civilization requires, then, a very flexible attitude toward the regions of the world. If we cannot identify the West with a fixed region of the world - as can be done when speaking of Chinese or Indian histories - then we are forced to seek some shared cultural, political, or economic identifier. Greco-Roman philosophy and Christianity are the obvious choices. As noted just above, the former survives in large part only through Islamic protection. The latter does become after Islamic conquests a primarily European endeavour (but there are now far more Christians outside Europe than within Europe). Yet it emerges and spreads while different universalist religions are appearing and spreading elsewhere in the world. We can and have usefully then compared these new religions.

For a variety of reasons, then, it makes sense to place "Western Civilization" within a global perspective. We could make similar arguments about "Chinese Civilization" or any other. In all cases, one can identify both internal processes of change and important interactions with other parts of the world. One can also usefully compare the processes of change in different parts of the world. Our approach to the "Great Divergence," then, sensibly evaluated both internal and external factors that might have caused this.

\section{Questions}

1. Explore one or more of the questions in the paragraph that follows the table describing the challenges facing technological innovators.

2. Compare the challenges facing technological innovators in this chapter with those facing scientists in chapter 9. What are the similarities and differences in the challenges facing innovators in technology and science?

3. "Many scholars have thought that the only response to the claim that 'Europe was superior' was to argue that 'Europe was lucky' and thus sought to identify transitory and external causes of European success. The viewpoint of this book is that both propositions (superior or lucky) are nonsensical within an understanding that history always represents a complex interaction of causal forces." Explain.

4. This chapter has focused on a key historical controversy. Which explanation of the Great Divergence do you find most compelling? Why?

5. Why did we devote so much of the chapter to science? 


\section{Readings}

Appleby Joyce. 2013. Shores of Knowledge: New World Discoveries and the Scientific Imagination. New York: W.W. Norton. [Argues that the discovery of the Americas, by exposing Europe to new ideas and new data, and questioning the wisdom of ancient texts (that had been ignorant of these continents) encouraged the Scientific Revolution in Europe.]

Cook, Harold. 2007. Matters of Exchange: Commerce, Medicine, and Science in the Dutch Golden Age. Yale University Press. [Recounts many cases of medical, botanical, and chemical knowledge "translated" to European environments from India, Southeast Asia, Taiwan, and Japan by the Dutch East India Company.]

Duchesne, Ricardo. 2011. The Uniqueness of Western Civilization. Leiden: Brill. [Provides a passionate defence of Western exceptionalism, disagreeing with many who downplay the role of Europe in world history.]

Dudley, Leonard, 2017. The Singularity of Western Innovation: The Language Nexus. New York: Palgrave Macmillan. [This book argues that vernacular languages were standardized earlier in Europe than elsewhere, and that this facilitated communication in general and scientific and technological innovation in particular.]

Ede, Andrew, and Lesley B. Cormack. 2016. A History of Science in Society: From Philosophy to Utility. 3rd ed. University of Toronto Press.

Feldhay, Rivka, and F. Jamil Regep, eds. 2017. Before Copernicus: The Cultures and Contexts of Scientific Learning in the Fifteenth Century. Montreal \& Kingston: McGill-Queen's University Press.

Juma, Calestous. 2016. Innovation and Its Enemies: Why People Resist New Technologies. New York: Oxford University Press. [Discusses various examples of resistance to technological innovation in history (from self-interest or fear of the unknown), including Islamic opposition to the printing press, and contemporary resistance to genetically modified foods.]

Kuran, Timur. 2011. The Long Divergence: How Islamic Law Held Back the Middle East. Princeton University Press.

Lee, John S. 2018. The Medieval Clothier. Woodbridge, UK: Boydell Press. [Though focused on England, this book provides a detailed examination of the economic and social role of entrepreneurs and workers in cloth production as this moved into the countryside.]

Mokyr, Joel. 1991. The Lever of Riches: Technological Creativity and Economic Progress. Oxford University Press. [This book investigates a variety of factors that may have made Europe more innovative than Asia.]

Mokyr, Joel. 2016. The Culture of Growth: The Origins of the Modern Economy. Oxford University Press. [This book argues for a strong connection between Enlightenment and Industrial Revolution. See also Margaret C. Jacob, 2014, The First Knowledge Economy: Human Capital and the European Economy, 1750-1850, Cambridge University Press.]

Parthasarathi, Prasannan. 2011. Why Europe Grew Rich and Asia Did Not: Global Economic Divergence 1600-1800. Cambridge University Press.

Pomeranz, Kenneth. 2000. The Great Divergence: China, Europe, and the Making of the Modern World Economy. Princeton University Press. [Both Parthasarathi and 
Pomeranz argue that Europe was not far ahead in any important way in the eighteenth century.]

Prak, Maarten, and Jan Luiten van Zanden. 2013. Technology, Skills and the Pre-Modern Economy in the East and the West. Leiden: Brill.

Renn, Jürgen, ed. 2012. The Globalization of Knowledge in History. Berlin: Max Planck Institute. [This book stresses the role of the conflict between Church and state in Europe in allowing an independent science to develop.]

Roy, Tirthankar, and Giorgio Riello, eds. 2019. Global Economic History. London: Bloomsbury Academic [Focuses on identifying causes of the Great Divergence.]

Statman, Alexander. 2019. "The First Global Turn: Chinese Contributions to Enlightenment World History." Journal of World History 30:3, September 2019, 363-92.

Theodoridis, Dimitrios, Paul Warde, and Astrid Kander. 2018. "Trade and Overcoming Land Constraints in British Industrialization: An Empirical Assessment." Journal of Global History, 13:3, 328-51.

Yazdani, Kaveh. 2017. India, Modernity and the Great Divergence: Mysore and Gujarat (Seventeenth to Nineteenth C.). Leiden: Brill. 


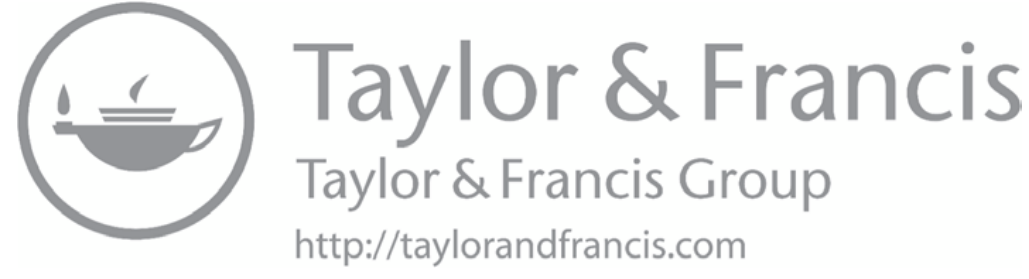


The nineteenth and twentieth centuries 


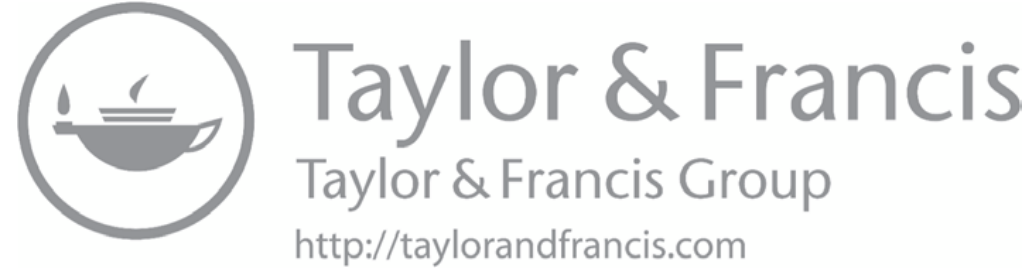




\section{Key thematic}

\section{transformations of \\ the long nineteenth century}

\section{Guiding questions}

Can we observe common trends across multiple regions in the century and a bit after about $1790 \mathrm{CE}$, and if so why, and what effects did they have?

Why, how, where, and when did European powers - and Japan colonize during this time period? What were some of the key effects of colonization?

How, why, and when were transport costs decreased and transport speeds increased during this period? What effects did these changes have?

Why, how, where, and when did working and middle classes emerge? Who constituted these classes? What political, cultural, and economic influences did they exert?

How, why, where, and when did science come to exert a much stronger influence on technology than before? What were the key developments in scientific understanding?

How, why, where, and when was agriculture transformed such that a much more substantial non-agricultural population could be supported across an expanding number of countries?

Relationship to other chapters: Chapter 23 follows most obviously from chapter 18 in its discussion of transport. We 
have devoted attention throughout this book to expanded trade links and information flows, for these serve to connect the regions of the world. The dramatic change in this period involves a reduction of both travel times and costs with the introduction of railways and steamships (and new canals and bridges). These advances are best understood in comparison with preceding transport technology and infrastructure. We have traced political developments through many preceding chapters; for the nineteenth century, we address two critical transformations: colonization in this chapter and revolution in chapter 25. Colonization will exert important influences on both colonizers and colonized in later chapters; we will return most explicitly to the causes and effects of colonization when we discuss decolonization in chapter 30 . We can best appreciate the dramatic change in speed of scientific discovery and its impact on the wider world by comparing nineteenthcentury science to the science of earlier centuries. Likewise, we can best appreciate unprecedented increases in agricultural productivity comparatively. Transport times continue to drop and agricultural productivity continues to rise in the twentieth century; we thus resume discussion of these trends, and their effects, in later chapters. Our discussion of working and middle classes suggests that something qualitatively new emerges during this period; this new world can be contrasted to earlier societies characterized by a large peasantry, small elite, and much lower proportions of workers or merchants or managers. These new classes remain important in the twentieth century: They encourage political, cultural, and social changes that many later chapters address.

Historians have long spoken of a "long nineteenth century" stretching from the American and French Revolutions of the late eighteenth century to World War I in the twentieth century. Such a characterization is a little bit Eurocentric. Within Europe, the period from the end of the Napoleonic Wars to World War I is mostly peaceful: The wars associated with the unification of Germany and Italy (chapter 21) were far less severe than the wars of the preceding and succeeding centuries. However, the late nineteenth century would witness a European scramble for colonies in Africa and parts of Asia: From the 
perspective of these regions, then, the later nineteenth century is an entirely different time period from the earlier nineteenth century. This epoch of colonization will be one of our main concerns in this chapter.

We discussed the Great Divergence in chapter 22. Whatever one's view of the difference between average incomes in Europe and other continents in 1800 , it is clear that these were radically different by 1914 . We trace the technological and institutional (and transport infrastructure and technology) trajectory of European economic growth from the late eighteenth century, and how this slowly spread to other continents, in chapter 24 . Here again, the long nineteenth century appears not as a century of stability but of significant transformations within some regions and resulting transformations of relations between regions. There is, not surprisingly, a connection between Europe's rising economic influence and its increased political control.

We will in chapter 25 place the American and French Revolutions in a global context. We will note important differences between the influences on, the course of, and the effects of the two. We will engage with a series of revolutions that followed in other countries over the nineteenth century.

These revolutions, though of considerable importance, may not be the most important political changes of the nineteenth century. We have discussed the institutions of slavery and serfdom in several preceding chapters. Through much of human history, some humans were either enslaved or enserfed. Over the course of the nineteenth century, both slavery and serfdom were abolished throughout almost the entire world. Though illegal slavery continues to this day in some parts of the world, the end to legal slavery and serfdom must qualify as one of the most important transformations in human history. It is the subject of chapter 26.

The nineteenth century marks important transformations in areas such as gender roles, the environmental impact of human activity, the role of the state, and population growth. Since each of these transformations continues into and generally accelerates in the twentieth century, we will discuss nineteenth- and twentieth-century developments together in chapters 27 and 31. In this chapter, we will investigate remarkable nineteenth-century changes in the organization of work, transport systems, the world of science, and agriculture.

We will address a diverse range of themes in this chapter. Colonization was a rather intense form of societal interaction. Transport improvements also intensified contact between societies. We will later discuss how institutions governing both work and science were transmitted across societies. With respect to agriculture, natural fertilizers became an important trade item in the late nineteenth century. We consider how technologies, institutions, and scientific insights built on what had gone before, but do not employ formal evolutionary analysis; we do, though, have cause more than once to mention the emergence of evolutionary theory itself. 


\section{Colonization}

Politics

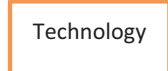

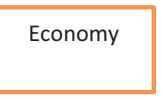

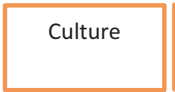

Social Structure

We discussed in chapter 19 how European countries came to colonize the Americas and parts of Africa and Asia from the fifteenth century. While Europe had colonized almost all of the Americas at one time, most of Africa and Asia remained independent as the nineteenth century began. Europeans had found it relatively easy to colonize the Americas as epidemic disease decimated local populations. Smallish trading posts had long served European trading interests in Asia and Africa; European countries had only slowly expanded their territorial holdings over time to secure supplies of raw materials. In Africa, European susceptibility to tropical disease had long discouraged movement beyond coastal trading posts.

By 1914, though, European states had colonized most of Asia and almost all of Africa. Indeed Europe - or former European colonies in the Americas controlled an astounding 84 per cent of the earth's surface by 1914. In Asia, the Europeans faced and conquered large states. The British took Malaya (for tin and rubber) and Burma (now Myanmar, for rice, timber - especially teak, jade, ivory, and oil). The French expanded in Vietnam, Cambodia, and Laos (for rice and rubber). The Dutch extended their hold on Indonesia (adding rubber, tin, and tobacco to long-standing spice, sugar, coffee, and tea exports). The Germans came later and took eastern New Guinea and some Pacific Islands. Europeans did not officially conquer China but the Qing Empire, after a series of military engagements with European gunboats, would grant European powers spheres of influence in which they could trade unimpeded. Thailand also managed

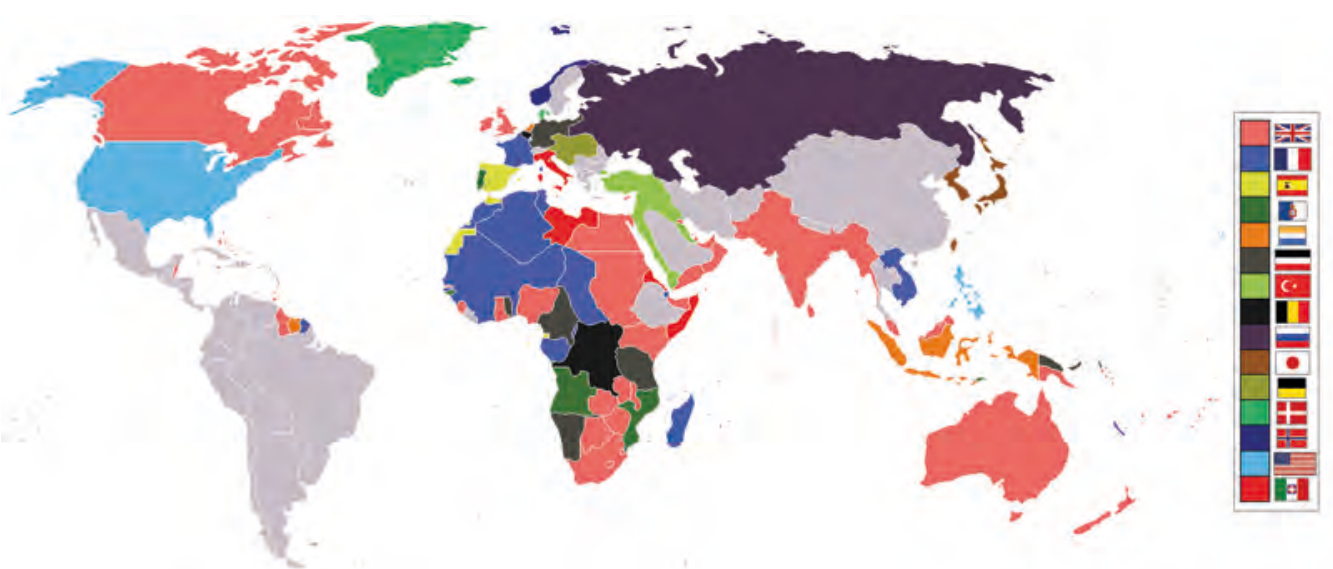

Map 23.1

European colonies in 1914

Map by Andrew092 1, CC BY 3.0 


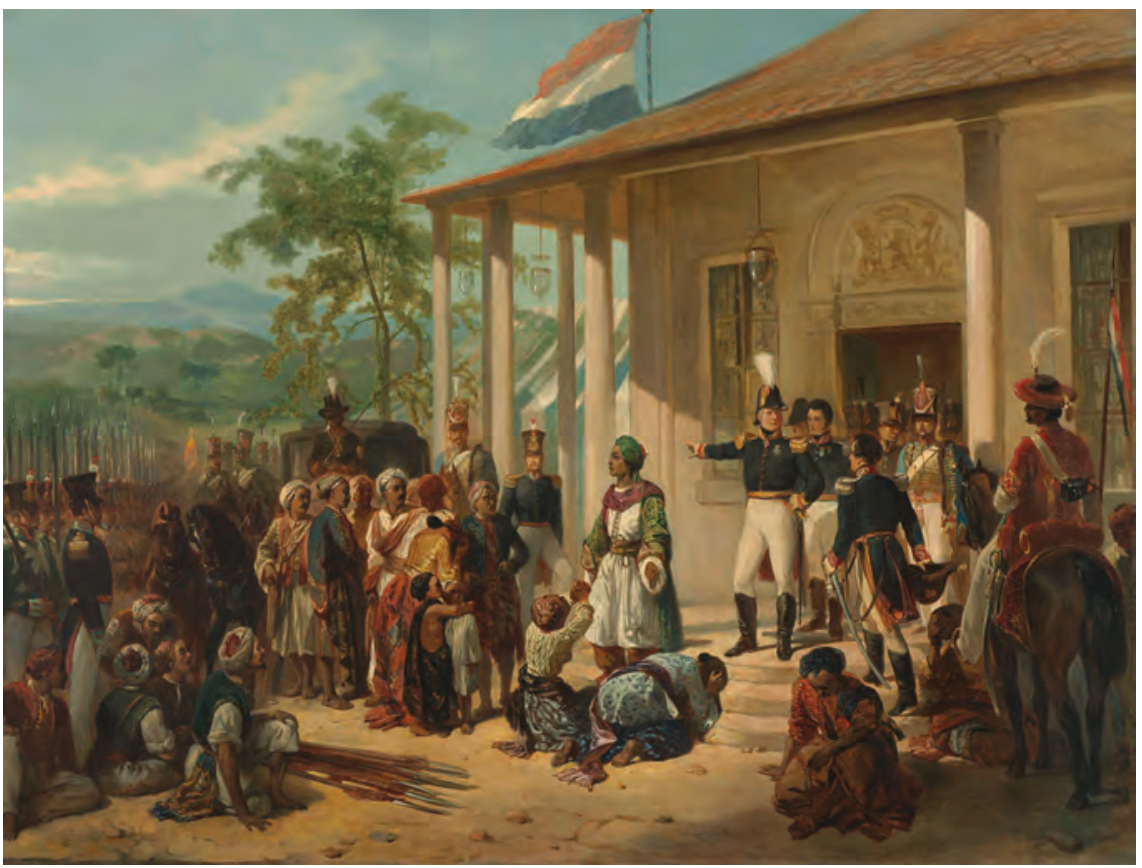

Illustration 23.1

The Submission of Diepo Negoro to Lieutenant-General Hendrik Merkus Baron de Kock, 28 March 1830, which ended the Java War (1825-30), Nicolaas Pieneman, 1835, Rijksmuseum

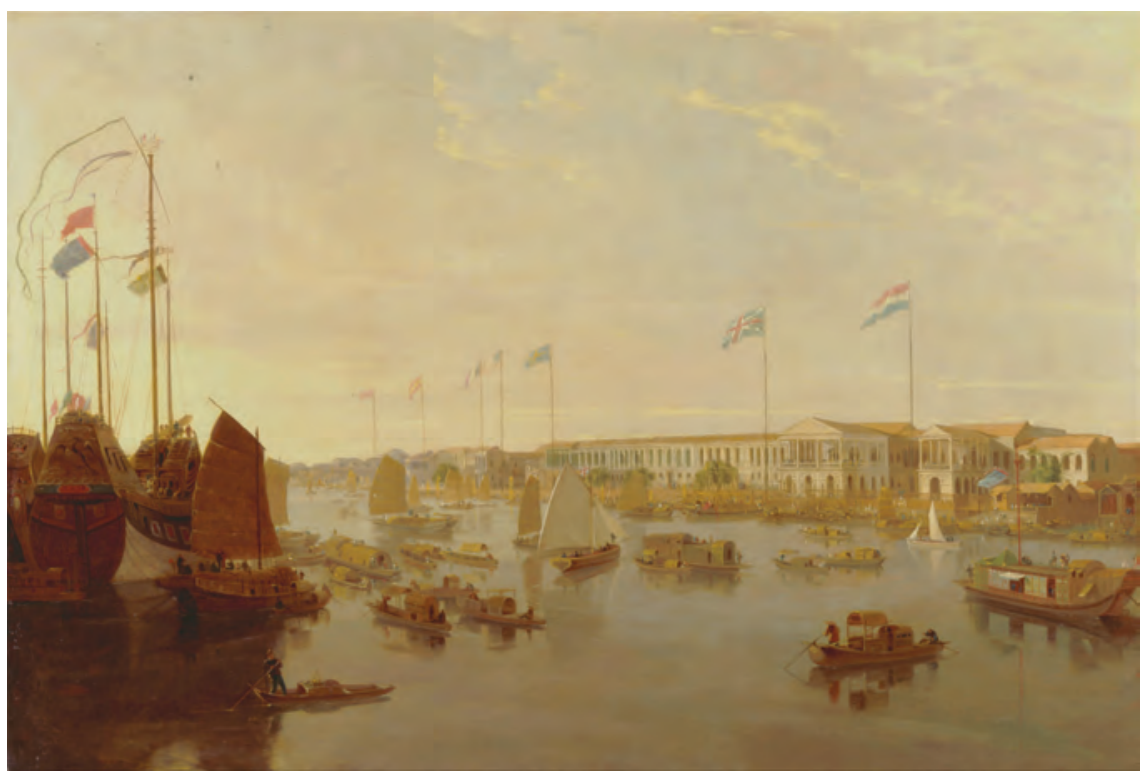

Illustration 23.2

The European Factories, Canton, William Daniell, 1806, Yale Center for British Art. These were the official trading outposts of European countries in China before most gained larger spheres of influence 
to retain its independence by skilfully playing European powers against each other: The French and British came to accept it as a useful buffer between their respective colonies.

In Africa, Europeans generally had to deal with a larger number of small polities. They also had to face desert, rainforest, and un-navigable rivers. Intrepid explorers had only mapped the interior of the continent in the late eighteenth and early nineteenth centuries. As late as 1875, Europeans held just a few coastal enclaves. Europeans were attracted inland by ivory (used mainly for piano keys and billiard balls), peanuts, and tropical woods, but would then develop coffee, sugar, and rubber plantations. (Africa had grown peanuts for centuries, but these expanded in importance after Columbus; in the nineteenth century, peanut oil was an important lubricant and ingredient in soap before synthetic lubricants replaced it.) Cloves were important in eastern Africa. Southern Africa might have declined when its role in supporting shipping between Asia and Europe was eclipsed by the Suez Canal after 1869 (see below), but substantial deposits of gold and diamonds were discovered from the 1870s. The British attempted to link their colonies in South Africa and Egypt (aspiring to an all-British Cape to Cairo route). The French would come to dominate West Africa and hoped to link possessions there with the small French outpost on the Red Sea to the east. The French and British almost fought a war in central Africa in 1898 because of their competing east-west versus north-south visions, but the French backed down. It should be noted that neither vision made a great deal of economic sense since the vast bulk of colonial trade moved directly to the coast rather than overland across the continent. The Portuguese expanded their holdings in Angola and Mozambique that dated from the time of early Portuguese exploration (chapter 19). Again, the Germans came late and occupied what are now Namibia and Tanzania. The Italians attacked Ethiopia, the only remaining independent state in Africa (excluding Liberia, a small state in West Africa founded by freed slaves), but were defeated in 1896 by an Ethiopian army trained in the use of European weapons. To limit conflict between European powers, these agreed at the Berlin conference of 1884-5 that each could exercise power inland where they had a coastal presence. They could then each focus military efforts on suppressing local rebellions: Germany killed some 70,000 rebels in what is now Tanzania between 1905 and 1907.

The most horrific story of African colonization involved the Belgian king Leopold II in the Congo. From 1871, he had employed the explorer Henry Stanley to establish trading stations along the Congo River. Stanley negotiated treaties with hundreds of local chiefs that gave him and Leopold power over a vast area. The colonizers then forced the local population onto rubber plantations and into (especially copper) mines. The colony would become infamous for its brutality. Overseers killed or maimed workers for not producing enough or for resisting authority. Some ten million Congolese died. In 1908, an embarrassed Belgian state would take official control from Leopold, though rule remained harsh. 


\section{BOX 23.1 \\ THE HISTORY OF RUBBER}

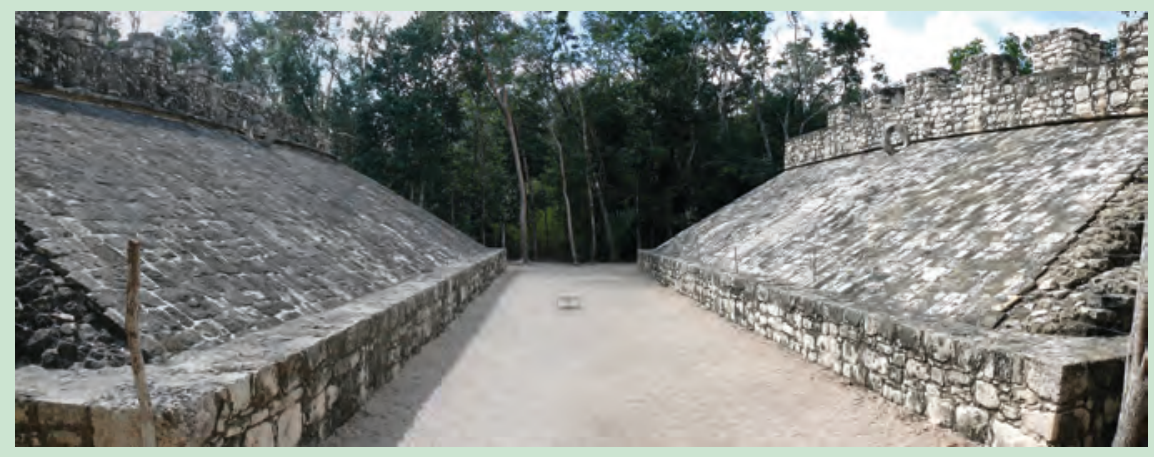

Illustration 23.3

Mayan ball court at Coba in the Yucatan of Mexico. Note the rings on the side that the ball was to be sent through

Rubber trees are indigenous to the Amazon basin of South America and tropical Central America. The Mayans (or their predecessors) had developed a rubber ball from the sixteenth century BCE. They mixed the rubber with juice from local plants to strengthen it and then wound strips of rubber around a harder core to create balls. A substance that was solid but flexible naturally intrigued the Spanish. Both Columbus and Cortés carried rubber balls to Spain where they amazed the royal court when bounced. Though ball games had been known in Eurasia for millennia, these had employed balls made of straw or animal intestines or other materials with far less flexibility. Mesoamerican ballplayers were taken to Spain to display their skills. In the eighteenth century, European scientists explored the nature of rubber - though the understanding of its peculiar chemical bonds would be possible only after a more general understanding of chemical bonds developed in the late nineteenth century. In 1844, Charles Goodyear, a self-taught chemist in the United States, developed the vulcanization process: The addition of substances such as sulphur made rubber products more durable. Rubber had previously melted in the heat and become brittle in the cold. We can see vulcanization as an improvement on Mayan techniques of ball manufacture. Rubber use spread after vulcanization. With the development of tyres for bicycles and then automobiles in the late nineteenth century, the market exploded. Brazil had long maintained a monopoly on the Hevea trees that produced the best natural rubber - though Congolese vines could also be used. Profits from the rubber trade financed an elaborate opera house with imported Alsatian marble and British steel - in Manaus in the Amazon in the 1880s (it is claimed that the famed opera singer Caruso sang there). Seeds of the Hevea tree were taken to Britain in 1876 where they were grown at the Kew Botanical Gardens and then distributed to British colonies in Southeast Asia. Tyre manufacturers Michelin and Firestone would later 
establish plantations in Vietnam and Liberia respectively. Local labour was often forced to work on rubber plantations. Large numbers of Chinese and Indian indentured servants moved to Malaya and Indonesia to work on rubber plantations. Tyre manufacture would become one of the leading industries of the twentieth century. Though the international spread of rubber production lowered its cost, it was nevertheless expensive to produce. As rubber tyres became important militarily, many states in temperate climates came to worry about the supply of a product grown only in the tropics. Scientists created the first synthetic rubber in 1909 in Germany. Both Germany and the United States would invest heavily in research on synthetic rubber during the World War II. In the later twentieth century, attention turned to recycling used tyres: There are many millions of these in the world, and they are toxic if burned.

This brief history of rubber touches every inhabited continent and involves a range of actors in key roles: rulers, ballplayers, explorers, scientists, indentured servants, and industrialists among them. It also spans multiple themes: We have touched upon culture, politics, technology and science, non-human environment, economy, individual differences, and population and health above. It thus exemplifies the interactions across societies, themes, and different types of human agent that characterize world history as a whole.

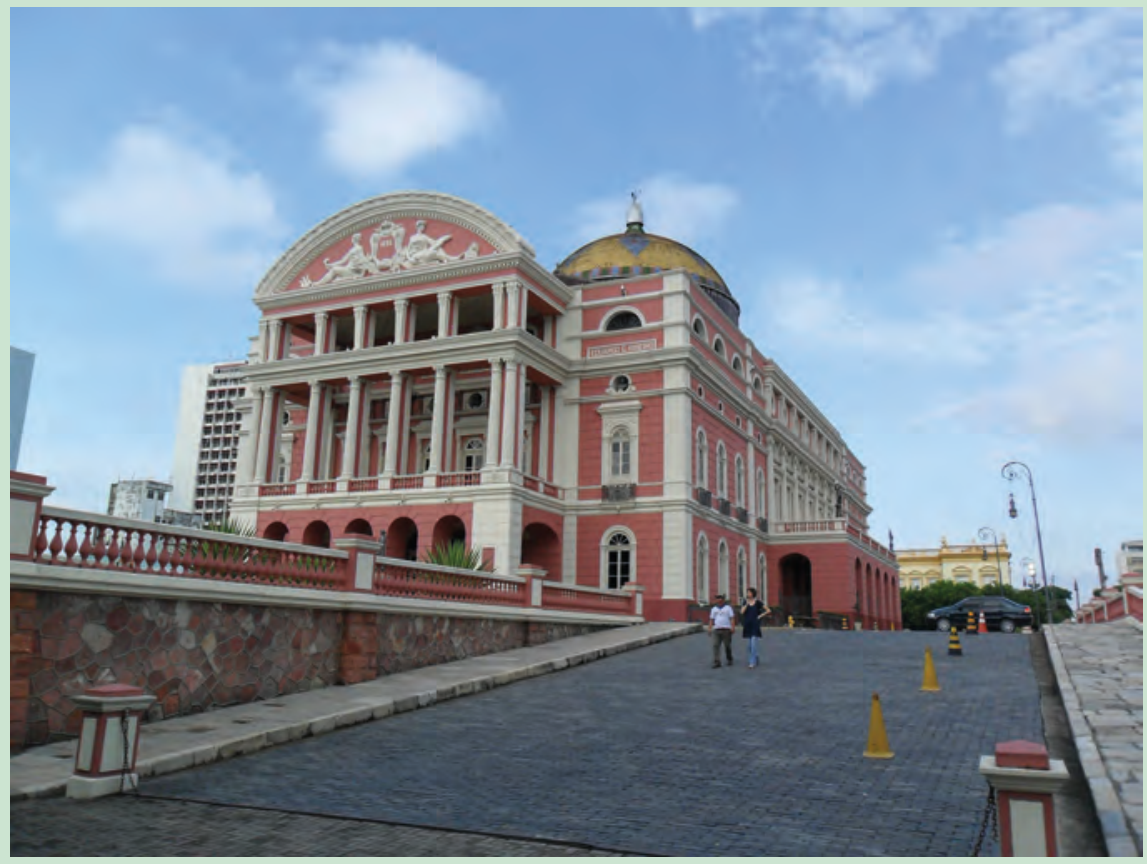

Illustration 23.4

Manaus Opera House, Brazil, built 1884-99 
As in Africa, there had been limited direct colonization of Pacific Islands before the late nineteenth century, though Europeans had often insisted on trading rights. The French turned a protectorate into a formal colony in eastern Polynesia in 1880, and Britain made Fiji a colony in 1874. Germany took several Marshall Islands in the 1870s. The Berlin conference of 1884-5 addressed Pacific Islands as well, and in the next decades these were annexed by the United States and European countries. Tonga remained nominally independent though Britain protected it.

A set of technological innovations facilitated European conquests. One was quinine. The Incas had long appreciated the medicinal properties of the cinchona tree, and Jesuits had taken cinchona to Europe in the sixteenth century. It was used medicinally in Europe after that; it is said that Charles II of Britain was cured of malaria with cinchona. Quinine, the medicinal element of cinchona, was isolated in 1820 and named after the Inca word for the tree. Its production would allow Europeans - who had no immunity to malaria - to survive in the tropics. It would remain the leading anti-malarial medication until the 1940s when drugs with fewer side effects were developed.

We discussed in chapter 18 how and why Europe had pulled ahead of the rest of the world with respect to gunpowder weapons. Over the nineteenth century, as Europe industrialized and developed steam engine technology, it also pulled ahead in the construction of naval ships. Iron ships could carry much larger cannons than wooden ships and were better able to withstand attack. The combination of better guns and stronger ships led to "gunboat diplomacy": The appearance of powerful ships in coastal ports, with cannons that could destroy local shipping and fortifications, forced local rulers throughout Asia and Africa to make political concessions. When Europeans needed to march inland, improvements in machine guns allowed small numbers of European troops to massacre large numbers of local forces. The Maxim gun from the 1880s could fire eleven bullets per second with considerable accuracy. The British used machine guns against the Mahdi (an Islamic revival state) in Sudan in 1898 (The Mahdi had beaten the British in 1885); they lost 40 soldiers while killing 11,000 and injuring 16,000 . The military advantage was in most cases far greater than that exercised by Europeans in the Americas centuries earlier.

These technologies allowed European conquest. But why did Europe want colonies? Economic growth in Europe and the development of new technologies during the Second Industrial Revolution increased European demand for a host of

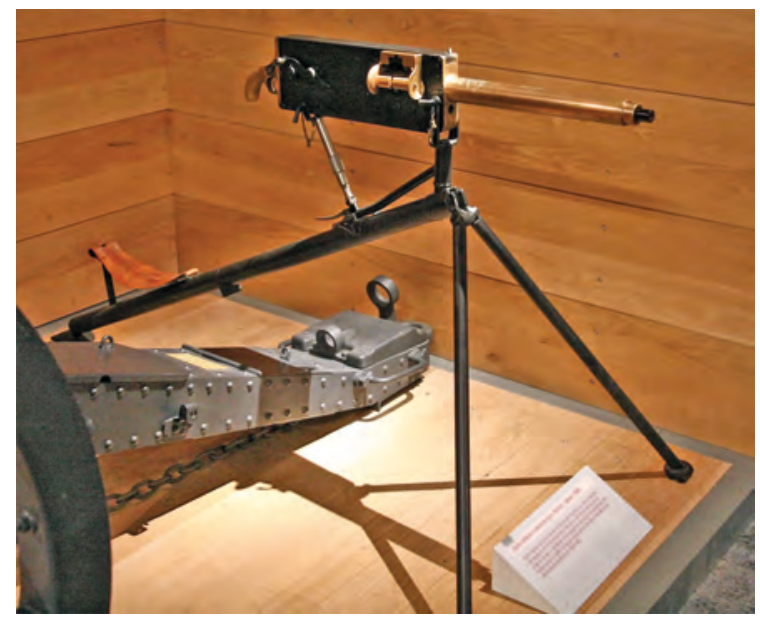

Illustration 23.5

Maxim gun from 1895, the Royal Artillery Museum; there were also larger calibre Maxim guns 
plant and mineral resources. Many Europeans believed that guaranteed access to both raw materials and markets was critical to economic success. The development of steamships and railways increased the potential advantages of both. However, the fact that European countries continued to prosper after shedding colonies in the twentieth century (chapters 29 and 30) suggests that colonies were far from economically necessary. As was the case with empires earlier in history, colonial expansion may have served the interests of European elites more than the average European. Elites could, as always, benefit from the theft associated with conquest, including gaining rights to mine or establish plantations without compensating locals (much). The average European may have gained little to compensate for the military costs associated with empire (though European states might have maintained reasonably large militaries to scare each other anyway). The European soldiers and administrators that served in colonies did generally benefit from both higher wages and the ageold opportunities for abuse of power over locals. However, these numbers were often small relative to both local and imperial populations: There were only ever a little over a thousand British occupying the top spots in the Indian bureaucracy, and some 80,000 British troops exercising control over 300,000 local troops. It was much less expensive to employ locals when possible in both

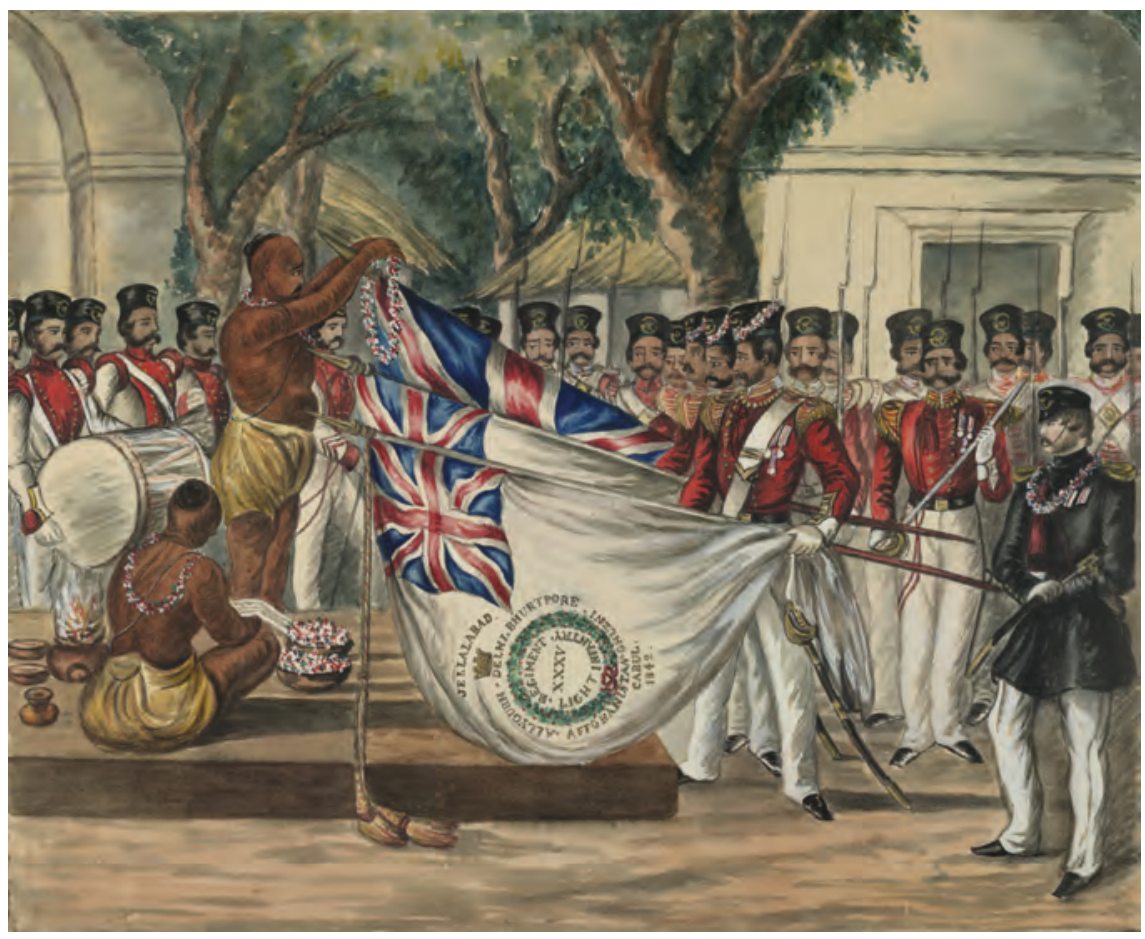

Illustration 23.6

Hindu priest placing garlands on the flags of the 35th Bengal Light Infantry (c. 1847), British Library, CC0 
bureaucracy and army. The British may have drawn on the Chinese example in establishing an examination system to recruit Indians into the civil service (and later employed examinations in Britain itself); these jobs became quite prestigious.

Colonization took on a momentum of its own. European nationalists came to see another critical motive for taking a new colony: to prevent other states from getting it. We have seen that the French and British had competing ambitions. The Germans and Italians shortly after unification would accept the logic of empire espoused by the French, British, and Dutch and scrambled to take whatever lands these had not yet taken. In this last stage of colonization, the potential economic value of a particular colony might matter very little.

We have seen that empires throughout history have not just relied on brute force but also always sought to justify their rule. European empires were no different. Indeed, these not only wished to provide some rationale to those they colonized but needed to satisfy those back home that colonies were both just and worthwhile. Since European nation states were themselves justified in terms of ideas of popular sovereignty (chapter 21), it was no simple task to explain colonial subjugation. However, European rulers could appeal to nationalist sentiment, which itself often reflected ideas of ethnic superiority. Europeans argued that European civilization was superior. (This is one reason we have generally avoided the use of the word "civilization" in this book.) Local peoples in Asia and Africa were judged unable or unprepared to operate mature economies or polities. Europeans then had a responsibility to bring "civilization" to such people. Such an idea may seem laughable in the context of the glories of Asian and African societies through the millennia that we have encountered throughout this book. Such histories were less well known in the nineteenth century, and were simply overlooked in the celebration of evidence of European superiority in industrial and military technology. Rudyard Kipling's poem "The White Man's Burden” of 1899, in which he celebrated the civilizing mission of Europeans (his particular focus at the time was United States intervention in the Philippines), is now decried as racist but captured much (but far from unanimous) popular feeling of the time.

Europeans often argued that it was only proper to ensure that the resources of colonial lands were properly utilized. They often suggested that these resources were unused or unowned by local people, ignoring complaints of locals who were pushed off lands or coerced into working in mines. Though local residents would often participate in ownership or management of plantations and mines, Europeans tended to occupy the best positions in most ventures.

There was tension within these ideas of European superiority. As Kipling's title hinted, it was common to think that there was some genetic advantage of White over Black or Asian peoples. Though arguments of cultural superiority dominated in the early days of colonization, ideas of racial superiority became more common over time. Such ideas were often associated with misapplications of Darwinian theories of evolution: European conquest appeared then as merely another case of the survival of the fittest. Yet ideas of European superiority 


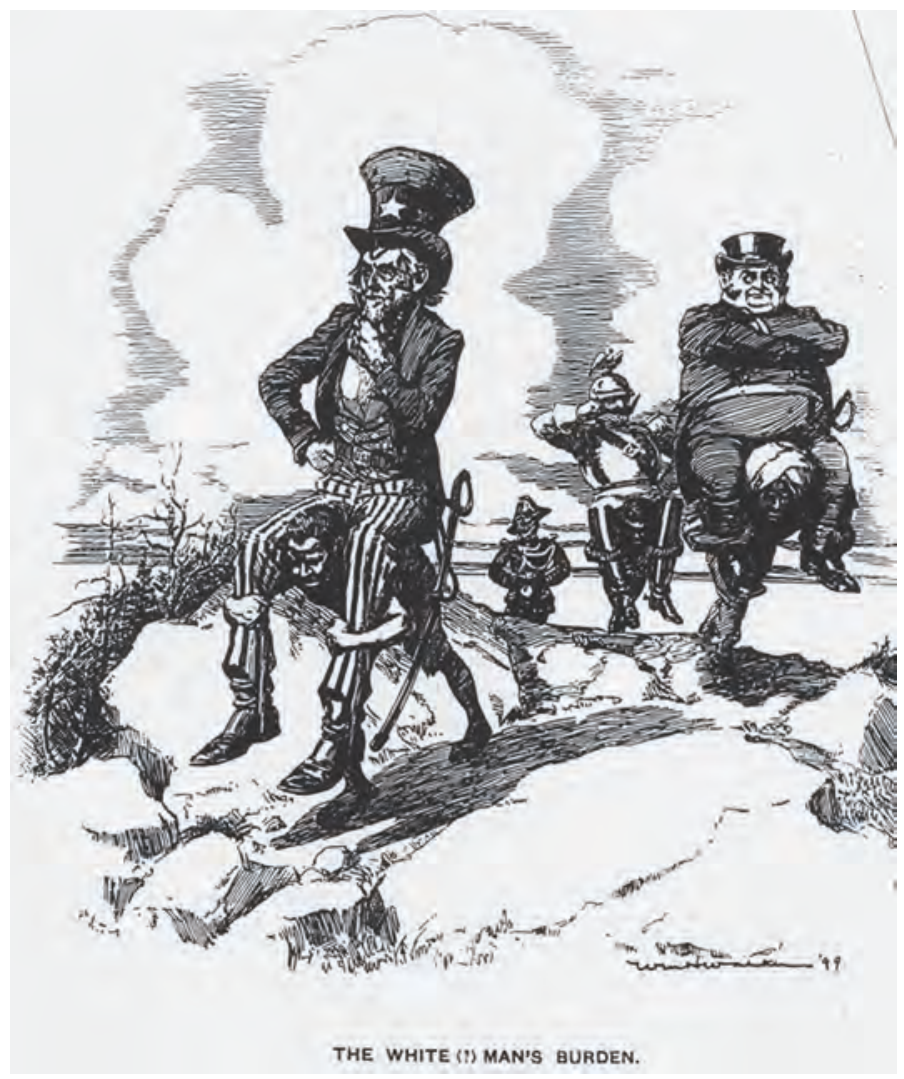

Illustration 23.7

Sarcastic take on the poem "The White (?) Man's Burden," shows the "White" colonial powers being carried as the burden of their "Coloured" subjects, Life, March 16, 1899

often remained grounded in history rather than genes: Europeans had merely developed better values and institutions. If this were the case, then one might hope that Asians and Africans could be educated to match European achievements. Colonial governments often voiced such intentions. As we shall see in chapter 30, though, colonies were generally woefully unprepared for independence after generations of colonial rule. Colonial powers made limited efforts to educate locals - beyond perhaps a narrow group that might be useful in local bureaucracy. The civilizing mission took second place to the benefits of the social and economic subordination that the idea of European superiority could so easily justify.

As we shall see in chapter 30 , members of colonized societies often spoke eloquently about the inequities of colonial rule. The debate about the justice of colonialism was thus global in scope. Opponents of colonization within colonizing nations interacted with opponents based in the colonies. Advocates of colonialism had to react to arguments coming from the colonies. Steamships and telegraph cables meant that colonial arguments - and descriptions of acts of colonial repression - reached audiences in colonial countries quickly. 


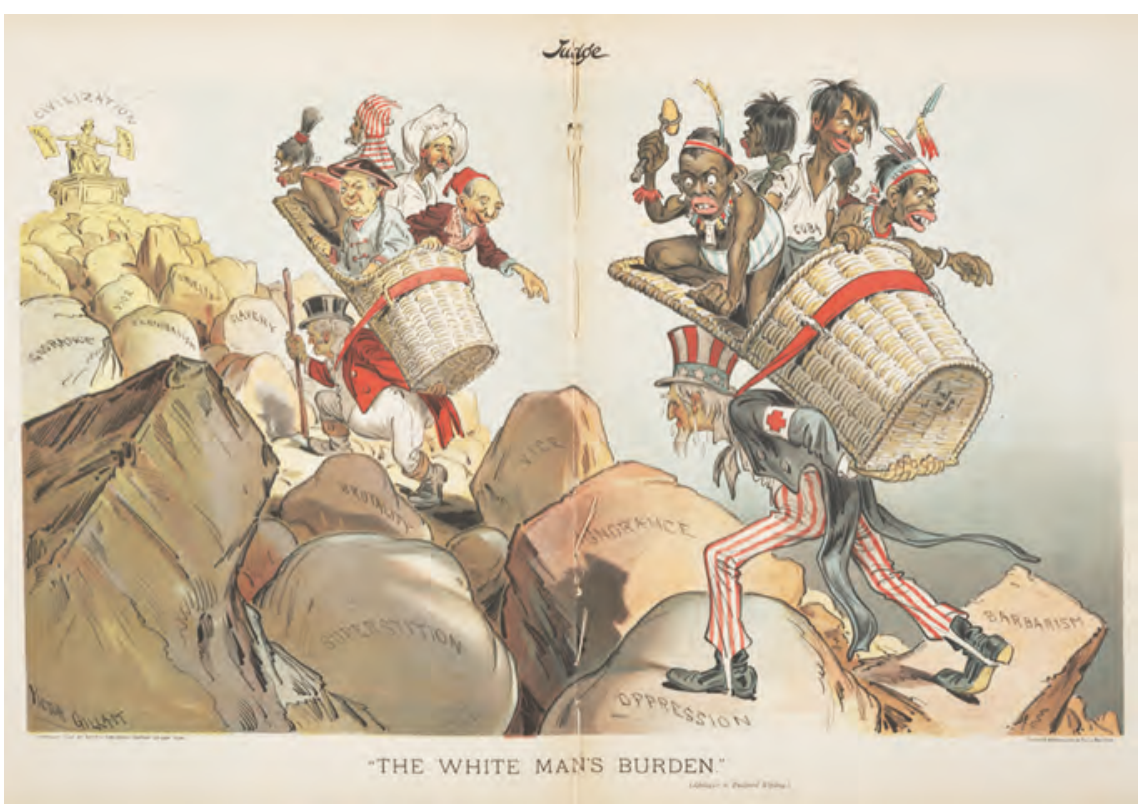

Illustration 23.8

A more supportive take: "The White Man's Burden (Apologies to Rudyard Kipling)" Judge, April 1, 1899, The Ohio State University Billy Ireland Cartoon Library \& Museum

What were the effects of colonies? As noted above, the economic benefits to European countries may have been modest. This may especially have been the case for the last colonies taken just for the sake of having colonies. Competition for colonies may have inflamed nationalist sentiments in Europe, setting the stage for World War I (chapter 28): In particular, German nationalists grated at the fact that the French and British had much more extensive empires. This encouraged them to think of colonizing parts of Europe itself. Cultural effects may have been far greater: Europeans would often absorb cuisine and arts and crafts from their colonies: The first curry restaurant in London opened in 1809.

The effects on the colonized were much greater. Some benefits may have occurred with the transmission of European technologies and the identification of trade opportunities. Colonizers often introduced new crops such as tea and rubber from one colony to another, albeit under harsh conditions of production and with local farmers pushed off their land. Hospitals and schools were established. Though these might mostly serve the European population or a small local elite, some transfer of medical technologies and science occurred. (European governesses for local elite children were another avenue by which European science and attitudes were transmitted to local elites.) In some cases, European colonization ended warfare among small local polities. Europeans built thousands of kilometres of railways in their colonies, though these often merely linked mines and ports. Colonizers introduced legal systems, though these generally favoured Europeans. 
The negative effects of colonization were immense. Colonization often displaced existing and effective political and economic institutions. Though European colonizers, like past empires, often governed through local elites, Europeans generally occupied the top positions in colonial bureaucracies. Even just a few decades of colonization, then, could destroy local institutions but not prepare locals to run the new institutions. There was also significant environmental damage from mines and plantations; imperial authorities did from the 1920s undertake some efforts to protect wildlife and forests and waterways, but often without consulting the locals most affected by these plans. In addition to these longer-term costs, there were the short-term costs always associated with power disparities in a society: discrimination, lack of freedom, and death or assault at the hands of the military or police. Scholars estimate that some 30-50 million people died in famines in the late nineteenth century that colonial authorities largely ignored.

We noted back in Box 6.2 that social stratification always has a gender component. We saw in chapter 19 that colonial authorities in the Americas worried about sexual relationships between European men and local women. Though women increasingly accompanied officials to European colonies in the nineteenth century, there was still a severe gender imbalance in European populations in the colonies. European men then took local concubines. Colonial authorities generally tried and failed to limit such sexual interactions. Different colonies made different accommodations. In French West Africa, children of mixed parentage could not inherit land from their mothers or marry locals. They became a local trading elite that operated between Europeans and locals. Yet in various colonies the British, French, and Dutch recognized some marriages between European men and local women, and were willing to grant citizenship to small numbers of children of such marriages. (The French in 1928 would allow all children of mixed parentage to apply for French citizenship.) These were still generally prevented from reaching the very top levels of colonial governance, the appointment of James Douglas as the first governor of the then-small colony of British Columbia in 1851 being a notable exception. European men often treated local concubines poorly but at other times fought for the recognition of their rights and their children's rights. Colonial authorities were generally even more hostile to relationships between European women and local men, though many European women travelled to North Africa or the Caribbean for this purpose in the late nineteenth century. Local men sometimes sought to limit local female interaction with colonizers, but sometimes saw advantages in their sisters or daughters forging connections with rulers. Muslim men often disparaged Europeans for not secluding their women, while western men often disparaged men on Pacific Islands for not limiting the sexual behaviour of local women (we have seen that Pacific men may have valued the strategic importance of relations between local women and European men).

European authorities often legislated European family law, limiting polygamy, allowing widow remarriage, banning child marriage, encouraging 
marital choice, and allowing women to hold property; they often encouraged some education of girls. Such laws were important in justifying colonialism in Europe. Local women in the colonies often welcomed these initiatives but sometimes saw them as interference with the traditional local family.

\section{PRIMARY DOCUMENTS: IN DEFENCE OF COLONIALISM}

Jules Ferrey, twice Prime Minister of France, addressed the French Chamber of Deputies, March 28, 1884. This is an excerpt from his speech:

In the area of economics, I am placing before you, with the support of some statistics, the considerations that justify the policy of colonial expansion, as seen from the perspective of a need, felt more and more urgently by the industrialized population of Europe and especially the people of our rich and hardworking country of France: the need for outlets [for our exports].

Is this a fantasy? Is this a concern for the future? Or is this not a pressing need, one may say a crying need, of our industrial population? I merely express in a general way what each one of you can see for himself in the various parts of France. Yes, what our major industries, irrevocably steered by the treaties of 1860 into exports, lack more and more are outlets. Why?

Because next door Germany is setting up trade barriers; because across the ocean the United States of America have become protectionists, and extreme protectionists at that; because not only are these great markets ... shrinking, becoming more and more difficult of access, but these great states are beginning to pour into our own markets products not seen there before.

$\cdots$

Gentlemen, we must speak more loudly and more honestly! We must say openly that indeed the higher races have a right over the lower races ....

I repeat, that the superior races have a right because they have a duty. They have the duty to civilize the inferior races ....

In the history of earlier centuries these duties, gentlemen, have often been misunderstood; and certainly when the Spanish soldiers and explorers introduced slavery into Central America, they did not fulfil their duty as men of a higher race .... But, in our time, I maintain that European nations acquit themselves with generosity, with grandeur, and with sincerity of this superior civilizing duty.

I say that French colonial policy, the policy of colonial expansion, the policy that has taken us under the Empire to Saigon, to Indochina, that has led us to Tunisia, to Madagascar - I say that this policy of colonial expansion was inspired by... the fact that a navy such as ours cannot do without safe harbours, defences, supply centres on the high seas .... Are you unaware of this? Look at a map of the world.

...

And that is why we needed Tunisia; that is why we needed Saigon and Indochina; that is why we need Madagascar... 
It is noteworthy that he begins by outlining economic motives for colonialism - motives that we might now question - but then asserts a right and then a duty to colonize what he calls "lower races." He appreciates that colonial power can be abused but argues that French colonies are reasonably run. He closes by claiming that a network of colonies is needed for military reasons.

What reasons do you suspect were most important to him and why?

\section{South Africa and Algeria}

As noted above, Europeans had generally only had small coastal possessions in Africa until the late nineteenth century. There were two exceptions. One was South Africa. The Dutch had established a thriving colony there in the mid seventeenth century to provide supplies to ships sailing between Europe and Asia. The soil and temperate climate of the region proved quite amenable to a variety of European crops (including grapes for wine). The Indigenous peoples of the area had not previously had access to these crops and had not developed agriculture. The Dutch East India Company had not intended a large colony for it wished only to supply its ships. They forced prisoners from both Europe and Java to work in their new colony but also encouraged some free migration. The European population would spread along the coast and begin to explore inland. The British would take over the colony, at first temporarily when the Netherlands fell to the French in the Napoleonic Wars, but would then establish permanent control. The British also showed little interest in the colony at first. Nevertheless, they, like the Dutch before them, followed a policy of pushing local Black populations off the best farmland so that Europeans could take this.

This land policy continued after the British abolished slavery in all of their domains in 1837 (chapter 26). The British established an elected assembly, but Blacks could not vote. Nevertheless Dutch settlers (the Boers) chafed at British rule - and the imposition of English as the official language - and trekked inland from 1834-41, pushing local Blacks onto reservations. The Zulu people under their leader Shaka raised an army of 40,000 that fought in formation with spears. This army pushed other tribes out of the area, but the resulting Zulu kingdom would fall to the British in 1879. British interest in the region rose dramatically with the discovery of diamonds in 1867 and gold in 1886. Thousands of British miners moved in. Taxation in cash forced many Blacks to work in mines (in the lower-paying jobs). Mines also employed tens of thousands of indentured servants from China until White miners went on strike about these taking the best jobs. Political conflict between the inland Boer republics and the British colony led to the Boer War of 1898-1901, which the British won. Though there was a tacit agreement between Boers and British not to employ Black troops, both sides did so; ten thousand died in a British internment camp. The British established a dominion with assemblies dominated by Whites from 1913. 
French soldiers landed in Algeria in 1827 to confront pirates operating along the coast. From 1830, the French established a colony in the coastal region of Algeria. French troops would kill some 825,000 Algerians - over 10 per cent of the population - over the next decades. From 1848, coastal Algeria was treated not as a colony but as part of France - but only European settlers were recognized as citizens. As in South Africa, Europeans found the fertile agricultural lands along the Mediterranean attractive - but here there was already a farming population that they had to remove from the land. There would be half a million people of European heritage in Algeria by 1900 (about 13 per cent of the population); their descendants would leave Algeria at independence in the 1950s (chapter 30).

\section{Japan and colonialism}

American gunboats had forced Japan to open itself to international trade in 1853-4 (Americans had previously gained some familiarity with Japan by whaling in adjacent seas). In the ensuing years, it seemed that Japan might follow the Chinese trajectory of becoming subject to foreign domination. The Meiji Restoration of 1868 - in which the emperor assumed nominal power from the Tokugawa shoguns - was motivated in large part by a desire to avoid foreign colonization. Japanese leaders built on a long tradition of central governance and a shared language and culture to urge nationalism but also the adoption of Western technologies. As we have seen in earlier chapters, Japan had in preceding centuries borrowed many ideas from China while remaining culturally and politically distinct. Japan would send students to study abroad and import experts to transmit military and industrial technology. It modelled its army on Germany's and its navy on Britain's. It encouraged railways, telegraphs, mining, and industry - and Western laws, attire and the seven-day week. It had already achieved literacy rates similar to Europe's in 1868 but by 1905 over 90 per cent of both boys and girls would attend primary school. It developed modern universities also from 1886.

Along with technology, Japanese leaders would absorb the idea that a strong country needed colonies. The desire to avoid colonization segued quickly into a felt need to colonize. Japan occupied Okinawa and the Ryukyu Islands to the south from 1879. They then took Taiwan (and the Liaodong peninsula and Pescadore islands) from China in 1895, and effectively gained control over Korea (and treaty rights like Europeans within China itself). The Japanese at the time employed ships bought from the British. Still, European powers forced the Japanese out of Liaodong. This led to conflict with Russia, and a Japanese victory against the Russian navy that surprised many in 1904-5. Japanese colonial ambitions would then be important in the world wars of the twentieth century (chapter 28).

Japan could not justify its conquests in quite the same language employed by Europeans. It would often describe itself as saving Asians from European dominance. Yet it came to celebrate the superiority of Japanese ways - despite 
centuries of applauding Chinese practices. Japan would treat those it colonized similarly to how Europeans behaved in their colonies. Japan killed most of the aboriginal population in Taiwan, and established separate school systems for Chinese and Japanese there. As in European colonies, they also built much useful infrastructure.

\section{American imperialism?}

Much ink has been spilled over the question of whether the United States has been an imperial power. Unfortunately, much of this has reflected terminological confusion. Some writers define imperialism loosely to mean any sort of self-interested interference by a powerful country in the affairs of a less powerful country. Others define imperialism more narrowly as explicit territorial conquest. These different definitions can guide different answers to the question. This book has favoured the more precise definition of empire for reasons of clarity. It may indeed be somewhat hypocritical for a country to decry imperialism of the narrow sort while pursuing imperialism of the broader kind, but these imperialisms are nevertheless quite distinct in both how they operate and how they affect the weaker country. Our definition has not guided us to ignore the other ways in which powerful states influence the less powerful, but rather to treat these as important processes in their own right.

The United States has a long history of opposing the narrow sort of imperialism. The American Revolution was justified in this way. In 1823 the Monroe doctrine would warn European states that the United States would oppose colonial endeavours in the Americas (most Spanish and Portuguese colonies were gaining independence at that time; chapter 25). In the twentieth century, the United States would urge the break-up of the defeated Ottoman and Austrian Empires after World War I (chapter 28) and support the more general move toward decolonization after World War II (chapter 30). Yet as we saw in chapter 19, much of the territory of the United States was gained by conquest or purchase. This process would continue in the late nineteenth century. The United States bought Alaska from Russia in 1867. It established a protectorate over the Hawaiian kingdom in 1875, and accepted the invitation of the American plantation owners there to assume full control after these had overthrown the queen in 1893. The Americans fought the Spanish-American War of 1898 as an anti-colonial venture. When American troops stayed in the Philippines rather than delivering the promised independence, a Filipino revolt cost 200,000 Filipino lives. Debate raged in the United States (to an even greater extent than in European democracies such as Britain) as to whether a democracy could have colonies. As Americans settled in large numbers in Alaska and Hawaii, these came to be recognized as states. The Philippines gained autonomy in 1935 and independence in 1946 - somewhat earlier than most European colonies gained independence (chapter 30). Puerto Rico and a smattering of small Caribbean and Pacific islands have remained American territories, generally with local support. 


\section{Mining}

We mentioned mining several times above. We have seen in earlier chapters that even in ancient times valuable minerals might be carried hundreds or even thousands of kilometres. In the early years of European industrial revolutions (chapter 24), minerals were generally obtained in Europe itself. However, over the course of the nineteenth century, a global trade in key minerals emerged. Tin would become especially important as tin cans were used to preserve food from about 1810. Copper would gain increased importance in wiring as electrification spread.

We wondered for the ancient period how minerals were discovered and mined within territories characterized by less sophisticated economic and political institutions (chapter 5). Likewise in the nineteenth century, mineral deposits that might be of limited interest to the local economy had to be identified and extracted. Mining is an expensive and technically demanding pursuit. Financial institutions in London and other centres funded mines and the railways to reach them (with occasional bouts of senseless speculation as investors struggled to value distant mines). Mines were designed and managed by a mobile community of mining engineers, many from the United States (Herbert Hoover, later president during the Great Depression, had been one).

Mining tasks are often dangerous and dirty. Local workers often had to be encouraged to work in mines. In some locales they were forced. Elsewhere, mines enticed them with higher wages than they could earn in agriculture. The need to pay colonial taxes encouraged many toward mine work. Local workers often suffered racist disdain. Workers in Africa often migrated long distances to work in mines, a practice that disrupted family life.

Advocates of nineteenth-century colonization argued that only colonial governments could adequately protect Western capital and skilled workers, provide clear property rights over expensive mines, and organize the necessary local labour force. However, independent Latin America, and Chile in particular, was also an important site of mining investment. This global trade in minerals has expanded in the decades following decolonization - though mining companies have often been accused of bribing local governments.

Most of the world's tin came from what are now Malaysia and Indonesia in the late nineteenth century; Bolivia and Nigeria produced smaller quantities. Southeast Asian tin was highly valued for its purity, which allowed tin coatings to be very thin. Surface tin deposits had been harvested for centuries, and Chinese merchants financed some mining activities in the area. As the easiest deposits were mined out, it became necessary either to dig deeper or harvest inferior ores. The British and Dutch strengthened their control over Malaya and the tin islands of Indonesia respectively as mining operations increased. Processes that scarred the local environment were employed. Hydraulic mining washed entire hillsides through sluices and into local waterways, often clogging these. Dredging in deltas deposited tons of soil and rock from deep below the 


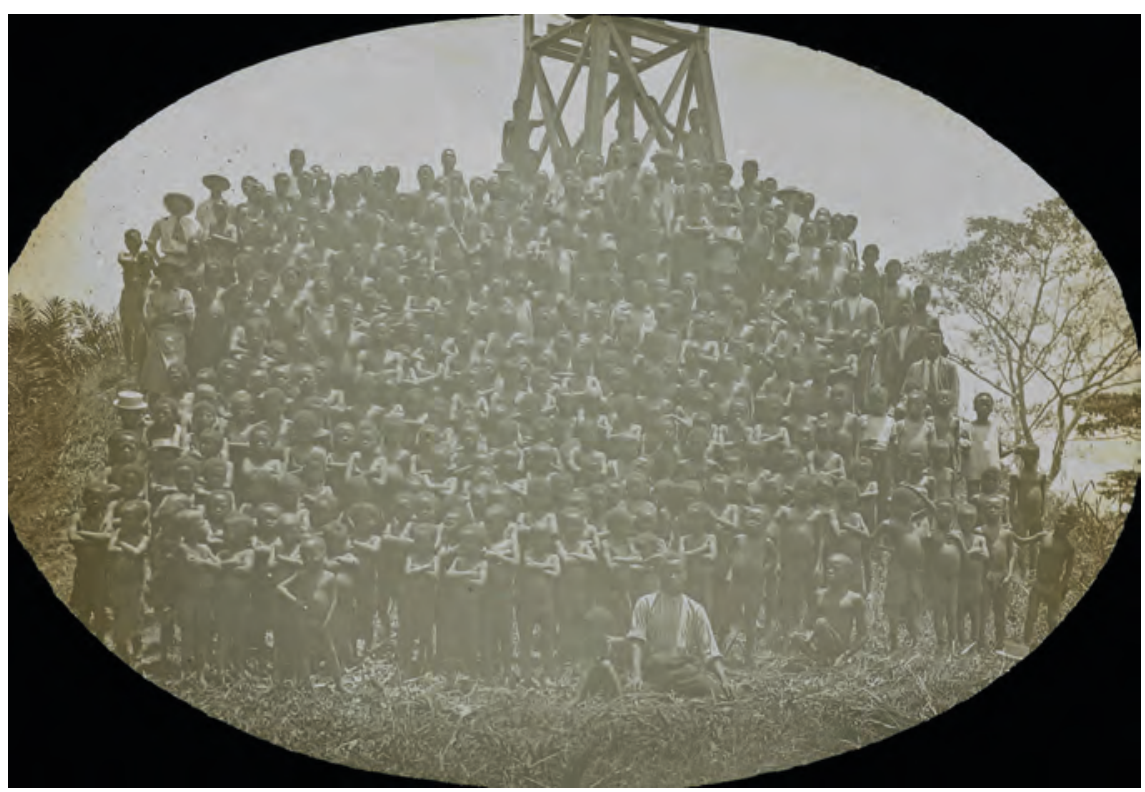

Illustration 23.9

Mine workers, Belgian Congo, 1900-15, photographer unknown

surface on fertile topsoil. The consumers of food packaged in tin cans were generally unaware of where the tin came from or how it was obtained.

\section{Transport}

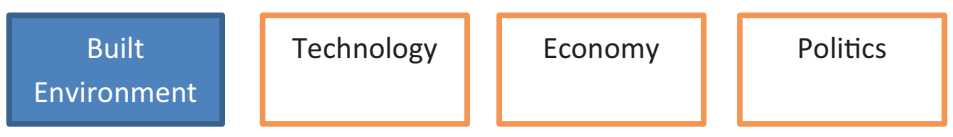

We have mentioned transport improvements above, and we will do so again in many later chapters. They are a crucial element in nineteenth (and twentieth)-century history, facilitating trade, investment, migration, and the spread of ideas. The railway (termed railroad in the United States) was invented at the very start of the nineteenth century in Britain. The railway was an unforeseen result of industrialization, wedding advanced steam engine and ironproduction technology with rails first employed to get coal out of mines. Railways soon became a critical means of transporting both goods and people. They could excel at the tasks previously played by both road and water transport (Box 5.3). Railways were often less expensive than transportation by water and yet faster than transport by road. Like roads, they could extend almost anywhere - though railways faced challenges in climbing steep hills. Railways in Britain would expand from under 160 kilometres in 1830 to over 10,000 by 1860. The figures for the United States were 65 kilometres in 1830, 28,900 in 


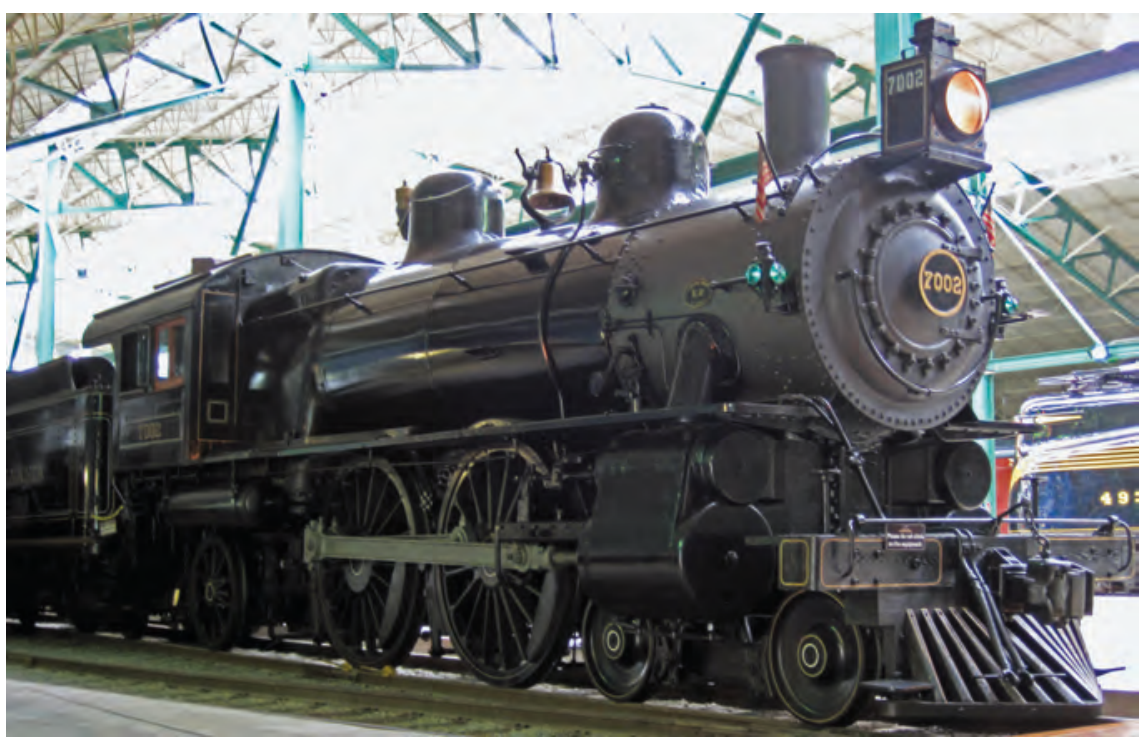

Illustration 23.10

Steam locomotive built in 1902 in Pennsylvania, Railroad Museum of Pennsylvania Photograph by James St. John, CC BY 2.0

1860 , and 163,000 in 1890 , peaking at 254,000 in 1916 . Worldwide there were around 800,000 kilometres of railway by 1900 , and some construction occurred in virtually every country. Railways added speed and convenience where good systems of road and water transport existed, and opened regions such as the western plains of North America or parts of the Russian steppe that were not well served by roads or water transport.

Though the initial purpose of the new transcontinental railways in North America was to connect North American farms or mines to urban or foreign markets, they came to play a role in global commerce. Indeed, for the last decades of the nineteenth century and first decades of the twentieth century, the fastest trains in both Canada and the United States carried raw silk from Japan to cities such as New York for use in textile manufacture. Given the high price of silk at the time (and thus costs of insuring it), it made sense to ship it by (faster) rail rather than by sea. Only when silk prices fell due to the development of synthetic fibres in the interwar period (prices also fell in part because of the Great Depression) did this traffic shift to the Panama Canal (below). Silk trains were famous not just for top speeds of well over 90 kilometres per hour but also for the speed with which engines were changed and wheels lubricated along the way. Silk trains also had special carriages designed to take corners fast.

Steamships were developed in both Europe and the United States soon after the railway. By 1900, there would be 4,000 steamships on the Mississippi River alone. The paddlewheels used on calm rivers and lakes were ill-suited to the waves of the open ocean. Screw propellers allowed ocean travel. Costs of shipping and travel times across the Atlantic fell dramatically. It could take 
two years for a return trip from Britain to India by sail; with steam, it took four months.

Though steamships were faster than sail, they were still limited to much the same routes. The Suez Canal of 1859-69 would allow ships to move directly from the Mediterranean to the Red Sea and thence to the Indian Ocean. The canal eliminated the millennia-old challenge of trans-shipment between these bodies of water. (Recall that a much smaller canal for much smaller boats had been temporarily built in classical times by the Persian emperor Darius between the Nile and the Red Sea; chapter 9.) The Suez Canal is over 160 kilometres long, and thus it was an impressive engineering feat - guided by French engineers and financed by Europeans but relying on Egyptian labour and government sponsorship. Forced labour was employed at first, and thousands of Egyptians died during construction, but heavy machinery was later employed. The flat terrain meant that a flat canal could be built that did not require ships to be raised and lowered in locks. The return trip from Britain to India fell to four weeks.

Fuelled by success in Suez, French investors and engineers hoped to build a canal across Panama, linking the Pacific and Atlantic Oceans. However, Panama was a far greater challenge. Much greater changes in altitude had to be managed. The tropical rainforests hosted both yellow fever and malaria that decimated the workforce. The United States would take over from the French. The United

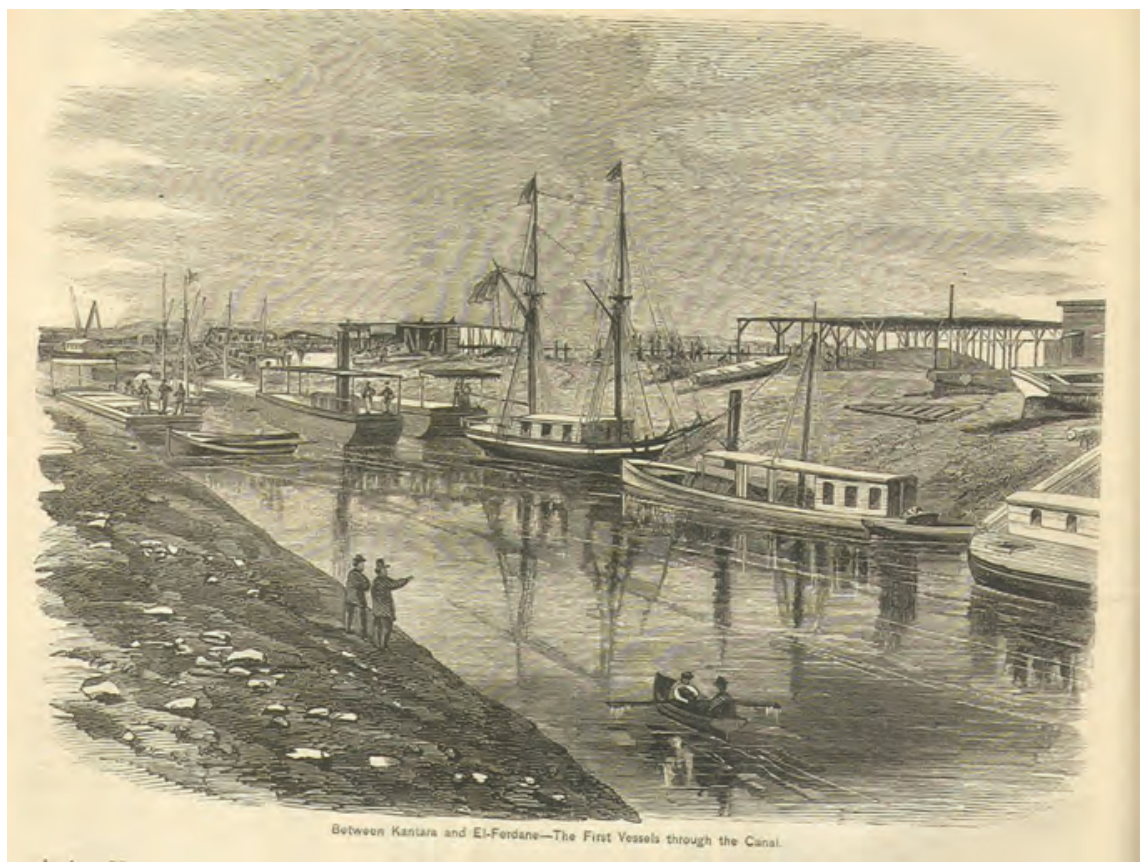

Illustration 23.11

"Suez Canal, between Kantara and El-Fedane. The first vessels through the Canal." Appleton's Journal of Popular Literature, Science, and Art, 1869 


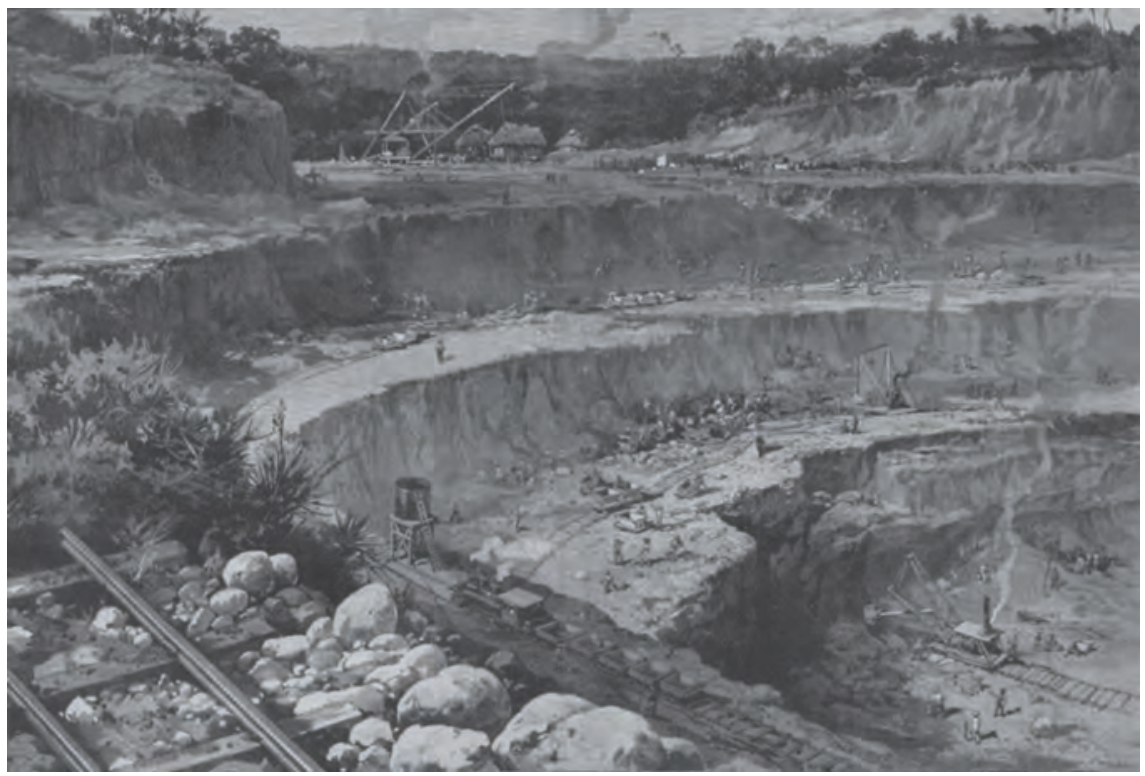

Illustration 23.12

“The Panama Canal - The Great Culebra Cut," Charles Graham (1852-1911), Harper's Weekly, July 4, 1885

States would encourage Panamanian independence from Columbia to gain political authority over the Canal Zone (the United States would return the Canal Zone to Panama in 1979). Construction took ten years and was completed only in 1914. Though shorter than Suez, the Panama Canal required locks to raise ships 26 metres at both ends; Gatun Lake in central Panama was flooded to allow large ocean-going vessels to travel across the lake between the two canal segments. (It is a very peculiar sight to this day to observe huge ocean-going ships cross this smallish lake.)

Though grains were the primary agricultural trade good by volume, consumers increasingly wanted to eat meat and a variety of fruits and vegetables. Even steamships using canals could not get meat or fruits or vegetables to distant markets fast enough. The answer was refrigeration. A variety of improvements in the design and insulation of ships would allow perishable crops to be transported long distances. In 1874, frozen beef was shipped from the United States to London. The challenges of refrigerating rail cars were more significant: There were barely 1,000 of these in the United States in 1880 but 70,000 by 1900 . These developments in refrigeration encouraged ranching in the Americas and Australia (which was largely settled after refrigeration), and fruit and vegetable exports from many regions of the world.

The volume of world trade increased ten times between 1850 and 1913. Its composition changed just as dramatically. This was no longer a luxury trade. Instead, bulky raw materials and basic consumption items now dominated 
world trade. Most of the trade occurred between advanced economies, and especially between Europe and the Americas.

Railways and steamships also served administrative purposes. States were better able to govern distant territories if they could quickly transport news and officials. The British could better rule India when messages took weeks rather than years. The telegraph - one of the first applications of the new scientific understanding of electricity - would from the 1830s lead to a further dramatic reduction in message time. When telegraph cable first came to India in 1879 , it took only five hours for a message to reach Britain. A telegraph cable was laid across the Atlantic from Ireland to Newfoundland in 1866. Wireless transmission between Ireland and Newfoundland by Marconi in 1901 would further reduce the cost and time involved in international communication. The telegraph was not well suited to large documents, though, which usually were still sent by ship or rail. And telegrams were expensive: Only a few details of the massive volcanic eruption in Krakatoa in Indonesia in 1883 were therefore reported in newspapers elsewhere in the world at the time; it would take years for Europeans to connect the dark skies and unusual weather they experienced to that eruption.

Postscript: The first oceanic copper cables could only transmit telegrams. From the 1950s, coaxial cables could carry voice but with limited capacity. Capacity expanded a thousand-fold with fibre optic cables from the 1980s. Even in an age of satellites, these still carry the bulk of international communications. These cables are very secure at the bottom of oceans but face damage from ship anchors as they approach the shore. There have also been protests about bulky cables spoiling a shoreline, so most now land in inaccessible areas. Cables are often buried near and along the coastline. Every transoceanic cable ever laid rests at the bottom of the ocean.

As steamships, railways, and telegraphs tied the world together, there was pressure to standardize the treatment of time itself. The European calendar with twelve months and weeks of seven days was adopted through much of the world, at least by merchants engaged in international trade. (A lunar calendar would also survive in China and much of the Islamic world.) So too was the idea of twenty-four hours of sixty minutes. Until late in the century, though, each city set its clocks so that noon occurred when the sun was at its highest. Such a practice wreaked havoc on railway timetables. International agreement on the present system of twenty-four time zones, each separated by an hour from neighbouring zones (with some exceptions) was achieved in 1884, though some states were slow to adopt it. 


\section{BOX 23.2}

\section{THE GLOBALIZATION OF TIMEKEEPING}

Before the development of railroads, different towns or regions tended to set their own times. Railroads urged the standardization of times to make their schedules easier to manage and comprehend. Emerging nation states - including Germany after unification and India after independence - came to see a standardized national time as a symbol of the nation (with only a handful of nation states being too large to easily encompass just one time zone). As global trade and travel expanded, the idea of an international system of time zones was urged. By the 1880s, the idea of twenty-four time zones, separated by an hour, was accepted internationally, though it would take many decades before all countries adopted a time that differed from Greenwich Mean Time by a number of hours (or in some cases half hours). There was simultaneously a push for standardization of calendars. Along with merchants, scientific associations played a leading role here: It was easier to compare a range of statistics across countries if these measured weeks and months in the same fashion. Given the military and economic dominance of Western Europe and the United States, standardization came to occur around the Gregorian calendar used there - though many parts of the world would maintain different calendars locally while adopting Western dates when trading. There were many efforts in the late nineteenth and early twentieth century to revise the Gregorian calendar: to make months the same length (often by having thirteen months of twenty-eight days) and to have the same date fall on the same day every year (by adding a spare day to annual calendars outside the rotation of weeks and months). Such innovations were occasionally debated in legislatures and international organizations but were never adopted.

\section{Changes in work}
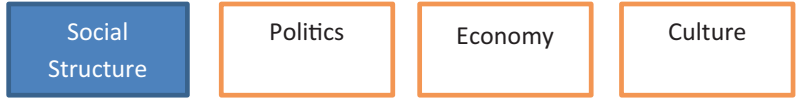

Built

Environment

We will in chapter 24 discuss how modern factory-based industry spread through the world. We should appreciate that most humans were still engaged in agriculture in 1900, and that the service sector would eclipse manufacturing in most of the world in the twentieth century. Nevertheless, the spread of factories transformed the world of work. Most obviously, a workforce of paid and supervised industrial workers emerged which slowly eclipsed industrial production by skilled artisans in homes or small workshops. As urbanization proceeded (chapter 31), these workers organized to fight for better working conditions and political rights.

Britain, as the home of the first Industrial Revolution, witnessed some of the earliest and nastiest confrontations between labour and political and economic elites. After numerous violent strikes, the rights to unionize and strike gained 


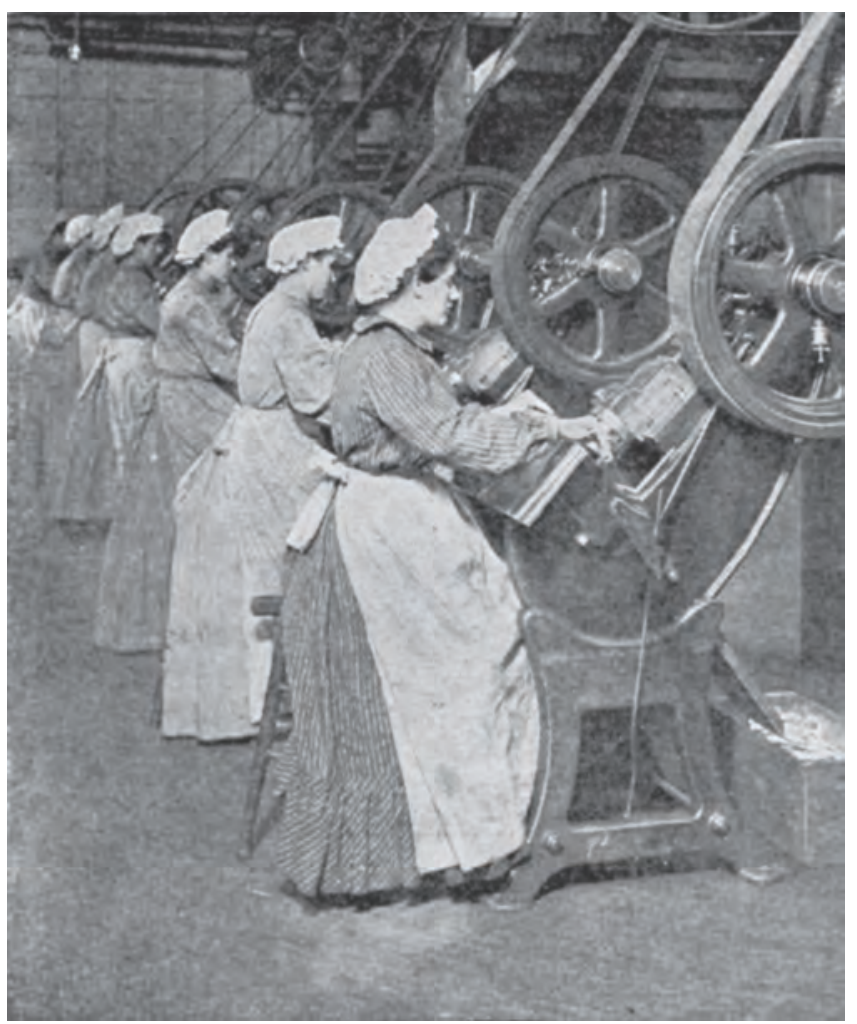

Illustration 23.13

American women making metal cans for Heinz before 1909

legal recognition in 1871. A series of Reform Acts expanded the electorate: By 1867 , a third of males could vote, including many industrial workers; the electorate doubled again in 1884. Workers also agitated for public education, though employers and the state also saw advantages in this: It was enacted in 1870.

States tended to introduce legal rights for workers, political rights, and social policies such as public education as industrialization spread to other countries. Though some countries copied British precedents, sometimes in a deliberate attempt to catch up, there were violent confrontations in many countries as armies, police, or private security forces ended strikes with force. As in earlier cities, rulers had to worry about the potential for rebellion of urban masses but these urban masses were now larger and had a collective experience of supervised wage labour. Worker rebellions in 1848 in many European countries (chapter 25) were brutally repressed: 10,000 were killed in Paris alone. Yet countries such as Germany would later introduce social policies to satisfy industrial workers. Karl Marx and other writers grappled with this dramatic change in the composition of the labour force (we discuss communism in chapter 27); their calls for radical political and economic reform encouraged a mixture of violent repression and enlightened social policy from governments around 
the world (we discuss social policy in chapters 27 and 29). Marx's ideas would inspire revolutions in Russia in 1917 and China in 1949, among others (see chapter 27). However, these revolutions did not occur as he predicted in the most advanced economies.

One common trend worldwide was a reduction in the length of the working week. In the early Industrial Revolution, workers worked six days a week for ten to sixteen hours a day. French workers agitated successfully for a maximum of twelve hours after 1848. Workers in many countries then agitated for five days of eight hours, a goal that would be widely achieved in the early decades of the twentieth century (after which workweeks largely stabilized). As workweeks shortened, entertainments such as theatres and amusement parks grew in importance. The working class now had time to both participate in sports and watch these (while children in school had more opportunity for sports than previous generations in factories). Since the social changes that facilitated organized sports originated in Europe, and especially Britain, sports such as soccer and cricket and rounders (the antecedent of baseball) and rugby (the forerunner of American and Canadian football) came to dominate global sporting events. North America played a subsidiary role, with baseball and football developed in the northern United States, and hockey and basketball in Canada (Europeans had borrowed polo from Asia). Wrestling proved very popular in the Middle East and India. Men outside Europe and North America and especially colonial men - may have seen sport as a venue for displaying masculinity in a world dominated militarily by Europe. In any case, cricket became popular in South Asia, baseball in Japan, and soccer in Latin America. At the end of the nineteenth century, the modern Olympic movement would also originate in Europe. It was hoped that international sports could lessen international tensions, just as the ancient Olympics was intended to encourage Greek solidarity (chapter 10).

As just noted, one fundamental characteristic of the new working class was that it was supervised in large industrial establishments. Along with the working class, then, a new managerial class emerged. Though there had long been state bureaucrats in history, there had not previously been large numbers of managers of industrial establishments. Railways, shipping companies, and water and sewer systems also required management. The number of lawyers expanded with increased government activity and regulation as well as clarification of land titles. Financial institutions would grow in size and complexity, and marketing would become an important profession in its own right. Engineering would blossom as a profession. As health, education, and the service sector more generally grew in size through the late nineteenth and twentieth centuries, this "middle class" - neither manual labourers nor owners of large enterprises - increased in size. The middle class had been a small minority in previous human societies but would come to be larger than the working class in many countries during the nineteenth century.

This middle class too would agitate for democracy in many countries. Its political goals were sometimes the same as the working class: Both fought for 
education and healthcare. However, the middle class might often align with the upper class in seeking protection of property. Nevertheless, the existence of a growing middle class likely served to weaken tensions between the working class and the industrial elite. As public education spread and the service sector grew, there was considerable mobility between the working class and middle class. Economically, the growing middle class - along with increased wages in the working class - provided a market for a wide range of goods and services (including the entertainments noted above). The middle class was also an essential market for the global trade in consumer goods that expanded through the century (chapter 24). Ironically, though most of the middle class had official workweeks similar to those in the working class, they often needed to be available outside of work hours - some would then see their actual workweeks increase in the late twentieth century as mobile phones and the internet made them available at all times. Culturally, middle-class values and standards of dress tended to be considered dominant, at least by members of the middle class. The middle class tended to stress "respectability," which in turn meant education, ethical behaviour, belief in hard work and advancement through merit, and appreciation of family. There was always a tension between valuing family and valuing merit: Should the middle class hire its own children or ensure that working-class children had access to quality education?

In the colonies, ethnic minorities that intervened between locals and colonial administrators or merchants often dominated the middle class. These colonial middle classes were often less self-confident than the middle classes of independent states. The "divide-and-rule" strategies often pursued by colonial governments could inspire distaste for successful minority groups that worked with colonial authorities.

Most agrarian societies in human history had been characterized by a large mass of peasants and a small elite. The development of a large middle class could work to reduce stark differentials in the distribution of both incomes and power. It appears that the upward trend in income inequality which we have observed through much of human history (albeit interrupted by periodic shocks) was attenuated in the second half of the nineteenth century (and may even have been reversed a little) in many/most countries as the middle class expanded in size and middle-class incomes rose. However, the late nineteenth century was also a period in which some industrialists and financiers amassed unprecedented fortunes, ensuring that measures of wealth or income inequality would not fall too far. We have discussed above the role that the middle class (and working class) played in agitating for political change, and will return to a discussion of the role of the middle class in encouraging democracy in chapter 27 (and will discuss the relationship between income inequality and democracy again in chapter 32 ).

We can also speak of certain classes that declined in importance in the nineteenth century. However, the international experience of class decline is far more diverse. Landowners lost social status and political influence in much of western Europe, but not farther east. The samurai in Japan had maintained 
a ceremonial social status after they lost their military function to peasant infantries but would lose their special legal status after the Meiji Restoration of 1868. The Chinese scholar elite would remain influential into the twentieth century. The British would decide to rule through Indian princes in the late nineteenth century, enhancing their status and power.

The large factories of the nineteenth century changed the landscape along with the world of work. Some of these factories still operate, others have been transformed into warehouses or retail space or even apartment buildings, and a very few have been appreciated as examples of industrial design. The vast majority have been torn down.

\section{PRIMARY DOCUMENTS: TESTIMONY OF A YOUNG FEMALE FACTORY WORKER IN 1832}

The British Parliament held an inquiry regarding conditions in the textile mills in 1832. This is the testimony of Elizabeth Bentley. [Parliamentary Papers, 1831-2, XV, 195.]

What age are you? - Twenty-three.

Where do you live? - At Leeds.

What time did you begin to work at a factory? - When I was six years old.

At whose factory did you work? - Mr. Busk's.

What kind of mill is it? - Flax-mill.

What was your business in that mill? - I was a little doffer.

What were your hours of labour in that mill? - From 5 in the morning till 9 at night, when they were thronged.

For how long a time together have you worked that excessive length of time? - For about half a year.

What were your usual hours when you were not so thronged? - From 6 in the morning till 7 at night.

What time was allowed for your meals? - Forty minutes at noon.

Had you any time to get your breakfast or drinking? - No, we got it as we could.

And when your work was bad, you had hardly any time to eat it at all? - No; we were obliged to leave it or take it home, and when we did not take it, the overlooker took it, and gave it to his pigs.

Do you consider doffing a laborious employment? - Yes.

Explain what it is you had to do? - When the frames are full, they have to stop the frames, and take the flyers off, and take the full bobbins off, and carry them to the roller; and then put empty ones on, and set the frame going again. 
Does that keep you constantly on your feet? - Yes, there are so many frames, and they run so quick.

Your labour is very excessive? - Yes; you have not time for any thing.

Suppose you flagged a little, or were too late, what would they do? - Strap us.

Are they in the habit of strapping those who are last in doffing? - Yes.

Constantly? - Yes.

Girls as well as boys? - Yes.

Have you ever been strapped? - Yes.

Severely? - Yes.

Could you eat your food well in that factory? - No, indeed I had not much to eat, and the little I had I could not eat it, my appetite was so poor, and being covered with dust; and it was no use to take it home, I could not eat it, and the overlooker took it, and gave it to the pigs.

You are speaking of the breakfast? - Yes.

How far had you to go for dinner? - We could not go home to dinner.

Where did you dine? - In the mill.

Did you live far from the mill? - Yes, two miles.

Had you a clock? - No, we had not.

Supposing you had not been in time enough in the morning at these mills, what would have been the consequence? - We should have been quartered.

What do you mean by that? - If we were a quarter of an hour too late, they would take off half an hour; we only got a penny an hour, and they would take a halfpenny more.

The fine was much more considerable than the loss of time? - Yes.

Were you also beaten for being too late? - No, I was never beaten myself, I have seen the boys beaten for being too late.

Were you generally there in time? - Yes; my mother had been up at 4 o'clock in the morning, and at 2 o'clock in the morning; the colliers used to go to their work about 3 or 4 o'clock, and when she heard them stirring she has got up out of her warm bed, and gone out and asked them the time; and I have sometimes been at Hunslet Car at 2 o'clock in the morning, when it was streaming down with rain, and we have had to stay until the mill was opened.

How accurate do you think this testimony is? Would such testimony affect public attitudes toward industrialization? Toward treatment of workers? 


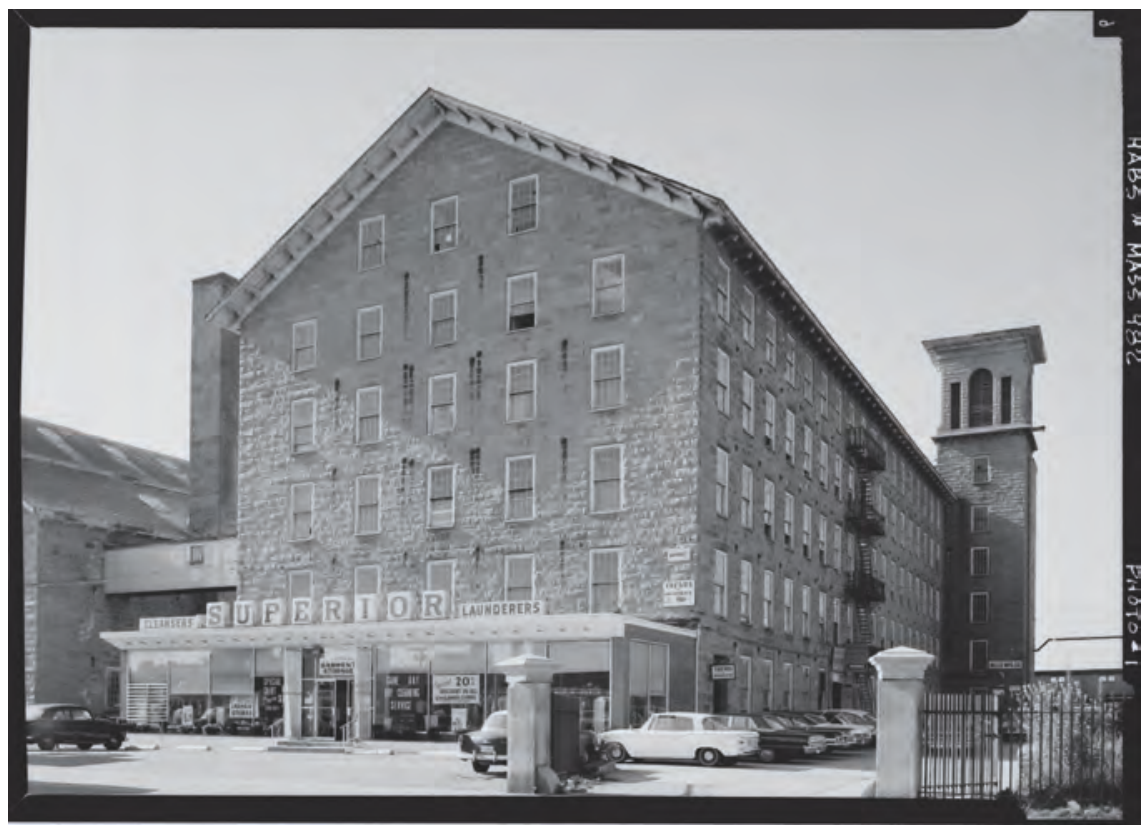

Illustration 23.14

Durfee Mills No.1, Fall River, MA. One of many mills that survive in a town that was once the largest textile producer in the United States with over 100 mills. Many of these mills look very similar to this one. Many have been refurbished as homes, offices, and even medical clinics. This is one of several in Fall River on the National Register of Historic Places. The river and falls that gave the city its name have disappeared

\section{Science}

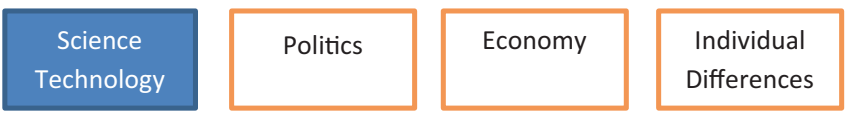

Though discoveries continued in long-standing scientific pursuits such as astronomy, the nineteenth century is especially notable for the development of new lines of scientific enquiry. Electricity had been known for centuries (as static electricity mainly; Benjamin Franklin had established in the eighteenth century that lightning was electrical) but not systematically studied. Scientific experiments would pave the way for the telegraph in the 1830s and the generation, transmission, and application of electrical power late in the century (chapter 24). Even more surprisingly, the scientific study of electrical waves would set the stage for radio transmission at the very end of the century (above). This is indeed one of the great examples in history of scientific curiosity having unimagined practical implications.

The discovery early in the century that electrical currents could stimulate chemical reactions supported a theory that electrical charges held chemicals 
together. This, in turn, encouraged the development of atomic theory and, late in the century, the development of the periodic table of elements (in which each chemical element was identified with a specific number of protons). This enhanced understanding of chemistry would also stimulate a range of industrial innovations late in the nineteenth century (again, chapter 24). Artificial dyes would revolutionize textile manufacture from 1856, and similar techniques of investigating the qualities of different chemicals would soon thereafter be applied to pharmaceuticals. (German chemists isolated cocaine from the coca plant in the 1860s and patented it as a medicine; it would gain popularity as a recreational drug thereafter, with mixed consequences for coca farmers in Latin America.)

Improved microscopes allowed the recognition of cells as the building block of all organisms. Whereas biology had before the nineteenth century consisted of atheoretical classifications of species, cell biology (and evolutionary theory, developed late in the nineteenth century; see chapter 1,2 ) would guide a more theoretical investigation of the nature of biological systems. This would support medical advances in the twentieth century (and a new field of biotechnology later in the twentieth century after the nature of DNA was recognized). The first significant development came in 1874 when Louis Pasteur discovered that heating food products could kill disease-causing germs.

Science also gained a new institutional structure. The foundation of the École Polytechnique from 1794 in revolutionary France made France the leading scientific nation for decades; prominent scientists gathered there and taught promising students. German states built upon this idea, founding

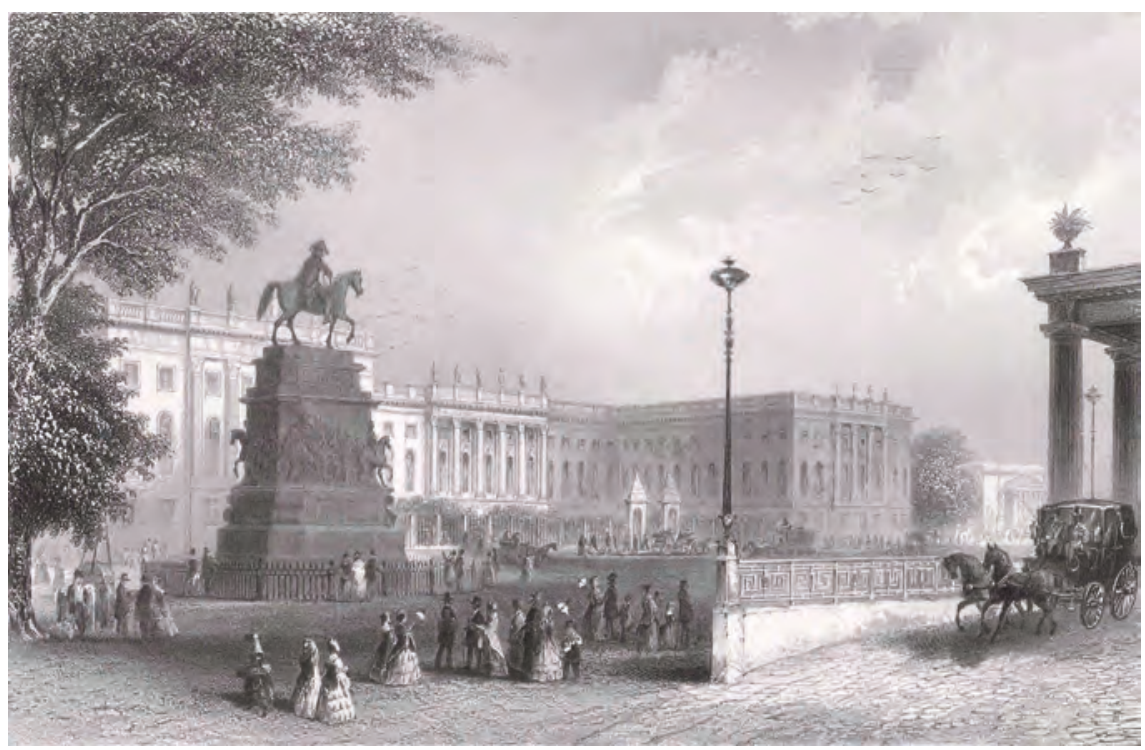

Illustration 23.15

Humboldt University, Berlin, c. 1850 
the modern research university from 1810. Professors in these institutions combined research and teaching. The teaching laboratory was developed, as was the graduate research seminar in which professors and students shared ideas. Though we saw that universities had played a role in the discoveries of the Scientific Revolution (chapter 22), the medieval European university had de-emphasized natural science. Medieval universities also had stressed teaching over research (often teaching classical theories of nature). The research university was so different from preceding universities that there was a discussion of inventing a new word to describe it. German scientists also advised German industry. As this collaboration began to bear fruit, other countries copied the idea of the research university. Still, late in the century American, Japanese, and other students flocked to Germany for the best scientific training available.

Postscript: The United States would copy the idea of the German research university and rise to global dominance in the twentieth century. The German scientific effort would suffer in the aftermath of both world wars, especially the second, as leading scientists moved to both the United States and the Soviet Union.

We will re-encounter German research universities when we discuss the Second Industrial Revolution in chapter 24. We can note here that they played a critical role in building on the discoveries of Pasteur. Robert Koch and his students in north Germany would use microscopes to identify the causal agents of tuberculosis, cholera, typhoid, diphtheria, gonorrhoea, and several other diseases in the late nineteenth and early twentieth centuries. This involved isolating a pathogen associated with some disease and infecting another animal with that pathogen. His student Petri developed the sterilized dish for isolating pathogens that still bears his name. Japanese student Shibasaburo Kitasato discovered the causal agent for bubonic plague. These various efforts reflected a desire for medical practices grounded in evidence. They set the stage for the development of a variety of pharmaceuticals in the twentieth century that would attack these various pathogens.

Research universities are now so familiar that it may be surprising that they are only a couple of centuries old. It is easy also to take the manner of their organization for granted. Since science was opening up exciting new lines of enquiry, each with its own theories and instruments, these new research universities were organized from the start around disciplines. While universities had long taught courses on a variety of subjects, the goal had always been a general education. The scientists we met in chapter 22 had broad training and often explored a wide range of questions. The new research universities stressed disciplinary specialization for both professors and students. They would no longer be generalists but physicists, chemists, or biologists - and increasingly a particular kind of chemist or biologist. The social sciences, too, would split 
into a handful of disciplines in the late nineteenth and early twentieth century: Political economy would become economics and political science, while anthropology and sociology would develop as new lines of enquiry (the ethnographic research of anthropologists, at first in the colonies and Latin America, later among workers at home, attracted great popular attention). Since most early professors relied on student tuition for their income, they each advertised the specialized courses that they could teach. Disciplinary specialization in the growing number of high schools duplicated and reinforced specialization in universities: Many university graduates went on to teach chemistry or physics in high school. The development of specialized education faculties in universities only slowly weakened this link between disciplinary specializations at the two levels.

The disciplinary nature of universities would be matched by the foundation of disciplinary scholarly organizations within and then across countries in the last decades of the nineteenth century and early decades of the twentieth. Physicists would attend conferences with other physicists and publish in scholarly journals edited by physicists. These distinct disciplines would each develop a unique jargon to go with their specialized theories and methods. It became increasingly difficult, therefore, for a chemist to understand a physicist.

This disciplinary specialization would be transcended in another novel institution of the late nineteenth century: the industrial research laboratory (chapter 24). Industrialists financed and organized these laboratories with the express purpose of developing novel technologies. The German chemical and pharmaceutical firm Bayer would establish such a laboratory in 1874; the American electrical innovator Edison would create a laboratory from 1876 that would first work on electric lighting and later develop phonographs and movies. Since scientific input was necessary for innovation in chemistry, electronics, and elsewhere, these laboratories generally employed an interdisciplinary mix of engineers and scientists from multiple disciplines. Engineering, it might be noted, had become an important discipline in its own right, charged with employing scientific theories and methods to identify best practices in the world of technology.

The appreciation within industrial research laboratories that complex realworld challenges called for an interdisciplinary approach would feed back into the university very slowly. To be sure, some educators had decried increasing specialization from the very beginning. There would be many experiments with interdisciplinary teaching programmes within research universities in the early twentieth century. However, it was only in the postwar period that the advantages - and feasibility - of both interdisciplinary teaching and research were widely appreciated. Research universities by then had solidified an administrative structure grounded in disciplines. It has proven no easy task to institutionalize interdisciplinarity. Though interdisciplinary teaching and research programmes are now common (and we discussed some of the advantages of interdisciplinary analysis in chapter 1), university decision-making tends still to be dominated by disciplines. 
The development and worldwide spread of both research universities and industrial research laboratories meant a dramatic increase in the number of scientists in the world. Marked increases in economic productivity allowed societies to support these large numbers (chapters 24, 29). Their training reflected massive investments in public education and research universities. The vast majority of the scientists that have ever lived have lived in the last decades. This increase in the numbers of scientists, and the fact that they had both time and equipment to devote to research, led to a dramatic increase in research output. Moreover, recall that scientific and technological advance is always a collaborative effort. The increased size of the scientific community increased the potential for useful collaboration. One challenge, though, has been keeping track of the flow of information: Scientists must struggle to ensure that they keep abreast of discoveries elsewhere and that others in turn recognize their discoveries.

We can mention a few of the key developments in twentieth-century science here. Some late-nineteenth-century physicists thought that the most important discoveries in their field had all been made, but physics would be revolutionized in the next century by the theory of relativity (mass and energy are related, and time is not absolute), the investigation of subatomic particles and radioactivity (shedding of particles by heavy elements), and quantum theory, which sees particles and waves as not distinct. Nuclear bombs and nuclear power were the most obvious impacts but nuclear medicine has been important, and there are potential implications in electronics and computing. The discovery of genes and the decoding of DNA in the late twentieth century advanced biological understanding and created a whole biotechnology sector. Much of chapters 2 and 3 in this book were possible only because of advances in cosmological and biological understandings in the twentieth century.

Scientific discoveries have been concentrated in those countries that could best finance research universities and industrial research laboratories. Only very late in the nineteenth century did the idea of the research university spread much beyond Europe and former European settler colonies. Nevertheless, there have always been important contributions from other countries: Charles Darwin interacted a great deal with scientists in Chile as he developed his theory of evolution. Moreover, the leading universities and laboratories have attracted people from throughout the world. There are thousands of Indian scientists and engineers in Silicon Valley in California, for example. As more countries have financed research universities, the location of scientific discovery has become more diffuse.

We noted in chapter 9 the importance of libraries for sustained scientific and philosophical advance. In the nineteenth century, national libraries, archives, and museums were widely seen as a symbol of a modern nation state. The Louvre had become a public museum during the French Revolution, and other countries soon copied this idea - with colonial powers filling their museums with artefacts from their colonies. There was less publishing and therefore fewer and smaller libraries in the Islamic world - a dramatic change from 


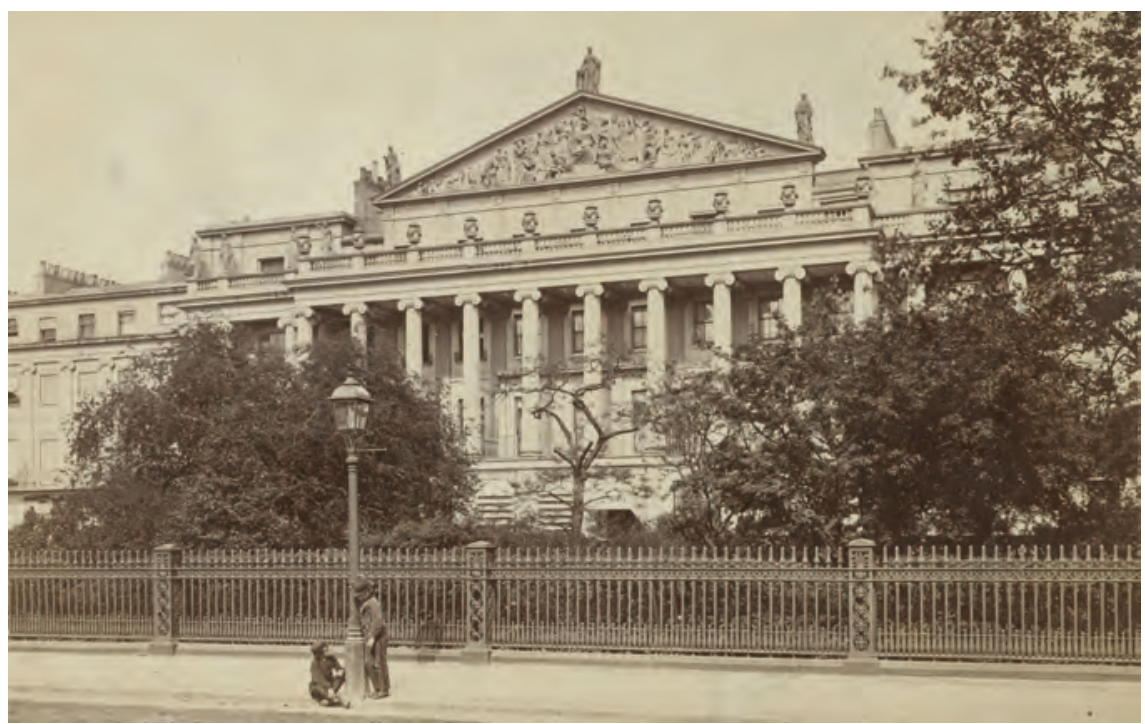

Illustration 23.16

The British Museum, between 1870 and 1900, Rijksmuseum, CC0

earlier centuries in which the Islamic world housed the greatest libraries in the world (chapter 12). Though nationalism inspired these new national libraries and museums, they tended to collect globally. Charities and local governments developed local libraries and museums as well. The new research universities developed libraries for the use of instructors and students; these had to cope with an increasing volume of scientific publications. Both scientists and the public gained better access to information through these various developments. As the number and size of these institutions increased, specialized professions of librarian, archivist, and curator emerged, each with their own formal educational expectations.

Postscript: Museums around the world face demands from former colonies and from Indigenous people for the return of artefacts taken in previous eras of power imbalance. Many such items have been returned. However, concerns are often voiced about whether poor communities have the resources to care for such artefacts. There have been unfortunate cases of theft and vandalism in many countries. There is a larger issue of how we in the present day should address past injustices.

There had been a long tradition of archiving in the Ottoman Empire and especially China. Yet new dynasties in China often destroyed the records of previous dynasties once their official histories were written. State library collections were destroyed and rebuilt some fourteen times between the Han and the nineteenth century. Other regions of the world lacked any 
sustained attempt to maintain either archives or libraries. These institutions are today expected to be permanent in much of the world. Moreover, digitization may provide a further source of confidence that written records will be retained in perpetuity.

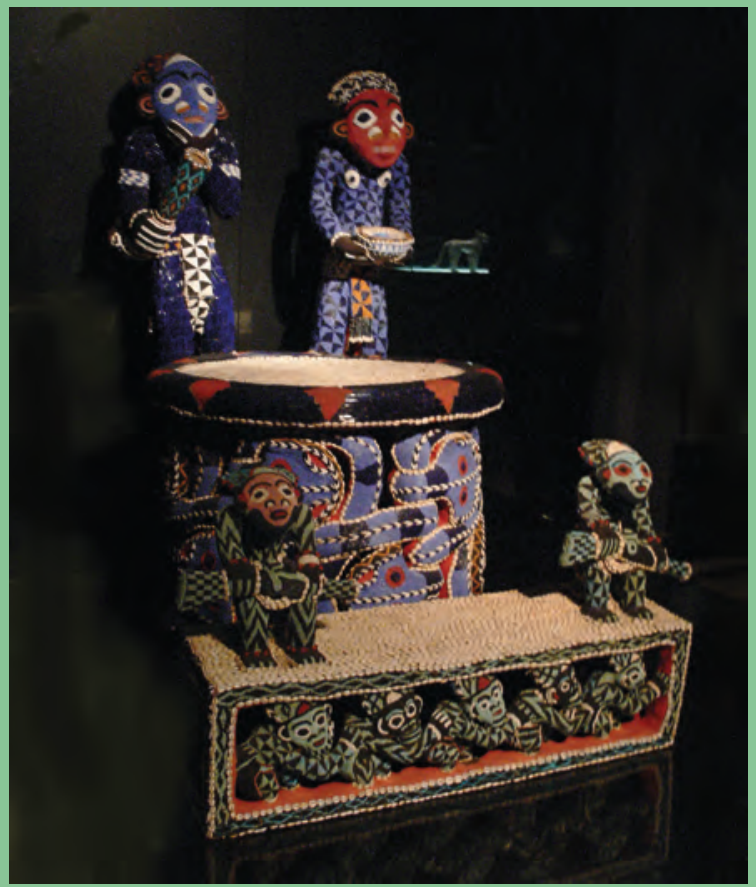

Illustration 23.17

Royal throne mandu yenu, Bamum, Cameroon, 19th century, Ethnological Museum, Berlin

\section{Agriculture}

Economy

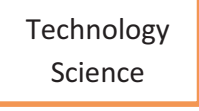

Politics

A variety of advances in science, transport, and industry would increase agricultural productivity over the nineteenth century, feeding the increased population of that century (chapter 31 ). A gradually warming climate aided agricultural output.

Guano is a corruption of the Inca word "huanu." The Inca had used these bird droppings from islands off the Peruvian coast as fertilizer for centuries. In the 1830s, scientific understanding of plant growth combined with reduced transport costs and increased population to create a market in Europe for guano 


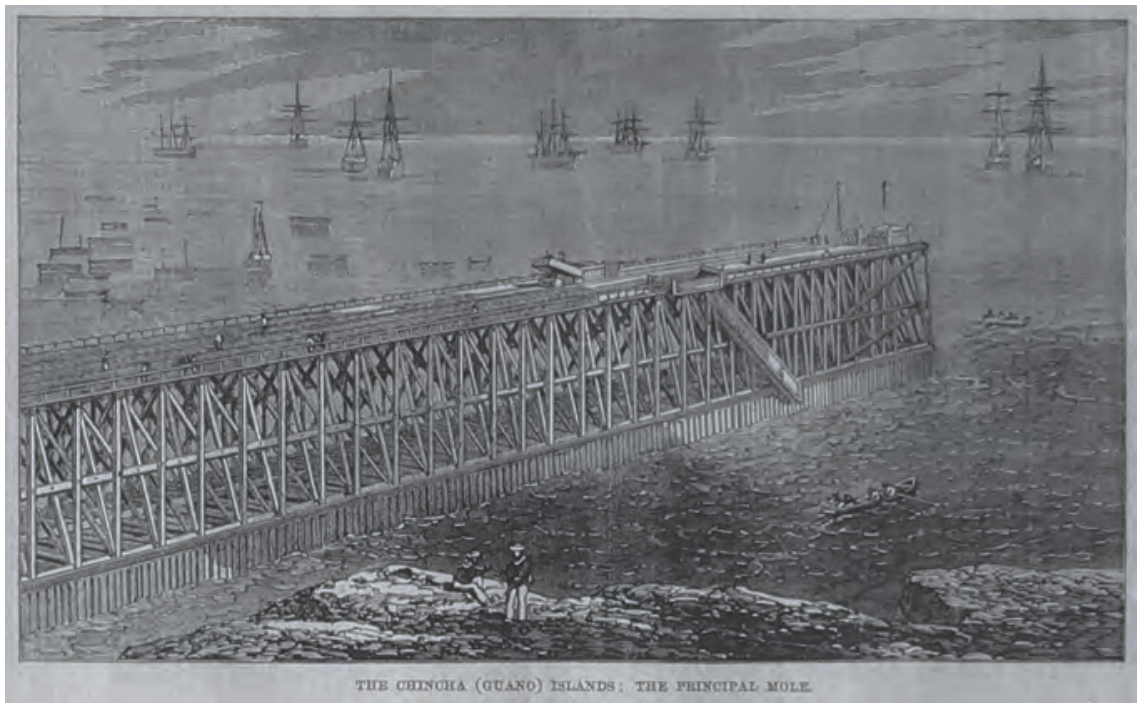

Illustration 23.18

Loading Guano, The Illustrated London News, February, 21, 1863

from off the South American west coast. The Peruvian government earned significant royalties from guano exports (though working conditions were decried), but defaulted in 1876 when guano exports fell.

Europeans turned to nitrates on the border between Peru and Chile. Chile won a war in 1879-83 for control of these - a rare war among Latin American nation states. At the end of the century, phosphate deposits were discovered in the Pacific Island of Nauru; these would make Nauru one of the wealthiest places on earth for a while - though the island faced problems as the resource ran out around 2000.

Increased scientific understanding of the role of nitrogen, phosphate, and other chemicals in agriculture stimulated a search for synthetic fertilizers. In the very early twentieth century, Fritz Haber, a professor at a German research university in Karlsruhe, developed methods for fixing nitrogen from the air. There would be many other developments in synthetic fertilizers and also pesticides and herbicides.

As industrial capabilities expanded (chapter 24), a set of improved agricultural implements was developed. Better seed drills allowed seeds to be distributed evenly. Reapers allowed farmers to harvest a much more extensive crop than they could by hand. These implements were at first pulled by animals but would in the twentieth century be wedded to internal-combustion tractors.

We mentioned advances in refrigeration above. There were other advances in food preservation. Napoleon had offered a prize for superior means of feeding an army on the move. The winner recognized that foods did not spoil if tightly sealed. A canning industry would develop with improved seals and coatings 
that did not interfere with taste. Aided by Pasteur's understandings late in the century, it became increasingly possible to transport foods long distances and store them for months or years. (Freeze-drying from the early twentieth century had even better implications for the taste and texture of stored foods.) Although not strictly agricultural innovations, these developments had a significant effect on the quantity and variety of foods available to consumers.

There were also important institutional developments. Large companies emerged to buy and transport agricultural goods. They sometimes engaged in production - as in the plantations run in tropical countries by fruit companies. Exchanges for commodity trading developed in several countries. Finally, the number of restaurants in the world proliferated as incomes rose and workers gained free time.

\section{Questions}

1. What is your favourite empire in world history? You can evaluate empires in terms of how interesting they were or what effects they had. Or do you think empires are evil?

2. We have explored the nature of technological innovation in other chapters. Would humans have developed synthetic rubber if not familiar with natural rubber?

3. Diagram the influences on and effects of either the industrial working class or the middle class.

4. Could research universities have developed in a non-specialized manner as industrial research laboratories did? What would such universities have looked like?

5. What special challenges faced colonial bureaucrats (local or from the colonial power) in this period?

\section{Readings}

Bell, Duncan. 2016. Reordering the World: Essays on Liberalism and Empire. Princeton University Press.

Benton, Lauren, and Richard J. Ross, eds. 2013. Legal Pluralism and Empires, 15001850. New York University Press. [This book traces the importance of intermediaries between imperial ruler and ruled such as magistrates, local or religious elites, and bureaucrats (and the legal institutions in which they operated); the intent is to show how these exerted agency.]

Crowley, John E. 2011. Imperial Landscapes: Britain's Global Visual Culture, 17451820. New Haven, CT: Yale University Press. [Discusses how European artists painted colonial landscapes for home audiences; argues this was a primary reason for increased interest in landscape painting.]

James, Lawrence. 2017. Empires in the Sun: The Struggle for the Mastery of Africa. New York: Pegasus. 
Maurer, Noel, and Carlos Yu. 2011. The Big Ditch: How America Took, Built, Ran, and Ultimately Gave Away the Panama Canal. Princeton University Press.

Miran, Jonathan. 2009. Red Sea Citizens: Cosmopolitan Society and Cultural Change in Massawa. Bloomington, IN: Indiana University Press. [This book documents the cosmopolitan nature of trading centres. This city in modern Eritrea was more closely linked to the Islamic world than to Ethiopia.]

Ogle, Vanessa. 2015. The Global Transformation of Time 1870-1950. Cambridge, MA: Harvard University Press. [Addresses the transformations in Box 23.2.]

Osterhammel, Jürgen. 2014. The Transformation of the World: A Global History of the Nineteenth Century. Princeton University Press.

Repko, Allen F., and Rick Szostak. 2020. Interdisciplinary Research: Process and Theory. 4th ed. Thousand Oaks, CA: Sage. [This book discusses some of the history of disciplines and interdisciplinarity - see also their Introduction to Interdisciplinary Studies 3rd ed., 2020, with Michelle Buchberger - and identifies best practices for interdisciplinary research and teaching.]

Rizzo, Tracy, and Steven Gerontakis. 2017. Intimate Empires: Body, Race, and Gender in the Modern World. Oxford University Press.

Ross, Corey. 2017. Ecology and Power in the Age of Empire: Europe and the Transformation of the Tropical World. Oxford University Press.

Tully, John. 2011. The Devil's Milk: A Social History of Rubber. New York: Monthly Review Press.

Ward, Kerry. 2008. Networks of Empire: Forced Migration in the Dutch East India Company. New York: Cambridge University Press.

There was a forum in the Journal of Global History in March 2015 on mining, and one in July 2016 devoted to the cultural life of ships. A special issue of the Journal of Global History in July, 2015, explored the relationship between capitalism and the communications revolution. It traced how businesses and governments collaborated in developing transport infrastructure. Another forum in the Journal of Global History in March 2016 addressed botany and noted that there was an extensive international exchange of biological specimens from the early nineteenth century. A special issue of the Journal of Global History in July 2013 examined the international diffusion of sports. There was a forum in the Journal of World History in September 2015 on networks within the British Empire. [The Journal of Global History tends to focus on the last two centuries (reflecting a guiding interest in "globalization") whereas the Journal of World History addresses all time periods.] 


\section{Industrial revolutions}

\section{Innovation, factories, and economic growth}

\section{Guiding questions}

What was the Industrial Revolution? Why, when, where, and how did it occur? How and why were the main elements of the Industrial Revolution transmitted across countries and regions (and when were they transmitted where)? What was the Second Industrial Revolution?

What were some of the key effects of the Industrial Revolution?

On regional income differentials? On population growth? On society more generally?

Relationship to other chapters: This chapter connects to chapter 22 as both address the divergence in economic performance between Europe and the rest of the world in the nineteenth century. This chapter has a narrower temporal focus and looks at particular countries more than did chapter 22. As in chapter 22, it draws on many preceding discussions of economic growth, institutions, and other phenomena. The Industrial Revolution would have many impacts, but most obviously it would generate (almost) unprecedented rates of economic growth. The effects of the Industrial Revolution reverberate throughout the rest of the book, but most obviously when we address economic and technological developments in the twentieth century in chapter 29, and when we look at urbanization in chapter 31 and environmental issues in chapter 27. Economic growth would free humanity from Malthusian constraints but 
encourage fertility control; we resume our discussion of population trends in chapter 31.

We trace in this chapter the spread of "industrial revolutions" across the globe. We shall see that the main characteristics of these revolutions are rapid technological innovation, the introduction of factories, and unprecedented (as far as we know) rates of economic growth. We will draw comparisons across states and regions to comprehend why industrial revolutions occurred in some places before others. We will also discuss how certain technologies, and the institution of the factory, built on what had gone before. Although we focus our attention on economy and technology, we will find that politics much influenced these, as did the environment (both natural and built), and culture. Later in the chapter, we will discuss effects on population and culture (and social structure).
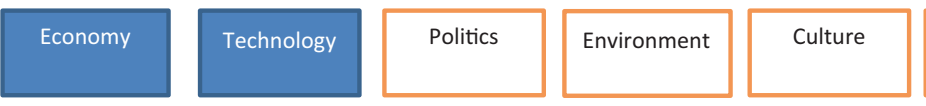

Population

\section{The British Industrial Revolution}

As we noted in chapter 22, there has been an unfortunate ideological element in discussions of the "Great Divergence." The same is unfortunately true of treatments of the British Industrial Revolution. We saw in chapter 22 that economic growth rates became much higher in the nineteenth century than they had (almost) ever been before. The world would be transformed by unprecedented economic growth in the nineteenth and twentieth centuries, with some regions of the world growing much faster than others. Some scholars view the British Industrial Revolution from 1750 as having triggered the nineteenthcentury acceleration in growth rates, and as therefore being equivalent in importance to the Agricultural Revolution that we discussed back in chapter 4 . Other scholars argue that nothing particularly noteworthy happened in Britain in the century after 1750 . As in the broader "Great Divergence" debate, they wish to downplay the importance of any development unique to (part of) Europe.

The reader should recall that the agricultural revolution occurred independently in many places. There were, that is, multiple agricultural revolutions. But the world had become much more interconnected by 1750 . It may be (or not) that many countries were ready for an independent industrial revolution soon after 1750. However, all later industrializers would borrow ideas from the British directly or indirectly. If we accept that the Industrial Revolution was historically important, we must recognize that there was only one First Industrial Revolution. It is quite possible that later episodes of industrialization in other countries would not have occurred without the British example - just 


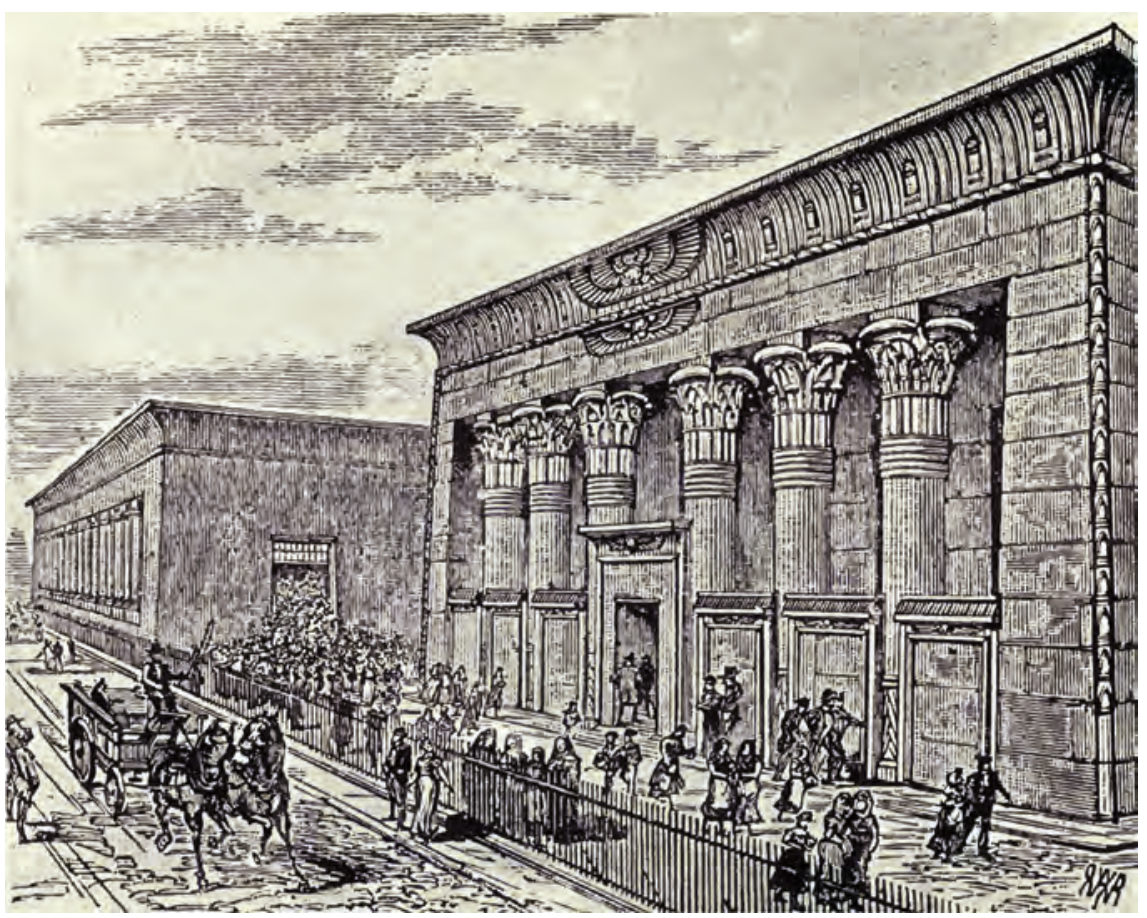

Illustration 24.1

Marshall's flax mill, Holbeck, Leeds c. 1800, from The Story of Leeds by J.S. Fletcher, 1919

as the idea of agriculture spread geographically after the original agricultural revolutions.

Arguments in favour of an important British Industrial Revolution generally point to three characteristics of the century after 1750 in Britain:

- $\quad$ The emergence of the modern factory. There had of course been centralized workplaces previously in history: Shipbuilding and sugar refining and pottery kilns were among the industrial processes that required gathering many workers in one place. Moreover, rulers throughout the world had often advertised their wealth and encouraged the arts through workshops focused on luxury good production. However, in the decades after 1750 many British entrepreneurs gathered dozens or hundreds of workers together in one place to produce textiles or metal goods that for centuries had been produced in homes or small artisanal workshops. Such centralized workplaces would grow in importance over time, first in Britain and then throughout the world. We indeed tend to think in the twentyfirst century that "industry" is something that happens in "factories." We should appreciate that this is instead a - revolutionary - transformation of the last centuries with its roots in mid-eighteenth-century Britain. Factories transformed the relationship between employers and employees, not to 
mention the lives of the working class and an emerging managerial class (chapter 23). Factory labour was generally, though not always, supervised, whereas workers in their own homes naturally were not. Among other things, workers (and managers) were now required to adhere to a strict schedule.

- An increase in the rate of technological innovation. This is of course somewhat harder to measure. It is easy to point to many significant technological innovations. The improved steam engine associated with James Watt in the 1780s would become over time a vital power source for both factories and transport. It would allow the development of railways and steamships in the early nineteenth century. There were a set of discoveries (and marketing plans) associated with Josiah Wedgwood from the 1770s that allowed the mass production of quality ceramics. Several innovations in textile spinning, weaving, dyeing, and printing from the 1770 s dramatically lowered the cost while increasing the quality of first cotton and then woollen and linen textiles. A set of developments in the refining and processing of wrought iron and steel also lowered prices while increasing quality. There is also evidence from the British patent office of a general increase in innovative activity from about 1770 . The rate of innovation would continue to expand globally over the next centuries. The British Industrial Revolution serves then as a watershed moment in human history. For most of the previous history of humanity (with perhaps rare exceptions such as Song China; chapter 15), technological innovation was rare enough that people generally died in a world that looked much like the world of their birth. The Industrial Revolution introduces the modern era where technological innovation is frequent, and we have come to expect major transformations in the world around us.

- An increase in economic growth rates. For Britain as a whole, economic growth of about 3 per cent per year became common after about 1820 . If we focused our analysis on regions in which industry came to concentrate, such as Lancashire with its cotton mills, we would likely find increased economic growth rates in the later eighteenth century. As the ideas of the British Industrial Revolution spread to other lands, growth rates of 3 per cent or higher were observed at first in a handful of countries in the mid nineteenth century (see below); by the late twentieth century every country in the world had experienced at least some years of rapid economic growth. Global economic output would increase by eight times in the nineteenth century (versus perhaps five times in the previous millennium). Importantly, economic growth now occurred faster than population growth, and so the Malthusian population dynamics we first addressed in chapter 4 ceased to operate: Humans could now continually increase output per person. World population roughly doubled in the nineteenth century, a rate of growth that would have driven incomes below subsistence in preceding centuries but instead was dwarfed by economic growth. It is one of the ironies of history that Malthus expounded his 
theory in Britain just before economic growth accelerated. As with factories and technological innovation, the British Industrial Revolution stands as an inflection point between a human past in which economic growth was sluggish at best and a lengthy period of economic growth that has touched every corner of the world, albeit unequally.

There were other interesting characteristics of the Industrial Revolution. As noted above, industrial activities became concentrated in particular regions: cotton in Lancashire, wool in Yorkshire, pottery in Staffordshire, many metal goods in the area around Birmingham (a small town in 1750 transformed within decades into a major industrial centre). In addition, specialized firms came increasingly to build industrial machinery rather than workers themselves making their own. We will address these characteristics briefly below. However, scholars have devoted most attention to explaining the three characteristics listed above.

We should not move on without stressing that the Industrial Revolution was not without its costs. Early factories, and especially industrial cities, were heavily polluted. The average heights of the British fell in the early nineteenth century, indicating that they were suffering from disease or undernourishment or perhaps overwork as children. Many workers lost their jobs as machines replaced them. The standard of living of the average worker likely fell for a couple of decades before rising. Factories existed for decades before Parliament introduced basic health and safety regulations. It was well into the nineteenth century before unions were legalized to protest abuses of managerial power.

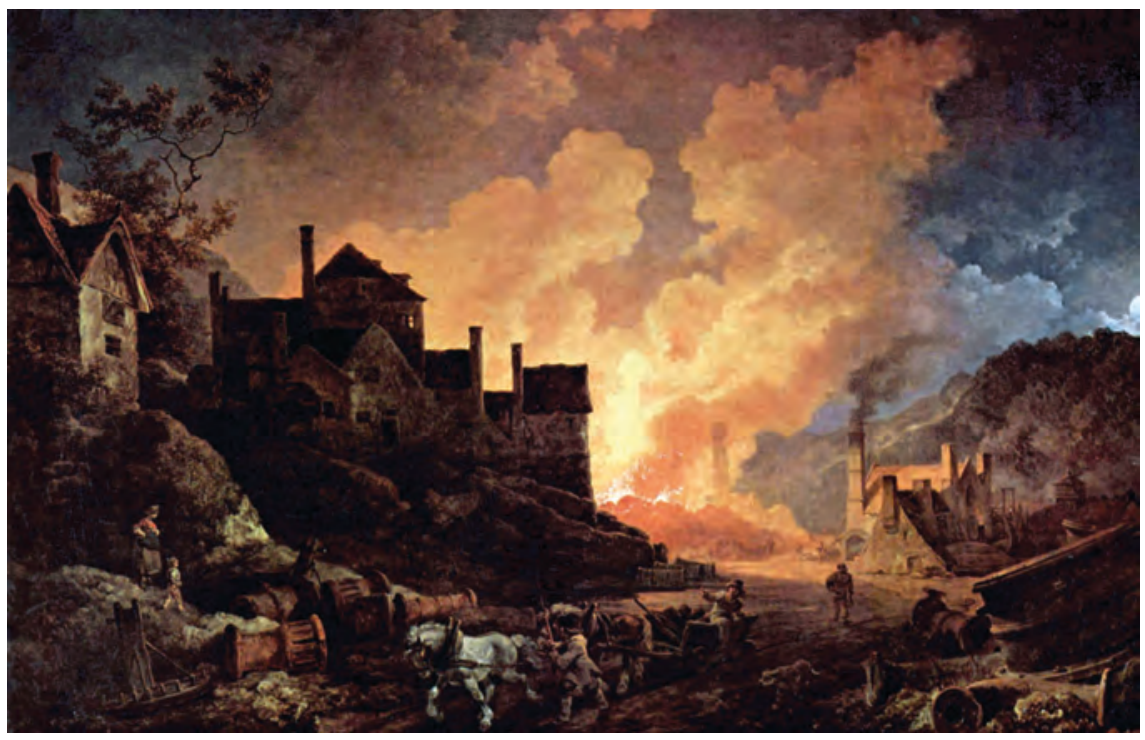

Illustration 24.2

Coalbrookdale by Night, Iron production at Coalbrookdale, Shropshire (Madely Furnaces), by Philipp Jakob Loutherbourg, 1801 
As we shall see below, many workers came to perform one simple repetitive task: This sort of industrial boredom was fairly novel in history, but would become common worldwide in the next centuries.

\section{PRIMARY DOCUMENTS: [PETITION] TO THE MERCHANTS, CLOTHIERS AND ALL SUCH AS WISH WELL TO THE STAPLE MANUFACTORY OF THIS NATION, 1784.}

The Humble ADDRESS and PETITION of Thousands, who labour in the Cloth Manufactory.

SHEWETH, That the Scribbling-Machines have thrown thousands of your petitioners out of employ, whereby they are brought into great distress, and are not able to procure a maintenance for their families, and deprived them of the opportunity of bringing up their children to labour: We have therefore to request, that prejudice and self-interest may be laid aside, and that you may pay that attention to the following facts, which the nature of the case requires.

The number of Scribbling-Machines extending about seventeen miles south-west of LEEDS, exceed all belief, being no less than one hundred and seventy! and as each machine will do as much work in twelve hours, as ten men can in that time do by hand, (speaking within bounds) and they working night-and day, one machine will do as much work in one day as would otherwise employ twenty men.

How are those men, thus thrown out of employ to provide for their families; - and what are they to put their children apprentice to, that the rising generation may have something to keep them at work, in order that they may not be like vagabonds strolling about in idleness? Some say, Begin and learn some other business. - Suppose we do; who will maintain our families, while we undertake the arduous task; and when we have learned it, how do we know we shall be any better for all our pains; for by the time we have served our second apprenticeship, another machine may arise, which may take away that business also; so that our families, being half pined while we are learning how to provide them with bread, will be wholly so during the period of our third apprenticeship.

But what are our children to do; are they to be brought up in idleness? Indeed as things are, it is no wonder to hear of so many executions; for our parts, though we may be thought illiterate men, our conceptions are, that bringing children up to industry, and keeping them employed, is the way to keep them from falling into those crimes, which an idle habit naturally leads to.

These things impartially considered will we hope, be strong advocates in our favour; and we conceive that men of sense, religion and humanity, will be satisfied of the reasonableness, as well as necessity of this address, and that their own feelings will urge them to espouse the cause of us and our families.

Do we hear similar complaints today about technology? Why or why not? What do you think of the workers' petition? 


\section{Relationships among factories, technology, and economic growth}

It seems unlikely that the three key transformations identified above were unrelated. Historians have often argued that new technologies encouraged the development of factories. It was impracticable to place a steam engine or a giant spinning machine with hundreds of spindles into someone's home. This dynamic surely becomes important from the late eighteenth century, and remains important to this day: We associate industry with factories in large part because we think of large machines. However, the very earliest centralized workshops emerged from the 1750s, decades before the revolution in industrial machinery. They employed at first the same technology as was used in people's homes. Therefore, there must have been some other reason for factories (see below). Moreover, once these were in place, they encouraged technological innovation. Much of the innovation of the Industrial Revolution involves connecting machines together and attaching these to an external power source. Innovators were unlikely to develop factory technology when industry was pursued in workers' homes and workers powered their own machines. Once factories became common, innovators naturally turned their attention to technologies well suited to this new environment. These technologies would

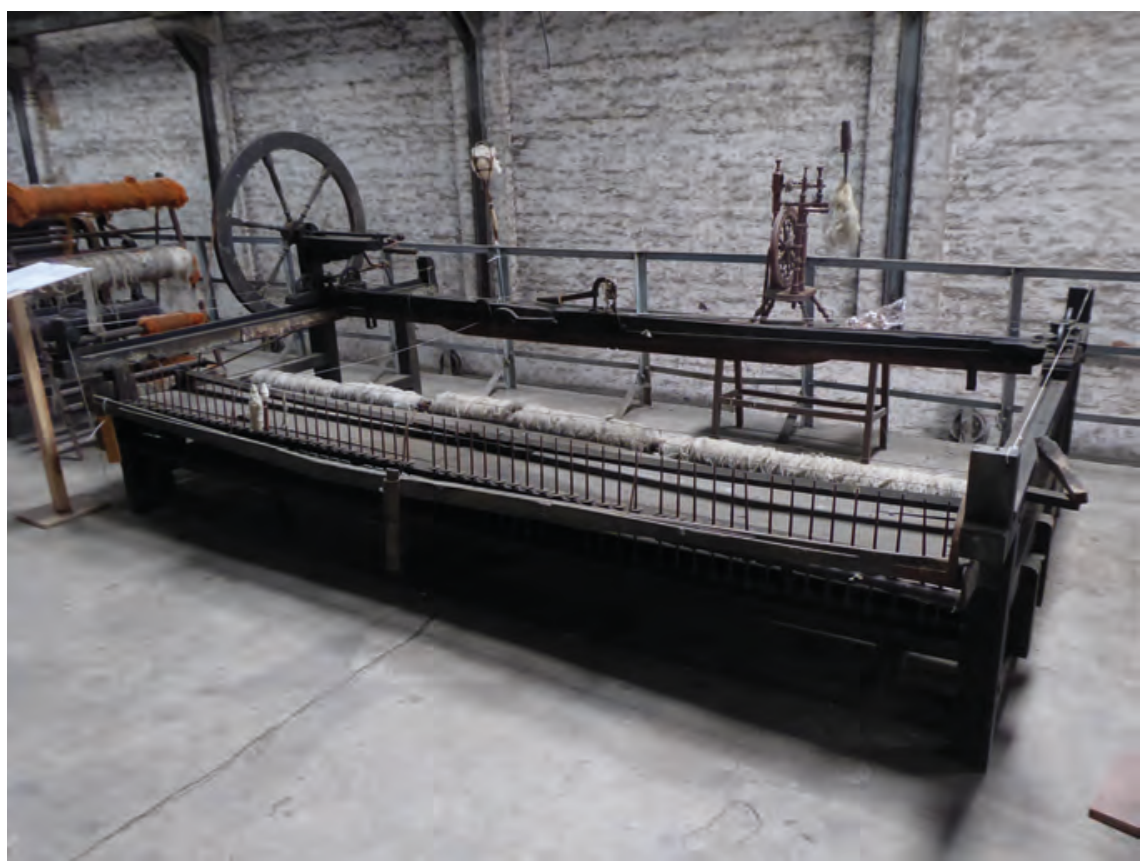

Illustration 24.3

Crompton's Mule, about 1800. One of the key advances in spinning technology, it was designed for use in factories, Bolton Museum and Archive Service

Photograph by Okapi07, CC BY-SA 4.0 
then further encourage the use of factories. We then have a dynamic where factories and innovation encourage each other.

Factories and new technology then combined to increase industrial productivity. Increased productivity encouraged increased per capita incomes. However, this took a while: Factories emerge from the 1750s, and technological innovation accelerates from the 1770s, but per capita incomes rise only from the 1820s. Why does it take decades for factories and increased technological innovation to affect (our estimates of) British levels of per capita output? The main answer is that it took a long time for these ideas to spread. Steam engines provided less than one per cent of industrial power in Britain in 1800. The vast bulk of the industrial labour force still worked at home - though work at home increasingly supplied inputs to work in factories. Only as factories were built and new technologies adopted could per capita incomes rise. As noted above, this likely occurred well before 1820 in regions of industrial concentration. The fact that Britain was at war with France through much of the period of the French Revolution and Napoleon (1789-1815; see chapter 25) may also have

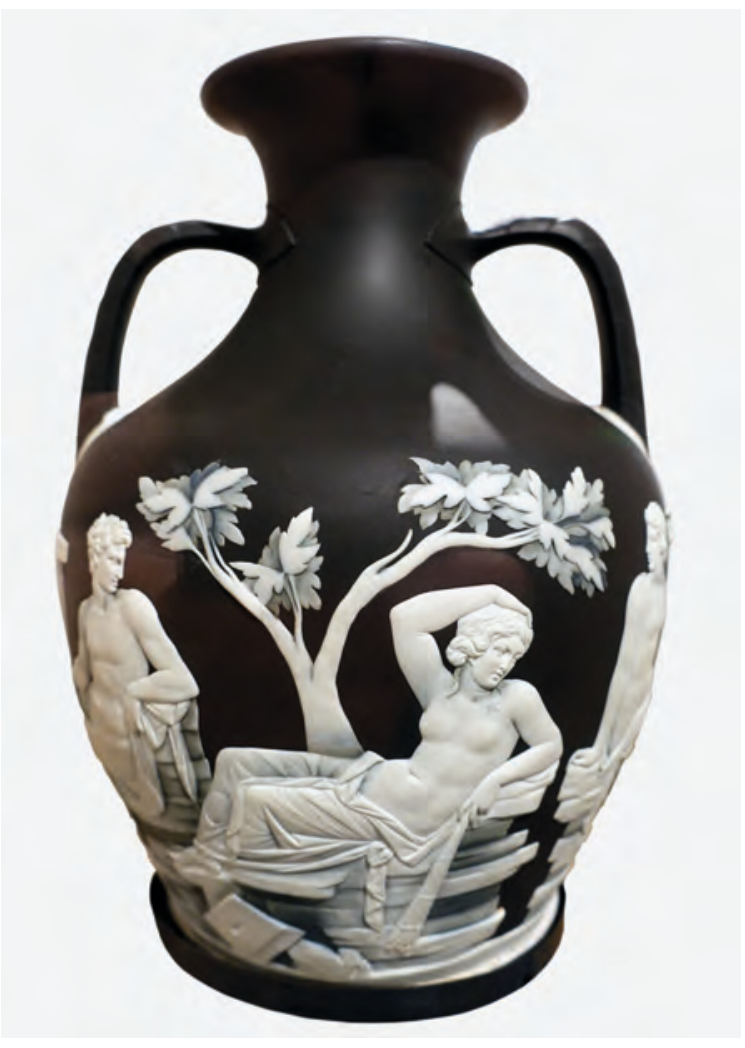

Illustration 24.4

Portland vase, about 1795, copy of classical vase by Josiah Wedgwood and Sons Ltd., Fogg Art Museum, Harvard

Photograph by Daderot, CC0 served to delay the eventual increase in per capita incomes.

Economic growth, once it began to accelerate, was self-reinforcing in important ways. Industrialists reinvested their profits into bigger factories and more complex technology. As we shall see, this would create a challenge for countries trying to catch up to Britain, for they had to leap to a much more expensive technology and more complex workplace environment.

\section{Why? The European context}

We discussed in Chapter 22 the timing and reasons for European economic divergence from Asia. If one thinks that Europe had developed better technologies or institutions or cultural attitudes before 1750, then the British Industrial Revolution likely owes much to these general European advantages. If one thinks instead that Europe only pulled ahead in the nineteenth century, then the British Industrial Revolution becomes potentially a cause more than an effect of the Great Divergence. We will not repeat the discussion of chapter 22 in this chapter 
but instead focus on Britain-specific influences on industrialization. We will note from time to time how these connect to issues discussed in chapter 22.

We should stress, of course, that Britain and Europe did not operate in isolation from trends elsewhere in the world. In particular, we might note that Indian cottons and Chinese porcelain had established in the previous centuries that there was a large, growing market for these goods in particular, and consumer goods more generally. Josiah Wedgwood, the well-known pottery producer of the Industrial Revolution, was undoubtedly quite conscious of the Chinese example. It may be that Asian exemplars inspired many more British entrepreneurs. We also noted in chapter 18 that imports of coffee, tea, and cocoa may have inspired Europeans to work harder in order to afford such goods; imports of cotton and porcelain may have had a similar impact.

\section{Politics and property rights}

We have seen in chapter 21 that Britain was one of the first countries in the world in which an elected assembly with an extensive electorate gained authority over matters of taxation and law. Since landowners and merchants dominated Parliament, that body naturally stressed protection of property and adjudication of contract disputes. Such protections may have encouraged investments in factories and machines, as well as the internal and external trade that created markets for industrial products. Britain was also one of the first states to establish a patent office - but in practice, innovators during the Industrial Revolution often had great difficulty enforcing patent rights. When workers agitated against the adverse effect that some new technologies had on their jobs, Parliament strongly supported the rights of industrialists to employ whatever techniques they chose - fourteen workers were hanged in 1813 for destroying machines. Parliament only belatedly moved to limit child labour in factories. Autocratic governments elsewhere might have been far less enthused about such potentially destabilizing social change. Still, the legal situation of British industrialists may not have been that much better than elsewhere: As we have seen, autocrats could often provide reasonably secure property rights. Moreover, a century elapsed between the Glorious Revolution (in which Parliament solidified power) and Industrial Revolution. It is perhaps unlikely then that Parliamentary authority alone induced the Industrial Revolution.

\section{The British Empire}

We noted above that the developments observed in factories, technology, and economic growth were unlikely to be coincidental. We might apply the same argument to the twin facts that Britain industrializes while developing a vast global empire. In a World History text such as this, it is all too tempting to attribute the British Industrial Revolution to the British Empire. However, it proves difficult in practice to attribute the former to the latter. Most importantly, there is a timing problem: British expansion in the Americas long 
preceded the Industrial Revolution, and Britain lost its major colonies there in the American Revolution (chapter 25) just as the Industrial Revolution was beginning. British expansion in Asia and especially Africa mostly occurred well after the Industrial Revolution. British superiority in steamships and weaponry was critical to this later imperialism: It is thus far easier to trace a connection from industrialization to empire than the reverse.

One mechanism by which imperialism might have supported industrialization is through profits. Britons might have made extraordinary profits in the Empire and reinvested these in industry. While some Britons did make vast sums in the Empire, it is not clear that average returns were ever much, if at all, higher than investment returns at home or elsewhere in Europe. Moreover, those who invested abroad were not the same people that invested in industry: Industrial firms grew as profits in industry were reinvested. There may be an indirect link, though, if foreign profits were an important source of investment in the transport infrastructure that supported industrialization (see below).

Foreign markets might also have been important. To be sure, Caribbean slave plantations provided a mass market for the least expensive textiles - cotton being preferred for working in the heat. Nevertheless, most early British industrialists sold the bulk of their output within Britain. Exports comprised less than 20 per cent of industrial production at the beginning of the Industrial Revolution, though this figure rose to 40 per cent in 1800. (Cotton manufacturers benefited from British restrictions on the import of cotton cloth from India.) Likewise, industry obtained most raw materials locally. Britain imported iron ore in large quantities, but from Scandinavia rather than the Empire. Raw cotton

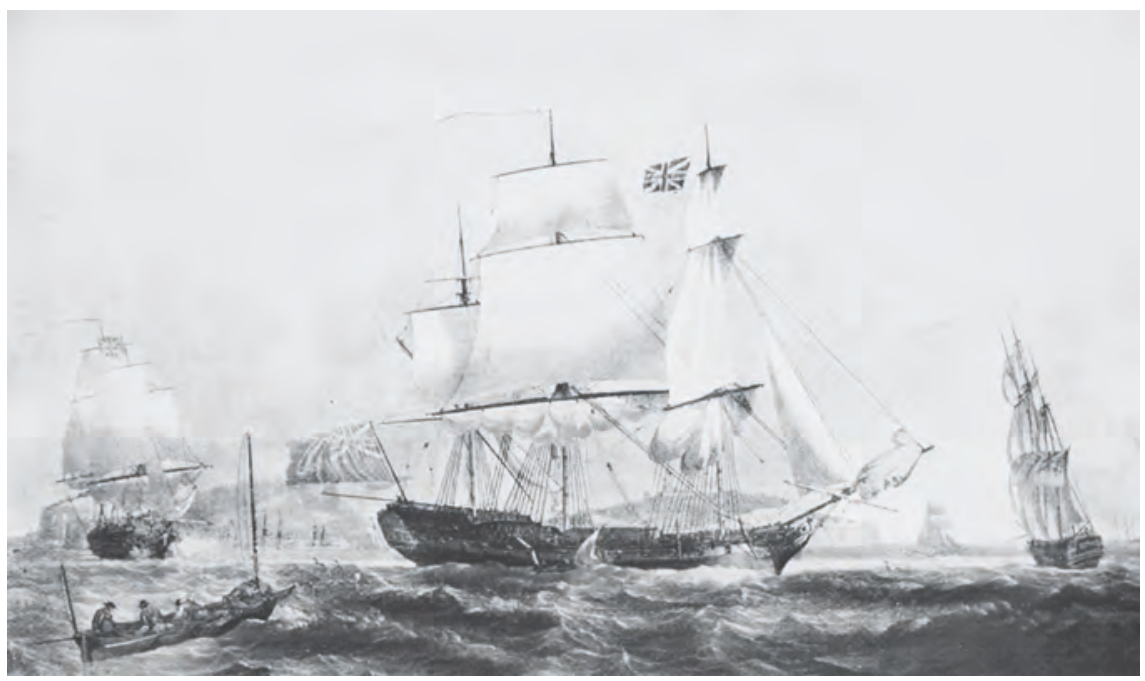

Illustration 24.5

An East Indiaman, 1788, Arthur Hamilton Clark 
was imported, and the cotton industry was the first to be transformed by new technology and factories. However, the primary source of cotton was the United States. Overall, foreign trade as a whole, within or beyond the Empire, accounted for just a few per cent of British economic activity.

Food deserves special attention. As we have stressed throughout this book, societies can only support a sizeable non-agricultural population if they can feed these. Agricultural productivity did advance in Britain - though again more just before and after the Industrial Revolution than during, it seems. British farmers were able to focus increasingly on producing food since coal was replacing wood as a fuel and imported cotton substituted for local fibres such as wool. In the early decades of the Industrial Revolution, Britain provided most but far from all of the food needed by its industrial workforce. As the Industrial Revolution proceeded, Britain imported food in large amounts from Ireland and later from North America, Australia, and India. The Industrial Revolution would surely have slowed if not stopped but for this ability to import food. Note, though, that much of this food came from outside the Empire.

\section{Transport}

Ideally, we wish to identify a unique characteristic of Britain that itself changed in important ways just before or during the Industrial Revolution and that exerted clear influences on one or more of factories, innovation, and economic growth. One candidate here is the British internal transport system (the subject, the author confesses, of his $\mathrm{PhD}$ dissertation and first book). Recall that in the pre-railway era both land and water transport were important: Water transport was much less costly, but land transport was quicker, more reliable, and far more extensive (Box 5.3). In 1750, Britain had the best internal network of both roads and waterways in the world. This network had improved dramatically in the previous century. Parliament had approved hundreds of acts that allowed turnpike road, river improvement, and canal companies to do three critical things: expropriate land if necessary (with compensation decided by a jury if there was disagreement), borrow money to finance construction, and then charge tolls to pay off the debt and finance maintenance. By 1770 at the latest, England had a national network of roads suitable for wheeled traffic that linked virtually every town (recall that horses can pull several times as much in a wagon as they can carry on their backs). Navigable river mileage had doubled in the century before 1750; in succeeding decades, canals would link these rivers to connect major raw material deposits to industrial areas and urban markets. Autocratic governments elsewhere in the world hesitated to interfere with the landholdings of local elites. Ironically then, British confidence in property rights may have exerted their most significant effect by allowing forced confiscation of property. Governments elsewhere also struggled to finance infrastructure investment: Loans and then tolls instead funded British transport infrastructure. 


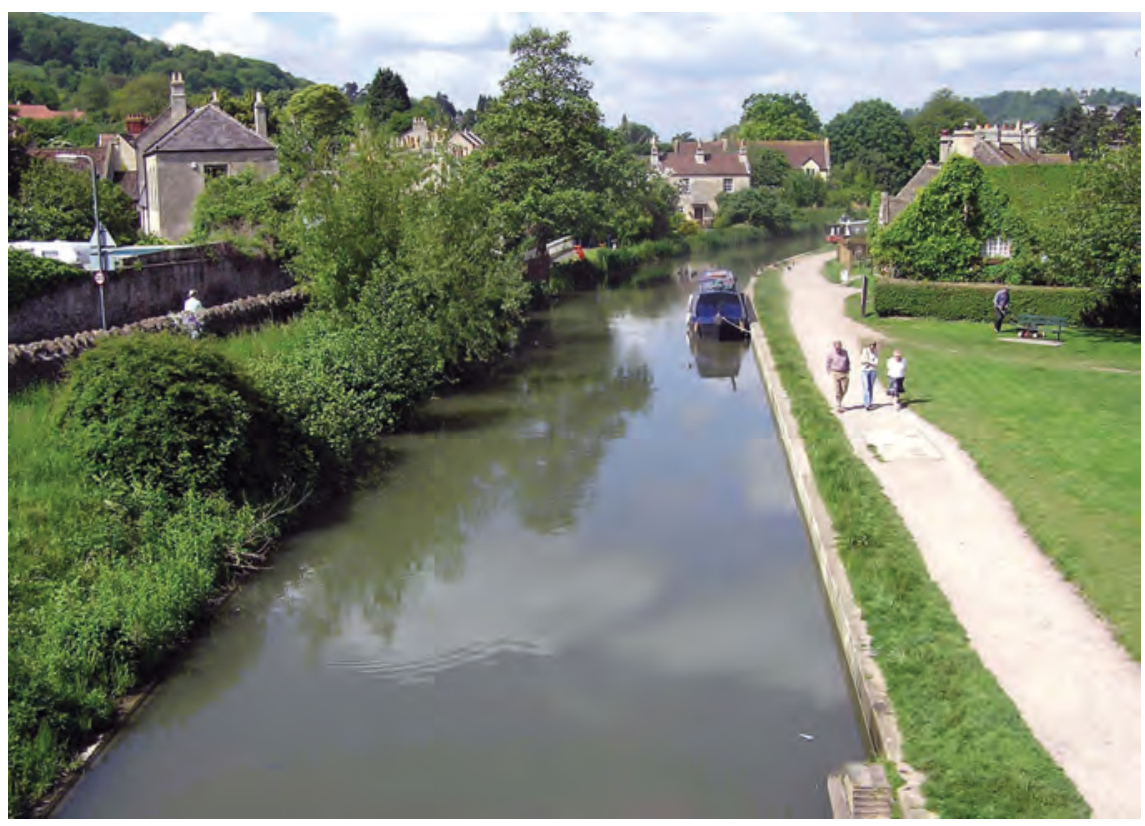

Illustration 24.6

Kennet and Avon Canal at Bathampton, built in the early 19th century

Turnpike trusts and river and canal companies all took a corporate form. Corporations were by this time familiar in Britain (Box 15.1). Yet families rather than corporations financed factories during the early Industrial Revolution. However, the widespread use of the corporate form in transport - which would continue with the more expensive railways - would set the stage for its later use in industry as the scale of industrial firms grew.

With a national road network came a national system of "common carriers" so that industrialists could dispatch goods by wagon anywhere in the country. In the decades around 1750, entrepreneurs changed the way that they sold products. Rather than travelling between fairs and markets with goods loaded on packhorses, they came to send out either catalogues or sales representatives with samples, receive orders, and dispatch these by carrier. However, consumers or storeowners who order by sample expect to receive a good that is very similar to what they ordered. Standardized output was hard to achieve if workers each worked in their own homes. To take advantage of this new method of distributing their goods, then, entrepreneurs had a strong incentive to gather workers and supervise them. They also then had an incentive to mechanize production so that they could further standardize output.

Transport improvements encouraged factories in other ways. If an employer did not receive raw materials on time, and his workers worked in their own 
homes for piece rates (workers in their homes necessarily were paid for what they produced rather than by the hour), those workers just did something else with their time. Factory workers generally had to be paid no matter what. Due to the speed and reliability of the road network, industrialists need not hold large inventories to ensure regular production. As transport costs fell, entrepreneurs also used a more extensive range of materials in production: Belt buckles were adorned with various gemstones, for example. This increased the danger that workers would embezzle, but embezzlement was easier to prevent if workers were gathered and supervised. Increased travel - as stagecoach rides became both smoother and quicker - meant that national fashion tastes changed frequently; it was easier to respond to such changes if workers were collected and supervised. Lower transport costs also made it easier to feed a concentrated workforce, whether the food was domestic or foreign. This became particularly important as steam engines freed factories from the necessity of waterpower so that factories and workers could cluster in cities. Lower transport costs also allowed industrialists to serve a broad geographical market, and so expand their scale of output (though they might have satisfied this by leaving workers in their homes).

Transport improvements also facilitated technological innovation in a variety of ways. Those smooth and fast stagecoaches encouraged innovators and entrepreneurs to communicate: It is clear that these did visit each other's establishments. We have often noted when discussing technology in this book that innovation is generally a collaborative effort. By facilitating collaboration, transport improvements facilitated innovation. There was also an indirect effect: Lower transport costs encouraged regional specialization. The wool industry of Yorkshire could only invade markets in other regions if Yorkshire goods could be transported there cheaply. Once a particular industry became concentrated in a specific region, it became easier for potential innovators not just to interact but also to appreciate the potential benefits of innovation. Regional specialization may have been especially important in the emergence of specialized machine manufacturers: It made sense to build machines for the woollen industry when surrounded by wool factories. Note that these machinemaking firms had an obvious financial incentive to make steady improvements in the devices that they sold. Last but far from least, regional specialization allowed workers to specialize: Adam Smith, the founder of modern economics, would celebrate in 1776 a pin factory in which each worker performed one simple task among dozens, and recognized that this was only possible because the factory could serve an extensive market. Smith stressed that this "division of labour" lowered costs because workers became good at one thing and did not have to move between tasks. However, there was a further impact on innovation: Once workers came to perform just one task rather than many, it was much easier to see both the benefits and feasibility of mechanizing particular tasks. 
In addition to indirect effects on economic growth via factories and technology, transport improvements had a direct impact on incomes by lowering the cost of moving goods to consumers. We have often seen in this book how increased trade benefited the masses. Recall how Roman peasants had better roof tiles and pottery than did post-Roman peasants (chapter 10). These benefits were important in the age of turnpike roads and canals, but they would expand further with the development of railways.

\section{PRIMARY DOCUMENTS: TRANSPORT AND INDUSTRIALIZATION}

We can gain some sense of the transformation wrought by transport improvements from the comments of contemporaries. Carl Moritz visited England in 1782, and was distressed by the speed of stagecoaches:

From Oxford to Birmingham is sixty-two miles; but all that was to be seen between the two places was entirely lost to me, for I was again mewed up in a post-coach, and driven along with such velocity from one place to another, that I seemed to myself as doing nothing less than travelling ... The journey from Northampton to London I can hardly call a journey, but rather a perpetual motion, or removal from one place to another in a closed box.

Richard Whitworth, in his pamphlet, The Advantages of Inland Navigation, 1766, 2-3:

It is an undoubted certainty that all passage and communications bring trade into a country, and into those parts where not the least idea of traffic, among the inhabitants ever went further, or was brought to a higher pitch, than a coat to cover them, and bread to satisfy their hunger; these are the people who will soon feel the great advantage from inland navigation, who have hitherto been bred up for no other use than to feed themselves, and I may number them as some millions that have thus been buried alive, as it were, from the public service; what advantage will not accrue to this nation when so many millions will be allured out into the world of nature, robust by nature, and become useful to the state, by the temptation of gain.

Figure 24.1 is a detailed flowchart of the various ways that transport improvements influenced factories (captured as "Increased Scale of Production") and technological innovation. We outlined the four stages of technological innovation at the bottom in chapter 5 .

\section{Other factors}

Scholars have long debated the causes of the Industrial Revolution. We can briefly appreciate several other factors that they have suggested as important: 


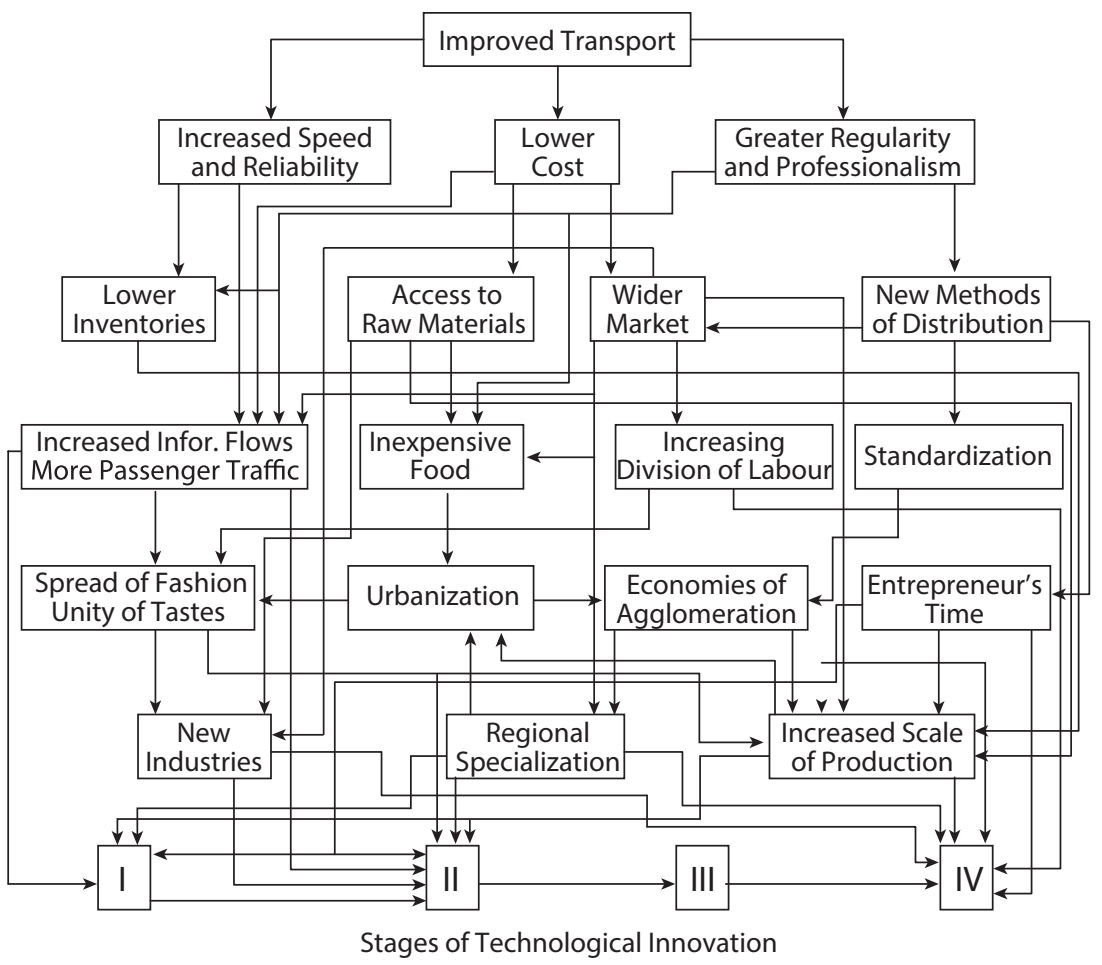

Figure 24.1

Transport improvements and the Industrial Revolution

- Britain had good supplies of coal and decent supplies of iron ore. This raw material abundance was undoubtedly helpful. Yet Britain would import large amounts of iron ore and all of the cotton for its cotton factories. Many other countries also had substantial coal and iron ore resources. These were of little value unless accessible by (especially) water transport. China certainly had coal and iron ore (though perhaps not as fortuitously situated), but had not developed the same access to these (chapter 22). Some have suggested that Britain had to utilize coal because of a wood shortage. It then benefited from a resource disadvantage, since coal turned out to have greater technological potential in iron manufacture. However, Britain's encroachment on forestland was similar to that in many other countries (including China). Moreover, a wood shortage might be an effect of earlier industrialization. In any case, coal would come to power steam engines and then be a major source of mechanical energy as well as a source of heating. Indeed, in the nineteenth century, coal would provide mechanical power to British and world industry at levels many times that ever achieved by wind and water power; humans ceased to rely on renewable energy sources but became dependent upon non-renewable energy. 
Some scholars would emphasize this increased reliance on non-renewable energy as a vital characteristic of the Industrial Revolution, stressing the role played by non-renewable energy sources in increasing average incomes.

- Many of the early industrial workers were single women and children. These were less expensive than men. Social relations and cultural attitudes that allowed women and children to work outside the home aided industrialization, though perhaps to a limited degree.

- The direct influence of science on the technology of the time was limited. Advances in iron manufacture occurred through trial and error with a limited understanding of the chemical reactions involved. The scientific understanding of vacuums may have aided steam engine technology, but that understanding was centuries old. However, there may have been a critical indirect effect through the adoption of the scientific method. As we saw in chapter 22, European scientists had come in previous centuries to stress careful experimentation or observation, and especially the careful recording of observations. The technological innovations of the Industrial Revolution period also generally involved an extensive trial-and-error process in which different "recipes" for iron refining or pottery kilns were tried and the results carefully recorded, or slightly different designs of textile machinery carefully evaluated. Though technological innovation historically has generally proceeded through trial and error, the innovators of the Industrial Revolution were likely more careful in their practice. European scientists interacted with technological innovators. The French Royal Society of scientists published an extensive multi-volume encyclopedia of industrial technologies with the explicit goal of encouraging improvement. In Britain, innovators such as James Watt mingled with scientists in societies that promoted the scientific method.

- Britain had become a more flexible society socially than many in the world, though likely not the most flexible. Successful industrialists could buy land and be accepted into the rural aristocracy. The children of aristocrats would seek industrialists' children as marriage partners. The son of the cotton manufacturer Robert Peel would become Prime Minister. While it was useful that there were social rewards for wealth obtained in any manner, it was less wonderful that some successful industrialists devoted their profits to land rather than industry.

- $\quad$ Some have argued that Britain had developed a system of apprenticeship that meant that British industrialists had more and better skilled workers to draw upon.

- Britain had not been successfully invaded since 1066. Though it had experienced civil wars, it could nevertheless be that entrepreneurs were more willing to invest in factories there than in lands often ravaged by warfare. 


\section{Challenges facing agents: Industrialists (factory owners)}

Since the industrialist must buy raw materials and sell a finished output, the industrialist must face all of the challenges faced by merchants (chapter 5). Note that a factory is hard to hide and so the industrialist must be particularly wary of confiscation by the state. The industrialist will necessarily always have raw materials and finished goods on hand and must worry about theft - not least theft by employees. The industrialist also must correctly gauge market demand before producing large numbers of a particular good.

The industrialist faces some new challenges. One is the need to finance the upfront cost of buildings and machinery. It may only be possible to pay for these over a period of decades: The industrialist needs much longer-term finance than even longdistance merchants required.

The industrialist must also somehow coordinate the efforts of factory workers. This generally requires supervision. Also, the industrialist must ensure that supplies of all necessary materials are always at hand lest the workforce be underemployed.

\section{The second generation of industrializers}

The British attempted to ban the export of industrial technology. However, these efforts barely slowed the spread of technology to other lands. Within a few decades, textile industries throughout the world would adopt new spinning and weaving machinery. Railways were built on all continents within decades of their invention in Britain. Movement of people was generally necessary for the successful transmission of technology: It was not enough to send blueprints and instructions. Many British entrepreneurs moved to other countries, and other countries sent spies into Britain.

The new technology affected four countries most profoundly in the middle decades of the nineteenth century: France, Belgium, and Germany in Europe, and the United States of America. These countries also experienced rapid economic growth. These were each countries with close economic ties to Britain - but the same was true of Portugal or much of Scandinavia, whose performance was less impressive at the time. Each of these four countries had significant coal deposits (which were soon reached by railway or water transport where this did not already exist) - but again many countries with coal failed to achieve much industrialization at the time. Industrialization in both France and Germany concentrated in the coalfield that spanned northeastern France, Belgium, and western Germany. (Note that we speak of Germany as a country for convenience here; we saw in chapter 21 that Germany was unified only in 1871.) 


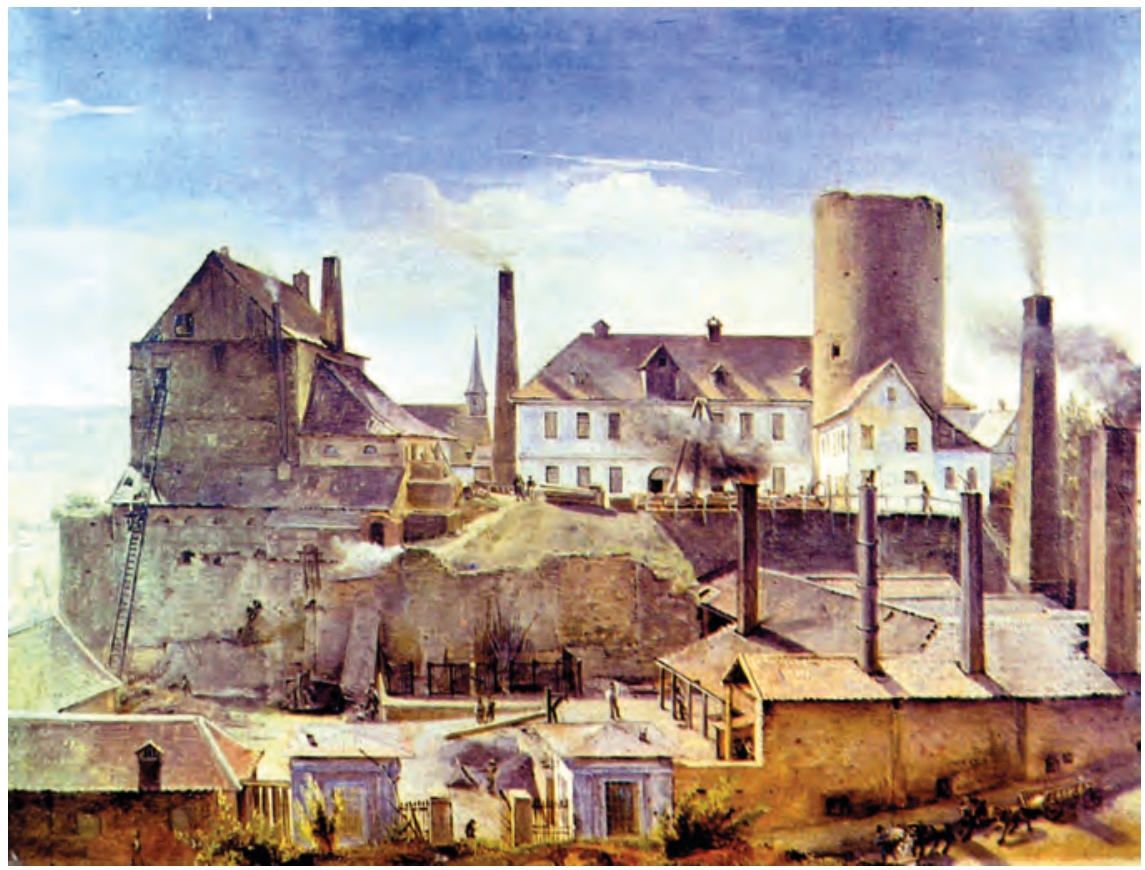

Illustration 24.7

The Factory Mechanische Werkstätten Harkort \& Co., Zurg Wetter (Wetter Castle), Germany ca. 1834, Alfred Rethel

Railways were an important part of the story of economic growth in these countries. To be sure, the cost savings over water transport where this was feasible could be moderate. However, railways combined low cost with speed and reliability, and railways could be built almost anywhere. Railways opened vast swathes of the interior of the United States. Railways accelerated the dynamic effects associated above with earlier advances in roads and waterways. By expanding market size, railways encouraged the development of large industrial establishments. Travelling salespeople became even more common, increasing the need for standardization of industrial output. Industrialists became even more confident of their ability to obtain supplies in a timely fashion, further tempting them to gather workers together. Railways also directly stimulated local iron industries with their demand for rails and railway locomotives and cars.

There were notable differences among these four countries. Growth in both population and per capita incomes occurred much faster in Germany than France over most of the nineteenth century. The French outnumbered the Germans at the time of Napoleon and likely had higher incomes; the Germans far outnumbered the French by 1900 and had higher incomes and a much bigger iron industry. Many commentators in France and elsewhere worried about the potential repercussions of this change in the balance of power in Europe. It would certainly play a role in fostering the world wars of the twentieth century (chapter 28). One characteristic of German development was the early 


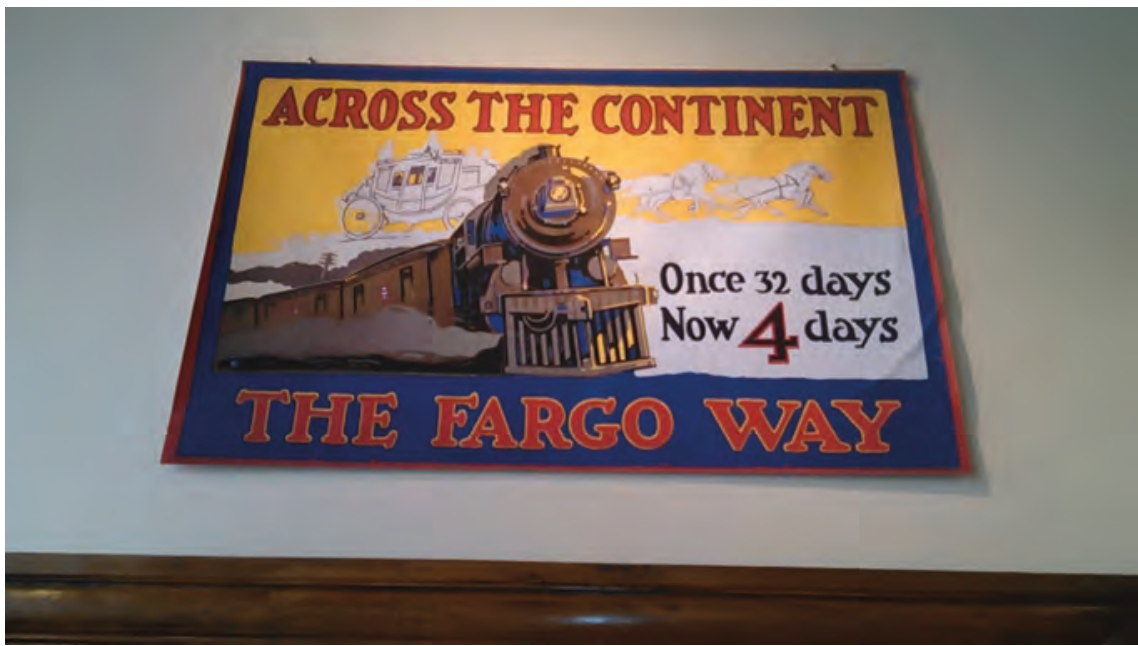

Illustration 24.8

Poster celebrating reduction in travel times by train, Wells Fargo Museum, Phoenix, AZ

appearance of investment banks: banks willing to make longer-term loans than merchants had required in the past. These became closely associated with large industrial concerns in some but not all industries. Large family firms instead dominated French industry: These were arguably conservative in investment strategies (lest risky expansion wipe out the family fortune) and perhaps less competently managed because of the emphasis on family. Economic historians hotly debate the quantitative importance of these institutional differences.

The United States would gain fame for the "American System of Manufactures." This was in turn associated with the idea of "interchangeable parts." The parts of a gun might be manufactured so precisely, for example, that the handle from one would fit flawlessly with the barrel from another. This precision mainly reflected mechanization: Lathes, for instance, could achieve standardization that work by hand could not. We have discussed the incentives for standardization above. Why did manufacturers in the United States push this farther and faster (on average, it should be stressed) than those in Europe?

\section{BOX 24.1}

\section{INTERNATIONAL EXPOSITIONS}

The American System of Manufactures caught the attention of the world at the London "Crystal Palace" Exhibition of 1851. This is widely considered to be the first ever "World's Fair." Its purpose was to gather in one place the advanced manufactures of the world. It stimulated technological transmission and found great favour with the public. Similar fairs 
would occur every few years after 1851 in the United States or Europe. The Eiffel Tower was built in Paris in 1889 for such a World's Fair. The 1939 Fair in New York expanded the scope of these expositions beyond current manufactures toward "the world of tomorrow" (the 1939 theme) or other social themes. Later in the century these fairs, while maintaining themes, came to be dominated by national pavilions in which nation states advertised their culture and politics and landscape as well as technology. The fair in Montreal in 1967 was styled "Expo 67," and since then, the word "Expo" has generally replaced "World's Fair."

Many political leaders came to see such fairs as an excellent opportunity to advertise the host city and nation - though it is not clear that such expositions have more than a transitory effect on such reputations. The temporary uptick in tourist visitation was another perceived benefit; though there were concerns that the cost of hosting often outweighed this benefit. (Similar considerations arose regarding the hosting of international sporting events.) As cities came to compete globally to host such expositions, an international organization was established in 1928 to allocate fairs to interested cities. The Bureau of International Expositions allows only one major international Expo every few years, but also certifies smaller Expos with a regional or topical focus.

The 1851 Exposition also displayed artworks from around the world. This encouraged considerable cross-fertilization of artistic styles. It became more and more common after that for artists from one part of the world to visit artists elsewhere - a behaviour of course facilitated by decreased costs of transport. Art galleries, especially in Europe and North America, increasingly displayed art from many regions of the world.

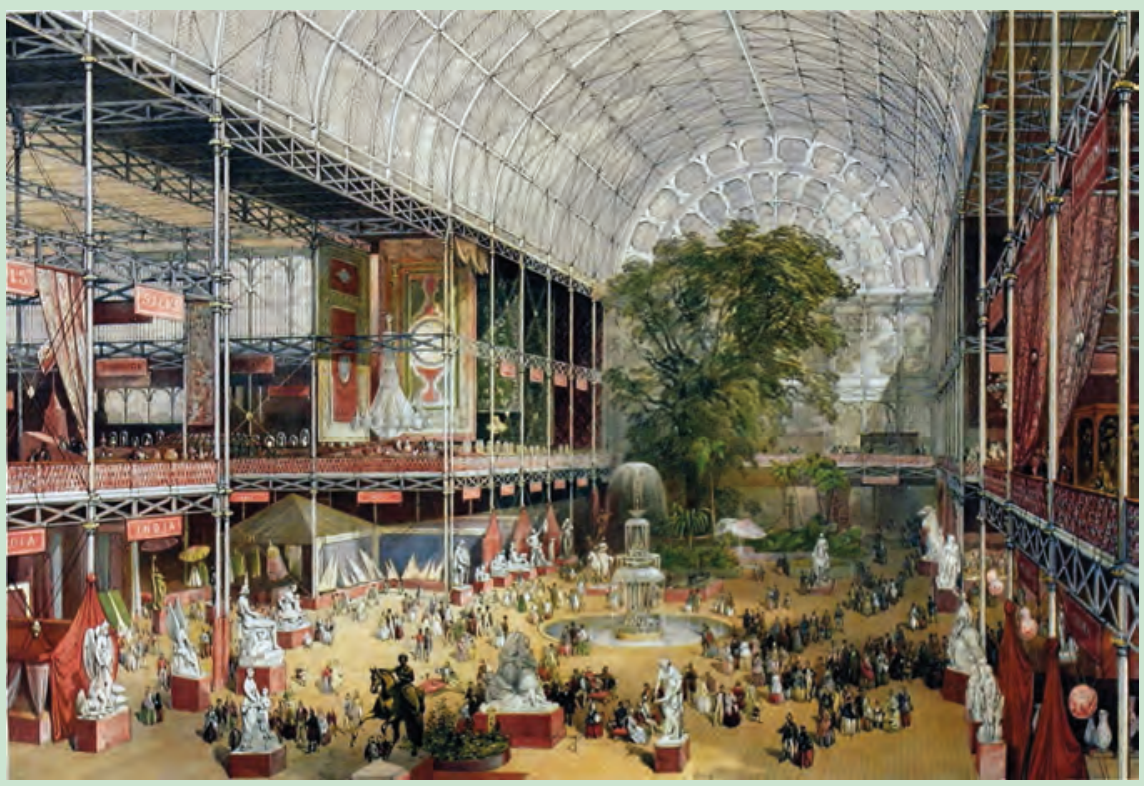

Illustration $\mathbf{2 4 . 9}$

The Transept from the Grand Entrance (Crystal Palace), souvenir of the Great Exhibition, by J. McNeven, William Simpson (lithographer), Ackermann \& Co., 1851. Note that the iron and glass construction of the Crystal Palace was itself remarkable for the time 
Higher wages in the United States - the availability of free land encouraged high wages - may have encouraged mechanization. Nevertheless, the abundance of raw materials may have been of even greater importance: Lathes and other machines were far more wasteful of wood and metal than was hand production.

\section{The Second Industrial Revolution}

A set of significant innovations in the 1870s and 1880s has been widely termed the Second Industrial Revolution. Though less "revolutionary" than the First Industrial Revolution, it is nevertheless true that the vast bulk of technological innovation since the 1880s has built on the technologies of the Second Industrial Revolution.

There are three critical areas of innovation in the Second Industrial Revolution. One is internal combustion. Internal-combustion engines were first developed in Germany, and were utilized as industrial power sources from the 1880s. Innovators would explore new uses for these in locomotion after that: Internal combustion provided much greater power per unit of weight than steam engines ever could.

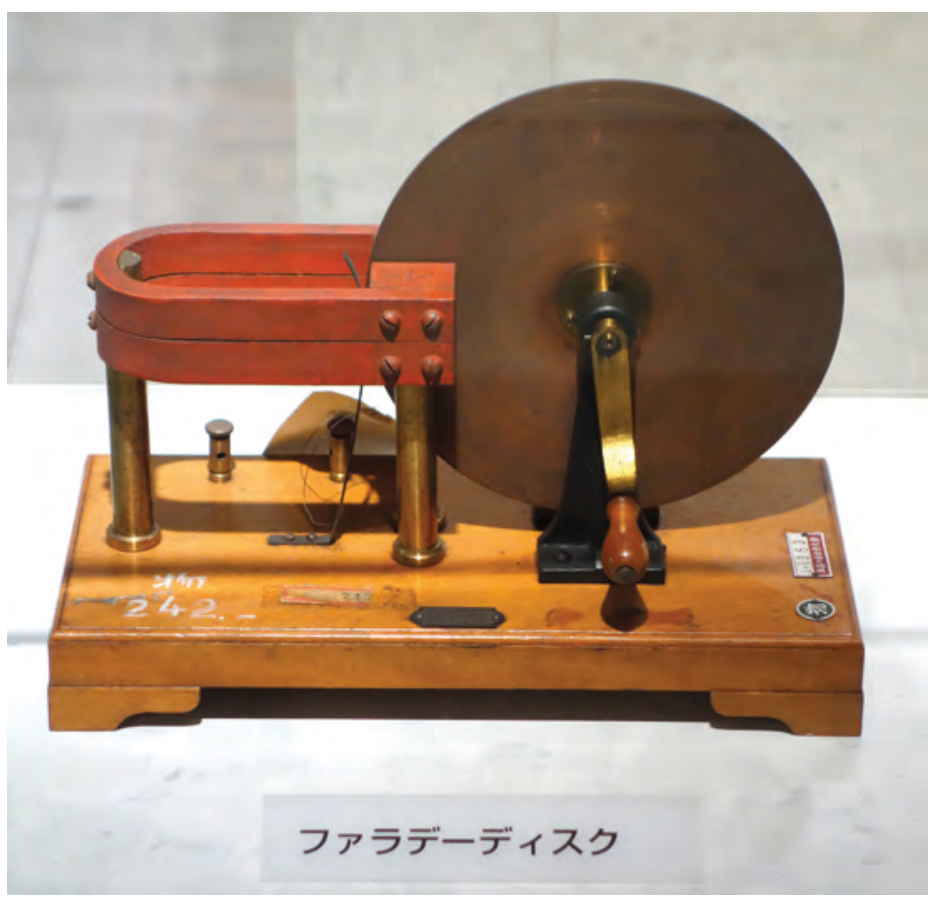

\section{Illustration 24.10}

Model of Faraday's disk, the first electric generator, invented by British scientist Michael Faraday in 1831, The National Museum of Nature and Science, Tokyo, Japan Photograph by Daderot, CC0 
A second area involved the harnessing of electricity. Electricity had received considerable scientific attention in the eighteenth and nineteenth centuries. Only in the 1880s, though, was electricity commercialized. The crucial development here occurred in New York: Thomas Edison developed systems for generating, transmitting, and metering electricity, and a practical light bulb that would be the first major application of electric power. He established a profitable company that allowed New York homes, offices, and factories to have a cleaner and more reliable source of lighting than gas lighting could provide. Once such a system was in place, innovators developed a variety of other applications for electricity.

The third area was chemicals. There had been important advances in dyeing and bleaching during the First Industrial Revolution, but these had occurred by trial and error. By the late nineteenth century, industrial chemists could draw upon a much greater scientific understanding of chemical reactions. The immediate result was a range of superior and inexpensive synthetic dyes that would replace a set of natural dyes employed for centuries or millennia. Then developments in pharmaceuticals began to follow. The stage was set for synthetic fibres and plastics in the twentieth century (we will trace how the Second Industrial Revolution unfolded in the early twentieth century in chapter 29). Germany and the United States were the early leaders in this new chemical industry.

Some scholars would also include steel-making in discussions of the Second Industrial Revolution. Steel had been produced for thousands of years, but it had always been very expensive. We now know that steel is a compound of iron with about a 2 per cent carbon content. This compound is harder and carries an edge better than iron itself. Soldiers had long prized steel for swords, but it had generally been too expensive for agricultural implements. Advances during the First Industrial Revolution had extended the uses of steel somewhat, but steel-makers still transformed iron in small batches. Only in the late nineteenth century did it become possible to produce steel directly from iron ore in large furnaces. Bessemer achieved success in Britain, but the technology soon spread globally though it had to be adapted to the chemical composition of different ores.

Were there commonalities across the three or four areas of the Second Industrial Revolution? One commonality was size: These new technologies generally required much larger industrial establishments than those of the early First Industrial Revolution. They only made sense in an era of massive industrial establishments - which were usually though not always run by corporations. A second commonality was science. Advances in both electrification and chemicals relied heavily on advances in scientific understanding over the preceding century. Innovation still involved a costly process of experimentation (Edison and his associates spent years developing filaments for light bulbs), but science provided useful directions to experimenters and identified possibilities. Increased understandings of chemical reactions also aided advances in steelmaking, though to a lesser extent; scientists would struggle to explain after the fact why certain smelting technologies worked on some ores but not others. 
Internal-combustion technology benefited from an increased understanding of combustion. The coincidental timing of these three or four different types of innovation might then in part be attributed to the development of institutions for financing and organizing large industrial establishments, and in part to advances in science resulting from the Scientific Revolution (chapter 22) and the research university (chapter 23).

We saw above that the technology of the First Industrial Revolution was closely associated with the factory. For the Second Industrial Revolution, the institutional counterpart was the industrial research laboratory. Through most of human history, innovators had usually operated on a small scale: They worked away in their own private homes or workshops, though they networked with others. Large firms, seeking new products and processes, and recognizing the importance of both science and careful experimentation, came during the Second Industrial Revolution to establish research facilities (chapter 23). These facilities were generally interdisciplinary: They employed a mix of scientists, engineers, and technicians. Edison's research laboratory would not only continue to develop his integrated system but would be responsible for the first

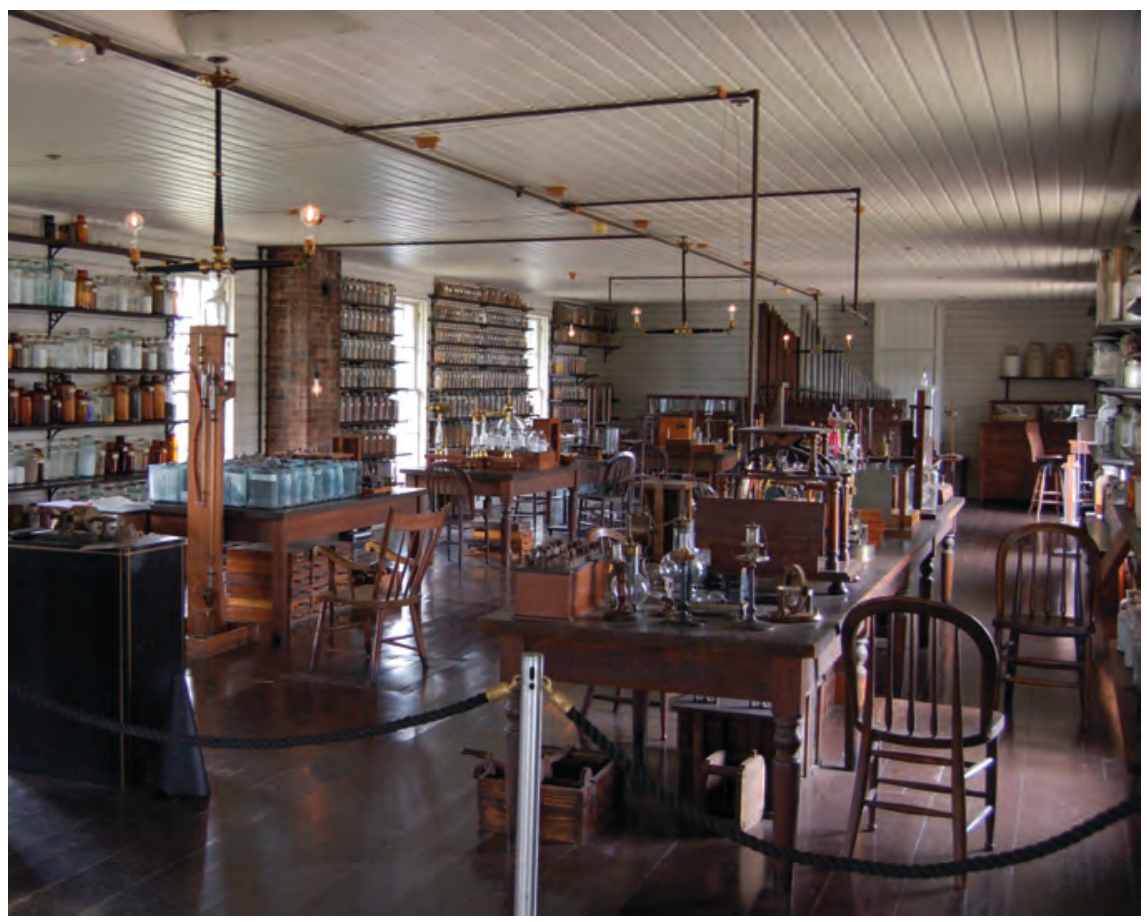

Illustration 24.11

Replica of the second storey of Thomas Edison's Menlo Park (New Jersey) laboratory (Greenfield Village, Michigan). Edison became known as the "Wizard of Menlo Park" when he and his team invented the phonograph in this building in 1877. He and his team there then developed the electric light bulb, and a system for generating, transmitting, and metering electricity to power these

Photograph by Andrew Balet, CC BY-SA 2.5 
phonographs and moving pictures. Chemical firms such as Bayer in Germany and DuPont in the United States would investigate a range of new chemical possibilities. Managers would struggle to figure out how to manage the inherently unpredictable process of technological innovation. Industrial research laboratories would grow in size and extent over the next century, and are probably responsible in the early twenty-first century for over half of the technological innovation in the world - but an important place remains for the independent innovator in at least some sectors of the economy.

\section{The third generation of industrializers}

In the last decades before the First World War, many more countries experienced rapid rates of economic growth. Most European countries - including Russia can be included in this group, as well as Canada, Australia, Argentina, and Japan. Almost all countries of the world experienced some growth in per capita incomes, but at far lower rates than among the first three generations of industrializers. The result, then, was that Europe and European settler economies on average achieved per capita incomes several times those of most countries in Asia or Africa by 1914. This is the most dramatic period of the Great Divergence that we sought to comprehend in chapter 22. The glaring exception to the Great Divergence was Japan, which alone among Asian states managed to achieve similar growth rates to those in Europe. The Japanese exception shows that a close cultural bond with Europe was not essential to economic growth in this period - but the fact that Japan was an exception suggests that it was not easy for countries to industrialize without strong connections to Europe. Of course, Japan differed from much though not all of the rest of Asia by not being colonized; this allowed it to pursue independent economic policies.

Second-generation industrializers had consciously sought to "catch up" to Britain. Third-generation industrializers had a different option. At least potentially, they could latch on to the new technologies of the Second Industrial Revolution at their inception. This may have been especially important for countries with little coal but abundant hydroelectric power potential, including Japan and (especially northern) Italy. Japan would be one of the earliest adopters of electricity, and would become a world leader in the development of electric railway systems for its growing cities. Italy would be the home of one of the first successful automobile manufacturers. Nevertheless, the industries of the First Industrial Revolution remained important: Japan would develop extensive textile manufacture, for example. Railway construction continued to be an important characteristic of economic development.

The Russian state was heavily involved in industrialization. It focused its efforts on both railway construction and the development of steel mills. There was only 1,000 kilometres of railway in Russia in 1860 but 58,000 by 1900 . The 9,000-kilometre Trans-Siberian railway was completed in 1904 - just decades after transcontinental railways spanned the much shorter distances across the 
United States and Canada. The Trans-Siberian crossed parts of Manchuria controlled by Russia at the time. Russia taxed the peasantry heavily to finance these investments. The early Soviet Union would continue these policies in its first decades after the Russian Revolution of 1917. Though the actions of the Russian and Soviet states reflect a long history of autocracy in Russia (chapter 21), they also reflect the challenges of being a late industrializer. Firms in Britain had often expanded slowly by reinvesting profits. Later industrializers struggled to finance much bigger factories than those of the early First Industrial Revolution. Russia lacked firms and financial institutions that could readily build railways or steel mills without state support. Russia also invested in education, and in a legal system to support commerce, but provided no political rights or social policies for the growing industrial workforce.

India had readily adopted in the early nineteenth century the technological advances in iron production associated with the First Industrial Revolution. However, as the scale - and scientific content - of iron technology advanced in the late nineteenth century, the Indian iron industry fell behind. There was only one modern steel mill in India in 1913 (and only one in China and none in Africa). India would nevertheless embrace railway technology. The British had feared that this new technology might offend traditionalists, but there was little objection. Indeed, this was a general outcome in the world: There were some objections to the noise, pollution, and economic transformation associated with railways (Ecuador's government forcefully resisted protests in the late nineteenth century), but in general, people welcomed railways for the ease of movement of both goods and people that they provided.

The American Admiral Perry had forced the reclusive Japanese state to open itself to international trade in 1853. The Meiji Restoration of 1868, which ended the power of autocratic shoguns in favour of the emperor and an

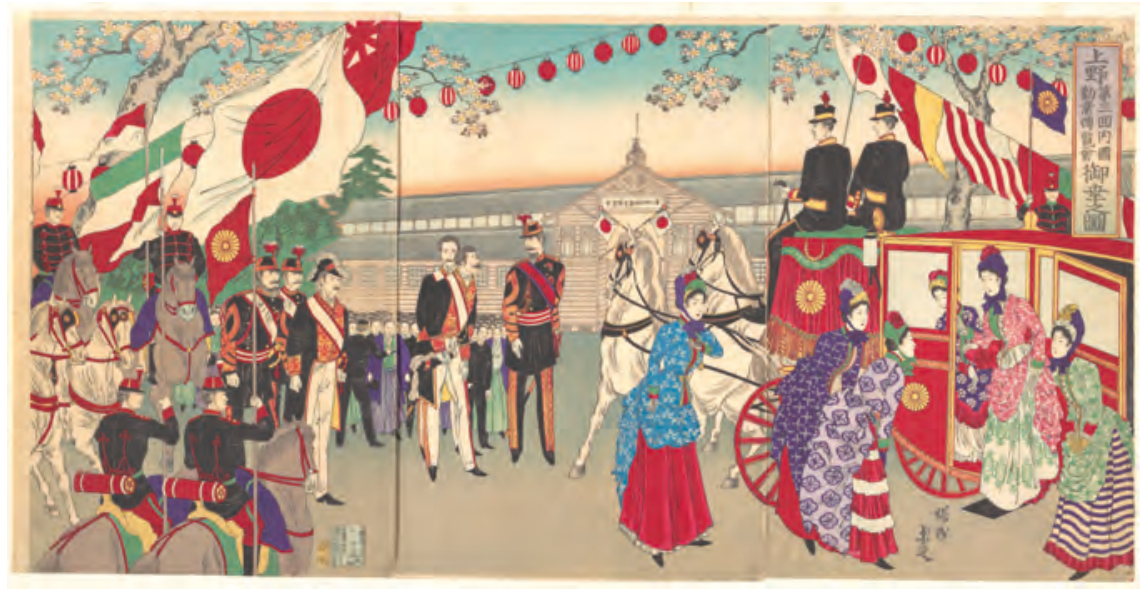

Illustration 24.12

Visit of the Empress of Japan to the Third National Industrial Exhibition, Ueno Park, Tokyo, 1889, by Yoshu Chikanobu, Metropolitan Museum of Art 
elite-dominated legislature, was in large part guided by a desire to pursue industrialization to match Western military might. The Japanese invested heavily in education and sent numerous students abroad to learn the latest science and technology. It also invested heavily in railways. The success of Japanese industrialization and technological borrowing would be reflected not only in rapid economic growth but in a naval defeat of Russia in 1905 (they had come into conflict in Manchuria). Though Russia was one of the least economically advanced European nations, and had to send its navy halfway around the world to fight the Japanese, the defeat of a European navy by an Asian navy was still a surprise to many observers. (Not everyone was surprised: The American writer Mencken predicted a Japanese victory while the Russian fleet was on its way.) The Japanese victory encouraged opponents of colonization, especially elsewhere in Asia. At the same time, it increased pressure for political change in Russia.

\section{Demographic changes associated with industrialization}

These industrial revolutions had far-reaching demographic implications. New machines and fertilizers increased agricultural output, while steamships and railways made it easier to move food between continents. The population of Europe would rise from 105 million in 1700 to 390 million in 1900. There were even more dramatic increases in population in the Americas. The population there grew from 13 to 145 million over the same period, reflecting both high birth rates and the migration of 50 million (chapter 31), mostly from Europe (making Europe's population growth even more astonishing). Mortality rates fell in Europe as nutrition improved and as certain diseases like cholera were tackled by the provision of clean drinking water to urban populations (advances in medical knowledge and pharmaceuticals had a limited impact before the twentieth century; again see chapter 31). Birth rates soared as young people were enabled to get a job and form families earlier. The average marriage age in Britain fell from 30 in the seventeenth century to 23 in 1800. Though premarital sex was far more common than we might think - British records show that one-fifth of British brides were pregnant before 1700 and two-fifths after - birth rates were far higher among the married.

This population growth would slow in the late nineteenth century, and birth rates would fall to or below replacement rates (that is, the level of about 2.1 births per adult woman that is required to maintain a stable population through time) in the twentieth century in most developed countries, including Japan. Just as with rapid economic growth (above), Malthus had not foreseen such a transformation. Throughout human history, increases in incomes had induced increases in population. Yet as incomes continued to rise - and at unprecedented rates - most people decided to have smaller families. Historians 


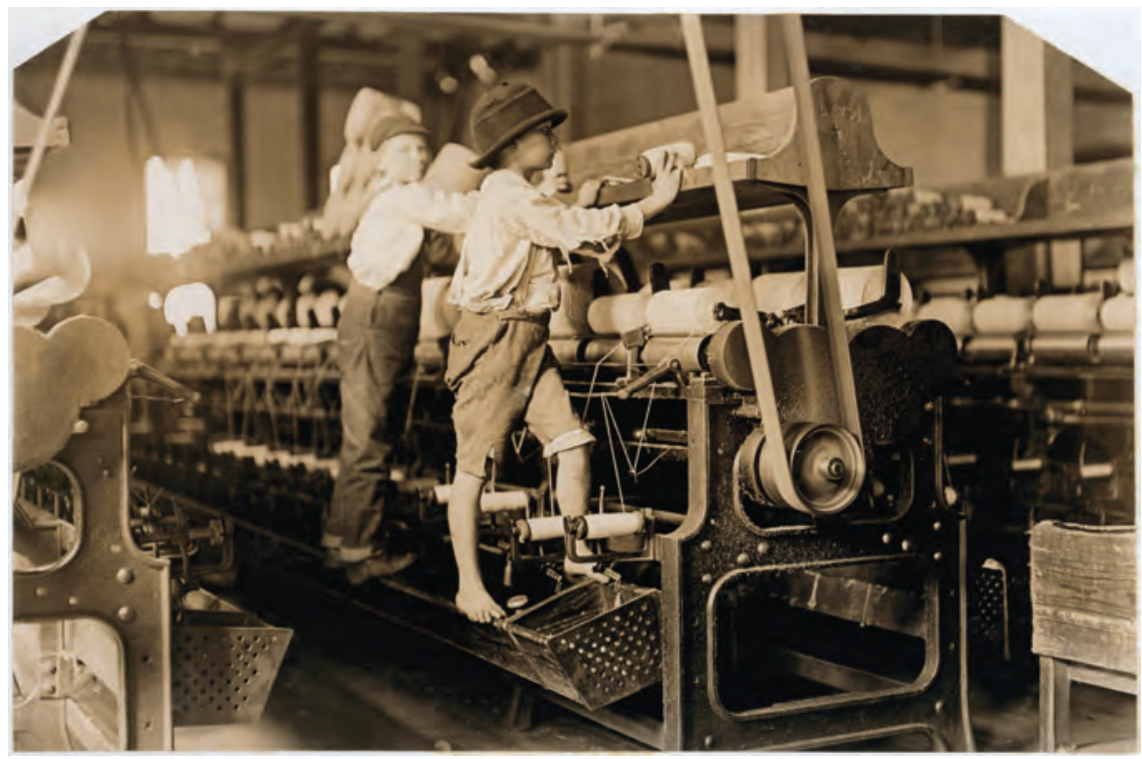

\section{Illustration 24.13}

Bib Mill no. 1, Macon Georgia, by Lewis Hine, 1909. Young children still managed complex and dangerous machinery

have described this experience of first rapid population growth followed by low fertility and population stability or decline as the "demographic transition."

Why did fertility fall despite rising incomes? The likely answer reflects a handful of changes associated with industrialization. One such transformation was urbanization. The steam engine freed industrialists from locating near sources of water power. They found it convenient to cluster in towns or cities where they could share access to workers, suppliers, machine repair specialists, financial institutions, and eventually advertisers. The railway and steamship meant that it was easier to feed urban populations than ever before. As agricultural productivity expanded through the nineteenth century, more and more of the population became urban. Britain had shown the way: About half of its population was urban already in the late eighteenth century. On farms, there are many useful tasks that small children can perform (as my mother never ceased to remind me as a child). Workers in their own homes could also have their children perform useful tasks such as sweeping debris or loading thread. The earliest factories employed child labour - often, entire families worked as a unit - but the British banned such labour, and many other industrializing nations never allowed it. Child labour would peak in the United States in the 1910s and then decline sharply. Therefore, for urban industrial families, children were far more expensive than for rural families: They were a net cost rather than a contributor to family production.

Moreover, these families had other things on which to spend their income. We have recorded a slow increase in average incomes through much of world 
history. Nevertheless, in 1700 the typical European family still had a meagre set of personal belongings: one or two changes of clothing, some pottery, and so on. Dramatic improvements in industrial productivity exposed the working class to a range of new consumption opportunities: They could eat off nice plates, and wear comfortable and colourful cottons. There was a corresponding change in attitudes from focusing on subsistence to becoming "consumers." We saw in chapter 22 that farmers and workers were increasing their non-food consumption in most of the world before 1800. The widespread purchase of many non-food items then accelerated as real incomes rose in the nineteenth century.

The cost of raising children increased as systems of public education expanded. The First Industrial Revolution had not required a very educated workforce. As technology became more complex over the nineteenth century and firms grew in size and complexity - and especially with the Second Industrial Revolution - industrialists and workers both urged governments to provide education. This was one area in which Japan after the Meiji Restoration of 1868 sought to duplicate Western efforts: The state mandated primary education for both boys and girls from 1872. Workers who could read instructions and perform basic mathematical calculations were far more productive. Also, there was an increased demand for advanced training in science, engineering, and management itself. The enhanced value of education may have given some advantage to Protestant countries within Europe: Children had often learned to read there so that they could read the Bible. This tradition of literacy may be the primary reason why Scandinavian countries would grow faster than most countries within the third generation of industrializers. In any case, once states came to require that children go to school, and as average years in school steadily expanded, children became ever more a cost rather than a potential revenue source.

Before the Industrial Revolutions, most children had tended to follow their parents' occupations. Most people were farmers, and their children would become farmers. Artisans would often train their children as artisans. Industrialization broke this link for many: Children would no longer inherit a family farm, and no longer work beside their parents. They would instead seek their own jobs as they grew into adulthood. This fact had temporarily caused birth rates to rise in many countries as children left home and married early. The longer-term effect was to cause parents to worry about their children's future. They could no longer teach them how to farm or spin while working but had to encourage children not just to attend school but pay attention and prepare for some occupation. This task became even more challenging as industrialization created a dizzying array of new occupations: managers, technicians, railway workers. Parents might reasonably decide to have fewer children and help them with their homework (or later pay for tutors) - especially if the parents themselves were educated. They were tempted, that is, to emphasize child quality over quantity. 
Children had long been useful not just when they were young but again later when parents were old. Children were, through most of human history, the pension plans of the elderly. People too old to maintain themselves were reliant on their children for support. With industrialization, workers were able to save for retirement. Financial instruments were developed to facilitate this over time (see Box 15.1). In the nineteenth and especially twentieth centuries, firms and governments developed pension plans. Germany was a leader here, seeing the provision of social policies such as state pensions as a way of satisfying urban workers without extending political power to these. Many other countries would not provide state pensions until the Great Depression or early postwar period (chapter 29). In any case, as opportunities to self-finance one's retirement increased, the importance of having children declined.

Parents desirous of having children to look after them in their old age had long had to worry about high rates of infant and child mortality. Through most of human history, between a quarter and a half of babies would die before reaching adulthood. The decreases in mortality noted above were concentrated in childhood: Better nutrition of mothers and children, and the decreased incidence of disease, combined to reduce rates of infant and child mortality dramatically. It is now a tragedy in rich countries when a child dies; mere centuries ago it was just a part of life everywhere. One result of declining child mortality was that parents determined to have a couple of children around in their old age might have three rather than seven.

\section{Challenges facing agents: Modern parents}

Through much of history, parents had to worry that their children would die. Most though far from all - parents did not have to worry too much about their children's future: Children would learn to farm or pursue a trade while working with their parents. A significant minority of children did, of course, join the army, migrate from farm to city, or in other ways leave the life of their parents behind. The modern parent can only rarely bequeath an occupation to a child. They must hope that the child gains the skills required for some occupation. In a world of myriad occupations, children will often pursue a path with which parents have little familiarity. Parents in many parts of the world, especially Asia, have recourse to tutors to help their students in school. Parents everywhere trust much of their children's preparation in life to teachers over whom they have limited influence.

The challenge then is to provide one's children with skills to prosper in one or more occupations. This is not a challenge that most parents can undertake alone.

For a variety of reasons related to industrialization, then, the cost of having children rose while the benefits of doing so fell. There was also, in most countries, an important change in the power relations within families. As women gained employment opportunities in industry, and then gained educations, they gained power both to choose marriage partners and to exercise authority within a marriage. Since women bear the costs of pregnancy and breastfeeding, 
they likely on average evaluate the costs and benefits of children differently from men. Increased female influence within families likely encouraged smaller families.

Of course, the will to have fewer children needed to be coupled with the ability to achieve that end. It is important to appreciate that the demographic transition began at a time when birth control technologies were still quite limited. Primitive condoms made of animal intestines were known from at least the sixteenth century in Europe (seemingly earlier in China, and there is debate as to whether they were known in ancient societies). Linen condoms treated with chemicals were popular in the eighteenth century, condoms made from rubber appeared in 1855, and the first latex condoms emerged in the 1920s: These were both cheaper and more comfortable. Diaphragms were invented and improved from the 1860s. It is likely, though, that women bore the greatest responsibility for birth control and relied on a variety of natural substances such as moss to prevent insemination well into the twentieth century. Accurate knowledge of the female menstrual cycle, and thus that sex was riskier at some times than others, spread from the middle decades of the nineteenth century. As millions of observant Catholics can attest, timing sexual intercourse is an error-prone method of birth control at the level of families, but it can have a significant impact on societal birth rates if pursued by an entire population. Doctors had (correctly) advocated breastfeeding since at least the sixteenth century to reduce the chances of pregnancy. Of course, couples could abstain if determined not to have more children. Religious leaders often opposed contraception, but were often ignored. (The pope decreed that the fetus had a soul at the moment of conception in 1869, against centuries of thinking that the soul entered the fetus three months later; this decision would shape debates over abortion in succeeding centuries.)

The demographic experience of the first three generations of industrializers was duplicated elsewhere in the twentieth century as industrialization and economic growth spread. Europe had largely completed the demographic transition by 1914 while most of Africa and Asia was just beginning. However, the fall in mortality in Asia and Africa would be much more dramatic as developments in medical understanding and pharmaceuticals advanced. Mortality rates in India fell from 5 per cent in 1900 to 2.7 per cent in 1950 to 1.5 per cent in 1970. Much of the world, therefore, experienced dramatic population growth in the twentieth century. India's population grew from 100 million in 1700 to about 300 million in 1900, but then grew to exceed 1.2 billion by 2015. Estimates of the population of China place it at approximately 150-200 million in 1700, and 400 million in 1900; it exceeded 1.35 billion by 2015. In the 1970s there was widespread concern that population growth would outstrip the earth's carrying capacity: We can only feed so many, even with advanced agricultural technology. However, birth rates in most of the world have fallen toward replacement rates in recent decades. The population will continue to grow in countries such as India just because they have young populations at or entering prime childbearing years. And some 


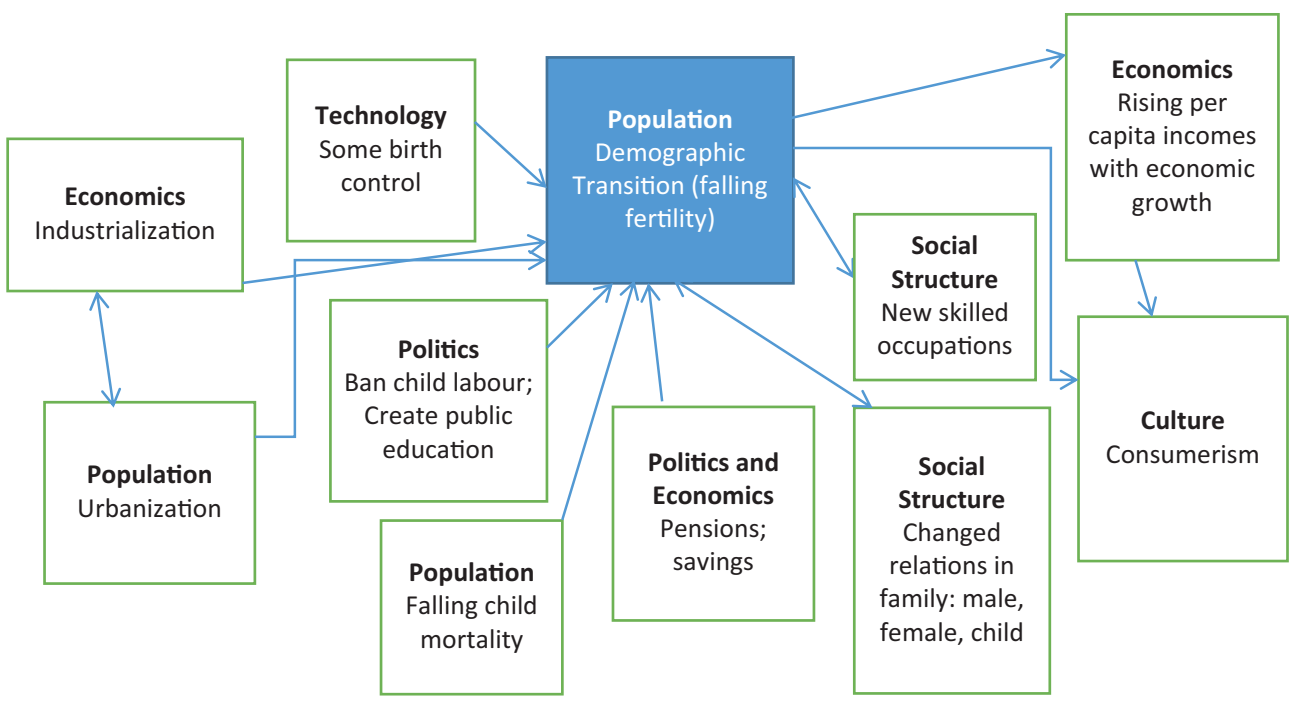

Figure 24.2

Influences on and effects of the demographic transition

\section{BOX 24.2}

\section{HISTORY OF CHILDHOOD AND AGEING}

There has been growing interest in the history of childhood in recent decades. There is now a scholarly association and journal devoted to this field. One challenge is that children themselves leave few records through most of history. Historians must then interpret what adults say about them. (Notably, adults have often worried about the next generation, especially in times of societal change.) We know much more at present about childhood in the last couple of centuries in Europe and North America, but the scholarly understanding of childhood in other times and places is growing.

One side effect of the demographic transition was an increase in the proportion of the population that were considered aged (with 60 often serving as the point at which people were deemed to be old). The most obvious impact of this development was in social policy: Societies around the world struggled to provide pensions, hospitals, and homes for the aged (chapter 27). There were differences, of course, in the degree to which societies expected families to care for the elderly. There was increased recognition of the challenges families faced, especially as twentieth-century medical advances meant that many people continued to live long after they had lost the mental or physical capability to care for themselves.

Through most of human history, ageing had been relatively rare. Societies might revere small numbers of elderly for their wisdom - though of course there were important societal differences in how much respect the aged received. Respect was the typical attitude 
in hunter-gatherer bands. Agriculture had conflicting effects: The aged no longer had to keep up with mobile hunters or gatherers, and often exercised power through control of land. On the other hand, social stratification may have decreased the respect accorded to the aged simply by creating alternative loci of power in societies. The respect accorded the elderly declined in the nineteenth century. This may have in part reflected the demographic transition: The elderly were no longer a rarity to be treasured but a commonplace that burdened both families and states. Yet rapid social change - and especially progressive political movements (chapters 25,27 ) - also played a role: The elderly's experience no longer seemed so valuable to the young when they were both reacting to social change and striving to direct this.

It is less clear how well the elderly were cared for historically, especially as they became physically or mentally limited. Societies that were facing difficulty in feeding the young might easily view the old as an economic burden. As incomes began to rise across the world from the early nineteenth century, it became feasible to care for a large but unproductive elderly population.

Most societies celebrated the role of grandparents. We see this in Europe from the late eighteenth century. Notably, over the course of the nineteenth century, grandparents ceased to be viewed as an austere source of authority but instead were expected to "spoil" their grandchildren. Grandparents, then, came to set an example of the less strict treatment of children that parents themselves followed in the later twentieth century.

In the twenty-first century, care for the elderly remains an important public policy issue. Many countries will see a significant increase in the proportion of the population over 60 in the next decades (chapter 31). Many countries face challenges in financing the pensions they have promised. In addition, healthcare costs are rising with ageing. These economic concerns should not blind us to the broader social question: What role should the elderly play in society? Is their wisdom still valued and valuable in a world of change? Can/should we better connect the very old with the very young? The Covid-19 pandemic that began in 2019 shone a bright light on severe shortcomings in the care of the elderly in many countries.

Islamic and African countries have not seen birth rates fall so far - likely in large part because women there are less educated, have lower labour force participation, and are less influential within families. The earth's population will continue to rise for decades but may level off before the end of the twentyfirst century. Many countries, including Japan, are indeed grappling with the somewhat novel challenges of population decline and population ageing.

The changes above also affected how children were treated. We noted in chapter 4 that strict child discipline was the norm in agricultural societies though younger children especially had some latitude for play. Industrialization and urbanization, and especially the extension of public education, meant that childhood became a more protracted process in which children operated much of the time separately from parents and were engaged in different pursuits from adults. When children were at home, parents could lavish more attention on them because they had fewer children and these were less likely to die. The 
general cultural outcome was to rethink the nature of childhood. Rather than urging children to behave like adults, adults recognized childhood and adolescence as special periods of personality development. Parents increasingly treated children with both affection and respect. Governments, which through most of human history had left childrearing to families (bestowing complete authority on parents, except in the societally important area of inheritance), began to intercede by providing education, legislating against child labour, and eventually (usually well into the twentieth century) even placing limits on corporal punishment by parents. Less happily, a set of childhood mental challenges, including what we now call attention deficit disorder, anorexia, and depression, received scientific attention from the nineteenth century. Though it is hard to say for sure, these mental challenges appear to have become more prevalent in the modern era.

\section{Social and cultural changes associated with industrialization}

We have touched on some critical social changes already: urbanization, education, and changing relationships between both men and women, and parents and children. We mentioned above another important transformation between employers and employees in that the former increasingly came to supervise the latter. We discussed the growth of both the working class and middle class in chapter 23.

One other noteworthy social transformation in the nineteenth century was that standards of attire converged across both classes and nations. Convergence is first observable among middle-class men, but working-class men and upperclass men then came to dress much like middle-class men. This transformation was generally a voluntary response to the increased availability of mass-produced clothing and a shared aspiration toward clothing that seemed "modern," and at least somewhat comfortable. However, colonial governments encouraged Western attire among bureaucrats. The modernizing Meiji government in Japan in 1894 ordered bureaucrats to wear Western clothing. Women's fashion displayed far more regional diversity, but here too there was marked convergence in styles of dress across classes and countries. The typical practice by which women in tropical countries did not cover their breasts largely disappeared because of missionary preaching, gawking tourists, and the more general convergence in attire. Within countries, the decreased ability to identify someone's class by their clothing served to lessen class identification and conflict. Though Western fashion was most widely adopted, especially among men, the shawl and kimono - but not the sari - were adopted by many Western women in the late nineteenth century in rejection of less comfortable Western clothing.

Industrialization - and the political changes associated with the rise of the nation state (chapter 21) - caused people around the world to reflect on change. 


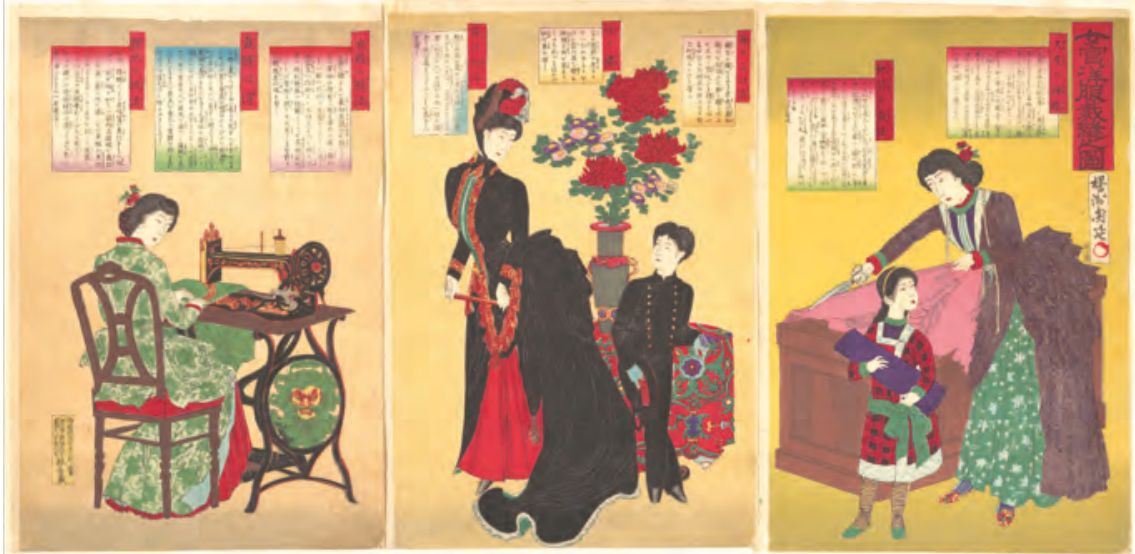

Illustration 24.14

Japanese court ladies sewing Western clothing, 1887. Woodblock print by Yoshu Chikanobu, Metropolitan Museum of Art

Many began to consider the possibility that there was progress in human societies, an idea only intermittently espoused before. In Europe, such ideas were often associated with a sense that Europe was more progressive than other places, and speculation on whether others could or would catch up. Everywhere, such ideas caused many to question inherited traditions that had failed to predict these changes and often seemed not to accord well with these new realities. These ideas of human progress would be crushed in much of the world by the horrors of the world wars (chapter 28) and the Great Depression (chapter 29) but would be revived in the postwar era - with less ethnocentrism. To a lesser but significant degree, many people in the late twentieth and early twenty-first centuries also came to doubt that there was progress in human affairs. We will in chapter 32 explore whether it makes sense to speak of human progress.

\section{Questions}

1. You are now able to compare the Song economic experience (chapter 15) with the British Industrial Revolution. Does it make sense to speak of a Song Industrial Revolution?

2. One of the critical-thinking skills that students should develop involves thinking about how an author's arguments may reflect their point of view. How might this author's own research on the Industrial Revolution have shaped his analysis of the Great Divergence?

3. Diagram the influences on and main effects of the First or Second Industrial Revolution.

4. Does it make sense to compare the Industrial Revolution to the Agricultural Revolution? 
5. Would you prefer to work in a factory, in your home, or on a farm in the time of the First Industrial Revolution?

\section{Readings}

Allen, Robert C. 2011. Global Economic History: A Very Short Introduction. Oxford University Press. [Most of the book focuses on why some countries industrialized before others.]

Beauchamp, Christopher. 2015. Invented by Law: Alexander Graham Bell and the Patent That Changed America. Cambridge MA: Harvard University Press. [This book argues that patent law played an important role in many of the innovations of the Second Industrial Revolution, including electric lighting and advances in rubber manufacture.]

Cross, Gary, and Rick Szostak. 2018. Technology and American Society: A History, 3rd ed. New York: Routledge. [Discusses both industrial revolutions.]

Griffin, Emma. 2013. Liberty's Dawn: A People's History of the Industrial Revolution. New Haven, CT: Yale University Press. [Draws on the autobiographies of workers to argue that the Industrial Revolution increased personal freedoms: where to work, who and when to marry, and freedom of speech.]

Hoffman, Philip T. 2020. "The Great Divergence: Why Britain Industrialised First." Australian Economic History Review 60:1, 1-22.

Jacob, Margaret C. 2014. The First Knowledge Economy: Human Capital and the European Economy, 1750-1850. Cambridge University Press. [Argues from the extensive correspondence of key innovators that a scientific spirit infused technological innovation and entrepreneurship in Britain but not elsewhere in Europe. See also Mokyr Joel, The Culture of Growth: The Origins of the Modern Economy. Oxford University Press, 2016.]

Stearns, Peter. 2010. Childhood in World History. 2nd ed. New York: Routledge.

Stearns, Peter. 2013. The Industrial Revolution in World History. 4th ed. Boulder CO: Westview Press.

Szostak, Rick. 1991. The Role of Transportation in the Industrial Revolution, Montreal: McGill-Queen's University Press.

Troyansky, David G. 2016. Aging in World History. New York: Routledge.

Wrigley, E. A. 2016. The Path to Sustained Growth: England's Transition from an Organic Economy to an Industrial Revolution. Cambridge University Press. [This book stresses the importance of the transition from wood to coal as a fuel.]

There was a forum on World Expositions in World History Connected, October, 2016. [The forum suggested that students might pursue individual or group projects around World Fairs, such as imagining how a particular country or ethnic group should (versus did) present itself. Many references are provided to a literature on such fairs.] The World History Bulletin, Spring 2017, focused on international "megaevents" including both sporting events and expositions. 


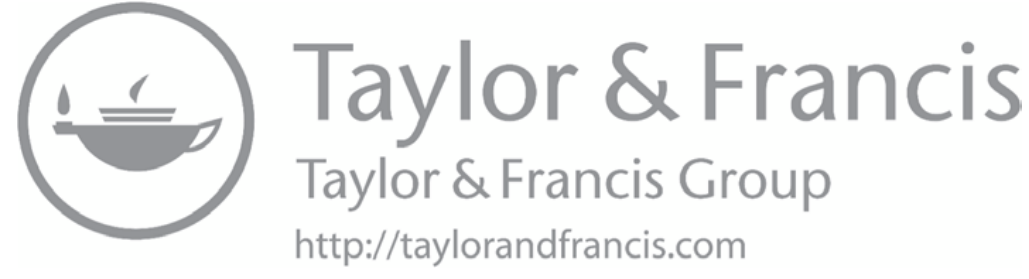




\section{Political revolutions around the world}

\section{A diverse set of experiences with important commonalities}

\section{Guiding questions}

Why, where, and when do we observe so many political revolutions and revolts in this period? How were revolutionary ideas transmitted across countries?

Why were the outcomes of revolutions and rebellions so different across countries?

Who guides revolutions and how do they unfold through time?

What effects did (certain) revolutions have?

Relationship to other chapters: Though we have not often had cause to discuss revolutions previously in this book, we draw useful comparisons to earlier episodes of violent political change and internal disagreement regarding how power should be distributed and exercised. We also draw upon previous discussions of the financial challenges faced by all states in raising enough in taxes to finance a military and bureaucracy. We also reflect upon the ideas of popular sovereignty associated with the rise of nation states in chapter 21. Most of our attention in this chapter will be on the American and French Revolutions, in part because these were among the earliest revolutions, but mostly because they have had the most significant historical impact. The United States - and to a lesser but substantial extent France - would be among the 
most influential nations over the next centuries: They will play critical roles in particular in the World Wars of chapter 28 and the Great Depression of chapter 29. More generally, many later democracies would copy the democratic ideals and institutions of both revolutions (chapter 27). The discussions of other revolutions and revolts are important in reminding us of the quite diverse outcomes that revolutions can achieve. These other revolutions or revolts were also important in the histories of different regions.

Political revolutions involve a change not just in rulers but also in the institutions of rule: The resulting institutional changes usually have lasting consequences. Revolutions are also in general quite unpredictable. Revolutionaries may (or not) have a clear idea of the new institutions that they wish to create: They may focus on replacing the old. Even if they do have a vision of the future, periods of dramatic change call for sudden and pragmatic decisions that may alter the shape of the state from what revolutionaries initially envisaged. Moreover, diverse elements often compete for power within revolutionary movements. A small group that exercises dominant physical power can come to shape postrevolutionary institutions; they may then gain support or at least acquiescence from people who just want an end to political uncertainty. Many revolutionaries end up quite disappointed with the changes that they helped bring about. In studying revolutions, then, we need to appreciate the forces driving the desire for change, but also understand that historical contingency can have a significant effect on the path the revolution takes.

The revolutions of the eighteenth and nineteenth centuries generally reflected the ideals of popular sovereignty that had emerged over the previous centuries. These ideals guided revolutionaries to create institutions that reflected the popular will. However, as we saw in chapter 21, arguments for popular sovereignty could support a wide range of institutions from democracy to autocracy. Despite this disagreement regarding desirable political outcomes, revolutionaries often reflected Enlightenment-inspired confidence that humans were capable of designing superior institutions.

Two of the most studied revolutions in human history - the American and the French - occurred in the last decades of the eighteenth century. These would, in turn, inspire many revolutions and failed revolutions in other countries and continents over the next centuries - several of which we address below. As arguably two of the most important revolutions in world history, they will receive much of our attention. We shall also see that the Haitian Revolution likely had a much greater influence than is generally appreciated. We can note here that all three reflected in part the growing cost of military conflict in general and the expenses of the Seven Years War (1756-63) in particular. France and Britain (and allies) fought throughout the world in that war - with Britain 
gaining dominance in Canada and India as a result. Both countries incurred massive debts - but little booty - in fighting this war, which they struggled to pay off. It was in part because of the costs of this war, and the fact that some of it occurred in the Americas (in the United States it is often called the French and Indian War), that the British sought to raise new tax revenue from their American colonies. It was in part the costs of the same war - and further expenses incurred in aiding American revolutionaries against the British - that drove the French king to call the Estates General together to request an increase in taxes. As we shall see, both actions triggered revolutionary acts. These revolutionary disruptions, combined with revolutionary rhetoric, encouraged the Haitian Revolution.

While our central focus in this chapter is on the political institutions of governance, we shall find that a variety of other themes influence these. We have already alluded to cultural attitudes and philosophical beliefs. We will see that various changes in the economy are also of critical importance. Individual personalities influence the unpredictable course of political revolutions. Social stratification could both encourage and shape revolutions. Religion loomed large in some revolutions but not others. Technology and health occasionally play a role. Various political phenomena, including war and public opinion, also influence revolutions. Though styled "revolutions," the political transformations we address in this chapter necessarily built evolutionarily on pre-existing political institutions. We shall find that revolutions were more successful when they could focus on changing institutions of governance alone rather than attempting to refashion a range of political and economic institutions suddenly. We shall see that both ideas and institutions flowed between different states, but often with different consequences in different places.

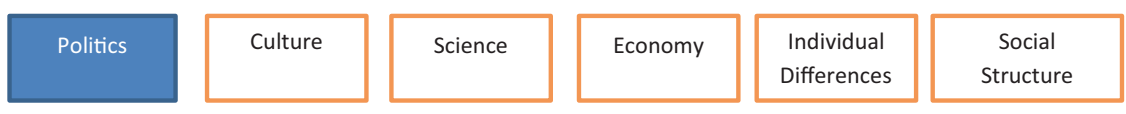

\section{The American Revolution}

The American Revolution is perhaps the most celebrated of historical events at least in the United States. A World History text can strive to put the event in some comparative perspective.

\section{To revolt or not}

A first point that we might make here is that Britain was far from the worst imperial master in world history. Compared, say, to the Haitian slaves that would rebel in Haiti a couple of decades later, the free American population was not particularly ill-treated. Indeed, the American colonies were prospering in the eighteenth century. Each of the thirteen American colonies possessed an elected legislature - legislatures that would themselves declare independence 
from Britain. These legislatures had control of most day-to-day governmental decision-making in the colonies, though they could not pass laws that contravened imperial statutes. Local assemblies appointed the vast bulk of colonial bureaucrats. Britain itself had only about 200 bureaucrats in North America on the eve of the Revolution - and these often struggled unsuccessfully to obtain permanent funding from local assemblies. Though the British army was an important presence in some colonies, British bureaucratic power was severely curtailed in most, and relied on a shared sense of purpose and cultural affinity with colonists.

In Britain itself, royalty had already ceded much of its power to Parliament, and there was a set of legal protections against the exercise of arbitrary power by any king (chapter 21). These protections might be of limited use to conquered peoples in India or Africa, but American settlers had access to British courts. The British Parliament and British people indeed worried about the inherent dichotomy between democratic governance at home and colonial rule abroad: How could a state that prized democracy not allow self-governance to all its citizens? The answer with respect to India or Africa was often to deny the capability of locals for self-government, but Britain could not easily apply this rationale to settler colonies such as the United States. Indeed, many British politicians would agree with American revolutionaries about the inequities of imperial rule.

To be sure, the British Empire, like all empires, intended to make a profit. The British government imposed taxes on its American colonists that they hoped

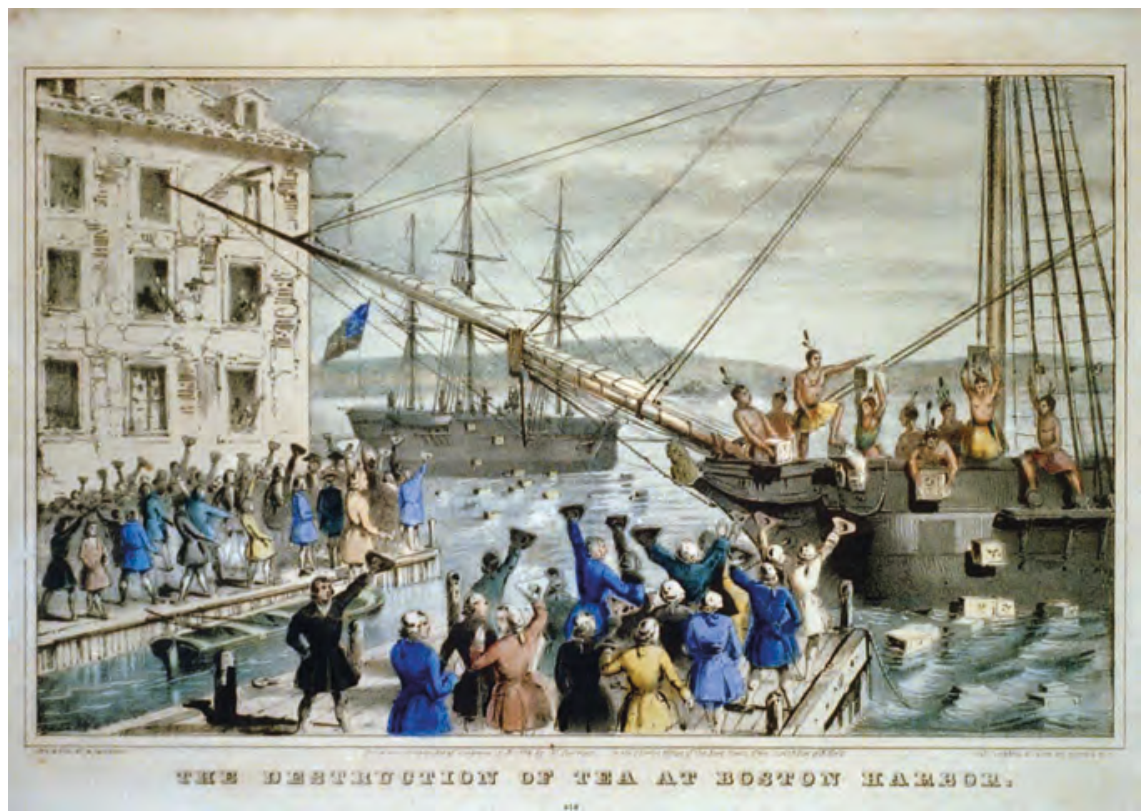

Illustration 25.1

The Destruction of Tea at Boston Harbor, Nathaniel Currier lithograph, 1846 
would more than pay for whatever military and administrative costs the British might incur in operating these colonies. The Americans could rightly complain of "taxation without representation" since the British Parliament rather than their own legislatures imposed such taxes. Here we can see a conflict at the heart of British rule in North America: The creation of local legislatures celebrated democratic principles but to survive the Empire had to impose taxes whether local legislatures liked these or not. In Britain itself, the British Parliament had long since gained power over taxation. It is no coincidence that the first celebrated act of the American Revolution - the Boston tea party, in which rebels attacked a ship carrying imported tea - was a protest against newly imposed British taxes on tea.

The Revolution reflected for the most part a calculation that the colonists could govern themselves at a lower cost than the British charged them for the privilege. There were also other important inconveniences of colonial rule. In particular, the British insisted on the right to billet soldiers in local homes, a practice that locals naturally objected to.

The Seven Years War (1756-63), often called the French and Indian War in the United States, had resulted in the defeat of the French and their Indigenous allies in North America. The British had also taken Florida from Spain. The British had thus removed the most significant military threats to the American colonies. Yet the British felt that they should maintain a significant military presence in North America. They then raised taxes on tea, stamps for legal documents, sugar, and imports in general.

The American public disagreed about whether British taxation warranted a revolution. Many Americans supported the safer option of remaining British. Some did so out of attachment to the British crown. Others were much more pragmatic: The British Empire was a known entity, and the future political contours of an independent polity or polities in North America were unclear. Though the horrors of the French Revolution lay ahead, Americans could well worry about the inherent uncertainty associated with revolution. Revolutionary leaders would indeed haggle over the constitutional details for many years after the first shots were fired. Though Britain surrendered in 1781, the new country only proclaimed its constitution in 1787 and ratified it in 1789. World history is littered to this day with examples of revolutionaries fired by the noblest of motives who led their nations into chaos rather than renaissance. Supporters of the Empire could also worry about losing the benefits of British protection: American ships would indeed suffer increased attacks by pirates once they could no longer rely on the protection of the British navy abroad. (The new US Marine Corps would soon attack pirates in Tripoli in Africa, an act memorialized in the Marine Corps hymn.) Finally, yet importantly, people could reasonably worry about the costs of war itself. Like most wars, the American Revolutionary War was often brutal, with atrocities - massacres of civilians, rape, extra-judicial hangings, and the frequent (non-lethal but humiliating) practice of tarring and feathering opponents - committed by both sides. Civilians needed to take sides and were 
often punished severely if they found themselves under the control of the opposing army.

A mix of values and self-interest also guided those who favoured revolution. Ideals of popular sovereignty were critical. Nevertheless, we can compare revolutionaries to the local elites that had throughout history risen to wrest power from the imperial centre. Moreover, those who fought for principles had to make one essential compromise: Though the northern states would soon abolish slavery (chapter 26), the revolution relied heavily on the support and actions of southern slave holders. Those who believed in the cause of liberty accepted the denial of freedom to millions of slaves.

What would have happened in the absence of revolution? The fate of Canada is suggestive: It would achieve political independence from Britain gradually over the next century or so. It also would inherit a set of democratic institutions from Britain, and would follow Britain itself in reducing the power of the monarchy to virtual figurehead status. It would also spread from Atlantic to Pacific Oceans. Economically, Canada would maintain per capita incomes just below those of the United States through the next two centuries. We should be careful of relying on this comparison too much for the simple reason that the history of Canada was always closely tied to that of the United States and may have been entirely different in the absence of the American Revolution. The United States may itself have had a quite different history if not independent. In particular, one can legitimately wonder if the British would have purchased the Louisiana territory from the French as the newly formed United States soon would, or would have fought so zealously against Spain and Mexico for territory to the west. Nevertheless, Canada's history suggests that the alternative to revolution would not have been too horrible for the people of the United States.

One question often engaged by historians involves the effect of the revolution on the history of slavery. We will find in the next chapter that Britain would abolish slavery decades earlier than the United States, and peacefully. It is possible that without the revolution, slavery would have ended earlier in North America than it did - and that North America might have avoided the horrors of the American Civil War. Alternatively, it could be that Britain itself might have delayed the abolition of slavery if the revolution had not succeeded: Southern plantation owners were not without friends and influence in the British Parliament. Slave owners in the British Caribbean exerted far less influence after the loss of their American allies. Moreover, the British move toward abolition of slavery was motivated in part to allow the British to assert some moral superiority over their former colonies.

\section{The twenty colonies}

Histories of the American Revolution focus naturally on the thirteen British colonies that would together form the United States. We should stress that there were, in fact, twenty British colonies in North America at the time (and eleven 
additional island colonies in the Caribbean: Some of these voted support for the revolutionaries, but the British navy tightly controlled these islands). These twenty colonies had generally been established by private companies under a royal charter. These had often created assemblies as part of a strategy of attracting settlers. The British had slowly taken over governance from most of these private companies (as they had done in India; chapter 20).

One of these colonies, the Hudson's Bay Company territory, was still governed from London as a corporation primarily interested in the fur trade. Granted by the king rights over all lands draining into Hudson's Bay way back in 1670, the business of the company proceeded directly from London to forts along Hudson's Bay and inland rivers, and so had little contact with the rest of North America (beyond buying food from Indigenous peoples of the Plains). It is hardly surprising that this royally proclaimed company remained loyal to the British crown. Likewise, it is not surprising that Newfoundland, transitioning in the late eighteenth century from a fishery run from England to a fishery based in Newfoundland itself, but still exporting the bulk of its fish to Europe via English ports (chapter 19), remained loyal to the British crown. Newfoundlanders could reasonably have still viewed themselves more as Europeans than North Americans. In any case, Newfoundland did not yet possess a legislature that could have declared independence.

There is also Prince Edward Island, which had a small assembly created in 1774. East and West Florida (the latter mostly in the modern states of Mississippi and Alabama), which like Quebec had been gained in the Seven Years War, was mostly Catholic (though many English Protestant residents moved to Spanish territories after the British took over), and remained loyal. There were small battles there during the war between revolutionaries and royalists, followed by a Spanish invasion of West Florida. Britain would cede Florida back to the Spanish after the war.

The two other British colonies - Quebec and Nova Scotia - were both recent results of British conquest: Britain took Quebec from the French only in 1760 and Nova Scotia, again from the French, in 1713. The British promised the small French Canadian population in Quebec that the British Empire would forever respect their language, religion, and land tenure system. American revolutionaries attempted on more than one occasion to encourage French Canadians to join them in revolt. However, most French Canadians felt safer within a British Empire they had come to know, however briefly, than to throw in their lot with a group of English-speaking and mostly Protestant revolutionaries. Besides, they lacked a legislature that could debate independence: The British would grant an assembly to its Quebec colony only in 1840 and only after a substantial influx of English-speaking settlers. (There was some speculation in Quebec during the revolution that the British might give Quebec back to France just as they returned Florida to Spain but the British decided to maintain control.)

That leaves Nova Scotia, which deserves more attention as the only British colony in North America with a legislature that voted to remain a part of the 


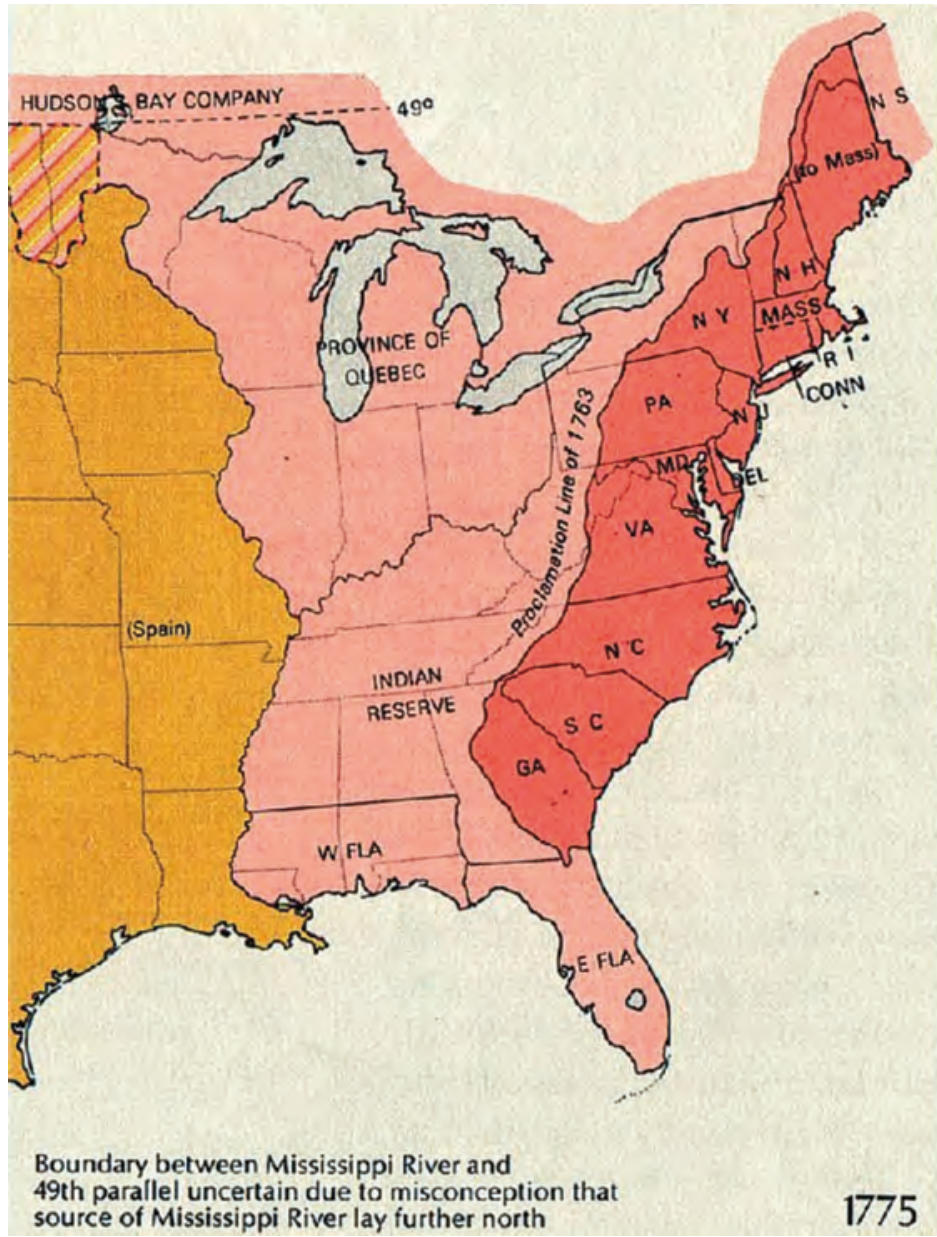

Map 25.1

British colonies in continental North America, 1775. The colonies coloured red rebelled but the others (and Newfoundland and Prince Edward Island, not on this map) did not

British Empire. Like Newfoundland, it was a land of relatively recent European settlement that traded more with Europe than with other parts of North America.

\section{Forging a new state}

Local militia skirmished with British troops near Lexington outside Boston in 1775. The American Declaration of Independence followed on July 4, 1776. This was a declaration of popular sovereignty: People had the right to change government if dissatisfied and especially if unjustly treated. The revolutionaries benefited from the support of many other European states (France, Netherlands, Spain, several German states) over the next years of war: These allies were 
generally more interested in weakening Britain than in supporting the revolution. The success of the American Revolution must be understood within the context of the ongoing competition among European nation states (chapter 21), for without foreign support, the revolution might well have failed. Even with foreign backing, there were many points between 1775 and 1781 when it appeared that the revolutionaries might lose, but British forces surrendered in 1781 .

While the battles of the American Revolution proceeded, leaders from the thirteen colonies met to imagine what a newly independent land might look like. These colonies were themselves proudly independent and were almost as suspicious of each other as of the British. Most colonies were jealous of their legislative powers and wanted a central government with limited authority. The original articles of confederation envisioned a remarkably weak central government. However, a shared desire for mutual defence encouraged the creation of a central government with some authority. One perhaps unlikely outcome of the negotiations over the shape of the American constitution was that these colonies eventually agreed to a central government that would grow over time to become arguably the most powerful government in the world. In addition to pursuing a shared defence, there was a desire to create a large market for internal trade (in part to replace trade they might lose with Britain), and so the central gov-

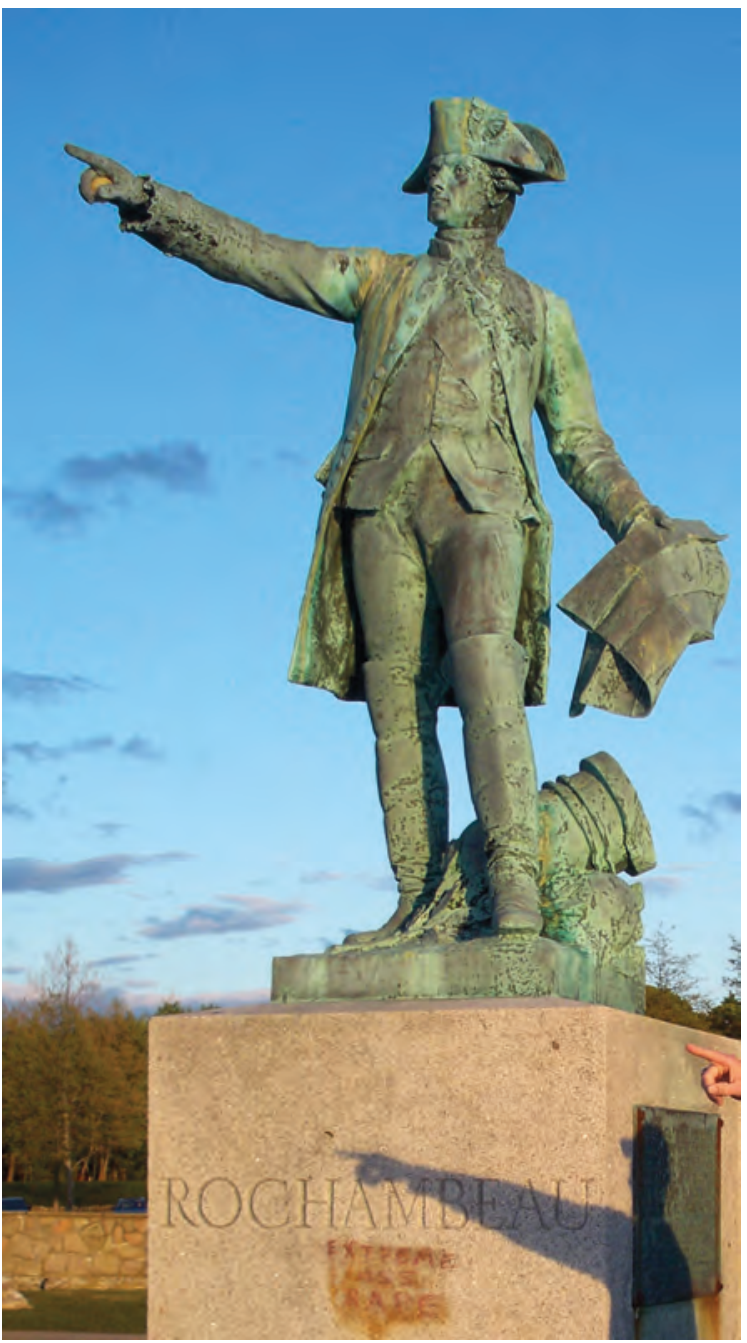

\section{Illustration 25.2}

Statue of Rochambeau, commander of French troops during the American Revolution, Newport, RI. The French had wintered in Newport before joining General Washington's forces in Virginia and at the siege of Yorktown that ended the war Ahodges7, CC BY-SA 3.0 ernment gained broad powers to legislate in favour of "inter-state commerce." This was hardly an inevitable outcome of negotiations among the colonies. While there were reasons for centralization, we should realize that this was a debate, and not one which one side was necessarily destined to win. Principal actors had diverse motives. For example, the revolutionary general and first president George Washington held land in the west and so had a personal interest in a strong and expansive central state. Moreover, the drafters of the 
constitution could not foresee how clauses regarding inter-state commerce would allow the federal government to expand its authority over the next centuries. Governments absorbed only a few per cent of economic output in the late eighteenth century but would extend their activities dramatically in the twentieth century (chapter 27). The constitution allowed the federal government later to exercise power in areas such as social policy that the drafters of the constitution could barely imagine. Even the vexed issue of slavery would become a matter in which the central government would eventually exert a powerful influence (chapter 26).

State legislatures maintained the same format as colonial legislatures. The vast bulk of British laws remained in place, excluding only those that granted powers to British king and British Parliament. This institutional inheritance deserves emphasis: The revolutionaries did not have to create an entire system of laws and courts but could focus their energies entirely on structuring a government without a king. The main innovation was then the creation of a central and democratic government. This, famously, would involve "checks and balances": the executive, legislature, and judiciary were each granted separate powers that limited the ability of any group to use power for their own purposes. The newly agreed constitution would soon be amended to include a Bill of Rights that guaranteed a set of fundamental rights to all. The independent judiciary could thus limit the ability of either legislature or executive to mistreat any individual or group. Special note might be made here of the freedom of religion: Diverse Christian denominations had settled the United States, and so revolutionary leaders appreciated early the need for mutual respect - but this legal protection would then apply to atheists and a variety of non-Christians in succeeding centuries. These rights were not inviolate: Later American governments would intern loyal Japanese civilians during World War II and commit torture. Even at the time, these rights did not apply to slaves and Native Americans - and the latter were summarily removed from their ancestral lands. And women had far fewer rights than men. Still, the Bill of Rights and the broader contours of the Constitution provided the average White American male with greater protection against tyranny than almost any other people on the globe. Slowly over time, others would gain these rights. These innovations were not guaranteed when the revolution began - the focus was on removing the king - but emerged as the difficult task of imagining a new government unfolded.

Historians hail the framers of the American Constitution for exercising a considerable degree of judgement. They point to multiple reasons for this. One is certainly the simple fact of education: Many of the framers of the Constitution were familiar with the works of a range of liberal philosophers mostly from Europe - who had long debated the form that democratic governance should take. Of likely even greater importance was the practical experience they had of elected legislatures. The American colonies had possessed elected legislatures for decades. The framers of the Constitution had ample knowledge of how democracy works in practice - and importantly that 
it can easily yield bad decisions. As Winston Churchill would famously say in the twentieth century, democracy is the worst form of governance except for all the rest. The framers of the Constitution knew all too well that voters acted on limited information and often elected representatives with severe flaws in character. They knew that fear or avarice rather than reason might guide legislators. They knew, that is, that people might need protection from those they elected just as they needed protection from the power of kings. They were guided to allocate power sparingly, limiting the ability of any person or group to exercise power against any other person or group. The careful delineation of "checks and balances" between executive, legislative and judicial authorities is not something that the drafters of the Constitution would likely have engaged without personal experience of the possibilities for abuse of elective power.

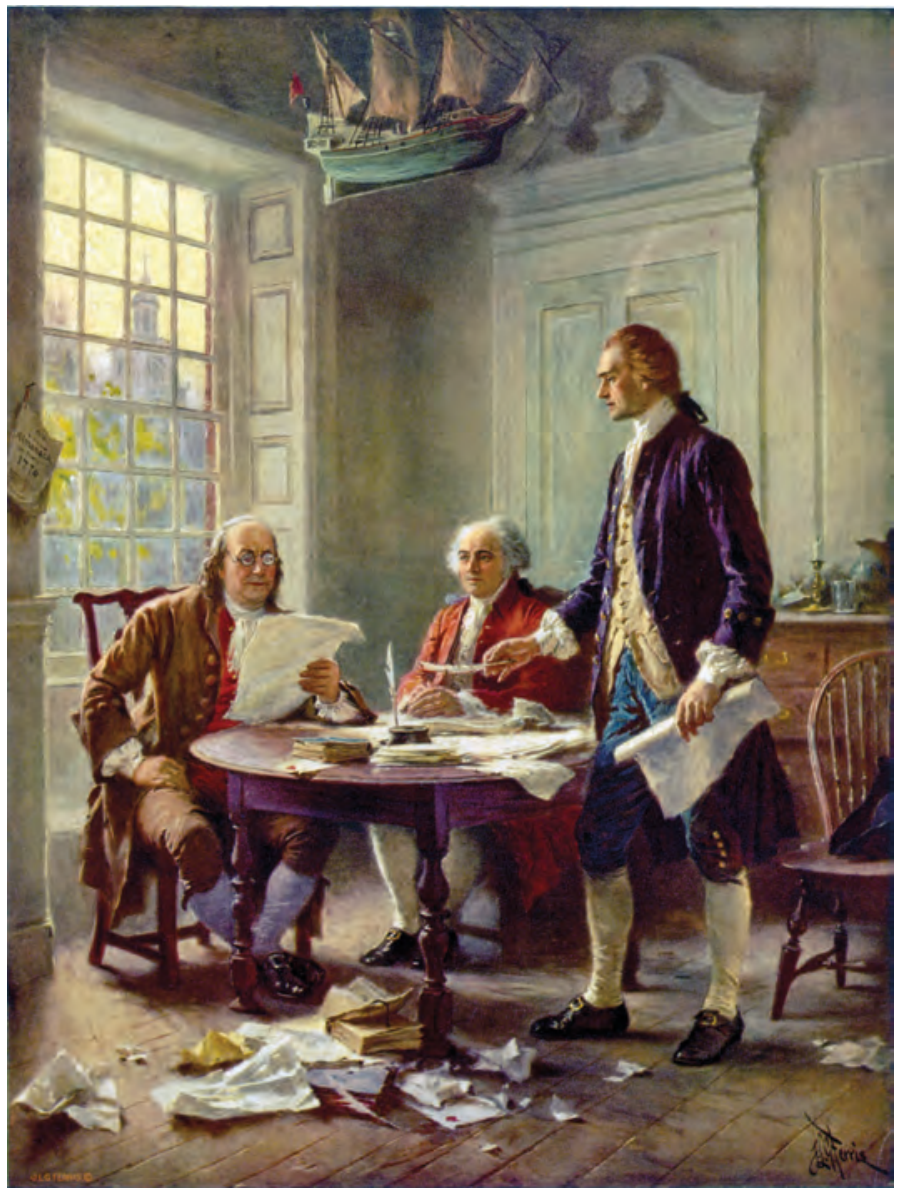

Illustration 25.3

Writing the Declaration of Independence, 1776 Jean Leon Gerome Ferris 1863-1930. Thomas Jefferson (right), Benjamin Franklin (left), and John Adams (centre) meet at Jefferson's lodgings, Virginia Historical Society 
An obvious comparison is with the French Revolution (below), which would start in 1789, the year of ratification of the American Constitution. The French revolutionaries had little experience of legislative debate: The king had just convened the Estates General - in which nobility, church leaders, and commoners met separately in three houses - for the first time in a century and a half. Convinced that any government would be an improvement on the arbitrary power exercised by the king (and hereditary nobility), the revolutionaries endowed their legislature with absolute power. As that legislature struggled to establish its authority against an international alliance of royal powers, it descended into "the Terror" in which fellow revolutionaries were sentenced to death on suspicion of disloyalty to the revolution. After years of the worst form of political instability, in which political actors needed to fear for their very lives, Napoleon was able to achieve dictatorial powers by promising political stability and some respect for human rights. The legacy of the French Revolution is therefore far more ambiguous than the legacy of the American Revolution: The path to democracy in France would unfold over centuries, punctuated by periods of empire and monarchy. The French Revolution did succeed in replacing an absolute monarchy but failed to establish a replacement form of democratic governance that would not regularly be replaced by some degree of authoritarian rule.

The American revolutionaries had several key advantages over later French revolutionaries. They had legislative experience. They had international support, whereas the French Revolution soon faced foreign invasion. They had a strong familiarity with Enlightenment thinking. They could focus on the important task of designing a new central government whereas the French revolutionaries grappled also with economic reforms such as the abolition of feudalism. Finally, continuity in governance at the state level provided a stability that the French revolutionaries lacked.

The world has witnessed hundreds of revolutions since those of America and France. In most, though not all, of these there was a desire to replace kings or dictators with democratic governance. Only a minority have succeeded in this aim, at least at first. Sometimes failure reflected the staying power of authoritarian elites, who employed their economic or military power to frustrate revolutionary goals. However, often the challenge is democracy itself. Democracy is much less pleasing in practice than in theory. The first post-revolutionary legislatures are often disappointments. Citizens may reasonably conclude that some autocrat might better govern them. This is especially likely if constitutional protections or checks and balances are not put in place at the start that limit the powers of elected legislators. Democratic governments need to be carefully designed. Even then, it may take decades before people become accustomed to rule by politicians. The American population had decades of experience of politicians and legislatures at the time of the revolution. This experience may have contributed to the election of relatively honest and farsighted leaders in the early years of the United States. 
We should distinguish institutions that are enshrined in law from those exercised in practice. It would become commonplace in the twentieth century for dictators to hold sham elections or proclaim bogus human rights protections. Pretending to democratic institutions is easy. It is harder to make these real. The success of the United States and other long-standing democracies depends on most of those in positions of power playing by the rules. Armies, in particular, need to support even officials they dislike. Laws must guide judges more than political pressure or bribery. Legislators must at least appear to care for the common good.

Perfection here may be unachievable. Certainly, even the United States has not been immune to bribery, or to government officials acting outside of their own laws. Nevertheless, the survival of democracy depends on confidence that such behaviours operate within severe limits, and that most people most of the time are treated justly by their government. This confidence has grown over time in most democracies (though we will wonder in chapter 32 if people may come to take democratic freedoms and responsibilities for granted over time). The early days of a democracy are its most challenging as the corrupt and power-hungry test the boundaries of new institutions. We need not lionize, but should celebrate, those revolutionaries who sacrificed personal power to establish institutions that limited their own power as well as the power of those who followed.

It is worth noting in this respect that George Washington was often urged to proclaim himself king of this new land. He refused. We can applaud him for this. Nevertheless, his decision also reflected the wishes of those he had fought with who were mostly determined not to replace one king with another.

The democracy established by the revolution was far from perfect. Only men of property could vote. However, some northern states abolished slavery soon after the revolution. Vermont would establish universal male suffrage in 1800, shortly followed by other states. (Women would only gain the vote after World War I; chapter 28.) The reasonably equal income distribution that characterized the early United States, especially in the north, likely aided this further democratization: There was no entrenched landed aristocracy that could fear and work against extending the vote to mere peasants.

\section{The French Revolution}

As with many previous states, the decline of the French monarchy in the late eighteenth century had financial roots. Massive debts had been incurred in fighting (and often losing) wars in the previous centuries, most recently the Seven Years War and the American Revolution. Half of the French budget was debt payments. The nobility and church were exempt from taxes. The French state had attempted some financial innovations in areas of government debt and paper currency, but these had not solved its financial difficulties. Therefore, 
King Louis XVI gathered the Estates General for the first time in 150 years to ask for more money, likely in return for ceding some power.

The French Revolution was more revolutionary than its American counterpart in that it sought a dramatic change in laws and social relations. It occurred in a much larger country (24 million, compared to just 4 million in the new United States) and one with limited democratic experience and an entrenched aristocracy and church. Like the American and Glorious Revolutions (in Britain), it was triggered by the king's need for funds. The nobles wanted an assembly that they could control and that would guarantee private property and free speech; the Third Estate (that is, all who were not noble or clergy) wanted a single assembly that they could dominate numerically. Arguing that they were not only the bulk of the population but paid the taxes, the Third Estate seceded from the Estates of the nobility and clergy. When the king closed their deliberations, they met at an indoor tennis court and pledged to continue until they had drafted a new constitution. At the same time, mobs in Paris and elsewhere gathered to complain about taxes and high bread prices (the harvest of 1788 had been particularly bad). They borrowed the American call of "No taxation without representation" and sang American revolutionary songs. They stormed the Bastille, a jail and armoury, killing some soldiers and the mayor of Paris. The king then agreed that the new "National Assembly" should draft a constitution. This National Assembly abolished feudalism and compulsory tithes (taxes) to the Church, proclaimed popular sovereignty and equal rights, and called for meritocracy and transparency in government, freedom of religion, and due process in law. A mob comprised mainly of women attacked the royal palace at Versailles and forced the king and his family to Paris. The National Assembly of 1789-91 drafted a constitution for a constitutional monarchy where males with property but of any religion could vote - about half the male population. More controversially, it confiscated Church property.

The king and his family attempted to flee in 1791 and were imprisoned in their Parisian palace. Thousands of aristocrats fled. Austria (Leopold the Austrian king was the brother of the French queen) urged other European monarchies to oppose the French revolutionaries; the new French legislature declared war in 1792. As noted above, some of the difference in outcomes between the French and American Revolutions may reflect the fact that the French revolutionaries came under foreign attack whereas the American revolutionaries received substantial international support.

When the war went badly at first, mobs stormed the palace. The monarchy was terminated. A new legislature was elected with universal male suffrage. It voted 361:359 to execute the king and his family. The closeness of this vote was symbolic of the intense debates that convulsed this new Assembly as it tried to plot a course forward. It should be stressed that the debate was far from (just) a philosophical discourse on the merits of the monarchy but was mainly a debate about this particular king and queen, who had been ridiculed and accused of corruption and wasteful spending in popular (often banned) literature for decades. The Paris communal government was even more radical than 
the National Assembly: It arrested thirty of the more moderate legislators from the National Assembly.

The Assembly then created a Committee of Public Safety that would execute some 40,000 for treason during what is now known as the Reign of Terror. This killing spree was aided by the technological innovation of the guillotine, whose sliding blade beheaded the condemned. The Committee also instituted a national draft to combat other states (conscription had been common in China for millennia, but European states had relied first on feudal levies and later on mercenaries). The Committee decided that feudal lords should receive no compensation for their former feudal landholdings. In addition, it abolished slavery in the colonies. Women could inherit and divorce, but not vote. The Committee's leader, Robespierre, was himself killed before the Terror ended. The public at first supported the killing of alleged traitors, especially those who had been powerful previously, but became appalled as the numbers of those killed grew.

The Assembly then attempted to establish a directory of propertied men elected by the Assembly to exercise executive power, but these elections were often disputed, and the army was called in to quell mob violence. Napoleon Bonaparte - promoted on merit to general in the king's army by the age of 24 , but a supporter of the revolution - was proclaimed First Consul in 1799 and Emperor in 1804. Democracy was terminated, but equal rights and the end of feudalism were embraced. Napoleon pursued a sort of benevolent despotism, arguing that authoritarian power was necessary to

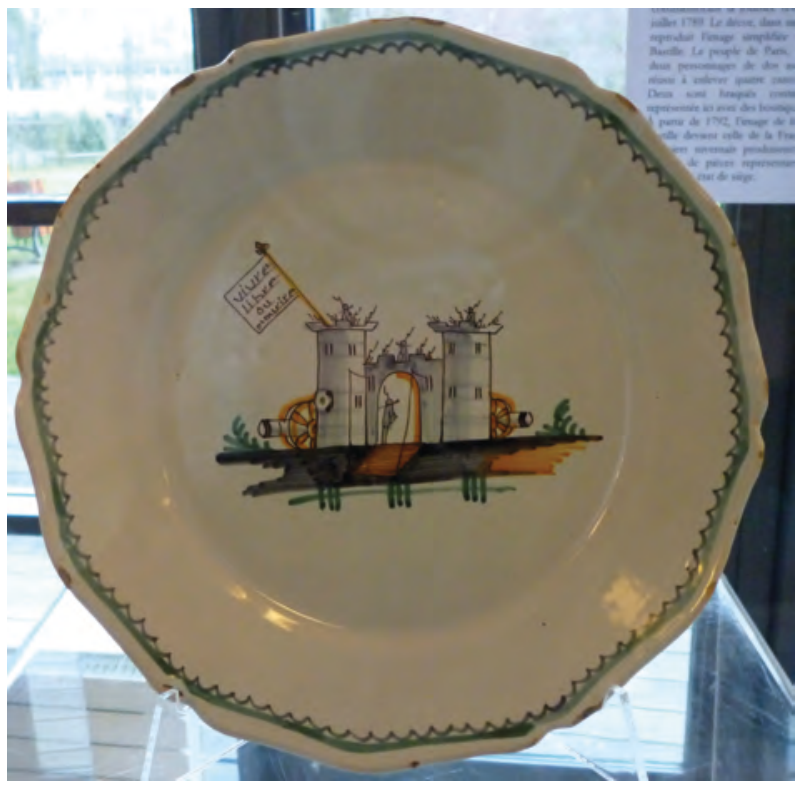

Illustration 25.4

"Vivre libre or mourir" means "live free or die." This plate from 1792 or 1793 is a call for vigilance during the French Revolutionary Wars, Musée des Beaux-Arts de Bernay Photograph by Stanzilla, CC0

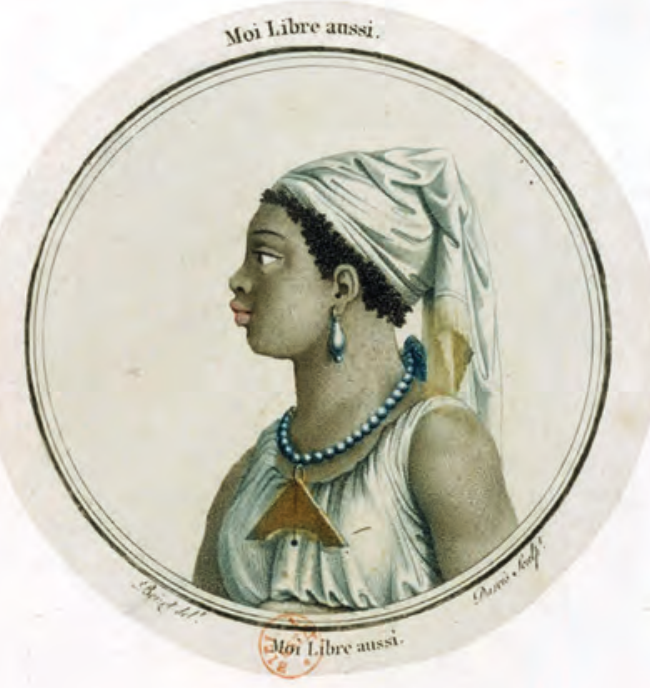

Illustration 25.5

Engraving representing a former Black slave dressed in a revolutionary outfit. The caption reads "Moi Libre aussi" (I am free too), Louis Boizot, 1792 
push forward the goals of the revolution. This included bureaucratic restructuring to stress merit in hiring and promotion, and a new law code that established due process. He reached an agreement with the Church whereby the state kept Church land but paid clergy of all faiths. He also removed ghetto walls in cities he conquered. Napoleon would spread by force across Europe the ideals of individual rights. Locals tired of autocratic government often welcomed him. However, he restricted free speech, reintroduced slavery, and reasserted male power in the home. He also frequently ruled through local elites.

Napoleon faced an alliance of most other European states but won many victories due to a combination of brilliant strategy and inspired troops. He was able to keep paying his massive army because of the booty and then tax revenues gained in these various conquests. He came to control most of continental western Europe. However, Napoleon overstretched - perhaps in part because conquests provided a significant revenue stream - losing 400,000 troops in Russia, and was defeated in 1814 and again in 1815 (after briefly escaping from custody). He also lost 50,000 troops trying to regain and reenslave Haiti (see below). The French monarchy would be restored, but as a constitutional monarchy that governed in concert with an elected assembly. Over the next centuries France would cycle between monarchy, republic, and empire (Napoleon's nephew would be elected president and later become

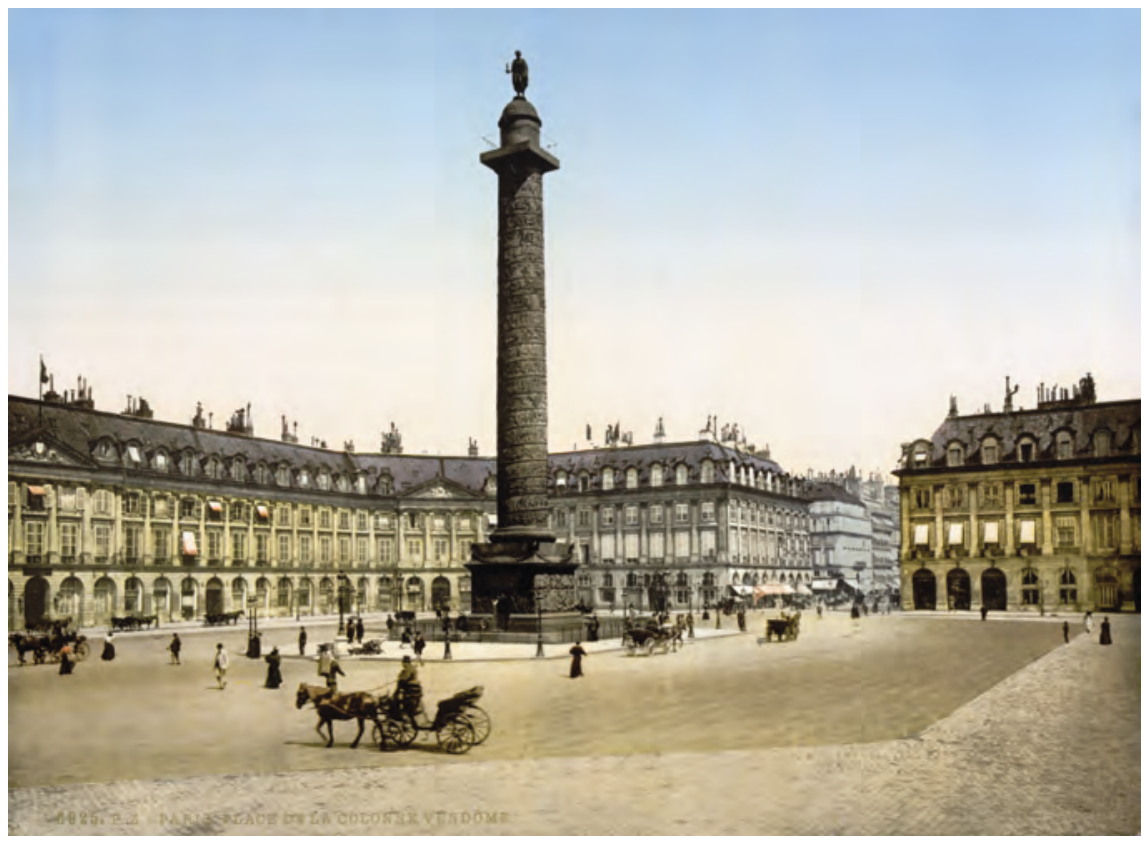

Illustration 25.6

Column in Place Vendome, c. 1890. Vendome Column was erected in 1805 using melted Austerlitz cannons to celebrate Napoleon, then redesigned during the royal restoration, again, and again in the 1870s 


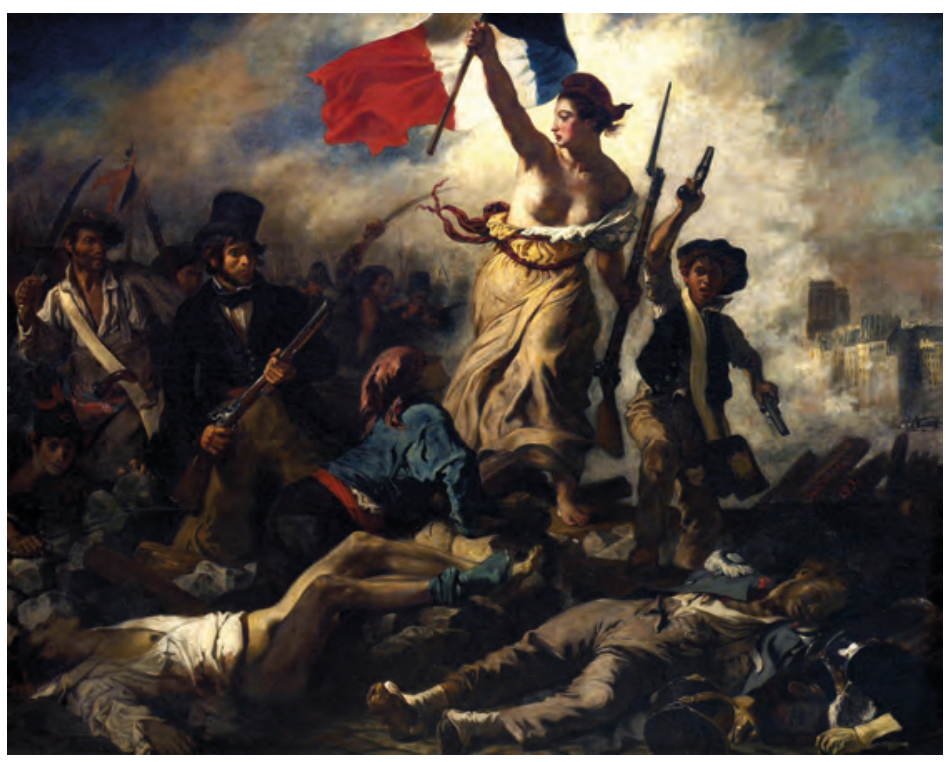

Illustration 25.7

Liberty Leading the People, by Eugene Delacroix, 1830, celebrating the Second French Revolution of that year, which displaced one constitutional monarch with another

emperor in mid century), before eventually settling on a strong presidential form of democracy after the World War II.

It is useful to compare the French and American Revolutions again. The French Assembly had passed its own Declaration of the Rights of Man and of Citizens in 1789, but it did not establish judicial institutions that could prevent the legislature from ignoring those rights. The French revolutionaries never solved the challenge of distinguishing executive, judicial, and legislative powers. An all-powerful legislature could too easily ignore human rights. Once a government starts executing its enemies, citizens are guided to seek stability at all costs.

Why did the French revolutionaries develop less successful institutions? The revolutionaries suffered both from limited experience with democratic debate and from a threatening foreign environment. They also faced a far more demanding agenda: They could not focus like the Americans on designing a new government as they had pressing decisions to make regarding feudalism, bureaucracy, foreign relations, and economic regulations. These other issues divided legislators - often along class or occupational lines - and made constitutional consensus far harder to achieve, while encouraging mutual suspicion.

Many revolutionaries in other lands in the nineteenth and twentieth centuries would face similar challenges - of lacking democratic experience and needing to remodel many institutions quickly, and often of foreign interference. Very few of these have been able to escape the French slide into authoritarian government. 


\section{PRIMARY DOCUMENTS: DECLARATION OF THE RIGHTS OF MAN AND OF CITIZENS, 1789}

We provide here a selection of articles:

Article I - Men are born and remain free and equal in rights. Social distinctions can be founded only on the common good.

Article II - The goal of any political association is the conservation of the natural and imprescriptible rights of man. These rights are liberty, property, safety and resistance against oppression.

Article III - The principle of any sovereignty resides essentially in the Nation. No body, no individual can exert authority which does not emanate expressly from it.

Article IV - Liberty consists of doing anything which does not harm others: thus, the exercise of the natural rights of each man has only those borders which assure other members of the society the enjoyment of these same rights. These borders can be determined only by the law.

Article VIII - The law should establish only penalties that are strictly and evidently necessary, and no one can be punished but under a law established and promulgated before the offense and legally applied.

Article IX - Any man being presumed innocent until he is declared culpable, if it is judged indispensable to arrest him, any rigor which would not be necessary for the securing of his person must be severely reprimanded by the law.

Article $\mathbf{X}$ - No one may be disturbed for his opinions, even religious ones, provided that their manifestation does not trouble the public order established by the law.

Article XI - The free communication of thoughts and of opinions is one of the most precious rights of man: any citizen thus may speak, write, print freely, except to respond to the abuse of this liberty, in the cases determined by the law.

Article XIII - For the maintenance of the public force and for the expenditures of administration, a common contribution is indispensable; it must be equally distributed between all the citizens, according to their ability to pay.

Article XV - The society has the right of requesting account from any public agent of its administration.

The preamble speaks of "natural, unalienable and sacred" rights and consisting of "simple and incontestable principles." While similar in many ways to the US Declaration of Independence, note how it attacks feudalism by urging that all offices be available to all, and that all pay taxes according to ability. Note also how it reflects ideas of popular sovereignty and refers to the nation as the source of all authority.

Can you see how the declaration reflects some of the abuses of authority of the previous regime? 


\section{Haiti}

The revolution in what is now Haiti was the only successful slave revolt in history. It was also the only successful revolution in the Caribbean. The halfisland was the home of sugar, cotton, coffee, and tobacco plantations responsible for a third of France's foreign trade. Indeed, the colony at one time produced a third of the world's sugar and half of its coffee. France earned more from this one colony than Britain derived from the thirteen colonies that revolted from 1776. Haiti before the revolution had half a million Black slaves, 40,000 White settlers, and another 30,000 free Blacks (some of whom owned slaves). There were many communities of escaped slaves in the mountains, known as maroons, who often attacked slave plantation owners. The French had sent a few hundred free Blacks to help in the American Revolution. When the French Revolution began, White settlers and free Blacks disagreed over who should have power in Haiti, with civil war ensuing in 1791. The Haitian elite shared with American revolutionaries a desire to escape from colonial restrictions on trade that forced colonies to trade directly with the colonial power. There had long been trade between Haiti and the North American continent that was at times illegal and at times grudgingly accepted by Britain and France (New Englanders made rum from Haitian molasses). Slave masters also feared the

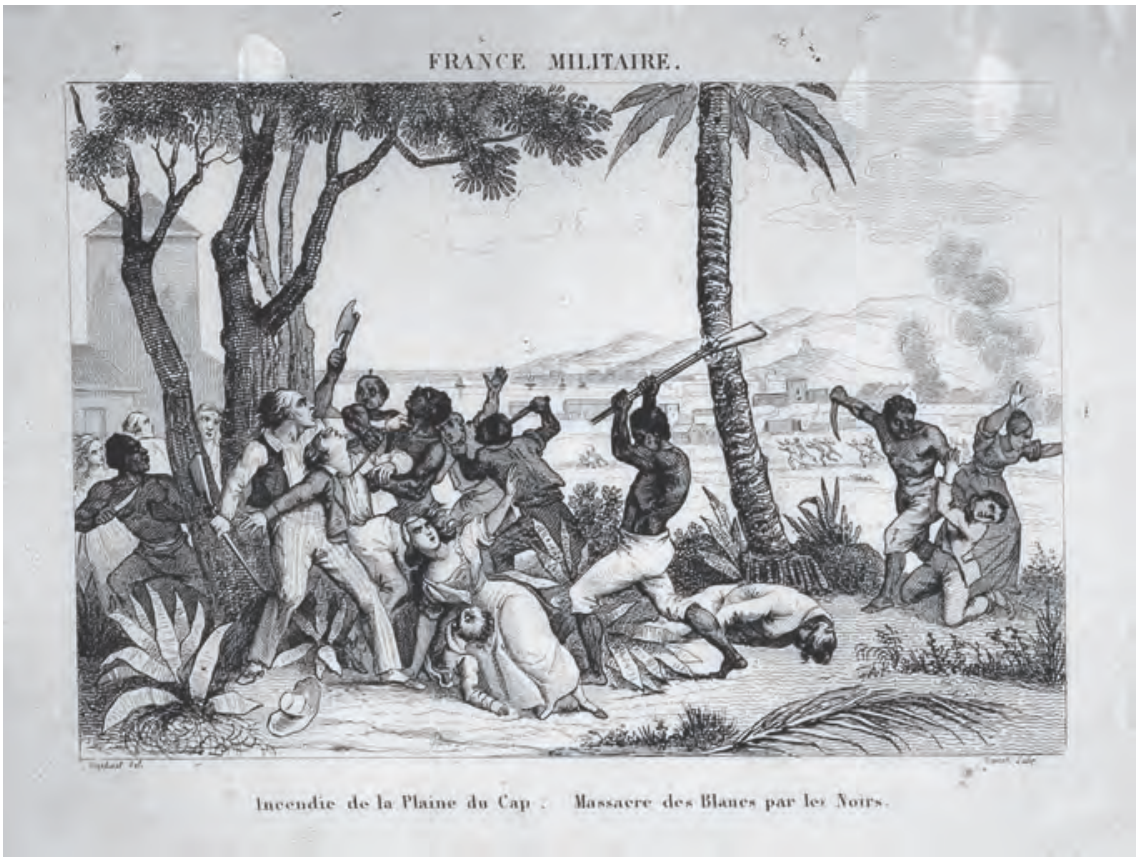

\section{Illustration 25.8}

"Burning of the Plaine du Cap - Massacre of Whites by the Blacks." On August 22, 1791 , slaves set fire to plantations, torched cities and killed White plantation masters. France Militaire, 1833 
abolitionist sentiments of some French revolutionaries. Slaves then revolted in Haiti, killing White settlers. Free Blacks also sought power. Many of the rebel leaders claimed that they were part of the American expedition (we have no records of who was part of the force sent to aid the American Revolution): We can speculate both that they learned revolutionary ideas while there and then chafed at their limited power upon return.

The revolutionary government in Haiti did not immediately seek independence from revolutionary France but did abolish slavery in 1793. This was arguably the most sudden abolition in history (see chapter 26) for abolition was rarely advocated just a couple of years previously. Abolition in Haiti encouraged the revolutionary government in France to abolish slavery throughout the French Empire in 1794 (see above). Note that a handful of Black Haitians served in the French National Assembly in the 1790s. While France pursued its own revolution, both the Spanish and British attempted unsuccessfully to invade Haiti. The American revolutionaries Washington and Jefferson sent arms to Haitian planters to support them against slave rebels. American writers of the time often heaped racist abuse on the leaders of the Haitian Revolution.

Francois-Dominique Toussaint (who added L'Ouverture or "opening" to his name because of his skill in breaching enemy lines) had been a free Black since 1776 but was the son of slaves, and managed to unite some 20,000 disciplined troops behind him. He controlled most of Haiti by 1797 and issued a constitution in 1801 that granted equality and citizenship to all residents (but he had not freed the slaves when he had earlier invaded the Spanish half of the island, and had worried that sudden abolition had crippled the Haitian economy).

Napoleon would reinstate slavery in 1802 and send an expedition to Haiti. Some 50,000 French troops and 18 generals would die of disease and battle in the Haitian campaign, which was the most massive seaborne expedition the French had ever launched. Haitian losses were likely much higher. Napoleon's army captured Toussaint and sent him to France, where he died in jail. However, the French army could not retake Haiti, and Haiti declared independence in 1804 as only the second independent republic in North America. Haitian troops were poorly armed and likely far less well trained than the French forces. Supporters in the United States shipped some arms to Haitian forces. Haitian success was in part due to the French army succumbing to tropical diseases, and in part because freed slaves in Haiti fought with determination not to be re-enslaved.

Haitian troops declared Jean-Jacques Dessalines, the commander of Haitian forces, emperor for life. He at first promised equality for all but soon began to massacre White settlers - except some who had cooperated with the revolution - and political opponents. He would, in turn, be overthrown in what would become a long succession of autocratic rulers, some of whom declared themselves kings. Though Haitian revolutionaries had imbibed many of the ideas of the American and French Revolutions, they had no experience of democracy - and limited education. Force of arms had achieved a significant societal change - the abolition of slavery - and force alone would determine power in Haiti going forward. 
France did not recognize Haitian independence until 1825. France sent gunboats that forced the Haitian government to pay crippling reparations to former planters. Other nations were even more hostile. The United States would be the last major nation to recognize independent Haiti in 1862. Though the Haitians had not achieved the democratic reforms of the American Revolution, they had achieved a crucial social reform with the abolition of slavery. It could be argued, indeed, that the Haitian Revolution was more revolutionary than its American and French counterparts. It certainly challenged entrenched economic interests to a far greater degree. Those states that continued to enforce slavery viewed the abolition of slavery as a threat. Yet while the United States refused to recognize the new land, it soon resumed trade with Haiti. Ex-slaves managed to produce coffee exports on small plots of land with which to purchase a variety of manufactured goods from the United States.

The Haitian Revolution forced intellectuals in Europe and America to grapple with issues of slavery in particular and human rights more generally. Historians now widely credit the Haitian Revolution with a formative role in the move toward abolition (chapter 26) and the general recognition of universal human rights (chapter 27). Unfortunately, very few documents have survived from Haitian revolutionaries themselves so that we could know how they both read and contributed to these debates. Displaced slave holders often moved to other Caribbean islands where they became a strong voice in favour of slavery.

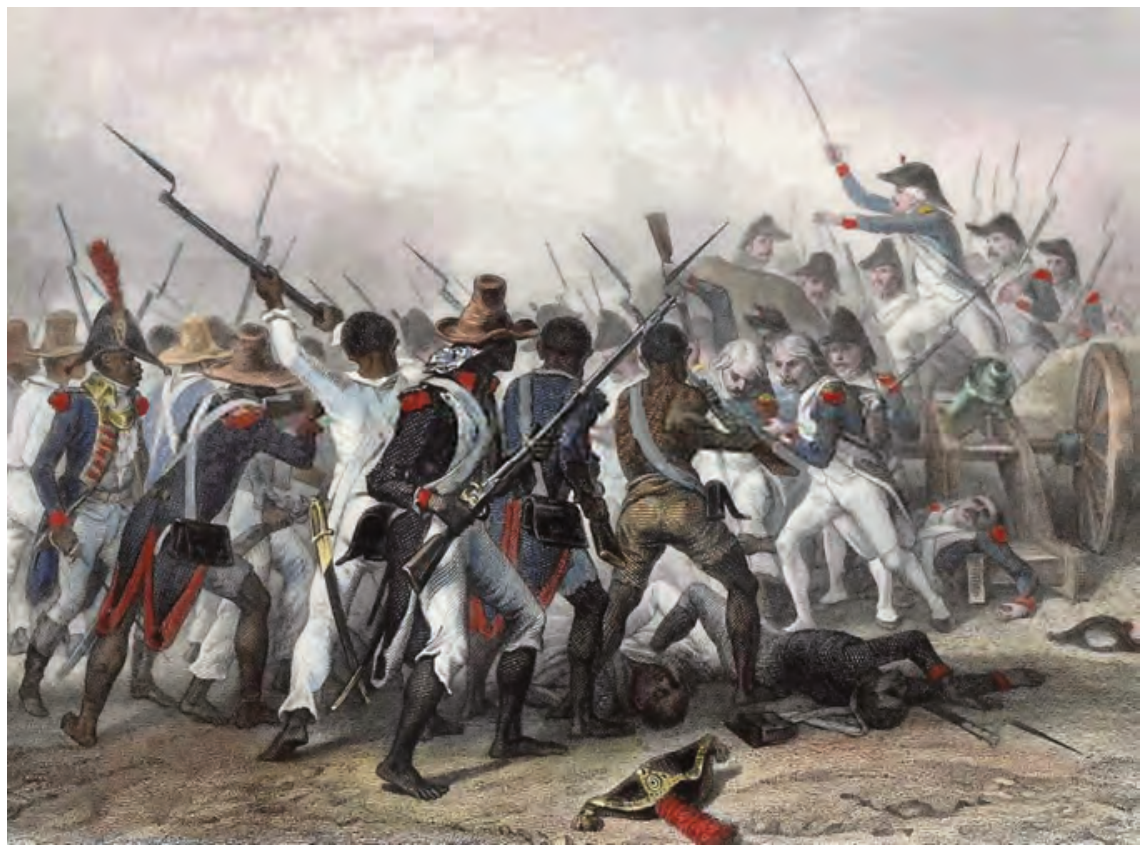

Illustration 25.9

Battle of Vertieres, 1803, Auguste Raffet 


\section{PRIMARY DOCUMENTS: THE HAITIAN DECLARATION OF INDEPENDENCE OF 1804}

This document emphasizes slavery from its opening lines:

It is not enough to have expelled the barbarians who have bloodied our land for two centuries; it is not enough to have restrained those ever-evolving factions that one after another mocked the spectre of liberty that France dangled before you. We must, with one last act of national authority, forever assure the empire of liberty in the country of our birth; we must take any hope of re-enslaving us away from the inhuman government that for so long kept us in the most humiliating torpor. In the end we must live independent or die.

Can you see how such a document might justify authoritarian rule? Can you see how revolutionary sentiment might have focused on the singular issue of slavery in a land where almost 90 per cent of the population had been enslaved?

Postscript: Though the Haitian Revolution had important impacts on the world, Haiti itself has had a troubled history since then. Economically, Haiti has long been the most impoverished country in the western hemisphere. Politically, it has been plagued by dictatorships and civil wars; efforts to institute democracy are recent and tentative. The Haitian revolutionaries had no experience of democracy, and so autocracy associated with military force immediately became the norm for governance on the island. The disdain that other states showed for independent Haiti - including a two-decade occupation by US Marines from 1915 - likely encouraged an authoritarian and militarist form of governance.

\section{Latin American revolutions}

During the period 1810-26, almost all Spanish colonies in the Americas revolted successfully, the principal exceptions being Cuba and Puerto Rico. These revolutions espoused liberal ideals, and particularly the idea of popular sovereignty, and echoed the arguments of American and French revolutionaries. Nevertheless, the outcomes of the French and Haitian revolutions frightened many revolutionaries. Local elites of Spanish or mixed ancestry who had long dominated local economies generally led the revolts. These had in the eighteenth century worked with Spain and Portugal to crush popular rebellions in Brazil, Bolivia, and Peru. The desire to maintain elite power generally overwhelmed liberal ideals. Creoles - those of Spanish or mixed 


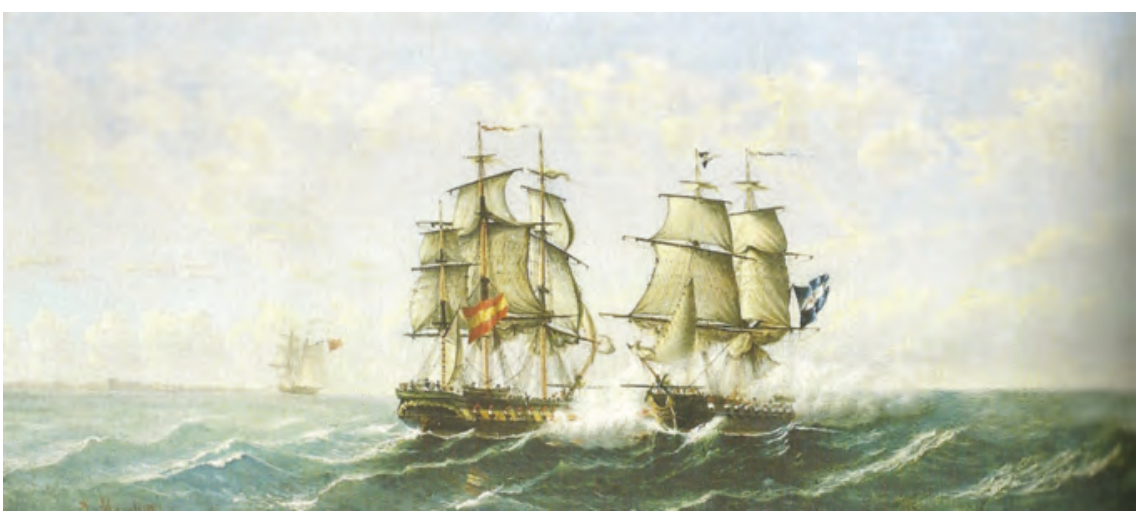

Illustration 25.10

Naval battle, 1810, Rio de la Plata, Argentina, Jose Murature, 19th century

ancestry - grated at the exercise of power by those from Spain, but were often even more fearful of extending political power to large Black or Indigenous populations. These elites often also pursued a strict Catholicism. Nevertheless, they distinguished themselves from leaders in Spain by being more open to recognizing Indigenous and Black people as citizens. This at first may have simply been a strategic argument to increase Latin American representation in the Spanish Cortes (legislative assembly) but would become a key argument for revolution.

The revolutions gained momentum while Napoleon controlled Iberia. Local assemblies were established at this time that urged greater autonomy but remained loyal to the crown. Spain was weak even before this but would be even weaker after, suffering an internal rebellion in 1820. Spain tried to reassert control in its colonies after the fall of Napoleon but with limited resources and success. The Creole revolutionary Simón Bolívar attempted to forge a united state in South America, and temporarily united Colombia, Venezuela, and Ecuador, but his generals would divide this territory up roughly along the lines of Spanish administration. The result was a set of nation states that characterize the region to this day, at a time when most of Africa and much of Asia was still colonized and much of Europe still dominated by multi-ethnic empires. Most of the nation states in the world in 1830 were in Latin America, and these states would do much to encourage the global evolution toward a system of nation states (chapter 21). As in post-independence Africa a century later (chapter 30), the borders set by the colonial powers at the time of independence were mostly respected. The main exceptions occurred when Chile took land from Bolivia (rendering it landlocked to this day) and Peru in the 1870s, when the United States encouraged an independence movement in Panama (from Colombia) in 1898 in order to gain permission to build the Panama Canal, and when Central American states seceded from Mexico (below).

Though revolutionary armies had enticed slaves with promises (which they generally honoured) of freedom, the results of revolution were dictatorships 
and entrenched economic inequality. Dictators - autocratic rulers who unlike kings lack a historical claim to legitimacy - were rare outside Latin America between the fall of Napoleon and the rise of communism, but were prevalent within. Governance by military dictators proved highly unstable in many countries: There were sixty coups or revolts in Bolivia alone during the nineteenth century. Corruption was also typical: This reflected both political inexperience and venal dictatorship. Of course, elites were better able to bribe officials than were the poor, and thus corrupt governments tended to reinforce inequality.

In the latter decades of the nineteenth century, most Latin American countries introduced assemblies (this occurred earlier in Colombia), but voting rights were generally restricted to elites. This limited introduction of elections coincided with the development of export markets for minerals and agricultural products, and massive immigration from Iberia and Italy. Immigration, by transforming the ethnic mix in several countries, may have caused elites to be less fearful of democratization. Foreign investors often financed exports and transport infrastructure: These, though quite happy to work with dictators, may have encouraged the greater stability that democratization might bring.

Though Latin American countries would belatedly transition to democratic governance in the later twentieth century, they still have on average the most unequal income distributions in the world (and often corrupt judiciaries and limited tax collection that caters to elite interests; as we have seen, the task of developing democratic institutions is far from simple). The economic inequality that existed in most states at the time of independence discouraged the immediate transition to democracy that Bolívar and others had sought. Most agriculture occurred on large haciendas, generally owned by Creoles. Plantation economies, with a small, wealthy elite and a large group of poor workers or slaves, encouraged a form of politics that maintained the power and status of the narrow elite. Military strongmen exercised authority in collaboration with the elite. As noted above, economic inequalities often reflected ethnic divisions. Economic self-interest could thus combine with ethnic identification in discouraging elite support for democratization.

Plantations in many countries reflected the nature of sugar or cotton or tobacco production: These crops were well suited to large estates with lots of unskilled but arduous labour. Yet even grain and cattle production, handled in most of Canada and the United States by family farms (though slaves worked on grain farms in Virginia), was operated in Argentina in large estates. The difference reflects in large part the different institutions employed regarding land ownership by Spain and Britain in their colonies. Though elite groups had restricted voting rights to men of property in the early United States, these were a much more substantial proportion of the population, at least in the northern states. It thus proved far easier to gradually extend voting rights in the United States than in Latin America.

One exception to the combination of autocracy and income inequality was Paraguay. It pursued both democracy and policies of land redistribution in the 
nineteenth century. Surrounding elite-dominated states invaded Paraguay in 1865-70, killing most of the adult male population.

The fact that Spain had preferred to send Spaniards to administer its colonies would complicate life after independence. Unlike American revolutionaries, Latin American revolutionaries had little experience of either elected assemblies or service at senior levels of the bureaucracy. There was a further challenge of institutional inheritance. These newly independent states tended to maintain most Spanish laws - just as American revolutionaries had maintained British laws. If we accept that British institutions were supportive of economic growth (chapter 24), then the United States of America may have started with a significant economic advantage in addition to its greater political experience.

The revolutionary experience of Mexico reflected an intense competition for power between elites and those who sought more inclusive forms of government. Mass revolts at first guided the Mexican Revolution, but Creole elites would nevertheless gain control at independence. They declared a republic with very limited voting rights. Mexico would be unstable for many decades after that, and would lose territories in Central America and to the United States (see above). Central America declared its independence from Mexico soon after Mexican independence and then fragmented into the states that exist to this day. When the Indigenous Benito Juárez gained power in Mexico and sought to establish universal male suffrage (and was applauded by liberals in the United States and elsewhere, including the Italian revolutionary Garibaldi), Mexican elites invited French troops to establish a European monarchy in Mexico. However, Archduke Maximilian from Austria was killed within years of being installed as ruler. French intervention in the Americas was possible because the Civil War was convulsing the United States at the time; there was American opposition to Maximilian after the end of the Civil War. After the death of the reinstated Juárez, a dictatorship of Porfirio Díaz would last from 1876 until 1911, to be replaced in yet another revolution by the one-party state of the Institutional Revolutionary Party (PRI). Some 1 million of Mexico's 15 million people died in the revolution from 1911 until 1920. Though the PRI held elections, it would not lose a national election until 2000. The PRI would, though, extend education and provide periodic land reforms.

\section{PRIMARY DOCUMENTS: EXCERPTS FROM THE MEXICAN CONSTITUTION}

The Constitution of 1917 remains in force. It is a very long document, and many articles expressed aspirations that remain to be achieved. We quote here from article 3 , which proposed a free and secular public education, and article 123, which expressed some very progressive thoughts on labour relations. 
Article 3: Instruction is free. ... No religious body, nor a minister of any religious sect, will be allowed to establish or direct schools of primary education.

Article 123 has many subsections:

1. Eight hours will be the maximum limit of a day's work

2. The maximum limit of night work will be seven hours...

3. For children over twelve and under sixteen years of age the maximum limit of a day's work shall be six hours. The labour of children under twelve years of age may not be the object of a contract.

Section 4 insists on one day off per week, section 5 limits physical labour for a pregnant woman and calls for one month of paid maternity leave after birth, chapter 6 calls for a minimum wage that can supply the basic necessities of life, and section 7 insists on the same wage for the same work regardless of gender or ethnicity. Later sections speak to unions and bargaining.

How can you place this document in the context of the tensions we have described in Latin American nation-building? What were the intentions of those who drafted the document?

The case of Costa Rica deserves particular attention here. It was a land of small farmers at the time of its independence. Though the development of coffee exports over the next decades would create a wealthy elite and force Indigenous peoples off their lands, the coffee elite often cooperated with existing small landholders. Though Costa Rica experienced several dictators in its early history, it also experienced efforts to develop assemblies and an independent judiciary. It has been governed democratically since 1869 , with only a couple of brief periods of civil conflict. It took the unusual step of abolishing its army after a civil conflict in 1948. To be sure, this decision was made suddenly by a weak government facing financial difficulties - but it was only possible because there was no strong military tradition in Costa Rica and no segment of the population that thought their interests were tied to the military. It was also only possible because treaties among Central American nations in 1902, 1907, and again in 1923, as well as a non-aggression treaty between the United States and fourteen Latin American countries in 1933, had decreased Costa Rica's need for self-defence. However, note that other Central American governments did not enact a similar policy since dictators there relied on army support. The savings on military expenditure allowed Costa Rica to spend more on health and education than its neighbours. Its democratic institutions have survived the development and decline of an unequal banana export sector, and deep divisions over the gradual extension of social welfare schemes to poorer Costa Ricans. Though many individual actors demonstrated both courage and judgement in creating and maintaining Costa Rica's democracy, arguably they were successful in large part because Costa Rica had more egalitarian economic and social relations than other Latin American countries. 


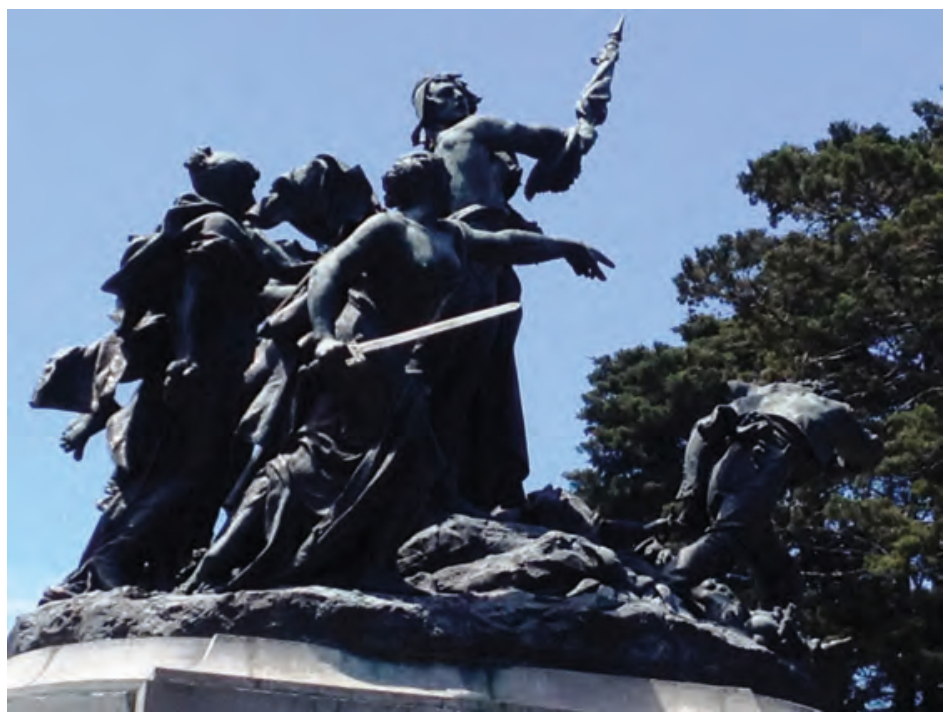

Illustration 25.11

National monument of Costa Rica, Louis-Robert Carrier-Belleuse, 1891

The path to independence in Brazil was entirely different from that in the former Spanish colonies. The Portuguese royal family escaped to Rio de Janeiro with British help in 1807 when Napoleon invaded Iberia. It stayed thirteen years and raised the status of Brazil to equivalence with Portugal itself. Brazil thus briefly headed the Portuguese Empire. When the king returned to Portugal, local elites insisted on the maintenance of Brazil's enhanced status. The king left a son, Pedro, behind, who with Creole instigation declared Brazilian independence in 1822 .

\section{The Sokoto caliphate}

The revolutions above sought to replace autocratic rule. Some resulted in an increased exercise of popular sovereignty while others transferred power to different elites or autocrats. The Sokoto caliphate was created in West Africa in 1804 because of a revolution against autocratic rule in the area. Usman dan Fodio, a Fulani religious scholar, led the revolution. He objected primarily to the constant warfare in the region and the resulting enslavement of millions of Muslims. He railed against a practice forbidden by the Quran, and was attacked in turn by the king of the state of Gobir. Dan Fodio thus cast the revolution as a holy war against un-Islamic practices. He sought to replace existing kings in the region with a polity that would respect Islamic principles. The resulting caliphate would come in the next decades to encompass some ten million people ranging from modern-day Burkina Faso across northern Nigeria to Cameroon. It was, though, a loose federation in which local emirs retained considerable power. Still, the 


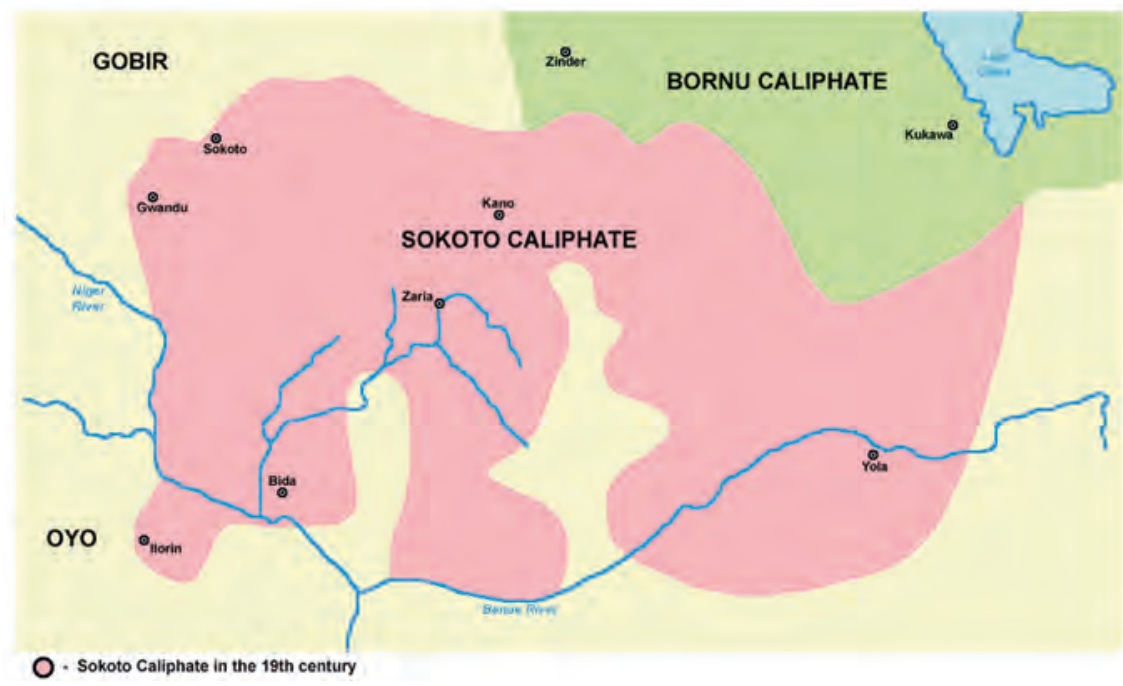

Map 25.2

The Sokoto caliphate in the late 19th century Map by PANONIAN, CCO

caliphate did succeed in ending internal warfare and the enslavement of Muslims. The Fulani conquerors intermarried with local Hausa elites. The caliphate also encouraged trade within and beyond the region. Indeed plantations exported cotton, indigo, and other crops. Ironically, perhaps, the number of slaves in the region grew as these plantations expanded. Slaves were sometimes purchased from other lands or obtained in warfare against non-Muslim lands. Locals were often accused of pursuing pagan practices in order to justify their enslavement. There were at least one million slaves and perhaps well over two million in the later decades of the caliphate. The caliphate was likely second only to the United States in terms of total slaveholding in the mid nineteenth century - but slavery was much less harsh in the caliphate. Slave masters were expected to introduce slaves to Islam. Slaves were supposed to be freed if they converted. Slave masters often treated slaves as part of an extended family.

The Sokoto caliphate was prosperous both politically and economically. It was perhaps the greatest African empire of the nineteenth century, and it entrenched Islam across a broad region of West Africa. Its decentralized political structure may have lessened its ability to cope with European colonialism (chapter 23). In 1903, the caliphate would fall to the British after decades of both internal strife and attacks by the French and British.

\section{Other revolutions and revolts}

There were definite connections among the many revolutions discussed above. There would then be no more successful revolutions (if we define success 
in terms of replacing autocracy) until the twentieth century, though failed revolutions would occur with some regularity in the nineteenth century. The idea of popular sovereignty - and perhaps especially the American cry of "No taxation without representation" - resonated globally. Yet each revolution naturally combined these general principles with more local concerns (such as whether the Qing in China had lost the Mandate of Heaven).

In 1820, only five years after Napoleon's defeat, there were revolts in both the Kingdom of the Two Sicilies in Italy and in Spain. In both places, revolutionaries called for a constitutional monarchy with elected assemblies. In both places, kings promised reforms. Yet in both places kings successfully appealed to foreign kings for support. Austrian and northern Italian troops crushed the Italian uprisings, and 100,000 soldiers of the restored French monarchy defeated the Spanish revolutionaries. These events established that the large and well-trained armies created during the Napoleonic Wars could defeat revolutionary movements.

In 1848, there were revolts in some fifty countries in Europe and Latin America. Uprisings in one country inspired uprisings elsewhere. There were some international linkages also among revolutionaries, who shared some goals and strategies, though each revolt was guided internally. Nationalism joined popular sovereignty and in some places socialism in motivating revolutionaries. As in 1820, there were general calls for elections and reduction in the powers of kings in Europe or military dictators in Latin America. In many countries, though, organizations of workers fought also for reforms in social policy and the legal recognition of unions, and sometimes for societal ownership of industry. There was thus often a loose alliance of middle-class and workingclass revolutionaries. Their aims overlapped but were far from identical. We can therefore attribute these many revolutions in large part to the rising importance of both the middle and working classes (chapter 23). The economic dislocation associated with particularly bad harvests in 1846 may in part explain the simultaneous occurrence of so many revolts.

States crushed most of these revolts, as had happened in the 1820s, killing or deporting many revolutionaries. They were guided both by naked selfinterest (as often were revolutionaries) and by terror at the instability that had characterized the French Revolution (a feeling reinforced by intense debates among revolutionary factions in many countries). Nevertheless, the revolts inspired autocrats to consider social policies that might temper revolutionary fervour. The Austrian Empire was harsh in its treatment of nationalist revolts but did soon after move to abolish feudalism (chapter 26).

Some revolts led to changes in institutions of governance. In France, the uprising toppled the constitutional monarchy established after Napoleon's defeat and elected Napoleon's nephew as president of a new republic; he would declare himself Emperor Napoleon III four years later. Denmark established a constitutional monarchy in response to revolutionary demands. Though there were no revolts in Britain's Canadian colonies in 1848 (there had been in 1837), the revolutions in Europe and Latin America arguably encouraged Britain to grant a considerable degree of self-rule to these. 
Attempted revolutions also occurred in Asia in the mid nineteenth century. The Taiping Rebellion from 1851 in China resulted in some 20 million deaths. Rebellion leaders blended Christian and Buddhist ideas, and redistributed land and enshrined women's rights in territories they controlled. The early leader Hong Xiuquan - after having failed the examinations to become a bureaucrat earlier in life - proclaimed that he was the brother of the Christian Jesus and had been sent by god to liberate China from the foreign Qing and antiquated Confucian principles. He attracted followers in large part because the Qing had been humiliated in wars with Europeans and had struggled to address local famines. As often in history, early successes attracted more followers. He and his followers came to control a territory in southern China with some 30 million people. Civil conflict engulfed almost every Chinese province. The Taiping were eventually defeated, in large part as the Chinese gentry rallied to the defence of the Qing state to avoid losing their lands and treasured Confucian beliefs. The rebellion inspired a deep suspicion of Christianity. It also further weakened the Qing state.

The Indian "Mutiny" of 1857-9 began as a mutiny of East India Company troops and employees but soon expanded into a more general

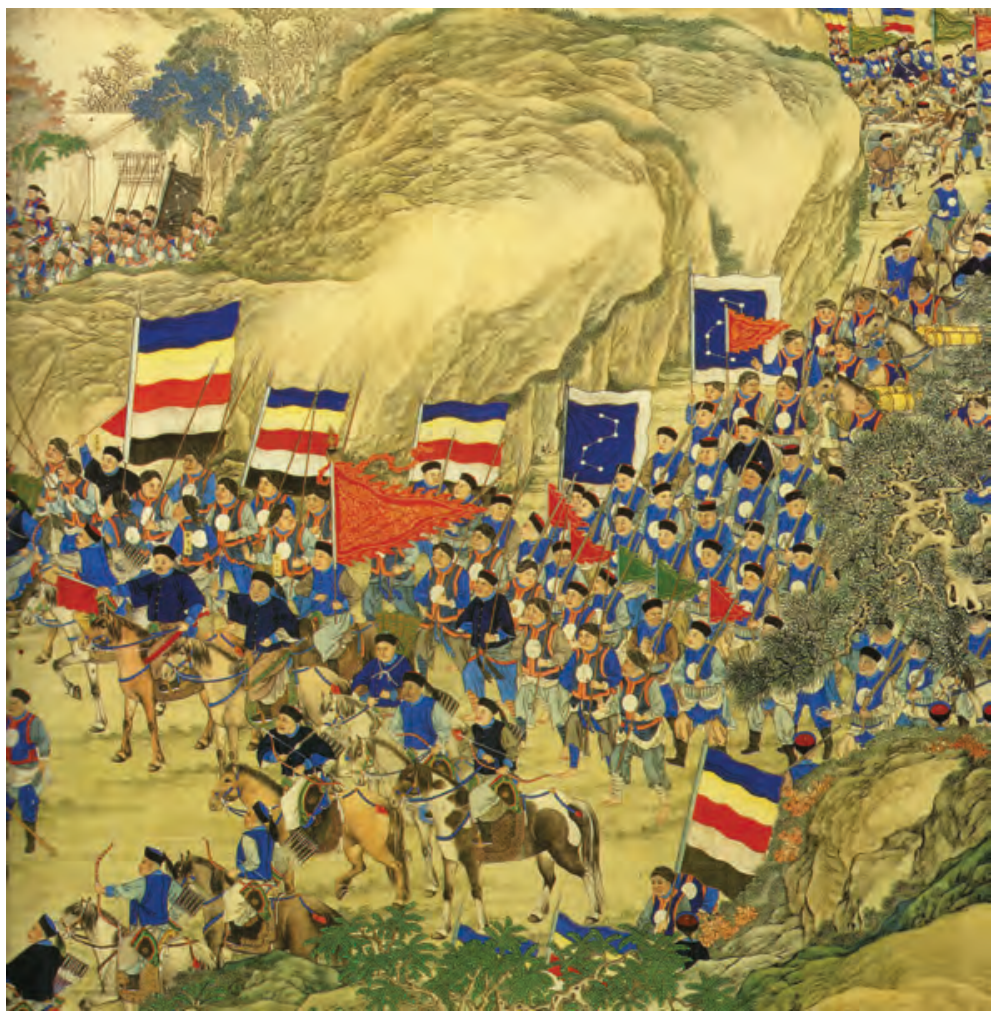

Illustration 25.12

Suppression of the Taiping Rebellion, unknown, c. 1860 


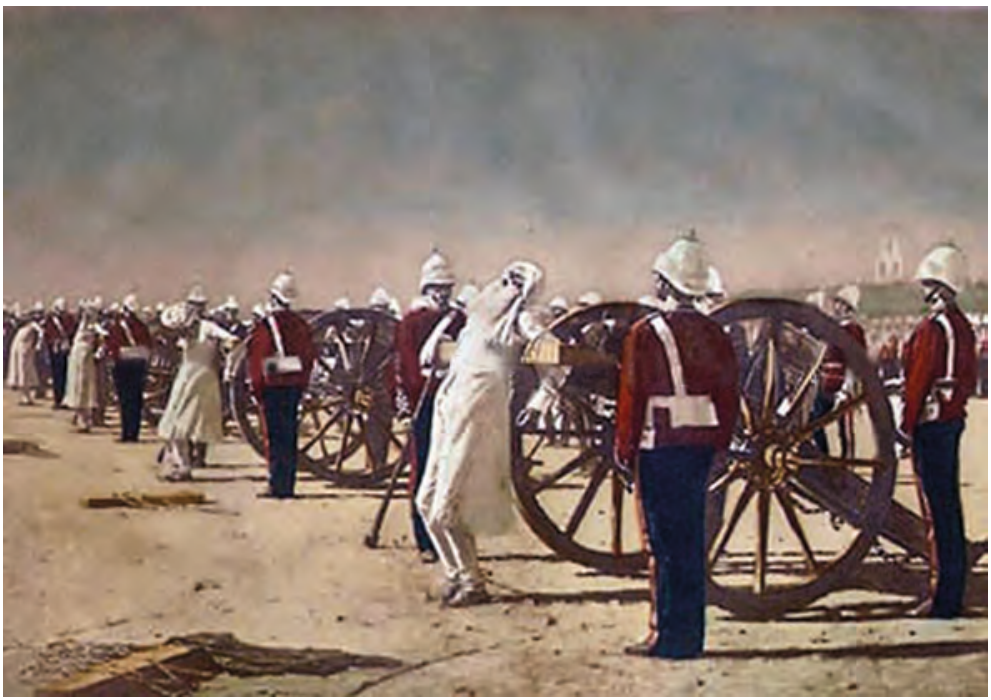

Illustration 25.13

Execution by cannon of Indian soldiers who rebelled during the "Mutiny", 1857-9

rebellion against Company rule. The spark for the initial mutiny was that Indian soldiers were required to bite open cartridges greased with animal fat from both cows and pigs - offensive in turn to Hindu and Muslim religious beliefs. The wider conflict is now generally termed a rebellion or even the First Indian War of Independence. As in the American Revolution, the Indian population was divided between those who remained loyal to Britain and those who sought independence. Yet there were general concerns about both economic policy (especially from those who disliked British land reforms) and cultural policy (especially how history was taught in schools). The conflict was particularly brutal. Some 6,000 of the 40,000 Britons in India were killed, while (at least) hundreds of thousands of Indians died. Tales of torture and sexual assault enraged both sides. British troops at times took no prisoners and raped local women as an act of retribution for attacks on the families of British officials. This rebellion inspired the British government to take India from the East India Company. Queen Victoria of Britain would promise Indians that they would be granted the same rights as British subjects; the British would often be reminded of this unkept promise in succeeding decades. Though each of these Asian rebellions had important effects, neither of them erased absolute government.

The next major revolution - that is, one that overthrew a government would occur in Russia in the early twentieth century (see chapter 27). In Russia in 1905, China in 1911, and both Iran and the Ottoman Empire in the first decades of the twentieth century, revolutionaries tried to replace autocrats with democracy. All would fail to establish a stable democracy - though Turkey 


\section{BOX 25.1}

\section{REVOLUTIONARY COMMONALITIES}

There have been many dozens of revolutions in the last couple of centuries. Yet autocratic governments have also often survived attempted revolutions. Can we identify the historical conditions under which revolutions are most likely to both occur and succeed? It seems that revolutions happen in response to a confluence of influences. This should hardly be a surprise at this point in the book for we have seen that major transformations in history generally reflect multiple influences. What, then, are the set of forces that tend both to generate revolutions and to increase chances of success?

- Economic circumstances are important. Harvest failures can encourage revolt among the hungry. Concerns over government taxation also loom large.

- Revolutionaries must imagine that they might succeed. Signs of weakness or conflict among ruling elites can be important here. Sadly, one lesson that autocrats might take from history is that efforts to compromise may be interpreted as signs of weakness. We might advise autocrats to display real respect for democratic principles, rather than seeming to cave in against their will.

- Revolutions require that many people act in concert in a dangerous project with unpredictable outcomes. Ideas thus matter. Revolutionary leaders must generate a shared sense of purpose.

- A shared sense of injustice must, in turn, support these ideas. Large numbers of people must have come to doubt that their ruler deserves to rule. Here the lesson for autocrats is more benign: By reducing injustice, they can mitigate revolutionary fervour.

- Revolutions do not occur in a vacuum but reflect the ties that existing rulers have to other states. Revolutions are more likely if rulers are not internationally popular. Revolutionaries also may attract support - or opposition - from other states.

would achieve democracy later. In all these cases, we can point to some mix of entrenched income inequalities and limited experience of democracy. Both Russian and Iranian autocrats had experimented with elected assemblies but only briefly.

\section{Questions}

1. We identified above some factors that jointly encourage successful revolution. Can you identify factors that together encourage democratic outcomes from revolution?

2. Describe the challenges facing revolutionary leaders or followers.

3. Diagram the influences on and effects of revolution.

4. What insights do we gain by comparatively discussing revolutions?

5. What advice does history provide to potential revolutionaries? 


\section{Readings}

Armitage, David, and Sanjay Subrahmanyam. 2010. The Age of Revolutions in Global Context. New York: Palgrave Macmillan.

Brown, Matthew. 2015. "The Global History of Latin America." Journal of Global History 10:3, 365-86. [Argues that Latin America deserves more attention than it generally receives in world history. Mentions its importance with respect to revolutions and nation states in particular.]

Dubois, Laurent. 2004. Avengers of the New World: The Story of the Haitian Revolution. Harvard University Press. [See also his The Aftershocks of History, 2011, which traces how the experience of the Revolution has contributed to Haiti's oftenunfortunate political and economic history since that time. His co-authored Slave Revolution in the Caribbean, 1789-1804: A Brief History with Documents (New York: Bedford/St. Martin's, 2006) contains a translation of the entire Haitian Declaration of Independence.]

Ellis, Joseph. The Quartet: Orchestrating the Second American Revolution 1783-1789. Knopf. [Discusses the development of the US Constitution after hostilities had ended.]

Goldstone, Jack. 2014. Revolutions: A Very Short Introduction. Oxford University Press. [Explores the factors that combine to encourage successful revolutions.]

McPhee, Peter. 2016. Liberty or Death: The French Revolution. New Haven, CT: Yale University Press. [This book addresses multiple influences on and effects of that revolution.]

Joanna Neilson, "Nineteenth-Century Constitutions: A Teaching Guide," World History Connected, http://worldhistoryconnected.press.uillinois.edu/16.1/ neilson.html, provides links to various constitutions in English. She suggests asking students to compare the role of individuals, and the nexus of power, across constitutions. Other comparisons are possible. 


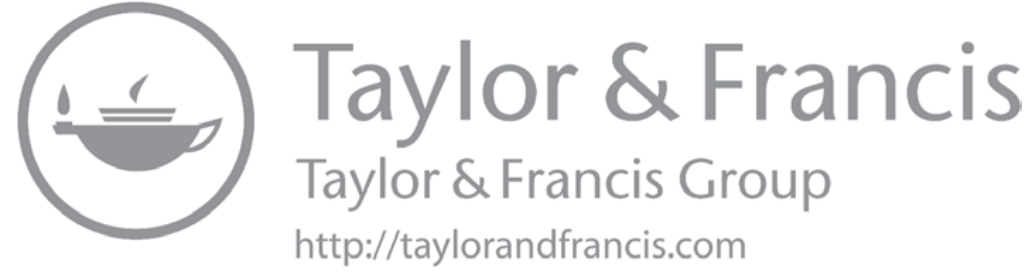




\section{A unique historical transformation}

\section{The abolition of slavery and serfdom}

\section{Guiding questions}

Why and how were the slave trade, slavery, and serfdom abolished? When and where did this occur and how did ideas about abolition spread across states? Who were some of the critical actors in abolition?

How did abolition affect other sorts of coerced labour?

What were the effects of abolition? On economy and polity? On freed slaves and serfs? On former masters? On cultural attitudes surrounding race?

Relationship to other chapters: We have discussed slavery in many chapters but especially 9 and 19. We have addressed feudalism in many places, but especially chapter 13 . We naturally benefit from understanding why slavery and serfdom existed in the first place when we attempt to understand abolition. Moreover, we can only appreciate the enormity of this historical transformation once we recognize how common practices of slavery and serfdom had been through history. There are important connections between abolition and the political and economic developments we have discussed in chapters 22, 24, and 25, and the cultural attitudes associated with these. We will not often explicitly mention abolition in later chapters but can appreciate here that a society characterized by free labour is 
quite different culturally and politically from a society of slaves or serfs. In particular, the student can reflect on whether international consensus around human rights (chapter 27) would have occurred in the absence of abolition.

Slavery was prevalent in human societies from before humans developed writing systems that could describe it. It would linger well into the twentieth century in a few countries - and continues illegally in some places even in the twenty-first century. However, states would outlaw slavery through almost the entire world during the nineteenth century. At the same time, and for similar reasons, the last European countries that still tied peasants to the land abolished serfdom. Though serfdom was less common across time and place than slavery, it had characterized much of European agriculture for well over a thousand years (chapter 13).

The abolition of slavery and serfdom is one of the most remarkable transformations in all of world history. Before the nineteenth century, some proportion of the population was either enslaved or enserfed across the vast majority of human societies. Slave masters had rights to sell, beat, and coerce their slaves. Landowners had a more limited set of powers over serfs but could direct their labour much of the time and could even in some places sell them to other landowners. After the nineteenth century, it was generally no longer legal for one person to control the entire life of another. Former slaves and serfs gained rights to move and to choose whom they worked for - though in practice many people might face a very limited set of opportunities. In this important way, life since the nineteenth century has been different from life before the nineteenth century. With the abolition of slavery and serfdom, ideas of universal human rights (chapter 27) could become widely shared.

The fact that slavery and serfdom largely disappeared at the same time, and across a wide range of polities, suggests that there were common sources of change. As we shall see, ideas were of critical importance: Many people came to view slavery and serfdom as simply unacceptable. Philosophers espoused these ideas, and societal values changed to incorporate them. Yet ideas do not generally emerge in a vacuum: We must be careful also to explore economic and political considerations. Nevertheless, once some societies pursued abolition, and proclaimed their ethical superiority for having done so, governments in other lands felt immense pressure to do the same.

Though the abolition of slavery and serfdom was a revolutionary transformation in human relations, we should not imagine that the situation of freed slaves or serfs improved dramatically overnight. Most of them continued to work for the same employers under conditions that were only slightly better. In many post-slave societies, racist attitudes or laws served to limit the economic and political opportunities available to freed slaves. It would take generations of struggle to widen the set of economic and political opportunities. Moreover, 
some less severe forms of servitude - notably indentured service in which a worker promised to work for an employer for several years - continued after the abolition of slavery. Indeed in many places indentured servitude replaced slavery.

We have already suggested a handful of key themes: politics, culture, science (philosophical evaluation of slavery), and economy. We will note some critical individuals along the way. Of course, slavery and serfdom are instantiated in social structure. We will stress throughout this chapter how the idea and practice of abolition spread across borders. The primary reference to evolutionary analysis occurs when the misguided use of this supports racism.

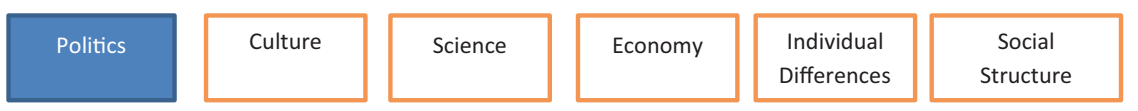

\section{The abolition of slavery}

From the vantage point of the twenty-first century, it is hard not to see the political debates regarding the abolition of slavery as a straightforward battle between good and evil. There can be little doubt that the best of motives usually guided those arguing for abolition: The existence of slavery offended them, and they felt that its repeal was an ethical necessity for a society that deemed itself "civilized." They had no apparent personal stake in abolition. Those who opposed abolition often - though far from always - did benefit economically from slavery. People of deep religious conviction often spearheaded the abolition movement. Yet, as we shall see, these movements dissipated quickly after achieving the well-defined goal of legally abolishing slavery. There was far less sustained effort toward the much more complicated task of ensuring that freed slaves had economic opportunities or political rights.

The abolition of slavery provides clear evidence that ideas matter. We cannot emphasize too much that abolition occurred while slavery was still profitable. A heated historiographical debate unfolded for decades from the 1960s regarding the profitability of slavery. Some had argued that slavery was becoming unprofitable in the nineteenth century while others claimed that it was still quite profitable. If the former case was correct then one might attribute abolition to a cultural reflection of economic forces: Slavery disappeared because it no longer made sense. The consensus today, though, is that slavery was still quite profitable at the time. It is indeed hard to understand why the debate over abolition was so heated in most lands if this were not so. In the United States, the development of the cotton gin from 1794 - which dramatically lowered the cost of separating cotton fibres from cottonseeds - and the use of slave labour encouraged an increase in cotton exports from 100,000 bales in 1801 to 5.4 million bales in 1859 (and a trebling of the number of slaves over that period). Cotton became the most important export of the United States by the 1830s. Slaves in the United States in 1860 were responsible for 60 per cent of 
the country's exports and 80 per cent of world cotton production. The value of the millions of slaves held in the United States exceeded the value of all railroads and industrial establishments in 1860.

In some parts of the world, the plantations that grew such crops would disappear with the abolition of slavery, while in other places they would survive only through introducing somewhat less severe mechanisms of labour coercion. Where plantations disappeared, as in much of Jamaica, the exports that had emanated from those plantations also disappeared. Consumers of sugar and other tropical goods lost a significant source of supply. Abolition imposed economic costs on society in terms of both decreased production and consumption - and the tax revenue that plantations provided. In many societies, the state not only suffered a decrease in taxes but paid landowners to compensate them for the loss of their slaves. Abolitionists, then, had to convince states to do something that was not in their narrow economic interest. The dedication and rhetorical skill of abolitionists convinced enough of the population/electorate of the value of their cause that political leaders found it advisable to consent to abolition. Voters and politicians voted - and in many countries autocrats acted - against their selfish interests in the service of an ethical principle.

Nevertheless, the economic cost was not severe in most places. Despite the growth in production of cotton (and sugar and tobacco), agriculture as a whole was becoming less important economically as the nineteenth century proceeded in most of the world. With industrialization and economic growth (chapter 24), slavery accounted for an ever-smaller proportion of total incomes. It might still be costly to abolish slavery, but that cost was more easily borne. Indeed, life would go on after abolition in most countries much as it had before. There would be no huge worldwide economic contraction in the aftermath of abolition, though some localities in which slavery was particularly important did witness an economic decline.

The growth sectors of the economy in the nineteenth century - industry most obviously but also trade and other services - rarely had recourse to slave labour. Indeed merchants and industrialists celebrated the value of free markets, including free markets for labour. Working conditions in the new factories might be harsh but factory owners did not have to coerce workers into taking factory jobs. Most merchants and industrialists, therefore, had little economic stake in slavery (especially after trade in slaves was abolished; see below), and might find it an embarrassment to the free market ideology that justified their own activities. If located near large slave populations, some industrialists might see potential benefits in an expanded free labour force that they might employ. As the abolitionist movement gained traction, these industrialists and merchants came to fear the economic dislocation that might result from sustained conflict over abolition. The slave revolt in Haiti, and the political and economic dislocation that ensued there (chapter 25), served as a warning. Slave revolts in Jamaica in the 1820s and Virginia in 1831, though suppressed, indicated that slavery did not guarantee a steady supply of raw materials. These revolts 
gave merchants and industrialists a financial incentive to support a peaceful transition toward abolition. Yet this incentive might have seemed small in the absence of objections in principle to slavery.

We should also appreciate that states had to expend resources in order to maintain slavery. Slave owners relied on the state to capture and return escaped slaves. In the United States, it has been estimated that about $\$ 4.5$ million was spent on slave patrols in 1850, a sum equal to 0.3 per cent of total income in the southern states or about a dollar per non-slave person in the south. This amount can be seen as a sizeable public subsidy to the slave owners. Those who did not directly benefit from slavery would see some economic benefit from abolition.

Some historians have made an intriguing argument: It is slavery's expansion in the preceding centuries rather than abolition in the nineteenth century that is the historical "surprise." We have indeed traced a general process of increased freedoms - to trade, to innovate, to marry - in the early modern period. The economic transformations of the previous centuries had been associated with the development of markets for both land and labour. In many parts of the world, including western Europe and China, labour had become freer than it had ever been. We have also discussed how emerging nation states sought direct relationships with citizens rather than through the mediation of property owners (chapter 21). Why, then, had slavery expanded in scope so dramatically at a time when most of the world's labour force was gaining increased freedom, and when the ideologies of both free markets and nationalism were gaining ground?

Can we seek a shared explanation of both "surprises"? If we understand why slavery expanded before the nineteenth century, we might also better understand abolition. Why, then, had the practice of slavery expanded so much in preceding centuries? We saw back in chapter 9 that the advantages of slavery may often be moderate - except when there are resources located far from any labour supply. The Atlantic slave trade reflected the fact that there was a large mass of land in the Americas well suited to cotton, sugar, and other crops, but a limited local labour force because of the mass deaths among Indigenous populations (chapter 19). Encouraging voluntary migration toward such plantations was hard. The expansion of slavery then reflected a historically unprecedented mismatch between land and labour. By the nineteenth century, though, there was a local labour force comprising both the slaves themselves (who would most often continue to work on the same plantations after abolition, at least for a while) and an influx of immigrants from Europe (chapter 31). The agricultural products that slaves mostly produced were much less dependent on slave labour than they once had been.

There has been a rather intense historical controversy in recent decades regarding just how important slavery was to the rise of capitalism or to the Industrial Revolution. Participants in this debate do not always carefully define either "capitalism" (as we did in chapter 18) or "Industrial Revolution" (as we did in chapter 24). The debate hinges on a set of empirical questions, many of 
which we touched upon in those chapters, such as how vital slave-produced goods were as industrial inputs, how important slave plantations were as a market for mass-produced goods, and where the profits from slavery went. Our analysis above suggests that slavery's importance (whatever this is estimated to be) in the sixteenth through nineteenth centuries reflected not so much the inherent logic of capitalism but rather the pursuit of profit in conjunction with an unprecedented mismatch between land and labour. For present purposes, the important point is that by the nineteenth century, slavery was much less important to the world economy than it had been. Even if one thinks that slavery contributed to the rise of capitalism or industrialization, it is clear that slavery could disappear in the nineteenth century without seriously injuring either capitalism in general or industrialization in particular.

\section{Europe}

Not surprisingly, the first dramatic successes in abolishing slavery occurred in Europe and parts of Asia. Slavery was not important within Europe itself but only in European colonies. Indeed, slavery's expansion in previous centuries may have owed much to the fact that it occurred out of sight of Europeans until abolitionists brought freed slaves to Europe to tell their tales. Colonial slave owners had political influence in Europe, but this was declining with industrialization. It is perhaps no coincidence that it was Britain, the first nation to have an industrial revolution, which was the first in Europe to move to abolish slavery. It was much easier to urge abolition in a society with few local slaves

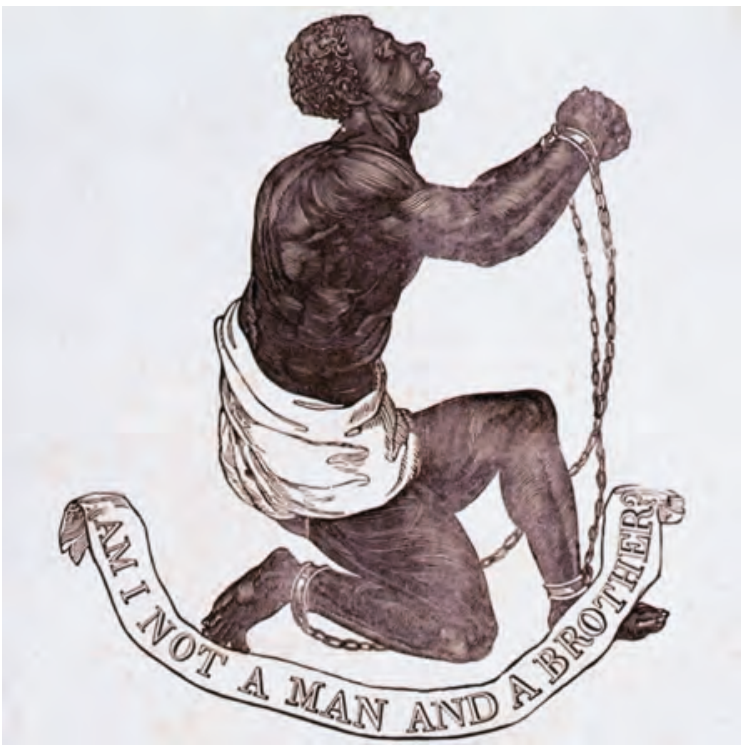

Illustration 26.1

The official medallion of the British Anti-Slavery Society, Josiah Wedgwood, and others, 1795 and in which slavery provided a declining contribution to national income.

The role of freed slaves within the abolitionist movement deserves emphasis. There were organizations of freed slaves in London and other cities who advocated for abolition. These could and did describe the horrors they had suffered: being beaten and separated from their families; in some cases being captured in Africa in the first place. Their eloquent denunciation of slavery provided the most unequivocal evidence possible against racist justifications of slavery. The most notable of these freed slaves was Olaudah Equiano (about 1745 to 1797). There is some debate as to whether he was born in Nigeria or South Carolina, but it is clear that he was sold multiple times and moved between the Caribbean and United States. He 
described the use of a metal device that prevented recalcitrant slaves from speaking or eating. A Quaker merchant eventually employed him as a trader and allowed him to buy his freedom in 1766. He worked on his own account as a merchant and seaman while lobbying for abolition. He adopted Christianity and quoted biblical passages in decrying slavery. He married in England and had two daughters, but suffered psychologically throughout his life.

It is also not surprising that abolitionists successfully attacked the slave trade before slavery itself. Turning free people into slaves and carrying them thousands of miles from their families seemed more evil than maintaining slavery where it already existed. Moreover, the slave trade had become less critical for the practice of slavery. In the sixteenth century, one could not have slavery without a slave trade. At the time of abolition, there were 4 million slaves in the United States, over 2 million in Brazil, and many more in various Caribbean islands. Slave owners in the United States had long relied on local reproduction more than imports to maintain slave populations. In other parts of the Americas, harsh working conditions and unequal sex ratios had militated against reproduction; in those lands support for the slave trade tended to continue. Denmark was the first country to ban the Atlantic slave trade in 1803, but both the British in 1807 and Americans in 1808 soon followed. The British navy intercepted slave ships and destroyed slave-trading ports along the African coast in the succeeding decades. This was a costly endeavour for which the British benefited only very indirectly through weakening other countries that wished to continue the slave trade. Still, hostility to Napoleon in part motivated British actions. Other nations soon followed in banning the slave trade - France in 1815 (in the last days of Napoleon as he sought to burnish his liberal reputation), the Netherlands in 1817, and Spain in 1845 - though an illegal trade continued until 1867 when the British navy finally erased it. The leaders of African states that engaged in the slave trade urged Europeans to continue it: The Assante king in West Africa offered to pay the British to maintain the slave trade.

The British then abolished slavery itself in 1833. The British colonial secretary claimed at the time that the decision reflected the "liberal and humane spirit of the age." The British compensated slave owners from the public treasury with expenditures of 20 million pounds, and required that emancipated slaves continued to work for former masters for four to six years. The typical result in Jamaica was that former slaves received small plots of land. They tended to focus on growing food rather than sugar for export. In India, however, colonial authorities faced a welter of different types of coerced labour relations in different regions. There was, of course, a long tradition of slavery within Muslim communities (chapter 12), but also in some regions such as Kerala it was common among Hindu communities for members of lower castes to be bought and sold. A very lengthy process of negotiating with local elites meant that India did not entirely abolish slavery until 1856. The British would abolish slavery slowly over the next decades in other Muslim colonies. 


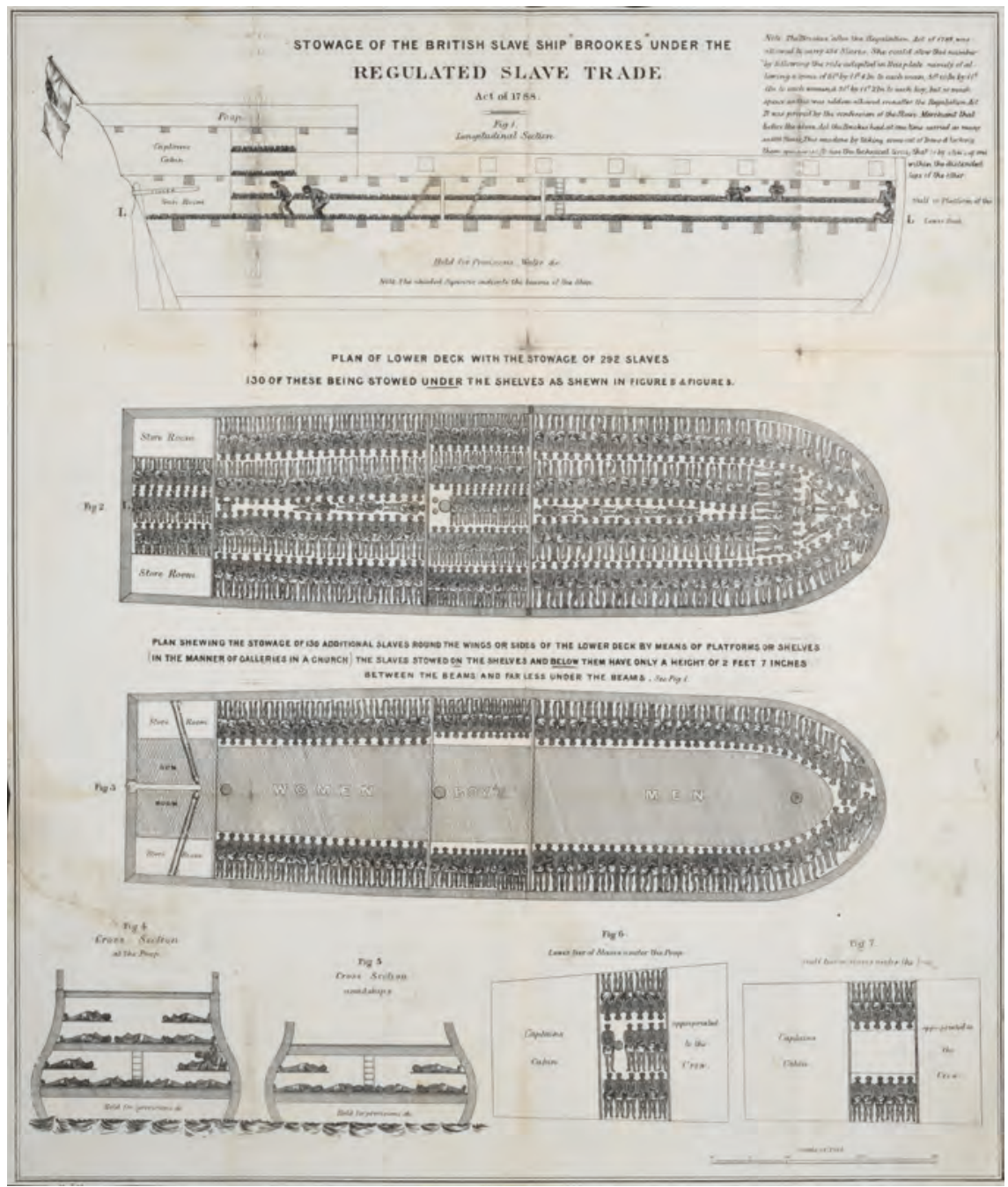

Illustration 26.2

Plan of slave ship

Library of Congress Prints and Photographs Division

The British naturally gloated of their moral superiority. From 1833, then, nationalism often served as an important force in abolition movements elsewhere: No country wished to seem morally inferior to another. As the economic forces unleashed by the British Industrial Revolution spread, other European countries followed Britain in abolishing slavery. France had briefly abolished slavery during the French Revolution but Napoleon had reinstated it. The slave revolution in Haiti - the only case in the world where slavery ended by revolt rather than legislation by the state - owes much to this reversal of French policy (chapter 25). Slave owners from Haiti migrated to both Jamaica and Cuba where they often urged stricter forms of coercion. France would 


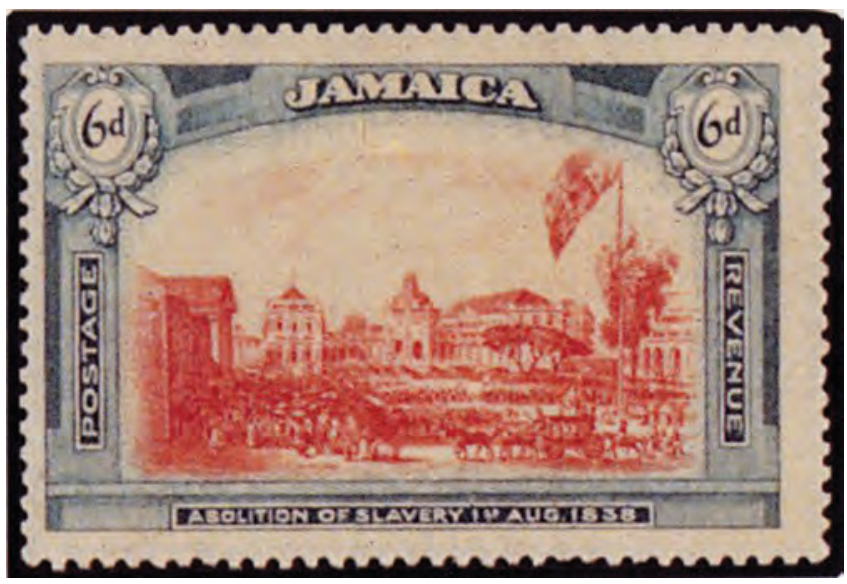

Illustration 26.3

Jamaican stamp from 1921 celebrating the abolition of slavery. Note that the stamp celebrates 1838, when the period of indenture to former slave masters ended in Jamaica

abolish slavery for good in 1848 (one of the first acts of the Second Republic under Napoleon III). The Dutch followed in 1863, citing the British example. The Dutch could apply rising profits from indentured service on Indonesian plantations to fund the compensation of slave owners in Surinam.

Abolitionists had often argued that only a small elite benefited from slavery. Yet abolition in Europe was generally associated with some form of compensation for slave owners. This was in part merely political compromise: Slave owners still had some political influence and so could negotiate a kind of abolition that did not impoverish them. Political change is often only possible in the world if the losers gain compensation. Yet slave owners could also appeal to a general acceptance of property rights and a widespread opposition to states suddenly appropriating private property. Society might have come to judge slavery as abhorrent but also loathed the idea of someone suddenly losing property by state fiat without compensation.

\section{The Americas}

Several of the new nation states in the Americas abolished slavery before Britain did. This simple fact is not widely appreciated. There had been very little slavery in most of the Spanish colonies in South or Central America at the time of independence (chapter 25). Indigenous peoples had been coerced into labour in Peruvian and Mexican silver mines in earlier centuries (chapter 19), and there was a severe power imbalance between large landlords and the rural peasantry (chapter 25), but there was far less explicit slavery in non-Caribbean Spanish colonies than in either Brazil or the United States. Most of the Spanish nonCaribbean colonies abolished slavery at the time of independence, though they 
did not always formalize this decision for decades. In Argentina, for example, the future children of slaves gained freedom in 1813, though only the first constitution of 1853 formally abolished slavery. Chile abolished slavery outright in 1823. Gran Colombia - comprising Colombia, Venezuela, Panama, and Ecuador - freed the future children of slaves in 1821 and developed a plan to compensate the owners of existing slaves. These various countries acted in accord with the anti-abolitionist sentiment of the times. It cost little for revolutionaries to abolish slavery in countries with few slaves. Abolition was a means of establishing some egalitarian credentials (and rewarding slaves that supported the revolution) without having a severe material impact on the vast majority of influential landowners.

Abolition was a more challenging prospect in lands with large numbers of slaves. In Caribbean colonies, colonial powers would make decisions about slavery, albeit with input from powerful slaveholding interests. English, French, and Dutch colonies would see slavery abolished as their respective empires pursued abolition (see above). Though Denmark had been the first to outlaw the Atlantic slave trade, it would only abolish slavery in its colonies in 1848. The last outpost of slavery in the Caribbean was Spanish Cuba, where abolition occurred only in 1886.

\section{PRIMARY DOCUMENTS: DECREE OF SIMÓN BOLÍVAR EMANCIPATING SLAVES IN 1816}

Simón Bolívar, revolutionary leader, issued this decree on June 2, 1816:

Considering that justice, policy, and the country imperiously demand the inalienable rights of nature, I have decided to formally decree absolute freedom for the slaves who have groaned under the Spanish yoke during the three previous centuries. Considering that the Republic needs the services of all her children, we must impose on these new citizens the following conditions: Every healthy man between the ages of fourteen and sixty shall appear in the parish church of his district to enlist under the flag of Venezuela, within twenty-four hours of the publication of this decree. Old men, women and children, and all invalids shall be exempt from this day forth from military service as well as from any domestic or field service in which they were previously employed for the benefit of their masters. The new citizen who refuses to bear arms in fulfilment of the sacred duty to defend his freedom shall be subject to servitude, not only for himself but also for his children under the age of fourteen, his wife, and his aged parents....

(translated by David Bushnell, El Libertador:Writings of Simón Bolívar, Oxford University Press, 2003)

What do you think of this decree? Is it really emancipation? What conflicting pressures might Bolívar have faced? Does your evaluation change if you know that Bolívar had often railed against slavery (even though his parents held slaves) or that he had been sheltered in Haiti after his first failed revolutionary attempt against the Spanish? 


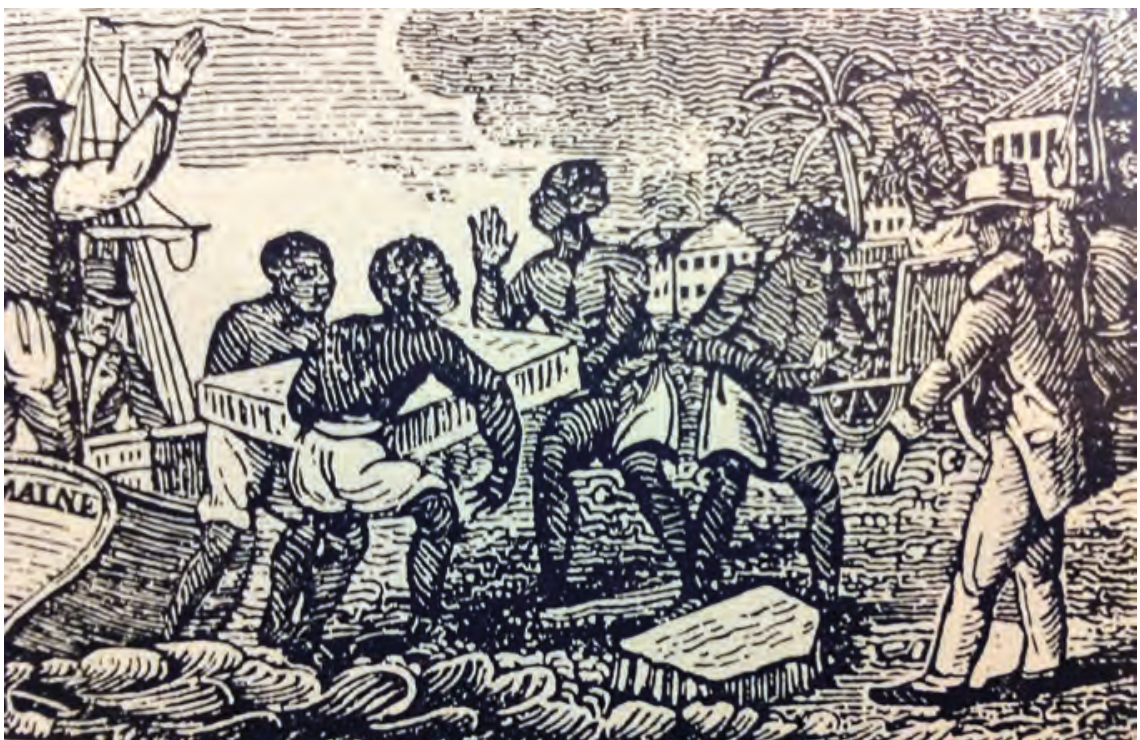

Illustration 26.4

"Slaves Unloading Ice in Cuba," 1832, from Samuel Griswold Goodrich, A Pictorial Geography of the World

The United States and Brazil were by far the largest slave-owning countries in the Americas: As noted above, there were 4 million slaves in the former and well over 2 million in the latter at the time of abolition. Both the United States of America and the United States of Brazil were federal states. In both countries, some states would abolish slavery long before others. In the United States, the most northern states - like the former Spanish colonies noted above - had few slaves and so tended to abolish slavery soon after the United States gained independence. They were among the first polities in the world to do so, decades before either Britain or Argentina. Massachusetts abolished slavery in 1783. Other northern states pursued abolition more gradually from about the same time (Pennsylvania from 1780), first freeing the future children of slaves and moving to full abolition over decades. A gradual process of abolition that began with the unborn was a political compromise that had a limited immediate financial impact on those who did own slaves. Whether gradual or immediate, we can again see the power of ideas at work: Abolitionists widely cited the principles of equal rights elucidated in the American Declaration of Independence of 1776 . Northern slave holders did not receive compensation.

Quakers were the dominant force in the abolitionist movement in the late eighteenth century but gradually drew others into the campaign. The abolitionists tended to focus on the cruelty of slavery. They appealed to the emotions as well as or more than to abstract conceptions of human rights. Harriet Beecher Stowe's novel Uncle Tom's Cabin of 1852 captured the harshness of slavery and inflamed 
the public debate. Abolitionists were generally unable to agree, though, on what role freed slaves should play in society.

\section{PRIMARY DOCUMENTS: AFRICAN SLAVERY IN AMERICA}

Thomas Paine (later a leading revolutionary) wrote this open letter decrying slavery in 1774:

To Americans:

That some desperate wretches should be willing to steal and enslave men by violence and murder for gain, is rather lamentable than strange. But that many civilized, nay, Christianized people should approve, and be concerned in the savage practice, is surprising; and still persist, though it has been so often proved contrary to the light of nature, to every principle of Justice and Humanity, and even good policy, by a succession of eminent men, and several late publications.

Our Traders in MEN (an unnatural commodity!) must know the wickedness of the SLAVE-TRADE, if they attend to reasoning, or the dictates of their own hearts: and such as shun and stiffle all these, wilfully sacrifice Conscience, and the character of integrity to that golden idol.

The Managers the Trade themselves, and others testify, that many of these African nations inhabit fertile countries, are industrious farmers, enjoy plenty, and lived quietly, averse to war, before the Europeans debauched them with liquors, and bribing them against one another; and that these inoffensive people are brought into slavery, by stealing them, tempting kings to sell subjects, which they can have no right to do, and hiring one tribe to war against another, in order to catch prisoners. By such wicked and inhuman ways the English are said to enslave towards one hundred thousand yearly; of which thirty thousand are supposed to die by barbarous treatment in the first year; besides all that are slain in the unnatural ways excited to take them. So much innocent blood have the managers and supporters of this inhuman trade to answer for to the common Lord of all!

Many of these were not prisoners of war, and redeemed from savage conquerors, as some plead; and they who were such prisoners, the English, who promote the war for that very end, are the guilty authors of their being so; and if they were redeemed, as is alleged, they would owe nothing to the redeemer but what he paid for them.

Note that he exaggerates the peacefulness of Africa before Europeans. He goes on to maintain that the Bible does not condone the practice of slavery, and that Americans who complain of British authority cannot then embrace the enslavement of others. Unlike many abolitionists, he addresses the question of how to treat former slaves: Healthy slaves should receive land or jobs. What do you think of this open letter?

As the United States expanded westward, northern and southern states argued over whether to allow slavery in new territories. A compromise of 1820 prohibited slavery only north of the latitude of 36'30". Abolitionists in the 
north agitated for an end to slavery throughout the United States. This proved a challenging and dangerous task: Abolitionists were threatened, beaten, and had their homes and businesses attacked. Many abolitionists risked their lives smuggling slaves out of the south; the fugitive slave law of 1850 required that slaves be returned to their owners, and so slaves were smuggled into Canada (in which the British had eliminated slavery by 1838). As abolition proceeded in Europe and much of Latin America (above), abolitionists found it increasingly offensive that it continued in parts of the United States. Yet slavery was still profitable and an important part of the - still primarily agricultural - economy of southern states.

One can imagine a different history for the United States in which the economic importance of slavery receded even in the south, allowing a gradual process of abolition with compensation in the late nineteenth century. This is indeed the history of Brazil. Abolitionist sentiment had emerged later in Brazil than elsewhere in the Americas. Still, by 1850, Brazil had followed the lead of other nations in banning the Atlantic slave trade - though it did not enforce the ban effectively for many years. Many slaves gained freedom after fighting for Brazil in its war against Paraguay in the late 1860s. Large-scale immigration from Spain and especially Italy in the 1860s and 1870s provided a new source of inexpensive labour. More importantly, perhaps, these immigrants had absorbed abolitionist sentiments in their European homelands. These joined a growing body of freed slaves in urging abolition. In 1871, the children of slaves received their freedom. A massive drought in the late 1870s in the northeastern states where slavery was concentrated induced many slave owners to sell their slaves in southern states. This sparked an increase in abolitionist sentiment in

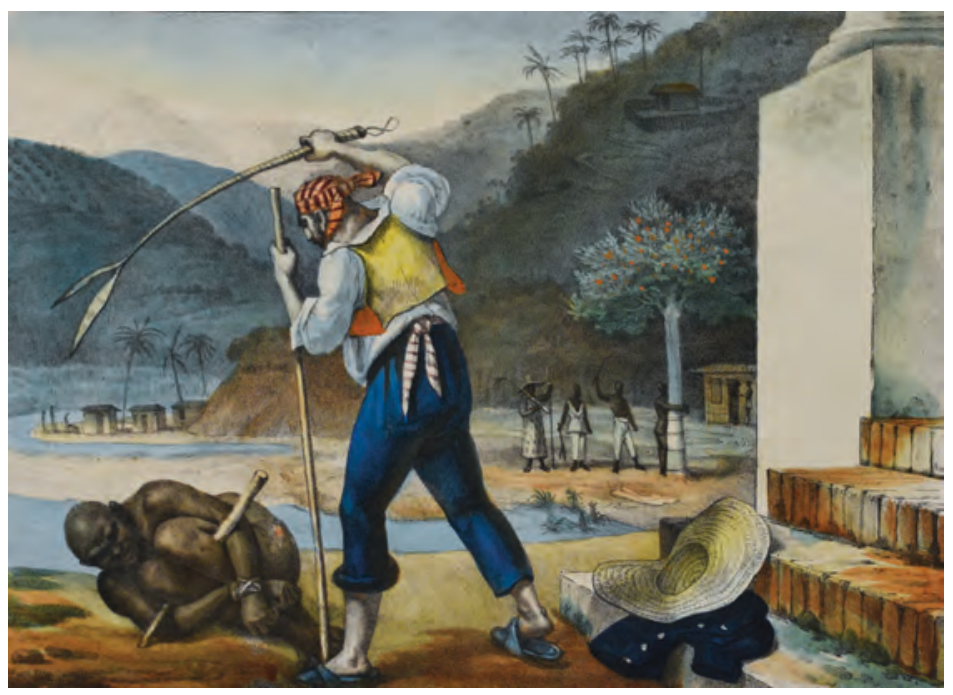

Illustration 26.5

“'Overseers Punishing Slaves on a Rural Estate," Jean-Baptiste Debret, Voyage

Pittoresque et historique au Bresil. 1834-9 


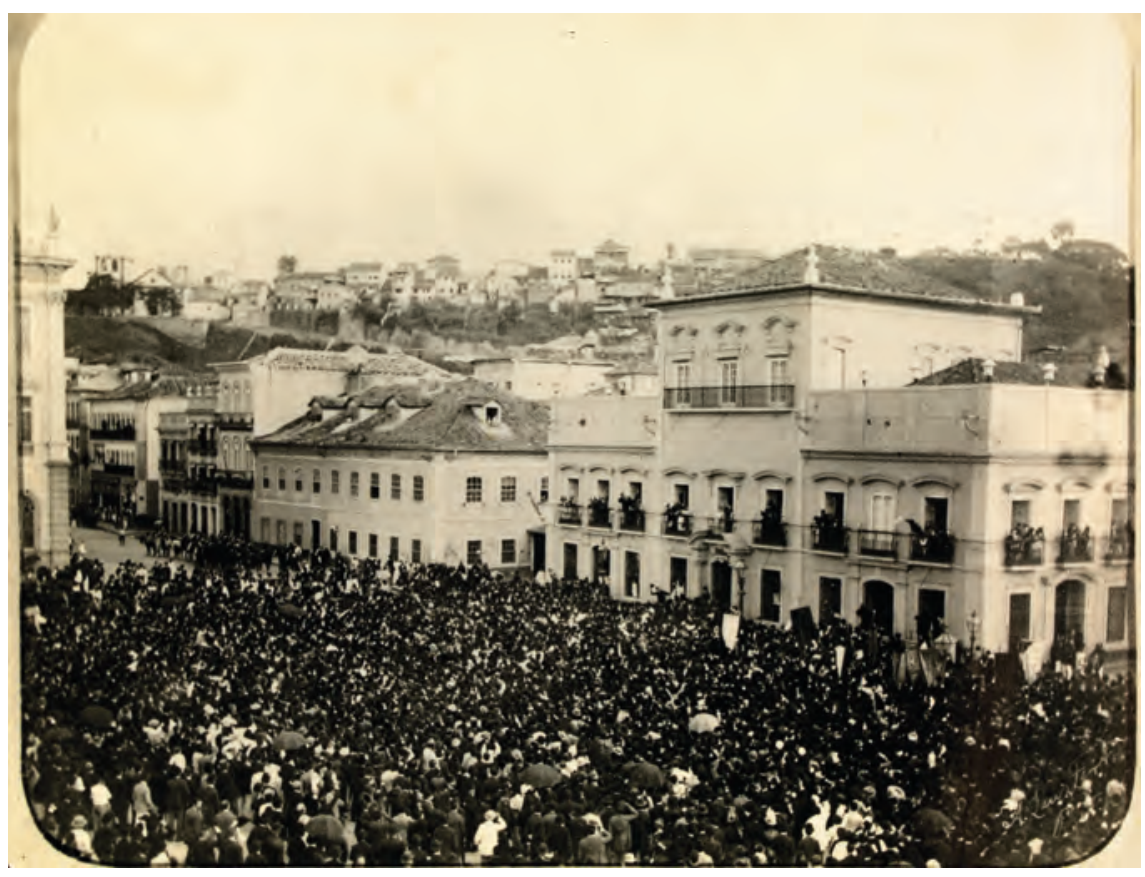

Illustration 26.6

Abolition of slavery by Princess Isabel of Brazil, 1888

Photograph by Marc Ferrez

southern Brazil. In 1885, all slaves over 60 were freed in Brazil. In one of the last acts of the Brazilian monarchy, slavery was abolished entirely in 1888 the final act of abolition in the Americas. By this point in time, abolition was politically popular and slave owners had limited influence. Nevertheless, the Brazilian government provided them with compensation.

The history of the United States was quite different. Slave owners in the southern states feared that - despite the compromise of 1820 - slave-owning states would be increasingly outnumbered as the United States expanded. The southern states, therefore, decided to secede from the United States in 1861. Though slavery was not the only issue involved - many southerners, especially those who did not own slaves, supported states' rights over federal encroachment - there can be little doubt that slavery was the main motive for secession. The United States government decided to oppose secession. The result was the Civil War - the only war fought anywhere over slavery. As with many/most wars, it would prove far more horrific than either side might have imagined at the outset. It was the first war that employed a machine gun on both sides - the Gatling gun in this case. It would also witness the first submarines, though these were quite ineffective. With hindsight, the Civil War should have served as a warning of the horrors of modern warfare. The Civil War lasted until 1865 , and over 600,000 soldiers were killed. While the two sides proved equally matched at the outset, the north's industrial advantage would prove decisive 
in the longer term. The northern states possessed some 90 per cent of American industry at the time. The north was also able to limit the amount of aid that the southern states received from European powers eager to weaken the United States.

The northern states at first declared that their goal was merely to save the union. Only in 1863, as the death toll mounted, and as escaped and freed slaves became an important component of northern armies, did President Abraham Lincoln issue the emancipation proclamation that declared an end to slavery in the secessionist states. This proclamation discouraged European powers from providing aid to the south for they had all previously abolished slavery themselves. In addition, it encouraged slave revolts in the south. The handful of northern states that had not yet abolished slavery did so somewhat later. In 1865 , the United States officially abolished slavery.

In 1862, Lincoln's government had abolished slavery in the District of Columbia, the small area surrounding the capital city of Washington. A few hundred slave owners received compensation of about $\$ 300$ per slave. Lincoln proposed compensated abolition to the southern states as well but they rejected this. Because of the Civil War, slavery would be abolished in the south without compensation.

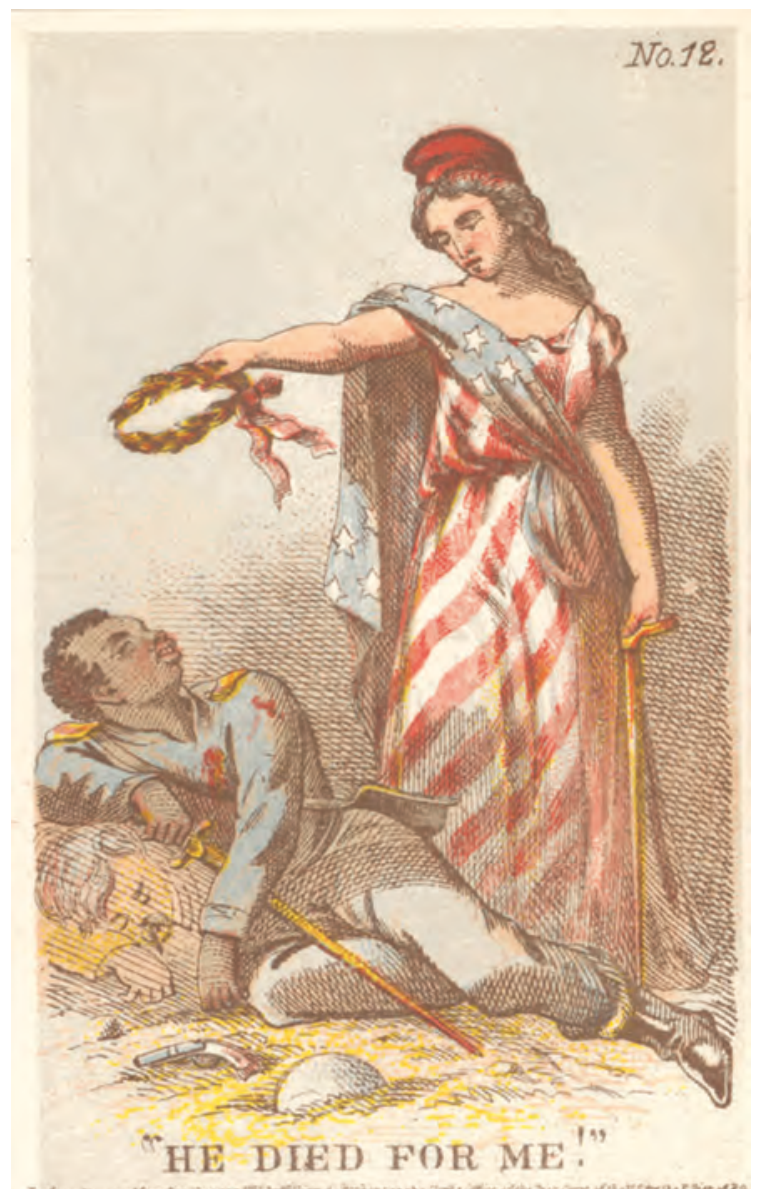

Illustration 26.7

"He Died for Me!" Liberty stands over the dead body of an African American Union soldier, collecting card c. 1863

\section{Elsewhere}

We have seen above that it was easiest to abolish slavery in places where it had ceased to be of great economic importance. Japan had officially abolished slavery in the sixteenth century, and it disappeared in the seventeenth century. The Portuguese had traded in Japanese slaves, and Japanese objection to this trade encouraged the official abolition of slavery in Japan in 1590. Abolition also served to strengthen the power of the central state relative to slave-owning lords. The Japanese would much later abolish slavery in Korea in 1894 when they established a colony there - though they would themselves coerce Korean labour on public works. 


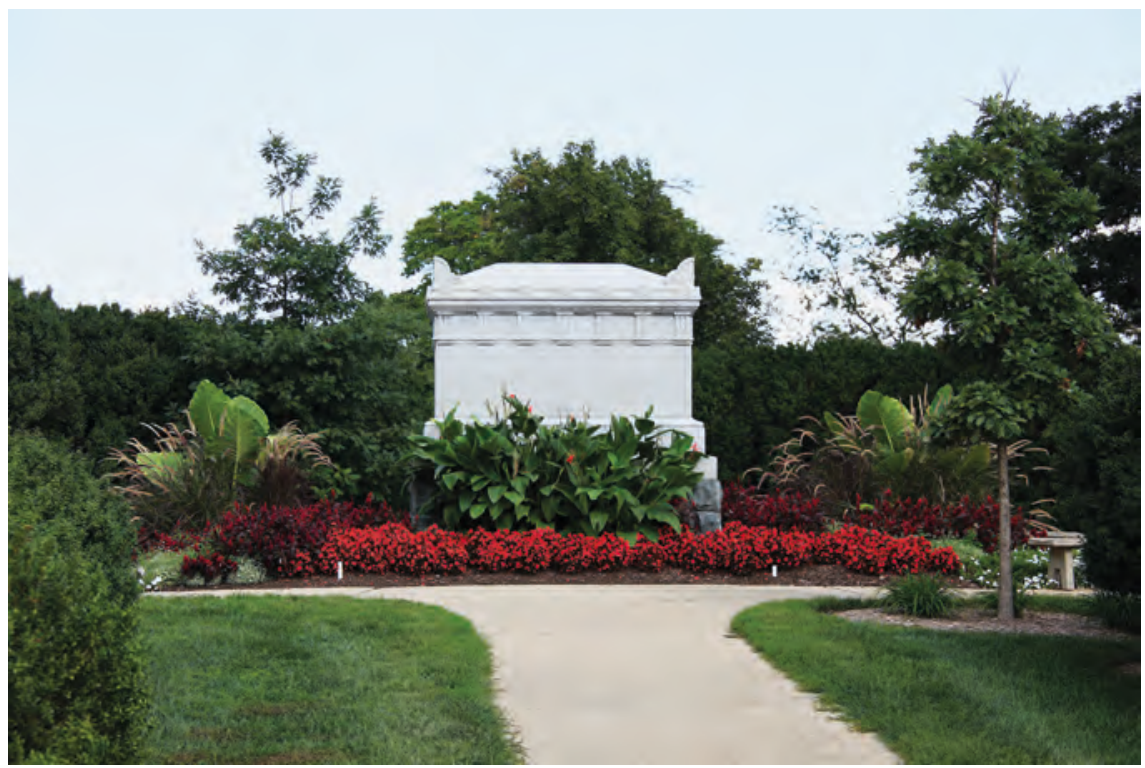

Illustration 26.8

Civil War Unknowns Memorial, Arlington National Cemetery Photograph by Tim1965, CC BY-SA 3.0

The Qing dynasty in China pursued compulsory military service but tried to weaken the power of lords over the peasantry. Though the Qing were not always able to enforce edicts that gave peasants the rights to move and change employers, it is likely the case that Chinese peasants were generally much freer than slaves or serfs by the nineteenth century. In the 1660s, laws forbade beating and selling slaves; in 1685, the Manchus freed their own slaves; and in the 1720s, all slaves were supposed to be released. The new Chinese Republic would abolish slavery in 1911 though it would persist in some regions.

In Thailand (then called Siam), slavery was abolished in stages. In 1874, all slaves born after 1868 gained their freedom. This was a somewhat more liberal version of the practice of freeing the unborn that had been pursued widely in the Americas. In 1897, it became illegal to sell slaves. Finally, in 1912 slavery itself was abolished. In Thailand, as in Brazil, the royal decisions to gradually eliminate slavery were encouraged by a wave of immigration (in this case from China; see chapter 31 ) that served to provide an alternative source of inexpensive labour.

Tunisia would be the first Muslim polity to abolish slavery in the 1840 s. It hoped that this decision would gain political friends in Europe and in particular discourage French threats of colonization. Tunisia abolished slavery while the French themselves were still debating abolition. Elsewhere in the Islamic world, slavery expanded in importance in the late nineteenth century. In Egypt, a massive expansion in cotton production and export relied heavily on slaves. They would also work in the construction of the Suez Canal, though this 
practice would offend European investors. Egypt placed some limits on the sale (but not purchase) of slaves in 1877 , but slavery would only disappear officially under British influence in 1896. The legality and morality of slavery would be debated well into the twentieth century in the Islamic world. Turkey would outlaw slavery in the 1920s after the fall of the Ottoman Empire. Iran, Iraq, Afghanistan, and Morocco also only officially abolished slavery in the 1920s. Saudi Arabia, Yemen, and the United Arab Emirates would follow in the 1960s, and Oman in 1970. Mauritania would be the last to abolish slavery in 1981. In these various countries, arguments that slavery was offensive and anachronistic competed against observations that it was countenanced by Quranic texts and over a thousand years of Islamic practice. Many Islamic thinkers have argued that god had always wanted freedom for all, and had merely countenanced slavery as a necessary evil at the time; many argue for an important difference between approving and allowing such a practice.

Postscript: There have been media reports in the early twenty-first century that slavery is still practised in some parts of the world, including some parts of Mauritania. Weak governments are unable or unwilling to enforce laws against it. The African Union rebuked Mauritania in 2018 for failing to stamp out slavery, though it arguably still exists in Sudan, the Central African Republic, and Niger. And perhaps parts of Southeast Asia.

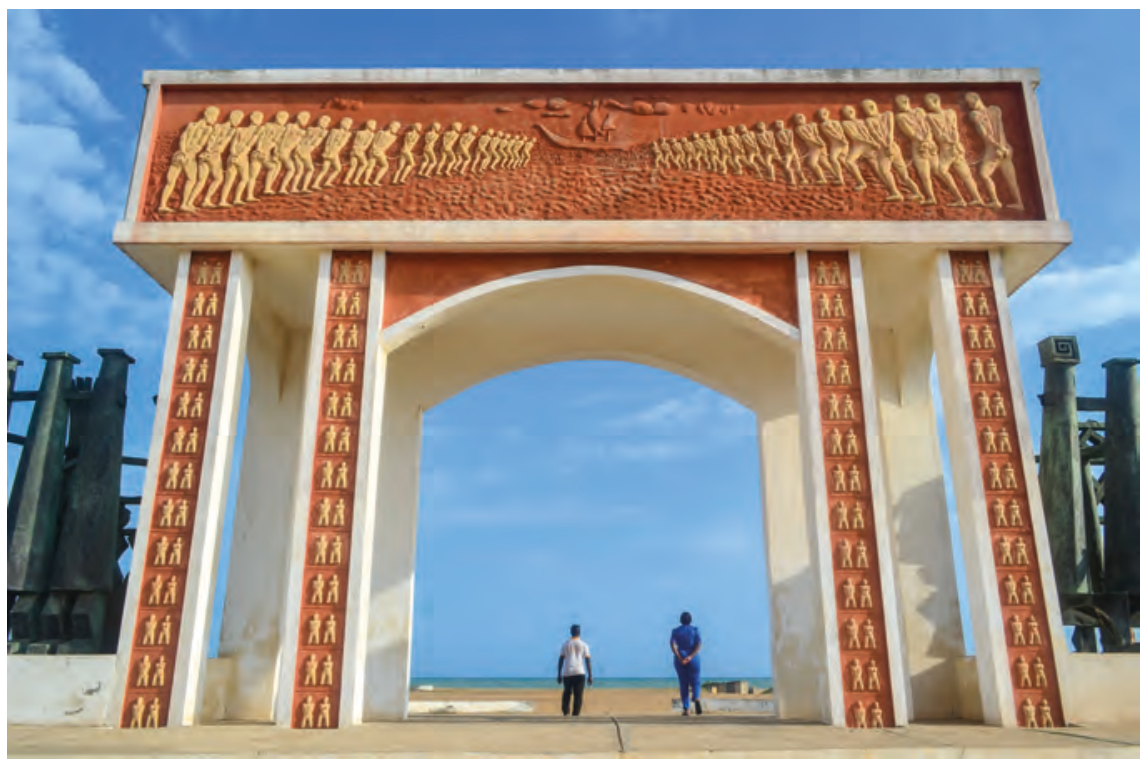

Illustration 26.9

The Door of No Return, Monument to the slave trade in Ouidah, Benin Photograph by jbdodane, CC BY 2.0 


\section{The aftermath of abolition}

The experience of freed slaves differed across countries but was nowhere wonderful. Some abolitionists urged governments to ensure that freed slaves had economic opportunities and political influence. However, abolitionist movements generally lost their zeal after abolition. The political compromises involved in abolition usually left freed slaves with limited power or opportunity.

In the United States, the victorious north tried to pursue both economic and political advancement for freed slaves in the defeated south. Numerous Blacks were elected to state legislatures and Congress in the years after the Civil War, and many were appointed judges. However, within decades, southern elites had introduced restrictions on voting - such as tests that non-literate former slaves could not pass - that prevented most freed slaves from voting. There was also physical intimidation of Black candidates and voters. Discrimination against Blacks then limited economic opportunities. Thousands of people - three-quarters of them Black - would be lynched over the next decades. Though lynching was illegal, authorities rarely investigated or prosecuted these crimes. The threat of lynching discouraged not only political activity but also economic advancement among freed slaves. The victims of lynching were often accused of having sex with White women, but the victims were often Blacks who were relatively prosperous. Many Blacks would migrate north to take industrial jobs. They would often find that they were discriminated against there in employment, housing, and access to education. Only in the 1960s would the United States outlaw political and economic discrimination based on race. Though a large Black middle class has emerged, average incomes remain much lower among Blacks than Whites, and Blacks are far more likely to be imprisoned or killed by police.

Slaves accounted for about half of private wealth in the south before the Civil War. Abolition without compensation reduced wealth dramatically. However, it had much less impact on the incomes of plantation owners. Though they received no financial compensation for the abolition of slavery, they did retain ownership of their land (unlike in Jamaica, where much property was divided among freed slaves). There were promises made by government officials and generals during the war that freed slaves would receive " 40 acres and a mule," but the government only rarely kept these promises as southern elites introduced discriminatory policies against former slaves (and wartime land redistribution was usually reversed after the war). Some freed slaves moved to vacant lands, but most freed slaves continued to work on plantations. The most common institutional form was sharecropping in which workers and landowners shared the output. As cotton output rebounded after the Civil War, plantation owners prospered while the workforce generally remained impoverished. Indeed, landowners often regained their wealth as the price of their land rose dramatically due to the combination of cheap labour and high cotton prices.

In Brazil, there was not the same sharp racial divide. Centuries of sexual relations between Blacks and Whites and Indigenous peoples bequeathed 
a much more varied racial profile. Brazil would, as a result, be much more open socially. Nevertheless, economically there would be as strong a correlation between skin colour and income as in the United States. Lighter-skinned Brazilians would dominate politics and the economy.

It is perhaps useful to think of degrees of freedom. Though freed slaves often lived little better than they had as slaves, abolition gave them and their descendants some freedom of choice. Slowly over the decades, there would be economic and political successes that were only possible because of abolition.

In Trinidad and elsewhere, plantation owners reacted to the end of slavery by importing large numbers of indentured servants from Asia. These workers agreed to work for a particular number of years for a specific employer. Employers promised transport from Asia and wages in return. Indentured servants often ended up working in conditions that were little if any better than those of the slaves they replaced. Far from home and tied to a particular employer, these workers had limited recourse against mistreatment by their employers. Though recruiters in China and India often misled such workers, we should appreciate that workers entered indentured servitude arrangements voluntarily over a period of several decades. Many were able to save money while indentured with which they could afford to get married and perhaps buy land (or a business) of their own back home.

Some abolitionists in both Europe and the United States financed the return of some freed slaves to Africa. Liberia and Sierra Leone became refuges for freed slaves. We can applaud this resettlement for its intent to reverse the original enslavement of Africans, but can question its scepticism that freed slaves would or even should have a place in the Americas. Freed slaves, though never

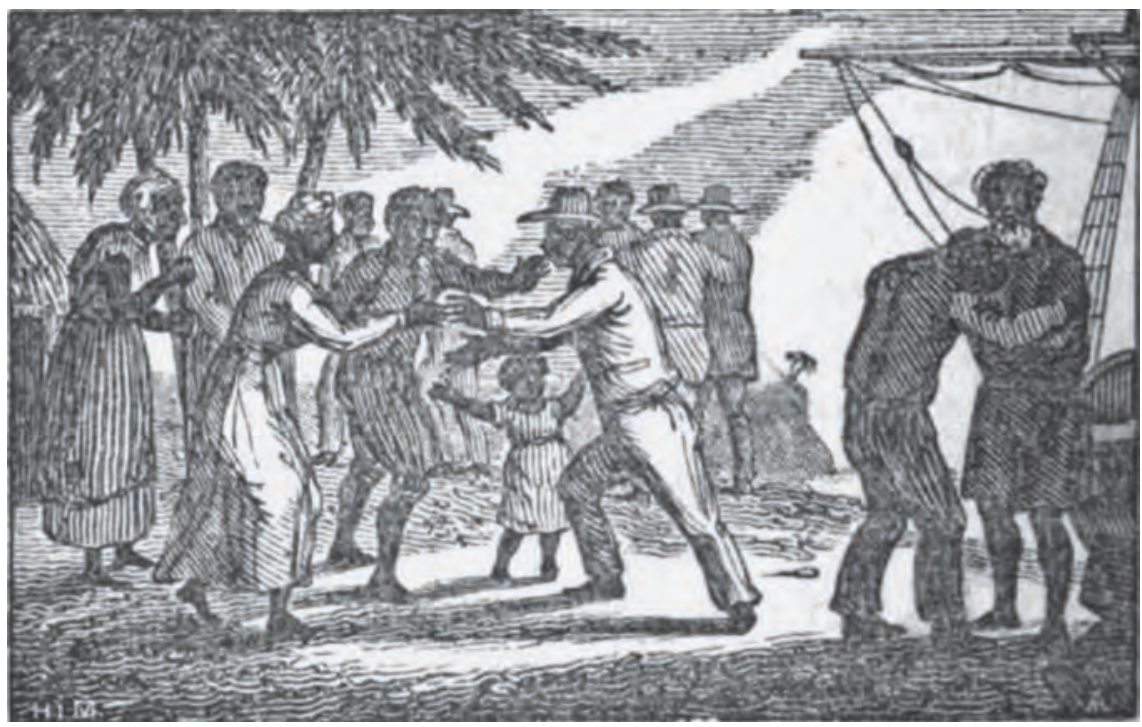

Illustration 26.10

Liberated slaves arriving in Sierra Leone, 1835, by Samuel Griswold Goodrich 


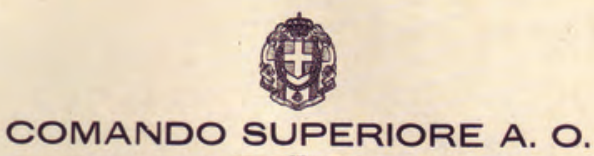

\section{BANDO DI SOPPRESSIONE DELLA SCHIAVITU' INTIGRE'}

Genti del Tigrè,

U D I T E :

Voi sapete che dove sventola la Bandiera d'Italia ivi è la libertà.

Perciò nel vostro Paese la schiavitù, sotto qualunque forma, ì soppressa.

Gli schiari che sono attualmente in Tigrè sono liberi ed è vietata la compera e la vendita degli schiavi.

Chi contravverrà alle disposizioni del presente bando sarà severamente punito, siccome traxgressore agli ordini del Governo.

Dato ad Adua il 14 ottobre 1935-XIII E.F.

(3 tekemt 1928)

DE BONO

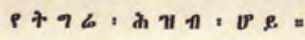

i) 9 .

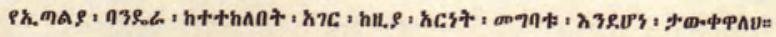

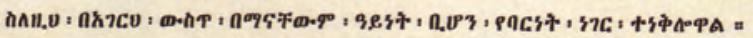

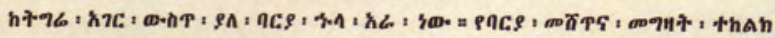

A०PA $=$

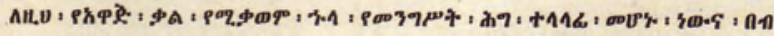

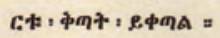

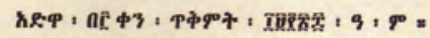

\& $ค$ ?

\section{Illustration 26.11}

Italian poster opposing slavery in Tigre, Ethiopia, 1935. European powers used their opposition to slavery as one excuse for colonization in Africa

comprising more than a couple of per cent of the population in either Liberia or Sierra Leone, would exercise political power there for much of the twentieth century.

Though the African states that had profited from the slave trade had worried about the economic effects of abolition, exports of other goods such as palm oil, peanuts, rubber, and cocoa soon replaced the revenues they had earned from slaving. Those groups in Africa that had tended to be victims of slavery gained 
a greater sense of security - though the internal African slave trade continued (as did the slave trade to the Islamic Middle East for some decades). Indeed, the new export crops were generally produced on slave plantations. Ironically, European powers would use the abolition of slavery in Europe as an excuse for colonizing Africa itself in the late nineteenth century. Europeans would be guilty of many types of coerced labour in their African colonies (chapters 23 and 30) but would celebrate the fact that they abolished formal slavery as they conquered. Even in 1935, fascist Italy would advertise its abolition of slavery in newly conquered Ethiopia.

\section{Other types of coerced labour}

We have had cause to mention indentured servitude a few times above. Since such contracts were voluntary, non-hereditary, and limited in term, they are generally far more benign than outright slavery. Indentured servitude had been important in early colonial America: Migrants from Europe had often financed the cost of sailing across the Atlantic by contracting to work for a particular employer for several years. It became less common by the 1820 s as transport costs fell. Notably, there was an extensive debate in the United States at the time as to whether one could "freely" enter an indentured servitude contract. Indentured servitude would then blossom worldwide in the late nineteenth century as plantation agriculture expanded in many lands where slavery was now illegal. Though indentured servitude can be mutually beneficial, it could also be abusive. Millions of indentured servants migrated from Asia, and especially India, throughout the world but especially to plantations in the Caribbean, Malaysia, and Fiji.

Postscript: Indigenous peoples or freed slaves in places such as Guyana, Trinidad, and Fiji often viewed imported Indian labour with hostility. Ethnic-based political parties have been typical in all these lands, though ethnic cooperation has increased in recent years. There is an evolving sense of transnational "coolie" identity among this Indian diaspora, often encouraged by Indian nationalists.

The "temporary foreign worker" programmes of many contemporary developed countries bear important similarities to the indentured servitude of the nineteenth century. Though both employees and employers can benefit, it is also easy for employers to take advantage of workers who are legally bound to a particular employer. There have been many cases of underpayment and sexual abuse.

Many parts of the world, and especially the Middle East, still practise indentured servitude. Oil-exporting countries often import people 
from poorer countries to perform manual labour. Remittances from such workers are important sources of income for their families and even the broader economies of countries such as the Philippines. Again, there have been many cases of abuse.

Note that the immigration rules of the receiving countries, rather than the need to finance transport costs, drives these contemporary forms of labour service.

There are many other types of coerced labour. One is debt bondage, where one person pays off debts owed to another by working for that other person. As we saw in chapter 9 , many people enter slavery historically because of inability to pay debts. We have seen in many chapters how peasants in many times and places might remain tied to particular landowners through debt bondage. And as mining expanded in the nineteenth and twentieth centuries (chapter 23), many miners also found themselves bound to a particular mine by debts incurred to the company-owned store. As with indentured servitude, we can see how working off debts could often be advantageous for both lender and borrower. Nevertheless, if the borrower is never able to pay off debts, the relationship seems less beneficial. Debt bondage would disappear with the development of personal bankruptcy laws.

We should also mention criminals. European empires had often transported criminals - including those sentenced for acts of rebellion - to distant parts of the Empire. Such criminals could then build public works such as forts or ports or roads. This practice predated the abolition of slavery. The British had transported criminals to their American colonies in the eighteenth century, and then after the American Revolution transported some to Australia. This policy would not have made economic sense if these prisoners did not work at their destination. As with slaves, such criminals could provide labour in lands without a local labour supply. The practice of transporting criminals continued after the abolition of slavery. The British used criminals from their Indian colonies to build infrastructure in Penang and other parts of their Southeast Asian colonies.

It is also worth noting that early factory owners could physically chastise workers in many countries. Workers fought for laws against this practice from the late nineteenth century.

In addition to the various institutions (including serfdom below) that could tie workers to employers, we should also recognize the various systems that could force workers to work for the state. We have seen examples of such labour often in this book. We have seen above cases such as Qing China, where the desire to outlaw private labour coercion was associated with an insistence on state coercion. Again, state coercion might have important advantages in allowing public works that the state would otherwise have difficulty financing. 


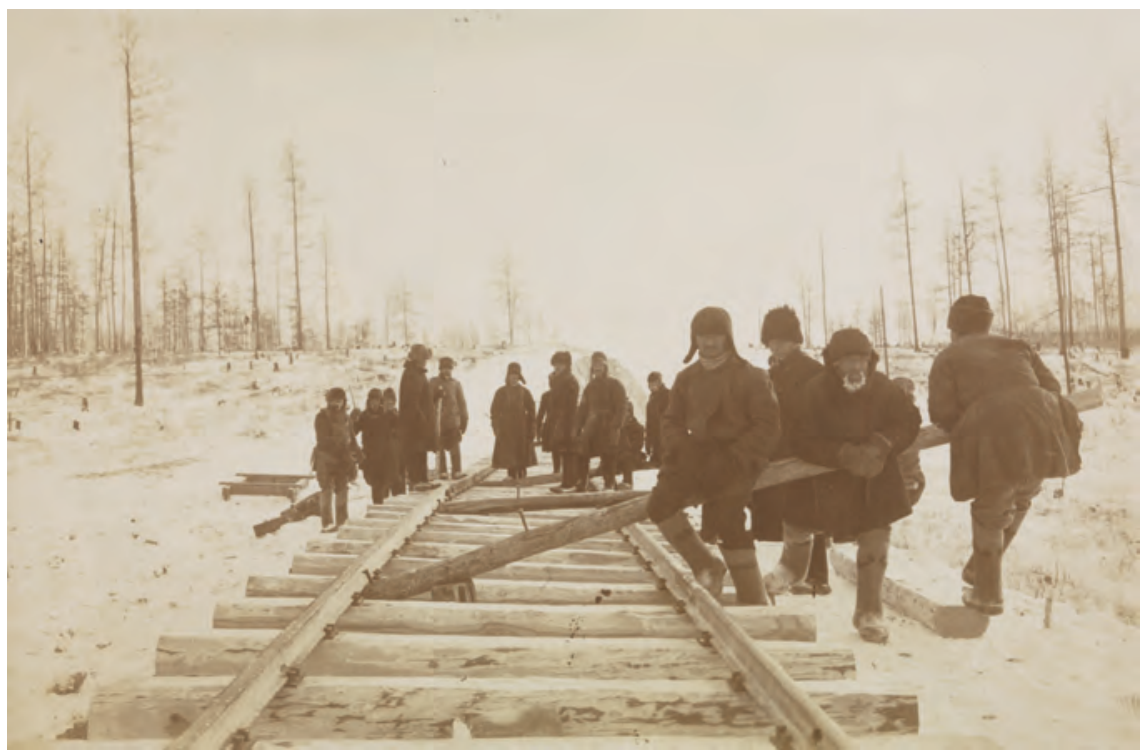

Illustration 26.12

Convicts performing railroad construction in Siberia, 1913, Fridtjof Nansen

However, states have often employed coerced labour on monuments to their own glory. In addition, states have at many times and places forced workers into military service: States have often exaggerated the public value of their military adventures.

Most states in the world have turned away from forced non-military labour since the nineteenth century: It is usually both easier and less controversial simply to hire workers in monetized economies with large labour forces. Totalitarian states - including Nazi Germany, the Soviet Union, and Communist China - have been the most prominent exceptions to this general trend in the twentieth century. They have each at times forced millions of people to work for the state. Two other exceptions deserve note: Many countries require a year or two of service from young adults, and many still expect prisoners to perform forced labour.

\section{Racism}

There can be little doubt that racist attitudes hardened during the period in which people from Africa toiled as slaves in the Americas. Racist attitudes served as a justification for slavery in a land that otherwise proclaimed equal rights for all, just as the earlier denigration of Indigenous peoples had helped to justify colonization of the Americas. Abolitionists instead appreciated the essential humanity of slaves. Yet the attempt to resettle slaves in Africa attests that they were less sure that freed slaves could or should be welcomed into the broader society of the Americas. 
Though it is hard to measure attitudes accurately, it may well be that racist attitudes further hardened after the abolition of slavery. Though racism provided an excuse for slavery, it was not necessary for slavery to function: The rules of slavery itself could keep slaves in their place. With abolition, landowners who wished to maintain an inexpensive labour force could benefit from racist discrimination that deprived former slaves of alternative employment opportunities. Poor Whites could see an advantage in discrimination that prevented former slaves from competing with them. Of course, economic motives for racism and discrimination exist in any society where some jobs are better than others (though some employers may find it financially advantageous to hire those who are discriminated against elsewhere). However, these motives were particularly strong in the bitter aftermath of the Civil War (or after abolition elsewhere) as landowners sought to maintain the value of their landholdings.

Although slavery was condemned, ideas of racial distinctiveness remained strong during the late nineteenth century. Theories of Darwinian evolution were twisted to suggest that some races had advanced further than others. Even those who hesitated to claim that one race was superior to another might be willing to accept that they were somehow different. Post-slave societies tended to accept racial discrimination. Britain, which had played a critical role in the global abolition of slavery, would agree in 1910 that White settlers in South Africa should exercise political control over the much larger Black population and occupy most of the best land.

Once people had accepted racist ideas, they could apply these to any group with different physical appearance. Indeed, racism toward one group almost always led to racism toward multiple groups. Australia, Canada, the United States, and others would introduce race-based immigration policies in the later nineteenth century aimed at limiting immigration from Asia. The post-Civil War experience (above) was used to suggest that racial diversity was incompatible with democracy. Though China and Japan objected to such policies in the Americas, the idea of immutable racial differences took hold in Asia as well. Local traditions, such as the Japanese idea of divine descent or Hindu notions of pollution, could be adapted to racist purposes.

Different racial stereotypes served different purposes. Indigenous people in the Americas and Australia were portrayed as lazy: They could then be pushed off traditional lands and maintained on reserves. It was not necessary to decry their intelligence or even to police their sexual interactions with the White majority for it was their land rather than their labour that was sought. Blacks, on the other hand, were seen as ideally suited to brute labour. In the United States, children of mixed race were disparaged and treated as Black. This increased the pool of cheap labour while discouraging inter-racial relationships; such an attitude was not feasible in Brazil with its long history of racial interaction. Asians and Jews were seen as dishonest in order to exclude them from economic opportunity. In Asia itself, denigration of Whites could encourage nationalist struggle while acceptance of White racism toward Blacks could support a sense of racial superiority. 
Anti-Semitism appeared to be declining in Europe in the middle of the nineteenth century but intensified later in the century. Increased anti-Semitism may have reflected both the increase in racism more generally and the tensions associated with the economic and political transformations we have detailed in chapters 21, 24, and 25. Notably, many Jews fled Europe for the Ottoman Empire in the late nineteenth century. When areas of the Balkans gained independence from the Ottomans, both Muslims and Jews were mistreated (as had been the case centuries earlier after the Christian conquest of Spain). At the Congress of Berlin in 1878, European powers urged the Balkan states to tolerate religious minorities.

From about 1900, though, racist ideas began to recede. The Japanese suggested an ideal of non-racial discrimination for the new League of Nations in 1919, though this was unsuccessful. Racist views were advanced by fascists in the 1930s, especially in Germany, but these were found more offensive by other countries than they might have been decades earlier. The excesses of Nazi Germany during the World War II (chapter 28) would further discredit racism. The decolonization movement postwar (chapter 30) was in part a denial of racist sensibilities. From the 1960s, many countries abandoned racist immigration policies and reduced racial discrimination internally. Though racism remains a potent force in most states, racially mixed neighbourhoods, schools, and workplaces have become common.

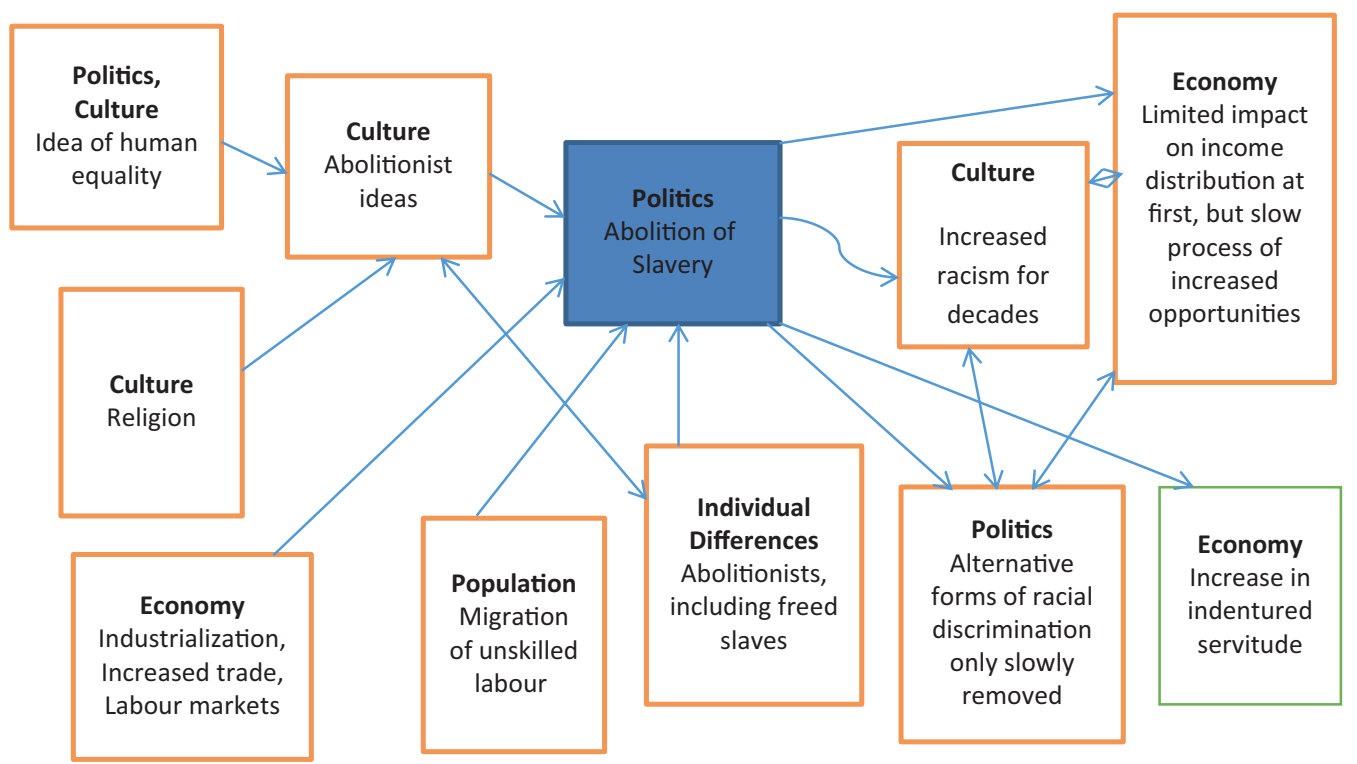

Figure 26.1

Influences on and effects of the abolition of slavery

Note the two-way arrows on the right side of the diagram. Cultural, economic, and political discrimination were mutually reinforcing 


\section{The abolition of serfdom}

We saw in chapter 13 that serfdom had weakened across western Europe over the centuries. It was a system well suited to autonomous local communities, and so its economic rationale dissipated with urbanization and trade. Still, these economic transformations had to be enshrined politically. The French Revolution ended feudalism in France, and Napoleon erased feudalism in much of western Europe. States would not generally restore it after Napoleon's defeat. Prussia abolished serfdom in 1807. Farther east, though, a different kind of feudalism had strengthened in the early modern period just as feudalism was weakening in the west. The growing cities of the west received food from farms in the east. Proprietors and rulers in the east found it convenient to tie peasants to the land in order to maximize profits from food exports. Peasants might otherwise move to new lands coming available as the agricultural frontier pushed into areas previously dominated by nomads (chapter 19).

Just as the abolition of slavery had been encouraged by the ideals of equality voiced during the American and French Revolutions, elites in eastern Europe could hardly ignore these revolutionary ideals or the disappearance of serfdom in western Europe. Lords in the east might be especially conscious of the fact that serfdom had ended in France without compensation to large landholders. They might then be quite open to a compensated abolition of serfdom. However, the political and economic challenges of ending serfdom were great. We noted above that there were 4 million slaves in the United States. There were at the same time over 20 million serfs in Russia. Whereas the majority of American slaves worked on reasonably small plantations, it was common for Russian estates to have hundreds or even thousands of serfs. The Russian rulers had co-opted the support of large landowners over the centuries by agreeing to enforce feudalism (chapter 19). Though the tsars were militarily dominant, they could not just ignore the preferences of wealthy landholders. Moreover, serfdom, like slavery, was still profitable. The situation was similar in the Austrian Empire where the landholding elite wielded even greater political influence.

As with slavery, economic transformations encouraged the abolition of serfdom. Both Russia and Austria had reason to worry about the rapid industrialization that was proceeding in western Europe. The Russians were defeated in the Crimean War of 1853-6 by an alliance of Britain, France, and the Ottoman Empire. Like the slightly later and even more brutal American Civil War (above), the Crimean War had demonstrated the importance of modern industry to modern warfare: Troops moved by steamship and railways, and exploding shells were employed as armaments. Though serfs sometimes worked in factories in Russia, there was a general appreciation that industrialization was more likely to proceed with free labour.

The German philosopher Hegel coined the term "intelligentsia" but it was applied first to writers and speakers in Poland and then Russia who commented 
on matters of political import. Unlike educated people in many western European countries who might channel their efforts into democratic politics, the eastern intelligentsia could only hope to influence autocratic rulers through the force of their arguments. In Austria and especially Russia, this intelligentsia argued that serfdom was both ethically reprehensible and a barrier to the economic development (and resulting military prowess) that was taking place in the west.

The Austrian Empire took the first steps toward abolition of serfdom in the late eighteenth century, but abolition itself occurred only in 1848. In Russia, abolition happened in 1861, only five years after the end of the Crimean War. It came only in 1864 in the Russian-ruled Grand Duchy of Poland. (The presentday territory of the state of Poland experienced abolition at four separate dates: in 1807 in lands controlled by Prussia, 1848 in territories controlled by Austria, 1861 in areas ruled directly by Russia, and 1864 in the Grand Duchy.) Tsar Alexander II said in 1861 that it was better to achieve abolition from above than to wait for a peasant rebellion to force abolition from below. His aunt apparently played a critical role in encouraging him to move toward abolition. Many nobles had mortgaged their serfs to pay debts in preceding decades; they may have become particularly open as a result to abolition with compensation.

Both Austria and Russia felt the need to compensate powerful landholders for the loss of their rights to both serf labour and the plots of land granted to former serfs. In Austria, the state provided compensation. Russia lacked the resources to pay for the freedom of over 20 million serfs - even if many of these laboured for the state itself. The state decreed that serfs had to pay off a debt to their former masters in return for their freedom and plots of land. This reform unfolded in different ways in different regions - Polish serfs gained larger parcels of land, for example, because of greater fears of rebellion there but meant that most serfs continued to work for their local lords much as they had before. Indeed the final vestiges of feudalism were only removed - and full mobility of the peasantry achieved - in Russia in 1906 in the aftermath of the revolution of 1905 .

Unlike slaves, the vast majority of serfs lived where their families had lived for generations. It was rare though possible for serfs to be sold between estates in some places. Serfs generally had their own plots of land as well as obligations to work on the lord's land. Serfs were tied not just to their lords but also to a village community that tended to make joint decisions about what might be planted and when. In Russia, serfs gained explicit ownership of about half the land at the time of abolition. They generally paid off their debts by continuing to work on the other half of the land that the lord had retained. The serf's plots of land were generally small and were devoted to feeding their families. Food was exported from the properties owned by the lords. The combination of small parcels and indebtedness may have limited the market involvement of Russian peasants and their ability to migrate to the cities. Village-level decision-making may have discouraged peasants from experimenting with new crops. Though 


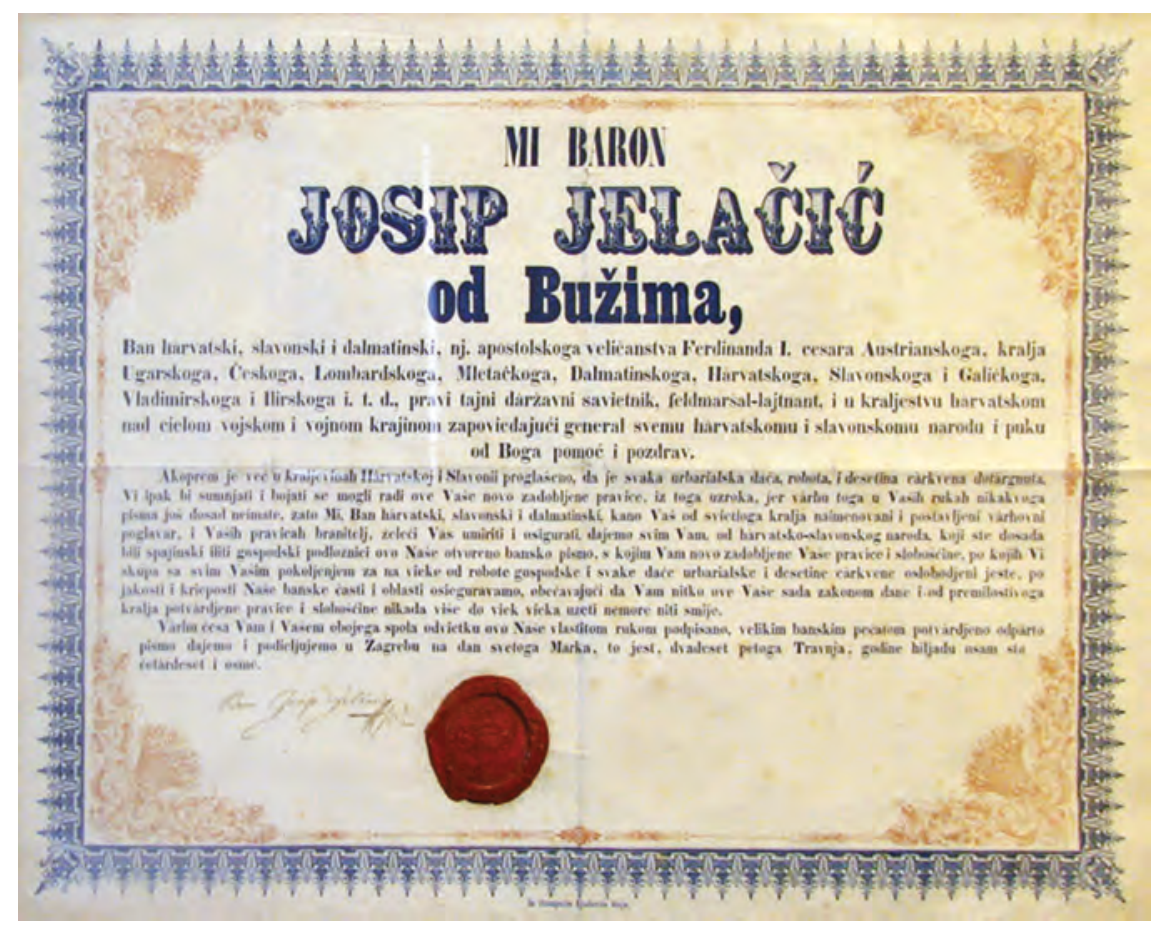

Illustration 26.13

The proclamation by Baron Josip Jelaćić abolishing serfdom in the Kingdom of Croatia, dated April 25, 1848

the state would officially abolish village-level decisions in 1907, the long tradition of collective decision-making may have encouraged later experiments with collective farming in the Soviet Union.

Serfs were in general treated better than slaves, both before and after abolition. Since serfs gained ownership of land with the elimination of serfdom, they were generally better off than most ex-slaves. The small size of these plots, though, and in Russia the debts owed to large property owners, still limited the incomes of ex-serfs.

As we described it in chapter 13, European feudalism involved a specific form of both economic and political organization. Scholars have debated for many years whether feudalism existed elsewhere in the world. We can ignore a debate that often revolves around differing understandings of the word "feudalism" (recall our discussion of the importance of clarity in terminology in chapter 1). We can appreciate that systems with some degree of resemblance to feudalism were abolished even later than in Russia: in Georgia between 1864 and 1871, in Iceland in 1894, in Afghanistan in 1923, and in Bhutan and Tibet in 1959. These countries had each been slow to industrialize and were isolated geographically from global intellectual currents. As with slavery, then, arguably the last vestiges of feudalism survived well into the twentieth century. 


\section{The abolition of seigneurial tenure in Quebec in 1854}

Though deservedly overshadowed by the Russian decision to emancipate serfs in 1861, the abolition of the last vestiges of feudal tenure in Quebec in presentday Canada in 1854 is a curious historical episode that can provide a valuable comparative perspective. If feudal relations could survive until 1854 in a society that was economically advanced and somewhat democratic, then the Russian and Austrian Empires were not quite so out of step with the rest of the world as they might appear. Also, the fact that a legislature rather than an autocrat abolished feudalism in Canada allows us to trace the path of political decisionmaking in detail.

To be sure, the system of seigneurial tenure as practised in Quebec had never had the more restrictive practices associated with European feudalism. French Canada was always a colony with lots of land and few people (in large part because the French, unlike the British, did not allow religious or ethnic minorities to migrate to their colonies). The local lords - the seigneurs - needed to compete for peasants - the censitaires - to work the land. It was not possible for the limited French state presence in Canada to coerce peasants onto the land as the Russian state did. The censitaires were never in practice bound to the land; they always had the potentially lucrative option of engaging in the fur trade (at least until this became consolidated out of Hudson's Bay after 1810). The social gulf between censitaires and seigneurs was never as great in Quebec as the gulf between peasant and lord in pre-Revolutionary France.

Still, the system was feudal in form. Censitaires owed an annual payment to the seigneur, had to pay a tax of one-twelfth of the value of their land if they sold their rights to another, and had to grind their grain in the seigneur's mill (though many seigneurs failed in their duty to build such a mill). The Quebec landscape to this day reflects the long thin fields extending back from navigable rivers that were put in place by seigneurs and censitaires during Quebec's days as a French colony.

The British gained Quebec as a colony from the French in 1763. They quickly decided that it would be easier to govern their new territory if they did not interfere with local language or religion or land tenure. By this time, feudalism had effectively disappeared in Britain itself as lords bought out the rights of peasants in order to operate large farms. Ironically, feudalism would end suddenly in France itself with the French Revolution (chapter 25) - with lords uncompensated for their losses. However, seigneurial tenure would remain in Quebec, for Quebec was no longer a French colony.

Britain belatedly endowed its colony of Canada - comprising at the time only parts of present-day Ontario and Quebec - with a legislature in 1840. Britain was motivated in part by the influx of many English-speaking settlers into the colony during and after the American Revolution. The British decided to institute freehold tenure in newly settled lands. However, they left it up to 
the new legislature to address the situation of seigneurial tenure where this continued in place. The new legislature required a double majority: Legislators from both present-day Ontario and Quebec had to agree to any new law. The English legislators of Ontario made it clear that they found seigneurial tenure an embarrassment and a mild inconvenience but were unwilling to subsidize its demise. The possibility of voluntary commutation mitigated the economic costs of seigneurialism: Seigneurs and censitaires could agree to end seigneurial tenure on a particular plot of land - much as lords and peasants had agreed over the preceding centuries in England to end feudal relations on particular estates. Voluntary commutation was pursued as the cities of Montreal and Quebec City expanded into feudal lands, and as railways were built. Since seigneurial tenure did not limit labour mobility and had only a slightly negative impact on investment - censitaires might be less likely to invest because of the percentage of the sale price the lord would receive if they later sold their land - its economic cost was likely minimal. Both seigneurs and censitaires were well represented in the Quebec delegation in the legislature and acted to ensure that abolition did not occur at their expense. With both Britain and Ontario refusing to pay for abolition, and both censitaires and seigneurs refusing to suffer financially, abolition was politically challenging. In the end, a deal was done in which all censitaires and most if not all seigneurs benefited financially: The former gained ownership of their land at the cost of paying the amount they had traditionally paid to seigneurs into an annuity to compensate seigneurs; the latter gained ownership over all unceded lands. The seigneurs were supposed to have divided their entire estates among censitaires but many seigneurs had not done so; the unceded land was increasingly valuable as Canada's population expanded. How could both benefit? The underrepresented urban working class in Montreal was subject to new taxes in order to finance the end of seigneurial tenure. If not for this political compromise in 1854, Canada might have had the dubious distinction of being the last polity in the world to abolish European-style feudal tenure. The lesson that we might draw is that even with democratic decision-making, politicians might long delay the abolition of feudalism due to the simple challenge of finding a politically feasible funding formula. It is notable that in Canada, as in Russia, this involved continued payments from the former censitaires or serfs to the former feudal lords.

\section{Questions}

1. Why have historiographic debates regarding slavery been so heated, both in the 1960s and more recently?

2. It is not clear why racist attitudes may have declined from about 1900. Can you identify possible reasons?

3. What are the challenges facing freed slaves? 
4. Why did abolitionists not ensure that freed slaves had economic and political opportunities?

5. Identify fundamental similarities and differences between the abolition of slavery and serfdom.

\section{Readings}

Abruzzo, Margaret. 2011. Polemical Pain: Slavery, Cruelty, and the Rise of Humanitarianism. Baltimore, MD: Johns Hopkins University Press.

Anderson, Clare, ed. 2018. A Global History of Convicts and Penal Colonies. London: Bloomsbury Academic. [Shows how convict labour was employed across empires and continents to build key imperial infrastructure.]

Burnard, Leonard, and Giogio Riello. 2020. "Slavery and the New History of Capitalism." Journal of Global History 15:2, 225-44. [Argues that claims that slavery was central to capitalism are exaggerated.]

Eddie, S.A. 2013. Freedom's Price: Serfdom, Subjection, and Reform in Prussia 16481848. Oxford University Press. [Takes a favourable view of feudalism but still argues that peasants benefited from abolition.]

Garrigus, John D., and Christopher Morris, eds. 2010. Assumed Identities: The Meanings of Race in the Atlantic World. College Station: Texas A\&M University Press. [The last chapter describes the life of a Black slave named Rosalie from what is now the Dominican Republic who achieved freedom twice and lived a fascinating international life. Some of her descendants became prosperous merchants.]

Lake, Marilyn, and Henry Reynolds. 2008. Drawing the Global Colour Line: White Men's Countries and the International Challenge of Racial Equality. Cambridge University Press.

Osterhammel, Jürgen. 2014. The Transformation of the World: A Global History of the Nineteenth Century. Princeton University Press.

Parry, Mark. 2016. "Shackles and Dollars: Historians and Economists Clash Over Slavery." Chronicle of Higher Education, December 8, 2016. [This review provides an excellent overview of the recent debate, noted above, regarding the role of slavery in the rise of capitalism or the Industrial Revolution.]

Percy, Michael B, and Rick Szostak. 1992. "The Political Economy of the Abolition of Seigneurial Tenure in Canada East." Explorations in Economic History 29:1, 51-68.

Rönnbäck, K. 2018. "on the Economic Importance of the Slave Plantation Complex to the British Economy During the Eighteenth Century: A Value-Added Approach." Journal of Global History 13:3, 309-27.

Suzuki, Hideaki. 2016. Abolitions as a Global Experience. National University of Singapore Press.

Wolfe, Patrick. 2016. Traces of History: Elementary Structures of Race. London: Verso.

Wright, Robert E. 2017. The Poverty of Slavery: How Unfree Labour Pollutes the Economy. New York: Palgrave Macmillan. 


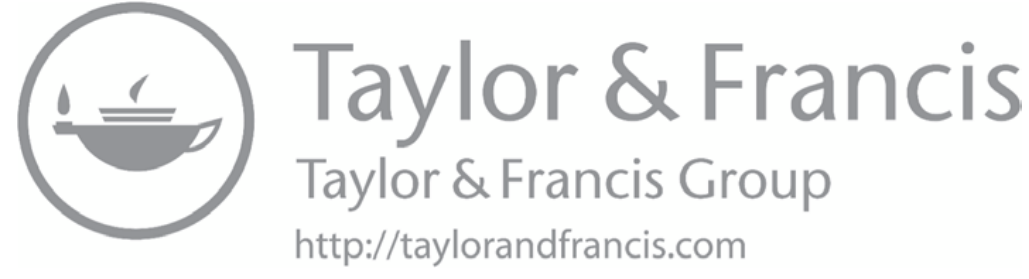




\section{Key thematic transformations of the twentieth century}

\section{Guiding questions}

Why, how, when, and where did bureaucracies grow in size?

What new roles did states take on and why?

Where and why was democracy established in this period?

Where and why was communism established in this period? Where and why did it decline?

When and why did international organizations develop and what role have they played? How and why did international consensus on human rights emerge and what has been the effect of this?

What were the critical environmental challenges of the twentieth century and how have these been addressed?

Why, where, and when did agnosticism and atheism become more prevalent?

How, why, when, and where did social stratification decrease especially with respect to gender?

Relationship to other chapters: As with chapters 14, 18, and 23, this chapter addresses several different trends (or events) that characterize a particular time period. Many of the topics discussed in here are political (politics also features prominently in discussions of war and decolonization and migration in later chapters). These topics each build on our discussion in chapter 22 of how nation states gained increased taxation and administrative capability. These abilities would balloon in the 
twentieth century, allowing states to engage in a broader range of activities. Some states would seek to dominate economic decision-making. We noted in chapter 22 that only some of the new nation states were democratic; we discuss here how democratic ideas spread in the late twentieth century. Increased state power enhanced the value of international cooperation, but world wars (chapter 28) would make this point powerfully. Our discussion of religious agnosticism builds on preceding discussions not only of religion but also of science and law. Our examination of the environment reflects the industrialization of chapter 24 but also our more general understanding of political decision-making. The discussion of social stratification requires an understanding of preceding trends in technology, economy, politics, and values. We often continue our discussion of processes into the twenty-first century; this sets the stage for a discussion in chapter 32 of how humanity might approach the future.

The twentieth century is the most ambiguous of the time periods generally employed in World History. Historians are reasonably comfortable with discussions of ancient, classical, and (less so) early modern periods. And the idea of a "long nineteenth century" stretching from the Industrial, American, and French Revolutions through until World War I has considerable appeal (though we saw in chapter 23 that it fits some parts of the world better than others). As we have seen in preceding chapters, it made sense to trace such themes as economic growth and political revolution through that time period. The twentieth century seems to have less coherence as a historical epoch. As a result, World History courses often neglect the period: Coming at the end of the course, instructors can be tempted to treat it superficially. Such a strategy would impede our ability to draw lessons from history: We need to appreciate how the themes we have been following unfolded through the most recent century.

We should nevertheless appreciate that historical analysis becomes harder in many ways as we approach the present. Most obviously, it is too early to assess the effects of recent developments. It can also be harder to detect the causes of recent transformations, and even to appreciate what are the most important events and processes. It takes time for the historical community to achieve consensus, even about what the important questions are. The fact that we have far more documentary evidence for the recent past is a mixed blessing, for the sheer mass of material can quickly overwhelm us.

We noted in chapter 1 that this book would be flexible in its treatment of time periods. We appreciated that boundaries between time periods were always 
fuzzy and reserved the right to follow particular themes across any temporal boundaries that we might employ. We noted that some themes might exhibit a dramatic change in a specific period of time while other themes displayed continuity instead. It is useful to survey briefly how each of our themes fares in the twentieth century. This will clarify both the nature of the century and how we will cover it in this book. We will indeed find that some themes exhibit change while others exhibit continuity. The boundary between nineteenth and twentieth centuries is then more meaningful for some themes than for others. We will also find that some themes play out quite differently in the second half of the century than they do in the first. In some of these cases, themes follow a similar trajectory in the later twentieth century to that experienced in the nineteenth century: In such cases, the wars and Depression of the early twentieth century appear as a temporary aberration that interrupted longerterm processes. The stark differences between the two halves of the twentieth century are of course one key reason why historians have often found the century analytically inconvenient.

With respect to politics, the steady extension of the nation state in the twentieth century such that nation states came to characterize almost the entire planet continues a process begun centuries earlier and addressed in chapter 21 . Yet this process intertwines with a dramatically new historical transformation: widespread decolonization concentrated in three decades after 1947. Decolonization will be the focus of chapter 30. As we saw in chapter 21, there is a connection between nation states and democracy. We will discuss later in this chapter how democracy ebbed and flowed through the century, with dramatic extensions of democracy in the last decades. Two alternative political ideologies - fascism and communism - became of great importance early in the century but their influence had dissipated by the end of the century. They serve as reminders that events and processes that are of critical importance at one point in time may appear later in history to have had little direct impact on longer-term historical trajectories. We discuss fascism in chapter 28 and communism below.

Wars - both hot and cold - are a crucial characteristic of the century. These are the subject of chapter 28 . These wars - and the danger of nuclear obliteration also encouraged the proliferation of international organizations devoted directly or indirectly to the pursuit of peace and cooperation (see below). Wars were horrific in part because of the expanded role of the state: We discuss later in this chapter how and why the size of the state grew. Although the size of the state had been expanding for centuries (chapter 21), this trend would accelerate dramatically in the twentieth century. This allowed some governments to be more tyrannical than had previously been possible. Yet the century would also witness a dramatic expansion of human rights (see below).

The expansion of legal rights was matched in the social and cultural realms by increased respect for diversity. Here too we see a dramatic acceleration of trends from earlier centuries. Women gained legal rights across a wide range of states (but not quite all) and came to pursue the full variety of occupations 
(again not quite everywhere). Ethnic and religious minorities also received increased legal and social acceptance in most of the world. In many parts of the world, rigid class or caste hierarchies became more flexible. Very late in the century, people with different sexual orientations or gender identities also came to receive legal protection and social acceptance in some parts of the world. Another notable cultural change involved the rise of agnosticism and atheism in some parts of the world (see below). Cultural diversity came to be lauded almost everywhere in the second half of the century. As the twenty-first century begins, one fundamental question - perhaps the ethical challenge of our time - is how the laudable respect for cultural diversity can coexist with shared societal respect for values such as honesty and responsibility on which societal cohesion has depended for millennia. (We return to that question in chapter 32.)

Diversity came to characterize the world of art as well. Through most of human history, a dominant artistic style characterized each time and place. From the nineteenth century, different art styles competed for audiences. Economic growth and population growth largely shaped this new world of artistic diversity: The much broader audience for works of art meant that there was room for competing styles. These styles borrowed from each other, creating much greater opportunities for artistic evolution than in previous eras. The increased global flow of ideas was also important. This meant that artists could reach global audiences. (We discuss the global art market briefly in chapter 29.)

With respect to the economy, the main story was the continuation of the modern economic growth that had begun with the Industrial Revolution (chapter 24). Virtually every corner of the world experienced growth in the twentieth century. Indeed many countries, especially but not entirely in East Asia, would experience decades of growth at rates much higher than were ever seen in Europe or North America. We discuss the conditions under which developing countries have and might catch up to rich countries in chapter 29; The Great Divergence (chapter 22) might slowly give way to convergence. (We discuss some of the economic challenges faced by former colonies in chapter 30.) However, the growth experience of the twentieth century was quite uneven across both time and space. We discuss the Great Depression and postwar Golden Age in chapter 29. We also in that chapter address consumer culture and social policy (an interaction between politics and economy), which expanded dramatically in the later twentieth century. We also address the question of how historically novel was twentieth-century "globalization." Various technological developments are discussed in chapter 29, for they were important in generating economic growth (and military technology is addressed in chapter 28); advances in science were addressed in chapter 23.

Population growth accelerates through most of the century, but there are definite signs of deceleration and even decline in most but not quite all parts of the world by century end (with some countries as a result experiencing a dramatic ageing of their populations). Migration flows had expanded dramatically in the middle of the nineteenth century and would remain high through 
much of the twentieth century and into the twenty-first - though war and Depression would interrupt these flows. Urbanization would accelerate quite dramatically through the twentieth and twenty-first centuries. Humans now for the first time in history inhabit an urbanized planet: More than half of all humans live in cities. These dramatic changes in population, migration, and urbanization - and some related issues in the area of human health - are the focus of chapter 31 .

Human impact on the environment expanded markedly as industrial production grew and as agriculture expanded into areas of forest. Climate change, reduced biodiversity, and limited supplies of natural resources have all emerged as concerns. These environmental impacts are not new but reflect a dramatic acceleration of processes underway in previous centuries (see below).

Costs of transport continued to fall through the twentieth century, encouraging the migration and trade noted above. In part, this was through the spread and improvement of existing technologies such as railways and ships. However, much of it reflected new technologies such as automobiles, trucks, and airplanes. The humble shipping container would have a huge impact on shipping costs in the last half of the century (see chapter 29).

We will engage a wide range of themes in the remainder of this chapter. We will throughout discuss how societies exchanged and often synthesized both ideas and institutions. We apply evolutionary analysis briefly to political revolutions, bureaucracy, laws, and religious beliefs.

\section{Democratization}

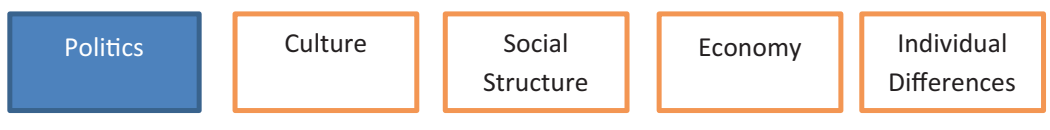

We addressed democracy in chapter 21 . Democracy had spread slowly through the nineteenth century. France switched between democracy, empire, and kingdom as the century progressed. In the aftermath of the First World War, Germany became a democracy with the king's abdication, but weak democratic institutions in concert with the challenges of reparations payments and the Great Depression led to a return to autocracy under the Nazis (chapter 28). After the Second World War, though, democracy became firmly established in most of Western Europe, as well as in Japan - at first under the tutelage of Allied occupation.

Decolonization (chapter 30) created an opportunity for the spread of democracy as dozens of new states emerged. Colonial powers did indeed often bequeath authority to elected governments (but often decamped with little succession planning). In India, democracy - vibrant but often ugly and with a brief period of martial law - has survived in one of the two most populous countries in the world since independence in 1947. Elsewhere, though, the 
military soon supplanted elected governments: The army was often the only strong institution in these countries. Even while Europe itself was democratizing in the nineteenth century, it had often dismantled in its colonies precolonial institutions of political consultation. We will trace the decolonization experience of many countries in chapter 30.

From the 1970s, though, democracy spread. There were 45 democracies in the world in 1970, but 120 in 2010, accounting for 60 per cent of the world's states. Democracy in 1970 concentrated in Europe and lands of European settlement. In 2010, democracy was common in almost every region of the world. Spain and Portugal and Greece became democratic in the 1970s, in large part to gain access to the European Union (the death of dictators such as Franco in Spain was also crucial as was the dedication of his chosen successor to democracy). Eastern Europe would become democratic with the fall of communism after 1989 - though democratic institutions are still weak in some of those countries. By the 1990s, almost all of Latin America was democratic. South Korea, the Philippines, and Indonesia also moved toward

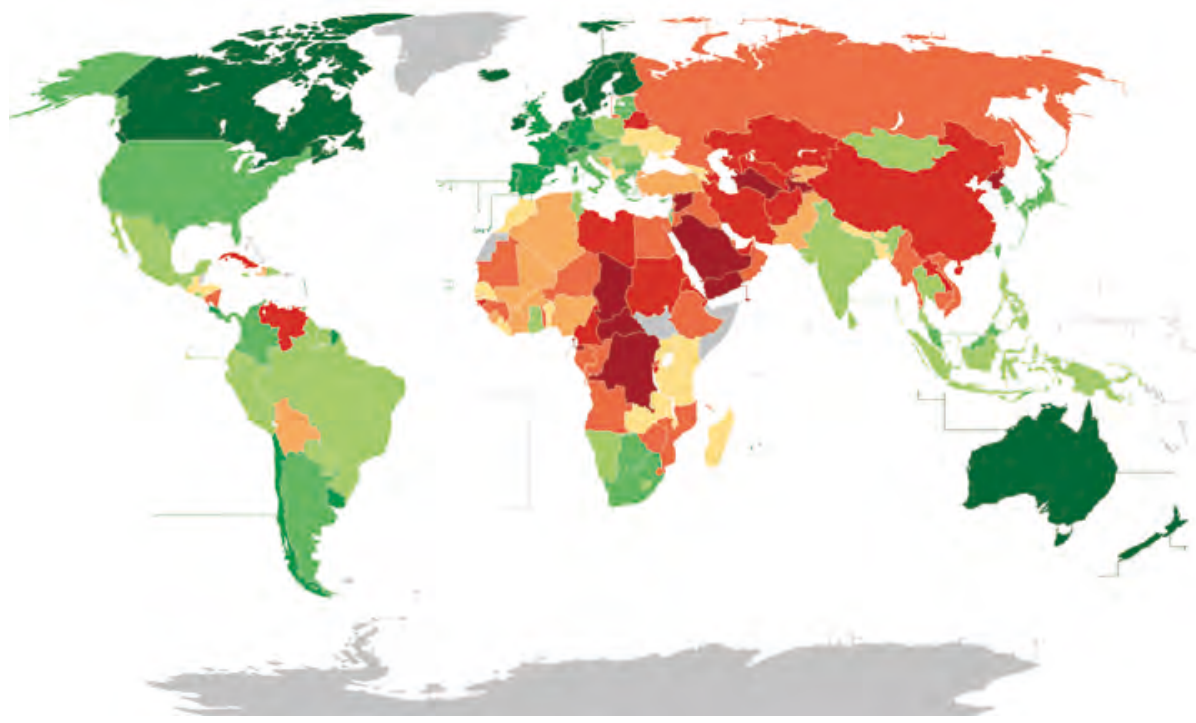

$$
\begin{aligned}
& \text { Full democracies } \ 9.01-10 \rrbracket 8.01-9 \\
& \text { Flawed democracies } \square 7.01-8 \rrbracket 6.01-7 \\
& \text { Hybrid regimes } \square 5.01-6 \square 4.01-5 \\
& \text { Authoritarian regimes } \square 3.01-4 \llbracket 2.01-3 \rrbracket 0-2
\end{aligned}
$$

\section{Map 27.1}

Countries rated by "degree of democratization," 2018, Economist Intelligence Unit Map from BlankMap-World6 by Canuckguy, derivative work by WeifengYang, CC BY-SA 4.0 
democracy in this period. The transition in Africa was latest and the most fragile. But by 2000, half of African countries were democratic. That proportion rose in the next few years, but there has been some backsliding since. Zambia was in 1991 one of the first African countries to see power transferred peacefully from one elected leader to another; in 2016, however, the incumbent retained power in a very narrow electoral contest marred by intimidation, government domination of public broadcasting, and accusations of vote fraud. Many countries have not yet firmly established the institutions required for widespread confidence in electoral results. Violence and allegations of electoral fraud have marred recent elections in Kenya, Uganda, and elsewhere. Massive corruption among both politicians and bureaucrats is too prevalent in many states that hold elections; citizens do not then gain the potential benefits of democracy.

Why did democracy spread through much of the world in a couple of decades? The end of the Cold War (chapter 28) was important: The Soviet Union and Western countries had long supported dictators in many parts of the world. The rise of a middle class demanding both freedoms and superior economic management was also important. Increased education was likewise critical: Literate and numerate citizens were better able to monitor governments and push for reforms. Rapid urbanization (chapter 31) also helped for it is much easier to organize political opposition in cities: City-dwellers have proven far more willing to support opposition voices in fledgling democracies. Activists fought for democracy in each country - often heroically against authoritarian rulers who tortured and killed opponents. We should also not neglect the importance of example: Once some Latin American countries became democratic, and especially as these achieved economic growth, it became ever harder for generals in other countries to oppose elections. The same power of example later spread across Africa.

It should be stressed that democracy was achieved in especially sub-Saharan Africa when these countries were much poorer than European countries had been when they became democratic a century or two earlier. Though levels of urbanization were higher in Africa, there was generally a much lower level of education than had been the case in the earliest modern democracies.

The trend toward democracy seemed inexorable in the 1990s. Even the remaining autocrats tended to hold sham elections. However, progress has slowed. Democratic institutions have arguably weakened in both Russia and Venezuela, though elections continue. The economic success of China has been cited as an exemplar by autocrats, especially in Central Asia (where autocrats gained power as the Soviet Union collapsed) - and China has supported autocratic governments in many parts of the world. The Middle East has remained stubbornly autocratic. Attempts by the United States and Britain to install a democratic government in Iraq have had limited success: The state has been weak and riven by ethnic and religious conflict. The "Arab Spring" of 2011 saw public demonstrations for political change in several countries. Some states 
crushed demonstrations brutally. Elections in Egypt produced an Islamist government that showed little respect for minority rights; many were relieved when the army again asserted authority (and even won an election). In Syria and Libya, the result has been brutal civil wars. Only in Tunisia has democracy prospered since 2011, but a series of terrorist acts has sorely tested that democracy.

Though the trend toward democracy may have stalled, there has nevertheless been a definite trend away from military coups. There were some fifteen of these a year on average in the 1960s but under five a year in the 2000s. When states slide from democracy to autocracy, it is often because elected leaders dismantle democratic institutions. Military leaders are often elected (especially in countries where the military is still the most developed arm of the state), but it is no longer widely felt that armies are good economic or political managers. The likelihood of a coup falls significantly as average income increases in a country: Citizens are then more satisfied, and institutions are stronger. Coups are also less likely when economies are growing, presumably because existing governments are more popular.

We can identify a handful of factors that have limited successful transitions to democracy. Naturally, authoritarian governments themselves often act to prevent democratization - but these can be overthrown. Countries with substantial natural resources provide a temptation to autocratic and corrupt rule

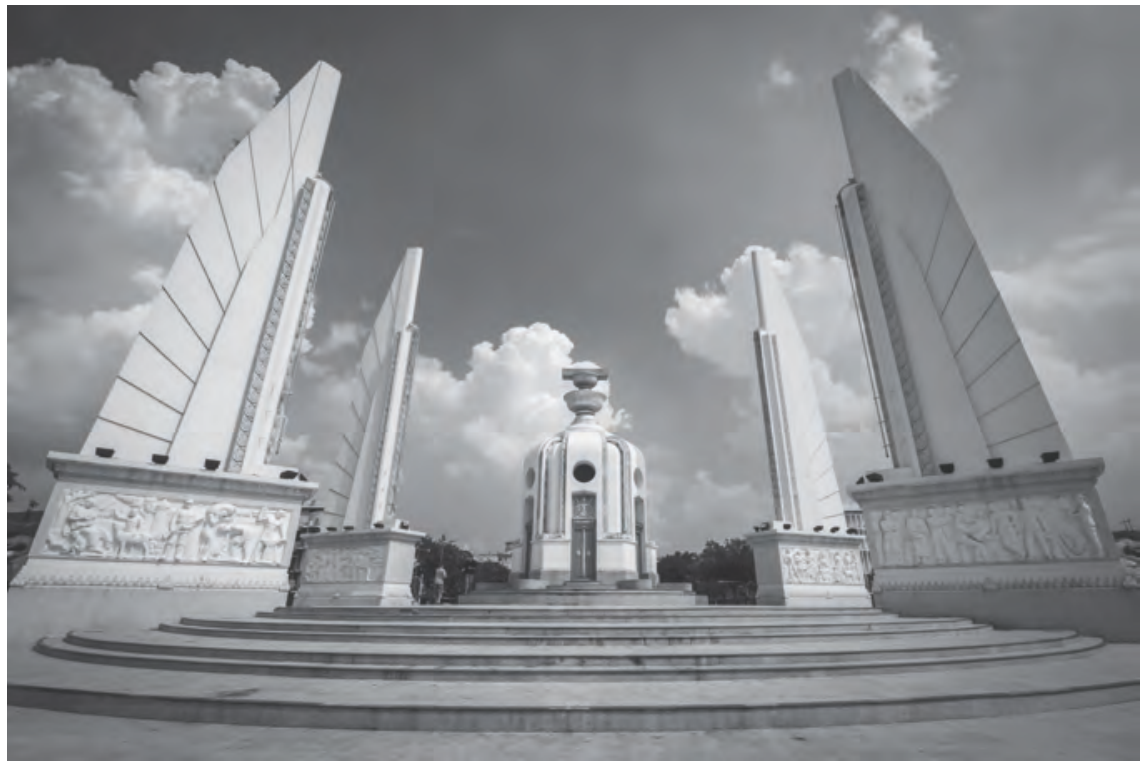

Illustration 27.1

Democracy Monument, Bangkok. Army coups have disrupted Thailand's democracy on many occasions

Photograph by Manoonp, CC BY-SA 4.0 
for it is then easy for even an incompetent dictatorship to profit from resource extraction. Authoritarian rulers often dismiss democracy as a Western cultural artefact. It is difficult to ascertain how much autocrats believe this, or how many of their citizens accept this line of argument. A lack of democratic traditions can be problematic when a transition to democracy is attempted; it is hard to establish democratic institutions where these have never existed. As we have often noted in this book, it is not enough to just create new rules. These need to be enforced by bureaucrats and armies and broadly supported by the population. These outcomes are hard to achieve if democracy is unfamiliar. Even if countries have not (often) held elections, though, it is easier to establish democracy if there is an independent judiciary and a competent and reasonably honest and independent bureaucracy. Many but not all autocrats fail to develop such institutions. Civil society - the existence of organizations that urge a range of government policies or pursue community-level development - can also facilitate democracy by creating both a sense of community and experience in political debate. Yet autocrats often attempt to ban such organizations. Freedom of the press can be critical; yet in many countries autocratic leaders have muzzled or intimidated the news media. Ethnic or religious diversity has proven a challenge in many countries, especially in Africa: Democracy depends on some sense of shared national purpose (chapter 21). Islamic fundamentalism has also proven a challenge. Even if only a minority of a population favours clerical rule, this can threaten the search for consensus on political institutions. Disdain for religious freedom can also limit the achievement of the constitutional protections on which democracy depends. We noted above that the Cold War encouraged developed countries to support "their" autocrats elsewhere; sadly, many wealthy democracies may now be tempted to support autocrats that pledge to fight terrorism or engage in peacekeeping missions in troubled parts of the world. The examples of Libya and Syria and Iraq warn of the dangers of failed attempts to move from autocracy toward democracy.

For those who believe that democracy is the best form of governance, the transition toward democracy in recent decades is one of the most fundamental transformations in human history. Yet in addition to backsliding in some of the world's newest democracies, there have been worrisome developments in some of the world's oldest democracies. Political debate has become toxic in many places: The compromise and mutual respect on which democracy depends are seen as a sign of weakness. Politicians with an authoritarian message have gained political support; some want to weaken constitutional protections for minorities. Many citizens feel - with much justification - that politicians are corrupt and that the rich benefit disproportionately from democratic processes (this dissatisfaction grows when income distribution worsens, as it has in many countries in recent decades). We will return in chapter 32 to the question of democracy, drawing lessons from history for democratic prospects moving forward. 


\section{Communism}

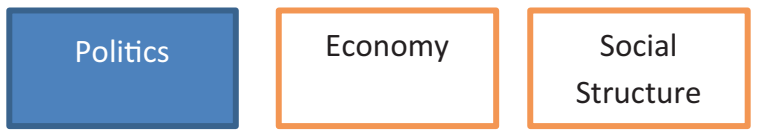

As noted above, we lack the historical perspective to appreciate which events and processes of the twentieth century will have the most significant impact on the future. Our understanding of path dependence (chapter 2) guides us to address communism here - for it affected the outcomes of wars (chapter 28) and the process of decolonization (chapter 30) among other historical processes. But it may well be that communism - which was a dominant historical force through the middle decades of the twentieth century - will prove to have had only a temporary impact on history. To be sure, there are still nominally communist governments in Asia and Cuba, but these have largely abandoned the economic principle of state ownership on which communist ideology rests. The state still owns more productive enterprises than is now common in noncommunist states, but private enterprise and market exchange and foreign investment are now accepted. These states have recognized, that is, that private markets have some advantages in meeting consumer needs and encouraging innovation. The question going forward in these countries is what will happen politically. Communism has also meant political domination by the communist party: Will this form of autocracy survive a dramatic change in economic institutions, or will a rising middle class clamour for the rule of law and a greater say in political decisions?

The original rise of communism in Russia in 1917 is an object lesson in the limited foresight of human actors. Tsars might have been able to retain some power if they had moved earlier and more resolutely toward political and economic reforms. Though the tsars had freed the serfs, expanded the rail network, and encouraged steel production, Russia proved no match for the German army in World War I: Its industrial production was half that of Germany despite having 2.5 times the population. The tsars had created an elected assembly in 1905 after widespread rioting, but only one-eighth of the population could vote. This gave the councils (soviets) established by industrial workers a great deal of democratic legitimacy, at least in their eyes. The elites that displaced the tsar early in 1917 (after more riots and an army mutiny) could likewise have moved more quickly. The communist revolution months later succeeded in large part due to communist promises to withdraw from World War I and to pursue land reform. If the previous government had pursued these actions, the communists might have found little support. German leaders, who weakened the tsarist state by attacking it, and then transported the communist leader Vladimir Lenin from exile in Switzerland to destabilize their foe, ended up fomenting a revolution that they certainly did not want. With the Russian army weak and in mutiny, a small and well-organized group could seize power unless the 
population felt some strong attachment to existing authorities. Many Russian people supported or at least did not oppose the communist takeover because they disliked the existing regime; they ended up with a government that was even more repressive. As we have had cause to note before (chapter 25), it is challenging to predict the path that a revolution will take. Peasants were suspicious but attracted to the idea of land redistribution; within about a decade they would be forced into collective farms. (Peasants played an even more critical role in the communist victory in China in 1949; there also the promised land redistribution would be soon followed by forced collectivization; chapter 28.) A majority of Russians in 1917 likely favoured liberal democracy - this was certainly the wish expressed by elected assemblies - but they were not organized to achieve this. Even many of the communist revolutionaries fought for ideals of worker democracy that were cast aside after the revolution. Still, we should appreciate that millions supported the revolution in the hope that it would usher in a more just and secure society.

The communists took over government offices, power stations, and railways. They then won a civil war over three years against a divided opposition. The rest of the world mostly opposed the communists but did little to stop them due to exhaustion from World War I. The communists executed 200,000 "traitors," including the entire royal family, to limit opposition. The

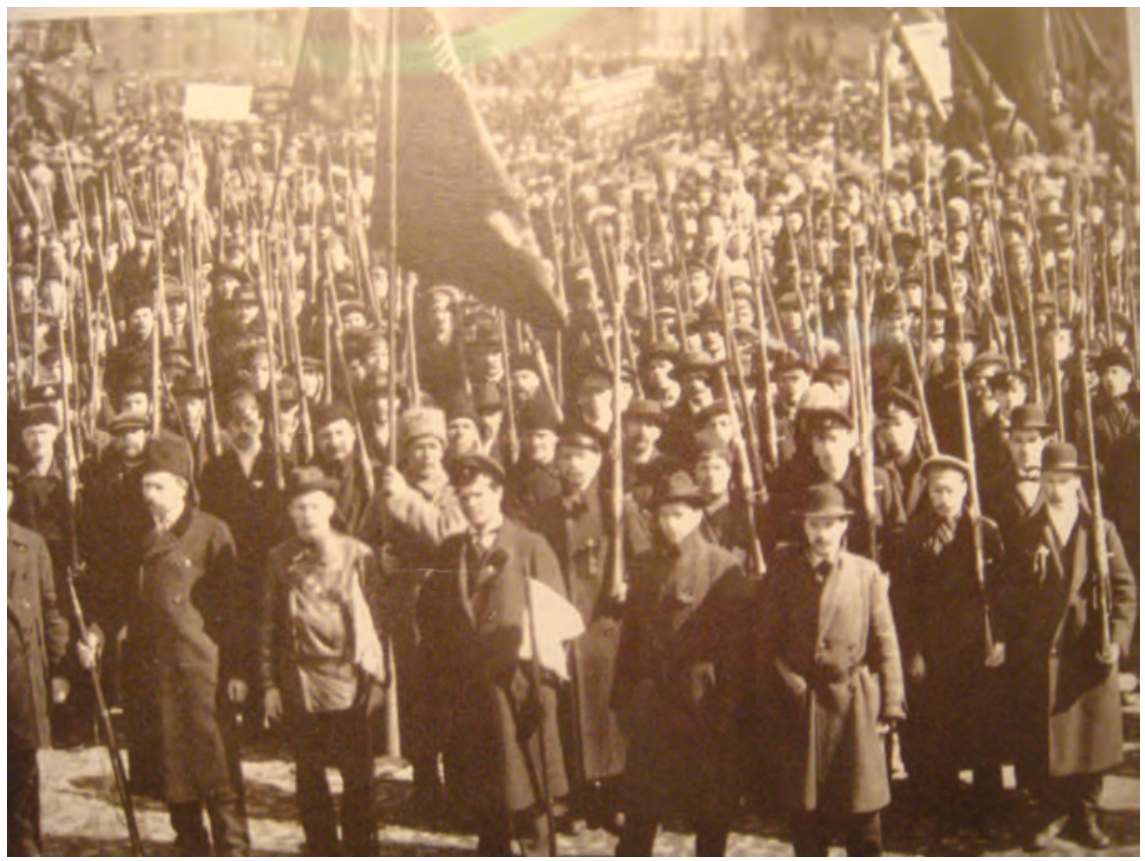

Illustration 27.2

Members of one of the first communist Red Guard regiments, Petrograd, autumn 1917 


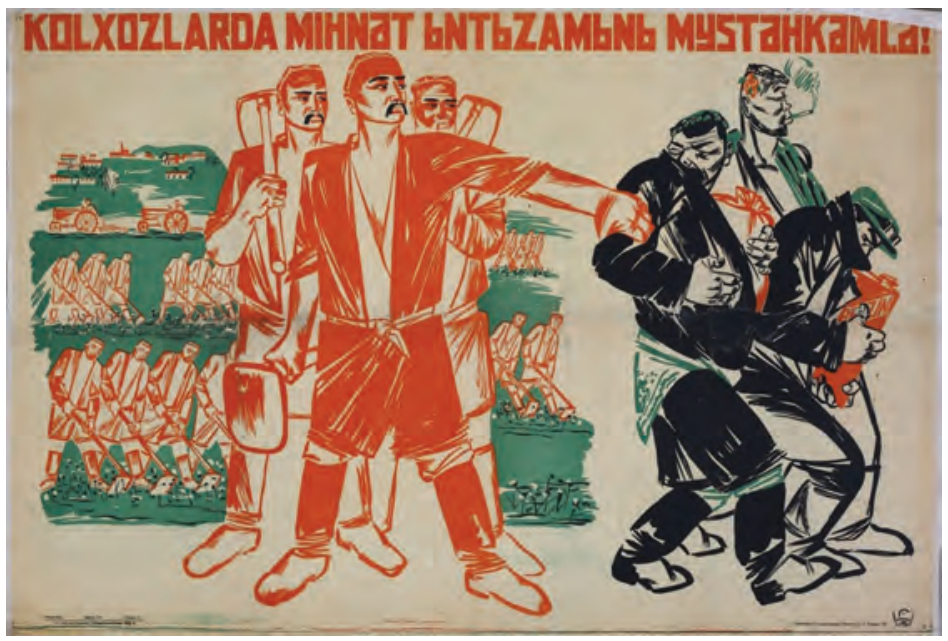

Illustration 27.3

"Strengthen working discipline in collective farms," Uzbek-language poster, Tashkent, 1933, Mardjani Foundation

Soviet Union had some early economic success - albeit at great personal cost, especially when agriculture was collectivized - by directing the development of steel mills and railways. During the Great Depression, as output in market economies fell dramatically (chapter 29), Soviet planning attracted admirers around the world. Soviet steel output increased from 4 to 18 million tons between 1928 and 1940, and electricity output from 5 to 48 billion kilowatthours. The Soviet Union would provide a far better response to the German army in the Second World War than the tsars had in the First. However, in the postwar world, central planning had trouble delivering a range of consumer goods. The Soviet Union could still master large organized projects such as building atomic bombs or launching satellites, but the Soviet economy was far less innovative on average.

The government of the Soviet Union attempted both political and economic reforms in the 1980s as it became clear that Western economies were outperforming the Soviet Union. As these reforms unfolded, the Soviet Union itself dissolved into its constituent states (see chapter 28). Though the weaknesses of the Soviet economy and polity had been evident for some time, the speed with which the Soviet Union fell apart was very surprising, both inside and outside that country. The result of the dissolution of the Soviet Union, and its withdrawal from Eastern Europe, has been a set of democracies with market economies in Eastern Europe (which all, we should note, suffered a wrenching economic transition to a market economy). Farther east, a set of autocracies emerged in Central Asia, and some fragile democracies in between in Russia and the Ukraine. In Eastern Europe, as elsewhere in the world, higher incomes and historical memories of democracy and the rule of law proved 
conducive to a successful transition to democracy (though authoritarianism and corruption remain problematic in some of these countries - especially where there is ethnic diversity).

What lessons can we draw from the experience of communism? One simple but valuable lesson might be that it is dangerous to invest too much power in any one group of people. Though previous empires might have claimed to own all of the land, they generally lacked the bureaucratic capability to dominate both polity and economy. The communist party was able to exercise supreme political and economic control - though a thriving black market emerged to supply goods that the state could not. This all-powerful state would kill millions of people and banish millions more to prison camps in Siberia as a result of savage battles for power within the party and against any who opposed the party (a fact later admitted by the Soviet state itself).

Some have also drawn a lesson that government ownership of any sort is a mistake. Such a lesson seems to fail a simple historical test: Democratic, marketoriented states have continued to be active in the production of a range of goods and especially services. We might reasonably conclude that states and markets likely have different strengths and weaknesses (a conclusion in accord with many theories in both economics and political science). We might also appreciate that path dependence has likely limited the range of economic institutions experimented with historically. There may be alternative institutional forms, such as labour-managed firms, that could exercise a more significant role in economic production if more widely supported economically and culturally.

We might place the eventual failure of communist economies within our broader historical understanding of rulers and bureaucracy. Rulers, we have seen, have always had difficulty getting bureaucrats to do as they wished. The Soviet Union gave bureaucrats the responsibility to organize the entire economy. Even with the latest communications technology and monitoring devices, it was impossible for rulers to micro-manage millions of detailed production decisions. The result was widespread corruption and mismanagement. Firms and farms regularly missed quotas because workers shirked or sold on the black market. Enterprises produced goods and services of poor quality as the state struggled to police quality (something that market exchange handles better, though regulation of unobservable health and safety characteristics is still required). Although bureaucrats had incentives to think of clever ways to subvert the system, they had little incentive to encourage innovations that would expand production or improve quality. (Yugoslavia and China have experimented with having state-owned firms compete in markets, but even then, managers may not face the same incentive to innovate as owners of private firms.) In democratic countries, citizens can monitor and even sometimes sue bureaucrats; in the Soviet Union, there was no external check on bureaucratic abuse. 


\section{PRIMARY DOCUMENTS: DECLARATION OF THE RIGHTS OF WORKING AND EXPLOITED PEOPLE}

In January of 1918 the All-Russian Central Executive Committee adopted this declaration, drafted by Vladimir Ilyich Lenin, the revolutionary leader. It would later be endorsed by the constituent assembly.

\section{CHAPTER ONE}

1. Russia is proclaimed a Republic of Soviets of Workers', Soldiers', and Peasants' Deputies. All central and local authority is vested in these Soviets.

2. The Russian Soviet Republic is established on the basis of a free union of free nations, a federation of National Soviet Republics.

\section{CHAPTER TWO}

The Constituent Assembly sets for itself as a fundamental task the suppression of all forms of exploitation of man by man and the complete abolition of class distinctions in society. It aims to crush unmercifully the exploiter, to reorganize society on a socialistic basis, and to bring about the triumph of Socialism throughout the world. It further resolves:

1. In order to bring about the socialization of land, private ownership of land is abolished. The entire land fund is declared the property of the nation and turned over free of cost to the toilers on the basis of equal right to its use. All forests, subsoil resources, and waters of national importance as well as all live stock and machinery, model farms, and agricultural enterprises are declared to be national property.

2. As a first step to the complete transfer of the factories, shops, mines, railways, and other means of production and transportation to the Soviet Republic of Workers and Peasants, and in order to ensure the supremacy of the toiling masses over the exploiters, the Constituent Assembly ratifies the Soviet law on workers' control and that on the Supreme Council of National Economy.

3. The Constituent Assembly ratifies the transfer of all banks to the ownership of the workers' and peasants' government as one of the conditions for the emancipation of the toiling masses from the yoke of capitalism.

4. In order to do away with the parasitic classes of society and organize the economic life of the country, universal labour duty is introduced.

5. In order to give all the power to the toiling masses and to make impossible th $e$ restoration of the power of the exploiters, it is decreed to arm the toilers, to establish a Socialist Red Army, and to disarm completely the propertied classes.

\section{CHAPTER THREE}

1. The Constituent Assembly expresses its firm determination to snatch mankind from the claws of capitalism and imperialism which have brought on this 
most criminal of all wars and have drenched the world with blood. It approves whole-heartedly the policy of the Soviet Government in breaking with the secret treaties, in organizing extensive fraternization between the workers and peasants in the ranks of the opposing armies and in its efforts to bring about, at all costs, by revolutionary means, a democratic peace between nations on the principle of no annexation, no indemnity, and free self-determination of nations.

2. With the same purpose in mind the Constituent Assembly demands a complete break with the barbarous policy of bourgeois civilization which enriches the exploiters in a few chosen nations at the expense of hundreds of millions of the toiling population in Asia, in the colonies, and in the small countries. The Constituent Assembly welcomes the policy of the Soviet of People's Commissars in granting complete independence to Finland, of removing the troops from Persia and allowing Armenia the right of self-determination. The Constituent Assembly considers the Soviet law repudiating the debts contracted by the government of the Tsar, landholders, and the bourgeoisie a first blow to international banking and finance-capital. The Constituent Assembly expresses its confidence that the Soviet Government will follow this course firmly until the complete victory of the international labour revolt against the yoke of capital.

\section{CHAPTER FOUR}

1. Having been elected on party lists made up before the November Revolution, when the people were not yet in a position to rebel against the exploiters whose powers of opposition in defence of their class privileges were not yet known, and when the people had not yet done anything practical to organize a socialistic society, the Constituent Assembly feels that it would be quite wrong even technically to set itself up in opposition to the Soviet.

2. The Constituent Assembly believes that at this present moment of decisive struggle of the proletariat against the exploiters there is no place for the exploiters in any organ of government. The government belongs wholly to the toiling masses and their fully empowered representatives, the Soviets of Workers', Soldiers', and Peasants' Deputies.

3. In supporting the Soviet and the decrees of the Soviet of People's Commissars, the Constituent Assembly admits that it has no power beyond working out some of the fundamental problems of reorganizing society on a socialistic basis.

4. At the same time, desiring to bring about a really free and voluntary, and consequently more complete and lasting, union of the toiling classes of all nations in Russia, the Constituent Assembly confines itself to the formulation of the fundamental principles of a federation of the Soviet Republics of Russia, leaving to the workers and peasants of each nation to decide independently at their own plenipotentiary Soviet Congresses whether or not they desire, and if so on what conditions, to take part in the federated government and other federal Soviet institutions. 
What justifications are given for ignoring the elected congress? What happened later to the promises of national self-determination made in this document? What do you think of the document as a whole? What does it tell you about the unpredictability of revolutionary processes?

Note: Students and instructors might also read Aleksandra Kollontai's visionary "Soon," written in 1919, which imagined the happy communist future to be achieved by 1970 (www.marxists.org/archive/ kollonta/1922/soon.htm), and reflect on the difficulties of predicting a revolutionary future.

\section{The growth of the state}

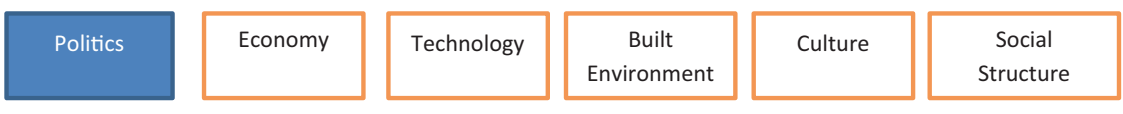

It is perhaps ironic that while communism as an ideology has declined, the size of the state has expanded dramatically across the world in the last century. In 1900, states typically absorbed some 10 per cent of economic activity. This was a level occasionally reached and even surpassed by Chinese empires thousands of years ago. Nevertheless, most states through much of history had probably taxed more like 5 per cent (often less) of total output. The 1900 level of taxation and spending represented, then, a significant but neither dramatic nor unprecedented level of state activity. Nevertheless, it had allowed many governments to make expensive investments in railways and roads and ports, as well as schools. By 2000, however, governments in at least the developed world had expenditures that averaged more like 50 per cent of economic activity. This must rank among the most significant historical transformations of the century.

Though states can borrow, they can for the most part only spend money in the end if they can pay for that spending with taxes. We have often marvelled in this book at the ability of empires in the distant past to tax individual incomes. Recall that the Egyptians and Chinese and Indians (and others) did not just levy taxes on land but estimated the productivity of different plots of land and collected taxes accordingly. In 1900, most states in the world relied instead on taxes on trade: tariffs on international trade and sales taxes on domestic commerce. Though trade had expanded through history, and especially in the nineteenth century, governments would always be limited in scope if they relied entirely on taxing trade.

The growth of the state depended critically on the introduction of income taxes. Britain had experimented with income taxes during the Napoleonic Wars, and instituted these more formally from the mid nineteenth century. The United States had experimented during the Civil War, but only established a constitutional right to levy income taxes from 1913. Many other countries 
developed income taxes during the First World War: Canada, for example, introduced a "temporary" income tax in 1917, but the challenge of paying off wartime debts meant that the tax remains in place.

States often only levied these early modern income taxes on those earning high incomes. The United States in 1913 collected income taxes from only about 10 per cent of the population. Over time, as incomes rose and bureaucratic capabilities increased - through better communications technology, typewriters, business machines, and then computers - it became feasible to levy income tax on everyone. One of the challenges faced by the poorest countries in the world today is that they struggle to collect income taxes: Incomes are far lower there and bureaucratic capabilities limited.

Governments spent some of this increased tax revenue in ways that had become familiar in the nineteenth century. They continued to support transport infrastructure (taxes on fuel would fund most road construction in most countries for many decades). Governments spent increased amounts on education as educational expectations increased: Whereas in the mid nineteenth century most people only pursued an elementary education, in the twentieth century first high school and then post-secondary education would become increasingly common. A public policy interest in education - both to fill skilled jobs and to generate a body of educated voters - meant that much of this advanced education was government funded, though post-secondary students paid tuition in most countries. Police forces had also become familiar globally in the nineteenth century - Meiji Japan modelled its national police force on that of France - and would expand with urbanization and increased government capability in the twentieth century.

In the past, states had occasionally pursued what we now call social policy. The Chinese Empire had in particular stockpiled grain to relieve harvest shortfalls (and the Inca stored potatoes and corn). In the early modern period, Britain and some other countries had "Poor Laws" which required local authorities to look after the very poor (though the able poor were often forced to work). States, as we have seen, sought to justify their rule, and taking care of the poorest might seem a good strategy. Some rulers might internalize these self-justifications and act out of real compassion toward the poor. States especially if there were large urban centres - might also help the poor in order to avoid public unrest or rebellion: the Roman Empire famously gave free bread to the Roman masses. Yet most states in history controlled a small proportion of total economic output: Even if selfless, they might have been unable to prevent severe poverty (the Qin and Inca may be exceptions here.) In general, then, families needed to deal with the challenges of illness, old age, and unemployment on their own. Some families had access to guilds (or later unions) or other sorts of association that might provide limited sorts of insurance for illness or early death.

Government provision of social services had begun to expand in the nineteenth century. This was in part because governments had increased financial capability, and partly because local communities could not cope 
with social needs as societies became more complex. Famously, the German state introduced industrial accident insurance in 1871, health insurance in 1883, and an old-age state pension system from 1889 in an attempt to gain public support for autocratic government, and to limit the rising support for socialist political parties. France would develop the first system of unemployment insurance in 1905. A handful of other European countries introduced social policies before World War I. So too did Chile and Uruguay, though such policies were unusual at this time in Latin America. Such programmes would become increasingly common almost everywhere in the twentieth century. The Great Depression was an important watershed here: Countries around the world struggled to care for masses of unemployed (in part to avoid social unrest or even revolution). The United States, for example, would introduce insurance systems to provide for both unemployment and old age during the 1930s, while also funding programmes of direct relief to the unemployed. In the postwar economic boom - with rising incomes and low levels of unemployment through much of the world - such programmes tended to be formalized and expanded. As memories of the Depression faded and unemployment grew from the 1970s, the consensus around social policy began to fray. Notably, the consensus has frayed fastest in countries characterized by racial, ethnic, or religious stratification: Those of high incomes dislike supporting members of other groups. Whereas in the 1930s it was widely assumed that people would work if they could find a job, there was widespread concern from the 1970s that some people would cease looking if supported by the state. The United States and other countries moved to limit the time that any person could receive social assistance: This did succeed in pushing some people into employment, but others fell deeper into poverty.

Voters sometimes assume that social assistance absorbs a majority of government expenditures. However, the vast bulk of government spending goes toward programmes that serve the entire population: roads, schools, military (see chapter 28 for some discussion of military expenditure), police, fire, sewers, and healthcare. Medical care came to have a significant impact on health outcomes in the twentieth century as a range of pharmaceuticals and medical procedures were developed. Most rich countries moved to provide free medical care to all citizens over the course of the century, while others only protected the poor and the old. The justification was similar to that provided for employment insurance: that a caring society should help the unfortunate.

The introduction of new programmes was often intensely debated, and usually only occurred if the public was mobilized in support of new initiatives. In the early postwar decades, the combination of prosperity and vivid memories of the Depression encouraged substantial political consensus toward the expansion of social policy. The introduction of social security in 1935 in the United States was controversial; by 1960, it was taken for granted. In the twenty-first century, hardly anyone suggests scrapping it. The first provincial experiment 
with public healthcare in Canada from the 1940s was extremely controversial; by the 1960s, all political parties supported the idea. In the twenty-first century, there is some debate in Canada regarding the private provision of some health services but no direct suggestion by any major party to move away from public payment for healthcare.

As noted above, states may institute social policy out of compassion, selfjustification, or fear of social unrest. The threat of communism, and agitation by labour unions, played an important role in the adoption of social policy in much of the world in the interwar and early postwar years. The decline of both communism and union power has likely played some role in decreasing state support for some programmes in some countries in more recent decades. Culture also looms large: Voters are willing to support social policy if they identify with the recipients. The Depression sent a powerful signal that misfortune could befall almost anyone. If those with steady incomes come to view the very poor as different, and especially as lazy or undeserving, then support for social policy withers. It can, of course, be in the interest of the richest taxpayers to encourage such attitudes. Social divisions by ethnicity or class increase the likelihood that the poor will be viewed with suspicion. Recall that we need social policy more than previously in history because complex modern societies cannot rely on local communities to care for the unfortunate: But this very complexity increases the likelihood that we will view the poor negatively. Modern states may nevertheless at times justify social policy for pragmatic reasons: addressing poverty may reduce crime and healthcare costs, and even yield tax increases if states can successfully move some of the very poor into the labour force.

Social policy has often focused in history on male "breadwinners." As both single motherhood and two-income families have become more common in many countries, social policy has often become more gender neutral. Yet there are substantial cross-country differences in attitudes toward female work, which are then reflected in social policy. Provision of public day care is one key area in which there are substantial cross-country differences. Parental leaves after childbirth or adoption are increasingly common in the world: In some countries mothers are emphasized while in other countries both parents are eligible.

The first advances in modern social policy occurred in Europe and North America, countries that were rich, complex, and urbanized. New countries created by decolonization (chapter 30) often experimented with social policy despite being much poorer. They were often limited in social spending by their limited tax revenues. International aid agencies sometimes supported social spending, in part from a belief that educated and well-fed people would be more productive, but other times were more concerned that governments balance budgets than encourage growth. Cold War tensions often encouraged spending on armies rather than social policy. Widespread corruption was a further drain on state resources. In many of these new countries, ethnic rivalries also limited societal consensus around social policy. 
Postscript: The intense debate regarding social security in the 1930s encouraged selection in the United States and elsewhere of an option whereby the next generation finances the public pensions of the retiring generation. This meant that the initial cost of the programme was low, and so it was easier to achieve political approval. Eight decades later, that choice of institutional format leads to concerns about the ability to fund the retirement of the baby-boom generation (those born between 1945 and 1960). Though support for social security is now widespread, politicians have avoided facing up to future funding challenges.

Why did these new expenditures absorb the increased capacity of states to tax? Sceptics can well imagine that self-aggrandizing states and politicians naturally sought to tax and spend as much as they could. However, increased government spending reflected a couple of significant trends. One was rising incomes. As noted above, this made it easier to tax. As incomes rise, people devote a lesser proportion of their incomes to the necessities such as food and clothing and shelter, goods that (non-communist) states have played a limited role in providing to most people. People want to spend more of their income on education and healthcare and travel, and politicians could make a compelling case for public provision of schools and hospitals and roads. A second trend has involved technological innovation. It has become much less expensive (controlling for inflation) to produce a variety of goods and services over the last centuries due to assembly lines, continuous processing, electrification, and automation (see chapter 29). We still mainly deliver education in a very similar fashion to the way it always has been, with teachers addressing small groups of students. One-on-one diagnoses characterize healthcare, but these often lead to ever-more-expensive prescriptions or treatments. The costs of education and healthcare have therefore risen relative to the costs of a range of other goods and services. (We will discuss in chapter 32 whether newer technologies might reverse this trend going forward.)

A significant increase in taxes and spending was perhaps inevitable. Even politicians who decry these trends rarely imagine scaling the state back below 30 per cent of economic output. Yet our experience of the ruler's challenge throughout this book guides us to doubt that all of this massive increase in government spending has been desirable. Bureaucrats will inevitably have designed programmes to their own tastes. Rulers will have struggled to manage an increasing array of government initiatives. Special interests will have encouraged programmes that benefit themselves at the expense of others. Once a programme is in place, those who benefit from it will fight to keep it; they will likely be more influential politically than the mass of taxpayers who suffer only slightly from any one ill-advised programme. The fact that new programmes were often introduced - sometimes supposedly temporarily - during unusual 


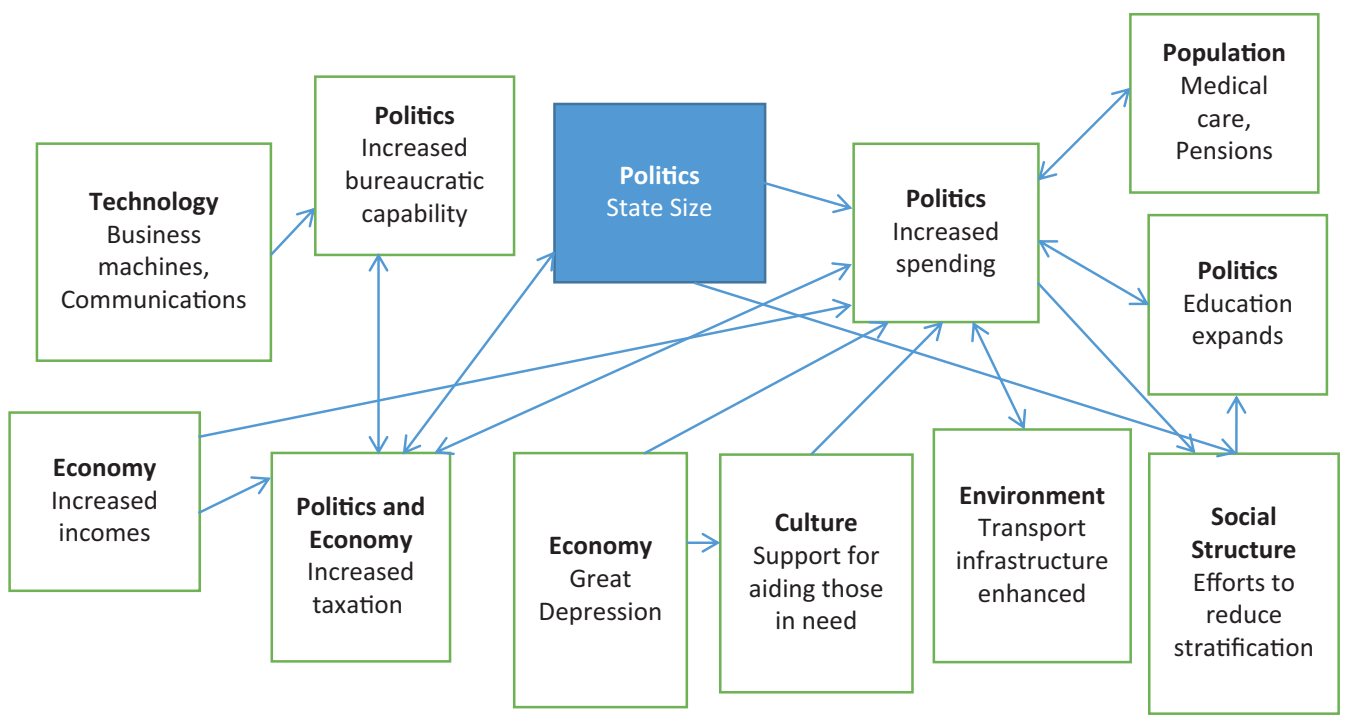

Figure 27.1

Influences on and effects of expanded state size

Note: The prevalence of two-way arrows signals that this was a process in which increased tax revenues and increased spending on new and enhanced programmes were mutually reinforcing. We have not cluttered the diagram with all types of government spending; we might note here the dramatic increases in military expenditure observed during the Cold War and since (chapter 28) that have also only been possible because of increased revenues.

periods such as World War or Great Depression increases the likelihood that programmes will be inherited that are less than ideal for periods of peace and stability. In sum, we have not had ideal conditions for generating institutional mutations, selecting among these, or transmitting these through time.

Political debate often seems a contest between those who lionize all government programmes and those who hate all government programmes. History can encourage a more nuanced understanding, and perhaps attention to the careful evaluation of each government programme (see chapter 32).

We should take care that our discussion of the challenges of bureaucracy does not lead us to demonize public servants (or bureaucrats within large corporations for that matter). Modern bureaucracies function differently from ancient bureaucracies in several important ways. As noted above, technologies (including advanced accounting techniques) have increased the ability of governments to ensure that bureaucrats are performing their jobs appropriately. The bureaucracies created in the nineteenth and twentieth centuries were generally dedicated to principles of generality - that the state should treat all citizens in precisely the same way - and efficiency that earlier bureaucracies might only dream of. National censuses and the regular auditing of government accounts had become common practices already in the nineteenth century (and a considerable international consensus developed on how to operate a census in the middle of that century). It was still common in the eighteenth 
century for public offices to be sold, and purchasers clearly viewed such offices as an opportunity for private gain; by the late nineteenth century merit-based appointments and expectations of public service dominated. The growing middle class (chapter 23) rather than the elite took most bureaucratic posts, and corruption received official and cultural disdain. Increased tax revenues allowed bureaucrats in many countries to earn a regular and reasonable salary such that corrupt practices could be discouraged. Indeed, one complaint often levelled against bureaucrats is that they show no flexibility but instead stick to rules that by their nature cannot ideally suit every situation. Cultural attitudes have also changed. As bureaucracy has become more meritocratic - not only are there civil service exams and interviews in many countries but educational systems prepare the majority of the population to be able to apply for these bureaucrats have come to develop a

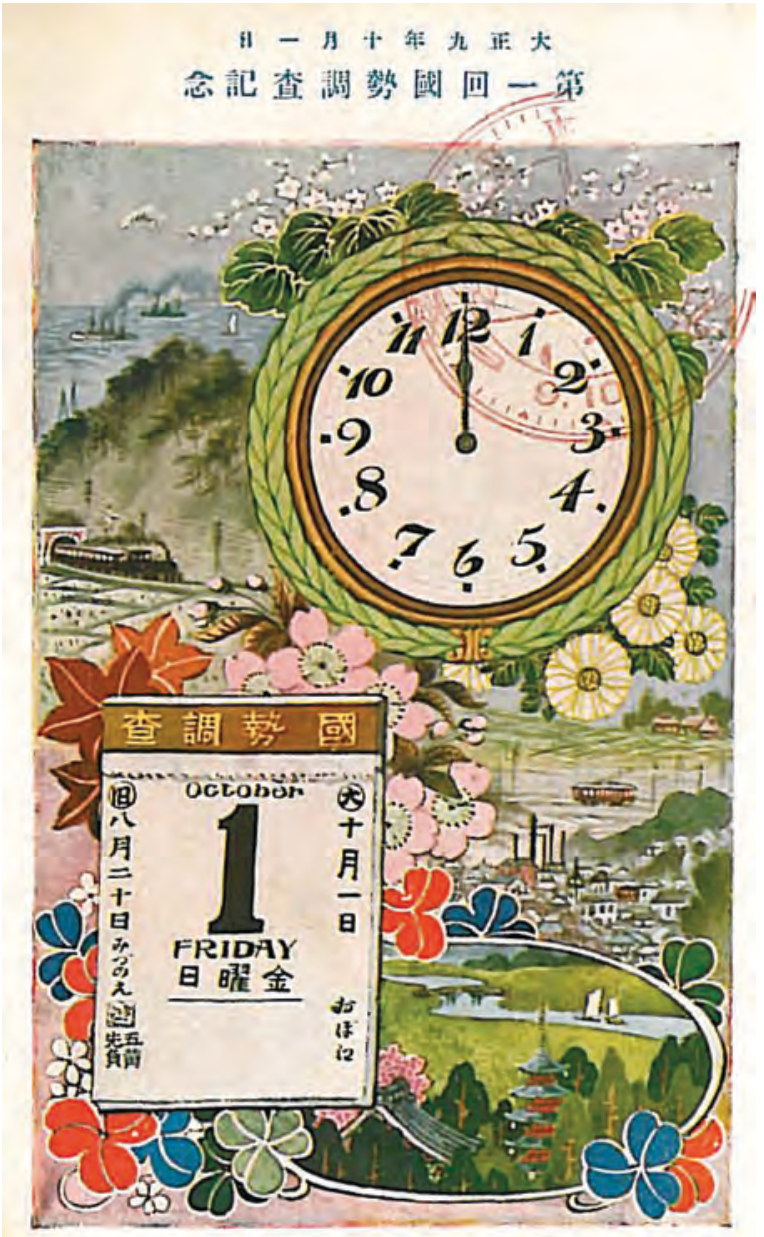

Illustration 27.4

Historical postcard commemorating the Japanese Empire's first census, October 1, 1920 sense of duty rather than entitlement. Most public servants - at least those not confined to boring routines - have some sense of public service. Democratization has helped here: Bureaucrats can more easily be inspired to public service if they believe that the state they serve is legitimate and itself pursues the public good. We noted in chapter 9 that the arbitrary power of rulers over bureaucrats might encourage bureaucratic theft and insubordination: Bureaucrats with legal protections against abuses of power by superiors are better able to focus on their official duties. Bureaucratic institutions themselves have evolved in ways that punish at least some kinds of wrongdoing and reward certain types of ethical behaviour (but only rarely reward innovation or even whistle-blowing on the corrupt practices of others). None of these changes eliminates corruption or empire-building, but they do limit such behaviours.

The experience of China is noteworthy. In the eighteenth century it likely still had the most rational and effective bureaucracy in the entire world. By the late nineteenth century, the Chinese bureaucracy would be widely mocked for corruption and incompetence. In part, this mockery simply 


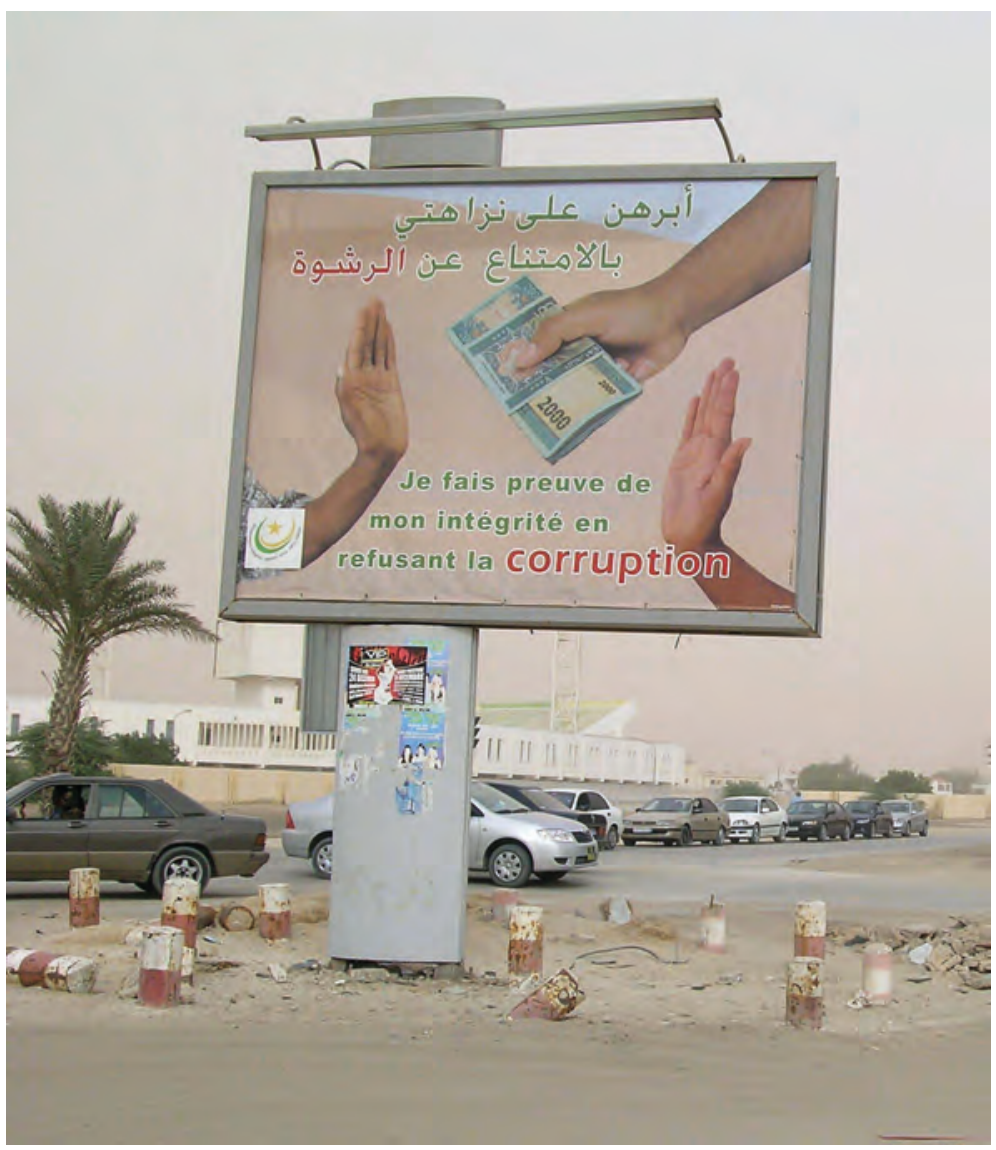

Illustration 27.5

Billboard urging refusal of corruption (in Arabic and French), Nouakchott, Mauritania, 2007

Photograph by c.hug (Cropped from Flickr), CC BY-SA 2.0

reflected European and Japanese arrogance as they recorded military victories. Nevertheless, there were real problems. The focus of Chinese examinations on Confucian classics did not prepare bureaucrats to react to the dramatic political and economic changes of the nineteenth and early twentieth centuries. Confucian conceptions of a social hierarchy may have militated against the egalitarian ethos developed elsewhere. The very fact that the Chinese bureaucracy had existed for millennia militated against new bureaucratic practices (recall our discussion of path dependence in chapter 2; the Ottomans also struggled to change bureaucratic traditions by replacing patronage with merit over the nineteenth century). Tax revenues had stagnated, preventing the Chinese state from developing new programmes or discouraging corruption among poorly paid bureaucrats. The Qing, desperate for money, even began to sell bureaucratic offices. And the courtly intrigue that characterized the last decades of Qing rule meant that the bureaucracy often received limited or 
contradictory instructions. At the same time that the Chinese bureaucracy was declining in effectiveness, Meiji Japan built a modern bureaucracy based on European precedents with an explicit goal of catching up to the West economically. Scholars debate whether such a path was open to China if its rulers had behaved differently. Decades later, the Chinese economic miracle must owe something to bureaucratic competence, and many scholars speculate that the success of the modern Chinese bureaucracy owes much to China's historical experience of bureaucracy.

\section{Challenges facing agents: Bureaucrats}

The bureaucrat operates within a hierarchy. They often must supervise subordinates while being overseen by either other bureaucrats or rulers (or owners of a company). They face the ruler's challenge of encouraging subordinates to do as they wish, but bureaucratic institutions limit the rewards, punishments, and monitoring mechanisms that they can employ. In pleasing superiors, there is a distant analogy to the slave's challenge of negotiating work conditions and salary within a power relationship.

The bureaucrat hopes to pursue the state's (or company's) goals both for personal advancement and for public service motives. (They may often find that the strategies that best serve one purpose do not best serve the other; they may self-deceive in such cases.) They may sometimes disagree with particular goals or means. They may be frustrated by the rules they are expected to follow but limited in their ability to persuade superiors or the state (company) to alter these. They may have some scope for flexibility (if so, they can be a source of evolutionary mutations in rules). Change across a bureaucracy may occur frustratingly slowly.

\section{International organizations}
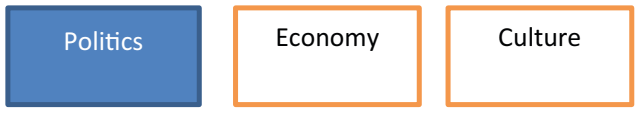

\section{Technology}

The expansion in size of national governments was matched by an increase in number, size, and influence of international organizations. Some of the same trends were at work. National governments could more easily fund the work of international organizations as their own revenues increased. Concern for the less fortunate within countries supported some concern for the less fortunate internationally. But perhaps the two most significant forces at work were rising concern with maintaining world peace and the increased importance of international trade and investment. Concerning the first, the shocking experiences of world war were transformative. For the second, the imposition of trade restrictions during the Great Depression, widely perceived by both politicians and experts to have exacerbated the Depression, spurred postwar efforts at trade negotiation.

The treaties that ended World War I established the League of Nations. Though there had been efforts toward creating international institutions that 
would work toward peace in the nineteenth century (chapter 28), the League of Nations was supposed to be a permanent organization with a dedicated bureaucracy and some powers to intervene in international disputes. In part because the United States refused to participate, and in part because its members gave the League limited resources and authority, it did little more than complain about aggressive acts during the interwar period. In the aftermath of World War II, a more powerful and inclusive United Nations (UN) was established. Polls in the United States at the time found that some 85 per cent of the population supported a strong UN. Its General Assembly would eventually welcome almost every state in the world and engage in debates not just on peace but on other matters of international interest, such as gender rights and environmental amelioration (some of these issues, such as overfishing in international waters, or the spread of epidemics across borders, themselves reflect trends in economic activity and travel). The 1992 convention on chemical weapons induced many countries to destroy these. The UN would establish over time a number of subsidiary organizations: the World Health Organization (WHO) from 1948 (which declared smallpox beaten in the 1970s but has been criticized for its handling of some pandemics); the United Nations Educational, Scientific and Cultural Organization (UNESCO; which among other things designates sites of global historical importance); the Food and Agriculture Organization (FAO); UNICEF (family welfare); International Labour Organization (ILO; monitors labour conditions, advocates for worker rights); and the Commission on Human Rights.

The UN Security Council would provide a venue in which the leading military powers, plus a smattering of other states chosen by the General Assembly, could discuss possible interventions in international conflicts. Though consensus was not possible on disputes guided by Cold War considerations, the

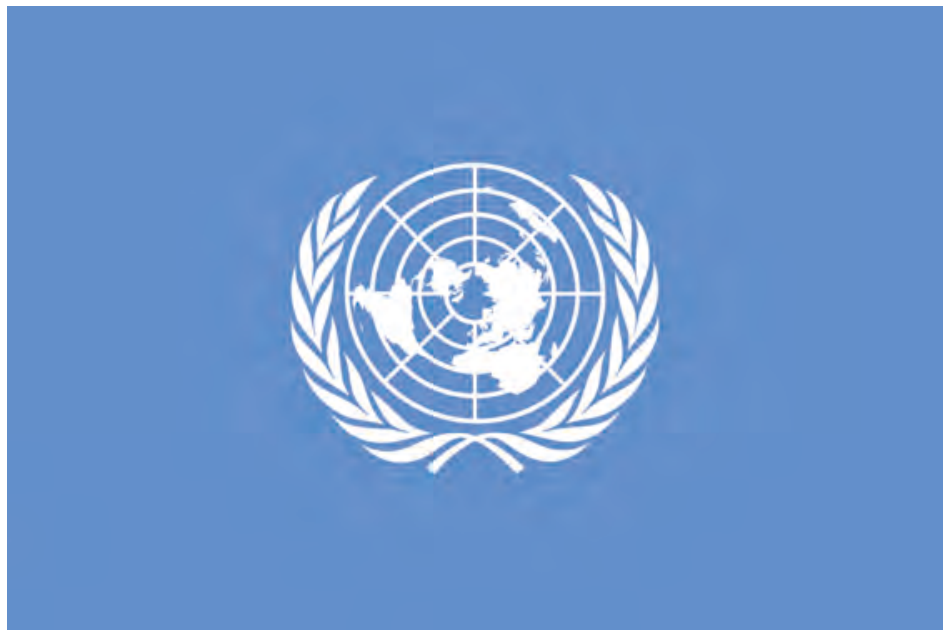

Illustration 27.6

United Nations flag 


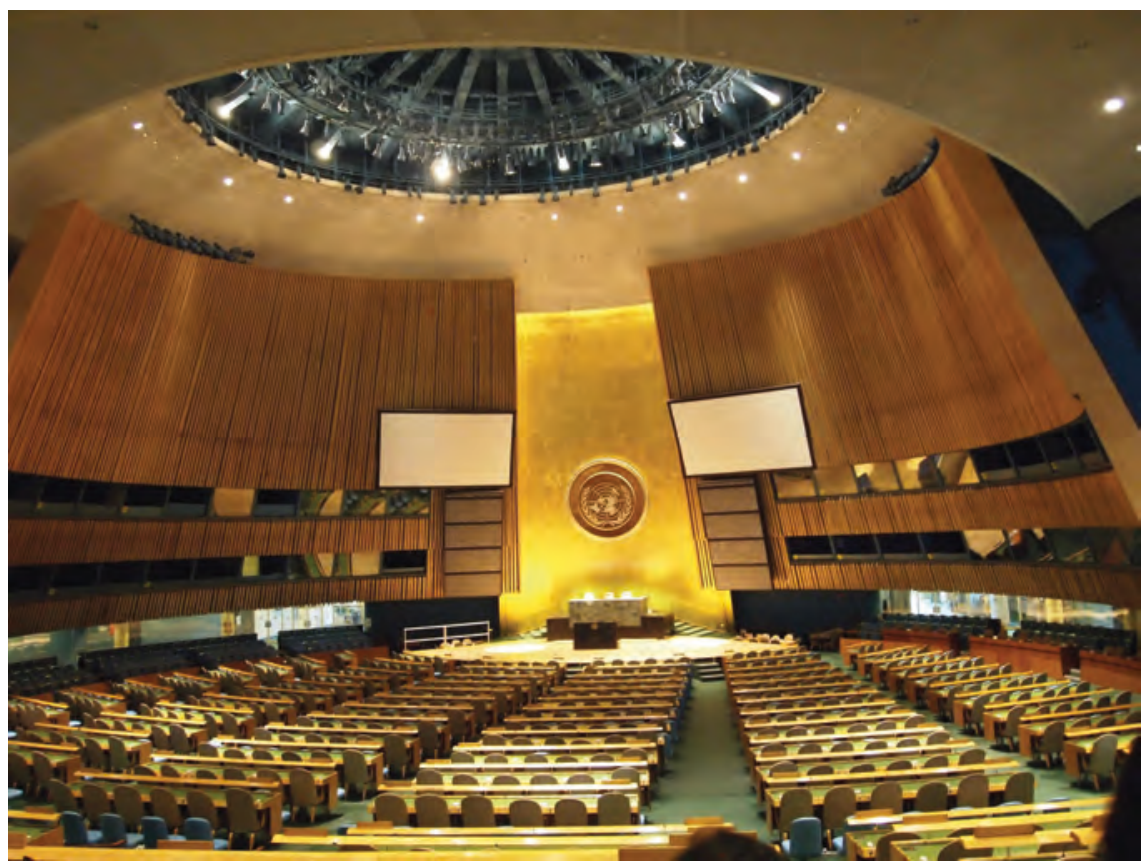

Illustration 27.7

United Nations General Assembly, 2012. Architects were advised that there might one day be as many as seventy members of the United Nations. Almost 200 countries are now represented in the General Assembly

Photograph by Lowlova, CC BY-SA 3.0

Security Council would nevertheless address a variety of conflicts. The idea of peacekeeping forces was developed after the aborted British-French-Israeli invasion of the Suez Canal in 1948 to separate Israeli and Arab forces (Canadian diplomat and future Prime Minister Lester Pearson received the Nobel Peace Prize for his advocacy of this and later peacekeeping forces). Since then the UN has sponsored some seventy peacekeeping missions, involving hundreds of thousands of troops; almost sixty of these have occurred since the fall of the Soviet Union. These have not always been successful: There were far too few peacekeepers on the ground in Rwanda in the 1990s to prevent genocide, for example. Yet states would hardly have engaged in these expensive and dangerous missions if not for a sense that they often do reduce the incidence or extent of war and its consequences for civilians. International peacekeepers maintained peace in Cyprus for decades. Despite their noble purpose, peacekeeping troops have often looted and raped, as armies long have in war zones. (Despite efforts in many countries to recruit women, and recognition that protecting families is a crucial objective of peacekeeping, women have comprised only a few per cent of peacekeeping forces.) In recent decades the UN has even intervened in civil wars, deciding that the responsibility to protect innocent civilians (R2P; officially recognized in 2005) can supersede its general 
respect for national sovereignty. An organization of nation states hardly took such a decision lightly, but it reflects an increased appreciation of human rights (see below). Peacekeeping has become more challenging: It is one thing to separate competing armies but quite another to prevent the activities of guerrilla armies or terrorists (again see chapter 28).

The United Nations itself has been criticized for over-bureaucratization and poor financial management. It is perhaps not surprising that these "ruler's challenges" have arisen in an organization loosely managed by member states: No one member state has a powerful incentive to ensure that the UN spends its money wisely. A policy of hiring from all member countries is laudable in many ways but interferes with the pursuit of a professionalized bureaucracy.

At about the same time that the United Nations emerged, a set of organizations were developed to address economic matters. The International Monetary Fund (IMF) deals with international financial transactions and especially exchange rates among different currencies; the World Bank finances projects in developing countries, and the General Agreement on Tariffs and Trade (GATT) - which would later become the World Trade Organization (WTO) - negotiates international trade agreements. Rich market-oriented countries at first dominated the membership in these three organizations, but almost all states are now members (though voting powers in the IMF are still heavily weighted toward the original members). The GATT and WTO would supervise several rounds of global reductions in trade barriers, playing an important role in the postwar expansion of international trade (chapter 29). In recent decades, WTO negotiations have become more challenging and controversial as trade in services and the international harmonization of regulations have become central topics of negotiation. The World Bank borrows money on capital markets - with an excellent credit rating because of promises from member countries to cover its debts - and lends the money to poor countries for infrastructure projects (with outstanding loans of US\$190 billion in 2016). It also provides direct aid with the profits it makes on loans (US\$500 million in 2016). The IMF initially managed a system of fixed exchange rates where every state set its currency relative to gold or the United States dollar. From the 1960s, though, states moved toward flexible exchange rates, a movement that became firmly established when the United States itself freed its currency from gold in 1971. Flexible exchange rates have proved far more volatile than economists had predicted (in large part as flows of short-term capital have had a more significant effect on exchange rates than more stable movements in trade or long-term investment). The IMF makes loans to governments facing difficulties in managing their currency or government budget. It was envisioned to spare indebted countries from interference in governance by lenders (as had happened to the Ottomans and Qing, among others; chapter 20). It has been criticized, though, for requiring developing countries to balance their budgets in order to receive loans. Yet when wealthier countries such as Greece needed IMF support in the twenty-first century, they argued with some success that moving immediately to a balanced budget would worsen their economic 
performance and then limit future tax revenues. The IMF has been encouraged to take a longer-term perspective on economic policy.

In addition to these international organizations of states, a vast host of international organizations - of workers, women, or environmentalists or indeed of almost every group with shared interests or concerns - have emerged over the last century. In some cases, these continue the efforts of nineteenth-century (and even eighteenth-century) organizations; it is a mistake to think that international civil society is entirely new. Nevertheless, the numbers and influence of such organizations have expanded dramatically. In part, these organizations of people reflect the expansion of the organizations of states discussed above: These groups seek to influence the deliberations of the UN or IMF or WTO, and these are more likely to respond to international sentiment. However, such groups of individuals also lobby national governments and were expanding in size and influence even during the interwar period. Enhanced global communications make it easier to attract an international membership and deploy these effectively in both online petitions and on-site demonstrations (which are now a characteristic of almost any international gathering). The spread of democracy (above) also facilitates international discussions of controversial issues. Also, growth in average incomes has freed more people to engage in such activities. Environmental issues, social issues, political reform, and economic development are among the top foci of such organizations.

\section{Rights}
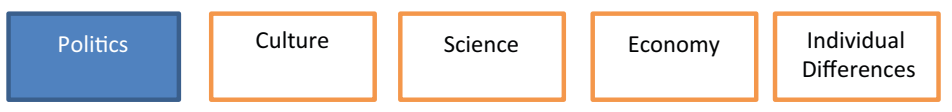

Social Structure

We mentioned human rights briefly above. The League of Nations had recognized rights only vaguely. A motion by Haitian delegates to recognize individual rights had failed in the mid 1930s. Nevertheless, the treaties that created new nation states in Eastern Europe after World War I had enjoined these to respect minority rights.

The original United Nations charter affirmed the idea of fundamental human rights. Then in 1948, the UN issued the Universal Declaration on Human Rights - though it was only in the 1970s that this was fully ratified. The Declaration decries executions without due process, torture, slavery, and racial, sexual, or religious discrimination. Historians disagree about the degree to which this Declaration was novel as opposed to being a continuation of past trends. One can find references to human rights in various documents in human history. We have seen that states have often justified their rule in terms of ethical principles. Nevertheless, appeals to rights are just one type of rules-based ethics, and rules-based ethics are only one of five broad categories of ethical argument (chapter 8). States generally developed systems of laws in an evolutionary fashion rather than trying to ground these in any particular 
ethical principle. While the UN Declaration falls within a long tradition of ethical declarations by official bodies, it has several important elements of novelty: It prioritizes rights as a type of ethical argument; it maintains that one set of rights has general validity; and it suggests that both national and international laws should reflect these rights. Whereas Enlightenment thinkers had often posited that rights were a result of an implicit contract between individuals and a state, the UN Declaration described a universal obligation of all states. The range of rights listed in the Declaration is also noteworthy: In particular, states in Europe had come to accept the principle of freedom of religion after centuries of war between Protestants and Catholics, but this was and is viewed with suspicion in some parts of the world (see below).

The institutional novelty of the Declaration was possible because of a widespread desire to avoid a repeat of World War II, and a general feeling that doing so required some agreed principles of (at least) international affairs. War crimes trials were being undertaken against German and Japanese authorities; these presumed that there were international principles to be applied. (The idea of international war crimes tribunals would be set aside during the Cold War but would be applied to conflicts in Yugoslavia, Rwanda, and elsewhere from the 1990s, though with mixed results.) We can usefully recall here that states have often pursued wars through human history to steal or subjugate. Recognizing individual rights might then remove critical incentives to war - but only if an international body with some power might respect or enforce such rights. Though we have seen that the Declaration differed in important ways from preceding ethical principles, it was nevertheless true that international consensus was only possible because opinion leaders in many lands connected the idea of universal rights to local traditions of ethical behaviour.

An agreement might only have been possible in the few years between the end of the war and the intensification of the Cold War. However, such a declaration could not be imagined into being overnight. Instead, it built upon the efforts of many people and international organizations in preceding decades. In particular, it had to be determined which rights to include. International women's groups had been advocating for the recognition of gender rights for decades. Much of the world had achieved freedom of the press as newspapers spread in the nineteenth century. Anti-colonial advocates had been urging an end to racial and ethnic discrimination. Humanitarian efforts after both world wars invoked fundamental rights to life and liberty. Advocacy groups complained successfully when a first draft of the Declaration was weak.

Though guided by a sincere desire to prevent another world war, member states may have under-appreciated the importance of such a declaration. Colonial powers might have proven less willing to accept the idea of human rights if they had fully appreciated that such principles would be used in the next decades to unravel colonial empires (chapter 30).

The UN Declaration recognized women's rights long before most member states would do so. The United Nations would thereafter document educational and employment discrimination against women. Member states could then be 


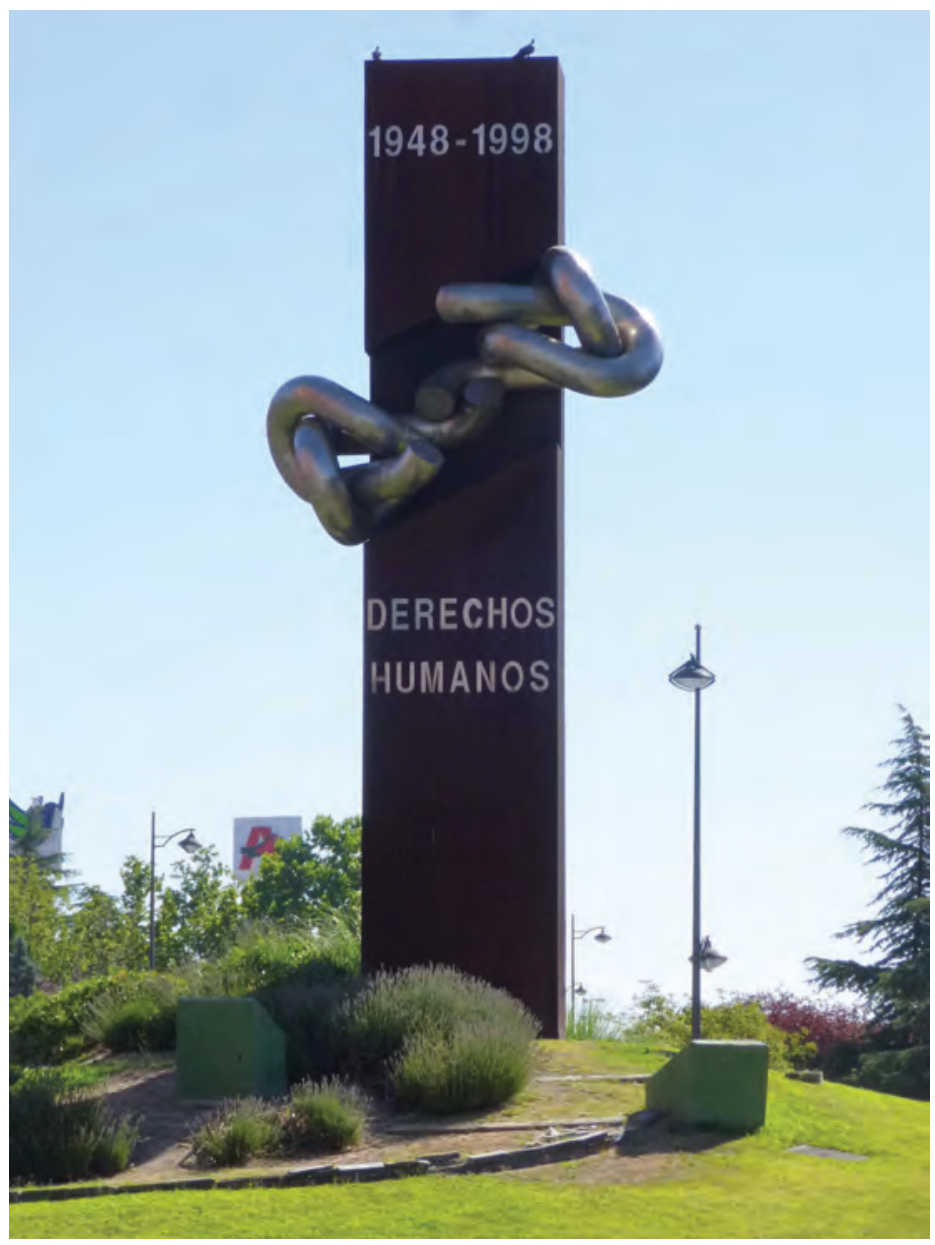

Illustration 27.8

Monument to Human Rights, Leganés, Spain Zarateman, CC0

embarrassed into reducing discrimination. More recently, the UN has engaged issues of reproductive rights, violence against women, and international sex trafficking. Countries can declare exemptions from particular elements of the Declaration (Saudi Arabia objected to the right to marry across religious boundaries, apartheid South Africa to racial rights): These exemptions have been more common for gender rights than any other rights. Yet the vast majority of countries have not exempted themselves.

There are, we should note, critiques of the Declaration. Some of these focus on particular rights that were included or excluded. Others focus on the broader question of national self-determination. Some countries have argued that gender rights, or even the whole idea of individual rights, are a Western imposition on local cultural traditions - an argument that neglects the international advocacy that led to the Declaration (and similar and sometimes 
stronger declarations by regional organizations such as the African Union or Organization of American States). Even those who recognize the value in at least some international rights debate how far the UN should go in encouraging these within member states.

\section{PRIMARY DOCUMENTS: THE UNIVERSAL DECLARATION OF HUMAN RIGHTS}

The UN Declaration borrowed heavily from previous texts, notably in:

Article 3. Everyone has the right to life, liberty, and security of person.

The most controversial clause was:

Article 18. Everyone has the right to freedom of thought, conscience, and religion; this right includes freedom to change his religion or belief, and freedom, either alone or in community with others and in public or private, to manifest his religion or belief in teaching, practice, worship and observance.

Also notable was:

Article 16. (1) Men and women of full age, without any limitation due to race, nationality or religion, have the right to marry and to found a family. They are entitled to equal rights as to marriage, during marriage and at its dissolution. (2) Marriage shall be entered into only with the free and full consent of the intending spouses. (3) The family is the natural and fundamental group unit of society and is entitled to protection by society and the State.

And then the Declaration added a set of rights concerning economic well-being:

Article 23. (1) Everyone has the right to work, to free choice of employment, to just and favourable conditions of work and to protection against unemployment. (2) Everyone, without any discrimination, has the right to equal pay for equal work. (3) Everyone who works has the right to just and favourable remuneration ensuring for himself and his family an existence worthy of human dignity, and supplemented, if necessary, by other means of social protection.

(4) Everyone has the right to form and to join trade unions for the protection of his interests.

Article 24. Everyone has the right to rest and leisure, including reasonable limitation of working hours and periodic holidays with pay.

Article 25. (1) Everyone has the right to a standard of living adequate for the health and well-being of himself and of his family, including food, clothing, housing and medical care and necessary social services, and the right to security in the event of unemployment, sickness, disability, widowhood, old age or other lack of livelihood in circumstances beyond his control. 
(2) Motherhood and childhood are entitled to special care and assistance. All children, whether born in or out of wedlock, shall enjoy the same social protection.

Article 26. (1) Everyone has the right to education. Education shall be free, at least in the elementary and fundamental stages. Elementary education shall be compulsory. Technical and professional education shall be made generally available, and higher education shall be equally accessible to all on the basis of merit. (2) Education shall be directed to the full development of the human personality and to the strengthening of respect for human rights and fundamental freedoms. It shall promote understanding, tolerance, and friendship among all nations, racial or religious groups, and shall further the activities of the United Nations for the maintenance of peace. (3) Parents have a prior right to choose the kind of education that shall be given to their children.

Can you see how the diverse states of the world could only have approved this document at a particular historical moment?

\section{Social change}

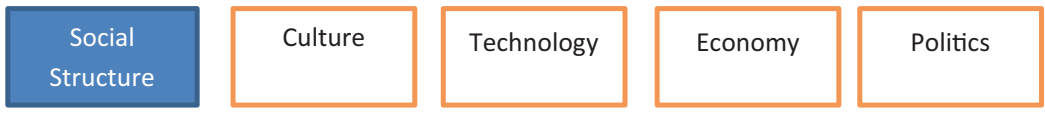

We have seen that rights were enshrined internationally at a particular historical moment but because of a century or more of organized advocacy. That advocacy, in turn, reflected improvements in incomes, education, communications, and political freedoms. Yet these rights also reflected and then reinforced changes in social structure. Most dramatically, women came to play a much more significant role both economically and politically. Ethnic and religious minorities also gained incomes and influence. Strict barriers between workers and employers weakened in most countries. Late in the period, many but far from all countries banned discrimination based on sexual orientation.

Women had always been economically important in hunter-gatherer, agricultural, and nomadic families. Female employment was significant in the earliest factories in many countries - though women often performed the lowest-paid jobs. The fact that the word "spinster" became synonymous with "unmarried woman" provides some indication that the possibility of industrial employment increased the ability of women to guide their own life choices. As urbanization proceeded (chapter 31), though, many women came to focus on household tasks such as cooking and cleaning. Since such duties were unpaid, they were generally associated with low social status - though of course, certain cultural attitudes hailed the undeniable importance of good food and clean homes. Poorer women did earn some money by performing laundry or sewing for single men, or by taking in boarders, but this was generally only a fraction 
of household income. As the germ theory of disease became widely known in the late nineteenth century, increased pressure was placed on women within the home.

A variety of new household technologies in the early twentieth century would relieve much of this pressure. Laundry had long been a backbreaking task - though admittedly most people had only one or two sets of clothes through most of human history. The automatic washing machine transferred a day of weekly drudgery into an hour or two of less strenuous labour. The vacuum cleaner made housecleaning much quicker and better. Electrical appliances such as toasters, and a range of frozen and prepared foods (and microwave ovens much later in the century), facilitated cooking. These innovations, though, only created a potential for women to leave the home; other changes were necessary for activation of this potential. Indeed, in the early twentieth century the immediate reaction to the new household technology involved increased emphasis on the importance of quality parenting (a matter we briefly engaged in chapter 24).

We should not leave the impression that married women had performed housework alone. In the late nineteenth and early twentieth century, middleclass families tended to have servants. This reflected in part heightened concerns with cleanliness associated with the germ theory of disease. Such servants were predominantly women. Though some women made a career in domestic service, it was more common for young women to work in service until they married. They might then both save some money toward that marriage while practising housekeeping skills. It was entirely possible then for the occupation of domestic service to reinforce traditional gender roles - though a woman bringing her own money into a marriage likely wielded some influence as a result. Domestic service would become much less common in the twentieth century as household technology improved and as young women gained alternative employment prospects (though temporary recourse to nannies, often from abroad, would become familiar decades later).

A host of technological and institutional changes acted to create job opportunities outside the home for women. We noted the expansion of both healthcare and education above - and how these have become significant components of government expenditure. Most societies could easily assign the roles of nurse and teacher to women, as they each reflected caring and child-raising roles long associated with women. Besides, women were generally cheaper to hire than men. Mechanization in industry - steam and later electric power, assembly lines, and forklifts - removed the necessity for human strength in a range of industrial jobs, but women would only slowly enter industrial occupations long associated with men (they would, though, find some early opportunities such as painting in their homes the buttons produced by men in factories). The growing service sector would provide far greater opportunities. In addition to teaching and nursing, women could become secretaries. The job of secretary had been a male preserve through most of the nineteenth century. But the occupation exploded in numbers into the early twentieth century as 


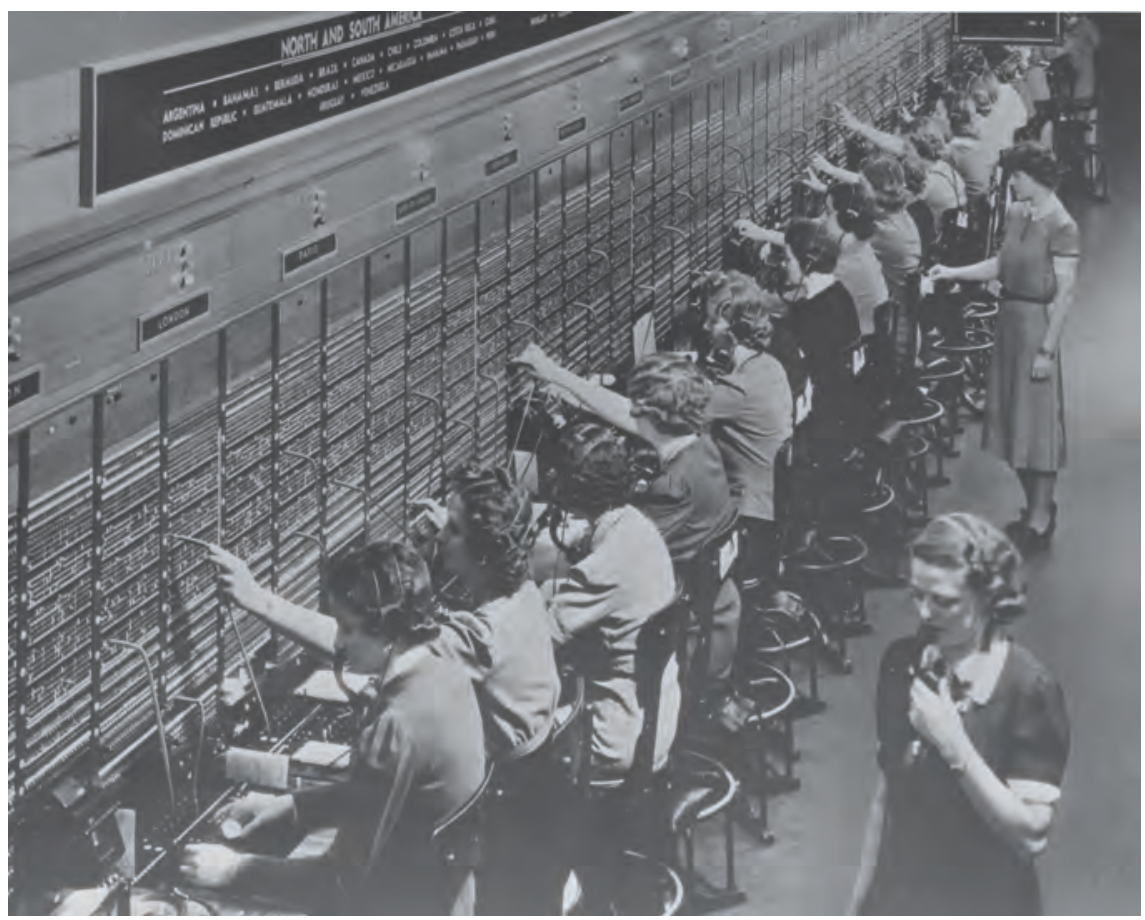

Illustration 27.9

Photograph of women working at a Bell System international telephone switchboard during World War II

the service sector expanded and as typewriters, telephones, and other business machines were introduced. Businesses and governments found that women could perform secretarial jobs as well as men, but for a lower wage. And since secretaries were generally subservient to male bosses, this type of employment was not a direct insult to cultural ideas of male dominance - though concerns were voiced about the possible sexual improprieties that might result from men and women working together in an office. Able secretaries often became indispensable and thus influential, though their salaries might not adequately reflect this influence. Women also worked as receptionists, telephone operators, and store clerks, occupations that could draw on cultural expectations of female sociability.

The First and Second World Wars would each pull women out of the home to perform a variety of jobs usually pursued by men. In each case, most but not quite all of them returned to the home when the war ended. The Great Depression had an adverse effect on female employability, as both governments and businesses reserved jobs for male "breadwinners." (Fascists, unlike communists, urged women to stay at home.) In the postwar period, though, female labour force participation would expand in most countries. Yet there were important cross-country differences. German women were more likely to stay home than British women. Women remained secluded in several Islamic 


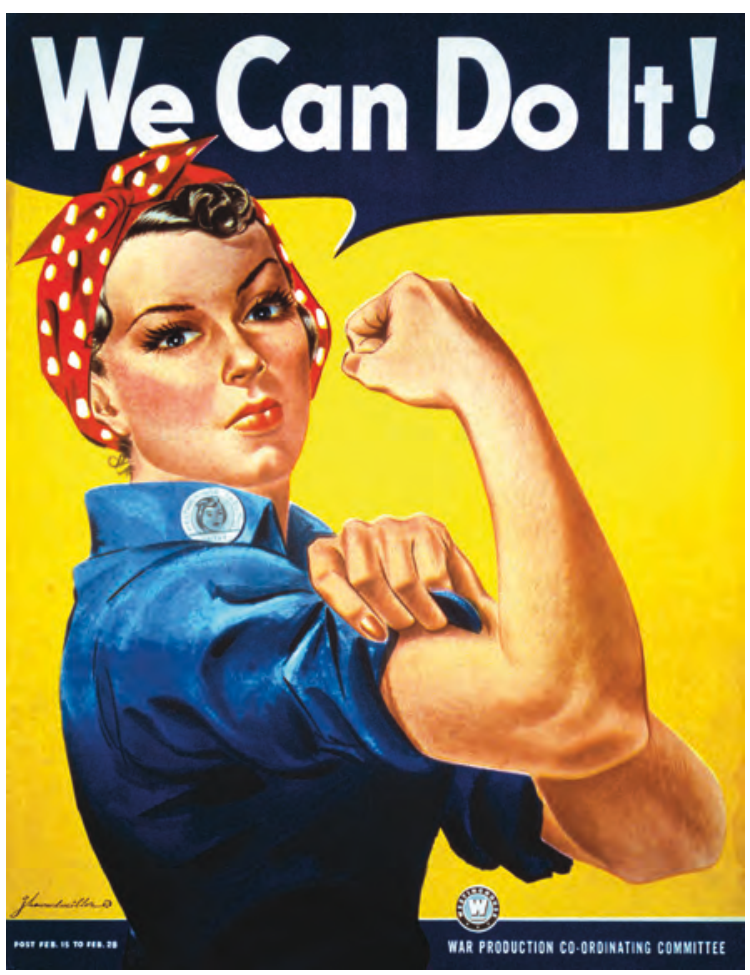

Illustration 27.10

Poster, War Production Board, United States, compiled 1942-3

countries. In most countries, though, female labour outside the home became an accepted fact. Husbands came to value the increased family income which could be devoted to new consumer goods such as televisions - and slowly began to bear a share (though still not an equal share) of housekeeping. Middle-class fathers accepted that their daughters would leave home for school and work before marriage (as working-class fathers had long accepted as their daughters entered domestic service).

Though women first entered the paid labour force in large numbers in segmented occupations - teachers, nurses, and secretaries - this transformation set the stage for further advances. Once societies accepted that women would work outside the home, it became harder to justify occupational discrimination. Many women fought lonely battles for acceptance in different jobs across diverse countries over a period of decades, but received support from an international community of activists. Also, women organized to fight for the same wages as men, and for protection from sexual harassment in the workplace.

Women exercised increased power far beyond the workplace. The development of the birth control pill in the 1960s (following decades of advocacy of birth control by women's groups) - coupled with an increasingly educated, employed, and therefore independent female population - changed sexual practices in most countries. (Some historians argue that nineteenth-century 
advances in condoms and diaphragms - discussed briefly in chapter 24 - had played an essential early role in female emancipation.) Women asserted a right to make their own decisions about sex - though cultural values still often viewed female sexual activity with some suspicion. Female performers in the new domains of movies and radio and recorded music - even males playing female roles in interwar Indian cinema - both reflected and reinforced cultural changes in attitudes toward women. Changes in fashion occurred globally which signalled the increased freedom of women - though women disagree about whether all fashion trends were beneficial.

The economic and cultural changes noted above were associated with political reforms. Women drew on principles of popular sovereignty to argue that women should be able to vote. After decades of advocacy, women gained the right to vote in New Zealand in 1893, Finland in 1908, and the Netherlands, Norway, Iceland, and Denmark just before World War I. An international women's suffrage (voting) alliance was formed in 1902 (but faced internal challenges associated with racist attitudes). Women began to gain the vote during World War I in Canada. Several more countries, including the United States and Germany, would introduce female voting shortly after the war. Though some countries lagged - Switzerland until 1971 - women have the right to vote in all twenty-first-century democracies. As women voted, they came slowly to be elected as well. Women tend still to be a minority among elected officials except in countries where institutions require a rough balance. Yet women have now been elected heads of government across dozens of countries - though there are still only a handful of states where more than one woman has accomplished this feat. Women have also been prominent in acts of civil disobedience - as when Rosa Parks refused to give her seat on a public bus to a White passenger in Montgomery, Alabama, in 1955.

As noted above, the change in gender roles has been uneven globally. Countries that did not educate their women needed to employ men as teachers or nurses. The militarization that followed independence in many former colonies (chapter 30) often encouraged a reassertion of male power, despite the fact that women had usually played a key role in independence movements. In Japan, legal equality - and the adoption of western fashions - have nevertheless been associated with a more limited economic and political role for women than was common in western Europe. Women had long been influential in West Africa, and this remained the case: Women were often particularly active there in trade. Indian women gained equal rights at independence in 1947, but women have been socially subordinated in many regions and castes. In both India and China, parents who wished for a son may have killed a hundred million female babies. As a result, both societies have a very skewed gender balance. Social tensions may well arise as millions of men are unable to find a mate. Parents have sold millions of girls into prostitution worldwide. While women in more affluent countries now focus on issues of violence and reproductive rights, women in many developing countries are still fighting for access to education and employment. 


\section{BOX 27.1 \\ A THIRD GENDER}

In many North American Indigenous groups, people thought to have the spirits of both genders performed particular religious rituals that invoked this dual nature. Several other historical societies also had people in the role of some third gender - Sulawesi, Oman, India, and parts of Polynesia among them. Note that these various societies tended to approve only one of the possible array of alternative gender identities. There is, of course, both scholarly and popular interest in connecting these experiences to the experience of those who do not identify as either male or female today. We should note, though, that there is a debate about whether their own societies honoured or disdained them. Though early explorers were often fascinated by such people, colonial powers often vilified them for they offended their sense of order.

We have focused on women's rights here but could have told a broadly similar story concerning all types of social division. In some cases, change has been very recent. Homosexuality was still against the law in Canada in the 1960s. A majority of Americans still opposed inter-racial dating in a survey in 1987. Segregated seating by caste at upper-caste Indian weddings was still prevalent in 1990 but is rare decades later. Though change has been global, these transitions toward respect for diversity are incomplete: Homosexuality is still illegal in many African countries, and women lack rights in parts of the Middle East that are taken for granted in the rest of the world. The "Black Lives Matter" protests in many parts of the world in 2020 reflected the fact that there are still huge racial differences in incomes, power, and treatment by legal authorities in many countries. These protests tended to be multiracial, indicating widespread social disdain for racism.

\section{Religion}

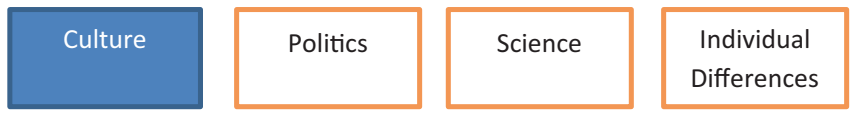

The most important religious trend in the twentieth century has likely been the increased incidence of atheism or agnosticism or simple non-religiosity. Difficulties in defining these terms or distinguishing among them - confidence that there is no god, scepticism about the existence of god, or just lack of interest - make it hard to measure trends here. Besides, in countries with intense cultural or institutional pressure toward religion, many people pretend to religious belief. Nevertheless, it seems likely that some 10 to 20 per cent of 
the world's population in 2000 did not follow any formal religion. The percentage in 1900 would have been far lower than this.

There had been some moves away from religion in previous centuries. The French Revolution had attacked the power of the Catholic Church. Later regimes would soften this policy but would institutionalize the idea that France was a secular state. Religious toleration was accepted during the American Revolution and would become a standard policy in the nineteenth century. Russia was the last sizeable European state to accept the principle of toleration in 1905. Turkey would officially embrace secularism when founded after World War I. Qing China was also generally tolerant though it sometimes restricted Islamic activity. European colonial governments were also generally tolerant. They often sought the support of local religious leaders. They allowed Christian missionaries if these did useful things like operate schools and hospitals but restricted their activities if they criticized colonial governance or threatened social peace. (Christian missionaries were successful in Africa but converted only one or two per cent of the population in India or China.) One exception to this general trend was Meiji Japan, which declared Shinto the state religion. As the political and social penalties for non-conformity to the local faith decreased in much of the world in the nineteenth century, there was some increase in the incidence of agnosticism. This trend accelerated in the twentieth century.

The trend toward agnosticism has differed dramatically by region. A handful of countries - China, Germany, and the Netherlands among them - have a majority of the population without formal religious affiliation. In China (and elsewhere in East Asia), this result reflects the unique historical evolution of Chinese religion. As we saw in chapter 11, the border between philosophy and theology in China was fuzzy, and people often prayed to ancestors without recourse to a formal religious doctrine. Outside of East Asia, Europe is by far the most non-religious region. North America has a lower proportion of non-religious than Europe despite similar cultural roots: Arguably, the early acceptance of religious freedom in the United States allowed various denominations to cater to different religious preferences whereas official state religions in many European countries were not universally satisfying. Nonreligious people are even less common in South America, still less so in Africa, and least common in the Middle East. Again, though, we must appreciate that many people may pretend to belief in countries where this is expected.

The widespread rise of agnosticism and atheism is often attributed to science: Science provides explanations of how the universe and humanity came to be that do not rely on godly intervention (Chapters 2 and 3). Modern medicine allows humans to understand and have some degree of control over what appeared through most of history to be random attacks of illness, rather than attributing these to godly displeasure or the actions of witches or demons. However, to attribute agnosticism entirely to science would be to ignore the diverse roles that religion plays in human life. Religion has also been a source of group solidarity historically; here it may be nationalism and ideologies such as communism that have supplanted the role of religion. Science provides 


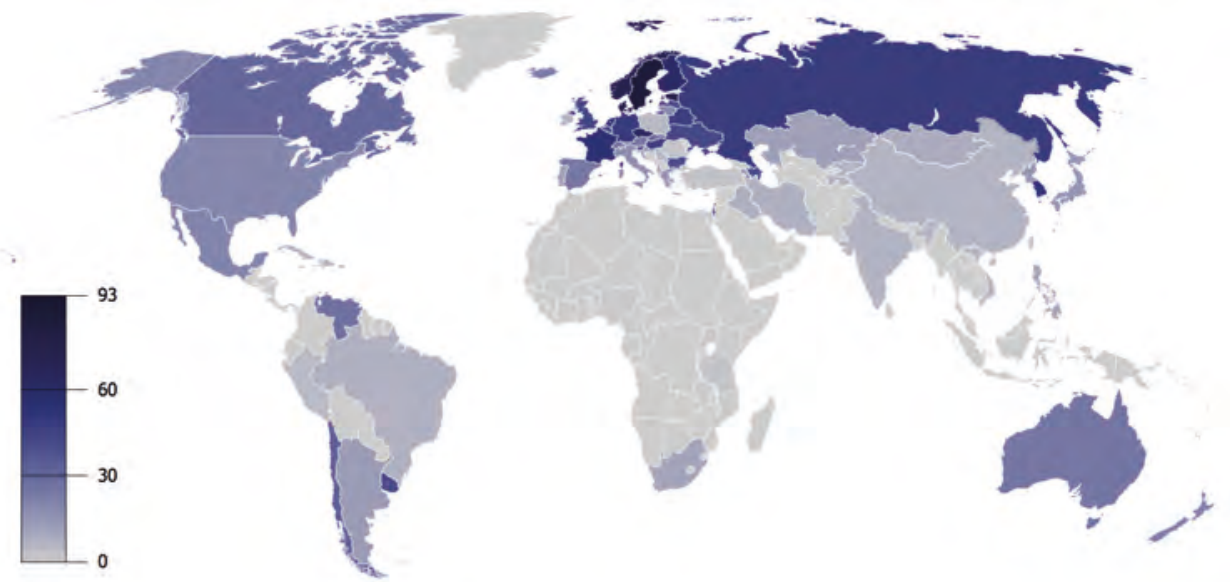

Map 27.2

Proportion of atheists and agnostics by country, 2006. Note that China is characterized as more religious on this map than in our discussion above

a limited basis for the ethical attitudes that religion has encouraged across human societies (chapters 8 and 11). The development of institutions may be critical here. States that have well-respected legal systems may have less need for religious belief. It may be no coincidence that areas of the world such as the Middle East that have retained the highest rates of religious observance are also regions that have been characterized by autocracy and corruption. The disdain that some Islamic fundamentalists direct at democratic institutions and constitutional protections may, in fact, encourage religious belief, if not quite for the reasons they imagine. Yet societies have everywhere relied on a mix of rules and beliefs to encourage trust and cooperation. Agnostic or atheistic societies should be wary of relying only on laws; they likely need also to promote secular ethics.

In addition to connections to ethics and group solidarity, declining religious observance can have a couple of practical effects. Religious buildings are expensive to maintain: Sometimes state funding is provided if these are seen as historical monuments, but less noteworthy buildings are often renovated for commercial, residential, or educational use. Religions have played an important role in charity throughout history; secular agencies are not always available, at least immediately, to provide the same services.

Organized religions have responded to declining attendance in many countries. In particular, they have often connected themselves to nationalism. Iranian nationalism is encouraged by Shia leaders, while Italian priests celebrate Italy's historic role in the Catholic Church. Religions have utilized new communications technologies to reach broader audiences. Some religions have attempted to renew their attention to matters of an ethical nature, but there has been a tendency to focus on issues of sexual behaviour (we discussed changing cultural attitudes toward sex above). 
Religions have also become more "organized" over the nineteenth and twentieth centuries. They developed extensive bureaucracies at the same time as states and many private firms did. Many religions organized schools - often with financial support from the state - and needed a bureaucracy to manage these. The missionary efforts of Christian churches also required bureaucratic support: They had to raise funds, train missionaries, and finance missions. These missionary efforts inspired a response among Islamic, Hindu, and Buddhist leaders; these religions then organized too. All of these religions sought to clarify their teachings and practices and root out unorthodox beliefs. The Roman Catholic Church would celebrate papal infallibility from 1870; this served to encourage uniform practices throughout the Catholic Church. Leading schools of theology and standard school curricula developed within the Islamic world. Syncretic practices nevertheless survived, especially in Africa and the Pacific.

In some countries, though, new religions or denominations have gained ground at the expense of old. By some calculations, the Mormon Church is the fastest growing religion in the world. The Baha'i faith also emerged in the nineteenth century; though persecuted in its native Iran, Baha'is would prosper in the United States and elsewhere. Pentecostalism, a Christian denomination that stresses personal experience of god over doctrine, was established in 1906 in Los Angeles; it now has 500 million followers around the world. It has been especially successful in poor neighbourhoods. In some countries, it has absorbed elements of shamanistic practices.

Most noticeably of all, in many Islamic countries, fundamentalist groups have emerged as an important political and social force, reacting to political corruption at home and the limited economic and political power of Islam globally. The Iranian Revolution of 1979 signalled that a religious state was feasible (though many/most Iranians chafe at religious authority). Many Sunnis were inspired to attempt to imitate this Shia state, and perhaps even restore the Sunni caliphate abolished as the Ottoman Empire imploded (some Sunnis had yearned for a new caliphate as soon as the Ottoman caliphate disappeared). World history suggests that such a project will be challenging: Caliphs have generally not ruled in strict accord with Islamic principles, religious bureaucracies across all religions have often been corrupt and autocratic, and all faiths have been fraught with doctrinal disputes. Yet a vocal minority (especially if armed) that favours a religious state can prove a challenge to efforts by others to establish democratic institutions. While a couple of majority-Islamic states are now democratic, the vast majority are autocratic and corrupt: As we saw above, such a political environment encourages religious fervour.

Global surveys suggest that the worldwide trend toward non-belief may have stalled in the early twenty-first century. This may reflect the efforts of both old and new religions to gain adherents. Another factor here may be that religious people have more children than the non-religious. Political instability in many parts of the world may also have encouraged many to return to religious certainty. 


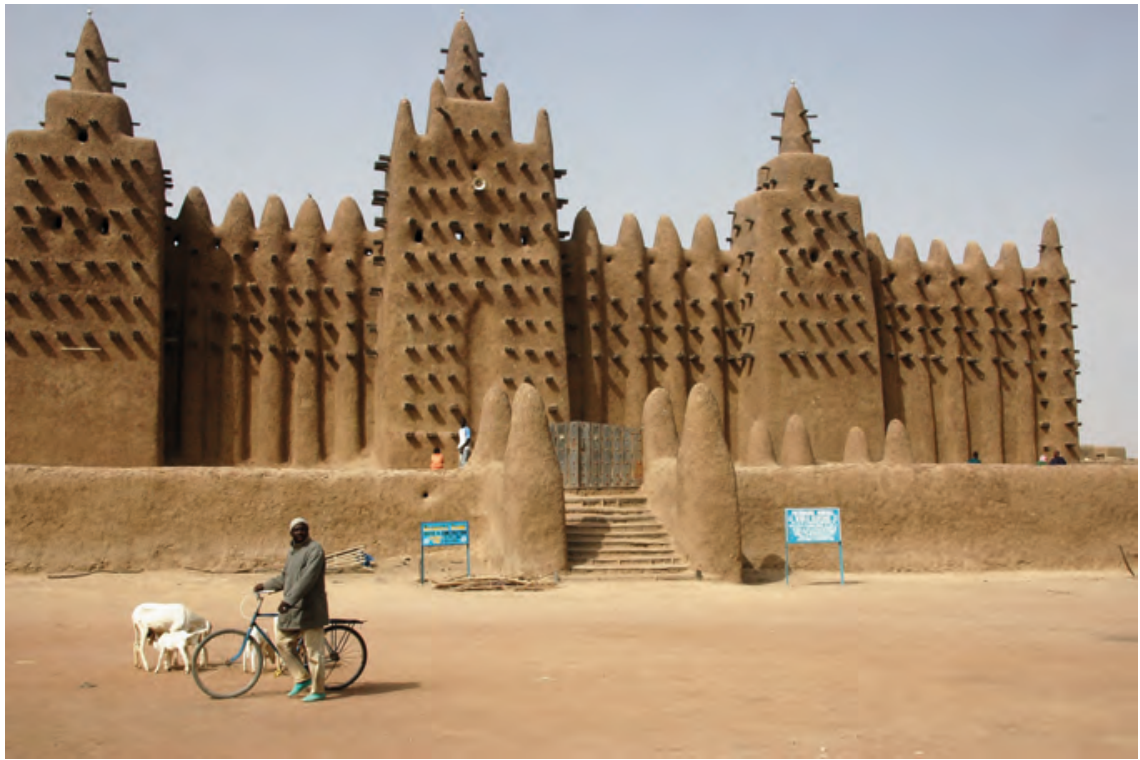

Illustration 27.11

Great Mosque of Djenne, Mali; built under French rule in 1907. There is debate about the degree of French influence and the degree to which it followed the lines of an earlier mosque dating from the 13th century. It is the largest mud brick building in the world

Photograph by Ruud Zwart, CC BY-SA 3.0

\section{Environment}
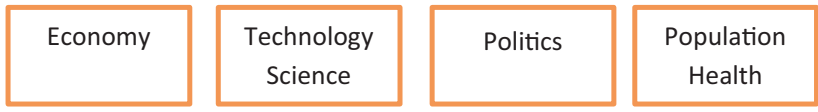

Human effects on the environment are certainly one of the most critical historical transformations of the twentieth century - and may prove to have the most significant impact on the future of humanity. The space devoted to environmental history here is not proportionate to the inherent interest of the topic, in large part because the issues are so well known.

Humans have always affected their environment, even in the days of huntergatherers. The twentieth century was different in two ways. First, the rate of environmental impact expanded dramatically. For most types of pollution, the majority of emissions in all of human history have occurred since 1900. Second, these environmental impacts were often global in scope, complicating the development of strategies to address them. Yet we should appreciate that there have been some successes in environmental policies, both locally and internationally.

As industrial production expanded, and new chemical processes were developed, humans spewed vast quantities of chemicals into the air and 
water. The development of the automobile would exacerbate these problems. Automobiles would become the primary source of air pollution in the United States and many other countries. Some of this pollution had severe local effects. Twenty people died in Donora, Pennsylvania, in 1948 because of thick smog, and hundreds more were hospitalized. In London in 1952, as many as 4,000 may have died from smog. Water pollution was often less evident than air pollution. However, the Cuyahoga River in Cleveland, Ohio, burst into flames on several occasions, most famously in 1964. Events such as these spurred governments in rich countries to enact laws and regulations that protected the air and water. These have hardly eliminated air or water pollution. Nevertheless, the Cuyahoga is no longer flammable, and Londoners can see where they are going. Economists have detected an inverted-U relationship between average incomes and pollution levels across many countries: Pollution expanded as

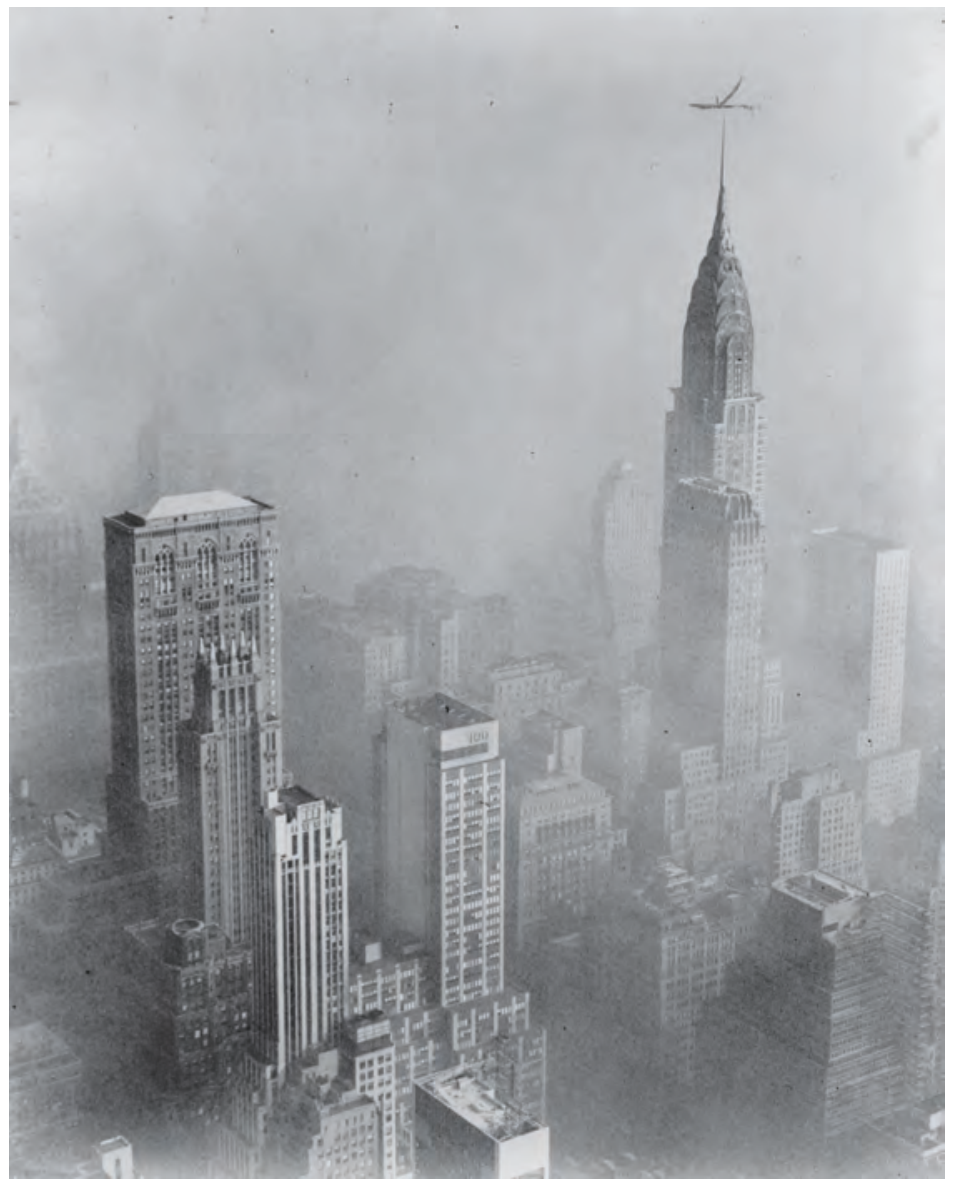

Illustration 27.12

Smog in New York City, 1953. View from Empire State Building toward Chrysler Building

Photograph by Walter Albertin 
countries first industrialized but as incomes rose, countries introduced environmental regulations that reduced (at least some kinds of) pollution. However, this relationship is far from an iron law. Even in affluent countries, citizens are often willing to sacrifice environmental quality to protect jobs. Developing countries may be tempted to incur significant ecological damage as industries seek to avoid rich-world regulations. Autocratic governments have often proved especially willing to sacrifice the environment: This was indeed one of the major criticisms of communist regimes (though China has recently made strides in attacking if not yet reducing air and water pollution).

Scientists came to appreciate in the middle decades of the twentieth century that the ozone layer was eroding. Depletion of the ozone layer allows ultraviolet light to reach the earth's surface at levels that can trigger cancers in humans and also hurt animal and plant life. Scientists identified the cause of ozone depletion as chemical reactions involving chlorofluorocarbons (CFCs), substances employed and emitted into the atmosphere as refrigerants, solvents, and propellants. In the Montreal Protocol of 1987, the principal CFC-producing countries agreed to cut CFC production. But as evidence of ozone depletion mounted in the next years, it was decided to eliminate CFC use - except in minimal amounts in asthma inhalers. Happily, reasonable substitutes were found for CFCs, though significant research expenditures were required to develop these. The ozone layer remains robust in tropical and temperate latitudes but has declined precipitously toward the poles. There may have been some moderate increase in diseases such as eye cataracts worldwide due to ozone depletion. A marked increase in skin cancer at the southern tip of Chile may also be due to ozone depletion. One can draw a positive lesson from the ozone story: International cooperation did address a severe environmental problem. Yet there are also warning signs: Earlier action would have been even better, and the earth continues to suffer from ozone depletion.

We have often suggested in this book that changes in climate have had significant political and economic (and other) impacts. Over the last century, average global temperatures have risen by about 1 degree Celsius. The rate of change has increased during that period such that scientists estimate it to be well above a tenth of a degree per decade. Sea levels have risen by over $2.5 \mathrm{~mm}$ per year in recent decades. Glaciers and Arctic ice have been receding for decades. Examination of polar ice cores suggests that this rate of increase in global temperatures is unprecedented, at least in the last ten thousand years or so. Yet satellites have detected no increase in the heat emitted by the sun over the last decades. Nor can the slow natural changes in the earth's orbit explain the rise in global temperatures. The vast majority - well over 95 per cent - of natural scientists accept that global warming has been caused by human-generated emissions into the atmosphere of carbon dioxide, methane, and other substances that act to reduce the amount of heat that escapes from the earth into space. The fact that agriculture is now a small fraction of global economic activity tempers the economic impact - though Inuit hunters and farmers in some regions have likely suffered. Most scientists suspect that an 
increase in the frequency and severity of storms reflects climate change: If so, climate change has destroyed many billions of dollars' worth of property. Climate change may also be responsible for more frequent droughts in several parts of the world. Future costs are expected to be much higher if global temperature continues to increase, and dramatic changes are possible if global weather patterns change or if massive ice sheets slide into the ocean. Though it is hard to predict regional weather patterns, it is quite likely that regional impacts will differ, which may encourage increased migration of human populations. Countries that still rely heavily on agriculture, or that have much land near sea level, will likely suffer most. We will return to the challenge of addressing climate change in chapter 32 , but can note here that this will be much more difficult to resolve than ozone depletion, as it is much harder to substitute for fossil fuels than for CFCs.

Postscript: The hydrofluorocarbons (HFCs) that replaced CFCs turned out to contribute to climate change. An international agreement was reached in 2016 to phase out HFCs, but it was not entirely clear how easily this could be done.

Industry and automobiles were not the only environmental challenge of the twentieth century. Cities, farms, and plantations expanded into areas of forest. Though humans have been chopping down forests for thousands of years - and tropical countries criticized for allowing rapid depletion of tropical forests can reasonably retort that many temperate countries had devastated their forests centuries ago - it is nevertheless true that most of the forest destruction that has occurred in human history has happened in the last 150 years. Since forests store carbon, this reduction in forests has exacerbated the global warming that has resulted from burning oil and coal in industry and transport. Since tropical forests contain the vast majority of the earth's organisms, reducing these forests has also reduced the planet's biodiversity. And since humans have a limited understanding of how ecosystems work - that is, how the interactions among a variety of organisms and natural processes lead to environmental stability - it is hard to predict the environmental effects of decreased biodiversity. Moreover, humans have found medicinal uses for thousands of plants over the millennia; there are concerns that we are destroying organisms before we can identify their potential usefulness.

We can briefly note a host of other environmental concerns. Fertilizers and pesticides may destroy the fertility of our soils over time. Some worry that we will soon run out of many vital natural materials. Irrigation systems are yet again increasing salt levels in our soils. Chemicals in our air and food threaten human fertility itself. Fish farming may be squeezing out and spreading disease to indigenous fishes. Each of these concerns involves potentially huge impacts 
on the future economic well-being of humanity. We return to a discussion of environmental policy in chapter 32 .

\section{Questions}

1. We began the chapter by reviewing how we would address each of our themes in the twentieth century. For any of the themes that we promised to follow in chapter 1, trace how this book has treated these during each time period.

2. Diagram the influences on and effects of increased levels of agnosticism or atheism.

3. We will revisit the prospects for democracy in chapter 32. But the reader can usefully reflect here on how (and whether) democracy should be encouraged. That is, under what conditions are transitions to democracy most successful?

4. (How) have the challenges faced by women evolved over the last centuries?

5. What are the challenges faced by the manager of a non-governmental organization?

6. How much attention should be devoted to communism in a world history book?

\section{Readings}

Crooks, Peter, and Timothy H. Parsons. 2016. Empires and Bureaucracy in World History: From Late Antiquity to the Twentieth Century. New York: Cambridge University Press.

Davies, Thomas. 2014. NGOs: A New History of Transnational Civil Society. New York: Oxford University Press. [Traces the history of NGOs to the late eighteenth century; suggests that these had an impact on critical issues such as the abolition of slavery. See also Shaping the Transnational Sphere: Experts, Networks and Issues from the 1840s to the 1930s, edited by Davide Rodogno, Bernhard Struck, and Jakob Vogel, New York: Berghahn Books, 2014, which provides a detailed analysis of several NGOs in the nineteenth and twentieth centuries.]

Decker, Alicia C., and Andrea L. Arrington. 2015. Africanizing Democracies, 1980Present. New York: Oxford University Press. [A textbook that explores thematically the move toward democracy in Africa since 1980.]

Dickinson, Edward Ross. 2018. The World in the Long Twentieth Century: An Interpretive History. Berkeley, CA: University of California Press.

Finkel, Alvin. 2019. Compassion: A Global History of Social Policy. London: Macmillan.

Ishay, Micheline R. 2008. The History of Human Rights: From Ancient Times to the Globalization Era. Berkeley, CA: University of California Press. [The book seeks to identify precursors in mostly European history for the rights adopted by the United Nations in 1948.] 
Jackson, Simon, and Alanna O'Malley, eds. 2018. The Institution of International Order: From the League of Nations to the United Nations. Abingdon, UK: Routledge.

Kaplan, Temma. 2015. Democracy: A World History. Oxford University Press.

Pons, Silvio. 2014. The Global Revolution: A History of International Communism. Oxford University Press.

Quataert, Jean H. 2010. "The Gendering of Human Rights in the International Systems of Law in the Twentieth Century," in Michael Adas, ed., Essays on Twentieth-Century History. Philadelphia. PA: Temple University Press.

Reynolds, David. 2001. One World Divisible: A Global History since 1945. New York: Norton.

Smith, Bonnie G. 2010. "Women in the Twentieth-Century World," in Michael Adas, ed., Essays on Twentieth-Century History. Philadelphia, PA: Temple University Press.

Tucker, Richard P. 2010. "A Century of Environmental Transitions," in Michael Adas, ed., Essays on Twentieth-Century History. Philadelphia, PA: Temple University Press.

Van Leeuwen, Marco H.D. 2016. Mutual Insurance, 1550-2015: From Guild Welfare and Friendly Societies to Contemporary Micro-Insurers. Basingstroke, UK: Palgrave Macmillan. [Discusses how local communities, guilds, unions, and friendly societies had provided some kinds of social insurance in the early modern period.]

Weitz, Eric D. 2019. A World Divided: The Global Struggle for Human Rights in the Age of Nation-States. Princeton University Press.

There was a symposium in the Journal of Global History in July 2014 on social policy movements across borders. There are several articles in that journal in March 2017 that address international organizations and international law. A forum in the Journal of World History, June 2019, addressed Afro-Asian internationalism in the 1950s and 1960s, treating both state and non-state interactions. 


\section{Devastation and fear}

\section{War in the twentieth century}

\section{Guiding questions}

Why was war so destructive in the twentieth century? Why, how, where, and when did particular wars occur? What were the main effects of these wars? What trends if any can we discern in the prevalence and intensity of war?

What is fascism? Why, when, where, and how did fascist governments emerge? What was the connection between fascism and war?

What is terrorism? When, where, and why has it been pursued?

Relationship to other chapters: The chapter naturally builds on earlier discussions of war and military technology. It also builds on our understanding in chapter 27 of the increased ability of states to tax, spend, and organize vast armies and bureaucracies and on our discussions of nation states and nationalism from chapter 21. Our examination of fascism connects to our discussion of communism in chapter 27. We shall see in chapter 30 that there are important connections between world wars, cold war, and decolonization. We shall see that there are also connections to economic developments in chapter 29 and population growth in chapter 31 .

Our primary thematic focus in this chapter is political, but one can hardly discuss war in the twentieth century without addressing in some depth the economic and technological capabilities of combatants. We must also investigate the cultural attitudes that at times encouraged war and at others favoured peace. As always, social structure played a crucial role in warmongering, especially 
the presence of ethnic minorities within nation states. The massive death tolls associated with these wars mean that population is also an important theme. The decisions of particular individuals were likely of critical importance. We will naturally compare attitudes and capabilities across combatants. We will, of course, address the effects of war on various states and their citizens. We apply evolutionary analysis sparingly; we will again see how this was misused to support racist ideas.

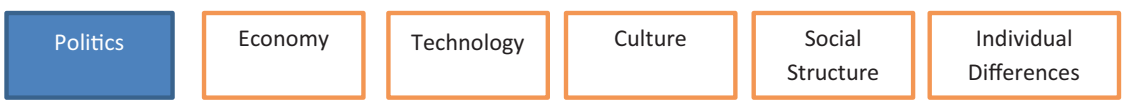

\section{Prelude to war}

The twentieth century opened with many predictions that the age of war was over, especially in Europe. There were many reasons for this optimism. Arbitration of international disputes had become increasingly common. The American Civil War and the Crimean War had demonstrated the horror of war with modern weaponry: This would surely cause leaders to hesitate before starting another war. Moreover, machine guns and submarines were far deadlier than those used in the Civil War, and soon would be joined by aircraft and tanks (and much better capacities for mass production of weapons and the ships and trucks and railcars to move these around). Though there had been wars in Europe associated with the unification of Germany and Italy (chapter 21), these had been small-scale affairs compared to the ravages of the Napoleonic Wars. The last century, and especially the last decades, had been relatively peaceful. Some countries - Switzerland and to a lesser extent Sweden - had been officially recognized as neutral in the treaties that ended the Napoleonic Wars and had largely escaped conflict after that: They might set an example for the rest of the world. There had been wars of colonization, but the mismatch in weaponry between Europeans and the colonized had limited fatalities, at least among the conquerors. In any case, with the world mostly colonized, it seemed there was limited scope for future wars.

There was also philosophical justification for optimism. Enlightenment thinkers had often addressed the pursuit of peace. Voltaire had thought that religious toleration was the key to peace. Rousseau had instead argued that the popular will was generally opposed to war: The replacement of kings with assemblies was necessary to achieve peace. Adam Smith and Jeremy Bentham thought that trade could substitute for war: Countries could gain the goods and markets they sought without violence, and as incomes rose, people would become less willing to risk war. Bentham also argued that war failed consequential calculation: Total suffering far exceeded the gains that victors might extract. Such an argument also suggested that allowing the general population increased power would reduce the impulse to war. Socialist thinkers attributed 
war to class antagonism and predicted its demise if workers gained power. As religious toleration, popular assemblies, and trade expanded, it seemed that war must become less likely. More generally, as economic growth and technological innovation proceeded in the nineteenth century, many thinkers celebrated human progress (again especially in Europe); it was natural to associate the attainment of peace with these general signs of progress. The World Wars would shatter confidence in human progress for many decades.

There had been some concrete achievements during the nineteenth century. Henri Dunant, a Swiss citizen who had witnessed the war between Italy and Austria, urged the development of protocols regarding the treatment of prisoners and the wounded. The first Geneva Convention of 1864 addressed these matters. Japan would sign and largely adhered to it when it invaded Manchuria in China in the 1890s. The Geneva Convention would be revised many times, notably to ban chemical weapons in 1925 after their (brief) use in World War I. (Britain and Germany developed chemical weapons during World War II but did not deploy them from fear of reprisal.) The International Red Cross also emerged in the late nineteenth century to provide (among other things) concrete aid to wounded soldiers and civilians. Of course, such initiatives reduced the horror of war rather than war itself, but they did reflect a widespread revulsion at the effects of warfare with advanced weaponry.

Many international organizations emerged in the late nineteenth century that advocated for peace. These often had links to women's groups or worker organizations. Some had religious support. Their efforts to encourage states to pursue peace had limited success. States did see some value in international arbitration and settled some dozens of small border (including rights over fishing) disputes in this way. An international court was established for such purposes in 1899 after what may have been the first ever peace conference not called to end a war. The modern Olympic Games from 1896 were designed in part to allow a peaceful outlet for nationalist sentiment. The Nobel Peace Prize was created in 1901 to encourage efforts toward peace.

Of course, history also suggested that states would not so easily achieve peace. The French Revolution (chapter 25) had reflected Enlightenment ideals in endorsing peace. Nevertheless, it would soon turn to war (at first under attack), introduce conscription (the "draft"), and not allow conscientious objection. (American revolutionaries had also jailed conscientious objectors for up to two years.) Napoleon would use Enlightenment ideals to justify aggressive war throughout Europe and into Egypt. The idea of conscription - novel in Europe if not elsewhere - meant that armies became much larger than ever before. Though endorsed in France by a popular assembly, autocratic rulers soon employed conscription in response, supported by notions of popular sovereignty and the nation state (chapter 21). Advances in agricultural productivity that liberated men from food production facilitated mass mobilization. In addition, nation states usually possessed enhanced bureaucratic capability: The rulers of the sixteenth century might only have extracted some 5 per cent of 
national income and so were limited in their ability to finance war, whereas Nazi Germany and the Soviet Union would be able to devote over half of national income to the war effort.

Ideas of popular sovereignty and the nation state might lead to mutual respect for borders that reflected cultural/linguistic boundaries but - especially in a world where ethnic groups were often interspersed - could also lead to popular support for wars of conquest. Nazi Germany would seek to conquer all lands with a German population, while Russia would claim a responsibility to protect all Slavic peoples from non-Slavic domination. In Asia, Japan would appeal to Asian nationalism as it attacked European colonies.

The scramble for colonies in the late nineteenth century had accentuated national hostility within Europe. Germany and Italy, only unified in the 1870s, found that there was little unconquered colonial territory remaining. If other European powers were correct in the belief that colonies were essential to political power and economic prosperity (an idea we can now disdain), then Germany and Italy might have to wrest territory from these other states. Japan, which also came late to the race for colonies, would make a similar argument. Some have even suggested that the brutality of colonial repression set the stage for the carnage of the World Wars, but it may be better to see the barbaric treatment of civilians during both colonial expansion and World Wars as expressions of nationalism coupled with bureaucratic and technological capability. Many see the Italian conquest of Ottoman Libya in 1911 as a precursor to World War: It, in turn, reflected nationalism, colonialism, the emergence of new powerful states in Europe, and the continuing decline of the Ottoman Empire.

\section{World War I}

The First World War lasted from August 1914 to Nov 11, 1918. At its peak, twenty-two nations, including the United States and Japan, were allied against Germany, Austria-Hungary, Bulgaria, and the Ottoman Empire. Britain and France drew heavily on their colonial possessions: Britain had 1.4 million soldiers from India alone, and France fielded 600,000 colonial soldiers, in part to replace/punish French soldiers and to patrol the occupied borderlands after the war. The wartime experiences of these colonial soldiers would have dramatic and adverse effects on colonial attitudes toward Europe. German colonies and Ottoman possessions became targets for attack (see below). African troops commanded respectively by British and German officers battled in Cameroon. Some 15,000 German troops in Africa used guerrilla tactics to tie up Allied soldiers. As many as 150,000 African soldiers would die serving Britain, France, and Belgium in Europe. Even Chinese labour contributed to the war effort. This truly was a "world war" that affected all continents.

During the war some 15 million soldiers and 20 million civilians died. Another 20 million soldiers were injured. This was by far the highest death and 


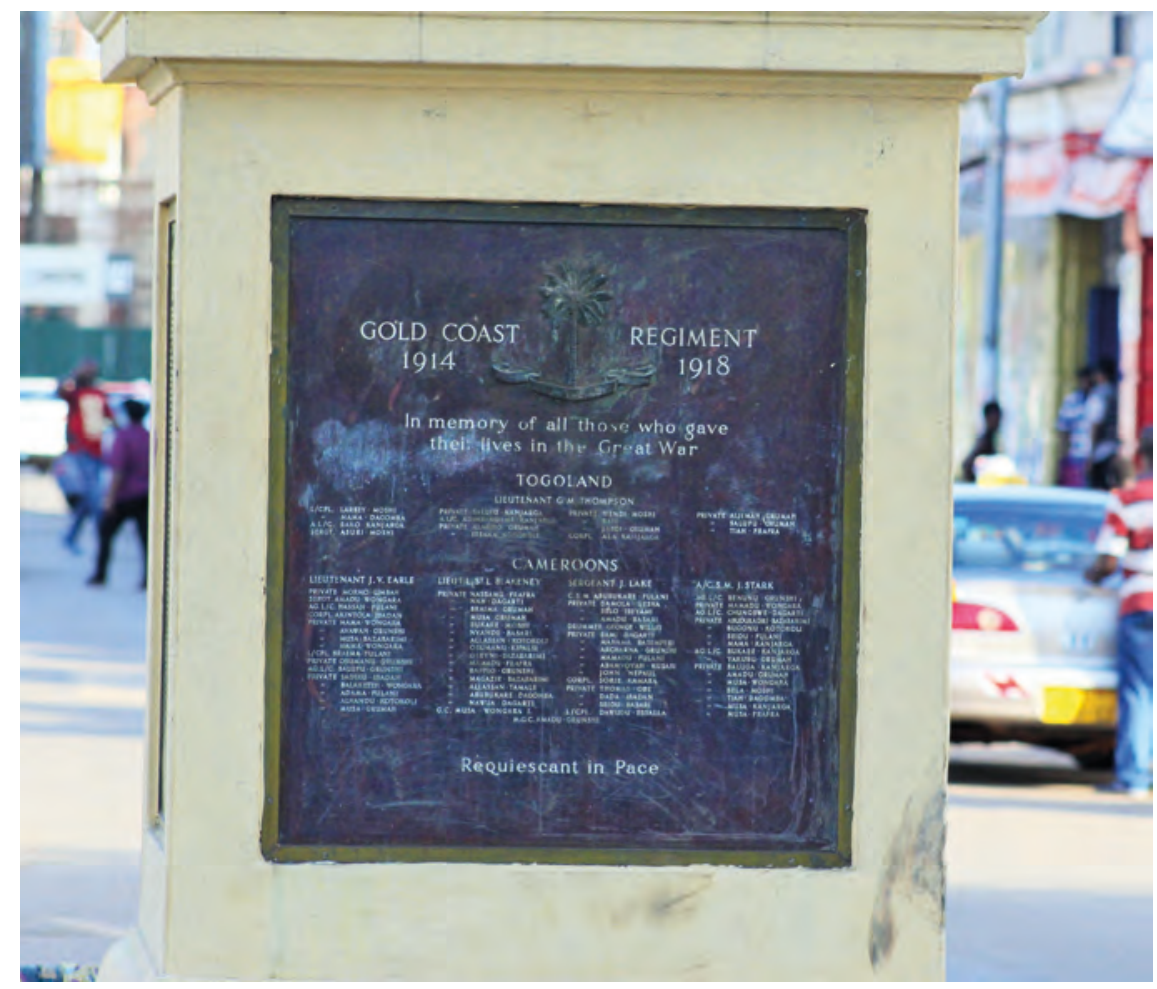

Illustration 28.1

World War I memorial in Kumasi, Ghana

Photograph by Celestinesucess, CC BY-SA 4.0

casualty toll in human history. Many imagined - again incorrectly - at war's end that war was now too horrific for governments ever again to contemplate.

We noted above that the ability of governments to finance war had increased through time. Some scholars consider World War I to be the first "total war" due to the large proportion of total income that was devoted to it. There was rationing of consumer goods, wage and price controls, and direct control of the production of weaponry in countries on both sides of the war. Conscription was common, even in the colonies and overseas dominions (This would result, for example, in a political crisis in Canada when French Canadians objected to being forced to serve under British generals.) There was also widespread censorship and propaganda on both sides: To question the war effort was treachery.

The war started with the assassination of Archduke Franz Ferdinand, the heir to the Austrian crown, in Sarajevo (in present-day Bosnia), by a Serbian nationalist angry at the Austrian seizure of Serb territories. Victories over the Ottomans in the Balkan Wars of 1912-3, which had seen the Ottomans lose almost all their European lands, had stoked Serbian nationalism. Yet this assassination was just the trigger for war. Nationalism and a changing power balance 


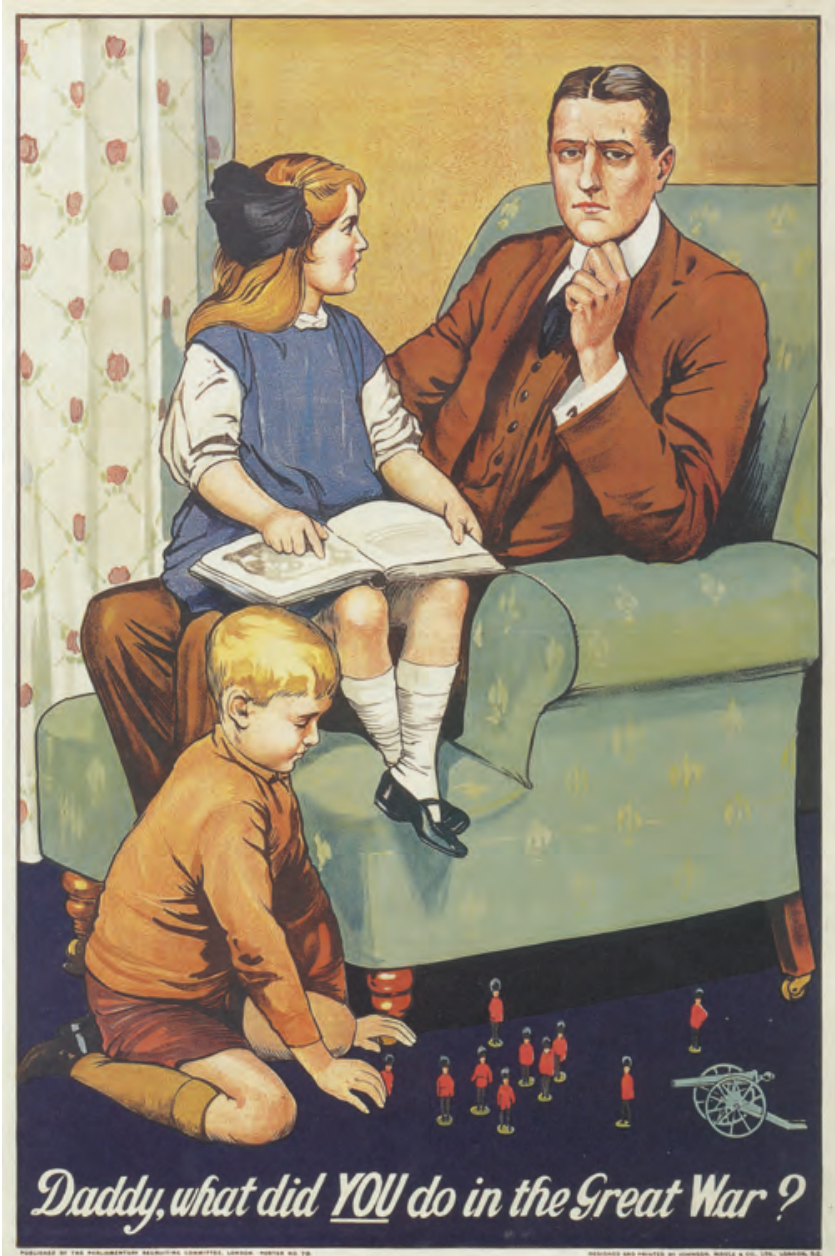

\section{Illustration 28.2}

"Daddy, what did YOU do in the Great War?" by Savile Lumley (active 1910-50); a British propaganda poster from World War I, commissioned by the British Parliamentary Recruiting Committee. Published in Britain, 1915

in Europe, as well as particular decisions by particular states, provide deeper explanations for war.

As noted in chapter 21, the ideal of national sovereignty posed a challenge to the multi-ethnic empires of Eastern Europe. Western European powers had supported some independence movements against the Ottomans, and tried to limit the ability of either Russia or Austria-Hungary to capitalize on these. Newly independent states had their own nationalist ambitions. Serbs in AustriaHungary wanted to unite with the newly independent state of Serbia. Russia supported the aspirations of other Slavic peoples, though it often had strategic motives for destabilizing its Ottoman and Austrian opponents. The Germans, in turn, had encouraged Moroccan independence from France. 
As Germany's population and economy grew rapidly in the nineteenth century, the balance of power in Europe altered. At the time of Napoleon, there were more French than Germans in Europe and their average incomes were likely higher. A century later, there were many more Germans than French, and German average incomes had pulled ahead. Germany had also developed superior steel and chemical industries. Germany could potentially field a more massive army than France and provide it with the best modern weaponry. Germany had already demonstrated its superiority in the Franco-Prussian War of 1870, which had both allowed the creation of the German state and transferred the mixed-ethnicity territories of Alsace and Lorraine from France to Germany.

France reacted to the increasing power of Germany by allying with Britain and Russia. As noted above, Russia had long opposed Germany and Austria, and it saw itself as the protector of the Slavic populations that each ruled. It had fought Britain and France in Crimea in the 1850s but now saw Germany as a far greater threat. Britain and Germany engaged in a dramatic naval arms race from 1898 in which each launched dozens of new and larger battleships (these were often called "dreadnoughts" in Britain): The British would have forty-nine battleships on the eve of World War I and Germany twenty-nine. Notably, the public broadly supported this arms race in both countries as each had come to view the other as a threat to its security. The growing power of Germany alarmed Britain, and British threats to blockade German access to the sea in the event of war frightened Germany in turn. Britain also formed an alliance with Japan against Germany from 1902; Japan would protect British ships against German submarines in the Mediterranean and elsewhere during the war. Germany responded to the alliance of Britain (and Japan), France, and Russia by strengthening its ties to Austria-Hungary and reaching out to the Ottomans. Prussia and Austria had warred in 1866 as part of the process of German unification but now celebrated a common German heritage. The Ottomans had for centuries battled the Austrians for territory in the Balkans (laying siege to Vienna in the sixteenth and seventeenth centuries), but in recent decades had been losing ground to Russia in both the Balkans and Caucasus, and had objected to British and French support for independence movements within Ottoman territories. Ottoman leaders judged the alliance as an opportunity to reverse some of these territorial losses. Germany saw the Ottomans as particularly valuable because the Ottoman Empire could serve as a gateway to Asia. Germany had come very late to the European rush to colonize the world and so had far fewer and smaller colonies than Britain or France (or even Portugal). It hoped to build a railway connecting Berlin and Baghdad to reach Asian markets. It was also interested in secure supplies of oil, which were beginning to be discovered in the Middle East and Iran. The German government was inspired to pursue war by the growing strength of Germany, while the Ottomans were tempted to war by their increasing relative weakness. In both cases, it was changes in relative power, with roots in differential economic performance, which encouraged war. 
The French in allying with Russia and Britain had hoped to deter war. Yet these two competing alliances ensured that if a war began it would quickly explode in size. Austria reacted to the assassination of Franz Ferdinand by declaring war on Serbia. Russia responded by declaring support for Serbia. Germany demanded that Russia stop mobilizing; it then declared war when Russia refused. The Germans demanded that France announce its intentions and declared war on France when they did not answer. The French appealed to Britain for support, but the British government debated this. When the Germans invaded Belgium, the British declared war.

Nevertheless, why start? With the advantage of hindsight, both sides should have avoided World War I at (almost) any cost. It would lead to the collapse of the Austro-Hungarian, Ottoman, and Russian empires, and force the abdication of the German king. As noted above, Europe had been relatively peaceful for decades. Diplomacy had managed changes in relative power. Yet Kaiser (king) Wilhelm of Germany wanted to assert German power in the world and came to believe that he could win a war on two fronts. German military strategists developed the "Schlieffen Plan" for a two-front war. This plan urged a rapid attack in the west while the Russians mobilized. There may have been no peaceful means of satisfying the Kaiser's overconfidence and urge to power. Nevertheless, the actions of Britain, France, and Russia in the Balkans and Caucasus had undoubtedly facilitated Germany's pursuit of an alliance.

The historiography of World War I has changed dramatically over the years - an important reminder of the dangers of writing a history of the recent past before historical consensus has time to emerge. The question of "Was it Germany's fault?" usually answered affirmatively, dominated historiography in the first decades after the war. Over time, historians devoted more attention to the more general trends surveyed above such as nationalism and industrialization. More recently, many historians have urged a view that the war was mostly the result of historical contingency: a series of unfortunate events and decisions in the 1910s, including the Balkan Wars and archduke assassination. Diplomacy might otherwise have continued to substitute for war.

Leaders on both sides predicted a brief war - though it is clear that many advisers on both sides feared a long and horrific contest. Leaders do not appear to have appreciated how the machine gun would change warfare. Machine guns had improved over the decades since their development during the Civil War in the United States as they were applied extensively in colonial conquests. Machine guns facilitated rapid victories if only one side possessed them, but would turn out to lead to a long and bloody conflict if both sides had them. In 1914, after a rapid advance through Belgium, the German army became bogged down in northeastern France. The French and British on one side and the Germans on the other dug a series of trenches extending 650 hundred kilometres in length. Blistering machine gun fire met soldiers venturing "over the top" to attack the other side. Over time, both sides built barricades of barbed wire between the trenches to further slow enemy advance. There were some experiments with tanks from 1916, but these were not yet capable 


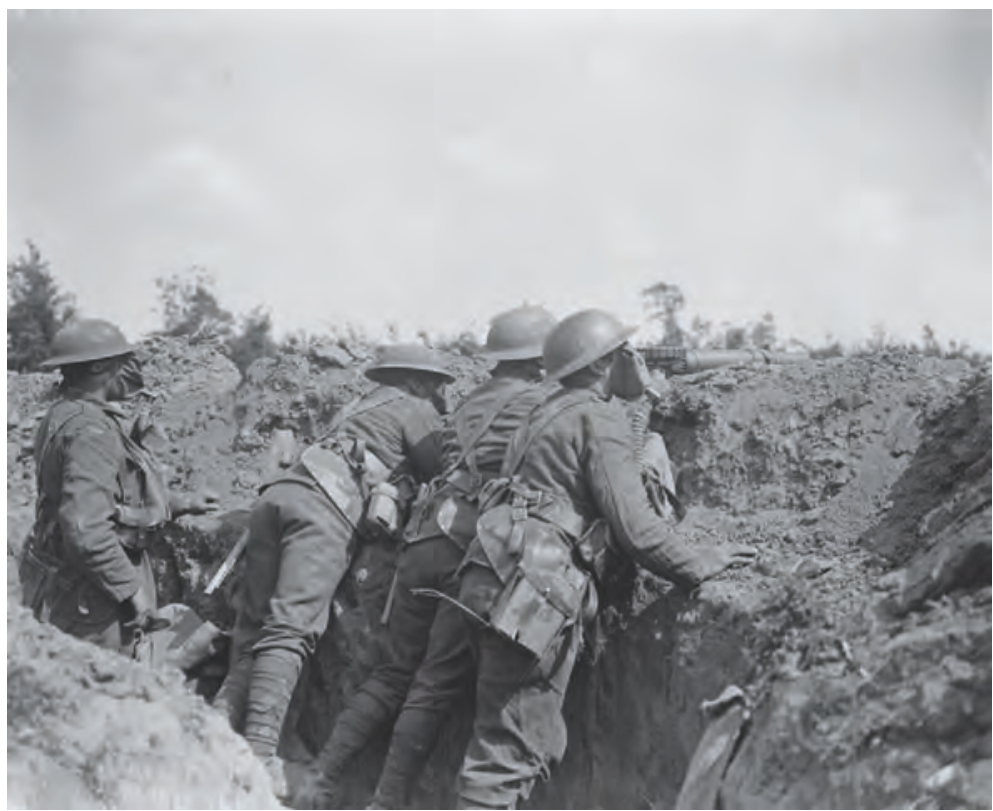

Illustration 28.3

Lewis gunners of the 12th Royal Scots wear box respirators during a gas attack on a front line trench, Meteren

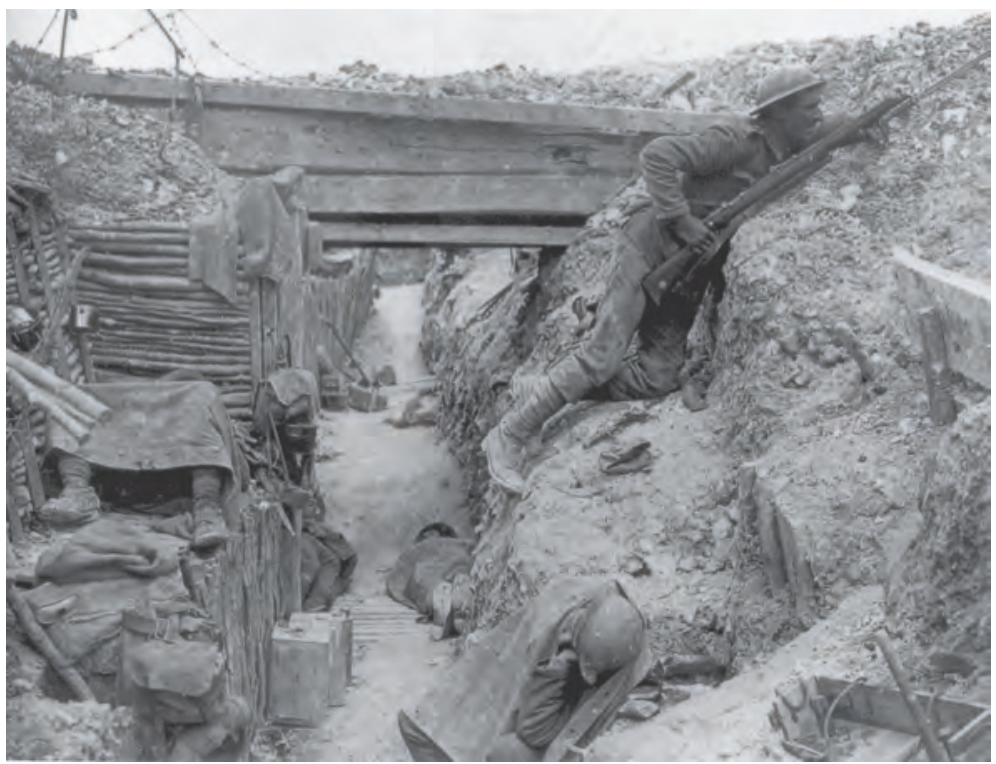

Illustration 28.4

A German trench occupied by British soldiers, July 1916 during the Battle of the Somme 
of advancing across rough terrain under enemy fire. Both sides introduced airplanes: These were useful for spying but had limited capability as bombers. The result was a defensive stalemate that lasted more than three years. Attacks by either side generally meant massive casualties: 350,000 on each side when the Germans attacked Verdun; over half a million on each side when the Allies attacked the Somme. Note that these losses were higher than the entire size of the Roman army at its peak. In addition to deaths by machine gun fire, the trenches themselves proved muddy, rat-ridden, and unhealthy. The Germans briefly experimented with the use of poisonous mustard gas, which killed or injured hundreds of thousands, but it proved hard to control and the Allies soon donned protective masks.

\section{PRIMARY DOCUMENTS: GAS ATTACK 1916}

Arthur Empey was an American who joined the British army after the sinking of the Lusitania (below). He provides an eyewitness account of a gas attack:

We had a new man at the periscope, on this afternoon in question; I was sitting on the fire step, cleaning my rifle, when he called out to me: "There's a sort of greenish, yellow cloud rolling along the ground out in front, it's coming-"

But I waited for no more, grabbing my bayonet, which was detached from the rifle, I gave the alarm by banging an empty shell case, which was hanging near the periscope. At the same instant, gongs started ringing down the trench, the signal for Tommy to don his respirator, or smoke helmet, as we call it.

Gas travels quietly, so you must not lose any time; you generally have about eighteen or twenty seconds in which to adjust your gas helmet.

A gas helmet is made of cloth, treated with chemicals. There are two windows, or glass eyes, in it, through which you can see. Inside there is a rubber-covered tube, which goes in the mouth. You breathe through your nose; the gas, passing through the cloth helmet, is neutralized by the action of the chemicals. The foul air is exhaled through the tube in the mouth, this tube being so constructed that it prevents the inhaling of the outside air or gas. One helmet is good for five hours of the strongest gas. Each Tommy carries two of them slung around his shoulder in a waterproof canvas bag. He must wear this bag at all times, even while sleeping. To change a defective helmet, you take out the new one, hold your breath, pull the old one off, placing the new one over your head, tucking in the loose ends under the collar of your tunic.

For a minute, pandemonium reigned in our trench, - Tommies adjusting their helmets, bombers running here and there, and men turning out of the dugouts with fixed bayonets, to man the fire step.

Reinforcements were pouring out of the communication trenches.

Our gun's crew was busy mounting the machine gun on the parapet and bringing up extra ammunition from the dugout. 
German gas is heavier than air and soon fills the trenches and dugouts, where it has been known to lurk for two or three days, until the air is purified by means of large chemical sprayers. We had to work quickly, as Fritz generally follows the gas with an infantry attack. A company man on our right was too slow in getting on his helmet; he sank to the ground, clutching at his throat, and after a few spasmodic twistings, went West (died). It was horrible to see him die, but we were powerless to help him. In the corner of a traverse, a little, muddy cur dog, one of the company's pets, was lying dead, with his two paws over his nose.

It's the animals that suffer the most, the horses, mules, cattle, dogs, cats, and rats, they having no helmets to save them. Tommy does not sympathize with rats in a gas attack.

At times, gas has been known to travel, with dire results, fifteen miles behind the lines.

A gas, or smoke helmet, as it is called, at the best is a vile-smelling thing, and it is not long before one gets a violent headache from wearing it.

I trained my machine gun on their trench and its bullets were raking the parapet. Then over they came, bayonets glistening. In their respirators, which have a large snout in front, they looked like some horrible nightmare.

All along our trench, rifles and machine guns spoke, our shrapnel was bursting over their heads. They went down in heaps, but new ones took the place of the fallen. Nothing could stop that mad rush. The Germans reached our barbed wire, which had previously been demolished by their shells, then it was bomb against bomb, and the devil for all.

Suddenly, my head seemed to burst from a loud "crack" in my ear. Then my head began to swim, throat got dry, and a heavy pressure on the lungs warned me that my helmet was leaking. Turning my gun over to No. 2 , I changed helmets.

The trench started to wind like a snake, and sandbags appeared to be floating in the air. The noise was horrible; I sank onto the fire step, needles seemed to be pricking my flesh, then blackness.

I was awakened by one of my mates removing my smoke helmet. How delicious that cool, fresh air felt in my lungs.

A strong wind had arisen and dispersed the gas.

They told me that I had been "out" for three hours; they thought I was dead.

The attack had been repulsed after a hard fight. Twice the Germans had gained a foothold in our trench, but had been driven out by counter- attacks. The trench was filled with their dead and ours. Through a periscope, I counted eighteen dead Germans in our wire; they were a ghastly sight in their horrible-looking respirators.

I examined my first smoke helmet, a bullet had gone through it on the left side, just grazing my ear, the gas had penetrated through the hole made in the cloth.

Out of our crew of six, we lost two killed and two wounded.

That night we buried all of the dead, excepting those in No Man's Land. In death there is not much distinction, friend and foe are treated alike. 
After the wind had dispersed the gas, the R.A.M.C. got busy with their chemical sprayers, spraying out the dugouts and low parts of the trenches to dissipate any fumes of the German gas which may have been lurking in same.

“Gas Attack, 1916," EyeWitness to History, eyewitnesstohistory.com (1999)

How do you evaluate this source? What effect does it have on the reader?

Germany had more success in the east, killing or capturing millions of poorly armed Russians (after some early Russian successes that inspired the Germans to shift some troops from the western front). The western allies lost 145,000 soldiers (many from Australia and New Zealand and India) at Gallipoli in the Ottoman Empire in a failed attempt to open a route through the Bosporus and the Black Sea to ship supplies to the Russians. The British later allied with Arab groups to push the Ottomans to quit the war in 1918. But by this time the Russian state had collapsed in 1917 in reaction to the enormous losses sustained in the war, and the Bolshevik revolutionaries had withdrawn from the war (the Bolshevik leader Vladimir Lenin had been transported by Germany from exile in Switzerland to foster disorder in Russia).

War extended deep into the Atlantic. As promised, the British had blockaded shipping to Germany, even though intercepting neutral ships violated rules of international law. This blockade proved particularly crucial because of trench warfare. As it became clear that a quick victory was not possible in the trenches, the path to victory seemed to involve draining one side of men or bullets or food. The British blockade limited German capacity to both arm and feed its soldiers in the trenches. Over a half million German civilians may have starved to death during the war because of the blockade (Germany had become a food importer before the war, and mass mobilization limited its ability to increase local production). This would be an understandable source of German enmity toward Britain after the war - though the Germans also displayed a callous disregard for civilian suffering and starvation in occupied Belgium and the Netherlands. The Germans responded with submarines that torpedoed commercial shipping in the Atlantic: They hoped to limit supplies to the British and French armies. In 1917, the Germans sank the steamship Lusitania, causing 1,200 civilian deaths. This action, plus the discovery of a German telegram to Mexico urging the Mexicans to reclaim territories lost in previous decades to the United States, prompted American entry into the war. The combination of fresh troops on one side with the shortage of material on the other finally broke the trench warfare stalemate.

The Kaiser would abdicate in 1918 when informed that the military situation was hopeless. The abdication had the unfortunate consequence that elected officials would negotiate the terms of the German surrender. The Nazi leader Adolf Hitler (see below) would later seize upon this fact to argue that 


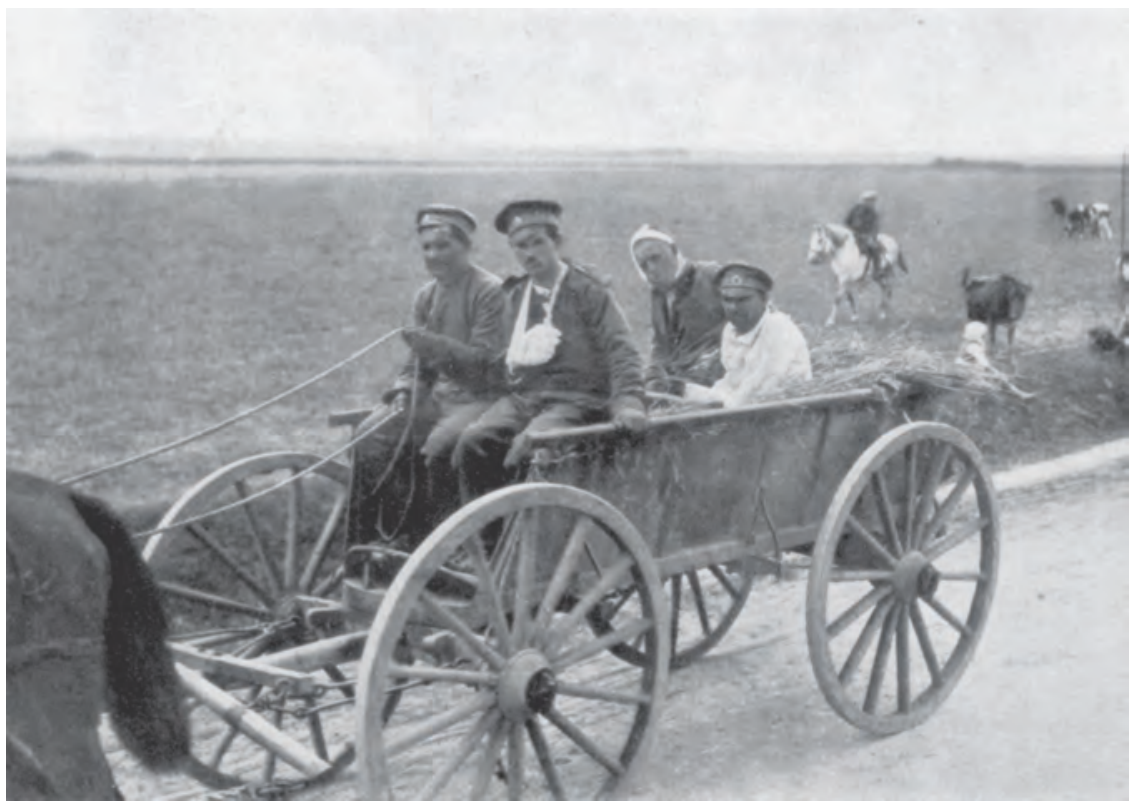

Illustration 28.5

Russian wounded in peasant carts due to a shortage of vehicles, George H. Mewes

democracy would not adequately support the German nation. Though war on the western front ended in 1918, battles continued in the Middle East into 1922 and 1923. The Japanese would fight the new Soviet Union in Siberia until 1922.

The victors would apply the principle of national sovereignty in Eastern Europe after the war. Poland, Czechoslovakia, Hungary, Romania, and Yugoslavia (the last an amalgam of Slavic and Islamic peoples judged too small and two intermingled at the time to each have their own state) were created from territories taken from Germany, Austria, Russia, and the Ottomans. Austria, shorn of its empire, became a majority-German state, but was forbidden to merge with Germany in fear that such a state would be too powerful. Some one-quarter of the population of these new states were ethnic minorities. These new states were "required" by the peace treaties to respect their minorities. They had to agree to constitutional protections for minorities in order to be recognized.

As noted in chapter 21, the principle of the nation state had long clashed with the urge to colonize. Britain, France, and Japan sought to maintain the new colonies they had gained during the war. Britain and France had taken German colonies in Africa, and Japan had done the same in the Pacific and China. Britain and France had worked with Arab nationalists to take much of the Ottoman Empire. The United States, along with an important segment of public opinion in Britain and France, was opposed to further colonization. The newly elected German government asked to have its colonies returned, but the victors rebuffed this proposal. The compromise reached was the idea of a 
"League of Nations Mandate": These new colonies were officially temporary holdings ceded in trust to the colonial powers until the locals were ready for self-governance. Arabs in the Middle East to whom the Allies had promised independence in return for support against the Ottomans viewed this compromise with particular hostility. They noted a stark difference in treatment compared to the former Ottoman territories in Europe that became independent states (see chapter 21 for a discussion of the creation of Turkey).

Britain had also made promises of independence in India in 1917 to ensure support for the war, but the British soon backed away from these promises. India had broadly supported the war effort - though neither the British nor the Indian nationalists mentioned this fact much after the war. The British had asked Indian nationalists to put aside controversial issues during the war, and they had largely agreed. India exported grain to Europe during the war despite famine at home. Indian troops and workers saw service in Europe as a chance to show the British their capabilities. Indian forces would suffer even greater losses than Australian and New Zealand troops in the ill-fated attack on Gallipoli (see above). The Indian government over a century later belatedly began erecting a monument to its First World War sacrifices. After the war, the British not only reneged on promises of increased local authority but tightened restrictions on freedom of speech.

Its allies had promised the Japanese that it could keep the Pacific islands it claimed from Germany. The Japanese had declined to send troops to Europe but had usefully sent naval patrols into the Indian and Atlantic Oceans, and even the Mediterranean, during the war. Japan would have little influence at the peace conferences that ended the war. Australia and New Zealand urged Britain to keep both Germany and Japan out of the South Pacific. The Australians also appealed to the Americans directly on this matter, though Britain still officially represented them in foreign affairs. In the end, both Australia and New Zealand would receive League of Nations mandates over islands they had occupied in the South Pacific, and Japan would gain no islands south of the Equator.

The war encouraged change even in victorious nations. Women had during the war taken on a variety of industrial jobs previously reserved for men. The female contribution to the war effort gave a considerable boost to decades of advocacy of women's right to vote. Women over 30 gained the vote in Britain in 1918, and all adult women in 1928. Women won the right to vote in the newly democratic Germany and Austria in 1918-9 and in the United States in 1920. Wartime mobilization of men and factory work by women also decreased the exercise of parental control: Juvenile delinquency rose during wartime, and many young adults embraced sexual freedom. One common fear during the war was that women in factories might interact sexually with colonial men of colour also employed there.

We noted above that the war in part reflected a change in the balance of power in the world. The war itself shifted economic and military power away from Europe. Much of Europe was devastated. Even victorious Britain and France had borrowed billions to prosecute the war. Europe imported vast quantities of food 
during the war and remained dependent on such imports for years after. The king of Afghanistan would declare war on Britain in 1919: Though defeated in battle, the Afghans won full independence from Britain due to British exhaustion from four years of war. To be sure, Europe would maintain colonies in Asia and Africa for a few more decades (chapter 30). But the United States - which had not been directly attacked (beyond some submarine activity in coastal waters) but instead had developed both its industrial and agricultural potential during the war - emerged from the war as clearly the economically and militarily strongest nation in the world. In four short years, it was transformed from a large net debtor in the world (for Europeans had financed American railways and industry in preceding decades) to a major creditor. A similar transformation occurred in the status of Canada, Australia, and Japan. These too had contributed in important ways to the war effort but had not themselves suffered severe attack. Latin American countries had been less directly involved in the war but had also responded to the new markets for especially agricultural goods that the war generated.

An influenza epidemic in 1918-9 killed at least 20 million people worldwide. It is likely that this death toll was enhanced both by wartime international movements of people and by the malnourishment that affected many world populations because of the war. The disease ravaged both Europe and North America. Yet the highest death toll by far was in India, where as many as 12 million died. Moreover, the highest death rates occurred on Pacific Islands, where a quarter of the population may have perished. In the United States (and likely elsewhere), mortality was much higher in cities with higher levels of air pollution.

\section{Linking the World Wars}

The First World War - or "Great War" at the time - was often called "the war to end all wars." It was widely thought that the massive death and destruction of that war would deter any leader from starting another. There were some efforts to work toward peace in the years after the war. Governments had often jailed or censored peace advocates during the war, but these found a new audience after the war ended. The Kellogg-Briand Pact of 1925 pledged states to nonaggression; it eventually had sixty-four signatories. In the Naval Conference of 1921, the United States, Britain, and Japan agreed to accept a 5:5:3 ratio of battleship tonnage, with limits on the size of the largest battleship. France and Italy accepted even lower quotas. Dozens of ships were destroyed because of the agreement. A further deal in 1930 covered smaller cruisers and submarines. Japan pulled out of the agreements in 1936. An even larger conference in 1932 included the Soviet Union and Germany; Franco-German rivalry proved an obstacle, and then the new Nazi government withdrew Germany in 1933.

The peace treaties that ended the war - and especially the Treaty of Versailles that dealt with Germany - were not conducive to ensuring future peace. 
France had insisted on exacting large reparation payments from Germany at the war's end. This was a common practice: Reparations had featured in the treaties that ended many previous wars. Reparation payments might be seen as part of a millennia-long tradition of the victors of war exacting booty from the vanquished. The Germans were required to pay some US\$33 billion over a period of years. This was a much more substantial sum than in any previous war. The victors justified the high figure in terms of the extensive damage done during the war and the belief that Germany had been the primary instigator of the war. These reparation payments proved difficult for Germany to pay. The new democracy that succeeded the Kaiser's abdication in Germany undoubtedly suffered in popular support as it struggled to make payments. Germany was also required to cede Alsace and Lorraine to France and to lose territory in the east to newly created nations such as Poland and Czechoslovakia. Although these treaty conditions are understandable, they differ dramatically from the much less punitive treatment of Germany after the even greater horrors of the Second World War (see below). Some in the west - such as the economist John Maynard Keynes in Britain - prophetically criticized the treaty for hobbling economic recovery in Germany and encouraging German animosity toward the Allies.

\section{PRIMARY DOCUMENTS: TREATY OF VERSAILLES}

The treaty is the length of a small book. A couple of the most notable Articles address the issue of war crimes:

Article 227. The Allied and Associated Powers publicly arraign William II of Hohenzollern, formerly German Emperor, for a supreme offense against international morality and the sanctity of treaties.

Article 228. The German Government recognizes the right of the Allied and Associated Powers to bring before military tribunals persons accused of having committed acts in violation of the laws and customs of war. Such persons shall, if found guilty, be sentenced to punishments laid down by law. This provision will apply notwithstanding any proceedings or prosecution before a tribunal in Germany or in the territory of her allies.

The German Government shall hand over to the Allied and Associated Powers, or to such one of them as shall so request, all persons accused of having committed an act in violation of the laws and customs of war, who are specified either by name or by the rank, office or employment which they held under the German authorities.

The Treaty goes on to describe how international tribunals would be established. Yet the Dutch would refuse to surrender the Kaiser, and Germany would later insist on trying its own soldiers. Only a few were ever tried: Germany complained that just its soldiers were accused, while others complained that German courts were lenient. 
The Treaty also addressed the question of war guilt and reparations:

Article 231. The Allied and Associated Governments affirm and Germany accepts the responsibility of Germany and her allies for causing all the loss and damage to which the Allied and Associated Governments and their nationals have been subjected as a consequence of the war imposed upon them by the aggression of Germany and her allies.

Article 232. The Allied and Associated Governments recognize that the resources of Germany are not adequate, after taking into account permanent diminutions of such resources which will result from other provisions of the present Treaty, to make complete reparation for all such loss and damage.

The Allied and Associated Governments, however, require, and Germany undertakes, that she will make compensation for all damage done to the civilian population of the Allied and Associated Powers and to their property during the period of the belligerency of each as an Allied or Associated Power against Germany by such aggression by land, by sea and from the air, and in general all damage as defined in Annex 1 hereto.

Note that the Treaty recognized to some degree the limited capacity of Germany to pay reparations. What do you think of these elements of the treaty?

The peace treaties also called for the establishment of the League of Nations. Peace activists had long advocated an international organization that could settle inter-state disputes through consultation. The idea received particularly strong support from American president Woodrow Wilson during peace negotiations. However, the American Congress refused in the end to join the League: The lesson drawn by many Americans from the war was that it was best to avoid foreign entanglements. The League, in any case, suffered from a lack of power. It could call for economic sanctions but could not initiate armed interventions. The League would complain when Japan invaded China and when Italy invaded Ethiopia in the 1930s (massive technological superiority meant that 275,000 Ethiopians died but only 2,000 Italians), but both Japan and Italy just ignored it.

The Indian independence leader Mohandas (Mahatma) Gandhi's message of non-violence (see chapter 30) resonated globally in the aftermath of the war. Many saw it as a rebuke of feeble attempts by Western nations to ensure peace. Gandhi spoke of the damage done to millions in war by the "few who abuse the power of their convictions." We have seen above that the leaders of many states, and especially Germany, chose national glory over the health and welfare of soldiers and civilians. Despite the horrors of war, a new set of leaders would emerge that would make the same choice. They would convince themselves that a great victory was both possible and justified. They joined a long human tradition of leaders convincing themselves of the value of horrific policies. 
Once such leaders were in place (see the discussion of fascism below), it was difficult to maintain peace in the world. When Nazi Germany announced in 1938 the intention of invading those parts of Czechoslovakia with a large German-speaking population, the leaders of Britain and France flew to Munich for talks with the Nazi leadership (Italy was also represented). They reached an agreement that accepted this annexation of Czech territory - over the strong objection of the elected Czech government - in return for a promise from Germany of no further aggression. The agreement was popular at the time, for it seemed to protect the peace. Indeed, the British Prime Minister Neville Chamberlain famously hailed it as ensuring "peace in our time." Yet there were concerns about the ethics of sacrificing Czech territory in response to threats of invasion. There were also concerns that such behaviour would encourage a German belief that its opponents lacked the stomach for another war. In early 1939, Germany would invade the rest of Czechoslovakia and then Poland. France and Britain would declare war on Germany in response (and Winston Churchill, who had disparaged the Munich accord, would replace Chamberlain as Prime Minister).

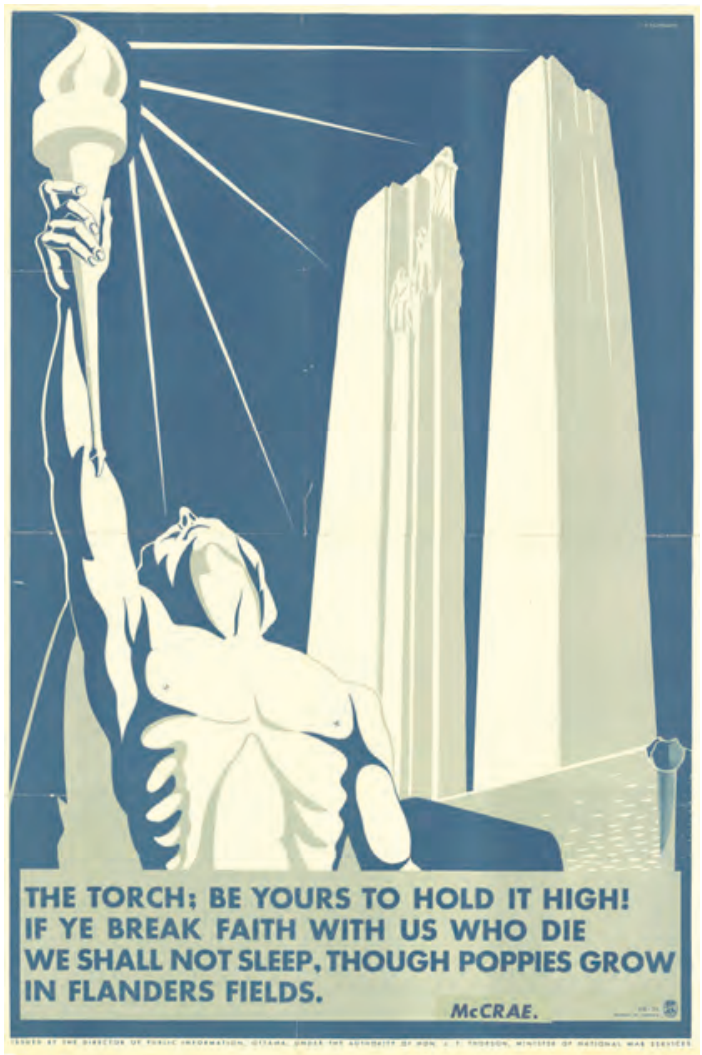

Illustration 28.6

Canadian recruitment poster in World War II cites poem and memorial from World War I
The Munich accord has become an emblem of "appeasement." Any time that a leader (especially a democratic leader) seems less than resolute in the face of aggressive acts by other states, analogies with Munich are drawn. Of course, analogies are only valuable if there are strong similarities between cases. Historians now appreciate that the Nazi German regime was extreme in many ways, and had a powerful desire to annex much of Eastern Europe. It may be that a clear sign that France and Britain were ready to fight would have changed German offensive plans. It may also be that there was no action short of going to war that would convince Germany that its plans for expansion would face determined opposition. The German army once again had a strategy for winning a two-front war - a plan that would succeed for the first years of war - and may well have started the war even in the face of clear Anglo-French resistance.

Some see the Second World War as a direct continuation of the First, since it reflected the same animosities and involved very similar alliances, and because the peace treaties that ended the First War stoked anger that paved the way for the Second. 
However, there were reasons for hope in the 1920s. In particular, democracy seemed to be gaining favour in Germany. It may then be that without the economic shock of the Great Depression (chapter 29) - by far the worst economic calamity ever - the Second World War might not have occurred. Hitler would likely not have gained power in Germany if not for unemployment rates of 25 per cent or more there during the Depression. In Japan, also, the Depression weakened the authority of the elected Assembly and encouraged the colonial ambitions of military leaders - who were emboldened to invade China against the express wishes of elected officials (the military at the time reported to the emperor rather than the Assembly). These had taken a lesson from the German defeat in the First World War that they needed a guaranteed source of raw materials in Manchuria, and provoked war there from 1931. The high tariffs that were widely introduced during the 1930s (chapter 29) likely served to encourage a belief in many countries that they needed to gain colonies rather than relying on international trade for materials and markets.

\section{Fascism}

Given the levels of death and destruction of the First World War, how could any leader contemplate another war? As noted above, many people reasonably anticipated at war end that no future leader could imagine that the benefits of a war against well-armed foes could exceed the costs. The rise of fascism would overturn this optimistic prediction. Fascism was a political philosophy that exalted the nation, justified autocracy in the service of the nation state, was willing to exercise force to get and maintain power, and glorified the military. The twin beliefs that the nation had a shared purpose and that the nation needed to be strong against others (including internal minorities) served to support the idea of a strong leader who would face no limitations on their power. Economic activity might be mostly private within a fascist state but should support state objectives.

Fascism itself had many causes. It built upon the nationalist sentiments that had developed over preceding centuries (chapter 21). In Germany especially, there was misguided theorizing of genetic superiority (which in turn reflected a misapplication of evolutionary theory and also the eugenics movement, which argued that humans, like plants and animals, should be bred for superior qualities). The First World War caused many to question inherited values. Democratic institutions were weak in many countries and seemed ill-equipped to deal with the political and economic challenges of the 1920s and 1930s. The communist revolution in Russia in 1917 seemed to provide a promising alternative method of political and economic organization. Communist and socialist parties fared well at the polls in many western European countries in the 1920s. Fascism was in many ways a reaction to communism. Communism gained power in Russia through force of arms rather than force of the public will. Fascists too would employ force in their march to power. Fascists in Italy 
and Germany would manipulate democratic institutions to their ends but gain power without achieving an electoral majority; fascists in Spain would engage in military action against the elected government. While fascism earned significant popular support in all three countries, in each case millions of people supported fascism - and millions more did not bother to oppose it - either because it seemed the lesser of two evils compared to communism or because the fascist exercise of violence cowed them. Fascist leaders, once in power, then exercised this power to erase any opposition.

The fascist leader in Italy, Benito Mussolini, used paramilitary units to threaten, beat, and kill opponents, especially socialists. The Italian fascists claimed to have killed 3,000 people in Italy 1920-2. When the fascists marched on Rome in 1922, the king invited Mussolini to be Prime Minister despite having gained just 7 per cent of the vote in the previous election. The fascists then won 60 per cent of the vote in 1924, though there may have been widespread electoral fraud. Many may have voted for the fascists out of fear - just as people throughout history had resigned themselves to autocratic rule by those who could wield violent authority. The fascists thereafter abolished other parties and unions and censored the press. They jailed or killed opposition figures. Mussolini forced business-labour collaboration and - famously - used force to make the trains run on time (though his success in this respect has likely been exaggerated). The fascists also built roads and drained swamps.

Mussolini's success gained the attention of Hitler in Germany. Hitler had been exhilarated by World War I and then scandalized by the terms of the peace. He blamed this on the new democracy that had accepted the terms of the peace, and on groups such as Jews, the rich, and the socialists, who he thought were too powerful and had questionable values. As in Italy, many preferred his armed thugs to both socialist demonstrations and ineffective government. He launched a coup in 1923 and was jailed for nine months when this failed. He would then pursue, like Mussolini, a mixed strategy of electoral competition and paramilitary intimidation. He never won a majority in the Reichstag but became Chancellor in 1933 because his party was the largest. The Nazi party grew in popularity during the Great Depression as Hitler blamed the widespread suffering on foreigners and minorities while promising remedies. He then used censorship and intimidation to gain 44 per cent of the vote in 1933, and after that used threats to win majority support in the legislature for emergency dictatorship powers. He not only banned other parties but also killed those he did not trust within his own party in 1934, and killed or jailed opposition leaders. He then stripped Jews of citizenship and government jobs. As in Italy, he launched an extensive public works programme. The Nazis ignored peace treaties that limited the size of the German military as they expanded the army.

The path to authoritarian rule was different in Japan. Japan had elections but also maintained an ideal of rule by the emperor. The armed forces held the positions of minister of war and the navy. The army could simply ignore an elected assembly that wished to pursue peace. Violence again played an 
important role in silencing those who opposed increased militarism. Two prime ministers, a finance minister, and a banker were assassinated 1930-6.

In Spain, the army staged a coup when socialists were elected to government. Italy sent 50,000 troops in support of General Franco's coup, and Germany's new air force gained valuable field experience. The bombing of Guernica - captured by Picasso in a famous painting - would provide a foretaste of the destruction that aircraft could perpetrate upon civilians. While the Spanish fascists received foreign support, the elected government received little. Russia sent some advisers. Western democracies did nothing, through a mixture of war-weariness and suspicion of socialism. Their inaction may have encouraged other fascist states in a belief that democracies would not oppose them. There were volunteer brigades from western countries; writers such as Ernest Hemingway and George Orwell chronicled their exploits. Some 600,000 people died over three years (1936-9) in the Spanish Civil War. The fascist Franco regime would thereafter employ repressive techniques to silence all opposition.

Postscript: Amazingly, Spain would transform peacefully to democracy after Franco's death in 1975. Franco restored the Spanish monarchy, and the new king supported democracy, speaking out forcefully against one coup attempt. Spain wished to join the European Union and needed to adopt democracy to achieve this goal. Finally yet importantly, communism was far less popular in the 1970s than it had been in the 1930s.

We have noted in chapters 21 and 27 that successful democracy requires a set of constitutional guarantees and a bureaucracy, judiciary, and military devoted to the protection of democracy. Fascism emerged in states where democracy was new and weak. The national army played a crucial role in instituting fascism in both Spain and Japan. Fascists quickly co-opted the military in Germany and Italy. In none of these countries could judges or legislators prevent the fascist overthrow of democratic institutions.

Though fascism encouraged war, this was still not inevitable. Fascist Spain would refuse to join the Second World War - despite Germany and Italy having aided Spanish fascists militarily in seizing power. As a result, fascist rule would continue into the 1970s in Spain. Italy had been cautious in its foreign military adventures before it allied with Germany. Germany calculated that it could benefit from foreign war. In the view of German military leaders, tanks and airplanes would mean that rapid military advance, even against machine gun fire, was entirely feasible. The Second World War would not become bogged down in trenches like the First. Though it proved reasonable to imagine in 1938 that the next war would be quite unlike the last, it would turn out that two sides which both possessed tanks and planes could again fight for years, this time with even greater destruction. With the advantage of hindsight, we can again recognize that German leadership seriously overestimated their chances 
of winning a war. Though the Nazis in Germany had proclaimed themselves a bulwark against communism, their war in Eastern Europe would allow the Soviet Union to spread communist rule after the war, even into Germany.

This new military equipment was expensive. We noted above that modern state bureaucracies and economies were able to mobilize a far larger share of national output for war than had been possible centuries earlier. By 1937, Germany and Japan (and the Soviet Union in response) were already devoting a quarter of total output to military expenditure. Western democracies found it much harder to divert that much effort to the military until after they had declared war. Some have argued that fascist Germany and Japan pursued war in part to justify these massive military expenditures.

\section{World War II}

It is common in the west to date the Second World War from 1939 until 1945. However, it arguably began in 1937 with the Japanese invasion of China or even earlier with the Japanese invasion of Manchuria from 1931. As with the Spanish Civil War, the Japanese attack provided a sense of how horrific modern warfare could be. Aerial bombardment of Chinese cities killed tens of

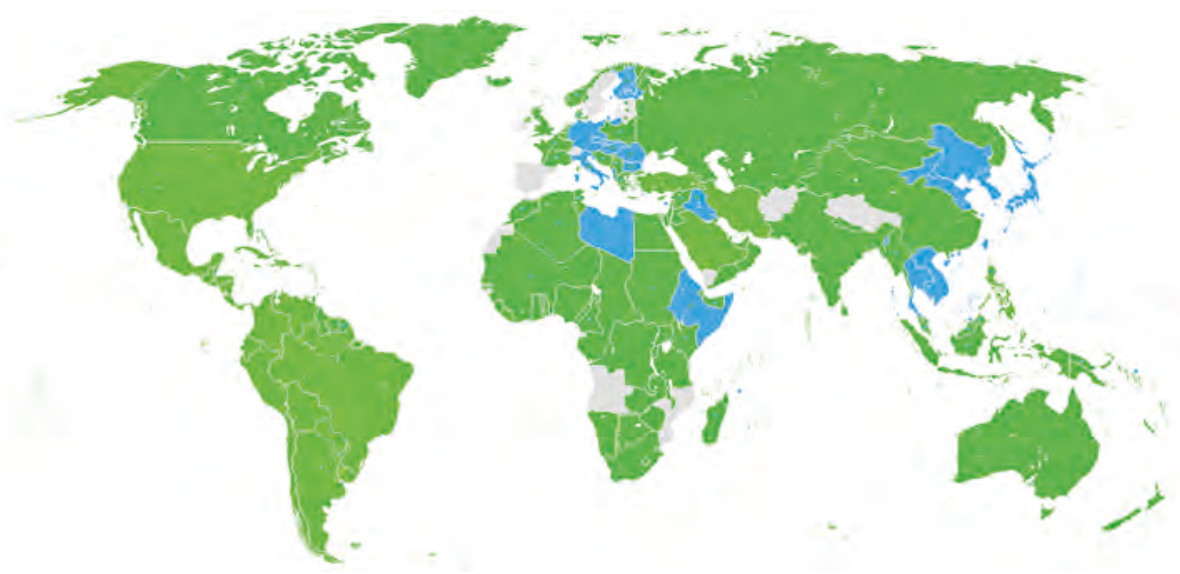

Map 28.1

Combatants in World War II

Dark Green: Allies before the Japanese attack on Pearl Harbor, including colonies and occupied countries. Light Green: Allied countries that entered the war after the attack on Pearl Harbor. Blue: Axis powers and their colonies or countries that had to choose a side in order to stay independent. Grey: Neutral countries during WWII. Dark green dots represent countries that initially were neutral but during the war were annexed by the USSR. Light green dots represent countries that later in the war changed from the Axis to the Allies. Blue dots represent countries either being conquered by the Axis powers, becoming puppets of those (Vichy France and several French colonies)

Map by Joaopais, Roxanna, CC-BY-SA-3.0 
thousands. In Nanjing, Japanese soldiers murdered hundreds of thousands of civilians and raped tens of thousands of women. This attack is known as the "Rape of Nanjing."

If the First World War was remarkable for the stability of battle lines on the western front, the Second World War was instead remarkable for the speed of early victories in all theatres of war. Just as machine guns had encouraged trench warfare in 1914, new technologies of tanks and airplanes were critical to rapid military advance in the 1930s.

The Japanese advanced deep into China. Western powers objected to Japanese aggression but again did little to deter it. The communist and nationalist forces in China united in opposition, but conflicts on the ground limited their cooperation (chapter 30). The communists gained public support by guerrilla tactics against the Japanese, and by introducing land reform in areas they controlled. As the Russians had against both Napoleon and the Kaiser, the Chinese armies retreated into the interior, scorching the earth behind them. After early victories, the Japanese found themselves contending with difficult terrain and a very broad front. Though the eventual defeat of Japan would come from Allied action in the Pacific (see below), it is important to appreciate that almost 2 million Japanese troops were tied up in China at the end of the war.

Japan signed a treaty with Germany and Italy in 1940 but did not coordinate military action with them. The three countries would nevertheless be known as the Axis powers thereafter. Indeed, Japan entered a non-aggression treaty with the Soviet Union in 1941 (after one aborted attack on the Soviets) just as Germany began to attack the Soviet Union. Japan would then look south to attack European colonies in Southeast Asia (see below).

Even before Nazi Germany had threatened parts of Czechoslovakia in 1938, it had defied the peace accords of World War I by remilitarizing the Rhineland in 1936 and absorbing Austria in 1938. Germany introduced a national draft in 1935. Hitler argued that he was just reuniting the German people, an act that nationalist thinkers elsewhere could appreciate. However, Germany soon took all of Czechoslovakia and threatened Poland. Britain and France had promised to defend Poland. Germany and the Soviet Union signed a treaty in 1939 that promised peace and secretly divided eastern Europe between them. Germany and the Soviet Union then conquered and divided Poland within a month in late 1939: Polish cavalry was no match for German tanks. The Soviet Union also took the Baltic states and parts of Finland and Romania. (Italy conquered Albania at this time.) The Germans would ally with Hungary, Romania, and Bulgaria (who also then became Axis powers), and invade Greece and Yugoslavia.

The western front was mostly quiet in 1939 despite declarations of war on both sides. Then Germany occupied Denmark and Norway in 1940 (the latter with the aid of Vidkun Quisling, a Nazi sympathizer, whose name would become synonymous with "traitor"), and then invaded France through Belgium and the Netherlands. France surrendered in June of 1940. The conquest of France took only a month. The French had built a system of defensive fortresses along the 


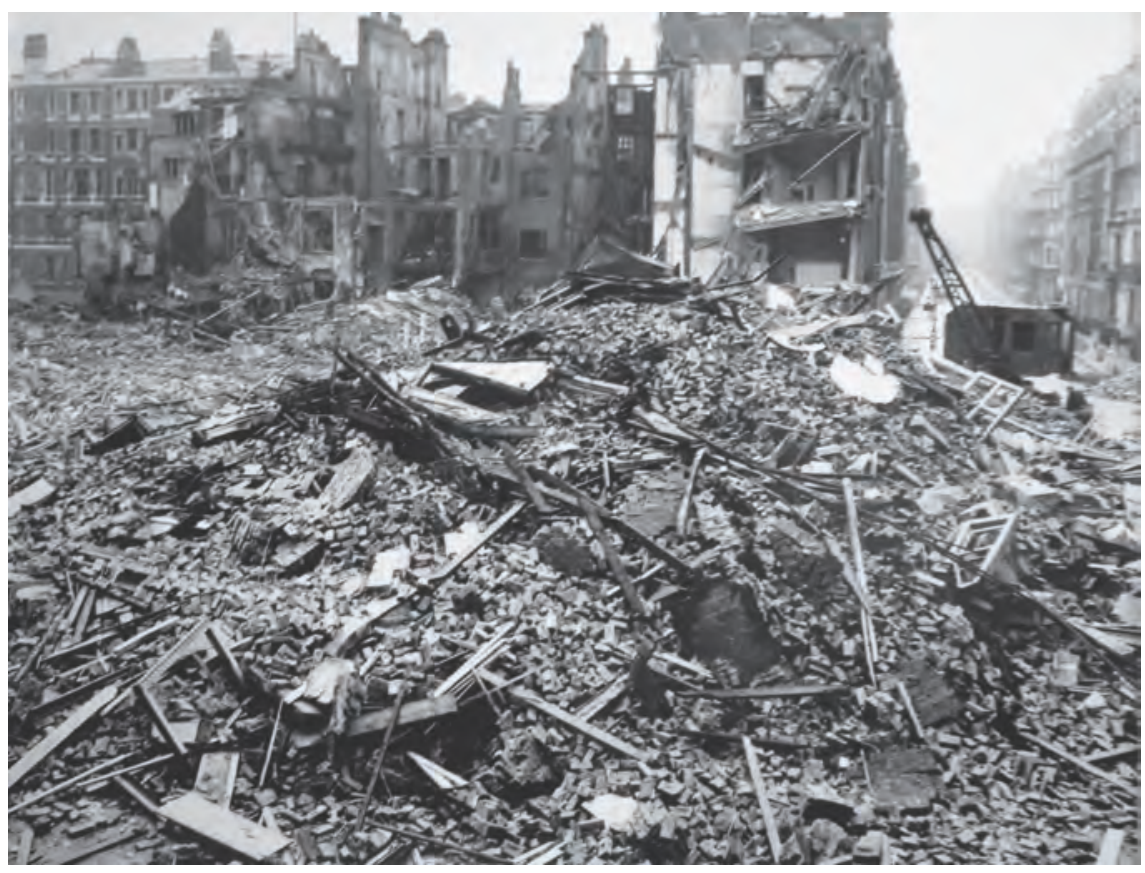

Illustration 28.7

Damage from German bombs, London

City of Westminster Archives, CC BY-SA 3.0

German border, but the Germans went to the north of these through Belgium and the Ardennes forest that the French had thought impassable.

The stunning German advance across France had encircled hundreds of thousands of British, French, and Belgian troops. Over 300,000 soldiers evacuated to Britain through the port of Dunkirk in just a few days: British naval destroyers were joined by a massive flotilla of small merchant and fishing vessels. There is speculation that the Germans did not bomb the evacuation as they might have in the hope that Britain would withdraw from the war. It is also possible that the Germans were just not confident of their own success and feared being cut off from supplies if they advanced fast enough to prevent the evacuation. Britain would vow to fight on. German bombs then killed some 40,000 British civilians over the next five years. The Germans late in the war would develop rockets that could reach London from bases across the English Channel, but their launch sites were soon overrun by Allied troops. (German rocket technology and scientists would be actively borrowed and developed by both the United States and Soviet Union after the war for both space exploration and military purposes.)

After its stunning successes in the west, Germany decided to attack the Soviet Union in 1941. With the advantage of hindsight, we can question this decision: Germany by the end of 1940 had successfully conquered several countries to its west, north, and east, and had a peace accord with the Soviets 
to the east. It might reasonably anticipate that the British would soon abandon their opposition, especially if Germany focused its resources in their direction. Germany might then have held on to its vast territories of conquest. However, these early victories whetted Germany's appetite for more, and they could reasonably imagine that the Soviets would mount an even less effective defence than the French and British had. They might have been especially attracted to Soviet oilfields. They had not learned from the experiences of Napoleon and Kaiser Wilhelm - or the contemporaneous challenges faced by Japan in China that victory was hard to achieve across the vast Russian front. The attack, with 3.6 million soldiers and 3,700 tanks, began in June of 1941 and by December German troops were at Moscow. The Soviets killed or wounded hundreds of thousands of German soldiers along the way. A severe winter hurt the unprepared German forces. The Germans advanced southward in 1942 seeking oil

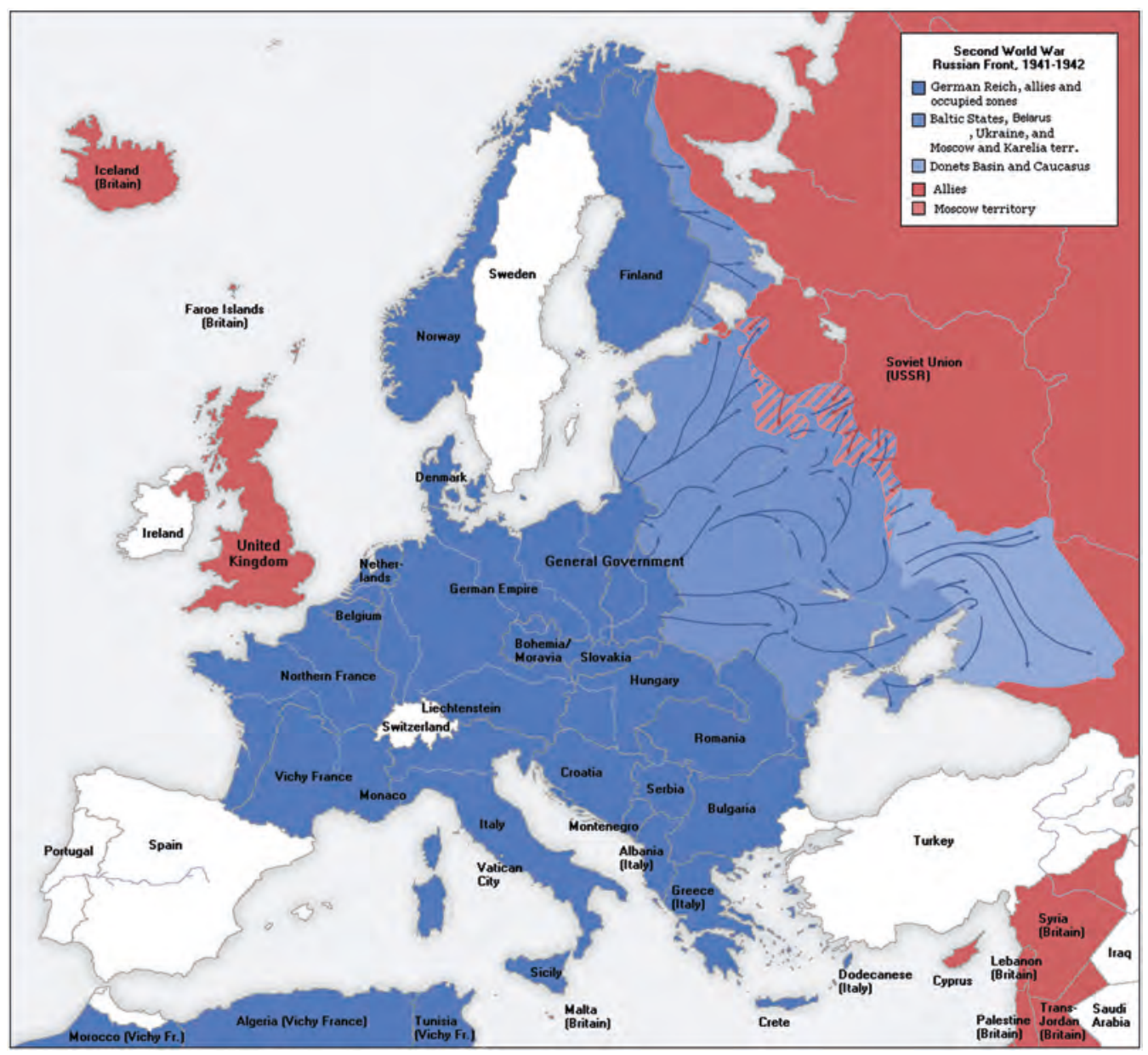

Map 28.2

The German Empire and the Eastern front, 1941-2

Map by San Jose and ArmadniGeneral, CC BY-SA 3.0 
and other resources but were stopped at Stalingrad after a lengthy siege. The Soviet Union then began to receive supplies from its new ally, the United States. (American and Canadian troops constructed the Alaska Highway in a matter of months - along pretty much its present route - to support airlifts of supplies and planes to Russia and to bolster Alaskan defences against the Japanese.)

As in the First War, the United States had stayed neutral at first. It had then increasingly provided aid to Britain. Also, the United States impounded Japanese assets as Japan moved into Southeast Asia. The Japanese decided to pursue a surprise attack on the United States, and tried to devastate the US Navy with a bombing raid on Pearl Harbor in Hawaii on December 7, 1941. The Japanese sent 300 planes to Pearl Harbor; they killed 2,000 military personnel and destroyed several ships and 180 planes. The attack could have been much worse: three aircraft carriers were away on patrol, and the Japanese attacking force suffered from miscommunication. The United States immediately declared war on Japan. Germany and Italy declared war on the United States four days later.

Brazil had also been neutral in the early years of the war. It severed diplomatic relations with Germany, Italy, and Japan early in 1942. German and Italian submarines in the next months sank thirty-one Brazilian merchant ships. Brazil responded by declaring war in August 1942. It would be the only independent

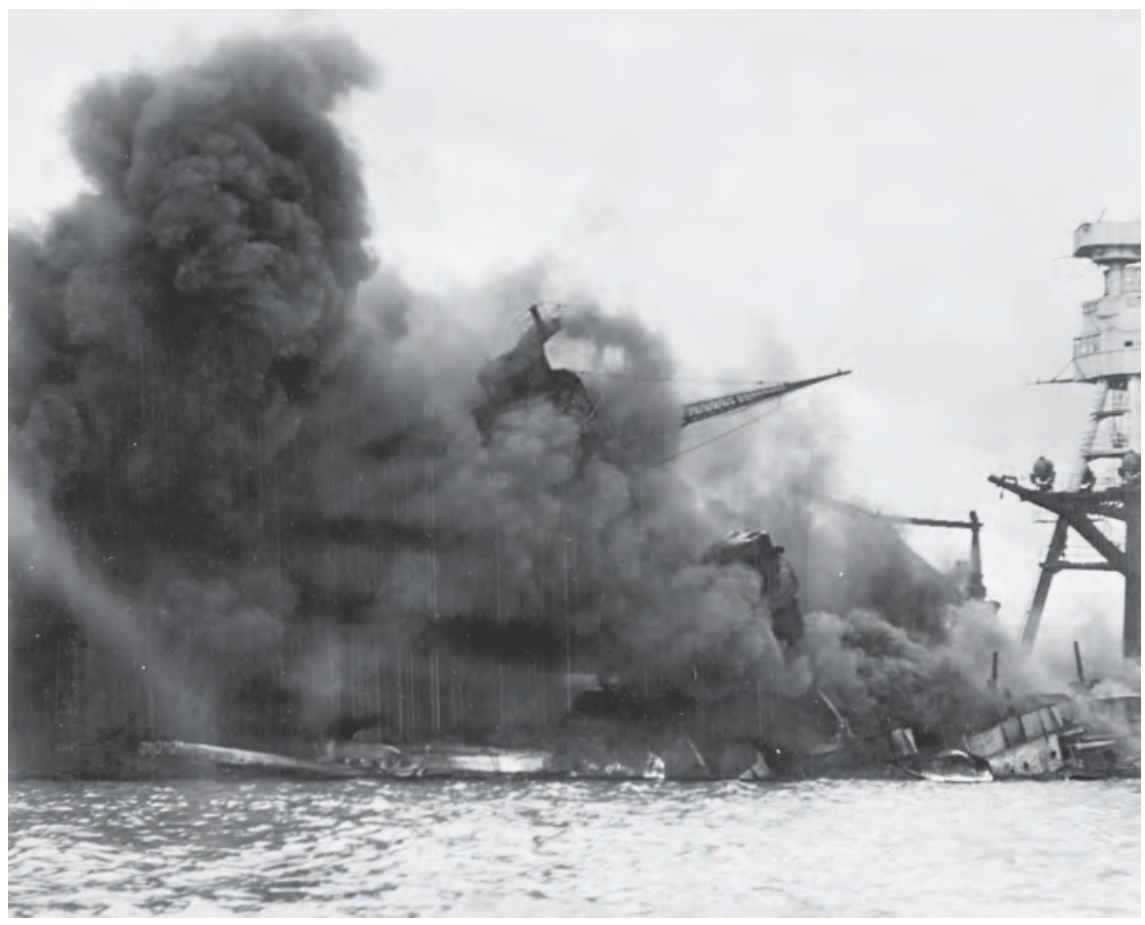

Illustration 28.8

Attack on the USS Arizona at Pearl Harbor 
state in Latin America to send troops to Europe during the war: Some 25,000 would serve in Italy in 1944-5.

Japan had many successes after Pearl Harbor in Southeast Asia, defeating American troops in the Philippines, and British forces in Singapore, Malaya, and Burma. These various conquests together took only a matter of months. They gave the Japanese access to Indonesian oil and Malaysian aluminum and rubber (though they had traded for such goods with the United States before attacking it). The Japanese even took some Aleutian islands in Alaska, the only conquest of domestic territory suffered by the United States in either war. The Japanese appealed to a sense of pan-Asian nationalism in the lands they conquered but soon lost local sympathy through brutal repression. Still, they recruited 100,000 Indian prisoners of war into the Japanese army.

We noted above how Germany and Japan devoted much of their economic output to preparation for war. The Soviet Union would be able to match German expenditures on military goods. The United States, with a much larger economy, could outspend everybody. The United States would spend three times as much as Germany and eight times as much as Japan on weapons, and had two and

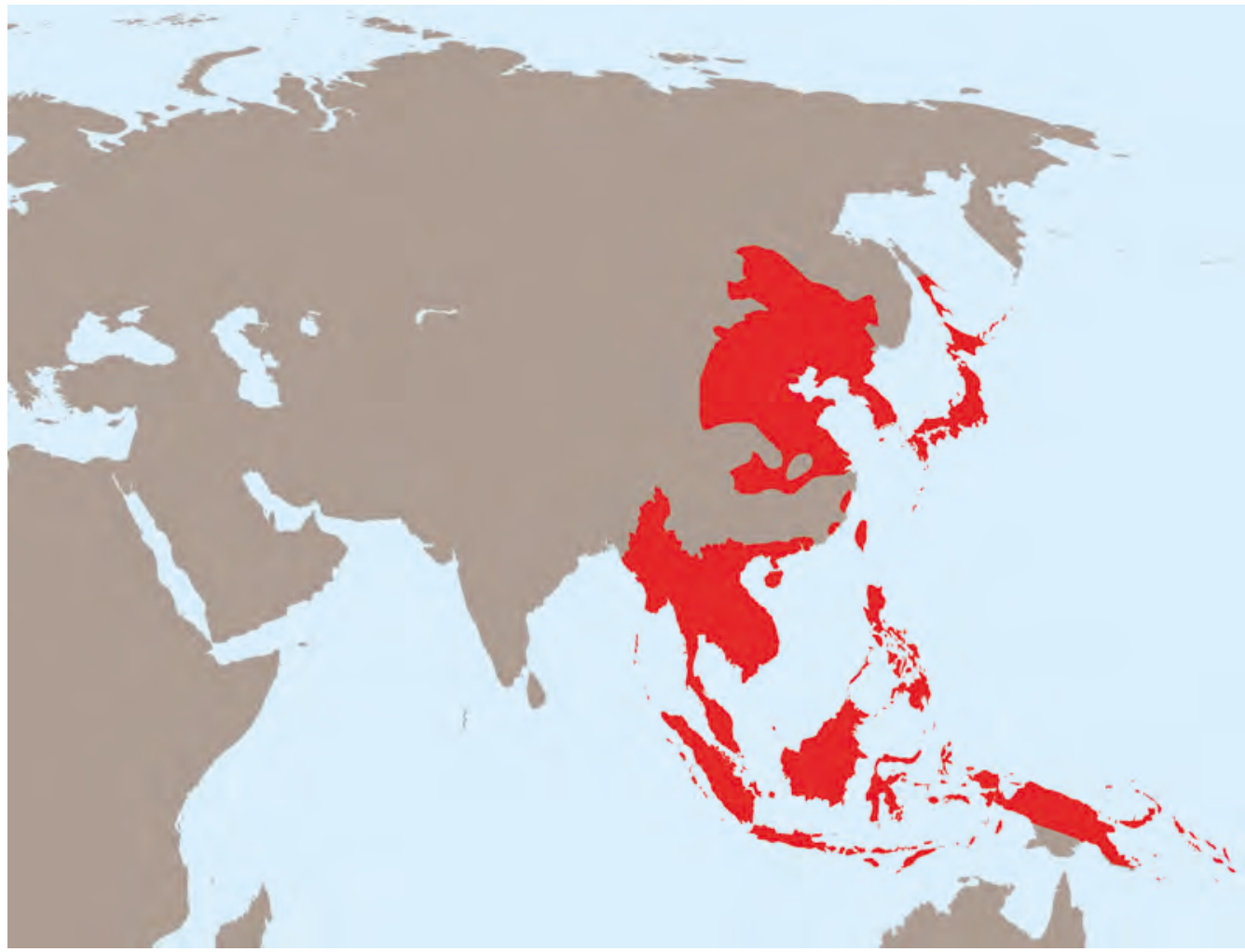

Map 28.3

The Japanese Empire in 1942

Map by Mackay, derivative work by TastyCakes, CC BY-SA 3.0 
a half times the number of planes as Germany and three times those of Japan by 1944. This massive air advantage aided in both the bombing of cities and in strafing soldiers on the ground before and during battles. Moreover, the Allies could draw on a much larger number of engineers and scientists, and as a result generally had the better technology: Though both sides developed radar during the war, the Allied technology was far superior.

We have often noted in earlier chapters the importance of plunder in war-making. Though Germany could not match the industrial might of the United States, it was able to extract considerable resources from the lands it conquered. In western countries with sophisticated institutions such as France and Belgium, the Germans may have obtained some half of economic output through systems of direct and indirect taxation and forced labour. In some of the lands conquered to the east, Germany relied instead on old-fashioned looting alongside the use of forced labour.

The Germans and Japanese (and to a far lesser extent Italy) had to rule vast territories for years. As empires had for centuries, they often tried to co-opt local elites. Local elites and businesses justified their collaboration as offering local control that benefited local populations. They would often be decried after the war for aiding the enemy. For the Germans and Japanese, local authority limited the resources that the victors had to devote to administration. In areas of particular strategic importance, Germany and Japan instead exercised direct military control. Slave labour was a common practice in such territories. The Germans in France installed the Vichy regime in the southern third of the country under General Philippe Pétain, a French hero of the First World War, but they maintained direct control of the French coastline.

There were resistance efforts that committed sabotage or espionage in most conquered territories. Those caught resisting were often tortured and killed in particularly nasty ways. The resistance effort with the greatest long-term effect was that of the communist Tito in Yugoslavia. He would amass 250,000 guerrilla fighters and would receive aid from the Allies in fighting German troops in the mountainous Yugoslav terrain. He would form the postwar government in Yugoslavia, but pursue independent economic and foreign policies from other communist nations.

Germany would suffer 6 million casualties as the Soviets pushed them back. The eastern front was for years by far the most important theatre of the European war: This is where the vast bulk of German troops were stationed and where by far the highest losses were incurred. Losses were even heavier on the Soviet side, but the Soviet Union had a much larger population. Eventually, though, Allied troops would force Germany to fight on other fronts as well. The Allies pushed Germany out of Africa (where they had earlier replaced Italian troops) and then forced Italy to surrender and switch sides in 1944. They then fought German troops for control of the Italian peninsula for a year. However, they could not attack Germany itself through the Alps. On June 6, 1944, Allied troops landed successfully in Normandy on the north coast of France in what is 
still by far the most massive troop landing in world history. Some 4,000 ships, 10,000 airplanes, and 130,000 soldiers were involved on the first day, and a million troops landed within the first month. Troops reached Paris by August and entered Germany in September. Germany surrendered on May 8, 1945, with Russian troops already in Berlin.

Only slowly in the postwar years did British wartime success in cracking German codes become widely known. The British had employed thousands at Bletchley Park to decipher German messages. It managed during the war to keep Germany from appreciating that it could read German messages: It acted only occasionally on the information received in order not to arouse German suspicions - often allowing its own soldiers to be attacked as a result. After the war, the thousands of cryptographers were sworn to secrecy. Only as wartime documents were declassified decades later did the truth come out. Of particular note, it was a half-century after the end of the war before it was recognized that the British had effectively created the world's first computer - called Colossus during the war: That accolade had long been ascribed to the American ENIAC of 1946. Some analysts suspect these efforts may have shortened the war by years. In particular, detailed knowledge of German defences and plans aided the Normandy landings.

The war in the Pacific had a turning point in the battle over Midway in 1942, where the Allies learned of Japanese plans by cracking their code. They pushed the Japanese out of islands in the Pacific and Philippines before attacking Okinawa and Iwo Jima from which Japan itself could be bombed. It took three months to take the island of Okinawa as the Japanese had developed a network of tunnels from which they could unleash machine gun fire. American ships and submarines also destroyed Japan's ability to import supplies from Southeast Asia. The Japanese introduced kamikaze pilots who crashed their planes into enemy ships (though this was an expensive strategy) and ordered their troops to fight to the death. Tokyo was firebombed in March 1945 with 100,000 deaths and the destruction of a quarter of its buildings. Then on August 6 and 9, American planes dropped atomic bombs on Hiroshima and Nagasaki. Of 245,000 people in Hiroshima at the time, 75,000 died immediately and 100,000 later while still others suffered genetic defects or cancer. Japan surrendered days later. The Soviet Union declared war against Japan on August 8, invading Manchuria and Korea, and would regain some islands lost to Japan in 1905. Allied troops delivered food to Japan soon after its surrender for the country was facing mass starvation.

Some Pacific islands had had limited contact with outsiders before the war. Such islanders were naturally amazed when thousands of troops and massive quantities of supplies suddenly arrived. Both Japanese and American soldiers shared supplies with locals, in part in trade for local knowledge of food and hiding places. These supplies often included manufactured goods with which locals were unfamiliar. When the troops just as suddenly disappeared at war end, "cargo cults" emerged on many islands in which locals performed rituals intended to draw further supplies of goods to the islands. 


\section{Wartime atrocities}

The German Special Forces killed 1.4 million Jews and Roma (and some Slavs) in occupied territories before 1941. The German leadership then decided to build concentration and death camps in Poland, in which victims were gassed to death. Six million Jews and millions of others - Roma, gays, the disabled, those who resisted - were killed there. Few in any of the countries involved objected, though some heroic individuals hid Jews. Mass killings have occurred often in history. Yet as we saw in chapter 17, these often served a strategic role in encouraging submission by other groups. The Nazi slaughter of groups they disdained was instead deliberately secretive. Western allies and the Catholic Church both knew what was going on: The pope considered condemning the atrocity but feared that he would only encourage worse behaviour. The Allies considered leaflet drops and sabotage of rail lines to the gas chambers and the death camps themselves but in the end did little. There is debate as to whether such actions would have either discouraged the Nazis or induced public objection (35 million Germans, including Hitler, were Catholic); they might also have encouraged Jews and others to flee or fight.

In any case, the Holocaust was something qualitatively different in history though feelings of hatred or disdain had undoubtedly guided mass killings in the past. If so, this new horror can in part be attributed to extreme nationalism that provided a much more powerful rationale for killing others. Nor should we neglect the role of technology: Railways, machine guns, and poison gas dramatically reduced the cost of mass murder. Earlier regimes simply could

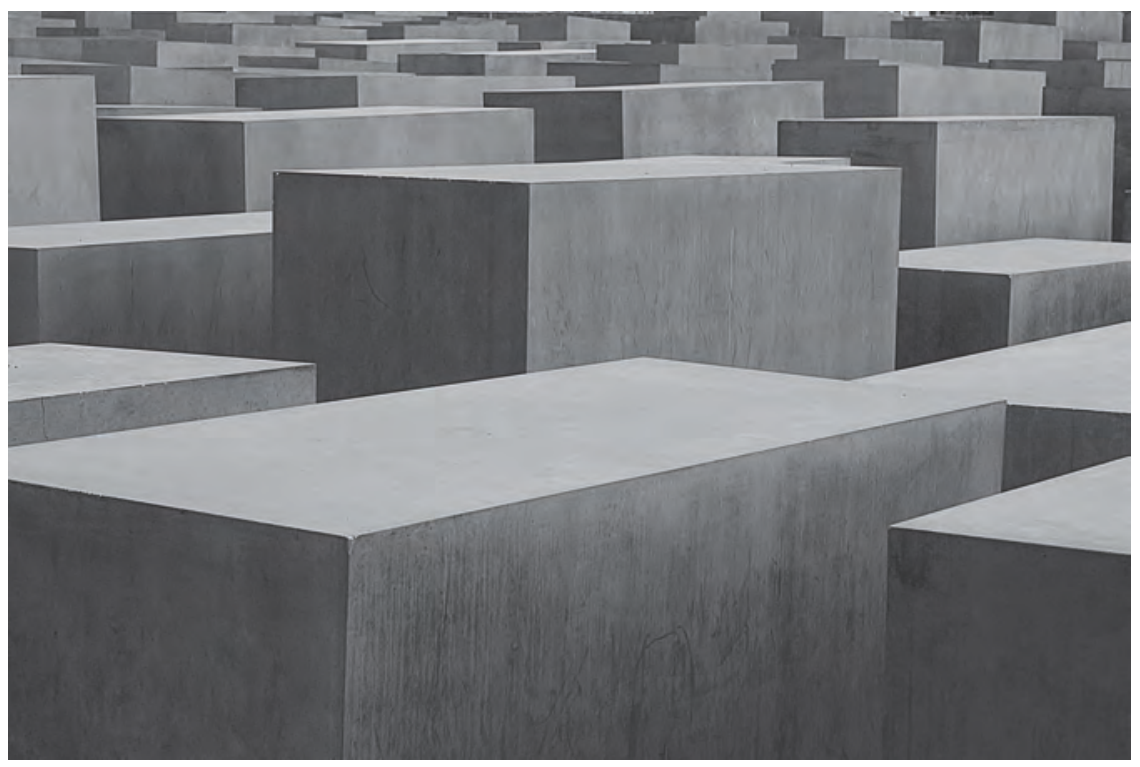

Illustration 28.9

Berlin Holocaust Memorial 
not murder millions so quickly. One constant in history, though, was human psychology: Rulers could often justify mass murder and soldiers would willingly follow orders to do so. Soldiers in many but far from all countries now receive training to ignore orders that violate the Geneva Conventions.

Rape and kidnapping of women have characterized war from the beginning. This was institutionalized on a grand scale during the Second World War. Japanese troops forced some 300,000 women into brothels. The Japanese have at times claimed that these women volunteered - though millions of Japanese soldiers would clearly have overwhelmed existing supplies of prostitutes - and only officially recognized some limited culpability in 2016 (though there had been some unofficial and private payments to victims beforehand). Eighty per cent of these women were from Korea - though there were large numbers from other Asian countries, as well as the Dutch population in Indonesia. This forced prostitution was a reaction to the mass rapes in Nanjing, which had embarrassed Japanese military leaders, but it resulted in another, though hidden, atrocity. The Japanese often killed these women if they caught sexually transmitted diseases or tried to escape. Such women were often shunned after the war in their home countries. This meant that widespread knowledge of their plight occurred only decades later with a Korean report of 1990. Even then, the phrase "comfort women" that has been used to describe them fails to capture the nature of mass forced prostitution/rape.

\section{Allied bombing}

Debate rages decades later about the ethics and strategic value of Allied bombing campaigns in both Germany and Japan. The German and Italian and Japanese bombing that preceded this is more generally disdained since the motives of the perpetrating governments themselves are now generally renounced. The bombers of the time had far less accuracy than the guided missiles of the early twenty-first century - which still often miss targets and cause massive civilian damage. Although bombers might target military installations, the more common outcome of bombing raids was the mass destruction of urban areas. The Allies largely destroyed German cities such as Hamburg as they advanced to the east. Allied bombers reduced Dresden to rubble in 1943, with some 50,000 civilian deaths. A similar story unfolded in Japan. Indeed, as we saw above, it was bombing rather than advancing ground troops that resulted in the surrender of Japan itself (though that bombing was only possible once nearby islands were taken at high cost). These urban bombing raids were intended to sow discord by inflicting massive civilian damage.

As for the atomic bombs, it is now clear that these were not ready while the war was still ongoing in Europe. The fact that they were used only against the Japanese need not then reflect racist motives. Nevertheless, reports of Japanese brutality against both civilians and Allied prisoners encouraged leaders to consider the nuclear option. These leaders also argued that even greater death totals would have occurred if Allied troops had been forced to take Japan. This is 


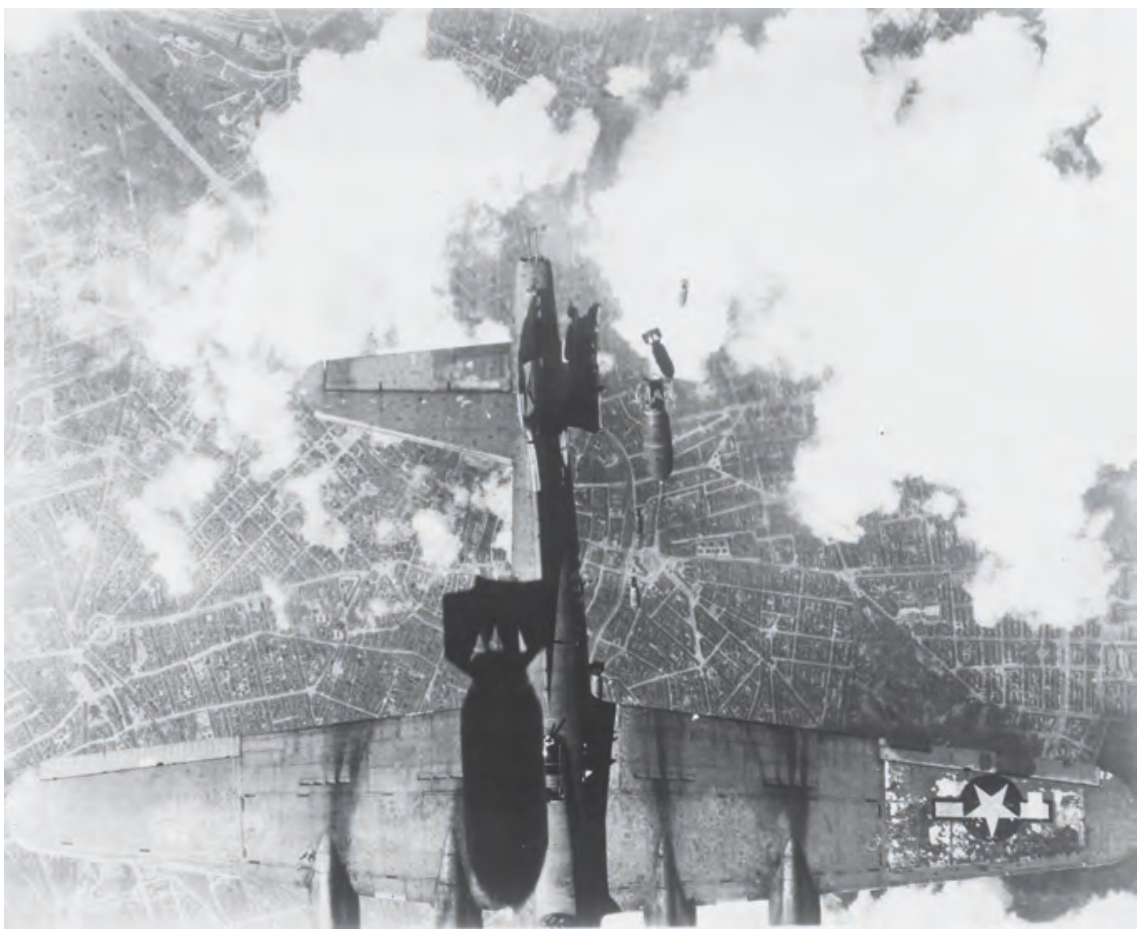

Illustration 28.10

Bombing of Berlin by the US Air Forces c. 1942, US National Archives and Records Administration

almost certainly true. However, there is a question about whether the Japanese were already preparing to surrender. One sticking point for the Japanese was a desire to maintain the millennia-long imperial dynasty. The Allies saw the emperor as a source of authoritarian rule and wanted abdication. In the end, they would compromise and allow the emperor to continue in a mostly ceremonial role much like the British royal house. It is not clear if such a compromise might have been possible without the atomic bombs (or at least one of them). Some have suggested that American leaders wanted to justify the two billion dollars spent on developing the atomic bomb. (This research would also lead to nuclear energy, though this has proven a controversial but significant source of power in the postwar world.)

It has also become clear - from government documents declassified decades later - that the Allies were motivated at least in part by a desire to frighten the Soviets - and to end the war before the Soviets could make substantial territorial gains in the east. As the war ended, western Allies recognized that their wartime ally would likely be a postwar adversary: Its economic and political systems were anathema to them (and vice versa). If this were the real motive for the atomic bombs, it failed: The Soviet Union forced communist governance onto the areas of Eastern Europe it occupied (despite having promised Western 


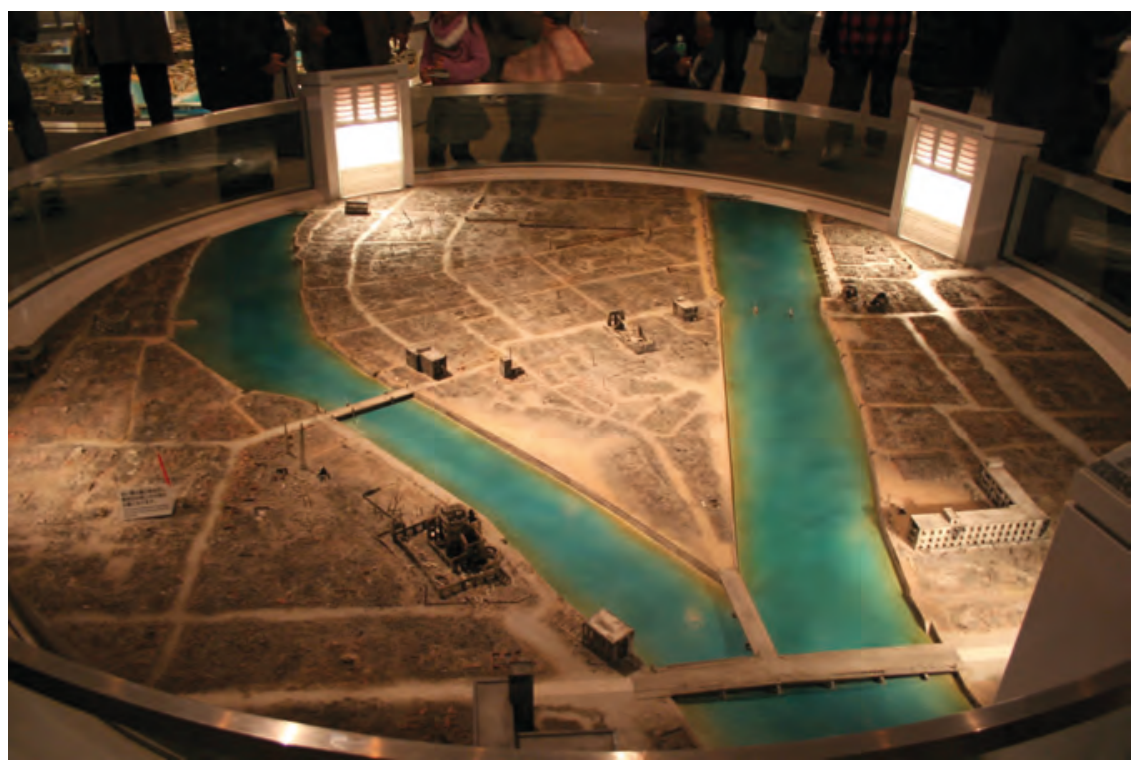

Illustration 28.11

Hiroshima after the atomic bomb. Model in the Hiroshima Peace Memorial Museum Photograph by Derek Springer, CC BY-SA 2.0

leaders that it would allow self-determination) and developed its own atomic capability within four years.

\section{The impacts of war}

The death toll from the Second World War was much higher than that of the First World War. A standard figure is 60 million in total, but this number can rise toward 100 million if the earlier Japanese conquests in China are included (some 24 million lives were lost between 1931 and 1945 in China) as well as deaths from disease during and right after the war. Even 60 million would represent about 3 per cent of the world population at the time. Over 20 million were soldiers - and millions of these died as prisoners of war. The Soviet Union (20 million) and China (at least 15 million) suffered the highest overall casualties.

A brief recap of the Second World War cannot possibly capture what it was like to live through it. As with any story, it is ruined a bit if you know the ending. Much of the world was gripped for years with a fear of their side losing. It was clear that defeat would be costly: The Germans, Italians, and Japanese governed conquered territories efficiently and brutally. The Soviet Union would be brutally repressive also in the lands they came to control. Civilians on both sides in all theatres of war had to worry about aerial bombardment. Mass conscription meant that few families anywhere were untouched by the war. Only eleven countries in the entire world managed to avoid direct involvement in 
the war: Sweden, Switzerland, Portugal, Spain, Iceland, Ireland, Greenland, Afghanistan, Mongolia, Yemen, and Tibet. Even these suffered as war raged nearby and disrupted trading networks - though they might also benefit by exporting food and war materials to belligerents.

A brief recap necessarily also elides over personal stories. Some generals made good decisions: Even westerners came to admire the strategies of German troops in North Africa. The Allied landings in Normandy were well planned and executed. The later decision to accelerate the conquest of Germany by landing paratroopers in the Netherlands failed. Such a massive war naturally meant thousands of acts of heroism: saving colleagues on the battlefield, rescuing civilians, sheltering Jews. It also provided opportunities for all of humanity's lesser impulses. Prisoners were tortured, undernourished, enslaved, and killed. Soldiers raped millions of women. They also looted businesses, homes, and museums.

After the war, millions of Germans, Poles, Japanese, and others returned to devastated lands. Germans (some 10 million) and Japanese retreated from the lands they had conquered which were now parts of other states. Poles moved as the borders of Poland shifted westward. The Soviet Union also moved people they distrusted from the Baltics and Central Asia to Siberia, replacing these with Russians and Ukrainians. Hundreds of thousands of Jews migrated to Palestine. Various other ethnicities fled the enlarged Soviet Union or responded to border changes.

American forces occupied Japan until 1952. The United States wanted to punish war criminals, invigorate democracy, restore the economy, and gain an ally against the Soviet Union (and later communist China). Twenty-five Japanese officials were tried, seven executed, and 200,000 banned from office. The occupation terminated state support of the Shinto religious establishment. It demobilized the Japanese army. Japan then became an unusual sort of ally, allowing American bases on Japanese islands (and serving as a supply base during the Korean War; see below) but not contributing its own troops to foreign wars for decades. Universal suffrage was established in 1947, with women gaining the vote. An independent judiciary was also introduced, and human rights guarantees instituted. Land was redistributed. The Zaibatsu - large conglomerates that had dominated the economy and were seen as antithetical to democracy - were disbanded, though less powerful Keiretsu conglomerates would replace these. Unions and strikes were legalized but then controlled as some unions displayed communist sympathies. Education expanded and employed new textbooks with a less nationalistic tone - by the end of the century, almost all Japanese children finished high school and a third attended university. In sum, the allies not only re-established democracy itself but also encouraged the constitutional protections, economic relationships (that is, not extreme inequalities), and perhaps cultural attitudes that support democracy elsewhere. Though Japanese democracy has often been corrupt, and was long dominated by one party, the country has avoided authoritarianism in succeeding decades. Its economy would grow rapidly for most of the rest of 
the twentieth century (by 10 per cent per year on average in the 1950 s and 1960s), and it became a technological leader in areas such as automobiles and consumer electronics. The Japanese government has not fully acknowledged its wartime guilt as German governments have.

The war destroyed a quarter of German houses and half of its infrastructure. Germany would be divided into four zones for occupation by Soviet, British, French, and American troops (The Soviets had balked at French participation, and so the French zone was taken from the British and American portions). The capital, Berlin, though deep within the Soviet zone, was also divided into four zones. The Nuremberg war crimes trials had an international panel of jurists; they tried twenty-two German leaders, executed twelve, and sentenced seven. These trials were controversial but are generally hailed for establishing legal duties in war. They, in turn, drew inspiration from the Geneva Conventions and other international agreements that had in previous decades tried to create rules for behaviour during wartime. It was noted at

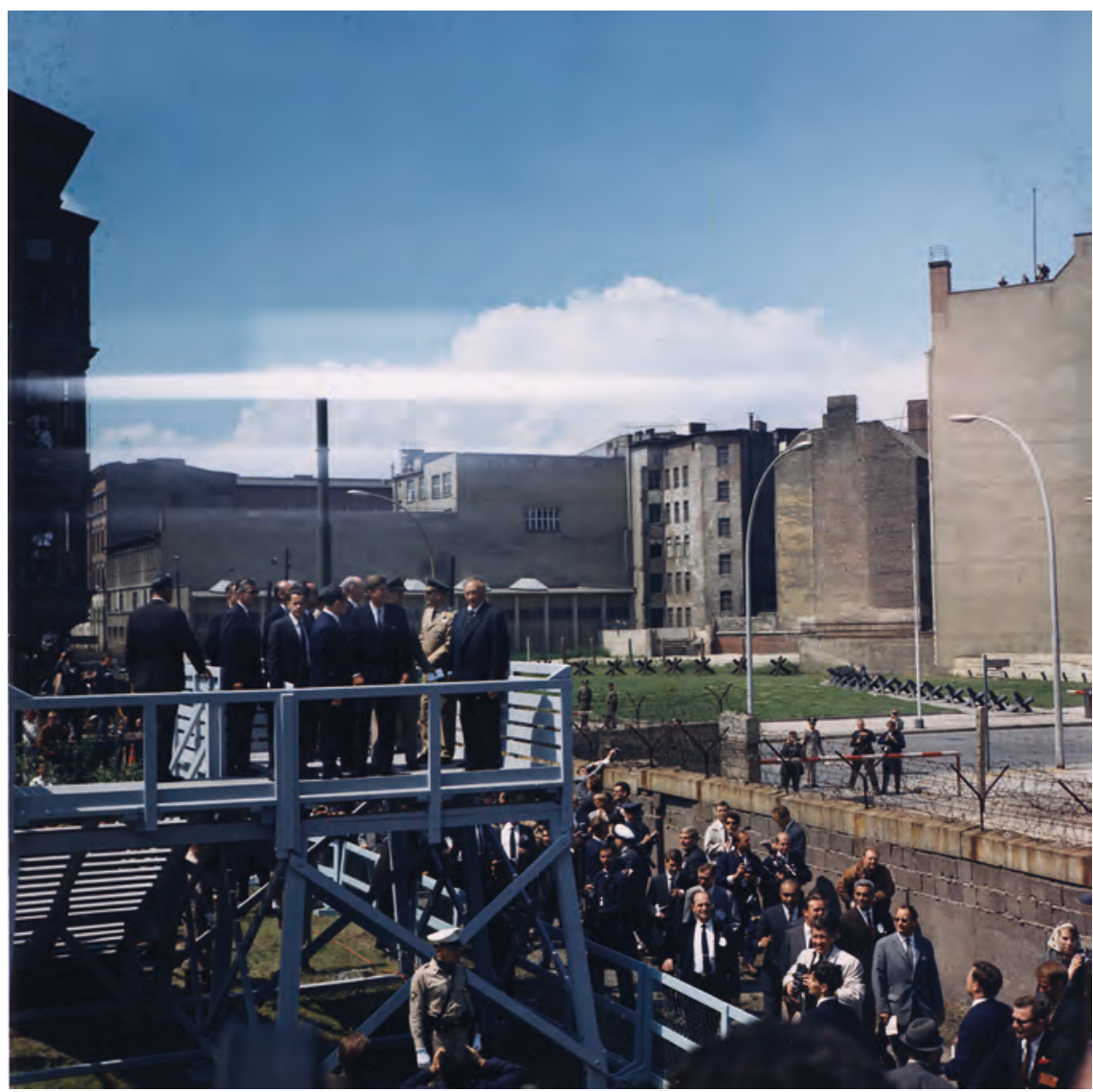

Illustration 28.12

“US President Kennedy Looks over the Berlin Wall, 1963," photo by Robert L. Knudsen 
the time, and has generally been the case since, that only those on the losing side are prosecuted. This may have the unfortunate side effect of encouraging the guilty to cling to power to avoid prosecution. The Soviets would install a communist regime in its eastern third of the country. As in Japan, the Western powers would encourage a set of institutions that supported democracy and market economies in the western zone. The Soviets sought reparations whereas Western powers were primarily concerned with reinvigorating the German economy. The Soviets would in 1948 refuse to allow land transport between the west and the western sectors of Berlin (in response to Western powers having merged their three zones economically). Western air forces responded with a massive airlift of supplies: Planes landed every few minutes at a small urban airport for eleven months until the Soviets relented in 1949 and allowed land traffic to resume. In the next decades, West Berlin would be a magnet for some 3.5 million Germans and others who disliked life under communist rule; the East German government would build a wall around it in 1961 to limit

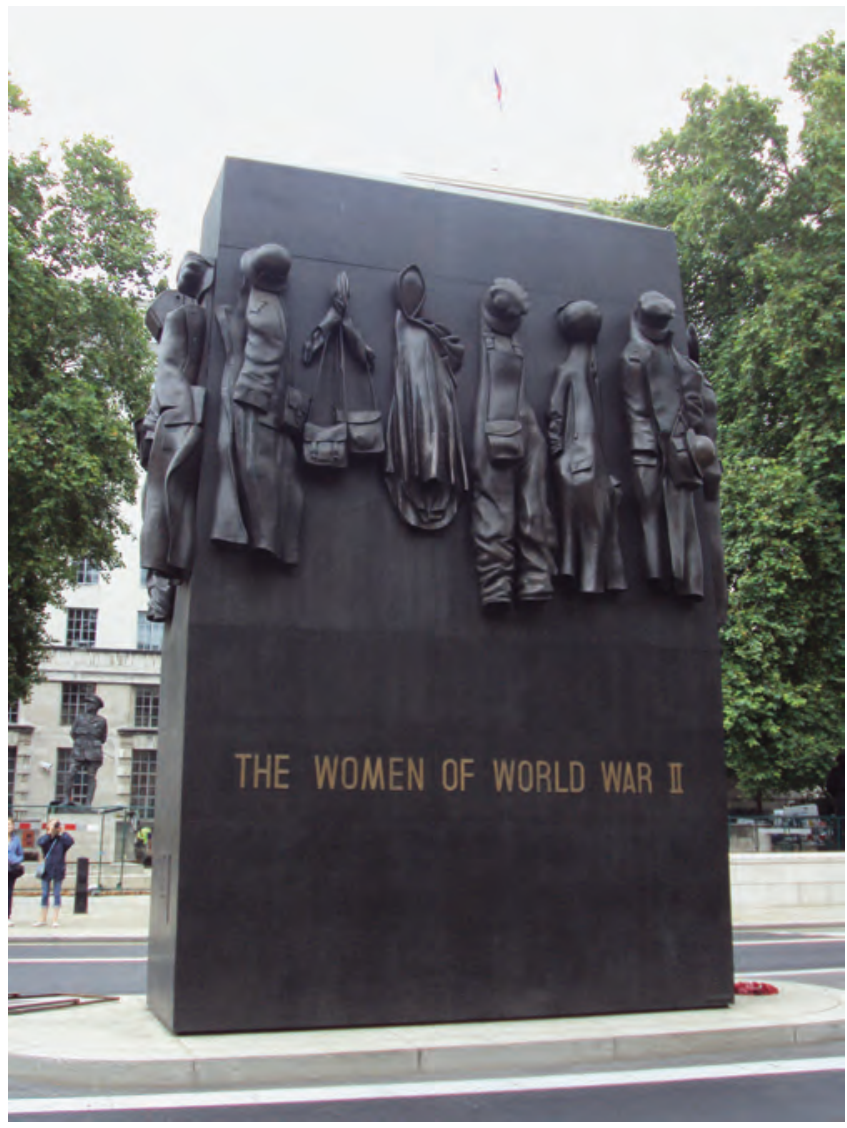

Illustration 28.13

Women of World War II Monument, London Rept0nlx, CC BY-SA 3.0 
migration. The destruction of that wall in 1989 was an important symbol of the end of communism in Eastern Europe.

We noted above that women's work during the First War aided suffrage movements after that. The even greater female effort in the Second War further challenged ideas of gender roles in many countries. In addition to extensive industrial labour, Chinese communists (but not nationalists), and to a lesser extent the Soviets, British, and Americans found supporting military roles for women during the war. However, the Axis powers all resisted female participation: This may be further evidence of how gender stratification often interacts with other types of stratification (fascist ideology tended to stress martial men and homebound women). Many women continued in "male" jobs after the war in Allied nations. The fact that women could obviously perform "male" jobs likely encouraged writers such as Simone de Beauvoir and Betty Friedan in the early postwar decades to write of cultural stereotypes and discrimination: Culture and institutions rather than physiology decreed women's roles.

\section{The Cold War}

The second "war to end all wars" segued all-too-smoothly into what has come to be known as the Cold War. Wartime collaboration between Western democracies and the Soviet Union had been pragmatic: The West was at war with Axis nations, and the Soviet Union bore the brunt of that war for years. The Soviets had at first aligned with Nazi Germany in the war, building on a shared distaste of Western democracies, and only aligned with those democracies after Germany had invaded the Soviet Union. As the war turned, some German officials tried to reach out to the West for a pact against the Soviets. Western powers instead sought to limit communist absorption of Eastern Europe by agreement with the Soviet Union: The Soviets would violate this agreement and install communist regimes by 1948 in the lands they occupied. The Soviets were guided in part by the memory of efforts by fourteen Western nations to overturn the Russian Revolution in 1917. At war's end, the former Allies disagreed about how to proceed in Germany, Eastern Europe, China, and Korea. As we shall see in chapter 30, the Soviet Union and the United States would also disagree with Britain and France about the future of colonial empires, and the difficult path to statehood of newly independent nations would be complicated by Cold War efforts to draw these into alignment with either communism or anti-communism. (Yet it would be a mistake to view decolonization entirely through the lens of the Cold War; we therefore treat them separately.) The United States, Canada, and many Western European countries would form the North Atlantic Treaty Organization (NATO) in 1949, promising mutual defence in the event of foreign attack. The Soviet Union and Eastern European countries would form the Warsaw Pact alliance in 1955.

Despite disagreements that spanned the globe, armies of Western democracies would only directly confront troops from the Soviet Union or communist 


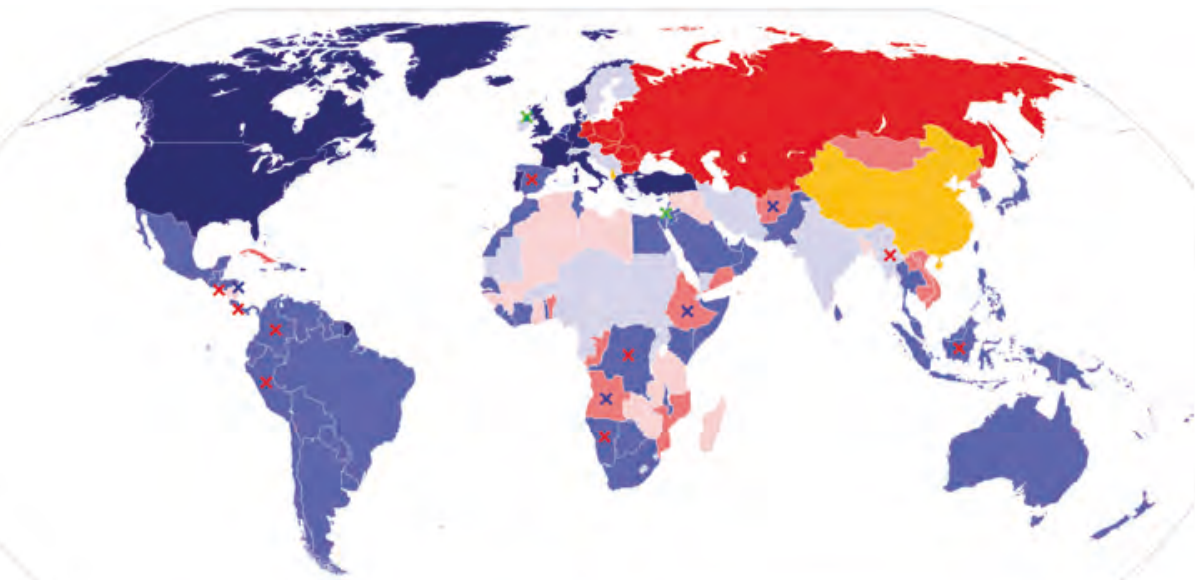

Map 28.4

The Cold War as of 1980

NATO and allies are in blue; the Warsaw Pact and allies are in red. Conflicts are marked by an $\mathrm{x}$ Map by Chabacano, based on Sancebau, CC BY-SA 3.0

China once, in the Korean War that soon followed the Second World War (see below). Other conflicts would be fought indirectly by financing and advising the armies of poorer countries. There are two compelling reasons for the "coldness" of the Cold War. First was widespread recognition of the tremendous costs of modern warfare. The Soviet Union would not soon forget 20 million deaths and massive wartime destruction. Citizens of Western democracies likewise wished to avoid another such calamity. Both sides could easily countenance distant wars mostly fought by others, but had good reason to steer away from all-out confrontation. Second, and connected to the first, was the development of atomic weapons. This gave both sides a genuinely terrifying offensive capability. Despite ongoing efforts to develop appropriate defences, both sides could assume that they would suffer massive damage in any nuclear conflict. Indeed, such a conflict could easily destroy life as we know it.

The two essential characteristics of the Cold War, then, were proxy wars in poorer countries and a massive arms buildup throughout the world. Almost a trillion dollars were spent annually on the military worldwide by the 1980s. The Soviet Union and the United States (and to a lesser extent Britain and France, later joined by a handful of other countries) developed nuclear arsenals that could destroy the world many times over. They also encouraged and financed military expenditures in other countries. The creation of dozens of new countries (chapter 30) might always have been associated with increased military spending as new countries tended to think a national military an essential emblem of nationhood, but Cold War conflicts encouraged and allowed 
(through foreign military aid) many countries to militarize to a much greater extent.

The arms race had a spillover effect on space technology: Rockets could blast straight up as well as sideways, and the global battle for prestige and leadership could take place in deep space as well as on the ground. The Soviet Union scored first, with the first earth orbit in 1957 and first human in space in 1961. The United States followed quickly, pledged to reach the moon by the end of the 1960s, and did.

There was also an important spillover concerning computerization. Universities and private firms lobbied successfully for the development of "early warning" systems that might detect and destroy incoming missiles. Such initiatives were politically popular (especially given early overestimates of Soviet strike capability), but many scientists doubted that it would ever prove possible to destroy all, or even most, incoming missiles. In the West, these systems depended on far more sophisticated computerization than their counterparts in the Soviet Union. IBM (International Business Machines) in particular benefited from large government contracts for computer development. Scientists enjoyed a burst of prestige during the early Cold War, though this would dissipate as science proved unable to solve the nuclear threat, and as expert advice regarding the war in Vietnam (below) was disparaged. Given the inability to defend against a nuclear attack, many officials recommended a pre-emptive first strike on the enemy, but neither Western nor Soviet leaders followed this advice.

As with the Second World War, the drama of the Cold War is diminished because we know how it unfolded - though tensions remain between the West and both Russia and China. There has not been a nuclear attack since Hiroshima and Nagasaki - but the millions of people guided through nuclear attack drills and encouraged to build atomic fallout shelters in the 1950s and 1960s could not know that. When the United States employed a naval blockade to prevent the Soviets from installing nuclear missiles in Cuba in 1962, the world held its breath - but in the end, an agreement was reached in which the Soviets did not arm Cuba and the Americans withdrew missiles from Turkey and pledged not to invade Cuba. Understandable concerns regarding placement of nuclear weapons in nearby countries would fade with the development of both long-range missiles and nuclear submarines: No country is today immune from foreign nuclear attack.

Fear of nuclear obliteration was not the only way in which the Cold War affected individual lives in the developed world. The development of an atomic bomb by the Soviets in 1949 caused many to suspect espionage, as did their development of a hydrogen (fusion) bomb in 1953 just one year after the American development in 1952. There was indeed some spying, though its extent was much less than was feared at the time; the Soviets had a substantial scientific and engineering capacity, and the example set by the United States guided Soviet research efforts. The fact that each side was engaged in a worldwide game of secret diplomacy, involving efforts to support or undermine 


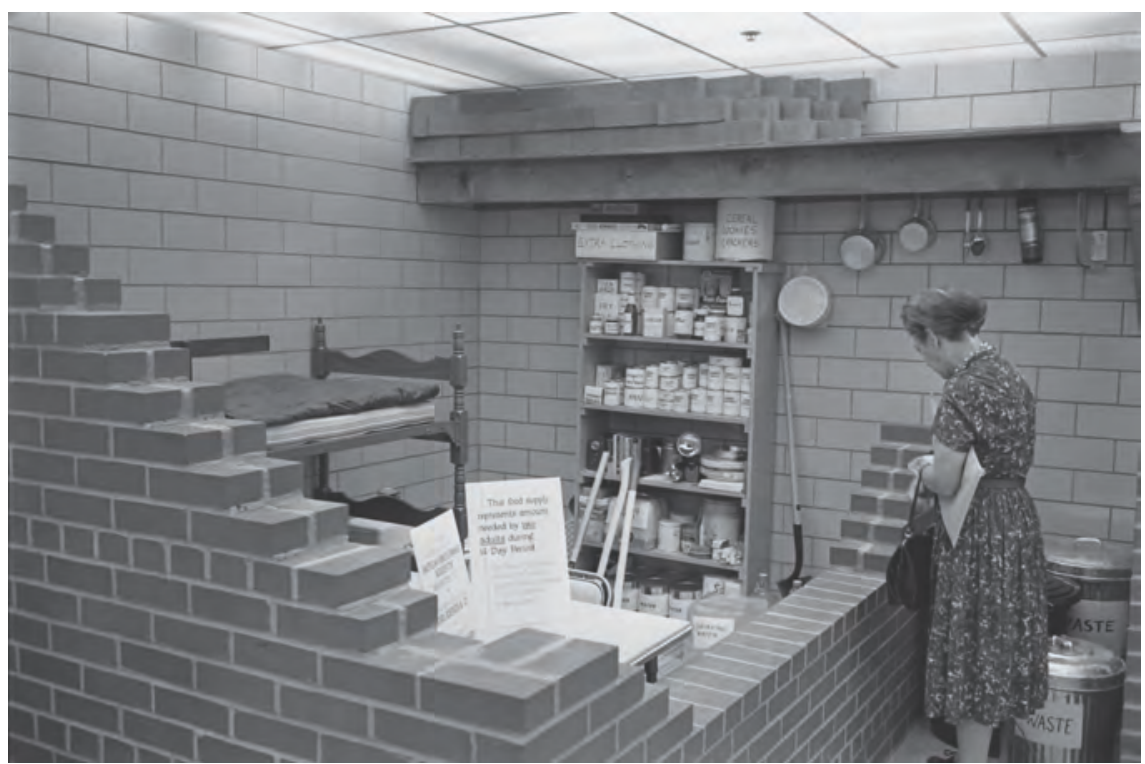

Illustration 28.14

Woman in bomb shelter, 1961. Note canned foods and bunk bed Library of Congress Prints and Photographs Division

foreign governments, provided a further cause for suspicion: The Soviet Union certainly wanted to know what initiatives Western countries were pursuing elsewhere (and vice versa). The Soviet Union and China would develop secret police forces that imprisoned or tortured those suspected of seeking to undermine the communist state in any way. Western democracies were far less draconian in their approach to those suspected of communist sympathies. Nevertheless, in the 1950s, thousands of individuals came under suspicion in the United States and were fired from government jobs or blacklisted from employment in the entertainment sector or universities. Such individuals had limited ability to defend themselves: Having suspicious friends or having ever been members of dubious organizations was evidence enough. False accusations were common, and there was fear and recrimination among many progressives with no communist sympathies. Though this anti-communist "witch hunt" is now generally regarded as excessive and misguided, questions as to how a democratic society should best balance democratic freedoms with defence against an aggressive and undemocratic foe continue to the present day.

Eastern Europeans occasionally challenged Soviet influence. The Soviets repressed agitation for democracy in East Germany in 1953. Soviets troops invaded Hungary in 1956 when the Hungarian army joined massive street protests urging democracy and independence from the Soviet Union, and the Hungarian leader Imre Nagy announced withdrawal from the Warsaw Pact. A dozen years later in 1968 Soviet troops invaded Czechoslovakia when its government led by Alexander Dubček promised "socialism with a human 
face," including decentralization, more press freedom, freedom of speech, and freedom to travel. In both cases, Western nations protested but refrained from providing concrete support. The fiction that Eastern European governments were independent of Soviet control was set aside.

Though Western nations quietly accepted the Soviet domination of the countries absorbed right after the Second World War, they reacted more actively when the Soviet Union or China attempted to expand communist rule into new areas. They worried that new states were like "dominoes": every new state that turned communist would encourage neighbouring states to "fall" also. In Korea in the 1950s, Vietnam in the 1960s and 1970s, and Afghanistan in the 1980s, Western countries would actively support non-communist forces.

Despite these conflicts, the United States and the Soviet Union managed from the 1970s to reach agreements on arms limitation. Though they each retained arsenals capable of destroying the earth many times over, these agreements did cause the destruction of thousands of weapons, while many more were never built. Such agreements reflected the recognition both that these weapons were hugely expensive and that there was a non-trivial possibility that they might be employed by accident. The sudden dissolution of the Soviet Union after 1989 raised a new fear: that some of these weapons might fall into the hands either of other authoritarian regimes or of terrorist groups. Western nations reached

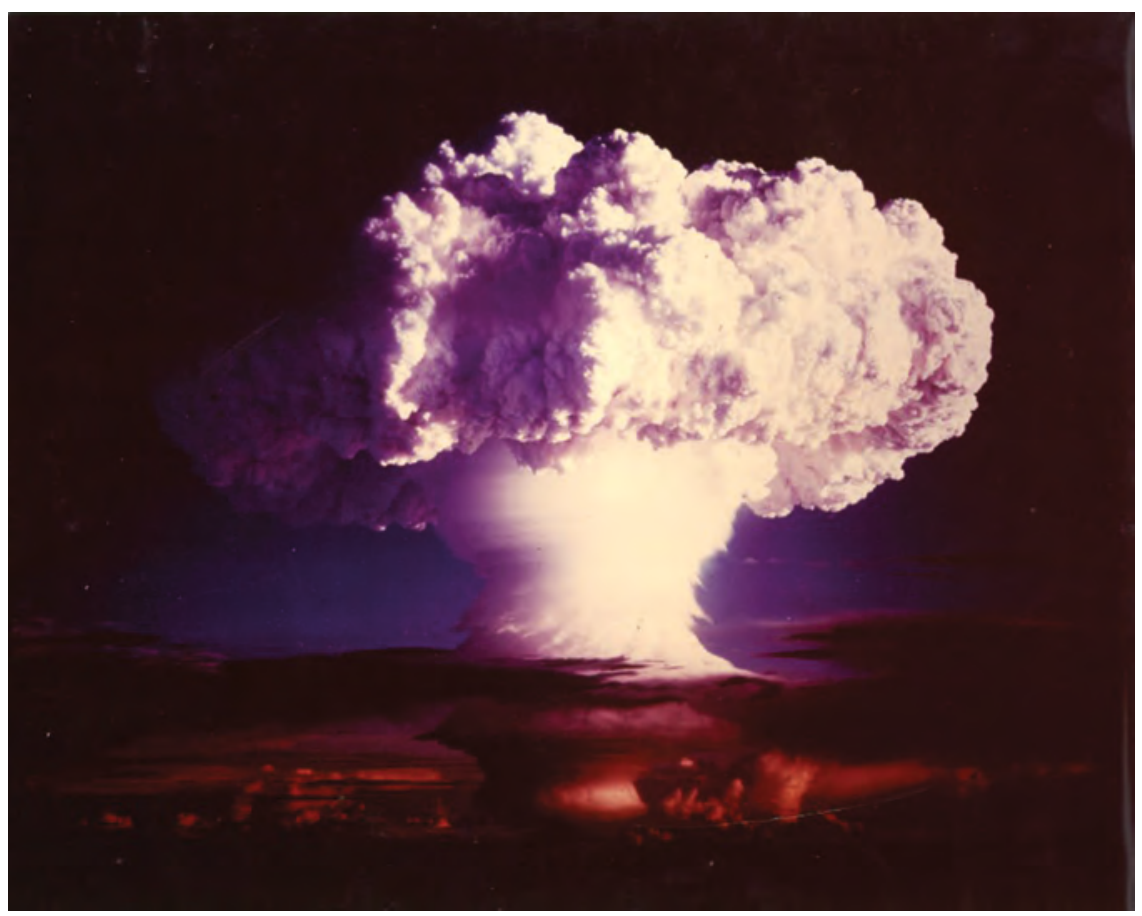

Illustration 28.15

The first test of a hydrogen (fusion) bomb, on the island of Elugelab in Enewetak Atoll, in the Pacific Ocean 
an agreement with the other former members of the Soviet Union to allow Russia alone to maintain a nuclear capability - Russia promised at the time never to employ military force against Ukraine - and Western funds were used to transport or destroy weapons in these other countries. Fears have remained that Russia itself may not guard its arsenal as well as it should.

Immense public pressure had forced the Soviet Union and the United States to reach a nuclear test ban treaty in 1963, in recognition of the detrimental effect of atomic radiation from atomic tests. In the previous decades, atomic bombs had been exploded on land and at sea (the United States and France both used Pacific islands as test sites) as part of military experimentation; Soldiers were occasionally stationed nearby precisely to monitor the health effects.

The Irish foreign minister advanced the idea of nuclear non-proliferation in 1958: The hope was that nuclear capability could be limited to a handful of countries. Finland was the first to sign the non-proliferation accord in 1968. Canada was an early adopter and one of the few at the time that could likely have developed a bomb if it wished. By 2002, almost the entire world had signed - but several countries had developed nuclear capability along the way. Signatories agreed to facilitate the peaceful use of nuclear technology in signatory countries. India, Pakistan, and Israel did not sign. North Korea left the treaty in 2003, and it has since claimed nuclear capability. Treaties in 2016 attempted to limit Iran's ability to build a bomb while allowing it to pursue nuclear power. South Africa dismantled its bomb programme in the 1990s after the extension of the franchise to the Black population.

Though our focus here has been on military conflict, we should note that both sides also attempted to exercise "soft power": to win the hearts and minds of the peoples of poorer countries so that they would be attracted to either a Soviet or an American way of life. This activity involved direct propaganda drafting messages that celebrated one side and denigrated others - but also more subtle efforts to expose other lands to the cultures of the Soviet Union or United States (or their allies). The most important of these initiatives historically may be efforts to spread the Russian and English languages. Such efforts had particular appeal in newly independent states that lacked a majority indigenous language. The United States by the 1960s was teaching English directly to a million students annually and reaching many more through the production of televised courses and textbooks (which provided subtle messages about the superiority of American society along with grammar and vocabulary). Such efforts played a significant role in the spread of English as a global language. Such efforts also built on the fact that English had become a standard trading and administrative language through much of Asia and Africa in the nineteenth century due to British trade and colonization.

Cold War adversaries also engaged in a wide range of covert activities. They employed vast networks of spies. They intrigued to encourage military coups. These secret actions were not always in close accord with the ideals that the two sides claimed to support. The United States encouraged the military coup against the democratically elected Salvador Allende in Chile in 1973, an act 
that the American electorate likely would not have endorsed. Even Congress objected to President Ronald Reagan's support of the Contra guerrillas in Nicaragua, financed with clandestine arms sales to Iran. Such activities naturally engendered widespread distrust in other lands of both American and Soviet governments.

The Cold War seemed like it might never end. However, the Soviet Union unravelled with amazing speed in the late twentieth century. Though the Soviet economy had impressed the world in the 1930s, it had for decades failed to impress its citizens. The Soviets could organize massive steel mills but found it harder both to provide a range of consumer goods and to encourage widespread industrial innovation (though it could achieve technological success as in space exploration when it focused resources on this). Economic dissatisfaction combined with an increased disdain for governmental corruption and incompetence. Later leaders had exposed the horrors of Joseph Stalin's leadership during and after the war when the state killed tens of millions and sent millions more to prison camps. Though these later leaders sought to distance themselves from Stalin, knowledge of these atrocities raised questions about the power of the Soviet state.

Economic and political dissatisfaction also grew in the Eastern European states that had fallen under Soviet influence after the Second World War. Citizens there disdained both their local governments and Soviet domination. The "Solidarity" labour union in Poland - the first independent labour union in a Soviet-bloc country - mobilized almost ten million workers to press for economic and political reforms (including the right to strike and freedom of speech) through the 1980s. Though Soviet tanks had brutally repressed movements for change in Hungary in 1956 and Czechoslovakia in 1968, efforts to restrain Solidarity were more limited.

Mikhail Gorbachev became the leader of the Soviet Union in 1988, promising both economic and political reform. He announced that the Soviet Union would no longer interfere in the affairs of Eastern Europe. This announcement encouraged political transformation in Poland and elsewhere toward democratic elections and market economies. Poland held elections in 1989-90 in which Lech Walesa, the Solidarity leader, became President. Elections were held in Czechoslovakia, Hungary, and Bulgaria in 1990. As in Poland, the sudden and generally non-violent transition to democracy in these countries could build on the heroic efforts of dissidents in previous decades to agitate for political change and develop nascent networks of change agents. The Romanian government resisted change but fell to a popular revolt in which the president, Nicolae Ceaușescu, was killed in 1989: He had been addressing a crowd on national television when protestors engulfed the square. The East German government dismantled the Berlin Wall in 1989, and held elections: The new government soon agreed to merge with West Germany.

Soviet citizens were unimpressed by Gorbachev's attempts to reform the Soviet economy and polity. Such changes would take time, and he had unleashed expectations of dramatic change that neighbouring countries had 
quickly realized. The Baltic states of Estonia, Latvia, and Lithuania, which the Soviet Union had absorbed during and after the war, soon also declared independence. When the newly elected Russian Parliament declared independence in 1991, the Soviet Union came to an end.

It seems quaint now, but it was widely felt at the end of the Cold War that the world was entering a period of peace and stability. The Cold War had dominated international relations for decades. It would soon turn out that there were ethnic, religious, and other antagonisms in the world that would quickly replace the Cold War as drivers of hostility and war. This new reality was brought home in the 1990s when Yugoslavia - a country with a milder form of communism and with independence from the Soviet Union, but which was still caught up in the transition of Eastern Europe - was convulsed by a civil war in which various war crimes were committed.

\section{The Hot Cold War}

Though the Soviet Union and the United States avoided direct confrontation, they each supported armed conflicts in many parts of the globe. The death toll in these various conflicts ran well into the millions: 9 million were killed in Korea, Vietnam, and elsewhere in East Asia; 2.5 million died in South Asia, and 3.5 million in Africa and 0.5 million in the Middle East between 1945 and 1983. (Note: We discuss Vietnam in chapter 30.) The death toll would be much higher if we included the earlier decades of civil war in China between communist forces aided by the Soviet Union and nationalist forces aided by the west.

\section{Korea}

Japan had colonized Korea from 1910. As Japan was defeated in the Second World War, Korea was occupied by both American and Soviet troops. As in Europe, they disagreed about how Korea should be governed. They officially split the Korean peninsula into two countries in 1948. In 1950, the North launched a surprise attack with 100,000 troops, capturing Seoul, the capital of the south. This attack was apparently not just a surprise to those attacked but to both the Soviet Union and China, which supported the communist regime in the north. The United Nations (with the Soviets absent, and the noncommunist Taiwanese representing China) sanctioned a response led by the United States but involving several other countries, which soon pushed back and took Pyongyang, the capital of the north. The new Chinese communist government then protested. Receiving no response, they sent 300,000 troops into Korea. Three million died over the next years, mostly civilians, as the war settled around the original border. The Chinese had an advantage in numbers, but they were less well armed. Technologically, the war is noteworthy for the widespread use of helicopters. A ceasefire of 1953 has lasted for two-thirds of a century. At the time, many Western intellectuals predicted that North Korea 
would outperform South Korea economically. However, the South would soon embark on decades of economic growth, and would in time transition from autocracy to democratic rule. China felt that the Soviet Union should have provided more support in the war, and relations between the two states deteriorated as a result.

\section{Cuba}

Fidel Castro took power in Cuba in 1959. His original goals, beyond ousting a corrupt dictator, were unclear, and at least some of his key supporters expected democratic reforms. He promised healthcare, education, land reform, limits on foreign investment, and full employment. He also pledged to hold elections but then declared that he obviously had public support. He at first said he was not communist, but soon nationalized foreign property. He collectivized land rather than redistributing it. However, he did deliver on promises to improve healthcare and education. The United States backed a group of exiled Cubans that invaded but failed at the Bay of Pigs in 1961: They had mistakenly presumed that they would immediately attract public support. The United States also cut off trade, which was critical for Cuba since it exported sugar to the United States. Cuba increasingly turned to the Soviet Union, which bought half their sugar and provided military and other aid. The United States blockaded the island in 1962 to prevent installation of nuclear missiles there, and the world feared nuclear conflict (see above). The Cubans after that provided support to communist insurgencies elsewhere in Latin America as well as Africa. The Cuban economy suffered severely with the dissolution of the Soviet Union: Cuba for a while traded doctors for Venezuelan oil. A vocal exile community in Florida long supported the economic sanctions against Cuba, though the United States traded openly with many regimes with similar records of political repression.

\section{Indonesia}

The Indonesian dictatorship led by Suharto killed hundreds of thousands of alleged communists in 1965-6. The Indonesian state repressed knowledge of these killings for decades. Indeed, Indonesia still shies away from discussing these state-sponsored murders, though it is now possible to talk about them publicly.

\section{Afghanistan}

The Soviet Union invaded Afghanistan in 1978 at the invitation of coup leaders and installed an even more pro-Soviet government. It would leave in 1988 after suffering 15,000 deaths to its troops. The United States at the time provided military and financial support to opposition groups, including the Taliban, who would thrive after foreign troops left. The United States would invade Afghanistan in 2001 when the Taliban provided bases for Islamic terrorists. 


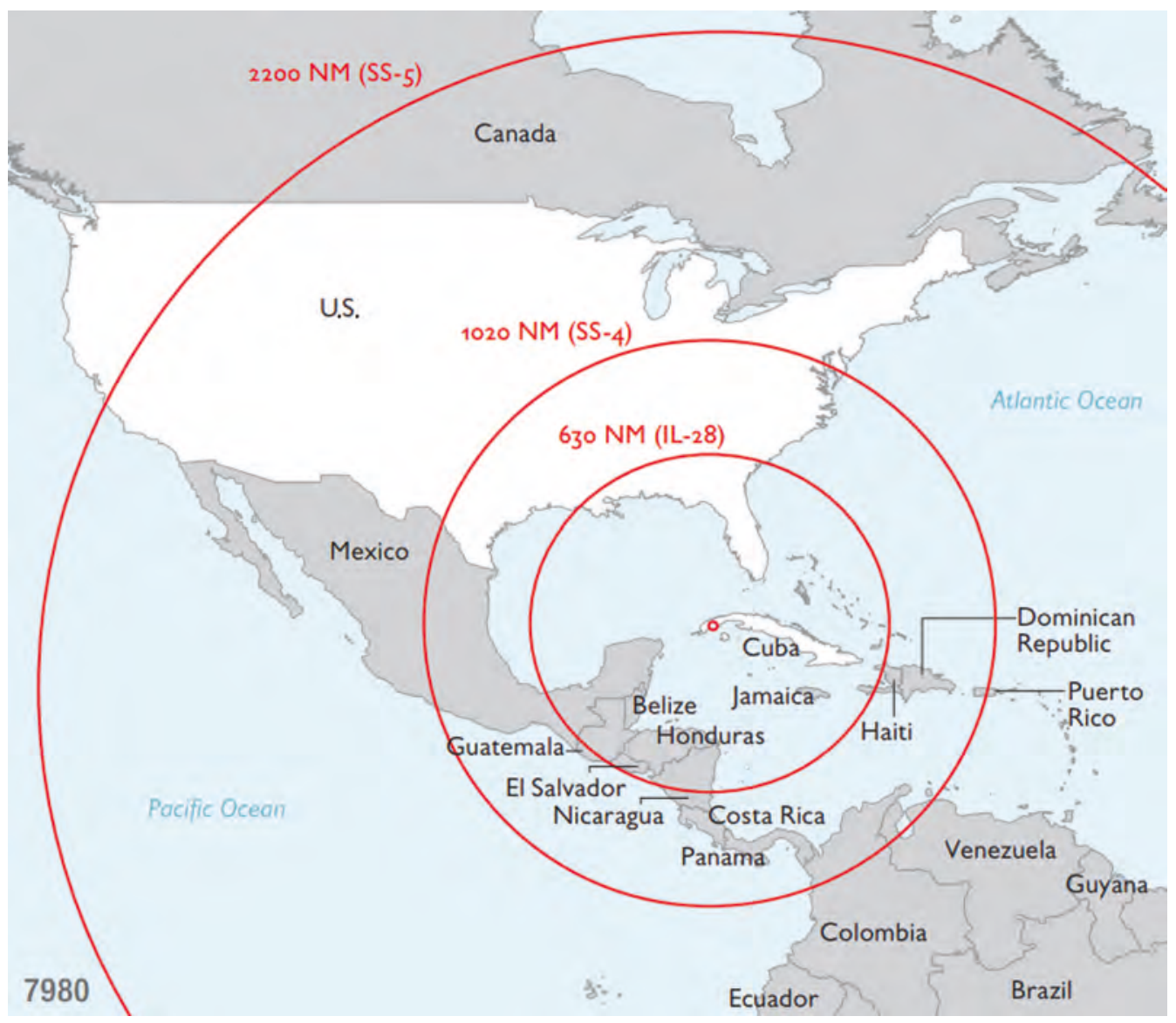

\section{Map 28.5}

Estimated range in nautical miles (NM) of the three missile types installed in Cuba in 1962: Il-28/

Beagle, SS-4/Sandal, and SS-5/Skean

The early support of the Taliban was hardly the only time during the Cold War when a strategy of aiding those who opposed the Cold War adversary backfired. Both sides supported dictators who were brutally repressive, earning the enmity of local people in the process.

\section{Terrorism}

In the early twenty-first century, terrorism - that is, violent attacks on civilians in pursuit of some political or religious objective - has become associated with radical Islam. It is important to appreciate, then, that many people in many countries have committed terrorist acts for a wide variety of stated purposes in the last century. Northern Ireland was the scene of many terrorist acts over a period of several decades. Less frequents acts of terrorism occurred in dozens of 
other countries. Moreover, terrorism has a long historical pedigree: The Jewish Zealots of biblical times killed Roman citizens to discourage Roman rule (and then committed mass suicide rather than allow Roman soldiers to take their families). Though terrorism has existed for millennia, advances in weaponry and transport and communications technology mean that individual terrorist acts can kill many more people, that terrorists can potentially strike anywhere in the world, and that the horror of terrorist acts will almost immediately grace television and computer screens around the globe.

Scholars debate the causes of terrorism. A sense of injustice is important. This can be encouraged by poverty (though many terrorists have been well off financially, and many more receive financial support from the wealthy) or the imposition of disdained institutions whether by an autocrat or by a democratic majority. Terrorists must believe that their acts are justified and that violence is the best means of pursuing their goals. Terrorism is often associated with a sense of powerlessness.

Since terrorists believe that terror may accomplish certain goals, it is worth asking if terrorism works. The world historian can usefully engage this question. The Jewish Zealots not only died themselves but also encouraged the Romans to disperse the Jewish people. The goals of terrorists were eventually achieved in places like Northern Ireland and South Africa, where Catholics and Blacks respectively won democratic powers. Arguably, though, terrorist acts delayed more than accelerated the tortuous path to political compromise in both lands. Terrorism did attract international attention in both cases. Yet Protestants in Northern Ireland and Whites in South Africa could reasonably disdain negotiating with terrorists. Catholics and Blacks had to assure others that they were willing to set their guns aside before negotiation was possible. Since terrorist organizations are generally loosely organized (to avoid capture), such commitments are difficult to make. In South Africa, it was international pressure more than anything that encouraged the White minority to accept majority rule. International pressure increased with violent acts by the South African government against peaceful protestors. (There is also a Cold War element to the story: The Communist party had been an important player in Black resistance in South Africa, and both internationally and locally it became easier to countenance majority rule after the collapse of the Soviet Union.)

What about Palestine? Israeli governments to this day cite security concerns as a justification for an armed presence in the West Bank and occasional military incursions into Gaza and Lebanon. If not for decades of terrorist attacks, Israel would have trouble justifying these behaviours both internationally and domestically. Many Israeli citizens are troubled by its treatment of the West Bank and Gaza. Israel is a proud democracy, but it has denied political autonomy to Palestinians in the West Bank. These (unlike Arabs within Israel proper) cannot vote in Israeli elections, but Israel circumscribes the powers of the Palestinian Authority. A similar discomfort guided European states to divest themselves of colonies in the postwar decades (chapter 30). Decades of peaceful 
protests might well have worked better for the Palestinians than decades of terrorist attack.

Terrorists in Ireland, South Africa, and Palestine had clear political goals. Other terrorists - the extreme right wing in the United States, the extreme left wing in Germany or Italy, or the more recent wave of Islamic terrorism - have had goals that are more diffuse. Much that they see around them appals them, and they seek mainly to sow discord and fear. Such acts certainly do engender fear, but also may stiffen the resolve of the terrorized. If Islamic terrorists wish to discourage Western interference in Islamic countries, they may well achieve the opposite outcome.

Terrorism is often but far from always associated with religion (and not just Islam: an extreme Buddhist sect released poisonous gas in a Tokyo subway in 1995). Religion is particularly important in cases of suicide terrorism for the terrorist then believes that they will be rewarded in the afterlife (Buddhist self-immolation has been an important form of protest in Tibet and Southeast Asia). Religious terrorism can be seen as part of a very long history of religious justification of violence - and assurances from religious authorities that martyrs will benefit in the next life. As often in history, religious faith can be a powerful motivator, but not always toward positive outcomes. We have noted many times that conflicts between denominations are often more vicious than disputes between religions. This has been true of Islamic terrorism too: Though attacks on Western targets receive far more media coverage in the

\section{BOX 28.1}

\section{ETHICAL REFLECTION}

We discussed in chapter 8 the five types of ethical analysis, and noted that these are interrelated. The historical question of whether terrorism works has significant implications for whether terrorism can be justified ethically. Note in this respect that people viewed as terrorists by some may be celebrated as "freedom fighters" by others. A consequential justification of terrorism depends directly on whether terrorism works. If a terrorist act is more likely to hurt than help the cause it supports, then it cannot be justified consequentially. In the case of terrorism, consequential evaluation is also critical to the other types of ethical evaluation. One might argue that compassion toward the downtrodden trumps compassion toward the innocent victims of terrorist acts, but this argument from values fails if the terrorist act will not in fact aid the oppressed at all. Likewise, one might argue that some rule such as justice for all trumps the right to life of the victims of terrorism, but again this argument fails if the terrorist act will not move the world toward justice. Terrorists may appeal to the traditions of a particular ethnic or religious group, but such an argument depends on the terrorist act advancing the group's interests. An intuitive feel that one is justified in striking out must - if one's action will do no good - reflect anger or vengeance or jealousy or some other harmful emotion rather than an intuitive feel for social justice. 


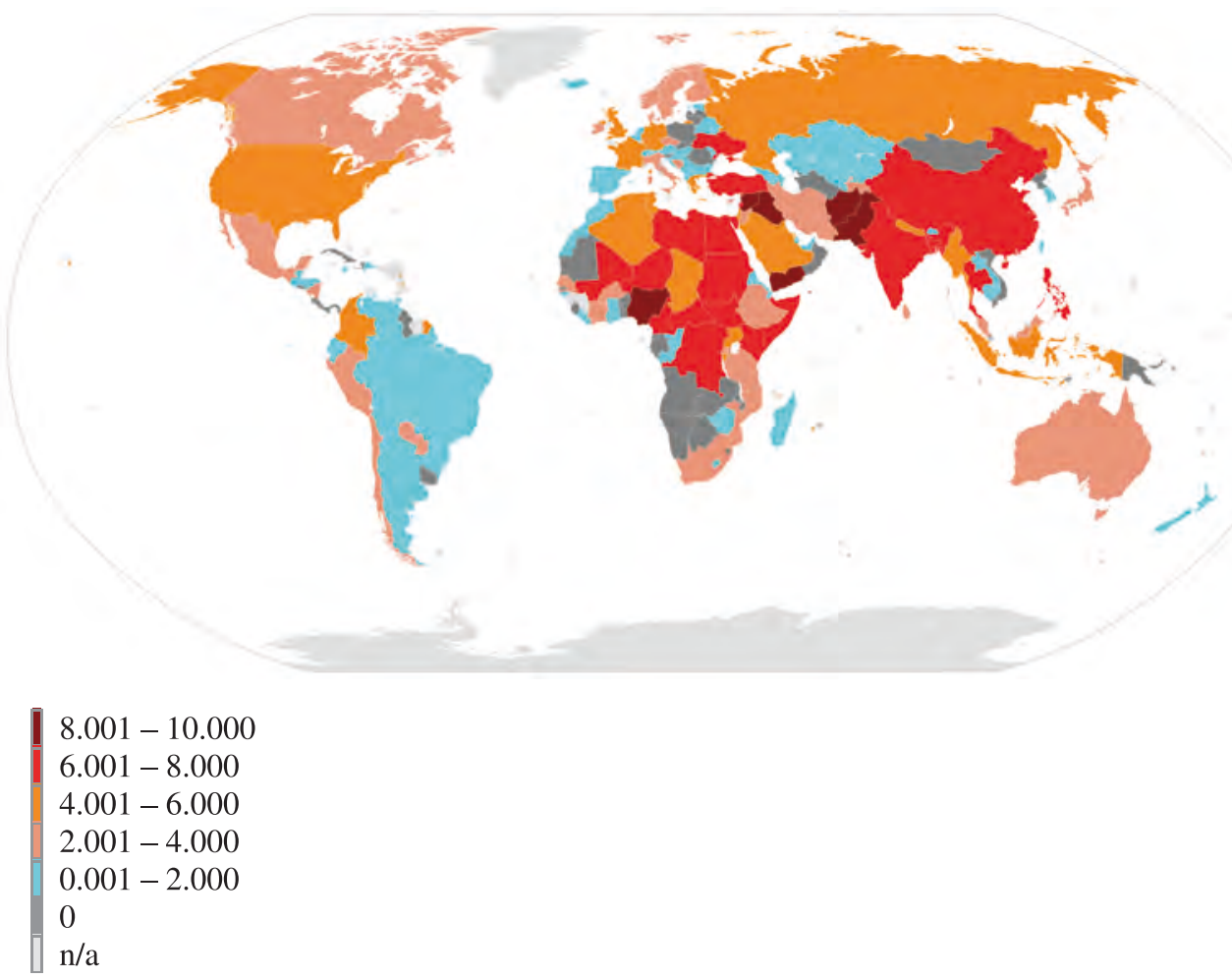

Map 28.6

Global Terrorism Index by country, 2016

Note that many countries in Africa and Asia have been more affected by terrorism than most countries in Europe or the Americas

West, there have been even more attacks on targets within Islamic countries. Such attacks are aimed both at other denominations and at secularists. Note in the latter regard that there are often religious motives behind the political aim of replacing corrupt or incompetent governments. Yet as noted in chapter 27, the existence of corrupt governments may serve to strengthen religious belief.

\section{New wars}

Some analysts identify a new type of war: This generally occurs within rather than between states and focuses on plunder rather than permanent territorial gains. The "blood diamonds" of Africa, sold by insurgent armies, are emblematic of this sort of conflict. Attacks on Nigerian oil facilities provide another example. When states lack military capability and there are resources to seize or ransom, armed attacks are an all-too-common outcome. Such wars can last, sometimes intermittently, for decades. These wars may be novel in their "asymmetry": The insurgents generally are far smaller in numbers and lack the 
same technology as the armies they confront. However, war for the sake of plunder has been typical throughout human history. Indeed many territorial conquests in history were likely afterthoughts to wars that had plunder as the primary goal.

\section{Trends in war-making}

We began the chapter by reviewing misguided reasons for optimism regarding peace as the twentieth century began. As the twenty-first century proceeds, there are again reasons for optimism, but also causes for doubt. One worldhistorical insight involves the question of whether the frequency of war has decreased over time. The answer depends crucially on how war is defined (1,000 deaths per year is a standard cut-off, and involving troops from at least two countries) and what time period one examines. The frequency of war may have declined from the sixteenth century, but this decline may have reversed in recent decades. Has the size of these wars increased or decreased? This answer again depends crucially on time period. The World Wars were by far the most devastating wars in human history - certainly in absolute numbers and almost certainly relative to world population (the Mongol conquests are comparable in the latter regard but took place over a much longer timeframe). Wars since have been much smaller.

Switzerland and Sweden could be viewed as harbingers of peace a century ago. Today much of the world can celebrate a half-century of peace. There has not been a war that directly involved great powers since Korea in the 1950s. This is the most prolonged period without "great power" conflict in several centuries. Europe and Japan have each largely escaped inter-state conflict civil war in the former Yugoslavia, and Russian occupation of parts of Ukraine and Georgia being the main exceptions. The Americas have been mostly untouched by war for even longer. Some analysts suspect that the costs of a war between states with sophisticated weaponry now exceed the benefits by a large margin; history warns us, though, that leaders may miscalculate these costs and benefits.

We discussed in chapter 27 how the United Nations, and especially its peacekeeping forces, has acted to limit conflict. Beyond the UN, there are thousands of non-governmental organizations (NGOs) devoted to the pursuit of peace, and there has been a blossoming of Peace Studies programmes in universities. It remains to be seen whether these movements will have more impact than the peace movements before World War I (above), but there are at least a growing number of people worldwide dedicated to and trained for the pursuit of peace.

As a century ago, there are also worrying signs. Authoritarian governments in China and Russia each press territorial claims against neighbours. Military technology continues to advance in ways that increase destructive capability 
and encourage arms races. Several countries devote tens of billions of dollars annually to military spending. The increased number of sovereign states in the world - many as we shall see in chapter 30 with somewhat arbitrary borders has increased the potential number of conflicts. With few exceptions, new countries have seen military expenditure as a critical component of statehood. Economic growth and the development of state bureaucracies mean that states can increasingly finance weapons and wars. Perhaps most troubling is the increased importance of guerrilla armies that strike from jungles or mountains: These have proven difficult to either defeat or defend against, and many military strategists have come to doubt the possibility of winning wars against them (though economic pressure may at times succeed).

President Dwight D. Eisenhower - previously the general that led the Normandy invasion in World War II - warned in the 1950s of a vast "militaryindustrial complex." He worried that weapons manufacturers benefited financially from war, and he urged democratic vigilance to ensure that private interests did not foment war. Throughout the world, many localities depend economically on weapons manufacture or military bases. Such locations cannot be enthusiastic about peace. Weapons manufacturers exert political influence through donations, bribes, or political pressure in many countries. The very size of military expenditures in the world today means that it is in the interest of many to advocate violent solutions to international disputes. As we have seen throughout history, such people may, of course, provide entirely different justifications for such advocacy, even to themselves.

\section{Questions}

1. Diagram the influences on and effects of fascism. Did fascism have longterm effects?

2. Ditto for the Cold War.

3. What role should the student of World History play in debates about terrorism or nuclear weapons?

4. In what ways was war different in the twentieth century than before?

5. Can you identify positive outcomes from the World Wars?

\section{Readings}

Black, Jeremy. 2015. A Century of Conflict: War, 1914-2014. Oxford University Press. [See also his Rethinking World War Two: The Conflict and Its Legacy, London: Bloomsbury, 2015. He argues that allied victory was due to historical contingency and resource superiority. He surveys how the war has been viewed differently in different countries since.]

Brose, Eric Dorn. 2010. A History of the Great War: World War One and the International Crisis of the Early Twentieth Century. New York: Oxford University Press. 
Carmichael, Cathie, and Richard C. Maguire, eds. 2015. The Routledge History of Genocide. New York: Routledge. [The book mostly focuses on the twentieth century, but provides a mix of thematic essays and case studies.]

Dickinson, Edward Ross. 2018. The World in the Long Twentieth Century: An Interpretive History. University of California Press.

Hecht, Gabrielle, and Paul N. Edwards. 2010. "The Technopolitics of Cold War: Toward a Regional Perspective," in Michael Adas, ed., Essays on TwentiethCentury History. Philadelphia, PA: Temple University Press. 271-314. [Focuses on the technological elements of the Cold War.]

Heuser, Beatrice. 2010. The Evolution of Strategy: Thinking War from Antiquity to the Present. New York: Cambridge University Press. [This book discusses how military strategy expanded from a focus on the battlefield in early days to stress how threats of force could achieve various political aims. The modern idea of "total war" came to be justified consequentially: Civilian deaths were necessary to quickly achieve a just peace.]

Jacoby, Tim. 2016. "Global Fascism: Geography, Timing, Support, and Strategy." Journal of Global History 11:3, 451-72. [Explores the meaning of fascism, and its temporal and global extent.]

Judge, Edward H., and John W. Langdon. 2018. A Hard and Bitter Peace: A Global History of the Cold War, 3rd ed. Lanham MD: Rowman \& Littlefield.

Leonhard, Jörn. 2018. Pandora's Box: A History of the First World War. Translated by Patrick Camiller. Cambridge, MA: Belknap Press of Harvard University Press.

Morrow, John H. Jr. 2010. "The Impact of the Two World Wars in a Century of Violence," in Michael Adas, ed., Essays on Twentieth-Century History. Philadelphia, PA: Temple University Press. 161-212.

Pine, Lisa. 2019. Debating Genocide. New York: Bloomsbury Academic.

Sanborn, Joshua. 2014. Imperial Apocalypse: The Great War and the Destruction of the Russian Empire. Oxford University Press.

Scherner, Jonas, and Eugene N. White, eds. 2016. Paying for Hitler's War: The Consequences of Nazi Hegemony for Europe. New York: Cambridge University Press. [Discusses how and how much Nazi Germany expropriated from the lands it conquered.]

Stearns, Peter N. 2014. Peace in World History. New York: Routledge.

Storey, William Kelleher. 2009. The First World War: A Concise Global History. Lanham, MD: Rowman \& Littlefield.

There was a Forum in the Journal of Global History, July 2017 that examined fascism in a global context, comparing the imperial expansion of Germany, Italy, and Japan, and exploring ways in which they learned from each other. There was a Forum in World History Connected, June 2017 on the comparative study of genocide. A review essay in the Journal of World History, June 2019, addresses several books about World War II. 


\section{The worst of times and the best of times}

\section{The Great Depression and postwar recovery}

\section{Guiding questions}

What was the Great Depression? When, where, and why did it happen? What were its effects?

What was the postwar Golden Age? When, where, and why did it happen? What were its effects?

Can we detect general trends in economic growth through the twentieth century? How and why did growth rates differ across countries or regions?

How and why did consumer tastes diffuse across societies?

What is "globalization"? Did "globalization" increase in intensity? Why or why not?

Relationship to other chapters: We build of course on our general understandings of economic growth but especially chapter 24. We connect the Great Depression to some of the technological developments addressed in that chapter. The Great Depression encourages fascism and so contributes to World War II (chapter 28). Our discussion of globalization compares the twentieth-century experience with earlier exchanges of goods and ideas. Economic growth influences and is influenced by the trends in population addressed in chapter 31 . This chapter provides insight into why some 
countries are richer than others in the twenty-first century and why all countries are richer than they were a century ago.

Our primary thematic focus in this chapter is economic: We will address both the worst and best of times economically in modern human history. We will find that technology and politics influenced economic performance in important ways. The economy in turn has a significant impact on culture, population, and individual well-being. We will also discuss the relationship between economy and social structure. We will compare economic circumstances across states and also explore how economic performance in one country influenced economic performance elsewhere. We will later also both compare and trace connections across countries in both culture and population movements. Though the word "revolution" appears far more often than "evolution" in this chapter, a crucial part of our understanding of both the Depression and postwar growth involves the evolution of technologies associated with the Second Industrial Revolution of the late nineteenth century.
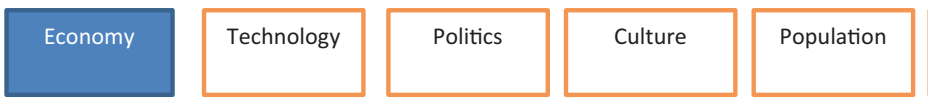

Individual Differences

\section{The Great Depression}

The term "Great Depression" had been applied to economic downturns in the 1870s and 1890s. But the Great Depression of the 1930s was much much worse: It was longer, deeper, and more geographically extensive than any other economic contraction in the modern world, and likely in all of world history. In many countries, including the United States, per capita incomes were still lower in 1939 than they had been in 1929. Unemployment rates reached 25 per cent or more in the United States, Canada, and much of Europe, and were still above 10 per cent as World War II began in 1939 leading to some speculation that the Depression would have continued for some years if not for the onset of war. The Great Depression touched almost every country in the world.

Economists generally think that the Great Depression began in those countries that had become the richest in the world over the previous century: Europe and former European settler societies, and especially the United States. As these economies contracted, they reduced their imports from the rest of the world. As we shall see, the Depression was associated with financial crises in the rich world, and this resulted in a precipitous decline in foreign investment. Poorer countries then suffered simultaneously from declines in export demand and investment flows. The one region of the world that largely escaped the Great Depression was the Soviet Union: It had largely cut itself off from foreign trade and investment flows in the aftermath of the 1917 revolution, and was pursuing 
a course of state-managed investment in industry during this period. The relative success of the Soviet Union during this unusual period would encourage many countries to pursue trade restrictions and state ownership well into the postwar period. Indeed, trade restrictions by both developed and developing countries during the 1930s - in the hopes of protecting local jobs from foreign competition - led to a further reduction in international trade and likely caused global economic output to shrink by an additional few percentage points.

In the United States, output and employment fell by perhaps a third from 1929 to 1933 . There was then a recovery to 1937 , but then another sharp recession and recovery from 1938. Canada and several other countries followed a broadly similar pattern. In Germany, the decline started a bit later but was perhaps even sharper. Britain was unusual: Its economy had struggled while others were growing in the 1920s but then it suffered less than others in the 1930s.

The Great Depression occurred at a time when most states had rudimentary programmes to aid the unemployed. Many states introduced unemployment insurance, public works programmes, and welfare systems during the 1930s; they would often strengthen these during years of postwar prosperity (below). In the meantime, the unemployed often had to rely on charity: Long lines at "soup kitchens" which doled out free food were characteristic of the times. Young men often rode illegally on freight trains in search of work. Families too migrated in search of work but often found that jobs were difficult to find

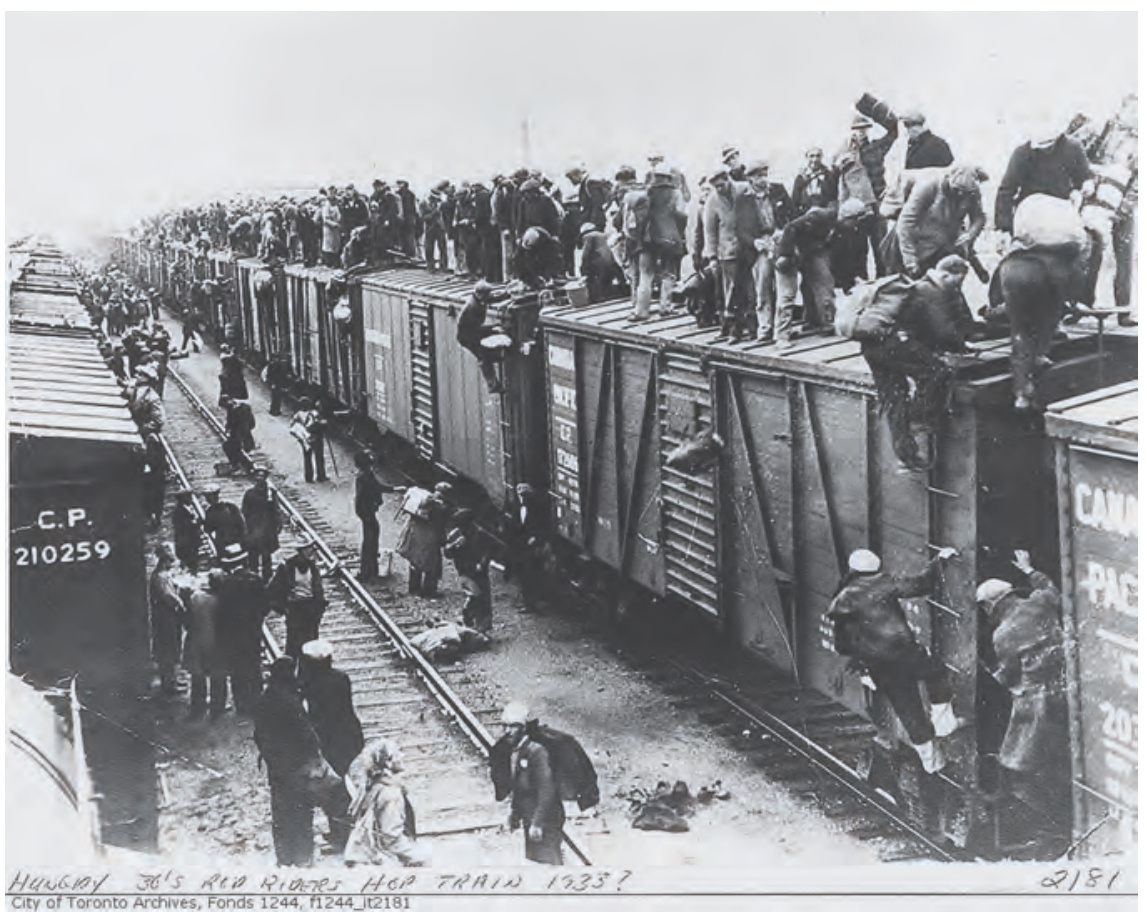

Illustration 29.1

Unemployed Canadian men hop a freight train, c. 1933 
everywhere. This migration was almost entirely internal to states as most countries restricted immigration to protect local jobs. The severe psychological and social costs of the Depression are indicated by an increase in suicides and a decrease in marriages and births. Since the Soviet Union largely escaped the ravages of the Depression, there were fears of communist revolutions or electoral victories in many Western democracies. In the end, though, it was fascism rather than communism that succeeded in the 1930s (chapter 28). The social programmes introduced in the 1930s may have spared many countries from political tumult.

Why did the Great Depression occur? This question has vexed economists as well as historians for decades. Economic theory, in general, suggests that markets - including markets for labour and capital - should move reasonably quickly toward equilibrium prices (or wages or interest rates) in which there is a balance between the amount that people want to buy and sell. A period of years in which many workers are unemployed, and people wish to save far more than they want to invest, challenges economic theory.

In the depth of the Depression, many commentators naturally wondered if they were witnessing the death of capitalism itself. Perhaps there was an internal logic to capitalism whereby the ability to produce outstripped the ability to consume? Or maybe Marx was right, and growth in profits destabilized the system so that it was no longer possible to consume all that could be produced (since the rich save a much higher share of income than the poor)? Income inequality had indeed increased in most of the world during the 1920s. With hindsight, we can appreciate that consumption per person would multiply many times in the postwar world: Inhabitants of every country in the world have an average standard of living unimaginable to their great-grandparents of the 1930s. And capitalism has stumbled through many financial crises since the 1930s, but none have been associated with a worldwide Depression as severe as that of the 1930s.

The English economist John Maynard Keynes suggested in 1936 that economic theory could be adjusted to explain the Depression. He argued that equilibrating mechanisms were weak at the level of the aggregate economy. Investors could become fearful, in which case desired investment could fall below desired savings for years. These unspent funds would mean that demand for labour would be insufficient to achieve full employment (if wages did not drop precipitously). Economists were sceptical at first but came to appreciate that Keynes' adjustments to economic theory were far less threatening than alternative interpretations that the entire market system was collapsing. As the economics profession became highly mathematical in the postwar years, "Keynesian" models proved a good fit for the fairly mild business cycles of the first postwar decades (below). Economists urged postwar governments to spend during recessions to compensate for declines in private investment, and many felt that the problem of business cycles had been solved. Economists were disappointed by the fact that Keynesian models could not "explain" the much more severe business cycles of the 1930s, but this fact was largely ignored 
until the 1970s when economists again had trouble applying their models to observed business cycles.

The Keynesian theory could not explain why investment (or perhaps consumption) demand could have fallen so far and so fast at the start of the Depression. There seemed no reason for this. Commentators, including leading economists, had predicted in 1929 a future of unending growth. From the 1960s an alternative theory was put forward that pointed away from investment toward changes in the money supply. It was argued that if the supply of money declined, economic activity would decrease. And it was suggested that the Federal Reserve System in the United States had reduced the supply of money. Over the next decades, this argument would be expanded to implicate the international "Gold Standard" by which most of the world's currencies were fixed to the price of gold. It would be argued that this system forced many countries to decrease money supply in efforts to maintain the fixed value of their currency. But this new "monetarist" theory also faced both theoretical and empirical challenges. Theoretically, a fall in money supply might just cause a fall in prices: A smaller amount of money could then finance the same level of output. The velocity of circulation - the number of times a dollar (or other unit of currency) is spent in a year - might also rise so that a smaller amount of money could purchase the same output. In the United States, all four variables money supply, prices, velocity of circulation, and output - fell by about a third between 1929 and 1933. If prices had fallen but the velocity of circulation had not, output would not have fallen even when money supply did. Moreover, the money supply is not dictated by the Federal Reserve (or the central banks of other countries) but also reflects decisions of individual banks regarding their willingness to make loans. If I deposit money in a bank, and the bank lends that money to someone else, both of us think we have money to spend. The decline in money supply might then be a result of economic decline rather than a cause: Banks might merely have seen few promising investment opportunities that they were willing to finance through loans. Moreover, it is not clear that the Federal Reserve itself acted in a very contractionary fashion, at least before 1931 when the Depression was already well underway. Indeed the first monetarist theories had suggested only that the Federal Reserve had exacerbated an already severe downturn; later monetarists then searched for signs of Federal Reserve tightening in the late 1920s.

Economists debated Keynesian versus monetary explanations for decades. By the 1980s, there was a widespread appreciation that neither of these, nor even both in combination, could satisfactorily explain the Depression. The bulk of economists preferred to focus on postwar data rather than the enigma of the Depression. Others suggested new approaches. One approach effectively involved Depression denial: If the millions of desperate Americans - a similar argument could be made in other countries - receiving government relief in return for building post offices or trails in national parks were treated as employed, estimated unemployment rates during the 1930s could be cut in half. But this "employment" was created in desperation by governments worried 
about the social and political costs of double-digit unemployment. Another approach focused on the motives of banks: These might have become more conservative as their balance sheets worsened with bad loans. The Depression, once begun, would then intensify. But one still needed to understand the initial downturn and also why banks might have become quite so pessimistic. Still another approach - which gained considerable popularity - emphasized expectations (this approach echoes some of Keynes' ideas): If investors became pessimistic in 1929, then a decline in investment would follow. But, as noted above, the mood at the time seems to have been generally optimistic.

Given that the Depression was far worse than any other economic contraction before or since, it might make sense to look outside of economic theory for one-time shocks that exacerbated the Depression experience. One possible candidate here is the First World War. Keynes himself had warned before the Depression began that the economic repercussions of the treaties that ended that war (chapter 28) might be severe. Germany struggled to pay the reparations demanded by the French. Various governments and the banks with which they interacted became enmeshed in an unsustainable flow of funds. Investment flows from the United States into Germany maintained this system during the 1920s, but Keynes felt that failure was inevitable. Once flows of investment from the United States collapsed after 1929, Germany was unable to finance transfers to other European states. Banks across much of Europe failed as a result. So the Depression in Europe might have been aggravated by the treaties that ended

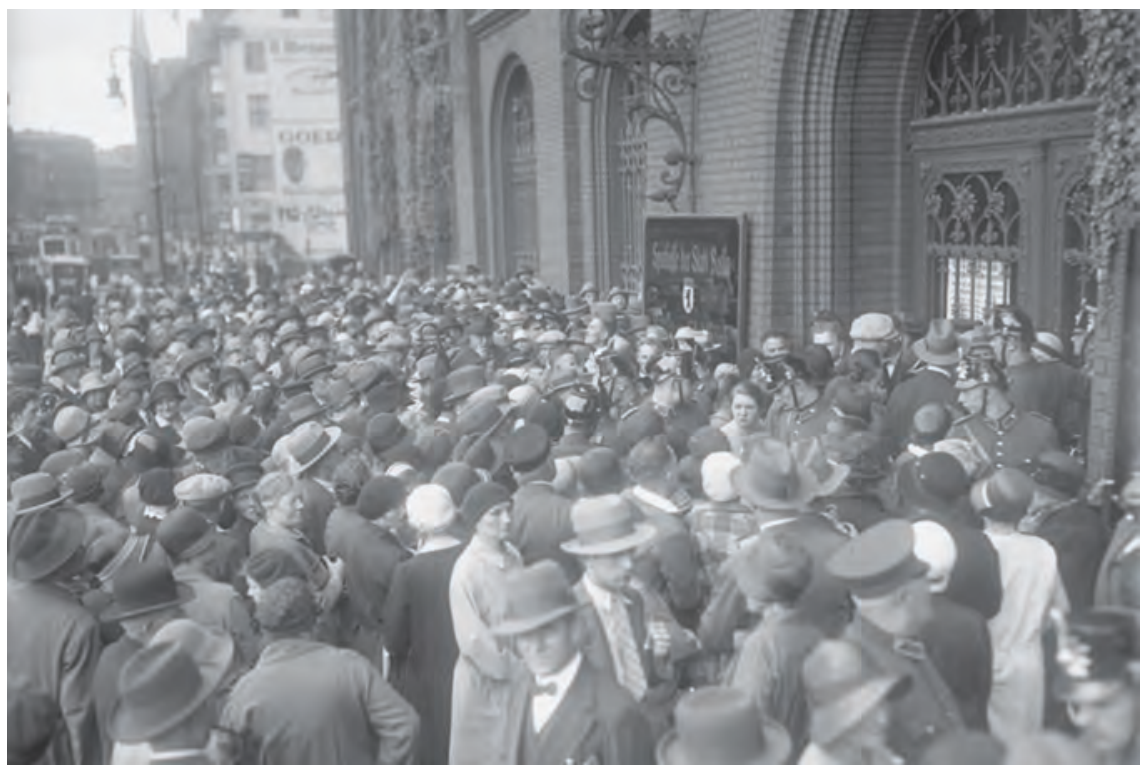

Illustration 29.2

Depositors of the Savings Bank of Berlin attempt to withdraw their savings after learning of the collapse of the Darmstadt and National Bank, July 13, 1931

Bundesarchiv, Bild 102-12023 / Georg Pahl / CC-BY-SA 3.0 
World War I. But it would be harder to blame the severity of Depression outside Europe on those treaties.

Another candidate for exacerbating shock, and one that can more readily explain global Depression, is technological change. The idea that technology might have caused some or all of the Great Depression was first suggested during the Depression itself and has been periodically revived since. The author of this book has been a proponent of the technological argument. He takes the liberty of reviewing that argument in the next section. But he notes here that this argument fits well with the general approach of this book: that major events and processes in world history generally reflect the interaction of multiple themes. Economists have struggled with limited success to explain the Depression with recourse only to narrowly economic variables. The logic of world history suggests that the most severe economic calamity in history may reflect multiple causes, including influences from non-economic variables. Moreover, the trajectory of technological innovation during the interwar period is of intrinsic interest to the world historian - just as we have discussed important innovations in several other chapters of this book - even if one is sceptical that this was important in generating the Great Depression.

\section{PRIMARY DOCUMENTS: THE DEPRESSION EXPERIENCE}

Studs Terkel performed some 400 interviews for his An Oral History of the Great Depression, Pantheon, 1970. Virginia Durr, who later became a civil rights activist, described the shame and humiliation people experienced (pp. 461-2):

It was a time of terrible suffering. The contradictions were so obvious that it didn't take a very bright person to realize something was terribly wrong.

Have you ever seen a child with rickets? Shaking as with palsy. No proteins, no milk. And the companies pouring milk into gutters. People with nothing to wear, and they were ploughing up cotton. People with nothing to eat, and they killed the pigs. If that wasn't the craziest system in the world, could you imagine anything more idiotic? This was just insane.

And people blamed themselves, not the system. They felt they had been at fault: ... "if we hadn't bought that old radio" ... "if we hadn't bought that old secondhand car." Among the things that horrified me were the preachers-the fundamentalists. They would tell the people they suffered because of their sins. And the people believed it. God was punishing them. Their children were starving because of their sins.

People who were independent, who thought they were masters and mistresses of their lives, were all of a sudden dependent on others. Relatives or relief. People of pride went into shock and sanatoriums. My mother was one.

Up to this time, I had been a conformist, a Southern snob. I actually thought the only people who amounted to anything were the very small group which I belonged to. The 
fact that my family wasn't as well off as those of the girls I went with-I was vice president of the Junior League - made me value even more the idea of being well-born ...

What I learned during the Depression changed all that. I saw a blinding light like Saul on the road to Damascus. (Laughs.) It was the first time I had seen the other side of the tracks. The rickets, the pellagra-it shook me up. I saw the world as it really was ...

The Depression affected people in two different ways. The great majority reacted by thinking money is the most important thing in the world. Get yours. And get it for your children. Nothing else matters. Not having that stark terror come at you again ...

And then there was a small number of people who felt the whole system was lousy. You have to change it. The kids come along and they want to change it, too. But they don't seem to know what to put in its place. I'm not so sure I know, either. I do think it has to be responsive to people's needs. And it has to be done by democratic means, if possible.

Did she underestimate the degree to which people wished to "change the system"?

\section{Technological innovation and the Great Depression}

We have noted in preceding chapters that technological innovation has often been associated in history with fears that jobs would be lost. In the very long term, this has not occurred: The vast majority of the adult human population is still employed despite millennia of technological innovation. Why? Much of humanity today is involved in the production of goods and services that have not always existed: Airplane trips, computer software, video games, automobiles, television, plastics, and pharmaceuticals have been developed in the last decades or centuries but have become important sources of employment. We might then usefully distinguish two types of technological innovation: "product innovation," which introduces new goods or services, and "process innovation," which develops improved ways of producing existing goods or services. The airplane is an example of product innovation, the assembly line an example of process innovation.

It is not difficult theoretically to argue that product innovation will generally lead to an increase in investment and employment over the next few years: Factories or offices have to be built and workers hired to produce the new good or service. An exception may occur if the new good or service substitutes for an older good or service that employed a more substantial number of workers (though it may take some years for the old industry to die). In such a case, product innovation mimics process innovation: Consumers are satisfied, but fewer workers are required. It is also easy to show theoretically that process innovation can lower employment, since fewer workers are generally needed 


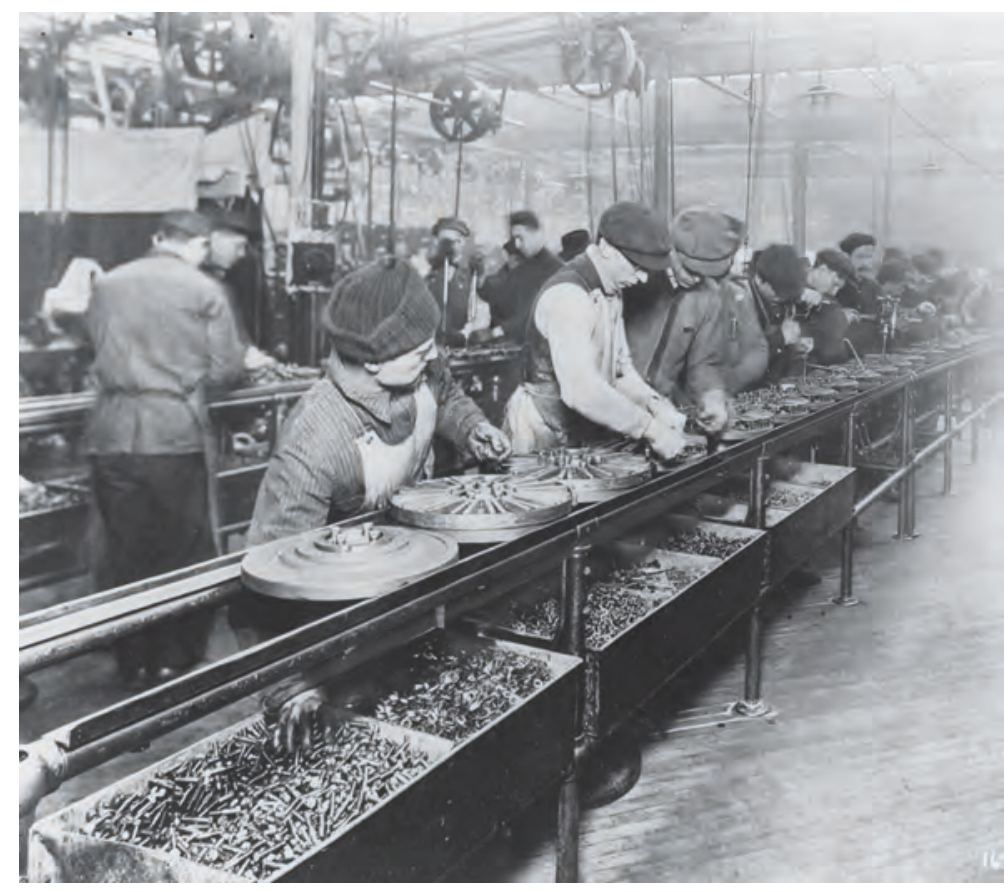

Illustration 29.3

Workers on the first moving assembly line put together magnetos and flywheels for Ford, Highland Park, Michigan, 1913

to produce the same output. The exception will occur where process innovation so reduces the price of a good that there is an expansion in production that more than compensates for the decreased workforce per unit of output. The assembly line indeed so lowered the cost of automobiles in the 1920s that employment in automobile production (and then servicing and road construction) rose dramatically in several of the most advanced economies. But more often process innovation will mean that fewer workers are required to produce a particular good or service. In sum, it is theoretically entirely possible that a period in which there is a great deal of process innovation but limited product innovation will witness high levels of unemployment. This was indeed the case in the late 1920s and early 1930s. We shall see that the technological experience of the 1930s also helps us understand why investment levels were so low in the 1930s.

What happened with respect to product and process innovation in the years right before the Great Depression and in the early years of the Depression itself? The period from 1925 to 1935 is very clearly a period of abundant process innovation and limited product innovation. The main new product in this period is the electric refrigerator of 1931. Notably, factories were built and workers hired to produce hundreds of thousands of these in the United States and elsewhere in the depths of the Depression. It would seem that other new products could 
have been similarly financed if these had existed, with a resulting increase in employment.

We discussed the Second Industrial Revolution in chapter 24. One analysis of technological innovation in the first half of the twentieth century suggested that the zipper was the most important innovation that could not be traced to one or more elements of the Second Industrial Revolution. In other words, technological innovation in the early twentieth century focused almost entirely on further developing the ideas of the Second Industrial Revolution. Recall that there were three critical areas of innovation: chemicals, electrification, and internal combustion. All three of these types of technology yielded significant new products in the early 1920s and late 1930s but little in between. For internal combustion, the automobile became a major industry in the 1920s, but the airplane only did so from the late 1930s (the DC-3 of 1936 was the big innovation, lowering costs per passenger mile to a fraction of their previous level). For electronics, the radio was popularized from the early 1920s, but television only became technically feasible in 1939. One of the most important areas of chemical innovation in the twentieth century was synthetic fibres: Rayon, the first semi-synthetic fibre, was manufactured widely from the early 1920s, but nylon, the first fully synthetic fibre, was invented only in 1939. The lack of new products between 1925 and 1935 may merely reflect, then, an unfortunate coincidence in how the potential of the three main strands of the Second Industrial Revolution was realized through time.

We also noted in chapter 24 that the Second Industrial Revolution was associated with the rise of the industrial research laboratory. Arguably, in their

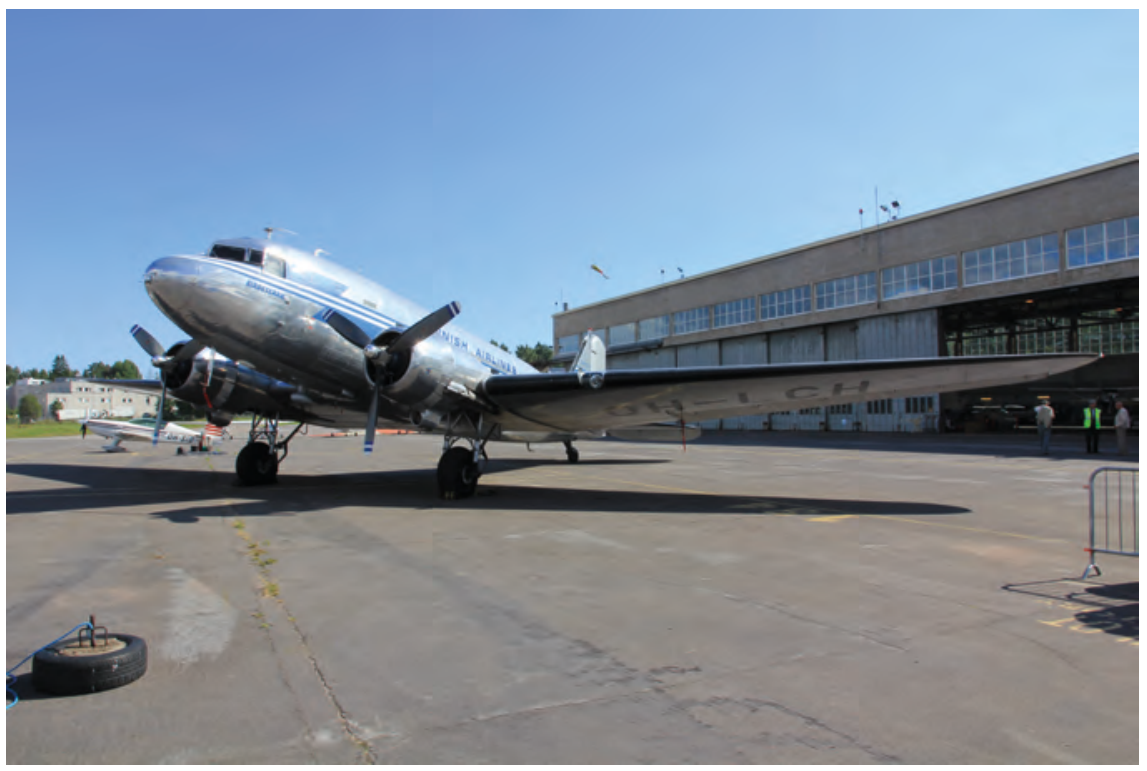

Illustration 29.4

DC-3 flown by Finnish Airlines 
early decades these focused primarily on minor product innovations and process technology. Such innovations were much easier to manage bureaucratically: Firms could identify explicit goals and monitor progress toward these. Industrial research laboratories only slowly attempted major product innovation because it was hard to predict the exact nature of a new product and thus much harder to evaluate whether progress was being achieved. Nylon, and to a lesser extent television, resulted from lengthy and expensive experimentation within industrial research laboratories, while the airplane benefited from extensive military finance of research. If industrial research laboratories had turned toward major product innovation earlier, the Great Depression might have been much less severe.

Each of the three strands of the Second Industrial Revolution was also associated with significant process innovations in the interwar period. These are of great historical importance regardless of their immediate effect on the Depression. The most famous is the assembly line. This had roots in the disassembly lines developed by meatpacking plants in the late nineteenth century (as refrigerated transport by rail and sea created new markets for cut meat) in which carcasses on hooks moved past workers who each took a different cut. But Henry Ford's determination to lower the price of cars brought the assembly line to fruition in the early twentieth century. In the 1920s, as noted above, the assembly line would render the automobile an item of mass consumption. Assembly lines would be employed in that decade in the production also of radios and washing machines and indeed any mass-produced good that required assembly.

What of homogenous goods such as petrol or paint? These required not assembly but a formulaic combination of ingredients, often associated with chemical reactions, to produce homogenous outputs. The key innovation here was "continuous processing": Rather than produce outputs one batch at a time, ingredients would be continually mixed to provide a steady output. This new technology also spread widely in the 1920s.

Last but not least was electrification. Before electricity, industrial machinery had to be connected by belts to large steam or internal-combustion engines. Large factories were necessarily dirty and noisy, and belt placement rather than product flow dictated decisions regarding machine placement. Complex gearing was required to allow different machines to run at different speeds. Electrification allowed machines to be placed and operated independently, while dramatically reducing the noise and dirt associated with factories. In the United States and several other countries, the 1920s was the decade in which most industry electrified.

Assembly lines, continuous processing, and electrification are perhaps the three most significant process innovations of the twentieth century - challenged only by computer automation in the later part of the century. The fact that all three of these innovations were adopted primarily in the decade preceding the Depression is notable. They collectively increased labour productivity by several per cent annually in the 1920s. And this productivity advance continued 
in the 1930 s as the managerial challenges associated with these new technologies were addressed. Note that some investment was required in the 1920s to introduce these new processes, but that little investment was required in the 1930s to achieve further labour-saving advances (there were also developments in new cutting tools, embodying new chemical compounds such as tungsten carbide, that allowed machines to run much faster but also required very little investment). As with product innovation (above), there was one main exception to the limited need for investment in new processes in the 1930s: New rolling mill technologies for steel-making were widely adopted in the early 1930s, again establishing that funds for investment were available when a profitable investment opportunity appeared.

Postscript: There are concerns in the early twenty-first century that artificial intelligence may replace a host of office jobs that focus on pattern recognition of various types. That is, computers may prove able to mine data to make a host of decisions now made by people. We will discuss in chapter 32 whether we should encourage product innovation to absorb these displaced workers.

The sharpness of the decline into Depression is a challenge for all attempts to explain it, including the economic theories surveyed above. One might have expected the technological forces outlined above to result in a gradual increase in unemployment through the late 1920s rather than a sudden decline after 1929. One partial explanation is what economists call labour hoarding: Firms do not immediately lay off workers just because they can but re-evaluate their employment decisions when facing financial challenges. Many firms kept redundant workers on the payroll during the 1920s but dumped these when their financial situation worsened in the 1930s.

We might also have expected investment to decline more gradually than it did. In capital markets, though, financial speculation masked the mismatch between desired saving and desired investment in the late 1920s. That is, individuals and firms with money on hand but no sound investment ideas ended up instead temporarily bidding up the price of land and corporate stocks. There was a real estate frenzy, especially in Florida. While the stock market crash of October 1929 is often thought in the popular imagination to have triggered the Great Depression, economists have long doubted that link. It may be best to see the preceding rise in stock prices as speculative activity and the crash as a belated recognition by the market of a lack of profitable investment opportunities. Firms in the automobile and other industries had begun to fear in the late 1920s that they were over-selling their markets: Once almost every middle-class family had a durable automobile, sales were bound to fall. But competition for market share prevented the industry from trying to even out sales. And 
attempts to introduce annual model changes so that consumers would trade in their cars came too late. Sales of automobiles started to fall precipitously early in 1929, months before the stock market crash.

Note that the technological explanation of the Great Depression is compatible with the explanations propounded by economists. Indeed an appreciation of technological trends in the interwar period potentially solves some of the puzzles in economic accounts of the Depression. We can understand the fall in investment from 1929 as representing (at least in part) a limited number of investment opportunities. With no new products or processes, what opportunities would investors have pursued in 1930, especially in the United States? There was also a fall in consumer expenditure associated with saturation of markets for automobiles and other new goods: Almost every middleclass family bought a car and a radio in just a few years in the 1920s, and these were built to last. The fact that banks reduced their loans may reflect a lack of compelling investment opportunities more than anything else. Though aggregate expectations were positive in 1929, there was industry-specific pessimism regarding market saturation and a lack of new products. Investments real investments in factories, offices, and machines, as opposed to financial speculation - are primarily guided by industry-specific expectations.

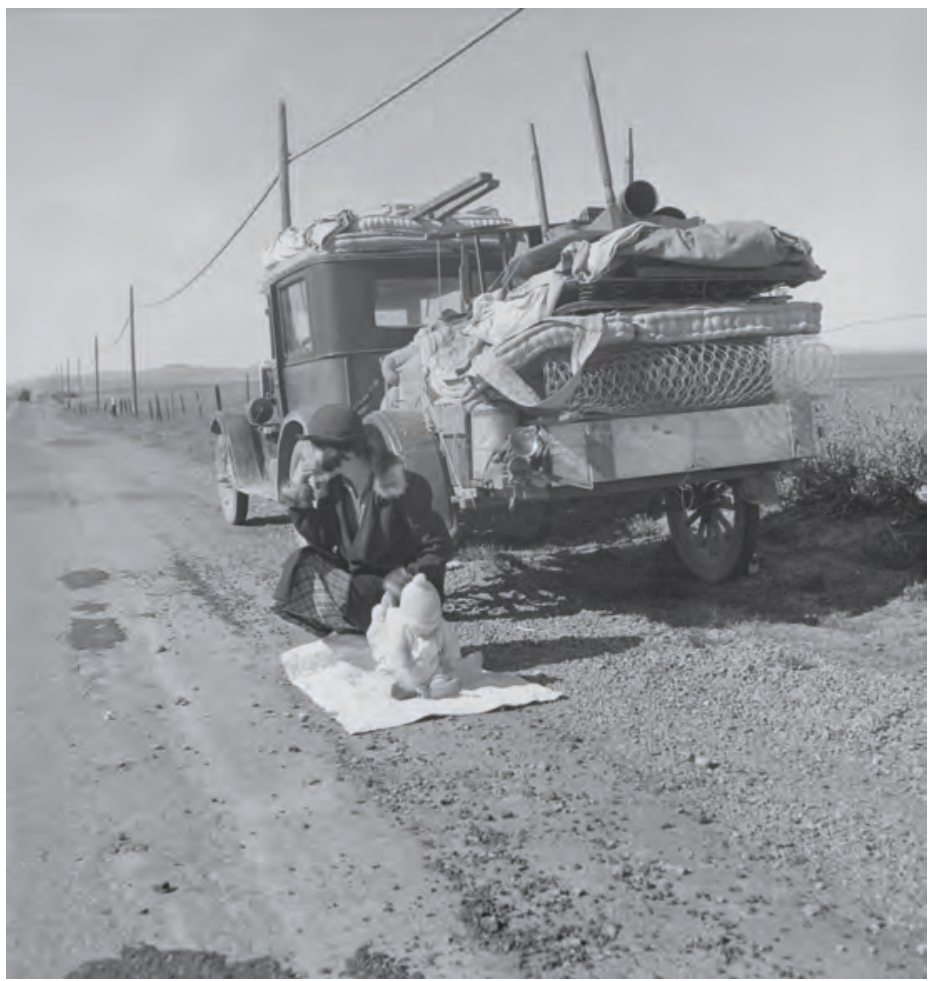

\section{Illustration 29.5}

Missouri family moving in search of work, 1937. This is a famous photograph by Dorothea Lange, labelled "Broke, baby sick, and car trouble!" 


\section{BOX 29.1}

\section{HISTORIOGRAPHY}

We have only rarely in this book reviewed in as much detail as we have above how the historical understanding of a particular event or process evolves. Students are often horrified to discover that our understanding of the past can change. They often mistakenly jump to the view that history is merely a matter of opinion (a view, it must be admitted, that a minority of scholars also hold). To be sure, historical understanding can be biased: The increased preference of economists for mathematical exposition in the postwar period steered them away from hard-to-quantify variables such as technological innovation, and a general belief that markets work may have encouraged some economists to either deny the Great Depression or blame it on the Federal Reserve. We have in earlier chapters seen how debates about the Great Divergence and Industrial Revolution have been tinged by attitudes toward the "Rise of the West." But historical research does gradually - by amassing argument and evidence narrow the plausible range of interpretation, and often eventually yields considerable consensus regarding the most likely explanation of key events or processes.

This book has hopefully communicated some of the joy of history. It has tried to provide some structure to World History but still allow the uniqueness of particular events, processes, and people to shine through. To the joy of history as a subject can be added the pleasure of history as a professional process. Students should appreciate that historical controversies are not never-ending but that through comparing competing arguments and evidence, the community of historians sharpens its understanding of history. Students should partake in this process in their own written work. They should enjoy both reading and writing careful arguments grounded in evidence, and critically evaluating the flaws in their own work as well as the work of others.

One historiographic challenge is that the interests of historians have changed through time. In the nineteenth century, most history was political history. In recent decades many historians have engaged issues of gender or race or the environment. The danger is that each succeeding generation of historians sees history differently. But this need not be. Our analysis in chapter 1 suggested that there are a manageable number of key themes that interact through history. Environmental history is not then a substitute for but rather a complement to political history. We can, by studying all themes and how these interact, aspire to a more comprehensive understanding of history. In the case of the Great Depression, one can imagine a future in which both historians and economists appreciate the interplay between technological and economic processes (and perhaps others; we mentioned the First World War above).

\section{The postwar Golden Age}

Though the 1920s had been prosperous in much of the world, the decade had been preceded by a short but steep recession at the end of the First World War. That experience, plus the Depression itself, led to widespread pessimism at the 
end of the Second World War. However, the next two and a half decades would witness unprecedented economic growth in much of the world, accompanied by mild business cycles and generally low rates of unemployment, at least among developed countries. Growth was particularly rapid in most of Europe, North America, Australia, and Japan in this period, but almost every country in the world saw a significant increase in per capita incomes. This period is often termed the Golden Age.

Why was this period so prosperous? In part, we can credit postwar decisions. Leaders had drawn some useful lessons from the interwar experience. Germany was not required to pay enormous reparations as after the First War. Indeed, the United States and Canada would provide extensive government funding to encourage the rehabilitation of the West German economy, along with those of other Western European nations, in the years after the war. Though these billions of dollars in foreign aid - the US Marshall Plan involved \$23 billion were small relative to European GDP, they may have been important in two ways: They helped cash-strapped governments to finance education and rebuild infrastructure destroyed during the war, and they provided foreign exchange with which to purchase American technology. Japan also received billions of dollars in American aid. The newly created IMF allowed countries to protect the value of their currency without being forced to reduce the money supply. Trade liberalization was pursued under the General Agreement on Tariffs and Trade (chapter 27). Rather than suddenly demobilize millions of soldiers, several countries developed systems of finance for ex-soldiers to pursue post-secondary education. This undoubtedly prevented a sharp rise in unemployment.

Postwar growth also in part reflected pent-up demand from decades of Depression and war. The automobile market may have been saturated in the United States in 1929, but auto factories operated below capacity in the 1930s and then were used to produce tanks and aircraft during the war. There was, therefore, a ready market for automobiles and a range of other consumer goods after 1945. The baby boom further accentuated consumer demand. Marriage rates fell during the Depression in most countries, and then the war separated millions of men of prime parenting age from spouses and

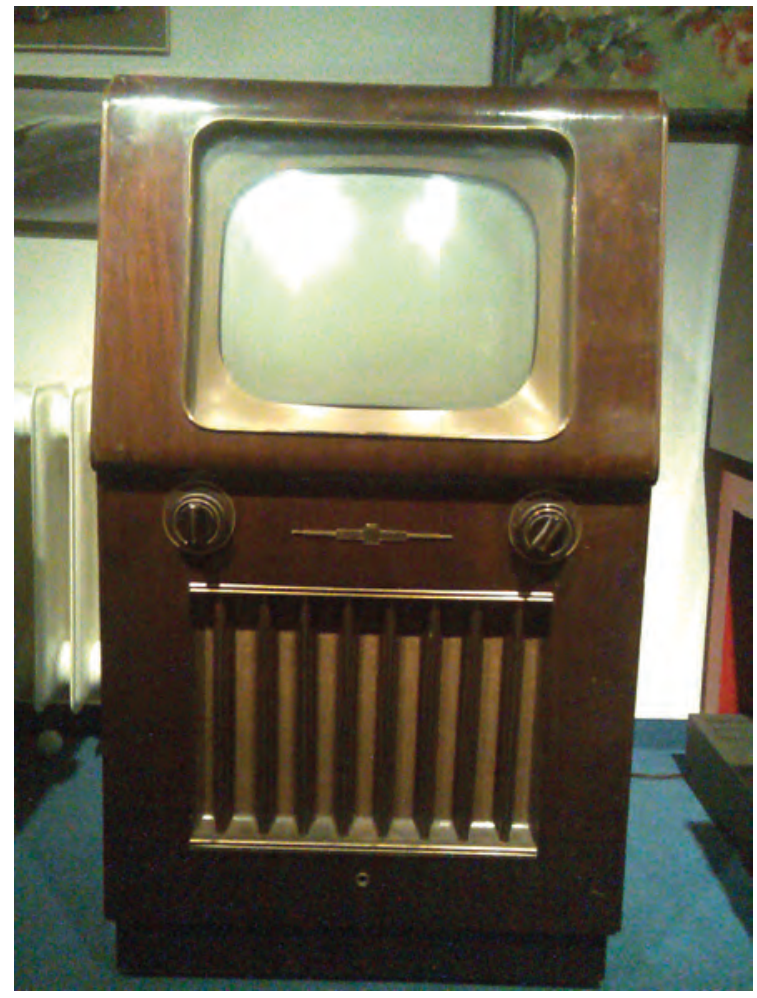

Illustration 29.6

Television receiver Nordmende Panoramic 54 360, 1953/54, with seventeen vacuum tubes 
girlfriends. Birth rates rose dramatically after 1945 across the developed world and remained high until at least 1960. As most modern parents can attest, having children increases one's consumption expenditures considerably.

Just as we can provide a technological explanation of the Great Depression, we can recognize an important role for technology in the Golden Age. The new products of the late 1930s - airplanes, television, and synthetic fibres especially - blossomed in the early postwar years. Aircraft production had focused on military uses during the war, and nylon had been devoted to parachute cords more than stockings. A range of synthetic fibres was developed in the early postwar period, and a wide range of plastics joined these. There were also significant developments in pharmaceuticals, and investment and employment grew there as well.

One irony of the Golden Age is that growth rates were particularly high in Germany and Japan, the losers of the war. As noted above, these were not penalized but instead encouraged in the early postwar years. The emergence of the Cold War (chapter 28) may have been important here, as both West Germany and Japan were seen in the West as strategic bulwarks against the Soviet Union and (after 1949) communist China. Yet there may also have been internal forces at work. We have seen throughout history that elite groups manage over time to gain advantages at the expense of the broader polity and economy. The Second World War discredited elites in both Germany and Japan and led to a radical restructuring of institutions of government. The result may

\section{BOX 29.2}

\section{REVISITING THE RULER'S CHALIENGE}

We have noted throughout this book that rulers everywhere face challenges from local elites. These elites find ways over time to gain control of economic resources that might otherwise be employed by the state. One controversial explanation of Japanese and German postwar economic success is that the war devastated previous elite groups and replaced the institutions that these had managed to put in place. New institutions were introduced in both countries that affected not only political decision-making but also economic activity. The intervention of the occupying forces - who wished to see economic growth as a counterweight to communist expansion, but were fearful of a return to authoritarianism in the lands they had just fought a long war against - may have further encouraged the introduction of institutions that facilitated economic growth for all more than they enhanced the profits of a few. Though the argument has a compelling logic, it is challenging to evaluate its empirical importance: How much does a particular institution contribute to economic growth? In particular, there has been much debate about the degree to which the Japanese state guided economic development: It did encourage some critical technological developments but also many failed initiatives. 
have been an institutional structure mainly oriented toward economic growth rather than rewarding particular elites.

As noted above, economists had become confident in the 1960s that economies could be guided to achieve steady growth with mild recessions. This confidence would be shaken in the 1970s when most world economies experienced both double-digit unemployment and inflation (even though unemployment is expected to place downward pressure on wages and prices). The unemployment of the 1970s can be attributed to the same sort of forces blamed for the Depression above, including a (less dramatic than in the 1930s) downturn in the rate of product innovation. The inflation and some of the unemployment reflected in part the formation of the OPEC oil cartel in the early 1970s and a resultant spike in the price of oil. Yet there may be a deeper reason why the 1970s mimicked in milder form the Depression experience of high unemployment and severe cycles. Some contemporary economists now appreciate that modern economies appear to be characterized by alternating periods of a decade or two in length: Periods of rapid growth and mild business cycles alternate with

\section{BOX 29.3 \\ THE CAUSES OF ECONOMIC GROWTH}

It is beyond the scope of this book to engage in a detailed theoretical discussion of the causes of economic growth. We have at times discussed particular episodes of economic growth, especially in this chapter and chapters $4,15,22$, and 24 . We have along the way engaged at some length with most of the phenomena identified by economic theory as affecting growth: technology (influenced by science in recent centuries), economic and political institutions, trade, networks, transport infrastructure (and resulting division of labour), education, population, health, geography, investment, and work effort. Figure 29.1 shows how these various phenomena interact with each other in affecting growth rates. Figure 29.1 is somewhat different from the other figures in this book, as it is borrowed from a previous publication and focuses only on the causes of economic growth (various effects of growth have been addressed in other chapters). It is a bit more detailed than other diagrams, reflecting the myriad influences on economic growth. We have discussed briefly above how aggregate economic growth interacts both with business cycles and the performance of individual sectors of the economy. We have not often used the word "entrepreneurship" in this book but have appreciated at many places that artisans, builders, merchants and industrialists and often bureaucrats as well - must identify economic opportunities and devote time and energy and money to developing these opportunities. Economists debate how much "entrepreneurship" differs across societies: When we see more investment in one society than another is this because there are more opportunities or more entrepreneurs (we discussed this debate briefly in chapter 24 regarding France and Germany). But undoubtedly different cultural attitudes toward risk or ambition or hard work might exert important influences 
on entrepreneurship. Such cultural attitudes are hard to identify, however, and may themselves reflect how many economic opportunities there are: Ambition is likely to be shunned if there are few opportunities to exercise this. The supply of entrepreneurs will also reflect social structure: Are some groups prevented from economic activity, or are some groups driven toward economic activity by being prevented from owning land or participating in government?

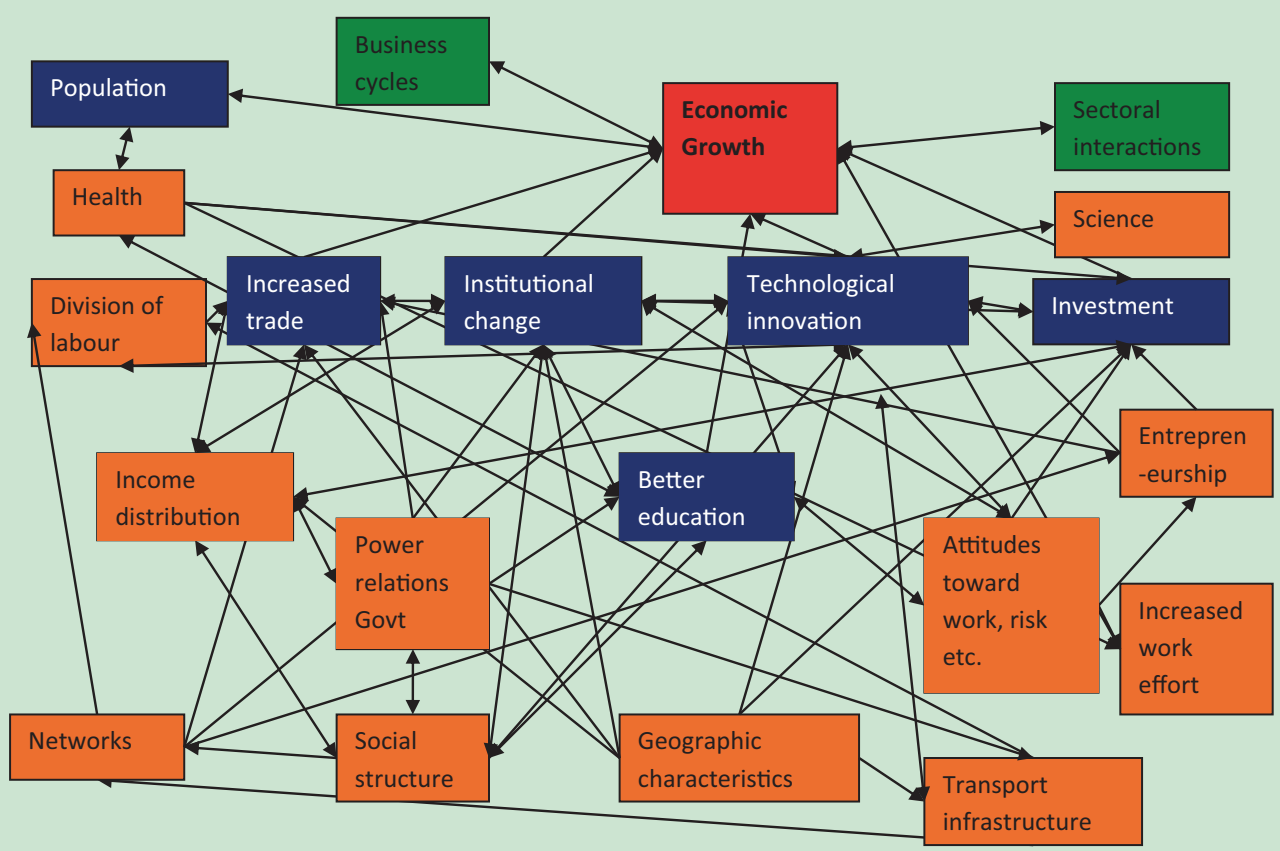

Figure 29.1

Influences on economic growth

Source: Repko, Szostak, and Buchberger (2020)

periods of sluggish growth or decline and severe cycles. The Golden Age may have been particularly wonderful because of pent-up demand and technology, but we can perhaps reasonably anticipate some periods of steady growth with mild recessions in future, but punctuated by periods of slower growth and higher unemployment. The 1990s and very early 2000s were such a period of sustained growth.

\section{Social policy revisited}

We recognized in chapter 27 that social policy expanded during both Depression and Golden Age. We can now place that vital transformation in context. 
When the Great Depression hit, most countries had few programmes in place to support the unemployed - though some countries such as Germany had developed some social policies, including public pensions, in the nineteenth century to appease the demands of urban workers. It often fell to local authorities or charities to provide aid, and these had limited resources. The unemployed struggled to feed and clothe and shelter themselves. As noted above, birth rates and marriage rates plummeted. Suicide rates rose. Indigent men travelled in search of work, often riding freight trains illegally. Though some men cycled in and out of part-time employment, many remained unemployed throughout the 1930s. For these, wartime enlistment into the army might seem a godsend. The hardest hit were the old and young: Those who lost their jobs in their 50s struggled to find another. Those who were entering the workforce in 1929 struggled to land their first job. Women were often laid off to save jobs for men. The reader can usefully speculate on what their life might have been like if they had turned 18 in 1929 and struggled to find work for a decade.

The rise of Nazism in interwar Germany (chapter 28) owed much to the dislocation of the Depression. And as noted above, the relative success of the Soviet Union during the 1930s inspired emulation, especially in developing countries. The Nazis appealed to a fear of communism in their electoral campaigns. Yet there were no (non-fascist) revolutions against capitalism in the developed world. Though economic calamity has often encouraged political revolution in

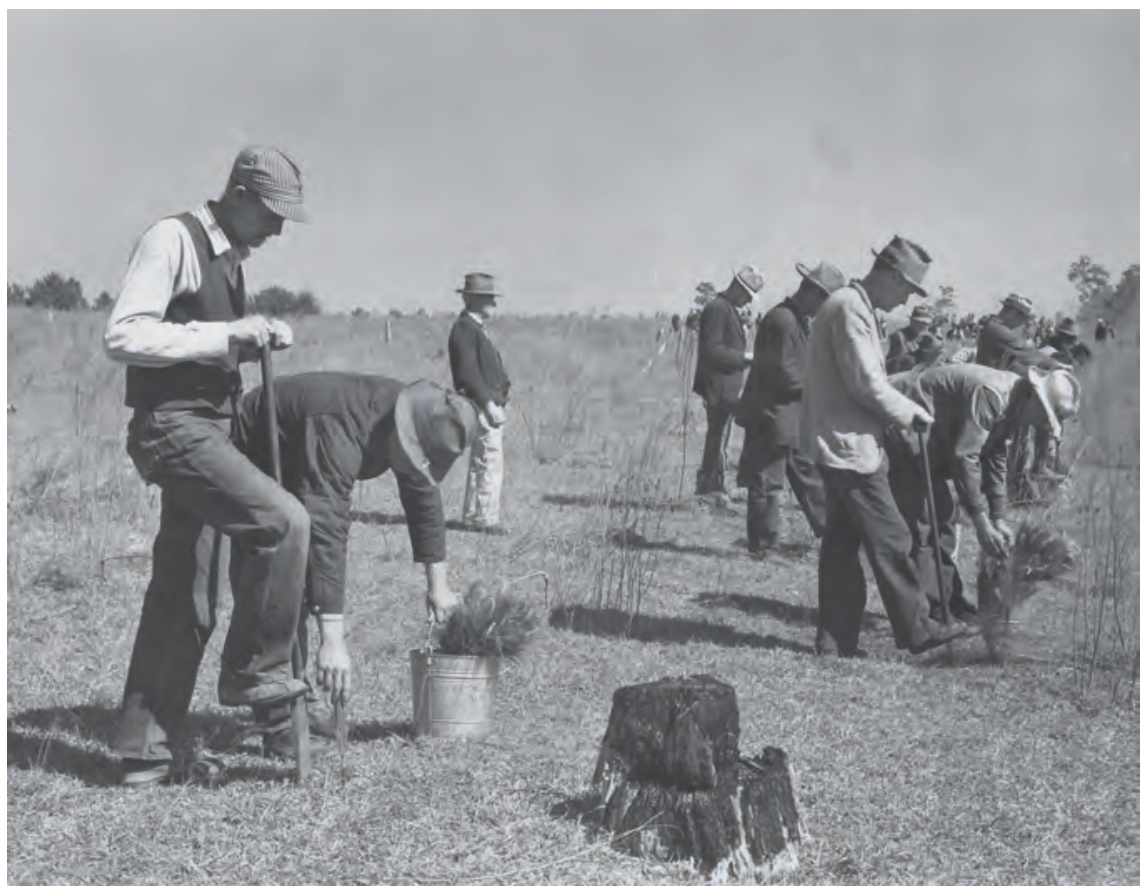

Illustration 29.7

Relief workers with the Civilian Conservation Corps plant trees in the United States, around 1933 


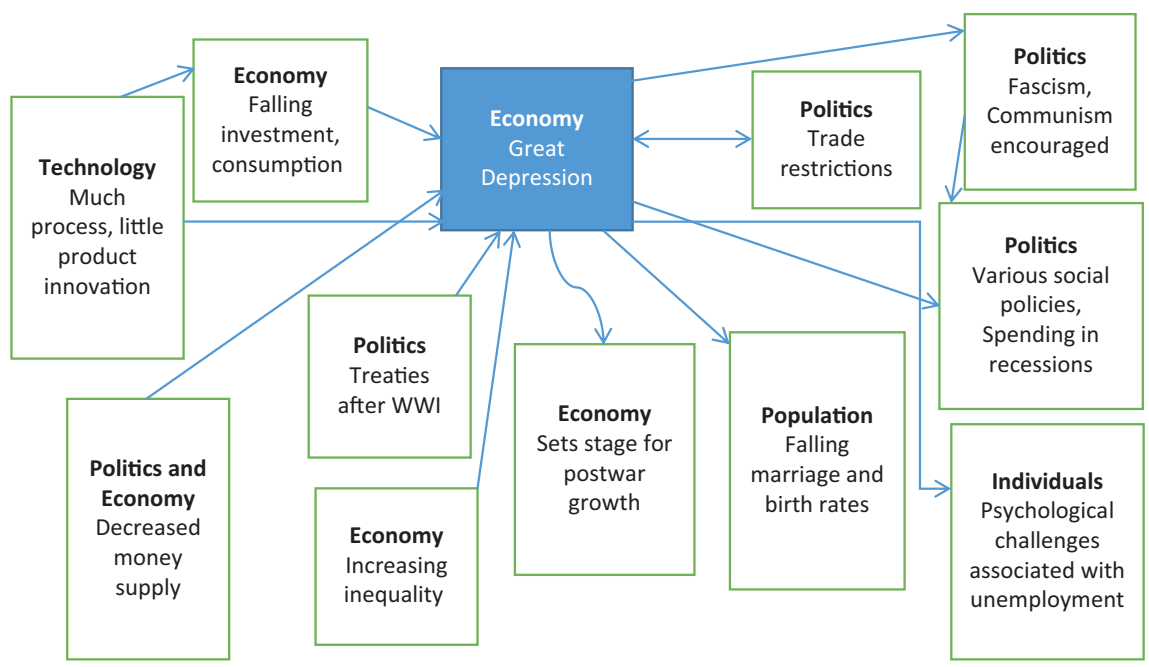

Figure 29.2

Influences on and effects of the Great Depression

world history, the unemployed primarily focused on survival rather than protest in the 1930s. Recall from Box 25.1 that revolutions occur only when several contributory factors are in place. The political stability of the 1930s in large part reflected the fact that governments everywhere struggled to put a basic social safety net in place. In some countries, such as the United States and Germany, this involved public employment programmes. Other countries favoured direct payments to the unemployed.

The growth experienced during the Golden Age made it easy to either enhance programmes established during the Depression or create new ones. Public pensions, unemployment insurance, and formal welfare programmes became common across the developed world. The Great Depression had established beyond doubt that individuals could suffer unemployment through no fault of their own. It was expected that individuals receiving government support would endeavour to find work. Over the next decades, as memories of the Depression faded, and as instances of abuse of these systems emerged, public support for such programmes receded (chapter 27). Those in positions of power and influence may also have felt less need to appease the poor as memories of the Depression faded and the fears of communist revolution disappeared.

\section{Convergence}

There are two sound theoretical arguments commonly made by economists as to why we might expect developing countries to grow faster than rich countries so that their per capita incomes would "converge" toward those of rich 
countries. The first focuses on investment. One of the reasons that poor countries are poor is that they lack capital: Poor country workers work with fewer and worse machines. This should mean that the rate of return on investment is higher in developing countries (because workers in rich countries already have expensive devices to work with). We might then anticipate funds to flow from rich to poor countries, causing the latter to grow faster. There has indeed been considerable foreign investment from rich to poor countries through much of the postwar period, but also some periods in which more money flowed the other way.

The second reason is technological. Developing countries are poor in part because they use older technologies. They can achieve growth by borrowing technology from rich countries. Rich countries, however, since they are already employing recent technology, are forced to innovate to adopt better technology. So the rate of technological advance can potentially be much higher in developing countries.

We might make similar arguments concerning the other proximate causes of growth in Figure 29.1. It may be easier to improve institutions or educational systems in developing countries than in rich countries because these institutions are often less well developed and ideas can be borrowed from other countries. But all of these theoretical possibilities will only be observed if developing countries are able and willing to absorb capital or technology or develop better institutions and schools.

Has there been convergence in the postwar world? If one looks at just the prosperous countries in the world - say, the membership of the Organisation for Economic Co-operation and Development - there has been convergence, in large part as European countries caught up to the per capita incomes of the United States and Canada. If one looks at all of the present members of the United Nations, then there is little evidence that developing countries have grown faster. But this result is mostly driven by the sluggish growth experience of many (often small) African states through much of the postwar period. If we weight countries by population in our calculations, then the rapid growth experienced by China and India in recent decades generates a substantial degree of convergence.

Although there is some evidence that developing countries can, in fact, converge or catch up to rich countries, the postwar record of growth is quite diverse. Some countries in Asia - first Japan, then South Korea, Taiwan, and Singapore, more recently China, India, and much of Southeast Asia - have experienced rapid growth. Indeed, they achieved growth rates that were higher and sustained longer than had ever been the case in Europe or North America. These countries generally exported labour-intensive goods such as textiles in their early days but progressed to more sophisticated manufactures. (India has been unusual in exporting services to a far greater extent than manufactured goods; facility with the English language coupled with advances in computer and communications technology have enabled this.) The economic rise of first Japan and more recently China has often been viewed with trepidation 
in Western countries, though Western consumers certainly benefited from Japanese quality improvements in automobiles and electronics (which were then copied by Western firms). Other countries in Asia, such as the Philippines, had much less impressive growth rates until very recently. African incomes generally grew more slowly than incomes in the rest of the world, but grew faster in many countries in the early twenty-first century, in part as the prices of commodity exports rose. Latin American economies on average performed far better than Africa but less spectacularly than Asia. Differences in institutional structures may be the main reason for cross-country differences in economic performance (and we will discuss one important source of institutional differences when we discuss decolonization in the next chapter - and say a bit more about the institutional experience of particular countries), though geographical and climatic differences may also be significant: Tropical countries, small countries, and landlocked countries are observed to grow more slowly on average.

One group of countries that has converged in recent decades is the formerly communist countries in Eastern Europe. Though incomes collapsed in the transition from communism to capitalism, growth has been rapid since. Membership in the European Union has given most of these countries access to one of the largest markets in the world, and encouraged the widespread adoption of Western economic institutions. These institutions have been supported culturally by citizens who self-identify as European and wish to catch up economically to Western Europe. It may have helped that decades of communism had destroyed the elites that had previously governed these countries, and created a well-educated population with an egalitarian ethos.

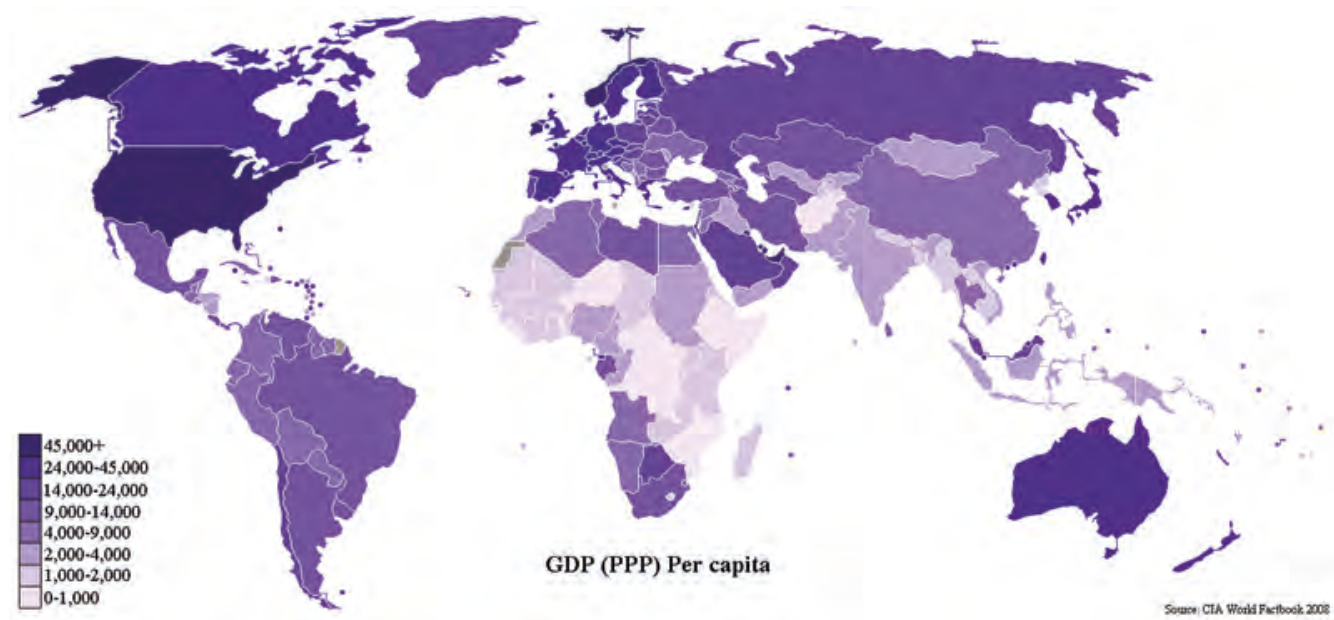

Map 29.1

GDP per capita by country, 2007

Map by Sbw0lf, CC BY-SA 3.0 


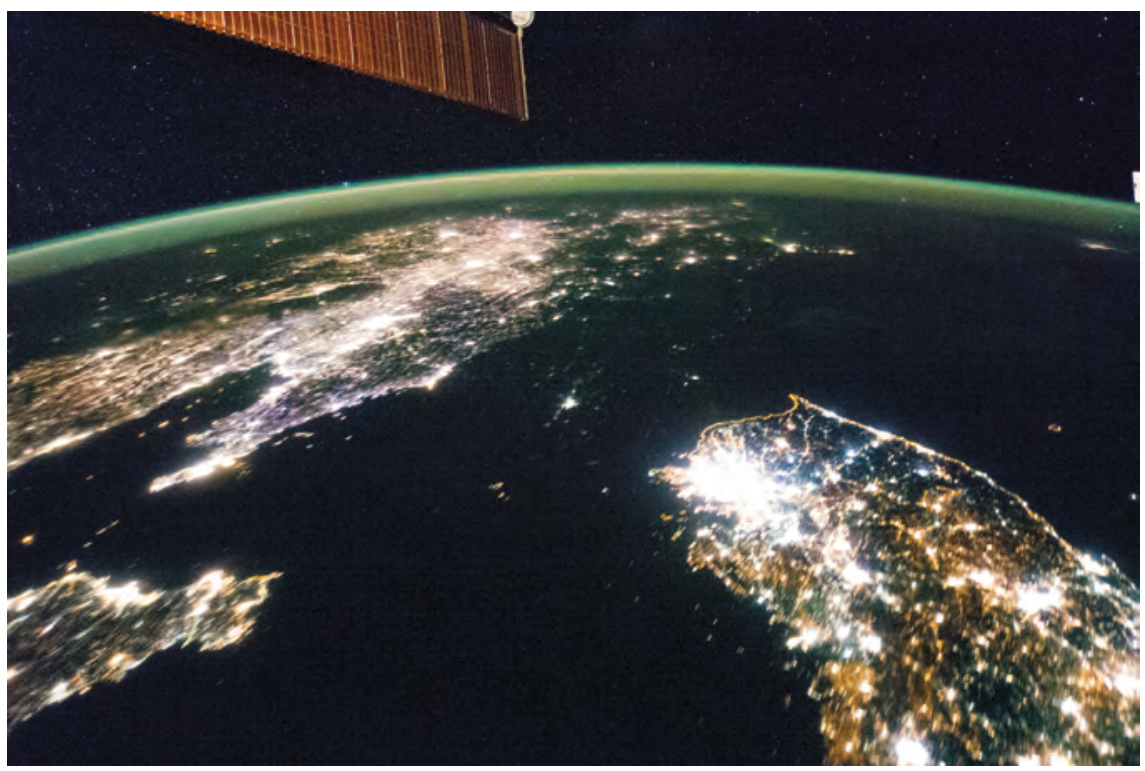

Illustration 29.8

Korean peninsula at night, from International Space Station, 2008. The stark institutional differences between North and South Korea are reflected in relative use of electric lighting

Nevertheless, corruption has been a problem in some countries. Farther east, most of the republics of the former Soviet Union have been governed autocratically since the fall of communism. These have generally been less willing or able to introduce trustworthy institutions.

Economic inequalities within countries are also significant. Income inequality is connected with social stratification: Countries with unequal incomes also tend to be countries with huge differences in social status by occupation. And income inequality is often reflected in elite political domination. Latin America has had among the worst levels of economic inequality in the world for centuries; that inequality long encouraged autocratic forms of government dominated by a narrow elite (chapter 25) and complicates democratic governance to this day. Economists have detected a small but important negative influence of inequality on economic growth: This may reflect the advantages of a middle-class mass market in stimulating investment in industry and services, but also perhaps the ability of narrow elites in unequal countries to enact economic regulations that serve their narrow interests at the expense of the broader population. Income inequality fell in most countries during the Depression, world wars, and Golden Age but has risen in most countries since. Technological change has played a significant role here in mechanizing some skilled occupations. And trade liberalization, while allowing some developing countries to catch up, has hurt the incomes of unskilled labour 


\section{BOX 29.4 AIR CONDITIONING}

One simple but now common technology may be critically important in narrowing the income gap between tropical and temperate countries: air conditioning. It is well known that human (and animal) work effort suffers in very hot and humid temperatures. While air conditioning has a limited effect in agriculture (but note that grain farmers in rich countries have air-conditioned farm machinery), it can revolutionize productivity in factory and office. Economic historians have conjectured that much of the economic development in the southern states of the United States in the postwar era was due to air conditioning. More recently, air conditioner use has expanded dramatically in China as that country has expanded its industrial sector. Perhaps the most dramatic story is in the Persian Gulf, where population has expanded from one million to twenty million in the last decades, and airconditioned homes and offices are ubiquitous. Air conditioning is becoming common in India, Indonesia, and other warm countries, but remains relatively rare in much of Africa. Yet air conditioning is not an unmixed blessing. It is an important source of climate change though increases in air conditioner efficiency and changes in the coolant used may mitigate this effect. The global stock of air conditioners was 1.6 billion in 2016, and was predicted at the time to grow by another billion in the next decade or so.

in rich countries. Profit levels have generally been high through much of the postwar period, allowing those who already have money to amass more. The highest-earning one per cent of the population has tended to do particularly well in recent decades. This may reflect in large part the expansion in global trade and investment. Executives in large multinational corporations are rewarded lavishly - though it is far from clear that these rewards reflect their contribution to corporate profits. The most popular entertainers have also seen earnings skyrocket as movies and music are marketed worldwide - though recent technological changes have created intellectual property challenges for musicians in particular. The most dramatic worsening of income distribution in recent decades has occurred in China, where movement toward a market economy has transformed one of the more equal (but poor) economies in the world in 1970 into one of the more unequal economies in the world in the early twenty-first century. This transformation should remind us that institutional choices made by governments have an important effect on inequality. We should also note that economic growth has occurred so rapidly that hundreds of millions of Chinese have been lifted out of extreme poverty (chapter 30) despite the severe worsening in income inequality. That is, the poor have seen their incomes grow, but not anywhere near as fast as those now at the top of the income distribution. 


\section{BOX 29.5}

\section{THE HISTORICAL EXPERIENCE OF INEQUALITY}

We saw way back in chapter 5 that income inequality had likely increased through most of the history of agrarian or nomadic societies - but with occasional setbacks, notably the fall of the Roman and Han Empires, and the Great Plague of the fourteenth century. (There were also more local shocks that temporarily reduced inequality in particular times and places.) Inequality then increased again for many centuries but appears to have stabilized or fallen in many countries in the late nineteenth century, perhaps because of the rapid increase in the size of the middle class (chapter 23). We identified certain critical sources of increased inequality in chapter 5: political consolidation, expansion in trade, increased scale of land ownership. We noted there and in chapter 10 the importance of inheritance: Parents could pass landholdings in particular but wealth more generally to their children. Wealth could then be accumulated over generations. And that wealth could then be invested to generate even more wealth. The existence of profitable investment opportunities would then allow the rich to grow richer while most of humanity struggled to finance basic consumption.

Capitalism (chapter 18) and industrialization (chapter 24) allowed industrialists and merchants to join landowners and political authorities in the economic elite. Wealth could now be reinvested by elites in industry and commerce as well as in land (to a greater degree than in most previous societies).

The period from 1914 to about the 1970s would serve as a whole as another shock to levels of inequality (with the 1920s providing a brief exception in which inequality rose). We saw in chapter 28 that both world wars were hugely expensive and hugely destructive. Many industrialists and merchants suffered as their buildings were bombed to rubble. More generally, though, the rich suffered as governments everywhere financed the wars through unprecedented levels of taxation and unforeseen levels of inflation (which destroyed financial fortunes in many countries). Some countries also introduced price and rent controls or even nationalized private property. (Most previous wars in world history had not had anywhere near as significant an economic impact and served mainly to merely transfer resources between losing and winning elites.) We have seen above that the Depression was also devastating: While the poor suffered grievously, the rich had more to lose financially. The net effect of both wars and Depression was a dramatic decrease in elite wealth holdings and thus the incomes that could be earned from these. The share of national income earned by the top one per cent fell by a dramatic two-thirds in Japan between 1914 and 1945, by about one-half in France, and by a third in the United States.

Inequality continued to decline in the first postwar decades in most countries. Tax rates on the highest incomes remained high. Economic elites only slowly built or rebuilt large fortunes to invest. As we saw above, a range of social programmes were put in place which transferred income to both the least fortunate and the broader working and middle classes. Wages rose with increased educational attainment and technological advances which increased the productivity of unskilled labour - and because labour forces grew very slowly as a result of low birth rates during Depression and war. Many scholars posited the time 
that there might be a natural tendency for inequality to lessen in modern economies as incomes of working and middle classes rose.

Across the globe, though, measures of inequality have tended to rise since the 1970 s or 1980s. Though land consolidation is no longer a significant contributor to increased inequality in most countries, some of the other mechanisms observed historically are still at work. Trade has expanded dramatically; we saw above that this has enhanced elite incomes more than the incomes of workers (though the entire population has benefited on average). Wealth is transmitted across generations and reinvested. There have also been institutional changes such as decreases in both the top rates of income tax and inheritance tax in many countries. These reflect in part the political influence that the rich have tended to exert in most human societies. But these changes in taxation also are in part a reflection of a world which is linked economically but governed by nation states: Nation states fear that the wealthy will just leave if taxed too highly. International cooperation in taxation policy has proven a challenge. Technological innovation has also played a significant role: The jobs previously held by unskilled workers were often mechanized, causing the incomes of some of the poorest to fall further.

Increased inequality - and especially the incomes and wealth of the top one per cent has become an important political issue in many countries. Beyond questions of fairness, people worry that increased inequality may hamper economic growth and interfere with democracy. World history can usefully inform these debates. One clear lesson from history is that institutions matter: Most obviously, taxation and social policy affect income distribution. Levels of corruption also matter because today, as in the past, those with political connections are often able to profit from these. Economic and political elites are closely intertwined in many countries. This suggests that addressing inequality institutionally is both desirable and difficult. The historical record suggests that significant decreases in income inequality are relatively rare and have always been associated with calamities such as epidemics, the collapse of large states, world wars, or depression. The pessimist can doubt that the political influence of economic elites can be challenged in times of peace and stability in a manner that would markedly affect the degree of income inequality in a society. Last but not least, we should not forget that trends in technology have come to play an important role in the distribution of income: Whereas it has been the working class that has been most affected by technology in the past centuries, it is entirely possible that it is the middle class that will be most affected going forward as computers develop the capacity to make decisions on the basis of machine learning and the analysis of massive data sets.

An even scarier historical lesson is that states have often suffered and fallen through history as economic elites came to control a larger part of the economy. If emperors can succumb to the efforts of economic elites, are democratic governments immune? We have noted above that economic elites may over time shape democratic institutions to serve their purposes. Such efforts weaken public support for democracy. History tells us that elites often suffer when states collapse - but this may not dissuade elites from weakening the states on which they depend in the pursuit of their own narrow interests. 


\section{Consumer culture}

As incomes have risen in many developing countries, consumers have tended to embrace a broadly similar set of consumer goods to those long consumed in rich countries: automobiles, refrigerators, televisions, and so on. Often Western "luxury" brands of clothing or handbags or cosmetics or beverages or even consumer electronics have become very popular in these countries, serving as a signal of newfound prosperity.

Many commentators worry about the spread of "consumerism" and especially conspicuous consumption: the purchase of goods that primarily serve to advertise one's income. Some note that increased purchases of such products do not seem to have made the average Western consumer happier in recent decades. Others worry about cultural homogenization: that the acquisition of similar goods encourages similar cultural attitudes. But it is not clear that buying a car or refrigerator has a huge impact on culture (beyond, perhaps, increasing interest in economic growth itself). Local cuisines and dances and music have generally proven robust. The international spread of restaurant chains and beverage companies, and the development of global markets for music and movies (though less so television) is seen as a threat by some and as enhancing personal choice and cross-cultural understanding by others.

It would be a mistake to think that American or European consumer culture has spread inexorably and exclusively through the rest of the world. Latin American countries developed shopping centres before Europeans in the early postwar decades (in part as automobile usage was higher for a while). The Brazilian soap operas that are now exported worldwide emerged in the early postwar period, largely independently from American soap operas. The Japanese Pokémon was homegrown but soon transfixed American children in the 1990s - as have Japanese manga and animé and Korean K-pop music more recently. Mexicans adopted the idea of comic books from the United States in the 1930s, but Mexican comics were often infused with anti-American sentiments. And multinational firms recognized very early on that they needed to tailor advertising messages - and often products themselves - to local cultures. McDonald's changed its menu as it expanded internationally, offering wine in some countries and emphasizing vegetarian fare in India. We discussed the importance of syncretism with respect to religion in chapter 11: Syncretism was also common with respect to consumerism as goods and services from one country were reinterpreted or transformed in another to accord with local tastes.

Though we can debate how great the cultural impact of expanded trade has been on poorer countries in general, the effect on Indigenous peoples has often been catastrophic. Indigenous peoples throughout the world have attempted to adjust to dramatic economic transformation in just a couple of generations. The cultural attitudes generally associated with hunting and gathering are quite different from those associated with modern industrial societies. Recall that 
culture tells us how to behave and how to expect others to act. Cultures also tells us what is important in life. Dramatic changes in culture are destabilizing because one no longer knows what to expect or how to behave, and one may question the very meaning of life. Individuals with a distinct role in a small society may wonder about their place in a larger world that is different in many ways. Indigenous peoples have struggled to adapt to modern realities, and have wondered how much of their cultural inheritance they can and should maintain.

\section{Globalization}

We have not used the word "globalization" much in this book. As noted in chapter 1, the word conflates increased international interactions in the economic, political, and cultural spheres, tends to imply that these are closely intertwined, and also tends to suggest that globalization is novel, important, and troublesome. We have found it more useful to address particular elements of globalization separately. We addressed consumer culture briefly above. We have discussed political interactions in chapters 25,26 , and 30 . We can focus here on expanded trade and investment in the postwar world.

About one-fifth of global economic output is traded internationally. This proportion can be viewed as big or small depending on your perspective: It means that four-fifths of global production is consumed domestically. The onefifth figure for trade is, though, the highest proportion ever achieved in world history. Trade expansion at a rate of several per cent per year postwar has been facilitated by international treaties, especially those associated with the General Agreement on Tariffs and Trade (1948-95) and the World Trade Organization from 1995 (see chapter 27). Both GATT and WTO have sponsored years-long rounds of negotiation which have resulted in decreases in both tariff and nontariff barriers to trade: Tariffs are effectively taxes on goods crossing borders, while non-tariff barriers come in a variety of forms. The most controversial non-tariff barriers are rules governing methods of manufacture: These may be seen as a trade barrier by some but as worthy protections of health or safety or environment by others. The GATT and WTO have generally insisted that member countries charge the same tariff rates against all others: The pre-GATT world was one where countries often had a bewildering array of different tariff rates on goods from different countries. But GATT and WTO have made two exceptions. One is to allow a generally lower level of tariffs on goods from developing countries. The other has been to allow regional trade agreements. The most successful of these has been what is now the European Union, which began in the aftermath of the war (to achieve peace through economic prosperity and trade) as an agreement governing coal and steel among six countries and has over the decades achieved a mostly free flow of goods and people across twenty-eight countries (though the United Kingdom voted to leave in 
2016 and left in January 2020). But almost every region of the world has some sort of - often multiple and overlapping - trade deal.

In addition to reductions in trade barriers, there has been a reduction in transport costs. Ships have become bigger, lowering the per-unit costs of transport. Railroads and highways have been extended - though parts of the world, such as much of sub-Saharan Africa, are still poorly served by these. The most significant single innovation in goods transport since World War II has been the humble container. It is now a cornerstone of shipping, railways, and road transport worldwide. Though efforts toward containerization can be traced from the dawn of railways, the modern standardized container emerged in the United States only in the 1950s. It then took a decade for international agreement on a standard size, and then for purpose-built ships to be constructed. Its adoption was often vehemently imposed by labour unions in shipyards, for containerization greatly facilitated the loading and unloading of vessels. Costs of trans-shipment fell to 5 per cent of the previous level. Employment in ports is today a fraction of its 1960 level despite a dramatic increase in cargo. Sailors too have been affected: They now spend far less time in foreign ports. And new ports with room for massive cranes and storage yards (like Oakland) grew to replace smaller ports (like San Francisco) - and some of the romance of ports was lost as one could no longer see what was being unloaded. On the other hand, many cities have transformed old ports into waterfront esplanades and parks which have become entertainment and restaurant centres.

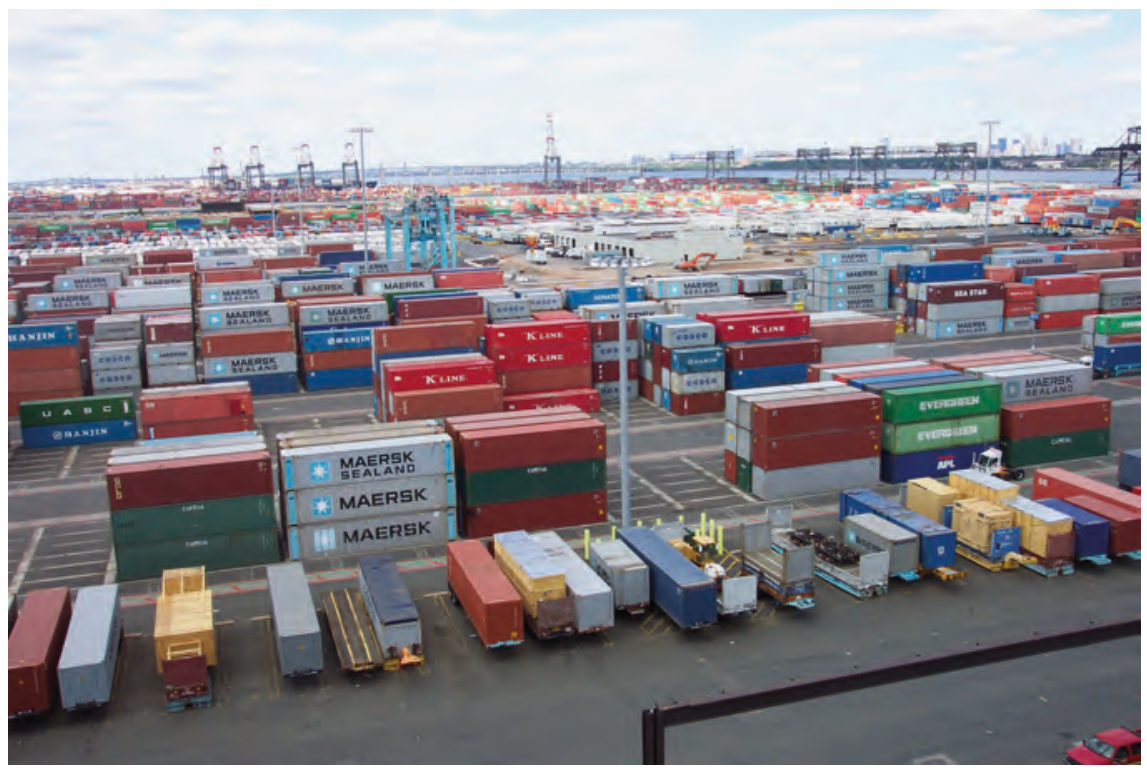

Illustration 29.9

Shipping containers at the terminal at Port Elizabeth, New Jersey 
Goods often are transferred between ship, rail, and truck multiple times in a journey: Containers allow these transfers to occur with much less effort. Manual labour was replaced by massive cranes that could move containers off and on ships and by large forklifts in rail depots. Computer programs were devised to inform the order in which thousands of containers were to be offloaded from a single vessel. One challenge has been with border crossings; border agents generally examine the paperwork associated with containers and search only those containers that attract suspicion. For many goods, the cost of global transport has fallen below one per cent of the cost of production of the good. International trade in almost every commodity is now possible.

In addition to the changes in transport itself, there have been even more dramatic changes in communications. With the internet, people anywhere in the world can find out the prices of goods elsewhere in the world (though, as with trade, the vast bulk of internet traffic is domestic). Small flower producers in East Africa can find out on their mobile phones what price flowers are selling for in various European cities. (Many developing countries that had not developed sophisticated telephone networks have seen rapid adoption of mobile phones). Telephones, email, and videoconferencing have each in their own way facilitated trade by making it easier for merchants and others to converse. Face-to-face conversations are still important, but these too have been aided by a fall in the cost of air transport as bigger, faster, and more efficient aircraft have been developed (and as governments have encouraged competition in the air transport industry).

Most trade in the world still occurs between high-income countries. But trade between rich and poor countries has grown. Such trade is more likely when countries share a language, currency, or colonial past. Inexpensive textiles are now rarely produced in the rich world but are imported from countries such as Bangladesh. As noted above, trade expansion has shifted many low-skilled jobs from rich to poor countries. These low-paying jobs then allow some of the poorest people in the world to better support their families - but there have been notable concerns regarding the health and safety and environmental standards of factories in developing countries. It is often forgotten that developing countries have used their income from exports to increase their purchases of more complex products - which involve more recent technology and more capital and skilled labour in production - from rich countries. One public policy challenge in rich countries is to train workers so that they can move from less-skilled toward more-skilled jobs.

One reasonably novel characteristic of contemporary trade is the existence of long supply chains. Different stages of production may occur in several different countries, or the components of a complex machine such as an airplane may be manufactured in several different countries. Indeed, by 2005 over half of trade in goods was trade in intermediate products rather than raw materials or finished goods. The service sector has been even more affected by international supply chains: In 2005 almost three-quarters of service trade 
was in intermediate services. Call centres and software support are among the tasks that may be performed in quite different countries. These international supply chains allow firms to perform tasks that require particular materials or particular types of labour where these are abundant. They lower costs of production and serve, among other things, to move unskilled jobs toward poorer countries.

A global market has emerged for works of art. To be sure, local tastes are still important, and most art is still sold locally. Nationalism is still an important theme in the world of art - and newly independent states (chapter 30) have often witnessed a revival of local art forms. But collectors and galleries on all continents actively pursue works of art from other regions of the world. The global art market had been growing for centuries (chapter 18) but this process intensified in the nineteenth and especially twentieth centuries with expanded tourism and communications. The increased secularization of art both reflected and encouraged this global market. Artists themselves became better acquainted with the works of other lands - in much of the world they could view artworks from distant lands in local galleries. And artists came to produce with an eye to global markets. These markets were no longer dominated by a narrow ruling elite. (This mass market, along with some degree of mechanization and mass production, has led some to decry a decrease in artistic quality.) Increasing incomes have allowed this global art market to fragment: Whereas historically there was one dominant art style in most human societies, there are now many different styles. These different styles are regularly fused. The Impressionists of late-nineteenth-century Europe were already explicitly borrowing from

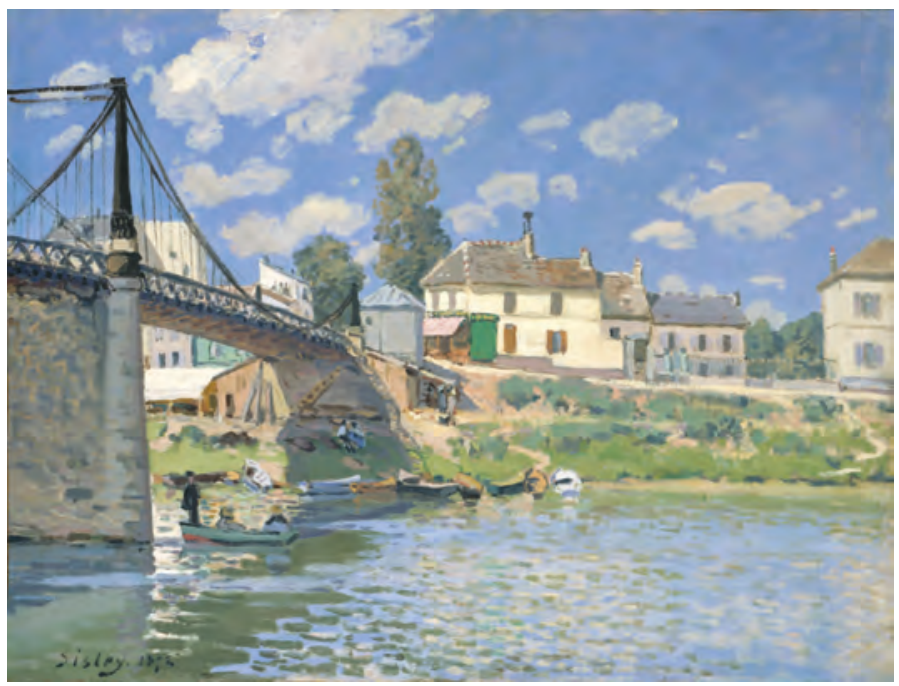

Illustration 29.10

Early Impressionist art: Alfred Sisley, The Bridge at Villeneuve-la-Garenne, 1872, Metropolitan Museum of Art 
Japanese printmakers, Islamic designs, and the arts of traditional societies. In the twentieth century, individual artworks can easily reflect influences from several once-distinct artistic traditions. And it may no longer be possible for any work of art anywhere to reflect only one artistic tradition.

Postscript: The development of the camera in the late nineteenth century encouraged many (but far from all) artists thereafter to move away from representational art toward a variety of types of non-representational art, including cubism, which used geometrical forms and multi-dimensional perspectives, and abstract art. The contemporary global but diverse art market takes a variety of approaches to the representation of subject matter.

Opera provides a useful example of artistic globalization. Opera was developed in sixteenth-century Italy but only became the most prestigious form of theatre in the early nineteenth century in Europe itself. By the end of that century, there were elaborate opera houses on every continent, and famous opera singers toured internationally. The Ottoman Empire and Brazil were particularly appreciative of opera. Many cities around the world still possess elaborate nineteenth-century opera houses. For some reason, the most famous operas are still those of the nineteenth or earlier centuries. There was an independent development of Chinese opera, but this never gained the same international acclaim.

Trade liberalization has often been politically unpopular in recent decades. Street protests surrounded a WTO meeting in Seattle in 1999 and have since become a regular feature of such meetings. Some of the hostility to trade may reflect the fact that it is generally much easier to identify those who have or will suffer from trade than those who benefit. In particular, consumers often fail to appreciate how much less expensive a variety of goods and services are either because these are produced in other countries or because local firms were forced to cut costs to compete with imports. Even less obvious are the technological advances associated with trade, but we have often seen in this book how technologies are transmitted and improved because of trade. This does not mean that every trade deal deserves applause. Developing countries with limited bureaucracies may be hampered in their ability to negotiate such accords. And some developing countries - like many of the states we have encountered in history - are highly dependent on tariff revenue because they lack the bureaucracies to effectively tax production. As noted above, governments must be careful not to sacrifice health or safety or the environment in the pursuit of freer trade. And there are legitimate concerns about the greater uncertainty in unemployment that comes with global trade: Workers may suddenly find that their jobs have moved elsewhere in the world (though changes in technology 


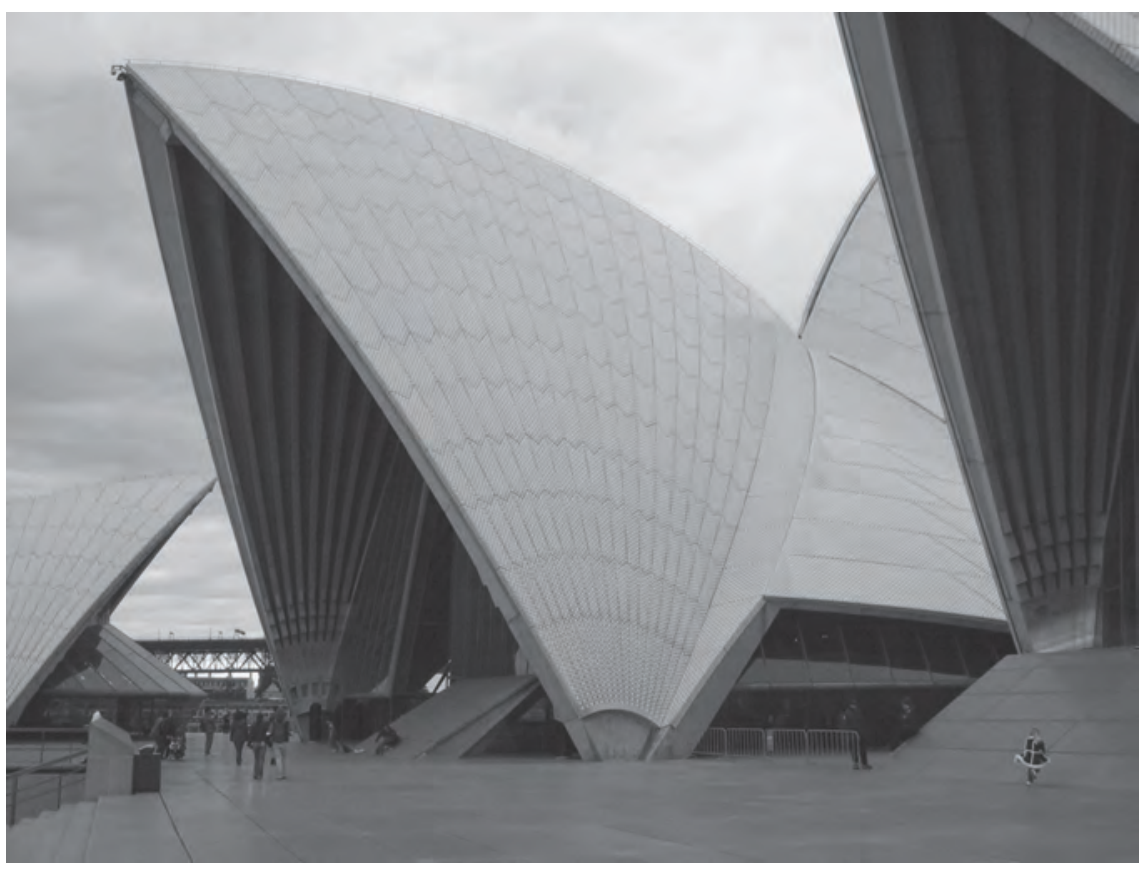

Illustration 29.11

Sydney Opera House

may be an even greater cause of employment instability). Many governments have programmes to aid such workers in transition, but these are rarely enough.

Much of the hostility to recent trade negotiations reflects the fact that these increasingly engage trade in services rather than goods. And here the main barriers to trade involve not tariffs but government regulations regarding how and by whom such services can be produced. Think here of the voluminous regulations of financial services that exist in the world. Liberalizing trade in services requires that countries harmonize regulations, sacrificing some degree of national sovereignty. Nationalists (chapter 21) have for this reason often opposed this sort of trade liberalization. We will discuss how these concerns might be addressed in chapter 32 .

There are also legitimate concerns regarding increased investment flows. These flows come in two broad types. Short-term international investments in financial instruments such as stocks or bonds have proven highly volatile. As several Asian countries discovered during the 1997 financial crisis, if investors become just a little concerned about a particular country, tens or hundreds of millions of dollars can suddenly move elsewhere, with dramatic effects on exchange rates and money supply and the availability of funds for investment. (Note that financial markets seem to exhibit herd behaviour such that actions by a few investors can encourage many to follow.) It is these short-term investment flows that cause exchange rates in the world to fluctuate far more 
than trends in international trade itself would justify. Some economists have suggested - controversially to be sure - a tax of some sort on such international transactions to decrease this volatility. But even these economists appreciate the potential advantage of funds flowing quickly into a country with much growth potential.

The other type of investment is longer-term. Multinational corporations (MNCs) build factories or offices or stores or mines in more than one country. Though only a tiny proportion of the world's firms operate internationally (about 1 per cent for the United States), many of these firms are huge. Such companies can produce goods or services where these are cheapest to produce. They can also potentially cut costs by serving a global market (if costs per unit fall as more is produced). But in achieving these gains, MNCs necessarily increase the volatility of employment, for they may reallocate production across the countries in which they operate. And they achieve through advertising a greater homogeneity in consumer purchases than might otherwise exist - though it must be stressed that consumers see value in international brands (and we saw above that MNCs tailor products to different markets). On the other hand, MNCs often transmit technologies to poorer countries and provide training to local workers.

The primary concern with MNCs, though, is the power imbalance between these and governments. Many MNCs have bigger budgets than many small developing countries. Many developing countries have limited or corrupt bureaucracies. MNCs may bribe or lie to government officials to obtain mining concessions for far less than these are worth. They might also encourage such governments to relax health and safety and environmental regulations or labour laws (though on average MNCs are observed to pay higher-thanaverage wages in developing countries). MNCs have often, especially in Latin America, collaborated with governments to push peasants off land to make room for coffee or banana plantations. The average person in a developing country may then benefit less from trade and investment than they should, and may even be made to suffer. Plantations have often also been responsible for extensive deforestation. Though the greatest dangers lie with respect to developing countries, even rich countries may face challenges in dealing with MNCs. Local politicians often provide subsidies to MNCs to keep or create local jobs. The very flexibility of MNCs increases their bargaining power. MNCs may also call on political and even military support from their home countries as they negotiate with foreign countries. The United States intervened militarily in Central America and the Caribbean at the urging of MNCs on several occasions in the late nineteenth century. And last but not least, MNCs can use various accounting procedures to ensure that most of their taxable income is taxed in countries with low tax rates.

The very largest MNCs have grown in both size and market power (that is, domination of particular sectors) in recent decades. Such firms often serve 


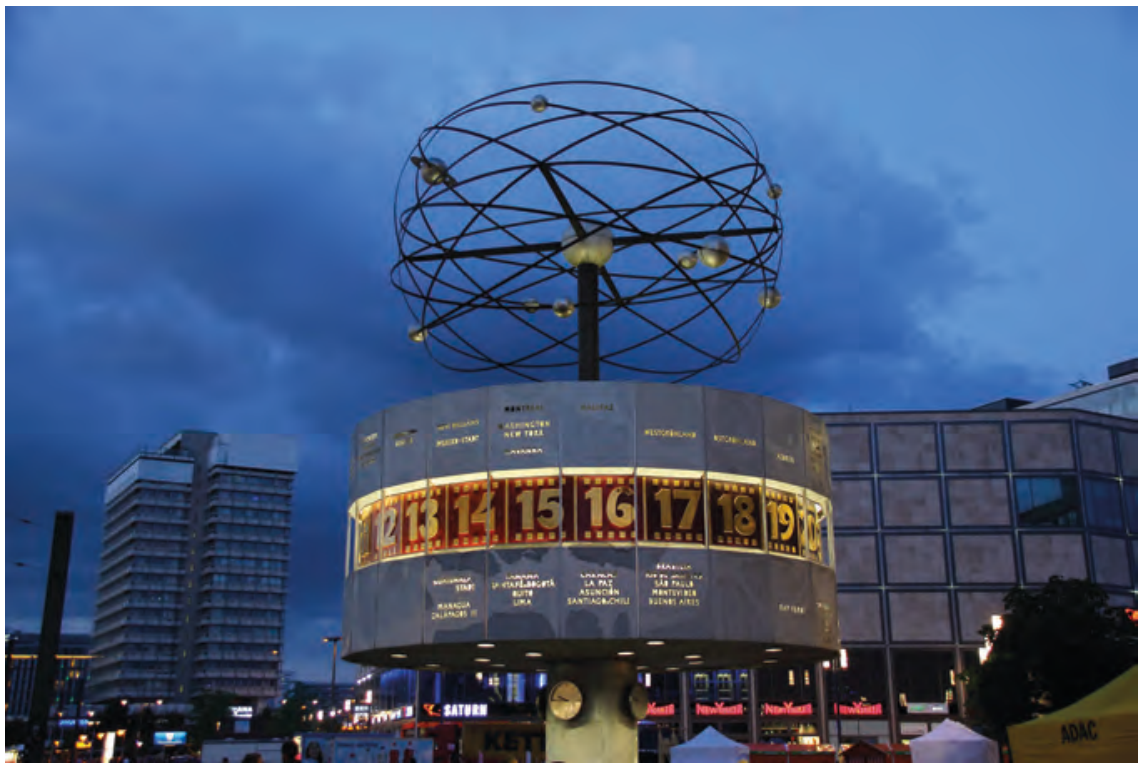

Illustration 29.12

World Clock, Berlin, from 1969, shows the time in 148 world cities

the entire world. In some sectors - such as social media - there are network effects such that consumers are drawn to firms that already have many clients. In sectors characterized by continual technological innovation, only one or a few firms can support the research laboratories necessary to remain at the technological forefront. Though such firms may perform valuable research and facilitate international collaboration, they exacerbate the challenges associated with MNCs.

There have been reactions to each of these challenges. Many rich countries now have laws against bribery, and firms and managers have been prosecuted for bribing other governments. A variety of non-government organizations (NGOs) track and expose the behaviour of MNCs: Many MNCs have in response come to protect their brand by encouraging better treatment of their workers in other countries (whether these are directly employed or through subcontractors). NGOs also encourage greater financial transparency from both MNCs and governments. Governments are trying to change regulatory practices and put pressure on low-tax jurisdictions, to reduce opportunities for tax avoidance - but also sometimes are tempted to cut their own tax rates. Environmental and labour rights clauses are increasingly standard in trade agreements. Trade treaties sometimes - and again controversially - limit the ability of any level of government to subsidize local production. 


\section{BOX 29.6}

\section{THE HISTORICAL NOVELTY OF GLOBALIZATION}

One fundamental question in the literature on globalization is whether we are witnessing something dramatically new, or whether globalization has a long historical pedigree. Of course, some aspects of postwar economic globalization are new: the specific technology of containers or the internet, or global supply chains in which MNCs or others may pass raw materials through production processes in several countries before marketing a finished good. On the other hand, raw materials from one place have been used in manufactures in another for millennia. And we have traced in this book numerous advances over the millennia in transport and communications technology. Still, the very fact that each one of us can instantaneously observe what is happening in the rest of the world may be considered a revolution in global perceptions with implications far beyond trade and investment.

Intercontinental trade was, in general, a luxury trade through most of human history. But bulk cargoes of timber and wheat and cotton have moved thousands of kilometres for centuries. The novelty today is that almost any good can be shipped long distances. Containers lower the cost of smallish shipments of anything non-perishable. And advances in refrigeration and aircraft speed allow food and flowers to be sent thousands of kilometres. And advances in telecommunications technology mean that services too can now be traded internationally - India, in particular, has benefited from an ability to provide English-language customer service and technical support services, though several African countries and the Philippines are trying to expand in these areas.

International trade relative to global GDP surpassed its 1910 peak by 1970 and has expanded reasonably steadily ever since. Trade had been growing through most of the nineteenth century, but then was interrupted by world wars and fell sharply during the Great Depression. On the other hand, international migration levels are far lower than they were in the late nineteenth century (chapter 31).

Even small amounts of trade can have important cultural and economic impacts. We have described in earlier chapters how Indian cottons and Chinese porcelain inspired industrial innovation in Europe, and how imports of coffee, tea, and cocoa changed daily life in Europe and may have encouraged people to work harder.

We can marvel at our ability to fly anywhere in the world in a matter of hours. But we should also appreciate that the earliest steamships reduced trans-Atlantic passage to two weeks in each direction (six days by 1900) from three weeks eastward and six westward by sail. Sailing speeds themselves had been rising through history (with significant advances in the eighteenth and nineteenth centuries as prevailing winds and ocean currents were better mapped and this information shared internationally). The railway led to a similar revolution in travel times by land, and this also followed advances in the speed of coach travel. 
We have spent much of this book describing the incidence and effects of cross-cultural contact. Stories, songs, and art styles have moved cross-culturally for millennia. Central Asian dancers were famed in China during the days of the old Silk Road. But before modern streaming services and global movie distribution, it was not possible for the same performer to be seen in multiple countries at the same time. Cultural transmission can now be instantaneous. As noted above, this allows some entertainers to serve a global audience.

We spoke of MNCs above. Only recently has the ratio of foreign direct investment to global GDP risen above levels achieved in the late nineteenth century. Those earlier investment flows financed railways and steel and cotton mills and electrification. On the other hand, contemporary levels of short-term capital flows are dramatically higher than ever seen before: There is far more money in the world and international financial institutions facilitate its rapid transmission. The minute-by-minute volatility in exchange rates is something that economic agents had not had to deal with until the last decades.

Hopefully, you were not expecting to be able to give a straightforward "yes" or "no" answer on the final exam.

\section{PRIMARY DOCUMENTS: SOME QUOTES ABOUT GLOBALIZATION}

In an era of globalization, people recognize that they are part of a global society, but they have no idea how to make such a society work. So far, no unified vision or leadership has emerged to guide us in this endeavour. We have not yet found a way to expand the spiritual ideals of democracy so that they pertain to every human being, every animal, and every plant. Until we do, human civilization and the Earth's ecosystem will continue to be in peril.

Victor Shamas, The Way of Play: Reclaiming Divine Fun \& Celebration

In today's globalized world nothing is sure. Routines are falling; stereotypes are breaking. Life has never been as piquant as it is now. So go out of your way; leave your cocoon. Do that crazy thing and be happy you did it.

Ogwo David Emenike

Globalization has made national boundaries more porous but not irrelevant. Nor does globalization mean the creation of a universal community.

Joseph S. Nye Jr.

Do these (and other) authors have a shared vision of globalization? Do they understand the term in the same way? 


\section{Questions}

1. Identify where each phenomenon in Figure 29.1 was addressed in this book. Was each arrow in that diagram discussed someplace?

2. Should we expect a repeat of the Golden Age? What about the Great Depression?

3. Why is so much attention paid to globalization by both scholars and others? Why do they often find it useful not to define the term?

4. Can economic change happen "too fast"?

5. What lessons might you draw from this chapter that would help you guide your own future?

\section{Readings}

Bernanke, Ben S. 2000. Essays on the Great Depression. Princeton University Press.

Crafts, Nicholas, and Gianni Toniolo. 1996. Economic Growth in Europe since 1945. New York: Cambridge University Press.

de Zwart, Pim, and Jan Luiten van Zanden. 2018. The Origins of Globalization: World Trade in the Making of the Global Economy 1500-1800. Cambridge University Press.

Fitzgerald, Robert. 2015. The Rise of the Global Company: Multinationals and the Making of the Modern World. Cambridge University Press.

Levinson, Marc. 2016. An Extraordinary Time: The End of the Postwar Boom and the Return of the Ordinary Economy. New York: Basic Books.

Piatkowski, Marcin. 2019. Europe's Growth Champion: Insights from the Economic Rise of Poland. Oxford University Press.

Rothermund, Dietmar. 1996. The Global Impact of the Great Depression. New York: Routledge.

Scheidel, Walter. 2017. The Great Leveler: Violence and the History of Inequality from the Stone Age to the Twenty-First Century. Princeton University Press. [The general argument of the book is that inequality has risen through most of history, but has been reduced very occasionally by shocks such as societal collapse or epidemics - or the world wars of the twentieth century.]

Szostak, Rick. 1995. Technological Innovation and the Great Depression. Boulder, CO: Westview Press.

Szostak, Rick. 2005. "Evaluating the Historiography of the Great Depression: Explanation or Single-Theory Driven?" Journal of Economic Methodology 12:1, 35-61.

Szostak, Rick. 2009. The Causes of Economic Growth: Interdisciplinary Perspectives. Berlin: Springer. [Surveys the causes of growth; the flowchart used in this chapter, though based on that book, was first employed in Allen Repko, Rick Szostak and Michelle Buchberger, Introduction to Interdisciplinary Studies, Thousand Oaks, CA: Sage, 2014 (2nd ed. 2016).] 
Van Bavel, Bas. 2016. The Invisible Hand? How Market Economies Have Emerged and Declined since AD 500. Oxford University Press. [This book argues that both economic and political inequality rises with economic growth and the expansion of markets for land and capital (which allow more rapid accumulation of wealth). This in turn leads to societal decline. Case studies of the Abbasid Empire, Renaissance Italy, and the early modern Low Countries are provided, and comparisons drawn to the contemporary United States.]

Woodard, James P. 2012. "Consumer Culture, Market Empire, and the Global South." Journal of World History 23:2, 375-98.

There was a Review Essay in the Journal of Global History in March 2014 which explored "what can modern art offer to global history?" 


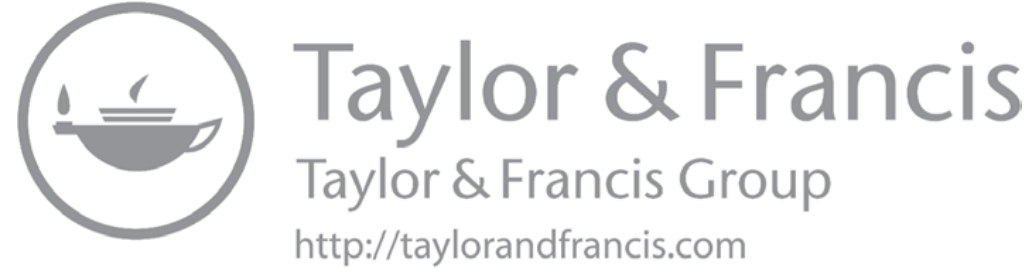




\section{An unprecedented development}

\section{Postwar decolonization}

\section{Guiding questions}

Why, where, and when were colonies divested throughout the world in the decades after the Second World War?

How did divestiture happen?

What were the effects of decolonization?

Relationship to other chapters: We need to understand why colonizers took colonies in the first place before we can appreciate why decolonization occurred - and particularly to understand how remarkable this historical experience of divestiture was. This chapter reflects our discussion of many past empires, but especially discussions of colonial acquisitions in chapters 19 and 23. We shall find that world wars and cold war (chapter 28) played significant roles in both triggering and shaping the process of decolonization. We noted in chapter 21 a conflict between the practice of colonization and the emerging ideal of public sovereignty; this conflict would also prove vital in driving decolonization. Our discussion in chapter 21 of the challenges of forging multi-ethnic nation states will inform treatments in this chapter of many newly independent states that have struggled to achieve a sense of national identity. Our discussions of the modern state in chapter 27 and of colonial practices in chapter 23 inform us of the challenges that a new state with limited bureaucratic capability must face. 
Our primary thematic focus in this chapter is political. Both economic considerations and cultural values inevitably influenced the decision to divest colonies. The decision would, in turn, have significant implications for social structure in newly independent nations. We will employ evolutionary analysis in investigating the difficulties experienced in the transition from colony to independent state. We shall find that the idea of decolonization spread reasonably quickly across most colonizers and colonies, with the result that most of the world came to comprise independent nation states within just a couple of decades. We will compare how decolonization proceeded across former colonies. We will see that certain individuals played a critical role in shaping the decolonization process within and often across colonies.

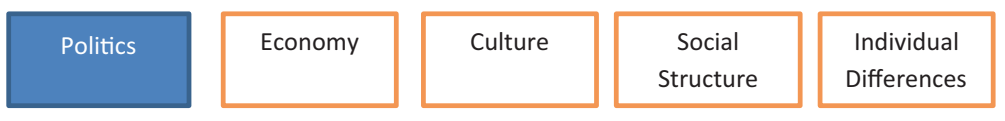

\section{A unique historical transformation}

We have devoted much of this book to discussing empires. We have seen many empires rise and fall. But we have never yet described a moment in time when several empires decided to divest themselves of colonial possessions voluntarily. However, this is precisely what would happen in the two decades after the end of the Second World War. Britain, France, Belgium, and the Netherlands would abandon most of their colonial possessions in this period - including those that they had obtained from Germany, Italy, Japan, and the Ottoman Empire in the aftermath of the two world wars. They would maintain only some small islands in the Pacific and Indian Oceans and Caribbean that were judged too small for independence. Portugal would be a laggard, divesting its three African colonies only in 1975.

\section{Influences on the timing and nature of decolonization}

As often in this book when an important and unusual transition occurs, we can identify multiple reasons for decolonization. One of these was the increasing belief in the "nation state" as the best form of government (chapter 21). This not only encouraged the colonized to press for independence but caused citizens of the colonizing countries to question both the justice and advisability of imperial possessions. Nationalist sentiment had spread in Asia especially after the First World War. Though decolonization would occur when European citizens themselves tired of the imperial adventure, this only happened after decades of agitation by the colonized themselves. Increasingly the efforts of the colonized were coordinated: When the British closed a Nigerian newspaper in 1945, the British Foreign Office received condemnation from throughout Africa and the Caribbean as well as from organizations representing Black people in Canada and the United States. 
In colonizing nations, there had always been an inconsistency between celebrating national sovereignty at home and crushing sovereignty abroad. Democratic institutions were critical in allowing this public questioning of the empire to be readily reflected in public policy. Britain, France, Belgium, and the Netherlands each had democratic institutions that could question imperial policy and vote to change it. Portugal was instead a dictatorship, and Portuguese decolonization would come suddenly in the aftermath of a coup in Portugal itself. A dictator that oppressed at home could more easily justify repression abroad.

One counter-argument against decolonization often employed in Europe was that many of their colonies were ethnically or religiously diverse. European governments worried - with much cause, as we shall see - that independent colonies would experience sectarian strife or mistreatment of minorities. Most European colonies had not previously existed as states, and so lacked the shared history on which European nation states at least claimed to be grounded. Colonial rule might be justified then as a way of limiting ethnic hostilities. But colonial repression struck many as a weak solution to ethnic diversity. It was hoped that local peoples could find their way to some enlightened solution, a result that would be achieved only rarely in the early days of decolonization.

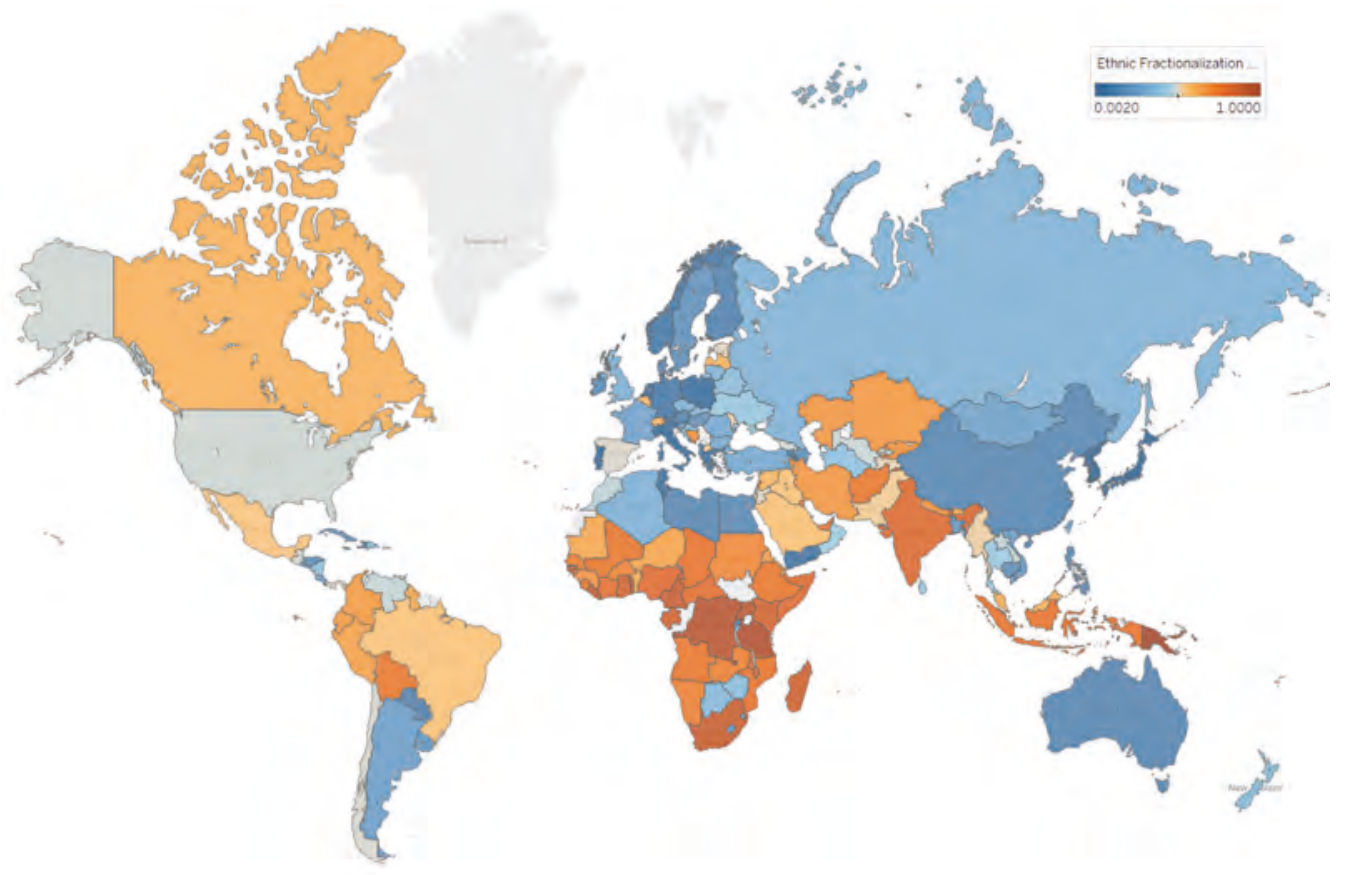

Map 30.1

Countries ranked by ethnic and cultural diversity. Note that - for historical reasons former colonies in Africa and Asia are far more diverse than former colonial powers Map by Jroehl, CC BY-SA 4.0 
The fact that colonial powers came themselves to question the value and virtue of colonies meant that decolonization often occurred reasonably peacefully. This sort of peaceful transition is almost unheard of historically: Imperial decline has generally been associated with foreign invasion or internal rebellion. There were notable exceptions to the pattern of peaceful transition: Bloody wars occurred in Algeria and Vietnam. And lands that escaped all-out civil war still generally experienced some episodes of violence in the last decades of colonial power. As we shall see, many countries were ill-prepared for independence; in some of these there would be violent transfers of power in the decades after independence.

It is no coincidence that this wave of decolonization followed the Second World War. This war, even more than the first, destroyed European claims to represent a superior civilization. Empires employed colonial armies widely in the war (chapter 28): The million colonial soldiers who served in Europe were horrified at what they saw but gained valuable military experience in the process. The colonized often agitated against conscription during the war. Colonial powers often made promises to their colonies during the war in order to maintain their allegiance and support. And colonial powers focused on fighting in Europe often had to loosen their grip on power in their colonies during the war. This was especially the case for France, Belgium, and the Netherlands, all occupied by German troops during the war. In Southeast Asia and the Pacific, Japanese conquests during the war had toppled colonial regimes and established without doubt that Asians could defeat Europeans militarily. At the end of the war, colonial powers were crippled financially and focused on rebuilding at home. They were forced in such a circumstance to reflect on the costs of empire. When faced with colonial rebellion or civil disobedience, they had to ask themselves if they wanted more military conflict.

The Second World War also changed the relative power of various states. The European colonizers were eclipsed by two powers that had played a critical role in winning the war: the United States and the Soviet Union. Both of these countries railed against colonialism - while each proving all too willing to interfere in the internal politics of former colonies. They nevertheless provided intense international pressure for decolonization. The United States, in particular, wielded considerable influence over colonial powers as it helped them rebuild their economies (chapter 29).

Though the Second World War served as a trigger mechanism, decolonization would not have occurred so suddenly if not for the widespread efforts in previous decades to make a case for decolonization. This case was made on many fronts and in many ways. Some argued forcefully within the legislatures of the colonizers for decolonization, and raised public awareness of both the inequities and costs of empire. In the colonies too, powerful orators and writers increased public awareness. In some countries, notably India, acts of civil disobedience weakened colonial authority. In many countries, violent attacks on government officials and armies occurred. Advocates 
of decolonization could everywhere note that the colonizer's rhetoric of a civilizing mission was often at odds with strategies of maintaining authority through the use of repression and racialized governance. Colonizers often complicated the task of forming cohesive states by pitting ethnic groups against each other in their colonies.

We have noted many times in this book how particular empires declined as they were no longer able to finance necessary military expenditures. As people in colonies agitated for independence, the cost of maintaining colonies rose while the benefits of doing so fell. Both peaceful and violent protests were costly to police, and protests disrupted the flows of taxes and export goods on which colonies depended. Nevertheless, we should be careful of assuming either that the colonial powers could not have maintained colonies or that these had suddenly become unprofitable. European powers could likely have hung on for decades longer in many colonies if they had wished - the fact that Portugal, one of Europe's smaller and poorer countries, maintained large colonies in Africa into the 1970s provides clear evidence of this. As for profitability, we have wondered more than once in this book if only a ruling elite rather than the ruler's compatriots benefited from empire. There have been empirical estimates that doubt that the average British citizen ever benefited much if at all from the British Empire. Postwar struggles for independence may then have served to increase the awareness of average citizens that they might be better off without colonies. The embarrassment they suffered from repressive acts in response to colonial resistance would be a further encouragement to decolonize. Note that we have an argument for the importance of democratic institutions again: Portuguese colonies may have benefited the Portuguese elite rather than the Portuguese people, but the latter lacked decision-making power.

Historically, empires often declined not long after they ceased to expand. In such cases, we have suspected that the booty from conquest had never been replaced as a source of government revenue. European empires ceased to expand only once they established colonies (or spheres of influence in the case of China) across almost all of Africa and Asia. There was nowhere to expand further. Since European colonizers focused more on long-term resource extraction than short-term theft (though the national

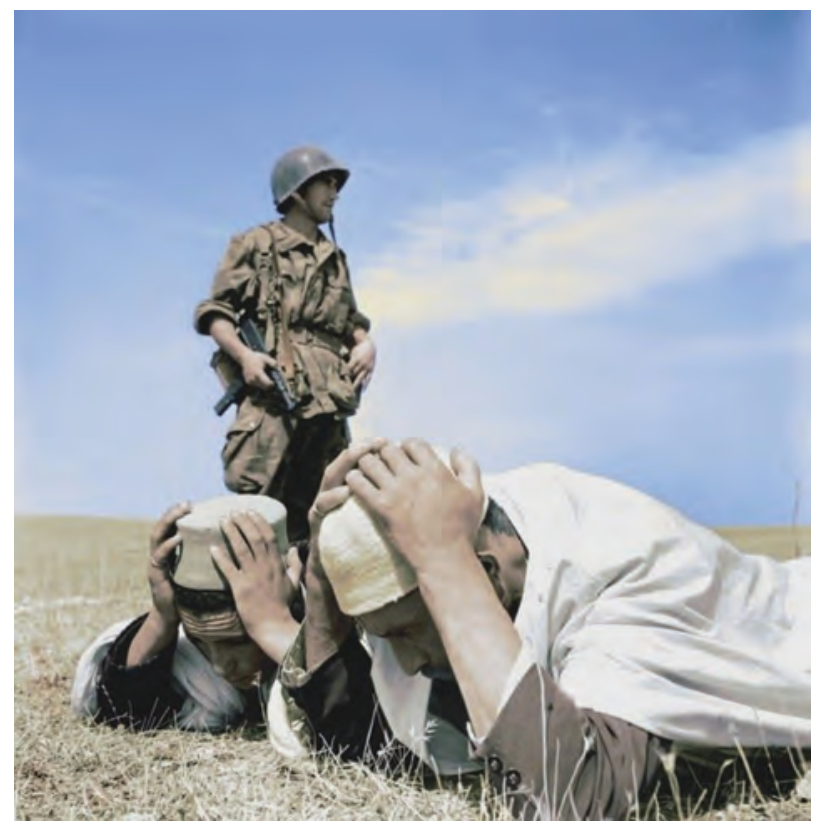

Illustration 30.1

French soldier with Algerian prisoners 


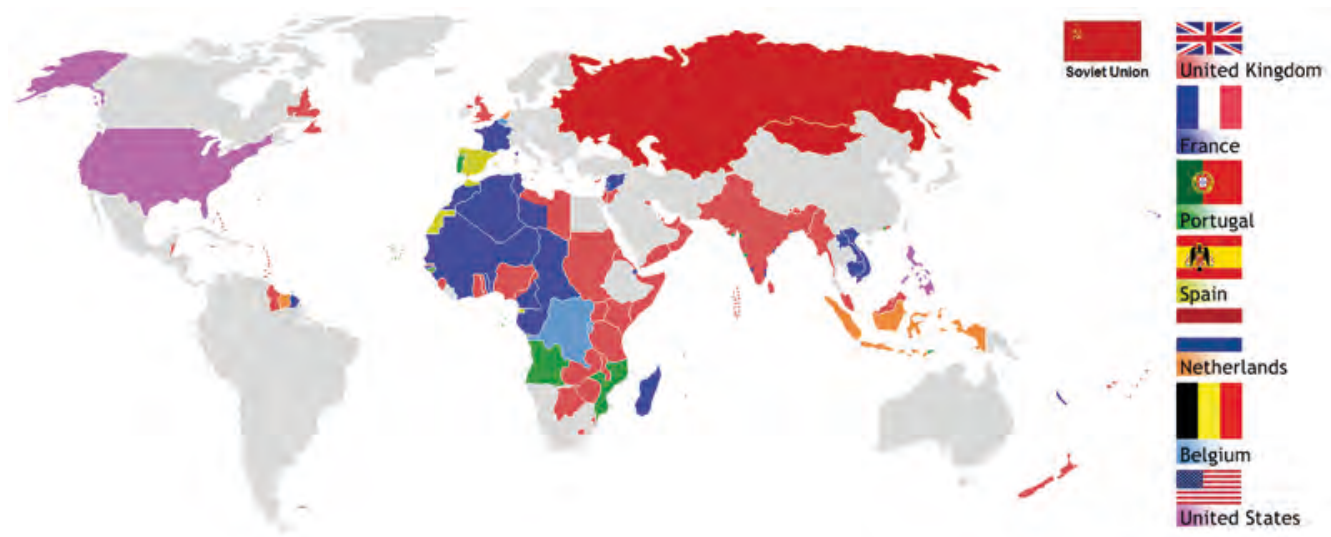

Map 30.2

Colonies in 1945

Map by AniRaptor2001, CC BY-SA 3.0

museums of most European states provide considerable evidence of the latter), the profitability of European empires may not have declined much when these empires ceased to expand.

Former colonies generally continued similar trade relations after independence. Colonial powers continued to obtain raw materials at affordable prices without bearing the costs of governing other lands. All too often, weak governments in former colonies were bribed or coerced to establish rules on foreign investment or labour relations that served the interests of foreign investors. Looked at in this way, the failure of colonizers to prepare their colonies for independence might seem sinister rather than incompetent. But such a view requires that the colonizers were more aware that decolonization was coming than appears to have been the case.

In any case, once decolonization began, it took on a momentum of its own. Indeed, it came to seem inevitable. The largest of all colonies, British India, was among the first to be granted independence in 1947. This development naturally encouraged those pursuing decolonization elsewhere. Other colonial powers - except Portugal - also came to conclude that it was time to step away from colonies. One of the greatest surprises here was the French decision to leave Algeria in 1962. The French in the 1950s had greeted Algerian independence movements with armed resistance. They resisted in part because Algeria was officially a part of France (albeit with limited voting rights for nonEuropean residents), and in part because a million Europeans lived there and considered it home (some 80 per cent of the "European" population had been born in Algeria). These Europeans owned a third of the land. President Charles de Gaulle - a hero of the French resistance during the Second World War was installed as president of France in late 1958 with extensive powers and was widely expected to prosecute the Algerian war but instead soon recognized Algerian independence. 


\section{Challenges in transition}

Though we can marvel at the suddenness of decolonization, it would also prove problematic. European powers had often proclaimed a civilizing mission in their colonies but had generally done very little to prepare their colonies for independence. They had replaced pre-existing governance structures and co-opted local elites into their own ruling structure in ways that gave these elites little popular support. They had generally educated only a small proportion of the local population and reserved all senior posts in government bureaucracies for people from the colonial power itself. Most importantly, perhaps, they had provided for elected assemblies in only a minority of colonies. As a result of these colonial policies, most newly independent nations had minimal experience of governance, and were poorly prepared to create either political decision-making systems that reflected the public will or the complex government bureaucracies that modern states require. The world had become a more complicated place during the decades of colonial rule, but colonial peoples had only rarely had the opportunity to learn how to govern a modern state.

An evolutionary analysis may once again be useful. Revolutionary America (chapter 25) could move more smoothly into independence. The new country maintained most British laws. Americans had long debated in assemblies and held senior positions in local governance. There was a significant body of educated people well-schooled in political philosophy. Though the changes to governance structures were indeed revolutionary, much of day-to-day governance evolved smoothly from one regime to the next. This sort of smooth transition was not possible in most postwar colonies for the simple reason that colonial powers had systematically excluded local peoples from senior levels of government. When the colonizers left, there was in many countries no capacity to maintain institutional structures (which in any case were tainted by association with colonial policies). Political instability and a slow process of institutional restructuring was a likely outcome in such a situation.

It is useful to reflect on how history might have unfolded if colonial powers instead had devoted decades to slowly transferring power: educating more than a narrow elite, promoting locals to senior bureaucratic and judicial positions, enhancing the powers of local assemblies. Many countries might then have been spared decades of military dictatorship, civil strife, and massive corruption. India provides a compelling case in this respect, as it was arguably far better prepared for independence than any other colony. The British had announced some intention of eventually providing independence in 1917 during the First World War. They did establish regional assemblies with powers over education and public works, and a national assembly which could debate the shape that an Indian state might take. Though millions would die in the partition between India and Pakistan in 1947, and later in the separation of Bangladesh from Pakistan, and corruption has been problematic in all three countries, India at least has provided a stable democratic government across a vast and diverse 


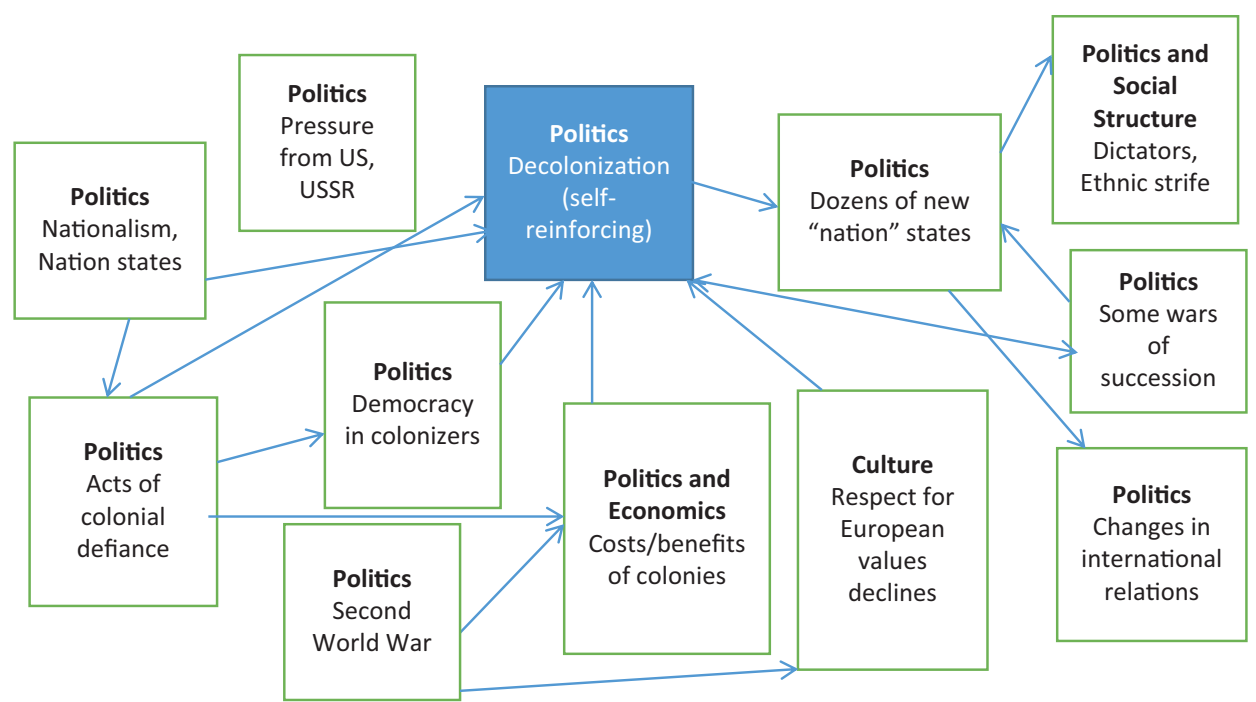

Figure 30.1

Influences on and effects of postwar decolonization

Note: This diagram likely underestimates the importance of a general change in attitudes, and perhaps also of economic considerations. The creation of new states would also, of course, have a host of cultural and economic effects in those states.

land for decades. Its bureaucracy has been extensive and capable if often too interfering and riddled with corruption.

\section{The Middle East}

The very earliest episodes of decolonization occurred in the Middle East. That region still struggles with the aftermath of decisions made by colonial powers at the time. Lebanon gained independence from France in 1943 (though European troops remained until 1945) and Syria in 1946. Jordan gained independence from Britain also in 1946. Iraq had been granted independence even earlier in 1932. The British and French had held these former Ottoman lands since the end of World War I as League of Nations "mandates." Though they had governed them much like other colonies, there had been an international expectation that they would gain independence. Nationalist leaders in these mandates had appealed to the League for independence in the 1920s (such appeals were rare in other colonies before the 1940s). Though the League had no power to force Britain or France to change their attitudes, these countries did listen more to local leaders in these mandates than they did in most other colonies. In both Iraq and Jordan, the British had fostered a local monarchy (as they did in Saudi Arabia). The Iraqi monarchy would be overthrown in 1956, to be replaced by a series of military dictators. The United States and Britain attacked Iraq in 2002 and a shaky democracy riven by ethnic and religious 
disputes was installed. Jordan remains a monarchy; it has created an elected assembly with limited powers. The French established an assembly in Lebanon that declared independence. Lebanon has remained democratic through much of its history but has struggled to contain conflicts between Shia, Sunni, and Christian populations - especially given a massive influx of Palestinian (and more recently Syrian) refugees. When European troops left Syria in 1946, it was left in the hands of an assembly established during the Mandate. There would be a series of military coups from 1949 (triggered by Syria's loss in its war with Israel; see below) to 1961 when the Assad dictatorship was installed. Civil war has engulfed Syria since 2011.

The most momentous colonial decision involved Palestine. Jewish settlers had begun moving into biblical lands from the late nineteenth century in response to European anti-Semitism. There had been pogroms in Russia in which mobs attacked Jewish homes, businesses, and people. Many Jews had viewed Western Europe as much safer: They had, for example, been granted equal citizenship during the French Revolution, and Napoleon had extended this freedom across much of Western Europe. Jews had become much more integrated into broader European society. In particular, they were freed from a legal requirement to live in separate ghettoes. However, the trial for treason in France of a Jewish army officer on seemingly trumped-up charges in the 1890s exposed lingering antiSemitism there. Though there was an obvious religious motive for resettling the lands of the Old Testament, the early Jewish migrants to Palestine were often quite secular in orientation and more focused on a Jewish ethnic identity. There were enough Jewish settlers in Palestine that British officials promised them a homeland in 1917 in the very early days of the Mandate - yet they made a similar promise to the Palestinian Arabs who also lived there. Jews and Arabs jostled for power against the British in ensuing decades. As Europeans began to devolve power in the Middle East after the Second World War, Palestine received a new influx of Jewish settlers in the aftermath of the Holocaust (chapter 28). The British asked the United Nations for guidance, and the UN voted to partition Palestine into Jewish and Arab states. This plan was accepted by most Jewish settlers but rejected by most Arabs in Palestine and in the newly created Arab states surrounding it. The Jewish settlers declared unilateral independence within more generous borders, and war ensued during which many Palestinians fled (debate rages to this day as to whether they fled of their own accord or were pushed by Jewish troops). The result was the creation of the state of Israel with a large Jewish majority and the displacement of millions of Palestinians. Jordan would occupy the area known as the West Bank (of the Jordan River), and so no Palestinian state was created. In 1967, Israel would take the West Bank in another war against Arab countries. It has allowed Palestinians there a limited authority, but stations troops throughout the West Bank and has allowed Jewish settlers to move on to some of the best land. The plight of the Palestinians has garnered substantial international support, but their recourse to acts of terrorism has blunted this (chapter 28). The Jewish state has also received significant support from the influential Jewish diaspora, especially in 


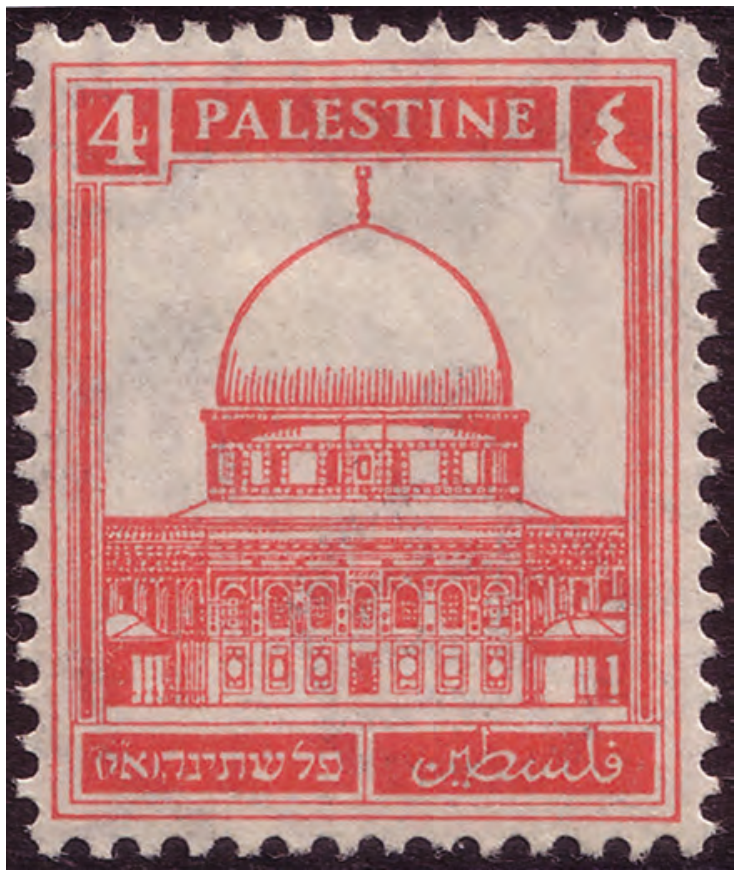

Illustration 30.2

Palestine Mandate stamp, 1927; note the use of English, Hebrew, and Arabic scripts the United States, and also from many (especially evangelical) Christians in part from remembrance of the Holocaust and in part from reverence for the Old Testament of the Bible. Within Israel and in the countries that support it, a debate has raged about the conflict between the democratic values espoused by the Israeli state and its authoritarian rule of the West Bank. This debate might have unfolded differently if not overshadowed by security considerations. That is, democratic Israel might have "decolonized" as European countries had if not concerned for its own safety. (Note, though, that arguments that colonies are expensive to administer can be blunted by a belief that Israel needs a large army for defence anyway.) The Palestinian situation has undoubtedly served to cause many Arabs and Muslims more generally to be hostile toward the West and (not for the first time) to detect hypocrisy in the preaching of democratic ideals.

Egypt had been nominally independent since 1922 but did not control the Suez Canal. An army coup in 1952 forced British troops out and nationalized the canal, but Israel, Britain, and France then invaded the canal area in 1956 and bombed Egypt. The United States and the Soviet Union both demanded that they leave. Embarrassed by international opprobrium, the three countries did return the canal to Egyptian control. Egypt for a while collaborated with the Soviet Union but then later with the United States. A popular revolt in 2011 deposed the military in favour of an elected assembly. But when Islamist parties won that election and designed a constitution with Islamic elements, there was widespread condemnation by secularists and religious minorities. This unrest has allowed the army to reassert authority. These events serve as a reminder that democracy is challenging in its early days and that constitutional protections of minorities and of fundamental rights are a crucial component of democratic governance.

\section{Asia}

The Japanese military victory over Russia in 1905 was an inspiration to independence leaders throughout Asia. Sun Yat-sen in China, Mohandas Gandhi in India, and Kemal Ataturk in Turkey were among those who celebrated the 


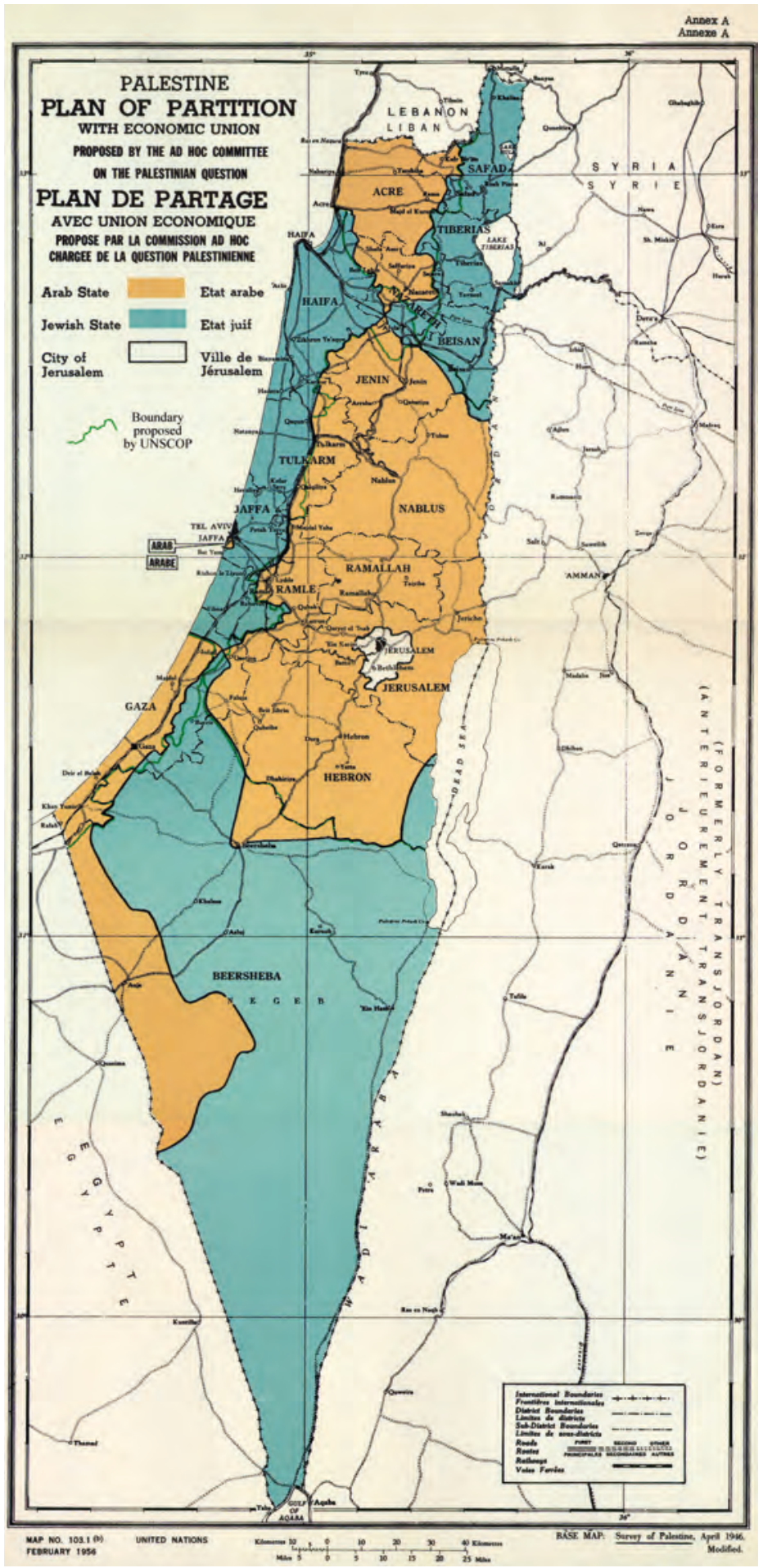

Map 30.3

UN plan for the partition of Palestine, 1948 


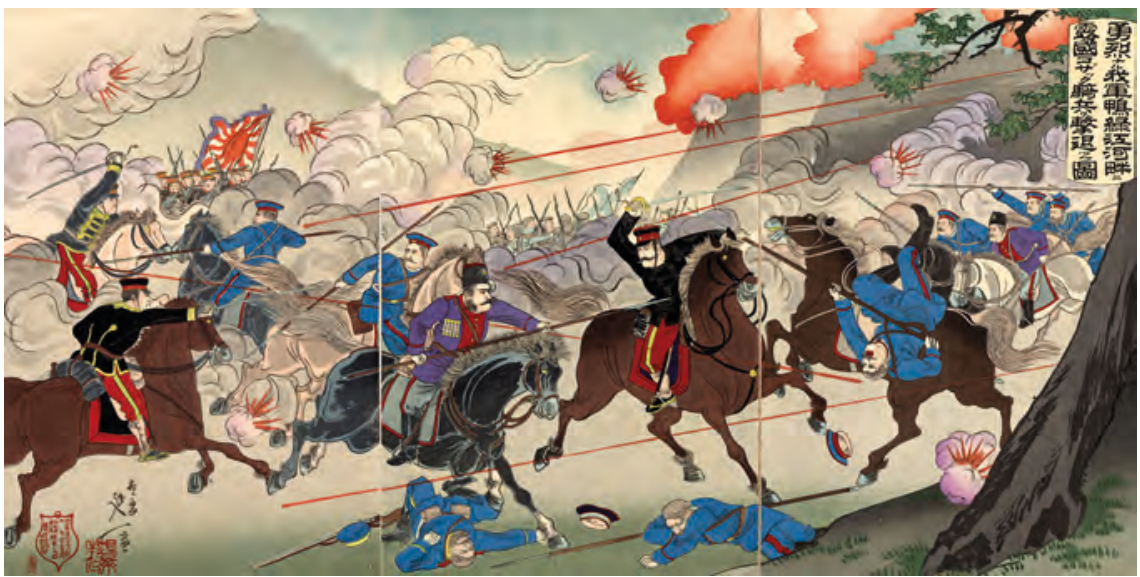

Illustration 30.3

"Picture of Our Valorous Military Repulsing the Russian Cossack Cavalry on the Bank of the Yalu River" by Watanabe Nobukazu (1874-1944), March 1904, Sharf Collection, Museum of Fine Arts, Boston

fact that Asian armies could defeat European armies. Chinese intellectuals, in particular, flocked to Japan to learn the lessons of Japanese success. Japanese conquests of European colonies in Southeast Asia and the Pacific during the Second World War would further incite national aspirations in Asia - though Japanese rule was generally brutal (some Japanese would later blame this brutality on the European colonial example they had absorbed). In about ninety days in 1941, Japan took the Philippines, Indonesia, Hong Kong, Singapore, Malaya, Burma (modern Myanmar), and French Indochina. Japanese thinkers had detected hypocrisy in Western democratic rhetoric and had urged a strong authoritarian state in order to battle European powers who did not act democratically outside their own borders. Though Japan itself would establish strong democratic institutions under American occupation after the war (chapter 28), many of the Asian independence movements that were inspired by earlier Japanese successes would pursue authoritarian forms of government.

This "awakening" of Asian thought is an important characteristic of the twentieth century. Thinkers and political leaders throughout the continent would seek to fashion a path forward that was not dependent on European institutions or values. While there was a shared determination to reject Western ideals somehow, the precise choices made would differ. Decades of experience with elected assemblies encouraged Indian independence leaders to mostly embrace democracy while elsewhere this was often viewed as an un-Asian charade. Communist ideas, despite their Western roots, would be embraced in some countries, brutally exterminated in others (like Indonesia; chapter 28), and have limited impact elsewhere. Western individualism and science were often also viewed with suspicion. Authoritarian leaders were not alone in arguing that an Asian tradition of community spirit was best expressed by an authoritarian 
government that would represent the community's shared desires. However, in both China and Japan, the ideas of Wang Yangming (chapter 18) were often drawn upon to suggest that individualism could be usefully combined with a strong sense of duty.

Many Asian thinkers sought to resuscitate Asian philosophies such as Confucianism. Others - following the reformers in Japan - sought some combination of ancient ways and modern practices. The rejection of Western values was most notable in Islamic countries where thinkers such as Mohammad Iqbal (1877-1938) argued that only an Islamic state could respond to European power. (His writings and speeches would inspire the movement to carve a Muslim-majority state out of British India.) There were also advocates of Western-style democracy in the Islamic world, but these views were often seen as traitorous. Colonial powers had only rarely and belatedly instituted democratic institutions in their Muslim-majority colonies (and these often had little power). In Saudi Arabia, the Saud monarchy would establish a close relationship with Wahhabi (a branch of Sunni Islam) clerics, receiving justification for their autocratic rule in return for instituting Islamic laws. Elsewhere, though, new Islamic-majority states were characterized by the mixture of democratic assemblies and military dictatorships that we have already seen in the Middle East. Pakistan and Bangladesh would establish democratic institutions, but military coups often disrupted these. Malaysia has been nominally democratic since independence but with one party exercising control until 2018 (and

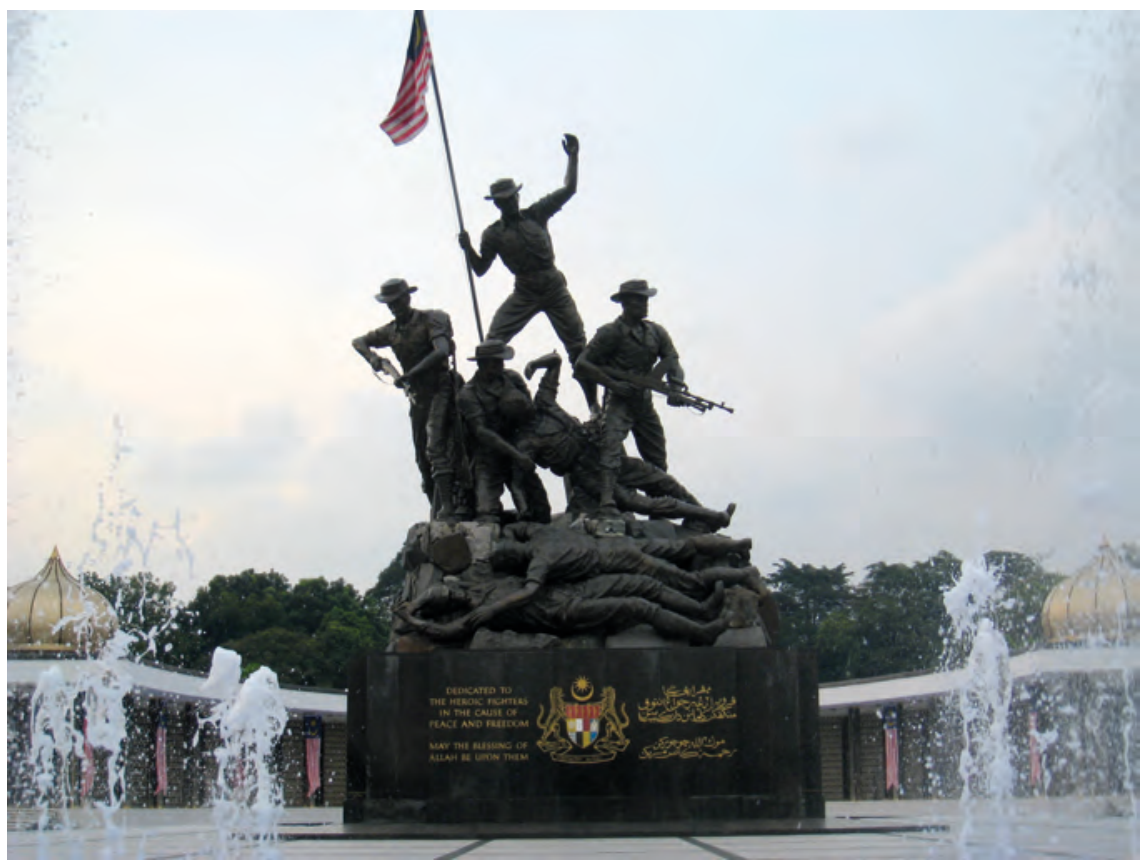

Illustration 30.4

Tugu Negara, National Monument to Malaysian independence, Kuala Lumpur 


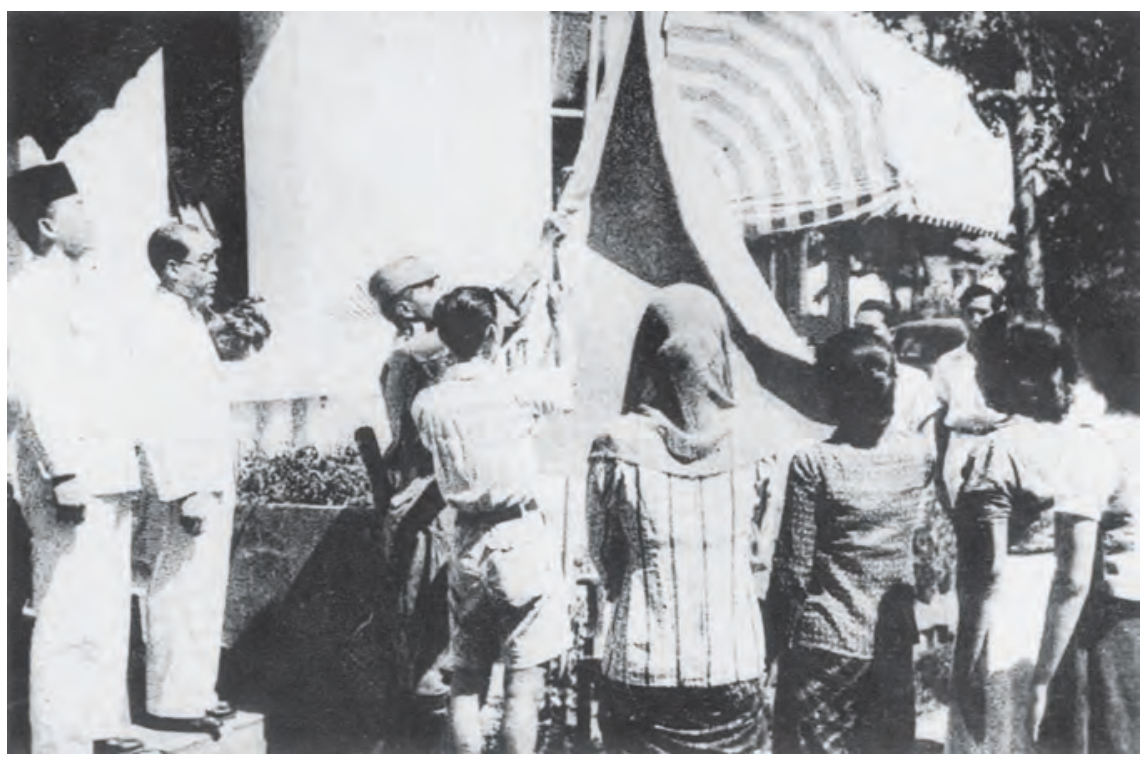

Illustration 30.5

Ceremony of raising the flag of Indonesia moments after the reading of the Indonesian Declaration of Independence, Aug 17, 1945

often accused of vote-rigging and coercive practices such as jailing opponents). Indonesia has been a dictatorship for most of its independence (which it gained in 1949 after a lengthy insurgency and with intense international pressure on the Dutch to withdraw), but has established democratic institutions in recent decades.

The leaders of most new Islamic-majority states were not interested in developing a theocratic government. Indeed leaders such as (especially) Ataturk in Turkey but also Muhammad Ali Jinnah in Pakistan and Gamal Abdel Nasser in Egypt thought that a secular state would be more conducive to economic growth. Most sought, though, to accommodate Islamist sentiments. Most Islamists in turn soon abandoned a dream of pan-Islamic unity under a new caliphate, and worked to reform institutions within particular Islamic countries. As noted in chapter 21, the idea of the nation state was one Western idea that proved irresistible elsewhere (though, as we have indicated, China had achieved a kind of nation state status long before European nations). Corruption in government encouraged the growth of Islamist sentiments in many such countries (unaware, perhaps, that religious bureaucracies have often been as corrupt as secular bureaucracies in history). As urbanization and industrialization transformed economies, the resulting income inequalities and social transformations also encouraged some to embrace the idea of an Islamic state. Western support for Israel was a further encouragement to Islamist sentiments. The more recent "war on terror" has also been seen in some circles as just another attempt to impose Western rules and ideas. 
The appeal of religious authority extended far beyond Islamic-majority states. People disenchanted with local collaborator elites or post-independence corruption naturally turned to religious elites that shunned materialism. Gandhi in India was an important model for many Muslims. Though a devout Hindu (albeit one who sought to reform Hindu attitudes toward the untouchable caste), Gandhi dreamed of a secular Indian state in which Hindus, Muslims, and others would cohabit peacefully. His popularity owed much to the fact that he lived a simple life of moderation. He staunchly opposed the partition of India into Hindu-majority and Muslim-majority states and refused to celebrate Indian independence (which he had worked for decades to achieve) as a result. When religious rioting broke out at independence, he went personally to troubled areas and likely saved many lives by risking his safety and capitalizing on the personal respect he had among all groups. With such acts, he served as an exemplar of self-sacrifice for other lands.

\section{Iran}

The British supported the shah Reza Pahlavi in replacing his father on the Persian throne when they and the Soviets invaded Iran in 1941 during the war. They and the United States then supported an army coup in 1953 that replaced an elected and popular prime minister with communist sympathies who had nationalized the oil industry. The shah was very interested in economic development and encouraged industrialization and urbanization. He also developed an extensive secret police and imprisoned and tortured political opponents. In 1979 the shah was deposed when his army refused to fire on demonstrators. A theocratic government replaced him: Religious authorities determine who can stand for election to the country's parliament and also exercise final jurisdiction over many types of decision.

Though the Iranian Revolution of 1979 was a surprise to many, there are many characteristics of that revolution that we have observed elsewhere. Government repression and corruption can encourage a yearning for religious authority. So too can the social transformations associated with urbanization and economic growth. Western interference in the internal affairs of a government can encourage rejection of a broad range of Western ideals and practices. Perhaps most importantly, we should be reminded that revolutions are unpredictable. Many of those on the streets in 1979 were focused on removing the shah. Many would have preferred a typical democracy rather than a theocracy. However, after decades of the shah's repression, opposition forces were poorly organized. Islamists, though punished and exiled by the shah, were better prepared than any other group to seize power in the chaos of revolution (much as the communists were better organized than anyone else when the tsar fell in Russia in 1917). The new Islamic authorities soon instituted their own militias and secret police that have made it challenging to organize political opposition. Yet Iranian people have on many occasions indicated considerable interest in political reform. It remains difficult to ascertain precisely what form 
of government would be chosen if Iranians were free to do so. While some undoubtedly seek broader engagement with the wider world, it is clear that anti-Western sentiment is still influential.

\section{India}

In India, the railway network helped forge a sense of nationhood and facilitated the interaction of nationalist leaders. The British had encouraged the education of an Indian elite, who had naturally absorbed ideas of liberal democracy. The Indian National Congress from 1885 - inspired by anger that a famine relief fund had been used to fund British conquest of Burma - sought a gradual and peaceful transition to independence. However, the British, especially after the First World War, responded with repression.

We have discussed Gandhi's role briefly above. Mohandas Gandhi (18691948) was from a wealthy family and was educated as a lawyer in London. He moved to South Africa in 1893 to work as a lawyer, and became involved in the struggle against entrenched racism there. While there, he adopted the principles of non-violence and an austere lifestyle, wearing the clothes of a peasant. He had limited success in South Africa but achieved fame through his activities and writings. He returned to India in 1915 where various peasant and worker groups asked for his guidance. He expanded the appeal of the Congress Party beyond the elite, adding millions of peasant members, and came to argue against caste distinctions (despite being from the merchant caste). He urged non-violent protests against British goods and institutions, including schools. Some demonstrations nevertheless turned violent. The British responded harshly, killing 379 unarmed protestors in 1919 in Amritsar. Though the British prosecuted the officer in charge of this massacre, the event forever tarnished British rule in the eyes of many Indians. The British also created elected legislatures at the provincial level in 1919, and an expanded national legislature that was mostly elected. These provincial legislatures had authority over matters such as public works and education. The national legislature provided a locale for debating the form that an independent state might take. The British would, nevertheless, introduce laws limiting freedom of the press over the objections of the national legislature.

Gandhi is most famous for advocating non-violent protests. This was, Gandhi appreciated, a novel strategy. Empires - reliant on the use of force to maintain their power - had generally been toppled only through the exercise of violence. Gandhi appreciated that the world had changed. With the rise of both the idea of popular sovereignty and democracy, non-violent protests might embarrass a democratic colonizer into granting increased powers to the colonized. In 1920-2 he protested against British schools and courts by establishing local alternatives; he also urged opposition to British tariff policy by boycotting imports. Some Indians refused at the time to pay taxes to the colonial authority. In 1930-2 he led the "march to the sea" to produce salt in violation of a British monopoly. In 1942 he demanded independence in return 


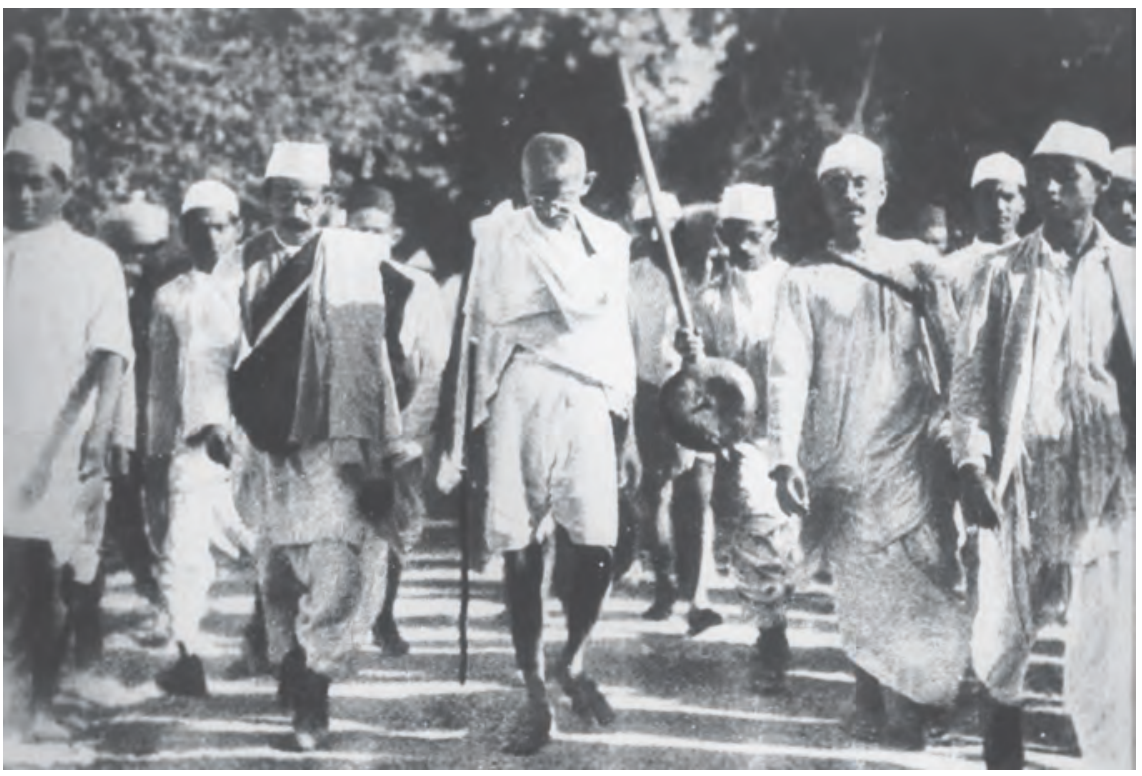

Illustration 30.6

Gandhi during the Salt March, March 1930

for military support in the war. Gandhi's advocacy of non-violence may have facilitated India's move to independence without the necessity of a violent attack on British authority. At the end of the Second World War the British government - after extensive public debate - decided that it would be prohibitively expensive and politically difficult to continue to suppress Indian independence. Gandhi's methods of non-violent protest would be copied elsewhere, notably by Martin Luther King in his marches for civil rights in the United States south in the 1960s, and in opposition to apartheid in South Africa over a period of decades (though violent resistance was also pursued there).

As noted above, Gandhi hoped for a secular India. Muslims doubted their future in a Hindu-dominated state and noted that Gandhi himself relied heavily on Hindu teachings. Muhammad Ali Jinnah formed the Muslim League in 1905 and argued for a separate Pakistan. The Depression had exacerbated ethnic tensions as Hindu landlords had turned indebted Muslim tenants off their lands. The British decided to accede to this wish. There were two Muslimmajority areas - present-day Pakistan and Bangladesh - but both had substantial Hindu minorities. And Muslims were a significant minority throughout the rest of India. The British established the borders between India and Pakistan in a matter of weeks. Six million people moved in each direction at partition. Between 200,000 and a million were murdered in religious rioting at the time. Pakistan became almost entirely Muslim while India retained a 10 per cent Muslim minority. The British had ruled much of India through local princes and gave these much say in which of the two countries their lands would inhabit. The Hindu prince of Kashmir in the northwest of India would opt to stay in 
India despite having a Muslim-majority population. War erupted over Kashmir in 1947 and again in 1965. A ceasefire line was drawn which remains tense to this day. A promised referendum has never been held. Violent uprisings and government repression continue to characterize the area - which has, among other things, limited the tourist traffic to one of the most beautiful places on earth. Religious tension has occasionally flared elsewhere in India: There was widespread rioting in 1992 when Hindus destroyed a mosque in Ajodhya that had centuries earlier been built on the site of a Hindu temple.

The British had not been the sole imperial power in India. Four small French colonial enclaves were ceded to India in 1954. India took two small Portuguese pockets in 1962. As well, the independent kingdom of Sikkim would decide to join India in 1975. Even within the area of British rule, various princely states had to be absorbed into the Indian state after independence.

Independent India abandoned Gandhi's suspicion of large-scale industry. Industry was seen as essential to India's political and economic development. But it long maintained his suspicion of foreign trade. And it would borrow from socialism the idea that the state should guide and regulate economic development. Provincial governments have pursued land reform (with compensation) to a limited but important extent. Developments in new seed varieties (mostly by Western scientists), especially for wheat and rice, coupled with improvements in irrigation and the use of fertilizers, allowed grain output to triple between independence and the mid 1980s. This meant that India could feed a rapidly growing population. Economic growth rates were sluggish for decades in India but have been much higher in recent times as India has embraced trade (and now provides services in computer software and customer relations throughout the English-speaking world, but has industrialized far more slowly than China) and reduced state regulation. India had intended to gradually phase out English as an official language after independence, but this proved impractical in a multilingual country. Facility with English has been advantageous to India's service sector.

India's federal structure has likely been critical in maintaining national unity. It has faced secessionist movements not only in Kashmir but in Assam in the northeast (whose people are ethnically more similar to the Chinese), Punjab (where Sikhs form a majority), and Tamil lands in the south. These groups could pursue distinct policies at the provincial level. However, provincial governments have introduced internal trade restrictions and differences in regulation that have limited national economic integration and prosperity. While the Congress party - and indeed the family of independence leader Jawaharlal Nehru - dominated national politics for decades after independence, a variety of other political parties have ruled at the provincial level. Indeed the first elected communist government in the world occurred in Kerala in 1957. In addition to its federal structure, India has benefited from a constitution that provided for an independent judiciary and protection of rights; these have generally been achieved despite decades of rule at the national level by 
one family (rights were briefly abrogated in 1975-7 when a national emergency was declared). A constitutional amendment in 1950 outlawed discrimination on the basis of religion or caste.

The constituent assembly that drafted the Indian constitution represented only 14 per cent of the population. But at the urging of Nehru, it opted to pursue universal suffrage. Though a laudable move, this meant that the vast bulk of the Indian electorate was inexperienced and poorly educated. This has arguably encouraged a clientistic approach to politics in which politicians provide financial rewards to particular groups rather than campaigning on the basis of policy. The social divisions associated with the caste system may also have encouraged this sort of campaigning. Politicians gained a reputation for corruption and acted to limit the independence of the bureaucracy. The Indian government expanded the size of the bureaucracy faster than the tax base: Poorly paid bureaucrats were often corrupt also. While some see the endemic corruption in modern India as a reflection of low pay and an inexperienced electorate, others point to a long history of corrupt imperial bureaucracies in India. Yet many countries have managed to transcend a history of corruption.

India would pursue a controversial policy of affirmative action after independence. Castes would jostle to be placed on lists of disadvantaged castes. As elsewhere in the world, it was often the most successful members of underprivileged groups that benefited the most from special access to schools or jobs. India would also address gender inequities by changing divorce laws for Hindus in 1955 and providing daughters with rights of inheritance in 1956. Yet women are often still viewed as a burden, especially in rural India, and the gender ratio has been badly skewed (in 1991, there were 927 women per 1,000 men), reflecting widespread abortion, infanticide, or malnourishment of girls. As in China, which also has a skewed gender distribution, there is concern about the social instability associated with tens of millions of young men who cannot find a female partner (this may contribute to the frequency of sexual assault in India, which has recently become a public issue after some horrific and wellpublicized attacks). The shortage of women has not served to transform their status.

India aided militarily the separation of Bangladesh from Pakistan in 1971. Bangladesh was/is quite distinct in both geography and language from Pakistan. The attempt to meld geographically separate lands into one country on the basis only of a shared religion may have made it more difficult for democracy to take root in Pakistan than it did in India. The religious identity of Pakistan and Bangladesh, in contrast to the secular identity of India, may also have challenged democratic ideals. It should also be noted that the British had been more hesitant to establish elected assemblies in areas where Muslims were a majority; the politicians and voters of the new state of Pakistan, therefore, had less experience of democracy. Though Pakistan and Bangladesh have often been ruled democratically, they have both often suffered from military coups. 


\section{China}

Europe never colonized China in the way that it had colonized other parts of Asia and Africa. The Qing Dynasty had remained in power until a popular revolt overthrew the emperor in 1911, but it had ceded control over several ports and government ministries to foreign powers over the years. Japan would after that colonize some parts of China, and China would emerge as a unified and independent state only in 1949.

As so often in history, the Chinese revolution of 1911 ushered in a period of political instability. Local warlords - often former imperial generals - held power in many regions of the country. This sort of temporary regional conflict among warlords had often characterized dynastic transition in China. Two groups - the Nationalists or Kuomintang, and the Communists - vied for power, but these had to engage with warlords, the Japanese, and continued interference by Western powers. The Japanese would take advantage of Chinese weakness and instability to invade Manchuria from the 1890s and establish a colony there in 1932 (with the deposed Chinese emperor serving as a puppet); the Japanese invaded deeper into China in 1937 (chapter 28). The Kuomintang government complained to the League of Nations about Japanese aggression, but the League did no more than criticize the Japanese. The Kuomintang and Communists would occasionally cooperate against the Japanese.

The leader of the Kuomintang, Chiang Kai-shek, had been trained in a Japanese military academy, participated in the 1911 revolt, and came to command the Chinese military academy. Like Sun Yat-sen, revolutionary leader and provisional president from 1911 to 1912, he became a Methodist Christian and married a Christian. He formed a "national" government that sought to extend control over areas dominated by warlords and communists. Despite political instability, there was a dramatic increase in foreign investment in railways, coal mines, and the cotton industry. Labour relations - as in many countries at the time - were hostile, and the government often acted to suppress strikes by workers. Chiang's government received business and foreign support. But his government was corrupt, and peasants and workers saw it as supporting landlords and firms. It killed thousands of communist sympathizers in the cities in the 1920s.

Mao Zedong (1893-1976) had lived a quite different life. He grew up on a farm but went to university. He joined the Communist party and organized industrial workers and then peasants. He came to argue that the Chinese peasantry could be the vanguard of a communist revolution in China if promised land redistribution and education; this was an important break from the traditional communist emphasis on organizing the working class. His views gained importance as the Kuomintang crushed urban sympathizers. He developed a local communist government on the border of Hunan and Jiangxi provinces that redistributed land, introduced new farming technologies, and spread education; he instructed his army to treat peasants well. Mao could not hold out when a million soldiers were sent against him by the Kuomintang. He instead 
launched the Long March in 1934; 80,000 soldiers left on a journey that took just over a year through difficult terrain and across nearly 10,000 kilometres to a new base to the northwest in Yan'an. Only 20,000 arrived, half of these having joined along the way. They would form the basis of the new movement, and Mao's position as its leader was solidified.

Though occasionally cooperating against the Japanese, the two forces focused on each other in the 1940s. The United States aided the Kuomintang, and the Soviet Union advised the Communists - but both countries became focused on the Second World War. The Communists developed a more disciplined army and greater peasant support. In 1949 they would emerge victorious, with Chiang and the remnants of the Kuomintang fleeing to the island of Taiwan. Taiwan would be ruled in an authoritarian manner for decades - with claims to being the legitimate government of all of China justifying representation by ageing Kuomintang operatives of mainland provinces in the Taiwanese government. Over time, democratic institutions were strengthened in Taiwan. It has had to avoid declarations of independence from mainland China for fear of inciting an invasion. Though the two Chinese states had little contact for decades, there are now extensive trade and investment ties between them.

After victory, the Communists redistributed land, expanded public education, and encouraged new technologies. As many as a million rural landlords were "tried" and killed. Most peasants gained land. The Communists also developed local networks to spy on and indoctrinate the population. At first, the Communists cooperated with some foreign businesses, but then they forced foreigners out in 1950, including missionaries. As earlier in the Soviet Union, the Communists emphasized investments in transport and heavy industry. Indeed, the Soviet Union helped draft the first five-year plan for economic development. The Communists hoped and claimed to increase industrial output 2.5 times in the first plan. They nationalized industry and collectivized rural farmland. The Communists also encouraged political expression for a while but then clamped down on those who opposed Communist rule. (They have been accused of plotting a trap to identify and then neutralize opposition by briefly allowing them to speak out.) The Great Leap Forward of 1957-60 was a plan to encourage small-scale industry, but instead as many as 20 million starved during this period (in part due to climatic challenges). The Cultural Revolution of 1966-9 attempted to restore revolutionary fervour: The army and students formed units of Red Guards that attacked those thought not to be ideologically pure. Many of those that came under attack were exiled to the countryside to perform peasant labour. Those accused of wrongdoing had little opportunity to defend themselves. Eventually, the army was turned on the Red Guards themselves as the latter seemed to become too great a threat to authority. In the 1970s there was an opening to foreign trade. The American President Richard Nixon visited in 1972 and normalized relations. Communist China was allowed to join the United Nations in 1971, replacing the fiction that the Kuomintang still ruled. 


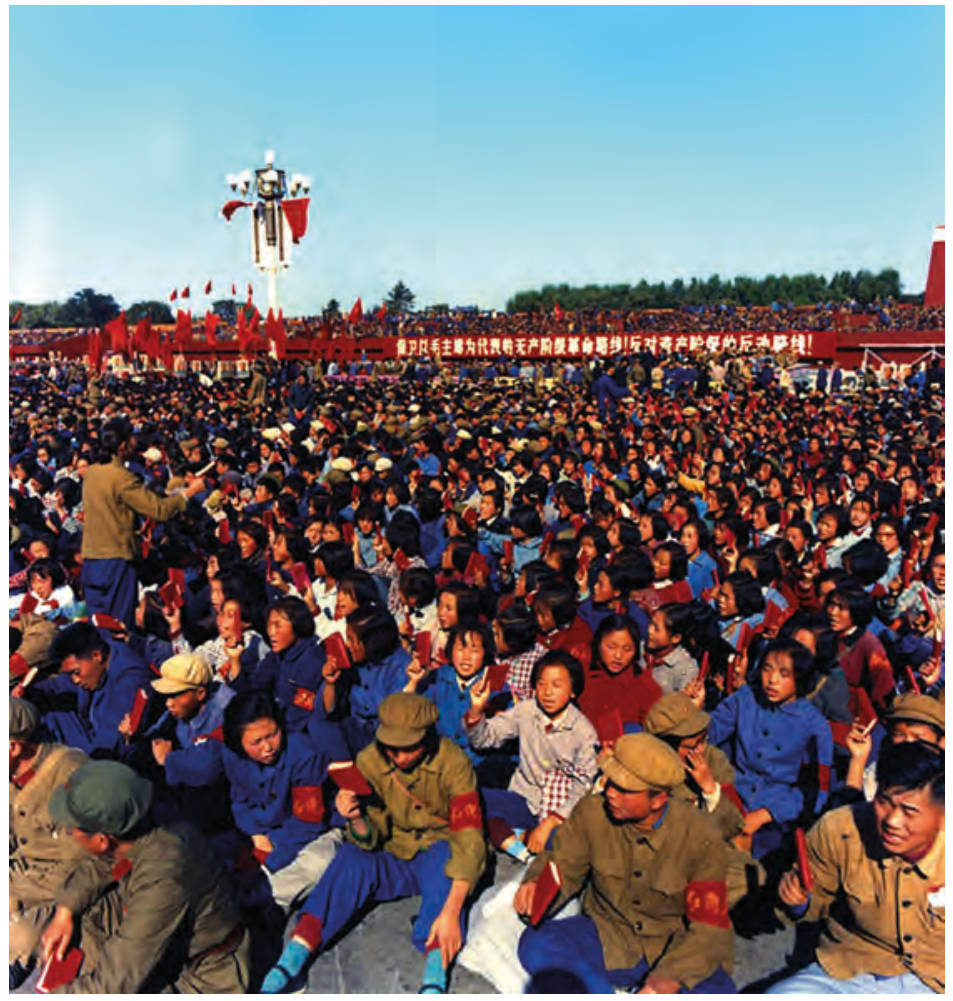

Illustration 30.7

Red Guards in Tiananmen Square, Beijing, 1966, during the Cultural Revolution

We have often in this book discussed the interplay between culture and institutions. The communist leader Mao often voiced the belief that if people operated under the right set of institutions for a while, they would change their cultural attitudes in accord with these. He was hardly the first ruler in history with such an aspiration. But the hope that peasants forced into collective farms would become devoted to the common good went unrealized: Agricultural productivity would leap decades later when farmers were allowed to sell their output individually. Of course, the institutions introduced by Mao were applied more corruptly than he might have wished, but the lesson would seem to be that we should be humble in trying to improve human nature through institutional reform.

China had taken Tibet in 1950, and brutally suppressed a revolt there in 1959. It encouraged the migration of ethnic Chinese into both Tibet and Xinjiang, a region in the northeast previously possessing a Muslim majority. China also extended its borders against India in a brief war in 1962, but took only what they had claimed despite an overwhelming victory. The Chinese government split with the Soviet Union in 1961, expelling all Soviet advisers. There were occasional border skirmishes in the following decades along the long border between the two states. 
Postscript: It is not entirely clear why China did not press further into India in 1962. The government may have responded to international pressure (including from the United States, even though there were no diplomatic relations between the two countries). The border between China and India remains disputed to this day - as does the maritime border between China and several East and Southeast Asian countries. A clash with Chinese soldiers resulted in twenty deaths in the Indian army in June of 2020 and raised fears of further conflict. The Chinese military is much more powerful than it was in 1962. But China is also now much more tied to international flows of trade and investment. Still, a better understanding of the 1962 war might be useful in ensuring continued peace in the region. In strictly economic terms, the disputed territories do not seem worth the risk of war. But nationalist sentiments may trump economic calculations - and politicians may cater to nationalism.

As in India, the new state introduced laws that strengthened the power of women. A new marriage law after 1949 forbade arranged marriages and bride purchase, but was not enforced at first due to an angry reaction by local male elites. More effective was a ban on foot-binding. Female rights to land were also instituted before this was collectivized.

After Mao's death, and as communist countries in Europe came to grapple with limits to their economic performance, communist China dramatically changed its economic policies. It allowed private investment across many sectors of the economy. And in a series of reforms, it gave individual peasants increased rights over land and the ability to sell their output in markets. The result was decades of economic growth at an annualized rate of 10 per cent or more. This, in turn, lifted hundreds of millions of Chinese out of poverty. It also transformed China from one of the most equal countries in the world into one of the most unequal in just a couple of decades.

\section{Vietnam}

Ho Chi Minh had led armed resistance against the Japanese during the Second World War, and he declared independence when the war was over. He had received some American aid during the war, but his appeal to the United States to stop the French from retaking control of Vietnam was ignored, in large part because of his communist sympathies. The Cold War (chapter 28) would complicate the decolonization process here and elsewhere as both sides enticed illprepared governments and treated them with hostility if they were thought to be leaning the wrong way.

The French retook Vietnam in 1947, but Ho used guerrilla tactics to oust them in 1954. Ho received aid from China after the communist victory there in 1949 


\section{BOX 30.1 POVERTY RATES}

What is poverty? This can roughly be defined as an inability to afford the basics of life: food, clothing, and shelter. How can poverty be measured? Different countries establish "poverty lines": estimates of how much money is required to live a basic existence in those countries. Notably, these poverty lines have been revised upward by far more than the rate of inflation in most developed countries over the years as people and governments have changed their view of minimal requirements for existence as economic growth has proceeded. It is now widely assumed that the poor should have some access to health and education and be able to participate in cultural activities.

For purposes of international comparison, a poverty line of US\$1 (or $\$ 1.25$ or $\$ 1.90$ ) a day is often employed. Such numbers seem bizarre to many from developed lands: One could hardly survive on a dollar a day in the United States. But for peasants in countries where households produce much of their own food, clothing, and shelter, such numbers can capture an important sense of the incidence of poverty.

What has happened to poverty rates over time? The World Bank maintains statistics from the 1980s. These show a dramatic decline in the percentage of people living in poverty (using the $\$ 1.90$ in 2012 benchmark) from 44 per cent in 1981 to 37 per cent in 1990 and an astonishing 12.7 per cent in 2012. This decline in the proportion of the world's population in poverty means that the absolute number of people living in poverty has also declined despite rapid population growth: There were 1.99 billion in poverty in 1981, 1.95 billion in 1990, and only 896 million in 2012. To be sure, this is still a large number. And progress has been far less dramatic if one uses a higher poverty line such as US\$3 per day: Clearly, hundreds of millions of people have been raised only slightly above the $\$ 1.90$ level. Nevertheless, this must be seen as one of the most important transitions in all of human history. Over a billion people have significantly better access to food, clothing, shelter, health, and education than would have been the case a mere generation ago.

The most dramatic decline in poverty occurred in China. Over 750 million people were lifted above the poverty line in China over the three decades from 1980. The Chinese poverty rate fell from around 80 per cent to under 10 per cent in those three decades. Important advances occurred elsewhere. Poverty rates in South Asia dropped from 58 per cent in 1981 to 18.7 per cent in 2012 as economic growth accelerated in India especially. Nevertheless, because of its large population, South Asia still hosts over 300 million of the world's impoverished. Growth rates have been sluggish in much of sub-Saharan Africa until the last decades, but poverty rates still fell to 42.6 per cent in 2012. Almost 400 million of the world's poor live in Africa.

Scholars have attempted to estimate global poverty levels before the 1980s. These estimates suggest that the dramatic changes witnessed since 1980 are an acceleration of a trend dating from the early nineteenth century. Some 90 per cent of the world's population may have lived below the poverty line in 1820 , but this rate would fall fairly steadily (except during the Great Depression) through the nineteenth and twentieth centuries. The key 
again would be the rapid economic growth that began with the British Industrial Revolution and spread to other countries. Since the world's population was growing even more rapidly than the proportion in poverty was falling, the absolute numbers of people in poverty rose through time, peaking at somewhere above 2 billion people just before the World Bank started collecting its data in 1981.

Let me briefly personalize this story. When I was a university student in the 1970s global poverty seemed to be a challenge that just kept getting worse. Few of us would have dared imagine that the number of people in poverty would be cut in half within our lifetimes. Though the challenge of global poverty remains, it must now seem beatable. If the economic growth rates that India and Africa often achieved in the last decade or two could continue for another decade or two, hundreds of millions more could be lifted out of poverty. The United Nations indeed has agreed to targets for global poverty reduction that are ambitious but not hallucinatory. We can well imagine a world where the worst kind of poverty ceases to exist.

Though economic growth by raising average incomes has been the key to poverty reduction, attention should also be paid to income distribution. There are dramatic differences both across time and across countries in how incomes are distributed. Historically, severe income inequality was likely prevalent in societies in which a small elite ruled over a large mass in poverty. In developed countries, income distributions became more egalitarian through much of the twentieth century but have become less equal in recent decades. The experience of poorer countries was more diverse, depending in part on economic institutions and government policies. Technological innovation and trade relationships also exert powerful influences on income distribution. Improvements in income distribution can lift people out of poverty. They may also encourage political stability, whereas worsening income distributions may invite calls for political change.

(see above). A peace conference in 1954 divided the country: Ho's communist dictatorship ruled the north, and a non-communist dictator ruled in the south. The people of Vietnam were not consulted as to their wishes but may well have supported Ho as the leader of the Vietnamese independence movement. The southern government never established widespread support and was harassed from the outset by communist insurgents aided by the north. The result was a long and bloody war with both sides supported by outside powers. The United States and allies such as Australia sent armies to aid the South Vietnamese. These were ill-prepared for the guerrilla warfare tactics they faced. As many as 58,000 American troops would die in Vietnam, along with some 200,000 local soldiers and perhaps a million civilians. The Americans and South Vietnamese often destroyed crops and villages in efforts to deprive guerrillas of a base; such tactics often turned locals against the southern government. The United States would abandon the war in 1975 and Vietnam would be unified under communist rule. Communist Vietnam has since - like China to the north - moved in the direction of economic liberalization (allowing private businesses and foreign trade and investment) while maintaining authoritarian political control. 
Vietnamese refugees scramble down a cargo net to a barge manned by men of the 1 st Battalion, 4 th Marines who served as a specially tailored security force. In the foreground is tiny Pawnec, which played a role throughout the evacuation of northern South Vietnam. Department of Defense Photo (USN) 1162058

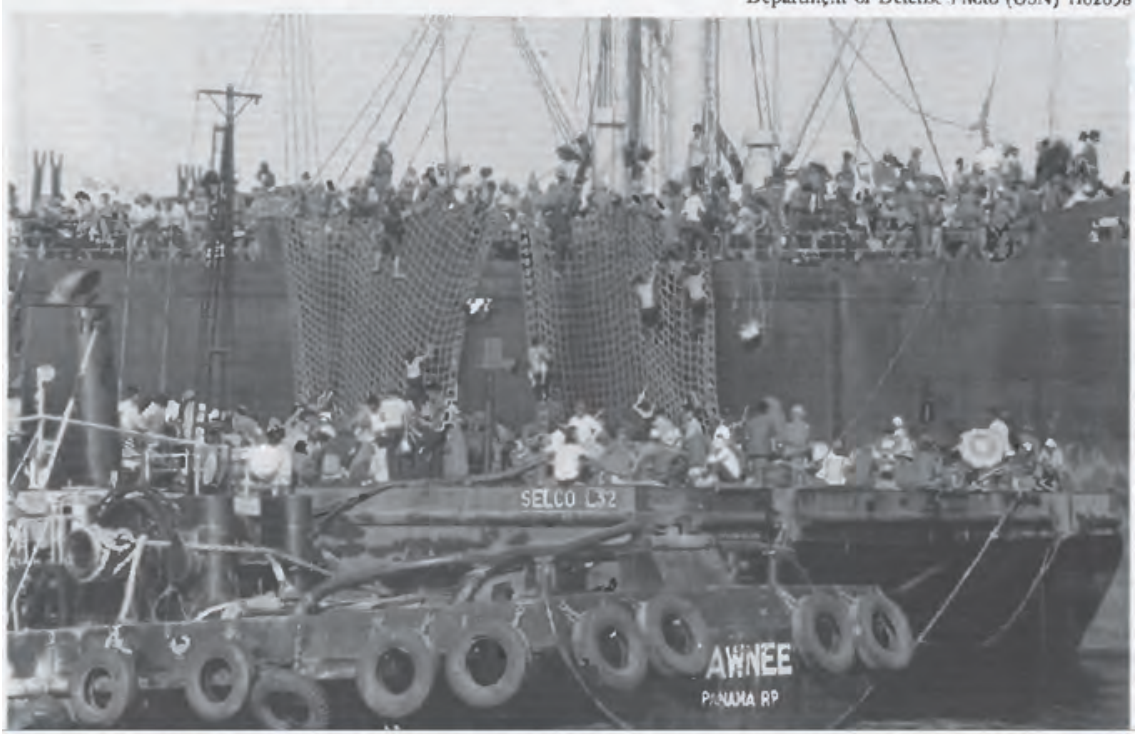

Illustration 30.8

Vietnamese refugees clamber down netting to a barge manned by US Marines during the evacuation of Saigon, 1975

There has been a rapprochement with the United States in recent years. As in China, it remains to be seen whether economic freedoms and a growing middle class will generate pressure for political reforms.

The Vietnam War had diverse impacts. It was politically divisive in the United States and led many there and elsewhere to question authority. It inspired or at least encouraged communist movements in diverse countries, including Mozambique, Angola, and Nicaragua. Yet the dire predictions at the start of the war - the "domino theory" had predicted that if one state was allowed to "fall" to communism, many others would fall in turn - proved unfounded. Though the Vietnam War had spilled over into neighbouring Laos and Cambodia, larger countries in the region such as Thailand and Indonesia remained resolutely opposed to communism (in part as a result of American aid programmes).

\section{Africa}

Africa was the last continent to be extensively colonized by Europeans. It was also the last to achieve independence. The vast majority of African countries gained their independence in the few years between 1956 and 1968. The only exceptions were Ethiopia, which became independent from Italy during the war in 1941, Libya, which was granted independence in 1951, Egypt (see above), 
South Africa (see below), the Portuguese colonies, which would be maintained until 1975, Spanish - now Western - Sahara, which gained independence of a sort in 1976, South Africa, Namibia, and Zimbabwe in the south (again see below), and a few small islands (and the coastal enclave of Djibouti). African colonies were, in general, the least prepared for independence. In many African countries, there was a tiny educated elite, and these had generally been barred from the highest levels of governance. Even in Algeria, there were only seventy non-European Algerians with a university education in 1954. In the Congo, the figure was less than ten at the time of independence. Europeans had for the most part not established assemblies in which political debate might pave the way for a shared vision of independence. Perhaps worst of all, European powers had created colonial borders that completely disregarded ethnic or linguistic divisions. State formation in Africa would have been problematic in any case: As we have seen in earlier chapters, the small-scale polities that had characterized much of African history had resulted in a far more diverse set of ethnic identities than characterized most other regions of the world. European powers often divided ethnic groups between states while constituting colonies in which groups with a history of suspicion or hostility were required to cohabit. Moreover, European powers often found it in their interest to encourage ethnic rivalries, for ethnic groups that disliked each other were less likely to combine against imperial authority.

Postscript: Though recognizing the arbitrary nature of colonial borders, the African Union (the association of African states) has accepted the inviolability of these borders to reduce the incidence of inter-state conflict. Though Africa has often been plagued by civil wars in the decades since independence, there have been few cross-border conflicts. The African Union is seeking to clarify precise borders in many cases where these are unclear: It hopes to complete this task by 2022 , several decades after independence.

With limited governmental capability and with deep ethnic rivalries, authoritarian governments and military coups became the norm in most newly independent African countries. One notable exception was Botswana, which has maintained democratic institutions through much of the independence period. This might be attributed primarily to the high degree of ethnic unity that characterizes Botswana.

Botswana also benefited from rich diamond mines. However, in Africa, independent countries that were rich in resources on average reported lower rates of economic growth for decades than countries that lacked minerals or oil. Resources often proved an invitation to corruption and civil wars over control of resources. Resource exports, by raising the value of a country's currency, also limited its ability to develop other export industries. And the variability 
in resource revenues ironically encouraged government debt as countries spent during good times and borrowed to maintain expenditures during bad. Resources, that is, were only beneficial for economic growth and political stability if associated with governmental institutions that ensured that resource revenues were shared widely and spent wisely. In the last decades of the twentieth century, such institutions were belatedly put in place in many African countries.

\section{BOX 30.2}

\section{RESOURCES AND ECONOMIC DEVELOPMENT}

We saw back in chapter 5 that resource exports in the ancient world were often associated with economic development. To be sure, there were examples then and later where advanced societies used conquest and coercion to extract resources for their own benefit - the Spanish silver mines in Mexico and Peru being the most obvious examples. But even in the Americas, resource exports would translate into economic development, notably in Canada and the United States. The fact that resource exports in postwar Africa were generally associated with below average growth rates is then a bit puzzling. Why might resource exports lead to economic development in some cases but not others?

We can first examine the economic linkages associated with resource exports. Are transport and merchant networks or trade institutions developed for resource exports that then also serve the broader economy? One criticism of colonial powers in Africa is that they only built transport links from mine to port. Are the raw materials processed near where they are mined (or grown)? This might lead to the development of a broader manufacturing sector as workers and managers gain appropriate skills. In days of high transport costs it generally made sense to process materials near mines if possible, but as transport costs have fallen through history this is often no longer the case.

Perhaps most importantly of all, how are the revenues associated with resource exports divided between exporting and importing societies? And how are the revenues that stay in the resource-exporting country utilized? One of the main economic critiques of colonialism is that colonial powers diverted revenues to the imperial centre. Newly independent countries were often poorly equipped to negotiate with multinational mining companies. The latter knew the value of resources far better than the former. Moreover, weak governments proved quite susceptible to bribery. The incomes from mining then flowed either out of the country or to luxury expenditures by the local political elite. Political instability was encouraged in some places as warlords fought over access to the riches associated with raw materials.

One crucial factor here is the distribution of power and income. In reasonably egalitarian societies - such as the frontier farmland of Canada and the United States - positive outcomes are quite possible from resource exports. In societies where both power and income are concentrated, corruption is likely as are expenditures on luxuries rather than the development of a mass market that might spur local industry and services. 
In the modern era, there are a couple of additional challenges associated with resource exports. To the extent that resource revenues flow into government coffers, they encourage governments to spend more than they tax. This can be good for economic development if they spend wisely on roads and schools. However, resource revenues are inherently unstable: Prices fluctuate far more than for manufactured goods, and mineral resources themselves tend to be developed unevenly through time. When resource revenues fall, governments often borrow: They have often not developed the ability to finance expenditures through taxes. The irony is that resource-rich countries are more likely to become severely indebted.

In the contemporary world of flexible exchange rates, resource exports have a further effect: They raise the value of the country's currency, making it harder to export anything else. This may hamper exports of industrial goods or services.

There is a final irony: At a time where the positive link between resources and economic development has weakened at the level of individual polities, concerns have emerged about the future at a global scale. Will we run out of certain resources? Are present patterns of resource use environmentally sustainable? We will return to these questions in chapter 32 .

African leaders often at first advocated pan-African unity rather than the development of nation states with colonialera borders. They occasionally even urged some sort of confederation with the colonial powers: They hoped then to benefit from European institutions while exercising autonomy. Léopold Senghor of Senegal first urged a confederation that included France, and then one for all of French West Africa. Similar arguments were made in Martinique and other Caribbean islands with more success: Small islands did remain part of France but on an "equal" constitutional footing (electing delegates to the French

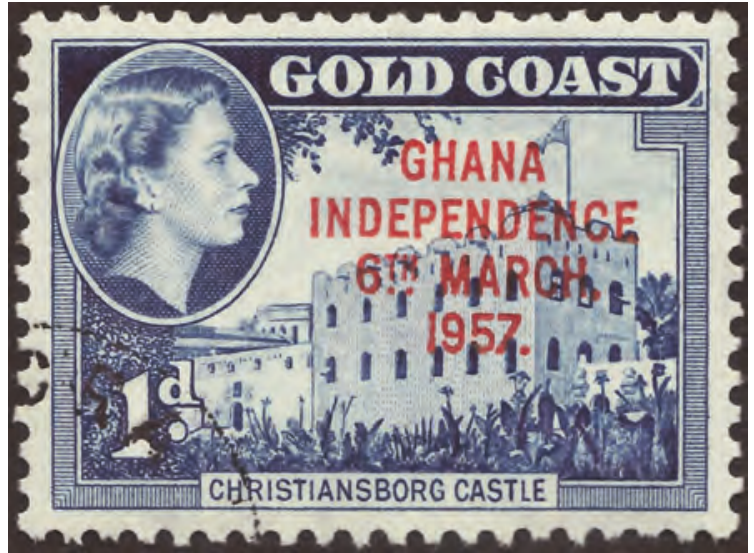

Illustration 30.9

Ghana independence date overprinted on Gold Coast (British colonial) stamp, 1957 National Assembly). Kwame Nkrumah and others made similar arguments regarding English West Africa. They worried that only a large state with diverse resources could thrive economically. And they stressed the shared experiences of African people. But the ideology of nation states proved too influential, especially as local elites in each colony sought power for themselves. Moreover, local groups had long held a strong sense of local identity (recall our discussion of the slave trade in chapter 19). Senghor would end up as the first president of Senegal, and Nkrumah as the first president of Ghana. 


\section{PRIMARY DOCUMENTS: A PLEA FOR AFRICAN UNITY}

Kwame Nkrumah, in I Speak of Freedom: A Statement of African Ideology (London: William Heinemann Ltd., 1961), pp. xi-xiv, outlined his vision of a united Africa:

For centuries, Europeans dominated the African continent. The white man arrogated to himself the right to rule and to be obeyed by the non-white; his mission, he claimed, was to "civilize" Africa. Under this cloak, the Europeans robbed the continent of vast riches and inflicted unimaginable suffering on the African people.

All this makes a sad story, but now we must be prepared to bury the past with its unpleasant memories and look to the future. All we ask of the former colonial powers is their goodwill and cooperation to remedy past mistakes and injustices and to grant independence to the colonies in Africa....

It is clear that we must find an African solution to our problems, and that this can only be found in African unity. Divided we are weak; united, Africa could become one of the greatest forces for good in the world.

Although most Africans are poor, our continent is potentially extremely rich. Our mineral resources, which are being exploited with foreign capital only to enrich foreign investors, range from gold and diamonds to uranium and petroleum. Our forests contain some of the finest woods to be grown anywhere. Our cash crops include cocoa, coffee, rubber, tobacco and cotton. As for power, which is an important factor in any economic development, Africa contains over 40 per cent of the potential water power of the world, as compared with about 10 per cent in Europe and 13 per cent in North America. Yet so far, less than 1 per cent has been developed. This is one of the reasons why we have in Africa the paradox of poverty in the midst of plenty, and scarcity in the midst of abundance.

Never before have a people had within their grasp so great an opportunity for developing a continent endowed with so much wealth. Individually, the independent states of Africa, some of them potentially rich, others poor, can do little for their people. Together, by mutual help, they can achieve much. But the economic development of the continent must be planned and pursued as a whole. A loose confederation designed only for economic cooperation would not provide the necessary unity of purpose. Only a strong political union can bring about full and effective development of our natural resources for the benefit of our people.

The political situation in Africa today is heartening and at the same time disturbing. It is heartening to see so many new flags hoisted in place of the old; it is disturbing to see so many countries of varying sizes and at different levels of development, weak and, in some cases, almost helpless. If this terrible state of fragmentation is allowed to continue it may well be disastrous for us all.

Critics of African unity often refer to the wide differences in culture, language and ideas in various parts of Africa. This is true, but the essential fact remains that we are all Africans, and have a common interest in the independence of Africa. The difficulties 
presented by questions of language, culture and different political systems are not insuperable. If the need for political union is agreed by us all, then the will to create it is born; and where there's a will there's a way.

...

We have to prove that greatness is not to be measured in stockpiles of atom bombs. I believe strongly and sincerely that with the deep-rooted wisdom and dignity, the innate respect for human lives, the intense humanity that is our heritage, the African race, united under one federal government, will emerge not as just another world bloc to flaunt its wealth and strength, but as a Great Power whose greatness is indestructible because it is built not on fear, envy and suspicion, nor won at the expense of others, but founded on hope, trust, friendship and directed to the good of all mankind.

Nkrumah goes on to argue that his plan is entirely feasible. Was it?

The wave of African independence began in North Africa. France granted independence peacefully to the Moroccan king and a Tunisian republic in 1956. (We saw above that Algeria only gained independence in 1962 after a bloody conflict.) Britain and Egypt granted independence to the majorityMuslim and Arabic Sudan in the same year. These countries were more prepared for independence than was sub-Saharan Africa but would be governed in an authoritarian manner for most of their history. The Moroccan monarchy has allowed some elected assemblies over the years. Tunisia has developed an impressive level of democratic governance since public demonstrations in 2011 toppled

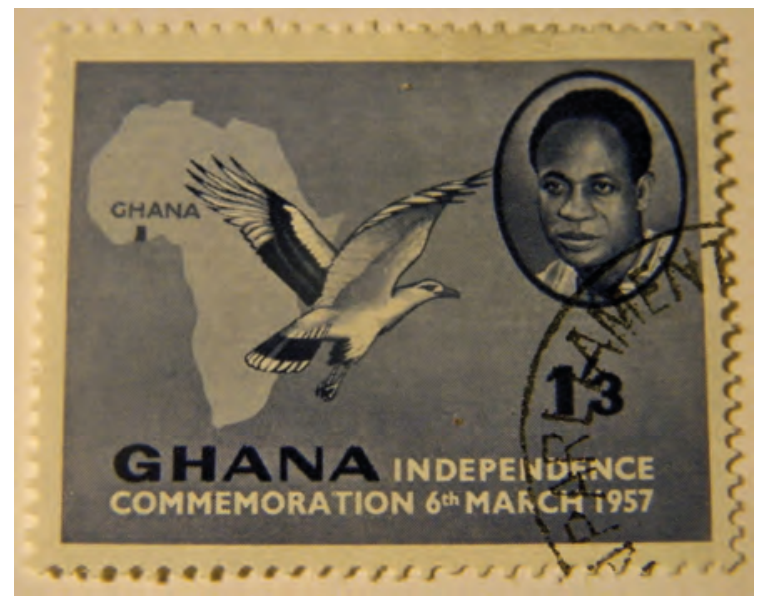

\section{Illustration 30.10}

Ghana soon issued its own stamp with a dove and a picture of Kwame Nkrumah in place of the British queen

Paasikivi, CC BY-SA 4.0 a dictatorship, but this government has struggled to ignite the economy and contend with Islamist terror attacks.

The first independent Black African state was Ghana, granted independence by Britain in 1957. Kwame Nkrumah had been one of the leading advocates of African independence for decades, and Nkrumah was elected as the first prime minister of Ghana. Ghana would then develop a presidential system, and Nkrumah was also elected the first president in 1960. He would be overthrown in a coup in 1966, and Ghana would be governed autocratically for most of the next decades before democracy was restored in 1992. 
The path to independence was particularly bleak in the Congo. The Belgian government established limited self-rule in 1957. When rioting began in 1959, the Belgians undertook to leave entirely in 1960. There was a tiny educated local elite and limited experience of democracy. Within days of independence, the army mutinied against the elected government (with foreign support as the elected government had communist sympathies), and the province of Katanga declared independence. Various developed countries then backed different sides in ensuing struggles for power. Morocco, France, and the United States helped Mobutu Sese Seko gain power in a coup in 1965. He would rule as dictator until 1997, amassing a personal fortune of $\$ 4-5$ billion.

The British, like the French in Algeria, found it harder to grant independence to colonies with sizeable European settler populations. In Kenya, the British militarily repressed an uprising primarily among the Kikuyu, who had been displaced by White settlers in previous decades. They imprisoned even peaceful nationalists and banned political parties. Though Britain won militarily

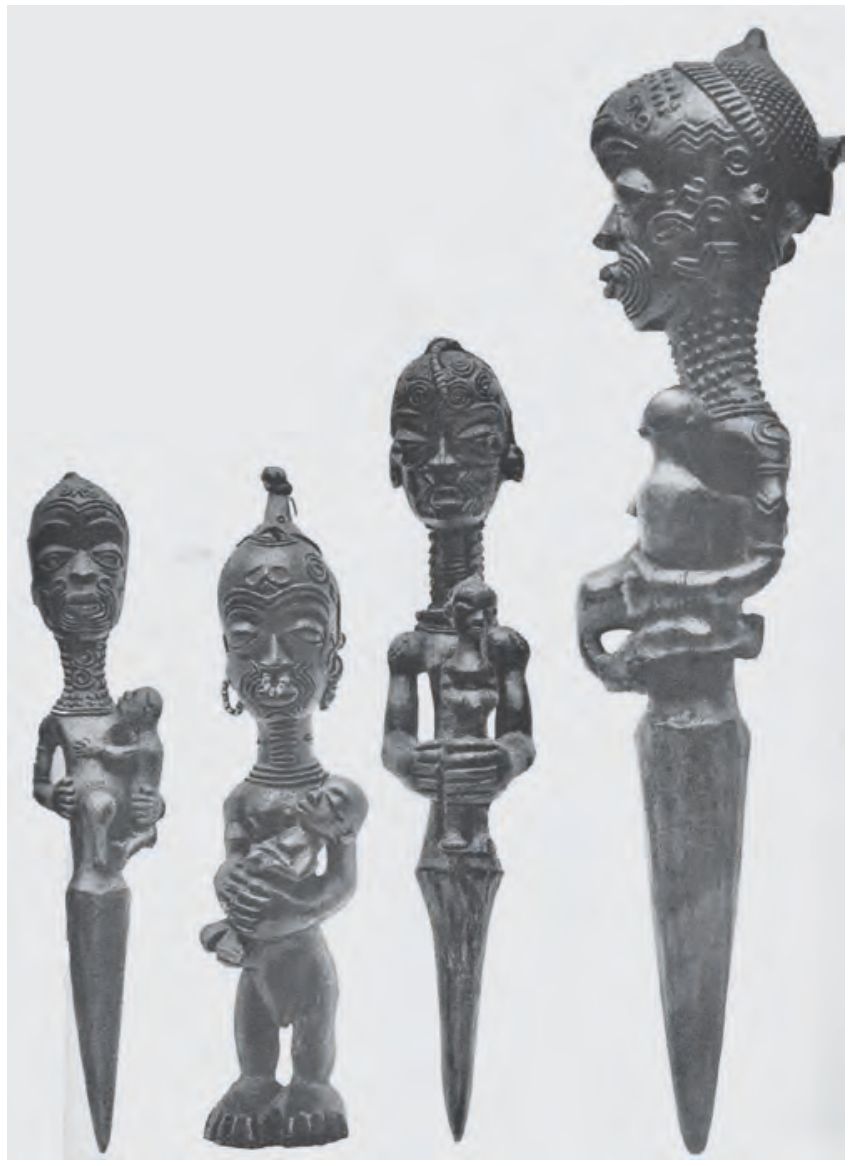

Illustration 30.11

Statuettes of the Kasai-Lulna people, Belgian Congo, 1942 
(employing airplanes to bomb resistance), it soon decided to legalize political parties and negotiated independence in 1963. Farther south, White elites in (what is now) Zimbabwe declared independence unilaterally and continued policies of racial dominance for decades. They drew on the example of South Africa, which had gained independence from Britain in 1910 and had instituted a set of laws which reserved political power and the ownership of most of the land for Whites. However, Whites were a much smaller proportion of the population in Zimbabwe. An armed resistance over a period of decades would force the government to hand authority back to Britain late in 1979 so that it could supervise elections in early 1980. The winner of that election, Robert Mugabe, would respond to protests among the Matabele ethnic group by sending in army units that massacred thousands; he then ruled autocratically for decades until ousted in 2017. In South Africa, Blacks would gain the vote in 1994, after decades of internal protest (including violent protests and acts of terrorism, but also civil disobedience) and international pressure. One contributing factor was that communism was no longer to be feared with the collapse of the Soviet Union (the communists had been a significant part of the resistance). The other was the magnanimous leadership of Nelson Mandela, who had been imprisoned for decades but urged racial harmony and non-vengeance. Still, it took four years from Mandela's release from jail and legalization of the African National Congress to negotiate a new constitution. Despite Mandela's example, South Africa has since experienced oneparty rule that has often shown disdain for basic rights and the independent judiciary.

As noted above, the Portuguese dictatorship held onto its three African colonies long after European democracies had granted independence to theirs. Armed insurgencies developed over the years in both Angola and Mozambique. These received support from the Soviet Union and Cuba. The Portuguese, in turn,

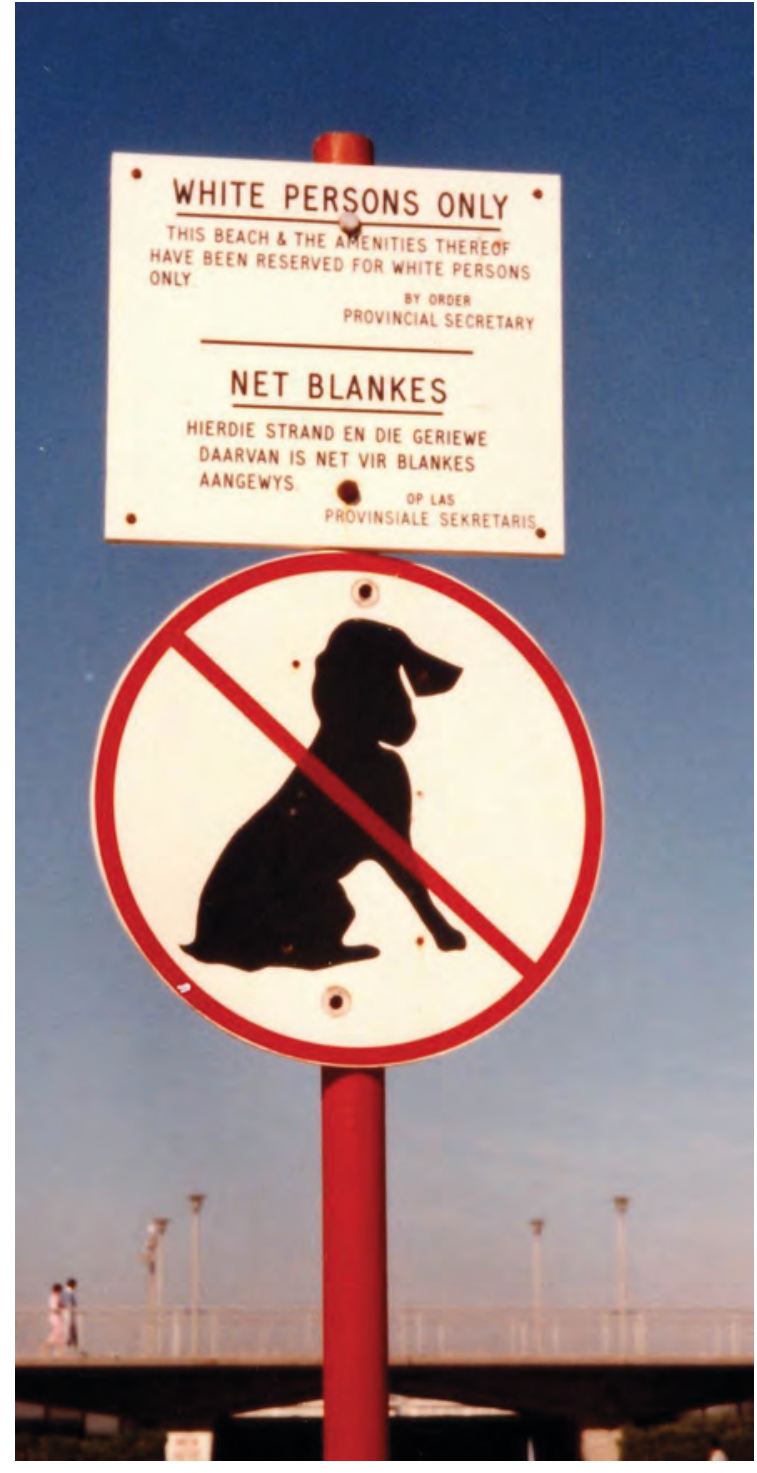

Illustration 30.12

Apartheid era sign Ullischnulli, CC BY-SA 3.0 
received support from South Africa. A coup in Portugal itself in 1974 led to a sudden granting of independence to Angola, Mozambique, and Guinea-Bissau. South Africa also held onto its mandate of (the former German colony of) Namibia until 1990, also facing an extensive armed insurgency.

We recognized above that independent African states were often ethnically divided. Particular attention might be devoted to the string of sub-Saharan countries that contain a Muslim-majority north and Christian/animistdominated south. In Sudan decades of civil war and international pressure led the Sudanese state to grant independence to South Sudan in 2011; that state has since been ravaged by civil war between tribal groups (fuelled in part by the desire to control valuable oil reserves). In Nigeria, Chad, and several other countries, the Islamic/Christian split has undoubtedly hobbled efforts to achieve a strong sense of national identity. This, in turn, has made it difficult to develop robust national institutions.

\section{Latin America}

The vast majority of Latin American countries had achieved independence from Spain or Portugal in the early nineteenth century (chapter 25). They were mostly ruled autocratically well into the twentieth century (we briefly discussed the exceptional case of democratic Costa Rica). Military coups had often interrupted experiments with democracy. In the postwar period, the governments of developed countries often interfered with these weak and authoritarian states. This political experience was similar in many ways to that of the newly independent states discussed above.

This interference - primarily by the British in the nineteenth century and the United States in the twentieth, though the latter proclaimed a "Good Neighbor" policy of non-interference in the 1930s - sometimes took the form of military intervention. More often it involved supporting one internal faction against others. This support naturally encouraged factions that would implement policies that the British or Americans favoured. During the Cold War, this meant supporting capitalists against communists. Earlier it generally meant helping those who would be open to foreign trade and foreign investment.

Economists still debate the precise relationship between trade, foreign investment, and economic growth. At least some types of foreign trade and foreign investment appear to encourage economic growth: The Chinese growth surge discussed above (and the less dramatic acceleration in India) was associated with increased openness to both trade and foreign investment. Foreign opposition to restrictions on trade and foreign investment may then have helped Latin American countries. Such restrictions might have served primarily to protect domestic producers with government connections from competition at the expense of the mass of local consumers and of economic growth. And limits on which goods can be traded or attempts to manage exchange rates provide a 
further opportunity for governmental corruption: Bribes can then be paid for preferential access to foreign products and currencies.

As we have noted throughout this book, information travels with trade. Countries that limited trade also tended then to limit technology transfer. And companies that face little competition - because their countries limit imports - have little incentive to innovate or indeed ensure that their firm is well managed. They are instead guided to ensure that friendly governments stay in power. Local companies and autocrats could easily abuse the rhetoric of economic nationalism for their own benefit.

Foreigners and local elites often combined, though, in activities that were far from benign. Local peasants were pushed off the land to make room for exportoriented plantations. Resources were sold for less than world market prices in return for bribes (recall from above that resource-rich countries in Africa were generally more corrupt). Rights of workers to organize and protest against unsafe, unsanitary or repressive working conditions were restrained forcefully. In various ways, then, corrupt local elites might cooperate with foreigners in activities that were not in the general interest of the citizens they ruled. Note that these "foreigners" were often foreign companies rather than foreign states. Yet these companies generally operated with the support of their home countries. Developed countries have only made significant efforts to limit bribery abroad by their companies in the most recent decades.

Recall that Latin American countries had often had very unequal income distributions rooted in the very uneven distribution of land during colonial times. Elites with a limited interest in the general well-being often ruled these countries. These elites had long used their political power to oppose efforts to redistribute land or that otherwise threatened their economic and political position. Colluding with foreign interests was a natural extension of these selfserving policies. By propping up these elites, foreign intervention may have slowed processes of democratization and economic growth.

Nevertheless, most Latin American countries transitioned to democracy in the last decades of the twentieth century. As with decolonization in Africa, successful democratization in one country encouraged democratization elsewhere. While most adult Latin Americans can now vote, democracies have been plagued by corruption.

\section{PRIMARY DOCUMENTS: THE PERUVIAN AMAZON RUBBER COMPANY}

In 1912, W.E. Hardenburg published an account of atrocities committed against Indigenous people by the Peruvian Amazon Rubber Company: The Putumayo: Devil's Paradise (London: T. Fisher Unwin). He reported that Indigenous people were punished physically if they did not deliver enough rubber to the company. Importantly, he compared these activities to those 
only recently exposed in the Belgian Congo. Though the company was Peruvian, its shares were traded in London. The British consul in Peru investigated and supported Hardenburg's testimony. Here Hardenburg enumerates the atrocities:

1. The Pacific Indians of the Putamayo are forced to work day and night at the extraction of rubber, without the slightest remuneration except the food necessary to keep them alive.

2. They are kept in the most complete nakedness, many of them not even possessing the biblical fig-leaf.

3. They are robbed of their crops, their women, and their children to satisfy the voracity, lasciviousness, and avarice of the company and its employees, who live on their food and violate their women.

4. They are sold wholesale and retail in Iquitos, at prices that range from $£ 20$ to $£ 40$ each.

5. They are flogged inhumanly until their bones are laid bare, and great sores cover them.

6. They are given no medical treatment, but are left to die eaten by maggots, when they serve as food for the chief's dogs.

7. They are castrated and mutilated, and their ears, fingers, arms, and legs are cut off.

The list, sadly, goes on, closing with \#13, that Indigenous people were shot or, worse, burned to death for the amusement of employees. Hardenburg worried that his report might seem incredible. Does it? Why did Hardenburg write this book and what effect do you think it had on readers?

\section{The Pacific}

The small size of most Pacific Islands had prevented European states from establishing elaborate colonial bureaucracies in the Pacific. When European powers divested themselves of colonies in Asia and Africa in the 1940s, 1950s, and 1960s, they held on to their smaller colonies in the Pacific (and some smaller Caribbean islands). In addition to a concern that these islands were particularly ill-prepared for independence, it was speculated that they might simply be too small ever to be capable of self-government.

We noted above that Indian independence encouraged decolonization efforts elsewhere in Asia and Africa. In the Pacific, examples would be set in the 1960s that would inspire decolonization throughout the region. New Zealand had received a League of Nations mandate over the former German colony in Samoa in the 1920s. New Zealand was ill-prepared to govern such a colony. It granted independence in 1962, though Samoa had a population of only about 100,000 . Then in 1968, phosphate-rich Nauru, with a population of a mere 6,000 , was granted independence by a joint Australian-British administration. When these states proved viable, further decolonization followed. The British in particular divested themselves of all of their Pacific colonies in the 1970s. The British had never placed a high value on Pacific possessions: They had 


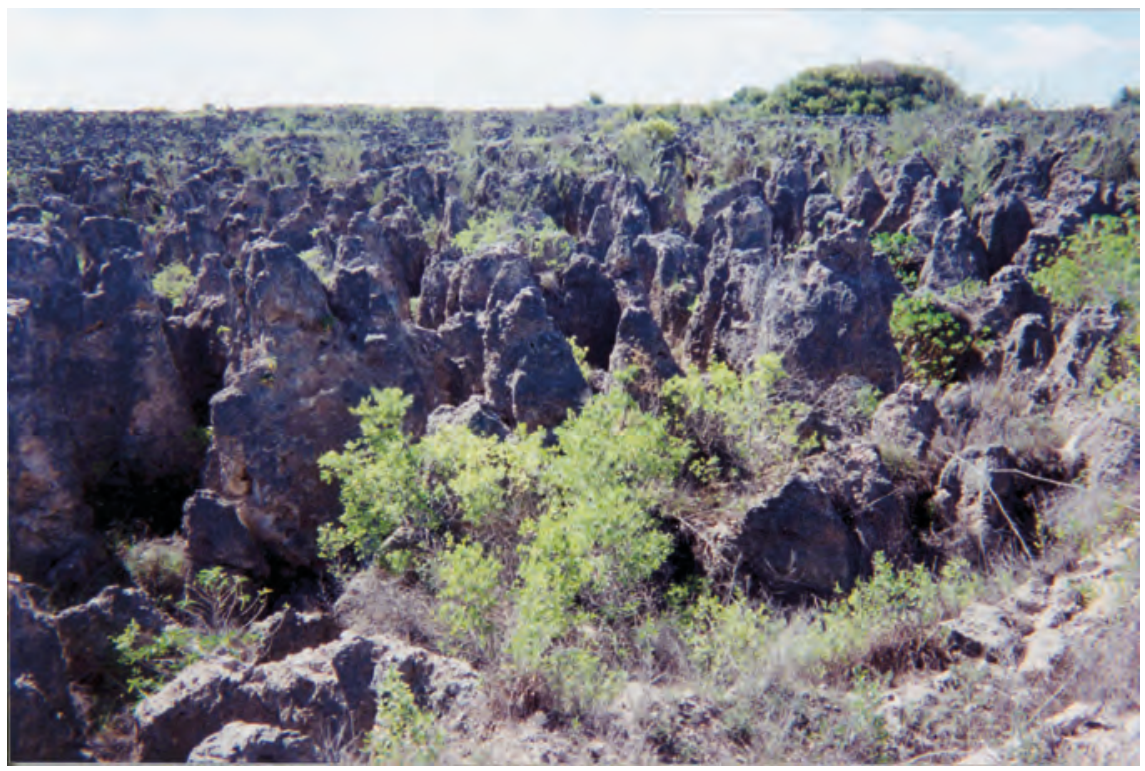

Illustration 30.13

Nauru phosphate fields

largely taken colonies at the urging of Australia and New Zealand (who wished to keep other European powers away), and to prevent Australian colonization (which the British feared would be harsh).

There had been some local agitation for independence in the preceding decades. Japanese victories in the Pacific during World War II had inspired some to oppose European powers, while the horrors of war (locals gained jobs on some islands but were forcefully evacuated on others) encouraged disdain for colonial authorities across much of the Pacific. As in Africa and Asia, a cross-colonial network of advocates of independence emerged. Yet European powers faced less pressure to leave than in larger colonies. As in Africa, European powers often drew borders that divided local communities. Independent New Guinea would face a secessionist movement on the mineral-rich island of Bougainville, which had a greater cultural connection to the neighbouring Solomon Islands. European colonies had imported large numbers of indentured plantation workers from India (sometimes China) into islands such as Fiji; such island states would have to cope with ethnic rivalries after independence (Fiji would experience civil strife and military coups as a result of ethnic divides). Though they might have learned from the postindependence experience of African and Asian colonies, European powers did little to prepare Pacific colonies for independence. Inspired in part by United Nations resolutions in favour of decolonization, European governments left in haste, pessimistic of the likely future stability of their former colonies. The constitutions drawn up by Europeans made little attempt to address issues of ethnic or regional diversity. 
The first colony to be divested, Papua (the western half of the island of New Guinea), was soon associated with Indonesia - as it had been before Indonesian independence from the Netherlands. It remains a part of Indonesia to this day, despite being culturally quite distinct, especially in the interior. The Americans held on to many small islands in the Pacific for strategic reasons, arguing that they were too small for independence. The United States used remote Pacific islands for nuclear tests, before such tests were banned by international agreements.

The French proved less eager to depart than the British and Dutch. In New Caledonia, the mining of nickel and the existence of significant European landholdings (many developed by Europeans who left Algeria after independence) provided both an economic rationale for staying and a vocal local population that opposed independence. The French government made money by administering New Caledonia, whereas British Pacific colonies each produced smallish losses. The French had extended limited self-government during World War II but withdrew this in 1956. All New Caledonians were, however, granted French citizenship in 1953. The French brutally suppressed revolts by the Indigenous Kanaks. As one of the largest islands in the Pacific, New Caledonia played a role for the French more like Australia than Nauru to the British. Indeed, 30,000 French convicts had been transported to New Caledonia in the nineteenth century, and their descendants (and those of their guards and provisioners) provided a large population with European roots. Haltingly, self-government has been introduced since the 1980s, but the largest political parties had until the twenty-first century all opposed outright independence. French (Eastern) Polynesia (often known by its most populous island Tahiti) has also remained a French possession: Economic motives were less compelling there, but romantic attachment to these widely dispersed and beautiful islands, and their value as a nuclear testing ground, encouraged the French to stay. Selfgovernment was gradually extended; in the twenty-first century, those who favour outright independence have often won elections.

\section{Questions}

1. We have covered in mere paragraphs (or less) the founding narratives of most of the countries in the world. Students can usefully explore the history of any newly independent country of their choice. The question to ask is how and to what extent did leaders in these countries manage to achieve results that were different from the general trends surveyed above?

2. Could (and should) European colonial powers have fostered a successful transition to democratic governance in more of their former colonies?

3. How important was the Cold War in encouraging autocracy in newly independent countries? 
4. How did limited human foresight or the human capacity for selfdeception encourage less-than-wonderful outcomes from the decolonization process?

5. Why do most former colonies still retain ties (such as through the British Commonwealth or Francophonie) with former colonizers?

\section{Readings}

Badia-Miró, Marc, Vicente Pinilla, and Henry Willebald, eds. 2015. Natural Resources and Economic Growth: Learning from History. New York: Routledge. [This book mostly supports the conjectures that resource abundance encourages corrupt and short-sighted government policies, and that good institutions can allow countries to benefit from resources. Detailed case studies highlight the difficulty of merely borrowing successful institutions from elsewhere.]

Bates, Robert H. 2017. The Development Dilemma: Security, Prosperity, and a Return to History. Princeton University Press. [Draws lessons from history that states are most likely to foster economic growth if those with power have a shared interest in growth-enhancing institutions (as England achieved long before France). Argues that colonization created states with ethnic and regional diversity that hamper the pursuit of growth.]

Buettner, Elizabeth. 2016. Europe after Empire: Decolonization, Society, and Culture. Cambridge University Press.

Butlin, Robin A. 2009. Geographies of Empire: European Empires and Colonies c. 18801960. New York: Cambridge University Press. [Chapters are organized thematically around subjects such as imperialism, migration, cartography, transport, or mining, but subchapters then address particular empires or regions or enterprises.]

Chappell, David A. 2013. The Kanak Awakening: The Rise of Nationalism in New Caledonia. Honolulu: University of Hawai'i Press, Pacific Islands Monograph Series, No. 27.

McIntyre, W. David. 2014. Winding Up the British Empire in the Pacific Islands. Oxford University Press. [Part of the Oxford History of the British Empire Companion Series.]

Mar, Tracey Banivanua. 2014. Decolonization and the Pacific: Indigenous Globalization and the Ends of Empire. Cambridge University Press.

Mishra, Pankaj. 2012. From the Ruins of Empire: The Revolt Against the West and the Remaking of Asia. London: Allen Lane.

Reeves, Mark L. 2016. "Teaching Decolonization beyond the Nation: The Case of West Africa." World History Connected 13:2.

Reynolds, Michael. 2011. Shattering Empires: The Clash and Collapse of the Ottoman and Russian Empires, 1908-1918. Cambridge University Press.

Streets-Salter, Heather, and Trevor R. Getz. 2016. Empires and Colonies in the Modern World: A Global Perspective. New York: Oxford University Press. 


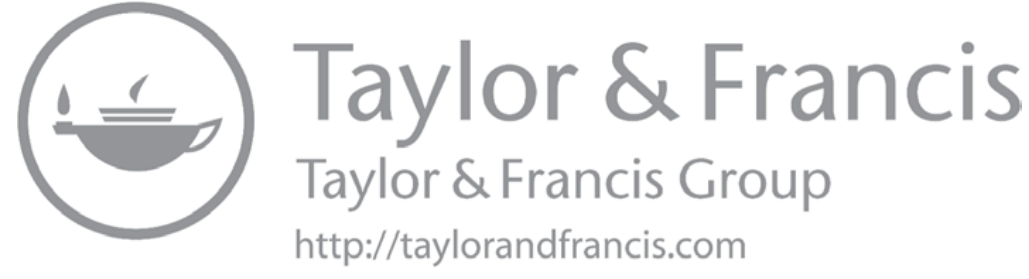




\section{Population movements}

\section{Dramatic changes in the numbers, location, and health of humans}

\section{Guiding questions}

Why, where, when, and how did population grow in the nineteenth and twentieth centuries? Where, when, why, and how did it then stabilize or even decline? What happened to life expectancy in different parts of the world and why?

When, where, why, and how did migration occur in the nineteenth and twentieth centuries? What were the main differences between nineteenth- and twentieth-century migration? Who were the migrants? What were the effects on both source and destination countries?

Why was there so much urbanization in this period? How did urbanization differ in this period from previous periods? How did urbanization unfold?

When and where did epidemics occur in this period? Can we detect a trend in the occurrence and seriousness of epidemics? What effects did epidemics have?

Why has obesity become a problem in almost all countries? How was the global challenge of obesity conditioned by earlier history?

Relationship to other chapters: This is the only chapter in this book devoted primarily to the theme of population and health. It continues a conversation about population carried through chapters 7, 14, and 18. Population, life expectancy, and levels of 
urbanization changed far more rapidly in this period than previously. It is useful to place developments in this period in the context of the much more gradual changes observed previously. We shall see that these dramatic developments reflected economic and technological developments that we have surveyed in earlier chapters such as chapter 24. Migration responded to both the economic and political divergences between states that we have discussed in several earlier chapters but especially $22,24,25,29$, and 30 . Epidemics are one topic addressed in this chapter that has occasionally received extended discussion in earlier chapters; it is important to compare experience across time periods in order to be able to reflect in chapter 32 on the possibility of future massive mortality such as occurred during the plagues of chapter 17 . We shall find that the modern challenge of obesity has deep historical roots, reflecting the lives of both hunter-gatherers and farmers.

We have traced population growth through every time period in this book. We have often discussed the key factors affecting both fertility and mortality. We have also often addressed human migration, both migration between regions and migration from country to city. However, we have not devoted a full chapter to population movements until now. Population growth accelerates dramatically in the nineteenth and twentieth centuries, but then population starts to stabilize or even decline in some countries late in the twentieth century. Both of these dramatic shifts deserve special attention. So do dramatic increases in life expectancy. International migration occurs in absolute numbers far higher than ever before in both centuries - and perhaps also as a proportion of total world population. These movements of hundreds of millions of people also deserve special attention. Then there is urbanization. As with population generally, trends in urbanization accelerated through the nineteenth and twentieth centuries - but here there is less indication that urbanization is slowing. We have often taken city size in this book as a rough proxy for the economic and political sophistication of a society (see Box 6.1): This link was broken in the twentieth century with the emergence of huge cities in some of the poorest countries in the world. These developments also demand our attention. We close the chapter with some discussion of equally dramatic changes in the health of human populations.

While population (including health) is our primary theme in this chapter, we shall find that these various changes in population are closely linked to changes in the economy, environment, science and technology, politics, and social structure. We will compare throughout the chapter developments in different parts of the world. In our discussion of migration, we will inevitably 
examine the influences of migration on both the lands migrants leave and the lands they settle in. Our primary applications of evolutionary analysis occur in the latter sections of the chapter when we discuss epidemics and especially obesity. Unusually, we engage biological evolution rather than social evolution in those sections.

\begin{tabular}{|c|c|c|c|c|c|}
\hline Population & Economy & Environment & $\begin{array}{c}\text { Science } \\
\text { Technology }\end{array}$ & Politics & $\begin{array}{c}\text { Social } \\
\text { Structure }\end{array}$ \\
\hline
\end{tabular}

\section{Population growth and stabilization}

Earth's population likely reached 1 billion only at the start of the nineteenth century in about 1804 - having risen slowly and irregularly from 6 million in the time of hunter-gatherers and 370 million at the end of the Great Plague of the fourteenth century. World population would then double to 2 billion in just 123 years to 1927. The population reached 3 billion in 1959 (after only 32 years), 4 billion in 1974 (15 years), 5 billion in 1987 (13 years), 6 billion in 1999 (12 years), and 7 billion only in 2012 (13 years). The population will likely continue to grow in future decades but at a decreasing rate: reaching 8 billion in about 2026 (after 14 years) and 9 billion in 2042 (after 16 years). World population may (or not) stabilize or decline sometime in the next century.

Why did population grow so fast for so long? As we discussed back in Box 4.1, the population has been limited through most of human history by the food supply. This link was broken in the nineteenth century as economic growth began to occur at faster rates than population could. The result, as we saw in chapters 24 and 29 especially, has been rising per capita incomes throughout the world. It is one of the ironies of history that Malthus designed his theory of population early in the nineteenth century just as it was ceasing to hold for reasons he could not yet foresee. It is even more ironic that the Qing scholar Hong Liangji (1746-1809) made similar arguments to those of Malthus at about the same time.

In chapters 24 and 29, we stressed developments in industry. We should recognize here that these developments - though only possible in the first place because farmers could feed industrial workers - had a feedback effect on agriculture itself. As industry produced better steel and machines, it transformed agricultural equipment: A variety of sharper and stronger and more precise tools for seeding, cutting, and processing crops were developed from the nineteenth century and were wedded to mechanical tractors in the early twentieth century and then combined in complicated planting and harvesting machinery later. Even more dramatically, the new chemical industries developed a set of artificial fertilizers and herbicides and insecticides. These had some worrisome environmental implications. Nevertheless, the combined effect of advances in machinery and chemicals was to allow fewer farmers to grow much more food. 


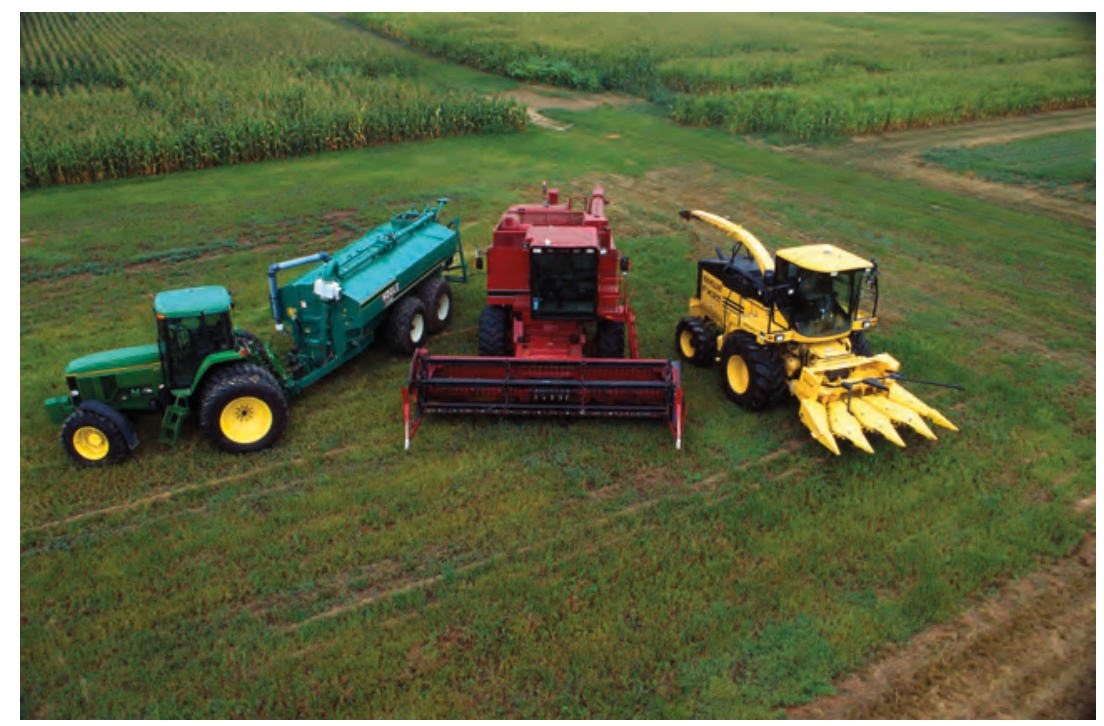

Illustration 31.1

Modern machines for fertilizing and harvesting

The seeds that farmers planted deserve particular attention. As we have seen since chapter 4 , farmers have for thousands of years acted to select seeds with desirable properties. As a result, the crops we grow today could generally not survive without human guidance. This has been especially the case as we select seeds that only work well in concert with modern fertilizers and insecticides. Before the nineteenth century, farmers had a limited scientific understanding of how selection worked. From the late nineteenth century, theories of genetic inheritance would inform efforts at agricultural selection. In the 1960s, the "Green Revolution" would involve the careful cross-breeding of plants with desired characteristics and selection on experimental farms of seeds with the highest potential - at least if applied in combination with the latest chemicals. New strains of wheat developed by Western scientists on Mexican farms would result in an increase in yields in many parts of India (and elsewhere) to five times the previous level. Similar advances were achieved for corn, rice, and other crops. Without such increases in food supply, the dramatic expansion in population observed in India and elsewhere would not have been possible. This concentrated research effort tended to focus on the crops grown in temperate climates. Tropical plants have seen increased attention in recent decades. The other recent development has been genetically modified foods: Researchers can now directly transfer genes from one organism to another rather than merely cross-breed compatible crops and hope for the desired combination; this approach has been controversial, especially when scientists transfer genes across entirely different organisms. 
Postscript: Scientists and governments have for some decades retained samples of the seeds that farmers used to plant in various parts of the world. If some disease were to strike the increasingly homogeneous and chemical-dependent crops grown in the world, farmers might employ these old seeds but with a resulting decline in agricultural output. In addition to a variety of national seed depositories, the Svalbard depository in that remote northern Norwegian archipelago collects seeds from around the world.

\section{BOX 31.1}

\section{THE CHANGING ROLE OF AGRICULTURE}

Through most of recorded human history, the majority of humans have engaged in agriculture. Levels of agricultural productivity have largely determined whether a society could support large numbers of bureaucrats or merchants or artisans. We have therefore devoted much attention to agriculture in this book. The combination of mechanization, advances in chemical fertilizers and pesticides, and then advances in breeding both plants and animals, resulted in dramatic increases in agricultural productivity in the nineteenth and twentieth centuries. These advances have been geographically uneven. Farmers in developing countries struggle to afford machinery, and often have plots of land too small to make such machines worthwhile. (In some cases this is because of land reforms that transferred property from large landowners.) Research on plant breeding has focused until very recently on temperate crops rather than tropical crops. In many developing countries, then, much of the population is still in agriculture and has low incomes. In rich countries, farmers generally comprise only a few per cent of the population. (Some rich countries, especially in Europe, came to rely heavily on food imports.) Farm incomes often fell relative to industrial and service sector incomes during the twentieth century, as increased agricultural productivity kept agricultural prices low. Food, which comprised the bulk of consumer expenditures for most people through most of human history, came to be just a fraction of spending for most people in the developed world. Overeating became a more significant problem than undernourishment for the first time in history (see below). Farmers in many countries exercised some political influence and were able to encourage public policies that supported farm incomes. They appealed to nationalist sentiment - and memories of food rationing during World Wars - in defence of a vibrant agricultural sector. These agrarian policies sometimes involve direct subsidies, and other times require government purchases at favourable prices. Many countries protect farmers from foreign competition through very high tariffs.

As we have seen, increased agricultural productivity allowed the human population to expand. In recent decades, though, food prices have occasionally risen dramatically. It 
seems that agricultural output may no longer be expanding faster than population. Further increases in population will only be possible if agricultural productivity continues to grow. This may require that poorer countries duplicate the productivity advances achieved in developed countries.

Of course, increased food supply only allowed the population to grow. Humans had to decide to take advantage of their higher incomes by having more children. Malthus, though he could not predict unimagined rates of economic growth, would not have been surprised at all that humans responded to increased incomes by increasing fertility rates far above mortality rates. Here too, though, Malthus would have been in for a big surprise. After some decades of rapid economic and population growth, fertility rates fell to levels at or below mortality rates in many countries. Malthusian theory, therefore, ceased to operate in the nineteenth century in two ways: economic growth faster than he could imagine, and conscious decisions to limit fertility to a degree that he had thought impossible.

We discussed this "demographic transition" in chapter 24. It is worth recalling that the costs of having children increased with urbanization and industrialization and occupational specialization, while the benefits fell with expectations that children would go to school rather than work and the emergence of private and public pensions that could take care of ageing parents. There were also significant changes in power dynamics within the family (and changes in associated cultural attitudes) as women were educated and gained paid employment. The demographic transition occurred first in Europe, parts of the Americas, Australia, and Japan: Many of these countries now have birth rates far below death rates. (Young couples in some of these countries have fewer children than they might like because of the high costs associated with housing and other child-related expenses.) It has since occurred throughout most of Asia - but populations will still rise for a while there just because there are large numbers of young people who will have children over the next decade or two. Fertility rates have fallen, but remain much higher than mortality rates, in the Middle East and parts of Africa. Some combination of cultural attitudes and the social position of women may be responsible for fertility rates not falling faster. It may well be that as educational attainment and employment of women expand in these countries, fertility rates will fall to levels observed elsewhere.

Mortality rates fell in the nineteenth century primarily because of improved nutrition and increased access to clean water and sewer systems in cities. In the twentieth century, modern medicine would further decrease mortality rates. The germ theory of disease from the late nineteenth century - only possible because of the use of sophisticated microscopes in laboratories - encouraged higher standards of cleanliness in homes and hospitals (the latter had previously 
been very unhealthy places indeed). It would set the stage for the development of drugs that would attack particular germs. Cleanliness in homes reflected the development of toilets, cheap floorings that could replace pounded dirt in the homes of the poor, and new devices such as vacuum cleaners and washing machines. Pharmaceuticals of various types prevented or cured a range of diseases: It is easy to forget now that simple scrapes could quickly lead to death in the era before antibiotics. Vaccinations eradicated diseases such as polio and smallpox that had terrorized humanity for millennia. Other diseases, such as measles, have been eliminated as long as enough of the population is vaccinated. Wealthy countries that were already experiencing low fertility developed most of these advances in medicine. Their transmission to poorer countries with high levels of fertility resulted in very rapid population growth rates there.

Divergent trends in fertility and mortality led to some important shifts in the distribution of the human population across continents. The most populous continent by far, Asia, showed little change: Indeed Asia has held between 55 and 65 per cent of the world's population for centuries. Europe's share of the global population fell from just below 25 per cent in 1900 (having risen from a little over 20 per cent in 1800) to barely 10 per cent in 2012 . North America would have seen a similar decline if not for massive immigration (see below): Its share of the global population was about 5 per cent in both 1900

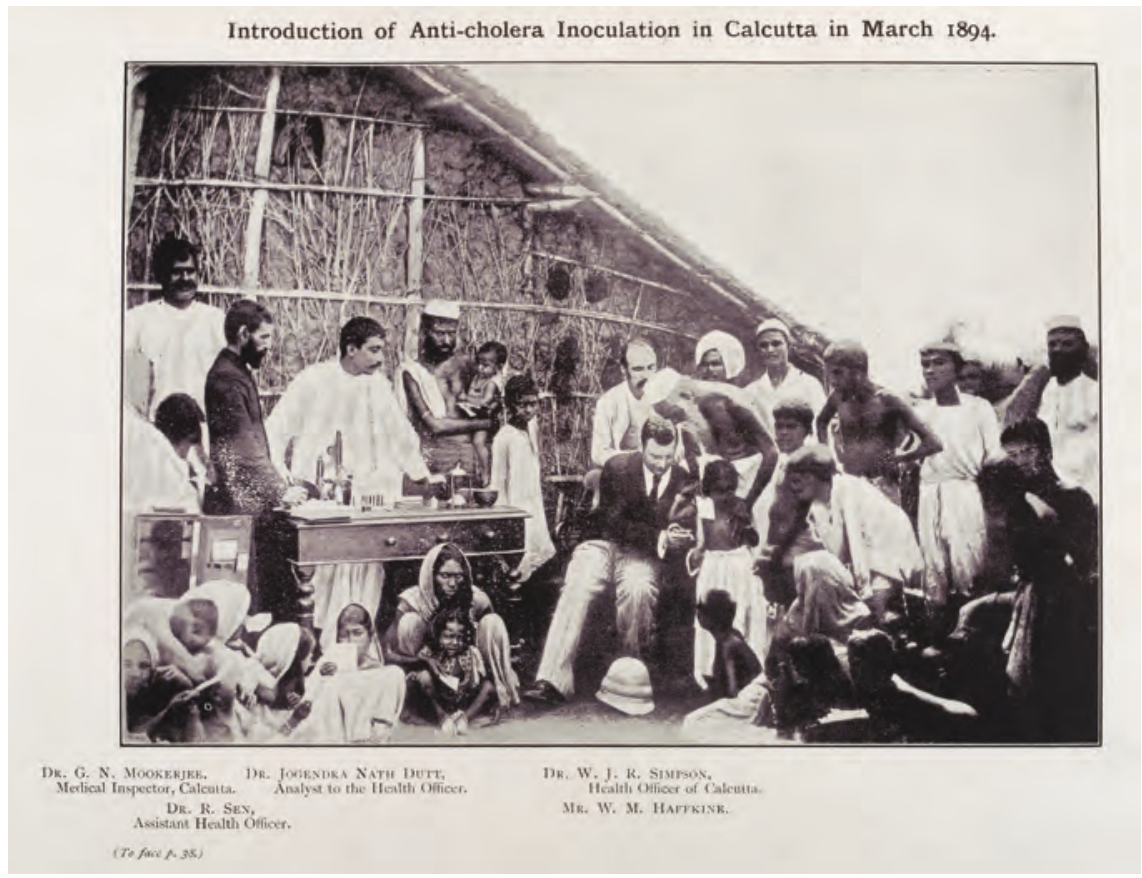

Illustration 31.2

Vaccination against cholera in Calcutta (Kolkata), 1894

Wellcome Images, CC BY 4.0 
and 2000 (rising from under 1 per cent in 1800), though this had risen toward 7 per cent in the middle of the twentieth century. Latin America's share increased from 2.5 per cent in 1800 to 4.5 per cent in 1900 to 8.5 per cent in 2000: It is once again far more populous than North America as it was centuries ago. The most dramatic change occurred in Africa, the most impoverished continent: Its share of the global population was barely 8 per cent in 1900 but almost 13 per cent in 2000 and over 15 per cent in 2015. This marks a return to Africa's previous position: It had likely held over 15 per cent of the world's population in 1700, and close to 20 per cent in 1600 .

There was also diversity in population growth rates within regions. British and German populations grew much faster than the French population in the nineteenth century. This upset the balance of power within Europe. The slow growth of the French population also meant that there were far fewer French emigrants: Canada, in particular, might have ended up with an entirely different ethnic mix if there had been more people leaving France in the nineteenth century. In Asia, parts of Indonesia and the Philippines saw particularly rapid growth while some regions of China and India saw little increase. Tahiti and New Caledonia experienced epidemics that killed much of their population in the late nineteenth century.

Global population growth rates peaked in the 1960s. There was considerable concern at the time about a "population explosion." Many feared that population would continue to expand exponentially beyond the planet's ability to sustain it. In other words, there were fears that deviations from Malthusian theory were temporary and that population would soon outstrip resources with disastrous results. As population growth rates fell through much of the world, and agricultural productivity advanced, these fears have receded. If the world's population were to stabilize at somewhere around 10-11 billion in the next century or so, it is entirely possible that this number could be fed, especially if agricultural technology continues to advance. One challenge here is that as incomes have risen, people have tended to prefer more meat in their diet, and meat is far more land-intensive per calorie or unit of protein than plants. However, in at least some developed countries young people have shown a much greater interest in vegetarian and vegan diets than their elders. And plant-based foods that mimic meat are becoming common items of consumption in many countries.

Food, though, is now only a small fraction of people's consumption expenditures in much of the world. Will there be enough oil or iron ore or the "rare earths" so essential to modern electronic devices to serve this larger population? In recent decades, humans have for the vast majority of natural resources discovered new supplies faster than we have utilized old supplies. Moreover, we have literally only scratched the earth's surface in our search for these - though of course costs rise as we dig deeper. At some point, of course, we must run out: There is only so much iron ore in the earth. It is hard to estimate whether that point will come in the next decades or far later. It is even harder to 


\section{BOX 31.2 \\ POPULATION AND ECONOMY IN A POST-MALTHUSIAN WORLD}

The Malthusian mechanisms outlined in chapter 4 posited a straightforward relationship between population growth and average incomes through most of human history. The demographic transition meant that a variety of other connections between population and economy became important. These might have operated in Malthusian times, but Malthusian forces overwhelmed them.

Population growth can have positive effects on the economy. It increases the size of both the market and the labour force. In some instances, it may encourage technological innovation: We saw in chapter 4 that population pressure may have inspired the development of agriculture itself. Yet population growth also has adverse effects. The existing agricultural land, capital stock, and public infrastructure must serve more people, pushing incomes down. Savings rates tend to fall as parents spend on children, leaving less money available for investment.

What is the net impact? Using twentieth-century data, economists have estimated that there is little impact if population growth rates are below about 2 per cent per year. However, those countries with higher population growth rates than 2 per cent probably see significantly lower rates of increase in per capita incomes as a result.

predict how fast technologies of resource substitution (especially toward renewable resources), recycling, and perhaps travel to other planets or asteroids will develop.

Along with concerns about resource availability, there are also concerns regarding environmental impact. We discussed the environment in chapter 27. We can note here that environmental challenges increase in intensity as the population expands. We can also note again that agricultural practices themselves raise environmental concerns: The chemicals we have been using may negatively affect soil fertility over time.

\section{Life expectancy}

Average life expectancy at birth is between 55 and 80 in almost all countries in the world in the early twenty-first century. Though there are still differences across countries, these are far smaller than cross-country differences in average incomes. There have been some periods of decline in some places - notably in Russia in the years following the break-up of the Soviet Union - but the general picture has been one of steady improvement (ignoring the impact of major wars). Improved nutrition, as agricultural output grew faster than 


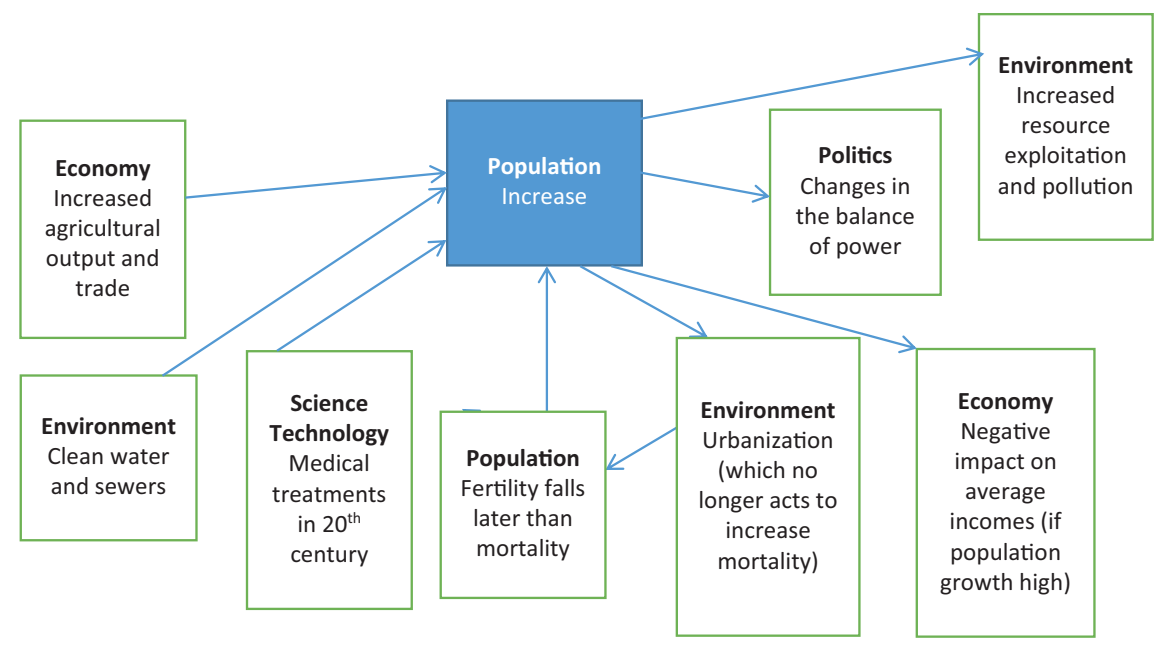

Figure 31.1

Influences on and effects of population growth

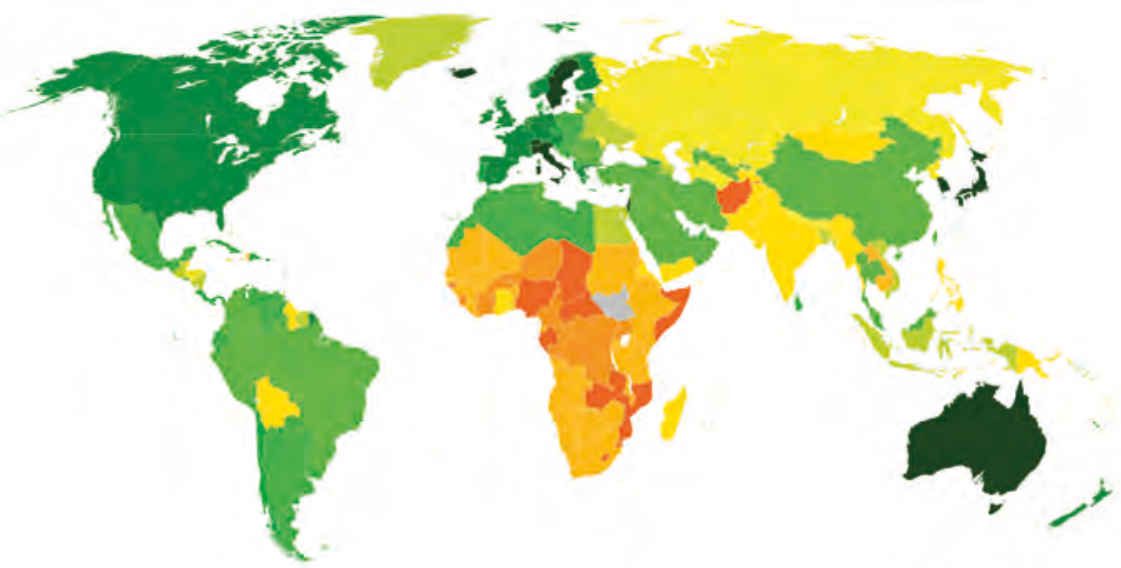

70 years and higher

82 years and higher 80-81 years

$78-79$ years

76-77 years

$74-75$ years

$72-73$ years

70-71 years

N/A

\section{Lower than 70 years}

65-69 years

60-64 years

$55-59$ years

$50-54$ years

$45-49$ years

40-44 years

35-39 years

lower than 35 years

\section{Map 31.1}

Life expectancy at birth by country, 2017 
population (above) and expanded trade increased dietary diversity, was one main reason. Another was improved public health as the world's cities built water and sewer systems (below). Moreover, in the twentieth century, medical science would develop treatments for a variety of diseases. Europe, North America, and Japan experienced a rapid expansion in life expectancy early in the twentieth century. In Latin America, the biggest advances occurred between 1930 and 1960. China and much of Africa saw dramatic improvement before 1980 .

In all cases, the most significant change occurred for infant and childhood mortality. Through much of human history, those who survived until 20 had a

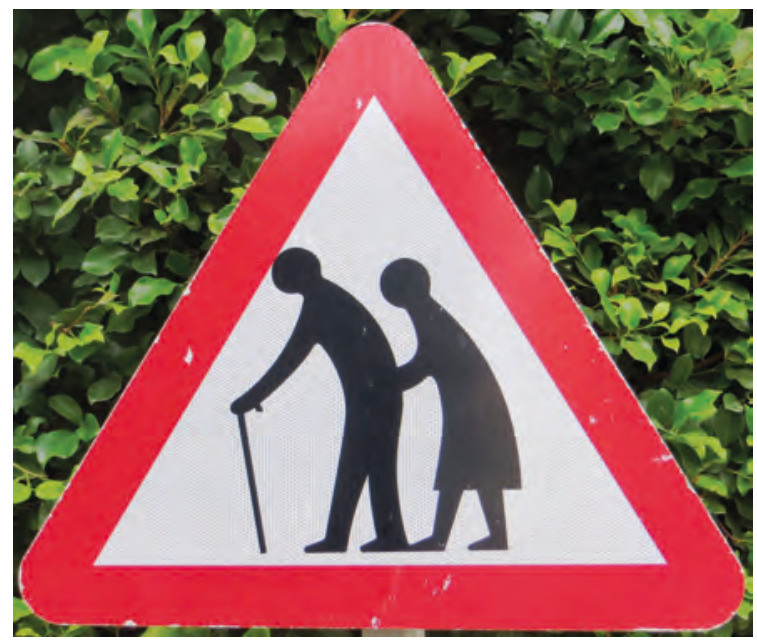

Illustration 31.3

Crossing sign, North Cyprus, 2013

Photograph by Dickelbers, CC BY-SA 3.0 good chance of surviving beyond 50 or so. However, infants and children were often undernourished and subject to a wide range of diseases. It is now a tragedy in most (but far from all) of the world when a young child dies; through most of human history, this was an unfortunate statistical regularity. This transformation has undoubtedly had a significant impact on the emotional relationship between parents and children, and also on the cultural expectations surrounding parenthood.

The combination of lower fertility and higher life expectancies means that many countries are experiencing something novel in human history: populations with a significant proportion of people over age 60. Changes in employment practices and pension policies mean that most of these people do not work. Advances in medicine mean that many live long after they are capable of looking after themselves. World history can provide little guidance on how to address the challenges of caring for large numbers of elderly - but we can note that societies throughout history have generally found an important role for (smaller numbers of) the old to play both in providing advice and in caring for young children.

\section{Migration}

Migration rates reached unprecedented levels in the decades between about 1830 and World War I. The World Wars and Great Depression interfered with migration patterns. However, very high levels of migration would then characterize the postwar period right up to the present. We discuss these two decadeslong periods of mass migration in turn. 


\section{Nineteenth-century migration}

Only about 3 million Europeans in total had migrated to the Americas before the 1820s (they and their descendants had very high average birth rates). However, some 55 million Europeans would do so between 1850 and 1930: About threequarters of these went to North America. Of course, migration levels were not steady across these decades: they rose from the 1840s through the 1870s, slowed in the 1870s and 1880s, reached unprecedented levels in the decades right before World War I, and revived a bit in the 1920s before collapsing in the 1930s. There was also considerable migration within Europe at the time as Italians, Poles, and Irish in particular migrated elsewhere.

It may surprise inhabitants of the Americas to learn that equally massive migrations were occurring in Asia at the same time. Some 50 million people moved into Southeast Asia in the century before 1940: They moved primarily from China, but there were also millions from India (Sri Lanka also received millions of migrants from India). Asia was even an important destination for Europeans: some 13 million migrated into Siberia. Some 30 million Chinese, and a couple of million each from Japan and Korea, migrated into Manchuria in the last decades of the nineteenth and first decades of the twentieth century. And a couple of million from each of China and India, and a half-million Japanese, migrated to Pacific Islands and the Americas. Asian migration to Latin America is often under-appreciated outside Latin America, but there are at least a million Peruvians of Chinese descent today and sizeable populations of Asian ancestry in Mexico, Chile, and Brazil, among other places.

It may be convenient to think of three similarly sized great migrations of about 50 million people: to the Americas, to Southeast Asia (and parts of South Asia, Australia, and the Pacific), and into north and northeastern Asia (Siberia and Manchuria). However, the first of these had a far more significant demographic impact: The gender balance was far more even in the migration to the Americas (25 to 50 per cent female over the years; often only 10 per cent in Asia) and the low population density there was conducive to high birth rates. Still, in Siberia and Manchuria, as in the Americas, migrants swamped the local population.

Why was there so much international migration after about 1830 ? One obvious reason is improvements in transport and communication. Steamships over time became faster and cheaper than sail. Ships carrying bulky raw materials in one direction were happy to transport migrants in the other. (There were, though, many shipwrecks, and problems with onboard sanitation, overcrowding, and food; regulations slowly addressed health and safety issues on board.) A million miles of railway were built by 1914 to carry migrants to and from their ships (or across Siberia and Manchuria). Lower transport costs not only influenced migration directly but also indirectly by creating opportunities in the mines, forests, farms, and cities that exported goods in world markets. Without growing international trade (chapter 27), there would not have been so many new jobs in previously underpopulated parts of the globe. 
Telegraphs and international mail delivery made migration less emotionally wrenching and allowed migrants to alert friends and family to opportunities abroad. This meant that migrants did not always move to the place with the best opportunities. Instead, we observe ethnic clustering, most apparent perhaps in ethnic farming towns in the Americas.

There was little in the way of migration controls during much of this period. Countries did not try to stop citizens from leaving or new people from arriving. The abolition of slavery and serfdom (chapter 26), and the expansion of free labour markets more generally, meant that workers did not need anyone's permission to move. Late in the nineteenth century, many receiving countries introduced race-based restrictions (chapter 26). These would be a harbinger of more widespread controls on international migration after 1920.

The growth in the population itself (above) influenced migration. More people meant more potential migrants. While incomes may always have been expanding globally faster than population, this was not the case in every locale: People moved from regions in which incomes were stagnating to regions where incomes were growing.

In chapters 22 and 24, we discussed how and why incomes grew much faster in some parts of the world than others during the nineteenth century. This divergence was perhaps the greatest single incentive to migration. Tens of millions of people calculated that they could gain much higher incomes if they migrated. However, economic motives did not act in isolation. We have also seen that a wide range of political regimes characterized the nineteenth century: Migrants often sought political freedoms. Nationalism and nation states often meant persecution of minorities: Migration would be especially attractive to these. Recipient countries might also discriminate, but as lands of immigrants they tended to be more open to cultural and ethnic diversity. Muslims fled Crimea and the Caucasus after Russian conquests. French and Poles left an expanding Germany. Jews fled persecution in Russia. A third of Lebanese farmers moved to Egypt or the United States between 1890 and 1920 due to civil conflict.

Migration to the Americas and Australia was encouraged by the availability of land to farm. In such places, migrants planted crops on lands previously devoted to hunting and gathering. Many of the 50 million migrants into Southeast Asia also headed toward new lands. New crops and transport infrastructure allowed agriculture to push higher up mountainsides and into the dense forest there. In Manchuria, farmers with new crops displaced nomads.

The development of urban labour markets was also of critical importance. Although histories of North America often emphasize those who moved to farm, most migrants settled in cities. Urbanization, industrialization, and the development of the service sector all encouraged migration, for these created urban jobs. There was, of course, a synergy between agricultural and industrial development: Increased rice production in Burma and Thailand fed workers in Malayan tin mines and rubber plantations. 


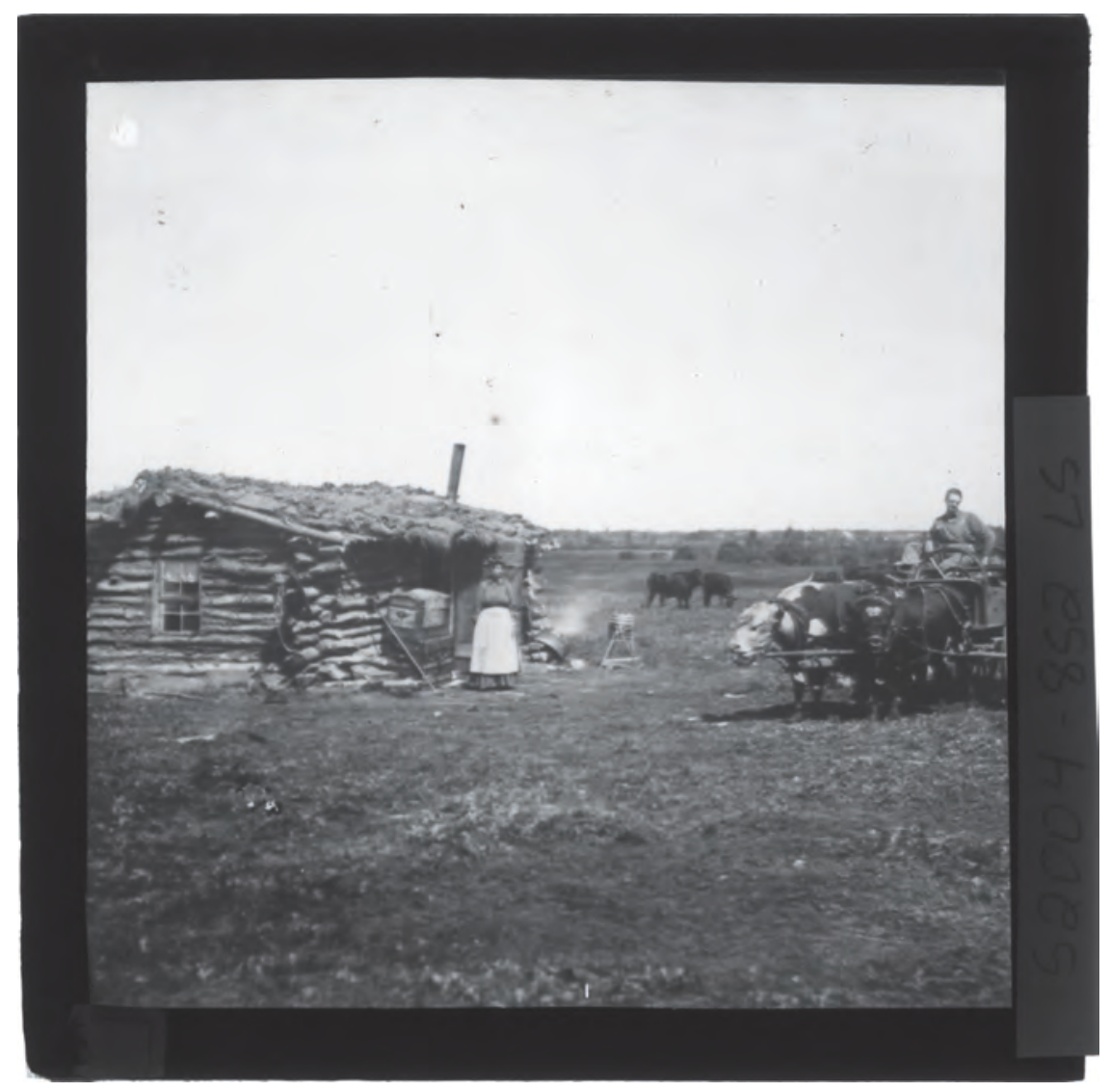

\section{Illustration 31.4}

Western Canadian farmhouse and animals, shortly after 1900

Photograph by Agnes Deans Cameron. Canadian Museum of History, CC BY-SA 4.0

The vast bulk of migration in this period was voluntary. However, the Russian state would forcibly move as many as 3 million Poles, Ukrainians, and Russians, many into Siberia. Greeks and Turks were moved forcibly in the interest of ethnic homogeneity after the creation of the Turkish state in the 1920s. More generally, millions moved as borders were redrawn and new states created in Eastern Europe after World War I.

Long-distance migrants often paid their passage by promising to work for a particular employer for several years upon arrival (though borrowing from family was likely more common). However, strict debts servitude, in which migrants were required to move and work to pay off pre-existing debts, was rare: It characterized perhaps one-tenth of Indian out-migration and 3 per cent of Chinese. Nevertheless, in some parts of the world, such as the Caribbean, such servants replaced slave labour - under scarcely better conditions - in the early decades after the abolition of slavery (chapter 26).

These mass migrations had dramatic impacts. Migration to the Americas, in concert with developments in international transport, transformed a region 
with a low population density into one of the wealthiest regions in the world. Manchuria came to look much like the rest of China. Malaya and Taiwan saw intense development of industry and agriculture. The economic effects elsewhere in Southeast Asia were less dramatic. Still, increased ethnic diversity would characterize the region. The regions that sent migrants often benefited from reduced population pressure on resources. It was also common for successful migrants to send part of their income back home, or even return with funds to invest.

\section{PRIMARY DOCUMENTS: THE DAWN OF RESTRICTIONS ON IMMIGRATION}

The Immigrant Restriction League was founded in Boston in 1894 and would agitate for immigration restrictions and especially a literacy test. Congress would introduce a literacy test in 1917. The League produced pamphlets, including "Present Aspects of the Immigration Problem" in 1894. This opened with some statistics, noting that immigration had been averaging over half a million people in recent years. It then proceeded:

it would be a stupendous task for this mass of people to be assimilated and Americanized even if they were of a related stock, such as the English, the Irish, the Germans, or the Scandinavians. When, however, we come to look at the races that have been contributing largely and in increasing proportion to our immigration during the last few years, we see how much more difficult the problem becomes. Our immigration has, until lately, chiefly been made up of the most intelligent and of the most desirable races of Europe, but recently the numbers have greatly increased of those who are without question the most illiterate and the most depraved people of that continent. [Provides figures on sources of immigrants]

...

One of the greatest [evils associated with such immigrants] is the enormous increase in the number of criminals in this country which can be traced directly to the growth of the lower foreign elements in our midst. According to the census of 1880 the foreignborn element, although constituting less than one-seventh of the population, furnished more than one-third of the paupers.

The census of 1890 shows that persons of foreign birth or parentage make up 38 per cent or somewhat over one-third of our total white population. THIS ONE-THIRD FURNISHES OVER ONE-HALF OF OUR CRIMINALS, TWO-THIRDS OF THE INMATES OF OUR REFORMATORIES, AND ABOUT TWO-THIRDS OF THE PAUPERS IN OUR ALMSHOUSES.

The illiteracy of the immigrants we are now receiving in great numbers is a source of imminent danger to the country. In every hundred foreigners over 16 years of age, who came here from February 1 to October 31, 1892, there were the following number of illiterates, according to the countries from which the immigrants came: England, ten; 
Ireland, eight; Wales, six; Germany, two, Scandinavian countries and Denmark, less than one; Russia, proper, twenty; Hungary, twenty-eight; Poland, fifty-six; Italy, sixty-six.

This danger from illiteracy is still further aggravated by the fact that many of our recent immigrants do not try to assimilate with us or become Americanized, but live in colonies by themselves, speaking their own language and keeping all their own customs, unaffected by the higher civilization around them.

... The serious riots that have taken place among the miners in Pennsylvania during the past winter were instigated and carried out by foreigners, principally Slavs, Huns, Italians, and Poles. In such disturbances as these the Anglo-Saxon element has been almost unanimously on the side of law and order. Such evils may be directly traced to the influence of the lower class of immigrants.

The pamphlet goes on to recommend a literacy test, a head tax of $\$ 25$ to $\$ 50$ per person, and a requirement for a certificate from American consular agents attesting that the potential immigrant is not ill or criminal.

Note how social distinctions associated with ethnicity become associated with distinctions based on occupation: Anglo-Saxon mine owners disagreed with ethnic miners. Note also that the author changes attitudes toward Germans here compared to above.

With the advantage of hindsight, how would you evaluate the concerns raised in this pamphlet?

\section{Twentieth-century migration}

Migration rates rose again from the 1950s. Absolute levels of migration were much higher in the 1990s - 80-90 million - than the 1910s, but similar relative to global population. That is, almost 2 per cent of the world's population moved in both decades. In the 1990s, the United States averaged 1 million immigrants annually and the European Union 1.5 million; Canada, Australia, and New Zealand between them took about 300,000. One million annually moved to the Middle East, including Israel. Argentina, Venezuela, South Africa, Singapore, Malaysia, and Japan also received sizeable numbers of migrants, generally from nearby countries. There were lesser but important flows between neighbouring countries in Southeast Asia, Africa, and the former Soviet Union. Since there was very little agricultural frontier left anywhere, these migrants predominantly sought employment in industry or services.

The sources of migrants had changed dramatically from the nineteenth century. Western Europe had almost ceased to be a source of migrants by the 1980 s - birth rates were low there and average incomes high. Eastern Europe which had generally prevented its citizens from leaving while under communist rule - witnessed a surge in migration after the fall of communism (chapter 27): Most went to Western Europe but many moved to the Americas. Communist China also limited emigration until the 1980s; this has surged since 
but is still far below the levels of the 1910s and 1920s (and tends to involve skilled rather than unskilled labour). South Korea was an important source of migration before the 1980s, but economic growth and democratization caused emigration to shrink after that. The Philippines, and to a lesser extent Indonesia and Vietnam, were important sources of migrants. Africa became a significant source of free migrants for perhaps the first time in world history. Latin America, which had received over 10 million migrants in the century before 1930, became a large net exporter in the postwar world: Migration from several other Latin American countries over time joined migration from Mexico and the Caribbean to the United States and Canada.

Migrants tended to move from countries with higher population growth rates to countries with low fertility. In other words, migrants moved from countries that had not yet completed the demographic transition to countries that had. In many recipient countries, the population would have fallen if not for immigration. Migrants also tended naturally to move toward countries with much higher average incomes. Yet migrants tended not to come from the very poorest countries but rather from the moderately poor: Such people could better gain information about migration opportunities and finance migration, and might possess some education. When people from the very poorest countries migrated, it was generally to nearby countries that might be only slightly more prosperous.

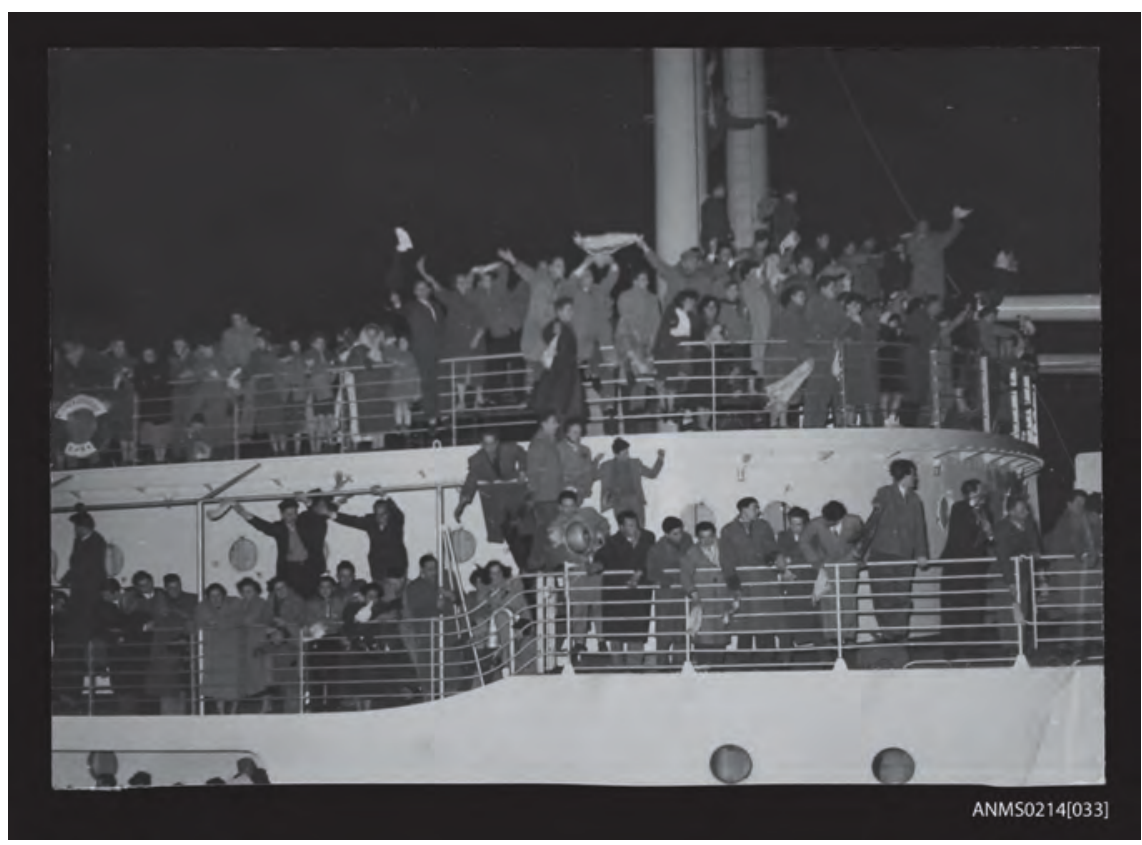

Illustration 31.5

Migrants on board Castel Verde departing Trieste, Italy, for Australia, 1953-4. There was out-migration from Western Europe in the earliest postwar decades 
The postwar wave of migrants would have to navigate a new world of immigration controls. States introduced passports and a variety of visas from the 1920s: Migrants either had to gain permission to enter a new country or evade border controls. However, many countries would relax immigration controls after the 1960s. In particular, changing public attitudes caused many countries to remove racial or ethnic preferences from immigration rules, leading to much more ethnic diversity in lands of high immigration such as Canada and the United States. The fact that Europe had become a net recipient of migrants as its birth rate fell meant that countries such as the United States now received most of their migrants from Asia.

The vast bulk of postwar migration was voluntary. Nevertheless, there were also sizeable movements of refugees because of wars and the collapse of empires. The Germans and Soviets forcefully moved 3 million Poles during the Second World War. Twelve million Germans and 10 million others moved as borders were redrawn after the war. Five million Japanese moved to Japan from former colonies after the war. Six hundred thousand Palestinians fled the creation of Israel in 1948; an equivalent number of Jews fled to Israel from hostile Arab states. Seven million Hindus and 7 million Muslims crossed the new border between India and Pakistan in 1947-8; another 8-10 million Hindus moved with the creation of Bangladesh in 1971. A million Europeans left Algeria as it gained independence in 1954-62. Six million left Afghanistan because of the Soviet invasion in the 1970s. Three million would move as Yugoslavia collapsed in the 1990s, and millions more when the Soviet Union disappeared. Wars in Rwanda, Iraq, Tibet, Lebanon, Syria, Libya, and Afghanistan - among many others - would also displace large numbers.

Wars have often caused civilians to flee in world history. Moreover, conquerors have often displaced the conquered. However, before the 1920s such refugees did not have to apply for visas to find a new place to live. The result of immigration restrictions has been that millions of refugees have had no place to go (there were 10 million of these in 2003 according to the United Nations, a number that increased with strife in various countries but especially Syria). Generations of Palestinians grew up in refugee camps. Uganda has served as host to refugees from a variety of African conflicts (often providing these with farmland).

Remittances from migrants to families back home have grown in size. For countries such as the Philippines, these can comprise several per cent of GDP. (Though both temporary and permanent migration from the Philippines blossomed in the twentieth century, "Manilamen" had been well known internationally from the late eighteenth century as seamen, pearl fishers, and mercenaries.) Yet several concerns have arisen. One involves the "brain drain": If the best-educated people leave a developing country, its development prospects may suffer. However, such migrants often form valuable international connections. Another concern involves flows of temporary migrants: "Guest workers" often have limited legal protections. They are usually tied to particular employers, who then have - and can abuse - immense power over them. 
As many as 130 million people lived outside of their country of citizenship in 2005.

In recipient countries, the big question has been whether the broader culture, economy, and polity can successfully absorb an increasingly diverse body of immigrants as earlier waves of migrants were absorbed. It is worth recognizing that English Protestants in the United States and Canada viewed nineteenthcentury waves of Poles or Jews or Italians with considerable suspicion (see primary document above). Yet over two or three generations, these groups integrated. (In Malaysia, on the other hand, political and social boundaries between Malay, Chinese, and Indian communities have remained a challenge). Immigrants tended to adopt the dominant language of their new land, and their children or grandchildren often married across ethnic and even religious lines. But not always: Migrants might maintain their languages and other customs if they concentrated in particular regions. Since it took generations for earlier immigrant populations to integrate, it is too early to tell what the future holds for the migrants of the 1990s. Will differences in race and religion prove harder to bridge than the divide between English Protestants and Polish Catholics? Will the greater ease of communication with families and friends back home discourage integration (but recall that migrants usually maintained some contact)? Moreover, what of illegal immigrants? These were not such a challenge in the nineteenth century due to the general absence of immigration controls. We return to some of these forward-looking questions in the next chapter.

There is also a question of the effect of migration on jobs and wages. In general, migration flows amounting to 2 per cent or less of the global population have modest effects. However, in particular regions and occupations, migrants may have a significant impact on labour markets. Local unskilled workers are especially likely to suffer. We should stress that migrants - like other people spend most/all of the income they receive: They create employment for others as they consume. In those cases where migrants take jobs that locals shun, or there is a shortage of local labour, local people will undoubtedly benefit economically from migration.

\section{Challenges facing agents: Migrants}

Migrants must usually find some source of income, and this usually means getting a job. In finding a job they face limited understanding of the job market in a new land (family or ethnic networks may be crucial here), communication challenges resulting from cultural and perhaps language differences, and perhaps overt discrimination. Those with skills face difficulties in having credentials recognized. (In an earlier time when migrants were farmers or nomads, the challenge would have involved adapting to local soils and climates and perhaps flora and fauna.)

Outside of work, migrants face further difficulties in adjusting to differences in culture, social structure (perhaps especially attitudes toward gender and family), climate, and politics. They will often have left friends and family behind (who they may urge to follow - this may lead to further challenges); they need to cope with a sense 
of loss while forming new social bonds. (This challenge lessened at times when large groups moved together.) They need to master new systems of health, government, police, transport, and so on. In particular, migrants need to find a place to live: When surveyed, migrants often list this as one of their primary challenges.

Migrants usually move to forge a better life for themselves and their families. Seeing to their children's education is then a critical challenge. Here too, a lack of familiarity with local customs, institutions, and especially language presents challenges.

\section{Urbanization}

In 1800, there were 6 million people in the ten largest cities in the world. This was not a dramatic change from classical times when Rome and Chang'an each had a million people. By 2000, though, there were about 200 million people in the world's ten largest cities. The size of the world's great cities had simply exploded. Cities of a million people are now nothing particularly special whereas just a couple of centuries ago they were still quite rare.

An equally dramatic story unfolded concerning the proportion of the world's population living in cities. This was just 3 per cent in 1800, 14 per cent in 1900, 30 per cent in 1950, and exceeded 50 per cent for the first time around 2010. We now for the first time in history live in an urbanized world. There is, of course, a link between these two stories: As world population grew (above), and this population became more urbanized, city sizes grew everywhere.

Though urbanization was a global process, it occurred unevenly. As trade expanded, port cities grew. Even more dramatically, railway junctions could become large cities in places where there was hardly any people before (Atlanta is a good example here). The growth in the size of governments (chapter 27) meant that capital cities often grew very fast. Many cities grew because of access to industrial raw materials. Some developed in response to increased tourism (see below), most notably in the case of seaside resorts. While most cities grew, some declined because they lost out to competitors or because of political changes.

In the nineteenth century, urbanization reflected economic development and political centralization as it always had: The biggest cities were in the wealthiest countries or those with the most extensive state bureaucracies or trade linkages. Britain would become the first country with a majority of its population urban in 1851 - reflecting the fact that both industry and trade concentrated in cities. In 1900, nine of the ten biggest cities in the world were in Europe or the United States: The tenth was Tokyo, in the Asian country that was first to achieve rapid economic growth (chapter 24).

The link between economic prosperity and city size would disappear in the twentieth century. Of the ten biggest cities in 1900, only New York and Tokyo 
remained on that list in 2000. They were joined by Mexico City, São Paulo, Delhi, and Shanghai (Lagos and Istanbul have since joined the list; note that these lists vary depending on how one establishes city boundaries). These latter cities are in countries with far lower average incomes than Europe or North America. Kinshasa has 10 million people despite being in one of the poorest and worst-governed countries in the world. Urbanization has proceeded far faster in developing countries in the last century than it had in Europe and North America when they were at similar levels of economic development. Mexico City grew from 3 million to over 10 million in just twenty-five years after 1950, São Paolo increased from 2.5 to over 10 million in the same time period, and Seoul went from 1 to about 7 million.

Why has urbanization proceeded so fast in poorer countries? Most obviously, the agricultural developments noted above have fed large urban populations.

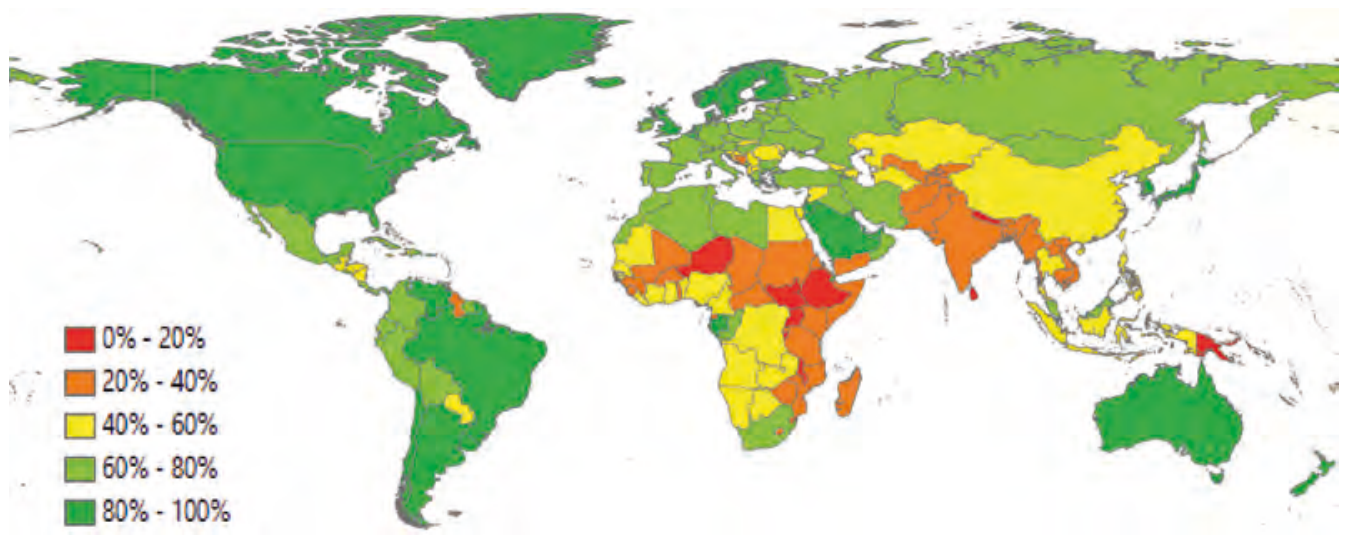

Map 31.2

Per cent of population that was urban, 2016

Map by Akantamn, CC BY-SA 4.0

\section{BOX 31.3}

\section{THE WORID'S BIGGEST CITIES}

Though the emergence of huge cities in developing countries was something new, dramatic changes in the global distribution of large cities was not. In 1300, six of the ten largest cities in the world were in China, along with Paris, Fez, Cairo, and Kamakura, Japan. By 1700, Istanbul was first and Isfahan third, with Delhi and Ahmadabad in seventh and eighth place, along with London, Paris, Beijing, and three Japanese cities. In 1800, the top ten comprised four Chinese cities, two in Japan, three in Europe (including Naples), and Istanbul on the border of Europe and Asia. 


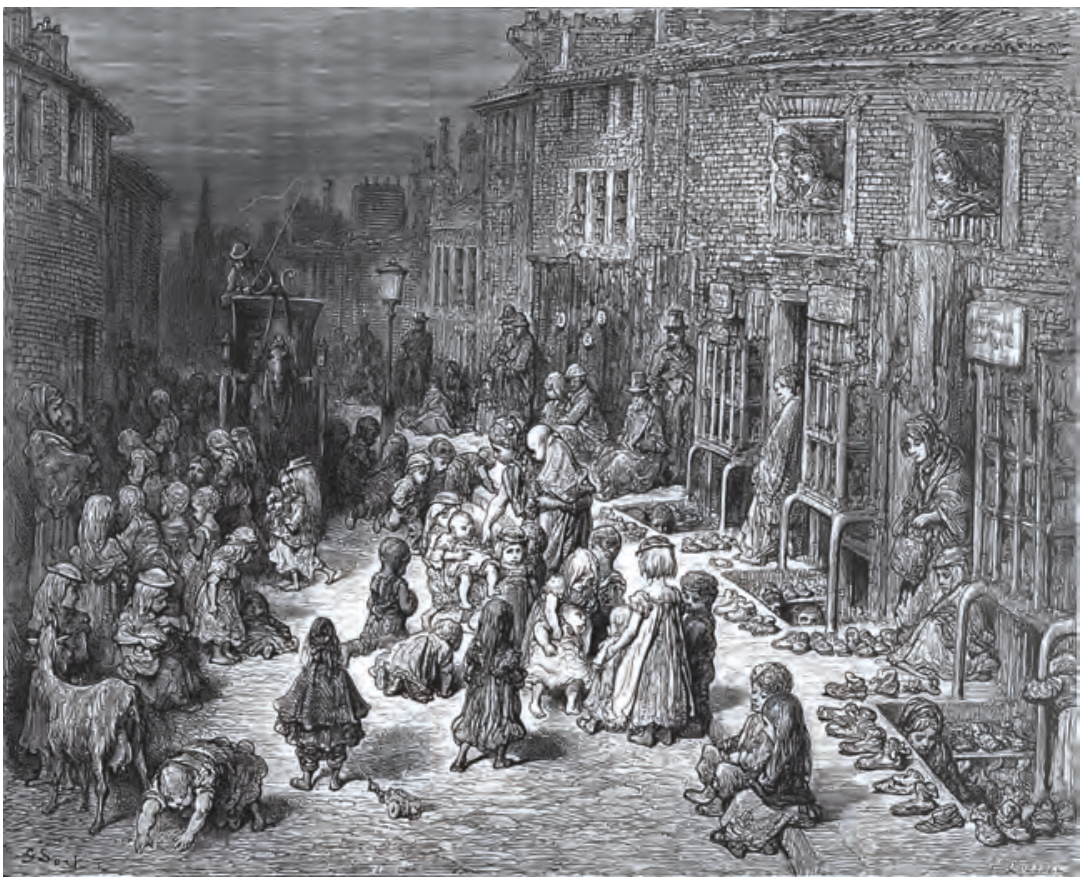

Illustration 31.6

Slum, Seven Dials area, London, Gustave Doré, 1872

Wellcome Trust, CC BY 4.0

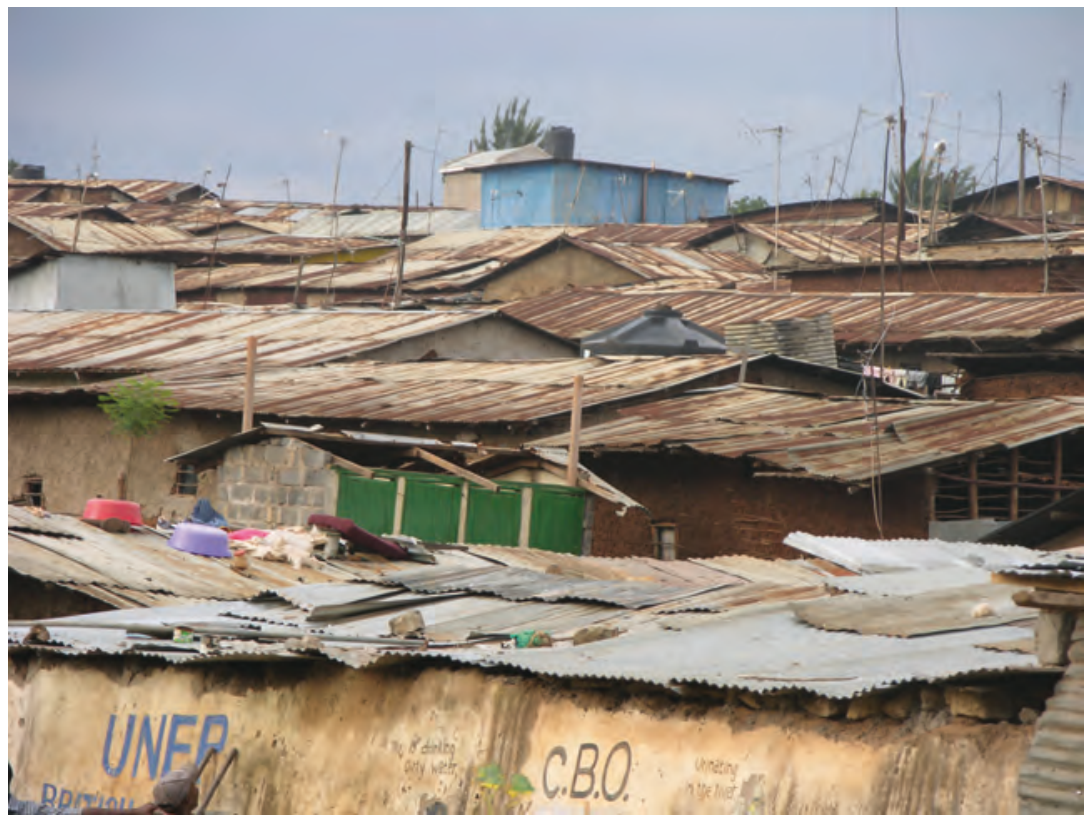

Illustration 31.7

Urban slum, twenty-first century, Nairobi 
Although transport infrastructure in developing countries often compares poorly with that in the rich world, automobiles, railways, and ships nevertheless allow food (and building materials) to move from a wide area to huge cities at a reasonable cost.

We have seen that social stratification has characterized cities since their earliest days. In the nineteenth century, class tensions, racism, and antiSemitism were quite visible in many of the world's cities. It is hard to compare the degree of stratification across times and places. What is clear is that large cities in developing countries all have massive slums in which large numbers of people live in dilapidated housing with limited access to clean water, sewage removal, garbage removal, and electricity. The scope of this urban poverty is something new in history. There are perhaps one billion slum-dwellers in the world, comprising almost a third of the total urban population.

\section{PRIMARY DOCUMENTS: THE SLUMS OF MANCHESTER}

Friedrich Engels, best-known for later collaborating with Karl Marx on The Communist Manifesto, went to England as an agent for his father's industrial enterprise. This is an excerpt from his The Condition of the Working-Class in England in 1844 (London: Swan Sonnenschein \& Co., 1892), pp. 45, 48-53.

Above Ducie Bridge, the left bank grows more flat and the right bank steeper, but the condition of the dwellings on both banks grows worse rather than better. He who turns to the left here from the main street, Long Millgate, is lost; he wanders from one court to another, turns countless corners, passes nothing but narrow, filthy nooks and alleys, until after a few minutes he has lost all clue, and knows not whither to turn. Everywhere half or wholly ruined buildings, some of them actually uninhabited, which means a great deal here; rarely a wooden or stone floor to be seen in the houses, almost uniformly broken, ill-fitting windows and doors, and a state of filth! Everywhere heaps of debris, refuse, and offal; standing pools for gutters, and a stench which alone would make it impossible for a human being in any degree civilized to live in such a district. ... Passing along a rough bank, among stakes and washing-lines, one penetrates into this chaos of small one-storied, one-roomed huts, in most of which there is no artificial floor; kitchen, living and sleeping-room all in one. In such a hole, scarcely five feet long by six broad, I found two beds - and such bedsteads and beds! - which, with a staircase and chimney-place, exactly filled the room. In several others I found absolutely nothing, while the door stood open, and the inhabitants leaned against it. Everywhere before the doors refuse and offal; that any sort of pavement lay underneath could not be seen but only felt, here and there, with the feet. This whole collection of cattle-sheds for human beings was surrounded on two sides by houses and a factory, and on the 
third by the river, and besides the narrow stair up the bank, a narrow doorway alone led out into another almost equally ill-built, ill-kept labyrinth of dwellings....

... Here, as in most of the working-men's quarters of Manchester, the pork-raisers rent the courts and build pig-pens in them. In almost every court one or even several such pens may be found, into which the inhabitants of the court throw all refuse and offal, whence the swine grow fat; and the atmosphere, confined on all four sides, is utterly corrupted by putrefying animal and vegetable substances....

Such is the Old Town of Manchester, and on re-reading my description, I am forced to admit that instead of being exaggerated, it is far from black enough to convey a true impression of the filth, ruin, and uninhabitableness, the defiance of all considerations of cleanliness, ventilation, and health which characterize the construction of this single district, containing at least twenty to thirty thousand inhabitants. And such a district exists in the heart of the second city of England, the first manufacturing city of the world. If any one wishes to see in how little space a human being can move, how little air - and such air! - he can breathe, how little of civilisation he may share and yet live, it is only necessary to travel hither. True, this is the Old Town, and the people of Manchester emphasize the fact whenever any one mentions to them the frightful condition of this Hell upon Earth; but what does that prove? Everything which here arouses horror and indignation is of recent origin, belongs to the industrial epoch.

What effect does this account have on you? What effect was the author hoping to achieve?

One common problem in these slums is limited property rights. Developing countries often lack bureaucracies that can establish land ownership. In some countries, traditions of communal ownership provide a further complication. Migrants to cities often build illegally. Since they then live in fear of ejection, they are discouraged from investing in their homes. Public land ownership is as unclear as private ownership: Not enough land is set aside for roads, parks, or other infrastructure. As slums have grown in recent decades, the average slumdweller is now much farther from an arterial road. Slums become overcrowded and difficult to provision or service (including by police). Some developing countries have moved in recent decades to provide slum-dwellers with security in their property. The early results seem to be that many are then able to improve the quality of their housing, and in some cases to gain access to water and power and sewers. These advances have proceeded much farther in Asia and Latin America than in Africa.

It is a mistake to see slums as pits of despair. Although the conditions are often horrific, and there is a shortage of good jobs, slum-dwellers are generally able to find work at least intermittently. Many operate small businesses. Many can provide their children with a better life. Government programmes that reward the poor for sending their children to school or getting them vaccinated have had impressive results in countries such as Brazil. In many 
slums, community groups have been able to provide health services and community protection.

Cities in rich countries were extremely unhealthy in the early decades of the nineteenth century. By the end of that century, though, they had developed systems of clean water and sewerage in recognition that dirty water transmitted diseases such as cholera. These practices (and efforts to combat air pollution) meant that cities no longer had much higher mortality levels than the countryside, as had been the case in almost all cities in previous world history. Cities also developed systems of urban roadways and railways (and later subways, from 1863 in London) to move people and goods throughout urban areas. Streets were lit by first gas (from 1807 in London) and then electricity. The slums of developing countries often still lack this basic infrastructure. Moreover, it can be difficult for slum-dwellers to gain access to food and other goods.

As cities have grown worldwide, urban planning has become an important profession. Planners routinely advocate the provision of green space. They worry about urban transport and whether jobs should be dispersed throughout the metropolitan area. One common concern is with retaining historical architecture. While communist city planners produced endless blocks of unattractive worker housing, they often did not tear down pre-communist urban cores: Some Eastern European cities, therefore, have particularly charming downtowns to this day. Some developing countries have planned new capital cities in part

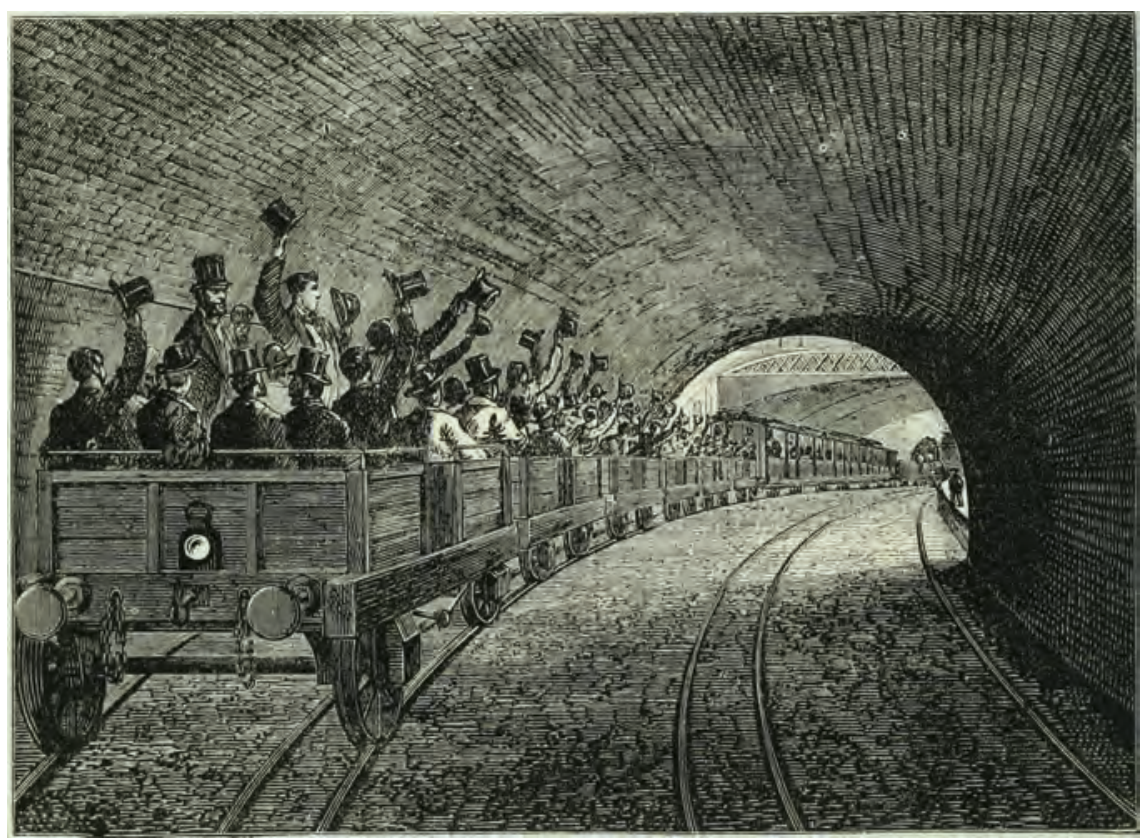

Illustration 31.8

London Underground, 1873 


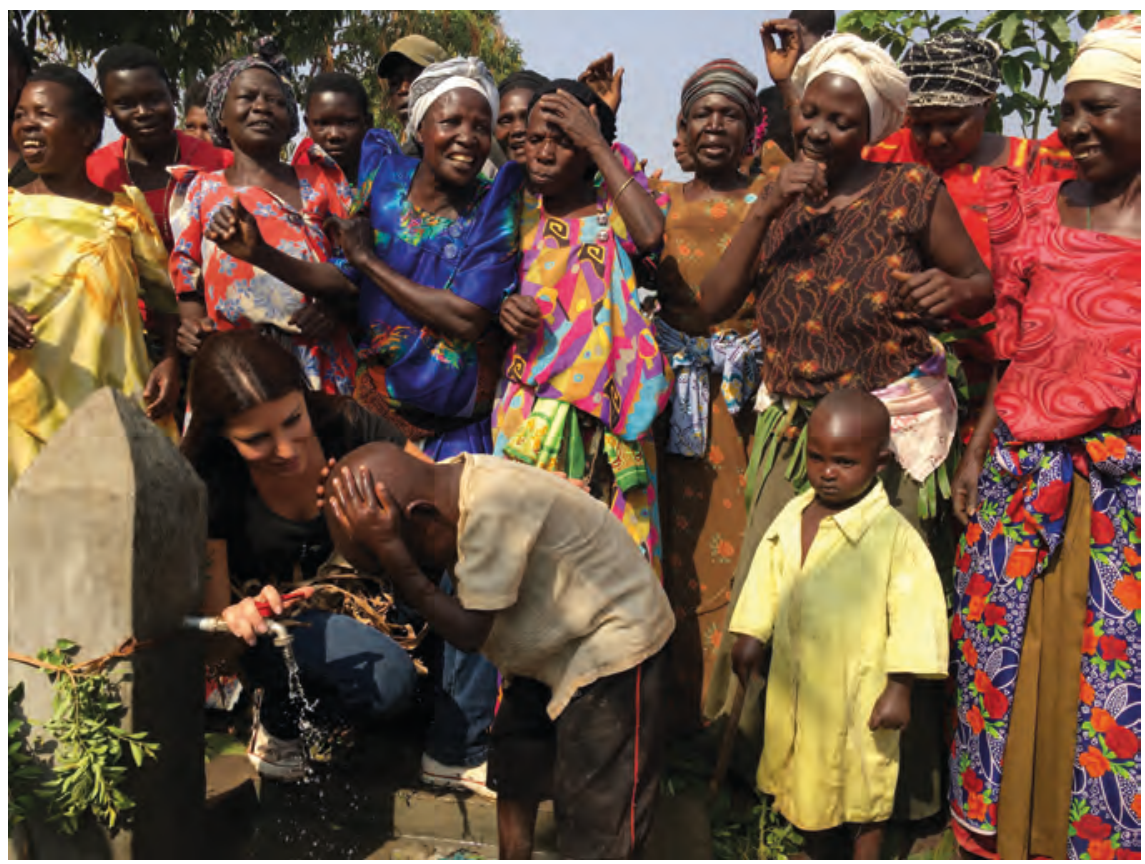

Illustration 31.9

Clean water flows for the first time in Nyanza, Uganda (part of greater Kampala), 2017. Perhaps a billion people worldwide still lack access to clean water Photograph by InnoAfrica, CC BY-SA 4.0

to relieve population pressure on previous capitals: Brasilia in Brazil, Abuja in Nigeria, and Islamabad in Pakistan, for example.

In rich countries, the twentieth century witnessed vast urban sprawl through the creation of suburbs. Urban railways and then automobiles allowed people to commute to work from previously unimagined distances. These suburbs were at first residential, with limited opportunities for working and even shopping. Over time, jobs and stores have left downtown cores as well - many cities have struggled to revitalize these. Suburbs were often racially segregated, but changes in both laws and cultural attitudes have reduced the degree of racial segregation. Race riots have nevertheless occurred in several countries. Suburbs tend to be segregated economically, with rich people living far from poor people. This degree of economic segregation was not possible in compact early cities. One pressing concern is whether suburbs escape paying taxes to support urban infrastructure on which all depend.

Cities with walls soon expanded far beyond the confines of these walls. Railways everywhere urged the removal of walls that stood in their way. These walls, in any case, provided limited defence against modern armaments. As a result, most of the walls that had characterized cities for millennia disappeared in the nineteenth century. Sometimes the occasional gate or section of wall remains as a monument to the past. However, entire city walls tend to remain 


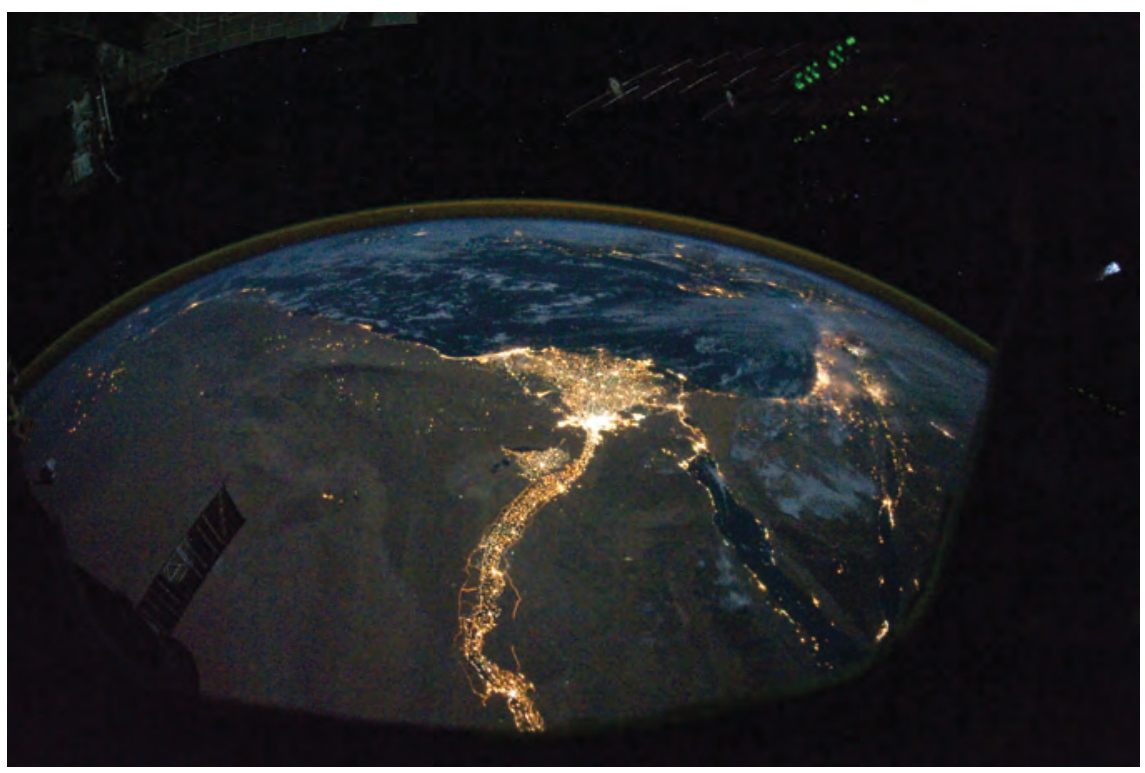

Illustration 31.10

Nile Delta (Cairo area) at night by satellite, 2010. Though cities in poor countries have sprawled less than those in rich countries, they have still expanded, often across very productive agricultural land

only in cities that did not experience much growth in the nineteenth or early twentieth centuries. Such cities often find that these walls are now an important tourist attraction.

Urbanization everywhere had a political impact. Urban masses could more easily riot, and so states needed to worry about urban concerns. Urbanites have been central to most revolutions in history. Colonial governments were particularly worried about urban discontent. As in ancient Rome, riots were more likely in times of economic downturn and tended to focus on wages or food prices (or working conditions) more often than changes to the political order. Nevertheless, urban workers can more easily organize unions and agitate for higher wages and better working conditions. In countries that hold elections, urban voters have generally been more willing to vote for politicians that promise institutional change. In South Africa and several other countries characterized by one-party rule centrally, opposition parties are elected more often in cities; urban politicians then argue against central policies. Cities have been the sites of extensive political debates wherever states have allowed these. In countries - especially in Africa - characterized by regions inhabited by distinct ethnic groups, large capital cities may serve as a place where political discussions and even party loyalties cross ethnic lines.

Urbanization has important cultural effects. The anonymity of the city likely encouraged increased individualism. Yet cities can also be sites of nationalist, ethnic, or religious fervour. The government buildings and railway stations of 


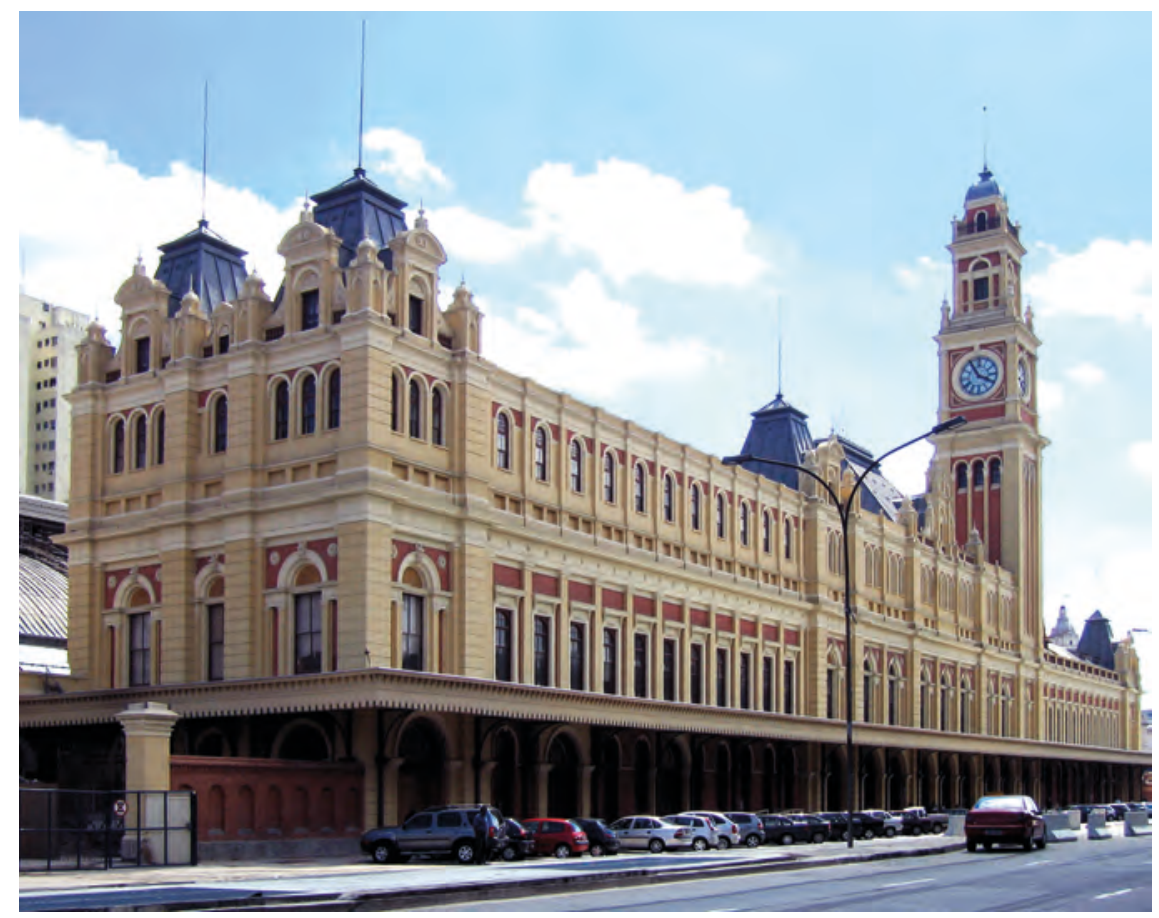

\section{Illustration 31.11}

Luz Station, São Paulo, Brazil. Many train stations are architecturally attractive, especially those built in the nineteenth century

these cities matched the size of earlier monuments, if not always the grandeur. Growing cities allowed growing audiences for theatres, art in general, music in particular, and a host of celebrations. Urban cultural attitudes are dominant in virtually every country in the world today, a dramatic change from mere centuries ago.

We have in this section recounted a number of effects of cities: supporting trade and industry, encouraging political reform and culture change, spreading disease, and more. We might add one more: innovation. We have often seen in this book that technological innovation is a collaborative effort. Growing cities would prove to have advantages in encouraging innovation, especially if the population had the sort of technical skills (and access to financing) required for innovation.

\section{Tourism}

The World Trade Organization estimates that there are some 900 million cases of international tourism annually. Tourism is now almost a stunning 10 per cent of global GDP. Still, some 90 per cent of the world's people never leave their country. There have long been tourists in world history: We described one 
example in ancient Egypt in chapter 7. However, before the late nineteenth century few could afford to be tourists. The elite "Grand Tour" of seventeenth and eighteenth century Europe by which young (especially British) aristocrats toured the great sites of the continent had spawned an increased interest in travel writing. Rising incomes and falling transport costs then encouraged a broader slice of the population to travel for pleasure. Industrialization and urbanization (and associated concerns regarding health) encouraged trips to the mountains and ocean. Excursion steamers for short trips emerged in the 1860s; the first purpose-built cruise ship was launched in 1901. These advances in transport have combined with curiosity regarding different cultures and landscapes. The source of tourists has changed as economic growth has spread through the world: Europeans, North Americans, and Japanese have been joined in recent decades by hundreds of millions of Chinese and other tourists. As those in the tourism business cater to a more diverse market than before, they need to grapple with differences in culture, income, and spending habits.

Locals rely on tourism in many parts of the world. In the Seychelles, tourism generates two-thirds of GDP. Yet tourism has challenges. One is maintaining the ecological wonders that attract tourists while dealing with garbage and sewage. Developing countries especially struggle with this. Another is that tourists often drink or do drugs excessively. Another is that tourists clog the beaches and museums so that locals feel squeezed out. Tourists may also not understand local driving rules or customs. Some localities, therefore, try to limit tourists. Santorini in Greece caps the daily influx at 8,000: Such locations may hope to charge higher entry prices if they restrict entry.

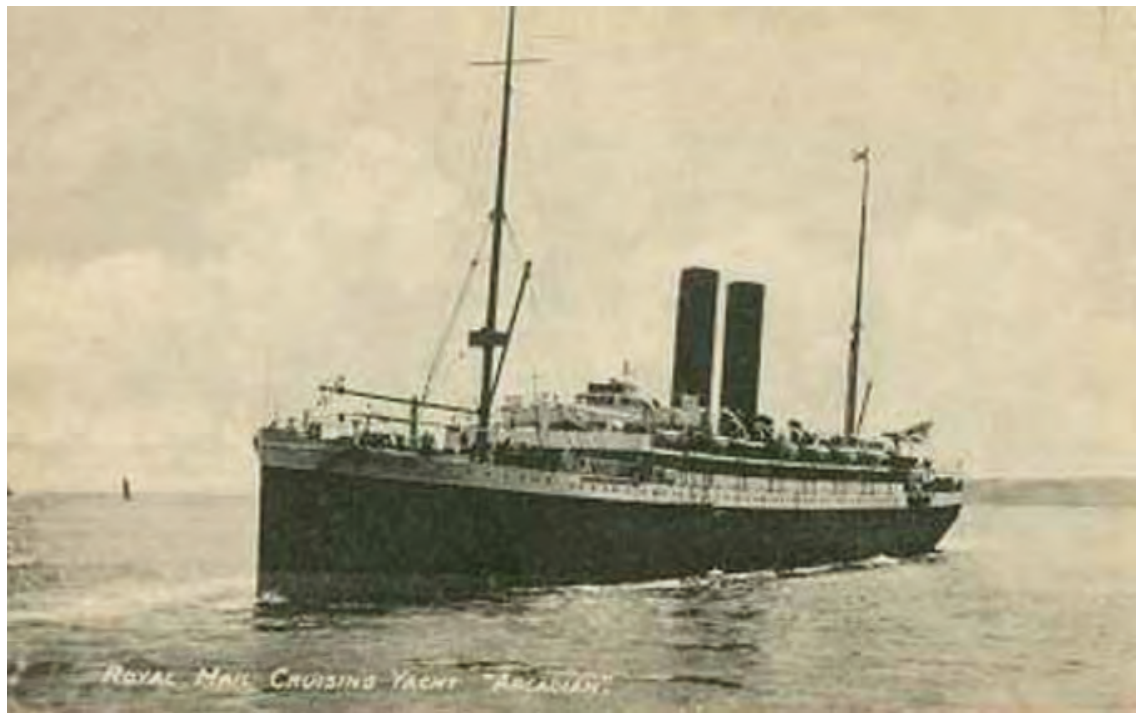

Illustration 31.12

British passenger liner built in 1899, renamed the Arcadian in 1910 as a cruise ship, torpedoed in World War I 


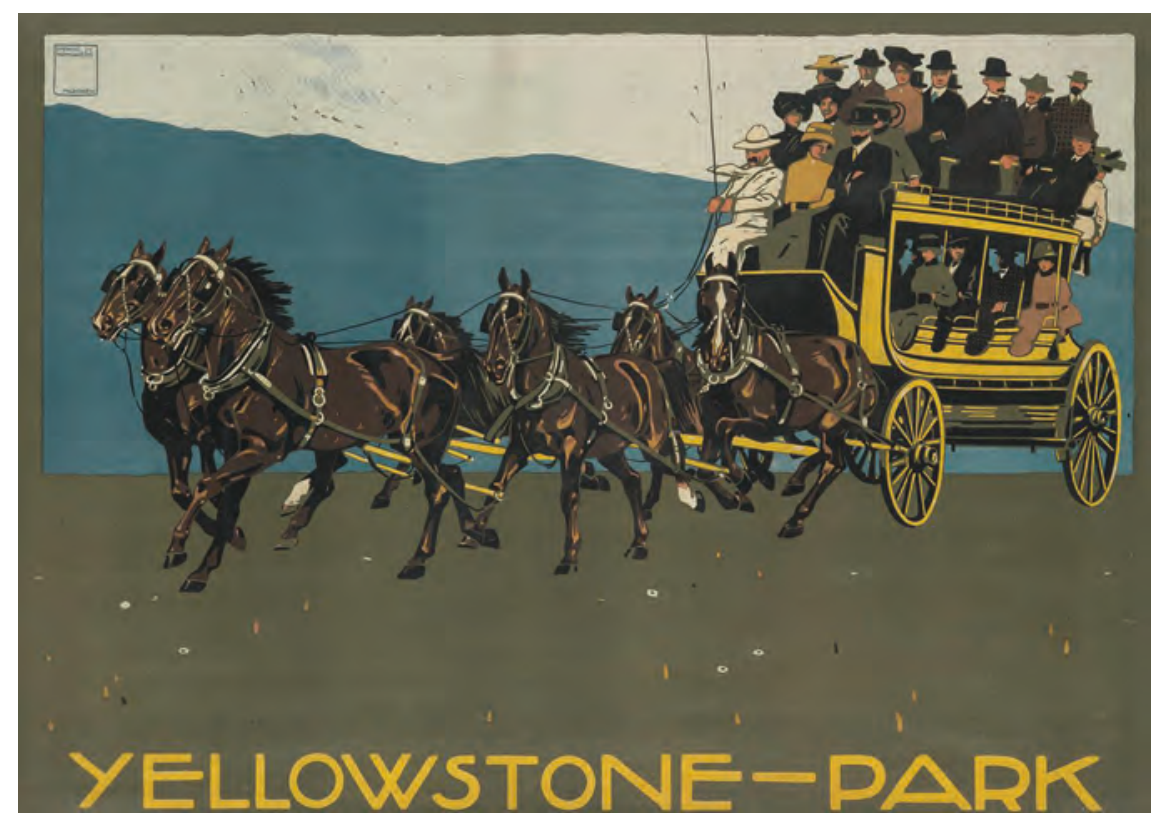

Illustration 31.13

Yellowstone Park poster, 1919

World history itself is a powerful magnet for tourists, who flock to famous castles, religious edifices, monuments, and museums throughout the world. Sadly, many UNESCO World Heritage sites (see Box 31.4) have been destroyed in recent years. Much destruction has been a side effect of war. Civil wars have turned cities such as Aleppo and Mosul, each with rich histories, to rubble. Besides, the chaos of war encourages vandalism and looting. Religious zealots have often destroyed monuments associated with other religions. These practices are hardly new. As long as there have been missiles, wars have destroyed monuments. Hakim, the caliph of Cairo, ordered the destruction of hundreds of Christian churches in the years around 1000 CE. During the religious wars in Europe, Protestants and Catholics each desecrated the churches of the other. However, modern explosives have increased dramatically both the physical damage associated with war and the ease with which individual monuments can be destroyed.

While tourism often provides much-needed jobs in poor localities, these jobs are often not well paid: house cleaners, waiters, taxi drivers. Tourism can generate some better opportunities also: guides, curators, scuba instructors. The Seychelles has banned large hotels in order to encourage small-scale locally owned inns. Recent internet innovations may allow locals increasingly to rent out rooms, but this may also lead to larger numbers of tourists than particular localities can handle (and raise the price of the local real estate). 


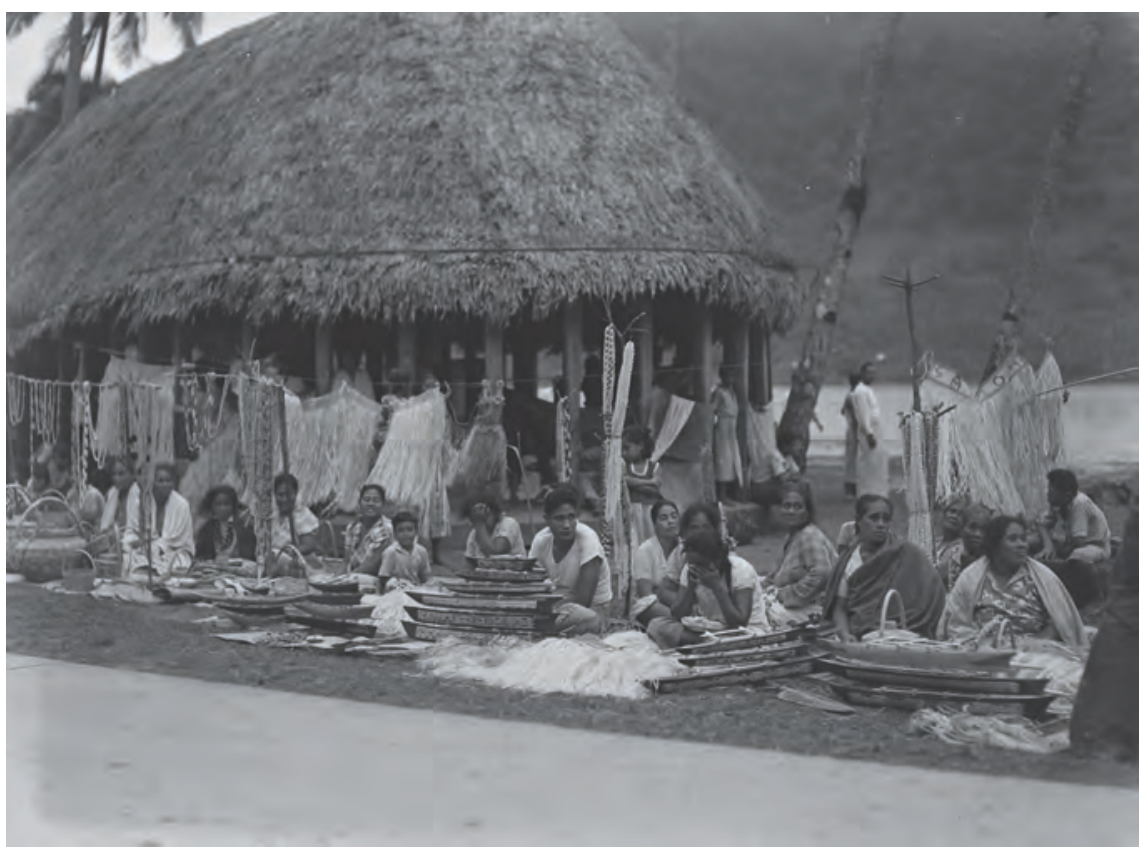

Illustration 31.14

Samoans displaying souvenirs in the 1950s

Auckland Museum, CC BY 4.0

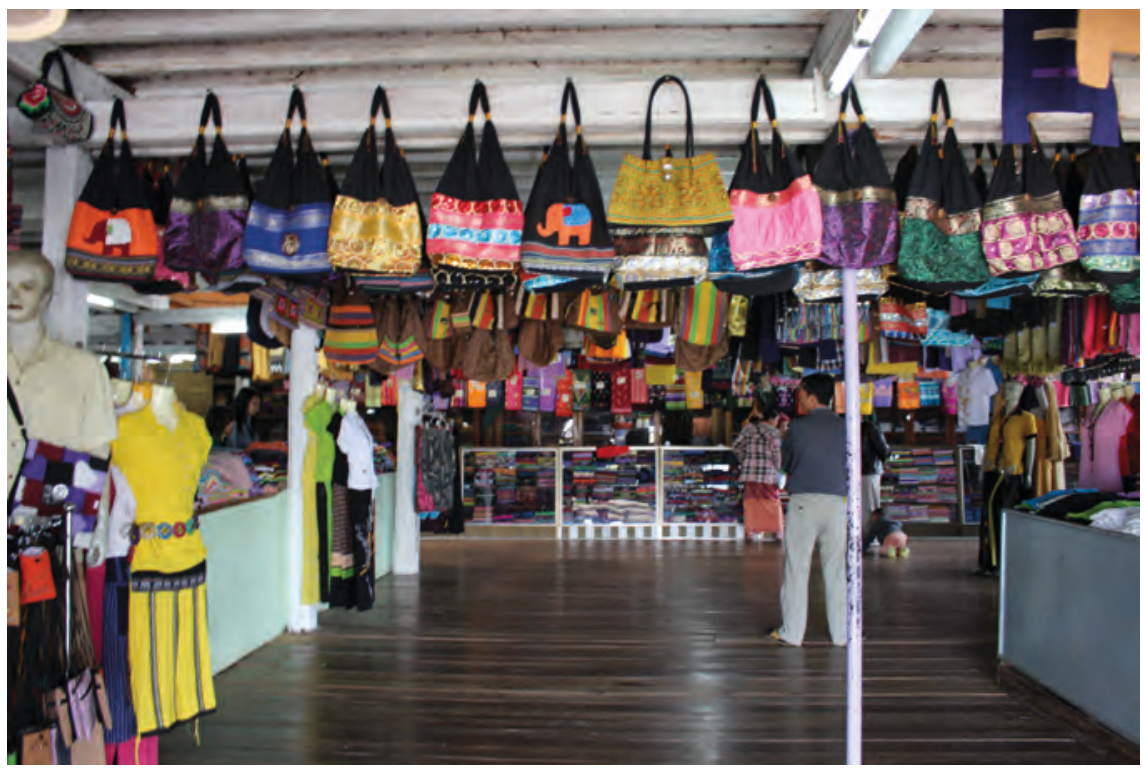

Illustration 31.15

Souvenir shop at Inle Lake, Myanmar, 2013 mydaydream89, CC0 


\section{BOX 31.4}

\section{MUSEUMS AND HISTORICAL SITES AND WORLD HISTORY}

An appreciation of world history can both encourage and enhance enjoyment from visiting museums and historical sites around the world. Such visits can deepen your historical understanding while allowing you to apply the insights you have gained from this book. You can compare your perceptions with the interpretations of local curators. You could even seek out the sources of the pictures that have graced this book.

TripAdvisor compiled a list of the top twenty-five museums in the world in 2014 (www. tripadvisor.com/TravelersChoice-Museums): It is heavily biased toward Europe and the United States, though this in part reflects the fact that museums there have artefacts from around the world. Yet TripAdvisor did celebrate both the Anthropology Museum in Mexico City with its incredible collections of Mayan, Aztec, and other objects, and the Museum of the Terra Cotta Warriors and Horses in Xian, China (but not what is likely the world's largest museum, the Palace Museum in the Forbidden City in Beijing) as well as a couple of art museums in South America. Yet one hardly needs to focus on just the greatest museums. One can now find excellent museums in almost every corner of the world. I quite liked the very new Biomuseo in Panama City, with impressive exhibits on various time periods (this is where I first learned of the possible climatic impact of the formation of the Isthmus of Panama).

As for historic sites, UNESCO's list of World Heritage Sites (http://whc.unesco.org/en/ list/) is an excellent place to start. Again, one can enjoyably stroll through countless less famous venues in every corner of the world. In some cases, one must creatively extrapolate from the rubble of past centuries. In others, one can experience carefully restored structures and even costumed interpreters. In all cases, it is useful to imagine yourself having lived in another time and place.

We discussed in Box 8.4 a set of reasons that one might appreciate any museum artefact or historic structure. Museum curators will only address some of these in an object's description. Visitors can ask themselves how they might have described a particular object if they were the curator.

Museums in former colonial powers are being pressed to return artefacts to their former colonies. Visitors to these museums can look for discussions of this issue. More generally, visitors can look for vestiges of imperialist attitudes in the way that such artefacts are described. Students of world history can strive to see objects as those who produced them would have seen them. Artefacts produced under colonial rule, or intended for European markets, often respond to European tastes and misunderstandings of local cultures.

\section{Epidemics and famines}

We briefly discussed decreased mortality above. Given the role that epidemics and famines have played in world history, it is worth paying a bit more attention to each of these. In the last centuries, across most countries, medicines and public health have eradicated or at least controlled many of the epidemics that 


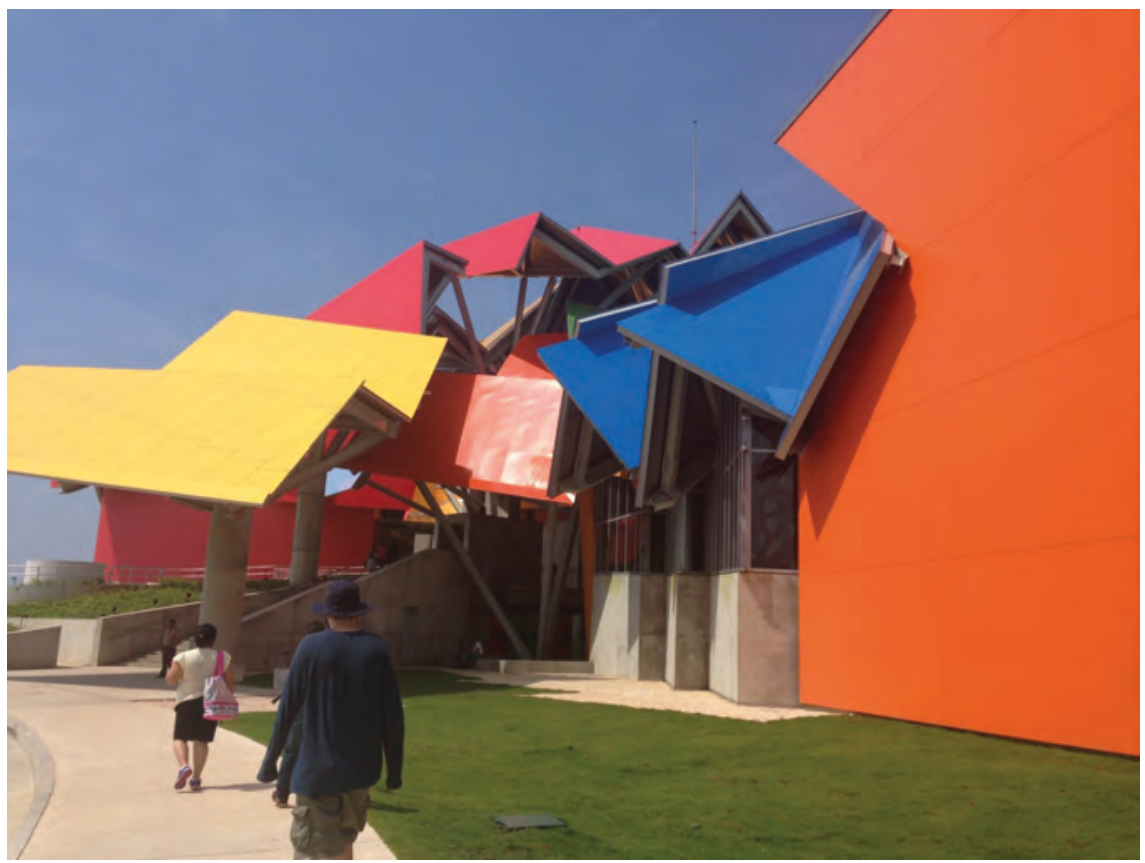

Illustration 31.16

Biomuseo, Panama City

Mireille Szostak

have long plagued (pun intended) humanity. Indeed, the first steps toward addressing smallpox, diphtheria, and scarlet fever occurred in the eighteenth century. The first successful vaccine in world history was developed in 1796 for smallpox. Scientists noticed that milkmaids exposed to cowpox did not catch smallpox; the word "vaccine" comes from the Latin for cowpox. Over the next centuries, improved methods for creating and storing vaccines were developed. From 1967, the WHO launched an international effort to vaccinate in areas reporting smallpox. In 1979, they declared the disease globally eradicated.

Vaccinations have addressed a range of diseases, including polio and measles. Polio is near eradication. Measles is under control, but epidemics can flare up among non-vaccinated populations. Vaccination has not been the only response to epidemic disease. Cholera is an interesting case. It was limited to the Ganges region until the nineteenth century. It spreads via human excrement in drinking water. When it became feasible for sick people to travel, the disease spread globally. When epidemics of cholera occurred in cities such as London, there was intense debate as to whether air or water transmitted it. Scientists eventually realized that the disease concentrated around drinking water systems. Cities worldwide were spurred to improve both water and sewage systems. A vaccination against cholera was also developed (see illustration 31.2 above). Cholera has effectively disappeared from the more affluent countries in the world. 


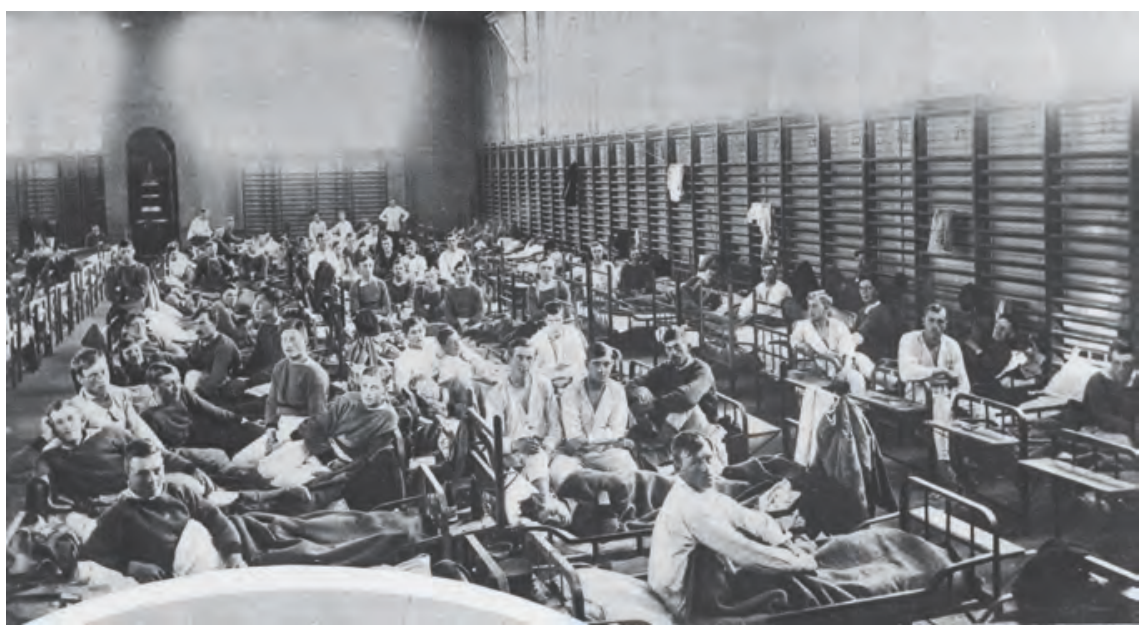

Illustration 31.17

Spanish flu patients hospitalized in a Swedish gym in 1918

There was an outbreak of bubonic plague in the late nineteenth and early twentieth centuries that did kill millions of people worldwide. However, it rarely progressed beyond port cities, as public health authorities controlled rat populations and then attacked the fleas carried by rats as scientists identified these as the carriers of the disease.

Though many historical diseases were conquered, new diseases emerged in the twentieth century. The most devastating was HIV/AIDS, which attacked the human immune system, rendering the infected defenceless against a range of diseases. Some 35 million people may have died worldwide from the disease. Scientists developed medicines that minimized the effects of the disease. There was a suspicion that governments and researchers might have addressed the condition more quickly if the majority of victims (at least in rich countries) had not been gay or drug users. There was also concern about the costs of these new drugs, but drug companies under intense pressure developed lowcost medications for use in poorer countries. Though AIDS has touched every country in the world, it has been most virulent in Africa.

Several other "new" epidemics occurred. These diseases generally emerged when a virus endemic in some animal species mutated such that it could afflict humans. Such mutated viruses often had very high mortality rates among humans or caused severe health concerns. Such diseases often spread rapidly around the world as infected individuals travelled by plane. It can take scientists years to develop vaccines or other medicines to address a new disease. While science and health authorities have controlled all such epidemics so far (often only by killing vast numbers of animals), the risk remains that some future epidemic may wreak widespread calamity. The Covid-19 pandemic that began in 2019 had a worse combination of lethality and transmission than any pandemic since at least the Spanish Flu of a century earlier (chapter 28); in the absence of 
a cure, health authorities throughout the world could only pursue quarantines, limits to social interactions, and the tracing of contacts with those known to be infected. Death rates among those contracting Covid-19 were about 2-3 percent in most countries, but some future disease might be even worse.

Though the WHO and other international agencies have done much to address both old and new epidemics, there have been concerns that the world is not doing as much as it could. There is still often less cooperation internationally than there could be, as some countries worry that reports of local epidemics will discourage tourism and investment. It is also often argued that it would be prudent to invest more in basic health facilities in poor countries particularly prone to epidemics. There may also be an exaggerated focus on "magic bullet" vaccines, when epidemics are often tamed by quarantines.

Famines in world history may often have reflected a simple shortage of food - though only rarely did the rich not have access to food. However, as we have noted above, in the twentieth century, food production has grown faster than population. There has always been enough food to feed everyone. Moreover, advances in transport mean that it is always feasible to transport food to the hungry: Planes can drop food where roads are non-existent. A famine in the twentieth century can only occur, then, if people in a particular region are unable to pay for food, and states are unwilling or unable to get food to them. Shockingly, when famines occur there is generally food nearby that the starving cannot access. The frequency of famines likely decreased in the twentieth century, but the century nevertheless witnessed some of the most extensive famines in world history. Millions died in various countries, including China, India, and Ethiopia. The United Nations and other international agencies strive to provide emergency food aid. One challenge here is that food aid may discourage local farmers from producing. Counter-intuitively, it may often be better to give the hungry money.

Until a couple of centuries ago, epidemics and famines were widely accepted as a fact of life. Colonial governments in particular, including the British in India, often did little to address famines. By the end of the twentieth century, people throughout the world had come to expect public policy and science to combat epidemics and famines.

\section{Obesity}

There is debate among medical professionals regarding whether obesity should be classed as a disease. Yet there is no doubt that obesity increases the chances of experiencing conditions such as heart attack and stroke, and that obesity lowers life expectancy. In the last decades, obesity has become a global health challenge with rates of obesity - and especially severe obesity - rising in virtually every country in the world. Just decades ago, obesity was a rich-world and especially North American - problem, but now poorer countries such as Mexico have some of the highest obesity rates in the world. There may be a link 
back to reduced rates of population growth as some scholars attribute lower sperm counts on many continents in part to obesity (chemicals released into the environment are likely also important). There are, it should be appreciated, still some billion people in the world that struggle to get enough food, but most humans today need instead to worry about overeating. Indeed, for the first time in history, more humans die from overeating than from eating too little.

The global challenge of obesity has obvious roots in world history. Most humans through most of history have not had easy access to (much) more food than that needed to satisfy basic nutritional requirements. Indeed, people often struggled, at least at times, to meet basic requirements. As we saw in chapter 24, modern economic growth has freed humanity for the first time from Malthusian constraints. We can note here that in addition to increasing our access to food, technological innovations over the last centuries have also reduced the work effort required of most humans, and thus the calories we burn in a day. It would seem that our bodies, evolving through millennia of inadequate or barely adequate food supplies, were ill-prepared for food abundance, especially relative to work effort. This may especially be the case for certain foods: We may all have a liking for sugar that developed when we had limited access to it, but which now guides us to overeat it.

Mere decades ago it was widely assumed that the "cure" for obesity was simply either to eat less or to exercise more. This simple assumption still guides the global diet industry. Healthcare professionals increasingly recognize, though, that the human body adjusts when we change our diets or exercise patterns. (They also, we might note, recognize a host of social, cultural, institutional, and other influences on obesity and have indeed produced a flowchart far more complicated than any we have presented in this book.) We may lose a few pounds at first when we diet or exercise, but our body then reacts hormonally to adjust how fast it burns calories. People then regain weight even while continuing to diet. It is not that they necessarily lack dedication or "fall off" their diets: Their bodies actively impede weight loss. Again, we can see world history at work. Through most of human history, when a human lost weight, it was usually involuntary and resulted from their inability to get as much food as they wanted. People experienced periods - after a hunt or harvest - when food was abundant and work requirements minimal, and other periods when food was scarce. The human body was selected to respond to periodic decreases in food intake by decreasing its food requirements and building up fat reserves if possible. The fact that human bodies in the twenty-first century react to dieting in a manner that frustrates efforts to lose weight likely reflects the fact that through most of human history, both food intake and work effort were highly variable. Human evolution, as we saw in chapter 3, is a prolonged process. (There may have been some changes during historical time; Aboriginal Australians, who likely suffered frequent food shortages, seem to have a much higher propensity to obesity than non-Aboriginal Australians - though this may reflect different social circumstances.) It will take humans millennia to evolve physiological mechanisms to cope with the challenge of abundance 


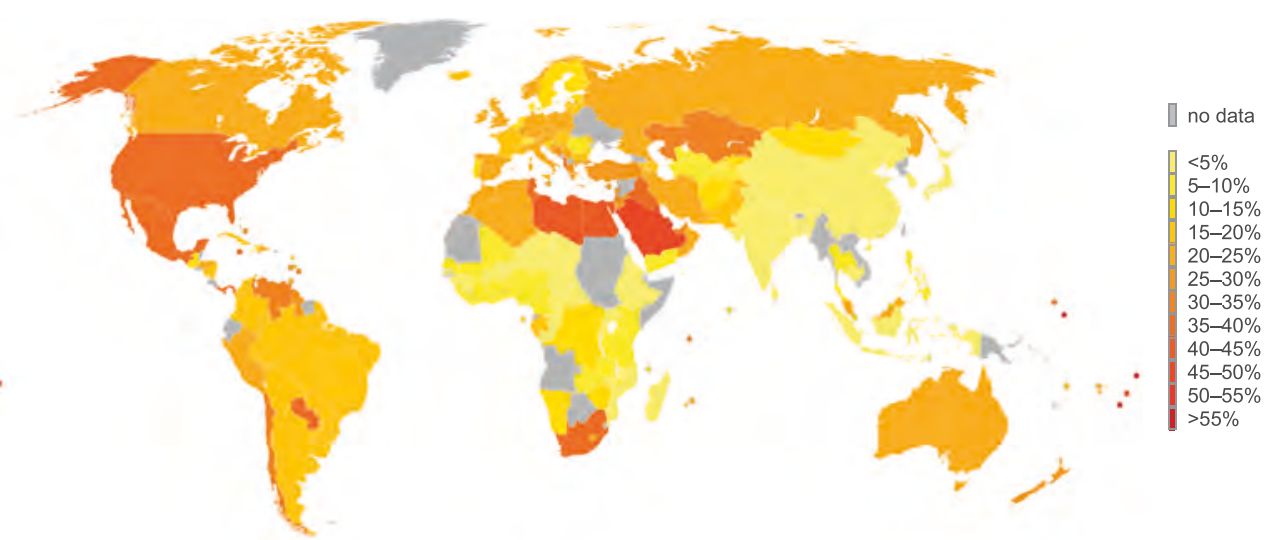

Map 31.3

World map of female obesity, 2016

Map by Lukepryke, CC BY-SA 4.0

(assuming that we have millennia and that abundance characterizes these). We can reasonably anticipate, then, that pharmaceuticals may be developed, and genetic interventions suggested, to try to induce our bodies to change the way they manage caloric intake. We will likely also see continued public policy interventions aimed at increasing the nutritional quality and reducing the sugar and other obesity-enhancing substances in our food.

\section{Questions}

1. Diagram the influences on and effects of either the nineteenth- or the twentieth-century migration experiences. (Note that many of the influences discussed for the nineteenth century still apply in the twentieth century.)

2. Have the challenges faced by farmers changed in the twentieth century?

3. Describe a museum or historic site that you have visited or hope to visit noting how the site or museum evoked some of the processes or events addressed in this book. What did you enjoy the most?

4. Compare the urbanization experience of poor and rich countries.

5. Describe the cultural effects of reduced child mortality.

\section{Readings}

Curran, Declan, Lubomyr Luciuk, and Andrew G. Newby, eds. 2015. Famines in European Economic History: The Last Great European Famines Reconsidered. New York: Routledge.

Goldin, Ian, Geoffrey Cameron, and Meera Balarajan. 2011. Exceptional People: How Migration Shaped Our World and Will Define Our Future. Princeton University Press. 
Lees, Andrew. 2015. The City: A World History. Oxford University Press.

Livi-Bacci, Massimo. 2012. A Concise History of World Population. Malden, MA: Wiley-Blackwell.

Moya, Jose C., and Adam McKeown. 2010. "World Migration in the Long Twentieth Century," in Michael Adas, ed., Essays on Twentieth-Century History. Philadelphia, PA: Temple University Press, 9-52.

Ó Gráda, Cormac. 2015. Eating People Is Wrong: Essays on the History and Future of Famine. Princeton University Press. [See also his Famine: A Short History, Princeton University Press, 2009.]

Packard, Randall. 2016. A History of Global Health: Interventions into the Lives of Other Peoples. Baltimore, MD: Johns Hopkins University Press.

Schayegh, Cyrus. 2017. The Middle East and the Making of the Modern World. Cambridge, MA: Harvard University Press. [Traces the interaction among urbanization, state formation, and international trade.]

Spodek, Howard. 2010. "Twentieth-Century Urbanization," in Michael Adas, ed., Essays on Twentieth-Century History. Philadelphia, PA: Temple University Press. 53-82.

Watts, Sheldon J. 2010. Disease and Medicine in World History. New York: Routledge. Zuelow, Eric G.E. 2015. A History of Modern Tourism. Basingstoke: Palgrave Macmillan. 
VII Drawing lessons 


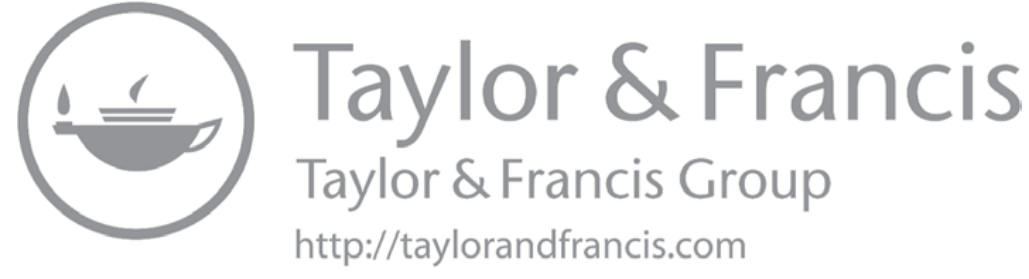




\section{Drawing lessons from history}

Why, how, and what

\section{Why to draw lessons}

Why study history? There are many reasons: It is enjoyable (hopefully) and gives us a sense of how we came to be where we are. Yet it is generally also hoped that by understanding the past, we could better guide our future. History should prepare us for action. In the famous phrase of philosopher George Santayana (1863-1952), "Those who cannot remember the past are condemned to repeat it."

\section{Some important general insights}

1. Humans are short-sighted. We often have in history set in motion processes that had results quite unforeseen and undesirable. We should then approach the task of better shaping our future with humility.

2. Humans have nevertheless accomplished much. We have over time developed exciting art forms, keen philosophical insights, better technologies and scientific understandings, superior political and economic institutions, and cultural values that embrace diversity. It would be a mistake to think that we cannot do good in the world (see our discussion of human progress below). Humility should not be an excuse for inaction.

3. Evolutionary analysis guides us to appreciate that the key to progress generally lies in selection more than mutation. While it is necessary to generate good institutions and technologies and values and art forms, it is even more important to carefully evaluate these in practice and discard those that do more harm than good.

4. Our study of revolutions suggests that humans are least able to guide big changes in desired directions. Nevertheless, our understanding of path dependence indicates that societies from time to time may need to change paths deliberately. We should act carefully but may at times need to act courageously. 


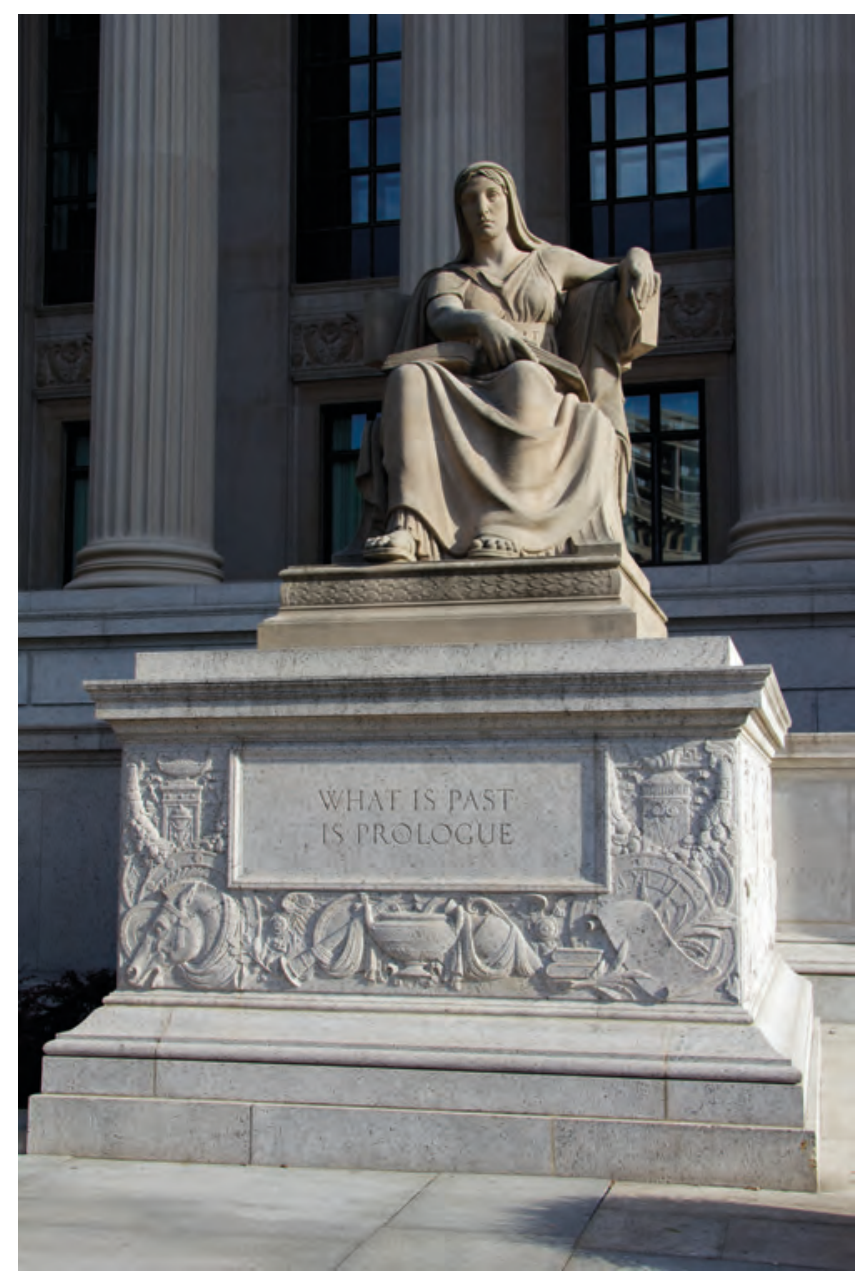

\section{Illustration 32.1}

What Is Past Is Prologue. Statue in front of National Archives building, Washington DC Photograph by Mike Peel (www.mikepeel.net), CC BY-SA 4.0

5. Both humility and evolutionary analysis guide us to seek to fashion a better world rather than leap to a supposedly perfect world. (Though we have not stressed these, history has been littered with utopian visions; these can be usefully consulted for ideas but should be treated with caution.)

6. The decline of all pre-modern societies should warn us away from complacency. Time after time, institutions that have served people reasonably well for centuries ceased to do so or were overturned. We may well have achieved a superior set of institutions today in much of the world, but both democracy and markets can generate undesirable outcomes. It takes a conscious effort to ensure that we do not lose what we already have. And then one of the advantages of comparative world history is to better allow us to recognize both the strengths and limitations of the societies that we inhabit. 
7. We have witnessed a tendency for economic inequality to increase through time, and for powerful economic interests to weaken the power of the state. The powerful may then undercut the very institutions on which their own success is based. It remains to be seen whether democratic governance can reduce or manage inequalities when millennia of autocratic empires have failed in the end to do so.

8. Ideas matter. If we are to consciously push for a better future, we need to have a good idea of what we want to achieve and why. In do-gooding, as in all other human activities, it is easy for us to self-justify actions that do not actually make the world a better place. We need to strive to evaluate the options before us dispassionately. (To be clear, then, we need to carefully assess mutations before applying these, and then re-evaluate them in practice.) Since ideas evolve, we should humbly appreciate that the set of ideas we have inherited from the past limits the ideas we develop for the future.

\section{Challenges in drawing lessons}

There are some crucial caveats we should keep in mind as we try to draw more precise lessons from history below:

1. Our understanding of history is limited, and there is controversy regarding many important historical events and processes. This book has employed strategies of interdisciplinary integration in addressing historical debates. We hope that we have provided a historical narrative that the reader can treat with confidence. Yet we have undoubtedly made mistakes along the way.

2. History is full of surprises: events that few, if any, saw coming. We can reasonably predict that the future will be full of surprises too. Indeed, evolutionary mutations are always somewhat unpredictable. We will be better able to respond to surprises if we know what our goals are and have an array of policies with which to respond to surprises (and the humility to know that sometimes doing nothing may be our best response).

3. History is complex. There is a large (but finite and countable) number of phenomena that influence each other. We may think that the primary influence on $\mathrm{X}$ is $\mathrm{Y}$ but be surprised when $\mathrm{Z}$ exerts an unprecedented influence next Thursday. We should try to understand the relationships among all our themes. In this way, we can make the future a bit less surprising.

\section{How to draw lessons}

Historical lessons are often drawn in terms of analogies. It is common when a politician seems to be appeasing another world leader to draw a comparison with Chamberlain in Munich agreeing to let Nazi Germany encroach on Czech 
territory (chapter 28). But then the question becomes in what ways does the contemporary crisis differ from the situation in Europe in 1938. Inevitably a host of similarities and differences can be identified. Though analogies have their place - they are rhetorically powerful, after all - the approach taken in this book and chapter is to focus on both evolutionary trajectories (if the selection environment seems to dominate evolutionary processes, we can carefully predict a future path of change) and particular relationships among phenomena. If, say, institutions for protecting property have been observed to exert a positive influence on trade and investment many times in the past, we can reasonably conjecture that they will do so again in future.

The trick is not to be seduced into over-emphasizing those evolutionary processes or thematic interactions that we think we understand well. As noted above, history is complicated, and we must always be alert to the possibility that other interactions will interfere with our confident predictions. This is, of course, one of the strengths of historical narrative: We should never lose sight of the inherent complexity and contingency of historical processes. The many diagrams that have characterized this text should remind the reader that history involves complex interactions among themes. Only by understanding the complex interactions that guide history can we hope to encourage those interactions that generate good outcomes and limit those interactions which produce adverse consequences.

\section{Institutions and values}

We have described the diverse challenges facing different types of human actors (rulers, merchants, farmers), and how these challenges have been addressed at different times and places. Some of these challenges emanate from the natural world: Farmers must find some way to restore nutrients to their fields. But most of the challenges we have discussed reflect human failings: Farmers need to defend their stored food from thieves, governments struggle to get bureaucrats to act in the state's interests, and merchants must protect themselves against both theft and deception. It is useful for us to at least reflect on how much more productive and happy humans could have been throughout history if humans were not so easily disposed to theft, deceit, and violence. And then we can usefully wonder if there are ways through culture and institutions to encourage more ethical behaviour going forward. Indeed, we have repeatedly seen in this book the advantages of institutions and values that limit theft and violence and deceit.

Those of us fortunate enough to live in countries at peace and with legal systems that enforce contracts and protect property (not least from the state itself) should appreciate how lucky we are. We can (most of us) live our lives without having to worry constantly about someone stealing our stuff or attacking our city. There are still, to be sure, injustices, both within such countries and beyond. We should strive to reduce opportunities for violence, theft, 
and deceit. But you might reasonably say that you did not have to read an entire world history text to learn that banal lesson.

We can stress, though, that humans have tried to address these challenges over time with a mixture of cultural attitudes and institutions. These can reinforce each other: A person facing an opportunity for theft can contemplate both how it is wrong and that it is dangerous. Institutions work best when supported culturally. The reader can reflect here on the difficulties of enforcing laws against drugs or littering if these are widely disparaged. One lesson of history would seem to be that we need both good values and good institutions. Certainly, polities of all types have worried about both institutions and values.

It has been easier to trace the development of institutions in this book. We can derive a crucial lesson: that we should always carefully design our institutions to reduce opportunities for deceit and theft. This result has not always been achieved, even in Western democracies. And historical analysis suggests why: People in positions of power can shape institutions that serve their narrow interests, and may wish to protect the gains they receive through theft or deceit. Good institutions are easiest to achieve when they serve those in power: A parliament of merchants and landowners could see advantages in protecting property and enforcing contracts. We need then to shape our political institutions in such a way that our economic and cultural institutions are not prisoners to the exercise of power. Family law should reflect the interests of children rather than those of divorce lawyers; financial institutions should not design financial regulations. Politicians financed by financial institutions have limited incentive to protect investors from deception and theft. (One lesson of the 2008 financial crisis would seem to be that buyers of securities are often unaware of both the management fees they are paying and the amount of risk they are incurring. Regulators need to take ignorance among supposedly sophisticated agents into account, and limit the likelihood of agents making decisions that are both individually and societally ill-advised.) And the average voter can hardly be expected to understand the thousands of pages of financial regulations in a complex modern economy. The political question is then how can we alter the political selection environment in order to select institutions that are in the public interest?

Nor should we forget that beliefs matter. The tremendous efforts of political and religious leaders to shape cultural attitudes through time would scarcely make sense if these did not matter at all (though these efforts might, I suppose, merely reflect self-deception). And then we can reasonably ask: Who advocates these days for honesty and responsibility and respect for others? Religious leaders often seem more interested in who is having sex with whom. And political leaders all too often are found to have broken any pledges to be honest they may have made. Multiculturalism has fostered a laudable respect in many lands for cultural diversity, but this has had the unfortunate side effect of leaving the impression in young minds that ethics is entirely a matter of choice. The ethical challenge of our times is to celebrate diversity without sacrificing values such as honesty and responsibility and respect that have been pursued across 


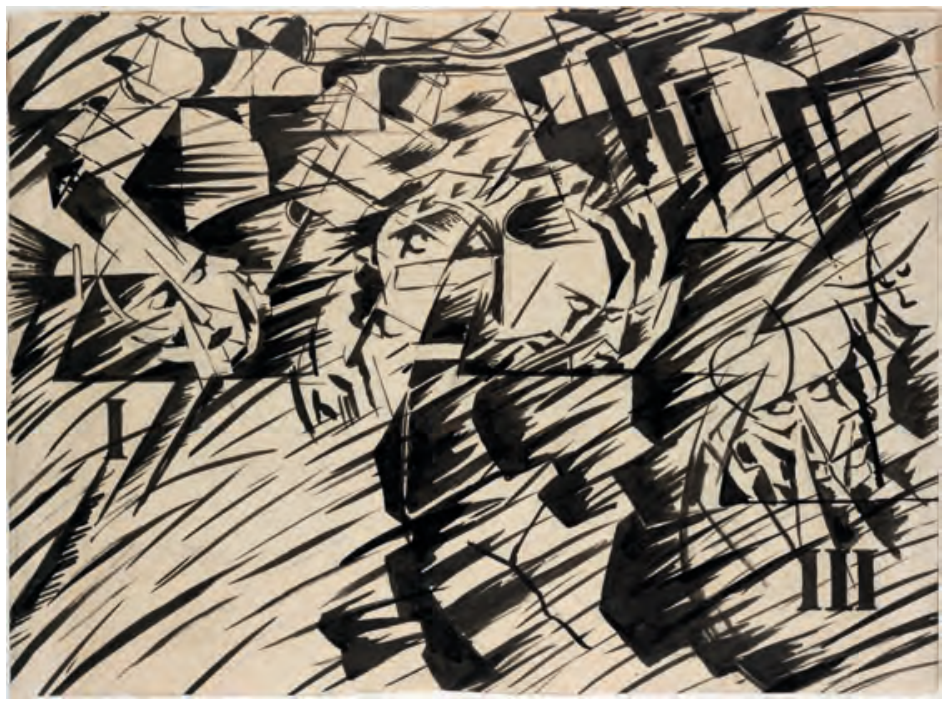

\section{Illustration 32.2}

Umberto Boccioni, States of Mind: Those Who Go, 1911. Boccioni was one of the founders of Futurism, an art movement that began in Italy in the early 20th century and celebrated speed and technology. This painting captures modern life's transitory nature, Metropolitan Museum of Art

human societies for millennia. History suggests that our institutions alone will not keep us honest unless our culture guides us there as well.

\section{Human nature}

If we appreciate that values are important, then we must ask which values a society might try to encourage. We have already mentioned honesty and responsibility, and will return to these in later sections.

Perhaps the most obvious lesson of history is that people have justified the most horrific acts: Thieves have justified theft, soldiers have justified rape and pillage, rulers have justified corruption and contempt and even genocide, and so on. Notably, people have continued to defend acts even when these have not been in their self-interest. Hollywood does us a disservice in portraying villains that are selfishly and purposely evil. The fact is that most of the evil in the world is perpetrated by people who consider themselves to be good (or at least not evil) and deceive themselves about the nature of their acts. Lack of self-knowledge is the greatest source of evil.

If this is true, then the one thing the world needs most is self-knowledge. This must seem, even after all these pages, to be a very squishy touchy-feely conclusion. Surely what the world needs is peace or environmental sustainability or cross-cultural understanding? Indeed, but our lack of self-knowledge leads us away from all these goals. We cannot reliably do good in the world 
without knowing ourselves. We are just too proficient at self-deception (having been selected for this; chapter 3). It is not surprising, then, that philosophers and religious figures across time and place have urged self-knowledge. We will address strategies for achieving self-knowledge below.

We should also stress humility. Caesar, Chinggis Khan, and Hitler all felt that they were destined for conquest, and pursued this goal single-mindedly without regard for the consequences. We can draw parallels with those today who join or lead cruel regimes that murder and torture with impunity. They all think that their cause is just and that the ends justify the means. What makes them feel that their view of the universe is so fantastic that it entirely negates the fundamental human rights of others? We could ask the same question of terrorists or revolutionaries (against societies that are not themselves manifestly evil). One answer often is religious. Once one accepts that absolute faith in some creed is a good thing, it is a small step for some people to use that complete faith as a rationale for torture and murder. Yet different leaders (and followers) use different religions to justify their similarly heinous acts. Humility would guide us to respect the opinions and rights of others, and to appreciate that we might have horrible subconscious motives for our actions.

Certainty, then, is dangerous. Doubt guides us to appreciate differing points of view, and to develop and examine arguments and evidence carefully. Doubt guides us to ask the all-important question: "What if I am wrong?" This simple question should decrease our willingness to engage in war or terror. Doubt need not mean indecisiveness. We can appreciate that we lack perfect information or insight and still act in the world. We will be guided, though, to respect the critiques of others, and to evaluate the results of our actions. It is often comforting to convince ourselves that we know certain things, but allowing doubt into our lives guides us to better decisions both for ourselves and for others.

We should also appreciate the deleterious effects of fear. Humans who are fearful are capable of justifying the most heinous acts. People who worry about how they are going to feed their families may support the most outrageous demagogue. People who are fearful of losing their jobs or falling to the bottom of the social hierarchy are much more open to racism or misogyny that will keep others in a state of inferiority. In times of civil strife, people bind themselves to warlords or armed groups that may commit atrocities against those they fear. We can urge people yet again toward self-knowledge: We may consciously reject our subconscious fears, or at least the actions we might be tempted to pursue because of these. We can also do much to reduce the sources of anxiety. We can try to reduce military strife and establish basic human rights protections so that people need not fear for their lives. We can reduce crime. We can develop social safety nets so that people need not fear falling into abject poverty. (The author quite likes both a guaranteed basic income and the idea of public works programmes for the unemployed. Public works programmes have been used in many times and places, and might provide an answer to those who feel that those on social assistance are lazy and are abusing the system.) 


\section{Do no harm}

One other historical lesson is often ignored: It is easier to do evil than good in the world. Theft, rape, murder: Any fool can perform such acts. It is far easier to destroy a benevolent polity or culture than to build it. Making the world a better place means encouraging better cultural attitudes or institutions or technologies. Our capacity for self-deception can too easily combine with our desire for self-efficacy in guiding us to justify harmful acts. We must, unfortunately, spend much of our time in this book discussing the actions of people who did more harm than good. But the fact that the world has improved in many ways (see below) signals that humans often also achieve good in the world. The humility and self-knowledge we urged in the previous section should guide each of us toward doing good rather than harm. Imagine the result if each of us somehow managed to make the world a better place.

\section{Human challenges}

It may be useful to briefly consider how we might wish to structure both institutions and values with respect to various historical actors in future in the light of historical experience:

- We want rulers - and bureaucrats - to face incentives to act in the public interest and penalties for behaving selfishly. We want to structure voting systems (which are, we might note, how democracies address the succession issue), government transparency, auditing, and other institutions to this end, and encourage values of honesty and social responsibility. We also want to limit the influence of economic elites on government policy: These elites may at times pursue the public interest (say, in fostering economic growth and employment) but will also pursue their narrow interests. This implicates transparency again but also campaign finance reform. These institutions (and other institutions described below) need to be buttressed by cultural attitudes: We should expect rulers to act in the public interest.

- We want merchants and industrialists (entrepreneurs more generally) to face strong incentives to do useful things, such as identify new trade opportunities, develop new products or services, improve quality, and lower costs. We want to discourage them from bribery, misleading workers or customers or shareholders, violating environmental regulations, co-opting government regulators into allowing private monopolies or other selfinterested practices, and deceiving other merchants. This calls for a careful set of regulations that would enable freedom to innovate but specifically punish particular practices. Corporate democratization and transparency are also vital. And we could usefully alter cultural practices to value not any profit-making enterprise but rather only those that provide consumers 
or other businesses with better goods or services while respecting environmental and labour policies.

- We likewise want builders to be discouraged from bribing or otherwise influencing local rulers (city officials etc.). Builders and rulers should be encouraged to work toward healthy living and working environments. A mix of city planning, urban democracy, zoning, and government transparency is desirable. Having spent much of this book marvelling at architectural achievements across diverse societies, we might wish to seriously consider encouraging the construction of aesthetically pleasing edifices. We should recognize as a society the importance of aesthetics.

- We want farmers to be protected from theft and to be guided to farm in environmentally sustainable ways. Human societies have displayed the capacity both to injure and to resuscitate the natural environment. We should carefully monitor soil quality to ensure that we are not slowly adding toxins. The same is true of fishers: these need to be protected from theft and encouraged toward environmental sustainability. This requires establishing clear property rights over particular fishing stocks so that some (governmental authority or) group of fishers has the incentive to manage these stocks in perpetuity.

- We want rulers and soldiers to respect the Geneva Conventions. Though the history of war crimes trials is controversial, it is hard to see a superior institutional structure. Such courts would benefit from greater international support. In the longer run, of course, we want peace. Democratization, peacekeeping, and international adjudication are among the (mutually supportive?) institutions here.

- We want managers to not abuse their power over employees. Mechanisms of employee grievance may be crucial here, but collaborative administrative structures where feasible can also be advantageous.

- We want to reward artisans both institutionally and culturally for producing goods that consumers want, and not misleading these. This calls for institutions and values similar to those urged above for merchants.

- We want voters to dispassionately evaluate their choices - both with respect to policy and the character of officials. Voters should punish unethical officials. Voters should carefully assess institutions both before and after these are introduced. This is a heavy burden. It can be lessened through increased transparency (so that voters can better monitor political behaviour), and better sources of unbiased information. Consultative fora, where randomly selected voters meet and are exposed to competing analyses of complex issues, can be useful in indicating how an informed electorate would choose to act. The first challenge is to get politicians to establish institutions that increase voter influence over them.

- We want priests to encourage values such as honesty, responsibility, and compassion that have been urged across human societies for millennia. Note that it is not always in the financial interest of priests to critique unethical behaviours of parishioners. We would like to discourage priests 
from encouraging hostility toward other religions (and perhaps promote openness to intermarriage). This again may conflict with financial incentives, and also with the priest's dedication to their faith. One fundamental question here is to what extent a secular state should seek to guide religious behaviour. (The growing population of agnostics and atheists may prefer to see priests disappear. They should then consider how cultural values are best encouraged in a secular society. This would likely involve an appreciation of the five types of ethical analysis engaged in chapter 8 , all of which can justify honesty, responsibility, and other desirable values. See below.)

\section{Cultural understanding}

We can learn two important things about cultural understanding from world history. First, cultural co-mingling has been common in human societies. The empires that have dominated political history have generally been multicultural and often religiously tolerant. And trade required many people to live in foreign communities and reach some level of trust and understanding with at least some of the locals.

Second, cultural difference has been generated by a shared and interlocking history. It would be a grave mistake to see today's cultures as reflecting independent historical developments. We can learn something of others by appreciating how other cultures emerged from our shared history. Moreover, all human cultures have changed dramatically through time in response both to societal interactions (most evident in the areas of cuisine and attire but extending far beyond that) and to changing political, economic, social, and technological (and other) circumstances. We should not reify existing cultures but anticipate further evolution. Last but not least, we have attempted to understand historical actors with reference to a common set of human challenges and temptations. We have hopefully learned to discern an underlying humanity not always a pretty one - beneath diversity. This should guide us to appreciate the similarities across world cultures as well as the differences.

These two lessons can guide us in solving the ethical challenge of our times (above) by both embracing diversity - which history asserts is a feasible societal attitude but one with problems as well as rewards - and a set of shared ethical principles.

\section{Toleration}

Many scholars have celebrated the toleration of differences that characterized many empires. Some have even urged a return to empire. It deserves to be stressed, then, that toleration was never absolute. Most obviously, empires insisted on loyalty. And while the Romans were happy to allow cultural 
diversity in general, they insisted not only that their own system of laws govern interactions across the empire but that local gods be absorbed into the Roman pantheon. More centrally, religions and states have each devoted tremendous efforts over the millennia to encouraging a set of ethical beliefs that would promote social harmony, a list that almost everywhere included honesty, charity, and responsibility to their families and the broader community or state. Toleration, then, was always coupled with the pursuit of some shared institutions and values.

Modern toleration often knows no bounds. When young children are surveyed regarding ethics in Western countries, they often respond that whatever any group says is okay. The value of toleration has squeezed out values such as responsibility and honesty. Modern societies might wish to reflect on the imperial compromise: Do they also wish toleration plus some set of shared values? At the very least they might want to reflect on how toleration needs to coexist with a shared respect for a country's principal political institutions: not every law, but democracy and human rights in general. The idea of popular sovereignty (chapter 21) depends on some shared appreciation of critical institutions and values.

Most historical empires ruled mostly agricultural populations. As service sectors grow, human interaction increases. The Romans were happy to leave groups in their place - though they established Roman garrisons in the provinces and drew local elites into Roman life. Modern societies may depend on much greater cross-group interaction and understanding. Curiosity and respect should infuse toleration. Multiculturalism is beneficial only when members of different groups interact, sharing their beliefs and customs.

Empires in polytheistic times sought syncretic religions. The rise of monotheism closed this avenue - though Ashoka (chapter 9) was not alone in still pursuing syncretism. Islamic Spain is notable for the fact that Christians, Jews, and Muslims interacted as friends and even occasionally intermarried. That apparently did not erase strong religious identities, for the Christians ejected Jews and Muslims once they could. Today's tolerant societies need to reflect on how to achieve cross-religious understanding, interaction, and respect. One fundamental proposition here is that parents in a multicultural society must anticipate that their children will want to have friends from other groups; this should not be discouraged. Indeed, a successful multicultural society may depend on parental acceptance of the fact that their children may live lives quite different from their own. Dating and intermarriage raise religious challenges of course, but potentially contribute to social harmony and understanding.

We traced in chapter 27 a variety of social changes over the last century or so, including the pursuit of gender equality. It is possible for a society to embrace cultural diversity and also these sorts of social change. This is likely best done by recognizing potential conflicts between the two: As we noted then, gender equality has been accepted more slowly by some groups than others. A first step here involves an honest appraisal of which cultural practices are reasonably innocuous and which actually impede the pursuit of social equality. Care must 
be taken not to be culturally biased in such an appraisal: High-heeled shoes might seem an unacceptable interference in female mobility to those not culturally accustomed to them. And then what? A societal conversation regarding values can be healthy, but the utmost care must be taken in prohibiting practices that are valued by any social group.

It may prove easier to achieve cultural understanding in the area of cultural expressions than in the area of beliefs or social practices. Indeed it is common to lament a "westernization" or "globalization" of standards of attire and preferences in music and dance (which often, it might be noted, reflect a synthesis of music and dance from different regions). We should certainly worry that advertising has driven much of this "globalization," and we can recognize that the self-knowledge urged above may be the best antidote to mindless consumer culture. But we might also appreciate that the conscious adoption of shared cultural expressions may reflect a subconscious yearning for shared values.

\section{Science}

We should celebrate our enhanced understanding of how the world works. In the realm of natural science and technology, we have clearly enhanced our ability to achieve human goals - both economic and aesthetic. Yet we should not value or fund natural science just because of its technological benefits. Humans appreciate astronomical understandings even if these lack obvious practical implications. And the links between science and technology are often unpredictable. Scientists in pursuing their curiosity have often had unexpected technological implications: Scientists trying to comprehend electricity discovered radio waves.

Natural science has provided over time a set of understandings of how our universe and our species emerged (chapter 2 and 3). Though scientists still struggle to understand how complex organs such as the eye appeared or what was "before" the Big Bang, these scientific understandings are widely respected. Science, then, can serve some of the roles of religion in explaining how the universe and world came to be (chapter 8). Philosophy has been less successful in serving other roles played by religion: putting meaning into our lives and identifying ethical precepts that can facilitate interpersonal interactions in a complex society. We will below explore whether history itself can inform the meaning of life. As for ethics, we have seen in chapter 8 and elsewhere that religions and philosophers have employed throughout world history each of five different types of ethical analysis. What is less obvious is that these five types of analysis often point in the same direction: honesty, personal and social responsibility, compassion, and other vital ethical dispositions can be justified with appeals to consequences, rules, virtues, intuition, and tradition. Note that the first three types of ethical analysis are mostly studied by philosophers, the fourth by psychologists, and the fifth by 
anthropologists: Only a historical and interdisciplinary approach connects all five. To be sure, none of these justifications - of any ethical value, from any of the five types of analysis - are perfect. One can always sketch a potential weakness in any ethical argument. Scientific understandings are not perfect either, but yet are powerful. If we can provide persuasive arguments in favour of, say, honesty, within all five types of ethical analysis, we can be very confident that humans should be encouraged toward honesty. It was suggested above that it may be of crucial importance moving forward to establish that our laudable acceptance of diversity is not at all incompatible with societal advocacy of crucial values such as honesty, responsibility, and self-knowledge. The fact that a value such as honesty can be justified in many ways and accords with the traditions of the vast majority of human societies suggests that we can indeed treasure both diversity and honesty. World history shows us the importance of such values and suggests that we can achieve them only if they are consistently advocated within a society.

In the area of social science and humanities, we are less confident of enhanced understanding than we are in natural science. The problem there is a less clear selection environment. Technologies have to work to be selected. The link between social science and social policy is both less strong and less evident than the connection between (at least some) natural science and technology. And the selection environment for social policy is itself less intense: It is not at all obvious that we routinely abandon bad policies and replace them with better ones. The success of a policy in meeting societal goals is undoubtedly one element of the selection environment for social policy, but it may take a back seat to political strategies, voter mobilization, and ideology. The success of a particular policy has an even more tenuous connection with selection among social science theories: A theory may be selected because it is convenient for social scientists, though its fit to an external reality undoubtedly plays some significant role in selection. As for philosophy, the fact that there are no "immaculate conceptions" (at least beyond Descarte's cogito) - philosophical statements for which it is not possible to sketch a reasoned disagreement - makes it seem to some that philosophy is just a game. Nevertheless, in both social science and philosophy, we can hope that the careful development of argument and evidence can increase our confidence in certain theoretical conjectures.

As noted above, selection environments in the social sciences are not flawlessly aligned with the public interest in identifying superior policies. Social science theories may be judged for "elegance" or especially their ability to provide research questions for academics desperate to publish, rather than whether they provide any insight into public policy. One institutional change that might be useful is to increase contact between policy-makers and social scientists by providing each with occasional secondments to the others' workplaces. At present, social scientists often lack a detailed understanding of the challenges that policy-makers face. The trickier question is whether there might be some useful role for non-academics in the evaluation of social science research. Governments have edged into this sphere by prioritizing some research funding for questions 
of particular public interest. Scientists worry with reason when this means decreased funding of research driven by the scientist's curiosity.

Then there has to be selection for sound public policy itself. One institutional innovation here would involve more careful evaluation. When programmes are introduced there should be a fairly precise set of expectations about what these will achieve. One clear lesson of history is that bureaucrats will pursue self-interest. And any public policy, once introduced, will benefit some people, and these will thereafter lobby for its maintenance even if its cost to the body of taxpayers in much greater than the benefits to these few (just as large landowners throughout history have protected their privileges). If each policy is not carefully and objectively (as much as possible) evaluated, we can expect a gradual accretion of programmes through time, not all of which will do more good than harm.

One key challenge to sound public policy is complexity itself. This challenge is best met through two strategies advocated in this book: organization and interdisciplinarity. Only by organizing our understandings can we hope to identify all of the thematic interactions relevant to a particular public policy challenge. And only by integrating these can we hope to determine the best set of interventions and minimize adverse side effects. Evaluations of public policies must be comprehensive, examining their influence on all phenomena.

There is also scope for improving the quality of conversations both within academia and in the wider world. We need to encourage conversations whose primary goal is to enhance understanding rather than achieve victory. In such discussions, participants make a real effort to appreciate the strengths as well as weaknesses in the arguments they oppose. And all participants seek a comprehensive understanding that combines the advantages of opposing arguments. Such conversations would be encouraged by a change in societal values toward open-mindedness: We often value the person with strong views rather than the person who revises their opinions in light of new information. Changes in institutions to reward open-minded dialogue should also be explored: The citizen's fora recommended above might prove useful here. We have discussed in this book several examples from quite different societies of advances in human understanding of philosophy or science that each reflected environments in which hundreds or thousands of individuals engaged in open and constructive dialogue; the trick is to scale this sort of helpful conversation so that hundreds of thousands or millions can somehow be involved.

Science in the past was often an elite activity. As science has become more important - due to its increased impact on technology - and political decisionmaking more democratic, it is essential that science be understood by the broader public. Voters must often evaluate advice from both natural and social scientists, and they are often frustrated when scientists disagree (but sometimes suspicious when they do not). Voters need to appreciate that scientists can never prove or disprove any scientific (or philosophical) statement. It is always possible to sketch an alternative explanation of any observation or experimental result. Even today one could argue for a complex set of orbits upon 
orbits by which the sun orbits the earth. However, scientists have amassed over time compelling argument and evidence such that the conjecture that the earth orbits the sun is now almost universally accepted. The same is true for a wide range of scientific conjectures. Voters should appreciate that scientific disagreement sets the stage for investigation and advance - as long as the selection environment chooses wisely. Voters should not see scientific disagreement as a reason to simply ignore scientific advice. While consensus is being pursued voters may wish to know what proportion of scientists favour a certain position: Over 95 per cent of climate experts believe in human-caused global warming, and over 90 per cent of economists agree that the best way of dealing with this is to put a price on carbon emissions. Of course all new ideas in science start out as minority opinions. We should not casually ignore theories posited by the vast majority of experts in a field, but nor should we be surprised if these theories change over time. (Note that the balanced approach urged toward science here - we

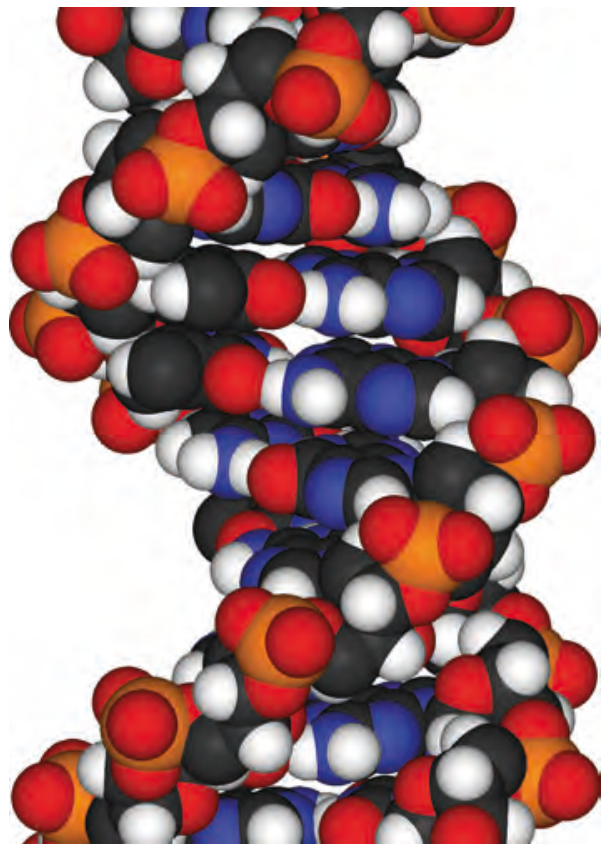

Illustration 32.3

Fragment of DNA model should neither reify nor ignore science, nor imagine that it is either entirely subjective or objective - accords with the philosophical approach outlined in the last sections of chapter 1.)

It could be that the internet will not only facilitate better public understanding of science but also public input into science. The public already plays an important role in spotting flora and fauna in biology and stars and planets in astronomy. Wikipedia - though not always an infallible source of information has demonstrated the possibility of public input into a wide range of subjects. Yet the internet may also serve to segment public understandings as people increasingly receive information only from sources that pursue particular points of view. Instead of one comprehensive public debate that draws in all relevant information and perspectives, we may instead see segmented communities that tell each other what they want to hear, select information that is conducive to these preconceptions, and demonize those with opposing views. The internet can be wonderful in allowing diverse viewpoints to be heard, but it may do more harm than good if we do not teach the young to value different points of view and to apply critical thinking skills to what they read.

\section{Technology}

One does not have to be a technological determinist (one who thinks that technology is the driving force in history) to appreciate the power of technological 
innovation in world history. To be sure, it has been a force for both good and evil. Yet the fact that billions can live lives of comfort, health, and abundance that previous generations would not have imagined is primarily - though not exclusively - because of technological innovation. We also suggested in chapter 29 that technological innovation can have significant effects on investment and employment in the shorter term. And so we must applaud those countless millions who have thought up something new, and recognize that innovation almost always requires hard work, risk-taking, lots of time, and a willingness to deviate from and even offend existing modes of thought. It makes sense for us to encourage technological innovation as we move forward, but perhaps also to guide innovation in specific directions and away from others: toward renewable energy and away from military attack technology perhaps. History tells us that specific strategies may prove useful:

- $\quad$ supporting (but not exclusively; see above) certain types of natural science research that are closely linked to technology

- $\quad$ encouraging widespread technical skills and literacy as innovation is (likely increasingly) a group effort

- encouraging cultural appreciation of innovation, creativity, and change (but recognizing that too much change is disorienting).

That being said, we should recall that technological innovation is unpredictable and that innovations often have unanticipated effects. It may be that we will develop technologies that will alleviate the challenges of climate change, but it would be irresponsible to depend on this outcome rather than take public policy steps to address the problem. And we should be prepared for social disruption: Just as past technologies have replaced some workers but increased average incomes in the long run, it is likely that future technologies will disrupt employment in both manufacturing and services. The goal should be a society which frees humans from the more tedious types of work in order to engage in creative enterprises.

Will technological innovation drive environmentally sustainable economic growth in the future? History provides reasons for both optimism and pessimism. The rate of innovation has expanded dramatically in the last centuries. However, some worry that humans have already discovered the best ideas and so rates of innovation must tail off. Yet there seem to be many reasonably novel technologies in their early stages: genetic engineering, artificial intelligence, driverless automobiles, and many more. These might all have dramatic impacts over the next decades and centuries. And innovations spread more rapidly throughout the world than ever before. While innovation may continue to generate economic growth, we should hardly presume this result, and should still develop institutions (pension plans, central bank policies, and so on) that are designed to cope with sluggish growth if necessary.

Technological innovation almost always disrupts work patterns. New ways of producing goods and services displace workers skilled in old ways. New 
products replace workers producing older goods. We saw in chapter 29 that (the wrong balance of types of) technological innovation can have a substantial negative influence on overall unemployment rates for a few years - though we are unlikely to ever again see an impact as severe as that during the Great Depression. Such effects are temporary. Technology also creates new jobs, and workers migrate to these. There are concerns these days that "deep learning" software, which allows computers to make complex decisions by analysing massive data sets, will allow machines to replace a wide range of service jobs. History tells us that the long-term effect of such a transformation (if it occurs) will likely be that people move to a set of new jobs (Mars transit pilots?). We may want to encourage the development of new goods and services in order to accelerate the creation of such new jobs. There may also be a further decrease in average work weeks: These had fallen for centuries until the late twentieth century in most countries. The effects on income distribution may prove troubling: Mechanization in the past has mostly replaced poorly paid unskilled labour but may now primarily affect well-paid workers. Workers - including university professors - can have legitimate concerns about how new technologies will affect their jobs. (One crucial concern is with how new technologies allow increased monitoring of a worker's movements, with positive implications for productivity but negative consequences for privacy.) Again, though, such technologies can create opportunities as well as challenges. Societies can potentially achieve better standards of health and education at lower cost. And both

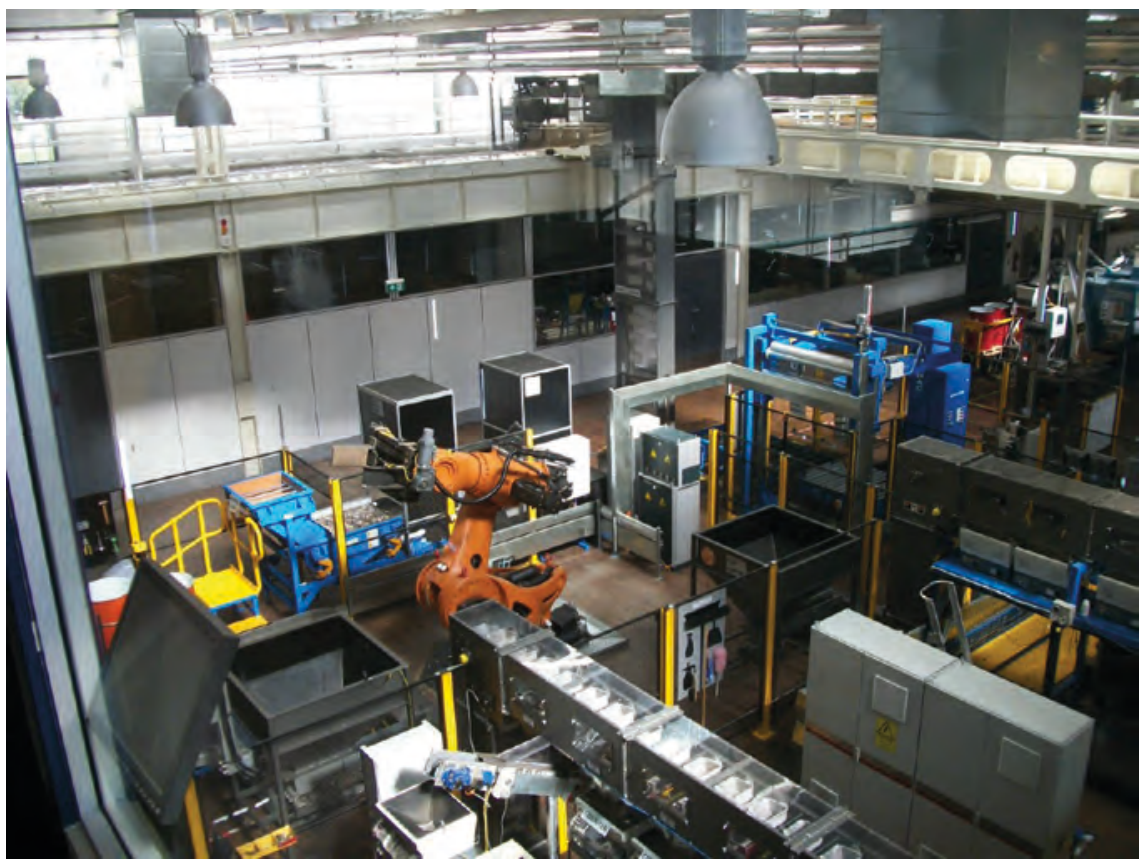

Illustration 32.4

Robot at Royal Australian Mint, Canberra 
teachers and doctors can shed the least exciting parts of their jobs - machines may come to guide rote learning and routine diagnoses - to focus on discussing complex issues with students (and guiding project-based learning) and devising comprehensive health strategies with patients.

\section{Energy, resources, environment}

We have been agnostic in our treatment of environmental history (see Box 7.1 in particular). Whether one leans toward those who argue that societies often destroy their environments or those who say that societies usually manage to avoid complete destruction, we can hardly doubt both that humans do environmental damage and that they take steps to minimize that damage. History can guide us to take care of local environments. But history is a limited guide to the contemporary challenge of climate change for the simple reason that it is "global" in impact. The past examples of environmental deterioration were local in scope: It was generally up to particular societies to manage their own environments. Today, we each mess up each other's environments. Every polity then worries that they bear all of the costs but receive only a fraction of the benefits from efforts to reduce climate change.

The history of international cooperation suggests that it will not be easy to reach agreement on dividing these costs. Nor will it be easy to monitor states' achievement of emissions targets or punish those who fail to achieve these. The economists' solution of putting a price on emissions - which is likely the strategy that minimizes the cost of any particular decrease in emissions - may also have political advantages: It might be far easier to agree on a global price and then monitor whether every state is imposing this. International negotiators have not pursued this avenue because of a general hostility to carbon taxes. There may be another way of moving forward: individual states pledging to impose a carbon tax of $\mathrm{X}$ per cent if, say, 80 per cent (by population or GDP) of other jurisdictions do the same (the Canadian province of Alberta, home of the Oil Sands, has made such a commitment recently). Environmental activists can then focus their energies on particular states. And these states might find it useful to avail themselves of another source of revenues. (Or they might pledge due to misplaced confidence that the 80 per cent threshold will not be achieved.) There are, it might be noted, ways to structure a set of carbon taxes and green incentives that can win the support of a business community that increasingly appreciates that something needs to be done and would like to be able to plan investments that will accord with future environmental policies.

It is a question of institutions. Haggling over the degree of carbon reduction each country must achieve is a challenging international task because it focuses everyone on the costs. One unsurprising lesson of institutional history is that it is easier to introduce institutions that seem to have benefits. Putting a price on carbon has a benefit for governments who collect the tax. Providing financial incentives to firms for reducing emissions can provide financial benefits (if 
they can cut emissions at a lower cost than the carbon price). As some countries introduce carbon taxes, and this brings benefits rather than an economic disaster, others will be more willing to follow.

Climate change is not the only environmental challenge. There are also concerns regarding resource use. Resource constraints in human history have generally been local as well - and humans have indeed mined local resources to exhaustion in many times and places. The possibility that we might run out of non-renewable energy resources (or other types of natural resources) at a global level is something new in human history. Moreover, estimates of how many resources remain in the earth or what the possible substitutions for these might be are far from precise. So a certain prudence might seem called for: to at least not subsidize resource extraction; to explore low-cost means of resource substitution and conservation; to encourage research into renewable energy sources; to develop less energy-intensive modes of production and transport. Note that while energy use per capita has increased through most of human history, there have been exceptions: If hunter-gatherers or early farmers used fire to burn vast areas, their per capita energy use might have been higher than most/all later peoples. We should not assume that the future necessarily involves even greater resource use per person.

There is one (renewable) resource for which increased international cooperation is clearly needed: Fishing in international waters needs somehow to be managed lest fish stocks be extinguished. If the bureaucratic reputation of the United Nations could be enhanced, an easy solution would be to endow

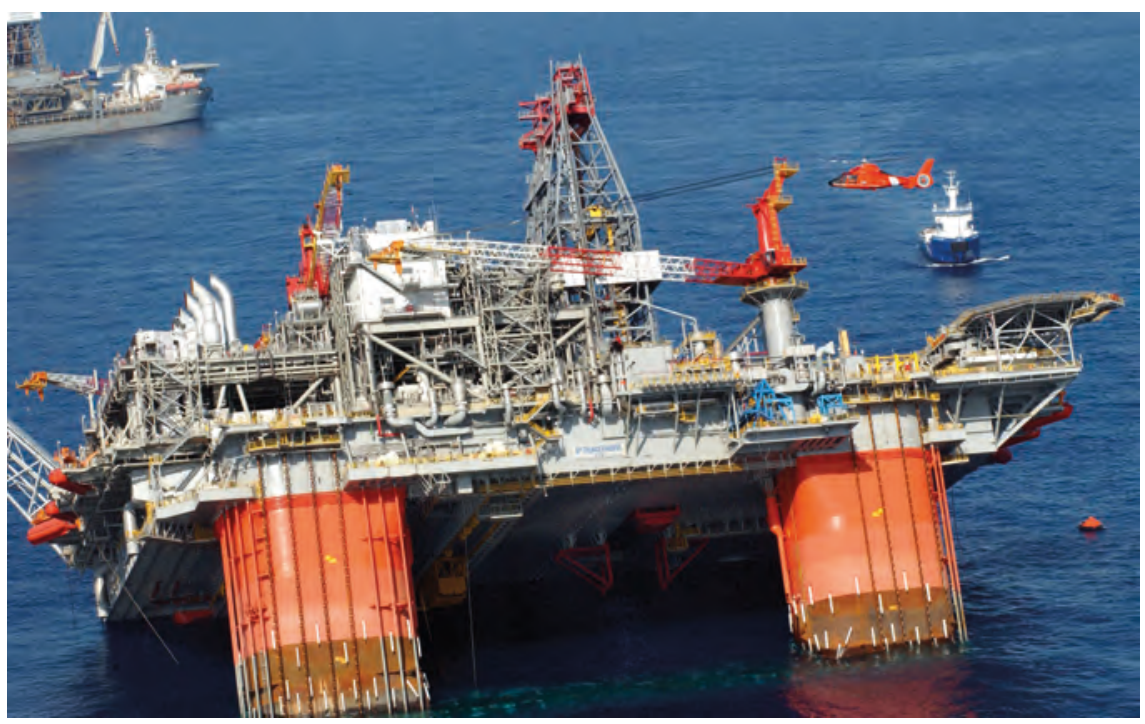

Illustration 32.5

Thunder Horse oil platform sinking, July 2005 near New Orleans. It was later righted and survived Hurricane Katrina

US Coast Guard 
it with property rights over this resource, giving it a financial incentive to manage it.

Concerning biodiversity, prudence is even more justified. It is estimated that 24 per cent of animals face extinction, 3 per cent of fish, 11 per cent of birds, and 10 per cent of plants. We live within complex ecosystems that we do not fully understand. And we have utilized a wide range of plants and animals in useful medicines (and other products). And we cannot yet genetically engineer species that we belatedly value after their extinction.

\section{Trade}

World history often celebrates trade: Trade was the primary conduit through which ideas were transmitted across societies for most of human history. Without trade, societies would have had much less influence on other societies. Trade gave consumers access to a broader range of goods. Trade gave farmers access to superior crops. Trade also had negative impacts: Forests might be destroyed or workers enslaved to facilitate trade.

Polls suggest that most people in the contemporary world understand that trade has important benefits but can also have undesirable impacts. Why then is there such hostility to trade deals (see chapter 29)? The simple answer here is that most of the easy gains from reducing tariffs on goods have been achieved. Trade negotiations increasingly address trade in services. Here the barriers are often not explicit tariffs but regulations regarding how services are to be provided. Every country has complex financial regulations, professional conduct guidelines, and intellectual property rules. In negotiating free trade in services, states must seek to harmonize these complex regulations and rules. Such efforts can be seen as attacks on sovereignty. Yet of course in any treaty that a country signs it must necessarily agree to abandon its right to act in ways contrary to the treaty. We have seen how ideas of popular national sovereignty have evolved over the last centuries. The question that needs to be asked with respect to trade negotiations is this: In what areas do we really need to protect national sovereignty? Perhaps a case can be made that each nation state will want an independent policy for the cultural sector. And social programmes can and should be protected (though societal preferences regarding these may not be so different). But do different states - at least those at similar levels of economic development - really need different sets of financial regulations? We have seen that institutions always function within a complex web of other institutions and cultural values. It may still be that states facing similar economic environments are best served by very similar economic regulations. (However, harmonization reduces the experimentation from which superior mutations might be selected.)

As trade negotiations focus on increasingly complex issues, they raise questions of democratic legitimacy. Citizens could potentially evaluate whether a set of tariff reductions was beneficial. It is much harder to evaluate 
the harmonization of regulations that are themselves hard to understand in the first place. We can hope - perhaps vainly - that elected politicians pursue our best interest when debating these regulations in our legislatures. However, when these rules are negotiated in secret internationally we can wonder whose interests are best served. Do some states negotiate better than others? Do firms influence trade negotiations more than workers? (Note that the latest trade agreements often give firms the right to sue governments that violate treaties. This is an important mechanism for ensuring that states obey the treaties they sign but can also obviously increase the power of firms.) Do our negotiators even know what is in our best interest? Going forward, then, we can wonder how to achieve democratic legitimacy with respect to complex regulations. Greater transparency in trade negotiations might be helpful. Better sources of dispassionate evaluation of complex issues would be helpful too.

Increased trade in both goods and services can only lower the costs paid by consumers if low-cost producers in some countries win markets from highercost producers in other countries. It is no surprise that firms and workers that lose in international competition complain. We have seen that states are often tempted to restrict trade (or innovation) to avoid potential unrest. The job losses associated with enhanced trade are unfortunately far more visible than either the job gains in export industries or the lower prices paid by consumers for goods and services produced at lower cost elsewhere. Trade restrictions would be less tempting if states provided better support for workers adversely affected so that these can move or retrain for jobs elsewhere. Note that in rich countries, increased trade tends to expand the number of skilled (and generally highly

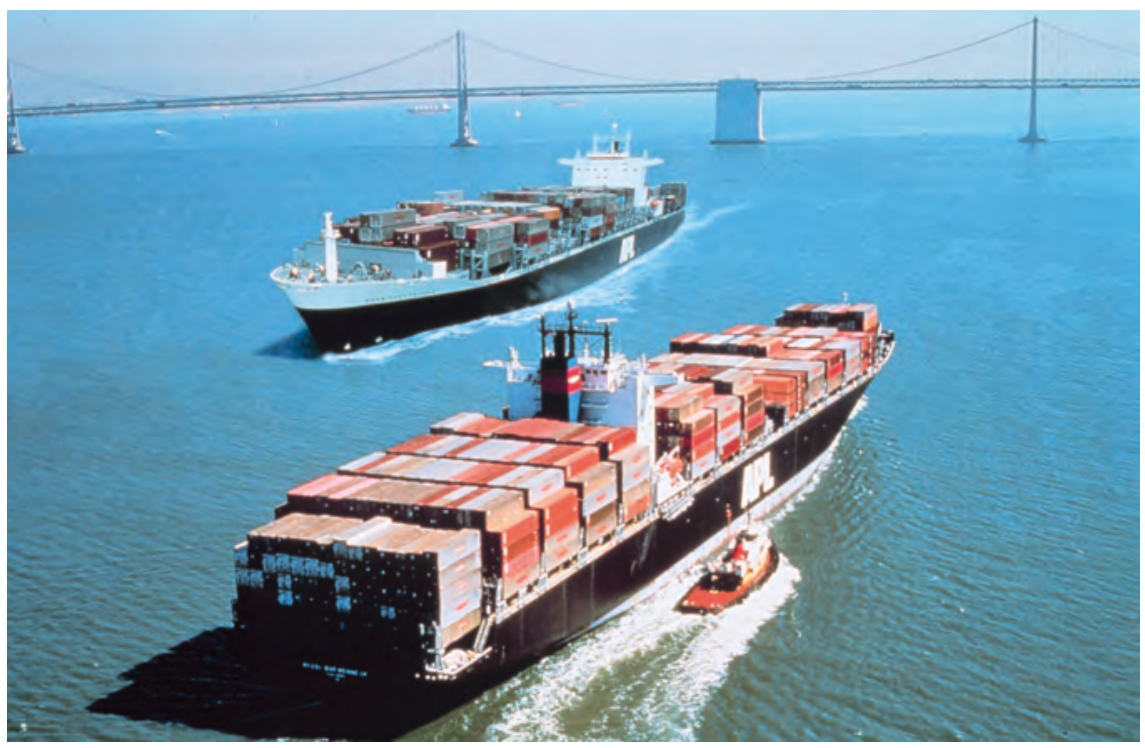

Illustration 32.6

Container ships in San Francisco, 1970s 
paid) jobs while reducing (but not eliminating) demand for unskilled workers. Theoretically, a combination of trade liberalization and retraining could make everyone better off. Note also that an interdisciplinary education may facilitate career changes of all types.

Trade remains an important conduit by which ideas move across borders though tourism and media now provide alternative conduits. A world that trades is arguably less likely to war - both because trade encourages understanding and because war will disrupt trade and this will be costlier the more that countries trade. Trade wars arguably encourage international hostility of other types. We might imagine, then, that trade brings political benefits alongside its economic benefits.

\section{Politics}

It would be a mistake to draw simple analogies between the financial difficulties that brought down most of the world's empires and the financial challenges that have often plagued modern states. For one, the share of taxes in GDP is much higher today: Agricultural productivity was too low through most of history to finance so much government expenditure. Second, many modern states have successfully paid down debts. We must look past the simple analogy to examine particular links. Have government bureaucrats or local elites managed to siphon off a higher and higher proportion of government expenditures in recent decades as they did in past societies? The answer is probably yes - bureaucrats like to expand their programmes, and special interests continuously seek favours from both bureaucrats and politicians - but recall that modern bureaucrats are better trained and selected and monitored than most bureaucrats in former empires. Some sort of mechanism that subjects government programmes to regular scrutiny may then be advisable. The simple fact is that citizens will support a state only if they believe that they receive more from the state than they pay in taxes. And they may, unfortunately, under-appreciate intangible benefits provided by many modern states such as the right to free speech. Taxpayers are understandably annoyed by examples of (apparent) wasteful or unfair government spending.

Though we should be careful of drawing lessons from the experience of ancient bureaucracies, we can note that governments have throughout history struggled to achieve their goals. Modern governments are engaged in a wide range of activities, and some of these - such as financial regulation - are incredibly complex. It is difficult for politicians, much less voters, to determine whether the right institutions are in place and whether these are appropriately enforced. Increased government transparency may allow interested voters to suggest better policies and practices in particular areas of government. The democratic challenge of our time is to encourage both politicians and voters toward careful consideration of well-defined policy options. As noted above, these need then to be carefully evaluated. 
The nation state is now assumed in international relations to be the "natural" form of governance. We have seen that it is a very recent historical creation and was, like any evolutionary development, not inevitable. Some have wondered if a return to empire might be a good idea in those parts of the world where ethnic diversity makes it difficult to achieve a stable nation state. We should not forget that - while many empires did set a useful precedent of cultural toleration - empires always, in the end, relied on force, and subject peoples often proved all too willing to throw off the yoke of empire when the opportunity arose. To be sure, many/most people in war-ravaged lands such as Somalia or Afghanistan might prefer an authoritarian peace to continued instability. We might nevertheless wonder if there is some way to achieve cultural toleration without resort to self-interested authoritarianism. One suggestion is some loose confederacy where each unit maintains its army and police, but there are rules that manage to limit internecine warfare. The Holy Roman Empire or early Swiss Confederacy might serve as examples. Such a system might not provide much guarantee of human rights within the units of the confederacy. And some international oversight might be required to maintain the peace. The alternative that most resembles the nation state would be a federal system with a centralized armed force but the devolution of many powers to local groups; this would require a strong sense of attachment to the institutions of the central state which might take some time to develop.

One lesson from history is that democratic institutions are most easily introduced slowly and depend upon constitutional protections of human rights (the United Kingdom has built up its protections of human rights slowly over the centuries; most other democracies have a constitution that serves this purpose). We do a disservice to the world when we over-sell democracy. The fact is that democracy is a frustrating form of governance: Dishonest, incompetent, and venal individuals are elected with some regularity. Such politicians often pretend that there are simple solutions to complex problems, and at other times avoid sensible policies that do not serve their personal interests. It can hardly be surprising that people who fight to achieve democracy in other lands are not always immediately impressed with the results. And if a constitution and bureaucracy - is not put in place that protects human rights, democratic leaders will often violate these. An independent judiciary - and especially one that protects freedom of speech and freedom of the press - can be particularly important in limiting the abuse of power by politicians. A return to authoritarianism may seem like a good idea to many if politicians abuse their power (the recent experience of Egypt leaps to mind here). And those with the ability to exercise force - including the state's own army - will always be tempted to take power if democracy is not entrenched. But democracy, once firmly established, does allow the public will to be reflected to a considerable extent in at least some of the state's institutions. And institutions can and have been put in place in many democracies that limit the opportunities for corruption. By recognizing both the strengths and weaknesses of democracy, we are better able both to improve existing democracies and to encourage democracy elsewhere. In 
both old and new democracies the focus should always be on achieving institutional mutations that promote selection for laws and regulations (that is, other institutions) that serve the public interest. As an example, this author would point to campaign finance reform: If the wealthy play a disproportionate role in determining electoral outcomes, we are likely to see significant deviations from the public interest in public policy.

There is scope also for less hypocritical foreign policies by democratic governments. When democracies support authoritarian regimes elsewhere, they invite local scepticism of democracy itself. There is some historical justification for trading with authoritarian countries: Economic advance may indeed encourage democratic reforms. World history can also justify an oftvoiced opinion that some autocrats are better than others, and that democracies are then justified in supporting relatively benign autocrats. But democratic governments should always make it clear that they promote democracy and human rights throughout the globe. A policy of propping up useful autocrats can be compared (carefully! - it is an analogy, after all) to a policy of bribing the "barbarians" at one's borders: It may make short-term sense but can have substantial long-term costs.

We have observed in much of history - and over the last decades - a worsening of income and wealth distributions in most societies such that a small minority controls an ever-larger share of national income and wealth. Such elites are commonly observed to use their economic influence to extract concessions from the state. In ancient empires this typically meant exemption from taxes on land. In the modern world it can mean a variety of tax breaks or government regulations. We have suspected that economic elites were responsible for the destruction of many empires in history: In extracting favours they weakened a state on which their own prosperity depended. The greatest single threat to modern democracy may be a perception that the institutions of the state are serving a small minority at the expense of the wider public. Addressing this threat may require both institutions that reduce economic inequalities and institutions that limit the influence of money on politics. It may also require a better public appreciation of the many expenditures that modern states undertake for the benefit of the entire population, such as schools and roads and hospitals.

A variety of policies have been advocated to reduce income inequality and poverty: changes in taxes and social policy most obviously but also shareholder democracy (to ensure that executive salaries reflect their contribution rather than their power), public works programmes for the unemployed, financial regulation (so that consumers are better aware of the risks associated with specific investment strategies), elimination of offshore tax havens, competition policy (to prevent firms from dominating markets or colluding), and limits to corruption (for some gain wealth through political connections and bribes). Some of these policies are controversial. Yet few would argue in principle against encouraging competition or fighting corruption. Unfortunately, history tells us 
that significant decreases in levels of inequality have tended to occur only as a result of some calamity (Box 29.5), and so we can imagine that it will be no easy task to change paths toward less inequality. Bold but sensible policies may have a better chance of success than the gradual tweaking of taxes and regulations.

It has been common in recent decades for politicians to gain considerable political followings by claiming outsider status and promising to reform their respective polities. Their popularity is sometimes misplaced. In particular, they often disdain the respect for the views of others that lies at the heart of democracy (we have urged a healthier response to dysfunctional debate above by encouraging better standards of discourse). Democracy depends on the virtues of tolerance and respect (and thus compromise), and we should be wary of politicians of any political stripe who treat the views of opponents with disdain. The popularity of such "reformers" does also reflect a popular understanding that some institutions are serving politicians and their friends better than the broader public. The challenge for voters is to back reformers who will actually make changes in the right direction. Voters must first of all gauge the integrity of reformers: History is full of demagogues who only claimed to have the people's interests at heart. Voters must also ask what particular institutional changes the reformer hopes to make, and then evaluate the likelihood that these will work. Perhaps the key lesson of history is the importance of somehow generating and selecting the right institutional mutations: We must, in particular, seek ways to get politicians to restrict political abuses and not reward their friends economically.

Given the difficulties in getting elected politicians to serve the public interest, there may be scope for resuscitating a practice pioneered by the ancient Greeks: choosing some officials by lottery. Such individuals would not be beholden to those who helped them get elected nor concerned with re-election. The question is whether they could quickly gain enough knowledge to be able to advocate for appropriate institutions. Here the availability of reasoned analysis of public policy options may be crucial.

Selection by lottery may help address a fear that politicians are no longer held to any standard of truth or justification for the policies they advocate. If every opinion is judged to be equally valid, then democratic debate is unlikely to yield better policies - and then suspicion of authority will only be enhanced. We discussed above how both scientific and public debate might be improved. Officials selected by lottery might serve as an essential voice of reason. They would have the time and incentive to evaluate the evidence dispassionately.

We have focused so far on democratic decision-making. Democracy also requires a state bureaucracy that can exercise the public will once this is revealed. The democratic challenge is to wield this necessary state power so that human rights are respected, and general prosperity encouraged. New technologies increase state power by facilitating surveillance of all human activities. This power can be employed to useful ends such as crime reduction, but it might also be abused. The abuse of state power can only be prevented through 
institutions that provide transparency in how power is exercised and a population that monitors state activity.

History is full of surprises. Most empires at the height of their power would likely have discounted predictions that they would disappear in a matter of generations. The "victory" of liberal democracies and market economies is very recent in the history of the world. They seem to many to be obviously the best form of governance. There is a danger that citizens do not always appreciate how successful democracies depend on many characteristics, including: respect for the views of others; freedom of speech and of the press; constitutional protections of human rights; constitutional limitations on the exercise of power by any individual or group; a strong sense of shared purpose; a shared belief that informed citizens can achieve desired outcomes; a belief that the state actually acts as the public would wish it too, at least much of the time; a feeling that the voices of all groups - classes, ethnicities, genders, and so on - are and should be heard; and a belief that it is possible to have reasoned discussions grounded in evidence that serve to identify superior public policies (that should then be carefully evaluated). There must be some general acceptance of the institutions of the state - and especially its laws and judges and electoral authorities - though individual institutions should be examined for possible improvements. It is easy to find examples where each of these pillars of democracy is questioned today - and arguably far more than they have been threatened for many decades. If toleration is seen as weakness, a constructive scepticism is replaced by blind belief, and identification with one group or individual supersedes the desire to forge better outcomes for all, then democracy is threatened, perhaps critically. Those who have never experienced authoritarian rule may chafe at the frustrations of democratic government the seemingly endless debate, the regularity with which fools and thieves are elected, the difficulty of keeping track of (much less forming educated opinions regarding) the myriad policies that modern states pursue - without fully appreciating the far greater frustrations of authoritarian rule.

Though democracy advanced in the late twentieth century, billions of people still live under authoritarian governments. Are revolutions against authoritarians a good idea? The historical record is decidedly mixed. The American Revolution - perhaps in large part because it changed the nexus of power but maintained most institutions - would generally be thought to have had beneficial effects (but we have seen that reasoned disagreement is possible on this point). Many other revolutions in human history resulted in massive bloodshed and ended in authoritarian government. So, at the very least, revolution must be seen as a risky proposition. Potential revolutionaries then have a special duty of self-knowledge for they can so easily do more harm than good. Where institutional evolution is possible, this may be the safest course. If dramatic change is necessary - and again great care needs to be taken in reaching such a conclusion - great care must also be taken to ensure that a better institutional structure can and will be put in place. It is unfortunately far easier to rail against present injustice than to build a better future. 


\section{War}

We can turn our attention now from domestic political institutions toward consideration of that frequent feature of world history: war. What is the future of war? On the one hand, the last century has witnessed the most devastating wars in human history, and deadly conflicts continue in many parts of the world and are threatened in many more. On the other hand, many parts of the world have lived in peace for decades, and there has been a downward trend in the frequency of wars both in the last decades and centuries. Nuclear deterrents may or may not keep the largest states from engaging in open confrontation. Changes in relative economic position (and population) have encouraged wars in the past; perhaps in an age where colonization is no longer seen as necessary for economic growth, this link can be broken in future. However, shortages in natural resources or drinking water might encourage military action, and any perceived unfairness in the rules of international relations or their application might be used to justify war. Convergence in average incomes across countries may lessen the chances of war - but in the meantime, increased awareness of international income differentials through global media may exacerbate tensions. Democracies are generally less likely to fight each other than are authoritarian states, and so the spread of democracy might decrease the likelihood of war in future (but the spread of democracy itself may at times be aided by the use of force). If most of the world were democratic, we might see a strengthening of institutions of global governance. Global governance will be encouraged by global challenges such as climate change and overfishing, and maybe even the discovery of extraterrestrial life. In the meantime, care must be taken to limit the rewards for belligerence to governments (and others) who are tempted to achieve their ends by violence.

Wars have often had primarily economic motives in the past, but nationalism and religious fervour have played a significant role. The future of war thus depends in part on addressing nationalism and religious conflict. This book has hopefully illustrated our common humanity, and the simple fact that humans have faced similar challenges and often responded in similar ways across time and space. One lesson we might learn is that humans have crosscutting identities: a merchant in one country may have much in common with a merchant in another who speaks a different language and follows a different religion. International organizations that link people with shared occupations or interests can serve as an antidote to ethnic and religious tension. Less obviously (and more controversially), a shared global consumer culture may help to remind people of their shared humanity.

If such cross-cutting connections are important, then the question should be engaged of whether individuals should be better able to choose their states (and vice versa) on the basis of cultural or institutional preferences. This idea might seem a natural extension of notions of popular sovereignty, but there are obvious practical difficulties in achieving it. Moreover, it threatens new grounds for international hostility. 


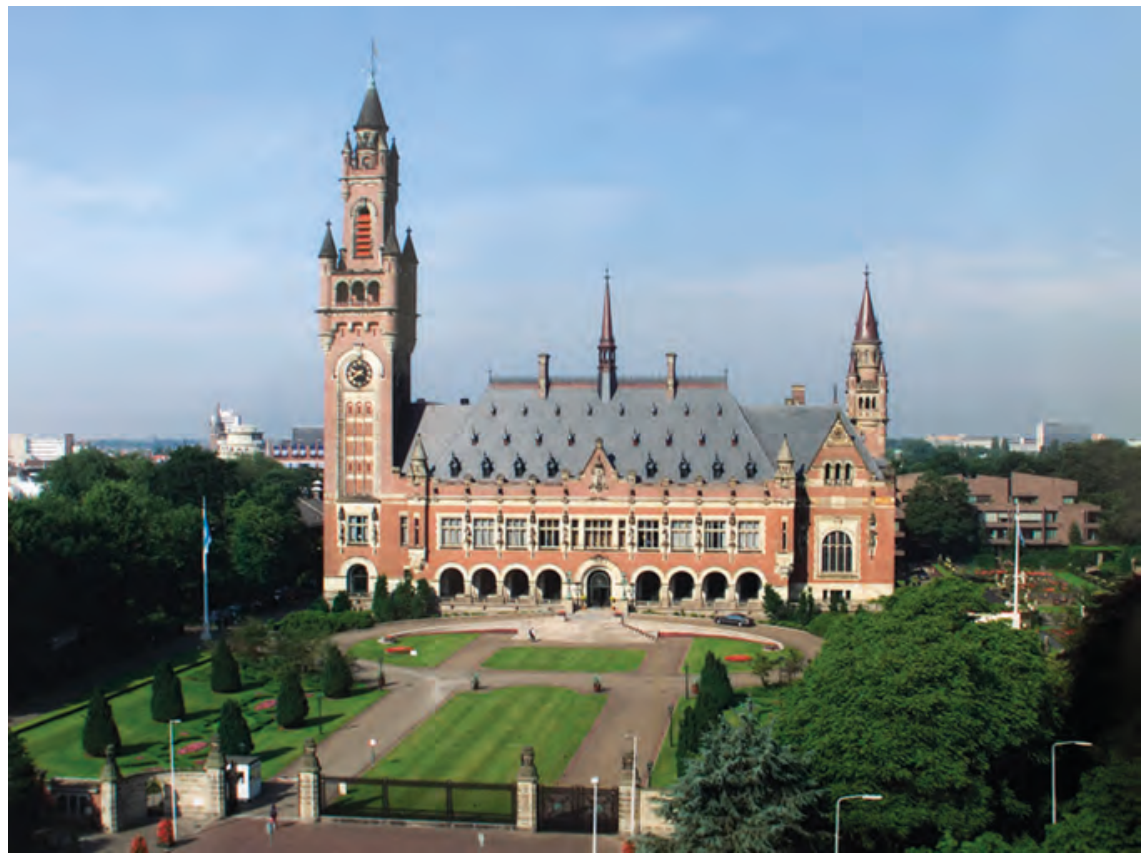

Illustration 32.7

The Peace Palace in The Hague, Netherlands, which is the seat of the International Court of Justice

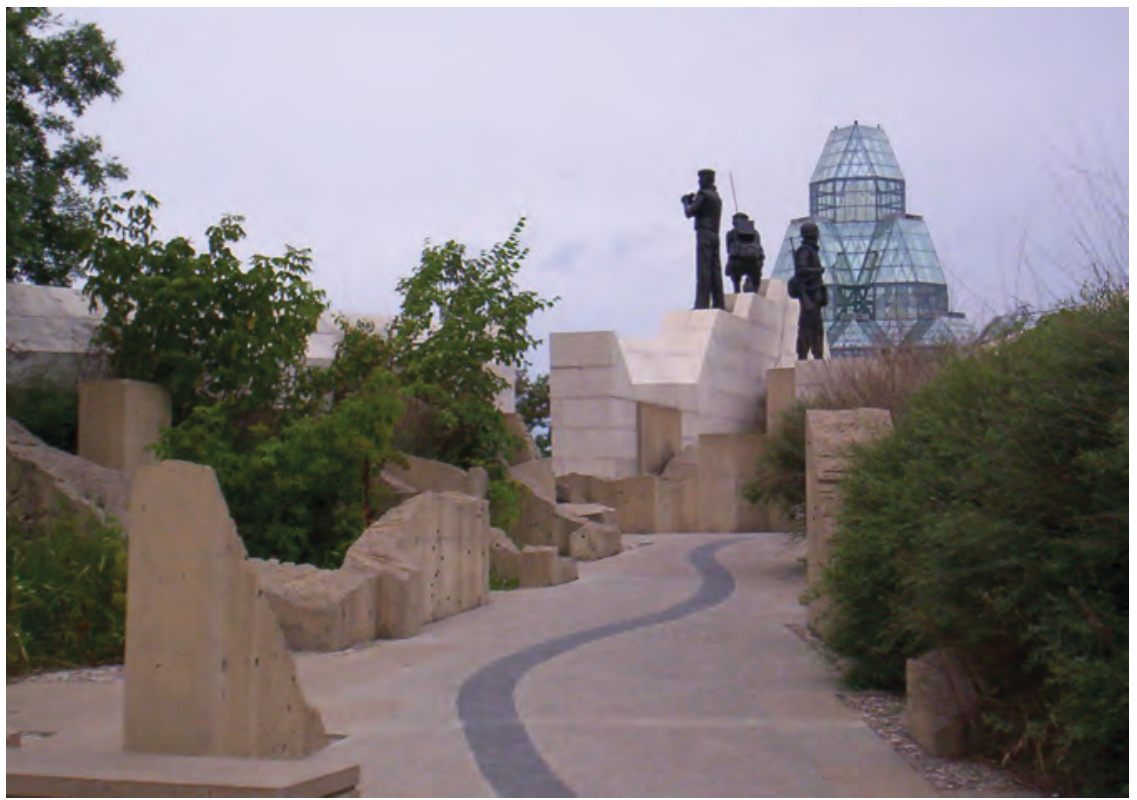

Illustration 32.8

Peacekeeping monument, Ottawa (National Gallery in background) 


\section{Changing paths}

Political revolutions are the most dramatic way in which a society may change evolutionary paths. There are other sorts of dramatic change that may prove beneficial and are far less risky. Societies throughout the world have created an array of social programmes over the years. Each is laudable in its way, and most do much good for the people they serve. Unfortunately, the result in most countries is a heap of overlapping programmes offered by different levels of governments that are difficult to navigate for those who need assistance. These programmes often also interfere with recipients' incentives to work. The bureaucrats running any particular programme, and those they serve, tend naturally to laud it. One institutional innovation would be to introduce a guaranteed annual income (sometimes called a basic income or negative income tax): All adults would receive money from the state if their incomes fell below a certain level. Such a programme would not replace all social programmes we would likely still want special initiatives for those with physical or mental impairments, for example - but could replace many of these. Notably, this idea has support from both conservative and progressive thinkers: This reflects the simple fact that we (almost) all want to help the less fortunate and to do this in the most effective way possible. It is not politically easy to replace a set of programmes offered by different levels of government with one streamlined scheme. But many academic estimates suggest that such a change would provide better service at lower cost. If true, then such a change would not only help the less fortunate but reinvigorate popular support for the society's institutions.

There is also support from some conservative and progressive thinkers for some sort of public works programmes for the unemployed such as have been pursued by many states in the past, notably during the Great Depression. Such programmes have a powerful advantage over welfare in that they cannot be (seen to be) so easily abused by the lazy. A combination of basic income and public works would provide a much more solid social safety net, and might well cost less than the existing mélange of programmes. It would also likely appear to be fair to a broad range of voters, including many that are suspicious of present welfare policies. Voters freed from the fear of falling into abject poverty might change their views on a host of public policies (we discussed fear above).

This example of changing paths is particularly important. Poverty and income inequality are associated with slower economic growth, crime, and political detachment and corruption. Institutional changes that alleviate the suffering of the least fortunate are then critical for both economy and polity. We noted the political dangers of increased inequality above. It should be appreciated that there are also economic costs. Though some inequality can be useful - for some people may choose to work harder than others - concentration of income and wealth within a small elite may slow economic growth, for 


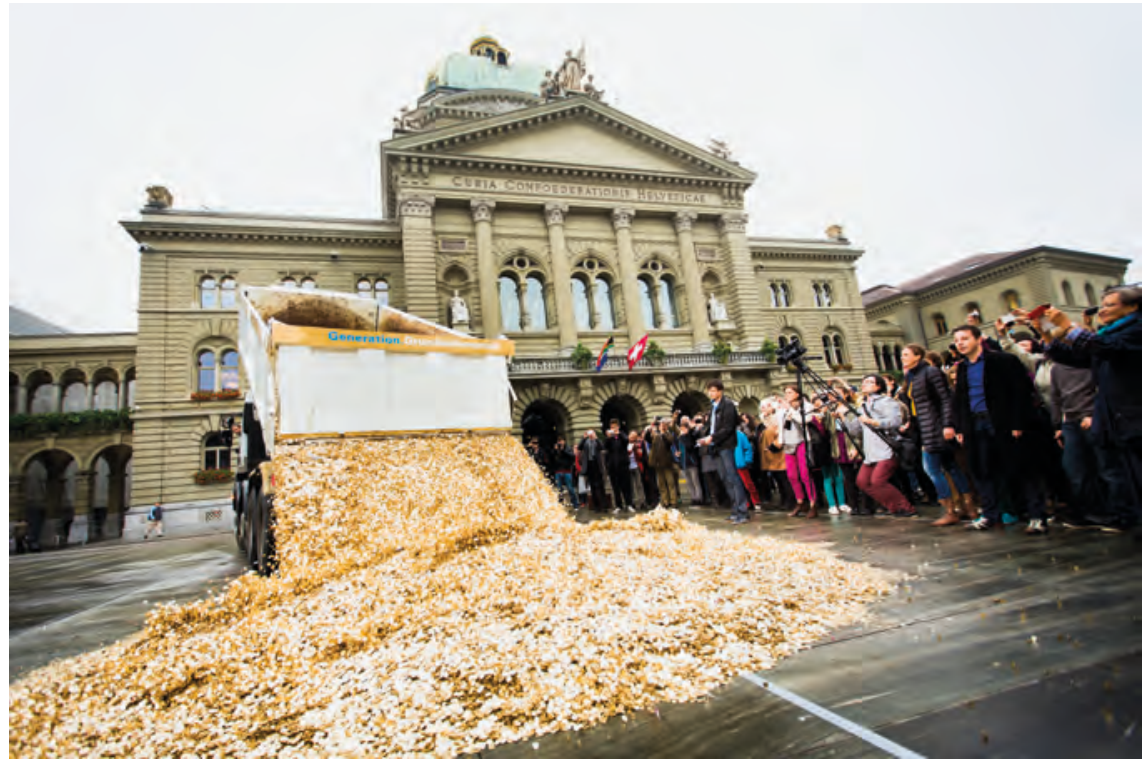

Illustration 32.9

Swiss activists dump 8 million coins - one per citizen - in a public square in Berne in 2013 as part of a failed campaign to require a basic income in the Swiss constitution

the very rich spend less of their income than the poor. Supporting the income of the least fortunate benefits local economies since the poor spend much of their income on food and shelter.

\section{Population}

Throughout most of the world, the fears of a global "population explosion" have dissipated in recent decades, as population growth rates have fallen or are falling to at or below the rates that maintain population stability in most countries. However, population growth rates remain high in some parts of the Middle East and Africa. There is considerable evidence that population growth rates above about 2 per cent per year are associated with slower rates of increase in per capita incomes. So these countries will likely suffer economically from rapid population growth. It could be that as women gain education and influence in these countries, birth rates will fall there as well. For the world, it is likely that we can absorb (feed and clothe and house) another billion or two people - unless climate change or some other ecological disaster dramatically reduces world agricultural output. New agricultural technologies including biotechnology and sensors that tell farmers the water and nutrient content of small sections of their fields so that they can target irrigation and fertilizer - may allow yet further advances in agricultural productivity. So also 
will the spread of the latest technologies from rich to developing countries, and increased research on tropical crops (chapter 31). The costs are falling for "vertical farming" in which crops can be raised indoors using artificial lighting for photosynthesis - though it remains to be seen if this technology will ever serve more than a luxury market.

Modern medicine has conquered - or at least dramatically reduced the incidence of - many diseases, including those that caused all of the major epidemics in world history (we think). Yet the combination of dense populations, close contact with animals in many parts of the world, and international air travel mean that global epidemics occur with some frequency. So far, the diseases that are most deadly have not been those that spread rapidly among humans. Yet the risk remains of a severe global calamity. Climate change and expansion into rainforests may increase the risk by bringing previously separate species into contact. Greater global cooperation in identifying and combatting the first outbreaks of new diseases would be salutary, as would efforts to combat conditions of poverty that are particularly conducive to the spread of disease. Enhancing public health provision in developing countries would improve many health outcomes while also allowing early detection of new epidemics. Sharing data between health authorities would also improve our ability to detect new threats. And while it is not possible to develop vaccines against unknown diseases, we can develop vaccines against known threats.

Postscript: The preceding paragraph was first drafted before the Covid19 pandemic that began in 2019. That pandemic has signaled the havoc that can be wreaked by a disease that is both easily transmitted and deadly. The world proved itself to be ill-prepared. Even the richest countries had failed to stockpile masks or ventilators. International cooperation was less wonderful than it could have been as rich countries engaged in bidding wars over available supplies. Will the world be better prepared for future pandemics?

In much of the world, we face a relatively novel situation of population decline and an ageing population as birth rates fall below mortality rates. History provides little guidance as to what such a society may look like. There will undoubtedly be costs imposed on those of working age in caring for the elderly, but this may also mean low rates of unemployment. There is some evidence from recent decades that the lowest birth rates occur in countries where women have entered the workplace in large numbers since it is difficult for parents to juggle work and parenting responsibilities; countries with policies that facilitate both careers and parenting may be able to achieve a stable population through time. 


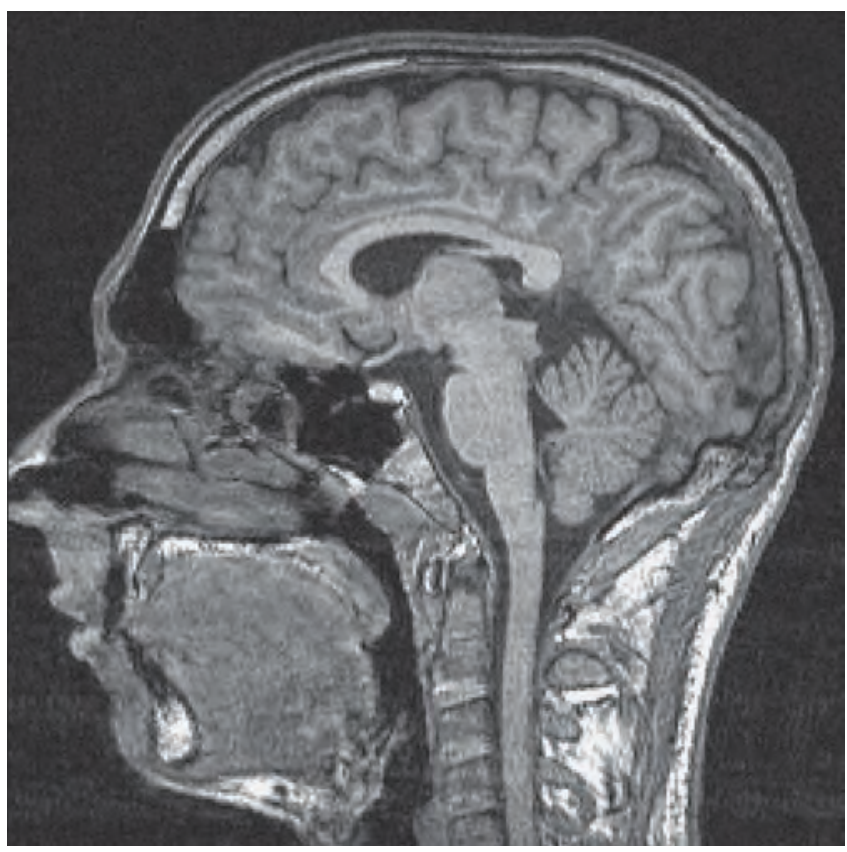

Illustration 32.10

Magnetic resonance imaging (MRI) scan of the human brain Erik1980, CC BY-SA 3.0

Will humanity continue to extend life expectancy? It is even possible that technologies may be developed that halt ageing. Humanity would have to change its attitudes toward careers and retirement in such a world. Religions might be transformed if lengthy life expectancies reduce concerns with an afterlife. What sorts of social conflicts might arise if only some can afford to extend their lives for decades?

\section{History itself}

We can briefly reflect on how History itself was pursued in the past and how it might be pursued in future:

- We can reasonably speculate that History has existed as long as humans have been able to talk. Humans have wished to understand where they came from and engaged early in both religious mythmaking about how the universe formed and oral history about the actions of (generally) their ancestors. Oral histories can of course change through time, and likely then come to resemble what the group wished had been their past.

- The advent of the written word made it much easier to record complicated histories. Written records also provided a documentary basis on which later historians could construct histories. 
- Political and religious authorities played a dominant role in constructing histories at many points in time. This was especially the case when only an elite was literate.

- Histories have generally been about one's own group, but there is a long minority tradition of what we would now call world history: Herodotus and Rashid al-Din are just two examples of attempts to record the history of all known groups.

- Though we can applaud all efforts at remembering, we can be especially appreciative of contemporary histories that are produced by the interaction of many different people from many different backgrounds, that strive to ground their accounts in historical evidence, that pursue contextualized generalizations (e.g., bad harvests encourage revolution, but especially in concert with other factors), and that try to place the history of any one group in global context.

- In teaching History, we should not shy away from complexity. We should embrace all possible thematic interactions. We should evaluate the strength of arguments and evidence for competing interpretations, and seek to integrate different perspectives. We should strive to draw lessons but be humble in doing so.

We are much better able to guide ourselves to a better future if we understand our past.

\section{Thoughts on our organizing structure}

It was argued in chapter 1 that we should seek an organizing structure for world history that has a potential place for the insights of every historian. We achieved this goal by outlining an exhaustive classification of individual and society-level phenomena and by stressing the importance of interactions among these. Though we have not, of course, been able to trace every possible interaction through history in this book, we have had cause to mention a large number of possible influences from one phenomenon or theme upon another. As stressed in chapter 1, it would be possible to find a place in our history for any interaction that any historian might uncover.

Providing an organizing structure for all of world history is itself a bold enterprise. But why stop there? If it is possible to organize all of history, then it must likewise be possible to organize all of human science inquiry. This, after all, is an attempt to understand the same set of phenomena and relationships. As the celebration of interdisciplinarity within world history (see chapter 1) testifies, there should be an interchange between historical research and human science research. World history can usefully be informed by other disciplines (though care must be taken to ensure that the historian does not become so enamoured of any theory that they misinterpret the historical record to fit). The human sciences, which tend to focus the vast bulk of their attention on the last couple 
of decades, will develop more accurate understandings if informed by history. As one key example, historians could usefully employ modern understandings of personality dimensions in their studies of historical figures, and psychologists could then draw upon historical analyses in appreciating the behaviours and effects of different personality types.

A historian interested in phenomenon $\mathrm{X}$ or how phenomenon $\mathrm{X}$ influences phenomenon Y could usefully learn what scholars in other disciplines have had to say about that phenomenon or relationship. And scholars in any human science discipline will likewise benefit from knowing both what historians and scholars in other human sciences have had to say about any phenomenon or relationship they wish to study. Although we have interacted less with the natural sciences (but see chapter 2 and frequent discussions after that of genes and environment), it is straightforward to extend this argument to them as well. Indeed, one of the purposes of Big History (chapter 2) is to link the natural and social sciences. The sort of coherent organization recommended in this book can achieve this goal better than a set of analogies.

We noted in chapter 1 that we are not able to follow all phenomena or relationships in our history. In part, this was because there is a lack of evidence for some of these. But in part, it reflects a lack of historical investigation. One purpose of an organizing system is to encourage historical research in areas that have received little attention; new insights can then be added to prior understandings. For example, we have suspected that art moves us to action; we could know much more about how this has occurred historically. The same logic can be extended beyond History to the entire scholarly enterprise.

Scholarship as a whole would benefit if our libraries - both digital and physical - were organized around phenomena and relationships. To be useful, this must be a universal or general classification of phenomena and relationships: We will benefit little if each discipline has a unique terminology. In practice, though, our libraries differ from the ideal in two crucial ways. Most obviously, different terminology is employed in different disciplines. Some scholars think that this is inevitable and even desirable, but arguably it is entirely feasible to develop a general classification of phenomena and relationships. Indeed some projects to do precisely that have advanced quite far (the author of this book should confess involvement in these projects and indeed authorship of such a classification). The second deviation is that we do not, in general, take a "synthetic" approach to classification that would facilitate a search by (phenomenon X)(exerts effect $\mathrm{N}$ on)(phenomenon Y). Rather we "enumerate" a significant subset of such possibilities, so that the user has to guess or ascertain how a library might name such a relationship. Synthetic approaches are often recommended within the theory of information science - and are increasingly feasible now that library catalogues are everywhere computerized - but the classification systems that dominate the world were created over a century ago and have proven challenging to revise. 


\section{World history and the meaning of life: How this book might shape your view of life}

The history of the world has evolved through the interactions of billions of individuals, each pursuing various goals and each faced by multiple challenges. We have in this book spoken a great deal about the challenges but less so about the goals - beyond the basic human needs for food and shelter, and certain psychological temptations that humans commonly face. We reflect here on what world history might tell us about the purposes of human life.

What lessons does world history suggest regarding the meaning of life? Our starting point in answering this question is a widely accepted view that there are multiple sources of meaning in life. We can, therefore, explore how history informs different sources of meaning. We will need to combine historical and philosophical understandings to do so.

It could well be that the search for meaning is far more important today than ever before. Though it is hard to know for sure, it is likely that most humans who struggled from day to day to feed their families did not devote enormous energies to reflecting on the meaning of life. They did have recourse to religion, as we have seen, and for a variety of reasons (chapter 8), but may have engaged in little metaphysical reflection beyond that. In small societies with strongly shared religious and cultural beliefs, most individuals may simply have accepted a communal sense of meaning. One needs leisure time to reflect on meaning. It may well be that it is abundant leisure time that drives some today to conjecture that life lacks meaning. It can be soul-destroying to have free time and nothing to do with it. Some people may then fill their days with activity simply to avoid reflecting on meaning. History may point us in better directions.

\section{We can achieve meaning by striving to make the world a better place}

\section{The role of individuals in history}

We have suggested above that our understanding of the past can inform our understanding of the future, albeit imperfectly. It follows that our understanding of the past may improve our ability to affect the future in desired directions, but that we should act with humility. We suggested then some strategies that humanity might pursue going forward. Our task here is to address the more general question of whether humans can achieve good in the world.

Does human agency matter in history? As noted in the first chapter, it is all too possible to tell a world history in which individuals fade into the background. Impersonal forces did indeed play a significant role in our history. 
Individuals often seemed caught in a web of forces beyond their control. Like the good people of Pompeii, they can only recoil in horror as the volcano Vesuvius moves against them. However, people usually have some choices, and these do have an impact on the course of history. We have traced in this book how various human actors addressed the challenges and opportunities they faced and examined the results of varying choices. We have also discussed at many points in this book the idea of path dependence (without always using the phrase): In the complex interplay of causal forces that is history, slight changes in culture or technology or institutions can change the path we collectively follow. History may at times seem like a giant boulder rolling down a hill. Nevertheless, humans can and do alter its course in many ways.

To be sure, humans do not always achieve the change in course they desire. Often we push in one direction only to achieve the precisely opposite result. Fascists railed against the dangers of communism but set the stage for communist expansion into (at least) Eastern Europe (though this expansion proved temporary itself within the long sweep of history). More generally, human actions often have unforeseen consequences. Early experimenters with radio waves never imagined public broadcasting. Early experimenters with fire did not imagine they were changing the future in myriad ways. Yet the mere fact that humans have imperfect foresight is a rationale for humility and experimentation rather than inaction.

Have humans been selfish or altruistic in history? The clear answer is both. Many actions in human history are best understood as selfish efforts to gain wealth or power or status. However, many others - remaining in a battle that seems to be lost, devoting time and energy to an innovation that one cannot expect to profit from, risking one's life to topple an autocrat - cannot easily be explained with recourse only to selfish motives. Happily, the multifaceted approach to meaning taken in this chapter urges individuals to be self-aware about their motives, and will have a place for both self-oriented and otheroriented behaviours.

\section{Human progress?}

It seems worthwhile to reflect on whether the combined efforts of billions of humans over the millennia have had any good effects. We could enhance our confidence that our actions today might lead to a better future if we could identify progress in the past course of human history.

Explorations of human progress have often fallen into the "big think" trap decried for world history itself in the first chapter: One or two aspects of our world are ascribed priority, and judgements reached about progress by looking only at them. Yet if history is seen correctly as the complex interaction of a large number of phenomena, it seems that we should seek a more diverse evaluation of human progress. It is too easy to focus on economic output and celebrate human achievement, or emphasize biodiversity and see only decline. Table 32.1 provides a survey of human progress by theme. 


\section{Table 32.1 Human progress by theme}

Genetic predisposition

Individual differences

Politics

Economy
Though human genes may not have not changed much in historical time, there has been an improvement in our actualization of inherent human capabilities through improvements in health and especially nutrition.

Moreover, we now appreciate that maternal health shapes human capabilities in significant ways. Our genes may have changed little, but hormones in healthy mothers activate these in better ways.

Contemporary studies of happiness suggest that the satisfaction of basic needs for food and shelter, health, and security are important contributors to happiness. It is not clear, though, that economic growth beyond fairly low levels has much effect on happiness. (There are, we should note, important concerns regarding the reliability of surveys of how happy people think they are since there is no way that individuals can objectively measure happiness.) We can identify progress in happiness over the last millennia as average incomes slowly increased, but perhaps not in the most recent decades in rich countries. It may well be that humans evolved to not be completely content: Our hunter-gatherer ancestors would have benefited from always striving for new accomplishments. There may then be a limit to human happiness (at least without pharmaceutical or genetic intervention). We can also identify progress regarding various individual-level phenomena: psychological understanding, freedom of choice, respect for diversity, and perhaps decreased anxiety levels - but regress concerning occupational over-specialization and the incidence of depression. Average scores on intelligence tests have risen in the last half-century by some 20 per cent in developed countries, reflecting improvements in nutrition, maternal health, and perhaps reduced lead levels in the environment.

There is in much of the world more freedom - religious, political, and occupational - than ever before. There is also more justice: limits on state power, courts that are independent, and so on. Most people would view democracy as superior to other forms of governance, albeit often frustrating in action. One can identify improvements in a range of institutions (but worry that there is nevertheless a confusing welter of institutions today). There have certainly been dramatic improvements in both public infrastructure (such as sewers) and education in most countries. With respect to war, the record is mixed: the last century or so has witnessed the worst wars ever, but we may now be seeing a decline in the incidence and severity of war. Certainly, attitudes against war have hardened (perhaps too much in some instances).

There has been dramatic growth in average incomes. Income distribution between countries worsened in the nineteenth century dramatically, but may now be improving. Income distributions within most countries have oscillated but have deteriorated in recent decades. The general tendency in history may be for income distributions to become more unequal, except during major shocks such as epidemics or the most severe wars. Leisure time has increased. (We saw above that this may be a mixed blessing.) Work fulfilment has improved for some but not others. There have been improvements in some economic institutions, but evident weaknesses in others. 
Art

Social structure

Technology and science

Health and population

Culture

Non-human environment
There has been increased artistic production, though this has become an increasingly specialized activity. It is not clear whether artistic quality has improved or declined. The total number of artistic inspirations has increased dramatically. Most people would view the contemporary diversity in artistic styles as a good thing. Nevertheless, we can worry that art has a less central role in modern society.

There is decreased gender stratification in most but not all countries. There has been a decrease in the importance of inherited status (though this trend is less visible in recent decades). It is harder to identify trends in ethnic or occupational stratification, though there are some signs of improvement.

We have developed technologies that allow both increased leisure and a more extensive range of goods and services. There is nevertheless cause to worry about the environmental, military, or social implications of many of these technologies. There have been dramatic advances in our understandings of natural (and social) processes, though further research may alter many of these.

The population has grown - though this is not entirely a good thing. Life expectancy is much higher in most parts of the world than a century or two ago. Rates of child mortality, in particular, have fallen dramatically. Nutrition is better and diseases less virulent - though there is always the possibility of a new epidemic.

Individuals can increasingly choose their cultural identity. However, sense of community (and shared stories) may be declining (though this may have a beneficial impact of reducing hostility between groups). Most people might applaud various cultural changes such as increased sexual freedom, support for ambition, curiosity, valuing romantic love, and religious freedom, but could reasonably worry that values such as honesty and responsibility are waning. Linguistic diversity is decreasing (though people disagree about the effects of this).

Transport infrastructure has improved, as has the human ability to predict and cope with natural disasters (though increased population densities work against this). Climate change and biodiversity are definite areas of regress. More local types of pollution have fallen in many countries. Human experience of nature has declined.

There has been human progress across each of the themes pursued in this book - but also important instances of regress. Some, though far from all, of the evaluations in Table 32.1 involve values about which people might disagree: Not all see increased personality diversity as an unmixed blessing, for example. Yet most people would probably agree regarding most types of human progress in the table. Where progress is observed, it is often (though again, not always) found over both more recent decades and centuries as well as over the millennia addressed in this book.

Did this progress result primarily from human actions or impersonal forces? Scholars have not often asked or investigated this question. However, if we 
accept the argument above that both have shaped history as a whole, then it seems likely that both have shaped human progress. Our discussion of the move toward gender equality in chapter 27 is indicative: Changes in technology were significant but so also were the tireless efforts of an international network of women and men advocating change. Moreover, of course, changes in technology themselves involve human agency.

\section{PRIMARY DOCUMENTS: OSWALD SPENGLER ON HUMAN PROGRESS}

Oswald Spengler, in The Decline of the West, 2 Vols., translated by Charles Francis Atkinson, (New York: Alfred A. Knopf, 1922), foresaw a bleak future:

The idealist of the early democracy regarded popular education as enlightenment pure and simple-but it is precisely this that smooths the path for the coming Caesars of the world. The last century [the nineteenth] was the winter of the West, the victory of materialism and scepticism, of socialism, parliamentarianism, and money. But in this century blood and instinct will regain their rights against the power of money and intellect. The era of individualism, liberalism and democracy, of humanitarianism and freedom, is nearing its end. The masses will accept with resignation the victory of the Caesars, the strong men, and will obey them. Life will descend to a level of general uniformity, a new kind of primitivism, and the world will be better for it ...

Why was Spengler so pessimistic (note when he was writing)? What does this quote tell you about how attitudes toward progress can change through history?

\section{Why bother?}

If one accepts that humans have and can act to achieve human progress, one might still wonder if this gives meaning to life. One potential objection is that there is unfairness in progress: Why should people lead better lives in the future than we do today? A variety of answers is possible: Parents, when given the opportunity, generally strive to give their children a better life; human compassion guides us to wish a better life for others; the individual human desire for betterment might reasonably be extended to the species as a whole.

A more metaphysical question is whether human progress is itself meaningful. I would suggest that this is a rich-world deceit. Parents huddled in refugee camps, or ravaged by civil war, or just struggling to nourish their children, would have little time for the argument that human progress is not worth fighting for. Human empathy should guide us to value progress. Even if we may struggle to put meaning in our own lives, we should respect the simple fact that others (and future generations will) find meaning in the help we might give them. 
This particular source of meaning in human life may diminish as progress proceeds, of course. Each improvement perhaps reduces the scope for further improvement. We might then agonize about regress in this source of meaning as human progress advances. Again, this is a conceit of the comfortable that should hardly excuse inaction in the world. We cannot know what humans are capable of if freed from some of the challenges we face in contemporary society. Nevertheless, we should seek additional sources of meaning in life beyond encouraging human progress. (The likelihood that humanity, like all other species, will one day disappear, though not perhaps for billions of years, should also guide us to seek other sources of meaning.)

\section{Can we do good?}

We have had many occasions in this book to examine human folly. Humans trying to achieve one end inadvertently act to achieve precisely the opposite. There is certainly a lesson here: By pursuing goal A you may energize those who oppose that goal. This will be especially the case if you pursue your goal in a way that seems arrogant or unfair. The lesson I would draw is that process is important, and that acting in a consultative manner that respects the interests and values of those you disagree with increases your chances of having lasting effects in the desired direction. Yet I appreciate that this may not be enough in all cases. In particular, it is entirely possible to merely make mistakes or misjudge the side effects of your actions. The lesson here is to be careful and humble. And self-aware: Humans have an amazing capacity to convince themselves that their selfish acts are socially beneficial.

It would be all too easy to draw a more negative conclusion: It is best not to bother because however well-intentioned one is, one can as easily do harm as good. It is hard to imagine a more powerful excuse for inaction in the world (though doubting the value of human progress would be as powerful). To be sure, humility, judgement, and self-awareness cannot provide complete assurance that one's well-intentioned acts will bring positive results. Yet the probabilities are then solidly in favour of good results.

It is, after all, much easier to do harm than good in the world. One can steal, lie, vandalize, assault, terrorize, or otherwise do harm to others without a great deal of planning or forethought or expertise. It is amazingly easy to hurt others. To help others requires an understanding of their needs and their situation. Part of the temptation to do harm in the world may indeed come from the fact that people want to make a difference and find that doing good is difficult. Our subconscious minds can condone such behaviour (convincing us, for, example, that those we hurt deserved it) or even convince us that we are indeed doing good. Yet if we accept that there has been progress in human history, then it would seem that we have nevertheless managed to do good more often than harm - or at least that our do-gooding has had more lasting effects than our do-harming. We might, as noted above, attribute progress primarily to impersonal forces in history. Yet despite our embrace of evolutionary 
analysis in this book, it is still the case that every good idea had to be put forward by someone.

We mentioned self-awareness briefly above. We saw in chapter 3 that humans were selected for an ability to self-deceive. We have seen in later chapters many examples of humans acting in ways that they would not have consciously approved, with generally deleterious results for both themselves and others. We have therefore urged self-knowledge at multiple points above. Philosophers across all major philosophical traditions have recognized the ethical importance of self-knowledge. Fortunately, there are conscious strategies that we can each pursue to enhance self-awareness. One is externally oriented. We will receive in our lives much advice and criticism from others. We should not automatically assume this advice is either good or bad: We will receive honest advice and dishonest advice, well-considered criticism and ill-advised criticism. It would be a mistake to ignore it all. One of the surest paths to self-ignorance is to react with anger to any external advice, discouraging even your closest friends and family from bothering to provide guidance. If we consciously analyse the advice and criticisms of others, we will often find that they expose some of our behaviours as less laudable than we had otherwise assumed. The internally oriented path to self-knowledge involves asking ourself regularly why we did what we did, why we felt the way we did, and what were the actual effects on others of our acts. This path to self-awareness takes time. It is natural for teenagers, with the physiological and psychological changes they undergo, not to know always why they act out or feel angry or sad. It is disappointing when someone in middle age still has no conscious understanding of why they do what they do or feel what they do, or how their behaviour affects others. A person who lacks self-knowledge will rarely do good in the world. Indeed, we have seen that people who thought they were justified have perpetrated most of the evil in the world.

It is reasonable to speculate that the evolutionary balance struck between honesty and dishonesty during the time of hunting and gathering is less well suited to complex societies: It is harder today to detect or discipline the dishonest acts of others. We can each reasonably expect, then, that external

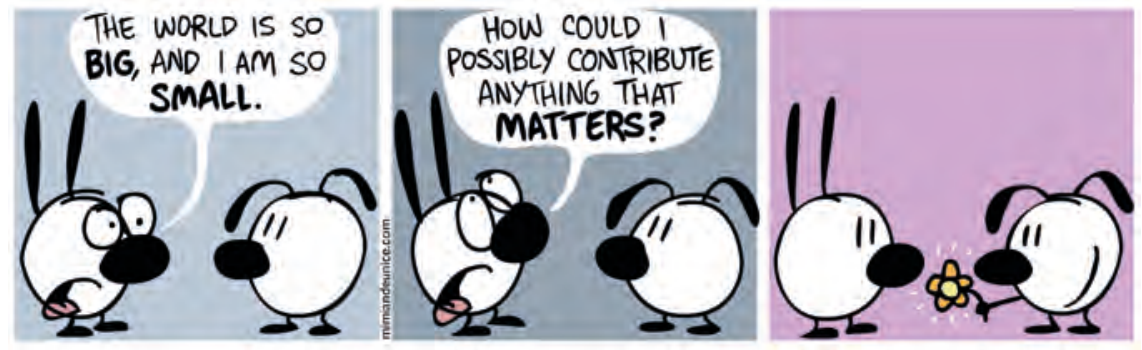

Illustration 32.11

Small things matter

Nina Paley, CC BY-SA 3.0 
sources of self-knowledge are less powerful than they were in small tight-knit societies. It is thus especially important - a critical ethical duty - that individuals actively pursue both external and internal sources of self-knowledge.

\section{We can achieve meaning through celebrating group identity (within a common humanity)}

Making the world a better place may seem a tall order - though only if we neglect the evolutionary insight that the world has, for the most part, improved through multiple small changes. It is therefore tempting to follow a thought process such as: "I am an X"; "The X are worthy"; "Therefore it is meaningful to be an X." There is a formidable logic to this source of meaning. And it resonates with the basic human tendency to identify with groups. It can complement our first source of meaning if it guides us to work toward the prosperity and happiness of group $\mathrm{X}$ in a way that is not harmful to the members of not-X. Humans can accomplish much when we jointly attach a shared meaning to certain activities. The danger, of course, is that a strong group identity can guide us toward acts of hostility toward non-members. It can also steer us away from self-knowledge by encouraging us to absorb without question all of group X's beliefs and practices.

World history provides a set of lessons that should guide us toward a positive type of group identity:

- Modern ethnic identities were primarily forged in the last centuries, often through deliberate efforts of states. Through most of history, ethnicity was much more fungible and human groups regularly intermingled.

- All human groups have done good and evil in history. All have contributed to world history.

- Individuals throughout history have had cross-cutting identities based on gender, occupation, interests, religion, and what we would now think of as ethnicity.

- Wars throughout history have often been justified in terms of ethnic superiority or some imagined slight. Most of these justifications appear suspect with the passage of time.

- Cultures evolve, and borrow from each other.

None of the above lessons need guide us to reject ethnic identity. History is lived in the present, and we can (or not) take pride in our group memberships. Such lessons should guide us, though, not to demonize the members of other groups. We should appreciate that we (and they) have both a right and responsibility to evaluate received traditions. We should understand that our children also have the right and duty to choose their groups and assess their cultural attitudes.

We should not allow the human tendency to identify with small groups to guide us away from appreciating the simple fact that we are all part of a common 


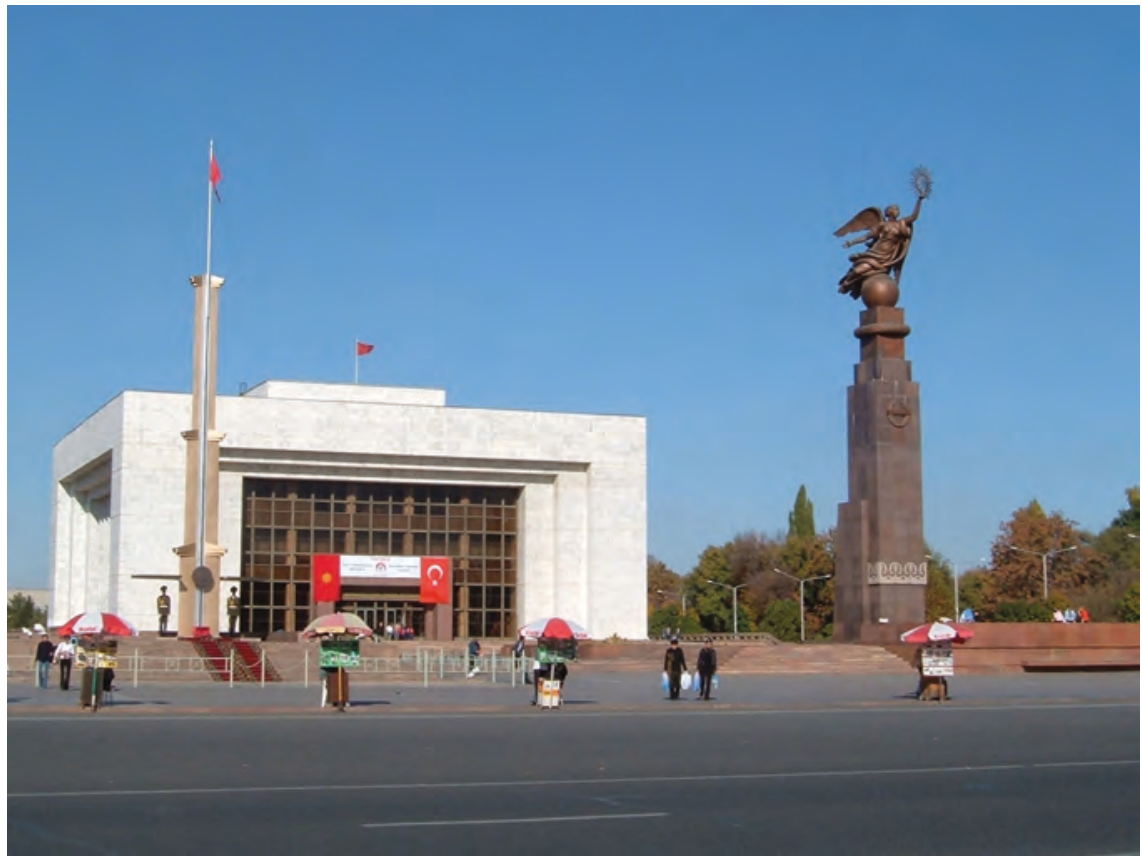

Illustration 32.12

National Historical Museum of the Kyrgyz Republic, Bishkek, Kyrgyzstan

humanity. We have intermarried, traded, fought, robbed, laughed with, and sometimes understood each other throughout history. We are all interrelated. We each (with the possible exception of smallish isolated Indigenous groups and then only if we limit the time scale of analysis) have ancestors from different historical human groups - for marrying out has been a common human practice for millennia. Most importantly, we each have thousands of ancestors and can be confident that some of them did wonderful things and others did awful things. If we have children, we can be likewise confident that our descendants will make their mark - and we can and should do what we can to guide them toward positive contributions.

\section{We can achieve meaning by celebrating personal identity}

One lesson we should draw from Table 32.1 is that we can make the world better in diverse ways. Each individual, then, is guided to look at their capabilities (and motivations) and opportunities and identify one or more contributions that they might strive to make. Since each individual is unique, each can fashion a unique set of strategies. Each can take legitimate pride in their uniqueness (though being weird for the mere sake of being weird is a questionable practice) and their strategies. 
We have noted above that humans have a cross-cutting set of identities. To the list of group affiliations, we can now add each individual's unique set of abilities and motivations. One of the tasks for world history going forward is to compare more explicitly the personalities and capabilities of historical actors - but we can already be confident that it has taken all kinds of people to fashion the world that we inhabit. In particular, the challenges addressed by different types of historical actor were distinct and so likely best addressed by people with different motivations and abilities. Great artists would often be poor merchants. We each have a unique contribution to make (or indeed a set of unique contributions to make). The key is to self-actualize ourselves in a way that ensures a positive contribution.

Recall that innovations of any kind are not just isolated acts of genius but always reflect group processes involving discussion and encouragement. Thus, simple actions like treating others with respect and engaging in constructive conversations can make the world a better place. As one forges one's identity, one can ask how this facilitates collaboration with others.

One challenge in developing and celebrating one's identity is precisely the cross-cutting identities noted above. While these cross-cutting identities support personal uniqueness, they may also generate conflicts that stand in the way of achieving personality coherence. Your desire to be a prosperous merchant may conflict with values held dear by your ethnic or religious group (the literature on economic development often references this particular challenge). While humans have likely always had complex identities, the complexity of individual identities has increased as the complexity of human societies has. This expands our opportunities for creativity if we successfully integrate our different identities into one. However, it can also lead to psychological challenges if we are effectively different people in different realms of our lives. Self-awareness is yet again of critical importance: We are far more likely to achieve coherence and creativity if we explicitly appreciate the conflicts we face.

As a naïve youth, I rebelled against the idea that we each had to find our own meaning in life: Surely, there was one meaning for all? I see past this false dichotomy now: We each seek meaning in a handful of broad ways, but are guided by individual personalities, capabilities, and circumstances to emphasize unique paths within these broad types of meaning. The idiosyncratic elements of meaning reflect the fact that personality diversity exists for a reason: Human societies function better with a variety of talents and attitudes.

\section{We can achieve meaning through balance}

We have followed the interactions among ten themes and dozens of subsidiary phenomena in this book because each has been important in human history. Though we should each follow our own path in life (see above), and this may involve some of us emphasizing art and others technology and others entrepreneurship (and some spending years writing a world history textbook), there is 
good reason to think that every human can find meaning in every one of our themes:

- Economically we can find meaning both in providing for our families and in helping the less fortunate to provide for theirs.

- Politically we can all find meaning in political activity at various levels (including volunteer organizations and school councils) with a focus on improving institutional quality and leadership practices.

- Demographically we can find meaning in raising children to be net contributors, and more generally in leaving a better world for our children.

- The prevalence of art in human history suggests that art plays a vital role in human lives; it could well be that we should all pursue artistic production (note that this tends to be common within traditional societies, and that young children everywhere enjoy drawing, sculpting, singing, and dancing) as well as artistic enjoyment.

- Socially we should find meaning in our various group identities in ways that are good for the world and do not interfere with self-actualization.

- This may involve consciously amending cultural practices. It will undoubtedly involve deliberately reflecting on what values are essential to both oneself and the world.

- Humans have lived closer to nature through much of history than do urbanized citizens of the twenty-first century. There is abundant psychological evidence that spending time in natural settings has positive effects on happiness. We might devote some of that time to caring for the natural environment.

- With respect to technology and science, we can all take pleasure in thinking of better understandings or ways to do things. Recall that technology evolves, and that we benefit from countless minor advances through the years. Technology is a broad enterprise, embracing recipes in cooking and videos on how to recharge dead batteries for electric drills (jiggling the charger plug in and out of the wall socket actually works!).

- As noted above, we can take justified pride in our individual differences (uniqueness) - while striving to avoid arrogance and narcissism.

- Genetically we can take pride in our common humanity.

Many of the above bullet points call for creativity. Two points seem relevant in this respect. First, scholars of creativity appreciate that persuasion is an important - for some scholars the most important - part of the creative process. It is not enough just to have good ideas: One must actively "sell" these ideas to others. Persuasion itself calls for creativity. Second, whether we succeed here is beyond our control, for no one person controls the selection environment. We should pursue a certain stoicism: If we have developed and advertised good ideas (with self-awareness!), we can laugh if the selection environment shuns 


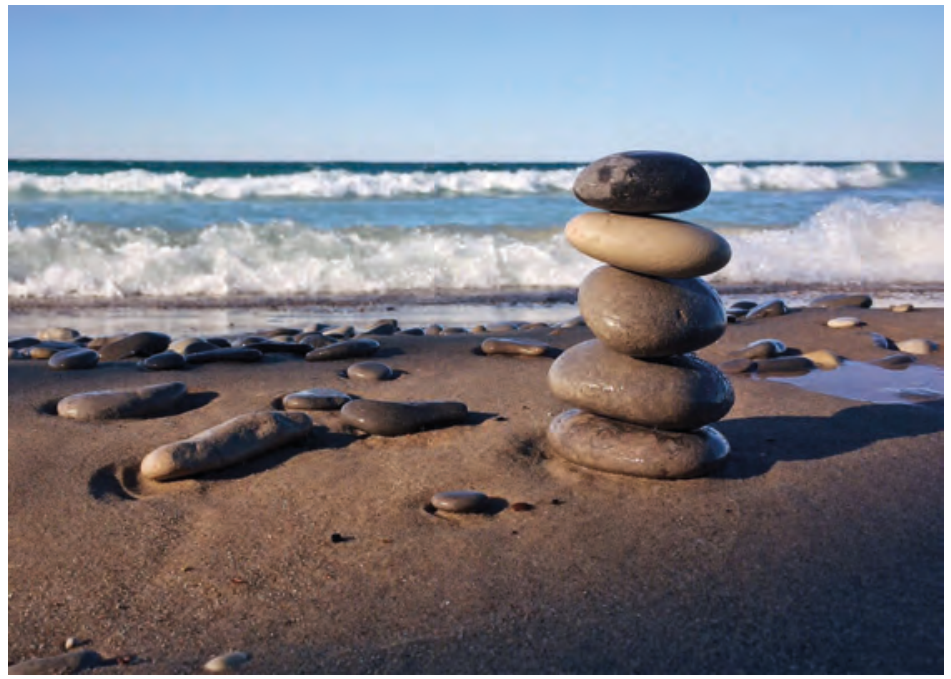

Illustration 32.13

Balance

these. One of life's challenges is to put energy into selling one's ideas but not be heartbroken if these efforts fail.

\section{We can achieve meaning through our own life story}

We have urged people above to identify their unique talents and motivations, yet also pursue balance in life. Each individual then lives a unique life and contributes in their own way. History provides valuable lessons here:

- $\quad$ Failure is a part of life. Even the most successful people in history - whether in war or technological innovation or art - failed along the way. They did not let failure stop them but instead learned from their failures and moved on.

- Human lives, like world history itself, are contingent. We each face surprises in life: some good, some bad. By honing our talents, we prepare ourselves to take advantage of opportunities. By reflecting on our misfortunes, we may find new meaning in life, both in overcoming these misfortunes in our own lives and in aiding others in similar situations. Some philosophers have urged us to celebrate life's challenges for these allow us to display our character and capabilities.

- We each build our own autobiography. Like any good story, it should have a guiding theme(s), but also twists and turns and places where we surprise ourselves. Our themes are not always evident at the start but are shaped by events.

- Though our autobiography should be unique, we can learn much from biographies of historical figures (and literary creations as well). 
- We deceive ourselves. We must be careful of dishonestly rewriting the history of our life. One important danger is that we refuse to admit mistakes: We may stay in a dysfunctional relationship because we refuse to admit that we chose badly. Another is that we feel a need to justify sacrifice: But just because we suffered does not mean it was worth it. We need to accept that our life may have some dead ends and lengthy detours.

It is not a problem if you do not know your life story at 20. Live. Be good. Take chances (but not stupid risks; learn to tell the difference). Bounce back from failure and misfortune. Enjoy yourself. Hone your talents. Find time for nature and art and the joy of a child's laughter. Know that life has good times and bad times - and that the bad times make the good times more meaningful. Value human relationships of all types. Know that you are part of a rich tapestry of life.

\section{We can find meaning in religion or philosophy}

Religions existed throughout human history. Humans have believed many things. They have prayed, killed, sacrificed, loved, explored, hated, and acted ethically and unethically, all in the name of one religion or another. Individuals throughout history and across societies have proclaimed absolute adherence to one faith or another. While different faiths may divide humanity, humanity seems to be united by its devotion to religion in general (though as noted in chapter 27, agnosticism has become increasingly common, and we may wonder how many humans in history were attached only nominally to a particular religion).

Though world history suggests that religion serves an important purpose(s) in people's lives, it also raises a troubling question. Different individuals throughout history have placed complete confidence in a wide variety of religions. How can anyone know that their confidence in their chosen religion reflects some eternal truth when billions have had the same precise confidence in entirely different sets of beliefs?

This question is exacerbated by the fact that religions evolve over time (albeit being constrained by holy texts) and are all characterized by denominational

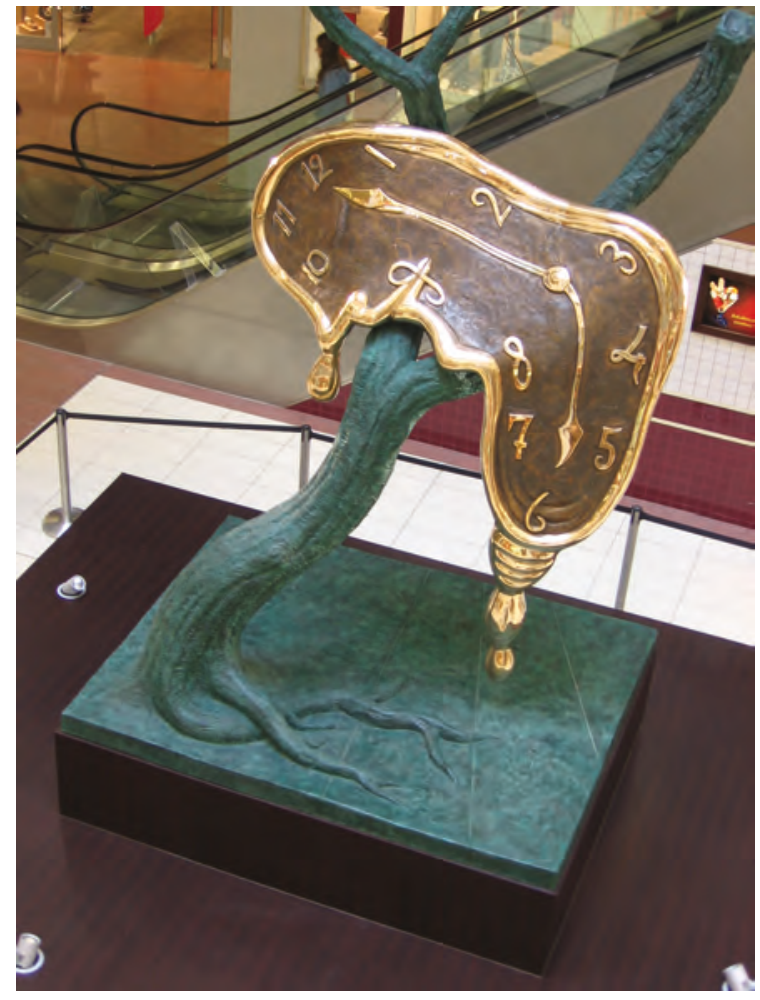

Illustration 32.14

Salvador Dali: Profile of Time. Sculpture at the entrance to the Sky Tower in Wrocław, Poland 
factionalism. Christian churches have made a series of at-the-time controversial decisions regarding the nature of Christ and the individual's relationship to god. A disagreement regarding religious and temporal leadership has divided Islam for over a thousand years. As noted in chapter 11, these doctrinal and denominational disputes are so heated precisely because they challenge the very idea of revealed truth.

There would seem to be three possibilities. One involves the recognition that our subconscious thoughts can convince our conscious minds of a wide variety of beliefs. Our subconscious minds may find it convenient to believe that there is an afterlife better than this one, or that we can (reasonably easily) influence rainfall and the other forces that buffet our lives; or that there is either justice in the cosmos or at least reasons for injustice. One might then conclude that if the billions of people convinced of beliefs contrary to your own have been fooled by their subconscious then perhaps you have too. Alternatively, one might conclude - either consciously or subconsciously - that god(s) will reward even misguided faith.

A second possibility is that one set of beliefs is indeed inspired by $\operatorname{god}(\mathrm{s})$. There may be no empirical means of distinguishing the true from false beliefs. One might strive to discern godly favouritism over time. We might all then mock the religions that have died out. We might think that longevity and extent of belief is a sign of godly favour. In doing so, we would have to accept that every religious community has suffered some grievous setbacks over time. Each of the major religions surveyed in chapter 11 was once popular in regions from which it has virtually disappeared.

A third possibility is that there are some universal truths in all/most religions. These may have been inspired by god(s): God(s) may care much more about the common purposes of religion than the differences in doctrine between religions. Alternatively, these commonalities may reflect the beneficial ways in which our subconscious minds do not just cater to our fears and prejudices but also contain understandings that we cannot adequately express in words. Ecumenicism sometimes just celebrates belief: It does not then matter what you believe as long as you believe something. More often, ecumenicism seeks to identify such commonalities. Most religions urge similar types of ethical behaviour, for example, and so perhaps these express some eternal truth. If so, being ethical would be meaningful.

There is one notable recent trend visible most starkly within Christianity. Many contemporary Christians reject the central tenet of early Christianity: that only those who believe will gain an afterlife. This attitude seems to be a reflection of cultural toleration within a multi-religious environment. It is perhaps a harbinger of a future of respect between faiths.

The perceptive reader will have noticed a subtle difference in the title of this section. This was at first unintentional. But it deserves note: Whereas the first five sources of meaning require active development by the individual, the most common approach to religion treats this instead as something which is taken as given by the individual. The individual is expected to pursue a set 
of correct beliefs and practices. However, there have been minority traditions across all religions - mysticism in Christianity, Sufism in Islam, Zen Buddhism, and Kabbalah in Judaism and beyond - that have emphasized personal experience over received wisdom. It could be that this more personal view of religion will prove conducive to recognizing the common elements across religions. We saw in chapter 12 that Sufis were often syncretic in their teaching.

We noted in chapter 27 an increase in non-religious attitudes over the last century or two. Those who eschew religion can potentially find sources of meaning in philosophy. Indeed, we have noted that the distinction between religion and philosophy is fuzzy, especially for Chinese Confucianism or Taoism. There is an entire field of philosophy, called metaphysics (referred to occasionally above), which explores questions of meaning. If this literature had solved the human quest for meaning for all time, we would know about it. Yet individuals seeking meaning can nevertheless find that reading works of metaphysics can complement the other sources of meaning outlined above.

\section{Questions}

1. You were invited in chapter 1 to reflect on what you hoped to learn from world history. It is now time to compare your results with your expectations. Do these differ, and if so why?

2. Do you agree with the lessons outlined above? Are there other lessons that you might draw from world history?

3. Which events or processes in history seem the most important to you?

4. How might the challenges facing artists be best addressed? What about migrants?

5. Singapore is an example of a contemporary democracy that has managed to focus on economic growth but with regrettable authoritarianism. How would you evaluate such an institutional compromise?

6. What meanings do you attach to your life?

7. Is understanding world history useful or important in your own search for meaning?

8. Will the future be better than the past?

9. Is it possible to achieve progress in future across all phenomena?

10. Can the roles of religion be served without religious doctrine?

\section{Readings}

This chapter has mostly integrated material from previous chapters. The last section on meaning also drew on some general philosophical insights and personal experiences. Students should be aware that there is a growing literature of "Futures Studies" or "Futurism" that attempts to predict future trends and suggests policy interventions. It is not always clear on what basis predictions are made. Sometimes 
fairly recent trends are extrapolated far into the future. The journal Futures is a good place to start. It often pursues interdisciplinary analysis. Readers can consult a wide range of works on metaphysics for a more in-depth appreciation of philosophical explorations of meaning.

Attewell, Steven. 2018. People Must Live by Work: Direct Job Creation in America, from FDR to Reagan. Philadelphia, PA: University of Pennsylvania Press. [Draws on the Depression experience to urge direct government employment of the unemployed on public works.]

Burke, Peter. 2012. A Social History of Knowledge, Volume II: from the Encyclopédie to Wikipedia. Cambridge: Polity Press. [Explores the possibility that the internet may lead to a democratization of scientific understanding.]

Csikszentmihályi, Mihaly. 1990. Flow: The Psychology of Optimal Experience. New York: Harper \& Row. [This classic book urges one to immerse oneself in various activities.]

Eichengreen, Barry. 2015. Hall of Mirrors: The Great Depression, the Great Recession, and the Uses - and Misuses - of History. New York: Oxford University Press. [Argues that better policies were pursued after 2008 than after 1929 to mitigate the economic effects of a financial crisis - but worries that as a result there was not the same concerted effort to develop better financial regulations.]

Frey, Carl Benedikt. 2019. The Technology Trap: Capital, Labour, and Power in the Age of Automation. Princeton University Press. [Draws on history to discuss whether workers will try to block the next round of labour-saving automation, and what the implications of artificial intelligence may be.]

Gregory, Brad S. 2012. The Unintended Reformation: How a Religious Revolution Secularized Society. Cambridge, MA: Belknap Press of Harvard University Press. [Argues that the Protestant Reformation led to religious pluralism and then secularism, and this, in turn, has led to a lack of societal consensus on ethical values. Seems to favour religious orthodoxy.]

Habermas, Jürgen. 2001. On the Pragmatics of Social Interaction, B. Fultner (trans.). Cambridge, MA: MIT Press. [This book addresses Habermas's pursuit of the "ideal speech situation" conducive to developing better societal understandings.]

Halden, Peter. 2012. "From Empire to Federation and Commonwealth: Orders in Europe 1200-1800," in Peter Fibiger Band and Dariusz Kolodziejczyck, eds., Universal Empire: A Comparative Approach to Imperial Culture and Representation in Eurasian History. Cambridge University Press. [Advocates confederation of multi-ethnic states, following the examples of the Holy Roman Empire and Swiss Confederation.]

Harari, Yuval Noah. 2016. Homo Deus: A Brief History of Tomorrow. Signal (Penguin Random House).

Laine, James W. 2014. Meta-religion: Religion and Power in World History. Berkeley, CA: University of California Press. [Laine worries about what if anything is replacing religion in the modern Western world as a source of legitimacy and identity, and what roles religion still plays today.]

Norberg, Johan. 2016. Progress: Ten Reasons to Look Forward to the Future. London: Oneworld Publishers. [As the title suggests, this book looks at only some types of progress, and its focus is on recent decades and centuries. It does a good job, though, in dispelling the notion that things have been getting worse.] 
Pinker, Steven. 2018. Enlightenment Now: The Case for Reason, Science, Humanism, and Progress. New York: Penguin. [Argues that there has been progress of many types in history.]

Szostak, Rick. 2012. Restoring Human Progress. Reading, UK: Cranmore. [I drew on this book (which is not easy to access through libraries) in developing Table 32.1. The book evaluates human progress with respect to about a hundred phenomena over three time periods: recent decades, recent centuries, and historical time.]

Szostak, Rick, Claudio Gnoli, and Maria López-Huertas. 2016. Interdisciplinary Knowledge Organization. Berlin: Springer. [This book explores the possibility and desirability of the sort of organizing structure outlined above.]

Tetlock, Philip, and Dan Gardner. 2015. Superforecasting: The Art and Science of Prediction. New York: Random House. [The book reports on an experiment in which thousands of volunteers made predictions on a variety of issues in world politics. Those who did best tended to be comfortable with complexity rather than following simple rules.]

For those who can read Japanese, www.historymining.org compares the sort of thematic interactions we have investigated in this book across time and place in an effort to guide policy-making. The Thucydides Trap Project explores sixteen historical cases of a change in the balance of power, suggesting that (as Thucydides had said of the rise of Athens versus Sparta in the fifth century вСE) war was inevitable in such situations before the twentieth century. See: http://belfercenter.ksg.harvard. edu/publication/25760/thucydides_trap_project.html 


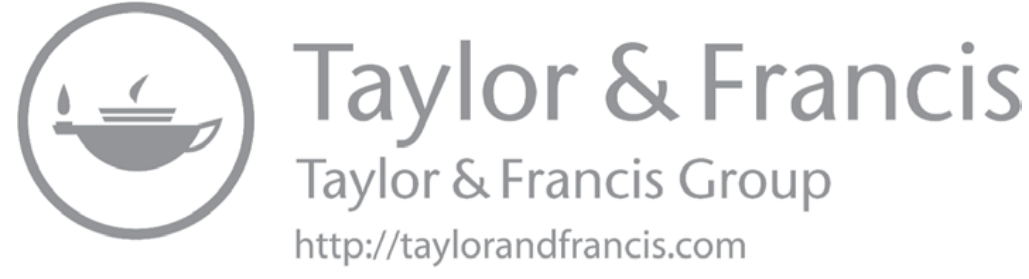




\section{Index}

abolition of slavery: in the

Americas 1103-9; in Asia

1109-10; in Brazil 1105,

1107-8; in Britain 1000,

1100-2; in the Caribbean

1098, 1101, 1104; in Denmark

1101, 1104; economic factors

1097-9; ethical arguments

for 1097, 1098, 1105-6; in

Europe 1100-3; in France

1102-3; freed slaves in

America 1112; freed slaves in

Brazil 1112-13; the Haitian

Revolution 1080, 1081, 1098,

1102; historical importance of

1096; impact on sub-Saharan

Africa 1114-15; indentured

servitude 1096-7, 1113, 1115-

16 ; influences and effects of

1119-20; in the Islamic world

1110-11; post-emancipation

conditions 1096-7, 1112-15;

racism following 1117-18;

resettlement of freed slaves

1113-14; Thomas Paine's letter

on 1106; in the United States

of America 1105-7, 1108-9

Afghanistan 1187, 1213, 1217-18

Africa: art of 332; Bantu language

families 122-4, 296; cowrie

shells as currency 176; early

religion in 318-19; hominid

migration from 98-101; iron

smelting 171; nomadism 146;

see also sub-Saharan Africa

agricultural technology:

crop selection 1308-9;

domestication of animals 157;

early technological innovation

117; in the Han Empire 436,

442; horse collars 436, 649;

of hunter gatherers 156; links

with language dissemination

121, 122-4, 148; metal plows
122-4, 140, 157; milling 649-

50; mouldboard plow 649; in

the nineteenth century 1022 ,

1307,1309 ; overview of $156-7$;

spread of 121

agriculture: agricultural

expansion in the Americas

844-6; changing role of

1309-10; climate change and

the transition to 118-19; the

Columbian Exchange 828-

9; crops 113-15; declining

economic importance of

1098; developments of

the nineteenth century

1021-3; development of

in the Middle East 118-20;

domestication of animals

113-14, 117, 134, 145-6,

827-8; ecological collapse

276-7; environmental impact

of 131; expansion of, post-

900CE 617; farm sizes 439-40;

farmers' challenges 131;

farmers' interactions with

nomads 146-8; fermentation

158-60, 650; fertilizers 1021-

2; food storage 115-16, 134;

formation of settlements

and 115; gender relations in

agricultural societies 140-2;

horticulture distinction 157;

human genetic inheritance

and 135-9; humankind's

aspirations for 1353; hunter

gatherers' transition to

115-17, 118-19; impact

on population densities

133; increased output and

trade opportunities 646-50;

influences on and effects of

143; intercontinental trade in

foodstuffs $608-9$; irrigation

$118,158,201,252,351,562$; land ownership 130, 133, 139-40; in the Ming dynasty 889; opportunities for trade 174; political organization and 130-1, 133, 139-40; in Polynesia 690-1; in the Qing dynasty 895; regional development of 120-30; role of women 117; serfs 559-60; socio-economic developments 133; soil health 132; in the Song dynasty 588, 663; in sub-Saharan Africa 709-12; taxation of farmers 133, 202, 215, 217; theft of agricultural produce 131, 133; toolmaking and 115-16; vegetable oils 160; war as a side-effect of 140, 141-2

airplanes: allied bombing in World War II 1201, 1203-5; bombing of Guernica 1193; in the Golden Age 1240; in World War I 1182; in World War II 1201

alcohol see fermentation

Alexander the Great 352, 360, 402

Algeria 1001, 1270

American Revolution: British administration 10634; compared with the French Revolution 1072, 1077; democracy in, postRevolution 1070-1, 1072, 1073, 1271; domestic concerns over 1065-6; impact on slavery 1066; state legislatures 1070-1; statebuilding 1068-73; taxation system 1064-5; the twenty colonies 1066-8

Americas: abolition of slavery 1103-9; African slaves in 834, 
836-9; agricultural crops 695; agricultural expansion 844-6; the Columbian Exchange 827-30; draft animals 694-5, 829; epidemic disease in 800, 801, 805, 806, 827-8; European conquest of 694 ; expulsion of indigenous peoples 845 ; migration to 844-6, 1316; name 809; political consolidation after 900CE 600; population growth 1050; settlement by religious minorities 844 ; silver from 806-7, 895, 955; Simon Bolivar's decree on emancipation 1104; Spanish conquest of 801-6; trade in 695; use of gunpowder weapons 774; see also Latin America; Mesoamerica; Mexico; North America; Peru; South America

Anatolia: Catalhoyuk, Turkey 203, 210, 236, 330; Hittites 256, 260,282 ; region of xliv 25960; trade with Mesopotamia 254, 256, 259, 287, 289; Turkic groups of 546

Arabia: exploration of the Atlantic coast of Africa 814; Mecca 479-81, 482, 505; nomadism of 482 ; trade in 504-5

architecture: of the Asian empires $854,868,878$; cannonresistant castle designs 773; of the Islamic Empire 517; of the Persian Empires 361; religious 328-9; of the Romans 415; stelae 258; see also monumental architecture

Arctic: earliest societies 286

Argentina: nation-building 921, 922

Aristotle 389, 392-3

Armenia: nation state formation 915, 916; trading networks 778

armies: of the ancient Greeks 399,402 ; of the Byzantine Empire 572; colonial soldiers 1176, 1268; conscription 1175, 1177; during colonialism 994-5; development of 238; early technological advances in 246-7; frontier troops 356 , 416, 424, 431, 438-40; gunpowder technology and army size 773, 775, 776; of the Gupta Empire 367; humankind's aspirations for 1353; importance of in empires 347, 354; iron weaponry 367,434 ; Islamic armies 508, 514-15; military leadership 243; Ottoman Janissaries 858-9, 864; of the Roman Republic 410-11, 412; of the Safavid Empire 871; of the Song dynasty 658, 665; see also military technology; weapons

art: artistic borrowing 788; artist's challenges 785; artistic representation in religions 490-1; of the Byzantine Empire 567, 572-3; cave drawings 81-2, 95-7, 656; Chinese landscape painting 665; cross-cultural transmission 784-8; cultural role of 97; in the earliest societies 81-2, 329-33; early humans capacity for 95-6, 97-8; economic aspect of 335; of Egypt 267, 331; evolution of 336-8; function of artistic illustrations 333-4; global art markets 785, 1255-6; of the Han Empire 430; of hominids 95-8; Islamic art 491, 533-5; of the Kushan Empire 369, 490; of the Mauryan Empire 365-6; of the Mongol Empire 741; of the Mughal Empire 854, 878-9, 880; opera 1256; of the Ottoman Empire 854; of the Persian Empires 360,361 ; of the Renaissance 655-6; of the Safavid Empire 854, 872-3; sculpture 99, 332-3; theme of 334-6; in the twentieth century 1130 artisans: apprenticeships 652; artisan guilds 651-3; challenges of 186; in early cities 205; early industrial production 186-7; humankind's aspirations for 1353

Ashurbanipal library 226, 228-9, 393

Asia: abolition of slavery 110910; Asian political thought 1274-7; colonialism in 988-90; decolonization in 1274-9; economic growth 1245-7; hominid migration to 98-101; indentured servitude 992, 1000, 1113, 1115-16; migration to 1316; see also South Asia; Southeast Asia astronomy: in ancient Greece 391, 394, 967; in the ancient world 389; in China 389; Earth's orbit of the Sun 966, 967-70; elliptical orbits 970-1; Galileo Galilei 968-9; Islamic 967; of the Mayans 391

Ataturk, Mustafa Kemal 916

Australia: aboriginal Australians 693-4; European exploration of 811; first humans in 101; lack of early agriculture 693; League of Nations Mandate 1186; as a penal colony 844 ; settlement of 846

Austria: abolition of serfdom 1121; Vienna 921

Austro-Hungarian Empire 913, 921 authoritarianism: in postcolonial Asia 1276-7

autocracies: democracy and 1133-5; in multi-ethnic states 935; popular sovereignty and 937; social stability under 1033; see also fascism

Axum Empire 369-70, 381

Aztecs: European conquest of 694; human sacrifice 699; social structure 698-9; Tenochtitlan 694, 696-7; trade 697-8; warriors 701

Aztec Empire: cacao consumption 780; Moctezuma 801-2; smallpox epidemic 801, 806, 827-8; 
Spanish conquest of 801-4, 805-6; Tenochtitlan 801-4

bacteria: cyanobacteria 53; ecological adaptation by 55 ; as first ever life forms 52-3, 54,60

Baha'i faith 766

Bangladesh 1277, 1281, 1283

Bantu 171

Batavia 821

Belgium: colonization of Congo 990; industrialization in 1041-3

Bering, Vitus 811-12; the Big Bang 40-2

Big History: overview of 38; revolutionary change $38-40$; timescales 40

biology: classification of species 975

boats: in ancient Egypt 177, 266; ancient Greek warships 401; boarding of ships 411; canoes 286, 687, 688; carracks 797; climate factors 383; development of 177; migration by early hunter gatherers 98, 100, 102, 104; Phoenician galleys 269; sea trade in the ancient world 380-3; sea trade, post-900 CE 607-8; settlement of the Americas 102, 129; settlement of Oceania 101, 102; shipping technology in the ancient world 381; shipwrecks 382; for trade 177, 180; troop transport during the Crusades 640; see also shipping technology

Bolivar, Simon 1083, 1084, 1104

Bonaparte, Napoleon 1075-6, 1175; attack on Egypt 864; conquest of Iberia 918

books: format of 394

Botswana 1291

Brazil: abolition of slavery $1105,1107-8$; freed slaves in 1112-13; independence 1087; Portuguese conquest of 808-9, 816; slaves in 834; in World War II 1198-9 bricks 164

British East India Company 820, 881-2, 883

British Empire: benefits of to British citizens 216-17; in relation to the Industrial Revolution 1033-5

British Industrial Revolution: economic growth 1028-9, 1031-2; the European context for 1032-3; factors for 10279, 1038-40; factory-based industry 1027-8, 1031-2; food production and imports 1035; the Great Divergence and 1026, 1032-3; historical importance of 1026-30; industrial machinery production 1029; negative impacts on workers 1029-30; political support for 1033; regional specialization 1029, 1037; in relation to the British Empire 1033-5; technological innovations 1028, 1031-2; transport factors 1035-8; unionization and 1009-10

Buddhism: Ashoka 364-5, 468; beliefs of 466-8, 469, 471; in China 470; doctrinal disputes 468-9; family values 467 , 487; the Happiness of the Dhammapada 471-2; India 529-30, 591-2; in Japan 586; in the Kushan Empire $368-9,468$, 492; life of the Buddha 466-7; Mahayana Buddhism 368-9, 469; in the Mauryan Empire 364-5, 468, 492; in the Mongol Empire $746,748-9$; in relation to Hinduism 367, 466, 467; spread of 388, 468-70, 492, 495; statues of Buddha 490; Sufist borrowings from 485; Theravada Buddhism 368-9, 468; Tibetan Buddhism 495, 589-90, 591, 749; warfare and 489

builders: building techniques and knowledge 621-2; challenges of 213; humankind's aspirations for 1353; of mills and bridges 650; of monumental architecture 212-13

buildings: prehistoric structures 89; see also monumental architecture

Burma: cultural consolidation 676-7; Pagan 599, 674-5; states during the middle ages 675-6

Byzantine Empire: armies 572; art of 567, 572-3; bureaucracy 565, 573; Constantinople 573; decline of 569-70, 571; as the Eastern Roman Empire 565; geography of 566; Greek fire 574; Hagia Sofia, Constantinople 567, 619, 620; the Huns and 573-4; Islamic conquests of 507-8, 566, 571, 574-5; under Justinian and Theodora 566-70; laws (Justinian's Code), 570; missionary activities 556; the Orthodox church and 474, 476, 566, 570; Ottoman victory over $857-8$; religions in 416; sport in 567-9; strategy of 570-1; trade in 380,572

Cabot, John (Giovanni Caboto) 839

cacao beans 780, 781

Caitanya, Sri Krishna 765, 768

Cambodia: Khmer kingdom of Angkor 492, 599, 619, 672-5; temple complexes 492, 619, 672-3

Canada: abolition of seigneurial tenure 1123-4; legislature 1123-4; nation-building 922-3; Nova Scotia 10678; political independence 1066, 1089; Prince Edward Island 1067; Quebec 1067; taxation 1142-3; see also Newfoundland

Canary Islands 797-8

capitalism: colonialism as a condition for 953; consumption patterns 790; 
cultural attitudes towards 789; definition of 786-7; dominance of 789 ; in the Early Modern period 787-9; the Great Depression and 1228; industrial firms 789-90

Caribbean: abolition of slavery 1098, 1101, 1104; epidemic diseases 800; indentured Asian servants 1113; region of xliv; slave labor 834, 836-7; spread of agriculture to 130 ; Tainos/Arawaks 800-1

Castro, Fidel 1217

cells: eukaryote cells 54; modern cells 52; multi-cellular life 601; RNA molecules 52

Central America: region of xliv Central Asia: Buddhism 470-1; earliest societies 282-3; the Islamic Empire 533; Khazars 462, 546, 556; nomadic conquests 542; nomadic/ agriculturalist relationships 540-2; nomad's military technology 542-3; nomads of the Eurasian Steppe 540-2; region of xliv; religious variety and syncretism 533; Seljuk Turks 546, 574; Turks 545-6; Uighurs 546-7, 734, 746

Charlemagne (Charles the Great) 493, 562, 629-30

childcare: cultural co-evolution and 78; in early agricultural societies 134-5; in early humans/hominids 105 ; hominid cognitive capabilities 75; see also families; parents

China: annexation of Tibet 1286 ; astronomy 389; Buddhism 470; bureaucracy of 1148-50; Chinese philosophy 421-4, 445, 972; communism 1284, 1285-6; compasses 607, 798; corporations 648; cowrie shells as currency 176, 377; cultural homogenization 576, 581-2; dialects 583; earliest societies 201, 203-4, 281-2; early law codes 231; early religion in 320-2; early writing in 226-7; economic liberalization 1287; examinations for bureaucrats 428-9, 576, 579, 581-2, 583, 658-9; the Forbidden City 867, 885; forced labor 280; Go (board game) 405; Grand Canal 576, 577, 885; gunpowder technology 772; heavenly mandate for rulers 280, 427, 429, 444, 448; hemp cloth 166; income distribution 1248; incursion into India 1286-7; influence in Tibet 589; influence in Vietnam 587-8; iron smelting 171, 282; Japan's invasion of Manchuria 1191, 1194-5, 1205, 1284; the Kuomintang 1284, 1285; lack of assemblies in 940-1, 957; land ownership 760-1; pagodas 618-19; $P^{\prime} a n \mathrm{Ku}$ creation myth 306 ; period of disunity 576-7; political consolidation 575 , 600; population 828-9, 955-6; population growth 1054; porcelain 614-16, 741, 784, 890, 1033; printing technology 653, 977; Qara Khitai (Western Liao) 547; Quanzhou 610; relations with the Portuguese 816; revolution of 1911, 1284; sea trade 382; shared language 916-17; silk 166; slavery in 207; spread of agriculture to 124-5; state capacity 930; Sui dynasty 385, 470, 577, 579; Taiping Rebellion 1090; Taoism 423, 429, 430, 447, 466, 470; trade 955, 956; universal supremacy concept 350; the Warring States period 345, 421, 424; women in post-colonial China 1287; Xia dynasty 277-8, 280; Xiongnu Empire 368, 384-5, 427-8, 544-5; Yuan dynasty 665-7, 733, 736-7, 747-8; see also Han Empire; Ming dynasty; Mongol Empire; Qin Empire; Qing dynasty; Shang dynasty; Song dynasty; Tang Dynasty; Zhou dynasty
Chinggis (Genghis) Khan 277-8, 352, 665, 722-4, 873

Christianity: artistic imagery 490-1; in the Axum Empire 370; beliefs of 472-3; in Constantinople 474, 476-7; co-option of pagan festivals 455; the Crusades 574, 637-40; doctrinal disputes 475-8; early Jewish Christians 473; Ethiopian Christianity 476; family values 487 ; five original centers of 474 ; in India 815-16; influences on 472; interactions with Islam 769 ; just war concept 489 90; life of Jesus Christ 472; Nestorian Christianity 475-6, 746; Orthodox Church 4768 ; in relation to economic growth 961; relationship with the state 474; in the Roman Empire 416, 473-5; spread of 474-5, 492, 493, 494; see also Roman Catholic church cities: city walls 1330-1; civilization and 200; trading cities 609-10

cities, early: Anyang, China 205, 206, 209, 278; Catalhoyuk, Turkey 203, 210, 236, 330; city states 215 ; destruction of 208; effects of 208-9, 223; emergence of 202-5; food and water supplies to 204-5; housing 214; income inequalities 206-7; of the Indus Valley 205-6, 272, 273; Jenne-Jeno 716; monumental architecture 209-11; nature of 205-7; occupational specialization 205; public health 208; Samarkand 385, 386-7, 609, 620; size factors 206; slavery in 207; trading cities 206; Uruk, Mesopotamia 253

city states: of ancient Greece 398-9

civilizations: cultural coherence in 200-1, 202; emergence of 201-2; key phenomena 200; term 200 
classical empires: Axum Empire 369-70, 381; bureaucracies 344-5, 353-4; communication in 353; control of armies 354; crime in 418; cultural and health impacts of trade on 388-9; cultural consolidation 348-9, 403; currency 377-8; decline of 355-7; defined 346-8; ethnic diversity of 346, 347, 348-9; expansion through conquest 347,352 ; financial institutions 378-80; frontier troops 356; functions of 351-2; hostile competition 350-1; justifications for 349-50; legal systems 351-2; monumental architecture of 351; political thought 443-6, 448-50; public rituals 350; relationships with local elites 354,355 ; return to 1367 ; role of religious beliefs 457-8; sea trade 380-3; taxation 344-5, 350, 352, 356; toleration in 1354-5, 1367; trade 344-5, 351, 377; transition from states 345-6; tribute relationships 347, 353; see also Gupta Empire; Han Empire; Kushan Empire; Mauryan Empire; Persian Empires; Qin Empire; Roman Empire climate: after 900сE 598-9, 600; of the Early Modern period 759, 761-2; impact on early sea trade 383

climate change: challenges of 1362-3; disruptive impact of 761-2; during human history 293-4; evolution of early life forms 60; global warming 1169-70; impact of the Columbian Exchange 827; impact on human evolution 76-7; Little Ice Age $555,759,827$; state decline and 277; and the transition to agriculture 118-19; in the twentieth century 1131 clothing: in ancient societies 166-7; early hominids 77, 88, 98-9, 100, 101; industrialization and

convergences in 1057

coal 61

coffee 779

cognitive sciences 96

Cold War: Afghanistan 1217-

18; arms build up 1210-11; atomic weapons 1210 , 1211, 1213-14; collapse of the Soviet Union 1138-9, 1215-16; computer technology 1211; conflicts over communist expansion 1213; covert activities 121112, 1214-15; Cuba 1211, 1217; in Eastern Europe 1212-13; ideologies of and decolonization 1209, 1287; Indonesia 1217; Korea 1213, 1216-17; proxy wars 1209-10, 1216; soft power tactics 1214 ; and the spread of democracy 1133, 1135; West Berlin 1208-9

collecting: global art markets 785 ; hobby of 784 colonialism: in Africa 990; in Asia 988-90; bureaucracy of 1271; colonial slave-owners 1100; colonial soldiers 1268; colonial states post-World War I 1185-6; effects of 9978; as essential for capitalism 953; institutional capacity for administration of 952; justification arguments 995-6, 999-1000; League of Nations Mandates 1185-6, 1272; the middle classes 1012; military costs of 994-5; motivations for 993-5, 1176; museum artefacts from 1019, 1020-1; opposition to 996; in the Pacific Islands 993; reevaluation of by colonizing nations 1267; in relation to economic growth 951-4; sexual relations with the locals 998-9; of sub-Saharan Africa 838, 900; technological innovations for 993; tensions with democracy 1064 Columbus, Christopher 798-801 commodities: commodity history 616; porcelain as 614-16; rubber 991-2

communism: in China 1284, 1285-6; Declaration of Rights of Working and Exploited People 1140-2; popularity of in Western Europe 1191; rise of in Russia 1136-7; rulers and bureaucracy 1139; state ownership principle 1136, 1139; in Vietnam 1287-90 computer technology 1201, 1211,1236

Confucianism: in China 442; in the Han Empire 424, 428-9, 442, 447; Mao Zedong and 447; neo-Confucianism 466, $470,655,658-9$; overview of 422-3; in the Tang Dynasty 580; Wang Yangming's reforms 766-7, 768-9; within political thought 447

Congo 990, 1296

consumerism: patterns of 1800 , 950, 1051-2, 1251-2

continents: defined xliii

Cook, James 811

Copernicus, Nicolai 967-8 corporations 761, 789, 865, 95960, 1036, 1258-9

Cortés, Hernan 801-4, 807

Costa Rica 1086

Crete: the Minoans 270-2; palace at Knossos 211

crime: in the classical empires 418; Code of Hammurabi 207, 231-3, 258-9; the Crusades 574, 637-40; in hunter gatherer societies 95 ; institutions and protection from 1348-9; role of the state and 249; theft of agricultural produce 131, 133

cuisine 14

culture: abstract thought capacity and 81; consumerism 1051-2, 1251-2; cultural consolidation after 900CE 603-6; cultural diversity 1129-30; cultural understanding 1354, 1356; defined 11; early human migration and 103; early 
humans/hominids $81-2$; evolutionary analysis of 20-3; expressions of 14 , 82-3; in human evolution $74,77-8$; impact of the Columbian Exchange 833-4; impact of intercontinental trade 388-9, 608; individual diversity within cultures 21; influences and effects of cultural consolidation 606; institutions and 11; institutions within 1349; mating and childcare within 78; stories 13; theme of 13-14; toleration 1354-6; urbanization and 1331-2; values of 13; see also religions Czechoslovakia 1190, 1195, 1212-13, 1215

Da Gama, Vasco 810, 814-15 decision-making processes: human nature and 221-2; kings and 219-21

decolonization: in Africa 1290-2, 1295-8; arguments for 12667, 1268-9; in Asia 1274-9; in China 1284-7; Cold War ideologies and 1209, 1287; coordinated agitation for 1266; counter-arguments against 1267; democracy in post-colonial states 1131-2; emergence of Asian political thought 1274-7; financial factors 1269-70; in India 1270, 1271-2, 1276, 1280-2; influences and effects of 1272; institutional weakness 1271-2; in Iran 1279-80; in Islamic states 1277-8; in Latin America 1298-300; in the Middle East 1272-4; nation state formation 1266 ; in North Africa 1295; in the Pacific Islands 1300-2; pan-African unity 1293-5; post-World War II 1268; reevaluation of by colonizing nations 1267; religious and ethnic diversity of the colonies 1267, 1291, 1298; transitional challenges 1268 , 1271-2; in Vietnam 1287-90 democracy: in ancient Greece 399, 448-50; autocracies and 1133-5; in Britain 1033, 1064; civil society organizations 1135; in classical political thought 448-50; colonialism by democratic states 1064; contemporary challenges for 1135; in Denmark 940; in England 939-40, 957; following the Cold War 1133, 1135; French national assembly 939; global spread of 1133; income inequality and 1012; institutions 937-8, 940, 941-2, 1135, 1193; in Latin America 1084, 1085, 1133, 1299; middle class agitation for 1011-12; within the nation state 937 ; in the Netherlands 940, 957; in post-colonial states 1131-2; in post-Revolution America 1070-1, 1072, 1073, 1271; in post-Revolution France 1072, 1077; in post-revolutionary societies 1072, 1271; Russian assemblies 938; Spain's transition to democracy 1193; in sub-Saharan Africa 1133; in the twentieth century 1129; urbanization and 1133; the voter's challenges 942; in Western Europe 1131; within world history 1367-8, 1369-70

Denmark: abolition of slavery 1101, 1104; democracy in 940; revolutionary demands in 1089

Diaz, Bartolemeu 799, 814 al-Din, Rashid 742, 747, 748 dinosaurs 61-4

diseases: animal-human transmission 134; bubonic plague 1338; cholera 1337; the Columbian Exchange 827-8; in early cities 208; epidemic disease in the Americas 694, 747, 800, 801, 805, 806, 827-8; epidemics
219, 569-70, 747; germ theory 1159, 1310-11; the Great Plague 743-6, 956; HIV/AIDS 1338; influenza epidemic, post-World War I 1187; new epidemics 1338-9, 1375; obesity 1339-41; pathogen isolation 1017; re-emergent diseases 745 ; syphilis 827 ; vaccinations against $1336-7$

Drake, Francis 811

Dutch East India Company 820

Early Modern period: capitalism 787-9; climate of 759, 761-2; cross-religious cooperation 769; differences in legal traditions 760-1; political consolidation 759-60; population growth 791 ; religious reform movements 764-9; Scientific Revolution of 966-7, 976-8; seventeenth century disruptions 761-3; sub-Saharan Africa 898-9

the Earth: climate of 46-7, 49, 50; environmental influences on human evolution 76-7; formation of 46-9; Gondwana 49; life on Earth 40, 51-4; tectonic plates 47-9, 64; underwater sea vents 51-3

East Asia: Jomon culture 152, 162; Portuguese exploration of 816 ; region of xliii; spread of agriculture to $124-5$

Eastern Europe: region of xliv ecological collapse: in earliest societies 276-7

economic growth in the twentieth century 1130; airconditioning and 1248; in Asia 1245-7; the British Industrial Revolution and 1028-9, 1031-2; causes of 1241-2; in the classical empires 356; colonialism and 951-4; of the Golden Age 1238-40; Gross Domestic Product (GDP) measurements 191-2; measurements 189-90; patterns of in 1800 950-1; 
post-Roman Empire 562-3; property rights and $957-8$; of the Qing dynasty 896; religious considerations 961; in the Song dynasty $661-5,896$; in the Soviet Union 1137-8; technological innovation and 952, 961-5; technological innovations for 1245,1248 ; theories of convergence 1244-7; trade, foreign investment and economic growth 1298-9; see also Great Divergence; income distribution; incomes

economics: business cycles 1228 9; explanations for the Great Depression 1228-30, 1236-7; of feudalism 562; Keynesian model 1188, 1228; monetarist theories 1229; population growth and 1313 economy: artistic production 335; economic development in ancient societies $167-8$, 289; economic institutions 153; goods and services 153; of hunter gatherers 91-2; theme of 152-3; unemployment 153; see also trade

Edison, Thomas 1018, 1046, 1047 education: in ancient Greece 408; for bureaucratic administration 344-5; by the Catholic Church 635; government funding for 1143, 1146; industrialization and 1052; early schooling 229; and the spread of democracy 1133; A Sumerian Schoolboy 229-30; see also universities Egypt: art of 267, 331; Coptic Church 476; creation myth 305; decolonization 1274; earliest societies 201, 203-4; early gender relations 233; early law codes 233 ; early religion in 313-16; forced labor 263, 264-5; hieroglyphs 225-6; the Hyksos 263; kings 263; linen 165-6; links with ancient Greece 400-1; links with Mesopotamia 261; links with Nubia 173-4, 218, 2656, 268; Mansa Musa's visit to Cairo 715; the Museum and Library at Alexandria 391, 393-4; Nile floodplain 118, 122, 260-1, 315-16; political organization 261-3; pyramids 210, 263-5, 313; scribes 267; slavery in 1110-11; spread of agriculture to 121-2; taxation 263, 264-5; trade 266 ; trade with nomads 1467 ; unification of 261 ; water transport 177, 266

electricity 1008, 1015, 1046, 1233-4

electrification 1235

elites: benefits of colonialism 994; benefit from conquest of neighbouring states 21617; corruption and foreign investment 1299; declines in 1012-13; during World War II 1200; in early cities 206-7; gender and social stratification 234; incomes of, pre-industrial revolution 189-90; local elites within empires 347, 354, 355, 434, 437-8, 440; luxury trade for 437; post-World War II in Germany and Japan 1240; power of rulers over 354; public perceptions of 417; in revolutionary Latin America 1082-3; Russian boyars 763; wealth of 788

emotions: human genetic inheritance and 82-3

England: Norman conquest 631; state formation 631; Stonehenge 211-12; the Enlightenment 910, 931, 9767, 1174, 1175

environment: environmental challenges 1362-4; forest destruction 1170; human effects on 1170-1; ozone layer 1169; pollution 1167-9; see also climate change epidemics see disease Equiano, Olaudah 1100-1 ethics: and the abolition of slavery 1097, 1098, 11056; challenges of 1349-50; chivalry 563; ethical analysis 324-5, 1356-7; ethics of duty 446; human behavior and 324; human rights arguments 1154-5; legacies of empires 446; links between philosophy and theology 409, 635-6; terrorist activities 1220; within religions 322-6, 457-8, 482

Ethiopia 370, 476

ethnic identities: autocracies and multi-ethnic states 935; decolonization and ethnic diversity 1267, 1291, 1298; ethnic self-determination in nation states 913-14; ethnic superiority in colonialism 995; group identities 1386-7; minority groups 904, 926; nationalism 934; in Russia 823; tensions over in empires 919-20; within the nation state 904, 928-9

ethnic identity: under classical empires 346, 347, 348-9; ethnic consolidation, post900CE 605; in the Mongol Empire 605; national identities and 605-6

Europe: abolition of serfdom 1120; abolition of slavery 1100-3; access to technology 955; adoption of tea, coffee and chocolate 779-81; adoption of the potato $830-1$; cannons 772-3; colonialism in Africa 990; colonialism in Asia 988-90; contract dispute resolution 760; the Crusades in 639; the Enlightenment 910, 931, 976-7, 1174, 1175; European slave trade 834, 836-7; exceptionalist arguments for European civilization 947, 948, 952, 957; family patterns, post-900 CE 623 ; in the fifteenth through eighteenth centuries 905-11; Gothic Churches 617-18, 621, 
634; gunpowder technology

774, 776-7, 951-2, 964; migrations into, post Roman Empire 548-9; nation state formation 911-15; political consolidation 905-6; political consolidation after $900 \mathrm{CE}$ 599-600; political institutions 906-7; population growth 1050; religious divisions 762 , 767-8; the Republic of Letters 973; spread of agriculture to 121; state formation 629-32; Thirty Years War 762, 769; trade expansion 642-6; trading cities 642, 644-6; witch hunts 770-1; see also Great Divergence; individual countries

evolutionary analysis: of art 336-8; of cultural values and practices 20-3; of institutions 289-93; inter-societal transmission 19; mutations $18,24,74$; overview of 18-20; progress within 24, 1345; of religions 307-9, 454-6, 499500; selection environments 18-19, 24; state formation 219-21; succession crises 866-8; of technology and science 192-7; transmission mechanisms 19; of war 246

evolutionary processes: bacteria

55; biological evolution 54-6; chemical evolution 52; defensive capabilities 60, 61; ecological limits on population 55; extinction of the dinosaurs 61-4; for human hunter gatherers 73-6; group selection 83, 84; harmful mutations 55, 57; increased complexity 197; path dependency 57-9, 61; revolutionary change 38-40; small vs. large mutations 57; social evolution via revolutions 57, 59; species adaptation 54-6

explorers: challenges of 690 factories: American System of Manufactures 1043; British Industrial Revolution and 1027-8, 1031-2; emergence of 1027-8; the factory owner's challenges 1040; physical punishment of workers 1116; of the Second Industrial Revolution 1046; transport improvements and 1038; workers in 1013-14

families: birth control technologies 1054, 1161-2; church inheritance patterns 634; decreasing fertility rates 1050-4; defined 14, 300; in the earliest societies 300-1; family formation 137; in hunter gather groupings 93; the modern parent's challenges 1053; nature of childhood 1056-7; nuclear families 623; polygamy 623; post-900CE 622-4; religion and the family unit 327; in the Roman Empire 417-18; as a social structure 14 ; see also childcare; parents

famines: in early agricultural societies 134; Malthusian theory and 137; in world history 1339

fascism: overview of 1191; path to World War II 1193-4; rise of, post-World War I 1191-3 fermentation 158-60, 650, 779 feudalism: abolition of serfdom 1096, 1122; abolition of serfdom in Austria 1121; abolition of serfdom in Eastern Europe 1120-2; abolition of serfdom in Russia 1121-2; abolition of serfdom in Western Europe 1120; chivalry concept and 563; decline of 564-5, 745-6; in Eastern Europe 565 ; economics of 562 ; economics of serfdom 1120; influences and effects of 563; the intelligensia's arguments against 1120-1; political structure 558-9, 561-2; in
Russia 1120; seigneurial tenure in Canada 1123-4; serfdom 559-61, 564; trade 563-4; women under 560-1, 563

financial institutions: in the ancient world 379-80; evolution of 648; first banks 379; government bonds 647; interest payments 378 ; international organizations 1153-4; in the Islamic Empire 526-7; in Mesopotamia 378; pension plans 1053; stock markets 647-8; tontines (pensions) 647; see also money

fishing: challenges of 152; early

fishing-based societies 151-2; fish stocks 1363-4; Jomon culture 162; overview of 112-13

food: adoption of new crops 528 ; beer 650 ; benefits of cut and cooked food $75,88,89$, 100; cacao beans 780, 781; coffee 779; the Columbian Exchange 828-9; cooking of grains 117; cooking using pottery pots 163-4; emergence of cities and 204; famines 1339; fermentation 158-60, 650; fish trade 839; food consumption and income 950, 1309, 1312; human consumption of 188 ; income allocation for 190-1; increased production postIndustrial Revolution 1035, 1307; nutrition and human nature 138; omnivore hunter gatherers 105; Portuguese influences on Asian cuisine 819; potatoes $830-1$; preservation of 1022-3; refrigeration 1007, 1233-4; rice 588, 663; of the Roman Empire 420; spices 382-3, $679,680-1,799$; storage $115-$ $16,162-3$; sugar cane 363 , $367,640,779,798,813$; tea 779-81; vegetable oils 160 fossilization 56

France: abolition of slavery 
1102-3; colonization of Algeria 1001; democracy in, post-Revolution 1072, 1077; Eiffel Tower 1044; feudalism in 564-5; within the Holy Roman Empire 629-30; industrialization in 1041-3; justification arguments for colonialism 999-1000; linguistic consolidation 910 11; national assembly 939; scientific enquiry 1016; Seven Years War 1032, 1062-3, 1065, 1073; state formation 630-1; in World War I 1179-80; in World War II 1195-6

French Revolution 911; compared with the American Revolution 1072, 1077; Declaration of the Rights of Man and of Citizens 1077, 1078; events of $1074-5,1089$; financial roots of 1073-4; legislatures 1072, 1077; the Reign of Terror 1072, 1075 fusion reactions $43-4,64$

galaxies 43

Galileo Galilei 968-9, 974

Gandhi, Mohandas (Mahatma) $489,1189,1279,1280-1$

the Ganges: agriculture in 363; the Ayrans 275, 363; caste system 275-7; migration to from the Indus Valley 274, 275, 345-6; nomad's conquest of 363; see also Mauryan Empire

gender relations: in ancient Greece 404; cultural variations in 1355-6; in early states 233-4; gender rights 1155-6; in the Gupta Empire 368; in hunter gatherer societies $77-8$, 93-4; in Islam 482-3, 487-8; in Japan 586-7; male warrior culture 141-2; organized sports and masculinity 1011; in the Persian Empires 360; rules of succession 281; social policy changes and 1145; and social stratification 234 ; third gender concept 1163; in times of war 245; and the transition to agriculture 140-2; see also women

genes see human genetic inheritance

Geneva Convention 1175

Germanic tribes: Franks 551; Goths 550, 551; migrations into Europe 548; Vandals 550; in the Western Roman Empire 549-51

Germany: bombing of in World War II 1203-5; during the Golden Age 1240-1; Hanseatic League 645-6; within the Holy Roman Empire 62930, 632; increased power of pre-World War I 1179, 1180; industrialization in 1041-3; post-war elites 1240; post-World War II 1207-9; repatriation payments postWorld War I 1187-8, 1230; research universities 1016-17; social service provisions 1144; state formation 912, 921; see also Nazi Germany; World War I; World War II

Ghana 813, 1295

Ghana Empire 532, 712-13 glass $164-5$

globalization: communication technologies 1254; criticism of trade liberalization 1256-7; global art markets 1255-6; international trade 1252 , 1254; international trade treaties 1252-3; long supply chains 1254-5; long-term investment flows 1258-9; opera 1256; power of multinational corporations 1258-9; quotes on 1261; reduced transport costs 1253-4; of services 1257; short-term investment flows 1257-8; wider historical context 1260-1

Golden Age: economic growth 1238-40; impact on social services provision 1244; impact of World War II 1239; technological innovation
1240; unemployment and inflation of the 1970s 1241-2

Goths 550, 551

gravity: formation of the stars 42-3, 45, 64; theory of 967

Great Britain: abolition of slavery 1000, 1100-2; administrative control in America 1063-4; Civil War 762-3, 939; code cracking during World War II 1201; coffee demand 779; colonization of South Africa 1000; Crimean War 1120; cultural exchange with India 883; democracy 1033, 1064; exploration of Australia and New Zealand 811; exploration of the Pacific 811; Hudson's Bay Company (HBC) 842-3, 1067; joint stock companies 820, 881-2, 883; Manchester slums 1327-8; organized sports 1011; at the outset of World War I 1179; Parliament 939-40, 957, 1033; population growth 1050; property rights 1033; railways 1004; relations with the Mughal Empire 881-2; road and waterway network 1035; rule in India 882-4, 918; Seven Years War 1032, 1062-3, 1065; taxation 940, 1142; tea plantations 780; trade in Asia 820-1; transportation of criminals 1116; in World War I 117980; in World War II 1196; see also American Revolution; British Industrial Revolution

Great Depression: causes 1226-7; economic theory and 1228 $30,1236-7$; impact of 1226 , 1227-8, 1231-2; impact on social services provision 1144, 1145, 1227, 1242-4; influences and effects of 1244; links to World War I 1230-1; links to World War II 1191; technological arguments for 1231, 1233-6, 1237; trade restriction policies 1150 , 1226-7 
Great Divergence: British Industrial Revolution and 1026, 1032-3; colonies and natural resources 951-4; demographic factors 955-6; European access to technology 955; explanations for 946-7, 948-9, 966; impact on the Ottoman Empire 865-8; income comparisons 949-51, 1048; political and economic institutions 956-61; political fragmentation and 957, 962, 963; religions 961; Scientific Revolution of 9667; technological innovation and 961-5; term 946; trade 954-5

Greece: nation state formation 914; population swap with Turkey 916; see also Crete

Greece, ancient: Aristotle 402, 404-7, 449, 635; armies 399,402 ; art of 331; city states 398-9; conflict with the Persian Empire 401-2; democracy 399, 448-50; earliest societies 269-70; early religion in 317-18; education 408; emergence of 345; financial institutions 379; gender relations 404; Homerian epics 270, 299; links with the Scythians 402 3; medicine 390-1; Museum and Library at Alexandria 391, 393-4; Mycenaeans 272; Olympic Games 404; patriotism 451; Pericles 399; philosophy 389, 404-9, 445, 635-6, 972; Plato 404-7, 449; Ptolemaic Empire 403-4; scientific practices 389-91, 967; seafaring prowess 400; Socrates 404-7, 408, 451, 457; Sparta 399, 402, 404; theatre 404; trade 400-1, 435; warships 401

Greenland 555

Gross Domestic Product (GDP): defined 191; within a historical framework 191-2; the Human Development
Index 192; limitations of 191

guilds: artisan guilds 651-3; knowledge transmission 9645; merchant guilds 645-6, 651 gunboat diplomacy 993 gunpowder technology: army use of $773,775,776$; for cannons 772-3, 816; in China 772, 774, 775, 776; European exploitation of 774, 776-7, 951-2, 964; global uses of 774; gunpowder empires 774, 850-1; impact on warfare 772 ; infantry firearms 772, 773; influences and effects of 777 ; links with state organisation and finances 776; nomads use of 776; of the Ottomans 774, 775, 850-1, 859; regional variations in 772

Gupta Empire: administration 367; armies 367; caste system 367; cotton cloth 363, 366-7; decline of 367 ; emergence of 366; gender relations 368; Hinduism 366, 367-8, 459, 492; legacy of 592; numeral system 366, 389, 977; sugar cane 363,367 ; trade 366

\section{Haiti 1076, 1080}

Haitian Revolution: and the abolition of slavery 1080 , 1081, 1098, 1102; Declaration of Independence 1082; events of 1079-80; French opposition to 1080, 1081; international hostility to 1081, 1082; post-revolution challenges 1082

Han Empire: agricultural technology 436, 442; art of 430; bureaucracy 4289, 434, 442-3; compared to the Roman Empire 431-6; Confucianism 424, 428-9, 442, 447; cultural consolidation 443; currency 436-7; decline of 434, 435, 438-41; emergence of 345 , 384-5, 424; examinations for bureaucrats 428-9; forced labor 435; Han Wudi 384,
427-8; heavenly mandate for rulers 427, 429, 444, 448; income inequalities 438; iron technology 429; land ownership 438-40; legal code 429; libraries 393; luxury trade 437; paper 429, 653-4, 977; political organization 427; reconstitution of the Chinese empire 441-3; road network 435; sea trade 382; Silk Road 384-5; taxation 436-7; trade 429-30, 436-8

\section{Hare Krishna 765}

Hawaii 925-6, 1002

head-binding 545

health: clean water 158; famines 1339; in early cities 208; of hunter gatherers 133-4, 138-9; impact of inter-empire trade 388-9; life expectancies 134, 135; long-distance trade and disease 208; malaria and quinine 993; overeating 1309, 1340; reduced food diversity's impact on 133-4, 138-9; scurvy 809, 810; state funding for healthcare 1146; theme of 299; see also diseases

Hinduism: the Bhagavad Gita 446, 459, 765; Buddhism as a reaction to $367,466,467$; caste system 459, 591, 881; creation myth 306-7; in the Gupta Empire 366, 367-8, 459, 492; Hindu-Muslim conflict in India 1281-2; in India 591, 671-2; merchants' spread of 388 ; polytheism 458; representations of the gods 490; rise of 591; Sri Krishna Caitanya 765, 768; the Upanishads 320, 323, 4589, 591, 883; the Vedas 274, 277, 319-20, 363, 458, 591; warfare and 489

history: challenges of 1347 ; comparisons across societies 432-3; historiography 1238; how to draw lessons from 1347-8; human progress and 1345-7; Ibn Khaldun's theory of 547-8; modernist 
approaches 25; postmodernist approaches 24; scholarship on 24-5; world history and 1376-7

Hitler, Adolf 921, 1184-5, 1191, 1192

Hittites 256, 260, 282

Ho Chi Minh 1287-9

Holy Roman Empire 493, 562, 629-30, 762

hominids: art of 95-8;

Australopithecus Afarensis 701; biological evolution into hunter gatherers 73-6; control of fire 89-91; effects on the natural environment 105; evolution of modern humans 70; gender relations 93; group size/brain size connection 72 ; homo genus 71-2, 77, 103-4; homo sapiens $70,72-3,77$, 79-80, 88, 94, 100-1, 103-4; language capacity 71,72 , 81, 106; migration of 101-3; neanderthals 73, 77, 79, $82,88,93,95,103-4$; from primates 64 ; tool-making 71, 72, 85-8; see also hunter gatherers

horses: agricultural uses for 145 , 157; in the Americas 829; in Central Asia 275, 282, 540, 541-2; domestication of 148 , 282; horse collars 436, 649; horseshoes 649; stirrups 543, 726

horticulture 157

housing: in early cities 214 Hudson's Bay Company (HBC) 842, 1067

human genetic inheritance: altruism 83, 107-8, 324; artistic evolution 81-2; bipedalism 70, 75-6; capacity for self-deception 84-6, 95, $107,1351,1352$; cultural co-evolution and 74 , 77-8, 81-2; emotions 82-3; environmental influences 76-7; evolution as hunter gatherers 73-6; evolutionary psychology 74; Family Tree of the Human Race
64; group identity 932-3; impact of agriculture on 135-9; increased brain size 74-5; individual differences within 78; key characteristics of human evolution 69-70, 73-4; lactose tolerance 76, 134, 135-8, 146; language development 71, 72, 74-5, 78-81, 82; theme of 107-8

human nature: group identities 1386-7; humankind's aspirations for 1352-4; importance of self-knowledge 1350-1, 1385; institutions to modulate 1348-50; selfjustification 1351, 1352; see also individual differences human progress: achievability of 1384-6; balanced perspectives 1388-90; evaluations of 1380 3; motivations for 1383-4; philosophical ideas of 1057-8; by theme $1380-2$; in the twentieth century 1129-30; within world history 1345-7 human rights: gender rights 1155-6; peacekeeping missions to protect 1152-3; Universal Declaration on Human Rights 1154-8; universal human rights concept 1096

Hungary 1212-13, 1215; the Huns 544-5, 550, 551, 573-4 hunter gatherers: aboriginal Australians 693-4; agricultural technology of 156; cave drawings 81-2, 95-7, 656; challenges of 106; childcare 105; earliest religions 81-2, 304, 310, 311-12; economic activities 91-2; evolution of 73-6; fishing 152; gender relations 77-8, 93-4; group identity $932-3$; health of 133 4, 138-9; human capacity for self-deception 95; incomes 92 3 ; inter-societal connections 106; as omnivores 105; path dependency and 74; population densities 99-100, 104-5, 133; short-distance trade 92; social structure 94-5; tool-making 88-9; transition to agriculture 115-17, 118-19; wars $94-5,236$

Ibn Battuta 532, 609, 610-12, 613-14, 624

Ibn Fadlan 553-4

Ibn Khaldun 229-30, 542, 547-8, 851

Iceland: Viking migration to 554-5

imperialism: in American foreign policy 1002; defined 1002; national sovereignty and 911

Inca: quipu 223-4, 225

Inca Empire: Atahualpa 805; Cuzco 705; European conquest of 694; guano 10212; lack of writing system 694, 704, 805; llamas 694-5, 705; Macchu Picchu 707; overview of 706-7; potatoes 830; quipus 704-5; smallpox epidemic $805,806,827-8$; society of 703-4, 707-9; Spanish conquest of 805-6; trade 706; transport infrastructure 705-6 income distribution: in ancient Greece 399; in early societies 189-90; in hunter gatherer societies 92-3; global economic convergence 1247-8; historical experience of inequalities in 1249-50; human sacrifice 206-7; income inequalities in classical empires 438; inequality in early cities 206-7; and migration 1317; overview of 152-3; postGreat Plague 745-6; poverty reduction 1289; slave incomes 372-3, 376; within world history $1368-9$

incomes: allocations for food 190-1; under classical empires 188; consumption patterns 790, 950; early income taxes 1142-3; of elites 189-90; growth in 156, 187-9, 190; income distribution 189-90, 192; income inequality and 
democracy 1012; income inequality in Latin America 1084; of merchants $184-5$; of the middle classes 1012; overview of 152-3; poverty lines 1288-9; rises in 1146; wage rates in 1800 949-50; see also Great Divergence

India: abolition of slavery 1101 ; British rule in 882-4, 1008; Buddhism 529-30, 591-2; cannons in 774; caste system 367, 445, 591; Charvaka 460; China's incursion into 1286 7; Chola 679-80; Christian communities in 815-16; classification of species 975 ; colonial bureaucracy 994-5; cotton 1033; culture exchange with Britain 883; the Deccan 774; decolonization 1270 , 1271-2, 1276, 1280-2; democracy 941, 1131; early religion in 319-20; ethics of duty 446; Hindu identity 918; Hindu-Muslim conflicts 920, 1281-2; Hinduism 591, 671-2; the Indian Mutiny 1090-1; indigo dye 581; industrialization in 1049; influences on Vietnam 588-9; influenza epidemic, post-World War I 1187; iron industry 1049; Islam 484, 493, 593; Islamic art influences in 535; under the Islamic Empire 529-30; Jainism 591; kingdoms of, post-Guptas 592-3; legal system 883; Nalanda university 529-30, 580, 592; opposition to British rule 918; partition 1281; period of disunity 590 1; population growth 1054; post-900CE 600, 670-2; postcolonial political structure 1282-3; stupas 366; Taj Mahal 535 ; women in post-colonial India 1283; during World War I 1186; see also Gupta Empire; Indus Valley; Mauryan Empire; Mughal Empire
Indian Ocean: Portuguese exploration of 815-16

individual differences: genetic diversity and 10; human agency 1379-80; individual diversity within cultures 21 ; individual historical actors 108-9; live your best life 1390-1; personality diversity 108; schemas for 108; theme of 108-9; through religion/ philosophy 1391-3; within human evolution 78; world history and the meaning of human life 1379; see also human nature; human progress

individualism: in religion 767 , 768

Indo-European language: Hittites 260 ; spread of $124,148,282-$ 3, 295, 296

Indonesia: Papua 1302; under Suharto 1217, 1278; tin mines 1003; Wajorese trade network 778

Indus Valley: art of 331; cities 205-6, 272, 273; civilization of 272-4; cotton cloth 166 , 274; decline of 273-4, 276, 345 ; earliest societies 201, 203-4; gender relations 233-4; migration to the Ganges 274, 275, 345-6; Mohenjo Daro 273 ; spread of agriculture to 124; trade 274-5; writing in 227,272

industrialization: demographic changes 1050-7; free markets for labor 1098-9; humankind's aspirations for 1352-3; mining 1003; nature of childhood 1056-7; need for educated workforces 1052; second generation industrializers 1041-5, 1048; smaller family sizes 1050-4; third generation industrializers 1048-9; urbanization 1009, 1051; the working week 1011; see also British Industrial Revolution; Second Industrial Revolution
Industrial Revolution: links with the Scientific Revolution 977-8;

industry: best practice manuals 963-4; capitalism and 78990; cottage industry 789-90, 958; of the Song dynasty 664; see also British Industrial Revolution; guilds; Second Industrial Revolution

infrastructure development: of the Song dynasty 661-2

institutions: control and design of 1349; cultural support for 1349; decline in religious belief 1165; defined 11, 289-90; of democracy 9378, 940, 941-2, 1135, 1193; economic institutions 153; elected assemblies 906-7; and environmental challenges 1362-3; European political institutions 906-7; for the human good 1348-50; institutional capacity for colonial administration 952; institutional evolution 28993; institutional weakness in post-colonial societies 1271 2; political and economic institutions during the Great Divergence 956-61; religious 309; revolutionary institutional change 1062; state capacity 929; trade institutions 643-4; see also economic growth; financial institutions; laws; states; universities

international organizations: civil society organizations 1154; emergence of 1150; financial institutions 1153-4; International Red Cross 1175; League of Nations 1150-1, 1189; non-governmental organizations 1259; peace organizations 1175, 1222; United Nations (UN), 1151-3 International Red Cross 1175 inter-societal connections: hunter gatherers 106 Iqbal, Muhammad 915-16, 1277 
Iran 1166, 1279-80

Iranian Revolution 1279-80

Iraq 1272-3; prehistoric burial 81; see also Mesopotamia

Ireland 831

iron production: in Africa 709, 837; for agricultural productivity 363 , 695; by Bantu agriculturalists 122-4, 171 ; in the Ganges region 363; in the Han Empire 429; in India 1049; metal plows 140,171 ; for weaponry 367 , 434

Islam: abolition of slavery in Islamic states 1110-11; appropriate behaviors for merchants 481-2, 488, 528; and Arabia's nomadic culture 482; artistic imagery 491; beliefs of 483 ; doctrinal disputes 482, 485; gender relations 482-3, 487-8; hajj 610; Hindu-Muslim conflict in India 1281-2; in India 484, 493, 593; interactions with Christianity 769; Islamic fundamentalism 1135, 1166; libraries 1019-20; life of Muhammad 479-81, 505; in the Mongol Empire 747-9; monumental architecture 619-20, 621; Muhammad's contested succession 485 , 513; nationalism and 915-16; Plato's influence on 449; post-colonial Islamic states 1277-8; the Quran 481-3, 534; relationship with the state 484; religious authorities 761, 941; religious toleration 483-5, 493; the Shafiite on the treatment of Jews and Christians 853-4; the Sokoto Caliphate revolution 1087-8; in Southeast Asia 528, 677; in Spain 535, 620; spread of 4834, 492, 493, 494, 783; Sufism 485, 494, 764-5; Sunni/Shia split 485, 513; syncretic Islam in West Africa 532; warfare and 490; in the Western Roman Empire 557; women in 482-3
Islamic Empire: Abbasids 490, 515-17, 523-4, 640; architecture 517, 619-20, 621; armies 508, 514-15; art of 533-5; Baghdad c. 1000 ce 520-1; Cairo 522-3; in Central Asia 533; conquest of the Byzantine Empire 507-8, 566, 571, 574-5; contested succession 513; cosmopolitanism of 523-4; the Crusades in 640; early characteristics of 505-6; financial institutions 526-7; in India 529-30; limited expansion 508-11, 512-13; literature 517-18, 602, 608; Mamluks 531; mathematics 518, 521, 977; nomadism's influences on 506; paper 522, 653-4; political organization 511-13; scientific practices 518-19, 521-2, 967; slavery 527; in Spain 483-4, 493, 515, 523; technology 522; trade 526-8, 606; Umayyads 514-15; in West Africa 531-2; women in 525-6

Israel 1219-20, 1273-4

Israeli-Palestinian conflict 1273-4

Italy: conquest of Libya 1176; early city states 631, 632; fascism in 1191-2; political consolidation 912-13; Venice $526,640,642,643,647$

Jainism 459-60, 591

Japan: abolition of slavery 1109; adoption of western technologies 1001; agriculture 585-6; Asian decolonization and 1274-6; attack on Pearl Harbor 1198; authoritarian rule in 1192-3; bombing of in World War II 1201, 1203-5; Buddhism 586; Chinese influence 586-7, 669; colonialism of 1001 ; contract dispute resolution 760; control of conquered territories during World War II 1200; economic growth 1048; electricity in 1048; during the Golden Age 1240-1; gender relations 586-7; gunpowder weapons 774, 775; industrialization in 1049-50; invasion of Manchuria 1191, 1194-5, 1205, 1284; Jomon culture 152, 162; Meiji Restoration 1012-13, 1049-50, 1057; nation state formation 917 18; naval victory over Russia 1050; political consolidation 668-9; population 1056; post-900CE 668-70; postwar elites 1240; post-World War II 1206-7; printmakers 784-5; relations with the Portuguese 816 ; resistance to the Mongols 665, 668; Shinto 485-6, 586; Shintoism 1164; state formation, post-900CE 599-600; Tale of Genji 587, 602; textiles 1048; Tokugawa shoguns 668-9, 774, 1001; in World War I 1179, 1186; in World War II 1194-5, 1199, 1201, 1203, 1205, 1284; written script of 586-7; Yamamoto emperors 586 joint stock companies: British 820; formation of 819 , 820; military expenses 819-20

Jordan 1272-3

Judaism: anti-Semitism 1119; creation myth 305-6; early Jewish Christians 473; emergence of 460-1; the Holocaust 1202-3; Jewish settlers in the Middle East 1273-4; the Kabbalah 765; links with early Levantine religions 316; monotheism 461; political thought 450-1; under the Roman Republic 412; Sabbatai Sevi of Izmir 765; warfare and 489; wider influence of 462 ; written texts 462

Kenya 1296-7

Keynes, John Maynard 1188, 1228, 1230 
Korea: Chinese influence in 5834; conflicts over communist expansion 1213, 1216-17; gunpowder weapons 775; Jang Bogo 585; Koryo dynasty 584-5, 599; Mongol Empire in 665; political consolidation 917; political consolidation after 900ce 599-600; Silla rulers 584

Korean War 1136, 1206, 120910, 1216-17

Kush kingdom 218, 263, 268

Kushan Empire: art of 369, 490; Buddhism 368-9, 468, 492; emergence of 368, 403, 545; expansion 385

land ownership: British property rights 1033; in China 760-1; concentration of 189 ; early law codes 231; farm sizes 43940; inheritance systems 142 , 525-6, 623; in Newfoundland 841 ; property rights and economic growth 957-8; in sub-Saharan Africa 710; under the Tang Dynasty 578-9; and the transition to agriculture 130, 133, 139-40

languages: abstract thought capacity and 82, 84; Bantu language families 122-4, 296, 717; Chinese dialects 583; Cold War soft power tactics 1214; defined 14; in early humans/hominids 71 , $72,81,82,106$; evolution of cognitive capabilities 74-5; families of 296; in human evolution 73 ; influences on and effects of 80-1; linguistic consolidation 933-4; linguistic standardization, post-900CE 604; links with the spread of agriculture 121, 122-4, 148; links with tool-making 79, 87; Martin Luther's translation of the Bible 767; migrations into Europe 549; national identity and 910-11; spread of 296; see also Indo-European language
Latin America: decolonization in 1298-300; democratic institutions 941; earliest societies 285-6; elites and foreign investment 1299; fishing 151; metallurgy 170; nation state formation 918-19; nomadism in 146; pyramids 210; region of xliv; spread of agriculture to 128 30; transition to democracy 1133, 1299; see also Americas; Mesoamerica

Latin American Revolutions: in Brazil 1087; in Costa Rica 1086; democracy in 1084, 1085, 1299; elites in 1082-3; income inequalities 1084; in Mexico 1085; nation states 1083; in Paraguay 1084-5; plantation economies 1084; post-revolution dictatorships 1083-4; process of 1082-3

laws: bankruptcy laws 761, 958-9, 1116; of the Byzantine Empire 570; of the Chinese empires 429; of classical empires 351-2; Code of Hammurabi 207, 231-3, 258-9, 287, 300; contract disputes 760, 782-3, 959; in the Early Modern period 760-1; in early states 233 ; emergence of formal laws 231; Hammurabi Code 359, 461; in India 883; inheritance laws 960; Justinian's Code (Byzantine) 570; links between law and commerce 760; of the Ottomans 761, 860; of the Persian Empires 359; for the protection of trade 286-7; public/private law distinction 760, 960; role of local administrators 232; of the Roman Republic 412; state vs. religious authority within 761

League of Nations 1150-1, 1189

League of Nations Mandate 1185-6, 1272

Lebanon 1273
Levant: architecture of 211, 214; Christianity 268; city states 268-9; early religion in 316; Judaism 268; Phoenician alphabet 227-8, 269, 407; Phoenicians 269; region of xliv

libraries of the ancient world 392-4; Ashurbanipal library 393; Islamic 1019-20; of Islamic Cordoba 523; Museum and Library at Alexandria 391, 393-4; scientific enquiry and 1019-21

literacy: Europe's Republic of Letters 973; growth in, post900CE 604; reading of religious texts 961 ; role in religious reforms 768

literature: The Divine Comedy (Dante) 656, 657; of the Islamic Empire 517-18, 602, 608; of the Ming dynasty 889-90; regional vernacular literature 604,656 ; of the Renaissance 656; Tale of Genji 587, 602; see also stories

Luther, Martin 767, 768

Lydia, Anatolia 354, 357-8, 377

Magellan, Ferdinand 809-10

Magyars 554

Malaysia 1003, 1277-8

Mali Empire: expansion of 532; Jenne-Jeno 716; Mansa Musa 532,715 ; overview of 713-14; Timbuktu 532, 609, 620, 714, 716

Malthus, Thomas Robert 136-7, 1307, 1310, 1313

mammals 63-4

Manchuria 1191, 1194, 1205

Manchus 762, 893

Manicheanism: beliefs 478; in Central Asia 470-1; critical texts 478; decline of 479; life of Mani 478, 479; Parable of the Pearl-borer 480; spread of 478-9

Mao Zedong: Confucianism and 447, 1284-5, 1286

Marx, Karl 1010-11

mathematics: in the Islamic 
Empire 518, 521, 977; numerals of the Gupta Empire 366, 389, 977

Mauryan Empire: administration 363-4; art of 365-6; Ashoka 364-5, 446; Buddhism 3645, 468, 492; Chandragupta Maurya 363; decline of 365; emergence of 345-6, 363; Pataliputra 363; spy networks 363-4

Mayans 391, 486

Mazzini, Giuseppe 936

medicine: in ancient Greece 390-1 merchants: advice for merchants in the Mongol Empire 739-40; Aztec 697-8; Buddhism and 388,469 ; challenges of 181 , 286, 643; cultural suspicion of $183-5$; in early cities 205 ; fairs 643-4; guilds 645-6, 651; humankind's aspirations for 1352-3; Islamic guidelines for 481-2, 488, 528; Muslim merchants, post-900CE 607; in the Persian Empires 359; relationship with states $180-2$, 183-4, 288-9; Silk Road trade networks 386; the spread of religions and 388, 469, 488, 494-5; state protection of 180-2, 286-7; trade networks, post-900 CE 607 ; trading networks 182-3, 287, 383-4, $488,643,820$; wealth of 788 ; writing for record-keeping 224-5, 378

Mesoamerica: art of 332; ball games 991; cacao beans as currency 175, 284; earliest societies 201, 203-4; early religion in 322; head-binding 545; Maya 276, 283-5; Mayans 391, 486; metallurgy 170; monumental architecture 620; Olmecs 283, 285, 545; political consolidation after 900CE 600; pyramids 210, 284-5; region of xliv; spread of agriculture to $127-8$; Teotihuacan 370, 695-6; Toltecs 696; writing 283, 285; writing systems $694,697,703$; see also Aztecs

Mesopotamia: Akkadian empire 254-5; art of 331; Assyrian Empire 253, 256-7; city states 445; Code of Hammurabi 207, 231-3, 258-9, 287, 300; cotton cloth 166; cuneiform 226, 228; decline of 345; earliest societies 201-2; Epic of Gilgamesh 222, 241, 253, 317,461 ; finance system 378 ; gender relations 233 ; Hammurabi Code 359, 461; inscriptions 257-8; links with Egypt 261; palace at Mari 211; political organization 252-3; region of xliv; religion 253 , 316-17; slavery in 207; stelae 258; Sumer/Babylonia 253, 255-6; taxation in 253-4; trade with Anatolia 254, 256, $259,287,289$; trade with the Indus Valley 274-5; trading networks 182; Ur III 255, 378; urban gardens 211, 255; Uruk 253; use of bricks 164; water supplies 252; ziggurats 210 11, 264

metals: in the ancient world 167, 169; bronze 167, 169-70, 207, 279-80; causes and effects of 172 ; copper 167, 168; environmental impact of 172; gold trade in Africa 532; iron 171; nomad manufacturing 147; plows 122-4, 140, 157; precious metals 172, 175-6; silver from the Americas 8067,895 , 955; smelting 167 , 170-1; steel 171; trade in 1678; World War I 1184

Mexico: Catholic missionaries during the conquest of the Americas 808; Mexican Constitution 1085-6; Mexican Revolution 1085; red dye production 829-30; Spanish colonial rule in 807-8; trade route to the Philippines 811 ; Zacatecas silver mines 806-7; see also Aztec Empire middle classes: clothing 1057; in the colonies 1012; emergence of 1011; incomes of 1012; political aims 1011-12, 1133; revolutionaries 1089; socioeconomic values of 1011-12; supervisory roles 1009, 1011, 1027-8

Middle East: autocracies and democracies in 1133-4; colonial states post-World War I 1185-6; the Crusades in 574, 637-9; decolonization 12724; early agriculture in 118-20; early regional centers 120 ; Jericho 203, 236; nomadism in 146; organized sports 1011; region of xliv

migration: to the Americas 844-6, 1316; to Asia 1316; cultural factors for hominid migration 103; due to social conflicts 95; during the ancient period 295; during the Great Depression 1227-8; of early humans/hominids 101-3; economic incentives 1317; effects of 1322-3; of Germanic tribes into Europe 548 ; human adaptability 102 3 ; immigration controls 1317, 1319-20, 1322; of individuals/ family units 297 ; involuntary 1318,1322 ; map of early human migration 102; mass migrations 296-7; the migrant's challenge 1323-4; motivations for 298-9; in the nineteenth century 1316-19; of nomadic groups 144-5; of nomads into Europe 548-9; to the Pacific Islands 295-6; and the spread of religions 495; transport for 1316-17; in the twentieth century 1130-1, 1320-3

military coups 1134

military technology: cannons 631, 742; chemical weapons 1175, 1182-4; composite bows $542-3,573,722,726$, 822; contemporary challenges for 1222-3; European advantages in 865-6; Greek fire 574; gunpowder 741-2; 
of the Huns 573-4; modern industry's role in 1120; rocket technology 1196; of the Spanish conquest of the Americas 801, 805-6; of the twentieth century 1174; use in colonialism 993; in World War I 1180-4; in World War II 1193-4; see also armies; gunpowder technology; weapons

Ming dynasty: agriculture 889; bureaucracy $884,887-8$, 889; Catholic missionaries 889; decline of 762,893 ; the Forbidden City 885; Great Wall 888; gunpower technology 772, 774, 775; Hongwu's founding of 884 ; literature 889-90; naval expeditions 890-2; social structures 889; succession crises 885; taxation 884-5; trade 890, 891-2; war over Taiwan 774; women's roles 889,890

mining: in Africa 1000, 1003; in colonial regions 994 , 995, 1003; copper 1003; establishment of 167, 1003; global trade in minerals 1003; tin 1003

monarchies: rise of European monarchies 631-2; sacred monarchy concept 444-5, 446; succession crises 634; see also rulers

money: cowrie shells 176, 377; currency production, post900CE 608; in the feudal system 563-4; first coins 354, 377-8; gold and silver for 172, 175-6, 377; in the Han Empire 436-7; paper currency 608,662 ; role of 176; in the Roman Empire 436-7; trade and 377-8; within empire bureaucracy 354

Mongol Empire: account of a visit to 729; administration of 734-5, 738, 742-3; advice for merchants trading in 739-40; art of 741; Buddhism 746,
748-9; in China 665; Chinggis (Genghis) Khan 352, 665, 722-4, 730, 873; destruction of 737; emergence of 542; ethnic identity strategies 605; fear as a weapon 727, 730; gunpowder technology 772 ; influences and effects of 729 ; Islam 747-9; in Japan 665, 668; Karakorum 738; Khubilai 733, 736, 747; in Korea 665; later khans of 731-3; logic of slaughter 730-1; Marco Polo at the court of $610,613,734$, 736; military strength 722 , 726-7; military technology 722, 726; political strategies 727-9; religion in 733, 746-9; security 738 ; taxation 734 , 737; trade 737-8, 741; transfer of knowledge in 741-3; transport infrastructure 737-

8; Uighurs in 546-7, 734, 746; women in 735-6

monumental architecture: Borobudur temple 681-2; builders of 212-13; building techniques and knowledge 621-2; of the classical empires 351; Coliseum, Rome 351, 415; cultural role of, post900CE 617-22; earliest forms of 119-20; early monumental architecture 209-11; Gothic Churches 617-18, 621, 634; Great Wall of China 888; Hagia Sofia, Constantinople 567, 619, 620; influences and effects of 622; of the Islamic world 619-20, 621; Macchu Picchu 707; in the Middle Ages 601; pagodas 618-19; pyramids 620; pyramids, Egypt 210, 263-5, 313; pyramids, Mesoamerica 210, 284-5; Stonehenge, England 211-12; Taj Mahal 854, 880; temple complexes 492, 619, 672-3; ziggurats 210-11, 264

Mormonism 766

Mughal Empire: under Akbar 874-7, 878-9; architecture 854,878 ; art of $854,878-9$,
880; Babur's founding of 873-6; and the British 881-2; bureaucracy 851,875 ; collapse of 850 ; decline of 878,882 ; Delhi 880; as a gunpowder empire 774, 775, 850-1, 873-4; marriage practices 856; population 855 ; religious toleration 769, 852-3, 875-7; sciences in 854; succession crises 851, 877; Taj Mahal 854, 880; taxation 851-2, 875, 880; trade 851-2, 877; Turkic origins 851 ; women's roles 880

museums 1019-21, 1336

Mussolini, Benito 1191-2

Nanak 765, 769

Napoleon see Bonaparte nation states: border controls 913; cross-national cooperation 912, 913; cultural identity 910-11; decolonization and nation formation 1266; definition of 904-5; democratic 937; in Eastern Europe 1177-8, 1185; empires and 919-20; ethnic identities 904, 928-9; ethnic self-determination 913-14; of Europe, fifteenth to eighteenth centuries 905-11; external expansion 911; influences and effects of 926; influences and effects of expanded state size 1147; of Latin America 1083; linguistic consolidation 910-11, 912, 913, 933-4; minority groups 904, 926; national identity within 910; national sovereignty and imperialism 911; the Pacific 924-6; popular sovereignty and 910-11; in post-colonial Africa 1291; post-revolution America 1068-3; post-World War I, 915; as a problematic concept 904; role of libraries and museums 1019-21; settler societies 921-3; state capacity 929-31; sub-Saharan Africa 
923-4; system of 926; taxation 911; in the twentieth century 1129

national identities: ethnic identity and 605-6

nationalism: cultural nationalism 932; ethnic identities 934; Giuseppe Mazzini 936; group identity 932-3; Islam and 915-16; in the Ottoman Empire 865; motivations for colonial expansion 995; popular sovereignty and 931-2; as a problematic concept 935-6; religions and 1165; religious identity 933; socialism and 933; state capacity and 930; support for wars of conquest 1176; warfare and 249; warmongering and 934-5 natural resources: in Africa 12912; coal 953, 1039-40, 1041; economic development and 1292-3; resource availability and population growth 1312 13, 1363

naval engagements: attack on Hormuz 255; Battle of Lepanto 773, 860-1; battleships, post-World War I 1187; Japanese victory over Russia 1050; Ming naval expeditions 890-2; naval blockades in World War I 1184; at the outset of World War I 1179; of the Ottoman Empire 860-1; of the Roman Republic 411-12; use of cannons 773

Nazi Germany: advance across France 1195-6; annexation of Czechoslovakia 1190, 1195; attack on the Soviet Union 1196-8, 1200; control of conquered territories during World War II 1200; fascism in 1191; the Holocaust 1202-3; invasion of Poland 1195; Munich accord 1190; rocket technology 1196

the Netherlands: abolition of slavery 1103; Batavia 821; colonization of South Africa 1000; democracy 940, 957; in the Early Modern period 764; revolt against the Spanish 764; trade 764, 820-1; war over Taiwan 774

New Guinea: contact with European explorers 846; spread of agriculture to $125-7$, 204; successionist movement 1301; tribal leadership structures 214

New Zealand: governance of Samoa 1300; James Cook's exploration of 811; League of Nations Mandate 1186; settlement of 688,846

Newfoundland: Admiral System 839-40; Beothuk 841; fisheries 831, 839, 1067; land ownership 841; loyalty to the British crown 1067; the Vikings in 555

Newton, Isaac 970-1, 975, 976

Nkrumah, Kwame 1293, 1294-5

nomadic conquests 505,542 ; nomadic groups of Central Asia 544-7; overview of 112-13, 143-5; political organization 543, 544, 549; population densities 144 ; relationships with sedentary societies 540-2; religion 322; Scythians 402-3; social structure 148-50; tool-making 147

nomadism: in Arabia 482;

Ayrans' conquest of the Ganges 363; causes and effects of 151; challenges of 150-1, 543-4; domestication of animals 144, 145-6; emergence of 145-6; ethnic identities 928; in the Eurasian Steppe 144-5, 540-2, 822; gunpowder weapons 776; horses of 145-6, 148, 363, 540, 541-2; Indo-European language dissemination 148; interactions with farmers 146-8; lactose tolerance 76, 134, 135-8, 146; long-distance trade 144, 146-7; migrations into Europe 548-9; military technology 542-3

non-human environment: the individual and 10; theme of 49-50

North Africa: region of xliv

North America: Cahokia 702-3; the Columbian Exchange 827-30; fish trade 842; fur trade $823,824,842-3$; in the Middle Ages 701-3; organized sports 1011; see also Canada; Newfoundland; United States of America

North Atlantic Treaty Organization (NATO) 1209

Nubia: kingdom of Kush 218, 263, 268; links with Egypt 173-4, 218, 265-6, 268

Oceania: early human migration to 101

Olympic Games 404, 1011, 1175

Ottoman Empire: architecture 854,868 ; art of 854 ; Battle of Lepanto 773, 860-1; Blue Mosque 854 , 868; bureaucracy $851,859,862-4$; as a caliphate 861-2; Christian and Jewish merchants 865 ; conquest of Constantinople 574, 857-8; decline of 850, 864-5, 914$15,920,1176$; emergence of $600,856-7,858$; ethnic selfdetermination movements 914-15; expansion in Europe 859-60; financial challenges 864,865 ; as a gunpowder empire 774, 775, 850-1, 859; Istanbul 868-9; Janissaries 858-9, 864; legal system 761, 860; local elites 862-3, 864, 865; marriage practices 855-6; nationalism in 865 ; navy 860 1 ; population 855 ; reforms in 864-5; relations with the Safavids 870-1; religious toleration 769, 852-3, 869; sciences in 854 ; succession crises 851, 866-8; taxation 851-2, 862-3; trade 851-2, 865 ; trade routes 822,861 ; Turkic origins 851 ; victory 
over the Byzantines 857-8; World War I, 866, 867, 915, 1179; Young Turks 864-5

oxygen: the Cambrian explosion and 60; oxygen emitting bacteria $53-4$

Pacific: European exploration of 811-12; nation states 924-6; Spanish exploration of 809-10

Pacific Islands: colonialism in 993; decolonization 13002; Hawaii 925-6; human migration into 295-6; influenza epidemic, postWorld War I 1187; region of xliv; spread of agriculture to 125-7; Tonga 924-5; trade 812-13; trade potential of 812 Paine, Thomas 1106

Pakistan 1277, 1281, 1283; see also Indus Valley

Palestine 1219-20, 1273-4

Panama 806-7

Panama Canal 1005

paper 429, 522, 653-4, 977

Paraguay 1084-5

parents: challenges of 1053; child mortality 134, 135; in early agricultural societies 134-5

path dependencies 57-9, 61, 74, 442

peasants: behavior of 142-3; in early societies 202; forced labor in ancient Egypt 263, 264-5

Persia: Isfahan 854; Safavids 600; see also Safavid Empire

Persian Empires: administration 357, 358-9, 360, 361, 362, 600; Alexander the Great's conquest of 360,402 ; art of 360,361 ; conflict with ancient Greece 401-2; cultural and religious tolerance 35960; Cyrus 357-8, 359-60, 362; Darius 357, 358, 359-60, 380, 446,464 ; emergence of 345 , 346, 357-8, 362; financial institutions 378-9; gender relations 360; infrastructure development 359; Islamic conquest of 507-8; legal systems 359; overview of 357 ;
Parthians 360-1; public rituals 350; Sassanids 357, 360, 361, 402, 410, 479; slavery 360, 362 ; spy networks 358, 362; trade 359; Zoroastrianism 361, 463-4

Peru: Catholic missionaries during the conquest of the Americas 808; colonial rule in 807-8; Inca Empire 1021-2, 1299300; Potosi silver mines 806-7 petroleum 61

Philippines: Spanish colonial rule in 810-11

philosophy: Aristotle 404-

7; Chinese 421-4, 445;

Confucianism 422-3, 424, 428-9, 442, 447, 465-6; Greek philosophy 389, 404-9, 445 , 635-6; ideas of human progress 1057-8; interaction amongst philosophers 407-8, 421-2; in the Islamic Empire 519; Legalists 424, 446, 447; links between philosophy and theology 409, 635-6, 655, 657; for personal meaning 1393; Plato 404-7; Socrates 404-7; Taoism 423, 429, 430, 447, 466, 470; Thomas Aquinas 635-6, 655, 976; Zhu $\mathrm{Xi} 658-9$; see also political thought

Phoenicians 227-8, 269

Pizarro, Francisco 805, 807

planets: cores of 46 ; formation of 44-6

Poland 1195, 1215

political institutions: theme of 248-9

political leadership: bands 104, 106, 141; big men systems 214-15; chiefdoms 141, 215; decision-making processes 219-21; in early states 141 ; family lineages 141,215 ; of nomads 543, 544, 549; polity 141; tales of 725; tribes 141 political organization: of the Abbasids 515-16; of ancient Greece 399; the bureaucrat's challenges 1150; of early humans/hominids 80; of early Islamic states 511-13; in early states 141, 218; in Egypt 261-3; of feudal systems 558-9, 561-2; in the Han Chinese empire 427; humankind's aspirations for 1353; in Mesopotamia 252-3; in Polynesia 691; in the Roman Empire 412-14; in the Roman Republic 410; rulers' claims to universalism 857 ; selection by lottery 1369 ; spread of agriculture and 130-1, 133, 139-40; theme of 248-9; transport for 179; war as a side-effect of 140; within world history 1366-70

political thought: classical political thought 443-6, 448-50; Jewish thought 450-1; patriotism 451; role of bureaucracy 447-8

pollution: in early cities 208

Polo, Marco: as a merchant 737; at the Mongol court 610, 613, 734, 736; travel writing 610, 613, 738, 799

Polynesia: agriculture and fishing 690-1; canoes 687, 688; Easter Island 692-3; political organization 691; religions 691 ; settlement of $102,686-$ 90; social stratification in 688, 691 ; society 687 ; trade 687 , 691

popular sovereignty: and the idea of the nation state 910-11; political thought on 910; revolutionary change and 1062; support for wars of conquest 1176

population: agricultural capacity and 1374-5; agriculture's impact on 133-5, 136-7; of the Asian empires 855; challenges of an aging population 1055-6, 1375; childhood 1056-5; of China 828-9, 955-6; dynamics of population growth 294-5; of the Early Modern period 791; ecological limits on population 55; economic 
factors 1313; fertility rates 1050-4, 1310; global average life expectancy 1313-15; global patterns 1054-6, 1311-12, 1374; growth and stabilization 1307; human fertility 93, 105, 134-5, 140-1; in hunter gatherer societies 104-5; impact of the Columbian Exchange 828-9, 831; influences and effects of the demographic transition 1057, 1310; influences and effects of population growth 1313; Malthusian theory of 136-7; migration and population densities 99-100, 105 ; mortality rates $1310-11$; of the Mughal Empire 855; nomadic population densities 144; of the Ottoman Empire 855; post-900CE 624-5; resource availability $1312-13$, 1363; of the Safavid Empire 855; during the Song dynasty 661 ; theme of 299; in the twentieth century 1130-1

Portugal: colonization of Brazil 808-9, 816; exploration of the Atlantic coast of Africa 81314; exploration of the Indian Ocean 815-16; exploration south 799; influences on Asian cuisine 819; sugar plantations 813; trading posts in Asia 816-19

pottery $107,173,414$; Ottoman ceramic tiles 868; Persian ceramics 872

priests: challenges of 310 primary documents 229-30 printing press: role in religious reforms 767, 768

Protestantism: Martin Luther 767,768 ; rise of 767-8; witch hunts 771

psychology: evolutionary psychology 74; human capacity for self-deception 84-6, 107; justification for war 238-9

public opinion: theme of 249
Qin Empire: book burning 393, 425, 447; bureaucracy 424-5, 576; common written script of $421,425-7,443,576,583$; currency 377; emergence of 345 , 346, 424; legal code 429; Legalist practices 424,446 , 447; Terracotta Army 425; unified state $425-7$

Qing dynasty: abolition of slavery 1110; agriculture 895; bureaucracy $893-5,1148-50$; decline of 896-8, 917, 1284; economic development 896; expansion into Central Asia 825-6; expansion southwest 826; formation of 762,893 ; gunpowder weapons 774,776 ; the Manchus and 762, 893; multiculturalism of 895 ; trade 895; women's roles 898

racism: anti-Semitism 1119; following the abolition of slavery 1117-18; racial stereotyping 1118; in the twentieth century 1119; workplace discrimination 1118

radio 1015

refrigeration 1007, 1233-4

regimes of order 39

religions: abstract thought capacity and 81, 84; in Africa 318-19; architecture of 3289; artistic expression 81-2; artistic representation 490-1; atheism and agnosticism 1163-5, 1166; of the Axum Empire 370; canon development 487; in China 320-2; of the classical empires 347 , 457-8; community cohesion through 326-7; creation myths 305-7; crossreligious cooperation in the Early Modern period 769; cultural consolidation after 900CE 603-4; defined 13, 305; deism 456; doctrinal disputes 308-9; early fertility figures 82 , 99, 311, 327; in Egypt 322-4; ethics within
322-6, 457-8, 482; evil devil concept 456; evolutionary analysis 454-6, 499-500; evolutionary processes $307-9$; explanations for the universe 40 ; faith 489 ; the family unit and 327; in Greece 317-18; historical analysis of 309-13; humankind's aspirations for 1353-4; human sacrifice 206-7; of hunter gatherers 81-2, 304, 310, 311-12; impact of the Columbian Exchange 833-4; impact of printing on $767,768,961$; in India 319-20; individualism in 767,768 ; institutions of 309; justifications for empires 349; justification for kingly rule 221; in the Levant 316; links between philosophy and theology 409, 635-6, 655, 657,976 ; magic in relation to 323; in Mesoamerica 322; in Mesopotamia 253, 316-17; in the Mongol Empire 733, 746-9; monotheism 454, 455, 976; nationalism and 933, 1165; new religions 1166; new religions and the state 456-7; nomadic religions 322; omniscient god concept 455-6; organization of 1166; of the Persian Empires 359-60; personal choice 498; for personal meaning 1391-3; Polynesia 691; polytheism 313, 454, 455; prehistoric burial 81 , 82 ; reform movements 764 9; relics 491, 636; religious divisions in Europe 762, 767-8; religious reforms of the Early Modern period 764-9; religious terrorism 1220-1; religious toleration 1164 , 1355; religious toleration in the Asian empires 769, 852-3, 869, 875-7; responses to the Great Plague 746; in Rome 318 ; sacrifice in early religions 327-8; settlement by religious minorities in the 
Americas 844; of slaves in the Americas 838; in sub-Saharan Africa 900; tensions with science 391 ; ties with states 312-13; wars and 489-90; written texts 456 , 478; the written word and 308; see also individual religions

religions, spread of; Buddhism 468, 469-70, 492, 495; by conquest 493; Christianity 474-5, 492, 493, 494; conversion of rulers 492, 552; financial institutions 495; Hinduism 388; Islam 483-4, 492, 493, 494; Manicheanism 478-9; migration 495; missionaries and merchants 388, 469, 488, 494-5; overview of 497-9; political conflict 494; regional factors 496-7; religions of the world 491; rulers religious tolerance 493; syncretism 495-6

Renaissance: art of 655-6; emergence of 656-7; links with ancient Greece and Rome 654-5; literature 656; women in 656

reproduction: of the earliest lifeforms 54; lifespans and 54; sexual reproduction 55 revolutions: and the establishment of democracy 1072, 1271; commonalities of 1092; the Indian Mutiny 1090-1; institutional change 1062; Iranian Revolution 1279-80; the Kingdom of the Two Sicilies 1089; Marxist 1011; of the nineteenth century 1088-91; Russian Revolution 1136-7; as social evolution 57, 59; the Sokoto Caliphate 1087-8; Taiping Rebellion 1090; of the twentieth century century 1091-2; see also American Revolution; French Revolution; Haitian Revolution; Latin American Revolution

Roman Catholic Church: attacks on homosexuality 634; authority of 633-4, 637; clash with Galileo 968-9; corruption in 767, 768; development of 474; educational role 635; missionaries during the conquest of the Americas 808; monasteries 634-5; monumental architecture 634; Papal Bull of Innocent VIII on Witchcraft 771; political autonomy of 633; popular faith 636; priestly celibacy 474, 487, 623, 634; reforms in 767; relations with Christian rulers 630

Roman Empire: bureaucracy 434, 442; Christianity 416, 473-5; Cicero 413, 446, 450; Coliseum 351, 415; compared to the Han Empire 431-6; concrete 415; cuisine 420; currency 436-7; daily life 418-20; decline of 415-16, 434, 435, 438-41; early religion in 318 ; emergence of $345,346,411$, 412; failure to reconstitute 441-3; family life 417-18; financial institutions 379-80; Germanic tribes and 549-51; income inequalities 438; land ownership 438-40; land reforms 416-17, 438-9; luxury trade 437; political organization 412-14; pottery 414; road network 414, 435; Roman identity in 348; scientific practices 391 ; slave population $373,374,375$, 379-80, 416-17, 434; soldiers 244; stoicism 407, 446; taxation 436-7; trade 414, 436-8; women's lives 418

Rome, Republic of: armies 410-11, 412; citizenship 410; democratic elements of 450 ; legal systems 412 ; navy 41112; political organization 410 rubber 991-2, 1299-300

rulers: absolutism 907; autocracies 935, 937; challenges of 222, 1240; claims to universalism 857; in the classical empires 347,350 ; communist 1139; courtly life, post-900 601-2, 632; in Egypt 263; heavenly mandate for rulers in China 280, 427, 429, 444, 448; human nature and 221-2; humankind's aspirations for 1352; justification for 221, 326; justification for war 238-9, 245; in Mesopotamia 252-3; relationships with local elites 354, 355; religions and 444-5, 492, 493, 552; rise of 219-21; rules of succession 281; secluded rulers 867 ; succession crises 866-8; within democracies 937-8; see also monarchies

Russia: abolition of serfdom 1121-2; assemblies 938; boyars 763; Crimean War 1120; expansion of 822-3; feudalism in 565, 763, 822-3, 1120; fur trade 823-4, 842; gunpowder weapons 776; Japanese naval victory over 1050; Mongol rule in 749-50; Muscovy 750-1; nuclear capabilities 1213-14; political consolidation 907-9; railways 1048-9; religious and ethnic identities 823; rise of communism 11367; Romanov dynasty 763; Russian Revolution 1136-7; share of Central Asia 825; Siberian territories 811-12, 823-4; state industrialization in 1048-9; support for Serbia 1177, 1178, 1180; in World War I 1179-80, 1184; see also Soviet Union

Safavid Empire: architecture 854; armies 871; art of 854, 872-3; bureaucracy 851 ; decline of 871-2; as a gunpowder empire 774, 775, 850-1, 871; Isfahan 872; marriage practices 856 ; population 855 ; relations with the Ottomans 870-1; religious 
toleration 852-3; sciences in 854; under Shah Abbas 871; Shah Ismail's founding of 870 ; Shia identity 870 ; succession crises 851,871 ; trade and taxation 851-2; trading networks 778; Turkic origins 851 ; women's roles 873

Sahel: region of xliv

Samoa 1300

Sargon 243, 254-5

sciences: in the ancient world 389-91; in the Asian empires 854; biology 1016; causal empiricism 970, 973-4; chemistry 1015-16, 1046; the Church's criticism of 968-9, 972-3; decline in religious belief 1164-5; developments of the nineteenth century 1015-16; developments of the twentieth century 1019; disciplinary specialization 1017-18; in early states 235 ; Enlightenment thinkers 976-7; Europe's Republic of Letters 973; evolutionary processes of 192-7; the Four Elements (Pliny) 390; human understanding of the world 1356; industrial research laboratories 1018 , 1047-8, 1234-5; intellectual interaction and discourse 407-8; in the Islamic Empire 518-19, 521-2; in the Mongol Empire 741; nonEuropean antecedents 977; numbers of scientists 1019; public understanding of 1358-9; research universities 1016-17, 1018, 1019; role of libraries and museums 1019-21; science clubs 974-5; scientific communities 9715; scientific enquiry 1015, 1040; Scientific Revolution of the Early Modern period 966-7, 976-8; the scientist's challenges 391; in the Second Industrial Revolution 1046-7; social aspect of advancement 971; social sciences 1357-8; in the Song dynasty 659-60; tensions with religious belief 391; see also astronomy sculpture: Easter Island heads 692-3; statues of Buddha 490, 784; Terracotta Army 425

Second Industrial Revolution: factories 1046; industrial research laboratories 1047-8; scientific advances 1046-7; steelmaking 1046; technological innovations of 1045-6, 1234

Serbia 1177, 1178, 1180

Shang dynasty: Anyang 205, 206, 209, 278; art of 331; bronze 207; early monumental architecture 211; heavenly mandate for rulers 280; irrigation 280; military technology 247; overview of 278; Zhou's conquest of 278

Shia Islam: hereditary kingship 870; in Safavid Persia 870

Shintoism 485-6, 586, 1164 shipping technology: cannons onboard ships 816; containerization 1253-4; iron ships 993; for ocean travel 797; refrigeration 1007; regional variations 964; steam engine technology 993, 1028; steamships 1005, 1008, 1260, 1316

Sigurdsson, Harald 556-7

Sikhism: development of 765; Nanak 765, 769

Silk Road: Buddhism and 469; China's new Silk Road 825-6; cities of 386-7, 547; disease transmission along 388-9; in the Early Modern period 817; under the Han Empire 3845; under the Mongols 737; revival in, post-900ce 606; trade networks of 386 slavery: African states involved in 835, 899, 1101; in America 1097-8; in the ancient world 370-1, 373-6; banning of the slave trade 1101 ; colonial slave-owners 1100; compensation for slave owners 1103; in early cities 207; economics of 372-3, 1099; European slave trade 834, 836-7; expansion of prenineteenth century 1099-100; forced labor under the Song dynasty 660; Ghaznavid elite 529, 531; Haitian slave revolt 1079-80; impact of the American Revolution 1066; internal slavery in sub-Saharan Africa 710, 711, 798, 834, 836; in the Islamic Empire 527; malaria transmission 828; Mamluks 531; maroon societies 838; modern day slavery 1111 ; occupations of 371,376 ; in the Persian Empires 360, 362; profitability of the slave trade 838-9; in the Roman Empire 373, 374, 375, 379-80, 41617, 434; skills of African slaves 837; Slav slave trade 553-4; the slave's challenges 375; in the Sokoto Caliphate 1088; in the southern United States 372; for sugar plantations 813 , 836-7; syncretic religions 838; in warfare 238; from warfare 373,374

Slavs 548, 551-4, 575

Smith, Adam 956

social policies: during the Golden Age 1244; impact of the Great Depression 1144, 1145, 1227, 1242-4; links with social sciences 1357-8; social programs 1351, 1373-4; of states 1143-6, 1227, 1242-4

social sciences: phenomena of 11, 1357-8

social structure: of the Aztecs 698-9; caste system 880-1; within classical empires 445 ; comparisons across societies 432-3; ethnic divisions 14; gender and stratification 234; hunter gatherer groups 94-5; of the Ming dynasty 889; theme of 14-15; see also families socialism: nationalism and 933 
solar system: diagram of 46 ; the Moon 47; the Sun 44-5, 46

soldiers: challenges of 244 ; in the Roman army 244; and social mobility 244

Song dynasty: agricultural developments 588, 663; armies 658, 665; Chinese landscape painting 665 ; decline of 661 ; economic growth 661-5, 896; examinations for bureaucrats 658-9; forced labor 660; gunpowder technology 772; industrial output 664; infrastructure development 661-2; neo-Confucianism 466, 470, 655, 658-9; pagodas 618-19; paper currency 608, 662; population 661; printing technology 653, 977; sciences 659-60; taxation 660-1, 885; trade $662-3$

Songhay Empire 714

South Africa 815, 1000, 1219, 1297

South America: Chimu state/ society 703; early states of 703; political consolidation after 900ce 600; see also Inca Empire

South Asia: region of xliv; spread of agriculture to 124

Southeast Asia: Borobudur temple 681-2; Chinese and Indian influences on 678 ; cultural consolidation 676-7, 917; Funan 587; geography of 587; the Islands during the Middle Ages 678-82; the mainland in the Middle Ages 672-8; Melaka 528, 587, 609, 677, 679, 809, 816; migration to 587 ; political consolidation after 900CE 599-600; Portuguese trading posts $816-19$; region of xliii; religions 676, 677; sea trade in the ancient world 381-2; spice trade 382-3, 679, 680-1, 799,821 ; spread of agriculture to 125 ; Srivijaya kingdom 679-80; state formation and dissolution 675-6; trade 677-8; warfare 678; in World War II 1199, 1201; see also individual countries

Soviet Union: collapse of 1138 9, 1215-16; Declaration of Rights of Working and Exploited People 1140-2; economic growth 1137 8; ethnic identities 920; formation of 1136-7; during the Great Depression 1226-7; invasion of Afghanistan 121718; invasion of Poland 1195; post-World War I 1208-9; post-World War II 1209; space technology 1211; in World War II 1196-8, 1200, 1204-5; see also Cold War; Russia

Spain: Charles V 905;

circumnavigation of the globe 809-10; colonial rule in the Americas 807-8; conquest of the Aztecs 801-4; conquest of the Incas 805-6; the Crusades in 639; Dutch revolt against 764; fascism in 1191-2, 1193; the Inquisition 764, 767-8; Islam in 483-4, 493, 515, 620; Islamic Cordoba 523, 535, 630; silver from the Americas 806-7; sponsorship of Columbus's voyages 799, 800; transition to democracy 1193 Spice Islands 809, 811, 816, 820 sports: in the ancient world 405; ball games in Mesoamerica 991; in the Byzantine Empire 567-9; Olympic Games 404, 1011, 1175; organized sports 1011

Sri Lanka: tea plantations 780

stars: brown dwarf stars 44; formation of 42-4, 46; orbital planets of 44-6; supernovas $43,45,46$

states: bureaucratic capabilities and 1146-50; civilization and 200; communist 1136, 1139; cultural consolidation after 900CE 603-6; defined 141; European state formation 629-32; government spending 1143, 1144, 1146; Islamic religious states 1166 ; maximization of gunpower technology 776; nationstate concept 349; and the new religions 456-7; political consolidation after 900CE 599-601; political consolidation during the Early Modern period 759-60; protection of merchants 180-2, 286-7; relationship with merchants 180-2, 183-4, 288-9; rules of succession 281; social policies 1143-6, $1227,1242-4$; taxation by in the twentieth century 1142-3, 1146; term 904-5; transition into empires 345-6; see also nation states

states, early: alliances between 218; causes and effects of 235; city states 215 ; decline of 219 ; ecological collapse and 276-7; effects of 223; emergence of 215; emergence of kings 219-22; ethnic diversity 208, 215; gender relations 233-4; growth through conquest 216-17, 245-6; laws 231-3; limitations on expansion 217; nature of 218-19; political organization in 218; protective role of 351; science in 235; taxation of farmers 133, 202, 215,217 ; ties with religions 312-13; tribute payments 217-18, 238; warfare in 236-7; written record-keeping 223-4, 225,378

steam engines: significance of 193

stories: creation stories/myths 38; Epic of Gilgamesh 222, 241, 253, 317, 461; Homerian epics 270,299 ; impact of writing on 228; intergenerational transmission of values 13 , 22-3; tales of leadership 725; travel narratives 299; travel writing 819; see also literature sub-Saharan Africa: agriculture 709-12; Assante 899; 
colonization of 838 , 900, 990; decolonization 1290-2, 12958 ; democracy in 1133; during the Early Modern period 8989; East Africa 717; East African city states 899; Ghana Empire 712-13; Great Zimbabwe 600, 620,717 ; gunpowder weapons 774; Igbo 899; income growth 1246; internal slave trade 798, 834, 836; internal slavery 710,711 ; iron production 709, 837; kingdoms of 714; Kongo Empire 600, 717, 814; land ownership 710; Mali Empire 713-14; mining in 1000, 1003; natural resources 1291-2; Ndonga 900; panAfrican unity 1293-5; political consolidation after $900 \mathrm{CE}$ 600; population densities 710, 711, 712; Portuguese exploration of West Africa 813-14; post-emancipation conditions 1114-15; region of xliv; religion 900; resettlement of freed slaves 1113-14; social structure 710; Sokoto Caliphate 899, 1087-8; Songhay Empire 714, 782, 834; South Africa 815 ; spread of agriculture to 122-4; states involved in the slave trade 835,899 ; trade 531-2, 607, 709, 711, 712, 716; trans-Sahara trade 781-3; Uganda 899; Wangara trade network 783; women in 712;

Xhosa 899; see also individual countries

Suez Canal 990, 1006, 1110-11, 1274

sugar $779,798,813$

Sumatra: Srivijaya kingdom 679-80

Sumeria: A Sumerian Schoolboy 229-30

Sun Tzu: The Art of War 241-2

Syria: country 1273; Palmyra 383-4, 386-7

Taiwan 1285

Taliban 1217-18
Tang Dynasty: ban on external travel 580; Confucianism 580; conquest of Korea 584; cultural achievements 581; decline of 583; expansion of 578; indigo dye 581; land ownership 578-9; military rebellions 580; religion in 470; Tang Taizhong 577-8; taxation 578 ; trade in $580-1$, 582-3; wider influence of 581; Wu Zitian 579

taxation: advent of writing and 223-4; of American colonies 1064-5; in ancient Egypt 263, 264-5; in Britain 940; bureaucratic capabilities and $1143,1146-50$; in the classical empires 344-5, 350, 352, 356; General Agreement on Tariffs and Trade 1153, 1239, 1252-3; in the Han Empire 436-7; income taxes 1142-3; of merchants 180, 182; in Mesopotamia 253-4; in the Ming dynasty $884-5$; in the Mongol Empire 734, 737; in the Mughal Empire 851-2, 875, 880; nation states 911; in the Ottoman Empire 851-2, 862-3; in the Roman Empire 436-7; of the Song dynasty $660-1,885$; state capacity and 929-30, 931; state taxation of farmers 133, 202, 215, 217; during the Tang Dynasty 578; in the twentieth century state 1142-3; for wars 1175-6; within world history 1366-7; World Trade Organization 1153,1256

tea $779-81$

technology: airconditioning 1248; artisan guilds 652; assembly lines 1232,1233 , 1235; best practice manuals 963-4; birth control technologies 1054, 11612; bricks 164; the British Industrial Revolution and 1028, 1031-2; cities as sites of innovation 1332; compasses 607, 798; computer technology 1201, 1211, 1236; continuous production 1235; domestic innovations 1159 ; in early warfare $246-7$; electrification 1235; European access to 955 ; evolutionary processes of 192-7; glass 164-5; of the Golden Age 1240; human innovators 193-4; impact on economic convergence 1245, 1248; industrial research laboratories 1018, 1047-8, 1234-5; innovation, four stages of 194; innovation within world history 1359-62; the innovator's challenges 9656; innovations for colonial expansion 993; internal combustion engines 1045; of the Islamic Empire 522; loss of jobs due to 1030, 13602; metals 167-72; military applications in civilian life 246; paper 429, 522, 653-4, 977; pottery 161-4; printing press 653-4, 741, 977; product and process innovation 1232-3; radio 1015; the Scientific Revolution 977-8; the Second Industrial Revolution and 1045-6, 1234; shipping technology in the ancient world 381; space technology 1211 ; technological arguments for the Great Depression 1231, 1233-6, 1237; technological innovation and economic growth 952, 961-5; technological innovation due to improved transport 1037; telegraph 1008, 1015; textiles 165-7; theme of 173; trade and technological innovation 161; transfer of 523; and the transition to agriculture 117; transmission of 1041; see also agricultural technology; shipping technology; tool-making temples: in early cities 206,210 terrorism: ethical debates 1220 ; 
goals of 1218-20; IsraeliPalestinian conflict 1219-20; in Northern Ireland 1218, 1219; religious terrorism 1220-1; in South Africa 1219 textiles: American cotton industry 1097-8; artisan guilds 651-3; cotton cloth 166, 274, 363, 366-7, 1033, 1034-5; in early societies $165-$ 7 ; indigo dye 581; in Japan 1048; of the Ottomans 868; of the Persians 872; red dye production 829-30; silk trains 1005; textile mills 1013-14; wool 166

Thailand: abolition of slavery 1110; cultural consolidation 676-7, 917; states during the middle ages 675-6

theory of relativity 42

Tibet 1286; Chinese influence in 589; Tibetan Buddhism 495, 589-90, 591, 749

time: circularity in 41 ; entropy

38-9, 41, 43; global

standardization of 1008;

history of timekeeping 666; as infinite 41

Timur 751-2, 851, 863-4, 869, $870,873,878$

Tonga 924-5

tool-making: Acheulian tools 86, 88, 91, 100; adaptation to environments 87-8; batons 89 ; benefits of cut and cooked food 75,89 ; brain size and 74, 86, 87, 88, 100; by early hominids 71,72 , 85-8; evolution of manual dexterity 74-5, 76; food preparation 88,89 ; hunter gatherers 88-9; links with language development 79, 87; by nomads 147; occupational specialization in 94; and the transition to agriculture 115-16

tourism: challenges of 1333, 1334; growth in 1332-3; historical sites for 1334, 1336 trade: access to the Indian Ocean 816-19; across the
Sahara 387, 781-3; in Africa 531-2, 709, 711, 712, 716;

American cotton industry 1097-8; in the Americas 695; in ancient Greece 400-1, 435; in the ancient world 380-4, 388-9; Aztecs 697-8; of the Byzantine Empire 380, 572; causes and effects of 185; in China 955, 956; in Chinese porcelain 615-16, 741, 784, 890; in the classical empires 344-5, 351, 377; in coffee, tea and cacao 779-81; contract dispute resolution 782-3; the Crusades and 640; decline in Central Asia 825; doubleentry bookkeeping 646; early financial institutions 378-80; in early societies $167-8,173-4$, 289; in early states 223; in Egypt 266; expansion in 174-5; expansion in Europe 642-6; expansion in, post900CE 606-9; in the feudal system 563-4; fish trade 839 , 842; fur trade 823-4, 842-3; General Agreement on Tariffs and Trade 1153, 1239, 1252, 1364-5; global trade increases 1007, 1033; gold and silver for 175; Great Divergence 954-5; in the Gupta Empire 366; in the Han Empire 429-30, 436-8; Hanseatic League 645-6; impact of the Industrial Revolution 1036-7; importance of 152; of the Incas 706; increased agricultural output and trade opportunities 646-50; Indus Valley 274-5; institutions for, Middle Ages 643-4; international trade levels 1252 ; introduction of currency 3778; in the Islamic Empire 5268, 606; links between law and commerce 760; long-distance trade and disease 208, 747; merchant trading networks 182-3; in Mesopotamia 254; in metals 167-8; in the Ming dynasty $890,891-2$; in the
Mongol Empire 737-8, 741; in the Mughal Empire 851-2, 877; the Netherlands 764 , 820-1; nomad's control of 144, 146-7; in the Ottoman Empire 822, 851-2, 861, 865; overview of 175 ; Pacific Islands 812-13; in the Persian Empires 359; in Polynesia 687, 691; in the Qing dynasty 895; in the Roman Empire 414, 436-8; by railway 1005 ; in rubber 991-2; short-distance trade by hunter gatherers 92; slave trade 834-9, 1101; in the Song dynasty 662-3; in Southeast Asia 677-8; specialization of occupations 133; spice trade 383,679 , 680-1, 799, 821; spread of religions through 388 , 488, 494-5; state formation and 631; as a substitute for war 1174; during the Tang Dynasty 580-1, 582-3; and technological innovation 161 ; trade restriction policies 1226-7, 1365-6; trade, foreign investment and economic growth 1298-9; trading cities 206, 609-10; trading networks 777-8; and the transition to agriculture 174; transmission of ideas 289; transport and 177-80; Wangara trade network 783; within world history 1364-6; World Trade Organization 1153, 1252-3, 1256; see also merchants; Silk Road

transport: characteristics of 180 ; land transport 177-9, 180; occupational specialization and 179-80; role in political organization 179; rubber tires 991-2; and the spread of information 180; trade and 177-80; using animals 178, 179; wheeled transport 178 , 180; see also boats

transport infrastructure: administrative benefits of 1008; British Industrial 
Revolution and 1035-8; in the classical empires 351 ; corporations in 1036; creation of 50; globalized timekeeping 1008; Grand Canal, China $576,577,885$; impact on factories 1038; impact on technological innovations 1037; in the Inca Empire 705-6; for migration 131617; in the Mongol Empire 737-8; Panama Canal 1005, 1006; in the Persian Empires 359; railways 1042, 1048-9; reduced transport costs 1253 4; road and waterway network 1035; in the Roman Empire 414; and the settlement of the Americas 845-6; Suez Canal 990, 1006, 1110-11, 1274; in the twentieth century 1131 ; see also shipping technology

Tunisia 1110

Turkey: Catalhoyuk 203, 210, 236, 330; nation state formation 916; Young Turks 864-5

Turks: groups of 545-6

unions 1009-10, 1089

United Nations (UN) 1151-3, 1154-8

United States of America (USA): abolition of slavery 1105-7, 1108-9; American Constitution 1069-1; American System of Manufactures 1043; annexation of Hawaii 926, 1002; attack on Pearl Harbor 1198; Bill of Rights 1070; Civil War 1108-9; communist witch hunts 1212; cost of slave patrols 1099; cotton industry 1097-8; electricity in 1046; entry in World War I 1184, 1187, 1189; freed slaves in 1112; freedom of religion 1070; Immigration Restriction League 1319-20; as an imperialist power 1002; industrialization in 1041-5; influenza epidemic,
post-World War I 1187; nation-building 921-2; railways 1004, 1042; slave population 1097-8; slavery in 372 ; social security policy 1146; space technology 1211; state legislatures 1070-1; state of Alaska 1002; taxation 1142-3; Vietnam War 1289-90; see also American Revolution; Cold War universities 635; disciplinary specialization 1017-18; German research universities 1016-17; interdisciplinary teaching 1018; research universities 1016-17, 1018, 1019; scientific innovation and 974

Ur III 255

urban planning: in early cities 209; general 1329-30 urbanization: city sizes 1324, 1325; cultural impact of 1331-2; due to industrialization 1009, 1051; economic development and 1324-5; political impact 1331; rates of 1324 ; as sites of innovation 1332; and the spread of democracy 1133; suburbs 1330; urban poverty 1327-9; see also cities

values: cultural values $13-14,20-$ 3 ; institutions as reflections of 1348-50; religious 13; stories for the transmission of 13 , 22-3

Vespucchi, Amerigo 809

Vietnam: communism 1287-90; cultural consolidation 676-7, 917; Dai Vet 599, 674-5; decolonization 1287-90; Indian influence in 588-9; rice cultivation $587-8,663$; states during the middle ages 675-6

Vietnam War 1211, 1213, 1216, 1289-90

Vikings: migrations to the East 556-7; migrations to the West $554-5$
Wang Yangming 766-7, 768-9 war: The Art of War (Sun Tzu) 77-8, 210; conquest for state growth 216-17, 245-6; contemporary challenges for 1222-3; Crimean War 1120; decisive battles 511; defined 235-6; destruction of early cities 208; destruction of historical sites 1334; in the Early Modern period 772; in early states 236-7; English Civil War 762-3, 939; evidence of early warfare 236; evolutionary analysis 246 ; experiences of 237-41, 242; farmer/nomad conflicts 147; funding for 1175-6, 1177, 1194, 1199-200, 1222-3; in hunter gatherer societies $94-5$, 236; influences on and effects of 247; just war concept 450, 489-90; justifications for 238-9, 245; Korean War 1136, 1206, 1209-10, 1216-17; Malthusian theory and 137; military leadership 243; motives for 238-9, 249; nationalism 934-5; nationalist motives for 249, 1176; new wars 1221-2; peace following 774-5; political philosophy legacies of empires 446; the pursuit of peace 1174-5; religions and 489-90; and the settlement of the Americas 845; social differentiation and 245; Seven Years War 1032, 1062-3, 1065, 1073; sexual assault of women 1195, 1203; terror tactics 410-11; Thirty Years War 762, 769; trade as a substitute for 1174; and the transition to agriculture 140, 141-2; in the twentieth century 1129; United Nations peacekeeping missions 11513 ; wartime atrocities 1065-6, 1152, 1203; within world history 1371; war crimes tribunals 1155, 1188, 1207-8; see also World War I; World War II 
weapons: atomic weapons 1201 , 1203-5, 1210, 1211, 1213-14; bronze 169 , 170, 246, 247; iron production 367,434 ; iron weaponry 171, 246-7; machine guns 993, 1180; muskets 773; see also military technology

whaling 812

William the Conqueror 631 witch hunts 770-1

women: beliefs of 481 ; domestic servants 1159; during and after World War I 1186; during and after World War II 1209; expanded employment opportunities 1159-61; family structures, post-900CE 624; fashion 1057; female slaves 375 ; fertility of 93 , 105, 122-4, 134-5; under feudalism 560-1, 563; gender and social stratification 234 ; household labor of 1158-9; impact of industrialization on 1053-4; in the Islamic Empire 525-6; marriage practices of the Asian empires 855-6; migration to North America 844; in the Ming dynasty 889, 890; in the Mongol Empire 735-6; in the Mughal Empire 880; new religions and 487; in nomadic cultures 149-50; in the Qing dynasty 898; in postcolonial China 1287; in postcolonial India 1283; in the Renaissance 656; right to vote $1162,1186,1209$; role in the transition to agriculture 117; in the Roman Empire 418; in Safavid Persia 873; sexual assault of 239, 1195, 1203; social changes for 1158, 1162; in sub-Saharan Africa 712; trading alliances via marriage 821; witch hunts 770-1; see also gender relations work: artificial intelligence in 1236; debt bondage 1116; division of labor 1037; factory workers 1013-14; forced labor 1116-17; free markets for labor 1098-9; indentured servants 992 , 1000, 1096-7, 1113, 1115-16; labor hoarding 1236; leisure time 1011; managerial classes 1009; Marxism 1010-11; need for educated workforces 1052; negative impacts of the Industrial Revolution 102930; racist discrimination 1118 ; strikes 1009-10; technology and the loss of jobs 1030 , 1360-2; transportation of criminals 1116; unions 1009-10, 1089; women's opportunities for 1159-61; the working week 1011

workers: challenges of unskilled workers 420

working classes: clothing 1057; consumption patterns 1051-2; leisure time 1011; revolutionaries 1089; supervision of 1009, 1011, 1027-8; unionization and 1009-10; the working week 1011

world history: and General Education 8; both/and perspectives 26-7; field of 6-9; human history within history of the universe 40; idea of Western civilization 978-9; the individual within 10 ; interdisciplinary approaches 25-6; key themes 10-13, 25, 1377-8; multiple perspectives in $27-8$

World War I: alliances of 1176, 1179-80; casualties 1176-7, 1182; chemical weapons 1182-4; colonial soldiers 1176; colonial states following 1185-6; conscription 1177; end of 1184-5; European political context for 1177-8; German motivations for 1179, 1180; global impact of 1186-7; the Great Depression and 1230-1; historiography of 1180; Japan 1179, 1186; military technology 11804; nation state formation
915; nation state formation in Eastern Europe 1177-8, 1185; naval blockades 1184; Ottoman Empire 866, 867, 915, 1176, 1179; peace treaties following 1187-9; in relation to World War II 1190-1; Treaty of Versailles 1187-9; women's roles 1186 World War II: aftermath of 1205-6; allied bombing 1201, 1203-5; attack on Pearl Harbor 1198; Axis controlled territories 1200; battle over Midway 1201; Brazil in 1198-9; British code cracking 1201; casualties of 1200, 1201, 1205; colonial soldiers 1268; eastern front 1196-8, 1200, 1204-5; final years 1200-1; funding for 1194, 1199-200; Germany's annexation of Czechoslovakia 1190, 1195; the Golden Age following 1239; the Great Depression and 1191; the Holocaust 1202-3; invasion of Poland 1195; Japan in China 1194-5, 1205, 1284; Japan's comfort women 1203; local elites during 1200; military technology 1193-4; Munich accord 1190; in relation to World War I 1190-1; rise of fascism and 1193-4; western front 1195-6; women during and after 1209

World's Fairs 1043-4

writing: alphabets 227-8; in China 226-7; civilization and 200; common written script of the Qin Empire 421, 425-7, 443, 576, 583; and the communication of stories 228 ; Egyptian hieroglyphs 225-6; in the Indus Valley 227, 272; Japanese script 586-7; libraries 228-9, 392-4; in Mesoamerica 283, 285, 694, 697, 703; Mesopotamian cuneiform 226, 228; Phoenician alphabet 227-8, 269, 407; for recordkeeping 223-5, 378; religious 
written texts 456,462 , 478 ; role of monasteries 634-5; and the transmission of ideas 392-3; transmission of religions

308; Uighur writing system 734

Xiongnu Empire 368, 384$5,427-8,544-5$
Yemen: Judaism's influence in 462

Yuan dynasty $665-7,733$, 736-7, 747-8

Yugoslavia 1200, 1216

Zhou dynasty: bronze 207, 279-80; conquest of the Shang 278; decline of 345,424 ; early monumental architecture 211; early writing in 227 ; heavenly mandate for rulers 280 , 427; irrigation 280; military technology 247; state 279

Zimbabwe 1297

Zoroastrianism: beliefs of 463; in the Persian Empires 361, 463-4; wider influence of 464-5, 472 
$\Rightarrow$ Taylor \& Francis Group

an informa business

\section{Taylor \& Francis eBooks}

\section{www.taylorfrancis.com}

A single destination for eBooks from Taylor \& Francis with increased functionality and an improved user experience to meet the needs of our customers.

$90,000+$ eBooks of award-winning academic content in Humanities, Social Science, Science, Technology, Engineering, and Medical written by a global network of editors and authors.

TAYLOR \& FRANCIS EBOOKS OFFERS:
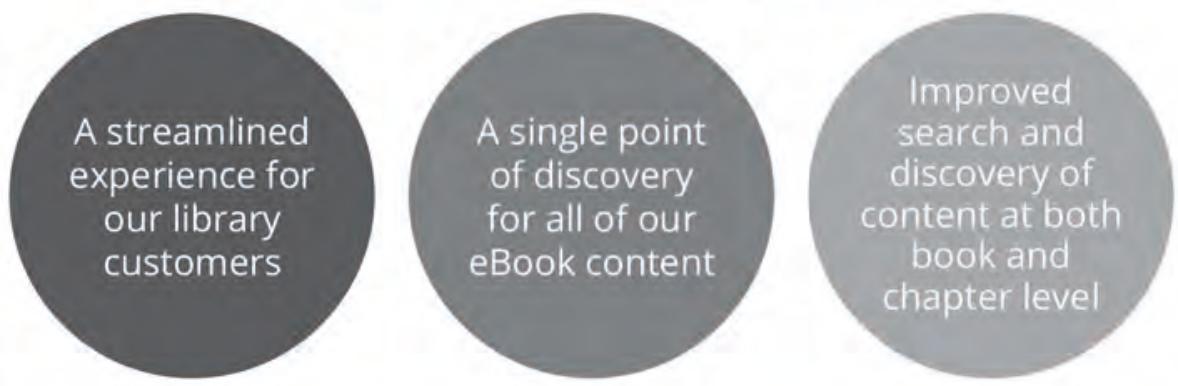

\section{REQUEST A FREE TRIAL support@taylorfrancis.com}

